\title{
MINUTES OF THE 42nd GENERAL ASSEMBLY OF THE EUROPEAN ASSOCIATION FOR THE STUDY OF DIABETES
}

\author{
held in Copenhagen, Denmark, on 16 September 2006 at 18.15 \\ Present: \\ Drs. \\ E. \\ J. \\ D. L. \\ E. \\ V.

Ferrannini
Škrha
Eizirik
Gale
Jörgens \\ (President) \\ (Honorary Treasurer) \\ (Honorary Secretary) \\ (Editor-in-Chief, Diabetologia) \\ (Executive Director)
}

The President welcomed everyone to the 42nd General Assembly.

\section{MINUTES, 41 st GENERAL ASSEMBLY 2005}

Since there were no comments, the minutes were approved unanimously and officially signed as a correct record.

\section{REPORTS}

\section{a) President}

Dr. Ferrannini reported that a teleconference had taken place with the European Medicines Agency (EMEA) to discuss the format of the meeting in London at the EMEA offices on 22 September 2006. The EASD committee for this meeting includes Drs. C. Bailey, A. Barnett, B. Charbonnel, S. Del Prato, E. Ferrannini, R. Heine and R. Holman, U. Smith and E. Standl.

The President reported on the meeting held in Vienna, Austria on 15-16 February 2006 organised by the Austrian Ministry of Health (OBIG) which had been attended by representatives from EASD, WHO and patient organisations. As a result of the meeting the recommendation had been made to set up a working group to take initiatives to increase the awareness of diabetes.
The President reported that 'The case for a UN resolution' had been prepared by the IDF to highlight the need for a United Nations Resolution on diabetes in order to focus attention on the need to stop the growing diabetes epidemic.

Dr. Ferrannini reported that ISPAD was a small, but enthusiastic organisation which had recently held a very well-attended meeting in Cambridge, UK. Following information that pediatricians are not attending the EASD Annual Meetings, discussions to organise a pediatric track in the programme of the Annual Meeting have been put forward. Dr. DahlJørgensen has also suggested organising a joint EASD/ ISPAD Postgraduate Course on Pediatric Diabetes in Europe in 2008.

The President reported that a joint statement with ADA on guidelines on the treatment of type 2 diabetes was simultaneously published in August in both Diabetologia and Diabetes Care. Drs. R.J. Heine and R. Holman lead an ad-hoc committee from the EASD which also included Dr. S. del Prato and Dr. U. Smith.

More details on EASD and EFSD activities were reported in the President's Address given before the Minkowski Lecture, which is available on the internet under: http://www.easd-lectures.org/copenhagen/index. php?menu=lectures\&id=66 and in his speech before the 38th Claude Bernard Lecture, available under: http://www.easd-lectures.org/copenhagen/index. php? menu=lectures\&id $=72$ 


\section{b) Honorary Treasurer}

Dr. Škrha reported that the income of the Association had increased in 2005 due to the registration fees for the Annual Meeting 2005 being collected directly by the EASD office. Expenses increased due to an increase in the costs paid for the Annual Meetings, with costs for the Munich Meeting in 2004 being paid in 2005. Regarding expenditure there are no significant differences between the years 2004 and 2005. The Honorary Treasurer said that after having personally checked the accounts of EASD, he was satisfied with the accounting. He summarised by saying the accounts of EASD were secure and correctly kept in accordance with non-profit legislation.

Dr. Škrha thanked the EASD staff for their friendly assistance and the members of the Executive Committee for the fruitful collaboration.

The President thanked Dr. Škrha for his diligence.

\section{c) Honorary Auditors}

The President asked the Honorary Auditors to formally discharge the accounts. Drs. Tiedge and Katsilambros confirmed that the accounts had been checked carefully and were in perfect order. Dr. Ferrannini asked for the vote to accept the accounts.

The accounts were accepted unanimously.

\section{d) Honorary Secretary}

Dr. Eizirik reported that 1931 abstracts had been received, with the highest number coming from USA and UK, closely followed by Germany and Italy.

For the Annual Meeting in 2006, 97 (70\%) of the 138 applications for Travel Grants received were accepted (total value Euro 60.280). From the 34 received, 15 Stayment Grants were awarded.

Dr. Eizirik closed his report by thanking all members of the EASD staff for their outstanding help and support with the organisation of the EASD Annual Meetings.

\section{e) Editor-in-Chief, Diabetologia}

Dr. Gale reported that the submission rate had doubled from 900 to 1800 per year, resulting in a doubling of the number of pages. Acceptance rate is $20 \%$, with the Impact Factor remaining steady at 5.4. Dr. Gale said there had been an improvement in the processing of the papers, with 33 days being needed for the first decision and 65 days from acceptance to online publication. Dr. Gale pointed out that the journal was being sent to members on time i.e. on $20^{\text {th }}$ of the month preceding the month of the journal.

\section{f) Chair, Postgraduate Education Sub-committee}

Dr. Ferrannini announced that due to a family emergency Dr. Pieber had had to return home earlier than expected. Dr. Pieber has also stepped down from his position as Chair of the Postgraduate Education Sub-Committee.

\section{ELECTIONS}

\section{a) President extension until 2008}

The extension of the President's term of office until 2008 was unanimously approved with one abstention.

\section{b) Editor-in-Chief extension until 2008}

The extension of the Editor-in-Chief's term of office until 2008 was unanimously approved with one abstention.

\section{c) Vice President 2006-2009}

The Council's election of Dr. C. Boitard (F) was unanimously approved with one abstention.

\section{d) Honorary Treasurer 2006-2009}

The Council's election of Dr. G. Spinas $(\mathrm{CH})$ was unanimously approved.

\section{e) Honorary Secretary Adjunct 2006-2007}

The Council's election of Dr. M. Stumvoll (D) was unanimously approved.

\section{f) Chair, Postgraduate Education Sub-committee 2006-2009}

The Council's election of Dr. J. Nolan (IRE) was unanimously approved with one abstention.

\section{g) Council Members 2007-2010}

The following Council Members were unanimously elected by the General Assembly:

Drs. F. Ashcroft (UK)

F. Bosch (E)

V. Petrenko (L)

A. Siebenhofer (A). 


\section{STUDY GROUPS}

Dr. Eizirik announced that Dr. A. Cuesta and colleagues had proposed creating a new Study Group: European Group for the Study of Genetics of Diabetes. The Council unanimously agreed with the creation of the Study Group. The Honorary Secretary announced that Dr. A. Ceriello and colleagues had proposed creating a new Study Group: EASD Study group on Diabetes and Cardiovascular Disease. The Council unanimously agreed with the creation of the Study Group.

The General Assembly unanimously approved the creation of the two new Study Groups.

\section{HONORARY MEMBERSHIP}

Drs. E. Cerasi and J. Roth were unanimously elected by the General Assembly.

\section{ANY OTHER BUSINESS}

There was no other business.

Dr. Ferrannini thanked the outgoing members of the Executive for their dedication and friendly cooperation. He presented Drs. Škrha and Standl with the Albert Renold Medal.

Dr. Ferrannini also thanked the outgoing members of the Council: Drs. I. Gourieva, S. Lenzen, C. Sanjeevi and J.-L. Selam.

Dr. Ferrannini thanked the industry for their support. He also expressed his sincere gratitude to Dr. J Nerup for his outstanding contribution to the organisation of the 42nd EASD Annual Meeting. He again thanked Dr. D. L. Eizirik and the members of the Programme Committee for their hard work with regard to the scientific programme. The President warmly thanked the ladies working in the EASD secretariat for their kind and efficient help.

Dr. Ferrannini brought the General Assembly to a close at 19:15. 


\section{Agenda for the 43rd General Assembly of the European Association for the Study of Diabetes}

to be held in Amsterdam, The Netherlands on 20 September 2007 at 18:15 hours

\section{Minutes of the 42nd General Assembly, Copenhagen, Denmark 2006}

\section{Reports}

a) President

b) Honorary Treasurer

c) Honorary Auditors

d) Honorary Secretary

e) Editor in Chief, Diabetologia

f) Chair, Postgraduate Education Sub-committee

\section{Elections}

a) President 2008-2011

b) Honorary Secretary 2007-2010

c) Editor-in-Chief 2008-2009

d) Council Members 2008-2011
Dr. E. Ferrannini

Dr. J. Škhra (2003-2006)

Dr. G. Spinas (2006-2009)

Dr. N. Katsilambros

Dr. M. Tiedge

Dr. D. L. Eizirik

Dr. E. Gale

Dr. J. Nolan in place of

Dr. E. Ferrannini

in place of

Dr. D. L. Eizirik

Extension:

Dr. E. Gale

in place of

Dr. K. Dahl Jørgensen (Norway)

Dr. R. J. Heine (The Netherlands)

Dr. B. Mankovsky (Ukraine)

Dr. S. Sasson (Israel)

\section{Study Groups}

\section{Honorary Membership}

6. Any other business 


\title{
$43^{\text {rd }}$ EASD Annual Meeting of the European Association for the Study of Diabetes
}

\author{
Amsterdam, The Netherlands, 18 - 21 September 2007
}

\author{
Abstracts \\ Index of Oral Sessions \\ OP 1 Prediction of diabetes \\ OP 2 Lipoproteins and vascular disease \\ OP 3 Brain metabolism \\ OP 4 Molecular regulation of beta cell function and \\ survival \\ OP 5 Endothelial biology in diabetes \\ OP 6 Predictors and markers of cardiovascular disease \\ and mortality \\ OP 7 Body weight regulation \\ OP 8 Insulin therapy: physiology and pharmacokinetics \\ OP 9 Insulin secretion in vivo \\ OP 10 Immunopathogenesis of type 1 diabetes: from mice \\ to men \\ OP 11 Nephropathy: mechanisms \\ OP 12 Prevalence of childhood diabetes and mortality in \\ type 2 diabetes \\ OP 13 Thiazolidinediones \\ OP 14 Genome-wide association scans \\ OP 15 Signal transduction and metabolism in liver \\ OP 16 Mechanisms of beta cell dysfunction and death \\ OP 17 Cardiovascular disease: role of glycaemia and other \\ factors \\ OP 18 Models of care \\ OP 19 GLP-1 based therapies \\ OP 20 Pregnancy: screening, epidemiology and outcomes \\ OP 21 Adipocyte derived peptides \\ OP 22 Signal transduction in metabolism \\ OP 23 Peripheral neuropathy: novel therapies \\ OP 24 Zinc transporter SLC30A8: candidate gene for beta \\ cell function \\ OP 25 Lifestyle \\ OP 26 Inflammation in metabolism \\ OP 27 Psychological aspects of diabetes \\ OP 28 Imaging beta cell signal transduction \\ OP 29 Genetics of type 2 diabetes \\ OP 30 Mitochondrial function \\ OP 31 Hypoglycaemia \\ OP 32 Diabetes in children and adolescents in all its forms \\ OP 33 Adipocytes \\ OP 34 Regulation of insulin exocytosis \\ OP 35 Mechanisms of cardiovascular complications: \\ animal models \\ OP 36 Nephropathy: treatment \\ OP 37 Novel therapies \\ OP 38 Insulin therapy: clinical studies \\ OP 39 Retinopathy: clinical observations \\ OP 40 Islet cell differentiation and neogenesis
}

OP 41 Novel biomarkers for coronary vascular disease

OP 42 Metabolism in vasculature

OP 43 Metformin and sulfonylureas

OP 44 Treatment of type 1 diabetes in pregnancy

OP 45 Incretin effects: clinical

OP 46 Genetics of type 1 diabetes mellitus

OP 47 Adiponectin and diabetic complications

OP 48 Making the best use of diabetes technology

\section{Index of Poster Sessions}

PS 1 Obesity as a predictor of type 2 diabetes

PS 2 The spectrum of diabetic disorders

PS 3 Genetics and epidemiology of metabolic syndrome, stroke and hypertension

PS 4 Genome-wide association scans and bioinformatic tools for identifying candidate pathways

PS 5 Genetics and epidemiology of type 1 diabetes and LADA

PS 6 Type 2 diabetes genes

PS 7 Updates on the type 2 diabetes genes TCF7L2 and PPARgamma

PS 8 Epidemiology: treatment

PS 9 Diabetes mortality and hospitalisation in different populations

PS 10 Cardiovascular risk factors

PS 11 Gender, intrauterine environment and risk of diabetes

PS 12 Social and economic complications of diabetes

PS 13 Environmental factors in diabetes aetiology

PS 14 Genetics and epidemiology of diabetic nephropathy

PS 15 Classification of IGT, type 2 diabetes and metabolic syndrome

PS 16 Diabetes prevalence and incidence

PS 17 Circulating risk factors

PS 18 Genetics of beta cell function and insulin resistance

PS 19 Gluco- and lipotoxicity as mediators of beta cell degeneration

PS 20 ER stress, ROS and FFA-induced beta cell degeneration

PS 21 Putative mediators of beta cell damage in type 2 diabetes

PS 22 Beta cells - a choice of death

PS 23 Beta cell dysfunction and death following different insults

PS 24 The role of cytokines and chemokines on pancreatic islet physiology and pathology 
PS 25 Immunoregulation and novel interventions in diabetes secondary to beta cell loss

PS 26 Immunopathogenesis of animal and human diabetes

PS 27 Immunology and prediction of type 1 diabetes

PS 28 Pancreas and islet transplantation

PS 29 Regulation of glucagon synthesis and release

PS 30 Transcriptional regulation in beta cells

PS 31 Beta cell neogenesis and replication

PS 32 Mediators of insulin secretion

PS 33 Intracellular signaling for insulin secretion

PS 34 Metabolic regulation of insulin secretion

PS 35 Stem cells and extracellular matrix in beta cell growth

PS 36 Structural and functional aspects of beta cell regulation

37 The signal transduction of cytokine-induced beta cell degeneration

38 Signal transduction I

39 Signal transduction II

40 Transcription factors in metabolism

41 Incretin effects: clinical

42 Incretin effects: experimental I

43 Incretin effects: experimental II

44 Insulin secretion in vivo

45 Adiponectin

46 Insulin sensitivity

47 Carbohydrate metabolism: humans

48 Adipose tissue biology and treatment

49 Adipokines: effects of weight loss

50 Animal models of insulin resistance

51 Metabolism: children

52 Inflammatory markers

53 Obesity therapy

54 Insulin action in the critically ill

55 ER stress and insulin resistance

56 Other hormones

57 DPP-4

58 Incretin release

59 Adipocyte differentation

60 Insulin and vasculature

61 Non-alcoholic steatohepatitis

62 Oxidative stress

63 Fetal programming

64 Nitric oxide

65 Adipocytes

$66 \mathrm{RBP} 4$ and resistin

67 Mitochondria

68 Carbohydrate metabolism - glucose transport

69 Iron / anaemia

70 Lipid metabolism

71 Polycystic ovary syndrome

72 Nutrition

73 Exercise

74 Hypoglycaemia

75 Lifestyle

76 Antiobesity drugs

77 Obesity-related disorders

78 GLP-1 receptor agents
PS 79 Type 2 diabetes: mechanisms

PS 80 Type 2 diabetes: clinical

PS 81 Metformin and sulphonylurea

PS 82 DPP-4 inhibitors: session I

PS 83 DPP-4 inhibitors: session II

PS 84 Body weight regulation

PS 85 Prediction, detection and biomarkers

PS 86 Novel therapies, drugs

PS 87 Novel therapies, plant-derived

PS 88 Diabetes in childhood

PS 89 Care and treatment of diabetes in pregnancy

PS 90 Diabetes in pregnancy: education and diet

PS 91 Causes and consequences of gestational diabetes

PS 92 PumPS and analogues

PS 93 Pharmacokinetics and physiology of insulin therapy

PS 94 Novel insulins

PS 95 Insulin analogues in type 2 diabetes

PS 96 Pre-mixed insulins

PS 97 Insulin therapy in routine practice and special situations

PS 98 Devices and information technology

PS 99 Continuous glucose monitoring systems

PS 100 Health care delivery: national variations in practice

PS 101 Health care delivery: keeping up with the guidelines

PS 102 Costs of diabetes

PS 103 Socio-economic aspects of diabetes

PS 104 Psychological aspects

PS 105 Education and psychological aspects

PS 106 Neuropathy: diagnosis and prevalence

PS 107 Nephropathy: assessing renal function

PS 108 Eye and brain

PS 109 Neuropathy: cardiovascular links

PS 110 Complications: role of glycation

PS 111 Diabetic foot: natural history and intervention

PS 112 Nephropathy: clinical treatment

PS 113 Diabetic foot: skin and bone

PS 114 Complications: new markers and inflammation

PS 115 Neuropathy: symptoms and treatment

PS 116 New mechanisms in diabetic retinopathy

PS 117 Nephropathy and end-stage renal failure

PS 118 Hypertension: clinical management

PS 119 Vascular biology: in vitro studies

PS 120 Endothelium, arterial stiffness and platelets: human studies

PS 121 Glycation and vascular disease

PS 122 Cardiovascular complications: animal studies

PS 123 Cardiac complications I

PS 124 Cardiac complications II

PS 125 Epidemiology of cardiovascular risk factors in diabetes

PS 126 Cardiovascular risk in diabetes: prospective studies

PS 127 Lipids, lipoproteins and macrovascular disease

PS 128 Biomarkers for cardiovascular disease

PS 129 Genetic determinants of dyslipidaemia and cardiovascular disease

PS 130 Cardiovascular complications: therapeutic interventions 


\section{OP 1 Prediction of diabetes}

\section{1}

\section{Predicting diabetes: performance of six diabetes risk questionnaires in} the Hoorn Study

M. Alssema ${ }^{1}$, G. Nijpels ${ }^{1}$, L. M. Bouter ${ }^{1}$, C. D. A. Stehouwer ${ }^{2}$, R. J. Heine ${ }^{3}$, J. M. Dekker';

${ }^{1}$ EMGO Institute, VU University Medical Center, Amsterdam,

${ }^{2}$ Department of Internal Medicine, Academic Hospital Maastricht,

${ }^{3}$ Department of Endocrinology / Diabetes Center, VU University Medical

Center, Amsterdam, The Netherlands.

Background and Aims: Several investigators have developed simple risk questionnaires not requiring blood testing to detect undiagnosed prevalent type 2 diabetes (DM2) or to predict incident DM2. Until now, no extensive external validation of available risk questionnaires with oral glucose tolerance test (OGTT) defined incidence of DM2 has been made. We evaluated the performance of existing diabetes risk questionnaires in detection of undiagnosed DM2 and in prediction of incident DM2.

Materials and Methods: Four risk questionnaires for prevalent DM2 using routinely available data were found: the Danish diabetes risk score, the Cambridge risk score, the Rotterdam risk score and a symptom-risk questionnaire (SR-Q) from the Hoorn Study. Two risk questionnaires for incident DM2 were found; the ARIC and the Finnish diabetes risk score. These risk scores were evaluated in the Hoorn Study, which is a populationbased prospective cohort study with repeated OGTT measurements. OGTTdata were used for definition of prevalent (150 cases out of 2379) and 6.4-year incident DM2 (134 cases out of 1353).

Results: The area under the ROC curve was generally higher for prevalent DM2 (range from 0.69 to 0.76 ) than it was for incident DM2 (range from 0.63 to 0.69 ). Although the performance of the risk scores was very similar, the ARIC risk score performed best for both prevalent $(0.76[0.73-0.80])$ and incident DM2 (0.69 [0.64-0.73]) (Figure).

Conclusion: Available risk questionnaires developed in Caucasian populations are less accurate in prediction of incident DM2 as compared to detection of prevalent undiagnosed DM2. The slightly better performance of the ARIC risk questionnaire might be due to the fact that it was developed on incident DM2, defined by the OGTT. Because of the high cardiovascular risk constituted by post-load OGTT levels, for primary prevention, it is probably important to develop a diabetes risk questionnaire based on OGTT data.
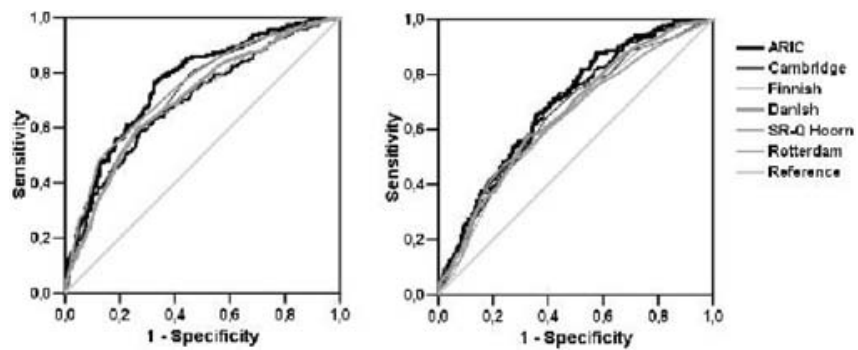

Fig 1. ROC curves for prediction of prevalent (left) an incident (right) type 2 diabetes

Supported by the Dutch Diabetes Research Foundation

\section{2}

Additive effects of adiponectin and C-reactive protein in predicting the risk of type 2 diabetes: a 10 -year prospective study

K. S. L. Lam ${ }^{1,2}$, N. M. S. Wat ${ }^{1}$, A. W. K. Tso ${ }^{1}$, C. H. Y. Fong ${ }^{1}$, A. Xu ${ }^{1,2}$;

${ }^{1}$ Department of Medicine, ${ }^{2}$ Research Centre of Heart, Brain, Hormone, and Healthy Aging, The University of Hong Kong, Hong Kong, China.

Background and Aims: Both serum adiponectin, an adipokine with insulinsensitizing and anti-inflammatory action, and high sensitivity C-reactive protein (hsCRP), a marker of chronic low-grade systemic inflammation, have been reported to predict the development of type 2 diabetes. Here we examined, in a 10-year prospective study, whether these two biomarkers had an additive effect on predicting the long-term risk of type 2 diabetes. Materials and Methods: Serum adiponectin and hpCRP were measured in 417 non-diabetic Chinese subjects from the Hong Kong Cardiovascular Risk Factor Prevalence Study, who had returned for their 10-year follow-up in 2005/06. Of these, 76 had developed DM, according to the 1998 WHO diagnostic criteria. Adiponectin was measured using an in-house ELISA assay and hsCRP was measured using a particle-enhanced immunoturbidimetric assay. The association of adiponectin and hsCRP, alone or in combination, with the 10-year risk of diabetes was investigated.

Results: Compared to the 341 subjects who remained non-diabetic at year10 , subjects who had developed diabetes consisted of more men $(\mathrm{p}<0.003)$. They had higher baseline BMI, waist circumference, mean arterial blood pressure, plasma glucose (fasting and 2-hour post-OGTT), fasting insulin, HOMA-insulin resistance index (HOMA-IR), triglycerides, LDL-cholesterol and hsCRP levels (all $\mathrm{p}<0.001$ except for LDL-cholesterol with $\mathrm{p}<0.05$ ). They also had lower baseline serum adiponectin and HDL-cholesterol levels (both $\mathrm{p}<0.001)$. In a stepwise multiple logistic regression analysis model including sex, age, BMI, fasting insulin, presence of metabolic syndrome according to NCEP criteria, adiponectin and hsCRP, only baseline adiponectin (adjusted OR 0.44; 95\% CI 0.27-0.74; $\mathrm{p}=0.002$ ), hsCRP (adjusted OR 1.43; 95\% CI $1.10-1.84 ; \mathrm{p}=0.007$ ) and metabolic syndrome (adjusted OR 2.72; 95\% CI $1.55-4.78 ; \mathrm{p}=0.001$ ) were independently predictive of diabetes at 10 years. The addition of baseline adiponectin or hsCRP to a model consisting of sex, age and BMI significantly improved the prediction of diabetes risk, as reflected by the likelihood ratios in logistic regression analysis $(\mathrm{p}=0.001$ for adiponectin and $p=0.019$ for hsCRP). Furthermore, the combined addition of adiponectin and hsCRP to the model provided significantly greater improvement than the addition of adiponectin alone ( $\mathrm{p}=0.048)$ or the addition of hsCRP alone $(\mathrm{p}=0.003)$.

Conclusion: Serum adiponectin and hsCRP were both independent predictors of the 10-year risk of diabetes in this Chinese cohort. Their usefulness as biomarkers for predicting the development of type 2 diabetes were over and above those of conventional risk factors including sex, age and BMI, and appeared to be additive when these two new biomarkers were used together.

Supported by the STR seeding fund on Healthy Aging of the University of Hong Kong and the Innovation \& Technology Fund

\section{3}

Comparison of a risk score based on non-invasive measurements and fasting glucose for prediction of type 2 diabetes risk in the EPICPotsdam Study

M. B. Schulze ${ }^{1}$, K. Hoffmann ${ }^{1}$, H. Boeing ${ }^{1}$, C. Thamer ${ }^{2}$, H.-U. Häring ${ }^{2}$, A. Fritsche ${ }^{2}$, H. Al-Hasani ${ }^{3}$, H.-G. Joost ${ }^{3}$;

${ }^{1}$ Department of Epidemiology, German Institute of Human Nutrition Potsdam-Rehbruecke, Nuthetal, ${ }^{2}$ Department of Internal Medicine IV, University of Tübingen, ${ }^{3}$ Department of Pharmacology, German Institute of Human Nutrition Potsdam-Rehbruecke, Nuthetal, Germany.

Background and Aims: The German Diabetes Risk Score (DRS) is a tool for screening of large populations for individuals at high risk of developing type 2 diabetes based on non-invasive measurements. However, it remains unclear whether its prediction is comparable to or could be enhanced by other screening methods, in particular fasting plasma glucose (FPG).

Materials and Methods: In the prospective EPIC-Potsdam Study, 9,711 men and 15,402 women aged 35-65 years and free of diabetes were followed for incident type 2 diabetes with 849 detected incident cases of physician confirmed type 2 diabetes over an average of 7 years of follow-up. Among the 7588 participants with fasting blood samples at baseline, there were 200 incident cases, and we randomly selected 647 fasting participants as subcohort for a case-cohort with all participants having FPG $<126 \mathrm{mg} / \mathrm{dl}$ at baseline. We evaluated the predictive power of the German Diabetes Risk Score, FPG, and the combination of both to identify individuals at high risk to develop type 2 diabetes through receiver operator characteristic (ROC) curve analysis based on logistic regression models with the area under the curve (AUC) being a measure of the discriminatory power.

Results: The German Diabetes Risk Score, consisting of information on age, waist circumference, height, history of hypertension, physical activity, smoking, and consumption of red meat, whole-grain bread, coffee, and alcohol, performed similarly to fasting glucose (ROC AUC: 0.845 and 0.852 , 
respectively). A model consisting of the German Diabetes Risk Score plus FPG performed better (ROC AUC: 0.905$)$. Sensitivity and specificity were $85.0 \%$ and $68.2 \%$ for Score Points $>=500,93.0 \%$ and $23.7 \%$ for FPG $>=80$ $\mathrm{mg} / \mathrm{dl}, 81.5 \%$ and $67.7 \%$ for FPG $>=90 \mathrm{mg} / \mathrm{dl}$ and $50.0 \%$ and $93.0 \%$ for FPG $>=100 \mathrm{mg} / \mathrm{dl}$. The corresponding sensitivity and specificity for $>=500$ Score Points plus FPG $>=80 \mathrm{mg} / \mathrm{dl}$ were $81.0 \%$ and $73.2 \%$, and for $>=500$ Score Points plus FPG $>=90 \mathrm{mg} / \mathrm{dl} 73.0 \%$ and $83.9 \%$, respectively.

Conclusion: The German Diabetes Risk Score (http://drs.dife.de/en) predicts incident type 2 diabetes similarly well as FPG. In addition, FPG as a second screening step in addition to the German Diabetes Risk Score enhances the discriminatory power.

Supported by Federal Ministry of Science, Germany, European Union, German Cancer Aid, European Community

\section{4}

Atrial natriuretic peptide correlates with insulin resistance/impaired glucose tolerance in cardiopathic patients affected by valvulopathy

P. C. G. Lucotti, L. D. Monti, E. Setola, E. Galluccio, M. G. Pala,

A. Rossodivita, S. Costa, M. Oldani, G. La Canna, A. Castiglioni, E. Bosi, O. Alfieri, P. Piatti;

Medicine Divison, Diabetology, Endocrinology and Metabolic Disease Unit, Scientific Institute San Raffaele, Milan, Italy.

Background and Aims: Few data are available concerning insulin resistance and glucose tolerance in patients affected by cardiac valvulopathy. The pathogenetic aspects involved in such alterations could be linked with the metabolic effects of myocardial-secreted peptides in condition of myocardial damage or dysfunction, as atrial natriuretic peptide (ANP) that has been shown to exert a potent lipolytic effect. The aim of the present study was to correlate ANP levels with glucose tolerance, insulin resistance, NEFA and inflammation markers.

Materials and Methods: Thirty patients (Group 1) with aortic/mitral valve disease before valve replacement and 58 patients (Group 2) submitted to aortic/mitral valve replacement were compared to 58 normal subjects (Group 3). A standard OGTT was performed in the three groups. None of the patients of group 1 and 2 had known diabetes while all the patients showed a normal ejection fraction and, at coronarography before intervention, the coronaries were free from atherosclerotic lesions.

Results: In group 1, according to glycemic levels after OGTT, 39\% of the patients had IGT and $25 \%$ had a diabetic response. Similar results were found in group 2 where $43 \%$ of the patients had IGT and $19 \%$ with a diabetic response while the normal subjects were NGT. Mean HbAlc levels were significantly higher in Group 1 and 2 compared to normal subjects. Insulin sensitivity measured during OGTT (ISI index) showed a marked insulin resistance in Group 1 as in Group 2 compared to normal subjects, independently from the degree of glucose tolerance (diabetic response: $4.4 \pm 1.1$ and $2.1 \pm 1.2$; IGT: $5.8 \pm 0.7$ and $5.6 \pm 0.7$; NGT: $15.5 \pm 2.2$ and $12.4 \pm 3.1$ for group 1 and 2 vs group 3: $\left.21.7 \pm 1.910^{-4} \mathrm{dl} \mathrm{kg}^{-1} \mathrm{~min}^{-1} / \mu \mathrm{Uml}^{-1}, \mathrm{p}<0.0001\right)$. Interestingly, ANP levels were significantly higher in group 1 and $2(148.9 \pm 22.4$ and $67.9 \pm 1.2 \mathrm{pg} / \mathrm{ml})$ than in normal subjects $(32.4 \pm 1.4 \mathrm{pg} / \mathrm{ml} ; \mathrm{p}<0.001)$. In accordance with a possible lipolytic effect of ANP, NEFA levels were significantly increased in group 1 and 2 , in the fasting state $(0.81 \pm 0.04$ and $0.50 \pm 0.03$ vs $0.38 \pm 0.02$ $\mathrm{mmol} / \mathrm{l}, \mathrm{p}<0.001$ ) and during OGTT (at $60 \mathrm{~min}: 0.36 \pm 0.04$ and $0.20 \pm 0.01$ vs $0.15 \pm 0.01 \mathrm{mmol} / \mathrm{l}, \mathrm{p}<0.002$ ). ANP levels significantly correlated with NEFA levels, $\mathrm{HbAlc}$ and ISI index.

Conclusion: In conclusion, chronic valvular disease, in the absence of atherosclerosis, seems to be characterized by high ANP levels which, in turn, determine a lipolytic effect, increasing NEFA levels. This mechanism could explain the pathogenesis of insulin resistance and impaired glucose tolerance in this class of patients. Furthermore, patients with chronic valvular disease represent a new class of patients at high risk for developing type 2 diabetes and they need to be inserted in programs for early diagnosis and treatment of this disease.
0005

Novel biomarkers identified by protein profiling: potential new diagnosis and therapeutics for type 2 diabetes

L. Liu ${ }^{1}$, Z. Xie ${ }^{1}$, S. Zangen ${ }^{2}$, R. Manteiga ${ }^{1}$, S. Hacohen ${ }^{2}$, W. W. Cheatham ${ }^{3}$, I. Raz ${ }^{2}$, C. Gelber';

${ }^{1}$ SCIENCE, ATCC, Manassas, United States, ${ }^{2}$ Diabetes Unit, Hadassah Hebrew University Medical Center, Jerusalem, Israel, ${ }^{3}$ Research, MedStar Research Institute, Washington, DC, United States.

Background and Aims: The pre-diabetic state can be present for 10 or more years prior to detection of glycemic disorders. Treatment of pre-diabetics can suspend or prevent Diabetes; yet few pre-diabetics are identified and treated since no simple laboratory test exists to determine the actual risk of an individual to develop Type 2 Diabetes (T2D). Due to the delay in diagnosis, diabetes complications have already advanced and thus, the future risk of further complication and derailment is severely increased.

Materials and Methods: We applied various proteomics approaches to identify disease-specific biomarkers in serum samples from Cohen Diabetic (CD) Rat Model, T2D patients and matched healthy individuals. The Cohen diabetic (CD) rat is comprised of 2 strains manifesting many features common to T2D in humans. The sensitive strain (CDs) develops diabetes within 30 days of a high sucrose/copper poor diet (HSD), while the resistant strain (CDr) retains normal blood glucose when fed with the same diet. Neither strain shows any signs of diabetes when provided regular rodent diet (RD). CD rat serum samples were profiled using Surface-Enhanced Laser Desorption/ Ionization mass spectrometry, two-dimensional liquid chromatography and 2-dimensional gel electrophoresis. The protein markers were sequenced using liquid chromatography tandem mass spectrometry (LC/MS/MS).

Results: A constellation of differentially expressed proteins emerged when profiled proteins from normal serum were compared with diabetic samples. A panel of 27 differentially expressed biomarker \{Apolipoproteins (C-III precursor, $\mathrm{C} 2, \mathrm{~A}-1, \mathrm{E}$, and $\mathrm{H}$ ) and liver regeneration-related protein)\}, inflammatory mediators (Complement component C9), protease inhibitors (Thrombin, Serpina 3M, LMW T-kininogen I precursor, inter- $\alpha$-inhibitor $\mathrm{H} 4$ heavy chain and Serpinc 1), proteins associated with insulin signaling pathway ( $\alpha-2$-HS-glycoprotein, pregnancy-zone protein, Fetuin $\beta$ and Spin 2a) and several novel and unknown proteins. A predicted protein of the Serpin family was found to be upregulated in $\mathrm{CDr}$ and share homology with the Cterminal fragment of Serpina 3M (D3). WB using a D3 specific polyclonal serum showed that the full length protein, $\sim 50 \mathrm{kDa}$, is expressed in the liver and secreted into the serum of $\mathrm{CDr}$ and $\mathrm{CDs}$ rats, while the $\mathrm{D} 3$ polypeptide fragment was detected in the serum of CDr only. To investigate the relevance of this biomarker to human T2D, a Western Blot was performed on human serum samples reacted with D3 specific polyclonal serum. A doublet protein bands with MW of $60 \sim 80 \mathrm{kDa}$ was shown to be differently expressed with a $\sim 65 \mathrm{kD}$ band emphasized in T2D patients whereas the $\sim 75 \mathrm{kDa}$ band is emphasized in healthy individuals. In addition, a smaller fragment $(\sim 20 \mathrm{kDa})$ reacting with D3-specific serum appears to be downregulated in diabetic patients as compared with healthy individuals.

Conclusions: In this study, we demonstrated that using proteomics profiling of normal and T2D serum samples we identified a constellation of differentially expressed protein/peptide markers, some of which were novel, associated with inflammation, insulin signaling, lipid metabolism, and several unknown. Their potential use for development of early diagnosis or therapeutics of T2D is investigated.

\section{6}

Quantification of a subset of 48 blood-borne protein biomarkers in a case controlled study can distinguish between converters to diabetes type 2 within 5 years and matched non-converters

M. P. McKenna ${ }^{1}$, R. Gerwien ${ }^{1}$, E. Moler ${ }^{1}$, J. Kolberg ${ }^{1}$, M. Urdea ${ }^{1}$, V. Lyssenko ${ }^{2}$, T. Tuomi ${ }^{3}$, B. Isomaa ${ }^{4}$, L. Groop ${ }^{2}$;

${ }^{1}$ Research, Tethys Bioscience, Emeryville, United States, ${ }^{2}$ Clinical Science, Diabetes and Endocrinology, Lund University, Malmo, Sweden, ${ }^{3}$ Department of Medicine, Helsinki University Hospital, Finland, ${ }^{4}$ Folkhalsan Ostanlid, Malmska Municipal Health Care Center and Hospital, Jakobstad, Finland.

Background and Aims: The Botnia Study is a family based study initiated in 1990 to identify genes predisposing individuals to type 2 diabetes. From this 
cohort we have compared individuals who did and did not convert to diabetes to determine whether or not a panel of serum protein biomarkers can be used to predict the future development of type 2 diabetes within 5 years.

Materials and Methods: Molecular Counting Technology (MCT), a new ultrasensitive protein detection method, was employed to quantify 42 proteins in small volumes (on average $1.3 \mu \mathrm{L} /$ assay) of 444 blood samples from the Botnia cohort. The nested case/control study involved baseline blood samples collected from 148 subjects who developed diabetes during a $7 \mathrm{yr}$ follow-up period and matched 2:1 (296 subjects) for BMI, family history, age, gender, and glucose tolerance status with non-converters. Baseline test results for the subjects (e.g. BMI, age, SBP, DBP, smoking history, fasting glucose, 2 hour glucose, total cholesterol, HDL cholesterol, triglycerides, free fatty acids (FFA), serum insulin (at -10, 0, 30, 60, 120 min of OGTT) , etc.) were available and used in conjunction with the new protein information. A total of 48 molecular biomarkers and 5 clinical parameters were tested. Sixteen statistical predictive models and marker search algorithms were used for the analysis and were compared based upon Akaike Information Criterion (AIC) and the c-statistic.

Results: Seven of the 48 biomarkers quantifications (including both proteins and other blood borne markers) provided the majority of the statistical distinction noted for the two populations: seven biomarkers were selected in the majority of models. In this analysis only subjects who converted within 5 yrs $(n=60)$ and non-converters with at least 8 years of follow-up $(n=177)$ were included. Consistent with the initial matching of subjects, BMI, family history, age and glucose were not significant factors. The best models generated from repeated 10 -fold cross-validations involved only protein markers (c statistic $=0.73-0.78$ ). Although individual proteins had $\mathrm{p}$-values as low as $10^{-4}$ individually, the concentration of no single biomarker of the 48 studied was capable of distinguishing the converter from non-converter populations as well as statistical models constructed from multiple proteins. Existing risk indices based upon age, gender, family history, SBP, HDL and FGT were also significantly less useful for segmenting the two closely matched groups (c statistic $=0.58-0.63$ ). The seven protein biomarkers in the new panel are related to glucose and lipid metabolism, inflammation, and the coagulation and complement cascades.

Conclusion: A panel of biomarkers may provide a new tool for identifying individuals at future risk of developing T2D with a sensitivity and specificity of approximately $80 \%$.

\section{OP 2 Lipoproteins and vascular disease}

\section{7}

Parabolic relation between plasma triglycerides and LDL-cholesterol in the general population and patients with type 2 diabetes mellitus 6-year follow-up results from the Hoorn study

M. Brouwers ${ }^{1}$, J. Dekker ${ }^{2}$, A. Georgieva ${ }^{1}$, M. van Greevenbroek ${ }^{1}$, C. van der Kallen ${ }^{1}$, R. Heine ${ }^{2,3}$, T. de Bruin ${ }^{4}$, C. Stehouwer ${ }^{1}$;

${ }^{1}$ Internal Medicine, University Hospital Maastricht, The Netherlands, ${ }^{2}$ EMGO Institute, VU University Medical Center, Amsterdam,

The Netherlands, ${ }^{3}$ Endocrinology/Diabetes Center, VU University Medical Center, Amsterdam, The Netherlands, ${ }^{4}$ Translational Genetics and Medicine, GlaxoSmithKline, Research Triangle Parc, United States.

Background and Aims: Patients with type 2 diabetes mellitus often present with moderately elevated plasma triglycerides and fairly normal LDLcholesterol levels. Of interest, lowering of plasma triglycerides, e.g. by lifestyle intervention or fibrates, is often accompanied by an increase in LDLcholesterol in these patients.

In the present study, we hypothesized that plasma triglycerides and LDLcholesterol are related in a parabolic fashion, i.e. at low triglycerides $(<1.5$ $\mathrm{mmol} / \mathrm{L}$ ) an increase in plasma triglycerides is accompanied by an increase in LDL-cholesterol, whereas a further increase in plasma triglycerides is followed by a decline in LDL-cholesterol levels. This hypothesis was based on the fact that triglycerides are mainly transported by VLDL2 particles at low plasma triglycerides, whereas VLDL1 particles are abundant at high triglycerides levels. The former particle is mainly degraded into buoyant LDL particles, the latter into small-dense LDL.

Materials and Methods: Data were obtained twice with an interval of six years in 1343 subjects derived from the general population (the Hoorn study). Three groups were defined: one with triglycerides $<1.5 \mathrm{mmol} / \mathrm{L}$ at both time-points (group A), one group with triglycerides $<1.5 \mathrm{mmol} / \mathrm{L}$ at one and $>1.5 \mathrm{mmol} / \mathrm{L}$ at the other measurement (group B), and one group with triglycerides $>1.5 \mathrm{mmol} / \mathrm{L}$ at both measurements (group C). The diabetic state was ascertained by an oral glucose tolerance test.

Results: In a mixed linear model for repeated measurements, a positive relation between triglycerides and LDL-cholesterol was observed for males in group $A(\beta=0.5, p<0.001)$ and group $B(\beta=0.2, p<0.001)$, whereas a negative relation was found for males in group $C(\beta=-0.2, p=0.003)$ (Figure 1a). The regression slopes did not differ between subjects with and without type 2 diabetes mellitus. In the female subgroup, the relation was similar, but not significantly negative in group $C(\beta=-0.1, p=0.4)$ (Figure $1 b)$. Again, the results were not different for female diabetic patients.

Conclusion: The present parabolic relation between triglycerides and LDLcholesterol provides more insight in the complex relation of clinically relevant cardiovascular risk markers and shows that one cannot interpret one without the other. (Fig. 1)

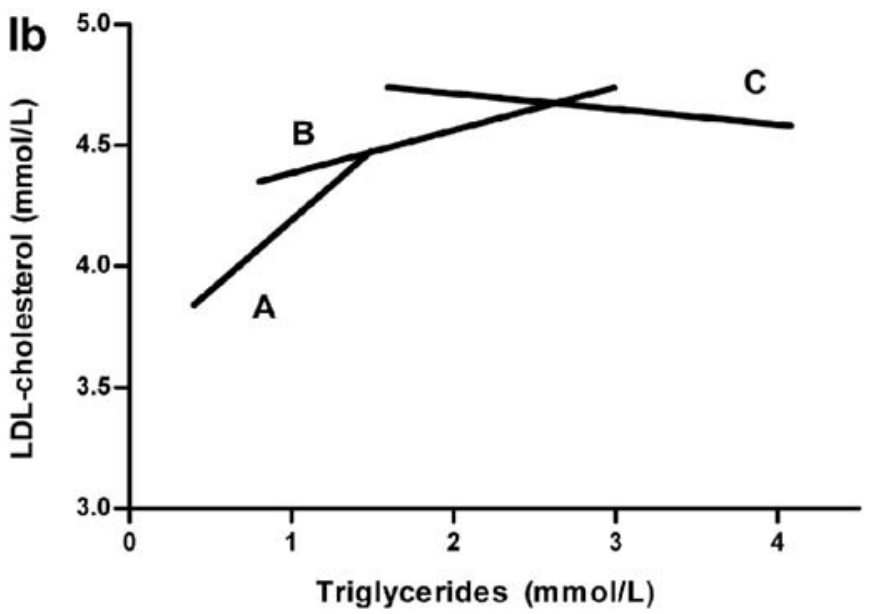

Fig. 1

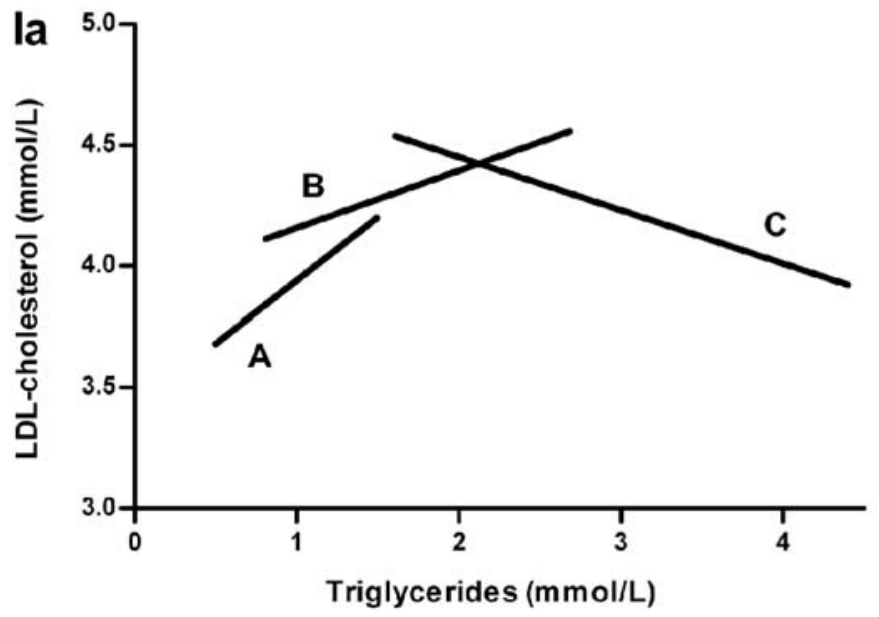




\section{8}

Hypertriglyceridaemic waist predicts coronary heart disease in the EPIC-Norfolk Prospective Population Study

B. J. Arsenault ${ }^{1}$, I. Lemieux ${ }^{1}$, J.-P. Després ${ }^{1}$, N. J. Wareham ${ }^{2}$, R. Luben ${ }^{3}$, J. J. P. Kastelein ${ }^{4}$, K.-T. Khaw ${ }^{3}$, S. M. Boekholdt';

${ }^{1}$ Québec Heart Institute, Hôpital Laval Research Centre, Canada,

${ }^{2}$ Medical Research Council Epidemiology Unit, University of Cambridge, United Kingdom, ${ }^{3}$ Department of Public Health and Primary Care, Institute of Public Health, Cambridge, United Kingdom, ${ }^{4}$ Department of Vascular Medicine, Academic Medical Center, Amsterdam, The Netherlands, ${ }^{5}$ Department of Cardiology, Academic Medical Center, Amsterdam, The Netherlands.

Background and Aims: The combination of an elevated waist circumference and triglyceride (TG) levels, the so-called hypertriglyceridemic waist (HTGW) phenotype, could represent an inexpensive and practical method that could be performed on a regular basis by health professionals in order to identify patients carrying features of the metabolic syndrome. The objective of the present study was to investigate whether the HTGW phenotype is associated with an increased risk of CHD in apparently healthy men and women.

Materials and Methods: A prospective case-control study nested in the EPIC-Norfolk cohort was performed. Cases were apparently healthy men and women aged 45 to 79 years who developed fatal or nonfatal CHD $(n=927)$, and were matched by age, gender and enrollment time to 1642 controls who remained free of CHD. HTGW was defined as waist girth $>90 \mathrm{~cm}$ and TG levels $>2.0 \mathrm{mmol} / \mathrm{L}$ for men and as waist girth $>85 \mathrm{~cm}$ and TG levels $>1.5$ $\mathrm{mmol} / \mathrm{L}$ for women.

Results: Men with CHD had higher waist girths $(98 \pm 10$ vs. $95 \pm 9 \mathrm{~cm}$, $\mathrm{p}<0.001)$ and TG levels [1.9 (1.4-2.7) vs. $1.7(1.2-2.3) \mathrm{mmol} / \mathrm{L}, \mathrm{p}<0.001]$ compared to CHD-free controls. Women with $\mathrm{CHD}$ also had higher waist girths ( $87 \pm 11$ vs. $83 \pm 10 \mathrm{~cm}, \mathrm{p}<0.001)$ and TG levels [1.8 (1.3-2.4) vs. 1.5 (1.1-2.1) $\mathrm{mmol} / \mathrm{L}, \mathrm{p}<0.001]$ compared to CHD-free controls. Men with the HTGW phenotype had unadjusted hazard ratio (HR) for future CHD of 1.66 (95\% CI, 1.33 to $2.07 ; \mathrm{p}<0.0001$ ) whereas women had unadjusted HR for future CHD of 2.39 (95\% CI, 1.76 to 3.24; $\mathrm{p}<0.0001$ ). After adjustments for the Framingham Risk Score (FRS), men with an elevated waist girth and elevated TG had OR of 1.29 ( $95 \%$ CI, 1.01 to $1.64 ; \mathrm{p}=0.04$ ) compared to men with low waist and low TG levels. After adjustments for the FRS, women with an elevated waist girth and elevated TG had OR of 1.70 (95\% CI, 1.25 to 2.78; $\mathrm{p}=0.002$ ) compared to women with low waist and low TG levels.

Conclusion: The HTGW phenotype predicts the risk of CHD even after control for traditional risk factors in both men and women of in a European cohort representative of a contemporary Western population.

EPIC-Norfolk is supported by programme grants from the Medical Research Council UK and Cancer Research UK and with additional support from the European Union, Stroke Association, British Heart Foundation, Department of Health, Food Standards Agency

\section{9}

Postprandial changes in LDL composition, oxidisability and particle size and endothelial function following three consecutive meals in men with and without type 2 diabetes

P. G. Scheffer ${ }^{1}$, M. E. Tushuizen ${ }^{2}$, R. P. Vermue ${ }^{1}$, C. Rustemeijer ${ }^{2}$,

R. J. Heine ${ }^{2}$, M. Diamant ${ }^{2}$;

${ }^{1}$ Metabolic Laboratory, Clinical Chemistry, ${ }^{2}$ Endocrinology and Diabetes

Center, VU University Medical Center, Amsterdam, The Netherlands.

Background and Aims: Type 2 diabetes (DM2) is associated with low-grade inflammation and oxidative stress, leading to cardiovascular damage. We studied whether postprandial hyperglycaemia and hypertriglyceridaemia, both of which have been linked to oxidative stress and inflammation, affect the low-density lipoprotein (LDL) composition, size and susceptibility to oxidation in men with and without DM2.

Materials and Methods: Nine men with DM2 (mean \pm SD: age $55.7 \pm 4.2$ yrs; HbAlc $7.5 \pm 1.3 \%$; BMI $32.7 \pm 2.8 \mathrm{~kg} / \mathrm{m}^{2}$ ), and 7 age-matched healthy men were given 3 standardized high-fat mixed meals $(900 \mathrm{kcal} ; 50 \mathrm{~g}$ fat, $50 \mathrm{~g}$ carbohydrate, $35 \mathrm{~g}$ protein) as breakfast $(\mathrm{t}=0,09.00 \mathrm{a} . \mathrm{m}$.), lunch ( $\mathrm{t}=4 \mathrm{~h}, 01.00$ p.m.) and dinner ( $t=8 \mathrm{~h}, 05.00$ p.m.). Blood sampling was performed before the meals, and at $12 \mathrm{~h}(09.00$ p.m), and $16 \mathrm{~h}(01.00$ a.m.) after breakfast. Flowmediated dilation (FMD) of the brachial artery was measured by ultrasound before each blood collection. LDL was isolated by ultracentrifugation and the mean LDL particle size was measured by high performance gel-filtration chromatography. The susceptibility of LDL to in vitro oxidation, expressed as lag time, was determined by monitoring the conjugated dienes formation.

Results: Area-under-the-curve for plasma glucose and triglycerides (TG) concentrations were higher in DM2 $(\mathrm{P}<0.05)$. DM2 had a most marked rise in plasma glucose and TG at $\mathrm{t}=12 \mathrm{~h}$ after breakfast $(\mathrm{P}<0.01$ and $\mathrm{P}<0.05$, respectively). At baseline, DM2 relative to controls had smaller LDL particles $(21.58 \pm 0.49$ and $21.90 \pm 0.40 \mathrm{~nm}$, respectively; $\mathrm{P}<0.05)$ and in agreement a lower cholesterol-to-protein content in their LDL $(3.9 \pm 0.5$ and $4.2 \pm 0.2 \mathrm{mmol} /$ g, respectively; $\mathrm{P}<0.05)$. FMD at baseline was lower in DM2 compared to controls ( $4.0 \%$ and $7.5 \%$, respectively; $\mathrm{P}<0.01$ ), and decreased postprandially in both groups, reaching the lowest value at $\mathrm{t}=4 \mathrm{~h}$. Although not significant, the LDL TG/cholesterol ratio was 31\% higher in DM2 at baseline. LDL was postprandially enriched in TG-to-cholesterol ratio in DM2 $(18 \% ; \mathrm{P}<0.01)$ and controls $(13 \% ; \mathrm{P}<0.05)$ reaching a maximum concentration $12 \mathrm{~h}$ after breakfast. No postprandial changes were observed in LDL particle size, but LDL was more prone to oxidation in the postprandial state (Figure). In particular, LDL lag time was significantly decreased at $\mathrm{t}=4 \mathrm{~h}$ and $\mathrm{t}=8 \mathrm{~h}$ after breakfast (both $\mathrm{P}<0.05$ ), and subsequently returned to baseline levels in healthy men, whereas LDL oxidisability increased further until $\mathrm{t}=12 \mathrm{~h}$ in DM2.

Conclusion: In DM2, an increase in susceptibility of LDL particles to oxidation was found in the postprandial state, which was paralleled by endothelial dysfunction. The physiological exposure to 3 consecutive meals and the prolonged study-period allowed to uncover the full scope of alterations in LDL composition and function in DM2.

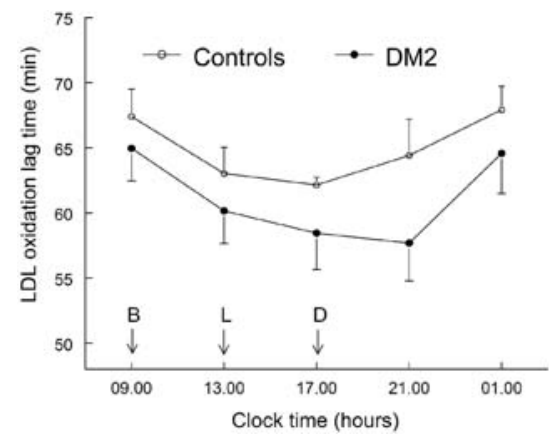

0010

Carotid intima-media thickness and apoB/apoA-I ratio in middle-aged patients with type 2 diabetes

E. M. Dahlén ${ }^{1}$, T. Länne ${ }^{2}$, J. Engvall ${ }^{3}$, T. Lindström ${ }^{4}$, E. Grodzinsky ${ }^{5}$,

F. Nyström², C. J. Ostgren ${ }^{1}$;

${ }^{1}$ Linköpings universitet, Dept. of Health and Society, ${ }^{2}$ Linköpings universitet, Dept. of Medicine and Care, ${ }^{3}$ Dept. of Clinical Physiology, Linköping University Hospital, Linköping, Sweden, ${ }^{4}$ Dept. of Endocrinolgy, Linköping University Hospital, ${ }^{5}$ County Council of Östergötland, Research and Development dept. of Local Health, Linköping, Sweden.

Background and Aims: Hypercholesterolaemia, and elevated levels of LDL-cholesterol in particular, is a strong risk factor for atherosclerotic cardiovascular mortality. Recently, the balance between the proatherogenic apolipoprotein B (apoB) and the antiatherogenic apolipoprotein a-I (apoA-I), has been suggested to be more predictive in the evaluation of cardiovascular risk. Intima-media thickness (IMT) of the carotid arteries is used as a surrogate and intermediate endpoint of early atherosclerosis. The aim of this study was to explore the association between the IMT and the apoB/apoAI ratio compared to serum triglycerides, total cholesterol, LDL- and HDLcholesterol in middle aged patients with type 2 diabetes.

Material and Methods: We analysed data from 249 consecutive patients with type 2 diabetes (152 men and 97 women) aged 55-66 years (mean age 60.8 years) who participated in the ongoing observational CARDIPP (Cardiovascular Risk factors in Patients with Diabetes - a Prospective study in Primary care) study. Nurses in primary care measured blood pressure and anthropometric measurements. Blood samples were taken for analyses of serum lipids and $\mathrm{HbAlc}$. Blood samples were also frozen for later analysis of apoB, apoA-I and C-Reactive Protein (CRP). The carotid ultrasosnographic 
investigations were performed at the University Hospital of Linköping and Länssjukhuset Ryhov, Jönköping, Sweden. The intima-media thickness of the carotid arteries, were evaluated by a Philips P700 ultrasound device (Philips Ultrasound, Santa Ana, California, United States) with either a 5 $\mathrm{MHz}$ or a $3.5 \mathrm{MHz}$ transducer. A longitudinal image was frozen in diastole, and a section of $10 \mathrm{~mm}$ measured manually by tracing a cursor along the echo edges.

Results: In a bivariate correlation analysis, only apoB/apoA-I ratio $(r=0.203$, $\mathrm{p}=0.002), \operatorname{apoB}(\mathrm{r}=0.156, \mathrm{p}=0.018)$, age $(\mathrm{r}=0.139, \mathrm{p}=0.034)$ and treatment with statins $(\mathrm{r}=-0.171, \mathrm{p}=0.033)$ correlated with IMT. Serum triglycerides, Total cholesterol, LDL-cholesterol, LDL-/ HDL- cholesterol ratio, CRP, HbA1c and systolic blood pressure were not significantly correlated to IMT. When further exploring the strength of the association between IMT and apoB/ apoA-I ratio in a multiple linear regression with IMT as dependent variable including apoB/apoA-I ratio, gender, age, smoking status, HbA1c, CRP, LDLand HDL-cholesterol and LDL-/HDL-cholesterol ratio, serum triglycerides, treatment with statins and mean systolic blood pressure, only the apoB/apoAI ratio remained significantly associated with IMT $(\mathrm{p}=0.041)$.

Conclusion: We conclude that there is a strong association between the apoB/apoA-I ratio and IMT, in middle aged patients with type 2 diabetes. The association is independent of conventional lipids and lipoproteins, inflammatory markers, glycaemic control and use of statins.

Supported by the Medical Research Council of Southeast Sweden

\section{1}

Matrix $\gamma$-carboxyglutamic acid protein (MGP) levels in type 2 diabetic patients with or without chronic kidney disease (CKD). Their correlation with insulin resistance and $L p(a)$ levels

S. Antonopoulos ${ }^{1}$, M. Mylonopoulou ${ }^{2}$, S. Mikros ${ }^{1}$, E. Konstantellou ${ }^{3}$, G. Papadakis ${ }^{2}$, A. Melidonis ${ }^{1}$, N. Tentolouris ${ }^{4}$, N. Katsilambros ${ }^{4}$;

${ }^{1}$ Diabetes Center, Tzanio General Hospital, Piraeus, ${ }^{2}$ Nephrology Dept, Tzanio General Hospital, Piraeus, ${ }^{3}$ Biochemistry Dept, General Hospital, Nikaia, ${ }^{4} 1$ st Dept of Propaedeutic Medicine, University Medical School, Athens, Greece.

Background and Aims: Matrix GLA Protein (MGP) was the first in vivo inhibitor of vascular calcification discovered, expressed in abundance in atherosclerotic plaques. Results of the Framingham Study in 2006 correlated higher levels of MGP with low HDL-cholesterol and high triglycerides levels, age, systolic hypertension, smoking, obesity and high Framingham score. Homeostasis Model Assessment (HOMA) is an efficient and practical estimator of Insulin Resistance (IR). Lipoprotein (a) is a well established independent risk factor for Cardiovascular Disease (CVD). It is a strong promoter of atherogenesis and thrombogenesis and a strong predictor of advanced atherosclerosis, independent of LDL cholesterol. Our aim was to study the differences in MGP, HOMA and Lp(a), in type 2 diabetic patients with or without stage 4 Chronic Kidney Disease (CKD).

Materials and Methods: We studied 100 patients divided in 4 groups. Group 1: 25 patients (age $67 \pm 9$ years, 11 men) with T2 DM and stage 4 CKD, Group 2: 25 diabetic patients $(67 \pm 7$ years, 10 men) without renal disease Group 3: 25 stage 4 CKD patients ( $65 \pm 7$ years, 14 men) without diabetes, and Group 4 : 25 healthy individuals ( $65 \pm 3$ years, 10 men). Serum MGP was measured by enzyme-linked immunosorbent assay (ELISA, Biomedica, Vienna, Austria). We explored possible correlations among MGP, HOMA and Lp(a).

Results: MGP was significantly elevated in diabetic patients with stage $4 \mathrm{CKD}$ compared to non-diabetics with kidney disease $(\mathrm{p}=0,002)$. Accordingly, HOMA- IR was significantly elevated in group 1 compared to all other patient groups $(p=0,003)$. Lp (a) was found to be significantly elevated in group 1 patients compared to group $2(\mathrm{p}=0,007)$ and group $4(\mathrm{p}<0,001)$ patients. There was no significant difference in Lp (a) between uremic patients with (group 1) or without (group 3) diabetes. Moreover, after controlling for group differences, MGP was found to be independently and significantly correlated with $\operatorname{Lp}(\mathrm{a})(\mathrm{p}=0,09)$ as well as with HOMA - IR $(\mathrm{p}=0,003)$.

Conclusion: The results indicate that the atherogenic process and the subsequent vascular calcification, as expressed by MGP and Lp(a) levels, play an important role as CVD risk factors in patients with CKD with or without diabetes. The patients with diabetes and concomitant pre - ESRD display an even greater danger for future cardiovascular complications compared to non-diabetics with CKD and diabetics without CKD. The high levels of the protective MGP in these patients might reflect the negative feedback process because of the intense calcification on their atheromatous vessel walls. MGP was also independently correlated with serum levels of Lp(a) and HOMA IR, suggesting the in vivo correlation of the vascular calcification process with atherogenesis and with the Insulin Resistance pathophysiologic spectrum.

\section{2}

Fibroblast cholesterol efflux to plasma from metabolic syndrome subjects is not impaired

R. P. F. Dullaart ${ }^{1}$, A. K. Groen ${ }^{2}$, G. M. Dallinga-Thie ${ }^{3}$, R. de Vries ${ }^{1}$, W. J. Sluiter ${ }^{1}$ A. van $\mathrm{Tol}^{1}$;

${ }^{1}$ Endocrinology, University Medical Center, Groningen, ${ }^{2}$ Laboratory of Experimental Hepatology, Academic Medical Center Amsterdam, ${ }^{3}$ Laboratory of Vascular Medicine, Erasmus University Medical Center, Rotterdam, The Netherlands.

Background and Aims: It is unknown whether the ability of plasma to stimulate cellular cholesterol efflux, an early step in the anti-atherogenic reverse cholesterol transport pathway, is abnormal in metabolic syndrome (MetS) subjects.

Materials and Methods: In 76 subjects with and 94 subjects without MetS (NCEP, ATP III criteria), we determined plasma (apo)lipoproteins, pre $\beta$ HDL (formation), phospholipid transfer protein (PLTP) activity, cholesterol esterification (EST), cholesteryl ester transfer (CET), adiponectin, serum paraoxonase-1 (arylesterase) activity, and the ability of plasma from each subject to stimulate cholesterol efflux out of cultured fibroblasts obtained from a single donor.

Results: Apolipoprotein E, PLTP activity, EST and CET were higher $(p=$ 0.04 to $<0.001)$, whereas adiponectin and paraoxonase- 1 activity $(p<0.01)$ were lower in Mets subjects. Pre $\beta$-HDL and pre $\beta$-HDL formation were not different between subjects with and without MetS. Cellular cholesterol efflux to plasma from Mets subjects was slightly higher $v s$. plasma from subjects without MetS ( $8.5 \pm 0.90 v s .8 .8 \pm 1.00 \%$ per $4 \mathrm{~h}, p=0.05)$, but the difference was not significant after age, sex and diabetes adjustment. Cellular cholesterol efflux was positively related to pre- $\beta$ HDL formation, EST, PLTP activity and apolipoprotrein $\mathrm{E}(p<0.05$ for all by multiple linear regression analysis), without an independent association with MetS.

Conclusion: No defect in the ability of plasma from MetS subjects to promote fibroblast cholesterol efflux was found, despite low HDL cholesterol. Higher cholesterol esterification, PLTP activity and apolipoprotein E levels may contribute to maintenance of cholesterol efflux in MetS.

Supported by the Dutch Diabetes Research Foundation 


\section{OP 3 Brain metabolism}

\section{3}

Localization and processing of the Amyloid Precursor Protein are regulated by the interaction with PED/PEA-15

A. P. M. Barbagallo, P. Iardino, G. Perruolo, A. Ilardi, I. Esposito,

A. T. Alberobello, A. Perfetti, A. Cassese, F. Giacco, F. Beguinot, P. Formisano; DIP. Biologia e Patologia Cellulare e Molecolare \& IEOS-CNR, University of Naples, Italy.

Background and Aims: Increasing evidence indicates that insulin plays a physiological role in brain, in particular in cognitive function and memory performance. Recent data suggest the association between insulin-resistance and cognitive decline. Alterations of genes involved in insulin signalling have also been found in patients with Alzheimer Disease (AD) and other neurodegenerative disorders. PED/PEA-15 (Phosphoprotein Enriched in Diabetes/ Phosphoprotein enriched in astrocytes) is commonly overexpressed in individuals with type 2 diabetes and its overexpression induces insulinresistance both in vitro and in vivo. PED/PEA-15 is physiologically expressed at high levels in brain, and in particular we now show that its expression is enhanced in the hippocampus, prefrontal and frontal cortex, brain structures with an important role in learning and memory. We hypothesize that PED/ PEA-15 could represent a link between insulin-resistance and cognitive impairment.

Materials and Methods: To explore the function of PED/PEA-15 in brain, a yeast two-hybrid system has been used to search for proteins that specifically interact with PED/PEA-15 in brain. Further studies have been performed in the neuroblastoma cell line, N2a.

Results: By yeast two-hybrid system we identified the Amyloid Precursor Protein (APP), a protein involved in the pathogenesis of $\mathrm{AD}$, as a PED/PEA15 molecular interactor. We confirmed the interaction between PED/PEA15 and APP in intact cells and in mouse brain by co-immunoprecipitation and pull down experiments. We then evaluated the effect of this interaction on APP proteolytic processing by measuring the amount of soluble APP fragment (sAPP) secreted in the culture medium. In cells transfected with APP, the simultaneous overexpression of PED/PEA-15 determined a 5-fold increase of sAPP secretion in the culture medium compared to control cells, without modifying precursor expression. APP plasma membrane expression is reduced in PED/PEA-15 overexpressing cells compared to control cells. Inhibition of conventional PKC isoforms (cPKCs) by Bisindolylmaleimide (100nM) caused a 50\% decrease of PED/PEA-15-dependent sAPP secretion and reverses PED/PEA-15 effects on APP expression onto the plasma membrane, while the ERK1/2 inhibitor PD98059 (50 microM) did not alter these effects.

Conclusion: Our data show that PED/PEA-15 overexpression enhances sAPP secretion by increasing APP processing via a PKC-dependent mechanism. Modulation of APP processing in neurons may be involved in cognitive functions.

\section{4}

Insulin signaling, glucose transporters and glycogen content in the cerebral cortex from undernourished suckling rats

F. Escrivá, E. Fernández, L. de Miguel, C. J. Martínez-Honduvilla,

C. Álvarez;

Bioquímica y Biología Molecular II, Facultad de Farmacia,

Universidad Complutense, Madrid, Spain.

Background and Aims: The developing brain is vulnerable to nutrient insufficiency because of its high needs for both energy generation and synthetic purposes. Undernutrition during critical periods of brain growth increases the risk of neurological disorders. Malnourished children have low intellectual performance; however, most of them are economically disadvantaged, a condition that influences intellectual aptitudes and masks the direct effects of poor nutrition on developing brain metabolism. Animal models applied to search for definite effects of food restriction on brain metabolism could be useful to reveal possible relationships between undernutrition and longterm damage in this organ. The brain contains a small amount of glycogen, mainly in astrocytes; its precise role is not well understood. Some studies suggest that glycogen provides glucose when needed. Indeed, little is known about the role of insulin in the regulation of brain glycogen metabolism. We have shown that circulating glucose and insulin are reduced in suckling undernourished rats, but they have improved insulin sensitivity in peripheral tissues. The manner in which insulin signalling in the brain is affected by early undernutrition has not been systematically studied. Since suckling constitutes a critical phase of rat brain growth, the purpose of this study was to investigate glucose transporters, glycogen content and insulin signalling in the cortex of undernourished 10-day-old rats.

Materials and Methods: Wistar rats were submitted to a $60 \%$ restriction of the commercial diet from $16^{\text {th }}$ day of pregnancy and during lactation. Offspring from restricted dams and those from dams that received ad libitum the same diet were studied at age 10 day. Suckling rats, injected or not with i.p. insulin, were killed by decapitation. Cerebral cortices was rapidly removed. Glycogen was measured from glucose derived by amyloglucosidase hydrolysis. Microvessels of the blood-brain barrier were isolated by attachment to glass beads. Total membrane fraction and whole lysates were also obtained. Endothelial and astroglial GLUT-1, neuronal GLUT-3 and proteins associated with insulin signaling (receptor, Akt, GSK3) were analyzed by Western.

Results: Accumulated glycogen in the cortex from undernourished rats was $30 \%$ higher than control values. Undernutrition led to an increase in glial GLUT-1; however, neither endothelial GLUT-1 nor neuronal GLUT-3 were altered. The content of insulin receptor did not differ between control and restricted rats. The degrees of insulin receptor, Akt and GSK $3 \alpha / \beta$ phosphorylation were not affected by i.p. exogenous insulin. Nevertheless, phospho-GSK $3 \alpha / \beta$ was markedly elevated in undernourished rats.

Conclusion: The increases in GSK3 phosphorylated and glycogen content observed in undernourished suckling rats raise the possibility that undernutrition enhances insulin sensitivity via enhancing some step of the signaling cascade in the cortex. It suggests that a fraction of the blood glucose taken up by astrocytes was directed to glycogen synthesis, perhaps depressing the amount of glucose utilized by energy generation or synthethic purposes. Further studies are needed to verify if these changes that coincide with the critical period of brain growth, when energy demand is high, can lead to deleterious consequences on brain development.

Supported by the Ministerio de Ciencia y Tecnología and ISCIII-RETIC RD06, Spain

\section{5}

Brain insulin signaling regulation by leptin and serotonin. Potential impact on the development of metabolic diseases

K. Gerozissis ${ }^{1}$, S. M. Banas ${ }^{1}$, F. Berthou ${ }^{2}$, V. Bailleux ${ }^{2}$, C. Rouch ${ }^{1}$, M. Taouis ${ }^{2}$; ${ }^{1}$ Laboratoire de physiopathologie de la nutrition, Université Paris, France, ${ }^{2}$ Neuroendocrinologie Moléculaire de la Prise Alimentaire, Université ParisSud, Orsay, France.

Background and Aims: The central nervous system has a primary role in the control of energy and glucose homeostasis and insulin, in intraction with leptin and serotonin, is a major regulator of these processes. Disruption of physiological signaling leads, along with peripheral insulin resistance, to central insulin resistance that is directly involved in metabolic diseases (MD). This study focuses on the interaction of the above negative regulators with brain insulin signaling.

Materials and Methods: $\underline{I n}$ vivo experiments. Adult male Wistar rats, received icv, $1 \mathrm{nmol}$ of LY294002, a phosphatidylinositol 3 kinase (PI3K) inhibitor and one hour later, ip, $5 \mathrm{mg} / \mathrm{kg}$ of dexfenfluramine $(\mathrm{dF})$, a stimulator of the serotonergic system. Food intake (FI) was measured during the dark phase, after four hours and overnight, starting $30 \mathrm{~min}$ following $\mathrm{dF}$ injection. Cellular approach. In differentiated human neuroblastoma (SH-SY5Y) cells expressing endogenous long isoform leptin receptor and insulin receptor (IR). We have previously shown that, leptin or insulin activate STAT-3 (Signal Transducer and Transcription Factor 3) and MAPK (mitogen-activating protein kinase) phosphorylation, and PI3K activity. SH-SY5Y cells were incubated for 10 min, in presence of leptin $(15 \mathrm{nM})$ or insulin $(100 \mathrm{nM})$, and serotonin $(10$ microM). Phosphorylated STAT-3 and MAPK were detected using specific antibodies.

Results: FI was dramatically reduced by dF. Associated to dF, LY294002 largely restored FI, suggesting that PI3K is involved in the serotonergicdependent FI inhibition, as it is involved in leptin- and insulin-dependent anorexic effect.

In SH-SY5Y cells, where leptin pre-treatment was shown to abolish insulin effect and vice versa, serotonin increased basal MAPK phosphorylation. 
Furthermore, serotonin clearly increased MAPK phosphorylation in response to insulin and leptin. Preliminary results indicate that STAT-3 phosphorylation in response to insulin or leptin is also positively affected by the addition of serotonin.

Conclusion: The present studies indicate that serotonin, insulin and leptin at the neuronal and brain level, share several signaling pathways such as STAT3 , MAPK and IRS/PI3K. In addition to leptin and insulin, serotonin involves the PI3K pathway in its anorectic action. Whether this action is direct on IR, IRS or PI3K, or indirect by increasing extracellular brain insulin release, needs further investigation. These data suggest a complex interaction in the brain between serotonin, leptin and insulin signaling pathways. Thus modifications of leptin and/or serotonin levels in the brain, observed in situations favoring metabolic disturbances, may impair brain insulin efficiency and contribute to the central insulin resistance, a potential determinant step on the development of peripheral insulin resistance that leads to MD, including T2D.

\section{6}

\section{Evidence of GLP-1 effects on human brain glucose delivery and metabolism \\ O. Schmitz ${ }^{1}$, S. Lerche ${ }^{1}$, B. Brock ${ }^{1}$, J. Rungby ${ }^{1}$, H. E. Boetker ${ }^{1}$, N. Moeller ${ }^{2}$, A. Rodell ${ }^{3}$, J. J. Holst ${ }^{4}$, A. Gjedde'; \\ ${ }^{1}$ Department of Pharmacology, Aarhus University, ${ }^{2}$ Medical Research Laboratory, Aarhus University Hospital, ${ }^{3} \mathrm{PET}$-center, Aarhus University Hospital, ${ }^{4}$ Department of Medical Physiology, Copenhagen University, Denmark.}

Background and Aims: Glucagon-like-peptide-1 (GLP-1) has many effects regarding glucose homeostasis. GLP-1 receptors are broadly represented in the brain. Recent research in rodents has suggested a potentially protective effect of GLP-1 in the brain. The mechanism behind this effect is unknown. The possible role of GLP-1 in the human brain glucose metabolism is not well characterized. To gain more insight into changes of glucose delivery and/or metabolism, which may explain neuroprotective effects, we explored glucose transport and consumption in 10 healthy men in a randomized, doubleblinded placebo-controlled cross-over experimental design.

Materials and Methods: The acute effect (independent of insulin) of GLP1 on glucose uptake in the brain was visualized by Positron Emission Tomography (PET) during a pituitary-pancreatic normoglycaemic (plasma glucose $\approx 4.5 \mathrm{mM}$ ) clamp with 18 -fluoro-deoxy-glucose (FDG) as a tracer of glucose.

Results: The insulin infusion rate was $0.12 \mathrm{mU} / \mathrm{kg} / \mathrm{min}$, growth hormone and glucagon were replaced to baseline levels. Intact GLP-1 levels were $51 \pm 3$ (mean \pm SEM) vs. $0.95 \pm 0.1 \mathrm{pmol} / 1$ (GLP-1 vs. placebo). In total cerebral grey matter, GLP-1 reduced cerebral glucose uptake by $30 \%, 0.99 \mathrm{micromol} / \mathrm{cm}^{3} /$ $\min \pm 0.09$ (placebo) vs. $0.70 \pm 0.04$ (GLP-1) $(\mathrm{P}=0.05)$. In the cerebral cortex, reduction was $29 \%, 0.98 \pm 0.09$ vs. $0.69 \pm 0.04(\mathrm{P}=0.06)$, in white matter $26 \%$, $0.50 \pm 0.04$ vs. $0.37 \pm 0.02(\mathrm{P}=0.03)$, in thalamus $31 \%, 1.11 \pm 0.12$ vs. 0.77 $\pm 0.06(\mathrm{P}=0.05)$, in striatum $28 \%, 0.98 \pm 0.09$ vs. $0.70 \pm 0.05(\mathrm{P}=0.06)$, in cerebellar cortex $30 \%, 1.12 \pm 0.12$ vs. $0.82 \pm 0.06(\mathrm{P}=0.06)$ and in brainstem $33 \%, 0.76 \pm 0.07$ vs. $0.50 \pm 0.03(\mathrm{P}=0.02)$. The same regions showed a uniform trend towards reduced cerebral glucose metabolism. As a result, the intra cerebral glucose concentration remained the same in all regions, with and without GLP-1.

Conclusion: For the first time, we demonstrate that a hormone involved in postprandial glucose regulation also limits glucose delivery to brain tissue and hence provides a possible explanation for the connection between plasma glucose and brain glucose regulation. As GLP-1 reduces glucose uptake across the intact blood brain barrier at normal glycaemia, GLP-1 may also protect the brain by limiting intra cerebral glucose fluctuation when plasma glucose is increased.

Supported by Lundbeck Foundation, Danish Diabetes Association, Novo Nordisk Foundation

\section{7}

Insulin-stimulated brain glucose uptake is enhanced in subjects with impaired glucose tolerance (IGT)

K. A. Virtanen ${ }^{1}$, J. Hirvonen ${ }^{1}$, M. Bucci ${ }^{1,2}$, M. Honka ${ }^{1}$, S. Nesterov ${ }^{1}$, P. Iozzo $^{1,3}$, J. O. Rinne ${ }^{1}$, P. Nuutila ${ }^{1,4}$;

${ }^{1}$ Turku PET Centre, University of Turku, Finland, ${ }^{2}$ Alma Mater Studiorum, University of Bologna, Italy, ${ }^{3}$ Institute of Clinical Physiology, CNR

- National Research Council, Pisa, Italy, ${ }^{4}$ Department of Medicine, Turku University Hospital, Finland.

Background and Aims: Central nervous system regulates hepatic glucose metabolism. However, cerebral blood flow, cerebral glucose uptake and hepatic glucose uptake has not been studied earlier simultaneously in same subjects.

Materials and Methods: We studied 8 subjects with impaired glucose tolerance (IGT) and 10 normal subjects in random order twice, during the fasting state and euglycemic hyperinsulinemia $(1 \mathrm{mU} / \mathrm{min} / \mathrm{kg}$ clamp) in separate days. Cerebral and hepatic glucose uptake was measured using $\left[{ }^{18} \mathrm{~F}\right]$-fluoro-deoxyglucose $\left(\left[{ }^{18} \mathrm{~F}\right] \mathrm{FDG}\right)$ and PET, and cerebral blood flow was quantified using $\left[{ }^{15} \mathrm{O}\right] \mathrm{H}_{2} \mathrm{O}$ and PET. Hepatic glucose uptake rate was determined using graphical analysis, and cerebral blood flow and glucose uptake were determined using statistical parametric mapping (SPM). Results: Insulin had no effect on global or regional perfusion either in healthy or IGT subjects. During the fasting state, cerebral glucose uptake was $8.5 \%$ lower in IGT than in normal subjects $(\mathrm{p}=\mathrm{ns})$. Insulin stimulation did not change cerebral glucose uptake in normal subjects but enhanced glucose uptake by $13.5 \%$ in IGT subjects as compared to fasting values $(\mathrm{p}<0.05)$. This increment was most prominent in amygdala, hippocampus, and orbitofrontal cortex. In the same subjects, hepatic glucose uptake was stimulated $(+25 \%)$ by insulin in normal subjects as compared with fasting values $(\mathrm{p}=0.02)$ but not in IGT group.

Conclusion: Insulin-stimulated cerebral glucose uptake is up-regulated in IGT subjects. The liver of IGT subjects reflects insulin resistance.

Supported by Maud Kuistila Memorial Foundation

\section{8}

Brain unsaturated fatty acid (FA) uptake is increased in obesity and reversed by weight reduction

P. Nuutila, A. Viljanen, J. Hirvonen, K. Virtanen, A. Karmi, V. Oikonen, J. Kemppainen, T. Viljanen, M. Haaparanta, K. Någren, O. Solin, P. Iozzo; Turku PET Centre, University of Turku, Turku, Finland.

Background and Aims: Free fatty acids (FFA) are taken up by the brain, but their role in obesity and metabolic syndrome is poorly understood. The aim of this study was to test whether brain fatty acid uptake is enhanced in obese subjects having metabolic syndrome (MBO), and whether weight reduction modifies it.

Materials and Methods: A total of seven healthy subjects (age $42 \pm 11$ year, BMI $26.8 \pm 2.5 \mathrm{~kg} / \mathrm{m}^{2}$ ) and $22 \mathrm{MBO}$ patients (age $43 \pm 8$ year, BMI $33.5 \pm 4.1 \mathrm{~kg} /$ $\mathrm{m}^{2}$ ) were studied during fasting conditions using PET with $\left[{ }^{11} \mathrm{C}\right]$-palmitate and $\left[{ }^{18} \mathrm{~F}\right]$ fluoro-6-thia-heptadecanoic acid (FTHA). In mitochondria, $\left[{ }^{18} \mathrm{~F}\right]-$ FTHA is trapped after initial steps of oxidation. Sixteen MBO subjects were restudied after 6 weeks of very low calorie diet intervention. The fractional tracer uptake (FUR) was calculated voxel-wise, and an automated ROI-analysis was performed to derive regional FUR estimates. FUR was multiplied with serum FFA concentration to yield FFA uptake $\left(\left[{ }^{18} \mathrm{~F}\right]\right.$-FTHA) and nonoxidised retention $\left(\left[{ }^{11} \mathrm{C}\right]\right.$-palmitate). The results were analyzed using repeated-measures analysis of variance (rmANOVA).

Results: The cerebral global fatty acid uptake derived from $\left[{ }^{18} \mathrm{~F}\right]-\mathrm{FTHA}-$ PET was $50 \%$ higher in $\mathrm{MBO}$ compared with controls $(\mathrm{p}=0.012)$. The mean percentual increment was $+130 \%$ in the white matter and $+47 \%$ in the gray matter and uniform across brain regions. In the $\mathrm{MBO}$ group, weight reduction $(-11 \pm 2.5 \mathrm{~kg})$ decreased fatty acid uptake by $17 \%(\mathrm{p}=0.01)$. In line with this, fatty acid retention measured using $\left[{ }^{11} \mathrm{C}\right]$-palmitate was $+86 \%$ higher in the MBO group ( $\mathrm{p}=0.017)$, but did not change following weight reduction.

Conclusions: Fatty acid uptake and accumulation in the brain appears to be increased in MBO; and decreases after weight reduction, which seems to primarily affect the oxidative fate of fatty acids. These results could contribute to altered brain white mater volume in obesity and may have consequences on the regulation of peripheral metabolism.

Supported by Novo Nordisk Foundation 


\section{OP 4 Molecular regulation of beta cell function and survival}

\section{9}

\author{
Epigenetic regulation of PGC-1 $\alpha$ in human type 2 diabetic islets and \\ effect on insulin secretion \\ T. S. Rönn ${ }^{1}$, C. Ling ${ }^{1}$, S. del Guerra ${ }^{2}$, R. Lupi ${ }^{2}$, C. Granhall ${ }^{1}$, H. Luthman ${ }^{1}$, \\ P. Masiello ${ }^{2}$, P. Marchetti ${ }^{2}$, L. Groop ${ }^{1}$, S. del Prato ${ }^{2}$; \\ ${ }^{1}$ Clinical Sciences, Diabetes and Endocrinology, Lund University, Malmö, \\ Sweden, ${ }^{2}$ Endocrinology and Metabolism, Metabolic Unit, University of \\ Pisa, Italy.
}

Background and Aims: Insulin secretion in pancreatic islets is dependent upon mitochondrial function and production of ATP. The transcriptional co-activator peroxisome proliferator activated receptor- $\gamma$ coactivator- $1 \alpha$ (PGC-1 $\alpha$ ) is a master regulator of mitochondrial genes and its expression is decreased and related to impaired oxidative phosphorylation in muscle from patients with type 2 diabetes (T2D). Also, we recently showed that PGC- $1 \alpha$ mRNA was reduced by $90 \%(\mathrm{p}<0.005)$ in T2D human pancreatic islets and correlated with reduced insulin secretion. Furthermore, a polymorphism (Gly482Ser polymorphism) in the PGC-1 $\alpha$ gene was associated with markedly reduced PGC- $1 \alpha$ mRNA levels $(\mathrm{p}<0.0001)$ and insulin secretion $(\mathrm{p}<0.05)$ in T2D islets. In order to study potential mechanisms leading to impaired PGC$1 \alpha$ expression and insulin secretion we measured DNA methylation of the PGC-1 $\alpha$ promoter in human islets and studied whether experimental (siRNA) down-regulation of PGC-1 $\alpha$ in human islets influences insulin secretion.

Materials and Methods: Human pancreatic islets from 10 T2D (age: $65.1 \pm$ 2.6 years, $\operatorname{sex}[\mathrm{M} / \mathrm{F}]: 5 / 5, \mathrm{BMI}: 26.9 \pm 1.1 \mathrm{~kg} / \mathrm{m}^{2}$ ) and 10 non-diabetic (ND; age: $54.3 \pm 4.0$ years, sex[M/F]: 7/2, BMI: $25.6 \pm 1.2 \mathrm{~kg} / \mathrm{m}^{2}$ ) multi-organ donors were analysed for DNA methylation at 4 target sites in the PGC-1 $\alpha$ promoter region using bisulfite sequencing. mRNA levels were quantified by Real-Time Quantitative RT-PCR. PGC- $1 \alpha$ silencing RNA (siRNA) was used for experimental down-regulation of PGC-1 $1 \alpha$ expression in human islets and insulin secretion studies were performed with 3.3 resp. $16.7 \mathrm{mmol} / \mathrm{l}$ glucose. Differences in PGC-1 $\alpha$ mRNA levels, percentage of DNA methylation and insulin-secretion between the different groups studied were analyzed using non-parametric Mann-Whitney exact test. Correlations were calculated using Spearman correlation coefficient.

Results: There was an approximately 2 -fold increase in DNA methylation in the $P G C-1 \alpha$ promoter of T2D compared with ND human islets $(10.5 \pm 2.7$ $\%$ vs. $4.7 \pm 0.9 \%$; $<0.04)$. The degree of DNA methylation showed a trend to an inverse correlation with the PGC- $1 \alpha$ mRNA level $(r=-0.48 ; \mathrm{p}=0.07)$. Transfection with PGC- $1 \alpha$ siRNA resulted in a $45 \%$ reduction in PGC- $1 \alpha$ mRNA level, $32 \%$ reduction in insulin mRNA level, and a $41 \%$ reduction in insulin secretion (measured as insulin stimulation index, i.e. incremental folds above baseline insulin release), $\mathrm{p}<0.05$.

Conclusion: Expression of PGC-1 $\alpha$ in human islets is subject to DNA methylation which, in turn, could influence insulin secretion.

Supported by SweGene, the Diabetes programme at Lund Universtiy, Ake Wiberg and Hedlunds foundations.

\section{0}

\section{Nuclear cytosolic translocation of PTB1 in human islets of type 2 diabetic patients \\ K.-P. Knoch ${ }^{1}$, F. Ehehalt ${ }^{2}$, K. Erdmann ${ }^{1}$, S. Kersting ${ }^{2}$, H. Bergert ${ }^{2}$, M. Jäger ${ }^{1}$,} D.-D. Dittert ${ }^{3}$, E. Kuhlisch ${ }^{4}$, S. Bornstein ${ }^{5}$, E. Bonifacio ${ }^{6}$, H. Saeger ${ }^{2}$, M. Solimena ${ }^{1}$;

${ }^{1}$ Experimental Diabetology, Medical School, Dresden, ${ }^{2}$ Visceral, Thoracic and Vascular Surgery, Medical School, Dresden, ${ }^{3}$ Pathology, Medical School, Dresden, ${ }^{4}$ Institute for Medical Informatics and Biometrics, Medical School, Dresden, ${ }^{5}$ Clinic III, Medical School, Dresden, Germany, ${ }^{6}$ Immunology of Diabetes Unit, San Raffaele Scientific Institute, Milan, Italy.

Background and Aims: The rapid biosynthesis of insulin secretory granules (SG) is regulated by glucose. Previously we have shown that in rat islets and cultured insulinoma INS-1 cells glucose and glucagon-like peptide 1 (GLP-1) rapidly increase SG biogenesis through the activation of a posttranscriptional pathway involving the nucleocytosolic translocation of polypyrimidine tract-binding protein 1 (PTB1). In this study we compared the glucose- stimulated insulin turnover and the translocation of PTB1 in human islets isolated from patients with or without type 2 diabetes (T2D) who underwent pancreatectomy because of pancreatic diseases.

Materials and Methods: Islets were isolated from the macroscopically healthy resection margins of pancreatic tissue from 5 patients with T2D and 6 matched non-diabetic (ND) patients. Insulin content and insulin secretion were measured before and after islet stimulation with $25 \mathrm{mM}$ glucose for 2 hours. The subcellular localization of polypyrimidine tract-binding protein 1 (PTB1) was analyzed by western blotting and confocal microscopy.

Results: Glucose stimulated insulin secretion was decreased, albeit not significantly, in T2D islets compared to ND islets. Strikingly, stimulation increased the total amount of insulin (islet insulin content + secreted insulin) in each islet preparation from ND patients, but not from T2D subjects. Furthermore, stimulation decreased the nuclear levels of PTB1 in ND islets, but not in T2D islets. Confocal microscopy supported the retention of PTB1 in stimulated T2D islets.

Conclusion: These results point to impaired glucose-stimulated rapid turnover of insulin as a specific trait of T2D islets. Nuclear retention of PTB1 suggests that reduced insulin biosynthesis contributes to this impairment, and thus to deficient insulin secretion. Overall, these data highlight the importance of investigating the post-transcriptional mechanisms regulating insulin biosynthesis and degradation to gain insight into the pathogenesis of T2D.

Supported by BMBF Gemany

\section{1}

The effects of E-cadherin engagement on human beta-cell survival and function

D. Bosco, P. Morel, D. Matthey-Doret, T. Berney;

Surgery, University of Geneva, Switzerland.

Background and Aims: E-cadherin is a cell-to-cell adhesion molecule that has been shown to be involved in islet cell differentiation and aggregation, as well as in the maintenance of islet architecture. In this work, we aimed to assess whether E-cadherin plays a role in the function and survival of adult human beta-cells. This task is rendered difficult as it is challenging to distinguish between effects that are mediated directly by E-cadherin ligation versus the effects that are downstream of this ligation, such as cell-to-cell communication via gap junctions to the cell membrane. To overcome this problem we used a recombinant protein consisting of the ectodomain of human E-cadherin fused to the Fc region of IgG (E-cadh-Fc) as a model matrix for single cell adhesion to specifically study the effects mediated by E-cadherin ligation,

Materials and Methods: Human islets were isolated from pancreases harvested from multiorgan cadaveric donors. Islet cells were obtained by Accutase treatment and incubated in suspension for $24 \mathrm{~h}$ in CMRL medium at low density to limit cell-to-cell aggregation and to allow cell recovery. Cells were then seeded on microscopic slides coated with E-cadh-Fc. Controls include uncoated glass slides and glass slides coated with analogous fused proteins containing the ectodomains of human $\mathrm{N}$-cadherin $(\mathrm{N}-\mathrm{cadh}-\mathrm{Fc})$ or human P-cadherin (P-cadh-Fc). In every experiment, beta-cells were detected using immunofluorescence for insulin. Cells were attached on the different substrates for $24 \mathrm{~h}$ and apoptosis was assessed by TUNEL. To assess insulin secretion, cells were attached for $1 \mathrm{~h}$ and were submitted to a reverse hemolytic plaque assay (RHPA). Hemolytic plaques that developed around single beta-cells or beta-cell pairs were analysed after $1 \mathrm{~h}$ stimulation with $16.7 \mathrm{mM}$ glucose. Data are expressed as means \pm SEM of 6 independent experiments and statistically analysed by Student's $t$ test.

Results: When attached on uncoated glass slides, $14.6 \pm 3.9 \%$ of single betacells were TUNEL positive. This percentage significantly $(\mathrm{p}=0.02)$ decreased to $3.9 \pm 1.1 \%(\mathrm{p}=0.02)$ for beta-cells aggregated into pairs and to $5.6 \pm 1.0$ for single beta-cells attached on E-cadh-Fc ( $\mathrm{p}=0.05$ vs control). Neither N-cadh$\mathrm{Fc}_{\mathrm{c}}$ nor P-cadh-Fc affected the percentage of apoptotic cells when compared to control. By RHPA, it was observed that $11.4 \pm 2.3 \%$ of single beta-cells attached on control glass were surrounded by a hemolytic plaque with a total plaque development (for 100 beta-cells) of $14900 \pm 3700 \mu \mathrm{m} 2$. These values were increased to $28.4 \pm 7.6 \%$ ( $\mathrm{p}=0.05$ vs control) and $73800 \pm 30400 \mu \mathrm{m} 2$ ( $\mathrm{p}=0.08$ vs control) for beta-cell pairs. When attached on E-cadh-Fc, $25.5 \pm$ $4.7 \%$ ( $\mathrm{p}=0.02$ vs control) of single beta-cells were surrounded by a hemolytic plaque and the total plaque development was $54400 \pm 12400 \mu \mathrm{m} 2(\mathrm{p}=0.01$ vs control). 
Conclusion: These results indicate that cell-to-cell contact at least in part via E-cadherin engagement improves human beta-cell survival and secretion.

\section{2}

Role of glucokinase in the regulation of $\beta$ cell mass under insulin resistant conditions

Y. Terauchi ${ }^{1}$, A. Nakamura ${ }^{1}$, I. Takamoto ${ }^{2}$, N. Kubota ${ }^{2}$, T. Kadowaki ${ }^{2}$;

${ }^{1}$ Department of Endocrinology and Metabolism, Yokohama City University, ${ }^{2}$ Department of Metabolic Diseases, University of Tokyo, Japan.

Background and Aims: Glucokinase (Gck) functions as a glucose sensor for insulin secretion, and in mice fed standard chow, haploinsufficiency of $\beta$ cellspecific glucokinase $\left(\mathrm{Gck}^{+/-}\right)$led to impaired insulin secretion to glucose, but the animals had a normal $\beta$ cell mass. When fed a high-fat (HF) diet, wildtype mice showed marked $\beta$ cell hyperplasia, whereas $\mathrm{Cck}^{+/-}$mice showed insufficient $\beta$ cell hyperplasia, decreased $\beta$ cell replication and impaired upregulation of insulin receptor substrate (Irs)-2 in islets, despite the presence of a similar degree of insulin resistance. Thus, Gck and Irs-2 are required for $\beta$ cell hyperplasia to occur in response to HF diet-induced insulin resistance. In the present study, we investigated the role of Gck in the regulation of $\beta$ cell mass under various insulin resistant conditions.

Materials and Methods: We generated an animal model with insulin resistance and impaired insulin secretion by crossing $\mathrm{Irs}^{-1^{-/-}}$and $\mathrm{Gck} \mathrm{H}^{+/-}$mice, and investigated glucose tolerance and $\beta$-cell mass in the $\mathrm{Irs}-\mathrm{1}^{-{ }_{-}} \mathrm{Gck} \mathrm{k}^{+-}$mice and Irs-1/- mice on the standard-chow diet or HF diet. To evaluate the role of Gck in pregnancy, we investigated glucose tolerance and $\beta$-cell mass in pregnant wild-type, $\mathrm{Gck}^{+/-}$and Irs-2-/- mice.

Results: On the standard-chow diet, Irs-1 $1^{-/-}$mice showed normal glucose tolerance and Irs- $1^{-1-} G c k^{+/}$mice exhibited severe diabetes. After 20 weeks on the HF diet, Irs-1-1- mice developed only mild diabetes as a result of compensatory hyperinsulinemia, whereas Irs- $1^{-1-} G c k^{+/-}$mice demonstrated the exacerbation of diabetes because of a lack of compensatory hyperinsulinemia, despite the similar degrees of obesity and insulin resistance shown by both HF diet-fed groups. On the standard-chow diet, the proportion of the area of $\beta$ cell to the total area of the pancreas ( $\beta$ cell mass) was $1.19 \pm 0.06 \%$ in Irs- $1^{-1-}$ mice and $0.53 \pm 0.13 \%$ in wild-type mice of the same genetic background. On the HF diet, $\beta$ cell mass was $1.88 \pm 0.12 \%$ in Irs $1^{-/-}$mice, indicating $\beta$ cell hyperplasia to compensate for HF diet-induced insulin resistance. By contrast, $\beta$ cell mass was $1.17 \pm 0.21 \%$ in Irs- $1^{-/-} G c k^{+-}$mice on the standard-chow diet, but there was no significant difference between those on the standard-chow diet and those on the HF diet. There were significantly fewer insulin and PCNA double-positive cells in the Irs-1-1-Gck $\mathrm{Gc}^{+/-}$mice on the HF diet than in the Irs-1 $1^{-}$ ${ }^{1-}$ mice on the HF diet. Body weight was significantly increased by 70 to $80 \%$ in both $G c k^{+/}$and wild-type mice on day 17.5 of pregnancy, but fed glucose level was significantly lower in pregnant $G c k^{+/-}$mice than in non-pregnant $G c k^{+/-}$ mice. $\beta$-cell mass was significantly increased by $70 \%$ and $67 \%$, respectively, in pregnant wild-type and $G c k^{+/-}$mice, compared with non-pregnant ones. There was little difference in $\beta$ cell size between the two pregnant groups. In Irs$2^{-/-}$mice, marked aggravation of glucose intolerance and lack of compensatory $\beta$ cell hyperplasia was seen on the HF diet, whereas fed glucose level was not increased and $\beta$ cell mass was increased during pregnancy.

Conclusion: Haploinsufficiency of Gck led to insufficient $\beta$ cell hyperplasia on the HF diet, but to the compensatory increase in $\beta$ cell mass under Irs-1deficient or pregnancy-associated insulin-resistant conditions. These results suggest the existence of Gck-dependent and Gck-independent pathway in the regulation of $\beta$ cell hyperplasia to occur in response to insulin resistance.

\section{3}

Glucose tolerance and insulin secretion in pancreatic beta cell specific Mn-SOD knockout mice

M. Tsuda ${ }^{1}$, T. Adachi ${ }^{2}$, T. Shimizu ${ }^{3}$, T. Shirasawa ${ }^{3}$, K. Tsuda ${ }^{1}$;

${ }^{1}$ Laboratory of Metabolism, Graduate School of Human and Environmental Studies, Kyoto, ${ }^{2}$ Department of Genomic Drug Discovery Science, Graduate School of Pharmaceutical Sciences, Kyoto, ${ }^{3}$ Research Team for Molecular Biomarkers, Tokyo Metropolitan Institute of Gerontology, Tokyo, Japan.

Background and Aims: Oxidative stress is well known as the cause of aging, cancer, arteriosclerosis, and/or diabetic complications, but the detailed mechanisms are not cleared. Manganese superoxide dismutase (MnSOD) reduces superoxide in mitochondrial matrix. We have generated the pancreatic beta-cell specific Mn-SOD knockout (beta-KO) mice. The aim of this study is to investigate glucose tolerance and insulin secretion in the betaKO mice.

Materials and Methods: Twelve- and 24-week-old beta-KO or age-matched Mn-SOD flanked loxp (F/F) male mice were used. Body weights and nonfasting plasma glucose and insulin level were measured. For the intraperitoneal glucose tolerance test (ipGTT), the mice were fasted for over $14 \mathrm{hr}$ and blood was drawn from the tail vein at $0,15,30,60$, and 120 min after intraperitoneal injection of glucose $(2 \mathrm{~g} / \mathrm{kg}$ body weight). Then, plasma glucose levels were measured. On the pancreatic perfusion, the insulin secretion level was measured. Perfusion flow with $3.3 \mathrm{mM}$ glucose concentration was performed for $30 \mathrm{~min}$. Then, perfusion flow with $11.1 \mathrm{mM}$ glucose concentration was performed for $30 \mathrm{~min}$, and thereafter, with $3.3 \mathrm{mM}$ glucose concentration for $10 \mathrm{~min}$. For check of viability, perfusion flow with $11.1 \mathrm{mM}$ glucose and $10 \mathrm{mM}$ arginine concentration was performed for $10 \mathrm{~min}$. Then, beta-KO and $\mathrm{F} / \mathrm{F}$ mouse islets were isolated, and stimulated with $5.5 \mathrm{mM}$ or 11.1 $\mathrm{mM}$ glucose concentration for $30 \mathrm{~min}$ in $37^{\circ} \mathrm{C}$. Moreover, protein contents of beta-KO and $\mathrm{F} / \mathrm{F}$ mouse islets were measured, and the insulin secretion level of beta-KO and $\mathrm{F} / \mathrm{F}$ mouse islets was corrected using each protein content. The morphological observation of pancreas was performed. Two types of stains were performed. Staining for succinate dehydrogenase (SDH) activity was performed for analysis of mitochondrial functions. Furthermore, immunostaining was performed using insulin and glucagon antibody for analysis of morphology of pancreatic islets.

Results: Body weights were significantly lower in beta-KO than F/F mice at both 12 and 24 weeks of age. Non-fasting plasma glucose and insulin levels were not significantly different between beta-KO and $\mathrm{F} / \mathrm{F}$ mice. The plasma glucose level in ipGTT was significantly higher in beta-KO than F/F mice. On the panceratic perfusion with $11.1 \mathrm{mM}$ glucose concentration, the insulin secretion level was significantly lower in beta-KO than $\mathrm{F} / \mathrm{F}$ mice. The insulin secretion level per islet was significantly higher in beta-KO than $\mathrm{F} / \mathrm{F}$ mice, but the insulin secretion level per protein was significantly lower in beta-KO than F/F mice. Mitochondrial SDH activity was lower in beta-KO than F/F mice. Hyperplasia was observed in pacreatic islets of beta-KO mice.

Conclusion: Beta-KO mice exhibited glucose intolerance, suggesting that insulin secretion capacity of beta-KO mice was decreased. We surmise that mitochondrial Mn-SOD in beta-cells is one of the important enzymes for insulin secretion.

\section{4}

Reduced sensitivity to oxidative stress in beta cells of $K_{\text {ATP }}$ channeldeficient Sur1KO mice

M. Düferr, B. Gierr', P. Krippeit-Drews' ${ }^{1}$, L. Aguilar-Bryan², J. Bryan², G. Drews ${ }^{1}$;

${ }^{1}$ Pharmazeutisches Institut, Universität Tübingen, Germany,

${ }^{2}$ Pacific Northwest Research Institute, University of Washington, Seattle, United States.

Background and Aims: In beta cells of wildtype (WT) mice the opening of $\mathrm{K}_{\mathrm{ATP}}$ channels and subsequent membrane hyperpolarization contribute to the impairment of insulin secretion induced by oxidative stress. The aim of the present study was to investigate whether beta cells of $\mathrm{K}_{\text {ATP }}$ channel-deficient, Sur1KO mice are less sensitive to $\mathrm{H}_{2} \mathrm{O}_{2}$ than WT cells.

Materials and Methods: Membrane potential $\left(\mathrm{V}_{\mathrm{m}}\right)$ recordings were performed in the perforated-patch configuration; $\left[\mathrm{Ca}^{2+}\right]_{c}$ was determined using fluorescence techniques; insulin secretion was monitored by radioimmunoassay.

Results: In islets of WT mice stimulated with $15 \mathrm{mM}$ glucose insulin secretion was dose-dependently reduced by $\mathrm{H}_{2} \mathrm{O}_{2}$ between 0.025 and $0.5 \mathrm{mM}$ $(\mathrm{n}=7-10)$. By contrast, insulin release from Sur1KO islets was not affected by $\mathrm{H}_{2} \mathrm{O}_{2}$ concentrations up to $100 \mu \mathrm{M}(\mathrm{n}=9)$, but was decreased by 0.25 and 0.5 $\mathrm{mM} \mathrm{H}_{2} \mathrm{O}_{2}(\mathrm{n}=9)$. The $\mathrm{IC}_{50}$ for $\mathrm{H}_{2} \mathrm{O}_{2}$ was threefold higher in Sur1KO vs WT islets $(0.46 \pm 0.10 \mathrm{mM}$ vs $0.16 \pm 0.02 \mathrm{mM}$, respectively). As insulin secretion is critically determined by membrane potential-regulated changes of $\left[\mathrm{Ca}^{2+}\right]_{\mathrm{c}}$ we investigated whether $\left[\mathrm{Ca}^{2+}\right]$ and membrane potential were less sensitive to $\mathrm{H}_{2} \mathrm{O}_{2}$ in SurlKO vs WT beta cells. $10 \mu \mathrm{M} \mathrm{H}_{2} \mathrm{O}_{2}$ stopped $\left[\mathrm{Ca}^{2+}\right]_{c}$ oscillations in WT beta cells stimulated with $15 \mathrm{mM}$ glucose and reduced $\left[\mathrm{Ca}^{2+}\right]_{c}$ to basal values $(n=9)$, while oscillations persisted in Sur1KO beta cells $(n=8)$. In isolated WT beta cells 10 and $100 \mu \mathrm{M} \mathrm{H}_{2} \mathrm{O}_{2}$ hyperpolarized $\mathrm{V}_{\mathrm{m}}$ by $15.5 \pm 1.3$ 
$\mathrm{mV}(\mathrm{n}=11)$ or $17.8 \pm 1.9 \mathrm{mV}(\mathrm{n}=4)$, respectively, which drastically reduced or terminated action potentials within 5 min after $\mathrm{H}_{2} \mathrm{O}_{2}$ application; AP frequency $=112 \pm 8.1 \mathrm{APs} / \mathrm{min}$ in $15 \mathrm{mM}$ glucose, $\mathrm{n}=13$, vs $16.1 \pm 9.3(\mathrm{n}=9)$ or $23.0 \pm 23.0(\mathrm{n}=4) \mathrm{APs} / \mathrm{min}$ in 10 or $100 \mu \mathrm{M} \mathrm{H}_{2} \mathrm{O}_{2}$, respectively. 10 or 100 $\mu \mathrm{M} \mathrm{H}_{2} \mathrm{O}_{2}$ did not change $\mathrm{V}_{\mathrm{m}}$ or the action potential frequency in Sur1KO beta cells $(n=6-10)$. Higher $\mathrm{H}_{2} \mathrm{O}_{2}$ concentrations $(1 \mathrm{mM})$ produced a large, irreversible increase of $\left[\mathrm{Ca}^{2+}\right]_{c}$ that was not blocked by L-type $\mathrm{Ca}^{2+}$-channel inhibitors in WT $(n=5)$ or Sur1KO beta cells $(n=4)$.

Conclusion: Elimination of $\mathrm{K}_{\text {ATP }}$ channels significantly reduced the $\mathrm{H}_{2} \mathrm{O}_{2}-$ mediated impairment of insulin secretion. Electrical activity and $\mathrm{Ca}^{2+}$ influx, preconditions for normal exocytosis, persist in Sur1KO beta cells under conditions of oxidative stress, but are disrupted in WT cells. High $\mathrm{H}_{2} \mathrm{O}_{2}$ concentrations, $>100 \mu \mathrm{M}$, that affect $\left[\mathrm{Ca}^{2+}\right]_{\mathrm{c}}$ in WT and Surl $\mathrm{KO}$ beta cells inhibit insulin secretion independent of $\mathrm{K}_{\mathrm{ATP}}$ channels. As the lower concentrations of $\mathrm{H}_{2} \mathrm{O}_{2}$ are more relevant in the immune response leading to beta cell failure during development of type 1 diabetes mellitus, modulation of $K_{\text {ATP }}$ channels could be a promising target to protect beta cells against oxidative stress.

Supported by Deutsche Forschungsgemeinschaft, U.S. National Institute of Health and Juvenile Diabetes Research Foundation

\section{OP 5 Endothelial biology in diabetes}

\section{5}

Exendin-4 rapidly increases nitric oxide production, promotes proliferation and protects against glucolipoapoptosis in human coronary artery endothelial cells

Ö. Tütüncï̈ ${ }^{1}$ D. Nathanson ${ }^{2}$, Å. Sjöholm², T. Nyström ${ }^{2}$, Q. Zhang ${ }^{1}$;

${ }^{1}$ Karolinska Institutet, Research Center, Stockholm South Hospital,

${ }^{2}$ Karolinska Institutet, Internal Medicine, Stockholm South Hospital, Sweden.

Background and Aims: Endothelial dysfunction plays an important role in macrovascular complications in diabetes. We recently showed that the glucagon-like peptide-1 receptor is expressed in human coronary artery endothelial cells (HCAEC) and that GLP-1 improves endothelial dysfunction in type 2 diabetic patients with coronary artery disease. Exendin- 4 is a stable GLP-1 receptor agonist and has been approved for clinical use against type 2 diabetes in Europe and the U.S. The aim of the present study was to investigate the effects of exendin- 4 on neogenesis and apoptosis under normal and hyperglucolipidemic conditions (simulating the diabetic milieu) in HCAEC cells in vitro.

Materials and Methods: HCAEC cells were cultured in the endothelial growth medium in the presence of $5 \mathrm{mM}$ glucose. Phosphorylation and expression of endothelial nitric oxide synthase (eNOS) were examined by Western blotting using anti-phospho-eNOS and anti-eNOS antibodies, respectively. $\beta$-actin examined in same blots acted as loading control. Realtime production of NO in single living HCAECs was monitored by a highly sensitive non-fluorescent cell-permeable NO probe, DAF-2DA. In the cell, the product reacts rapidly with $\mathrm{NO}$ in the presence of $\mathrm{O}_{2}$ to form a highly fluorescent compound. ${ }^{3} \mathrm{H}$-thymidine incorporation was assayed in 96-well plates as a measure of DNA synthesis, and caspase- 3 activity was evaluated using ELISA kit as a measure of apoptosis. DNA synthesis and apoptosis were examined after $48 \mathrm{~h}$-incubation with the reagents. Cell death was assessed using Trypan-blue inclusion.

Results: Incubation of HCAEC cells with exendin- 4 from 1 to $10 \mathrm{nM}$ for $10 \mathrm{~min}$ resulted in a dose-dependent increase of eNOS phosphorylation to an extent comparable with that of GLP-1 and acetylcholine. Continuous treatment of the cells with exendin- 4 for $48 \mathrm{~h}$ resulted in increased expression of eNOS protein. Similar to the acute effects of the peptide, long-term treatment with exendin- 4 also enhanced the phosphorylation state of the enzyme even when normalized for eNOS expression. In cells pre-loaded with the NO probe DAF-2DA, addition of exendin-4 $(10 \mathrm{nM})$ resulted in a rapid increase in NO production in single living HCAECs. Additionally, exendin-4 promoted DNA synthesis in HCAEC after $48 \mathrm{~h}$ incubation in $5 \mathrm{mM}$ glucose, evaluated by thymidine incorporation and neogenesis, confirmed by an increased cell number. Incubation of the cells with high glucose or palmitate provoked apoptosis and cell death, both of which were completely prevented by exendin- 4 .

Conclusions: Our study reveals salutary effects of exendin-4 on cell regeneration and protection from glucolipoapoptosis in human coronary artery endothelial cells, effects that are associated with increased eNOS activity and NO production. These novel findings add yet other beneficial properties of exendin-4, increasing its clinical utility in type 2 diabetic patients in whom endothelial dysfunction is a salient feature that adversely affects their survival. Elucidation of the mechanisms underlying these beneficial vascular effects of exendin- 4 may be exploited when intervening pharmacologically against vascular dysfunction in diabetes and may form the basis of improved incretin enhancers that hold the potential to prevent vascular lesions in diabetic patients.

Supported by the EFSD/Servier European Research Programme on Vascular Complications of Type 2 Diabetes 


\section{6}

Interaction of C-reactive protein with leptin impairs its ability to stimulate eNOS through alpha1AMP activation in human aortic endothelial cells

F. Andreozzi, C. Procopio, E. Laratta, P. Iania, I. Laino, I. Presta, D. Scarpelli, G. Ruscio, M. L. Hribal, G. Sesti;

Department of Experimental and Clinical Medicine, University of Catanzaro-Magna Græcia, Italy.

Background and Aims: Obesity is associated with leptin-resistance, increased circulating C-reactive protein (CRP) levels and endothelial dysfunction. Recent evidence suggests that leptin can modulate vascular tone through local mechanisms involving NO release. Vasorelaxant responses to $\mathrm{ACh}$ are markedly attenuated in mesenteric microvessels from ob/ob mice while leptin replacement reverts this phenotype. Leptin selectively stimulates phosphorylation and activation of the alpha2 catalytic subunit of AMPK in skeletal muscle resulting in stimulation of fatty acid oxidation in muscle by inhibiting acetyl-CoA carboxylase (ACC) activity. In human endothelial cells, activation of AMPK induces eNOS phosphorylation at Ser ${ }^{1177}$ and NO production. We hypothesized that leptin may exert vasodilator action by stimulating phosphorylation and activation of eNOS in vascular endothelium increasing production of NO via a AMPK-dependent pathway.

Materials and Methods: Human Aortic Endothelial Cells (HAEC) were treated with leptin alone $(10 \mathrm{ng} / \mathrm{ml}$ for $20 \mathrm{~min})$, or after stimulation with AICAR ( $2 \mathrm{mM}$ for $1 \mathrm{~h}$ ) or 5-Iodotubercidin ( 0.2 micro for $3 \mathrm{~h})$. Smart pool siRNAs were used to knockdown expression of human alpha1- or alpha2AMPK in transient trasfection assay. eNOS Activity Assay kit was used to measure eNOS activity by monitoring the conversion of $\left[{ }^{3} \mathrm{H}\right]$ Arginine to Citrulline after leptin stimulation.

Results: Exposure of cultured human aortic endothelial cells (HAECs) to leptin resulted in phosphorylation of both $\mathrm{Thr}^{172}$ AMPK and $\mathrm{Ser}^{79}$ ACC. Similar results were observed with AICAR. Both leptin and AICAR stimulates Ser ${ }^{1177}$ eNOS phosphorylation. AMPK chemical inhibitor 5-Iodotubercidin was able to revert the stimulatory effect of leptin. To confirm the role of AMPK in mediating vasodilator action of leptin, we used siRNAs to specifically knockdown either the alpha1 or alpha 2 isoform of AMPK catalytic subunit. In transient transfection assays, selective siRNAs reduced by $80 \%$ the abundance of both alpha1 and alpha2 AMPK isoforms. The stimulatory effect of leptin on Ser $^{1177}$ eNOS was blocked by alpha1AMPK siRNA, but not alpha2AMPK siRNA, indicating that leptin stimulates eNOS through alpha1AMPK. To reproduce these results in vivo, we acutely treated C57BL/6J mice with human leptin. In aortas of mice, leptin stimulated both $\mathrm{Thr}^{172}$ AMPK and Ser ${ }^{1177}$ eNOS phosphorylation. Recent studies have suggested that a potential mechanism contributing to leptin resistance in vivo is the inhibitory action of CRP, which binds to leptin and attenuates its physiological functions. To test this phenomenon in vitro, we pre-incubated human leptin in the presence of human CRP for 30min before treating HAECs. We observed that leptin preincubated with CRP was unable to stimulate both $\operatorname{Thr}^{172}$ AMPK, Ser ${ }^{79}$ ACC and Ser ${ }^{1177}$ eNOS phosphorylation.

Conclusion: These data suggest that leptin has novel vascular actions, since it acutely activates eNOS in HAECs through a signaling pathway that involves alAMPK. Moreover, the data confirm the cross-talk between leptin and the inflammatory protein CRP raising the possibility that disruption of leptinCRP interaction may be a new therapeutic objective for the treatment of obesity-related vascular complications.

\section{7}

Calreticulin induces dysfunctional vascular endothelial cell-extracellular matrix interactions under high glucose conditions

S. Sasson, Y. Riahi;

Department of Pharmacology, Hebrew University School of Medicine, Jerusalem, Israel.

Background and Aims: Recent studies show that hyperglycaemia increases the expression of the protein calreticulin in vascular cells in vitro and in vivo. Among other functions, calreticulin also controls cell-extracellular matrix (ECM) interactions by binding to and activating key proteins of the adhesion network to form focal ECM-contacts. We aimed at investigating whether overexpression of calreticulin contributes to dysfunctional VECECM interactions in diabetes.
Materials and Methods: Primary cultures of bovine aortic endothelial cells were prepared and exposed to 2 or $25 \mathrm{mmol} / \mathrm{l} \mathrm{D}$-glucose in complete Dulbecco's modified Eagle's medium (DMEM). Adenovirus expressing calreticulin and siRNA constructs were used to augment or silence calreticulin expression, respectively. Calreticulin mRNA and protein content in VEC were determined by real-time PCR and Western blot analyses. VEC proliferation, adhesion to matrix proteins, in vitro re-endothelisation potential, expression levels and tyrosine phosphorylation of adhesion proteins and binding of calreticulin to adhesion molecules were determined by using established assays and immunoprecipitation protocols.

Results: Hyperglycaemic conditions slow the rate of proliferation, motility and the ability of VEC to fill in 'scratches' to restore confluent and uninterrupted VEC monolayers in vitro. Our results show that these effects reflect an enhanced adherence of VEC to ECM components (e.g., fibronectin) under high glucose conditions in comparison with cells exposed to low glucose. This effect was not due to the slight increase in the osmotic pressure of the high-glucose DMEM, since supplementation of the medium with high Lglucose level had no significant effects in these assays. When exposed to high D-glucose level, but not to L-glucose, VEC nearly doubled calreticulin mRNA and protein content in comparison with VEC at low glucose. A central role of calreticulin in modifying VEC-ECM interactions was established following an adenovirus-mediated overexpression of the protein in VEC that had been maintained at $2 \mathrm{mmol} / \mathrm{l} \mathrm{D}$-glucose. The proliferation and re-endothelisation behaviour of these cells were similar to that of VEC that were maintained at $25 \mathrm{mmol} / \mathrm{l} \mathrm{D}$-glucose. Moreover, VEC, which were exposed to $25 \mathrm{mmol} / \mathrm{l}$ $\mathrm{D}$-glucose and in which calreticulin expression was silenced, acquired rates of proliferation and adherence capacity to ECM components characteristic of VEC incubated at $2 \mathrm{mmol} / \mathrm{l} \mathrm{D}$-glucose. Incubation of VEC at the high glucose level also increased the intracellular binding of calreticulin with the integrin-alpha receptor subunit. In parallel, it augmented the expression and tyrosine phosphorylation of paxillin and vinculin and of focal adhesion kinase (FAK). These proteins participate in the assembly of focal adhesion ECM-contacts. Similar interactions were observed in VEC overexpressing calreticulin. Conversely, silencing of calreticulin expression in these cells or transferring them to $2 \mathrm{mmol} / \mathrm{l} \mathrm{D}$-glucose significantly decreased the expression, tyrosine phosphorylation and association with calreticulin of these adhesion proteins.

Conclusions: We suggest that calreticulin recruits the adhesion machinery in VEC to form multiple and stronger focal contacts with the ECM. These interactions, which are enhanced under hyperglycaemic conditions, may impede the healing of lesions in the endothelial cell monolayer in blood vessels and contribute to diabetes-induced vasculopathy.

Supported by the Russel Berrie Fundation and D-Cure, Diabetes Care in Israel

\section{8}

Telmisartan inhibits AGEs-induced asymmetric dimethylarginine accumulation by suppressing receptor for AGEs -mediated reactive oxygen species generation

A. Hayashida, S. Ueda, S.-I. Yamagishi, H. Miyazaki, Y. Matsumoto, K. Fukami, S. Okuda;

Department of Medicine, Kurume University School of Medicine, Japan.

Background and Aims: There is a growing body of evidence that asymmetric dimethylarginine (ADMA), an endogenous nitric oxide synthase inhibitor, could play an important role in endothelial dysfunction and atherosclerosis. Advanced glycation end products (AGEs), the senescent macroprotein derivatives which are formed in increased amounts in patients with diabetes and/or chronic kidney disease, have been also shown to contribute to atherosclerosis. However, a functional relationship between ADMA and AGE levels has not been fully understood.

Materials and Methods: Fifteen patients with end-stage diabetic nephropathy(ESRD) $(24 \mathrm{~h} \mathrm{Ccr}<10 \mathrm{ml} / \mathrm{min}$, ranged $2.3-9.8 \mathrm{ml} / \mathrm{min}$ ) who did not undergo hemodialysis treatment were enrolled in this study. Serum levels of ADMA and AGE were measured by HPLC and ELISA, respectively. Flow-mediated vasodilation (FMD) of the brachial artery was measured to asses endothelial function. In vitro, AGE were prepared by incubating BSA with $0.1 \mathrm{~mol} / \mathrm{l} \mathrm{D}$-glyceraldehyde for 7 days. Reactive oxygen species (ROS) generation was measured by using the fluorescent probe CM- $\mathrm{H}_{2}$ DCFDA. RAGE expression was analyzed by western blotting.

Results: Plasma ADMA levels were inversely related to FMD $(\mathrm{p}<0.01)$ and were found to be significantly associated with plasma AGEs levels 
$(\mathrm{p}<0.05)$. Therefore, we next performed the in vitro-experiments to clarify the molecular link between ADMA and AGEs using cultured human endothelial cells (ECs). AGEs significantly decreased enzymatic activity of dimethylarginine dimethylaminohydrolase (DDAH), a rate-limiting enzyme for ADMA degradation and subsequently increased ADMA production by cultured ECs, both of which were completely blocked by an antioxidant, $\mathrm{N}$ acethylcysteine or telmisartan, an angiotensin II type-I receptor antagonist. Moreover, telmisartan was found to inhibit AGE-induced reactive oxygen species (ROS) generation by suppressing RAGE expression.

Conclusion: Our present study suggests that AGEs could inhibit DDAH activity and subsequently enhanced ADMA accumulation in ECs through RAGE-mediated ROS generation, thereby contributing to atherogenesis in patients with ESDN. Blockade by telmisartan of the AGE-RAGE interaction could play a protective role against endothelial dysfunction in ESDN by reducing ADMA.

\section{9}

Tumor necrosis factor modulates glycolysis, the glyoxalase system and induces the formation of specific methylglyoxal-modified proteins in aortic endothelial cells

M. Laga, F. Van Herreweghe, K. Vancompernolle;

VIB Dept. of Medical Protein Research, University of Ghent and VIB, Belgium.

Background and Aims: It is well established that the formation of intracellular methylglyoxal (MG)-derived Advanced Glycation End products (MG-AGEs) is one of the pathways that leads to hyperglycemic damage in endothelial cells. We have previously shown that Tumor Necrosis Factor (TNF) can induce the rapid formation of specific MG-derived AGEs in a tumor cell death model. Furthermore, these MG-AGEs are formed as a consequence of oxidative stress and involve the TNF-induced phosphorylation of glyoxalase I (GLO1). GLO1 is normally responsible for the detoxification of the cytotoxic glycolytic metabolite methylglyoxal. Because TNF can also contribute to the development of vascular complications in diabetes, the purpose of this study was to investigate whether TNF induces phosphorylation of GLO1, the formation of specific MG-derived AGEs and whether TNF modulates glycolysis and the glyoxalase system in aortic endothelial cells under normoand hyperglycemic conditions.

Materials and Methods: Primary bovine aortic endothelial cells (bAEC) were incubated with TNF for different time points. The TNF-induced phosphorylation of GLO1 was investigated by 2-dimensional gelelectroforesis combined with Western blotting using a polyclonal anti-glyoxalase antibody. The formation of specific MG-AGEs was investigated by two methods namely, by Western blotting using a monoclonal antibody (mAb6B) that recognizes a MG-derived argpyrimidine epitope and by in vivo cell labelling using ${ }^{14} \mathrm{C}-\mathrm{MG}$. The TNF-induced effect on glucose metabolism was examined by 2 parameters, namely by measuring the levels of fructose-2,6-bisphosphate (F2,6BP), an allosteric activator of PFK1, and by measuring glucose uptake using ${ }^{3} \mathrm{H}$-labelled 2-deoxyglucose. The TNF-induced effect on the glyoxalase system was investigated by measuring the end product D-Lactate and GLO1 activities.

Results: TNF induces a rapid phosphorylation of GLO1 in primary bovine aortic endothelial cells (bAEC). This is not accompanied by a decrease in GLO1 activities in TNF-treated cells. Instead, TNF induces a rapid increase in the concentrations of D-lactate. These data indicate that TNF increases the intracellular concentrations of MG, which are not caused by inhibition of the detoxification of MG through the glyoxalase system, but are presumably derived from the TNF-induced stimulation of glycolysis. Indeed, TNF induces a considerable increase in glucose uptake in bAEC and strongly increase the $\mathrm{F} 2,6 \mathrm{BP}$ levels, an allosteric activator of glycolysis. Furthermore, by using ${ }^{14} \mathrm{C}-$ MG, we could show that TNF induces the formation of specific MG-modified proteins in endothelial cells under normo- and hyperglycaemic conditions. It remains to be determined whether phosphorylated GLO1 mediates the formation of these specific MG-AGEs in endothelial cells.

Conclusion: Here we show for the first time that TNF induces increased intracellular levels of methylglyoxal and the formation of specific MGmodified proteins in aortic endothelial cells. This may be one of the underlying molecular mechanisms by which TNF contributes to the development of vascular complications in diabetes.

Supported by the EFSD/Servier European Research Programme on Vascular Complications of Type 2 Diabetes
0030

Reduction of both number and proliferative activity of human endothelial progenitor cells in obese patients

M. Artwohl ${ }^{1}$, K. Tobler ${ }^{1}$, M. Wolzt ${ }^{1}$, C. Maier ${ }^{1}$, B. Ludvik ${ }^{1}$, A. Freudenthaler ${ }^{1}$, D. Seidinger ${ }^{2}$, S. Steiner-Böker ${ }^{2}$, E. Nansalmaa ${ }^{1}$, A. Luger ${ }^{1}$, S. M. Baumgartner-Parzer';

${ }^{1}$ Medical University Vienna, Deptartment of Internal Medicine III, Division of Endocrinology and Metabolism, ${ }^{2}$ Medical University Vienna, Deptartment of Internal Medicine II, Division of Angiology, Austria.

Background and aims: Bone marrow-derived endothelial progenitor cells (EPCs) have only recently been described to circulate in human peripheral blood. Circulating EPCs are assumed to have a role in neoangiogenesis (new blood vessel formation) and vascular repair. Moreover, circulating numbers of EPCs have been shown to negatively correlate with risk factors for atherosclerosis and with disorders associated with vascular dysfunction. So far, it has been shown that the angiogenic activity of EPCs is reduced in obese, diabetic mice in comparison to lean, non-diabetic controls and that high concentrations of the adipose tissue-derived hormone leptin impair migration and tube formation of cultured human EPCs. On the basis of these findings the present study tested the hypothesis that number and proliferative activity of circulating human EPCs are different in obese versus lean control subjects.

Materials and Methods: Written informed consent was obtained from all subjects participating in this study. In brief, peripheral blood mononuclear cells (PBMCs) were isolated by density gradient centrifugation from whole blood obtained from obese- (BMI $=46 \pm 1$ [Range: 35-59], $n=30$ ) and lean subjects (BMI $=22 \pm 1$ [Range: 18-27], $\mathrm{n}=30$ ). $\mathrm{EPCs}$, expressing $\mathrm{CD} 34^{+} /$ $\mathrm{CD} 133^{+} / \mathrm{KDR}^{+}$, were quantified using FACS-analyses. In vitro, EPCs grew out from colonies and, as a result of selective culture conditions, differentiated into mature endothelial cells, which were characterized by expression of CD $31^{+}$(PECAM), CD144+ (VE-Cadherin), and CD146 ${ }^{+}$(S-endo). Protein expression was determined by Western blot analyses.

Results: Blood glucose, triglycerides, cholesterol, LDL, apolipoprotein B, and lipoprotein (a) were not different in lean versus obese subjects. However, obese patients had higher $(\mathrm{p}<0.001) \mathrm{HbA}(5.9 \pm 0.2 \%$ vs. $5.2 \pm 0.1 \%)$ and hsCRP $(1 \pm 0.1 \mathrm{mg} / \mathrm{dl}$ vs. $0.2 \pm 0.1 \mathrm{mg} / \mathrm{dl})$, but lower $(\mathrm{p}<0.001) \mathrm{HDL}(46.4 \pm 2.3 \mathrm{mg} / \mathrm{dl}$ vs. $65.3 \pm 1.9 \mathrm{mg} / \mathrm{dl})$ and apolipoprotein $\mathrm{A} 1(121.4 \pm 3.4 \mathrm{mg} / \mathrm{dl}$ vs. $152.3 \pm 4.5 \mathrm{mg} /$ dl) levels. Independent of the BMI, the number of CD $34^{+} / \mathrm{CD} 133^{+} / \mathrm{KDR}^{+}-$ EPCs was positively associated with HDL $(+1.8$ EPCs / 10mg/dl HDL $)$ and negatively with hsCRP ( $-3.2 \mathrm{EPCs} / 1 \mathrm{mg} / \mathrm{dl} \mathrm{CRP})$. Compared to their age and sex matched lean controls, obese patients had $38 \%(\mathrm{p}<0.05)$ less $\mathrm{CD} 34^{+} / \mathrm{CD} 133^{+} / \mathrm{KDR}^{+}-\mathrm{EPCs}$, reflecting a reduction by $1.4 \mathrm{EPCs}$ per $10 \mathrm{~kg} /$ $\mathrm{m}^{2}$. The proliferative capacity of cultured endothelial cells differentiated from the EPCs of lean control subjects was $20.3 \pm 3.3 \%$ per week, whereas it was only $9.6 \pm 2.2 \%$ in endothelial cells, differentiated from obese patients' EPCs. Reduced proliferative activity of obese patients' EPCs is reflected by decreased $(\mathrm{p}<0.05)$ protein expression of cyclins (cyclin D3: $-20 \pm 10 \%$, cyclin E: $-23 \pm 11 \%$, cyclin A: $-40 \pm 11 \%$, cyclin B: $-31 \pm 10 \%)$ and of the transcription factor E2F-1 $(-17 \pm 6 \%)$ as well as by increased $(\mathrm{p}<0.05)$ expression of the cell cycle inhibitor p $21^{\mathrm{WAF}-1 / \mathrm{Cip} 1}(+41 \pm 17 \%)$ in these cells.

Conclusions: Reduction of both the number and the proliferative capacity (as shown in in vitro experiments) of circulating endothelial progenitor cells, could well contribute to development and progression of vascular dysfunction in obese patients. 


\section{OP 6 Predictors and markers of cardiovascular disease and mortality}

\section{1}

$\mathrm{HbA}_{1 \mathrm{c}}$ in relation to 10-year fatal and non-fatal cardiovascular disease: The Hoorn Study

E. van 't Riet ${ }^{1}$, J. M. Rijkelijkhuizen' ${ }^{1}$, G. Nijpels' , C. D. A. Stehouwer ${ }^{2}$, L. M. Bouter ${ }^{1}$, R. J. Heine ${ }^{3}$, J. M. Dekker';

${ }^{1}$ EMGO-Institute, VU University Medical Centre, Amsterdam, ${ }^{2}$ Department of Internal Medicine, Academic Hospital Maastricht,

${ }^{3}$ Department of Endocrinology, VU University Medical Centre, Amsterdam, The Netherlands.

Background and Aims: Several large-scale studies have shown that in type 1 and type 2 diabetes patients $\mathrm{HbAlc}$ is a strong predictor of cardiovascular disease. In the general non-diabetic population, the association between $\mathrm{HbAlc}$ and both fatal and non-fatal cardiovascular disease are less clear. Therefore, we have investigated the associations between $\mathrm{HbAlc}$ and 10-year fatal and non-fatal cardiovascular disease and with all-cause mortality in people without diabetes in a population based study.

Materials and Methods: The Hoorn Study is a population-based cohort study with 2484 participants at baseline in 1989 to 1990 . The present study population comprised 1674 subjects without diabetes (known or newlydetected) at baseline and without missing data on $\mathrm{HbAlc}$, glucose levels and morbidity. Associations were analysed using Cox survival analysis, with $\mathrm{HbAlc}$ as a continuous variable. Adjustments were made for age, sex, hypertension, LDL cholesterol, triglycerides, waist-to-hip ratio and smoking. Results: The age- and sex-adjusted hazard ratios (with 95\% confidence intervals) for all-cause mortality, CVD morbidity, CVD mortality and CVD morbidity and mortality combined were 1.37 (1.05-1.80), 2.00 (1.56-2.57), $1.62(1.09-2.41)$ and 1.94 (1.54-2.43), respectively. After adjustment for traditional cardiovascular risk factors the association between $\mathrm{HbAlc}$ and CVD morbidity remained significant, with a hazard ratio of $1.66(1.28-2.13)$ Conclusion: This is the first study in which the association of HbAlc with cardiovascular disease in a population based study in people without diabetes was studied. Results showed that an increase of $1 \%$ of $\mathrm{HbAlc}$ is associated with an approximate $66 \%$ increase in risk of developing non-fatal cardiovascular disease, independent of other known cardiovascular risk factors.

$\mathrm{HbAlc}$ in relation to morbidity and mortality (hazard ratios with $95 \% \mathrm{CI}$ )

\begin{tabular}{lll}
\hline & $\begin{array}{l}\text { Model 1 } \\
\text { (age and } \\
\text { sex adjusted) }\end{array}$ & $\begin{array}{l}\text { Model 2 } \\
\text { (adjusted for age,sex, } \\
\text { hypertension, smoking, } \\
\text { LDL, triglycerides and } \\
\text { waist-to-hip ratio) }\end{array}$ \\
\hline All-cause mortality & $1.37(1.05-1.80)$ & $1.20(0.91-1.57)$ \\
Fatal CVD & $1.62(1.09-2.41)$ & $1.26(0.84-1.88)$ \\
Non-fatal CVD & $2.00(1.56-2.57)$ & $1.66(1.28-2.13)$ \\
Fatal and non-fatal CVD & $1.94(1.54-2.43)$ & $1.60(1.27-2.01)$ \\
\hline
\end{tabular}

0032

Fasting insulin and HOMA better predict cardiovascular events in non-diabetic women than in men

L. H. Oterdoom ${ }^{1,2}$, A. P. de Vries ${ }^{1,2}$, R. T. Gansevoort ${ }^{2}$, P. E. de Jong ${ }^{2}$, R. O. Gans ${ }^{1}$, S. J. Bakker';

${ }^{1}$ Department of Medicine, ${ }^{2}$ Department of Nephrology, University Medical Center Groningen, The Netherlands.

Background and Aims: Cardiovascular (CV) disease is the leading cause of death in diabetic and non-diabetic women. Almost all studies on fasting insulin as CV risk factor have been performed in non-diabetic men. Because diabetes mellitus is known to carry a greater CV risk in women than in men, we surmised that there may be a gender-difference in predictive properties of fasting insulin and Homeostasis Model Assessment (HOMA). We investigated prospectively whether fasting insulin and HOMA predict CV events in a nondiabetic general population, with an emphasis on a putative gender difference. Materials and Methods: Fasting insulin was assessed in 7134 non-diabetic, fasting subjects of the PREVEND study. Major adverse cardiovascular events (MACE) were defined as the combination of CV morbidity and CV mortality. $\mathrm{CV}$ mortality was defined according to the cause of death as coded by the Dutch Central Bureau of Statistics. Events were recorded prospectively from baseline survey (1997-1998) until October $31^{\text {st }} 2005$. HOMA was calculated as: [glucose $(\mathrm{mmol} / \mathrm{L}) \mathrm{x}$ insulin $(\mu \mathrm{U} / \mathrm{ml})$ ] / 22.5]. Gender interaction was tested by entering an interaction term of insulin $\mathrm{x}$ gender and HOMA $\mathrm{x}$ gender in Cox-Regression analyses. Subsequent analyses were performed for women and men separately.

Results: Characteristics of the population are shown in table 1. During a median [interquartile range] follow-up of 7.4 [7.1-7.6] years, MACE occurred in 129 women and in 322 men. There was significant gender interaction for fasting insulin and HOMA as predictor of MACE (both $\mathrm{P}=0.002$ ). The interaction indicated that with increasing insulin (fig 1) and HOMA, CV risk increased more in women than in men. After adjustment for smoking and BMI, the hazard ratio (HR $[95 \% \mathrm{CI}])$ of the $4^{\text {th }}$ vs the $1^{\text {st }}$ quartile of insulin remained significant in women $(1.9[1.0-3.5], \mathrm{P}<0.05)$, but not in men $(1.2$ [0.8-1.7], $\mathrm{P}=0.4$ ). Results were not materially changed for HOMA (data not shown).

Conclusion: It has not been investigated before whether fasting insulin and HOMA bear a different CV risk in women and men. We found significant effect-modification by gender for both fasting insulin and HOMA, indicating that insulin resistance parallels diabetes with regard to an increased CV risk in women compared to men. Our results indicate that specific attention for interventions can potentially provide greater benefit in insulin resistant women than in insulin resistant men. (Fig. 1)

\section{Table 1}

\begin{tabular}{llll}
\hline & Women & Men & P-value \\
\hline $\mathrm{N}=$ & 3646 & 3488 & - \\
Age, yr & $48 \pm 12$ & $50 \pm 13$ & $<0.001$ \\
Smoking, n (\%) & $1362(36.8)$ & $1315(37.2)$ & $<0.001$ \\
BMI, kg/m2 & $25.8 \pm 4.6$ & $26.2 \pm 3.6$ & $<0.001$ \\
Insulin, $\mu \mathrm{U} / \mathrm{mL}$ & $7.5[5.3-10.7]$ & $8.2[5.7-12.2]$ & $<0.001$ \\
HOMA & $1.5[1.0-2.3]$ & $1.7[1.2-2.7]$ & $<0.001$ \\
\hline
\end{tabular}

Supported by the Dutch Kidney Foundation
Fig. 1
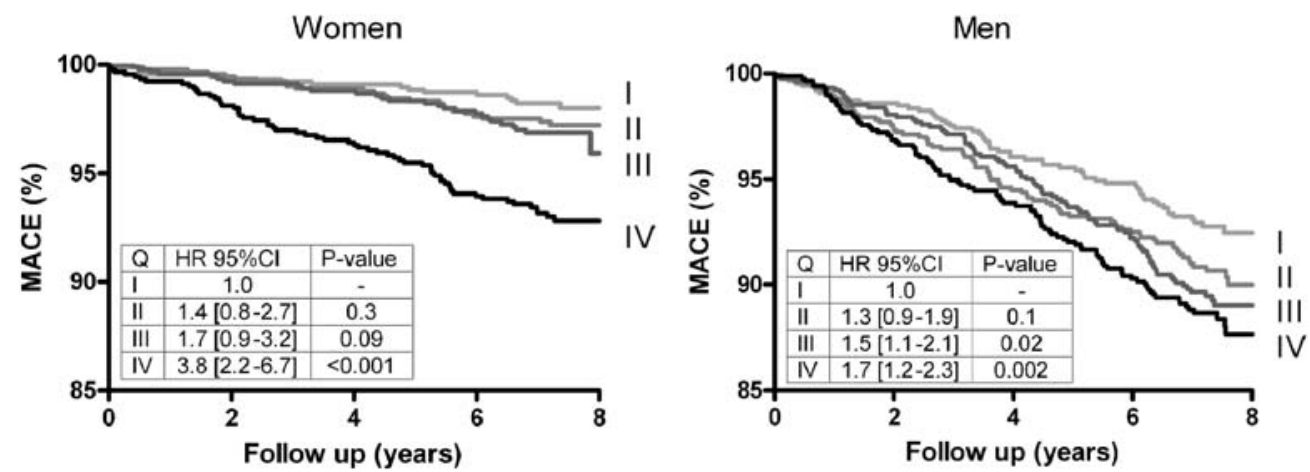


\section{3}

The NDR Risk Model - A novel tool for prediction of coronary risk in type 2 diabetic patients from the Swedish National Diabetes Register S. Gudbjornsdottir ${ }^{1}$, J. Cederholm², K. Eeg-Olofsson ${ }^{1}$, B. Eliasson ${ }^{1}$, P. M. Nilsson ${ }^{3}$;

${ }^{1}$ Goteborg University, Department of Medicine, ${ }^{2}$ Department of Public Health and Caring Sciences, Family Medicine and Clinical Epidemiology Section, Uppsala, ${ }^{3}$ Department of Clinical Sciences Medicine, Division of Medicine, Lund, Sweden.

Background and Aims: There are a number of models for cardiovascular risk prediction in type 2 diabetes, most notably the UKPDS Risk Engine, which was constructed from baseline data in 4540 newly detected type 2 patients followed for 10 years (less than one-third were on antihypertensive treatment and only few used lipid-lowering agents at baseline). However, more patients than previously are now under long-term drug treatment for risk factors, which is not fully accounted for in current risk models.

Material and Methods: We used prospective data from the NDR of Sweden (1997-98), comprising 21315 type 2 diabetic men and women (age 18-65 years, mean 57 years) followed for 6 years. Baseline risk factors included age, gender, diabetes duration, $\mathrm{HbA}_{1 \mathrm{c}}$, use of antihypertensive and lipid-lowering drugs, smoking and microalbuminuria. Outcome measures in Cox regression analysis were incident fatal or non-fatal coronary heart disease (CHD) events (myocardial infarction, unstable angina, or PCI/CABG intervention; data from the Swedish hospital discharge register).

Results: Based on 120603 person-years and $3162 \mathrm{CHD}$ events, independent risk ratios (95\% confidence intervals) for the included variables were in rank order: use of lipid-lowering drugs 1.520 (1.379-1.676), use of antihypertensive drugs 1.359 (1.260-1.466), smoking 1.279 (1.151-1.422), microalbuminuria 1.140 (1.054-1.234) and HbAlc 1.087 (1.060-1.115). All variables were significant ( $\mathrm{P}$-values $<0.001$ except microalbuminuria, $\mathrm{P}=0.0011$ ). Using these risk ratios and the ratios for age, sex and diabetes duration, an equation was created for estimation of the 10-year risk for fatal/non-fatal CHD.

This NDR Risk Model was applied in 20879 type 2 diabetic patients aged 18-65 years with clinical data from 2005 (mean diabetes duration 6.4 years; $62 \%$ on antihypertensive and $50 \%$ on lipid-lowering treatment). The mean calculated 10-year risk for fatal/non-fatal CHD was $22 \pm 9 \%$, compared to a calculated mean 10 -year risk of $16 \pm 10 \%$ for fatal/nonfatal myocardial infarction when using the UKPDS Risk Model in the same cohort.

Conclusion: Using a model based on data from the NDR, the 10-year risk of CHD in a cross-sectional national sample of type 2 diabetic patients was about $40 \%$ higher than the comparable risk found when the UKPDS Risk Engine was applied. The NDR Risk Model for type 2 diabetic patients should be validated in independent cohorts.

\section{4}

Do people with diabetes detected by a universal risk score have an increased risk of dying? The DETECT-2 study

D. Vistisen ${ }^{1}$, C. Glümer ${ }^{1}$, A. C. Jensen ${ }^{1}$, S. Colagiuri ${ }^{2}$, K. Borch-Johnsen ${ }^{1}$;

${ }^{1}$ Department of Epidemiology, Steno Diabetes Center, Gentofte, Denmark,

${ }^{2}$ Australian Centre for Diabetes Strategies, Prince of Wales Hospital,

Randwick, Australia.

Background and Aims: Several risk scores for type 2 diabetes have been developed. Whether they identify individuals at highest risk of death or complications is still uncertain. The aim of this study was to examine whether a universal risk score for detecting undiagnosed diabetes also identifies individuals with the highest mortality risk.

Materials and Methods: Results are based on follow-up data in the DETECT2 project. Five geographical regions were included, the age range was 30-74 years $(\mathrm{N}=44,513)$ with a total of 5,477 deaths over 412,840 person-years. The universal risk score includes information on age, sex, BMI, and systolic blood pressure. All individuals had an OGTT performed. Screen detected diabetes (SDM) was defined according to the 1999 WHO diagnostic criteria. The universal risk score includes information on age, sex, BMI and systolic blood pressure. A score above a predefined threshold was considered screen positive. Individuals were classified as true positive (TP), false positive (FP), false negative (FN) and true negative (TN) accordingly. All-cause mortality was measured and Poisson regression analysis was used to calculate mortality rates. For the regions, mean follow up time was between 5 and 12 years.
Results: Screen positives were significantly older, more obese, with higher BP and cholesterol levels. The fraction of men was higher, but with less current smokers when comparing with the screen negative group. Crude mortality rates were higher in the screen positive group. However, when adjusting for age and sex this excess mortality disappeared in all regions except Europe. For all regions, excess mortality for screen positives was found in the population below 60 years of age.

Conclusion: The universal risk score predicts diabetes but only excess mortality in the population below 60 years of age.

Rate ratios (RR) for all cause mortality by screening category and region

\begin{tabular}{llllll}
\hline Region & & TP & FP & FN & TN \\
\hline Europe & Crude RR & $3.0(2.6-3.4)$ & $1.7(1.6-1.8)$ & $2.2(1.8-2.6)$ & 1 \\
& RR adj. for & $2.0(1.7-2.2)$ & $1.2(1.1-1.3)$ & $2.0(1.6-2.4)$ & 1 \\
& age and sex & & & & \\
Africa & Crude RR & $5.5(3.5-8.8)$ & $2.9(2.0-4.2)$ & $5.1(2.6-10.2)$ & 1 \\
& RR adj. for & $1.2(0.7-2.0)$ & $0.6(0.4-1.0)$ & $4.4(2.2-8.9)$ & 1 \\
& age and sex & & & & \\
Japan & Crude RR & $4.3(1.5-12.5)$ & $1.5(0.9-2.5)$ & 0 & 1 \\
& RR adj. for & $2.1(0.7-6.5)$ & $0.7(0.4-1.4)$ & 0 & 1 \\
& age and sex & & & & \\
Pacific & Crude RR & $5.7(2.7-12.0)$ & $2.6(1.3-5.3)$ & $2.0(0.6-7.3)$ & 1 \\
Islands & RR adj. for & $1.8(0.7-4.6)$ & $1.0(0.4-2.5)$ & $2.5(0.7-9.2)$ & 1 \\
& age and sex & & & & \\
North & Crude RR & $3.1(2.3-4.1)$ & $2.4(2.0-2.8)$ & $3.7(2.4-5.5)$ & 1 \\
America & RR adj. for & $1.4(1.1-1.9)$ & $1.1(0.9-1.3)$ & $2.2(1.5-3.4)$ & 1 \\
& age and sex & & & & \\
\hline & & & & &
\end{tabular}

\section{5}

Type of preadmission antidiabetic treatment and mortality among patients hospitalized with myocardial infarction: a nationwide follow-up study

H. Thisted ${ }^{1,2}$, F. Søndergaard ${ }^{1}$, S. P. Johnsen ${ }^{1,3}$, J. Rungby ${ }^{2,4}$;

${ }^{1}$ Department of Clinical Epidemiology, Aarhus University Hospital,

${ }^{2}$ Department of Pharmacology, University of Aarhus,

${ }^{3}$ Center of Cardiovascular Research, Aalborg Hospital, Aalborg,

${ }^{4}$ Department of Endocrinology C, Aarhus University Hospital, Denmark.

Background and Aims: Improved glycaemic control may prevent cardiovascularcomplications in patients with type 2 diabetes mellitus. However, antidiabetic treatment may also be associated with adverse cardiovascular effects. Clinical studies have indicated an increased cardiovascular mortality among patients using some sulphonylureas as well as among patients using metformin. However, data are sparse and conflicting and we therefore sought to determine the impact of different types of antidiabetic treatment, including sulphonylureas, metformin and insulin, on mortality in patients with type 2 diabetes mellitus after hospitalization for myocardial infarction (MI).

Materials and Methods: We examined the mortality among users of the different antidiabetic therapies in a nationwide population-based followup study in Denmark. We identified 72,295 patients with a first-time hospitalization for MI in Denmark in the period 1996-2004 using data from the Danish National Patient Registry (DNPR). Among MI patients, data on diabetes and type of antidiabetic treatment were obtained from the DNPR and a national prescription database. Mortality was determined through the Civil Registration System. We estimated 30-day and 1-year hazard ratios (HRs) associated with the use of different antidiabetic treatments prior to MI adjusted for a range of prognostic factors including duration of diabetes, use of cardiovascular drugs and level of comorbidity using Cox regression analysis.

Results: We identified a total of 8,494 MI patients with type 2 diabetes mellitus. The overall 30 -day and 1-year mortalities were $22.2 \%$ and $36.6 \%$. We found no significant differences between the oral antidiabetic treatments, but the use of insulin was associated with an increased risk compared with the use of sulphonylureas (table). Adjusted HR for metformin was low, however non-significantly. Similar observations were evident during the 1year follow-up period. 
30-day HRs according to antidiabetic treatment

\begin{tabular}{lllll}
\hline Antidiabetic treatments & $\mathrm{N}$ & Deaths & Mortality & $\begin{array}{l}\text { Adjusted HRs } \\
(95 \% \mathrm{CI})\end{array}$ \\
\hline Sulphonylurea (mono) & 2,635 & 605 & $23.0 \%$ & 1.00 (reference) \\
Metformin (mono) & 502 & 67 & $13.4 \%$ & $0.90(0.70-1.17)$ \\
Insulin (mono) & 1,784 & 445 & $24.9 \%$ & $1.16(1.01-1.34)$ \\
Any combination & 1,314 & 259 & $19.7 \%$ & $1.09(0.94-1.27)$ \\
No pharmacotherapy & 2,098 & 475 & $22.6 \%$ & $1.02(0.89-1.16)$ \\
\hline
\end{tabular}

Conclusion: Mortality after MI remains high in patients with type 2 diabetes mellitus. Pre-MI antidiabetic treatments do not appear to be associated with substantial differences in mortality.

Supported by Danish Diabetes Association, Research Initiative of Aarhus University Hospital, University of Aarhus, Danish Medical Research Council, and Servier

\section{6}

Human atrial natriuretic peptide (ANP) gene and metabolic risk factors in the French population

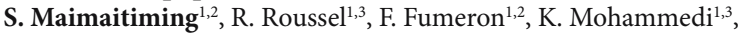

S. Hadjadj ${ }^{4}$, R. Aubert ${ }^{1}$, B. Balkau ${ }^{5}$, M. Marre ${ }^{1,3}$;

${ }^{1}$ INSERM, U 695, Paris, ${ }^{2}$ University Denis Diderot, Paris,

${ }^{3}$ AP-HP, Hôpital Bichat, Département d'Endocrinologie, Diabétologie, Paris, ${ }^{4}$ Diabetology, CHU, Poitiers, ${ }^{5}$ INSERM, U 258, Villejuif, France.

Background and Aims: The ANP plays an important role in blood pressure regulation, but also affects lipolysis in human adipocytes. This effect is reinforced by physical activity in overweight subjects. The gene coding for ANP (NPPA: natriuretic peptide precursor A) was thus considered as a candidate gene for susceptibility to hypertension and metabolic disorders. Our aim was to assess the effect of three NPPA polymorphisms, G664A (rs5063), 708C $>\mathrm{T}$ ( $\mathrm{rs5064}$ ) and 2238T $>\mathrm{C}$ ( $\mathrm{rs5065}$ ) on metabolic parameters levels and their interaction with overweight and physical activity in 5,212 French Caucasian subjects.

Materials and Methods: Subjects took part in DESIR (Epidemiologic Data on the Insulin Resistance Syndrome), a cohort of 2576 men and 2636 women from the middle-aged general population who were clinically and biologically evaluated at inclusion and at 3,6 and 9-year visits. These SNPs were identified as tag SNPs in the Hapmap project and identified most of haplotypes at the NPPA locus. All quantitative tests are adjusted for sex, age and body mass index. The genotypes were determined by the Taqman process.

Results: In the whole population, there was a marginally significant effect of the 708C $>$ T SNP on waist girth [CC: $83.5 \pm 0.1 \mathrm{~cm},(\mathrm{n}=4181), C T: 83.1$ $\pm 0.2(\mathrm{n}=865)$ and TT: $81.2 \pm 0.9(\mathrm{n}=51) p=0.04]$, HDL-C (CC: $1.63 \pm 0.01$ $\mathrm{mM}$, CT: $1.63 \pm 0.01$ and TT: $1.77 \pm 0.03, p=0.04)$ and ApoA1 levels (CC: $1.64 \pm 0.01 \mathrm{~g} / \mathrm{l}$, CT: $1.65 \pm 0.02$ and TT: $1.72 \pm 0.04, p=0.03)$. The $708 \mathrm{C}>\mathrm{T}$ SNP interacted with overweight on triglycerides (TG), waist girth and serum glutamic pyruvic transaminase (SGPT) $(p=0.002, p=0.03$ and $p=0.02$ respectively for interaction) and in overweight subjects, was associated with TG [CC: $1.51 \pm 0.03 \mathrm{mM},(\mathrm{n}=1834), C T: 1.37 \pm 0.07(\mathrm{n}=389)$ and TT: $1.13 \pm$ $0.31(\mathrm{n}=21), p=0.009$ ], waist girth (CC: $93.1 \pm 0.2 \mathrm{~cm}, \mathrm{CT}: 92.1 \pm 0.4$ and TT: $89.1 \pm 1.8, p=0.009$ ) and SGPT concentrations (CC: $31.8 \pm 0.4$, CT: $28.5 \pm$ 0.9 and TT: $26.9 \pm 4.2, p=0.006) .708 \mathrm{C}>\mathrm{T}$ TT carriers had lower triglyceride concentrations in the physically active overweight subjects $(p=0.01)$. The $2238 \mathrm{~T}>\mathrm{C}$ SNP interacted with overweight on waist girth and SGPT ( $p=0.08$ and $p=0.04$ respectively for interaction) and in overweight subjects, was associated with waist girth [TT: $93.1 \pm 0.2 \mathrm{~cm},(\mathrm{n}=1521)$, TC: $92.4 \pm 0.3$ $(\mathrm{n}=574)$ and CC: $91.1 \pm 0.9(\mathrm{n}=48), p=0.03)$ and SGPT concentrations (TT: $31.8 \pm 0.5$, TC: $29.5 \pm 0.8$ and CC: $26.1 \pm 2.7, p=0.01$ ). No association was found with $664 \mathrm{G}>\mathrm{A}$. Haplotype GTC, the only frequent haplotype with the minor allele of $708 \mathrm{C}>\mathrm{T}$ and $2238 \mathrm{~T}>\mathrm{C}$, was significantly associated with lower waist girth level $(p=0.007)$, TG $(p=0.02)$ and SGPT concentrations $(p=0.008)$ in overweight subjects.

Conclusion: NPPA tag SNPs and haplotypes may be associated with cardiovascular risk in the general French population through association with lipid levels, in particular in overweight subjects.

\section{OP 7 Body weight regulation}

\section{7}

Weight reduction modulates mRNA expression of pro-inflammatory molecules involved in NFkB activation and insulin signaling in subjects with the metabolic syndrome - the GENOBIN study

V. D. F. Mello ${ }^{1,2}$, M. Kolehmainen ${ }^{1}$, U. Schwab ${ }^{1,3}$, U. Mager ${ }^{1}$,

D. E. Laaksonen ${ }^{3}$, L. Pulkkinen ${ }^{1}$, L. Niskanen ${ }^{3}$, H. Gylling ${ }^{1,3}$, R. Rauramaa ${ }^{4,5}$, M. Uusitupa ${ }^{1}$;

${ }^{1}$ School of Public Health and Clinical Nutrition/Food and Health Research Centre, University of Kuopio, Finland, ${ }^{2}$ Internal Medicine, Federal

University of Rio Grande do Sul, Porto Alegre, Brazil, ${ }^{3}$ Institute of Medicine, Internal Medicine, Kuopio University Hospital, Finland, ${ }^{4}$ Kuopio Research Institute of Exercise Medicine, University of Kuopio, Finland, ${ }^{5}$ Department of Clinical Physiology and Nuclear Medicine, Kuopio University Hospital, Finland.

Background and Aims: Circulating mononuclear cells have been implicated in the low-grade inflammatory state of obese individuals and in the pathogenesis of insulin resistance. Previously we showed that weight loss altered gene expression of selected cytokines in peripheral blood mononuclear cells (PBMCs), which was associated with an improvement of insulin and glucose metabolism in subjects with the metabolic syndrome (MS). We sought to evaluate how weight loss would affect mRNA expression of genes related to immunological and endothelial function and pro-inflammatory mediators, such as nuclear factor-kappa-B (NFkB) inhibitor kinase beta (IKBKB) and suppressor of cytokine signalling-3 (SOCS3), all of which are related to NFkappa-B activation.

Materials and Methods: Altogether 34 overweight or obese (BMI: $32.6 \pm 3.1$ $\mathrm{kg} / \mathrm{m}^{2}$ ) subjects with the MS were randomized to either a weight reduction (WR) or a control group for 33 weeks. Subjects in the WR group had an intensive 12 -wk weight loss period followed by a 21 -wk period where the minimum aim was weight maintenance. The mRNA expression was studied using real-time PCR and TaqMan ${ }^{\circ}$ chemistry at baseline and wk 33 . Measures of insulin and glucose metabolism (intravenous and oral glucose tolerance tests) and body composition were also assessed.

Results:WR decreased the mRNA expression of intercellular cell adhesion molecule-1 (ICAM1) [median-14.4\% (interquartile range: $-23-0$ ), $p<0.01$ ], regulated on activation, normal $\mathrm{T}$ cell expressed and secreted (RANTES) [mean $-7.8 \%$ (95\% CI: $-20.1-4.6), \mathrm{p}<0.001$ ], IKBKB [median $-9.1 \%$ (interquartile range: $-24.4-20.6$ ), $\mathrm{p}<0.001$ ] and SOCS3 [median $-11.5 \%$ (interquartile range: $-26.7-3.6$ ), $p<0.001$ ]. The decrease in RANTES mRNA expression was correlated with an increase in the insulin sensitivity index $\left(S_{\mathrm{I}}\right)$, even after adjustment for changes in body weight $(\mathrm{r}=-0.50)$, body fat $\%(r=-0.53)$, or waist circumference $(r=-0.46, p<0.05$ for all). The change in RANTES mRNA expression also correlated with the change in ICAM1 $(\mathrm{r}=0.39, \mathrm{p}<0.05)$ and IKBKB mRNA expression $(\mathrm{r}=0.63, \mathrm{p}<0.01)$.

Conclusion: Weight loss decreased the mRNA expression of RANTES and SOCS3 in the PBMCs of subjects with the MS. The decrease in RANTES mRNA expression was associated with an improvement in $\mathrm{S}_{\mathrm{I}}$ and a decrease in ICAM1 and IKBKB mRNA expression. These results suggest that weight loss decreases expression of pro-inflammatory mediators associated with the NFkB activation and insulin signaling in the PBMCs of obese subjects with the MS, which is associated with an improvement in insulin sensitivity and endothelial function.

Supported by the Academy of Finland (to M.Kolehmainen, to M. Uusitupa), and from the Sigrid Juselius Foundation. V.D.F. Mello is supported by a CNPq grant from Brazil

\section{8}

Intravenous insulin affects hypothalamic insulin receptor substrates and inhibits appetite-stimulating neuropeptides at euglycaemia in normal rats S. D. Bouman ${ }^{1}$, L. Jessen ${ }^{1}$, P. J. Larsen ${ }^{2}$, J. Jelsing ${ }^{2}$, U. Ribel ${ }^{1}$, J. Sturis ${ }^{1}$,

C. L. Brand';

${ }^{1}$ Insulin Pharmacology, Novo Nordisk A/S, Maaloev,

${ }^{2}$ Basic Research Facilities, Rheoscience A/S, Roedovre, Denmark.

Background and Aims: Insulin has a signaling role in energy balance, with central insulin suppressing food intake and body weight. It has been suggested that peripheral insulin enters the brain and influences energy 
balance via direct impact on hypothalamic neurons involved in appetite regulation. The aim of our study was to investigate the effect of peripheral hyperinsulinemia on appetite-regulating neuropeptides at euglycemia in freely-moving rats.

Materials and Methods: Normal male Sprague-Dawley rats were implanted with permanent catheters via the jugular vein and carotid artery. A week later, the rats were fasted overnight and then placed in cages allowing free movement and stress-free infusions and blood sampling. The animals were divided into five groups, and received a continuous intravenous infusion with either vehicle for 5 hours, a low dose of insulin $\left(15 \mathrm{pmol} \times \mathrm{kg}^{-1} \times \mathrm{min}^{-1}\right)$ for 5 or 8 hours, or a high dose of insulin $\left(120 \mathrm{pmol} \times \mathrm{kg}^{-1} \times \mathrm{min}^{-1}\right)$ for 5 or 8 hours. Arterial blood samples were withdrawn at 10 -minute intervals for plasma glucose determination, and glucose was infused as needed to maintain plasma glucose at basal levels. The control group (infused with insulin vehicle) received saline instead of glucose. At the end of the infusion period the rats were sacrificed and their brains were removed and frozen. For analysis of neuropeptide changes, in situ hybridization on coronal cryostat sections was employed to quantify hypothalamic contents of mRNAs encoding neuropeptide $\mathrm{Y}(\mathrm{NPY})$, agouti-related peptide (AgRP), proopiomelanocortin (POMC), cocaine- and amphetamine-regulated transcript (CART), melaninconcentrating hormone $(\mathrm{MCH})$, and corticotropin-releasing hormone (CRH). Hypothalamic mRNAs for the insulin receptor substrates IRS-2 and IRS- 4 were also analyzed, to verify that insulin was directly involved in possible changes.

Results: A total of 46 rats were studied. All animals stayed normoglycemic throughout the studies, while plasma corticosterone indicated low stress levels. Plasma C-peptide (reflecting endogenous insulin secretion) was suppressed completely in the insulin-infused groups. Compared to the control vehicle group, insulin receptor substrates in the hypothalamus were affected by the insulin infusions, with a significant downregulation of IRS-4 in the arcuate nucleus $(\mathrm{p}<0.05)$, and a tendency to upregulation of IRS-2 in the ventromedial hypothalamus. Neuropeptides were also changed by insulin, which decreased mRNA contents for AgRP $(\mathrm{p}<0.05)$ and for NPY $(\mathrm{p}=0.07)$ in the arcuate hypothalamus, in agreement with the proposed body weight regulating role of insulin. CART showed a small tendency to upregulation in the arcuate nucleus. POMC, MCH and CRH were not different between groups. In general, effects were stronger after 8 hours of insulin infusion than after 5 hours.

Conclusion: This study shows that peripheral insulin changes hypothalamic insulin receptor substrates and decreases hypothalamic appetite-stimulating neuropeptides, under non-stressed and non-hypoglycemic conditions. Thereby our study provides the first experimental support on the neuropeptide level for the concept that peripheral insulin acts as an afferent signal in the regulation of energy balance. These data also suggest involvement of hypothalamic IRS-4 in insulin's effects, in addition to the known role of IRS-2.

\section{9}

Centrally-injected oleoyl-estrone reduces feeding and body weight in rats J. R. Vasselli ${ }^{1}$, P. J. Currie' ${ }^{1}$, T. Lukic ${ }^{2}$, A. G. Harris';

${ }^{1}$ Obesity Research Center, A University Hospital of Columbia University College of Physicians and Surgeons, New York, ${ }^{2} \mathrm{R} \& \mathrm{D}$, Manhattan Pharmaceuticals Inc., New York, United States.

Background and Aims: Oleoyl-estrone (O-E) is a synthetic form of oleoylestrone, a molecule that exists naturally in the body as an endogenous acylestrone expressed and released from adipose tissue in proportion to body fat mass. O-E is currently in Phase IIa clinical trials for the treatment of obesity and morbid obesity. Previous studies have shown that oral and intravenous administration of $\mathrm{O}-\mathrm{E}$ to rodents reduces both food intake and body weight. While O-E has anti-lipogenic effects, its site of action to produce reductions in feeding and body weight are not known. This study tests the hypothesis that $\mathrm{O}-\mathrm{E}$ acts centrally by determining the effects of intracerebroventricular administration of O-E on food intake (FI) and body weight (BW) in normal lean rats.

Materials and Methods: Young adult male and female Sprague-Dawley (S-D) rats were implanted unilaterally with a stainless steel guide cannula targeted to the lateral or $3^{\text {rd }}$ ventricles, respectively. Following recovery from surgery, the groups were microinjected with ascending doses of O-E dissolved in DMSO and diluted for injection with aCSF to a concentration of $0.5 \%$ DMSO. Single microinjections ( 4.0 and $2.0 \mu \mathrm{l}$ volumes, respectively) of O-E were given in ascending order, with FI and BW measured in the males at 24 and 48 hrs post-microinjection, and in females at 5, 24 and 48 hrs post-microinjection. $\mathrm{O}$-E doses tested were vehicle, 100, 250, and $500 \mathrm{ng}$. Microinjection tests were separated by a minimum of 3 days.

Results: In males, microinjection of O-E significantly and dose-dependently reduced BW at $24 \mathrm{hrs}$ post-microinjection $(\mathrm{p}<0.05)$, with a maximum reduction vs. pre-test BW baseline at $500 \mathrm{ng}$ of $-10.6 \mathrm{~g}$ (from a starting weight of $485 \mathrm{~g}$ ). Slight but non significant reductions of FI by O-E were noted in males. In females, microinjection of O-E significantly reduced BW at $5 \mathrm{hrs}$ post-microinjection at 250 and $500 \mathrm{ng}(\mathrm{p}<0.01)$, and at $24 \mathrm{hrs}$ postmicroinjection at $250 \mathrm{ng}(\mathrm{p}<0.01)$. A maximum $\mathrm{BW}$ reduction of $-5.1 \mathrm{~g}$ at $24 \mathrm{hrs}$ at $250 \mathrm{ng}$ was observed (starting weight of $295 \mathrm{~g}$ ). Also in females O-E reduced first 24 -hr post-microinjection FI at the 250 and $500 \mathrm{ng}$ doses $(\mathrm{p}<$ 0.05 ), with a maximum reduction vs. pre-test FI baseline of $-4.6 \mathrm{~g}$ at the 250 $\mathrm{ng}$ dose. FI remained reduced $(\mathrm{p}<0.01)$ at this level in the females at the 250 ng dose during the second 24-hr post-microinjection interval.

Conclusion: Acute cerebroventricular microinjections of O-E significantly and dose-dependently reduced BW in normal lean female and male S-D rats. In addition, O-E significantly reduced $24-\mathrm{hr}$ food intake in females on the first and second days following microinjection. Significant reductions of $\mathrm{BW}$ in females prior to reductions of FI indicate that O-E enhanced energy expenditure as well as inhibited feeding. Our data indicate that in addition to its demonstrated peripheral anti-lipogenic effects, O-E acts centrally to inhibit feeding and reduce body weight in rats.

Supported by Manhattan Pharmaceuticals Inc. to JRV

\section{0}

A common variant in the $F T O$ gene region is associated with $B M I$ in the general population and predisposes to adult and childhood obesity H. Lango ${ }^{1}$, M. N. Weedon ${ }^{1}$, N. J. Timpson ${ }^{2}$, E. Zeggini ${ }^{2}$, R. M. Freathy ${ }^{1}$, C. M. Lindgren ${ }^{2}$, J. R. B. Perry ${ }^{1}$, K. S. Elliott ${ }^{2}$, N. W. Rayner ${ }^{2}$, C. J. Groves ${ }^{2}$, G. Davey-Smith ${ }^{3}$, the UK Type 2 Diabetes Genetics Consortium, T. M. Frayling ${ }^{1}$, A. T. Hattersley ${ }^{1}$, M. I. McCarthy';

${ }^{1}$ Genetics of Complex Traits \& Diabetes Genetics, Peninsula Medical School, Exeter, ${ }^{2}$ Wellcome Trust Centre for Human Genetics \& OCDEM, University of Oxford, ${ }^{3}$ Department of Social Medicine, University of Bristol, United Kingdom.

Background and Aims: Obesity is a major risk factor for type 2 diabetes, and has a strong genetic component. However, there are no known examples of common genetic variants for which there is widely-replicated evidence of an effect on obesity. Using a genome-wide association study we aimed to identify common gene variants predisposing to obesity.

Materials and Methods: We used data from 394,000 SNPs in 1924 UK type 2 diabetes patients generated by The Wellcome Trust Case Control Consortium. We used data from 8 replication studies (mean age 28-74 years, mean BMI $22.7-27.2 \mathrm{~kg} / \mathrm{m}^{2}$ ) consisting of 28,165 adults with BMI available, including a further 3757 type 2 diabetic patients, and 7477 children with anthropometric measures at birth and ages 7 to 11 years.

Results: Variants in the FTO gene were most strongly associated $\left(\mathrm{P}=6 \times 10^{-6}\right.$ $-2 \times 10^{-5}$ ) with BMI in the genome wide study. SNP rs9939609 in intron 1 of FTO increased the risk of diabetes (OR $\left.1.23[1.18-1.32], \mathrm{p}=7 \times 10^{-14}\right)$ but this effect was attenuated $(p=0.44)$ when correcting for BMI. In the combined dataset each additional copy of the A-allele of rs9939609 is associated with a weight increase of $\sim 1.4 \mathrm{~kg}\left(\mathrm{P}=3 \times 10^{-35}\right)$. Sixteen percent of the population carrying two A-alleles has a $67 \%$ higher odds of being obese than the $35 \%$ of TT homozygote carriers $\left(\mathrm{P}=1 \times 10^{-14}\right)$. In children from the ALSPAC study, the A-allele was associated with BMI and obesity from age 7 years onwards, and with a specific increase in fat mass, as assessed at age 9 by DXA measures $\left(\mathrm{P}=6 \times 10^{-10}\right)$

Conclusion: Common variation in the FTO gene represents the first confirmed example of a common genetic effect on BMI and obesity in the general population. The association reflects a specific increase in fat mass. The function of the FTO gene is not known. Understanding how variation in the FTO gene region alters adiposity will provide insights into novel pathways involved in the control of adiposity.

Supported by Wellcome Trust, Diabetes UK 


\section{1}

Regional neuronal activation following calorific nutrient ingestion identified using functional magnetic resonance imaging - the case for a feeding network

Y. P. Samarasinghe ${ }^{1}$, S. Lee ${ }^{2}$, F. Zelaya ${ }^{2}$, L. J. Reed ${ }^{2}$, M.-M. Teh ${ }^{1}$,

P. Choudhary ${ }^{1}$, A. Pernet ${ }^{1}$, B. Wilson ${ }^{1}$, M. Brammer ${ }^{2}$, S. C. R. Williams ${ }^{2}$,

S. A. Amiel ${ }^{1}$;

${ }^{1}$ Diabetes Research Unit, King's College London School of Medicine,

${ }^{2}$ Institute of Psychiatry, Centre for Neuroimaging Sciences, London,

United Kingdom.

Background and Aims: Type 2 diabetes and obesity are lifestyle diseases resulting from disrupted energy homeostasis and appetite control. Although homeostatic control is maintained by the hypothalamus, the motivational and psychosocial aspects of eating are influenced by the higher cerebral centres. Studies of human brains using functional magnetic resonance imaging (fMRI) have identified low frequency fluctuations in baseline activity described as resting state networks (RSN), which are probably the brains anticipatory state. These distinct, interconnected areas coordinate a variety of functions including vision, language, auditory and motor processing. A RSN for feeding has not been described. To identify regional neuronal responses to feeding, we used fMRI to compare oral glucose with an isocalorific, noncarbohydrate fat.

Materials and Methods: Ten fasted, right handed, healthy volunteers (median(range)age: 22(20-55)yrs) underwent 2x40min continuous fMRI scans during which they drank $75 \mathrm{~g}$ glucose in $388 \mathrm{mls}$ water or $68 \mathrm{mls}$ peanut oil (fat) followed by $320 \mathrm{mls}$ water. Arterialised blood samples were obtained throughout. MR signal changes were correlated with a previously published model for hypothalamic activation after glucose ingestion. Brain regions were identified according to the power of the correlation $(\mathrm{p}=0.0005)$ and size of voxels $(>500)$. The average time series for the glucose and fat data for each of the regions identified was compared by ANOVA $(\mathrm{p}<0.05)$.

Results: We identified significant neuronal signal change at $10(+/-4) \mathrm{mins}$ after glucose ingestion in bilateral retrosplenium, left prefrontal cortex, insula, amygdala, posterior cingulate and cerebella cortical networks which was not different after the peanut oil ingestion $(p=0.06-0.57)$. A signal change in the orbitomedial frontal (OMF) cortex however, was significantly stronger after peanut oil vs glucose $(\mathrm{p}=0.009)$. Response amplitude increased over the experimental period to 10 minutes (paired t-test $\mathrm{p}<0.001$ ).

Conclusion: We have demonstrated an early (5-15mins) change in brain activation in a network of regions throughout the brain in response to both carbohydrate and fat ingestion, in isocalorific quantities and identical volumes. Regions identified have known associations to food seeking behaviour and appetite: insula cortex is activated in response to visceral stimulation; the amygdala with decrease in fear or greater reward; the prefrontal cortex by primary reinforcers (food and drink). The OMF is involved in sensory perception of modalities related to eating (taste, smell, visceral stimulation) and connects with the limbic structures above as well as the hypothalamus and brainstem. Its greater activation with the fat meal may relate to increased satiation attributed to fat or differences in satisfaction related to taste. These techniques will be useful in the further investigation of the central control of food intake and its contribution to obesity.

Supported by The Wellcome Foundation

\section{2}

Small changes in blood glucose in healthy subjects alter fMRI brain activation responses to appetising vs bland foods

A. Swamy ${ }^{1}$, S. Hoashi ${ }^{1}$, J. Shaw ${ }^{1}$, P. R. Corlett ${ }^{2}$, S. Sunram-Lea ${ }^{3}$, J. Foster ${ }^{4}$,

P. Fletcher ${ }^{2}$, M. L. Evans ${ }^{1}$;

${ }^{1}$ Dept of Medicine, University of Cambridge, United Kingdom,

${ }^{2}$ Dept of Psychiatry, University of Cambridge, United Kingdom,

${ }^{3}$ Dept of Psychology, University of Lancaster, United Kingdom,

${ }^{4}$ Dept of Medicine, University of Western Australia, Australia.

Blood glucose was first postulated as a physiological controller of appetite/ feeding over 50 years ago. Most recent work has concentrated on neuropeptide rather than substrate influences on feeding. Subtle changes in feeding behaviour particularly when presented with appetising food may contribute to human obesity. We therefore examined the effects of a small oral glucose load on patterns of brain activation following exposure to foods of differing palatabilities. We studied 7 healthy right handed volunteers (age $30 \pm 3$, BMI $23.9 \pm 0.67,5$ males, 2 females) on 2 occasions in random order Overnight fasted subjects had a venous cannula inserted in left hand and then underwent fMRI scanning (Siemens TIM TRIO 3 Tesla). Immediately prior to entering scanner, subjects drank $150 \mathrm{ml}$ containing either (1) 25g glucose (GLU) or (2) 5 aspartame tabs (CON). Subjects were blinded to study conditions with studies performed at least 1 week apart. During scans, subjects were presented with pictures comprising foods $[\mathrm{F}]$ (either appetising $[\mathrm{AP}]$ or bland $[\mathrm{BL}]$ ) or non-foods [NF]. Venous plasma glucose was monitored throughout (Analox GM9 analyser). Baseline glucose values were similar on both study days $(5.2 \pm 0.3 v s 5.2 \pm 0.2 \mathrm{p}=\mathrm{NS})$. On GLU days, there was a modest but significant rise in plasma glucose $(30 \min 7.0 \pm \mathrm{x} 0.2 v s 5.0 \pm 0.2 \mathrm{GLU} v s$ CON p < 0.01). Comparing F vs NF, presentation of food pictures resulted in marked activation in insula, caudate and putamen during both GLU and CON conditions. However, there were marked differences between study days when comparing AP and BL foods. Specifically, on CON days, there were no differences in activation between AP and BL (fig 2). In contrast, on GLU days, there was a significantly greater activation in insula and putamen when presented with appetising foods (figure and table below).

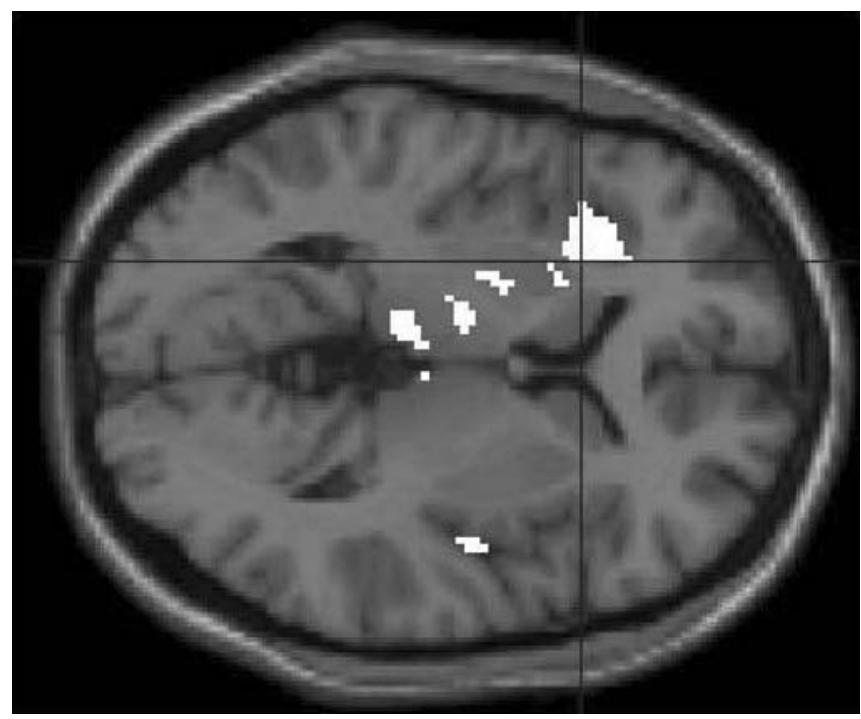

\begin{tabular}{lll}
$*$ & \multicolumn{2}{l}{ AP vs BL Effect size $(\mathrm{SE})$} \\
\cline { 2 - 3 } & Insula & Putamen \\
\hline $\mathrm{CON}$ & $-0.05(0.06)$ & $-0.03(0.07)$ \\
GLU & $0.14(0.06) *$ & $0.17(0.08) *$ \\
\hline
\end{tabular}

In summary, our data show that in the fasting state (CON), brain activation in response to bland and appetising foods was indistinguishable but this was reversed by a small oral glucose load. Our current study does not distinguish a direct effect of glucose from indirect effects mediated by insulin. Neverethless, our data suggest that small changes in blood glucose can alter patterns of brain activation in healthy subjects when presented with different food types.

Supported by JDRF 


\section{OP 8 Insulin therapy: physiology and pharmacokinetics}

\section{3}

Change in plasma glucose following exercise in type 1 diabetes: a comparison of three basal insulins

V. Arutchelvam ${ }^{1}$, T. Heise' ${ }^{2}$, S. Dellweg 2 , B. Elbroend ${ }^{3}$, I. Minns ${ }^{3}$, P. D. Home ${ }^{1}$; ${ }^{1}$ The Medical School, Newcastle University, United Kingdom,

${ }^{2}$ Profil Institut fuer Stoffwechselforschung, Neuss, Germany,

${ }^{3}$ Novo Nordisk, Crawley, United Kingdom.

Background and Aims: Exercise has complex effects on glucose production and utilization in particular in people on insulin therapy; both hyperglycaemia and hypoglycaemia can be exacerbated. Because of its large plasma depot insulin detemir would be expected to show fewer fluctuations in its effect during exercise than non-albumin bound insulins. We have therefore assessed the impact of physical exercise on the plasma glucose (PG) control of people with Type 1 diabetes, comparing three basal insulins (detemir, glargine, NPH).

Materials and Methods: In a two-centre randomised open-label threeperiod crossover study, participants were actively managed on each of the three insulins plus insulin aspart for a period of 4 weeks. At the end of each treatment period the participants performed an exercise study $6 \mathrm{~h}$ after injection of their last meal-time and basal insulin. Exercise was for $30 \mathrm{~min}$ at a target heart rate of 120 beats/min, with glucose monitoring every $10 \mathrm{~min}$ for $3 \mathrm{~h}$, and self-monitoring thereafter.

Results: Forty-seven out of 51 randomized subjects completed the study (age $39 \pm 10 \mathrm{yr}$, BMI $25 \pm 3 \mathrm{~kg} / \mathrm{m}^{2}$, diabetes duration $28 \pm 10 \mathrm{yr}$ ). Following exercise the differences in minimum PG, time to minimum PG, and area under the PG curve (AUC-PG ${ }_{0-150 \mathrm{~min}}$ ) did not differ significantly (Table). However, patient-related outcomes did differ, including number experiencing minor hypoglycaemia in the period $0-150$ min post-exercise and the number needing carbohydrate to prevent or treat hypoglycaemia. These were statistically significantly lower with both insulin detemir and NPH insulin compared to insulin glargine. Minimum post-exercise cortisol levels were significantly higher with glargine than with detemir, suggested this hypoglycaemia elicited a more pronounced counter-regulatory hormone.

Conclusion: Although decrements in PG post-exercise were similar between insulin detemir, NPH insulin, and insulin glargine, hypoglycaemia and carbohydrate snacking post-exercise was less frequent with insulin detemir and NPH insulin versus insulin glargine, confirmed by the counter-regulatory cortisol response. The findings suggest a more stable level of plasma free insulin with insulin detemir during and after exercise, consistent with the study hypothesis.

$0-150$ min post exercise

\begin{tabular}{llll}
\hline & detemir & NPH & glargine \\
\hline PG nadir (change from baseline, mmol/l) & $2.0 \pm 2.0$ & $2.1 \pm 1.8$ & $1.8 \pm 1.9$ \\
PG time to minimum (min) & $76 \pm 53$ & $82 \pm 53$ & $84 \pm 52$ \\
PG AUC (mmol/l.150-min) & $6.1 \pm 2.1$ & $5.7 \pm 2.1$ & $5.7 \pm 2.3$ \\
Cortisol minimum (nmol/l) & $163 \pm 58^{*}$ & $170 \pm 74$ & $192 \pm 74$ \\
Participants needing carbohydrate (\%) & $37 \%^{*}$ & $53 \%^{*}$ & $65 \%$ \\
Participants with hypoglycaemia (\%) & $21 \%^{*}$ & $27 \%^{*}$ & $57 \%$ \\
Mean $\pm \mathrm{SD} ;{ }^{*} \mathrm{p}<0.01$ vs. glargine & & &
\end{tabular}

Funded by Novo Nordisk

\section{4}

Brain responses to intravenous equipotent doses of the long-acting acylated insulin detemir and human regular insulin in humans P. Rossetti, F. Porcellati, N. Busciantella Ricci, P. Cioli, P. Candeloro, G. B. Bolli, C. G. Fanelli;

Internal Medicine, University of Perugia, Italy.

Background: Binding with the FFA myristic acid in B29 position, makes the long-acting insulin analog detemir more lipophylic than human insulin
(HI). In rat, and likely in humans detemir crosses the blood-to-brain barrier more, and concentrates in hypothalamus more than HI. Aim of the present studies was to assess the brain/hypothalamus responses to euglycemia and hypoglycemia in humans during detemir vs HI hyperinsulinemia.

Methods: Because data on i.v. detemir in humans are lacking, preliminary studies were devoted to finding equipotency between i.v. detemir and HI. The best approximation (based on total glucose infusion rate in euglycemia, GIR) was molar ratio detemir:HI 8:1. Ten normal, non-diabetic subjects (6 males, age $36 \pm 7$ years, BMI $\left.22.9 \pm 2.6 \mathrm{~kg} / \mathrm{m}^{2}\right)$ were studied on 4 occasions at random, during i.v. infusion for $5 \mathrm{~h}$ of either detemir $(48 \mu \mathrm{mol} / \mathrm{kg} / \mathrm{min})$ or $\mathrm{HI}(6 \mu \mathrm{mol} /$ $\mathrm{kg} / \mathrm{min}$ ) in euglycemia (plasma glucose, $\mathrm{PG}, 90 \mathrm{mg} / \mathrm{dl}$ ) or during stepped hypoglycemic clamps (PG 90, 78, 66, 54, and $42 \mathrm{mg} / \mathrm{dl}$ steps).

Results: Only data regarding euglycemic clamps are available. In euglycemia, GIR with detemir was lower than HI during the first $90 \mathrm{~min}(\mathrm{p}=0.007)$ but later was no different until the end of studies $(9 \pm 0.5$ vs $9.5 \pm 0.3 \mathrm{mg} / \mathrm{Kg} / \mathrm{min}$, $\mathrm{p}>0.2)$. The score of autonomic ( $2.1 \pm 0.6$ vs $1.8 \pm 0.3)$ and neuroglycopenic symptoms of hypoglycemia ( $0.7 \pm 0.2$ vs $0.7 \pm 0.1)$, and glucagon suppression were similar with detemir and $\mathrm{HI}(44 \pm 5 \mathrm{vs} 45 \pm 6 \mathrm{pg} / \mathrm{ml})(\mathrm{p}>0.2)$. Cognitive function, expressed as sum of $\mathrm{Z}$-scores was also similar with detemir vs $\mathrm{HI}$ $(-1.38 \pm 0.32$ vs $-1.55 \pm 0.41, \mathrm{p}>0.2)$.

Conclusion: This is the first study establishing equipotency between i.v. detemir and HI. The more lipophylic insulin detemir does not affect brain/ hypothalamus responses to hyperinsulinemia in euglycemia. However, responses to hypoglycemia with insulin detemir vs HI remain to be analyzed.

This is an investigator-initiated trial not supported by pharmaceutical companies.

\section{5}

Is insulin detemir hepatoselective when compared to NPH insulin using stable isotope methodology at plasma glucose concentrations of less than $4 \mathrm{mmol} / \mathrm{l}$ ?

F. J. Smeeton ${ }^{1}$, F. Shojaee-Moradie 2 , N. Jackson ${ }^{2}$, R. H. Jones ${ }^{2}$,

A. M. Umpleby' ${ }^{2}$, D. Russell-Jones ${ }^{1}$;

${ }^{1}$ Department of Diabetes and Endocrinology, The Royal Surrey County Hospital., ${ }^{2}$ The Wolfson Centre for Translational Research, The University of Surrey, Guildford, United Kingdom.

Insulin Detemir has shown a greater effect on suppression of endogenous glucose production (Ra) compared to NPH insulin in euglycaemic clamp studies in individuals without diabetes. There is also a lower incidence of hypoglycaemia when compared to NPH insulin in clinical studies when used to treat diabetes. Insulin Detemir is a basal insulin with the unique property of being bound to albumin. This may explain some of its observed effects by a more physiological action, with greater suppression of endogenous glucose production and lesser effect on peripheral glucose uptake.

Fourteen participants with type 1 diabetes were studied in a double blind, cross over trial after subcutaneous injection of Insulin Detemir compared to NPH insulin when fasted. A continuous infusion of $\left[6,6-{ }^{2} \mathrm{H}_{2}\right]$ glucose

Fig: Glucose Ra at time zero before injection of subcutaneous injection of either Insulin Detemir or NPH Insulin and at decreasing plasma glucose levels:

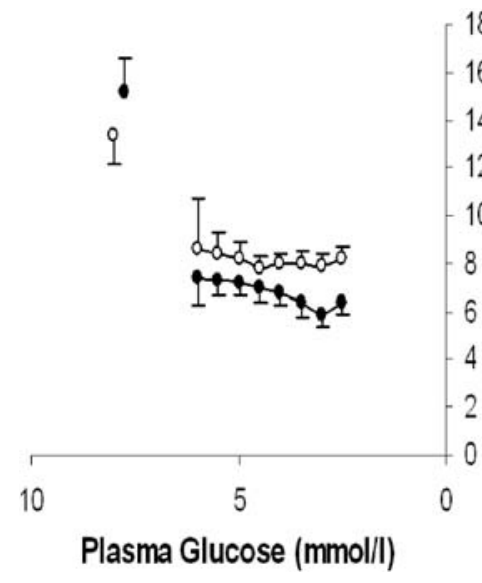


enabled endogenous glucose production $(\mathrm{Ra})$ and peripheral glucose uptake (Rd) to be calculated.

Table: Shows Glucose Ra when Ra NPH is compared to Ra Insulin Detemir:

\begin{tabular}{llll}
\hline $\begin{array}{l}\text { Plasma } \\
\text { Glucose } \\
\text { (mmol/l) }\end{array}$ & $\begin{array}{l}\text { Mean Glucose Ra } \\
\text { NPH (umol/kg/min) } \\
\pm \text { SEM }\end{array}$ & $\begin{array}{l}\text { Mean Glucose Ra } \\
\text { Insulin Detemir } \\
\text { (umol/Kg/min) } \pm \text { SEM }\end{array}$ & p value \\
\hline 6.0 & $8.59 \pm 0.57$ & $7.35 \pm 0.51$ & 0.03 \\
5.5 & $8.44 \pm 0.59$ & $7.24 \pm 0.51$ & 0.03 \\
5.0 & $8.22 \pm 0.48$ & $7.21 \pm 0.57$ & 0.08 \\
4.5 & $7.78 \pm 0.4$ & $6.94 \pm 0.47$ & 0.08 \\
4.0 & $8.0 \pm 0.47$ & $6.75 \pm 0.52$ & 0.03 \\
3.5 & $8.0 \pm 0.66$ & $6.34 \pm 0.57$ & 0.02 \\
3.0 & $7.84 \pm 0.85$ & $5.88 \pm 0.53$ & 0.01 \\
2.5 & $8.17 \pm 2.11$ & $6.37 \pm 1.13$ & 0.01 \\
\hline
\end{tabular}

No significant difference was observed between starting plasma glucose or Ra before administration of NPH or Detemir (plotted separately in the figure). Detemir had a greater effect on suppression of endogenous glucose output when compared to NPH insulin at plasma glucose concentrations between 4 and $2.5 \mathrm{mmol} / \mathrm{l}$. This provides further evidence that Insulin Detemir is hepatoselective.

Funded by Novo Nordisk

\section{6}

Head-to-head comparison of the long-acting insulin analogs glargine and detemir: effects on glucose and lipid metabolism in T1DM F. Porcellati, P. Rossetti, N. Busciantella Ricci, P. Lucidi, P. Candeloro, G. B. Bolli, C. G. Fanelli;

Internal Medicine, University of Perugia, Italy.

Aims: To compare head-to-head the effects of the long-acting insulin analogs glargine (GLA) and detemir (DET) on plasma glucose (PG) and lipid metabolism (plasma free fatty acids, FFA; $\beta$-OH-butyrate, $\beta$-OH-B), 24 subjects with T1DM ( $38 \pm 10$ years, BMI $22 \pm 1.6 \mathrm{~kg} / \mathrm{m}^{2}$, HbA1C $7.2 \pm 0.7 \%$, mean \pm SD) were studied.

Methods: All subjects underwent $24 \mathrm{~h}$ euglycemic clamp twice, after s.c. injection $0.35 \mathrm{U} / \mathrm{kg}$ GLA or DET, after 2 week treatment with either GLA or DET once/daily (randomized, double-blind, cross-over study). Primary endpoints were total AUC of G infusion rate (GIR), FFA and $\beta$-OH-B.

Results: Median time of end of action (time at which PG was $>8.3 \mathrm{mmol} / \mathrm{l}$ ) was $24 \mathrm{~h}$ and $17.5 \mathrm{~h}$ (GLA and DET, $\mathrm{p}<0.001)$.

\begin{tabular}{|c|c|c|c|c|c|}
\hline & Detemir & Glargine & $\begin{array}{l}\text { point } \\
\text { estimate } \\
(\%)\end{array}$ & $\begin{array}{l}95 \% \\
\text { confidence } \\
\text { interval }\end{array}$ & $P$ value \\
\hline $\begin{array}{l}\text { GIR AUC }_{0-24 \mathrm{~h}} \\
{[\mathrm{mg} / \mathrm{kg}]}\end{array}$ & $915 \pm 225$ & $1412 \pm 662$ & 70.3 & $53.4 ; 92.7$ & 0.015 \\
\hline GIR AUC $_{0-12 \mathrm{~h}}$ & $773 \pm 200$ & $807 \pm 352$ & 97.7 & $78.5 ; 121.6$ & 0.832 \\
\hline $\begin{array}{l}\text { GIR } \\
\text { AUC }_{12-\text { end of inf. }}\end{array}$ & $142 \pm 194$ & $605 \pm 390$ & 17.4 & $8.2 ; 36.7$ & 0.000 \\
\hline $\begin{array}{l}\text { FFA AUC } \\
{[\mathrm{mmol} / \mathrm{l}]}\end{array}$ & $11 \pm 1.7$ & $8 \pm 2.8$ & 131 & $117 ; 143$ & 0.001 \\
\hline FFA AUC $_{0-12 \mathrm{~h}}$ & $4.6 \pm 1.1$ & $34 \pm 1.6$ & 133 & $115 ; 147$ & 0.002 \\
\hline $\begin{array}{l}\text { FFA } \\
\text { AUC }_{12-\text { end }}\end{array}$ & $6 \pm 1.6$ & $4.4 \pm 1.3$ & 129 & $111 ; 144$ & 0.004 \\
\hline $\begin{array}{l}\text { of study } \\
\boldsymbol{\beta} \text {-OH-B AUC } \\
\\
24 \mathrm{~h} \\
{[\mathrm{mmol} / \mathrm{l}]}\end{array}$ & $22.9 \pm 8.9$ & $16.5 \pm 11.6$ & 139 & $110 ; 158$ & 0.014 \\
\hline $\begin{array}{l}\boldsymbol{\beta} \text {-OH-B } \\
\text { AUC }_{0-12 \mathrm{~h}}\end{array}$ & $6.4 \pm 1.4$ & $7.9 \pm 6.4$ & 91 & $61 ; 134$ & 0.608 \\
\hline $\begin{array}{l}\boldsymbol{\beta}-\mathbf{O H}-\mathbf{B} \\
\text { AUC }_{12-\text { end of study }}\end{array}$ & $15.6 \pm 78$ & $8.1 \pm 5.1$ & 152 & $128 ; 168$ & 0.001 \\
\hline
\end{tabular}

Conclusions: During the initial $12 \mathrm{~h}$ after s.c. injection, glucose metabolism is similarly controlled by GLA and DET, but DET has $~ 30 \%$ lower antilipolytic activity. During following $12-24 \mathrm{~h}$, DET has lower activity also on glucose metabolism, explaining earlier end of action and greater increase in $\beta-\mathrm{OH}-\mathrm{B}$ by end of study.

This is an investigator-initiated trial not supported by pharmaceutical companies.

\section{7}

Pharmacokinetics and pharmacodynamics of insulin VIAject ${ }^{\mathrm{TM}}$, insulin lispro and regular human insulin when injected subcutaneously immediately before a meal in patients with type 1 diabetes

S. S. Steiner ${ }^{1}$, L. Heinemann ${ }^{2}$, R. Pohl ${ }^{1}$, F. Flacke ${ }^{1}$, A. Pfuetzner ${ }^{1}$, P. Simms ${ }^{1}$, M. Hompesch;

${ }^{1}$ Biodel Inc., Danbury, United States,

${ }^{2}$ Profil Institute for Metabolic Research, Neuss, Germany,

${ }^{3}$ Profil Institute for Clinical Research, Chula Vista, United States.

Background and Aims: The aim of this study was to determine the action of VIAject ${ }^{\text {ts }}$ (a very rapid acting formulation of regular human insulin (RHI)), RHI, and insulin lispro (Lispro) on postprandial blood glucose (BG) excursions after a standard meal in patients with Type 1 diabetes.

Materials and Methods: BG of 9 patients (5 males and 4 females; age $40 \pm 10$ yrs, BMI $24.0 \pm 2.0 \mathrm{~kg} / \mathrm{m}^{2}$ ) were stabilized by means of a glucose clamp (target BG $120 \mathrm{mg} / \mathrm{dL}$ ) prior to meal ingestion. The glucose infusion was turned off prior to the standard meal and insulin dosing. Using a cross-over study design with fixed treatment order, the same patient specific dose of VIAject ${ }^{\text {tim }}$, Lispro or RHI was injected s.c. immediately before the meal. Subsequently, postprandial glucose excursions were continuously monitored for 8 hours and glucose infusion was re-initiated if $B G<60 \mathrm{mg} / \mathrm{dL}$. Plasma insulin levels were determined throughout the study.

Results: (mean $\pm \mathrm{SD})$

\begin{tabular}{llll} 
Pharmacokinetics & RHI & Lispro & VIAject $^{\mathrm{Tt}}$ \\
\hline Ins Tmax $(\min )$ & $143 \pm 29^{*}$ & $62 \pm 37$ & $43 \pm 36^{*}$ \\
\hline
\end{tabular}

${ }^{*} \mathrm{p}<0.001$, paired t-test

\begin{tabular}{llll} 
Pharmacodynamics (0-180 min.) & RHI & Lispro & VIAject $^{\text {tw }}$ \\
\hline BG TMax (min.) & $93 \pm 56^{\star}$ & $47 \pm 28$ & $41 \pm 26^{\star}$ \\
BG Max (mg/dL) & $185 \pm 44$ & $158 \pm 33$ & $157 \pm 27$ \\
BG Min (mg/dL) & $103 \pm 21$ & $73 \pm 31$ & $87 \pm 24$ \\
BG Max-BG Min (mg/dL) & $82 \pm 30$ & $84 \pm 11^{\star}$ & $70 \pm 18^{\star}$ \\
\hline
\end{tabular}

${ }^{*} \mathrm{p}<0.05$, paired $\mathrm{t}$-test

The total number of hypoglycemic events (hrs requiring glucose infusion) 3 to 8 hours post injection were 13 with RHI, 11 with Lispro and 4 with VJ. The mean total amount of glucose infused to prevent hypoglycemia during this time was six times higher for RHI and twice as much for Lispro than with VIAject $^{\mathrm{m} x}$. The areas above and below the normal glycemic target zone (BG AUC above 140 and below $80 \mathrm{mg} / \mathrm{dL}$ ) summed for all patients per group was 81,895 for RHI, 57,423 for Lispro and $38,740 \mathrm{mg} / \mathrm{dL}^{\star}$ min for VIAject ${ }^{\text {tw }}$.

Conclusion: VIAject ${ }^{\text {th }}$ was the fastest in reversing the rise in blood glucose following the standard meal. Patients treated with VIAject ${ }^{\text {tw }}$ experienced reduced post prandial glucose excursions. In contrast, RHI had the highest glucose excursion, which is consistent with its slower absorption rate. Variability of the glucose levels (mean difference between maximal and minimal values) was significantly less for VIAject ${ }^{\mathrm{m}}$ than Lispro, demonstrating VIAject's better glycemic control in these patients with Type 1 diabetes. 


\section{8}

Revisiting short-acting insulin analogues. In obese subjects with type 2 diabetes, are they short? A pharmacokinetics study

M. Gagnon-Auger, J.-P. Baillargeon, J. Ménard, P. Brassard, E. Martin, J.-L. Ardilouze;

Endocrine division, University of Sherbrooke, Canada.

Background and Aims: 1) Pharmacokinetics of short acting insulin (SAI) were established following small (4-10 IU) subcutaneous injections in normal weight healthy subjects, in lean subjects with type 1 diabetes and, rarely, in lean or moderately overweight subjects with type 2 diabetes (T2D). However, most patients using insulin are overweight and insulin resistant and receive much larger dosages. 2) Insulin absorption depends mainly on blood flow in adipose tissue (ATBF). However, in obese and insulin resistant subjects, ATBF is $40-70 \%$ lower than in healthy subjects and we showed that after subcutaneous injection of saline, ATBF decreases for a period which correlates with injected volume. This study aims to show that pharmacokinetics of SAI are delayed in obese subjects with T2D.

Materials and Methods: Plasma SAI (lispro) was measured by radioimmunoassay (RIA) method (Linco Inc) using a standard curve limited between 17,8 and $1780 \mathrm{pmol} / \mathrm{mL}$. In our hands, CV ranged between 2.5 and 8. We studied 6 healthy control subjects (age: $23.7 \pm 2.4 \mathrm{yrs}$, BMI $22.1 \pm 1.4 \mathrm{~kg} /$ $\mathrm{m} 2$ ) following subcutaneous injection of $10 \mathrm{IU}$ and 8 subjects with T2D (age: $60 \pm 6.8$ yrs, BMI: $37.6 \pm 6.6 \mathrm{~kg} / \mathrm{m} 2$, duration of diabetes $18.4 \pm 9.1$ yrs, duration of insulin therapy $4.6 \pm 4$ yrs, HbAlc $8.1 \pm 1.1 \%$ ) following $10 \mathrm{IU}$ and $30 \mathrm{IU}$ (random order).

Results: (see Fig) In controls, the curve shows expected results: Cmax $($ maximal concentration $)=552 \pm 55 \mathrm{pmol} / \mathrm{L}$, Tmax $($ time to reach maximal concentration) $=50.0 \pm 8.9 \mathrm{~min}, \mathrm{AUC}$ (trapezoidal method) $=79794 \pm$ 21420 pmol.min/L. In T2D subjects, following 10 IU lispro injection, Tmax was similar $(52.5 \pm 16.7 \mathrm{~min})$ to control $(\mathrm{p}=0.75)$, but $\mathrm{Cmax}$ was $42 \%$ lower $(353 \pm 96 \mathrm{pmol} / \mathrm{L}, \mathrm{p}<0.001)$. AUC was also lower in T2D subjects $(63735 \pm$ 12557 pmol.min/L, $\mathrm{p}=0.10$ ). In T2D subjects, following $30 \mathrm{IU}$, lispro peaked at $86.3 \pm 23.3 \mathrm{~min}, 36 \mathrm{~min}$ later than in controls after $10 \mathrm{IU}(\mathrm{p}=0.004)$ and Cmax was $879.5 \pm 281 \mathrm{pmol} / \mathrm{L}$. AUC was 2.8 times larger after $30 \mathrm{IU}(197155$ \pm 36552 pmol.min/L) than after $10 \mathrm{IU}$ injection ( $<<0.001)$, but only 2.5 times larger than in controls $(\mathrm{p}<0.001)$.

Conclusion: In healthy normal weight control subjects, our results reproduced data accepted and used in daily practice for insulin prescriptions. However, in a population of obese subjects with T2D, we showed for the first time that plasma levels of SAI are blunted (at low dosage) and severely delayed (at average dosage) when compared to the results of low dosage in non-obese controls. These data command for in depth exploration of pharmacokinetic and glucodynamic of SAI in obese subjects with T2D, so commonly treated by family practitioners and endocrinologists. (Fig. 1)

Funded by Diabète Québec

\section{OP 9 Insulin secretion in vivo}

\section{9}

Beta cell compensation for insulin resistance involves distinct mechanisms, which are differentially impaired in prediabetes A. Mari ${ }^{1}$, A. Tura ${ }^{1}$, G. Pacini ${ }^{1}$, J. Petrie ${ }^{2}$, M. Laakso ${ }^{3}$, J. Nolan ${ }^{4}$, A. Mitrakou ${ }^{5}$, E. Ferrannini ${ }^{6}$, \&. RISC investigators ${ }^{6}$;

${ }^{1}$ Institute of Biomedical Engineering, National Research Council, Padova, Italy, ${ }^{2}$ Medical School, Ninewells Hospital, Dundee, United Kingdom, ${ }^{3}$ Department of Medicine, Kuopio University Hospital, Finland, ${ }^{4}$ Department of Endocrinology, St James' Hospital, Dublin, Ireland, ${ }^{5}$ Diabetes Centre, Henry Dunant Charitable Foundation, Athens, Greece, ${ }^{6}$ Department of Internal Medicine, University of Pisa, Italy.

Background and Aims: In normal subjects, insulin secretion is upregulated in the presence of insulin resistance; however, the characteristics of this regulation are still debated.

Materials and Methods: In 1309 healthy subjects from the RISC study, we measured insulin sensitivity (IS, by euglycaemic clamp, as M/steady-state insulin) and $\beta$-cell function from a $2 \mathrm{~h}$ OGTT (by C-peptide deconvolution and modelling). Subjects (55\% women; age $44 \pm 8$ years; BMI $25 \pm 4 \mathrm{~kg} / \mathrm{m}^{2}$; mean \pm SD) had normal glucose tolerance (NGT) or impaired fasting or 2$\mathrm{h}$ post-OGTT glucose (IGM, $\mathrm{n}=152$, ADA 1997). Dynamic ß-cell function was quantified by a dose-response relating insulin secretion rate (ISR) to glucose concentration (dose-response slope=glucose sensitivity) and by a potentiation factor expressing a relative potentiation of the dose-response during the OGTT (characteristic parameter: $2 \mathrm{~h}$-to-basal ratio).

Results: In NGT subjects, IS was inversely related to basal and total ISR and ISR at $5 \mathrm{mM}$ glucose (centre-, sex- and age-partialled $r^{\prime}$ s of $-0.35,-038$ and -0.23 , respectively, all $p<0.0001$ ), as expected. In contrast, IS was only weakly related to $\beta$-cell glucose sensitivity $(\mathrm{r}=-0.04, \mathrm{p}=0.04)$ but positively related to the potentiation factor ratio (partial $r=0.14, \mathrm{p}<0.0001$ ). In comparison with NGT, IGM subjects had higher basal ISR (91[57] $v s 68[36] \mathrm{pmol}^{-1} \mathrm{~min}^{-1} \mathrm{~m}^{-2}$, median[interquartile range], $\mathrm{p}<0.0001)$, total ISR $(48[22]$ vs $39[17] \mathrm{nmol}$ $\left.\mathrm{m}^{-2}, \mathrm{p}<0.0001\right)$ and ISR at $5 \mathrm{mM}$ glucose (75[64] $v s 69[49] \mathrm{pmol}^{-1} \mathrm{~min}^{-1} \mathrm{~m}^{-2}$, $\mathrm{p}<0.0001)$. On the other hand, IS (93[73] vs 133[86] $\mu \mathrm{mol} \cdot \mathrm{min}^{-1} \cdot \mathrm{kg}_{\text {fim }}{ }^{-1} \mathrm{pM}^{-1}$, $\mathrm{p}<0.0001)$, B-cell glucose sensitivity (69[48] vs 118[82] $\mathrm{pmol}^{-1} \mathrm{~min}^{-1} \cdot \mathrm{m}^{-2} \mathrm{mM}^{-}$ $\left.\left.{ }^{1}\right), \mathrm{p}<0.0001\right)$ and potentiation $(1.7 \pm 0.8$ vs $2.1 \pm 1.5, \mathrm{p}<0.002)$ were impaired. By multivariate analysis of the whole dataset, the strongest determinants of glucose tolerance - expressed as the mean OGTT glucose levels - were glucose sensitivity, insulin sensitivity, the potentiation ratio and ISR at 5 $\mathrm{mM}$ glucose, (partial $r$ 's of $-0.33,-0.27,-0.21$ and -0.14 , respectively; total explained variance $37 \%, \mathrm{p}<0.0001$ ).

Conclusion: We conclude that insulin resistance upregulates the basal secretory tone (eg, ISR at $5 \mathrm{mM}$ glucose) but does not substantially

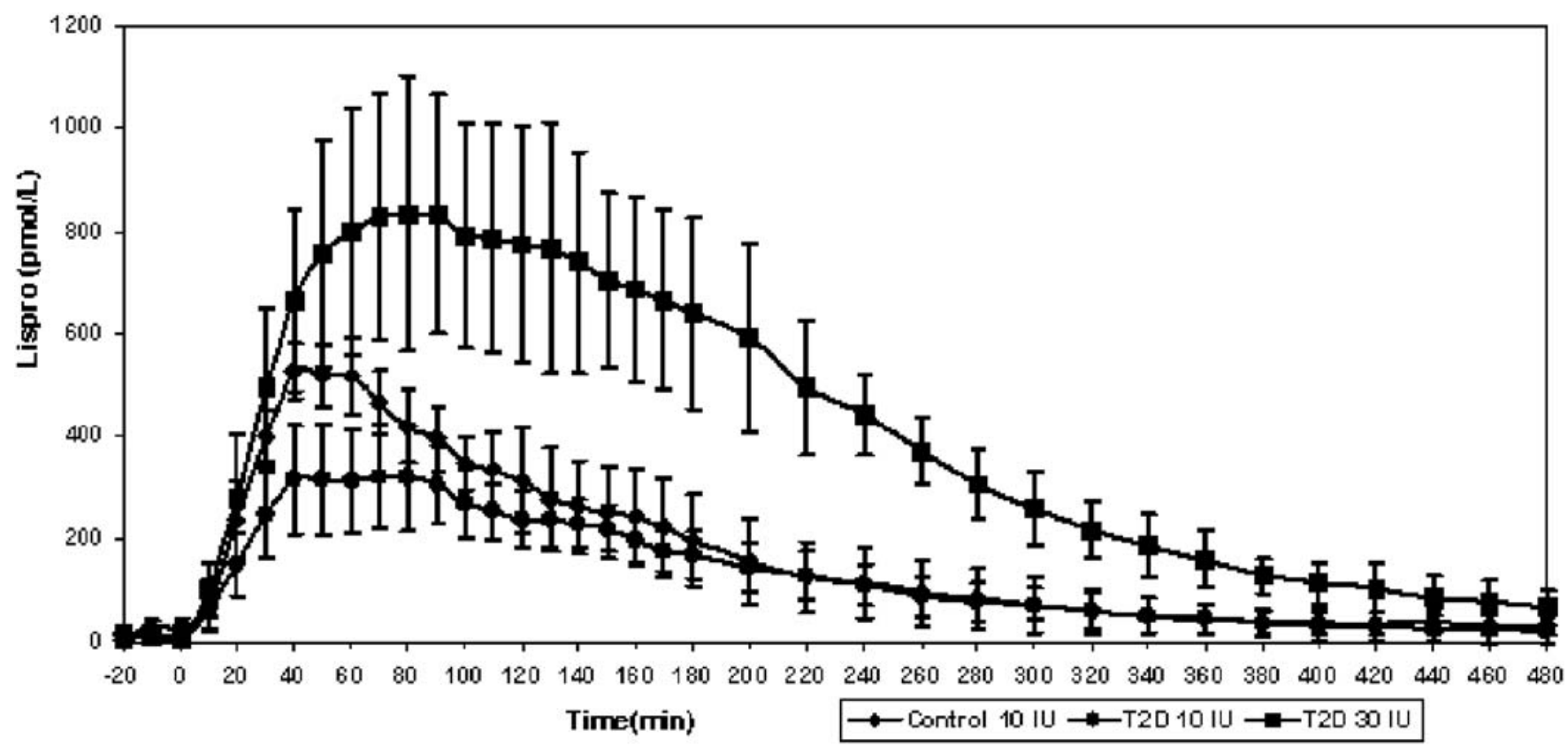

Fig. 1 
influence the dynamic secretion parameters (ß-cell glucose sensitivity and potentiation). Furthermore, $\beta$-cell function impairment in IGM concerns the dynamic parameters, while the regulation of the basal secretory tone is essentially intact. This suggests the existence of distinct B-cell mechanisms, of which those responsible for the dynamic secretory responses are selectively impaired in prediabetes.

Funded by EU and AstraZeneca

\section{0}

Potential signals for beta cell compensation for insulin resistance D. Stefanovski, O. Woolcott, M. Lottati, D. Zheng, L. N. Harrison, V. Ionut, S. P. Kim, I. Hsu, J. Chiu, K. Catalano, C. Kolka, J. M. Richey, R. N. Bergman;

Physiology and Biophysics, Keck School of Medicine of USC, Los Angeles, United States.

Background and Aims: Increased insulin is the primary mechanism of compensation for changes in insulin resistance which may occur due to environmental causes. Yet, the mechanism which is responsible for the compensatory hyperinsulinemia is not clarified. One obvious candidate is elevated glucose which could arise from reduced glucose tolerance secondary to insulin resistance. We asked if even subtle hyperglycemia accompanies the compensatory hyperinsulinemia of high fat feeding.

Materials and Methods: Male mongrel dogs $(n=9)$ were fed the hypercaloric high fat diet $(\sim 5400 \mathrm{kcal} / \mathrm{d}, 53 \% \mathrm{kcal}$ from fat) for 6 weeks. The Frequently Sampled Intravenous Glucose Tolerance (FSIGT) test was utilized to assess changes in glucose homeostasis at weeks 0 (pre-diet), 2, 4 and 6 (W:0; W:2; $\mathrm{W}: 4$; W:6, respectively). Basal glucose and FFAs were obtained at three time intervals during the day (8-8:30am, 6-8 pm, and 2-4 am) at W:0, and W:6. Results: Due to the diet, body weight was significantly increased by W:2 $(+2.5+/-0.33 \mathrm{~kg}, \mathrm{P}<.001)$ and remained elevated throughout the entire study (W: $6+1.79 \mathrm{~kg}, \mathrm{P}<0.001$ ). Within two weeks of fat feeding, insulin sensitivity $\left(\mathrm{S}_{\mathrm{I}}\right)$ dramatically decreased by $45 \%(5.6+/-0.9$ vs. $3.1+/-1.2$; W:0 vs. W:2 $\mathrm{P}=.04)$. At $\mathrm{W}: 6, \mathrm{~S}_{\mathrm{I}}$ decreased by $66 \%(1.9+/-1.3, \mathrm{P}=0.006)$. AIRg rose modestly by $\mathrm{W}: 2(14 \%, \mathrm{P}=\mathrm{NS})$, increasing by $46 \%(\mathrm{P}<0.02)$ and $59 \%(\mathrm{P}<0.001)$ at $\mathrm{W}: 4$ and $\mathrm{W}: 6$, respectively. Despite hyperinsulinemia, we observed constancy of the Disposition Index $(\mathrm{P}>0.13)$ over 6 weeks. Importantly, there was no increase in fasting glucose at any of the three time periods between weeks 0 and $6(\mathrm{P}>0.68, \mathrm{P}>0.56, \mathrm{P}>0.18)$. In contrast the fasting FFA levels were increased by $0.08+/-0.02 \mathrm{mM}(\mathrm{P}<0.001)$ at week 2 and remained elevated by week $6(\mathrm{P}<0.001)$. Also, we observed highly significant increase of FFA in the middle of the night by week $6(0.42+/-0.7$ to $0.75+/-0.04 \mathrm{mM}, \mathrm{P}<0.001)$. There was no significant correlation between the change of fasting levels of FFA from week 0 and the increase in insulin secretion, $\operatorname{AIRg}(\mathrm{R}=0.1, \mathrm{P}=\mathrm{NS})$. There was significant correlation between the change from week 0 in AUC of glucose after a glucose bolus, and $\mathrm{AIRg}(\mathrm{R}=-0.6, \mathrm{P}<0.001)$. In addition there was strong correlation between the change from week 0 in AUC of glucose and rise in fasting FFA $(\mathrm{R}=0.7, \mathrm{P}<0.001)$.

Conclusion: We propose that the change from week 0 in the AUC of glucose might be due to the elevated FFA in the portal, which impair insulin ability to suppress endogenous glucose production (EGP). Thus, postprandial glucose or 24-hour basal FFAs and not 24-hour basal glucose might be the signal for b-cell compensation induced by insulin resistance.

\section{1}

Relative contribution of insulin resistance and pancreatic beta cell dysfunction in the pathogenesis of impaired glucose tolerance in Friedreich ataxia

M. Cnop ${ }^{1,2}$, A. Begu ${ }^{3}$, C. Depondt ${ }^{4}$, M. Pandolfo ${ }^{4}$, F. Fery ${ }^{1}$;

${ }^{1}$ Division of Endocrinology, Erasmus Hospital, Universite Libre de Bruxelles, Belgium, ${ }^{2}$ Laboratory of Experimental Medicine, Universite Libre de Bruxelles, Belgium, ${ }^{3}$ Division of Nutrition, Metabolic Disorders and Endocrinology, CHU Timone, Marseille, France, ${ }^{4}$ Department of Neurology, Erasmus Hospital, Universite Libre de Bruxelles, Belgium.

Background and Aims: Friedreich ataxia (FA) is a rare neurodegenerative disease caused by a GAA trinucleotide repeat expansion in the frataxin gene. FA patients have an increased prevalence of diabetes, but the pathogenesis of this monogenic diabetes is poorly understood. Our aim was to study insulin secretion and insulin sensitivity in these patients.

Patients and Methods: We studied 13 non-diabetic FA patients $(6 \mathrm{~F} / 7 \mathrm{M})$ and 14 controls $(10 \mathrm{~F} / 4 \mathrm{M})$ matched for age (patients $32 \pm 2$ years vs controls $31 \pm 2$ years) and BMI $\left(22.8 \pm 1.2 \mathrm{vs} 23.3 \pm 1.2 \mathrm{~kg} / \mathrm{m}^{2}\right)$. Glucose tolerance was assessed by oral glucose tolerance testing. Frequently-sampled intravenous glucose tolerance tests were used to measure insulin secretion (acute insulin response to glucose, AIRg) and determine insulin sensitivity (insulin sensitivity index, SI) using the minimal model. The disposition index, a measure of beta cell adaptation to the prevailing insulin sensitivity, was calculated as AIRg x SI. Body composition was measured by injection of ${ }^{3} \mathrm{H}_{2} \mathrm{O}$ and resting energy expenditure by indirect calorimetry. The size of the GAA expansion in the frataxin gene was determined by PCR.

Results: Fasting glucose levels were similar in the FA and control groups $(90 \pm 3$ vs $89 \pm 2 \mathrm{mg} / \mathrm{dl}$ ). The 2 -hour glucose level during the oral glucose tolerance test was $143 \pm 9$ in patients vs $118 \pm 4 \mathrm{mg} / \mathrm{dl}$ in controls $(\mathrm{p}=0.01)$. The prevalence of impaired glucose tolerance and diabetes was $31 \%$ and $15 \%$, respectively, in FA patients, compared to $0 \%$ in the controls. FA patients were insulin resistant compared to controls (SI $21 \pm 3$ vs $36 \pm 3 \times 10^{-5} \mathrm{~min}^{-1} /(\mu \mathrm{U} / \mathrm{ml}), \mathrm{p}=0.001$ ). Body composition (fat-free mass $46 \pm 3 \mathrm{vs} 49 \pm 3 \mathrm{~kg}$ ) and basal energy expenditure $(28.6 \pm 1.0$ vs $28.3 \pm 0.9 \mathrm{kcal} / \mathrm{kg}$ lean mass $/ 24 \mathrm{~h})$ were similar in patients and controls. In the patients, SI correlated inversely with the number of GAA repeats $(r=-0.58, p<0.05)$. Insulin secretion was not different between the two groups (AIRg $70 \pm 10$ vs $62 \pm 10 \mu \mathrm{U} / \mathrm{ml}$ ). Hence, the disposition index tended to be lower in FA patients $\left(1488 \pm 231\right.$ vs $\left.2071 \pm 328 \times 10^{-5} \mathrm{~min}^{-1}\right)$.

Conclusion: While the FA patients we studied were young and lean, they had a high prevalence of impaired glucose tolerance. Compared to controls, the patients were insulin resistant, the severity of which correlated with the number of trinucleotide repeats. Their insulin secretion in response to glucose was not different from the control group. Thus, the expected compensatory increase in insulin secretion in response to insulin resistance did not occur. Our findings suggest that patients with FA have markedly impaired pancreatic beta cell function/adaptation.

\section{2}

Macrophage migration inhibitory factor (MIF) plays an important role in the maintenance of glucose homeostasis

V. Serre-Beinier, C. Toso, P. Morel, C. Gonelle-Gispert, D. Bosco, L. Bühler, T. Berney;

Surgical Research Unit, University Hospital Geneva, Switzerland.

Background and Aims: Macrophage migration inhibitory factor (MIF) is thought to be a key effector molecule of both the innate and antigenspecific immune system. While macrophages are the major source of MIF, MIF is also produced in pancreatic beta cells, adipocytes, liver, Leydig and ovarian follicular cells. MIF exerts a variety of biological functions, including macrophage activation, enhancement of adherence, phagocytosis, tumoricidal activity, and induction of nitric oxide $(\mathrm{NO})$ production. in vitro experiments, it has been shown that MIF localise within the secretory granules of pancreatic beta cells in islets and once released, regulates insulin secretion in an autocrine fashion. We aimed to examine whether MIF regulates glucose metabolism in vivo using MIF knockout (MIF-KO) mice.

Materials and Methods: Male MIF knockout (MIF-KO) mice in C57BL/6 background were studied at 1-2 months (Young mice), at 6 months and at 12 months of age (Old mice). Glucose stimulated insulin secretion was evaluated by an intra-peritoneal glucose tolerance test (IPGTT) and insulin secretion was measured by ELISA. In order to measure the peripheric insulin sensitivity of MIF-KO mice, an insulin tolerance test (ITT) was performed.

Results: The Area Under the Curve (AUC) glucose excursion to IPGTT was significantly lower in young male MIF-KO mice compared with controls C57BL/6 mice (WT) $(549 \pm 217$ vs $1305 \pm 305 ; \mathrm{p}<0.001 ; \mathrm{n}=7)$. Fasting plasma glucose and insulin were not significantly different between young male MIFKO and WT mice. ITT showed that MIF-KO mice had insulin sensitivity similar to that of WT mice.Young male MIF-KO demonstrated a reduction in body weight $(16.1 \pm 2.9 \mathrm{~g}$ vs $20.5 \pm 1.6 \mathrm{~g} ; \mathrm{n}=7 ; \mathrm{p}<0.01)$ compared to WT. At 6 months, MIF-KO mice showed an insulin response to IPGTT comparable to WT mice (AUC $1701 \pm 725$ vs $1256 \pm 186 ; \mathrm{n}=3$ ). The weight of the MIF-KO mice was similar to that of the WT $(30.4 \pm 1.8 \mathrm{~g}$ vs $29.4 \pm 0.8 \mathrm{~g} ; \mathrm{n}=4)$. Finally, old male MIF-KO mice showed a glucose intolerance with a higher glucose excursion to IPGTT evaluated by higher AUC compared to WT mice (1950 \pm 302 vs $954 \pm 223 ; \mathrm{p}<0.001 ; \mathrm{n}=5$ ). This was accompanied by a significant 
reduction in insulin response to IPGTT in MIF-KO mice compared to WT mice $(\mathrm{p}<0.001)$. These MIF-KO mice had also a significantly higher body weight ( $38 \pm 4.0 \mathrm{~g}$ vs $30.8 \pm 1.3 \mathrm{~g} ; \mathrm{p}<0.05 ; \mathrm{n}=5)$ compared to WT mice.

Conclusion: Deletion of MIF contributed to an impairment in glucose homeostasis with differences in glucose tolerance, insulin secretion and body weight. These changes are age-dependant. Data obtained on MIF-KO mice aged 12 months suggest that in humans, a progressive decrease in MIF secretion and/or MIF action within the insulin-secreting cells may contribute to beta cell dysfunction and the diminished insulin release associated with Type 2 diabetes.

\section{3}

Beta cell specific overexpression of glucagon receptors results in improved insulin secretion in response to glucose but impaired response to glucagon

M. S. Winzell ${ }^{1}$, J. Ahrén ${ }^{1}$, E. Renström ${ }^{2}$, B. Ahrén ${ }^{1}$;

${ }^{1}$ Clinical Sciences, Medicine, Lund University, Lund, Sweden, ${ }^{2}$ Clinical

Sciences, Endocrinology, Lund University, Malmö, Sweden.

Background and Aims: Glucagon is critical for blood glucose homeostasis. It is the major counter-regulatory hormone to insulin and it is released during hypoglycaemia, stimulating hepatic glucose output (HGO). Glucagon can stimulate insulin secretion but during hyperglycaemia, glucagon secretion is inhibited by factors secreted from the islet cells. In type 2 diabetes, the suppression of glucagon secretion is blunted resulting in increased HGO and elevated insulin secretion. It is possible that an impaired effect of glucagon on the beta-cells may be one mechanism behind the insulin secretory defect observed in type 2 diabetes. To investigate the effect of potentiated glucagon signalling for insulin secretion we examined mice with beta-cell targeted overexpression of glucagon receptors.

Materials and Methods: Mice with beta-cell specific overexpression of glucagon receptors (GR-tg) were generated on the C57BL/6J background. Insulin secretion was examined in male mice in intravenous glucose tolerance tests (IVGTT), as well as in isolated islets. The response to glucagon was examined both in vivo and in vitro. Exocytosis was examined in single betacells by capacitance measurements.

Results: Basal circulating glucose levels were reduced in GR-tg mice, while insulin and glucagon were similar to wild-type (wt) mice. After an IVGTT, the acute insulin response (AIR) was improved in GR-tg mice (AIR: 1045 $\pm 56 \mathrm{pM}$ vs. $520 \pm 85 \mathrm{pM}$ in wt $\mathrm{P}=0.006$ ). This resulted in improved glucose tolerance (KG(1-20min): $3.6 \pm 0.3$ vs. $2.5 \pm 0.2 \% / \mathrm{min}$ in wt, $\mathrm{P}=0.02)$. However, when glucagon was added to the IVGTT, the insulin response was significantly reduced compared to wt mice (AUC $(0-20 \mathrm{~min}): 37 \pm 5$ vs. $67 \pm 10 \mathrm{nM}^{*}$ min in wt, $\mathrm{P}=0.01)$. Despite increased insulin secretion after glucagon administration, the glucose elimination was slightly impaired in both wt and GR-tg mice (KG(1-20min): $2.4 \pm 0.5$ vs. $3.5 \pm 0.5 \% / \mathrm{min}$ in tg with or without glucagon, $\mathrm{P}>0.05)$ due to increased HGO. In isolated islets the insulin response to glucose was decreased in GR-tg ( $324 \pm 44$ in GR-tg vs. $524 \pm 54 \mathrm{pg} / \mathrm{h} / \mathrm{islet}$ in wt $(\mathrm{P}=0.008)$ at $11.1 \mathrm{mM}$ glucose). Insulin secretion in response to glucagon was reduced by $40 \%$ in GR-tg compared to wt islets ( $437 \pm 57$ vs. $770 \pm 100$ $\mathrm{pg} / \mathrm{h} /$ islet in wt, $\mathrm{P}=0.014$ ). Exocytosis was studied in perforated patch-clamp experiments where glucagon was demonstrated to increase capacitance in wt beta-cells ( $46 \pm 3$ vs $19 \pm 3 \mathrm{fF}, \mathrm{P}=0.001$ ) while there was no response to glucagon in GR-tg beta-cells ( $39 \pm 4$ vs. $31 \pm 3 \mathrm{fF}, \mathrm{P}>0.05$ ).

Conclusion: We demonstrate that insulin secretion in vivo is improved when GR are overexpressed in beta-cells, while the response to glucagon is blunted. In vitro, the inhibitory effect of glucagon on insulin exocytosis and secretion was confirmed. This illustrate that glucose and glucagon, when simultaneously elevated, inhibit insulin secretion through glucagon signalling in the beta-cells. Since GR-tg mice have improved glycaemia this study suggests that glucagon signalling in beta-cells are important for whole body glucose homeostasis and that this may be impaired in type 2 diabetes.

\section{4}

Chromogranin-B deficient mice are diabetic despite normal insulin granule biosynthesis

S. Barg ${ }^{1,2}$, S. Obermüller ${ }^{1}$, F. Calegari ${ }^{3}$, A. Lindqvist ${ }^{1}$, A. King ${ }^{4}$, I. Lundqvist ${ }^{1}$, A. Salehi ${ }^{1}$, J. Karanauskaine ${ }^{1,5}$, P. Rorsman ${ }^{6}$, P. Rosa ${ }^{4}$, W. Huttner ${ }^{7}$;

${ }^{1}$ Islet Cell Physiology, Lund University, Malmö, Sweden, ${ }^{2}$ Vollum Institute, OHSU, Portland, United States, ${ }^{3}$ Neurogenesis/Cell Biology/Development, Center for Regenerative Therapies Dresden, Germany, ${ }^{4}$ Department of Medical Pharmacology, University of Milan, Italy, ${ }^{5} \mathrm{Ocdem}$, University of Oxford, United Kingdom, ${ }^{6}$ OCDEM, University of Oxford, United Kingdom, ${ }^{7}$ Max Planck Institute for Cell Biology and Genetics, Malmö, Germany.

Background and Aims: Granins form a family of highly acidic proteins that are primarily found in the lumen of dense-core secretory granules of neuroendocrine cells and neurons. The precise function of the granins is still debated, but evidence points into four main directions: 1) granulogenesis factor, 2) facilitation of the sorting of secretory peptides, 3) structural and storage function within the granule lumen, and 4) hormonal/paracrine activities. The ubiquitous chromogranin $\mathrm{B}(\mathrm{CgB})$ is expressed in insulin containing granules of pancreatic B-cells.

Materials and Methods: We have generated, by homologous recombination in ES cells, a mice line that lacks chromogranin ( $\mathrm{CgB}$ k.o.) and then characterized the consequences of this mutation at the molecular, cellular and systemic levels, by in vitro and in vivo hormone release measurements, electron microscopy, electrophysiology/capacitance measurements, and live cell imaging in islets and other neuroendocrine tissues.

Results: $\mathrm{CgB}$ knockout animals exhibited a diabetic phenotype with impaired glucose tolerance, reduced stimulated secretion of insulin, glucagon and somatostatin, but elevated basal insulin and proinsulin release. Biogenesis, morphology and maturation of insulin granules was unaltered, while granule density was slightly reduced in adenocorticotropic cells. No differences were found with regard to the exocytotic machinery, $\mathrm{Ca}^{2+}$-handling, and release kinetics of individual insulin granules.

Conclusion: We conclude that chromogranin $\mathrm{B}$ is not required for biogenesis and maintenance of functional granules, but may promote the processing of insulin into its mature form.

A
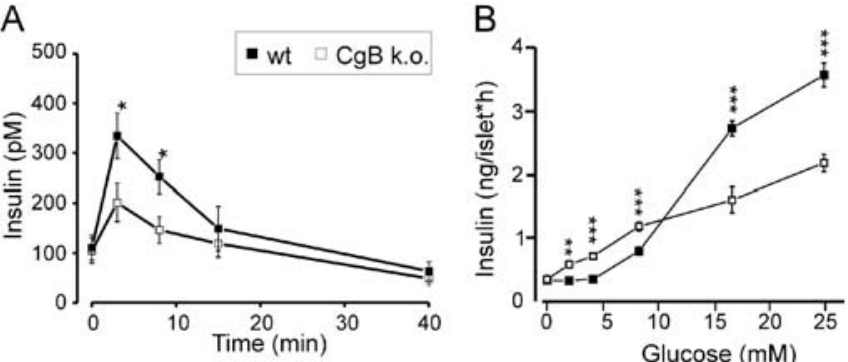

(A) Time course of insulin secretion in response to i.p. glucose $(3 \mathrm{~g} / \mathrm{kg}$ body weight) injection at time 0 ; wt (black squares, $n=12$ ) and k.o. (white squares, $\mathrm{n}=16$ ). ${ }^{\star *} \mathrm{P}<0.01 ;{ }^{\star} \mathrm{P}<0.05$.(B) Quantification of hormone release from isolated islets during $1 \mathrm{~h}$ incubations in various glucose concentrations $(0-25 \mathrm{mM}) ; \mathrm{n}=8$. ${ }^{\star * *} \mathrm{P}<0.001 ;{ }^{* \star} \mathrm{P}<0.01$.

Supported by Swedish Science Council, Diabetes Programme at Lund University, $E F S D, E M B O, D F G$, Fonds der Chemischen Industrie 


\section{OP 10 Immunopathogenesis of type 1 diabetes: from mice to men}

\section{5}

\begin{abstract}
Mitochondrial substitution strains increase diabetes and glucose intolerance and change the morphology and function of the islets L. Wester Rosenlöf ${ }^{1}, X$. Yu ${ }^{1}$, H. Weiss ${ }^{2}$, A. Jörns ${ }^{3}$, M. Tiedge ${ }^{2}$, S. Ibrahim'; ${ }^{1}$ Immunegenetics, University of Rostock, Germany, ${ }^{2}$ Medical Biochemistry and Molecular Biology, University of Rostock, Germany, ${ }^{3}$ Department of Clinical Biochemistry, Medical High school of Hannover, Germany.
\end{abstract}

Background and Aims: We have recently identified a mutation in the mitochondrial genome of the FVB/N mouse strain encoding for the ATP8 gene (a subunit of the ATP Synthase complex). The aim of our study was to characterize the impact of the ATP8 mutation on diabetes, glucose tolerance, islet morphology and insulin secretion. For that purpose Mitochondrial Substitution Strains (MSS) carrying the mutant FVB/N mitochondria were produced by repeated backcrossing (minimum12 generations) to different inbred strains. Four strains were used; two C57BL/6 strains carrying the $\mathrm{AKR} / \mathrm{J}$ or the $\mathrm{FVB} / \mathrm{N}$ mitochondria $\left(\mathrm{B}^{\mathrm{AKRmt}} \& \mathrm{~B}^{\mathrm{FVBmt}}\right.$ ) and two NOD/LtJ strains carrying the NOD/LtJ or FVB/N mitochondria (NOD \& NOD ${ }^{\mathrm{FVBmt}}$ ). The $\mathrm{B}^{\mathrm{AKRm}} / \mathrm{B} 6^{\mathrm{FVBmt}}$ mice are polymorphic in the ATP8 gene only, but the $\mathrm{NOD} / \mathrm{NOD}^{\mathrm{FVBmt}}$ have an additional polymorphism in the COX III gene.

Materials and Methods: Animals from the NOD and the NOD ${ }^{\mathrm{FVBmt}}$ strains (40-50 animals per group and sex) were monitored for body weight and blood glucose levels for 40 weeks. Intraperitoneal glucose tolerance test was performed on 12-13 weeks old $\mathrm{NOD} / \mathrm{NOD}^{\mathrm{FVBmt}}$ and $\mathrm{B} 6^{\mathrm{AKRmt}} / \mathrm{B}^{\mathrm{FVBmt}}$ mice. Pancreas sections of 12 and 16 weeks old NOD and $\mathrm{NOD}^{\mathrm{FVBmt}}$ mice were analyzed for islet morphology, immune cell infiltration and beta cell mass. Islets from prediabetic $\mathrm{NOD} / \mathrm{NOD}^{\mathrm{FvBmt}}$ mice were stimulated with $\mathrm{KCl}, 2.8$ $\mathrm{mM}$ and $16.7 \mathrm{mM}$ glucose and insulin secretion and content were analyzed by ELISA.

Results: Female NOD and $\mathrm{NOD}^{\mathrm{FVBmt}}$ mice showed a diabetes incidence of $65-70 \%$ and $88 \%$ respectively. Interestingly, male NOD and NOD ${ }^{\mathrm{FVBmt}}$ mice showed an incidence of $10-15 \%$ and $60 \%$ respectively. Diabetes onset was $\sim 20$ weeks for female NOD and NOD ${ }^{\mathrm{FVBmt}}$ mice, $30-35$ weeks for male NOD and 25 weeks for male NOD ${ }^{\mathrm{FVBmt}}$ mice. Both $\mathrm{NOD}^{\mathrm{FVBmt}}$ and $\mathrm{B}^{\mathrm{FVBmt}}$ mice showed higher blood glucose levels 60-120 minutes after glucose challenge $(p<0.05)$. In the pancreas of 12 week old mice a peri-insulitis was observed in normal NOD but not in NOD ${ }^{\mathrm{FVBmt}}$ mice. In 16 week old mice, both strains showed residual islets with very weak insulin staining. Glucose stimulated islets from $\mathrm{NOD}^{\mathrm{FVBmt}}$ mice showed lower insulin secretion, while the insulin content was much higher. $\mathrm{KCl}$ stimulation revealed no obvious defect in exocytosis pathways.

Conclusion: The ATP8 mutation increases diabetes susceptibility in the NOD strain, particularly in male mice. Once an immune cell infiltration of beta cell has started, the mutation leads to a faster destruction. The mutation also negatively affects glucose-stimulated insulin secretion in beta cells resulting in impaired glucose tolerance in prediabetic animals.

Supported by the Fp6 project MRTN-CT-2002-005693 EURO-RA

\section{6}

Toll-like receptor 4: Regulator of beta cell-directed immune reactivity and development of body weight in the non-obese diabetic mouse E. Gülden, S. Imamura, M. Ihira, H. Kolb, V. Burkart; Institute for Clinical Diabetes Research, German Diabetes-Center, Düsseldorf, Germany.

Background and Aims: Initiation and progression of beta cell-directed immune reactions underlying the pathogenesis of type 1 diabetes (T1D) are controlled by cells and mediators of the non-adaptive immune system. Recent findings have shown that the activities of non-adaptive immune cells, such as macrophages, strongly depend on the expression of toll-like receptors (TLR). Our previous studies demonstrated a critical role of TLR4 in the proinflammatory activation of macrophages. Based on these observations our present study was designed to investigate a potential regulatory effect of TLR4 on the development of beta cell-directed immunity and the pathogenesis of T1D.

Materials and Methods: Our studies were performed in mice with homozygous TLR4 expression $\left(\mathrm{TLR}_{4}^{+/+}\right)$or deficiency $\left(\mathrm{TLR} 4^{-/-}\right)$generated from backcrosses (>12 generations) of TLR4 deficient C57BL10/ScCr mice on the background of the non-obese diabetic (NOD) mouse. In these mice the development of diabetes and body weight was monitored and potential immuno-regulatory effects of TLR4 were assessed by investigating the responses of spleen cell populations to concanavalin A (Con A), interleukin2 (IL-2) and lipopolysaccharide (LPS).

Results: Female TLR4 ${ }^{-/}$mice show a significant acceleration of diabetes development compared to TLR $4^{+/+}$mice $(\mathrm{p}<0.05)$. At $120 \mathrm{~d}$ of age pancreata of TLR $4^{-/}$mice revealed significantly increased proportions of islets with advanced stages of immune cell infiltration compared to TLR $4^{+/+}$mice $(\mathrm{p}<0.05)$. These findings point to a dysregulation of beta cell-directed immune reactivity in TLR4 ${ }^{-/-}$mice. In fact, spleen cells of TLR4 ${ }^{-/-}$mice exhibit an aberrant reactivity towards immunologic stimuli. In response to Con A (2.5 $\mu \mathrm{g} / \mathrm{ml}, 3 \mathrm{~d}) \mathrm{TLR}^{+/+}$spleen cells showed a 4.5 times higher proliferation rate as TLR4 ${ }^{-/-}$cells $(\mathrm{p}<0.01)$. However, IL-2-stimulated $(50 \mathrm{ng} / \mathrm{ml}, 3 \mathrm{~d}) \mathrm{TLR}^{-/-}$ cells produced higher amounts of the beta cell toxic mediator tumor necrosis factor $\alpha(311 \pm 10 \mathrm{pg} / \mathrm{ml})$ than TLR4 ${ }^{+/+}$cells $(161 \pm 14 \mathrm{pg} / \mathrm{ml})(\mathrm{p}<0.001)$. To assess the cellular basis for the functional hyper-reactivity of TLR4 ${ }^{-1}$ cells $\mathrm{CD}^{+} / \mathrm{CD} 25^{+}$regulatory T-cells (Treg) were enriched from spleen cells. FACS analysis proved a purity of the Treg populations of $96.7 \%\left(\mathrm{TLR}^{+++}\right)$and 96.8 $\%\left(\right.$ TLR4 $\left.^{--}\right)$. As determined by conversion of the tetrazolium salt WST-1 the TLR4 ligand LPS $(5 \mu \mathrm{g} / \mathrm{ml}, 3 \mathrm{~d})$ induced a significant, 1.7 fold increased activation of TLR $4^{+/+}$cells compared to TLR $4^{-/-}$cells $(\mathrm{p}<0.001)$. Observations in T1D patients show an association of earlier disease onset and increased adiposity. Recent studies further identified components of the innate immune system, such as macrophages and TLR, in adipose tissue. Therefore, in a first approach to assess a possible effect of TLR4 on the increase of adipose tissue we monitored the development of body weight in female TLR $4^{+/+}$and $\mathrm{TLR}^{-/-}$mice. TLR4 ${ }^{-/-}$mice showed a significant acceleration of body weight development compared to TLR $4^{+/+}$mice $(\mathrm{p}<0.0001)$. Until the age of 21 weeks $\mathrm{TLR}^{-/-}$mice weighed $1.7 \pm 0.2 \mathrm{~g}(6.9 \pm 0.8 \%)$ more than TLR4 $4^{+/}$mice.

Conclusion: Our results in TLR4 ${ }^{-/-}$mice show an acceleration in weight gain and an immunologic hyper-reactivity, possibly due to an insufficient counter regulation in the absence of TLR4. These findings implicate that TLR4 is involved in the regulation of beta cell-directed immunity and body weight development thereby controlling the pathogenesis of insulin-deficiency diabetes in NOD mice.

Supported by Deutsche Forschungsgemeinschaft

\section{7}

Identification of a classII-RegII peptide complex in pancreatic lymph nodes that activates autoagressive T-cells in NOD mice

W. Gurr, M. Shaw, Y. Li, R. Sherwin;

Internal Medicine, Yale University, New Haven, United States.

Background and Aims: We have reported that RegII, a beta cell protein associated with islet regeneration, is a target of the autoimmune process leading to diabetes in NOD mice. According to this scenario RegII might be released locally in an attempt to regenerate islets, which in turn might activate RegII, specific autoreactive T-cells thereby promoting inflammation and further release of RegII. Our data indicate that an epitope within the N-terminal fragment of RegII (NtfrII) is specifically targeted by the autoimmune diabetogenic response. Since autoreactive CD4+ T-cells specific for islet antigens are activated in the pancreatic lymph nodes, we attempted to quantitate I-Ag7 NtfrII peptide complexes occurring at this site during the pathogenesis of T1DM.

Materials and Methods: To generate an anti I-Ag7 NtfrII peptide complexdirected immune response classII-mismatched, classI-matched mice were immunized with NtfrII-pulsed NOD spleen cells. To generate a phagedisplayed antibody library light and heavy chain variable fragments (Vl and $\mathrm{Vh}$ ) were amplified using cDNA derived from spleen cells of immunized mice as template and primers suitable for the assembling of a mouse $\mathrm{scFv}$ cassette in the orientation Vl-(G4S)4-Vh compatible with the phage-display vector pAK100. Phages were selected by a two-step process, first on NtfrII-pulsed and then on unpulsed NOD spleen cells. The selection/reinfection process was performed for three rounds after which phages were rescued from single colonies and analyzed for differential binding to NtfrII-pulsed vs. unpulsed NOD spleen cells. To quantitate I-Ag7 NtfrII peptide complexes pancreatic lymph nodes were removed and single cell suspensions were incubated with a phage displayed specific antibody or a mutated non-binding variant thereof. Bound phages were eluted and used to infect bacteria. A colony count was then obtained. 
Results: An antibody (termed D9) was obtained that showed binding to NtfrII-pulsed NOD spleen that was dependent on the pulse strength. Binding also occurred to NOD spleen cells pulsed with RegII indicating that the IAg7 NtfrII peptide complex recognized by D9 arises when full length RegII is processed. D9 did not bind to NtfrII-pulsed spleen cells from classIImismatched mice demonstrating its specificity. The peptide recognized by D9 in context of I-Ag7 was localized between positions 44 and 64 of NtfrII. Quantitation of the I-Ag7 NtfrII peptide complexes in pancreatic lymph nodes of NOD mice showed a progressive increase during the development of diabetes: (phages retrieved from 106 cells): at 6 weeks $54,000+/-4,100$, at 9 weeks $85,000+/-7,700$, at 14 weeks $105,000+/-9,300$. Counts for the mutated, non-binding D9 were $<18,000$ for all time points. To determine if the complex detected by the antibody $\mathrm{D} 9$ was recognized by autoagressive T-cells NOD mice were immunized with peptide 44-64 of RegII in alum. Adoptively transferred CD4+ T-cells from these mice targeted and destroyed islets of recipient NOD-SCID mice.

Conclusion: Antibodies recognizing $\mathrm{MHC}$ molecules presenting islet autoantigen-derived peptides were isolated from a phage-displayed antibody library. The antibodies provided a means to identify an epitope responsible for the activation of diabetes-related autoagressive T-cells and to quantitate the complexes presenting it. This approach might lead to the identification of new immune therapeutic targets to prevent the development of T1DM. Supported by the Juvenile Diabetes Research Foundation

\section{8}

Ii-key/MHC class II epitope hybrid technology to detect T cell response to insulin and GAD in type 1 diabetes

M. Vadacca ${ }^{1}$, G. Valorani ${ }^{2,3}$, R. Humphreys ${ }^{4}$, N. Kallinteris ${ }^{4}$, L. Valente ${ }^{1}$, G. D’Agostino ${ }^{5}$, P. Pozzilli' ${ }^{1}$, IMDIAB GROUP ${ }^{1}$;

${ }^{1}$ Diabetes Department, Università Campus Bio-Medico, Roma, Italy, ${ }^{2}$ Diabetes Department, University Queen Mary of London, United

Kingdom, ${ }^{3}$ Livio Patrizi, Foundation, Roma, Italy, ${ }^{4}$ Generex Biotechnology, Antigen Express, Boston, United States, ${ }^{5}$ Superiore di Sanità, Istituto, Roma, Italy.

Background and Aims: Type 1 diabetes (T1D) develops as a result of pancreatic beta-cell destruction. The immune response involves both cell mediated and humoral reactivity towards insulin GAD and IA-2. In T1D the specific epitopes recognized by pathogenic $\mathrm{T}$ cells remain poorly defined and the specific autoreactive $\mathrm{T}$ cells are difficult to detect. The design rationale for Ii-key/antigen hybrids was drawn from data prevously published and Xray crystallographic data indicating that the Ii-key binding site lies outside the antigenic peptide-binding trough of MHC class II molecules. In our previous work a series of hybrids has been synthesized containing Ii-key derivatives (beginning with LRMK, the shortest active derivative) coupled to insulin peptides. This offered us an efficient way to augment the binding of epitopes to the antigenic peptide-binding groove and thus enhances epitope immunogenecity. The main objective of the present study was to investigate the cellular immune response in T1D by characterising the cytokines secreted by peripheral blood mononuclear cells (PBMCs) in response to exposure to Ii-key/MHC class II GAD epitope hybrids.

Materials and Methods: Sequences of the human GAD were obtained from Genebank. The deduced protein sequence for Genbank was run through SYFPETHI (http://www.syfpeithi.de/Scripts/MHCServer.dll/Info.htm) and the top MHC II epitopes have been scored for each DR allele. Six peptide series (16 hybrids and six epitope-controls) were analyzed. The study was carried out in 8 recent onset T1D subjects ( 3 males) affected by T1D and in 7 normal subjects. Diagnosis was defined according ADA classification. ELISPOT assays were performed with BD Pharmingen set for IFN- $\gamma$ according to the manufacturer's instructions. Digitalized images of spots were analyzed with a Eli.Analyse 4.0 software.

Results: All assays were able to detect significant cytokine secretion in response to the polyclonal stimulators PMA/Ionomycin. Two out 8 patients $(25 \%)$ demonstrated a positive response to one or more Ii-Key/GAD65 hybrids. None of the control subjects showed a detectable response to stimulation with Ii-key hybrids or peptides.

Conclusion: The Ii-key hybrid technique is a new strategy for augmenting the potency of MHC class-II restricted epitopes resulting in a profound increase in $\mathrm{T}$ cell stimulation. Our previous study demonstrated that some Ii-key insulin hybrids, and not the epitope-peptide only, stimulate strong reactivity in T1D subjects. We have here shown a specific response to Ii-key/GAD65 hybrids only in T1D. The combined use of insulin and GAD key-hybrids might therefore assure a more selective and sensitive cytokine detection in response to antigen stimulation in T1D offering a new highly specific marker of cell mediated immunity in T1D.

Supported by Ag Exp, Boston

\section{9}

Human anti-IA-2 autoantibodies isolated from an immune library by phage display are able to bind to both major autoantigens: GAD65 and IA-2

L. Valera ${ }^{1}$, S. Peraldi-Roux ${ }^{2}$, B. Watelet-Jardin ${ }^{1}$, V. Lampasona ${ }^{3}$, C. Granier ${ }^{1}$, E. Bonifacio ${ }^{4}$, D. Laune ${ }^{1}$;

${ }^{1}$ MISCDIA, CNRS FRE 3009, Montpellier, France, ${ }^{2}$ CPID, CNRS UMR 5232, Montpellier, France, ${ }^{3}$ Immunology of Diabetes Unit, San Raffaele Scientific Institute, Milan, Italy, ${ }^{4}$ Diabetes research Institute, Munich, Germany.

Background and Aims: The selection of human antibodies directed towards major autoantigens, the glutamate decarboxylase GAD65 and the tyrosine phosphatase like protein IA-2, can provide useful tools to study humoral immune responses in autoimmunity of type 1 diabetes. The aim of our study was to isolate by phage display new human monoclonal autoantibodies to IA2 and characterize them in order to use these antibodies for an early diagnosis of type 1 diabetes.

Materials and Methods: We report here the molecular cloning and characterization of human anti-IA-2 Fab fragments generated from an immune library derived from the IgG repertoire of patients with type 1 diabetes mellitus. The phage screening was performed after three rounds of panning by enzyme-linked immunosorbent assay (ELISA) with coated IA-2. The specificity of binding and the localization of epitopes was analyzed in several ELISA formats and by Spot technology on cellulose membranes.

Results: We have been successful in selecting six new antibodies against human IA-2 using phage display. These antibodies interestingly had a dual specificity: they bind to both major autoantigens GAD65 and IA-2. After epitope analysis, we found that major epitopes recognized by these autoantibodies are present in the juxtamenbrane domain of IA-2 molecule. Moreover, the analysis of genes encoding these Fab revealed they had some genetical characteristics previously described for GAD65 antibodies and for autoantibodies associated to autoimmune thyroid diseases.

Conclusion: Our study demonstrates that anti-IA-2 antibodies can be isolated from a human immune library derived from patients with type 1 diabetes mellitus. This is the first report describing human antibodies recognizing the two main autoantigens of T1DM: GAD65 and IA-2. Thus, these bispecific autoantibodies could have some conformational epitopes in common, and their identification could be useful for the molecular modelization of both proteins and for the understanding of the induction of type 1 diabetes.

\section{0}

Specific immune response to GAD65 in type 1 diabetic children treated with GAD65 (Diamyd ${ }^{m \text { tw }}$ )

M. Faresjö, M. Hedman, R. Casas, S. Axelsson, J. Ludvigsson;

Department of Molecular \& Clinical Medicine, Division of Paediatrics, Linköping, Sweden.

Background and Aims: Type 1 diabetes (T1D) is associated with a defect regulation of the immune system and imbalance of T-helper (Th) cells resulting in destructive insulitis. Treatment with 20 microgram of GAD65 has indicated that beta-cell function can be preserved in LADA patients, and this effect has shown to be significant in T1D patients (10-18 years) still at 15 months follow-up. We hypothesise that treatment with GAD65 can induce GAD65-specific Th- and T-regulatory (Treg) cells, with a subsequent deviation towards a protective immune profile.

Materials and Methods: This phase II Diamyd ${ }^{\text {Tw }}$ study was designed as a randomised, double-blind, placebo-controlled, multi-centre trial including 70 T1D children ( 42 females, 28 males, $10-18$ years) diagnosed within the previous 18 months, fulfilling the criteria of fasting C-peptide $>0.1 \mathrm{nmol} / \mathrm{L}$ and presence of GAD65 autoantibodies $(>67.3 \mathrm{U} / \mathrm{mL})$ at the screening visit. Thirty-five patients were randomly assigned to 20 microgram of GAD65 (Diamyd ${ }^{\mathrm{m}}$, Diamyd Therapeutics AB, Stockholm, Sweden) and 35 patients to 
placebo (Diamyd $\mathrm{d}^{\mathrm{m}}$ bulk buffer alone) in a primary injection at day one and a booster of the same concentration four weeks later.

Peripheral blood mononuclear cells (PBMC), collected from 70 T1D children before and 15 months after the primary injection with GAD65 $\left(\right.$ Diamyd $\left.^{\mathrm{mw}}\right)$ or placebo and from a reference group (12 healthy children; 8 females, 4 males, 11-15 years), were stimulated with GAD65 (Diamyd $\mathrm{d}^{\mathrm{mix}}$ ) and phytohaemagglutinin (PHA) for 72 hours.

Secretion of cytokines (IL-5, -6, -10, -12, -13, -17 , IFN- $\gamma$ and TNF- $\alpha$ ) and chemokines (IP-10, MCP-1, MIP-1 $\alpha$, MIP-1 $\beta$ and RANTES) were detected in cell supernatants by multiplex fluorochrome technique (Luminex). Expression of FOXP3 mRNA was detected together with endogenous rRNA by multiplex real-time RT-PCR.

Correction with Bonferroni's was made to compensate for multiplex comparisons.

Results: The spontaneous secretion of cytokines and chemokines and FOXP3 mRNA expression did not differ between children treated with GAD65 or placebo either before or 15 months after treatment. Further, the mitogen PHA induced prominent response of cytokines and chemokines as well as FOXP3 mRNA in all children regardless of treatment.

However, after in vitro stimulation with GAD65, patients treated with Diamyd differed significantly from the placebo group, 15 months after the immune intervention. Thus, GAD65 induced higher secretion of Th1- (IFN$\gamma ; \mathrm{p}<0.0001$ ), Th2- (IL-5; $<<0.0001$, IL-13; $<<0.0001$ ), Th3- (IL-10 $\mathrm{p}<0.0001$ ), and Th17- (IL-17; $\mathrm{p}<0.0001)$ associated cytokines in Diamyd treated patients. Also, inflammatory cytokines (IL-6; $p=0.006$ and TNF- $\alpha$; $<<0.0001$ ) and chemokines (IP-10; $<<0.05$, MIP- $1 \alpha ; \mathrm{p}<0.05$ and MIP-1 $\beta ; \mathrm{p}<0.05$ ) were induced to a higher extent by stimulation with GAD65 15 months after Diamyd treatment. Increased FOXP3 mRNA expression by GAD65 stimulation was observed in T1D children treated with Diamyd $(\mathrm{p}<0.0001)$. Even compared to healthy children, GAD65-induced secretion of IL-5, -13, -17 and TNF- $\alpha$ and FOXP3 mRNA were higher in Diamyd treated children $(\mathrm{p}<0.0001)$.

Conclusion: Treatment with GAD65 causes a specific immune response to GAD65 remaining still after 15 months, which indicates that a specific cell population has been induced. This may explain the protective effect of GAD65 vaccination on beta-cell function.

Supported by Swedish Child Diabetes Foundation, Swedish Research Council and Diamyd Medicals.

\section{OP 11 Nephropathy: mechanisms}

\section{1}

EURAGEDIC study: identification of new candidate genes for diabetic nephropathy

N. Hager-Vionnet ${ }^{1,2}$, D. A. Trégouët ${ }^{1,2}$, L. Tarnow ${ }^{3}$, H.-H. Parving ${ }^{3}$, P.-H. Groop ${ }^{4,5}$, C. Forsblom ${ }^{4,5}$, S. Hadjadj ${ }^{6,7}$, M. Marre ${ }^{8,9}$, I. Gut ${ }^{10}$, R. Cox ${ }^{11}$, D. Gauguier ${ }^{12}$, G. Kazeem ${ }^{12}$, M. Farrall ${ }^{12}$, F. Cambien ${ }^{1,2}$, M. Lathrop ${ }^{10}$; ${ }^{1}$ UMR S 525, INSERM, Paris, France, ${ }^{2}$ Umr s 525, Université Pierre et Marie Curie-Paris6, France, ${ }^{3}$ Steno Diabetes Centre, Copenhagen, Denmark, ${ }^{4}$ Department of Medicine, Helsinki University Central Hospital, Finland, ${ }^{5}$ Biomedicum, Folkhälsan Institute of Genetics, Helsinki, Finland, ${ }^{6}$ Diabetology department, Poitiers Hospital, France, ${ }^{7}$ Erm 324, INSERM, Poitiers, France, ${ }^{8}$ Department of Diabetology, Bichat Hospital, Paris, France, ${ }^{9}$ U695, INSERM, Paris, France, ${ }^{10} \mathrm{CNG}$, CNRG, Evry, France,

${ }^{11}$ MRC Mammalian Unit, Mammalian Research Council, Didcot, United Kingdom, ${ }^{12}$ Wellcome Trust Centre for Human Genetics, University of Oxford, United Kingdom.

Background and Aims: Diabetic nephropathy (DN) is likely to be a complex genetic trait. We studied 144 candidate genes for DN to identify susceptibility genes. We report marginal and epistatic genetic effects identified in this dataset.

Materials and Methods: 437 SNPs were investigated for association with overt diabetic nephropathy (persistent albuminuria $>300 \mathrm{mg} / 24 \mathrm{~h}$ ) in a large Type 1 diabetes case/control (1176/1323) study from 3 European populations. Marginal and epistatic effects were investigated through a multi-stage strategy using Random Forest, DICE and haplotypic methods and were tested in men and in women separately. The consistency of the results was assessed by their homogeneity across genders and populations.

Results: Univariate effects - One univariate effect was identified in both gender populations. The T allele of the UNC13b_134327 polymorphism was less frequent in cases than in controls $(0.281 \mathrm{vs} 0.324)$, leading to a common Odds Ratio of 0.746 [0.624 - 0.892] ( $p=0.0013)$. Epistatic effects - Nine interactions between 2 SNPs were identified among which 3 were consistent in men and women. The effect of the T allele of the UNC13b_134327 SNP observed in univariate analysis was found to be modulated by the PARP1_46691 SNP. Carriers of the T allele at the UNC13b_134327 locus were at lower risk of nephropathy mainly in individuals homozygous for the $\mathrm{C}$ allele at the PARP1 46691 locus $(\mathrm{OR}=0.594[0.482-0.731, \mathrm{p}<10-6)$ and not in individuals carrying the PARP1_46691 T allele $(\mathrm{OR}=1.187[0.959-1.470], \mathrm{p}=0.116)$, the test for interaction being highly significant $(\mathrm{p}<10-4)$. Presence of the $\mathrm{C}$ allele at ATP1B2_3623 locus was associated with increased risk of nephropathy only in individuals homozygotes for the ADRA1D_30405 A allele (OR = 1.949 [1.563 -2.430] $\mathrm{p}<10-4)$ while no effect was observed in carriers of the ADRA1D_ 30405 T allele $(\mathrm{OR}=0.859$ [0.699 - 1.055], $\mathrm{p}=0.148)$. SLC12A3_53289 and EDN1_2688 were found to interact on the risk of nephropathy, homogeneously in men and women. Presence of the C allele at SLC12A3_53289 was associated with an OR of 0.397 [0.267 - 0.587] $(\mathrm{p}<10-4)$ in individuals homozygotes for the EDN1_2688 G allele while this OR was 0.957 [0.756 - 1.212] in carriers of the $\mathrm{T}$ allele, these two ORs being significantly different $(\mathrm{p}<10-3)$.

Conclusion: UNC13b is a strong candidate gene for DN and interacts with PARP1, possibly in the glucose-induced apoptotic pathway. SLC12A3 and EDN1 interact possibly in the regulation of renal sodium excretion and systemic blood pressure. The nature of the interaction between ATP1B2 and ADRA1D is unclear. Further studies are needed to identify the functional variants of those genes and the precise nature of their interactions in the pathogenesis of diabetic nephropathy.

Supported by EU contract QLG2-CT-2001-01669

\section{2}

Accelerated nephropathy in type 2 diabetic patients with type III hyperlipoproteinemia (apo E2/2 genotype), and the detrimental effects of their remnant lipoproteins on human mesangial cells

M. Saito, M. Eto;

Clinical Medicine, Ohu University, Koriyama, Japan.

Backgrounds and Aims: In 1977 apolipoprotein (apo) E2 was found to be a causal gene of type III hyperlipoproteinemia (HLP), which occurs in apo 
E2/2 genotype subjects and is characterized by increased plasma remnant lipoproteins. On the other hand, in 1994 we first found that apo E2 allele is a genetic factor to promote diabetic nephropathy. Now this finding has been accepted in the world, but the exact mechanism by which apo E2 promotes diabetic nephropathy remains unknown. Most studies on apo E2 allele and diabetic nephropathy have been conducted in the heterozygote apo E3/2 genotype patients partly because of the small sample size of the homozygote apo E2/2 genotype patients. It seems important to investigate the effect of type III HLP (apo E2/2 genotype) on diabetic nephropathy, but no information is available. Therefore, we investigated nephropathy in diabetic patients with type III HLP (apo E2/2 genotype), and effects of remnant lipoproteins from the patients on the human mesangial cells (HMCs).

Materials and Methods: Blood samples were collected after an overnight fast. The diagnosis of type III HLP was made according to the following criteria: increased plasma triglyceride and total cholesterol (chol); apo E2/2 genotype/ phenotype and high plasma level of remnant chol (normal range $<5.2 \mathrm{mg} /$ dl). Remnant-chol was measured by the method of Nakajima. Fifteen patients with type III HLP and type 2 diabetes were recruited from our hospitals. Overt nephropathy was diagnosed if they had persistent proteinuria, and/or plasma creatinine level $>1.4 \mathrm{mg} / \mathrm{dl}$. The histological study was performed using renal biopsy specimens in apo E2/2 genotype patient. Remnant lipoproteins were isolated from the type III HLP patients' plasma by ultracentrifugation. Cultured HMCs were incubated with the remnant liporoteins in the medium. After the incubation, expression of TGF-b mRNA and accumulation of cholesteryl esters (CE) by HMCs were estimated, and the related lipoprotein receptors in HMCs were examined by siRNA method.

Results: Mean age was 60.6 years, BMI 25.4, fasting plasma glucose 147 $\mathrm{mg} / \mathrm{dl}$, and HbAlc $8.1 \%$. The patients had increased plasma levels of triglyceride (mean $380 \mathrm{mg} / \mathrm{dl}$ ) and total chol (mean $260 \mathrm{mg} / \mathrm{dl}$ ), increased plasma remnant-chol level (mean $51.0 \mathrm{mg} / \mathrm{dl}$ ) and a high remnant-chol/TG ratio (mean 0.145). The patients had a short diabetes duration of 6.3 years (mean), but a high frequency (46.7\%) of overt nephropathy, demonstrating that type III HLP (apo E2/2 genotype) accelerated nephropathy. Histological findings of the kidney in apo E2/2 genotype patient were identical with those in diabetic nephropathy. Immunohistochemical analysis demonstrated a positive staining of apo $\mathrm{E}$ and apo $\mathrm{B}$ in the glomeruli, suggesting the accumulation of remnant lipoproteins. The effects of remnant lipoproteins on HMCs were estimated. The remnant lipoproteins from the patients stimulated the expression of TGF-b mRNA and the accumulation of CE by HMCs more significantly than those from the controls. The siRNA method showed that VLDL receptor but not LDL receptor was related to the accumulation of CE by HMCs.

Conclusion: The present study first demonstrated that type III HLP accelerates diabetic nephropathy. Increased plasma remnant lipoproteins may cause renal damage by stimulating the expression of TGF-b and the accumulation of CE by HMCs via VLDL receptor.

Supported by DVD

\section{3}

Ablation of galectin-3 gene accelerates lipid-induced glomerular injury: evidence for a relevant scavenging role of galectin-3

G. Pugliese ${ }^{1}$, S. Menini ${ }^{1}$, C. Ricci ${ }^{1}$, A. Scipioni ${ }^{1}$, V. Sansoni ${ }^{1}$, C. Pesce ${ }^{2}$,

F. Pugliese ${ }^{1}$, F. Pricci ${ }^{3}$, C. Iacobini ${ }^{1}$,

${ }^{1}$ Department of Clinical Sciences, La Sapienza University, Rome,

${ }^{2}$ DISTBIMO, University of Genoa, Italy, ${ }^{3}$ Department of Cell Biology

and Neurosciences, National Institute of Health, Rome, Italy.

Background and Aims: It has long been recognized that hyperlipidemia plays an important role in the initiation and progression of renal disease. High saturated fat and/or cholesterol intake in experimental animal models resulted in glomerulosclerosis and tubulo-interstitial injury, associated with renal accumulation of fatty acids and/or cholesteryl esters; lipid-lowering agents were effective in preventing these changes. Galectin-3, a multifunctional lectin with (anti)adhesive and growth-regulating properties, was recently identified as a receptor for advanced glycation endproducts (AGEs) and modified-LDL by promoting the uptake and removal of these potentially harmful molecules. Its expression was found to be increased in kidney and atherosclerotic lesions from both human patients and experimental animals. We previously showed that mice knockout (KO) for galectin-3 are more susceptible to develop glomerular disease induced by diabetes, AGEs and aging, as compared with the corresponding wild type (WT) mice. This study aimed at verifying the hypothesis that galectin-3 KO mice develop accelerated glomerular disease in response to high-fat diet (HFD)-induced hyperlipidemia.

Materials and Methods: Galectin-3 KO and coeval WT mice were fed a HFD $(15 \%$ fat $+1.25 \%$ cholesterol and $0.5 \%$ Na-cholate $)$; mice fed a normal-fat diet (NFD, 4\% fat) served as control. Metabolic, renal functional and structural parameters as well as renal/glomerular cell apoptosis and extracellular matrix accumulation, AGEs/AGE-receptors and oxidative stress were assessed in these animals.

Results: Body weights and blood glucose concentrations were not different among the experimental groups throughout the study, serum triglyceride and cholesterol levels increased $~ 3.5$-fold in both WT and KO mice, whereas serum insulin levels and HOMA-IR increased significantly in WT, but not in KO mice fed a HFD vs. the corresponding NFD-fed control animals. KO-HFD mice showed more pronounced changes in renal function and structure as compared with the corresponding WT mice, as evidenced by the significantly higher levels $(\mathrm{P}<0.001)$ of proteinuria $(8.9 \pm 1.2$ vs. $4.4 \pm 0.9$ $\mu \mathrm{g} / \mathrm{mg}$ creatinine), albuminuria ( $6.5 \pm 1.6$ vs. $3.8 \pm 0.8 \mu \mathrm{g} / \mathrm{mg}$ creatinine), glomerular sclerosis index, mean glomerular volume (189278 \pm 8073 vs. $\left.156024 \pm 7174 \mu^{3}\right)$ and area $\left(4043 \pm 116\right.$ vs. $\left.3503 \pm 108 \mu \mathrm{m}^{2}\right)$, mean mesangial area $\left(951.8 \pm 25.6\right.$ vs. $\left.788.4 \pm 35.5 \mu \mathrm{m}^{2}\right)$, fractional mesangial area ( $26.8 \pm 0.4 \mathrm{vs}$. $22.5 \pm 0.6 \%)$, glomerular cell apoptosis $(0.176 \pm 0.032$ vs. $0.120 \pm 0.029$ cells/ glom) and renal/glomerular protein and mRNA expression for fibronectin and collagen IV (). In addition, circulating AGE and isoprostane 8-epiprostaglandin F2 $\alpha$ levels, renal/glomerular content of the glycoxidation product $\mathrm{N}^{\varepsilon}$-carboxymethyllysine and the lipoxidation product 4-hydroxy-2nonenal, expression of RAGE, CD36 and MCP-1, activation of nuclear factor $\kappa \mathrm{B}$ and macrophage infiltration were higher and expression of SR-A1 was lower in HFD-fed KO vs. WT mice.

Conclusion: These data show that galectin-3 deficiency is associated with increased susceptibility to hyperlipidemia-induced renal disease, thus indicating a major role for galectin-3 in lipoprotein uptake and metabolism. Supported by the Ministry of University and Research of Italy

\section{4}

Inducible overexpression of angiopoietin-1 in podocytes results in albuminuria and foot process effacement in mice

L. Gnudi ${ }^{1}$, A. Dei Cas ${ }^{1}$, A. Hayward ${ }^{1}$, K. White ${ }^{2}$, B. Davis ${ }^{1}$, R. Bilous ${ }^{2}$,

S. Marshall ${ }^{2}$, G. Viberti ${ }^{1}$;

${ }^{1}$ Cardiovascular Division, King's College London,

${ }^{2}$ Diabetes and Metabolism, Newcastle University, United Kingdom.

Background and Aims: The Angiopoietin1/Angiopoietin2 system is important in wall vessel injury and repair. In the normal glomerulus angiopoietin-1 (Ang-1) is expressed while angiopoietin-2 (Ang-2) is absent. In diabetes there is an upregulation of both Ang-1 and Ang-2 expression with the latter being of a greater degree. To obtain insights into the role of Ang-1 in glomerular pathophysiology we overexpressed it in the podocytes of normal mice.

Materials and Methods: Podocyte-specific overexpression of Ang-1 and $\beta$-galactosidase cDNA was induced in mice using the Tet-On regulatable inducible system. The podocyte-specific promoter podocin was used to drive the expression of the reverse tetracycline transactivator. Mice were studied between 8 and 10 weeks of age. To activate the transgene, Doxycycline $(2 \mathrm{mg} /$ $\mathrm{mL}$ ) was administered with water for up to 10 weeks. The following groups of mice were studied: a) transgenic (TG) with doxycycline (DOX), b) TG without DOX, c) controls (C) with DOX and d) C without DOX.

Kidney cortex was collected and prepared for subsequent analysis for $\beta$ galactosidase staining, western immunoblotting, immunohistochemistry, and electron microscopy studies. Albumin concentration in the urine was determined by ELISA in $24 \mathrm{~h}$ collections.

Results: Podocyte positive $\beta$-galactosidase staining was present only in TG-DOX treated animals, indicating absence of leaky transgene activity. Western immunoblottting showed a $2-3$ fold increase $(p<0.05)$ in Ang-1 protein overexpression in the DOX treated TG mice only. Albuminuria was increased significantly by $2-3$ fold in the TG-DOX treated mice $(p<0.05)$ as early as 5 weeks after treatment with DOX. At 10 weeks VEGF expression was upregulated by $\sim 70 \%(\mathrm{p}<0.05)$ but no changes in nephrin expression was observed. Foot process effacement/fusion was increased by $30-4-\%$ in Ang- 1 overexpressing mice $(\mathrm{p}<0.05)$.

Conclusion: We have generated a transgenic mouse model of podocytespecific inducible Ang-1 overexpression. This resulted in induction of 
albuminuria, upregulation of VEGF, and podocyte foot processes effacement, suggesting a role of Ang-1 in glomerular capillary wall pathobiology. Supported by EFSD/BBSRC

\section{5}

PKC $\varepsilon$ is activated under high glucose conditions, and decreases the expression of bone morphogenetic protein 4 (Bmp4) through MAPK-dependent pathway in cultured mouse mesangial cells

S. Maeda ${ }^{1}$, N. Osawa ${ }^{1,2}$, T. Hayashi ${ }^{1,3}$, T. Uzu $^{2}$, T. Babazono ${ }^{3}$, Y. Iwamoto ${ }^{3}$, A. Kashiwagi ${ }^{2}$, R. Kikkawa ${ }^{2}$;

${ }^{1}$ Laboratory for diabetic nephropathy, SNP Research Center, RIKEN, Yokohama, ${ }^{2}$ Department of Medicine, Shiga University of Medical Science, Otsu, ${ }^{3}$ Diabetes Center, Tokyo Women's Medical University, Japan.

Background and Aims: Protein kinase C epsilon (PKCE) is a member of the novel PKC subgroups, and has been shown to be increased in the kidney of diabetic animals. The aim of the present study is to know the possible involvement of PKC $\varepsilon$ in the pathogenesis of diabetic nephropathy.

Materials and Methods: The activity of PKC $\varepsilon$ was measured using a synthetic substrate specific for PKC $\varepsilon$ in cultured mouse mesangial cells. The expressions of mRNA for bone morphogenetic protein 4 (Bmp4) and NADPH oxidase 4 (Nox4) were measured by real-time quantitative PCR using SYBR green detection in the cells infected with adenovirus vectors encoding PKC $\varepsilon$ or LacZ. We also examined the DNA binding activity of AP-1 in PKCE- or LacZoverexpressing cells by electrophoresis mobility shift assay (EMSA).

Results: To evaluate the role of $\mathrm{PKC} \varepsilon$ in diabetic renal disease, we first examined the activity of PKC $\varepsilon$ in mouse mesangial cells cultured under high glucose conditions (HG: 25mM D-glucose) for five days, and compared with those in the cells under normal glucose (NG: 5.6mM D-glucose), or conditions where osmolarity had been adjusted with mannitol (osmotic control [OC]: 5.6mM $\mathrm{D}$-glucose $+19.4 \mathrm{mM}$ mannitol). We found that the activity of PKCe in cells exposed to high glucose condition was significantly higher than those in the cells under NG or OC (HG: $3293.3 \pm 383.3$, NG: $1463.0 \pm 68.8$, OC: 1520.3 $\pm 143.5, \mathrm{pmol} / \mathrm{min} / \mathrm{mg}$ protein, mean $\pm \mathrm{sd}, \mathrm{p}<0.05)$, whereas the mRNA and protein expression of PKC $\varepsilon$ were not different among those cells. We next examined the several gene expressions in the cells overexpressing PKC\&, and found that the expressions of Bmp4 and Nox4 mRNA were significantly decreased in the PKCE-overexpressing cells compared with those in the LacZ-overexpressing cells (Bmp4: PKC $0.58 \pm 0.13$, LacZ $0.96 \pm 0.07$, Nox4: $\mathrm{PKC} \varepsilon 0.70 \pm 0.04$, LacZ $1.13 \pm 0.13$, mean $\pm \mathrm{sd}, \mathrm{p}<0.0001)$. The expressions of fibronectin, type 4 collagen $\alpha 1$ were not significantly different between the PKC $\varepsilon$-overexpressing and control cells. In contrast, the treatment of the cells with the broad PKC inhibitor, GF109203X (1 $\mu \mathrm{M})$ resulted in a significant increase of Bmp4 mRNA, whereas the treatment of Go $6976(10 \mu \mathrm{M}), \mathrm{PKC}$ inhibitor specific for $\alpha$ or $\beta$ isoform, did not affect the Bmp4 expression (GF109203X: $1.71 \pm 0.13$, Go 6976: $1.01 \pm 0.07$, fold increase, mean $\pm \mathrm{sd}$, $\mathrm{p}<0.0001$ GF109203X vs. control or Go 6976). The DNA binding of AP-1 was remarkably increased in PKC $\varepsilon$-overexpressing cells. Furthermore, PKC $\varepsilon$ induced decrease of the Bmp4 and Nox4 expression, and increase of DNAbinding activity of AP-1 could be reversed by the treatment of MEK inhibitor, U0126.

Conclusion: These results suggest that the increased activity of PKC $\varepsilon$ under high glucose condition might decrease the expression of Bmp4 and Nox4 through MAPK pathway, and contribute to the development and progression of diabetic nephropathy.

\section{6}

Short telomers - an independent predictor of mortality in diabetic nephropathy

L. Tarnow ${ }^{1}$, M. Lajer ${ }^{1}$, R. Nzietcheung ${ }^{2}$, A. Benetos ${ }^{2}$, H.-H. Parving ${ }^{3,4}$;

${ }^{1}$ Steno Diabetes Center, Gentofte, Denmark, ${ }^{2}$ Faculte de Medicine, INSERM

U684, Nancy, France, ${ }^{3}$ Department of Endocrinology, Rigshospitalet,

Copenhagen, Denmark, ${ }^{4}$ Faculty of Health Science, University of Aarhus,

Denmark.

Background and Aims: Telomeres are the repeat DNA sequences located at the end of chromosomes. Telomeres shorten at each cell division, and once shortened to a critical length cells are trapped into replicative senescence more likely to undergo apoptosis. Furthermore, mean length of the terminal restriction fragments (TRF) have been proposed to play a role in essential hypertension and atherosclerosis. Diabetes and diabetic nephropathy in particular are characterised by an increased risk of cardiovascular morbidity and mortality. This study investigated the prognostic value of telomere length in a large cohort of type 1 diabetic patients with and without diabetic nephropathy.

Material and Methods: Type 1 diabetic patients were included: 157 with overt diabetic nephropathy ( 89 men, age (mean(SD)) $41 \pm 9$ years, duration of diabetes $28 \pm 8$ years, GFR $75 \pm 32 \mathrm{ml} / \mathrm{min} / 1.73 \mathrm{~m}^{2}$ ) and a matched control group of 127 patients with longstanding type 1 diabetes and persistent normoalbuminuria (76 men, age $42 \pm 10$ years, duration of diabetes $27 \pm$ 8 years). Patients were followed in a prospective observational follow-up study (follow-up time: $9.3(0.0-9.5)$ years). Endpoints were all cause and cardiovascular mortality. Telomere length was measured as TRF from DNA samples extracted from white blood cells

Results: Mean telomere length did not differ between type 1 diabetic patients with and without diabetic nephropathy: $7.2(0.8) \mathrm{kb}$ versus 7.2 $(0.8) \mathrm{kb}$, respectively $(\mathrm{p}=0.8)$. TRF was similar in men and women but was inversely correlated with age and systolic blood pressure, $\mathrm{p}<0.05$. TRF was not correlated with $\mathrm{HbA}_{1 c}$ albuminuria, GFR, or presence of cardiovascular disease at baseline. During follow-up 38 patients with and 6 patients without nephropathy died, $\mathrm{p}<0.001$. Among patients with nephropathy 19 (36\%) patients with TRF in the shortest tertile, $13(24 \%)$ patients with TRF in the middle tertile, and $6(12 \%)$ in the longest TRF tertile died from any cause (log rank test $\mathrm{p}=0.02$ ). The effect of TRF persisted after adjustment for sex, age, systolic blood pressure, smoking and GFR in a Cox proportional regression model. This lower mortality was attributable to fewer cardiovascular deaths. Conclusion: Short telomere length is an independent predictor of the excess overall and cardiovascular mortality in type 1 diabetic nephropathy. 


\section{OP 12 Prevalence of childhood diabetes and mortality in type 2 diabetes}

\section{7}

Epidemiology of cystic fibrosis-related diabetes; results from a British cohort of children and adults

A. Adler ${ }^{1}$, B. Shine ${ }^{2}$;

${ }^{1}$ Diabetes, Addenbrooke's Cambridge University Hospitals, Cambridge, ${ }^{2}$ Clinical Biochemistry, John Radcliffe Hospital, Oxford, United Kingdom.

Background and Aims: Individuals with cystic fibrosis have a high prevalence of diabetes, but few estimates of incidence exist. Likewise, few prospective studies have identified risk factors for cystic fibrosis-related diabetes (CFRD). This study sought to determine incidence and risk factors for CFRD from a cohort of individuals with CF in the United Kingdom.

Materials and Methods: 8,029 individuals aged 0 - 64 years were identified from the UK CF Database, a registry of patients with CF coordinated by the UK Cystic Fibrosis Trust with data from over 60 specialist centres. Patient consent was obtained. Of the patients, 5,196 had no diabetes at baseline (1996-2004) and had at least one follow-up annual visit. Diabetes was defined as either a physician diagnosis of diabetes, a blood glucose of $>11.1$ $\mathrm{mmol} / \mathrm{l}$ two hours following an oral glucose tolerance test, or treatment with insulin or oral hypoglycaemic drugs. Follow-up was as calculated time from baseline to the first detection of new diabetes or censoring (death $(n=202)$, loss to follow-up or alive at last visit). Risk factors included clinical and anthropometric characteristics measured at baseline, and were analysed using Cox proportional hazards modelling.

Results: The median age was 12 years and BMI was $17.9 \mathrm{~kg} / \mathrm{m} 2.54 \%$ were male, and $96 \%$ were white. 526 patients developed diabetes during a total of 15,009 person years (median 2.8 years) of follow-up. The annual incidence was $3.5 \%$. Patients who developed diabetes were more likely to have a low body mass index (BMI), pulmonary infections, liver and pulmonary function abnormalities, require antibiotic treatment, have had an organ transplant, and use pancreatic enzyme replacement. In multivariate analyses, female sex (hazard ratio (HR) 1.59 (95\% CI 1.32 - 1.90), pulmonary infection with Pseudomonas aeruginosa $(1.28,1.06-1.56), \%$ predicted forced expiratory volume in 1 second $(1.021,1.017-1.026$ per $1 \%$ decrement), and portal hypertension $(2.0,1.2-3.1)$ were independently associated with incident diabetes controlling for age, BMI and history of organ transplantation. Conclusion: This study confirms the very high incidence of CFRD, and shows that pulmonary and hepatic complications precede the onset of diabetes. Supported by UK CF Trust

\section{8}

Prevalence and incidence of neonatal diabetes in children and adults in the largest international series: increased interest, extended definition, continued misclassification?

A. S. Slingerland ${ }^{1,2}$, S. E. Flanagan ${ }^{1}$, B. Shields ${ }^{1}$, A.-M. Patch ${ }^{1}$,

G. J. Bruining ${ }^{3}$, K. Noordam 4 , O. Cinek ${ }^{5}$, M. Malecki ${ }^{6}$, A. Gach',

W. Mlynarski ${ }^{7}$, Z. Sumnik ${ }^{5}$, I. Dzivite ${ }^{8}$, S. E. Ellard ${ }^{1}$, A. T. Hattersley ${ }^{1}$;

${ }^{1}$ Diabetes, Institute of Biomedical and Clinical Sciences, Peninsula Medical

School, Exeter, United Kingdom, ${ }^{2}$ Diabetes, Department of Cardiology,

Leiden University Medical Center, The Netherlands, ${ }^{3}$ Department

of Pediatrics, Maxima Medical Center, Veldhoven, The Netherlands, ${ }^{4}$ Department of Pediatrics, Radboud University Medical Centre, Nijmegen, The Netherlands, ${ }^{5}$ Motol University Hospital, the second medical school, Charles University, Prague, Czech Republic, ${ }^{6}$ Department of Metabolic Diseases, Jagilelonian University, Medical College, Krakow, Poland, ${ }^{7}$ Department of Pediatrics, Medical University of Lodz, 1st Institute of Pediatrics, Poland, ${ }^{8}$ Center for Child and Adolescent Endocrinology, University Children’s Hospital, Riga, Latvia.

Background and Aims: Recently, interest in 'neonatal' diabetes increased because a genetic diagnosis may allow patients to come off insulin, improve glycaemic control and possibly neurological features. We aimed to determine the anticipated increase in prevalence and incidence of permanent 'neonatal' diabetes in pediatric and adult care with the new definition.
Materials and Methods: The analysis included 293 (53\% male) referrals from 27 countries to the Exeter Laboratory in the period to December 2006. Diabetes was diagnosed before 6 months of life irrespective of current age, had not remitted at time of study. Data on country of referral, age of diagnosis, date of birth and sex were obtained from standardized forms. All referrals were tested for KCNJ11-mutations.

Results: Observed minimum prevalence of the five most representative countries was 1.17 (1.01-1.31) per million population, with estimated true prevalence twice as high. Seventy-five percent were aged below 16 years with a median (IQ) of 5.7(2.4-10.2) years. Prevalence was higher for the pediatric versus the adult age range $(0.78(0.54-1.31)$ vs $0.42(0-0.50) ; \mathrm{p}=0.009)$. Age of diagnosis was skewed to a median (IQ) of $6(1-13)$ weeks with $62 \%$ in the first 8 weeks. During 2000-4, minimum observed incidence was $2.95(0-49.1)$ per million live births.

Conclusion: Observed minimum prevalence adjusted for definition was 2 to 25 times higher than previously reported and estimated true prevalence even higher. Results indicate that diabetes diagnosed before 6 months of life is still misclassified and suboptimally treated calling for awareness in pediatric and adult care.

Supported by Exeter team

\section{9}

High- medium- and low-molecular weight adiponectin in children: Findings from a population-based cohort

R. Nishimura ${ }^{1}$, A. Morimoto ${ }^{1}$, T. Matsudaira ${ }^{1}$, Y. Miyashita ${ }^{1}$, T. Shirasawa ${ }^{2}$, E. Takahashi ${ }^{2}$, N. Tajima ${ }^{1}$;

${ }^{1}$ Department of Internal Medicine, Jikei University School of Medicine, ${ }^{2}$ Department of Public Health, Showa University School of Medicine, Tokyo, Japan.

Background and Aims: Adiponectin, which has drawn significant attention in clinical research, is a hormone released from adipocytes. Adiponectin is mainly classified into the high-molecular weight (HMW), hexameric medium-molecular weight (MMW) and trimeric low-molecular weight (LMW) adiponectin. In 2006, a number of clinical findings have been reported on the HMW adiponectin isoform, which indicated HMW, rather than total adiponectin levels is better for the prediction of insulin resistance, metabolic syndrome and coronary artery disease. However, there are little reports, to date, which have examined these adiponectin values in a population-based cohort of children.

Materials and Methods: A total of 760 children (374 male and 386 female fourth-graders aged 9-10 years old), who gave informed consent as well as their parents, participated in this study conducted in Ina, Saitama, Japan. These study subjects represented a nearly perfect population-based cohort, accounting for 98.7 / 99.0 (male / female) \% of the fourth-graders in the area. Blood samples were drawn from the subjects at the time of their health check-ups to measure their adiponectin isoform values using a commercially available ELISA kit (Daiichi Pure Chemical Co. Ltd., Tokyo, Japan). The correlation between HMW, MMW, and LMW adiponectin and total adiponectin, and between HMW, MMW, and LMW adiponectin and body mass index (BMI) were assessed using Spearman's correlation coefficient. Statistical analyses were made using SPSS program.

Results: The median (intraquartile range) of HMW, MMW, LMW and total adiponectin $(\mu \mathrm{g} / \mathrm{ml})$, and BMI $(\mathrm{kg} / \mathrm{m} 2)$ were $2.5(1.7-3.5), 1.8(1.5-2.3), 1.5$ (1.2-1.9) and $5.9(4.7-7.6)$, and $16.8(15.6-18.8)$ for males and $2.7(1.7-3.8)$, $1.8(1.5-2.3), 1.6(1.2-2.0)$ and $6.4(4.8-7.8)$, and $16.4(15.3-17.9)$ for females, respectively. There were no statistical differences in these values by sex except for BMI $(\mathrm{P}=0.002)$. HMW, MMW, and LMW adiponectin values were all found to be positively correlated with the total adiponectin value (Spearman's correlation coefficient: $\mathrm{r}=0.931, \mathrm{P}<0.001 ; \mathrm{r}=0.753, \mathrm{P}<0.001$; and $\mathrm{r}=0.562$, $\mathrm{P}<0.001$ for males, and $\mathrm{r}=0.936, \mathrm{P}<0.001 ; \mathrm{r}=0.743, \mathrm{P}<0.001$; and $\mathrm{r}=0.560$, $\mathrm{P}<0.001$ for females, respectively). HMW and MMW adiponectin showed significant negative weak correlation with the $\mathrm{BMI}(\mathrm{r}=-0.173, \mathrm{P}<0.001$ and $\mathrm{r}=-0.144, \mathrm{P}=0.005$ for males and $\mathrm{r}=-0.241, \mathrm{P}<0.001$ and $\mathrm{r}=-0.155, \mathrm{P}=0.002$ for females, respectively); however, LMW adiponectin showed no significant relationship with BMI at all $(\mathrm{r}=-0.005, \mathrm{P}=0.919$ for males and $\mathrm{r}=-0.091$, $\mathrm{P}=0.076$ for females, respectively).

Conclusion: The present study indicated that the HMW adiponectin negatively significantly correlated with BMI, which indicated that HMW 
adiponectin may be the active form of adiponectin in prepubertal children.

Supported by the Ministry of Education, Culture, Sports, Science and Technology, Japan and Basic Research

\section{0}

The National Diabetes Register of Denmark: mortality and relative mortality of diabetes patients

B. Carstensen ${ }^{1}$, P. Ottosen ${ }^{2}$, J. K. Kristensen ${ }^{3}$, K. Borch-Johnsen ${ }^{1}$;

${ }^{1}$ Epidemiology, Steno Diabetes Center, Gentofte,

${ }^{2}$ Research service, National Board of Health, Copenhagen,

${ }^{3}$ Dept. of General Practise, University of Århus, Denmark.

Introduction and Material: In 2006 a national register of diabetes patients in Denmark was established by the National Board of Health. It is based on existing registers and all data are linked over time and across different data sources by the unique civil registry number allocated to all Danish citizens.

The Danish Diabetes Register is routinely linked with the Danish civil registration system and the register of causes of death in Denmark, thus providing accurate follow-up data for the entire population of patients with diabetes in Denmark.

Studies validating this method have shown that using this type of record linkage is feasible and will capture more than $90 \%$ of all patients with diabetes and only include very few persons not verifiable.

Methods: Follow-up of the diabetes patients from date of inclusion till date of death or the end of 2005 was used to calculate the mortality as depending on age and duration of diabetes. Mortality rates were compared with mortality rates in the non-diabetic part of the population by computing the standardized mortality ratio.

Results: Mortality rates in diabetes patients are higher than in the nondiabetic part of the population, but increase slower by age. The standardized mortality ratio (relative risk of death) is about 4 in ages 50 and about 1.8 in age 80 , very similar between males and females. Thus the effect of diabetes on overall mortality is the same in males and females. Because of the very different age-distribution in males and females and in particular in persons with diabetes, an overall standardized mortality ratio will falsely show a higher relative mortality for males than for females due to age-confounding. Conclusion: Diabetes patients have a higher mortality than the general population, but the relative effect of diabetes is strongly decreasing by age. There is not difference in the relative mortality between males and females.

\section{1}

Undiagnosed diabetes - becoming a thing of the past? Data from the English longitudinal Study of Ageing and the Health Survey for England M. B. Pierce ${ }^{1}$, P. Zaninotto ${ }^{1}$, N. Steel ${ }^{2}$, J. Mindell';

${ }^{1}$ Epidemiology and Public Health, University College London, ${ }^{2}$ Department of Primary Care, University College London, Norwich, United Kingdom.

Background and Aims: Research findings on the prevalence of undiagnosed diabetes and the ratio of undiagnosed to diagnosed diabetes in England, are contradictory. Some studies show that there are as many people with undiagnosed as diagnosed diabetes, others suggest there are much fewer people with undiagnosed disease. None of these studies are current and nationally representative. The aim of the current study is to examine the prevalence and distribution of undiagnosed and diagnosed diabetes, in the population of England aged over 50 years.

Materials and Methods: This was a cross-sectional study carried out 2003/5. Participants were nationally representative samples of 7,657 respondents from the English Longitudinal Study of Ageing (ELSA) and 5818 people from the Health Survey for England 2003 (HSE), aged 50 years and over. Fasting blood glucose was measured in non-diabetic participants; 2683 of the ELSA participants and 448 of the HSE participants. The main outcome measures were:

a. Self-reported doctor diagnosed diabetes

b. Fasting blood glucose of $\geq 7 \mathrm{mmol} / 1$ amongst those without self-reported doctor diagnosis of diabetes.

Results: In ELSA the prevalence of undiagnosed diabetes was only $1.7 \%$ (95\%CI:1.2 to 2.2) and of diagnosed diabetes was 7.5\% (95\%CI to 6.9;8.2); the ratio of undiagnosed to diagnosed was 1:5 (1:3 in men and 1:10 in women).
The overall prevalence of diabetes (undiagnosed plus diagnosed) was $11.6 \%$ (95\%CI:10.4 to12.4) in men and 6.8\% (95\%CI:6.1 to 7.5) in women. Both undiagnosed and diagnosed diabetes were more common in men than in women $(\mathrm{p}<0.001)$. Results from HSE were similar. In ELSA undiagnosed diabetes was commoner in the poorest than the richest women $(2.1 \%$ vs. $0 \%$, $\mathrm{p}=0.012$ ). In some groups (the richest quintile of women, and those aged 75-79 years) we found no undiagnosed diabetes.

Conclusions: The prevalence of undiagnosed diabetes and the ratio of undiagnosed to diagnosed diabetes were substantially lower than previous estimates. This is probably due to improved clinical methods supported by recent policy initiatives. Our results support the notion that there is no need for a national diabetes screening programme in England and that it is likely that the Vascular Risk Assessment and Control Programme will improve detection in the more difficult-to-reach groups.

Supported by the U.S National Institutes of Aging and a consortium of English Government Departments

\section{2}

Association between serum heat shock proteins and anti-heat shock proteins levels with micro/macrovascular complications in type 1 diabetes: the EURODIAB Prospective Complications Study

D. Burt ${ }^{1}$, G. Bruno ${ }^{1}$, N. Chaturvedi' ${ }^{2}$, C. D. Stehouwer ${ }^{3}$, S. Pinach ${ }^{1}$, D. Witte ${ }^{2}$, J. H. Fuller' ${ }^{2}$ P. Cavallo Perin ${ }^{1}$, G. Gruden ${ }^{1}$;

${ }^{1}$ Department of Internal Medicine, University of Turin, Italy,

${ }^{2}$ Department of Epidemiology and Public Health, Royal Free and University College London Medical School, United Kingdom, ${ }^{3}$ Department of Internal Medicine, Academic Hospital Maastricht, The Netherlands.

Background and Aims: Heat Shock Proteins (Hsp) are a highly evolutionary conserved family of polypeptides with cytoprotective activity. Hsp normally function intracellularly; however cellular stresses can induce their release into the circulation and elicit an autoimmune response. There is considerable evidence for a relationship between circulating Hsp and anti-Hsp levels and cardiovascular disease (CVD) in the general population. People with type 1 diabetes are particularly at high risk of micro/macrovascular complications; however, no study has been designed to establish whether Hsp/anti-Hsp levels are markers of micro/macrovascular diseases in these patients.

Materials and Methods: We performed a cross-sectional nested case-control study from the EURODIAB Prospective Complications Study. A total of 531 type 1 diabetic patients were studied. Cases $(n=363)$ were defined as those with one or more complications of diabetes and control subjects $(n=168)$ those with no evidence of any complication. Hsp27, Hsp70, Hsp60, antiHsp60, and anti-Hsp70 serum levels were measured by ELISA. Total serum immunoglobulin levels were determined by immunonephelometry. Micro/ macrovascular complications (retinopathy, nephropathy, neuropathy, CVD), conventional and inflammatory risk factors were assessed.

Results: Hsp27, anti-Hsp60, and anti-Hsp70 were measurable in all samples, whereas Hsp60 and Hsp70 were detectable in less than 5\% of the patients. Hsp27 levels were positively correlated with AER $(\mathrm{p}=0.03)$ and higher mean values were found in people with nephropathy $(\mathrm{p}=0.07)$, proliferative retinopathy $(\mathrm{p}=0.01)$, and distal symmetrical polyneuropathy $(\mathrm{p}=0.005)$. Anti-Hsp60 levels were positively associated with anti-Hsp70 and IL-6. Anti-Hsp70 levels were negatively correlated with systolic and diastolic blood pressure $(\mathrm{p}=0.004$, $\mathrm{p}=0.001$, respectively), BMI $(\mathrm{p}=0.03)$, AER $(\mathrm{p}=0.01)$, creatinine $(\mathrm{p}=0.03)$, and LDL-cholesterol $(\mathrm{p}=0.05)$. Furthermore, mean anti-Hsp70 levels were significantly higher in females ( $\mathrm{p}=0.01)$, in normotensives $(\mathrm{P}=0.002)$, in patients without microvascular complications (retinopathy $\mathrm{p}=0.006$; nephropathy $\mathrm{p}=0.039$; neuropathy $\mathrm{p}=0.006$ ), and in those treated with ACE-inhibitors $(\mathrm{p}=0.005)$. In logistic regression analysis, with respect to values of anti-HSP70 in the lower quartiles, higher values were associated with a $38 \%$ risk reduction of micro/macrovascular complications $(\mathrm{OR}=0.62,95 \% \mathrm{CI} 0.33-1.18)$ and with a $45 \%$ risk reduction of developing nephropathy ( $\mathrm{OR}=0.55,95 \% \mathrm{CI} 0.32-0.95)$, independently of duration of diabetes, HbAlc, systolic blood pressure, smoking and TNF $\alpha$. On the contrary, anti-Hsp60 and HSP27 levels in the upper quartile conferred an increased risk of both complications (anti-Hsp60:OR=1.8, 95\% CI 0.95-3.3; Hsp27:OR=1.55, 95\% CI 0.84-2.86) and nephropathy (anti-Hsp60: $\mathrm{OR}=1.08,95 \%$ CI 0.63-1.84; Hsp27:OR=1.42, 95\% CI 0.83-2.4).

Conclusion: In this large cohort of European type 1 diabetic subjects, we demonstrate an independent association between micro/macrovascular complications and both anti-Hsp70 and Hsp27 circulating levels.

Supported by Compagnia San Paolo 


\section{OP 13 Thiazolidinedions}

\section{3}

Impact of PPAR-gamma agonists on adipose-tissue insulin resistance in patients with IGT or T2DM and non-alcoholic steatohepatitis (NASH)

A. Gastaldelli ${ }^{1}$, B. Balas ${ }^{2}$, J. Hardies ${ }^{3}$, S. A. Harrison ${ }^{4}$, R. Belfort ${ }^{2}$, K. Brown ${ }^{5}$, S. Schenker 5 , R. DeFronzo ${ }^{2}$, K. Cusi'2;

${ }^{1}$ Metabolism Unit, CNR Institute of Clinical Physiology, Pisa, Italy,

${ }^{2}$ Div. Diabetes, UTHSCSA, San Antonio, United States, ${ }^{3}$ Res. Imaging Ctr, UTHSCSA, San Antonio, United States, ${ }^{4}$ Gastroenterology, Brooke Army Medical Center, San Antonio, United States, ${ }^{5}$ Gastroenterology, UTHSCSA, San Antonio, United States.

Background and Aims: Hepatocellular lipids (Hep-L) accumulation is frequently observed in insulin-resistant states including type 2 diabetes mellitus. We have recently reported that pioglitazone (PIO) treatment improved glucose metabolism, steatosis and necro-inflammation in IGT/ T2DM patients and biopsy-proven non-alcoholic steatohepatitis (NASH). Because abnormal lipid metabolism/elevated plasma FFA are believed to play an important role in the development of hepatic steatosis (i.e., fat overflow to the liver) we examined the role of adipose tissue IR and impact of PIO therapy in this cohort of NASH pts.

Materials and Methods: Patients received a hypocaloric diet $(-500 \mathrm{kcal} / \mathrm{d})$ and were randomized (double-blind) to PIO $(45 \mathrm{mg} / \mathrm{d}$ ) or placebo (Pbo) for 6 months. Before and after treatment pts underwent: 1 ) liver biopsy; 2 ) liver fat measurement by magnetic resonance (Hep-L); 3) double-tracer 75g OGTT to assess glucose tolerance, glucose production (EGP) and clearance, and to estimate indexes of IR at the level of adipose tissue (Adip-IR=fasting plasma FFA $\mathrm{x}$ insulin conc [FPI]) and liver (Hep-IR=EGP x FPI). 15 control subjects (CT) were also studied in the same way (except for liver biopsy).

Results: Compared to CT, NASH had increased FFA ( $739 \pm 34$ vs. CT $596 \pm 44$ umol/l; $\mathrm{p}<0.001$ ), impaired FFA suppression during OGTT (360 \pm 18 vs $240 \pm 19 \mathrm{umol} / \mathrm{l} ; \mathrm{p}<0.001)$, severe Adip-IR ( $12.1 \pm 1.3 \mathrm{vs.} .7 \pm 0.6 \mathrm{mmol} / \mathrm{l} \cdot \mathrm{mU} / \mathrm{l}$, $\mathrm{p}<0.0003)$ and low adiponectin $(7.0 \pm 1.6$ vs. $13.2 \pm 2.1 \mathrm{mg} / \mathrm{l}<<0.001)$. Hep-L was $\sim 3$-fold higher in NASH $(\mathrm{p}<0.001)$ and correlated positively with AdipIR $(\mathrm{r}=0.35, \mathrm{p}<0.05)$ and negatively with adiponectin $(\mathrm{r}=-0.31, \mathrm{p}<0.03)$. Hep-IR was associated with high fasting and OGTT FFA $(r=0.30, p=0.02)$. Both Hep-IR and Adip-IR were strongly associated with reduced glucose clearance both fasting $(r=0.39$ and $r=0.37)$ and during OGTT $(r=0.44$ and $\mathrm{r}=0.40$, all $\mathrm{p}<0.002$ ). PIO significantly improved Hep-IR, glucose tolerance and clearance, steatosis and necro-inflammation (all $\mathrm{p}<0.01-0.001$ vs. Pbo). Compared to Pbo, FFA decreased by $\sim 15 \%$ with PIO ( $<<0.02)$, Hep-L by $~ 50 \%$ $(\mathrm{p}<0.001)$ and Adip-IR by $40 \%(\mathrm{p}<0.05)$. Hep-L decrease was associated with improved Adip-IR and OGTT glucose clearance $(\mathrm{r}=0.43$ and $\mathrm{r}=-0.55$, both $\mathrm{p}<0.02)$. Adiponectin increased $\sim 3$-fold $(\mathrm{p}<0.001)$, correlating strongly with a decrease in L-MRS ( $r=-0.57, \mathrm{p}<0.001)$, Hep-IR and Adip-IR $(r=-0.37$ and $\mathrm{r}=-0.54, \mathrm{p}<0.01)$ and enhanced OGTT glucose clearance $(\mathrm{r}=0.44, \mathrm{p}<0.004)$. Conclusion: Patients with IGT/T2DM and NASH showed severe Adip-IR which was associated with elevated liver fat, low adiponectin and hepatic and peripheral insulin resistance. Enhanced adipose tissue function (i.e., improved insulin sensitivity/increased adiponectin) after PIO treatment is likely to play a key role in the metabolic (i.e., hepatic/peripheral insulin sensitivity) and histologic (i.e., reduction in liver fat and associated necroinflammation) improvements associated with treatment.

Supported by the National Center for Research Resources(to the Frederic C. Bartter, General Clinical Research Center and its Imaging Core), Takeda Pharmaceuticals, and the Veterans Affairs Medical Research Fund

\section{4}

Greater reductions in C-reactive protein with rosiglitazone than with glyburide or metformin despite greater weight gain

S. M. Haffner ${ }^{1}$, S. E. Kahn², B. Zinman ${ }^{3}$, R. R. Holman ${ }^{4}$, G. F. Viberti', W. H. Herman ${ }^{6}$, J. M. Lachin ${ }^{7}$, B. G. Kravitz ${ }^{8}$, M. A. Heise ${ }^{8}$;

${ }^{1}$ Medicine/Clinical Epidemiology, University of Texas Health Science Center at San Antonio, United States, ${ }^{2}$ Department of Medicine, VA Puget Sound Health Care System, Seattle, United States, ${ }^{3}$ Medicine, Mount Sinai Hospital, Toronto, Canada, ${ }^{4}$ Diabetes Trials Unit, OCDEM, Churchill Hospital, Oxford, United Kingdom, ${ }^{5}$ Department of Diabetes \& Endocrinology, King's College London School of Medicine, United Kingdom, ${ }^{6}$ Internal Medicine - Metabolism, Endocrinology and Diabetes (MEND), University of Michigan, Ann Arbor, United States, ${ }^{7}$ The Biostatistics Center, George Washington University, Rockville, United States, ${ }^{8}$ GlaxoSmithKline, King of Prussia, United States.

Background and Aims: In ADOPT (A Diabetes Outcome Progression Trial), rosiglitazone (RSG) decreased time to monotherapy failure compared with glyburide (GLY) and metformin (MET) in patients with recently diagnosed type 2 diabetes. We have previously reported that obesity at baseline was the main determinant of C-reactive protein (CRP) in these patients. Previous small, short-term studies have shown that thiazolidinediones (TZDs) can reduce inflammation, as measured by high-sensitivity CRP. However, these studies were not able to determine whether increased weight gain with TZDs might preclude their effects on CRP.

Materials and Methods: Patients from the North American Cohort of ADOPT with a median follow-up of 4.0 years $(n=783)$ were examined. At baseline, the median BMI was $32.8 \mathrm{~kg} / \mathrm{m}^{2}$ and was similar in each treatment group. The median baseline CRP values were $4.5 \mathrm{mg} / \mathrm{l}, 3.7 \mathrm{mg} / \mathrm{l}$ and $4.2 \mathrm{mg} / \mathrm{l}$ for RSG, GLY and MET, respectively.

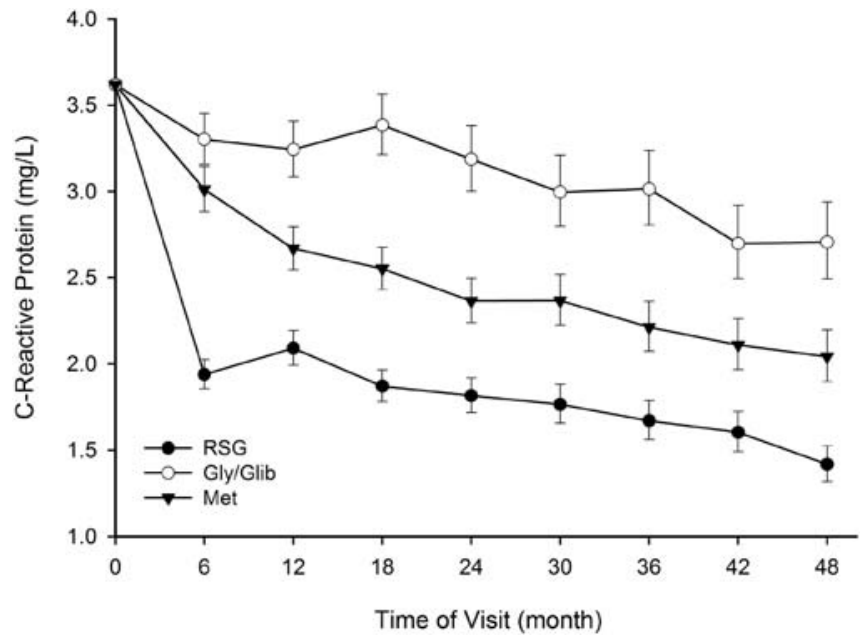

Results: While all treatments decreased CRP levels from baseline after 4 years, the reduction by RSG was $47.6 \%$ relative to GLY ( $95 \%$ CI $=57.8$, $35.0 ; \mathrm{p}<0.0001)$ and $30.5 \%$ relative to $\operatorname{MET}(95 \% \mathrm{CI}=43.3,14.9 ; \mathrm{p}=0.0004)$. However, RSG resulted in a $3.85 \mathrm{~kg}$ increase in weight compared with GLY $(95 \% \mathrm{CI}=2.39,5.31)$ and an $8.56 \mathrm{~kg}$ increase compared with MET $(95 \% \mathrm{CI}$ $=7.16,9.95)$ at 4 years.

Conclusion: Despite greater weight gain over the 4-year follow-up period, RSG more effectively reduced inflammation than did MET or GLY. Further study is required to clarify the mechanism involved.

Supported by GlaxoSmithKline 


\section{5}

Pioglitazone, oedema and congestive heart failure: a meta-analysis of randomised controlled trials

J. De Flines, A. J. Scheen;

Division of Diabetes, Nutrition \& Metabolic Disorders and Clinical

Pharmacology Unit, University of Liège, Belgium.

Background and Aims: Thiazolidinediones are associated with weight gain and peripheral oedema. In Europe, glitazones can not be prescribed in insulin-treated diabetic patients because of a suspected higher risk of congestive heart failure (CHF). The controversial results of the cardiovascular prevention trial PROactive with pioglitazone (PIO) raised further concern about the risk of CHF. The aims of the present meta-analysis were to evaluate the risk of oedema and $\mathrm{CHF}$ in randomised controlled trials (RCTs) with PIO and to compare the risk according to the type of therapy, i.e. PIO as monotherapy, added to another oral antidiabetic drug (OAD) or combined with insulin.

Materials and Methods: We performed an extensive search in the literature and selected all RCTs that randomised at least 50 patients and provided detailed figures on oedema incidence. We restricted our analysis to RCTs of at least 16-week duration with PIO therapy (15-45 mg/day) in order to have a chance to detect clinically meaningful differences in peripheral oedema, CHF and weight gain. The control group may receive either placebo or another OAD (except another glitazone). Odds ratio (OR) of oedema with PIO versus non-PIO were calculated for the pooled trials and analysed separately according to the associated therapy (diet, OAD or insulin). Data of the metaanalysis were also compared to those from the PROactive trial, which was analysed separately because of its specific design and population.

Results: Besides PROactive, we identified 25 RCTs (10 vs placebo, 14 vs another OAD, 1 vs insulin) that met the criteria used for selection : 12 with PIO added to diet alone, 10 with PIO added to one (8) or two (2) OADs, 2 with PIO combined with insulin, 1 with PIO added to any treatment, for a total of 5121 patients in the PIO cohort and 4567 patients in the non-PIO cohort. Overall, the OR of oedema was 2.64 (95\% CI 2.22, 3.15) in PIO compared to non-PIO groups $(\mathrm{p}<0.00001)$; $\mathrm{p}$ for heterogeneity $=0.08$. The respective OR were $2.37(1.85,3.02), 3.13(2.29,4.27), 2.73(1.77,4.23)$ for the three subanalyses of monotherapy, combined therapy, or insulin-treated groups (all $\mathrm{p}<0.00001$ ). Numbers of CHF cases were low, perhaps because of a careful patient selection, although underreporting could not be excluded. Nevertheless, overall $21 \mathrm{CHF}$ cases were specifically reported in the PIO group compared with only $5 \mathrm{CHF}$ cases in the non-PIO group: none in monotherapy, $17 / 2227$ patients in combined therapy and 4/521 patients with insulin + PIO. Weight gain averaged $2.35 \mathrm{~kg}$ in patients receiving PIO as compared to $-0.32 \mathrm{~kg}$ in patients not receiving PIO. In PROactive, despite a only slightly higher weight difference $(\mathrm{PIO}+3.6 \mathrm{~kg}$ vs placebo $-0.4 \mathrm{~kg}$ ), the incidence of CHF was much higher (281/2605 in the PIO group compared to $198 / 2633$ in the placebo group), possibly because of differences in the assessment of CHF but presumably also because of the specifically selected population at very high cardiovascular risk.

Conclusions: The present meta-analysis showed that PIO is associated with a 2.5 to 3 -fold increased risk of oedema, a risk apparently 33\% higher in combined therapy as compared to monotherapy. Astonishingly, the risk was not further aggravated in insulin-treated patients, but caution is recommended because of the limited number of trials in those patients. The rather low number of CHF cases reported in RCTs hinders any definite conclusion, although more CHF cases were noticed in the PIO group, confirming the data of the large PROactive trial.

\section{6}

Autonomic neuropathy predisposes to rosiglitazone-induced vascular leakage in insulin treated type 2 diabetes

C. J. J. Tack ${ }^{1}$, A. J. M. Rennings ${ }^{2}$, M. M. W. Stewart ${ }^{3}$, P. Smits ${ }^{4}$;

${ }^{1}$ Internal Medicine, Radboud University Nijmegen Medical Center, The Netherlands, ${ }^{2}$ Pharmacology-Toxicology, Internal Medicine, Radboud University Nijmegen Medical Center, The Netherlands, ${ }^{3}$ Glaxo Smith Kline, Harlow, United Kingdom, ${ }^{4}$ Pharmacology-Toxicology, Radboud University Nijmegen Medical Center, The Netherlands.

Background and Aims: Treatment with thiazolidinedione (TZD) derivatives is associated with fluid related complications, but the exact mechanism is unclear. One potential mechanism is that TZDs induce arterial vasodilatation resulting in both increased vascular leakage and blood pressure reduction, followed by reflex stimulation of the sympathetic nervous system and renal fluid retention. In subjects with autonomic neuropathy this counterbalance response is thought to be reduced. We investigated whether rosiglitazone (RSG) increases vascular leakage, especially in patients with autonomic neuropathy.

Materials and Methods: We conducted a randomized, double blind, placebo-controlled, parallel study in 40 patients with type 2 diabetes on insulin treatment. Both treatment groups, placebo and RSG, were stratified for sex and autonomic neuropathy assessed with the Ewing score $(<2.5$ or $\geq 2.5$ ). We investigated the effects of a 16-week treatment with RSG $4 \mathrm{mg}$ bid on vascular leakage (transcapillary escape rate of albumin, TERalb), body weight, extra cellular volume and total body water, plasma volume (PV), and hemodynamic changes.

Results: RSG improved glycemic control, decreased diastolic blood pressure $(-3.9 \mathrm{mmHg}, P=0.03)$, increased weight $(+2.0 \mathrm{~kg}, P=0.004)$, and increased $\mathrm{PV}(+241 \mathrm{ml}, P=0.02)$. RSG tended to increase vascular leakage ( $\triangle \mathrm{TER}$ alb $+1.08 \% / \mathrm{hr}, P=0.09$ ), but this effect was substantially higher in the subjects with Ewing scores $\geq 2.5(\Delta$ TERalb $+1.96 \% / \mathrm{hr}, P=0.03)$. In addition, the increase in vascular leakage during RSG treatment was highly correlated with the Ewing score at baseline $(\mathrm{r}=0.65, P=0.02)$. There was no correlation between Ewing score and the RSG-induced change in fluid parameters.

Conclusion: RSG increases vascular leakage especially in the co-existence of autonomic neuropathy in insulin treated patients with type 2 diabetes. Whether this in itself is sufficient to cause fluid retention is unclear, because autonomic neuropathy did not exaggerate the hemodynamic and fluid related effects of RSG. Nevertheless, autonomic neuropathy may represent a risk factor for edema formation in response to TZD therapy in insulin treated patients.

Supported by GlaxoSmithKline

\section{7}

Increased incidence of fractures in women who received rosiglitazone in ADOPT (A Diabetes Outcome Progression Trial)

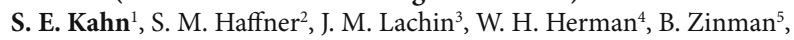
R. R. Holman ${ }^{6}$, M. A. Heise , R. P. Aftring ${ }^{7}$, G. F. Viberti ${ }^{8}$;

${ }^{1}$ Department of Medicine, VA Puget Sound Health Care System, Seattle, United States, ${ }^{2}$ Medicine/Clinical Epidemiology, University of Texas Health Science Center at San Antonio, United States, ${ }^{3}$ The Biostatistics Center, George Washington University, Rockville, United States, ${ }^{4}$ Endocrinology and Diabetes (MEND), University of Michigan, Ann Arbor, United States, ${ }^{5}$ Medicine, Mount Sinai Hospital, Toronto, Canada, ${ }^{6}$ Diabetes Trials Unit, OCDEM, Churchill Hospital, Oxford, United Kingdom, ${ }^{7}$ GlaxoSmithKline, King of Prussia, United States, ${ }^{8}$ Department of Diabetes \& Endocrinology, King's College London School of Medicine, United Kingdom.

Background and Aims: Fractures (Fx) occur more frequently in people with type 2 diabetes (T2DM) than in non-diabetics. More Fx adverse events (AE) were reported by subjects randomized to rosiglitazone (RSG) than either metformin (MET) or glyburide (GLY) in A Diabetes Outcome Progression Trial (ADOPT), a clinical trial of initial monotherapy in subjects with newly diagnosed T2DM (median follow-up $=4$ years). This increase in Fx AE occurred in women but not men.

Materials and Methods: AE in ADOPT were collected prospectively, but analysis of Fx was not prespecified. To explore the time-course of Fx AE in ADOPT and potential risk factors, we examined data from the 1840 women randomized.

Results: In ADOPT, at least one Fx was reported by 111 women (RSG $\mathrm{n}=60$, 9.3\%, 2.74/100 patient-years [PY]; GLY $\mathrm{n}=21,3.5 \%, 1.29 / 100$ PY; MET $\mathrm{n}=30,5.1 \%, 1.54 / 100 \mathrm{PY})$. The hazard ratio (95\% CI) for RSG vs GLY was $2.13(1.30,3.51)$ and for RSG vs MET was $1.81(1.17,2.80)$. The cumulative incidence for RSG, GLY and MET appeared similar for approximately 1 year post-randomisation with divergence of RSG in women but not men after that time. The majority of fractures observed in women who received RSG during ADOPT were in the upper arm (humerus, $n=5)$, hand $(n=8)$ or foot $(n=22)$. Overall, $76.6 \%$ of women were post-menopausal at baseline (BL). Among all women in ADOPT, those with Fx were older (58.8 \pm 9.6 years [mean $\pm S D$ ], range $37-76$ years vs $56.2 \pm 10.3$ years, $26-76$ years; $\mathrm{p}=0.01)$ and more were post-menopausal $(84.7 \%$ vs $76.1 \%, \mathrm{p}=0.04)$ than those without Fx. BL characteristics were compared within treatment groups (RSG: $n=645$, 
2187 PY; GLY: n=605, 1631 PY; MET: n=590, 1948 PY). Fx rates were higher in older women $(<65$ years vs $\geq 65$ years) taking RSG $(2.5 / 100$ PY vs $3.6 / 100$ PY) or GLY (1.1/100 PY vs 1.9/100 PY), but not MET (1.5/100 PY vs $1.6 / 100$ $\mathrm{PY}$ ). No other differences were apparent in BL characteristics (ethnicity, HbAlc, FPG, BP, weight, BMI, smoking and others) between women with Fx compared to those without Fx. No prior medical history or prior/concomitant medication use could be identified as a potential risk factor for Fx. The pattern of prior/concurrent $\mathrm{AE}$ of potential relevance to $\mathrm{Fx}$ was comparable among the treatment groups.

Conclusion: Further investigation into the risk factors for increased Fx in women is required to better understand the clinical implications of these findings.

Supported by GlaxoSmithKline

\section{8}

Rosiglitazone decreases serum bone-specific alkaline phosphatase activity in postmenopausal diabetic women

Z. Berberoglu ${ }^{1}$, A. Gursoy ${ }^{1}$, N. Bayraktar ${ }^{2}$, A. C. Yazici ${ }^{3}$, N. Tutuncu ${ }^{1}$, N. Demirag

${ }^{1}$ Endocrinology, Baskent University, ${ }^{2}$ Biochemistry, Baskent University, ${ }^{3}$ Biostatistics, Baskent University, Ankara, Turkey.

Background and Aims: The purpose of the present study was to evaluate the effect of rosiglitazone on bone metabolism and to assess the association between the changes in bone turnover parameters and plasma cytokine levels in postmenopausal diabetic women.

Material and Methods: This was an open-label, randomized, controlled trial of 12 -week duration. Fifty-six obese, postmenopausal women with newly diagnosed diabetes were studied. The nondiabetic control group comprised 26 healthy subjects matched for age and body mass index. All subjects were instructed to follow a weight-maintaining diet. Half of the diabetic subjects were randomly assigned to receive rosiglitazone ( $4 \mathrm{mg} / \mathrm{day})$, and the other half remained on diet alone. Before and after the intervention, metabolic bone markers and serum cytokine levels were assessed.

Results: Serum total alkaline phosphatase and bone-specific alkaline phosphatase activities (bsALP) were markedly lower 12 weeks after the initiation of rosiglitazone treatment $(82.7 \pm 21.9$ vs $69.2 \pm 16.8 \mathrm{IU} / \mathrm{L}$ for total ALP, respectively, $\mathrm{P}<0.001$ and $28.9 \pm 9.7$ vs $22.7 \pm 7.3 \mathrm{IU} / \mathrm{L}$ for bsALP, respectively, $\mathrm{P}<0.01$ ). On the other hand, the values of these parameters did not change in the other two groups. The differences in the changes in these parameters between the rosiglitazone and other two groups were significant ( $\mathrm{p}<0.05$ for both total ALP and bsALP, respectively). There were no significant changes in the osteocalcin levels among the three groups. No significant change was observed in the deoxypyridinoline levels in the rosiglitazone group. At the end of 12 weeks, patients in the three groups had significantly decreased interleukin-1 beta and tumor necrosis factor-alpha (TNF- $\alpha$ ) compared with baseline. The changes in bsALP levels showed a moderate negative correlation with the change in TNF- $\alpha$ after rosiglitazone and after diet in the diabetic control group $(r=-0.534, P=0.003$ and $r=$ $-0.376 ; P=0.049$, respectively).

Conclusion: These results suggest that rosiglitazone use is associated with reduced bone formation at earlier stages in postmenopausal diabetic women. However, the cytokine-lowering effects of rosiglitazone and lifestyle changes could reverse early inhibitory effect of rosiglitazone therapy on bone formation. Further studies will clarify the long-term effects of rosiglitazone therapy on bone loss and fracture.

\section{OP 14 Genome-wide association scans}

\section{9}

Genome-wide association data highlight an aetiological role for disturbances in cyclin-dependent kinase pathways in type 2 diabetes N. J. Timpson ${ }^{1,2}$, E. Zeggini ${ }^{1}$, M. N. Weedon ${ }^{3}$, C. M. Lingdren ${ }^{1}$,

T. M. Frayling ${ }^{3}$, K. S. Elliott ${ }^{1}$, H. Lango ${ }^{3}$, UK Type 2 Diabetes Genetics Consortium, Wellcome Trust Case Control Consortium, J. R. B. Perry $y^{3}$, N. W. Rayner ${ }^{1}$, R. M. Freathy ${ }^{3}$, J. C. Barret ${ }^{1}$, C. J. Groves ${ }^{1}$, A. D. Morris ${ }^{4}$, A. T. Hattersley ${ }^{3}$, M. I. McCarthy';

${ }^{1}$ Wellcome Trust Centre for Human Genetics \& OCDEM, Oxford University, ${ }^{2}$ Deparment of Social Medicine, Bristol University,

${ }^{3}$ Genetics of Complex Traits/Diabetes Genetics, Exeter University, ${ }^{4}$ Diabetes Research and Population Pharmacogenetics Gr,

Ninewells Hospital and Medical School, Dundee, United Kingdom.

Background and Aims: Large-scale genome-wide association (GWA) scans offer the potential to identify novel pathways involved in the aetiology of T2D.

Materials \& Methods: The Wellcome Trust Case Control Consortium recently completed a GWA scan (Affymetrix 500K chip) in 1924 UK T2D cases and 2938 controls. Association signals of interest were analysed further in up to 3757 additional T2D cases and 5346 controls from the UK. We also made collaborative use of GWA and replication data from two other studies conducted in European samples (the Diabetes Genetics Initiative and FUSION). The maximal combined data set available for analysis was 32,760 .

Results: Amongst several novel extensively-replicated association signals, two map close to or within genes implicated in the regulation of CDK (cyclindependent kinase) activity. A cluster of SNPs within the CDKAL1 gene on chr6 displaying T2D-associations in the WTCCC GWA scan (OR 1.20[1.10, $1.31], \mathrm{p}=2.5 \times 10-5)$, showed strong replication in the UK sets $(\mathrm{p}=8.4 \times 10-5$ : all UK data $\mathrm{p}=1.3 \times 10-8)$ and further support in the DGI and FUSION studies (combined evidence: OR 1.13 (1.09-1.17), $\mathrm{p}=1.4 \times 10-12)$. The function of the CDKAL1 product is unknown, but it is homologous to CDK5RAP1, a known inhibitor of CDK5, which a kinase previously-implicated in islet function. On chromosome 9, a second cluster of T2D-associated SNPs from the GWA scan (OR 1.22, 1.09-1.37; $\mathrm{p}=7.6 \times 10-4)$ was also confirmed in UK replication samples $(\mathrm{p}=1.7 \times 10-4$; combined $\mathrm{p}=4.9 \times 10-7)$ and generated a compelling signal across all studies (OR $1.20(1.15-1.25) \mathrm{p}=2.2 \times 10-15)$. Haplotype and conditional analyses within the region of association indicate that this signal is driven by an, as yet, untyped (non-HapMap) variant. The chr9 locus lies in sequence devoid of annotated genes, but is $\sim 150 \mathrm{~kb}$ from the genes encoding the CDK inhibitors, CDKN2A and CDKN2B. For both, overexpression of the murine homologue has been conclusively shown to disturb islet development and/or regeneration and to lead to diabetes. In humans, loss of function due to mutations in CDKN2A is a powerful contributor to the development of neoplasia.

Conclusion: Whilst further studies are required to establish a functional connection between the confirmed T2D-susceptibility variants identified and the genes described, our data provide the first indications that variation in CDK activity is a key pathogenetic mechanism in the development of T2D. This is most likely mediated through effects on islet development, regeneration and function. Since some of the same genes are also implicated in cancer, our data highlight potential shared aetiological pathways influencing both metabolic and neoplastic disease.

Supported by Wellcome Trust, European Commission, Diabetes UK, MRC 


\section{0}

Large-scale replication typing of modest signals from genome-wide association studies identifies additional type 2 diabetes susceptibility variants in the IGF2BP2 and VEGFA genes

E. Zeggini ${ }^{1}$, M. N. Weedon ${ }^{2}$, C. M. Lindgren ${ }^{1}$, T. M. Frayling ${ }^{2}$, K. S. Elliott ${ }^{1}$, H. Lango ${ }^{2}$, N. J. Timpson ${ }^{3}$, UK Type 2 Diabetes Genetics Consortium, Wellcome Trust Case Control Consortium, J. R. B. Perry ${ }^{2}$, N. W. Rayner ${ }^{1}$, R. M. Freathy ${ }^{2}$, J. C. Barrett ${ }^{1}$, C. J. Groves ${ }^{1}$, A. D. Morris ${ }^{4}$, A. T. Hattersley ${ }^{2}$, M. I. McCarthy ${ }^{1}$;

${ }^{1}$ WTCHG, University of Oxford, ${ }^{2}$ Genetics of Complex Traits/Diabetes Genetics, Peninsula Medical School, Exeter, ${ }^{3}$ Dept of Social Medicine, University of Bristol, ${ }^{4}$ Diabetes Research and Population Pharmacogenetics Groups, Ninewells Hospital and Medical School, Dundee, United Kingdom.

Background and Aims: Initial efforts to identify susceptibility variants from genome-wide association (GWA) scans have concentrated on variants which achieve highly significant results in initial analyses. This approach, as executed by the Wellcome Trust Case Control Consortium (WTCCC) and other groups, has already led to identification of several novel susceptibility genes for type 2 diabetes (T2D) including FTO, CDKAL1 and HHEX. However these variants, even combined, fail to account for the observed familiality of T2D, indicating that there are many more T2D susceptibility genes as yet unidentified, most likely to have small or modest effect size. We have combined GWA and large-scale replication data to detect additional T2D susceptibility variants.

Materials and Methods: Starting from GWA data (393,453 SNPs) for 1924 T2D cases and 2938 population controls, we sought to confirm signals of interest in up to 3757 additional cases and 5346 controls from the UK. We also accessed additional GWA and replication data through collaboration with other international consortia (DGI, FUSION). The maximal combined dataset included 32,760 samples.

Results: Proof of principle for the identification of T2D-susceptibility variants of lesser effect was provided by well-established variants in PPARG (P12A) and KCNJ11 (E23K, tagged by a close proxy $\left[\mathrm{r}^{2}=0.9\right]$ ). For both, GWA significance values ( $1 \mathrm{df}$ trend test) were modest (both $\mathrm{p}=0.0013$ ) and effects sizes consistent with previous reports. We used biological candidacy and statistical evidence across multiple GWA studies to prioritise additional variants for replication. SNPs within the IGF2BP2 gene (coding for a known regulator of insulin-like growth factor-2 translation), showed modest evidence of association in the WTCCC scan (maximal OR 1.15 [1.05-1.25], $\mathrm{p}=1.7 \times 10^{-3}$ ) with modest replication in the UK samples (all UK data, OR $\left.1.11[1.05-1.16], \mathrm{p}=1.6 \times 10^{-4}\right)$. However, evidence across all three studies (and replications) is overwhelming $\left(\mathrm{p} \sim 8.6 \times 10^{-16}\right)$. The signal maps to a $57 \mathrm{~kb}$ region spanning the promoter and first 2 exons of IGF2BP2. Similar analyses identified a further putative T2D-signal located $57 \mathrm{~kb}$ downstream of the VEGFA gene, encoding vascular endothelial growth factor A (maximal OR in UK data, $\left.1.11[1.05-1.17], \mathrm{p}=2.0 \times 10^{-4}\right)$, which was also detected in the DGI scan $\left(\mathrm{p}=4.4 \times 10^{-3}\right)$. Finally, we sought replication of the SLC30A8 association recently reported in a French GWA scan. The relevant SNP was poorlycaptured on the Affymetrix chip used by the WTCCC, but we obtained independent evidence rs13266634 in our UK datasets (OR 1.12 [1.04-1.19], $\left.\mathrm{p}=1.2 \times 10^{-3}\right)$ and when combining all three studies $\left(\mathrm{p} \sim 3.5 \times 10^{-7}\right)$.

Conclusion: By combining multiple GWA scans, and using large-scale replication samples, we have been able to extend the number of multiplyreplicated genes (or regions) with a confirmed role in T2D-susceptibility. Supported by Wellcome Trust, European Commission, Diabetes UK, MRC

\section{1}

The DGI genome-wide association study identifies three novel loci associated with type 2 diabetes

V. Lyssenko' ${ }^{1}$, R. Saxena ${ }^{2}$, P. de Bakker ${ }^{2}$, N. Burtt' ${ }^{2}$, B. Voight ${ }^{2}$, M. Orho-Melander ${ }^{1}$, J. Hirschhorn ${ }^{2}$, T. Tuomi ${ }^{3}$, B. Isomaa ${ }^{4}$, T. Hughes ${ }^{5}$, D. Altshuler ${ }^{2}$, L. Groop ${ }^{1}$;

${ }^{1}$ Department of Clinical Sciences- Diabetes \& Endocrinology, Lund University,University Hospital Malmö, Sweden, ${ }^{2}$ Broad Institute of Harvard and MIT, Genetic Analysis Platform, and Metabolism Initiative, Cambridge, Massachusetts, United States, ${ }^{3}$ Department of Medicine, Helsinki University Hospital, Finland, ${ }^{4}$ Folkhälsan Research Center, Folkhälsan Research Center, Helsinki, Finland, ${ }^{5}$ Novartis Institutes for BioMedical Research, Cambridge, Massachusetts, United States.

Background and Aims: Type 2 diabetes (T2D) is rapidly increasing worldwide thereby posing a severe burden on individual and public health. Although this increase can be ascribed to both genetic and environmental factors, the genetic factors have been largely restricted to a few candidate genes based upon our limited knowledge of the pathogenesis of the disease. In an attempt to identify underlying gene loci, the Diabetes Genetic Initiative (DGI) has recently completed a whole genome association study for T2D and 18-related traits (http://www.broad.mit.edu/diabetes). To replicate best signals associated with T2D in DGI and diabetes-related traits in conjunction with the results from companion whole genome scans (the FUSION study, the WTCCC, and UKT2D) as well as recently reported risk loci for T2D (Sladek et al.).

Material and Methods: DGI comprised of 1,464 Scandinavian participants with T2D and 1,467 matched control subjects who were genotyped for 500,568 SNPs of which 389,731 SNPs passed stringent quality control criteria (MAF $<1 \%,>95 \%$ call rate, HWE $p>10^{-6}$ ) using Affymetrix 500K Array. Three case-control samples of European ancestry (comprising a total of 10,850 individuals) were used to replicate original findings: (1) 2830 T2D patients from the Malmo Diabetes Registry and 3550 controls from the Malmo Diet and Cancer study in Southern Sweden (MDC-DR), (2) 1226 T2D patients of European ancestry from the United States matched to 1226 control individuals and 1009 T2D patients and 1009 control individuals from Poland (GCI US).

Results: In the initial replication in 10,850 individuals including 107 best associated signals we identified and confirmed three novel risk loci for T2D: a locus on chromosome 9 near the cyclin-dependent kinase inhibitor $2 \mathrm{~A}$ and 2B (CDKN2A/CDKN2B) (DGI 1.37, 95\%CI (1.18-1.59), $\mathrm{p}=3.6 \times 10^{-5}$; replication MDC-DR/GCI $1.16(1.09-1.27), \mathrm{p}=2.2 \times 10^{-5}$; and combined OR $\left.1.20(1.12-1.28), \mathrm{p}=5.4 \times 10^{-8}\right)$; the insulin-like growth factor 2 binding protein 2 (IGF2BP2), locus on chromosome 3 (DGI 1.12 (1.00-1.26); $\mathrm{p}=0.052$, MDCDR/GCI 1.19, (1.12-1.26), $\mathrm{p}=2.1 \times 10^{-9}$; and combined OR 1.17 (1.11-1.23), $\left.\mathrm{p}=1.3 \times 10^{-9}\right)$; and the cyclin-dependent kinase $5(\mathrm{CDK} 5)$ regulatory subunit associated protein 1-like 1( CDKAL1) on chromosome 6 (DGI 1.17 (1.041.31), $\mathrm{p}=7.5 \times 10^{-3}$; MDC-DR/GCI $1.07(1.01-1.13), \mathrm{p}=0.011$; and combined OR $\left.1.10(1.04-1.15), \mathrm{p}=4.1 \times 10^{-4}\right)$. We also replicated recently published by Sladek et al. risk locus for T2D, HHEX (DGI, OR 1.15 (1.04-1.29), $\mathrm{p}=0.01$; replication GCI $1.13(1.04-1.23), \mathrm{p}=6.0 \times 10^{-3}$; and combined OR $1.14(1.06-$ 1.22), $\left.\mathrm{p}=1.7 \times 10^{-4}\right)$.

Conclusions: Whole genome association studies can identify new loci increasing risk for T2D thereby providing novel clues into the pathogenesis of T2D. In our first replication effort, the CDKN2A/CDKN2B locus on chromosome 9, IGF2BP2, and CDKAL1 emerged as novel T2D loci. Supported by Novartis foundation 


\section{2}

A genome-wide association study identifies glucokinase regulatory protein (GCKR) locus as a novel regulator of blood triglyceride level M. Orho-Melander ${ }^{1}$, O. Melander ${ }^{1}$, V. Lyssenko ${ }^{1}$, R. Saxena ${ }^{2}$, P. de Bakker ${ }^{2}$, N. Burtt ${ }^{2}$, B. Voight ${ }^{2}$, J. N. Hirschorn ${ }^{2}$, T. Tuomi ${ }^{3,4}$, B. Isomaa ${ }^{4}$,

M.-R. Taskinen ${ }^{3}$, T. E. Hughes ${ }^{5}$, D. Altshuler ${ }^{6,2}$, L. Groop ${ }^{1,3} \&$ S. Kathiresan ${ }^{7,2}$ for the Diabetes Genetics Initiative (DGI),

${ }^{1}$ Clinical Sciences in Malmö, Lund University, Sweden, ${ }^{2}$ Genetic Analysis Platform, and Metabolism Initiative, Broad Institute of Harvard and MIT, Cambridge, United States, ${ }^{3}$ Department of Medicine, Helsinki University Hospital, Finland, ${ }^{4}$ Biomedicum, Folkhälsan Research Center, Helsinki, Finland, ${ }^{5}$ Novartis Institutes for BioMedical Research, Cambridge, United States, ${ }^{6}$ Center for Human Genetic Research and Department of Molecular Biology, Massachusetts General Hospital, Boston, United States, ${ }^{7}$ Cardiology Division, Massachusetts General Hospital, Boston, United States.

Background and Aims: The Diabetes Genetics Initiative (DGI) recently completed a genome-wide association study for type 2 diabetes (T2D) and 18 other traits including blood lipids. One polymorphism (rs780094) in the glucokinase regulatory protein gene $(G C K R)$ achieved genome-wide significance for triglyceride (TG) levels $\left(\mathrm{P}=3.7 \times 10^{-8}\right)$. In the liver, GCKR regulates the activity of glucokinase (GCK) and overexpression of GCK in rodents results in lower blood glucose and hypertriglyceridemia. We therefore sought to replicate association between rs780094 and TG levels in three independent Swedish cohorts. Given the known role of GCKR in glucose metabolism we also examined association of rs780094 to T2D and measures of glucose homeostasis.

Materials and Methods: The DGI study material of 2,931 participants from Finland and Sweden (m/f 1,448/1,482, age $62 \pm 11$ y, BMI $28 \pm 4 \mathrm{~kg} / \mathrm{m}^{2}$, TG $1.6 \pm 1.1 \mathrm{mmol} / \mathrm{l}, 50 \%$ with T2D) was genotyped using the Affymetrix $500 \mathrm{~K}$ SNP Array. In order to replicate the DGI finding, rs780094 was genotyped in 1) 5,190 individuals composing the Malmo Diet and Cancer Study Cardiovascular Arm (MDC-CVA: m/f 2,140/3,050, age 58 \pm 6 y, BMI $26 \pm 4 \mathrm{~kg} /$ $\mathrm{m}^{2}$, TG $1.3 \pm 0.7 \mathrm{mmol} / \mathrm{l}, 8 \%$ with T2D), 2) 5,152 patients with hypertension (NORDIL: $\mathrm{m} / \mathrm{f} 2,567 / 2,585$, age $60 \pm 7$ y, BMI $28 \pm 4 \mathrm{~kg} / \mathrm{m}^{2}$, TG $1.8 \pm 1.2 \mathrm{mmol} / \mathrm{l}$, $9 \%$ with T2D) and 3) 2,777 T2D patients from the Skania Diabetes Registry (DR: $\mathrm{m} / \mathrm{f} 1,636 / 1,141$, age $61 \pm 11 \mathrm{y}, \mathrm{BMI} 30 \pm 6 \mathrm{~kg} / \mathrm{m}^{2}$, TG $2.6 \pm 3.1 \mathrm{mmol} / \mathrm{l}$ ). SNP-phenotype association analyses were performed by multivariate linear regression using an additive genetic model and adjusting for age, sex and diabetes status.

Results: The initial association of rs780094 in GCKR was strongly replicated in all cohorts $\left(\mathrm{P}=8.7 \times 10^{-8}, 3.5 \times 10^{-10}, 1.2 \times 10^{-6}\right.$ in MDC-CVA, Nordil and $\mathrm{DR}$, respectively). The minor $\mathrm{T}$ allele (frequency $33.5-35.3 \%$ ) was associated with higher TG and the SNP explained 1.1, $0.4,0.7$ and $0.9 \%$ of the trait variance in DGI, MDC-CVA, NORDIL and DR, respectively. TG according to genotypes (CC, CT and TT) were: DGI $1.5 \pm 1.0,1.6 \pm 1.2$ and $1.8 \pm 1.1 \mathrm{mmol} /$ 1 ; MDC-CVA $1.3 \pm 0.7,1.4 \pm 0.7$ and $1.4 \pm 0.7 \mathrm{mmol} / 1$; NORDIL $1.7 \pm 1.0,1.8 \pm 1.5$, $2.0 \pm 1.0$, DR $2.4 \pm 3.4,2.7 \pm 3.0$ and $2.9 \pm 2.3 \mathrm{mmol} / \mathrm{l}$. In MDC-CVA the T allele was nominally associated with lower fasting blood glucose $(\mathrm{P}=0.02)$, lower HOMA-IR $(\mathrm{P}=0.01)$, and reduced risk for T2D $(\mathrm{P}=0.03)$. Accordingly, $\mathrm{T}$ allele carriers had lower fasting blood glucose $(\mathrm{p}=0.03)$ and a trend to lower risk for T2D in the NORDIL sample $(\mathrm{P}=0.06)$.

Conclusion: We have identified GCKR as a novel locus with a strong influence on plasma TG levels. Consistent with experimental data in rodents, GCKR variation was associated with opposite effects on TG and blood glucose levels.
0083

Type 2 diabetes whole genome association study in four populations: the DiaGen consortium

J. T. Salonen ${ }^{1}$, P. Uimari ${ }^{1}$, J.-M. Aalto ${ }^{1}$, M. Pirskanen ${ }^{1}$, B. Todorova ${ }^{1}$, T.-P. Tuomainen ${ }^{2}$, J. Luedemann ${ }^{3}$, M. Gibson ${ }^{4}$, N. Pendleton ${ }^{5}$, W. Mahoney ${ }^{6}$, D. Meyre ${ }^{7}$, J. Delplanque ${ }^{7}$, P. Froguel ${ }^{7,8}$, B. Yakir ${ }^{9}$, A. Darvasi ${ }^{10}$;

${ }^{1}$ Genetic Discovery Research, Oy Jurilab Ltd, Kuopio, Finland,

${ }^{2}$ School of Public Health and Clinical Nutrition, University of Kuopio, Finland, ${ }^{3}$ Institute of Clinical Chemistry and Laboratory Medicine, Ernst Moritz University of Greifswald, Germany, ${ }^{4}$ Vascular Research Group, University of Manchester, United Kingdom, ${ }^{5}$ Division of Medicine and Neurosciences-Hope, University of Manchester, Manchester, United Kingdom, ${ }^{6}$ Nanogen Inc, Seattle, United States, ${ }^{7}$ Institut Pasteur de Lille, France, ${ }^{8} \mathrm{Genomic}$ Medicine, Imperial College of London, United Kingdom, ${ }^{9}$ Genetic Discovery Research, Hebrew University of Jerusalem, Israel, ${ }^{10}$ Institute of Life Sciences, Hebrew University of Jerusalem, Israel.

Type 2 diabetes (T2D) is a common, polygenic chronic disease with high heritability. Population prevalence is $5-10 \%$ and consistently increasing. The purpose of this whole-genome association (WGA) study was to discover novel T2D-associated genes. The subjects were 500 T2D cases and 497 controls from two homogenous founder (Eastern Finns, Ashkenazi Jews) and two heterogeneous (English, Germans) populations. All cases had at least one affected family member. Each subject was genotyped with 315,917 single nucleotide polymorphism markers (SNPs) using the Illumina HapMap derived panel of tagging SNPs. The call rate was $99.5 \%$, reproducibility over 99.9\% and concordance with the reference methodology, the Centaurus, over 99.9\%. Following a permutation-based statistical correction for multiple testing, the only significant $\left(\mathrm{p}<2.3 \times 10^{-7}\right)$ gene identified in the WGA study was TCF7L2, which has already been discovered and confirmed as a T2D susceptibility gene. Subsequently, we selected ten SNPs in the most promising six chromosomal regions with nominal statistical significance (on their own or as part of a haplotype) for further replication study in an independent case-control set including 2573 T2D cases and 2776 controls from France. Two SNPs (rs1535435, rs9494266) in the same chromosomal region (6q23.3) were found significantly $(\mathrm{p}=0.00005, \mathrm{p}=0.0002)$ associated with T2D. The $\mathrm{p}-$ values for these SNPs from the pooled Mantel-Haenszel analysis of both the GWS and replication data were 0.000001 and $2.28 \times 10^{-7}$, respectively). These two SNPs were within a linkage disequilibrium block of $178 \mathrm{~kb}$ encompassing 19 introns of the AHI1 gene. We propose AHI1 as a novel T2D susceptibility gene. For the first time a WGA study is replicated in an independent population, providing strong evidence for the robustness of the WGA study approach.

Supported by TEKES, Finland

\section{4}

Genome wide association scan for type 1 diabetes susceptibility genes in a Danish population

C. A. Brorsson ${ }^{1}$, E. Swiergala ${ }^{2}$, R. Bergholdt ${ }^{1}$, K. Rapacki ${ }^{3}$, S. Purcell ${ }^{4}$, L. Field ${ }^{2}$, F. Pociot ${ }^{1}$;

${ }^{1}$ Steno Diabetes Center, Gentofte, Denmark, ${ }^{2}$ Department of Medical Genetics, University of British Columbia, Vancouver, Canada,

${ }^{3}$ Center for Biological Sequence Analysis, Technical University, Lyngby, Denmark, ${ }^{4}$ Center for Human Genetic Research, Massachusetts General Hospital, Boston, United States.

Background and Aims: Type 1 diabetes (T1D) is the result from an immunemediated destruction of the insulin producing pancreatic $\beta$-cells. The disease is believed to be influenced by interactions between multiple risk-conferring genes and environmental factors. In order to further investigate the genetic contribution to T1D we genotyped a Danish case-control material for 58000 SNP and tested these for association to disease.

Materials and Methods: We used Affymetrix GeneChip Human Mapping Array Xba to genotype each sample on a single array. The resulting genotype calls were analysed using the software PLINK. The first phase of the study comprised of thorough quality tests of the data for issues such as sample contamination, inbreeding, low genotyping rates, relatedness and population stratification which led to samples being excluded. SNPs were removed for failing HWE in controls, low genotyping rate, for being non-polymorphic and differences in genotyping success rates between cases and controls which 
contributed to significant false positive findings. After ensuring the highest quality of the data-set the remaining 52000 SNPs and 605 individuals (331 cases and 274 controls) were analysed using basic allelic and model-based tests of association corrected for multiple testing and using permutation with empirically corrected p-values. Furthermore we performed genome-wide sliding-window haplotype tests and a full pair-wise test for epistasis across the genome. A stratified analysis of cases from simplex vs. multiplex families was performed to identify possible differences between these groups. We also performed the same analyses for markers in 12 linkage regions previously reported in T1D.

Results: Every individual test confirmed the importance of the HLA-region on chromosome 6p21.3 for developing T1D. The combination of all tests identified markers in other chromosomal regions which could contribute to T1D development. HLA-region markers were also highly significant in both groups of cases after stratification. However, outside the HLA-region different markers on different chromosomes in the two groups showed the lowest pvalues, indicating differences in the genetic contribution between simplex and multiplex cases.

Conclusion: The HLA region is of major importance for the susceptibility to T1D. In combination these tests point to other chromosomal regions outside MHC contributing to T1D susceptibility in the Danish population. The most significant findings will be followed up in a larger case-control material consisting of 2000 cases and 2000 controls of Danish origin to confirm these present results. In addition this study has demonstrated the importance of high quality data for avoiding false positive findings when performing association analyses in complex diseases.

Supported by EFSD/JDRF/NovoNordisk

\section{OP 15 Signal transduction and metabolism in liver}

\section{5}

Nuclear FoxO1 controls and integrates key signaling pathways in hepatocytes

M. Naïmi ${ }^{1}$, N. Gautier ${ }^{1}$, C. Chaussade ${ }^{1}$, A. M. Valverde ${ }^{2}$, D. Accili ${ }^{3}$, E. Van Obberghen ${ }^{1}$;

${ }^{1}$ Inserm u145, IFR50, Nice, France, ${ }^{2}$ Consejo Superior de investigationes Cientificas C/Arturo Duperier 4, Instituto de Investigationes Biomedicas Alberto Sols, Madrid, Spain, ${ }^{3}$ Columbia University, College of Physicians and Surgeons, New York, United States.

Background and Aims: Insulin inhibits Forkhead O class (FoxO) transcription factors, which down-regulate the expression of genes involved in metabolism, cell cycle arrest, and apoptosis. After being phosphorylated by protein kinase $\mathrm{B}$ (PKB) on S253 in its DNA binding domain, FoxO1 is phosphorylated on T24 and additional sites. Together, these modifications trigger FoxO1 nuclear exclusion. During this process, FoxO1 is thought to retain some transcriptional activity and signaling potential.

Materials and Methods: To evaluate this FoxO1 action, both primary cultures of rat hepatocytes and mice hepatocytes lacking IRS-2 were infected with adenoviral constructs encoding wild-type FoxO1, a transactivation domain-deleted FoxO1 or a FoxO1-ADA mutant, this latter mutant being constitutively nuclear but displaying reduced DNA-binding potential due to a mutation of S253 to D.

Results: Adenoviral-mediated expression of FoxO1-ADA in hepatocytes activates $\mathrm{PKB}$ and MAPK pathways, more than wild-type or dominant negative FoxO1. PKB activation cannot be accounted for by a FoxO1mediated increase in upstream signaling components such as insulin receptor substrate 1 or 2 or by FoxO1-mediated down-regulation of Tribbles homolog 3. In contrast, $\mathrm{p} 38$ is required for mTORC2 activation and effects of FoxO1 on $\mathrm{PKB}$, at least in part.

Conclusion: FoxO1 phosphorylated on S253 turns on a feed-forward loop, relayed by p38 and acting to amplify both PKB activation and FoxO1 inhibition through subsequent phosphorylation events. To conclude, FoxO1 controls its own nuclear exclusion by a complex mechanism which involves the modulation of its DNA binding by insulin, p38 and mTORC2 activation, and PKB phosphorylation on S473.

\section{6}

Molecular mechanisms that coordinate regulation of liver metabolism D. A. Okar' 1 S. Baltrusch 2 , K. Zangger

${ }^{1}$ VA Medical Center, Department of Veterans Affairs, Minneapolis, United States, ${ }^{2}$ Institute of Clinical Biochemistry, Hannover Medical School, Germany, ${ }^{3}$ Institute of Chemistry/Organic and Bioorganic Chemistry, University of Graz, Austria.

Background and Aims: The liver has a major role in maintaining glucose homeostasis, especially in response to nutritional excursions such as meal consumption and fasting. We aim to reveal the molecular mechanisms by which hepatic fuel metabolism is regulated because these offer cellular targets for the development of novel diabetes and obesity treatments. The major research focus has been the bifunctional enzyme, 6-phosphofructo-2-kinase/ Fructose-2,6-bisphosphatase (PFK-2/FBP-2), and the regulatory metabolite fructose 2,6-bisphosphate $\left(\mathrm{F}-2,6-\mathrm{P}_{2}\right)$. We also describe the binding of glucokinase (GK) to the bisphosphatase domain of the bifunctional enzyme which activates both GK and PFK-2. Because changes in the phosphorylation state of Ser-32 in hepatic bifunctional enzyme is involved in modulating both the activities of the bifunctional enzyme $(\mathrm{K} / \mathrm{B})$ and the interaction with GK, understanding the molecular mechanism of this post-translational modification is a central aim of our research efforts. Until the recent report of a novel p53-related FBP-2, TIGAR [Bensaad, K, et al. 2006,Cell, 126:1], we had thought that the bifunctional enzyme was the sole enzyme involved in F-2,6- $\mathrm{P}_{2}$ metabolism. We have now begun to investigate the molecular mechanisms of TIGAR.

Materials and Methods: We used a combination of histidine-specific NMR spectroscopy, steady-state kinetics, point mutagenesis, fluorescent and radiolabeling, and affinity resin pull-down assays to elucidate molecular aspects 
of the bifunctional enzyme. Using the cardiac bisphosphatase domain as an NMR-accessible model, we have demonstrated the molecular basis for how post-translational modification of the bifunctional enzyme influences K/B. Using steady-state kinetics and affinity resin pull-down in conjunction with fluorescently labeled proteins, we probed the binding between GK and PFK2/FBP-2. Using new expression vectors, anion exchange, and size exclusion chromatography we have purified TIGAR from bacterial expression extracts.

Results: Differences in hydrogen bonding within the cardiac and hepatic FBP-2 domains during turnover correlate with the lower activity in the heart isoform. These are relevant to the question of post-translational control of the bisphosphatase activity because the heart domain has the serine site included and can be investigated by histidine-specific NMR. Further, we have revealed that bifunctional enzyme and glucokinase bind in a 1:1 complex that concomitantly activates GK and the PFK-2 activity. The conservation of the signature FBP-2 catalytic residues in TIGAR produces a very similar kinetic mechanism in which a stable His-P intermediate is formed.

Conclusion: The liver bifunctional enzyme coordinates glucose up-take with glycolysis in response to glucose, insulin, and glucagon via changes in the phosphorylation state of Ser-32 which alter activities and the interaction with GK. The coordinating elements are multi-modal, affecting sub-cellular location and catalysis, which may be related to the relevance of these mechanisms to the acute hepatic response to re-feeding after a fast. The in vivo results suggest other aspects of the metabolic coordinating role as it influences hepatic lipid metabolism. TIGAR and fructose 2,6-bisphosphate metabolism provide a potential link to apoptosis.

Supported by the Department of Veterans Affairs

\section{7}

HDAC3 and SMRT are required for inhibition of phosphoenolpyruvate carboxykinase expression by TNF- $\alpha$

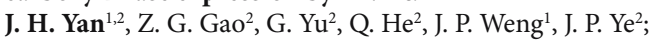

${ }^{1}$ Department of Endocrinology, the First Affiliated Hospital of Sun Yat-Sen

University, Guangzhou, China, ${ }^{2}$ Department of Antioxidant and Gene

Regulation, Pennington Biomedical Research Center, Louisiana State

University System, Baton Rouge, United States.

Background and Aims: Phosphoenolpyruvate carboxykinase (PEPCK) is a rate-limiting enzyme in the gluconeogenesis. Inhibition of PEPCK expression by tumor necrosis factor $\alpha$ (TNF- $\alpha$ ) occurs at transcriptional level, and contributes to the pathogenesis of hypoglycemia in endotoxin shock. In this study, the molecular mechanism underlying the inhibition was investigated in hepatoma cells.

Materials and Methods: Rat H4IIE cells were treated with cAMP $(200 \mu \mathrm{M})$ and TNF- $\alpha(20 \mathrm{ng} / \mathrm{ml})$ for induction and suppression of PEPCK expression. Transcriptional activation of the PEPCK gene promoter $(-490 /+100)$ was examined using rat PEPCK-Luc reporter in human HepG2 cells and ssIkB $\alpha$ HepG2 stable cell line in which super suppressor IkB $\alpha$ was consistently expression. Kinase-specific chemical inhibitors, including Aspirin (NF-kB), SP600125 (JNK), SB203580 (p38 MAPK kinase), PD98059 (MEK/ERK) and LY294002 (PI3K), were used to evaluate the pathways required for the TNF-inhibition of PEPCK. Nuclear/cytoplasmic shuttling of HDAC3 protein was analysed in H4IIE and ssIkB-HepG2 cells treated with cAMP and TNF$\alpha$. The chemical inhibitor of HDACs (Butyrate, TSA) and RNAi-mediated gene knockdown were used to determine the corepressor involved in the p65-mediated inhibition of PEPCK by TNF- $\alpha$. The interaction of CREB, HDAC3 and SMRT with the PEPCK gene promoter was investigated using ChIP asssay and qRT-PCR in H4IIE cells. The classical CRE element (CREB binding site at $-91 /-84$ ) was mutated in the rat PEPCK promoter through $\mathrm{T}$ to $G$ substitution.

Results: In H4IIE cells, cAMP-induced PEPCK expression was reduced in a time-dependent manner by TNF- $\alpha$ at both protein and mRNA levels. Two inhibitors, Aspirin and LY294002, which are known to block NF-kB activation, were able to block the TNF-inhibition. TNF- $\alpha$ induced the nuclear abundance of HDAC3. This change occurred with IkB $\alpha$ degradation in the cytoplsma. In HepG2 cells, the PEPCK-Luc reporter activity was increased 3 fold by cAMP and the induction was reduced more than $50 \%$ in the presence of TNF- $\alpha$ or $\mathrm{p} 65$ co-expression. In contrast, TNF- $\alpha$ activity was completely blocked in ssIkB $\alpha-\mathrm{HepG} 2$ stable cell line as TNF-induced nuclear translocation of NF-kB p65 was blocked. Inhibition of HDAC activity by the HDAC inhibitor or HDAC3 knockdown led to restoration of the PEPCK reporter activity in the presence of TNF- $\alpha$ or p65 cotransfection. Inhibition of SMRT by gene knockdown produced the same effect. In ChIP assay, the interaction of HDAC3 and SMRT with the PEPCK gene promoter was induced by TNF- $\alpha$. But CREB signal was reduced. The decrease in DNA-binding activity of CREB was not from inhibition of CREB phosphorylation or PGC-1 expression. CREB activity was inhibited by corepressor HDAC3 and SMRT in the PEPCK gene promoter. Point mutation suggested that the classical CRE element was required for TNFinhibition of PEPCK. This site was a target of TNF- $\alpha$ in the inhibition of PEPCK gene promoter.

Conclusion: Our data support that NF-kB p65 mediates TNF-inhibition of PEPCK transcription. The inhibition is dependent on the corepressor HDAC3-SMRT, and CREB is a target of the corepressor in hepatocytes. This study suggests a novel mechanism for TNF-inhibition of PEPCK expression and gluconeogenesis.

Supported by NIH and ADA to J Ye, and a grant from the National Natural Science Foundation of China and the Special Funds for Major State Basic Research Program of China to JP Weng

\section{8}

Transgenic mice overexpressing human fructose-1,6-bisphosphatase in the liver develop glucose intolerance in response to a high fat diet S. Visinoni, B. C. Fam, A. Blair, C. Rantzau, B. J. Lamont, R. Bouwman, J. Favaloro, J. Proietto, S. Andrikopoulos;

Department of Medicine (AH/NH), University of Melbourne, Heidelberg Heights, Australia.

Background and Aims: Fructose-1,6-bisphosphatase (FBPase) is postulated to be a key rate limiting enzyme in the gluconeogenic pathway. We have previously generated transgenic mice overexpressing human FBPase in the liver under the control of the transthyretin promoter. We reported that despite a 2-fold overexpression of liver FBPase, endogenous glucose production and glucose tolerance were normal. However, these mice also displayed transgene expression in the hypothalamus which may have affected the phenotype. We therefore generated a new liver FBPase transgenic mouse line in which the construct was flanked by insulator sequences to protect against site of integration effects. The aim of this study was to further characterise the glucose tolerance phenotype in the original model and the new insulated transgenic mice at two ages ( 24 weeks and 1 year old), when bred to homozygosity and in response to a $60 \%$ high-fat (HF) diet.

Materials and Methods: Glucose tolerance was measured by an intraperitoneal glucose tolerance test at a dose of $2 \mathrm{~g} / \mathrm{kg}$ for 120 minutes in both male and female transgenic and negative littermate control mice following an overnight fast. Plasma was collected for glucose and insulin measurements. Mice on the HF diet were fed from 12 weeks of age for the duration of 12 weeks.

Results: The insulated heterozygous transgenic mice had a 3-fold overexpression of total liver FBPase mRNA with concomitant increases in FBPase protein and enzyme activity levels $(n=4)$. Of importance, hypothalamic expression of FBPase mRNA was comparable in the insulated transgenic and control littermates $(n=3)$. There was no difference in glucose tolerance in the 24 week old insulated heterozygous transgenic mice compared to the negative littermates $(n=16)$. Similar findings were found in both the original transgenic and insulated transgenic mice at 1 year of age ( $n=7$ for each group) and the homozygote line (preliminary data $n=2$ ). Interestingly, the HF fed original and insulated transgenic mice were glucose intolerant compared to their negative littermates (original: $2451.6 \pm 136 \mathrm{mmol} / \mathrm{Lx} 120 \mathrm{~min}$; insulated: $2802.5 \pm 382 \mathrm{mmol} / \mathrm{Lx} 120 \mathrm{~min}$; control: $1974.3 \pm 106 \mathrm{mmol} / \mathrm{Lx} 120 \mathrm{~min} ; \mathrm{n}=6-$ 18 , p-value $<0.02$ ).

Conclusion: Overexpressing FBPase in the liver appears to have no effect on glucose tolerance in transgenic mice either at 24 weeks or 1 year of age or when bred to homozygosity. In contrast, under high fat feeding conditions, glucose tolerance was impaired. This suggests that FBPase is associated with glucose intolerance and may contribute to the chronic hyperglycaemia observed in Type 2 diabetic patients under conditions of excess fat intake. Supported by National Health and Medical Research Council Project Grant 145769 and Dora Lush Biomedical Postgraduate Research Scholarship 


\section{9}

In vivo phosphorylation of activating transcription factor 2 (ATF2) in insulin target tissues in mice: effects of insulin and of high-fat diet induced insulin resistance

B. Baan ${ }^{1}$, P. J. Voshol ${ }^{2}$, G. C. M. van der Zon ${ }^{1}$, J. Kriek ${ }^{1}$, E. Korsheninnikova ${ }^{1}$, J. A. Romijn' ${ }^{2}$ J. A. Maassen ${ }^{1}$, M. Ouwens ${ }^{1}$;

${ }^{1}$ Molecular Cell Biology, ${ }^{2}$ Endocrinology, LUMC, Leiden, The Netherlands.

Background \& Aims: We have identified activating transcription factor 2 (ATF2) as novel insulin signalling intermediate in cultured cells. Activation of ATF2 and its target genes has been implicated in the regulation of the expression of the genes for insulin and the key gluconeogenic enzyme phosphoenolpyruvate carboxykinase (PEPCK), but has also been linked to beta-cell apoptosis, inflammation and atherosclerosis. To further detail the role of ATF2 in insulin action, we studied the induction of ATF2phosphorylation by hyperinsulinemia in vivo, characterized insulin-regulated ATF2-target genes, and examined the effects of high-fat diet (HFD) induced insulin resistance on ATF2-activity in mice.

Materials \& Methods: Chow or HFD-fed mice were infused with insulin while maintaining euglycemia. After sacrifice, tissues were examined for ATF2 phosphorylation by Western blotting and immunohistochemistry and for induction of ATF2 target genes by real-time PCR.

Results: In vivo insulin infusion induced a transient phosphorylation of ATF2 in the liver, epididymal adipose tissue and pancreas. In the liver, hyperinsulinemia suppressed the expression of PEPCK, and increased the expression of the ATF2-target genes ATF3, c-jun, and Egr1. shRNA-mediated silencing of ATF2 in cultured cell lines prevented the induction of ATF2-target genes by insulin. In tissues from insulin-resistant HFD-fed mice, a sustained increase in ATF2-phosphorylation and expression of ATF3, c-jun, and Egr1 was found already in the fasting state. Remarkably, livers from HFD-fed mice remained responsive for the induction of ATF2 target genes in response to hyperinsulinemia, whereas suppression of PEPCK was blunted.

Conclusion: ATF2 has a dual role in insulin action in vivo as it is activated both by hyperinsulinemia and under conditions of HFD-induced insulin resistance. The increased ATF2 activity in HFD-fed mice suggests a state of chronic inflammation. The intact insulin-mediated induction of detrimental ATF2-target genes in livers from insulin-resistant mice may further accelerate the development of diabetes-associated complications.

Supported by the Dutch Diabetes Research Foundation

\section{0}

Hepatic regulation of insulin signal in IRKO models

B. Capuani ${ }^{1}$, G. Donadel ${ }^{1}$, L. Bova ${ }^{1}$, A. Biroccio ${ }^{2}$, B. Bartolini ${ }^{3}$, A. Urbani ${ }^{4}$, M. Federici ${ }^{1}$, P. Sbraccia ${ }^{1}$, D. Lauro ${ }^{1}$;

${ }^{1}$ Internal Medicine, University of Rome Tor Vergata, ${ }^{2}$ Clinical Biochemistry, Bambin Gesù Hospital, Rome, ${ }^{3}$ Biology, University of Tuscia, Viterbo,

${ }^{4}$ C.E.S.I., University of Chieti and Pescara, Chieti, Italy.

Background and Aims: Type 2 Diabetes Mellitus (T2DM) is associated with increased insulin resistance levels and hepatic glucose production and defect of glucose stimulated insulin secretion in pancreatic beta cells. The aim of this study is to investigate the role of liver in the pathophysiology of disglycaemia, insulin resistance and T2DM. It has been used on two experimental models of target knockout insulin receptor $\mathrm{IR}^{-/-}: 1$ ) transformed murine hepatocytes cell lines model - wild type $\mathrm{IR}^{+/+}$and $\mathrm{IR}^{-/-}$- to detect the modulation of insulin signaling in presence or absence of IR (basal conditions); 2) liver tissue from $\mathrm{IR}^{-/-}, \mathrm{IR}^{+/-}$and $\mathrm{IR}^{+/+}$to discover differences in insulin substrates expression and activation in diabetic ketoacidotic conditions (pathophysiological conditions).

Materials and Methods: Experimental models: in vitro culture cell lines of fibroblasts/hepatocytes from IRKO mice transformed by adenovirus SV40; ex vivo genetically modified IRKO mouse and littermate control IR+/-, IR $\mathrm{IR}^{+/+}$. The differences in proteins expression profile and insulin signaling has been analyzed and compared between $\mathrm{IR}^{+/+}, \mathrm{IR}^{+/-}$and $\mathrm{IR}^{-/-}$phenotypes Proteomic analysis: proteins from liver and cell lines has been extracted in $8 \%$ Urea/ $2 \%$ Chaps and separated in IEF using $18 \mathrm{~cm}$ Strips $\mathrm{pH} 3-10$. The proteins expression profile has been investigated by 2D-PAGE Silver Stain Analysis. The statistical analysis of 2D-GEL has been made by IMAGE MASTER 2D-PLATINUM. The proteins of interest have been identified by MALDITOF Mass spectrometry (MS). The amount of these proteins has been confirmed by Western Blot analysis in liver and cell lines. Real Time PCR: it was performed for genes of proteins differentially synthetized which were identified by 2D-PAGE and MS. A discrepancy between amount and relative mRNA expression of several proteins was detected. This could be due to a translation block then mTOR activation and expression has been investigated by Western Blot.

Results: Five proteins at present, of the 22 proteins differentially expressed, were identified: PRDX-6, HMGB-1, ANXA-1, Calreticulin and XRN-2. The different expression of these molecules has been confirmed in the two experimental models. It has been detected that in IRKO tissues were increased Calreticulin and ANXA-1 respect to heterozygous and wild type and HMGB1 was expressed only for $\mathrm{IR}^{-/-}$, differently XRN-2 was found only in $\mathrm{IR}^{+++}$, and $\mathrm{IR}^{+/}$. PRDX-6 is a molecule present normally in two forms, oxidated and native and in $\mathrm{IR}^{+/+}$cell line has be found in both forms differently in IR 1- cell line has been detected only the native form. In liver has been detected only oxidated PRDX-6 phenotype. Reduction of insulin signaling induces a decrease proteins and myotubules proteins expression that is associated with related increases in mRNA levels, but, differently, there is a normal production of albumin. Western Blot analysis shown that in $\mathrm{IR}^{-1-}$ PEPCK was not expressed and SREBP-1 was over expressed, indicating alterations in glucidic and lipidic metabolism, respectively.

Conclusion:

- Insulin modulates proteins involved in oxidative stress (PRDX-6), inflammatory response (HMGB-1 and ANXA-1), mRNA processing (XRN-2)

- IRKO models shown: increased transcription, translation block, decreased gluconeogenesis and increased cholesterol synthesis.

Supported by the University of Rome, Tor Vergata 


\section{OP 16 Mechanisms of beta cell dysfunction and death}

\section{1}

\author{
Molecular mechanisms implicated in free fatty acid-induced ER stress \\ and pancreatic beta cell apoptosis \\ L. Ladriere ${ }^{1}$, P. Hekerman ${ }^{1}$, D. A. Cunha ${ }^{1}$, A. Bazarra-Castro ${ }^{1}$, F. Ortis ${ }^{1}$, \\ D. L. Eizirik ${ }^{1}$, M. Cnop ${ }^{1,2}$; \\ ${ }^{1}$ Laboratory of Experimental Medicine, Universite Libre de Bruxelles, \\ ${ }^{2}$ Division of Endocrinology, Erasmus Hospital, Universite Libre de \\ Bruxelles, Belgium.
}

Background and Aims: Free fatty acids (FFA) cause pancreatic beta cell apoptosis and may contribute to beta cell loss in type 2 diabetes via the induction of endoplasmic reticulum (ER) stress. The molecular mechanisms implicated in FFA-induced ER stress are unknown.

Material and Methods: FACS-purified primary rat beta cells and INS-1E cells were cultured in the presence of oleate and/or palmitate, with or without salubrinal, a selective inhibitor of eIF2 $\alpha$ dephosphorylation. Gene expression was analyzed by real time PCR and protein expression by Western blot. ATF6/ XBP-1 and JNK activation was determined by luciferase reporters containing, respectively, unfolded protein and AP-1 response elements.

Results: Palmitate and oleate induced $59 \pm 2$ and $22 \pm 2 \%$ apoptosis in primary beta cells after $72 \mathrm{~h}(\mathrm{p}<0.01)$, while the equimolar combination of both FFA was not toxic. Palmitate induced a marked PERK and eIF2 $\alpha$ phosphorylation in INS-1E cells (up to 9 - and 4 -fold at $12 \mathrm{~h}$, respectively) compared to oleate (no detectable and 1.5-fold induction at $12 \mathrm{~h}$ ). Palmitate up-regulated ATF4 mRNA early (2-fold at 6-12 h, p<0.05), while oleate or the FFA combination induced ATF4 later (2-fold at $24 \mathrm{~h}, \mathrm{p}<0.005)$. Downstream of ATF4, ATF3 and CHOP mRNAs were markedly induced by palmitate (40- and 8-fold increased at $12 \mathrm{~h}, \mathrm{p}<0.01$ ), as compared to oleate (3- and 2 -fold induced, $\mathrm{p}<0.05$ ). Enhanced activation of the eIF2 $\alpha$ pathway by salubrinal $(75 \mu \mathrm{M})$ markedly potentiated FFA-induced apoptosis. In the IRE branch, XBP-1 splicing was earlier and more intense with palmitate than with oleate. Palmitate, but not oleate, activated the AP-1 reporter. The chemical JNK inhibitor SP-600125 $(25 \mu \mathrm{M})$ and the peptide JNK inhibitor L-TAT-JNKi $(1 \mu \mathrm{M})$ reduced palmitate-mediated AP-1 activation by $45 \%$ $(24 \mathrm{~h}, \mathrm{p}<0.05)$, and decreased palmitate-induced apoptosis in INS-1E cells by $20 \%(72 \mathrm{~h}, \mathrm{p}<0.05)$. All FFA conditions increased BiP expression ( 2 -fold from $12 \mathrm{~h}$ on, $\mathrm{p}<0.05$ ) and activated the unfolded protein response reporter comparably, suggesting that ATF6 activation was similar for all FFA. While high glucose (28 vs $11 \mathrm{mM}$ ) potentiated lipotoxicity in INS-1E cells, there was no consistent increase in FFA-induced ER stress signaling. In primary beta cells, palmitate induced ATF3 and CHOP mRNA and splicing of XBP1 after $24 \mathrm{~h}(\mathrm{p}<0.05)$ similarly at 10 and $28 \mathrm{mM}$ glucose. The non-metabolizable FFA analogues methyl-oleate and -palmitate induced neither ER stress nor beta cell apoptosis, indicating that FFA metabolization is required to activate the ER stress transducers.

Conclusion: Oleate and palmitate trigger a differential ER stress response in beta cells. All FFA activate the ATF6 branch, but palmitate activates IRE more potently. The resulting JNK activation by palmitate contributes to beta cell apoptosis. The marked activation of the PERK pathway by palmitate and the beta cell sensitization to both FFA by salubrinal suggest that this pathway plays an additional and important role in FFA-induced ER stress and beta cell apoptosis.

Supported by EuroDia LSHM-CT-2006-518153

\section{2}

EPA prevents palmitate-induced $\beta$ cell lipotoxicity through suppression of SREBP-1c

T. Kato, H. Shimano, M. Nakakuki, T. Matsuzaka, A. Takahashi, N. Yamada; Department of Internal Medicine (Endocrinology and Metabolism), Graduate School of Comprehensive Human Human Sciences and Center for Tsukuba Advanced Research Alliance, University of Tsukuba, Japan.

Background and Aims: Chronic uptake of excess fatty acids with resultant cellular accumulation of triglycerides is thought to be a part of molecular mechanism of $\beta$-cell failure, often referred as lipotoxicity. However, contribution of endogenous fatty acid synthesis to the $\beta$-cell lipotoxicity has not fully been studied. Sterol regulatory element-binding protein (SREBP)-1c is a transcription factor that controls hepatic lipogenesis. SREBP-1c is highly regulated by nutritional states, and inhibited by polyunsatulated fatty acids such as eicosapentaenoate (EPA). Studies with $\beta$-cells-specific transgenic mice demonstrated that over-expression of nuclear SREBP-1c causes impaired insulin secretion and glucose intolerance. In the current studies, effects of palmitate (PA) and EPA on insulin secretion of murine islets and involvement of SREBP-1c were investigated.

Materials and Methods: Pancreatic islets isolated from C57BL/6 mice or SREBP-1-null mice were incubated with palmitate (PA) or PA plus EPA. After the incubation, glucose-stimulated insulin secretion (GSIS) or potassiumstimulated insulin secretion (KSIS) were measured with cellular insulin contents. Expression profiles of various related genes were determined at mRNA and protein levels by real-time PCR and immunoblot analyses, respectively. Pancreatic islets were isolated from mice fed control, PA-rich, or PA plus EPA-diet, and diabetic KK-Ay mice treated or untreated with EPA. GSIS and KSIS of these islets were compared.

Results: Incubation of isolated islets from C57BL/6 mice with PA caused inhibition of both GSIS and KSIS in dose-dependent manner. Addition of EPA to PA treatment restored both inhibitions. Concomitantly, PA activated, and EPA suppressed both mRNA and nuclear protein of SREBP$1 c$, accompanied by reciprocal changes of SREBP-1c-target genes such as IRS-2 and granuphilin. In contrast, PA-inhibitions of GSIS and KSIS were completely cancelled in SREBP-1-null mice. Suppression of IRS-2/Akt pathway in insulin-signaling could be a part of the downstream mechanism for the SREBP-1c-mediated insulin secretion defect because adenoviral constitutive activation of Akt rescued PA-inhibition of insulin secretion. Uncoupling protein-2 also plays a crucial role in the PA-lipotoxicity as confirmed by knockdown experiments, although independent of SREBP1c. The similar PA-EPA regulation of insulin secretion was observed in islets from C57BL/6 mice pretreated with dietary manipulations. Finally, administration of EPA to KK-Ay mice ameliorated impairment of insulin secretion of their isolated islets.

Conclusions: EPA prevents PA-mediated insulin secretion defect through SREBP-1c inhibition, implicating a therapeutic potential for diabetes related to lipotoxicity.

\section{3}

Endoplasmic reticulum stress markers in pancreatic beta cells from type 2 diabetic subjects

P. Marchetti ${ }^{1}$, M. Masini ${ }^{1}$, M. Bugliani ${ }^{1}$, R. Lupi ${ }^{1}$, L. Marselli ${ }^{1}$, S. Del Prato ${ }^{1}$, G. Weir ${ }^{2}$, D. Eizirik ${ }^{3}$, M. Cnop ${ }^{3}$;

${ }^{1}$ Department of Endocrinology and Metabolism, University of Pisa, Italy, ${ }^{2}$ Joslin Diabetes Center, Harvard University, Boston, United States, ${ }^{3}$ Laboratory of Experimental Medicine, Free University of Brussels, Belgium.

Background and Aims: Pancreatic beta-cells have a highly developed endoplasmic reticulum (ER), due to their heavy engagement in insulin secretion. ER stress has been associated with beta-cell dysfunction and apoptosis in in-vitro and in-vivo studies. We studied some features of endoplasmic reticulum in beta-cells from human type 2 diabetic patients. Materials and Methods: Pancreatic samples and isolated islets from 22 nondiabetic (ND; age: $61 \pm 15$ yrs; body mass index, BMI: $25.8 \pm 3.4 \mathrm{~kg} / \mathrm{m}^{2}$; gender: $10 \mathrm{M} / 12 \mathrm{~F}$ ) and 9 matched type 2 diabetic (T2DM; age: $65 \pm 10$ yrs; BMI: $27.3 \pm 3.4 \mathrm{~kg} / \mathrm{m}^{2}$; gender: $7 \mathrm{M} / 2 \mathrm{~F}$ ) organ donors were evaluated regarding: glucose-stimulated insulin secretion; apoptosis, as assessed by electron microscopy (EM); morphological and morphometric ER assessment by EM; gene expression determination of ER stress markers in beta-cells prepared by laser capture microdissection and in isolated islets.

Results: Glucose-stimulated insulin release (stimulation index: ND, $2.3 \pm 0.8$ vs T2DM, $1.2 \pm 0.4)$ was lower $(\mathrm{p}<0.01)$ and beta-cell apoptosis (ND, $3.1 \pm 0.8$ vs T2DM, 6.6 $\pm 1.8 \%)$ higher $(\mathrm{p}<0.05)$ in diabetic than control islets. This was associated with increased ER density volume in T2DM $(1.7 \pm 0.1 \mathrm{ml} \%)$ compared to ND $(0.9 \pm 0.06 \mathrm{ml} \%)$ beta-cells $(\mathrm{p}<0.01)$. The expression of alpha-mannosidase was higher and that of UDP-glucose glycoprotein glucosyl transferase like 2 (UGCGL2) was lower in T2DM than ND betacell preparations, as assessed by microarray and/or real-time RT-PCR. The expression of immunoglobulin heavy chain binding protein $(\mathrm{BiP})$, protein disulfide isomerase (PDI), alpha-glucosidase, $\mathrm{X}$-box binding protein 1 (XBP1) and C/EBP homologous protein (CHOP) was similar in ND and T2DM beta-cells or isolated islets cultured at $5.5 \mathrm{mmol} / \mathrm{l}$ glucose (microarray and 
real-time RT-PCR results). However, when isolated islets were cultured for an additional $24 \mathrm{~h}$ at $11.1 \mathrm{mmol} / \mathrm{l}$ glucose, there was the induction of $\mathrm{BiP}$ (from $11 \pm 6.9$ to $36.4 \pm 14.5$, $\mathrm{p}<0.01$ ), XBP-1 (from $25.2 \pm 12.1$ to $119.8 \pm 54.5$, $\mathrm{p}<0.05$ ) and spliced XBP-1 (from $16.8 \pm 8.3$ to $56.6 \pm 37.2, \mathrm{p}=0.06$ ) in T2DM, but not in ND islets.

Conclusions: Beta-cells from T2DM subjects show modest alterations of ER when studied in pancreas samples or isolated islets maintained at physiological glucose concentration. A short period of exposure to increased glucose levels, however, induces the expression of ER stress markers in T2DM, but not in ND islet cells. These results suggest that T2DM beta-cells are more susceptible to ER stress induced by metabolic perturbations.

\section{4}

hFis1-induced mitochondrial fragmentation impairs glucose-stimulated exocytosis and causes apoptosis in INS-1E cells

K.-S. Park ${ }^{1}$, A. Wiederkehr ${ }^{1}$, Y. Mattenberger ${ }^{2}$, J.-C. Martinou ${ }^{2}$,

N. Demaurex ${ }^{1}$, C. B. Wollheim ${ }^{1}$;

${ }^{1}$ Cell Physiology and Metabolism, University Medical Center,

${ }^{2}$ Cell Biology, University of Geneva, Geneva, Switzerland.

Background and Aims: Mitochondria are dynamic organelles that continuously move, fuse and divide. In mammalian cells, several proteins mediating mitochondrial fission or fusion have been identified, the function of which is under investigation. Here, we elucidated the role of mitochondrial fission/fusion in metabolism-secretion coupling and cell survival in insulinsecreting cells.

Materials and Methods: To analyze mitochondrial morphology, we cotransfected INS-1E cells with mitochondrial fission/fusion genes and mitochondrial-targeted red fluorescent protein (mitoRFP). Hormone secretion was measured by co-transfection with human growth hormone (hGH) plasmid. Cytosolic calcium responses were studied by co-transfection with yellow cameleon $\left(\mathrm{YC}_{36}\right)$. To evaluate cytotoxic effects, INS-1E cells were infected with doxycycline-inducible adenovirus expressing mitochondrial fission/fusion genes.

Results: INS-1E cells displayed a dense network of elongated and interconnected mitochondria in $94 \pm 2 \%$ of mitoRFP transfected cells. Consistent with their proposed function, over-expression of hFis1 or Drp1 induced dramatic mitochondrial fragmentation in $64 \pm 6 \%$ and $28 \pm 6 \%$ of transfected cells, respectively. Dominant negative mutant of Mfn 1 (DNMfn1) also induced extensive mitochondrial fragmentation in $54 \pm 6 \%$ of the cells. Over-expression of hFis1 reduced glucose stimulated exocytosis of hGH (from $2.0 \pm 0.2 \%$ at $2.8 \mathrm{mM}$ to $4.1 \pm 0.3 \%$ at $16.5 \mathrm{mM}$; expressed as $\%$ content) compared to the transfection with empty vector (from $2.1 \pm 0.2 \%$ to $5.2 \pm 0.4 \%$ ). Intracellular hGH content was decreased by $35 \%$ in hFis1 overexpressing cells. Despite the similar mitochondrial phenotype, DN-Mfn1 over-expression did not attenuate, but slightly increased, the amount of hGH content and glucose-stimulated hGH release. Glucose activation of INS-1E cells results in depolarization of the plasma membrane and calcium influx. Over-expression of hFis1 reduced glucose-stimulated calcium responses by $64 \%$, while $\mathrm{KCl}(30 \mathrm{mM})$-evoked calcium responses were not affected. Adenoviral-driven expression of hFis1, but not of DN-Mfn1, showed marked cytotoxic effects as revealed by MTT assay and increased number of TUNELpositive cells.

Conclusion: These results show that hFis1 over-expression induces fragmentation of mitochondria, impairs glucose-stimulated hormone secretion, and elicits apoptosis in INS-1E cells. Because DN-Mfn1 overexpression also fragments mitochondria without impairing secretion or cell survival, we suggest that hFis1 induced mitochondrial dysfunction and cell death do not result from mitochondrial fragmentation itself, but instead from other mitochondrial processes.

Supported by the Swiss National Science Foundation
0095

The gap junctional protein Connexin $36(\mathrm{Cx} 36)$ protects pancreatic beta cells against cytotoxic attacks: a possible role in cytokine-mediated beta cell death

F. Allagnat ${ }^{1}$, P. Klee ${ }^{2}$, M. Peyrou ${ }^{2}$, D. Martin ${ }^{1}$, A. Charollais ${ }^{2}$, D. Caille ${ }^{2}$, J.-A. Haefliger ${ }^{1}$, P. Meda ${ }^{2}$;

${ }^{1}$ Department of Medicine, University Hospital, Lausanne, ${ }^{2}$ Department of Cell Physiology and Metabolism, University of Geneva School of Medicine, Switzerland.

Background and Aims: Type I diabetes develops when most of the insulinproducing cells have been killed by an autoimmune attack, leaving a few survivors which are unable to sustain an adequate insulin secretion and, hence, to control the levels of circulating glucose. The mechanisms whereby these cells survive for years to the immune attack that kills most of their islet partners is obscure. Herein, we investigated whether this mechanism implicates the gap junctional protein connexin36 (Cx36), a critical contributor to glucose-stimulated insulin secretion.

Materials and Methods: Using a variety of transgenic mice and in vitro models of islet cells, we investigated whether the levels of Cx36 expression influence an experimentally-induced $\beta$-cell apoptosis.

Results: We found that loss of Cx36 sensitizes the $\beta$-cells of KO-Cx36 mice, in a gene dosage-dependent way, to both streptozotocin- and alloxan-induced $\beta$-cell death. Conversely, over-expression of Cx36 protects the $\beta$-cells of transgenic RIP-Cx36 mice against the same drugs. In vitro, islets isolated from the latter animals were also protected against a cocktail of pro-inflammatory cytokines (IL-1 $\beta$ plus TNF $\alpha$ plus IFN $\gamma$ ), whereas islets isolated from KO-Cx36 mice were sensitized to the apoptosis induced by the same cytokines. Thus, loss of $\mathrm{Cx} 36$ sensitizes pancreatic $\beta$-cells to a variety of cytotoxic conditions in vitro and in vivo. We further investigated the effects of these pro-inflammatory cytokines on the endogenous Cx36 expression levels. We found that IL-1 $\beta$ plus TNF $\alpha$ plus IFN $\gamma$ strongly reduced the levels of Cx36 mRNA and cognate protein in the INS-1E and MIN6 insulin-secreting cell-lines, as well as in isolated mouse islets. To a lesser extent, IL-1 $\beta$ alone decreased the $\mathrm{Cx} 36$ levels in a dose-dependent manner, whereas TNF $\alpha$ and IFN $\gamma$ had no significant effect on the connexin. The effects of the 3 cytokines on Cx36 expression were prevented after inhibition of the AMPK by compound C, whereas metformin, which activates AMPK in $\beta$-cell, reproduced the effect of cytokines on Cx36 expression. The decrease in Cx36 levels observed under the latter conditions correlated with a decrease in gap junction coupling, as assayed by Lucifer Yellow transfer. AMPK phosphorylation was strongly increased by the cytokine treatment, suggesting that the activation of AMPK is central to the cytokine-mediated $\mathrm{Cx} 36$ decreased and $\beta$-cell apoptosis.

Conclusion: These results reveal an inverse relationship between the level of expression of $\mathrm{Cx} 36$ and the degree of $\beta$-cell apoptosis, which is controlled by AMPK activity. Since Cx36 protects pancreatic $\beta$-cells against a variety of cytotoxic conditions, the data suggest that the AMPK-dependent decreased in the levels of this connexin may contribute to the pathogenesis of type I diabetes.

Supported by Swiss National Science Foundation, Juvenile Diabetes Research Foundation, Placide Nicod Foundation, Octav and Marcella Botnar Foundation, and Novo Nordisk

\section{6}

Essential role for STAT1 in pancreatic $\beta$ cell death and autoimmune diabetes of NOD mice

M.-S. Lee, S. Kim, H.-S. Kim, K. W. Chung, H. S. Jung, M.-K. Lee, K.-W. Kim; Medicine, Samsung Medical Center, Seoul, Republic of Korea.

Background and Aims: We have reported important roles for STAT1 in the sensitization of pancreatic $\beta$-cells to cytokine-mediated apoptosis in vitro. However, in vivo evidence supporting the role for STAT1 in $\beta$-cell death and natural type 1 autoimmune diabetes has not been reported. Furthermore, papers showing protective role of STAT1 against insulinoma cell death or aggravation of autoimmune diseases by targeted disruption of STAT1 have been published, contrary to our in vitro data. Thus, the role for STAT1 in $\beta$ cell death and type 1 autoimmune diabetes in vivo remains unclear.

Materials and Methods: To resolve this issue, we produced NOD/ $S T A T 1^{--}$mice and studied in vivo role of STAT1 in $\beta$-cell death and autoimmune diabetes. 
Results: STAT1 ${ }^{-/}$islet cells were resistant to death by IFN- $\gamma /$ TNF- $\alpha$ or IFN- $\gamma /$ IL-1 $\beta$. Cytochrome $c$ translocation from mitochondria to cytosol by IFN- $\gamma /$ TNF- $\alpha$ was abrogated in STAT1 $1^{-/}$islet cells. The induction of XIAP protein by TNF- $\alpha$ was inhibited by IFN- $\gamma$ in $S T A T 1^{+/+}$islet cells but not in $S T A T 1^{-/}$islet cells. iNOS induction and NO production by IFN- $\gamma / \mathrm{IL}-1 \beta$ were impaired in STAT1 $1^{--}$islet cells. The development of diabetes and insulitis was completely abrogated in NOD/STAT1 $1^{-/}$mice. The incidence of diabetes after transfer of $\mathrm{CD}^{+}$diabetogenic $\mathrm{T}$ cells was also significantly decreased in those mice. In NOD/STAT1 ${ }^{-/-}$mice, priming of autoreactive T cells was not impaired but Th1 differentiation was impaired. A JAK2 inhibitor upstream of STAT1 attenuated islet cell death by IFN- $\gamma /$ TNF- $\alpha$ or IFN- $\gamma / \mathrm{IL}-1 \beta$, and delayed diabetes onset in NOD/BDC2.5-SCID mice.

Conclusion: These data demonstrating a critical role for STAT1 in $\beta$-cell death and autoimmune diabetes in vivo, suggest potential therapeutic values of STAT1 or JAK inhibitors in the treatment/prevention of autoimmune diabetes.

Supported by the Nano/Bio Science Program Grant and 21C Frontier Functional Proteomics Project (M.-S.L. is an awardee of the JDRF Grant and SRC Grants from the Korea Science)

\section{OP 17 Cardiovascular disease: role of glycaemia and other factors}

\section{7}

Glycaemic control associated with reductions in incidence of macrovascular events

J. M. Foody ${ }^{1}$, N. Rosenberg ${ }^{2}$, Y. Wang ${ }^{1}$, J. E. Thomas ${ }^{1}$;

${ }^{1}$ Internal Medicine/Section of Cardiology, Yale University School of Medicine, New Haven, United States, ${ }^{2}$ sanofi-aventis, U.S., Bridgewater, United States.

Background and Aims: The relationship between glycemic control and the risk of macrovascular events was examined in a retrospective analysis of patients with diabetes from the Integrated Health Care Information System database (IHCIS).

Material and Methods: Retrospective data of 69,418 selected patients with diagnosis of diabetes and at least $1 \mathrm{AlC}$ result were obtained from the IHCIS database. Patient data were examined 6 months prior to index A1C (I-A1C: The first $\mathrm{AlC}$ available from the beginning of plan enrollment) to determine baseline demographics, comorbidities, and health utilization characteristics. Survival analysis was conducted to examine the first occurrence of acute myocardial infarction (AMI), coronary artery bypass graft (CABG) surgery or stroke after I-A1C with patients being censored at the end of their health plan enrollment.

Results: Patients (54\% male, mean age 57y, and I-A1C 7.6\%) were stratified into 4 groups based on I-A1C: $<6.0 \%, 6.0-7.0 \%, 7.0-9.0 \%$, and $\geq 9.0 \%$. With a mean follow-up of 27 months from I-A1C, unadjusted incidence rates in the 4 groups were $76.5,80.5,81.7$ and 65.2 per 1000 patients/year, respectively. Elevated I-A1c was associated with increased incidence of macrovascular events even after adjustment for comorbidities, diabetes medications, selected lipid lowering and blood pressure control agents and laboratory values at baseline. Figure 1 shows the adjusted Kaplan-Meier curve. Cox proportional hazards regression model shows that elevated A1C was statistically significantly associated with higher risk of macrovascular events. Compared to $<6 \%$ group, hazard risk for AMI/CABG/stroke elevated $8 \%$ in $7-9 \%(\mathrm{p}<0.01)$ and $15 \%(\mathrm{p}<0.001)$ in $\geq 9 \%$ group. Subgroup analysis excluding prior $\mathrm{AMI} / \mathrm{CABG} /$ stroke showed almost identical survival pattern.

Conclusion: This analysis demonstrates that there is a high incidence of macrovascular events in diabetes patients with A1C $>7 \%$. Appropriate glycemic control may contribute to reducing the risks for macrovascular diseases in patients with diabetes.

Adjusted Kaplan-Meier Survival Curve on Time to AMI,CABG or Stroke in 4 A. IC Groups

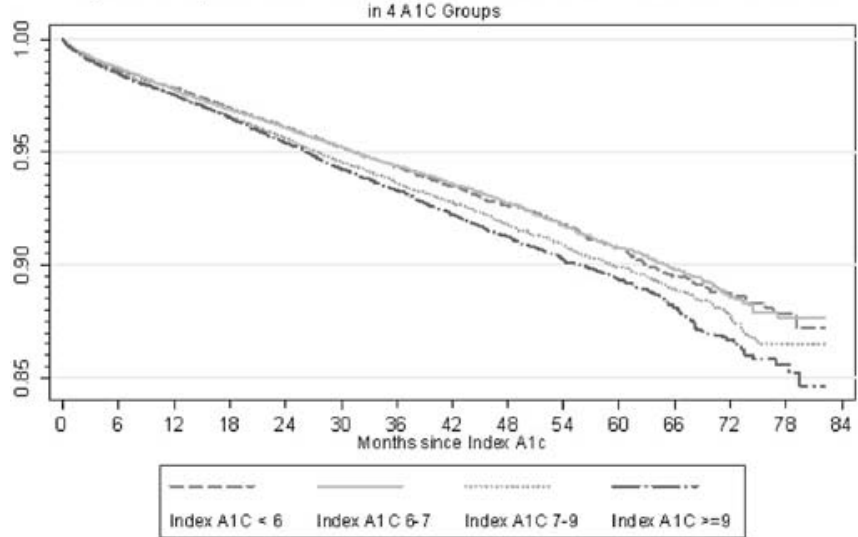

Fig. Note: Adjusted for Gender, Age, Baseline, AMI, Congestive Heart Failure, Periphral Vascular, and Cerebrovascular Diseases

Supported by sanofi-aventis U.S. 


\section{8}

Aggressive glycaemic control preserves cardiac function in patients with acute myocardial infarction and diabetes mellitus

L. G. Strongin, N. Beliaeva;

Medical Faculty, State Medical Academy, Nizhny Novgorod, Russian

Federation.

Background and Aims: The goal of the current study was to determine whether improved glycemic control, achived through an insulin infusion with a variable rate of insulin, has a beneficial effect on left ventricular ejection fraction ( LVEF), wall motion index score (WMIS) and mortality

Materials and Methods: A comparison of an intravenous insulin infusion regimen aimed to maintain the blood glucose level between 5 and 7,5 mmol/ $1(n=26)$, with routine antidiabetic control $(n=35)$ in the acute period of myocardial infarction (AMI) has been carried out in patients suffered from type 2 diabetes mellitus. Patients were excluded if an illness associated with markedly restricted life expectancy was present. A continuous infusion of

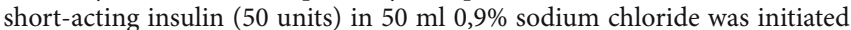
using a pump (Perfusor-FM, B. Braun, Melsungen, Germany). The infusion was continued for at least 24 hours. LVEF and WMIS were measured by echocardiography. Outcome variables were compared with the use of Student's t-test, the Chi-square test, the Mann-Whitney U test, Wicoxon test. Data are presented as medians with interquartile ranges.

Results: Echocardiography shows the improvement of regional function: the wall motion score improved from $1,25(1,12-1,7)$ to $1,18(1,06-1,25)$ in the insulin infusion group $(\mathrm{p}=0,01)$, and increased from $1,31(1,25-1,43)$ to $1,34(1,15-1,8)$ in the control group $(p=0,77)$. There was a significant improvement in the LVEF over 21-day period on $2,2 \%$ in the infusion group $(\mathrm{p}=0,04)$, versus $7 \%$ decrease of LVEF in the control group $(\mathrm{p}=0,1)$. Mortality within 6 month was 15,38 in the insulin - infusion group and 45,7 in the control group $(\mathrm{p}=0,02)$.

Conclusion: Tight glycemic control with insulin therapy following AMI improves left ventricular mechanics and survival in diabetic subjects.

\section{9}

The relationship between the prevalence of cardiovascular disease and the use of insulin among patients with type 2 diabetes mellitus - a large national observational study in a managed care setting P. Sun ${ }^{1}$, D. Buesching ${ }^{1}$, N. Engel-Nitz ${ }^{2}$, S. Martin ${ }^{1}$, V. Fonseca ${ }^{3}$;

${ }^{1}$ US Medical Division, Lilly Research Laboratories, Indianapolis, ${ }^{2} \mathrm{i} 3$ Innovus Health Economics \& Outcomes, i3 Magnifi, Eden Prairie, ${ }^{3}$ Endocrinolgy, Tulane University, New Orleans, United States.

Background and Aims: Cardiovascular disease (CVD) is the leading cause of mortality and major cause of morbidity in patients with type 2 diabetes (T2D). Although the etiological mechanisms responsible for CVD among T2D patients have been extensively examined, the association of insulin use with CVD risk among T2D patients has not been fully understood in a real world setting. To fill this gap, this study aims to examine whether T2D patients using insulin were more or less likely to have CVD than T2D patients not using insulin.

Materials and Methods: This retrospective observational study was based on a de-identified US national health claims database ( $\mathrm{N}:>70$ million lives). The study population included 357,655 T2D patients who continuously enrolled in health plans for at least 1 year in a period from 01/01/98 $-12 / 31 / 04$. CVD included unstable angina, myocardial infarction, stroke, other cardiac events, other cerebrovascular events, and were identified based on ICD-9CM codes. A logistic regression model was used to examine the odds ratio of having CVD between the patients who used insulin and patients who did not use insulin; and to control for age groups, gender, income, race, residential regions, provider specialties, various co-morbid conditions, medication history, baseline HbAlc level, and length of observation.

Results: With control of the confounding factors mentioned above, the odds ratio of having CVD between patients who used insulin and patients who did not use insulin (referent) was 0.66 ( $\mathrm{p}<0.0005,95 \%$ CI: $0.61538,0.72002)$. This odds ratio suggests that T2D patients who used insulin had $34 \%$ less odds of having CVD than T2D patients who did not use insulin.

Conclusion: With control of the 31 confounding factors, the odds of having any CVD among T2D patients using insulin were $34 \%$ lower than the odds among T2D patients not using insulin. Thus, the use of insulin is associated with a decrease of CVD. Further research in this area is imperative. Supported by an unrestricted grant from Lilly Research Laboratories

\section{0}

Skin autofluorescence as a marker of tissue AGE accumulation has additional value to the UKPDS risk-score in predicting cardiovascular disease in type 2 diabetes

H. L. Lutgers ${ }^{1,2}$, E. G. Gerrits ${ }^{2}$, H. J. Bilo ${ }^{2,1}$, A. J. Smit ${ }^{1}$;

${ }^{1}$ Internal Medicine, University Medical Center Groningen, ${ }^{2}$ Diabetes Center, Isala Clinics, Zwolle, The Netherlands.

Background and Aims: One of the pathogenetic mechanisms of accelerated atherosclerosis in type 2 diabetes mellitus (T2DM) is increased formation and accumulation of advanced glycation endproducts (AGEs). Some AGEs have characteristic fluorescent properties, which can be non-invasively measured as skin autofluorescence (AF). We have previously demonstrated the association and predictive value of skin AF with chronic diabetes complications. This study aims to assess whether skin AF is additive to classical risk factors, as combined in the UKPDS risk-engine, in predicting cardiovascular complications.

Materials and Methods: In 2001, 973 primary care T2DM-patients were included (mean age 66 yrs, mean $\mathrm{HbA1}$ c $7.0 \%$ and mean diabetes duration of $6.3 \mathrm{yrs}$ ). Skin AF, using a prototype of the current AGE reader, and UKPDS risk scores were assessed at baseline. Follow-up ended at January 2005. Primary endpoint was mortality or non-fatal cardiovascular event: coronary heart-, cerebrovascular-, or peripheral vascular disease. Patients were categorized in four groups, either: both AF and UKPDS risk score respectively above (1) and below (2) the median, and, AF (3) or UKPDS risk score (4) above the median (Fig 1, left panel), or: same categories but the UKPDS score set at clinically often used cutoff values of 10\% 10 year CVD risk (Fig 2, right panel).

Results: During a median follow-up of about 3.5 years, 6 patients were lost to follow-up. In the remaining 967 patients, there were 161 events. The calculated median UKPDS risk score for CHD and stroke in 3.5 years was

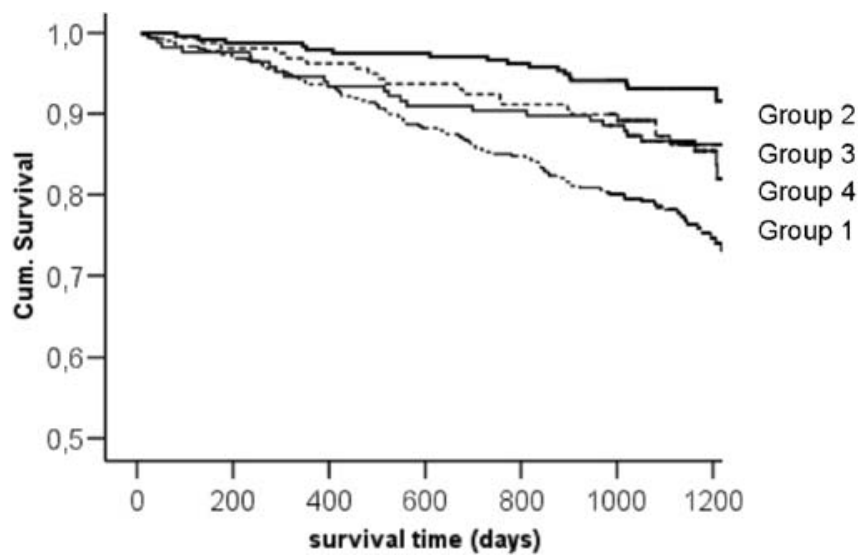

Fig 1

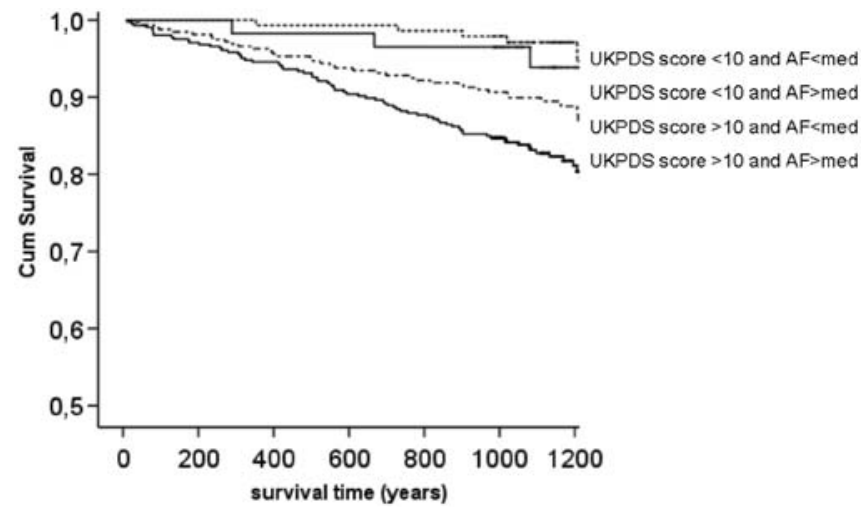

Fig 2 
$12 \%$. This expected CV-event rate of $12 \%$ was not significantly different from the $13 \%$ observed CV-event rate in this population. Median UKPDS risk score (10 years) was 30\%. Kaplan-Meier survival curves (left panel) showed significant differences between the groups, except between group 3 and $4(\mathrm{p}<0.05)$. When the UKPDS score was set at cutoff values of $10 \% 10$ year CVD risk, skin AF still provided additive information in the larger $(\mathrm{n}=$ $726)>10 \%$ UKPDS risk group, but not in the small $<10 \%$ UKPDS risk group (right panel).

Conclusion: Autofluorescence has additional value to the UKPDS risk-score in the risk assessment of cardiovascular complications in T2DM.

Supported by the Dutch Diabetes Research Foundation

\section{1}

Long-term effects of intensive multifactorial treatment on total and cardiovascular mortality in patients with type 2 diabetes

P. Gæde ${ }^{1}$, H.-H. Parving ${ }^{2}$, O. Pedersen ${ }^{1}$;

${ }^{1}$ Department of Diabetology, Steno Diabetes Center, Gentofte, Denmark,

${ }^{2}$ Department of Endocrinology, Rigshospitalet, Copenhagen, Denmark.

Background and Aims: Intensified multifactorial intervention in highrisk type 2 diabetes patients with the metabolic syndrome including microalbuminuria cuts the risk of cardiovascular events by half. We studied whether the use of intensive multitherapy as compared with conventional therapy in the Steno-2 study affected the 13 years' incidence of total and cardiovascular disease (CVD) mortality as well as a composite of CVD events.

Materials and Methods: 160 patients with type 2 diabetes and microalbuminuria were in the Steno-2 study randomly assigned intensified or conventional multifactorial treatment of multiple risk factors for an average of 7.8 years. All 130 surviving patients examined at end of the intervention trial were offered intensive multitherapy at Steno Diabetes Center and subsequently followed in the post-trial until December 31, 2006 when patients were re-examined after an average of 13.3 years.

Results: During the entire 13.3 year follow-up period 24 patients in the intensive therapy group died compared to 40 patients in the conventional therapy group. Intensive therapy reduced the risk for total mortality by $46 \%$ ( $95 \%$ confidence interval, 11 to $68 \%$; $\mathrm{P}=0.015$ ) corresponding to an absolute risk reduction of $30 \%$, while CVD mortality was reduced by 57 percent ( 6 to $81 \%, \mathrm{P}=0.036$ ), i.e. $13 \%$ in absolute terms and the risk for CVD events defined as a composite of CVD mortality, myocardial infarction, stroke, coronary bypass graft, revascularization, or amputation due to ischaemia was reduced by $59 \%$ ( 37 to $75 \%, \mathrm{P}=0.0003$ ) corresponding to an absolute risk reduction of $29 \%$. These differences in clinical endpoints between groups were achieved despite significant improvements in HbAlc, fasting levels of total-cholesterol, LDL-cholesterol, triglycerides, and systolic and diastolic blood pressure in the conventional therapy group during the observational post trial period to an extent where the two original treatment groups no longer differed significantly for any of these risk variables.

Conclusion: Intensive multifactorial intervention in at-risk patients with type 2 diabetes for an average of 7.8 years has long-term beneficial effects on total and CVD mortality as well as on a composite of CVD events.

\section{2}

Effects of EPA on coronary artery disease in patients with gluco-metabolism derangement: JELIS subgroup analysis S. Oikawa ${ }^{1}$, M. Yokoyama ${ }^{2}$, H. Origasa ${ }^{3}$, M. Matsuzaki ${ }^{4}$, Y. Saito ${ }^{5}$,

Y. Matsuzawa ${ }^{6}$;

${ }^{1}$ Division of Endocrinology and Metabolism, Nippon Medical School,

Tokyo, ${ }^{2}$ Department of Internal Medicine, Kobe University,

${ }^{3}$ Division of Clinical Epidemiology and Biostatistics, Toyama University,

${ }^{4}$ Department of Medicine and Clinical Science, Yamaguchi University, Ube,

${ }^{5}$ Department of Clinical Cell Biology, Chiba University,

${ }^{6}$ Department of Internal Medicine, Sumitimo Hospital, Osaka, Japan.

Background and Aims: The Japan EPA Lipid Intervention Study (JELIS), a large scale clinical study $(n=18,645)$, examined the preventive effects of eicosapentaenoic acid (EPA) against coronary artery disease (CAD). JELIS demonstrated that EPA significantly decreased the incidence of CAD by
19\% compared with non-EPA group (Lancet, in press). A later sub-analysis showed that EPA significantly decreased the incidence of CAD by $22 \%$ compared with non-EPA group in the impaired glucose metabolism (IGM) group $(n=4,565)$. The present study was the further sub-analysis of JELIS, and focused on the examination of correlation between fasting plasma glucose $(\mathrm{FPG})$ or hemoglobin $\mathrm{A}_{1 \mathrm{c}}\left(\mathrm{HbA}_{1 \mathrm{c}}\right)$ levels and $\mathrm{CAD}$ incidence, and the preventive effect of EPA was investigated in the subjects with IGM.

Materials and Methods: As described in the previous report, all subjects had hypercholesterolemia (TC $\geq 250 \mathrm{mg} / \mathrm{dL}$ ) treated with statin. Out of them, the subjects were intended as the impaired gluco-metabolism patients having diabetes mellitus at registration or with higher fasting plasma glucose levels $(F P G \geq 110 \mathrm{mg} / \mathrm{dL})$ and divided randomly into two groups treated with 1,800 $\mathrm{mg}$ /day of pure EPA. (EPA group) or without EPA (non-EPA group). CAD was monitored for 5 years. CAD was defined as major coronary events including sudden cardiac death, fatal and non-fatal myocardial infarction, unstable angina pectoris, coronary artery bypass surgery or cardiovascular reconstruction. CAD incidences were conducted according to FPG (EPA group, $\mathrm{n}=1,899$ and non-EPA group, $\mathrm{n}=1,862$ ) or $\mathrm{HbA}_{1 \mathrm{c}}$ (EPA group, $\mathrm{n}=1,540$ and non-EPA group, $\mathrm{n}=1,514)$ values at registration. According to the glycemic control criteria of Japan Diabetes Society, FPG was stratified into four levels, $<110,110-129,130-159$ and $\geq 160 \mathrm{mg} / \mathrm{dL}$. Using the stratum $<110$ $\mathrm{mg} / \mathrm{dL}$ in the non-EPA group as the referent, hazard ratios (HR) of CAD incidence were calculated in both EPA and non-EPA groups. HbA was also stratified into four levels, $<5.8,5.8-6.4,6.5-7.9$ and $\geq 8.0 \%$. Using the stratum $<5.8 \%$ in the non-EPA group as the referent, HR of CAD incidence were calculated in both EPA and non-EPA groups. The incidence of CAD was analyzed by the Cox proportional hazard model, adjusted for gender, age, smoking, prior CAD, and hypertension.

Results: As FPG increased in the non-EPA group, risk of CAD increased $(\mathrm{HR}=1.00,1.60,2.03$ and 2.51$)$. In the highest FPG stratum $(\geq 160 \mathrm{mg} / \mathrm{dL})$, the incidence of CAD was significantly high $(p=0.009)$. In the EPA group, there was no significant difference in the incidence of CAD ( $\mathrm{HR}=1.56,1.27$, 0.83 and 1.73). Also, as $\mathrm{HbA}_{1}$ increased in the non-EPA group, the risk of $\mathrm{CAD}$ increased ( $\mathrm{HR}=1.00,1.19,1.23$ and 2.66). In the highest $\mathrm{HbA}_{1 \mathrm{c}}$ stratum ( $\geq 8.0 \%$ ), the incidence of CAD was significantly high ( $p=0.003$ ). On the other hand in the EPA group, there was no significant difference in the incidence of $\mathrm{CAD}(\mathrm{HR}=0.50,1.12,0.99$ and 1.57). CAD risks were lower in all strata of EPA groups compared to non-EPA groups.

Conclusion: These results suggest that the administration of EPA ameliorates $\mathrm{CAD}$ in patients with gluco-metabolism derangement. 


\section{OP 18 Models of care}

\section{3}

Impact of self-monitoring of blood glucose on the management of patients with non-insulin treated diabetes: an open-label parallel group randomised trial

A. J. Farmer' ${ }^{1}$ A. N. Wade ${ }^{2}$, E. Goyder ${ }^{3}$, P. Yudkin ${ }^{1}$, A.-L. Kinmonth ${ }^{4}$, H. A. W. Neil';

${ }^{1}$ Department of Primary Health Care, University of Oxford, United Kingdom, ${ }^{2}$ Johns Hopkins Bayview Medical Center, Johns Hopkins University School of Medicine, Baltimore, United States, ${ }^{3}$ ScHARR, University of Sheffield, United Kingdom, ${ }^{4}$ General Practice and Primary Care Research Unit, University of Cambridge, United Kingdom, ${ }^{5}$ Division of Public Health and Primary Care, University of Oxford, United Kingdom.

Background and Aims: Self-monitoring of blood-glucose (SMBG) for noninsulin treated diabetes is costly but may lead to improved glycaemic control. Existing evidence is inconclusive, with previous trials using multi-component interventions. We aimed to determine whether self-monitoring, either alone or with additional instruction in incorporating the results into self-care, is more effective than usual care in improving glycaemic control and to explore the impact of monitoring in pre-defined patient groups.

Materials and Methods: Patients with non-insulin treated type 2 diabetes with $\mathrm{HbAlc}$ above $6.2 \%$ were enrolled into this three-arm trial based in 48 general practices. Patients were randomized to: (i) standardized usual care with three monthly measurement of HbAlc (control), (ii) SMBG with results interpreted by the research nurse in addition to usual care (less-intensive selfmonitoring), (iii) SMBG with training in interpreting and applying results of testing to enhance motivation and maintain adherence to diet, physical activity and medication regimens (more-intensive self-monitoring). An intention-to-treat analysis was performed with the primary outcome of 12 month $\mathrm{HbAlc}$ adjusted for baseline values. The primary outcome was also analysed for pre-specified sub-groups defined by duration of diabetes, use of oral antidiabetic drugs (OAD) and diabetes-co-morbidity.

Results: A total of 578 people were screened as potentially eligible, of whom 453 met the trial inclusion criteria. Characteristics of patients in the three randomised groups were similar at enrolment with mean (SD) HbAlc 7.5\% (1.1). The 12-month HbAlc did not differ between the patients receiving the control, the less-intensive and the more intensive self-monitoring interventions. $(\mathrm{p}=0.12)$. The mean difference between control and lessintensive self monitoring was $-0.14 \%(95 \% \mathrm{CI}-0.35$ to 0.07$)$ and between the control and more-intensive self-monitoring group $-0.17 \%$ (95\% CI -0.37 to 0.03 ). Change in $\mathrm{HbAlc}$ from baseline to 12 months by sub-group are shown in the table. $66 \%$ of those receiving the less intensive intervention and $52 \%$ of those in the more intensive intervention persisted in regular self-monitoring

Change in $\mathrm{HbAlc}(\%)$ from baseline to12-months by sub-group

\begin{tabular}{|c|c|c|c|c|}
\hline Mean $\mathrm{HbAlc} \%(\mathrm{SD})$ & $\begin{array}{l}\text { Control Group } \\
\text { No meter }\end{array}$ & $\begin{array}{l}\text { Less-intensive } \\
\text { self-monitoring }\end{array}$ & $\begin{array}{l}\text { More intensive } \\
\text { self-monitoring }\end{array}$ & $\begin{array}{l}\text { P value for } \\
\text { interaction }\end{array}$ \\
\hline \multicolumn{5}{|l|}{ Duration of diabetes } \\
\hline$\leq$ median & $0.01(1.03)$ & $-0.12(0.85)$ & $-0.16(0.73)$ & 0.82 \\
\hline$>$ median & $-0.01(1.01)$ & $-0.15(0.80)$ & $-0.18(0.73)$ & \\
\hline \multicolumn{5}{|l|}{ Baseline therapy } \\
\hline Diet only & $0.03(0.80)$ & $0.04(0.64)$ & $-0.09(0.72)$ & 0.90 \\
\hline $\begin{array}{l}\text { Oral hypoglycaemic } \\
\text { drug therapy }\end{array}$ & $-0.01(1.10)$ & $-0.20(0.87)$ & $-0.20(0.73)$ & \\
\hline \multicolumn{5}{|c|}{ Diabetes related co-morbidity } \\
\hline No & $-0.05(1.02)$ & $-0.19(0.88)$ & $-0.28(0.74)$ & 0.86 \\
\hline Yes & $0.20(1.02)$ & $0.05(0.56)$ & $0.16(0.56)$ & \\
\hline
\end{tabular}

Conclusion: Our results show no improvement in glycaemic control after one year in patients with non-insulin treated type-2 diabetes using SMBG. There was no evidence of significant glycaemic benefit in pre-defined sub-groups of patients, including those treated by diet or OAD. Current guidelines for the use of self-monitoring of blood glucose require review.

Supported by the NHS Health Technology Assessment Programme

\section{4}

The use of medication in a diabetes management system: an observational 8-year-follow-up study

L. Welschen, M. van Veen, S. Bot, J. Dekker, G. Nijpels;

General Practice, EMGO Institute, Amsterdam, The Netherlands.

Background and Aims: Type 2 diabetes is a progressive disease and in order to prevent complications, it seems that intensive medication treatment is inevitable. The aim of this study was to describe the changes in medication use of patients after their entry into a diabetes management system implemented in a region in The Netherlands in 1997.

Materials and Methods: An observational dynamic cohort study was performed from 1998 till 2005. Clinical data were collected every year as well as data on medication use. To evaluate patients' medication use following entry into the diabetes management system, a study cohort was defined. The measurements of every patient in their first year of entry into the system were set at $\mathrm{T}=1$. Follow-up measurements were defined as $\mathrm{T}=2$, for the second year, to $\mathrm{T}=8$, for the eighth year after entry into the system.

Results: The study cohort started with 4782 patients at $\mathrm{T}=1$. There were 440 patients with a follow-up of 8 years. Mean age of the patients was 62 years at $\mathrm{T}=1$ and $51 \%$ were male. Medication use increased during follow-up (Figure 1). At $\mathrm{T}=8,13.7 \%$ of the patients was using insulin, $26.9 \%$ used a combination of insulin and one or more oral hypoglycaemic agents, and $53.2 \%$ used only oral hypoglycaemic agents. Mean $\mathrm{HbA}_{1 c}$ decreased from $7.7 \%$ to $7.1 \%$ and remained stable. Mean systolic blood pressure increased from $143.3 \mathrm{mmHg}$ to $151.2 \mathrm{mmHg}$, despite an increase in the use of antihypertensive medicines from $49.5 \%$ to $68.2 \%$ of the patients. The level of cholesterol improved from $5.5 \mathrm{mmol} / \mathrm{l}$ to $4.8 \mathrm{mmol} / \mathrm{l}$.

Conclusion: Medication use of patients in a diabetes management system showed an incredible increase during 8 years of follow-up indicating that type 2 diabetes is a progressive disease. HbAlc showed a sharp decrease after entry in the system and stabilised during follow-up. Cholesterol levels improved every year while the control of blood pressure was still inadequate. An intensive medication treatment is needed for optimal diabetes control.

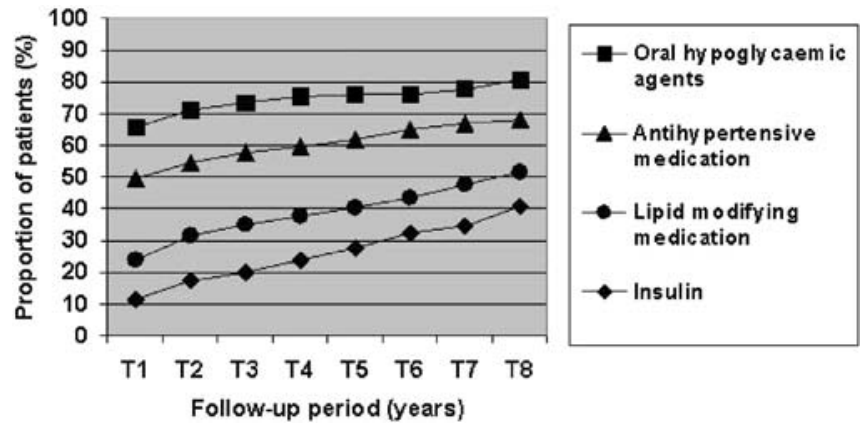

Fig 1. Use of medication of patients with type 2 diabetes during 8 years of follow-up

\section{5}

Practice nurses as main care-providers for patients with type 2 diabetes in a primary care setting: a randomized controlled trial

S. T. Houweling ${ }^{1}$, N. Kleefstra ${ }^{2,1}$, K. H. Groenier ${ }^{3}$, B. Meyboom-de Jong ${ }^{3}$, H. J. G. Bilo ${ }^{2,4}$;

${ }^{1}$ Medical Research Group, Langerhans, Zwolle, ${ }^{2}$ Diabetes Centre, Isala Clinics, Zwolle, ${ }^{3}$ General Practice, University of Groningen, ${ }^{4}$ Internal Medicine, University of Groningen, The Netherlands.

Background and Aims: In order to maintain quality of care for the increasing number of patients with type 2 diabetes (DM2), treatment of DM2 in the primary care setting is increasingly taken over by practice nurses (PNs). We investigated if this care provided by $\mathrm{PNs}$ was at least equal to the care provided by GPs.

Materials and Methods: In a randomized controlled study, we investigated the effects of medical treatment (including prescriptions according to protocol), quality of life (SF-36), diabetes related complaints (DSC-R), and patient satisfaction (PEQD) in patients treated by PNs compared to care provided by GPs; 206 patients from five general practices participated. The 
intervention group was treated by PNs using detailed protocols and the control group received "standard" care from their own GPs.

Results: After fourteen months, care in both groups was comparable according to the clinical parameters (HbAlc, blood pressure (BP) and lipid profile). In the group treated by the PNs, significantly more patients were referred to specialists according to protocol including the ophthalmologist $(71 \%$ vs. $37 \%(\mathrm{p}<0.01))$. Furthermore, more action was taken when the patient had significant foot problems $(57 \%$ vs. $27 \%(\mathrm{p}<0.01))$, and medication dosages were raised more often when considered necessary, except for lipid lowering drugs (hypoglycaemic agents: $83 \%$ vs. $50 \%(\mathrm{p}<0.01)$; antihypertensive agents: $50 \%$ vs. $29 \%(\mathrm{p}<0.01))$. The overall quality of life and the number of complications related to diabetes were equal in the two groups. Patients were more satisfied with the care received from the PNs than with the care provided by the GP (PEQD score: 66 vs. $52(\mathrm{p}<0.01))$.

Conclusion: This study allows the conclusion that PNs who work with strict protocols are capable to offer effective care to patients with DM2 in a primary care setting. PNs follow the quality guidelines, as formulated by the Dutch College of General Practitioners, more consistently than GPs, saving GPs time, whilst providing care of good quality.

\section{6}

Intensified multifactorial treatment of screen-detected type 2 diabetic patients: the ADDITION Netherlands randomised study, one-year results

P. G. H. Janssen ${ }^{1}$, K. J. Gorter ${ }^{1}$, R. P. Stolk², G. E. H. Rutten ${ }^{1}$;

${ }^{1}$ Julius Center for Health Sciences and Primary Care, University Medical Center, Utrecht, ${ }^{2}$ Department of Epidemiology, University Medical Center, Groningen, The Netherlands.

Background and Aims: To evaluate the effectiveness and feasibility of an intensified multifactorial treatment on cardiovascular risk factors in screendetected type 2 diabetic patients. The presented study is the Dutch part of the ongoing ADDITION study (Anglo-Danish-Dutch Study of Intensive Treatment in People with Screen-Detected Diabetes in Primary Care).

Materials and Methods: Screen-detected diabetic patients, assigned to intensive $(n=255)$ or routine treatment $(n=243)$ according to Dutch national guidelines, are followed during five years. Here we present first year results. Intensive treatment consisted of pharmacological treatment combined with structured lifestyle education and targeted on $\mathrm{HbA}_{1 \mathrm{c}}<7.0 \%$, blood pressure $<135 / 85 \mathrm{mmHg}$ (ACE prescribed above $120 / 80 \mathrm{mmHg}$ ), cholesterol $<3.5$ $\mathrm{mmol} / \mathrm{l}$. Acetylsalicylic acid was prescribed when antihypertensive agents were used. Analysis on ITT, clinical and biochemical variables are compared using ANCOVA with age, gender, BMI, and baseline values as covariates. SF-36 scores are compared between treatment groups (ANCOVA with adjustment for age, gender, and baseline values) and within groups (pairedsamples $t$-tests).

Results: At baseline, the two treatment groups were well matched. Table 1 summarizes changes after one year. Changes in SF-36 scores between baseline and end of the study did not differ significantly between the treatment groups.

Table 1 Changes in variables after one year in both treatment groups

\begin{tabular}{llll}
\hline & $\begin{array}{l}\text { Routine care } \\
(n=241)\end{array}$ & $\begin{array}{l}\text { Intensive } \\
\text { treatment } \\
(n=250)\end{array}$ & $\begin{array}{l}\text { Difference } \\
\text { between } \\
\text { groups }^{\mathrm{a}} p \\
\text { value }\end{array}$ \\
\hline BMI $\left(\mathrm{kg} / \mathrm{m}^{2}\right)$ & $-0.3 \pm 3.0$ & $-1.3 \pm 2.0$ & $<0.001$ \\
Systolic blood pressure $(\mathrm{mmHg})$ & $-19 \pm 22$ & $-34 \pm 22$ & $<0.001$ \\
Diastolic blood pressure $(\mathrm{mmHg})$ & $-8 \pm 10$ & $-12 \pm 11$ & $<0.001$ \\
Fasting blood glucose $(\mathrm{mmol} / \mathrm{l})$ & $-0.8 \pm 2.6$ & $-1.3 \pm 2.3$ & $<0.001$ \\
HbA $_{1 \mathrm{c}}(\%)$ & $-0.8 \pm 1.4$ & $-1.2 \pm 1.5$ & $<0.001$ \\
Cholesterol $(\mathrm{mmol} / \mathrm{l})$ & $-0.6 \pm 1.1$ & $-1.3 \pm 1.2$ & $<0.001$ \\
HDL-cholesterol $(\mathrm{mmol} / \mathrm{l})$ & $0.09 \pm 0.7$ & $0.03 \pm 0.2$ & 0.217 \\
LDL-cholesterol $(\mathrm{mmol} / \mathrm{l})$ & $-0.5 \pm 0.9$ & $-1.1 \pm 1.0$ & $<0.001$ \\
Triglycerides $(\mathrm{mmol} / \mathrm{l})$ & $-0.3 \pm 2.0$ & $-0.4 \pm 1.0$ & 0.111 \\
\hline
\end{tabular}

Data are means \pm SD unless otherwise indicated.

${ }^{a}$ Difference between treatment groups at end of study, adjusted for age, gender, BMI, and baseline value.
Within both groups General health, Vitality and Mental health, and in the intensive group also Physical functioning improved during follow-up.

Conclusion: Cardiovascular risk factors improved significantly more in the intensively treated group. Health-related quality of life was not influenced negatively by intensive treatment. Intensified multifactorial treatment of screen-detected type 2 diabetic patients in primary care is feasible. Supported by unrestricted grants of NovoNordisk, Glaxo Smith Kline and Merck

\section{7}

Diabetes type 2 - a chronic disease - how is the clinical practice after implementation of guidelines in Germany? The DIG (Diabetes in Germany) study baseline and 2 years' data

P. Ott, I. Benke, C. Koehler, M. Hanefeld;

Zentrum für Klinische Studien, GWT der Technischen Universität Dresden, Germany.

Introduction: As shown in the STENO 2 study the prevention of vascular complications requires an integrated approach with optimal control of the central components of the metabolic syndrome: blood glucose/HbAlc, lipids and blood pressure. The DIG study presents for the first time population based data on the quality of treatment of major coronary risk factors for type 2 diabetes in Germany for a two year period. It offers a clear and realistic picture of the quality of care that patients with type 2 diabetes receive by diabetologists and by general practitioners after the application of national and international guidelines.

Methods: 4020 patients with type 2 diabetes between 35 and 80 years old were recruited by 238 physicians from general and specialised practices across Germany. Medical history, risk factor profile and clinical data were registered. We analysed $\mathrm{HbAlc}$, fasting blood glucose (FBG), postprandial blood glucose (ppBG), blood pressure (RR), triglyceride (TG), HDL- and LDL-Chol at baseline and after 2 years and the administration of medication. 2538 patients were seen at 2 years follow-up.

Results: We found the following results at baseline and (after 2 years): HbAlc:7.0 (7.0) \%, FBG:7.5 (7.3) mmol/l, ppBG:9.0 (8.7) mol/l - p < 0.05, RR:138.7/81.4 (138.1/80.6) mmHg - p<0.001, TG:2.2 (2.2) mmol/l, HDLChol:1.3 (1.4 ) mmol/1 - p<0.001 and LDL-Chol:3.2 (3.0) mmol/l - $<<0.001$. The patients have after 2 years a significant better control of postprandial blood glucose, blood pressure as well as LDL- and HDL-Cholesterol as at baseline. We found an increase of patients with insulin (1054 at baseline - 1248 after 2 years) and an increase of antihypertensive drugs (for example ACE inhibitors and AT1 blocking drugs: 1453 - 1654) and statins (669- 972) too.

Conclusion: The level of HbAlc achieved in our study (7\%) demonstrates the effectiveness of the structured care system in place in Germany and the value of the application of national guidelines. In concordance with national and international recommendations we found an improvement in the control of blood pressure, lower levels of LDL-Cholesterol and increase in HDLCholesterol. This was achieved by a significant increase in insulin treatment, prescription of statins and antihypertensives drugs. Our data suggest a progress in the care for cardiovascular risk factors by implementation of national and international guidelines. But still action is necessary to target major cardiovascular risk factors.

Supported by Pfizer

\section{8}

Changing standards of diabetes care in general practice over the last six years: The impact of the National Service Framework and the General Medical Services contract

C. Hilton ${ }^{1,2}$, D. Russell-Jones ${ }^{1,2}$, C. Todd ${ }^{3}$, J. Williams ${ }^{1}$;

${ }^{1}$ Postgraduate Medical School, University of Surrey, Guildford,

${ }^{2}$ Cedar Centre, Royal Surrey County Hospital, Guildford, United Kingdom,

${ }^{3}$ PRIMIS+, Surrey PCT, Farnham, United Kingdom.

Background and Aims: The last six years have been a time of change for diabetes care in the UK, with systematic and structured changes to healthcare taking place. The National Service Framework (NSF) for diabetes was introduced in 2002 and set out key standards to improve overall quality of 
care. This was followed by the General Medical Services (GMS) contract (2004) which offered financial rewards for achieving high standards for chronic disease management. This study examines changing standards of diabetes care in general practice between 2001 and 2007.

Materials and Methods: This prospective longitudinal cohort study collected data in 2001, 2003 and 2007 from eight practices in the Guildford and Waverly area (population 74,225 in 2007). Pseudonymised data was extracted for all patients using MIQUEST queries to provide information on predefined indicators of quality of care. Data was analysed using SPSS 14.0.

Results: Between 2001 and 2007 the recorded incidence of diabetes rose from $1.94 \%$ to $2.69 \%$. Quality of care improved for all five indicators measured. There was a substantial improvement in average HbAlc between 2001 and $2003(8.15 \%$ to $7.54 \%, \mathrm{p}<0.001)$ and again between 2003 and 2007 (to $7.24 \%$, $\mathrm{p}<0.001)$. The proportion of patients having an $\mathrm{HbAlc}$ recorded at least once every 15 months rose from $78.2 \%$ in 2001 to $84.0 \%$ in $2003(\mathrm{p}<0.001)$ and to $91.7 \%$ in $2007(\mathrm{p}<0.001)$. The percentage of patients having an $\mathrm{HbAl} \mathrm{c}$ recorded every 3-6 months (in accordance with the NICE and ADA guidelines) also improved ( $\mathrm{p}<0.001)$. Average blood pressure fell from 143.5/79.0 in 2001 to $139.1 / 78.0$ in $2003(\mathrm{p}<0.02)$ and then to $136.4 / 76.9$ in $2007(\mathrm{p}=0.001)$. In $200187.5 \%$ of patients were having blood pressure checked 15 monthly, and this rose to $95.9 \%$ in $2007(\mathrm{p}<0.001)$. The percentage of patients receiving a cholesterol check at least once every 15 months rose from $61.2 \%$ to $91.5 \%$ between 2001 and 2007 ( $p<0.001$ ), but there was no trend towards improvement of cholesterol values. Over the course of the study there was an increase in 15 monthly recording of BMI from $68.0 \%$ to $89.5 \%(\mathrm{p}<0.001)$, although this was accompanied by a small increase in BMI from 28.6 to 29.2. $94.1 \%$ of patients had their smoking status recorded in 2001, and this rose to $99.7 \%$ in $2007(\mathrm{p}<0.001)$. In addition there was a marked drop in the number of recorded smokers from $25.0 \%$ in 2001 to $13.1 \%$ in 2007 ( $\mathrm{p}<0.001$ ).

Conclusion: Standards of care and data recording have improved substantially since 2001. By 2003 GPs were well placed to respond to the GMS contract, and standards have continued to rise with its implementation. In many cases this has led to a significant improvement in modifiable risk factors for the long term complications of diabetes.

\section{OP 19 GLP-1 based therapies}

\section{9}

DPP-IV inhibitors influence fat metabolism through modulation of NPY's paracrine antilipolytic action in adipose tissue

K. Kos, A. R. Baker, A. L. Harte, J. P. O’Hare, P. G. McTernan, S. Kumar; Diabetes and Endocrinology, Warwick University, Coventry, United Kingdom.

Background and Aims: Dipeptidyl-IV peptidase (DPP-IV) inactivates the incretin hormone glucagon-like peptide-1 (GLP-1). However, its action is not specific to GLP-1 and neuropeptide Y (NPY) is one of DPPIV's best substrates. This orexigenic hormone NPY is not only a central neurotransmitter but also found in cerebrospinal fluid and at lower levels in plasma. Recently we have demonstrated its secretion and presence of its receptors in human adipocytes. NPY(1-36) is truncated by DPP-IV to NPY(3-36); as a consequence its affinity changes from NPY receptor Y1, which is mediating the antilipolytic function of NPY, to receptor Y4 and Y5. As such, treatment of Type 2 diabetic patients with DPP-IV inhibitors, which are used as antihyperglyacemic agents, could affect NPY's regulation on fat metabolism.The aim was to investigate whether DPP-IV is expressed in adipose tissue (AT), whether it is associated with NPY receptor expression and whether it affects NPY metabolism.

Materials and Methods: Ex vivo human abdominal subcutaneous (AbdSc) AT was taken from women undergoing elective surgery (BMI: $27.5($ mean $\pm \mathrm{SD}) \pm 5 \mathrm{~kg} / \mathrm{m}^{2}$, Age: $43.7 \pm 10 \mathrm{yrs}, \mathrm{n}=18$ ). Isolated AbdSc adipocytes were treated with 1-100nM rhNPY with and without $1 \mathrm{M}$ of DPP-IV inhibitor (Sigma, Poole, UK); glycerol release was measured as an index of lipolysis and DPP-IV mRNA and NPY Y1 expression assessed in AbdSc AT.

Results: Treatment with NPY reduced glycerol release which was further blunted by co-incubation with DPP-IV inhibitor (baseline 234(mean \pm SE) $\pm 23 \mu \mathrm{mol} / \mathrm{l}$, NPY100: $187 \pm 30 \mu \mathrm{mol} / \mathrm{l}^{*}$; NPY100 with DPPIVinhibitor: $\left.121 \pm 14 \mu \mathrm{mol} / \mathrm{l}^{* *},{ }^{*} \mathrm{p}<0.01,{ }^{* *} \mathrm{p}<0.01, \mathrm{n}=8\right)$. DPP-IV is expressed in human subcutaneous tissue and omental AT and the relative DPP-IV mRNA expression was reduced in AbScAT taken from obese subjects versus lean subjects (obese: $77 \pm 6$ signal units (SU) versus lean: $186 \pm 29 \mathrm{SU}^{*}, \mathrm{n}=10$ ). NPY Y1 mRNA is expressed in all fat depots and is significantly higher in obese subjects, especially in AbdSc tissue (obese: 1944 $111 \mathrm{SU}$ versus lean: $711 \pm 112 \mathrm{SU}^{* *}, \mathrm{n}=10$ ).

Conclusion: The study of the paracrine effects of NPY in AT reveals that NPY is regulated by adipose tissue derived DPP-IV, which was previously thought to be predominantly derived from endothelial vascular cells. The endogenous AT-derived DPP-IV is reduced in obese subjects suggesting that DPP-IV inhibitors may have little effect on AT mass. This may explain the lack of weight loss observed in Type 2 diabetic patients treated with DPP-IV inhibitors. In contrast to this, fat accumulation in the lean may be increased through the enhanced antilipolytic effects of NPY. As we have previously shown NPY is further upregulated by in vitro insulin treatment which may have clinical significance in the hyperinsulinaemic patient.

\section{0}

Exenatide improved markers of hepatic function over 3 years in patients with type 2 diabetes

T. Okerson ${ }^{1}$, D. Maggs ${ }^{1}$, X. Guan ${ }^{1}$, C. Bowlus ${ }^{2}$, J. Holcombe ${ }^{3}$, M. Wintle ${ }^{1}$, L. Nielsen ${ }^{1}$;

${ }^{1}$ Amylin Pharmaceuticals, Inc., San Diego, United States, ${ }^{2}$ Department of Internal Medicine, University of California Davis, United States,

${ }^{3}$ Eli Lilly and Company, Indianapolis, United States.

Background and Aims: Type 2 diabetes (T2D), obesity, and nonalcoholic fatty liver disease (NAFLD) are emerging public health concerns that often co-exist with diabetes and obesity as the two most important risk factors for NAFLD. Because exenatide is associated with improvement in glycaemic control and weight reduction, we evaluated the effects of the incretin mimetic exenatide over $3 \mathrm{y}$ in T2D patients not achieving glycemic control with MET and/or a SU.

Materials and Methods: Patients were enrolled in 30-wk, placebo-controlled trials followed by open-label extensions $(64 \% \mathrm{M}, 58 \pm 10 \mathrm{y}, 99 \pm 18 \mathrm{~kg}$, BMI $\left.34 \pm 5 \mathrm{~kg} / \mathrm{m}^{2}, \mathrm{AlC} 8.2 \pm 1.0 \%[\mathrm{mean} \pm \mathrm{SD}]\right)$. In this ad-hoc analysis, 217 patients 
were evaluated for ALT and AST stratified by baseline ALT (normal: $\mathrm{F}<19$ IU/L, M $\leq 30 \mathrm{IU} / \mathrm{L}$ ) as surrogate markers of NAFLD.

Results: Patients completing $3 \mathrm{y}$ on exenatide had reductions in $\mathrm{AlC}$ $(-1.0 \pm 0.1 \% ; \mathrm{p}<0.0001)$, weight $(-5.3 \pm 0.4 \mathrm{~kg} ; \mathrm{p}<0.0001)$, fasting plasma glucose $(-1.3 \pm 0.2 \mathrm{mmol} / \mathrm{L} ; \mathrm{p}<0.0001)$, and ALT $(-4.5 \pm 1.1 \mathrm{IU} / \mathrm{L} ; \mathrm{p}<0.0001)$ $[$ mean $\pm S E]$. Exenatide was generally well tolerated. The correlation coefficient for ALT change vs. A1C change was $\mathrm{r}=0.25$, and for ALT change vs. weight change was $r=0.31$. Patients with normal baseline ALT $(21.3 \pm 0.5$ $\mathrm{IU} / \mathrm{L} ; \mathrm{n}=101 ;-0.9 \pm 0.1 \% \mathrm{AlC})$ had minimal increases in ALT $(+2.3 \pm 1.2 \mathrm{IU} / \mathrm{L}$; $\mathrm{p}=0.06)$ or AST $(+3.0 \pm 0.6 \mathrm{IU} / \mathrm{L} ; \mathrm{p}<0.0001)$. In contrast, patients with elevated baseline ALT $(38.4 \pm 1.5 \mathrm{IU} / \mathrm{L} ; \mathrm{n}=116 ;-1.0 \pm 0.1 \% \mathrm{~A} 1 \mathrm{C})$ had reductions in ALT $(-10.4 \pm 1.5 \mathrm{IU} / \mathrm{L} ; \mathrm{p}<0.0001)$ and AST $(-4.1 \pm 1.0 \mathrm{IU} / \mathrm{L} ; \mathrm{p}<0.0001)$. There was a difference in 3-y weight change for normal vs. elevated baseline ALT $(-4.4 \pm 0.5 \mathrm{~kg}$ vs. $-6.1 \pm 0.6 \mathrm{~kg} ; \mathrm{p}=0.03)$. Patients with elevated baseline ALT in the weight change quartile with the greatest weight loss $(n=29)$ had the greatest ALT reduction $(-16.3 \pm 3.1 \mathrm{IU} / \mathrm{L}$; baseline $37.2 \pm 2.9 \mathrm{IU} / \mathrm{L})$ coupled with $-2.0 \pm 0.2 \%$ A1C and $-13.9 \pm 0.7 \mathrm{~kg}$.

Conclusion: T2D patients treated with exenatide for 3 years had improvements in hepatic biomarkers, with reductions in $\mathrm{A} 1 \mathrm{C}$ and weight. These data support further investigations into possible improvements in NAFLD with exenatide treatment.

\section{1}

Sitagliptin, a selective DPP-4 inhibitor, and metformin have complementary effects to increase active GLP-1 concentrations E. M. Migoya ${ }^{1}$, J. L. Miller ${ }^{1}$, P. J. Larson ${ }^{1}$, M. R. Tanen ${ }^{1}$, D. Hilliard ${ }^{1}$, C. F. Deacon ${ }^{2}$, M. J. Gutierrez ${ }^{3}$, S. A. Stoch ${ }^{1}$, G. A. Herman ${ }^{1}$, P. P. Stein ${ }^{1}$, J. J. Holst ${ }^{2}$, J. A. Wagner ${ }^{1}$;

${ }^{1}$ Merck Research Laboratories, Rahway, United States,

${ }^{2}$ Department of Medical Physiology, University of Copenhagen, Denmark,

${ }^{3}$ Division of Comprehensive Neuroscience, Miramar, FL, United States.

Background: The potential complementary effects of sitagliptin (SITA) and metformin (MF) on the incretin axis (GLP-1 and GIP) were assessed.

Methods: A randomized, placebo ( $\mathrm{PBO}$ )-controlled, double-blind, 4-period crossover study was conducted in 16 healthy adults. In each 2-day treatment period, subjects received either SITA $100 \mathrm{mg}$ q.d., MF $500 \mathrm{mg}$ b.i.d., coadministration of SITA $100 \mathrm{mg}$ q.d. and MF $500 \mathrm{mg}$ b.i.d. or PBO. On Day 1, subjects received study medications in the AM and PM. On Day 2, subjects received study medications in the AM and, at 2 hours postdose, ate a standard meal (765 calories; $25 \%$ fat, $55 \%$ carbohydrate and $20 \%$ protein). Blood samples for active and total GLP-1 and GIP concentrations were collected pre- and at specified times post-meal.

Results: SITA alone and MF alone each increased post-meal incremental active GLP- 1 concentrations by 1.5 to 2 -times and in combination by $>4$-times. Active and total GLP-1 concentrations were increased to a similar extent by MF; SITA increased active but diminished total GLP-1 concentrations (table). Active GIP concentrations increased with SITA, but were unchanged with MF.

\begin{tabular}{lcllll} 
Treatment & $\mathrm{N}$ & \multicolumn{2}{c}{ Geometric } & \\
\cline { 3 - 6 } & & LS Means & $\mathrm{GMR}^{\dagger}$ & $95 \% \mathrm{CI}^{\ddagger}$ & p-value \\
\hline Active GLP-1 (pM) & & & & & \\
SITA & 16 & 16.37 & 1.95 & $(1.58,2.40)$ & $<0.001$ \\
MF & 16 & 14.81 & 1.76 & $(1.43,2.17)$ & $<0.001$ \\
SITA + MF & 16 & 34.68 & 4.12 & $(3.35,5.08)$ & $<0.001$ \\
PBO & 15 & 8.41 & & & \\
\hline Total GLP-1 (pM) & & & & & \\
SITA & 16 & 12.43 & 0.70 & $(0.58,0.85)$ & $<0.001$ \\
MF & 16 & 32.14 & 1.81 & $(1.49,2.20)$ & $<0.001$ \\
SITA + MF & 16 & 27.82 & 1.57 & $(1.29,1.90)$ & $<0.001$ \\
PBO & 15 & 17.77 & & &
\end{tabular}

${ }^{\dagger} \mathrm{GMR}=$ Ratio of the post-meal incremental geometric LS means. Reference: Pbo ${ }^{\ddagger} \mathrm{CI}=$ Confidence interval

LS means $=$ Least-squares means

Conclusions: Both SITA and MF increased active GLP-1 concentrations, but through different mechanisms. MF increased both active and total GLP-1 concentrations, suggesting an increase in release of GLP-1. SITA increased active GLP-1 concentrations, but not total, consistent with inhibition of degradation of the active hormone. The decrease in total GLP-1 with SITA is likely due to feedback inhibition of active GLP-1 on GLP-1 release. Combination of SITA and MF provided a complementary (more than additive) effect on active GLP-1 concentrations. This complementary effect may provide a unique benefit of combination therapy in patients with type 2 diabetes through substantial enhancement of the incretin axis. Supported by Merck

\section{2}

Pharmacokinetics and pharmacodynamic effects of oral GLP-1 and PYY3-36: a proof of concept study in healthy subjects

E. Arbit ${ }^{1}$, B. Poller ${ }^{2}$, C. Ganzoni ${ }^{3}$, S. Gass ${ }^{3}$, I. Gomez-Orellana ${ }^{1}$, J. Drewe ${ }^{2}$, C. Beglinger ${ }^{3}$;

${ }^{1}$ Emisphere technologies, Tarrytown, United States, ${ }^{2}$ Clinical Pharmacology, University Hospital, Basel, Switzerland, ${ }^{3}$ Gastroenterology, University Hospital, Basel, Switzerland.

Context: Oral formulations of GLP-1 and PYY3-36, two satiety peptides, were studied. An oral dosage form of these peptides would be preferable in many of the possible therapeutic indications.

Objectives: Our objective was to establish the pharmacological profile of increasing oral doses of GLP-1 and PYY3-36 in healthy volunteers. In addition, the pharmacological effects of GLP-1 were investigated. GLP-1 and PYY3-36 were delivered by the oral-enteric route by means of the eligenTM technology.

Setting: Single center escalating dose study with oral applications.

Subjects and Methods: In the first part, GLP-1 was given orally to 6 male subjects; the treatment consisted of one of the following oral doses of either GLP-1 $(0.5,1.0,2.0$ and $4.0 \mathrm{mg})$ or placebo. In the second part, PYY3-36 was given orally to another 6 healthy male subjects; the treatment consisted of one of the following oral doses of either PYY3-36 $(0.25,0.5,1.0,2.0$ and 4.0 $\mathrm{mg}$ ) or placebo.

Results: The oral administration of both peptides induced a rapid and dosedependent increase in plasma drug concentrations (linear dose-response relationship for GLP-1: $\mathrm{R}=0.96 ; \mathrm{P}=0.011$ and a curve linear dose response for PYY3-36). In addition, oral application of GLP-1 induced a potent effect ( $p<0.05$ vs placebo) on insulin release, whereas oral PYY3-36 induced a trend for inhibition of ghrelin secretion, which was significant ( $<<0.05$ vs placebo) for the highest dose.

Conclusions: This study showed for the first time that satiety peptides such as GLP-1 and PYY3-36, can be delivered orally in humans; GLP-1 was active with regard to stimulation of insulin release and PYY suppressed ghrelin levels after oral administration.

Cmax after oral application of peptides. Data are Mean (SEM)

\begin{tabular}{llllll}
\hline Doses & PLACEBO & $0.5 \mathrm{mg}$ Peptide & $1 \mathrm{mg}$ Peptide & $2 \mathrm{mg}$ Peptide & $4 \mathrm{mg}$ Peptide \\
\hline $\begin{array}{l}\text { Cmax, plasma } \\
\begin{array}{l}\text { GLP-1 } \\
\text { (pmol/L) }\end{array}\end{array}$ & $0.2(0.1)$ & $63.5(18.9)$ & $108.8(42.3)$ & $83.7(14.9)$ & $300.6(99.4)$ \\
$\begin{array}{l}\text { Cmax, plasma } \\
\text { PYY3-36 } \\
(\mathrm{pg} / \mathrm{ml})\end{array}$ & $113(2)$ & $257(59)$ & $450(169)$ & $418(149)$ & $630(150)$ \\
\hline
\end{tabular}

Supported by the Swiss National Science Foundation

\section{3}

Initial combination therapy with sitagliptin and metformin provides effective and durable glycaemic control over 1 year in patients with type 2 diabetes (T2DM): a pivotal phase III clinical trial D. E. Williams-Herman, J. R. Johnson, J. K. Lunceford; Merck Research Laboratories, Rahway, United States.

Background and Aim: Initial monotherapy is often unsuccessful at getting patients to glycemic goals, and due to the progressive nature of T2DM, patients require intensified treatment in order to maintain or achieve optimal glycemic control. Long-term efficacy and safety of initial combination 
therapy with sitagliptin and metformin was assessed in patients with T2DM and inadequate glycemic control (A1C 7.5\% to 11\%) on diet and exercise.

Methods and Materials: After completing an initial 24-wk placebo-controlled phase, patients continued in a 30-wk, double-blind, active-controlled phase. At $\mathrm{Wk} 24$, patients remained on their previous active treatments: sitagliptin $100 \mathrm{mg} / \mathrm{metformin} 2000 \mathrm{mg}$ (S100/M2000), sitagliptin $100 \mathrm{mg} / \mathrm{metformin}$ $1000 \mathrm{mg}$ (S100/M1000), metformin $2000 \mathrm{mg}$ (M2000), metformin $1000 \mathrm{mg}$ (M1000) (all as divided doses administered b.i.d.); and sitagliptin $100 \mathrm{mg}$ q.d. (S100). Patients on placebo were switched to M2000. This report presents 54wk results for patients who received active treatment throughout the study. Results: Of the 1091 patients who were randomized, 748 patients continued into the active-controlled phase: S100/M2000 n=157, S100/M1000 n=148, M2000 $\mathrm{n}=137, \mathrm{M} 1000 \mathrm{n}=122, \mathrm{~S} 100 \mathrm{n}=106$. In the all-patients-treated analysis, mean A1C changes from baseline were $-1.8 \%$ (S100/M2000), $-1.4 \%$ (S100/M1000), $-1.3 \%$ (M2000), $-1.0 \%$ (M1000), and $-0.8 \%$ (S100). With a mean baseline $\mathrm{A} 1 \mathrm{C}$ of $8.7 \%$ ( $8.5 \%$ to $8.8 \%$ across groups), the proportions of patients with an $\mathrm{A} 1 \mathrm{C}<7 \%$ at Wk 54 were $67 \%$ (S100/M2000), 48\% (S100/ M1000), 44\% (M2000), 25\% (M1000), and 23\% (S100). Glycemic response was generally durable over time across treatments. For the patients completing treatment through Wk 54, mean A1C changes from baseline were $-1.9 \%$ (S100/M2000), -1.7\% (S100/M1000), -1.6\% (M2000), -1.2\% (M1000), and $-1.4 \%$ (S100). The incidence of hypoglycemia $(1.1 \%-2.7 \%)$ was low across treatment groups. The incidences of gastrointestinal adverse experiences were similar for the combination groups compared with the respective metformin monotherapy groups.

Conclusions: In this study, the initial combination therapy of sitagliptin and metformin provided durable and substantial glycemic improvements and was generally well-tolerated over 54 wks in patients with T2DM.

Supported by Merck

\section{4}

PHX1149, a selective DPP4 inhibitor, improves postprandial blood glucose control in patients with type 2 diabetes

H.-P. Guler; Phenomix Protocol201 Study Group,

Phenomix Corp., San Diego, United States.

Background and Aims: GLP-1 and GIP are important mediators of blood glucose control that are secreted in the GI tract in response to a meal. They increase insulin secretion and decrease glucagon action. DPP4, an ubiquitous serine protease, degrades GLP-1 and GIP within a few minutes. Inhibitors of DPP4 prolong the half life of these two peptides; as a consequence, insulin secretion is increased and hepatic glucose production is suppressed. PHX1149 is a potent and selective, small molecule DPP4 inhibitor that is highly soluble in water and stays in the extracellular space. PHX1149 is excreted renally, is not metabolized, and its half-life is 10 to $13 \mathrm{hr}$.

Materials and Methods: This was a randomized, double-blind, placebocontrolled, multi-center 28 day study in subjects with type 2 diabetes mellitus. The primary efficacy endpoint was change in the area under the curve (AUC) of postprandial glucose values from Day 1 to Day 28 as measured after a test meal administered in the morning after fasting overnight. 174 subjects were enrolled into 3 active dose groups and one placebo group (1:1:1:1). Subjects had a mean age of 52 years, a mean BMI of $33 \mathrm{~kg} / \mathrm{m}^{2}$, and a mean baseline $\mathrm{HbA}_{1 \mathrm{c}}$ of $8.7 \%$. The majority (59\%) were female. Almost all of the subjects (93\%) were on a stable background therapy of metformin only, while $7 \%$ were on a combination of metformin and a glitazone. Multiple clinical study sites from the US, Mexico, and Australia participated.

Results: Abstract 0114

\begin{tabular}{lllll} 
& $\Delta$ AUC pp glucose $\left[\mathrm{mmol} / \mathrm{L}^{*} \mathrm{hr}\right]$ & $\Delta \mathrm{HbA}_{1 \mathrm{c}}[\%]$ & $\Delta$ AUC pp GLP-1 [pmol/L*hr] & Ex vivo DPP4 [\%] inhibition during test meal \\
\hline Placebo & $0.11 \pm 0.50$ & $-0.45 \pm 0.09$ & $3.90 \pm 2.83$ & - \\
$100 \mathrm{mg}$ & $-2.08 \pm 0.51, p=0.002$ & $-0.46 \pm 0.09$ & $11.63 \pm 2.86, p=0.053$ & 83 \\
$200 \mathrm{mg}$ & $-1.73 \pm 0.49, p=0.008$ & $-0.42 \pm 0.08$ & $16.42 \pm 2.72, p=0.001$ & 89 \\
$400 \mathrm{mg}$ & $-1.88 \pm 0.48, p=0.004$ & $-0.73 \pm 0.08$ & $15.75 \pm 2.71, p=0.0002$ & 96 \\
\hline
\end{tabular}

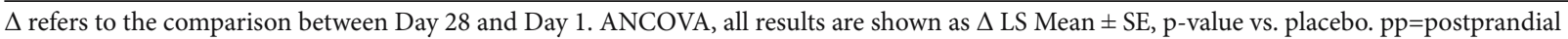

DPP4 inhibition remained high at $24 \mathrm{hr}$ post dose and was $\sim 80, \sim 70$, and adverse events were headache (10.3\%), followed by hyperglycemia $(8.6 \%)$, diarrhea (6.9\%), UTI (6.3\%), and nausea (5.7\%). Edema was reported in the placebo and drug treated subjects; there was no dose relationship in the drug treated subjects. Overall, there was no discernable pattern of drug related adverse events and drug treated patients were similar to placebo. $63.8 \%$ of all subjects reported at least one adverse event and all but 2 events were rated mild or moderate. Two serious adverse events were reported, both in the placebo group.

Conclusion: Patients in this study tolerated PHX1149 very well. No safety issues were identified. DPP4 inhibition during the meal test was near maximal and postprandial blood glucose control improved significantly. $\mathrm{HbAlc}$ in the high dose group was reduced significantly $(\mathrm{p}=0.019)$. GLP-1, the biological mediator was increased in all drug treated subjects. Studies in larger patient groups and over longer time periods will be conducted to further characterize this potential new diabetes drug in the class of DPP4 inhibitors.

Supported by Phenomix Corp. $50 \%$ in the 400,200 , and $100 \mathrm{mg}$ dose groups. The most frequently observed 


\section{OP 20 Diabetes and pregnancy: screening, epidemiology and outcomes}

\section{5}

An approach to translating results of the Hyperglycemia and Adverse Pregnancy Outcome (HAPO) study

D. R. Hadden ${ }^{1}$, B. Persson 2 , D. R. Coustan 3 , A. R. Dyer ${ }^{4}$, M. Hod ${ }^{5}$, L. P. Lowe ${ }^{4}$, B. E. Metzger ${ }^{6}$, J. N. Oats ${ }^{7}$, E. R. Trimble ${ }^{8}$; for the HAPO Study Cooperative Research Group,

${ }^{1}$ Regional Centre for Endocrinology and Diabetes, Royal Victoria Hospital, Belfast, United Kingdom, ${ }^{2}$ Pediatrics, Karolinska Hospital, Stockholm, Sweden, ${ }^{3}$ Maternal Fetal Medicine, Women and Infants Hospital of Rhode Island, Providence, United States, ${ }^{4}$ Department of Preventive Medicine, Northwestern University Medical School, Chicago, United States, ${ }^{5}$ Obstetrics and Gynecology, Rabin Medical Center, Petah-Tiqva, Israel, ${ }^{6}$ Division of Endocrinology, Metabolism and Molecular Medicine, Northwestern University Medical School, Chicago, United States, ${ }^{7}$ Women's Services, Royal Women's Hospital, Melbourne, Australia, ${ }^{8}$ Clinical Biochemistry, Queen's University of Belfast, United Kingdom.

Background and Aims: There is consensus that overt diabetes mellitus during pregnancy, whether or not accompanied by symptoms or signs of metabolic decompensation, is associated with a significant risk of adverse perinatal outcome; however, the risk of adverse perinatal outcome associated with degrees of glucose intolerance less severe than overt diabetes mellitus remains controversial.

Materials and Methods: Approximately 25,000 pregnant women at 15 collaborating centers in 9 countries [Bellflower CA, Chicago IL, Cleveland $\mathrm{OH}$, Providence RI (USA); Toronto ON (CA); Barbados WI; Manchester, Belfast (UK); Petah Tiqva, Beersheba (IS); Bangkok (Thailand); Brisbane, Newcastle (Australia): Singapore (Singapore); Hong Kong (China)] who met criteria for inclusion underwent a 75 gm oral glucose tolerance test (OGTT) at 24-32 wks gestation (mean $28 \mathrm{wks}$ ). Participants and caregivers remained blinded to results of OGTT unless 1 or more values fell outside pre-defined limits (fasting $>5.8 ; 2-\mathrm{hr}>11.1$; any value $<2.5 \mathrm{mmol} / \mathrm{L}$ ). Sociodemographic and health history data were collected via questionnaire and medical record abstraction. Maternal blood was obtained for measurement of serum Cpeptide and hemoglobin Alc $\left(\mathrm{HbA}_{1 \mathrm{C}}\right)$ during the OGTT, and cord blood for fetal serum C-peptide and plasma glucose at delivery. A heelprick capillary specimen was taken between one and two hours following delivery for neonatal plasma glucose. Neonatal anthropometrics were obtained within 72 hours, and follow-up data collected at 4-6 weeks post-delivery. The primary outcomes that are being assessed in relation to maternal glycemia are cesarean delivery, increased fetal size (reflected in macrosomia, large for gestational age or obesity), neonatal morbidity (hypoglycemia), and fetal hyperinsulinism.

Results: The analysis of associations between maternal glycemia and these outcomes is beginning at this time. Initial presentation of the findings from these basic epidemiological assessments will be made at the June 2007 Annual Scientific Meeting of the American Diabetes Association (a co-sponsor of the HAPO Study). In this presentation to the Scientific Meeting of EASD, we will highlight the primary findings as the foundation for the presentation of a strategy that can be used to translate the initial results into clinical applications.

Conclusions: This EASD presentation will represent an important contribution to the dialogue that will be needed to use the HAPO Study results to develop "outcome based" criteria for defining clinically significant levels of hyperglycemia in pregnancy.

Supported by NIH RO1 HD/DK 34242, NIH RO11 HD/DK 3424 and grant from $A D A$

Tab. 1 Abstract 0116

\begin{tabular}{|c|c|c|c|c|c|c|c|c|}
\hline & Japanese & Korean & Vietnamese & Other SE Asian & Filipino & Chinese & Indian & Other Asian \\
\hline N Deliveries & 869 & 1,132 & 1,734 & 622 & 8,146 & 2,841 & 2,136 & 5,010 \\
\hline Mean Maternal Age (yrs.) & 33.3 & 31.7 & 31.3 & 28.4 & 30.8 & 32.6 & 29.7 & 31.3 \\
\hline$\%$ with PGDM & 2.0 & 2.4 & 1.0 & 1.0 & 2.7 & 1.9 & 3.3 & 2.2 \\
\hline Age Adj. Prevalence PGDM & 1.5 & 2.1 & 1.1 & 1.1 & 2.8 & 1.8 & 4.1 & 2.2 \\
\hline$\%$ with GDM & 6.3 & 6.6 & 10.2 & 5.4 & 11.4 & 12.1 & 10.8 & 8.6 \\
\hline Age Adj. Prevalence GDM & 5.0 & 6.9 & 10.0 & 6.7 & 11.3 & 11.4 & 11.9 & 8.4 \\
\hline Trend PGDM & Increase & No change & No change & No change & Increase & Increase & Increase & Increase \\
\hline Trend GDM & No change & Decrease & No change & No change & No change & No change & No change & Decrease \\
\hline
\end{tabular}




\section{7}

Significant effect of maternal DM2 family history on the severity of gestational diabetes mellitus

E. Anastasiou, P. Tourli, K. Terzidis, E. Zapanti, E. Chatzimichelakis, M. Alevizaki;

1st Endocrine Section - Diabetes Centre, Alexandra Hospital, Athens, Greece.

Background: Most epidemiological studies among patients with DM2 report a higher frequency of diabetes history in the maternal rather than the paternal side. This difference has been attributed to a variety of factors including inter alia the influence of the intra-uterine environment. Data is scarce as to whether this pattern is found in women with Gestational Diabetes Mellitus (GDM).

Aim: The aim of this study is to assess the influence of parental family history of DM2 in a large sample of Greek pregnant women.

Patients and Methods: Over a period of 10 years, 6671 pregnant women without known DM underwent a 100g OGTT in the third trimester of pregnancy. For GDM diagnosis the ADA 2000 criteria were used. Among these, 3384 were found with 4 normal values in the OGTT (Group N), 1107 had one abnormal value (Group 1AV), and the remaining 2180 had two or more abnormal values and were diagnosed as GDM (Group GDM). GDM women were treated with diet; insulin was added when optimal glycemic BG thresholds were not achieved (fasting BG $<95 \mathrm{mg}$ and/or 1h-PP $<130 \mathrm{mg}$ ). Age, pre-pregnancy BMI, and family history of DM were recorded. For statistical analysis odds ratios (OR-95\%CI) were calculated, and Tarone's $\mathrm{x}^{2}$ for trends and stepwise logistic regression in combination with reverse Helmert contrasts were used.

Results: The DM2 family history per group and the odds ratio (crude and adjusted) for the onset of GDM are presented in the table below

\begin{tabular}{llllllll}
$\begin{array}{l}\text { Parental } \\
\text { DM History }\end{array}$ & $\begin{array}{l}\mathrm{N} \\
(\%)\end{array}$ & $\begin{array}{l}\text { 1AV } \\
(\%)\end{array}$ & $\begin{array}{l}\text { GDM } \\
(\%)\end{array}$ & $\begin{array}{l}\text { GDM vs N } \\
\mathrm{x}^{2}\end{array}$ & $\mathrm{p}$ & $\begin{array}{l}\text { OR(CI) } \\
\text { for GDM } \\
\text { onset }\end{array}$ & $\begin{array}{l}\text { OR(CI) } \\
\begin{array}{l}\text { BMI, Age } \\
\text { adjusted }\end{array}\end{array}$ \\
\hline Mother & 6.8 & 10.4 & 16.5 & 87.8 & $<0.001$ & $2.73(2.20-3.38)$ & $2.27(1.81-2.86)$ \\
Father & 8.7 & 10.4 & 13.3 & 19.1 & $<0.001$ & $1.61(1.30-1.99)$ & $1.41(1.15-1.80)$ \\
\hline
\end{tabular}

The severity of GDM was expressed in two ways: (i) according to the number of abnormal values in the OGTT, and (ii) according to the use of insulin for optimal glycemic control. The former (i) is presented in the following table:

\begin{tabular}{|c|c|c|c|c|c|}
\hline $\begin{array}{l}\text { OR(CI) for } \\
\text { DM2 History } \\
\text { adj.BMI,Age }\end{array}$ & $\begin{array}{l}\text { No abnormal } \\
\text { glucose } \\
\text { values }\end{array}$ & $\begin{array}{l}1 \text { abnormal } \\
\text { glucose } \\
\text { value }\end{array}$ & $\begin{array}{l}2 \text { abnormal } \\
\text { glucose } \\
\text { values }\end{array}$ & $\begin{array}{l}3 \text { abnormal } \\
\text { glucose } \\
\text { values }\end{array}$ & $\begin{array}{l}4 \text { abnormal } \\
\text { glucose } \\
\text { values }\end{array}$ \\
\hline In mother & 1 (base) & $\begin{array}{l}1.59 \\
(1.19-2.13)\end{array}$ & $\begin{array}{l}2.18 \\
(1.65-2.18)\end{array}$ & $\begin{array}{l}2.89 \\
(2.18-3.84)\end{array}$ & $\begin{array}{l}3.92 \\
(2.79-5.51)\end{array}$ \\
\hline In father & 1(base) & $\begin{array}{l}1.21 \\
(0.92-1.61)\end{array}$ & $\begin{array}{l}1.64 \\
(1.25-2.15)\end{array}$ & $\begin{array}{l}1.61 \\
(1.19-2.18)\end{array}$ & $\begin{array}{l}1.52 \\
(0.99-2.32)\end{array}$ \\
\hline
\end{tabular}

There was a significant correlation between increasing number of abnormal values in OGTT and maternal DM2 history $(\mathrm{p}<0.001)$, with no similar finding on the paternal side. Also (ii) insulin treated GDM women presented a significantly higher OR of maternal family history compared to paternal $\mathrm{OR}=3.30(2.48-4.38)$ vs $1.73(1.27-2.36)$ respectively, $\mathrm{p}<0.001$, Tarone's $\mathrm{x}^{2}$ test. On the contrary, no difference was found in women treated only with diet: $\mathrm{OR}=1.75(1.32-2.32)$ vs $1.55(1.19-2.02) \mathrm{p}=\mathrm{NS}$.

Conclusions: Maternal DM2 family history compared to paternal is a stronger independent risk factor for the occurrence of GDM. An interesting finding was that the maternal compared to paternal DM2 family history was significantly more frequent in the subgroup of GDM women with a more severe form of the disease, as expressed by a greater number of abnormal values in the OGTT and the need for insulin treatment

\section{8}

Should we see and treat all women with previous gestational diabetes (GDM) for GDM in their subsequent pregnancies without screening? C. S. Cotzias ${ }^{1}$, N. I. M. De-Mendonca ${ }^{1}$, T. J. J. Bracewell-Milnes ${ }^{1}$,

R. Kausahl' ${ }^{2}$, J. C. Girling ${ }^{1}$, M. Wu ${ }^{3}$;

${ }^{1}$ Obstetrics and Gynaecology, ${ }^{2}$ Endocrinology and Diabetes,

${ }^{3}$ Clinical Governance, West Middlesex University Hospital, Isleworth, United Kingdom.

Aim: To identify whether women with a history of GDM are demographically different from the newly diagnosed GDM population and whether they have different treatment needs and pregnancy outcomes.

To address whether seeing women with previous GDM early in a subsequent pregnancy is likely to offer better diabetic control.

Introduction: Throughout the literature, quoted recurrence rates of GDM in a subsequent pregnancy, range from about $30-70 \%$ depending on the population studied. In general, the heavier and less Caucasian the population the greater the recurrence rate. Our unit serves a multiethnic community with a high Asian prevalence and our incidence of non-Caucasian ethnicity is over $70 \%$ in women with GDM. As such, any women with a history of GDM are seen in the multi-disciplinary combined obstetric endocrine clinic early in their next pregnancy without further screening. They are reminded about the feto-maternal implications of GDM, the importance of the postnatal (PN) Oral Glucose Tolerance Test (OGTT) and the implications of GDM on their long term health. There is an emphasis on education, focussing on lifestyle changes, dietary control, Capillary blood glucose (CBG) monitoring and the importance of exercise is highlighted. Women unable to achieve target CBG levels with diet and exercise alone are commenced on insulin.

Methods: From 2000-2005 inclusive all women treated for GDM in the combined obstetric endocrine antenatal clinic were identified. A retrospective comparison was made between women treated for previous GDM with those newly diagnosed.

Results: From 2000-2005 inclusive there were 474 women treated for GDM in our unit. $419(88 \%)$ notes were reviewed, of which 123 women $(29 \%)$ had previous GDM and 296 (71\%) had not. Women with previous GDM were older (mean age 34 years compared to 32 years in the Newly Diagnosed Group (NDG) and heavier (median BMI $29 \mathrm{~kg} / \mathrm{m} 2$ compared to median BMI $27 \mathrm{~kg} / \mathrm{m} 2$ ). There was no difference in ethnicity between the two groups. More women with previous GDM had a first HBA1C $>=7(\mathrm{p}<0.005)$ and were more likely to need insulin in addition to diet and exercise to achieve target CBGs (82 / 123 (66.7\%) vs $139 / 296(47 \%) \mathrm{P}<0.00000) .48 / 82$ (58.3\%) of women with previous GDM who required insulin needed it before 28 weeks gestation (when routine GDM screening occurs). This is highlighted by a significantly earlier gestation at which insulin was started in the previous GDM group (median 25.1 weeks vs median 33.4 weeks $\mathrm{P}<0.0000)$ compared to the NDG. 66/123 (53.7\%) of previous GDMs returned for PN GTT compared to $188 / 296$ (63.5\%) of the NDG, and there was no significant difference in the rate of abnormal PN OGTT results. There were no differences in rates of mode of delivery, shoulder dystocia or serious peri-natal complication between the 2 groups.

Conclusion: For an older heavy multiethnic antenatal population we recommend seeing and treating all women with previous gestational diabetes (GDM) for GDM in their subsequent pregnancies without screening, as they seem to have earlier onset more insulin resistant carbohydrate intolerance. Supported by Novo Nordisk

\section{9}

A systematic review on perinatal outcome in women with type 1 vs those with type 2 diabetes mellitus

A. García-Patterson ${ }^{1}$, M. Balsells ${ }^{2}$, I. Gich ${ }^{3}$, R. Corcoy ${ }^{1}$; ${ }^{1}$ Servei d'Endocrinologia, Hospital de Sant Pau, Barcelona,

${ }^{2}$ Servei d'Endocrinologia, Hospital Mutua de Terrassa, Terrassa,

${ }^{3}$ Servei d'Epidemiologia Clinica, Hospital de Sant Pau, Barcelona, Spain.

Background and Aims: Increasing attention is paid to pregnancies of women with Type 2 diabetes mellitus (T2DM) due to their increasing prevalence and reports indicating that perinatal outcome can be worse than in women with Type 1 diabetes mellitus (T1DM). We aimed to perform a systematic review on perinatal outcome in women with T2DM vs T1DM.

Materials and Methods: A Medline search was performed using "diabetes AND pregnancy AND (pregestational OR type 1 OR type 2 OR insulin 
dependent OR non insulin dependent)" as query and limiting the search to human studies and the period 01/01/1995 - 31/12/2006. Abstracts and papers were reviewed by two independent reviewers and discrepancies settled with a third one. Whenever an abstract could fulfil the entry criteria, the original paper was reviewed. Entry criteria were papers providing original data of pregnancies in women with T1DM and T2DM, with 15 or more pregnancies for each type of diabetes, and reporting information on perinatal outcome. Comparison of background characteristics in women with T1DM and T2DM was performed with ANOVA (quantitative variables) and Mantel-Haenszel (categorical variables) tests. Comparison of outcomes was performed using meta-analysis tools (Review Manager software, v 4.2.10).

Results: Retrieved abstracts were 2781 and reviewed papers 107; 30 papers fulfilled entry criteria. Patients with T2DM were significantly older and heavier than those with T1DM, had higher rates of chronic hypertension, lower rates of prepregnancy care, less years of diabetes duration and better metabolic control throughout pregnancy. Women with T2DM had a higher risk of major congenital malformations (OR 1.33, 95CI 1.00-1.79, p 0.05) and similar risk of stillbirth (OR 1.11, 95CI 0.76-1.61), neonatal mortality (OR $1.54,95 \mathrm{CI} 0.88-2.70)$ and perinatal mortality (OR 1.32, 95CI 0.90-1.92) than women with T1DM.

Conclusion: In a systematic review of the last twelve years, pregnant women with T2DM had worse background characteristics and better metabolic profile than those with T1DM. Perinatal outcome in terms of fetal mortality and major congenital malformations was similar or worse.

\section{0}

Pregnancy outcomes in patients with microalbuminuria and their clinical status 3 years post partum

N. G. Asatiani ${ }^{1}$, R. B. Kurashvili ${ }^{1}$, M. G. Dundua ${ }^{1}$, E. L. Shelestova ${ }^{1}$, K. Pagava ${ }^{1}$, M. Hod ${ }^{2}$, Smirnov ${ }^{3}$, V. Vlasov ${ }^{3}$;

${ }^{1}$ Georgian Diabetes Center, Georgian Diabetes Center, Tbilisi, Georgia, ${ }^{2}$ Dep. of Obstetrics and Gynecology, Rabin Medical Center, Tel-Aviv, Israel, ${ }^{3}$ Novo Nordisk, Copenhagen, Denmark.

Background and Aims: Of the present work was to assess outcomes of pregnancies proceeding with microalbuminuria and clinical status of these women 3 years postpartum ( $3 \mathrm{yrsP})$.

Materials and Methods: In total 176 patients (pts) with type 1diabetes (T1DM) were enrolled in the study. Based on microalbuminuria (MicrA) levels in the $1^{\text {st }}$ trimester (tr) women were separated into 2 groups (Gr.). Gr.1 - 134 (76.2 \%) women with normal urinary albumin excretion (UAE), Gr.2 - $42(23.8 \%)$ women with MicrA (UAE<300 mg/24h). Pts were supervised throughout the pregnancies and delivery; repeated examinations were performed 3yrs PP. Pts with MacrA were not enrolled in the study.

Results: Mean HbAlc in the $1^{\text {st }}$ tr was $7.2 \pm 0.9 \%$ (Gr.1) and $6.9 \pm 1.09 \%$ (Gr.2), in the $3^{\text {rd }}$ tr it dropped to $5.5 \pm 0.64 \%$ (Gr.1) and $5.8 \pm 0.74$ (Gr.2), $\left(\mathrm{P}=0.000 ; \mathrm{P}=0.000\right.$, respectively). In Gr. 1 no MicrA was observed in the $1^{\text {st }}$ tr, though $12(8.9 \%)$ pts had it in the $3^{\text {rd }}$ tr; all Gr.2 pts had MicrA in the $1^{\text {st }} \operatorname{tr}, 6(14,2 \%)$ of them developed MacrA (UAE $>300 \mathrm{mg} / 24 \mathrm{~h}$ ) in the $3^{\text {rd }}$ tr. During pregnancy Gr.2 pts experienced gradual increase in daily amount of UAE: $~^{\text {st }} \operatorname{tr}-124.4 \pm 76.7 \mathrm{mg} / 24 \mathrm{~h} ; 3^{\text {rd }} \operatorname{tr}-289 \pm 143.3 \mathrm{mg} / 24 \mathrm{~h}$. No correlation between $\mathrm{UAE}$ and $\mathrm{HbAlc}(\mathrm{r}-0.363, \mathrm{P}=0.036)$ was revealed. In $\mathrm{Gr} .1$ and 2, respectively, we registered: preeclampsia - in $6.7 \%$ and $30.9 \%(\mathrm{P}<0.001)$; preterm delivery $-13.4 \%$ and $33.3 \%$ of women $(\mathrm{P}<0.001)$; mean infant's birth weight $-3435 \pm 320 \mathrm{~g}$ and $3287 \pm 398 \mathrm{~g}$. In Gr.1 - 3 (5.2\%) and in Gr.2 - 7 $(16.6 \%)$ newborns had respiratory distress syndrome. Repeated examinations 3yrsPP showed that $\mathrm{HbAlc}$ levels were statistically higher, that at term: 7.8 $\pm 1.04 \%$ - Gr.1 and $8.4 \pm 1.12 \%$ - Gr.2. Kidney function indices worsened 3yrsPP: Gr.1 - 21 (15.6\%) pts had MicrA; Gr.2 - 31 (73.8\%) pts had MicrA, 7 (16.6\%) pts had MacrA, and only 9.5\% had NormA. Three (7.1\%) Gr.2 pts are on regular hemodialysis; 1 (2.3\%) died of acute renal failure, and 1 had kidney transplantation.

Conclusion: If MicrA is present in the $1^{\text {st }}$ tr, pregnancy in T1DM pts more often ends in preeclampsia, preterm delivery and respiratory distress syndrom, than in NormA. In such pts no correlation between UAE and $\mathrm{HbAlc}$ is observed during pregnancy. 3yrsPP kidney function significantly deteriorates, including end-stage conditions, if pregnancy proceeded with MicrA.

\section{OP 21 Adipocyte derived peptides}

\section{1}

Vaspin improves insulin sensitivity in obesity

A. Nakatsuka, J. Wada, K. Murakami, S. Teshigawara, R. Yoshikawa, M. Kanzaki, A. Yasuhara, K. Shikata, H. Makino;

Department of Medicine and Clinical Science, Okayama University Graduate School of Medicine, Japan.

Background and Aims: Otsuka Long-Evans Tokushima fatty (OLETF) rat is an animal model of type 2 diabetes, characterized by abdominal obesity, insulin resistance, hypertension, and dyslipidemia. Differential screening of the genes up-regulated in visceral adipose tissues of obese OLETF rats and down-regulated in non-obese and diabetes-resistant Long-Evans Tokushima Otsuka (LETO) rats led to the identification of an insulin sensitizing adipocytokine in obesity; vaspin (visceral adipose tissue-derived serine protease inhibitor), which is a member of serine protease inhibitor (serpin) gene family. Rat, mouse and human mature vaspins are made up of 392, 394 and 395 amino acids, exhibit $\sim 40 \%$ homology with alphal-antitrypsin. Vaspin mRNA is exclusively expressed in visceral adipose tissue in genetically obese rats and also expressed in white adipose tissues of human and its expression is correlated with BMI and insulin sensitivity.

Materials and Methods: To explore the functional role of vaspin in metabolic syndrome, we prepared recombinant human vaspin protein and generated transgenic (Tg) mice overexpressing vaspin under control of AP2 promoter. Results: Under high fat-high sucrose (HF) chow, ICR mice administered with recombinant vaspin and $\mathrm{Tg}$ mice overexpressing vaspin revealed improved glucose tolerance and insulin sensitivity. Epidydimal fat and liver weight was reduced in vaspin $\mathrm{Tg}$ mice, and their body weight was $\sim 8 \%$ less than wild type (WT) mice under HF chow. Although serum adiponectin levels were similar, leptin levels were significantly higher in WT mice $(10.9 \pm 6.4 \mathrm{ng} / \mathrm{ml})$ compared with TG mice $(2.7 \pm 2.9 \mathrm{ng} / \mathrm{ml})$.

Conclusion: Vaspin improves insulin sensitivity by acting on insulin target organs and may be a new molecular target in the treatment of metabolic syndrome.

\section{Identification of vaspin (visceral adipose tissue-derived serine protease inhibitor)}
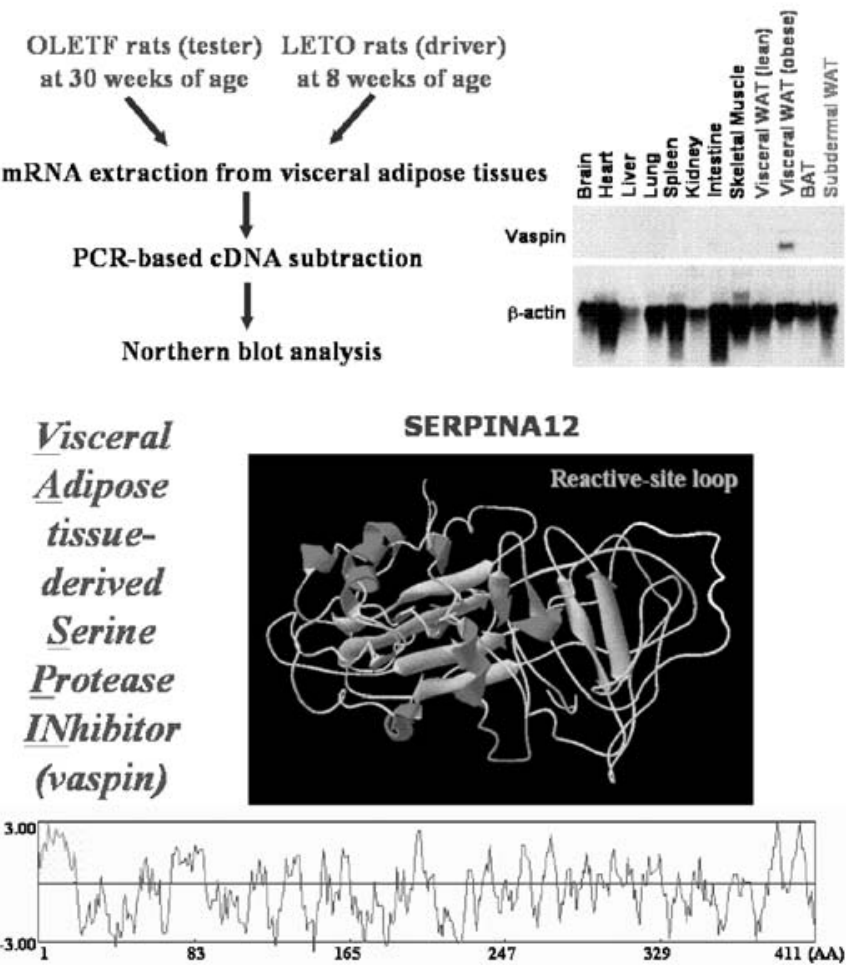


\section{2}

Chemerin is a novel adipokine associated with obesity and metabolic syndrome

K. Bozaoglu ${ }^{1}$, D. Segal ${ }^{1}$, K. Bolton ${ }^{1}$, J. McMillan ${ }^{1}$, J. Jowett ${ }^{2}$, P. Zimmet ${ }^{2}$, G. Collier ${ }^{1,3}$, K. Walder ${ }^{1,3}$;

${ }^{1}$ Metabolic Research Unit, Excercise and Nutrition Sciences, Deakin University, Waurn Ponds, Victoria, ${ }^{2}$ International Diabetes Institute, Caulfield, Victoria, ${ }^{3}$ ChemGenex Pharmaceuticals, Waurn Ponds, Victoria, Australia.

Background and Aims: Secreted proteins and cell surface receptors play crucial roles in intercellular signaling and homeostasis and can provide potential targets for developing novel therapies for diabetes. The aim of this study was to establish a novel signal sequence trap technology (SST) to identify secreted and membrane bound proteins that play a role in obesity and type 2 diabetes (T2D).

Materials and Methods: An SST was used to identify genes that encode secreted or membrane bound proteins from Psammomys obesus. Gene expression of chemerin and chemerin receptor (CMKLR1) were measured in adipose tissue of $P$. obesus and 3T3-L1 adipocytes by real time PCR. Serum chemerin levels were measured by ELISA in human plasma samples. Data analysis was performed using linear and multivariate regression.

Results: Using the SST, we identified the chemokine chemerin and have shown, for the first time, that both chemerin and its receptor CMKLR1 were highly expressed in adipose tissue of $P$. obesus. Furthermore, chemerin and CMKLR1 gene expression was significantly higher in adipose tissue of impaired glucose tolerant (IGT) and T2D P. obesus compared to normal glucose tolerant (NGT) animals $(\mathrm{p}=0.004, \mathrm{p}=0.01$ and $\mathrm{p}=0.009, \mathrm{p}=0.05$ respectively). Real time PCR analysis using fractionated mesenteric adipose tissue from $P$. obesus demonstrated that chemerin and CMKLR1 were predominantly expressed in adipocytes rather than stromal and vascular cells within adipose tissue $(\mathrm{p}=0.0001$ and $\mathrm{p}=0.01$ respectively). Chemerin gene expression was markedly induced during differentiation of 3T3-L1 adipocytes $(\mathrm{p}<0.001$ compared to day 0$)$, while CMKLR1 gene expression was downregulated ( $\mathrm{p}<0.001$ compared to day 0 ). Circulating chemerin levels in human plasma samples were not significantly different between NGT and T2D subjects. However, plasma chemerin levels were significantly higher in subjects with $\mathrm{BMI}>30 \mathrm{~kg} / \mathrm{m}^{2}$ compared with those with a $\mathrm{BMI}<25 \mathrm{~kg} / \mathrm{m}^{2}$ $(296.5 \pm 61.2$ vs $222.7 \pm 67.1, \mathrm{p}<0.001)$. After adjusting for age and gender, chemerin levels were significantly associated with measures of body fat (BMI, fat mass, weight and WHR) and metabolic syndrome related phenotypes (fasting glucose, fasting insulin, plasma triglycerides and blood pressure) in NGT subjects. After further adjustment for BMI, plasma chemerin levels were still independently associated with metabolic syndrome related phenotypes including systolic blood pressure $(\mathrm{p}=0.001)$ and plasma triglycerides $(\mathrm{p}=0.009)$ but not measures of insulin sensitivity or glucose homeostasis. When the data was adjusted for age, gender, BMI and triglycerides, chemerin levels were only associated with systolic and diastolic blood pressure $(p=0.002$ and $\mathrm{p}=0.004$ respectively).

Conclusion: Chemerin is a novel adipokine that exhibits increased gene expression in adipose tissue of obese animals. Subsequent characterization of circulating chemerin levels in human samples demonstrated that chemerin is strongly associated with key components of the metabolic syndrome. These finding suggests that chemerin is a novel adipokine and could be an important contributor to the development of the metabolic syndrome.

\section{3}

Adipocyte adhesion molecule (ACAM) inhibits adipocyte hypertrophy in obesity

K. Murakami, J. Wada, A. Nakatsuka, S. Teshigawara, R. Yoshikawa,

M. Kanzaki, A. Yasuhara, K. Shikata, H. Makino;

Department of Medicine and Clinical Science, Okayama University

Graduate School of Medicine, Japan.

Background and Aims: We identified adipocyte adhesion molecule (ACAM), a novel homologue of cortical thymocyte marker in Xenopus (CTX) gene family. ACAM CDNA was isolated during PCR-based cDNA subtraction and its mRNA up-regulated in white adipose tissues (WATs) of Otsuka LongEvans Fatty (OLETF) rats, an animal model of type 2 diabetes and obesity. ACAM with 372 amino acids has a signal peptide, V-type and C2-type immunoglobulin domains, single transmembrane segment, and a cytoplasmic tail; the amino acid sequence in rat is highly homologous to mouse (94\%) and human (87\%). ACAM mRNA was predominantly expressed in WATs in OLETF rats and increased with development of obesity until 30 weeks of age; that is the peak of body weight. Up-regulation of ACAM mRNAs in obesity was also ascertained in WATs of genetically obese $\mathrm{db} / \mathrm{db}$ mice, diet-inducedobesity ICR mice and human obese subjects. Several stably transfected CHO$\mathrm{K} 1$ cell lines were established and the quantification of ACAM mRNA and cell aggregation assay revealed that the degree of homophilic aggregation well-correlated with ACAM mRNA expression.

Materials and Methods: To explore the functional role of ACAM in the process of metabolic syndrome, we have generated ACAM transgenic $(\mathrm{Tg})$ mice under control of AP2 promotor.

Results: mRNA expression of ACAM was examined and Tg lines were classified as high- (H), medium- (M), and low- (L) expressing Tg mice. Under high fat high sucrose (HF) chow, the increase in body weight was significantly inhibited in ACAM Tg mice compared with wild type (WT) mice and the reduction rate was correlated with ACAM mRNA expression levels, i.e. $~ 9.2 \%$ reduction in $\mathrm{M}$-line and $\sim 56.5 \%$ in $\mathrm{H}$-line. In glucose tolerance tests, glucose levels were significantly lower in Tg mice (M-line) compared with WT mice. Daily intake of HF chow was similar in Tg and WT mice; however respiratory exchange ratio was increased in $\mathrm{Tg}$ mice compared with WT mice.

Conclusion: Adhesion process mediated by ACAM may have an inhibitory role in adipocyte hypertrophy in the status of obesity. ACAM is one of the candidates for the treatment of metabolic syndrome

\section{Hydrophilicity / Hydrophobicity Plot of ACAM}

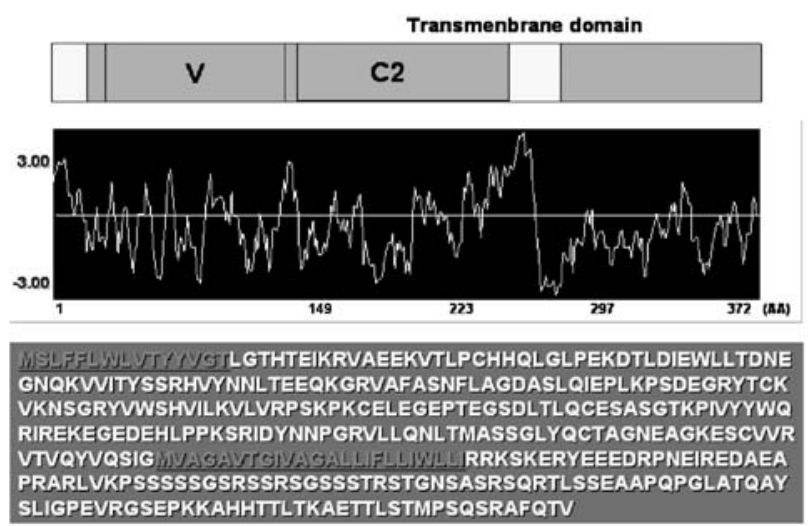

Cell Aggregation Assay

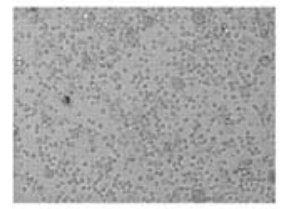

peDNA3.1

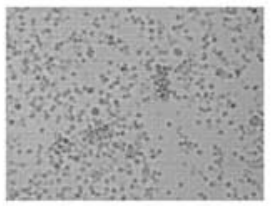

Low expression

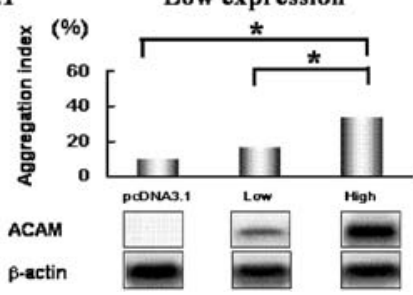

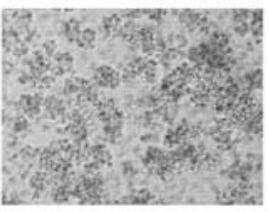

High expression 


\section{4}

The peripheral role of ghrelin: suppression of leptin secretion in human subcutaneous adipocytes

S. J. Goodyear ${ }^{1}$, N. F. da Silva ${ }^{1}$, K. Kos ${ }^{1}$, A. L. Harte ${ }^{1}$, E. Sung ${ }^{1}$, L. S. Wong ${ }^{2}$, J. P. O’Hare' ${ }^{1}$, S. Kumar' ${ }^{1}$, C. Nwokolo ${ }^{3}$, P. G. McTernan';

${ }^{1}$ Division of Clinical Sciences, Unit for Diabetes \& Metabolism, Clifford Bridge Road, Coventry, ${ }^{2}$ Division of Surgery, University Hospitals Coventry and Warwickshire NHS Trust, Clifford Bridge Road, Coventry,

${ }^{3}$ Department of Gastroenterology, University Hospitals Coventry and Warwickshire NHS Trust, Clifford Bridge Road, Coventry, United Kingdom.

Background and Aims: The anorexigenic leptin and orexigenic ghrelin, are peripheral signals that control appetite via the hypothalamic neuropeptide $Y$ (NPY) system. Ghrelin exists in an inactive form (desacetylated) and active (octanylated) which acts via the GHS-R1a. Although the central action of these peptides has been investigated, their peripheral actions remain unclear. Adipose tissue (AT) has been shown to produce an array of neuropeptides. Due to the importance of AT in the control of energy homeostasis we examined 1) AT expression of ghrelin; 2 ) the effect of human recombinant (hr) octanylated ghrelin (OctGhr) and desacetylated ghrelin (DesGhr) on leptin secretion; 3) and the mechanism of action, specifically NFKB activation.

Materials and Methods: Ex vivo human abdominal and omental AT was taken from women undergoing elective surgery (BMI:26.2(mean $\pm \mathrm{SD}) \pm 1.6 \mathrm{~kg} /$ $\mathrm{m}^{2}$, Age: $42.7 \pm 1.6 \mathrm{yrs}, \mathrm{n}=38$ ). Western blot procedures determined ghrelin protein expression. Isolated AbdSc adipocytes were treated with 1-100nM of desacetylated or octanylated ghrelin. Following 48 hours treatment, leptin secretion was measured by ELISA.

Results: Ex vivo human abdominal and omental AT was taken from women undergoing elective surgery (BMI:26.2(mean $\pm \mathrm{SD}) \pm 1.6 \mathrm{~kg} / \mathrm{m}^{2}$, Age:42.7 $\pm 1.6 y r s, n=38)$. Western blot procedures determined ghrelin protein expression. Isolated AbdSc adipocytes were treated with 1-100nM of desacetylated or octanylated ghrelin. Following 48 hours treatment, leptin secretion was measured by ELISA. Ghrelin was expressed in AT and adipocytes in a depot-specific manner. HrOctGhr (Control $5.2 \pm 1.4 \mathrm{ng} / \mathrm{mL}$; OctGhr: $1 \mathrm{nM}: 4.7 \pm 1.7 \mathrm{ng} / \mathrm{ml} ; 10 \mathrm{nM}: 5.0 \pm 1.6 \mathrm{ng} / \mathrm{mL}$ and $100 \mathrm{nM}: 4.4 \pm 1.3 \mathrm{ng} / \mathrm{ml})$ as well as DesGhr (1nM: $5.3 \pm 1.7 \mathrm{ng} / \mathrm{mL} ; 10 \mathrm{nM}$ : $4.9 \pm 1.4 ; 100 \mathrm{nM}: 4.1 \pm 1.5 \mathrm{ng} /$ $\mathrm{ml}$ ) reduced leptin secretion and remained significant after BMI adjustment for OctGhr ( $\mathrm{p}=0.029, \mathrm{n}=5)$. NFkB activation studies demonstrated a dosedependent increase with OctGhr (OctGhr 1nM 21\%, 100nM 66\%, p=0.01) and DesGhr (DesGhr: 1nM 29\%, 100nM 113\% p=0.03).

Conclusion: Centrally, leptin is known to antagonise ghrelin action. Our data suggests that both forms of ghrelin, partly produced in human adipose tissue, inhibit leptin production in human adipocytes, as part of a feedback loop. These findings implicate AT as a site of neuropeptide production and energy homeostatic regulation.

Supported by Glaxo SmithKline

\section{5}

IGFBP3 secreted by visceral adipose tissue mediates beta cell mass compensation for obesity

N. Palau, J. Altirriba, S. Piquer, R. Gomis, A. Barbera;

Endocrinology and Diabetes, Hospital Clinic/IDIBAPS, Barcelona, Spain.

Background and Aims: In obesity, an increase in insulin secretion and beta cell mass occurs to cope with the development of insulin resistance in order to maintain glucose homeostasis. This beta cell plasticity is essential to avoid the onset of hyperglycemia, however the molecular mechanisms that regulate this process still remain unclear. Besides storing energy, adipose tissue acts as an important endocrine organ secreting a number of adipokine with important effects in other organs. The aim of this study is to analyze the role of adipokines in the obesity-induced beta cell mass increase.

Materials and Methods: Wistar rats were fed with either standard chow (STD) or cafeteria diet (CAF) (65\% of the energy derived from lipids) for 30 days. Pancreas was removed to perform morphometric studies. Adipose tissue from inguinal subcutaneous (iSUB), epididymal (eWAT) and pancreatic visceral ( $p$ VIS) depots were excised and incubated for 4 hours in RPMI medium. This conditioned media was later used to culture INS1E cells and to assay its proliferation rate by $[3 \mathrm{H}]$-thymidine incorporation. Finally we carried out microarray gene profiling of pVIS from obese and lean animals using Affymetrix RAE 2302.0 GeneChip. Normalization and differential expression analysis were performed with RMA and LIMMA software and these results were validated by qRT-PCR in all three adipose depots.

Results: In CAF rats the morphometric and immunohistochemical analysis of the pancreas showed an increase in beta cell mass (STD $6.71 \pm 2.33 v s$. CAF $10.87 \pm 1.62 \mathrm{mg}, p<0.01$ ), hypertrophy (STD $119.3 \pm 6.6$ vs. CAF 128.7

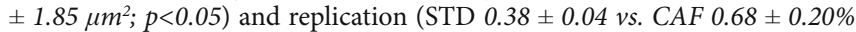
Ki67 + nuclei; $p<0.05)$. INS1E proliferation assays revealed a two fold increase in cells cultured with the obese pVIS conditioned media compared to the lean conditioned media $(p<0.05)$. This effect was only observed in visceral depot and it was suppressed by heat inactivation of conditioned medium, suggesting it was protein mediated. From all the genes differentially expressed in the pVIS arrays, 30 were secreted proteins and some of which are involved in proliferation processes. Using qRT-PCR, the expression of these proteins was determined in pVIS, eWAT and iSUB of lean and obese animals looking for adipokines which only show different expression levels in pVIS and could be responsible for the effect of pVIS conditioned media. This led to the identification of insulin-like growth factor binding protein 3 (IGFBP3) as a possible candidate of this effect. IGFBP3, described as a proliferation inhibitor, showed a two fold downregulation in the pVIS from obese rats without any change in its expression in iSUB and eWAT. Moreover, addition of a neutralizing antibody against IGFBP3 to lean pVIS conditioned medium resulted in an increase in INS1E proliferation rate by $66 \%(p<0.05)$. Finally, treatment of obese conditioned media with IGFBP3 protein had the inverse effect, and INS1E proliferation rate decreased by $66 \%(p<0,05)$.

Conclusion: Results presented indicate that, in obesity, visceral adipose tissue IGFBP3 downregulation is responsible for the increase in beta cell proliferation. This key finding will pave the way for understanding the mechanisms that regulate beta cell mass compensation for obesity.

Supported by grants from the Ministerio de Educación y Ciencia (Spain) and Red de Diabetes y Enfermedades Metabólicas Asociadas(REDIMET) from Instituto de Salud Carlos III, Ministerio de Sanidad y Consumo

\section{6}

Visfatin is regulated by rosiglitazone in type 2 diabetes mellitus and influenced by NF- $\kappa$ B and JNK in human abdominal subcutaneous adipocytes

K. C. McGee ${ }^{1}$, N. F. da Silva ${ }^{1}$, A. L. Harte ${ }^{1}$, S. J. Creely ${ }^{1}$, M. J. Hill' ${ }^{1}$, M. Khanolkar', M. Evans', M. V. Chittari'1, S. Kumar' ${ }^{1}$, P. G. McTernan ${ }^{1}$; ${ }^{1}$ Unit for Diabetes and Metabolism, University of Warwick, Coventry, ${ }^{2}$ Heart Research Institute, Cardiff University, Cardiff, United Kingdom.

Background and Aims: Visfatin, a novel adipocytokine shown to exert insulin mimetic effects, is elevated in the serum of Asian diabetics, suggesting a potential role for this protein in the pathogenesis of type 2 diabetes mellitus (T2DM) and cardiovascular disease (CVD). Whilst central adiposity is closely related to insulin resistance, the role of adipose tissue (AT) in the development of these conditions remains unclear.

Materials and Methods: For this study we investigated visfatin levels in Caucasian non-diabetics (ND) and type 2 diabetic (DB) subjects [ND BMI: 26.0 (mean SD) $\pm 2.7 \mathrm{~kg} / \mathrm{m}^{2}$; Age: $38.9($ mean \pm SD) \pm 12.9 yrs, $\mathrm{n}=23$; DB BMI: $35.9 \pm 7.2 \mathrm{~kg} / \mathrm{m}^{2}$; Age: $56.0 \pm 9.8 \mathrm{yrs}, \mathrm{n}=36$ ] and the influence of the insulin sensitizer, rosiglitazone (RSG). Further analysis examined the in vitro intracellular signalling of visfatin in cultured human Abd Sc adipocytes with the effect of insulin and RSG, as well as the influence of NF- $\mathrm{KB}$ and JNK inhibition on visfatin protein expression.

Results: In vivo studies showed T2DM subjects exhibited higher serum visfatin levels compared with $\mathrm{ND}$ subjects (T2DM: 21.8(mean \pm SEM) $\pm 3.6 \mathrm{ng} / \mathrm{mL}, \mathrm{ND}: 13.9 \pm 1.5 \mathrm{ng} / \mathrm{mL}, \mathrm{p}<0.05)$. We also examined visfatin levels in T2DM patients pre- and post-treatment with the insulin sensitizer, RSG. Post-RSG treated DB showed significant reductions in serum visfatin and insulin compared to pre-RSG treatment (pre-RSG: $10.77 \pm 1.47 \mathrm{ng} / \mathrm{mL}$, post-RSG: $7.45 \pm 1.60 \mathrm{ng} / \mathrm{mL}, \mathrm{p}=0.004)$. In vitro analysis determined that RSG, in combination with insulin, reduced visfatin expression, compared with insulin alone $(\mathrm{p}<0.001)$, in a similar manner to other cytokines, such as secreted TNF $\alpha$ and resistin $(\mathrm{p}<0.001)$. In determining the intracellular mechanism for activation of visfatin protein expression, in vitro studies showed inhibition of NF- $\kappa B$ caused visfatin expression to increase $(\mathrm{p}<0.01)$. In contrast, JNK blockade reduced visfatin protein expression $(\mathrm{p}<0.01)$; however, together, NF- $\mathrm{KB} / \mathrm{JNK}$ blockers caused a reduction in visfatin protein expression $(\mathrm{p}<0.01)$. 
Conclusion: In summary, RSG appears to reduce both circulating visfatin levels as well as regulating protein expression in human isolated adipocytes. Additionally, an NF- $\mathrm{KB} \&$ JNK mediated mechanism appears to contribute to the regulation of visfatin, which appears independent of TNF- $\alpha$ and IL-6 influence in human AT. Reduction of visfatin, together with other cytokines, during RSG treatment is a novel finding that may underlie the clinical metabolic effects of rosiglitazone.

Supported by the Department of Health

\section{OP 22 Signal transduction in metabolism}

\section{7}

Impaired signaling through the PI3K/Akt pathway precedes development of whole-body insulin resistance in young men with low birth weight

C. B. Jensen ${ }^{1}$, M. S. Martin-Gronert ${ }^{2}$, H. Storgaard ${ }^{1}$, S. Madsbad ${ }^{3}$, A. A. Vaag', S. E. Ozanne ${ }^{2}$.

${ }^{1}$ Steno Diabetes Center, Gentofte, Denmark, ${ }^{2}$ Department of Clinical Biochemistry, Cambridge University, United Kingdom,

${ }^{3}$ Department of Endocrinology, Hvidovre University Hospital, Denmark.

Background and Aims: Low birth weight (LBW), a surrogate marker of an adverse foetal environment, is linked to muscle insulin resistance, impaired insulin-stimulated glycolysis and future risk of type 2 diabetes (T2D). Yet, the underlying molecular mechanisms remain largely unknown. We have previously shown reduced protein abundance in skeletal muscle and subcutaneous fat of the p85 $\alpha$ regulatory and p110 $\beta$ catalytic subunits of

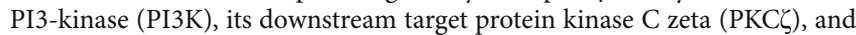
the major glucose transporter GLUT4, in young healthy men with LBW at baseline (fasting) conditions. The aim of this study was to investigate whether activation by insulin of its two main signalling pathways, the PI3K-AKT pathway, responsible for most of its metabolic actions, and the Ras-mitogenicactivated protein kinase (MAPK) pathway, which controls cell growth and differentiation, is altered in LBW subjects.

Materials and Methods: Vastus lateralis muscle was obtained by percutaneous biopsy from 20 healthy 19 -yr old men with birth weights $</=10^{\text {th }}$ percentile for gestational age (LBW) and 20 normal birth weight controls (NBW), matched for body fat, physical fitness and whole-body glucose disposal, before (BAS) and after a 4-hr hyperinsulinemic euglycemic clamp (INS). BAS and INS protein expression of IRS1, Akt1, p-Akt, PKCל, S6K1, p38 MAPK, JNK1, JNK2, and GLUT4, were determined by Western blotting.

Results: At BAS conditions, LBW subjects had lower $\mathrm{p} 85 \alpha, \mathrm{PKC} \zeta$, and GLUT4 (previously reported), and showed a trend for lower IRS1 ( 25\%, $\mathrm{p}=0.06)$, and higher $\mathrm{p}$-Akt $(\sim 27 \%, \mathrm{p}=0.07)$. BAS Akt1, S6K1, p38 MAPK, JNK1, and JNK2 were unaltered. In LBW subjects, insulin stimulation increased IRS1 by 2 -fold ( $\mathrm{p}=0.0008$, vs. no change in NBW; LBW-INS vs. NBW-INS, $p=0.15$ ), increased $p 85 \alpha$ by 2 -fold ( $p=0.00006$, vs. no change in NBW; LBW-INS vs. NBW-INS, $\mathrm{p}=0.001)$, increased PKC $\zeta$ by $60 \%$ ( $\mathrm{p}=0.03$, vs. 2 -fold in NBW, $p=0.0001$; LBW-INS vs. NBW-INS, $p=0.00006$ ), decreased Akt1 by $47 \%$ ( $p=0.002$, vs. $87 \%$ increase in NBW ( $p=0.0001$ ); LBW-INS vs. NBW-INS, $\mathrm{p}=0.00005$ ), increased $\mathrm{p}$-Akt by 3.6 -fold ( $\mathrm{p}=0.0001$, vs. 6.4 -fold in NBW ( $\mathrm{p}=0.00005)$; LBW-INS vs. NBW-INS, $\mathrm{p}=0.001$ ), increased S6K1 by $56 \%$ ( $\mathrm{p}=0.002$, vs. $29 \%$ in NBW ( $\mathrm{p}=0.12$ ); LBW-INS vs. NBW-INS, NS), and increased GLUT4 by $17 \%(\mathrm{p}=0.04$, vs. a $30 \%$ decrease in NBW ( $\mathrm{p}=0.002)$; LBW-INS vs. NBW-INS, $\mathrm{p}=0.01)$. JNK1, JNK2 and $\mathrm{p} 38$ MAPK were not significantly altered by insulin or LBW.

Conclusion: In 19-yr old healthy lean men matched for body fat, physical fitness and whole-body glucose disposal, LBW was associated with marked reductions of two of PI3K's main targets, PKC $\zeta$ and p-Akt. Moreover, despite significant reduction at baseline, the $\mathrm{p} 85$ regulatory subunit of PI3K was dramatically increased in LBW, as compared to NBW. Increased expression of $\mathrm{p} 85 \alpha$ is observed in various insulin resistant states, and excess has been shown to impair insulin signalling through the PI3K/AKT pathway. Thus, impaired signalling through the PI3K pathway in skeletal muscle, potentially through increased p85a expression precedes development of whole-body insulin resistance in subjects with LBW.

Supported by the Danish Research Agency

\section{8}

Role of decreased JNK and IRS-1 ser307 phosphorylation in the enhanced in vitro glucose transport activity of skeletal muscle from Zucker Diabetic Fatty rats following selective GSK-3 inhibition E. J. Henriksen, M. K. Teachey;

Department of Physiology, University of Arizona, Tucson, United States.

Background and Aims: Overactivity of glycogen synthase kinase-3 (GSK-3), a multifunctional serine/threonine kinase, is associated with insulin resistance 
of skeletal muscle glucose transport activity in type 2 diabetic rodents and humans. However, little is known regarding the potential molecular mechanisms underlying the involvement of GSK-3 in the etiology of this insulin resistance in type 2 diabetes. We therefore assessed the functionality of proximal and distal insulin signaling elements in skeletal muscle of male Zucker Diabetic Fatty (ZDF) rats, a model of type 2 diabetes, following acute in vitro exposure to a selective GSK-3 inhibitor. In addition, ser307 phosphorylation of insulin receptor substrate-1 (IRS-1), which has been implicated in the development of insulin resistance, was also determined in the absence or presence of the GSK-3 inhibitor.

Materials and Methods: Soleus strips isolated from 12-week-old male ZDF rats were incubated for $1 \mathrm{hr}$ in the absence or presence of a maximallyeffective concentration of insulin $(5 \mathrm{mU} / \mathrm{ml})$ without or with $1 \mu \mathrm{M}$ CT98014, a selective GSK-3 inhibitor. Glucose transport activity was assessed by 2deoxyglucose uptake, and phosphorylation of insulin receptor, IRS-1, Akt, GSK-3, and c-jun N-terminal kinase (JNK) was performed using standard immunoblotting methods.

Results: Insulin-stimulated GSK-3ß ser9 phosphorylation was significantly less $(35 \%, \mathrm{P}<0.05)$ in soleus muscle of $\mathrm{ZDF}$ rats compared to insulin-sensitive lean Zucker rats, documenting GSK-3 overactivity. In the absence of insulin, no effects of GSK-3 inhibition on ZDF muscle were detected. GSK-3 inhibition in ZDF muscle induced significant enhancement (28\%) of insulin-stimulated glucose transport activity that was associated with significant upregulation of tyrosine phosphorylation of insulin receptor (52\%) and IRS-1 (50\%) and with enhanced phosphorylation of Akt ser473 (48\%) and GSK-3ß ser9 (36\%). Moreover, selective GSK-3 inhibition caused a significant reduction in phosphorylation of IRS-1 ser307 (26\%) and JNK (31\%), a mediator of IRS-1 ser307 phosphorylation.

Conclusion: These results indicate that selective inhibition of GSK-3 activity in isolated skeletal muscle from type 2 diabetic ZDF rats enhances IRS-1dependent insulin signaling, possibly by a decrease in JNK activation and a diminution of the deleterious effects of IRS-1 ser307 phosphorylation.

Supported by a grant from the National Institutes of Health

\section{9}

Crosstalk of insulin-dependent serine phosphorylation sites Ser-302, Ser-307 and Ser-318 of IRS-1

C. Weigert, M. Kron, A. K. Pohl, H. Runge, H. U. Haring, E. D. Schleicher, R. Lehmann;

Internal Medicine, University of Tuebingen, Germany.

The diverse phosphorylation pattern of the multiple serine/threonine phosphorylation sites of IRS-1 suggest that they act as an orchestra rather than as solists. We aimed to investigate the interplay of the adjacent serine residues Ser302, Ser307, and Ser318 in insulin signal transduction. Phosphorylation kinetics were performed in L6 myotubes. For transient expression of IRS1 mutants and transfection of siRNA oligonucleotides C2C12 myoblasts were used. Studies on the insulin-stimulated phosphorylation of the three sites showed a sequential phosphorylation: a high basal phosphorylation of Ser302 (maximum after $10 \mathrm{~min}$ ), a maximum phosphorylation of Ser318 after $30 \mathrm{~min}$, and of Ser307 after $60 \mathrm{~min}$, respectively. We found three different kinases involved in this sequential phosphorylation: PKCzeta in early phosphorylation of Ser318, mTOR or a mTOR-dependent kinase in the later phosphorylation of Ser318, and JNK in phosphorylation of Ser307. The basal phosphorylation of Ser302 appeared to be important for the early phosphorylation of Ser318, which was clearly reduced after mutation of Ser302 to alanine. In contrast, the early phosphorylation of Ser318 appeared not to be necessary for phosphorylation of Ser307. Furthermore, PKCzeta was not necessary for insulin-induced JNK activation or JNK/IRS-1 interaction. Cells expressing a IRS-1 mutant simulating the early phosphorylation pattern (i.e. 302E,307A,318E) showed enhanced insulin-induced AKT phosphorylation. Thus, three adjacent serine residues in IRS-1 are phosphorylated by different kinases with different kinetics after insulin stimulation. Hence, the sequential phosphorylation of these sites exemplarily reflects the complex regulation in the orchestra of IRS-1 phosphorylation sites to mediate the activation and inhibition of insulin signaltransduction.

Supported by Landesstiftung Baden-Wurttemberg
0130

RNAi-based gene silencing of ribosomal protein S6 kinase 1 protects against leucine-induced insulin resistance in human skeletal muscle L. Al-Khalili ${ }^{1}$, A. Deshmukh ${ }^{1}$, F. Salehzadeh ${ }^{1}$, R. Fahlman ${ }^{1}$, K. S. Nair ${ }^{2}$; ${ }^{1}$ Molecular medicine and surgery, Karolinska Institutet, Stockholm, Sweden, ${ }^{2}$ Endocrinology Research Unit, Mayo Clinic School of Medicine, Rochester, United States.

Background and Aims: Excessive nutrients, including amino acids, impair insulin action in skeletal muscle. We tested the hypothesis that the branchedchain amino acid leucine induces insulin resistance in primary myotubes via a negative feedback mechanism involving ribosomal protein S6 kinase 1 (S6K1). Materials and Methods: primary human skeletal muscle cells were obtained from rectus abdominus muscle biopsies from healthy donors and the effect of S6K1 on glucose uptake and metabolism was determined by applying RNA interference (siRNA).

Results: siRNA against S6K1 reduced protein expression 64\%. Myotubes were incubated in the absence or presence of leucine $(5 \mathrm{mM})$ for 2 hours. Leucine treatment decreased basal glucose uptake $(22 \% ; P<0.05)$ and glucose incorporation to glycogen $(21 \%, P<0.05)$. Depletion of S6K1 restored basal glucose uptake and metabolism. Insulin increased glucose uptake and glycogen incorporation to glycogen 2.2 and 1.5 -fold, respectively $(P<0.05)$ and depletion of S6K1 enhanced insulin-stimulated glucose uptake $(54 \% ; P<$ 0.05). These finding provide evidence for a direct and essential role of S6K1 in the regulation of skeletal muscle glucose metabolism.

Conclusion: Depletion of S6K1 enhanced basal glucose utilization and protects against the development of insulin resistance in response to excessive nutrients caused by leucine. In conclusion, S6K1 plays an important role in nutrient sensing and the regulation of insulin action on glucose transport and metabolism in human skeletal muscle.

Supported by AFA/Hagelens, Swedish Medical Foundation, Erik and Edith Fernströms Foundation, and the Karolinska Institutet Foundation

\section{1}

Influences of hyperglycaemia, hyperinsulinaemia, and hepatic steatosis on the circadian clock in mouse liver

K. Shima ${ }^{1}$, H. Ando ${ }^{1}$, N. Matsuzawa ${ }^{1}$, T. Takamura ${ }^{1}$, A. Fujimura ${ }^{2}$,

S. Kaneko ${ }^{1}$;

${ }^{1}$ Department of Disease Control and Homeostasis, Kanazawa University Graduate School of Medical Science, Kanazawa,

${ }^{2}$ Department of Pharmacology, Jichi Medical University, Shimotsuke, Japan.

Background and Aims: Major components of energy homeostasis, including feeding behavior and glucose and lipid metabolism, are subjected to circadian rhythms that are regulated by a circadian clock system composed of transcriptional/translational feedback loops involving a set of clock genes. Some of these circadian rhythms are known to be attenuated in obese diabetic patients, and we showed that the rhythmic expression of clock genes is diminished in the liver and visceral adipose tissue of obese diabetic $\mathrm{KK}-\mathrm{A}^{\mathrm{y}}$ mice (Endocrinology 2005). Moreover, mice lacking the molecular clock are obese and develop a metabolic syndrome. Therefore, impairment of the molecular clock in peripheral tissues may be involved in the pathophysiology of obese type 2 diabetes. However, it remains to be determined which pathological alteration is associated with the decreased molecular clock function. To address this issue, we investigated the effects of hyperglycemia, hyperinsulinemia, and steatosis on daily mRNA expression profiles of the clock genes in mouse liver.

Materials and Methods: Male C57BL/6J, db/db, and ob/ob mice were maintained under a condition with controlled temperature and humidity and a 12-h light/12-h dark cycle for more than 2 weeks. A subset of C57BL/6J (B6) mice were given a high-fat diet $(60 \mathrm{kcal} \%$ fat, $1.27 \%$ cholesterol, and $0.5 \%$ sodium cholate) for 5 weeks. At 10 weeks of age, animals were sacrificed to obtain blood and liver samples at the following zeitgeber times (ZT): 0, 6, 12, and 18, in which ZT 0 is defined as lights on and ZT 12 as lights off. Transcript levels of clock genes (Clock, Bmal1, Per1, Per2, Cry1, Cry2, Dbp) in each sample were determined by real-time quantitative PCR.

Results: The $\mathrm{db} / \mathrm{db}$ mice developed obesity (mean body weight $45.4 \mathrm{~g}$; B6, $26.1 \mathrm{~g}$ ), severe hyperglycemia (440 mg/dl; B6, $151 \mathrm{mg} / \mathrm{dl}$ ), and mild hyperinsulinemia $(12.0 \mathrm{ng} / \mathrm{ml} ; \mathrm{B} 6,2.6 \mathrm{ng} / \mathrm{ml})$ at 10 weeks of age. The ob/ob strain developed more severe obesity $(52.4 \mathrm{~g})$ and hyperinsulinemia (86.8 $\mathrm{ng} / \mathrm{ml})$, but less degree of hyperglycemia $(272 \mathrm{mg} / \mathrm{dl})$, than $\mathrm{db} / \mathrm{db}$ mice. All 
of the clock genes examined exhibited significant 24-h rhythmicity not only in control B6 mice, but in both the obese diabetic strains. In accord with our previous findings in KK- $\mathrm{A}^{y}$ mice, the phase of molecular clock was not shifted, but the peak transcript levels of most clock genes were significantly attenuated in both $\mathrm{db} / \mathrm{db}$ and ob/ob mice. Interestingly, the rhythmic mRNA expression of these genes was mildly dampened in $\mathrm{db} / \mathrm{db}$ mice and greatly attenuated in ob/ob mice. Although severe fatty liver was developed in the mice fed a high-fat diet, as well as $\mathrm{db} / \mathrm{db}$ and ob/ob mice, hepatic steatosis hardly affected the rhythmic expression of clock genes in the liver.

Conclusion: In the mouse models of obese diabetes, obesity and/or hyperinsulinemia appeared to be more closely associated with the impaired function of molecular clock than hyperglycemia. The rhythmic expression of clock genes was attenuated in the fatty liver of genetically obese models, but not in a non-obese dietary model. Although further studies are needed to clarify the causal relationship between the function of molecular clock and the development of obesity/hyperinsulinemia, current irregular lifestyle patterns might have led to the worldwide increase in obesity/insulin resistance partly through the impairment of molecular clock.

\section{2}

Rhythmic mRNA expression of clock genes is attenuated in peripheral leukocytes of type 2 diabetic patients

H. Ando ${ }^{1}$, T. Takamura ${ }^{1}$, N. Matsuzawa ${ }^{1}$, K. Shima ${ }^{1}$, S. Kaneko ${ }^{1}$, A. Fujimura ${ }^{2}$;

${ }^{1}$ Department of Disease Control and Homeostasis, Kanazawa University Graduate School of Medical Science, ${ }^{2}$ Department of Pharmacology,

Jichi Medical University, Shimotsuke, Japan.

Background and Aims: Various physiological processes exhibit circadian rhythmicity, which is generated at the cellular level by the core oscillators that are composed of transcriptional/translational feedback loops involving a set of clock genes. Recent studies have suggested that mice lacking the molecular clock are obese and develop a metabolic syndrome. Moreover, we have shown that the rhythmic expression of various genes, including the genes related to molecular clock, metabolism, and cellular processes, is attenuated in the liver and visceral adipose tissue of genetically obese diabetic mice (Endocrinology 2005; Biochem Biophys Res Commun 2006). Therefore, impairment of the molecular clock in peripheral tissues may be involved in the pathophysiology of type 2 diabetes and/or metabolic syndrome. However, it remains to be determined whether the rhythmic expression of clock genes is altered in human subjects with these clinical conditions. To address this issue, we investigated daily mRNA expression profiles of the clock genes in peripheral leukocytes of type 2 diabetic patients.

Materials and Methods: Six non-diabetic subjects (5 males, 23-42 years) and 8 patients with type 2 diabetes ( 5 males, $45-73$ years) were enrolled in this study after written informed consent was obtained from each participant. We excluded subjects who experienced jet lag, shift work, or an acute illness during the 2 weeks preceding the experiment. None of the subjects had insomnia, or were taking psychotropic drugs during the one month preceding the study. The collection of peripheral blood was performed at 9:00, 15:00, 21:00, and 3:00 $\mathrm{h}$ beginning at 9:00 $\mathrm{h}$. Immediately after sampling, leukocytes were isolated and stabilized using the LeukoLock system. Transcript levels of clock genes (CLOCK, BMAL1, PER1, PER2, PER3, CRY1, CRY2) in each sample were determined by real-time quantitative PCR. The protocol was approved by the relevant ethics committee and was conducted in accordance with the Declaration of Helsinki.

Results: The mRNA expression of PER1 exhibited a significant 24-h rhythm $(F=7.7, P<0.001$, one-way ANOVA) with a peak at 9:00 $\mathrm{h}$ and a trough at 21:00. In addition, the mRNA expression of PER3 and BMAL1 showed 24$\mathrm{h}$ rhythmicity. These significant rhythms were observed not only in whole subjects, but in diabetic patients $(P E R 1, F=10.6, P<0.0001$; BMAL1, $F=3.0$, $P<0.05)$. However, the peak levels of PER1 and BMAL1 were significantly decreased by $31 \%$ and $19 \%$, respectively in diabetic patients, compared with non-diabetic subjects.

Conclusion: The rhythmic expression of clock genes in peripheral tissues appears to be dampened not only in obese diabetic mice, but also in patients with type 2 diabetes. Further clinical studies are needed to clarify the causal relationship between the function of molecular clock and the development of type 2 diabetes.

Supported by a Grant-in-Aid from the Ministry of Education, Culture, Sports, Science and Technology of Japan

\section{OP 23 Peripheral neuropathy: novel therapies}

\section{3}

Aldosterone blockade attenuates diabetic neuropathy

in streptozotocin-induced diabetic rats

Y. Takeda, H. Takata, A. Zhu, T. Yoneda, M. Mikiya Usukura, Y. Yamamoto, S. Karashima, M. Yamagishi;

Internal Medicine, Graduate School of Medical Science, Kanazawa

University, Japan.

Background and Aims: Blockade of angiotensin II (Ang II) using ACE inhibitor or type 1 angiotensin II receptor blocker (ARB) is effective to attenuate diabetic neuropathy. We have reported that not only Ang II but also aldosterone plays a key pathophysiological role in the progression of diabetic renal disease. However, little information is available about the potential benefits of specific mineralocorticoid receptor (MR) blocker, eplerenone, on diabetic neuropathy. We understood this study to determine whether MR exists in the peripheral nerve and to investigate the effects of eplerenone on nerve conduction velocity (NCV) and morphometric change in streptozotocin (STZ)-diabetic rats and compared with the effects of ARB, candesartan.

Materials and Methods: The expression of MR mRNA in the rat peripheral nerve and kidney was measured using a real time PCR. The protein expression was done by Western blot analysis. The NCV of STZ-diabetic rats (DM, $\mathrm{n}=10), \mathrm{DM}$ rats treated with eplerenone $(100 \mathrm{mg} / \mathrm{kg}$ in rodent chows, $\mathrm{n}=10)$ or candesartan $(0.2 \mathrm{mg} / \mathrm{kg} /$ day, intraperitoneally, $\mathrm{n}=10)$ for 8 weeks was measured. Mean myelinated nerve fiber area and myelin area were measured in each experimental group.

Results: The MR mRNA expression was detected in rat peripheral nerves and the mRNA levels were almost equal to those in the kidney. The MR protein expression was clearly demonstrated using a Western blot analysis. The NCV of DM rats was $32 \mathrm{~m} / \mathrm{s}$ (mean), and the treatment with eplerenone or candesartan significantly improved NCV $(39 \mathrm{~m} / \mathrm{s}, 46 \mathrm{~m} / \mathrm{s}$ respectively) $(\mathrm{p}<0.05)$. The decreased fiber and myelin sizes seen in DM rats were significantly improved by the treatment with eplerenone or candesartan $(\mathrm{p}<0.05)$.

Conclusion: These results suggest that aldosterone blockade may be effective treatment for diabetic neuropathy. Not only Ang II but also aldosterone may possess some pathophysiological roles for the diabetic neuropathy.

\section{4}

Peripheral nerve protective effect of COMP-angiopoietin-1 in $d b / d b$ diabetic mice

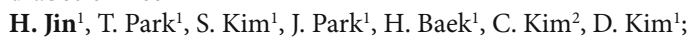
${ }^{1}$ Division of Endocrinology and Metabolism, Department of Internal Medicine, Chonbuk National University Medical School, Jeon Ju, ${ }^{2}$ Division of Endocrinology and Metabolism, Department of Internal Medicine, Bu Cheon Se-Jong Hospital, Bu Cheon, Republic of Korea.

Background and Aims: Recently vascular and inflammatory processes have been reported as underlying pathogenesis of diabetic neuropathy. Angiopoietin1 (Ang1) plays essential roles in regulating vascular growth, development, maturation, permeability, and inflammation. We investigated the effect of cartilage oligomeric matrix protein (COMP)-Ang1 which is a soluble, stable, and potent Ang1 variant on peripheral nerve of diabetic animal model.

Materials and Methods: $d b / d b$ diabetic mice were treated with recombinant adenovirus expressing either COMP-Ang1 or LacZ for 8 weeks. After that, the measurement of intraepidermal nerve fiber and dermal vascular changes in the foot dorsum skin was performed.

Results: COMP-Ang1 significantly decreased blood glucose level during fasting state and after intraperitoneal glucose challenge. In addition, COMPAng1 significantly improved the intraepidermal nerve fiber density $(8.95 \pm$ 3.30 vs $3.57 \pm 0.73, P<0.05)$ and total skin capillary length $(2.79 \pm 0.99$ vs $2.04 \pm 0.58, P<0.05)$.

Conclusion: We demonstrated that COMP-Ang 1 can preserve intraepidermal nerve fiber and capillary vessels in the foot dorsum skin of $d b / d b$ diabetic mice through its diverse effects. Although the exact mechanism of COMPAng1 will need to be investigated in the future, we propose that COMP-Ang1 can be effective agent to prevent or treat diabetic peripheral neuropathy. 


\section{5}

Lack of diabetic peripheral neuropathy progression after three years in patients with mild DPN: experience from the Ruboxistaurin clinical trial E. J. Bastyr, III, C. Cheng, K. L. Price, K. A. Kles, T. Deal, V. Skljarevski, A. Lledo;

Eli Lilly and Company, Indianapolis, United States.

Background: The time course of progression of mild diabetic peripheral neuropathy (DPN) to clinically meaningful changes is unknown. The objective of this analysis was to evaluate the rate of progression of mild DPN in metabolically well-controlled placebo-treated patients in a clinical trial setting.

Design: Baseline to endpoint mean change was analyzed in placebo-treated patients studied for up to 3 years in a randomized, double-blind, placebocontrolled trial that was stopped for futility at 2 years. This trial evaluated the effect of ruboxistaurin, a PKC $\beta$ inhibitor, on a composite measure of nerve function (Neuropathy Impairment Score of the Lower Limbs +4 nerve attributes + vibration detection threshold; NIS [LL] +4+VDT). Mild DPN were identified by sural sensory nerve action potential amplitude $>1 \mu \mathrm{V}$. Change in nerve function as measured by composite score, the neurological examination as measured by the NIS(LL) (range $0-88$ points), and electrophysiology measured by nerve conduction studies were assessed using last observation carried forward.

Results: 348 placebo-treated patients ( $49 \%$ female, 44.9 years) with diabetes (48\% type 1 ) had a mean $\mathrm{HbA}_{1 \mathrm{c}}$ of $7.5 \%$.

\begin{tabular}{llll} 
Measure & & & \\
\hline & Baseline & Endpoint & Change at study end \\
\hline NIS(LL)+4+VDT (pts, n.d.) & 10.12 & 9.50 & $-0.73^{*}$ \\
VDT (JND) & 19.39 & 19.17 & $-0.26^{*}$ \\
NIS(LL) (pts) & 4.73 & 4.46 & $-0.39^{*}$ \\
$\begin{array}{l}\text { Peroneal Nerve Conduction } \\
\text { Velocity (m/sec) }\end{array}$ & 43.11 & 42.78 & $-0.31^{\star *}$ \\
$\begin{array}{l}\text { Peroneal Conduction Velocity } \\
\text { and Amplitude (mV) }\end{array}$ & 4.61 & 4.39 & $-0.18^{* *}$ \\
$\begin{array}{l}\text { Peroneal F Wave Latency } \\
\text { (msec) }\end{array}$ & 51.59 & 51.73 & 0.13 \\
\hline
\end{tabular}

${ }^{*} \mathrm{p}<0.05$ improved, ${ }^{* *} \mathrm{p}<0.05$ worsened, $\mathrm{JND}=$ just noticable difference; n.d.=normal deviates; $\mathrm{m} / \mathrm{sec}=$ meters $/$ second; $\mathrm{msec}=$ milliseconds; $\mathrm{mV}=$ millivolts; pts=points

There was statistically significant improvement in a composite of nerve function (NIS[LL] +4+VDT), VDT and the neurological examination(NIS[LL)). There was no statistical change in electrophysiology attributes of latencies (distal onset and $\mathrm{F}$ wave), while there was a statistically significant worsening of peroneal and tibial conduction velocities and amplitudes. There was a statistically significant worsening of $\mathrm{HbA}_{1 c}(0.31 \%)$. No change in DPN measures reached clinical significance. Intervention with ruboxistaurin did not result in a statistically significant impact on the progression of DPN.

Conclusion: Small changes in progression of neuropathy were observed, therefore in placebo-treated patients with good glycemic control, clinically significant changes in standard measures evaluating disease state progression may require greater than 3 years.

Supported by Lilly Research Laboratories

\section{6}

Chronic treatment with aldose reductase inhibitor zopolrestat suppressed sorbitol, but not fructose, in sural nerves of patients with diabetic neuropathy

P. J. Oates ${ }^{1}$, S. S. Klioze ${ }^{2} \&$ the Zopolrestat Diabetic Neuropathy Study Group.

${ }^{1}$ Cardiovascular Metabolic and Endocrine Diseases, ${ }^{2}$ Clinical Development, Pfizer Global Research \& Development, Groton, United States.

Background and Aims: Metabolism of glucose to sorbitol by aldose reductase (AR) has been implicated in the pathogenesis of diabetic neuropathy, and AR inhibitors (ARIs) have effected dose-dependent reductions in nerve sorbitol
(S) and downstream metabolite fructose (F) and caused improvements in human nerve function and structure. However, the optimal degree of suppression of $\mathrm{S}$ and $\mathrm{F}$ in diabetic human nerve with chronic ARI treatment is unknown. In part to help answer this question, we studied over a period of 18 months the effect of two doses of ARI zopolrestat $(\mathrm{Z})$ on sural nerve polyol content and on nerve function and structure, and on neural signs and symptoms.

Materials and Methods: Protocol 078-107 was a multicenter, doubleblind, placebo-controlled, parallel-group study of 436 type 1 and 2 diabetic patients with moderate peripheral symmetrical diabetic polyneuropathy. The study included a subset of 234 patients who completed 18 months of treatment with placebo (P) or Z, $250 \mathrm{mg} /$ day (LZ) or $500 \mathrm{mg} /$ day (HZ). Nerve conduction velocity (NCV) and amplitude (AMP) for six motor or sensory nerve segments and $\mathrm{HbA}_{1 \mathrm{c}}$ were measured at $0,3,6,12$ and $\sim 18$ months by standard electrophysiological and analytical methods. Quantitative sensory threshold (QST) (vibration; great toe; Vibratron II), neurological signs and symptoms, RR-variation and Quality of Life (QOL) were also assessed at periodic intervals. Sural nerve biopsies were obtained at baseline and at 18 months $(n=172)$, and nerve glucose $(\mathrm{G})$, myo-inositol $(\mathrm{MI}), \mathrm{S}$ and $\mathrm{F}$ were measured. Nerve fiber density (NFD) was determined in chemically-fixed biopsy segments by standard morphometric methods. Data: mean \pm SEM (n); units: $\mathrm{NCV}, \mathrm{m} / \mathrm{sec} ; \mathrm{S}$ and $\mathrm{F}, \mu$ mole/g protein.

Results: $\mathrm{HbA}$ values $(\sim 8.3 \%)$ remained unchanged in all groups, and $\mathrm{Z}$ was generally well tolerated. Modest treatment-related improvements in NCV were seen from 6 to 18 months. The average NCV change from baseline to 18 months for all 6 nerve segments $(n=69-84$ patient NCVs per nerve type per group) were: $\mathrm{P},-0.09 \pm 0.11$ (6); $\mathrm{LZ},+0.49 \pm 0.15$ (6); $\mathrm{HZ},+0.41 \pm 0.18$ (6) $(p=0.031$, ANOVA). No treatment-related effects were found for AMP, QST, RR-variation, NFD, signs, symptoms or QOL. While $\mathrm{S}$ was unchanged in $\mathrm{P}$ $(0.50 \pm 0.09(60)$ at baseline vs. $0.44 \pm 0.04(60)$ at $18 \mathrm{mo}$. (NS)), S was reduced $76 \%$ in LZ (0.46 \pm 0.07 (56) vs. $0.11 \pm 0.03(56))$, and by $80 \%$ in $\mathrm{HZ}(0.46 \pm 0.07$ (56) vs. $0.09 \pm 0.02(56))(p<0.0001, \mathrm{LZ}$ or $\mathrm{HZ}$ vs. baseline). In all groups, $\mathrm{F}$ was unchanged (P, $2.3 \pm 0.1(60)$ vs. $2.4 \pm 0.1(60)$ at $18 \mathrm{mo}$. (NS); LZ, $2.6 \pm 0.2$ (56) vs. $2.7 \pm 0.2(56)(\mathrm{NS})$; $\mathrm{HZ}, 2.6 \pm 0.2(56)$ to $2.6 \pm 0.2(56)(\mathrm{NS})$ ), as were $\mathrm{G}$ and MI.

Conclusion: The present clinical results are consistent with preclinical data that show 1) ARIs dose-dependently suppress sciatic nerve $S$ and $F$ with suppression of F occurring at doses 3 -fold higher than needed to reduce $\mathrm{S}$; and 2) only modest NCV effects occur at ARI doses that lower S without substantially lowering F. The results imply that sorbitol-associated osmotic stress is unlikely to be a key pathogenic factor in human diabetic nerve. Potential therapeutic benefits from ARIs capable of a greater degree of nerve polyol suppression remain to be explored.

\section{7}

Pooled analysis of 7 randomized, controlled trials of pregabalin for the treatment of painful diabetic peripheral neuropathy (DPN): efficacy, safety, tolerability

R. Freeman ${ }^{1}$, J. Rosenstock ${ }^{2}$, B. Emir ${ }^{3}$, E. Durso-Decruz 3 ;

${ }^{1}$ Beth Israel Deaconess Medical Center, Harvard Medical School, Boston, ${ }^{2}$ Diabetes and Endocrinology Dept, Dallas Diabetes and Endocrine Center, ${ }^{3}$ Pfizer Global Pharmaceuticals, New York, United States.

Background and Aims: This abstract aims to more fully describe pregabalin for the treatment of painful DPN across its effective dosing range (150-600 $\mathrm{mg} / \mathrm{d}, \mathrm{BID}$ or TID). To this end, we pooled data from 7 randomized controlled trials of 5-13 weeks' duration.

Materials and Methods: 1510 patients comprised the pooled ITT population: 557 received placebo (PBO), and 953 received pregabalin (176, 266, and 511 received 150,300 , and $600 \mathrm{mg} / \mathrm{d}$ ). The primary efficacy measure in each trial was endpoint (EP) mean pain score (MPS) from patients' daily pain diaries (11-point numeric rating scale [NRS]).

Results: Reductions in MPS from baseline (BL) to EP were significantly larger in pregabalin-treated patients than in those receiving PBO: $150 \mathrm{mg}$ / d, -2.05 ( $P=.0065) ; 300 \mathrm{mg} / \mathrm{d},-2.36(P<.0001) ; 600 \mathrm{mg} / \mathrm{d},-2.75(P<.0001)$; $\mathrm{PBO},-1.49$. Proportions of responders (patients with $\geq 50 \%$ reduction in MPS from BL to EP) were also greater than PBO: $150 \mathrm{mg} / \mathrm{d}, 27 \%(P=.0574)$, $300 \mathrm{mg} / \mathrm{d}, 39 \%(P<.0001) ; 600 \mathrm{mg} / \mathrm{d}, 47 \%$ ( $P<.0001)$; PBO, 22\%. Pregabalin was associated with significantly greater improvement in sleep interference (11-point NRS) from BL to EP than was PBO: $150 \mathrm{mg} / \mathrm{d},-1.92(P=.0033)$; $300 \mathrm{mg} / \mathrm{d},-2.32(P<.0001) ; 600 \mathrm{mg} / \mathrm{d},-2.62(P<.0001) ; \mathrm{PBO},-1.32$. On the 
Patient Global Impression of Change, $65 \%, 74 \%$, and $80 \%$ of patients treated with pregabalin 150,300 , and $600 \mathrm{mg} / \mathrm{d}$ rated themselves as improved vs $54 \%$ of PBO patients $(P<.0001$ for 300 and $600 \mathrm{mg} / \mathrm{d}$ vs PBO). Six of the trials included the SF-36 as a measure of health status. Significant improvements were seen on 5 of $8 \mathrm{SF}-36$ domains among the $300 \mathrm{mg} / \mathrm{d}$ pregabalin group and on 6 of 8 domains among the $600 \mathrm{mg} / \mathrm{d}$ group. Most treatment-emergent AEs were mild to moderate in intensity and of limited duration. Dizziness and peripheral edema were the most common in the $600-\mathrm{mg} / \mathrm{d}$ group, occurring in $34 \%$ and $19 \%$ of patients vs $6 \%$ and $8 \%$ of $\mathrm{PBO}$ patients.

Conclusion: In a population of over 1500 patients, treatment with pregabalin across its effective dosing range was associated with significant improvement in pain and pain-associated sleep interference, as well as improvement in global health status, in patients with painful DPN.

Supported by Pfizer, Inc.

\section{8}

Antioxidant treatment with $\alpha$-lipoic acid in diabetic polyneuropathy: a 4-year randomised double-blind trial (NATHAN 1 Study)

D. Ziegler' ${ }^{1}$, P. A. Low ${ }^{2}$, A. J. M. Boulton 3 , A. I. Vinik ${ }^{4}$, R. Freeman', R. Samigullin ${ }^{6}$, H. Tritschler ${ }^{6}$, U. Munzel ${ }^{6}$, J. Maus ${ }^{6}$, K. Schuette ${ }^{7}$, P. J. Dyck ${ }^{2}$; ${ }^{1}$ Institute for Clinical Diabetology, German Diabetes Center, Düsseldorf, Germany, ${ }^{2}$ Department of Neurology, Mayo Clinic, Rochester, United States, ${ }^{3}$ Department of Medicine, Manchester Royal Infirmary, United Kingdom, ${ }^{4}$ Strelitz Diabetes Research Institutes, Eastern Virginia Medical School, Norfolk, United States, ${ }^{5}$ Beth Israel Deaconess Medical Center, Harvard Medical School, Boston, United States, ${ }^{6}$ Meda Pharma, GmbH \& Co. KG, Bad Homburg, Germany, ${ }^{7}$ Ergomed, GmbH, Frankfurt, Germany.

Background and Aims: To evaluate the efficacy and safety of $\alpha$-lipoic acid over 4 years in diabetic patients with mild to moderate distal symmetric polyneuropathy.

Materials and Methods: In this multicenter, randomised, double-blind, parallel-group clinical trial 460 diabetic patients with stage 1 or stage $2 \mathrm{a}$ polyneuropathy were randomly assigned to oral treatment with $\alpha$-lipoic acid $600 \mathrm{mg}$ qd (ALA; $\mathrm{n}=233)$ or placebo $(\mathrm{n}=227)$ for 4 years following a 6-week placebo run-in phase. Primary outcome measure was a composite score including the Neuropathy Impairment Score of the lower limbs and 7 nerve function tests (NIS[LL]+7 tests). Secondary outcome measures included the Total Symptom Score (TSS), Neuropathy Symptoms and Change (NSC), NIS, NIS[LL], individual NIS components, motor and sensory nerve conduction attributes, and quantitative sensory testing (QST). Data analysis was based on the intention to treat.

Results: The demographic variables and the outcome measures at baseline were comparable between the groups as were the HbAlc levels during followup. The NIS[LL]+7 tests composite score decreased after 4 years vs baseline by $0.45 \pm 0.37$ (mean \pm SEM) in the ALA group and increased by $0.34 \pm 0.35$ points in the placebo group $(\mathrm{p}=0.105)$. The NIS and NIS[LL] improved by $0.68 \pm 0.44$ and $0.34 \pm 0.30$ points on ALA and worsened by $0.61 \pm 0.46$ and $0.43 \pm 0.31$ points on placebo, respectively ( $\mathrm{p}=0.028$ and $\mathrm{p}=0.051$ ). The NIS[LL] muscular weakness subscore improved by $0.21 \pm 0.11$ points on ALA and deteriorated by $0.17 \pm 0.15$ points on placebo $(\mathrm{p}=0.045)$. The NSC score for weakness severity improved by $0.05 \pm 0.03$ points on ALA and worsened by $0.04 \pm 0.03$ points on placebo ( $\mathrm{p}=0.008$ ). No significant differences between both groups after 4 years were noted for the nerve conduction parameters and QST. The rates of adverse events were comparable between the groups during the study. Serious adverse events and deaths occurred in $88(38.1 \%)$ and $3(1.3 \%)$ patients in the ALA group and $63(28.0 \%)$ and $6(2.7 \%)$ patients in the placebo group, respectively.

Conclusion: In conclusion, 4-year treatment with $\alpha$-lipoic acid in mild to moderate diabetic polyneuropathy is well tolerated and improves some neuropathic deficits and symptoms, but not nerve conduction. Future longterm trials should not anticipate significant progression of polyneuropathy in diabetic patients.

Supported by Meda Pharma, Bad Homburg, Germany

\section{OP 24 Zinc transporter SLC30A8 - candidate genes for beta cell function}

\section{9}

\begin{abstract}
A bioinformatic approach to dentification of novel type 1 diabetes autoantigens
\end{abstract}

J. C. Hutton, J. M. Wenzlau, K. Juhl, S. Sarkar, L. Yu, G. S. Eisenbarth, J. Jensen, H. W. Davidson;

Barbara Davis Center for Childhood Diabetes, University of Colorado Health Sciences Center, Aurora, United States.

Background and Aims: Circulating autoantibodies provide important predictive markers for the underlying autoimmunity that may precede clinical disease by many years at a time when therapeutic intervention may be effective. Currently islet cytoplasmic autoantibodies (ICA), insulin, the $65 \mathrm{kD}$ form of glutamate decarboxylase (GAD65) and the protein tyrosine phosphatase IA2 (PTPrN) can detect up to $90 \%$ or more of patients at risk of developing disease. However it is clear that this list is not exhaustive and other candidates remain to be discovered.

Material and Methods: The Novartis custom oligonucleotide array representing 79 different human tissues was initially queried to determine which genes in the pancreatic islet dataset showed a significantly different signal from the median value of all other tissues (ANOVA cutoff $<0.0002$ ). The subset of these transcripts that displayed a signal 5 fold greater than that of the median of all tissues was filtered to remove transcripts showing low signal strengths $(<200)$ and those that were expressed at higher levels in pancreas versus islets. The 140 genes that met these criteria were then used to interrogate the Unigene EST expression database to determine the frequency with which each gene was transcribed in 52 different human tissues. The frequency of appearance of cDNA for each gene in the pancreas versus all other tissue was used as an index of tissue specificity of mRNA expression.

Results: A list of 51 candidate islet autoantigens that was compiled list included major known targets of diabetes autoantibodies above as well other putative targets (heat shock protein 90B, carboxypeptidase E, islet glucose 6 phosphatase related protein (IGRP), islet amyloid polypeptide (IAPP), pancreatitis associated protein (humanReg3a /Mouse Reg2) and ICA69 (islet cell antigen 1). Thirteen gene products have been screened to date in immunoprecipitation assays resulting in the detection of one major and one minor target. 60 to $80 \%$ of newly-diagnosed patients with type 1 diabetes were seropositive with the former molecule and in combination with other autoantibodies detected in up to $98 \%$ of the individuals. Longitudinal studies of high-risk individuals showed that high levels of ZnT8 autoantibodies emerged from 3-4 years of age and generally persisted until onset of disease in the ensuing 1-7 years.

Conclusion: The combined bioinformatics / molecular engineering strategies used to select and develop assays for candidate autoantigens in combination with genetic screening raises to potential of future pediatric screening for type 1 diabetes susceptibility in the general population.

Supported by Childhood Diabetes Foundation, NIDDK

\section{0}

Identification of SIc30A8 as a major humoral autoantigen in type 1 diabetes and a predictive diasease marker J. M. Wenzlau, O. Moua, L. Yu, M. Rewers, G. S. Eisenbarth, J. C. Hutton, H. W. Davidson;

Barbara Davis Center for Childhood Diabetes, University of Colorado Health Sciences Center, Aurora, United States.

Background and Aims: A novel $\beta$-cell specific target of diabetic autoimmunity in humans was identified using a combination of bioinformatics and molecular engineering to design sensitive and specific assays for circulating autoantibodies. The molecule, Slc30A8, is a 6 transmembrane spanning islet-specific member of the large family of cation-efflux ion transporters that is localized to insulin secretory granule of the beta cell. The present study examines the value of measurement of Slc30A8 autoantibodies in the prediction of clinical disease in genetically at-risk individuals 
Material and Methods: Autoantibody assays were conducted using radioimmunoprecipitation assays based on in vitro translation of fragments of the $\mathrm{COOH}$ terminal cytoplasmic domain of Slc30A8 and a N-terminal/Cterminal fusion protein alongside similar assays for insulin, GAD2 and IA2 autoantibodies. Subjects included 223 new-onset patients ranging from 1-40 years of age, age matched controls and first degree relatives and 10 subjects from the Diabetes Autoimmunity Study in the Young (DAISY) from whom sera was obtained from age 9 months to the appearance of disease.

Results: Assays were initially developed to a series of fragments of variable length from the $\mathrm{NH}_{2}$ and $\mathrm{COOH}$ terminal regions and segments joining the transmembrane segments. Autoreactivity to aa1-74 of the N-terminal measured as the percentage specific binding broadly correlated with that of the full-length sequence $\left(\mathrm{R}^{2}=0.605, \mathrm{P}<0.001, \mathrm{n}=186\right)$ but the assay showed relatively low sensitivity ( $8.0 \%$ sensitivity, $98 \%$ specificity, $n=223$ ). A COOH terminal construct spanning aa268-369 produced a robust and sensitive assay (50\% sensitivity, $98 \%$ specificity) with an acceptable background (250-350 $\mathrm{cpm})$. A synthetic molecule that combined both $\mathrm{N}$ and C-term sequences in a single chain construct separated by a Gly3 linker performed almost as well as the C-term construct in terms of specificity and background and in fact complemented the assay such that the assays in combination detected $63 \%$ of new-onset diabetic subjects. When used in combination with the classical biochemical autoantibody markers on a panel of 223 new onset subjects, the number of antibody negative individuals was reduced from 13 to 4 and the number who tested positive for 2 or more autoantibodies was increased from $71.7 \%$ to $82.0 \%(\mathrm{n}=223, \mathrm{P}=0.013)$.

High levels of Slc30A8 autoreactivity did not occur in DAISY subjects until $\geq 3$ years of age. In 2 cases, autoreactivity arose within a year preceding clinical diabetes, in other cases Slc30A8 autoantibodies were present up to 7 years prior to disease.

The question of the predictive value of autoantibodies was examined in serum samples from 88 DAISY subjects by assaying Slc30A8 autoreactivity in the first sample to test positive for a single biochemical antibody. Of this group, $36.8 \%(7 / 19)$ of individuals with Slc30A8 antibodies progressed to clinical diabetes compared to $7.3 \%(5 / 69)$ of those without $(\mathrm{P}<0.0032$-tailed Fisher exact test).

Conclusion: Slc30A8 is a major target of diabetic autoimmunity whose measurement adds power to the risk prediction in pre-diabetes based on autoantibody number especially in older individuals in whom insulin autoantibodies wane with age. Slc30A8, unlike GAD and IA2, has a high B-cell specificity that may be advantageous in monitoring recurrent autoimmunity and possibly $\beta$-cell mass in type 1 diabetic subjects.

Supported by Childhood Diabetes Foundation, NIDDK

\section{1}

The decline in circulating Slc30A8 autoantibodies track falling post-prandial C-peptide responses in the years following type 1 diabetes onset in human subjects

M. Walter ${ }^{1}$, T. Kaupper ${ }^{1}$, A. Ziegler ${ }^{1}$, H. W. Davidson ${ }^{2}$, J. M. Wenzlau², J. C. Hutton ${ }^{2}$.

${ }^{1}$ Diabetes Research Institute, Munich, Germany,

${ }^{2}$ Barbara Davis Center for Childhood Diabetes, University of Colorado Health Sciences Center, Aurora, United States.

Background and Aims: Type 1 diabetes onset in man is typically followed by a honeymoon period with progressive increase in insulin requirements and difficulty of metabolic control. The cellular counterpart of this phenomenon is postulated to be continuing attrition of beta cell mass by cell mediated autoimmunity ultimately leading to complete loss of insulin secreting capacity or achievement of a steady state of beta cell loss and regeneration. Circulating autoantibodies do not necessarily reflect these changes since patients receive autoantigens exogenously (insulin) or immunological memory is maintained by sources of autoantigens located outside of the pancreas (GAD, IA2 and IA2beta). The recent discovery of a major new beta cell specific humoral autoantigen, Slc30A8 provides an opportunity of monitoring changes in beta cell-directed autoimmunity and potentially beta cell mass in this context.

Material and Methods: Sera was obtained from 21 new-onset patients within 2 months of diagnosis and thereafter at 3 monthly intervals for 2 years. Cpeptide responses $120 \mathrm{~min}$ following to a standard meal were measured as an index of endogenous secretory capacity and compared to circulating titers of antibodies directed towards GAD2 (Glutamate decarboxylase 65kD isoform), IA2 (PTPrN) and Slc30A8 using radioimmunoprecipitation assays based on in vitro translation of encoded cDNAs and molecularly engineered constructs.

Results: C-peptide responses generally declined in all patients; in 2 individuals by less than $20 \%$ and in 8 more by than $80 \%$ over the 2 year follow-up. At the outset $76.2 \%$ of patients were seropositive for GADA, $85.7 \%$ for IA-2, $66.7 \%$ for Slc30A8 C-term and $71.2 \%$ for the Slc30A8 N-term/C-term fusion protein construct. All patients had at least one positive autoantibody, 2 patients had GADA only and 1 had IA2 as the only autoantibody. GADA were highly erratic with levels increasing up to 6 fold in 5 patients and decreasing up to 8 fold in the remaining 9. Two individuals who were seronegative at the outset developed low GADA during the course of follow-up. With one exception, IA2 antibodies fell and all individuals showed a progressive decline in Slc30A8 levels that were highly correlated both to disease duration and to the level of C-peptide. Overall the observed annual decline in the $120 \mathrm{~min}$ C-peptide of $33.4 \pm 19.9 \%$ /year was matched by a $27.7 \pm 9.3 \% / y r$ decline in Slc30A8 autoantibodies and a $19.9 \pm 15.7 \% / y r$ change in IA2 autoantibodies (all data; mean \pm SD, $\mathrm{n}=15$ ).

Conclusion: Slc30A8 autoantibody levels calculated as a proportion of the levels at onset of disease provide a potential index of the decline in functional islet mass post disease onset.

Supported by Childhood Diabetes Foundation, NIDDK

\section{2}

The zinc transporter $\mathrm{ZnT}$ 8 protects cultured beta cells from oxidative stress-induced cell death

F. Chimienti, S. Libert, T. Pointecouteau, P. Jolivet, R. Garcia-Cuenca; Mellitech, SCIB/DRFMC, CEA Grenoble, France.

Background and Aims: To date, the zinc transporter ZnT8 has been shown to be expressed at the protein level solely in the secretory vesicles of pancreatic beta cells. ZnT8 overexpression amplified insulin release in response to stimulation with high levels of glucose in a beta cell model. Moreover, a genome-wide association study recently strongly associated SNP in SLC30A8, the gene encoding for ZnT8, with type 2 diabetes risk. Undoubtedly, zinc, insulin and glucose homeostasis thus appear to be closely linked. In many cell types, cellular zinc is described as an inhibitor of apoptosis, while its depletion induces cell death in many cell lines; we have previously shown that ZnT8 overexpression significantly protected INS-1E cells from zinc-depletion-induced cell death. Herein we studied the effects of ZnT8 overexpression on oxidative stress-induced cell death in the MIN6 cell line, and show a protective effect against $\mathrm{H} 2 \mathrm{O} 2$ or xanthine/xanthine oxidase-induced cell death. This protective effect was, at least in part, mediated by overexpression of metallothionein, a small, cysteine-rich, metalbinding protein whose antioxidant role is well documented. Although further studies remain necessary, these results may be of importance for preventing islet cell destruction and prolonging islet graft survival after transplantation in patients with type 1 diabetes mellitus (T1DM).

Materials and Methods: MIN6 cells were transfected with a ZnT8-EGFP expression plasmid. EGFP-positive cell population was selected and used for further experiments. Parental and ZnT-8 overexpressing MIN6 cells were treated with indicated doses of hydrogen peroxyde or xanthine/xanthine oxidase before cell death quantification by MTT assay. Apoptosis and necrosis were quantified using a dual-color flow cytometric procedure with fluorescein labeled annexin- $\mathrm{V}$ and propidium iodide. Total or differential cell extracts were used for western-blot experiments or HPLC/MS-MS analysis of 8oxodGuo levels. Metallothionein was observed by confocal immunofluorescence microscopy on fixed cells, and "free" cellular zinc levels were measured with a fluorescent probe using a pZnmeter.

Results: ZnT8 overexpression significantly protected MIN6 cells from both hydrogen peroxyde- and xanthine/xanthine oxidase -induced cell death. The free zinc levels were higher in ZnT8 overexpressing cells, which also have a higher zinc accumulation capacity. Higher free zinc levels leaded to upregulation of metallothioneins, which functions at a central node in cellular signaling by both redistributing cellular zinc to apoproteins and interconverting redox and zinc signals, including nitric oxide (NO) signals. Indeed, we also observed that ZnT8 overexpression reduces cellular nitrosative stress markers.

Conclusion: The zinc transporter ZnT-8 plays an important role in the multiple regulation pathways of insulin synthesis/secretion, and we show here that it can also significantly protect beta cells from oxidative stressinduced cell death. This suggests that modulation of zinc homeostasis- 
regulating proteins may offer a new target pathway to achieve requirements for preventing islet cell destruction and prolonging islet graft survival after transplantation.

\section{3}

\begin{abstract}
A dominant autoantibody epitope in Slc30A8 maps to a conserved secondary structure in the $\mathrm{COOH}$ terminus

H. W. Davidson, J. M. Wenzlau, O. Moua, G. S. Eisenbarth, J. Hutton; Barbara Davis Center for Childhood Diabetes, University of Colorado Health Sciences Center, Aurora, United States.
\end{abstract}

Background and Aims: The newly discovered type 1 diabetes autoantigen Slc30A8 is a multispanning transmembrane protein that resides in the insulin secretory granule. As such it is presumably incorporated into the plasma membrane upon activation of insulin secretion and may be a beta cell surface marker. A critical immunological question is whether it is contacted by circulating autoantibodies in normal tissue or is only exposed following cell disruption. A more general question is what structural features distinguish it from the many thousand proteins that are expressed in the beta cell to which the immune system is tolerized. Slc30A8 is a member of an exceptionally large gene family (10 genes in man; 1500 overall including invertebrates, plants, prokaryotes and archaebacteria) which further raises the question as to whether molecular mimicry plays a role in its recognition as an antigen. To address these questions we investigated the location of the major epitopes in the structure that were recognized by circulating autoantibodies in human type 1 diabetic subjects.

Material and Methods: Autoantibody assays were conducted using radioimmunoprecipitation assays based on in vitro translation of fragments of Slc30A8 cloned into eukaryote expression vectors. Such constructs included truncated versions of the protein, chimeric constructs between mouse and human Slc30A8 and point mutations that changed individual amino acids in the sequence.

Results: Truncation mutants identified the 104aa COOH terminus (aa265369) as containing a major epitope. Further deletions and the use of synthetic peptides narrowed the region to a conformational epitope between aa280349. Mouse / human chimeras identified a cluster of acidic and basic residues as critical residues, surrounding lysine 340 , an amino acid that is deleted in the non-immunogenic mouse sequence. Secondary structural prediction and application of molecular threading algorithms (PHYRE) provided a plausible model for the epitope in a 86aa dimeric alpha helix beta barrel structure $(75 \%$ precision; E value 0.88 ) that is common to a number of proteins of unrelated protein sequences that share a common property of binding heavy metals. The probable intracellular localization is in the cytoplasmic membrane leaflet similar to other major membrane associated autoantigens IA2, IA2 beta and GAD2.

Conclusion: We have derived a plausible model for major antibody recognition site in Slc30A8 that makes important predictions regarding the relationship of the antigen to proteins of differing primary sequence. Such models can be further refined through crystallographic analysis and to direct futher mutagenesis experiments aimed at exploring the nature and extent of the antibody binding surface .

Supported by Childhood Diabetes Foundation, NIDDK

\section{4}

The long-acting GLP-1 analogue liraglutide regulates zinc transporter mRNA in $\beta$ cells

N. Magnusson ${ }^{1}$, L. Bjerre Knudsen 2 , L. Wogensen ${ }^{3}$, B. Brock ${ }^{4}$, O. Schmitz L. Egefjord ${ }^{1}$, J. B. Jeppesen ${ }^{1}$, J. Rungby';

${ }^{1}$ Pharmacology and Endocrinology C, University of Aarhus, ${ }^{2}$ Diabetes Biology \& Pharmacology, Novo Nordisk, Måløv, ${ }^{3}$ Biochemical Pathology, University of Aarhus, ${ }^{4}$ Pharmacology and Pharmacy, University of Aarhus, ${ }^{5}$ Pharmacology and Endocrinology M, University of Aarhus, Denmark.

Background and Aims: Zinc transporters (ZnT)-proteins regulate zinc flux into $\beta$-cell vesicles. $\beta$-cells depend on zinc for crystallization of insulin. Zinc efflux, which is also regulated by ZnTs controls $\beta$-cell mass and glucagon secretion. Recently, the zinc transporter gene ZnT8 was identified as a novel risk factor for type 2 diabetes. We examined whether glucose levels, zinc depletion, GLP-1 or liraglutide affect ZnT mRNA expression.

Materials and Methods: The rat $\beta$-cell line INS1-E was exposed to 2, 5 or 16 $\mathrm{mM}$ glucose for $24 \mathrm{hrs}$. For zinc depletion the INS1-E cells were exposed to $100 \mu \mathrm{M}$ DEDTC for $24 \mathrm{hrs}$. GLP-1 and liraglutide was added at 5 or $16 \mathrm{nM}$ glucose (GLP-1 1 or $10 \mathrm{nM}$; liraglutide 10 or $100 \mathrm{nM}$ ) for $24 \mathrm{hrs.} \mathrm{ZnT} \mathrm{mRNA}$ was measured by RT-PCR. Insulin was measured by Elisa. Regulations of genes were considered significant with p-values for relative expressions < 0.05 .

Results: Relative to $5 \mathrm{mM}$ glucose, $2 \mathrm{mM}$ glucose caused a down-regulation of ZnT-3 and an up-regulation of most other ZnT mRNA. $16 \mathrm{mM}$ caused ZnT3 up-regulation and down-regulation of other ZnT mRNA. Results were significant for ZnT3, ZnT5 and ZnT8, all transporters with high expression levels in $\beta$-cells. Zinc chelation lowered insulin expression and content and insignificant - insulin secretion along with a ZnT3 up-regulation. Liraglutide augmented glucose stimulated ZnT3 expression (1.73 fold increase), stimulated insulin expression and eliminated the glucose dependant downregulation of ZnT7 and ZnT8.

Conclusion: ZnT mRNA in $\beta$-cells responds to changes in glucose and zinc, supporting the hypothesis that ZnTs participate in insulin processing and that zinc trafficking in $\beta$-cells is regulated. Furthermore, long-term (liraglutide) but not short-term (GLP-1) stimulation of the GLP-1 receptor has significant influences on ZnT mRNA expression indicating that this system is a potential target for pharmacological manipulation. (Fig. 1)

Supported by Novo Nordisk/The Danish Diabetes Association

Fig. 1 Abstract 0144

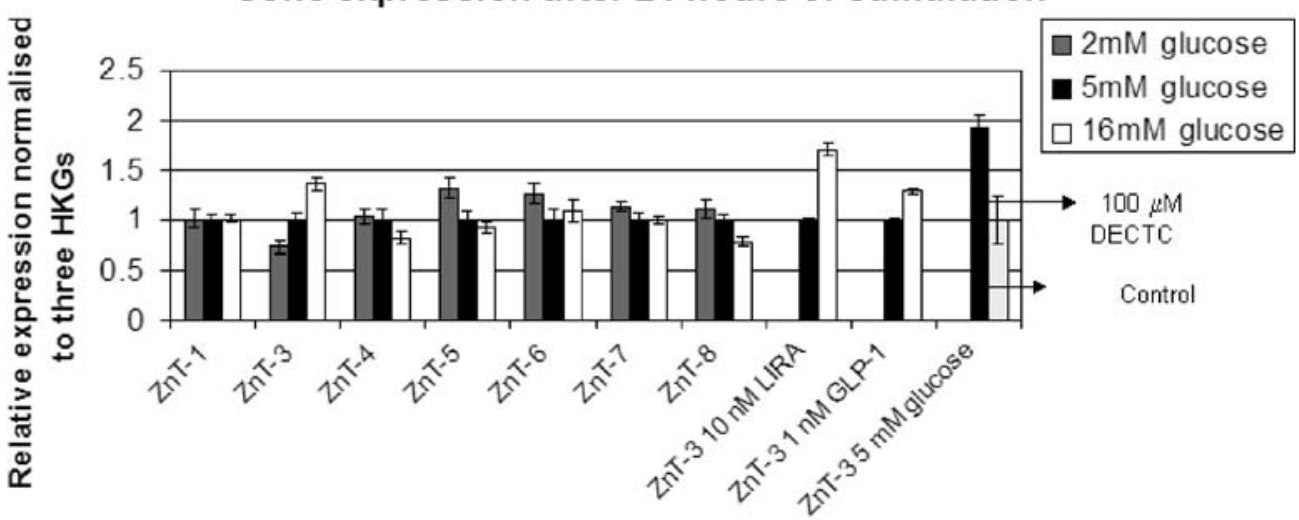




\section{OP 25 Lifestyle}

\section{5}

Dietary unsaturated fat reduces the postprandial glycaemic index, reduces hepatic lipogenesis but increases adipose tissue lipogenesis and reduces inflammation in diabetic pigs

S. J. Koopmans ${ }^{1}$, R. Dekker ${ }^{1}$, T. Schuurman ${ }^{1}$, M. Ackermans ${ }^{2}$, H. Sauerwein ${ }^{3}$;

${ }^{1}$ Animal Sciences Group, Wageningen University and Research Center, Lelystad, ${ }^{2}$ Clinical Chemistry, Academic Medical Center, Amsterdam, ${ }^{3}$ Endocrinology and Metabolism, Academic Medical Center, Amsterdam, The Netherlands.

Background and Aims: Dietary carbohydrates and fats may affect glycaemic control, insulin sensitivity, lipogenesis and inflammation in diabetic subjects. We investigated the long-term effects of diets, supplemented with starch (S), unsaturated fat (UF) or saturated fat (SF), on pre- and postprandial hyperglycaemia and lipidaemia, on insulin sensitivity, muscle and liver lipid content, retroperitoneal fat weight and plasma C-reactive protein (CRP) concentrations in diabetic pigs.

Materials and Methods: Eighteen domestic (Landrace x Yorkshire, D-line) pigs $(\sim 30 \mathrm{~kg})$ were treated with streptozotocin $(140 \mathrm{mg} / \mathrm{kg})$ and, according to the severity of hyperglycaemia, balanced over 3 diet groups. Mixed meals were fed twice daily (at a gross energy level of $25.10^{6} \mathrm{~J} /$ day) for a duration of 10 weeks. Supplemental S, UF, or SF content was $40 \%$ of total dietary energy. Permanent blood vessel catheters were used for blood sampling at meal tolerance tests and at hyperinsulinaemic euglycaemic clamps with 6,6${ }^{2} \mathrm{H}$-glucose infusion.

Results: Diabetic pigs were non-ketotic and required no insulin therapy to maintain body anabolism, final body weights were $\sim 50 \mathrm{~kg}$. Fasting plasma insulin $(\sim 4 \mathrm{mU} / \mathrm{L})$, glucose $(\sim 15 \mathrm{mmol} / \mathrm{L})$ and triglyceride $(0.5 \mathrm{mmol} / \mathrm{L})$ concentrations were similar among diet groups but fasting plasma NEFA concentrations were higher $(\mathrm{p}<0.05)$ in the SF group $(1.1 \pm 0.2 \mathrm{mmol} / \mathrm{L})$ compared to the $S$ group $(0.6 \pm 0.2 \mathrm{mmol} / \mathrm{L})$ but not to the UF group $(0.8 \pm 0.2$ $\mathrm{mmol} / \mathrm{L}$ ). Postprandial area under the curves (AUC) for glucose but not for insulin and triglyceride responses were lower $(\mathrm{p}<0.01)$ in the UF group (529 \pm 67 AUC) compared to the S group (1121 \pm 135 AUC) but not to the SF group (785 \pm 51 AUC). Postprandial plasma CRP concentrations were lower $(p<0.05)$ in the UF group $(60 \pm 3 \mathrm{mg} / \mathrm{L})$ compared to the SF group $(83 \pm 3 \mathrm{mg} /$ L) but not to the $S$ group $(79 \pm 3 \mathrm{mg} / \mathrm{L})$. Fasting hepatic glucose production $(\sim 6 \mathrm{mg} / \mathrm{kg} . \mathrm{min})$, insulin inhibited hepatic glucose production $(\sim 1 \mathrm{mg} /$ $\mathrm{kg} . \mathrm{min})$ and insulin stimulated whole body glucose uptake $(\sim 8 \mathrm{mg} / \mathrm{kg} \cdot \mathrm{min})$ were similar among diet groups. Liver weight was higher $(p<0.001)$ in the SF group $(1.47 \pm 0.09 \mathrm{~kg})$ compared to the $S$ and UF groups $(1.11 \pm 0.03 \mathrm{~kg})$. Triglyceride concentration in the liver was higher $(\mathrm{p}<0.01)$ in the SF group $(48.7 \pm 12.2 \mathrm{~g} / \mathrm{kg})$ compared to the $S(20.7 \pm 3.3 \mathrm{~g} / \mathrm{kg})$ and UF groups $(26.9 \pm 1.1$ $\mathrm{g} / \mathrm{kg})$. Triglyceride concentration in muscle was higher $(\mathrm{p}<0.01)$ in the SF group $(20.9 \pm 4.2 \mathrm{~g} / \mathrm{kg})$ compared to the $S$ group $(8.5 \pm 1.4 \mathrm{~g} / \mathrm{kg})$ but not to the UF group $(12.9 \pm 3.4 \mathrm{~g} / \mathrm{kg})$. Retroperitoneal fat weight was higher $(\mathrm{p}<0.05)$ in

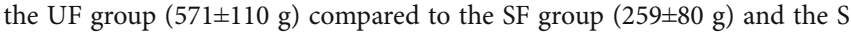
group (135 $\pm 57 \mathrm{~g})$.

Conclusion: In diabetic pigs, dietary UF but not SF had a lower postprandial glycaemic index compared to dietary $\mathrm{S}$, but hepatic and whole body insulin sensitivity were not affected by the diets. Pigs on dietary UF showed lower liver weights and liver triglyceride concentrations, higher retroperitoneal fat weight and lower plasma CRP concentrations compared to pigs on dietary SF. This indicates that dietary UF improves lipogenesis in adipose tissue but reduces hepatic lipogenesis and reduces inflammation.

Supported by SenterNovem

\section{6}

Effect of viscosity of oat fibre on gastrointestinal responses in healthy humans

K. R. Juvonen ${ }^{1}$, M. Salmenkallio-Marttila ${ }^{2}$, L. Lähteenmäki ${ }^{2}$, D. E. Laaksonen ${ }^{3}$, K.-H. Herzig ${ }^{4,5}$, M. I. J. Uusitupa ${ }^{1}$, K. S. Poutanen ${ }^{1,2}$, L. J. Karhunen ${ }^{1}$;

${ }^{1}$ Food and Health Research Center, School of Public Health and Clinical Nutrition, University of Kuopio, ${ }^{2}$ VTT, Biotechnology, Espoo,

${ }^{3}$ Department of Internal Medicine, Kuopio University Hospital,

${ }^{4}$ Department of Biotechnology and Molecular Medicine, A.I. Virtanen

Institute for Molecular Sciences, Kuopio,

${ }^{5}$ Department of Physiology, Oulu University, Finland.

Background and Aims: Viscosity slows gastric emptying and may exert a beneficial influence on appetite. Viscous fibres, including oat fibre, have favourable effects on carbohydrate and lipid metabolism. However, the effect of viscosity in liquid meals in relation to satiety-related gastrointestinal responses has been less well investigated, and was therefore examined in this study. Materials and Methods: Twenty healthy, normal-weight subjects (4 men/16 women aged 22,6 $\pm 3,25$ years, body mass index (BMI) $21,6 \pm 1,32 \mathrm{~kg} / \mathrm{m}^{2}$ ) ingested one of two isocaloric ( $1250 \mathrm{~kJ}$; carbohydrates $57.9 \mathrm{~g}$, protein $7.8 \mathrm{~g}$, fat $3.3 \mathrm{~g}$, fibre $10.2 \mathrm{~g}) 300 \mathrm{ml}$ beverages with differing viscosities in a randomised order: a beverage with viscous oat fibre and a beverage with oat fibre with no viscosity (viscosity eliminated by beta-glucanase enzyme). Blood samples were drawn from fasted subjects before and 15, 30, 45, 60, 90, 120 and 180 min after the tested beverages. The blood samples were analyzed to determine plasma glucose, ghrelin, peptide YY, glucagon-like peptide-1 and serum insulin concentrations. Gastric emptying was assessed using the paracetamol absorption test.

Results: The oat fibre beverage in which viscosity was eliminated induced greater increases in concentrations of postprandial glucose $(\mathrm{P}<0.001)$, insulin $(\mathrm{P}=0.008)$, glucagon-like peptide- $1(\mathrm{P}=0.037)$ and peptide $\mathrm{YY}(\mathrm{P}=0.051)$ and a greater decrease in postprandial ghrelin $(\mathrm{P}=0.009)$ than the beverage with viscous oat fibre. Gastric emptying was also accelerated after the non-viscous oat fibre beverage $(\mathrm{P}=0.034)$.

Conclusion: Reducing viscosity of oat fibre in liquid meal accentuates shortterm postprandial gastrointestinal responses, implying the importance of viscosity in producing the beneficial effects of oat fibre on glucose and insulin metabolism and gastrointestinal physiology.

Supported by The Technology Development Center of Finland (Tekes), Atria Finland Ltd, Fazer Bakeries Ltd, Ingman Foods Oy Ab, Raisio Plc, Valio Ltd, Oy Sinebrychoff Ab, and The Academy of Finland

\section{7}

Effect of moderate alcohol intake on patients with type 2 diabetes; multi-center, randomised, diet-controlled intervention study I. Shai ${ }^{1}$, J. Vainstein ${ }^{2}$, I. Harman-Bohem ${ }^{3}$, I. Raz ${ }^{4}$, A. Rudich ${ }^{5}$, M. J. Stampfer ${ }^{6}$; ${ }^{1}$ Epidemiology, Ben Gurion University, Beer-Sheva, Israel, ${ }^{2}$ Diabetes Clinic, Wolfson Medical Center, Holon, Israel, ${ }^{3}$ Diabetes Unit, Soroka Medical Center, Beer-Sheva, Israel, ${ }^{4}$ Diabetes clinic, Hadassah Hebrew University Medical Center, Jerusalem, Israel, ${ }^{5}$ Biochemistry, Ben Gurion University, Beer-Sheva, Israel, ${ }^{6}$ Epidemiology, Harvard School of Public Health, Boston, United States.

Introduction: Recent studies suggest that diabetic patients may benefit from the effects of moderate alcohol consumption, but the glycemic effects of such intervention are unclear. Using an interventional approach, we aimed to address the effect of daily moderate alcohol intake on glycemic control in the fasting and postprandial states in type 2 diabetic abstainers, and to determine adverse effects of such intervention.

Methods: One hundred and nine abstainer patients (41-74 yrs old) with established type 2 diabetes were randomised to receive $150 \mathrm{ml}$ wine (intervention) or non-alcoholic diet beer (control) during 3 months of a dietcontrolled, multi-center randomized intervention trial. The beverages were consumed during dinner. Diet and alcohol consumption were monitored. Results: Within the alcohol intervention group, fasting plasma glucose (FPG) decreased from $139.58 \mathrm{mg} / \mathrm{dL}+/-41.0$ to $118.01+/-32.5$ ) after 3 months (Delta $=-21.55 \mathrm{mg} / \mathrm{dL}, \mathrm{Pv}=0.015$ as compared to delta of the controls). However, alcohol consumption had no effect on 2-hour postprandial glucose levels $(\mathrm{Pv}=0.966)$. Patients in the intervention group with higher baseline 
$\mathrm{HbAlc}$ levels had greater reduction in FPG (age-adjusted correlation $=-0.33$, $\mathrm{Pv}=0.045)$. No significant changes were observed in the levels of Bilirubin, Alkaline Phosphatases, ALT or AST, nor were their reported adverse effects. However, participants in the intervention group reported an improvement in the ability to fall asleep ( $\mathrm{Pv}=0.001$ as compared to the controls).

Conclusions: Among abstainers who are type 2 diabetic, initiation of moderate daily alcohol consumption results in decreased FPG but not post-meal glucose. Patients with higher HbAlc may benefit more from the favorable effect of alcohol on glycemic control. Longer studies are needed to determine the efficacy of initiating consumption of moderate alcohol intake among abstainers with type 2 diabetes.

Supported by ClinicalTrials.gov Identifier: NCT00295334 Ben-Gurion University, IDRG, Tishbi Wines, Israel and Admiral Wine Imports, US

\section{8}

\section{Accuracy of meal carbohydrate content estimation by type 1 diabetics under CSII and MDI}

G. Freckmann, I. S. Koch, A. Baumstark, N. Jendrike, A. Abicht, C. Haug; University of Ulm, Institute for Diabetes-Technology, Germany.

Background and Aims: Accurate estimation of the carbohydrate (CHO) content of meals is a prerequisite for making appropriate insulin therapy decisions. Diabetic patients who are skilled in estimating the $\mathrm{CHO}$ content of food stuffs are more likely to calculate an appropriate insulin dose for a specific meal. The goal of this study was to determine how well a group of type 1 diabetic patients could estimate the $\mathrm{CHO}$ content of multiple meals and quantitate how much their estimates deviated from the actual $\mathrm{CHO}$ content of the meal.

Materials and Methods: Seventyfour type 1 diabetic patients, (36f, 38m, age $43.9 \pm 12.3$ years [mean \pm SD], HbAlc $7.2 \pm 1.2 \%$, duration of diabetes $20.6 \pm 11.8$ years, BMI $25.9 \pm 3.8 \mathrm{~kg} / \mathrm{m} 2$, insulin therapy: CSII 48 , MDI 26) participated in the study. Twentyfour different test meals ( 8 breakfasts, lunches and dinners, respectively; 13 cold meals, 11 warm meals) of variable $\mathrm{CHO}$ content (min. $54.6 \mathrm{~g}$, max. 164.1g) were prepared and presented in random order to the patients. They were asked to estimate the $\mathrm{CHO}$ content of each meal in their preferred carbohydrate estimation unit. 12 subjects used $\mathrm{KE}(=10 \mathrm{~g} \mathrm{CHO})$ and 62 used $\mathrm{BE}(=12 \mathrm{~g} \mathrm{CHO})$. The estimated unit was transformed into grams $\mathrm{CHO}$ and compared to the $\mathrm{CHO}$ calculated for each meal from the nutrition labeling of the package or the nutritional software DGE-PC vers. 2.9.1.007.

Results: The patients' estimation of the test meal $\mathrm{CHO}$ content was generally lower than the actual $\mathrm{CHO}$ content of the meals. When compared as percent of the calculated $\mathrm{CHO}$ content, the mean estimates of the 74 patients were $74.8 \% \pm 11.8 \%$ (median $74.0 \%$, min. $53.2 \%$, max. $126.9 \%$ ). The mean relative estimate of warm meals $(71.8 \% \pm 13.4 \%)$ was significantly $(\mathrm{p}<0.001)$ lower than the relative estimate of cold meals $(77.4 \% \pm 12.5 \%)$. The relative $\mathrm{CHO}$ estimation of test meals showed negative correlation to the carbohydrate content $(\mathrm{r}=-0.78, \mathrm{p}<0.001)$, that is, larger meals were underestimated more than smaller meals.

Conclusion: These results show that the patients in this study generally underestimated the $\mathrm{CHO}$ content of the test meals. Warm meals were more difficult to estimate and especially large test meals tend to be underestimated. Bias and variation in $\mathrm{CHO}$ counting should be considered in intensified insulin therapy and in the development of algorithms for semi-closed loop insulin infusion.

\section{9}

Fuel metabolism during exercise in eu- and hyperglycaemia in subjects with type 1 diabetes mellitus

S. Jenni ${ }^{1}$, S. Allemann ${ }^{1}$, M. Ith ${ }^{2}$, C. Oetliker ${ }^{1}$, S. Wuerth ${ }^{3}$, C. Boesch ${ }^{2}$, L. Tappy ${ }^{4}$, P. Diem ${ }^{1}$, E. R. Christ ${ }^{1}$, C. Stettler ${ }^{1}$;

${ }^{1}$ Div. of Endocrinology, Diabetes and Clinical Nutrition,

University Hospital, Bern, ${ }^{2}$ Magnetic Resonance Spectroscopy and

Methodology - Department of Clinical Research, University, Bern,

${ }^{3}$ Institute of Sport and Social Sciences, University, Basel,

${ }^{4}$ Dept. of Physiology, University, Lausanne, Switzerland.

Background and Aims: Little is known about the effect of blood glucose levels on local muscle fuel metabolism during exercise in individuals with type 1 diabetes mellitus (T1DM). Preliminary data revealed similar exercise capacities in eu-compared to hyperglycaemic conditions. The aim of the present study was to assess whether the consumption of intramyocellular lipids (IMCL) and of intramyocellular glycogen was different during aerobic exercise in eu-compared to hyperglycaemic clamp conditions.

Methods and Material: Seven male individuals with T1DM under continuous subcutaneous insulin infusion (CSII) were studied on two occasions. After discontinuation of CSII on the evening before each trial, a variable i.v. insulin infusion was started to reach normal glucose levels overnight. The participants were kept fasting. At 8 a.m. the next day blood glucose was clamped to euglycaemic (target level $5 \mathrm{mmol} / \mathrm{l}$ ) or hyperglycaemic levels (target level 11 $\mathrm{mmol} / \mathrm{l}$ ) in a randomised, single blinded, cross-over design. Insulin infusion was kept constant according to the patient's CSII basal insulin rate. After stable clamp conditions were reached, IMCL and intramyocellular glycogen (M. quadriceps) were measured using magnetic resonance spectroscopy. The participants then cycled for 120 minutes at a constant power corresponding to $55-60 \%$ of their $\mathrm{VO}_{2 \max }$ determined earlier. Respiratory exchange ratio (RER) was measured by spirometry. Directly after the exercise IMCL and intramyocellular glycogen were again measured at the same site using magnetic resonance spectroscopy. Results of the two tests were compared using Student's paired t-test.

Results: Mean age of the participants was $33.5 \pm 6.3$ years (mean \pm standard deviation), mean body mass index was $24.1 \pm 1.2 \mathrm{~kg} / \mathrm{m}^{2}$. Diabetes duration was $20.1 \pm 9.4$ years and $\mathrm{HbA}_{1 \mathrm{c}}$ reflected good glycaemic control $(6.7 \pm 0.5 \%)$. Euglycaemic clamps were at a mean blood glucose of $5.0 \mathrm{mmol} / \mathrm{l}$, whereas mean glycaemic level was at $10.9 \mathrm{mmol} / \mathrm{l}$ in hyperglycaemic clamps (difference $5.9 \mathrm{mmol} / \mathrm{l}, p<0.0001$ ). Insulin levels did not differ between eu- and hyperglycaemic clamp conditions. RER was significantly lower in eu- compared to hyperglycaemic conditions ( 0.84 and 0.93 , respectively, $p$ $=0.034$ ), reflecting a higher net lipid oxidation rate in euglycaemic exercise condition. The decline in IMCL during exercise tended to be increased in the eu-compared to the hyperglycaemic exercise condition $(16.2 \%$ and $11.5 \%$, respectively, $p=0.18$ ). In contrast, intramyocellular glycogen consumption was similar in the eu- and the hyperglycaemic condition $(31.2 \%$ and $35.2 \%$, respectively, $p=0.56$ ).

Conclusion: In individuals with T1DM performing aerobic exercise lipid oxidation appears to play a predominant role in euglycaemia and IMCL are suggested to be the main fuel source. In contrast, during hyperglycaemia energy production appears to rely stronger on carbohydrate oxidation. Of note, the latter did not result in an increased consumption of intramyocellular glycogen thereby suggesting that the increased levels of blood glucose provided the main energy substrate in hyperglycaemia.

Supported by unrestricted grants from the Swiss Diabetes Foundation, the Oetliker-Stiftung für Physiologie, NovoNordisk, Swiss National Foundation

\section{0}

Objectively measured sedentary time and light-intensity physical activity are independently associated with components of the metabolic syndrome: the AusDiab study

G. N. Healy ${ }^{1}$, D. W. Dunstan ${ }^{2}$, J. E. Shaw ${ }^{2}$, P. Z. Zimmet ${ }^{2}$, N. Owen'; ${ }^{1}$ School of Population Health, The University of Queensland, Brisbane, ${ }^{2}$ Epidemiology, International Diabetes Institute, Melbourne, Australia.

Background and Aims: Physical activity and sedentary behaviour are key modifiable risk factors for components of the metabolic syndrome. However, studies typically have used physical activity variables derived from imprecise self-report measures. We examined the associations of objectively-measured sedentary time and light-intensity physical activity with components of the metabolic syndrome in Australian adults without known diabetes.

Materials and Methods: 70 men and 108 women (mean age 53.4, range 30 to 87 ) were recruited from the 2004-2005 Australian Diabetes, Obesity, and Lifestyle (AusDiab) study. Physical activity was measured by Actigraph accelerometers worn during waking hours for seven consecutive days, and divided into sedentary time (counts/minute $<100$ ), light-intensity (counts/ minute 100-1951), and moderate-to-vigorous intensity (counts/minute $>1952$ ). An oral glucose tolerance test was used to obtain fasting and 2-hr plasma glucose, serum-triglycerides, and HDL-C. Waist circumference and blood pressure were measured at the testing site. Components of the metabolic syndrome were dichotomised according to the current IDF definition, and reported as central obesity, raised triglycerides, blood pressure, fasting plasma glucose, and 2-hr plasma glucose, and reduced HDL-C. 
Results: In logistic regression analyses adjusted for age, sex, time accelerometer worn, income, education, and moderate-to-vigorous intensity activity, each additional hour/day of sedentary time was associated with an increased risk of central obesity $(\mathrm{OR}=1.40, \mathrm{p}=0.017)$; while each hour/day of light intensity activity was associated with a reduced risk $(\mathrm{OR}=0.69, \mathrm{p}=0.009)$. When waist circumference $(\mathrm{cm})$ was included in the model, each hour/day of sedentary time was associated with an increased risk of raised triglycerides $(\mathrm{OR}=1.44$, $\mathrm{p}=0.046)$, raised $2 \mathrm{-hr}$ plasma glucose $(\mathrm{OR}=1.72, \mathrm{p}=0.052)$, and reduced HDL-C (OR=1.56, $\mathrm{p}=0.009)$; each hour/day of light-intensity activity was associated with a significantly lower risk of raised triglycerides $(\mathrm{OR}=0.66$, $\mathrm{p}=0.028)$, raised 2 -hr plasma glucose $(\mathrm{OR}=0.55, \mathrm{p}=0.036)$, and reduced HDL-C (OR=0.65, $\mathrm{p}=0.012)$. The associations of sedentary time and lightintensity activity with raised blood pressure and fasting blood glucose were not statistically significant $(\mathrm{p}>0.1)$.

Conclusion: These findings provide some of the first evidence from objective activity data that sedentary time is unfavourably associated with components of the metabolic syndrome, particularly central obesity, triglycerides, $2-\mathrm{hr}$ plasma glucose, and HDL-C, and that light-intensity activity is beneficially associated. Importantly, these associations were independent of moderateto-vigorous intensity activity. The findings suggest that substituting lightintensity activity for television viewing or other sedentary time may be a practical and achievable strategy to improve metabolic risk in adults. Supported by Queensland Government Growing the Smart State PhD fund, NHMRC

\section{OP 26 Inflammation in metabolism}

\section{1}

Changes in gut microflora are responsible for high-fat diet-induced diabetes through a mechanism associated with endotoxaemia P. D. Cani ${ }^{1}$, A. M. Neyrinck ${ }^{1}$, K. M. Tuohy ${ }^{2}$, F. Fava ${ }^{2}$, G. R. Gibson², C. Knauf ${ }^{3}$, R. G. Burcelin ${ }^{3}$, N. M. Delzenne ${ }^{1}$,

${ }^{1}$ Unit PMNT, Université catholique de Louvain, Brussels, Belgium,

${ }^{2}$ Food microbial sciences unit, The University of Reading, United Kingdom, ${ }^{3}$ IFR31, I2MR, Toulouse, France.

Background and Aims: Diabetes and obesity are two metabolic diseases characterized by insulin resistance and a low grade inflammation. Recently, a very encouraging and innovating hypothesis has been proposed, where a specific gut microbial community would favor the occurrence of the metabolic diseases. However, germ free mice fed a high-fat (HF) obesitogenic diet did not gain weight -whereas HF diet promotes obesity in animals with conventional florasuggesting that indeed, a bacterially related factor is responsible for HF dietinduced obesity. In the light of the role of gut microbiota for the development of metabolic diseases, we recently reported that HF feeding was associated with a higher plasma endotoxin (LPS) concentration and a lower Bifidobacterium spp. caecal content in mice. We reported that metabolic endotoxemia increased the inflammatory tone, triggered body weight gain and shortened the onset of HF induced-insulin resistance and obesity. Thus we tested the hypothesis that modulating endotoxemia by changing gut microbiota profile could be a potent strategy to control the development of metabolic diseases.

Materials and Methods: Since, bifidobacteria have been reported to reduce intestinal endotoxin levels and to improve mucosal barrier function, we specifically increased gut bifidobacteria content of high-fat (HF) diet treated mice by adding prebiotic in the diet (oligofructose, HF-OFS). We correlated microflora changes, which occur through 14 weeks HF diet, control to the occurrence of metabolic disturbances.

Results: HF feeding significantly reduced the levels of bifidobacteria, a dominant member of the intestinal microbiota, as compared to normal chow fed mice (CT). As expected, HF-OFS fed mice totally restored the number of bifidobacteria. HF feeding significantly increased endotoxemia which was normalized to CT when the mice were treated with HF-OFS. To identify whether one specific gut bacteria is involved in the determination of endotoxemia, we performed multiple correlations analysis between the major Gram positive and Gram negative bacteria present in the caecal content of mice. Endotoxemia significantly and negatively correlates with Bifidobacterium spp. but no relationship can be shown between endotoxemia and any other bacterial group. Finally, in HF-OFS treated mice, endotoxemia negatively correlates with an improved glucose tolerance, glucose-induced insulin-secretion, a lower fat mass and body weight, and normalized inflammatory tone. Whereas, Bifidobacterium spp. significantly and positively correlates with these factors.

Conclusion: The modulation of gut microbiota by increasing bifidobacteria reduces endotoxemia and improves metabolic parameters in high-fat diet fed mice. Together, these findings suggest that gut microbiota contributes to the patho-physiological regulation of endotoxemia, and sets the tone of inflammation for the occurrence of diabetes and obesity. Thus it would be useful to develop specific strategies for modifying gut microbiota in favour of bifidobacteria to promote health in the context of metabolic syndrome. PDC is Postdoctoral Researcher from the FNRS, Belgium

\section{2}

JNK in macrophages plays a key role in the development of obesity-induced insulin resistance

G. Solinas ${ }^{1}$, C. Vilcu' ${ }^{2}$ J.-L. Luo ${ }^{3}$, W. Naugler ${ }^{3}$, S. Grivennikov ${ }^{3}$, J. G. Neels ${ }^{2}$, G. K. Bandyopadhyay', J. M. Olefsky², M. Karin ${ }^{3}$;

${ }^{1}$ Dept. of Medicine, Division of Physiology, University of Fribourg, Switzerland, ${ }^{2}$ Dept. of Medicine, Division of Endocrinology and Metabolism, University of California, San Diego, CA, United States, ${ }^{3}$ Dept. of Pharmacology, University of California, San Diego, United States.

Background and Aims: c-Jun N-terminal Kinases (JNKs), which respond to several pro-inflammatory cytokines such as TNF-alpha and IL1, and metabolic stress such as ROS, ER-stress and long-chain saturated fatty acid were proposed to be key players in the development of obesity-induced 
insulin resistance. $j n k 1^{-/-}$and $j n k 1^{+-j} j n k 2^{-/-}$mice are resistant to obesity and to obesity-induced glucose intolerance; moreover, acute inhibition of JNK improved insulin sensitivity in obese mice. The current molecular mechanism for JNKs in insulin resistance is based on the direct phosphorylation of IRS2 Ser307 and the equivalent IRS2 site Thr287. Phosphorylation of these sites is believed to decrease the affinity of IRS1-2 for the activated insulin receptor. An increasing body of evidence supports the hypothesis that a chronic low-grade inflammation is involved in the pathogenesis of obesity-induced insulin resistance. Since JNKs are known to regulate the expression of proinflammatory genes, and since macrophages were proposed to play a major role in the development of insulin resistance, we have tested the role of JNKs in the hematopoyetic tissue using bone marrow transplantation technology. Materials and Methods: To study the role of JNK in the hematopoyetic tissue we have performed bone marrow transplantation in lethally irradiated (10 Gy) mice. We have generated $w t$ mice with $j n k 1^{-/-}$bone marrow; $j n k 1^{-/-}$mice with $w t$ bone marrow and as control we have used $w t$ mice reconstituted with $w t$ bone marrow after the lethal irradiation. Body weight was measured weekly to determine growth curves. Adiposity was measured by MRI and used to determined lean body mass. GTTs, ITT and hyperinsulinemic euglycemic clamps were performed to measure insulin sensitivity. Macrophage number in adipose tissue was measured by immunohystochemistry and expression of pro-inflammatory cytokines was measured in tissues and macrophages cell cultures by real time PCR.

Results and Conclusions: In this study we have tested the role of JNKs in the hematopoyetic tissue using bone marrow transplantation technology. We show that $j n k 1^{-/-}$mice transplanted with $w t$ bone marrow are resistant to diet-induced obesity and have improved glucose tolerance, suggesting that the resistance to obesity observed in $j n k 1^{-1-}$ is due to a tissue of nonhematopoyetic origin. We have discovered that high fat diet-fed $w t$ mice transplanted with $j n k 1^{-/-}$bone marrow, have improved glucose tolerance and are less insulin resistant than control mice despite having similar body weight and adiposity. Functional studies in isolated macrophages show that JNKs act directly in these cells. In conclusion, we propose a new molecular mechanism for the diabetogenic action of JNK1 where in addition to its action in insulin sensitive tissues, JNK1 also regulates the function of hematopoyetic cells, in particular macrophages, and therefore is directly involved in the low-grade chronic inflammation associated with obesity.

GS was supported by a Mentor-Based Post-Doctoral Fellowship by the ADA

\section{3}

The effects adiposity, diabetic status and depot-specificity on the activation of NFKB on JNK in human abdominal adipose tissue C. M. Kusminski, N. F. da Silva, A. E. Fowler, S. J. Creely, A. L. Harte, A. R. Baker, T. S. Billyard, J. P. O’Hare, S. Kumar, P. G. McTernan; Unit of Diabetes and Metabolism, Warwick Medical School, University of Warwick, Coventry, United Kingdom.

Background and Aims: Central obesity is closely associated with sub-clinical inflammation, insulin resistance and type 2 diabetes mellitus (T2DM); however the precise underlying mechanisms of such associations are unclear. Recent rodent studies have highlighted $\mathrm{NF \kappa B}$ and $\mathrm{c}$-Jun $\mathrm{N}$ terminal kinase (JNK) as key mediators linking insulin action and inflammation. Therefore the aims this study were to firstly, investigate inflammation within human adipose tissue; in particular, the innate immune signalling pathway in relation to NFאB and JNK activation in abdominal subcutaneous (AbdSc) and omental (Om) human adipose tissue. Secondly, to determine the influence of increasing adiposity and type 2 diabetic status on the level of activation of NFkB and JNK within AbdSc and Om AT depots. Finally, to examine the effects of TNF $\alpha$, a known mediator of inflammation, on the level of NFKB and JNK activity in isolated human AbdSc adipocytes.

Materials and Methods: Human adipose tissue was collected from patients (age: $44.7 \pm 9.3 \mathrm{yrs}$; BMI: $27.9 \pm 7.3 \mathrm{.kg} / \mathrm{m}^{2}$ ) undergoing liposuction surgery. A total of 32 non-diabetic adipose tissue samples were obtained and sub-divided into AbdSc $(\mathrm{n}=22)$ and Omental $(\mathrm{n}=10)$ depots. Western blot procedure was used to analyse the expression of key components of the NFkB and JNK signalling pathways. Additionally, isolated human AbdSc adipocytes were treated with human recombinant TNF- $\alpha(1-50 \mathrm{ng} / \mathrm{ml})$. Finally, the levels of active NFKB or phosphospecific JNK in both AT depots were assessed using assay-based measures.

Results: Our ex vivo analysis firstly demonstrated that the protein expression levels of several intracellular signalling molecules, in particular, IKK $\beta$, IKK $\gamma$,
$\mathrm{I} \kappa \mathrm{B} \alpha$ and $\mathrm{I} \kappa \mathrm{B} \alpha-\mathrm{P}$ were increased in $\mathrm{Om} \mathrm{AT}$ in comparison to AbdSc AT $(p<0.01)$. Furthermore, the ratio of active NFKB to total NFKB expression (NFKB ratio) was upregulated in Om AT compared with AbdSc, in both lean and obese subjects $(p<0.05)$. In contrast, phosphospecific JNK1 was increased by 5.8 -fold in the AbdSc depot $(\mathrm{p}<0.01)$ when compared with $\mathrm{Om}$ AT. Finally, in vitro data revealed that isolated AbdSc adipocytes treated with $\mathrm{TNF} \alpha$ exhibited a significant increase in NFKB activity in comparison with control; such enhanced activity further increased over time $(2-48 \mathrm{hr}, \mathrm{p}<0.05)$. In contrast to rodent studies, JNK activity in response to TNF- $\alpha$ treatment, remained unaltered.

Conclusions: Our findings suggest distinct inflammatory signalling pathways, with increased $\mathrm{NF \kappa B}$ activity in human Om AT and increased JNK activity in AbdSc AT. Additionally, NFKB was shown to be influenced by obesity and diabetic status, whilst TNF $\alpha$ treated AbdSc adipocytes led to activation of $\mathrm{NF \kappa B}$ and not JNK. Taken together, NFKB may play a more predominant central role in inflammatory-related metabolic disease in comparison to JNK, suggesting a potential role for $\mathrm{NF \kappa B}$ as a key therapeutic target. Supported by the University of Warwick, UK

\section{4}

Regulation of adipokines by hormones and inflammation in human omental adipocytes

E. Maury, K. Ehala-Aleksejev, L. Noël, S. M. Brichard; Endocrinology and metabolism, Catholic University of Leuven, Brussels, Belgium.

Background and Aims: We have identified six adipokines that are oversecreted by omental adipose tissue (OAT) in obesity: 3 chemokines [Growth-Related Oncogen factor (GRO), Regulated upon Activation Normal T cells Expressed and Secreted (RANTES), Macrophage Inflammatory Protein-1ß (MIP-1ß)], 1 interleukin (IL-7), 1 tissue inhibitor of metalloproteinases (TIMP-1) and 1 growth factor (thrombopoietin, TPO). Enhanced expression of these adipokines in adipocytes did correlate with several features of the metabolic syndrome. The aim of the present study was to examine whether the abnormal hormonal milieu and/or the chronic inflammation state that prevail in obesity could contribute to such dysregulation of adipokine production. We thus examined the effect of some hormones (insulin, glucocorticoids and catecholamines) and the proinflammatory cytokine, TNF $\alpha$ on these adipokines in omental adipocytes.

Materials and Methods: OAT was obtained from patients undergoing elective abdominal surgery. Preadipocytes were isolated and differentiated into mature adipocytes in vitro. The adipocytes were cultured in a chemically defined medium. After 12 days of differentiation, $100 \mathrm{nM}$ insulin, $1 \mu \mathrm{M}$ dexamethasone, $10 \mu \mathrm{M}$ isoproterenol (a $\beta$-adrenergic agonist) or 10-100 nM $\mathrm{TNF} \alpha$ were added to the medium for up to $24 \mathrm{~h}$. Adipokine expression levels were measured by real-time RTQ-PCR.

Results: Treatment of omental adipocytes with insulin, dexamethasone or isoproterenol induced a significant, but modest increase in GRO and TPO mRNA levels (from 1.3- up to 2-fold) without affecting the other adipokines studied. The second messenger of the $\beta$-adrenergic agonist, cAMP produced qualitative similar effects to those of isoproterenol. By contrast, the addition of TNF $\alpha$ to the medium induced a strong rise in the expression of all the adipokines (except for TPO). This rise occurred in a dose-dependent fashion and reached an $\sim 600$ fold increase for some of them (i.e. RANTES).

Conclusion: Compared to hormones such as insulin, glucocorticoids and catecholamines, TNF $\alpha$ appears to be a main contributor to the enhanced expression of the investigated adipokines in human omental adipocytes.

\section{5}

Macrophage-adipocyte cross-talk in the initiation of obesity-related insulin resistance and type 2 diabetes: role of adiponectin I. T. Y. Lau ${ }^{1}$, H.-Y. Ye ${ }^{1}$, A. Xu ${ }^{1,2}$, K. S. L. Lam ${ }^{1,2}$;

${ }^{1}$ Department of Medicine, The University of Hong Kong,

${ }^{2}$ Research Centre of Heart, Brain, Hormone, and Healthy Aging,

The University of Hong Kong, China.

Background and Aims: Recent studies suggest that, in obesity, chronic inflammation of adipose tissues (AT) with macrophage infiltration and 
enhanced expression of pro-inflammatory cytokines plays a significant role in the development of obesity-related metabolic disorders, such as type 2 diabetes. We have previously demonstrated that adenovirus-mediated over-expression of adiponectin, an insulin-sensitizing adipokine with antiinflammatory action and reduced expression in obesity, results in a marked down-regulation of several inflammation-related genes, including F4/80 (a macrophage marker) and monocyte chemotactic protein-1 (MCP-1), in the white AT of db/db diabetic mice (Lam \& Xu, Curr Diab Rep 2005;5:254-9). As MCP-1 has been suggested to contribute to macrophage infiltration of AT in $\mathrm{db} / \mathrm{db}$ mice (Kanda et al, JCI 2006;116:1494-505), we hypothesize that the reduced expression of adiponectin in obesity may play an important role in initiating the macrophage-adipocyte cross-talk which leads to obesity-related insulin resistance and diabetes. This study aims at determining the time course and magnitude of macrophage infiltration and inflammatory activation in $\mathrm{AT}$, in relation to changes in the AT levels of adiponectin, glucose tolerance and insulin sensitivity, in $\mathrm{db} / \mathrm{db}$ diabetic mice.

Materials and Methods: At the age of 5, 6, 7, 8, 9, 10, 11, 12 weeks, male db/ $\mathrm{db}$ mice and lean controls were tested for blood glucose (non-fasting, fasting, and in response to ip. insulin tolerance test and glucose tolerance test), insulin and lipid levels. The mRNA expression level of adiponectin, MCP-1 and F4/80 were determined by real-time PCR in epididymal fat pads obtained after sacrifice of mice at different ages. Immunohistochemistry was performed to assess the severity of macrophage infiltration in AT. Adiponectin and MCP-1 were also measured by ELISA in medium obtained after the fat pads were incubated for 24 hours.

Results: Compared to lean controls, $\mathrm{db} / \mathrm{db}$ mice had significant increases in the weight of AT in 3 depots (epididymal, perirenal and abdominal subcutaneous) at 5 weeks, accompanied by significant hyperinsulinaemia and hypercholesterolaemia, and a trend towards impaired responses during insulin tolerance test, compared to lean controls. A significant $80 \%$ reduction in adipose tissue adiponectin mRNA expression, accompanied by significant reductions in adiponectin in the explant mediium, was also seen at 5 weeks, when no significant increase was found in F4/80 mRNA expression or macrophage infiltration of epididymal AT. There was also no significant increase in the release of MCP-1 during AT explant incubation at 5 weeks. Greater weight than controls, accompanied by impaired glucose tolerance, was seen from 6 weeks. Non-fasting hyperglycaemia, compared to lean controls, became evident at 8 weeks. By 10 weeks, fasting hyperglycaemia was established.

Conclusion: These data suggest that reduced adiponectin expression precedes the macrophage infiltration in $\mathrm{AT}$ of $\mathrm{db} / \mathrm{db}$ mice and may contribute, at least in part, to the macrophage infiltration and inflammatory gene activation in white AT in obese animals. Hyperinsulinaemia precedes macrophage infiltration of AT which may, nevertheless, contribute to progressive insulin resistance and development of diabetes in the $\mathrm{db} / \mathrm{db}$ mice.

Supported by RGC grant.

\section{6}

C-reactive protein expression in human monocytes/macrophages and endothelial cells: regulation by inflammatory cytokines and metabolic factors relevant to type 2 diabetes

L. Li, G. Renier;

Vascular Immunology Laboratory, CHUM Research Centre, Notre-Dame Hospital, Department of Medicine, University of Montreal, Canada., Canada.

Background and Aims: C-reactive protein (CRP) is a predictor of future cardiovascular events and diabetes and, may be causally involved in the pathogenesis of atherosclerosis. Besides being synthesized by the liver, CRP is produced by non hepatic cells, including vascular cells. CRP released from these cells may contribute to arterial inflammation and expression of this protein in atherosclerotic plaque. Besides resident vascular cells, inflammatory cells, especially monocytes, may represent a significant source of CRP in humans. While substantial expression of CRP has been documented in peripheral blood mononuclear cells, no studies have determined the level of CRP expression in human monocytes/macrophages. In the present study, we sought to determine the constitutive CRP expression by human monocytes/ macrophages and the regulation of this parameter by inflammatory cytokines and metabolic factors relevant to diabetes. The modulatory effect of metabolic factors on vascular CRP expression was further investigated in human aortic endothelial cells (HAECs).
Materials and Methods: Highly purified monocytes (85-90\%) were isolated from healthy control subjects. Differentiation of monocytes into macrophages was achieved by culturing the cells in RPMI supplemented with $20 \%$ autologous serum for 8 days. HAECs (passage 3 to 5) were grown to confluence in endothelial growth medium-2. Human monocytes were exposed to $25 \mathrm{ng} / \mathrm{ml}$ interleukin-1 $\beta$ (IL-1 $\beta$ ) and $10 \mathrm{ng} / \mathrm{ml}$ interleukin-6 (IL-6) or $100 \mu \mathrm{g} / \mathrm{ml}$ glycated bovine serum albumin (BSA-AGE) for 24 or 48h. Confluent HAECs were incubated with $40 \mu \mathrm{g} / \mathrm{ml}$ oxidized low-density lipoprotein (oxLDL) or $100 \mu \mathrm{g} / \mathrm{ml} \mathrm{BSA-AGE} \mathrm{for} \mathrm{3,} \mathrm{6,} \mathrm{12,} \mathrm{and} \mathrm{24h.} \mathrm{At} \mathrm{the} \mathrm{end}$ of these incubation periods, cells were lysed and CRP mRNA and protein expressions were analyzed by RT-PCR and Western-blot, respectively.

Results: Human monocytes and macrophages constitutively express CRP. Treatment of human monocytes with IL-1 $\beta$ plus IL- 6 for 24 or $48 \mathrm{~h}$ enhanced CRP both at the gene and protein levels (CRP mRNA expression (\% of control values) $24 \mathrm{~h}$ : control:100 \pm 2 , IL-1 $\beta+\mathrm{IL}-6: 162 \pm 40$; CRP protein expression $(\%$ of control values): $48 \mathrm{~h}$ : control:100 \pm 2 , IL-1 $\beta+$ IL-6:180 \pm 37 ). Inflammatory cytokines induced, to a similar extent, CRP protein expression in human macrophages. Increased CRP protein levels were also observed following exposure of human monocytes to BSA-AGE (CRP protein expression (\% of control values): $48 \mathrm{~h}$ : control:100 \pm 2, BSA-AGE:222 \pm 11 ). Finally, upregulation of CRP gene and protein expression was documented in HAECs exposed to BSA-AGE and oxLDL. Induction of endothelial CRP by these factors was time-dependent, with maximal effect occurring around $12 \mathrm{~h}$ and $24 \mathrm{~h}$, respectively.

Conclusion: Our results demonstrate for the first time that CRP is expressed by human monocytes and macrophages under resting conditions. They further establish the role of inflammatory and metabolic factors relevant to diabetes in the regulation of CRP expression in human monocytes/macrophages and endothelial cells. Increased monocyte and vascular CRP expression may contribute, at least in part to the accelerated atherosclerosis associated with human diabetes. Further research is needed to evaluate whether monocyte CRP expression may provide cardiologists with a more precise and specific biomarker for assessing cardiovascular risk in patients with diabetes. 


\section{OP 27 Psychological aspects of diabetes}

\section{7}

Health-related quality of life in patients with type 2 diabetes: an independent predictor of mortality

N. Kleefstra ${ }^{1,2}$, G. W. D. Landman ${ }^{3}$, S. T. Houweling ${ }^{2}$, L. J. Ubink-Veltmaat ${ }^{4}$, B. Meyboom-de Jong ${ }^{5}$, K. H. Groenier ${ }^{5}$, H. J. G. Bilo ${ }^{1,6}$;

${ }^{1}$ Diabetes Centre, Isala Clinics, Zwolle, ${ }^{2}$ Medical Research Group, Langerhans, Zwolle, ${ }^{3}$ Internal Medicine, Isala Clinics, Zwolle, ${ }^{4}$ t Veen, Huisartsenpraktijk, Hattem, ${ }^{5}$ General Practice, University of Groningen, ${ }^{6}$ Internal Medicine, University of Groningen, The Netherlands.

Background and Aims: Hypertension and dyslipidemia are well known predictors of mortality in type 2 diabetes (T2DM) and should be treated. However, other factors like health related quality of life (HRQOL), which is negatively associated with having diabetes, are separate treatment objectives. This study investigates the relation between HRQOL and total mortality in T2DM.

Materials and Methods: The ZODIAC study started in 1998. A group of 1145 patients with T2DM was seen annually, and data on mortality were assessed 5.8 years later on average. The Rand-36 was used to measure HRQOL. It contains nine health dimensions; and two summary measures can be calculated: the Physical Component Summary (PCS) and Mental Component Summary (MCS). The range of the scale is from 0-100, a higher score meaning better HRQOL. Ten variables were selected for their possible confounding effects in the relationship between mortality and HRQOL: smoking (yes or no), age, sex, duration of diabetes, HbAlc, Cockcroft, BMI, blood pressure, cholesterol/HDL ratio and macrovascular complications. To investigate whether HRQOL was associated with mortality, Cox proportional Hazard modeling was used.

Results: 335 patients died. Date of death could not be traced in 6 patients. Cause of death could not be confirmed for 20 patients. Data on HRQOL were available of $1000(87 \%)$ patients, of whom 857 (75\%) were complete. Mean age was 68.2 years, with a median diabetes duration of 6 years. After adjustment for other known risk factors, HRQOL measured with the PCS was inversely associated with mortality (HR: $0.979,95 \%$ CI: 0.966 to 0.992 ) (Figure: Kaplan- Meier curve PCS: below median versus above median) and MCS was not (HR $1.00895 \%$ CI: 0.994 to 1.022). 145 (32.4\%)) of 448 patients with PCS scores below median and 69 of 449 (15.4\%) above median died; ratio 2.1. Every 1 point decrease on the PCS scale led to an increase of the hazard of mortality of $2.1 \%$, on average. For separate RAND-36 dimensions, significant relationships were found for "Physical Functioning" (HR 0.989 95\%CI: 0.982 to 0.996 ) and "General Health Perception: (HR 0.982 95\% CI: 0.971 to 0.994$)$.

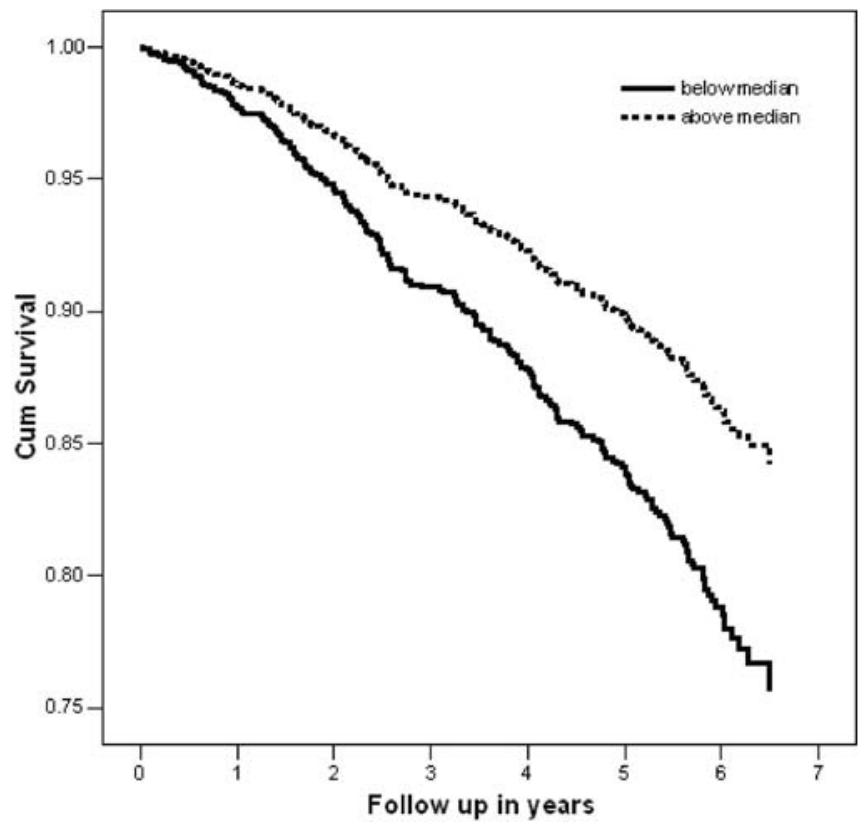

Fig. 1
Conclusion: Worse HRQOL (adjusted for well-established risk factors) is associated with a higher mortality in patients with type 2 diabetes after a follow-up of six years. Patients with HRQOL (PCS) below median versus above median have a 2.1 -fold increased mortality risk. (Fig. 1)

\section{8}

Quality of life and treatment satisfaction in adults with T1DM treated with CSII or MDI

A. Nicolucci ${ }^{1}$, A. Maione ${ }^{1}$, M. Franciosi ${ }^{1}$, R. Amoretti ${ }^{2}$, E. Busetto ${ }^{3}$, F. Capani ${ }^{4}$, D. Bruttomesso ${ }^{5}$, P. Di Bartolo ${ }^{6}$, A. Girelli ${ }^{7}$, F. Leonetti ${ }^{8}$, L. Morviducci ${ }^{9}$, P. Ponzi ${ }^{3}$, E. Vitacolonna ${ }^{10}$;

${ }^{1}$ Clinical Pharmacology and Epidemiology, Consorzio Mario Negri Sud, S. Maria Imbaro ${ }^{2}$ Diabetes Unit, Azienda Ospedaliera S. Giovanni Addolorata, Roma, ${ }^{3}$ Economics, Fondazione Medtronic Italia, Sesto San Giovanni ${ }^{4}$ Medicine and Ageing, University "G. D'Annunzio", Chieti, ${ }^{5} \mathrm{Clinical}$ and Experimental Medicine, University of Padova,

${ }^{6}$ Diabetes Unit, AUSL Provincia di Ravenna, ${ }^{7}$ Diabetes Unit, Spedali Civili di Brescia, ${ }^{8}$ Clinical Sciences, La Sapienza University, Roma,

${ }^{9}$ Diabetes Unit, San Camillo Hospital, Roma, ${ }^{10}$ Medicine and Ageing, University “G. D’Annunzio", S. Maria Imbaro, Italy.

Background and Aims: Many clinical trials have investigated and demonstrated the efficacy of Continuous Subcutaneous Insulin Injection (CSII) vs. Multiple Daily Injection (MDI) in individuals with Type 1 Diabetes (T1DM). However, the evidence regarding the quality of life benefits of CSII is conflicting. The aim of this case-control trial was to compare the $\mathrm{QoL}$ and treatment satisfaction in a large sample of individuals with T1DM treated either with CSII or MDI.

Materials and Methods: Sixty-two Italian diabetes clinics enrolled 1341 consecutive patients (age 18-55 years), of whom 481 were treated with CSII (cases) and 860 with MDI (controls). During a routine visit, patients completed the Diabetes Specific Quality of Life Scale (DSQOLS), the Diabetes Treatment Satisfaction Questionnaire (DTSQ) and SF-36 Health Survey. All the scores ranged from 0 to 100 , with higher scores indicating better QoL or higher satisfaction with therapy.

Results: The proportion of women was higher among cases than controls ( $57 \%$ vs. $46 \%$; $<<0.0001$ ). In comparison with controls, cases also had a longer diabetes duration $(18 \pm 10$ years vs. $15 \pm 10$ years, $p<0.0001)$ and suffered more often from eye complications ( $32 \%$ vs. $22 \%, \mathrm{p}<0.0001$ ) and renal complications $(11 \%$ vs. $6 \%, p<0.0007)$. In the control group, $90 \%$ of patients were treated with glargine-based MDI regimens. Groups did not differ with respect to age, school education, occupation and HbAlc levels. Multiple logistic regression, adjusted for socio-demographic and clinical characteristics, showed that, as compared with cases, controls had a more than threefold risk of having a DTSQ score in the lowest quartile (OR=3.3; 95\%CI 2.5-5.0) and a 70\% higher likelihood of having a score in the lowest quartile for the "diet restrictions" and "fear of hypoglycaemia" DSQOLS scores (OR=1.7; 95\%CI 1.3-2.0 for both scores). No difference emerged between the two groups as for SF-36 scores. Results were confirmed when CSII was compared separately with glargine- or NPH-based MDI regimens.

Conclusion: This is the first large scale, office based study describing how CSII affects quality of life and treatment satisfaction when compared with either glargine-based or NPH-based MDI regimens in adults with T1DM. The study documents that, despite more severe diabetes, patients treated with CSII are more satisfied of their treatment, perceive a greater lifestyle flexibility, and show less fear of hypoglycemia.

Supported by Fondazione Medtronic Italia

\section{9}

Rates and risks for suicide attempt in outpatients with diabetes mellitus: results from the Dutch Screening for Depression in Diabetes (DDD) Study F. Pouwer ${ }^{1}$, B. Kilic ${ }^{1}$, P. H. L. Geelhoed-Duijvestein ${ }^{2}$, F. J. Snoek ${ }^{1}$;

${ }^{1}$ Diabetes Psychology Research Group, VU University Medical Center, Amsterdam,

${ }^{2}$ Internal Medicine, Westeinde Hospital, The Hague, The Netherlands.

Background and Aims: There is convincing evidence of an increased risk for depression in diabetes. However, it is unclear whether this translates into 
an elevated risk of suicide attempt. We therefore aimed to: 1) determine the number and percentage of outpatients with diabetes mellitus that report a history of attempted suicide 2) to study current suicide ideation in this particular group, and 3) to explore clinical and socio-demographic factors associated with a history of suicide attempt.

Materials and Methods: Depressive symptoms, including current suicide ideation were assessed in 519 outpatients with diabetes (19-95 years of age) using the Patient Health Questionnaire (PHQ-9). Information regarding type of diabetes, diabetes duration and complications was obtained from the patient's medical charts. Patients with and without a history of suicide attempt were compared using Chi-square tests for categorical variables and Student's t-tests for continuous variables. To explore the strength of the association between sociodemographic/clinical factors and history of suicide attempt univariate logistic regression analyses were used.

Results: 33 patients $(6.4 \%)$ of 519 reported that they had ever attempted suicide. The most robust predictors of suicide attempt were: history of admission to a psychiatric hospital because of depression (OR 13.5, 95\% CI 5.3-34.8), depression treatment in the past (OR 5.1, 95\% CI 2.9-9.0), admission to a hospital because of diabetic coma (OR 3.2, 95\% CI 1.0-9.8), being partially/fully disabled (OR 3.0, 95\% CI 1.4-6.2), having cardiovascular disease (OR 2.5, 95\% CI 1.1-5.5), and for men: impotence (OR 8.4, 95\% CI 1.6-44.6). Almost three quarter (73\%) of the diabetes patients who had ever attempted to commit suicide reported to have had suicidal thoughts during the last two weeks.

Conclusion: Prior research in the general Dutch population showed that the lifetime prevalence of suicide attempt is $2.3 \%$. The prevalence rate of $6.4 \%$ in the present sample suggests that a history of attempted suicide is relatively common among outpatients with diabetes. Health care providers should be particularly alert for suicide ideation in patients with a history of inpatient treatment for psychiatric problems and in patients with complications of diabetes.

Supported by the Dutch Diabetes Research Foundation

\section{0}

Interaction of depressive symptoms, diabetes-related distress and glycaemic control in type 2 diabetic patients

M. Pibernik-Okanovic ${ }^{1}$, D. Begic ${ }^{2}$, S. Szabo ${ }^{1}$, I. Renar ${ }^{1}$, Z. Metelko ${ }^{1}$;

${ }^{1}$ Outpatient Clinic, Vuk Vrhovac Institute,

${ }^{2}$ Psychiatric Clinic, Rebro University Hospital, Zagreb, Croatia.

Background and Aims: Psychological well-being and diabetes selfmanagement have been shown to be interdependent. However, associations between psychological symptoms and glycemic control are not quite consistent. This study was aimed at examining patterns of glycemic control in emotionally burdened subgroups versus symptom-free individuals with type 2 diabetes.

Materials and Methods: A random sample of 470 type 2 diabetic patiens (aged $58 \pm 8 \mathrm{yrs}, 52 \%$ female, with diabetes duration of $9 \pm 6 \mathrm{yrs}, 33 \%$ insulin treated) were assessed for depression and diabetes-related distress by using the Center for Epidemiologic Studies-Depression (CES-D) and the Problem Areas in Diabetes (PAID) scales. Relying on the standard cut offs (CES-D $>16$, PAID $>40$ ), four discrete subgroups were analysed: patients with severe depressive symptoms and high diabetes distress, patients with severe depressive symptoms only, patients with high diabetes-related distress only and symptom-free patients. The indicators of glycemic control, as determined by $\mathrm{HbA1C}$ values, were analysed at the point of psychological assessment and one year later. Pearson's correlation coefficients were used to determine univariate associations between glycemic control, disease-related and psychological variables. Analysis of covariance was used to compare glycemic control among the four groups while controlling for the diseaserelated variables.

Results: Twelve percent of the examined patients suffered from severe depressive symptoms combined with high diabetes-related distress, $10 \%$ reported severe depressive symptoms, 22\% expressed high distress and $56 \%$ were symptom-free. The entire group's glycemic control was associated with diabetes duration $(r=0,14 \mathrm{p}<0.05)$, diabetes therapy $(r=0.17 \mathrm{p}<0.05)$, body mass index $(r=0.12 \mathrm{p}<0.05)$, the CES-D scores $(r=0.10 \mathrm{p}<0.05)$ and the PAID scores $(r=0.27 \mathrm{p}<0.05)$. After controlling for diabetes duration, diabetes therapy and body mass index, the four groups differed with respect to glycemic control $(\mathrm{F}=8.25 \mathrm{p}<0.0001)$. The Tukey 's HSD for unequal $\mathrm{N}$ indicated that $\mathrm{HbA1C}$ values were significantly higher in the depressed-distressed group as compared to depressed and symptom-free groups $(8.6 \% \pm 1.6$ vs $7.3 \% \pm 1.3$ and $7.6 \% \pm 1.4 ; \mathrm{p}=0.0003$ and $\mathrm{p}=0.005$, respectively), whereas the distressed group had higher $\mathrm{HbAlC}$ values than the depressed group $(8.1 \pm 1.7$ vs $7.3 \% \pm 1.3$ $\mathrm{p}=0.05)$. After a one-year follow-up, glycemic control remained associated with depressive symptoms and diabetes-related distress after controlling for diabetes-related variables $(\mathrm{F}=6.94 \mathrm{p}=<0.0001)$. Between-group differences were significant for depressed-distressed vs depressed group $(8.2 \% \pm 1.7$ vs $7.3 \% \pm 1.3 \mathrm{p}=0.005)$. While the distressed group improved glycemic control during the follow-up period $(8.2 \pm 1.7$ to $7.6 \pm 1.3 \mathrm{p}=0.0004)$, the depresseddistressed and depressed groups $\operatorname{did}$ not $(8.6 \% \pm 1.6$ vs $8.2 \% \pm 1.8 \mathrm{p}=0.11$ and $7.3 \pm 1.3$ vs $7.3 \pm 1.3 \mathrm{p}=0.95$ respectively).

Conclusion: Depressive symptoms and diabetes-related distress were both cross-sectionally and longitudinally shown to adversely interact with glycemic control. Patients suffering from severe depressive symptoms and high diabetes-related distress seem to have the greatest difficulties in achieving good glycemic control. Screening for both may be recommended in order to provide appropriate psychological help and improve glycemic control. Supported by the Ministry of Science of the Republic of Croatia

\section{1}

Cognitive dysfunction in type 2 diabetes mellitus: comparison with pre-diabetic stages

E. van den Berg ${ }^{1}$, G. Biessels ${ }^{1}$, G. Nijpels ${ }^{2}$, J. M. Dekker²;

${ }^{1}$ Department of Neurology G03.228, University Medical Center Utrecht,

${ }^{2}$ Institute for Research in Extramural Medicine (EMGO Institute), VU

University Medical Center, Amsterdam, The Netherlands.

Background and Aims: Type 2 diabetes (DM2) is associated with cognitive impairments and an increased risk of dementia. DM2 is preceded by a prolonged 'pre-diabetic' stage characterised by insulin resistance and vascular and metabolic risk factors such as hypertension and obesity. The concept of the 'metabolic syndrome' (MS) entails the clustering of these risk factors and as such can be viewed as a pre-diabetic stage. We hypothesise that the cognitive impairments associated with DM2 develop in this pre-diabetic stage, before the actual onset of the hyperglycaemia, and that vascular and metabolic factors related to the MS are the prime determinants of these impairments.

Materials and Methods: Participants were recruited through the 'Hoorn study', a longitudinal cohort study in which detailed metabolic and vascular data were collected in 1989, 1996, and 2001. For the current examination in 2005/2006 three groups were selected according to the risk factor profile at the 2001 survey: control participants (0-1 MS risk factor (ATPIII-criteria); $\mathrm{n}=115)$, MS without DM2 ( $\mathrm{n}=98$ ), and recent onset DM2 (onset after 1996; $\mathrm{n}=64)$. Performance on 12 neuropsychological tasks, covering 6 cognitive domains, was expressed in standardized z-scores.

Results: Age, sex distribution and education were similar across the groups. The number of MS risk factors was similar in the MS and the DM2 group. We observed small differences between groups on cognition (abstract reasoning: control $0.04 \pm 0.10$, MS $-0.002 \pm 0.11, \mathrm{DM} 2-0.06 \pm 0.13, \mathrm{p}=0.84$; memory: $-0.01 \pm 0.05,0.04 \pm 0.05,-0.10 \pm 0.06, \mathrm{p}=0.2$; information processing speed: $0.13 \pm 0.08,-0.11 \pm 0.09,-0.18 \pm 0.11, \mathrm{p}=0.04$; attention and executive functions: $0.08 \pm 0.06,-0.05 \pm 0.07,-0.17 \pm 0.08, \mathrm{p}=0.04$; visuoconstruction: $0.16 \pm 0.10$, $-0.04 \pm 0.10,-0.21 \pm 0.13, \mathrm{p}=0.06$; language: $0.11 \pm 0.10,0.01 \pm 0.10,-0.22 \pm 0.13$, $\mathrm{p}=0.11$ ). The MS group had an intermediate position between the DM2 and control group. As expected, the effects in the early DM2 group were smaller than in previous studies with advanced DM2.

Conclusion: This first analysis of our study population suggests that the cognitive impairment that is associated with DM2 already develops in prediabetic stages and progresses gradually thereafter. Future analyses will address longitudinal associations between vascular and metabolic risk factors and cognition, to identify determinants of cognitive dysfunction in the whole population.

Supported by the Dutch Diabetes Research Foundation 


\section{2}

\begin{abstract}
A Diabetes and Psychological Treatments Study (ADaPT): preliminary results from a randomised controlled trial

K. Ismail ${ }^{1}$, S. M. Thomas ${ }^{2}$, T. Chalder ${ }^{1}$, C. Dickens ${ }^{3}$, F. Creed ${ }^{3}$, E. Maissi ${ }^{1}$, J. Bartlett ${ }^{4}$, U. Schmidt ${ }^{1}$, A. Patel ${ }^{1}$, J. Treasure ${ }^{1}$;

${ }^{1}$ Psychological Medicine, Institute of Psychiatry, London,

${ }^{2}$ Diabetes Centre, Guy's and ST Thomas' NHS Trust, London,

${ }^{3}$ Psychiatry, University of Manchester, ${ }^{4}$ Medical Statistics, London School

of Hygiene and Tropical Medicine, London, United Kingdom.
\end{abstract}

Background and Aims: Despite intensive medical regimes, suboptimal glycaemic control is common in people with type 1 diabetes. The pooled evidence that psychological treatments are effective in improving glycaemic control is limited. We conducted a multicentre randomised controlled trial to test first, whether motivational enhancement therapy (MET) was more effective than usual diabetes care in improving glycaemic control and second, whether MET plus cognitive behaviour therapy (CBT) was more effective than usual diabetes care in improving glycaemic control in adults with type 1 diabetes and persistent suboptimal glycaemic control.

Materials and Methods: Six nurses were trained to deliver diabetes specific MET and CBT using a standardised manualised training and competency ratings. Trial criteria were adults (18-65 years) with type 1 diabetes (duration $>2$ years); local residents of the 9 study hospitals in South London and Manchester, United Kingdom; no advanced diabetes complications or severe mental illness; not pregnant; at least one glycated haemoglobin $\left(\mathrm{A}_{1 \mathrm{c}}\right)$ in past 12 months plus current $A_{1 c} \geq 8.2$ and $\leq 15.0 \%$. After baseline assessments, participants were randomised to 4 sessions of MET over 2 months or 4 sessions of MET plus 6 sessions of CBT over 6 months or usual diabetes care using computerised proportional minimised randomised blocks and allocation concealment. The main outcome was $\mathrm{A}_{1 c} 12$ months later (technicians blind to allocation) adjusted for baseline $\mathrm{A}_{1 c}$.

Results: Using clinic registers, around 2000 people with type 1 diabetes were screened and 344 were randomised 117, 106 and 121 were assigned to MET $(\mathrm{n}=117)$, MET plus CBT $(\mathrm{n}=106)$ and to usual care $(\mathrm{n}=121)$ respectively. The follow up rate for $A_{1 c}$ was $88 \%(n=305) 12$ months later. The average age was 36.0 years (interquartile range (IQR) 28.1-43.8); duration of diabetes was 17.9 years (IQR 10.6-24.8); A 9.4\% (IQR 8.8-10.2); 60.5\% were female; $80.2 \%$ were white; $69.5 \%$ were employed; $17.9 \%$ had a depressive disorder. The nurses had reasonable competency in delivering MET (Motivational Interviewing Competency Scale empathy score 5.2 (SD 0.68) and CBT (Cognitive Therapy Rating Scale-R score 4.5 (SD 9.7). In the preliminary analysis, the adjusted mean 12 month $\mathrm{A}_{1 \mathrm{c}}$ was $0.2 \%$ ( $95 \%$ confidence interval $-0.2 \%$ to $0.5 \%$ ) lower in the MET group compared to the usual diabetes care group but this difference was not statistically significant. There was statistically significant evidence that the adjusted mean 12 month $A_{1 c}$ was $0.5 \%$ (95\% confidence interval $0.1 \%$ to $0.8 \%$ ) lower in the MET plus CBT group compared to the usual care group ( $\mathrm{p}=0.008)$.

Conclusion: In summary in this group of difficult to control diabetes nursedelivered MET plus CBT was associated with a clinical and statistically significant improvement in glycaemic control but MET alone was not. Supported by NHS Health Technology Assessment Programme

\section{OP 28 Imaging beta cell signal transduction}

\section{3}

Glucose-stimulated oscillations of cAMP in insulin-secreting $\beta$ cells O. Dyachok, J. Sågetorp, E. Gylfe, A. Tengholm; Department of Medical Cell Biology, Uppsala University, Sweden.

Background and Aims: Rise of glucose is a primary physiological stimulus for insulin secretion. It results not only in increase of the cytoplasmic $\mathrm{Ca}^{2+}$ concentration $\left(\left[\mathrm{Ca}^{2+}\right]_{i}\right)$ but also in a modest rise of the cAMP concentration in pancreatic islets/ $\beta$-cells. It is well documented that cAMP amplifies $\mathrm{Ca}^{2+}$ dependent insulin release. Whereas $\mathrm{Ca}^{2+}$ signalling has been extensively characterized and found to involve oscillations important for the temporal control of insulin secretion, the kinetics of glucose-triggered cAMP signals is still unknown.

Materials and Methods: MIN6 $\beta$-cells were transfected with a translocation reporter based on modified protein kinase A subunit fused to the cyan and yellow fluorescent proteins (CFP and YFP). Changes in CFP/YFP fluorescence ratio depending on cAMP in the submembrane space $\left([\mathrm{cAMP}]_{\mathrm{i}}\right)$ were recorded with evanescent wave microscopy. Simultaneous recording of $[\mathrm{cAMP}]_{\mathrm{i}}$ and $\left[\mathrm{Ca}^{2+}\right]_{\mathrm{i}}$ was performed in cells loaded with the fluorescent indicator fura- 2 .

Results: Reminiscent of the $\left[\mathrm{Ca}^{2+}\right]_{\mathrm{i}}$ response, MIN6 $\beta$-cells reacted to rise of glucose from 3 to $11 \mathrm{mM}$ with a modest temporary decrease of [cAMP] followed by rapid increase and pronounced oscillations with nadirs well above basal [cAMP]. The oscillatory patterns varied among cells with frequencies ranging from 0.07 to $1.8 \mathrm{~min}^{-1}$. The glucose-induced [cAMP] oscillations were abolished by preventing adenylyl cyclase activation with 3 $\mu \mathrm{M}$ adrenaline or noradrenaline or by enzyme inhibition with $50 \mu \mathrm{M}$ of 2,5'dideoxyadenosine. The amplitude of the [cAMP] oscillations was reduced by the $\mathrm{Ca}^{2+}$ channel blocker methoxyverapamil or removal of extracellular $\mathrm{Ca}^{2+}$. Some cells showed synchronized oscillations of $[\mathrm{cAMP}]_{i}$ and $\left[\mathrm{Ca}^{2+}\right]_{i}$.

Conclusion: Contrary to previous reports of a modest glucose-induced increase of total cAMP in $\beta$-cells we found that the sugar induces pronounced $[\mathrm{cAMP}]_{\mathrm{i}}$ oscillations in the submembrane space, where the nucleotide has important effects on ion channel activity and exocytosis. These oscillations and the interplay between $\mathrm{Ca}^{2+}$ and cAMP at the membrane should be important determinants for pulsatile release of insulin.

Supported by EFSD/MSD European Studies on Beta Cell Function and Survival and O.E. och Edla Johanssons vetenskapliga stiftelse

\section{4}

Generation of sub-membrane cyclic AMP signals by $\mathrm{Ca}^{2+}$ and ATP in insulin-secreting cells

J. Sågetorp, O. Dyachok, E. Gylfe, A. Tengholm;

Department of Medical Cell Biology, Uppsala University, Sweden.

Background and Aims: cAMP is an important regulator of insulin secretion from pancreatic $\beta$-cells. Glucose stimulation of $\beta$-cells results in elevation of cAMP. The present study aimed to determine how the cAMP concentration beneath the plasma membrane $\left([\mathrm{cAMP}]_{\mathrm{i}}\right)$ is affected by the cytoplasmic $\mathrm{Ca}^{2+}$ concentration $\left(\left[\mathrm{Ca}^{2+}\right]_{\mathrm{i}}\right)$ and by stimulation of metabolism.

Materials and Methods: $[\mathrm{cAMP}]_{i}$ was recorded in MIN6 $\beta$-cells with a translocation reporter based on engineered protein kinase A subunits fused to the cyan and yellow fluorescent proteins (CFP and YFP). Changes in $\mathrm{CFP} / \mathrm{YFP}$ ratio depending on $[\mathrm{cAMP}]_{\mathrm{i}}$ were recorded with evanescent wave microscopy, and $\left[\mathrm{Ca}^{2+}\right]_{\mathrm{i}}$ measured with ratiometric fluorescence imaging and the fura-2 indicator.

Results: Plasma membrane depolarization with $30 \mathrm{mM} \mathrm{K}^{+}$in the presence of $3 \mathrm{mM}$ glucose and $250 \mu \mathrm{M}$ diazoxide induced a prompt rise of [cAMP], that was reversed when the $\mathrm{K}^{+}$concentration was normalized. Elevation of the glucose concentration to $11 \mathrm{mM}$ in cells hyperpolarized with diazoxide did not significantly change [cAMP $]_{i}$. However, the presence of high glucose amplified the [cAMP] $]_{i}$ response to depolarization with $\mathrm{K}^{+}$in the presence of diazoxide $(153 \pm 40 \%$ increase of response, $n=6, p<0.02)$. When the glucose concentration was increased from 3 to $11 \mathrm{mM}$ in the presence of $30 \mathrm{mM} \mathrm{K}^{+}$and $250 \mu \mathrm{M}$ diazoxide there was a pronounced rise of [cAMP] $]_{\mathrm{i}}(104 \pm 20 \%, \mathrm{n}=10$, $\mathrm{p}<0.001$ ), sometimes with superimposed oscillations (frequency $=0.19 \pm 0.02$ 
$\left.\min ^{-1} ; \mathrm{n}=8\right)$. A similar, but transient $[\mathrm{cAMP}]$, elevation was induced by $2 \mathrm{mM}$ $\alpha$-ketoisokaproic acid (KIC). The [cAMP $]_{i}$-elevating effects of glucose and KIC in depolarized cells were not paralleled by increases of $\left[\mathrm{Ca}^{2+}\right]_{\mathrm{i}}$. Instead, rise of glucose or addition of KIC induced a transient lowering of $\left[\mathrm{Ca}^{2+}\right]_{\mathrm{i}}$ from the level obtained with $\mathrm{K}^{+}$depolarization.

Conclusion: Rise of $\left[\mathrm{Ca}^{2+}\right]_{\mathrm{i}}$ is sufficient to trigger elevation of $[\mathrm{cAMP}]_{\mathrm{i}}$ in $\beta$-cells. In the presence of elevated $\left[\mathrm{Ca}^{2+}\right]_{i}$ metabolically generated ATP is a potent stimulus for cAMP production. The interplay between $\mathrm{Ca}^{2+}$, ATP and cAMP in the sub-membrane space may help to explain the initiating and amplifying pathways of glucose-induced insulin secretion.

Supported by EFSD/MSD European Studies on Beta Cell Function and Survival

\section{5}

Pulsatile insulin release from single $\beta$ cells critically depends on cAMP O. Idevall Hagren, A. Tengholm;

Medical Cell Biology, Uppsala University, Sweden.

Background and Aims: Insulin is secreted in distinct pulses from pancreatic $\beta$-cells following elevation of the plasma glucose concentration. The triggering signal for insulin granule exocytosis is a rapid increase in the cytoplasmic $\mathrm{Ca}^{2+}$ concentration $\left(\left[\mathrm{Ca}^{2+}\right]_{i}\right)$. cAMP is known to amplify glucose-induced insulin secretion by potentiating the $\mathrm{Ca}^{2+}$-dependent exocytosis. However, the kinetics of this process is unknown and the aim of the present study was to investigate the relationship between cAMP signals and pulsatile insulin secretion in single $\beta$-cells.

Materials and Methods: Insulin secretion from single MIN6 $\beta$-cells was monitored with a fluorescent translocation biosensor (GRP1-GFP ) reporting formation of phosphatidylinositol-3,4,5-trisphosphate ( $\mathrm{PIP}_{3}$ ) induced by autocrine activation of insulin receptors on the $\beta$-cell surface. Changes in cAMP concentration beneath the plasma membrane $\left([\mathrm{cAMP}]_{\mathrm{i}}\right)$ were measured with evanescent wave microscopy and a translocation biosensor based on fluorescence-tagged subunits of protein kinase A (PKA).

Results: Elevation of the glucose concentration from 3 to $11 \mathrm{mM}$ induced a rise of the plasma membrane $\mathrm{PIP}_{3}$ concentration $(161 \pm 14 \%$ increase in GRP1-GFP fluorescence) followed by pronounced oscillations from a slightly elevated level (frequency $=0.22 \pm 0.02 \mathrm{~min}^{-1} ; \mathrm{n}=39$ cells). Simultaneous recording of $[\mathrm{cAMP}]_{\text {, }}$ revealed that each rise of $\mathrm{PIP}_{3}$ was preceded by an increase of [cAMP]. Inhibition of adenylate cyclases with 2,5'-dideoxyadenosine suppressed the PIP oscillations in a concentrationdependent manner. Conversely, elevation of $[\mathrm{cAMP}]_{i}$ with forskolin or IBMX induced an immediate stimulation of PIP formation ( $87 \%$ of the cells) followed by sustained elevation (22\%) or continued oscillations $(65 \% ; n=67$ for IBMX). The time-integrated GRP1-GFP signal was significantly higher in the presence of IBMX than with $11 \mathrm{mM}$ glucose alone $(102 \pm 38 \%$ increase, $\mathrm{n}=26, \mathrm{P}<0.02$ ). Whereas the PKA inhibitor $\mathrm{H}-89$ was without effect on the glucose- and IBMX-induced PIP ${ }_{3}$ signals, the effect of cAMP elevation was mimicked by the Epac-activating nucleotide analogue 8-pMeOPT-2'-O-MecAMP.

Conclusion: Insulin is released in pulses from individual $\beta$-cells. This rhythmicity is intimately controlled by changes in cAMP concentration in the sub-membrane space. The results reinforce the role of Epac in insulin granule exocytosis.

Supported by EFSD/MSD European Studies on Beta Cell Function and Survival

\section{6}

Visualization of single insulin granules in insulin-producing cells by selective photoactivation

S. Baltrusch, S. Lenzen;

Institute of Clinical Biochemistry, Hannover Medical School, Germany.

Background and Aims: Intracellular transport and exocytosis of insulin is a prerequisite for glucose-induced insulin secretion in pancreatic beta cells. The mechanism of insulin storage and release requires proper packaging of the insulin containing dense core vesicles, their translocation, docking and fusion to the plasma membrane. Targeting the granule membrane as well as the cargo of the secretory granules by fluorescent proteins has facilitated detailed knowledge inside these processes during recent years. While total internal reflection fluorescence microscopy, which allows fluorescence excitation with $100 \mathrm{~nm}$ from the plasma membrane, promotes the analysis of docking and fusion of single insulin granules, tracking single insulin granules within the cytoplasm by real-time fluorescence microscopy is so far less accessible. The aim of this study was to visualize single insulin granules in insulin-producing cells with selective photoactivation.

Materials and Methods: Plasmids were generated for expression of fusion proteins of the granule membrane resident phosphatase phogrin or the granule cargo protein neuropeptide Y (NPY) with the photoactivatable fluorescent protein PA-GFP-A206K as well as with the fluorescent protein mCherry. Insulin-producing MIN6 cells were either co-transfected with plasmids expressing Phogrin-PA-GFP-A206K and NPY-mCherry or with plasmids expressing Phogrin-mCherry and NPY-PA-GFP-A206K and cultured for $48 \mathrm{~h}$ in DMEM medium with $25 \mathrm{mmol} / \mathrm{l}$ glucose. Cells were imaged with an Olympus Fluoview1000 confocal microscope, equipped with a second scan unit for simultaneous photoactivation.

Results: In transfected MIN6 cells NPY-mCherry and Phogrin-mCherry showed distinct colocalization after fixation and immunostaining with insulin, which indicates proper targeting of the generated fusion constructs. Living cells co-transfected with Phogrin-PA-GFP-A206K and NPY-mCherry or with Phogrin-mCherry and NPY-PA-GFP-A206K showed a comparable pattern of punctuated red fluorescence. Overall illumination with a 405 $\mathrm{nm}$ laser diode provoked activation of PA-GFP-A206K. The resulting green fluorescence of Phogrin-PA-GFP-A206K and NPY-PA-GFP-A206K was with $\geq 70 \%$ colocalized with the red fluorescence of NPY-mCherry and PhogrinmCherry, respectively. Region selected $405 \mathrm{~nm}$ laser light exposure for less than $1 \mathrm{sec}$ resulted in $\geq 50 \%$ of the experiments in visualization of three or $\geq 20 \%$ of a single granule by green fluorescence. After treatment with $25 \mathrm{mmol} / \mathrm{l}$ glucose the marked granules moved significantly faster than in MIN6 cells treated with $3 \mathrm{mmol} / \mathrm{l}$ glucose. Acquisition of several layers of the MIN6 cell after photoactivation revealed a deeper spatial insight in the granule movement. Interestingly, in the generated 3-D images the changes in the granule membrane during movement illuminated by Phogrin-PA-GFPA206K could be clearly distinguished from the granule cargo visualized by NPY-mCherry.

Conclusion: Visualization of single insulin granules by targeting of the granule membrane or its cargo with a photoactivatable protein in combination with a dual scanner confocal microscope is a useful tool to analyze the mechanism of intracellular granule transport. This technique may open the perspective to further elucidate the regulation of different pools of granules within the beta cell also with respect to the secretory defects in type 2 diabetes.

\section{7}

ATP, mitochondrial calcium and mitochondrial $\mathrm{pH}$ responses during nutrient activation of the pancreatic $\beta$ cell

A. Wiederkehr ${ }^{1}$, K.-S. Park ${ }^{1}$, O. Dupont ${ }^{1}$, N. Demaurex ${ }^{1}$, T. Pozzan ${ }^{2}$, C. B. Wollheim ${ }^{1}$,

${ }^{1}$ Department of Cell Physiology and Metabolism, University of Geneva, Switzerland, ${ }^{2}$ Department of Biomedical Sciences, University of Padua, Italy.

Background and Aims: Nutrient activation of the pancreatic $\beta$-cell results in a large number of signals and metabolic changes that stimulate insulin secretion. Here we have studied cytosolic ATP and mitochondrial calcium signals as well as mitochondrial $\mathrm{pH}$ changes following the stimulation of $\beta$ cells with glucose in order to gain insight into the molecular mechanisms of $\beta$-cell activation.

Materials and Methods: For the measurement of mitochondrial $\mathrm{pH}$, the $\mathrm{pH}$ sensitive probe mitochondrial AlpHi (mtAlpHi) was cloned into an adenovirus vector. INS-1E cells and freshly isolated rat islets were infected with adenoviruses carrying mtAlpHi or the previously described adenoviruses carrying Luciferase (ATP) or mitochondrial Aequorin (calcium). Fluorescence respectively luminescence of the probes was analysed 2-3 days after infection.

Results: ATP or the connected ATP/ADP ratio is a key regulator of insulin secretion. Consistent with earlier studies we find that glucose stimulation increases ATP levels by $25-35 \%$ over the signals measured in non-stimulated $\beta$-cells. In INS-1E cells, ATP as well as mitochondrial calcium were found to oscillate with a periodicity of 2-3 minutes. The delay of onset of the signal and the frequency of oscillations was indistinguishable suggesting a causal link between the two parameters. In rat islets very similar ATP oscillations were observed however glucose stimulation only resulted in a single 
mitochondrial calcium rise not followed by calcium oscillations. In both systems removal of extracellular calcium prevented mitochondrial calcium signals whereas ATP oscillations persisted. Together the results demonstrate that calcium oscillations are not required to drive ATP oscillations. In contrast the absence of extracellular calcium strikingly affected the observed nutrient dependent net increase in ATP levels. During the initial 4 minutes ATP rose equally in the absence and presence of calcium. Thereafter ATP levels increased steadily in control islets but remained close to constant in the absence of calcium influx. Re-addition of calcium restored normal rates of ATP synthesis. Mitochondrial metabolism and calcium signals are intimately linked to changes in mitochondrial $\mathrm{pH}$. To study how mitochondrial $\mathrm{pH}$ relates to calcium and ATP signals in $\beta$-cells we have employed organelle targeted mtAlpHi. MtAlpHi fluorescence was $\mathrm{pH}$ sensitive in both INS-1E and rat islet $\beta$-cells. Titration of the probe in situ showed that mitochondrial $\mathrm{pH}$ is fairly low $(<7.3)$ in the $\beta$-cell when compared with published values for mitochondrial $\mathrm{pH}$ in other cell types. Glucose activation consistently resulted in alkalinisation of the mitochondrial matrix. Matrix $\mathrm{pH}$ increased gradually to reach a new steady-state 10-15 minutes after glucose addition. The $\mathrm{pH}$ increase following glucose was quite large ranging from $0.2-0.6 \mathrm{pH}$ units.

Conclusion: Our results illustrate the calcium dependence of ATP synthesis in $\beta$-cells during a second phase of the nutrient response. Furthermore we add mitochondria matrix $\mathrm{pH}$ to the list of parameters that respond to nutrients. Matrix $\mathrm{pH}$ changes are likely to modulate mitochondrial metabolism thereby the insulin secretory response of the $\beta$-cell.

Funded by the Swiss National Science Foundation and EuroDia

\section{8}

The lipase inhibitor, orlistat blocks glucose-induced $\mathrm{Ca}^{2+}$ oscillations and insulin secretion from pancreatic $B$ cells; effects partially reversed by fatty acid

J. T. Deeney, L. Liu, R. Corkey, B. E. Corkey;

Department of Medicine, Boston Medical Center, Boston, United States.

Background and Aims: Glucose-induced insulin secretion is impaired under conditions in which orlistat is used to inhibit lipolysis in rat pancreatic islets. Our goal was to elucidate the mechanism by which the lipase inhibitor, orlistat blocks insulin secretion.

Methods: Insulin secretion from INS-1 (823/13) clonal pancreatic B-cells and dissociated rat pancreatic islets was measured by RIA. Intracellular $\mathrm{Ca}^{2+}$ was monitored using fura-2 loaded cells visualized on a Zeiss microscope attached to a time-sharing filter based fluorometer (Ionoptix Corp., MA).

Results: Orlistat $(6-50 \mu \mathrm{M})$, a non-specific lipase inhibitor, inhibited glucose-induced insulin secretion from INS-1 cells in a concentrationdependent manner. Addition of oleate $(0.2 \mathrm{mM}, 4: 1$ molar ratio to BSA) partially reversed the orlistat effects on secretion. Intracellular $\mathrm{Ca}^{2+}$ was then monitored to determine if inhibition of insulin secretion was mediated by impaired $\mathrm{Ca}^{2+}$ homeostasis. Orlistat $(100 \mu \mathrm{M})$ inhibited glucose-induced $\mathrm{Ca}^{2+}$ oscillations and addition of oleate reversed this inhibition. Orlistat also reduced intracellular $\mathrm{Ca}^{2+}$ elevated using $\mathrm{KCl}$ in the presence of glucose and diazoxide $(400 \mu \mathrm{M})$. This reduction was also reversed by the addition of oleate. Preincubation of INS-1 cells with orlistat $(100 \mu \mathrm{M})$ inhibited KCl-induced plasma membrane depolarization by $30 \%$ as monitored with the fluorescent indicator bis-oxonol. Similar effects on glucose-induced insulin secretion and $\mathrm{Ca}^{2+}$ homeostasis were found in dissociated rat islet cells using higher concentrations of orlistat $(200 \mu \mathrm{M})$.

Conclusion: Fatty acids provide lipid signals required for both normal $\mathrm{Ca}^{2+}$ homeostasis and insulin secretion. Thus, impaired glucose-induced insulin secretion due to inhibition of lipolysis can be attributed to both the $\mathrm{K}_{\text {ATP }^{-}}$ dependent triggering pathway as well as the $\mathrm{K}_{\mathrm{ATP}}$-independent amplification pathway of secretion.

Supported by DK35914

\section{OP 29 Genetics of type 2 diabetes}

\section{9}

High-density association mapping and comprehensive tagging of the type 2 diabetes linkage region on chromosome 1q in 4 European populations

I. Prokopenko' ${ }^{1}$, E. Zeggini ${ }^{1}$, N. W. Rayner ${ }^{1}$, C. J. Groves ${ }^{1}$, R. L. Hanson ${ }^{2}$, B. D. Mitchell ${ }^{3}$, M. Vaxillaire ${ }^{4}$, S. E. Hunt ${ }^{5}$, L. R. Cardon ${ }^{1}$, P. Froguel $^{6}$, S. C. Elbein ${ }^{7}$, A. R. Shuldiner, ${ }^{3}$, P. Deloukas ${ }^{5}$, M. I. McCarthy';

${ }^{1}$ WTCHG, University of Oxford, United Kingdom, ${ }^{2}$ NIH/NIDDK, Phoenix, United States, ${ }^{3}$ School of Medicine, University of Maryland, Baltimore, United States, ${ }^{4}$ Institut de Biologie de Lille Génomique et Physiologie Moléculaire des Maladies Métaboliques, Institut Pasteur, Lille, France, ${ }^{5}$ Wellcome Trust Sanger Institute, Hinxton, United Kingdom,

${ }^{6}$ Division of Medicine, Imperial College, London, United Kingdom, ${ }^{7}$ Division of Endocrinology and Metabolism, University of Arkansas, Little Rock, United States.

Background and Aims: The International Type 2 Diabetes 1q Consortium has undertaken sequential high-density linkage disequilibrium mapping of the extensively-replicated 1q linkage region in the search for the variants responsible.

Materials and Methods: We have extended our existing coverage of the $22.7 \mathrm{Mb}$ candidate region (147.0-169.7Mb, NCBI35) by genotyping 1536 additional SNPs in 2198 individuals (1000 cases, mainly probands from extended families, and 1198 matched controls) from four European-descent populations (Amish, French, UK and Utah). The new combined dataset includes 5285 SNPs passing stringent quality control criteria (density 4.3kb) and increases coverage of common variation from $\sim 60 \%$ to over $80 \%\left(\mathrm{r}^{2} \geq 0.8\right.$, pairwise) based on HapMap CEU data. Population-specific single-point analyses and Mantel-Haenszel-based meta-analysis across 4 populations were performed testing additive and dominant/recessive models.

Results: Thirteen SNPs showing highly significant associations with T2D (combined $\mathrm{p}<5 \times 10^{-5}$ ) were identified by Mantel-Haenszel model-based metaanalysis. The minor allele frequencies observed in each single population were high for all 13 SNPs, ranging from 0.19 to 0.45 . This increased density of the genotyping performed in this study provides increased support for the two principal association signals in the region. The first cluster (9 SNPs) falls into a region of extended $\mathrm{LD}$ at $\sim 152.1 \mathrm{Mb}$ that includes the gene encoding liver pyruvate kinase, $P K L R$. The strongest association in $P K L R$ was observed for rs3020781 (OR for C allele 1.33 [95\%CI 1.16-1.54], additive $\mathrm{p}=1.1 \times 10^{-6}$ ). The second cluster (4 SNPs) resides within NOS1AP (coding for nitric oxide synthase 1 (neuronal) adaptor protein [CAPON]) at $\sim 158.8 \mathrm{Mb}$. The strongest association here was observed with allele $\mathrm{T}$ of rs7538490 under the dominant model (OR=1.53 [95\%CI 1.28-1.81], $\left.\mathrm{p}=1.8 \times 10^{-6}\right)$.

Conclusion: Extended association analyses in the 1q candidate region for T2D have identified several common polymorphisms within 2 genes, PKLR and NOS1AP, sharing the same association pattern in 4 different populations of European origin.

Supported by NIDDK

\section{0}

The genetic variation in the IDE (insulin degrading enzyme) gene is associated with impaired insulin metabolism and increased risk of type 2 diabetes

N. N. Rudovich ${ }^{1,2}$, O. Pivovarova ${ }^{1,2}$, E. Fisher ${ }^{3}$, M. Osterhof ${ }^{1,2}$, M. Moehlig ${ }^{1,2}$, J. Spranger ${ }^{1,2}$, P. Slominski ${ }^{4}$, M. Schulze ${ }^{3}$, H. Boeing ${ }^{3}$, A. F. H. Pfeiffer ${ }^{1,2}$; ${ }^{1}$ Clinical Nutrition, DIFE Potsdam-Rehbrücke, Nuthetal, Germany, ${ }^{2}$ Endocrinology, Diabetes and Nutrition, Campus B. Franklin, Charite University Medicine, Berlin, Germany, ${ }^{3}$ Epidemiology, DIFE PotsdamRehbrücke, Nuthetal, Germany, Institute of Molecular Genetic, Russian Academy of Science, Moscow, Russian Federation.

Background and Aims: Evidence for linkage of type 2 diabetes to a region on chromosome 10q23-q25 has been provided in several populations. Among genes in this region is IDE encoding insulin degrading enzyme which serve as a major enzyme responsible for insulin proteolysis. IDE knockout mice show a diabetic phenotype with hyperinsulinemia. Thus, the aim of the present study was to examine the genetic associations between IDE SNPs, insulin 
metabolism and the risk for T2DM in the Metabolic Syndrome BerlinPotsdam (MESYBEPO) and European

Prospective Investigation into Cancer and Nutrition-Potsdam (EPICPotsdam) study populations.

Materials and Methods: 12 SNPs from IDE gene were selected and genotyped in the MESYBEPO cohort $(\mathrm{n}=1023(\mathrm{men}=32.6 \%)$; subjects with $\mathrm{T} 2 \mathrm{DM}=235, \mathrm{IGT}+\mathrm{IFG}=312, \mathrm{NGT}=475)$. The $\mathrm{IDE}$ variant $(94214145 \mathrm{~T}>\mathrm{C}$, rs1887922) was additionally genotyped in a nested case-cohort population of incident diabetics of the EPIC-Potsdam cohort $(n=801$ cases; $n=2248$ controls).

Results: We compared genotype frequencies in subjects with T2DM with those with NGT and found an association for rs1887922 (odds ratio 1.58 [95\% CI: 1.03-2.2]; $\mathrm{p}=0.036$ ) in women in MESYBEPO. Adjusting for the effect of age strengthened the association (recessive model: $p=0.009$ ). In nondiabetic women, the risk genotype was associated with a reduction in insulin clearance derived from oral glucose tolerance tests $(p=0.043)$. In the prospective EPIC-Potsdam population the same marker was associated with increased risk of T2DM in the additive inheritance model (RR 1.31 [95\% CI: $1.11-1.55] ; \mathrm{p}=0.0015$ ).

Conclusion: We demonstrate an association between an IDE SNP and type 2 diabetes in the cross-sectional MesyBepo cohort. This relation to diabetes risk is confirmed in the independent, prospective EPIC-Potsdam cohort. Therefore, this study provides convincing evidence that genetic predisposition to disturbances in the insulin degradation may play a role in the development of T2DM.

\section{1}

Common variation in the FTO (fused toes) gene is strongly associated with adiposity measures in the Northern Finnish Birth Cohort of 1966 A. J. Bennett ${ }^{1}$, M. N. Weedon ${ }^{2}$, E. Zeggini ${ }^{1}$, R. M. Freathy ${ }^{2}$, C. M. Lindgren ${ }^{1}$, U. Sovio ${ }^{3}$, N. J. Timpson ${ }^{4}$, A. Ruokonen 5 , H. Martikainen ${ }^{5}$, A. Pouta ${ }^{5}$, A.-L. Hartikainen ${ }^{5}$, T. M. Frayling' 2 , A. T. Hattersley ${ }^{2}$, M.-R. Järvelin ${ }^{3,5}$, M. I. McCarthy ${ }^{1}$;

${ }^{1}$ DRL, Oxford Centre for Diabetes, Endocrinology \& Metabolism/Wellcome Trust Centre for Human Genetics, Oxford, United Kingdom, ${ }^{2}$ Genetics of Complex Traits \& Diabetes Genetics, Peninsula Medical School, Exeter, United Kingdom, ${ }^{3}$ Department of Epidemiology and Public Health, Imperial College London, London, United Kingdom, ${ }^{4} \mathrm{MRC}$ Centre for Causal Analyses in Translational Epidemiology, University of Bristol, United Kingdom, ${ }^{5}$ Depts of Clinical Chemistry, Obstetrics and Gynecology, Public Health Science \& General Practice, University of Oulu, Finland.

Background and Aims: In large-scale association studies conducted in over 30,000 individuals from multiple populations, we have recently shown that a cluster of variants mapping within the FTO (fused toes) gene on chromosome 16 is strongly associated with adiposity. Genotypes at these SNPs are associated with $\sim 3 \mathrm{~kg}$ difference in adult body weight (attributable to effects on fat mass), and up to $\sim 1.7$ fold difference in risk of obesity (BMI $>30 \mathrm{kgm}^{-2}$ ). To understand the biochemical and longitudinal consequences of variation at this locus, we typed a representative SNP from this cluster (rs9939609) in 4593 individuals from the Northern Finland Birth Cohort of 1966.

Materials and Methods: We sought evidence for associations with longitudinal growth parameters (birth weight, measures of adiposity at age 14 and 31) and metabolic biochemistry available at age 31. Analyses used linear regression assuming an additive model, with stratification by gender and adjustment for possible confounding factors.

Results: No significant associations were seen for early growth variables including birth weight or ponderal index. At age 14, BMI was significantly associated with genotype with each copy of the risk-allele increasing BMI by $0.1 \mathrm{kgm}^{-2}(\mathrm{P}=0.04)$. The association with BMI was more striking at age 31 $\left(0.4 \mathrm{kgm}^{-2}\right.$ per allele, $\left.\mathrm{P}=5 \times 10^{-5}\right)$ and clearly reflected a difference in weight rather than height. Adiposity-associated FTO genotypes showed modest but non-significant associations with triglycerides (between-homozygote groups difference $+0.04 \mathrm{mmol} / \mathrm{l}$ ) and HDL-cholesterol (between-homozygote groups difference $-0.03 \mathrm{mmol} / \mathrm{l})$. These findings were consistent in magnitude with the effects attributable to the observed between-genotype differences in BMI (e.g. within-cohort regression indicates that a $0.8 \mathrm{kgm}^{-2}$ difference in BMI is associated with a $0.05 \mathrm{mmol} / \mathrm{l}$ difference in TG, in line with the $0.04 \mathrm{mmol} /$ 1 difference observed at FTO). Although variants in this gene are strongly associated $\left(\mathrm{P}<10^{-10}\right)$ with T2D in case-control studies, we found no significant relationship with fasting glucose in this study.
Conclusion: In this cohort, as with others we have studied, FTO genotypes are strongly associated with adult BMI. This association with weight was not evident at birth, but was apparent by age 14. In this young adult population, the effects of this gene on continuous metabolic phenotypes are weak, though consistent in direction and size with the observed effect of FTO variation on BMI.

Supported by Wellcome Trust

\section{2}

A genome wide scan for linkage in families with early onset maturity-onset diabetes of the young suggests a potential role for genes on chromosomes $2 p \mathbf{p q}, 4 \mathrm{q}$ and $10 \mathrm{q}$ in glucose homeostasis C. M. Lindgren ${ }^{1,2}$, B. Barrow ${ }^{2}$, D. Hammersley ${ }^{2}$, M. Shepherd ${ }^{3}$, K. Elliott ${ }^{1}$, N. W. Rayner', S. Ellard ${ }^{3}$, A. L. Gloyn²;

${ }^{1}$ Wellcome Trust Centre for Human Genetics, University of Oxford, ${ }^{2}$ Diabetes Research Laboratories, Oxford Centre for Diabetes Endocrinology \& Metabolism, University of Oxford, ${ }^{3}$ Institute of Biomedical \& Clinical Science, Peninsula Medical School, Exeter, United Kingdom.

Background: Maturity-onset diabetes of the young (MODY) is an autosomal dominantly inherited early onset subtype of non-insulin dependent diabetes. To date MODY causing mutations in six different genes have been described. However, these genes only explain around $87 \%$ of cases and previous studies have suggested that the remaining cases are heterogeneous. We hypothesised that amongst these, patients with a very young age of diagnosis $(<15 \mathrm{yrs})$ may represent a genetically homogenous group and could thus facilitate identification of novel genes. The aim of this study was to identify potential novel loci for further gene candidacy testing for young onset MODY.

Methods: We performed an autosomal genome wide linkage scan in 40 individuals ( 20 with diabetes) from 5 families with $>6$ subjects with onset of diabetes $\leq 15$ years. Samples were genotyped using the Illumina IV linkage panel. Extensive quality checks were performed including HapMap concordance rates $(0.2 \%)$, within genotyping concordance rates $(0.4 \%)$, and checking of Mendelian inconsistencies. A total of 4,846 markers were subsequently used for linkage analysis using non-parametric linkage analysis as well as a parametric autosomal dominant model taking linkage disequilibrium into account $\left(\mathrm{r}^{2}>0.1\right)$ as implemented in Merlin. Genedropping simulations of the data set were used to generate genome-wide empirical values.

Results: The highest NPL score 3.75 ( $\mathrm{p}_{\text {nominal }}<0.0001$, HLOD 1.5), was observed on chromosome 10q. Three additional regions showed an NPL score $>3.00$ on chromosomes $2 p$ (NPL 3.38, $p_{\text {nominal }}<0.0005$, HLOD 0.83 ), $3 q$ (NPL 3.10, $\mathrm{p}_{\text {nominal }}=0.0010$, HLOD 1.81) and $4 \mathrm{q}$ (NPL 3.00, $\mathrm{p}_{\text {nominal }}<0.0015$, HLOD 1.43). About 145 genes map to the 1 NPL drop off region of these 4 loci of interest, including several strong biological candidates, potassium channels and cell signalling components.

Conclusions: Our genome wide scan has identified novel loci on chromosomes $2 \mathrm{p}, 3 \mathrm{q}, 4 \mathrm{q}$ and $10 \mathrm{q}$ which potentially harbour novel genes involved in the pathogenesis of early onset MODY. Utilizing families with an early age of diagnosis in combination with advanced bioinformatics to prioritise candidacy, is a strategy to facilitate identification of novel disease loci by linkage mapping.

Supported by Diabetes UK

\section{3}

Common variants in the TCF7L2 gene increase future risk of type 2 diabetes by causing impaired incretin and $\beta$ cell function M. Sjögren ${ }^{1}$, M. Orho-Melander ${ }^{1}$, P. Almgren ${ }^{1}$, K.-F. Eriksson ${ }^{2}$, Å.-L. Lethagen ${ }^{1}$, G. Berglund ${ }^{2}$, T. Tuomi ${ }^{3}$, P. Nilsson ${ }^{2}$, L. Groop ${ }^{1,3}$, V. Lyssenko ${ }^{1}$;

${ }^{1}$ Diabetes \& Endocrinology, Lund University, Malmö, Sweden, ${ }^{2}$ Medicine, Lund University, Malmö, Sweden,

${ }^{3}$ Department of Medicine, Helsinki University Central Hospital, Finland.

Background and Aims: Genetic variants in the gene encoding for the transcription factor-7-like 2 (TCF7L2) have been associated with type 2 diabetes (T2D) as well as with impaired $\beta$-cell function but the mechanisms have remained obscure. The aim of this study was to prospectively study the 
ability of common variants in the TCF7L2 gene to predict future T2D and to explore the mechanisms by which they would increase risk for the disease.

Materials and Methods: 7051 Swedish individuals from the Malmoe Prospective Study followed for up to 24 years and 2651 Finnish individuals from the Botnia Prospective Study followed for up to 8 years were genotyped for three SNPs (rs7903146, rs12255372 and rs10885406) in the TCF7L2 gene; part of them underwent extensive metabolic studies.

Results: The CT/TT genotypes of SNP rs7903146 strongly predicted future T2D in two independent cohorts (Swedish: OR 1.58, p $<0.0001$; Finnish OR 1.61, $\mathrm{p}=0.007)$. Carriers of the risk CT/TT genotypes had lower insulin secretion during OGTT (insulinogenic index, $\mathrm{p}=0.0006$; disposition index, $\mathrm{p}=0.005)$ than carriers of the CC genotypes in the Malmoe study. In the Botnia prospective study, converters carrying the CT/TT genotypes had a more severe deterioration in insulin secretion over time (insulinogenic index, $\mathrm{p}=0.034$; disposition index, $\mathrm{p}=0.003$ ) as compared to the CC carriers. The risk CT/TT genotypes were also associated with impaired acute insulin response to glucose and arginine (AIR) at both $14 \mathrm{mmol} / \mathrm{l}(\mathrm{p}=0.02)$ and 28 $\mathrm{mmol} / \mathrm{l}(\mathrm{p}=0.009)$ of glucose as well as with enhanced rate of basal hepatic glucose production $(3.2 \pm 0.8$ vs. $2.8 \pm 0.3, p=0.01)$. Further refinement of the TCF7L2 locus into HapA and HapB haplotypes suggested a stepwise decrease in AIR $(p=0.03)$ being lowest in HapB, and increase in hepatic glucose production $(\mathrm{p}=0.03)$ being highest in HapB. Additionally, the CT/TT carriers demonstrated a significant incretin defect estimated as AUCinsulinOGTT / AUCinsulin IVGTT $(\mathrm{p}=0.0003)$ as well as using the formula: incretin effect $=$ $100 \% \times$ (AUCi OGTT-AUCi IVGTT)/AUCi OGTT) $(\mathrm{p}=0.004)$ in individuals with hyperglycemia; the incretin defect was more severe in carriers of HapB than in HapAB or HapA ( $p=0.004)$ carriers. The glucagonotropic effect of GIP was enhanced in TT carriers as demonstrated by a more steep correlation between fasting GIP and glucagon concentrations in TT than in CC genotype carriers $(\mathrm{r}=0.59, \mathrm{p}=0.009, \mathrm{n}=21$ and $\mathrm{r}=0.05, \mathrm{p}=0.8, \mathrm{n}=28)$. Also, the insulinotropic effect of GIP was attenuated in TT vs CC carriers as judged from the correlation between $2 \mathrm{hr}$ GIP and $2 \mathrm{hr}$ insulin (TT and CC, $\mathrm{r}=0.55$, $\mathrm{p}=0.004, \mathrm{n}=28$ and $\mathrm{r}=0.35, \mathrm{p}=0.2$ ).

Conclusion: The increased risk of T2D conferred by variants in the TCF7L2 gene involves impaired $\beta$-cell function which may partially be due to impaired incretin effect.

\section{4}

TCF7L2 gene increase risk for future type 2 diabetes:

study on diabetic isolated islets

R. Mancarella ${ }^{1}$, R. Lupi ${ }^{1}$, V. Lyssenko ${ }^{2}$, C. Ling ${ }^{2}$, S. Del Guerra ${ }^{1}$,

M. Bugliani ${ }^{1}$, S. Torri ${ }^{1}$, M. Galli ${ }^{1}$, V. D’Aleo ${ }^{1}$, P. Marchetti ${ }^{1}$, L. Groop ${ }^{2}$,

S. Del Prato'

${ }^{1}$ Endocrinology and Metabolism - Metabolic Unit, University of Pisa, Italy,

${ }^{2}$ Diabetes and Endocrinology, University of Lund, Malmö, Sweden.

Background and Aims: Identification of susceptibility genes for type 2 diabetes has not yet been very successful. Most recently, however, two single nucleotide polymorphisms (SNPs) (rs7903146 and rs12255372) in the transcription factor-7-like 2 (TCF7L2) gene were found to be strongly associated with T2DM in several populations. Increased T2DM risk in TCF7L2 variant carriers has been claimed to be associated with impaired insulin secretion. Moreover, TCF7L2 has been implicated in pro-glucagon processing, opening the possibility that TCF7L2 variants may simultaneously affect insulin and glucagon secretion. Therefore, have been performed to assess TCF7L2 expression in T2DM and non-diabetic human islets and to correlate it with pancreatic hormone release.

Materials and Methods: Human pancreatic islets were obtained from 15 non-diabetic (10M/5F, 59 yrs, BMI 26) and 7 T2DM (4M/3F, mean age 68 yrs, mean BMI $28 \mathrm{~kg} / \mathrm{m} 2$ ) multi-organ donors. Upon isolation, islets were cultured free floating in M199 culture medium at $5.5 \mathrm{mmol} / \mathrm{l}$ glucose. Within 3 days from isolation $24 \mathrm{~h}$ basal release of insulin and glucagon was determined in the media (IRMA and RIA technique, respectively), and islets were used for RNA extraction. Human TCF7L2 mRNA levels were quantified by RealTime Quantitative RT-PCR. Finally, non-diabetic islets were transfected with plasmid DNA containing the TCF7L2 gene and glucose-mediated insulin response determined.

Results: TCF7L2 mRNA levels were 5-fold higher in T2DM than in nondiabetic islets ( $12.5 \pm 2.1$ vs. $2.9 \pm 0.4 ; \mathrm{p}=0.0002)$. Basal insulin release was lower ( $209 \pm 9$ vs. $2534 \pm 105 \mu \mathrm{U} / \mathrm{ml} ; \mathrm{p}=0.0002$ ) whereas glucagon release was higher ( $55 \pm 3$ vs. $8 \pm 0.4 \mathrm{pg} / \mathrm{ml}$; $=0.0002$ ) in T2DM islets than in those of non- diabetic donors. Co-staining of TCF7L2 with RhodZin showed localization of TCF7L2 to $\beta$-cells. Although the expression of the insulin gene was markedly decreased in islets from T2DM subjects compared with non-diabetic donors $(0.5 \pm 0.1$ vs $3.8 \pm 0.5 ; \mathrm{p}=0.003)$ there was a positive correlation between the mRNA levels of TCF7L2 and insulin $(r=0.76, p=0.001)$. Intriguingly, the insulin gene mRNA correlated inversely with glucose-stimulated insulin release (SI) after normalization for total insulin content $(\mathrm{r}=-0.63, \mathrm{p}=0.02)$. In order to establish to which extent those correlations reflected a cause-effect relationship, islets from non-diabetic pancreas were transfected with TCF7L2 gene resulting in $+121 \pm 52 \%$ gene over-expression. In these islets, glucosemediated insulin response was significantly impaired as indicated by a $35 \%$ reduction in the Stimulation Index $(1.34 \pm 0.28$ vs. $1.83 \pm 0.3, \mathrm{p}<0.05)$. No relationship was seen between TCF7L2 and glucagons expression in human islets.

Conclusions: TCF7L2 gene expression is increased in islets from T2DM pancreases. Moreover, over-expression of TCF7L2 gene in non-diabetic islets is associated with impaired glucose-mediated insulin response. These results support the hypothesis that the increased T2DM risk associated with TCF7L2 gene could be mediated by defects of islet function. 


\section{OP 30 Mitochondrial function}

\section{5}

Loss of PGC-1 $\beta$ results in reduced exercise performance, ultrastructural alterations and mitochondrial dysfunction in skeletal muscle C. Zechner ${ }^{1}$, L. Lai ${ }^{1}$, T. C. Leone ${ }^{1}$, P. J. Schaeffer ${ }^{1,2}$, D. P. Kelly ${ }^{1,3}$; ${ }^{1}$ Center for Cardiovascular Research and Cardiovascular Division, Department of Internal Medicine, Washington University in St. Louis, United States, ${ }^{2}$ Department of Zoology, College of Arts and Sciences, Miami University, Oxford, United States, ${ }^{3}$ Departments of Pediatrics and Molecular Biology \& Pharmacology, Washington University in St. Louis, United States.

Background and Aims: Altered mitochondrial function is one of the earliest defects in diabetic skeletal muscle. Dysregulation of the transcriptional coactivators peroxisome-proliferator activated receptor gamma coactivator (PGC) $-1 \alpha$ and $\beta$ in muscle has been associated with insulin resistance. Previous studies have shown that PGC- $1 \alpha$ deficiency results in muscle mitochondrial dysfunction and reduced exercise performance in mice. However, the effects of PGC-1 $\beta$ deficiency on muscle metabolism and function have been examined in less detail. The aim of our study was to determine the skeletal muscle phenotype of PGC-1 $\beta$ deficient mice.

Materials and Methods: The PGC- $1 \beta$ gene was disrupted to generate mice with generalized PGC-1 $\beta$ deficiency (PGC-1 $\beta$ KO mice). Weight-, age- and sex-matched wild type (WT) mice were used as controls.

Results: Crosses of heterozygous (HET) PGC- $1 \beta$ KO mice revealed that KO mice were born at reduced Mendelian frequency (WT: 26.8\%; HET: $56.0 \%$; KO: $17.2 \% ; \mathrm{n}=366 ; \mathrm{p}<0.01$ ). When exercised on a treadmill at low speed $(10 \mathrm{~m} / \mathrm{min}$ for $1 \mathrm{~h}$, then $2 \mathrm{~m} / \mathrm{min}$ increment every $15 \mathrm{~min}$; level), PGC-1 $\beta$ KO mice only showed a mild performance deficit (WT: $204 \pm 10.02 \mathrm{~min}$; KO: $159 \pm 3.31 ; \mathrm{p}<0.05)$. In contrast, the running performance of PGC-1 $\beta$ KO mice was drastically reduced compared with WT mice (WT: $18.9 \pm 4.86$ min; KO: $3.9 \pm 1.07$ min; $\mathrm{p}<0.05)$ when exercised at high speed $(35 \mathrm{~m} / \mathrm{min}$; level). Mitochondrial respiration studies performed on isolated mitochondria from mouse hindlimb revealed a reduction in state 3 respiration in the KO (pyruvate: $-27.9 \%, \mathrm{p}<0.05$; palmitate: $-15.2 \%, \mathrm{p}=0.057$ ). Electron microscopic analysis of $\mathrm{KO}$ soleus muscle revealed the presence of a subset of mitochondria with vacuolar degeneration and degenerative features including "swirl-like" structures. While mtDNA was reduced by $12.9 \%$ in the soleus of the KO $(\mathrm{p}<0.05)$, the RNA expression of genes for fatty acid oxidation (acyl-CoA oxidase, medium chain acyl-CoA dehydrogenase, and muscle carnitine palmitoyl transferase I), glucose metabolism (glucose transporter 4 and pyruvate dehydrogenase kinase 4) and several mitochondrial genes (citrate synthase, cytochrome c oxidase (COX) 2 and ATP synthase $\beta$ ) was unchanged. However, PGC-1 $\alpha$ expression was increased in the soleus of the KO by $42 \%(\mathrm{p}<0.05)$, suggesting a compensatory response.

Conclusions: PGC-1 $\beta$ is necessary for full exercise performance and mitochondrial function in skeletal muscle. Loss of PGC-1 $\beta$ does not result in downregulation of a subset of previously described targets, possibly due to compensatory PGC- $1 \alpha$ upregulation. However, PGC- $1 \alpha$ cannot fully compensate for the loss of PGC- $1 \beta$, indicating that PGC- $1 \alpha$ and $\beta$ have overlapping as well as distinct regulatory roles.

Supported by DFG grant to C. Zechner and NIH grant to D. P. Kelly

\section{6}

Changes in resting and maximal mitochondrial ATP production in type 2 diabetes; non-invasive investigation by ${ }^{31} \mathrm{P}$-magnetic resonance spectroscopy

M. I. Trenell ${ }^{1}$, K. Hollingsworth 2 , E. Lim ${ }^{1}$, J. Gerrard ${ }^{1}$, R. Taylor ${ }^{1}$; ${ }^{1}$ Clinical Medical Sciences, ${ }^{2}$ Clinical Laboratory Sciences, Newcastle University, United Kingdom.

Background and Aims: Defects in mitochondrial function may be central to the development of insulin resistance and type 2 diabetes. Defects in mitochondrial function reduce the ability of muscle to metabolise fatty acids. The associated accumulation of fatty acids in skeletal muscle, in turn, impedes insulin action through inhibition of insulin action in skeletal muscle. It is possible that reduced basal ATP use in people with type 2 diabetes represents reduced insulin-stimulated ATP demand. In recovery from aerobic exercise ATP production is maximal stimulated, independent of insulin. As such, quantification and comparison of basal ATP use with maximal stimulated ATP production in recovery from aerobic exercise may provide more specific information on mitochondrial function in people with type 2 diabetes than either value in isolation. In the present study we evaluated whether there were changes in basal ATP use and maximal ATP production in type 2 diabetes. This abstract reports baseline data of an ongoing study which observes the effects of increased physical activity upon mitochondrial function and glucose control in people with type 2 diabetes.

Materials and Methods: Volunteers with type 2 diabetes and normal controls underwent assessment of mitochondrial ATP production at rest in the soleus muscle. $\mathrm{A}^{31} \mathrm{P}$-Magnetic resonance spectroscopy saturation transfer technique was developed for use at 3 Tesla (coefficient of variation $=4.6 \%$ ). Basal ATP flux was assessed by observing the change in inorganic phosphate following saturation of $\gamma$ ATP. Maximal oxidative ATP production was then assessed in recovery from 3 minutes of plantar flexion exercise at $30 \%$ maximal voluntary contraction. Maximal mitochondrial ATP production was calculated from the recovery rate of adenosine diphosphate from contraction.

Results: At rest, oxidative ATP production was reduced by approximately $27 \%$ in people with type 2 diabetes in comparison with control ( $8.1 \pm 1.6$ vs. 11.1 $\left.\pm 0.5 \mu \mathrm{mol} \cdot \mathrm{g} \mathrm{muscle}{ }^{-1} \cdot \mathrm{min}^{-1}, P<0.05\right)$. There were no significant difference in resting phosphocreatine $(31.1 \pm 0.6$ vs. $33.3 \pm 1.2 \mathrm{mM})$, adenosine diphosphate $(9.5 \pm 0.4$ vs. $9.7 \pm 0.1 \mu \mathrm{M})$ and $\mathrm{pH}(7.04 \pm 0.01$ vs. $7.06 \pm 0.01)$ between the groups $(P>0.05)$. Maximal oxidative ATP production, reported as the recovery half time of adenosine diphosphate was similar between groups $(22.7 \pm 5.1$ vs. $25.6 \pm 3.8 \mathrm{sec}, P>0.05)$. There was no difference in end exercise phosphocreatine breakdown during exercise (7.2 \pm 1.7 vs. $8.2 \pm 1.1 \mathrm{mM}, P>$ $0.05)$ or end exercise $\mathrm{pH}(7.04 \pm 0.02$ vs. $7.02 \pm 0.02, P>0.05)$, suggesting that the end exercise metabolic origins were similar between groups.

Conclusion: Our data reveals that basal oxidative ATP use is reduced in people with type 2 diabetes. However, the decrease in basal ATP is not accompanied by a decrease in contraction induced maximal oxidative ATP production.

Supported by the William Leech Foundation

\section{7}

In vivo and ex vivo quantification of mitochondrial function in type 2 diabetic patients and first-degree relatives E. Phielix ${ }^{1}$, V. Schrauwen-Hinderling ${ }^{1}$, M. Mensink ${ }^{1}$, M. Hesselink², P. Schrauwen ${ }^{1}$;

${ }^{1}$ Human Biology, ${ }^{2}$ Movement Sciences, Maastricht University, The Netherlands.

Background and Aims: Skeletal muscle insulin resistance is an early hallmark in the development of type 2 diabetes. Recently, mitochondrial dysfunction has been implicated in the development of muscle insulin resistance and type 2 diabetes. Here, we examined if mitochondrial function is impaired in diabetic patients and in first-degree relatives of type 2 diabetic patients, a population at increased risk of developing type 2 diabetes.

Methods: So far, 9 diabetic patients (T2D), 12 normoglycemic first-degree relatives (FDR) and 16 controls (C) were included (age (y) 60.4 $\pm 5.1,59.8 \pm 3.1$ and 59.9 \pm 2.7 ; BMI $\left(\mathrm{kg} / \mathrm{m}^{2}\right): 28.9 \pm 2.3,31.3 \pm 4.0$ and $28.2 \pm 2.4 ; \mathrm{VO}_{2} \mathrm{max}(\mathrm{ml} /$ $\mathrm{kg} / \mathrm{min}): 27.6 \pm 7.6,30.3 \pm 7.3$ and $31.5 \pm 7.7$ respectively). Insulin sensitivity was measured with a hyperinsulineamic euglyceamic clamp. In vivo mitochondrial function was determined by measuring post-exercise $\mathrm{PCr}$ recovery half-time ( $\mathrm{PCr}_{\mathrm{t} 1 / 2}$ ) using 31-Phosphorus Magnetic Resonance Spectroscopy ( $\left.{ }^{31} \mathrm{P}-\mathrm{MRS}\right)$. Ex vivo mitochondrial function was analysed in permeabilized muscle fibers isolated from needle biopsies from the vastus lateralis using high-resolution respirometry. ADP-stimulated state 3 respiration (oxygen consumption upon glutamate+succinate addition), non-ADP stimulated state 4 respiration, FCCP-driven maximal respiratory capacity (state $3 \mathrm{u}$ ) and respiratory control ratio (RCR; state 3/state 4 ), were determined in the presence and absence of palmitoyl-carnitine. The RCR reflects the tightness of coupling between the ATP production via oxidative phosphorylation and the respiration-driven proton pumping via the electron transport chain.

Results: Preliminary results show lower insulin sensitivity in T2D compared to C (GIR (umol $/ \mathrm{kg} / \mathrm{min}$ ): $16.4 \pm 6.3$ vs. $25.9 \pm 9.6, \mathrm{p}<0.05$, respectively), with intermediate values in FDR $(20.0 \pm 8.4, \mathrm{p}=\mathrm{n} . \mathrm{s}$ for control or diabetic patients). Ex vivo mitochondrial oxidative capacity showed a (tendency to) reduced RCR for T2D compared to C and FDR (1.78 \pm 0.4 vs $2.44 \pm 0.6$ and $2.28 \pm 0.5, \mathrm{p}<0.05$ and $\mathrm{p}=0.07$, respectively), implicating less tightly-coupled mitochondria. Furthermore, T2D showed a lower FCCP-driven maximal 
respiratory capacity compared to $C(40.3 \pm 8.7$ vs $55.1 \pm 15.1, \mathrm{p}<0.05)$, with again intermediate values for FDR (45.5 $\pm 15.6, \mathrm{p}=\mathrm{n} . \mathrm{s})$. Addition of palmitoylcarnitine significantly lowered RCR in both $\mathrm{C}$ and FDR, which may indicate fatty acid-induced uncoupling; however, this effect of palmityol-carnitne was absent in T2D. ADP-stimulated state 3 respiration $(\mathrm{R}=-0.586, \mathrm{p}<0.01)$ and maximal respiratory capacity $(\mathrm{R}=-0.461, \mathrm{p}<0,054)$ (tended to) correlated strongly with $\mathrm{PCr}_{\mathrm{t} 1 / 2}$, which is a measure of in vivo mitochondrial oxidative capacity.

Conclusions: We show that ex vivo oxidative capacity of permeabilized fibers is reduced in T2D, with intermediate values in FDR. Importantly, these values are not yet corrected for mitochondrial content and may thus reflect reduced mitochondrial density in T2D. Nevertheless, we also found that RCR, which is independent of mitochondrial content, was reduced in T2D suggesting that their mitochondria are less well coupled. Finally, the strong correlation between ex vivo and in vivo mitochondrial function indicates that the metabolic abnormalities observed ex vivo truly result in a reduced in vivo mitochondrial oxidative capacity.

Supported by Diabetes Foundation The Netherlands (DFN)

\section{8}

Early or advanced stage type 2 diabetes is not accompanied by in vivo skeletal muscle mitochondrial dysfunction

J. J. Prompers ${ }^{1}$, H. M. M. De Feyter ${ }^{1}$, N. M. A. van den Broek ${ }^{1}$,

S. F. E. Praet ${ }^{2}$, K. Nicolay ${ }^{1}$, L. J. C. van Loon $^{2}$;

${ }^{1}$ Biomedical NMR, Eindhoven University of Technology, Eindhoven,

${ }^{2}$ Department of Movement Sciences, Nutrition and Toxicology Research

Institute Maastricht (NUTRIM), Maastricht University, The Netherlands.

Background and Aims: Several lines of evidence support a potential role of skeletal muscle mitochondrial dysfunction in the pathogenesis of insulin resistance and/or type 2 diabetes. However, it remains to be established whether mitochondrial dysfunction represents either cause or consequence of the disease. We examined in vivo skeletal muscle mitochondrial function in early and advanced stages of type 2 diabetes, with the aim to gain insight in the proposed role of mitochondrial dysfunction in the development of insulin resistance and/or type 2 diabetes.

Materials and Methods: 11 long-standing, insulin treated type 2 diabetes patients (T2D), 11 subjects with impaired fasting glucose, impaired glucose tolerance and/or recently diagnosed diabetes (IHG), and 12 healthy, normoglycaemic controls (NGT), all matched for age and body composition and with similar habitual physical activity levels, were studied. In vivo mitochondrial function of the vastus lateralis muscle was evaluated from

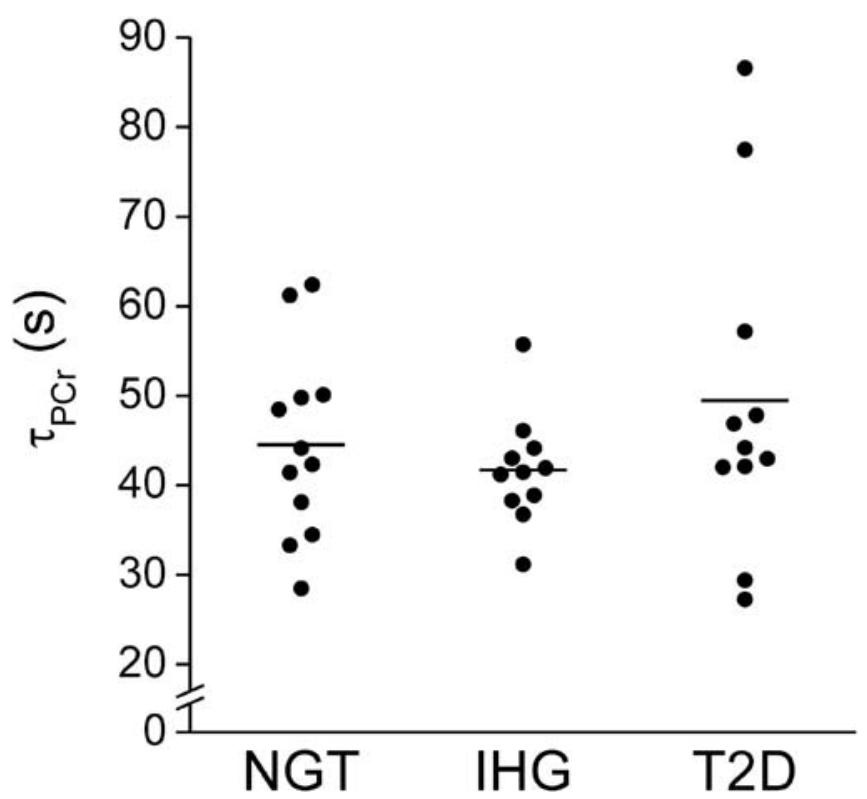

Fig. 1. $\tau_{\mathrm{PCr}}$ in NGT, IHG and T2D groups was not significantly different. Bars indicate the mean values for the $\mathbf{3}$ groups and filled circles represent individual data points.

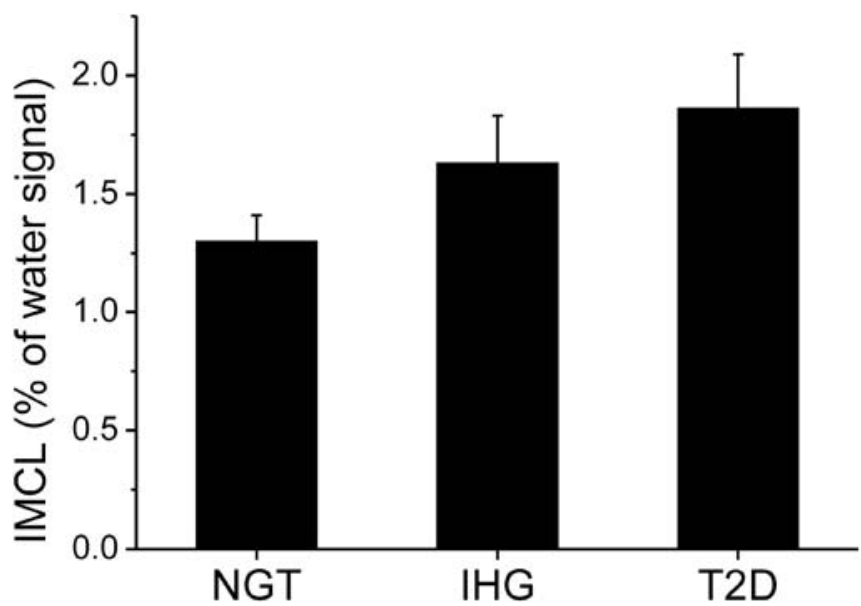

Fig. 2. IMCL content in NGT, IHG and T2D groups was not significantly different.

post-exercise phosphocreatine (PCr) recovery kinetics using ${ }^{31} \mathrm{P}$ magnetic resonance spectroscopy (MRS). Intramyocellular lipid (IMCL) content was assessed in the same muscle using single-voxel ${ }^{1} \mathrm{H}$ MRS.

Results: ${ }^{31} \mathrm{P}$ MRS parameters for mitochondrial function, i.e. the PCr recovery time constant $\left(\tau_{\mathrm{PC}}\right.$; Figure 1$)$, ADP recovery time constant and maximum aerobic capacity, did not differ between groups $(\mathrm{P}=0.4, \mathrm{P}=0.14$ and $\mathrm{P}=0.11$, respectively). IMCL content also did not differ significantly between groups $(\mathrm{P}=0.13$; Figure 2).

Conclusion: Subjects with impaired fasting glucose, impaired glucose tolerance and/or recently diagnosed diabetes as well as long-standing, insulin treated type 2 diabetes patients do not show any in vivo skeletal muscle mitochondrial dysfunction, when compared to healthy, normoglycaemic controls matched for age and body composition and with similar habitual physical activity levels. These findings imply that mitochondrial function does not necessarily represent either cause or consequence of insulin resistance and/or type 2 diabetes. Impairments in oxidative metabolism in type 2 diabetes patients as observed in previous studies might be secondary to a more sedentary lifestyle and/or impaired insulin signalling.

\section{9}

Short-term elevation of plasma free fatty acids (ffa) decreases skeletal muscle mitochondrial membrane potential in healthy glucose tolerant subjects

D. Tripathy ${ }^{1}$, A. Chavez-Velazquez ${ }^{1}$, R. Jani ${ }^{1}$, M. A. Abdul-Ghani ${ }^{1}$, V. Frohlich ${ }^{2}$, S. Padma ${ }^{1}$, C. P. Jenkinson ${ }^{1}$, F. Folli ${ }^{1}$, R. A. DeFronzo ${ }^{1}$; ${ }^{1}$ Division of Diabetes, Dept of Medicine, University of Texas Health Science Center, San Antonio, ${ }^{2}$ Cellular \& Structural Biology, University of Texas Health Science Center, San Antonio, United States.

Background and Aims: Elevated FFA play a pivotal role in the pathogenesis of insulin resistance. Experimental elevation of FFA produces insulin resistance in a dose dependent manner. Prolonged lipid infusion to cause a physiologic elevation in plasma FFA reduce expression of genes involved in mitochondrial function. Since FFA-induced insulin resistance typically is seen within 2-4 hrs, we examined whether mitochondrial dysfunction plays a role in FFA-induced impairment in insulin action.

Materials and Methods: 8 healthy NGT subjects ( 4 male / 4 female, age $=34 \pm$ $3 \mathrm{y}, \mathrm{BMI}=24.8 \pm 1.0 \mathrm{~kg} / \mathrm{m}^{2}, \mathrm{FPG}=90 \pm 2,2 \mathrm{~h}$ Glucose $94 \pm 2$ ), without family history of T2DM, received a lipid infusion $(60 \mathrm{ml} / \mathrm{h})$ for $8 \mathrm{hrs}$ and euglycemic insulin clamps $\left(80 \mathrm{mU} / \mathrm{m}^{2} \mathrm{~min}\right)$ with vastus lateralis biopsies before and after 6 hrs of lipid infusion which raised basal FFA from $576 \pm 60$ to $1382 \pm$ $201 \mu \mathrm{m}$. Mitochondrial membrane potential $(\Psi)$, which is related to state of energization of mitochondria, was determined in skeletal muscles ex vivo with tetramethyl rhodamine ester (TMRE), which accumulates in mitochondria in response to change in $\Psi$. Mitochondrial mass was determined by Mitotracker Red. TMRE and Mitotracker fluorescence was quantitated with confocal laser microscopy. Skeletal muscle mitochondria morphology was examined by EM and mitochondrial DNA copy number was determined by RT-PCR before and after 6 hrs of lipid infusion. 
Results: Mitochondria membrane potential $(\Psi)$ decreased by $43 \%$ from $0.550 \pm 0.06$ to $0.283 \pm 0.03$ arb units, $\mathrm{p}=0.02$, after $6 \mathrm{hrs}$ of lipid infusion and the decrement in $\Psi$ correlated with plasma FFA concentrations both at baseline $(\mathrm{r}=0.888, \mathrm{p}=0.008)$ and at the end of lipid infusion $(\mathrm{r}=0.823, \mathrm{p}=0.02)$. Mitochondrial mass (Mitotracker Red) did not change following lipid infusion $(0.527 \pm 0.2$ to $0.449 \pm 0.07$ arb units, $\mathrm{p}=\mathrm{ns})$. Mitochondrial DNA copy number declined from $649 \pm 112$ to $511 \pm 106$ copies/genome, $\mathrm{p}=0.02$. There was no change in mitochondrial morphology assessed by electron microscopy.

Conclusion: Short-term physiologic increase in plasma FFA conc induces mitochondrial dysfunction in skeletal muscle. This may be an important mechanism by which elevated FFA inhibit basal and insulin stimulated ATP synthesis and induces skeletal muscle insulin resistance in subjects with type 2 diabetes.

Supported by CREF Award, VA

\section{0}

Effect of rosiglitazone treatment on in vivo mitochondrial function in type 2 diabetic patients

M. Mensink ${ }^{1}$, V. B. Schrauwen-Hinderling ${ }^{1,2}$, M. E. Kooi ${ }^{2}$,

M. K. C. Hesselink ${ }^{1}$, P. Schrauwen ${ }^{1}$;

${ }^{1}$ Departments of Human Biology and Movement Sciences, Nutrition and Toxicology Research Institute NUTRIM, Maastricht University, The Netherlands, ${ }^{2}$ Dept of Radiology, University Hospital Maastricht, The Netherlands.

Background and Aims: Mitochondrial dysfunction has been implicated in the development of insulin resistance and type 2 diabetes mellitus. Using phosphorus Magnetic Resonance Spectroscopy (31P-MRS) we demonstrated that in vivo mitochondrial function is indeed reduced in diabetic patients. Rosiglitazone improves glycemic control and enhances insulin sensitivity. One of the underlying mechanisms could be a direct mitochondrial effect, as is suggested by studies in adipocytes. We recently showed that rosiglitazone increased the expression of PGC- $\alpha$ and PPAR $\beta / \delta$ in skeletal muscle of type 2 diabetic patients. In the present study we examined whether rosiglitazone treatment is also associated with an improved in vivo mitochondrial function in skeletal muscle.

Materials and Methods: Eight overweight subjects with type 2 diabetes mellitus were treated with rosiglitazone for 8 weeks. Before and after treatment, fasting plasma glucose, insulin and free fatty acids (FFA) levels, insulin sensitivity (hyperinsulinaemic-euglycaemic clamp), and mitochondrial function were determined. To evaluate in vivo mitochondrial function, the time to recover half of the phosphocreatine level after exercise was determined by 31P-MRS (PCr half-time). MRS measurements were performed on a $1.5 \mathrm{~T}$ whole body scanner. A time-serie of 120 spectra was acquired $(\mathrm{TR}=4 \mathrm{~s})$ during a protocol consisting of 2 minutes of rest, 5 minutes knee-extension exercise and 5 minutes recovery. Spectra were fitted with AMARES in the jMRUI 3 software package. PCr recovery time-course was fitted with a mono-exponential curve and the recovery half-time was calculated.

Results: Eight weeks treatment reduced plasma glucose $(9.3 \pm 0.7$ to $8.8 \pm 0.7$ $\mathrm{mmol} / \mathrm{L})$, insulin $(16.1 \pm 2.9$ to $13.4 \pm 1.8 \mathrm{mU} / \mathrm{L})$ and $\mathrm{FFA}(430.6 \pm 29.8$ to $398.5 \pm 41.9 \mu \mathrm{mol} / \mathrm{L})$, although these differences did not reach statistical significance $(\mathrm{P}>0.05)$. The glucose infusion rate (GIR) during the last $30 \mathrm{~min}$ of hyperinsulinemia was increased from $19.9 \pm 2.8$ to $24.8 \pm 2.1 \mu \mathrm{mol} / \mathrm{kg} / \mathrm{min}$ $(\mathrm{P}<0.05)$, indicating a reduction in insulin resistance. Rosiglitazone treatment did not significantly reduce PCr recovery half-time: $23.8 \pm 3.5$ to $20.0 \pm 1.7 \mathrm{~s}$ $(\mathrm{P}=0.23)$. Interestingly, the changes in $\mathrm{PCr}$ half-time correlated with changes in fasting plasma insulin level $(\mathrm{R}=0.70, \mathrm{P}=0.049)$, and tended to correlate with fasted plasma glucose $(\mathrm{R}=0.60, \mathrm{p}=0.077)$. Changes in $\mathrm{PCr}$ half-time however, did not correlate with changes in insulin sensitivity $(\mathrm{R}=-0.10, \mathrm{P}=0.80)$.

Conclusion: Although the data should be interpreted with some care regarding the limited number of subjects, rosiglitazone improved insulin sensitivity, while in vivo mitochondrial function was not (yet) improved. Moreover, changes in insulin sensitivity were not correlated with changes in mitochondrial function, suggesting that an improved mitochondrial function is not a prerequisite for rosiglitazone-enhanced insulin sensitivity. Interestingly, in vivo mitochondrial function improved the most in subjects who showed the largest reduction in plasma insulin and glucose levels. This could suggest that mitochondrial function can be affected by the degree of hyperglycemia or hyperinsulineamia, leaving the option of mitochondrial dysfunction being a consequence rather than cause of the diabetic state.

\section{OP 31 Hypoglycaemia}

\section{1}

The ADA definition of hypoglycaemia: quantitatively examined and challenged

S. G. H. Swinnen ${ }^{1}$, P. Mullins ${ }^{2}$, M. Miller ${ }^{3}$, J. B. L. Hoekstra ${ }^{1}$, F. Holleman ${ }^{1}$; ${ }^{1}$ Dept. of Internal Medicine, Academic Medical Centre, Amsterdam, The Netherlands, ${ }^{2}$ Dept. of Statistics, University of Auckland, New Zealand, ${ }^{3}$ Sanofi Aventis, Bridgewater, United States.

Background and Aims: In 2005 the ADA defined hypoglycaemia as an event with a measured plasma glucose (PG) concentration $\leq 3.9 \mathrm{mmol} / \mathrm{l}$, as glucose counterregulation is activated at this level, and because antecedent hypoglycaemia of $\leq 3.9 \mathrm{mmol} / \mathrm{l}$ reduces sympathoadrenal and glucagon responses to subsequent hypoglycaemia. Although standardized reporting of hypoglycaemia is desirable, we fear that the ADA definition will limit attempts of both patients and doctors to achieve current $\mathrm{HbAlc}$ goals, while avoiding hypoglycaemia. The aim of this study was to investigate the relationships between hypoglycaemia and $\mathrm{HbAlc}$ when using various PG cut-off levels for hypoglycaemia.

Material and Methods: Data from 2 randomised clinical trials, examining insulin glargine dose titration in 12138 patients with type 2 diabetes uncontrolled by oral antidiabetic agents, were combined. In both studies hypoglycaemic events and HbAlc were prospectively recorded. The proportion of patients experiencing hypoglycaemia and the event rate during the final 12 weeks of the trials were plotted against endpoint $\mathrm{HbAlc}$, constructing various curves representing pre-defined PG cut-off levels. Hypoglycaemia included all symptomatic and asymptomatic episodes with a measured PG concentration $<3.9 \mathrm{mmol} / \mathrm{l}$ and all severe events, defined by $3^{\text {rd }}$ party assistance.

Results: 10502 patients with end of study HbAlc recorded 21265 hypoglycaemic episodes, including 253 severe, 9074 symptomatic and 11938 asymptomatic events. Comparing a hypoglycaemia cut-off level of $<3.1$ $\mathrm{mmol} / \mathrm{l}$ to the ADA definition more than doubles the percentage of affected patients at $\mathrm{HbAlc} 6.9-7.2 \%$ (17.6\% vs. $42.6 \%$ ) (Fig.). Considering event rates the discrepancy is even larger ( 0.5 vs. 2.5 events per 100 patient days), as the number of events per patient also increases with higher cut-offs. There were 4419 events with PG $<3.1 \mathrm{mmol} / 1$ in 1785 patients. Raising the cut-off level to $<3.9 \mathrm{mmol} / \mathrm{l}$ adds another 16839 events in a total of 3961 patients. The majority of these events occurred in patients who had also experienced hypoglycaemia below $3.1 \mathrm{mmol} / \mathrm{l}$ (9795 events in 1531 patients), versus those who had not (7044 events in 2176 patients). Of the severe hypoglycaemic events, 201 occurred in patients who had at some other time had an event $<3.1 \mathrm{mmol} / \mathrm{l}$. Additionally, we found that further reduced cut-off levels (e.g. $2.7 \mathrm{mmol} / \mathrm{l}$ ) conceal the increase in hypoglycaemia frequency expected with improved glycaemic control.

Propartion of patients with hypoglycaemia for various $P G$ cut-off levels

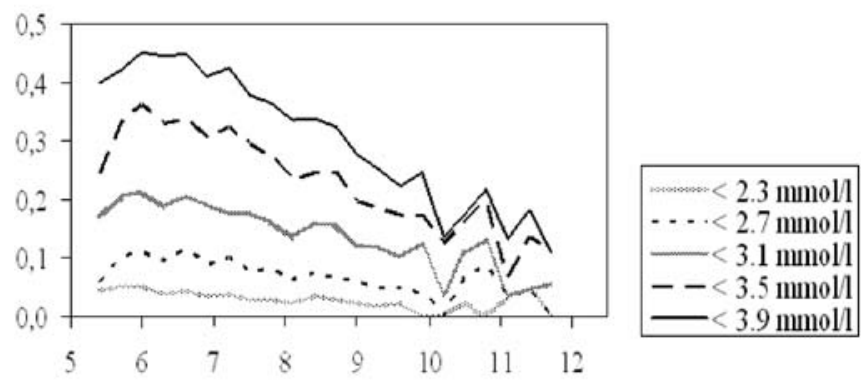

Endpoint HbAlc

Conclusion: The frequency of hypoglycaemia is highly dependent on the PG concentration used for its definition. Raising the PG cut-off level from 3.1 to $3.9 \mathrm{mmol} / \mathrm{l}$ mainly identifies more episodes of clinically irrelevant hypoglycaemia. Standardized definitions of hypoglycaemia should be supported by quantitative examinations of hypoglycaemia rates, considering the clinical effects of the proposed cut-off value.

Supported by Sanofi Aventis 


\section{2}

\section{ICV catalase increases counterregulatory responses to hypoglycaemia} in rats

C.-Y. Yueh ${ }^{1}$, S. Moore ${ }^{1}$, M. Osundiji ${ }^{1}$, J. Shaw ${ }^{1}$, L. Heisler ${ }^{2}$, M. L. Evans ${ }^{1}$;

${ }^{1}$ Dept of Medicine, University of Cambridge, ${ }^{2}$ Dept of Clinical Biochemistry, University of Cambridge, United Kingdom.

Although chronic increases in oxidative stress have been causally implicated in the complications of diabetes, there is growing evidence that a short term rise in reactive oxygen species (ROS)- the products of oxidative stress- might play a physiological role in nutritional signalling. One ROS species in particular, hydrogen peroxide (PX), has also been implicated as a brain neurotransmitter. We hypothesised that hypothalamic nutrient-sensing neurons might detect a falling blood glucose by sensing the corresponding fall in hypothalamic PX, leading on to triggering of counterregulatory hormone $(\mathrm{CRH})$ responses. Specifically, we anticipated that brain delivery of the anti-oxidant PXcatabolising enzyme catalase (CAT) during hypoglycaemia would cause PX levels to fall still faster and lead to an earlier triggering of $\mathrm{CRH}$.

We studied overnight-fasted 300g healthy male Sprague Dawley rats with implanted vascular catheters and a single third ventricle (ICV) cannula. Rats underwent $210 \mathrm{~min}$ ICV infusion of either CAT $(4.14 \mathrm{U} / \mathrm{min}, \mathrm{n}=8)$ or vehicle $(\mathrm{CON}, \mathrm{n}=8) .90 \mathrm{~min}$ after starting ICV infusions, a stepped hyperinsulinaemic (20 mU/kg/min Humulin S) hypoglycaemic clamp study was started, with plasma glucose lowered sequentially in 40 minute steps to 5, 3.5 and $2.5 \mathrm{mM}$ respectively. We measured plasma insulin, glucose and, for $\mathrm{CRH}$, glucagon $(\mathrm{GN})$ and adrenaline (AD). Plasma glucose thresholds for initiation of $\mathrm{CRH}$ for each rat were identified as the point at which responses rose more than $2 \mathrm{SD}$ above starting levels. Baseline data before ICV infusions and at start of clamp studies were identical in both groups. During clamp studies, insulin rose and plasma glucose fell equally in both CAT and CON groups (table below).

\begin{tabular}{llllllll}
\multicolumn{3}{l}{ Glucose $(\mathrm{mM})$} & & & \multicolumn{2}{c}{ Insulin $(\mathrm{ug} / \mathrm{l})$} \\
\cline { 2 - 4 } \cline { 7 - 8 } & $0 \mathrm{~min}$ & $40 \mathrm{~min}$ & $80 \mathrm{~min}$ & $120 \mathrm{~min}$ & & 0 min & $40 \mathrm{~min}$ \\
\hline CON & $6.3+0.3$ & $5.0+0.2$ & $3.5+0.1$ & $2.7+0.1$ & & $1+0$ & $30+5$ \\
CAT & $6.3+0.2$ & $4.9+0.3$ & $3.4+0.1$ & $2.5+0.1$ & & $1+0$ & $30+5$ \\
\hline
\end{tabular}

During hypoglycaemia, CRH rose in both groups (figure below). In keeping with our hypothesis, the plasma glucose level at which AD responses were triggered was significantly higher in CAT than CON $(3.8 \pm 0.4$ vs. $2.8 \pm 0.4$ $\mathrm{mM}$; $\mathrm{p}<0.05) \mathrm{GN}$ also tended to rise earlier in CAT although differences failed to reach significance $(3.9 \pm 0.4$ vs. $3.1 \pm 0.4 \mathrm{mM} ; \mathrm{P}=\mathrm{NS})$. In summary, brain delivery of catalase during hypoglycaemia altered the thresholds for $\mathrm{CRH}$ so that defensive hormonal responses were triggered at a higher plasma glucose level than controls. These data are consistent with our hypothesis that hypothalamic glucose-sensing neurones may sense changes in plasma glucose at least in part by sensing local changes in ROS.

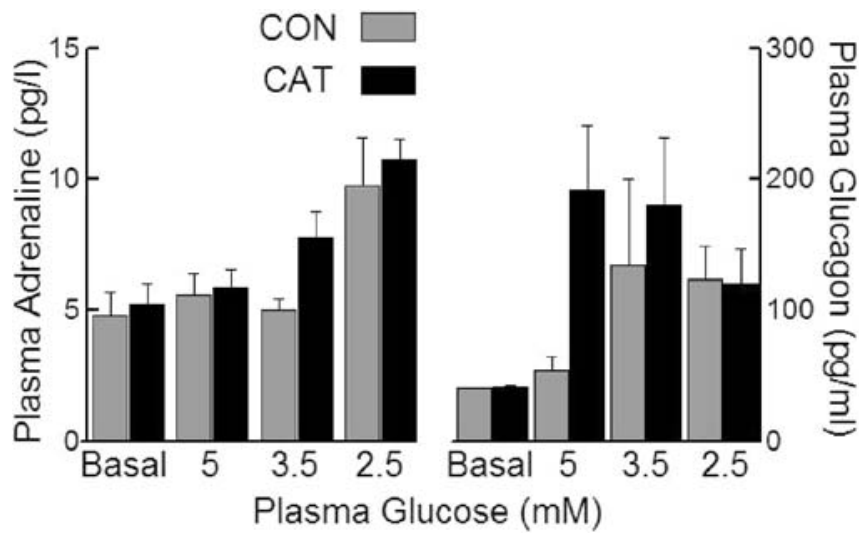

\section{3}

Increased hexokinase activity in brain following recurrent hypoglycaemia

M. A. Osundiji, J. Shaw, S. Moore, S. Hoashi, C.-Y. Yueh, M. L. Evans; Dept of Medicine, University of Cambridge, United Kingdom.

Background and Aims: Brain is critically dependent on circulating glucose as a fuel source. As blood glucose levels fall, defensive counterregulatory responses are triggered and, if blood glucose falls low enough, cognitive function will become impaired. Recurrent insulin-induced hypoglycaemia as experienced by some diabetic patients can alter glucose thresholds for counterregulatory responses (CR) and perhaps also for cognitive impairment, suggesting that brain can adapt to exposure to hypoglycaemia and/or insulin and that these adaptations have clinical consequences. Following glucose transport into neurones, phosphorylation by hexokinase(s) (HK) is rate limiting for flux of glucose metabolites into downstream intracellular pathways to provide energy to support metabolism. We hypothesised, therefore, that brain might adapt to antecedent insulin-induced hypoglycaemia by an upregulation of $\mathrm{HK}$.

Materials and Methods: Pilot studies using microarray suggested significant upregulation of $\mathrm{HK}$ expression in hypothalamus following recurrent hypoglycaemia. Consequently, we examined chronically catheterised (right jugular vein) adult male Sprague Dawley (SD) rats. Animals were exposed to 1 to 4 days of insulin injections (10,8,6 and $5 \mathrm{U} / \mathrm{kg}$ on days 1 to 4 respectively). The daily insulin dose was reduced to avoid profound hypoglycaemia as CR impairment developed. Plasma glucose was assayed using Analox GM-9 analyser over 90 minutes. At the end of studies, rats were euthanised and brains removed rapidly for ex vivo measurement of $\mathrm{HK}$ at $0.5 \mathrm{mM}$ glucose utilising a reporter assay which couples glucose phosphorylation with oxidation of glucose-6-phosphate.

Results: Despite reducing doses of insulin, nadir plasma glucose levels were significantly lower in rats exposed to four days relative to one-day of hypoglycaemia (table 1) in keeping with the development of defective CR following exposure to hypoglycaemia. Consistent with our hypothesis, recurrent insulin-induced hypoglycaemia significantly increased $\mathrm{HK}$ activities in both hypothalamus and brain stem over a period of 1 to 4 days.

\begin{tabular}{lllll} 
& Day 1 $(\mathrm{n}=5)$ & Day 2 $(\mathrm{n}=3)$ & Day 3 $(\mathrm{n}=2)$ & Day 4 $(\mathrm{n}=5)$ \\
\hline $\begin{array}{l}\text { Basal plasma glucose } \\
(\mathrm{mM})\end{array}$ & $9.5 \pm 0.4$ & $9.3 \pm 0.4$ & $8.8 \pm 0.2$ & $9.2 \pm 0.2$ \\
$\begin{array}{l}\text { Nadir plasma glucose } \\
(\mathrm{mM})\end{array}$ & $3.6 \pm 0.3$ & $2.9 \pm 0.4$ & 3.6 & $\begin{array}{l}{ }^{*} 2.7 \pm 0.1 \\
(\mathrm{P}=0.03)\end{array}$ \\
$\begin{array}{l}\text { Hypothalamic HK } \\
(\mathrm{mU} / \mathrm{mg}\end{array}$ & $0.16 \pm 0.02$ & $0.31 \pm 0.06$ & $0.37 \pm 0.06$ & $\begin{array}{l}{ }^{*} 0.57 \pm 0.11 \\
(\mathrm{P}=0.03)\end{array}$ \\
$\begin{array}{l}\text { tissue weight) } \\
\begin{array}{l}\text { Brain stem HK } \\
\text { (mU/mg }\end{array}\end{array}$ & $\mathrm{ND}$ & $0.1 \pm 0.02$ & $0.182 \pm 0.08$ & ${ }^{*} 0.252 \pm 0.03$ \\
tissue weight) & & & & $(\mathrm{P}=0.005)$ \\
\hline
\end{tabular}

${ }^{*}=\mathrm{P}<0.05$ vs first day of data, $\mathrm{ND}=$ no data

Conclusion: These findings suggest that brain adapts to recurrent hypoglycaemia by increasing $\mathrm{HK}$ activity in both hypothalamus and brain stem. We speculate that this increase in HK may, at least in part, contribute to (1) CR failure as a "maladaptive response" and/or (2) protect against hypoglycaemia-induced cognitive dysfunction as a protective response. Supported by JDRF, DUK, DRWF 


\section{4}

Relationship between prior mild-moderate hypoglycaemic events and the risk of subsequent severy hypoglycaemia in patients with type 2 diabetes mellitus treated with basal insulin according to fasting BG targets: a time-dependent covariate analysis

A. Zisman ${ }^{1}$, M. Miller ${ }^{2}$, R. Tanenberg ${ }^{3}$;

${ }^{1}$ Endocrine, Diabetes \& Metab, Univ of Miami Miller School of Med, Miami, United States, ${ }^{2}$ sanofi-aventis U.S., Bridgewater, United States, ${ }^{3}$ Division of Endocrinology, Brody School of Medicine, Greenville, United States.

Background and Aims: Patients with T2DM often do not meet recommended glycosylated hemoglobin A1C (A1C) goals $(<7.0 \%)$. Studies have shown that adjusting basal insulin dose to achieve these goals may be limited by hypoglycemic episodes. The aim of this study is to examine the role of prior episodes of moderate hypoglycemia (MMH) and mild hypoglycemia (MH) on the subsequent risks for severe hypoglycemia $(\mathrm{SH})$ and moderate/severe hypoglycemia (MSH) within the context of titration target.

Methods: This analysis of a 24-wk, randomized study investigated the effect of titration algorithms for insulin glargine (GLAR) on SH event rates and glycemic control in patients with T2DM ( $\mathrm{N}=4988)$. Treatment included oncedaily GLAR, titrated weekly according to algorithm group (defined by fasting blood glucose [FBG] goals $=80,90,100,110$, or $120 \mathrm{mg} / \mathrm{dL}$, respectively). Time to first occurrence of $\mathrm{SH}$ and of MSH were studied with and without a prior occurrence of $\mathrm{MMH}$ or $\mathrm{MH}$ respectively.

Results: Baseline characteristics (mean A1C, 9.2\%; body mass index, $34.7 \mathrm{~kg} /$ $\mathrm{m}^{2}$ ) and study completion (88.40-90.57\%) were similar among titration groups. A Cox proportional hazards model revealed that the risk for a first occurrence of an $\mathrm{SH}$ event declined as titration targets increased. Every 10$\mathrm{mg} / \mathrm{dL}$ increase in the target yielded an estimated $28.5 \%$ reduction in risk (slope coefficient for log hazard, $-0.034 ; P<0.001$ based on $107 \mathrm{SH}$ events). This methodology revealed that prior occurrence of an $\mathrm{MMH}$ event substantially increased the risk of an $\mathrm{SH}$ event in all study arms and reduced the rate of decline in risk with increasing titration target (slope coefficient of log hazard $=-0.014, \mathrm{P}>0.4$, based on $59 \mathrm{SH}$ events with prior $\mathrm{MMH}$ ). In patients without prior $\mathrm{MMH}$, the decline in risk of $\mathrm{SH}$ event with increasing titration target remained statistically significant (slope coefficient for log hazard $=-0.034$, $\mathrm{P}<0.005$ based on $48 \mathrm{SH}$ events). Also, prior occurrence of $\mathrm{MH}$ increased the risk of MSH over 9 fold, but the effect of titration target was not altered.

Conclusion: Prior occurrence of MMH increases risk of an SH event and weakens the decline in SH event risk with increasing targets. Prior occurrence of a mild event does not weaken this effect of titration target on the risk of MSH. Patients experiencing MMH may need to adjust their individual diabetes care plan, but they should not automatically alter titration targets to avoid later SH event. However, avoidance of MSH following a mild event would entail a less aggressive titration target.

This new analysis supports the previous conclusion of the GOT study that targeting an FBG goal of $100-110 \mathrm{mg} / \mathrm{dL}$ would yield a beneficial balance between glycemic goals and hypoglycemia. (Tab. 1)

Editorial support provided by sanofi-aventis U.S.

\section{5}

Insulin binding to antibodies is a risk factor for inexplicable severe hypoglycaemia in children with type 1 diabetes mellitus

O. Seewi ${ }^{1}$, C. Jaeger ${ }^{2}$, R. G. Bretzel ${ }^{2}$, E. Schönau ${ }^{1}$;

${ }^{1}$ Paediatric Diabetology, University of Cologne, Germany,

${ }^{2}$ Medical Department III, University of Giessen, Germany.

Background and Aim: Type 1 diabetic patients differ with regard to both, the formation of circulating insulin antibodies, and the incidence of severe hypoglycaemia (SH). Aim of the study: to assess the association of insulin binding to antibodies with the incidence of $\mathrm{SH}$.

Patients and Methods: In a cross sectional study, 73 children with type 1 diabetes mellitus (median age 14 years, duration of diabetes 6 years) were investigated, 22 of whom ever, and 51 never had experienced SH. Of the patients with SH, 16 had experienced SH deemed inexplicable, and 6 had experienced SH which deemed explicable (by missed meals, unplanned physical exercise etc.). Insulin binding was measured by radioimmunoassay, and expressed as ratio bound/unbound insulin; a binding $>15 \%$ was considered relevant insulin binding (RIB).

Results: A total of 38 patients displayed RIB (17 of whom had experienced $\mathrm{SH}$ ), and 35 patients did not display RIB (5 of whom had experienced $\mathrm{SH}$; $\mathrm{p}=0.0055$, Fisher's exact test). Patients with RIB were younger ( 13 versus 15 years, $\mathrm{p}=0.006$ ) than patients without RIB. Of the 16 patients with inexplicable $\mathrm{SH}, 15$ displayed RIB, compared to 2 of the 6 patients with explicable SH $(\mathrm{p}=0.009)$. The association of any $\mathrm{SH}$, and of inexplicable $\mathrm{SH}$, with RIB was significant (odds ratio 4.8(95\%CI 1.5-15.2), and 22.1(95\%CI 2.7-179.6), $\mathrm{p}<$ 0.006). Patients with/without RIB, or with/without $\mathrm{SH}$, were comparable regarding sex, duration of diabetes, number of insulin injections per day, HbAlc and C-peptide levels (ANOVA).

Conclusion: Insulin binding to antibodies $>15 \%$ appears to be a strong risk factor for inexplicable severe hypoglycaemias in type 1 diabetic children. Supported by Stiftung "Das zuckerkranke Kind"

\section{6}

Hypoglycaemia does not increase long-term mortality or morbidity in diabetic patients with myocardial infarction: a report from the DIGAMI 2 trial

L. G. Mellbin ${ }^{1}$, K. Malmberg ${ }^{1}$, A. Waldenström² ${ }^{2}$ H. Wedel ${ }^{3}$, L. Rydén ${ }^{1}$; ${ }^{1}$ Department of Cardiology, Karolinska Institutet, Stockholm, Sweden, ${ }^{2}$ Department of Cardiology, University Hospital, Umeå, Sweden, ${ }^{3}$ Nordic School of Public Health, Nordic School of Public Health, Göteborg, Sweden.

Background and Aims: Tight, insulin-based glucose control is recommended to diabetic patients (DM pat) with acute myocardial infarction (AMI). A potential draw-back would be if insulin induced hypoglycaemia (HG) had a negative impact of future prognosis.

Materials and Methods: 1253 pat (mean age 68 years; 67\% males) with type $2 \mathrm{DM}$ and suspect AMI were followed for a median of 2.1 years. 947 were randomised to insulin infusion during at least 24 hours while 306 were treated routinely. HG (blood glucose $<3.0 \mathrm{mmol} / \mathrm{L}$ with or without symptoms) was recorded during hospitalisation. Unadjusted and adjusted (age, sex, smoking, previous MI and heart failure, renal function, duration of DM, coronary interventions, blood glucose at randomisation) Hazard Ratios (HR) and 95\% Confidence Intervals (CI) for cardiovascular events (CVE = death, MI or stroke) during follow up were calculated.

Results: 153 pat (12.2\%) experienced HG. During the first 24 hours 111 (11.7\%) insulin-treated pat experienced HG (symptomatic 23.4\%) compared

Tab. 1

\begin{tabular}{lllllll}
$\begin{array}{l}\text { Treatment Group } \\
\text { (Target FBG, } \mathbf{m g} / \mathbf{d L})\end{array}$ & \multicolumn{2}{l}{ SH Event Occurrence Hazard (SE) events/sbj-y } & & Risk Ratios \\
\cline { 2 - 4 } & All & No prior MMH event & With prior MMH event & & $\begin{array}{l}\text { Prior event/ } \\
\text { no prior event }(95 \% \text { CI) }\end{array}$ & $P$ value effect of prior event \\
\hline 80 & $0.088(0.014)$ & $0.072(0.040)$ & $0.144(0.080)$ & $2.01(1.03,3.91)$ & 0.039 \\
90 & $0.063(0.012)$ & $0.043(0.025)$ & $0.122(0.068)$ & $2.86(1.28,6.37)$ & 0.010 \\
100 & $0.038(0.009)$ & $0.034(0.021)$ & $0.062(0.039)$ & $1.82(0.67,4.94)$ & 0.238 \\
110 & $0.047(0.010)$ & $0.037(0.022)$ & $0.093(0.055)$ & $2.54(1.05,6.17)$ & 0.039 \\
120 & $0.019(0.007)$ & $0.014(0.010)$ & $0.046(0.032)$ & $3.25(0.81,13.07)$ & 0.096 \\
\hline
\end{tabular}

$\mathrm{CI}=$ confidence interval 
with $3(1.0 \%)$ pat on routine treatment (symptomatic 33.3\%). As outlined in the figure HG was not an independent predictor of subsequent CVE.

Conclusion: Insulin induced HG was not a significant risk factor for subsequent CVE in AMI-pat with type 2 DM. Hypoglycaemia seem to identify patients at high risk for other reasons.

\section{Endpoint: Death}

Unadjusted: Hypoglycemia (0/1)

Adjusted : Hypogycemia (0/1)

Unadjusted. Hypoglycemia with symtom (a/1)

Adjusted : Hypogycemia with symtom (0/1)

Endpoint: CV Death

Unadjusted: Hypoglycemia $(0 / 1)$

Unadjusted: Hypoglycemia (0/1)

Unadjusted: Hypoglycemia with symtom (a/1)

Adjusted Hypoglycemia with symtom (Q/1)

Endpoint: Death/StrokeReinfarction Unadjusted: Hypoglycemia (0/1)

Adjusted : Hypogycemia (0/1)

Unadjusted: Hypoglycemia with symtom $(0 / 1)$

Adjusted : Hypogycemia with symtom (0/1)

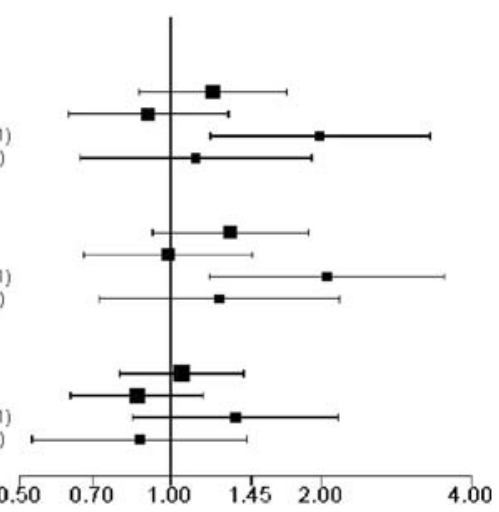

Supported by the Swedish Heart-Lung Foundation, AFA Insurance and by unconditional research grants from Aventis Sweden and Novo Nordisk Denmark

\section{OP 32 Diabetes in children and adolescents in all its forms}

\section{7}

Differential relationship between family relationships and $\mathrm{HbA}_{1 \mathrm{c}}$, by insulin regimen in adolescents with type 1 diabetes

T. Skinner ${ }^{1}$, C. de Beaufort ${ }^{2}$, P. G. F. Swift ${ }^{3}$, F. Cameron ${ }^{4}$; Hvidoere Childhood Diabetes Study Group,

${ }^{1}$ Psychology, University of Wollongong, Australia, ${ }^{2}$ DECCP, Clinique pédiatrique de Luxembourg, Luxembourg, ${ }^{3}$ Paediatrics, Leicester Royal Infirmary, United Kingdom, ${ }^{4}$ Dep of Endocrinology \& Diabetes, Royal Chidlren's Hospital, Melbourne, Australia.

Background and Aims: To establish whether the relationship between family interaction and diabetes outcome, varies as a function of insulin regimen Material and Methods: Cross-sectional study of adolescents with type 1 diabetes and their parents was conducted in 21 paediatric diabetes centres across 19 countries. Demographic data, details of insulin regimens, severe hypoglycemic events and number of episodes of diabetic ketoacidosis were recorded. Adolescents completed the Diabetes Quality of Life (DQOLY-SF), the Diabetes Family Responsibility Questionnaire (DFRQ) and the HBSC Well-Being questionnaire. Parents completed the DFRQ and the WHO-5 Well-Being questionnaire. A capillary blood sample was drawn from each patient and analyzed centrally for HbAlc.

Results: 2269 adolescents (age 14.5 SD 2.0; duration 6.1 SD 3.5, 49\% female) participated, and the insulin regimens were classified as BD Insulin $(\mathrm{n}=459$ ) basal bolus regimen $(\mathrm{n}=938)$, CSII pump $(\mathrm{n}=336)$. Those on TD regimens $(n=68)$ or who could not be readily classified into one of these insulin regimens $(n=468)$ are not included in this analysis. Mothers of adolescents on CSII pumps had more positive mood than all other regimens, followed by basal bolus and $\mathrm{BD}$ regiments $(\mathrm{F}=5.3 ; \mathrm{p}<.001)$, whereas adolescents on basal bolus and CSII regimens reported lower well-being than individuals on $\mathrm{BD}$ regiments $(\mathrm{t}=2.49 ; \mathrm{p}<.05)$. Adolescents' perception of parental overinvolvement in care was related to $\mathrm{HbAlc}$, emotional well-being, diabetes related worry and impact of diabetes in all insulin regimen groups. However, for parent-adolescent disagreement on diabetes care responsibility, there was no relationship with $\mathrm{HbAlc}$ for those on $\mathrm{BD}$ or Basal Bolus regimens, but there was for those using CSII pump ( $\mathrm{F}=7.79 ; \mathrm{p}<.001)$. Controlling for diabetes centre, age, duration and gender effects does not affect the pattern of these results.

Conclusion: Family interaction may be of more importance for achieving optimal outcomes for individuals using CSII, than in other insulin regimen groups.

Supported by Novo Nordisk

\section{8}

Continuous subcutaneous insulin infusion (CSII) of insulin aspart vs. insulin lispro in paediatric subjects (ages 4 to 18 years) with type 1 diabetes (T1D)

C. Ternand ${ }^{1}$, S. A. Weinzimer ${ }^{2}$, L. M. B. Laffel ${ }^{3}$, C. Howard ${ }^{4}$, C.-T. Chang ${ }^{4}$, D. J. Becker ${ }^{5}$;

${ }^{1}$ Endocrinology, University of Minnesota Children's Hospital, Minneapolis, ${ }^{2}$ Pediatric Endocrinology, Yale University School of Medicine, New Haven, ${ }^{3}$ Pediatric, Adolescent and Young Adults, Joslin Diabetes Center, Boston, ${ }^{4}$ CMR, Novo Nordisk Inc., Princeton, ${ }^{5}$ Dept. of Pediatric Endocrinology, Children's Hospital of Pittsburgh, United States.

Background and Aims: Insulin aspart and insulin lispro are rapid-acting insulin analogues currently indicated for administration via multiple daily injection (MDI) therapy in children and adolescents, and for use in MDI and CSII therapy in adults with T1D. The aim of this study was to compare the safety and efficacy of insulin aspart vs. insulin lispro administered via CSII in paediatric subjects ( 4 to 18 years) with T1D.

Materials and Methods: This was a 16-week, open-label, randomised, parallel-group, 45-site, U.S. clinical trial. Subjects ages $4-18$ years, diagnosed with T1D $\geq 1$ year and previously treated for $\geq 3$ months with analogue insulin CSII therapy were randomised 2:1 to receive aspart or lispro for 16 weeks via insulin pump ( $\mathrm{N}=198$ aspart, $\mathrm{N}=100$ lispro). Standard diabetes safety and efficacy measurements were collected over 13 visits. 
Results: Aspart was non-inferior to lispro (as measured by change in $\mathrm{HbA}_{1 \mathrm{c}}$ from baseline to end-of-study [EOS]) $(95 \% \mathrm{CI}[-0.27,0.07])$. $\mathrm{HbA}_{1 \mathrm{c}}$, change in $\mathrm{HbA}_{1 \mathrm{c}}$, and fasting plasma glucose (FPG) values were not significantly different between aspart and lispro (Table). The percentage of subjects who achieved their age-specific 2006 ADA paediatric $\mathrm{HbA}_{1 c}$ targets $(<8.5 \%$ for subjects $<6$ yrs; $<8 \%$ for subjects $6-18$ yrs) was insignificantly higher at baseline, but significantly greater in the aspart group vs. the lispro group at Week 16. At Week 16, daily weight-adjusted insulin dose was significantly lower for subjects treated with aspart, as compared to lispro.

\begin{tabular}{|c|c|c|c|c|}
\hline & & $\begin{array}{l}\text { Aspart } \\
\mathrm{N}=198\end{array}$ & $\begin{array}{l}\text { Lispro } \\
\mathrm{N}=100\end{array}$ & p-value \\
\hline \multirow[t]{2}{*}{$\mathrm{HbA}_{1 c}($ mean $\pm \mathrm{SD}), \%$} & Baseline & $8.04 \pm 0.94$ & $8.15 \pm 0.84$ & 0.301 \\
\hline & EOS & $7.88 \pm 0.93$ & $8.07 \pm 0.85$ & 0.238 \\
\hline $\begin{array}{l}\triangle \mathrm{HbAlc} \\
(\mathrm{LS} \text { mean } \pm \mathrm{SE}), \%\end{array}$ & $\begin{array}{l}\text { Baseline } \\
\text { to EOS }\end{array}$ & $-0.15 \pm 0.05$ & $-0.05 \pm 0.07$ & 0.241 \\
\hline $\mathrm{FPG}($ mean $\pm \mathrm{SD}), \mathrm{mmol} / \mathrm{l}$ & EOS & $9.25 \pm 3.74$ & $10.01 \pm 4.59$ & 0.113 \\
\hline $\begin{array}{l}\% \text { subjects at age-specific } \\
\text { HbAlc goal }\end{array}$ & $\begin{array}{l}\text { Screening } \\
\text { Week } 16\end{array}$ & $\begin{array}{l}50.3 \\
59.7\end{array}$ & $\begin{array}{l}40.4 \\
43.8\end{array}$ & $\begin{array}{l}0.138 \\
0.014\end{array}$ \\
\hline $\begin{array}{l}\text { Weight-adjusted insulin } \\
\text { dose, } \mathrm{U} / \mathrm{kg} / \text { day }\end{array}$ & Week 16 & $0.86 \pm 0.24$ & $0.94 \pm 0.23$ & 0.018 \\
\hline
\end{tabular}

There were no statistically significant differences between treatment groups in major hypoglycaemic events (requiring third-party assistance) per subject year (aspart, 0.42; lispro, 0.3 [p=0.482]) or minor hypoglycaemic events $(\mathrm{PG}<3.1 \mathrm{mmol} / \mathrm{l})$ per subject year (aspart, 77.18; lispro, $65.95[\mathrm{p}=0.129]$ ). The incidence of hyperglycaemia episodes (PG $>16.67 \mathrm{mmol} / \mathrm{l}$ ) recorded as adverse events (AEs) was $10.6 \%$ and $17.0 \%$ in the aspart and lispro treatment groups, respectively. The percentages of subjects experiencing at least 1 AE were similar between treatment groups (aspart, 81.8\%; lispro, 83.0\%). Treatment with either aspart or lispro did not adversely affect physical examination findings, vital signs, or laboratory parameters.

Conclusion: Insulin aspart is as effective and safe as insulin lispro for CSII use in paediatric subjects $4-18$ years of age, with approximately $60 \%$ of insulin aspart-treated youth achieving age-specific $\mathrm{HbA}_{1 \mathrm{c}}$ goals.

Supported by Novo Nordisk Inc.

\section{9}

Lower within-subject variability in pharmacokinetic profiles of insulin detemir in comparison to insulin glargine in children and adolescents with type 1 diabetes

T. Danne ${ }^{1}$, L. Endahl ${ }^{2}$, H. Haahr ${ }^{2}$, N. Datz ${ }^{1}$, C. Nestoris ${ }^{1}$, L. Westergaard ${ }^{2}$, O. Kordonouri ${ }^{1}$;

${ }^{1}$ Diabetes Centre for Children and Adolescents, Kinderkrankenhaus auf der Bult, Hannover, Germany, ${ }^{2}$ Novo Nordisk A/S, Bagsvaerd, Denmark.

Background and Aims: Insulin detemir has previously been shown to be associated with a lower within-subject variability compared to other basal insulin preparations in adults with type 1 diabetes. This randomised, double-blind, cross-over trial compared the within-subject variability of pharmacokinetic profiles of insulin detemir and insulin glargine in children and adolescents with type 1 diabetes.

Materials and Methods: Thirty-two children and adolescents with type 1 diabetes (19 girls and 13 boys, $8-17$ years of age, BMI: $21.4 \pm 2.9 \mathrm{~kg} / \mathrm{m}^{2}, \mathrm{HbA}_{1}$ : $7.9 \% \pm 1.0 \%)$ were randomised to two s.c. doses $(0.4$ units $/ \mathrm{kg})$ of each insulin. There were two dosing visits. Insulin detemir and insulin glargine were injected 24 hours apart at each of two dosing visits. Insulin concentrations were measured at frequent intervals for 16 hours post dosing. Carry-over effect was eliminated due to specific insulin assays without cross-reaction.

Results: Insulin detemir showed significantly less within-subject variability compared to insulin glargine (3.1 and 2.9 fold lower \%CV for AUC $(0-16 \mathrm{~h})$ and Cmax, respectively), see table below. Similar findings were observed when insulin profiles for children and adolescents were analysed separately. No safety concerns were raised during the trial.
Within-Subject Variability (\%CV)

\begin{tabular}{llll}
\hline & Insulin Detemir & Insulin Glargine & $P$-values \\
\hline AUC $(0-16 \mathrm{~h})$ & $10 \%$ & $31 \%$ & $<0.0001$ \\
Cmax & $12 \%$ & $35 \%$ & $<0.0001$ \\
\hline
\end{tabular}

Conclusion: In conclusion, within-subject variability in pharmacokinetic properties was significantly lower for insulin detemir than for insulin glargine in children and adolescents with type 1 diabetes. This indicates a less variable absorption with insulin detemir, which is expected to be associated with a more predictable therapeutic effect in this population.

Supported by Novo Nordisk A/S

\section{0}

Continuous glucose monitoring (CGMS) enhances the detection of cystic fibrosis related diabetes (CFRD) in children with cystic fibrosis

S. M. P. O'Riordan ${ }^{1,2}$, E. F. Roche ${ }^{1,2}$, S. George ${ }^{1,2}$, H. M. V. Hoey ${ }^{1,2}$, C.

Costigan $^{3}$;

${ }^{1}$ Paediatric Diabetes and Endocrinology, The National Childrens Hospital, AMNCH, DUBLIN, ${ }^{2}$ Paediatric Daibetes and Endocrinology, The University of Dublin, Trinity College, Dublin, ${ }^{3}$ Paediatric Diabetes and Endocrinology, Our Ladys Hospital for sick children, Crumlin, Dublin, Ireland.

Background and Aims: Cystic Fibrosis Related Diabetes (CFRD) and impaired glucose intolerance (IGT) are now frequent complications with improved survival in children with cystic fibrosis (CF). The increased morbidity and mortality associated with CFRD emphasizes the need for accurate screening. Many of the screening tools for the diagnosis of CFRD rely on standarised thresholds in the oral glucose tolerance test (OGTT) derived from epidemiological studies based on non-CF populations for the diagnosis of type 1 and 2 diabetes. Current screening tools are inadequate for the diagnosis of CFRD in children. Aims: to evaluate continuous glucose monitoring (CGMS) in CF children; compare results with OGTT and HbAlc and determine if CGMS enhances the detection of CFRD.

Materials and Methods: $101 \mathrm{CF}$ children had paired screening with OGTT \& CGMS: patients commenced CGMS after 1 hour calibration, underwent OGTT and continued CGMS for 48 hours. Assessment was repeated after 6 months. CGMS analysis: All cases were individually analysed by experienced physicians in the management of diabetes in children. All CGMS glucose values were carefully analysed for mean glucose, variability (MAGE, MODD \& CONGA), duration and degree of hyperglycaemia. Clinical assessment was considered essential and individualised in every case.

Results: Children were categorized as normoglycaemic (NGT), IGT and CFRD. 101 children with CF were screened at baseline and 100(99\%) underwent repeat assessment at 6 months. Paired screening identified 12 new cases of CFRD. Insulin therapy was commenced in 12 cases following assessment of OGTT, CGMS and clinical status of each child.

Screening children with CF with OGTT and CGMS at Tme 0 \& 6 months

\begin{tabular}{lllllll}
\hline $\begin{array}{l}\text { Glucose } \\
\text { tolerance } \\
\text { category }\end{array}$ & $\begin{array}{l}\text { Baseline } \\
\text { OGTT }\end{array}$ & $\begin{array}{l}\text { Baseline } \\
\text { CGMS }\end{array}$ & $\begin{array}{l}\text { Baseline } \\
\text { Paired } \\
\text { Diagnosis }\end{array}$ & $\begin{array}{l}\text { 6mths } \\
\text { Followup } \\
\text { OGTT }\end{array}$ & $\begin{array}{l}\text { 6mths } \\
\text { Followup } \\
\text { CGMS }\end{array}$ & $\begin{array}{l}\text { 6mths } \\
\text { Followup } \\
\text { Paired } \\
\text { Diagnosis }\end{array}$ \\
\hline NGT & 71 & 60 & 65 & 70 & 65 & 61 \\
IGT & 14 & 12 & 11 & 15 & 14 & 11 \\
CFRD & 16 & 29 & $24 \dagger$ & 15 & 21 & $28 \dagger$ \\
Totals & 101 & 101 & 101 & 100 & 100 & 100 \\
\hline
\end{tabular}

Correlation coefficient $\mathrm{r}=0.88, \mathrm{p}<0.001 \dagger$

Over a total 12 months time period $8 \%$ converted from NGT to IGT; $88 \%$ were unchanged and $5 \%$ progressed from IGT-CFRD. CGMS correlates well with OGTT at all 5 time points ( $r=0.75-0.88)$. CGMS correlated less well with $\mathrm{HbAlc}((\mathrm{r}=0.27, \mathrm{p}>0.05)$ mean $5.5 \%$ range $3.8-9.0 \%) .20 \%$ of cases had abnormal HbAlc results; $50 \%$ of these were defined as NGT on OGTT and CGMS screening.

Conclusion: HbAlc and OGTT alone are not sensitive screening tools in CF. CGMS enhances early detection of abnormalities of glucose tolerance by revealing important trends that facilitate diagnosis. This report highlights the need to redefine the diagnostic criteria for CFRD, as HbAlc and OGTT are 
missing up to $75 \%$ and $27 \%$ of glucose intolerance in CF children, respectively. We propose annual paired screening OGTT \& CGMS in all children with CF over 10 years if there is any clinical suspicion of diabetes development. Supported by the Diabetes Federation of Ireland and Cystic Fibrosis Association of Ireland

\section{1}

The evaluation of bone mineral density in diabetic children with celiac disease and the influence of gluten free diet and diabetes control on bone mineralisation

J. Nazim ${ }^{1}$, J. Starzyk ${ }^{1}$, E. Czerwiński²;

${ }^{1}$ Endocrinology Department, Polish-American Children's Hospital, ${ }^{2}$ Radiology Department, Jagiellonian University, Medical College, Kraków, Poland.

Background and Aims: The prevalence of celiac disease in children with type 1 diabetes greatly exceeds the prevalence of this disease in general population. Celiac disease in diabetic kids is usually asymptomatic and untreated, may lead to serious complications; one of these is osteopenia. The aim of the study was to determine whether bone mineralisation is reduced in diabetic children and adolescents with celiac disease and the influence of gluten free diet on bone mineral density (BMD).

Materials and Methods: We measured lumbar spine (LS) BMD in 15 children and adolescents aged 5.1-17.8 years (median $10.1 \mathrm{yr}$ ) with type 1 diabetes of median duration 4.1 years $(0.4-13.1$ years) and celiac disease. Control group comprises 10 diabetic children without celiac disease. BMD was assessed by dual-energy $\mathrm{x}$-ray absorptiometry (DXA) at diagnosis of celiac disease, one and two years thereafter. Celiac disease was diagnosed on the basis of intestinal biopsy specimen microscopic examination in children positive for antiendomysial antibodies. All children had silent or subclinical form of the disease. Glycemic control was estimated using glycated hemoglobin measurement by HPLC method (BioRad).

Results: At diagnosis of celiac disease BMD Z-score was reduced in diabetic kids compared to control group $(-0.1$ to -3.6 , mean $-1.2 \pm 0.8$ SD vs -0.7 to 0.9 , mean $-0.4 \pm 0.3 \mathrm{SD}$ ) in spite of the similar diabetes control. Four kids with osteoporosis (Z-score $<-2 \mathrm{SD}$ ) had the worst mean year HbAlc in comparison with 4 children with osteopenia (Z-score -1SD to -2 SD) and patients with normal BMD values ( $8.7 \%$ vs $8.3 \%$ vs $7.4 \%)$. One and two years of gluten free diet improved BMD Z-score in 10 children, in three patients with osteoporosis who were not adherent to the diet, BMD decreased from 0.3 to 0.9 SD despite suplementation of vitamin $\mathrm{D}$ and calcium.

Conclusion: Children with type 1 diabetes should be screened towards celiac disease. Gluten free diet and good glycemic control favoured the accrual of bone mass in diabetic children with celiac disease.

\section{2}

Insulin omission and glycaemic control in 2062 adolescents with diabetes in 21 international centres

S. E. Skovlund ${ }^{1}$, C. de Beaufort ${ }^{2}$, T. Skinner ${ }^{3}$, P. Swift ${ }^{4}$; on behalf of the

Hvidøre Study Group ${ }^{5}$

${ }^{1}$ DAWN - Diabetes Attitudes, Wishes and Needs, Novo Nordisk, Denmark,

${ }^{2}$ Clinique Pédiatrique, Centre Hospitalier de Luxembourg, Luxembourg,

${ }^{3}$ School of Psychology, University of Wollongong, Australia,

${ }^{4}$ Children's Hospital, Leicester Royal Infirmary, United Kingdom,

${ }^{5}$ Centre Differences Study, Hvidøre Study Group, Bagsvaerd, Denmark.

Background and Aims: Large proportion of adolescents with diabetes fail to achieve optimal glycaemic control despite availability of effective medicine. The aim of this study was to examine the extent of self-reported omission of insulin associated with weight concern and the relationship to glycaemic control in a large international cohort of adolescents with type 1 diabetes.

Materials and Methods: Questionnaires were completed by adolescents and their parents/carers in 21 international centres. Questionnaires investigated age, gender, diabetes duration, insulin regimens, insulin adjustments and glycaemic targets as well as psychosocial and service provision factors. HbAlc (DCCT adjusted) was measured centrally.

Results: Questionnaires were completed by 2062 adolescents (age $14.4 \mathrm{y}+/-$ $2.3 \mathrm{y}$, male $50.6 \%$, diabetes duration $6.1 \mathrm{y} \pm 3.5$ ). Different insulin regimens were used both within and between the 21 centres. Mean $\mathrm{HbAlc}=8.2 \% \mathrm{SD}$ 1.4 , with significant influence of age $(\mathrm{r}=0.1 ; \mathrm{p}<0.001)$, gender (females $8.3 \%$ \pm 1.5 , males $8.1 \% \pm 1.3 ; \mathrm{t}=3.0 ; \mathrm{p}<0.005)$, diabetes duration $(\mathrm{r}=0.29 ; \mathrm{p}<0.001)$. $91.7 \%$ of girls and $93 \%$ of boys stated they miss insulin to control weight "once a month", $5.1 \%$ and $4.2 \%$ respectively stated they never miss insulin to control weight. $2.5 \%$ and $1.9 \%$ respectively stated they omitted insulin once a week and $0.7 \%$ and $0.9 \%$ respectively stated they did that every day. Multiple regression analysis showed that omission of insulin to control weight was significantly associated with poor $\mathrm{HbAlc}(\beta=.103, \mathrm{t}=2.558, \mathrm{p}=0.011)$ after controlling for age and gender effect.

Conclusion: Self-reported omission of insulin to control weight in both female and male adolescents was common in this large international cohort. It was associated with poorer glycaemic control. These findings suggest that concerns about weight gain associated with insulin therapy may have significant short and long-term clinical implications. The findings suggest that screening for insulin omission related to weight control might be warranted in paediatric care. Appropriate intervention may involve education, counselling and adjustment of treatment regimens.

Supported by Novo Nordisk 


\section{OP 33 Adipocytes}

\section{3}

Depot-related differences in insulin action and signaling, gene expression, and adiponectin secretion in human adipocytes differentiated in vitro from precursor stromal cells

S. Perrini ${ }^{1}$, L. Laviola ${ }^{1}$, A. Natalicchio ${ }^{1}$, A. Cignarelli ${ }^{1}$, M. Melchiorre ${ }^{1}$, F. De Stefano ${ }^{1}$, C. Caccioppoli ${ }^{1}$, M. R. Orlando ${ }^{1}$, G. Garruti ${ }^{1}$, M. De Fazio ${ }^{2}$, V. $\mathrm{Memeo}^{2}$, R. Giorgino ${ }^{1}$, F. Giorgino'

${ }^{1}$ Endocrinology \& Met. Diseases, ${ }^{2}$ General Surgery, University of Bari, Bari, Italy.

Background and Aims: Visceral and subcutaneous fat depots possess different metabolic properties, that can be demonstrated both in wholetissue specimens and isolated adipocytes. In this study, we have investigated whether human adipocytes obtained from visceral and subcutaneous fat depots retain distinct properties also when they are differentiated in vitro from precursor stromal cells.

Materials and Methods: Paired visceral and subcutaneous biopsies were obtained from 15 non-obese, non-diabetic subjects undergoing open abdominal surgery. Precursor cells were isolated, grown to confluence, and differentiated in vitro into adipocytes. Terminal differentiation was assessed by visual inspection, Oil-O-Red staining, and gene expression of adipocytespecific proteins using real-time PCR. Intracellular insulin signaling was studied by immunoblotting with specific antibodies.

Results: Differentiated adipocytes showed marked up-regulation of GLUT4, $\operatorname{PPAR} \gamma, \mathrm{C} / \mathrm{EBP} \alpha, \mathrm{aP} 2$, and adiponectin mRNA levels compared to the preadipocytes, whereas GLUT1 expression did not change during adipogenesis. mRNA levels of $\mathrm{C} / \mathrm{EBP} \alpha$, adiponectin and aP2 were found to be higher in subcutaneous than in visceral adipocytes. By contrast, basal adiponectin secretion in the culture medium was 3 -fold higher in visceral than in subcutaneous adipocytes. When compared to subcutaneous adipocytes, visceral adipocytes also showed $50 \%$ higher rates of insulin-stimulated glucose transport, measured by determining 2-deoxy-D-glucose uptake rates. Intracellular insulin signaling was next investigated following stimulation with $10 \mathrm{nM}$ insulin for 5 to $120 \mathrm{~min}$. Insulin-induced phosphorylation of the insulin receptor, IRS-1/2, Akt and Erk-1/2 appeared to be more rapid and tended to decrease at earlier time points in visceral than in subcutaneous adipocytes, in which phosphorylation of these signaling proteins in response to insulin was more gradual and persistent.

Conclusion: Subcutaneous and visceral adipocytes, also when differentiated in vitro from precursor stromal cells, retain differences in gene expression, adiponectin secretion, and insulin action and signaling. This suggests that the precursor cells resident in the visceral and subcutaneous fat depots possess inherent and specific metabolic characteristics that are expressed upon completion of the differentiation programme.

\section{4}

Effect of beta-adrenergic stimulation on whole-body and regional abdominal subcutaneous adipose tissue lipolysis in lean and obese men J. W. E. Jocken ${ }^{1}$, G. H. Goossens ${ }^{1}$, A. van Hees ${ }^{1}$, K. N. Frayn ${ }^{2}$, M. van Baak ${ }^{1}$, M. Pakbiers ${ }^{3}$, W. H. M. Saris ${ }^{1}$, E. E. Blaak ${ }^{1}$;

${ }^{1}$ Human Biology, Maastricht University, The Netherlands,

${ }^{2}$ Oxford Centre for Diabetes, Endocrinology and Metabolism,

Oxford University, United Kingdom,

${ }^{3}$ Nuclear Medicine, University Hospital Maastricht, The Netherlands.

Obesity is characterized by increased triacylglycerol (TAG) storage in adipose tissue. There is evidence for a blunted catecholamine-induced lipolytic response in adipose tissue of obese subjects. In vitro studies suggest that the site of this catecholamine resistance is abdominal subcutaneous adipose tissue. We investigated for the first time in vivo whole-body and regional abdominal subcutaneous adipose tissue glycerol metabolism during $3 \mathrm{~h}$ and $6 \mathrm{~h}$ [2H5]glycerol infusion. Arterio-venous differences were measured in 13 lean and 10 obese male subjects after an overnight fast and during intravenous infusion of the non-selective beta-adrenergic agonist isoprenaline (20ng. kg FFM-1. min-1). Lean and obese subjects showed significant but comparable glycerol uptake by adipose tissue $(9.7+/-3.4$ vs. $9.3+/-2.5 \%$ of total release, $\mathrm{P}=0.92$; $\mathrm{P}<0.05$ compared to zero). Second, obese subjects showed an increased whole-body lipolytic response, due to their increased fat mass. In contrast, obese subjects showed a blunted fasting lipolysis (glycerol Ra: 7.3+/-0.6 vs. $13.1+/-0.9$ micromol. kg FM-1. min-1, $\mathrm{P}<0.01$ ) and lipolytic response per unit fat mass (delta total glycerol release: $140+/-71$ vs. $394+/-112$ nmol. $100 \mathrm{~g}$ tissue -1 . min $-1, \mathrm{P}<0.05$ ) compared with lean subjects. Finally, net TAG extraction was increased in the obese during beta-adrenergic stimulation. In conclusion, a blunted adipose tissue TAG mobilization and increased TAG extraction during beta-adrenergic stimulation may be important factors in the development or maintenance of increased TAG stores in obesity. Supported by NWO-ZonMW

\section{5}

Proteomic analysis reveals differential expression of chaperones and redox proteins in human adipose tissue in response to rosiglitazone treatment

M. Ahmed ${ }^{1,2}$, F. Karpe ${ }^{1}$;

${ }^{1}$ Oxford Centre for Diabetes, Endocrinology \& Metabolism, University of Oxford, United Kingdom,

${ }^{2}$ Department of Physiology, Ibrahim Medical College, Dhaka, Bangladesh.

Background and Aims: Increased reactive oxygen species (ROS) production and ER stress are positively linked with obesity, insulin resistance and type 2 diabetes. In adipocytes, elevated levels of fatty acids and glucose increase oxidative stress and cause dysregulated production of adipokines suggesting the redox state in adipose tissue as a potentially useful therapeutic target. Rosiglitazone is an insulin sensitizing drug with adipose tissue as its primary target. We, therefore, used the unique potential of the two-dimensional based proteomics and explored the synchronized changes in expression of antioxidants and chaperones in rosiglitazone-treated human adipose tissue. Materials and Methods: Six healthy moderately obese men aged $42 \pm 2$ years were treated with rosiglitazone $4 \mathrm{mg} \mathrm{BD}$ for 14 days and adipose tissue biopsies were taken at day 0 (without medication), day 1 and day 14 . Plasma samples were analyzed for glucose, lipids and insulin to verify global insulin sensitization. Adipose tissue protein profiles were obtained by twodimensional gel (2-DG) electrophoresis. Spot identification was made by tandem mass spectrometry.

Results: Short-term treatment with rosiglitazone improved glycemic control and reduced plasma insulin and NEFA levels. In adipose tissue, rosiglitazone significantly $(\mathrm{p}<0.01)$ reduced the expression of peroxiredoxin- 2 whereas superoxidedismutase $[\mathrm{Mn}]$ showed increased expression. Therewas no significant change in the expression of catalase. Another antioxidant, ferrtin light chain appeared as a new spot ( 3 fold change) in response to rosiglitazone. One of the transferrin spots showed significant $(\mathrm{p}<0.05)$ increase on day 14 . The expression of aldehyde dehydrogenase, which protects against lipid peroxidation-induced stress and insulin resistance, showed 1.5 fold rise but no significant association. Proteins involved in ER stress like endoplasmin (HSP90B1/GRP94), GRP78, and GRP58 showed reduced expression indicating proper protein folding and reduced ER stress. Both HSP27, which prevents activation of IKK- $\beta$ and HSP20 (HSPb5), which promote survival of cells subjected to different form of stresses, were significantly over-expressed in rosiglitazone treated adipose tissue. Conclusion: Thus, some of the major changes observed among the mentioned proteins suggest rosiglitazone reduces NF- $\mathrm{\kappa B}$ activation and ROS production, corrects protein folding and reduces ER stress, regulates cytoskeletal assembly as well as enhances adipocyte survival. Such orchestrated changes in expression of multiple proteins may help to explain mechanisms behind improvement of insulin sensitivity in response to rosiglitazone treatment. Supported by Wellcome Trust

\section{6}

In human adipocytes exposed to high glucose and insulin levels, IRS-1 displays altered phosphorylation and may be targeted for degradation F. Renstrom ${ }^{1}$, M. Lundgren ${ }^{1}$, S. Lindmark ${ }^{1}$, J. W. Eriksson ${ }^{2,3}$;

${ }^{1}$ Medicine, Umea University Hospital, Sweden, ${ }^{2}$ Lundberg Laboratory for Diabetes Research, Sahlgrenska University Hospital, Gothenburg, Sweden, ${ }^{3}$ AstraZeneca R\&D, Molndal, Sweden.

Background: In human subcutaneous adipocytes exposed to high insulin and high glucose in combination, IRS-1 depletion occurs as a secondary 
phenomenon after the development of a defect in insulin-stimulated glucose transport. Insulin-stimulated phosphorylation of IRS-1 at $\mathrm{Tyr}^{612}$ leads to activation and propagates the insulin signal, whereas $\mathrm{Ser}^{312}$ phosphorylation is implicated in IRS-1 deactivation and in insulin-induced degradation of IRS-1. In the present study we aimed to investigate whether alterations in $\mathrm{Tyr}^{612}$ and $\mathrm{Ser}^{312}$ phosphorylation are seen in this model of cellular insulin resistance.

Methods: Abdominal subcutaneous needle biopsies were performed on 17 healthy nondiabetic subjects $(7 \mathrm{M} / 10 \mathrm{~F}$, age $45 \pm 4$, BMI $25 \pm 1)$. Isolated adipocytes were cultured for a total of $24 \mathrm{~h}$ in $6 \mathrm{mM}$ glucose with or without addition, during the final 2, 6 or the entire $24 \mathrm{~h}$, of high insulin alone $\left(10^{4}\right.$ $\mu \mathrm{U} / \mathrm{mL}, \mathrm{n}=7$ ) or insulin in combination with high glucose (final conc $20 \mathrm{mM}$, $\mathrm{n}=10$ ). Then cells were washed and treated with or without $10^{3} \mu \mathrm{U} / \mathrm{mL}$ insulin for 15 or $10 \mathrm{~min} .{ }^{14} \mathrm{C}$-glucose uptake in intacts cells was measured, and IRS-1 (total as well as phospho-Tyr ${ }^{612}$ and phospho-Ser ${ }^{312}$ ) was assessed in cellular lysates with immunoblotting using specific antibodies.

Results: Pretreatment with high insulin + glucose for $2 \mathrm{~h}$ or more was associated with a reduced (by $\sim 40-50 \%, \mathrm{p}<0.05$ ) insulin-stimulated glucose uptake capacity. In parallel, there was an increased basal Ser ${ }^{312}$ phosphorylation of IRS-1 (by $\sim 80 \%, \mathrm{p}<0.05)$. In accordance with this, a $\sim 50 \%$ decrease $(\mathrm{p}<0.05)$ in the protein level of IRS- 1 was evident after 6 and $24 \mathrm{~h}$ pretreatments. High insulin levels alone induced a smaller increase in $\mathrm{Ser}^{312}$ phosphorylation (by $\sim 40 \%, \mathrm{p}<0.05$ ) and did not consistently alter IRS-1 protein content. However, high insulin levels for $2 \mathrm{~h}$ or longer produced an impaired insulinstimulated glucose uptake capacity (by $30-40 \%, \mathrm{p}<0.05$ ). Moreover, basal $\mathrm{Tyr}^{612}$ phosphorylation was increased by $>400 \%(\mathrm{p}<0.05)$ following insulin pretreatment with or without high glucose. No change in subsequent insulinstimulated $\mathrm{Tyr}^{612}$ phosphorylation was seen, but accordingly the relative effect of acute insulin stimulation was attenuated.

Conclusion: High glucose and insulin levels in combination can enhance IRS-1 phosphorylation at $\operatorname{Ser}^{312}$ and this can contribute to impaired insulin signalling and is likely to target IRS- 1 to a degradation pathway. These findings in human adipocytes may be of relevance for adipose tissue insulin resistance in type 2 diabetes.

Supported by Swedish Research Council, Swedish Diabetes Association, AstraZeneca R\&D, Västerbotten County Council

\section{7}

Gender differences in insulin sensitivity also include differences in expression of IRS-1 in subcutaneous adipocytes

F. Pellme, A. Hammarstedt, U. Smith, P.-A. Jansson;

The Lundberg Laboratory for Diabetes Research, Goteborg University, Sweden.

Background and Aims: We have previously shown in male subjects that low adipocyte IRS-1 expression is related to impaired insulin sensitivity in muscle, hypertriglyceridemia, hypoadiponectinemia and atherosclerosis, supporting a significant role of adipose tissue in the Insulin Resistance Syndrome. We have now evaluated gender differences in IRS-1 expression, metabolic profile and circulating markers of endothelial function in non-diabetic first degree relatives of type 2 diabetic patients (FDR).

Materials and Methods: We recruited by advertisement 125 FDR of both genders (Age: $42 \pm 7$ vs. $39 \pm 7$ yrs (ns) (Mean \pm SD); BMI: $25.5 \pm 2.5$ vs. $24.7 \pm 3.6 \mathrm{~kg} / \mathrm{m} 2, \mathrm{p}<0.05$; Waist/Hip-ratio: $0.92 \pm 0.06$ vs. $0.81 \pm 0.06, \mathrm{p}<0.0001$; Cigarette pack years: $3.1 \pm 5.7$ vs. $2.4 \pm 5.1$ yrs $(n s)$ in male $(n=44)$ and female $(\mathrm{n}=77)$ FDR, respectively). All subjects underwent a modified euglycemic hyperinsulinemic clamp ( $40 \mathrm{mU} / \mathrm{m} 2 / \mathrm{min})$, monitoring of blood pressure and venous blood sampling. On a separate day a subcutaneous needle biopsy was performed and isolated fat cells were lysed and IRS-1 protein expression quantified by Western blot using rhIRS- 1 as reference.

Results: Adipose cell IRS-1 protein content was significantly lower in male FDR $(0.24 \pm 0.09$ vs. $0.34 \pm 0.19$ arbitrary units, $\mathrm{p}<0.005)$, as well as adiponectin (7.2 \pm 2.7 vs. $11.7 \pm 4.7 \mu \mathrm{g} / \mathrm{ml}, \mathrm{p}<0.0001)$, insulin-mediated glucose uptake $(11.8 \pm 3.0$ vs. $14.3 \pm 4.1 \mathrm{mg} / \mathrm{kg} / \mathrm{LBM}, \mathrm{p}<0.01)$, Acute Insulin Response ( $550 \pm 530$ vs. $1100 \pm 1300 \mathrm{mU} / \mathrm{l}, \mathrm{p}<0.01)$, FFA ( $460 \pm 170$ vs. $600 \pm 240 \mu \mathrm{mol} / \mathrm{l}$, $\mathrm{p}<0.001)$ and HDL-Cholesterol $(1.26 \pm 0.29$ vs. $1.67 \pm 0.37 \mathrm{mmol} / \mathrm{l}, \mathrm{p}<0.0001)$ whereas male FDR exhibited significantly higher fP-Glucose $(4.89 \pm 0.63$ vs. $4.75 \pm 0.31 \mathrm{mmol} / \mathrm{l}, \mathrm{p}=0.05), \mathrm{HbAlc}(4.4 \pm 0.3$ vs. $4.1 \pm 0.2 \%, \mathrm{p}<0.0001)$, Triglycerides $(1.37 \pm 0.79$ vs. $0.97 \pm 0.44 \mathrm{mmol} / \mathrm{l}, \mathrm{p}<0.005)$, LDL-Cholesterol ( $3.1 \pm 0.8$ vs. $2.6 \pm 0.7 \mathrm{mmol} / \mathrm{l}, \mathrm{p}<0.001)$, Systolic Blood Pressure ( $123 \pm 13$ vs. $116 \pm 11 \mathrm{~mm} \mathrm{Hg}, \mathrm{p}<0.01)$, Diastolic Blood Pressure (77 \pm 7 vs. $73 \pm 8 \mathrm{~mm} \mathrm{Hg}$, $\mathrm{p}<0.01), \operatorname{ALT}(0.50 \pm 0.23$ vs. $0.35 \pm 0.28 \mu \mathrm{kat} / \mathrm{l}, \mathrm{p}<0.001)$, ICAM-1 ( $218 \pm 51$ vs. $182 \pm 36 \mathrm{ng} / \mathrm{ml}, \mathrm{p}<0.0001)$ and E-Selectin ( $47 \pm 19$ vs. $34 \pm 12 \mathrm{ng} / \mathrm{ml}, \mathrm{p}<0.001)$. The differences remained after adjustments for age and BMI. No gender differences appeared for subcutaneous fat cell size, fs-Insulin, hsCRP, IL-6 and VCAM-1 (not shown).

Conclusion: In conclusion, our data shows that non-diabetic male FDR are less sensitive to insulin and have a less favourable metabolic and vascular risk profile compared to female premenopausal FDR. Reduced adipocyte IRS-1 content may be a predictor of the metabolic syndrome in male FDR.

\section{8}

Adipose tissue expression of the glycerol channel Aquaporin-7 gene is altered in severe obesity but not in type 2 diabetes

J. J. Vendrell ${ }^{1}$, V. Ceperuelo-Mallafré ${ }^{1}$, M. Miranda ${ }^{1}$, N. Vilarrasa ${ }^{2}$, A. Megía ${ }^{1}$, C. Gutiérrez ${ }^{1}$, J. M. Fernández-Real ${ }^{3}$, J. M. Gómez², E. Caubet ${ }^{4}$, M. R. Chacón ${ }^{1}$;

${ }^{1}$ Research Unit, University Hospital Joan XXIII, Tarragona, ${ }^{2}$ Endocrinology Unit, University Hospital of Bellvitge, Barcelona, ${ }^{3}$ Diabetes Unit, University Hospital “Dr. Josep Trueta”, Girona, ${ }^{4}$ Surgery service, Hospital Sta. Tecla, Tarragona, Spain.

Background and Aims: Aquaporin-7 is required for efflux of glycerol from adipocytes and influences whole-body glucose homeostasis in animal studies. $A Q P 7$ gene contains a putative peroxisome proliferator response element (PPRE) and a putative insulin response element (IRE) in the promoter region. Adipose tissue $A Q P 7$ expression is regulated by fasting-refeeding, insulin, dexamethasone, $T N F \alpha$, isoproterenol and PPAR $\alpha-\gamma$. Studies on AQP7 expression in human adipose tissue are scarce but suggest that $A Q P 7$ gene is less expressed in subcutaneous adipose tissue of obese compared to lean men subjects. Three missense mutations (R12C,V59L and G264V) have been identified in human subjects in a Japanese cohort. Functional analysis showed that the permeability of water and glycerol is disturbed in Xenopus oocytes expressing G264V mutant protein. The aim was to test the hypothesis that $A Q P 7$ gene expression levels may be affected by presence of obesity and type 2 diabetes in humans.

Materials and Methods: Subjects: 47 non-diabetic and 56 type 2 diabetic patients. sMCP-1, sTNFR1, sTNFR2 and sIL-6 measured by ELISA. Plasma glycerol levels were analysed. Subcutaneous adipose tissue gene expression of: AQP7, MCP-1, IL-6, TNF $\alpha, P P A R \gamma$ and SREBP1c genes were measured by real time PCR. Determination of G264V mutation of the $A Q P 7$ gene in 178 Caucasian subjects, these included 127 non-diabetic subjects, and 51 type 2 diabetic subjects.

Results: Severely obese subjects showed significantly lower $A Q P 7$ expression levels compared with lean and non-severely obese $(p<0.001)$. Moreover, circulating glycerol concentration was significantly lower $(\mathrm{p}=0.003)$ in severely obese subjects ( $\mu \mathrm{M}$ Glycerol in lean: $361.26 \pm 165.61$; non-severely obese: $451.52 \pm 180.22$ and severely obese subjects: $233.53 \pm 78.10$ ); however no correlation between plasma levels and $A Q P 7$ adipose tissue expression was observed. AQP7 expression was negatively related with pro-inflammatory genes (MCP-1: $\mathrm{r}=-0.203, \mathrm{p}=0.044$ and TNF $\alpha: \mathrm{r}=-0.209, \mathrm{p}=0.036)$ and was positively associated with PPAR $\gamma$ mRNA expression levels $(\mathrm{r}=0.265, \mathrm{p}=0.012)$. In linear regression analysis, including sex and presence of type 2 diabetes as confounding variables, $A Q P 7 \mathrm{mRNA}$ expression was positively dependent on $\operatorname{PPAR} \gamma(\mathrm{B}=0.248, \mathrm{p}=0.002$, in women and $\mathrm{B}=0.321, \mathrm{p}=0.004$, in men) and negatively associated with $T N F \alpha$ mRNA in men $(B=-0.329, \mathrm{p}=0.005)$. AQP7 expression did not show differences regarding the presence of type 2 diabetes. Frequency of the G264V mutation was $8 \%$. No association between G264V and obesity, type 2 diabetes, $A Q P 7$ expression or plasma glycerol levels were observed.

Conclusion: Expression of $A Q P 7$ is down-regulated in severe obesity. The expression of this glycerol channel is not affected by type 2 diabetes. The lack of correlation between gene expression and glycerol levels in the human adipose tissue may be explained by the existence of an alternative glycerol channel in the human adipocyte.

Supported by grants FIS 04/0377, FIS 05/1994 and REDIMET 


\section{OP 34 Regulation of insulin exocytosis}

\section{9}

The transcriptional repressor REST impairs insulin secretion by controlling the expression of proteins of the exocytotic pathway D. Martin ${ }^{1}$, F. Allagnat ${ }^{1}$, R. Regazzi ${ }^{2}$, A. Abderrahmani ${ }^{2}$, G. Waeber ${ }^{1}$, P. Meda ${ }^{3}$, P. Maechler ${ }^{3}$, J.-A. Haefliger';

${ }^{1}$ Department of Medicine, University Hospital, Lausanne,

${ }^{2}$ Department of Cell Biology and Morphology, University of Lausanne, ${ }^{3}$ Department of Cell Physiology and Metabolism, University Medical Center, Geneva, Switzerland.

Background and Aims: The transcriptional repressor RE-1 silencing transcription factor (REST) restricts some neuronal traits to neurons and pancreatic $\beta$-cells, by silencing the expression of these target genes in other cell types. To evaluate the contribution of neuronal traits to the control of insulin release, we repressed their expression by ectopically expressing REST in $\beta$-cells.

Materials and Methods: The secretory response of INS-1E and primary $\beta$ cells were evaluated after transduction of cells with adenoviruses encoding REST. The consequences of a chronic downregulation of the neuronal traits were analyzed, in vivo, using transgenic mice expressing REST in $\beta$-cells.

Results: As a consequence of REST expression, the capacity of INS-1E cells to secrete insulin in response to glucose, $\mathrm{KCl}$, and an analogue of Leucine was impaired. In mice islets expressing REST, the first and second phase of insulin secretion were decreased in response to glucose. Analysis of intracellular signaling leading to $\mathrm{Ca} 2+$ influx suggested perturbed vesicular trafficking or fusion. Consistent with this hypothesis, we identified functional REST binding sites (RE-1) in genes encoding key proteins of the machinery of exocytosis such as SNAP25, Synaptotagmin (SYT) IV, VII, IX or complexin II. Accordingly, their expression was strongly silenced after ectopic REST expression, and a small interfering RNA strategy underlined the crucial role of REST target genes by uncovering the implication of Syt IV and Syt VII in the control of insulin release. We further studied the functional importance of neuronal traits, in vivo, by generating transgenic mice expressing REST in $\beta$-cells. Whereas these mice featured morphologically normal islets and $\beta$-cells, they exhibited defects in insulin secretion and intolerance to glucose. Islets isolated from these mice showed a decreased $\beta$-cell secretory function, as revealed by glucose perifusion, which was associated with a decreased expression of the identified target genes participating to exocytosis.

Conclusion: These data document the functional importance of neuronal traits in pancreatic $\beta$-cells, providing evidences that their downregulation by the transcriptional repressor REST is detrimental for the exocytotic machinery integrity, thus contributing to $\beta$-cell dysfunction and impaired glucose homeostasis.

Supported by Swiss National Science Foundation, Juvenile Diabetes Foundation International, Placide Nicod Foundation, Octav and Marcella Botnar Foundation, Novartis Foundation

\section{0}

Knockdown of a downstream effector of the small G-protein Rac 1 by siRNA affects glucose metabolism and insulin secretion in INS-1 $\beta$ cells J. Zhang, R. Luo, F. Xie, G. Li;

National University Medical Institutes, National University of Singapore, Singapore.

Background and Aims: Stimulation of insulin secretion by glucose and other secretagogues from pancreatic islet $\beta$-cells is mediated by multiple signaling pathways. Our previous work has shown that the small G-protein Racl is involved in glucose- and forskolin-stimulated insulin secretion, probably at the level of recruitment of secretory granules through actin cytoskeletal network reorganization. In this study the possible role of two downstream effectors of Rac-1, type I $\alpha$ phosphatidylinositol-4-phosphate 5-kinase (PIP5K$\mathrm{I} \alpha)$ and P21-activated kinase- $\alpha$ (PAK- $\alpha)$, in this scenario was investigated. Materials and Methods: RNA interference technique was used to knock down PIP5K-I $\alpha$ and PAK- $\alpha$ in INS- $1 \beta$-cells. Cellular F-actin was assessed by using FITC-phalloidin staining under confocol microscopy. Membrane potential was measured by a voltage-sensitive fluorescent probe, bisoxonol. Insulin secretion upon various conditions was measured by radioimmunoassay. Glucose metabolism was determined by a tetrazolium (MTS) reduction assay. Results: Reverse transfection of siRNA duplexes targeting PIP5K-I $\alpha$ reduced its mRNA level by $75 \%$ as assessed by real-time RT-PCR. PIP5K-I $\alpha$ knockdown seemed not to affect cell growth and demise, assessed by flow cytometry. Dramatic changes in cell morphology, disruption of cellular F-actin structure and decrease of F-actin content were observed in the knock-downed cells. The increment of high $(16.7 \mathrm{mM})$ glucose-stimulated insulin secretion was markedly reduced by $40 \%(\mathrm{P}<0.01)$ in PIP5K-I $\alpha$ knock-downed cells. Interestingly, the basal insulin release rate in these cells at $2.8 \mathrm{mM}$ glucose was augmented more than double $(\mathrm{P}<0.01)$. In addition, PIP5K-I $\alpha$ knockdown increased glucose metabolism at $2.8 \mathrm{mM}$, but significantly inhibited (by 29\%) glucose metabolism at $16.7 \mathrm{mM}(\mathrm{P}<0.01)$. Similarly, the resting membrane potential of knock-downed cells was in a more depolarized status. In contrast to PIP5K-I $\alpha$, knockdown of PAK- $\alpha$ in INS-1 cells appeared no significant effects on morphology, glucose metabolism and insulin secretion.

Conclusion: These results indicate that PIP5K-I $\alpha$ is implicated in not only the distal steps (actin cytoskeleton reorganization and granule recruitment) but also the proximal events (membrane depolarization and glucose metabolism) in the cascade leading to insulin secretion in $\beta$-cells. Supported by Singapore NMRC/0803/2003

\section{1}

Expression of mutant Huntingtin disrupts post-Golgi transport in the 832/13 Ins- 1 cell line K. Bacos ${ }^{1}$, R. Smith ${ }^{1}$, D. Soulet ${ }^{1}$, S. Obermüller ${ }^{2}$, A. Lindqvist ${ }^{2}$, M. Björkqvist ${ }^{1}$, P. Klein ${ }^{1}$, P. Brundin ${ }^{1}$, H. Mulder ${ }^{1}$, J.-Y. Lii ${ }^{1}$; ${ }^{1}$ Experimental Medical Science, Lund University, Lund, Sweden, ${ }^{2}$ Clinical Science, Lund University, Malmö, Sweden.

Background and Aims: Huntington's disease (HD) is a severe progressive neurodegenerative disorder. Aside from the neurological symptoms, the prevalence of diabetes is significantly higher among HD patients than in the general population. HD is caused by expansion of CAG-repeats $(>37)$ in the IT15 gene, resulting in an elongated polyglutamine domain in the protein huntingtin. The mutation has been suggested to have several cellular effects; for instance, intracellular trafficking has been shown to be affected in neuronal HD models. We have previously shown that the R6/2 HD mouse model develops diabetes due to a severe degranulation of $\beta$-cells and reduced exocytosis of insulin. The aim of the current study was to further characterise this $\beta$-cell perturbation.

Materials and Methods: We used confocal microscopy to study morphology and insulin distribution in primary R6/2 $\beta$-cells. We studied the effects of expressing normal (17Q) and mutant huntingtin (69Q) in clonal 823/13 Ins-1 cells, using recombinant adenoviruses. Intracellular transport in general and that of insulin vesicles were studied by coexpressing VSVG-YFP or IAPP-GFP with the huntingtin mutants. Fluorescence recovery after photobleaching (FRAP) and inverse FRAP were used to determine pre- and post-Golgi transport.

Results: We observed an altered localisation of insulin in the mouse $\beta$-cells, where proinsulin/insulin co-localized with the Golgi apparatus and not the mature insulin granule marker phogrin. Furthermore, we observed that mutant huntingtin impaired glucose stimulated insulin secretion already 48 hours after transduction of 832/13 Ins-1 cells (17Q 47.6 $\pm 9.0 ; 69 \mathrm{Q} 27.7 \pm 5.2 \mathrm{ng} / \mathrm{mg} /$ $\mathrm{h}, \mathrm{p}=0.03)$. The defect occurred despite unaltered insulin content in the cells (17Q 2.3 $\pm 0.01 ; 69 \mathrm{Q} 2.6 \pm 0.4 \mu \mathrm{g} / \mathrm{mg}, \mathrm{p}>0.05$ ). Using a VSVG-YFP construct we found that delivery of the protein to the plasma membrane was delayed in cells expressing mutant huntingtin compared to cells expressing normal huntingtin (ratio of membrane and cytoplasm fluorescence, 17Q 1.5 $\pm 0.1 ; 69 \mathrm{Q} 1.2 \pm 0.1$, $\mathrm{p}=0.018$. A combination of FRAP and inverse FRAP showed that while ERGolgi transport is unaffected, post-Golgi transport in the $69 \mathrm{Q}$ huntingtin cells is slower than in $17 \mathrm{Q}$ huntingtin cells (two-factor anova, $\mathrm{p}=0.03$ ). Moreover, we found a reduction in peak insulin granule speed and a substantial loss of insulin granules moving directionally at high speeds.

Conclusion: Taken together these results suggest that expression of mutant huntingtin perturbs the intracellular trafficking in Ins-1 cells and that this may, at least in part, underlie the defects in insulin secretion observed in the same cells.

Supported by Swedish Research Council, Swedish Society for Medicine, Crafoord Foundation, Hedlund Foundation, Greta and Johan Kock Foundation, Segerfalk Foundation 


\section{2}

Ral GTPases as potential regulators of insulin secretion S. Ljubicic, P. Bezzi, R. Regazzi;

Department of Cellular Biology and Morphology, University of Lausanne, Switzerland.

Background and Aims: RalA and RalB form a special branch within the Ras monomeric GTPase family and have been implicated in the modulation of a variety of cellular processes including exocytosis and endocytosis. The main goal of this project was to investigate the possible role of Ral GTPases in the control of insulin release from pancreatic beta cells.

Materials and Methods: The experiments were performed in the insulinsecreting cell lines INS1E and MIN6B1. Expression of Ral GTPases was measured by RT-PCR and Western Blotting analysis. Activation of Ral GTPases in living cells was assessed by Fluorescence Resonance Energy Transfer (FRET) using a construct including a YFP-labeled RalA and a CFPlabeled Ral effector. The role of Ral GTPases in insulin exocytosis was assessed by transiently overexpressing wild type or constitutively active mutants of RalA and RalB or by reducing the endogenous levels of each of these GTPases by RNA interference.

Results: We found that INS1E and MIN6 cells express both RalA and RalB. Incubation of these cells with $30 \mathrm{mM}$ potassium or $20 \mathrm{mM}$ glucose, 0.01 $\mathrm{mM}$ Forskolin and $0.1 \mathrm{mM}$ IBMX led to a rapid activation of Ral GTPases. This activation was prevented by the addition of the $\mathrm{Ca} 2+$ channel blocker nifedipin $(0.01 \mathrm{mM})$ suggesting that it is probably a consequence of the increase in cytoplasmic free $\mathrm{Ca} 2+$ concentration following depolarisation of beta cell membrane. Reduction of the endogenous level of RalA or RalB by RNA interference resulted in a significant decrease in secretagogue-induced insulin release, indicating that these small GTPases are required for beta cell exocytosis. However, excessive amounts of Ral GTPases were also deleterious for insulin secretion. In fact, overexpression of wild type or constitutively active mutants of Ral GTPases led to a reduction in insulin exocytosis. This suggests that the level of Ral GTPases has to be maintained in a narrow range to insure an optimal secretory response.

Conclusion: Ral GTPases are activated by insulin secretagogues and participate in the control of insulin exocytosis. Future experiments will need to elucidate the mechanisms leading to the activation of these GTPases and to precisely define their mode of action.

Supported by the Swiss National Foundation

\section{3}

Islet brain 1 is required for glucose-induced insulin secretion and is regulated by the cAMP signaling pathways

M. Ferdaoussi ${ }^{1}$, G. Niederhauser ${ }^{1}$, M. Cornu ${ }^{2}$, J.-Y. Yang ${ }^{3}$, C. Widmann ${ }^{3}$, B. Thorens ${ }^{2}$, G. Waeber ${ }^{1}$, A. Abderrahmani ${ }^{1}$;

${ }^{1}$ CHUV, DBCM, Lausanne, ${ }^{2} \mathrm{CIG}$, UNIL, Lausanne, ${ }^{3} \mathrm{DBCM}$, DP, Lausanne, Switzerland.

Background and Aims: The cAMP signalling pathways are involved in several physiological functions of pancreatic $\beta$-cells, including the control expression of genes, potentiation of nutrients-induced insulin secretion and the cellsurvival/apoptosis. Herein, we investigated whether Islet Brain 1 (IB1), a key modulator for the $\beta$-cell tasks, might be a target of cAMP signalling.

Materials and Methods: Expression of IB1 was monitored by real-time PCR, western Blotting and a luciferase gene reporter construct under the control of IB1 promoter. Silencing of IB1 expression was performed by using interference RNA strategy. The insulin levels were quantified by ELISA kit according to the manufacturer's protocol (SPI-BIO).

Results: Inspection for putative binding sites of transcription factors within human, rat and mouse promoter region of the gene encoding IB1, revealed the presence of a cAMP-response element (CRE) that is highly conserved among these species. Electromobility shift assays using nuclear extracts from the insulin-producing INS-1E cells demonstrated the ability of this element to bind CRE binding proteins. The implication of the cAMP pathways in the regulation of IB1 expression was then addressed. Real-time PCR and western blotting experiments performed with extracts of rat isolated islets, INS-1E or MIN6 cells cultured in the presence of cAMP-raising agents such as forskolin (F), IBMX (I) or GLP-1, showed a 2-3 fold rise in mRNA and protein levels of IB1. This induction reached a peak at $16 \mathrm{hrs}$ and declined at $48 \mathrm{hrs}$. Incubation of transiently transfected INS-1E cells also increased by 2 fold the luciferase activity of the $731 \mathrm{bp}$ sequence of the human IB1 promoter linked to a luciferase gene, indicating that I/F-mediated effects occur at transcriptional levels. The PKA inhibitor H89 prevented the augmentation of IB1 induced by cAMP-raising agents. Because cAMP signalling is involved in the potentiation of glucose-induced insulin-secretion, we evaluated the potential contribution of IB1 in this $\beta$-cell task. Inhibition of IB1 expression levels with the use of interference RNA strategy drastically decreased glucose-induced secretion. The introduction of an IB1-encoding plasmid that was resistant to siRNA restored the capacity of the cells to secrete in response to glucose.

Conclusion: The data indicates that the potent effects of cAMP/PKA pathways in the control of insulin secretion and cell survivals are in part mediated through induction of IB1.

Supported by FNS and Botnar Fondation

\section{4}

Expression of the synaptogenic adhesion molecules neurexin, neuroligin and SynCAM in islet $\beta$ cells

A. T. Suckow ${ }^{1}$, D. Comoletti ${ }^{2}$, M. Waldrop ${ }^{1}$, P. Taylor ${ }^{2}$, S. D. Chessler ${ }^{3}$;

${ }^{1}$ Medicine, ${ }^{2}$ Pharmacy, ${ }^{3}$ Endocrinology, University of California, San Diego, La Jolla, United States.

Background and Aims: The insulin secreting $\beta$-cells share many features with the neurons of the central nervous system. For example, $\beta$-cells express high levels of the major inhibitory neurotransmitter GABA and all of the machinery necessary for GABAergic signaling. $\beta$-cells also express many of the scaffolding and synaptic vesicle proteins important for neurotransmitter release, several of which have also been implicated as key components of the insulin secretory machinery. Recently, three families of neuron-specific proteins have been identified that seem to be key drivers of synaptogenesis in the central nervous system: the neurexins, the neuroligins and SynCam. The neurexins and neuroligins are cell-surface, synaptic adhesion molecules that interact with each other across the synaptic cleft, seeding the local assembly of the submembrane protein complexes that enable synaptic neurotransmission. Coculture experiments demonstrate that neuroligin-2 and a variant of neurexin- $1 \beta$ are important for inhibitory synapse formation. Since neuroligin-2 is expressed exclusively at inhibitory synapses in the brain and $\beta$-cells express all of the machinery necessary for GABA secretion, we hypothesized that neuroligin- 2 and its heterophilic binding partner neurexin are expressed in $\beta$-cells. It is also postulated that these molecules are important for the assembly of the insulin secretory apparatus since they can induce assembly of the machinery necessary for neurotransmitter release in neurons.

Materials and Methods: To determine whether neurexin, neuroligin and SynCAM family members are expressed in islets, a combination of RT-PCR, immunohistochemistry and western blot analysis were used. The effect that overexpression of neuroligin family members have on insulin secretion were assessed by transfecting each of the neuroligin family members into the rat $\beta$-cell line INS- 1 and by quantifying the levels of secreted insulin under basal glucose conditions using an insulin radioimmunoassay.

Results: Here, we demonstrate expression of all neurexin and neuroligin family members at both the RNA and protein levels. Neurexin-1 and neuroligin- 2 were detected exclusively in $\beta$-cells via immunohistochemistry. SynCAM was also detected in $\beta$-cells Additionally, overexpression of all neuroligin family members increased insulin secretion in INS-1 cells. When insulin secretion was compared in cells transfected with neuroligins relative to mock-transfected cells, $52 \%, 60 \%, 135 \%$ and $148 \%$ increases in insulin secretion were observed in NL-1, $-2,-3$ and -4 transfected cells.

Conclusion: The neurexins and their trans-synaptic binding partners, the neurexins, as well as SynCam are expressed on the $\beta$-cell surface. Neuroligin-neurexin interactions may play a role in the formation of the submembrane insulin sectretory apparatus analagous to their role in synaptic neurotransmission. These findings may have important implications for determination of how specific $\beta$-cell plasma membrane regions may be primed for insulin and GABA exocytosis.

Supported by the NSF Graduate Research Fellowship Programme 


\section{OP 35 Mechanisms of cardiovascular complications: animal models}

\section{5}

Reversible and irreversible changes in arterial gene expression after initiation of hyperglycaemia in pIns -MYCER mice in vivo

S. Zervou, S. Pelengaris, D. Epstein, M. Khan;

Biological Sciences, University of Warwick, Coventry, United Kingdom.

Background and Aims: Hyperglycaemia has long been a prime suspect for accelerated CVD in diabetes, yet intervention studies have been disappointing, with most convincing reduction in CVD risk achieved by targeting blood pressure or lipids rather than glucose. We must first expand knowledge regarding the pathogenic effects of hyperglycaemia (independent of other confounding atherogenic features of the metabolic syndrome) and the potential reversibility of such effects when glucose is normalised in vivo. In this study, we sought to investigate the immediate and early effects of hyperglycaemia, on gene and protein expression in the arteries of reversible 'diabetes-inducible' pIns-MYCER ${ }^{\text {TAM }}$ mice in vivo. Following which we examined to what extent the consequences of transient relatively brief exposure to hyperglycaemia in vivo were reversible following restoration of normal ambient glucose levels.

Materials and Methods: In pIns-MYCER ${ }^{\mathrm{TAM}}$ mice, c-Myc activation by administration of the specific-activating ligand, 4-hydroxytamoxifen (4OHT), induced rapid sub-total $\beta$-cell ablation by apoptosis resulting in an entirely predictable timecourse of initiation and progression of hyperglycaemia. Importantly, in these models deactivation of c-Myc results in recovery from diabetes by re-differentiation of surviving beta cells and by regeneration of new beta cells.

The effects of hyperglycaemia were investigated after a series of early and later time-points ( $\mathrm{n}=3$ for each). Blood glucose, LDL and HDL cholesterol were recorded for all mice. Mouse aortas were isolated and processed for extraction of either RNA, or for fixation and immunohistochemistry. Global mouse gene expression arrays (Affymetrix) were used to study immediate and early changes in gene expression in the aortic wall following the onset of hyperglycaemia in vivo. Microarray data were verified using real-time RTPCR with the Roche ${ }^{\otimes}$ Light Cycler. Changes in mRNA levels were detected, as the result of hyperglycaemia in the mouse artery. Immunohistochemistry was also performed to monitor altered protein expression for a group of cell adhesion molecules, as early indications of vascular effects due to hyperglycaemia.

Results: Several genes were found to increase with the onset of high glucose and decrease to initial levels when hyperglycaemia was corrected, including known-atherosclerosis related genes such as VCAM-1, MMP-9, MMP-2, $v W F, V E G F$ a, insulin-related IRS2 and IGF1, and others involved in cell turnover such as $p 16^{\text {Inkta }}$ and caspase 12, the hyperlipidaemia-related Apo AII, and those implicated in energy homeostasis such as leptin and adiponutrin. Surprisingly, given the transitory and brief period of hyperglycaemia, several genes elevated as a result of hyperglycaemia do not reverse when blood glucose is corrected, including those encoding receptors for LDL, Leptin and GLP1. We have confirmed some of these results at the protein level by means of immunohistochemistry of mouse aorta.

Conclusion: As anticipated, hyperglycaemia results in rapid alteration in gene expression in the aorta in vivo, some of which fail to reverse after normalisation of blood glucose levels. The obvious potential ramifications of these results to diabetes are that normal glycaemia should be achieved as rapidly and completely as possible; with this in mind our important findings are being examined at a functional level. Supported by the Wellcome Trust, UK
0206

Dietary fish-oil preserves cardiac contractile function by upregulation of UCP3 in a rat model of high-fat diet-induced glucose intolerance and cardiomyopathy

M. Ouwens ${ }^{1}$, C. E. van den Brom ${ }^{2}$, J. Kriek ${ }^{1}$, G. Schaart ${ }^{3}$, M. K. Hesselink ${ }^{3}$, P. Schrauwen ${ }^{3}$, R. Vlasblom ${ }^{2}$, M. Diamant ${ }^{4}$;

${ }^{1}$ Molecular Cell Biology, LUMC, Leiden, ${ }^{2}$ Laboratory for Physiology,

VUMC, Amsterdam, ${ }^{3}$ Human Biology, Maastricht University,

${ }^{4}$ Endocrinology/Diabetes Centre, VUMC, Amsterdam, The Netherlands.

Background \& Aims: Myocardial insulin resistance and lipotoxicity, leading to mitochondrial dysfunction and oxidative stress, have been implicated in the development of diabetic cardiomyopathy (DCM), a common complication in type 2 diabetes mellitus. Uncoupling protein 3 (UCP3) has been proposed to protect against lipid-induced mitochondrial damage, either by exporting excessive fatty acid anions from the mitochondrial matrix and/or by reducing mitochondrial ROS production and lipid peroxide formation, thus being an important protective mechanism in diabetes-related organ damage. We studied whether diet-induced alterations in myocardial insulin sensitivity and in vivo cardiac function in rats could be ascribed to changes in UCP3 protein expression.

Materials \& Methods: Male Wistar rats, fed isocaloric low-fat- (LF), high-fat(HF), or high-fat fish-oil-enriched (FISH) diets, underwent an oral glucose tolerance test (oGTT) at day 25, and in vivo echocardiography at day 27. Rats were sacrificed at day 28 for analysis of myocardial protein expression and phosphorylation.

Results: No significant differences in body weight gain, fasting plasma glucose and insulin levels were found among the diet-groups. However, compared to LF-diet fed rats, HF- and FISH-diet fed rats showed glucose intolerance in the oGTT $(P<0.05)$. Also insulin-mediated activation of the PKB/Akt-pathway was $40-50 \%$ lower in hearts from both HF- and FISHdiet fed rats, compared to LF-diet fed hearts $(P<0.05)$. Interestingly, cardiac contractile function, expressed as fractional shortening and ejection fraction, was similar in FISH- and LF-diet fed rats, but was decreased in HF-diet fed rats $(P<0.05$ versus FISH). Myocardial protein levels of UCP3 were 1.9-fold increased in FISH-diet fed rats compared to LF-diet fed rats $(P<0.01)$, but failed to rise significantly in HF-diet fed rats.

Conclusion: FISH-oil enriched diet induced myocardial UCP3 expression while preserving cardiac contractile function in rats. We propose that dietary FISH-oil prevents lipotoxic heart disease by upregulation of UCP3, rather than by improving myocardial insulin signalling, in a rat model of dietinduced glucose intolerance.

Supported by the Dutch Diabetes Research Foundation

\section{7}

Evaluation of the effects of fenofibrate and metformin in an experimental model of diabetic cardiomyopathy (DCM)

M. Beylot ${ }^{1,2}$, F. Forcheron ${ }^{1}$, A. Basset ${ }^{2}$, P. Abdallah ${ }^{3}$, P. Del Carmine ${ }^{3}$, C. Metais ${ }^{1}$, G. Bricca ${ }^{1}$;

${ }^{1}$ Faculte RTH Laennec, ERI 22, LYON, ${ }^{2}$ Faculté Rockefeller, ANIPHY, Lyon, ${ }^{3}$ Faculté RTH Laennec, INSERM U499, LYON, France.

Background and Aims: Diabetes can induce, independently of hypertension and myocardial ischemia, a specific diabetic cardiomyopathy (DCM) characterised by increased triglycerides (TG) stores and appearance of fibrosis in left ventricle (LV). The mechanisms leading to DCM remain to be precised and no specific treatment has been defined. Cardiac specific overexpression of ppar alpha results in a cardiopathy resembling DCM suggesting that ppar alpha agonists, such as fenofibrate used for the treatment of hpertriglyceridemic diatic patients, could have unfavorable effect on DCM. Therefore we evaluated the action of fenofibrate on an experimental model of DCM, the Zucker diabetic rat (ZDF). We evaluated also the effect of metformin, widely used in the treatment of type 2 diabetes.

Materials and Methods: ZDF $(n=35)$ and control (Lean, $L, n=15)$ rats, all receiving a high fat diet, were studied at 7, 14 and 21 weeks. After the initial study at 7 weeks, $10 \mathrm{ZDF}$ received no treatment, 10 received metformin and 10 fenofibrate (oral administrations) until final studies at 14 or 21 weeks. $\mathrm{L}$ rats received no treatment. Each study comprised measurements of plasma glucose, TG, insulin levels, energy intake and expenditure (indirect calorimetry) and (for 5 rats of each group at 7,14 and 21 weeks) sampling 
of heart (measurement of TG content and relevant mRNA concentrations, histology).

Results: ZDF rats were, compared to L, obese, hypertriglyceridemic and insulin-resistant at 7 weeks, type 2 diabetic at 14 weeks and diabetic with insulin deficiency at 21 weeks. Plasma TAG levels were always high at 14 and 21 weeks $(\mathrm{p}<0.001)$. ZDF had, at 7 weeks, an increase in LV weight $(\mathrm{p}<0.05)$ and TG content $(1.0 \pm 0.1$ vs. $0.2 \pm 0.1 \mu$ moles/g $\mathrm{p}<0.01)$ with some fibrosis. At 14 and 21 weeks, $L V$ fibrosis and TG content $(8.0 \pm 1.7$ and $3.7 \pm 0.6$ $\mu$ moles/g respectively, $\mathrm{p}<0.05$ vs $\mathrm{L}$ ) increased in ZDF rats. Fenofibrate and metformin did not change significantly the evolution of body weight nor energy intake, energy expenditure, evolution of diabetes. Plasma TG were decreased by metformin at $14(\mathrm{p}<0.01$ vs. untreated ZDF) and $21(\mathrm{p}<0.05)$ weeks, but only at 21 weeks $(\mathrm{p}<0.05)$ by fenofibrate. LV TG content was decreased by metformin at $14(1.2 \pm 0.2 \mu$ moles/g, $\mathrm{p}<0.01$ vs untreated ZDF) and $21(0.6 \pm 0.1 \mathrm{p}<0.01)$ weeks and by fenofibrate at 14 weeks only $(2.4+0.3$ $\mathrm{p}<0.01$ ). Fibrosis was reduced by both compounds at 14 and 21 weeks. FAS, ACC1, LCAD, FAT, LPL mRNA were comparable in ZDF and L rats and were not modified by either compounds. Fenofibrate increased Acyl-CoA oxidase mRNA at 14 weeks $(\mathrm{p}<0.05)$ while metformin decreased Acyl-CoA synthase mRNA ( $<<0.01$ at 14 and 21 weeks). Surprisingly the receptors of adiponectin, AdipoR1 and R2, mRNA were increased by metformin at 14 and 21 weeks $(\mathrm{p}<0.01)$

Conclusion: In this model fenofibrate had no adverse effect on DCM but on the contrary rather favorable actions. Metformin had a clear beneficial effect in reversing both fibrosis and TG accumulation. This data suggests that both compounds could be useful for the prevention and treatment of DCM in humans.

Supported by the Fondation de France

\section{8}

UCP blockade decreases disturbances of contractive reactions of heart and vessels with type 1 experimental diabetes mellitus

O. D. Prysyazhna, V. F. Sagach;

Blood circulation, Bogomolets Institute of Physiology, Kyiv, Ukraine.

Background and Aims: Disorders of mitochondrial function play a significant role in the development of complications of diabetes mellitus according to modern conception. The increased expression of uncoupling proteins (UCP) is one of the most common problems of mitochondrial functioning. An increase of UCP expression leads to the proton leak growth and the reduction of the effectiveness of the mitochondrial respiratory chain work. Such changes lead to reduction of ATP synthesis and increase of the oxidative stress. The purpose of this work is to investigate the influence of inhibitor UCP genipin introduction on complications of diabetes mellitus such as contractive reaction of myocardium and vessels in rats with streptozotocininduced diabetes mellitus.

Materials and Methods: Streptozotocin $50 \mathrm{mg} / \mathrm{kg}$ was intraperitoneally injected to male rats of the weight $180-220 \mathrm{~g}$ to reproduce experimental model of diabetes mellitus I type. Glucose level control was carried out with glucometer Medisens. The level of blood glucose in diabetic rats was $17,5 \pm 0,9 \mathrm{M} / \mathrm{l}$ and in control rats $-6,4 \pm 0,5 \mathrm{M} / 1$. Endothelium-dependent reactions of aorta and coronary vessels, left ventricular diastolic stiffness and force-loading dependence (Franc-Starling curve) in rats with experimental diabetes mellitus after the single intraperitoneal introduction $10 \mathrm{mg} / \mathrm{kg}$ of UCP blocker genipin were studied.

Results: Experimental diabetes mellitus leads to appearance of endothelial dysfunction that is revealed by the essential disturbance of endotheliumdependent reactions of both the aorta and the coronary vessels. In addition, the essential increase of left ventricular diastolic stiffness (considered as end-diastolic pressure rise/load increase) and reduction of Franc-Starling curve phase plateau extension were found at diabetes. The genipin injection, that was done one-hour before the beginning of the experiment, brought restoration of disrupted reactions. Furthermore, dramatical decrease of myocardial oxygen consumption was found after the genipin injection.

Conclusion: This data points to the role of UCP expression growth in the appearance of cardio-vascular complications of type I diabetes mellitus. Thus, the application of UCP blockers is the forward-looking method of angioprotection and curing the vascular complications of diabetes mellitus.

\section{9}

Blockade of angiotensin type 2 receptors decreases diabetes-associated atherosclerosis

A. Koitka, P. Koh, K. Jandeleit-Dahm, M. E. Cooper, T. J. Allen; Diabetes Complications Laboratory, Baker Heart Research Institute, Melbourne, Australia.

Background: Activation of the renin angiotensin system has been described in various pathological conditions, including diabetic complications such as atherosclerosis. Angiotensin II acts through two receptors, type 1 angiotensin (AT1) and type 2 (AT2) receptors. Most of the known actions of angiotensin II (Ang II), including vasoconstriction and fibrosis, are considered to be due to AT1 receptor activation. Recent data suggest that the AT2 receptor subtype participates in the regulation of cell growth and inflammation. Therefore, the aim of this study was to investigate the effect of chronic AT2 receptor subtype blockade (AT2RB) by PD123319 in an in vivo model of diabetes-associated atherosclerosis.

Methods: Control and streptozotocin-induced diabetic apolipoprotein E (ApoE)-deficient mice received PD123319 (5 mg. kg-1.day-1 via Alzet osmotic minipump) or no treatment (buffer alone) for 20 weeks ( $\mathrm{n}=8 /$ group). Aortic plaque deposition was assessed by Sudan IV staining and subsequent en face quantification. Markers of inflammation were measured using realtime RT-PCR and immunohistochemistry.

Results: Diabetes was associated with a greater than five-fold increase in plaque area (Table1) as well as increased complexity in the aorta in association with a significant increase in aortic AT2 receptor expression $(1.2+/-0.1$ in control mice vs. $6.7+/-0.3$ in diabetic mice, $\mathrm{p}<0.01)$, collagen content, macrophage and alpha-smooth muscle actin-positive cell infiltration, as well as increased monocyte chemoattractant protein-1 (MCP1) and vascular cell adhesion molecule-1 (VCAM-1) expression. The increase in plaque area with diabetes was attenuated by approximately $50 \%$ with the AT2RB in diabetic mice but not in control mice. The benefits of the AT2RB were seen at all three sites of the aorta (Table1). This occurred despite similar glycated haemoglobin $(16.3+/-1.4 \%$ in diabetic mice vs. $16.9+/-1.2 \%$ in AT2RB-treated diabetic mice) and similar blood pressure $(135+/-10 \mathrm{mmHg}$ in diabetic mice vs. $129+/-5 \mathrm{mmHg}$ in AT2RB-treated diabetic mice). Mean +/- SEM shown, ${ }^{*} \mathrm{p}<0.01$ vs. control; $\ddagger \mathrm{p}<0.05, \dagger \mathrm{p}<0.01$ vs. diabetic.

Conclusions: Diabetes-associated atherosclerosis is ameliorated by AT2 receptor blockade, independent of glucose or blood pressure. This suggests that the atherosclerotic effects of Ang II are at least partly mediated via the AT2 receptor subtype. The specific role of the AT2 receptor in diabetes-associated atherosclerosis remains to be confirmed using a non-pharmacological strategy such as similarly designed experiments in recently generated diabetic ApoE/AT2 double-knockout mice.

Quantification of plaque area (\%)

\begin{tabular}{lllll}
\hline Group & Total & Arch & Thoracic & Abdominal \\
\hline Control mice & $2.2+/-0.3$ & $5.3+/-1.0$ & $0.8+/-0.1$ & $2.2+/-0.4$ \\
$\begin{array}{l}\text { AT2RB-treated } \\
\text { control mice }\end{array}$ & $2.9+/-0.3$ & $6.2+/-1.5$ & $1.6+/-0.2$ & $2.3+/-0.5$ \\
$\begin{array}{l}\text { Diabetic mice } \\
\text { AT2RB-treated }\end{array}$ & $14.4+/-2.1^{*}$ & $32.3+/-2.7^{*}$ & $11.0+/-2.8^{*} 8.8+/-1.8^{*}$ \\
diabetic mice & $7.1+/-0.5 \dagger$ & $13.3+/-2.3 \dagger$ & $5.0+/-0.9 \neq$ & $5.2+/-$ \\
\hline & & & & $0.9 \ddagger$ \\
\hline
\end{tabular}

Supported by the Juvenile Diabetes Research Foundation and ALFEDIAM

\section{0}

Coronary and aortic vascular pathology in a diabetic porcine model M. van den Heuvel ${ }^{1}$, W. Kerver ${ }^{1}$, S. Kazim ${ }^{1}$, O. Sorop ${ }^{1}$, R. Dekker², S. J. Koopmans' ${ }^{2}$ D. J. Duncker ${ }^{1}$, W. J. van der Giessen ${ }^{1}$,

H. M. M. van Beusekom ${ }^{1}$;

${ }^{1}$ Experimental Cardiology, Erasmus Medical Center, Rotterdam, ${ }^{2}$ Animal Sciences Group, University of Wageningen, Lelystad, The Netherlands.

Background and Aims: Cardiovascular disease is a major cause of morbidity and mortality in patients with diabetes mellitus (DM). They are prone to diffuse and accelerated forms of atherosclerosis. In coronary artery disease, this increases the likelihood of revascularization therapy, even with use of 
drug eluting stents (DES). While DES effectively reduce in-stent neointimal formation, DM still remains an independent risk factor for restenosis possibly because of exacerbated endothelial dysfunction.

We used a porcine model of streptozotocin induced DM to examine the vascular healing response to coronary DES implantation. In addition we studied the effects of diet and commonly used oral anti-diabetic combination therapy on early aortic plaque formation.

Materials and Methods: DM was induced by a slow and titrated infusion of streptozotocin $( \pm 130 \mathrm{mg} / \mathrm{kg})$ to give subtotal pancreatic beta-cell destruction. DM was allowed to settle and stabilize for a few weeks before the experiments started.

1) Paclitaxel eluting stents (PES) were implanted in coronary arteries under guidance of quantitative coronary angiography. PES were placed in both control pigs $(\mathrm{C}, \mathrm{n}=5)$ and diabetic pigs $(\mathrm{D}, \mathrm{n}=5)$ for 5 weeks to study maximal intimal thickening in response to stenting.

2) For aortic plaque formation, both DM (D) and control (C) pigs were fed a cafetaria diet (saturated fat, cholesterol and sucrose rich, $C D, n=8$ ) or a healthy diet (unsaturated fat, carbohydrate rich, $\mathrm{HD}, \mathrm{n}=7$ ) for 8 weeks. One group of DM pigs on CD was treated with oral anti-diabetics (metformin 1g/ day + pioglitazone $20 \mathrm{mg} /$ day, $\mathrm{TD}, \mathrm{n}=8$ ). Histology, immunohistochemistry and Sudan IV fat staining were performed. Morphometry and planimetry were analysed with a computerised system.

Results: Postprandial (3 h) glucose levels were significantly increased in DM pigs in both experiments (D vs C: $24 \pm 11$ vs. $6 \pm 1 \mathrm{mmol} / \mathrm{l}, \mathrm{p}<0.01$; $D$-CD vs. C-CD: $26 \pm 15$ vs. $7 \pm 1 \mathrm{mmol} / \mathrm{l}, \mathrm{p}<0.05)$. Histology and immunocytochemistry showed no clear Langerhans islets in diabetic pancreas sections, only single beta cells, with an $88 \%$ reduced insulin staining $(p<0.01)$. Therefore, a poorly controlled disease state of DM was created. Liver sections of diabetics on CD showed a marked steatosis (D-CD vs. C-HD: $23 \pm 10$ vs. $0 \pm 0 \%, \mathrm{p}<0.01$ ), a DM type 2 feature. 1) Five weeks after PES placement, an increased neointimal response was found in DM pigs ( $D$ vs C: $2.9 \pm 2.9$ vs. $1.7 \pm 0.3 \mathrm{~mm}^{2}, \mathrm{p}=0.05$ ). Interestingly, the aortic endothelium in diabetics showed an increased percentage of active von Willebrand factor (vWf) (D vs. C: $40 \pm 18$ vs. $22 \pm 8 \%$, $\mathrm{p}<0.03)$. 2) A CD for 8 weeks induced aortic fatty streak formation in diabetics leading to an increased percentage of early plaque area as compared to both controls on HD and diabetics on HD (D-CD vs. C-HD/D-HD: $16 \pm 15 \%$ vs. $0.1 \pm 0.1 \%, p=0.03)$. Anti-diabetic combination therapy did not affect post-prandial $(3 \mathrm{~h})$ glucose levels, the percentage of liver steatosis or aortic fatty streak formation (D-TD vs. D-CD: $23 \pm 17$ vs. $26 \pm 15 \mathrm{mmol} / \mathrm{l} ; 12 \pm 11 \mathrm{vs}$. $23 \pm 10 \% ; 7 \pm 12$ vs. $16 \pm 15 \%)$.

Conclusion: This DM porcine model shows 1) an increased neointimal response to injury (PES) 2) accelerated diet-induced liver steatosis and atherogenesis, which are unresponsive to anti-diabetic combination therapy. A specific feature, endothelial dysfunction with a prothrombotic status (increased vWf), is likely to play a role in the cardiovascular pathology of DM.

\section{OP 36 Nephropathy: treatment}

\section{1}

Study on effects of polysaccharides extracted from the fruits of Lycium barbarum on reducing blood glucose and preventing diabetic nephropathy

G. Sun ${ }^{1}$, Y. Zhang 1 , S. Wang ${ }^{1}$, J. Huang ${ }^{1}$, L. Zhang ${ }^{2}$, Z. Sun ${ }^{2}$, P. Huang ${ }^{3}$, J. Schrezenmeir";

${ }^{1}$ Nutrition and Food Hygiene, Southeast University, School of Public Health, Nanjing, China, ${ }^{2}$ Nutrition and Food Hygiene, Southeast University, School of Clinical Medicine, Nanjing, China, ${ }^{3}$ Nutrition and Food Hygiene, Southeast University, School of Basic Medical Sciences, Nanjing, China, ${ }^{4}$ Nutrition, The Federal Research Centre for Nutrition and Food, Institute for Physiology and Biochemistry of Nutrition, Kiel, Germany.

Background and Aims: Lycium barbarum polysaccharides (LBP) extracted from Lycium barbarum has been reported to decrease the blood glucose level and improve the insulin sensitivity of the diabetic model animals. However there is no report concerning the diabetic complications. The aims of the present study are to attempt to clarify whether LBP can prevent the occurrence or reduce the progression of diabetic nephropathy.

Materials and Methods: Diabetes was induced by the injection of small dose streptozotocin (STZ) combined with high-fat feeding consisting of $10 \%$ sucrose, $10 \%$ lard, $10 \%$ plant oil and $5 \%$ casein. 32 diabetic rats were randomly allocated to four groups: diabetic control, LBP low dose $(20 \mathrm{mg} / \mathrm{kg} /$ d), LBP medium dose $(40 \mathrm{mg} / \mathrm{kg} / \mathrm{d})$, and LBP high dose $(80 \mathrm{mg} / \mathrm{kg} / \mathrm{d})$ group. The experiment lasted 30 days. The levels of glucose, lipids, insulin, advanced glycosylation endproducts (AGEs), creatinine, superoxide dismutase (SOD) and malondialdehyde (MDA) in serum and creatinine in urine, hemoglobin$A_{1 c}\left(\mathrm{HbA}_{1 c}\right)$, and interleukin-8 (IL-8) expression of renal cortex were determined.

Results: At the end of the experiment the fasting blood glucose of rats in diabetic control group, LBP low, medium, and high dose groups were $23.86 \pm 4.62,8.22 \pm 1.63,6.85 \pm 2.13$, and $7.14 \pm 1.05 \mathrm{mmol} / \mathrm{l}$ respectively and the differences between diabetic control group and three LBP treated groups were significant $(p<0.05)$. LBP increased the level of insulin in serum and the insulin sensitivity index (ISI) of the diabetic rats. Correspondinly islet cell mass was higher in the LBP group compared to the model control. The mean AGEs level in serum of LBP high dose group rats was the lowest among all groups which was $2.73 \pm 0.84 \mu \mathrm{g} / \mathrm{ml}$, and that of diabetic control group was the highest $(5.22 \pm 0.80 \mu \mathrm{g} / \mathrm{ml})$. The $\mathrm{HbA}_{1 \mathrm{c}}$-values of the three LBP treated groups $(6.3 \pm 1.3 \%, 5.8 \pm 1.1 \%$ and $5.2 \pm 0.9)$ were lower than $(p<0.05)$ that of the diabetic control group $(7.7 \pm 1.3 \%)$. LBP decreased the 24 hours urine volumes and creatinine levels in serum and increased the endogenous creatinine clearance of diabetic rats. The histopathological examination of their kidneys confirmed lower progression of nephropathy in the LBP groups. LBP decreased the IL-8 mRNA expression and all the LBP treated groups had significant difference comparing to the diabetic control group $(p<0.05)$. The levels of SOD and MDA in serum of LBP high dose group were $226.5 \pm 12.7$ $\mathrm{NU} / \mathrm{ml}$ and $8.5 \pm 0.8 \mathrm{nmol} / \mathrm{ml}$ which were significantly different from that of diabetic control group $(180.6 \pm 37.6 \mathrm{NU} / \mathrm{ml}$ and $11.7 \pm 3.9 \mathrm{nmol} / \mathrm{ml})$. However LBP had no effect on the lipids in serum. LBP had protecting effects on the islets particularly the $\beta$-cells.

Conclusion: LBP decreased the blood glucose level, improved the insulin sensitivity, ameliorated the kidney function and histopathology in diabetic rats. The mechanism might include reducing glycemia, oxidative stress, formation of AGEs, IL-8 expression in the kidney, and promoting regeneration of the pancreas islets. LBP may be a candidate for treating diabetes and preventing the development of diabetic nephropathy.

Supported by Nanjing Scientific and Technological Bureau and DAAD 


\section{2}

Heat shock protein expression in early diabetic nephropathy F. Barutta ${ }^{1}$, S. Pinach ${ }^{1}$, J. Forbes ${ }^{2}$, R. Chiarle ${ }^{3}$, S. Giunti ${ }^{1}$, M. Arnstein ${ }^{2}$, P. Cavallo Perin ${ }^{1}$, G. Camussi ${ }^{1}$, M. E. Cooper ${ }^{2}$, G. Gruden ${ }^{1}$;

${ }^{1}$ Department of Internal Medicine, University of Turin, Italy, ${ }^{2}$ Diabetes and Metabolism Division, Baker Heart Research Institute, Melbourne, Australia, ${ }^{3}$ Department of Biomedical Sciences and Human Oncology, University of Turin, Italy.

Background and Aims: Heat Shock Proteins (Hsp), a highly evolutionary conserved family of intracellular proteins, are induced by cellular stresses and play an important role in cytoprotection. In diabetic nephropathy the metabolic and the haemodynamic insults result in osmotic, oxidative, and haemodynamic stress on target cells, thus representing potential Hsp inducers. Furthermore, the phosphorylated form of Hsp25 (P-Hsp25) plays a key role in cytoskeleton remodelling and has been implicated in the pathogenesis of proteinuric conditions. Our aim was to study both Hsp expression and distribution in the diabetic kidney in early experimental diabetes.

Materials and Methods: Diabetes was induced in eight-week-old male Sprague-Dawley rats by tail-vein injection of streptozotocin at a dose of $50 \mathrm{mg} / \mathrm{kg}$ in citrate buffer, following an overnight fast. Control rats were injected with citrate buffer alone. Diabetic $(\mathrm{DM} n=6)$ and control (ND $n=6)$ animals were euthanised 4 weeks following induction of diabetes; kidneys were removed, fixed in $10 \%$ formalin, and embedded in paraffin. Hsp25, PHsp25, Hsp60, and Hsp70 expression was studied by immunohistochemistry on $4 \mu \mathrm{m}$ kidney sections. Briefly, sections were dewaxed and rehydrated, and antigens demasked by boiling in citrate buffer. After quenching for endogenous peroxidase activity and blocking of both endogenous avidinbinding activity and non-specific binding sites, sections were incubated with either specific anti-Hsp or nonimmune isotypic control antibodies for 1 hour at room temperature. The signal was detected using the LSAB+ system-HRP and quantification of the percentage area of staining/number of positive cells was performed in 11 randomly selected renal tubulointerstitial fields and 20 glomeruli for each section in a blinded manner by two independent observers. To identify Hsp-positive cells within the glomeruli, sections were double stained by immunofluorescence with specific glomerular cell markers.

Results: In control animals there was a progressive increase in Hsp25, Hsp60, and Hsp70 expression along the corticopapillary axis. In the medulla Hsp25, $\mathrm{Hsp} 60$, and Hsp70 expression was significantly greater in diabetic rats than in control animals, particularly in the outer medulla (Hsp60: DM 36.07 \pm 0.10 vs. ND 4.7 $\pm 1.73, \mathrm{p}<0.01$; Hsp70: DM $36.2 \pm 3.24$ vs. ND $24.1 \pm 1.46, \mathrm{p}<0.05$; Hsp25: DM $22.23 \pm 2.55$ vs. ND 9.97 $\pm 5.12, \mathrm{p}<0.01)$. Within the glomeruli, cells staining positively for Hsp60 and Hsp70 were almost absent in control animals, while 1 or $2 \mathrm{Hsp} 70$-positive cells per glomerulus were found in the diabetic rats $(\mathrm{P}<0.001)$. There was no significant difference in glomerular Hsp25 expression between diabetic and control rats; whereas P-Hsp25 expression was significantly greater in the diabetic rats (DM $10.3 \pm 0.5$ vs. ND $3.9 \pm 0.3, \mathrm{p}<0.001)$. Furthermore, double staining showed that podocytes were the predominant glomerular cell type overexpressing P-Hsp25.

Conclusion: In early experimental diabetes there is an overexpression of Hsp25, Hsp60, and Hsp70 in the medulla, possibly due to osmotic stress. The overexpression of P-Hsp25 in the diabetic glomeruli may be implicated in podocyte functional and structural abnormalities.

Supported by Compagnia San Paolo

\section{3}

Effect of all-trans retinoic acid on treatment of type 2 diabetes

C. Kim ${ }^{1}$, J. Kang ${ }^{1}$, S. Lee ${ }^{1}$, S. Ihm ${ }^{1}$, H. Yoo ${ }^{1}$, H. Jung' 2 J. Nam², C. Ahn²; ${ }^{1}$ Internal Medicine, Hallym University Sacred Heart Hospital, Anyang-si, Gyeonggi-do, ${ }^{2}$ Internal Medicine, Yonsei University College of Medicine, Seoul, Republic of Korea.

Background and Aims: Diabetic nephropathy is the most common cause of end-stage renal disease, and with increasing number of diabetes patients and old people, over $40 \%$ of ESRD patients have this disease. Hyperglycemia, advanced glycation end-product, increased polyol pathway, oxidative stress, activation of transforming growth factor- $\beta 1$ (TGF- $\beta 1$ ) are interrelated in the pathogenesis of diabetic nephropathy. All-trans retinoic acid (ATRA) is reported to suppress interstitial proliferation and glomerular inflammation, and prevent renal damage in diabetic rats. Moreover, retinoic acid is reported to block lipid peroxidation in streptozocin-induced diabetic rats, and other studies also support the protective effect of retinoic acid in progression of type 2 diabetic nephropathy. Therefore, in this study, we examined the effect of ATRA in improving diabetic nephropathy by measuring the amount of urine albumin excretion (UAE) after administrating ATRA to Otsuka LongEvans Tokushima Fatty (OLETF) rat. And in rat mesangial cell (RMC), we administered ATRA to see its inhibitory action on production of TGF- $\beta 1$. Moreover, we examined changes in protein kinase $\mathrm{C}(\mathrm{PKC})$ and reactive oxidative stress (ROS) after the administration of ATRA.

Materials and Methods: We orally administered $10 \mathrm{mg} / \mathrm{kg}$ body weight ATRA to 20 OLETF rats for 16 weeks. And, we administered vehicle to 20 OLETF rats and 10 Long-Evans-Tokushima-Otsuka (LETO) rats for the same period. At 44 weeks of age albumin excreted into the urine was measured by an immunoturbidmetric method. After incubation of quiescent RMC with $10^{-8}, 10^{-7}, 10^{-6}, 10^{-5} \mathrm{M}$ ATRA for the given period $(6,24,48 \mathrm{hrs})$ in a media containing 30 or $5 \mathrm{mM}$ glucose. We measured the concentration of TGF- $\beta 1$ with the quantitative sandwich enzyme immunoassay technique. PKC assay were performed using SDS-PAGE and immunoblotted with an anti-PKC isoforms. And, we examined changes in DCF-sensitive reactive oxidative stress (ROS) after the administration of ATRA.

Results: Sixteen weeks after the administration of ATRA, daily UAE of the ATRA-treated OLETF rats was lower than that of non-treated OLETF rats ( $0.07 \pm 0.03$ vs. $0.17 \pm 0.15 \mathrm{mg} / \mathrm{mgCr}, P=0.008)$. Also, a decrease of fasting glucose in serum was observed in ATRA-treated OLETF rats when compared with non-treated OLETF rats $(133.1 \pm 16.2$ vs. $148.5 \pm 21.3 \mathrm{mg} / \mathrm{dL}, P=0.014)$. ATRA-treated OLETF rats showed decreased total cholesterol, triglyceride, and LDL-cholesterol levels compared with not-treated OLETF rats. In RMC, treatment with ATRA showed dose and time-dependent decreases in TGF- $\beta 1$ levels, PKC activity and DCF-sensitive cellular ROS.

Conclusion: In this study, we demonstrated that the administration of ATRA resulted in amelioration of UAE, glucose, and lipid profile in OLETF rats. Moreover, ATRA treatment suppressed TGF- $\beta 1$ synthesis induced by high glucose stimulation. Also high glucose-induced ROS and PKC increments in RMCs are effectively suppressed by ATRA. It is of interest that ATRA treatment suppressed UAE and TGF- $\beta 1$ synthesis, which was mediated by significant reduction of $\mathrm{PKC}$ activity and ROS production. Our results suggest that ATRA has a potentially therapeutic role for diabetic nephropathy.

\section{4}

Edaravone protects from endothelial barrier injuries under diabetic condition

K. Sarai, Y. Shikata, K. Shikata, K. Ohmori, N. Watanabe, J. Wada, H. Makino;

Department of Medicine and Clinical Science,, Okayama University Graduate School of Medicine, Okayama, Japan.

Background and Aims: The primary pathway involved in enhanced endothelial barrier permeability is paracellular transport of fluid and macromolecules through intercellular gaps following the disruption of intercellular adherens junction and focal adhesion complexes. An increase in vascular permeability is a fundamental feature of inflammation and essential component of macrophage migration. Edaravone is a potent scavenger of hydroxyl radicals. It also improves in endothelial-dependent vasodilation in smokers, suggesting the protective effect on endothelial cell monolayers. These observations suggest the protective effect of edaravone on systemic vascular endothelial disorders including diabetes mellitus. We have reported the pivotal role of macrophages in the progression of diabetic nephropathy, as a major consequent of vascular disorder. To elucidate the effectiveness of edaravone against the progression of diabetic nephropathy, we investigated the protective effects of edaravone on endothelial barrier dysfunction in diabetes mellitus using experimental diabetic rats and cultured glomerular endothelial cells.

Materials and Methods: 1) Glomerular endothelial cells (GE cells) were preincubated with hyperglycemic $(30 \mathrm{mM})$ media for $48 \mathrm{~h}$ and then treated with $9 \mathrm{mM}$ edaravone for $30 \mathrm{~min}$ before co-culture with THP-1 cells. To monitor the effects of edaravone on adhesion of THP-1 cells on GE cells, numbers of CD34 positive cells were counted using immunfluorescent microscopy. 2) To investigate the renoprotective effect of edaravone in experimental diabetic rats, we induced diabetes in 6 week-old male Sprague-Dawley (SD) rats by intravenous injection with streptozotocin followed by intraperitoneal injection with edaravone $(3 \mathrm{mg} / \mathrm{Kg})$ for 10 weeks. Blood glucose, urinary creatinine and urinary albumin excretion $(24 \mathrm{~h})$ were monitored. We also 
evaluated the histological changes, infiltration of macrophages (ED1- positive cells), and expression of TGF-b and type IV collagen in renal glomeruli using harvested kidneys.

Results: 1) Immunfluorescent microscopy and co-culture study revealed that stress fiber formation and intercellular gap were observed and the number of THP-1 cells on a GE cellmonolayer was increased under the high glucose condition. Furthermore, THP-1 cells were chiefly observed at cell-cell contact area of GE cells, suggesting the migration process out of vascular lumen in vivo. On the other hand, edaravone induced cortical actin ring enhancement instead of stress fiber and intracellular gap formation in GE cells and reduced the number of THP-1 cells significantly. 2) Edaravone improved glomerular hyperfiltration and reduced urinary albumin excretion in experimental diabetic rats. Immunohistochemical studies revealed that the number of ED1 positive cells and expression of type IV collagen in glomeruli were significantly decreased by edaravone.

Conclusion: In the present study, edaravone revealed the protective effects against renal injuries in diabetic rats. Morphological study using co-culture system of both GE cells and THP-1 cells suggested that the endothelial barrier enhancement in a GE cell monolayer resulting in the inhibition of macrophage migration into gromeruli might be essential for edaravone to exert a renoprotective effect in diabetic rats. Edaravone may be beneficial for diabetic vascular complications via novel protective effects on endothelial barrier functions.

Supported in part by a Grant-in-Aid for Scientific Research from the Ministry of Education, Science, Culture, Sports, and Technology of Japan

\section{5}

Continuous subcutaneous insulin infusion is more effective than multiple daily insulin injection in preventing albumin excretion rate increase in type 1 diabetic patients

G. Lepore ${ }^{1}$, A. R. Dodesini ${ }^{1}$, D. Bruttomesso ${ }^{2}$, S. Costa ${ }^{2}$, M. Bonomo ${ }^{3}$, E. Meneghini ${ }^{3}$, A. Corsi ${ }^{1}$, I. Nosari ${ }^{1}$, R. Trevisan ${ }^{1}$;

${ }^{1}$ Diabetes Unit, A.O.Ospedali Riuniti, Bergamo, ${ }^{2}$ Unit for Metabolic Diseases, University of Padua, ${ }^{3}$ Unit of Diabetology, A.O.Niguarda Cà Granda, Milan, Italy.

Background: Although the DCCT trial has demonstrated the importance of intensive management of diabetes in reducing microalbuminuria in patients with type 1 diabetes, there are still little data as to whether continuous subcutaneous insulin infusion (CSII) may provide greater benefit than multiple daily insulin injection (MDI). Recent meta-analysis also indicates that CSII provides a glycemic control slightly superior to MDI.

Aims: To compare the effect of CSII vs MDI on albumin excretion rate in a large group of type 1 diabetic patients.

Material and Methods: Design: a 3-year prospective observational followup study. Patients: 110 type 1 diabetic patients treated with CSII $(52 \mathrm{M} / 58 \mathrm{~F}$, age $40 \pm 10$ yrs, diabetes duration $19 \pm 9$ years, $\mathrm{HbA}_{1 \mathrm{c}}$ at entry $8.8 \pm 1.5 \%$ ) were compared with 110 patients treated with MDI matched at baseline for age, sex, diabetes duration and $\mathrm{HbA}_{1 \mathrm{c}}(52 \mathrm{M} / 58 \mathrm{~F}$, age $41 \pm 13$ yrs, diabetes duration $20 \pm 11 \mathrm{yrs}, \mathrm{HbA}_{1 \mathrm{c}} 8.9 \pm 1.4 \%$ ). At entry in each group 90 patients had normal albumin excretion rate and 20 had persistent microalbuminuria. All of them were treated with maximal tolerated dose of ACE-inhibitors.

Setting: Diabetes Unit - Hospital of Bergamo, Unit for Metabolic Diseases - University of Padua and Unit of Diabetology - Niguarda Hospital of Milan , Italy.

Main outcomes measurements: albumin excretion rate (AER), individual measures of GFR (estimated by MDRD formula), $\mathrm{HbA}_{1 \mathrm{c}}$ and $\mathrm{BP}$ were assessed every four months. Data is shown as mean $\pm \mathrm{SD}$, otherwise stated.

Results: In CSII group $\mathrm{HbA}_{1 c}$ was lower than in MDI group after 1 year (8.1 \pm 0.9 vs. $8.6 \pm 1.2 \% ; p<0.005)$ and after three years of follow-up $(8.1 \pm 0.9$ vs. $8.4 \pm 1.3 \%$; $p<0.005)$. Blood pressure and estimated GFR were similar in the two groups during the whole study period. AER [median (range)], similar at baseline [6.0 $(1-165) \mu \mathrm{g} / \mathrm{min}$ in CSII group vs. $4.6(1-111)$ in MDI group, NS, did not change significantly in both groups at 1 year. On the contrary AER was significantly lower in the patients treated with CSII both at the second year $(p<0.05)$ and at the third year follow-up [4.8 $(1-109) \mu \mathrm{g} / \mathrm{min}$ vs $6.4(1$ $-178), p<0.001]$. This significant difference between CSII and MDI groups was observed even when normo- and micro-albuminuric patients were analysed separately. Of note in microalbuminuric patients, despite $\mathrm{HBA}_{1 \mathrm{c}}$ in CSII group was similar (during the follow-up) to that of MDI group ( $8.6 \pm 1.2$ vs. $8.9 \pm 1.7 \%$, NS), AER decreased from $38(21-165) \mu \mathrm{g} / \mathrm{min}$ at entry to $19(3-109)(p<0.01)$ in CSII group, while it increased from $35(20$ - 111) to $42(4-178)$ in MDI group. In particular 9 patients out of 20 regressed to normoalbuminuria in CSII group, whereas only 2 out of 20 regressed to normoalbuminuria in MDI group. Conclusion: This data suggests that CSII therapy may be useful in decreasing the progressive increase in albumin excretion rate in type 1 diabetic patients. Since the decrease in $\mathrm{HbA}_{1 c}$ in CSII group was small when compared to that observed patients treated with MDI, the suggestion is that a lower glucose variability may be the reason for the difference in AER between the two insulin treatments.

\section{6}

Effects of long-term fenofibrate treatment on markers of kidney function in type 2 diabetes: FIELD Helsinki substudy

A. Hiukka ${ }^{1}$, E. Leinonen ${ }^{1}$, C. Forsblom ${ }^{2,3}$, P.-H. Groop ${ }^{2,3}$, M.-R. Taskinen'; ${ }^{1}$ Department of Medicine, Helsinki University Central Hospital and Biomedicum, ${ }^{2}$ Department of Medicine, Division of Nephrology, Helsinki University Central Hospital, ${ }^{3}$ Folkhalsan Institute of Genetics, Folkhalsan Research Center, Biomedicum Helsinki, Helsinki, Finland.

Background: Fenofibrate was associated with less albuminuria progression in the FIELD study. We explored the changes in commonly available markers of kidney function during fenofibrate treatment in a FIELD Helsinki cohort not using additional statin.

Subjects and Methods: 170 patients with type 2 diabetes recruited to the FIELD study in Helsinki were randomly assigned to micronised fenofibrate $200 \mathrm{mg} /$ day or placebo in a double-blind design for 5 years. In this substudy, we measured plasma creatinine (P-Crea), overnight urine albumine excretion rate (nU-Alb), diurnal proteinuria and creatinine (dU-Prot and U-Crea), and calculated creatinine clearance (Crea-Clearance), and estimated glomerular filtration rate (eGFR) by the Cockroft-Gault equation. For urinary albumin/ creatinine ratio (Alb/Crea) we used the spot sample data obtained in the main FIELD study. Mann-Whitney U-test was used to compare the change from baseline between the groups.

Results: Due to intensified treatment during the study, mean blood pressure (from 144/88 to $137 / 80 \mathrm{mmHg}, \mathrm{p}<0.001$ ) and mean fasting plasma glucose (from 8.0 to $7.5 \mathrm{mmol} / \mathrm{L}, \mathrm{p}=0.007$ ) decreased in both groups and $\mathrm{HbA}_{1 \mathrm{C}}$ remained at $7.2 \%$. Medications were similar between the groups, except for the study drug. S-Crea increased with fenofibrate but $\mathrm{U}$-Crea remained comparable between the groups, resulting in decreases in both Crea-Clearance and eGFR in the fenofibrate group. Alb/Crea and dU-Prot remained unchanged, whereas $\mathrm{nU}$-Alb showed a minor decrease in both groups $(\mathrm{p}=0.004)$.

Conclusion: Fenofibrate is known to reversibly raise both P-Crea and U-Crea levels. We are the first to show the contemporary decrease in Crea-Clearance and eGFR. Such changes will complicate the clinical surveillance of kidney function during fenofibrate treatment. In contrast to the main FIELD study, we did not find evidence for a beneficial effect of fenofibrate on albumin excretion rate.

\begin{tabular}{|c|c|c|c|c|c|}
\hline & \multicolumn{2}{|l|}{ Baseline } & \multicolumn{2}{|l|}{$5^{\text {th }}$ year } & \multirow[t]{2}{*}{ P value } \\
\hline & Placebo & Fenofibrate & Placebo & Fenofibrate & \\
\hline $\begin{array}{l}\text { P-Crea, } \\
\mu \mathrm{mol} / \mathrm{L}\end{array}$ & $\begin{array}{l}73 \\
66-78\end{array}$ & $\begin{array}{l}73 \\
68-85\end{array}$ & $\begin{array}{l}74 \\
63-85\end{array}$ & $\begin{array}{l}87 \\
75-101\end{array}$ & $<0.001$ \\
\hline $\begin{array}{l}\text { U-Crea, } \\
\mathrm{mmol} / \mathrm{L}\end{array}$ & $\begin{array}{l}5.4 \\
4-7.4\end{array}$ & $\begin{array}{l}5.7 \\
4.7-7.9\end{array}$ & $\begin{array}{l}5.4 \\
3.9-7.3\end{array}$ & $\begin{array}{l}6.1 \\
4.3-7.6\end{array}$ & 0.855 \\
\hline $\begin{array}{l}\text { Crea-Clearance, } \\
\mathrm{mL} / \mathrm{s} / 1.73 \mathrm{~m}^{2}\end{array}$ & $\begin{array}{l}1.78 \\
1.59-1.98\end{array}$ & $\begin{array}{l}1.70 \\
1.45-1.97\end{array}$ & $\begin{array}{l}1.73 \\
1.40-2.12\end{array}$ & $\begin{array}{l}1.59 \\
1.28-1.70\end{array}$ & 0.051 \\
\hline $\begin{array}{l}\text { eGFR, } \\
\mathrm{mL} / \mathrm{s}\end{array}$ & $\begin{array}{l}1.92 \\
1.57-2.18\end{array}$ & $\begin{array}{l}1.81 \\
1.55-2.13\end{array}$ & $\begin{array}{l}1.62 \\
1.39-2.05\end{array}$ & $\begin{array}{l}1.46 \\
1.09-1.70\end{array}$ & $<0.001$ \\
\hline $\begin{array}{l}\mathrm{nU}-\mathrm{Alb} \\
\mu \mathrm{g} / \mathrm{min}\end{array}$ & $\begin{array}{l}6.5 \\
5-11\end{array}$ & $\begin{array}{l}6 \\
4-11.5\end{array}$ & $\begin{array}{l}4 \\
2-11\end{array}$ & $\begin{array}{l}4 \\
2-13\end{array}$ & 0.958 \\
\hline $\begin{array}{l}\text { dU-Prot, } \\
\mathrm{mg} / 24 \mathrm{~h}\end{array}$ & $\begin{array}{l}105 \\
82-190\end{array}$ & $\begin{array}{l}123 \\
78-184\end{array}$ & $\begin{array}{l}100 \\
70-15\end{array}$ & $\begin{array}{l}110 \\
73-190\end{array}$ & 0.287 \\
\hline $\begin{array}{l}\mathrm{Alb} / \mathrm{Crea} \\
\mathrm{mg} / \mathrm{mmoL}\end{array}$ & $\begin{array}{l}1.0 \\
0.7-2.3\end{array}$ & $\begin{array}{l}1.1 \\
0.6-2.8\end{array}$ & $\begin{array}{l}1.1 \\
0.4-2.9\end{array}$ & $\begin{array}{l}1.0 \\
0.0-3.3\end{array}$ & 0.136 \\
\hline
\end{tabular}

Data presented as median (IQR). P value based on comparing the percentage changes from baseline with Mann-Whitney U-test.

Supported by the Helsinki University Central Hospital Research Foundation 


\section{OP 37 Novel therapies}

\section{7}

Isosteviol as novel oral anti-diabetic agent for treatment of type 2 diabetes and the metabolic syndrome - $\mathrm{a}$ in vivo study on diabetic KKAy-mice

P. B. Jeppesen, I. Nordentoft, R. Abudula, J. Hong, K. Hermansen; Dept. of Endocrinology and Metabolism C, Aarhus University Hospital, Aarhus Sygehus THG, Denmark.

Background and Aims: Isosteviol (ISV) is a unique novel chemical entities being the backbone in a group of substances with proven anti-diabetic capabilities in both man and rodent. In this present study we investigate if ISV possesses beneficial effects on the metabolism and gene expression in the diabetic KKAy-mouse.

Materials and Methods: $20 \mathrm{KKAy}$-mice, age 5 weeks, were divided into 2 groups and treated for 9 weeks with; A: standard chow diet (control); B: standard chow diet $+0.02 \mathrm{~g} / \mathrm{kg}$ BW of ISV; D: 20 normal C57BL-mice fed with standard chow diet (control to A). Hormones and lipids were measured from blood sample at start and end of the treatment period. Fasting blood glucose as well as BW was measured 3 times during the study. At the end of the study mRNA was purified from Islets and different key genes was investigated as expression level compared to the control KKAy group.

Results: No difference in fasting plasma glucose or insulin was seen at the onset of the study. After 9 weeks of treatment, ISV exerted beneficial effects on metabolism in the diabetic KKAy-mice i.e.: 1.) reduction in p-glucose (A vs. B by $59 \%(\mathrm{p}<0.01))$ and 2 .) decreased $\mathrm{p}$-insulin concentration, indicating increased insulin sensitivity, (A vs. B by $62 \%$ reduction $(\mathrm{p}<0.05)$ ), respectively. At the end of the study there was a marked decrease in the glucose insulin index for the ISV group vs. KKAy control (6932 vs.1596 unit, p<0.01). P-TG concentration was significantly lowered for the groups treated with ISV by $74 \%(\mathrm{p}<0.01)$. BW was reduced significantly for the ISV group by $13 \%$. Islets from group B vs. A experienced an increased expression of PDX1, Beta2, GLUT2, Ins1 and decreased expression of C/EBPalpha and 11beta-HSD-1 $\mathrm{p}<0,05)$, respectively

Conclusion: This study demonstrates that ISV may be used as a new insulin sensitiser in the treatment of type 2 diabetes. ISV co-regulate many of the target genes. The large increase in GLUT2 expression indicates improved glucose sensitivity. The down regulation of 11 beta-HSD- 1 by ISV is potentially very interesting since 11 beta-HSD-1 expression and activity are increased in islets of diabetic ZDF rats and Troglitazone concomitantly prevents both the increase in 11beta-HSD-1 and diabetes development. ISV comprises a new class of anti-diabetic agents addressing several aspects of the abnormal metabolism accompanying type 2 diabetes.

Supported by the Danish Medical Research Council

\section{8}

CS-917, a fructose 1,6-bisphosphatase inhibitor, reduces plasma glucose by suppressing hepatic glucose production, whereas metformin stimulates gastrointestinal glucose uptake

A. Okuno, J. Tanaka, K. Takahashi, J. Ogawa, Y. Hagisawa, S. Kanda, T. Yoshida;

Biological Research Laboratories II, Daiichi Sankyo Company, Limited, Tokyo, Japan.

Background and Aims: CS-917 is a novel inhibitor of fructose 1,6bisphosphatase, a rate-limiting enzyme in gluconeogenesis (GNG). In-vivo studies in Goto-Kakizaki (GK) rats have shown that CS-917 reduces glucose production, while metformin (MET) appears to accelerate glucose utilisation. Thus, CS-917 and MET appear to have different mechanisms of action. In this study, we evaluated the effect of both agents on glucose uptake/release in GK rats in vivo, and in human cells in vitro.

Materials and Methods: Plasma levels of glucose (PG) and lactate were measured 3 hours after oral administration of CS-917 (15mg/kg) or MET $(300 \mathrm{mg} / \mathrm{kg})$ to fasted and non-fasted GK rats. Blood samples were taken from the hepatic vein, portal vein and abdominal aorta. The difference between the PG and lactate levels in blood entering and leaving the liver and the gastrointestinal tract was calculated as a measure of glucose uptake/release. The impact of CS-917 $(0.003-100 \mu \mathrm{M})$ or MET $(0.3-1000 \mu \mathrm{M})$ was also investigated in vitro. In primary human hepatocytes, GNG was determined by measuring glucose concentration in the culture medium. Similarly, using colon carcinoma (Caco-2) cells, glycolysis was determined by measuring glucose utilisation, lactate concentration and the appearance of anionic metabolites of ${ }^{14} \mathrm{C}$-glucose in the culture medium.

Results: CS-917 $(15 \mathrm{mg} / \mathrm{kg})$ reduced hepatic glucose production and inhibited hepatic lactate clearance in fasted rats (see Table). MET (300mg/ $\mathrm{kg}$ ) increased gastrointestinal glucose uptake and increased gastrointestinal lactate production in non-fasted rats. CS-917 $(0.01-1 \mu \mathrm{M})$ inhibited hepatocyte GNG in a dose-dependent manner $\left(\mathrm{IC}_{50} \mathrm{~s}=0.041-0.061 \mu \mathrm{M}\right)$. In contrast, MET $(0.3-100 \mu \mathrm{M})$ did not inhibit hepatocyte GNG. MET (100$1000 \mu \mathrm{M})$ stimulated glycolysis in Caco-2 cells in a dose-dependent manner. CS-917 $(10-100 \mu \mathrm{M})$ did not alter glucose utilisation by Caco-2 cells or the appearance of anionic metabolites.

Glucose and lactate release/uptake in vivo following oral administrtion of CS-917 or MET

\begin{tabular}{llll}
\hline & Control & CS-917 & MET \\
\cline { 2 - 4 } & $\mathrm{mg} / \mathrm{dL}$ & & \\
\hline Liver (fasted rat) & $(\mathrm{n}=9)$ & $(\mathrm{n}=10)$ & $(\mathrm{n}=10)$ \\
Glucose release & $72 \pm 10$ & $8 \pm 2^{*}$ & $89 \pm 10$ \\
Lactate uptake & $14.2 \pm 2.5$ & $-3.2 \pm 1.4^{*}$ & $17.5 \pm 2.0$ \\
GI tract (non-fasted rat) & $(\mathrm{n}=9)$ & $(\mathrm{n}=9)$ & $(\mathrm{n}=10)$ \\
Glucose uptake & $23 \pm 6$ & $13 \pm 5$ & $53 \pm 4^{*}$ \\
Lactate release & $4.1 \pm 1.0$ & $-2.4 \pm 1.9^{*}$ & $17.9 \pm 1.5^{*}$ \\
\hline
\end{tabular}

$\mathrm{GI}=$ gastrointestinal; mean \pm standard error; ${ }^{\star}=\mathrm{P}<0.05$ versus control (t-test).

Conclusion: These results suggest that CS-917 and MET reduce PG through different mechanisms of action. CS-917 appears to suppress hepatic glucose production by directly inhibiting GNG, while MET stimulates gastrointestinal glucose uptake. Thus, CS-917 offers a novel mode of action that warrants further investigation for the treatment of hyperglycaemia in type II diabetes.

\section{9}

Pharmacokinetics and pharmacodynamics of a single oral dose of the insulin analog IN-105, in tablet form, in normal healthy volunteers, in the presence of food

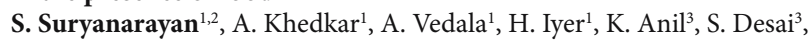
S. Savale ${ }^{3}$, A. Atignal';

${ }^{1}$ Research and Development, Biocon Limited, Bangalore, India, ${ }^{2}$ Visiting Faculty, Diabetes \& Endocrinology Division, Department of Molecular Medicine and Surgery, Karolinska Institute,, Stockholm, Sweden, ${ }^{3}$ Human Pharmacology Unit, Clinigene Limited, Bangalore, India.

Background and Aims: IN105 is a modified insulin analog that is more resistant to proteolytic digestion compared to normal insulin and was found to be orally bioavailable in animal models and has the advantage of coming in through the portal vein. The aim was to study the safety, pharmacodynamics and pharmacokinetics of IN105 in Normal Healthy volunteers.

Materials and Methods: In the first of 2 Phase I studies, escalating doses, starting from $5 \mathrm{mg}, 10$ and $15 \mathrm{mg}$ of IN105 were given to each of 12 overnight fasted, Normal healthy male volunteers (age range: 18 to 45 , fasting glucose $70-110 \mathrm{mg} / \mathrm{dl} \&$ weight within $10 \%$ of ideal body weight from the table of desirable weights of adults - Life Insurance Corporation of India,), in consecutive study periods, spaced by a week each, to determine the Pharmacokinetics and Pharmacodynamics of the product. Subjects whose blood glucose fell by more than $30 \%$ in a given period were maintained on the same dose of IN105 for subsequent periods. 11 volunteers completed the study, with the cause of 1 dropout unrelated to drug administration. In the 2nd Phase 1 study, tablets delivering 5 or $10 \mathrm{mg}$ of IN105 were given 20min, 10min and 2 minutes before a high carbohydrate breakfast, to overnight fasted subjects, in consecutive study periods to determine food effects on the absorption and action of IN105. These studies were preceded by a screening period which was used to determine a subject's response to a $5 \mathrm{mg}$ dose of IN105 under fasted conditions, and based upon whether their blood glucose fell by more than $30 \%$, they either remained on a $5 \mathrm{mg}$ dose or escalated to a $10 \mathrm{mg}$ dose for the subsequent food effects study periods. The plasma 
insulin levels achieved with 10mg of IN105 from the first Phase 1 study were considered adequately high to allow any food effects to be observed. 14 normal healthy male volunteers (inclusion criteria as for the first Phase 1 study), participated in this study, with 1 dropout with a cause unrelated to drug administration. Immunoreactive insulin levels in the blood were measured along with the C-peptide and glucose levels. Mass spectrometry was also used to verify the presence of intact IN105 in the Plasma and distinguish it from human Insulin.

Results: IN105 in a tablet form was found to be safe in Normal Healthy volunteers. The most common adverse event in the first study was hypoglycemia, all of which was found to be drug related $(n=7)$. In both studies, IN105 levels were detected in blood within a few minutes of dosing. IN105 levels peaked at approximately 20 minutes and the maximum pharmacodynamic effects appear at approximately 40 minutes post dosing. IN105 is absorbed when taken 2 minutes before a meal also. However, the best absorption occurs when taken 20 minutes before a meal. There is pharmacokinetic dose response with increasing dosing of IN105 and there is a predictable pharmacodynamic response of individual subjects to a given dose, over the periods studied.

Conclusion: IN-105 is safe and has pharmacodynamic and pharmacokinetic response of a rapid acting insulin.

\section{0}

Safety and preliminary efficacy of PPM-204, a novel pan-PPAR agonist, in treatment-naive type 2 diabetic subjects

A. Katz ${ }^{1}$, K. Weisel ${ }^{1}$, L. Hickey ${ }^{1}$, B. Tammara ${ }^{1}$, G. Skoglund ${ }^{2}$, W. Spinelli ${ }^{3}$, K. Nolop ${ }^{4}, X$. Meng';

${ }^{1}$ Early Development \& Clinical Pharmacology, Wyeth Research, Collegeville, United States, ${ }^{2}$ Wyeth Nordic, Wyeth Research, Solna, Sweden,

${ }^{3}$ Medical Research, Wyeth Research, Collegeville, United States,

${ }^{4}$ Development, Plexxikon, Berkeley, United States.

Background and Aims: The safety and anti-hyperglycemic effects of PPM204, a novel pan-PPAR agonist, were studied in treatment-naïve type 2 diabetic subjects.

Materials and Methods: Eighty-seven treatment-naïve type 2 diabetic subjects (53 male and 34 female, with the following baseline characteristics \{mean \pm SD\}: age $56.6 \pm 8.4$ years, BMI $30.8 \pm 3.9 \mathrm{~kg} / \mathrm{m}^{2}$, and fasting glucose $158.4 \pm$ $43.5 \mathrm{mg} / \mathrm{dL}$ ) were randomized to receive one of three oral doses of PPM-204 $(25,75$, or $225 \mathrm{mg} / \mathrm{d})$, pioglitazone $30 \mathrm{mg} / \mathrm{d}$, or placebo once daily for 28 days. Four-hour oral glucose tolerance tests (OGTT) were performed before and after 28 days of treatment. Other efficacy and pharmacodynamic parameters included fasting glucose and insulin, adiponectin, and fructosamine. Safety evaluation included edema assessment, body weight, total body water (TBW) and extracellular fluid (ECF) using deuterium and $\mathrm{NaBr}$.

Results: Time-adjusted area under the curve (AUC/T) for glucose and insulin during a four-hour OGTT decreased in a dose-dependent manner. Mean change from baseline in AUC/T for glucose was $-1.4,-10.4$, and $-36.5 \mathrm{mg} / \mathrm{dL}$ in the 25,75 , and $225 \mathrm{mg} / \mathrm{d}$ groups respectively, 11.9 for placebo, and -11.8 for pioglitazone. Mean change from baseline in AUC/T for insulin was -46.6, -28.3 , and $-58.6 \mathrm{pmol} / \mathrm{L}$ in the 25,75 , and $225 \mathrm{mg} / \mathrm{d}$ groups, respectively, -6.9 for placebo, and -10.7 for pioglitazone. After 28 days of treatment, fasting glucose decreased significantly in the $225 \mathrm{mg} / \mathrm{d}$ and the pioglitazone-treated groups and fasting insulin decreased significantly in the $225 \mathrm{mg} / \mathrm{d}$ group. Adiponectin increased in a dose-proportional manner to approximately $57 \%$ and $195 \%$ in the 75 and $225 \mathrm{mg} / \mathrm{d}$ groups and by $93 \%$ in the pioglitazone group. Fructosamine did not change significantly in any of the groups.

No subjects were discontinued because of adverse events (AEs) and no serious AEs occurred in the study. Four mild AEs of edema were reported (one in the placebo group, two in the $75 \mathrm{mg} / \mathrm{d}$, and one in the $225 \mathrm{mg} / \mathrm{d}$ ). No dose-dependent trends in AEs, vital signs, or ECGs occurred in the study. Mean body weight remained unchanged in all dose groups. TBW and ECF did not change significantly in any of the groups. Hemoglobin and hematocrit decreased in the $225 \mathrm{mg} / \mathrm{d}$ group by approximately $6 \%$ and $5 \%$, respectively. In the $225 \mathrm{mg} / \mathrm{d}$ cohort, serum creatinine increased by approximately $11 \%$, urea increased by approximately $27 \%$, and creatine kinase did not change significantly.

Conclusion: PPM-204, given once daily for 28 days, lowered glucose and insulin in a dose-dependent manner, and was safe and well tolerated in treatment-naïve type 2 diabetic subjects at doses of 25,75 , and $225 \mathrm{mg} / \mathrm{d}$.
Mean (SE) Change from Baseline in AUC/T for Glucose during OGTT

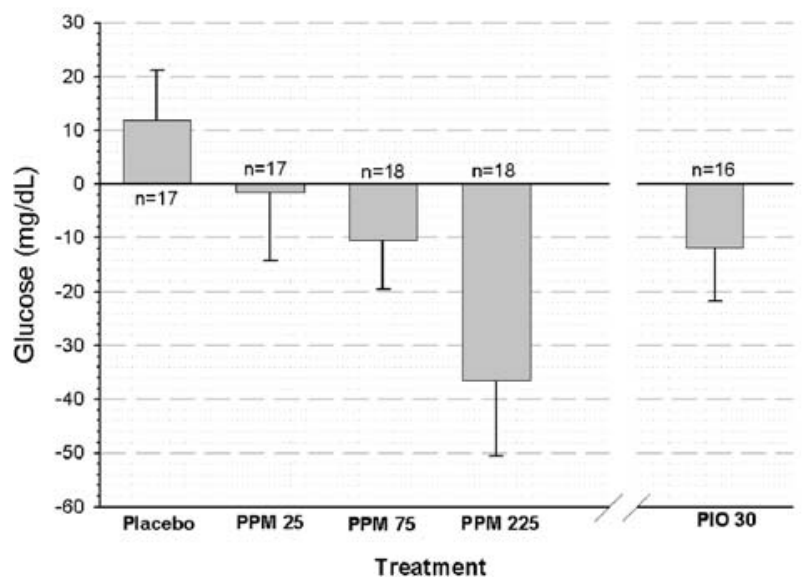




\section{OP 38 Insulin therapy: clinical studies}

\section{1}

Continuous subcutaneous insulin infusion therapy versus multiple daily injection therapy in patients with type 1 and type 2 diabetes mellitus: meta-analysis and systematic review

K. Horvath ${ }^{1}$, K. J. Jeitler ${ }^{2}$, A. Berghold ${ }^{3}$, T. W. Gratzer ${ }^{2}$, T. R. Pieber ${ }^{2}$, A. Siebenhofer ${ }^{1}$;

${ }^{1}$ EBM Review Center, Department of Internal Medicine, Medical University Graz, ${ }^{2}$ Institute of Medical Technologies and Health Management, Joanneum Research, Graz, ${ }^{3}$ Institute for Medical Informatics, Statistics and Documentation, Medical University Graz, Austria.

Background and Aims: Increasingly, continuous subcutaneous insulin infusion (CSII) is considered a valuable treatment option for patients with type 1 (T1DM) and type 2 (T2DM) diabetes mellitus in many health care systems; however, possible beneficial effects in terms of glycaemic control remain to be evaluated.

Materials and Methods: To compare the effects of CSII to multiple daily insulin injections (MDI) on glycaemic control and the risk of hypoglycaemic episodes in adult patients with T1DM and T2DM, a systematic literature search was conducted in the electronic databases MEDLINE, EMBASE and CENTRAL up to March 2007 for randomized controlled trials. A systematic review and meta-analyses were performed.

Results: T1DM: In 17 studies fulfilling the inclusion criteria, 908 patients were included. 11 Studies were conducted before the year 1990 and only three after the year 2000. Study periods lasted from a minimum of 5 weeks to a maximum of 2 years. Our meta-analysis found a between-treatment difference of $-0.63 \%$ $\mathrm{HbAl} / \mathrm{HbAl}$ in favour of CSII vs. MDI therapy (although a high degree of heterogeneity was detected). Median rates of mild or overall hypoglycaemic events per patient per time period were available from seven studies and were comparable between the different interventions: 1.9 (0.9 to 3.1) vs. 1.7 (1.1 to 3.3) events/patient/week for CSII and MDI therapy respectively. Incidence of severe hypoglycaemia was generally low and statistically different in two trials favouring CSII treatment. Total daily insulin requirements were found to be lower with CSII than with MDI therapy.

T2DM: Both studies fulfilling our inclusion criteria were conducted after the year 2000 and included a total of 234 patients. Follow-up was 24 and 52 weeks. In both trials CSII treatment resulted in a statistically not significant reduction of $\mathrm{HbAlc}$ of $-0.1 \%$ when compared to treatment with MDI. Also, the incidence of mild hypoglycaemic events was comparable between the intervention groups (1.1 [CSII] vs.1.2 [MDI] events/patient/week in one and 0.8 [CSII] vs. $1.2[\mathrm{MDI}]$ events/patient/30 days in the other trial). No severe hypoglycaemic episodes were detected in one study and a rate of 0.1 [CSII] vs. $0.2[\mathrm{MDI}]$ events/patient/year in the other.

Conclusion: In this most extensive and up-to-date meta-analysis, CSII therapy in patients with T1DM resulted in a greater reduction of $\mathrm{HbAlc}$, seemingly without a higher rate of hypoglycaemia. This confirms current indications for CSII in patients with frequent hypoglycaemia with MDI. In T2DM patients, CSII provides similar glycaemic control when compared to MDI therapy. The rates of hypoglycaemic episodes were comparable between the two insulin treatment strategies, indicating that CSII therapy is safe. The benefit of CSII for T2DM patients cannot be documented in terms of improved $\mathrm{HbAlc}$ for an average patient. Supported by "Roche Diagnostics"

\section{2}

Superior glycaemic control, less nocturnal hypoglycaemia and less weight gain with insulin detemir relative to NPH insulin in subjects with T1DM treated for 24 months using a treat-to-target concept P. C. Bartley ${ }^{1}$, M. Bogoev ${ }^{2}$, J. Larsen 3 , S. C. Tamer 3 , A. Philotheou ${ }^{4}$; ${ }^{1}$ Department of Medicine, University of Queensland, Stones Corner, Australia, ${ }^{2}$ Clinic of endocrinology and metabolic diseases, Diabetes Center, Skopje, Macedonia, ${ }^{3}$ Novo Nordisk A/S, Bagsvaerd, Denmark,

${ }^{4}$ Faculty of Health Sciences, University of Cape Town, South Africa.

Background and Aims: This 24-month, multi-national, open-label, parallel group trial investigated the long-term efficacy and safety of insulin detemir
(IDet) and NPH insulin (NPH), both in combination with mealtime insulin aspart (IAsp), in subjects with T1DM using a treat-to-target concept. Materials and Methods: A total of 497 men and women (men: 55\%, mean age: 35 yrs, duration of diabetes: 13 yrs, BMI: $24.7 \mathrm{~kg} / \mathrm{m}^{2}, \mathrm{HbA}_{1 \mathrm{c}}$ : $\left.8.3 \%\right)$ were randomised 2:1 to IDet $(n=331)$ or NPH $(n=166)$. Basal insulin was initiated once daily (evening) at comparable doses in both groups. Subjects were individually titrated based on self-measured plasma glucose (PG) levels aiming for pre-breakfast and pre-dinner PG targets $\leq 6 \mathrm{mmol} / \mathrm{L}(108 \mathrm{mg} /$ $\mathrm{dL}$ ) using simple algorithms with specific dose adjustments. A second basal morning dose was added if the average fasting $\mathrm{PG} \leq 6.0 \mathrm{mmol} / \mathrm{L}$, but the average pre-dinner PG remained $\geq 6.0 \mathrm{mmol} / \mathrm{L}$ after optimisation of the evening and bolus insulin doses.

Results: After 24 months, $\mathrm{HbA}_{1 c}$ was superior with IDet compared to NPH: (IDet: 7.36, NPH: 7.58\%, mean difference (IDet-NPH): $-0.22 \%$ point, [95\% CI: $-0.41 ;-0.03 \%]$ ) with reductions of 0.9 and $0.7 \%$ points, respectively. A higher proportion of subjects treated with IDet reached an $\mathrm{HbA}_{1 \mathrm{c}} \leq 7 \%$ in the absence of confirmed hypoglycaemia during the last month of treatment (IDet: $22 \%$ vs. NPH $13 \%, p=0.019$ ). The risk of major and nocturnal hypoglycaemia was respectively $69 \%$ and $46 \%$ lower with IDet than with $\mathrm{NPH}(p<0.001)$. Fasting PG (FPG) was lower with IDet (IDet: 8.35, NPH: 9.43 $\mathrm{mmol} / \mathrm{L}, p=0.019)$ as was within-subject variability in FPG as estimated by the SD (IDet: 2.18 vs. NPH: $2.52 \mathrm{mmol} / \mathrm{L}, p<0.001$ ), whereas variation in predinner PG was comparable in the two groups (IDet: 2.50, NPH: $2.46 \mathrm{mmol} /$ $\mathrm{L}, p=\mathrm{NS})$. The shape of the 9-point PG profiles differed between treatments $(p=0.004)$ with higher pre-dinner and lower FPG concentrations with IDet. Body weight gain was lower with IDet than with NPH (IDet: $1.7 \mathrm{~kg}$, NPH: 2.7 $\mathrm{kg}, p=0.024$ ), while hip/waist ratio and skinfold thickness were comparable in the two groups (NS). Insulin antibodies (IDet-specific and cross-reacting) increased in the IDet group during the first 64 weeks, but decreased slightly during the last 40 weeks. The overall safety profile was similar in the two groups, but hypoglycaemia was reported as serious adverse events (SAEs) in a higher proportion of subjects on NPH, while withdrawal due to adverse event were slightly more frequent with IDet (3.9\% vs. $0.6 \%$ ) and included 4 deaths (all considered as unlikely related to trial drug). SAEs considered possibly or probably related to trial drugs were reported in $4.2 \%$ vs. $6.7 \%$ of subjects on IDet and NPH, respectively.

Conclusion: Long-term (24 months) treatment with the insulin analogues IDet+IAsp was superior to NPH+IAsp in reducing $\mathrm{HbA}_{1 \mathrm{c}}$, with added benefits of less hypoglycaemia at night and less weight gain. This treatment regimen was safe and the antibody development stabilised shortly after 1 year. Supported by Novo Nordisk A/S

\section{3}

Intraperitoneal versus subcutaneous insulin administration in type 1 diabetes: preliminary results of a prospective randomized cross-over trial E. van Ballegooie ${ }^{1,2}$, S. J. J. Logtenberg ${ }^{3}$, N. Kleefstra ${ }^{3,1}$, S. T. Houweling ${ }^{1}$, K. H. Groenier ${ }^{4}$, H. J. G. Bilo ${ }^{3,5}$;

${ }^{1}$ Langerhans, Medical Research Group, Zwolle,

${ }^{2}$ Internal Medicine, Bethesda General Hospital, Hoogeveen, ${ }^{3}$ Diabetes Centre, Isala Clinics, Zwolle, ${ }^{4}$ General Practice, UMCG, Groningen, ${ }^{5}$ Internal Medicine, UMCG, Groningen, The Netherlands.

Background and Aims: As part of an ongoing cross-over trial to compare the effects of intraperitoneal (IP) insulin to subcutaneous (SC) insulin in poorly controlled type 1 diabetes $\left(\mathrm{HbA}_{1 \mathrm{c}}>7.5 \%\right.$ and/or $\geq 5$ hypoglycaemic events/week), preliminary data on glycaemic control, quality of life (QOL) and treatment satisfaction (TS) of 25 subjects enrolled thus far are reported. Materials and Methods: At the end of a 3 month run-in phase, subjects are randomised to receive insulin either by IP (implantable pump; MIP 2007C, Medtronic) or SC administration. After a 6 month treatment phase, subjects crossed over to the other treatment mode. Glycaemic control was assessed with $\mathrm{HbA}_{1 \mathrm{c}}$, frequency of hypoglycaemic events and time spent in euglycaemia. QOL was assessed with the SF-36 and the WHO-5. TS was assessed with the DTSQ. Data of the $1^{\text {st }}$ trial phase were analyzed with t-test and general linear model.

Results: Enrolment started March 2006. Baseline characteristics: 11 males, 14 females; mean age $43.8 \pm 11.6$ years; mean diabetes duration $22.2 \pm 10.6$ years; mean $\mathrm{HbA}_{1 \mathrm{c}} 8.5 \pm 1.1 \%$; $\mathrm{HbA}_{1 \mathrm{c}}>7.5 \%$ in 20 subjects; hypoglycaemic events $\geq 5$ /week in 14 subjects. By March 2007, 19 subjects had completed the $1^{\text {st }}$ treatment phase. One subject is lost to follow-up (IP-group) due to reasons unrelated to the study. There were no significant differences at the start of 
the $1^{\text {st }}$ phase between groups (table). $\mathrm{Hba}_{1 \mathrm{c}}$ decreased during the $1^{\text {st }}$ half of the treatment phase and during the total treatment phase with IP insulin with $0.59 \%$ and $0.26 \%$ respectively, compared to $0.08 \%$ and $0.06 \%$ with SC insulin respectively (differences non-significant). Time spent in euglycaemia increased after 3 months IP insulin with $18.2 \%$ compared to a decrease with SC insulin of 3.8\% (mean difference $22.1 \%$; $95 \%$ CI 5.1, 39.0; $\mathrm{p}=0.01$ ). At the end of the treatment phase the difference did not reach statistical significance. However, after 6 months, WHO-5 scores had increased significantly in the IP-group compared to the SC-group (mean difference 21 points; $95 \%$ CI 6.1, 35.6; $\mathrm{p}=0.01$ ); differences in SF-36 summary scores did not reach statistical significance. DTSQ scores increased 8.4 points $(95 \%$ CI $4.8,12.1 ; \mathrm{p}<0.001)$ in the IP-group compared to the SC-group. Perceived hypo- and hyperglycaemic events decreased significantly in the IP-group compared to the SC-group ( $\mathrm{p}=0.020$ and $\mathrm{p}=0.024$, respectively).

Conclusion: Interim data suggest that IP insulin infusion tends to improve glycaemic control and increases time in euglycaemia, TS and QOL in otherwise poorly controlled type 1 diabetes. Further evaluation, especially after termination of the cross-over phase will determine whether these effects are sustainable and significant at the individual level.

Patient characteristics at start first treatment phase

\begin{tabular}{lll}
\hline & $\begin{array}{l}\text { IP Insulin } \\
\text { in 1 }\end{array}$ phase & $\begin{array}{l}\text { SC Insulin } \\
\text { in 1 } \mathbf{1}^{\text {st }} \text { phase }\end{array}$ \\
\hline Number of subjects (M/F) & $3 / 6$ & $4 / 6$ \\
Age (years) & $45.1 \pm 11.1$ & $41.9 \pm 12.1$ \\
Diabetes duration (years) & $22.3 \pm 9.9$ & $24.0 \pm 12.5$ \\
HbA1c (\%) & $8.3 \pm 1.0$ & $8.4 \pm 1.7$ \\
Number of hypoglycaemic events per & $4.0 \pm 2.2$ & $4.0 \pm 2.7$ \\
week (n) & & \\
Time in euglycaemia (\%) & $42.1 \pm 10.3$ & $47.8 \pm 15.8$ \\
BMI (kg/m2) & $27.2 \pm 5.3$ & $27.1 \pm 4.5$ \\
DTSQ score (range 0-36) & $24.5(20.3-26.9)$ & $25.5(19.0-26.8)$ \\
WHO-5 score (range 0-100) & $48(36-68)$ & $40(25-66)$ \\
SF-36: Physical Component Summary & $35.8 \pm 10.8$ & $42.3 \pm 8.2$ \\
SF-36: Mental Component Summary & $47.8 \pm 11.6$ & $43.2 \pm 12.8$ \\
\hline
\end{tabular}

\section{4}

PREDICTIVE 303 Study: Implementation and outcomes of a simplified self-titration dosing guideline for insulin detemir in patients with type 2 diabetes

L. Meneghini ${ }^{1}$, C. Koenen ${ }^{2}$, W. Weng ${ }^{3}$, J.-L. Selam ${ }^{4}$;

${ }^{1}$ Diabetes Research Institute, University of Miami Miller School of Medicine, United States, ${ }^{2}$ Novo Nordisk A/S, Bagsvaerd, Denmark, ${ }^{3}$ Novo Nordisk Inc., Princeton, United States, ${ }^{4}$ Endocrinology, Diabetes Research Center, Tustin, United States.

Background and Aims: The PREDICTIVE 303 study (N=5604) was part of the global PREDICTIVE study to evaluate the efficacy and safety of insulin detemir. The PREDICTIVE 303 study utilised a randomised controlled design to compare a simplified patient self-adjusted dosing algorithm (G1) to a standard-of-care physician-driven adjustments (G2), using insulin detemir in predominantly primary care settings over a period of 6 months. The primary objective of the study was to show that G1 was non-inferior to G2 in terms of $\mathrm{HbA}$ reduction from baseline.

Materials and Methods: Detemir was started once daily at bedtime as an add-on therapy to any other glucose-lowering regimens, or as a replacement of previous basal insulin in patients with type 2 diabetes. Study sites were randomised to either G1 or G2. Patients from G1 sites adjusted their detemir dose every 3 days based on mean fasting blood glucose (FBG) values using a simplified algorithm: mean $\mathrm{FBG}<4.4 \mathrm{mmol} / \mathrm{L}$, reduce dose by $3 \mathrm{U}$; between $4.4-6.1 \mathrm{mmol} / \mathrm{L}$, no change; $>6.1 \mathrm{mmol} / \mathrm{L}$, increase by $3 \mathrm{U}$. Detemir dose for patients from G2 was adjusted by physicians according to the standard of care.

Results: Mean $\mathrm{HbA}_{1 \mathrm{c}}$ decreased from $8.5 \%$ at baseline to $7.9 \%$ at 26 weeks for $\mathrm{G} 1$, and from $8.5 \%$ to $8.0 \%$ for $\mathrm{G} 2$ (between group $\mathrm{p}=0.0106$ ). FBG values decreased by 1.8 and $1.2 \mathrm{mmol} / \mathrm{L}$ for G1 and G2, respectively (between group $\mathrm{p}<0.0001$ ). The mean daily detemir doses at 26 weeks for the patients in G1 and G2 groups were 0.7 and $0.5 \mathrm{U} / \mathrm{kg}$, respectively. Body weight change from baseline at 26 weeks $(\triangle \mathrm{BW})$ was minimal $(0.1 \mathrm{~kg}$ for $\mathrm{G} 1$ and $-0.2 \mathrm{~kg}$ for $\mathrm{G} 2$, between group $\mathrm{p}=0.0676$ ). When patients were stratified into quintiles according to baseline BMI, mean weight changes were: $+0.97,+0.31,-0.19$, -0.41 , and $-0.45 \mathrm{~kg}$ in the BMI quintiles: $\leq 25,>25$ and $\leq 30,>30$ and $\leq 35$, $>35$ and $\leq 40$, and $>40 \mathrm{~kg} / \mathrm{m}^{2}$, respectively. At 26 weeks, $88 \%$ of all patients remained on once-daily detemir therapy. The rates of hypoglycaemia (event/ patient/year) were calculated based on episodes reported for the four weeks prior to each visit. The rates decreased significantly from 9.0 at baseline to 6.4 at 26 weeks for G1, and from 9.5 to 4.9 for G2 (p $<0.0001$ vs. baseline for both groups).

Conclusion: Better glycarmic control was archieved in the patient selftitration group than in the physician-driven titration group. The majority of the patients were managed on once-daily detemir therapy. The use of detemir in this predominantly primary care setting resulted in significant improvements in glycaemic control without weight gain in the overall population. The simplified patient-driven titration algorithm appears to be safe and effective compared to physician-driven insulin dose adjustments. 


\section{OP 39 Retinopathy: clinical observations}

\section{5}

The distribution of macular hard exudates is related to future visual acuity in subjects with type 2 diabetes

I. M. Stratton ${ }^{1}$, P. H. Scanlon ${ }^{2}$, R. R. Holman ${ }^{1}$, R. J. Stirling ${ }^{3}$, H. L. Lipinski S. J. Aldington ${ }^{3,2}$

${ }^{1}$ Diabetes Trials Unit, Oxford Centre for Diabetes, Endocrinology and Metabolism, Oxford, United Kingdom,

${ }^{2}$ Ophthalmology, Cheltenham General Hospital, United Kingdom,

${ }^{3}$ Metabolic Medicine, Imperial College London, United Kingdom.

Background and Aims:To examine whether the geographical distribution of hard exudates (HE) in the macula field in subjects with type 2 diabetes (T2DM) is related to subsequent visual acuity (VA).

Materials and Methods: The UK Prospective Diabetes Study (UKPDS) was a randomised clinical trial of therapies of newly diagnosed T2DM in 5102 subjects who had four field retinal photographs taken at entry and then at 3 yearly intervals. Photographs were graded using the ETDRS system. Those subjects with HE in the macular field were retrieved, digitised, and diagonal lines superimposed intersecting at the foveal centre, defining nasal, superior, temporal and inferior regions, each split into inner and outer areas, and centre. HE in each of these 9 fields were recorded as none, definite HE ETDRS standard level 2, level 3, level 4 or worse. VA was measured with LogMAR charts ( 5 letters per line). Association of HE score by region with VA 3 years after $\mathrm{HE}$ appearance was analysed using mixed models. These adjusted for age, gender, biochemical variables and duration of diabetes. Levels of $\mathrm{HE}$ in each sector were included in the model in a step-wise fashion, and estimates obtained for the influence of HE in each sector on subsequent VA.

Results: Of 4738 who ever had a photograph of sufficient quality taken, 746 subjects had HE recorded at level 2 or higher at any time (15.7\%). Photographs for those with at least 2 good quality photographs three years apart and VA assessed at coincident visits were used in this analysis.In 168 eyes in 132 patients with newly emergent HE (i.e. with at least one earlier photographs with no evidence of HE) the distribution of HE was non uniform (table 1), Chisquared on 24 degrees of freedom $=145, p<0.0001$. In 255 subjects contributing 1 or 2 eyes over up to 21 years (833 eyes), HE level 3 or worse was associated with reduced LogMAR letter count 3 years later, in IN field 7.9 (2.6 ) (mean (s.e.)) letters were lost, II field 7.9 (3.0) and OI field 4.9 (2.4). Conclusion: The geographical distribution of HE is not uniform across the macula field. Those with HE in IN, II and OI sectors of the macula field had worse vision 3 years later than those with HE elsewhere. The distribution of $\mathrm{HE}$ in diabetic retinopathy may be an important indicator of reduced visual acuity. (Fig. 1)
Regional distribution and severity of $\mathrm{HE}$ ( $\mathrm{n}=$ no of eyes, \%=proportion)

\begin{tabular}{lllll}
\hline Region & $\begin{array}{l}\text { Grade 0 } \\
(\%)\end{array}$ & $\begin{array}{l}\text { Grade 2 n } \\
(\%)\end{array}$ & $\begin{array}{l}\text { Grade 3 n } \\
(\%)\end{array}$ & $\begin{array}{l}\text { Grade 4 n } \\
(\%)\end{array}$ \\
\hline Centre (C) & $112(56.7 \%)$ & $44(26.2 \%)$ & $12(7.1 \%)$ & $0(0 \%)$ \\
Inner superior (IS) & $122(72.6 \%)$ & $28(16.7 \%)$ & $16(9.5 \%)$ & $2(1.2 \%)$ \\
Outer superior (OS) & $97(57.7 \%)$ & $33(19.5 \%)$ & $31(18.9 \%)$ & $7(4.2)$ \\
Inner temporal (IT) & $114(67.9 \%)$ & $26(15.5 \%)$ & $27(16.0 \%)$ & $1(0.6 \%)$ \\
Outer temporal (OT) & $74(44.3 \%)$ & $42(25.2 \%)$ & $38(22.8 \%)$ & $13(7.8 \%)$ \\
Inner Inferior (II) & $126(81.0 \%)$ & $17(10.1 \%)$ & $14(8.3 \%)$ & $1(0.6 \%)$ \\
Outer Inferior(OI) & $116(69.5 \%)$ & $25(15.6 \%)$ & $18(10.8 \%)$ & $7(4.2 \%)$ \\
Inner nasal (IN) & $137(81.5 \%)$ & $17(10.1 \%)$ & $12(7.1 \%)$ & $2(1.2 \%)$ \\
Outer nasal (ON) & $144(85.7 \%)$ & $10(6.0 \%)$ & $10(6.0 \%)$ & $4(2.4 \%)$ \\
\hline
\end{tabular}

Supported by Diabetes Research and Wellness Foundation

\section{6}

Flicker-induced vasodilation of retinal arteries decreases with increasing stages of diabetic retinopathy

A. Mandecka ${ }^{1}$, J. Dawczynski², M. Blum ${ }^{3}$, N. Müller ${ }^{1}$, C. Kloos ${ }^{1}$, W. Vilser ${ }^{4}$, G. Wolf ${ }^{1}$, U. Müller ${ }^{1}$;

${ }^{1}$ Internal Medicine III, Friedrich Schiller University Jena, ${ }^{2}$ Department of Ophthalmology, Friedrich Schiller University Jena, ${ }^{3}$ Department of Ophthalmology, Helios Klinikum Erfurt, ${ }^{4}$ Imedos, Jena, Germany.

Background and Aims: Stimulation of the retina with flickering light increases retinal arterial and venous diameter in animals and humans. Nitric oxide appears to be a mediator of the retinal vasodilation to flicker. The reduction of flicker induced retinal vasodilation is considered as a vascular/ endothelial dysfunction. We investigated the response of retinal vessels on flickering light in patients with diabetes mellitus in different stages of diabetic retinopathy (DR).

Materials and Methods: We studied 54 healthy volunteers (age 41 \pm 16 ), 68 type 1 diabetic patients (age $47 \pm 15$, duration of diabetes $17 \pm 10$ years) and 172 type 2 diabetic patients (age $61 \pm 10$, duration of diabetes $11 \pm 7$ years). The diameter of major temporal retinal arteries and veins was measured continuously online with a Dynamic Vessel Analyzer (IMEDOS GmbH Jena, Germany). Each test consisted of an 80 -s baseline plus three 20 -s periods of flicker stimulation followed by an 80 -s period of observation. The fundus examination was performed after induction of mydriasis with tropicamide $1 \%$ eye drops. The diabetic retinopathy was classified using ETDRS-criteria as no DR (NDR), mild nonproliferative DR (mild NPDR), moderate nonproliferative DR (MNPDR) and severe nonproliferative DR (SNPDR).

Results: In retinal vessels, the response to stimulation with diffuse luminance flicker was significantly diminished in diabetic patients compared to healthy volunteers. In healthy controls, flicker stimulation increased the retinal arterial diameter by $3.58 \pm 2 \%$ and in diabetic patients by $2.17 \pm 2.4 \%(\mathrm{p}<0.001)$. This response was significantly decreased regardless of type of diabetes. In non-diabetic controls flicker stimulation increased venous retinal diameter by $4.5 \pm 2.3 \%$ and by diabetic patients by $3.6 \pm 2.2 \%$ ( $\mathrm{p}=0.008)$.
Fig. $1 \mathrm{a}$ and $1 \mathrm{~b}$

Example scanned right macular image with grid applied and identity of regions within overlay grid (for right eye)
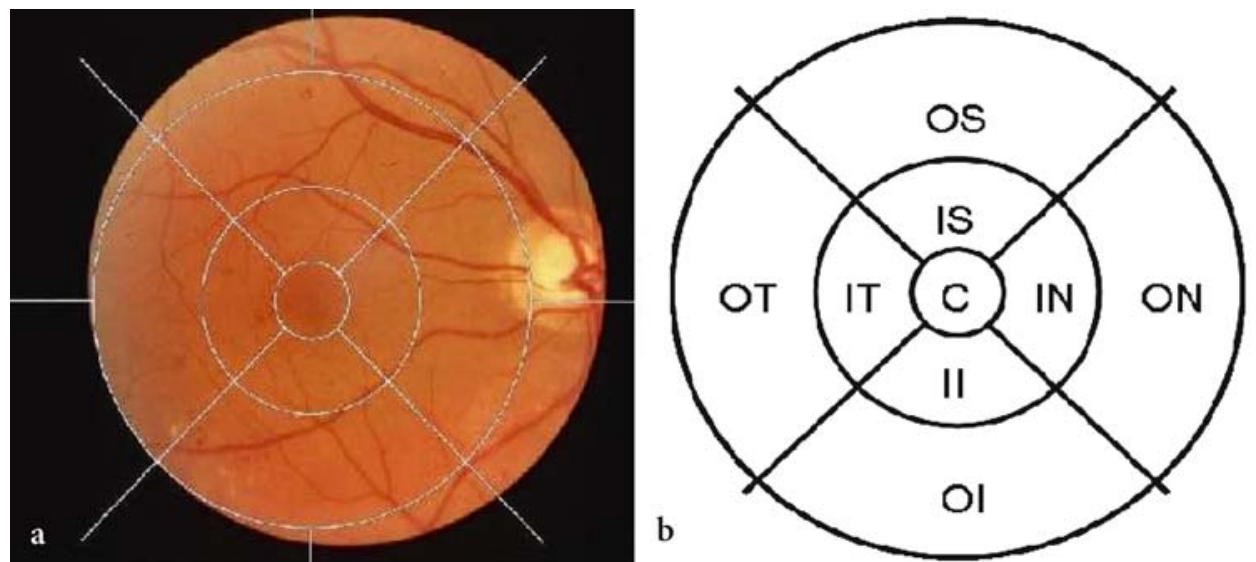
The retinal arterial diameter change was $3.5 \pm 2,2.6 \pm 2.4,2 \pm 2.7,1.6 \pm 2.1$ and $1.4 \pm 1.8 \%$ in the control group, NDR group, mild NDR group, moderate NDR and severe NDR group, respectively. The retinal arterial response in all diabetic patients without retinopathy was significantly decreased in comparison to healthy subjects $(P=0.003)$. Despite of no significant difference in age between diabetic patients without retinopathy and moderate NDR group, the retinal response was, in patients with moderate NDR, significantly decreased $(P=0.048)$

The arterial and venous flicker response is also significantly decreased if approximately age-equivalent subgroups of diabetic patients and subjects 40 - 70 years of age were analyzed.

Conclusion: Flicker responses of retinal arteries and veins are abnormally reduced in patients with diabetes mellitus, regardless of the diabetes type. This decreased response deteriorated with increasing stages of diabetic retinopathy. The response was already low before the clinical appearance of retinopathy. The noninvasive testing of the function of retinal autoregulation using the Dynamic Vessel Analyzer offers promising perspectives to become a useful tool for the detection of diabetic vessel pathology.

\section{7}

Endothelial perturbation: A link between non-dipping and retinopathy in type 2 diabetes?

S. T. Knudsen ${ }^{1}$, P. Jeppesen ${ }^{2}$, C. A. Frederiksen ${ }^{2}$, N. H. Andersen ${ }^{3}$, T. Bek ${ }^{2}$, J. Ingerslev ${ }^{4}$, C. E. Mogensen ${ }^{5}$, P. L. Poulsen;

${ }^{1}$ Medical Dept. M/C (Diabetes \& Endocrinology), Aarhus University Hospital, ${ }^{2}$ Dept. of Ophthalmology, Aarhus University Hospital,

${ }^{3}$ Dept. of Cardiology, Aarhus University Hospital, ${ }^{4}$ Centre for Haemophilia and Thrombosis, Aarhus University Hospital, ${ }^{5}$ Medical Dept. M (Diabetes \& Endocrinology), Aarhus University Hospital, Denmark.

Background and Aims: Reduced diurnal blood pressure (BP) variation ("nondipping") is associated with both micro- and macrovascular complications in patients with type 2 diabetes. In non-diabetic subjects, non-dipping is associated with endothelial dysfunction. The relation between endothelial perturbation and diurnal BP variation in diabetic subjects has not previously been studied. We examined the relationship between non-dipping, markers of endothelial activation, and severity of retinopathy in patients with type 2 diabetes.

Materials and Methods: A total of 76 subjects, stratified to four gender-, age-, and duration-matched groups of each 19 subjects participated. Group A: non-diabetic subjects; Group B-D, type 2 diabetic subjects. Group B: no retinopathy; Group C: minimal background retinopathy; Group D: diabetic maculopathy. All subjects underwent 24-h ambulatory BP monitoring. Von

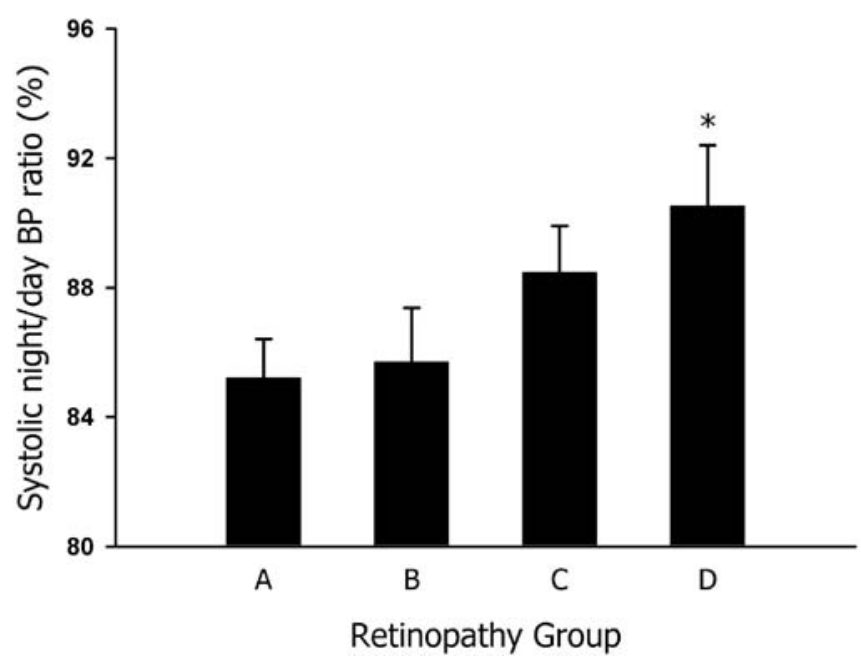

Fig. 1. Systolic night/day blood pressure ratio in the four groups. Group A: non-diabetic subjects. Group B: type 2 diabetic subjects without retinopathy. Group C: type 2 diabetic subjects with minimal background retinopathy. Group D: type 2 diabetic subjects with diabetic maculopathy.

Data are mean $\pm S E$. ${ }^{*} P<0.05$ vs. Group $A$ and $B$.

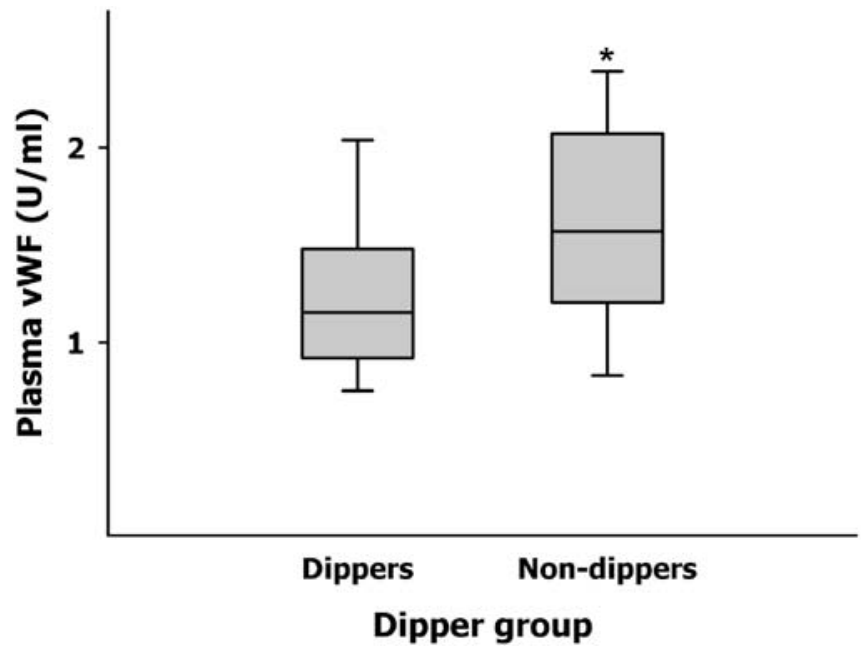

Fig. 2. Plasma levels of von Willebrand factor in systolic dipping and non-dipping type 2 diabetic subjects. Bars represent medians, and boxes indicate IQRs. ${ }^{\star} \boldsymbol{P}<0.01$ vs. dippers

Willebrand factor (vWF), fibrinogen, E-selectin, and ICAM-1 were measured in plasma.

Results: Systolic night/day BP ratio increased gradually in Groups A to D: $85.2 \pm 5 \%, 85.7 \pm 7 \%, 88.5 \pm 6 \%$, and $90.5 \pm 7 \%$, respectively, $P<0.05$. Among diabetic patients, non-dippers had significantly higher plasma levels of vWF and fibrinogen than dippers (median/interquartile range 1.7/1.4-2.1 vs. $1.2 / 0.9-1.5 \mathrm{U} / \mathrm{ml}, P<0.01$ and $3.6 / 3.6-3.7$ vs.2.9/2.5-3.6 g/l, $P=0.01$ ). Plasma levels of fibrinogen and ICAM- 1 were higher in diabetic than in non-diabetic subjects (3.3/2.6-4.0 vs. $2.5 / 2.4-2.8 \mathrm{~g} / \mathrm{l}$ and $218 / 192-268$ vs. $191 / 160-217$ $\mathrm{ng} / \mathrm{ml}$, respectively, $P<0.01$ for both), but did not differ significantly between retinopathy groups $(B-D)$.

Conclusion: Non-dipping is associated with elevated plasma levels of proteins related to endothelial cell activation as well as with retinopathy in subjects with type 2 diabetes. This finding suggests a possible mechanism linking nondipping with microvascular complications in these subjects.

Supported by the Sehested Hansen Foundation, Gärdejer af Stenløse Peder Laurits Petersens Legat, The Beckett Foundation, The Jochum Jensen Memorial Grant, The Velux Foundation, and The Danish Diabetes Association

\section{8}

Birth order is a predictor for proliferative retinopathy in type 1 diabetic siblings (The FinnDiane Study)

K. Hietala ${ }^{1,2}$, C. Forsblom ${ }^{1,3}$, P. Summanen ${ }^{2}$, P.-H. Groop ${ }^{1,3}$;

${ }^{1}$ Folkhalsan Institute of Genetics, Folkhalsan Research Center, Helsinki, ${ }^{2}$ Department of Ophthalmology, Helsinki University Central Hospital, ${ }^{3}$ Division of Nephrology, Department of Medicine, Helsinki University Central Hospital, Finland.

Background and Aims: Diabetic nephropathy and retinopathy (DRP) show familial clustering. Apart from conventional risk factors, such as blood pressure, glycemic control and duration of diabetes, it is not clear which nongenetic familial factors contribute to the risk of DRP. It can be hypothesized that the level of diabetes care of young children is likely to be affected by the number of siblings as well as by their birth order. Therefore, the aim was to study whether the number of siblings and their birth order is associated with the development of proliferative retinopathy (PDR) in type 1 diabetic (T1D) siblings.

Materials and Methods: The FinnDiane-study has so far examined around 4800 patients with T1D. Among them we found 199 families with at least two siblings with T1D. The ophthalmic records were obtained from 388 /417 (93 $\%$ ) patients and fundus photographs from 262/388 (68\%) patients of 196/199 families. In families with more than two siblings the siblings were paired using all possible combinations. A total of 208 sibpairs were thus formed. Four twin pairs were excluded from the calculations. The DRP-status was classified based on fundus photographs and/or repeated clinical ophthalmic examinations using a modified ETDRS-grading scale. Due to clustered data (sib pairs) with a binary outcome measure (PDR yes/no) odds ratios were 
estimated using generalized estimating equations with logit link function. A Kaplan-Meier model was used for survival analysis.

Results: The mean age at onset of T1D was 15.6 (SD 11.6) years and duration of T1D was 25.5 (12.1) years. Male/female ratio was 216/172. PDR occurred in $116 / 388$ patients $(29.9 \%)$. The median number of siblings in the family was 4 (IQR 3-6). There was an increased risk of PDR for the younger siblings (OR 1.5, 95\% CI 1.07-2.2, $\mathrm{p}=0.022$ ) which increased further (OR 2.2, 1.2 4.0, $\mathrm{p}=0.01$ ) when adjusted for $\mathrm{HbA}_{1 \mathrm{c}}$, blood pressure, age at onset and triglycerides. Number of children in the family $(\mathrm{p}=0.78)$ and sex of siblings $(\mathrm{p}=0.35)$ had no effect in this analysis. A higher risk for younger siblings was shown as well by survival analysis (LogRank $\mathrm{p}=0.006$ ).

Conclusion: The birth order, but not the number of siblings appears to predict the development of PDR in sib pairs implicating a possible role for social factors in the pathogenesis of PDR.

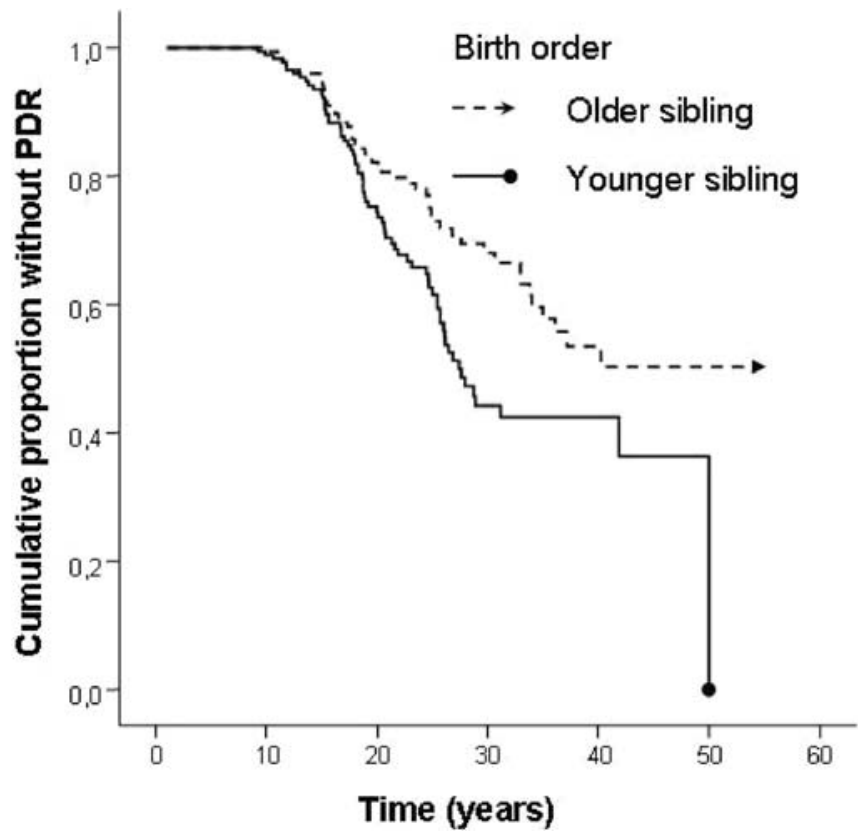

\section{OP 40 Islet cell differentiation and neogenesis}

\section{9}

Proteomic approach to identify novel soluble factors secreted by pancreatic stellate cells with paracrine effects on ARIP ductal cell model M. Lucas ${ }^{1}$, E. Mato 2 , S. Barceló ${ }^{1}$, A. Novials ${ }^{2}$, R. Gomis ${ }^{1}$.

${ }^{1}$ Endocrinology and diabetes, IDIBAPS-Hospital Clinic, Barcelona, ${ }^{2}$ Diabetes Unit, Sardà Farriol Foundation, Barcelona, Spain.

Background and Aim: Active pancreatic stellate cells (PaSCs) secrete many soluble factors important in pancreas physiology. Most of them are associated with fibrosis and matrix turnover. Other roles including paracrine effects on progenitors have not been investigated. In pancreas, there is no consensus on the nature of the adult pancreatic stem cells or where these progenitors are located. Several authors have proposed that a subpopulation of ductal cells has the capacity of transdifferentiation into endocrine phenotype, but the external and morphogenic signals that determine their differentiation are still unknown. The aim of this study was to analyse the secretome of PaSC in order to identify novel soluble factors that could have paracrine effects on ductal cells.

Materials and Methods: PaSC were isolated and expanded from pancreas of lactating rats. The culture medium of PaSC (conditioned medium) was collected and used to treat ARIP ductal cells for 24 or 72 hours. Proliferation and viability assays were performed by BrdU and MTT, and gene expression of CK19 (ductal marker) and PDX1 (endocrine marker) were quantified by Real-Time PCR. The soluble factors of the conditioned medium were identified by proteomics using two-dimensional electrophoresis (2D) coupled to mass spectrometry MALDI-TOFF and peptide mass fingerprint (Aldente (Expasy)). Identified proteins (focus proteins) were validated by RT-PCR, and mapped onto Ingenuity Pathways. Also ELISA assay was performed.

Results: PaSC conditioned medium inhibited proliferation of ARIP cells $40 \%$ as compared to control media without affecting cell viability. Additionally, conditioned media-treated cells showed 5.4-fold PDX1 mRNA levels and 0.48-fold CK19 mRNA levels compared to control cells, being these differences statistically significant (Student's t-test). 112 protein spots identificated in the conditioned medium were then selected for analysis, and 72 of these were successfully identified. 19 of them were soluble factors (with signal peptide), and were included in clusters of development/differentiation, chaperones, proteases, immune response, transport related proteins, GTPases, extracellular matrix and retinol related proteins. Proteomic data mining by Ingenuity Pathway Analysis displayed several significant networks. One of them highlighted TGF $\beta 1$ as a non-focused protein. Presence of this factor in the conditioned medium was confirmed by ELISA immunoassay $(48.95 \pm 5.18 \mathrm{pg} / \mathrm{mL})$, and ARIP cells were shown to express TGF $\beta 1$ receptors. To evaluate the importance of TGF $\beta 1$ in this cellular model, we blocked their effects with a pan-neutralizing antibody. The results showed that the rate of proliferation was recovered to initial levels, but TGF $\beta 1$ had no effect on the PDX1 expression increase. Finally, the secretome identificated three novel proteins: PEDF, LIF and Wnt5b, all of them involved in differentiation and developmental processes in different biological systems.

Conclusion: We characterised for the first time the PaSC secretome. This secretome inhibits proliferation and induces changes of gene expression in ARIP ductal cell line. TGF $\beta 1$ secreted by PaSC has the antiproliferative effect, whereas novel soluble factors identificated such as PEDF, LIF and Wnt5b, could be involved in the PDX1 and CK19 transcription changes Supported by the Spanish Ministerio de Sanidad y Consumo, ISCIII, REDIMET

\section{0}

Hedgehog signaling during expansion and beta cell differentiation of human pancreatic islet-derived mesenchymal (hPIDM) cells

R. Gallo ${ }^{1}$, V. Tellone ${ }^{1}$, G. Bernardi ${ }^{1}$, P. Marchetti ${ }^{2}$, M. Masini², E. Ferretti ${ }^{3}$, A. Gulino ${ }^{3}$, F. Dotta ${ }^{1}$;

${ }^{1}$ Dept. Internal Medicine and Endocrine Sciences, University of Siena, ${ }^{2}$ Dept. Endocrinology and Metabolism, University of Pisa, ${ }^{3}$ Dept.

Experimental Medicine and Pathology, University of Rome, Italy.

Background and Aims: Hedgehog (Hh) signalling is a key regulator of cell fate and differentiation, as well as of stem/progenitor cell maintenance 
and self-renewal in many organs. "In vitro" generation of regulated insulinproducing cells from stem/progenitor cells is crucial to develop a proper celltherapy as a cure for type 1 diabetes. In the light that Hh signalling plays an important role both in endocrine pancreas development and in the regulation of insulin secretion in beta cell lines, we have studied the expression of $\mathrm{Hh}$ pathway components during expansion and beta-cell differentiation of primary precursor cells derived from human pancreatic islets.

Materials and Methods: Human Pancreatic Islet-Derived Mesenchymal (hPIDM) cells were obtained by plating human pancreatic islets in tissue culture dishes with RPMI 1640 medium containing 10\% FBS. hPIDM cells, cultured for 21 days in serum-free RPMI, were differentiated into a pancreatic endocrine phenotype. Origin and phenotype of proliferating and differentiated hPIDM cells were studied by immunofluorescence, electron microscopy, FACS, and Real-Time-PCR analysis. To study the Hh signalling we evaluated, by real time RT-PCR, expression of target genes Gli1 and Gli2, activators of Hh pathway, and of repressor Gli3, in human islets and in proliferating as well as differentiated hPIDM cells. The effect of Hh signalling on proliferation of hPIDM cells was evaluated using cyclopamine, a known inhibitor of Hh pathway.

Results: Human pancreatic islets attached within $24 \mathrm{hrs}$ in "in vitro" culture, showing a central "core" of aggregated cells surrounded at the periphery by a migrating population of flattened epithelial cells. Double immunofluorescence analysis, for Ki-67, nestin, C-peptide and PDX-1, after 3 and 6 days of culture, showed proliferating cells deriving from $\beta$-cells. These cells were maintained in culture for up to 20 passages and approximately $10^{10}$ cells were obtained from each original islet seeded. FACS, immunofluorescence and Real-TimePCR revealed a transition from endocrine to mesenchymal phenotype of such proliferating cells (hPIDM). These cells, in serum free RPMI, generated "islet-like" aggregates showing C-peptide and glucagon induction, by immunofluorescence analysis, and beta-cell like secretory granules, by electron microscopy. Real-Time-PCR confirmed an acquired endocrine pancreatic phenotype of these aggregates. In addition, Gli1 and Gli2 mRNA levels were highly upregulated, paralleled by down-regulated Gli3, in hPIDM cells vs. human islets, in accordance with an activation of Hh signalling in hPIDM cells. Interestingly, in hPIDM-derived islet-like aggregates, an inhibition of the Hh pathway was confirmed by reduction of Gli1 and Gli2 and induction of Gli3 expression. Cyclopamine administration decreased the proliferating rate of hPIDM cells in a dose-dependent manner.

Conclusion: Taken together, these results indicate a direct involvement of Hh signalling in the $\beta$-cell-mesenchymal transition occurring in "in vitro" cultured human islets. Based on these data Hh signalling could contribute either to stimulate proliferation, or to maintain a "precursor" phenotype in the hPIDM cells.

Supported by the Ministry of the Health

\section{1}

A CK19+ population of insulin-producing surrogate cells generated from mouse embryonic stem cells

O. Naujok $^{1}$, F. Francini ${ }^{1}$, A. Jörns ${ }^{2}$, S. Lenzen ${ }^{1}$;

${ }^{1}$ Institute of Clinical Biochemistry, Hannover Medical School, Germany,

${ }^{2}$ Center of Anatomy, Hannover Medical School, Germany.

Background and Aims: Embryonic stem (ES) cells are a potential source for insulin-producing cells, which have the potential to differentiate into tissues from all three germ layers. The scarcity of donor islets of Langerhans for therapy of type 1 diabetes mellitus has focused research on the development of surrogate cells with characteristics similar to those of pancreatic beta cells. It was therefore the aim of this study to develop a differentiation protocol, which drives differentiation of ES cells towards insulin-producing cells and to combine this with a technique for separation of these insulin-positive cells.

Materials and Methods: A $2.9 \mathrm{~kb}$ promoter fragment of the Krt19 gene (cytokeratin 19) was cloned to drive the expression of eYFP in stably transfected ES-D3 cells. eYFP+ and eYFP- cells were FACS sorted on d12, $\mathrm{d} 19$ and $\mathrm{d} 26$ of differentiation and gene expression was examined by qPCR analyses. The differentiation stages were additionally studied by electron microscopy (EM) and immunohistochemistry.

Results: The pCK19-eYFP construct was successfully integrated into ES cells. Analysis of the gene expression of the endogenous cytokeratin 19 revealed a 4 to 7 fold higher cytokeratin 19 expression in eYFP+ cells when compared to eYFP- cells. Carbonic anhydrase 2, as a second marker for ductal cell progeny, showed a 2 fold increase in eYFP+ cells at day 12 and a 9 fold increase in expression in eYFP+ cells at day 26 when compared to eYFP- cells. Quantitative analysis of gene expression revealed a low expression of insulin in eYFP+ and eYFP- cells up to day 19. A significant 80 fold increase in insulin gene expression was observed in eYFP+ cells on day 26 as compared to day 12 and day 19. Compared with eYFP- cells insulin gene expression on day 26 was higher by a factor of 38 , whereas the level of insulin gene expression in eYFP- cells did not change significantly. Glucagon gene expression was not detectable at day 12 and day 19. While glucagon gene expression remained barely detectable at day 26 in eYFP+ cells, it increased to appreciable levels in eYFP- cells 12 times higher than in eYFP+ cells. Ultrastructure of differentiated eYFP+ cells, in contrast to eYFP- cells, exhibited clear signs of differentiation comprising subcellular organelles for synthesis, processing, storage and release of insulin. Moreover, eYFP + cells showed positive staining for C-peptide and insulin in the cytoplasm providing evidence for ongoing active pre-proinsulin biosynthesis and for insulin processing, while CK19cells were negative for C-peptide.

Conclusion: Cytokeratin 19 is a marker for pancreatic duct cells. Duct cells have been considered to be a pool for newly derived beta cells during organogenesis of the pancreas. Following this assumption, we have set up a separation strategy using FACS sorting of fluorescent eYFP+ and nonfluorescent eYFP- cells. Gene expression analysis of the endogenous CK19 revealed, that this gene is predominantly expressed in the FACS-sorted eYFP+ population. Thus, our selection procedure purifies ductal progenitor cells and separates them successfully from an eYFP-, non-ductal cell population. A detailed molecular, biochemical and morphological analysis revealed that eYFP+ cells represent a population of differentiated ES cells, which express the insulin prohormone gene, and process, store and release insulin.

\section{2}

Generation and characterization of human beta cells long term cultures

L. Labriola ${ }^{1}$, M. G. Peters ${ }^{2}$, K. Krogh ${ }^{1}$, I. Stigliano ${ }^{2}$, M. C. C. Machado ${ }^{3}$,

E. Bal de Kier Joffé 2 , L. Puricelli ${ }^{2}$, M. C. Sogayar ${ }^{1,4}$;

${ }^{1}$ NUCEL- Cell and Molecular Therapy Center, USP, São Paulo -SP, Brazil,

${ }^{2}$ Institute of Oncology, UBA, Buenos Aires, Argentina,

${ }^{3}$ Surgery, School of Medicine, University of São Paulo, Brazil,

${ }^{4}$ Biochemistry, Chemistry Institute, USP, São Paulo, Brazil.

Background and Aims: The use of primary beta-cells in biochemical and molecular research is limited by the availability of pancreatic endocrine tissue. The in vitro culture of insulinomas, which are rare pancreatic tumors arising from beta-cells, provides an attractive tool to study cell proliferation and insulin synthesis and secretion. However, only few human beta-cell 1 lines have been described, with long-term passage resulting in loss of insulin secretion. Therefore, we set out to establish and characterise human insulinoma cell cultures.

Materials and Methods: Three ex-vivo primary cultures (APM, VGA and CPR) were obtained from independent donors after surgical resection and tissue processing. In order to further characterise these cultures, we assessed insulin and C-peptide levels by electrochemiluminescence. Specific antigenic characteristics were determined by immunofluorescence (IF) and Western blotting (WB) assays. Beta-cell markers were examined by Real-time RTPCR. We also examined serum starvation and Doxorrubicin-induced apoptosis, tumorigenicity by in vivo assays, cell migration and invasion by wound healing, Transwell and zymography assays.

Results: Ex-vivo primary cultures obtained from independent human insulinomas (APM, and CPR cultures) and VGA cultures derived from a human nesideoblastosis case, were cultured up to passage number 15 (approximately 20 weeks), presenting a similar doubling time (40h). APM cells were 1.5 times more sensitive to apoptosis induced by serum starvation than the other cell lines, while only CPR cells were sensitive to Doxorubicin (48h treatment, $0.5-12 \mathrm{uM}$ ), with an IC50 of 5.1uM. We observed that these cell cultures produce and secrete human insulin and C-peptide, but none of them display glucose-induced insulin secretion. IF assays revealed that the APM, CPR and VGA cultures express Chromogranin A, Insulin, C-peptide, Glut2 and PDX-1, while no positive staining was detected for Glucagon, Desmin, Vimentin and CK19, confirming the beta-cell nature of these cell cultures. Furthermore, using IF and WB, we determined that all of these cultures express betal-integrin and beta-catenin, but only APM was positive for E-cadherin. All beta cell lineages survived an anchorage independent culture forming spheroids, while only APM proliferated under these conditions. VGA cells presented the highest motility, as demonstrated 
by wound healing assays (VGA: $30 \%$ of wound coverage vs. $17 \%$ displayed by the other cell lines, at $18 \mathrm{~h}$ post-healing). All cell lines secreted similar MMP9 activity while no MMP2 was detected. However, none of them were able to invade an extracellular matrix substrate, as shown by Transwell assays.

Finally, in vivo tumorigenesis assays in nude mice showed no tumor formation when VGA cells were tested and mice were examined three times a week for up to 60 days after cell suspension inoculation.

Conclusion: We have developed three human beta cell lines that maintain the characteristic antigenic and insulin secretion profiles of the original tumors. Although the behavior of these cell lines does not perfectly mimic the primary beta-cell physiology, they are extremely valuable tools for the study of molecular events underlying beta-cell function and dysfunction.

Supported by FAPESP, CNPq, FINEP, PRP-USP, CABBIO-CBAB

\section{OP 41 Novel biomarkers for coronary vascular disease}

\section{3}

The inflammation marker YKL-40 is elevated in patients with type 2 diabetes and is associated with an increased mortality rate in normal individuals after adjustment for risk factors of cardiovascular events C. N. Rathcke ${ }^{1}$, I. Raymond ${ }^{2}$, C. N. Kistorp ${ }^{1}$, J. Faber ${ }^{1}$, H. Vestergaard ${ }^{1}$; ${ }^{1}$ Dep. of Endocrinology, Herlev Hospital, Herlev, ${ }^{2}$ Dep. of Cardiology, Gentofte Hospital, Gentofte, Denmark.

Background and Aims: Patients with type 2 Diabetes (T2D) have an increased mortality due to cardiovascular disease (CVD). We have previously found that the inflammation marker YKL-40 is elevated in patients with T2D and is correlated with insulin resistance and concentrations of fatty acids and triglycerides, but not with CRP nor with parameters of the glycaemic profile. Furthermore, patients suffering from acute coronary syndrome have elevated levels of YKL-40. All together this indicates a specific association to the accelerated atherosclerosis in patients with T2D. In the present study we focus on normal individuals without any history of CVD to state the baseline correlation between levels of YKL-40 and the development of CVD and the mortality status. The follow-up period was 5 years.

Materials and Methods: Blood and urine samples were provided from 658 normal individuals aged 50 to 89 years recruited from general practices in a community of Copenhagen, Denmark. 32 participants were excluded because of prevalent heart failure, renal insufficiency and lack of followup data including a total of 626 participants in the study. Participants were examined between September 1998 and January 2001 and were followed up until December 2003 with respect to mortality status and the development of CVD. Median follow-up period was $5.0(0.17-5.28)$ years. Codes of diagnosis for CVD were pre-specified according to the International Classification of Diseases (ICD-10) and were cross-checked with the discharge registry of The Danish National Board of Health, which records all primary hospital discharge diagnoses in Denmark. Deaths from CVD were ascertained from central registers in the Danish National Board of Health and verified by study physicians from death certificates.

Results: Concentrations of YKL-40 were significantly elevated in patients with T2D compared with healthy individuals $(149,14 \mathrm{ng} / \mathrm{ml}$ vs. 100,14 $\mathrm{ng} / \mathrm{ml}, \mathrm{p}<0,0001)$. The absolute unadjusted increase in mortality risk for patients with YKL-40 > median $(59,50 \mathrm{mg} / \mathrm{ml})$ vs. YKL- $40 \leq$ median was $15,4 \%(24 \%$ vs. $9 \%, \mathrm{p}<0,0001)$. After adjustment for the cardiovascular risk factors of age, sex, smoking, diabetes, hypertension and ischemic heart disease, total cholesterol, CRP and serum creatinine the hazard ratio

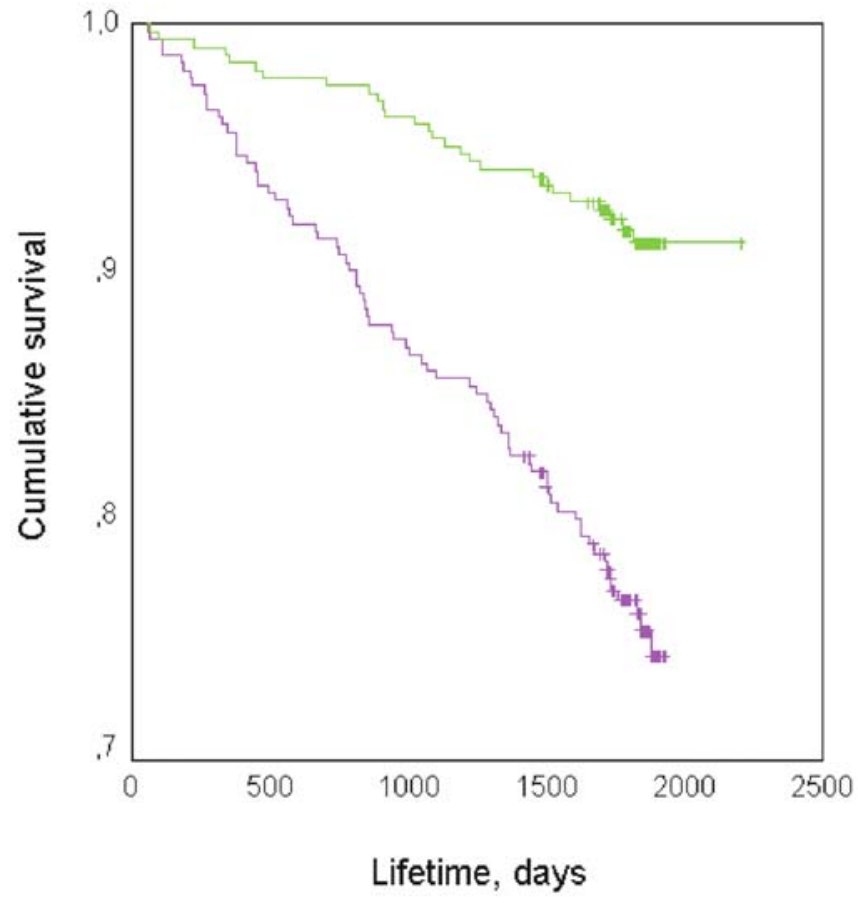


of mortality for values of YKL-40 > median $(59,5 \mathrm{ng} / \mathrm{ml})$ was $1,63(95 \%$ confidence interval (CI) 1,30-2,01). Additional adjustment for urinary albumin/creatinine ratio and NT-proBNP did not attenuate the predictive value of YKL-40 significantly (HR, 1,54; CI, 1,21-1,95, p<0,0001). YKL-40 was not found to be a specific predictor of coronary heart disease, stroke or cardiovascular death.

Conclusion: YKL-40 is elevated in patients with T2D and is an independent predictor of death of all causes without specific prediction of macrovascular disease or cardiovascular death.

\section{4}

Adam 17 is elevated in pre-diabetic and diabetic patients

M. Cardellini, R. Menghini, S. Rizza, R. Lauro, M. Federici;

Department of Internal Medicine, University of Rome "Tor Vergata”, Italy.

Background and Aims: The Regulated Membrane Proteolysis Network (RMPN) is a system that controls the shedding of several cell surfaceanchored molecules such as cytokines, growth factors and receptors and is modulated by metalloproteases belonging to the ADAMs family, particularly ADAM17. This system sustains chronic inflammation that may play a role in the pathogenesis of insulin resistance and related endothelial dysfunction. To investigate if an activation of the RMPN is related to the glucose metabolism impairment we studied two different cohorts: 1) healthy first degree relatives of type 2 diabetic patients (FDRs) and 2) atherosclerotic patients (diabetics, DM2, or nondiabetics, ATS) who underwent to carotid endoarteriectomy.

Materials and Methods: In the first cohort (35 healthy FDRs) we evaluated insulin sensitivity by euglycemic-hyperinsulinemic clamp study, the carotid artery intima-media thickness, as an early manifestation of atherosclerosis, and the endothelial dysfunction by flow mediated vasodilation (FMD). Monocytes were isolated from peripheral blood in order to assess expression of LOX-1, CCR-2, ADAM17 and TIMP-3 genes and some soluble molecules, such as sICAM-1, sVCAM-1, sTNFRI, sRIL-6 and IL-6, were measured to evaluated the membrane proteolytic cleavage through elisa assays.

In the second cohort $(\mathrm{n}=70, \mathrm{DM} 2=21, \mathrm{ATS}=49)$ we analyzed expression of ADAM17 in the atherosclerotic plaques obtained from carotid endoarteriectomy.

Results: In FDRs, both insulin sensitivity $(r=-0,381 p=0,04)$ and intimamedia thickness $(r=0,545 p=0,003)$ were significantly correlated with plasmatic levels of soluble receptor of interleukin 6 (sR-IL6), a specific substrate for ADAM17. Next, we dichotomized FDRs in insulin sensitive and insulin resistant using the glucose disposal rate. We observed that ADAM17 expression was significantly increased in insulin resistant $(n=17)$ compared with insulin sensitive $(n=18)$ subjects $(p=0,03)$.

In the second cohort, we found that the expression of ADAM17 is higher in plaques from diabetic patients than in non-diabetic patients $(\mathrm{p}=0,05)$ and it correlates with HOMA-IR $(\mathrm{r}=0.42, \mathrm{p}<0.04)$ and fasting plasma glucose $(\mathrm{r}=0.315, \mathrm{p}<0.02)$.

Conclusions: Our data suggest that ADAM17 expression and activity is increased in prediabetic subjects showing early atherosclerosis and in patients affected by atherosclerosis and diabetes compared with normoglucose tolerant atherosclerotic patients.

\section{5}

Inverse correlation between activated protein $\mathrm{C}$ generation and carotid atherosclerosis in type 2 diabetic patients

K. Matsumoto ${ }^{1}$, Y. Yano ${ }^{1}$, R. Araki ${ }^{2}$, M. Suematsu-Urawa ${ }^{1}$, H. Akatsuka ${ }^{1}$, T. Suzuki ${ }^{1}$, Y. Sasaki ${ }^{1}$, N. E. Bruno ${ }^{1}$, A. Katsuki ${ }^{1}$, E. C. Gabazza ${ }^{3}$,

Y. Sumida ${ }^{1}$;

${ }^{1}$ Division of Diabetes,Metabolism and Endocrinology, Mie University Graduate School of Medicine, Tsu, Mie, ${ }^{2}$ Department of Internal Medicine, Mie National Hospital, Tsu, Mie, ${ }^{3}$ Department of Immnology, Mie University Graduate School of Medicine, Tsu, Mie, Japan.

Background and Aims: Activated protein C (APC), generated on endothelial cells after cleavage of protein $\mathrm{C}$ by the thrombin-thrombomodulin complex, is a key regulator of the clotting system and immune responses. Previous studies have shown that less numbers of and less activity of thrombomodulin on endothelial cells from vascular areas with atherosclerosis have decreased APC generation. These observations suggest that insufficient APC generation is associated with more progressive forms of atherosclerosis. This study aimed at studying the relationship between the degree of atherosclerosis as measured by the intima-media thickness (IMT) of carotid artery and APC generation in type 2 diabetic patients.

Materials and Methods: This study comprised 87 type 2 diabetic patients and 35 normal subjects. The degree of APC generation was assessed by the plasma level of activated protein C-protein C inhibitor complex (APC$\mathrm{PCI}$ ). APC-PCI, a marker of ongoing protein $\mathrm{C}$ activation, was measured by enzyme-linked immunoassay. The mean IMT of carotid artery was measured by ultrasonography. The plasma levels of thrombin-antithromobin complex (TAT) and platelet-derived growth factor (PDGF) were measured by enzymelinked immunoassays.

Results: The plasma level of TAT, a sensitive marker of coagulation activity was significantly increased in diabetic patients compared with normal subjects $(1.28 \pm 0.88$ vs. $2.00 \pm 1.06 \mathrm{ng} / \mathrm{ml}$; mean $\pm \mathrm{SD}, \mathrm{p}<0.01)$. This observation confirmed the existence of hypercoagulability in diabetic patients. The plasma level of APC-PCI $(1.79 \pm 0.77$ vs. $1.01 \pm 0.56 \mathrm{ng} / \mathrm{ml}$; $\mathrm{p}<0.001)$ was significantly lower in type 2 diabetic patients than in normal subjects. The mean IMT $(0.669 \pm 0.140$ vs. $0.881 \pm 0.242 \mathrm{~mm} ; \mathrm{p}<0.01)$ was significantly increased in type 2 diabetic patients as compared to normal subjects. Univariate analysis showed a significant and inverse correlation between the plasma level of APC-PCI and the mean IMT $(r=-0.32, \mathrm{p}<0.005)$, and multivariate regression analysis showed that both were significantly and independently correlated $(\mathrm{p}<0.05)$. Moreover, the plasma level of APC-PCI was significantly and inversely correlated with PDGF $(r=-0.30, p<0.01)$ in all diabetic patients. PDGF participates in the pathogenesis of vascular remodeling and atherosclerosis. We have reported that APC directly inhibits the secretion of PDGF from macrophages, endothelial and epithelial cells. Thus, decreased APC generation may favor increased production and secretion of PDGF and this may explain the inverse correlation between the plasma levels of PDGF and APC-PCI in type 2 diabetic patients.

Conclusion: These results suggest that decreased APC generation is associated with vascular atherosclerotic changes in type 2 diabetic patients.

Supported by the Suzuken Memorial Foundation

\section{6}

Visceral fat thickness measured by ultrasonography is associated with carotid intima-media thickness even among diabetic men with normal waist circumference

S.-K. Kim, S.-W. Park, Y.-W. Cho;

Department of Internal Medicine, College of Medicine, Pochon CHA University, Sungnam, Republic of Korea.

Background and Aims: The simple way of assessing visceral fat accumulation is measuring waist circumference. However, there might be substantial variations in the visceral fat amount among persons with similar waist circumference because waist circumference itself could not discriminate between visceral fat and subcutaneous fat. In this study, we investigated whether measurement of visceral fat amount by ultrasonography may have an additional role in assessing atherosclerotic burden in men with type 2 diabetes.

Materials and Methods: Two-hundred and thirteen Korean men with type 2 diabetes mellitus were participated in this study. Abdominal obesity was defined as waist circumference $\geq 90 \mathrm{~cm}$, which is an Asian criterion for abdominal obesity. Visceral fat thickness (VFT) defined as the distance between the anterior wall of the aorta and the internal face of the rectoabdominal muscle was measured by ultrasonography, and $47.6 \mathrm{~mm}$ of VFT was selected as the cutoff for excess accumulation of visceral fat (visceral obesity). Atherosclerotic burden was assessed by a measure of carotid arterial intima-media thickness (IMT).

Results: Among subjects with abdominal obesity ( $\mathrm{n}=97), 20$ subjects $(21 \%)$ had no visceral obesity (Group 1). In contrast, among the subjects without abdominal obesity $(\mathrm{n}=116), 55$ patients $(47 \%)$ showed visceral obesity (Group 2). The subjects in group 2 showed higher VFT compared to those in group 1 $(58.4 \pm 10.5$ vs. $37.8 \pm 11.5 \mathrm{~mm}, \mathrm{P}<0.001)$, even though subjects in group 2 had significantly lower waist circumference $(85.1 \pm 3.1$ vs. $94.7 \pm 5.4 \mathrm{~cm}, \mathrm{P}<$ 0.001). There were no differences in age, HbAlc, total cholesterol, and HDLcholesterol concentrations between two groups. However, maximal carotid IMT $(0.93 \pm 0.35$ vs. $0.72 \pm 0.23 \mathrm{~mm}, \mathrm{P}<0.001)$ and mean carotid IMT $(0.73$ 
\pm 0.22 vs. $0.63 \pm 0.176 \mathrm{~mm}, \mathrm{P}<0.001)$ were significantly increased in group 2 compared with group 1 .

Conclusion: About half of diabetic men with normal waist circumference had visceral obesity when assessed by ultrasound-measured VFT. Those who had visceral fat accumulation showed a higher carotid IMT regardless of their waist circumference. In addition to waist circumference, simultaneous measurement of VFT may provide an additional role in assessing atherosclerotic burden in men with type 2 diabetes.

\section{OP 42 Metabolism in vasculature}

\section{7}

Insulin receptor substrate 1 and 2 are key regulators of vasoactive responses in skeletal muscle arteries

W. Bakker ${ }^{1}$, P. Sipkema ${ }^{1}$, C. D. A. Stehouwer ${ }^{2}$, V. W. M. van Hinsbergh ${ }^{1}$, E. C. Eringa ${ }^{1}$;

${ }^{1}$ Laboratory for Physiology, Institute for cardiovascular research, Amsterdam,

${ }^{2}$ Internal Medicine, Academic Hospital Maastricht, The Netherlands.

Background and Aims: Insulin actions in vasoreactivity and glucose uptake are regulated by insulin receptor substrates (IRSs). In the microcirculation insulin exerts vasodilator effects through activation of Akt and Nitric Oxide and vasoconstrictor effects through activation of endothelin-1. IRS1 and IRS2 are thought to be involved in the development of insulin resistance and the metabolic syndrome, which is associated with a diminished activity of insulin to stimulate vasodilation. The aim of this study was to determine the exact role of IRS1 and IRS2 in vasoactive responses in micro- and macrocirculation of skeletal muscle.

Material and Methods: To investigate insulin-mediated vasoreactivity and maximal passive response, we used gracilis and femoral artery of IRS1 KO, IRS2 KO mice and their wild type (WT) littermates. The arteries were isolated and studied in a pressure myograph under physiological conditions.

Results: In the WT mice, the maximal inner diameters of the gracilis and femoral artery were $128 \mu \mathrm{m} \pm 4$ and $311 \mu \mathrm{m} \pm 9$ respectively. Furthermore, insulin induced an endothelin-1 dependent vasoconstriction and an Aktdependent vasodilator response in the gracilis artery of WT mice. Mice lacking IRS1 were insulin resistant and mice lacking IRS2 were diabetic. In the IRS1 KO and IRS2 KO mice, the maximal diameters of the gracilis and femoral artery were reduced by $24 \% \pm 1$ and $15 \% \pm 5$ for IRS1 KO and $20 \% \pm 3$ and $14 \% \pm 2$ for IRS2 KO respectively, compared to their WT littermates. Furthermore, insulin had no effect on the diameter, but showed an impaired vasoconstriction and a reduced Akt-dependent vasodilator response in the gracilis artery of both IRS1 and IRS2 KO mice.

Conclusions: Genetic deletion of either IRS1 or IRS2 results in a reduction of the maximal diameter of both micro- and macrocirculation of skeletal muscles, which implies that IRS1 and IRS2 regulate structural remodelling of skeletal resistance arteries. In addition, both IRS1 and IRS2 seem to be involved in both vasodilator and vasoconstrictor effects of insulin in the microcirculation of skeletal muscle. This suggests that IRS1 and IRS2 are key regulators in insulin dependent vasoactive responses in skeletal muscle arteries.

\section{8}

Insulin-like effect of adiponectin to vasodilate against endothelin-1-mediated vasoconstriction in muscle C. T. Bussey, S. Rattigan, M. G. Clark, S. M. Richards; Muscle Diabetes Research Group, Menzies Research Instutite, Hobart TAS, Australia.

Background and Aims: We recently reported that insulin opposes vasoconstriction due to endothelin in the constant-flow pump-perfused isolated rat hindlimb preparation, possibly by acting through nitric oxide release. Insulin has not been found to dilate against any other vasoconstrictor in perfused hindlimb, making endothelin unique in this respect. Adiponectin exhibits a number of actions similar to insulin, for example stimulation of glucose uptake in incubated muscle, and reduction of glucose release from hepatocytes. A number of recent studies have also indicated that adiponectin acts acutely to increase nitric oxide production by the endothelium, and it has been shown to relax isolated aortic rings pre-constricted with prostaglandin F2. These data suggest that adiponectin may act in a similar manner to insulin to vasodilate against endothelin-1. However, to date no study has examined the vasoactivity of adiponectin in an intact vascular system. The aim of this study was therefore to determine whether adiponectin opposes the vascular actions of endothelin-1 in an intact perfused muscle system.

Materials and Methods: Adiponectin was expressed in E. coli and purified using an N-terminal His tag. SDS-PAGE gels showed the recombinant adiponectin to be mainly the trimer or low molecular weight form. 
Adiponectin (final concentration $10 \mathrm{ug} / \mathrm{ml}$ ) was infused for $20 \mathrm{~min}$ in the constant flow pump-perfused isolated rat hindlimb. Infusion of endothelin$1(1 \mathrm{nM})$ commenced $10 \mathrm{~min}$ after the adiponectin infusion started, and continued for 10min after adiponectin infusion ceased, to examine reversal of adiponectin-induced changes. Perfusion pressure, oxygen consumption and release of lactate into the venous effluent were monitored.

Results: Endothelin-1 infusion increased perfusion pressure by $25 \mathrm{mmHg}$ and exhibited a type A vasoconstrictor effect by increasing oxygen uptake and lactate release. Infusion of adiponectin reduced the endothelin-1induced perfusion pressure increase by approximately $60 \%$, blunted the increase in oxygen consumption and almost completely abolished the lactate release caused by endothelin-1. Removal of adiponectin during continued endothelin-1 infusion did not result in an immediate rapid rise in perfusion pressure or lactate release, suggesting that the actions of adiponectin do not reverse rapidly.

Conclusion: To our knowledge, this is the first time that adiponectin vasoactivity has been demonstrated in an intact vascular system. We have shown that adiponectin has similar actions to insulin in opposing vasoconstriction due to endothelin-1 in the isolated perfused rat hindlimb. This suggests that in addition to its effects on skeletal myocyte glucose uptake, adiponectin may also be involved in the vascular control of muscle insulin action.

\section{9}

Oleate induces an insulin resistance "selective" for the PI3-K/Akt pathway in cultured human and rat vascular smooth muscle cells P. Del Mese, I. Russo, G. Doronzo, L. Mattiello, M. Viretto, G. Anfossi, M. Trovati;

Department of Clinical and Biological Sciences, University of Turin, Orbassano, Italy.

Background and Aims: Increased Free Fatty Acids concentrations (FFAs) are a link between obesity and insulin resistance, because FFAs interfere with insulin signaling, inducing the so called "lipid toxicity". Relevant targets of insulin action are Vascular Smooth Muscle Cells (VSMC): the FFA influence on insulin signaling in these cells, however, is not known. We aimed at investigating whether oleate (a 18-carbon cis-monosaturated FA) influences the insulin-induced activation of the Phosphatydil Inositol 3-kinase (PI3-K)/ Akt/p70S6K and of the Mitogen-Activated Protein Kinase (MAPK) pathways in VSMC from humans and from lean and obese Zucker rats.

Materials and Methods: Protein expression and phosphorylation of Akt, p70S6K, ERK1/2 and JNK1/2 were evaluated by Western blots after $6 \mathrm{~h}$ incubation with $2 \mathrm{nM}$ insulin in aortic VSMC from humans, lean insulin sensitive and obese insulin resistant Zucker rats, in the absence and in the presence of a 30-min preincubation with oleate $100 \mu \mathrm{M}(\mathrm{n}=8)$

Results: Data are expressed as mean \pm SEM. In human VSMC: i)oleate did not induce phosphorylation of Akt, p70S6K, ERK1/2, and JNK1/2; ii)the insulininduced Akt phoshorylation $(162.3 \pm 2.5 \%$ of baseline, $\mathrm{p}=0.0001)$ was blunted in the presence of oleate $(103 \pm 3.1 \%$ of baseline); iii)the insulin-induced p70S6K phosphorylation $(175.6 \pm 1.9 \%$ of baseline, $\mathrm{p}=0.001)$ was blunted in the presence of oleate ( $101.4 \pm 3.5 \%$ of baseline); iv)the insulin-induced ERK1 and ERK2 phosphorylation $(206.8 \pm 4.6 \%$ and $177.1 \pm 3.1 \%$ of baseline, respectively, $\mathrm{p}=0.0001$ for both) was not modified in the presence of oleate (202.1 $\pm 2.4 \%$ and $170.5 \pm 4.1 \%$ of baseline, ns vs insulin alone); v)the insulininduced JNK1 and JNK2 phosphorylation $(240.2 \pm 2.6 \%$ and $192.6 \pm 3.0 \%$ of baseline, respectively, $\mathrm{p}=0.0001$ for both) was not modified in the presence of oleate ( $229.3 \pm 2.8 \%$ and $195.5 \pm 1.9 \%$ of baseline, ns vs insulin alone). In VSMC from lean and obese Zucker rats, oleate did not induce phosphorylation of Akt and ERK1/2. Insulin-induced phosphorylation of Akt and ERK1/2 was smaller in VSMC from obese than in those from lean Zucker rats $(\mathrm{p}=0.0001$ for Akt, $\mathrm{p}=0.003$ for ERK1, $\mathrm{p}=0.001$ for ERK2). Both in lean and in obese Zucker rats, oleate reduced the insulin-induced phosphorylation of Akt ( $p=0.001$ for both), but not that of ERK1/2. In the presence of insulin+oleate, no significant difference was observed between Akt phosphorylation in lean and obese Zucker rats.

Conclusions: In human VSMC, oleate influences insulin signaling by inhibiting the PI-3K/Akt/p70S6K pathway without affecting the MAPK pathway, thus inducing a "selective" insulin resistance. Furthermore, our study confirms our previous observation that in cultured VSMC from obese Zucker rats the insulin-induced activation of both PI3-K and MAPK pathways is similarly impaired: however, when VSMC are incubated "in vitro" with oleate at concentrations occurring "in vivo" in insulin resistant states, we can observe the "selective" inhibition of the insulin activated PI-3K pathway that has been described "in vivo" or "ex vivo" in insulin resistant states. This "selectivity", therefore, seems not to be an intrinsic characteristic of these cells but a phenomenon induced by circulating factors increased in insulin resistant states, such as FFAs.

Supported by the Italian Ministry of University

\section{0}

The TACE/TIMP3 dyad regulates the inflammatory infiltrate caused by obesity in vascular and metabolic tissues

M. Federici ${ }^{1}$, R. Menghini ${ }^{1}$, S. Menini ${ }^{2}$, R. Amoruso ${ }^{1}$, L. Fiorentino ${ }^{1}$, M. Serino ${ }^{1}$, G. Pugliese ${ }^{2}$, R. Khokha ${ }^{3}$, R. Lauro';

${ }^{1}$ Dept. of Internal Medicine, University of Tor Vergata, Rome, Italy,

${ }^{2}$ Dept. of Clinical Sciences, University of La Sapienza, Rome, Italy,

${ }^{3}$ Dept. of Medicine, Ontario Cancer Institute, Toronto, ON, Canada.

Background and Aims: We recently observed that a reduction in Tissue Inhibitor of Metalloproteinase-3 (TIMP-3) couples the onset of type 2 diabetes to vascular inflammation in Insulin Receptor heterozygous knockout mice. TIMP3 is the major inhibitor of TNF-a Converting Enzyme (TACE or ADAM17) as well as cognate proteases such as ADAM10,12,15, all acting via a process called REGULATED MEMBRANE PROTEOLYSIS to shed from plasma membrane several growth factor and cytokines. This shedding process generates soluble factors with paracrine/endocrine activity. ADAMs were hypothesized to play a role in inflammatory and metabolic disorders, but no mechanistic evidences were provided.

Materials and Methods: First, we treated 3T3F442A preadipocytes with free fatty acids (FFA) to study TACE activation. Next, to test the therapeutic role of the TACE/Timp3 dyad in metabolic and vascular complications of obesity, we studied WT, Insr+/-, Timp3-/-, Tace+/-, double Insr+/-/Timp3-/- and double Insr+/-/Tace+/-, all fed a HFD.and

Results: We found in preadipocytes that FFA caused TACE serine phosphorylation $(\mathrm{p}<0.01$ versus basal) and increased TNF-alpha shedding ( $p<0.01$ versus basal), an effect abolished by TACE inhibitor, TAPI-1. Then, in the same cell model we observed TACE overexpression is coupled to 8 -fold increase of MCP1 expression ( $\mathrm{p}<0.01$ versus mock cells). Effect of HFD in animal models revealed that both glucose tolerance and insulin sensitivity were significantly reduced in double Insr+/-/Timp3-/- compared to other genotypes $(p<0.001$ for both intraperitoneal glucose and insulin tolerance test). We found by immunohistochemistry analysis that HFD caused: increased MCP1 expression and macrophage accumulation in multiple organs including WAT, liver, aorta and kidney in Insr+/-/Timp3-/- mice but not in Insr+/-/Tace+/- mice, while WT, Insr+/-and Timp3-/- mice had an intermediate phenotype. In the liver of Insr+/-/Timp3-/- we found increased steatohepatisis (grade 3) compared with WT, Insr+/-and Timp3-/- mice (all grade 1) and Insr+/-/Tace+/- mice (grade 0). In the aorta of Insr+/-/Timp3I- we observed presence of fatty streaks and increased RAGE expression. No morphological alterations were found in WT, Insr+/-and Timp3-/- mice and Insr+/-/Tace+/-. A similar pattern of mixed inflammation/lipotoxicity was found in the kidney with the following grading: Insr+/-/Timp3-/- > WT > Insr+/-/Tace+/-.

Conclusion: Our results suggest that the TACE/Timp3 pathway integrates signals derived from High Fat Diet to trigger the development of inflammatory burden associated to diabetes, atherosclerosis, glomerulopathy and steatohepatitis. Our results suggest that TACE inhibitors should be tested for treatment of obesity-related metabolic and vascular diseases.

Supported by EFSD/SERVIER GRANT 2005 


\section{OP 43 Metformin and sulfonylureas}

\section{1}

\section{Metformin activates the AMP-activated protein kinase in hepatocytes by changes in adenine nucleotides \\ B. Guigas ${ }^{1,2}$, M. Foretz $^{3}$, X. Stephenne ${ }^{4}$, E. Sokal ${ }^{4}$, B. Viollet ${ }^{3}$, L. Hue ${ }^{2}$; ${ }^{1}$ Dept. of Molecular Cell Biology, Leiden University Medical Center, The Netherlands, ${ }^{2}$ Hormone and Metabolic Research Unit, Université catholique de Louvain and Christian de Duve Institute of Cellular Pathology, Brussels, Belgium, ${ }^{3}$ Department of Genetics, Development and Molecular Pathology, Institut Cochin, Paris, France, ${ }^{4}$ Laboratory of Paediatric Hepatology and Cell Therapy, Université catholique de Louvain and Cliniques St Luc, Brussels, Belgium.}

Background and Aims: The precise mechanism of action of metformin, one of the most prescribed drugs for type 2 diabetes, still remains incompletely elucidated. During the last years, metformin has been shown to activate the AMP-activated protein kinase (AMPK), which is considered as a cellular energy sensor. This remarkable effect, which was observed in several tissues, could explain some of the cellular effects of metformin. However, the exact pathway(s) involved in AMPK activation by the drug are still elusive. Indeed, while metformin has been previously shown to induce specific inhibition of the mitochondrial respiratory-chain complex 1 in the liver, early works performed on various cell lines suggested that the biguanide activated AMPK without increasing AMP-on-ATP ratio. The aim of this study was to test the hypothesis that AMPK activation by metformin was associated with modification of adenine nucleotides in liver cells.

Materials and Methods: Hepatocytes were isolated from both rat and mouse livers and from human liver segments unused for transplantation. Primary cultured hepatocytes were treated with increasing dose of metformin (100 $\mu \mathrm{M}-1000 \mu \mathrm{M}$ ) for $24 \mathrm{~h}$ following determination of AMPK activity (after both PEG fractionation and immunoprecipitation of $\alpha 1$ and $\alpha 2$ subunits separately) and expression, ACC phosphorylation and intracellular concentrations of adenine nucleotides. The mitochondrial oxygen consumption rates were measured on both intact and permeabilized hepatocytes isolated from wildtype and AMPKal $\alpha 2$ liver specific knockout mice and incubated for $30 \mathrm{~min}$ with metformin.

Results: Here we report that metformin concentrations higher than 250 $\mu \mathrm{M}$ activated AMPK in primary cultured hepatocytes from both rat and human, this effect being clearly associated with an increase in AMP-on-ATP ratio. Interestingly, human hepatocytes exhibited very low level of AMPK $\alpha 2$ expression and activity (less than $2 \%$ of the total activity) compared with rats and metformin only activated this AMPK isoform. In addition, we also demonstrated that the specific inhibitory effect of metformin on the mitochondrial respiratory-chain complex 1 evidenced in hepatocytes from wild-type mice was still present in mice lacking both liver $\alpha 1$ and $\alpha 2$ AMPK catalytic subunits.

Conclusion: We conclude that (i) AMPK activation by metformin is associated with significant modifications of adenine nucleotides in hepatocytes and that (ii) inhibition of the respiratory-chain complex 1 by metformin is independent of AMPK.

Supported by the European Union FP6 programm (Exgenesis). B. Guigas is recipient of the ICP- "Michel de Visscher" Fellowship

\section{2}

Impact of metformin treatment on glycaemic control and cardiovascular risk-factors in patients with poorly controlled type 1 diabetes (T1DM)

P. K. Jacobsen ${ }^{1}$, S. S. Lund ${ }^{1}$, L. Tarnow ${ }^{1}$, A.-S. Astrup ${ }^{1}$, A. C. Alibegovic ${ }^{1}$, P. Hovind ${ }^{1}$, L. Pietraszek ${ }^{1}$, M. Frandsen ${ }^{1}$, I. Parving ${ }^{1}$, P. Rossing ${ }^{1}$,

H.-H. Parving ${ }^{2}$, A. A. Vaag';

${ }^{1}$ Steno Diabetes Center, Gentofte, ${ }^{2}$ Dep of Medical Endocrinology, University of Copenhagen; Rigshospitalet, Denmark.

Background and Aims: Despite intensive insulin-treatment many patients with T1DM have longstanding poor glycaemic control. Metformin is an oral hypoglycaemic agent that improves insulin-action in patients with type 2 diabetes. We aimed to investigate the effect of one year treatment with metformin versus placebo in patients with T1DM and persistent poor glycaemic control.
Materials and Methods: One hundred patients with T1DM, preserved hypoglycaemic awareness and poor glycaemic control $\left(\mathrm{HbA}_{1 c} \geq 8.5 \%\right)$ during one year prior to enrolment were included in a one month run-in period on placebo treatment. Hereafter patients were randomised (baseline) to receive treatment with either metformin (1g twice-daily) or placebo (double-masked) for 12 months. Patients continued ongoing insulin-therapy and usual outpatient clinical care. Changes in insulin doses were allowed. The primary endpoint was baseline adjusted changes in $\mathrm{HbA}_{1 c^{\circ}}$. Patients were evaluated at baseline and after 12 months.

Results: At enrolment mean (SD) $\mathrm{HbA}_{1 c}$ was: metformin ( $\mathrm{n}=49$ ): $9.48 \%$ (0.99); placebo ( $\mathrm{n}=51)$ : $9.60 \%(0.86)$. After 12 months the mean $(95 \% \mathrm{CI})$ baseline-adjusted change in $\mathrm{HbA}_{1 \mathrm{c}}$ was: metformin: $-0.10 \%(-0.32 ; 0.12)$; placebo: $-0.23 \%(-0.45 ;-0.01)$. Mean $(95 \% \mathrm{CI})$ baseline-adjusted differences after 12 months during metformin versus placebo: $\mathrm{HbA}_{1 c} 0.13 \%(-0.19 ; 0.44)$, $\mathrm{p}=0.422$; Total daily insulin-dose: $-5.6 \mathrm{U} /$ day $(-8.4 ;-2.7), \mathrm{p}<0.001$; Bodyweight: $-1.74 \mathrm{~kg}(-3.32 ;-0.17), \mathrm{p}=0.030$; Fasting LDL-cholesterol: -0.27 $\mathrm{mmol} / \mathrm{l}(-0.56 . ; 0.33), \mathrm{p}=0.080$ (in subjects with stable lipid-lowering therapy: $\mathrm{p}=0.018$ ). The reported number of minor and major hypoglycaemic events was not significant different between treatments. Mean (SD) compliance with the study-medication was: metformin: $85.7 \%$ (17.5); placebo $86.5 \%$ (15.7). In subgroups of patients with baseline $B M I \geq 25, \geq 27$ or $\geq 30 \mathrm{~kg} / \mathrm{m}^{2}$ or with baseline $\mathrm{HbA}_{1 \mathrm{c}}$ above the median $\left(\mathrm{HbA}_{1 \mathrm{c}}>9.15 \%\right)$ there was no significant change in $\mathrm{HbA}$ after one year between treatments or within the metformin treated group alone. Adjustment for baseline insulin-dose did not change the between treatment effect on $\mathrm{HbA}$ substantially.

Conclusion: In patients with poorly controlled T1DM, metformin treatment does not improve glycaemic control after one year. However, metformin might have other potential beneficial effects on CVD risk-factors such as insulin-dose, body-weight and LDL-cholesterol.

Supported by the Danish Diabetes Association

\section{3}

Impact of chronic metformin and sulfonylureas treatment in diabetic patients developing an acute myocardial infarction

M. Zeller ${ }^{1}$, N. Danchin ${ }^{2}$, J.-M. Pernes ${ }^{3}$, P. Steg ${ }^{4}$, D. Carrie ${ }^{5}$, P. Quandalle ${ }^{6}$, G. Amat ${ }^{7}$, E. Durand ${ }^{2}$, J.-P. Cambou ${ }^{8}$, G. Mulak ${ }^{8}$, T. Simon';

${ }^{1}$ Cardiology, CHU Dijon, ${ }^{2}$ Cardiology, HEGP, Paris,

${ }^{3}$ Cardiology, Hôpital Privé Antony, ${ }^{4}$ Cardiology, CHU Bichat, Paris,

${ }^{5}$ Cardiology, CHU Toulouse, ${ }^{6}$ Cardiology, $\mathrm{CH}$ Roubaix,

${ }^{7}$ Cardiology, CH Vichy, ${ }^{8}$ Société Française de Cardiologie,

${ }^{9}$ Pharmacology, CHU St Antoine, Paris, France.

Background and Aims: Little information is available on the impact of chronic oral antidiabetic medications on outcomes in patients developing an acute myocardial infarction. Aim: To assess early and 6-month mortality according to the prior use of metformin and sulfonylureas in diabetic patients admitted for AMI in a nationwide French registry.

Materials and Methods: The FAST-MI registry included consecutive patients admitted for ST-elevation or non-ST elevation myocardial infarction $\leq 48$ hours of symptom onset, in 223 French intensive care units over 2 months from October 2005. In-hospital and 6-month outcome was compared for all 1,316 diabetics. In diabetic patients, 4 groups were defined according to their antidiabetic treatment before admission: no SU and no metformin (S- M-, $\mathrm{n}=594)$, SU alone (,$+ \mathrm{n}=264)$, metformin alone $(\mathrm{M}+, \mathrm{n}=257)$, and combination of $\mathrm{SU}$ and metformin $(\mathrm{S}+\mathrm{M}+\mathrm{n}=201)$.

Results: Age was $71 \pm 12$ for M-S-, $68 \pm 10$ for $\mathrm{M}+, 71 \pm 11$ for S+ and $67 \pm$ 11 years for $\mathrm{S}+\mathrm{M}+(\mathrm{p}<0.001)$. GRACE risk score was higher in $\mathrm{S}-\mathrm{M}-(173 \pm$ $39)$ and $S+(168 \pm 38)$, compared with $M+(163 \pm 36)$ and $M+S+(162 \pm 36)$ $(\mathrm{p}=0.001)$. HbAlc (available in half of the patients) was higher in $\mathrm{M}+\mathrm{S}+(8.0$ $\pm 1.6 \%)$ compared with S-M- $(7.5 \pm 1.6), \mathrm{S}+(7.3 \pm 1.4)$ or $\mathrm{M}+(7.4 \pm 1.4)$ $(\mathrm{p}<0.005)$ and entry glycaemia was also higher $(2.39 \pm 1.18 \mathrm{~g} / \mathrm{L})$ vs. $\mathrm{S}+(2.10$ $\pm 0.98), \mathrm{M}+(1.90 \pm 0.67)$ or $\mathrm{S}-\mathrm{M}-(2.07 \pm 1.18)(\mathrm{p}<0.001)$.

Five-day mortality was $6.1 \%(\mathrm{M}-\mathrm{S}-), 3.5 \%(\mathrm{M}+), 2.3 \%(\mathrm{~S}+)$ and $0.5 \%(\mathrm{M}+\mathrm{S}+)$ $(\mathrm{p}<0.001)$. Six-month survival was $80 \%, 86 \%, 87 \%$ and $95 \%$, respectively $(\mathrm{p}<0.001)$ (Figure). Using Cox multivariate analysis, the odds ratio for 6month mortality compared to the M-S-group were: 0.84 (95\%CI: $0.56-1.25$, $\mathrm{p}=\mathrm{NS})$ for $\mathrm{M}+, 0.65$ (95\%CI: $0.43-0.97, \mathrm{p}<0.05)$ for $\mathrm{S}+$ and $0.27(95 \% \mathrm{CI}$ : $0.13-0.54, \mathrm{p}<0.001)$ for $\mathrm{M}+\mathrm{S}+$.

Conclusion: In this real world registry, the combined use of sulfonylureas and metformin was associated with improved early and 6-month survival after acute myocardial infarction. Similar outcomes were observed for patients on 
metformin or sulfonylureas alone, but using multivariate adjustments, only sulfonylureas alone was significantly associated with improved survival.

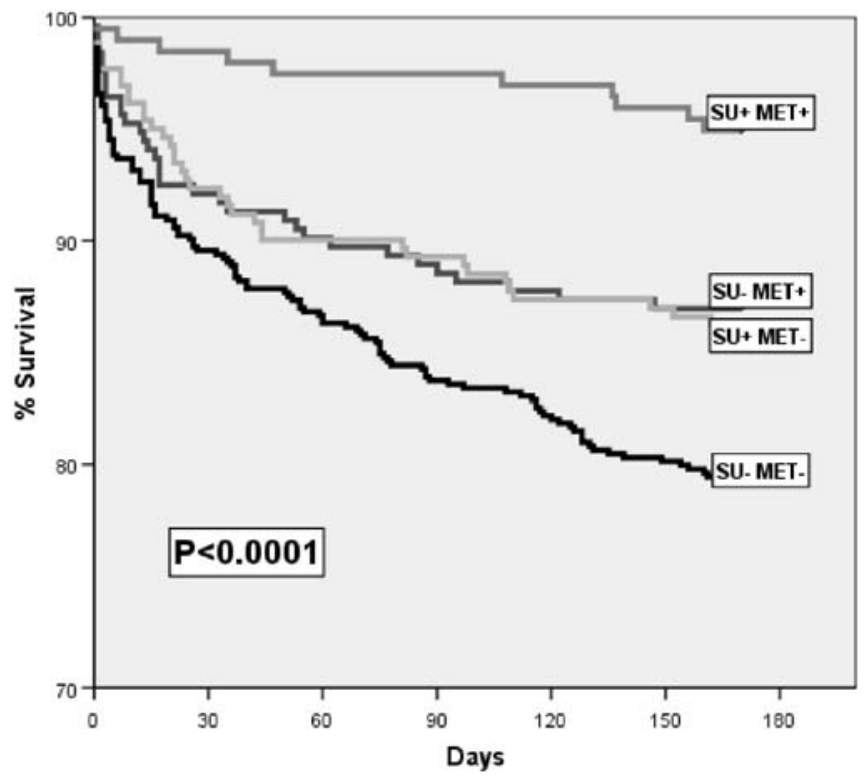

Supported by Pfizer, Servier, CNAM

\section{4}

Treatment with insulin secretagogues and cancer-related mortality in type 2 diabetic patients. A cohort study

M. Monami ${ }^{1}$, C. Lamanna ${ }^{1}$, M. Petracca ${ }^{2}$, D. Balzi ${ }^{3}$, I. Dicembrini ${ }^{2}$,

N. Marchionni ${ }^{1}$, C. M. Rotella ${ }^{2}$, E. Mannucci' ${ }^{1}$;

${ }^{1}$ Critical Care Medicine and Surgery, Unit of Gerontology - University

of Florence, Italy, ${ }^{2} \mathrm{Clinical}$ Pathophysiology, Section of Endocrinology

- University of Florence, Italy,

${ }^{3}$ Local Health Unit 10, Epidemiology Unit, Florence, Italy.

Background and Aims: Recent evidence suggests that some hypoglycemic treatments could affect the incidence of malignancies. In particular, metformin has been associated with a reduced incidence of cancer. Conversely, sulfonylureas and insulin have been found to be associated with increased cancer-related mortality; insulin treatment, in particular, seems to be associated with a higher incidence of colorectal cancer. This study was aimed at the assessment of cancer-related mortality in type 2 diabetic patients treated with different hypoglycemic drugs.

Materials and Methods: A retrospective observational cohort study was performed on a consecutive series of 3002 type 2 diabetic outpatients. Cancerrelated death was identified through the City Registry Office

Results: During a mean follow-up of $4.3 \pm 2.5$ years, 87 cases of cancerrelated death were recorded, with a yearly incidence rate of $0.70 \%$. Patients receiving secretagogues $(n=1241)$ showed a significantly higher mortality than the rest of the sample (unadjusted OR [95\% CI] 1.76 [1.15-2.69], $\mathrm{p}=0.009$ ), which was maintained after adjustment for sex, age, duration of diabetes, HbA1c, BMI, Charlson's Comorbidity Scale (CCS), smoking status and alcohol abuse (HR 2.29 [1.21-4.02], $\mathrm{p}=0.003$ ). Conversely, no significant association of cancer-related mortality was observed with insulin sensitizers $(n=1302)$ or exogenous insulin $(n=764)$. In order to exclude the possible effect of drug interactions, a separate analysis was performed in patients treated with only one hypoglycemic agent. When compared with those receiving no drug treatment for diabetes $(n=727)$, patients on secretagogue monotherapy $(\mathrm{n}=372)$ showed a significantly higher cancer-related mortality (unadj. OR 2.29 [1.10-4.82]; $\mathrm{p}=0.028$ ). Insulin treatment $(\mathrm{n}=421)$ was also associated with a higher cancer-related mortality (OR 2.26 [1.10-4.69]; $\mathrm{p}=0.28)$. After adjustment for age, sex and CCS, both insulin secretagogues (HR 2.25 [1.10-4.78]; $\mathrm{p}=0.034$ ) and insulin (HR 2.11 [1.01-4.50], $\mathrm{p}=0.048$ ) were associated with a significantly higher cancer-related mortality in comparison with patients receiving no drug treatment. Among patients on secretagogue monotherapy, glyburide was associated with a higher cancerrelated mortality (unadjusted OR 4.41[1.23-15.90]; $\mathrm{p}=0.023$ ) in comparison with subjects receiving other drugs of the same class, which was confirmed at multivariate analysis after adjustment for age, sex, and CCS (HR 4.31 [1.1616.00]; $\mathrm{p}=0.029)$. Conversely, monotherapy with insulin sensitizers $(n=444)$ was not associated with significant differences in cancer-related mortality in comparison with patients receiving no hypoglycemic drugs (OR 0.77 [0.282.15], $\mathrm{p}=0.621$ ).

Conclusion: Insulin secretagogues and, to a lesser extent, exogenous insulin, appear to be associated with increased mortality for cancer, even after adjustment for multiple confounders. This issue deserves further investigation through epidemiological studies on larger samples of patients. 


\section{OP 44 Treatment of type 1 diabetes in pregnancy}

\section{5}

Hypoglycaemia during first trimester of pregnancy in type 1 diabetes M. García Domínguez, L. Herranz de la Morena, L. Sáez de Ibarra, E. Moya Chimenti, L. Pallardo Sánchez;

Servicio de Endocrinología y Nutrición, Hospital Universitario La Paz, Madrid, Spain.

Background and Aims: It is widely assumed that women with type 1 diabetes are at increased risk of hypoglycaemic episodes, particularly of severe hypoglycaemia, during the first trimester of pregnancy. Our objective was to investigate frequency and characteristics of hypoglycaemia, during first trimester of pregnancy, in women with type 1 diabetes.

Materials and Methods: We conducted a longitudinal survey, comparing glycaemic parameters between preconception and first trimester of pregnancy, in 64 women with type 1 diabetes (mean age $31.9 \pm 2.9$, mean diabetes duration $13.8 \pm 7.2$ ). who were attended at the Diabetes and Pregnancy Unit from preconception throughout pregnancy. All women were requested to perform home blood glucose monitoring using the same type of glucose meter. Glucose readings stored in the meter were downloaded to a computer at each visit. The mean number of glucose readings/day was $4.9 \pm 1.3$ during preconception and $4.8 \pm 0.9$ during first trimester. Severe hypoglycaemia was defined as any hypoglycaemic episode for which external help was required. Frequency of non-severe hypoglycaemia (percentage of glucose readings below $50 \mathrm{mg} / \mathrm{dl}$ ) and nocturnal hypoglycaemia (proportion of all hypoglycaemic episodes that were nocturnal), mean glucose levels, mean standard deviation of mean glucose and mean bedtime glucose levels were obtained from the glucose readings. HbAlc was measured during preconception and at 12 weeks gestation.

Results: Both during preconception and first trimester, only 5 women ( 7.8 $\%)$ were affected by severe hypoglycaemia. Only two patients $(3.1 \%)$ had severe hypoglycaemia during preconception and during first trimester. Frequency of non severe hypoglycaemia did not differ significantly between preconception and first trimester $(4.3 \pm 3.7$ vs. $4.6 \pm 2.7 \%$; $\mathrm{p}=0.557)$. Nocturnal hypoglycaemia was significantly higher during first trimester than during preconception $(27.8 \pm 27.4$ vs. $14.2 \pm 26.2 \%$; $=0.000)$. HbAlc $(6.7 \pm$ 0.7 vs. $6.4 \pm 0.6 \%, p=0.000)$, mean glucose levels $(140.7 \pm 18.6$ vs. $136.1 \pm$ $15.3 \mathrm{mg} / \mathrm{dl}, \mathrm{p}=0.011)$ and mean standard deviation of mean glucose $(69.5 \pm$ 13.5 vs. $65.2 \pm 11.2 \mathrm{mg} / \mathrm{dl}, \mathrm{p}=0.000$ ) were significantly lower during first trimester. Mean bedtime glucose levels $(164.3 \pm 33.8 \mathrm{vs.} 164.9 \pm 23.8 \mathrm{mg} / \mathrm{dl})$ and mean daily insulin dose $(0.62 \pm 0.15$ vs. $0.64 \pm 0.15 \mathrm{U} / \mathrm{kg})$ did not differ significantly.

Conclusion: Women with type 1 diabetes who attend a preconception programme are not at increased risk of severe hypoglycaemia during the first trimester of pregnancy. The overall frequency of non-severe hypoglycaemia is not different during preconception and first trimester; however, a shift towards nocturnal hypoglycaemia during the first trimester of pregnancy is observed. This trend does not seem related to bedtime glucose levels nor to a change in insulin dose.

\section{6}

Foetal outcomes associated with glargine's use during pregnancy: the Italian National Survey

E. Torlone, G. Di Cianni, M. Bonomo, G. Capuano, A. Di Benedetto, M. Dolci, I. Franzetti, M. Imbergamo, V. Manicardi, D. Mannino, A. Napoli, L. Sciacca, L. Tonutti, E. Vitacolonna, A. Lapolla;

on behalf of the Italian Diabetes and Pregnancy Study Group, Italian Society of Diabetology, Roma, Italy.

The long-acting insulin analog glargine (IAG) is largely used as a basalbolus regimen in type 1 diabetes mellitus (T1DM), however its use is not still recommended during pregnancy. We promoted a national, multicentre, retrospective study to determine the rate of congenital malformation in a large group of offspring of mothers with T1DM who were treated with IAG before and during pregnancy. The observation's period included all pregnancies ended before August 31 2006. We collected data on 107 TIDM pregnancies from 29 Italian centres and evaluated both maternal data and foetal outcomes. The rate of congenital malformation (CM) was compared with published data. The mean maternal age was $30.4 \pm 4.7$ years, diabetes duration $15.8 \pm 7.7$ years, White's Classes $\mathrm{B}=23 \%, \mathrm{C}=25 \%, \mathrm{D}=35 \%, \mathrm{R}=16 \%, \mathrm{RF}=1 \%$ ). IAG was started $10.3 \pm 6.9$ months before conception as a basal-bolus regimen with an average dose of $19.2 \pm 7.5 \mathrm{IU} /$ die. The pregnancy was unplanned in $92 \%$ of cases and the first diabetes assessment occurred at $8.7 \pm 4.1$ weeks of pregnancy (Mean $\mathrm{HbAlc} 7.7 \pm 1.3 \%$, range 5.3-13.8\%). IAG was continued until the end of pregnancy in $38.3 \%$ of case, whereas it was stopped during the first trimester of gestation in $61.7 \%$. Results: Glycemic control improved during the whole pregnancy with a mean $\mathrm{HbAlc}$ of $6.5 \pm 0.9 \%$ at the end of pregnancy. Spontaneous and elective abortions occurred in 5 and 2 women respectively. 100 babies were delivered at $36.7 \pm 2.1$ week (pre-term deliveries $30.0 \%$, caesarean sections $75.5 \%$ ) with mean birth weight of $3298.0 \pm 717.4$ g (LGA 42.7\%). CM were reported in 5 newborns (5.0\%), involving cardiovascular $(n=2)$, genitourinary system $(n=2,1$ minor $)$, and syndactylia $(n=1)$. The rate of CM is not different from that reported in other studies regarding pregnancies of T1DM women treated with other insulins.

Conclusions: Our survey, performed in a large cohort of T1DM pregnant women, suggest that insulin glargine does not seem to affect embryo-foetal development.

\section{7}

Use of insulin aspart from pregnancy planning may reduce the incidence of severe hypoglycaemia - data from the insulin aspart pregnancy study S. Heller ${ }^{1}$, E. Mathiesen ${ }^{2}$, P. Damm ${ }^{3}$, M. Hod ${ }^{4}$, A.-S. Pade Hansen 5 ,

K. Kanc Hanzel';

${ }^{1}$ Clinical Sciences Centre, University of Sheffield, United Kingdom,

${ }^{2}$ Dept. Endocrinology, Rigshospitalet, Copenhagen, Denmark,

${ }^{3}$ Obstetric Clinic, Rigshospitalet, Copenhagen, Denmark,

${ }^{4}$ Perinatal Division, Tel Aviv University, Petah-Tiqva, Israel,

${ }^{5}$ Novo Nordisk A/S, Bagsvaerd, Denmark.

Background and Aims: Planning of pregnancy in order to achieve optimal glucose control has been shown to reduce malformations and early fetal losses in pregnant women with type 1 diabetes. Whether pregnancy planning also reduces the risk of severe hypoglycaemia is unknown. In this controlled trial,

Tab. 1 Abstract 0247

Severe and Nocturnal (0:00-06:00) hypoglycaemia. Rate is episodes/exposure year.

\begin{tabular}{|c|c|c|c|c|c|c|}
\hline \multicolumn{7}{|c|}{ All severe hypoglycaemic episodes, number (rate) } \\
\hline \multirow[t]{2}{*}{ Population based on time of randomization } & \multicolumn{2}{|c|}{ Planning phase } & \multicolumn{2}{|c|}{ Pregnancy } & \multicolumn{2}{|c|}{ Post partum } \\
\hline & IAsp & $\mathrm{HI}$ & IAsp & $\mathrm{HI}$ & IAsp & $\mathrm{HI}$ \\
\hline Prior to pregnancy & $13(0.9)$ & $34(2.4)$ & $15(0.6)$ & $60(1.9)$ & $1(0.2)$ & $17(2.2)$ \\
\hline Early pregnancy & - & - & $98(1.7)$ & $114(2.2)$ & $37(2.6)$ & $16(1.5)$ \\
\hline
\end{tabular}

Nocturnal severe hypoglycaemic episodes, number (rate)

\begin{tabular}{|c|c|c|c|c|c|c|}
\hline \multirow[t]{2}{*}{ Population based on time of randomization } & \multicolumn{2}{|c|}{ Planning phase } & \multicolumn{2}{|c|}{ Pregnancy } & \multicolumn{2}{|c|}{ Post partum } \\
\hline & IAsp & $\mathrm{HI}$ & IAsp & $\mathrm{HI}$ & IAsp & $\mathrm{HI}$ \\
\hline Prior to pregnancy & $5(0.3)$ & $21(1.5)$ & $3(0.1)$ & $31(1.0)$ & $1(0.2)$ & $5(0.7)$ \\
\hline Early pregnancy & - & - & $23(0.4)$ & $28(0.5)$ & $9(0.6)$ & $6(0.6)$ \\
\hline
\end{tabular}


women were randomised to insulin aspart or human insulin. The primary endpoint was severe hypoglycaemia (requiring third party assistance) during pregnancy.

Materials and Methods: Mealtime insulin aspart (IAsp) was compared with human insulin (HI) in 99 subjects (IAsp:44, HI:55), randomised prior to known pregnancy and 223 subjects (IAsp:113, HI:110) randomised in early pregnancy. NPH insulin was used as basal insulin throughout. Severe hypoglycaemia was recorded prospectively before pregnancy (where possible), during pregnancy and post-partum. Subjects were followed throughout pregnancy with one visit per trimester and at 6 weeks postpartum.

Results: The observed rates of severe hypoglycaemia in subjects treated with IAsp pre-pregnancy were consistently lower than for $\mathrm{HI}$ treated subjects for the periods before pregnancy, during pregnancy and post pregnancy. The risk of severe hypoglycaemia was evaluated by the relative risk (RR) using a gamma frailty model. The estimated risk was $77 \%$ lower for IAsp than for HI during pregnancy (RR: 0.33; 95\% CI: [0.09; 1.27], non-significant, $\mathrm{p}=0.11$ ). The estimated risk for the period prior to pregnancy was $66 \%$ lower $(\mathrm{RR}=0.34$; 95\% CI: $[0.07 ; 1.71]$, non-significant, $\mathrm{p}=0.19)$. Post partum, the estimated risk was $92 \%$ lower (RR: $0.08,95 \%$ CI: [0.01; 0.84], $\mathrm{p}=0.04$ ). Overall, nine subjects in each treatment group had 15 (IAsp) and 60 (HI) episodes during pregnancy, suggesting that the observed differences were mainly due to a lower number of recurrent episodes of severe hypoglycaemia for IAsp treated subjects. There was a trend for the observed rate (episodes/exposure year) of severe hypoglycaemia during pregnancy for subjects treated with IAsp pre-pregnancy (0.6) to be lower than for subjects who initiated IAsp early in pregnancy (1.7). This was also true for severe nocturnal hypoglycaemia during pregnancy (0.1 vs. 0.4$)$. No subjects experienced hypoglycaemic coma during the trial. During pregnancy mean $\mathrm{HbA}_{1 \mathrm{c}}$ was the same in both treatment groups, $6.0 \%$ in the second trimester and $6.2 \%$ in the third trimester.

Conclusion: These results suggest that the use of insulin aspart from the planning phase of pregnancy might reduce the incidence of severe hypoglycaemia before, during and after pregnancy, although the statistical power to establish this was limited by the relatively small numbers. (Tab. 1) Supported by Novo Nordisk A/S

\section{8}

No increase in insulin antibodies during pregnancy in type 1 diabetes treated with insulin aspart

E. Mathiesen ${ }^{1}$, D. McCance ${ }^{2}$, P. Damm ${ }^{3}$, R. Kaaja ${ }^{4}$, M. Hod ${ }^{5}$, F. Dunne ${ }^{6}$, L. Jensen ${ }^{7}$, H. Mersebach;

${ }^{1}$ Dept. Endocrinology, Rigshospitalet, Copenhagen, Denmark,

${ }^{2}$ Regional Centre for Endocrinology and Diabetes, Royal Victoria Hospital,

Belfast, United Kingdom, ${ }^{3}$ Obstetric Clinic, Rigshospitalet, Copenhagen,

Denmark, ${ }^{4}$ Naistenklinikka, Helsinki University Hospital, Finland,

${ }^{5}$ Division of Maternal Fetal Medicine, Rabin Medical Center, Petah-Tiqva,

Israel, ${ }^{6}$ Dept. Medicine, Galway University College Hospital, Ireland,

${ }^{7}$ Novo Nordisk A/S, Bagsværd, Denmark.

Background and Aims: The development of insulin antibodies (AB) during pregnancy was assessed in women with type 1 diabetes, as part of the largest $(n=322)$ randomised controlled study of a rapid acting insulin analogue in pregnancy to date. The potential transfer of insulin and $A B$ to the child was assessed by analysis of umbilical cord blood.

Material and Methods: Mealtime insulin aspart (IAsp) was compared with human insulin (HI) in subjects who were pregnant or planning pregnancy at enrolment. NPH was used as basal insulin in both groups. Insulin AB were measured in a sub-group of 97 subjects (IAsp:50; HI:47). Antibodies were detected by radio-immunoassay, and insulin levels by ELISA. Maternal AB were assessed at baseline and gestational week (GW) 36. Cord blood samples were analysed for $\mathrm{AB}$ (IAsp:36; HI:29) and insulin levels (IAsp:14; HI:16) post delivery.

Results: Antibody levels specific to HI or IAsp remained low throughout pregnancy. A small but significant decrease was observed in maternal crossreacting $\mathrm{AB}$ levels from baseline to GW $36(\mathrm{p}=0.012)$. No correlation was observed between total daily insulin dose and the level of cross-reacting $\mathrm{AB}$ at GW 36 in either treatment group. There was a significant correlation between the level of cross-reacting $\mathrm{AB}$ in cord blood and maternal cross-reacting $A B$ measured at GW $36\left(\mathrm{R}^{2}=0.91 \mathrm{p}<0.0001\right)$. The analysis of insulin in cord blood detected IAsp in one sample $(22.9 \mathrm{pmol} / \mathrm{L})$, however the validity of this sample was questionable, as the HI level was unphysiologically high (10950 $\mathrm{pmol} / \mathrm{L}$ ), and it was omitted from analysis. Levels of IAsp were below the detection limit in all other samples analysed. HI levels in cord blood were widely distributed in both treatment groups, median levels were $67.5 \mathrm{pmol} /$ $\mathrm{L}$ (IAsp) and $286.5 \mathrm{pmol} / \mathrm{L}$ (HI), with an estimated ratio (IAsp/HI) of 0.40 , suggesting less hyperinsulinaemia in infants from the IAsp group $(\mathrm{p}=0.073)$. Conclusions: Levels of specific and cross-reacting $\mathrm{AB}$ were similar between IAsp and HI groups, and did not increase during pregnancy. The significant correlation between the level of cross-reacting $\mathrm{AB}$ in cord blood and maternal cross-reacting $\mathrm{AB}$ indicated a possible transfer of maternal $\mathrm{AB}$ across the placenta. On the contrary, cord blood analysis provided no clear evidence of transfer of IAsp across the placenta.

Changes in maternal insulin $\mathrm{AB}$ levels during pregnancy. ${ }^{*}$ Relative $\%$ antibody-bound radioactivity .

\begin{tabular}{|c|c|c|c|c|c|c|c|}
\hline \multirow[t]{2}{*}{ Treatment } & \multirow[t]{2}{*}{ Antibody } & \multicolumn{2}{|c|}{ Baseline } & \multicolumn{2}{|c|}{ GW 36} & \multicolumn{2}{|c|}{ Change } \\
\hline & & $\mathrm{n}$ & $\begin{array}{l}\text { Median* } \\
(\%)\end{array}$ & $\mathrm{n}$ & $\begin{array}{l}\text { Median* } \\
(\%)\end{array}$ & $\mathrm{n}$ & $\begin{array}{l}\text { Median* } \\
(\%)\end{array}$ \\
\hline IAsp + & IAsp-specific & 34 & 0.3 & 46 & 0.2 & 30 & -0.1 \\
\hline \multirow[t]{2}{*}{$\mathrm{NPH}$} & HI-specific & 34 & -0.1 & 46 & -0.1 & 30 & 0.0 \\
\hline & Cross-reacting & 34 & 6.9 & 46 & 3.7 & 30 & -2.2 \\
\hline $\mathrm{HI}+$ & IAsp-specific & 33 & 0.3 & 37 & 0.3 & 23 & -0.1 \\
\hline \multirow[t]{2}{*}{$\mathrm{NPH}$} & HI-specific & 33 & -0.1 & 37 & 0.0 & 23 & 0.0 \\
\hline & Cross-reacting & 33 & 8.6 & 37 & 4.1 & 23 & -2.7 \\
\hline
\end{tabular}

Supported by Novo Nordisk A/S 


\section{OP 45 Incretin effects: clinical}

\section{9}

Four weeks of near-normalization of blood glucose restores the insulin response to GIP and improves the insulin response to GLP-1 in patients with type 2 diabetes

P. V. Højberg ${ }^{1,2}$, T. Vilsbøll², M. Bache ${ }^{1}$, F. K. Knop ${ }^{2}$, R. Rabøl' ${ }^{1}$, T. Krarup², J. J. Holst ${ }^{3}$, S. Madsbad ${ }^{1}$;

${ }^{1}$ Endokrinology, Hvidovre Hospital, Hvidovre,

${ }^{2}$ Internal Medicin, Gentofte Hospital, Denmark, ${ }^{3}$ Medical Physiology,

Panum Institute, University of Copenhagen, Denmark.

Background and Aims: The incretin effect is attenuated in patients with type 2 diabetes mellitus, primarily because of a defect in beta-cell responsiveness to glucose-dependent insulinotropic polypeptide (GIP), while insulin response to glucagon-like peptide-1 (GLP-1) stimulation is relatively more preserved. The aim of the present study was to investigate whether 4 weeks of nearnormalization of blood glucose (BG) could improve the insulin responses to GIP and GLP-1 in patients with T2DM.

Materials and Methods: Eight patients with T2DM in poor glycemic control (BMI: $31.1 \pm 3.9 \mathrm{~kg} / \mathrm{m}^{2}, \mathrm{HbA}_{1}: 8.6 \pm 1.3 \%$, mean BG: $13.8 \pm 4.3 \mathrm{mM}$ (three days seven points glucose profiles); mean $\pm \mathrm{SD}$ ), were investigated before and after four weeks of near-normalization of BG using insulin treatment. $\mathrm{HbA}_{1 c}$ after four weeks of insulin treatment was $7.5 \pm 1.1 \%$, and mean BG was $7.4 \pm 1.2 \mathrm{mM}$. Before and after insulin treatment the patients underwent three hyperglycemic clamps $(15 \mathrm{mM})$ in random order, with infusion of either GLP-1 (1/2 pmol/ $\mathrm{kg} / \mathrm{min})$, GIP $\left(1 \frac{1}{2} \mathrm{pmol} / \mathrm{kg} / \mathrm{min}\right)$ or saline during 120 minutes. Insulin responses were evaluated as the incremental area under the plasma insulin curve from 0-120 minutes. For comparison eight control subjects were evaluated.

Results: Before and after near-normalization of $\mathrm{BG}$, the insulin responses were: during GIP infusions $(4.9 \pm 1.1 \mathrm{nM} / 120$ min vs. $20.7 \pm 10.4, \mathrm{P}<0.04)$; during GLP-1 infusions $(6.2 \pm 1.5 \mathrm{nM} / 120 \mathrm{~min}$ vs. $23.2 \pm 12.5, \mathrm{P}<0.008)$ and during saline infusions $(3.0 \pm 0.8 \mathrm{nM} / 120 \mathrm{~min}$ vs. $10.2 \pm 4.1, \mathrm{P}<0.008)$. Corresponding values in the control subjects were $148.9 \pm 32.1 ; 175.6 \pm 36 ; 57.3 \pm 12.5 \mathrm{nM} / 120$ min, respectively (GLP-1 vs. GIP; NS; GLP-1/GIP vs. saline; P< 0.05)

Conclusion: The results indicate that near-normalization of blood glucose for four weeks improves beta-cell responsiveness to both GLP-1, GIP and glucose per se with a factor 3-4. Nevertheless the insulin responses are still only $10-20 \%$ of those observed in the control subjects.

Supported by Novo Nordisk

\section{0}

Mechanisms of exenatide attenuation of postprandial hyperglycaemia in T2DM subjects

E. Cersosimo;

Medicine/Diabetes, University of Texas, San Antonio, United States.

Background and Aims: Chronic exenatide therapy markedly attenuates the rise in postprandial hyperglycemia and causes a modest reduction in fasting glucose concentration. Our aim was to examine the mechanisms responsible for these beneficial effects on glucose homeostasis

Materials and Methods: Six T2DM subjects $(45 \pm 8 \mathrm{y} ; 2 \mathrm{~F} / 4 \mathrm{M} ; \mathrm{BMI}=31 \pm 2$ $\left.\mathrm{kg} / \mathrm{m}^{2}, \mathrm{FPG}=171 \pm 18 \mathrm{mg} / \mathrm{dl}, \mathrm{HbA}_{1 \mathrm{c}}=8.1 \pm 0.8 \%\right)$ taking metformin $(\mathrm{n}=3)$ or a sulfonylurea $(n=3)$ received a mixed meal tolerance test (MMTT) before (PRE) and (POST) 2 weeks after exenatide, $5 \mu \mathrm{g}$ sc bid during the first week and $10 \mu \mathrm{g} \mathrm{sc}$ bid during the second week. Exenatide was not given on the day of the POST study. The mixed meal contained $600 \mathrm{cal}$ and 75 grams of glucose labeled with $100 \mu \mathrm{Ci}$ of [1]- ${ }^{14} \mathrm{C}$-glucose. Three hours prior to and for 6 hours after the ingestion of the meal subjects received a primed continuous infusion of 3-[ $\left.{ }^{3} \mathrm{H}\right]$-glucose. The total rate of appearance of glucose in the systemic circulation ( $\mathrm{RaT})$, the rate of oral glucose appearance $(\mathrm{RaO})$, endogenous (EGP) glucose production and splanchnic glucose uptake (SGU=75 grams minus $\mathrm{RaO}$ from 0-360 min) were measured with the dual isotope technique, and hepatic insulin sensitivity (HIS) was calculated as [100/EGPxFPI]. Plasma glucose, insulin, glucagon, and glucose kinetics were measured for 6 hours after the meal was consumed.

Results: In the PRE study, FPG ( $171 \pm 18 \mathrm{mg} / \mathrm{dl})$ increased to a mean of $225 \pm 23$ $\mathrm{mg} / \mathrm{dl}$ during the MMTT, insulin increased from $11 \pm 2$ to $15 \pm 3 \mu \mathrm{U} / \mathrm{ml}$, and glucagon remained unchanged ( $58 \pm 8$ vs. $57 \pm 7 \mathrm{pg} / \mathrm{ml}$ ). Following 2 weeks of exenatide (POST study), FPG decreased to $132 \pm 13 \mathrm{mg} / \mathrm{dl}$ and mean PG during the MMTT declined to $173 \pm 16 \mathrm{mg} / \mathrm{dl}$ (both $\mathrm{p}<0.01 \mathrm{vs.}$ PRE). Insulin increased from $7 \pm 3$ to $18 \pm 4 \mu \mathrm{U} / \mathrm{ml}$ and glucagon decreased from $66 \pm 6$ to $60 \pm 8$ $\mathrm{pg} / \mathrm{ml}$ (both $\mathrm{p}<0.01$, PRE vs. POST); body weight did not change significantly (87.4 \pm 6.2 to $86.6 \pm 4.8 \mathrm{~kg}, \mathrm{p}=\mathrm{NS})$. After exenatide, basal EGP decreased from $2.3 \pm 0.1$ to $1.8 \pm 0.2 \mathrm{mg} / \mathrm{kg} \cdot \mathrm{min}(\mathrm{p}<0.05)$, and the incremental area under the curve (AUC) for $\mathrm{RaO}$ over 6-hour post-meal was reduced by $\sim 50 \%$, $(\mathrm{p}<0.01)$. In the PRE study $43.3 \pm 4.2$ grams of the ingested glucose appeared in the systemic circulation over 6 hours compared with $23.2 \pm 4.9$ grams in the post-exenatide study $(\mathrm{p}<0.01)$. Thus exenatide induced a $\sim 60 \%(32.7 \pm 4.2$ vs. $52.5 \pm 4.9$ grams, $\mathrm{p}<0.01$ ) increase in $\mathrm{SGU}$ (plus delayed gastric emptying), and HIS increased $\sim 50 \%$ from $4.3 \pm 0.4$ to $6.7 \pm 0.6(\mathrm{mg} / \mathrm{kg} \cdot \mathrm{min})^{-1}(\mu \mathrm{U} / \mathrm{ml})^{-1}$ $(\mathrm{p}<0.05)$. In summary, exenatide treatment of T2DM subjects for two weeks: i) decreased FPG by inhibiting basal EGP; ii) attenuated the postprandial glycemic excursion by enhancing the suppression of endogenous glucose production during the meal and augmenting splanchnic glucose uptake; iii) increased insulin secretion ( $\Delta$ Insulin/ $\Delta$ Glucose); and iv) suppressed glucagon levels during the meal.

Conclusion: Chronic exenatide therapy (exenatide was not given on the day of MMTT and plasma exenatide levels were undetectable) profoundly influences splanchnic (hepatic) glucose handling by enhancing hepatic insulin sensitivity and augmenting splanchnic glucose uptake of ingested glucose. The role of delayed gastric emptying is yet undetermined. Glucagonlike-peptide 1 may represent the long-searched for "gut factor".

Supported by Amylin/Lilly Alliance

\section{1}

One year treatment with exenatide improves $\beta$ cell function and glycaemic control in metformin treated type 2 diabetes patients M. C. Bunck ${ }^{1}$, M. Diamant ${ }^{1}$, A. Cornér ${ }^{2}$, B. Eliasson ${ }^{3}$, J. L. Malloy ${ }^{4}$, R. M. Shaginian ${ }^{5}$, W. Deng' ${ }^{4}$, D. M. Kendall', M.-R. Taskinen'2, U. Smith ${ }^{3}$, H. Yki-Jarvinen ${ }^{2}$, R. J. Heine';

${ }^{1}$ Department of Endocrinology, VU University Medical Center, Amsterdam, The Netherlands, ${ }^{2}$ Department of Medicine, Helsinki University Hospital, Finland, ${ }^{3}$ Lundberg Laboratory for Diabetes Research, Sahlgrenska University Hospital, Goteborg, Sweden,

${ }^{4}$ Amylin Pharmaceuticals, Inc., San Diego, United States,

${ }^{5}$ Eli Lilly and Company, Nederland, Houten, The Netherlands.

Background and Aims: Traditional blood glucose lowering therapies do not sustain adequate glycaemic control in the majority of patients. Preclinical and clinical studies with exenatide, an incretin mimetic have shown improvements in $\beta$-cell function. In this investigator designed study, we compared the effect of one year exenatide vs. insulin glargine on $\beta$-cell function, measured with an euglycaemic-hyperinsulinaemic $(5 \mathrm{mM})$ followed by a hyperglycaemic (15 $\mathrm{mM}$ ) clamp combined with additional arginine stimulation.

Materials and Methods: Sixty-eight metformin-treated patients with DM2 (mean \pm SD: age $59 \pm 8$ y; HbAlc $7.5 \pm 0.8 \%$; BMI $31 \pm 4 \mathrm{~kg} / \mathrm{m} 2$; body weight $91.5 \pm 13.1 \mathrm{~kg}$ ) were randomised to exenatide ( $\mathrm{n}=36 ; 5 \mu \mathrm{g}$ BID for 4 weeks, $10 \mu \mathrm{g}$ BID with treat to HbAlc target $\leq 7 \%$, maximum dose $20 \mu \mathrm{g}$ TID thereafter) or insulin glargine ( $n=32$; treat to fasting plasma glucose (FPG) target $<5.6 \mathrm{mmol} / \mathrm{L}$ ). $\beta$ cell function was assessed as the week 52 to baseline ratio in glucose-potentiation $(15 \mathrm{mM})$ of arginine stimulated C-peptide secretion during the first 10 min following the arginine bolus. Additional endpoints included first and second phase C-peptide secretory response after IV glucose, HbAlc, body weight and safety.

Results: Interim analysis from 48 of the 68 randomised subjects showed arginine stimulated C-peptide secretion to be $125 \%$ [95\% CI 0.91 to 1.64 , $\mathrm{p}<0.0001]$ greater after 1 year treatment with exenatide $(\mathrm{n}=26)$ as compared to insulin glargine $(n=22)$ (mean ratio relative to baseline, for exenatide and insulin glargine \pm SEM: $2.45 \pm 0.16$ vs. $1.15 \pm 0.06$, respectively). First phase glucose-induced C-peptide secretion was 52\% [95\% CI 0.27 to 0.80 , $\mathrm{p}<0.0001]$ greater after 1 year of exenatide compared to insulin glargine therapy (exenatide $1.76 \pm 0.12$ vs. insulin glargine $1.21 \pm 0.09$ ). Second phase C-peptide secretion increased $175 \%$ [95\% CI 1.31 to $2.28, \mathrm{p}<0.0001$ ] more with exenatide $(2.94 \pm 0.21)$ than glargine $(1.14 \pm 0.06)$. After 1 year, both therapies reduced $\mathrm{HbAlc}$ similarly: exenatide by $-0.77 \pm 0.13 \%$, and glargine by $-0.94 \pm 0.17 \%$ difference $0.29 \%$ [ $95 \%$ CI -0.09 to $0.66 \%, p=0.13$ ]. Exenatide significantly reduced body weight compared to insulin glargine $(-3.88 \pm 0.8 \mathrm{~kg}$ vs. $+1.4 \pm 1.1 \mathrm{~kg}$ respectively; difference $-5.2 \mathrm{~kg}[95 \% \mathrm{CI}-8.0$ 
to $-2.4 \mathrm{~kg}, \mathrm{p}=0.0005])$. The adverse event rate was comparable to previous exenatide trials: 17 of the $36(47 \%)$ exenatide treated patients experienced mild to moderate nausea while none of the glargine treated patients did. Hypoglycaemic events were more common in the glargine treated group than in the exenatide treated group ( $25 \%$ vs. $14 \%$ respectively).

Conclusion: One year of exenatide therapy, as compared to insulin glargine, markedly improved different indices of $\beta$-cell function, i.e. glucose and arginine induced insulin secretion, along with similar glycaemic improvement. In addition, patients treated with exenatide lost weight whereas patients treated with glargine gained weight.

Supported by Amylin Pharmaceuticals, Inc. \& Eli Lilly and Company

\section{2}

Treatment with glucagon-like peptide 1 leads to blood glucose normalization in patients with type 2 diabetes after coronary artery bypass surgery

B. Gallwitz ${ }^{1}$, K. Müssig $^{1}$, A. Öncü ${ }^{1}$, P. Lindauer ${ }^{1}$, A. Heininger ${ }^{2}$, A. Fritsche ${ }^{1}$, H. Aebert ${ }^{3}$, J. J. Holst ${ }^{4}$, K. Unertl², H.-U. Häring ${ }^{1}$;

${ }^{1}$ Department of Endocrinology, Diabetology, Nephrology, Angiology, and Clinical Chemistry, University Hospital of Internal Medicine, Tübingen, Germany, ${ }^{2}$ Department of Anesthesiology and Intensive Care Medicine, University Hospital of Tübingen, Germany, ${ }^{3}$ Department of Thoracic, Cardiac, and Vascular Surgery, University Hospital of Tübingen, Germany, ${ }^{4}$ Department of Medical Physiology, The Panum Institute, University of Copenhagen, Denmark.

Background and Aims: Hperglycemia, a common feature of the critically ill, has been associated with a poor outcome after major surgery in patients with type 2 diabetes. Intensive insulin therapy (IIT) improves the outcome of prolonged critically ill patients, but the broad clinical application is limited by its practicability and the risk of hypoglycemia. The incretin hormone glucagon-like peptide-1 (GLP-1) only stimulates insulin secretion under hyperglycaemic conditions. Furthermore, it suppresses glucagon secretion, slows gastric emptying, induces satiety, and improves beta cell function as well as left ventricular and systemic hemodynamics in type 2 diabetes. Therefore, the glucose-lowering effect of GLP-1 was studied in patients with type 2 diabetes after coronary artery bypass surgery.

Materials and Methods: Twenty patients with type 2 diabetes who had undergone coronary artery bypass surgery were randomised to receive either IIT according to the intensive care unit algorithms (ten men; age, $67.8+/-2.3$ yrs; body mass index, $30+/-1 \mathrm{~kg} / \mathrm{m}^{2}$; glycosylated hemoglobin, $7.1 \%+/-0.3 \%$ ) or a continuous intravenous GLP-1 infusion ( $3.6 \mathrm{pmol} \mathrm{x} \mathrm{kg} \mathrm{x} \mathrm{min)} \mathrm{within} \mathrm{the}$ first 12 hours postoperatively (eight men, two women; age, $62.5+/-2.2 \mathrm{yrs}$; body mass index, $28+/-1 \mathrm{~kg} / \mathrm{m}^{2}$; glycosylated hemoglobin, $6.6 \%+/-0.3 \%$ ). Blood was drawn in 2-hr intervals for measurement of glucose (glucose oxidase), insulin, C-peptide, glucagon, and GLP-1 (specific immunoassays). Statistics were done with repeated-measures analysis of variance and Duncan's post hoc tests.

Results: During the intravenous infusion of GLP-1, plasma glucose concentrations were significantly lowered due to stimulation of endogenous insulin secretion and inhibition of glucagon secretion. There were no statistical differences in blood glucose between both treatment groups. No hypoglycemic events were recorded during the experiments. The incidence of complications were comparable in both groups (SIRS, duration of antibiotic treatment and duration of ICU-treatment).

Conclusion: With GLP-1 a normalization of glucose metabolism is feasible under ICU-conditions.

\section{OP 46 Genetics of type 1 diabetes mellitus}

\section{3}

Characterization of antibody positive diabetes across the age groups of 15-59 years

E. Bakhtadze, C. Cervin, E. Lindholm, G. Sundkvist, L. Groop; Diabetes and Endocrinology, Lund University, Malmö, Sweden.

Background and Aims: Type 1 diabetes in children is characterised by autoimmune destruction of pancreatic $\beta$-cells and presence of certain highrisk HLA genotypes, the Ins VNTR polymorphisms in the insulin gene and a polymorphism in the PTPN22 gene. In adults, the same situation is often referred to LADA or latent autoimmune diabetes in adults. However, the phenotype of autoimmune diabetes across different age groups is not well established. Our aim was therefore to genetically and metabolically characterise autoimmune diabetes across the age groups of 15-59 years.

Materials and Methods: We determined the HLA-DQB1 genotypes, the Ins VNTR polymorphisms as well as the PTPN22 polymorphisms and measured GADA, and IA-2A antibodies and fasting C-peptide concentrations in 1,869 younger (15-34 years old) and 1,619 elderly (40-59 years old) Swedish diabetic patients.

Results: Altogether, $67 \%$ of the young and $13 \%$ of the elderly patients were $\mathrm{Ab}+$. There were more females among elderly than among younger $\mathrm{Ab}+$ patients $(49 \%$ vs. $39 \% ; \mathrm{p}=0.009)$. Elderly $\mathrm{Ab}+$ patients had significantly $(\mathrm{p}<0.0001)$ higher BMI $(26 \pm 5 \mathrm{vs.} 23 \pm 4 \mathrm{~kg} / \mathrm{m} 2)$, more often a family history of diabetes (63\% vs. $23 \%$;), were less often treated with insulin ( $60 \%$ vs. $93 \%$ ) and had higher C-peptide concentrations $(0.41 \pm 0.48$ vs. $0.30 \pm 0.26 \mathrm{nmol} / \mathrm{l})$. BMI correlated positively $(\mathrm{p}<0.0001)$ with $\mathrm{C}$-peptide concentrations in young $(r=0.29)$ and elderly $(r=0.46) \mathrm{Ab}+$ patients. $r$-value significantly differ between them $(p=0.005)$. The frequencies of HLA-DQB $1{ }^{*} 0302 / X$ was higher among young than elderly $\mathrm{Ab}+$ patients $(26 \%$ vs. $15 \%$; $\mathrm{p}=0.013)$, whereas, that of HLA-DQB1 ${ }^{*} 0201 /{ }^{*} 0302$ was similar $(27 \%$ vs. $27 \%$; $p=0.93)$. Elderly $\mathrm{Ab}+$ patients with Ins VNTR AA ( 0.27 vs. $0.48 \mathrm{nmol} / \mathrm{l} ; \mathrm{p}=0.01)$ and Ins VNTR CC ( 0.33 vs. $0.68 \mathrm{nmol} / \mathrm{l} ; \mathrm{p}=0.0009)$ genotypes had lower C-peptide concentrations than non-risk Ins VNTR genotype carriers.

Elderly Ab- patients had significantly $(\mathrm{p}<0.0001)$ more often a family history of diabetes ( $70 \%$ vs. $45 \%)$, were less frequently treated with insulin $(23 \%$ vs. $60 \%)$ and had higher C-peptide concentrations $(0.97 \pm 0.55 \mathrm{vs} .0 .79 \pm 0.54 \mathrm{nmol} /$ 1) than younger $\mathrm{Ab}$ - patients. BMI correlated positively $(\mathrm{p}<0.0001)$ with $\mathrm{C}$ peptide concentrations among both young $(r=0.63)$ and elderly $(r=0.44) \mathrm{Ab}$ patients, but $r$ value did not differ. The frequency of the Ins VNTR CC (class IIIA/IIIA) genotype was higher in young than elderly $\mathrm{Ab}$ - patients (66\% vs. $60 \% ; \mathrm{p}=0.036)$. Young Ab- patients with risk HLA-DQB1 (0.63 vs. $0.84 \mathrm{nmol} /$ $1 ; \mathrm{p}=0.0007)$ and PTPN22 CT or TT ( 0.64 vs. $0.83 \mathrm{nmol} / \mathrm{l} ; \mathrm{p}=0.002)$ genotypes had lower C-peptide concentration than non-risk HLA-DQB1 and PTPN22 CC genotype carriers. However, elderly Ab- patients with risk HLA-DQB1 (0.86 vs. $0.97 \mathrm{nmol} / \mathrm{l} ; \mathrm{p}=0.009$ ) had lower C-peptide concentrations than non-risk HLA-DQB1 genotype carriers. In linear regression analysis factors associated with decreased C-peptide concentration were: in $\mathrm{Ab}+$ patients, decreased BMI $(p<0.0001)$, older age at onset $(p=0.012)$ and absence of family history of diabetes $(\mathrm{p}=0.031)$ and in $\mathrm{Ab}$ - patients, decreased BMI $(p<0.0001)$, younger age at onset $(\mathrm{p}<0.0001)$ and risk HLA-DQB1 $(\mathrm{p}=0.02)$, PTPN22 CT or TT ( $\mathrm{p}=0.008)$ genotypes.

Conclusion: Despite a marked increase in BMI with age, $\mathrm{Ab}+$ patients aged between 15-59 years show similar reduction in $\beta$-cell function and similar frequency of high-risk HLA-DQB1 genotypes as well as of Ins VNTR polymorphisms. Obesity is thus not an uncommon corollary of autoimmune diabetes in adults. Absence of islet antibodies makes younger and elderly diabetic patients more alike.

Supported by the Swedish Research Council (including a Linne grant) 


\section{4}

The PTPN22 1858T gene variant is associated with reduced residual insulin secretion and worst metabolic control in type 1 diabetes A. Petrone ${ }^{1}$, M. Spoletini ${ }^{1}$, M. Capizzi ${ }^{1}$, S. Zampetti ${ }^{1}$, S. Zavarella ${ }^{1}$, C. Venditti ${ }^{1}$, C. Foffi ${ }^{1}$, I. Alemanno ${ }^{1}$, IMDIAB Group ${ }^{2}$, P. Pozzilli ${ }^{2}$, R. Buzzetti ${ }^{1}$;

${ }^{1}$ Clinical Sciences, "Sapienza” University, Rome,

${ }^{2}$ Endocrinology, Campus Biomedico, Rome, Italy.

Background and Aims: Different factors (i.e. genetic background) may be responsible for the heterogeneity of beta-cell function detectable at onset of type 1 diabetes (T1DM). The C1858T missense variation of the Protein Tyrosine Phosphatase Non receptor (PTPN22) gene, which encodes for a powerful inhibitor of T-cell activation, known as LYP, has been recently reported to be associated with T1DM and other autoimmune diseases. A recent study has provided evidence that the PTPN22 C1858T variant is a marker of disease progression from pre-diabetes to clinical disease. The present study was designed to investigate whether PTPN22 gene is associated with a different degree of beta-cell loss at diagnosis and can influence the residual beta-cell function in the course of the 12 months after disease onset. Materials and Methods: $\mathrm{N}=120$ consecutive T1DM subjects with age ranged from 5 to 36 years (mean age $14.9 \pm 7.8$ ) were evaluated. The C1858T SNP was genotyped using the fluorogenic 5' nuclease assay (ABI, Foster City, USA). Insulin requirements, glycated haemoglobin (HbAlc) and C-peptide levels were determined at diagnosis and every 3 months for 12 months, in all subjects. HbA1c was measured by a column assay (Bio-Rad) and C-peptide levels by a radioimmunoassay (Bio-Rad). The variance of the investigated variables, during follow up, was analyzed using the general linear model repeated-measures procedure. The model includes "between-subjects" factors that divided the population into groups according to their genotype to investigate their effect on variance, using age and gender as covariates.

Results: C-peptide levels of subjects carrying the $1858 \mathrm{~T}$ susceptible variant of PTPN22 gene were significantly lower than those of T1DM patients homozygous for C1858 from time of disease onset through follow up $(\mathrm{p}=0.01)$. Moreover the levels of HbA1c in T1DM subjects carrying the $1858 \mathrm{~T}$ variant of PTPN22 gene were significantly higher compared to T1DM homozygous for C1858 ( $\mathrm{p}=0.02)$. The trend in the pattern of C-peptide and HbAlc levels in the two groups, stratified according to the $1858 \mathrm{~T}$ variant was similar during the follow-up. The insulin requirement at diagnosis and after 3, 6, 9 and 12 months did not show any significant difference between subjects with or without the $1858 \mathrm{~T}$ variant at PTPN22 locus. Additionally we observed a correlation between C-peptide levels and age at diagnosis in T1DM subjects from time of disease onset through follow-up $(p=0.016)$.

Conclusion: The implication of our study is that PTPN22 T1858 individuals had experienced a more destructive beta-cell damage in the pre-diabeticperiod and maintain significant lower levels of C-peptide compared to the C1858 homozygous worsening the metabolic control (HbA1c levels). The present results could be useful for designing trials aimed to prevent the progression of beta-cell destruction.

\section{5}

The +49AG variant of the CTLA4 gene (IDDM12) is associated with ketoacidosis at disease onset and with anti-GAD antibody titres during disease progression in children with newly diagnosed type 1 diabetes

L. B. Nielsen ${ }^{1}$, H. Mortensen ${ }^{1}$, S. Poerksen ${ }^{1}$, P. Hougaard ${ }^{2}$, H.-J. Aanstoot ${ }^{3}$, K. Robertson ${ }^{4}$, C. Pfleger ${ }^{5}$, N. Schloot ${ }^{5}$, S. Gammeltoft ${ }^{6}$, L. Hansen ${ }^{7}$; ${ }^{1}$ Dep Paediatrics, Glostrup University Hospital, Glostrup, Denmark, ${ }^{2}$ Dep Statistics, University of Southern Denmark, Odense, Denmark, ${ }^{3}$ Paediatrics, DIABETER, Rotterdam, The Netherlands,

${ }^{4}$ Paediatrics, Royal Hospital for Sick Children, Glasgow, United Kingdom, ${ }^{5}$ Diabetes Research Institute, Heinrich-Heine University, Dusseldorf, Germany, ${ }^{6}$ Biochemistry, Glostrup University Hospital, Glostrup, Denmark, ${ }^{7}$ Novo Nordisk A/S, Bagsværd, Denmark.

Background and Aims: The cytotoxic T lymphocyte antigen 4 (CTLA4) gene is associated with common autoimmune disorders like type 1 diabetes (T1D), rheumatoid arthritis, and Grave's disease. In the Japanese population the $\mathrm{G}$ allele of the $+49 \mathrm{AG}$ variant on the CTLA4 gene tend to associate with higher levels of GAD autoantibodies and also shown association ketoacidosis at disease onset. The aim of this study was to investigate the impact of three common genetic variants of the CTLA4 gene, Thr17Ala/+49AG (rs231775), CT60 (rs3087243), and JO30 on disease severity and autoimmune activity in children and adolescents the first 12 months after diagnosis of T1D.

Materials and Methods: The study is a multicenter longitudinal investigation with 18 participating paediatric centres from 15 countries in Europe and Japan (84\% Caucasians). Clinical information and blood samples were collected from 275 children less than 16 years at diagnosis of T1D and during the following 1, 6, and 12 months. PCR-RFLP analysis was performed for genotyping the population for the three variants on the CTLA4 gene.

Results: In accordance with previous investigations in Japanese populations we find an association between carriers of the G allele (alias Ala phenotype) of the +49AG variant and high levels of GAD autoantibodies 12 month after disease onset $(\mathrm{p}=0.03)$. In addition we find a significant association between the GG genotype carriers and diabetic ketoacidosis at onset $(\mathrm{OR}=2.2,[1.04-$ 4.67]). We did not find significant associations to the CT60 and JO30 variants with any of the clinical variables investigated.

Conclusion: These findings substantiate the involvement of the CTLA4 gene in the pathogenesis of T1D, linking the +49AG variant to a more severe onset of T1D and a more aggressive autoimmune activity during disease progression.

Supported by the Danish Diabetes Association

\section{6}

SUMO4 M55V, in IDDM5 locus, polymorphism increases susceptibility to type 1 diabetes in HLA-DR3 and DR4 positive Swedish patients S. K. Sedimbi, X. Luo, C. B. Sanjeevi;

Department of Molecular Medicine and Surgery, Karolinska Institutet, Stockholm, Sweden.

Background and Aims: Small ubiquitin-related modifier 4 (SUMO4) is a protein encoded by SUMO4 gene in the IDDM5 locus in chromosome $6 \mathrm{q} 25$. It encodes the SUMO4 protein, suggested to be a post-translational modifier of the inhibitor for nuclear factor-kB (IkB). A nonsynonymous SNP (rs237025; 163A $>\mathrm{G}$ ) causing a methionine-to-valine substitution at codon 55 (M55V) in SUMO4 protein was reported to be associated with T1DM in Asians but not in Caucasians. The current study aims to test the significance of M55V association to T1DM in a large cohort of Swedish Caucasians and to test the whether M55V is associated in those carrying high-risk HLA class II molecules, MICA alleles (5 and 5.1) and autoantibodies (GAD 65 and IA-2). Materials and Methods: The study included 673 T1DM patients and 535 age and sex matched healthy controls ( $0-35$ years) collected as a part of two major studies (The Swedish Childhood Diabetes Study and The Diabetes Incidence Study (DIS) in Sweden). HLA and MICA typing was performed using PCRSSOP, and autoantibodies were measured for glutamic acid decaboxylase isoform 65 (GAD 65) and islet antigen-2/ tyrosine phosphatase (IA-2) using radio ligand binding assay, previously. PCR-RFLP using Sequence Specific Primers was done to identify the genotype and allele variations in SUMO4. Results: Our data suggests that SUMO4 M55V is not associated with susceptibility to T1DM in Swedish Caucasians. When we stratified our patients and controls based on heterozygosity for HLA-DR3/DR4 and SUMO4 genotypes (AA or AG or GG), SUMO4 GG further increased the relative risk conferred by HLA-DR3/DR4 to T1DM [from OR=5.77, 95\% CI= 4.22-7.88, $\mathrm{P}=<0.0001$ (HLA-DR3/DR4 alone) to $\mathrm{OR}=8.34,95 \% \mathrm{CI}=4.07-17.13, \mathrm{P}=$ $<0.0001$ (HLA-DR3/DR4 with SUMO4 GG)], whereas SUMO4 AA decreased the relative risk [from $\mathrm{OR}=6.91,95 \% \mathrm{CI}=4.93-9.67, \mathrm{P}=<0.0001$ (HLA-R3/ DR4 alone) to $\mathrm{OR}=3.66,95 \% \mathrm{CI}=2.22-6.02, \mathrm{P}=<0.0001$ (HLA-DR3/DR4 with SUMO4 AA)]. SUMO4 M55V was no associated with autoantibody positivity to either GAD65 or IA-2 as well as high-risk MICA alleles 5 and 5.1 in T1DM patients.

Conclusion: We conclude that SUMO4 M55V is associated with T1DM in those carrying high-risk HLA-DR3 and DR4, but not by itself.

Funded by grants from Swedish Medical Research Council, Swedish Diabetes Association, Bran Diabetes Fund, Svenska Diabetes Stiftelsen and Karolinska Institute. SKS was funded by a scholarship from SwedishInstitute 


\section{OP 47 Adiponectin and diabetic complications}

\section{7}

\begin{abstract}
Adiponectin and coronary artery disease risk in apparently healthy men and women: the EPIC-Norfolk Prospective Population Study

M. Cote ${ }^{1}$, A. Cartier ${ }^{1}$, I. Lemieux ${ }^{1}$, J.-P. Despres ${ }^{1}$, N. J. Wareham ${ }^{2}$,

J. J. P. Kastelein ${ }^{3}$, K.-T. Khaw ${ }^{4}$, S. M. Boekholdt';

${ }^{1}$ Quebec Heart Institute, Hopital Laval Research Centre, Quebec, Canada, ${ }^{2}$ Medical Research Council Epidemiology Unit, University of Cambridge, United Kingdom, ${ }^{3}$ Department of Vascular Medicine, Academic Medical Center, Amsterdam, The Netherlands, ${ }^{4}$ Department of Public Health and Primary Care, Institute of Public Health, University of Cambridge, United Kingdom, ${ }^{5}$ Department of Cardiology, Academic Medical Center, Amsterdam, The Netherlands.
\end{abstract}

Background and Aims: Several recent studies have reported a relationship between adiponectin levels and cardiovascular disease. Increased plasma adiponectin levels have generally been associated with a lower risk of myocardial infarction among men without previous cardiovascular disease, independently of traditional cardiovascular risk factors. However, inconsistent results have been reported among prospective studies regarding the ability of adiponectin to predict cardiovascular risk. The purpose of the present study was to evaluate in a prospective case-control analysis nested in the European Prospective Investigation into Cancer and Nutrition (EPIC)-Norfolk cohort, the association between adiponectin levels and the development of future coronary artery disease (CAD) in healthy men and women aged 45 to 79 years who developed fatal or nonfatal CAD during follow-up.

Material and Methods: 1034 cases were matched to 1920 controls who remained free of $\mathrm{CAD}$, by age, gender and enrolment time. Baseline nonfasting plasma adiponectin concentrations were determined by ELISA.

Results: Adiponectin levels were lower in cases than in matched controls $(10.1 \mathrm{vs.} 10.7 \mathrm{ug} / \mathrm{ml}$; for cases and controls, respectively, $\mathrm{p}=0.005)$. Significant differences in HDL cholesterol levels (1.37 vs. $1.27 \mathrm{ug} / \mathrm{ml}, \mathrm{p}<0.0001)$ and waist circumference ( 90.9 vs. $94.0 \mathrm{ug} / \mathrm{ml}, \mathrm{p}<0.0001$ ) were observed between cases and controls, respectively. The unadjusted odds ratio for future CAD according to adiponectin levels (as continuous variable, per adiponectin unit) was $0.76(95 \% \mathrm{CI}, 0.64-0.90 ; \mathrm{p}<0.001)$. However, after adjustment for body mass index, waist circumference and established cardiovascular disease risk factors, this relationship was no longer significant. Furthermore, stratification of men and women on the basis of low vs. high adiponectin (median value: $8.2 \mathrm{vs.} 12.2 \mathrm{ug} / \mathrm{ml}$, for men and women, respectively) and low vs. high waist circumference (median value: 95.9 vs. $82.9 \mathrm{~cm}$, for men and women, respectively) indicated that risk of CAD was more closely related to the presence of a high waist circumference than to low adiponectin levels. A similar analysis revealed that low HDL cholesterol levels $(<1.15 \mathrm{mmol} / \mathrm{l}$ vs. $<1.45 \mathrm{mmol} / \mathrm{l}$, for men and women, respectively) were predictive of an increased CAD risk in both men and women, irrespective of adiponectin levels.

Conclusion: These results suggest that though low adiponectin levels are associated with an increased CAD risk in men and women they not appear to provide additional predictive value beyond established cardiovascular risk factors such as elevated waist circumference and low HDL cholesterol levels.

\section{8}

Serum adiponectin predicts development of ESRD and the ADIPOQ prom2G/A polymorphism is associated with diabetic nephropathy in patients with type 1 diabetes

A. Jorsal ${ }^{1}$, L. Tarnow ${ }^{1}$, M. Lajer ${ }^{1}$, H.-H. Parving ${ }^{2,3}$, P. Rossing ${ }^{1}$;

${ }^{1}$ Steno Diabetes Center, Gentofte,

${ }^{2}$ Department of Medical Endocrinology, Rigshospitalet, Copenhagen,

${ }^{3}$ Faculty of Health Science, University of Aarhus, Denmark.

Background and Aims: Patients with diabetic nephropathy have a high risk of end-stage renal disease (ESRD) and cardiovascular morbidity and mortality. Adiponectin is a hormone exclusively produced by adipocytes and possesses anti-inflammatory, anti-atherogenic and cardioprotective properties. Adiponectin levels in patients with type 1 diabetes are increased compared with healthy subjects and increased levels are associated with microangiopathy in type 1 diabetes. The aim of this study was to investigate serum levels of adiponectin and 8 frequent polymorphisms in the adiponectin gene in relation to diabetic nephropathy, ESRD, cardiovascular events, and mortality in type 1 diabetic patients.

Materials and Methods: Our study was a prospective observational follow-up study; including 438 type 1 diabetic patients with overt diabetic nephropathy and a matched control group of 442 patients with longstanding type 1 diabetes and persistent normoalbuminuria. The patients were followed for $8.1(0.0-$ 12.8) years (median [range]). Cases with diabetic nephropathy included 267 men and 171 women; age $42.3 \pm 10.4$ years [mean $\pm S D$ ], duration of diabetes $28.4 \pm 8.8$ years, GFR $66 \pm 28.1 \mathrm{ml} \mathrm{min}^{-1}$ per $1.73 \mathrm{~m}^{2}$. Normoalbuminuric controls included 233 men and 207 women; age $45.4 \pm 11.5$ years, duration of diabetes $27.7 \pm 10.1$ years.

Results: Serum adiponectin was measured in 199 patients with diabetic nephropathy. 12 patients (12\%) with adiponectin levels below the median and 28 patients $(30 \%)$ with levels of adiponectin above the median reached ESRD during follow-up (hazard ratio 3.44 [1.77-6.78], $p<0.001$; covariateadjusted (sex, age, systolic $\mathrm{BP}, \mathrm{HbA}$, GFR, and cholesterol at baseline) hazard ratio 2.44 [1.17-5.08], $p=0.017$ ). Furthermore, 8 variants in the adiponectin gene were analyzed. The A-allele of ADIPOQ_prom2G/A (rs17300539) was associated with diabetic nephropathy (A-allele frequency: $9.4 \%$ versus $6.6 \%$ in cases and controls, respectively, $p=0.014)$. None of the adiponectin polymorphisms was associated with serum levels of adiponectin or renal/ cardiovascular outcome in patients with type 1 diabetes.

Conclusion: In conclusion, the ADIPOQ_prom2GA polymorphism is associated with diabetic nephropathy and higher serum adiponectin levels predict development of ESRD in Danish Caucasian patients with type 1 diabetes.

The EURAGEDIC study was supported by the European Commission

\section{9}

Serum adiponectin and progression of diabetic nephropathy in type 1 diabetic patients (The FinnDiane Study)

M. T. Saraheimo ${ }^{1,2}$, C. Forsblom ${ }^{1,2}$, L. Thorn ${ }^{1,2}$, J. Waden ${ }^{1,2}$, N. Tolonen ${ }^{1,2}$, J. Frystyk ${ }^{3}$, A. Flyvbjerg ${ }^{3}$, P.-H. Groop ${ }^{1,2}$;

${ }^{1}$ Folkhälsan Institute of Genetics, Folkhälsan Research Centre, Helsinki, Finland, ${ }^{2}$ Department of Medicine, Helsinki University Central Hospital, Division of Nephrology, Finland,

${ }^{3}$ Aarhus University Hospital, Medical Department M (Diabetes and Endocrinology) and the Medical Research Laboratories, Denmark.

Background and Aims: Serum adiponectin concentrations are increased in type 1 diabetic patients with nephropathy. The mechanisms responsible for the increase are still unclear, but adiponectin has been suggested to mitigate renal tubular injury and thus to prevent further progression of diabetic nephropathy. Adiponectin has also been suggested to reduce both micro- and macrovascular complications, but in contrast to this high, rather than low, adiponectin levels have been associated with increased mortality in patients with chronic kidney disease. The aim of this study was to elucidate whether serum adiponectin is associated with progression of diabetic nephropathy in type 1 diabetic patients.

Materials and Methods: This is a prospective follow-up study, which is part of the nationwide Finnish Diabetic Nephropathy Study (FinnDiane). 1385 type 1 diabetic patients were followed for $5.0 \pm 2.2$ years. Patients were divided at baseline into three groups according to their urinary albumin excretion rate (AER) in three consecutive overnight or 24 hour urine collections: 882 patients with normoalbuminuria [NA](AER $<20$ $\mathrm{ug} / \mathrm{min}), 229$ with microalbuminuria $[\mathrm{MI}](20 \mathrm{ug} / \mathrm{min}<\mathrm{AER}<200 \mathrm{ug} / \mathrm{min})$ and 274 with macroalbuminuria [MA] (AER $>200 \mathrm{ug} / \mathrm{min})$. Progression of albuminuria was the main outcome. Adiponectin was measured by a timeresolved immmunofluorometric assay (TR-IFMA), and the values were logtransformed and adjusted for age, BMI and sex before analysis.

Results: 165 patients (69 NA, $37 \mathrm{MI}$ and $59 \mathrm{MA}$ ) progressed either to the next albuminuria level or ESRD. No difference in adiponectin concentrations was observed between progressors and non-progressors in NA (11.3 \pm 5.4 vs. $11.9 \pm 5.4 \mathrm{mg} / \mathrm{l}, \mathrm{p}=0.4)$ or MI (12.0 \pm 6.0 vs. $12.4 \pm 6.1 \mathrm{mg} / \mathrm{l}, \mathrm{p}=0.7)$ patients. In the MA patients, progression to ESRD was associated with higher adiponectin in the entire group $(22.9 \pm 12.1 \mathrm{vs.} 16.0 \pm 8.2 \mathrm{mg} / \mathrm{l}, \mathrm{p}<0.001)$ and also when men $(19.8 \pm 11.2$ vs. $13.7 \pm 6.7 \mathrm{mg} / \mathrm{l}, \mathrm{p}<0.001)$ and women $(28.5 \pm 11.9$ vs. $18.7 \pm 8.9$ $\mathrm{mg} / \mathrm{l}, \mathrm{p}<0.001$ ) were analyzed separately. Progression to ESRD was associated with $\mathrm{HbA}_{1 \mathrm{c}}$, S-creatinine, AER and triglycerides, but not with age, waist-hip 
ratio and blood pressure. In a multiple logistic regression analysis, adiponectin, $\mathrm{HbA}_{1 \mathrm{c}}$ S-creatinine and triglycerides were independent predictors of ESRD $\left(R^{2}=0.315\right)$, while gender did not enter the model.

Conclusion: High serum adiponectin levels predict the progression from macroalbuminuria to end-stage renal disease in type 1 diabetic patients.

\section{0}

Low and middle molecular weight adiponectin isoforms and polymorphisms of the adiponectin gene are associated with the incidence of nephropathy in type 2 diabetic subjects

F. Fumeron $^{1,2}$, R. Aubert ${ }^{1}$, R. Jaziri ${ }^{1,2}$, F. Péan ${ }^{1}$, S. Maimaitiming ${ }^{1,2}$, N. Bellili ${ }^{1,2}$, R. Roussel ${ }^{1,2}$, S. Hadjadj ${ }^{3}$, M. Marre ${ }^{1,2}$;

${ }^{1}$ INSERM, U695, Paris, ${ }^{2}$ Faculté Xavier Bichat, Université Paris France,

${ }^{3}$ Endocrinologie, Centre Hospitalier Universitaire, Poitiers, France.

Background and Aims: Low adiponectin levels are found in type 2 diabetes and coronary heart disease, while high levels are associated with diabetic nephropathy. Adiponectin circulates in various isoforms: low (LMW), middle (MMW) and high molecular weight (HMW). It has been suggested that the amount of HMW isoform is the most important correlate of insulin sensitivity. Nevertheless, only few studies measuring isoforms have been performed with respect to clinical phenotypes. Our aim was to assess the association between total plasma adiponectin and adiponectin multimers at entry, and 4-year incidence of myocardial infarction (MI) and/or renal disease (RD) in type 2 diabetic subjects from the DIABHYCAR prospective study. The association of adiponectin (total and isoforms) with three adiponectin gene (ADIPOQ) SNPs $(-11391 \mathrm{G}>\mathrm{A},+45 \mathrm{~T}>\mathrm{G},+276 \mathrm{G}>\mathrm{T})$ was also investigated.

Material and Methods: Participants were the 3123 French type 2 diabetic subjects with micro- or macro-albuminuria from the DIABHYCAR study. Plasma total adiponectin and multimers were assayed by ELISA (ALPCO Diagnostics ${ }^{\mathrm{NI}}$ ). These measurements were performed on plasma samples drawn at the beginning of follow-up in all patients with incident MI $(n=95)$ or incident $\mathrm{RD}(\mathrm{n}=75)$ and in controls matched for sex, age, MI and genotypes. Results: In people with incident RD, the MMW and LMW isoform concentrations at entry were significantly higher than in matched controls $1.66 \mu \mathrm{g} / \mathrm{mL}$ vs $1.37 \mu \mathrm{g} / \mathrm{mL}, \mathrm{p}=0.018$ and $2.20 \mu \mathrm{g} / \mathrm{mL}$ vs. $1.94 \mu \mathrm{g} / \mathrm{mL}, \mathrm{p}=0.048$, respectively). Total adiponectin and all isoforms concentrations were lower in subjects with incident MI, but none of these differences was significant. Nevertheless, when excluding subjects with incident RD, HMW levels were lower in MI $(2.16 \mu \mathrm{g} / \mathrm{mL}$ vs. $2.62 \mu \mathrm{g} / \mathrm{mL}$, with a borderline significance $(\mathrm{p}=0.07)$. We had previously shown that the $-11391 \mathrm{G}>\mathrm{A}$ and $+45 \mathrm{~T}>\mathrm{G}$ ADIPOQ SNPs were associated with the risk to develop renal disease (RD) in the DIABHYCAR study. These SNPs were also associated with adiponectin levels. Total, HMW and MMW adiponectin concentrations were higher in -11391A carriers than in -11391 GG homozygotes $(7.42 \mu \mathrm{g} / \mathrm{mL}$ vs. $5.72 \mu \mathrm{g} /$ $\mathrm{mL}, \mathrm{p}<0.0005 ; 3.55 \mu \mathrm{g} / \mathrm{mL}$ vs. $2.33 \mu \mathrm{g} / \mathrm{mL}, \mathrm{p}<0.0005 ; 1.69 \mu \mathrm{g} / \mathrm{mL}$ vs. $1.36 \mu \mathrm{g} /$ $\mathrm{mL} \mathrm{p}=0.025$, respectively). In subjects with $+45 \mathrm{GG}$ genotype, all adiponectin related concentrations were higher than in $+45 \mathrm{~T}$ carriers $(8.89 \mu \mathrm{g} / \mathrm{mL}$ vs. $5.90 \mu \mathrm{g} / \mathrm{mL}, \mathrm{p}=0.011 ; 3.10 \mu \mathrm{g} / \mathrm{mL}$ vs. $2.48 \mu \mathrm{g} / \mathrm{mL}, \mathrm{p}=0.008 ; 2.05 \mu \mathrm{g} / \mathrm{mL}$ vs $1.40 \mu \mathrm{g} / \mathrm{mL}, \mathrm{p}=0.013 ; 2.52 \mu \mathrm{g} / \mathrm{mL}$ vs $1.95 \mu \mathrm{g} / \mathrm{mL}, \mathrm{p}=0.019$, for total, HMW, MMW, LMW adiponectin, respectively). There was no interaction of genotypes with sex or disease on adiponectin related measurements.

Conclusion: In type 2 diabetic subjects, an elevation of MMW and LMW adiponectin isoforms precedes the onset of RD. Moreover, the genotypes associated with elevated levels of adiponectin are those associated with the incidence of $\mathrm{RD}$. These results support the hypothesis of a causal role of elevated adiponectin levels in diabetic nephropathy.

Supported by grants from Association Française des Diabétiques (AFD) and ALFEDIAM-SANOFI-AVENTIS

\section{OP 48 Making the best use of diabetes technology}

\section{1}

RCT of continuous glucose monitoring devices on $\mathrm{HbA}_{1 \mathrm{c}}$ - The MITRE Study

S. Newman ${ }^{1}$, S. Hurel' , D. Cooke ${ }^{1}$, L. Steed ${ }^{1}$, J. Ahlquist ${ }^{3}$, J. Weaver ${ }^{4}$, D. Kerr ${ }^{5}$, M. Barnard ${ }^{6}$, A. Casbard', S. Meredith 7 , A. Nunn'; ${ }^{1}$ CBSSM, UCL, London, ${ }^{2}$ Diabetes \& Endocrinology, UCLH, London, ${ }^{3}$ Endocrinology, Southend Hosp., Southend, ${ }^{4}$ Diabetes \& Endocrinology, QEH, Newcastle,

${ }^{5} \mathrm{BDEC}, \mathrm{RBCHT}$, Bournemouth, ${ }^{6}$ Diabetes \& Endocrinology, Whittington Hosp., London, ${ }^{7} \mathrm{CTU}, \mathrm{MRC}$, London, United Kingdom.

Background \& Aims: Minimally invasive continuous glucose monitoring devices have been developed on the assumption that periods of detailed continuous information will lead to improved metabolic control. This RCT was designed to evaluate the efficacy of two such devices in poorly controlled, insulin-requiring people with diabetes.

Materials and Methods: 102 patients were randomly assigned to the CGMS (Minimed), 100 to the Biographer (Animas), 102 to Standard Care and 100 to an Attention Control group. The last group was to control for the impact of increased levels of contact with health care professionals in the 2 device groups. HbAlc was assessed at 3 (excluding Standard Care), 6, 12 and 18 months. The primary outcome was the mean \% change in $\mathrm{HbAlc}$ from baseline. In addition the proportion of patients in each group showing a $12.5 \%$ reduction in baseline $\mathrm{HbAlc}$ was calculated.

Results: The mean percentage change in $\mathrm{HbAlc}$ from baseline for each of the 4 groups and $95 \%$ CIs are displayed for each timepoint. Only those patients for whom an $\mathrm{HbAlc}$ was available in a specified window of time, around the scheduled research visit, have been included. Mean HbA1C declined from baseline in all groups, although the effect waned with time. At 18 months the decline ranged from a little over 1\% (Biographer) to between 4 and $5 \%$. No significant differences were found between the groups at any time-point. A per-protocol analysis, specifying a minimal amount of use of the two monitors, produced similar results. The proportion of patients showing a reduction of $12.5 \%$ from their baseline $\mathrm{HbAlc}$ at each timepoint is displayed (see table). At all timepoints the proportion with a relative reduction of at least $12.5 \%$ ranged from 15\% (Biographer) to 29\% (CGMS). No differences were found between the groups at any time point.

Conclusion: These results suggest that the effectiveness of the 2 monitors is no different to standard care or treatment of a similar intensity. A secondary analysis, where minimum usage of the devices was required, indicated no advantage of the devices on $\mathrm{HbAlc}$. All groups showed a sustained reduction in HbAlc suggesting that trial participation lead to improved metabolic control. This may have obscured any effects of the devices. (Tab. 1)

\section{$\mathrm{HbA1c} \%$ - Relative change from baseline Mean $(95 \% \mathrm{Cl})$}

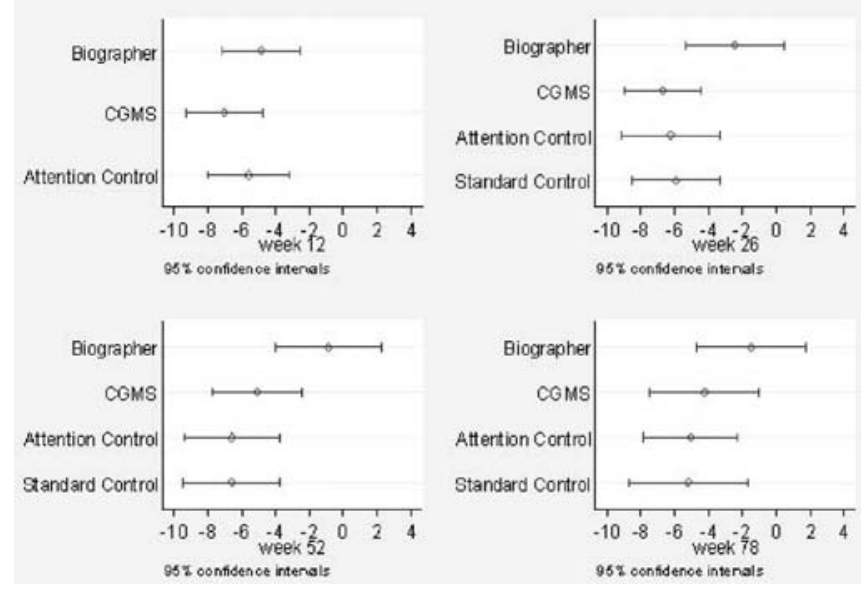


Tab. 1 Abstract 0261

Proportion of Patients Achieving a 12.5\% Reduction in HbAlc from Baseline

\begin{tabular}{lllll}
\hline Month & $\mathbf{0 - 3}$ & $\mathbf{0 - 6}$ & $\mathbf{0 - 1 2}$ & $\mathbf{0 - 1 8}$ \\
\hline Biographer & $18 \%$ & $21 \%$ & $20 \%$ & $15 \%$ \\
CGMS & $29 \%$ & $27 \%$ & $24 \%$ & $27 \%$ \\
Attention Control & $22 \%$ & $24 \%$ & $28 \%$ & $27 \%$ \\
Standard Care & N/A & $24 \%$ & $27 \%$ & $24 \%$ \\
Total & $23 \%$ & $24 \%$ & $25 \%$ & $23 \%$ \\
\hline
\end{tabular}

Supported by HTA

\section{2}

Increased postprandial hyperglycaemia due to rising preprandial glucose: Results from a 40-day home use study using the FreeStyle Navigator ${ }^{\oplus}$ Continuous Glucose Monitoring System

T. C. Dunn, K. Rebrin, T. A. Peyser;

Abbott Diabetes Care, Alameda, United States.

Background and Aims: Continuous glucose monitoring provides patients with both more glucose values and glucose trend information to improve glycemic control. Meal-related glycemic profiles were collected from a 40-day home use study with the FreeStyle Navigator" System ("CGM", investigational device) and analyzed for the effect of premeal glucose and trend on postmeal glycemic state.

Materials and Methods: Subjects (T1DM or T2DM, $\mathrm{n}=122$ ) were masked to the sensor data and alarms for the first 20 days, and unmasked thereafter. The system stored glucose values every 10 minutes and meal times were entered by the participant, resulting in 3,505 analyzable profiles. The premeal trend was determined in the $40 \mathrm{~min}$ premeal. The premeal and $3 \mathrm{hr}$ postmeal glucose for each profile were categorized as hypoglycemic $(<3.89 \mathrm{mmol} / \mathrm{L})$, euglycemic $(3.89-10 \mathrm{mmol} / \mathrm{L})$, or hyperglycemic $(>10 \mathrm{mmol} / \mathrm{L})$. Statistical analyses were performed for the categories of hyperglycemia, hypoglycemia and trend stability. Stable trend was defined within -1.7 to $1.7(\mathrm{mmol} / \mathrm{L}) / \mathrm{hr}$. Results: Premeal, the rates of hypoglycemia, euglycemia, and hyperglycemia were $4 \%, 63 \%$ and $33 \%$, while postmeal the rates were $4 \%, 50 \%$ and $45 \%$, respectively (Table 1). For the entire study, the rate of postmeal hyperglycemia was found to vary due to premeal trend, with rates of $48 \%$ hyperglycemia for unstable premeal trend, compared to $44 \%$ for stable trend $(\mathrm{p}=0.019)$. During the masked phase $(\mathrm{n}=1999)$, the effect of premeal trend on postmeal hyperglycemia was significant $(\mathrm{p}=0.016)$, with $49 \%$ postmeal hyperglycemia for unstable premeal trends compared to $44 \%$ for stable premeal trends. However, during the unmasked phase $(\mathrm{n}=1506)$, this effect disappeared and rates of $46 \%$ and $44 \%$ were not different $(\mathrm{p}=0.260)$. This suggests that even in this study, in which patients were given no explicit instructions on modifying therapy based on CGM, patients made improvements in their treatment based on the additional data.

A major finding of this study is that patients do not adequately treat (or compensate) for hyperglycemia at mealtimes. When the premeal glucose trend was stable, patients who were hyperglycemic remained hyperglycemic after the meal in $66 \%$ of the cases. Also, there was a significant difference in glycemic outcome depending on the glucose trend at the start of the meal. For

\begin{tabular}{|c|c|c|c|c|c|c|}
\hline \multirow[b]{2}{*}{$\begin{array}{c}\text { Premeal } \\
\text { Glycemic } \\
\text { Range }\end{array}$} & \multirow[b]{2}{*}{$\begin{array}{c}\text { Premeal } \\
\text { Glycemic } \\
\text { Trend } \\
(\mathrm{mg} / \mathrm{dL}) / \mathrm{min}\end{array}$} & \multirow[b]{2}{*}{$\begin{array}{c}\text { Premeal } \\
\text { Glycemic } \\
\text { Trend } \\
\text { (mmol/L)/hr }\end{array}$} & \multicolumn{3}{|c|}{ Postmeal Glycemic Range } & \\
\hline & & & $\begin{array}{c}<3.89 \\
\mathrm{mmol} / \mathrm{L} \\
<70 \mathrm{mg} / \mathrm{dL} \\
4 \%\end{array}$ & $\begin{array}{c}3.89 \text { to } 10 \\
\mathrm{mmol} / \mathrm{L} \\
70 \text { to } 180 \\
\mathrm{mg} / \mathrm{dL} \\
50 \%\end{array}$ & $\begin{array}{c}>10 \\
\mathrm{mmol} / \mathrm{L} \\
>180 \\
\mathrm{mg} / \mathrm{dL} \\
45 \%\end{array}$ & $\mathrm{n}$ \\
\hline \multirow{8}{*}{$\begin{array}{c}>10 \mathrm{mmol} / \mathrm{L} \\
>180 \mathrm{mg} / \mathrm{dL} \\
33 \%\end{array}$} & All & All & $2 \%$ & $34 \%$ & $64 \%$ & 1160 \\
\hline & $>2$ & $>6.7$ & $4 \%$ & $28 \%$ & $68 \%$ & 57 \\
\hline & 1 to 2 & 3.3 to 6.7 & $0 \%$ & $32 \%$ & $68 \%$ & 145 \\
\hline & 0.5 to 1 & 1.7 to 3.3 & $2 \%$ & $33 \%$ & $65 \%$ & 190 \\
\hline & -0.5 to 0.5 & -1.7 to 1.7 & $2 \%$ & $32 \%$ & $66 \%$ & 592 \\
\hline & -0.5 to -1 & -1.7 to -3.3 & $3 \%$ & $43 \%$ & $54 \%$ & 102 \\
\hline & -1 to -2 & -3.3 to -6.7 & $9 \%$ & $44 \%$ & $47 \%$ & 66 \\
\hline & $<-2$ & $<-6.7$ & $25 \%$ & $25 \%$ & $50 \%$ & 8 \\
\hline \multirow{8}{*}{$\begin{array}{c}3.89 \text { to } 10 \\
\mathrm{mmol} / \mathrm{L} \\
70 \text { to } 180 \\
\mathrm{mg} / \mathrm{dL} \\
63 \%\end{array}$} & All & All & $5 \%$ & $59 \%$ & $37 \%$ & 2202 \\
\hline & $>2$ & $>6.7$ & $4 \%$ & $44 \%$ & $52 \%$ & 25 \\
\hline & 1 to 2 & 3.3 to 6.7 & $6 \%$ & $44 \%$ & $50 \%$ & 115 \\
\hline & 0.5 to 1 & 1.7 to 3.3 & $5 \%$ & $55 \%$ & $41 \%$ & 217 \\
\hline & -0.5 to 0.5 & -1.7 to 1.7 & $4 \%$ & $60 \%$ & $36 \%$ & 1413 \\
\hline & -0.5 to -1 & -1.7 to -3.3 & $3 \%$ & $60 \%$ & $37 \%$ & 295 \\
\hline & -1 to -2 & -3.3 to -6.7 & $6 \%$ & $62 \%$ & $32 \%$ & 117 \\
\hline & $<-2$ & $<-6.7$ & $15 \%$ & $80 \%$ & $5 \%$ & 20 \\
\hline \multirow{8}{*}{$\begin{array}{c}<3.89 \\
\mathrm{mmol} / \mathrm{L} \\
<70 \mathrm{mg} / \mathrm{dL} \\
4 \%\end{array}$} & All & All & $11 \%$ & $59 \%$ & $29 \%$ & 143 \\
\hline & $>2$ & $>6.7$ & - & - & - & 0 \\
\hline & 1 to 2 & 3.3 to 6.7 & $0 \%$ & $0 \%$ & $100 \%$ & 1 \\
\hline & 0.5 to 1 & 1.7 to 3.3 & $40 \%$ & $40 \%$ & $20 \%$ & 5 \\
\hline & -0.5 to 0.5 & -1.7 to 1.7 & $8 \%$ & $60 \%$ & $31 \%$ & 91 \\
\hline & -0.5 to -1 & -1.7 to -3.3 & $16 \%$ & $55 \%$ & $29 \%$ & 31 \\
\hline & -1 to -2 & -3.3 to -6.7 & $0 \%$ & $77 \%$ & $22 \%$ & 9 \\
\hline & $<-2$ & $<-6.7$ & $33 \%$ & $50 \%$ & $17 \%$ & 6 \\
\hline
\end{tabular}

Shaded rows have $n<35$ ( $<1 \%$ of the 3505 total observations)

Tab. 2. Frequency of postmeal glycemic range by premeal glycemic range and premeal glycemic trend for the entire study

example, with premeal euglycemia and rapidly rising trend $(>3.3(\mathrm{mmol} / \mathrm{L}) /$ $\mathrm{hr}$ ), postmeal hyperglycemia rates were $50 \%$, as opposed to only $33 \%$ with stable or falling trend.

Conclusion: These findings represent an important opportunity for additional intervention by HCPs to help patients improve glycemic control using CGM, and specifically trend arrows. As CGM becomes more widely used, HCPs may encourage patients to treat hyperglycemia more aggressively, especially when the glucose trend indicates a rising premeal glucose. This may enable patients to reduce exposure to post-prandial hyperglycemia in the short term and to reduce A1C in the long-term. (Tab. 2)

Supported by Abbott Diabetes Care

\section{3}

Improved glycaemic control in intensively treated subjects with type 1 diabetes using Accu-Chek ${ }^{\circledast}$ advisor insulin guidance software S. Garg, S. L. Ellis, C. Beatson, P. Gottlieb, R. Gutin, T. Bookout, C. Figal, B. Snyder;

Young Adult Clinic, Barbara Davis Center for Childhood Diabetes, Aurora, United States.

Background and Aims: Day to day management of insulin could be significantly improved with the availability of computerized insulin algorithms for home use. This study was designed to evaluate the effect of
Fig. 1a-b Abstract 0263
$1 b$

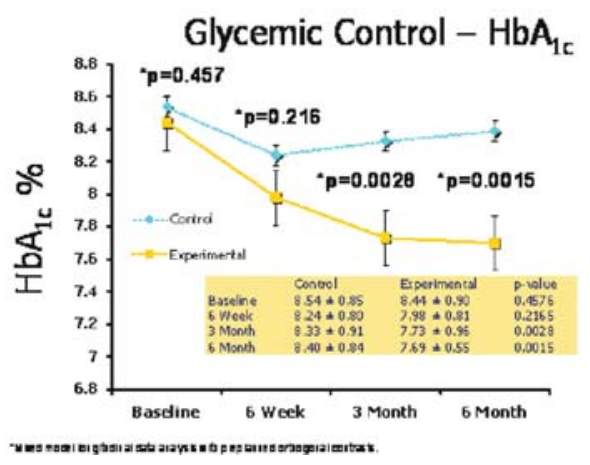

Glycemic Control at 3 and 6 Months Mean of Glucose Readings

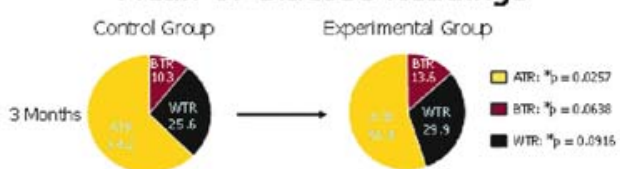

6 Mont

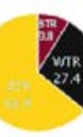

nemi kan sin tost 
the Accu-Chek Advisor Insulin Guideance Software on glycemic control in patients with type 1 diabetes.

Materials and Methods: This was a one year open label randomised study of 123 subjects assigned to either the Accu-Chek ${ }^{\bullet}$ Advisor Insulin Guidance Software program for personal data assistant (PDA) or the control group. The study included adults $18-60$ yrs with type 1 diabetes and Alc values between $7.5-11 \%$. The primary endpoint of the study was a difference in the A1c values. Data for blood glucose (BG) values, testing frequency, glucose excursions, hypoglycemic events (all, nocturnal and severe), insulin dose, weight and BMI, were recorded.

Results: All baseline demographics were similar in the two groups. The mean Alc values were significantly lower at 3 and 6 months in the experimental group (Figure 1a). Mean change in A1c at 6 months from baseline was $-0.8 \%$ vs. $-0.1 \%$ in the experimental and control groups, respectively $(\mathrm{p}=0.0015)$. Within target range (WTR) glycemia was significantly increased in the experimental group ( $\mathrm{p}=0.0076$; Figure $1 \mathrm{~b}$ ). Body weight, total, basal and bolus insulin dosages were similar in the two groups. Severe hypoglycemic events and BG testing frequency were higher in the experimental group. Conclusion: This is the first report that shows improved glycemic control can be maintained over 1 year in patients with type 1 diabetes by using the Accu-Chek ${ }^{\oplus}$ Advisor Insulin Guidance Software with no change insulin dose or weight. Day to day management of glycemic control can be significantly improved using computerized insulin dosing software. (Fig. 1a-b)

Supported by Roche Diagnostics

\section{4}

Task delegation and computerised decision support improve cardiovascular risk for type 2 diabetes patients. A randomised controlled trial in primary care

F. G. W. Cleveringa, K. J. Gorter, M. van den Donk, G. E. H. Rutten;

General Practice, Julius Centre for Health Science and Primary Care,

Utrecht, The Netherlands.

Background and Aims: In The Netherlands most type 2 diabetes (DM2) patients are treated in primary care. Coronary heart disease (CHD) causes $70 \%$ of the mortality and reducing cardiovascular risk is a priority. However, implementing guidelines in primary care appears to be difficult, and at least $30 \%$ of DM2 patients do not meet the strict targets for good glycaemic and cardiovascular control. Most interventions aiming to improve diabetes care showed improvements in the process of care, but not in patient outcome. The Diabetes Care Protocol (DCP) combines task delegation, computerised decision support, and benchmark information, but it is not known whether this will improve patient outcome.

Aim: To study the effect of DCP on cardiovascular risk factors for DM2 patients in primary care.

Materials and Methods: 48 primary care practices participated in a cluster randomised trial. 26 practices started with DCP (intervention group) and 22 practices continued usual care (control group). A practice nurse registered all clinical parameters in the DCP software. Mean changes between intervention and control group for $\mathrm{HbAlc}$, systolic and diastolic blood pressure, total cholesterol and HDL-cholesterol were calculated (independent samples ttest) and corrected for clustering (generalized linear models). For patients without a history of cardiovascular disease (CVD) we also calculated the change in 10 year UKPDS CHD risk estimate (www.dtu.ox.ac.uk).

Results: 2390 patients participated, 1363 intervention group and 1027 control group. At baseline the clinical parameters were comparable. Total cholesterol, systolic and diastolic blood pressure improved significantly more in the intervention group compared with the control group, but the change in $\mathrm{HbAlc}$ was not significant. In patients without a history of cardiovascular disease the difference in 10 year UKPDS CHD risk estimate was $2.1 \%$ better in the intervention group. (Tab. 1)

Conclusion: Delegation of routine diabetes care to a practice nurse combined with computerised decision support and benchmark information improved the (intermediate) patient outcome of diabetes care significantly. This was also translated in improvement of the10 year UKPDS CHD risk score. Supported by Pfizer B.V.

Tab. 1: Baseline values and one year change in both groups after the DCP intervention

\begin{tabular}{|c|c|c|c|c|c|}
\hline & \multicolumn{2}{|c|}{ Intervention group $n=1363$} & \multicolumn{2}{|c|}{ Control group $n=1027$} & \multirow{2}{*}{$\begin{array}{l}\text { Difference between groups } \\
\text { with correction for clustering } \\
(95 \% \mathrm{CI})\end{array}$} \\
\hline & $\mathrm{t}-0 \pm ; \mathrm{SD}$ & Mean change $t-12 \pm ; S D$ & $\mathrm{t}-0 \pm ; \mathrm{SD}$ & Mean change t-12; SD & \\
\hline $\mathrm{HbAlc}(\mathrm{mmol} / \mathrm{l})$ & $7.0 \pm 1.1$ & $-0.2 \pm 1.0$ & $6.9 \pm 1.0$ & $-0.1 \pm 0.8$ & $-0.10(-0.23 ; 0.03)$ \\
\hline Systolic blood pressure (mm Hg) & $149 \pm 22$ & $-6.7 \pm 19.4$ & $148 \pm 20$ & $-0.8 \pm 19.4$ & $-4.7(-8.1 ;-1.2)$ \\
\hline Diastolic blood pressure (mm Hg) & $83 \pm 11$ & $-3.3 \pm 10.7$ & $83 \pm 10$ & $-1.0 \pm 10.3$ & $-2.1(-0.5 ;-3.8)$ \\
\hline Total cholesterol (mmol/l) & $5.0 \pm 1.0$ & $-0.4 \pm 1.0$ & $4.9 \pm 1.0$ & $-0.2 \pm 0.9$ & $-0.20(-0.33 ;-0.07)$ \\
\hline HDL cholesterol (mmol/l) & $1.37 \pm 0.36$ & $0.017 \pm 0.21$ & $1.32 \pm 0.36$ & $0.024 \pm 0.33$ & $0.011(-0.052 ; 0.031)$ \\
\hline Patients without a history of CVD & \multicolumn{2}{|c|}{ Intervention group $n=612$} & \multicolumn{2}{|c|}{ Control group n=393 } & \\
\hline 10 year UKPDS CHD risk (\%) & $22 \pm 16.5$ & $-2.1 \pm 8.1$ & $21 \pm 15.0$ & $-0.1 \pm 6.9$ & $-2.1(-3.5 ;-0.6)$ \\
\hline
\end{tabular}




\section{PS 1 Obesity as a predictor of type 2 diabetes}

\section{5}

Problems and their resolves in diabetes screening

I. V. Misnikova, A. V. Dreval, I. A. Barsukov;

Endocrinology, Moscow Regional Resaerch Clinical Institute, Russian

Federation.

Background and Aims: WHO recommends oral glucose tolerance test (OGTT) as being single best choice, but if it is not possible to perform OGTT fasting plasma glucose (FPG) alone may be used for epidemiological purposes. Aim of study was to estimate influence of OGTT exclusion from diabetes screening to prevalence of hyperglycemic state.

Materials and Methods: Study was population-based screening for IFG, IGT and T2D. Personal data of participants were obtained from municipal medical insurance data of two Moscow County districts (100 666 inhabitants). 8000 subjects (age 18-90 years) was randomised. Invitation letters and information brochures were sent to them. 2638 subjects agreed to took part in the study. All participants gave their informed consent and study was approved by the ethic committee of the Moscow Region Research Clinical Institute. FPG was measured in all participants. A standard $75 \mathrm{~g}$ OGTT was performed using HemoCue analyzers in subjects previously undiagnosed with diabetes (2508 subjects). Diagnosis of IFG, IGT, T2D followed the 1999 World Health Organization (WHO) criteria. Of the inhabitants, 1,63\% subjects had previously been diagnosed with $\mathrm{T} 2 \mathrm{D}$ and were excluded from subsequent analyses.

Results: In total, 2188 subjects ( $87,24 \%)$ had normal glucose tolerance (NGT). The figures for IFG were 58 subjects (2,31\%), for IGT 120 subjects $(4,78 \%)$, for IFG + IGT 44 subjects $(1,75 \%)$ and finally T2D was diagnosed among 98 participants $(3,9 \%)$. When we excluded the results of OGTT, prevalence of T2D decreased to $3,15 \%$ (79 subjects). The figure of IFG became 112 subjects $(4,47 \%)$. The amount of subjects with NGT increased up to 2317 subjects $(92,38 \%)$. In the structure of early abnormalities of glucose homeostasis the main part occupied by IGT (54,5\%), IFG $26,13 \%$ and IFG+IGT 19,82\% . We estimated the necessity of OGTT in groups with different FPG level. Among subjects with FPG $<4 \mathrm{mmol} / \mathrm{l}$ IGT was not diagnosed at all. Prevalence of IGT among subjects with FPG $4-4,5 \mathrm{mmol} / 1$ is very low $(0,28 \%)$. There are no patients with T2D among subjects with FPG $<4,7 \mathrm{mmol} / \mathrm{l}$. Prevalence of T2D among subjects with FPG $4,7-5,5 \mathrm{mmol} / \mathrm{l}$ is very low $(0,16 \%)$.

Conclusion:

1) Hyperglycemic abnormalities are revealed by OGTT more often $(12,76$ $\%$, include T2D 3,9\%) than by measurement FPG only $(7,62 \%$ include T2D 3,15\%).

2) Exclusion of OGTT from screening will result in undiagnosing of main part of early abnormalities of glucose homeostasis (54,05\%).

3) The exclusion of OGTT from screening of subjects with FPG $<4,7 \mathrm{mmol} / \mathrm{l}$ does not influence on prevalence of T2D.

\section{6}

Predictors of incident diabetes mellitus in Basrah, Iraq A. A. Mansour;

Medicine, Basrah College of medicine, Basrah, Iraq.

Background: New-onset diabetes was associated with a 90\% increase in risk of all-cause mortality and a $120 \%$ increase in risk of cardiovascular mortality compared with study participants without diabetes. The aim of this study was to study prospectively the predictors of incident diabetes mellitus in Basrah, Iraq with special emphasis on predictive performance of four anthropometric variables of obesity, body mass index (BMI), waist circumference (WC), waist-to-hip ratio (WHpR) or waist-to height ratio (WHtR).

Material and Methods: Total of 13730 (7101men and 6629 women) cohort free of diabetes at baseline were followed for a mean of 5 years (January 2001 to end of December 2006).

Results: There were $935(6.80 \%)$ cases of incident diabetes (513 men and 422 women). All anthropometric indices (BMI, WC, WHpR WHtR) were higher among those with incident diabetes $(\mathrm{P}<0.001)$. In both sexes WHpR has the strongest associations with incident diabetes that was gender insensitive (AUC $=0.74$ in males and 0.72 in females) followed by WC then BMI which has the weakest association with incident diabetes. On multivariable logistic regression, only hypertension ( $\mathrm{OR}, 1.66 ; 95 \% \mathrm{CI}, 1.41$ to 1.96 ; $\mathrm{p}<0.001$ ) was associated with incident diabetes. All anthropometric indices were significantly associated with incident diabetes except WHtR. No association between incident diabetes and gender, age, stroke, and ischemic heart disease.

Conclusion: In both sexes WHpR has the strongest associations with incident diabetes, followed by WC then BMI which has the weakest association with incident diabetes while WHtR has no association. Hypertension is the only non anthropometric variable associated with incident diabetes.

\section{7}

Diabetes is associated with inflammation independent of obesity: a community-based sample of routine care patients

K. L. Pedula, G. A. Nichols, T. A. Hillier;

Science Program Department, Kaiser Permanente Center for Health Research, Portland, United States.

Background and Aims: Inflammation has been shown to be associated with cardiovascular disease (CVD), the leading cause of death among persons with type 2 Diabetes (T2DM). Additionally obesity, a risk factor for T2DM, has been linked to inflammation. The objectives of this study were to assess the relationship between inflammatory markers and T2DM and to determine if this relationship persists after adjusting for obesity and other comorbidities.

Materials and Methods: Among a large USA health plan sample with electronic medical records for all laboratory tests, we identified persons who had a complete blood count $(\mathrm{CBC})$ that included both a white blood cell (WBC) count and platelet (PLT) test in 2005. These tests are widely available as part of routine outpatient care and are known to be early markers of low-level inflammation. CBC tests ordered during a hospitalisation or for an emergency care visit were excluded. Also excluded were persons with diagnoses of cancer or HIV. Patients were stratified into 2 groups: all persons with T2DM; and a random sample of persons without diabetes matched on age, gender, and body mass index (BMI) to the T2DM group. Using electronic medical records, we linked individuals to other comorbidities (e.g., diagnosis of CVD, hypertension, dyslipidemia). We then used multivariable models to compare WBC and PLT between T2DM and controls across the levels of BMI while adjusting for comorbidities.

Results: A total of 17,036 patients were included in the final analyses. Average age was $64.8( \pm 12.3)$ years and $49 \%$ were male. BMI averaged 32.6 $( \pm 7.3) \mathrm{kg} / \mathrm{m}^{2}$ with $13 \%$ of matched pairs having a BMI $>40 \mathrm{~kg} / \mathrm{m}^{2}$. Relative to controls, average WBC counts were significantly higher in T2DM cases across all BMI levels (see Table). Average PLT levels were only marginally associated with diabetes status. Adjustment for hypertension, CVD, and dyslipidemia attenuated these associations slightly, but the relationships remained significant. Within the T2DM group, anti-hyperglycemic therapy (insulin or oral hypoglycemic agents) was not associated with WBC counts or PLT levels

Conclusion: Inflammatory markers are consistently higher in persons with T2DM relative to controls, irrespective of obesity status. Further investigation is necessary to assess the contribution of hyperglycemia and other diabetes characteristics to this increased inflammation in order to identify possible mechanisms for reducing the burden of CVD in T2DM.

\begin{tabular}{|c|c|c|c|c|c|c|}
\hline \multirow[t]{2}{*}{ BMI $\left(\mathrm{kg} / \mathrm{m}^{2}\right)$} & \multicolumn{3}{|c|}{$\begin{array}{l}\text { Mean White Blood } \\
\text { Cell Count }\left(\times 10^{9} / \mathrm{L}\right)^{*}\end{array}$} & \multicolumn{3}{|c|}{$\begin{array}{l}\text { Mean Platelet Count } \\
\left(\begin{array}{l}\text { ( 10 10 } / \mathrm{L})^{*} \\
\end{array}\right.\end{array}$} \\
\hline & T2DM & Control & p-value & T2DM & Control & p-value \\
\hline$<25$ & 7.7 & 7.0 & $<0.001$ & 258.3 & 251.5 & 0.076 \\
\hline $25-30$ & 7.5 & 6.9 & $<0.001$ & 243.6 & 247.0 & 0.087 \\
\hline $31-40$ & 8.3 & 7.3 & $<0.001$ & 247.8 & 251.4 & 0.028 \\
\hline$>40$ & 8.5 & 7.7 & $<0.001$ & 268.6 & 270.0 & 0.678 \\
\hline
\end{tabular}

${ }^{*}$ Adjusted for age and gender

Supported by a contract with Bristol-Myers Squibb 


\section{8}

Abdominal obesity: a predictor of type 2 diabetes and cardiovascular disease in a primary care cross sectional study

S. Massano-Cardoso ${ }^{1}$, E. Mendes ${ }^{2}$, D. Carvalho ${ }^{3}$; on behalf of the W-Risk investigators,

${ }^{1}$ Instituto de Higiene e Medicina Social, Faculdade de Medicina de Coimbra, Coimbra, ${ }^{2}$ APMCG, Associação Portuguesa dos Médicos de Clinica Geral, Lisboa, ${ }^{3}$ Endocrinology, Diabetes and Metabolism, Faculdade de Medicina/ Hospital de S. João, Porto, Portugal.

Background and Aims: There is not enough evidence on prevalence of abdominal obesity (AO) associated with diabetes in Portugal. A descriptive analysis of data from a Primary Care Physician (PCP) population aged $\geq$ 18 years in Portugal was used to assess how AO is associated with diabetes, cardiovascular risk (CVR) factors and presence of a CV disease.

Materials and Methods: W-Risk (Body Weight Deviations and Risk Situations) was a non-interventional cross-sectional study conducted in 2005. A standardised questionnaire was completed in 14,695 subjects consulting their PCP $(n=1,500)$. Demographic data, weight, waist circumference (WC), CVR factors, physical exercise and concomitant treatment were collected. AO is defined as a WC in women $\geq 80 \mathrm{~cm}$ and in $m e n \geq 94 \mathrm{~cm}$. The prevalence was standardised to the general population.

Results: A total of 14,695 subjects were included with a mean age $55.5 \pm 16.2$; $65.8 \%$ were female.

Prevalence of $\mathrm{AO}$ was $66.3 \%$ in men and $82.9 \%$ in women. The prevalence of diabetes was $9.2 \%$, being $10.5 \%$ in men and $7.6 \%$ in women. The prevalence of diabetes was stepwise increased through the gender specific quartiles of $\mathrm{AO}$ as shown in table 1 :

\begin{tabular}{lllll} 
Sex & $1^{\text {st }}$ Quartile AO & $2^{\text {nd }}$ Quartile AO & $3^{\text {rd }}$ Quartile AO & $4^{\text {th }}$ Quartile AO \\
\hline Males & $8.9 \%$ & $15.2 \%$ & $23.3 \%$ & $30.3 \%$ \\
Females & $4.2 \%$ & $10.1 \%$ & $16.3 \%$ & $24.2 \%$ \\
\hline
\end{tabular}

The age adjusted odds ratios of different CVR factors and respective CIs (95\%) for $\mathrm{AO}$ versus normal WC are shown in table 2 :

\begin{tabular}{lll} 
Variable & Women & Men \\
\hline Diabetes & $3.3(2.5-4.5)$ & $2.3(1.9-2.8)$ \\
Hypertension & $2.8(2.2-3.6)$ & $2.8(2.4-3.3)$ \\
Hypertriglyceridaemia & $2.8(2.2-3.6)$ & $2.8(2.4-3.3)$ \\
Total Hypercholesterolaemia & $1.8(1.6-2.1)$ & $1.7(1.5-1.9)$ \\
High LDL & $1.7(1.4-2.0)$ & $1.8(1.5-2.0)$ \\
Low HDL & $1.9(1.6-2.3)$ & $1.8(1.6-2.1)$ \\
Smoking & $0.8(0.6-1.06)$ & $0.5(0.45-0.66)$ \\
Physical exercise & $0.8(0.7-0.9)$ & $0.8(0.7-0.9)$ \\
\hline
\end{tabular}

The relative risk of diabetes and $\mathrm{CV}$ disease comparing the $4^{\text {th }} \mathrm{AO}$ quartile to $1^{\text {st }}$ is shown in table 3 :

\begin{tabular}{lll} 
& Males & Females \\
\hline Diabetes & $3.4(2.4-4.7)$ & $5.8(3.9-8.6)$ \\
Angor & $3.2(1.6-6.2)$ & $3.9(1.8-8.3)$ \\
Myocardial Infarction & $3.2(1.5-6.6)$ & $2.2(0.4-12.1)$ \\
Stroke & $2.1(0.8-5.5)$ & $3.1(0.96-10.0)$ \\
\hline
\end{tabular}

Conclusion: Our results show that in Portugal, in PCP practice, and using IDF criteria for defining $\mathrm{AO}, \mathrm{AO}$ is highly prevalent, especially in women. Subjects with $\mathrm{AO}$ are more likely to have diabetes, hypertension and hypertriglyceridaemia in both gender. Identification of subjects who have AO may provide opportunities to early identify patients who are likely to have diabetes and CVR.

Supported by sanofi-aventis

\section{9}

Comparison of anthropometric indicators for obesity as predictors of diabetes incidence in Mauritius

R. Nyamdori ${ }^{1,2}$, Q. Qiao ${ }^{1,2}$, S. Söderberg ${ }^{3,4}$, J. Pitkäniemi ${ }^{1}$, P. Zimmet ${ }^{4}$, J. Shaw ${ }^{4}$, G. Alberti ${ }^{5}$, V. Pauvaday ${ }^{6}$, P. Chitson ${ }^{6}$, J. Tuomilehto ${ }^{1,2}$;

${ }^{1}$ Public Health, University of Helsinki, Finland, ${ }^{2}$ Health Promotion and Chronic Diseases Prevention, National Public Health Institute, Helsinki, Finland, ${ }^{3}$ Medicine, Umeå University Hospital, Sweden, ${ }^{4}$ International Diabetes Institute, Caulfield, Victoria, Australia, ${ }^{5}$ Endocrinology and Metabolic Medicine, Mint Wing, St Marys Hospital, London, United Kingdom, ${ }^{6}$ Ministry of Health and Quality of Life, Port Louis, Mauritius.

Backgrounds: Obesity is a risk factor for diabetes and CVD. The definition of obesity is important for the purpose of intervention. There is controversy regarding which anthropometric measures best defines obesity and conveys highest risk for diabetes.

Aims: To compare different anthropometric indicators of BMI, waist circumference (WC), WHR, and waist to height ratio (WSR) as predictors of diabetes incidence in Mauritian Indians and Creoles living in Mauritius.

Methods: Three population-based surveys were conducted in Mauritius in 1987, 1992, and 1998. According to the participation in these surveys, participants were divided into 4 subgroups as shown in table 1 .

Results: Hazard ratios (HRs) for diabetes incidence were estimated applying an interval censored survival analysis using age as timescale for each ethnic group based on baseline obesity indicators. All models in the data analysis were adjusted for cohort and baseline fasting glucose. A paired homogeneity test was performed in order to test equality of regression coefficients in the survival analysis between BMI and WC (or WHR or WSR). All linear hypotheses concerning the regression coefficients were tested using $\mathrm{R}$ program 2.2.1 for diabetes incidence. We chose 5\% significance level. A significant result from the paired homogeneity test indicates that the two indicators have a different effect on incident diabetes.

The number of participants and the incidence rates are shown in table 1.

\begin{tabular}{llll} 
Cohorts & $\begin{array}{l}\text { Men } \\
\%(\text { number) }\end{array}$ & $\begin{array}{l}\text { Women } \\
\% \text { (number) }\end{array}$ & $\begin{array}{l}\text { Total } \\
\% \text { (number) }\end{array}$ \\
\hline $1987-1992$ & $11.5(393)$ & $7.6(380)$ & $9.6(773)$ \\
$1992-1998$ & $11.3(575)$ & $11.8(686)$ & $11.6(1261)$ \\
$1987-1998$ & $13.8(58)$ & $20.3(74)$ & $17.4(132)$ \\
$1987-1992-1998$ & $22.9(844)$ & $20.1(993)$ & $21.4(1837)$ \\
Total & $16.6(1870)$ & $15.2(2133)$ & $15.9(4003)$ \\
\hline
\end{tabular}

Table 1. Diabetes incidence (\%) and the total number of non diabetic individuals at baseline in four subgroups (cohorts)

The HR for diabetes incidence was slightly lower for BMI than for central obesity indicators but their $95 \%$ CIs overlapped (table 2).

\begin{tabular}{llllll} 
& \multicolumn{2}{l}{ MAURITIAN INDIAN } & & \multicolumn{2}{l}{ CREOLES } \\
\cline { 2 - 3 } \cline { 5 - 6 } & Men & Women & & Men & Women \\
\hline BMI & $1.64(1.48-1.82)$ & $1.54(1.38-1.72)$ & & $1.99(1.68-2.35)$ & $1.44(1.22-1.70)$ \\
WC & $1.74(1.56-1.93)$ & $1.56(1.40-1.74)$ & & $2.14(1.81-2.54)$ & $1.49(1.26-1.76)$ \\
WHR & $1.66(1.51-1.81)$ & $1.56(1.42-1.71)$ & & $2.01(1.74-2.32)$ & $1.51(1.31-1.73)$ \\
WSR & $1.80(1.62-2.00)$ & $1.60(1.44-1.77)$ & & $2.27(1.92-2.69)$ & $1.46(1.24-1.71)$ \\
\hline
\end{tabular}

Table 2 HRs ( $95 \%$ CIs) for diabetes incidence corresponding to a one SD increase in obesity indicators, estimated separately for each indicator

Paired homogeneity tests showed BMI did not differ from central obesity indicators except for in Mauritian Indian men where WSR was better than BMI $(p=0.03)$ with regard to the prediction of incident diabetes.

Conclusion: BMI was as strong as central obesity indicators in the prediction of diabetes incidence in this population. 


\section{0}

Body mass index is strongly associated with impaired insulin sensitivity in Europeans from 19 countries, independent of age, sex, social class and smoking habits: the RISC Study

N. M. Lalic ${ }^{1}$, P. M. Nillson ${ }^{2}$, J.-A.. Nillson ${ }^{2}$, R. Gabriel ${ }^{3}$, C. Anderwald ${ }^{4}$, B. Balkau ${ }^{5}$ the RISC Investigators,

${ }^{1}$ Institute for Endocrinology, School of Medicine, Belgrade, Serbia,

${ }^{2}$ Department of Clinical Sciences, Lund University, Malmö, Sweden,

${ }^{3}$ Unidad de Investigacion, Hospital Universitario La Paz, Madrid, Spain,

${ }^{4}$ Department of Internal Medicine 3, Endocrinology and Metabolism,

General Hospital, Vienna, Austria, ${ }^{5}$ Inserm U 780 - IFR 69, Epidemiological and Biostatistical Research, Villejuif, France.

Background and Aims: Impaired insulin sensitivity (IS) is associated with a large number of cardiovascular risk factors, including obesity and smoking. As subjects from low social class tend to be more obese and smoke more, it is of interest to test whether impaired IS differs with social class. This study was aimed to analyse the relationship among IS, obesity, social background parameters and smoking habits in European healthy individuals.

Materials and Methods: We investigated 1284 subjects from 19 academic centres all over Europe as part of the ongoing EGIR-RISC Study. These subjects were selected as healthy and with a low cardiovascular risk profile. At baseline a clinical examination was carried out (weight, height, waist circumference, blood pressure) together with a self-administered questionnaire (social background, lifestyle). All subjects underwent a standardised euglycaemic hyperinsulinemic clamp for measurement of SI, reflected by the M/I level $(\mathrm{mg} / \mathrm{kg} / \mathrm{min} /$ insulin level during the last $40 \mathrm{~min}$ of clamp).

Results: There were in total 575 men and 709 women, stratified for occupational category: non-manual $(n=969 ; 75.5 \%)$, self-employed $(n=66$; $5.1 \%)$ and manual $(\mathrm{n}=249 ; 19.4 \%)$. Mean $\mathrm{M} / \mathrm{I}$ was $14.1+/-7.1 \mathrm{mg} / \mathrm{kg} / \mathrm{min} / \mathrm{nM}$. No significant difference was seen in $\mathrm{M} / \mathrm{I}(\mathrm{p}=0.094)$, between occupational groups adjusted for age $(p=0.41)$, sex $(p<0.001)$, body mass index (BMI) $(\mathrm{p}<0.001)$, and study centre $(\mathrm{p}<0.001)$, in general or stratified for gender. The same results were obtained with waist circumference replacing BMI. No interaction with waist and social class was noticed $(\mathrm{p}=0.69)$. However, the BMI correlated significantly with $\mathrm{M} / \mathrm{I}(\mathrm{p}<0.001)$, but was not associated with smoking, after adjusting for all other factors including social class. BMI differed between manual and non-manual female workers ( 25.5 vs. $24.7 \mathrm{~kg} /$ $\mathrm{m}^{2} ; \mathrm{p}=0.045$ ), but not between corresponding categories of male workers. Conclusion: In this study, obesity is found to be strongly associated with impaired IS independently of other covariates, especially of social background and smoking habits in healthy subjects across Europe. The results imply that prevention of obesity should therefore be the main objective to prevent impairment of IS in all subjects at risk, irrespective of their social background.

RISC is supported by the European Union and by AstraZeneca

\section{1}

Body mass index is an important risk factor for developing type 2 diabetes in Japanese

J. Yatabe, H. Sanada, M. S. Yatabe, Y. Kusano, S. Hashimoto, T. Watanabe; Internal Medicine III, Fukushima medical University, Japan.

Background and Aims: Some prospective studies have examined the relationship between obesity/overweight and the incidence of type 2 diabetes in Japan. The purpose of this study is to establish causal connections among individuals who are overweight and the onset of diabetes in a retrospective cohort study.

Materials and Methods: Our investigation included 1,044 men and 608 women, aged 33 to 75 years at baseline who had a voluntary complete medical checkup. The participants were followed for 10 years. Onset of diabetes was identified by an annual 75-g oral glucose tolerance test (75g-OGTT). Participants with fasting plasma glucose $\geq 126 \mathrm{mg} / \mathrm{dl}, 2$-h glucose level in a $75 \mathrm{~g}$-OGTT $\geq 200 \mathrm{mg} / \mathrm{dl}$ and/or those receiving medical treatment for type 2 diabetes during the previous year were counted as new-onset diabetics. We used the $\chi^{2}$-test to compare relevant characteristics of participants at baseline and to estimate the odds of developing type 2 diabetes based on risk factors. We applied the Cox proportional hazards model to assess the independent contribution of BMI to the risk for developing type 2 diabetes.
Results: During the follow-up period, we diagnosed 95 men and 53 women with newly-onset type 2 diabetes. A Kaplan-Meier survival curve for 10 years showed the cumulative probability of developing type 2 diabetes was higher in participants who were moderately (BMI $25.0-27.4 \mathrm{~kg} / \mathrm{m}^{2}$ ) or considerably overweight/obesity $\left(\mathrm{BMI} \geq 27.5 \mathrm{~kg} / \mathrm{m}^{2}\right)$ (Figure). The analysis indicated that $\mathrm{BMI}$ is a strong risk factor (odds ratio: 5.61, $\mathrm{p}<0.01$ ). Elevated TC (odds ratio: 1.51, $\mathrm{P}<0.05$ ), TG (odds ratio: 4.54, $\mathrm{P}<0.01$ ), HOMA-IR (odds ratio: $2.88, \mathrm{P}<0.01$ ), and decreased HDL-C (odds ratio: $2.41, \mathrm{P}<0.01$ ), but not fasting plasma glucose were associated with increased risk of new-onset type 2 diabetes. In the Cox proportional hazards model, the risk of diabetes mellitus increased with increasing BMI even after adjusting for age, sex, blood pressure, hyperlipidemia, and insulin resistance. In the final model, setting BMI less than 25 as a reference group, the odds ratio of diabetes mellitus was 3.66 for those with a BMI of 25-27.4 and this increased to 4.18 for participants with a BMI of 27.5 or higher (Table).

Conclusion: BMI is an independent and dose-dependent risk factor for type 2 diabetes in overweight Japanese patients. Management of body mass is therefore important for the prevention of type 2 diabetes.

Figure

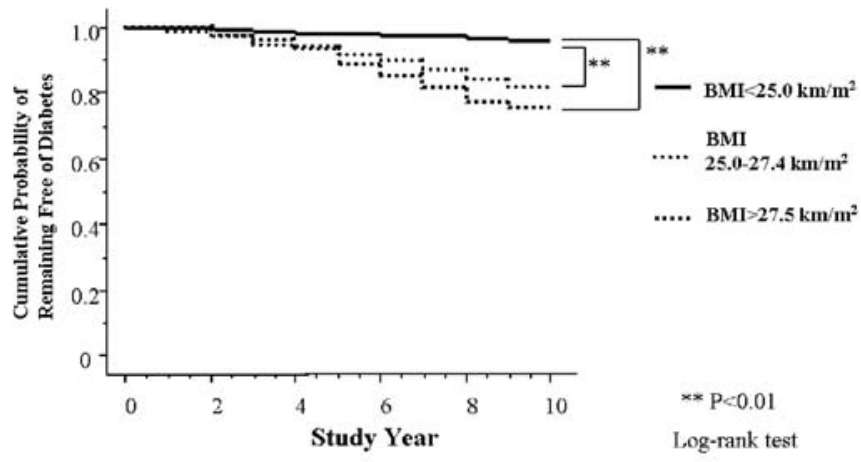

(Kaplan-Meier method)

Table

Cox's proportional hazards model of the incidence of type 2 diabetes mellitus over 10-years according to BMI.

\begin{tabular}{|c|c|c|c|c|}
\hline & Model 1 & Model 2 & Model 3 & Model 4 \\
\hline & Adjusted (+) & Adjusted (+) & Adjusted (+) & Adjusted (+) \\
\hline & & +SBP,DBP & + SBP,DBP & +SBP,DBP \\
\hline $\begin{array}{l}\text { BMI } \\
\left(\mathrm{kg} / \mathrm{m}^{2}\right)\end{array}$ & & & +TC, HDLC, TG & $\begin{array}{r}+\mathrm{TC}, \text { HDLC, TG } \\
+ \text { HOMA-IR }\end{array}$ \\
\hline$<25.0$ & 1.00 & 1.00 & 1.00 & 1.00 \\
\hline \multicolumn{5}{|l|}{$(n=1193)$} \\
\hline $25.0-27.4$ & $4.61 * *$ & $4.46^{* x}$ & $3.60 * *$ & $3.66^{* *}$ \\
\hline$(n=341)$ & $(3.21-6.64)$ & $(3.10-6.43)$ & $(2.48-5.22)$ & $(2.52-5.32)$ \\
\hline$>27.5$ & $6.38 * *$ & $6.16^{* x}$ & $4.23 * *$ & $4.18 * *$ \\
\hline$(n=118)$ & $(4.08-9.99)$ & $(3.92-9.66)$ & $(2.68-6.70)$ & $(2.64-6.62)$ \\
\hline
\end{tabular}

Adjusted (+): Adjusted for age and sex.

$A n: p<0.01$

\section{2}

Combined information from type 2 diabetes susceptibility genes, implications for risk prediction and treatment outcome: the Hoorn study L. M. 't Hart' ${ }^{1}$ J. M. Dekker ${ }^{2}$, E. C. van Hove ${ }^{1}$, E. Reiling ${ }^{1}$, G. Nijpels ${ }^{2}$, R. J. Heine ${ }^{2}$, J. A. Maassen ${ }^{1,2}$;

${ }^{1}$ Molecular Cell Biology, Leiden University Medical Center, Leiden, ${ }^{2}$ EMGO Institute, Vrije Universiteit Medical Center, Amsterdam, The Netherlands.

Background and Aims: Progress in identifying susceptibility genes for T2DM has been slow and for the few genes identified so far the associated risks are generally low. In this study we evaluated whether combinations of several different susceptibility genes improve disease prediction in the Dutch Hoorn study cohort. Furthermore we evaluated whether this kind of information can be used in treatment of subjects with type 2 diabetes mellitus. 
Materials and Methods: Single nucleotide polymorphisms (MAF $>10 \%)$ in four different genes (PPARG (P12A), KCNJ11 (E23K), TCF7L2 (rs4506565) and $A B C C 8$ (rs1799854) were genotyped in 1055 participants of the Hoorn study (505 T2DM, 550 NGT). These four genes were chosen based on the fact that they are either A: unambiguously associated with T2DM in several large population-based studies and meta-analysis or B: associated with the disease in our Hoorn study cohort and at least one other Dutch cohort (ABCC8). Response to treatment was analysed in 156 subjects with T2DM who participated in a two-year intervention study aiming at improved glycaemic control. Subjects were stratified according to risk allele count prior to the analysis (low $<2$, medium 3-5, high $>6$ risk alleles). The difference between fasting glucose levels before and after the study was used as outcome variable.

Results: The observed single gene effects were comparable to those in literature for Caucasian populations (odds ratios ranging from 1.1 to 1.4). Four gene risk allele counts were made for each individual and compared between cases and controls. The odds of having T2DM increased by 1.23 (95\% CI 1.12-1.35) with each additional risk allele. Comparing the extremes of the distribution ( $0-1$ risk alleles $(2 \%)$ versus 7 or 8 risk alleles $(4 \%)$ even increased the odds to 9.7 (95\% CI 2.5-37.2). We next evaluated whether there are differences in response to intensified diabetes treatment between those with a low $(n=15)$, medium $(n=109)$ or high number of risk alleles $(n=32)$. Baseline characteristics (glucose, insulin, $\mathrm{HbAlc}$, duration of diabetes, use of medication etc) were not significantly different between the three groups. After the 2 year intervention study those with a high number of risk alleles showed significantly more often and the largest response to the intensified diabetes treatment and education program (delta fasting glucose $-0.6 \pm 0.3$, $-0.5 \pm 0.3$ and $+0.6 \pm 0.4 \mathrm{mmol} / \mathrm{l}$ for high, medium and low resp, $\mathrm{p}=0.02$ and $\mathrm{p}=0.04$ for high or medium vs. low). As expected the use of medication increased during the intervention but this could not explain the observed differences in response.

Conclusion: We conclude that combined information from type 2 susceptibility genes can be used to identify subgroups with markedly different risks of developing type 2 diabetes. Furthermore our data suggest that this type of information can also be used in treatment and programs aiming at prevention or delay of diabetes and its complications in those with the largest a priori risk. Additional larger studies, including pharmacogenetic, intervention and prevention studies are needed to confirm and substantiate our results.

Supported by grants from ZonMW-RIDE program and the Dutch Diabetes Research Foundation

\section{PS 2 The spectrum of diabetic disorders}

\section{3}

The prevalence of the mitochondrial DNA 16189 variant in Czech diabetic and non-diabetic population and its associations with thinness, birth weight and insulin resistance but not with type 2 diabetes M. Vankova, P. Lukasova, J. Vcelak, D. Vejrazkova, H. Kvasnickova, K. Vondra, B. Bendlova;

Dept. of Molecular Endocrinology, Institute of Endocrinology, Prague 1, Czech Republic.

Background and Aims: Type 2 diabetes (T2D) is a multifactorial disorder in which variants in mitochondrial DNA (mtDNA) could play a role. Some studies have shown that the mode of inheritance of T2D performs an excess of maternal transmission, but only a minority of mtDNA mutations have been identified in diabetics. A common mtDNA variant 16189 is positively associated with insulin resistance, T2D or metabolic syndrome in different populations. In this study we investigated whether 16189 variant is associated with risk diabetogenic parameters in Czech population.

Materials and Methods: 267 T2D patients (60.5 \pm 8.5 years) and 159 unrelated non-diabetics, including 71 healthy offspring of T2D and 88

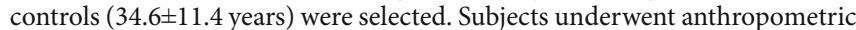
measurement, fasting biochemical examination and non-diabetics also OGTT and ivITT. Detailed anamnestic data were recorded by questionaire. The 16189 variant was identified by PCR-RFLP (MnlI) of blood DNA. All samples positive for the 16189 variant were sequenced. Statistical analyses were performed using NCSS 2004 software.

Results: In contrast to other published studies, the variant 16189 was found more commonly in non-diabetics (13.8\%, but in controls $21.6 \%)$ than in the diabetic cases $(9.7 \%)$. Frequence of variant between diabetics and controls was significantly different $(\mathrm{p}=0.004)$. Most diabetics reported having a maternal family history rather than paternal ( $49.8 \%$ vs. $30.6 \%)$, but frequency of variant in diabetics with maternal and paternal family history of diabetes is similar. Age of T2D diagnosis is not dependent on presence of the variant. Diabetics with the variant had significantly higher triglycerides than those with wild type $(p=0.01)$ and higher cholesterol $(p=0.05)$. Non-diabetics with the variant had higher stimulated $\mathrm{C}$ peptide than those with wild type $(\mathrm{p}=0.04)$ and higher growth hormone in $60 \mathrm{~min}$ OGTT $(\mathrm{p}=0.01)$. Fasting glucose, insulinemia, indices of insulin resistance and beta cell function and blood pressure were not different between groups. Non-diabetic women with the variant had significantly less subcutaneous fat $(\mathrm{p}=0.02)$ and higher muscle/fat mass ratio $(\mathrm{p}=0.04)$ than those with wild type and lower BMI $(\mathrm{p}=0.05)$. Birth weight was significantly lower in subjects with variant $(\mathrm{p}=0.04)$ than in wild types.

Conclusion: The frequence of 16189 variant in Czech diabetic patients is similar to other published Caucasian populations, while the frequence of variant in non-diabetics is markedly higher than those other Caucasians and this difference is more apparent in controls, i. e. healthy subject without T2D in parents. We demonstrated a significant association between the variant and thinness in non-diabetic women and also with lower birth weight. We did not confirm any association with T2D or clear association with metabolic syndrome (except for triglycerides in causes). However, higher C peptide during OGTT in non-diabetics with the variant suggests possible beginning of the insulin resistance. The finding of much higher growth hormone in 60 min OGTT in non-diabetics with the variant is of metabolic interest. Supported by IGA MHCR NR/7809-5

\section{4}

Diabetes due to the $\mathrm{mt} 3243 \mathrm{~A}>\mathrm{G}$ mutation among young adult diabetic subjects in Sri Lanka - prevalence and clinical heterogeneity P. Katulanda ${ }^{1,2}$, D. R. Matthews ${ }^{3}$, C. Groves ${ }^{1}$, A. Barrett ${ }^{1}$, M. I. McCarthy ${ }^{1}$, A. L. Gloyn ${ }^{1}$;

${ }^{1}$ Diabetes Research Laboratories, Oxford Centre for Diabetes Endocrinology and Metabolism, Oxford, United Kingdom, ${ }^{2}$ Dept of Clinical Medicine, Faculty of Medicine, Colombo, Sri Lanka, ${ }^{3}$ Oxford Centre for Diabetes Endocrinology and Metabolism, University of Oxford, United Kingdom.

Background and Aims: The maternally inherited mt3243 mutation is associated with a variable clinical phenotype including diabetes and deafness 
(MIDD). A genetic diagnosis can assist with clinical management and genetic counselling. MIDD has been reported to account for $0.5-3 \%$ of diabetes among other populations, however the frequency of this mutation in Sri Lanka (SL) is not known. The aim of our study was to determine the prevalence and clinical characteristics of patients with the mt3243 mutation in a large cohort of SL patients with young onset diabetes.

Materials and Methods: DNA was available from 1007 subjects (age of diagnosis of diabetes $16-40$ yrs, age at recruitment $\leq 45$ yrs). The cohort was screened by quantitative real time PCR on an ABI 7900HT system using sequence specific TaqMan probes. Samples with heteroplasmy $\geq 5.0 \%$ were considered positive.

Results: 9 (4 males) mutation positive subjects were identified (prevalence $0.9 \%$ ). Their mean characteristics were; \% heteroplasmy 35.2 (SD 23.0; range 13-87), age at diagnosis 26.0 (SD 4.8) vs. 31.9 yrs (SD 5.6) in non-mutation carriers (NMC) ( $\mathrm{p}=0.002)$, BMI 18.6 (SD 2.6) vs. 25 (SD 15.0) $\mathrm{kgm} 2(\mathrm{p}<$ 0.0001 ). Maternal family history of diabetes was present among $66.0 \%$ of carriers vs. $42.0 \% \mathrm{NMC}(\mathrm{p}=\mathrm{ns})$, a personal history of hearing impairment was seen among $33.0 \%$ of carriers vs. $6.3 \% \mathrm{NMC}(\mathrm{p}=0.017)$. One mutation positive subject $(11.1 \%)$ has the metabolic syndrome vs $60.0 \%$ of NMC. Insulin treatment from diagnosis was used in $4(44.0 \%)$ of carriers compared to $16.0 \%$ of NMC ( $p=0.04)$. Clinical neuropathy was present in $22.2 \%$ of carriers compared to $3.0 \%$ of NMCs $(\mathrm{p}<0.001)$. Glomerular filtration rate of $<60 \mathrm{ml} / \mathrm{min} / \mathrm{m}^{2}$ was present in $44.4 \%$ compared to $5.0 \%$ NMCs ( $\mathrm{p}=0.001$ ).

Conclusion: In this large cohort of young-onset diabetic subjects from SL the $\mathrm{mt} 3243 \mathrm{~A}>\mathrm{G}$ mutation was detected in $0.9 \%$ of patients. Those with the mutation have a higher prevalence of neuropathy and low renal function. Our study demonstrates that a maternal family history of diabetes and either a personal and/or family history of deafness distinguish less than half $(44.0 \%, 4 / 9)$ of patients with MIDD from Sri Lankan subjects with youngonset diabetes. Therefore, additional characteristics such as BMI, absence of metabolic syndrome and age of diagnosis of diabetes should be considered when referring patients for genetic testing.

Supported by Oxford Centre for Diabetes Endocrinology \& Metabolism, Department of Clinical Medicine University of Colombo

\section{5}

A complex investigation of maternally inherited diabetes and deafness (MIDD) including clinical characteristics, C-peptide secretion, HLA-DR and -DQ status and autoantibody pattern

N. Hosszúfalusi ${ }^{1}$, V. Karcagi ${ }^{2}$, R. Horváth ${ }^{2}$, E. Palik ${ }^{1}$, J. Varkonyi ${ }^{1}$, K. Rajczy ${ }^{3}$, Z. Prohaszka ${ }^{1}$, I. Karadi ${ }^{1}$, L. Romics ${ }^{1}$, P. Panczel ${ }^{1}$;

${ }^{1}$ 3rd Dept. of Internal Medicine, Semmelweis University,

${ }^{2} \mathrm{Dpt}$, Johann Béla National Epidemiology Center, ${ }^{3} \mathrm{Dpt}$, National Institute of Hematology and Immunology, Budapest, Hungary.

Background and Aims: A point mutation of mitochondrial DNA (A3243G) is associated with maternally inherited diabetes and deafness (MIDD). The misclassification of MIDD was reported previously. Our aim was to characterise MIDD with detailed investigation.

Materials and Methods: Six patients with MIDD and one mutationpositive relative with normal glucose tolerance (NGT) from two families were examined. The A3243G and the HLA-DR, -DQ status were tested in leukocytes. Fasting serum C-peptide was measured in all subjects and compared with controls having NGT $(n=14)$. C-peptide response to an intravenous glucose load (IVGTT) was investigated in the diabetic patients not treated with insulin $(n=3)$ and in the mutation-positive healthy subject and compared with the controls. Clinical characteristics and the presence of islet cell specific (ICA, GAD, IA-2) and mitochondrial autoantibodies were also tested. All data are presented in mean \pm SD.

Results: The A3243G heteroplasmy value varied between $5-30 \%$ in the carriers. Apart from the subject with NGT each A3243G carrier had hearing impairment. The A3243G carriers were smaller (height: $158.3 \pm 10.8 \mathrm{~cm}$ vs. $171.8 \pm 10.2 \mathrm{~cm}$ ) and had lower body mass index (BMI: $20.53 \pm 2.05 \mathrm{~kg} /$ $\mathrm{m}^{2}$ vs. $\left.27.16 \pm 3.62 \mathrm{~kg} / \mathrm{m}^{2}\right)$ compared with the controls $(\mathrm{p}=0.018, \mathrm{p}=0.0009$, respectively). All mutation-positive subjects carried the HLA-DR1-DQ5 haplotype, and either the -DQ5 or the -DQ6 allele. The fasting serum Cpeptide levels of the A3243G carriers varied between $0.79-6.57 \mathrm{ng} / \mathrm{ml}$ and did not differ from the controls $(2.19 \pm 2.01 \mathrm{ng} / \mathrm{ml}$ vs. $2.08 \pm 0.73 \mathrm{ng} / \mathrm{ml}$, respectively). All A3243G carriers had measurable fasting C-peptide levels including the two diabetic patients with long disease duration. A missing first phase and a decreased second phase of insulin response was detected in the
A3243G carriers compared with controls based on the curves of serum Cpeptide measured during an IVGTT $(\mathrm{p}<0.0001)$. The same abnormality was detected in the A3243G carrier with NGT. However, the serum C-peptide levels of the $\mathrm{A} 3243 \mathrm{G}$ carriers did not differ from the controls at $120 \mathrm{~min}$ during the IVGTT $(2.59 \pm 0.20 \mathrm{ng} / \mathrm{ml}$ vs. $2.37 \pm 0.53 \mathrm{ng} / \mathrm{ml})$. Circulating islet cell antibodies (ICA) were present in three patients with MIDD. GAD, IA-2 and mitochondrial antibodies were not found. The diagnosis of MIDD was delayed in each case. The disease was previously considered as type 1, LADA or type 2 diabetes.

Conclusion: A missing first phase and a decreased second phase of C-peptide response to intravenous glucose load was characteristic for A3243G carrier status in our study. However, the fasting and the 120 min serum C-peptide levels did not differ from the controls. Patients with MIDD having long diabetes duration still had measurable fasting C-peptide. Circulating ICA was present is some patients but GAD, IA-2 and mitochondrial antibodies were not detected. All subjects had HLA-DR1-DQ5 haplotype, and either DQ5 or -DQ6 alleles. The diagnosis of MIDD was delayed in each case. The referred cases demonstrate that the clinical features of diabetes are sometimes not enough for the proper etiologic classification.

\section{6}

Clinical and genetic study of Thai patients with fibrocalculous pancreatic diabetes

T. Snabboon ${ }^{1}$, W. Plengpanich ${ }^{1}$, S. Sunthornyothin ${ }^{1}$, R. Rerknimitr ${ }^{1}$,

P. Kongkam ${ }^{1}$, S. Suwanwalaikorn ${ }^{1}$, V. Vorasuk Shotelersuk²;

${ }^{1}$ Internal medicine,

${ }^{2}$ Pediatrics, Chulalongkorn University, Bangkok, Thailand.

Background: Fibrocalculous pancreatic diabetes (FCPD) is classified as a secondary cause of diabetes mellitus (DM) resulting from pancreatic exocrine dysfunction. Recently, mutations in the SPINK1 gene have been reported in patients with FCPD.

Objective: To perform a detailed clinical and molecular analysis of patients with FCPD from thirteen unrelated Thai families.

Material and Methods: Genomic DNA was prepared from 13 probands and 10 family members. All exons of the SPINK1 gene of all probands were amplified by polymerase chain reaction, and directly sequenced. Clinical courses of pancreatic disease and DM of patients and their family members were reviewed.

Results: The age of onset of DM in our patients is ranged from 15 to 32 years old. All of the 13 probands had large intraductal pancreatic calculi; however, 4 of them did not have symptoms of abdominal pain or exocrine pancreatic dysfunction. Sequencing analysis of the SPINK1 gene successfully identified mutations in 8 probands. The heterozygous mutations for $\mathrm{N} 34 \mathrm{~S}$, IVS3 $+2 \mathrm{G}>\mathrm{A}$ and $206 \mathrm{C}>\mathrm{T}$ were found in five, two and one probands, respectively. The SPINK1 206C $>\mathrm{T}$ (T69I) mutation has not been previously reported. In addition, a novel heterozygous for IVS1-4C >A polymorphism was identified in remaining five probands with no definite pathogenic mutations in the coding regions.

Conclusions: Of the 13 probands with FCPD, 8 were found to carry mutations in the SPINK1 gene, suggesting this gene is responsible for the majority of FCPD in Thai patients. In addition, a novel SPINK1 gene mutation has been identified in our study.

Supported by the Anandamahidol research fund of Chulalongkorn University

\section{7}

A case of hepatocyte nuclear factor-1 alpha diabetes (the new mutation in mody 3 )

M. D. Jesic ${ }^{1}$, S. Sajic ${ }^{1}$, M. Maringa ${ }^{2}$, M. M. Jesic ${ }^{3}$, D. D. Micic ${ }^{4}$; ${ }^{1}$ Endocrinology, University Childrens Hospital, Belgrade, Serbia, ${ }^{2}$ Genetic, IntegraGen GmbH, Bonn, Germany,

${ }^{3}$ Neonatology, University Childrens Hospital, Belgrade, Serbia, ${ }^{4}$ Center for Metabolic Disorders in endocrinology, Institute of Endocrinology, Diabetes and Diseases of Metabolism, Belgrade, Serbia.

Background and Aims: MODY3 belongs to monogenic forms of diabetes mellitus and is caused by monoallellic mutation in gene for transcription factor HNF-1alpha, essential for regulation of beta-cell function. Clinical 
presentation of MODY3 is similar to that of type 1 diabetes. Although MODY 3 patients are not threatened by ketoacidosis, good metabolic control is important for prevention of chronic diabetic complications.

Materials and Methods: We present a 37-year old female (BMI $23.8 \mathrm{~kg} / \mathrm{m}^{2}$ ) with insulin treated diabetes mellitus since age 14 and a family history of diabetes (father died in August 2006. and also had insulin treated diabetes mellitus since age 16). By the end of 2006 in her daughter aged 10.5 years (BMI $20 \mathrm{~kg} / \mathrm{m}^{2}$ ) metabolic glucose disorder was revealed. Genetic testing for MODY due to "vertical line diabetes" was done by analyzing maternal and daughter's DNA. All of the exons were polymerase chain amplified on the intron sequences. The obtained genetic fragments were then sequenced. When in euglycemia, glucagon (1 mg i.v.) stimulation test was done in the mother. Oral tolerance test to glucose with insulinemia and blood analysis for antibody presence to portions of pancreatic cells and their products were done in the daughter.

Results: In the daughter and her mother we identified identical mutation that has not been described in the literature until now - TCF 1 exon 2 c.368 $>C$ p.Leu 123 pro heterozygote mutation sequence HNF - 1 alpha (TCF 1). Maternal glucagon testing showed preserved endogenic insulin secretion 23 years after diagnosed diabetes mellitus (glycemia 0. min. $6.5 \mathrm{mmol} / \mathrm{l}$ insulin $3.46 \mathrm{mUI} / \mathrm{mL}$, glycemia $6 . \mathrm{min} .7 .1 \mathrm{mmol} / \mathrm{l}$ - insulin $8.21 \mathrm{mUI} / \mathrm{mL}$ ). Following these findings, the mother was gradually transferred from insulin to sulphonylurea therapy - gliklazid $(30 \mathrm{mg} /$ daily), and after 2 months her HbAlc decreased from $6.7 \%$ to $6.1 \%$. In the daughter we disclosed a marked insulinopenia (in the $60^{\text {th }}$ min the maximal insulin rate was $18.6 \mathrm{mUI} / \mathrm{mL}$ at glycemia $16.9 \mathrm{mmol} / \mathrm{l})$, while antibodies (GAD, anti IA2, antiinsulinic) were negative. Therefore, we attempted treatment with small doses of sulphonylurea - gliklazid ( $20 \mathrm{mg} /$ daily). After 3 months the impression was that the treatment gave good results; glycemia was practically normalised, there was no hypoglycemia, and after initial $7.9 \%$ her HbAlc decreased to $5.8 \%$

Conclusion: Treatment with small doses of sulphonylureas can be successful, not only in children with MODY3, but also in vertical line diabetic relatives previously on long-term insulin therapy; however, its long-term safety and efficacy will have to be confirmed. Early diagnosis of HNF-1alpha diabetes is important in planning treatment, monitoring complications and genetic counseling of affected families.

\section{8}

Pancreatic exocrine deficiency is common in MODY 3

M. Vesterhus ${ }^{1}$, S. Johansson ${ }^{2}$, H. Rader ${ }^{1}$, A. Molven ${ }^{3}$, P. R. Njolstad ${ }^{2}$;

${ }^{1}$ Dept. of Pediatrics, Haukeland University Hospital, Bergen,

${ }^{2}$ Dept. of Clinical Medicine, University of Bergen,

${ }^{3}$ The Gade Institute, University of Bergen, Norway.

Background and Aims: Exocrine pancreas dysfunction is seen in 10-30\% of patients with type 1 and 2 diabetes, and is also found in patients with MODY5. The molecular factors involved are not understood. We have recently identified a novel syndrome of autosomal dominantly inherited diabetes and exocrine pancreas dysfunction due to mutations in the carboxyl ester lipase (CEL) gene. We wanted to investigate the prevalence of pancreatic exocrine dysfunction in the Norwegian MODY3 population and its relationship to common variants of $C E L$.

Materials and Methods: An invitation letter and a tube for stool sampling was sent to all 119 patients registered with a genetically verified diagnosis of MODY 3 in the Norwegian MODY Registry. The response rate was $60.5 \%$, and 70 patients ( 22 men, 48 women; mean age 40.4 years) were included. Pancreatic exocrine deficiency measured as fecal elastase deficiency (FED) was defined as moderate if faecal elastase-1 (E1) was less than $200 \mu \mathrm{g} /$ $\mathrm{g}$ in two tests and severe if less than $100 \mu \mathrm{g} / \mathrm{g}$ in at least one of two tests. Increased fecal fat excretion was defined as more than $7 \mathrm{~g} /$ day in a $72-\mathrm{h}$ stool collection.

Results: We found a prevalence of moderate and severe FED of $2.9 \%(2 / 70)$ and $8.6 \%(6 / 70)$, respectively, in the MODY3 patients. Six patients with FED returned a 72-hour stool collection and all had increased fecal fat excretion (mean $13.5 \pm 7 \mathrm{~g} /$ day ) The patients with FED were significantly older than the group with normal E1 (mean age 55, 68 and 38 years for patients with severe FED, moderate FED or normal elastase, respectively), and had significantly longer duration of diabetes (33,36, and 19 years, respectively). There was no difference in BMI or age at diagnosis of diabetes. Fecal fat excretion did not correlate with either BMI or fecal elastase within the group of patients with exocrine deficiency, but the number was small. The CEL gene was successfully sequenced in 50 (of the 70 ) patients. Of these, 10 patients (20\%) had single base insertions. CEL insertions were found in $33.3 \%(2 / 6)$ of the patients with FED who were sequenced, compared to $18.2 \%(8 / 44)$ of patients with no insertions ( $\mathrm{OR}=2.25$, not significant). No patients had deletions in the CEL gene. Age at diagnosis of DM and BMI were similar between groups.

Conclusion: We found a prevalence of pancreatic exocrine deficiency of $11.4 \%$ in the Norwegian MODY3 population, similar to findings in type 1 and 2 diabetes patients. Those patients with FED who delivered stool samples all had increased faecal fat excretion, underscoring the potential clinical importance of the exocrine defect. CEL insertions showed a tendency to correlate with exocrine deficiency, supporting previously published findings in a cohort of type 1 and 2 diabetic patients.

Supported by Helse Vest, Norway

\section{9}

Diagnosis and treatment of permanent neonatal diabetes mellitus: US experience

J. Stoy ${ }^{1}$, S. A. W. Greeley ${ }^{2}$, L. H. Philipson ${ }^{1}$;

${ }^{1}$ Department of Medicine,

${ }^{2}$ Department of Pediatrics, University of Chicago, Chicago, United States.

Background and Aims: Mutations in the genes KCNJ11 and ABCC8 are important causes of permanent neonatal diabetes. Here we report 8 patients initially diagnosed with type 1 diabetes (T1D) but who have diabetes due to a mutation in KCNJ11. Recent reports have shown these patients can be successfully switched from insulin to oral sulfonylurea therapy.

Materials and Methods: The patients were from across the US and we used Oragene ${ }^{\text {tws }}$ DNA Self-Collection kits to obtain a saliva sample for DNA. KCNJ11 was amplified in two segments using PCR and both strands sequenced.

Results: Eight out of 18 patients diagnosed with diabetes before 6 months of age had a KCNJ11 mutation. The median age at diagnosis of diabetes in the 8 patients was 4 weeks ( 0 to $26 \mathrm{wk}$ ) with median birth weight $2732 \mathrm{~g}$ (1531 to $2920 \mathrm{~g}$ ) corresponding to the $7^{\text {th }}$ percentile for male patients and the $14^{\text {th }}$ percentile for females.

We identified 4 different mutations: $\mathrm{H} 46 \mathrm{Y}$ in a mother and son; $\mathrm{R} 201 \mathrm{H}$ in 4; and R201C and V59M in 1 patient each. Three of these patients were admitted to the University of Chicago General Clinical Research Center for transition from insulin to glyburide. Case 1, a 6 year-old (yo) female diagnosed with $\mathrm{T} 1 \mathrm{D}$ at 1 month (mo) had the R201C mutation. Transition to oral glyburide resulted in cessation of insulin by day 14 . The initial post-insulin glyburide dose was $1.2 \mathrm{mg} / \mathrm{kg} /$ day, but subsequently decreased to $1.0 \mathrm{mg} / \mathrm{kg} /$ day due to occasional modest hypoglycemia. Pre-transition HbAlc was $9.3 \%$ and decreased to $7.4 \%$ by 1 mo and $6.1 \%$ at 2 mo post-insulin cessation. This patient also had celiac disease with positive tissue transglutaminase antibodies. Case 2 , a 3 yo female diagnosed at 6 mo with diabetic ketoacidosis (DKA) had the $\mathrm{R} 201 \mathrm{H}$ mutation. She transitioned off insulin (day 4 ) with a lower daily dose of glyburide $(0.8 \mathrm{mg} / \mathrm{kg} /$ day $)$; current glyburide dose is $0.95 \mathrm{mg} / \mathrm{kg} /$ day. Her parents reported a yellowish discoloration of her teeth which improved after instructions to not chew the glyburide pills. Her father had GAD-antibody positive T1D diagnosed at 29 yo. Case 3 , a 2.5 yo male diagnosed at 5 mo in severe DKA had the V59M mutation. He had developmental delay consistent with the intermediate DEND (Developmental delay, Epilepsy and Neonatal Diabetes) syndrome. Initial glyburide dose was $1.0 \mathrm{mg} / \mathrm{kg} /$ day (off insulin day 5); current glyburide dose is $0.83 \mathrm{mg} / \mathrm{kg} /$ day. Pre-transition HbAlc was $8.5 \%$ and decreased to $6.9 \%$ by 1 mo. The remaining patients are being transitioned to glyburide using an outpatient protocol. A 5 yo female (R201H) has successfully transitioned with a glyburide dose of $0.90 \mathrm{mg} / \mathrm{kg} /$ day divided in 5 doses. Her pre-transition HbAlc was $7.8 \%$ and decreased to $6.4 \%$ by 2 mo. The others are in progress (a 36 yo mother and 16 yo son with $\mathrm{H} 46 \mathrm{Y}$ and 14 and 17 yo males with R201H). One of these had a mild self-resolving transaminitis at $3 \mathrm{wk}$ of glyburide therapy.

Conclusion: These findings confirm that patients with diabetes due to a KCNJ11 mutation have a low birth weight and a variable age at diagnosis of diabetes from $<1$ wk to 6 mo. The results show that salivary samples can be used to obtain an appropriate DNA sample for genetic analysis even in young children. Oral sulfonylurea therapy has allowed for life-changing modification of diabetes management in these children. Since we estimate only a small fraction of cases in the US have been discovered there is a need to increase efforts to find all cases in order to improve control and quality of life of all who could potentially benefit from sulfonylurea therapy. 


\section{0}

Comparison of prioritisation strategies for selecting subjects with apparent type 1 diabetes for MODY gene sequencing

K. R. Owen ${ }^{1}$, M. P. Selwood ${ }^{2}$, D. Swan ${ }^{1}$, A. J. Farmer ${ }^{2}$, M. I. McCarthy ${ }^{1}$; ${ }^{1}$ Diabetes Research Laboratories, University of Oxford,

${ }^{2}$ Primary Health Care, University of Oxford, United Kingdom.

Background and Aims: Young adults with diabetes represent a difficult diagnostic challenge. Differential diagnosis includes type 1 (T1DM), type 2 and rare genetic subtypes. It can be particularly difficult to differentiate T1DM from Maturity-onset diabetes of the young (MODY) because they present in similar ways, but those misdiagnosed as T1DM often have the most to gain from making a correct aetiological diagnosis due to the potential to stop insulin treatment. The standard criteria used for diagnosing MODY are onset of diabetes $\leq 25 \mathrm{yrs}$ and autosomal dominant family history (FH) but it is not known whether selection criteria based on pathophysiology are superior. We aimed to test this hypothesis.

Materials and Methods: We examined a community-ascertained population diagnosed with diabetes $\leq 45$ yrs. Type 1 diabetes was defined as permanent insulin treatment since diagnosis $(n=51)$. Available data included $F H$, anthropometry, GAD antibodies and fasting biochemistry. Subjects were first selected from the group using standard MODY criteria. We then selected subjects on the basis of residual insulin secretion $\geq 3$ years post-diagnosis (c-peptide detectable and HOMA\% B > 20). We compared the individuals identified by these methods.

Results: 8 cases (16\%) were selected who fitted standard MODY criteria. However all 8 had typical T1 features: all GAD antibody +ve, median [IQR] c-peptide undetectable [0-0.15] nmoll ${ }^{-1}$ and HOMA\%B 1.4 [1.2-4.3] ).

3 cases were identified using pathophysiological criteria. All 3 were GAD antibody -ve. One was found to have features inconsistent with MODY (insulin resistance). The remaining 2 had evidence of residual $\beta$-cell function 10 and 26 years post-diagnosis with HOMA\% B values of 33 and 22 respectively. They were older at diagnosis, but were otherwise indistinguishable from the main T1DM cohort. They have been referred for genetic testing (involving sequential re-sequencing of the HNF-1 $\alpha$ and $4 \alpha$ genes).

Conclusion: In summary, in a cohort with apparent T1DM, using traditional MODY criteria alone to identify candidates for testing has poor specificity. Using an alternative approach based on pathophysiology identified 2 individuals ( $4 \%$ of the cohort) with features consistent with a transcription factor mutation. Our pilot data suggests that diagnostic pathways for MODY based on pathophysiology are feasible.

Supported by NHS R\&D, Oxford Health Services research Committee

\section{1}

The prevalence estimation of PNDM in Poland: the relative contribution of mutations in KCNJ11 and $\mathrm{ABCC} 8$ genes

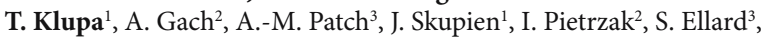
A. Hattersley ${ }^{3}$, W. Mlynarski ${ }^{2}$, M. T. Malecki ${ }^{1}$, J. Sieradzki';

${ }^{1}$ Department of Metabolic Diseases, Jagiellonian University, Krakow, Poland, ${ }^{2}$ Clinic of Pediatrics, Institute of Pediatrics, Medical University of Lodz, Lodz, Poland, ${ }^{3}$ Institute of Biomedical and Clinical Science, Peninsula Medical School, Exeter, United Kingdom.

Background and Aims: Mutations in the KCNJ11 and ABCC8 genes encoding the Kir6.2 and SUR1 subunits of the beta cell KATP channel, respectively, have recently been shown to be a common cause of permanent neonatal diabetes mellitus (PNDM). The aim of the study was to estimate the prevalence of PNDM in Poland and to determine the relative contribution of mutations in the KCNJ11 and ABCC8 genes to its development.

Materials and Methods: To dissect the genetic background of PNDM in Poland, the Nationwide Registry was established in 2004. The patients with the permanent diabetes diagnosed before 6 months of age were recruited through contact with physicians and direct appeals for patients through the national media. The search for the presence of mutations in the KCNJ11 and ABCC8 genes is being done by direct sequencing.

Results: The Polish Nationwide Registry of PNDM currently includes 40 patients meeting PNDM criteria. This corresponds to the prevalence of 1/950, 000 in the general population of Poland. However, if we consider the population below the age of 18 , the prevalence was about $1 / 300000$. This difference may reflect a more efficient screening in the pediatric population, alternatively, a lower survival of adult PNDM patients, or both. So far, the KCNJ11 gene has been sequenced in 39 PNDM subjects with mutations found in 16 subjects. Seven subjects carried the R201H mutation, three had the V59M substitution, two patients were carriers of the R50Q, the H46L, the G53D, the $\mathrm{E} 229 \mathrm{~K}$ and the $\mathrm{K} 170 \mathrm{~N}$ mutation, respectively. 14 patients were switched to sulphonylurea (SU) compounds, however 2 subjects were transferred back to insulin due to socioeconomic reasons or health problems unrelated to diabetes (hepatitis C). For 2 individuals the transfer is in process. So far, the ABCC8 gene has been sequenced in 8 PNDM patients, in whom KCNJ11 mutations were excluded. So far, 3 PNDM patients were identified to be ABCC8 gene mutation carriers. They were carriers of the F132V, the R826W substitutions, one patient was a carrier of two, N23H and R286W, mutations. In spite of a long exposure time to large doses of SU, we were not able to transfer off insulin the carrier of the F132V mutation. The carrier of two mutations, $\mathrm{N} 23 \mathrm{H}$ and R286W, is sufficiently controlled on diet alone. The R826W mutation carrier remains yet to be exposed to SU.

Conclusion: We report that PNDM frequency may be much higher than previous estimates. About $40 \%$ of PNDM cases may be explained by KCNJ 11 mutations. ABCC8 mutations seems to be common in the KCNJ11 negative patients. 


\section{PS 3 Genetics and epidemiology of metabolic syndrome, stroke and hypertension}

\section{2}

The clinical value of alanine aminotransferase as predictor of the metabolic sydrome in an overweight/obese paediatric population M. van Vliet ${ }^{1}$, M. Diamant ${ }^{2}$, I. A. von Rosenstiel ${ }^{1}$;

${ }^{1}$ Paediatrics, Slotervaart Hospital Amsterdam, ${ }^{2}$ Endocrinology, VU University Medical Centre, Amsterdam, The Netherlands.

Background and Aims: Among children, the global prevalence of obesity and components of the metabolic syndrome (MetS), including glucose intolerance, insulin resistance (IR), dyslipidaemia, and hypertension is increasing at an alarming rate. However, the occurrence and clinical significance of non-alcoholic fatty liver disease (NAFLD), a feature that may underlie the abnormalities of the MetS, has not as yet been established in paediatrics. Alanine aminotransferase (ALT), a valid marker of NAFLD, is associated with the components of the MetS, but is not considered as part of its definition. In the present study, we examined the association between ALT values and the features of the metabolic syndrome in a cohort of overweight and obese children aged 3 to 17 years.

Material and Methods: In total, 298 overweight and obese children (according to age-adjusted criteria; mean age 10.5 \pm 3.3 years) who were referred to an outpatient clinic of a community hospital in Amsterdam (period 2004-2006), underwent an oral glucose tolerance test (OGTT). Additionally, anthropometric parameters and fasting blood samples were collected to assess lipid profiles and liver enzymes. Metabolic syndrome and insulin resistance were defined according to criteria of the American Academy of Paediatrics. Participants were divided into two groups according to ALT values based on established criteria: those with ALT $\leq 30 \mathrm{U} / \mathrm{L}$ (normal group) and with ALT $>30 \mathrm{U} / \mathrm{L}$ (high group).

Results: The population consisted of 165 boys (55\%) and 133 girls (45\%) of multiethnic origin: $64(21 \%)$ were of Caucasian and $234(79 \%)$ of nonCaucasian origin (76 (26\%) Turkish, 67 (22\%) Moroccan, 32 (11\%) Surinam and $59(20 \%)$ children of other origin. Insulin resistance was present in $35 \%$, dyslipidaemia in $51 \%$, hypertension in $25 \%$ and metabolic syndrome in $28 \%$ of the children, showing no raised occurrence in the non-Caucasian group. Elevated ALT levels were found in $16.4 \%(n=49)$ of the study population (23.0\% boys, $8.3 \%$ girls, $\mathrm{p}<0.001)$. The age- and gender-adjusted BMI was significantly higher in the high versus normal ALT group (BMI 173\% $( \pm 21)$ vs. $162 \%( \pm 23), p=0.002)$. The prevalence of IR and MetS was significantly higher in the high ALT group compared to the normal ALT group $(\mathrm{p}<0.001$ and $\mathrm{p}<0.02$, respectively). ALT was positively correlated with low HDL levels (odds ratio (OR) 1.02 (CI95\% 1.003-1.037), $\mathrm{p}=0.02$ ), and high triglyceride levels (OR 1.02 (CI95\% 1.005-1.04), $\mathrm{p}=0.013$ ). Overall, no difference in ALT was found between the Caucasian and non-Caucasian group.

Conclusion: In an overweight/obese multiethnic paediatric urban population, IR and MetS and its associated abnormalities are highly prevalent. Additionally, a high percentage of elevated ALT, suggesting NAFLD, was found, especially in boys. Elevated ALT appeared to be strongly associated with IR and MetS. Longitudinal large-scaled studies should establish the predictive value of these findings, in particular of elevated ALT, in the development of type 2 diabetes and cardiovascular disease in children and adolescents.

\section{3}

Associations of sitting time with components of the metabolic syndrome in adults without diagnosed diabetes - The AusDiab Study

D. W. Dunstan ${ }^{1}$, N. Owen ${ }^{2}$, J. Salmon', G. Healy ${ }^{2}$, K. Ball ${ }^{3}$, J. E. Shaw ${ }^{1}$, P. Z. Zimmet';

${ }^{1}$ Epidemiology and Clinical Research, International Diabetes Institute, Caulfield, ${ }^{2}$ School of Population Health, The University of Queensland, Brisbane, ${ }^{3}$ School of Exercise and Nutrition Sciences, Deakin University, Burwood, Australia.

Background and Aims: Several studies have shown that sedentary behaviour may be an important contributor to poor health outcomes. However, most studies have focused on leisure time behaviours such as time spent watching television. Less is known about the association of sedentary behaviours in both leisure and non-leisure time on metabolic outcomes. We have examined the associations of self-reported time spent sitting and components of the metabolic syndrome in Australian adults without diabetes.

Materials and Methods: The Australian Diabetes, Obesity and Lifestyle Study (AusDiab) is a nationally representative population-based survey of 11,247 adults aged $\geq 25$ years with baseline measurements obtained between 1999 and 2000 . The present analyses include a total of 5,749 adults who attended the 5-year follow-up and who were free from diagnosed diabetes. Fasting measures of plasma glucose, serum triglycerides and HDL-C and $2 \mathrm{hrPG}$ were obtained from an oral glucose tolerance test. Self-reported time spent sitting (hrs/day) was assessed using an interviewer-administered questionnaire. Waist circumference, height and resting blood pressure were measured at the testing site. The log transformation of the triglyceride measure was used in the analysis. All regression models were adjusted for age, education, employment status and physical activity time. Data are presented as regression coefficients (95\% CI).

Results: The mean (SD) sitting time (hrs/day) for women and men was 5.1 (2.6) and 5.5 (2.7) respectively. In both men and women, sitting time was positively associated with $2 \mathrm{hrPG}$, log-transformed triglycerides, and waist circumference and inversely associated with HDL-C (P value range: $0.001-$ $0.01)$. The mean differences between the top tertile and bottom tertile were: $2 \mathrm{hrPG}$ [men: $0.2 \mathrm{mmol} / \mathrm{l}$ ( 0.01 to 0.40$)$, women $0.3 \mathrm{mmol} / \mathrm{l}(0.14$ to 0.44$)$ ], log-transformed triglycerides [men 0.1 ( 0.05 to 0.16 ), women 0.1 (0.06 to $0.14)$ ], waist [men $2.4 \mathrm{~cm}$ (1.3 to 3.4), women $3.3 \mathrm{~cm}$ (2.2 to 4.4)] and HDL-C [men $-0.06 \mathrm{mmol} / \mathrm{l}(-0.09$ to -0.03$)$, women $(-0.05 \mathrm{mmol} / \mathrm{l}(-0.09$ to -0.02$)]$. Systolic and diastolic blood pressure were positively associated with sitting time in women only (both $\mathrm{P}=0.01$ ). No association was observed with FBG. The associations observed for $2 \mathrm{hrPG}$ and log-triglycerides in women and logtriglycerides and HDL-C in men remained significant $(\mathrm{P} \leq 0.003)$ following further adjustment for waist circumference. In regression models containing both weekday and weekend sitting time as covariates, weekday sitting time was associated with log-triglycerides in men and women (both $\mathrm{P}<0.01$ ) and HDL-C and waist in men $(\mathrm{P}<0.001)$. Weekend sitting time was associated with 2 hrPG in both men and women (both $\mathrm{P}<0.05)$ and log-triglycerides $(\mathrm{P}$ $=0.04)$ and waist $(\mathrm{P}=0.001)$ in women.

Conclusion: These findings highlight the unique deleterious relationships of sedentary behaviour (indicated by sitting time) and components of the metabolic syndrome, independent of physical activity time. Population strategies to reduce the risk of developing the metabolic syndrome should focus not only on increasing physical activity, but also on reducing sedentary behaviours, such as time spent sitting.

\section{4}

Relationship between polymorphism in the aldosterone synthase gene (CYP11B2) and risk for type 2 diabetes and the metabolic syndrome in a French population: THE DESIR STUDY

N. Bellilii ${ }^{1}$, R. Roussel ${ }^{1,2}$, R. Jaziri ${ }^{1}$, S. Maimaitiming ${ }^{1}$, B. Balkau ${ }^{3}$, J. Tichet ${ }^{4}$, F. Fumeron ${ }^{1}$, M. Marre ${ }^{1,2}$;

${ }^{1}$ INSERM, U695, Paris, ${ }^{2}$ Assistance Public-Hôpitaux de Paris, Service d'Endocrinologie, Diabétologie, Nutrition, Groupe Hospitalier Bichat Claude Bernard, Paris, ${ }^{3}$ INSERM, U258-IFR69, Villejuif, ${ }^{4}$ Institut Régional Pour la Santé, IRS, Tours, France.

Background and Aims: The aldosterone synthase (CYP11B2) is an enzymatic mitochondrial complex catalysing the key step in the synthesis of aldosterone. It plays a major role in regulating blood pressure and may affect glucose homeostasis. CYP11B2 affect gene expression and thus might modulate cardiovascular risk factors. We investigated the impact of the -344 $\mathrm{T} / \mathrm{C}$ and $5945 \mathrm{G} / \mathrm{A}$ polymorphisms of CYP11B2 gene in the DESIR study for the risk of type 2 diabetes (T2D), hypertension and Metabolic Syndrome (MS IDF definition).

Materials and Methods: 2576 men and 2636 women from the general population, aged 30-65 years, took part in the DESIR (Epidemiologic Data on Insulin Resistance Syndrome) study, from 1994 to 2003 and were evaluated every three years. Genotyping for $-344 \mathrm{~T} / \mathrm{C}$ and $5945 \mathrm{G} / \mathrm{A}$ in CYP11B2 was done by high-throughput techniques (Molecular Beacon ${ }^{\circ}$ and TaqMan, Abgene for $-344 \mathrm{C} / \mathrm{T}$ and $5945 \mathrm{G} / \mathrm{A}$ respectively). Chi ${ }_{2}$ test and ANOVA were used to test the effects of the genotypes with adjustment for age in the whole population and after stratification according to gender.

Results:Allelic frequencies were $55 \%$ and $45 \%$ for $\mathrm{T}$ and C respectively and $71 \%$ and $29 \%$ for G and A respectively. Distributions of genotypes were in 
Hardy-Weinberg equilibrium. The -344C/T SNP was associated with the presence of hypertension in women at inclusion: 194 (35.6\%), 290 (28.4\%) and $107(25 \%)$ for TT, TC and CC respectively, $\mathrm{p}=0.008$. It was also associated with the incidence of T2D in men at the end of study: 29 (5.3\%), 78 (8.2\%) and $33(9 \%)$ for TT, TC and CC respectively, $\mathrm{p}=0.029$. The $5945 \mathrm{~A} / \mathrm{G}$ polymorphism was associated with the incidence of T2D in all participants: 93 (5\%), 101 (6.4\%), 7 (2.3\%) for GG, GA and AA respectively, $p=0.008$. In men, the $5945 \mathrm{G} / \mathrm{A}$ SNP was associated with the prevalence of hypertension at baseline: $412(50.4 \%), 314(43 \%)$, and $62(33.8 \%)$ for GG, GA and AA respectively, $\mathrm{p}=0.050$, and incidence of hypertension: $354(43.3 \%), 325$ (44.7\%), 52 (33.8\%) for GG, GA and AA respectively, $\mathrm{p}=0.044$. The $5945 \mathrm{G} / \mathrm{A}$ was associated with the incidence of MS in men at last visit: 220 (28.5\%), 177 (27.3\%) and 24 (18.6\%) for GG, GA and AA respectively, $\mathrm{p}=0.037$.

Conclusion: In this large prospective follow-up study, we found that genetic variation in the Aldosterone Synthase gene affects risk for T2D, hypertension and MS. This data provides a rationale to target this gene for pharmacological manipulations aimed at reducing cardiovascular risk.

\section{5}

TNF- $\alpha-308$ G/A and NOS3 rs3800787 SNPs interaction with features of the metabolic syndrome (The Segovia Study)

J. L. González-Sánchez, M. T. Martinez-Larrad, M. J. Martínez-Calatrava,

C. Zabena, M. Serrano-Ríos;

Internal Medicine II, Hospital Clínico San Carlos, Madrid, Spain.

Background and Aims:To determine whether the $-308 \mathrm{G} / \mathrm{A}$ polymorphism of the TNF- $\alpha$ gene has a gene-gene interaction with NOS3 rs3800787 SNP on the association of both gene on insulin resistance and other features of the metabolic syndrome (MS) in a representative sample of the Spanish population, we genotyped 751 unrelated subjects recruited from a crosssectional population-based epidemiological survey in Segovia, Spain (The Segovia Study Group).

Materials and Methods: Anthropometric parameters: BMI, waist to hip ratio (WHR). OGTT, fasting and 2 hrs glucose, serum insulin, adiponectin levels; lipid profile. Plasma soluble TNF- $\alpha$ receptor 2 (sTNFR2), PAI-1 levels were measured. Insulin resistance was assessed by HOMA-IR. Categories of glucose tolerance (ADA Clinical Practice Recommendations 2006). MS was defined according to the 2005 IDF criteria.

Results: TNF- $\alpha$ genotype distribution: -308 G/G 73.1\%, -308 G/A 24.8\%, -308 A/A 2.1\%. Subjects carrying the -308 A allele had higher 2-hours postload glucose and insulin concentrations as compared to $-308 \mathrm{G} / \mathrm{G}$ homozygotes. Patients with type 2 diabetes carrying the $-308 \mathrm{~A}$ allele had higher sTNFR2 and lower adiponectin levels than $-308 \mathrm{G} / \mathrm{G}$ homozygotes. Plasma levels of sTNFR2 were positively correlated with BMI, and WHR among type 2 diabetic subjects. Interestingly diabetic subjects carrying the -308A allele had higher sTNFR2 levels, WHR, insulin 2 hours, PAI levels, and lower adiponectin concentrations as compared with $-308 \mathrm{G} / \mathrm{G}$ homozygous. As regard to the rs3800787 SNP of NOS3 gene, the C allele was associated with a higher OR for MS (OR 1.44, 95\% CI 1.02-2.03) and for decreased HDL-cholesterol (OR $1.71,95 \%$ CI 1.11-2.63). Moreover C allele carriers had higher mean of fasting glucose, and HOMA-IR than those with the $-308 \mathrm{G} / \mathrm{G}$ genotype. No gene-gene interaction on features of MS was detected between both SNPs.

Conclusion: Our findings indicate than both SNPs are individually associated with features of MS. TNF- $\alpha-308 \mathrm{~A}$ allele could be associated with a higher risk for abdominal obesity, but not for type 2 diabetes, whereas the $\mathrm{C}$ allele of NOS3 gene could potentially confer a higher risk for MS as a categorical variable.

\section{6}

Association of variants of neuropeptide $\mathrm{Y}$ receptors with susceptibility to obesity and type 2 diabetes

C. C. Elbers ${ }^{1,2}$, J. V. van Vliet-Ostaptchouk ${ }^{3}$, N. C. Onland-Moret ${ }^{1,2}$,

M. H. Hofker ${ }^{3}$, Y. T. van der Schouw ${ }^{2}$, C. Wijmenga ${ }^{1}$;

${ }^{1}$ Complex Genetics Group, Biomedical Genetics, UMC Utrecht,

${ }^{2}$ Julius Center for Health Science and Primary Care, UMC Utrecht,

${ }^{3}$ Molecular Genetics, Maastricht University, The Netherlands.

Background and Aims: In a previous study we used bioinformatics tools to identify candidate genes for both obesity and T2D. One interesting group of genes, neuropeptide Y receptors (NPYRs), were further investigated. NPYRs are known to be involved in the regulation of eating behaviour. The aim of the study is to investigate the role of genetic variation in five NPYRs (NPY1R, NPY2R, NPY3R, NPY4R, NPY5R) for susceptibility to T2D and obesity. Materials and Methods: Tagging single nucleotide polymorphisms (SNPs) were selected for each gene and genotyped in 502 T2D cases and 920 unrelated controls (the Breda study). In addition 364 healthy males (the Hamlet study) were genotyped to study the effect of the tag SNPs on different measures of obesity.

Results: The minor alleles of variants in NPY5R and NPY1R were underrepresented in T2D cases (odds ratio (OR) 0.88, 95\% CI 0.75-1.04 for rs11724320 in NPY5R and OR $0.86,95 \%$ CI $0.72-1.03$ for rs9764 in NPY1R) compared to controls in the Breda study. We also saw significantly fewer individuals with one or two copies of the minor allele of rs11724320 in NPY5R in the T2D group (OR 0.79, 95\% CI 0.63-0.99). Experiments to establish the effect of genetic variants in NPYRs on measures of obesity in the Hamlet study are currently ongoing.

Conclusion: Our data may suggest that variants in NPY1R and NPY5R are protective against T2D in a Dutch population.

Supported by SenterNovem (IOP genomics grant IGE05012)

\section{7}

A putative role of variations in the endocannabinoid receptor 1 gene (CNR1) in stroke

C. $\operatorname{Roos}^{1}$, M. Benzinou ${ }^{2,3}$, O. Melander ${ }^{1}$, M. Sjögren ${ }^{1}$, P. Almgren ${ }^{1}$, M. Svensson ${ }^{1}$, L. Rosberg ${ }^{1}$, B. Isomaa ${ }^{4}$, G. Berglund ${ }^{1}$, T. Hedner ${ }^{5}$, L. Groop ${ }^{1,6}$, P. Frougel ${ }^{2,3}$, M. Orho-Melander ${ }^{1}$;

${ }^{1}$ Clinical Sciences, Diabetes and Endocrinology, Lund university, Malmö, Sweden, ${ }^{2}$ CNRS 8090-Institute of Biology, Pasteur Institute, Lille, France, ${ }^{3}$ Genomic Medicine, Hammersmith Hospital, Imperial College London, United Kingdom, ${ }^{4}$ Folkhälsan Research Center, Genetic Institute, Helsinki, Finland, ${ }^{5}$ Clinical Pharmacology, Sahlgrenska Academy, Göteborg, Sweden, ${ }^{6}$ Medicine, Helsiniki University Central Hospital, Finland.

Background and Aims: The endocannabinoid (EC) system plays an important role in the regulation of food intake, energy metabolism, atherogenesis and stroke. Treatment with EC receptor 1 (CB1) antagonists reduce food intake, improve glucose metabolism and modulate lipid levels suggesting them as potential drugs in treatment of cardio- and cerebrovascular diseases. Animal studies have demonstrated the importance of ECs in cerebral regulation during and after ischemia and addressed the beneficial effect of $\mathrm{CB} 1$ antagonists in reducing the infarction size. A large-scale genetic analysis of the CB1 gene (CNR1) has recently revealed a few variations in CNR1, among them rs806381, in association with obesity and type 2 diabetes (T2D). Herein we challenge the question if rs 806381 is associated with or predicts morbidity and mortality to stroke in three large Scandinavian cohorts.

Materials and Methods: The rs806381 was genotyped on ABI7900 in three large cohorts: 1) 4,660 Finnish individuals participating in the Botnia T2D family study and followed for a mean time of 11 years (Botnia: $\mathrm{m} / \mathrm{f}$ $2,146 / 2,514$, age $58 \pm 14 \mathrm{y}, \mathrm{BMI} 27 \pm 4 \mathrm{~kg} / \mathrm{m}^{2}, 34 \%$ with T2D). During the follow-up time $23.4 \%$ of the individuals died, of these 125 due to stroke [including both thrombotic stroke (TS) and cerebral haemorrhage (CH)]. 2) 5,190 Swedish individuals composing the population based cohort of Malmo Diet and Cancer Study Cardiovascular Arm (MDC-CVA: m/f 2,140/3,050, age $58 \pm 6 \mathrm{y}$, BMI $26 \pm 4 \mathrm{~kg} / \mathrm{m}^{2}, 8 \%$ with T2D) were followed for a mean time of 10.5 years. During this time, 167 individuals had stroke ( $133 \mathrm{TS}$ and $20 \mathrm{CH}$ ) and 13 patients died of stroke. 3) A cohort of 5,152 Swedish patients with hypertension (NORDIL: $\mathrm{m} / \mathrm{f} 2,567 / 2,585$, age $60 \pm 7$ y, BMI $28 \pm 4 \mathrm{~kg} / \mathrm{m}^{2}, 9 \%$ with T2D). In NORDIL, 162 individuals had experienced stroke (136 TS and $19 \mathrm{CH}$ ) and of these 18 had died of stroke. Analyses were performed using Cox- or logistic regression adjusted for age, sex and diabetes status, or by $\mathrm{chi}^{2}$ tests. Survival analyses in Botnia families were stratified for sex and by using a robust variance estimate adjusted for within family dependence by treating each pedigree as an independent entity.

Results: The frequency of the rs806381 minor G-allele was 34.5, 32.9 and $33.4 \%$ in Botnia, MDC-CVA and NORDIL, respectively. The rs806381 GGgenotype predicted stroke mortality in Botnia T2D families (HR=2.2 [1.43.5], $\mathrm{p}=0.00050$ ). In line with this, the GG-genotype was a predictor of stroke mortality $(\mathrm{HR}=3.9[1.0-14.7], \mathrm{p}=0.049)$ and of $\mathrm{CH}(\mathrm{HR}=3.9$ [1.3-11.2], $\mathrm{p}=0.012$ ) but not of TS (HR=1.2 [0.7-1.9], $\mathrm{p}=0.47)$ in MDC-CVA. Finally, the GA and GG genotypes were more common among patients with stroke 
compared to subjects without ( 66.0 vs. $55.1 \%, \mathrm{p}=0.0060$ ) in the NORDIL cohort. This association was significant for TS ( 66.2 vs. $55.2 \%, \mathrm{p}=0.011)$ but not for the small number of $\mathrm{CH}$ patients (68.4 vs. $55.4 \%$, $\mathrm{p}=0.36$ ).

Conclusion: We demonstrate that variation in CNR1 is associated with stroke and predicts stroke morbidity and mortality.

\section{8}

Impaired glucose tolerance is a risk factor for stroke in a Japanese sample - the Funagata Study

T. Oizumi ${ }^{1}$, M. Daimon ${ }^{1}$, Y. Jimbu ${ }^{1}$, K. Wada ${ }^{1}$, W. Kameda ${ }^{1}$, S. Susa ${ }^{1}$, H. Yamaguchi ${ }^{1}$, H. Ohnuma ${ }^{1}$, M. Tominaga ${ }^{2}$, T. Kato ${ }^{1}$;

${ }^{1}$ Department of Neurology, Hematology, Metabolism, Endocrinology and Diabetology (DNHMED), ${ }^{2}$ Laboratory Medicine, Yamagata University School of Medicine, Yamagata, Japan.

Background and Aims: Abnormal glucose tolerances such as impaired glucose tolerance (IGT) as well as diabetes are well-established risk factors for cardiovascular disease (CVD). CVD includes several medical conditions, such as stroke and coronary heart disease (CHD), and thus, the observed risk for CVD represents a risk of the combined outcomes of these medical conditions. Both diabetes and IGT seem to be well-established risk factors for CHD. Furthermore, the risk of diabetes for stroke has also been shown in many studies. However, IGT has not been established as a risk factor for stroke. A high prevalence of abnormal glucose tolerance, including IGT, in patients who have had a recent ischemic stroke has been reported, thus, IGT may also be a risk factor for stroke. Increased risks for future stroke in patients with transient ischemic attacks or minor ischemic stroke as well as in those with CHD have been reported; therefore, at least in some high-risk populations, IGT seems to be a risk factor for stroke. We, here, conducted a cohort study consisting of the participants of the Funagata study, a community-based study, to determine whether or not IGT is a risk factor for stroke.

Materials and Methods: The incidence of stroke and CHD of a cohort population ( $\mathrm{n}=2938$ ) consisting of participants of the Funagata study held in 1990-1997 was assessed by studying questionnaires, death certificates, and residence transfer documents through 2002. Glucose tolerance at the baseline was classified by the criteria of the 1998 World Health Organization (WHO) (normal glucose tolerance (NGT) $(\mathrm{n}=2189)$, IGT $(\mathrm{n}=320)$, and diabetes $(n=286))$. The cumulative incidences among the groups were compared using the Kaplan-Meier product-limit method, and Cox's proportional hazard model was adopted.

Results: During the 147-month (mean 116.5) follow-up, 158 (NGT, IGT and diabetes: 94,35, and 29) and $94(54,16$, and 24) participants experienced a stroke and CHD, respectively. The stroke risk was significantly higher in the participants with IGT (age-, sex-, and hypertension-adjusted hazard ratio [HR]:1.51, 95\% CI: 1.02-2.24, $\mathrm{p}=0.039)$ and tended to be higher in those with diabetes (HR:1.47, 95\% CI:0.96-2.25, 0.079) than it was in those with NGT. There was no significant association between IGT and the risk of CHD (HR: $1.21,95 \%$ CI:0.69-2.13, 0.509), while diabetes was a significant risk for CHD (HR:1.97, 95\% CI: 1.18-3.28, 0.010).

Conclusion: Impaired glucose tolerance is an independent risk factor for future stroke in a Japanese population. (Fig. 1)

\section{9}

A functional promoter polymorphism of $X B P 1$ gene is associated with younger age of onset of type 2 diabetes and susceptibility to hypertension T. Kakimoto ${ }^{1}$, H. Furuta ${ }^{1}$, T. Shimada ${ }^{1}$, Y. Furukawa ${ }^{1}$, S. Matsuno ${ }^{1}$, A. Kusuyama ${ }^{2}$, M. Nishi ${ }^{2}$, H. Sasaki ${ }^{1}$, T. Sanke ${ }^{3}$, K. Nanjo ${ }^{1}$;

${ }^{1}$ The First Department of Medicine, ${ }^{2}$ Department of Metabolism and Clinical Nutrition, ${ }^{3}$ Department of Clinical Laboratory Medicine, Wakayama Medical University, Wakayama city, Japan.

Background and Aims: The X box-binding protein 1 (XBP1) is a transcription factor that modulates endoplasmic reticulum (ER) stress through transcriptional regulation of proteins, such as molecular chaperones. ER stress inhibits insulin receptor signaling through activation of c-Jun $\mathrm{N}$-terminal kinase (JNK) and subsequent serine phosphorylation of insulin receptor substrate-1, and overexpression of XBP1 improved these disorders. XBP1 heterozygous mice exhibited increased ER stress, hyperactivation of JNK, reduced insulin receptor signaling, systemic insulin resistance and glucose intolerance. The $-116 \mathrm{C} / \mathrm{G}$ polymorphism in the promoter region of XBP1 gene has been reported to be associated with bipolar disorder. The XBP1 expression is positively regulated by itself and the $-116 \mathrm{C} / \mathrm{G}$ polymorphism exists in the putative XBP1 binding site. It has also been reported that XBP1-dependent transcription activity of the promoter with the $-116 \mathrm{G}$ allele was lower than that of the $\mathrm{C}$ allele and induction of XBP1 expression after ER stress was markedly reduced in the lymphocytes derived from subjects with the $\mathrm{G}$ allele. In this study, we examined the role of the $-116 \mathrm{C} / \mathrm{G}$ polymorphism in type 2 diabetic patients.

Materials and Methods: A total of 813 Japanese subjects were studied (407 type 2 diabetic patients and 416 non-diabetic controls). The DNAs were obtained from peripheral blood. The $-116 \mathrm{C} / \mathrm{G}$ polymorphism was genotyped by PCRRFLP method using a restriction enzyme, BstE II. The genotype distribution obtained was in compliance with the Hardy-Weinberg equilibrium.

Results: The frequency of G/G, C/G and C/C genotypes was $49 \%, 41 \%$ and $10 \%$ for type 2 diabetic patients, and $47 \%, 44 \%$ and $8.7 \%$ for non-diabetic controls, respectively. No significant difference was observed between these two groups $(\mathrm{P}=0.58)$. We next divided the patients into two groups $(\mathrm{G} / \mathrm{G}$ vs. $\mathrm{C} / \mathrm{G}+\mathrm{C} / \mathrm{C}$ ) and compared their clinical characteristics. Age of onset in the patients with $\mathrm{G} / \mathrm{G}$ genotype $(46.7 \pm 10.2$ years, mean $\pm \mathrm{SD})$ was younger than that in $\mathrm{C} / \mathrm{G}+\mathrm{C} / \mathrm{C}$ group $(49.0 \pm 9.9$ years $)(\mathrm{P}=0.026)$. Furthermore, the prevalence of hypertension was higher in the patients with $\mathrm{G} / \mathrm{G}$ genotype $(60 \%)$ than that in $\mathrm{C} / \mathrm{G}+\mathrm{C} / \mathrm{C}(48 \%)(\mathrm{P}=0.014)$. However, no association was found between the $-116 \mathrm{C} / \mathrm{G}$ polymorphism and other characteristics (max body mass index, dyslipidemia, retinopathy and nephropathy).

Conclusion: Thus, this data suggest that the $-116 \mathrm{C} / \mathrm{G}$ polymorphism is associated with younger age of onset of diabetes and susceptibility to hypertension in Japanese type 2 diabetic patients.

Supported by Grant-in-aid for Scientific Reserch from the Ministry of Education, Culture, Sports, Science and Technology of Japan

\section{0}

Two-hour glucose predicts the development of hypertension over five years: the AusDiab study

E. L. M. Barr ${ }^{1}$, E. J. Boyko ${ }^{1,2}$, P. Z. Zimmet ${ }^{1}$, J. E. Shaw ${ }^{1}$;

${ }^{1}$ Epidemiology, International Diabetes Institute, Melbourne, Australia,

${ }^{2}$ Medicine, University of Washington, Seattle, United States.

Background and Aims: Elevated 2-hr plasma glucose (2hPG) concentration from an oral glucose tolerance test (OGTT) predicts a higher risk of subsequent

\section{$\begin{array}{lll}\text { Fig. } 1 & \text { CVD } & \text { Stroke }\end{array}$}

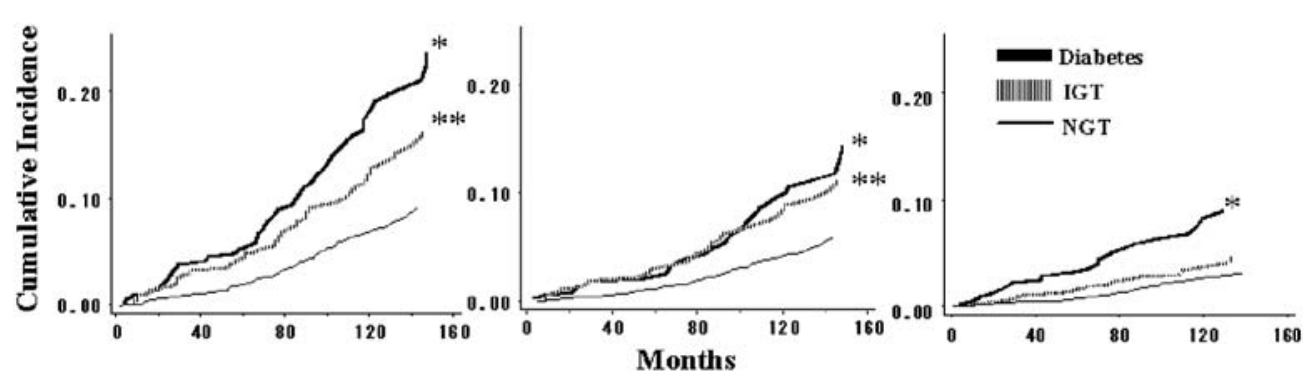


cardiovascular disease and is more strongly related to this outcome than is fasting plasma glucose (FPG). Mechanisms underlying these associations are not well understood. A recent clinical trial among persons with IGT (STOPNIDDM) demonstrated that a reduction in $2 \mathrm{hPG}$ was accompanied by lower blood pressure values and a lower risk of hypertension compared to controls. We examined the association between glucose measurements and risk of hypertension in a prospective observational study.

Materials and Methods: The Australian Diabetes, Obesity, and Lifestyle Study (AusDiab) examined 4,413 persons without hypertension at baseline (1999-2000) and at a 5 year follow-up examination (2004-5). Measurements at both time points included blood pressure (mean of 2 readings), a $75 \mathrm{~g}$ OGTT, fasting insulin, anthropometrics, dietary and alcohol intake based on the Cancer Council of Victoria food frequency questionnaire, and physical activity based on the Active Australia questionnaire. Hypertension was defined as a systolic pressure $\geq 140$ or a diastolic pressure $\geq 90 \mathrm{mmHg}$ or antihypertensive medication use. HOMA-S was calculated as a measure of insulin sensitivity using the HOMA2 calculator. Differences in BMI and waist circumference between baseline and follow-up were calculated. Multivariable linear and logistic regression methods were used to estimate associations between glycaemia and hypertension risk at 5 years as a binary outcome and systolic and diastolic blood pressure as continuous outcomes while adjusting for covariates and cluster sampling.

Results: After additional exclusions for pregnancy, inadequate fasting, and missing blood pressure measurements, 4,306 subjects remained (mean baseline age 47.7 years, $56.9 \%$ female), of whom $14 \%$ developed hypertension over 5 years. Higher FPG and 2hPG were significantly related to greater risk of hypertension in univariate analyses [odds ratio for a 1 standard deviation increase (95\% confidence interval) in FPG 1.28 (1.19 to 1.38); 2hPG 1.36 (1.27 to 1.46 ). The risk associated with $2 \mathrm{hPG}$ remained after adjustment for age, gender, FPG, BMI (baseline and difference), waist circumference (baseline and difference), education, exercise, alcohol intake, and smoking [1.15 (1.05 to 1.25)], but the association between FPG and hypertension diminished [1.06 (0.94 to 1.20)]. Further adjustment for HOMA-S did not change these findings. The relationship between $2 \mathrm{hPG}$ and hypertension was linear and apparent throughout the range of glucose values. The same results were seen after exclusion of persons on antihypertensive medication at 5 years or those with diabetes at baseline. Higher baseline 2hPG was more strongly associated with an increase in systolic than in diastolic blood pressure over 5 years.

Conclusion: Higher 2hPG predicted hypertension occurrence over 5 years. This association was not explained by adjustment for other factors measured in this study related to hypertension or glycaemia, and was present even within the normal range for $2 \mathrm{hPG}$. If this association is demonstrated in other studies, interventions aimed at reducing the $2 \mathrm{hPG}$ level may potentially yield additional benefits through reduction of hypertension incidence.

Supported by National Health and Medical Research Council (NHMRC) project grant, Bayer HealthCare, NHMRC/National Heart Foundation joint postgraduate scholarship

\section{PS 4 Genome-wide association scans and bioinformatic tools for identifying candidate pathways}

\section{1}

Genome-wide scan of type 2 diabetes-related quantitative traits in French-Canadian hypertensive families

J. Vcelak ${ }^{1,2}$, O. Seda ${ }^{1}$, P.-L. Brunelle ${ }^{1}$, A. Gurau ${ }^{1}$, E. Merlo ${ }^{1}$, D. Gaudet ${ }^{3}$, F. Gossard ${ }^{1}$, T. A. Kotchen ${ }^{4}$, A. W. Cowley ${ }^{5}$, J. Tremblay ${ }^{1}$, P. Hamet ${ }^{1}$; ${ }^{1}$ Research Centre, CHUM, Montreal, Canada, ${ }^{2}$ Department of Molecular Endocrinology, Institute of Endocrinology, Prague, Czech Republic, ${ }^{3}$ Genomic Medicine Center, Hospital Complex of Sagamie, Chicoutimi, Canada, ${ }^{4}$ Department of Medicine, Medical College of Wisconsin, Milwaukee, United States, ${ }^{5}$ Department of Physiology, Medical College of Wisconsin, Milwaukee, United States.

Background and Aims: The identification of genomic determinants responsible for common multifactorial diseases like type 2 diabetes mellitus (T2DM) and hypertension is facilitated in large families from relatively genetically-isolated populations with a demonstrated founder effect. The French-Canadian population from the Saguenay-Lac-St-Jean (SLSJ) region of Quebec (Canada) lives in a relatively geographic and genetic enclave unique in its detailed genealogical annotation originating from ancestors of French descent who migrated to this region from the end of the 17th century. The aim was to analyse the genomic components of complex traits of insulin resistance, known to be prevalent in hypertensive subjects.

Materials and Methods: 108 families were primarily selected on the basis of having $\geq 2$ siblings with primary hypertension and dyslipidemia. Additional selection criteria were the absence of gross obesity, T2DM and renal dysfunction. Other siblings, parents and children who did not necessarily fulfil these criteria were then also enrolled in the study. 664 subjects were examined under a wide spectrum of biochemical parameters, particularly fasting glucose $\left(\mathrm{G}_{\mathrm{o}}\right)$ and fasting insulin $\left(\mathrm{I}_{\mathrm{o}}\right)$ for this study. Bioimpedance (RJL Sorba System) was gauged to quantify water distribution and body fat mass, supplemented by complete anthropometric measurements. 204 selected subjects without any contraindications were invited for additional extensive phenotyping after the withdrawal of their antihypertensive and lipidlowering medications. For better assessment of insulin sensitivity and glucose effectiveness, the intravenous glucose tolerance test (ivGTT) was performed and evaluated using Bergman's minimal model (MINMOD software).

Genotyping: More than 400 microsatellite markers and 58000 SNPs (Affymetrix 50K Chip, Xba240) covering the entire genome were used.

Statistical analysis (software): Genome-wide linkage and haplotype identification (SOLAR, MERLIN) were undertaken with genome-wide association (FBAT, NCSS).

Results: The table following the abstract shows a summary with the chromosomal localisation of the highest statistical results for the genomic components of complex traits of insulin resistance and T2DM in hypertensive families from SLSJ.

Conclusion: These results suggest that it is possible to distinguish the genetic determinants of two major features abnormal glucose metabolism: impaired insulin secretory capacity and systemic insulin resistance. (Tab. 1)

Supported by DGDG (Genome Québec and Genome Canada), Cardiogen (IRSC), IGA MH CR NR/7809-5 and SCORE (NIH)

Tab. 1

Explanatory note: $\mathrm{Chr} .=$ chromosome; $\mathrm{cM}=$ centi-Morgan; $\mathrm{LOD}=$ logarithm of the odds

\begin{tabular}{|c|c|c|c|c|c|}
\hline & Chr. & cM & PHENOTYPE & Stats & Program \\
\hline Linkage & 1 & 187 & HOMA-IR $=\left(\mathrm{I}_{\mathrm{o}} \times \mathrm{G}_{\mathrm{o}}\right) / 22.5$ & $L O D=2.97$ & SOLAR \\
\hline Linkage & 3 & 145 & $\mathrm{Si}$ (insulin sensitivity index - Minimal model) & $L O D=2.30$ & SOLAR \\
\hline Linkage & 1 & 171 & HOMA-B $=\left(\mathrm{I}_{\mathrm{o}} \times 20\right) /\left(\mathrm{G}_{\mathrm{o}}-3.5\right)$ & $L O D=2.14$ & SOLAR \\
\hline Association & 1 & 179 & Sg (glucose effectiveness index - Minimal model) & $p=0.000004$ & FBAT \\
\hline Association & 1 & 239 & $\mathrm{Si}$ (insulin sensitivity index - Minimal model) & $p=0.000028$ & FBAT \\
\hline Association & 5 & 131 & HOMA-B $=\left(\mathrm{I}_{\mathrm{o}} \times 20\right) /\left(\mathrm{G}_{\mathrm{o}}-3.5\right)$ & $p=0.000276$ & FBAT \\
\hline Association & 17 & 14 & HOMA-IR $=\left(\mathrm{I}_{\mathrm{o}} \times \mathrm{G}_{\mathrm{o}}\right) / 22.5$ & $p=0.000446$ & FBAT \\
\hline Association & 11 & 12 & AIRg (insulin secretory response to glucose index - Minimal model) & $p=0.000922$ & FBAT \\
\hline Association & 1 & 252 & Insulin $\left(I_{o}\right)$ & $p=0.001107$ & FBAT \\
\hline Association & 1 & 17 & Si dynamic (ivGTT - new insulin sensitivity index; Pillonetto 2006) & $p=0.024853$ & FBAT \\
\hline
\end{tabular}




\section{2}

The recently identified type 2 diabetes gene CDKAL1 is widely expressed and its expression in pancreatic beta cells is affected by glucose concentration

V. Steinthorsdottir ${ }^{1}$, I. Reynisdottir ${ }^{1}$, G. Thorleifsson ${ }^{1}$, S. Ghosh ${ }^{1}$, R. Benediktsson ${ }^{2}$, G. Sigurdsson ${ }^{2}$, A. Kong ${ }^{1}$, M. Gurney ${ }^{1}$, J. R. Gulcher ${ }^{1}$, U. Thorsteinsdottir ${ }^{1}$, K. Stefansson ${ }^{1}$;

${ }^{1}$ deCode genetics, Reykjavik, ${ }^{2}$ Dept. of internal medicine, Landspitali Haskolasjukrahus, Reykjavik, Iceland.

Background and Aims: In our genome wide association study on type 2 diabetes (T2D), a variant we had previously identified in TCF7L2 stood out as the most significant finding. In addition, a variant of the CDKAL1 gene was found to be associated with increased risk of T2D in a nearly recessive manner, with genotype odds ratio for the homozygous carrier 1.45 and 1.55 for individuals of European and Asian ancestry respectively. The function of the CDKAL1 gene product was unknown but it is similar to another protein, CDK5RAP1, an inhibitor of the CDK5/p35 complex in neuronal tissue. This complex is also expressed in pancreatic beta cells and, in the presence of its active form, insulin expression is decreased under glucotoxic conditions. This led to the suggestion that CDKAL1 may be an inhibitor of the CDK5/ p35 complex in beta cells, similar to the role of CDK5RAP1 in neuronal tissue. Furthermore, we have shown that the risk variant of CDKAL1 is associated with reduced insulin secretion and this effect is mostly seen for the homozygote where a $24 \%$ reduction in insulin response is observed compared to the heterozygous carriers or non-carriers. This is in line with the nearly recessive mode of inheritance observed for this variant with respect to disease risk. The aim of this study was to gain insight into the functional role of CDKAL1 by analyzing its expression, particularly in pancreatic beta cells. Materials and Methods: To determine tissue distribution of CDKAL1 human cDNA libraries from various tissues, including colon, liver, pancreas, small intestine and brain, were screened for CDKAL1 expression by PCR. The rat pancreatic beta cell line INS-1 was cultured in the presence of variable glucose concentration, ranging from $2.5-30 \mathrm{mM}$, to evaluate whether CDKAL1 expression is regulated by glucose concentration. The mRNA expression of CDKAL1 was evaluated using rat specific TaqMan probes. As controls, mRNA expression of insulin and beta actin was similarly measured.

Results: CDKAL1 mRNA expression was detected in all the human tissues tested, including the pancreas. Expression of CDKAL1 in rat INS-1 cells varied according to glucose concentration in the culture medium with reduced expression detected under glucotoxic conditions compared to normal glucose concentration.

Conclusion: CDKAL1 expression in pancreatic beta cells is sensitive to glucose concentration. It is possible that this response to glucose may be affected in individuals carrying the variant of CDKAL1 that is associated to T2D. This could explain the reduced insulin secretion observed for these individuals in response to an oral glucose tolerance test.

\section{3}

Polymorphisms near the HHEX gene are associated with type 2 diabetes in the Dutch Breda cohort

N. C. Onland-Moret ${ }^{1,2}$, J. V. van Vliet-Ostaptchouk ${ }^{3,4}$, C. C. Elbers ${ }^{1,2}$, T. W. van Haeften ${ }^{5}$, M. H. Hofker ${ }^{3,4}$, C. Wijmenga ${ }^{1,6}$;

${ }^{1}$ DBG-Dept. of Medical Genetics, University Medical Center Utrecht, ${ }_{2}^{2}$ Julius Center for Health Sciences and Primary Care, University Medical Center Utrecht, ${ }^{3}$ Department of Pathology and Laboratory Medicine, University Medical Center Groningen, ${ }^{4}$ Dept. Molecular Genetics, Maastricht University, ${ }^{5}$ Dept. of Internal Medicine, University Medical Center Utrecht, ${ }^{6}$ Department of Genetics, University Medical Center Groningen and University of Groningen, The Netherlands.

Background and Aims: Recently, three novel susceptibility loci that contribute substantially to type 2 diabetes risk were identified in a large genome-wide scan in French individuals. One of the new loci contains the Hematopoietically Expressed Homeobox (HHEX) gene encoding a transcription factor. In this study, we aimed to replicate this finding in a Dutch cohort.

Materials and Methods: The two most associated common variants rs7923837 and rs1111875 located near the HHEX gene were genotyped in 501 unrelated type 2 diabetes patients and in 920 healthy control subjects, using logistic regression analysis, where individuals homozygous for the common allele were the reference group. Haplotype analysis was performed using the COCAPHASE package of UNPHASED program.

Results: For both rs7923837 and rs1111875 variants the carriers of major allele had a significantly increased risk for type 2 diabetes compared with non-carriers with an odds ratio (OR) of 0.64 (95\% CI 0.44-0.92) $(\mathrm{P}=0.017)$ and $0.60(0.43-0.84)(\mathrm{P}=0.003)$.

The population-attributable risks for diabetes due to the at-risk alleles of rs7923837 and rs 1111875 were estimated to be $7.3 \%$ and $11.4 \%$, respectively. Since the HHEX gene is involved in the Wnt signaling pathway, as is TCF7L2, the major determinant of type 2 diabetes risk, we examined a possible genegene interaction using the case-only design. The analysis revealed that amount of minor homozygotes for both rs7923837 and rs1111875 was significantly increased in the at-risk TT genotype carriers for rs7903146 in the TCF7L2 $(\mathrm{P}=0.007$ and $\mathrm{P}=0.037$, respectively). The analysis of haplotypes derived from the three SNPs showed that the haplotype carrying three protective alleles was significantly more frequent in controls compared with patients $(24.4 \% \mathrm{vs}$ $16.8 \%$, respectively, $\mathrm{P}=5.4 \times 10-5$ ).

Conclusion: These data provide replicating evidence that variants near HHEX contribute to the risk of type 2 diabetes in a Dutch population and suggest the possible interaction between the HHEX and the TCF7L2 genes.

Supported by Dutch Diabetes Foundation, European Vascular Genomics Network and SenterNovem (IOP Genomics IGE05012)

\section{4}

T1DBase: integration of public and private data in a type 1 diabetes community website

E. M. Hulbert ${ }^{1}$, E. C. Adlem², D. B. Burdick ${ }^{1}$, J. E. Allen ${ }^{2}$, V. M. Cassen ${ }^{1}$, O. S. Burren², K. F. Friery ${ }^{1}$, G. E. Dolman², B. C. Healy ${ }^{2}$, L. S. Wicker ${ }^{2}$, J. A. Todd ${ }^{2}$, N. Goodman' ${ }^{1}$, L. J. Smink ${ }^{2}$;

${ }^{1}$ JDRF Center for Bioinformatics, Institute for Systems Biology, Seattle, United States, ${ }^{2}$ Juvenile Diabetes Research Foundation/Wellcome Trust Diabetes and Inflammation Laboratory, University of Cambridge, United Kingdom.

Background and Aims: T1DBase (http://T1DBase.org) is a website and database that supports the type 1 diabetes (T1D) research community, including the type 1 Diabetes Genetics Consortium (http://tldgc.org). The site integrates public and user-contributed data to let researchers share data with collaborators and analyze their own data in the context of public information.

Methods: T1DBase integrates many datasets including: annotated genomes for human, mouse, and rat; T1D susceptibility regions; SNPs from dbSNP and HapMap which have been mapped to NCBI human builds 34, 35 and 36; gene association from the Genetic Association Database; gene models; NOD mouse congenic strains; the Beta Cell Gene Expression Bank, which combines functional annotation with beta cell expression under various conditions (with D. Eizirik); tissue-specific gene expression; T1D microarray data; protein interactions; and biological pathways. Tools include: GBrowse genome browser; search optimized for gene names and other biological identifiers; Beta Cell Gene Atlas, which compares gene expression across pancreatic tissues; Microarray Viewer; T1DMart, a BioMart-style query tool for marker and genotyping data; Gene Dossier, which scores a list of genes for T1D relevance and provides an overview of data we have about each gene; BLAT, to map sequences to genomes; EMBOSS, a sequence manipulation suite of tools; Connect-the-Dots, to translate gene identifiers; Cytoscape and the Cytoscape Pathway Builder; and Gaggle, a suite of interactive data analysis tools. Poster Pages are user contributed web documents that can be used to distribute experimental results, explain findings, or review a topic. Posters Pages are created using a wiki editor, and are easily configurable. Current Poster Pages include supplemental data and descriptions of genotyping data, a summary of a gene family and its contribution to T1D, and the methods description for the Beta Cell Gene Atlas. Private Poster Pages also exist where collaborators share experimental results and hypotheses. Users can save lists of genes or markers in folders which can be saved for future visits, shared among collaborators, or made public. In addition, T1DBase provides folders based on published T1D studies. Folders act as the starting point for the various tools. As a user works through tools on the website, genes can be added and removed from the current working list then sent to the next tool. Public data on T1DBase is freely available, but access to user contributed data is controlled by the contributor. Registered users can securely view private data including folders and Poster Pages. On request, we can add private data, 
such as gene models, candidate genes, genotypes, or microarray results which authorized users can analyze and view in combination with public data. Conclusion: T1DBase combines public and private datasets in a T1D relevant manner, providing an integrated and up-to-date view. Users can incorporate their own data and view it in the context of other available data, despite the fact that public source databases may be out of sync. Users who choose to make their contributed data public enrich T1DBase for other users. Supported by JDRF

\section{5}

Biological pathway analysis for type 2 diabetes using genome-wide association data

M. N. Weedon ${ }^{1}$, J. R. B. Perry ${ }^{1}$, E. Zeggini' ${ }^{2}$ H. Lango ${ }^{1}$, C. M. Lindgren ${ }^{2}$,

R. Freathy ${ }^{1}$, K. S. Elliot ${ }^{2}$, B. Shields ${ }^{1}$, N. J. Timpson ${ }^{2}$, N. W. Rayner ${ }^{2}$,

C. J. Groves ${ }^{2}$, A. T. Hattersley ${ }^{1}$, M. I. McCarthy ${ }^{2}$, T. M. Frayling';

${ }^{1}$ Genetics of Complex Traits, Peninsula Medical School, Exeter,

${ }^{2}$ Wellcome Trust Centre for Human Genetics \& OCDEM,

University of Oxford, United Kingdom.

Background and Aims: The underlying pathophysiology of type 2 diabetes is still unknown. Recently, using a genome-wide association (GWAS) approach, nine common variants have been identified that predispose to type 2 diabetes. We hypothesized that a large number of risk variants of smaller effect remain to be found. One approach to prioritizing follow-up of a GWAS is to identify biological pathways where there was a significant excess of associations compared to that expected under the null distribution. This approach may also provide a global insight into the genetic basis of T2D.
Materials and Methods: The Wellcome Trust Case Control Consortium (WTCCC) recently completed a GWAS comparing 1924 UK type 2 diabetes patients and 2938 UK population controls using the Affymetrix GeneChip Human Mapping 500k Array Set. We used data from the WTCCC, and the Kyoto Encyclopedia of Genes and Genomes (KEGG) to test 198 human pathways. We totaled the trend test chi-sq for all SNPs in all genes (as defined by the NCBI and $25 \mathrm{~kb}$ flanking sequence) in a KEGG defined pathway. This observed chi-sq total was compared to that of a distribution of 100,000 permuted chi-sq totals from an equivalent-sized random selection of genic SNPs from across the genome.

Results: The pathways showing the strongest association were the WNT signaling $(\mathrm{P}<0.00001)$, Adherens Junction $(\mathrm{P}<0.00001)$, and cell cycle $(\mathrm{P}$ $<0.00001)$ pathways. The WNT signaling and Adherens Junction pathway associations remained even when TCF7L2 variants were removed from the analyses $(P=0.03)$. The cell cycle pathway association was not explained by the recently identified CDKN2A/B or CDKAL1 signals $(P=0.00002)$. There was no evidence for other candidate pathways, for example oxidative phosphorylation $\mathrm{P}=0.99$.

Conclusion: In conclusion, we have identified biological pathways likely to be involved in the etiology of type 2 diabetes. Genes in these pathways should be prioritised in genome-wide association follow-up studies. Supported by Wellcome Trust

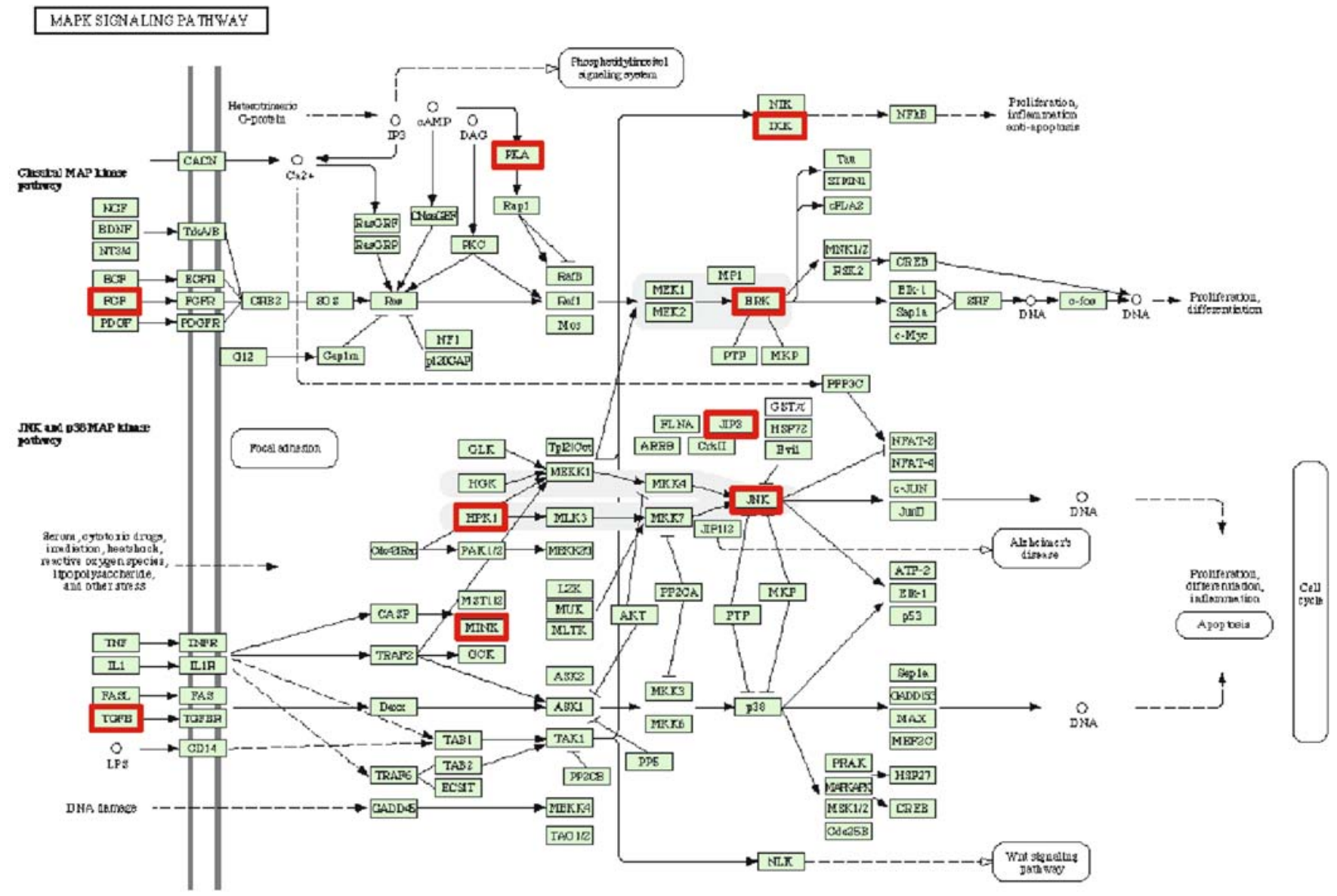

Figure. MAPK pathway from Kyoto Encyclopedia of Genes and Genomes (KEGG).

Genes surronded in bold are significantly over-expressed in high-IMT ( $4^{\text {th }}$ quartile) vs low-IMT ( $1^{\text {t }}$ quartile) group.

Fig. 1 Abstract 0296 


\section{6}

Whole genome expression profiling in lympho-monocytes identifies a significant correlation between over-expression of MAPK pathway and degree of IMT in healthy young volunteers

D. Ardigò ${ }^{1}$, L. Franzini ${ }^{1}$, E. Derlindati ${ }^{1}$, R. Navon ${ }^{2}$, I. Steinfeld ${ }^{2}$, Z. Yakhini ${ }^{2}$, A. Dei Cas $^{1}$, V. Spigoni ${ }^{1}$, S. Valtuena ${ }^{1}$, I. Zavaroni ${ }^{1}$;

${ }^{1}$ Internal Medicine, University of Parma, Italy,

${ }^{2}$ Agilent Laboratories, Agilent Technologies, Tel Aviv, Israel.

Background and Aims: Preliminary data show significant differences in gene expression profile (GEP) of circulating leukocytes in presence of several cardiovascular (CV) factors, such as smoking, hypercholesterolemia, hypertension, and insulin resistance. However, no data are available investigating whether GEP correlates with markers of vascular damage, such as intima-media thickness (IMT).

Material and Methods: To address this aim, IMT and GEP were evaluated in a group of 48 apparently healthy young-adults [21M/27F, age $36 \pm 8 \mathrm{y}$ ], volunteering for $\mathrm{CV}$ risk assessment. IMT was measured by ultrasound examination with an automated edge-detection system (Carotid Analyzer, MIA-LLC, US), whereas GEP of peripheral blood mononuclear cells (PBMC) was assessed using whole genome dual-dye arrays from Agilent. Enrolled subjects were free of diabetes, CV and chronic inflammatory diseases, and did not take pharmacological medications. Measurements were also made of multiple anthropometric, metabolic, and hemodynamic variables.

Results: Subjects were stratified in quartiles of IMT and clinical characteristics compared by one-way ANOVA with linear contrast. An increase in IMT was significantly correlated with several established CV risk factors, including increased age ( $\mathrm{p}=0.023)$, male gender $(0.008)$, smoking $(0.008$ for pack-years of cigarettes), BMI (0.027), waist (0.001), plasma glucose (0.010) and LDLcholesterol concentration (0.014), and decreased HDL-cholesterol (0.002). A total of 320 genes resulted differentially expressed (174 up- and 146 downregulated) in subjects with high IMT ( $4^{\text {th }}$ quartile, IMT from 0.9 to 1.05 $\mathrm{mm}$ ) compared to low IMT ( $1^{\text {st }}$ quartile, 0.6 to $0.75 \mathrm{~mm}$ ), using Bonferronicorrected t-test with a level of significance of $p<0.001$. The Gene Ontology (GO) analysis on differentially expressed genes identified the presence of a significant over-representation of several biological process terms related to the MAPK system (protein kinase cascade $\mathrm{p}=0.0048$; stress-activated protein kinase signaling pathway 0.0051 ; JNK cascade 0.0051 ; MAPKKK cascade 0.029 ), and the MAPK signaling pathway resulted the most represented pathway within KEGG and Biocarta encyclopedias ( $\mathrm{p}=0.00059$, figure). We also found significant direct correlation between the expression level of several MAPK-related genes and IMT in the entire sample set by Bonferronicorrected ANOVA and Pearson's correlation analysis $(\mathrm{p}<0.001)$.

Conclusion: In conclusion, the presence of vascular damage (as assessed by increased IMT) is associated with a significantly different gene expression profile in circulating mononuclear cells, whose primary signature is an overexpression of several genes related to the MAPK pathway, a crucial crosspoint for inflammation, cell differentiation and survival. (Fig. 1)

Supported by the EU commission as part of the "Multi-Knowledge project"

\section{PS 5 Genetics and epidemiology of type 1 diabetes and LADA}

\section{7}

Family history of diabetes is a strong risk factor for LADA and may be mediated by reduction of insulin secretion. Results from the Nord-Trøndelag Health Study V. Grill' ${ }^{1}$, K. Midthjell ${ }^{2}$, S. Carlsson ${ }^{3}$;

${ }^{1}$ University Hospital of Trondheim, Dept.Cancer Research and Molecular Medicine, Norway, ${ }^{2} \mathrm{HUNT}$ Research Centre, Dept.Community Medicine and General Practice, Verdal, Norway,

${ }^{3}$ Division of Epidemiology, Stockholm City Council, Sweden.

Aim: To investigate the association between family history of diabetes (FHD) and prevalence and incidence of LADA compared to traditional type 1 and type 2 diabetes.

Materials and Methods: Cross-sectional data from 64,498 men and women of the Nord-Trøndelag Health Study including 128 cases of LADA; 1,134 cases of type 2 and 123 cases of traditional type 1 diabetes. In addition, prospective data on 46,210 subjects, including 80 cases of LADA, observed between 1984-86 and 1995-97 were available. Patients with LADA had antibodies against GAD and were insulin independent at diagnosis. Information on FHD was obtained by questionnaire.

Results: FHD was associated with a 4 times (Odds Ratio (OR) $=3.88$, 95\% $\mathrm{CI}=2.73-5.52)$ increased prevalence of LADA. Corresponding estimates for type 2 diabetes and traditional type 1 diabetes were 4.2 (95\% CI=3.72-4.75) and 2.78 (95\% CI=1.89-4.10), respectively. LADA patients with FHD had lower levels of C-peptide (541 vs. $715 \mathrm{pmol} / \mathrm{l}$ ) and were more often insulin treated ( $47 \%$ vs. $31 \%$ ), than patients without FHD. Similar associations with FHD were found in type 2 diabetes. The occurrence of LADA was twice as high in those with male compared to female relatives with diabetes $(95 \%$ $\mathrm{CI}=1.07-3.66$ ). Prospective data indicated that subjects with siblings with diabetes were 2.5 times as likely to develop LADA during 11-years of followup compared to those without (95\% CI=1.39-4.51).

Conclusion: This study indicates that FHD is a strong risk factor for LADA and that the influence of family history may be mediated through a heritable reduction of insulin secretion.

\section{8}

Prevalence of metabolic syndrome in latent autoimmune diabetes in adults (LADA): data from a multicenter investigation (LADA China)

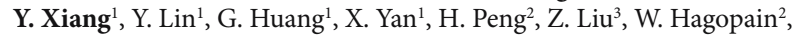
R. D. G. Leslie ${ }^{4}$, Z. Zhou ${ }^{1}$;

${ }^{1}$ Diabetes Center, Institution of Metabolism and Endocrinology of Central South University, Changsha, China, ${ }^{2}$ Diabetes Immunology Imunogenetics, Pacific Northwest Research Institute, Seattle, United States, ${ }^{3}$ Division of Endocrinology and Metabolism, Department of Internal Medicine, Charlottesville, United States, ${ }^{4}$ Dept of Diabetes and Metabolic Medicine, St. Bartholomew's Hospital, London, United Kingdom.

Background and Aims: Metabolic syndrome (MS) is prevalent in type 2 diabetics and general population in China. This analysis is aimed at investigating the prevalence of MS in Chinese LADA patients according to IDF criteria.

Materials and Methods: Patients in this analysis were recruited from LADA China Study 1, which was a multicenter investigation of 46 hospitals from 25 cities throughout China. We discriminate LADA patients from more than 5000 newly diagnosed type 2 diabetics by antibodies to glutamic acid decarboxylase (GADA) assays. GADA were tested centrally using a validated radioimmune precipitation assay, with $82 \%$ sensitivity and $98 \%$ specificity in the workshop (DASP2005). Those who at least accept serum triglycerides and high density lipoprotein cholesterol determination were included into this analysis. Results: Accoring to the IDF 2005 criteria, prevalence of MS in Chinese LADA patients was $43.8 \%$ (123/283). This prevalence was significantly lower than $58.4 \%(2394 / 4097)$ in type 2 diabetics (data also from LADA China Study 1). Compared with males, more female LADA patients had MS [56.5 $\%(61 / 108)$ vs. $36.0 \%(63 / 175), P<0.05]$. LADA with higher GADA index $(\mathrm{GADA} \geq 0.3)$ had significantly lower prevalence $[18.9 \%$ (14/74) vs.52.6 $\%(110 / 209), P<0.01]$ of MS than the patients with lower GADA index 
$(0.3>\mathrm{GADA} \geq 0.05)$. Based upon Waist Circumference (WC) in IDF criteria as cutoff point, prevalence of LADA in newly diagnosed type 2 diabetics was significantly higher in males whose waist circumference (WC) were less than $90 \mathrm{~cm}$ than those whose WC were over $90 \mathrm{~cm}(8.3 \%$ vs. $4.7 \%, P<0.01)$. The similar difference was also found between female patients with WC below $80 \mathrm{~cm}$ and upon $80 \mathrm{~cm}(7.5 \%$ vs. $5.2 \%, P<0.05)$.

Conclusion: MS is frequently found in Chinese LADA patients. Preliminary data showed that prevalence of MS in LADA is associated with gender, GADA titer and central obesity. The further study is needed.

We acknowledge all the members of LADA China Study Group. The study is supported by the European Foundation of Study of Diabetes (EFSD).

\section{9}

LADA China Study 1: prevalence of latent autoimmune diabetes in adults among Chinese newly diagnosed type 2 diabetics

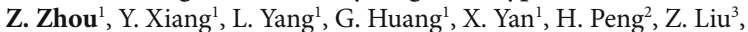
W. Hagopian², R. D. G. Leslie

${ }^{1}$ Diabetes Center, Institution of Metabolism and Endocrinology, Changsha, China, ${ }^{2}$ Diabetes Immunology Immunogenetics, Pacific Northwest Research Institute, Seattle, United States, ${ }^{3}$ Division of Endocrinology and Metabolism, Department of Internal Medicine, Charlottesville, United States, ${ }^{4}$ Dept of Diabetes and Metabolic Medicine, St. Bartholomew's Hospital, London, United Kingdom.

Background and Aims: Latent autoimmune diabetes in adults (LADA) and in the young (LADY) is prevalent in Caucasian populations. No data has been collected prospectively in large-scale from different parts of China. LADA China Study 1 is aimed at defining the prevalence of LADA and LADY among type 2 diabetics in 46 centers from 25 cities throughout China.

Materials and Methods: This Study started in June 2006 and finished in January 2007. Entry criteria include diagnosis of diabetes at age over 15 years, duration of less than 1 year, and non-insulin requirement for the first 6 months of diagnosis. Antibodies to glutamic acid decarboxylase (GADA) were tested centrally using a validated radioimmune precipitation assay, with $82 \%$ sensitivity and 98\% specificity for GAD65Ab in the workshop (DASP2005).

Results: The positivity for GADA was found in $6.2 \%$ (313/5041) of patients initially diagnosed T2DM, with significant difference to other large-scale investigations, such as UKPDS $(9.8 \%, 361 / 3672, \mathrm{P}<0.01)$, ADOPT $(4.2 \%$, $170 / 4066, \mathrm{P}<0.01)$, and Ehime $(3.8 \%, 188 / 4980, \mathrm{P}<0.01)$. There was no geographic difference between north China $(6.9 \%, 154 / 2230)$ and south China $(5.7 \%, 159 / 2811)$ in this study. Gender difference between males $(6.5 \%, 195 / 3004)$ and females $(5.8 \%, 118 / 2037)$ was not found as well. The GADA positivity was $11.7 \%(28 / 239)$ in group aged $15 \sim 29 \mathrm{yr}$, which was higher than 30 44yr group [vs.7.4\% (108/1462), $P<0.05$ ], 45 59yr group [vs.5.1\% (111/2168), $\mathrm{P}<0.01$ ], and $\geq 60 \mathrm{yr}$ group [vs. 5.8\% (66/1142), $\mathrm{P}<0.01$ ]. The positivity in 30 44yr group was higher than $45 \sim 59 \mathrm{yr}$ group $(\mathrm{P}<0.01)$ but without any differences with $\geq 60 \mathrm{yr}$ group.

Conclusion: We conclude that LADA and LADY are prevalent among newly diagnosed phenotypic type 2 diabetic patients in China, and that LADY is more common than LADA in Chinese. The study is ongoing.

We acknowledge all the members of LADA China Study Group. The study is supported by the European Foundation of Study of Diabetes (EFSD).

\section{0}

Serum concentrations of adiponectin, interleukin - 6 and tumor necrosis factor - alpha in latent autoimmune diabetes of adults (LADA)

E. Hatziagelaki, K. Kantartzis, M. Panagiotidou, I. Garofallakis, Th. Economopoulos, S. A. Raptis;

2nd Dept. of Internal Medicine, Research Institute and Diabetes Center, Athens University. "Attikon" University General Hospital, Athens, Greece.

Background and Aims: Adipokines play a critical role in generating or maintaining insulin resistance in both subjects with type 2 diabetes and non-diabetics. Adiponectin is inversely and interleukin (IL) -6 and tumor necrosis factor - alpha (TNF-a) are positively associated with body mass index (BMI) and insulin resistance. In type 1 diabetes, plasma adiponectin concentrations were found to be higher than in healthy subjects, but the physiologic relevance of this finding it still unknown. IL-6 and TNF-a were reported to be higher than in healthy subjects mainly in newly diagnosed type 1 diabetes, suggesting a role of them in its pathogenesis. The role of adipokines in the Latent Autoimmune Diabetes of Adulthood (LADA), which shows characteristics of both type 1 (progressive autoimmune-mediated destruction of $\beta$-cells) and type 2 diabetes (insulin resistance), has not been investigated yet.

Materials and Methods: In a cross-sectional setting, we measured fasting adiponectin, IL-6 and TNF-a levels and explored their relationships to metabolic parameters, as insulin sensitivity (estimated by homeostasis model assessment - HOMA-IR) and BMI and to $\beta$-cell function in patients with LADA $(\mathrm{n}=31)$, with type 1 diabetes $(n=41)$ and in healthy controls $(n=72)$. Patients with LADA were identified by their age at diagnosis of diabetes ( $>30$ years), a type 2 diabetes phenotype and the presence of GAD 65 and IA-2 antibodies.

Results: Patients with LADA had lower, compared to patients with type 1 diabetes, plasma adiponectin levels, adjusted for gender, age and BMI ( $\mathrm{p}=0.006$ ), and comparable levels to healthy controls. Serum IL-6 concentrations, adjusted as above, significantly differed between the three groups (ANOVA, $\mathrm{p}<0.0001$ ), being in LADA patients lower than in type 1 diabetics $(p=0.045)$ and higher than in healthy controls $(p<0.0001)$. TNF-a serum levels were not different in the three groups. No association of IL-6 or TNF-a with adiponectin levels were found in either of the groups. Also, there was no association of IL- 6 or TNF-a levels with diabetes duration. In LADA, adiponectin was significantly associated with female sex $(p=0.012)$ and negatively with BMI (adjusted for sex and age $\mathrm{p}=0.033$ ) and HOMAIR (adjusted $\mathrm{p}=0.005$ ), whereas in patients with type 1 diabetes only with female sex $(p=0.03)$. Serum IL- 6 concentrations were correlated with BMI in LADA diabetes (adjusted $\mathrm{p}=0.05$ ) and healthy controls (adjusted $\mathrm{p}=0.0004$ ), but not with HOMA-IR in either group. IL-6 in type 1 diabetes and TNF-a in all groups showed no correlation with these parameters. A highly significant inverse association of adiponectin, but not IL- 6 and TNF-a levels with fasting $\mathrm{C}$-peptide concentrations was found in LADA (adjusted $\mathrm{p}=0.0011$ ) and in type 1 diabetes $(\mathrm{p}=0.0009)$, but not in healthy controls $(\mathrm{p}=0.68)$.

Conclusion: Serum concentrations of adiponectin and IL-6 in LADA are higher than in healthy controls and lower than in type 1 diabetes. While the association of adiponectin levels with C-peptide concentration implies that hypoinsulinemia is a principal reason for this increase, the increase of IL-6 levels possibly reflects the underlying inflammatory process. However, levels of both adipokines were also affected by adiposity and especially these of adiponectin inversely correlated with insulin resistance, suggesting a physiologic role similar to healthy subjects. As in healthy controls, in LADA patients TNF-a seems to have a paracrine effect that is more important than the systemic effect.

\section{1}

Latent autoimmune diabetes in adults should be suspected in Korean diabetic patients with low C-peptide level and without metabolic syndrome

S.-H. Lee, J.-H. Kim, M.-J. Kang, H.-S. Kwon, S.-J. Yoo, K.-H. Yoon, B.-Y. Cha, H.-Y. Son;

Division of Endocrinology and Metabolism, Department of Internal Medicine, Kangnam St.Mary's Hospital, Seoul, Republic of Korea.

Background and Aims: Latent autoimmune diabetes in adults (LADA) is a subgroup of diabetes which resembles the clinical characteristics of type 2 diabetes (T2DM) but positive for islet-related autoantibodies. We aimed to establish the prevalence and characteristics of LADA and compare it with T2DM in Korean patients.

Materials and Methods: From 2003 to 2006, anthropometric measurements and biochemical studies including IA-2 antibodies and glutamic acid decarboxylase $(\mathrm{GAD})$ antibodies were done in 938 adults who visited a tertiary care institution for the management of diabetes. Type 1 diabetes $(n=49)$ and other types of diabetes $(n=9)$ were excluded in this study. The diagnosis of LADA was made with the fulfillment of i) onset of diabetes at age $\geq 30$, ii) presence of circulating islet autoantibodies, iii) lack of requirement of insulin at least 6 months after diagnosis.

Results: The prevalence of LADA was $4.8 \%(\mathrm{n}=45)$. Among them, 32 were positive for GAD antibody, 8 were positive for IA-2 antibody, and 5 were positive for both antibodies. These patients had a significantly lower Cpeptide level $(0.81 \pm 0.76$ vs. $1.62 \pm 1.44 \mathrm{ng} / \mathrm{mL} ; \mathrm{p}<0.0001)$, waist circumference ( $83.8 \pm 9.6$ vs. $88.1 \pm 10.2 \mathrm{~cm} ; \mathrm{p}=0.02)$, waist-hip-ratio $(0.91 \pm 0.07$ vs. $0.94 \pm 0.08$; $\mathrm{p}=0.04)$ and triglyceride $(115.2 \pm 61.2$ vs. $163.1 \pm 130.6 \mathrm{mg} / \mathrm{dL} ; \mathrm{p}<0.0001)$ 
than patients with T2DM. They also had a significantly lower prevalence of hypertension ( $35.5 \%$ vs. $55.1 \%$; $\mathrm{p}=0.01)$ while the rate of insulin treatment $(37.8 \%$ vs. $25.5 \% ; \mathrm{p}=0.01)$ and history of weight loss and/or $3 \mathrm{p}$ sign $(43.2 \%$ vs. $26.9 \% ; \mathrm{p}=0.01$ ) were higher than that of T2DM. LADA constituted $10 \%$ among the patients without metabolic syndrome, while it constituted $3.2 \%$ among the patients with metabolic syndrome.

Conclusion: LADA takes a considerable proportion in Korean diabetic patients, and they are characterised by lower parameters of insulin resistance than T2DM. Therefore, LADA should be suspected in patients with low Cpeptide level, especially in those without metabolic syndrome.

\section{2}

\section{Latent autoimmune diabetes in adults (LADA)} in the UK South Asian population

A. Britten ${ }^{1}$, K. Jones ${ }^{1}$, C. Törn², M. Hillman², B. Ekholm², S. Kumar ${ }^{3}$, A. Barnett ${ }^{1}$, A. Kelly ${ }^{1}$;

${ }^{1}$ Medicine, Birmingham University, United Kingdom,

${ }^{2}$ Medicine, University Hospital, Lund, Sweden, ${ }^{3}$ Diabetes and Metabolism, Warwick Medical School, Coventry, United Kingdom.

Background and Aims: Type 2 diabetes is five-fold more common among UK-resident South Asians than indigenous white Caucasians. In many populations, a subset of patients initially diagnosed with type 2 diabetes show evidence of a slowly-evolving autoimmune form of diabetes, known as Latent Autoimmune Diabetes in Adults (LADA). The prevalence of LADA in South Asian populations is not well established and the disease is poorly characterised in this ethnic group.

The aim of this study was to investigate the frequency of LADA in UK-resident South Asians of Punjabi origin and to characterise the clinical phenotype and genetic basis of the disease in this population.

Materials and Methods: 500 individuals with type 2 diabetes and 206 control subjects of Punjabi origin were recruited. The subjects were analysed for circulating autoantibodies to GAD65 and IA-2 and genotyped for alleles of the HLA-DRB1, $-D Q A 1,-D Q B 1$ and MIC-A genes and the INS-VNTR.

Results: Antibodies to GAD65 and IA-2 were detected in 13/500 (2.6\%) individuals with type 2 diabetes (eight GAD65-positive [1.6\%], six IA-2positive [1.2\%], one GAD65/IA-2 double-positive) and 8/206 (3.9\%) control subjects (three GAD65-positive [1.5\%] and five IA-2-positive [2.4\%]). There was no significant difference in the antibody titre between the diabetic and control subjects.

At the HLA locus the $D R B 1^{*} 04, D Q A 1^{*} 03$ and $D Q B 1^{*} 0302$ alleles were increased in frequency among the IA-2-positive diabetic subjects compared with those lacking this marker ( $\mathrm{p}=0.020, \mathrm{p}=0.066$ and $\mathrm{p}=0.015$ ). A similar trend was also observed in the control group. The $D R B 1^{*} 03, D Q A 1^{*} 05$ and $D Q B 1^{*} 02$ alleles were more frequent among the GAD65-positive diabetic subjects than the antibody-negative subjects $(\mathrm{p}=\mathrm{NS})$, but no such trend was observed in the control group.

There was no association between INS-VNTR genotypes and antibody positivity. The MIC-A6 allele was significantly less frequent among the IA2 -positive diabetic subjects compared with the antibody-negative diabetic subjects $(\mathrm{p}=0.044)$, but no association was seen between antibody positivity and the MIC-A5.1 allele.

Mean weight and body mass index (BMI) were significantly lower in the antibody-positive diabetic subjects compared with the antibody-negative diabetic subjects (weight, $70.1 \mathrm{~kg}$ vs. $77.3 \mathrm{~kg}, \mathrm{p}=0.029 ; \mathrm{BMI}, 25.9 \mathrm{~kg} / \mathrm{m}^{2}$ vs. $28.7 \mathrm{~kg} / \mathrm{m}^{2}, \mathrm{p}=0.032$ ). IA-2-positive diabetic subjects had a significantly lower mean $\mathrm{HbA}_{\mathrm{c}}$ level than IA-2-negative subjects (5.6\% vs. 7.0\%, $\mathrm{p}=0.046$ ).

Insulin treatment was more common in the antibody-positive group. Of the GAD65-positive diabetic subjects, six (75\%) were treated with insulin and two (25\%) with oral hypoglycaemic agents (OHA). Of the IA-2-positive diabetic subjects, two (33\%) were treated with insulin, three $(50 \%)$ with OHAs and one (16.7\%) with diet. The latter four individuals had a duration of diabetes of less than four years. In contrast, among the antibody-negative diabetic subjects, only 87 (18.2\%) were treated with insulin.

Conclusion: LADA is uncommon in the Punjabi population living in Birmingham, UK. The presence of islet autoimmunity was associated with a similar HLA profile to that reported in other populations, but no associations with the INS-VNTR or the MIC-A5.1 allele were observed. Autoantibodypositive diabetic individuals had a similar clinical phenotype to that reported in other ethnic groups.

Supported by Diabetes UK

\section{3}

Low risk HLA genotype is associated with less destruction of beta cells persisting 12 months after diagnosis

M. Spoletini ${ }^{1}$, A. Petrone ${ }^{1}$, M. Capizzi ${ }^{1}$, S. Zavarella ${ }^{1}$, S. Zampetti ${ }^{1}$, G. Leto ${ }^{1}$, I. Alemanno ${ }^{1}$, IMDIAB Group ${ }^{2}$, P. Pozzilli², R. Buzzetti';

${ }^{1}$ Clinical Sciences, "Sapienza” University, Rome, Italy,

${ }^{2}$ Endocrinology, "Campus Biomedico", Rome, Italy.

Background and Aims: The role of HLA genes in the susceptibility to type 1 diabetes (T1DM) is well known, however remains to be established whether the degree of beta-cell destruction depends on different HLA genetic risk. The aim of this study was to evaluate the influence of $\mathrm{DRB1}^{*}$ and $\mathrm{DQB1}{ }^{*}$ genes on the rate of beta-cell loss in a prospective series of $n=120$ consecutive newly-diagnosed T1DM subjects in the course of 12 months after disease onset.

Materials and Methods: $\mathrm{N}=120$ consecutive T1DM subjects with age ranged

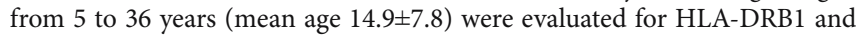
DQB1. The HLA genotypes were classified in three risk categories, based on the absolute risk values previously estimated in the Italian population: high risk for $\mathrm{DRB} 1{ }^{*} 03-\mathrm{DQB} 1 * 0201 / \mathrm{DRB} 1^{*} 04-\mathrm{DQB} 1^{*} 0302$ genotype (DRB1 ${ }^{*} 04$ different from 0403, 06, 11); moderate risk for $\mathrm{DRB} 1{ }^{*} 04-\mathrm{DQB} 1{ }^{*} 0302 /$ DRB $1 * 04-\mathrm{DQB} 1{ }^{*} 0302, \mathrm{DRB} 1{ }^{*} 03-\mathrm{DQB} 1{ }^{*} 0201 / \mathrm{DRB} 1{ }^{*} 03-\mathrm{DQB} 1{ }^{*} 0201$, $\mathrm{DRB}^{*} 04-\mathrm{DQB} 1 * 0302 / \mathrm{X}$ (X different from $\mathrm{DRB} 1^{*} 02,03$, DRB1 ${ }^{*} 04-$ $\mathrm{DQB1}^{*} 0302\left(\mathrm{DRB}^{*} 04\right.$ not $\left.0403,06,11\right)$ or $\left.\mathrm{DQB1}^{*} 0602\right)$ genotypes and $\mathrm{DRB} 1^{\star} 03 / \mathrm{X}$ (X different from DRB $1^{\star} 02,03, \mathrm{DRB} 1^{\star} 04-\mathrm{DQB} 1^{\star} 0302$ (DRB ${ }^{\star} 04$ not $0403,06,11)$ or DQB1 $\left.{ }^{\star} 0602\right)$; low risk for the remaining genotypes. Insulin requirements, glycated haemoglobin (HbAlc) and C-peptide levels, were determined at diagnosis and every 3 months for 12 months, in all patients. $\mathrm{HbAlc}$ was measured by a column assay (Bio-Rad) and C-peptide levels by a radioimmunoassay (Bio-Rad). The variance of investigated variables during follow up was analyzed using the general linear model for repeated-measures procedure.

Results: Baseline C-peptide in T1DM subjects with low HLA genetic risk showed a similar trend but significantly higher levels compared to patients with moderate and high HLA genetic risk from time of diagnosis up to 12 months ( $p=0.007$ and $p=0.0002$, respectively). We also observed a significant positive correlation between C-peptide levels and age at diagnosis from the time of disease onset through the 12 months of follow up ( $\mathrm{p}=0.016$ ).

Conclusion: Both HLA markers and age at onset of T1DM are independent factors which influence beta-cell destruction from the time of disease onset up to 12 months of follow-up. These results are useful for designing trials for future therapies aimed to prevent the progression of beta-cell destruction in recent onset T1DM.

\section{4}

Chemokine CXCL10 gene polymorphisms are associated with type 1 diabetes

E. Ramos-Lopez, W. Bartsch, P. Brück, H. Kahles, M. Penna-Martinez, J. Herwig, K. Badenhoop;

Endocrinology, Diabetes and Metabolism, University Frankfurt am Main, Germany.

Background and Aims: CXCL10 and CXCL9, two CXCR3 chemokines belong to a group of structurally related molecules, which regulate cell trafficking of diverse cells including activated T-helper 1 lymphycytes (TH1cells), natural killer cells and monocytes. In patients with type 1 diabetes high levels of CXCL10 also known as chemokine interferon $\gamma$ inducible protein (IP-10) have been found. Additionally, in a mouse model of virus induced diabetes CXCL10 was found as the key mediator to induce insulitis and T-cell mediated destruction of $\beta$-cells. Therefore we investigated the role of CXCL10 and CXCL9 gene polymorphisms in German type 1 diabetes families. Materials and Methods: German type 1 diabetes families [181, (573 subjects)] were genotyped for the CXCL10_89 and 120 families (360 subjects) for the CXCL10 90 polymorphism within the CXCL10 gene as well as 182 families (552 subjects) for the CXCL9_99 polymorphism within the CXCL9 gene, using real time PCR. Genotype-, haplotype-frequencies and linkage disequilibrium (LD) were estimated using Unphased version 2.403. A corrected $P(P \mathrm{c})<0.05$ was considered significant.

Results: The allele " $G$ " of the CXCL10_89 polymorphism was more often transmitted to the affected offspring (103 times vs. 73 times, $P=0.023$ ) as well 
as the haplotypes CXCL10_89G-CXCL10_90T (72. times vs. 43 times, $P=$ 0.006), while the haplotype CXCL10_89A-CXCL10_90T was less frequently transmitted (47 times vs. 69 times, $P=0.041$ ). No differences were observed in the transmission of the CXCL9_99 polymorphism.

Conclusion: CXCL10 gene polymorphisms but not CXCL9 are significantly associated with type 1 diabetes in the German population. Functional studies are underway to put these findings into physiological contexts.

\section{5}

Genetic analysis of putative loci located in regions 1q42, 2q34-q35, 16q22-q24 and 5q33.1-q34.1 in Russian families with type 1 diabetes V. V. Nosikov ${ }^{1}$, A. Chernyshova ${ }^{1}$, L. I. Zilberman ${ }^{2}$, T. L. Kuraeva ${ }^{2}$, N. M. Tsitlidze², V. A. Peterkova², I. I. Dedov²;

${ }^{1}$ Molecular Diagnostics and Genomic Fingerprinting, National Research Centre "GosNII genetika", ${ }^{2}$ Department of Juvenile Diabetes, Endocrinology Research Centre, Moscow, Russian Federation.

Background and Aims: Type 1 diabetes mellitus (T1DM) is a complex disorder with multiple genetic loci. In the current study, we have performed the sibpair analysis to localize minimal regions of linkage with T1DM in regions 1q42, 2q34-q35, 16q22-q24 and 5q33.1-q34.1.

Materials and Methods: All families in this study (35 affected and 66 discordant sibpairs) are Caucasian Russians with nondiabetic parents. Polymorphous markers were amplified using PCR and primer sequences from GenBank. Combined transmission/disequlibrium test (TDT) and sib TDT (S-TDT) were used for data analysis.

Results: To minimize a region of linkage with T1DM on chromosome 16q22-q42 we used five polymorphous microsatellites. Only slight linkage evidence has been obtained for markers D16S3098, D16S422, D16S3118 and D16S3073. The peak of linkage has been obtained for D16S750 marker (MLS $\left.=1.63, p<0.03 ; Z^{\prime}=2.20, p<0.03\right)$ located between D16S3118 and D16S3073 markers. The MLS and $Z^{\prime}$ values are significantly lower in Russian families in comparison with families from United Kingdom (Mein et al, Nature Genetics, $19,297)$ but the peak of linkage locates in the same area of chromosome 16. To estimate a level of linkage with T1DM of interleukin 12B gene (IL12B) located in 5q33.1-q34.1 region we have used two markers: C1159A SNP in 3'untranslated region of $I L 12 B$ gene and polymorphous microsatellite D5S2060 located at a distance $0.54 \mathrm{Mb}$ from $I L 12 B$ gene. No linkage evidence has been obtained for these markers in Russian families with T1DM. To minimize a region of linkage with T1DM on region 1q42 we have studied a linkage with T1DM of five polymorphous markers: D1S1617, D1S1668, D1S103, D1S225 and D1S1644. MLS values was higher 2.50 for all these markers and the peak of linkage has been obtained for D1S1617 marker (MLS $=3.42, p<0.01$; $\left.Z^{\prime}=3.63, p<0.01\right)$ located between D1S1644 and D1S1668 markers. The position of maximal linkage, MLS and Z' values completely consist with the data obtained earlier by Concannon et al (Nature Genetics, 19, 292-296). To minimize a region of linkage with T1DM on region 2q31-q34 we have studied a linkage with T1DM of five polymorphous markers: D2S137, D2S164, D2S143, D2S301 and D2S157. The peak of linkage has been obtained for D2S137 marker (MLS $=3.18, p=0.008 ; Z^{\prime}=3.23, p<0.005$ ).

Conclusion: The genetic analysis of loci located in regions $1 \mathrm{q} 42,2 \mathrm{q} 34-\mathrm{q} 35$, 16q22-q24 and 5q33.1-q34.1 in Russian families with T1DM has shown the strong linkage of $1 \mathrm{q} 42$ and $2 \mathrm{q} 34$-q35 regions and the relatively slight linkage of 16q22-q24 region with T1DM.

Supported by the Russian Foundation for Basic Research

\section{PS 6 Type 2 diabetes genes}

\section{6}

Gene expression and genetic association studies suggest a role for the Parkinson's gene PINK1 in the pathogenesis of type 2 diabetes P. W. Franks ${ }^{1,2}$, C. Scheele ${ }^{3}$, R. J. F. Loos ${ }^{2}$, A. R. Nielsen ${ }^{4}$, B. K. Pedersen ${ }^{4}$, N. J. Wareham ${ }^{5}$ J. A. Timmons ${ }^{6,7}$;

${ }^{1}$ Genetic Epidemiology \& Clinical Research Group, Umea University Hospital, Sweden, ${ }^{2}$ Genetics Group, MRC Epidemiology Unit, Cambridge, United Kingdom, ${ }^{3}$ Department of Cellular and Molecular Biology, Karolinska Institute, Stockholm, Sweden, ${ }^{4}$ Department of Infectious Diseases, University of Copenhagen, Denmark, ${ }^{5} \mathrm{MRC}$, MRC Epidemiology Unit, Cambridge, United Kingdom, ${ }^{6}$ Programme for Genomics and Bioinformatics, Karolinska Institute, Stockholm, Sweden, ${ }^{7}$ School of Life Sciences, Heriott-Watt University, Edinburgh, United Kingdom.

Background and Aims: PTEN induced putative kinase 1 (PINK1) locates to the mitochondria and mutations at the PINK1 locus cause mitochondrial dysfunction, energy depletion and autosomal recessive Parkinson's disease.

Materials and Methods: We measured PINK1 transcripts levels in skeletal muscle and adipose tissue of 53 adults $(61.1 \pm 9.1 \mathrm{yrs})$ : 12 were non-obese $(\mathrm{BMI}<27)$ with normal glucose tolerance $(\mathrm{NGT}), 14$ were obese $(\mathrm{BMI}>35)$ with NGT, 13 were non-obese/type 2 diabetics (T2D), and 14 were obese/ T2D. We also tagged the PINK1 gene in a1703 adults $(55.9 \pm 10.0 \mathrm{yrs})$ and tested associations for the 10 PINK1 tagging SNPs (derived via pair-wise tagging in the CEU HAPMAP panel) and indirect measures of lipid and glucose oxidative capacity.

Results: In the expression study, 3 transcripts were examined (PINK1, naPINK1, DDOST), which were all down-regulated by $\sim 30 \%$ in T2D skeletal muscle vs. the NGT control muscle (PINK1: $\mathrm{p}<0.05$, naPINK1: $\mathrm{p}<0.05$, DDOST: $p<0.001)$. In obese vs non-obese NGT individuals, PINK1 expression was unaltered, while expression of both naPINK1 and DDOST were reduced (naPINK1: $\mathrm{p}<0.01$, DDOST: $\mathrm{p}<0.01$ ). In the genetic studies, the tagging SNPs were simultaneously regressed against the metabolic traits with adjustment for age, sex, exam date, and BMI using stepwise regression models. Modest evidence for association was observed for all traits: fasting glucose (rs3131713: $\mathrm{p}=0.098), 2 \mathrm{hr}$ glucose ( $\mathrm{rs} 10799655: \mathrm{p}=0.042)$, LDL (rs650616: $\mathrm{p}=0.097$ ), total cholesterol ( $r$ 650616: $\mathrm{p}=0.021$ ), triglycerides (rs3738136: $\mathrm{p}=0.014 ; \mathrm{rs} 2298299: \mathrm{p}=0.067)$, fasting NEFA ( $\mathrm{rs650616:p=0.04)}$ and $\mathrm{VO}_{2} \max$ ( $\mathrm{rs} 12410193$ : $\mathrm{p}=0.025$ ). Owing to the modifying effects of obesity on PINK1 transcript levels observed in the expression studies, we tested for interaction between BMI and each of the associated genotypes on their respective phenotypes. For rs650616, a strong interaction with BMI was observed on NEFA levels ( $\mathrm{p}=0.0076$ ), where the minor allele was associated with higher NEFA in non-obese $(\mathrm{p}=0.0008)$, but in obese, the minor allele tended to associate with lower NEFA levels $(\mathrm{p}=0.065)$. A tendency for interaction between rs12410193 and BMI on $\mathrm{VO}_{2} \max$ was also observed $(\mathrm{p}=0.079)$, where the minor allele was associated with higher $\mathrm{VO}_{2} \max$ in non-obese $(\mathrm{p}=0.007)$ but not in obese $(\mathrm{p}=0.75)$ individuals.

Conclusion: In skeletal muscle of obese or diabetic individuals, PINK1 transcript levels are significantly down-regulated compared with the levels in tissue of non-obese NGT individuals. Furthermore, sequence variation across the PINK1 gene influences levels of post-challenge glucose, lipids, and maximal oxidative capacity. Obesity modifies these relationships, such that the genetic associations for NEFA and $\mathrm{VO}_{2}$ max are strong in non-obese individuals and weak or absent in obese. We conclude, therefore, that the PINK1 gene may be involved in the pathogenesis of T2D, possibly through defective lipid and glucose oxidation.

Supported by Danish NRC, Danish MRC, UK MRC (NJW), Swedish Diabetes Association, Novo Nordisk 


\section{7}

Minor contribution of Smad7 and KLF10 variants to genetic susceptibility of type 2 diabetes

B. Neve ${ }^{1,2}$, R. Gutierrez-Aguilar ${ }^{1}$, Y. Benmezroua ${ }^{1}$, B. Balkau ${ }^{3}$, M. Marre ${ }^{4}$, N. Helbecque ${ }^{5}$, G. Charpentier ${ }^{6}$, R. Sladek ${ }^{7}$, P. Froguel $^{1,2}$;

${ }^{1}$ Cnrs, umr8090, Institute of Biology, Lille, France, ${ }^{2}$ Genomic Medicine, Imperial College, London, United Kingdom, ${ }^{3}$ Inserm U780-IFR69, and University Paris-Sud, Villejuif, France, ${ }^{4}$ EA3516, Xavier Bichat, Faculty of Medicine, University Paris VII, Paris, France, ${ }^{5}$ Inserm, u744, Pasteur Institute, Lille, France, ${ }^{6}$ Department of Diabetes, Sud Francilien Hospital, Corbeil-Essonnes, France, ${ }^{7}$ Departments of Human Genetics and Medicine, Faculty of Medicine, McGill University and Genome Quebec Innovation Centre, Montreal, France.

Background and Aims: It has been shown that transgenic mice over expressing Smad7 in pancreatic cells developed overt type 2 diabetes (T2D). Both KLF11, which gene variants have been shown to be involved in genetic susceptibility to T2D, and the highly homologous KLF10 regulate expression of Smad7. If the TGFbeta-induced transcription factor KLF10 and KLF11 affect transcriptional regulation of the same set of genes (including TGFbeta signaling pathway inhibitor Smad7), we may expect they contribute similar to the development of T2D. This study aimed to assess the genetic contribution of SMAD7 and KLF10 genes to susceptibility to T2D.

Materials and Methods: We screened both genes to identify rare variants and frequent variants by direct sequencing and genotyped those variants. Rare variants were analyzed for cosegregation with T2D in the pedigrees of the proband families. We assessed T2D association of frequent gene variants in a two-stage design case/control study. We compared the allelic distribution of 6 frequent Smad7 SNPs and 6 frequent KLF10 SNPs between 358 T2D and 352 normoglycaemic subjects. Then, statistically significant differences in allele distribution were confirmed in a population sample of 2066 T2D and 1107 normoglycaemic subjects.

Results: The combined analysis of the case/control studies showed two variants with modest T2D association. The Smad7 intron 2 variant showed a significant $\mathrm{T} 2 \mathrm{D}$ association under a recessive model $(\mathrm{P}=0.007$, Odds ratio $=$ $0.62,95 \%$ confidence interval $=0.44-0.88 ; \mathrm{P}=0.03$ when adjusting for age, sex and BMI in a nominal regression model). The KLF10 variant located in the 3-UTR showed a significant T2D association under a recessive model ( $\mathrm{P}$ $=0.009$, Odds ratio $=0.81,95 \%$ confidence interval $=0.69-0.95 ; \mathrm{P}=0.04$ when adjusting for age, sex and BMI in a nominal regression model).

Conclusion: The observed modest T2D association for Smad7 and KLF10 gene variants suggests that they may contribute to a particular genetic background that increases the susceptibility to development of T2D. Further investigations are needed to assess whether the potential effects of Smad7, KLF10 and KLF11 gene variants on TGF-beta signaling is the pathogenic mechanisms that contributes to development of T2D.

Supported by Grant of European Regional Development Fund (FEDER) and Region Nord-Pas de Calais (ARCir) to BN and PF. RGA is supported by a SFERE-CONACYT grant and BN was supported by a VIP-grant from the Imperial College

\section{8}

Evidence that the LARS2 gene represents a novel type 2 diabetes mellitus susceptibility gene

E. Reiling ${ }^{1}$, G. C. M. Janssen ${ }^{1}$, P. J. A. van den Broek ${ }^{1}$, E. C. van Hove ${ }^{1}$, J. A. Maassen ${ }^{1,2}$, L. M. 't Hart';

${ }^{1}$ Molecular Cell Biology, Leiden University Medical Center, Leiden,

${ }^{2}$ EMGO institute, VUMC, Amsterdam, The Netherlands.

Background and Aims: It is known that mitochondrial dysfunction can lead to an increased risk for type 2 diabetes mellitus (T2DM). LARS2 encodes for the mitochondrial leucyl tRNA synthetase (LeuRS), which is essential for mitochondrial protein synthesis. Previously we have shown that an H324Q polymorphism associates with an enhanced risk for T2DM in populations from the Netherlands, Denmark and the UK. We now performed an extensive functional study in order to find the pathogenic mechanism of the H324Q variant.

Materials and Methods: We used recombinant wild type and mutant LeuRS for measuring enzyme activity and misacylation in vitro with ${ }^{3} \mathrm{H}$-labeled leucine and isoleucine. LeuRS expression levels and mitochondrial function were assessed in heterozygous patient fibroblasts.

Results: First we assessed the LeuRS expression levels in patient fibroblasts, but observed no differences. We therefore measured the enzyme activity of both wild type and mutant LeuRS in vitro, but no significant differences were observed. Next the misacylation rate was measured. We found an approximately 3 -fold increased misacylation rate $(\mathrm{p}<0.001)$. An increased misacylation is likely to induce a higher error rate in mitochondrial protein synthesis, resulting in impaired ATP synthesis. AMPK and its downstream target ACC are phosphorylated by an increased AMP / ATP ratio, which results in a switch to fatty acid oxidation. We therefore analyzed the phosphorylation status of ACC as a readout of possible energy depletion in patient fibroblasts. Our results indeed suggest that ACC phosphorylation is increased in H324Q carriers, which indicates an impaired ATP production.

Conclusion: In conclusion we have shown that the LARS2 gene represents a T2DM susceptibility gene in several Caucasian populations. The H324Q variant results in an approximately 3 -fold increased misacylation rate and an increased ACC phosphorylation. This indicates that mitochondrial dysfunction due to the H324Q variant in LeuRS results in impaired ATP synthesis which enhances susceptibility to develop type 2 diabetes.

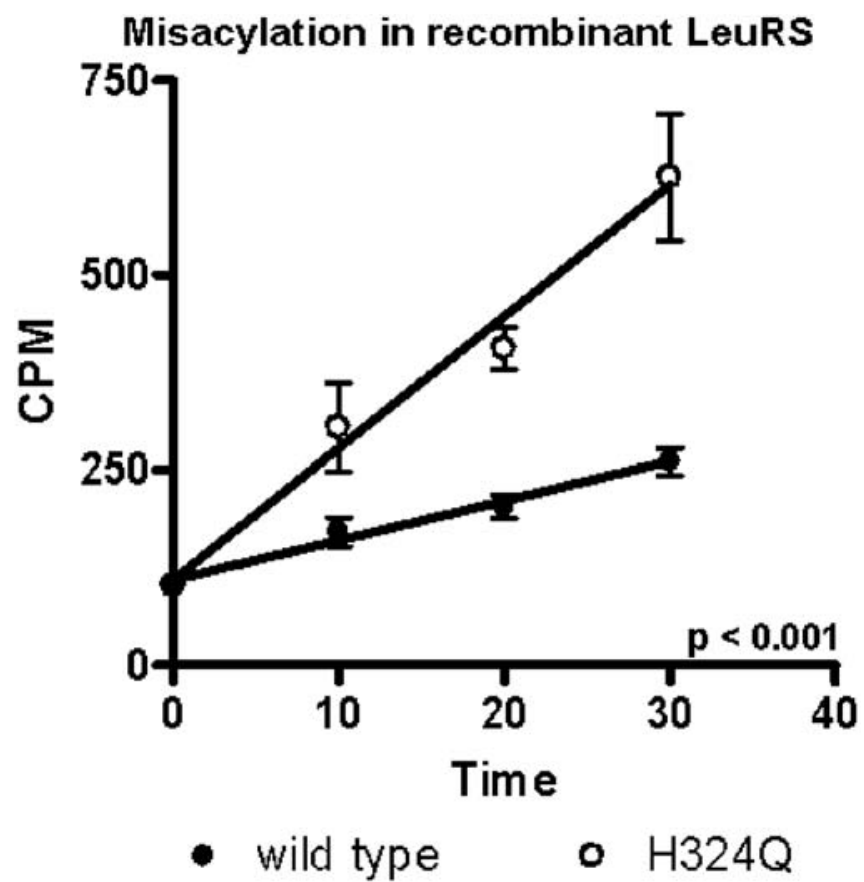

Supported by ZonMW RIDE programme

\section{9}

Effects of genetic variation in the human retinol binding protein-4 gene (RBP4) on insulin resistance and fat depot specific mRNA expression A. Tönjes ${ }^{1}$, M. Geyer ${ }^{1}$, N. Klöting ${ }^{1}$, T. E. Graham ${ }^{2}$, J. Berndt ${ }^{1}$, Y. Böttcher ${ }^{1}$, B. Enigk ${ }^{1}$, M. R. Schön ${ }^{3}$, B. B. Kahn ${ }^{2}$, M. Blüher ${ }^{1}$, M. Stumvoll ${ }^{1}$, P. Kovacs ${ }^{1}$; ${ }^{1}$ IIIrd Medical Department, University of Leipzig, Germany, ${ }^{2}$ Division of Endocrinology, Diabetes, and Metabolism, Beth Israel Deaconess Medical Center, Boston, United States, ${ }^{3}$ Department of Surgery, University of Leipzig, Germany.

Objective: Serum Retinol Binding Protein 4 (RBP4) is a new adipocytederived signal that may contribute to insulin resistance. Therefore, the RBP4 gene represents a plausible candidate gene involved in susceptibility to type 2 diabetes mellitus (T2DM) and related traits.

Research design: In this study, the RBP4 was sequenced in DNA samples from 48 non-related Caucasian subjects. Five novel and 3 known single nucleotide polymorphisms (SNPs) were identified. Four SNPs, representative of their linkage disequilibrium groups were further genotyped in 504 diabetic and in 681 non-diabetic subjects. 
Results: In non-diabetic subjects, 1 haplotype [C-G-T-T] was associated with significantly higher glucose infusion rate and lower waist-to-hip ratio (adjusted $P<0.05$ ). This haplotype also showed association with significantly lower mRNA levels in visceral adipose tissue in a subgroup of non-diabetic subjects with measurements of RBP4 mRNA expression in visceral and subcutaneous fat depots. In addition, three SNPs (rs3758358, rs3758539, rs10882283) were significantly associated with circulating free fatty acids (FFA) $(P<0.05)$, which was reflected in subsequent haplotype analyses, where 1 haplotype was significantly associated with FFA and 2 other haplotypes were associated with body mass index $(P<0.05)$. However, no association of T2DM with any of the genotyped SNPs or their haplotypes was found. Conclusions: Our data indicate a minor role of RBP4 genetic variation in susceptibility to insulin resistance, possibly through an effect on RBP4 expression.

\section{0}

Genetic polymorphisms explain variation in the ability to regulate fat oxidation

E. Corpeleijn ${ }^{1}$, L. Petersen ${ }^{2}$, T. Sørensen ${ }^{2}$, W. H. M. Saris ${ }^{1}$, O. Pedersen ${ }^{3}$, P. Froguel ${ }^{4}$, E. E. Blaak ${ }^{1}$; the NUGENOB consortium,

${ }^{1}$ Department of Human Biology, Nutrition and Toxicology Research Institute Maastricht (NUTRIM), Maastricht University, The Netherlands, ${ }^{2}$ Institute of Preventive Medicine, Danish Epidemiology Science Centre, Copenhagen University Hospital, Denmark,

${ }^{3}$ Steno Diabetes Center, Copenhagen, Denmark, ${ }^{4}$ CNRS UPRES A8090, Institut Biologie de Lille, Institut Pasteur de Lille, France.

Background and Aims: A low fasting fat oxidation and a disability to stimulate fat oxidation in response to a high fat diet are predisposing factors for obesityand type 2 diabetes. In the present study, the variation in fat oxidation rates was studied in relation to 42 common single nucleotide polymorphisms (SNP's) in 26 candidate genes in overweight subjects. The selected genes were associated with the regulation of lipid and glucose metabolism, regulation of adipocyte differentiation and function, production of adipocytokines, hypothalamic regulation of appetite and efficiency of energy expenditure.

Materials and Methods: In 722 overweight to obese subjects (541 women, $\mathrm{BMI}=35.8 \pm 4.8$, age $=36.6$ (range 20 to 51 ) and $181 \mathrm{men}, \mathrm{BMI}=34.9 \pm$ 4.8 , age $=38.7$ (range 21-51)) from 8 European centres, whole body energy expenditure and fat oxidation were measured with indirect calorimetry one and a half hours before and three hours after a high fat load containing 95 energy $\%$ of fat ( $60 \mathrm{E} \%$ saturated fat, energy content equals $50 \%$ of estimated resting energy expenditure). Fasting and postprandial fat oxidation were expressed as \% of average energy expenditure during fasting or postprandial, respectively. DNA was extracted from buffy coats and genotyped with the LightCycler assay, TaqMan assay or direct sequencing. Only statistically significant results $(\mathrm{P}<0.05)$ are presented. Results are explorative and are not corrected for multiple testing.

Results: After adjustment for centre and gender, a lower fasting fat oxidation was related to the $-178 \mathrm{~A}>\mathrm{C}$ SNP of CD36, a fatty acid transporter, and the T690S C>G SNP of protein convertase subtilisin/kexin type 1 (PCSK1), which plays a key role in regulating insulin biosynthesis and can cleave proopiomelanocortin. Fasting fat oxidation was higher in subjects with the $17 \mathrm{C}>\mathrm{G}$ SNP of sterol regulatory element binding transcription factor 1 (SREBF1/SREBP1), a gene regulator involved in cholesterol and fatty acid biosynthesis pathways, and the $1044 \mathrm{~A}>\mathrm{G}$ SNP of ectonucleotide pyrophasphatase/phosphodiesterase 1 (ENPP1), which may interact with insulin receptor beta-subunit autophosphorylation. The ability to increase fat oxidation after a high fat load was reduced in subjects with the $1431 \mathrm{C}>\mathrm{T} \mathrm{SNP}$ of peroxisome proliferative activated receptor gamma isoform 2 (PPARG2), an important gene in adipocyte function, and increased with the $-174 \mathrm{G}>\mathrm{C}$ SNP of IL-6, a pro-inflammatory cytokine associated with insulin resistance, and the $-829 \mathrm{a} A>\mathrm{G}$ SNP of WW domain containing adaptor with coiled-coil (WAC), involved in adipocyte function. Further adjustment for fat mass, HOMA index for insulin resistance and circulating free fatty acids did not change the outcome.

Conclusion: Variants of genes which are associated with fatty acid uptake, lipid storage, fatty acid oxidation and inflammation may explain part of variation in fasting fat oxidation (e.g. SREBF1, CD36, ENPP1, PCSK1, PGC$1 a$ ) as well as the ability to increase fat oxidation in response to a high fat load (e.g. IL-6, PPARG2, WAC) in human adiposity.

Supported by the European Community

\section{1}

FABP2 promoter polymorphism may exert its functional impact on glucose metabolism due to variability in GIP secretion

D. Rubin, U. Helwig, J. Schrezenmeir;

Institute of Physiology and Biochemistry of Nutrition, Federal Research Centre for Nutrition and Food, Kiel, Germany.

Background and Aims: The intestinal fatty acid-binding protein gene is proposed as a candidate gene for diabetes because the protein it codes is involved in fatty acid absorption and metabolism. Variation in the intestinal fatty acid binding protein (FABP-2) promoter is associated with an increase in postprandial triglycerides and a decrease in insulin sensitivity in the presence of the rare Thr54Thr exon polymorphism. This study investigated the functional impact of the FABP2 promoter haplotype and Ala54Thr exon polymorphism on the postprandial enterocrine response to a mixed meal to eluciate the underlying mechanisms for this association.

Materials and Methods: 167 men from the Metabolic Intervention Cohort Kiel (MICK) were genotyped for the FABP2 promoter haplotype and Thr54Ala exon polymorphism by the TaqMan method. Glucose dependent insulinotropic polypeptide (GIP), insulin, glucose and triglycerides were measured after ingestion of a standardized mixed meal.

Results: $28.5 \%$ of subjects were homozygous carriers of the rare FABP2 promoter haplotype $(n=37)$. Homozygous carriers of rare FABP2 promoter haplotype showed significantly lower postprandial GIP levels (AUC: 22.4 vs. $25.5 \mathrm{ng} / \mathrm{ml}, p=0.04)$, lower postprandial insulin sensitivity (HOMA) $(p=0.01)$ and higher insulin secretion (HOMA $\beta$-cell) $(p=0.02)$ when compared to carriers of the common FABP2 promoter haplotype. This effect was independent of the common A54T FABP2 exon polymorphism.

Conclusion: The rare FABP2 promoter haplotype may exert its deleterious effects on insulin sensitivity and triglyceride metabolism by decreased postprandial secretion of GIP.

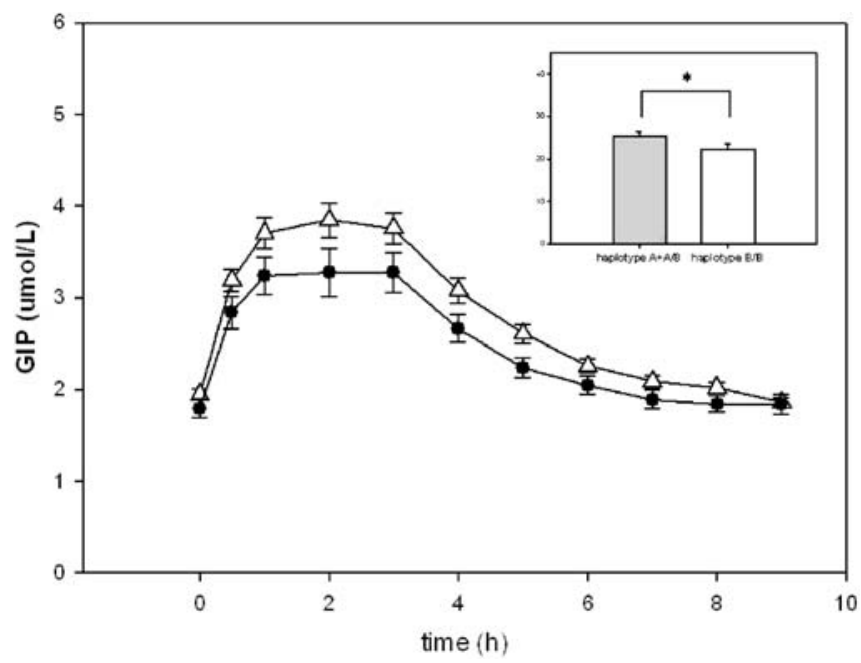

Supported by MN 0313437A

\section{2}

Low-density lipoprotein receptor-related protein (LRP5) C/T polymorphism in the exon 18 in relation to the beta cell secretion B. Bendlová ${ }^{1}$, M. Vankova ${ }^{1}$, P. Lukasova ${ }^{1}$, A. Zemanova ${ }^{1}$, K. Zajickova $^{2}$, D. Vejrazkova ${ }^{1}$, J. Vcelak ${ }^{1}$, K. Vondra ${ }^{2}$, J. Vrbikova ${ }^{2}$;

${ }^{1}$ Department of Molecular Endocrinology, ${ }^{2}$ Department of Clinical Endocrinology, Institute of Endocrinology, Praha 1, Czech Republic.

Background and Aims: Low-density lipoprotein receptor-related protein 5 (LRP5) acts as a membrane co-receptor in the Wnt signaling pathway and thus affects bone accrual and development. Data from knock-out mice have shown that LRP5 could play a role in the lipid and glucose metabolism. LRP5 might modulate the glucose-induced insulin secretion through the insulin signaling cascade and the hepatocyte nuclear factors regulation. Diabetes mellitus type 2 (DM2) as well as polycystic ovary syndrome (PCOS) are often 
accompanied by the defect in insulin secretion. The aim of the presented study is to compare the frequency of $\mathrm{C} / \mathrm{T}$ (c.4037: A1330V) polymorphism in the exon 18 of LRP5 gene in the sets of DM2 and PCOS patients, offspring of DM2 and healthy controls without the family history of DM2 and to evaluate beta-cell secretion and lipid levels of the study subjects in the relationship to the described polymorphism.

Materials and Methods: The study involved 1122 people. Detailed biochemical and anthropometric characteristics of 368 patients with DM2 (M/F 129/239; age 61.0 $\pm 7.6 / 59.0 \pm 8.7$ years; BMI 29.8 $\pm 4.4 / 31.8 \pm 5.6 \mathrm{~kg} /$ $\mathrm{m}^{2}$ ); 297 women with PCOS defined according to ESHRE consensus (age $27.1 \pm 6.6$ years; BMI $\left.26.7 \pm 6.5 \mathrm{~kg} / \mathrm{m}^{2}\right) ; 165$ offspring of DM2 (OD) (56/109; age $39.8 \pm 10.7 / 37.0 \pm 11.7$ years; BMI $\left.26.8 \pm 4.4 / 25.1 \pm 4.2 \mathrm{~kg} / \mathrm{m}^{2}\right)$ and 292 controls (C) (97/195; age 29.0 $\pm 8.8 / 29.3 \pm 10.2$ years; BMI $23.8 \pm 3.0 / 22.5 \pm 3.6$ $\mathrm{kg} / \mathrm{m}^{2}$ ) were collected. Oral glucose tolerance test (oGTT) with sampling for blood glucose, insulin, C-peptide and proinsulin in 0, 30, 60, 120, 150 and $180^{\text {th }}$ minute was done (except of DM2 patients). Total cholesterol, HDLcholesterol and triglycerides were determined in basal blood sample. The $\mathrm{C} /$ $\mathrm{T}$ polymorphism in the LRP5 gene was assessed by PCR with a mismatched primer introducing a restriction site for AdeI enzyme. Statistics was done using NCSS 2004 and Statgraphics (chi-square, Mann-Whitney, ANCOVA). Results: The genotypic distribution (CC/CT/TT) was not significantly different between DM2, PCOS, OD vs. C: DM2 - 72.8/23.6/3.5; PCOS - 80.1/18.5/1.4; OD - 76.9/21.2/1.8; C - 77.7/21.3/1.0 [\%] though higher frequency of $\mathrm{T}$ allele carriers in DM2 group was found (still NS). T allele carriers had higher levels of fasting and stimulated C-peptide and insulin in comparison with CC homozygotes, the significance was achieved in healthy controls without family history of DM2 (AUC - the area under the oGTT curve: AUC Cpeptide, $\mathrm{p}=0.004$; AUC insulin, $\mathrm{p}=0.02$ ). There was no difference in insulin sensitivity and lipid spectra among CC/CT/TT genotypes.

Conclusion: Our study suggests that the polymorphism C/T (A1330V) of the LRP5 gene is associated with higher fasting and stimulated insulin and C-peptide levels. LRP5 seems to be another candidate gene for the beta-cell secretion.

Supported by IGA MHCR NR/7809-5

\section{3}

Association between the ACE gene I/D polymorphism and insulin sensitivity in healthy subjects

F. Bonnet ${ }^{1}$, S. Patel ${ }^{2}$, I. M. Ibrahim ${ }^{2}$, M. Laville ${ }^{1}$, B. Balkau ${ }^{3}$, M. Walker ${ }^{2}$; ${ }^{1}$ Centre for Research in Human Nutrition. INSERM U449, Dept of Endocrinology, Lyon, France, ${ }^{2}$ Newcastle University, School of Clinical Medical Sciences, United Kingdom, ${ }^{3} \mathrm{U}$ 258, INSERM, Villejuif, France.

Background and Aims: Angiotensin-converting enzyme (ACE) is a key enzyme in the renin-angiotensin system (RAS) modulating the synthesis of angiotensin II and inactivation of bradykinin. The ACE gene has an insertion/ deletion (I/D) polymorphism, with the $\mathrm{D}$ allele being associated with higher ACE levels. Recent studies suggested that the blockade of RAS is associated with a reduced incidence of type 2 diabetes. However, data about the potential influence of the ACE I/D genotype on insulin resistance have been so far contrasting and have included a limited number of subjects and/or only used surrogate markers of insulin sensitivity. We investigated the relationship between the ACE I/D polymorphism and insulin sensitivity, as assessed by the euglycemic clamp, in a cohort of healthy subjects participating in the RISC study (Relationship between Insulin Sensitivity and cardiovascular disease study).

Materials and Methods: Healthy subjects aged 30-60 yrs were recruited at 19 centres in 14 European countries. Each subject underwent anthropometric and metabolic assessment, including a $75-\mathrm{g}$ oral glucose tolerance test (OGTT). Whole body insulin sensitivity (M/I value) was assessed by an hyperinsulinemic-euglycemic clamp and the ACE I/D polymorphism was genotyped using a PCR based method. Variables with a skewed distribution were log-transformed before analysis.

Results: The study cohort consists of 1286 subjects (579 men and 707 women) aged $43.8 \pm 8.3$ yrs, with a mean BMI of $25.5 \pm 4.0 \mathrm{~kg} / \mathrm{m} 2$. Age, BMI, waist, fat-free mass and physical activity did not differ by ACE genotype. Fasting glucose and insulin were similar among genotypes, but $2 \mathrm{~h}$ glucose levels were higher in DD than in ID and II subjects (DD:5.9 \pm 1.7 ; ID: $5.7 \pm 1.5$; II: $5.5 \pm 1.5 \mathrm{mmol} / \mathrm{l}, \mathrm{p}=0.002$ after adjustment for sex, age, centre, BMI and waist). Subjects with the DD genotype were more likely to have impaired glucose tolerance than those with the ID and II genotypes (13.1\% vs. $8.7 \%$, $\mathrm{p}=0.02)$. Insulin sensitivity was lower in subjects with the DD genotype than in those with the II genotype [135.7 $\pm 63.4 \mathrm{vs.} 147.4 \pm 65.1(\mu \mathrm{mol} / \mathrm{min} / \mathrm{kgFFM}) /$ $(\mathrm{nmol} / \mathrm{l}), \mathrm{p}=0.02]$. There were no associations between the ACE genotype and plasma adiponectin level nor the insulinogenic index from OGTT. In multiple regression, after correction for age, sex, centre, BMI and waist, the ACE I/D genotype was independently related to insulin sensitivity ( $M / I$ value) and to the $2 \mathrm{~h}$ glucose levels with increased insulin sensitivity and a lower $2 \mathrm{~h}$ plasma glucose level among those with the II genotype, as compared to those with the DD or I/D ACE genotype.

Conclusion: ACE I/D polymorphism is associated with whole body insulin sensitivity and with $2 \mathrm{~h}$ glucose levels in healthy subjects but is independent of circulating adiponectin levels. These findings confirm potential interactions between the renin-angiotensin system and glucose metabolism.

Supported by the European community

\section{4}

A meta-analysis of QTL for diabetes-related traits in rodents H. Al-Hasani ${ }^{1}$, C. Schmidtt ${ }^{1}$, N. P. Gonzaludo ${ }^{1}$, S. Dahm ${ }^{2}$, J. Schuchhardt ${ }^{3}$, F. Kleinjung ${ }^{3}$, S. Wuschke ${ }^{1}$, H.-G. Joost ${ }^{1}$;

${ }^{1}$ Pharmacology, German Institute of Human Nutrition, Nuthetal,

${ }^{2}$ Epidemiology, German Institute of Human Nutrition, Nuthetal, ${ }^{3}$ Bioinformatics, Microdiscovery GmbH, Berlin, Germany.

Background and Aims: Cross-breeding experiments with different mouse and rat strains have successfully been used by many groups to identify genetic loci that predispose for insulin resistance and diabetes. In order to provide a statistical assessment of these QTL as a basis for a systematic investigation of candidate genes, we have performed a meta-analysis of genome-wide linkage scans for diabetes-related component traits.

Materials and Methods: From a total of 44 published rodent cross-breeding experiments (30 for mouse, 14 for rat), we compiled a list of 170 QTL for blood glucose, insulin levels, and glucose tolerance. Collectively, these studies include data from 39 different parental mouse and rat strains and $>11,000$ individual animals. The peak markers for 134 mouse QTL and 36 rat QTL were mapped to the mouse chromosomes using NCBI's UniSTS and a ratmouse synteny database. The results of the studies were analysed using the truncated product method (TPM).

Results: The analysis revealed significant evidence (LOD score $>4.3$ ) for linkage of blood glucose, insulin levels, and glucose tolerance to 26 different segments on 15 different mouse chromosomes. Of these, 15 segments have also been found significantly linked with body weight and body fat. Conversely, 11 segments show linkage with diabetes but not with obesity-related traits, respectively, indicating the presence of distinct body-weight dependent and independent consensus regions for diabetes component traits. The most prominent regions with linkage for glucose, insulin, and glucose tolerance (LOD scores 10.5 - 17.4) on mouse chromosomes 2, 4, 7, 9, 11, 13, and 19 cover only about $10 \%$ of the mouse genome but contain $>30 \%$ of all known QTL for blood glucose, insulin levels, and glucose tolerance. On the other hand, for $>30 \%$ of the mouse genome no QTL were found to map within the designated region. For a systematic investigation of candidate genes, we performed an extensive analysis of the haplotype structure of the consensus QTL regions by integrating high-density SNP maps of multiple parental strains using a Hidden-Markov model. In addition to $>30$ previously known genes involved in insulin action, including DPP4 (dipeptidyl-peptidase 4), FOXA2 (forkhead box A2), SCD1 (stearoyl-Coenzyme A desaturase 1), STAT3 (signal transducer and activator of transcription 3), and TCF7L2 (transcription factor 7-like 2), we identified several novel candidate genes that may regulate blood glucose, insulin levels, and glucose tolerance.

Conclusion: Our results demonstrate the presence of numerous distinct consensus QTL regions with highly significant LOD scores that control glucose homeostasis. Our study may provide guidance for a further systematic investigation of candidate genes within the consensus QTL regions and presents a new resource for the identification of positional candidates in QTL studies.

Supported by EUGENE2, LSHM-CT-2004-512013 


\section{PS 7 Updates on the type 2 diabetes genes TCF7L2 and PPARgamma}

\section{5}

Variant of Transcription Factor 7-like 2 (TCF7L2) gene and the risk of posttransplant diabetes mellitus (PTDM) in renal allograft recipients E. Kang ${ }^{1,2}$, M. Kim ${ }^{3}$, Y. Kim ${ }^{3}$, K. Hur ${ }^{1}$, S. Han ${ }^{1}$, S. Chun ${ }^{1}$, C. Ahn ${ }^{1}$, B. Cha ${ }^{1}$, S. Kim ${ }^{3}$, H. Lee ${ }^{1}$;

${ }^{1}$ Internal Medicine, ${ }^{2}$ Brain Korea 21 for Medical Science,

${ }^{3}$ Surgery, Yonsei University College of Medicine, Seoul, Republic of Korea.

Background and Aims: Posttransplantation diabetes mellitus (PTDM) is a major complication after kidney transplantation. Defect in insulin secretion plays a pivotal role in the pathogenesis of PTDM. Transcription factor 7-like 2 gene (TCF7L2) polymorphism was reported to be associated with type 2 diabetes and possibly associated with insulin secretory defect. The aim of this study was to investigate the association between genetic variations in the TCF7L2 and PTDM in renal allograft recipients.

Materials and Methods: A total of 516 unrelated renal allograft recipients without previously known diabetes were enrolled. We genotyped six single nucleotide polymorphisms (SNPs) (rs11196205, rs4506565, rs12243326, rs7903146, rs12255372, and rs7901695) in the cohort consisting of 120 PTDM patients and 396 No-PTDM patients. The genotyping of TCF7L2 polymorphism was performed using real-time PCR.

Results: Rs4506565, rs7901695, and rs7903146 were in complete linkage. Rs7903146 genotype distribution was as follows: CC $94.4 \%$ and CT 5.6\%. Patients with CT genotype at rs7903146 was significantly associated with PTDM (odds ratio 2.477, 95\% CI: 1.148-5.346, p=0.017). Effect of genotype remains significant after multivariate logistic regression for PTDM (odds ratio $2.716,95 \%$ CI: $1.120-6.586, \mathrm{p}=0.011$ ).

Conclusion: These data suggest evidence that TCF7L2 rs7903146 genetic variation is associated with increased risk of PTDM in renal allograft recipients.

Supported by Korean Society of Endocrinology (2007)

\section{6}

Common variants of the TCF $7 L 2$ gene are associated with increased risk of type 2 diabetes in a UK-based South Asian population

S. Rees ${ }^{1}$, S. Bellary ${ }^{1}$, A. Britten ${ }^{1}$, A. Dixon ${ }^{1}$, P. O’Hare ${ }^{2}$, S. Kumar ${ }^{2}$,

A. Barnett ${ }^{1}$, A. Kelly ${ }^{1}$;

${ }^{1}$ Medicine, Birmingham University, ${ }^{2}$ Diabetes and Metabolism,

Warwick Medical School, Coventry, United Kingdom.

Background and Aims: The South Asian population is genetically heterogeneous, comprising of individuals originating from India, Pakistan and Bangladesh. The risk of type 2 diabetes (T2DM) in this ethnic group is high; almost 6-fold greater in UK-based South Asian populations than in the local white Caucasian population. Despite this, there have been relatively few genetic association studies carried out in South Asian populations. Recently, the transcription factor 7-like 2 (TCF7L2) gene has emerged as a forerunner in the search for T2DM susceptibility genes, with significant associations being reproduced in a large number of independent studies. Only two of these studies, however, have investigated populations of South Asian origin, and neither has studied a cohort originating from the north of the Indian subcontinent. In this study we investigated the association of common polymorphisms within the TCF7L2 gene with T2DM in a well characterized UK-based South Asian population of Punjabi origin.

Materials and Methods: Subjects with T2DM $(\mathrm{N}=831)$ and non-diabetic control subjects $(\mathrm{N}=436)$ of Punjabi ancestry were recruited as part of the United Kingdom Asian Diabetes Study (UKADS). We genotyped four SNPs (rs7901695, rs7903146, rs11196205 and rs12255372) that showed association with T2DM in a previous study by Grant $e$ t al. Genotyping was carried out using TaqMan SNP Genotyping assays. Alleles and genotypes were tested for association with T2DM using standard $\mathrm{X}^{2}$ analyses. Association between genotypes and continuous variables was tested using analysis of variance. The significance of the relationship between odds ratio (OR) and minimum age threshold was determined using linear regression. All statistical analyses were performed in SPSS 13.0.
Results: In agreement with previous studies, the minor allele of each SNP was significantly associated with T2DM. The strongest association was seen for the variant rs11196205, with an OR of 1.51 (95\% CI $1.17-1.93$, $\left.p=1.1 \times 10^{-3}\right)$. Genotypes of the variants studied were not associated with any clinical, biochemical or morphological characteristic in either cases or controls. The inclusion of young individuals in a control group can interfere with association tests, as these subjects may develop T2DM later in life. To examine this potential effect, we compared allelic ORs calculated using subsets of our control group defined by different minimum age thresholds. There was a highly significant, positive relationship between the control group age cut-off and the OR of the association test ( $\mathrm{p} \leq 6.3 \times 10^{-9}$ for all SNPs); an increase of minimum age from 35 to 47 years resulted in an average increase in OR of 0.18 .

Conclusion: In conclusion, our results add to the rapidly expanding body of evidence that implicates TCF7L2 as an important risk factor for T2DM in multiple ethnic groups. This study also highlights the importance of applying a minimum age threshold when recruiting control subjects from a population at high risk of disease. Including young subjects could significantly dilute the genetic effects observed and have implications for the power to detect an association, especially when investigating polymorphisms with lower ORs than TCF7L2.

\section{7}

Gestational diabetes is associated with a common variant of the transcription factor 7 like 2 (TCF7L2) gene

A. Katsarou, K. Lynch, N. Shaat, R. Håkansson, A. Nilsson, B. Lernmark, C.-D. Agardh, Å. Lernmark;

Department of Clinical Sciences/Malmö, Lund University, Malmö, Sweden.

Background and Aims: Recent studies have reported that variants in the transcription factor 7 like 2 gene confer risk for type 2 diabetes. The aim of the study was to investigate whether the rs7903146 variant of the TCF7L2 gene that confers risk for type 2 diabetes also confers risk for gestational diabetes or type 1 diabetes.

Materials and Methods: We genotyped the rs7903146 variant of the TCF7L2 in 883 mothers with gestational diabetes (GDM), 194 mothers with type 1 diabetes (T1D) and 1367 healthy controls using TaqMan allelic discrimination assay. The serum blood samples from all women were taken at the time of delivery and were collected as dried blood spots. GAD65, IA-2 autoantibodies and antibodies against insulin were determined in cord blood taken at delivery in standard radioligand binding assays.

Results: The T allele of the rs7903146 was positively associated with gestational diabetes (OR 1.4 [1.2-1.6], $\mathrm{p}<0.0001$ ). The heterozygous CT-genotype and the homozygous TT-genotype carriers had an 1.4-fold increased risk (95\%CI $1.2-1.6, \mathrm{p}=0.0003)$ and an 1.9-fold increased risk (95\%CI $1.4-2.6 \mathrm{p}<0.0001)$ respectively, compared to the wild type CC-genotype carriers in GDM women. No significant difference was found between the three genotype frequencies of the rs7903146 in women with type 1 diabetes. Approximately $61 \%$ and $6 \%$ of children born to T1D and GDM mothers, respectively, were positive for autoantibodies against GAD65, IA-2 and antibodies against insulin in the cord blood. The association between TCF7L2 and gestational diabetes remained statistically significant even after adjusting for age, gestational week at birth and parity and after excluding the cord blood antibody positive GDM women.

Conclusions: The rs790346 variant of the TCF7L2 gene is associated with an increased risk for gestational diabetes but not for type 1 diabetes. The same variant has been shown to confer risk for type 2 diabetes in several studies. Gestational diabetes is defined by a similar pathogenetic background with type 2 diabetes, since it is characterized mainly by insulin resistance. It is, therefore, possible that these two diseases share also a common genetic background.

Supported by the Swedish Research Council (14064) and the National Institutes of Health (DK26190) 


\section{8}

Expression of the transcription factor 7-like 2 (TCF7L2) gene in adipocytes is down regulated by insulin both in vitro and in vivo M. Ahlzén, L. Johansson, C. Cervin, M. Ridderstråle; Clinical Sciences - Diabetes and Endocrinology, Lund University, Malmö, Sweden.

Background and Aims: Variants in the gene coding transcription factor 7 like 2 (TCF7L2, former TCF-4) have recently shown strong association to type 2 diabetes. Two risk haplotypes have been defined, HapA was associated with increased body mass index (BMI) and $\mathrm{HapB}_{\mathrm{T} 2 \mathrm{D}}$ was associated with decreased BMI. TCF7L2 may play a role in both glucose homeostasis and adipogenesis, thus suggesting a connection between expression of TCF7L2 variants and BMI. Our aim was to characterize the TCF7L2 mRNA expression and the regulation of the expression in adipose tissue.

Materials and Methods:We quantified TCF7L2 mRNA levels in cultured human adipocytes incubated with glucose, palmitate or oleate with or without insulin, and in biopsies from visceral (VAT) and subcutaneous (SAT) adipose tissue of 38 obese non-diabetic subjects using Real Time PCR. Two variants of TCF7L2, rs10885406 and rs7903146 (representing HapA and HapB respectively) were genotyped. The influence of haplotype and different clinical traits on expression levels were investigated.

Results: In vitro insulin decreased the TCF7L2 mRNA expression in a nonglucose dependent fashion by approximately two thirds $(p<0.05)$. When adipocytes were incubated with free fatty acids, the inhibitory effect of insulin was diminished by half with palmitate $(p=0.064)$ and by a third with oleate $(p=0.027)$. In the biopsy study we found no correlation between TCF7L2 mRNA expression and measures of obesity, except for a weak trend between SAT TCF7L2 expression and homeostasis model assessment for insulin resistance ([HOMA] $\mathrm{r}=0.40, p=0.058)$. TCF7L2 expression levels were higher in VAT compared to SAT $(p=0.00029)$. Similar results were found when restricting analysis to HapA carriers $(\mathrm{n}=28, p=0.00038)$ while non-HapA carriers showed no difference in TCF7L2 expression between VAT and SAT $(\mathrm{n}=5, p=0.88)$.

Conclusion: TCF7L2 expression is inhibited by insulin and seems to increase in subjects that are insulin resistant and in those carrying the HapA haplotype. These findings further emphasize the role of TCF7L2 in both glucose homeostasis and adipogenesis.

\section{9}

The Pro12Ala PPARgamma-2 polymorphism is associated with insulin resistance, but not with adiposity or dislipidaemia in a non-diabetic population from Gran Canaria

A. M. Wägner ${ }^{1,2}$, M. Boronat ${ }^{1}$, F. Varillas ${ }^{3}$, J. C. Rodríguez ${ }^{4}$, A. Caballero ${ }^{4}$, F. J. Nóvoa ${ }^{1}$;

${ }^{1}$ Endocrinology, Hospital Universitario Insular de Gran Canaria, Las Palmas de GC, Spain, ${ }^{2}$ Endocrinology, Steno Diabetes Center, Gentofte, Denmark, ${ }^{3}$ Endocrinology, Hospital General de Fuerteventura, Puerto del Rosario, Spain, ${ }^{4}$ Research Unit, Hospital Dr Negrín, Las Palmas de GC, Spain.

Background and Aim: The Pro12Ala polymorphism in the peroxisome proliferator-activated receptor (PPAR) gamma-2 gene is associated with type 2 diabetes and insulin resistance. However, results on its effects on obesity and dislipidaemia are inconsistent. The aim of this study was to assess the effect of the Pro12Ala polymorphism on insulin resistance, adiposity and dyslipidaemia in a population-based sample.

Patients and Methods: For this cross-sectional, population-based study, the Pro12Ala polymorphism of the PPARgamma-2 gene was genotyped in a sample of 907 people (583 with normal glucose tolerance (NGT), 205 with impaired glucose regulation (IGR, including impaired glucose tolerance and impaired fasting glucose) and 119 with type 2 diabetes (DM)) from the city of Telde (Gran Canaria, Canary Islands, Spain). Due to the low frequency of 12Ala homozygotes $(\mathrm{N}=5)$, the latter were pooled with heterozygotes for analysis. Anthropometric parameters included BMI and waist and hip girth; total body fat was estimated by bio-impedance; insulin resistance was estimated using the homeostasis model of assessment (HOMA); triglycerides, total cholesterol and HDL cholesterol were measured using enzymatic methods. Comparison between groups was performed using chi-square for frequency comparisons, and Student's $\mathrm{t}$ and Mann-Whitney's $\mathrm{U}$ tests for quantitative variables.
Results: $12 \%$ of NGT, $7.8 \%$ of IGR and $10.9 \%$ of DM were carriers of the 12Ala allele (ns). Among the non-diabetic subjects, Ala carriers showed less insulin resistance (HOMA of $1.30(0.32-6.70)$ vs $1.57(0.04-12.16), \mathrm{p}=$ 0.017 ), but similar triglycerides, HDL cholesterol, BMI ( $27.2 \pm 4.3$ vs $28.1 \pm 5.0$ $\mathrm{Kg} / \mathrm{m} 2)$, waist girth $(94.1 \pm 12.3$ vs $95.7 \pm 13.4 \mathrm{~cm})$, waist/hip ratio $(0.93 \pm 0.08$ vs $0.92 \pm 0.08)$ and total body fat $(29.4(12.8-44.6)$ vs $31.1(7.2-56.4) \%)$.

Conclusions: In this Canarian, population-based study, the Pro12Ala PPARgamma-2 polymorphism is associated with insulin resistance, but not with adiposity or dislipidaemia.

\section{0}

Association of SNPs in the PPARG gene with the development of type 2 diabetes and their interaction with physical activity. The Finnish Diabetes Prevention Study

T. O. Kilpeläinen ${ }^{1}$, T. A. Lakka ${ }^{1,2}$, D. E. Laaksonen ${ }^{1,3}$, L. Jaana ${ }^{4}$,

J. G. Eriksson ${ }^{4,5}$, T. T. Valle ${ }^{4}$, H. Hämäläinen ${ }^{6}$, P. Ilanne-Parikka ${ }^{7,8}$,

S. Keinänen-Kiukaanniemi ${ }^{9,10}$, V. Lindi ${ }^{11}$, J. Tuomilehto ${ }^{4,5}$, M. Uusitupa ${ }^{11}$, M. Laakso ${ }^{3}$;

${ }^{1}$ Institute of Biomedicine, Physiology, University of Kuopio,

${ }^{2}$ Kuopio Research Institute of Exercise Medicine, ${ }^{3}$ Department of Medicine, Kuopio University Hospital, ${ }^{4}$ Department of Health Promotion and Chronic Disease Prevention, Diabetes Unit, National Public Health Institute, Helsinki, ${ }^{5}$ Department of Public Health, University of Helsinki, ${ }^{6}$ Research Department, Social Insurance Institution, Turku, ${ }^{7}$ Finnish Diabetes Association, Tampere, ${ }^{8}$ Research Unit, Tampere University Hospital, ${ }^{9}$ Department of Public Health Science and General Practice, Unit of General Practice, Oulu University Hospital, ${ }^{10}$ Department of Sports Medicine, Oulu Deaconess Institute, ${ }^{11}$ School of Public Health and Clinical Nutrition, Clinical Nutrition, University of Kuopio, Finland.

Background and Aims: The Ala12 allele of the Pro12Ala polymorphism in the peroxisome proliferator-activated receptor gamma (PPARG) gene was associated with an increased risk of type 2 diabetes (T2D) in the Finnish Diabetes Prevention Study (DPS). However, most other studies indicate that the Ala12 is protective against T2D. Furthermore, some data suggest that the association of the Pro12Ala polymorphism on glucose and insulin metabolism may be altered by physical activity. We studied the associations of seven single nucleotide polymorphisms (SNPs) in the peroxisome proliferator-activated receptor gamma $(P P A R G)$ gene with the conversion from impaired glucose tolerance (IGT) to type 2 diabetes (T2D), and the interactions of the SNPs with physical activity.

Materials and Methods: Overweight individuals with IGT who participated in the Finnish Diabetes Prevention Study (DPS) $(n=479)$ were followed on average 4.2 years. Physical activity was assessed yearly with a 12-month validated questionnaire.

Results: In Cox regression analyses, the rare alleles of rs17036314 and rs1801282 (Pro12Ala) predicted conversion to T2D ( $p=0.038$ and $p=0.037$, respectively), but only rs17036314 predicted T2D after adjustment for baseline fasting glucose $(p=0.030)$. The change in the total amount of physical activity, stratified by median, modified the association of rs17036314 and rs1801282 (Pro12Ala) with the risk of T2D during the intervention ( $p=0.002$ and $p=0.033$, respectively, for interaction between physical activity change and genotype); an increase in physical activity seemed to remove the effect of the risk alleles. The distinct rs1152003 polymorphism interacted with the study group on the conversion to T2D ( $p=0.027)$, and tended to increase the risk of T2D in the intervention group $(p=0.050)$. No interaction between rs1152003 and the change in physical activity was found.

Conclusion: The rs17036314, rs1801282 (Pro12Ala) and rs1152003 were associated with the risk of T2D in the DPS. Increased physical activity seemed to decrease the effect the of risk alleles of rs17036314 and rs1801282 on the conversion to T2D. The effect of rs1152003 was modified by other lifestyle changes or the lifestyle intervention as a whole.

Supported by the Ministry of Education of Finland and the Academy of Finland 


\section{1}

Is the Pro12Ala polymorphism of the peroxisome proliferator-activated receptor gamma gene associated with type 2 diabetes? A meta-analysis I. Vardarli;

Johannes Gutenberg Universität, Mainz, Germany.

Background and Aims: Type 2 diabetes (T2DM) is one of the major public health problems. Many candidate genes for T2DM were investigated. Conflicting findings have been published concerning the asscociation of the Pro12Ala polymorphism of the peroxisome proliferator- activated receptor gamma (PPAR $\gamma$ ) gene with type 2 diabetes. To give an update on this issue a meta-analysis was performed in this study.

Materials and Methods: Electronic databases (PubMed, EMBASE, Cochrane Library) were searched (last search February 3rd, 2007) for studies and reviews dealing with the Pro12Ala polymorphism of the PPAR $\gamma$ gene and T2DM using a systematic strategy. Reference lists of all identified studies were also searched. Efforts were made to identify non published studies. During meetings scientists investigating this issue were contacted and programs and presentations were screened. Additionally, the scientific sessions abstracts archive (2003-2006) of the American Diabetes Association (ADA) was searched. Studies were selected following a priori defined inclusion criteria. Identified data were systematically collected and assessed. Quality of the studies were evaluated using a quality score. Pooled estimates were obtained using the DerSimonian and Laird random effects model. Estimates are given as odds ratio (OR) for the Ala allele (Ala/Ala + Pro/Ala vs. Pro/Pro), and for the Pro allele (Pro/Pro + Pro/Ala vs. Ala/Ala) with the $95 \%$ confidence interval (CI). For the estimation of heterogeneity chi-square test was used. Publication bias was tested by using funnel plot, the Begg's and Egger's methods. To explain the heterogeneity, subgroup analyses (e.g. for body mass index (BMI) in cases and definition of controls by oral glucose tolerance test (OGTT)) and meta-regression were performed. For the statistical analyses RevMan 4.2.8, Stata 9 and PS (power calculation) software were used.

Results: 405 studies were identified, of those 31 underpowered case-control studies with 33 datasets with a total of 29,284 subjects $(14,771$ cases, 14,513 controls) with various ethnic groups investigating the genotype distribution of the Pro12Ala polymorphism of the PPAR $\gamma$ gene in patients with T2DM and non-diabetic controls (datasets with subjects declared to be exclusively with impaired glucose tolerance (IGT) or impaired fasting glucose (IFG) were excluded) fulfilled the inclusion criteria and were selected for inclusion in this study. There is a significant heterogeneity $(\mathrm{P}<0.00001)$ between the studies. The random effects meta-analysis showed a significant association between the Ala allele and T2DM (OR 0.86; 95\% CI 0.75-0.97; $\mathrm{P}=0.02)$. There was no evidence for publication bias. In subgroup analyses OR for the Ala allele was lower for mean $\mathrm{BMI}<30 \mathrm{~kg} / \mathrm{m}^{2}$ in cases. The meta-regression showed that mean BMI $(<30 / \geq 30)$ of cases $(\mathrm{P}=0.004)$ and definition of controls by OGTT $(\mathrm{P}=0.032)$ are effect modifiers which affect the pooled estimate of OR for the Ala allele. Genotype information for Ala/Ala were available for 21 of 33 datasets. In those, for the Pro allele the random effects model showed a significant association with T2DM (OR 1.52; CI 1.17-1.98; $\mathrm{P}=0.002$; test for heterogeneity: $\mathrm{P}=0.59$ ).

Conclusion: There is an association between the Pro12Ala polymorphism of the PPAR $\gamma$ gene and T2DM. BMI in cases and definition of controls by OGTT are effect modifiers. Sufficiently powered studies on this issue should be stratified by individual BMI of cases, and non-diabetic controls should be defined by OGTT.

\section{PS 8 Epidemiology: treatment}

\section{2}

Results of the International Diabetes Management Practices Study (IDMPS), year 2: pattern of care in patients with type 1 diabetes

J. C. N. Chan; on behalf of the IDMPS Group,

Department of Medicine and Therapeutics, The Chinese University of Hong Kong, Shatin, Hong Kong.

Background and Aims: Despite the findings of the DCCT, comparing intensive to conventional treatment, and the recommendations of the ADA, EASD and IDF, a large number of patients with diabetes are not well controlled, emphasizing the need for implementation of measures designed to improve the quality of diabetes care. In this regard, our aim was to gather data on current practices in the management of patients with diabetes worldwide and to evaluate the degree of compliance with international guidelines.

Material and Methods: The IDMPS is an international, multicenter, observational study performed in 27 countries within Africa, Asia, Eastern Europe, the Middle East and Latin America. Data are being collected from patients with Type 1 and Type 2 diabetes ( $\geq 18$ years), seen in current medical practice in 1-year cycles (a 2-week cross-sectional recruitment period, followed by a 9-month longitudinal period for Type 2 patients) for 5 years. Here, we report results for patients with Type 1 diabetes collected during the second cross-sectional period (Year 2) in 8 countries. In addition to monitoring $\mathrm{HbA}_{1 \mathrm{c}}$ and $\mathrm{FBG}$, information about insulin self-injection, selftitration, diabetes education and frequency of visits to their physician was collected. Data were analyzed using standard descriptive methods.

Results: 940 patients with Type 1 diabetes (50.5\% men; mean age: $34.1 \pm 12.6$ years) were recruited during November and December 2006. The mean BMI was $24.0 \pm 3.9 \mathrm{~kg} / \mathrm{m}^{2}$ for men and $24.0 \pm 4.8 \mathrm{~kg} / \mathrm{m}^{2}$ for women. In the past 3 months, patients had visited a specialist in diabetes $1.3 \pm 1.4$ (median: 1.0) times and a GP 1.0 \pm 1.5 (median: 0; range: $0-12$ ) times. Diabetes education was provided by diabetes educators to $52.2 \%$ of patients, $2.3 \pm 2.1$ times (median: 2.0) within the last 12 months. Overall, $86.8 \%$ of patients had $\mathrm{HbA}_{1 \mathrm{c}}$ tested at least once with a mean of $2.0 \pm 1.1$ (median: 2.0) tests within the past year; the mean time elapsed since last $\mathrm{HbA}_{1 \mathrm{c}}$ measurement was $3.8 \pm 7.7$ (median: 1.0) months. The mean last available $\mathrm{HbA}$ was $8.3 \pm 2.0 \%$. $24.4 \%$ of patients had an $\mathrm{HbA}_{1 \mathrm{c}}<7 \%, 27.6 \%$ between $7 \%$ and $8 \%$, and $48 \%>8 \%$. Self-injection of insulin was reported in $93.5 \%$ of patients and self-titration in $66.2 \% .28 .7 \%$ of patients were considered to be on target according to the physician, and among these the mean $\mathrm{HbA}_{1}$ was $7.0 \pm 1.6 \%$. Laboratory testing for FBG was performed 5.8 10.4 (median: 3.0) times/year. The mean last available FBG (laboratory) was $163.0 \pm 78.6 \mathrm{mg} / \mathrm{dL}$ and $17.2 \%$ had a FBG $\leq 100 \mathrm{mg} / \mathrm{dL} ; 75.4 \%$ of patients had a glucometer at home, and $76.7 \%$ selfmonitored their blood glucose (SMBG). FBG was monitored by SMBG 15.9 \pm 15.1 (median: 10.0) times/month and post-prandial glucose $12.8 \pm 15.8$ (median: 7.0) times/month. The use of SMBG was associated with a higher number of patients reaching target according to the investigator $(84.6 \% v s$. $15.4 \%)$. SMBG and the investigator's statement that the patient had reached target were associated with a better glycemic control in terms of laboratory $\mathrm{HbA}_{1 \mathrm{c}}$ and $\mathrm{FBG}$ values.

Conclusion: Our data show that patients with Type 1 diabetes are poorly controlled (in terms of $\mathrm{HbA}_{1 \mathrm{c}}$ and $\mathrm{FBG}$ ), despite being monitored regularly for their condition. This suggests that improvement of education and commitment of both patients and their providers is necessary to achieve a more interactive and effective care provision to these patients.

Supported by an unrestricted grant from sanofi-aventis

\section{3}

Results of the International Diabetes Management Practices Study (IDMPS), year 2: pattern of care in patients with type 2 diabetes H. Ilkova;

Department of Internal Medicine, Division of Endocrinology, Istanbul University Cerrahpasa Medical Faculty, Istanbul, Turkey.

Background and Aims: Despite the recommendations of the ADA, EASD and IDF, a large number of patients with diabetes are not well controlled, emphasizing the need for implementation of measures designed to improve the quality of diabetes care. In this regard, our aim was to gather data on 
current practices in the management of patients with diabetes worldwide and to evaluate the degree of compliance with international guidelines.

Material and Methods: The IDMPS is an international, multicenter, observational study performed in 27 countries within Africa, Asia, Eastern Europe, Middle East and Latin America. Data are being collected from patients with Type 1 and Type 2 diabetes ( $\geq 18$ years), seen in current medical practice in 1-year cycles (a 2-week cross-sectional recruitment period, followed by a 9-month longitudinal period for Type 2 patients) for 5 years. Here, we report results for patients with Type 2 diabetes collected during the second cross-sectional period (Year 2 ) in 8 countries. To measure the degree of glucose metabolism control, $\mathrm{HbA}_{1 c}$ and fasting blood glucose (FBG) values were collected. Data were analyzed using standard descriptive methods.

Results: 4312 patients with Type 2 diabetes ( $47.8 \%$ men; mean age: $58.3 \pm 11.2$ years), were recruited during November and December 2006. The mean BMI was $27.4 \pm 4.4 \mathrm{~kg} / \mathrm{m}^{2}$ for men and $28.4 \pm 5.6 \mathrm{~kg} / \mathrm{m}^{2}$ for women. In the past 3 months, patients had visited a specialist in diabetes $1.5 \pm 1.7$ (median: 1.0) times and a GP $0.9 \pm 1.5$ (median: 0; range: $0-20$ ) times. In $23.6 \%$ of patients, $\mathrm{HbA}_{1 \mathrm{c}}$ was never tested; $76.4 \%$ of patients had $\mathrm{HbA}_{1 c}$ tested at least once with a mean of $2.0 \pm 1.2$ (median: 2.0 ) tests within the past year; the mean time elapsed since the last $\mathrm{HbA}_{1 \mathrm{c}}$ measurement was $2.8 \pm 5.5$ (median: 1.0) months. The mean last available $\mathrm{HbA}$ was $8.0 \pm 2.0 \%$. $32.7 \%$ of patients had an $\mathrm{HbA}_{1 c}<7 \%, 26.5 \%$ between $7 \%$ and $8 \%$, and $40.8>8 \%$. Laboratory testing for FBG was performed $5.3 \pm 7.3$ (median: 4.0) times/year. Mean FBG (laboratory) was $158.8 \pm 64.0 \mathrm{mg} /$ $\mathrm{dL}$ and $12.7 \%$ had a FBG $\leq 100 \mathrm{mg} / \mathrm{dL}$. Among patients receiving insulin (alone or in combination with oral glucose lowering drugs (OGLD)), $67.0 \%$ had a glucometer at home, $68.6 \%$ self-monitored their blood glucose (SMBG), and FBG was being monitored by SMBG $11.3+10.7$ (median:8.0) times/month and post-prandial glucose $7.9 \pm 9.3$ (median: 4.0 ) times/month. $37.3 \%$ of patients were considered to be on target according to the physician's opinion; among these $79.9 \%$ were tested and had a mean $\mathrm{HbA}_{1 c}$ of $6.7 \pm 1.3 \%$.

Conclusion: Our data show that $1 / 3$ of patients with Type 2 diabetes are controlled (in terms of $\mathrm{HbA}_{1 \mathrm{c}}$ ) as shown in DECODE and NHANES studies. This highlights that a more interactive and effective care provision has to be actively pursued to ameliorate the glycemic control of patients with Type 2 diabetes.

Supported by an unrestricted grant from sanofi-aventis

\section{4}

Results of the International Diabetes Management Practices Study (IDMPS), year 2: compliance with international guidelines of the management of patients with type 2 diabetes

J. J. Gagliardino;

Faculty of Medical Sciences, CENEXA (UNLP-CONICET), La Plata, Argentina.

Background and Aims: Despite the recommendations of the ADA, EASD and IDF, a large number of people with diabetes are not well controlled. In this regard, our aim was to gather data on current practices in management of people with diabetes worldwide and to evaluate the degree of compliance with international guidelines.

Material and Methods: The IDMPS is an international, multicenter, observational study performed in 27 countries within Africa, Asia, Eastern Europe, the Middle East and Latin America. Data are being collected from people with Type 1 and Type 2 diabetes ( $\geq 18$ years), seen in current medical practice in 1-year cycles (a 2-week cross-sectional recruitment period, followed by a 9-month longitudinal period for Type 2 patients) for 5 years. Here, we report results for people with Type 2 diabetes collected during the second cross-sectional period (Year 2) in 8 countries. In addition to measuring the degree of glucose metabolism control, information about patient education, care delivery and monitoring were collected. Data were analyzed using standard descriptive methods.

Results: 4312 people with Type 2 diabetes ( $48 \%$ men; mean age: $58.3 \pm 11.2$ years), were recruited during November and December 2006. The mean BMI was $27.4 \pm 4.4 \mathrm{~kg} / \mathrm{m}^{2}$ for men and $28.4 \pm 5.6 \mathrm{~kg} / \mathrm{m}^{2}$ for women. In the past 3 months, patients had visited a specialist in diabetes $1.5+1.7$ (median: 1.0) times and a GP $0.9 \pm 1.5$ (median: 0; range: $0-20$ ) times. Diabetes education was provided by diabetes educators to $35 \%$ of patients ( $49 \%$ of insulin-treated patients), $2.9 \pm 4.1$ (median: 2.0) times within the last 12 months, and was individual in $72.1 \%$ of patients being trained. Only $76 \%$ of patients had $\mathrm{HbA}_{1}$ tested at least once with a mean of $2.0 \pm 1.2$ (median: 2.0) tests within the past year. The mean $\mathrm{HbA}_{1 c}$ was $8.0 \pm 2.0 \%$. Moreover, only $33 \%$ of patients had an
$\mathrm{HbA}_{1 \mathrm{c}}<7 \%$. Laboratory testing for FBG was performed 5.3 \pm 7.3 (median: 4.0) times/year. The mean FBG (laboratory) was $158.8 \pm 64.0 \mathrm{mg} / \mathrm{dL}$ and only $13 \%$ of patients had a FBG $\leq 100 \mathrm{mg} / \mathrm{dL}$. Among patients receiving insulin (alone or in combination with oral glucose lowering drugs (OGLD)), $67 \%$ had a glucometer at home and $69 \%$ self-monitored their blood glucose (SMBG); FBG was monitored by SMBG $11.3 \pm 10.7$ (median: 8.0 ) times/month and post-prandial glucose 7.9 \pm 9.3 (median: 4.0 ) times/month. Target treatment levels were achieved for blood pressure in $20 \%$ of patients, for LDL $(<100 \mathrm{mg}$ / $\mathrm{dL})$ in $33 \%$, for $\mathrm{HDL}(>40 \mathrm{mg} / \mathrm{dL})$ in $62 \%$, and for triglycerides $(<150 \mathrm{mg} /$ $\mathrm{dL}$ ) in $54 \%$. The percentage of patients screened for diabetes complications is given in the Table.

Table. Frequency of screening for diabetes complications.

\begin{tabular}{lll}
\hline & $\begin{array}{l}\text { Screened at least once } \\
\text { within the past year }\end{array}$ & Never tested \\
\hline Lipid abnormalities & $61 \%$ & $15 \%$ \\
Cardiovascular disease & $51 \%$ & $21 \%$ \\
Microalbuminuria & $47 \%$ & $33 \%$ \\
Retinopathy & $47 \%$ & $29 \%$ \\
Neuropathy & $47 \%$ & $36 . \%$ \\
Diabetic foot ulcer & $46 \%$ & $38 \%$ \\
\hline
\end{tabular}

Conclusion: Our data show that the current management practices of people with Type 2 diabetes poorly complies with most international guidelines. This suggests that improvement of education and commitment of both patients and their care providers is necessary to achieve a more interactive and effective care provision to these patients.

Supported by an unrestricted grant from sanofi-aventis

\section{5}

Results of the International Diabetes Management Practices Study (IDMPS), year 2: baseline characteristics and treatment regimens in patients with type 1 diabetes

P. Aschner; on behalf of the IDMPS group,

Oficina de Investigaciones, Universidad Javeriana, Bogota, Colombia.

Background and Aims: Despite the findings of the DCCT, comparing intensive to conventional treatment, and the recommendations of the ADA, EASD and IDF, a large number of patients with diabetes are not well controlled, emphasizing the need for implementation of measures designed to improve the quality of diabetes care. In this regard, our aim was to gather data on current practices in the management of patients with diabetes worldwide and to evaluate the degree of compliance with international guidelines.

Material and Methods: The IDMPS is an international, multicenter, observational study performed in 27 countries within Africa, Asia, Eastern Europe, the Middle East and Latin America. Data are being collected from patients with Type 1 and Type 2 diabetes ( $\geq 18$ years), seen in current medical practice in 1-year cycles (a 2-week cross-sectional recruitment period, followed by a 9-month longitudinal period for Type 2 patients) for 5 years. Here, we report results for patients with Type 1 diabetes collected during the second cross-sectional period (Year 2) in 8 countries. Data were analyzed using standard descriptive methods.

Results: Overall, 940 patients $(50.5 \%$ men) with Type 1 diabetes were recruited during November and December 2006. The mean age was $34.1 \pm 12.6$ years, mean BMI $24.0 \pm 3.9 \mathrm{~kg} / \mathrm{m}^{2}$ for men and $24.0 \pm 4.8 \mathrm{~kg} / \mathrm{m}^{2}$ for women, and mean disease duration $11.8 \pm 9.0$ years. The mean last available $\mathrm{HbA}_{1}$ was $8.3 \pm 2.0 \% ; 24.4 \%$ of the patients had an $\mathrm{HbA}_{1 c}<7 \%$. Mean last available FBG (laboratory) was $163.0 \pm 78.6 \mathrm{mg} / \mathrm{dL}$. The mean systolic/diastolic blood pressure was 120/74 mmHg. Target treatment levels were achieved for LDL $(<100 \mathrm{mg} / \mathrm{dL})$ in $43.3 \%$ of patients, for HDL $(>40 \mathrm{mg} / \mathrm{dL})$ in $75.8 \%$, and for triglyceride $(<150 \mathrm{mg} / \mathrm{dL})$ in $81.0 \%$ of patients. The mean time since start of insulin therapy was $10.8+8.5$ years and the mean daily dose was $0.75+0.30$ $\mathrm{U} / \mathrm{kg}$. A basal + prandial insulin regime was used in $56.1 \%$ of patients with a mean daily dose of $0.82 \pm 0.29 \mathrm{U} / \mathrm{kg}(0.39 \pm 0.21 \mathrm{U} / \mathrm{kg}$ for basal insulin and $0.43 \pm 0.21 \mathrm{U} / \mathrm{kg}$ for prandial insulin); mean $\mathrm{HbA}_{1 \mathrm{c}}$ was $8.3 \pm 2.0 \%$. Premixed insulin alone was used in $22.3 \%$ of patients with a mean daily dose of $0.68 \pm 0.27$ $\mathrm{U} / \mathrm{kg}$; mean $\mathrm{HbA}_{1 \mathrm{c}}$ was $8.3 \pm 2.1 \%$. Only $14.0 \%$ of patients were using basal insulin alone (mean daily dose: $0.53 \pm 0.28 \mathrm{U} / \mathrm{kg}$; mean $\mathrm{HbA}_{1}: 8.5 \pm 2.1 \%$ ) and 
$0.7 \%$ prandial insulin alone (mean daily dose $0.49 \pm 0.19 \mathrm{U} / \mathrm{kg}$; mean $\mathrm{HbA}_{1 \mathrm{c}}$ : $7.9 \pm 1.4 \%)$. Among patients with $\mathrm{HbA}_{1 c}<7 \%, 9.4 \%$ were treated with basal insulin alone, $61.3 \%$ with basal+prandial insulin, $0.6 \%$ with prandial insulin alone and $23.2 \%$ with premixed insulin alone.

Conclusion: The analysis of data from the second cross-sectional period of the IDMPS (Year 2), shows that the majority of Type 1 patients analyzed did not achieve the recommended treatment goals for $\mathrm{HbA}_{1 \mathrm{c}}$ and FBG. Thus, measures designed to improve these results, probably related to overall education of patients and members of the health care team, are urgently needed to reduce the current and future increases in the demand for care and the costs resulting from this poor diabetes control worldwide. Supported by an unrestricted grant from sanofi-aventis

\section{6}

Results of the International Diabetes Management Practices Study (IDMPS), year 2: baseline characteristics and treatment regimens in patients with type 2 diabetes

S. H. Baik;

Division of Endocrinology, Department of Internal Medicine, Korea University Guro Hospital, Seoul, Republic of Korea.

Background and Aims: Despite the evidence provided by the UKPDS and the recommendations of the ADA, EASD and IDF, a large number of patients with diabetes are not well controlled. In this regard, our aim was to gather data on current practices in the management of patients with diabetes worldwide and to evaluate the degree of compliance with international guidelines.

Material and Methods: The IDMPS is an international, multicenter, observational study performed in 27 countries within Africa, Asia, Eastern Europe, the Middle East and Latin America. Data are being collected from Type 1 and Type 2 diabetic patients ( $\geq 18$ years) seen in current medical practice in 1-year cycles (a 2 -week cross-sectional recruitment period, followed by a 9-month longitudinal period for Type 2 patients) for 5 years. Here, we report results for patients with Type 2 diabetes collected during the second cross-sectional period (Year 2) in 8 countries. Data were analyzed using standard descriptive methods.

Results: Overall, 4312 patients with Type 2 diabetes ( $47.8 \%$ men), were recruited during November and December 2006. The mean age was $58.3 \pm 11.2$ years, mean BMI $27.4 \pm 4.4 \mathrm{~kg} / \mathrm{m}^{2}$ for men and $28.4 \pm 5.6 \mathrm{~kg} / \mathrm{m}^{2}$ for women and mean disease duration $8.3 \pm 7.5$ years. The mean last available $\mathrm{HbA}$ was $8.0 \pm 2.0 \%$ and $32.7 \%$ of patients had an $\mathrm{HbA}_{1 c}<7 \%$. The mean last available FBG (laboratory) was $158.8 \pm 64.0 \mathrm{mg} / \mathrm{dL}$. Mean systolic/diastolic blood pressure was $134 / 80 \mathrm{mmHg}$. Target treatment levels were achieved for LDL $(<100 \mathrm{mg} / \mathrm{dL})$ in $32.5 \%$ of patients, for HDL $(>40 \mathrm{mg} / \mathrm{dL})$ in $62.0 \%$, and for triglyceride $(<150 \mathrm{mg} / \mathrm{dL})$ in $53.8 \%$. A regimen of diet and exercise was reported in $3.6 \%$ of patients (mean $\mathrm{HbA}_{1}: 7.4 \pm 1.7 \%$ ). Among patients treated with oral glucose lowering drugs (OGLD) only (66.9\%; mean $\mathrm{HbA}_{1}$ : $7.8 \pm 1.9 \%)$, combined therapy was more frequent than monotherapy $(59.7 \% v s$. $40.3 \%$ ), the preferred combination being sulphonylureas + metformin (more than $80 \%$ ), and $10.4 \%$ of patients received more than two OGLD. Among patients treated with both OGLD and insulin (15.7\%; mean $\mathrm{HbA}_{1 c}: 8.7 \pm 2.1 \%$; mean insulin dose: $0.46 \pm 0.28 \mathrm{U} / \mathrm{kg}$ ), the mean time since start of insulin therapy was $3.0 \pm 3.8$ years. The association of OGLD and premixed insulin (mean daily dose: $0.56 \pm 0.20 \mathrm{U} / \mathrm{kg}$ ) was observed in $37.6 \%$ of OGLD+insulin treated-patients, and that of OGLD and basal insulin in $60.2 \%$ (of whom $10.2 \%$ had prandial+basal insulin; mean daily dose of basal insulin alone: $0.33 \pm 0.20$ U/kg; basal+prandial: $0.38 \pm 0.20 \mathrm{U} / \mathrm{kg}$ ). Among patients treated with insulin only (13.8\%; mean $\mathrm{HbA}_{1}: 8.4 \pm 2.1 \%$; mean insulin dose: $0.75 \pm 0.30 \mathrm{U} / \mathrm{kg}$ ), premixed insulin alone was used in $49.2 \%$ of patients, basal insulin alone in $26.3 \%$, basal+prandial insulin in $18.5 \%$ and prandial insulin alone in $2.4 \%$. The percentage of patients reaching target $\mathrm{HbA}_{1 c}<7 \%$ was $37.2 \%$ in patients receiving OGLD alone, $16.9 \%$ in those receiving OGLD+basal insulin, $19.6 \%$ in those receiving OGLD+basal+prandial insulin and $21.3 \%$ in those receiving OGLD+premixed insulin.

Conclusion: Our data show that the majority of Type 2 patients analyzed did not achieve the recommended treatment goals for $\mathrm{HbA}$ and FBG. Thus, measures designed to improve these results are urgently needed to reduce the current and future increases in the demand for care and the costs resulting from this poor diabetes control worldwide.

Supported by an unrestricted grant from sanofi-aventis
0327

Association between mean daily dose of insulin in adult type 1 patients and diabetes prevalence in 24 Ukrainian regions

M. D. Khalangot ${ }^{1}$, V. I. Kravchenko ${ }^{1}$, M. D. Tronko ${ }^{1}$, Y. B. Kulchinska ${ }^{1}$, A. M. Vaiserman';

${ }^{1}$ Epidemiology, Institute of Endocrinology and Metabolism, Kiev, ${ }^{2}$ Modelling of Aging, Institute of Gerontology, Kiev, Ukraine.

Background and Aims: We investigated whether insulin requirement in type 1 diabetes (T1D) patients can change systematically and is influenced by the same factors that determine the regional differences in T1D prevalence. For this purpose, we have compared the rate of insulin requirement among T1D adults in 24 Ukrainian regions.

Materials and Methods: Data consists of prevalent cases of T1D in Ukraine by the end of 2006. The completeness of the Ukrainian T1D register is estimated to be $91,1 \%$. Cases are restricted to persons over 14 years at the moment of being added to the Ukrainian register, diagnosed with T1D before age $30(n=26$ 796). Prevalence of T1D was calculated based on the official statistical data. $95 \%$ confidence intervals were determined through arcsine transformation. Data about HbA1c levels were available for the 1129 persons included in T1D register.

Results: Differences in T1D prevalence among 24 Ukrainian regions were obtained $(\chi 2=648.30, \mathrm{p}<0,001)$. In the "minimal" regional cluster (MIC) the prevalence rate was $0.60(0.58-0.62) \%$; in "intermediate" - $0.68(0.67-0.69)$ $\%$; in "maximal" (MAC) - $0.86(0.83-0.89) \%$. For 23633 patients with data about the daily insulin dose (DID) available, the mean disease duration (DD) was $14.86 \pm 10.75$ years. For patients with $\mathrm{DD}$ up to 15 years $(\mathrm{n}=13$ 677), DID is increasing linearly with DD (see figure): DID $=0.73 \times \mathrm{DD}+$ $43.74(\mathrm{R}=0.899, \mathrm{p}<0.001)$. The subsequent increase of $\mathrm{DD}$ is not followed by DID rate alteration. The median insulin doses standardized according to DD were lowest in the MIC and highest in the MAC populations: 45.89 (45.28-47.19) and 56.59 (53.33-57.88) units/24h, respectively; $\mathrm{p}<0,01$. The level of HbAlc in the MAC T1D patients was significantly higher than those in the MIC $(9.52 \pm 2.24 \%, n=240$, and $8.57 \pm 3.29 \%, n=111$, respectively; $\mathrm{p}<0.01)$. A positive linear relationship between DID and T1D prevalence in 24 Ukrainian regions was detected.

Conclusion: The obtained linear relationship between DD and DID might be caused by reduction of islet function in T1D patients. Our findings are consistent with those in the literature which noted an inverse linear relation between the residual beta-cell secretion and the duration of type 1 diabetes. It can be suggested that residual insulin secretion in T1D is associated with the regional T1D prevalence.

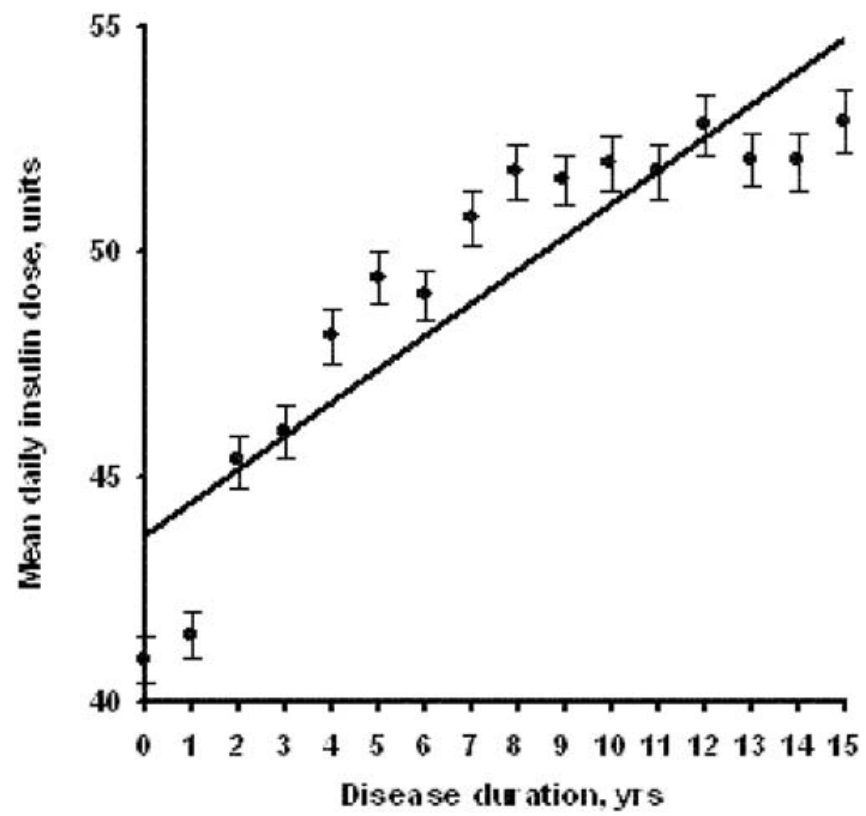

Fig. 1. Insulin daily doses (mean \pm SE) in type 1 diabetes patients $(n=13677)$ with disease duration up to $15 \mathrm{yrs}$.

Supported by National diabetes mellitus plan 


\section{8}

Which patients are suitable for continuous subcutaneous insulin infusion (CSII) in Japanese type 1 diabetes mellitus?

K. Taki, R. Nishimura, Y. Miyashita, K. Fujimoto, N. Tajima;

Diabetes and Endocrinology, Tokyo Jikei University school of Medicine,

Tokyo, Japan.

Background and Aims: Not many patients with type 1 diabetes mellitus were treated with continuous subcutaneous insulin infusion (CSII) in Japan. In this study, we attempted to determine which patients were suitable for CSII in Japanese type 1 diabetes mellitus.

Materials and Methods: Since March 2005, a total of 21 patients with type 1 diabetes mellitus had been introduced to CSII in our university hospital. We have divided these 21 cases into a CSII effective group, whose difference in Hemoglobin A1c (HbA1c) before introduction of CSII and that after three month was greater than the median, and a CSII ineffective group, whose difference in HbAlc was less than the median. Body mass index (BMI) $(\mathrm{kg} /$ $\mathrm{m} 2$ ), age at diagnosis of type 1 diabetes mellitus (years), the duration of type 1 diabetes mellitus (months), age at introduction of CSII (years), Urine C peptide ( $\mu \mathrm{g} /$ day), HbAlc $(\%)$, total cholesterol $(\mathrm{mg} / \mathrm{dl})$, triglycerides $(\mathrm{mg} /$ dl), and total daily insulin dosage (IU) were compared between the CSII effective group and the CSII ineffective group. Statistical analyses (t-tests) were performed using SPSS software.

Results: There were 10 cases in the CSII effective group and 11 in the CSII ineffective group. The mean \pm standard deviation of HbAlcbefore introduction of CSII in the CSII effective group $(9.6 \pm 2.4 \%)$ was significantly higher than that of the CSII ineffective group $(7.7 \pm 6.8 \%)(\mathrm{p}<0.001)$. No significant differences existed for the other parameters between the CSII effective group and the CSII ineffective group - BMI: $22.0 \pm 3.8 / 23.0 \pm 4.6 \mathrm{~kg} / \mathrm{m} 2(\mathrm{p}=0.62)$, the duration of type 1 diabetes mellitus: $105.8 \pm 94.1 / 131.9 \pm 112.4$ (months) ( $\mathrm{p}=0.33$ ), age at diagnosis of type 1 diabetes mellitus $30.5 \pm 15.3 / 30.7 \pm 20.8$ (years), age at introduction of CSII: $39.3 \pm 13.1 / 41.8 \pm 13.3$ (years) $(\mathrm{p}=0.74)$, Urine C peptide: $11.8 \pm 9.7 / 11.9 \pm 5.4 \mu \mathrm{g} /$ day $(\mathrm{p}=0.40)$, total cholesterol: $194.0 \pm 45.9 / 169.0 \pm 39.7$ $\mathrm{mg} / \mathrm{dl}(\mathrm{p}=0.61)$, triglycerides: $99.7 \pm 33.6 / 76.0 \pm 31.0 \mathrm{mg} / \mathrm{dl}(\mathrm{p}=0.87)$, and total daily insulin dosage: $41.3 \pm 13.3 / 40.9 \pm 12.9 \mathrm{IU} /$ day $(\mathrm{p}=0.73)$.

Conclusion: Our results suggest that type 1 diabetes mellitus patients in Japan with poor glycemic control using multiple daily insulin injection therapy should try CSII to improve their HbAlc levels.

\section{9}

Glycaemic control goal attainment among type 2 diabetic patients who initiated oral combination therapy in Europe

G. Krishnarajah ${ }^{1}$, F. Alvarez-Guisasola ${ }^{2}$, S. Tofe Povedano ${ }^{3}$, Q. Zhang ${ }^{1}$, G. Nocea Pulfer ${ }^{4}$, D. Yin ${ }^{1}$, A. Pfeiffer ${ }^{5}$;

${ }^{1}$ Outcomes Research, Merck \& Co, White house station, United States, ${ }^{2}$ Centro de Salud La Calzada II, Gijón, Spain, ${ }^{3}$ Hospital Son Dureta, Palma de Mallorca, Spain, ${ }^{4}$ Outcomes Research and Pricing Support, Merck Sharp \& Dohme de España S.A, Madrid, Spain, ${ }^{5}$ Charité Unversity Medical School Berlin, Campus Benjamin Franklin, Berlin, Germany.

Objective: To assess the level of adequate glycemic control in the real life practice settings in European countries in adult patients with type 2 diabetes mellitus (T2DM) who added a sulfonylurea (SU) or glitazone (PPAR) to metformin(MF) monotherapy.
Materials and Methods: Retrospective clinical chart review and patient survey at the point of visit (June 2006-Feb 2007) were conducted in7 countries (Finland, France, Germany, Poland, Norway, Spain, and United Kingdom). Patients were aged $\geq 30$ years at time of T2DM diagnosis and added a SU or PPAR to previous MF mono-therapy irrespective of whether those drugs were discontinued afterwards. Patients with gestational DM or T1DM were excluded. All patients gave informed consent. The information of each patient on $\mathrm{HbA} 1 \mathrm{c}$, medication use and co-morbid conditions were extracted from their clinical charts, for up to 7 months baseline period (before SU or PPAR addition) and for a minimum of one year follow-up period (between therapy addition and date of survey). Glycemic goal attainment at $\mathrm{HbAlc}<$ $6.5 \%$ was assessed according to the IDF (2005) recommendations using the last available $\mathrm{HbA1c}$ value during follow-up.

Results: 2039 patients (75.3\% initially added SU to MF and 24.7\% added PPAR) were included with mean age 62.9 yrs (SD 10.5), average 8.1 yrs (SD 5.1) of T2DM duration, $54.7 \%$ males, and $96.9 \%$ Caucasians. (Tab. 1) Conclusions: In this study involving seven European counties, after adding a SU or PPAR to their MF monotherapy, approximately $74 \%$ of patients with T2DM failed to attain glycemic control goal. More tolerable and effective oral hypoglycemic therapies may be needed to help these patients attain and maintain goal.

\section{0}

An evaluation of glycaemic control following switching from soluble regular human insulin to either insulin lispro or other analogue insulins in people with type 1 diabetes in UK general practice

C. D. Poole ${ }^{1}$, C. J. Currie ${ }^{2}$, A. J. Tynan ${ }^{3}$, A. Simpson ${ }^{4}$;

${ }^{1}$ Healthcare Research, $360^{\circ}$ Research, Penarth, United Kingdom,

${ }^{2}$ Department of Medicine, Cardiff University, United Kingdom,

${ }^{3}$ European Health Outcomes Research, Eli Lilly and Company Limited,

Windlesham, United Kingdom, ${ }^{4}$ European Medical Department, Eli Lilly and Company Limited, Windlesham, United Kingdom.

Background and Aims: Switching treatment can occur for a variety of reasons. A number of short acting insulin (SAI) treatments are available and used mainly to improve prandial glucose control. The purpose of this study was to evaluate the impact of switching from an insulin regimen that included soluble regular human insulin (RHI) to a similar regimen that included either insulin lispro (IL) or an alternative short acting insulin analogue (IA) as short acting insulin analogue (SAIA) therapies.

Materials and Methods: Data were extracted from the General Practice Research Database (GPRD), a resource that describes the primary care histories of around $7 \%$ of the UK population. Among all subjects with type 1 diabetes (T1DM) who switched between either RHI or SAIA, the vast majority (96.6\%) had been given RHI as their first prescription for short-acting insulin, and thus formed the focus of this study. Subjects with T1DM were selected having been treated with RHI for at least one year prior to switching to one SAIA, and had continued for at least one year with no other change in insulin therapy over these two years. Interpolated paired-sample comparisons of HbAlc were made for the four quarterly periods post-switching.

Results: 438 people switched from RHI to IL (RHI-IL) and 477 patients switched from RHI to IA (RHI-IA). The patient characteristics in each group with T1DM were as follows for RHI-IL and RHI-IA, respectively: age, 38.2 and 39.0 years $(\mathrm{p}=0.428)$; percent male, $53.4 \%$ and $58.7 \%(\mathrm{p}=0.110)$. In both groups $\mathrm{HbA} 1 \mathrm{c}$ decreased significantly from baseline to Q1 $(-0.12 \%, \mathrm{p}<0.001)$;

Tab. 1

\begin{tabular}{llllll} 
Country & $\mathrm{N}$ & $\begin{array}{l}\text { HbAlc at baseline (\%) } \\
\text { mean } \pm \mathrm{SD}^{*}\end{array}$ & $\begin{array}{l}\text { HbAlc during follow-up } \\
(\%) \text { mean } \pm \mathrm{SD}^{*}\end{array}$ & $\begin{array}{l}\text { Mean HbA1c change from } \\
\text { baseline (\%) mean } \pm \mathrm{SD}^{*}\end{array}$ & $\begin{array}{l}\text { Goal attainment rate during follow-up } \\
\text { at HbAlc }<6.5 \% \%^{*}\end{array}$ \\
\hline Total & 2039 & $8.1 \pm 1.4$ & $7.2 \pm 1.1$ & $0.90 \pm 1.5$ & $25.8 \%$ \\
Finland & 195 & $7.8 \pm 1.2$ & $7.2 \pm 1.2$ & $0.6 \pm 1.3$ & $29.7 \%$ \\
France & 163 & $8.1 \pm 1.4$ & $7.1 \pm 1.0$ & $1.1 \pm 1.7$ & $28.8 \%$ \\
Germany & 410 & $7.8 \pm 1.4$ & $7.1 \pm 1.0$ & $0.7 \pm 1.3$ & $24.6 \%$ \\
Norway & 64 & $8.6 \pm 1.8$ & $7.4 \pm 1.1$ & $1.2 \pm 1.9$ & $17.2 \%$ \\
Poland & 296 & $7.8 \pm 1.1$ & $7.1 \pm 1.1$ & $0.7 \pm 1.2$ & $28.0 \%$ \\
Spain & 502 & $8.0 \pm 1.5$ & $7.1 \pm 1.3$ & $0.8 \pm 1.6$ & $30.5 \%$ \\
United Kingdom & 409 & $8.6 \pm 1.4$ & $7.3 \pm 1.2$ & $1.3 \pm 1.4$ & $17.9 \%$ \\
\hline
\end{tabular}

${ }^{*}$ Indicates significant difference among countries, at $\mathrm{p}<0.05$ 
Q2 $(-0.18 \%, \mathrm{p}<0.001)$; Q3 $(-0.27 \%, \mathrm{p}<0.001)$; and Q4 post-switching ($0.30 \%, \mathrm{p}<0.001)$. Patients switched to lispro showed a trend towards greater improvement in glycaemic control than those switched to other analogue insulins (Q3 average improvement $-0.38 \%$ vs. $-0.20 \%$, respectively $[\mathrm{p}=0.065$ ]; Figure]).

Conclusion: Patients with T1DM treated in UK general practice showed significant improvement in glycaemic control when switched from soluble regular human insulin to short-acting insulin analogues. There was evidence of insulin lispro being more effective in improving glycaemic control than other insulin analogue therapies, though this study was under-powered $(60 \%)$ to detect a statistically significant difference at the $5 \%$ level.

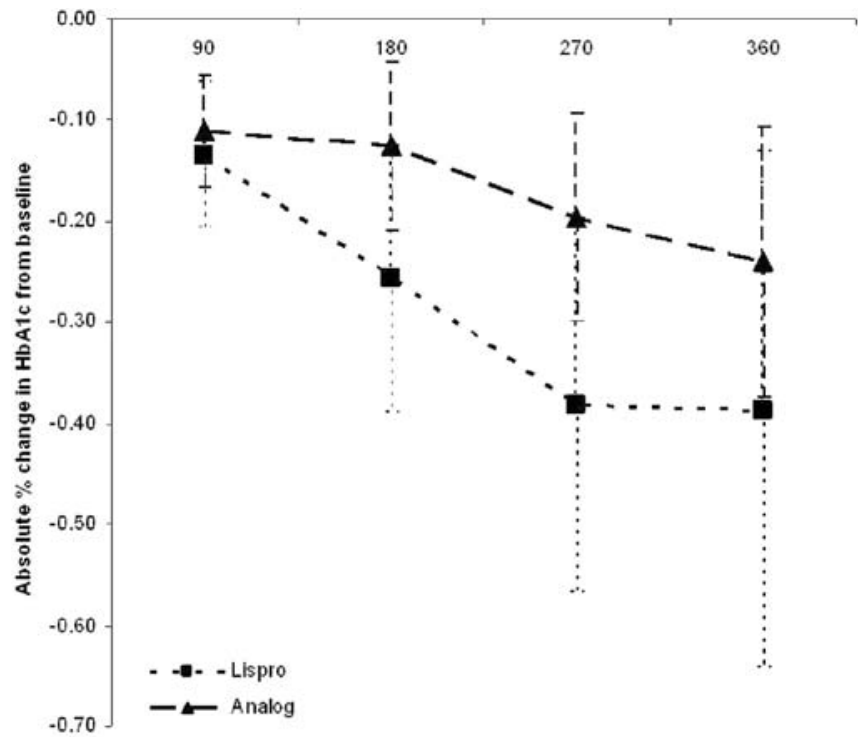

Days after SAIA initiation

Supported by Eli Lilly and Company Limited

\section{1}

Compliance to treatment and reaching metabolic goals are better in OAD treated diabetics than insulin treated diabetics in Turkish population

S. Ucak, E. Satir, D. Tural, T. Kilicdagi, K. Aydin, S. Dal, I. Hacibekiroglu, A. Cifcibasi, T. Hacibekiroglu, O. Basat, Y. Altuntas;

Endocrinology \& Metabolism, Sisli Etfal Research and Training Hospital, Istanbul, Turkey.

Background and Aims: The aim of this study was to assess the success rates of reaching HbAlc goals in different treatment groups.

Materials and Methods: This was a single-centre, retrospective study including 3000 patients admitted to our diabetes outpatient clinic as a primary care unit after year of 2005. All data obtained from patients files which were archived in our outpatient clinic. Duration of disease, type of diabetes, HbAlc levels (3 months intervals in a year), type of treatment were evaluated. All parameters were also evaluated at the end of a year long follow up. $\mathrm{HbAlc}$ levels were compared at the beginning and the end of the study. Rate of patients reaching the HbAlc goals according to IDF, AACE and ADA were determined. A p value less than 0.05 was considered significant.

Results: A total number of 3000 diabetic patients were recruited for the study. $3.1 \%$ of patients had type 1 diabetes mellitus and $96.9 \%$ had type 2 diabetes mellitus. Female rate was $58.2 \%$ while male rate was $41.8 \%$. There were 4 different treatment groups.1-Diet group $(\mathrm{n}=140), 2-\mathrm{OAD}$ group (excluding glitazones) $(\mathrm{n}=1238), 3$-insulin group $(\mathrm{n}=765)$, and 4 - insulin + OAD group $(\mathrm{n}=812)$. According to IDF and AACE criteria rate of patients reaching $\mathrm{HbAlc} \leq 6.5 \%$ was $32 \%$ in total group. At the end of one year it was $45 \%$. In diet group $92 \%$ of patients and one year later $100 \%$ of patients reached the goals. In OAD group The rate was $61 \%$ and next year it was $69 \%$. In insulin group $15 \%$ of patients and one year later $33 \%$ of patients reached the goal HbA1c levels. In insulin + OAD group rates were $10 \%$ and $21 \%$ respectively. All these changes were statistically significant $(p<0.05)$. According to ADA criteria patients reaching $\mathrm{HbAlc}$ levels $\leq 7 \%$ were $40 \%$ in total group and the end of one year $59 \%$. In diet group $95 \%$ of patients and one year later $100 \%$ of patients reached the goals. In OAD group the rate was $75 \%$ and next year it was $83 \%$. In insulin group $23 \%$ of patients and one year later $52 \%$ of patients reached the goal $\mathrm{HbAlc}$ levels. In insulin $+\mathrm{OAD}$ group rates were $16 \%$ and $32 \%$ respectively. All these changes were statistically significant $(\mathrm{p}<0.05)$. In $\mathrm{OAD}$ group number of visits were decreasing while $\mathrm{HbAlclevels}$ were increasing $(\mathrm{r}=-0.2, \mathrm{p}=0.001)$. In insulin group similar correlation was observed between number of visits and HbAlc levels $(r=-0.2, p=0.005)$.

Conclusion: A big number of patients with diabetes mellitus seem to reach $\mathrm{HbAlc}$ goals in different treatment groups. Especially the rate was greater in OAD group than insulin group. This might be due to the fact that patients under OAD treatment have a shorter duration of diabetes and their better compliance to therapy regimen. 


\section{PS 9 Diabetes mortality and hospi- talisation in different populations}

\section{2}

Diabetes mortality in Serbia 1980-2005: characteristics and trends I. Rakocevic, D. Miljus, J. Malogajski, S. Plavsic, J. Jankovic, N. Mickovski, S. Zivkovic, S. Savkovic;

Department for prevention and control of noncommunicable diseases, Institute of Public Health of Serbia, Belgrade, Serbia.

Background and Aims: In 1980 there were 960 deaths (2\%) for which diabetes was certified as the underlying cause of death in Serbia. Diabetes related death rate had been steadily increasing since that period. Today diabetes is the $5^{\text {th }}$ leading cause of death and the $5^{\text {th }}$ leading cause of burden of disease in the country. The purpose of this work is to determine the characteristics and trends of diabetes mortality among the Serbian population from 1980 through 2005 and to provide suggestions to health policy-makers.

Materials and Methods: Deaths caused by diabetes mellitus occurred during the period 1980-2005 were obtained. For each gender the following parameters were calculated: crude mortality rates and standardized rates by means of the direct method using the World population as standard, for the 25 years studied. Trend analysis in mortality was performed using a regression model. Standardized rates were adjusted to a regression straight line regarding the death year and theoretical rates were calculated. With these calculations the percentages in changes of mortality rates were estimated and a projection has been calculated until the year 2015 .

Results: The significant increasing trend of diabetes mortality in Serbian population during the 25 -yr study period is observed $(\mathrm{p}<0.001)$. Standardized mortality rates due to diabetes mellitus had increased from 9.37/100,000 to $13.48 / 100,000$ in males and from $10.78 / 100,000$ to $13.40 / 100,000$ among females over the study period. From 1980 to 2005 females had a higher mortality than males, however diabetes mortality rates among males increased significantly (43.8\%) compared to females (24.4\%), with annual percentage change for both genders 1.3. Regression-based linear trends in diabetes mortality indicate an even growth in all age groups, although the annual percentage change is most prominent in the $75-79 \mathrm{yr}$ age group for females $(2.1 \% / \mathrm{yr})$ and in the $80-84$ yr age group for males $(2.8 \% / \mathrm{yr})$. Projections of diabetes mortality trends into the year 2015 show linear increase for both genders.

Conclusion: Our results indicate that diabetes mortality has been markedly increasing in the Serbian population, primarily in males and persons aged 65 years or more. These findings could be related to population aging, increased prevalence of obesity and sedentary lifestyle. It also highlights the need for targeted diabetes prevention and treatment. Three main strategies are proposed to deal with the problem: develop national policies, strategies and plans for prevention and care; promote and implement community participation in prevention and care; specific monitoring of health and quality of care in elderly people with diabetes.

\section{3}

Mortality risk in children and young adults with type 1 diabetes: the population-based registry of the Province of Turin, Italy G. Novelli ${ }^{1}$, G. Bruno ${ }^{1}$, F. Cerutti ${ }^{2}$, A. Soddu ${ }^{1}$, S. Piano ${ }^{1}$, C. Tringali ${ }^{1}$, B. Aimone ${ }^{1}$, P. Cavallo Perin ${ }^{1}$, G. Pagano ${ }^{1}$.

${ }^{1}$ Internal Medicine, University of Turin,

${ }^{2}$ Dept. of Pediatrics, University of Turin, Italy.

Background and Aims: Studies performed in Northern European countries have reported two- fold higher mortality risk in young people with type 1 diabetes than in general populations. As most of early diabetes-related deaths (diabetic ketoacidosis, hypoglycemia) could have been prevented, monitoring the number of these cases over time is relevant to assess whereas quality of diabetes care should be improved. We have assessed mortality risk in an italian incident cohort with type 1 diabetes onset in period 1974-2000.

Materials and Methods: Two lists of incident people with type 1 diabetes in the Province of Turin, Italy were employed: 1) the hospital-based cohort of cases aged 0-14 years in the period from January 1, 1974 to December 31, 1983 , identified through the regional paediatric hospital $(n=157$, completeness of ascertainment 95\%);2) the population-based cohort of incident cases aged 0-29 years in the period from January 1, 1984 to December 31, 2000
( $\mathrm{n}=1056$, completeness of ascertainment $99 \%$ in age-group $0-14$ yrs; $97 \%$ in age group 15-29). The relevant time scale for the analysis was time since diabetes onset to death or to 31 December 2003. Information about deaths and the causes of death was obtained from the demographical files of towns of residence, hospital discharges and autopsy records. Only three patients were lost to follow-up. Mortality rates were calculated dividing the number of deaths occurring during the study period by the number of person-years of observation. Standardized mortality ratios (SMRs) for all-cause mortality were computed using the Italian population as standard, by 5-yrs age-group, sex, and calendar period (1974-83, 1984-88, 1989-93, 1994-98 and 1999-2003). Results: The study base included 1213 incident cases in period 1974-2000; at the onset of the disease, 677 were aged $0-14$ yrs and 536 were aged $15-29$ yrs. During mean follow-up of 15.8 yrs (range 1.96-29.9), there were 19 deaths in 15967.9 person-years of observations, giving an all-cause mortality rate of 1.19/1000 person-years (95\% CI 0.76-1.87). With respect 9.54 expected deaths, we observed 19 cases, giving an SMR 1.96 (1.25-3.08), with a tendency toward lower excess in incident cases in childhood (SMR=1.84, 95\% CI 0.92-3.67) than in adulthood ( $\mathrm{SMR}=2.06,95 \%$ CI 1.14-3.73), although not statistically significant. Mean duration of diabetes was 13.4 (SD 7.4, range 2.0-24.4) in people who died and 13.3 (7.4) in those who survived. In no case death occurred at the time of diabetes onset or in childhood. Out of 19 deaths, 8 were clearly diabetes-related (diabetic ketoacidosis, hypoglycemia, end-stage renal disease).

Conclusion: this report of the population-based registry of the Province of Torino points out that children and young adults with onset of diabetes in period 1974-2000 experienced a two-fold higher mortality risk than Italian people of similar age and sex. The impact of the disease on survival might be declining over time, and monitoring the number of these events over time is crucial as these data may serve as a reflection of the quality of health care in the community.

\section{4}

All-cause mortality in hospitalized patients with type $\mathbf{2}$ diabetes mellitus P. S. Koureta, A. Angelis, A. Kramvis, A. E. G. Alaveras;

1st Medical Department, Red Cross Hospital, Athens, Greece.

Background and Aims: Many clinicians believe that diabetes mellitus exerts an excess risk of death among those patients need to be hospitalized for any reason. Although certain complications, such as cardiovascular disease are certainly more common among diabetic patients, others like common infections and inhospital mortality related to those complications are not well studied.

Materials and Methods: A retrospective cohort study was conducted, comparing all patients with any common infection hospitalized in our clinic during a decade, (1990-2000), to matched patients hospitalized for any reason other than infection. The risk ratios of death attributable to diabetes mellitus, as well as to infectious or cardiovascular disease between those with and without diabetes were calculated, using multiple logistic regression analysis models. The results were adjusted for possible confounding factors (age, sex and history of cancer).

Results: The cohort consisted of 4959 patients, 2583 hospitalized for any common infection (491(19.01\%) with diabetes and 2092 without) and 2376 matched for age and sex patients, hospitalized for any reason other than infection (337(14.18\%) with diabetes and 2039 without). The risk of acquiring an infectious disease was $47 \%$ higher for diabetic patients compared to those without diabetes (OR=1.47 95\% CI 1.23-1.77 $\mathrm{p}<0.001)$.There were $96(11.59 \%)$ deaths among the 828 diabetic patients and $406(9.83 \%)$ deaths among 4131 non-diabetic patients. The overall risk of death, for patients hospitalized for any reason, did not differ between patients with and without diabetes (OR=0.91 95\% CI 0.53-1.56). The risk of death did not differ between diabetic and non-diabetic patients with concomitant cardiovascular disease (interaction $\mathrm{OR}=0.9895 \% \mathrm{CI} 0.58-1.66$ ) or any common infection (interaction $\mathrm{OR}=1.2395 \%$ CI $0.71-2.16$ ). Generally, the risk of death was about six times higher for patients hospitalized with cardiovascular disease $(\mathrm{OR}=5.695 \%$ CI $3.69-8.59 \mathrm{p}<0.001)$ and about two times higher for patients hospitalized with any common infection (OR=2.1 95\% CI 1.57-2.74 p<0.001), compared to patients hospitalized for any other reason.

Conclusion: Although patients with diabetes mellitus are more prone to complications such as common infections, they do not seem to suffer an excess risk of dying when they need to be hospitalized for any reason, even for those diabetic patients with concomitant cardiovascular disease or any common infection. 


\section{5}

Changes in mortality pattern over the last six decades in type 2 diabetes patients with onset between 30 and 60 years

S. Ioacara ${ }^{1}$, C. Ionescu-Tirgoviste ${ }^{1}$, E. Farcasiu ${ }^{1}$, O. Bradescu ${ }^{1}$, C. Guja ${ }^{1}$, M. Pallayova ${ }^{2}$;

${ }^{1}$ "N. Paulescu" Institute of Diabetes, Bucharest, Romania,

${ }^{2}$ Physiology and Sleep Laboratory, PJ Safarik University, Kosice, Slovakia.

Background and Aims: To analyze the changes in age at onset, disease duration, age at death and cause of death in patients with noninsulintreated Type 2 Diabetes Mellitus (T2DM) deceased between 1943 and 2005.

Materials and Methods: We performed a retrospective study in noninsulintreated T2DM patients, registered at Bucharest Diabetes Centre, Romania and deceased between 1943 and 2005. We analysed 5856 deaths, 3299 (56.3\%) males (M) and 2557 (43.7\%) females (F). For each patient, the age at diabetes onset, disease duration, cause of death and sex were recorded. Body mass index (BMI) at onset was obtained for a subgroup of 2632 cases. Statistical analysis used (non)parametrical tests as apropriate.

Results: The major changes in mortality according to 6 "decades" of year of death are presented in table 1.

Overall, comparing with females, males had a significantly lower age at onset $(52.1 \pm 6.0$ years $M$ vs. $53.0 \pm 5.7$ years $F, p<0.001)$, age at death $(65.0 \pm 9.0$ years $\mathrm{M}$ vs. $65.6 \pm 8.7$ years $\mathrm{F}, \mathrm{p}=0.005)$ and $\mathrm{BMI}\left(27.1 \pm 4.3 \mathrm{Kg} / \mathrm{m}^{2} \mathrm{M}\right.$ vs. $28.8 \pm 5.4$ $\left.\mathrm{Kg} / \mathrm{m}^{2} \mathrm{~F}, \mathrm{p}<0.001\right)$; there was no difference by gender for disease duration (12.8 \pm 8.2 yrs.). The major causes of death during the 1996-2005 period were: cardiovascular diseases $(60.8 \%$, from which $18.6 \%$ stroke), malignancies (17\%), diabetes $(6.8 \%)$, gastrointestinal and hepatic diseases $(6.2 \%)$, infectious diseases $(4.9 \%)$, chronic renal failure $(1.2 \%)$, diabetes coma $(0.1 \%)$ and others (3\%). The percentage of infectious diseases related deaths decreased 3.4 times from 1943 to 2005, while cardiovascular diseases related deaths rose 1.5 times during the same period.

Conclusion: Comparing the 1996-2005 with the 1943-1955 period, we found no significant change in age at onset, 8.2 years increase $(\mathrm{p}<0.001)$ in mean disease duration at death (also clearly showed by the Kaplan-Meier survival curves, Log rank test $\mathrm{p}<0.001), 7.8$ years increase $(\mathrm{p}<0.001)$ in mean age at death and $3.3 \mathrm{Kg} / \mathrm{m}^{2}$ increase in mean BMI at onset. Males have a significantly lower age at death than females, not because of a lower survival period but because of a lower age at diabetes onset. The percentage of infectious diseases related deaths decreased 3.4 times from 1943 to 2005, while cardiovascular diseases related deaths rose 1.5 times during the same period.

\section{6}

Insulin titration; gaining an understanding of the burdern of type 2 diabetes in Europe (INSTIGATE): number of French patients admitted in hospitals at time of insulin initiation

M. A. Charles ${ }^{1}$, S. Tcherny-Lessenot ${ }^{2}$, J. Pentel ${ }^{2}$, C. Salaun-Martin², L. Timlin ${ }^{3}$, S. Cleall ${ }^{3}$, F. Fagnani ${ }^{4}$;

${ }^{1}$ INSERM, U780, Villejuif, France, ${ }^{2}$ Lilly France, Suresnes, France, ${ }^{3}$ EHOR, Eli Lilly and Company, Windlesham, United Kingdom,

${ }^{4}$ D, Cemka Eval, Bourg La Reine, France.

Background and Aims: INSTIGATE study provides data from clinical practice on people with Type 2 diabetes who have not achieved adequate glycaemic control and are being initiated on insulin therapy. Objective of this analysis is to describe baseline characteristics of French patients enrolled in the study in terms of demographics, diabetes history, glycaemic control, and resource utilization.
Materials and Methods: 580 physicians (470 diabetologists and 110 primary care physicians), selected by a stratified random procedure respectively from a database of 1000 diabetologists and a database of 300 primary care physicians high prescribers of insulins, were solicited to participate in the study. Among them, 65 physicians (50 diabetologists and 15 primary care physicians) accepted to participate. To date, 41 physicians (34 diabetologists (18 in hospitals and 16 in private practice) and 7 primary care physicians) have enrolled at least one patient. Data on the 178 patients enrolled in France were analyzed.

Results: Enrolled patients (male 57\%) had a mean age of $64.8 \pm 11.2$ (SD) and mean BMI of $29.4 \pm 6.2(\mathrm{SD})$. They had been diagnosed diabetes $13.0 \pm$ 8.1 (SD) years ago with a mean $\mathrm{HbAlC}$ at diagnosis of $8.9 \pm 2.3(\mathrm{SD})$. At time of insulin initiation, $50 \%$ of patients were inpatients. In the previous 6 months, $28.2 \%$ were admitted at hospital at least once, mean duration of stay was $6.4 \pm 2.7$ days for acute complications and $4.1 \pm 3.5$ days for longterm complications, $87.4 \%$ consulted their primary care physician, $75.3 \%$ consulted their endocrinologist. In the previous 12 months, patients received one antidiabetic medication in $15.2 \%$ of cases, 2 in $40.4 \%, 3$ in $30.3 \%$ and more than 4 in $9.6 \%$. The most frequent treatment observed was progression from 1 to 2 oral therapies and then to insulin for $23.0 \%$ of patients. Other treatments frequently observed were: progression from double oral therapy to insulin in $18.4 \%$, progression from 1 to 2 and to 3 oral agents and then to insulin $12,6 \%$, and progression from single oral therapy to insulin in $12.1 \%$. Conclusion: In our sample of French patients enrolled in INSTIGATE study, half of patients were admitted to the hospital to initiate insulin therapy. There are a number of treatment pathways followed by patients prior to insulin initiation.

Supported by Eli Lilly and Company

\section{7}

Patients with type 2 diabetes in hospitals. Therapy and diabetic complications assessment of 11.197 in-patients from 153 medical centres in Poland

B. Kozlowska-Boszko, T. Lietz, M. Wronka, P. Markiewicz;

Medical Department, Bayer Schering Pharmaceuticals, Warsaw, Poland.

Background and Aims: The aim of the study was to assess the clinical profile of type 2 diabetes (T2D) patients hospitalized for various reasons in Polish medical centres. We evaluated type and quality of antidiabetic treatment before the admission, type of diabetic complications and reasons for hospitalization.

Materials and Methods: This non-interventional, cross-sectional study, involving 11197 in-pts (46\% men, 54\% women, age $61 \pm 11$ and $62 \pm 11$ yrs, respectively), was conducted between April 2005-March 2007 in 153 medical centres in Poland. For each patient information on demografic data, diabetes diagnosis and complications was documented by the physician using the standardized individually-numbered case-report form. Information about treatment prior to hospital admission was also collected. A biometric evaluation was carried out according to a statystical analysis plan at the end of the study.

Results: Diabetes and diabetes-related complications were the main reason for hospitalization and involved $75,5 \%$ of all admission causes. The prevalence of cardiovascular complications was significantly higher than that of microvascular complications $(64,6 \%$ vs $49,7 \%$; $\mathrm{p}<0,05)$. The most prevalent cardiovascular diseases (CVD) included hypertension, coronary heart disease and congestive heart failure which were responsible for hospital admission of $37,2 \%, 20,2 \%$ and $13,5 \%$ of all pts, respectively. In patients with early diagnosed T2D ( $<1$ year before hospitalization) the prevalence of

Table 1

\begin{tabular}{lllllll}
\hline Year of death & $1943-1955$ & $1956-1965$ & $1966-1975$ & $1976-1985$ & $1986-1995$ & $1996-2005$ \\
$\mathrm{n}$ & 278 & 387 & 1605 & 1706 & 715 & 1165 \\
Age at onset (years) & $53.1 \pm 5.9$ & $51.8 \pm 6.2^{*}$ & $52.7 \pm 5.8^{\mathrm{Ns}}$ & $52.2 \pm 6.1^{*}$ & $52.3 \pm 6.0^{\mathrm{NS}}$ & $52.7 \pm 5.5^{\mathrm{Ns}}$ \\
Disease duration (years) & $6.0 \pm 3.8$ & $11.6 \pm 7.2^{* *}$ & $11.3 \pm 7.2^{* *}$ & $13.5 \pm 8.1^{* * *}$ & $15.4 \pm 8.6^{* * *}$ & $14.2 \pm 9.1^{* * *}$ \\
Age at death (years) & $59.2 \pm 7.2$ & $63.4 \pm 8.4^{* *}$ & $63.9 \pm 8.0^{* *}$ & $65.8 \pm 8.9^{* * *}$ & $67.8 \pm 8.9^{* * *}$ & $66.9 \pm 9.4^{* * *}$ \\
BMI $\left(\mathrm{Kg} / \mathrm{m}^{2}\right)$ & - & $25.7 \pm 3.4$ & $27.2 \pm 4.6^{\mathrm{Ns}}$ & $28.0 \pm 4.6^{* * *}$ & $28.3 \pm 5.0^{* * *}$ & $29.0 \pm 6.8^{* * *}$ \\
\hline
\end{tabular}

${ }^{\text {Ns }}$ not significant, ${ }^{*} \mathrm{p}<0.05,{ }^{* *} \mathrm{p}<0.01,{ }^{* * *} \mathrm{p}<0.001$ vs. first available "decade" 
CVD was significantly higher than the prevalence of microvascular diabetic complications ( $42 \%$ vs $21 \%$; $<<0,05$ ). Despite the marked increase in the rate of microvascular complications observed in pts with advanced T2D (diagnosis $>5$ yrs before hospitalization), CVD remained the most prevalent complication $(73 \%$ vs $66 \%$; $<00,05)$. The quality of diabetes control was rated by doctors as "good" only in 5,3\% of all patients, independent of time of T2D diagnosis. Sulphonylureas and metformin were used prior to hospital admission by $55 \%$ and $37 \%$ of patients, respectively. The usage of alphaglucosidase inhibitor, acarbose, was low (7,8\%), however, it was significantly higher in pts with advanced diabetes in comparison with pts with early T2D diagnosis $(10,9 \%$ vs $4,4 \%$; $p<0,05)$.

Conclusion: Our study shows that CVD is the leading cause of hospital admissions of diabetic patients in Poland. Hypertension, coronary heart disease and congestive heart failure are the main reasons for hospitalization. The prevalence of CVD is very high even in patients with early diagnosed T2D. In Poland sulphonylureas and metformin are most often prescribed oral hypoglycemic drugs, however the quality of diabetes control remains low, even in doctor's opinion.

Supported by Bayer Schering Pharmaceuticals

\section{PS 10 Cardiovascular risk factors}

\section{8}

Cardiovascular disease prediction and type 2 diabetes: Framingham risk versus metabolic syndrome criteria

J. Saumell' ${ }^{1}$ J. J. Cabre², J. L. Piñol ${ }^{3}$, M. Baldrich², F. Martin², B. Costa ${ }^{4}$,

J. Basora ${ }^{3}$;

${ }^{1}$ Basic Health Area Reus- $4,{ }^{2}$ Basic Health Area Reus-1,

${ }^{3}$ Central Service Primary Care Reus,

${ }^{4}$ Basic Health Area Reus-2, Catalan Health Institute, Reus, Spain.

Background and Aims: Is not well established if the Metabolic Syndrome (MS) is better predictive of cardiovascular disease (CVD) than other methods, like Framingham risk score (FRAM). So, the objective is comparing the MS criteria (according to NCEP-ATPIII) with the Framingham risk score in the prediction of CVD and of type 2 diabetes (T2D).

Materials and Methods: Prospective cohorts study of 8 year follow-up (19982005), in two Basic Areas of urban Health (43.000 individuals). Randomized sample of subjects older than 40 years, registering sociodemographic data and those precise for the calculation of the FRAM and MS approaches (NCEPATPIII) (range 0-5 components); cardiovascular events and incident cases of T2D. The statistical tests (ROC curves and areas under the curve (AUC)) were carried out separately for genders (because the MS criteria differs).

Results: 943 individuals between $40-75$ years (applicable margin to FRAM) (588 women). The MS was good predictor of CVD in men and women (AUC $0.63 / 0.68$, respectively) and of incident cases of T2D in women (AUC 1.0, front 0.45 in men). On the other hand, the FRAM was better predictor of CVD in men (AUC 0.72 front 0.68 in women) and only predicted well new cases of T2D in women (AUC 0.87 front 0.22 in men).

Conclusion: The presence of MS is good predictor of CVD and of new cases of T2D, but mainly of these last. Although the MS doesn't predict the CVD the same as the FRAM, it's useful like easy tool of detection of subjects at high risk.

\section{9}

Results of the International Diabetes Management Practices Study (IDMPS), year 2: cardiovascular risk factors in patients with type 2 diabetes

N. Hancu; on behalf of the IDMPS Group,

Clinical Center of Diabetes, Nutrition and Metabolic Diseases, Iuliu

Hatieganu University of Medicine, Cluj-Napoca, Romania.

Background and Aims: Despite the recommendations of the ADA, EASD and IDF, a large number of patients with diabetes are not well controlled. In addition, patients with Type 2 diabetes are exposed to arterial hypertension and dyslipidemia and thus a further increased cardiovascular risk. We aimed to gather data on current practices in the management of patients with diabetes worldwide and to evaluate compliance with international guidelines.

Material and Methods: The IDMPS is an international, multicenter, observational study performed in 27 countries within Africa, Asia, Eastern Europe, the Middle East and Latin America. Data are being collected from patients with Type 1 and Type 2 diabetes ( $\geq 18$ years) seen in current medical practice in 1-year cycles (a 2-week cross-sectional recruitment period, followed by a 9-month longitudinal period for Type 2 patients) for 5 years. Here, we report preliminary results from patients with Type 2 diabetes collected during the second cross-sectional period (Year 2) in 8 countries. To measure the degree of glucose metabolism control, $\mathrm{HbA}_{1 c}$ and $\mathrm{FBG}$ values were collected in addition to information on blood pressure and lipid level control. Data were analyzed using standard descriptive methods.

Results: 4312 patients with Type 2 diabetes ( $47.8 \%$ men; mean age: $58.3 \pm 11.2$ years) were recruited during November and December 2006. The mean last available $\mathrm{HbA}_{1}$ was $8.0 \pm 2.0 \%$. In addition, only $32.7 \%$ of patients had an $\mathrm{HbA}_{1 \mathrm{c}}<7 \%$, although all patients were receiving some form of anti-diabetic therapy; $37.3 \%$ of patients were considered to be on target according to the physician's opinion, and among these the mean $\mathrm{HbA}_{1 c}$ was $6.7 \pm 1.3 \%$. The mean values of other cardiovascular risk factors were as follows: systolic/ diastolic blood pressure: 134/80 mmHg; LDL: $133.2 \pm 132.6 \mathrm{mg} / \mathrm{dL}$ (median: 115.0); HDL: $48.6 \pm 31.0 \mathrm{mg} / \mathrm{dL}$ (median: 44.0 ); triglycerides: $179.5 \pm 167.9 \mathrm{mg} /$ dL (median: 141.0). Among 3342 patients with SBP $\geq 130 \mathrm{mmHg}$ and/or DBP $\geq 80 \mathrm{mmHg}, 1044$ were not reported by the physician as having hypertension 
and 2298 were reported as having hypertension, of whom 2222 were receiving antihypertensive treatment (Table). Similar results were found for patients with dyslipidemia (Table).

Table.

Percentage of Type 2 diabetic patients with other cardiovascular risk factors

\begin{tabular}{llll}
\hline $\begin{array}{l}\text { Risk factor } \\
(\text { no. of patients }\end{array}$ & $\begin{array}{l}\text { Not } \\
\text { diagnosed }\end{array}$ & \multicolumn{2}{l}{ Diagnosed } \\
\cline { 2 - 4 } & Treated & Not treated \\
\hline Hypertension & $31.2 \%$ & $66.5 \%$ & $2.3 \%$ \\
LDL $>100 \mathrm{mg} / \mathrm{dL}(\mathrm{n}=15342)$ & $27.8 \%$ & $60.1 \%$ & $12.1 \%$ \\
$\mathrm{HDL}<40 \mathrm{mg} / \mathrm{dL}(\mathrm{n}=916)$ & $25.6 \%$ & $63.5 \%$ & $10.9 \%$ \\
Triglycerides $>150 \mathrm{mg} / \mathrm{dL}(\mathrm{n}=1400)$ & $16.4 \%$ & $68.1 \%$ & $15.5 \%$ \\
\hline
\end{tabular}

*based on physicians' reporting; ${ }^{* *}$ for whom information on physician classification was available ${ }^{* *} S B P \geq 130 \mathrm{~mm} \mathrm{Hg}$ and/or $D B P \geq 80 \mathrm{mmHg}$ The percentage of patients reaching the triple target of $\mathrm{HbA}_{1 c}<7 \%$, blood pressure $<130 / 80 \mathrm{mmHg}$ and $\mathrm{LDL}<100 \mathrm{mg} / \mathrm{dL}$ was only $1.7 \%$.

Conclusion: Our data show that patients with Type 2 diabetes are poorly controlled, in terms of $\mathrm{HbA}_{1 \mathrm{c}}$ and other cardiovascular risk factors, despite having had full access to specific drugs treating all these conditions. This suggests that more appropriate guidance, education and commitment are necessary to optimize the effective use of drug therapy by patients with Type 2 diabetes worldwide.

Supported by an unrestricted grant from sanofi-aventis

\section{0}

Increased prevalence of cardiovascular disease and risk biomarkers in patients with unknown type 2 diabetes visiting cardiology specialists - results from the DIASPORA Study

T. Schöndorf ${ }^{1}$, E. Karagiannis ${ }^{2}$, E. Erdmann ${ }^{3}$, G. Lübben² ${ }^{2}$ B. Jenke ${ }^{1}$,

M. Löbig ${ }^{4}$, T. Forst ${ }^{1}$, A. Pfützner ${ }^{1}$;

${ }^{1}$ IKFE Institute for Clinical Research and Development, Mainz, Germany,

${ }^{2}$ Diabetes, Takeda Pharma, Aachen, Germany,

${ }^{3}$ Cardiology, University Hospital, Cologne, Germany.

Background and Aims: Patients with Type 2 Diabetes suffer from excess cardiovascular risk and mortality. Early improvement of glycemic control and treatment of metabolic and vascular insulin resistance is required to improve patients' prognosis.

Materials and Methods: We performed this analysis of the patient population participating in the cross-sectional DIASPORA trial to investigate, whether knowledge about the glycemic disease status of patients visiting a specialist cardiology ward is associated with a different prevalence of macrovascular disease and plasma concentrations of several cardiovascular risk markers. The participating sites were asked to include 30 consecutively appearing patients at risk or confirmed of having CAD, without any other exclusion criteria. Patients with known diabetes mellitus were documented and patients with unknown glycemic status were asked to participate into a $75 \mathrm{~g}$ oral glucose tolerance test (OGTT). A blood sample was drawn for assessment of cardiovascular risk parameters (hsCRP, lipids, IL-6, MMP-9, MCP-1, sCD40L, ICAM, VCAM, E-selectin). Prevalence of macrovascular diseases (infaction (MI), stroke, angina pectoris (AP), peripheral or coronary artery disease) was assessed by a standardised questionnaire.

Results: A total of 539 patients with complete data sets could be included into the analysis ( 355 women, 184 men, age $(\operatorname{mean} \pm \mathrm{SD})$ : $68 \pm 7 \mathrm{yrs})$. Patients without diabetes (NoD; $\mathrm{n}=184$ ) had a lower prevalence of macrovascular disease $(58 \%)$ than patients with previously unknown diabetes (NEWD; $\mathrm{n}$ $=84,73 \%, \mathrm{p}<0.05$ vs. non-diabetic group) or patients with impaired glucose tolerance or impaired fasting glucose (IGT, $\mathrm{n}=115,70 \%, \mathrm{p}=0.077$; patients with known diabetes: OLDD; $\mathrm{n}=156,65 \%$, n.s.). An impaired laboratory risk marker profile was seen in patients with unknown diabetes or IGT vs. the nondiabetic group for hsCRP (e.g. NEWD: $3.6 \pm 2.6 \mathrm{mg} / \mathrm{l}$ vs. NoD: $2.8 \pm 2.1 \mathrm{mg} / \mathrm{l}$, $\mathrm{p}<0.05)$; MMP-9 ( $637 \pm 529$ vs. $441 \pm 302 \mathrm{ng} / \mathrm{ml}, \mathrm{p}<0.001)$; VCAM ( $810 \pm 319$ vs. $707 \pm 235 \mathrm{ng} / \mathrm{ml}, \mathrm{p}<0.005)$; E-selectin ( $62 \pm 29$ vs. $43 \pm 19 \mathrm{ng} / \mathrm{ml}, \mathrm{p}<0.001)$, which was accompanied by a significantly higher prevalence of MI and AP (NEWD/ IGT vs. NoD: $\mathrm{p}<0.05$ in both cases). No significant differences between the groups were seen for IL-6, ICM, sCD40L, MCP-1, or the lipid values.

Conclusion: Patients visiting cardiology specialists with previously unknown deterioration of glycemic control (diabetes or IGT) had a higher prevalence of macrovascular disease and an impaired laboratory risk marker profile as compared to patients with treated diabetes or without the disease. Our study confirms the importance of early diagnosis and appropriate treatment of diabetes patients suffering from macrovascular disease.

Supported by Takeda Pharma

\section{1}

Risk of coronary artery disease associated with initial sulphonylurea treatment of type 2 diabetes: a matched case-control study

S. M. Sadikot;

Jaslok Hospital \& Research Centre, Mumbai, India.

Background and Aims: Although glycemic control reduces cardiovascular risk, there have been concerns about reducing blood sugar with sulphonylureas. These agents are not entirely specific for beta cells, but also bind to ATP sensitive potassium channel receptors in the heart that could prevent ischemic preconditioning. Sulfonylureas are frequently prescribed as initial monotherapy or with the addition of metformin for up to 10 years, and may influence the risk of coronary events. However, the possible association between sulphonylurea treatment at diagnosis of diabetes and the subsequent development of CAD has not been studied. Further, newer sulphonylureas have a lower binding affinity to cardiac ATP sensitive potassium channel receptors and may not harm the heart. The aim of this CAD risk matched, case control study was to assess the association between different initial sulfonylurea treatments and the subsequent development of acute coronary events in type 2 diabetic patients.

Material and Methods: In a multicenter, retrospective, matched, case control study conducted in India, on type 2 diabetic outpatients with a complete case record going back to the date of diagnosis, with no history of CAD at the first clinical examination, the estimated 20 year CAD risk at diagnosis was calculated using the UKPDS risk engine. Cases were defined as patients who developed CAD during the period between the diagnosis of type 2 diabetes and the date of selection. Controls were identified as those patients free of CAD up-to the date of selection. The matching variable was the 20-year CAD risk score at diagnosis of diabetes. The odds-ratio of the retrospective frequency with which cases and controls received glibenclamide, glipizide, glimepiride, gliclazide or metformin as the first drug at diagnosis, or as the second add on drug was used to estimate the relative risk of CAD associated with the initial use of each drug.

Results: In 2733 patients free of CAD at diagnosis of type 2 diabetes, 76 developed stable angina, unstable angina or myocardial infarction. The remaining 2657, provided 152 matched controls. The hazard of developing CAD ( $95 \% \mathrm{CI}$ ) increased by 2.4 fold ( 1.3 to $4.3, \mathrm{P}=0.004)$ with glibenclamide, 2 fold ( 0.9 to $4.6, \mathrm{P}=0.099)$ with glipizide, and 2.9 fold (1.6 to $5.1, \mathrm{P}=0.000$ ) with either. The hazard decreased 0.3 fold ( 0.7 to $1.7, \mathrm{P}=0.385)$ with glimepiride, 0.4 fold ( 0.7 to $1.3, \mathrm{P}=0.192$ ) with gliclazide, and 0.4 fold ( 0.7 to $1.1, \mathrm{P}=0.09$ ) with either. There was no risk of CAD with metformin (Table 1).

Conclusion: Initiating treatment of type 2 diabetes with glibenclamide and glipizide is associated with increased risk of CAD in comparison to gliclazide

Table 1: Risk of developing coronary artery diseases with initial oral hypoglycaemic treatments in type 2 diabetes

\begin{tabular}{|c|c|c|c|c|c|}
\hline Initial drug & & $\begin{array}{l}\text { Cases with } \\
\text { CAD }(n=76)\end{array}$ & $\begin{array}{l}\text { Controls without } \\
\text { CAD }(n=152)\end{array}$ & $\begin{array}{l}\text { Odds ratio } \\
(95 \% \mathrm{CI})\end{array}$ & $\mathrm{P}$ \\
\hline Glibenclamide & $\begin{array}{l}\text { Yes } \\
\text { No }\end{array}$ & $\begin{array}{l}31 \\
45\end{array}$ & $\begin{array}{l}34 \\
118\end{array}$ & $2.4(1.3-4.3)$ & 0.004 \\
\hline Glipizide & $\begin{array}{l}\text { Yes } \\
\text { No }\end{array}$ & $\begin{array}{l}12 \\
64\end{array}$ & $\begin{array}{l}13 \\
139\end{array}$ & $2.0(0.9-4.6)$ & 0.099 \\
\hline Glimepiride & $\begin{array}{l}\text { Yes } \\
\text { No }\end{array}$ & $\begin{array}{l}7 \\
69\end{array}$ & $\begin{array}{l}20 \\
132\end{array}$ & $0.7(0.3-1.7)$ & 0.385 \\
\hline Gliclazide & $\begin{array}{l}\text { Yes } \\
\text { No }\end{array}$ & $\begin{array}{l}11 \\
65\end{array}$ & $\begin{array}{l}33 \\
119\end{array}$ & $0.6(0.3-1.3)$ & 0.192 \\
\hline $\begin{array}{l}\text { Older agents } \\
\text { (Glibenclamide } \\
\text { or Glipizide }\end{array}$ & $\begin{array}{l}\text { Yes } \\
\text { No }\end{array}$ & $\begin{array}{l}43 \\
33\end{array}$ & $\begin{array}{l}47 \\
105\end{array}$ & $2.9(1.6-5.1)$ & 0 \\
\hline $\begin{array}{l}\text { Newer agents } \\
\text { (Gliclazide or } \\
\text { Glimepiride) }\end{array}$ & $\begin{array}{l}\text { Yes } \\
\text { No }\end{array}$ & $\begin{array}{l}18 \\
58\end{array}$ & $\begin{array}{l}53 \\
99\end{array}$ & $0.6(0.3-1.1)$ & 0.09 \\
\hline Metformin & $\begin{array}{l}\text { Yes } \\
\text { No }\end{array}$ & $\begin{array}{l}19 \\
57\end{array}$ & $\begin{array}{l}37 \\
115 \\
\end{array}$ & $1.0(0.5-2.0)$ & 0.913 \\
\hline
\end{tabular}


or glimepiride. If confirmed, this may be important because present guidelines do not distinguish between individual sulfonylureas. (Tab. 1)

Supported by Serdia Pharmaceuticals (India) Pvt. Ltd.

\section{2}

Longitudinal study of primary and secondary medication adherence for cardometabolic therapies associated with type 2 diabetes

A. J. Karter' ${ }^{1}$, M. M. Parker ${ }^{2}$, H. H. Moffet ${ }^{2}$, J. Y. Liu' ${ }^{2}$, A. T. Ahmed²;

${ }^{1}$ Division of Research, Kaiser Permanente, Bainbridge Island,

${ }^{2}$ Division of Research, Kaiser Permanente, Oakland, United States.

Background and Aims: Medication non-adherence is well-documented, but may be underestimated. Pharmacy databases reveal medication dispensing (utilization) but generally not prescribing data. Thus, "primary nonadherence" (i.e., prevalence of patients who never fill a new prescription) could not be obtained from electronic pharmacy records alone. Further, refill-based adherence measures (e.g., proportion of days not covered) generally require at least two dispensings for the index to be stable, and thus excluded those who haven't filled a new medication at least twice ("early non-persistence"); therefore only "secondary adherence" (ongoing use with multiple fills) is typically reported.

Materials and Methods: We sought to identify all new prescriptions (no evidence of use in the previous 2 years) for antihyperglycemic, antilipemic and antihypertensive medications recorded by providers in an electronic medical record system from January 1, 2006 to June 30, 2006, among 210,000 diabetics members of an ethnically-diverse, managed care population (Kaiser Permanente). Using a "new user" design, we estimated medication adherence longitudinally, evaluating primary non-adherence, early non-persistence, and secondary adherence.

Results: We identified 49,403 new prescriptions ordered for 36,695 diabetic patients. $5.2 \%$ of these patients never filled their first new prescriptions during the study period (primary non-adherence). Among those $94.8 \%$ of patients who did fill their first prescription, $23.8 \%$ never refilled the prescription (early non-persistence). $26.1 \%$ of persistent patients (two or more dispensings) had poor secondary medication adherence $(<80 \%$ of time with sufficient medication supplies).

Conclusion: While a small proportion of patients fail to fill their first prescription, a substantial proportion of primary adherent patients fail to pick up their first refill (i.e., early non-persistent). Thus, approximately onequarter of patients fail to transition to being ongoing medication users. This form of nonadherence has been inadequately captured in previously published pharmacy-based studies of medication adherence. Further research and intervention development needs to include this at-risk group of nonadherent patients.

Supported by U.S. National Institutes of Diabetes and Digestive and Kidney Diseases and U.S. National Institute of Child Health and Human Development

\section{3}

Significant impact of the presence of type 2 diabetes on the cardiovascular risk conferred by the plasminogen activator inhibitor-1 $-6755 \mathrm{G} / 4 \mathrm{G}$ polymorphism

A. Muendlein ${ }^{1}$, C. H. Saely ${ }^{1,2}$, T. Marte ${ }^{1,2}$, P. Rein ${ }^{1,2}$, F. Schmid ${ }^{1,2}$, L. Koch ${ }^{1,2}$, P. Langer ${ }^{1}$, S. Aczel ${ }^{1,2}$, H. Drexel ${ }^{1,2}$;

${ }^{1}$ VIVIT Institute, Feldkirch, Austria,

${ }^{2}$ Internal Medicine, Academic Teaching Hospital Feldkirch, Austria.

Background and Aims: The -675 GG/4G polymorphism of the plasminogen activator inhibitor-1 (PAI-1) gene has been associated with increased plasma levels of PAI-1 and, in some studies, with increased vascular risk. Hardly any data on the vascular risk conferred by this polymorphism are available for patients with type 2 diabetes (T2DM). We therefore aimed at investigating i) the association of this polymorphism with angiographiaclly diagnosed coronary artery disease (CAD) and ii) its impact on future vascular events in patients with T2DM and in nondiabetic individuals.

Materials and Methods: Genotyping was performed in 672 consecutive Caucasian patients (463 men and 209 women, mean age $62 \pm 10$ years) undergoing coronary angiography for the evaluation of stable CAD. Prospectively, we recorded vascular events over 4 years.
Results: The prevalence rates of the $5 \mathrm{G} / 5 \mathrm{G}$, the $5 \mathrm{G} / 4 \mathrm{G}$, and the $4 \mathrm{G} / 4 \mathrm{G}$ genotypes were $17.6 \%, 52.7 \%$, and $29.7 \%$ in patients with T2DM $(n=148)$ and $23.5 \%, 48.3 \%$, and $28.2 \%$ in non-diabetic subjects $(\mathrm{n}=524)$. In non-diabetic subjects the homozygote PAI-1 4G4G genotype after adjustment for age, gender, hypertension, smoking, BMI, LDL cholesterol, and HDL cholesterol was significantly associated with significant stenoses (adjusted odds ratio (OR) 1.77 [95\% CI 1.13-2.78], $\mathrm{p}=0.013$ ), whereas no such association was observed in patients with T2DM (OR 0.81 [0.34-1.93]; $\mathrm{p}=0.635)$. An interaction term T2DM x 4G4G genotype was significant $(\mathrm{p}=0.026)$, indicating that the association of the polymorphism with CAD was significantly stronger in non-diabetic subjects than in patients with T2DM. Also prospectively, the 4G4G genotype conferred an increased risk of vascular events in non-diabetic subjects but not in T2DM patients, with hazard ratios of 1.66 [1.05-2.64], $\mathrm{p}=0.031$ and $0.72[0.33-1.60], \mathrm{p}=0.423$, respectively (adjusted for age, gender, hypertension, smoking, BMI, LDL cholesterol, and HDL cholesterol). Again, an interaction term T2DM x 4G4G genotype was significant $(\mathrm{p}=0.042)$.

Conclusion: We conclude that presence of T2DM significantly modulates the vascular risk conferred by the PAI-1 -675 5G/4G polymorphism.

\section{4}

The M55V polymorphism in SUMO4 gene is associated with macrovascular complications in type 2 diabetes

T. Shimada ${ }^{1}$, Y. Furukawa ${ }^{1}$, H. Furuta ${ }^{1}$, S. Matsuno ${ }^{1}$, A. Kusuyama ${ }^{2}$, M. Nishi ${ }^{2}$, H. Sasaki ${ }^{1}$, T. Sanke ${ }^{3}$, K. Nanjo ${ }^{1}$;

${ }^{1}$ The First Department of Medicine, ${ }^{2}$ Department of Metabolism and Clinical Nutrition, ${ }^{3}$ Department of Clinical Laboratory Medicine, Wakayama Medical University, Wakayama City, Japan.

Background and Aims: The small ubiquitin-like modifier (SUMO) family has emerged as one of key regulators of cellular stress responses. SUMO4, a novel member for the family, conjugates with inhibitor- $\kappa \mathrm{B} \alpha(\mathrm{I} \kappa \mathrm{B} \alpha)$ and negatively regulates the nuclear factor- $\kappa \mathrm{B}(\mathrm{NF}-\mathrm{\kappa B})$ signaling pathway. A single amino acid substitution, Met to Val at codon 55 (M55V), of SUMO4 has been identified and it has been reported that the Val type has lesser effect on suppression of NF- $\kappa B$ transcriptional activity than the Met type. Because the NF- $\kappa B$ activation is a key event in the development of diabetic vascular complications, the M55V polymorphism may be associated with increased risk of diabetic vascular complications through higher NF- $\mathrm{KB}$ activation. We examined the role of the $\mathrm{M} 55 \mathrm{~V}$ polymorphism on vascular complications in type 2 diabetic patients.

Materials and Methods: The SUMO4 M55V polymorphism was genotyped using TaqMan SNP genotyping assay system. We examined the association between the $\mathrm{M} 55 \mathrm{~V}$ polymorphism and the prevalence of macro- and microvascular complications in 423 Japanese type 2 diabetic patients. The diagnosis of coronary heart disease (myocardial infarction and angina pectoris) was based on symptoms and ischemic changes in an electrocardiogram, either at rest or exercise test, or on findings from coronary angiography. Cerebral infarction was diagnosed by a history of acute neurological deficits, together with positive findings on computed tomography and/or magnetic resonance imaging of the brain. Patients with $\geq$ grade 2 on the La Fontaine classification, with overt proteinuria and/or an elevated serum creatinine level $(\geq 1.2 \mathrm{mg} /$ $\mathrm{dl}$ ), and with $\geq$ simple retinopathy were assumed to have peripheral vascular disease, nephropathy and retinopathy, respectively.

Results: We found the $\mathrm{V}$ allele was more frequent in the patients with the history of coronary heart disease than in those without it $(p=0.033)$. The V allele was also frequent in the patients with the history of cerebral infarction than in those without it $(p=0.016)$. In the logistic regression analysis, the risk of coronary heart disease and cerebral infarction was significantly higher in patients with the $\mathrm{V} / \mathrm{V}$ genotype than in those with the M/M genotype (odds ratio [OR] 2.76, 95\% confidence interval [CI] 1.12-6.78, $p=0.027$ for coronary heart disease and OR $4.12,95 \%$ CI $1.49-11.36, p=0.006$ for cerebral infarction, respectively). Adjustment for sex, duration of diabetes, smoking status, hypertension status and dyslipidemia status did not appreciably change the associations (OR 2.85, 95\% CI 1.08-7.49, $p=0.034$ for coronary heart disease and OR 3.83, 95\% CI 1.25-11.74, $p=0.019$ for cerebral infarction, respectively). No significant differences were observed for other diabetic vascular complications such as peripheral vascular disease, nephropathy and retinopathy according to genotypes and alleles.

Conclusion: Our results suggest that the SUMO4 M55V polymorphism is associated with increased risk of coronary heart disease and cerebral infarction in type 2 diabetic patient.

Supported by Grant-in-Aid for Scientific Research on Priority Areas 


\section{PS 11 Gender, intrauterine environment and risk of diabetes}

\section{5}

Type 2 diabetes eliminates the relative protection against death in women G. W. D. Landman ${ }^{1}$, N. Kleefstra ${ }^{2,3}$, S. J. J. Logtenberg' ${ }^{2}$, H. J. G. Bilo ${ }^{2,4}$, K. H. Groenier

${ }^{1}$ Internal Medicine, Isala Clinics, Zwolle, ${ }^{2}$ Diabetes Centre, Isala Clinics, Zwolle, ${ }^{3}$ Langerhans, Medical Research Group, Zwolle,

${ }^{4}$ Internal Medicine, University of Groningen,

${ }^{5}$ General Practice, University of Groningen, The Netherlands.

Background and Aims: Several studies have suggested that, unlike in the general population, diabetic women have an even higher relative mortality rate than diabetic men. At the same time, the specific risk factors that are associated with mortality in both sexes are mostly comparable. We aimed to examine mortality rates of diabetic women and men compared to the general population and to identify gender specific risk factors for all cause mortality in a cohort of patient with type 2 diabetes mellitus (T2DM) in the Netherlands.

Materials and Methods: The ZODIAC study started in 1998. A group of 1145 patients with T2DM was seen annually, and data on mortality were assessed 5.8 years later on average. Mortality rates in male and female patients were evaluated using standardized mortality ratios (SMR) and the association of mortality with established risk factors was evaluated with the Cox Proportional Hazard Ratio. The following variables were selected in the model as possible risk factors: Smoking (yes or no), age, duration of diabetes, HbAlc, eGFR (Cockcroft), body mass index (BMI), hypertension (yes or no), Cholesterol/HDL ratio and macrovascular complications (yes or no).

Results: The study population consisted of 658 women and 487 men, a total of 335 patients died. SMR for all cause mortality was 2.15 (CI 1.85-2.49) in women and 1.75 (CI 1.48-2.05) in men. In diabetic women mortality was associated with age (HR 1.11; CI 1.09-1.14), glycaemic control (HbAlc) (HR 1.25; CI 1.12-1.41) and with macrovascular complications (HR 1.91; CI 1.36-2.70). In diabetic men mortality was associated with age (HR 1.06; CI 1.03-1.09), smoking (HR 1.78; CI 1.23-2.57), eGFR (HR 0.97; CI 0.96-0.99), and macrovascular complications (HR 1.51; CI 1.00-2.28).

Conclusion: Having diabetes appears to eliminate the relative protection women have on mortality compared to men. Our results reemphasize the magnitude of the association between diabetes and mortality. There were comparable risk factors in both genders, however, glycaemic control in women, smoking and eGFR in men appeared to be gender specific in this Dutch cohort of patients with T2DM.

These results confirm that the intensity of diabetes management should not be gender specific; women with diabetes mellitus type 2 should be treated (as aggressively) as men.

\section{6}

Increased risk of type 2 diabetes in men when compared to premenopausal as well as postmenopausal women, aged 35-66 years A. Hilding ${ }^{1}$, A.-K. Eriksson ${ }^{2}$, S. Efendic ${ }^{1}$, C.-G. Östenson ${ }^{1}$;

${ }^{1}$ Dept. of Molecular Medicine and Surgery, The Endocrine and Diabetes Unit, ${ }^{2}$ Dept. of Medicine, Unit of Clinical Epidemiology, Karolinska Instititet, Stockholm, Sweden.

Background and Aims: Prevalence of newly diagnosed type 2 diabetes (T2D) is two-fold higher in men than in women in a diabetes screening program in Stockholm, Sweden. We have studied the impact of menopause on this gender difference.

Materials and Methods: Healthy Swedish men $(\mathrm{n}=3,128)$ and women (n $=4,821$ ), aged $35-56$ years, living in the Stockholm area, participated in a baseline investigation, performed 1992-1994 and 1996-1998, respectively. Approximately $50 \%$ had a family history of diabetes (FHD). They all underwent an oral glucose tolerance test (OGTT) and responded to a questionnaire regarding e.g. lifestyle and for women menopausal status and hormone replacement therapy (HRT). Of those not having newly diagnosed T2D at baseline (65 men and 63 women) and still living in the same area, $87 \%$ of the men $(2,383 / 2,746)$ and $76 \%$ of the women $(3,329 / 4,365)$ were reinvestigated with a new OGTT and questionnaire ten or eight years later, respectively. Newly diagnosed T2D at the follow-up examination was found in 99 men and 58 women. Odds ratios (OR) for T2D was assessed, adjusted for major confounders, e.g. age, FHD, BMI, physical activity and smoking. Results: At both baseline and follow-up the prevalence of newly diagnosed T2D was higher in men than in women, $2.1 \%$ and $4.2 \%$ respectively for men vs. $1.3 \%$ and $1.7 \%$ respectively for women. At baseline the female study sample comprised both premenopausal and postmenopausal (median duration two yrs) women. Menopausal status was however not associated with T2D, adjusted OR was 0.81 (95\%CI: 0.33-1.95) when comparing preand postmenopausal women. At baseline a similar increased risk of T2D was found in men when compared to premenopausal women, $\mathrm{OR}=1.89$ (1.06-3.36) as when compared to postmenopausal women, OR=1.93 (1.053.54). The OR for T2D associated with male sex was 1.70 (0.81-3.57) when compared to postmenopausal women not treated with HRT. At follow-up the majority of the women, now aged 46-63 yrs, were postmenopausal (median duration six yrs), and only a limited number of cases were identified among the premenopausal. Thus, comparison between men and postmenopausal women at follow-up again showed an increased risk of T2D in men, OR=2.33 (1.49-3.67). Specifically, when comparing men with the subgroup of postmenopausal women not treated with HRT OR=1.72 (1.00-2.98).

Conclusion: Our data in Swedish middle-aged subjects indicate that menopausal status has no obvious influence on the risk of T2D in women, and that men are at an increased risk as compared to women, irrespective of menopausal status.

Supported by Stockholm County Council, Swedish Council for Working Life and Social Research, Novo Nordisk Scandinavia

\section{7}

Perinatal mortality, preterm birth and macrosomia in offspring of women with type 1 diabetes in Norway

I. Eidem ${ }^{1,2}$, L. C. Stene ${ }^{1,2}$, T. Henriksen ${ }^{3}$, K. F. Hanssen ${ }^{2,4}$, S. Vangen ${ }^{1,5}$, G. Joner ${ }^{2,6}$;

${ }^{1}$ Division of Epidemiology, Norwegian institute of Public Health, ${ }^{2}$ Diabetes Research Centre, Aker and Ullevål University Hospitals, ${ }^{3}$ Department of Obstetrics and Gynaecology, the National Hospital, ${ }^{4}$ Department of Endocrinology, Aker University Hospital,

${ }^{5}$ Department of Obstetrics and Gynaecology, Ullevål University Hospital, ${ }^{6}$ Department of Paediatrics, Ullevål University Hospital, Oslo, Norway.

Background and Aims: The St. Vincent declaration (1989) stated that within a five-year period, the risk of adverse pregnancy outcome among women with type 1 diabetes should be lowered to a level equal to the background population. Recent European studies have shown that this goal has not yet been met. The objective of the study is to compare pregnancy outcome in women with type 1 diabetes with the Norwegian background population. Materials and Methods: By a record linkage of the Norwegian Childhood Diabetes Registry (NDR) of Norway and the Medical Birth Registry of Norway, we designed a population based cohort study with a highly reliable type 1 diabetes diagnosis from the NDR. From 1975 to 1998, 1025 births of women with type 1 diabetes occurred in our cohort. From 1999 to 2004, 419 births occurred.

Results: We present preliminary results for perinatal mortality (stillbirth after 28 completed weeks of pregnancy or postnatal death up to 7 days after birth), preterm birth (<37 completed weeks of gestation) and macrosomia (birth weight $>=4500 \mathrm{~g}$ ). Among women with type 1 diabetes the perinatal mortality for the period $1975-98$ was $1.9 \%$ compared with $0.89 \%$ in the background population RR (relative risk) 2.2 (95\% CI: 1.4-3.4). For the period 1999-2004 the perinatal mortality was $1.7 \%$ among women with type 1 diabetes and $0.55 \%$ in the background population RR 3.1 (95\% CI: 1.5-6.5). The risk of preterm delivery $1975-98$ was $27.4 \%$ among women with type 1 diabetes and $6.5 \%$ in the background population RR 4.2 (95\% CI: 3.8-4.7). For the period 1999-2004 the risk was $27.6 \%$ among women with type 1 diabetes and $7.7 \%$ in the background population RR 3.6 (95\% CI: 3.1-4.2). The risk of macrosomia was for the period 1975-98 8.1\% among women with type 1 diabetes and 3.3\% in the background population RR 2.4 (95\% CI: $2.0-$ 3.0). For the period $1999-2004$ the risk was $12.2 \%$ among women with type 1 diabetes and 4.6\% in the background population RR 2.7 (95\% CI: 2.1-3.5).

Conclusion: Based on our results, Norwegian women with type 1 diabetes still have a higher risk of perinatal mortality, preterm birth and macrosomia in their babies compared to women in the background population.

Supported by Helse øst, Norway 


\section{8}

Gestational hypertension and the offspring's manifestations of metabolic syndrome

S. Miettola ${ }^{1}$, A.-L. Hartikainen ${ }^{2}$, A. Bloigu ${ }^{3}$, A. Ruokonen ${ }^{4}$, M.-R. Järvelin ${ }^{5,6}$, A. Pouta ${ }^{7}$

${ }^{1}$ Department of medicine, University of Oulu, Finland,

${ }^{2}$ Department of Obstetrics and Gynecology, University of Oulu, Finland, ${ }^{3}$ Department of Public Health, National Public Health Institute, Oulu, Finland, ${ }^{4}$ Department of Clinical Chemistry, University Hospital of Oulu, Finland, ${ }^{5}$ Department of Epidemiology and Public Health, Imperial College of London, United Kingdom, ${ }^{6}$ Department of Public Health Science and General Practise, University of Oulu, Finland, ${ }^{7}$ Department of Child and Adolescent Health, National Public Health Institute, Oulu, Finland.

Background and Aims: The impact of de novo hypertension in pregnancy, i.e. pre-eclampsia (PE) and transient gestational (nonproteinuric) hypertension (GH), on the long-term outcome of offspring is poorly understood. There are no previous studies on the association between maternal GH and offspring's metabolic outcomes. Three previous studies have shown that children born to mothers with PE had slightly more elevated blood pressures (BP) than the reference group.

Material and Methods: The data for the study were obtained from the Northern Finland 1986 Birth Cohort (NFBC 1986) and included all singleton pregnancies of the 9247 mothers and their offspring born in 1985-86. The clinical follow-up of the offspring was conducted at age 16 years with $74 \%$ response rate $(\mathrm{N}=6798)$. We allocated the mothers into three different groups: 1) GH group with $\mathrm{BP}>140 />90 \mathrm{mmHg}(\mathrm{n}=267) 2) \mathrm{PE}$ group with $\mathrm{BP}$ $>140 />90 \mathrm{mmHg}$ and proteinuria $(\mathrm{n}=182) 3)$ reference group with normal $\mathrm{BP}(\mathrm{n}=7006)$.

Results: Offspring of the mothers with GH had higher BP compared to the reference group (Table 1). They also had higher fasting cholesterol and apolipoprotein B levels. No difference was seen in HOMA-S levels. Adjustments for birth weight, gestational age and current BMI did not change these differences. There were no differences in the BP, lipid, glucose or insulin levels between the offspring of the PE mothers and the reference group.

Conclusions: The features of metabolic syndrome were clearly seen in the offspring of GH mothers. This risk group should be noted already in childhood by planning preventive strategies already before potential clinical manifestation of metabolic syndrome.

Table 1. Metabolic outcome measures between the offspring of mothers with GH and the reference offspring.

\begin{tabular}{llll}
\hline Variable & $\begin{array}{l}\text { Reference }(\mathrm{n}=7006) \\
\text { Mean }(95 \% \mathrm{CI})\end{array}$ & $\begin{array}{l}\text { GH }(\mathrm{n}=267) \\
\text { Mean }(95 \% \mathrm{CI})\end{array}$ & $\begin{array}{l}\text { GH vs. Reference } \\
\text { P value* }\end{array}$ \\
\hline SBP, mm Hg & $115(115-115)$ & $120(118-122)$ & $<0.001$ \\
$\mathrm{DBP}, \mathrm{mm} \mathrm{Hg}$ & $67(67-68)$ & $71(70-72)$ & $<0.001$ \\
$\mathrm{MAP}, \mathrm{mm} \mathrm{Hg}$ & $83(83-84)$ & $87(86-88)$ & $<0.001$ \\
$\mathrm{BMI}, \mathrm{kg} / \mathrm{m}^{2}$ & $21.1(21.0-21.2)$ & $21.6(21.1-22.1)$ & 0.088 \\
$\mathrm{LDL}, \mathrm{mmol} / \mathrm{L} \#$ & $2.17(2.16-2.19)$ & $2.27(2.19-2.36)$ & 0.054 \\
$\mathrm{HDL}, \mathrm{mmol} / \mathrm{L} \#$ & $1.38(1.37-1.39)$ & $1.40(1.36-1.45)$ & 0.494 \\
$\begin{array}{l}\text { Cholesterol, } \\
\text { mmol/L\# }\end{array}$ & $4.18(4.16-4.20)$ & $4.35(4.24-4.47)$ & 0.007 \\
$\begin{array}{l}\text { Triglycerides, } \\
\text { mmol/L\# }\end{array}$ & $0.75(0.74-0.76)$ & $0.74(0.70-0.79)$ & 0.871 \\
$\begin{array}{l}\text { Lipoprotein, } \\
\text { apo A1, g/L }\end{array}$ & $1.36(1.36-1.37)$ & $1.38(1.35-1.41)$ & 0.484 \\
$\begin{array}{l}\text { Lipoprotein, } \\
\text { apo B, g/L }\end{array}$ & $0.67(0.66-0.67)$ & $0.70(0.67-0.72)$ & 0.021 \\
HOMA-S & $87.16(86.09-88.23)$ & $90.00(84.66-95.34)$ & 0.520 \\
\hline
\end{tabular}

\#geometric mean (95\% confidence interval); ${ }^{*}$ Dunnett test

Supported by the University of Oulu, Department of Medicine

\section{9}

Socio-economic position at birth, but not birth weight, is independently related to metabolic syndrome at age $\mathbf{5 0}$

N. Unwin, M. Pearce;

Institute of Health and Society, Newcastle University, United Kingdom.

Background and Aims: The risk of cardiovascular disease in middle to older age has been associated with measures of socio economic status throughout the life course. It has been suggested that associations found with anthropometric measures at birth are strongly confounded by factors related to socio-economic status in early and later life. However, this remains controversial and difficult to test given the rarity of data sets that contain all the variables of interest at different stages of life. Our aim was to investigate the relationship between metabolic syndrome at age 50 and socioeconomic status at different points in the life course, and to examine potential determinants of any associations found.

Materials and Methods: We used data from a cohort of 1142 births in May and June 1947 in the North of England on whom detailed social and anthropometric data were collected up to the age of 15 . Birth weights were standardised for gestational age and gender. At age 50 years, 412 study members (180 men and 212 women, representative of the original cohort) attended for a clinical examination, which included anthropometry, blood pressure, fasting lipids and glucose, and questionnaire data on diet, smoking, alcohol consumption and physical activity. Data on social class (SC) were available at birth, and ages 25 and 50 years, and divided into three categories (social class $1 \& 2 ; 3$ non-manual and manual; $4 \& 5$ and unemployed). Metabolic syndrome (MS) was defined following the IDF 2005 definition. Results are described for men and women together, with any differences between them highlighted.

Results: MS was present in $27.6 \%$ (33.7\% men and $22.9 \%$ women, $\mathrm{p}=0.018$ ). It was strongly related to low SC at birth $(11.6 \%, 26.3 \%$ and $34.9 \%$ across the three groups respectively, $\mathrm{p}$ for trend $=0.004)$, not at age $25(\mathrm{p}=0.5)$ and associated with SC at age $50(22.9 \%, 29.1 \%$ and $37.0 \%, p=0.02)$. The relationship of MS with SC at birth was largely independent of SC at 50: unadjusted odds ratio for SC 3 vs SC 1 at birth 4.3 (95\% CIs 1.6 to 12.1); OR adjusted for SC at age 50, 3.7 (1.3 to 10.6). There was no evidence of a relationship between social mobility between birth and 50 (up or down) and risk of MS. Of the components of MS, low SC at birth was associated with higher waist circumference, triglycerides and low HDL cholesterol, but not with fasting glucose or blood pressure. There was no relationship $(p>0.2)$ between birth weight and MS at age 50. Low SC at birth was not associated with birth weight, but was associated with earlier age of weaning (means across the three sc groups: 168 days, 159 and 151, p for trend 0.02). Low SC at birth was associated with smoking at age 50 , total pack years smoked and with low levels of physical activity at age 50, but not with measures of diet. However, controlling for all of these factors significantly related to SC at birth had limited impact on the relationship with metabolic syndrome at age 50 (adjusted OR for MS, lowest compared to highest SC 3.4, 1.2 to 9.8).

Conclusion: SC at birth, but not birth weight, was strongly, and independently, associated with the risk of metabolic syndrome at age 50 . This may represent the influence of early life experience on programming future metabolism, or the fact that low SC in infancy is associated with the accumulation of risk over the life course. Our data largely support the former hypothesis, given the independent association of SC at birth with MS at age 50. Supported by Wellcome Trust

\section{0}

Association of intrauterine exposure to maternal diabetes and obesity and type 2 diabetes in youth: SEARCH Case-Control (SEARCH CC) Study

D. Dabelea ${ }^{1}$, E. J. Mayer-Davis ${ }^{2}$, A. Pande ${ }^{2}$, R. B. D’Agostino Jr. ${ }^{3}$, A. D. Liese ${ }^{2}$, K. Narayan ${ }^{4}$, R. F. Hamman';

${ }^{1}$ Preventive Medicine and Biometrics, University of Colorado Health Sciences Center, Denver, United States, ${ }^{2}$ Epidemiology and Biostatistics, University of South Carolina School of Public Health, Columbia, ${ }^{3}$ Public Health Sciences, Wake Forest University School of Medicine, Winston-Salem, ${ }^{4}$ Epidemiology, Emory University School of Public Health, Atlanta, United States.

Background and Aims: Intrauterine exposure to maternal diabetes is associated with an increased risk for type 2 diabetes (T2DM) in the offspring. 
Several studies have also shown an independent association of maternal obesity with excessive fetal adiposity, but there are limited data on longterm consequences on offspring T2DM risk. We hypothesized that exposure to maternal DM and maternal obesity in utero would be independently associated with increased T2DM in African American (AA), Hispanic (H), and non-Hispanic white (NHW) youth.

Materials and Methods: We used data from SEARCH CC, an ancillary study to SEARCH for Diabetes in Youth. Seventy-nine youth with T2D age 10-24 yr attended a research visit at the Colorado and South Carolina sites, where nondiabetic control youth $(\mathrm{n}=190)$ were also recruited. Information on in utero exposure to maternal diabetes was recalled by biological mothers. Maternal pre-pregnancy body mass index (BMI) based on self-reported weight and measured height was used to estimate in utero exposure to overweight/ obesity (BMI $\geq 25)$.

Results: Prevalence (\%) of in utero exposure to diabetes was higher among youth with T2DM than among controls ( $30.4 \mathrm{v} 6.3, \mathrm{p}<0.0001)$, and it was similarly higher across racial/ethnic groups $(30.2 \mathrm{v} 3.8$ for AA; $35.7 \mathrm{v} 6.5$ for $\mathrm{H} ; 27.3 \mathrm{v} 7.5$ for NHW; respectively, $\mathrm{p}=0.7$ for interaction between race and DM exposure). In multiple logistic regression, after adjusting for offspring age, sex and race/ethnicity, in utero exposure to maternal DM [Odds Ratio (OR) $5.7(95 \% \mathrm{CI}=2.4-13.4)]$ and in utero exposure to maternal obesity [2.8 (95\% CI=1.5-5.2)] were independently associated with T2DM in the offspring. Adjustment for other potential confounders (paternal DM, birth weight, breastfeeding, maternal age at index pregnancy, education, income, smoking and alcohol consumption during pregnancy, and current BMI) did not substantially alter these associations. When current offspring BMI $\mathrm{z}$-score was added to the model, the OR for the association between in utero exposure to DM and offspring T2DM was unchanged. However, the OR for the association between in utero exposure to maternal obesity and offspring T2DM was attenuated toward to the null value $(\mathrm{OR}=1.1 ; 95 \% \mathrm{CI}=0.5-2.4)$, suggesting complete mediation through obesity development in offspring of obese mothers.

Conclusion: Exposure to maternal DM in utero is strongly associated with T2DM in youth in all ethnic groups, including African Americans, Hispanics, and non-Hispanic White youth, with a magnitude of effect similar to that seen among Pima Indians. Exposure to maternal obesity in utero is independently associated with T2DM, but this association is completely mediated through development of obesity in the offspring.

Supported by R01 DK59184

\section{PS 12 Social and economic complications of diabetes}

\section{1}

Depressive symptoms and risk of type 2 diabetes and prediabetes in a prospective study of middle-aged Swedish men and women C.-G. Östenson ${ }^{1}$, A.-K. Eriksson ${ }^{1}$, F. Granath ${ }^{2}$, A. Hilding ${ }^{1}$, S. Efendic ${ }^{1}$, A. Ekbom²;

${ }^{1}$ Molecular Medicine and Surgery,

${ }^{2}$ Medicine, Karolinska Institutet, Stockholm, Sweden.

Background and Aims: Depression is suggested to be a risk factor involved in the development of type 2 diabetes (T2D). The aim of the present study was to analyse depressive symptoms in association with T2D and prediabetes. Material and Methods: The study group consisted of 5432 subjects that were followed-up in a prospective population-based cohort of 6725 Swedish middle-aged persons with normal glucose tolerance (NGT) in five municipalities (Stockholm Diabetes Prevention Programme). Glucose tolerance was measured by OGTT at baseline and $51.9 \%$ of study persons had a family history of diabetes by study design. Depressive symptoms at baseline were measured by 6 questions with four answering alternatives (ranging from "never" to "frequently"), concerning sleep difficulties, apathy, anxiety, depression, tiredness and back/shoulder pain during the last twelve months. Each answer was given 1-4 points and a summed index score for all 6 questions (reliability measured by Cronbach's alpha 0.75 for men and 0.78 for women) was created and divided into quartiles, where the lowest scoring quartile was considered unexposed to depressive symptoms. Glucose tolerance at follow-up 8-10 years later was measured by OGTT. Odds ratios (OR:s) for a state of prediabetes (impaired fasting glucose, IFG; impaired glucose tolerance, IGT; and IFG + IGT) or T2D at follow-up, were estimated in association to depressive symptoms at baseline and controlled for potential baseline confounders.

Results: At follow-up, 111 men and 60 women with baseline NGT had developed T2D and a total of 255 men and 181 women were classified with a state of prediabetes. About $60 \%$ of T2D cases were detected by OGTT and $40 \%$ were self-reported to have occured at any point during the follow-up period. The mean index sum of depressive symptom points for men were $11.2 \pm 3.3$ and for women 13.4 \pm 3.6 . In men, age-adjusted OR:s for T2D in association with depressive symptoms were 3.6 (CI 1.9-6.7) for the upper quartile and 1.8 $(1.0-3.4)$ for middle quartiles $\left(2^{\text {nd }}+3^{\text {rd }}\right.$ quartiles $)$. Corresponding estimates for pre-diabetes in men were $1.7(1.2-2.5)$ in the upper quartile and $1.0(0.7-$ $1.4)$ in middle quartiles. When adjusted for BMI, family history of diabetes, smoking, physical activity at leisure time and socio-economic position the OR:s for T2D in men were $2.6(1.3-5.0)$ and $1.5(0.8-2.9)$, respectively for upper and middle quartiles and for pre-diabetes $1.5(1.0-2.2)$ and $1.0(0.7-$ 1.4). In women, age-adjusted OR for T2D was 0.9 (0.4-1.7) for the upper quartile and $0.8(0.4-1.4)$ for the middle quartiles. OR:s for prediabetes were $1.8(1.1-2.9)$ and $1.6(1.0-2.4)$ for upper and middle quartiles, respectively. When adjusting for all named potential confounders OR for T2D in women was $0.6(0.3-1.2)$ in the upper quartile and $0.7(0.4-1.4)$ in middle quartiles and for prediabetes $1.4(0.8-3.3)$ and $1.5(0.9-2.3)$ for upper and middle quartiles, respectively.

Conclusions: The results indicate that depressive symptoms increase the risk of T2D and pre-diabetes in Swedish middle-aged men. No association between depressive symptoms and T2D was present in women in this study, although there was a weak association to prediabetes.

Supported by Stockholm County Council, Swedish Council of Working Life and Social Research, Novo Nordisk Scandinavia, Glaxo Smithkline Sweden 


\section{2}

Trends in mental health in the diabetes population of Alberta, Canada, 1996-2005

L. C. Brown ${ }^{1,2}$, L. W. Svenson ${ }^{1,3}$, C. A. Beck ${ }^{4,5}$, S. U. Vermeulen ${ }^{2}$, J. A. Johnson ${ }^{1,2}$

${ }^{1}$ School of Public Health, University of Alberta, Edmonton,

${ }^{2}$ Alliance for Canadian Health Outcomes Research in Diabetes, Institute of Health Economics, Edmonton,

${ }^{3}$ Health Surveillance Branch, Alberta Health and Wellness, Edmonton,

${ }^{4}$ Department of Community Health Sciences, University of Calgary,

${ }^{5}$ Department of Psychiatry, University of Calgary, Canada.

Background and Aims: Research demonstrates an association between diabetes and several mental illnesses, including depression, bipolar disorder, and schizophrenia. Comorbid mental illness has been shown to increase the risk of complications, functional disability, healthcare expenditures, and mortality in people with diabetes.

Materials and Methods: Diagnostic codes (ICD-9-CM/ICD-10-CA) were extracted from population-based administrative health databases maintained by the Alberta Ministry of Health and Wellness for affective disorders, anxiety disorders, and organic and non-organic psychoses among individuals 20 years of age and older from the province of Alberta, Canada. Individuals with diabetes were identified using the algorithm from the National Diabetes Surveillance System (NDSS). Descriptive analyses present trends over time (1996-2005) and average annual prevalence of mental illnesses for people with and without diabetes. Rates were age-standardized using the 2001 Alberta population as the reference.

Results: People with diabetes had a higher prevalence of all mental illnesses compared to people without diabetes, and this finding was consistent over the examined time period (1996-2005). Affective and anxiety disorders were more than $30 \%$ higher $(\mathrm{p}<0.05)$ in people with diabetes, but the excess was limited to people under the age of 50 . The prevalence of non-organic psychoses were more than 2.0 times higher $(\mathrm{p}<0.05)$ in people with diabetes, with increasing frequency in people with diabetes in the past five years. The increased risk of psychoses in diabetes was apparent across all ages $(p<0.05)$. Conclusion: People with diabetes had a higher prevalence of affective disorders, anxiety disorders, and organic and non-organic psychoses compared to their non-diabetic counterparts. Given the increased prevalence of mental illness in people with diabetes, research should focus on strategies to minimize complications and mortality in this population with comorbidity. Supported by Alberta Health and Wellness

\section{3}

Prescriptions costs and socioeconomic differences in prevalence of diabetes: the population-based Torino Study

G. Bruno ${ }^{1}$, L. Karaghiosoff ${ }^{2}$, F. Pomero ${ }^{1}$, O. Segre ${ }^{1}$, C. Zucco ${ }^{1}$, F. Merletti ${ }^{3}$, G. Costa' ${ }^{2}$, R. Gnavi ${ }^{2}$

${ }^{1}$ Internal Medicine, ${ }^{2}$ Public Health,

${ }^{3}$ Cancer Epidemiology Unit, University, Torino, Italy.

Background and Aims: Current comprehensive economic data on the costs of diabetes are required for policy decisions to optimise resource allocation and to evaluate different approaches for disease management. No populationbased data are available at present in Italy. As part of a surveillance programme based on administrative data, we have assessed variations in prevalence of diabetes by age, sex and socio-economic status and prescription costs in an Italian population-based cohort.

Materials and Methods: People with diabetes residents of Torino on July 31, $2003(n=897,743)$ were identified through three data sources: the regional register of persons with diabetes, the file of persons discharged from hospitals with a diagnosis of diabetes (ICD9-CN = 250) and the file of persons who received at least two prescriptions of antidiabetic drugs $(\mathrm{ATC}=\mathrm{A} 10)$. Data sources were linked to the Torino population register to obtain individual data on educational level and census tract median income. Missing cases were estimated by using the capture-recapture method. All prescriptions registered in the 12-months period August 1, 2003- July 31, 2004 were examined to compare prevalence of treatment and costs in diabetic (n. 34 420 ) and non-diabetic people (n. 867500 ). All rate ratios were age and sexstandardized.
Results: Diabetes prevalence adjusted for undercount was 4.91\% (95\% CI 4.69-5.22) among men and 4.68\% (4.41-5.08) among women. Prevalence ratios between low and high socio-economic level were 1.54 (1.48-1.59) in men, and $2.42(2.32-2.54)$ in women. Results were not modified by age; indeed, prevalence ratios were higher in low than in high socioeconomic level and in women than in men in all age groups $(<65,65-74,>74 \mathrm{yrs})$. In most categories prevalence of treatment was higher among diabetic people than among those without diabetes, with a prevalence ratio $=3.67$. The highest prevalence was found for cardiovascular drugs (particularly for expensive drugs such as ACE-inhibitors, ARB and calcium-antagonists), which were prescribed to $79.9 \%$ of diabetic people.

Conclusion: this study shows: 1) feasibility of a surveillance program of diabetes using routinely collected data; 2) increasing temporal trend in prevalence; 3 ) socio-economic inequalities in risk of the disease, particularly in women, in all age groups; 4) four-fold higher prescriptions cost in diabetic than in non-diabetic people.

Supported by Piedmont Region

\section{4}

Lifetime risk of diabetes: the AusDiab study

A. Peeters ${ }^{1}$, D. J. Magliano ${ }^{1,2}$, W. J. Nusselder ${ }^{3}$, E. L. M. Barr ${ }^{2}$, D. Liew ${ }^{1}$, P. Z. Zimmet ${ }^{2}$, J. E. Shaw ${ }^{2}$;

${ }^{1}$ Epidemiology \& Preventive Medicine, Monash University, Melbourne, Australia, ${ }^{2}$ International Diabetes Institute, Caulfield, Australia,

${ }^{3}$ Public Health, Erasmus MC, Rotterdam, The Netherlands.

Background \& Aims: Incidence rates for diabetes are increasing rapidly worldwide with a doubling in prevalence in some countries such as Australia since the 1980s. There has been no estimate of the implications of this on the lifetime risk of developing diabetes, or duration of life that a population can expect to live with and without diabetes. The aim of this paper is to estimate the lifetime risk of diabetes, the number of years lived free of, and the number of years lived with diabetes for the Australian adult population from the year 2000.

Material and Methods: Data from the Australian, Diabetes, Obesity and Lifestyle (AusDiab) study (a national population based study of 11,247 adults aged $\geq 25$ ) were used to estimate the age-specific incidence rate of diabetes. Presence of diabetes was assessed based on a single oral glucose tolerance test. Age-specific mortality rates for the diabetic and non-diabetic populations were estimated by combining national mortality rates with the age-specific prevalence of diabetes and relative risk of mortality in people with diabetes from AusDiab. These three transition rates were used to construct multi-state life tables, to simulate the progress of a cohort of 25 year old Australians. From these life tables, residual lifetime risk of diabetes, average life years lived free of diabetes, and average life years lived with diabetes were derived. Life tables were also constructed for a cohort of people with diabetes from age 25 , from which life expectancy of a diabetic population was derived.

Results: Within the AusDiab population, the observed incidence of diabetes was 7.8 per 1000 person years. The crude relative risk of mortality associated with diabetes was 4.2 , but this varied by age, with relative risks decreasing with increasing age. If the rates of mortality and diabetes incidence observed in the year 2000 continued we would expect $20 \%$ of 25 year olds to develop diabetes by age 65 , and $37 \%$ to develop diabetes at some time throughout their life. A cohort of twenty-five year old Australians has a remaining life expectancy of 54 years (until age 79) - with 47 of these expected to be lived free of diabetes and seven with diabetes. Due to their increased risk of mortality, the diabetic population has a decreased life expectancy. A 55 year old person with diabetes can expect to live four years less than a person free of diabetes at age 55 , and 10 years less than a 55 year old person who remains diabetes-free throughout life.

Conclusion: Based on the mortality and diabetes incidence rates observed in the year 2000, diabetes will exert a significant burden on the future health of Australians, with more than a third of individuals developing diabetes within their lifetime. An appreciation of the implications of the current trends is imperative for appropriate public health and clinical responses. Supported by the National Health \& Medical Research Council and in part by a VicHealth research fellowship 


\section{5}

The challenges of diabetes care in changing times

T. Dugal;

Diabetes and Endocrinology, St Georges Hospital,Tooting, London, United Kingdom.

Background and Aims: Provision of specialist services in primary care has been shown to be associated with reduced admission rate. With the drive to get diabetes care in the community, integration of primary and secondary health care to facilitate patient care is an important step in improving patient outcomes. We present a baseline and follow up audit of 26 practices in South West London. Data was collected from computerised diabetes registers on a template. Development of structured professional education to facilitate patient repatriation through a pioneering programme, Community Rolling Education Programme (CD-REP) was also initiated to enhance patient care and awareness. The introduction of recent Department of Health "Payment by results" and "Quality outcomes framework" scores has contributed to improvement of care in primary care. This audit looks at the impact of all these factors on care of patients with diabetes in community.

Materials and Methods: Data of 4167 patients was collected over fifteen months.2250 males, mean age 62 years and 1917 females, mean age 65 . Prevalence of diabetes matched national average of $3 \%$. Ethnicity of the population was mixed. $48 \%$ were on oral hypoglycaemics, $22 \%$ diet controlled and $16 \%$ on insulin. The body mass index (BMI) was normal $(<25)$ in $25 \%$ and $63 \%$ were pre-obese or obese. $67 \%$ patients had blood pressure within the guidelines $(<145 / 85)$. $44 \%$ had $\mathrm{HbA1C}$ less than $7.4 \%$ and $12 \%$ had more than $10 \%$. Total cholesterol (TC) was below target in $57 \%$. HDL cholesterol was $>1 \mathrm{mmol} / \mathrm{L}$ in $82 \%$ and $\mathrm{LDL}$ was $>3 \mathrm{mmol} / \mathrm{L}$ in $41 \%$. Primary care had $44 \%$ patients, while $33 \%$ were managed in shared care; in $22 \%$ care was not recorded. Mean age was 64 years in practice only and 61years in shared care. $8 \%$ patients had T1 diabetes (T1DM) and 92\% T2 diabetes (T2DM). Only $3 \%$ of T1DM was managed in community, $97 \%$ was in shared care. $51 \%$ of T2DM were managed in shared care and $49 \%$ in practice only. $75 \%$ of diet controlled patients were in primary care, $60 \%$ of patients on oral hypoglycaemics were in shared care. $95 \%$ patients on insulin were in shared care.

Results: It was noted that surveillance of patients with diabetes in the community improved with more patients reaching targets for blood pressure and $\mathrm{TC}$ although no change in $\mathrm{HbAlC}$ was noted. There was variation by where the care was delivered. HbA1C was $7.6 \%(7.5-7.7)^{*}$ in practice and 8.2 $(8.1-8.3)^{*}$ in shared care. Mean systolic pressure was $139 \mathrm{mmHg}(138-140)^{*}$ in practice patients and $137 \mathrm{mmHg}(136-138)^{*}$ in shared care. The outcomes monitored over two years in a sub group of patients transferred from shared care to primary care showed an increase in $\mathrm{HbAlC}$ from 6.9\% (S.D0.94) to 7.1 (S.D1.2), creatinine increased from 86.2 (S.D 22.700 to 93.5 (S.D 29.00, TC 4.3 (S.D 0.78 ) to 4.2 (S.D 0.69). The number of referrals to secondary care also fell by $16 \%$ in this period.

Conclusion: The quality of care has improved in the community but tighter glucose control is needed. Specialist backup and support should be available to primary care to have seamless diabetes care and cannot be undermined. This role needs to be developed further also for exchange of expertise, patient repatriation and education in the community. Department of health targets have also meant better care of diabetes in the community. Further and on going assessment of the changing patterns of care are needed.

\section{6}

The impact of diabetes mellitus on the health of the Taiwanese elder population

T.-Y. Tai ${ }^{1}$, W.-C. Chang ${ }^{2}$;

${ }^{1}$ Director General Office, Taipei Jen Chi Relief Institution,

${ }^{2}$ Division of Gerontology Research, National Health Research Institutes,

Taipei City, Taiwan.

Background and Aims: According to the National Health Insurance (NHI) claim data, the prevalence rate of DM in Taiwan read 3.8\% in 2002 and is increasing at an average rate of $0.2 \%$ every year. The health care expenditure spent on diabetes patients accounts for approximately $11.5 \%$ of the total NHI cost. In this study, we aim to examine the relationships between diabetes and other health outcomes of Taiwanese old people.

Materials and Methods: A national-representative, multistage stratified systematic sample of 2353 individuals aged 65 and over from the 2005
National Health Interview Survey in Taiwan is analyzed. Among them, 401 cases $(17 \%)$ are diabetic. Mean \pm SD of age for non-diabetic and diabetic subjects are $739 \pm 6.5$ and $740 \pm 6.5$ respectively. The analyzed health outcomes include stroke, TIA, kidney diseases, uremia, joints replacement, fall experience, fracture experience, urine incontinence, feces incontinence, use of audiphone, glaucoma, ablepsia, teeth function, daily functional status, use of emergency room, hospitalization, use of dental services, body mass index, depressive symptoms, cigarette smoking, betel quid chewing, physical laboring, and exercise. Chi-square test and logistic regression were applied to examine their correlations.

Results: The analyses of chi-square tests show that diabetes patients report a significantly higher rate in terms of stroke, TIA, kidney diseases, joints replacement, fall experience, urine incontinence, stool incontinence, daily functional disability, obesity, depressive symptoms, emergency room and hospitalization utilization, and an obviously lower rate in terms of physical laboring work and dental services utilization. When sex and the other aforementioned significant variables are controlled, the result shows that DM patients have higher odds of TIA (Odds ratio=1.60, 95\% CI 1.07-2.40), kidney diseases (Odds ratio $=1.62,95 \%$ CI 1.08-2.42) and lower odds of engaging in physical laboring work (Odds ratio $=0.66,95 \%$ CI $0.47-0.95$ ).

Conclusion: Findings show that DM does have negative impacts on old patients' health and lifestyle. It is very important to enhance self-management in diabetes patients so as to prevent from advanced health decline. 


\section{PS 13 Environmental factors in diabetes aetiology}

\section{7}

The Environmental Determinants of Diabetes in the Young (TEDDY) Study

M. Rewers; on behalf of the TEDDY INVESTIGATORS,

Barbara Davis Center for Childhood Diabetes, University of Colorado, School of Medicine, Denver, United States.

Background and Aims: The incidence of type 1 diabetes (T1D) has doubled in the US and Europe during the past 20 yrs, likely due to changes in early childhood infectious or dietary exposures. To determine the cause of T1D, the TEDDY consortium follows intensively large cohorts of genetically susceptible infants born in Finland, Sweden, Germany and the U.S. (Georgia, Florida, Washington and Colorado).

Materials and Methods: The study aims to screen for HLA-DR,DQ genotypes associated with T1D over 360,000 newborns and to enroll 7,800 high-risk infants in intensive follow-up including measurement of autoantibodies to insulin, GAD65 and IA-2, infectious exposures and dietary biomarkers every 3 months. Monthly stool samples, questionnaires and diary kept by parents provide additional exposure data. Candidate environmental exposures assessed prospectively by repeated measurements are formally evaluated by time-to-event analyses using as the endpoints development of islet autoantibodies and progression to T1D.

Results: Between Sept. 2004 and March 2007, 146,900 newborns from the general population, including 2,320 first degree relatives of T1D patients, were screened for HLA-DR,DQ genotypes associated with T1D. Of the 7,040 eligible high-risk infants found so far, 2,900 have been enrolled into follow-up. The prevalence of the highest-risk HLA-DR3/4,DQB1 ${ }^{*} 0201 / 0302$ genotype in general population newborns screened by TEDDY does not correlate with previously published T1D incidence gradient from high-risk Scandinavians to moderate-risk US non-Hispanic whites (NHW) to low risk US Hispanics and Germans (Table). There is a significant heterogeneity, by population, in the prevalence of the HLA-DR3/4,DQB1*0201/0302 genotype $(\mathrm{p}<0.0001)$ and in additional elevated risk genotypes that TEDDY is screening for. Examples of environmental exposures studied and their cumulative incidence in infants enrolled in TEDDY so far $(n=2,507)$ are shown in the Table. Only in Finland BCG vaccination is given shortly after birth, later in the first year of life in Sweden and rarely in the other populations. The exposure to cow's milk in infancy is also dissimilar by the study population $(\mathrm{p}<0.01)$.

Conclusion: There are major major inter-population differences in the prevalence of candidate T1D genetic and environmental risk factors within the U.S. and Western Europe.

Prevalence (\%) of selected candidate risk factors

\begin{tabular}{llllll}
\hline Population & Finland & Sweden & $\begin{array}{l}\text { US } \\
\text { NHW }\end{array}$ & $\begin{array}{l}\text { US } \\
\text { Hispanics }\end{array}$ & Germany \\
\hline T1D incidence $/ 100$ 000/yr & 41 & 39 & 28 & 14 & 14 \\
HLA-DR3/4,DQB1 ${ }^{* 0302[\%]}$ & 1.9 & 3.4 & 2.3 & 1.9 & 1.8 \\
BCG vaccination at birth [\%] & 30 & 0.5 & 0.1 & 0 & 0.5 \\
$\begin{array}{l}\text { Fed cow's milk by 3 months } \\
\text { of age [\%] }\end{array}$ & 72 & 71 & 73 & 90 & 53 \\
\hline
\end{tabular}

Supported by NIH, NIDDK
0358

The development of humoral immunity against insulin after rotavirus infection in infancy

J. Aarnisalo ${ }^{1,2}$, M. Mäkelä ${ }^{1,2}$, J. Marttila ${ }^{1,2}$, R. Veijola ${ }^{3}$, O. Simell ${ }^{4}$, M. Knip ${ }^{5,6}$, O. Vaarala ${ }^{7}$, J. Ilonen $^{1,8}$;

${ }^{1}$ Immunogenetics Laboratory, University of Turku, ${ }^{2}$ Department of Virology, University of Turku, ${ }^{3}$ Department of Pediatrics, University of Oulu, ${ }^{4}$ Department of Pediatrics, University of Turku, Finland, ${ }^{5}$ Department of Pediatrics, University of Tampere, ${ }^{6} \mathrm{Hospital}$ for Children and Adolescents, University of Helsinki, ${ }^{7}$ Laboratory of Immunobiology, The National Public Health Institute, Helsinki, ${ }^{8}$ Department of Clinical Microbiology, University of Kuopio, Finland.

Background and Aims: Failure in the development of tolerance to dietary bovine insulin has been implicated to be of importance in the initiation of type 1 diabetes (T1D) associated autoimmunity. We studied the association between rotavirus infections and elevated bovine insulin-binding antibodies in a large cohort of prospectively observed children with HLA-conferred susceptibility to T1D.

Materials and Methods: Serum bovine insulin-binding IgG antibodies, rotavirus IgG and IgA class antibodies and respiratory syncytial virus (RSV) IgG antibodies were measured at the age of 3, 6, 12, 18 and 24 months in prospective series of 100 children who seroconverted to positivity for at least two of the T1D-associated autoantibodies (ICA, IAA, GADA, IA-2A) during prospective observation. Controls $(n=483)$ matched for HLA-DQB1 risk alleles were also studied. The effect of early rotavirus and RSV infection on the subsequent development of antibodies to bovine insulin and beta-cell autoantibodies was analysed.

Results: Rotavirus infection before the age of 6 months was associated with increased levels of bovine insulin-binding antibodies at the age of 6 and 12 months ( $\mathrm{p}=0.037$ and 0.077 , respectively, MWU-test). Rotavirus infection occurring by 12 months of age was associated with increased bovine insulin antibody levels at the age of 18 months ( $\mathrm{p}=0.045)$ and by 18 months of age with increased bovine insulin antibody levels at the age of 18 months $(\mathrm{p}=0.012)$. In addition, among children infected with rotavirus during the first 6 months of life the seroconversion rate for any of the four autoantibodies and for IAA, in particular, by the age of 2 years was higher compared to uninfected children ( $\mathrm{p}=0.011$ and 0.046 , respectively, Fisher's exact test). In contrast, we observed no effect of early RSV infection on bovine insulin antibody levels.

Conclusion: These data confirm our previous findings on the effect of early rotavirus infections on the development of bovine insulin humoral immunity. Moreover, the results suggest a role of early rotavirus infection in the development of T1D-associated autoimmunity. Early rotavirus infection may increase gut permeability and thus break tolerance to dietary bovine insulin and other beta-cell antigens in genetically susceptible children. Supported by the Juvenile Diabetes Research Foundation, Sigrid Juselius Foundation

\section{9}

Enterovirus RNA in serum of children with newly diagnosed type 1 diabetes and islet cell autoantibody-positive subjects in a population with a low incidence of type 1 diabetes

O. Diaz-Horta ${ }^{1}$, L. Sarmiento ${ }^{2}$, E. Cabrera-Rode ${ }^{1}$, I. Cuba ${ }^{1}$, G. Molina ${ }^{1}$, M. Fonseca ${ }^{2}$, A. Diaz ${ }^{3}$, P. Gonzalez ${ }^{4}$, L. Lekuleni² ${ }^{2}$ L. Heng-Hung²; ${ }^{1}$ Immunology and Genetics on Diabetes, National Institute of Endocrinology, ${ }^{2}$ Enterovirus, Institute of Tropical Medicine "Pedro Kouri", ${ }^{3}$ Endocrinology, Hospital Univeritario "Eliseo Noel Caamaño", ${ }^{4}$ Endocrinology, Hospital "William Soler", Havana, Cuba.

Background and Aims: The penetrance of type 1 diabetes mellitus (T1DM) in a genetically susceptible population is largely determined by environmental influences amongst which the human enteroviruses are prominent putative factors. The aim of this study was to determine the occurrence of enterovirusRNA in serum of children with type 1 diabetes at onset and ICA-positive subjects in a population with low incidence of type 1 diabetes and high circulation of enteroviruses.

Materials and Methods: Serum samples were collected children with newly diagnosed type 1 diabetes $(n=34)$; islet autoantibody-positive $(n=32)$ and negative $(n=31)$ first-degree relatives of type 1 diabetic patients; and control 
subjects $(n=194)$. Enteroviral RNA was assessed using a highly sensitive reverse transcriptase- polymerase chain reaction method.

Results: The frequency of positive signals corresponding to enteroviral sequence amplification was higher in newly diagnosed T1DM children $(9 / 34,26.5 \%)$ and islet autoantibody-positive first-degree relatives $(5 / 32$, $15.6 \%)$ than in their corresponding matched controls $(2 / 68,2.9 \%, p=0.0007$ and $0 / 64,0.0 \%, p=0.0033$, respectively). The presence of enteroviral RNA appeared to be associated with severe diabetic ketoacidosis at onset $(\mathrm{pH}<7.1$, $p=0.0328)$ ) and high ICA titres ( $\geq 20$ JDF units, $p<0.05$ ).

Conclusion: Despite there is a high circulation of enteroviruses and a low type 1 diabetes incidence in the Cuban population, the presence of enteroviral RNA is associated with type 1 diabetes and $\beta$-cell autoimmunity similarly to European countries in which this scenario is inverted.

Supported by Ministry of Health, Vice-Ministry of Science and Technology

\section{0}

Enterovirus genomes in the blood in children with T1DM at disease onset and one year later

G. Bianchi ${ }^{1}$, A. Salvatoni ${ }^{1}$, A. Baj ${ }^{2}$, L. Nespoli ${ }^{1}$, A. Toniolo ${ }^{2}$, A. De Paoli ${ }^{1}$; ${ }^{1}$ Pediatric Department, ${ }^{2}$ Microbiology, Università dell'Insubria, Varese, Italy.

Background and Aims: Enteroviral (EV) infections have been proposed as one triggering environmental factor leading to T1DM. The mechanism(s) through which EV collaborate to beta cell destruction is still unclear. Human EV have long been known as agents of acute diseases, chronic infections being rarely documented. Objectives and hypotheses: To assess whether T1DM were associated with chronic EV infection, we evaluated the presence of EV RNA genomes both at the time of clinical onset and one year later.

Materials and Methods: Blood samples of 68 T1D patients (38 boys; age 218 years) have been investigated. Detection of EV genomes was performed by RT-PCR using different primer pairs directed to conserved genomic regions. Sensitivity of the amplification methods in use was 10 genome equivalents per reaction tube. Direct sequencing of purified amplicons allowed identifying $\mathrm{EV}$ at the species level.

Results: At onset, EV genomes were detected in 46/68 patients (67\%). No viral genomes were detected in 18 healthy control children. Direct amplicon sequencing showed that viruses of the HEV-B and HEV-C species were particularly prevalent. Retesting positive patients one year after clinical onset showed that EV genomes were present in only $1 / 46$ children.

Conclusion: The results confirm the temporal association of EV infection with the onset of T1DM. We were unable to show persistent EV infection in the blood of most pediatric patients. This, however, does not exclude chronic infection of pancreatic islets.

\section{1}

Epigallocatechin 3-gallate protects pancreatic Ins-1 cells against 2,3,7,8-tetrachlorodibenzo-p-dioxin-induced acute toxicity

V. De Tata, L. Martino, M. Novelli, M. Masini, P. Masiello;

Dept. of Experimental Pathology \& B.M.I.E., University of Pisa, Italy.

Background and Aims: The increasing prevalence of type 2 diabetes prompted several scientist to suggest that environmental contaminants, in addition to obvious nutritional habits, could increase the risk of diabetes development. In this context, dioxins are considered the only environmental contaminants so far identified that could exert widespread effects in the general population. Several epidemiological studies have demonstrated a positive correlation between dioxin exposure and glucose metabolism disorders including diabetes. In order to clarify the biological basis of this correlation we have previously demonstrated that 2,3,7,8-tetrachlorodibenzo-p-dioxin (TCDD) is highly toxic for INS- 1 cells and that, even at very low doses, markedly impairs glucose-stimulated insulin secretion. The aim of this research has been to investigate the protective effect of the green tea polyphenol epicallocatechin 3-gallate (EGCG) against the TCDD-induced toxicity in INS-1 cells.

Materials and Methods: INS-1 cells were exposed for $1 \mathrm{hr}$ to TCDD alone or in the presence of different concentrations $(0-200 \mu \mathrm{M})$ of EGCG. We have then assayed: 1) cell survival; 2) the morphological alterations by electron microscopy; 3) the mitochondrial membrane potential by cytofluorimetry; 4) the variations in the intracellular calcium concentration by fluo-3/AM-based fluorimetry; 5) glucose-stimulated insulin secretion.

Results: Our results show that EGCG was able to significantly increase the survival of INS-1 cells after $1 \mathrm{hr}$ incubation with TCDD. This protective effect was clearly dose-related and was evident, and quantitatively remarkable for the higher TCDD doses (cell survival was 2.5- and 6-fold higher in $200 \mu \mathrm{M}$ EGCG-treated cells for 25 and $50 \mathrm{nM}$ TCDD, respectively; $\mathrm{p}<0.01$ ). The ultrastructure of TCDD-exposed INS-1 cells was also well preserved in the presence of EGCG. One of the most interesting consequences of TCDD treatment was a remarkable activation of autophagy, indicated by the presence of several large autophagic vacuoles containing identifiable organelles, such as mitochondria or degraded membranes. In EGCG-protected cells no autophagic vacuoles were found and interstingly numerous apoptotic bodies were evident in the cytoplasm of surviving cells. EGCG had no effect on the TCDD-induced increase in the intracellular calcium concentration, but partially prevented the TCDD-induced mitochondrial depolarization. EGCG was also able to prevent completely the TCDD-induced impairment of glucose-stimulated insulin secretion.

Conclusions: Our research clearly shows that EGCG is highly effective in preventing the TCDD-induced toxicity in INS-1 cells, in terms of both cell survival and preservation of their physiological properties. This protective effect could be related to the reported inhibitory effect of EGCG on the Aryl Hydrocarbon Receptor (AhR). EGCG indirectly inhibits AhR by binding the accessory protein Hsp90 and thus probably blocking the TCDD-induced activation of the MAPKs signal transduction pathway. Supported by M.I.U.R.

\section{2}

A cross-sectional study of the association between persistent organic pollutants and glucose intolerance and insulin M. E. Jørgensen ${ }^{1}$, K. Borch-Johnsen ${ }^{2}$, P. Bjerregaard ${ }^{1}$;

${ }^{1}$ Centre for Health Research in Greenland, National Institute of Public Health, Copenhagen, ${ }^{2}$ Steno Diabetes Centre, Gentofte, Denmark.

Background: Experimental evidence supports the hypothesis that persistent organic pollutants (POPs) may cause type 2 diabetes. However, epidemiological studies do not convincingly support an association. The Inuit population in Greenland, which is highly exposed to POPs due to a high intake of sea mammals and fatty fish, has experienced a rapid increase in diabetes prevalence over the last 30 years. Thus the aim was to study the association between POPs and diabetes and impaired glucose regulation in a population-based design.

Methods: From 1999 to 2002 the Greenland population study was carried out among adult Inuit living in Greenland. The examination included a $75 \mathrm{~g}$ OGTT, anthropometric measurements, a structured interview, and blood tests. P-glucose, s-insulin, lipids were analysed, and PCB153 and p,p'-DDE were analysed in a sub-group as biomarkers of POP exposure. Associations were adjusted for age, sex, waist circumference, HDL cholesterol and triglycerides.

Results: Data on POPs were available on 692 individuals, 305 men (mean age 50 years) and 387 women (mean age 49 years). The table shows mean levels of POPs (geometric means) according to glucose tolerance status. (Tab. 1)

Tab. 1

\begin{tabular}{|c|c|c|c|c|c|c|c|}
\hline & $\mathrm{N}(\%)$ & $\begin{array}{l}\text { PCB153 } \\
\text { ng/g lipid }\end{array}$ & $\begin{array}{l}\text { Mean PCB153 ng/g lipid, } \\
\text { adjusted }\end{array}$ & p-value & $\begin{array}{l}\text { p,p'-DDE } \\
\text { ng/g lipid }\end{array}$ & $\begin{array}{l}\text { Mean p,p'-DDE ng/g lipid, } \\
\text { adjusted }\end{array}$ & $p$-value \\
\hline Diabetes & $71(10.3)$ & 777 & 752 & 0.63 & 1494 & 1445 & 0.52 \\
\hline Non-diabetes & $621(89.7)$ & 653 & 722 & & 1240 & 1358 & \\
\hline IGR & $254(36.7)$ & 774 & 731 & 0.82 & 1443 & 1361 & 0.90 \\
\hline Non-IGR & $438(63.3)$ & 600 & 722 & & 1153 & 1370 & \\
\hline
\end{tabular}


There was no association between POPs and fasting glucose or between POPs and fasting insulin or HOMA index of insulin resistance.

Conclusions: No association was seen between POPs and glucose intolerance or markers of insulin resistance. Prospective studies are needed to clarify whether POPs are involved in glucose intolerance or insulin resistance. Supported by Danish Medical Research Council, Greenland Medical Research Council and Commission for Scientific Research in Greenland

\section{3}

Whole grain, bran, and germ intake and risk of type 2 diabetes R. M. Van Dam ${ }^{1,2}$, J. S. L. De Munter ${ }^{3}$, D. Spiegelman ${ }^{4,5}$, M. Franz $^{1}$, F. B. $\mathrm{Hu}^{1,2}$;

${ }^{1}$ Department of Nutrition, Harvard School of Public Health, Boston, United States, ${ }^{2}$ Channing Laboratory, Department of Medicine, Brigham and Women's Hospital, and Harvard Medical School, Boston, United States, ${ }^{3}$ Institute of Health Sciences, Vrije Universiteit Amsterdam, The Netherlands, ${ }^{4}$ Department of Epidemiology, Harvard School of Public Health, Boston, United States, ${ }^{5}$ Department of Biostatistics, Harvard School of Public Health, Boston, United States.

Background and Aims: Control of body weight by balancing energy intake and energy expenditure is of major importance for the prevention of type 2 diabetes, but the role of specific dietary factors in the etiology of type 2 diabetes is less well established. We evaluated intakes of whole grain, bran and germ in relation to risk of type 2 diabetes in prospective cohort studies. Materials and Methods: We followed 161,737 U.S. women of the Nurses' Health Studies (NHSs) I and II, without history of diabetes, cardiovascular disease, or cancer at baseline. The age at baseline was $37-65$ years for NHSI and 26-46 years for NHSII. Dietary intakes and potential confounders were assessed using regularly administered questionnaires. We documented 6,486 cases of type 2 diabetes during 12-18 years of follow-up. Other prospective cohort studies on whole grain intake and risk of type 2 diabetes were identified using searches of MEDLINE and EMBASE up to January 2007, and data were independently extracted by 2 reviewers.

Results: The median whole grain intake in the lowest and highest quintile of intake was 3.7 and $31.2 \mathrm{~g}$ per day for NHSI and 6.2 and $39.9 \mathrm{~g}$ per day for NHSII. After adjustment for potential confounders, the relative risks for the highest as compared with the lowest quintile of whole grain intake was 0.63 (95\% confidence interval [CI] 0.57-0.69) for NHSI and 0.68 (95\% CI $0.57-0.81$ ) for NHSII (both: P-value, test for trend $<0.0001$ ). After further adjustment for body mass index, these relative risks were 0.75 (95\% CI $0.68-$ 0.83 ; P-value, test for trend $<0.0001)$ and $0.86(95 \%$ CI $0.72-1.02$; P-value, test for trend 0.03) respectively. Associations for bran intake were similar to those for total whole grain intake, whereas no significant association was observed for germ intake after adjustment for bran. Based on pooled data for 6 cohort studies including 286,125 participants and 10,944 cases of type 2 diabetes, a 2 serving per day increment in whole grain consumption was associated with a $21 \%(95 \%$ CI $13 \%-28 \%)$ decrease in risk of type 2 diabetes after adjustment for potential confounders and body mass index.

Conclusion: Whole grain intake was inversely associated with risk of type 2 diabetes, and this association was stronger for bran than for germ. Findings from prospective cohort studies consistently support increasing whole grain consumption for the prevention of type 2 diabetes.

Supported by NIH research grants

\section{PS 14 Genetics and epidemiology of diabetic nephropathy}

\section{4}

Association studies on DDOST, PRKCSH and LGALS3 in Finnish type 1 diabetic patients (the FinnDiane Study)

A. Hoverfält ${ }^{1,2}$, J. M. Söderlund ${ }^{1,2}$, R. Sallinen ${ }^{1,2}$, J. Vesa ${ }^{1,2}$,

K. Pettersson-Fernholm ${ }^{1,2}$, R. Kilpikari ${ }^{1,2}$, C. Forsblom ${ }^{1,2}$, M. Wessman $^{1,2}$, P.-H. Groop ${ }^{1,2}$,

${ }^{1}$ Folkhälsan Institute of Genetics, Folkhälsan Research Center, Biomedicum Helsinki, Finland, ${ }^{2}$ Department of Medicine, HUCH, Division of Nephrology, Helsinki, Finland.

Background and Aims: Chronic hyperglycaemia results in formation and accumulation of advanced glycation end-products (AGEs) in diabetes. AGEs can directly disturb normal function of proteins/macromolecules although the impairment caused by AGEs is mainly receptor mediated. The genes encoding AGE-receptor 1 (DDOST on chromosome 1p36.1), 2 (PRKCSH on 19p13.2), and 3 (LGALS3 on 14q21-q22) have been suggested to play a role in the pathogenesis of diabetic micro- and macrovascular complications. The aim of this case-control study was to examine whether single nucleotide polymorphisms (SNPs) in the DDOST, PRKCSH, and LGALS3 genes are associated with type 1 diabetes (T1D) or with its micro- and macrovascular complications.

Materials and Methods: 1273 well-phenotyped Finnish T1D patients were studied and grouped into patients with normoalbuminuria $(n=457)$, microalbuminuria $(n=271)$, macroalbuminuria $(n=364)$, and end-stage renal disease $(n=181)$ based on their urinary albumin excretion rate in at least two out of three timed urine collections. Furthermore, 702 Finnish non-diabetic subjects served as controls for the T1D patients. Four SNPs (rs631357, rs640742, rs2070660, rs686658) in the DDOST gene, five SNPs (rs34100, rs34088, rs313624, rs11557488, rs311778) in the PRKCSH gene, and six SNPs (rs8007614, rs3825613, rs1009977, rs4644, rs10498475, rs2274273) in the LGALS3 gene were chosen based on the HapMap data. Ten out of the 15 SNPs studied were tagSNPs. Genotyping was performed using the ABI Prism 7900 Sequence Detection System. Association with categorical variables was tested using the $\chi^{2}$-test and with continuous variables using the one-way ANOVA. Statistical analyses were performed using the SPSS 12.0.1 for Windows.

Results: All SNPs were in Hardy-Weinberg equilibrium. No association between the SNPs and T1D, diabetic nephropathy or related quantitative variables (blood pressure, estimated GFR, serum creatinine, triglycerides, total cholesterol, HDL cholesterol, glycated hemoglobin A1c) was observed. Furthermore, no association with retinopathy, coronary heart disease, acute myocardial infarction or stroke was observed.

Conclusion: These preliminary data do not indicate the involvement of the DDOST, PRKCSH and LGALS3 genes with T1D or with the diabetic microand macrovascular complications.

\section{5}

ACE I/D polymorphism predicts end stage renal disease and or mortality in type 1 diabetic patients except for those with already advanced nephropathy: the follow up of the Genesis/Genediab Studies M. Fysekidis ${ }^{1,2}$, S. Hadjadj ${ }^{3}$, R. Roussel ${ }^{1,2}$, N. Bellilii' ${ }^{2}$, S. Madani ${ }^{2}$, F. Fumeron ${ }^{2}$, H. Leblanc ${ }^{4}$, J. Gautier ${ }^{4}$, L. Weekers ${ }^{5}$, B. Bauduceau, G. Charpentier ${ }^{7}$, M. Virally ${ }^{8}$, F. Alhenc-Gelas ${ }^{9}$, P. Lefebvre ${ }^{5}$, M. Marre ${ }^{1,2}$; ${ }^{1}$ Diabetology, Bichat Hospital, APHP, Paris, France, ${ }^{2}$ Inserm, U695, Paris, France, ${ }^{3}$ Division of Diabetes,, CHU, Poitiers, France, ${ }^{4}$ Diabetology, St Louis Hospital, APHP, Paris, France, ${ }^{5} \mathrm{CHU}$ du Sart Tilman, Division of Diabetes, Liège, Belgium, ${ }^{6}$ Department of Endocrinology, Army Hospital, St Mande, Begin, France, ${ }^{7}$ Diabetology, CHU Sud Francilien, Corbeil-Essones, France, ${ }^{8}$ Diabetology, Lariboisiere Hospital, APHP, Paris, France,

${ }^{9}$ U652, INSERM, Paris, France.

Background and Aims: Angiotensin Converting Enzyme (ACE) activity controls renal hemodynamics and its insertion /deletion (I/D) polymorphism was reported to affect development and progression of diabetic nephropathy in type 1 diabetes(T1D).We tested its impact on the risk for End Stage Renal Failure (ERSD) and mortality in prospective follow up of two cohorts of 856 T1D patients from France and Belgium (Genesis/Genediab). 
Materials and Methods: Of the 533 participants of the Genediab study 332 agreed in prospective follow up as well as 524 out of 588 of the Genesis study. They were originally included between 1994 and 2000 on the basis of having insulin treatment before the age of 35 years and backround retinopathy.They had a follow up untill 31/12/2006 for the occurrence of ESRD or death. The tested hypothesis was that the 182 participants with II genotype will display less events than the others. Survival analysis was performed and adjustements were made using the Cox model.

Results: At baseline 463 subjects had no nephropathy, 222 incipient, 205 established and 186 advanced (plasma creatinine $>150 \mu \mathrm{mol} / 1$ ) nephropathy. Age, sex ratio, $\mathrm{HbAlc}$ and diabetes duration were similar between groups. There was an expected association between nephropathy stage and ACE I/D polymorphism at baseline ( $\chi^{2}$ for trend II vs ID/DD :6,6. $\left.\mathrm{p}<0,05\right)$. During follow up 141 events occurred the odds ratio for participants with II genotypes vs others was 0,55(95\% CI 0,32-0,94). However, only 23 events occurred in participants without nephropathy at baseline, 13 with incipient nephropathy,34 those with established and 79 among those with advanced nephropathy(nephropathy stage effect $\mathrm{p}<<0,0001$ ). When both nephropathy stage and ACE I/D genotype effects entered together in the Cox model the II genotype effect disappear OR 0.820 ( $95 \%$ CI $0,45-1,51 ; \mathrm{p}=0,48)$ due to the lack of II genotype effect among the participants with advanced nephropathy at the baseline. Treatment with ACE inhibitors did not alter the result.

Conclusion: The ACE II genotype protects from the development and progression of diabetic nephropathy, and from the risk for ESRD and mortality as well. However its effect disapears in people with already advanced nephopathy.

\section{6}

Evaluation of the association between adiponectin promoter polymorphisms and nephropathy in type 1 diabetes

D. Zhang, J. Ma, K. Brismar, S. Efendic, H. F. Gu;

Molecular Medicine and Surgery, Rolf Luft Center for Diabetes Research,

Karolinska Institutet, Karolinska Hospital (Solna), Stockholm, Sweden.

Background and Aims: Recent reports have demonstrated that the adiponectin promoter single nucleotide polymorphism (SNP) -11391G/A is associated with nephropathy in type 1 diabetic (T1D) patients in the Danish, but not in the French, Finnish and Swedish populations. The present study aims at evaluation of the association between adiponectin promoter SNPs and nephropathy in T1D.

Materials and Methods: Three adiponectin promoter SNPs, including $11426 \mathrm{~A} / \mathrm{G},-11391 \mathrm{G} / \mathrm{A}$ and $-11377 \mathrm{C} / \mathrm{G}$, were genotyped with dynamic allele specific hybridization (DASH). The subject material includes 1177 (female 599/male 578) American T1D patients with and without diabetic nephropathy (DN). All are European descents and belong to the Genetics of Kidneys in Diabetes (GoKinD) study.

Results: The G allele of SNP-11377C/G was found to be associated with $\mathrm{DN}$ in the female T1D patients ( $\mathrm{P}=0.022, \mathrm{OR}=1.352$, CI $95 \% 1.044-1.752)$. The haplotype A-G-G constructed with three studied promoter SNPs is also associated with $\mathrm{DN}$ in female T1D patients $(\mathrm{P}=0.011, \mathrm{OR}=1.417$, CI $95 \%$ 1.149-1.685). It has previously demonstrated that transcriptional stimulatory protein (SP1) has stimulatory effects on adiponectin promoter activity. We have extended this finding: first, we found four binding sites of SP1 in the adiponectin promoter; second, the G allele of SNP-11377C/G alters the sequence for one of SP1 binding sites.

Conclusion: The adiponectin promoter polymorphisms are associated with DN in T1D in the GoKinD material. SNP - 11377C/G may play a role in the regulation of adiponectin promoter activity.

The GoKinD study sample collection is supported by the Juvenile Diabetes Research Foundation in collaboration with the Joslin Diabetes Center and George Washington University, and by the United States Centers for Diabetes Control and Prevention

\section{7}

The prevalence of diabetic foot in patients with diabetic nephropathy A. Y. Tokmakova, G. Y. Strakhova, G. R. Galstyan;

Diabetic Foot Care Department, National Research Centre for Endocrinology, Moscow, Russian Federation.

Background and Aims: The aim of this study was to investigate the prevalence of diabetic foot syndrome in diabetic population with diabetic nephropathy in Moscow.

Materials and Methods: 346 diabetic patients with diabetic nephropathy were recruited (220 women and 126 men, mean age of $60.37 \pm 13,93$ yrs, diabetes duration $14.68 \pm 9,16$ yrs, HbAlc $8.43 \pm 1,6 \%)$. They were divided into 3 groups: the $1^{\text {st }}$ group included 136 patients with microalbuminuria; the $2^{\text {nd }}$ group - 110 patients with proteinuria and the $3^{\text {rd }}$ group - 100 patients with chronic renal failure. There was no statistical difference between groups $(p>0.05)$ in sex, age, duration of diabetes, HbAlc level. The proportion of patients with type 1 diabetes in the $3^{\text {rd }}$ group was significantly greater at $37 \%$ compared with $14 \%$ in the $1^{\text {st }}$ group and $15 \%$ in the $2^{\text {nd }}$ group $(\mathrm{p}<0.001)$. We evaluate the prevalence of foot ulcers, Charcot osteoarthropathy, amputations (minor/major) and degree of peripheral vascular disease (PVD).

Results: Results are reported in the table 1 . There was significant difference of foot ulcers in patients with proteinuria and chronic renal failure than in patients with microalbuminuria ( $64 \%$ and $58 \%$ vs. $32 \%$, p $<0.0001$ ). The ratio of neuropathic to ischemic ulcers didn't differ significant between the groups. The total number of amputations in the $2^{\text {nd }}$ and the $3^{\text {rd }}$ groups was also significantly higher than in the $1^{\text {st }}$ group $(\mathrm{p}<0.05$ and $\mathrm{p}=0.0001$, respectively). The presence of Charcot osteoarthropathy was significantly higher in the $3^{\text {rd }}$ group ( $\mathrm{p}<0.001$ vs the $1^{\text {st }}$ group, $\mathrm{p}<0.05$ vs. the $2^{\text {nd }}$ group). When type 1 patients were excluded, the number of ischemic ulcers in the $3^{\text {rd }}$ group increased up to $51.4 \%$ and the number of major amputations - to $50 \%$, but these differences were not significant.

Conclusion: Diabetic patients with chronic renal failure have extremely high risk for developing Charcot osteoarthropathy. The prevalence of foot ulcers was higher in patients with proteinuria than in patients with microalbuminuria $(\mathrm{p}<0.0001)$ and renal failure $(\mathrm{p}>0.05)$. There was a trend to more severe PVD in patients with more severe diabetic nephropathy.

Table 1.

\begin{tabular}{llll}
\hline & $\begin{array}{l}\text { Group 1 } \\
(\mathbf{n}=136)\end{array}$ & $\begin{array}{l}\text { Group 2 } \\
(\mathbf{n}=110)\end{array}$ & $\begin{array}{l}\text { Group 3 } \\
(\mathbf{n}=100)\end{array}$ \\
\hline Neuropathic foot ulcers & $30(68,2 \%)$ & $45(64,3 \%)$ & $36(62,1 \%)$ \\
Ischemic foot ulcers & $14(31,8 \%)$ & $25(35,7 \%)$ & $22(37,9 \%)$ \\
Minor amputations & $11(78,6 \%)$ & $24(72,7 \%)$ & $15(68,2 \%)$ \\
Major amputations & $3(21,4 \%)$ & $9(27,3 \%)$ & $7(31,8 \%)$ \\
Charcot osteoarthropathy & $5(3,7 \%)$ & $8(7,3 \%)$ & $17(17 \%)$ \\
\hline
\end{tabular}

\section{8}

Close similarities in urinary albumin excretion between type 2 diabetes and LADA versus type 1 diabetes - results from the Nord-Trøndelag Health Study 1995-97 (HUNT2)

M. Radtke ${ }^{1}$, T. I. Lund Nilsen ${ }^{2}$, K. Midthjell ${ }^{3}$, V. Grill ${ }^{1}$;

${ }^{1}$ Department of Cancer Research and Molecular Medicine, Faculty of Medicine, Norwegian University of Science and Technology, Trondheim, ${ }^{2}$ Department of Public Health, Faculty of Medicine, Norwegian University of Science and Technology, Trondheim, ${ }^{3}$ HUNT Research Centre, Faculty of Medicine, Norwegian University of Science and Technology, Verdal, Norway.

Background and Aims: Microalbuminuria is considered the earliest sign of microvascular damage in diabetes, but it is not clear whether a particular type of diabetes influences urinary albumin excretion. We wanted to compare urinary albumin excretion in type 1, type 2 diabetes and late autoimmune diabetes in the adults (LADA) as part of an all-population epidemiological survey.

Materials and Methods: Albumin/Creatinine ratio was measured in three urine samples from all diabetic $(n=1525)$ and hypertensive non-diabetic $(n=5436)$ subjects and from a $5 \%$ random sample $(n=2104)$ from the large $(n=64931)$ Nord-Trøndelag Health Survey, performed 1995-96. 
Results: Mean Albumin/Creatinine ratio $[\mathrm{mg} / \mathrm{mmol}](\mathrm{CI})$, adjusted for age, gender, smoking, blood pressure, cholesterol/HDL-cholesterol ratio, BMI and diabetes duration was highest in the LADA $1.32(1.10-1.58)$ and the type 2 diabetes group $1.25(1.16-1.3)$, and non-significantly lower in the type 1 diabetes group $1.05(0.84-1.31)$, the hypertensive group 0.99 (0.96-1.02) and lowest in the random selected group 0.95 (0.90-0.99). Dichotomizing for $\mathrm{HbAlc}$ or hypertension revealed effect modifications. Thus, lower albumin excretion in type 1 diabetes was restricted to the $50 \%$ of subjects with lower $\mathrm{HbAlc}$ (tendency) or lower blood pressure(significant).

Conclusion: A higher albumin excretion in type 2 diabetes and LADA than in type 1 diabetes indicates closer resemblance of LADA to type 2 than type 1 in terms of complications. It may also indicate that albumin excretion in type 2 diabetes is increased by factors additional to the standard ones for microalbuminuria and cardiovascular disease.

Supported by Central Norway Regional Health Authority and Norwegian University of Science and Technology

\section{9}

Progression of glucose tolerance in kidney transplant recipients: pre- and post-transplantation OGTT analysis

F. Moreau ${ }^{1}$, S. Caillard ${ }^{2}$, L. Eprinchard ${ }^{2}$, M. Pinget ${ }^{1}$, T. Hannedouche ${ }^{2}$,

B. Moulin ${ }^{2}$, L. Kessler ${ }^{1}$;

${ }^{1}$ Endocrinology and Diabetology, ${ }^{2} \mathrm{Nephrology}$ and Renal Transplantation,

University Hospital, Strasbourg, France.

Background and Aims: Post-transplant diabetes mellitus increases the morbidity of kidney transplant recipients. Transplant patients developing diabetes are vulnerable to the usual metabolic complications but also to a higher risk of graft failure and cardiovascular diseases. Identify subjects with risk of new-onset diabetes after transplantation is of great importance. We evaluated glucose tolerance in kidney transplant patients before and after kidney transplantation.

Materials and Methods: OGTT were performed consecutively in all patients on waiting list for kidney transplantation with fasting blood glucose $<6.9$ $\mathrm{mmol} / \mathrm{L}$ and at least 6 months after kidney transplantation.

Results: From Jan 2005 to March 2007, OGTT were performed in 154 patients. Pre-transplant OGTT revealed 4 cases of diabetes $(2,6 \%)$ and 33 cases of impaired glucose tolerance (21\%). 83 patients underwent a kidney transplantation. Post-transplant OGTT was performed in 38 of these 83 patients. Post-transplant OGTT showed that $50 \%$ of patients had glucose metabolism abnormalities: $26 \%(10 / 38)$ were diabetic and $24 \%(9 / 38)$ had impaired glucose tolerance while $66 \%(25 / 38)$ had normal OGTT and $34 \%$ (13/38) impaired glucose tolerance before transplantation. $4 / 10$ of these new-onset post-transplantation diabetes occurred in subjects with pre-transplantation impaired glucose tolerance. Diabetes and impaired glucose tolerance were observed in $24 \%$ and $16 \%$ of cases respectively after transplantation in patients with normal glucose tolerance before transplantation $(n=25)$. Mean age of patients who developed diabetes and impaired glucose tolerance was 53 and 55 years old respectively, mean BMI was 25 and $27 \mathrm{~kg} / \mathrm{m}^{2}$ respectively. Among patients with pre-transplant impaired glucose tolerance $(n=13), 33 \%$ have post-transplant diabetes and $33 \%$ have impaired glucose tolerance after transplantation. Patients with pre-transplant impaired glucose tolerance who developed post-transplant diabetes have a mean BMI of $29 \mathrm{~kg} / \mathrm{m}^{2}$ before transplantation.

Conclusion: One third of kidney recipients with pre-transplant impaired glucose tolerance had post-transplant diabetes mellitus. These results suggest that OGTT should be performed before transplantation in all patients in order to detect patients with high risk of post-transplant diabetes and to adapt the immunosuppressive therapy.

\section{PS 15 Classification of IGT, type 2 diabetes and metabolic syndrome}

\section{0}

The risk of diabetes in the metabolic syndrome is extremely high if the appropriate reference category is used

E. Bonora ${ }^{1}$, S. Kiechl ${ }^{2}$, J. Willeit ${ }^{2}$, R. C. Bonadonna ${ }^{1}$, M. Muggeo ${ }^{1}$;

${ }^{1}$ Endocrinology and Metabolic Diseases, University of Verona, Italy, ${ }^{2}$ Neurology, University of Innsbruck, Austria.

Background and Aims: The Metabolic Syndrome is a risk factor for type 2 diabetes (T2DM). However, previous studies demonstrated that any component of the Metabolic Syndrome can predict T2DM. Among subjects without the Metabolic Syndrome many have one or two components of the syndrome, and this may lead to an underestimate of the true diabetes risk in these subjects. In the present study we assessed the risk of T2DM in subjects with the Metabolic Syndrome using different reference categories.

Materials and Methods: We calculated the 10-yr risk of T2DM in 857 subjects aged 40-79 years from the general population living in Bruneck, north-eastern Italy. Diabetes was diagnosed according to ADA 1997 criteria (fasting glucose only). The Metabolic Syndrome was diagnosed by NCEPATP III criteria.

Results: Incident cases of T2DM were 76 . In the 169 subjects with the Metabolic Syndrome at baseline the sex- and age-adjusted odds ratio (OR) for T2DM was $4.4(95 \%$ CI 2.7-7.2, $\mathrm{p}<0.001)$. As compared to subjects without any metabolic components of the Metabolic Syndrome the ORs in those with $1,2,3,4 / 5$ components were $6.4(0.8-49.6, \mathrm{p}=0.08), 18.5$ (2.5-139, $\mathrm{p}=0.005), 22.2(2.9-172, \mathrm{p}=0.003)$ and $119(15.1-942, \mathrm{p}<0.001)$, respectively (overall $\mathrm{p}<0.001$ ). Among components of the Metabolic Syndrome, IFG was the strongest risk factor (sex- and age-adjusted OR 12.2, 7.1-20.5, $\mathrm{p}<0.001$ ), but also high waist circumference $(5.4,3.0-9.5, \mathrm{p}<0.001)$, hypertension $(4.4$, $2.1-9.0, \mathrm{p}<0.001)$, high triglycerides $(1.7,1.1-2.8, \mathrm{p}=0.03)$ and low HDLcholesterol $(1.8,1.0-3.1, \mathrm{p}=0.05)$ were predictors of T2DM. The risk of T2DM was very high in subjects with the Metabolic Syndrome also when insulin sensitivity was normal (quartiles 1-3 of HOMA-IR)(ORs 12.3, 1.4$109, \mathrm{p}=0.024$ in those with 3 , and $38.0,3.1-471, \mathrm{p}=0.005$ in those with $4 / 5$ abnormalities vs. those with no abnormality).

Conclusion: The risk of T2DM in subjects with the Metabolic Syndrome is very high when the reference category is composed by subjects without any metabolic abnormality. The risk is high also when insulin resistance was estimated to be normal.

\section{1}

Characteristics of glucose intolerance in the Japanese subjects with and without lowering of early-phase insulin responses after glucose load (GTT)

Y. Sakamoto ${ }^{1,2}$, Y. Inoue ${ }^{2}$, H. Matsumoto ${ }^{2}$, K. Oono ${ }^{2}$, Y. Tokumasa ${ }^{2}$;

${ }^{1}$ Diabetes center, Eastern Japan Medical Center, Ebina-City,

${ }^{2}$ Ichihara-City Medical Association, Japan.

Background and Aims: The aim of this study was to clarify the characteristics of glucose intolerance after glucose load in the Japanese subjects with and without lowering of early- phase of insulin responses after glucose load.

Materials and Methods: We did a screening test of 22228 general healthy subjects aged over 40 years old. In the 1st. screening test, The subjects were screened for the criteria of glucosuria, FPG levels $(110 \mathrm{mg} / \mathrm{ml})$ and/or HbA1c levels $(5.6 \%)$. In the second test (75g OGTT), GTT data from 143 cases applying for a close examination among the positive subjects in the 1st. test were analyzed in detail. Based on the results of Insulinogenic index (30 min): (Ins 30min-Ins 0min)/Glu30min-Glu 0min) from75g OGTT, the subjects were classified into the following 3 groups: Low Respoder group (Insulinogenic index: less than 0.4), Moderate Responder group (Insulinogenic index: 0.4less than 1.0) and High Responde group (Insulinogenic index: not less than 1.0). Normal glucose tolerance (NGT), isolated impaired fasting glucose (IFG: FPG between $110 \mathrm{mg} / \mathrm{dl}$ and $125 \mathrm{mg} / \mathrm{dl}, 2 \mathrm{hPG}$ less than $140 \mathrm{mg} / \mathrm{dl})$, isolated IGT (FPG less than $110 \mathrm{mg} / \mathrm{dl}$ and $2 \mathrm{hPG}$ between $140 \mathrm{mg} / \mathrm{dl}$ and $199 \mathrm{mg} / \mathrm{dl}$ ), isolated IGT with fasting hyperglycemia (IFG/IGT: FPG between $110 \mathrm{mg} / \mathrm{dl}$ and $125 \mathrm{mg} / \mathrm{dl}$, $2 \mathrm{hPG}$ between $140 \mathrm{mg} / \mathrm{dl}$ and $199 \mathrm{mg} / \mathrm{dl}$ ) and DM groups. The DM group is further divided into 2 groups, isolated FPG-DM group (FPG not 
less than $126 \mathrm{mg} / \mathrm{dl}$ and $2 \mathrm{hPG}$ less than $200 \mathrm{mg} / \mathrm{dl}$, 2hPG-DM group (2hPG not less than $200 \mathrm{mg} / \mathrm{dl}$ ).

Results: 1) In the GTT study, Low Responder subjects was found in $63.6 \%$, Moderate Responder was $24.5 \%$ and High Responder was $11.9 \%$. Mean value of Insulinogenic index were as follows: (Low R:0.194, Moderate R:0.588 High $\mathrm{R}: 1.588)$. 2) The incidence of the $2 \mathrm{hPG}$-DM subjects was found in $26.4 \%$ in the Low R group, $5.7 \%$ in the Moderate group and no case in the High R group. The incidence of the NGT subjects were as follows: (in the Low R: $34.1 \%$, in the Moderate R: $48.6 \%$ and in the High R: 64.7\%). The incidence of the isolated FPG-DM subjects were as follows: (in the Low R: $3.3 \%$, in the Moderate R: 5.7\% and in the High R: 9.1\%). The incidence of the isolated IGT: (in the Low R: $19.8 \%$, in the Moderate R: $22.9 \%$ and in the High R: $27.3 \%$ ). The incidence of the isolated IFG: (in the Low R: $4.4 \%$, in the Moderate R: $2.9 \%$ and in the High R: $18.2 \%$ ). The incidence of the IFG/IGT: (in the Low R: $12.1 \%$, in the Moderate R: $14.3 \%$ and in the High R: $0 \%$ ).

Conclusion: In the Japanese subjects, the incidence of the 2hPG-DM subjects was much lower in the High Respoder group compared to that in the Moderate and Low responder groups. And the incidence of the isolated FPG-DM subjects ( $4.2 \%$ in all subjects) was much lower than that of $2 \mathrm{hPG}$ DM subjects (18.2\% in all subjects). The pattern of insulin secretion in the Japanese type 2 diabetic subjects is characterized by marked low responses in the early- phase insulin secretion after glucose load.

Supported by Ichihara-City

\section{2}

Which of the criteria is more accurate ADA(IFG100) or IDF(IFG110) for the prediction of IGT1 and IGT2 at high risk group?

The RISK Study Results III

T. Yilmaz 1 , M. Sargin 2 , B. Olmez ${ }^{3}$, S. Karadeniz ${ }^{4}$, B. Oguzhan ${ }^{5} \&$ the RISK Study Group,

${ }^{1}$ Endocrinology and Metabolism Dept., Istanbul Medical Faculty, Istanbul University, ${ }^{2}$ Endocrinology and Metabolism Dept., Kartal Educ. and Res.

Hosp., Istanbul, Turkey, ${ }^{3}$ Novartis Pharma, Istanbul, Turkey,

${ }^{4}$ Medical Faculty, Istanbul Science University,

${ }^{5}$ Diabetes Centers, Turkish Diabetes Foundation, Istanbul, Turkey.

Background and Aim: To compare the prevalance of impaired fasting glycemia (IFG) according to the ADA and IDF criteria for the prediction of impaired glucose tolerance (IGT) 1-h and 2-h glucose values in asymptomatic individuals with high risk characteristics for type 2 diabetes (ADA criteria).

Materials and Method: The population based, cross-sectional RISK study includes 13.140 individuals randomly selected from 191 centers in Turkey. 75-g oral glucose tolerance test (OGTT) and body mass index (BMI) were evaluated. 1.h/2.h postprandial glucose levels between $140-199 \mathrm{mg} / \mathrm{dl}$ were considered as IGT1 and IGT2, respectively in individuals with OGTT in nondiabetic range.

Results: The prevalance of IFG according to ADA criteria (FBG $\geq 100 \mathrm{mg}$ / $\mathrm{dl}$, IFG100) was higher than IDF criteria (FBG $\geq 110 \mathrm{mg} / \mathrm{dl}$, IFG110) in the study population $(39.7 \%$ versus $18.7 \%, \mathrm{p}<0.001)$. The prevalance IFG100 and IFG110 were higher in subjects aged $\geq 45$ yrs than in subjects aged $<45 \mathrm{yrs}$ and with high risk characteristics for type $2 \mathrm{DM}$ according to ADA criteria $(39.7 \%$ and $18.7 \%$ vs $33.8 \%$ and $12.1 \%, \mathrm{p}<0.001, \mathrm{p}<0.001$, respectively). The OGTT revealed IGT1 in $79.1 \%$ of subjects with IFG110, $72 \%$ with IFG100, $65.8 \%$ with IFG90 and IGT2 in $58.9 \%, 47.1 \%, 36 \%$, respectively. The prevalances of IFG110 and IFG100 were $\% 10.8$ and $25 \%$ in subjects with BMI $<25 \mathrm{~kg} / \mathrm{m}^{2}$, $12.5 \%$ and $34.7 \%$ in subjects with BMI $25-30 \mathrm{~kg} / \mathrm{m}^{2}, 14.8 \%$ and $36.3 \%$ in subjects with $\mathrm{BMI} \geq 30 \mathrm{~kg} / \mathrm{m}^{2}$, respectively.

Conclusion: In our study population consisting of people at risk for type 2 diabetes, the rate of IFG100 was 2.1 times higher than IFG110. On the other hand, the prevalances of either IGT1 or IGT2 were higher in IFG110 group compared to IFG100 group. The concordance between IFG110 and IGT1 or IGT2 is more prominent than IFG100 and IGT1 or IGT2. According to our results, the predictive value of IFG110 is higher than of IFG 100 for glucose intolerance.

Supported by Novartis Pharma

\section{3}

Associations between insulin resistance, beta cell function and metabolic markers in a non-diabetic adult population

M. H. Hedman ${ }^{1}$, B. Lindahl' ${ }^{2}$, S. Söderberg ${ }^{3}$, G. Hallmans ${ }^{4}$, O. Rolandsson ${ }^{1}$; ${ }^{1}$ Department of Public Health and Clinical Medicine, Family Medicine,

${ }^{2}$ Department of Public Health and Clinical Medicine, Behavioural Medicine, ${ }^{3}$ Department of Public Health and Clinical Medicine, Cardiology, ${ }^{4}$ Department of Public Health and Clinical Medicin, Nutritional Research, Umeå, Sweden.

Background and Aims: Insulin resistance and beta cell dysfunction are major characteristics of T2DM. We aimed to characterize a non-diabetic population by the associations between diffferent levels of insulin resistance (IR) and beta cell function (B\%) and their relation to metabolic markers. We hypothesized that subjects with high IR and low B\% would have more metabolic pertubations than other groups.

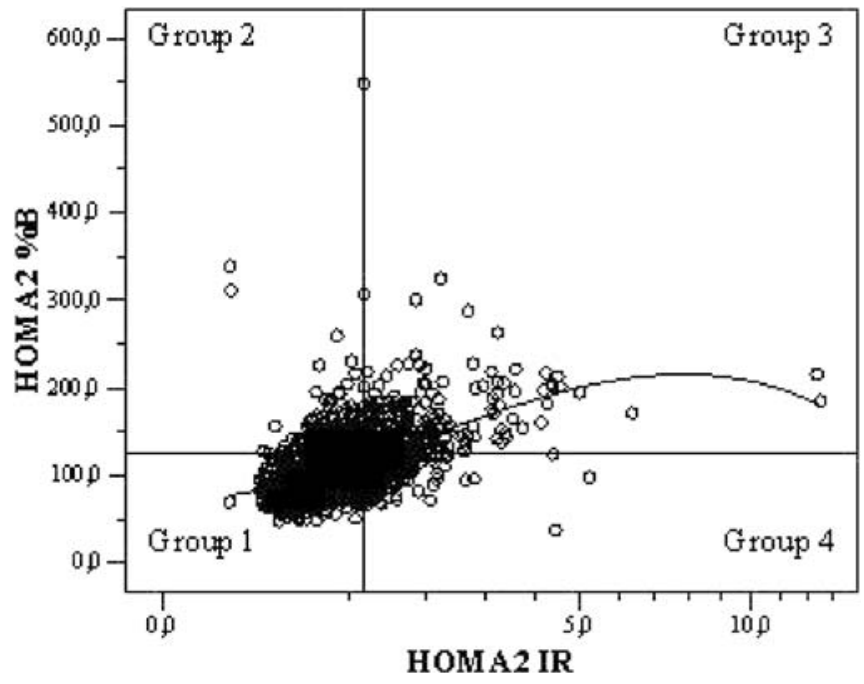

Fig. 1

Tab. 1 Abstracts 0373

\begin{tabular}{llllll}
\hline & Group 1 $\mathrm{p}=1037$ & Group 2 $\mathrm{p}=181$ & Group 3 $\mathrm{n}=224$ & Group 4 $\mathrm{n}=183$ & $\mathrm{p}$-values \\
\hline Sex $(\mathrm{M} / \mathrm{F})$ & $514 / 523$ & $83 / 98$ & $104 / 120$ & $81 / 102$ & $\mathrm{n} . \mathrm{s}$ \\
Age & $42.0(10.2)$ & $41.7(10.5)$ & $44.7(11.0)$ & $45.6(10.7)$ & $\mathrm{p}<0.01$ \\
fP-glucose mmol/L & $5.2(0.4)$ & $4.6(0.5)$ & $5.2(0.5)$ & $5.7(0.4)$ & $\mathrm{p}<0.01)$ \\
Body Mass Index & $24.3(3.0)$ & $25.8(3.9)$ & $28.4(4.6)$ & $26.1(3.8)$ & $\mathrm{p}<0.01$ \\
Cholesterol mmol/L & $5.9(1.3)$ & $5.8(1.4)$ & $6.3(1.2)$ & $6.1(1.2)$ & $\mathrm{p}<0.01$ \\
Triglycerides mmol/L & $1.0(0.1-13.6)$ & $1.2(0.1-4.2)$ & $1.7(0.1-11.0)$ & $1.2(0.3-8.9)$ & $\mathrm{p}<0.01$ \\
Adiponectin mg/L & $8.3(1.1-27.5)$ & $7.7(1.7-22.5)$ & $6.6(1.4-18.9)$ & $7.5(1.8-21.1)$ & $\mathrm{p}<0.01$ \\
Leptin mg/L & $5.0(0.6-32.7)$ & $6.7(0.6-49.1)$ & $10.7(0.8-44.4)$ & $7.5(0.6-74.0)$ & $\mathrm{p}<0.01$ \\
IGT \% & 7.3 & 6.1 & 9.4 & 9.3 & $\mathrm{n}$ \\
IFG \% & 2.8 & 0.0 & 4.5 & 12.6 & $\mathrm{p}<0.01$ \\
\hline
\end{tabular}

Table 1: Distribution of men and women, mean(SD) for age, fP-glucose, BMI and cholesterol, and median(min-max) for triglycerides, adiponectin and leptin in groups 1-4, respectively. Percentages of subjects with IGT and IFG, respectively. P-values for non-specified differences between the groups. 
Materials and Methods: 1625 (M/F 782/843, mean age 42.8 SD10.4) nondiabetic Swedish subjects participated in a health program. Data on fasting plasma glucose (fPG), 2 hour plasma glucose (2hPG), cholesterol, triglycerides, BMI and blood pressure were collected. Additional analyses included insulin, C-peptide, adiponectin and leptin. HOMA2 method was used to calculate B\% from C-peptide data and IR from insulin data. By dichotomizing IR and $\mathrm{B} \%$ by their $75^{\text {th }}$ percentiles, four groups with combinations of IR and $\mathrm{B} \%$ were defined (figure 1).

Results: IR correlated with $\mathrm{B} \%(\mathrm{r}=0.535, \mathrm{p}<0.01$; figure 1$)$. Women had higher IR $(\mathrm{p}<0.01)$ and $\mathrm{B} \%(\mathrm{p}<0.05)$ than men. IR $(\mathrm{r}=0.180, \mathrm{p}<0.01)$, but not $\mathrm{B} \%$ was correlated with age. Differences between groups $1-4$ are presented in table 1. Bonferroni post hoc test showed that subjects in group 3 and 4 were older than in 1 and 2. fPG levels were highest in group 4. BMI, cholesterol, triglycerides and leptin values were highest in group 3 , while adiponectin was lowest in the same group. IFG but not IGT was more frequent in group 4. Conclusion: As found in previous studies the association between IR and $\mathrm{B} \%$ was hyperbolic. In contrast to our hypothesis, the group with high IR and high $\mathrm{B} \%$ had more metabolic perturbations than the other groups. This could reflect the natural course of the decreasing beta cell function and increasing insulin resistance towards fulminant T2DM. (Tab. 1)

\section{4}

Heterogeneity of clinically classified type 2 diabetes mellitus: implication to epidemiological studies

F. C. F. Otieno ${ }^{1}$, G. Huho' ${ }^{2}$, A. Amayo

${ }^{1}$ Clinical Medicine and Therapeutics, University of Nairobi,

${ }^{2}$ Clinical Chemistry, University of Nairobi, Kenya.

Background. Diabetes is a heterogeneous disorder broadly classified as type 1 and 2 . Type 2 diabetes is equally heterogeneous with obese and non-obese types. Lately, an addition of LADA, that is phenotypically type 2 at onset, is complicating the picture in clinical practice. These clinical varieties of type 2 diabetes vary in their cardiovascular risk loading, therapeutic considerations and consequently their response to the chosen therapies. Primary prevention studies $(1,2,3)$ lend credence to differential response to the various therapeutic interventions on type 2 diabetes, at least, by the weight categories. (4)

Subjects and Methods: Ambulatory patients with clinical type 2 diabetes mellitus (classified by age of onset and BMI only), in equal numbers obese $\left(\mathrm{BMI}>25 \mathrm{Kg} / \mathrm{M}^{2}\right)$ and non-obese $\left(\mathrm{BMI} \leq 25 \mathrm{Kg} / \mathrm{M}^{2}\right)$ were randomly sampled. The patients enrolled were limited to those with $\leq 5$ years duration of diabetes. Socio-demographic data and diabetes history were obtained. Clinical examination, laboratory tests of GAD antibody and fasting C-peptide assays, among other tests, were done.

Results: 124 subjects were seen and their fasting C-peptide and GAD antibody status were used to help re-categorise them as types 1A, 1B, LADA or type 2 diabetes.

Table 1. Demographic characteristics of study subjects.

\begin{tabular}{lll}
\hline & $\mathrm{BMI}\left(\mathrm{Kg} / \mathrm{M}^{2}\right) \leq 25$ & $>25$ \\
\hline Gender \% & $\mathrm{F}-46.6 \%$ & $54.6 \%$ \\
Mean (SD)age (yrs) & $47.2(13.2)$ & $47.6(10.5)$ \\
Mean (SD) age at diagnosis (yrs) & $45.2(13.2)$ & $45.1(10.3)$ \\
Mean (SD) duration of diabetes (months) & $25.4(21.7)$ & $29.6(19.9)$ \\
$\begin{array}{l}\text { Mean (SD) fasting blood glucose } \\
\text { (mmol/L) }\end{array}$ & $8.09(4.4)$ & $8.17(4.8)$ \\
Current Treatment (\%pts) & $41.7 \%$ & \\
Insulin only & & $25.4 \%$ \\
Insulin \&OHA & $10 \%$ & \\
OHA only & $38.3 \%$ & $14.3 \%$ \\
Diet only & $10 \%$ & $54 \%$ \\
Family History, $1^{\text {st degree relative with }}$ & $43.0 \%$ & $6.3 \%$ \\
diabetes & & $40.6 \%$ \\
Mean BMI Kg/M $\mathrm{M}^{2}$ & $22.45(2.4)$ & \\
\hline OHA Orl & &
\end{tabular}

OHA =Oral hypoglycaemic agents
TABLE 2: AETIOLOGICALLY CLASSIFIED DIABETES IN THE STUDY

AETIOLOGIC TYPE 1A TYPE 1B TYPE 2 LADA

TYPES/

CLINICAL

\begin{tabular}{llllll}
\hline BMI KG/M & Non-obese $\leq 25$ & $4(6.7 \%)$ & $13(21.6)$ & $39(65 \%)$ & $4(6.7 \%)$ \\
& Obese $>25$ & 0 & $2(3.1 \%)$ & $59(91.2 \%)$ & $3(4.7 \%)$ \\
\hline
\end{tabular}

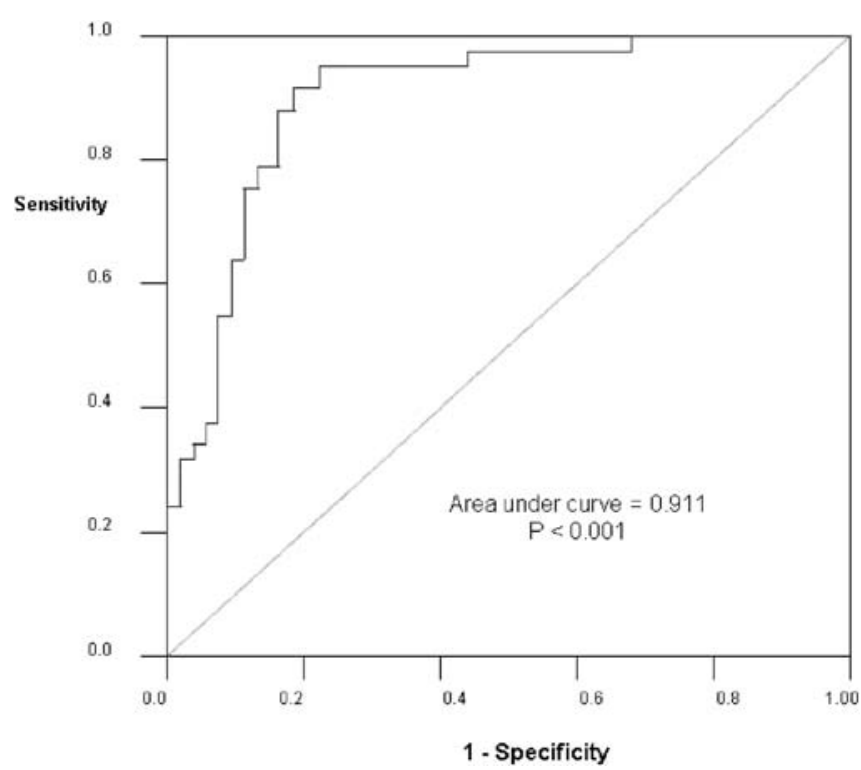

Figure 1. ROC-Curve to illustrate utility of BMI in identifying aetiologic type 2 diabetes in the study subjects

BMI cut-off of $25 \mathrm{Kg} / \mathrm{M}^{2}$ had sensitivity of $71.4 \%$ and specificity of $94.5 \%$ for detecting aetiologic type 2 diabetes in study subjects.

Conclusion: Clinical categorisation of the study patients having type 2 diabetes using BMI was useful. However, the patients with $\mathrm{BMI} \leq 25 \mathrm{Kg} / \mathrm{M}^{2}$, $17 / 124(13.7 \%)$ were re-classified as type 1 diabetes and $4 / 124(3.2 \%)$ as LADA on laboratory testing, suggesting that the lower BMI $\left(\leq 25 \mathrm{Kg} / \mathrm{M}^{2}\right)$ group was the more heterogeneous group. Both groups had similar proportions of patients with LADA.

BMI appears to be a major indicator of heterogeneity.

Supported by Diabetic Clinic Staff, Kenyatta National Hospital

\section{5}

Development and validation all-event risk score in patients with type 2 diabetes - Hong Kong Diabetes Registry

X.-L. Yang ${ }^{1}$, W.-Y. So ${ }^{1}$, A. P. S. Kong ${ }^{1}$, G. T. C. Ko ${ }^{2}$, R. C. W. Ma ${ }^{1}$, C. S. Cockram ${ }^{1}$, C.-C. Chow ${ }^{1}$, P. C. Y. Tong ${ }^{1}$, J. C. N. Chan ${ }^{1}$;

${ }^{1}$ Department of Medicine and Therapeutics, The Chinese University of Hong Kong, Shatin, ${ }^{2}$ Hong Kong Institute of Diabetes and Obesity, The Chinese University of Hong Kong, Shatin, Hong Kong.

Background: Patients with Type 2 diabetes are at increased risk of coronary heart disease, heart failure, stroke, peripheral arterial disease, end-stage renal disease and premature death (all-event endpoint). It is unknown whether these events as a whole can be predicted using a single risk score.

Methods: A prospective cohort of 6392 Chinese patients with type 2 diabetes who did not have history of the all-event endpoint at enrolment was used in the analysis. The data were randomly assigned to a training and a test dataset. In the training dataset, stepwise Cox regression $(\mathrm{p}=0.05)$ was used to select predictors. In the test dataset, calibration was checked using the life table method and discrimination was checked using the area under the receiver's operating characteristic curve (aROC). Risk factors were linear-transformed using spline Cox models before model development.

Results: Age, sex, blood haemoglobin $(\mathrm{Hb})$, spot urinary albumin:creatinine ratio (ACR), estimated glomerular filtration rate (eGFR), $\mathrm{HbA}_{1 \mathrm{c}}$, current smoking and use of insulin were selected as predictors in the training dataset. The all-event risk score was compiled as follows: $0.0389 \times$ age (year) -0.5295 $\times \operatorname{sex}(1$ if female $)+0.0620 \times\left|\mathrm{HbA}_{1 c}-6.5\right|(\%)-0.2344 \times$ blood $\mathrm{Hb}(\mathrm{g} / \mathrm{dL})$ 
$+0.2249 \times\left\{\log _{10}(1+\mathrm{ACR})\right\}^{2}-1.4493 \times \log _{10}(\mathrm{eGFR})+0.3638 \times$ current smoking ( 1 if yes) $+0.5235 \times$ use of insulin ( 1 if yes). The 5 -year probability of the all-event endpoint was: $0.9019^{\operatorname{EXP}(\mathrm{RISK} S \mathrm{SCORE}+3.8424)}$. The predicted all-eventfree probability was within the $95 \%$ confidence intervals of the observed all-event-free rate over a 5-year period. With follow-up time and censoring status considered, the adjusted aROC was 0.803 over the 5 -year observation period. At the cutoff point of $\geq-3.6024$, the sensitivity was $71.1 \%$ and the specificity was $71.0 \%$.

Conclusions: All-event endpoint can be predicted and the developed allevent risk score performed well in the Hong Kong Chinese patients with type 2 diabetes. External validation in other Chinese type 2 diabetic populations is needed before clinical use.

Partially supported by a Merck Sharp \& Dohme (MSD) University Grant and the Hong Kong Foundation for Research and Development in Diabetes, established under the auspices of the Chinese University of Hong Kong

\section{PS 16 Diabetes prevalence and incidence}

\section{6}

The prevalence of undiagnosed type 2 diabetes mellitus in Ukraine B. Mankovsky; Diabetology, Center for Endocrinology, Kiev, Ukraine.

Background and Aims: Diabetes mellitus represents the world-wide epidemics. However, the prevalence of diabetes mellitus in Ukraine is significantly lower than in neighboring countries. The number of registered patients with diabetes is 1,063 million subjects with the population of the country are 48 million. However, the real prevalence of diabetes in the country remains unknown.

Materials and Methods: To estimate the real prevalence of undiagnosed type 2 diabetes mellitus we performed screening of subjects who have some traditional risk factors for diabetes but who did not have any history of hyperglycemia. We examined 4640 subjects aged from 30 to 80 years old, 1098 men $(23,66 \%)$ and 3542 women $(76,34 \%)$ living at 8 different regions of the country. After getting informed consent the plasma glucose was measured either at fasting or random condition. In case of increased plasma glucose level another test was performed at fasting. The statistical analysis was done using the statistical package SPSS-13.

Results: We found that type 2 diabetes mellitus was newly diagnosed in 635 subjects (13.69\%), impaired fasting glucose was registered in 1008 persons $(21.72 \%)$. The risk factors for type 2 diabetes mellitus revealed by regression analysis were age $(\mathrm{OR}-2.64, \mathrm{p}<0.001)$, history of myocardial infarction (OR-1.73, $\mathrm{p}<0.001)$, arterial hypertension (OR-0.71, $\mathrm{p}<0.001)$, male gender (PR - 0.52, $\mathrm{p}<0.001$ ), BMI more than $27 \mathrm{~kg} / \mathrm{m}^{2}$ (OR-1.47, $\mathrm{p}<0.001$ ), family history of diabetes (OR-1.23, 0.05) and in women - the history of the birth of the baby with body mass of more than $4 \mathrm{~kg}(\mathrm{OR}-1.2, \mathrm{p}<0.1)$. The prevalence of type 2 diabetes mellitus in subjects younger than 45 year old was $6.53 \%$, in those aged $45-55$ year old $-11.67 \%$, in subjects aged $56-65$ year $-16.83 \%$ and in those older than 65 years was $18.71 \%$. The risk factors for impaired fasting glucose were arterial hypertension (OR-2,5), history of myocardial infarction $(\mathrm{OR}-2,5)$ and stroke $(\mathrm{OR}-2,5)$, age $(\mathrm{OR}-1,98)$, obesity $(\mathrm{OR}-1,75)$ and male gender (OR-1,65), $\mathrm{p}<0.05$ for all factors. There were some interregional differences in the prevalence of newly-diagnosed diabetes mellitus. Conclusion: The real prevalence of type 2 diabetes mellitus in Ukraine is significantly higher that registered and active diagnostics of the disease based on an assessment of risk factors is urgently needed to prevent the late diagnostics and complications of the disease. Unrestricted educational grant from Servier

\section{7}

The prevalence of diabetes in a large representative Hungarian population sample

E. P. Vamos ${ }^{1}$, M. Novak' 2 , M. Kopp 2 , I. Mucsi ${ }^{1,2}$;

${ }^{1}$ First Department of Internal Medicine, Hungary, ${ }^{2}$ Institute of Behavioral

Sciences, Semmelweis University, Budapest, Hungary.

Background and Aims: Although the dramatic effect of diabetes on public health is well-recognized, there is no reliable data concerning the prevalence of diabetes in Hungary. The few studies examining the incidence or prevalence of diabetes in this country focused on specific age groups or certain geographic areas. The aim of our study was to estimate the overall prevalence of diabetes in the Hungarian general population.

Materials and Methods: Hungarostudy 2002 was a cross-sectional survey conducted on a large, nationally representative sample of the Hungarian population over the age of 18 . The survey's aim was to extensively examine the mental and physical health status, health behavior and psycho-social risk factors of the Hungarian general population. Study participants were randomly selected from the National Population Register by a clustered, stratified sampling procedure. The identified individuals were homeinterviewed by trained nurses. Socio-demographic, lifestyle, health behavior, self-rated health data was obtained during the structured interview. Selfreported information on diabetes and comorbidity was also tabulated.

Results: A total of 12653 patients were enrolled in the survey, refusal rate was $17.7 \%$. The mean age of the population was $47.6 \pm 18$ with a range of $18-102$, 
$45 \%$ were male. The overall crude prevalence of diabetes was $6.6 \%$. There was no gender difference regarding diabetes ( $6.5 \%$ men, $6.7 \%$ women, NS). Prevalence increased sharply with age $(1.1 \%, 7.2 \%$ and $15.4 \%$, respectively, in age groups 18-39, 40-65 and over 65 years). Forty-eight percent of diabetic subjects vs $29.4 \%$ of non-diabetics had less than 10 years in education $(\mathrm{P}<0.0001)$. A wide geographic variation in the prevalence of diabetes $(3.9 \%$ - 9.0\%) was observed. Higher BMI was associated with higher prevalence of diabetes (underweight 1.8\%, normal $3 \%$, overweight $7.5 \%$, obese $13.9 \%$ ). Prevalence of comorbid conditions was significantly higher in diabetic individuals compared to non-diabetics; hypertension (59.5 vs. $19.2 \%$ ), history of stroke (11.2 vs 3.6\%), history of myocardial infarct (7 vs $2.1 \%$ ) and renal failure (5.7 vs $2.2 \%$ ), $\mathrm{P}<0.0001$ for all comparisons.

Conclusion: Based on this self-reported data the prevalence of diabetes in Hungary is higher than previous estimations and extrapolation from international data predicted. Since up to $50 \%$ of the diabetic patients may be undiagnosed, the true burden of diabetes is obviously even larger.

Supported by NKFP 1/002/2001, UNDP HUN/00/002/A/01/99, OTKA T29067 (1999) and OTKA T-32974 (2000) grants

\section{8}

Prevalence of impaired fasting glucose, impaired glucose tolerance and newly-diagnosed diabetes in subjects at risk of developing type 2 diabetes T. Tankova, N. Chakarova, M. Lazarova, I. Atanassova;

Clinical Center of Endocrinology, Medical University, Sofia, Bulgaria.

Background and Aims: The aim of the present study is to evaluate and compare the prevalence of impaired fasting glucose (IFG), impaired glucose tolerance (IGT) and newly-diagnosed diabetes in subjects at risk of developing diabetes according to $2003 \mathrm{ADA}$ and $2006 \mathrm{WHO}$ criteria.

Materials and Methods: 1029 subjects (467 males and 562 females), of mean age $49.3 \pm 18.1$ years and mean BMI $29.8 \pm 7.9 \mathrm{~kg} / \mathrm{m}^{2}$, with at least two risk factors for diabetes were enrolled. Subjects' risk was assessed by means of two questionnaires - about main diabetes risk factors and individual risk index FINDRISK. Glucose tolerance was studied during OGTT. Plasma glucose was measured at 0 and 120 minute by a dehydrogenase method (DiaSys; Glucose - GD). Categories of glucose tolerance were defined according to 2003 modified ADA and 2006 WHO criteria.

Results: Using 2003 ADA criteria, 46.6\% of subjects demonstrated normal glucose tolerance (NGT), while the rest $53.4 \%$ showed different abnormalities - $28.4 \%$ appeared to be with IFG, $8.6 \%$ - with IGT and $16.4 \%$ - with newly-diagnosed diabetes (NDD). The group with NGT demonstrated mean individual risk index FINDRISK 9.98 \pm 4.1 , the IFG group $-12.21 \pm 4.3$ $(\mathrm{p}<0.001$ vs NGT), the IGT group $-13.22 \pm 4.4(\mathrm{p}<0.0001$ vs NGT) and the newly-diagnosed diabetes group $-14.5 \pm 4.07$ ( $\mathrm{p}<0.0001$ vs NGT; $\mathrm{p}<0.01$ vs IFG and IGT groups). When applying $2006 \mathrm{WHO}$ criteria, $63.8 \%$ of subjects appeared to be with NGT, $11.2 \%$ - with IFG, $8.6 \%$ - with IGT and $16.4 \%$ - with newly-diagnosed diabetes. The group with NGT demonstrated mean individual risk index FINDRISK 10.42 \pm 4.23 , the IFG group $-12.99 \pm 4.08$ $(\mathrm{p}<0.0001$ vs NGT), the IGT group $-13.22 \pm 4.4(\mathrm{p}<0.0001$ vs NGT) and the diabetes group $-14.5 \pm 4.07$ ( $p<0.0001$ vs NGT; $p<0.01$ vs IFG and IGT groups). The prevalence of newly-diagnosed diabetes appeared to be higher in males at risk as compared to females $(19.0 \%$ vs $14.2 \%)$; the other forms of glucose intolerance being similar in both sexes. Significant positive correlation was established between the individual risk index FINDRISK and both fasting plasma glucose $(\mathrm{r}=0.73, \mathrm{p}<0.001)$ and 2-hour plasma glucose $(\mathrm{r}=0.67, \mathrm{p}<0.001)$.

Conclusions: The prevalence of IFG, IGT and newly-diagnosed diabetes in individuals at risk of developing type 2 diabetes is rather high with either of the criteria being used. When comparing 2003 ADA with 2006 WHO criteria the main difference appears to be in the prevalence of IFG, being 2.5 times higher with ADA criteria. The progression from NGT to IFG, IGT and diabetes correlates well with the number of risk factors and the individual risk index. Therefore, on the basis of the present results we may conclude that screening for diabetes should be performed in subjects with an individual risk index FINDRISK higher than 10.

\section{9}

Fasting plasma glucose, glycated haemoglobin $A_{1 c}$ and incidence of diabetes in a Japanese population: the Funagata Diabetes Study T. Nakagami ${ }^{1}$, M. Tominaga ${ }^{2}$, R. Nishimura ${ }^{3}$, M. Daimon ${ }^{4}$, T. Oizumi ${ }^{4}$, N. Yoshiike ${ }^{5}$, N. Tajima ${ }^{3}$;

${ }^{1}$ Diabetes Center, Tokyo Women's Medical University, Tokyo, ${ }^{2}$ Department of Laboratory Medicine, Yamagata University School of Medicine, Yamagata, ${ }^{3}$ Division of Diabetes, Metabolism and Endocrinology, Department of Internal Medicine, Jikei University School of Medicine, Tokyo, ${ }^{4}$ Department of Neurology, Hematology, Metabolism, Endocrinology and Diabetology, Yamagata University School of Medicine, Yamagata, ${ }^{5}$ International University-Business Coordination Center, National Institute of Health and Nutrition, Tokyo, Japan.

Background and Aims: The Ministry of Health, Welfare and Labour, Japan has introduced fasting plasma glucose (FPG) and glycated hemoglobin Alc (HbAlc) measurements to screen undiagnosed diabetes (DM) in the general health check-up system for adults aged 40-75 years old. In this health checkup system, FPG or $\mathrm{HbAlc}$ measurement is mandatory. Then, people with FPG of 5.6-6.9 mmol/l or HbAlc of 5.2-6.0\% take life-style counseling whereas people with $\mathrm{FPG} \geq 7.0 \mathrm{mmol} / \mathrm{l}$ or $\mathrm{HbAlc} \geq 6.1 \%$ take medical care by physicians. In this study, we assess the predictability of FPG and $\mathrm{HbAlc}$ for incident cases of DM and the relevance of the FPG and HbAlc cut-offs to undergo life-style counseling, by using data from Funagata town inhabitants.

Subjects and Methods: In the population-based cohort, $\mathrm{HbAlc}$ and fasting and 2-hour plasma glucose on an oral glucose tolerance test (OGTT) were determined in 1995-1997. A total of 1,188 Funagata-town inhabitants aged 35-89 years old and free of DM was confirmed their glycemic status by medical history of DM during 5 years and a repeated OGTT in 2000-2002 if people had no treatment of DM during 5 year period (participation rate: 60.3\%). DM was diagnosed according to 1999-World Health Organization criteria when an OGTT would be applied.

Results: During the period, 57 subjects (4.8\%) developed DM. The area under the receiver operating characteristic curve for incident of DM was similar between HbA1c $(0.785$ [95\% CI: 0.714-0.855]) and FPG $(0.786$ [0.719-0.852]). The FPG cut-off point of $5.6 \mathrm{mmol} / \mathrm{l}$ gave a sensitivity of $61.4 \%$, a specificity of $82.5 \%$, and a positive predictive value of $15.0 \%$. The corresponding values for HbAlc cut-off point of $5.2 \%$ were $73.7 \%, 71.8 \%$, and $11.7 \%$, respectively. Both FPG and $\mathrm{HbAlc}$ were positive, continuous, and independent predictors for incident of DM (both p-values $<0.001$ ). Hazard ratios adjusting for age, sex, and body mass index (95\% confidence interval) for incident of DM corresponding to FPG of 5.0-5.5, 5.6-6.0, 6.1-6.9 mmol/l at baseline were: 1.55 (0.63-3.82), 4.61 (1.96-10.85), and 13.12 (5.14-33.48), whereas those corresponding to HbAlc of $4.9-5.99,5.0-5.19,5.2-6.0$, $\geq 6.1 \%$ at baseline were: $0.33(0.07-1.66), 1.16(0.39-3.46), 4.68(2.05-10.70)$, 34.27 (9.46-124.08), respectively. Identified cases by FPG $5.6 \mathrm{mmol} / \mathrm{l}$ or HbAlc 5.2\% at baseline had worse cardiovascular risk profiles than those unidentified, although the difference was not statistically significant.

Conclusion: The cut-off point of FPG and HbAlc for life-style counseling according to the guideline recommended by Ministry of Health, Welfare and Labour, Japan was associated with increased risks of incident of DM. These cut-off points seemed to be effective to identify future DM in the next 5 years, however it seemed to be expensive. Further analyses focusing on mortality and the cost-effectiveness of the screening system will be needed.

Supported by a grant for the Study Group of Regional Differences in Risk Factors of Life-style Diseases and Methods for Continuous Monitoring (for N.Y.), from the Japanese Ministry of Health, Welfare and Labour

\section{0}

High prevalence of abnormal OGTT in patients with chronic hepatitis B or chronic hepatitis $\mathrm{C}$

A. Mavrogiannaki, B. Karamanos, E. Manesis, C. Demponeras, J. Koskinas, G. Papatheodoridis, A. Archimandritis;

2nd Academic Department of Internal Medicine, Diabetes Center, University of Athens, Hippokration Hospital, Greece.

Background and Aims: The association of glucose intolerance with chronic hepatitis $\mathrm{B}(\mathrm{CHB})$ or $\mathrm{C}(\mathrm{CHC})$ remains controversial, despite the large number of studies, which however have not used Oral Glucose Tolerance Test (OGTT) as criterion of glucose intolerance and the comparisons were 
not done with matched controls. The aim of the present study is to compare the prevalence of glucose intolerance, documented by OGTT, among patients with $\mathrm{CHB}$ or $\mathrm{CHC}$ and perfectly matched control groups.

Materials and Methods: 187 patients with $\mathrm{CHB}$ or $\mathrm{CHC}$, consecutively examined at the outpatient liver clinic, were compared with 187 subjects from the general population, matched for age, sex and Body Mass Index. OGTT was performed in all (except known diabetics).

Results: The prevalence of known diabetes did not differ between the groups of $\mathrm{CHB}$ or $\mathrm{CHC}$ and their controls $(7.2 \%$ vs $11.1 \%, \mathrm{P}=0.389$ and $7.1 \%$ vs $7.5 \%, \mathrm{P}=0.894$, respectively). However, abnormal OGTT was higher in $\mathrm{CHB}$ patients compared with their control-group ( $23.4 \%$ vs $8.3 \%, \mathrm{P}=0.013)$. Fasting blood glucose $(80.9 \pm 2.3$ vs $77.3 \pm 3.8 \mathrm{mg} / \mathrm{dl}, \mathrm{P}=0.398)$, insulin resistance, by HOMA model, $(3.7 \pm 0.4$ vs $3.3 \pm 0.4, \mathrm{P}=0.144)$ and family history of diabetes (29.9\% vs $16.7 \%, \mathrm{P}=0.081)$ did not differ between these two groups. CHC patients compared with their controls had higher prevalence of abnormal OGTT ( $30.5 \%$ vs $9.2 \%, \mathrm{P}=0.000$ ). Fasting blood glucose was higher in $\mathrm{CHC}$ patients than in controls $(84.9 \pm 1.7$ vs $76.8 \pm 1.6 \mathrm{mg} / \mathrm{dl}, \mathrm{P}=0.001)$. Insulin resistance ( $3.4 \pm 0.3$ vs $3.1 \pm 0.2, \mathrm{P}=0.721)$ and family history of diabetes ( $25.7 \%$ vs $16.3 \%, \mathrm{P}=0.123)$ did not differ between these two groups. The majority of patients, both $\mathrm{CHB}$ and $\mathrm{CHC}$, with abnormal OGTT had normal fasting blood glucose $(69.8 \%$ and $54.5 \%$, respectively), thus the diagnosis would had been missed, if only fasting blood glucose had been used. Conclusion: Increased prevalence of abnormal OGTT is documented not only in chronic hepatitis $\mathrm{C}$, but also in chronic hepatitis B patients, compared with perfectly matched control groups, reported for the first time. In patients with chronic hepatitis $\mathrm{B}$ or $\mathrm{C}$ the diagnosis of any degree of glucose intolerance requires OGTT, especially if diabetogenic treatments are planned.

\section{1}

Chlamydia pneumoniae and Chlamydia trachomatis antibodies are associated with symptoms of polycystic ovary syndrome: a new aetiology for the syndrome?

L. C. Morin-Papunen ${ }^{1}$, A. Torvinen ${ }^{1}$, A. Bloigu ${ }^{2}$, S. Franks ${ }^{3}$, A. Ruokonen ${ }^{4}$, L. Törmäkangas ${ }^{5}$, M.-R. Järvelin ${ }^{6}$, M. Paldanius ${ }^{5}$, M. Leinonen ${ }^{5}$, P. Saikku ${ }^{5}$, A. Pouta ${ }^{2}$;

${ }^{1}$ Department of Obstetrics and gynecology, University Hospital of Oulu, Finland, ${ }^{2}$ Department of Child and Adolescent Health, National Public Health Institute, Oulu, Finland, ${ }^{3}$ Institute of Reproductive and Developmental Biology, Imperial College London, United Kingdom, ${ }^{4}$ Department of Clinical Chemistry, University Hospital of Oulu, Finland, ${ }^{5}$ Department of Microbiology, National Public Health Institute, Oulu, Finland, ${ }^{6}$ Department of Epidemiology and Public Health, Imperial College London, United Kingdom.

Background and Aims: Chronic inflammation has been shown to be involved in the pathogenesis of insulin resistance, type 2 diabetes, and atherosclerosis. All chlamydial species have a tendency to cause persistent infections with subclinical chronic inflammation. C. pneumoniae infections have been associated with e.g. atherosclerosis and C. trachomatis infections with e.g. infertility and ectopic pregnancies. Polycystic ovary syndrome (PCOS) has been linked to insulin resistance, hyperinsulinemia and low-grade chronic inflammation. Our hypothesis was that the systemic inflammation induced by chlamydial infections might contribute to the development of PCOS. We therefore investigated, if chlamydial antibodies and slightly elevated Creactive protein (hsCRP) levels are associated with symptoms of PCOS in Northern Finland Birth Cohort 1966 (NFBC)

Materials and Methods: Members of NFBC $(n=5889)$ have been studied longitudinally from fetal period, with the most recent assessment occurring at age 31 , when a postal questionnaire including questions about hirsutism and oligomenorrhoea was sent to all women. 4523 women returned the questionnaire. Those who reported both symptoms were defined as cases $(n=$ 576). The presence of C. pneumoniae (IgG titers $\geq 32$ ) and for $C$ trachomatis (IgG titers $\geq 8$ ) was measured by microimmunofluorescence (MIF) in 96 cases and 2879 controls, and 96 cases and 853 controls, respectively. Samples for plasma glucose, serum insulin and high-sensitivity C-reactive protein (hsCRP) were collected.

Results: Both C. pneumoniae and C. trachomatis antibodies were more commonly present in PCOS patients (OR 1.58 with 95\% CI 1.05-2.38 and 2.11 with $95 \%$ CI 1.19-3.74 respectively) than in the controls. When adjusted for smoking, socioeconomic status, alcohol consumption and BMI, these differences remained significant. Also elevated hsCRP levels (upper quartile) were a significant risk factor for PCOS (OR 1.53 (1.00-2.35)) without adjustment. The simultaneous presence of $C$. pneumoniae or C. trachomatis antibodies with elevated hsCRP levels further strengthened the association (OR 2.25 (1.19-4.24) and 3.96 (1.49-10.55, respectively).

Conclusion: Our findings suggest the association between symptoms of PCOS and chlamydial antibodies. Interestingly, this association was found with both $C$. pneumoniae and C. trachomatis antibodies. This association was strengthened by the simultaneous presence of elevated hsCRP as a marker of low grade inflammation. This is a novel finding suggesting that chronic inflammation, associated with chlamydial infections, could contribute to the pathogenetic processes leading to decreased insulin sensitivity and consequently to the metabolic and hormonal disorders of the syndrome.

\section{2}

Prevalence of diabetes mellitus, pre-diabetes and the underlying risk factors in Sri Lanka - Sri Lanka Diabetes and Cardiovascular Study D. R. Matthews ${ }^{1}$, P. Katulanda ${ }^{1,2}$, G. R. Constantine ${ }^{2}$, M. Wijesuriya ${ }^{3}$, M. I. McCarthy ${ }^{1}$, R. D. Seneviratne ${ }^{4}$, M. H. R. Sheriff';

${ }^{1}$ Oxford Centre for Diabetes Endocrinology and Metabolism, University of Oxford, Oxford, United Kingdom, ${ }^{2}$ Dept of Clinical Medicine, Faculty of Medicine, Colombo, Sri Lanka, ${ }^{3}$ Sri Lanka Diabetes Association, National Diabetes Centre, Rajagiriya, Sri Lanka, ${ }^{4}$ Dept of Community Medicine, Faculty of Medicine, Colombo, Sri Lanka.

Background and Aims: South Asian populations are among the worst affected in the current pandemic of diabetes due to the ethnic susceptibility. Impaired fasting glucose (IFG) and impaired glucose tolerance (IGT) increase the risk of diabetes and are termed pre-diabetes. We aimed to determine the prevalence of diabetes and pre-diabetes and the underlying risk factors among different socio-demographic groups in Sri Lanka.

Materials and Methods: In this nationally representative prevalence survey, 5000 subjects above 18 years of age were selected by a multi stage random cluster sampling technique. A structured questionnaire was used to record detailed interview data and physical examination findings. Fasting and post-glucose load plasma glucose was estimated. New cases of diabetes, IGT and IFG were diagnosed according to the ADA criteria. Standardization was performed using UN population estimates 2004. Prevalence and the continuous variables are reported with confidence intervals (CI) and standard deviations (SD) respectively.

Results: Altogether 4464 subjects participated (response 89\%); males $40 \%$; mean age 46 years (SD 15). The crude prevalence of diabetes was $11.5 \%$ (10.6 - 12.4) and $40 \%$ of these were newly diagnosed. The overall age and sex standardized prevalence of diabetes was 10\% (9.1-10.9); Male $9.2 \%$ $(7.9-10.5)$ and Female $10 \%(8.9-11.1)(\mathrm{p}=\mathrm{ns})$. The prevalence in the urban, rural and plantation sectors were $17.3 \%(14.7-19.9), 10.6 \%$ (9.6-11.6) and $3.8 \%(0.8-6.8)$ respectively $(\mathrm{p}<0.0001)$. The overall prevalence rates of prediabetes by sector were urban $13.9 \%$ (11.5-16.3); rural 12.3\% (11.2-13.4) and plantations 5.1\% (1.6-8.6). The prevalence of individual components of prediabetes: IFG, IGT and both IFG and IGT were 4.3\% (3.7-4.9), 6\% (5.3-6.7) and $2 \%(1.6-2.4)$ respectively. Mean age in years (54.7 SD12.5 vs 44.7 SD 15 ), BMI (23.8kg/m3 SD4 vs $21.5 \mathrm{~kg} / \mathrm{m} 3 \mathrm{SD} 4.2)$, waist circumference $(84.7 \mathrm{~cm}$ SD 10.8 vs $76.3 \mathrm{~cm}$ SD11.5) and systolic blood pressure (138mmHg SD 24 vs $127 \mathrm{mmHg}$ SD20) were significantly higher $(\mathrm{p}<0.001)$ among diabetic subjects compared to others. The prevalence rates of diabetes among sufficiently active, moderately active and insufficiently active subjects were $8.1 \%(7.1-$ 9.1), $15.7 \%(13.8-17.6)$ and $23.4 \%(17.1-29.7)$ respectively. The prevalence among those with and without family history were $21.5 \%(18.8-24.2)$ vs $8.2 \%$ (7.3-9.1) $\mathrm{p}<0.001$; in those with and without acanthosis nigricans were $4.3 \%$ $(3.7-4.9)$ vs $14.3 \%(9.2-19.4) \mathrm{p}<0.001)$ and in those who had and hadn't been in overseas were $19 \%(15.2-22.8)$ vs $10.7 \%(9.7-11.7) \mathrm{p}<0.001$.

Conclusion: There is a high prevalence of diabetes and pre-diabetes in urban, rural and plantation sectors in Sri Lanka. Diabetes is positively associated with obesity, hypertension, family history, urban living, overseas stay and acanthosis nigricans. It was negatively associated with physical activity. High prevalence of diabetes indicate the need for urgent primary urgent strategies specifically targeting modifiable risk factors.

Supported by National Science Foundation Sri Lanka, Oxford Centre for Diabetes Endocrinology and Metabolism 


\section{3}

Diabetic patients detected in a population screening programme have similar outcomes to clinically diagnosed patients from a matched unscreened population

M. Rahman ${ }^{1}$, S. Hennings ${ }^{2}$, N. J. Wareham ${ }^{2}$, S. J. Griffin ${ }^{2}$;

${ }^{1}$ Department of Diabetes and Endocrinology, Northwick Park Hospital, London, ${ }^{2}$ Epidemiology Unit, Medical Research Council, Cambridge, United Kingdom.

Background and Aims: There is continuing uncertainty regarding population screening for type 2 diabetes. Screening may be cost-effective if patients detected and treated early have better outcomes than those detected clinically. We compared outcomes for diabetic patients in a population that had been offered 5-yearly screening by OGTT with clinically diagnosed diabetic patients in a matched population that had not been offered screening.

Materials and Methods: The Ely Study is a population-based, parallel group, cohort study. Individuals aged 40-64 years in 1990 were identified from the register of a single general practice serving the city of Ely. A third of individuals without known diabetes (screened group, SG) were selected at random for offer of 5-yearly screening with OGTT. A further third were randomly selected and invited for OGTT and outcome measurement 10 years later (unscreened group, UG). All diabetic patients from both groups were invited for outcome assessment undertaken by staff unaware of their study group status. Analysis was restricted to those patients still registered at the practice.

Results: Of 220 eligible diabetic patients, 166 (75.5\%) attended for testing with no significant difference in attendance between the groups (99 [78.6\%] SG vs $67(71.3 \%$ ] UG; $p=0.2)$; there was no significant difference in age, gender and social deprivation between attenders and non-attenders. The patients were middle-aged (mean age $67 \pm 7$ years), not deprived (mean Townsend score $-1.3 \pm 1.9$ ), and $51 \%$ were male. Patients in the screened group were slightly older than the unscreened group (mean age in years: $68.4 \pm 6.7$ vs $66.1 \pm$ 6.2 , respectively; $\mathrm{p}=0.02$ ), but there was no significant difference in duration of diabetes $(6.2 \pm 5.1$ years vs $5.1 \pm 6.4$ years, respectively; $\mathrm{p}=0.2)$. Clinical and functional outcomes were not significantly different between groups (see table; values as mean $\pm \mathrm{SD}$, unless stated otherwise).

Conclusion: Differences in health outcomes among diabetic patients in a screened population compared with an unscreened population were small ten years after commencement of screening. This study cannot exclude the possibility that early detection may reduce long term risk of complications, but stronger evidence of net benefit is needed before population screening can be recommended.

\begin{tabular}{llll} 
Outcome & $\begin{array}{l}\text { Screened } \\
\text { Group }\end{array}$ & $\begin{array}{l}\text { Unscreened } \\
\text { Group }\end{array}$ & p value \\
\hline BMI $\left(\mathbf{k g} / \mathbf{m}^{2}\right)$ & $30.2 \pm 5.0$ & $29.5 \pm 4.6$ & 0.4 \\
HbA $_{1 \mathrm{c}} \mathbf{( \% )}$ & $7.1 \pm 1.9$ & $7.5 \pm 1.6$ & 0.2 \\
Systolic BP (mmHg) & $133 \pm 15$ & $132 \pm 14$ & 0.4 \\
Diastolic BP (mmHg) & $80 \pm 10$ & $79 \pm 10$ & 0.5 \\
Microalbuminuria \% $(\boldsymbol{n})$ & $17.5(22)$ & $17.0(16)$ & 0.9 \\
Neuropathy \% $(\boldsymbol{n})$ & $34.9(44)$ & $41.5(39)$ & 0.7 \\
Retinopathy \% $(\boldsymbol{n})$ & $45.2(28)$ & $58.5(55)$ & 0.1 \\
Ischaemic heart disease \% $(\boldsymbol{n})$ & $31.9(30)$ & $24.6(31)$ & 0.2 \\
SF-36 physical function & $67.2 \pm 29.4$ & $69.5 \pm 30.6$ & 0.5 \\
SF-36 general health & $59.4 \pm 12.6$ & $60.1 \pm 13.5$ & 0.4 \\
SF-36 mental health & $78.4 \pm 16.5$ & $79.8 \pm 16.3$ & 0.6 \\
\hline
\end{tabular}

Supported by NHS R\&D Eastern

\section{PS 17 Circulating risk factors}

\section{4}

Adiponectin and proinflammatory state in type 1 diabetic patients with and without metabolic syndrome

R. Z. Timar, V. Serban, L. Diaconu, A. Vlad, V. Botea, B. Timar, C. Mazilu; Diabetes Clinic, University of Medicine and Pharmacy "Victor Babes",

Timisoara, Romania.

Background and Aims: The metabolic syndrome (MS) is a condition characterized by a clustering of clinical and biochemical proatherogenic abnormalities. MS is an important risk factor for cardiovascular disease and premature mortality in patients with type 1 or type 2 diabetes mellitus (DM) and in nondiabetic persons. Adiponectin is a $30 \mathrm{kDa}$ protein hormone secreted almost exclusively by adipocytes with anti-atherogenic, anti-inflammatory and insulin-sensitizing properties.

The aims of our study were to estimate the prevalence of MS in patients with type $1 \mathrm{DM}$ and to assess plasma levels of adiponectin and proinflammatory state in type 1 diabetic patients with and without MS.

Materials and Methods: The study enrolled 72 patients with type $1 \mathrm{DM}, 38$ men $(52.8 \%)$ and 34 women $(47.2 \%)$, with the mean age of $41.3 \pm 7.4$ years and the mean duration of DM $13.9 \pm 4.8$ years. The MS was defined according to the IDF criteria. In these patients we assessed plasma levels of adiponectin, leptin, interleukin 6 (IL-6), tumor necrosis factor $\alpha$ (TNF- $\alpha$ ), high-sensitive $\mathrm{C}$ reactive protein (hs-CRP), fibrinogen. Linear relationships between key variables were tested by Pearson's correlation coefficient.

Results: The MS was present in 21 patients $(29.2 \%)$ with type $1 \mathrm{DM}$. The prevalence of MS was higher in women (32.35\%) than in men (26.31\%). Serum adiponectin levels were markedly higher in type 1 diabetic patients without MS $(12.4 \pm 1.6 \mu \mathrm{g} / \mathrm{mL}$ in males, and $14.1 \pm 1.3 \mu \mathrm{g} / \mathrm{mL}$ in females) than in type 1 diabetic patients with MS $(6.6 \pm 0.5 \mu \mathrm{g} / \mathrm{mL}$ in males and $8.3 \pm 0.8 \mu \mathrm{g} / \mathrm{mL}$ in females), $\mathrm{p}<0.001$. In patients with MS adiponectin was negatively correlated with body mass index (BMI), waist to hip ratio (WHR), leptin, IL-6, TNF- $\alpha$, hs-CRP and fibrinogen. We did not found a correlation between adiponectin and proinflammatory state in type 1 diabetic patients without MS.

Table 1. Adiponectin and proinflammatory state in type $1 \mathrm{DM}$ with and without MS

\begin{tabular}{llll}
\hline Type 1 DM & MS & Without MS & p \\
\hline Number $(\mathrm{M} / \mathrm{F})$ & $10 / 11$ & $28 / 23$ & \\
Adiponectin $(\mu \mathrm{g} / \mathrm{mL})$ & $7.1 \pm 0.7$ & $13.2 \pm 1.5$ & $\mathrm{p}<0.001$ \\
Leptin $(\mathrm{ng} / \mathrm{mL})$ & $12.4 \pm 2.6$ & $7.3 \pm 2.4$ & $\mathrm{p}<0.001$ \\
TNF- $\alpha(\mathrm{pg} / \mathrm{mL})$ & $8.3 \pm 1.5$ & $6.2 \pm 1.3$ & $\mathrm{p}<0.001$ \\
IL-6 $(\mathrm{pg} / \mathrm{mL})$ & $4.4 \pm 0.7$ & $3.1 \pm 0.6$ & $\mathrm{p}<0.001$ \\
hs-CRP $(\mathrm{mg} / \mathrm{L})$ & $7.3 \pm 0.9$ & $5.3 \pm 0.7$ & $\mathrm{p}<0.001$ \\
Fibrinogen $(\mathrm{mg} / \mathrm{dL})$ & $342.3 \pm 45.6$ & $231.8 \pm 39.7$ & $\mathrm{p}<0.001$ \\
\hline
\end{tabular}

Data are mean \pm SD. $\mathrm{p}$ was calculated with unpaired Student`s $\mathrm{t}$ test.

Conclusion: MS is more prevalent in individuals with type $1 \mathrm{DM}$ than in general population and increases the cardiovascular risk of these patients. Hypoadiponectinemia and increased plasma concentrations of acute-phase proteins are present in adult patients with type $1 \mathrm{DM}$ and MS.

Adiponectin may be enhanced in type 1 diabetic patients without MS as a physiological counterregulatory response to mitigate endothelial damage and vascular damage.

\section{5}

Leptin and progression to type 1 diabetes in children with islet autoimmunity

M. M. Lamb ${ }^{1}$, X. Yin ${ }^{1}$, D. Dabelea ${ }^{1}$, M. Rewers ${ }^{1,2}$, J. Norris ${ }^{1}$;

${ }^{1}$ Preventive Medicine and Biometrics, University of Colorado at Denver and Health Sciences Center, Denver, United States,

${ }^{2}$ Barbara Davis Center for Childhood Diabetes, Aurora, United States.

Background and Aims: Increased body fat and insulin resistance in persons with islet autoimmunity (IA) may accelerate $\beta$-cell loss and speed progression to type 1 diabetes (T1D). We examined whether levels of leptin (a marker of 
fat mass), adiponectin (a marker of insulin sensitivity) and body mass index zscores (a marker of excess weight relative to others of the same age and gender) differed between children with IA who did or did not progress to T1D.

Materials and Methods: Since 1993, the Diabetes Autoimmunity Study in the Young (DAISY) has prospectively followed children at increased risk for T1D based on HLA-DR,DQ genotype or family history of T1D. IA (insulin, GAD65 or IA-2 autoantibodies present on $\geq 2$ consecutive visits) developed in 57 pre-pubertal subjects (21 girls age $1-11$ and 36 boys age $1-12$ ). Upon IA development, subjects had clinic visits every 3-6 months, at which height, weight, and non-fasting blood samples were collected. Eighteen subjects progressed to T1D. Leptin and adiponectin were measured in non-fasting plasma, and hemoglobin Alc (HbAlc) was measured in whole blood collected at first IA positive visit (baseline age 3.6 years) and within one year of T1D diagnosis (mean age 6.9 years) for those that progressed to T1D, and at first IA positive visit (baseline age 5.2 years) and most recent visit (mean age 7.8 years) for non-progressors.

Results: At baseline, leptin levels did not differ between those children who progressed to diabetes and those who did not progress. However, leptin was marginally significantly lower in children who progressed to T1D at last follow-up. Adiponectin levels did not differ between progressors and nonprogressors at either time point. Body mass index (BMI z-score) at baseline did not differ between progressors and non-progressors, but was significantly lower in progressors than non-progressors at last follow-up. HbAlc was lower in progressors than non-progressors at baseline, but significantly higher in progressors than non-progressors at last follow-up (Table). All analyses were adjusted for age.

Conclusion: These results suggest that progression from IA to overt T1D is not related to decreasing insulin sensitivity, as marked by adiponectin. The lower leptin levels and lower BMI z-scores at last follow-up suggest less excess weight in progressors. The strong rise in $\mathrm{HbAlc}$ levels and the decline in leptin levels in children progressing to T1D may reflect insulinopenia preceding diagnosis.

\begin{tabular}{|c|c|c|c|c|c|c|c|c|}
\hline \multirow{2}{*}{$\begin{array}{l}\text { Varaible } \\
\text { (mean) }\end{array}$} & \multicolumn{4}{|c|}{ Baseline } & \multicolumn{4}{|c|}{ Last Follow-up $\dagger$} \\
\hline & $\mathrm{N}$ & $\begin{array}{l}\begin{array}{l}\text { Pro- } \\
\text { gressors }\end{array} \\
\end{array}$ & $\begin{array}{l}\begin{array}{l}\text { Non- } \\
\text { progressors }\end{array} \\
\end{array}$ & $\mathrm{p}$-value ${ }^{*}$ & $\bar{N}$ & $\begin{array}{l}\begin{array}{l}\text { Pro- } \\
\text { gressors }\end{array} \\
\end{array}$ & $\begin{array}{l}\begin{array}{l}\text { Non- } \\
\text { progressors }\end{array} \\
\end{array}$ & p-value ${ }^{\star}$ \\
\hline $\begin{array}{l}\text { Leptin } \\
\end{array}$ & 57 & 2.4 & 3.1 & 0.867 & 57 & 1.8 & 4.2 & 0.075 \\
\hline Adiponectin & 57 & 17.7 & 17.1 & 0.867 & 57 & 16.6 & 17.3 & 0.497 \\
\hline BMI z-score & 40 & 0.7 & 0.6 & 0.839 & 55 & -0.1 & 0.5 & 0.048 \\
\hline HbAlc & 54 & 4.8 & 5.0 & 0.061 & 56 & 6.5 & 5.1 & $<0.0001$ \\
\hline
\end{tabular}

*p-value adjusted for age

tlast follow-up represents the visit just prior to or at diagnosis in progressors, and the most recent visit for non-progressors

Supported by NIH R01-DK49654, NIH R01-DK32493

\section{6}

The genetic variation in the tenomodulin gene is associated with serum levels of systemic immune mediators - the Finnish Diabetes Prevention Study

A.-M. Tolppanen ${ }^{1}$, L. Pulkkinen ${ }^{1}$, C. Herder ${ }^{2}$, W. König ${ }^{3}$, J. Lindström ${ }^{4}$, J. Tuomilehto ${ }^{4}$, M. Uusitupa ${ }^{1}$;

${ }^{1}$ Department of Public Health and Clinical Nutrition and Food and Health Research Centre, University of Kuopio, Finland, ${ }^{2}$ German Diabetes Clinic, German Diabetes Center, Leibniz Center at Heinrich Heine University, Düsseldorf, Germany, ${ }^{3}$ Department of Internal Medicine II-Cardiology, University of Ulm Medical Center, Germany, ${ }^{4}$ Department of Epidemiology and Health Promotion, Diabetes Unit, National Public Health Institute, Helsinki, Finland.

Background and Aims: We have reported that single nucleotide polymorphisms (SNPs) of the X-chromosomal tenomodulin gene (TNMD) have gender-specific associations with the risk of type 2 diabetes (T2DM), central obesity and impaired glucose metabolism. The mRNA levels of TNMD decrease along with weight reduction and correlate with insulin sensitivity and serum and mRNA levels of inflammatory markers. Our aim was to investigate the associations of TNMD SNPs with systemic immune mediators in the Finnish Diabetes Prevention Study (DPS).

Materials and Methods: The baseline concentrations of serum immune mediators of 522 DPS participants (BMI>25, IGT) were analysed. Seven
SNPs were genotyped with Taqman allelic discrimination method. The linkage disequilibrium was calculated by Haploview. Associations of SNPs with immunomarkers were analyzed with general linear model univariate analysis (SPSS 14.0). Data split according to the median 2-hour plasma glucose concentration (2h-pGlu; $8.72 \mathrm{mmol} / \mathrm{l}$ ) was analyzed to address the genotype effects in different glucose tolerance categories. Q-value was used for multiple corrections ( $\mathrm{q}<0.1$ reported).

Results: Rs1155974 and rs2073163, associated with increased T2DM risk in men in our previous study, were associated with serum amyloid A concentrations (SAA) in all men ( $\mathrm{p}=0.01$ and 0.016 respectively) and in the group with higher $2 \mathrm{~h}$-pGlu ( $\mathrm{p}=0.021$ and 0.021 respectively). The same markers were associated with concentrations of soluble intercellular adhesion molecule ( $\mathrm{p}=0.025$ and 0.033 , respectively). Interestingly, the genotypes associated with higher risk for T2DM also had higher serum concentrations of these mediators. The genotype differences of rs11798018 and rs2073162 regarding IL-6 levels ( $\mathrm{p}=0.049$ and 0.035 , respectively) and those of rs2073162 and rs4828038 regarding macrophage inflammatory protein $1 \alpha$ levels (MIP- $1 \alpha ; p=0.024$ and 0.006 , respectively) were statistically significant only in the group with higher $2 \mathrm{~h}$-pGlu. Rs4828037 and rs5966709 associated with regulated on activation normal T-cell expressed and secreted concentrations (RANTES) in all men ( $\mathrm{p}=0.02$ and 0.023 , respectively) and in the group with higher $2 \mathrm{~h}$-pGlu ( $\mathrm{p}=0.006$ for both markers). In women rs 4828037 was associated with SAA ( $p=0.008, q=0.081$ ) in the group with higher 2h-pGlu while rs11798018 associated with IL-6 levels in all women $(\mathrm{p}=0.024)$. In addition, rs4828037 and rs5966709 were associated with MIP$1 \alpha$ in all women ( $\mathrm{p}=0.045$ and 0.022$)$ and in the group with higher $2 \mathrm{~h}-\mathrm{pGlu}$ ( $p=0.013$ and $0.007, q=0.081$ for both). rs5966709 was also associated with RANTES ( $p=0.031$ ) in all women while the genotype effect of rs 1155974 and rs2073162 were statistically significant only in the group with higher $2 \mathrm{~h}$-pGlu ( $\mathrm{p}=0.018, \mathrm{q}=0.081$ for both).

Conclusion: These results confirm our previous observation on the connection between TNMD and low-grade inflammation. They also suggest that the putative link between TNMD and T2DM may be mediated via the effects on systemic immune mediators.

Supported by the Academy of Finland, Sigrid Juselius Foundation, Juho Vainio Foundation and EVO-fund of the Kuopio University Hospital from Ministry of Health and Social Affairs

\section{7}

A strong association between serum levels of monocyte chemoattractant protein 1 (MCP-1) and insulin resistant syndrome

A. Fukami, H. Adachi, S.-I. Yamagishi, M. Enomoto, A. Satoh, K. Furuki, Y. Shigetoh, M. Otsuka, S. Kumagae, T. Imaizumi;

Department of Internal Medicine, Division of Cardio-Vascular Medicine,

Kurume University School of Medicine, Kurume, Fukuoka, Japan.

Background and Aims: Inflammation plays an essential role in the atherosclerotic process. MCP-1 is a chemokine responsible for the recruitment of monocytes to sites of inflammation. Several human studies suggested that an elevated MCP-1 concentration was associated with a higher prevalence of the atherosclerosis. Recently, MCP-1 has been shown to contribute to macrophage infiltration into adipose tissue and insulin resistance in obesity in animal model. However, data for an epidemiological study of the association between serum levels of MCP-1 and insulin resistance have not been available on a general population. We investigated whether serum levels of MCP-1 are associated with insulin resistance syndrome in a general population.

Materials and Methods: We performed a health examination in a small fishing community in Uku Island, Nagasaki, Japan. A total of 642 Japanese residents ( 231 men and 411 women, mean age $65.2 \pm 9.8$ ) received the examination. The subjects' medical history, use of alcohol, and smoking habits were ascertained by a questionnaire. Data for fasting blood samples including serum levels of MCP-1 was obtained from 622 subjects ( 226 men, 396 women).

Results: Uni- and multi-variate analysis were performed to obtain correlates of serum levels of MCP-1. The mean levels of MCP-1 were $189.3 \pm 4.9 \mathrm{pg} / \mathrm{ml}$. The serum levels of MCP-1 were higher in men than in women. MCP-1 levels increased with age. Elevated MCP-1 levels were associated with waist circumference $(p<.0001)$, total protein $(p=0.009)$, insulin $(p<.0001)$, and HOMA-R $(\mathrm{p}<.0001)$ by multiple linear regression analysis adjusted for age and sex.

Conclusion: The present study reveals that serum levels of MCP-1 are strongly associated with insulin resistance syndrome in a general population. 


\section{8}

Association of C-reactive protein with type 2 diabetes: meta-analyses of prospective studies

C.-T. C. Lee ${ }^{1}$, S. Erqou ${ }^{2}$, S. J. Sharp ${ }^{3}$, A. I. Adler ${ }^{1,3}$;

${ }^{1}$ Diabetes Centre, Addenbrooke's Hospital, Cambridge, United Kingdom,

${ }^{2}$ Department of Public Health and Primary Care, University of Cambridge,

United Kingdom,

${ }^{3}$ MRC Epidemiology Unit, University of Cambridge, United Kingdom.

Background and Aims: Epidemiological studies suggest that elevated Creactive protein (CRP), a circulating marker for acute inflammation produced by the liver, increases the risk of type 2 diabetes. The mechanisms of this association have not been fully established. The heterogeneity (variation in relative risk estimates) among studies is yet to be explored systematically. We aimed to assess the potential associations between CRP and type 2 diabetes in prospective cohort and nested case-control studies.

Material and Methods: We conducted a literature-based meta-analysis of long-term ( $>3$ years follow-up) prospective studies of the association between CRP and type 2 diabetes published until March 2007. Studies were identified by searching MEDLINE with the terms C-reactive protein and type 2 diabetes, and by scanning relevant reference lists. In addition to information on the association between CRP and type 2 diabetes, information on age, sex, geographic location, study design, follow-up duration, adjustment for potential confounders and CRP assay method, was extracted to enable exploration of potential sources of heterogeneity. Individual studies were combined by using random effect model meta-analysis after appropriate conversion of the relative risk estimates.

Results: We identified 13 publications ( 8 prospective cohort studies, 5 nested case-control studies). A total of 1300 incident diabetes cases out of 22676 participants in prospective cohort studies and 1769 cases in nested casecontrol studies (2174 control participants) were reported. Assuming a loglinear association between CRP and risk of diabetes and an approximate normal distribution of CRP in the general population, comparison of individuals in the upper third of baseline CRP distribution with those in the bottom third yielded a combined adjusted relative risk estimate of 1.76 (95\% CI 1.56 - 1.99). Substantial heterogeneity between individual studies was found $\left(\chi_{12}^{2}=28.88, \mathrm{p}=0.004\right)$, possible reasons were explored.

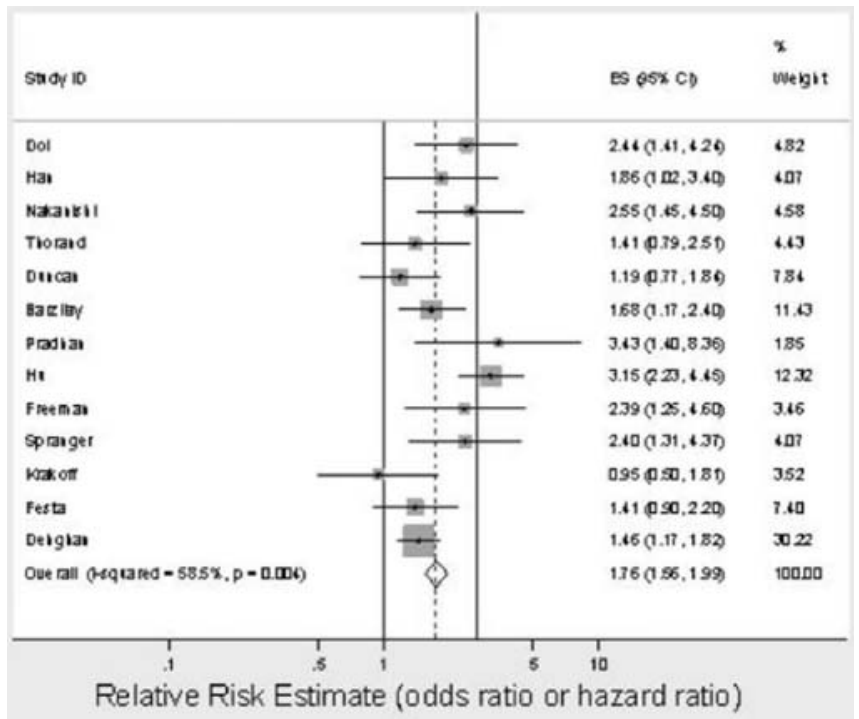

Conclusion: This meta-analysis suggests that elevated CRP level is associated with an increased risk of type 2 diabetes, independent of age and obesity. The existence of heterogeneity warrants thorough examination of possible reasons for the variability of study results, which would be useful in determining if combined analysis of studies is justified and in further characterization of any association. However, due to the relative small number of studies, further epidemiological data is needed to clarify the role of CRP in increasing the risk for type 2 diabetes and to elucidate the mechanisms of the association.

\section{PS 18 Genetics of beta cell function and insulin resistance}

\section{9}

Heritability of beta cell function in Dutch twins and siblings

A. M. C. Simonis-Bik ${ }^{1}$, E. M. W. Eekhoff ${ }^{1}$, R. J. Heine ${ }^{1}$, K. Kroon ${ }^{1}$,

G. Willemsen², E. de Geus' ${ }^{2}$, M. Diamant ${ }^{1}$, J. M. Dekker ${ }^{3}$, D. I. Boomsma²;

${ }^{1}$ Endocrinology, VU University Medical Center,

${ }^{2}$ Biological Psychology, VU University, ${ }^{3}$ EMGO Institute, VU University Medical Center, Amsterdam, The Netherlands.

Background and Aims: In type 2 diabetes mellitus two pathogenic factors play a crucial role in the development of hyperglycaemia: impaired insulin secretion and insulin resistance. Remarkably, only a few studies have addressed the heritability of beta-cell function.

Materials and Methods: We recruited 75 twin families from the Netherlands Twin Register to participate in a study of different aspects of beta-cell function. Only healthy monozygotic and dizygotic twins (age $20-45$ years) with additional siblings of the same sex were eligible The protocol consisted of a home visit for an Oral Glucose Tolerance Test (OGTT) and the completion of questionnaires about general health and food intake and two visits at the clinic. The first clinic visit involved a standardized mixed-meal test. During the second visit participants underwent a combined euglycaemic / hyperglycaemic clamp test modified with additional GLP-1 and arginine stimulation, to assess several aspects of beta-cell function in relation to the ambient insulin sensitivity.

Results: So far 62 families ( 29 all male, 33 all female) have completed the OGTT and the meal test and were analysed by Analysis of Variance (ANOVA) Mean age of the 157 participants is $31.2 \pm 6.0$ years (table 1 )

\begin{tabular}{llll} 
Characteristics & & & \\
\hline genetic relationship & monozygotic & dizygotic & sibs \\
\hline total number & 82 & 37 & 38 \\
sexe:male / female & $39 / 43$ & $16 / 21$ & $21 / 17$ \\
Age mean $( \pm$ S.D. $)$ & $30.6 \pm 4.4$ & $28.9 \pm 4.8$ & $34.9 \pm 8.3$ \\
OGTT fasting blgl.(mmol/l) & $4.72 \pm .45$ & $4.44 \pm .40$ & $4.61 \pm .48$ \\
OGTT 2 hours blgl (mmol/l) & $5.63 \pm 1.21$ & $4.99 \pm .99$ & $5.30 \pm 1.15$ \\
Before meal FBG ( mmol/l) & $4.40 \pm .41$ & $4.35 \pm .37$ & $4.43 \pm .42$ \\
2hours after meal BG(mmol/l) & $5,39 \pm, 67$ & $5,15 \pm, 51$ & $5,41 \pm, 52$ \\
FPG $(\mathrm{mmol} / \mathrm{l})$ & $4.68 \pm .49$ & $4.56 \pm .43$ & $4.83 \pm .48$ \\
HbA1c & $5.27 \pm .28$ & $5.21 \pm .24$ & $5.25 \pm .26$ \\
BMI $\left(\mathrm{kgr} / \mathrm{m}^{2}\right)$ & $23.81 \pm 3.37$ & $22.52 \pm 2.86$ & $25.23 \pm 3.82$ \\
\hline
\end{tabular}

blgl = capillary blood glucose; FBG = venous Fasting blood glucose; $\mathrm{FPG}=$ venous fasting plasma glucose; $\mathrm{BMI}=$ body mass index; WHR= waist/hip ratio

Table 1. The intra-class correlations for familial resemblance were obtained from the ANOVA and are presented in table 2 .If correlations in monozygotic(MZ)twins are larger than in dizygotic(DZ)twins and siblings, is this a first indication that genetic factors are of importance. A first estimate of heritability is obtained by doubling the difference in correlations between $\mathrm{MZ}$ twins and first degree relatives.

Table 2: MZ, DZ and sibling correlations and heritability estimates

\begin{tabular}{llll}
\hline Intra-class correlation & MZ pairs & DZ twins and sib pairs & heritability \\
\hline OGTT FBG & 0.631 & 0.397 & $47 \%$ \\
OGTT 2 hours BG & 0.408 & 0.385 & $4 \%$ \\
before meal FBG & 0.611 & 0.404 & $41 \%$ \\
2hours after meal BG & 0.457 & 0.030 & $46 \%$ \\
FPG & 0.797 & 0.399 & $40 \%$ \\
HbAlc & 0.803 & 0.387 & $83 \%$ \\
\hline
\end{tabular}

Conclusion: These preliminary results confirm the earlier suggested importance of heritability in $\mathrm{HbAlc}$ levels. It appears that genes play a less important role in FPG and post-challenge blood glucose.

Supported by Diabetes Fonds Nederland 


\section{0}

Screening for -30G $>$ A polymorphism in B-promoter of the GCK gene in the Czech diabetic and non-diabetic populations

P. Lukasova, M. Vankova, J. Vcelak, D. Vejrazkova, H. Kvasnickova, K. Vondra, B. Bendlova;

Laboratory of Molecular Endocrinology, Institute of Endocrinology, Prague, Czech Republic.

Background and Aims: The transition $\mathrm{G}>\mathrm{A}$ at position -30 of the GCK $\beta$ cell-specific promoter (rs1799884) is common within the Caucasian as well as Asian populations. Higher frequency of the minor allele A was detected in subjects with diabetes mellitus type 2 (DM2), gestational diabetes, metabolic syndrome and impaired glucose tolerance (IGT). The minor allele A was associated with elevated fasting and postprandial levels of glucose and with impaired glucose-stimulated insulin secretion (GSIR). Generally the effect of A allele was pronounced under a recessive model of inheritance - the dosage effect was observed. The aim of our study was to determine the genotypic distribution of the $-30 \mathrm{G}>\mathrm{A}$ polymorphism of the GCK gene in the Czech diabetic and nondiabetic populations and to assess possible association of the minor allele A carriership with biochemical and anthropometrical parameters.

Materials and Methods: Detailed biochemical and anthropometrical characteristics of diabetic groups: 338 patients with DM2 (M/F 124/224; age $60.8 \pm 7.95 / 58.7 \pm 9.27$ years; BMI $\left.29.7 \pm 4.44 / 31.9 \pm 5.59 \mathrm{~kg} / \mathrm{m}^{2}\right)$ and 158 gestational diabetics $(\mathrm{G})$ (age $32.3 \pm 4.90$ years; BMI $24.9 \pm 4.54 \mathrm{~kg} / \mathrm{m}^{2}$ ); and of nondiabetic groups: 141 healthy offspring of DM2 (HO) (M/F 52/89; age $40.5 \pm 9.62 / 37.9 \pm 12.59$ years; BMI $\left.26.6 \pm 4.29 / 24.8 \pm 4.02 \mathrm{~kg} / \mathrm{m}^{2}\right)$ and 256 control subjects $(\mathrm{K})(\mathrm{M} / \mathrm{F}$ 81/175; age $29.0 \pm 8.03 / 30.4 \pm 11.45$ years; BMI $\left.23.8 \pm 2.82 / 23.1 \pm 4.12 \mathrm{~kg} / \mathrm{m}^{2}\right)$ were collected. Except DM2 patients the oral glucose tolerance (OGTT) and insulin tolerance tests (ITT) were performed. For screening of $-30 \mathrm{G}>\mathrm{A}$ polymorphism in the B-promoter of the GCK gene the single strand conformation polymorphism (PCR-SSCP) method was used. The positive controls (GG, AG and AA genotypes) were used in every run. Statistical analysis: Chi-square test, Mann-Whitney test and ANCOVA (adjustment for age) (NCSS 2004).

Results: The genotypic distribution (GG/AG/AA) in diabetic and nondiabetic populations was not significantly different: DM2 - 70.9/25.9/3.2; $\mathrm{G}-65.2 / 33.5 / 1.3 ; \mathrm{HO}-63.8 / 31.9 / 4.3 ; \mathrm{K}-73.8 / 24.2 / 2.0$ [\%]. In control group the homozygotes for the minor allele A had significantly increased fasting glucose $(\mathrm{p}=0.025)$ and stimulated levels of glycaemia during OGTT ( $\mathrm{p}=$ 0.001). The homozygotes AA had also increased fasting (NS) and stimulated secretion of C-peptide ( $p=0.048)$ and of insulin (NS). Insulin sensitivity was decreased in AA subjects (HOMA R, $p=0.010$; Cederholm index, $\mathrm{p}=0.018$ ). The heterozygotes GA did not differ in biochemical and anthropometrical parameters from wild type homozygotes GG. In our study the minor allele A shows a dose effect (recessive model of inheritance). In groups of DM2, gestational diabetics and offspring no association with biochemical and anthropometrical parameters was found.

Conclusion: In control population in homozygous allele A carriers we observed increase of fasting and stimulated glucose levels but conversely to previous studies fasting and stimulated levels of C-peptide and insulin were in these subjects also increased. The impaired insulin sensitivity in AA homozygotes was observed. The occurrence of minor allele A demonstrated the recessive model of inheritance.

Supported by IGA MHCR NR/7809-5

\section{1}

Prevalence of glucokinase (GCK) mutations in people with elevated fasting glucose levels: implications for clinical trials

A. L. Gloyn ${ }^{1}$, M. van de Bunt ${ }^{1}$, I. Stratton ${ }^{2}$, L. Tucker ${ }^{2}$, L. Lonie ${ }^{3}$, S. Ellard ${ }^{4}$, R. R. Holman ${ }^{2}$;

${ }^{1}$ Diabetes Research Laboratories, Oxford Centre for Diabetes Endocrinology \& Metabolism, University of Oxford, ${ }^{2}$ Diabetes Trials Unit, Oxford Centre for Diabetes Endocrinology \& Metabolism, University of Oxford,

${ }^{3}$ Wellcome Trust Centre for Human Genetics, University of Oxford, ${ }^{4}$ Institute of Biomedical \& Clinical Science, Peninsula Medical School, Exeter, United Kingdom.

Background: There is considerable interest in large scale screening programmes and prospective studies to determine which factors influence whether individuals progress to diabetes. Within these programs there are likely to be individuals with permanent "fixed" defects in glucose sensing who are unlikely to progress to diabetes. Glucokinase (GCK) inactivating mutations cause a subtype of maturity-onset diabetes of the young (GCKMODY) with elevated fasting plasma glucose (FPG) levels (5.5-8.0 mmol/ L) but with normal 2 hour plasma glucose increments (2HPGI) after a $75 \mathrm{~g}$ oral glucose tolerance test (OGTT) $(<4.5 \mathrm{mmol} / \mathrm{L})$. Since the population prevalence of GCK mutations is unknown the number of individuals in these programmes with a "fixed" glucose sensing defect due to a GCK mutation is unknown. The aims of our study were to determine the prevalence of GCK mutations in a cohort selected for impaired glucose tolerance (IGT) and/or impaired fasting glucose (IFG) and to establish if these individuals could be distinguished from non-GCK-MODY subjects on clinical criteria alone.

Methods: We screened 662 people with FPG levels 5.5-7.7 mmol/L inclusive for GCK mutations by DHPLC analysis ( $98 \%$ sensitivity, $4 \%$ false-positive rate). Their mean (SD) FPG was 5.9 (0.7) mmol/L, 2HPGI 2.67 (2.3) mmol/L body mass index (BMI) $28.6(4.5) \mathrm{kg} / \mathrm{m}^{2}$ and age 52.1 (9.9) years. The assay was validated by testing 58 different heterozygous GCK mutations. Samples showing an abnormal chromatogram were sequenced on an ABI3700.

Results: Five pathogenic mutations and 11 non-functional polymorphisms were identified. The 5 individuals with GCK mutations were indistinguishable from the rest with mean (SD) FPG $6.2(0.8) \mathrm{mmol} / \mathrm{L}, 2$ HPGI 3.5 (1.5) $\mathrm{mmol} /$ L, BMI $29.8(5.2) \mathrm{kg} / \mathrm{m}^{2}$ and age 48.2 (8.9) years.

Conclusion: The prevalence of $<1 \%$ of GCK mutations in our cohort suggests that FPG screening may not greatly enhance identification of GCK-MODY individuals in this age group. Our data demonstrate that it is not possible to identify patients with GCK-MODY on clinical criteria alone in cohorts selected on the basis of IGT and/or IFG.

Supported by Diabetes UK

\section{2}

Insulin resistance and intrauterine growth retardation due to a novel balanced translocation $(46, t(7 ; 19)(\mathrm{p} 15.2 ; \mathrm{p} 13.2))$ which disrupts the insulin receptor (INSR) gene

S. G. I. Suliman ${ }^{1}$, J. Stanik ${ }^{2}$, N. Misovicova ${ }^{3}$, D. Gasperikova ${ }^{2}$, N. Wilson ${ }^{4}$, E. Edghill' ${ }^{5}$, V. Sandrikova ${ }^{6}$, K. Elliot ${ }^{4}$, L. Barak ${ }^{7}$, S. Ellard ${ }^{8}$, E. Volpi ${ }^{4}$, I. Klimes ${ }^{9}$, A. L. Gloyn ${ }^{1}$;

${ }^{1}$ Oxford Centre for Diabetes, Endocrinology and Metabolism, University of Oxford, United Kingdom, ${ }^{2}$ Slovak Academy of Sciences, Institute of Experimental Endocrinology, Bratislava, Slovakia, ${ }^{3}$ Clinical Genetics, Jessenius School of Medicine, Martin, Slovakia, ${ }^{4}$ Wellcome Trust Centre for Human Genetics, University of Oxford, United Kingdom, ${ }^{5}$ Molecular Genetics Laboratory, Peninsula Medical School, Exeter, United Kingdom, ${ }^{6}$ Pediatric Endocrinology, Prievidza Hospital, Prievidza, Slovakia, ${ }^{7}$ Children Diabetes Center at 1st Pediatric Dept., Comenius University School of Medicine, Bratislava, Slovakia, ${ }^{8}$ Molecular Genetics Laboratory, Peninsula Medical School, Exeter, United Kingdom, ${ }^{9}$ Institute of Experimental Endocrinology, Slovak Academy of Sciences, Bratislava, Slovakia.

Background and Aims: Balanced translocations have been pivotal in identifying genes and mutational mechanisms implicated in disease. We describe a family with early-onset diabetes, pre- and post-natal growth retardation, neonatal hypoglycaemia and hyperglycaemia later in life despite elevated insulin levels. The male proband developed hyperglycaemia aged 3 months; his mother developed insulin-treated diabetes aged 15 years. Karyotype analysis identified a balanced translocation [46, $\mathrm{t}(7 ; 19)(\mathrm{p} 15.2 ; \mathrm{p} 13.2)]$ co-segregating with the phenotype. We hypothesised that the break point of the balanced translocation disrupts a gene, a regulatory element or the relationship between them resulting in insulin resistance, intra-uterine growth retardation and dysglycaemia. The aim of our study was to identify the gene disrupted by the translocation and to determine whether a there was any loss of genetic material (microdeletion). Materials and Methods: Bio-informatic tools were used to identify genes located within the region of both break points. A gene prioritization tool (genesniffer) was used to prioritise genes in order of biological candidacy. Fluorescence in-situ hybridization (FISH) analysis was performed on patient metaphase arrested chromosomes using labeled bacterial artificial chromosomes (BACs). Multiple ligation probe amplification (MLPA) assay was performed to investigate microdeletions. Detailed biochemical analysis of patient plasma was performed.

Results: Bio-informatics identified 44 genes within the region of the break point on chromosome 19p and 23 genes on $7 p$. The most plausible biological 
candidate was the insulin receptor (INSR) gene on 19p. Mutations in INSR are implicated in defects of glucose homeostasis and intra-uterine growth retardation. FISH analysis using BACs containing genomic DNA for the entire coding region and known promoter regions of INSR demonstrated that the break point disrupts the INSR. Fine mapping using fosmids limited the break point to a region of $10.4 \mathrm{~Kb}$. MLPA analysis demonstrated a microdeletion involving exons 15 and 16. Sequencing of INSR in genomic DNA from both patients did not identify any mutations. Both patients have elevated insulin and c-peptide levels, an elevated insulin/c-peptide ratio (0.62 and 1.05 , normal $<0.1)$ and elevated adiponectin levels $(>44 \mathrm{ng} / \mathrm{ml})$ supporting the genetic diagnosis of an INSR defect.

Conclusion: This is the first demonstration of a cytogenetic defect causing disruption of INSR resulting in insulin resistance, pre-and post natal growth retardation.

Supported by Diabetes UK

\section{3}

Associations between insulin promoter factor 1 gene polymorphisms and proinsulin concentrations and type 2 diabetes mellitus

B. Zethelius ${ }^{1}$, N. Abrahamsson ${ }^{2}$, L. Berglund ${ }^{1}$, A.-C. Syvänen ${ }^{2}$, C. Berne ${ }^{2}$;

${ }^{1}$ Dept. of Public Health and Caring Sciences/Geriatrics,

${ }^{2}$ Dept. of Medical Sciences, Uppsala University Hospital, Sweden.

Background and Aims: Mutations in the insulin promoter factor 1 (IPF1), a transcription factor involved in the process of insulin gene expression is associated with MODY-4 and may predispose to type 2 diabetes mellitus (T2DM) in recent studies. As diminished early insulin response to oral glucose and fasting proinsulin are significant predictors of T2DM, we hypothesised associations between single nucleotide polymorphisms (SNPs) of the IPF-1 gene, quantitative traits and T2DM. The aim was to examine associations between IPF1 gene SNPs with fasting proinsulin and insulin concentrations, insulin at 30 minutes after oral glucose load and T2DM.

Materials and Methods: Nine SNPs selected from public databases, were determined using multiplex PCR at the Uppsala genotyping platform. Analyses were made in relation to proinsulin and insulin determinations in the ULSAM cohort (http://www.pubcare.uu.se/ULSAM/).

Results: In four SNPs, an association with proinsulin concentrations were observed ( $p$ for trend 0.003-0.04) and one SNP out of these, rs7331478, was associated with T2DM (p for trend 0.035); GG vs. TT, OR 1.37, 95\%CI, 0.71-2.67; GT vs. TT, OR 1.51, 95\%CI, 1.10-2.06; and as a recessive model; GG+GT v.s TT, OR 1.49, 95\%CI, 1.10-2.01, $\mathrm{p}=0.010$. For rs7331478, an association with proinsulin ( $8.7 \mathrm{vs} 6.5 \mathrm{pmol} / \mathrm{l}, \mathrm{p}=0.016)$ and an association with insulin at 30 minutes ( $215 \mathrm{vs} 260 \mathrm{pmol} / \mathrm{l}, \mathrm{p}=0.07$, borderline significant) was observed in GG-homozygotes as compared to TT-wildtype.

Conclusion: These results suggest that genetic variation in the IPF1 gene is associated with fasting proinsulin concentrations, with low insulin response to oral glucose and is predisposing to T2DM.

Supported by Wallenberg Consortium North

\section{4}

Common variants of pancreatic $\beta$ cell related transcription factor genes are not associated with young onset diabetic subjects of Bangladesh

Z. Hassan ${ }^{1}$, M. O. Faruque ${ }^{2}$, K. B. Biswas ${ }^{1}$, R. Islam ${ }^{1}$, K. Azad $^{3}$,

A. K. Azad Khan ${ }^{4}$, L. Ali ${ }^{2}$, G. A. Hitman';

${ }^{1}$ Physiology and Molecular Biology, BIRDEM, Dhaka, Bangladesh,

${ }^{2}$ Biochemistry and Cell Biology, BIRDEM, Dhaka, Bangladesh,

${ }^{3}$ Pediatrics, BIRDEM, Dhaka, Bangladesh, ${ }^{4}$ Gastroenterology, BIRDEM,

Dhaka, Bangladesh, ${ }^{5}$ Centre for Diabetes and Molecular Medicine,

Batrs and the London Queen Mary's School of Med and Dentistry, London, United Kingdom.

Background and Aims: Diabetes in the young Bangladeshis [including fibrocalculus pancreatic diabetes (FCPD)] presents with unique clinical and biochemical characteristics which create special problem in its classification. In an attempt to further characterize the disorders in these patients we have studied the common variants of transcription factors genes involved in pancreatic B cell differentiation and maturation.
Materials and Methods: Young (under 30 years) non-ketotic diabetes mellitus (YDM, $n=337)$, FCPD $(n=51)$ and age-matched healthy Controls $(n=332)$ were studied. Candidate gene markers TCF1 (A98V), NEUROD1 (A45T) and NEUROG3 (G167R and S199F)] were investigated by PCR-RFLP. Serum $\mathrm{C}$-peptide was measured by ELISA.

Results: Mean $( \pm$ SD) BMI of the Controls, YDM and FCPD was 19.7 \pm 3.2 , $18.30 \pm 4.9$ and $16.1 \pm 3.5$ respectively ( $<<0.001$ in YDM and FCPD vs Control). Both YDM and FCPD had significant low fasting C-peptide (nmol/l, medianrange) levels compared to Control [0.20 (0.20-1.33) in YDM and $0.12(0.023-$ $0.84)$ in FCPD vs 0.39 (0.16-1.44 in Control), pT (A98V): $0.821,0.168$ and 0.011 (homozygous wild, heterozygous variant and homozygous variant respectively) in YDM and $0.834,0.137$ and 0.020 in FCPD and 0.866, 0.142 and 0.003 in the Control; of NEUROD1 G>A (A45T) 0.764, 0.223 and 0.014 in YDM and $0.900,0.100$ and 0 in the FCPD vs $0.764,0.209$ and 0.027 in the controls; NEUROG3 G>A (G167R) 0.906, 0.086 and 0.008 in YDM and 0.941, 0.059 and 0 in FCPD and 0.940 and 0.060 in the Control and NEUROG3 C > T (S199F) $0.167,0.470$ and 0.363 in the YDM and $0.255,0.451$ and 0.294 in FCPD and $0.179,0.448$ and 0.343 in the Control. The genotype frequencies of the candidate gene markers in the YDM and FCPD groups did not show any significant difference compared to the controls. YDM and FCPD subjects with variant (heterozygous and homozygous together) vs wild genotype did not show statistical difference regarding C-peptide level in neither of the candidate gene [TCF1 (A98V), NEUROD1 (A45T) and NEUROG3 (G167R and S199F)] markers.

Conclusion: The data suggest that common variants of transcription factor genes involved in differentiation and maturation of pancreatic B cells are not associated with diabetes in the young Bangladeshi subjects.

Supported by grants from International Programme in the Chemical Sciences (IPICS) and Diabetic Association of Bangladesh (DAB) 


\section{PS 19 Gluco- and lipotoxicity as mediators of beta cell degeneration}

\section{5}

Structural requirements for the cytoprotective effects of monounsaturated fatty acids in pancreatic beta cells S. Dhayal, E. Diakogiannaki, H. J. Welters, N. G. Morgan;

Endocrine Pharmacology, Peninsula Medical School, Plymouth, United Kingdom.

Background and Aims: Saturated and monounsaturated fatty acids (FA) exert differential effects on $\beta$-cell viability. Long-chain saturated FAs (e.g. palmitate (C16:0)) are cytotoxic but the corresponding monounsaturates (e.g. palmitoleate $($ cis $\mathrm{C} 16: 1)$ ) are well tolerated. Furthermore, long-chain monounsaturates are directly cytoprotective to $\beta$-cells and can attenuate the loss of viability caused by multiple stimuli (including saturated FAs, proinflammatory cytokines and serum withdrawal). However, the structural features required to confer this $\beta$-cell cytoprotection remain unknown. It is also unclear whether a cell surface fatty acid receptor (e.g. GPR40) is involved or whether metabolism of the unsaturated fatty acids is required. The present study aimed to investigate these unresolved issues.

Materials and Methods: Clonal rat BRIN-BD11 $\beta$-cells were used as a model system and were treated with albumin-bound FAs in tissue culture. Caspase activation, cell viability and intracellular triacylglycerol (TAG) formation were studied in cells exposed to saturated and/or mono-unsaturated FAs.

Results: Palmitoleate dose-dependently $(0.05-0.25 \mathrm{mM})$ protected $\beta$-cells against cytotoxicity induced by palmitate or serum withdrawal and completely inhibited palmitate-induced caspase $3 / 7$ activation over $12 \mathrm{~h}$ incubation (palmitate: $14972 \pm 2793$ luminscence units; palmitate+palmitoleate: $596 \pm 62$ ). Oleate (cisC18:1) also abrogated $\beta$-cell cytotoxicity while elaidate (trans C18:1) was partially protective but less potent than oleate. Palmitelaidate (trans C16:1) failed to attenuate $\beta$-cell cytotoxicity at concentrations up to $0.25 \mathrm{mM}$. Monounsaturated FA analogues bearing esterified carboxyl groups (methyl-palmitoleate or methyl-oleate) afforded complete $\beta$-cell protection and there was no difference in potency between palmitoleate and methylpalmitoleate $\left(\mathrm{EC}_{50} \sim 20 \mu \mathrm{M}\right)$ suggesting that metabolism of unsaturated fatty acids is not required. In confirmation of this, methyl-palmitoleate failed to alter intracellular TAG formation in $\beta$-cells exposed to palmitate (control: $0.9 \pm 0.75 \mathrm{nmol} / 10^{6}$ cells; palmitate: $1.56 \pm 0.15$; palmitate+methyl palmitoleate: $1.50 \pm 0.05)$ under conditions when it exerted complete protection against cytotoxicity. The synthetic GPR40 agonist GW9508 was not cytoprotective in $\beta$-cells and it failed to influence the sensitivity of the cells to palmitoleate. Conclusion: The cytoprotective actions of monounsaturated FAs display clear structural requirements in $\beta$-cells, with chain length and the configuration of the double bond particularly important. Metabolism of the FAs is not essential and the results are consistent with the involvement of a specific FA binding site. However, no evidence was obtained to support a role for GPR40 in mediating the protective response.

Supported by Internationale Recherches Institute Servier

\section{6}

Proteomic analysis of palmitate-induced changes in insulin secreting INS-1E cells

M. Hovsepyan, P. Bergsten;

Medical Cell Biology, Uppsala University, Sweden.

Background and Aims: Individuals with type 2 diabetes mellitus have elevated blood levels of glucose and lipids. Such elevated levels of the nutrients have been postulated to cause impairment of insulin secretion from pancreatic $\beta$ cells. Chronic exposure to long chain fatty acids, in particular palmitate, has been linked not only to functional impairment of $\beta$-cells but also apoptosis. Different mechanisms for the detrimental effects of palmitate have been suggested including alterations in membrane fluidity, mitochondrial function and ceramide generation. Recently, it was suggested that palmitate-induced apoptosis involved the endoplasmic reticulum stress response. Although the latter and the other mechanisms are important contributing factors to explain how the fatty acid induces $\beta$-cell deterioration, they account for some aspects of palmitate-induced deterioration of $\beta$-cell function and mass. Given the central role of palmitate in $\beta$-cell glucose- and lipid-metabolism the aim of this study was to further explore mechanisms by which the fatty acid acts on the $\beta$-cell by identifying proteins exclusively regulated by palmitate.

Materials and Methods: INS-1E cells were cultured in RPMI 1640 medium containing $11 \mathrm{mM}$ glucose in the presence of $1 \%$ FCS for 24 hours. In addition, cells were cultured in medium supplemented with $0.5 \mathrm{mM}$ palmitate or oleate (complexed with albumin). After culture, cells were lysed and lysates of $250 \mathrm{ug}$ total protein (corresponding to approximately 1 million cells) were subjected to separation by iso-electric focusing using immobilized $\mathrm{pH}$-gradient strips $(11 \mathrm{~cm}, 3-10 \mathrm{NL})$, followed by size separation with $12.5 \%$ SDS-PAGE. Gels were stained by Coomassie and differential protein expression specifically induced by palmitate was analyzed. Proteins of interest separated by 2-DGE were exercised from the gel and identified by peptide mass fingerprinting using MALDI-TOF MS.

Results: INS-1E protein patterns obtained from INS-1E cultured in the presence of $11 \mathrm{mM}$ glucose alone or in combination with palmiatet or oleate contained more than 600 spots visualized by Coomasie staining. Total correlation between protein patterns derived from lysates of cells cultured under the same conditions was more than $95 \%$. Analysis revealed that 116 spots were significantly different in gels obtained from INS-1E cells cultured in the presence of palmitate or oleate compared to gels obtained from control cells. Further analysis revealed that out of these 116 spots only 9 spots represented proteins that were similarly regulated by palmitate and oleate. The number of spots that were specifically regulated by either oletate or palmitate was 58 and 49 , respectively. From the latter 49 spots, which represented proteins either up- or down-regulated by palmitate, 26 were chosen for identification work. Most of the up-regulated proteins were identified as chaperons and ERrelated proteins. In contrast, the down-regulated proteins were involved in a broad range of biological processes including structural proteins, glycolytic enzymes, lipid metabolism and protein degradation.

Conclusion: Whereas some $\beta$-cell proteins were jointly regulated by oleate and palmitate, most proteins were differentially expressed by either oleate or palmitate. These findings contribute to explain why the effect of palmitate on $\beta$-cell function and mass is different from that of oleate. From the identities of proteins specifically regulated by palmitate we have formulated novel hypotheses how palmitate may induce deterioration of $\beta$-cell function and mass.

Supported by EFSD/MSD, Swedish Medical Research Council and Swedish Diabetes Association.

\section{7}

PPAR-alpha controls the crossroad of lipid and glucose metabolism and protects INS-1E beta cells against lipotoxycity

F. Frigerio ${ }^{1}$, C. Bartley ${ }^{1}$, S. Mandrup ${ }^{2}$, P. Maechler ${ }^{1}$;

${ }^{1}$ Department of Cell Physiology and Metabolism, University of Geneva, Switzerland, ${ }^{2}$ Department of Biochemistry and Molecular Biology, University of Southern Denmark, Odense, Denmark.

Background and Aims: Type 2 diabetes is characterized by insulin resistance, impaired insulin secretion, and progressive loss of beta-cell function. Chronic elevation of circulating fatty acids may contribute to the phenomenon named lipotoxicity. Of interest in this context, the three peroxisome-proliferatoractivated receptor (PPAR) subtypes, nuclear lipid sensors, are able to react to metabolic changes by regulating gene expression. In particular, PPAR-alpha plays a crucial role in metabolic adaptation by inducing genes responsible for fatty acid oxidation. However, the role of PPARalpha in beta-cells remains poorly characterized. Here, we investigated the effects of PPAR-alpha overexpression in INS-1E beta-cells cultured in the presence of the fatty acid oleate.

Materials and Methods: INS-1E beta-cells were transduced with adenoviruses expressing PPARalpha and RXR before a 3-day culture period in the presence of $0.4 \mathrm{mM}$ oleate. Then, we measured key parameters involved in metabolism secretion coupling: mitochondrial membrane potential (rhodamine-123 fluorescence), cytosolic calcium levels (Fura-2 fluorescence), glutamate generation (enzymatic assay), gene expression (quantitative RT-PCR and immunoblotting), insulin secretion (radioimmunoassay), and glucose utilization (3H-glucose) and oxidation (14C-glucose).

Results: INS-1E cells cultured for 3 days in the presence of oleate increased basal insulin release by $80 \%$, while the secretory response to $15 \mathrm{mM}$ glucose was blunted compared to control cells (1.68-fold versus 4.56 -fold, respectively). PPAR-alpha overexpression partially rescued glucose stimulated insulin secretion (2.14-fold), revealing protection against fatty acid induced toxicity. Investigating metabolism secretion coupling parameters, mitochondrial 
membrane potential and cytosolic calcium rise were diminished by oleate treatment $(\mathrm{p}<0.01)$, while unchanged by PPAR-alpha overexpression. Elevation of cellular glutamate levels upon glucose stimulation was blunted in oleate treated INS-1E cells. PPAR-alpha restored glucose $(15 \mathrm{mM})$ induced glutamate rise $(+53 \% \mathrm{p}<0.002)$. This suggested that PPAR-alpha could promote anaplerotic pathways, necessary for glutamate formation. Such hypothesis was substantiated by the observed augmentation of pyruvate carboxylase expression in PPARalpha overexpressing cells, both at the mRNA (2.8-fold) and protein levels. The enhancement of anaplerotic pathways was corroborated by measurements of glucose utilization and oxidation. In oleate treated INS-1E cells overexpressing PPAR-alpha, glycolytic flux was increased versus glucose oxidation (1.44-fold versus oleate treatment), showing compensatory mechanisms to cataplerosis (i.e. glutamate generation) by enhanced anaplerosis (pyruvate carboxylase). The effects of PPAR-alpha on glucose metabolism paralleled increased fatty acids turnover, as demonstrated by enhanced FAT/CD36 expression.

Conclusion: Data show that PPAR-alpha stimulates both glucose and lipid metabolism in INS-1E beta-cells chronically exposed to oleate. These PPARalpha actions might explain the observed preservation of glucose stimulated insulin secretion in fatty acid treated cells.

Supported by the Swiss National Science Foundation

\section{8}

The role of $\mathrm{G}$ protein-coupled receptor 40 in lipoapoptosis in mouse beta line NIT-1

Y. Zhang, M. Xu, S. Zhang, L. Yan, C. Yang, W. Lu, Y. Li, H. Cheng;

Department of Endocrinology, The Second Affiliated Hospital,

Sun Yat-Sen University, Guangzhou, China.

Background and Aims: Elevated levels of circulating free fatty acids (FFAs) are tightly linked to Type 2 diabetes and FFAs exert divergent effects on $\beta$ cells, acute exposure to FFAs stimulates insulin secretion, whereas chronic exposure impairs insulin secretion and induces apoptosis (lipoapoptosis). The G protein-coupled receptor 40 (GPR40) is preferentially expressed in $\beta$ cells and is activated by a wide range of FFAs. GPR 40 has been shown to mediate the effects of FFAs on insulin secretion. However, it is uncertain whether GPR40 is implicated in mediating lipoapoptosis of pancreatic $\beta$ cells. The aim of this study is to determine whether GPR40 is implicated in FFAs mediated lipoapoptosis in NIT-1 cells.

Materials and Methods: We used siRNA approach to inhibit the expression of GPR40 in NIT-1 cells, then examined the effects of long-term incubation with saturated (palmitate) or unsaturated (oleate) fatty acids on apoptosis and function of NIT-1 cells transfected with GPR40 siRNA. Apoptosis was evaluated by TUNEL and flow-cytometry analysis (annexin V/PI staining). And we detected the activation of the phosphatidylinositol 3-kinase (PI3K) and mitogen-activated protein kinases (MAPK) pathway to address the possible mechanisms.

Results: Results showed that palmitate induced $\beta$ cell apoptosis, which was GPR40 independent; whereas oleate protected NIT-1 cells from palmitateinduced lipoapoptosis, which was mediated at least in part through GPR40. Moreover, by detect the activation of the PI3K and MAPK pathway, we found that oleate promoted the activation of extracellular signal-regulated protein kinase (ERK) MAPK pathway mainly via GPR40, leading to the antilipoapoptotic effect on NIT-1 cells.

Conclusion: It was suggested that GPR40 might be implicated in the control of $\beta$ cell mass plasticity and GPR40 probably provide a link between obesity and Type 2 diabetes.

Supported by research grants from Natural Science Foundation of Guangdong Province, China

\section{9}

Analysis of specific arachidonic acid induced changes in gene and protein expression in a clonal rat pancreatic beta cell line

D. Keane, P. Newsholme;

UCD School of Biomolecular and Biomedical Science, Conway Institute, Dublin, Ireland.

Background and Aims: Diabetes Mellitus is a complex disorder characterized by hyperglycemia and dyslipidaemia. The metabolic abnormalities associated with type 2 diabetes mellitus can be attributed to failure of the pancreatic beta cells to secrete sufficient insulin. Type 2 diabetes mellitus is often coupled with obesity. Therefore it is possible that increased circulating fatty acid levels are responsible in part for the beta cell dysfunction in type 2 diabetes. It is well established that chronic exposure of cultured beta cells to saturated fatty acids such as palmitic acid (PA) results in a reduction in insulin secretion and an increase in beta cell death. Recent studies have shown that the degree of saturation and chain length are very important when assessing the effects of fatty acids on the beta cell. For example, in contrast to the negative effects of PA, studies from our own lab have demonstrated that chronic exposure of cultured beta cells to the polyunsaturated fatty acid arachidonic acid (AA) does not alter subsequent acute insulin secretion nor does it promote cell death. Other authors have demonstrated protective effects of some monounsaturated or polyunsaturated fatty acids against the negative effects of PA. We wished to explore aspects of AA action at the gene and protein level.

Materials and Methods: Clonal BRIN-BD11 beta cells were cultured in the presence or absence of $100 \mu \mu \mathrm{A} \alpha$ for $24 \mathrm{hr}$. Microarray gene expression profiling of BRIN BD11 cells exposed for 24 hours to $100 \mu \mathrm{M}$ AA was performed. Protein was also isolated from BRIN-BD11 cells for NADPH oxidase protein expression utilising a specific anti-P47-phox polyclonal antibody in Western blot based analysis. Reactive oxygen species (ROS) generation was measured using flow cytometry.

Results: Culture for $24 \mathrm{hr}$ in $100 \mu \mathrm{M}$ AA compared to control resulted in significant (using the Bioconductor software package) changes in 3 genes. Stearoyl-CoA dehydrogenase and ATP-binding cassette, sub-family G (WHITE) member 1 expression were downregulated 3.87 ( $p$ value 0.001 ) and 1.7 fold ( $\mathrm{p}$ value 0.03 ) respectively. On the other hand Enoyl Coenzyme A hydratase 1 , expression was upregulated 1.73 fold ( $\mathrm{p}$ value 0.02 ) relative to control. In contrast to PA, $24 \mathrm{hr}$ exposure of BRIN-BD11 cells to $100 \mu \mu \mathrm{A} \alpha$ led to no significant changes in both P47-phox protein expression and ROS generation.

Conclusion: AA dependent beta cell specific effects appear to be mediated through alteration in signal transduction. We have determined that $\beta$-cell ROS production was regulated by type and degree of saturation of the various fatty acids tested.

Supported by Health Research Board Ireland

\section{0}

Glucose signaling via Akt in beta-cells: Rab-GAP AS160 is expressed in pancreatic islets, and is regulated by glucose

K. Bouzakri, K. Rickenbach, P. A. Halban;

Genetic Medicine and Development, University of Geneva Medical Center, Switzerland.

Background and Aims: PKB/Akt plays a central role in beta-cells but little is known regarding downstream Akt substrates in these cells. Recently a substrate of Akt (Rab-GAP AS160) was shown to be involved in insulin modulation of GLUT4 trafficking in skeletal muscle and adipose tissue. Our aim was to investigate the potential role of AS160 in beta-cells.

Materials and Methods: AS160 mRNA expression was measured in human pancreatic islets. Islets of Langerhans were isolated by collagenase digestion of pancreas from adult male C57BL/6 mice and beta-cells were sorted by fluorescence-activated cell sorter (FACS). Proliferation was measured following BrdU incorporation and apoptosis was assessed by TUNEL assay and detection of active caspase-3. Insulin secretion was quantified by radioimmunoassay (RIA). For knockdown experiments transformed mouse insulin secreting MIN6B1 cells were transfected with pSUPER-GFP plasmid encoding a shRNA against IRS-2, AS160 or a negative control. Cells were cultured for 72 hours before selection of GFP-positive cells by fluorescenceactivated cell sorting. Sorted cells were then used to perform the various experiments.

Results: We first establish by RT-PCR that AS160 mRNA is expressed in human pancreatic islets, but due to limited material functional studies were performed using mouse cells. AS160 was phosphorylated after $10 \mathrm{~min}$ glucose stimulation $(16.7 \mathrm{mM})$ in primary mouse beta-cells. Using siRNA to knockdown IRS-2 and a specific inhibitor of PI3-Kinase (LY-294002), we demonstrated in MIN6B1 cells that glucose action on Akt and AS160 phosphorylation was IRS-2/PI3-Kinase dependent. Stimulation of cells with 2-deoxyglucose or pretreatment with diazoxide to prevent glucose-stimulated $\mathrm{Ca} 2+$ influx showed that neither metabolism of glucose nor increased cytosolic $\mathrm{Ca} 2+$ were required for glucose-stimulated Akt and AS160 phosphorylation. 
In MIN6B1 cells, 90\% knockdown of AS160 (confirmed by western blot) resulted in a 4 -fold increase of basal insulin secretion whereas glucose mediated insulin release was abolished, directly demonstrating a dual role for AS160 in these two components of insulin secretion. Furthermore, glucose increased MIN6B1 cell proliferation by $2.42 \pm 0.13$-fold but was no longer able to do so in cells lacking AS160 and/or IRS-2. Apoptosis was increased by $3.5 \pm 0.7$-fold in standard culture conditions ( $25 \mathrm{mM}$ glucose and $15 \% \mathrm{FCS}$ ) and $1.54 \pm 0.03$-fold in deprived conditions ( $5 \mathrm{mM}$ glucose and $1 \%$ FCS $)$ in cells lacking IRS-2 and/or AS160 compared to control cells.

Conclusion: Our results demonstrate that Rab-GAP AS160 is expressed in pancreatic beta-cells and phosphorylated after glucose stimulation via IRS-2/PI3-Kinase pathway. Moreover, AS160 silencing demonstrated its implication in beta-cell survival and glucose-mediated proliferation, as well as the regulation of insulin secretion.

Supported by JDRF and EFSD/Eli Lilly Research Fellowships

\section{1}

Effects of C75, a fatty acid synthase (FAS) inhibitor, on insulin secretion and metabolism in rat pancreatic islets and clonal $\beta$ cells

S. G. Straub, G. W. G. Sharp;

Molecular Medicine, Cornell University, Ithaca, United States.

Background and Aims: C75, a synthetic compound that potently inhibits fatty acid synthase, has potential clinical use both as an antitumoral agent and in the fight against obesity. Also, it is well known that the level of expression and activity of FAS is markedly different in primary and clonal $\beta$-cells, and in rodent models of diabetes. Consequently, the aim of this study was to investigate the effects of FAS inhibition on $\beta$-cell function in pancreatic islets from Sprague Dawley (SD) and obese Zucker fa/fa rats, and the clonal INS $832 / 13$ cell line.

Materials and Methods: Pancreatic islets were isolated by collagenase digestion and studied under both static incubation and perifusion conditions. Insulin was assayed by RIA. Glucose (G) oxidation was measured as $14 \mathrm{CO} 2$ production from 14C-labeled glucose; FAS activity by incorporation of $3 \mathrm{H}-$ acetic acid.

Results: In islets from SD rats, C75 inhibited $16.7 \mathrm{mM}$ G-induced insulin release in a concentration-dependent manner with maximal inhibition at 25 $\mu \mathrm{g} / \mathrm{ml}$. A time-course study revealed a pronounced reduction of stimulated insulin release by $93 \%$ in the presence of the compound, which affected both first and second phases. G oxidation at $16.7 \mathrm{mM}$ was inhibited by $30 \%$. In $2.8 \mathrm{mM} \mathrm{G}$, palmitate oxidation was inhibited by $50 \%$ with no further reduction in $16.7 \mathrm{mM} \mathrm{G}$. We next studied the ability of C75 to inhibit FAS in the G-responsive INS 832/13 cell line. $16.7 \mathrm{mM} \mathrm{G}$ alone induced a marked enhancement of FAS activity and increased incorporation of label in all of the four lipid subclasses studied. C75 (again $25 \mu \mathrm{g} / \mathrm{ml}$ ) strongly reduced the incorporation in all four. Remarkably, in contrast to the results in rat islets, C75 augmented insulin secretion in the presence of $2.8 \mathrm{mM} \mathrm{G}, 16.7 \mathrm{mM} \mathrm{G}$, and $20 \mathrm{mM} \mathrm{BCH}$ despite inhibition of G oxidation. As in islets from SD rats, C75 inhibited G-stimulated insulin release in islets of lean Zucker rats but enhanced insulin secretion in islets from Zucker fa/fa rats despite a decrease in glucose oxidation.

Conclusion: FAS is the enzyme responsible for the synthesis of long-chain fatty acids through the condensation of malonyl CoA and acetyl CoA using NADPH as a cofactor. Clonal $\beta$-cells, like the INS $832 / 13$ cell line, have ample expression of this enzyme. Therefore, it seems likely that the enhanced secretion seen in these cells in response to C75 is due to an accumulation of either malonyl CoA or NADPH or both, as they have been implicated as key players in glucose stimulus-secretion coupling. In contrast, treatment with C75 in SD rats, which show very low levels of FAS activity, is not expected to result in a significant buildup of these signaling molecules. As a result we see inhibition of secretion despite inhibition of palmitate oxidation. Obese Zucker fa/fa rats reportedly show an appreciable increase in anaplerotic input and malonyl CoA generation and therefore inhibition of FAS could augment the levels further and thereby enhance secretion. FAS has an important role in the control of insulin secretion in obese animals.

\section{2}

Prevention of mitochondrial fission protects beta cells from glucolipotoxicity

A. J. A. Molina ${ }^{1,2}$, G. Twig ${ }^{1}$, J. Wikstrom ${ }^{1}$, H. Mohamed ${ }^{1}$, B. Corkey ${ }^{2}$, O. Shirihai ${ }^{1}$;

${ }^{1}$ Pharmacology, Tufts University, Boston,

${ }^{2}$ Obesity Research Center, Boston University, United States.

Long term exposure to high levels of glucose and free fatty acid have been shown to result in impaired mitochondrial function, impaired insulin secretion, and beta cell apoptosis. To date, the mechanism by which increased nutrients lead to mitochondrial dysfunction remains unclear. Using confocal imaging technology, we studied the effect of glucolipotocity (20 $\mathrm{mM}$ glucose $+0.4 \mathrm{mM}$ palmitate) on mitochondrial architecture in INS1 cells. We found that GLT results in fragmentation of mitochondrial networks that begins as early as 5 hours of exposure. Mitochondrial networks have been shown to be essential for metabolic activity as they allow the sharing of matrix and membrane components across the mitochondrial population. We hypothesized that the GLT induced fragmentation is due to reduced mitochondrial fusion and increased fission. To test this, we used matrix targeted Photoactivatable-GFP, a probe that allows for the photo-labeling and tracking of individual mitochondria within the intact cell. We found that 18 hour treatment of INS1 cells with GLT arrested mitochondrial fusion, a finding that likely explains the resultant fragmentation. In order to determine the functional significance of this finding we inhibited mitochondrial fission by using shRNA. We found that Fis1 knockdown resulted in restoration of mitochondrial networks and also prevented GLT-induced cell death. These findings suggest that a decrease in mitochondrial fusion activity plays a role in the detrimental effects of chronic exposure to high levels of glucose and FFA.

Supported by NIH

\section{3}

Over-expression of CD36 in insulin producing cells reduces fatty-acidinduced insulin secretion and glucose-regulation of fatty-acid oxidation T. Wallin ${ }^{1}, \mathrm{Z} . \mathrm{Ma}^{1}$, H. Wang ${ }^{2}$, M. Iezzi ${ }^{2}, \mathrm{H}$. Ogata ${ }^{3}$, I. Jörgensen ${ }^{3}$,

C. Wollheim ${ }^{2}$, A. Björklund ${ }^{1,2}$;

${ }^{1}$ Molecular Medicine and Surgery, Karolinska Institutet, Stockholm, Sweden, ${ }^{2}$ Cell Physiology and Metabolism, University Medical Center, Geneva, Switzerland, ${ }^{3}$ Cancer Research and Molecular Medicine, Norwegian University of Science and Technology, Trondheim, Norway.

Background: CD36 facilitates uptake of fatty acids in muscle and adipose tissues. CD36 is present in beta cells and could potentially play a role for fatty acid metabolism and signalling.

Aim: To investigate the impact of over expressing CD36 on beta cell functioning.

Results and Methods: In INS-1 cells (Tet-On system) doxycyclin (DOX, 75, 250 and $500 \mathrm{ng} / \mathrm{ml}$ ) increased CD36 protein by respectively 7.5, 13.4 and 27 times (Western blotting). DOX-induced CD36 was localized to the cell membrane (immunofluorescence). In acute experiments $50 \mu \mathrm{M}$ palmitate in $30 \mathrm{~min}$ final incubations increased glucose-induced insulin both at 2.5 and $21.5 \mathrm{mM}$ glucose $(\mathrm{G}), \mathrm{p}=0.05, \mathrm{n}=4$. This effect of palmitate was abolished by DOX. After long term exposure $(60 \mathrm{~h})$ to $50 \mu \mathrm{M}$ palmitate the DOX-induced cells displayed decreased glucose- induced insulin release $(\mathrm{p}<0.05, \mathrm{n}=9)$ during final incubations at 2.5 and $21.5 \mathrm{mM} \mathrm{G}$. Culture with $100 \mu \mathrm{M}$ oleate gave similar results. DOX increased 5 min uptake of oleate and palmitate. Oxidation of oleate at high glucose was increased: the \% oxidized at high glucose compared to low glucose was $9.5 \pm 1.9$ without DOX and $14.3 \pm 2.7$ $\%$ with DOX, $\mathrm{p}=0.022, \mathrm{n}=7$. DOX did not induce apoptosis (assessed by annexinV and DNA laddering). Neither oil red O staining, nor measurements of total triglycerides, nor thin layer chromatography with $\mathrm{C} 14$ oleate or palmitate indicated that CD36 over-expression increased cellular lipids.

Conclusions: Increased access to fatty acids intracellularly by a fatty acid transporter exerts important effects on beta cell function and metabolism. Supported by Swedish Society of Medicine 


\section{PS 20 ER stress, ROS and FFA- induced beta cell degeneration}

\section{4}

Palmitate-induced manifestations of the unfolded protein response in pancreatic $\beta$ cells; dependency on glucose

E. Sargsyan, E. M. Sol, P. Bergsten;

Medical Cell Biology, Uppsala University, Sweden.

Background and Aims: Elevated levels of glucose and lipids are detrimental for $\beta$-cell function and mass. The reason for this has been attributed the fact that $\beta$-cells preferentially metabolize glucose in the presence of elevated levels of glucose and fatty acids. Inhibition of carnitine-palmitoyl transferase 1 (CPT-1) via glucose-derived elevation of malonyl-CoA has been proposed to be a mechanism of how combined elevated levels of glucose and fatty acids negatively affect the $\beta$-cell. Recent studies have demonstrated that endoplasmic reticulum (ER)-stress may also play a role. In the present study, short- and long-term effects of palmitate (PA) on the unfolded protein response (UPR) were studied in the presence of different glucose concentration, i.e. under conditions that direct metabolism towards accumulation or oxidation of PA. The aim of the study was to investigate to what extent PA activates the ER-stress response and if the activation depends on the ambient glucose concentration, i.e. if PA by itself is sufficient to elicit the response or if elevated glucose is required to obtain the full manifestations of the UPR.

Materials and Methods: INS-1E cells were cultured for 3 or 24 hours with $0.5 \mathrm{mM}$ PA at 3,11 or $20 \mathrm{mM}$ glucose in the presence of $10 \%$ FCS. After culture, cellular protein and mRNA were isolated and used for measurements of transcript and/or protein levels of p-eIF2 $\alpha$, ATF4, CHOP, GADD34, XBP1, EDEM, ATF6, BiP and CPT1. Total protein level in the wells was used as a measure of $\beta$-cell number.

Results: B-cell mass was only reduced in INS-1E cells cultured for 24 hours in the presence of PA and high glucose. In such cells CPT1 transcript as well as protein levels were lower compared to cells cultured in the presence of PA and low glucose. Phosphorylation of eIF $2 \alpha$ is an early manifestation of ER-stress. Indeed, increased levels of p-eIF $2 \alpha$ were already detected in cells cultured for 3 hours in the presence of PA. In addition, p-eIF2 $\alpha$ was higher when the glucose concentration during culture was increased. Transcript levels of ATF4, CHOP, GADD34 and BiP, which are downstream factors of p-eIF $2 \alpha$, required 24 hours culture in the presence of PA to be up-regulated. Their up-regulation was not glucose-dependent. When corresponding protein levels were measured for $\mathrm{CHOP}$ and $\mathrm{BiP}$ no changes were observed, however. Enhanced splicing of XBP1 was observed in cells cultured for 24 hours in PA irrespective of the glucose concentration. EDEM, its downstream target involved in protein degradation, was not affected at the transcript level, however. Examination of the ATF6 pathway demonstrated lack of active form of this transcription factor in PA-treated cells at all glucose concentrations. Conclusion: PA exerts more harmful effects on $\beta$-cell mass when combined with high glucose probably due to lowered oxidation of the fatty acid. PA rapidly elicits initial stages of the UPR, which are augmented by high glucose concentrations. It appears that PA causes partial activation of UPR, which does not lead to up-regulation of chaperones and proteins involved in protein degradation and apoptosis. The role of UPR as mediator of apoptosis in INS$1 \mathrm{E}$ cells cultured in the presence of PA is therefore unlikely.

Supported by EFSD/MSD, Swedish Medical Research Council and Swedish Diabetes Association

\section{5}

The mono-unsaturated fatty acid, palmitoleate, attenuates the expression of the ER stress-associated proteins, ATF4 and CHOP-10, in pancreatic beta cells exposed to palmitate or tunicamycin

E. Diakogiannaki, H. J. Welters, N. G. Morgan;

Biomedical \& Clinical Science, Peninsula Medical School, Plymouth, United Kingdom.

Background and Aims: Saturated and mono-unsaturated fatty acids (FA) exert differential effects on $\beta$-cell viability with long-chain saturated FAs (e.g. palmitate (C16:0)) causing loss of viability whereas mono-unsaturates (e.g. palmitoleate $($ cis $\mathrm{C} 16: 1))$ are well tolerated. Furthermore, long-chain monounsaturates are directly cytoprotective to $\beta$-cells exposed to saturated fatty acids. The molecular basis of this differential response is poorly understood but morphological alterations seen in FA-treated cells suggest that changes in the activation state of endoplasmic reticulum (ER) stress pathways may be involved. Therefore, the aim of the present work was to investigate the effects of FA on the signalling cascades associated with ER stress in pancreatic $\beta$-cells.

Materials and Methods: The rat pancreatic $\beta$-cell line BRIN-BD11 was used and cells were exposed to FAs as their albumin complexes, under serum free conditions. Electron microscopy and Western blot analysis were employed to investigate the morphological changes and altered ER stress pathways respectively, in FA-treated cells.

Results: Palmitate $(\mathrm{C} 16: 0 ; 0.05-0.25 \mathrm{mM})$ reduced beta-cell viability in a time-dependent manner (6-24h) and caused dramatic alterations to the cell morphology, including marked distension of ER membranes. Palmitoleate (C16:1) antagonized the cytotoxicity of palmitate completely such that the cells remained viable and could proliferate but it only partially alleviated the morphological changes seen in the ER. The PERK signalling pathway of ER stress was activated dose-dependently $(0.025-0.15 \mathrm{mM})$ in palmitatetreated cells as indicated by increased expression of the transcription factor ATF4. By contrast, ATF4 was not induced in cells exposed to palmitoleate (densitometry for ATF4: control - 1.0 unit; $0.025 \mathrm{mM}$ palmitate - 3.7 units; $0.025 \mathrm{mM}$ palmitoleate - 1.1 unit). The pro-apoptotic factor CHOP-10/ GADD153 was also increased by palmitate but not by palmitoleate. GRP78 expression was not up-regulated in palmitate- or palmitoleate-treated cells. Palmitoleate markedly attenuated the induction of ATF4 and CHOP10 caused by palmitate and it also reduced the induction of these factors in response to tunicamycin, an agent which induces ER stress by inhibiting protein glycosylation. Consistent with these results palmitoleate protected $\beta$-cells against the cytotoxicity caused by tunicamycin $(1 \mu \mathrm{g} / \mathrm{ml}$ tunicamycin $22.7 \% \pm 1.3$ dead cells; tunicamycin + palmitoleate: $15.4 \% \pm 1.4$ dead cells; $\mathrm{p}<0.05)$.

Conclusion: The mono-unsaturated fatty acid, palmitoleate inhibits the induction of markers of ER-stress in $\beta$-cells exposed to either palmitate or tunicamycin. Thus, palmitoleate may exert its protective actions on cell viability by reducing the pro-apoptotic response associated with ER stress although this is not accompanied by complete normalisation of the morphological changes seen in the ER of palmitate-treated cells. Supported by Boehringer Ingelheim Pharma GmbH \& Co

\section{6}

Counteraction of glucolipotoxicity in INS-1E cells by carnitinepalmitoyltransferase 1 over-expression involves reduction in phosphorylation of ER-stress marker eIF2 $\alpha$ E. M. Sol ${ }^{1}$, E. Sargsyan ${ }^{1}$, G. Akusjärvi ${ }^{2}$, P. Bergsten ${ }^{1}$; ${ }^{1}$ Department of Medical Cell Biology, ${ }^{2}$ Department of Medical Biochemistry and Microbiology, Uppsala University, Sweden.

Background and Aims: Chronic exposure of elevated levels of glucose and free fatty acids are correlated with dysfunctional pancreatic $\beta$-cells and development of type 2 diabetes. The mitochondrial protein carnitine palmitoyltransferase 1 (CPT1) has an important role in the oxidation of fatty acids by transporting them into the mitochondria. To examine if enhanced fatty acid oxidation is beneficial for $\beta$-cells exposed to elevated concentrations of glucose and fatty acids, we performed regulated over-expression of CPT1 in INS-1E cells cultured in the presence of palmitate and $20 \mathrm{mM}$ glucose. After culture, glucose-stimulated insulin secretion (GSIS) and total protein were measured in INS-1E cells with different amounts of CPT1.

Materials and Methods: An adenoviral construct was designed for Tet-ON regulated over-expression of liver type of CPT1 (Ad-Tet-ON-CPT1) and used for infecting INS-1E cells. Subsequently, cells were cultured for 48 hours in the presence of $10 \%$ serum, $0.5 \mathrm{mM}$ palmitate and 5.5 or $20 \mathrm{mM}$ glucose. CPT1 expression was induced by adding 0.2 or $1 \mu \mathrm{M}$ doxycycline to the culture medium. After culture, INS-1E cells were used for determination of GSIS, which was measured at basal $(3 \mathrm{mM})$ and stimulatory $(20 \mathrm{mM})$ glucose in the presence or absence of palmitate. Total protein levels in the wells were also measured by using DC protein assay. Expression of CPT1 and endoplasmic reticulum (ER)-stress markers phosphorylated eIF2 $\alpha, \mathrm{CHOP}$ and $\mathrm{BiP}$, were examined using western blot analysis.

Results: INS-1E cells cultured in the presence of $20 \mathrm{mM}$ glucose and palmitate demonstrated impaired insulin release with elevated basal insulin secretion and perturbed insulin secretion at stimulatory glucose 
concentration irrespective of whether palmitate was absent or present during the measurements. In contrast, cells over-expressing CPT1 6-fold demonstrated lowered basal insulin release and improved GSIS when palmitate was present during the release measurements. If palmitate was omitted during release measurements, GSIS was not improved by overexpressing CPT1. Total protein was increased with elevated CPT1-levels. Since total protein rise could reflect decrease in apoptosis, phosphorylation of the early marker of ER-stress eIF2 $\alpha$ was measured. ER-stress is proposed to be one of the underlying mechanisms of fatty acid induced pancreatic $\beta$-cell apoptosis and phosphorylation of eIF2 $\alpha$ an initial step in the development of ER-stress response. Phosphorylated eIF2 $\alpha$ was decreased in cells cultured at high glucose concentration and palmitate and over-expressing CPT1. The down-stream ER-stress markers $\mathrm{CHOP}$ and $\mathrm{BiP}$ were, however, not affected by enhanced levels of CPT1.

Conclusion: The study demonstrates that over-expression of CPT1 is counteracting glucolipotoxocity in INS-1E cells. Enhanced levels of CPT1 improves GSIS, reduces insulin secretion at low glucose, increases total protein content possibly reflecting attenuation of apoptosis, and finally alleviates ER stress by down-regulating p-eIF2 $\alpha$.

Supported by EFSD/MSD, Swedish Medical Research Council and Swedish Diabetes Association

\section{7}

Fatty acid-induced lipid handling and apoptosis in MIN6 cells is determined by fatty acid saturation and chain length

K. Thörn, P. Bergsten;

Medical Cellbiology, Uppsala Universitet, Sweden.

Background and Aims: Prolonged exposure to fatty acids impairs the function of the pancreatic $\beta$-cell and causes apoptosis. Most work has been performed using palmitate as a model of saturated fatty acids and oleate as its monounsaturated counterpart. To explain the decline of $\beta$-cell function after fatty acid exposure endoplasmic reticulum (ER)-stress was shown to be induced by palmitate but not by oleate. The reason for observed differences between effects on $\beta$-cell function and mass of different fatty acids is to a large extent still unclear, however. The aim of the present study was to investigate the regulation of genes involved in intracellular lipid handling and ER-stress when the $\beta$-cell is exposed to fatty acids of different chain length and degree of saturation.

Materials and Methods: MIN6 cells were cultured in absence or presence of $0.5 \mathrm{mM}$ palmitate, stearate, palmitoleate or oleate for 48 hours. After culture, glucose-stimulated insulin secretion and apoptosis was measured. RNA was isolated and used to determine transcriptional regulation of genes involved in intracellular fatty acid handling by qPCR. Protein was isolated and separated by SDS-PAGE and immunoblotted with antibodies directed towards proteins involved in ER-stress response.

Results: Insulin secretion at stimulating $(20 \mathrm{mM})$ glucose concentrations from MIN6 cells cultured in presence of saturated fatty acids (SFAs) palmitate or stearate was significantly reduced. On the other hand, stimulated insulin secretion was not affected when cells were cultured in the presence of monounsaturated fatty acids (MUFAs) palmitoleate or oleate. Cells treated with either of the MUFAs displayed no difference in apoptosis compared to control cells. In contrast, apoptosis was significantly increased in cells cultured in the presence of either of the SFAs with the effect of palmitate being $45 \%$ higher than stearate. To determine if this difference could be attributed to changes in proteins involved in intracellular lipid handling, mRNA levels of SCD1, SCD2, DGAT1, DGAT2, CPT1 and FAS were determined. SFAs regulated the genes differently compared to MUFAs except for CPT1 which was similarly regulated by all fatty acids. No differences in expression depending on fatty acid chain length were found except for FAS. Palmitate exclusively up-regulated levels of FAS indicating increase in de novo synthesis of fatty acids. Since palmitate caused a higher degree of apoptosis than stearate, we examined if this could be attributed to differences of the SFAs to induce ERstress response. Although both SFAs increased phosphorylated (P) eIF2 $\alpha$, phosphorylation was more enhanced after palmitate treatment. While palmitate-induced increase of P-eIF2 $\alpha$ translated into increased levels of the pro-apoptotic protein $\mathrm{CHOP}$, stearate-induced increase of P-eIF2 $\alpha$ failed to induce CHOP.

Conclusion: Fatty acids affect the $\beta$-cell differently depending both on chain length and saturation. Whereas degree of saturation was important for insulin secretion and in regulating genes involved in lipid handling, chain length affected apoptosis in cells exposed to SFAs. The difference in apoptosis observed when cells were cultured in the presence of palmitate or stearate may be accounted for by selective palmitate-induction of pro-apoptotic protein CHOP.

Supported by EFSD/MSD, Swedish Medical Research Council and Swedish Diabetes Association

\section{8}

Palmitate, but not oleate, induces endoplasmic reticulum (ER) stress and impairs ER to Golgi trafficking independent of de novo ceramide synthesis

A. Preston ${ }^{1}$, E. Gurisik ${ }^{1}$, A. Busch ${ }^{1}$, M. Fuller ${ }^{2}$, P. Meikle ${ }^{2}$, R. Laybutt ${ }^{1}$, T. Biden ${ }^{1}$;

${ }^{1}$ Diabetes and Obesity, Garvan Institute of Medical Research, Sydney, ${ }^{2}$ Lysosomal Diseases Research Unit, Children Youth and Women's Health Service, Adelaide, Australia.

Background and Aims: Type 2 diabetes (T2D) is characterised by $\beta$-cell dysfunction which correlates with increases in plasma lipids and $\beta$-cell apoptosis. The ER is a major site of synthesis for protein and complex lipids such as ceramide, a species implicated in $\beta$-cell apoptosis. We have previously shown that ER stress occurs in $\beta$-cells of human T2D and animal models of the disease. Moreover, we showed that ER stress was necessary for full lipo-apoptosis in vitro. Therefore, we sought to determine the mechanisms whereby fatty acids induce ER stress. As fatty acids are important regulators of vesicular transport, our aim was to investigate links between vesicular trafficking, ER stress and apoptosis in fatty acid treated MIN6 cells.

Materials and Methods: Apoptosis was measured by ELISA. Total ceramide was measured by mass spectrometry and de novo synthesis was determined by incorporation of ${ }^{3} \mathrm{H}$-serine into ceramide as assessed by thin layer chromatography. To study vesicular trafficking, we transfected MIN6 cells with GFP-tagged vesicular stomatitis virus temperature sensitive mutant ts045 (VSVG-GFP), which is reversibly misfolded and retained in the ER at $40^{\circ} \mathrm{C}$ and moves out of the ER and into the Golgi complex when temperature is reduced to $32^{\circ} \mathrm{C}$. Cells expressing VSVG-GFP were incubated with $0.92 \%$ BSA either alone or coupled to $0.4 \mathrm{mM}$ palmitate or oleate for $48 \mathrm{~h} .12 \mathrm{~h}$ before the end of the incubation, cells were shifted to $40^{\circ} \mathrm{C}$ and then $32^{\circ} \mathrm{C}$ for $20 \mathrm{~min}$. VSVG-GFP trafficking was assessed by confocal microscopy. Cells were fixed and immunocytochemistry was performed using markers of the ER and Golgi complex. Trafficking was also measured by resistance of VSVG-GFP to endoglycosidase $\mathrm{H}$ (Endo $\mathrm{H}$ ), a characteristic of secretory products in the Golgi complex. Membranes were isolated following fatty acid treatment, incubated with Endo H and separated by SDS-PAGE. Western blotting for GFP was performed to identify changes in VSVG-GFP mobility and resistance to Endo $\mathrm{H}$.

Results: Confocal microscopy showed that VSVG-GFP was retained in the $\mathrm{ER}$ at $40^{\circ} \mathrm{C}$. Time course analysis revealed that with lowering the temperature to $32^{\circ} \mathrm{C}$, VSVG-GFP in control and oleate treated cells was efficiently transported to the Golgi complex. However in the palmitate treated cells, a proportion of VSVG-GFP exhibited delayed trafficking and was retained in the ER. Endo $\mathrm{H}$ treatment of isolated membranes revealed trafficking of VSVGGFP due to the temperature dependant shift was $90 \%$ reduced $(\mathrm{p}<0.05)$ in palmitate treated cells compared to control, whereas no significant alteration was observed in oleate treated cells. No change in total ceramide levels was observed with palmitate treatment when compared to control treated cells as assessed by mass spectrometry $(8.1 \pm 0.6$ vs. $8.3 \pm 0.8 \mathrm{nmol} / \mathrm{mg}$ protein respectively). Moreover, concentrations of cycloserine which completely abolished de novo ceramide synthesis did not inhibit lipoapoptosis.

Conclusion: These results indicate that treatment of MIN6 cells with the saturated fatty acid palmitate but not the unsaturated fatty acid oleate causes impairment of vesicular trafficking from ER to Golgi. This defect in trafficking is associated with ER stress and $\beta$-cell apoptosis and may contribute to the development of T2D. This blockade in trafficking is not due to increases in de novo ceramide synthesis.

Supported by the National Health and Medical Research Council, Australia 


\section{9}

The role of reactive oxygen species (ROS) for lipotoxicity in insulin producing cells

W. Gehrmann, M. Elsner, S. Lenzen;

Institute of Clinical Biochemistry, Hannover Medical School, Germany.

Background and Aims: Elevated levels of free fatty acids (FFA) cause $\beta$-cell dysfunction and apoptosis in type 2 diabetes, but the molecular mechanisms of lipotoxicity are only partially understood. It is unclear if increased reactive oxygen species (ROS) formation or long chain free fatty acid binding receptors GPR40 and GPR120 are involved in lipotoxicity. The aim of the present study was to analyse the toxicity of different saturated and unsaturated fatty acids in insulin producing cells and the possible molecular mechanisms, particularly with regard to the role of elevated ROS formation and an uncoupling of the respiratory chain.

Materials and Methods: To investigate the cytotoxic effects of different fatty acids upon insulin-producing RINm5F cells in vitro viability was measured by MTT assay after exposure to saturated and unsaturated fatty acids for $24 \mathrm{~h}$. A FACS-based caspase- 3 assay was performed to determine cell death mode of lipotoxicity. To assess the role of ROS formation for lipotoxicity we analysed the cell viability of RINm5F cells overexpressing antioxidative enzymes after palmitic acid treatment. The mitochondrial membrane potential was determined by staining with JC-1. The effect of FFA on the mRNA expression level of uncoupling protein 2 (UCP2) and the expression levels of GPR40 and GPR120 were determined by quantitative RT-PCR. Electron microscopy analyses were performed to detect morphological changes due to FFA treatment.

Results: The saturated fatty acids palmitic $\left(\mathrm{C} 16: 0, \mathrm{EC}_{50}=113 \mu \mathrm{M} \pm 6\right)$ and stearic acid $\left(\mathrm{C} 18: 0, \mathrm{EC}_{50}=80 \mu \mathrm{M} \pm 5\right)$ showed toxic effects on insulin producing cells whereas the unsaturated fatty acids oleic (C18:1), palmitoleic (C16:1), linoleic (C18:2), $\gamma$-linolenic (C18:3) and arachidonic acid (C20:4) were not toxic up to supraphysiological concentrations. Moreover, unsaturated fatty acids had a significant protective effect against saturated fatty acid induced toxicity at equimolar concentrations. The caspase- 3 signal pathway was activated in RINm5F cells due to palmitic acid treatment. Only the cytosolically located antioxidative enzymes catalase and CuZnSOD showed protective effects against palmitic acid induced toxicity, whereas the mitochondrially located antioxidative enzymes mito-catalase and MnSOD had no protective effect. The mitochondrial membrane potential was enhanced by palmitic acid incubation $(31 \% \pm 6$ less green fluorescence measured by JC- 1$)$ whereas the UCP-2 expression level was unaltered. The FFA binding receptors GPR40 and GPR120 in RINm5F cells were not detectable by quantitative RT-PCR. In electron microscopy analyses we found damage to the endoplasmic reticulum induced by palmitic acid whereas the mitochondria were intact. Incubation with oleic acid led to the formation of lipid droplets in the cytosol.

Conclusion: The presented data indicate that only ROS formation in the cytosol and not in the mitochondria is important for the toxicity of saturated fatty acids. This is in agreement with the morphological changes of the endoplasmic reticulum and not of the mitochondria observed by electron microscopy and the unaltered UCP-2 expression level due to palmitic acid treatment. The FFA binding receptors GPR40 and GPR120 are probably not involved in lipotoxicity.

\section{0}

Insulin secretion defects of human type 2 diabetic islets are corrected by preculture with the radical scavenger IAC

R. Lupi ${ }^{1}$, S. Del Guerra ${ }^{1}$, R. Mancarella ${ }^{1}$, L. Valgimigli ${ }^{2}$, M. Paolini ${ }^{2}$,

A. Soleti ${ }^{3}$, M. Bugliani ${ }^{1}$, M. Martano ${ }^{3}$, F. Filipponi ${ }^{4}$, U. Boggi ${ }^{4}$, S. Del Prato ${ }^{1}$, P. Marchetti ${ }^{1}$;

${ }^{1}$ Endocrinology and Metabolism, Metabolic Unit, University of Pisa, Italy, ${ }^{2}$ Pharmacology, University of Bologna, Italy,

${ }^{3}$ Medestea Research, Medestea, Torino, Italy, ${ }^{4}$ Oncology, Transplantation and Advanced Technologies in Medicine, University of Pisa, Italy.

Background and Aims: Oxidative stress is a putative mechanism leading to beta-cell damage in Type 2 diabetes. In the present study, we investigated the effects of the new antioxidant, bis(1-hydroxy-2,2,6,6-tetramethyl-4piperidinyl)decandioate di-hydrochloride (IAC) on the function of isolated human pancreatic islets from Type 2 diabetic and non-diabetic subjects, matched for age and body mass index.
Materials and Methods: The islets were obtained from 8 non-diabetic and 5 Type 2 diabetic multiorgan donors (age, $49 \pm 16$ and $65 \pm 9.5$ years; gender, 4 males $/ 4$ females and 3 males/2 females; body mass index, $25.6 \pm 2.5$ and $27.5 \pm 4.0 \mathrm{~kg} / \mathrm{m}^{2}$ ), cultured free-floating at $37{ }^{\circ} \mathrm{C}$ for $24 \mathrm{~h}$ in M199 culture medium either plain or containing 10 or $100 \mu \mathrm{mol} / 1 \mathrm{IAC}$. Oxidative stress was demonstrated by measuring nitrotyrosine concentration (Elisa technique), and by electron paramagnetic resonance (EPR). Functional (glucosestimulated insulin secretion) and molecular (qualitative and quantitative RTPCR) studies were also performed.

Results: Nitrotyrosine concentrations were significantly $(\mathrm{p}<0.01)$ higher in Type 2 diabetes $(12.4 \pm 0.9 \mathrm{nmol} / \mathrm{l})$ than in control $(6.6 \pm 0.3 \mathrm{nmol} / \mathrm{l})$ islets. In addition, increased oxidative stress in diabetic islets was demonstrated by EPR, which showed a 2-fold increase of nitroxide levels. As expected, glucose-stimulated insulin release from Type 2 diabetic islets was significantly lower than from non-diabetic islets (Stimulation Index, SI $2.0 \pm 0.4$ and $0.9 \pm 0.2$ respectively, $\mathrm{p}<0.01)$. Pre-culture of Type 2 diabetic islets with 10 or $100 \mu \mathrm{mol} / \mathrm{l}$ IAC caused a significant reduction $(\mathrm{p}<0.01$ by ANOVA) of nitrotyrosine levels ( $\mathrm{nmol} / \mathrm{l}$ ), which decreased from $12.4 \pm 0.9$ to $9.3 \pm 0.5$ at 10 $\mu \mathrm{mol} / \mathrm{l} \mathrm{IAC}$ ( $\mathrm{p}<0.05 \mathrm{vs}$ non-treated by the Bonferroni test) or to $10.2 \pm 1.4$ at $100 \mu \mathrm{mol} / \mathrm{l} \mathrm{IAC}$ ( $\mathrm{p}<0.05 \mathrm{vs}$ non-treated by the Bonferroni test). These changes were accompanied by improved glucose-stimulated insulin secretion, with SI values significantly increasing to $1.5 \pm 0.4$ and $1.6 \pm 0.5$ with 10 and $100 \mu \mathrm{mol} /$ 1 IAC, respectively. Finally, mRNA expression of catalase and glutathione peroxidase was significantly higher in Type 2 diabetic than control islets, and $24 \mathrm{~h}$ exposure to IAC normalized the expression of the two ROS scavenging enzymes. This was accompanied by a significant increase of the expression of insulin, which was more than $50 \%$ lower in diabetic islets as compared to control cells prior to IAC pre-culture, and increased approximately $30 \%$ afterwards.

Conclusion: The present study supports the concept that therapy with antioxidants could be an interesting adjunctive pharmacological approach to the treatment of Type 2 diabetes. In this regard, the protection determined by IAC suggests the potential usefulness of this compound. Supported by Medestea Research, Medestea, Torino, Italy

\section{1}

Evolution with age of antioxidant capacity in rat pancreatic islets. Early programming by maternal diet

N. H. Theys, B. Reusens, C. Remacle;

Institute of Life Sciences, Université Catholique de Louvain,

Louvain-la-Neuve, Belgium.

Background and Aims: In Type 1 as well as in Type 2 diabetes, $\beta$-cell function is progressively disturbed through a complex process during which the cells are exposed to reactive oxygen species (ROS). An increase in ROS production may be a harsh challenge since the level of antioxidant enzymes activities is low in pancreatic islets. We have shown that the vulnerability of the foetal and adult $\beta$-cells against ROS can be influenced by the nutritional status of the mother during gestation and lactation. Indeed, islets from foetal and adult progeny of dams fed a low protein diet present a higher rate of apoptosis after cytokines or ROS attacks. In this context, our first aim was to study the evolution of antioxidant capacity during post-natal development. Our second aim was to investigate whether maternal low protein diet may programme the antioxidant activity in islets and liver of the progeny.

Materials and Methods: Wistar rats were fed a control diet containing $20 \%$ protein ( $\mathrm{C}$ group) or an isocaloric low protein diet (LP) containing $8 \%$ protein during gestation only (LPR group) or until weaning (LPT group). At birth (LPR group) or after weaning (LPT group), offspring received the control diet. The antioxidant capacity was studied at 5 days, 28 days, 6 weeks, and 3 months in male rats.

Results: In C group, superoxide dismutase (SOD) and catalase (CAT) activities were much lower in islets than in liver at each age. In the liver, SOD activity increased significantly at 6 weeks $(\mathrm{p}<0.05)$ compared to younger ages and remained stable thereafter. In contrast, islets showed a higher SOD activity at 5 days $(\mathrm{p}<0.05)$ compared to later ages. In the liver, CAT activity was significantly decreased by $50 \%$ at 28 days $(\mathrm{p}<0.01), 6$ weeks $(\mathrm{p}<0.05)$ and 3 months $(\mathrm{p}<0.05)$ compared to 5 days whereas in islets, no significant change was observed with age and this activity was as low as $2 \%$ of that in the liver. Plasma lipid hydroperoxide levels were decreased in 3 month-old LPT offspring $(\mathrm{p}<0.05)$ whereas the total peroxyl-radical trapping potential tended to be lower. In liver, SOD activity was reduced at 
5 days in LPR offspring $(\mathrm{p}<0.05)$ compared to $\mathrm{C}$ offspring. At 28 days, this difference was no more observed, and at 3 months, LPT animals showed an increased SOD activity compared to $\mathrm{C}$ and LPR rats $(\mathrm{p}<0.01)$. In islets, SOD activity was lower at 5 days in LPR offspring $(\mathrm{p}<0.001)$ compared to $\mathrm{C}$ islets but not in LPT progeny. At 3 months, SOD activity in islets from $\mathrm{C}$ and LPR animals was similar, whereas it was significantly increased in LPT rats $(\mathrm{p}<0.01)$. In comparison to controls, CAT activity was significantly higher in liver $(\mathrm{p}<0.05)$ of LPR and LPT offspring already from 28 days. In islets, CAT activity was so low for many samples that it couldn't be detected, particularly in LPT islets.

Conclusion: In C offspring, at all ages studied, antioxidant enzyme activities in islets were much lower than those in liver. Then, already at birth, the endocrine pancreas seems to be particularly vulnerable to oxidative stress. Maternal LP diet modulated the activity of liver and islets antioxidant enzymes. In islets as well as in liver, SOD activity was higher in adult LPT animals and could correspond to a compensatory effect due to early malnutrition. In contrast, CAT activity completely collapsed in islets of LPT offspring. The modulation of antioxidant activities can not explain the increased apoptosis rate observed previously in LP islets but could program antioxidant capacity in ways unfavourable for the future of the progeny.

\section{PS 21 Putative mediators of beta cell damage in type 2 diabetes}

\section{2}

High density lipoprotein protects human and mouse pancreatic islets from high glucose and interleukin-1 beta induced apoptosis S. Rütti ${ }^{1}$, J. Ehses ${ }^{1}$, L. Rohrer ${ }^{2}$, R. Prazak ${ }^{1}$, A. von Eckardstein ${ }^{2}$, M. Y. Donath ${ }^{1}$;

${ }^{1}$ Endocrinology and Diabetology,

${ }^{2}$ Institute of Clinical Chemistry, University Hospital, Zürich, Switzerland.

Background and Aims: Increasing evidence indicates that decreased functional insulin-producing $\beta$-cell mass is a hallmark of type 2 diabetes. Lipoproteins have been shown to affect rodent $\beta$-cell survival. We tested the putative protective role of high density lipoprotein (HDL) on human and mouse $\beta$-cell turnover and function.

Materials and Methods: Human islets, obtained from cadaveric organ donors and isolated mouse islets were cultured for 2 or 4 days with varying concentrations of HDL alone as well as in the presence of high glucose or interleukin-1 beta (IL-1 $\beta$ ). Proliferation was assessed by Ki-67 staining and apoptosis by TUNEL staining.

Results: High density lipoprotein, isolated from the plasma of healthy donors by sequential ultracentrifugation, increased 2 -fold the basal proliferation of human islet cells $(n=4, p<0.05)$ and decreased 2-fold their basal apoptosis $(\mathrm{n}=6, \mathrm{p}<0.05)$. Furthermore, HDL $(1 \mathrm{mM})$ protected mouse and human islets from IL-1 $\beta$ and high glucose induced apoptosis (for IL-1 $\beta, n=3, p<0.05$ ). $\beta$ cell function, ascertained by glucose stimulated insulin secretion, was not affected by HDL. High density lipoprotein was unable to protect from high glucose or IL-1 $\beta$ induced functional impairment. Finally, we found that HDL increased islet FLIP mRNA expression $(\mathrm{n}=4, \mathrm{p}<0.05)$ alone as well as in the presence of high glucose or IL-1 $\beta$.

Conclusion: These results indicate that HDL induces human islet cell proliferation and protects human and mouse islet cells from glucose and IL$1 \beta$ induced apoptosis, possibly via regulation of FLIP expression, without affecting $\beta$-cell secretory function.

\section{3}

Molecular mechanism of pioglitazone effect on the pancreatic beta cell preservation in diabetic $d b / d b$ mice: evidence for direct action as PPAR $\gamma$ agonist

Y. Kanda, F. Kawasaki, M. Hashiramoto, K. Nakashima, M. Matsuki,

K. Kaku;

Diabetes and Endocrine Division, Kawasaki Medical School,

Kurashiki-shi, Japan.

Background and Aims: We have previously reported that pioglitazone (Pio) preserves the pancreatic beta-cell morphology and function in obese diabetic animal model $d b / d b$ mice. The aim of this study is to clarify the molecular mechanism for preventive effect of Pio on pancreatic beta cell damage in $d b / d b$ mice by directly analysing gene expression in pancreatic beta-cells using Laser Capture Microdissection (LCM) and real-time RTPCR method.

Materials and Methods: Male BKS.Cg- $+\operatorname{Lepr}^{d b} /+\operatorname{Lepr}^{d b} / \mathrm{Jcl}(d b / d b)$, BKS.Cg$\mathrm{m}+/+\operatorname{Lepr}^{d b} / \mathrm{Jcl}^{*}(d b /+)$ and lean littermate (+/+) mice were devided into Piotreated and -untreaed (control) groups $(\mathrm{n}=5)$. Pio was administered orally for two weeks from 10 weeks of age $(30 \mathrm{mg} / \mathrm{kg}$ BW). BW, fasted plasma glucose (FBG), insulin (FIRI), adiponectin, TG and FFA were measured every week. To analyze gene expression profiles in beta cells, the islet core region was selectively obtained by LCM method at the age of 8 and 12 weeks. Primer pairs encoding genes associated with pancreatic hormones, cell proliferation, cell differentiation, apoptosis, cell cycle, oxidative stress, and organization construction were prepared, and real-time RT-PCR with Sybr Green was applied. Each gene expression was relatively quantified by the comparative $\mathrm{Ct}$ method with each result in the 18 SrRNA as a control. Immunoreactions of proliferating cell number antigen (PCNA) and 4-hydroxynonenal modified protein (4-HNE) in islet tissues were also assessed.

Results: BW, FBG, FIRI, HOMA-IR, TG and FFA were significantly increased and adiponectin was decreased in $d b / d b$ mice compared with those in other groups. These deranged metabolic markers were ameliorated by the treatment 
with Pio. Gene expression analysis of islet core tissues in $+/+$ mice revealed that beta-cells were selectively collected by LCM method. Gene expressions of PPAR $\gamma$ and insulin in beta-cells were decreased in all three groups of mice treated with Pio. Cycline E \& ERK1 gene expressions related with promotion of cell proliferation were significantly up-regulated in $d b / d b$ mice at 8 week, but down-regulated at 12 weeks of age. CAD gene expression related with promotion of apoptosis was also increased in $d b / d b$ mice with their weeks. In 12 week-old $d b / d b$ mice, treatment with Pio significantly up-regulated ERK1 gene expression ( 4 -fold, $\mathrm{p}<0.05)$, and down-regulated CAD gene expression $(1 / 3$-fold, $\mathrm{p}<0.05)$. On the other hand, anti-apoptotic bcl-2 gene expression was significantly increased by Pio treatment (4.6-fold, $\mathrm{p}<0.05)$. NADPH oxidase gene expression related with oxidative stress was markedly decreased $(\mathrm{p}<0.05)$, and anti-oxidative stress related GSHPx and SOD2 gene expression was significantly increased by Pio ( $3 \sim 4$-fold, $p<0.05$, respectively). Effects of Pio on gene expression demonstrated in $d b / d b$ were also observed in $d b /+$ and $+/+$ mice, but not so much significant compared with those in $d b / d b$. Morphometric results for cell proliferation (PCNA) and oxidative stress (4$\mathrm{HNE}$ ) observed in $d b / d b$ mice corresponded to the data of gene expression analysis.

Conclusion: The present results demonstrated the PPAR $\gamma$ expression in mouse beta-cells, which is regulated by Pio. Our results strongly suggest the possibility that Pio preserves the beta cell mass directly through a mechanism to modulate cell kinetics as a PPAR $\gamma$ agonist in addition to amelioration of metabolic disorder.

\section{4}

Beta cells of the diabetic Goto-Kakizaki rat exhibit an unexpected in vitro adaptation to oxidative stress- induced apoptosis

G. Lacraz, N. Kassis, F. Homo-Delarche, J. Coulaud, D. Bailbé, B. Portha; CNRS and University Paris Diderot, UMR 7059, Paris, France.

Background and Aims: Prolonged exposure to elevated plasma glucose levels exerts deleterious effects on $\beta$-cell function and ultimately leads to $\beta$ cell death. Considerable interest has recently been focused on the putative role of reactive oxygen species (ROS) upon deterioration of $\beta$-cell function/ survival in diabetes. Interestingly, Type 2 diabetic Goto-Kakizaki (GK) rats from a Japanese colony exhibit $\beta$-cell oxidative damage and we have reported an islet inflammatory process in our GK colony. We recently showed that insulin exocytosis by GK $\beta$ cells, when functionally activated (high glucose + acetylcholine $1 \mathrm{mM}$ ), exhibits an unexpected resistance to many pro-oxidant agents ( $50 \mu \mathrm{M} \mathrm{H} 2 \mathrm{O} 2,1 \mathrm{mM}$ streptozotocin, $1 \mathrm{mM}$ alloxan or $50 \mu \mathrm{M}$ ter-butylhydroperoxide) when acutely exposed. Here, we have further examined the delayed effects of a calibrated oxidative stress exposure, upon $\beta$-cell apoptosis response in vitro.

Materials and Methods: Wistar (W) or GK islets were cultured for $24 \mathrm{~h}$ or $48 \mathrm{~h}$ at $10 \mathrm{mM}$ glucose after acute exposure to vehicle, $50 \mu \mathrm{M} \mathrm{H} 2 \mathrm{O} 2$ alone or in combination with the antioxidant $\mathrm{N}$-acetylcysteine (1 mM NAC). Apoptosis within the $\beta$-cell population was monitored by DNA fragmentation (TUNEL method and insulin immunostaining). Stress gene expression study was performed by quantitative Real Time PCR.

Results: In the absence of $\mathrm{H} 2 \mathrm{O} 2$, GK $\beta$-cell apoptosis after $24 \mathrm{~h}$ and $48 \mathrm{~h}$ culture period was strongly increased (8.9 and 3.9 fold, respectively) as compared to W $\beta$ cells. Despite this pattern, $\mathrm{H} 2 \mathrm{O} 2$ did not further amplify apoptosis in GK $\beta$ cells, whereas it triggered a major apoptotic wave (12 and 5.6 fold, respectively) in $\mathrm{W} \beta$ cells. Stress gene analysis revealed that pro- (BAX, caspases 3-9) and anti-apoptotic gene (Bcl 2) in GK islets were similarly expressed under basal condition, as compared to $\mathrm{W}$ islets. However, several inflammatory responsive genes (cyclooxygenase 2, 12-lipooxygenase, Nuclear Factor $\kappa B$, Transforming growth Factor $\beta$ 1) were overexpressed up to 19 fold in GK islets. In addition, many antioxidant genes were increased (superoxide dismutase $1-2$, catalase, thioredoxin $1-2$, peroxiredoxin $1-2$, haeme-oxygenase 1), at the remarkable exception of glutathione reductase, which was decreased by 10 fold. Interestingly, treatment with the glutathione inductor agent NAC strongly lowered the $\mathrm{H} 2 \mathrm{O} 2$-induced apoptosis in GK $\beta$ cells to level similar to $\mathrm{W}$ control $\beta$ cells. Such a protective action of NAC suggests that the weak GK $\beta$-cell survival, as detected here under in vitro culture conditions, involves ROS production.

Conclusion: In conclusion, even if stressful culture conditions reveal that GK $\beta$ cells have a decreased survival capacity to adapt in vitro, there are still able to defend remarkably against the $\mathrm{H} 2 \mathrm{O} 2$-induced apoptosis. The pattern of stress gene expression and ROS production that accompanies the $\beta$-cell survival at the end of the $24 \mathrm{~h}$-culture period is currently investigated. Since an exaggerated cAMP production is a characteristic of the GK islet, we will also evaluate the putative contribution of the cAMP/PKA pathway to the GK $\beta$-cell resistance to the $\mathrm{H} 2 \mathrm{O} 2$-induced apoptosis.

\section{5}

IL-1ß-MyD88 signaling is central to islet chemokine secretion in response to metabolic stress: evidence from a spontaneous model of type 2 diabetes, the GK rat

J. A. Ehses ${ }^{1}$, M.-H. Giroix ${ }^{2}$, J. Coulaud ${ }^{2}$, S. Akira ${ }^{3}$, F. Homo-Delarche ${ }^{2}$, M. Y. Donath ${ }^{1}$;

${ }^{1}$ Div of Endocrinology \& Diabetes and the Center for Integrated Human Physiology, University Hospital of Zurich, Switzerland, ${ }^{2}$ Unité mixte de recherches (UMR) 7059, Paris University / D. Diderot, Paris, France,

${ }^{3}$ Department of Host Defense, Osaka University, Japan.

Background and Aims: Interleukin-1 $\beta$ (IL-1 $\beta$ ) plays an integral role in the deterioration of $\beta$-cell function in type 2 diabetes (T2D). We have recently shown that pancreatic islets in T2D are characterized by macrophage infiltration, and that increased glucose and palmitate concentrations stimulate the secretion of various chemokines from islets. The aim of the present study was to investigate a possible role for IL- $1 \beta$ in chemokine production by both C57BL/6 (B6) mouse islets and diabetic Goto-Kakizaki (GK) rat islets.

Materials and Methods: Pancreatic islets were isolated from male 8-10 week old B6, MyD88 +/-, MyD88 -/-, and IL-1 $\beta$-/- mice and plated on ECM matrix at 5 islets/well. All mice were backcrossed for 10 generations onto the B6 background prior to analysis. Islets from 3-month old male Wistar (the control strain from which the GK line originated) and GK rats were isolated and plated on $3 \mathrm{~cm}$ ECM dishes at 20 islets/dish. All islets were stimulated with test conditions for $48 \mathrm{~h}$ unless otherwise stated, and experiments performed in triplicate.

Results: In mouse islets, $2 \mathrm{ng} / \mathrm{mL}$ IL- $1 \beta$ mimicked the effects of elevated glucose and palmitate on islet KC, G-CSF, and IL- 6 secretion; an effect that was completely ablated in MyD88 $-/-$ mice $(\mathrm{p}<0.05, \mathrm{n}=3-5)$. Analysis of the kinetics of these effects indicated significant increases in chemokines already after $6 \mathrm{~h}$ of treatment $(\mathrm{p}<0.05, \mathrm{n}=3-5)$. To determine if these effects of glucose and palmitate were partially due to IL- $1 \beta$, both B6 and IL- $1 \beta$ -/- islets were treated with the IL-1 receptor antagonist, IL-1Ra. IL-1Ra inhibited the production of KC, G-CSF, and IL- 6 by $>50 \%$ in B6 islets $(\mathrm{p}<0.05, \mathrm{n}=3-10)$ with no effect on IL-1 $\beta-/-$ islets $(\mathrm{p}>0.05, \mathrm{n}=4-7)$. Thus, IL- $1 \beta$ signalling is required for elevated glucose and palmitate concentrations to stimulate islet chemokine secretion. Next, we evaluated this in 3-monthold GK rat islets. In this spontaneous T2D model, hyperglycemia starts at 1 month of age (weaning) and is then accompanied by progressive inflammatory cell infiltration. Compared to age-matched Wistar islets, GK islets secreted increased amounts of KC, IL-6, MCP- 1 and MIP- $1 \alpha$ on a per $\mathrm{mg}$ protein basis $(\mathrm{p}<0.05, \mathrm{n}=3-6)$. Treatment of islets with IL-1Ra significantly decreased the secretion of all these factors in vitro $(\mathrm{p}<0.05$, $\mathrm{n}=3$ ). We also analyzed cytokines and chemokines in Wistar and GK sera from 1 to 32 weeks of age. Certain cytokines (IL-6, IFN- $\gamma$, IP-10) spiked similarly in Wistar and GK sera around weaning. Others such as MCP-1 and MIP-1 $\alpha$, showed a progressive decline with age and, interestingly, their levels were significantly elevated in GK sera at 1 and 2 weeks of age $(p<0.05$, $\mathrm{n}=4-10$ animal samples).

Conclusion: IL- $1 \beta$ is a central mediator of islet chemokine secretion induced by elevated glucose and palmitate; an effect that is dependent on MyD88 signalling in mice. Further, diabetic GK rat islets, characterized by progressive macrophage and granulocyte infiltration, secrete elevated levels of KC, IL-6, MCP-1 and MIP-1 $\alpha$. In vitro treatment of these islets with IL-1Ra reduces the secretion of these inflammatory factors. Therefore, we propose that in vivo IL-1Ra treatment may prevent macrophage infiltration in islets in type 2 diabetes.

Supported by Swiss National Fund (SNF), University of Zürich, JDRF and EFSD/MSD 


\section{6}

Islet protein expression from type 2 diabetic donors correlating with impaired secretory response

H. K. Nyblom ${ }^{1}$, M. Bugliani ${ }^{2}$, P. Marchetti ${ }^{2}$, P. Bergsten ${ }^{1}$;

${ }^{1}$ Department of Medical Cell Biology, Uppsala University, Sweden,

${ }^{2}$ Department of Endocrinology and Metabolism - Metabolic Unit, University of Pisa, Italy.

Background and Aims: Islet dysfunction in type 2 diabetes mellitus is caused by alterations in multiple genes. This prompted the use of approaches capable of determining expression profiles rather than single gene products. The first aim was to protein profile islets obtained from type 2 diabetes mellitus and control donors. The second aim was to develop a protocol to discover and identify differentially expressed proteins relevant for the development of islet dysfunction in type 2 diabetes mellitus, by correlating changes in expression patterns to islet function studies.

Materials and Methods: Islets of Langerhans were isolated from five type 2 diabetes mellitus and ten control donors. The two groups were age and weight matched. Insulin release from isolated islets in response to glucose, glibenclamide and arginine was determined. Islet lysates corresponding to approximately 20 islets were applied onto strong anionic protein arrays (Q10) and analyzed by surface-enhanced laser desorption/ionization time-of-flight mass spectrometry (SELDI-TOF MS).

Results: Insulin release from control islets increased 4-fold when the glucose concentration was increased from 3 to $17 \mathrm{mM}$ glucose. Similar rises were observed when glibenclamide or arginine was added. In contrast, insulin release only increased 2 -fold from islets isolated from type 2 diabetes mellitus donors when either the glucose concentration was raised or glibenclamide or arginine was added. Protein profiles of islet lysates, from control or type 2 diabetes mellitus donors, showed about 110 peaks $(s / n=4$, peak percentage $=15 \%$ ). Although considerable variability in both insulin release and peak intensity existed within the two groups, 31 differentially expressed peaks $(\mathrm{p}<0.05)$ were detected. Individual insulin secretory results were plotted against corresponding peak intensities of the differentially expressed proteins. In seven of the differentially expressed proteins there was a correlation between mean peak intensities and the stimulation index for insulin release in the presence of glibenclamide.

Conclusions: Protein profiles of as little as 20 islets can be obtained by SELDITOF MS. The limited demand for islets made islet protein profiling from type 2 diabetes individuals possible. Differentially expressed islet proteins were discovered when control and type 2 diabetes mellitus islets were compared. The significance of some of these proteins for islet dysfunction is indicated by the correlation of the expression of these proteins to glibenclamide-induced insulin secretion.

Supported by the Swedish Diabetes Association

\section{7}

IL-1beta expression in human islets is induced by glucose and autostimulation, and increased in beta cells of individuals with type 2 diabetes

M. Boeni-Schnetzler ${ }^{1}$, L. Marselli ${ }^{2}$, G. Parnaud ${ }^{3}$, J. A. Ehses ${ }^{1}$, P. A. Halban ${ }^{3}$, G. C. Weir ${ }^{2}$, M. Y. Donath';

${ }^{1}$ Enocrinology and Diabetology, University Hospital of Zuerich, Switzerland, ${ }^{2}$ Joslin Diabetes Center, One Joslin Place, Boston, United States,

${ }^{3}$ Departement of Genetic Medicine and Development, CMU, Geneva, Switzerland.

Background and Aims: IL-1beta producing beta-cells were previously observed by immunostaining and by in situ hybridisation in pancreatic sections of patients with type 2 diabetes (T2D). Further, treatment of patients with T2D with an IL1 receptor antagonist (IL1Ra) improves insulin secretion and blood glucose levels. Exposure of human islets to high glucose levels increased production and release of IL-1beta in most but not all islet cell preparations. The aim of the present study was to substantiate the observations on IL-1beta expression in T2D and to understand the mechanisms of these variable effects of glucose on IL-1beta expression in vitro.

Materials and Methods: In vivo IL-1beta expression was analysed from gene profiles of near-pure populations of beta-cells obtained by laser capture microdissection (LCM) from frozen pancreatic tissue sections of cadavers from 4 subjects with T2D and 7 normal donors. In vitro Il-1beta expression was analysed in human islets cultured for 4 days with 5.5 or $33.3 \mathrm{mM}$ glucose, $200 \mathrm{pg} / \mathrm{ml}$ IL-1beta or $1 \mathrm{ug} / \mathrm{ml}$ IL1Ra. IL-1beta and IL1Ra mRNA levels were quantified using TaqMan analysis and measuring delta ct values relative to non-treated controls.

Results: Gene array analysis showed that IL-1beta mRNA expression was higher in each of the four samples of beta-cells within pancreas sections from T2D patients when compared to controls $(\mathrm{p}=0.014)$. RT-PCR confirmed increased IL- 1 beta mRNA levels in 3 of the 4 T2D patients while minimal expression of IL-1beta was observed in one out of 4 controls. In cultured human islets $33.3 \mathrm{mM}$ glucose induced IL-1beta mRNA levels in 6 out of 11 islet batches. The mean increase in all preparations was $2.15 \pm 0.46$ $(\mathrm{n}=11, \mathrm{p}<0.05)$ and $3.02 \pm 0.49(\mathrm{n}=6, \mathrm{p}<0.01)$ in those preparations that did respond. Islet preparations where glucose did not increase IL-1beta mRNA displayed higher basal IL-1beta mRNA levels than responsive preparations, and there was a significant negative correlation between basal IL-1beta mRNA levels and glucose-stimulated IL-1beta mRNA $\left(\mathrm{r}^{2}=0.526\right.$, $\mathrm{p}=0.0076$ ). IL1Ra mRNA levels of human islet preparations were decreased by $33.3 \mathrm{mM}$ glucose to $72.3 \pm 10.4 \%$ control $(\mathrm{n}=11, \mathrm{p}<0.05)$. Addition of IL1Ra strongly decreased basal and glucose stimulated IL-1beta mRNA levels $(\mathrm{p}<0.05)$, suggesting that IL-1beta mRNA levels are promoted by auto-stimulation. Confirming this, exogenous IL-1beta increased IL-1beta mRNA levels $6.3 \pm 2.18$ fold $(\mathrm{p}<0.001)$ and IL1Ra prevented IL-1beta autostimulation. IL-1beta induced auto-stimulation was also observed in sorted human beta-cells. Further, auto-stimulation of IL-1beta was inhibited by the NF-kappaB inhibitor Bay11-07082 to $58.9 \pm 12.4 \%$ of the control level $(\mathrm{n}=4)$.

Conclusion: IL-1beta mRNA levels of cultured human islets are induced by auto-stimulation and reduced by IL1Ra treatment. $33.3 \mathrm{mM}$ glucose increases IL-1beta mRNA levels by promoting auto-stimulation and by decreasing IL1Ra expression. Different islet preparations display variable basal IL-1beta mRNA levels and high basal IL-1beta expression may blunt the effects of glucose-stimulated IL-1beta. In beta-cells of individuals with T2D there is enhanced IL-1beta mRNA expression. This suggests a role for IL-1beta in mediating glucotoxicity in islets of T2D. Supported by JDRF

\section{8}

Evidence of inflammatory markers in beta cells of type 2 diabetic subjects

L. Marselli ${ }^{1}$, D. C. Sgroi ${ }^{2}$, J. Thorne ${ }^{3}$, S. Dahiya ${ }^{2}$, S. Torri ${ }^{1}$, A. Omer ${ }^{3}$,

S. Del Prato ${ }^{1}$, L. Towia ${ }^{4}$, H. H. Otu ${ }^{4}$, A. Sharma ${ }^{3}$, S. Bonner-Weir ${ }^{3}$,

P. Marchetti ${ }^{1}$, G. C. Weir ${ }^{3}$;

${ }^{1}$ Endocrinology and Metabolism, University of Pisa, Pisa, Italy,

${ }^{2}$ Massachusetts General Hospital, Harvard University, Boston, United States, ${ }^{3}$ Joslin Diabetes Center, Harvard University, Boston, United States, ${ }^{4}$ Beth

Israel Deaconess Medical Center, Harvard University, Boston, United States.

Background and Aims: Type 2 diabetes (T2D) is characterized by beta cell dysfunction associated with reduced beta cell mass. Among various mechanisms that may be responsible of these alterations, inflammation may play a role. To assess this possibility, gene profiling of beta cell enriched samples obtained from T2D donors were evaluated.

Materials and Methods: The samples were obtained using laser capture microdissection (LCM) from frozen pancreatic tissue sections of 4 T2D (age: $67.3 \pm 5.0$ years; BMI: $28.3 \pm 1.9 \mathrm{~kg} / \mathrm{m} 2(\mathrm{n}=3)$; diabetes duration: $5.7 \pm 2.5$ years $(\mathrm{n}=3)$ ) and 4 non-diabetic (age: $63.5 \pm 3.1$ years; BMI: $31.6 \pm 7.0 \mathrm{~kg} / \mathrm{m} 2$ ) cadaver donors. Extracted RNA was amplified by T7 RNA polymerase and hybridized to Human X3P Arrays (Affymetrix). Data were normalized using the DNA-Chip Analyzer (dChip) software. The program employs standard error to identify differentially expressed genes and uses the lower confidence bound (LCB) to estimate the significance. LCB cutoff of 1.2 was used to assess differentially expressed genes.

Results: In T2D 1532 genes were upregulated and 528 genes were downregulated. Samples from T2D pancreases showed increased expression of C-C and C-X-C inflammatory chemokines and some chemokine receptors. Chemokine genes that were most differentially expressed included: monocyte chemoattractant protein 4 (MCP-4) (LCB 2.8) and GRO beta (LCB 2.3). Other chemokines upregulated in T2D were monocyte chemoattractant protein 1 (MCP-1) (LCB: 2.0), GRO alpha (LCB: 1.5), eotaxin (LCB: 1.5) and stromal cell-derived factor 1 (SDF) (LCB: 1.4). Among the chemokine receptors, chemokine C-X3-C receptor 1 (CX3CR1) (LCB: 3.4), chemokine 
binding protein 2 (CCBP2) (LCB: 1.5) and chemokine C-X-C motif receptor 7 (CXCR7) were upregulated.

Conclusion: These results suggest that an inflammatory process is activated in the islets of subjects with T2D and that chemokines may have a fundamental role in its activation.

\section{9}

Human oxidised low density lipoprotein-particles cause a loss of insulin secretion by inducing the transcriptional repressor ICER

D. Favre ${ }^{1}$, G. Niederhauser ${ }^{1}$, F. Allagnat ${ }^{1}$, J.-A. Haefliger ${ }^{1}$, R. Regazzi ${ }^{2}$, G. Waeber ${ }^{1}$, A. Abderrahmani ${ }^{1}$;

${ }^{1}$ Service of Internal Medicine, CHUV-Hospital, ${ }^{2}$ Department of Cellualr Biology and Morphology, University of Lausanne, Switzerland.

Background and Aims: Inducible cAMP early repressor (ICER) is a transcriptional repressor that is induced in pancreatic $\beta$-cells challenged with a diabetic milieu including hyperglycaemia or non esterified fatty acids (NEFA). The sustained induction of ICER leads to impairment of nutrientsinduced insulin secretion. Like hyperglycaemia and NEFA, several studies including ours have shown that the pro-atherogenic oxidised low density lipoprotein-cholesterol (oxLDL) exerts deleterious effects on $\beta$-cells by increasing the rate of apoptosis and diminishing insulin synthesis. In this study, we investigated whether oxLDL affects nutrient-induced insulin secretion by increasing expression levels of ICER.

Materials and Methods: Mouse insulin-secreting MIN6 cells and rat isolated islets were exposed to $2 \mathrm{mM}$ of human native LDL or oxLDL-cholesterol for $72 \mathrm{hrs}$. Gene expression and insulin levels were monitored by Real-time PCR (Bio-Rad) and ELISA (SPI-BIO), respectively. Luciferase activities were measured by using an assay system (dual-Luciferase; Promega).

Results: Prolonged exposure of MIN6 or islets cells to oxLDL impaired insulin secretion in response to glucose whereas insulin secretion was preserved in the presence of native LDL. Similar results were obtained in MIN6 cells that were transiently transfected with the plasmid encoding the human Growth Hormone (hGH), a reporter gene monitoring the secretory activity of transfected cells independently of their capacity to produce insulin. In addition, the cells were unable to secrete in response to $\mathrm{KCl}$, a secretagogue that stimulates exocytosis by triggering the calcium influx. This result suggested a defect in the activity of the secretory machinery as observed in cells chronically cultured with elevated concentration in glucose or NEFA. In the latter condition, the secretory defect was in part due to a rise in the levels of the transcriptional repressor ICER. Real-time PCR experiments performed with total RNAs extracted from MIN6 cells or rat isolated islets cultured with oxLDL showed an increase in ICER mRNA levels. Transient transfection of the luciferase reporter construct under the control of ICER activity confirmed the induction of ICER expression in MIN6 cells incubated with oxLDL. Consistent with an increase in ICER activity, expression of target genes including granuphilin, Rab3a and Rab 27a was diminished in cells exposed to oxLDL. Finally, silencing of ICER with the use of an ICER antisense-encoding plasmid abolished the ICER activity induced by oxidised lipoproteins.

Conclusion: These data show that oxLDL induces a loss of insulin secretion by altering expression of genes of the secretory machinery through induction of ICER.

Supported by a grant from the Swiss National Foundation

\section{0}

Green tea extract epigallocatechin gallate preserves pancreatic islet structure, restores insulin secretion and ameliorates diabetes in $d b / d b$ mice

Å. Sjöholm ${ }^{1}$, H. Ghanaat-pour ${ }^{1}$, N. Kuehn ${ }^{2}$, S. Wolfram ${ }^{3}$;

${ }^{1}$ Department of Internal Medicine, Karolinska Institutet, Stockholm, Sweden, ${ }^{2}$ Frimorfo, Inc., Fribourg, Switzerland, ${ }^{3}$ Department of Human Nutrition and Health, DSM Nutritional Products Ltd, Basel, Switzerland.

Background and Aims: Green tea was suggested as a therapeutic agent for the treatment of diabetes more than 70 years ago, but the mechanisms involved in its antidiabetic effect still remain elusive. In this work, we attempted to address this issue by feeding the most abundant green tea catechin epigallocatechin gallate (EGCG), in comparison with the thiazolidinedione PPAR- $\gamma$ agonist rosiglitazone as positive control, to spontaneously diabetic $d b / d b$ mice.

Materials and Methods: Young (5 weeks old) diabetic $d b / d b$ mice were randomized and assigned into the following groups: Control, EGCG, rosiglitazone. Compounds were administered via the diet for 10 weeks. Glucose and insulin levels were determined during an OGTT after 5 and 10 weeks of treatment. Pancreata were sampled at end of the study for blinded histomorphometric analysis.

Results: In moderately diabetic ( 5 wk treatment) $d b / d b$ mice, EGCG lowered glucose levels (Fig 1A) and improved insulin sensitivity

(Fig 1B).
Fig $1 \quad 1 A$

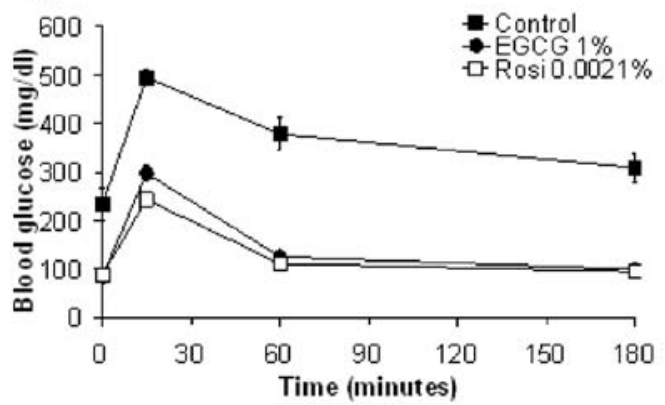

Fig 2 2A

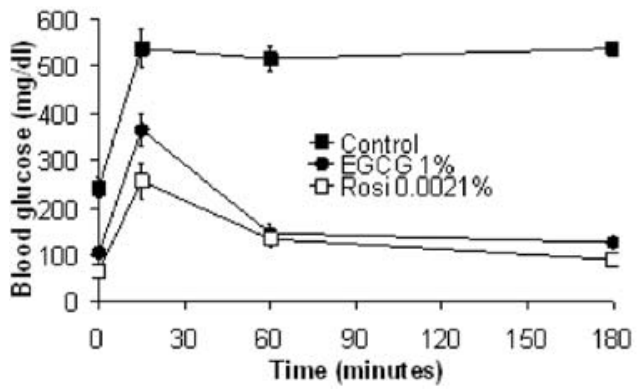

$1 B$

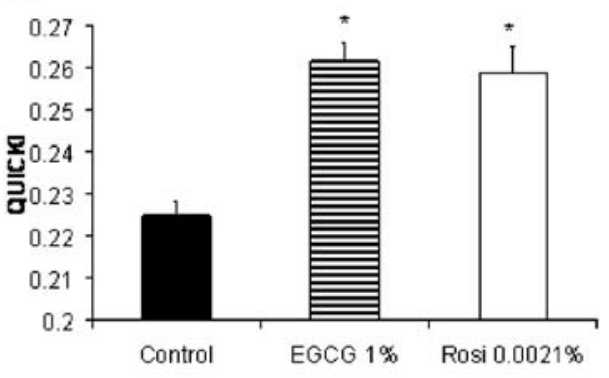

2B

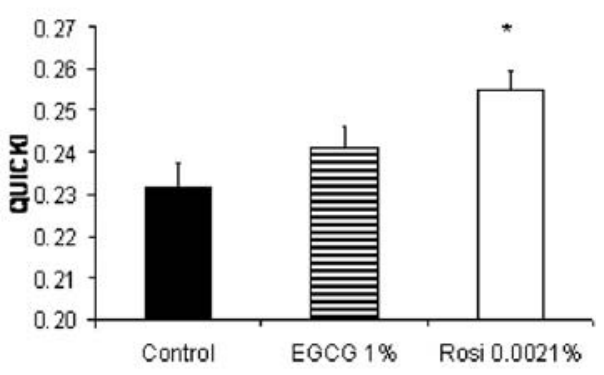


In severely diabetic (10 wk treatment) $d b / d b$ mice, EGCG enhanced glucose tolerance (Fig 2A) but did not any more enhance insulin sensitivity (Fig 2B).

EGCG supplementation reduced the number of pathologically changed islets of Langerhans (Fig 3B), increased the number (Fig 3A) and the size (Fig 3C) of islets, and improved pancreatic endocrine area (Fig 3).

Although EGCG was less potent than rosiglitazone, it exerted changes that were similarly beneficial. Thus, EGCG improved glucose tolerance and glucose-stimulated insulin secretion in $d b / d b$ mice and prevents pathological changes in islets linked to progression of type 2 diabetes.

Conclusion: This study shows that the green tea extract EGCG markedly preserves islet structure and enhances glucose tolerance in genetically diabetic mice. Dietary supplementation with EGCG could potentially contribute to nutritional strategies for the prevention and treatment of type 2 diabetes.

\section{PS 22 Beta cells - a choice of death}

\section{1}

Early mediators and downstream effectors of dsRNA-induced beta cell apoptosis: the role of IRF-3 and ER stress

J. Rasschaert ${ }^{1}$, Z. Dogusan ${ }^{1}$, M. García ${ }^{1}$, D. Flamez ${ }^{1}$, L. Alexopoulou ${ }^{2}$, M. Goldman ${ }^{3}$, C. Gysemans ${ }^{4}$, C. Mathieu ${ }^{4}$, C. Libert ${ }^{5}$, D. L. Eizirik ${ }^{1}$;

${ }^{1}$ Laboratory of Experimental Medicine, Université Libre de Bruxelles (ULB), Brussels, Belgium, ${ }^{2}$ Centre d'Immunologie de Marseille-Luminy, CNRS-INSERM-Université de la Méditerranée, Marseille, France, ${ }^{3}$ Insitute of Medical Immunology, Université Libre de Bruxelles (ULB), Charleroi, Belgium, ${ }^{4}$ LEGENDO, Katholieke Universiteit Leuven, Belgium, ${ }^{5}$ Department for Molecular Biomedical Research - VIB, Ghent University, Belgium.

Background and Aims: Viruses, or viral products such as double-stranded RNA (dsRNA), affect beta cell function and survival and trigger autoimmunity in type 1 diabetes by unknown mechanisms. During viral infection beta cells are exposed to both extra- and intracellular dsRNA. We have previously shown that the TLR3 pathway plays a crucial role in exogenous dsRNAinduced beta cell death, but the molecular mechanisms involved in internaldsRNA-induced beta cell apoptosis are TLR3-independent. We presently characterized the early mediators and downstream effectors of internaldsRNA-induced apoptosis.

Materials and Methods: FACS-purified rat beta cells and dispersed islet cells from wild type (wt) or IRF-3 $3^{-/-}$and type I IFN receptor ${ }^{-/}$(IFNAR1 ${ }^{--}$) mice were cultured for 5 days with or without transfected synthetic dsRNA (PICin; $10 \mu \mathrm{g} / \mathrm{ml}$ ). Viability was determined by the nuclear dyes HO 342 and propidium iodide, while production of IFN- $\beta$ was measured by ELISA. Gene expression was determined by real-time RT-PCR. Activation of NF- $\mathrm{kB}$ and eIF2- $\alpha$ was studied respectively by immunocytochemistry and Western blot using an antibody specific for the phosphorylated forms of STAT-1 and eIF2$\alpha$. Protein biosynthesis was determined using tritiated leucine incorporation and TCA precipitation.

Results: Exposure of dispersed islet cells from wt mice to internal PIC for 5 days increased 2 -fold apoptosis ( $50 \%$ vs $24 \%$ in control wt cells; $\mathrm{p}<0.005$; $\mathrm{n}=4$ ), while islet cells from IRF- $3^{-/-}$mice were completely resistant to PICininduced apoptosis ( $29 \%$ vs $28 \%$ in control IRF- $3^{-/-}$cells; $\left.n=4\right)$. PICin-induced $\mathrm{NF}-\kappa \mathrm{B}$ activation was unaffected in IRF- $3^{-/-}$islet cells when compared to wt cells. These data suggest a key role for IRF-3 in PICin-triggered beta cell apoptosis. IRF-3 signalling regulates type I IFNs production in other cell types, and we observed that PICin induced a 50-2000 fold increase in type I IFNs mRNA expression and a massive production of these cytokines in beta cells. Dispersed islet cells from IFNAR1 ${ }^{-/-}$mice were protected against PICin-
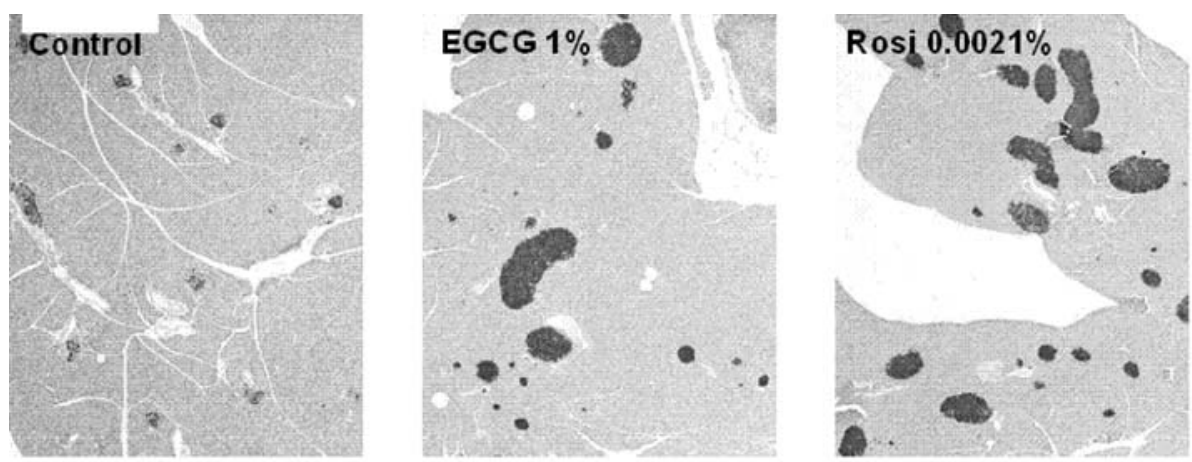

Fig 3
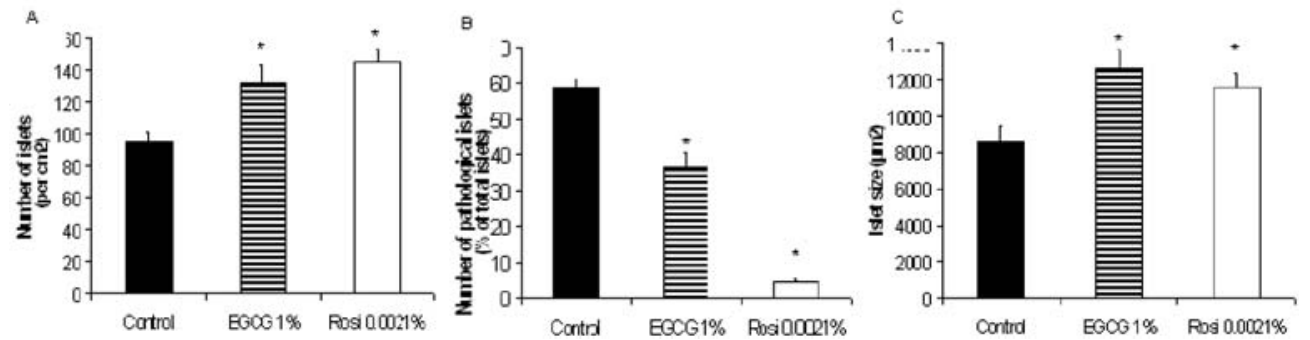
induced apoptosis, indicating that IFNs contribute to cell death in an autocrine manner. In purified beta cells PICin induced eIF- $2 \alpha$ phosphorylation and inhibited protein biosynthesis by $50 \%$, and it up-regulated expression of the ER stress markers ATF-4, CHOP and Bip, suggesting that activation of an ER stress response contributes to beta cell death.

Conclusions: a) The IRF-3 pathway plays a crucial role in internal dsRNAinduced beta cell apoptosis; b) Execution of PICin-mediated apoptosis depends on autocrine effects of type I IFNs and ER stress.

Supported by EFSD/Lilly European Diabetes Research Programme

\section{2}

17- $\beta$-Estradiol specifically induces apoptosis in cells expressing sulfonylurea receptor 1

S. Ackermann, S. Hiller, H. Osswald, A. Hambrock;

Department of Pharmacology and Toxicology, University of Tuebingen, Germany.

Background and Aims: Apoptosis of pancreatic $\beta$-cells is assumed to be involved in the pathophysiology of diabetes type 1 or 2 . Previously, we have shown that the insulinotropic sulfonylurea glibenclamide or the natural compound trans-resveratrol can induce enhanced $\beta$-cell apoptosis and that this effect is specifically linked to the expression of the sulfonylurea receptor isoform SUR1. SUR1 is the regulatory subunit of the pancreatic ATP-sensitive $\mathrm{K}^{+}$channel ( $\mathrm{K}_{\text {tp }}$ channel) which is essential for triggering insulin secretion in the $\beta$-cell. 17- $\beta$-Estradiol (E2) induces apoptosis in different experimental systems and is able to decrease $\mathrm{K}_{\text {ATP }}$ channel activity in $\beta$-cells. The aim of the present study was to investigate whether E2 stimulates $\beta$-cell apoptosis and whether this effect is mediated via SUR1.

Material and Methods: Cells from the $\beta$-cell lines HIT-T15 or RIN-m5F as well as HEK293 cells expressing different forms of SUR or sham-transfected HEK cells (ctr) were treated with E2, estrone (E1), which differs from E2 in lacking one hydroxyl group, or etoposide, a classical inducer of SURindependent apoptosis. In further experiments, cells were additionally supplemented with the phorbol ester TPA, an activator of protein kinase C. For determination of apoptotic parameters, cell detachment and viability were measured using a CASY Analyser, changes in nuclear morphology were assessed after Hoechst 33258 staining and caspase 3-like activity was quantified by cleavage of fluorescent DEVD-substrate.

Results: E2 treatment $(100 \mu \mathrm{M}, 24 \mathrm{~h})$ resulted in massive cell detachment and intensive nuclear condensation and fragmentation in HIT-T15-cells, RIN-m5F-cells or SUR1-expressing HEK cells, while HEK cells expressing SUR2A (cardiac isoform) or SUR2B (vascular isoform) as well as controlcells were much less affected. Differences between SUR1-cells and controlcells were already observed with lower E2 concentrations after longer treatment intervals (e.g. $>1 \mu \mathrm{M}, 4$ days). In the presence of E2 $(100 \mu \mathrm{M}, 24$ $\mathrm{h})$, the number of apoptotic nuclei in SUR1-cells was about 3-fold higher compared to SUR2A-, SUR2B- or control-cells (SUR1 vs. SUR2A/SUR2B/ ctr: $\mathrm{p}<0.001$; SUR2A/SUR2B vs. ctr: $\mathrm{p}>0.05$ ). E2-induced cell detachment and nuclear alterations were associated with a significant increase in caspase 3-like activity. The apoptotic potency of E2 was significantly higher than that of glibenclamide, resveratrol or etoposide. E1 induced apoptosis to a much smaller extent than E2. SUR1-specific apoptosis of E2-treated cells was abolished at the mutant SUR1(M1289T), in which a single amino acid in transmembrane helix 17 (TM17) was exchanged by the corresponding amino acid of SUR2B (SUR1(M1289T) vs. SUR1: p<0.001; SUR1(M1289T) vs. SUR2A/SUR2B/ctr: p>0.05). Co-treatment with TPA $(100 \mathrm{ng} / \mathrm{ml}, 24 \mathrm{~h})$ decreased E2-induced apoptosis in SUR1-cells about 1.5-fold (SUR1+E2 vs. SUR1+E2+TPA: $\mathrm{p}<0.01$ ). This effect of TPA was not observed in respective etoposide-treated cells.

Conclusions: Treatment with E2 specifically induces apoptosis in SUR1expressing cells (recombinant cells or clonal $\beta$-cells). Our results indicate that TM17 of SUR1 is important for E2-induced apoptosis and that protein kinase $\mathrm{C}$ may be involved in the respective signalling pathway. As E2 levels are elevated during pregnancy, E2-stimulated apoptosis in pancreatic $\beta$-cells might provide an interesting aspect concerning the etiology of gestational diabetes.

Supported by Deutsche Forschungsgemeinschaft

\section{3}

Endogenous IRS-2 expression and activation are responsible for $\beta$ cell survival during glucocorticoid excess

D. Avram ${ }^{1}$, F. Ranta ${ }^{1}$, S. Hopp ${ }^{1}$, A. M. Hennige ${ }^{1}$, F. Lang ${ }^{2}$, H.-U. Häring ${ }^{1}$, S. Ullrich ${ }^{1}$

${ }^{1}$ Internal Medicine IV, University of Tübingen, Germany,

${ }^{2}$ Institute of Physiology, University of Tübingen, Germany.

Background and Aims: IRS-2 is the main insulin receptor substrate responsible for cell differentiation and survival in insulin secreting cells. Previously we showed that stimulation of insulin secreting INS-1 cells with IGF-1 protects against glucocorticoid-induced cell death. IGF-1 increases IRS-2 tyrosine phosphorylation, an effect antagonized by the glucocorticoid dexamethasone (dex). The reduced IRS-2 phosphorylation is accompanied by reduced PKB phosphorylation and increased apoptosis. Surprisingly, IGF-1 protected against dex-induced cell death even in the presence of PI3 kinase inhibitors. The present study examined the underlying mechanism of protection against glucocorticoid toxicity.

Materials and Methods: INS- 1 cells were treated with $\operatorname{dex}(100 \mathrm{nM})$ and IGF$1(50 \mathrm{ng} / \mathrm{ml})$ under standard culture conditions. Apoptosis was quantified by DAPI and TUNEL staining. Proteins and protein phosphorylations were quantified by Western blotting, cellular mRNA concentrations by real time RT-PCR.

Results: During $4 \mathrm{~d}$ treatment with dex, IGF-1 inhibited cell death by $75 \%$ after $1 \mathrm{~d}$ and by $58 \%$ after $4 \mathrm{~d}$. In parallel, IGF-1 stimulated PKB phosphorylation in control as well as in dex-treated cells. In contrast, ERK phosphorylation induced by IGF-1 was inhibited by dex. When cells were simultaneously incubated with LY294002 $(10 \mu \mathrm{M})$, an inhibitor of PI3 kinase, IGF-1 induced phosphorylation of PKB was efficiently abolished while dex-mediated inhibition of ERK phosphorylation was counteracted. LY294002 treatment of the cells increased IRS-2 mRNA 2-fold in the absence and presence of IGF-1 and dex. IRS-2 protein was also increased in LY294002-treated cells. The IGF1 stimulated tyrosine phosphorylation of IRS-2 was detectable on Western blots only in cells treated with LY294002. When cell death was examined the MAP kinase inhibitor PD98059 $(10 \mu \mathrm{M})$ indeed counteracted the protective effect of IGF-1 against dex-induced cell death.

Conclusion: These data suggest that MAP kinase dependent protection against dex-induced cell death depends on sufficient amount of IRS- 2 and its stimulation.

\section{4}

Comparison of Quercetin and EGCG (epigallocatechin gallate) for the protection of hydrogen peroxide induced oxidative damage in INS-1 cell line

M. K. Kim ${ }^{1}$, H. Chung 2 , C. Yoon ${ }^{2}$, M. Kwon², S. Lee², J. Park²; ${ }^{1}$ Division of Endocrinology and Metabolism, Department of Internal Medicine, Maryknoll General Hospital, Busan,

${ }^{2}$ Division of Endocrinology and Metabolism, Department of Internal Medicine, Pusan Paik Hospital, Paik Memorial Institute for Clinical Research, Inje University, Busan, Republic of Korea.

Background and Aims: Chronic oxidative stress is not only involved in the pathogenesis of diabetic complications, but also play very important role in the development and the progression of type 2 diabetes mellitus via the deleterious effects on the pancreatic $\beta$-cells. Insulin resistance and the relative pancreatic $\beta$-cell dysfunction are the two main pathophysiologies of type 2 diabetes mellitus. The insulin resistance could be regarded as the prerequisite, while the $\beta$-cell dysfunction might be the key pathophysiology that determines the onset and the progression of type 2 diabetes mellitus. Effective protection of pancreatic $\beta$-cells from the oxidative stress induced damage in pre-diabetics and type 2 diabetic patients could enable to stop or even reverse the progression of worsening metabolic derangement. Quercetin and EGCG are natural anti-oxidants contained in the plants. Quercetin is very plentiful in grape, and EGCG is the main ingredient of green tea, with proven strong anti-oxidant properties. We wanted to confirm whether these 2 natural substances could protect the oxidative stress induced damage to INS1 cell line, and verify the detailed mechanisms of protection.

Materials and Methods: We cultured INS-1 cell line in RPMI medium. Hydrogen peroxide was used as an oxidative stressor. Quercetin or EGCG were added to the culture medium. Various kinds of experimental designs 
including the time sequences and the concentrations of each substance were employed to verify the possible differences between these 2 substances. The viabilities of the cells were verified by MTT assay. The activity of glutathione peroxidase was assessed by total glutathione quantification kit. Western blot and semi-quantitative RT-PCR for the catalase and SOD(superoxide dismutase) were performed. The phophorylation status of IRS2 and PI3K was verified also by Western blot and RT-PCR. To confirm the relative contributions of each protecting pathway, we performed knock out experiment using siRNAs. Functional status of INS-1 cell line was tested by GSIS(glucose stimulated insulin secretion).

Results: Both quercetin and EGCG effectively protected hydrogen peroxide induced damage in INS-1 cells verified by MTT assay. The protection by EGCG required pre-incubation of INS- 1 cells for at least 24 hours prior to the addition of hydrogen peroxide into the medium, while the protection exerted by quercetin did not. The expression and the activity of SOD and the glutathione peroxidase were significantly increased in quercetin group. The degrees of phosphorylation of IRS2 and PI3K were significantly enhanced in EGCG group. GSIS was well preserved in both groups.

Conclusion: In conclusion, both quercetin and EGCG effectively protected INS-1 cells from the oxidative stress induced by hydrogen peroxide. Quercetin exerted its effect mainly via the activation of anti-oxidant defense, while EGCG via anti-apoptotic pathway rather than anti-oxidant defense system. Further studies will be necessary for the more detailed action mechanisms and the possible clinical implications.

Supported by Korean Diabetes Association

\section{5}

Differential use of calcium stores in reactive oxygen species-dependent pancreatic beta cell death

D. Watson ${ }^{1}$, E. Kinsey ${ }^{1}$, S. Hazelwood ${ }^{1}$, J. Scarpello ${ }^{2}$, A. Loweth ${ }^{1}$;

${ }^{1}$ School of Life Sciences, Keele University, ${ }^{2}$ Dept of Diabetes \&

Endocrinology, University Hospital of North Staffs, United Kingdom.

Background and Aims: Beta cells' susceptibility to oxidant stress contributes to chronic glucose toxicity and beta cell loss in diabetes mellitus. We are currently investigating the mechanism(s) of this oxidant-induced cytotoxicity, particularly how reactive oxidant species (ROS) modulate calcium movements, since intracellular calcium increases promote cell death. The aim of this study was therefore to investigate the functional role of oxidant-induced mitochondrial calcium uptake and intracellular calcium movements in response to both hydrogen peroxide $\left(\mathrm{H}_{2} \mathrm{O}_{2}\right)$ and superoxide, the latter generated by the hypoxanthine/xanthine oxidase (HX/XO) system, in the rat pancreatic beta cell line, RINm5F.

Materials and Methods: Cell viability measurements, in response to $40 \mu \mathrm{M}$ $\mathrm{H}_{2} \mathrm{O}_{2}$ or $4 \mathrm{mM} / 0.2 \mathrm{mU} \mathrm{HX} / \mathrm{XO}$ for 1 hour, were performed using the MTTformazan reduction assay (results expressed as mean absorbance \pm SEM) and counting of detached, dead cell populations by Trypan blue staining and

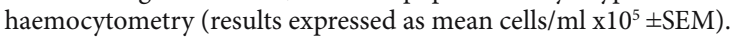

Results: The dependency of oxidant-induced cell death on intracellular calcium was investigated using the intracellular calcium chelator BAPTA$\mathrm{AM}$, which completely attenuated cell death in response to both ROS (dead cell numbers: control $1.20 \pm 0.2, \mathrm{H}_{2} \mathrm{O}_{2}^{*} 4.30 \pm 0.6, \mathrm{H}_{2} \mathrm{O}_{2}+\mathrm{BAPTA}^{*} 1.05$ $\pm 0.1, \mathrm{HXXO}^{*} 5.30 \pm 0.4, \mathrm{HXXO}+\mathrm{BAPTA}^{*} 1.30 \pm 0.2 ; \mathrm{p}<0.001$ vs. ${ }^{*}$ control or "treatment alone). Blockade of mitochondrial calcium uptake by the mitochondrial calcium uniporter inhibitor, ruthenium red (RR) attenuated $\mathrm{H}_{2} \mathrm{O}_{2}$ 's effect by $34 \%$ (MTT results: control $0.862 \pm 0.02$, RR $0.801 \pm 0.02$, $\mathrm{H}_{2} \mathrm{O}_{2}{ }^{*} 0.096 \pm 0.01, \mathrm{H}_{2} \mathrm{O}_{2}+\mathrm{RR}^{*} 0.354 \pm 0.03 ; \mathrm{p}<0.001{ }^{*} \mathrm{v}$. controls; ${ }^{*} \mathrm{vs}$. controls and $\mathrm{H}_{2} \mathrm{O}_{2}$ ), suggesting that mitochondrial calcium uptake is a necessary step in $\mathrm{H}_{2} \mathrm{O}_{2}$-induced cell death. By contrast, superoxide's cytotoxicity was found not to be dependent on mitochondrial calcium uptake and, indeed, inhibition of uptake with RR exaggerated the effect of superoxide (MTT: control 0.996 $\pm 0.06, \mathrm{RR} 0.909 \pm 0.03, \mathrm{HXXO}^{*} 0.698 \pm 0.04, \mathrm{HXXO}+\mathrm{RR}^{*} 0.464 \pm 0.02 ; \mathrm{p}<0.001$ ${ }^{*}$ vs. controls; "vs. controls and HXXO). We then investigated the effect of extracellular calcium influx on superoxide's effect using the extracellular calcium chelator, EDTA and the L-type calcium channel blocker, nifedipine. Both agents completely attenuated superoxide's effect although no effect was seen on $\mathrm{H}_{2} \mathrm{O}$-induced cell death (dead cell numbers: control $1.95 \pm 0.2$, EDTA $1.30 \pm 0.2$, nifedipine $2.10 \pm 0.1, \mathrm{HXXO}^{*} 5.30 \pm 0.2, \mathrm{HXXO}+\mathrm{EDTA}^{*}$ $1.60 \pm 0.3, \mathrm{HXXO}+$ nifedipine $^{*} 1.40 \pm 0.05, \mathrm{H}_{2} \mathrm{O}^{*} 4.10 \pm 0.3, \mathrm{H} 2 \mathrm{O} 2+\mathrm{EDTA}$ $4.40 \pm 0.1, \mathrm{H}_{2} \mathrm{O}_{2}+$ nifedipine $4.00 \pm 0.2, \mathrm{p}<0.001$ vs. ${ }^{*}$ controls, ${ }^{\#} \mathrm{HXXO}$ alone).
Conclusion: Our results show that an increase in intracellular calcium levels is necessary for superoxide- and $\mathrm{H}_{2} \mathrm{O}_{2}$-induced RINm5F cell death, although each species appears to have differential effects on calcium fluxes within the cell, since only $\mathrm{H}_{2} \mathrm{O}_{2}$-induced cell death was dependent on mitochondrial calcium uptake and superoxide depended only on extracellular calcium influx. It is tempting to speculate that $\mathrm{H}_{2} \mathrm{O}_{2}$ facilitates store-operated calcium release, generating 'hot-spot' calcium regions allowing uptake through the low affinity mitochondrial uniporter, whereas superoxide participates predominately in calcium influx from the extracellular medium, limiting the potential for mitochondrial uptake.

Supported by North Staffordshire Medical Institute Gallimore Research Award

\section{6}

\section{Oxidative stress is memorized by INS-1E beta cells resulting in mitochondrial dysfunction and impaired glucose stimulated insulin secretion}

N. Li, P. Maechler;

Department of Cell Physiology and Metabolism, University of Geneva, Switzerland.

Background and Aims: Mitochondria of pancreatic beta-cells serve as fuel sensors to integrate and generate metabolic signals, thereby coupling glucose recognition to insulin secretion. It has been proposed that mitochondrial defects, such as mutations caused by reactive oxygen species (ROS), might be associated with beta-cell failure in the course of diabetes. We have previously shown (J. Biol. Chem. 1999, 274:27905-27913) that a transient exposure of pancreatic beta-cells to oxidative stress interrupts the transduction of signals normally coupling glucose metabolism to insulin secretion. The aim of this study was to test the hypothesis if such a transient oxidative stress in insulin secreting cells could cause long-term impairment of mitochondrial function that normally couples glucose metabolism to insulin secretion.

Materials and Methods: Using INS-1E beta-cells, we studied the long-term impact of a single transient oxidative stress of $10 \mathrm{~min}$ exposure to $200 \mu \mathrm{M}$ $\mathrm{H} 2 \mathrm{O} 2$ applied 3 days before analyses. After this recovery period, we tested key parameters involved in metabolism secretion coupling in beta-cells, such as mitochondrial membrane potential (determined by rhodamine123 fluorescence), cytosolic ATP production (monitored through luciferase bioluminescence), oxygen consumption (performed on isolated mitochondria in an oxymeter), and glucose-stimulated insulin secretion (analysed by radioimmunoassay). In addition, western blot and real-time RT-PCR analyses were performed to detect possible underlying molecular mechanisms.

Results: Three days after one single transient oxidative stress, INS-1E betacells exhibited markedly reduced mitochondrial function and responses to glucose. Compared to non-stressed INS-1E beta-cells, we observed impaired mitochondrial membrane potential $(-55 \%$ upon glucose stimulation), reduced cytosolic ATP production ( $-59 \%$ upon glucose stimulation), decreased oxygen consumption levels $(-15 \%$ in response to succinate and $-60 \%$ in response to the combination of succinate and ADP, $\mathrm{P}<0.01$ in both cases), and blunted glucose-stimulated insulin secretion $(-51 \%, \mathrm{P}<0.01)$. These parameters correlated with down-regulation of subunits complex I $(-28 \%)$ and complex IV $(-23 \%)$ of the respiratory chain and a decrease in expression of genes responsible for mitochondrial biogenesis, such as TFAM $(-74 \%)$ and PGC-1alpha (-84\%).

Conclusion: INS-1E beta-cells could memorize one single transient oxidative stress as revealed by dysfunction of mitochondrial metabolism and glucosestimulated insulin secretion. The mechanisms underlying these long-term effects could be due to the alterations of enzyme subunits of the respiratory chain at the protein level, although not primarily at the mRNA level. Supported by FN

\section{7}

Involvement of glucokinase in glucose toxicity in insulin-producing beta cells

H. Schmitt, S. Lenzen, S. Baltrusch;

Institut of Clinical Biochemistry, Hannover Medical School, Germany.

Background and Aims: Metabolic stimulus-secretion coupling in pancreatic beta cells is dependent upon the glucose phosphorylating enzyme glucokinase 
(GK). Activation of GK is endogenously provoked by interaction with the bifunctional enzyme 6-phosphofructo-2-kinase/fructose-2,6-bisphosphatase (PFK2) and can be effectively achieved also by a new class of chemical compounds. GK activation as well as GK overexpression resulted in an increase in glucose-induced insulin secretion. Controversially, it was shown that GK overexpression causes a breakdown of glucose metabolism accompanied by glucose toxicity. The aim of this study was to elucidate whether GK mediates glucose toxicity in insulin-producing cells.

Materials and Methods: The recently developed RheoSwitch Mammalian Inducible Expression System, based on a synthetic inducer, exhibits in contrast to other available systems no cross talk with endogenous transcription factors. This system was adapted to generate stable insulin-producing RINm5F and MIN6 cells. Positive clones with precise regulation of GK or an EYFP-GK fusion construct were selected through resistance against G418 and verified by reporter gene assays. Cells were cultured at different glucose concentrations, GK enzyme activity was measured by a photometric assay, cell viability by MTT assay, mitochondrial membrane potential using JC1 and insulin secretion by a radioimmunoassay.

Results: Insulin-producing RINm5F cells with inducible GK or EYFP-GK overexpression were cultured for $48 \mathrm{~h}$ without or in the presence of $0,0.5$, $62.5,125,250$ and $500 \mathrm{nmol} / \mathrm{l}$ of the inductor RSL 1 in medium supplemented with 1,10 or $30 \mathrm{mmol} / \mathrm{l}$ glucose. Independent from the glucose concentration RINm5F-R-GK as well as RINm5F-R-EYFP-GK cells showed an increase in GK activity following the rising inductor concentration. Comparable results were also obtained for MIN6-R-EYFP-GK cells. Furthermore the 3 and 5 fold increase of GK enzyme activity in RINm5F-R-GK and RINm5F-R-EYFPGK cells after maximal induction correlates with the increase in GK protein determined by Western blot and fluorescence microscopy, respectively. The viability of RINm5F-R-GK and RINm5F-R-EYFP-GK cells after incubation at 1,10 or $30 \mathrm{mmol} / \mathrm{l}$ glucose was not impaired by switching on GK expression through treatment with the inductor RSL 1. Independently from the GK expression a non significant decrease in cell viability after $48 \mathrm{~h}$ exposure to high glucose ( $30 \mathrm{mmol} / \mathrm{l})$ was slightly obvious. RINm5F-R-GK and RINm5FR-EYFP-GK cells showed after maximal induction of GK expression an increase in insulin secretion by $80 \%$. Interestingly, insulin secretion decreased in the absence of GK expression in RINm5F-R-GK and RINm5F-R-EYFP-GK cells after $48 \mathrm{~h}$ exposure to high glucose $(30 \mathrm{mmol} / \mathrm{l})$ but not in the presence of GK. To further analyze the intermediary metabolism the mitochondrial membrane potential was measured in RINm5F-R-GK cells using JC1. Thereby a higher mitochondrial membrane potential was observed in the presence of GK after $48 \mathrm{~h}$ exposure to high glucose $(30 \mathrm{mmol} / \mathrm{l})$.

Conclusion: Our experiments based on a precisely regulated GK expression in insulin-producing cells do not support the hypothesis of GK as a precursor of cellular glucose toxicity. In contrast a beneficial effect on glucose-induced insulin secretion after GK expression was observed. Thus, toxic effects of chronically high glucose are likely not to be mediated by the metabolism of glucose.

\section{PS 23 Beta cell dysfunction and death following different insults}

\section{8}

Effects of controlled c-MYC activation on mouse pancreatic $\beta$ cell survival and function

S. M. A. Pascal ${ }^{1}$, S. Pelengaris ${ }^{2}$, M. Khan ${ }^{2}$, J.-C. Jonas ${ }^{1}$;

${ }^{1}$ Endocrinology and Metabolism, University of Louvain, Brussels, Belgium, ${ }^{2}$ Department of Biological Sciences, University of Warwick, United Kingdom.

Background and Aims: Increased expression of the transcription factor c-MYC may contribute to the alterations of $\beta$-cell differentiation, survival and function induced by high glucose concentrations. In support of this hypothesis, c-MYC overexpression in $\beta$-cells induces apoptosis more than proliferation and decreases preproinsulin gene expression, thereby reducing glucose-induced insulin secretion. In this study, we compared the effects of c-MYC activation and high glucose concentrations on key glucose stimulussecretion coupling events in mouse pancreatic islets.

Materials and Methods: pIns-c-MycER ${ }^{\mathrm{TAM}}$ mice express, under the control of the insulin promoter, c-MYC fused to the hormone binding domain of 4-OHtamoxifen (TAM)-responsive mutant estrogen receptor. Islets were isolated from heterozygous pIns-c-MycER ${ }^{\mathrm{TAM}+/-}\left(\mathrm{Myc}^{+}\right)$mice and their wild-type littermates (WT) (mixed C57BL/6J x CBA background). They were then cultured for 1wk in RPMI medium containing $5 \mathrm{~g} / \mathrm{l} \mathrm{BSA}$ and $10-30 \mathrm{mmol} / \mathrm{l}$ glucose (G10-G30), with eventual addition of $100 \mathrm{nmol} / \mathrm{l} \mathrm{TAM}$ or DMSO 1/1000 for the last 2-3 days. After culture, glucose-induced changes in mitochondrial membrane potential (rhodamine 123 fluorescence), cytosolic $\mathrm{Ca}^{2+}$ concentration $\left(\left[\mathrm{Ca}^{2+}\right]_{\mathrm{c}}\right)$ (Fura-PE3 spectrofluorimetry) and insulin secretion (RIA) were measured in perifused islets. Islet caspase activation was measured using FAM-VADfluoromethylketone. Results are means \pm SEM for at least 7 islets from 3 experiments. Statistical significance of differences between groups was assessed by unpaired Student's t-test or 2-way ANOVA and a test of Bonferroni.

Results: After culture in G10, acute stimulation of WT islets with increasing glucose concentrations (from 0.5 to 7,15 and $30 \mathrm{mmol} / \mathrm{l}$ ) led to a stepwise decrease in rhodamine 123 fluorescence (mitochondrial membrane hyperpolarisation) and a concentration-dependent rise in $\left[\mathrm{Ca}^{2+}\right]$ and insulin secretion. These glucose effects were unaffected by TAM treatment for 3 days. Compared with WT islets, the glucose-induced changes in mitochondrial membrane potential, $\left[\mathrm{Ca}^{2+}\right]_{\mathrm{c}}$ and insulin secretion were qualitatively similar in $\mathrm{Myc}^{+}$islets cultured in G10, but the maximal rate of glucose-stimulated insulin secretion was severely reduced $(1.0 \pm 0.3$ vs $6.9 \pm 1.5 \mathrm{pg} / \mathrm{min}$ per ng of islet DNA in WT, $P<0.01$ ). These islets also displayed a $\sim 1.6$ fold increase in caspase activation $(P<0.05)$ and a $\sim 60 \%$ reduction in insulin/DNA content ratio $(1.1 \pm 0.3$ vs $2.7 \pm 0.3 \mathrm{ng}$ insulin/ng DNA in WT, $P<0.01)$. TAM treatment of $\mathrm{Myc}^{+}$islets for 2-3 days further increased caspase activation $~ 3.5$ fold $(P<0.001)$, reduced their insulin/DNA content ratio by $\sim 55 \%(P=0.06)$, reduced rhodamine 123 loading, increased resting $\left[\mathrm{Ca}^{2+}\right]_{c}$ by $44 \pm 7 \mathrm{nmol} /$ $1(P<0.001)$, and reduced the maximal glucose-induced rise in $\left[\mathrm{Ca}^{2+}\right]_{c}$ (by $\sim 40 \%$ ) and in insulin secretion. Similar $\left[\mathrm{Ca}^{2+}\right]_{c}$ alterations were observed after overnight culture in the presence of $50 \mu \mathrm{mol} / \mathrm{l} \mathrm{H}_{2} \mathrm{O}_{2}$. In contrast, in both $\mathrm{Myc}^{+}$ and WT islets, 1wk culture in G30 instead of G10 induced a small increase in islet resting $\left[\mathrm{Ca}^{2+}\right]_{\mathrm{c}}$ and a shift to the left of the glucose-response curve for changes in mitochondrial membrane potential, $\left[\mathrm{Ca}^{2+}\right]_{\mathrm{c}}$ and insulin secretion without changes in maximal glucose effectiveness.

Conclusion: c-MYC activation triggers $\beta$-cell apoptosis and alterations of glucose stimulus-secretion coupling events that are better mimicked by $\mathrm{H}_{2} \mathrm{O}_{2}$ than high glucose concentrations.

Supported by the FRS-FNRS, Brussels, Belgium

\section{9}

Regulation of calcineurin (phosphatase 2B) activity and its role in apoptotic cell death in insulin secreting INS-1 cells

F. Ranta ${ }^{1}$, D. Avram ${ }^{1}$, F. Lang ${ }^{2}$, H.-U. Häring ${ }^{1}$, S. Ullrich ${ }^{1}$;

${ }^{1}$ Internal Medicine IV,

${ }^{2}$ Institute of Physiology, University of Tübingen, Germany.

Background and Aim: Calcineurin (PP2B), a $\mathrm{Ca}^{2+} /$ calmodulin-dependent serine and threonine phosphatase, regulates cellular functions as well as cell 
growth and survival. In insulin secreting cells PP2B has been found to increase apoptotic cell death by dephosphorylation of $\mathrm{BAD}$, a pro-apoptotic protein. Previously we described that dexamethasone (dex)-induced apoptosis is partially inhibited by calcineurin inhibitors FK506 and deltamethrin in insulin secreting INS-1 cells. The aim of the present study was to examine whether glucocorticoid treatment activates PP2B. Possible mechanisms of activation are the cleavage of PP2B by calpain that renders the enzyme activity independent of $\mathrm{Ca}^{2+}$ or stimulation of PP2B by HSP90 released from the activated glucocorticoid receptor.

Materials and Methods: INS- 1 cells were treated with $\operatorname{dex}(100 \mathrm{nM})$ for $1 \mathrm{~d}$ in standard culture conditions. Apoptosis was quantified by DAPI staining and TUNEL assay. Calcineurin activity was measured in cell homogenates either using pNPP (para-nitrophenylphosphate) or ${ }^{32} \mathrm{P}$-RII peptide as substrates. Calcineurin and HSP90 were quantified by Western blotting. Protein-protein interactions were examined by co-immunoprecipitation.

Results: FK506- and cyclosporine A-sensitive phosphatase activity was increased in cells treated with dex when measured with pNPP as substrate. Using RII peptide as substrate PP2B activity was significantly higher in homogenates of dex-treated cells at low, submaximal $\mathrm{Ca}^{2+}$ concentrations but not at high, $10 \mu \mathrm{M} \mathrm{Ca}^{2+}$ when the enzyme was maximally activated. In control and in dex-treated cells the uncleaved $59 \mathrm{kDa}$ protein but not a calpain-cleaved smaller protein band of $48 \mathrm{kDa}$ was detected by Western blotting. The calpain inhibitor ALLN $(1$ and $10 \mu \mathrm{M})$ did not protect against dex-induced apoptosis. Therefore, we examined whether the release of HSP90 from the stimulated GR might interfere with PP2B and stimulate the enzyme. In immunoprecipitates of INS-1 cell homogenates with calcineurin antibodies, calcineurin and HSP90 were detected indicating an interaction of these proteins.

Conclusion: These data suggest that dex-induced apoptosis in INS-1 cells may involve PP2B activation by HSP90 released from the stimulated GR complex.

\section{0}

The time course and mechanism of hypoxia-induced islet dysfunction K. Tomotsune ${ }^{1}$, Y. Ogawa ${ }^{1}$, M. Naraoka ${ }^{1}$, T. Kudo ${ }^{1}$, N. Hasegawa ${ }^{2}$, Y. Tando', J. Matsui ${ }^{1}$, N. Tamasawa ${ }^{1}$, T. Suda ${ }^{1}$;

${ }^{1}$ Department of Endocrinology, Metabolism and Infectious Disease, Hirosaki University, ${ }^{2}$ Department of Internal Medicine, Itayanagi Central Hospital, Aomori, Japan.

Background and Aims: In this study, we aimed to reveal the time course of deterioration of insulin (INS) secretion from the islets cultured in hypoxia $\left(1 \% \mathrm{O}_{2}\right)$, and whether this dysfunction was reversible or not. Furthermore, we investigated if this dysfunction was related with mitochondrial (M) activity or Beta-cell death.

Materials and Methods: The pancreatic islets isolated from SD rats were cultured in $1 \% \mathrm{O}_{2}$ for $0,6,12,24$, and 48 hours (h). Then, glucose stimulated INS secretion (GSIS) was examined by either static or perfusion method. As to static GSIS, islets were incubated with $50 \mathrm{mg} / \mathrm{dl}$ and $300 \mathrm{mg} / \mathrm{dl}$ glucose (glu50 and glu300) for 30 minutes (min) respectively. Then INS secretion ratio (ISR) calculated as the ratio of INS concentration in glu300 to glu50 was compared. As to perfusion, the experiments started with $10 \mathrm{~min}$ of perfusion with glu 50 , followed by $15 \mathrm{~min}$ perfusion with glu 300 , and finally $10 \mathrm{~min}$ with glu50, and the area under the curve (AUC) of 1st-phase GSIS and 2nd-phase GSIS was compared. Furthermore, the islets incubated in $1 \% \mathrm{O}_{2}$ for $6 \mathrm{~h}, 12 \mathrm{~h}$ and $24 \mathrm{~h}$ were cultured in normoxia for another $24 \mathrm{~h}$ to test the reversibility by re-oxygenation and AUC was assessed. To reveal the mechanism of islet dysfunction, we tried to evaluate $\mathrm{M}$ activity and islet cell death. $\mathrm{M}$ activity of the islets exposed to $1 \% \mathrm{O}_{2}$ was assessed by the alamarBlue assay. The islet cell death was assessed by apoptosis enrichment factor (ApEF) and necrosis enrichment factor ( $\mathrm{NcEF}$ ) determined by sandwich-enzyme immunoassay using antibody binding to DNA and histones.

Results: In static method, ISR significantly declined after $24 \mathrm{~h}$ in $1 \% \mathrm{O}_{2}(0 \mathrm{~h}$ : $3.11 \pm 3.20$, 6 h: $1.89 \pm 0.96$, $12 \mathrm{~h}: 1.82 \pm 1.20$, 24h: $0.94 \pm 0.45$, 48h: $0.94 \pm 0.32$ ). In perfusion method, AUC of 1st-phase GSIS significantly declined after $6 \mathrm{~h}$ $1 \% \mathrm{O}_{2}$ (0h: $12.67 \pm 4.07,6 \mathrm{~h}: 8.97 \pm 2.02,12 \mathrm{~h}: 6.28 \pm 1.47,24 \mathrm{~h}: 5.73 \pm 0.61,48 \mathrm{~h}$ : $3.96 \pm 0.55$ ), and AUC of 2nd-phase GSIS significantly declined after $12 \mathrm{~h}$ $1 \% \mathrm{O}_{2}$ (0h: $23.47 \pm 7.83$, 6h: $17.84 \pm 9.73,12 \mathrm{~h}: 11.60 \pm 3.02$, 24h: $10.77 \pm 2.15$, 48h: $7.67 \pm 1.74)$. In the re-oxygenation study, the following normoxia made deteriorated GSIS worse in $6 \mathrm{~h}$ and $12 \mathrm{~h}$ (1st-phase, $6 \mathrm{~h}: 4.93 \pm 0.91,12 \mathrm{~h}$ : $4.14 \pm 0.33$, 24h: $5.06 \pm 0.69$ / 2nd-phase, $6 \mathrm{~h}: 10.17 \pm 2.50,12 \mathrm{~h}: 8.64 \pm 1.07,24 \mathrm{~h}$ :
$10.50 \pm 1.80)$. In the alamarBlue assay, alamarBlue absorbance significantly declined after $48 \mathrm{~h} 1 \% \mathrm{O}_{2}(0 \mathrm{~h}: 95.52 \pm 12.33$, 6h: $99.71 \pm 11.26,12 \mathrm{~h}: 84.58 \pm 12.52$, 24h: $82.78 \pm 7.81,48 \mathrm{~h}: 74.06 \pm 10.86)$. In the assay of cell death, there was no significant difference between normoxia (ApEF, 6h:4.89 $\pm 1.95,12 \mathrm{~h}: 5.84 \pm 2.40$, 24h:6.85 $\pm 1.08, \quad 48 \mathrm{~h}: 5.28 \pm 0.56 \quad / \quad \mathrm{NcEF}, \quad 6 \mathrm{~h}: 3.75 \pm 1.10, \quad 12 \mathrm{~h}: 5.75 \pm 2.03$, 24h:11.21 $\pm 0.57,48 \mathrm{~h}: 11.24 \pm 0.65$ ) and $1 \% \mathrm{O}_{2}$ (ApEF, 6h:4.74 $\pm 2.17,12 \mathrm{~h}: 6.72$ $\pm 3.11,24 \mathrm{~h}: 8.83 \pm 4.45,48 \mathrm{~h}: 5.38 \pm 1.37$ / NcEF, 6h:3.90 $\pm 1.94,12 \mathrm{~h}: 5.92 \pm 1.64$, $24 \mathrm{~h}: 11.56 \pm 2.91,48 \mathrm{~h}: 11.24 \pm 0.65)$. Nevertheless, in the assessment of time course, NcEF but not ApEF was significantly increased after 24h cultured in either normoxia or $1 \% \mathrm{O}_{2}$.

Conclusion: In this study, the impairment of INS secretion was seen after $6 \mathrm{~h}$ exposure to $1 \% \mathrm{O}_{2}$. The declined INS secretion after $24 \mathrm{~h} 1 \% \mathrm{O}_{2}$ seen in static study could be explained by the loss of islet cells due to their necrosis, on the other hand, the diminution of 1st-phase INS exocytosis after $6 \mathrm{~h}$ culture in hypoxia seen in perfusion study might be caused due to independent mechanism from $M$ activity or islet cell death, because they did not decline at $6 \mathrm{~h}$.

\section{1}

\author{
Mitochondrial uncoupling protein UCP2 influences type 1 diabetes \\ development \\ Y. Emre ${ }^{1}$, C. Hurtaud ${ }^{1}$, M. Karaca ${ }^{2}$, T. Nubel ${ }^{1}$, F. Zavala ${ }^{3}$, D. Ricquier ${ }^{1}$; \\ ${ }^{1}$ Faculte de Medecine Paris 5 - Necker, CNRS UPR 9078, Paris, \\ ${ }^{2}$ Universite Paris 7, CNRS UMR 7059, Paris, \\ ${ }^{3}$ Universite Paris 5, Institut Necker, CNRS UMR 8147, Paris, France.
}

Background and Aims: Uncoupling protein UCP2 is a mitochondrial protein that controls mitochondrial production of reactive oxygen species (ROS). Besides the cells of the immune system, pancreatic beta-cells also exhibit a strong UCP2 expression. In beta-cells, UCP2 is reported to function as a regulator of insulin secretion. In macrophages, UCP2 controls MAPK activation by modulation of mitochondrial ROS. Lipopolysaccharide (LPS)stimulated Ucp2-KO macrophages exhibit quicker and stronger p38 and ERK activation compared to Ucp2-WT macrophages. Therefore, Ucp2-KO macrophages have increased nitric oxide $(\mathrm{NO})$ and cytokine productions. In this work, we investigated whether UCP2 is implicated in type 1 diabetes.

Materials and Methods: Ucp2-KO and Ucp2-WT male mice were injected with multiple low dose streptozotocin (MLDS). MLDS is an immunemediated murine diabetes model in which hyperglycemia and diabetes are achieved after five daily injections of subdiabetogenic doses of STZ.

Results: Occurrence of diabetes was accelerated in Ucp2-KO mice. Analysis of macrophages from MLDS-treated mice showed that Ucp2-KO macrophages had increased NO and IL-1beta productions compared to Ucp2-WT macrophages. In the same time, mononuclear infiltration of islets was stronger in Ucp2-KO mice. Interestingly, comparison of Ucp2+/+ and Ucp2-/- islets with the same minor degree of lymphocyte infiltration showed an increased number of macrophages in Ucp2-KO islets and was associated with increased NO damage inside Ucp2-/- islets.

Conclusion: We provide relevant data of the implication of UCP2 in MLDSinduced diabetes. It is suggested that increased activity of Ucp2-KO immune system, in terms of cytokine and NO production, leads to an increased inflammation and accelerates the occurrence of type 1 diabetes in Ucp2-KO mice.

\section{2}

Vitamin E homologs improve bioenergetics of porcine islets and INS- 1 cells under anoxia

G. Oz ${ }^{1}$, G. M. Wildey², S. Kendir³, K. K. Papas²;

${ }^{1}$ Department of Radiology, ${ }^{2}$ Department of Surgery,

${ }^{3}$ College of Education, Department of Curriculum and Instruction,

University of Minnesota, Minneapolis, United States.

Background and Aims: Pancreatic islet transplantation is a highly promising treatment for restoring normoglycemia in a subpopulation of patients with Typel diabetes. However, islet death induced by hypoxia is a major factor limiting viable islet yield and engraftment and ultimately the success rate of transplantations. We have recently observed that vitamin E homologs $(\alpha-$ and $\gamma$-tocopherol) offer substantial protection from anoxia (the most severe 
form of hypoxia) induced islet death. The aim of this study was to address the metabolic consequences of islet exposure to anoxia in the presence and absence of vitamin $\mathrm{E}$ homologs using ${ }^{31} \mathrm{P}$ and ${ }^{13} \mathrm{C}$ NMR spectroscopy. We utilized highly purified porcine islets that are a promising alternative to human islets that are in short supply, as well as INS-1 cells as a potentially faithful model for $\beta$ cell metabolism.

Materials and Methods: Porcine islets (30,000 islet equivalents per condition) and INS-1 cells were incubated in RPMI media supplemented with $11 \mathrm{mM}$ $\left[1,6-{ }^{13} \mathrm{C}_{2}\right]$ glucose for 6 hours under 3 conditions: 1 . Normoxia (95\% Air, $5 \%$ $\left.\mathrm{CO}_{2}\right) ; 2$. Anoxia $\left(95 \% \mathrm{~N}_{2}, 5 \% \mathrm{CO}_{2}\right)$; and 3 . Anoxia supplemented with $\alpha$ - and $\gamma$ tocopherol $(50 \mu \mathrm{M}$ each). At the end of the incubation cultures were extracted with perchloric acid using standard methods. Extracted metabolites were lyophilized and resuspended in $\mathrm{D}_{2} \mathrm{O}$ that contained reference compounds for ${ }^{13} \mathrm{C}$ and ${ }^{31} \mathrm{P}$ NMR. ${ }^{31} \mathrm{P}$ and proton decoupled ${ }^{13} \mathrm{C}$ NMR spectra were acquired using a $500 \mathrm{MHz}$ vertical bore magnet.

Results: ${ }^{13} \mathrm{C}$ label incorporation from glucose into glutamate, which is in exchange with the TCA cycle intermediate $\alpha$-ketoglutarate, was highly reduced under anoxia due to inhibition of oxidative metabolism in both islets and INS-1 cells. ${ }^{13} \mathrm{C}$ label incorporation into lactate, on the other hand, was increased indicating increased anaerobic glycolysis under anoxia. Although $\beta$ cells are known to have only low levels of lactate dehydrogenase, these data indicate that the Pasteur effect is operational in porcine islets and INS-1 cells. In addition, under anoxia in the presence of tocopherols, the $\left[3-{ }^{13} \mathrm{C}\right]$ lactateto- $\left[4-{ }^{13} \mathrm{C}\right]$ glutamate ratio was closer to the normoxic condition than in their absence for both islet and INS-1 cultures. Similarly, islets and INS-1 cells displayed an improved bioenergetic status in the presence than in the absence of tocopherols by both increased total nucleotide levels and ATP-to$\mathrm{ADP}$ ratios as measured by ${ }^{31} \mathrm{P}$ NMR.

Conclusions: Together with our prior data regarding protection of islets from anoxia induced death by tocopherols, these preliminary data indicate that islets and INS- 1 cells are capable of producing enough energy to survive a short $(6 \mathrm{~h})$ anoxic insult when supplemented with vitamin $\mathrm{E}$ homologs. The antioxidant supplementation likely prevents anoxia induced damage to islets possibly by directly or indirectly modulating the anaerobic and oxidative metabolic rates and preserving ATP levels. The findings implicate vitamin E homologs as promising candidates for islet protection from short and possibly longer exposures to oxygen deprivation.

Supported by the University of Minnesota Academic Health Center Faculty Seed Grant

\section{3}

Calcium elevations in MIN6 cells evoked by cytotoxic concentrations of human IAPP involve activation of the mechanosensitive ion channel Trpv4

F. M. Gribble ${ }^{1}$, S. Casas ${ }^{2,3}$, R. Gomis ${ }^{3}$, F. Reimann ${ }^{1}$, A. Novials ${ }^{2}$; ${ }^{1}$ Cambridge Institute for Medical Research and Department of Clinical Biochemistry, University of Cambridge, United Kingdom,

${ }^{2}$ Sarda Farriol Foundation, Institute of Diabetes, Barcelona, Spain,

${ }^{3}$ Endocrinology, IDIBAPS, Hospital Clínic, University of Barcelona, Spain.

Background and Aims: Islet amyloid polypeptide (IAPP) is the main component of amyloid deposits found in the islets of most type 2 diabetics. As islet amyloid colocalizes with areas of cell degeneration, the process of amyloidosis has been associated with the progressive loss of $\beta$-cell mass. Amyloid peptides elevate intracellular calcium levels $\left(\left[\mathrm{Ca}^{2+}\right]_{\mathrm{i}}\right)$ in neurones, which may play a role in cell apoptotis. The aim of this study was to investigate the effect of human IAPP (hIAPP) on $\left[\mathrm{Ca}^{2+}\right]_{\mathfrak{i}}$, morphology and apoptosis of pancreatic $\beta$-cells.

Materials and Methods: hIAPP aggregation was evaluated by examining the hIAPP peptide conformational state in culture media using transmission electron microscopy (TEM) or by the fluorescence of thioflavine T at $482 \mathrm{~nm}$. $\left[\mathrm{Ca}^{2+}\right]_{i}$ was monitored for $2 \mathrm{~h}$ as the $340 / 380 \mathrm{~nm}$ fluorescence ratio in fura-2 loaded MIN6-cells. Cell morphology was evaluated by TEM. Cell viability was assessed by FACS using propidium iodide. Expression of Trpv4 and Actb was analyzed by RT-PCR. Down-expression of Trpv4 was induced by siRNA for mouse Trpv4, using amylase siRNA as a control.

Results: TEM showed spontaneous aggregation of $10 \mu \mathrm{M}$ hIAPP. Prefibrillar hIAPP structures were first observed after $10 \mathrm{~min}$, with maximum formation by $30-45 \mathrm{~min}$. Fibrils were detected from $30-45 \mathrm{~min}$. Thioflavine T binding confirmed hIAPP fibril formation over time. hIAPP $(10 \mu \mathrm{M})$ induced an rise in $\left[\mathrm{Ca}^{2+}\right]_{\mathrm{i}}$ in 70/328 MIN6-cells, compared with 0/87 in control solution and $0 / 128$ with $10 \mu \mathrm{M}$ rat IAPP. Nifedipine $(5 \mu \mathrm{M}, \mathrm{NF})$ added at the start prevented any increase in $\left[\mathrm{Ca}^{2+}\right]_{i}(0 / 198)$, but its addition after $\left[\mathrm{Ca}^{2+}\right]_{i}$ changes were established only reversed the $\left[\mathrm{Ca}^{2+}\right]_{\text {i }}$ rise in $6 / 16$ cells. In $\mathrm{Na}^{+}$-free buffer hIAPP increased $\left[\mathrm{Ca}^{2+}\right]_{\mathrm{i}}$ in $7 / 85$ cells, but NF could no longer prevent the $\left[\mathrm{Ca}^{2+}\right]_{\text {is }}$ rise $(13 / 154$ cells, $p<0.01)$. This suggested the involvement of other $\mathrm{Ca}^{2+}$-channels, and we hypothesised that mechanosensitive trp channels might play a role. Trp channel inhibitors, gadolinium $(100 \mu \mathrm{M})$ or ruthenium red $(10 \mu \mathrm{M}, \mathrm{RR})$, abolished the $\left[\mathrm{Ca}^{2+}\right]_{\mathrm{i}}$ rise even in the absence of $\mathrm{Na}^{+}$. EM revealed extracellular insoluble hIAPP in close contact with cells. In these regions, the plasma membrane was irregular with deep invaginations at $24 \mathrm{~h}$ even with RR. hIAPP reduced cell viability to $63 \%(p<0.05)$, and this was partially reversed by inclusion of $\mathrm{RR}(79 \%$ vs $63 \%, \mathrm{p}<0.05)$. The mechanosensitive trp channel Trpv4 was found to be expressed in MIN6cells and mouse pancreas. siRNA against Trpv4 or amylase reduced the basal cell viability to $91 \%$, but viability was further reduced to $60 \%$ by hIAPP in the control group. Cells treated with Trpv 4 siRNA exhibited improved viability in the presence of hIAPP (77\%: $\mathrm{p}<0.05 \mathrm{vs}$ control).

Conclusion: Alterations in $\left[\mathrm{Ca}^{2+}\right]_{\mathrm{i}}$ play a role in $\beta$-cell cytotoxicity induced by hIAPP aggregation. Both L-type calcium channels and Trp channels play a role in the $\left[\mathrm{Ca}^{2+}\right]_{\mathrm{i}}$ rise. The mechanosensitive channel Trpv4 may sense membrane distortion induced by hIAPP aggregation, enabling $\mathrm{Ca}^{2+}$ entry, membrane depolarization and further activation of voltage gated $\mathrm{Ca}^{2+}$ channels. Pharmacological inhibition or decreased expression of Trpv4 prevented hIAPP-induced $\left[\mathrm{Ca}^{2+}\right]_{\mathrm{i}}$ changes and protected against its toxic effect on cell viability.

S.C. is recipient of a Juan de la Cierva contract from Ministerio de Educación $y$ Ciencia in Spain and thanks the award of an Albert Renold Fellowship from the European Foundation for the Study of Diabetes. F.M.G. is a Wellcome Trust Senior Research F

\section{4}

Presence of -132(G/A) mutation in the IAPP promoter gene increase synthesis and secretory granule content of IAPP in pancreatic MIN6-cells S. Casas ${ }^{1,2}$, R. Gomis ${ }^{1}$, A. Clark ${ }^{3}$, A. Novials ${ }^{2}$;

${ }^{1}$ Laboratory of Experimental Diabetes, IDIBAPS, Barcelona, Spain, ${ }^{2}$ Institute of Diabetes, Sardà Farriol Foundation, Barcelona, Spain, ${ }^{3}$ Oxford Centre for Diabetes, Churchill Hospital, Oxford, United Kingdom.

Background and Aims: Islet amyloid polypeptide (IAPP) is the component peptide of islet amyloid deposits in type 2 diabetic subjects. Increased human IAPP (hIAPP) production could play a role in islet dysfunction in type 2 diabetes. The -132 G/A mutation located within an activator domain of the hIAPP gene promoter was first identified in a cohort of Spanish type 2 diabetic patients. Higher fasting IAPP plasma concentrations were associated with the mutation in diabetic subjects suggesting that this mutation resulted in increased IAPP production. The aims of this study were to determine if pancreatic beta-cells expressing hIAPP under the mutant gene promoter had higher levels of hIAPP production and were less viable compared to those expressing wild type gene promoter.

Methods: Human IAPP cDNA (which includes the full coding sequence for proIAPP) was cloned into a pIRES-EGFP vector. The CMV promoter was replaced by either the wildtype or mutant hIAPP promoter. The plasmid constructs were transfected into MIN6-cells using liposomal lipid reagent. MIN6-cells treated with transfection reagent without DNA were included as control. Transfected cells were cultured for $96 \mathrm{~h}$ in 5.5 or $22.7 \mathrm{mM}$ glucose. Transfection efficiency was determined from percentage EGFP-positive cells using FACS. Morphology and hIAPP granule content was evaluated by quantitative morphometry and transmission electron microscopy using immunogold labeling with anti-human N-terminal-pro-IAPP antibody. Cell viability was assessed by FACS using propidium iodide straining. Expression of hIAPP mRNA was quantified by real-time RT-PCR.

Results: Eficiency of transfection with both wildtype or mutant construct was $85 \pm 5 \%$. Transfected cells (containing liposomes) showed normal ultrastructural cell morphology. When cultured at $22.7 \mathrm{mM}$ glucose, MIN6cells expressing hIAPP under mutant compared to wildtype gene promoter had increased gold particles/secretory granule $(0.33 \pm 0.03$ vs. $0.68 \pm 0.07$ particles $/ \mathrm{pm}^{2}$ vesicle, $p<0.001$ ). No differences were found at $5.5 \mathrm{mM}$ glucose. Increased expression levels of hIAPP mRNA were detected in MIN6-cells carrying mutant compared to wildtype construct. Those differences were higher in cells cultured at $22.7 \mathrm{mM}$ glucose $(1.33 \pm 0.41 v s .2 .56 \pm 0.33$ arbitrary units) than at $5.5 \mathrm{mM}$ glucose ( $1.01 \pm 0.23$ vs. $1.72 \pm 0.45$ arbitrary units). No 
changes in cell viability were observed in MIN6-cells transfected with mutant compared to wildtype construct at 5.5 or $22.7 \mathrm{mM}$ glucose.

Conclusions: The presence of the -132 (G/A) mutation of the hIAPP promoter induces hyperexpression of hIAPP in pancreatic beta-cells which is increased by glucose but not increased cell death. Increased hIAPP production in patients with the promoter polymorphisms could contribute to amyloidogenesis in type 2 diabetes.

Supported by FIS PI05/1215 from Ministerio de Sanidad y Consumo in Spain. S.C. acknowledges the receipt of a Juan de la Cierva contract from Ministerio de Educación y Ciencia in Spain

\section{5}

Fasting plasma glucose and haemoglobin $A_{1 c}$ predict baboon pancreatic islet amyloidosis: a novel non-human primate model for type 2 diabetes mellitus (t2dm)

R. Guardado-Mendoza ${ }^{1}$, A. Davalli ${ }^{2}$, A. O. Chavez ${ }^{1}$, E. Bazzigaluppi ${ }^{2}$,

C. Belloni' ${ }^{2}$ A. G. Comuzzie ${ }^{3}$, R. Bastarrachea ${ }^{3}$, A. Monroy ${ }^{1}$, E. Bosi ${ }^{2}$,

G. Halff, D. Triphaty ${ }^{1}$, E. J. Dick Jr. ${ }^{5}$, G. Hubbard ${ }^{5}$, R. A. DeFronzo ${ }^{1}$, F. Folli ${ }^{1}$;

${ }^{1}$ Diabetes Division, University of Texas Health Science Center, San

Antonio, United States, ${ }^{2}$ Department of Medicine, Istituto Scientifico San

Raffaele, Milano, Italy, ${ }^{3}$ Department of Genetic, Southwest Foundation for Biomedical Research, San Antonio, United States, ${ }^{4}$ Department of Surgery,

University of Texas Health Science Center, San Antonio, United States,

${ }^{5}$ Department of Pathology, Southwest Foundation for Biomedical Research,

San Antonio, United States.

Background and Aims: T2DM is characterized for insulin resistance (IR) and progressive beta cell dysfunction. Intra-islet accumulation of islet amyloid polypeptide deposits play a major role in the pathogenesis of beta cell dysfunction and death. Baboons share a high degree of genetic (98\%) and physiological similarity with humans. Therefore, we hypothesized that pancreatic islet amyloidosis (PIA) would be a major determinant of diabetes in baboons.

Materials and Methods: We analyzed histopathological, clinical and chemistry data in a colony of baboons who were followed over a 12 years period. PIA was diagnosed at necropsy by histological examination, and age/ gender/weight matched baboons without PIA were used as controls. Student $\mathrm{t}$ test and chi square test were used to compare variables, ROC analysis to evaluate the usefulness of fasting plasma glucose (FPG) and hemoglobin A1c (HbAlc) to predict the presence of PIA. Pearson correlation coefficient was calculated between variables of interest. $P$ values were considered significative when $<0.05$.

Results: 78 baboons were studied, $30(24 \mathrm{~F} / 6 \mathrm{M})$ with and $48(33 \mathrm{~F} / 15 \mathrm{M})$ without PIA. There were no significant differences in age ( $22.3 \pm 0.6$ vs $21.7 \pm$ $0.5, p 0.45)$, gender $(\mathrm{M} / \mathrm{F}$ ratio 0.25 vs $0.45, p 0.205)$ and weight $(26.3 \pm 1.2$ vs $26.3 \pm 1.1 \mathrm{~kg}, p 0.97)$. On the other hand, FPG $(158 \pm 17 \mathrm{mg} / \mathrm{dl} v s 88 \pm$ $3 \mathrm{mg} / \mathrm{dl}, p<0.0001)$, HbAlc $(9.6 \pm 2.0 \%$ vs $4.5 \pm 0.4 \%$, $p$ 0.01), frequency of glycosuria $(42.8 \%$ vs $0 \%, p<0.0001)$, proteinuria ( 47.6 vs $16.6 \%, p 0.019$ ) and infectious diseases ( 43.3 vs $20.8 \%, p 0.032)$ were significantly higher in baboons with PIA. The extent of PIA was evaluated semiquantitavely by two blinded experienced pathologists using a scale from 0 to 5 , where $0=$ no amyloid deposits and $5=$ amyloid involving $100 \%$ of the islets. The extent of PIA strongly correlated with FPG $(r=0.649, p<0.005)$. Finally, ROC analysis showed that the AUC for FPG to predict the pancreatic amyloidosis was 0.824 (CI 95\% $0.718-0.931, p<0.001$ ), and for FPG levels of 100, 120, and 140mg/ dl the sensitivity was 66,56 , and $40 \%$, and the specificity was $85.4,91.7$ and $97.9 \%$, respectively. For HbAlc, the AUC was 0.833 (CI 95\% 0.564-1.102, $p$ $<0.03$ ) and HbAlc levels of 5, 6 and $7 \%$ had a sensitivity of 83,75 and $66 \%$ and specificity of 44,89 and $100 \%$, respectively.

Conclusion: FPG and $\mathrm{HbAlc}$ concentrations were strongly associated with the presence of IAPP pancreatic deposits in baboons. Of note, the levels of FPG and HbAlc that predict, with very high specificity, the presence of PIA in this model are within the range of the early metabolic abnormalities associated with T2DM in humans. Furthermore, the extent of PIA was directly correlated with FPG and HbAlc. These data support the concept that the baboon is a novel and pertinent non-human primate model for the study of beta cell dysfunction/apoptosis and therapeutic interventions in T2DM.

\section{PS 24 The role of cytokines and chemokines on pancreatic islet physiology and pathology}

\section{6}

IL-1 ra increases size and function of islets of Langerhans in vivo A. Wojtusciszyn, M. Armanet, P. Morel, D. Bosco, T. Berney; Surgery, Geneva University Hospitals, Cell Isolation and Transplantation Center, Switzerland.

Background and Aims: IL- $1 \beta$ is a potent inhibitor of beta-cell function. A receptor antagonist of IL-1 (IL-1ra) has been shown to reverse this effect in vitro. We aimed to demonstrate whether IL-1ra has a beneficial effect on islets in vivo using IL-1ra KO and IL-1ra-overexpressing transgenic (hIL1raTg) mice.

Materials and Methods: Intraperitoneal glucose tolerance test (IPGTT) assay was performed on hIL1raTg, IL1-ra-KO and wild type (DBA1 strain) mice. Islets from these mice were isolated. The mean number of total islets (IT) and islet equivalent (IEQ) was evaluated. Insulin secretion was assessed on isolated islets by static incubation (SI) and on single beta-cells by reverse hemolytic plaque assay (RHPA). Static incubation was performed in absence or presence of cytokines (TNF $\alpha 1000 \mathrm{UI} / \mathrm{ml}$, IFN $\gamma 1000 \mathrm{UI} / \mathrm{ml}$ and IL1 $\beta 250$ $\mathrm{UI} / \mathrm{ml}$ ).

Results: When analysed by IPGTT, hIL1raTg mice presented better response to glucose, with an area under the curve (AUC) significantly lower compared to WT and KO mice ( $75 \pm 91$ vs $516 \pm 186$ and $541 \pm 246$ mM.min, respectively).The glycemic peak arose earlier and was also lower $(4.8 \pm 0.7$ vs $7.4 \pm 2.4$ and $9.0 \pm 2.4 \mathrm{mM}$ ) in hIL1 raTg compared to WT and KO mice. After islet isolation, IT, IEQ per pancreas and the mean size of islets was lower in KO mice compared to hIL1raTg and WT mice. Insulin secretion in response to glucose, assessed by SI and RHPA, was lower and more affected by cytokines in IL1-ra KO mice compared to other mice.

Conclusion: IL1-ra improves insulin secretion in vivo and plays a role in preserving islets against deleterious effect of cytokines. IL-1ra has an impact on pancreatic islet mass, suggesting that IL-1ra may be involved in the development and/or regeneration of islets.

\section{7}

Induction of chemokine CXCL1 by extracellular matrix and enhancement by IL-1 in rat pancreatic $\beta$ cells P. Ribaux ${ }^{1}$, J. A. Ehses ${ }^{2}$, E. B. Hammar ${ }^{1}$, J.-C. Irminger ${ }^{1}$, M. Donath ${ }^{2}$, P. A. Halban ${ }^{1}$;

${ }^{1}$ Department of Genetic Medicine and Development, University Medical Center, Geneva, Switzerland, ${ }^{2}$ Division of Endocrinology and Diabetes, University Hospital of Zürich, Switzerland.

Background and Aims: As we showed previously, the extracellular matrix (ECM) secreted by rat bladder carcinoma cells (804G-ECM) has positive effects on rat primary $\beta$-cell function and survival in vitro. The aim of this study was to define $\beta$-cell genes induced by this ECM with a specific focus on cytokines.

Materials and Methods: Rat primary $\beta$-cells were sorted by FACS and cultured in Petri dishes coated with poly-L-lysine (pLL) or 804G-ECM. Analysis of differential gene expression by oligonucleotide microarrays and RT-PCR was performed to identify cytokine mRNA induced by the 804G-ECM. The concentration of cytokines in the conditioned media was quantified by Luminex technology. Data are expressed as mean \pm SE for $n=$ 3 independent experiments.

Results: Five cytokines were overexpressed by $\beta$-cells plated for 4 hours on 804G-ECM compared to pLL: CXCL1, CXCL2, IP10, IL-1 $\alpha$ and IL-1 $\beta$. A time-course experiment indicated that maximal induction by $804 \mathrm{G}-\mathrm{ECM}$ occurred at 4 hours for CXCL1 (68.3-fold stimulation vs pLL), CXCL2 (14.5fold) and IP10 (6.7-fold) mRNA. IL- $1 \alpha$ and $\beta$ were expressed at low levels in cells on ECM with no expression detectable on pLL. Stimulation of CXCL1 release by $\beta$-cells on $804 \mathrm{G}$-ECM was confirmed at the protein level. Indeed, the concentration of CXCL1 secreted by 600000 cells $/ \mathrm{ml}$ after 24 hours of culture was $776 \pm 155 \mathrm{pg} / \mathrm{ml}$ (vs $35.4 \pm 5.6 \mathrm{pg} / \mathrm{ml}$ on $\mathrm{pLL}, \mathrm{p}<0.001)$. The absence of macrophages, a major source of cytokines, in the $\beta$-cell population 
was confirmed by immunofluorescence. Preventing the interaction of $\beta 1$ integrins and laminin-5 (a predominant component of $804 \mathrm{G}-\mathrm{ECM}$ ) with specific antibodies resulted in a $40-50 \%$ inhibition $(p<0.05)$ of CXCL1 mRNA expression after 6 hours of culture. NF- $\mathrm{kB}$ activation was shown to be indispensable, since the use of the NF- $\mathrm{KB}$ pathway inhibitor Bay 11-7082 (5 $\mu \mathrm{M})$ abolished the increased CXCL1 expression and secretion after 10 hours of culture on 804G-ECM. IL-1 ( $\alpha$ and/or $\beta$ ) secreted by cells plated on 804GECM was found to be a key soluble mediator, since treatment of cells with $1 \mu \mathrm{g} / \mathrm{ml}$ of the IL-1 receptor antagonist (IL-1Ra) significantly reduced both CXCL1 gene expression (by $67.8 \pm 5.9 \%, \mathrm{p}<0.001$ ) and secretion (by $65.4 \pm$ $6.3 \%, \mathrm{p}<0.001)$ after 6 hours of culture.

Conclusion: In the present study, we show that sorted rat primary $\beta$-cells express cytokines CXCL1, CXCL2, IP10, IL- $1 \alpha$ and IL-1 $\beta$ in response to ECM under conditions previously shown to improve function and survival. This effect of ECM is partly due to interaction between laminin-5 and $\beta 1$ integrins. Moreover, the production of CXCL1 is an NF- $\mathrm{KB}$ dependent event with amplification by IL-1 acting via a positive autocrine and/or paracrine feedback loop.

\section{8}

Lack of macrophage migration inhibitory factor (MIF) confers resistance to immunoinflammatory diabetes in mice

I. Stojanovic ${ }^{1}$, S. Lazaroski ${ }^{1}$, F. Nicoletti ${ }^{2}$, S. Stosic-Grujicic ${ }^{1}$;

${ }^{1}$ Department of Immunology, Institute for Biological Research, Belgrade,

Serbia, ${ }^{2}$ Department of Biomedical Sciences, University of Catania, Italy.

Background and Aims: Macrophage migration inhibitory factor (MIF) is implicated in the pathogenesis of several inflammatory/autoimmune disorders, including type 1 diabetes. Using a model of immunoinflammatory diabetes induced in susceptible mouse strains by multiple low doses of streptozotocin (MLD-STZ) we have recently shown that in vivo abrogation of MIF activity with pharmacological MIF inhibitor (S,R)-3-(4-hydroxyphenil)4,5-dihydro-5-isoxasole acetic acid methyl ester, or with neutralizing antiMIF antibodies, markedly reduced clinical and histopathological features of diabetes. Furthermore, MIF-deficient (MIF-KO) mice were also less susceptible to the MLD-STZ-induced hyperglycemia, insulitis and apoptosis within the endocrine pancreas than genetically matched wild type (WT) C57BL/6 mice. In all three in vivo approaches tested, negating the action of endogenous MIF has induced an immune deviation towards protective type $2 / 3$ response. The aim of this study was to further analyze the role of endogenous MIF in the pathogenesis of diabetes at the level of target tissue (pancreatic islets).

Material and Methods: Pancreatic islets from WT and MIF-KO mice were isolated by collagenase digestion method. $\beta$ cells were obtained after pancreatic islets digestion with trypsin. Both islets and $\beta$ cells were cultured 24 to $96 \mathrm{~h}$ in the presence or absence of combination of pro-inflammatory cytokines (IL$1+$ TNF- $\alpha+$ IFN- $\gamma-10 \mathrm{ng} / \mathrm{ml}$ each), or STZ $(2 \mu \mathrm{g} / \mathrm{ml})$. Evidence of apoptosis induction was indirectly obtained by determination of mitochondrial activity (MTT test). The concentration of phosphorylated form of I- $\kappa B$ (p-I-kB) was measured by Western blot. IL-1 $\beta$ expression was determined by reverse transcription-PCR, while its secretion was measured by ELISA.

Results: MIF-KO $\beta$ cells or pancreatic islets were more resistant to killing by cytokines (IL-1+TNF- $\alpha+$ IFN- $\gamma$ ) or STZ than WT counterparts. Furthermore, while cytokine-stimulated WT pancreatic islets showed significant NF- $\kappa B$ activation (judged by $\mathrm{p}-\mathrm{I}-\mathrm{\kappa B}$ ), MIF-KO islets had lower activity of NF- $\mathrm{KB}$. Finally, in STZ-stimulated MIF-KO islets both IL- $1 \beta$ mRNA expression and secretion were significantly down-regulated in comparison to the elevation seen in STZ-stimulated WT islets.

Conclusion: Our findings clearly show that the absence of MIF confers $\beta$ cell protection from the lethal insult of different agents, namely cytokines and STZ. This protection is probably mediated by suppression of NF- $\mathrm{KB}$ and subsequent expression and secretion of IL-1 $\beta$, a well-known mediator of $\beta$ cell death. According to both in vivo and in vitro observations, it can be hypothesized that MIF inhibition could be beneficial in diabetes treatment. Supported by the Ministry of Science of Serbia and research funds from the School of Medicine of University of Catania

\section{9}

Effects of anti-inflammatory cytokines on insulin-producing cells E. Gurgul-Convey, K. L. A. Souza, M. Elsner, S. Lenzen;

Institute of Clinical Biochemistry, Hannover Medical School, Germany.

Background and Aims: The role of pro-inflammatory cytokines, especially IL- $1 \beta$, in pancreatic beta cell death is well established. Little is known, however, about the relevance and influence of the so-called anti-inflammatory cytokines IL-4, IL-13 and IL-10 on beta cell survival and death pathways. The aim of this study was to clarify the effects of anti-inflammatory cytokines on pro-inflammatory cytokine-induced beta cell death and to investigate the mechanisms underlying these effects.

Materials and Methods: Insulin-producing RINm5F cells were incubated with pro-inflammatory cytokines (IL-1 $\beta$, TNF $\alpha$ and IFN $\gamma$ ) and/or antiinflammatory cytokines (IL-4, IL-13 and IL-10). Thereafter cell viability (MTT assay), transcription factor NFKB activation (SEAP-reporter gene assay), caspase-3 activity and inducible nitric oxide synthase (iNOS) expression (Real-Time PCR and Western Blot) were measured.

Results: It was found that the expression levels of anti-inflammatory cytokine receptors (IL-4Ralpha, IL-13R, IL-10R) in insulin-producing cells were 200 -fold lower than in the liver. Interestingly, pro-inflammatory cytokines, especially IL-1 $\beta$, elevated expression of these receptors $(6 \mathrm{~h}$ incubation: 2.5-fold IL-4R $\alpha, 2$-fold IL-13R, 2.2-fold IL-10R induction vs. untreated). Anti-inflammatory cytokines were not toxic to insulin-producing cells (72 $\mathrm{h}$ incubation, $60 \mathrm{ng} / \mathrm{ml}$, viability $>98 \%$ ). Co-incubations of pro- with antiinflammatory cytokines had a protective effect on beta cell viability $(72 \mathrm{~h}$ incubation, $60 \mathrm{U} / \mathrm{ml} \mathrm{IL}-1 \beta 70 \% \pm 2$ vs. $80 \%$ in cells treated with IL- $1 \beta$ and antiinflammatory cytokines; Cytokine Mix $45 \% \pm 4$ vs. $53 \%$ cells treated with IL$1 \beta$ and anti-inflammatory cytokines; $n=4$ in triplicates). Incubation with 60 $\mathrm{U} / \mathrm{ml}$ IL- $1 \beta$ activated caspase- 3 over 5 -fold vs. untreated cells. This induction was diminished by all anti-inflammatory cytokines (4-fold reduction). Antiinflammatory cytokines almost completely prevented an increase of caspase3 activity induced by a mixture of cytokines ( 8 h Cytokine Mix $422 \% \pm 25$, Mix + IL-4 $121 \% \pm 12$, Mix + IL-13 $189 \% \pm 19$, Mix + IL-10 $165 \% \pm 17$ $\mathrm{n}=4$ in triplicates). All tested anti-inflammatory cytokines significantly diminished IL-1 $\beta$-induced activity of NFkB, a transcription factor that plays a crucial role in the cytokine-induced beta cell death (6 h incubation, IL-1 $\beta$ 1.8 -fold increase \pm 0.2 , IL- $1 \beta+$ IL-4 1.2 \pm 0.1, IL- $1 \beta+$ IL-13 $1.4 \pm 0.1$, IL- $1 \beta+$ IL-10 $1.4 \pm 0.1 ; \mathrm{n}=6$ in duplicates). Anti-inflammatory cytokines decreased also IL-1 $\beta$-stimulated iNOS gene expression $(6 \mathrm{~h}$ incubation: $60 \mathrm{U} / \mathrm{ml} \mathrm{IL}-1 \beta$ 800 -fold \pm 109 , IL-1 $\beta+$ IL-4 $61 \pm 7$, IL- $1 \beta+$ IL-13 $65 \pm 20$, IL-1 $\beta+$ IL-10 133 \pm 40 ). Similar effects, although weaker, were observed on the iNOS protein level; anti-inflammatory cytokines tended to prevent a strong iNOS protein expression induced by IL-1 $\beta$ (ca. $30 \%$ reduction vs. IL-1 $\beta$ ).

Conclusion: Anti-inflammatory cytokines in insulin-producing cells 1. exert a protective effect against pro-inflammatory cytokine-induced toxicity (protection against cell death, decrease of iNOS expression, reduction of caspase-3 activity), and 2 . counteract the stimulatory effects of pro-inflammatory cytokines on the NFkB signal transduction pathway. Interestingly, our results also show a very low expression level of antiinflammatory cytokine receptors in insulin-producing cells, which can be however increased by pro-inflammatory cytokines thereby enabling protection against cell death.

\section{0}

Peroxiredoxin III prevents stress induced apoptosis in rat insulinoma cells RINm5F

G. Wolf, N. Aumann, M. Michalska, R. Walther;

Department of Medical Biochemistry and Molecular Biology,

University of Greifswald, Germany.

Background and Aims: Type 1 diabetes mellitus is characterised by a progressive autoimmune destruction of insulin-producing beta cells in the pancreas. Cytokines induce the synthesis of reactive oxygen and nitrogen species in macrophages and in their target cells. These insulin-producing beta cells are very sensitive to this attack due to their low content of antioxidant enzymes like superoxid dismutase or catalase. In particular the resulting mitochondrial damage gives an important signal to promote beta cell death. For this reason, there is considerable interest in peroxiredoxin III (Prx III, Mer5, SP-22, and AOP-1) and its function in pancreatic islets. Prx 
III is localised in mitochondria and belongs to a family of highly conserved thioredoxin-dependent peroxide reductases. The aim of our studies was to elucidate whether the Prx III-thioredoxin-system can contribute to prevent beta cell destruction.

Materials and Methods: Using immunohistochemistry, Northern and Western blot analyses, we determined the expression of Prx III in mouse pancreas and in the rat insulinoma cell line RINm5F. To further examine the role of this antioxidant enzyme in beta cell protection, we established stably transfected RINm5F cells, which can be induced by doxycyclin to overexpress or downregulate Prx III. These cells were treated with TNF- $\alpha$, IL-1 $\beta$, streptozotocin as well as alloxan and caspase activities were measured. After treatment with $\mathrm{H}_{2} \mathrm{O}_{2}$, we estimated the production of reactive oxygen species (ROS) by flow cytometry using the oxidation-sensitive fluorescent probe 5,6-carboxy-2 ', $7^{\prime}$-dichlorofluorescein-diacetate (DCFH-DA). Activation of iNOS and cleavage of PARP were detected by Western blots.

Results: The detection of Prx III in the pancreas revealed that this enzyme is expressed in the insulin producing $\beta$-cells. Our results demonstrate that after treatment with $\mathrm{H}_{2} \mathrm{O}_{2}$ the accumulation of ROS was reduced in Prx IIIoverexpressed cells and increased in Prx III-depleted cells. RINm5F cells can be protected against stress-induced apoptosis by enhanced amounts of mitochondrial Prx III. However increased apoptosis as revealed by measurement of caspase-3 and 9-activities, activation of iNOS and cleavage of PARP after treatment with different stressors has been observed if this enzyme is down-regulated.

Conclusion: The results demonstrate that the activation of the mitochondrial Prx III-thioredoxin-system may contribute to protect beta cells against oxidative stress and apoptosis occurring by autoimmune attack during pathogenesis of Type I diabetes.

Supported by the foundation "Das zuckerkranke Kind"

\section{1}

Do peroxiredoxins reduce the vulnerability of insulin-secreting cells to cytokines?

P. Romanus ${ }^{1}$, T. Bouckenooghe ${ }^{1}$, B. Vandewalle ${ }^{2}$, C. Remacle ${ }^{1}$, B. Knoops ${ }^{1}$,

B. Reusens';

${ }^{1}$ Institut of Life Sciences, Université Catholique de Louvain,

Louvain-la-Neuve, Belgium,

${ }^{2}$ U859, Faculté de Médecine Henri Warembourg, Lille, France.

Background and Aims: The autoimmune destruction of the insulinproducing $\beta$-cells in Type 1 Diabetes (T1D) is mediated in part by cytokines (IL-1 $\beta$, IFN- $\gamma$, TNF- $\alpha$ ) which are cytotoxic to $\beta$-cells via the production of peroxynitrite $\left(\mathrm{ONOO}^{-}\right)$. Both rodent and human islets are highly sensitive to $\mathrm{ONOO}^{-}$because of their low antioxidant enzymes expression. For this reason, there is a considerable interest in a newly discovered peroxidase family composed of six members, the peroxiredoxins (1-6). Moreover, the human $\operatorname{Prdx} 5$ which possesses a $\mathrm{ONOO}^{-}$reductase activity and a mitochondrial localisation could play a major protective role against this toxic nitrooxidant. Our goal was to investigate the regulation of the Prdx1, 2, 3, 5 and their cytoprotective function in $\beta$-cells exposed to cytokines.

Materials and Methods: Female rat islets, INS-1E and three human islets preparations were exposed during $8 \mathrm{~h}, 24 \mathrm{~h}$ and $48 \mathrm{~h}$ to cytokines in usual dosage for rat or human (IL- $1 \beta$, IFN- $\gamma$, TNF- $\alpha$ ). Expression of Prdx1,2,3,5, MnSOD and iNOS were investigated by Western blot analysis and by realtime RT-PCR. To further examine the role of Prdx 5 , we established transitory transfected INS-1E cells to induce overexpression of $\operatorname{Prdx} 5$, which was verified by Western blotting and immunohistochemistry. The viability of INS-1E cells treated by cytokines was determined by a MTT assay.

Results: In rat islets treated by cytokines, the Prdx 5 mRNA expression was 10 times overexpressed $(\mathrm{p}<0.01)$ after $24 \mathrm{~h}$ treatment while mRNA levels of the Prdx 1,2 and 3 were not modified. Such treated islets featured an increase in MnSOD and iNOS overexpression at the mRNA and protein level with a peak point at $8 \mathrm{~h}$. Surprisingly, Prdx 5 which was overexpressed at the mRNA level after $24 \mathrm{~h}$ treatment was the only Prdx significantly underexpressed by $80 \%(\mathrm{p}<0.05)$ at the protein level. In transfected INS-1E cells, an enhanced amount of mitochondrial Prdx5 reduced the cytoxicity by $20 \%(\mathrm{p}<0.05)$, after $24 \mathrm{~h}$ cytokines incubation. As for MnSOD and iNOS mRNA, in human islets preparation, Prdx 5 but not the Prdx3, was overexpressed after $24 \mathrm{~h}$ cytokines treatment. The protein level of Prdx5 in human islets did not seem to be modified during the time course study contrary to MnSOD which was overexpressed.
Conclusion: Prdx 5 overexpression conferred a protection against cytokines to the INS-1E cells. Surprisingly, in rat islets our data established for Prdx5 only, an obvious discrepancy between mRNA level which was markedly increased and protein level that was reduced. Interestingly, this discrepancy was also observed in human islets. Further investigations are therefore necessary to identify an hypothetical post-transcriptional mechanism or to find more evidence for proteasome involvement. Consequently, we propose that modulation of $\operatorname{Prdx} 5$ expression by cytokines would be in part implicated in the susceptibility of islets to $\mathrm{ONOO}^{-}$-induced $\beta$-cells death.

\section{2}

The influence of antioxidative enzyme overexpression on the expression of Bcl-2 members and their modulation through proinflammatory cytokines

I. Mehmeti, S. Lortz, S. Lenzen;

Institute of Clinical Biochemistry, Hannover Medical School, Germany.

Background and Aims:Type 1 diabetesis an autoimmune diseasecharacterized by the selective destruction of pancreatic $\beta$-cells. Proinflammatory cytokines are mediators of $\beta$-cell death in autoimmune diabetes mainly by the induction of signalling pathways leading finally to apoptosis. Proteins of the $\mathrm{Bcl}-2$ family are important for the regulation of the intrinsic pathway of apoptosis. This family of highly conserved molecules consists of antiapoptotic (e.g. Bcl-2, Bcl- $\mathrm{X}_{\mathrm{L}}$ ) and pro-apoptotic proteins (e.g. Bax, Bad, Bim, Bid) and acts primarily on mitochondria. This is mediated by the regulation of mitochondrial homeostasis, integrity and stability. Reactive oxygen species (ROS) generated during cytokine-mediated $\beta$-cell destruction are involved as additional mediators of programmed cell death in this intrinsic pathway. Therefore the aim of the present study was to quantify the expression of Bcl-2 family members in insulin-producing cells and to characterize the influence of antioxidative enzymes on the expression of Bcl-2 family members before and after cytokine stimulation.

Materials and Methods: RINm5F control cells and RINm5F cells overexpressing the antioxidative enzymes glutathione peroxidase (GPx), catalase (Cat), mitochondrially located catalase (MitoCat) and $\mathrm{Cu} / \mathrm{Zn}$ superoxide dismutase $(\mathrm{Cu} / \mathrm{ZnSOD})$ were exposed to IL-1ß or a cytokine mix (IL-1ß, TNF- $\alpha$ und IFN- $\gamma$ ) for $24 \mathrm{~h}$. The expression levels of the investigated Bcl-2-related proteins were assessed by RT-PCR and Western blotting.

Results: The expression of the anti-apoptotic proteins $\mathrm{Bcl}-2$ and $\mathrm{Bcl}-\mathrm{X}_{\mathrm{L}}$ as well as the pro-apoptotic proteins Bax, Bad, Bim and Bid could be detected and quantified in all RINm5F cell clones. Bax showed the highest expression of all investigated $\mathrm{Bcl}-2$ proteins and its expression was around 8 -fold higher compared with $\mathrm{Bcl}-2$. These findings could be confirmed in INS-1E cells. Cytokine exposure caused a significant reduction of Bcl-2 expression in control cells. This cytokine-mediated decrease of Bcl-2 expression was also observed in $\mathrm{Cu} / \mathrm{ZnSOD}$ and Cat transfected cells. In contrast to these findings, GPx and MitoCat cells showed no significant changes of the Bcl2 expression after cytokine treatment. Bax expression was decreased in all investigated cell clones after cytokine stimulation, except for $\mathrm{Cu} / \mathrm{ZnSOD}$ and MitoCat cells. Both clones exhibited a significant increase of the Bax expression level after exposure to cytokines. Because of the lower basal Bax expression in unstimulated MitoCat cells compared with controls cells, this cytokine-related increased Bax expression was still lower. The cytokine stimulation of control cells and cells overexpressing cytoprotective enzymes led to a significant increase of Bcl- $\mathrm{X}_{\mathrm{L}}$, Bim and Bid expression. However, Bad mRNA expression and the level of phosphorylated Bad were reduced in all investigated cell clones in the presence of cytokines.

Conclusion: Overexpression of the mitochondrially located $\mathrm{H}_{2} \mathrm{O}_{2}$ detoxifying enzymes GPx and MitoCat induced an increased expression of anti-apoptotic Bcl-2 and decreased expression of pro-apoptotic Bax after cytokine treatment. This observed shift of the Bcl-2/Bax balance in favour of the anti-apoptotic Bcl-2 can contribute to the observed higher resistance of these insulin-producing cells overexpressing hydrogen peroxide inactivating enzymes against cytokine mediated toxicity and indicates the involvement of mitochondria within the apoptotic process. 


\section{3}

Moderate over expression of UCP-2 in beta cells reduces ROS formation and protects against $\mathrm{H}_{2} \mathrm{O}_{2}$-but not cytokine-induced toxicity and aggravates toxicity due to NO

H. Ogata ${ }^{1}$, E. Petersen ${ }^{2}$, I. Jørgensen ${ }^{1}$, F. Skorpen ${ }^{2}$, Z. Ma ${ }^{3}$, A. Björklund ${ }^{3}$, K. W. Egeberg ${ }^{1}$, V. Grill ${ }^{1}$;

${ }^{1}$ Norwegian University of Science and Technology, Institute of Cancer Research and Molecular Medicine, Trondheim, Norway, ${ }^{2}$ Norwegian University of Science and Technology, Institute of Laboratory Medicine, Children's and Women's Health, Levanger, Norway, ${ }^{3}$ Department of Molecular Medicine and Surgery, Karolinska Institute, Stockholm, Sweden.

Background and Aims: Uncoupling protein 2 (UCP-2) exerts diabetogenic influence by decreasing efficiency of glucose metabolism to generate ATP and/or other signaling molecules for insulin secretion. A beneficial role coupled to protection against radical oxygen formation (ROS) may also be operative but is poorly documented.

Materials and Methods: We transfected INS-1 cells to obtain a tet-on UCP-2 inducible cell line. For $48 \mathrm{~h}$ induction by 0.1 or $1.0 \mu \mathrm{g} / \mathrm{ml}$ doxycyclin (dox) increased UCP-2 protein by respectively $178 \pm 29$ and $424 \pm 113 \%$. Flow cytometry (FC) and 3-(4,5,-dimethylthiazol-2-yl)-2,5-diphenyltetrazolium bromide (MTT) were used to test for functions and viability.

Results: UCP-2 induction by $1.0 \mu \mathrm{g} / \mathrm{ml}$ dox lowered mitochondrial membrane potential (MMP) modestly $(5.1+/-2.9 \%$, FC, rhodamine 123 , $\mathrm{n}=4, \mathrm{p}<0.02$ ) without affecting mitochondrial mass (FC, MitoTracker Green) and reduced basal ROS production ( $-26.6 \%$ of control fluorescence by 2,7 ' dichlorofluorescin diacetate (DCF), $n=18, \mathrm{p}=0.025$ ). Exposure overnight to a combination of cytokines (TNF $\alpha$, IL- $1 \beta$, IFN- $\gamma 10 \mathrm{ng} / \mathrm{ml}$ each) increased DCF 2.3 fold $(\mathrm{p}<0.002)$. This effect was partially abrogated by UCP-2 induction $(\mathrm{p}<0.02)$. The NO donor S-nitroso-N-acetyl penicillamine (SNAP) failed to reproduce the cytokine effect on ROS. In viability testing by MTT, UCP-2 induction protected against toxicity induced by $200 \mu \mathrm{M} \mathrm{H}_{2} \mathrm{O}_{2}(\mathrm{p}<0.01)$. However, UCP-2 induction did not prevent the decrease of MTT seen after 24 or $48 \mathrm{~h}$ exposure to cytokines, whereas induction significantly aggravated $(\mathrm{p}<0.05)$ the decrease in MTT seen after SNAP.

Conclusion: Moderate over expression of UCP-2 in beta cells reduces ROS formation and protects against $\mathrm{H}_{2} \mathrm{O}_{2}$ - but not cytokine-induced toxicity and aggravates toxicity due to NO. Hence UCP-2 is protective only during selected conditions.

\section{PS 25 Immunoregulation and novel interventions in diabetes due to beta cell loss}

\section{4}

Prevention of diabetes manifestation by treatment with the immunomodulator FTY720 in the IDDM rat, an animal model of human type 1 diabetes

A. Jörns ${ }^{1}$, A. Meyer zu Vilsendorf ${ }^{2}$, T. Taivankhuu ${ }^{3}$, S. Lenzen ${ }^{3}$;

${ }^{1}$ Institute of Clinical Biochemistry \& Center of Anatomy,

${ }^{2}$ Visceral- and Transplantation Surgery,

${ }^{3}$ Institute of Clinical Biochemistry, Hannover Medical School, Germany.

Background and Aims: The IDDM (LEW.1AR1-iddm) rat is an animal model of type 1 diabetes mellitus with a normal immune system. The aim of the treatment with FTY720 was to prevent in the IDDM rat either the clinical manifestation of diabetes or after onset of the disease to protect the remaining pancreatic beta cells from the autoimmune attack. FTY720 affects by interaction with the sphingosin-1-receptor the resting of all $\mathrm{B}$ - und $\mathrm{T}$ lymphocytes in the organ draining lymph nodes and leads to a reduction of the number of lymphocytes in the peripheral blood.

Materials and Methods: For the purpose of primary or secondary prevention the animals were treated with FTY720 $(1 \mathrm{mg} / \mathrm{kg}$ body weight) over 40 days. Gene (in situ und real time RT-PCR) and proteinexpression (immunohistochemistry and bead-based Multiplex-Assay) of Th1- und Th2cytokines, of the chemokine MCP-1 and the enzyme iNOS were performed in pancreatic biopsies and the pancreas-draining lymph nodes at the time point of diabetes manifestation, at the end of therapy, and 30 days after the end of treatment.

Results: Primary prevention with FTY720 led to a complete prevention of diabetes manifestation at day 60 in the spontaneously diabetic IDDM rats and also 30 days after the end of the treatment the animals remained normoglycaemic. At the time point of diabetes manifestation the pancreatic islets of the FTY720 treated animals showed no signs of beta cell apoptosis and of immune cell infiltration in contrast to a high rate of beta cell apoptosis accompanied by an immune cell infiltration of T-lymphocytes and macrophages in the spontaneously diabetic IDDM rats. Additionally, in the treated animals, there was no cytokine expression in the pancreatic parenchyma (IL- $1 \beta$, IFN- $\gamma$, TNF- $\alpha$, IL-4, IL-10). Interestingly, $60 \%$ of the treated animals showed signs of immune cell activation, as documented by an increase of the gene expression of Th1-cytokines and the beta cell apoptosis mediating enzyme iNOS in pancreas-draining lymph nodes as well as an enlarged Th1-cytokine protein expression in the peripheral blood. In the time course after the end of treatment only the pancreases of the animals with signs of immune cell activation showed in $5 \%$ of the pancreatic islets a macrophage dominated immune cell infiltrate which was restricted to the islet periphery. These infiltrated islets expressed the chemokine MCP1 in the beta cells. In contrast to the results in the primary prevention study, therapy with FTY720 after the clinical manifestation of disease had no preventive effect.

Conclusion: Primary prevention therapy before diabetes manifestation by the immunomodulator FTY720 prevented the disease. Even after the end of the therapy only a few islets showed minor sings of macrophage infiltration, but no lymphocyte infiltration and no loss of beta cell mass.

\section{5}

High-dose pravastatin prevents autoimmune diabetes in non-obese diabetic mice

S. Zhang, Z. Zhou, Y. Sun, P. Zhou, G. Huang, J. Peng, S. Sun, Y. Zhang; Diabetes Center, Institute of Metabolism and Endocrinology, Diabetes Center, Institute of Metabolism and Endocrinology, the Second Xiangya Hospital,, Changsha, China.

Background and Aims Statins are inhibitors of 3-Hydroxy-3-methylglutaryl$\mathrm{CoA}$ reductase and widely used as lipid-lowing agents, recently have been reported to possess favorable immunomodulatory properties. Therefore, we hypothesized that type 1 diabetes, which is thought to be a T cell-mediated, organ-specific autoimmune diseases, might benefit from statin treatment. 
Methods Female nonobese diabetic (NOD) mice were fed an approximate daily dose of $1 \mathrm{mg}, 10 \mathrm{mg}$ and $40 \mathrm{mg} \cdot \mathrm{kg}-1 \cdot$ day- 1 of pravastatin food admixture starting from 3-4 weeks of age, and the diabetes incidence were monitored up to 30 weeks. And the insulitis and apoptosis of islets $\beta$ cell were determined at the age of 12 weeks. Moreover, the secretion and mRNA expression of cytokine IFN- $\gamma$, IL-4 and IL-10 in vitro were measured. Furthermore, population of $\mathrm{CD} 4{ }^{+} \mathrm{CD} 25^{+}$regulatory $\mathrm{T}$ cells were observed.

Results We report in this study that although all doses of pravastatin appeared to decrease the incidence of diabetes at week 30, only the highdose pravastatin can prevent or delay the development of diabetes $(\mathrm{p}<0.01$, log-rank test). A significant suppression of severe insulitis was observed in NOD mice treated with high-dose pravastatin $(\mathrm{P}<0.05)$. Moreover, the group of mice treated with pravastatin at high but not medium or low doses displayed a significant lower level of IFN- $\gamma$ but significantly higher level of IL-4 comparing to the untreated group $(\mathrm{P}<0.05)$. At transcript level, the highdose pravastatin treatment significantly increased both IL-4 and IL-10 mRNA while decreasing IFN- $\gamma$ mRNA $(\mathrm{P}<0.05)$. And decreased the apoptosis of islets $\beta$ cell in mice treated with high-dose pravastatin $(\mathrm{p}<0.01)$. However, no significant changes in population of $\mathrm{CD} 4^{+} \mathrm{CD} 25^{+}$regulatory $\mathrm{T}$ cells were observed.

Conclusion In conclusion, we demonstrated that development of autoimmune diabetes in NOD mice can be arrested by treatment with high-dose pravastin at the early stage of disease, which is able to induce a Th2 immune response and decrease the apoptosis of islets $\beta$ cells. These findings suggest that highdose statins treatment at early time may benefit the prevention of autoimmune diabetes.

Supported by the National Natural Science Foundation of China

\section{6}

Activation of adenosine receptors protects against type 1 diabetes

J. G. Mabley ${ }^{1}$, Z. H. Nemeth ${ }^{2}$, D. Bleich ${ }^{2}$, B. Csoka ${ }^{2}$, P. Pacher ${ }^{3}$, S. Vizi ${ }^{4}$, E. A. Deitch ${ }^{2}$, C. Szabo ${ }^{2}$, B. N. Cronstein ${ }^{5}$, G. Hasko ${ }^{2,4}$;

${ }^{1}$ School of Pharmacy and Biomolecular Sciences, University of Brighton, United Kingdom, ${ }^{2}$ Department of Surgery, University of Medicine and Dentistry of New Jersey, Newark, United States, ${ }^{3}$ Laboratory of Physiological Studies, NIH, Bethesda, United States, ${ }^{4}$ Hungarian Academy of Sciences, Institute of Experimental Medicine, Budapest, Hungary, ${ }^{5}$ Division of Clinical Pharmacology, New York University School of Medicine, United States.

Background and Aims: There is evidence that adenosine receptors are promising targets in autoimmune disease. Adenosine is a potent endogenous autocrine anti-inflammatory and immunosuppressive molecule which binds to specific adenosine receptors of which there are 4 types; A1, A2a, $\mathrm{A} 2 \mathrm{~b}$, and A3. Activation of adenosine receptors decreases the release of proinflammatory mediators including cytokines and nitric oxide. The aim of this study was to determine whether adenosine receptor agonists could protect against Type I diabetes and the receptor subtype involved.

Materials and Methods: The non-selective adenosine receptor agonist 5'-Nethylcarboxamidoadenosine (NECA), 0.01 or $0.03 \mathrm{mg} / \mathrm{kg} / \mathrm{day}$, was used in these studies. Diabetes was induced in male CD-1 mice using multiple-lowdose-streptozotocin (MLDS) administration ( $40 \mathrm{mg} / \mathrm{kg}$ i.p. on 5 consecutive days). Treatment with vehicle, NECA or the other adenosine receptor agonists commenced on day 1 with blood glucose measured periodically over a 21 day period, with diabetes defined as blood glucose $>200 \mathrm{mg} / \mathrm{dL}$. On day 21 pancreas levels of insulin, chemokines and cytokines were measured using specific ELISA kits. Cyclophosphamide $(200 \mathrm{mg} / \mathrm{kg}$ ) was injected into 8 week old female NOD mice treated with vehicle or NECA; blood glucose levels and diabetes incidence were monitored over the following 21 days, pancreas biopsies taken on day 21 were used for histological analysis.

Results: NECA treatment significantly reduced MLDS-induced hyperglycemia on day 21 from $405 \pm 22$ to $180 \pm 26 \mathrm{mg} / \mathrm{dL}(\mathrm{P}<0.05)$, as well as diabetes incidence from $100 \%$ in the vehicle treated to $30 \%(\mathrm{p}<0.05)$. NECA treatment attenuated the MLDS-mediated loss of pancreas insulin content $(35 \pm 2$ vs. $72 \pm 10 \mathrm{ng} / \mathrm{mg}$ protein; $\mathrm{p}<0.05))$ as well as reducing pancreatic levels of inflammatory chemokines (MIP-1 from $2.87 \pm 0.91 \mathrm{pg} / \mathrm{mg}$ protein to $0.5 \pm 0.02 ; \mathrm{p}<0.05$ ) and cytokines (TNF from $1.5 \pm 0.6 \mathrm{pg} / \mathrm{mg}$ protein to $0.05 \pm 0.02$; IL-12 from $20 \pm 7$ to $5 \pm 0.1$; IFN from $0.4 \pm 0.1$ to $0.09 \pm 0.02$; $\mathrm{p}<0.05$ ). To determine which receptor subtype was involved we treated mice with the specific A1 receptor agonist CCPA $(0.1 \mathrm{mg} / \mathrm{kg})$, A2a receptor agonist CGS21680 $(0.1 \mathrm{mg} / \mathrm{kg})$ and A3 receptor agonist IB-MECA $(0.1 \mathrm{mg} / \mathrm{kg})$; all of which proved less effective than NECA at attenuating MLDS-induced hyperglycemia and diabetes incidence. Since no selective A2b receptor agonist was available we used the non-selective agonist NECA with the selective A2b receptor antagonist MRS 1754. MRS $1754(0.5 \mathrm{mg} / \mathrm{kg})$ administered $30 \mathrm{~min}$ prior to the NECA $(0.03 \mathrm{mg} / \mathrm{kg} /$ day $)$ prevented the protective effect of NECA on MLDS-induced hyperglycemia and diabetes incidence. NECA $(0.01 \mathrm{mg} /$ $\mathrm{kg}$ /day) also reduced hyperglycemia $(320 \pm 60 \mathrm{vs.} 150 \pm 20 \mathrm{mg} / \mathrm{dL} ; \mathrm{p}<0.05)$, diabetes incidence $(100 \%$ vs. $0 \% ; \mathrm{p}<0.05)$ and insulitis score $(1.3 \pm 0.7$ vs. $0.8 \pm 0.1 ; \mathrm{p}<0.05)$ on day 21 in the cyclophosphamide accelerated NOD mouse model of Type I diabetes.

Conclusion: These results demonstrate that activation of adenosine receptors can attenuate Type I diabetes and the A2b receptor plays the predominant role in this protective effect.

Supported by the National Institutes of Health, Hungarian Research Fund OTKA and Hungarian National R\&D Programme

\section{7}

NK cells in type 1 diabetes

H. Brauner ${ }^{1}$, M. Wareing ${ }^{2}$, M. Flodström-Tullberg' ${ }^{2}$ K. Kärre' ${ }^{1}$, P. Höglund ${ }^{1}$; ${ }^{1}$ IRIS Strategic Research Center, Microbiology, Tumor and Cellbiology, ${ }^{2}$ Center for Infectious Medicine, Department of Medicine, Karolinska Institutet, Stockholm, Sweden.

Background and Aim: Type 1 diabetes is generally thought to be a $\mathrm{T}$ cell-driven autoimmune disease. The Non Obese Diabetic (NOD) mouse develops a disease similar to human type 1 diabetes (T1D). NK cells are part of the early innate immune response and play an important role in fighting certain viruses, tumours and in rejection of bone marrow transplants. A role for NK cells in autoimmune disease has been suggested, but the literature is conflicting. This project aims at elucidating a possible role of NK cells in T1D.

Materials and Methods: 200ug of the antibody to IL-2R $\beta$ (TM $\beta 1$ ) was given i.p. once weekly to NOD mice and urine glucose measured. NK cells were sorted away from NOD splenocytes on the basis of their high DX5 expression and then transferred i.v. to NOD RAG-/- C $\gamma-/-$ mice. The influence of NK cells on T cell priming were investigated by transferring $\mathrm{T}$ cells labeled with the fluorescent dye CFSE to NOD mice treated with TM $\beta 1$ or PBS. CFSE distributes equally in divided cells, and was used to measure proliferation after 3 days. In order to investigate the phenotype of pancreatic NK cells without contamination of lymphocytes from blood or lymph nodes the mice were flushed with PBS via the heart and lymph nodes removed under microscopy.

Results: Most NK cells, but also some T cells express IL-2R $\beta$. Depleting NOD mice with an antibody to IL-2R $\beta$ (TM $\beta 1$ ) protects from T1D, also when administered only close to disease onset. To further address a possible role for NK cells T1D, NOD splenocytes with NK cells sorted away or still present were transferred to NOD recipient mice lacking T, B and NK cells (NOD RAG-/- C $\gamma-/-)$. Preliminary results show that NK cell removal led to less diabetes in the recipient after transfer, suggesting a pathogenic role for NK cells in this model. We currently address whether NK cells influence T cell priming in the pancreatic lymph node (PLN) or play a pathogenic role in the pancreas itself in the NOD model. Preliminary results suggest that NK cells do not affect the proliferation of T cells in the PLN. The possibility that NK cells may regulate cytokine production by these proliferating $\mathrm{T}$ cells is being investigated. Our results show that NK cells are present in the pancreas of NOD mice and that they display a distinct and more activated phenotype compared to spleen NK cells. A kinetic analysis revealed that pancreatic NK cells have acquired this activated phenotype already at 4 weeks of age and that the percent NK cells over T cells are higher in young NOD mice, suggesting an early role in the pathogenesis. Present investigations include the location and the functional properties of the pancreatic NK cells.

Conclusion: The role of NK cells in type 1 diabetes is still controversial. Our results from murine studies suggest that NK cells may play a pathogenic role in this disease, and that the effect of the NK cells may be in the pancreas itself.

Supported by the Swedish Diabetes Association 


\section{8}

Autoaggressiveness and regulatory potential in the LEW.1AR1-iddm rat, an animal model of human T1DM

T. Arndt ${ }^{1,2}$, A. Joerns ${ }^{3}$, H. Weiss ${ }^{4}$, M. Tiedge ${ }^{4}$, S. Lenzen ${ }^{1}$, H. J. Hedrich ${ }^{2}$, D. Wedekind ${ }^{2}$;

${ }^{1}$ Hannover Medical School, Institute of Clinical Biochemistry,

${ }^{2}$ Hannover Medical School, Institute of Laboratory Animal Science, ${ }^{3}$ Hannover Medical School, Centre of Anatomy, ${ }^{4}$ University of Rostock, Institute of Medical Biochemistry and Molecular Biology, Germany.

Background and Aims: The LEW.1AR1-iddm rat - an animal model of human Type 1 diabetes mellitus (T1DM) - originated through a spontaneous mutation within the congenic inbred strain LEW.1AR1. Adoptive transfers of lymphocytes have shown that both autoaggressive and protective cells can be transferred. The aim of this study was to characterise the T-cell subpopulations capable to induce and to prevent T1DM in LEW.1AR1-iddm rats.

Materials and Methods: T-cells were isolated by MACS separation using monoclonal antibodies. CD4+ or CD8+ T-cells from diabetic LEW.1AR1iddm rats were selectively transferred into athymic LEW.1AR1-Whnrnu rats. CD4+ and CD8+ T-cells from LEW.1AR1 rats were transferred into pre-diabetic LEW.1AR1-iddm rats. Purity of T-cell populations was varified by flow cytometry using a set of monoclonal antibodies: CD4 (OX-38) FITC and CD3 (G4.18) PE, (BD); CD8 (OX-8) FITC, CD8 (OX-8) PE, G4.18 PE, R73 FITC, CD45RA (OX-33) FITC, NKR (10/78) FITC, ED1 FITC (Serotec).

Results: After adoptive transfer of CD4+ T-cells from diabetic LEW.1AR1iddm rats into athymic LEW.1AR1-Whnrnu rats $60 \%$ of the recipients developed diabetes while CD8+ T-cells remained healthy. Only $10 \%$ of the athymic recipients became diabetic after transfer of both CD4+ and CD8+ T-cells. On the other hand, CD8+ T-cells adoptively transferred from T1DMresistant LEW.1AR1 rats into pre-diabetic LEW.1AR1-iddm rats were able to prevent T1DM development. The transfer of CD4+ T-cells alone as well as a combination of CD4+ and CD8+ T-cells had no effect on diabetes incidence in LEW.1AR1-iddm recipients.

Conclusion: Our results show that T1DM can be provoked by transfer of CD4+ T-cells from LEW.1AR1-iddm rats while T1DM can be prevented by transfer of CD8+ T-cells from LEW.1AR1 rats. These findings indicate that the mutation in the LEW.1AR1 strain leads either to a gain of autoaggressiveness or to a loss of diabetes protection in LEW.1AR1-iddm rats. In diabetic patients both $\mathrm{CD} 4+$ and $\mathrm{CD} 8+\mathrm{T}$-cell subpopulations have been discussed as lymphocytes bearing autoaggressive potential. But recently, it has been shown that in humans regulatory CD8+CD25+ T-cells are present which have a function similar to CD4+CD25+ T-cells. Thus, the LEW.1AR1-iddm model and its coisogenic LEW.1AR1 control strain provide a valuable experimental tool to characterise the regulatory function of CD8+ T-cells as well as the autoaggressive potential of CD4+ T-cells in T1DM development.

\section{9}

Recent-onset type 1 diabetes: clinical remission is associated with increases both in in CD25+ and CD 161+ regulatory subsets of T lymphocytes

T. Milicic ${ }^{1}$, N. M. Lalic ${ }^{1}$, A. Jotic ${ }^{1}$, I. Markovic ${ }^{2}$, B. Djuricic ${ }^{2}$, M. Zamaklar ${ }^{1}$, K. Lalic ${ }^{1}$, L. Lukic ${ }^{1}$, N. Rajkovic ${ }^{1}$;

${ }^{1}$ Institute for Endocrinology, Clinical Center of Serbia, Belgrade, Serbia, ${ }^{2}$ Institute for Biochemistry, School of Medicine, Belgrade, Serbia.

Background and Aims: Previous studies have reported an important role of the changes in CD4+ T cell subsets in the initial phase of Type 1 diabetes (T1D). However, the relevance of the changes in CD25+ and CD161+ subsets of the regulatory $\mathrm{T}$ cells, potentially exerting suppressive effect on the ongoing autoreactive response during the initial phase of T1D, has not been clarified. Therefore, the aim of this study was to analyze the percentage of regulatory CD25+ and CD161+ subsets of CD4+ T lymphocytes (a) during the first year of the clinical course of the disease comprising (1) insulin-requiring state (IRS) at the onset and (2) the state of clinical remission (CR) in 37 recentonset T1D patients (group A) as well as (b) in 20 healthy, age-matched control subjects (group B).

Materials and Methods: The CR was defined as optimal metabolic control without insulin lasting $>30$ days. The percentages of $\mathrm{CD} 3+$, total $\mathrm{CD} 4+$,
CD4+CD25+ and CD4+CD161+ T cell subsets were analyzed in peripheral blood by using two-color immunofluorescence staining and flowcytometry. Results: The percentages of $\mathrm{CD} 3+$, and total $\mathrm{CD} 4+\mathrm{T}$ cells did not differ significantly neither between IRS and CR in the group A, nor between the groups A and B. However, when the percentage of CD4+CD25+ Tlymphocytes was analyzed, we found that at onset of T1D, in IRS, it was found to be lower in group A vs group B (A/IRS: $2.32 \pm 0.31$ vs B: $5.10 \pm 0.70 \%, p<0.01)$. In contrast, in the state of $\mathrm{CR}$, in group A the percentage of CD4+CD25+ T cells increased and it became higher, reaching the values similar to those in group B (A/CR: $4.10 \pm 0.12 \mathrm{p}=\mathrm{NS}$ ), Moreover, the percentage of CD4+CD161+ T cells was found to be lower at IRS in group A vs group B (A/IRS: $3.35 \pm 0.68$ vs $\mathrm{B}: 6.08 \pm 1.12, \mathrm{p}<0.01)$, while in the state of $\mathrm{CR}$ it also increased and became similar to those in the group B (A/CR: $5.87 \pm 0.82, \mathrm{p}=\mathrm{NS})$.

Conclusion: Our results have demonstrated that in recent-onset T1D patients, the state of CR was associated with an increase in both CD25+ and CD161+ regulatory CD4+ $\mathrm{T}$ cell subsets. The results imply that the appearance of the CR might be strongly influenced on the level of the activity of the suppressive subsets of the regulatory $\mathrm{CD} 4+\mathrm{T}$ lymphocytes.

\section{0}

Abnormal monocyte-related biomarkers in type 1 diabetes; a study of identical twins

H. Beyan ${ }^{1}$, H. J. De Witt', R. D. G. Leslie ${ }^{1}$, H. A. Drexhage²;

${ }^{1}$ Centre for Diabetes and Metabolic Medicine (DMM), Institute of Cell and

Molecular Science, London, United Kingdom,

${ }^{2}$ Dept Of Immunology, Erasmus MC, Rotterdam, The Netherlands.

Background and Aims: Type 1 diabetes (T1DM) is due to an aggressive adaptive immune response but the innate immune system could be relevant since monocytes infiltrate the islets. Type 1 diabetes is considered to be an autoimmune disease, in which $\mathrm{T}$ cells specific for beta cell antigen (e.g. insulin and glutamic acid decarboxylase 65) target the islet, which finally results in the destruction of the beta cells. We have detected changes in monocytes in T1DM patients including a cluster of altered genes expression profiles compared with normal subjects. Our aim was to identify monocyterelated biomarkers in identical twin pairs discordant for T1DM (only one twin with diabetes) to determine which gene clusters were diabetes associated and which were associated with disease-risk.

Materials and Methods: We studied 14 identical twin pairs (all discordant for T1DM) 6 male; mean age of 36.5 (range17-53 years) with mean disease duration 20.5 (7-44 years) and 13 normal identical twin pairs, 7 male; mean age of 37 (23-55 years). Peripheral blood mononuclear cells (PBMC) were isolated using standard procedure on Ficoll. Monocytes CD14+ were isolated from frozen PBMC suspensions by MACS-CD14 separation following manufacture's instructions and RNA extracted by Qiagen (Qiagen, Hilden, Germany). Real-time quantitative RT- PCR was preformed using TaqMan 7700 for the following genes previously identified using Affymetrix systems: HSP70, MAPK6, PAC1, PTPN7, CCL2, CCL7, PTX3, COX-2, A20, EMP1, CDC42, STX1a, DHRS, FABP5 plus appropriate controls including Able gene (stable housekeeping gene).

Results: Abnormal expression levels of 11 monocyte genes were identified encompassing changes in monocyte pro-inflammatory features, MAP kinase pathway, cell adhesion/motility and immune regulation. In the diabetic twin a significantly abnormal level of gene expression was detected in the (e.g. FABP5, MAPK6, STX1a), whilst both diabetic and non-diabetic twins had altered expression of a different set of genes compared to controls (e.g.CCL2, CCL7, HSP70, SDR)(all differences $<0.02$ ). Gene expression levels were strongly correlated between twins of a pair and were not altered in patients with type 2 diabetes excluding a hyperglycaemic effect. This finding implies monocytes are altered in T1DM and we report for the first time the identification of monocyte molecular signature that may distinguish diabetic from non-diabetic twins.

Conclusion: In summary, these results indicate that there are monocyte signatures of altered gene expression levels which are associated with, on the one hand, familial, probably genetic, susceptibility to T1DM or, on the other hand, with T1DM. These signatures are distinct, reflect potential differences in altered monocyte function, and suggest targets for therapeutic intervention. Supported by JDRFI 


\section{1}

Expression of human insulin gene in gastrointestinal tracts X. Yancheng, N. Li, S. Jiazhong;

Department of Endocrinology, Medical College of Wuhan University, Wuhan China, China.

Background and Aims: Type 1 diabetes mellitus is the result of insulin deficiency caused by the autoimmune destruction of insulin-producing pancreatic $\beta$ cells. Development of gene therapy has also generated much hope and excitement for a possible "cure" for diabetes since insulin was first cloned and expressed in cultured cells in the late 1970s. The aim is to study the expression of human insulin genes in gastrointestinal tracts of rats with type 1 diabetes.

Materials and Methods: Plasmids isolated with a large-scale alkaline lysis procedure were purified. Experiments were carried out on 45 8-week-old male Wistar rats (180-190g). After an overnight fast, rats were given a single intraperitoneal injection of either $60 \mathrm{mg} / \mathrm{kg}$ streptozotocin in $0.01 \mathrm{M}$ citrate buffer. 40 rats with fast serum glucose level $>16.7 \mathrm{mmol} / \mathrm{L}$ were used in the study. Chitosan-DNA nanoparticles were transfected to diabetes rats by lavage and coloclysis respectively. 2 control groups were treated with Chitosan and normal sodium respectively. Fasting blood glucose and plasma insulin were measured. RT-PCR analysis was performed to confirm the expression of human insulin gene.

Results: Fasting blood glucose levels of the lavage group and coloclysis group decreased significantly compared with control groups $(\mathrm{P}<0.01)$. And plasma insulin levels of the lavage group and coloclysis group were much higher than those of the control groups accordingly $(\mathrm{P}<0.01)$. The human insulin gene mRNA was only detected in the lavage group and the coloclysis group.

Conclusion: Human insulin gene embedded with chitosan nanoparticle can be transfected to rats successfully by gastrointestinal tracts, indicating that chitosan is a promising non-viral vector. Compared with other non-viral vectors, chitosan has some merits as followed: (1) It has good biocompatibility, without any toxicity,and can be obtained economically. (2) We can regulate the transfection efficiency of chitosan and intaking of cells by changing molecular weight of chitosan, plasmid concentration, and the ratio of chitosan and plasmid. (3) After the plasmid is embeded in chitosan, it can resist degradation of nucleas.

\section{PS 26 Immunopathogenesis of animal and human diabetes}

\section{2}

Lowered expressions of the NF- $\mathrm{BB}$ family members in dendritic cells from NOD mice are associated with reduced expression of GATA-2 K. Takahashi ${ }^{1}$, Y. Oka ${ }^{2}$, J. Satoh ${ }^{1}$;

${ }^{1}$ Department of Diabetes and Metabolism, Iwate Medical University, Morioka, ${ }^{2}$ Department of Molecular Diabetes and Metabolism, Tohoku Univeresity, Sendai, Japan.

Background and Aims: Dendritic cells (DC) are central to the initiation of immune response and maintenance of self-tolerance. In type 1 diabetes, DC display defective phenotype and function in type 1 diabetes, and possibly play crucial roles in the pathogenesis of this disease. To characterise molecular changes in $\mathrm{CD} 11 \mathrm{c}^{+}$bone marrow (BM)-derived DC from NOD mice, a model of type 1 diabetes, we compared transcript profiles of these cells with those from the sister strain, NON mice. In the present study, we analaysed our microarray data focusing on the NF- $\mathrm{\kappa B} /$ Rel family members and the related molecules associated with DC development, function, and phenotype.

Materials and Methods: BM cells from 4-week-old female NOD and NON mice were cultivated in the presence of GM-CSF and IL- 4 over 5 days, and with LPS for following 2 days. The CD $11 \mathrm{c}^{+} \mathrm{DC}$ were then sorted by magnetic beads-conjugated anti-CD11 $\mathrm{c}^{+}$antibodies (MACS ${ }^{\mathrm{TM}}$, Miltenyi Biotech Inc, Bergisch Gladbach, Germany). RNA extracted from the CD11 $\mathrm{c}^{+} \mathrm{DC}$ from 3 independent mice $(100 \mu \mathrm{g} /$ mouse) was pooled, and used to prepare targets for hybridization to the GeneChip Mouse Genome 430A Arrays (Affymetrix, Santa Clara, CA).

Results: The DC from 4-wk-old NOD displayed reduced mRNA expressions of NF- $\mathrm{KB}$ components, p65, p50, p52, and RelB, compared to NON mice: the proportions of the each molecule relative to NON DC were 53.9, 54.1, 54.0, and $37.0 \%$, respectively. As expected, they showed lowered mRNA expressions of immunomodulatory molecules regulated by the NF- $\kappa B$ pathway, including IL- $1 \alpha$ (48.8\% vs NON DC), IL-1 $\beta$ (49.9\%), IL-6 (18.4\%), Flt-3L (41.0\%), CD80 (29.6\%), CD86 (51.1\%), 4-1BB (47.2\%) and CD40 (46.5\%), which is partially consistent with the previous reports indicating defective phenotype and function of NOD DC. The reduced expression of a proteasome subunit, beta type 9 (LMP-2) reportedly leads to defects in the NF- $\kappa B$ pathway in NOD mice, however, our microarray data were not in line with this observation: the expressions of all $26 \mathrm{~S}$ proteasome subunits, including LMP-2, and related chaperones were identical between NOD and NON DC (data not shown). The transcription factors for the NF- $\mathrm{KB}$ members, which showed reduced expressions, such as GATA-2 (45.6\% vs NON), heat shock factor 2 (22.6\%), and runt related transcription factor $1(44.3 \%)$, were noted as the more likely culprits.

Conclusion: The lowered expressions of NF- $\mathrm{KB}$ components, possibly due to defective expression of the upstream transcription factors, underlie the defective phenotype and function of DC from NOD mice, and could predispose to autoimmunity. It is of interest that the GATA-2 gene (Chr6, $38.5 \mathrm{cM})$ locates close to Idd20 (Chr6, $37.7 \mathrm{cM})$.

Supported by the Japan Society for the Promotion of Science

\section{3}

Suppressed Th1 and induced naive $\mathrm{T}$ cell phenotype in type 1 diabetic children

M. Hedman, M. Faresjö, S. Axelsson, J. Ludvigsson, R. Casas; Department of Molecular and Clinical Medicine, Pediatrics, Linköping, Sweden.

Background and Aims: Type 1 diabetes (T1D) is considered to be caused by an inflammatory process with selective destruction of the insulin producing beta-cells, mediated by $\mathrm{CD} 4^{+} \mathrm{T}$ helper $(\mathrm{Th})$ and $\mathrm{CD}^{+}$cytotoxic $\mathrm{T}(\mathrm{Tc})$ cells. Chemokines play important roles in inflammatory and immunological processes by their capacity to recruit selective subsets of leukocytes expressing their corresponding receptors. However, migration of inflammatory cells into tissues can itself be a cause and inducer of tissue damage. Identifying the signals controlling the recruitment of inflammatory cells into the pancreas might be a novel strategy to inhibit insulitis. This study aimed to determine the chemokine and chemokine receptor profile of $\mathrm{CD} 4^{+}$and $\mathrm{CD} 8^{+} \mathrm{T}$ cells in 
T1D children during the first 18 months after diagnosis in relation to healthy children.

Materials and Methods: Peripheral blood mononuclear cells (PBMC) were collected from 44 children (10-18 years of age; 16 boys and 28 girls) within 18 months after diagnosis of T1D and from 12 healthy children (11-15 years of age; 4 boys and 8 girls). The T1D children were grouped according to duration of disease at the time of blood sampling as follows: 0-4 months; 5-9 months, 10-14 months and 15-18 months duration. Expression of chemokine receptors (CCR7, CCR5, CXCR3, CXCR6, IL-18R and CCR4) on CD4 $4^{+}$and $\mathrm{CD} 8^{+}$lymphocytes were analysed by flow cytometry. Secretion of chemokines (IP-10, MCP-1, MIP-1alfa, MIP-1beta and RANTES) in cell supernatants after 72 hours cell culture was detected by multiplex fluorochrome technique (Luminex).

Results: The percentage of $\mathrm{CD}^{+}$cells expressing CCR7 $(\mathrm{p}<0.0001)$ and CD45RA $(p<0.005)$ was high among the T1D children, especially in those with less than nine months disease duration, in comparison to healthy children. There was a steady decline in expression of CCR7 and CD45RA with duration of disease and at 15-18 months duration the expression had decreased to levels almost comparable to healthy children. A positive correlation was observed between these naive receptors $(r=0.57 ; \mathrm{p}<0.0001)$ among the T1D patients. As expected, expression of the maturation marker CD45RO was inversely correlated to CD45RA on $\mathrm{CD}^{+}$cells $(\mathrm{r}=-0.78$, $\mathrm{p}<0.0001$ ). The percentage of cells expressing CD45RO was lower among T1D children during the first nine months after diagnosis in comparison to healthy children $(p<0.001)$, while the expression increased with longer duration of disease. The percentage of $\mathrm{CD}^{+}$cells expressing the Th1-associated receptors CXCR3 $(\mathrm{p}=0.001)$ and CXCR6 $(\mathrm{p}<0.01)$ was low in T1D patients compared to healthy children. CXCR3 correlated with another Th1-like receptor, CCR5 $(\mathrm{r}=0.45 ; \mathrm{p}<0.005)$ on $\mathrm{CD} 4^{+}$cells. In contrast, CCR7 was inversely correlated to CCR5 $(r=-0.45 ; \mathrm{p}<0.005)$ but positively with CXCR6 $(\mathrm{r}=0.46 ; \mathrm{p}<0.005)$ on $\mathrm{CD}^{+}{ }^{+}$cells. Secretion of the chemokines IP-10, MIP-1alfa and MIP-1beta were low in T1D children compared to healthy controls $(\mathrm{p}<0.001 ; \mathrm{p}<0.01$; $\mathrm{p}<0.01$, respectively).

Conclusion: Low expression of Th1-associated receptors together with an induced naïve Tc cell phenotype in the early phase after diagnosis suggests that the inflammatory process in the period close to diagnosis seems to recruit new cytotoxic $\mathrm{T}$ cells, which in turn could lead to enhancement of the inflammatory process. Increased knowledge of $\mathrm{T}$ cell phenotypes present in the disease process leading to T1D might lead to important clues in developing novel therapeutics for the prevention of disease.

Supported by Swedish Child Diabetes Foundation and Swedish Research Council

\section{4}

The human insulin B chain is covered with overlapping epitopes binding to protective (HLA-DQ6) and susceptible (HLA-DQ2/8) type 1 diabetes alleles

G. K. Papadopoulos ${ }^{1}$, A. K. Moustakas², R. A. Ettinger ${ }^{3}$;

${ }^{1}$ Laboratory of Biochemistry and Biophysics, Epirus Institue of Technology, Arta, Greece, ${ }^{2}$ Department of Organic Farming, Technological Educational Institute of Ionian Islands, Argostoli, Greece, ${ }^{3} \mathrm{RH}$ Williams Laboratory of Endocrinology, Univeristy of Washington, Seattle, United States.

Background and Aims: To scan the insulin B chain, already shown to be highly diabetogenic in NOD mice, for epitopes to the protective HLADQ0602 allele, besides the already-established InsB6-14 core nonamer.

Materials and Methods: The insulin B chain was scanned for possible core nonamers by testing via energy minimisation other possible nonamers for putative binding to HLA-DQ6. All candidate nonamers were then tested via energy minimisation software Discover (Accelrys, SanDiego CA) in a Silicon Graphics Octane II instrument, in order to determine possible fitiing of the peptide in the groove.

Results: The scanning of the molecule revealed two possible epitopes, B12-20 (VEALYLVCG) and B15-23 (LYLVCGERG), of which the former fitted much better into the respective groove upon energy minimisation. All five anchor residues satisfy the motif very well in the InsB12-20 epitope, while p6Gly is a very weak anchor in the InsB15-23 epitope. These two HLA-DQ0602 epitopes, along with the already identified InsB6-14 core nonamer, are overlapping to the established epitope InsB13-21 of the susceptibility allele HLA-DQ8cis, as well as -DQ8trans and -DQ2trans alleles, and the recently identified InsB8-16 HLA-DQ2cis epitope.
Conclusion: The sum total of the three epitopes that have affinity for the protective and biochemically very stable allele HLA-DQ0602, and overlapping epitopes associated with susceptibility to t1d, suggests that epitope stealing is one possible mechanism for protection from tld, to the extent that the insulin B chain will be shown to be as potently diabetogenic in man as in NOD mice.

Supported in part (to GKP) by a grant from $3^{\text {rd }}$ European Community Support Programme ARCHIMEDES I

\section{5}

Newly-diagnosed type 1 diabetic patients have distinct differences in the number and cell surface markers (TGF $\beta$, InsR) of regulatory T (Tregs) and effector $\mathrm{T}$ (Teff) lymphocytes compared to controls

S. A. Paschou ${ }^{1}$, G. Vartholomatos ${ }^{2}$, L. Dova ${ }^{2}$, N. Kolaitis ${ }^{3}$, E. Giotaki ${ }^{4}$, A. Tsatsoulis' 1 , G. K. Papadopoulos ${ }^{5}$;

${ }^{1}$ Endocrinology Clinic, University of Ioannina Regional Hospital, Greece, ${ }^{2}$ Haematology Laboratory, Molecular Biology Unit, University of Ioannina Regional Hospital, Greece, ${ }^{3}$ Haematology Laboratory, University of Ioannina Regional Hospital, Greece, ${ }^{4}$ Department of Nursing, Epirus Institute of Technology, Ioannina, Greece, ${ }^{5}$ Laboratory of Biochemistry and Biophysics, Epirus Institute of Technology, Arta, Greece.

Background and Aims: T regulatory lymphocytes (Tregs, CD $4^{+} \mathrm{CD} 25^{\mathrm{hi}}$ ) play an important role in tolerance and autoimmunity. Our aim was to investigate the expression of key markers on Tregs and T effector cells (Teff, $\mathrm{CD} 4^{+} \mathrm{CD} 25^{+}$) that may be the targets of Tregs, and to identify differences in the presence and intensity of certain markers between newly diagnosed type 1 diabetes (t1d) patients and controls.

Materials and Methods: Peripheral blood from 7 newly-diagnosed type 1 diabetes patients (ages $16+/-10.7,5 \mathrm{M} / 2 \mathrm{~F}$ ) and 22 healthy controls having no first or second degree relatives suffering from autoimmune diseases (ages $25.5+/-9.1,7 \mathrm{M} / 15 \mathrm{~F}$ ), was analysed via triple colour flow cytometry for characteristics of the Treg, and Teff populations. The cut-off line between Tregs and Teff was decided from the dot and contour plots, and confirmed by the higher expression of the transcription factor FoxP3 and the much lower membrane expression of IL-7R (CD127) in Tregs compared to Teff. The markers analysed for were TGF $\beta$, TGF $\beta$ RII, HLA-DR, HLA-DQ, IL-2R $\beta$ (CD122), and InsR (CD220).

Results: Newly-diagnosed type 1 diabetics had a significantly lower percent of Tregs (as percent of total CD4 ${ }^{+} \mathrm{T}$ cells) compared to controls: $1.02+/-0.27$ vs $2.29+/-0.56(\mathrm{p}=0.01)$. Where tested, Teff cells also expressed FoxP3 yet at lower percent and intensity compared to Tregs. Consistently, Treg cells from newly-diagnosed t1d patients exhibit to a lower extent than normals the markers of membrane TGF $\beta$ (mean $+/-$ SD, p value): $70.2 \%+/-12.9$ vs $90.9 \%$ +/- 4.8 ( $\mathrm{p}=0.02$ ), but at higher Mean Fluorescence Intensity (MFI): $198.2+/-$ 53.78 vs $154+/-119.1$ (p>0.05), and InsR (63.8\% +/- 15 vs. $83.2 \%+/-12.5$ $(\mathrm{p}<0.05)$ with MFI of $221+/-88$ vs. $96.5+/-22.1(\mathrm{p}<0.05)$. By contrast, a higher percent of Teff from tld express InsR compared to controls (24.14 vs. 17.7, $\mathrm{p}<0.05)$ with no significant differences in MFI; there is no significant difference in the percent $(55.6+/-10.5$ vs. $61.9+/-19.3, \mathrm{p}>0.05)$ or MFI of Teff expressing TGF $\beta$ RII between patients and controls. The majority of Teff cells from nearly all patients and controls express the activation markers CD122, HLA-DR, HLA-DQ, but with no significant differences between them.

Conclusion: Tregs that participate in the control of autoimmunity are significantly fewer in newly-diagnosed t1d patients compared to controls, and a lower proportion of them express TGF $\beta$ and InsR. Yet the intensity of expression of InsR in t1d Tregs is much higher, implying a compensatory mechanism for the substantial decrease in Treg numbers. By contrast more Teff cells from newly-diagnosed t1d patients express the InsR, a sign of the increased activity of such cells. If Teff in human t1d are unresponsive to Tregs acting via their membrane TGF $\beta$, this cannot be accounted for by lack of TGF $\beta$ RII in the former cells. The interplay of TGF $\beta$ and InsR in Tregs and Teff leading to $\mathrm{t} 1 \mathrm{~d}$, indicated here for the first time, must be further investigated. Supported by a grant (to GKP) from $3^{\text {rd }}$ European Community Support Programme ARCHIMEDES I 


\section{6}

Impaired immune activity is associated with increased expression of glucotransporter 4 (glut4) in natural killer (nk) cells of subjects in the prediabetic state

P. J. Piatkiewicz ${ }^{1}$, A. Czech ${ }^{1}$, J. Taton ${ }^{1}$, M. Kniotek ${ }^{2}$, A. Gorski²;

${ }^{1}$ Chair and Department of Internal Diseases and Diabetology,

Warsaw Medical University,

${ }^{2}$ Department of Clinical Immunology, Warsaw Medical University, Poland.

Background and Aims: Incorrect metabolic conditions (hyperglycemia) influence peripheral blood NK cells that are thought to represent the first line defense in the immune system. This relationship can be particularly expressed by glucose transporter proteins in these cells. The earlier obtained results revealed significant differences in glucose transport between NK cells of healthy subjects and prediabetic patients. The aim of this study was to evaluate the number and activity of NK cells obtained from prediabetic subjects in comparison with healthy control subjects and to determine the quantitative expression of insulin-dependent GLUT4 in these cells.

Materials and Methods: The study group included 6 patients with a diagnosis of the prediabetic state, according to $\mathrm{WHO}$ criteria, naive to any hypoglycaemic drugs. As a control group 6 carefully matched healthy subjects were enrolled. Peripheral blood mononuclear cells (PBMC) were isolated by Ficoll gradient centrifugation. The K562 human erythroleukemia cell line was used as the standard target for human NK cytotoxicity assay. K562 were washed in PBS and labelled with DIO (3,3-dioctadecyloxacarbocyanine perchlorate) for $20 \mathrm{~min}$ at $37^{\circ} \mathrm{C}, 5 \% \mathrm{CO}_{2}$. After two washes in PBS cells were adjusted to $10^{6} \mathrm{cell} / \mathrm{ml}$ in medium (RPMI $10 \% \mathrm{FCS}$ ). Target and effector cells were added to reach effector/target rations: 50:1, 12:1. Dead target cells were stained with PI (propidium iodide). After 4 hours of incubation data were collected for analysis on the Becton-Dickinson FACScalibur flow cytometer. Live target cells (T) were identified with strong green fluorescence, whereas dead target cells (Td) showed strong green and red fluorescence. The percentage of dead target cells (Td\%) was calculated as follows: \% $\mathrm{Td}=(\mathrm{Td} /$ T) $\mathrm{x} 100 \%$. Specific lysis was calculated as \%Td (cultured with effector cells) -Td (cultured without effector cells). Immunofluorescent phenotyping of NK $\left(\mathrm{CD} 16^{+}\right)$cells in (PBMC) was performed using specific murine anti-human CD16 PE-conjugated monoclonal antibodies. The data was analyzed using Cell Quest software.

Results: We determined the significant differences between the number $(\mathrm{P}<0.05)$ and activity of NK cells $(\mathrm{P}<0.01)$ obtained from prediabetic subjects and the control group. The prediabetics had an increased number but substantially decreased activity of NK cells when compared to healthy subjects $(12,83 \%$ and $3,3 \%$ respectively vs $9,80 \%$ and $9,2 \%)$. These abnormalities were associated with the higher level of GLUT4 protein in NK cells of prediabetic patients versus control group $(37 \pm 3,9 \%$ vs $13 \pm 2,1 \%)(\mathrm{P}<0.001)$, as shown in flow cytometry.

Conclusion: Increased number and decreased NK cells activity possibly reflect incorrect metabolic conditions of the prediabetic patients. Overexpression of GLUT4 may profoundly alter the immune response and be the cause of decreased NK cell cytotoxicity. We suggest that NK cell pathology shown in this study is the effect of glucotoxicity and predicts subsequent susceptibility to infections in Type 2 diabetes. NK-cells seem to be a promising model for studies on the impairment of immune activity in the prediabetic state and Type 2 diabetes.

\section{7}

Deficient inducibility of heat shock protein 70 in response to stress at the onset of type 1 diabetes

V. Burkart, L. Germaschewski, N. C. Schloot, K. Bellmann, H. Kolb; Institute for Clinical Diabetes Research, German Diabetes Center, Düsseldorf, Germany.

Background and Aims: Type 1 diabetes (T1D) is caused by the immunemediated destruction of autologous pancreatic beta cells. Animal models of the disease demonstrate a defective heat shock protein (hsp) -expression in beta cells resulting in decreased stress-resistance and increased susceptibility to immunological attacks. Furthermore, hsp are involved in the induction and maintenance of self-tolerance, which is severely disturbed in T1D as evident from the immune reactivity evolving against autologous beta cells. These findings strongly implicate that a defective hsp expression contributes to the progression of T1D. Therefore, our present study was designed to investigate the inducibility of hsp70, a prominent member of the hsp family, during the development of T1D.

Materials and Methods: Peripheral blood mononuclear cells (PBMC) were isolated from $8 \mathrm{~T} 1 \mathrm{D}$ patients at the time of diagnosis (before the initiation of insulin therapy) and $8 \mathrm{~d}$ after diagnosis and from 8 patients with a disease duration of $>8$ months ( $5.7 \pm 2.8$ years). Eight healthy controls were assigned to the 2 groups of patients. PBMC were exposed to heat shock conditions $\left(42.5^{\circ} \mathrm{C}, 60 \mathrm{~min}\right)$ followed by a recovery period of 6 or $18 \mathrm{~h}$. Hsp70 protein expression was analyzed by Western Blot; the resulting signals were quantified by chemiluminescence and expressed as Luminescence Units (LU).

Results: Hyperthermia strongly upregulated hsp70 protein expression in PBMC from healthy persons. Already $6 \mathrm{~h}$ after termination of the heat shock treatment Western blot analysis revealed high amounts of hsp70 (152 \pm 52 $\mathrm{LU})$ which remained stable until $18 \mathrm{~h}(159 \pm 51 \mathrm{LU})$. In contrast, PBMC from newly diagnosed T1D patients expressed significantly lower hsp70 levels (38 $\pm 31 \mathrm{LU}) 6 \mathrm{~h}$ after heat treatment $(\mathrm{p}<0.05)$. At $18 \mathrm{~h}$ after heat treatment, hsp70 levels increased to $131 \pm 40 \mathrm{LU}$, similar to the range of control PBMC. Eight d after diagnosis of T1D, when appropriate insulin substitution has normalized metabolic control, PBMC still showed depressed hsp70 inducibility. Compared to the control group hsp70 expression levels in PBMC of patients were reduced by about $50 \%$ at 6 and $18 \mathrm{~h}$ after heat exposure. After a disease duration of $>8$ months hyperthermia-induced hsp70 expression in PBMC of T1D patients was completely normalized. At 6 and $18 \mathrm{~h}$ after termination of the heat treatment hsp70 expression of PBMC from control subjects was in a range of $176-179 \mathrm{LU}$ and in PBMC from diabetic patients in a range of 172 $180 \mathrm{LU}$. To examine a potential impact of inflammatory mediators on stressinduced hsp70 expression PBMC of healthy controls were pre-incubated with interferon $\gamma$ (IFN $\gamma$ ) or transforming growth factor $\beta$ (TGF $\beta$ ) for $24 \mathrm{~h}$ prior to heat shock exposure. Pretreatment with the Th1-associated cytokine IFN $\gamma$ significantly diminished heat-induced hsp70 expression to $58 \pm 15 \%$ of the level observed in heat-treated PBMC without cytokine exposure $(\mathrm{p}<0.05)$. In contrast, the preferentially anti-inflammatory cytokine TGF $\beta$ significantly increased heat-induced hsp70 expression to $130 \pm 12 \%(\mathrm{p}<0.05)$.

Conclusion: Our findings show a deficient hsp70 response to stress in leukocytes of T1D patients at the time of disease manifestation. We conclude that the impaired stress-responsiveness in T1D is largely independent on the quality of the patients' metabolic control but is mediated by the Th1dominated inflammatory milieu prevailing at the onset of T1D.

\section{8}

Study of haematological parameters in type 1 diabetic patients and associated autoimmunity

N. Alonso' ${ }^{1}$, B. Soldevila ${ }^{1}$, E. Colomé ${ }^{1}$, M. Granada ${ }^{2}$, J. Juncà ${ }^{3}$, R. Puig ${ }^{1}$, I. Salinas ${ }^{1}$, E. Martínez-Cáceres ${ }^{4}$, A. Sanmartíi';

${ }^{1}$ Endocrinology and Nutrition, ${ }^{2}$ Clinical Biochemistry, ${ }^{3} \mathrm{Hematology,}$ ${ }^{4}$ Immunology (LIRAD-BST), Hospital Universitari Germans Trias i Pujol, Badalona, Spain.

Background and Aims: Type 1 diabetic patients (DM1) show an increased prevalence of associated organ specific autoimmune agression against other endocrine and nonendocrine tissues. Anemia of chronic disorders (ACD) is a frequent finding in systemic autoimmune diseases. It is characterizead by a relative deficit of erythorpoietin and a decrease of bone marrow erythropoietin activity due to an increase of proinflammatory citokines. The aims of the study were: 1 .-To study the hematological parameters of DM1 patients with associated autoimmunity (group A) and compare them with DM1 patients without associated autoimmunity (group B). 2.-To define the type of anemia present in patients with DM1 with associated autoimmunity. Materials and Methods: Transversal study of $227 \mathrm{DM} 1$ patients (51\% male); age: $33.81 \pm 10$ years; mean disease evolution time: $13 \pm 6$ years; all with normal renal and thyroid function and normoalbuminuria. The following parameters were measured: 1-Immunological: antithyroid peroxidase (aTPO), nuclear (ANA), antigastric parietal cell (CPG) and tissue transglutaminase (tTG) antibodies 2- Hematological: Hemoglobin (Hb), ferritin, cobalamin (RIA), erythropoietin (Epo) (RIA). 3-Inflammatory and biochemical: fibrinogen, Creactive protein (CRP) and $\mathrm{HbAlc}$.

Results: 1.- Prevalence of associated autoantibodies: 63\% of DM1 patients tested positive for 1 or more autoantibodies (group A): $21.9 \%$ aTPO $(17.3 \%$ females), $7.8 \%$ ANA (5.4\% females), $15.2 \%$ aCPG (9.8 females) and $6.2 \%$ tTG (4.5 females). 2.- Hematological parameters (group A vs group B): Hb 13.8 gr/ 
dL vs $14.8 \mathrm{gr} / \mathrm{dL}, \mathrm{p}<0.001$; ferritin $45(20-73) \mathrm{ng} / \mathrm{mL}$ vs 70 (36-115)ng/mL, $\mathrm{p}<0.001$; cobalamin $357(279-518) \mathrm{pg} / \mathrm{mL}$ vs $397(308-516) \mathrm{pg} / \mathrm{mL}$, NS; Epo $11.3(8.8-18.3) \mathrm{mU} / \mathrm{mL}$ vs $11.2(8.7-15.6) \mathrm{mU} / \mathrm{mL}$, NS. 3.- Inflammatory and biochemical parameters (group A vs group B): fibrinogen $332 \pm 88.21 \mathrm{mg} /$ $\mathrm{dL}$ vs $345.6 \pm 85.31 \mathrm{mg} / \mathrm{dL}$, NS; CRP $1(0.94-2.29) \mathrm{mg} / \mathrm{L}$ vs $1.2(0.54-2.99)$ $\mathrm{mg} / \mathrm{L}, \mathrm{NS} ; \mathrm{HbA1c} 7.53 \pm 0.97$ vs $7.56 \pm 1.38$, NS. 4.- Hematological parameters of anemic patients: $4.9 \%$ of patients presented anemia according to the OMS criteria $(\mathrm{Hb}<12 \mathrm{gr} / \mathrm{dL}$ in women and $<13 \mathrm{gr} / \mathrm{dL}$ in men): $3.1 \%$ women and $1.8 \%$ men. Of these, $54.3 \%$ tested positive for 1 or more autoantibodies $(50 \%$ aCPG, 33.3\% tTG). The results of hematological parameters in this group were: $\mathrm{Hb} 11.2 \pm 1.01 \mathrm{gr} / \mathrm{dL}$; ferritin 15 ( 7.7-251)ng/mL; cobalamin 323 (197406) pg/mL; Epo 61.4 (24.4-79.7) mU/mL; fibrinogen 317(245-500) mg/dL; CRP 0.3(0.2-13.9) mg/L.

Conclusion: Patients with type 1 diabetes mellitus and associated autoimmunity, principally of gastric origin, presented anemia most likely due to iron malabsorption and not to the possible proinflammatory state of a second autoimmune disease. This finding could be explained by the fact that the positivity of these autoantibodies responds only to a localized inflammation and not to a systemic inflammatory process.

\section{9}

Enhanced activation of innate immune system in subjects with associated type 1 diabetes mellitus and coeliac disease

S. Brugaletta ${ }^{1}$, C. Smaldone ${ }^{1}$, C. Fabiano ${ }^{2}$, F. Zaccardi ${ }^{2}$, A. Manto ${ }^{2}$,

T. Musella ${ }^{2}$, D. Pitocco ${ }^{2}$, F. Crea ${ }^{1}$, G. Ghirlanda ${ }^{2}$;

${ }^{1}$ Cardiology, ${ }^{2}$ Internal Medicine, Catholic University, Rome, Italy.

Background and Aims: Immune system seems to play a key role in the pathogenesis of type 1 diabetes (T1DM). T1DM is often associated with autoimmune diseases like as coeliac disease (CD). The aim of this study was to assess the effect of associated $\mathrm{CD}$ on both innate and adaptive immune system, by measuring serum level of c-reactive protein (CRP) and blood frequency of $\mathrm{CD} 4^{+} \mathrm{CD} 28^{\text {null }} \mathrm{T}$-cells and $\mathrm{CD} 4^{+} \mathrm{CD} 25^{+} \mathrm{T}$-cells, unusual populations of lymphocytes, involved in autoimmune regulation.

Materials and Methods: We enrolled 30 consecutive patients with T1DM and CD ( 16 males, mean age $28 \pm 5,6$ ); $C D$ was diagnosed by intestinal biopsy and every patient with $\mathrm{CD}$ was on a gluten-free diet. We also enrolled 19 patients with only T1DM ( 10 males, mean age $29 \pm 4,9$ yrs ) as control group. Demographic and clinical data were collected. Blood samples were taken at time of enrolment. Serum level of CRP was measured using a high sensitivity immuno-nephelometric assay. $\mathrm{CD} 44^{+} \mathrm{CD} 28^{\text {null }} \mathrm{T}$-cells and $\mathrm{CD} 4^{+} \mathrm{CD} 25^{+} \mathrm{T}$-cells frequency was measured by flow cytometry.

Results: Serum levels of CRP were significantly higher in patients with T1DM and CD than in patients of control group $(1,99 \pm 3,56$ vs $0,98 \pm 3,63 \mathrm{mg} / \mathrm{L})$ No significant differences in $\mathrm{CD} 4{ }^{+} \mathrm{CD} 28^{\text {null }} \mathrm{T}$-cells $(2,195 \pm 2,94$ vs $2,565 \pm 2,05 \%)$ and $\mathrm{CD} 4^{+} \mathrm{CD} 25^{+} \mathrm{T}$-cells $(1,56 \pm 0,64$ vs $1,43 \pm 0,73 \%)$ frequency and metabolic control (HbA1c 7, $1 \pm 0,4$ vs $7,2 \pm 0,3$ ) were found among the two groups.

Conclusion: Our study shows for the first time a higher serum level of CRP, marker of innate immune system activation, in patients with T1DM and CD than in those with only T1DM. This result show a possible enhanced activation of innate immune system in patients with associated T1DM and $\mathrm{CD}$, whereas it seems that cellular-mediated immune system isn't involved.

\section{0}

Residual beta cells and protracted islet autoimmunity after onset of type 1 diabetes in NOD mice: therapeutic opportunities for promoting beta cell regeneration and rescue from recurring immunity

S. Reddy, R. Chai, J. A. Rodrigues, T. H. Hsu, E. Robinson;

School of Biological Sciences, University of Auckland, New Zealand.

Background and Aims: During type 1 diabetes, the precise fate and characteristics of beta cells which remain after onset of disease are unclear. In this study, the extent of beta cell survival was assessed in diabetic NOD mice at various time-points during increasing duration of disease. Results were correlated with the severity of insulitis, the presence of alpha and delta cells and the expression of glucose transporter-2.

Materials and Methods: Pancreata from adult female non-diabetic NOD mice and diabetic NOD mice at diagnosis and at 1,2,3 and 4 weeks thereafter were obtained ( 5 mice per group and without insulin treatment). Sections of pancreas were analyzed by dual-label immunohistochemistry for the number and distribution of beta, alpha and delta cells per $\mathrm{mm}^{2}$ of islet surface area. The expression of glucose transporter- 2 in insulin immunoreactive beta cells was assessed by immunohistochemistry. The degree of insulitis per group was evaluated on a scale of $0-4$ in pancreatic sections stained by $\mathrm{H} \& \mathrm{E}$. Immunohistochemical and insulitis analyses were also correlated with the phenotypes of islet immune cell-types in sections of pancreas.

Results: The severity of insulitis showed considerable inter-islet variation within each study group. At onset of diabetes, there was a marked degree of insulitis which declined progressively from onset to 4 weeks of disease $(\mathrm{p}=0.0031)$. Although the mean number of beta cells declined sharply at onset of diabetes and remained low during the 4 -week study period, the differences between the various groups did not reach statistical significance $(\mathrm{p}=0.21)$. The mean number of intra-islet alpha and delta cells showed a significant increase with increasing duration of disease ( $\mathrm{p}=0.019$ for alpha cells; $\mathrm{p}=0.0009$ for delta cells). After diabetes onset, occasional islet cells showed co-expression of insulin and glucagon. Immunolabelling for glucose transporter-2 was undetectable in most most insulin-positive cells from 1 week of diabetes. CD4 and CD8 T cells and macrophages were the predominant islet inflammatory cells in the insulitis lesion and persisted until 4 weeks of diabetes, particularly in islets with residual beta cells or those with extensive insulitis. Diabetic islets without beta cells or those containing mostly alpha and delta cells showed either an absence or minimum insulitis.

Conclusion: In diabetic NOD mice, residual beta cells persist for at least 4 weeks after onset, with a concurrent increase in the number of intra-islet alpha and delta cells and a progressive decline in the severity of insulitis. The predominant phenotypes of the islet immune cells were CD4 and CD8 $\mathrm{T}$ lymphocytes and macrophages. The absence of glucose transporter-2 in most insulin-positive beta cells after diabetes onset, suggests that such beta cells may have an impaired response to rising hyperglycaemia. Although the low number of residual beta cells present soon after onset of type 1 diabetes may be amenable to therapeutic regeneration, additional approaches aimed at restoring glucose responsiveness of beta cells and preventing recurrent autoimmunity, may be required.

Supported by Auckland Medical Research Foundation and Child Health Research Foundation of New Zealand 


\section{PS 27 Immunology and prediction of type 1 diabetes}

\section{1}

Insulin autoantibody affinity as predictive marker for type $\mathbf{1}$ diabetes in young children with increased HLA-conferred disease susceptibility H. T. A. Siljander ${ }^{1,2}$, S. Simell ${ }^{3}$, A. Hekkala ${ }^{4}$, T. Salonsaari ${ }^{2}$, T. Simell ${ }^{3}$, O. Simell ${ }^{3}$, J. Ilonen ${ }^{5,6}$, M. Knip ${ }^{7,2}$;

${ }^{1}$ University of Helsinki, Hospital for Children and Adolescents/ Research Laboratory, ${ }^{2}$ Department of Paediatrics, Tampere University Hospital, ${ }^{3}$ Department of Paediatrics, University of Turku, ${ }^{4}$ Department of Paediatrics, University of Oulu, ${ }^{5}$ Department of Clinical Microbiology, University of Kuopio, ${ }^{6}$ Immunogenetics Laboratory, University of Turku, ${ }^{7}$ Research Laboratory, Hospital for Children and Adolescents, Helsinki, Finland.

Background and Aims: Insulin autoantibodies (IAA) are usually the first or among the first autoantibodies to appear during the prediabetic disease process. As transient and fluctuating positivity for IAA is common, the analysis of IAA affinity may help to differentiate type 1 diabetes (T1D)-related IAA positivity from non-progressive IAA positivity. To test this hypothesis we measured the IAA affinities of the first and last prediabetic IAA-positive samples in young children who progressed to overt T1D and in matched non-progressors.

Materials and Methods: In the Finnish Type 1 Diabetes Prediction and Prevention study (DIPP Study) newborn infants were screened for HLA risk genotypes, and those with high (HLA-DQB1*02/0302) or moderate disease risk (HLA-DQB1 ${ }^{\star} 0302$ without protective alleles) were observed for the development of diabetes-related autoantibodies (AABs) and clinical T1D. ICA were used for primary $\mathrm{AAB}$ screening. If a child tested positive for ICA, all his/her samples were analysed for IAA, GAD and IA-2 antibodies. DIPP children, who progressed to clinical T1D and tested positive for IAA before diagnosis, and IAA-positive non-diabetic control children matched for age, gender, HLA risk genotype and IAA titres were included in the present study. IAA were measured with a competitive radiobinding assay and the affinity assessed with GraphPad Prism 5.0.

Results: 64 IAA-positive children (38 males; 59.4\%) presented with T1D at a median age of 3.8 years (0.9-8.8 years). The median IAA affinity of the first IAA-positive sample was $1.35^{10} \mathrm{l} / \mathrm{mol}\left(2.4^{8}\right.$ to $\left.2.4^{11} \mathrm{l} / \mathrm{mol}\right)$ among cases compared to that of $1.32^{10} \mathrm{l} / \mathrm{mol}\left(1.4^{8}\right.$ to $\left.1.1^{12} \mathrm{l} / \mathrm{mol}\right)$ in controls $(\mathrm{p}=0.129)$. The IAA affinities were similar in the last samples analysed, the median affinity being $1.15^{10} \mathrm{l} / \mathrm{mol}\left(3.8^{8}\right.$ to $\left.5.5^{11} \mathrm{l} / \mathrm{mol}\right)$ among cases and $1.08^{10} \mathrm{l} / \mathrm{mol}\left(3.2^{5}\right.$ to $3.4^{12} \mathrm{l} / \mathrm{mol}$ ) among controls $(\mathrm{p}=0.496)$. The affinity values did not change over time in either of the groups ( $\mathrm{p}=0.847$ for cases and $\mathrm{p}=0.942$ for controls). The only difference observed was that the IAA titres increased during the observation period in the cases (median values 13.6 vs. $20.5 \mathrm{RU}, \mathrm{p}=0.011$ ), while the titres remained at a similar level among the controls (median value 15.0 vs. $11.9 \mathrm{RU}, \mathrm{p}=0.104)$.

Conclusion: Although previous studies have suggested that IAA affinity might be useful in the early identification of subjects en route to T1D, the present data indicate that IAA affinity is unable to differentiate between rapid progressors and subjects remaining unaffected or progressing slowly to clinical disease among young children from the general population carrying HLA-conferred susceptibility to T1D.

Supported by Special Public Grants for Medical Research at Tampere, Oulu and Turku University Hospitals, Academy of Finland, Juvenile Diabetes Research Foundation International, Novo Nordisk Foundation and Sigrid Juselius Foundation

\section{2}

Improved diabetes risk assessment in high risk relatives: role of autoantibodies to IA-2 $\beta$

P. Achenbach ${ }^{1}$, E. Bonifacio ${ }^{1}$, E. A. M. Gale ${ }^{2}$, P. J. Bingley ${ }^{2} \&$ the ENDIT Group,

${ }^{1}$ Immunology and Prevention, Diabetes Research Institute,

Munich, Germany,

${ }^{2}$ Clinical Science at North Bristol, University of Bristol, United Kingdom.

Background and Aims: Autoantibodies to IA-2 $\beta$ (IA2 $\beta A$ ) identify a subgroup of antibody positive relatives at increased risk. Our aim was to determine the role of IA2 $\beta$ A testing in diabetes prediction in a large, well-characterised cohort of ICA+ relatives followed for 5 years in the multinational intervention trial, ENDIT.

Materials and Methods: IA2 $\beta A$, IA2A, GADA and IAA at study entry were measured by radiobinding assay. FPIR and oral glucose tolerance were determined at baseline. Diabetes risk was assessed by survival analysis.

Results: Of $191 \mathrm{ICA}+/ \mathrm{IA} 2 \mathrm{~A}+$ participants (mean age 14.4 years $\pm 0.7,55 \%$ male), 107 developed diabetes and 84 remained non-diabetic during followup (5 year cumulative risk of diabetes $61 \%$ (95\% CI 53-69)). 137 participants were also GADA+/IAA+ (4Ab), 51 positive for one additional marker $(3 \mathrm{Ab})$ and 3 GADA-/IAA- (2Ab). IA-2 $\beta A$ were detected in 135 (71\%) ICA+/IA2A+ participants and were associated with increased 5 year diabetes risk (IA2 $\beta A+$ $68 \%(58-78)$ vs. $44 \%(30-58)$ in IA2 $\beta A-, p=0.0049)$. The effect was most marked in $4 \mathrm{Ab}+$ relatives (IA2 $\beta A+75 \%(64-86)$ vs. $53 \%(36-70)$ in IA2 $2 A$-, $\mathrm{p}=0.013$ ). Risk assessment was further refined by metabolic testing. Among $1004 \mathrm{Ab}+$ relatives with IA2 $\beta \mathrm{A}$, those with low FPIR $(n=45)$ had 5 year risk of $94 \%$ (84-100) compared with 55\% (35-74) with normal FPIR ( $\mathrm{p}<0.0001)$, and those with IGT $(n=21)$ had risk of $95 \%$ (86-100) vs. $71 \%$ (57-85) with normal glucose tolerance. Median time to diagnosis with $4 \mathrm{Ab} / \mathrm{IA} 2 \beta \mathrm{A}$ and low FPIR was 1.9 years $(1.2-2.7)$. Multivariate analysis confirmed IA2 $\beta$ A status, antibody number, FPIR and IGT as independent determinants of risk. Of all ENDIT participants who developed diabetes, $75 \%$ were IA2A+, $59 \%$ were IA $2 \beta A+, 64 \%$ had low FPIR and $22 \%$ had IGT.

Conclusion: IA2 $\beta A$ are associated with very high risk in ICA+ relatives with three biochemical antibodies and compare favourably with the IVGTT in identifying a subgroup at increased risk but with similar sensitivity. Combining IA2 $\beta$ A determination and IVGTT with standard antibody testing allows identification of relatives with $>90 \% 5$ year risk. IA $2 \beta A$ testing should be routinely added to the screening protocols of future intervention trials. Supported by JDRF, European Union, Novo Nordisk

\section{3}

Islet autoantibody development during follow-up of high-risk children from the general Norwegian population from three months of age: design and early results from the MIDIA study

L. C. Stene ${ }^{1,2}$, E. Wits $\emptyset^{1}$, P. A. Torjesen ${ }^{3}$, T. Rasmussen ${ }^{4}$, P. Magnus ${ }^{1}$, O. Cinek ${ }^{5}$, T. Wetlesen ${ }^{1}$, K. S. Rønningen ${ }^{1}$;

${ }^{1}$ Division of Epidemiology, Norwegian Institute of Public Health, Oslo, Norway, ${ }^{2}$ Diabetes Research Centre, Aker and Ullevål University Hospitals, Oslo, Norway, ${ }^{3}$ Hormone Laboratory, Aker University Hospital, Oslo, Norway, ${ }^{4}$ Division of Administration, Norwegian Institute of Public Health, Oslo, Norway, ${ }^{5}$ Motol University Hospital, 2nd Medical School, Charles University in Prague, Czech Republic.

Background and Aims: Limited data are available on pattern of type 1 diabetes associated autoantibody development from early age in healthy individuals who are not first-degree relatives of persons with type 1 diabetes. The aim was to describe the design of the MIDIA study and present serial islet autoantibody data from three months of age in children from the general population carrying the type 1 diabetes high-risk $H L A-D R B 1 * 0401$ $D Q A 1^{*} 03-D Q B 1^{*} 0302 / D R B 1^{*} 0301-D Q A 1^{*} 05-D Q B 1^{*} 02$ genotype.

Materials and Methods: MIDIA is an observational study with blood samples obtained from children at ages 3, 6, 9 and 12 months and annually thereafter. Plasma autoantibodies to insulin, glutamic acid decarboxylase and insulinoma-associated antigen- 2 were measured with radiobinding assays.

Results: About 25,000 general population newborns were genotyped (by Oct 2006), and among 526 children with the high-risk HLA genotype, 2104 samples were assayed for autoantibodies (range 1-13 samples per child, median 4). Median age at the most recent sample was 12 months (range 2.9 months to 5.3 years). Fourteen children were positive in at least two consecutive samples, including 12 who were positive for $\geq 2$ autoantibodies at least once, of which five developed type 1 diabetes at median age 15.3 months. Seven of 14 persistently positive children seroconverted before nine months, including two before six months of age. The cumulative probabilities of type 1 diabetes and multiple autoantibody positivity at five years estimated using Kaplan-Meier analysis were 2.6\% (95\% confidence interval: 1.0\%-6.4\%) and $7.3 \%$ (95\% confidence interval: $3.5 \%-12.4 \%)$, respectively. 
Conclusion: Persistent islet autoimmunity is not uncommon in the first year of life in children from the general population carrying the high-risk HLA genotype, and may develop as early as at six or nine months of age.

Funded by Research Council of Norway, Norwegian Diabetes Association, Novo Nordisk Fonden, Children with Diabetes Foundation, Roche Norway and Sweden to KSR; OC was funded by the Czech Ministry of Education

\section{4}

A novel auto-antibody for diagnosis of autoimmune diabetes mellitus N. Shehadeh ${ }^{1,2}$, S. Polak ${ }^{1}$, G. Wildbaum² ${ }^{2}$ N. Karin²;

${ }^{1}$ Pediatric Diabetes Unit, Rambam Medical Center, ${ }^{2}$ Faculty of Medicine, Technion - Israel Institute of Technology, Haifa, Israel.

Type 1 diabetes Mellitus (T1DM) is the result of organ-specific autoimmune destruction of the insulin secreting $\beta$-cells in the pancreatic islets of Langerhans. Although the critical events that trigger the autoreactive process in T1DM are not clear, destruction of insulin-producing $\beta$-cells appears to be mediated by the activation of autoreactive T-cells that recognize several islet $\beta$-cells antigens, including insulin, glutamic acid decarboxylase (GAD) 65 and 67 isotypes, heat shock ptotein 60 , and some uncharacterized $\beta$-cells antigens. The pro-inflammatory mediators in this process include two groups of related proteins: cytokines and chemokines. It had been demonstrated that in attempt to restrain autoimmunity, the immune system can selectively promote autoantibodies production in a selective manner to eliminate the "harmful" key mediator of the autoimmune process. In the first part of our research, we have evaluated the presence of a selective auto-antibody response to any of the $\mathrm{C}-\mathrm{C}$ chemokines that are expressed in the inflamed pancreas during the autoimmune process in new onset T1DM patients: 9 out of 10 newly diagnosed patient with T1DM developed a selective auto-antibody response to MIP-1 $\alpha$ (CCL3) and all were negative to other chemokines: MCP-1 (CCL2), MIP-1 $\beta$ (CCL4) as well as to IL-8 (CXCL8) that is exclusively expressed in humans. The second step was to evaluate the presence of auto-antibodies in blood samples taken from the following group of patients: 30 new onset T1DM patients (within one week of diagnosis), 38 patients with prolonged T1DM (> 5 years), 20 normal controls, 20 prediabetic subjects (first degree relatives of patients with T1DM with positive autoantibodies): Anti MIP1 $\alpha$ antibody was positive in 27/30 (90\%) of new onset diabetic patients, 19/20 (95\%) of prediabetic subjects and $1 / 20(5 \%)$ of normal controls (Figure 2). Comparing the presence of anti MIP1a antibodies with other T1DM related autoantibodies in the new onset group: Anti-Insulin, anti-GAD and ICA were positive in $70 \%, 60 \%$ and $63 \%$ respectively compared to $27 / 30$ (90\%) positivety to MIP1a. Moreover, MIP1 $\alpha$ auto-antibody was positive in $27 / 38$ (71\%) of patients with prolonged diabetes (more than 5 years duration). The current diagnosis of autoimmune diabetes is based on the presence of $\beta$ cell related auto-antibodies (Anti-Insulin., anti-GAD and ICA antibodies). A combined test of all 3 auto-antibodies covers $88 \%$ of patients. Therefore, an additional test as anti-MIP1 $\alpha$ will increase accuracy and reliability of the diagnosis of autoimmune diabetes. Moreover, anti-MIP1 $\alpha$ is positive in prediabetic patients and continues to be positive several years after the diagnosis of diabetes. We believe that this important autoantibody could serve as an excellent biomarker for the diagnosis of prediabetic patients, type 1 diabetic patients and autoimmune diabetes in adults and as an important immunological marker in prediction and prevention studies.

\section{5}

Distinct reactivity of cord blood mononuclear cells of newborns with risk of developing type 1 diabetes

K. Stechova ${ }^{1}$, K. Flajsmanova ${ }^{1}$, K. Bohmova ${ }^{1}$, Z. Hladikova ${ }^{1}$, M. Durilova ${ }^{1}$, I. Spalova ${ }^{2}$, D. Bartaskova ${ }^{3}$, P. Pithova ${ }^{3}$, M. Cerna ${ }^{4}$, K. Andelova ${ }^{5}$,

D. Chudoba ${ }^{6}$, M. Pechova ${ }^{7}$, M. Faresjo ${ }^{8}$;

${ }^{1}$ Paediatric Dpt., 2nd Medical Faculty of Charles University Prague, Czech Republic, ${ }^{2}$ Gynaecology and Obstetrics, 2nd Medical Faculty of Charles University Prague, Czech Republic, ${ }^{3}$ Internal Dpt., 2nd Medical Faculty of Charles University Prague, Czech Republic, ${ }^{4} \mathrm{NICU}$, Institute for the Care of Mother and Child, Prague Podoli, Czech Republic, ${ }^{5}$ Internal Dpt., Institute for the Care of Mother and Child, Prague Podoli, Czech Republic, ${ }^{6}$ Dpt.of biology and genetics, 2nd Medical Faculty of Charles University Prague, Czech Republic, ${ }^{7}$ Dpt.of biochemistry, 2nd Medical Faculty of Charles University Prague, Czech Republic, ${ }^{8}$ Div. of Paediatrics and Diabetes Research Centre, Faculty of Health Sciences, Linkoping, Sweden.

Background: Incidence of type 1 diabetes (T1D) is increasing worldwide. The highest increase of T1D incidence in the Czech Republic is observed in pre-school age children. For T1D there is still no potent tool to recognise early signs of cellular autoreactivity, which leads to beta cells damage and development of the disease. The naive immune system of the newborn (not yet influenced by external factors) can be used as an important model for studies of the pathogenesis of T1D.

Aim: To study the response of cord blood mononuclear cells to diabetogeneic autoantigen stimulation in babies with increased risk of developing T1Dby protein microarray.

Methods: Cord blood samples of 22 healthy children (mean gestational age $37+6$ ) born to a diabetic mother (marked as relatives) and 15 newborns with no family history of any autoimmune disease (controls, 38+1) were collected. Diabetes compensation of mothers was without problems (mean of their last GHBC was 5\%). Production of 23 cytokines and chemokines (including all important Th1-, Th2- and Th3- cytokines) by cord blood mononuclear cells were detected. Production of cytokines and chemokines, pre- and post stimulation with a mixture of diabetogenic autoantigens (including GAD65, IA2 and proinsulin derived synthetic peptides), were compared by protein microarray and ELISA. Higher basal as well as autoantigen induced production of GMCSF, GRO, GRO-alpha, IL-3, -7, -8, -15, MCP-2, MCP3 , MIG and RANTES were observed in the control group (at least $p=0,05$ ). The most evident differences between risk newborns and controls were observed in secretion of MCP- $2(p=0,009)$ and $\operatorname{GCSF}(p=0,03)$ by autoantigen stimulation after subtraction of basal secretion.

Conclusions: Basal as well as stimulated cytokine and chemokine production differed between newborns with and without family history of T1D. Neonates with family history of T1D showed in general a lower overall cytokine and chemokine production. This may indicate a less mature immune responsiveness in neonates with increased risk for T1D.

Supported by Proj.No 00064203 of the University Hospital Motol

\section{6}

GAD65 is released into the circulation by injured pancreatic islet $\beta$ cells and functions as a plasma marker of islet injury

S. D. Chessler ${ }^{1}$, M. A. Waldrop ${ }^{1}$, A. T. Suckow ${ }^{1,2}$;

${ }^{1}$ Department of Medicine, University of California, San Diego, La Jolla, ${ }^{2}$ Biomedical Sciences Graduate Program, University of California, San Diego, La Jolla, United States.

Background and Aims: GAD65 is a major autoantigen in autoimmune diabetes. The mechanism whereby autoreactivity to GAD65, an intracellular enzyme, is triggered is unknown, and it is possible that GAD65 may be released by injured pancreatic islet $\beta$ cells. There is a great need for methods by which to detect and monitor ongoing islet injury. While there are no serum markers of islet injury, tests based on the measurement of circulating, discharged enzymes to monitor tissue injury have become an integral part of clinical care and research. If GAD65 is released by injured $\beta$ cells and is furthermore able to reach the circulation, plasma GAD65 could function as a marker of $\beta$-cell injury. The aims of this study were:

1) to determine whether GAD65 is discharged by injured $\beta$ cells, whether discharged GAD65 reaches the circulation, and whether detection of plasma GAD65 may eventually provide a means by which to monitor $\beta$-cell injury. 
2) to provide general proof of principle that detection of discharged proteins in the circulation can provide a means by which to monitor islet injury.

3 ) to test the hypothesis that lethally and sub-lethally injured $\beta$ cells discharge GAD65 in vivo and that therefore such autoantigen release may possibly contribute to the pathogenesis of anti- $\beta$ cell autoimmunity.

Materials and Methods: A novel immunoassay was employed to determine plasma GAD65 levels in rats and mice treated with the $\beta$-cell-specific toxins alloxan and streptozotocin (STZ). Both diabetogenic, $\beta$-cell-lethal, and low, non-lethal doses of STZ were employed. Islet injury was assessed by immunohistochemistry and TUNEL staining. The half-life of GAD65 in the rat circulation was determined by measurement of GAD65 levels after injection of recombinant GAD65. Insulin and C-peptide levels were measured by ELISA.

Results: Alloxan and both low-dose and high-dose STZ induced GAD65 release into the circulation in a dose-dependent manner. GAD65 discharge was prolonged after low-dose treatment. Low-dose treatment also differed from high-dose in that it did not cause DNA strand breakage or alter islet morphology. In high-dose-treated rats, GAD65 release preceded hyperglycemia and, unlike insulin or C-peptide discharge, was consistently observed after toxin treatment. The half-time of elimination of GAD65 was $\sim 3 \mathrm{~h}$. In mice, which have very minimal islet GAD65 content, plasma GAD65 was not detected. In STZ-treated, $\sim 90 \%$ pancreatectomized rats, after an initial, abrupt period of $\beta$-cell injury, plasma GAD65 levels were markedly lower than in control rats.

Conclusion: Taken together, these data demonstrate that GAD65 can be released into the circulation by injured $\beta$ cells. Such autoantigen shedding may contribute to the pathogenesis of autoimmunity in the multiple lowdose STZ model and perhaps, more generally, in other forms of autoimmune diabetes. These results demonstrate that islet injury - at least in some circumstances - can be monitored by use of discharged, circulating proteins. This approach to the detection of tissue injury has proven to be of great utility with a variety of different pathogenic processes in different tissues. This is cause for optimism that this approach will be successful for detecting islet injury caused by processes such as insulitis, transplant rejection and virusinduced islet damage. GAD65 is the first confirmed biomarker of ongoing $\beta$-cell injury.

Supported by the Juvenile Diabetes Research Foundation and the National Institutes of Health

\section{7}

Determination of GAD65 in pancreatic islets and its in vitro and in vivo degradation kinetics in serum using a highly sensitive enzyme immunoassay

M. Schlosser ${ }^{1}$, U. Walschus ${ }^{1}$, I. Klöting ${ }^{2}$, R. Walther ${ }^{3}$;

${ }^{1}$ Department of Medical Biochemistry and Molecular Biology,

Ernst-Moritz-Arndt University Greifswald, Karlsburg,

${ }^{2}$ Department of Laboratory Animal Science, Ernst-Moritz-Arndt University Greifswald, Karlsburg,

${ }^{3}$ Department of Medical Biochemistry and Molecular Biology,

Ernst-Moritz-Arndt University Greifswald, Germany.

Background and Aims: Autoantibodies to GAD65 (GADA) are detectable in high prevalence at onset of T1DM but also in probands at risk of the disease. It has recently been proposed that the antigen itself might be useful as a biomarker of beta cell loss in probands following islet transplantation and in subjects at high risk for the disease. The aim of this study was to examine the GAD65 content of pancreatic islets from different species, the in vitro and in vivo half-life of GAD65 by determination of its degradation kinetics, and its release into circulation after streptozotocin-induced islet destruction.

Materials and Methods: GAD65 was measured using a sandwich ELISA based on two monoclonal GAD65-specific antibodies with different epitope specificities, one of them solid-phase bound for capturing GAD65 and a second biotin-labelled one for detecting bound GAD65. This assay allows the detection of GAD65 with a high sensitivity of $30 \mathrm{pg} / \mathrm{ml}$.

Results: GAD65 was found in quantities of $78 \mathrm{ng}$ (human), $43.7 \mathrm{ng}$ (LEW.1A rat) and $37.4 \mathrm{ng}(\mathrm{BB} / \mathrm{OK}$ rat) per 1,000 islets, respectively. No GAD65 was detectable in islets of $\mathrm{BALB} / \mathrm{c}$ and NOD mice. The in vitro half-life of porcine GAD65 at $37^{\circ} \mathrm{C}$ in human serum, plasma and blood was comparable and amounted to 1.27 hours (CI 95\%: 1.15-1.41), $1.32 \mathrm{~h}(1.23-1.41)$ and 2.16 h (1.75-2.66), respectively. Comparable results were found for human recombinant GAD65 at $37^{\circ} \mathrm{C}$ with individual serum samples of five healthy human blood donors (half-life $2.35 \mathrm{~h}$; CI: 2.03-2.72) as well as with a GADApositive serum from a patient with T1DM (half-life: $1.93 \mathrm{~h}$; CI: $1.55-2.41$ ). These results suggest that the GAD65 degradation kinetics is not affected by the presence of GADA. The recovery rate of GAD65 was 97\% in serum of healthy donors and $75 \%$ in the serum of the GADA-positive patient. After injecting 2,000 ng recombinant human GAD65 into LEW.1A rats, the in vivo half-life was $2.77 \mathrm{~h}$ (CI: 1.70-4.52). GAD65 was undetectable after 24 hours in these animals. Following experimental diabetes induction in LEW.1A rats by a single streptozotocin (STZ) application an increase of mean blood glucose levels from $7.7 \mathrm{mmol} / \mathrm{l}$ before STZ application to $8.2 \mathrm{mmol} / \mathrm{l}$ after $8 \mathrm{~h}, 20.4 \mathrm{mmol} / \mathrm{l}$ after $24 \mathrm{~h}$ and $23.9 \mathrm{mmol} / \mathrm{l}$ after $48 \mathrm{~h}$ indicates a loss of insulin production, however, no GAD65 was detectable in the circulation of animals. Thus, taking the assay's detection limit, the islet GAD65 content as well as the total blood volume into account, at least 13 islets in rat and 1,875 in human must be simultaneously destroyed to detect GAD65 in the circulation.

Conclusion: Therefore, these results should be taken into consideration in further studies aimed at examining the diagnostic relevance of GAD65 to detect graft rejection following islet transplantation. Additionally, studies involving the examination of sera from high-risk prediabetic subjects characterized by a genetic predisposition as well as multiple autoantibodies to islet antigens will help to assess the diagnostic relevance of GAD65 serum levels in the context of autoimmune diabetes.

\section{8}

Detection of free secretory IgA levels in type 1 diabetic patients

G. Nicoloff ${ }^{1}$, A. Blazhev ${ }^{1}$, A. Nikolov ${ }^{1}$, C. Petrova ${ }^{2}$;

${ }^{1}$ Department of Biology \& Pathological Physiology,

${ }^{2}$ Department of Pediatrics, Medical University, Pleven, Bulgaria.

Background and Aims: Secretory $\operatorname{IgA}(\mathrm{S}-\operatorname{Ig} \mathrm{A})$ is the main antibody secreted by the mucosa of the airways, gastrointestinal tract, and other mucosal tissues. A high proportion of Ig-producing cells in the mucosal lymphoid tissue is committed to the IgA isotype, and the majority of IgA in mucosa is derived from local synthesis. The importance of IgA in mucosal immunity is further supported by the presence of secretory component (SC) in SIgA. The SC is a soluble proteolytic cleavage fragment of the extracellular domain of membrane-bound polymeric Ig receptor. Addition of SC to IgA confers increased stability on the resultant S-IgA by helping to hold the IgA monomers together. However the effect of the addition of SC on the biologic activity of $\operatorname{IgA}$ is still unknown. The aim of our study was to detect the levels of free S-IgA in patients with Type 1 diabetes mellitus

Materials and Methods: To this end we used a method for detecting immune complexes CIF-ELISA. Isolation of a new glycoprotein complement inhibition factor (CIF) from the parasitic plant Cuscuta europea seed, which appears to bind specifically to complement component $\mathrm{C} 3$ has been provided an unique tool for the measurement of immune complexes by means of ELISA-type techniques (CIF-ELISA). Free Secretory IgA were detected by using a two-step method consisting of (i) CIF-ELISA to remove immune complexes followed by (ii) a modified ELISA for detection of free S-IgA. The baseline study population consisted of 61 patients with Type 1 diabetes mellitus (mean age $12.3 \pm 4$ years, diabetes duration $5.2 \pm 3.7$ years). The control group consisted of 20 healthy children. Thirty-two of 61 diabetics had vascular complications (group 1) and 29 were without vascular complications (group 2).

Results: Diabetics showed significantly higher levels of free S-IgA $(0,210 \pm 0,101$ vs. $0,159 \pm 0,036 ; \mathrm{p}=0,029)$ than controls. Patients without vascular complications did not show significantly higher levels of free $\mathrm{S}$ IgA $(0,184 \pm 0,076$ vs. $0,159 \pm 0,036 ; p>0,05)$ compared to the control group. Patients with vascular complications showed significantly higher levels of free S-IgA $(0,228 \pm 0,109$ vs. $0,159 \pm 0,036 ; p=0,008)$ than controls. Significantly higher levels of free S-IgA between both patients groups were not observed $(0,228 \pm 0,109$ vs. $0,184 \pm 0,076 ; p>0,05)$. Diabetics showed correlation with age $(\mathrm{r}=0,25 ; \mathrm{p}=0,042)$; duration $(\mathrm{r}=0,27 ; \mathrm{p}=0,05)$; fructose $(\mathrm{r}=0,26 ; \mathrm{p}=0,045)$ and puberty $(\mathrm{r}=0,329 ; \mathrm{p}=0,011)$. Group 1 showed correlation with age $(\mathrm{r}=0,33$; $\mathrm{p}=0,023)$ and puberty $(\mathrm{r}=0,46 ; \mathrm{p}=0,004)$ while Group 2 showed correlation with fructose $(r=0.379 ; p=0.036)$.

Conclusion: These findings show that free serum S-IgA levels are significantly higher in type 1 diabetic children than control group. Further prospective and longer studies of larger populations are needed to identify the role of free serum S-IgA in juvenile diabetes mellitus. 


\section{9}

Adiponectin levels decrease before clinical onset of type 1 diabetes but do not improve disease prediction in antibody-positive relatives

J. De Grijse ${ }^{1}$, I. Truyen ${ }^{1}$, C. Van Schravendijk ${ }^{1}$, D. De Smet ${ }^{1}$, K. Decochez ${ }^{1}$,

E. Vandemeulebroucke ${ }^{1}$, M. Giri ${ }^{2}$, B. Keymeulen ${ }^{1}$, C. Mathieu ${ }^{3}$, L. Van Gaal ${ }^{4}$, P. De Pauw ${ }^{1}$, I. Weets ${ }^{1}$, D. Pipeleers ${ }^{1}$, F. Gorus ${ }^{1}$; Belgian diabetes Registry

${ }^{1}$ Diabetes Research Center, Brussels Free University, ${ }^{2}$ Department of Endocrinology, University Hospital Ghent, ${ }^{3}$ Department of Endocrinology, University Hospital, Leuven, ${ }^{4}$ Department of Diabetology, Metabolism and Clinical Nutrition, University Hospital, Antwerp, Belgium.

Background and Aims: Insulin resistance has been proposed as risk factor for type 1 diabetes. We investigated whether decreased circulating levels of adiponectin - an abundant insulin sensitizer - can serve as additional predictive marker for type 1 diabetes in first degree relatives of known patients.

Materials and Methods: Adiponectin was measured yearly (ELISA, Linco) in 211 persistently islet antibody-positive ( $\mathrm{Ab}^{+}$; positive for IAA, ICA, IA-2A and/or GADA at entry and in a repeat sample) first degree relatives (0-39 years) of type 1 diabetic patients and in 211 age- and sex-matched persistently antibody-negative $\left(\mathrm{Ab}^{-}\right)$relatives. Adiponectin levels were correlated with demographic and anthropometric data, antibody status, random proinsulin/ C-peptide (PI/C) ratio and HLA-DQ genotype. During follow-up (median [interquartile range]: 81 [59-90] months), $37 \mathrm{Ab}^{+}$relatives developed type 1 diabetes.

Results: In the group of 422 relatives, baseline adiponectin correlated inversely with age and body mass index and was lower in males than in females especially after age $15(p<0.001)$. There was no correlation with antibody status or later development of diabetes. In $24 \mathrm{Ab}^{+}$relatives sampled fasted, adiponectin levels correlated significantly with homeostasis model assessment of insulin sensitivity (HOMA\%S; $p=0.006$ ). For 28 prediabetic relatives with at least 2 blood samples before clinical onset, adiponectin levels adjusted for age and sex decreased prior to diagnosis $(p=0.001)$ whereas no decrease occurred in 28 age- and sex-matched $\mathrm{Ab}+$ non-prediabetic relatives followed over a similar interval. Adiponectin increased again significantly after start of insulin therapy $(p=0.003)$ in those relatives for whom a sample after diagnosis was available $(\mathrm{n}=13)$. In $\mathrm{Ab}^{+}$relatives $(\mathrm{n}=211)$, a marked decrease in age- and sex-adjusted adiponectin levels during the first year after study entry could not predict type 1 diabetes nor complement risk assessment based on islet antibodies, $H L A-D Q$ genotype and pancreatic hormones in Cox regression analysis.

Conclusion: Adiponectin levels decrease shortly before clinical onset of type 1 diabetes, possibly reflecting insulinopenia, but do not contribute to disease prediction in $\mathrm{Ab}^{+}$relatives.

Supported by Flemish Research Council (FWO) and JDRF Center grant

\section{PS 28 Pancreas and islet transplantation}

\section{0}

Suppression of rejection for allogenetic islet transplantation in vivo by donor antigen-specific $\mathrm{CD}^{+} \mathrm{CD}^{+} 5^{+}$regulatory $\mathrm{T}$ cells M. Zhang, S. H. Xu, Y. Xu, C. P. Liu, X. D. Mao, C. Liu;

Department of Endocrinology, First Affiliated Hospital of Nanjing Medical University, Nanjing,Jiangsu, China.

Background and Aims: To investigate the potential effect of allogenetic antigen-specific $\mathrm{CD} 4^{+} \mathrm{CD} 25^{+}$regulatory $\mathrm{T}$ cells on the suppression of rejection for allogenetic islet transplantation in vivo.

Materials and Methods: Donor antigen-specific $\mathrm{CD} 4{ }^{+} \mathrm{CD} 25^{+}$regulatory $\mathrm{T}$ cells(Treg cells) were generated by the addition of multiple intravenous injections of ICR mice splenocytes in vivo. Spleen cells were then pooled and enriched for $\mathrm{CD} 4^{+} \mathrm{CD} 25^{+} \mathrm{T}$ cells by negative and positive selection by MACS in vitro, and were injected into diabetic BALB/cByJ mice. Six hundred ICR islets were allogeneically transplanted under the kidney capsule of streptozotocininduced diabetic BALB/cByJ mice with a single dose of as few as $8 \times 10^{5}$ of donor antigen-specific Treg cells $(n=7)$. Islet function was evaluated by in vivo glucose tolerance test. Grafts were harvested for histologic examination when rejection occured or 30 days after transplantation. Homing and distribution of antigen-specific Treg cells were investigated by CFSE and FACS.

Results: In vivo, the mean survival time of recipients with islets and Treg cells were $(34.57 \pm 17.15)$ days, whereas transplanted islets without Treg cells treatment survived a maximum of 13 days in control mice $(\mathrm{p}<0.01)$. At posttransplant day 30 , recipients of islets with Treg cells had similar responses to glucose challenge compared with nondiabetic $\mathrm{BALB} / \mathrm{cByJ}$ mice. On immunohistochemistry, the kidney capsules displayed higher insulin content and neoplastic microvessels than that in control group. The transferred antigen-specific Treg cells were resident in pancreatic node and mesenteric node.

Conclusion: Donor antigen-specific $\mathrm{CD} 4^{+} \mathrm{CD} 25^{+}$regulatory $\mathrm{T}$ cells prolong islet graft survival and normoglycemia in transplanted allogeneic diabetic mice. Therefore, donor antigen-specific $\mathrm{CD} 4{ }^{+} \mathrm{CD} 25^{+}$regulatory $\mathrm{T}$ cells might be used as adjunctive therapy in patients undergoing islet transplantation. Supported by Jiangsu Province 135 Projects

\section{1}

In vitro 1,25-dihydroxyvitamin $\mathrm{D}_{3}$-modulated dendritic cells: their altered $\mathrm{T}$ cell stimulatory capacity is reflected in an islet allotransplantation model E. Van Etten, G. B. Ferreira, L. Overbergh, C. Mathieu; LEGENDO, Katholieke Universiteit Leuven, Belgium.

Background: 1,25-dihydroxyvitamin $\mathrm{D}_{3}\left(1,25(\mathrm{OH})_{2} \mathrm{D}_{3}\right)$ is a potent immunomodulator with the in vivo capacity to prevent and attenuate the occurrence of autoimmune diabetes in NOD mice and to prolong islet allograft survival. On cellular level, $1,25(\mathrm{OH})_{2} \mathrm{D}_{3}$ exerts its effects mainly through interference with normal dendritic cell (DC) development and behavior. A therapy based on the transfer of DCs that have been treated in vitro with $1,25(\mathrm{OH})_{2} \mathrm{D}_{3}$ could bypass the hypercalcemia observed upon in vivo use of this compound, thus potentiating its clinical immunomodulatory applicability.

Aims: The aim of this study was to investigate in vitro and in vivo the $\mathrm{T}$ cellstimulatory capacity of in vitro $1,25(\mathrm{OH})_{2} \mathrm{D}_{3}$-modulated murine $\mathrm{DCs}$ as well as the outcome of DC transfer in murine allogeneic islet transplantation.

Methods and results: Murine bone marrow-derived mature DCs were generated ( 8 days GM-CSF + IL- 4 followed by 48 hours LPS + IFN- $\gamma$ ) in the continuous absence (ctr-DC) or presence of $10^{-8} \mathrm{M} 1,25(\mathrm{OH}) \mathrm{D}_{3}(1,25 \mathrm{D}$ DC). Analysis of surface marker expression (downregulated MHC II, CD80, CD86 and CD54; upregulated CD40, CCR5, F4/80 and DEC205) and cytokine production (inhibited IL-12; enhanced IL-10 and TNF- $\alpha$ ) confirmed the modulated phenotype of $1,25 \mathrm{D}$-DC. In vitro stimulation of T cells with $1,25 \mathrm{D}_{3}$-DC inhibited proliferation $(\mathrm{p}<0.05)$ and shifted cytokine expression towards IL- 10 and TGF- $\beta$ (both $p<0.05$ ). In islet allotransplantation (BALB/C islets in alloxan-diabetic C57BL/6 mice), triple transfer of alloislet-primed syngeneic $125 \mathrm{D}_{3}$-DC (days $-10,-3$ and 0 before transplantation) induced 
graft-specific anergy of recipient splenocytes. Although no robust tolerance to islet allografts could be induced (graft survival of $11.4 \pm 2.2$ days in $1,25 \mathrm{D}_{3} \mathrm{DC}$ treated recipients $(n=5)$ versus $9.0 \pm 1.0$ days in non-treated controls $(n=3)$, NS), primary-non-function of the graft occurring after alloislet-primed syngeneic ctr-DC treatment $(n=4)$ was prevented.

Conclusions: Together, these data provide evidence that in vitro generation of murine DCs in the presence of $1,25(\mathrm{OH})_{2} \mathrm{D}_{3}$ alters their T cell-stimulatory capacity. The in vivo data suggests that with further refinement of the DC transfer regimen possibly a prolonged allograft survival could be achieved, equivalent to or better that in vivo $1,25(\mathrm{OH})_{2} \mathrm{D}_{3}$ treatment without the accompanying calcemic effects.

Supported by a JDRF Postdoctoral Fellowship Grant

\section{2}

Long term survival of islet grafts by a novel approach of immunoisolation

B. J. de Haan, J. A. M. Kamps, M. M. Faas, P. de Vos;

Medical Biology, University Medical Center Groningen, The Netherlands.

Backgrounds and aims: The efficacy of immunoisolation in alginatePLL membranes in order to transplant pancreatic islets in the absence of immunosuppression has been demonstrated by several groups. Unfortunately, large-scale application is hampered by limited longevity of the encapsulated islet tissue. A crucial factor limiting survival is an inflammatory response in the immediate period after transplantation. Directly after implantation the number of macrophages is increased at the transplantation site. In response to islet-derived cytokines (MCP-1) these macrophages secrete high quantities of deleterious cytokines (IL-1 and TNF) which stimulate the islets to produce even more islet-derived cytokines with a harmful circle of activation as a consequence. In the first two weeks post transplantation period $60 \%$ of endocrine tissue is destroyed and the remaining cells are not capable to maintain long-term normoglycemia. In the present study we test in a xenograft murine transplantation model the efficacy of a novel encapsulation approach to overcome the tissue response and its consequences in the immediate transplant period.

Methods: We designed a double encapsulation system in which contains islets enclosed in an inner membrane and liposomes containing deoxyspergualin (DSG). Immunoisolated AO-rat islets were encapsulated in this novel system and transplanted into streptozotocin diabetic male BL-6 mice and subsequently transplantation survival was compared with traditional control capsules without DSG.

Results: Since the immediate tissue response after implantation occurs reportedly in the first 14 days after implantation we modulated the liposome composition as such that DSG was released over a period of 14 days. All transplanted animals became normoglycemic within 1 week after implantation. The islet grafts encapsulated in the conventional control capsules survived for 43 (median) days which is was much shorter as compared with the novel capsules containing the liposome enclosed DSG. These recipients are 83 (median) days normoglycemic and two are still ongoing and show no signs of graft failure.

Conclusions: Our study shows that inflammatory responses in the immediate post transplant period is crucial for the functional survival of immunoisolated islet graft. It also shows that temporary suppression of this response is a successful approach for prolongation of the graft survival.

\section{3}

Assembly of standardised pancreatic islets by the "Hanging Drop"technology: improved secretory function of small pseudoislets G. Cavallari ${ }^{1,2}$, R. Zuellig ${ }^{3}$, R. Lehmann ${ }^{3}$, M. Weber ${ }^{1}$, W. Moritz'; ${ }^{1}$ Clinic for Visceral and Transplantation Surgery, University Hospital, Zurich, Switzerland, ${ }^{2}$ Department of Surgery Intensive Care and Transplantation, Sant' Orsola-Malpighi University Hospital, Bologna, Italy, ${ }^{3}$ Division of Endocrinology and Diabetes, University Hospital, Zurich, Switzerland.

Background and Aims: Rejection and hypoxia are the main factors which limit islet engraftment in the recipient liver in the immediate posttransplant period. Recently we reported a negative relationship of graft function and islets size concluding that small islets are superior to large islets. Islets can be dissociated into single cells and reaggregated into so called "pseudoislets", which are functionally equivalent to intact islet. The aim of our study was develop a technique that enables to obtain pseudoislets of defined, preferably small dimensions.

Materials and Methods: Islets were harvested from Lewis rats by collagenase digestion and thereafter dissociated into single cells by trypsin treatment. Fractions with defined cell numbers were seeded into single drops onto cell culture dishes which were inverted and incubated for 5-8 days under regular cell culture conditions. Newly formed pseudoislets were analysed for dimensions, morphology, cellular composition and glucose stimulated insulin secretion (GSIS).

Results: The volume of reaggregated pseudoislets strongly correlated with cell number $(\mathrm{r} 2=0.995)$. Tissue loss could be minimized by performing reaggregation in presence of elevated glucose concentration $(11 \mathrm{mM})$ and the GLP-1 analogue Exendin-4 (100 nM). Morphology, cellular composition and architecture of reaggregated islets were comparable to intact islets. GSIS of small pseudoislets, composed of 250 islet cells, was increased by 2.7fold $(p<0.001)$ when compared to a total entity of freshly isolated islets. In small pseudoislets, insulin secretion at high glucose was stimulated by 10.6fold, while glucose stimulated insulin secretion in regular intact islets was enhanced only by 2.9 -fold $(\mathrm{p}<0.001)$.

Conclusion: With "hanging drop" culture method pseudoislets of standardized size and regular shape can be generated, which do not differ from intact islets in terms of cellular composition and architecture, but exhibit a markedly improved insulin secretory capacity. The concept of small pseudoislet could foster new approaches in islet cell replacement therapy. Supported by the Olga Mayenfisch and Hartmann-Müller Foundation

\section{4}

Different parameters of islet volume and size as predictors for islet transplantation outcome

P. A. Gerber ${ }^{1}$, P. Kugelmeier ${ }^{2}$, R. Zuelligin', V. Pavlicek ${ }^{1}$, M. Weber ${ }^{2}$, G. A. Spinas ${ }^{1}$, R. Lehmann ${ }^{1}$;

${ }^{1}$ Endocrinology and Diabetes, University Hospital Zurich, Switzerland, ${ }^{2}$ Transplantation Surgery, University Hospital Zurich, Switzerland.

Background and Aims: It has been previously shown that small islets show better function and survival in vitro as well as in vivo as compared to large islets both in animal models and human islet transplantation. In the last years it became increasingly clear that 5 years after islet transplantation, a majority of patients lose insulin independence. In the present study different parameters of islet transplantation were associated with beta cell function after transplantation to test which quality of transplanted islets best predicts the outcome of islet transplantation.

Materials and Methods: We correlated stimulated C-peptide levels 3 months after transplantation with the volume of transplanted islets (measured as islet equivalents IEQ, total number of transplanted islets, surface of islets, islet mantle volume, volume of islets with a diameter less than $150 \mu \mathrm{m}$, and the isolation index (IEQ / islet number)). All parameters are corrected by $\mathrm{kg}$ of recipient weight.

Results: The volume of transplanted islets did not correlate well with stimulated C-peptide levels after transplantation $\left(\mathrm{R}^{2}=0.22\right)$, nor did the surface of islets $\left(R^{2}=0.32\right)$ or the mantle volume of islets $\left(R^{2}=0.23\right)$. The correlation improved by correlation of stimulated C-peptide levels with the volume of islets with a diameter less than $150 \mu \mathrm{m}\left(\mathrm{R}^{2}=0.62\right)$ or the number of transplanted islets $\left(R^{2}=0.73\right.$, figure 1$)$, and the best prediction was achieved when we used the isolation index $\left(\mathrm{R}^{2}=0.78\right)$.

Conclusion: This study demonstrates that islet size is an important predictor for the outcome of islet transplantation assessed by stimulated C-peptide levels and that the isolation index, and more simple the islet number are the best predictors. The fact that islet surface and islet mantle volume did not predict transplantation outcome in contrast to islet number and the volume of small islets supports the hypothesis that in large islets the whole islet is destroyed by apoptosis/necrosis initiated by central hypoxia and that only the fraction with small islets determine the transplantation outcome. At our institution, the finding of the superiority of small islets led to the use of islet isolations for transplantation which so far have been considered insufficient for a good clinical outcome because of their low volume. The use of the islet number and isolation index for evaluation of islet preparations instead of IEQ increases the percentage of transplantable islet isolations. (Fig. 1) 


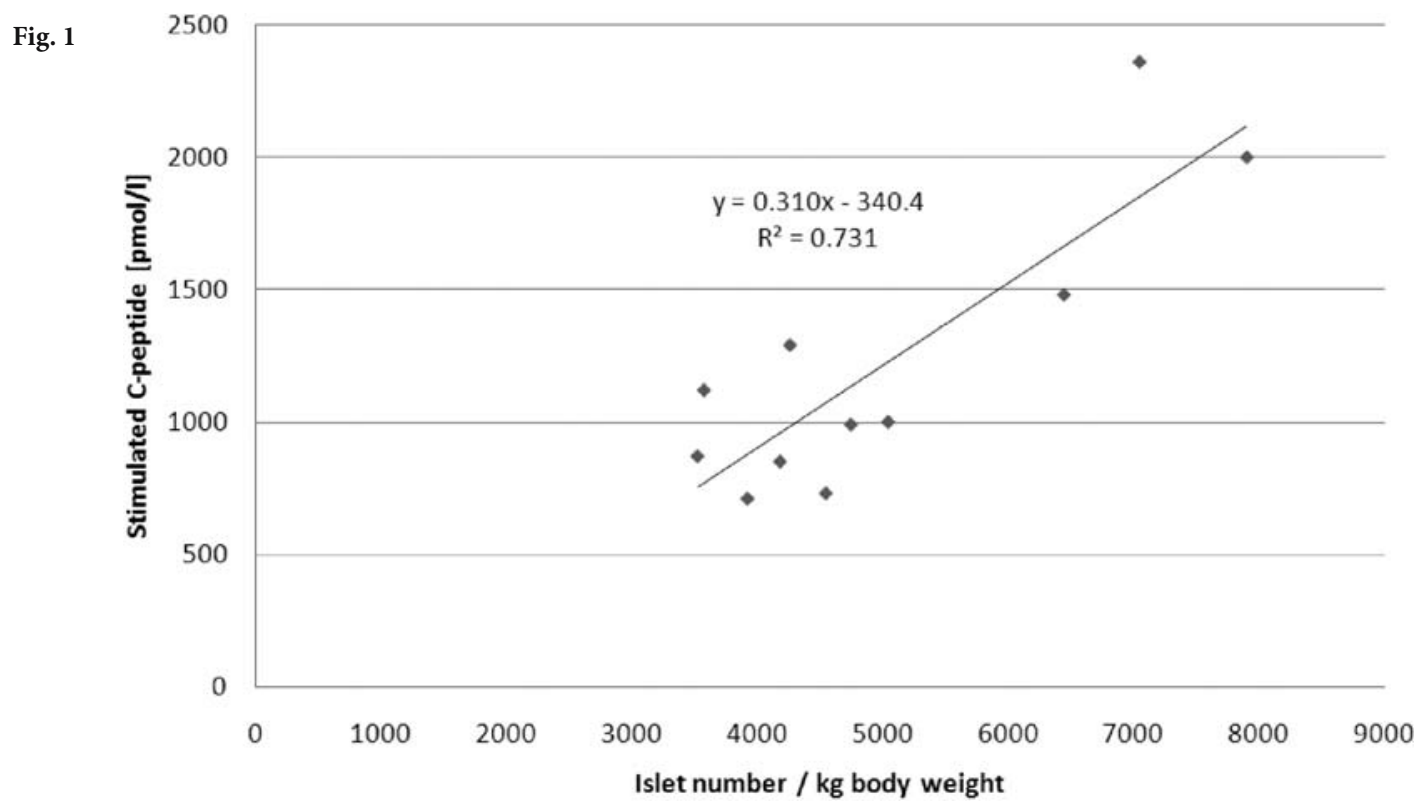

\section{5}

The deleterious effects of Cytomegalovirus on the functional survival of rat islet isografts

M. J. Smelt, M. M. Faas, A. de Haan, B. J. de Haan, P. de Vos;

Pathology and Laboratory Medicine, University Medical Centre Groningen, The Netherlands.

Background and Aims: Clinical application of islet transplantation has become a realistic option since the introduction of new immunosuppressive strategies, also known as the Edmonton protocol. However, the long term overall transplantation success rate is still unsatisfactory low. Graft failure is generally thought to be caused by metabolic exhaustion, alloreactivity and recurring autoreactivity. Recently, an association between Cytomegalovirus (CMV) infections and reduced graft function, i.e. allograft rejection, has been found in solid organ transplants, such as heart, lung, liver and kidney transplants. As the deleterious effects of viral infections on beta cells are well recognized, we hypothesize that viral infections, such as Cytomegalovirus, could also play an important role in islet graft failure. In this study, the effect of CMV infection on the survival and function of rat islet isografts was investigated.

Materials and Methods: STZ-diabetic AO rats received a isogenic islet transplant of $5 \mu \mathrm{l}$ under the kidney capsule and one day later, the animals received a rCMV infection of $2 \times 10^{5}$ pfu i.p.; non infected recipients of islet isografts served as controls. 4 weeks after transplantation IVGTT (bolus injection of $200 \mathrm{mg}$ D-Glucose) and MTT ( 2 gr. rat chow ( $53 \%$ carbohydrates, $20 \%$ protein and $5 \%$ fat) were performed and after 100 days, rats were sacrificed and pancreas, salivary glands and the transplant were retrieved for histological examination.

Results: All islet graft recipients became normoglycaemic after transplantation. Within 14 days after transplantation, the basal blood glucose levels were decreased from $28,26 \pm 5,67 \mathrm{mM}$ to $5,9 \pm 0,7 \mathrm{mM}$ in the control group $(\mathrm{n}=5)$ and from $27,68 \pm 2,93 \mathrm{mM}$ to $7,4 \pm 5,2 \mathrm{mM}$ in the CMV infected animals $(\mathrm{n}=5)$. The control animals became normoglycaemic at day $7 \pm 4$ after transplantation. The time to reach normoglycaemia was somewhat longer in the CMV infected animals, which became normoglycaemic at day $12 \pm 9$. Despite the longer period to reach normoglycaemia, no signs of CMV disease were seen, and weight gain was similar in the CMV infected animals compared to the control animals. The CMV infection had a profound effect on the function of the islets. CMV infected recipients showed a reduced glucose tolerance in both the IVGTT and MTT. During the IVGTT, CMV infected animals showed an Insulin Area Under Curve (AUC) of 192 $\pm 7,4$ A.U., which was 197,3 \pm 21 A.U. in the control animals. Glucose Area Under Curve (AUC $)$ was $1628 \pm 275$ A.U. in the CMV infected animals, compared to only 1152 96,5 A.U. in the control animals. During the MTT, CMV infected animals showed an $\mathrm{AUC}_{\mathrm{i}}$ of $142 \pm 9,4$ A.U., compared to $206 \pm 29$ A.U. in the control animals. AUC was $1118 \pm 249$ A.U. in the CMV infected animals, compared to $1024 \pm 35,3$ A.U. in the control animals.
Conclusion: These results show that CMV has a direct deleterious effect on islet graft function. Although the graft is able to maintain basal blood glucose levels within the normal range, the graft function is disturbed after CMV infection. Further research will focus on the mechanism by which CMV affects islet graft function and on the effect of CMV on islet allografts. Supported by the Diabetes Foundation

\section{6}

Immunological profile, choice of immunotherapy and CMV viraemia in pancreas transplant

E. Favaro ${ }^{1}$, L. Semperboni ${ }^{1}$, S. Pinach ${ }^{1}$, F. Giaretta ${ }^{1}$, M. Rossetti ${ }^{1}$,

R. Romagnoli' ${ }^{2}$ I. Miceli ${ }^{1}$, M. Cassader ${ }^{1}$, P. Cavallo Perin ${ }^{1}$, R. Quadri ${ }^{1}$, M. Zanone ${ }^{1}$;

${ }^{1}$ Dept of Internal Medicine, University of Turin, Italy,

${ }^{2}$ Liver Transplant, University, Turin, Italy.

Background and Aims: It has been suggested that anti-ATG (anti-thymocyte globuline) induction therapy increases Cytomegalovirus (CMV) infection risk, and newly acquired infection or reactivation from a latent state have been associated with increased rate of allograft rejection and incidence of other opportunistic infections and morbidity. We investigated the occurrence and effect of CMV infection after pancreas transplant, correlation with induction therapy, rejection episodes and autoimmune reactivity.

Materials and Methods: We followed 21 type 1 diabetic patients (mean age 42 years, mean duration of diabetes 29 years) recruited during a 7 year period, for a mean of 39 months after simultaneous pancreas-kidney (SPK), pancreasliver (1 case) or pancreas alone transplant(1). Induction therapy consisted of ATG in 1 patient and anti-CD25 in the others. All patients received tacrolimus, mycophenolate mofetil and short term steroids. Rejection episodes were treated with ATG and/or steroids. CMV antigenemia, number of positive leucocytes, CMV DNA level in plasma were monitored. Islet autoAbs were monitored before and after transplant in all recipients, and flowcytometric analysis of suppressor function of $\mathrm{CD} 4^{+} \mathrm{CD} 25^{+} \mathrm{T}$ cells was performed.

Results: Transplant was successful at follow up in all but 2 patients (the pancreas alone recipient, with loss of function after 1 year, and 1 SPK with partial loss of function). Overall incidence of CMV infection (all patients on ganciclovir prophylaxis) was $43 \%$, with different distribution between the ATG (induction or rejection treatment) group (75\%) and the anti-CD25 group (35\%). The rates of CMV infection according to the donor and the recipient serological status were: D-/R-0\%, D-/R+0\%, D+/R+67\% and D+/R-33\%. Rejection episodes (43\%) had a similar distribution between CMV-infected and non infected patients and CMV viraemia did not occurr earlier than in infected patients without rejection episodes. Both patients with partial or total graft loss were treated with ATG and were CMV infected. 14 patients (66\%) were anti$\mathrm{GAD} \mathrm{Ab}+, 2$ (9\%) anti-IA2 Ab+, 6 (3\%) ICA+ (5 also anti-GAD+). The Abs 
persisted after transplant, and 2 recipients became anti-GAD+. The pancreas recipient with graft loss became positive for all $3 \mathrm{Abs}$ after transplant, and had several episodes of detection of CMV early antigen and high CMV viraemia, before and after ATG treatment for rejection episodes. In this patient, by flowcytometric analysis, a higher proportion of the $\mathrm{CD} 44^{+} \mathrm{CD} 25^{+}$population coexpressed activation markers and intracellular CTLA-4, as described in recent-onset type 1 diabetic patients, possibly reflecting a disturbance of the balance, and deficiency in function in this population.

Conclusions: Although without statistical significance possibly related to the limited number of cases, ATG induction or rejection treatment increases CMV infection risk, and positive CMV donor status was associated with higher incidence of CMV viraemia. We did not detected more frequent $\mathrm{CMV}$ infection in rejection episodes, thus not corroborating the hypothesis of increased incidence of rejection episodes among CMV-infected allograft recipients. This may be related to the anti-CD25 induction therapy and to CMV prophylaxis CMV infection could however be linked to recurrence of islet autoimmunity in the patient with graft failure.

Supported by Regione Piemonte

\section{7}

Labeling of human pancreatic islets of Langerhans with Endorem ${ }^{\otimes}$ for non-invasive MRI imaging in vivo

M. L. Malosio ${ }^{1}$, A. Poletti ${ }^{1}$, A. Esposito ${ }^{2}$, C. Losio $^{2}$, L. Piemonti ${ }^{1}$,

R. Melzi ${ }^{1}$, R. $\mathrm{Nano}^{3}$, F. De Cobelli², P. Maffi ${ }^{3}$, E. Bonifacio ${ }^{1}$, A. Secchi ${ }^{3,4}$,

A. Del Maschio ${ }^{2,4}$;

${ }^{1}$ Immunology of Diabetes, Fondazione Centro San Raffaele del Monte Tabor,

${ }^{2}$ Diagnostic Radiology, Fondazione Centro San Raffaele del Monte Tabor,

${ }^{3}$ Islet Transplant Unit, Fondazione Centro San Raffaele del Monte Tabor,

${ }^{4}$ Vita-Salute San Raffaele University, Milano, Italy.

Background and Aims: Islet transplantation is one of the most promising and effective therapies for restoring normoglycemia in type 1 diabetic patients. Monitoring graft loss, caused either by immunological or non-immunological events, occurring in the first phase after transplantation and at later stages of patient's life is a very important issue. It is therefore of pivotal importance to develop a reliable non-invasive islets imaging modality for the follow-up of the graft fate and for studying the relationships between transplanted islet mass and insulin independence, graft function and diabetes complications. MRI represents an ideal tool for islet imaging because it provides high spatial and high contrast resolution images and can be transferred from animal models to the human clinical setting. Very promising results have been recently shown with islets of Langerhans, imaged using MRI (4.7T) after superparamagnetic iron oxide particles (SPIO) uptake. The aim of the project is to set up a protocol to label human islets in vitro with clinically approved SPIO $\left(\right.$ Endorem $\left.^{\circ}\right)$ and to visualize them in $1.5 \mathrm{~T}$ clinical and in $7.0 \mathrm{~T}$ research scanners after transplantation. :

Materials and Methods: Different labeling parameters using Endorem have been optimized in order to achieve the best-possible uptake preserving islet viability, insulin secretion, expression profile of relevant genes and proinflammatory status of islets. The persistence of Endorem -labeled islets and their ability to revert diabetes similarly to non- labeled is being tested both with mouse islets in syngeneic liver transplantation studies and with human islets transplanted into nude mice, made diabetic by streptozotocin injection. MRI $\left(\mathrm{T} 2^{*}\right)$ protocols, which can be translated into imaging of human islets in transplanted patients, are under development. :

Results: Iron labeled human islets do not show altered viability, reduced glucose induced insulin secretion or higher activation of pro-apoptotic pathways in vitro. Diabetic mice transplanted with 450 islets \pm SPIO became normoglicemic and islets incorporating iron could be persistently detected by MRI in mice many days after transplantation while non labeled islets could not be visualized. Monitoring persistence of hypointese islet signals is still ongoing. We have been able to visualize labeled islets in the mouse model both in 7.0 T research and in $1.5 \mathrm{~T}$ clinical scanners.

Conclusion: Our results, according to previous reports, provide the evidence that islets can be labeled with Endorem and can be imaged with MRI both with a $7.0 \mathrm{~T}$ research and also using $1.5 \mathrm{~T}$ clinical scanners. The future objectives of our research are to better define the behavior of Endorem ${ }^{\circ}$ labeled islets transplanted in animal models and to transfer the developed islets labeling and MRI protocols to humans.

Supported by JTD01/Telethon-JDRF (A.S.; E.B); CAR08.0548/Fondazione Cariplo (E.B.); 6-2006-1098 ECIT/JDRF (A.S.); CITR/NIH-NIDDK (A.S.);

\section{8}

Pancreatic islet allotransplantation improves the quality of life in brittle type 1 diabetic patients

L. Milliat-Guittard ${ }^{1}$, L. Kessler ${ }^{2}$, A. Wojtusciszyn ${ }^{3}$, T. Berney ${ }^{4}$, L. Badet ${ }^{5}$, F. Penfornis ${ }^{6}$, C. Thivolet ${ }^{7}$, E. Renard ${ }^{3}$, D. Bosco ${ }^{4}$, R. Baertschiger ${ }^{4}$, E. Morelon ${ }^{8}$, F. Bayle ${ }^{9}$, C. Colin ${ }^{1}$, P.-Y. Benhamou ${ }^{10}$;

${ }^{1}$ Department of Medical information, University Hospital, Lyon, France, ${ }^{2}$ Department of diabetology, University Hospital, Strasbourg, France, ${ }^{3}$ Department of Diabetology, University Hospital, Montpellier, France, ${ }^{4}$ Islet isolation laboratory, University Hospital, Geneva, Switzerland, ${ }^{5}$ Department of Urology, University Hospital, Lyon, France, ${ }^{6}$ Department of Diabetology, University Hospital, Besançon, France, ${ }^{7}$ Department of Diabetology, University Hospital, Lyon, France, ${ }^{8}$ Department of Nephrology, University Hospital, Lyon, France, ${ }^{9}$ Department of Nephrology, University Hospital, Grenoble, France, ${ }^{10}$ Department of Diabetology, University Hospital, Grenoble, France.

Background and Aims: The aim of this study was to evaluate the healthrelated quality of life in non uremic brittle type 1 diabetic patients after pancreatic islet transplantation in the Gragil 2 trial

Materials and Methods: From September 2003 to October 2005, 10 type 1 diabetic patients with severe hypoglycaemic events received $11089 \pm 505$ IEQ $/ \mathrm{kg}$ according to the Edmonton protocol. Patients answered to the 36item Short Form Health Survey (SF-36) and the Diabetes Quality Of Live (DQOL) questionnaires at the inclusion and 6 and 12 months after islet transplantation. SF-36 generic questionnaire assessed global health status while DQOL specific questionnaire analyzed five aspects related to diabetes: satisfaction, impact, social and vocational worry, diabetes worry, and global well-being according to a scoring scale from 0 (worse) to 100 (best).

Results: Twenty seven questionnaires were analysed. At six months posttransplantation, the physical evaluation score of SF-36 increased significantly from $36 \pm 9$ to $49 \pm 10(p=0.021)$ while the psychological evaluation score remained stable. The global perception of health state was improved at 6 and 12 months post-transplantation: $30 \pm 16$ vs. $78 \pm 30(p=0.01)$ and $79 \pm 17$ $(\mathrm{p}=0.016)$ respectively. At six months post-transplantation, DQOL revealed a significant decrease of the diabetes impact on the quality of life which was maintained at 12 months: $45 \pm 14$ vs. $68 \pm 13(\mathrm{p}=0.011)$ and $63 \pm 8(\mathrm{p}=0.018)$. While all patients had functional islets $(\mathrm{C}$ peptide $>0.5 \mathrm{ng} / \mathrm{ml})$ at 6 months post-transplantation and $8 / 10$ patients at one year, $8 / 10$ patients were insulinfree six months after transplantation and 5/10 one year after islet graft. Severe hypoglycaemic events disappeared in all patients. Severe adverse events requiring or prolonging hospitalization occurred in 3 patients.

Conclusion: Our data show that pancreatic islet transplantation improves quality of life in brittle type 1 diabetic patients despite a need for insulin therapy in $50 \%$ of patients. The disappearance of severe hypoglycaemic events could likely contribute to this beneficial aspect of islet transplantation. Supported by ALFEDIAM 


\section{PS 29 Regulation of glucagon synthesis and release}

\section{9}

Proglucagon processing can be altered to produce GLP-1 in pancreatic alpha cells

N. M. James ${ }^{1}$, L. E. Pritchard ${ }^{1}$, D. Livesey ${ }^{2}$, D. M. Smith², A. White ${ }^{1}$;

${ }^{1}$ University of Manchester, United Kingdom,

${ }^{2}$ Astrazeneca, Macclesfield, United Kingdom.

Background and Aims: The incretin hormone GLP-1 is primarily synthesised in the L-cells of the gut through the posttranslational cleavage of proglucagon by proprotein convertase 1 (PC1). It increases insulin secretion from the pancreas and augments beta cell mass. Pancreatic alpha cells also express proglucagon but it is normally cleaved by PC2, to yield glucagon. Emerging evidence suggests that alpha cells can adapt to produce GLP-1, possibly by upregulation of PC1. The aim of this study is to identify circumstances where processing of proglucagon can be switched to yield pancreatic GLP-1.

Materials and Methods: For regulation of GLP-1 and glucagon secretion, alpha TC1-6 cells were seeded at $1 \times 10^{5}$ cells per well and incubated overnight. After pre-incubation for 30 minutes in Krebs Ringer HEPES (KRH) buffer containing $25 \mathrm{mM}$ glucose, the cells were incubated in $\mathrm{KCl}(40 \mathrm{mM}) \mathrm{KRH}$ for 30 minutes or with different concentrations of glucose $(0-35 \mathrm{mM})$. Islets from PC2 knockout (PC2-/-), heterozygote (PC2-/+), and wild-type (PC2+/+) mice were isolated using collagenase and cultured at 20 islets per well for 2 days in RPMI (11 mM glucose). Glucagon was assessed by an enzyme immuno assay (CosmoBio, with a detection limit of $0.02 \mathrm{pmol} / \mathrm{ml}$ ). Active GLP-1 was measured by ELISA (Linco, with a detection limit of $10 \mathrm{fmol} / \mathrm{ml}$ )

Results: GLP-1 was released from alpha TC1- 6 cells over 30 minutes $(4.2 \pm 0.5$ $\mathrm{fmol} / 10^{5}$ cells; $\mathrm{n}=4$ ), but secretion was not stimulated by high or low glucose concentrations. However, glucagon secretion was stimulated from the alpha TC1-6 cells in response to low glucose $(0.3 \pm 0.07 \mathrm{pmol} /$ well at $0 \mathrm{mM}$ glucose vs. $0.1 \pm 0.03 \mathrm{pmol} /$ well at $35 \mathrm{mM}$ glucose; $\mathrm{n}=4, \mathrm{p}<0.05)$. To determine if GLP-1 was released from secretory vesicles, cells were stimulated with $40 \mathrm{mM}$ $\mathrm{KCl}$. GLP-1 levels didn't change, but glucagon release was stimulated $(0.1 \pm$ $0.03 \mathrm{pmol} /$ well at basal, vs. $0.2 \pm 0.02 \mathrm{pmol} /$ well at $40 \mathrm{mM} \mathrm{KCl} ; \mathrm{n}=4, \mathrm{p}<$ $0.05)$. To establish if proglucagon processing can be changed from glucagon to GLP-1 by decreasing PC2, islets from PC2 knockout mice were studied. Glucagon was virtually absent in PC2-/-, and decreased in PC2-/+ $(265.5 \pm$ $18.0 \mathrm{fmol} /$ islet; $\mathrm{n}=6)$ vs. $\mathrm{PC} 2+/+(453.3 \pm 43.0 \mathrm{fmol} / \mathrm{islet} ; \mathrm{n}=6)$, with a similar decrease in glucagon secretion from the islets. Conversely GLP-1 levels were increased in PC2-/ $-(4.7 \pm 0.3 \mathrm{fmol} /$ islet; $\mathrm{n}=6, \mathrm{p}<0.001)$ and PC2 $-/+(0.8 \pm$ $0.2 \mathrm{fmol} /$ islet, $\mathrm{n}=6)$ vs. $\mathrm{PC} 2+/+(0.3 \pm 0.1 \mathrm{fmol} /$ islet, $\mathrm{n}=6)$ when measured in total islet lysates, with similar results for its secretion. PC2-/- mice also displayed improved glucose tolerance following an intraperitoneal glucose tolerance test, (AUC $15.7 \pm 2.5 \mathrm{mmol} / \mathrm{h}, \mathrm{n}=6$ vs. $22.2 \pm 0.8 \mathrm{mmol} / \mathrm{h}, \mathrm{n}=7 ; \mathrm{p}$ $<0.01$, after a glucose load of $2 \mathrm{~g} / \mathrm{kg}$ ).

Conclusion: GLP-1 is released from the pancreatic alpha TC1-6 cell line. However, the GLP-1 does not appear to be in the regulated secretory vesicles, in contrast to glucagon, release of which is stimulated by low glucose and $\mathrm{KCl}$. The loss of PC2 from the alpha cell in PC2 - /- mice causes the expected loss of glucagon secretion, but interestingly, there is an increase in GLP-1 in islets. This switch from glucagon to GLP-1 may contribute to the improved glucose tolerance in these animals. Taken together these results indicate that GLP-1 can be released from the pancreatic alpha cell, although how the processing of proglucagon to glucagon and GLP-1 is regulated needs to be determined. The importance of local GLP-1 production in the islet remains unknown, however it may have a role in augmenting beta cell mass.

Supported by MRC and Astrazeneca
0480

Targeting the glucagon receptor for glycaemic control and beyond

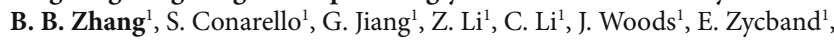
R. Bergeron ${ }^{1}$, R. Sinha Roy ${ }^{1}$, L. Zhu ${ }^{1}$, E. Muise ${ }^{1}$, J. Thompson ${ }^{1}$, K. Wong ${ }^{1}$, M. Charron ${ }^{2}, \mathrm{~J} . \mathrm{Mu}^{1}$;

${ }^{1}$ Merck Research Laboratories, Rahway, United States,

${ }^{2}$ Biochemistry, Albert Einstein College of Medicine, Bronx, United States.

Background and Aims: Glucagon signaling leads to stimulation of hepatic glucose output, providing a major counter regulatory mechanism for insulin. Elevated glucagon levels or altered insulin to glucagon ratios are associated with the development of type 2 diabetes. In contrast to its role in glycemia control, less is known about the importance of glucagon in adiposity and body weight control. It was reported previously that glucagon receptor knockout (KO) mice develop normally and have lower plasma glucose on a normal diet.

Materials and Methods: To further investigate the role of glucagon signaling in metabolic control and glucose homeostasis, wild type (WT) and KO mice were fed with high fat diet (HFD) to induce insulin resistance and injected with low dose streptozotocin (STZ) to induces beta cell damage. The animals were evaluated for changes in their metabolic phenotype as well as pancreatic islet morphology. Effects of deletion of the glucagon receptor gene on the ob/ ob background were also assessed in mice. To better understand the role of the glucagon receptor in energy homeostasis, we performed transcriptional profiling using tissues from HFD treated WT or KO mice.

Results: In comparison with the WT mice, KO mice exhibited decreased body weight and food intake, reduced plasma glucose levels, and improved glucose tolerance. Elevated glucagon-like peptide-1 (GLP-1) levels and reduced gastric emptying were also observed in KO mice. KO mice manifested reduced HFD-induced hyperinsulinemia and hyperleptinemia and were resistant to the development of hepatic steatosis. Moreover, STZ induced beta cell damage and hyperglycemia were observed in WT mice, but not in $\mathrm{KO}$ mice. Furthermore, glucagon receptor deletion in ob/ob mice led to reduced adiposity, improved glycemic control and increased energy expenditure. Transcriptional profiling of liver and adipose tissue from WT and KO mice on regular diet and HFD highlighted the role of glucagon signaling in the regulation of lipid metabolism and energy homeostasis. In WT mice, HFD feeding induced significant transcriptional changes in liver and white adipose tissue (WAT), most of which were not apparent in KO mice. The largest category of the differentially regulated genes in liver or WAT is related to lipid metabolism, including genes important for lipogenesis, fatty acid oxidation or lipolysis. For example, HFD treatment induced expression of CD-36, PPAR $\gamma$, acetyl-CoA dehydrogenase, and endothelial lipase in the liver of WT mice, but not in KO mice. Expression of adiponetin, adiponectin receptor 2, Glut 4 and ATGL was suppressed by HFD in the WAT of WT mice only. Furthermore, GCGR deletion resulted in upregulation of FGF21 (a recently identified metabolic regulator) in liver of $\mathrm{KO}$ mice; the change in the gene expression also correlated with circulating FGF21 polypeptide levels. Conclusion: These results imply that differential changes of the identified genes in WT and KO mice likely contribute to the lean phenotype of GCGR $\mathrm{KO}$ mice. Antagonizing the glucagon receptor will have beneficial effects that could extend beyond glycemic control to amelioration of multiple defects associated with metabolic syndrome.

\section{1}

Glucose-dependent short-term regulation of glucagon gene transcription in pancreatic alpha-cells

T. Moede, I. B. Leibiger, D. Kaiser, P.-O. Berggren, B. Leibiger; Department of Molecular Medicine \& Surgery, Karolinska Institutet, Stockholm, Sweden.

Background and Aims: The pro-glucagon gene is expressed in alpha-cell, intestinal L-cells and specific neurons, but due to different posttranslational processing only the pancreatic alpha-cell produces and secretes glucagon. The importance of both insulin and glucagon for glucose homeostasis necessitates a strict regulation of release and biosynthesis of both hormones. While a tight connection between insulin release and insulin gene transcription through a positive feedback involving the insulin receptor has been shown, little is know about a similar autocrine feedback mechanism in pancreatic alphacells on glucagon gene transcription. The aims of this study are therefore: 
1.) To study whether there is similar link between glucagon release and transcription.

2.) To investigate the signaling pathways involved in this feedback mechanism.

Materials and Methods: We employed mRNA analysis and online monitoring rat glucagon-promoter $(-775$ to $+7 \mathrm{bp})$ driven DsRed expression to determine glucagon gene transcription. Glucagon release was measured by RIA. Cells were stimulated by treatment with either various glucose concentrations ( 1 to $16.7 \mathrm{mM}$ glucose) for $15 \mathrm{~min}$ or with $200 \mathrm{pmol} / \mathrm{ml}$ glucagon at high, nonstimulatory glucose $(16.7 \mathrm{mM})$. To investigate the involvement of the glucagon receptor and various protein kinases, cells were incubated with pharmacological inhibitors or inhibitory peptides $30 \mathrm{~min}$ prior to and during the stimulation. All experiments were performed on the alpha-cell line alphaTC1-9.

Results: Stimulation of alphaTC1-9 cells with $1 \mathrm{mM}$ to $3 \mathrm{mM}$ glucose for 15 min resulted in a 2.7 to 3.6 -fold increase in glucagon release and in a 1.5 to 1.8 fold increased glucagon promoter activity measured as increase in DsRedfluorescence. Glucose concentrations that did not elicit glucagon release (5 to $16.7 \mathrm{MM}$ ) did not stimulate glucagon gene transcription. Addition of $200 \mathrm{pmol} / \mathrm{ml}$ exogenous glucagon at high, non-stimulatory glucose lead to a similar increase in glucagon gene transcription as stimulus-secreted glucagon. The stimulatory effect of either glucagon or low glucose was abolished when the cells were pre-treated with either $1 \mu \mathrm{M}$ of the glucagonreceptor blocking-peptide des-His1-[Glu9]-glucagon-amide or $20 \mathrm{nM}$ glucagon receptor antagonist II. Furthermore, $100 \mu \mathrm{M}$ of the protein kinase A inhibitor RPcAMPs also abolished the stimulatory effect of both glucagon and low glucose. These findings were confirmed on the RNA-level.

Conclusion: These results suggest, that glucose-dependent short-term regulation of glucagon gene transcription is dependent on a feedback mechanism involving secreted glucagon, the glucagon receptor and protein kinase A.

Supported by Novo Nordisk Fonden, Swedish Research Council Family Stefan Persson Foundation, Juvenile Diabetes Research Foundation

\section{2}

Pax6 positively regulates PC2 expression in glucagon expressing cells through the activation of $\mathrm{cMaf}$ and Beta2 proteins

L. S. Katz, E. Marthinet, Y. Gosmain, J. Philippe;

Diabetes Unit, Division of Endocrinology, Diabetes and Nutrition,

University Hospital, Geneva, Switzerland.

Background and Aims: Prohormone Convertase 2 (PC2) is the main processing enzyme in pancreatic $\alpha$ cells, where it processes proglucagon to produce glucagon. Pax6 is a master gene in the development of the pancreas, previously shown to regulate glucagon gene transcription. To understand the role of Pax6 in endocrine pancreas development and function we first investigate its target genes in $\alpha$ cells.

Materials and Methods: InR1G9 (hamster glucagon producing cells) clones expressing a dominant negative (DN) allele of Pax6 and control clones were studied by RT-PCR and western blot for expression of genes and proteins, respectively important for $\alpha$ cell differentiation and function. ChIP, EMSA, reporter assays and siRNA experiments were carried in InR1G9 cell culture, and when possible, also in the mouse glucagon producing cell line, $\alpha \mathrm{TC} 1$, or in a heterologous cell line, BHK, in order to characterize the interactions between cMaf, MafB, Beta2, and Pax6 transcription factors with the PC2 promoter.

Results: We report that glucagon and PC2 mRNA and protein levels decrease in Pax6-DN cells and cells transfected with siRNA for Pax6 compared to control cells. Pax6 activates glucagon gene transcription through the G1 and G3 promoter elements. However, no binding sites of Pax6 on the PC2 promoter were identified whereas we found MAF and Beta2 putative binding sites on the PC2 promoter. We confirmed the relevance of these sites by ChIP and EMSA assays, and demonstrated transactivation of the PC2 promoter by both $\mathrm{cMaf}$ and Beta2. In order to find the mechanisms by which Pax6 might regulate the PC2 gene, we measured cMaf, MafB and Beta2 mRNA in Pax6-DN clones. cMaf and Beta2, but not MafB were found to be decreased by 60 and 50\%, respectively and direct targets of Pax6 by ChIP, EMSA and transfection assays. Furthermore, overexpression of cMaf and Beta2 in Pax6-DN clones completely corrected the observed decrease in PC2 gene transcription.

Conclusion: This study establishes Pax6 as critical for glucagon synthesis by activating directly proglucagon gene transcription as well as indirectly through the activation of $\mathrm{cMaf}$ and Beta2, which also activate the glucagon gene and the PC2 promoter.

Supported by the Swiss National Fund

\section{3}

Postprandial lipaemia is associated with increased glucagon levels and inadequate glucose-induced suppression of glucagon secretion in healthy men

A. Niederwanger, M. Kranebitter, C. Ciardi, T. Tatarczyk, J. R. Patsch,

M. T. Pedrini;

General Internal Medicine, University of Innsbruck, Austria.

Background and Aims: A great wealth of studies has been published on the effect of lipids on insulin sensitivity and insulin secretion. The role of plasma lipids in the regulation of glucagon secretion, however, has received much less attention. Previous studies on the effect of lipids on glucagon secretion have led to conflicting results. Abnormalities in glucagon secretion have been implicated in the development of type 2 diabetes for a long time; some thirty years ago, Unger and Orci proposed that in addition to absolute or relative insulin deficiency, an elevated plasma glucagon level plays a key role in the pathogenesis of diabetes. More recent studies revealed that in addition to elevated plasma glucagon levels, reduced glucose-induced supression of glucagon secretion represents another feature of insulin resistant states. We have recently demonstrated that postprandial lipemia through elevation of triglyceride-rich lipoproteins induces insulin resistance in vitro in cultured skeletal muscle cells and in vivo in healthy male subjects independently of NEFA levels. The aim of the present investigation was to study the effect of postprandial lipemia on plasma glucagon levels as well as on glucose-induced suppression of glucagon secretion in vivo in healthy men. Our study design enabled us to assess the role of plasma NEFA levels in the regulation of glucagon secretion.

Materials and Methods: We performed a randomized crossover study involving 7 healthy volunteers to compare both basal glucagon secretion and glucose-induced suppression of glucagon secretion between the postabsorptive and the postprandial state. For glucose-induced suppression of glucagon secretion, two ivGTTs were performed, one postabsorptively and the other postprandially, i.e. $3 \mathrm{~h}$ after meal consumption. Two fat-enriched meals were employed with Meal 1 raising plasma levels of both triglycerides and NEFA, and Meal 2 raising triglycerides only.

Results: Basal glucagon levels rose from $80.7 \pm 18.2$ postabsorptively to $104.2 \pm 17.5 \mu \mathrm{g} / \mathrm{l}$ postprandially with Meal $1(\mathrm{P}=0.017)$ and from $86.1 \pm 16.2$ to $106.8 \pm 30.1 \mu \mathrm{g} / \mathrm{l}$ with Meal 2 ( $\mathrm{P}=0.028)$.

With glucose injection, glucagon levels immediately decreased with the percent decline not differing between the postabsorptive and the postprandial state. However, taking into account that glucose-induced insulin secretion increased postprandially much more than postabsorptively (by $205.5 \pm 145.4$ vs. $94.2 \pm 41.7 \mathrm{mU} / \mathrm{l}$ with Meal $1, \mathrm{P}=0.016$ and by $174.8 \pm 111.5$ vs. $68.5 \pm 30.3$ $\mathrm{mU} / \mathrm{l}$ with Meal 2, $\mathrm{P}=0.02$ ), the postprandial suppression of glucagon was inadequate.

Conclusion: Since postprandial lipemia induces a state of insulin resistance, our observations may be interpreted to suggest that either postprandial triglyceride-rich lipoproteins themselves or the acute state of insulin resistance induced by these lipoproteins are responsible for the observed changes in glucagon secretion during postprandial lipemia.

Supported by an FWF Grant to MT Pedrini

\section{4}

Effect of pioglitazone on glucagon secretion in persons with impaired glucose tolerance

V. Pauvaday ${ }^{1}$, G. Dunseath ${ }^{1}$, R. Peter ${ }^{1}$, R. Chudleigh ${ }^{1}$, O. Sawyer ${ }^{2}$, S. Luzio ${ }^{1}$, M. Evans' ${ }^{2}$, D. R. Owens';

${ }^{1}$ Diabetes Research Unit, Cardiff University,

${ }^{2}$ Diabetes, Llandough Hospital, Cardiff, United Kingdom.

Background and Aims: Postabsorptive plasma glucose concentrations are regulated not just by insulin secretion but also glucagon concentrations. Both fasting and postprandial glucagon concentrations are elevated in subjects with impaired glucose tolerance (IGT) compared to subjects with normal glucose 
tolerance. The aim of the study was to investigate the effects of pioglitazone on glucagon secretion in subjects with IGT.

Materials and Methods: 15 subjects ( 9 male) with IGT (WHO criteria) were recruited into this randomised, double-blind, placebo controlled study. All subjects undertook a standard 500kcal meal tolerance (MTT) and intravenous glucose tolerance (FSIVGTT) test on consecutive days following an overnight fast. Plasma glucose (PGs) and glucagon (PGn) were measured following the MTT. Insulin sensitivity (SI) was calculated from the FSIVGTT using the CIBA GEIGY programme. Following 6 months treatment with either placebo or Pioglitazone (Pio 30mg od) all tests were repeated.

Results: In the placebo group, subjects were aged (mean \pm SEM) $60.3 \pm 4.2$ years, with weight $75.7 \pm 7.1 \mathrm{~kg}$, BMI $27.4 \pm 1.6 \mathrm{~kg} / \mathrm{m}^{2}$ and $\mathrm{HbA}_{1 \mathrm{c}} 5.6 \pm 0.2 \%$ and in the Pio group age $61.7 \pm 3.3$ years, weight $74.8 \pm 3.9 \mathrm{~kg}$, BMI $27.8 \pm 1.1 \mathrm{~kg} / \mathrm{m}^{2}$ and $\mathrm{HbA}_{1 \mathrm{c}} 5.8 \pm 0.1 \%$. There were no significant differences between groups or between baseline and follow up. There was a significant decrease in fasting PGs following Pio ( $5.8 \pm 0.2$ vs $5.5 \pm 0.1 \mathrm{mmol} / \mathrm{L} ; \mathrm{p}=0.018$ ) compared to placebo $(6.0 \pm 0.2$ vs $5.6 \pm 0.2 \mathrm{mmol} / \mathrm{L} ; \mathrm{p}=0.398)$. However there were no differences in area under the prandial PGs profiles $\left(\mathrm{AUC}_{(\mathrm{PGs}-0-4 \mathrm{hr})}\right)$ after 6 months in either treatment group. There was a significant increase in SI following Pio $\left(0.53 \pm 0.26 \times 10^{-4} \mathrm{vs} .1 .06 \pm 0.351 / \mathrm{min}^{\star} \mathrm{microU} / \mathrm{ml} \mathrm{p}=0.043\right)$ with a significant decrease in the placebo treated group $\left(2.48 \pm 0.22\right.$ vs. $1.55 \pm 0.24 \times 10^{-4} 1 /$ $\left.\mathrm{min}^{\star} \mathrm{microU} / \mathrm{ml} \mathrm{p}=0.043\right)$. Compared to the change observed on placebo ( $82.5 \pm 8.7$ vs $88.8 \pm 6.9$ ), Pio significantly decreased $(\mathrm{p}=0.046)$ fasting glucagon concentrations after 6 months (124.8 \pm 22.4 vs $113.8 \pm 23.0 \mathrm{pg} / \mathrm{ml})$. There were no differences in $\mathrm{AUC}_{\text {(n) }}$ after 6 months in either the placebo (358.9 \pm 28 $.3 \mathrm{vs} .363 .4 \pm 19.1 \mathrm{pg} . \mathrm{h} / \mathrm{ml})$ or Pio $(479.3 \pm 64.1 \mathrm{vs} .445 .8 \pm 64.2 \mathrm{pg} . \mathrm{h} / \mathrm{ml})$ groups. Conclusion: In this small cohort of subjects with impaired glucose tolerance, pioglitazone improved insulin sensitivity and thereby lowered both fasting glucagon and glucose concentrations

Supported by Takeda

\section{PS 30 Transcriptional regulation in beta cells}

\section{5}

Liver $\mathrm{X}$ receptor activation in the zucker diabetic fatty rat increases beta cell mass and decreases beta cell apoptosis

B. Blondeau ${ }^{1}$, C. Peschard ${ }^{1}$, V. Peyrou ${ }^{2}$, J.-M. Luccarini' ${ }^{2}$, B. Husson², B. Breant ${ }^{1}$;

${ }^{1}$ UMRS 872 Cordeliers Research Center, INSERM, Paris, France,

${ }^{2}$ Research Center, Fournier Pharma Laboratories, Daix, France.

Background and Aims: Liver X receptors (LXR) are members of the family of nuclear receptors and involved in cholesterol synthesis, lipogenesis and inflammation. Moreover, in vivo, the activation of these receptors by specific ligands also reverses diabetes in mice and rats but their mechanisms of action, particularly on beta cells, is incompletely understood.

Materials and Methods: To gain further knowledge on LXR action, we have treated Zucker Diabetic Fatty (ZDF) rats at 8 weeks of age with the LXR agonist GW3965 and studied these rats during a 4-week treatment.

Results: We found that GW3965 treatment prevented the increase of glycaemia and $\mathrm{HbAlc}$ observed in the Vehicle-treated ZDF rats, maintained a high basal insulinemia and slightly increased plasma triglyceride levels. After 4 weeks of treatment with the agonist, the glucose response was greatly improved and insulin secretion dramatically enhanced upon an oral glucose tolerance test. At the end of the treatment, GW3965 significantly induced islet expression of PDX1, SREBP1c and SCD1 mRNA levels and tended to increase the pancreatic GK, MafA, Glut2 and FAS mRNA expressions. Histological and immunostaining studies revealed that islets from Vehicle-treated ZDF rats were disorganized with an irregular shape, obvious fibrosis and abnormal Glut2 staining while GW3965 treatment restored a regular shape and Glut2 staining without modification of fibrosis status. When compared to Lean controls, beta cell mass was increased in ZDF Vehicle-treated animals due to increased islet numbers/cm2 without modification of islet size. In GW3965treated ZDF rats, beta cell mass, islet density and islet size in the head of the pancreas were further enhanced when compared to Vehicle controls. Beta cell proliferation was unchanged between the two ZDF rats groups neither after 4 days nor at the end of the treatment. In contrast, beta cell apoptosis rate that was increased in Vehicle animals compared to Lean controls, was fully restored to basal levels in GW3965-treated ZDF rats.

Conclusion: In conclusion, LXR activation efficiently blocked the spontaneous development of diabetes in ZDF rats. Our thorough analysis of the pancreas reveals that GW3965 treatment is associated with an increased beta cell mass due to an increased islet density, suggesting islet neogenesis. Moreover, beta cell apoptosis is blunted in GW3965-treated ZDF rats, thus helping to maintain a sufficient beta cell mass to protect against diabetes. These data help understanding the mechanisms of action of LXR agonists which represent promising therapeutic targets for the treatment of type 2 diabetes.

\section{6}

PDX-1 interacts with 14-3-3epsilon in pancreatic beta cells S. Teller, N. Hai Ha, R. Walther;

Department of Medical Biochemistry and Molecular Biology, University Greifswald, Germany.

Background and Aims: 14-3-3epsilon belongs to the 14-3-3 protein family. These proteins are involved in various cellular processes. 14-3-3 proteins are adaptor proteins thought to alter the stability, activity and/or subcellular localisation of their target proteins via protein-protein interaction. PDX-1 is a transcription factor that is mainly expressed in beta and delta cells of the islets of Langerhans in the pancreas. It is required for normal pancreas development and for the proper differentiation of the pancreas. In beta cells, the glucose-induced insulin gene transcription comprises transactivation of PDX-1 via the phosphatidylinositol 3-kinase pathway. However, the detailed mechanism by which PDX-1 becomes activated and changes its localisation is still poorly understood.

Materials and Methods: MIN6 cells were grown to $80 \%$ confluence in DMEM containing $25 \mathrm{mM}$ glucose, $10 \%$ horse serum and 2,5\% FCS. Cells were washed twice and incubated in Krebs-Ringer buffer $(118 \mathrm{mM}$ $\mathrm{NaCl}, 4.75 \mathrm{mM} \mathrm{KCl}, 1.25 \mathrm{mM} \mathrm{CaCl}, 1.2 \mathrm{mM} \mathrm{MgCl}, 0,05 \%$ (w/v) BSA, 
$25 \mathrm{mM} \mathrm{NaHCO}_{3}, 10 \mathrm{mM}$ Hepes pH 7.4). Cells were then stimulated with $16 \mathrm{mM}$ glucose for $30 \mathrm{~min}$. SDS-PAGE, immunoprecipitation, Northern and Western blotting were performed using standard molecular biology protocols. Pulldown experiments were performed using bacterially expressed GST-PDX-1 protein as a bait in lysates of glucose stimulated MIN6 cells. ${ }^{35}$ S-labeled PDX-1 for GST pulldown experiments was produced by in vitro transcription/translation (TNT Quick Coupled Transcription/Translation Systems, Promega, Madison, USA).

Results: The starting point of our studies was the identification of phosphoproteins interacting with PDX-1 after stimulation of starved MIN6 cells with glucose. By in vitro GST pull-down assays followed by mass spectrometry, we identified 14-3-3epsilon as a protein binding to GSTPDX-1. This interaction was confirmed by GST pull-down assays using in vitro translated PDX-1 and GST-14-3-3epsilon. Furthermore, using deletion mutants of PDX-1, we could show that endogenous 14-3-3epsilon protein binds to the C-terminal part and the N-terminal transactivation domain but not to the homeodomain of PDX-1. PDX-1 was also co-immunoprecipitated with 14-3-3epsilon from MIN6 lysates. The interaction was diminished when the lysates were pre-treated with phosphatase. This indicates that the interaction between PDX-1 and 14-3-3epsilon requires phosphorylation. Experiments with kinase inhibitors showed that neither inhibitors of PKC nor inhibitors of CKII influence the interaction of PDX-1 and 14-3-3epsilon. Atypical PKC's were described as activators of PDX-1, whereas a glucose induced phosphorylation of PDX-1 by CKII was found by our group.

Northern blot experiments revealed that overexpression of 14-3-3epsilon increases the insulin-mRNA level in MIN6 cells. For further experiments, a siRNA sequence was determined which is capable to downregulate 14-33epsilon in beta cell lines. FRET analysis with YFP- and CFP-coupled fusion proteins were used to detect the localisation of PDX-1 and 14-3-3epsilon in MIN6 cells dependent on starvation or glucose stimulation.

Conclusion: Identification of the phosphorylation dependent interaction between PDX-1 and 14-3-3epsilon in pancreatic beta cells supports the hypothesis that 14-3-3epsilon plays a role in PDX-1 transactivation and the regulation of the insulin gene transcription.

\section{7}

Embryonic lethality and impaired beta cell replication by disruption of Reg receptor gene

S. Takasawa ${ }^{1}$, K. Nata ${ }^{1}$, I. Takahashi ${ }^{1}$, T. Ikeda ${ }^{2}$, N. Noguchi ${ }^{1}$, M. Asano ${ }^{3}$, K. Sugihara ${ }^{3}$, A. Yamauchi ${ }^{1}$, T. Yoshikawa ${ }^{1}$, N. J. Shervani ${ }^{1}$, A. Uruno ${ }^{1}$, M. Unno ${ }^{4}$, A. Sugawara ${ }^{1}$, H. Okamoto ${ }^{1}$;

${ }^{1}$ Advanced Biological Sciences for Regeneration, Tohoku University, ${ }^{2}$ Innovation Plaza Miyagi, JST,

${ }^{3}$ Institute for Experimental Animals, Kanazawa University, Kanazawa, Japan, ${ }^{4}$ Surgery, Tohoku University, Sendai, Japan.

Background and Aims: Reg (Regenerating gene) gene encodes a $16 \mathrm{kDa}$ secretory protein that induces pancreatic beta-cell proliferation as an autocrine/paracrine growth factor via Reg receptor (RegR). Accumulating in vitro and in vivo evidence using Reg transgenic and Reg knockout mice suggests that Reg protein is involved not only in regenerative cell proliferation of pancreatic islet beta-cells but also in the cell proliferation of a variety of tissues such as gastrointestinal cells, neural cells, and cardiovascular cells.

Materials and Methods: We produced mice with a targeted disruption of RegR by homologous recombination. Beta-cell replication was induced by partial pancreatectomy and BrdU incorporation and PCNA staining of insulin-positive cells were measured.

Results: The heterozygous mutant mice $\left(\operatorname{Reg} R^{+/-}\right)$had normal fertility and lifespans and maintained similar body weight and blood glucose levels to those of their wild-type cohorts. In addition, there was no difference in the pancreatic beta-cell mass between $R e g R^{+/-}$mice and the wild type mice, whereas the mRNA and protein levels of RegR in the tissues of $R e g R^{+-}$mice were about one half of those of the wild type mice. We then intercrossed $\operatorname{Reg} R^{+/-}$mice and found the total absence of $\operatorname{RegR}^{-/-}$pups, indicating that this genotype is embryonic-lethal. Timed pregnancies of heterozygotes revealed that $\operatorname{RegR}^{-/-}$mice die between 8.5 and 9.5 days post coitum. As Reg knockout mice showed the decreased beta-cell regenerating activity, we induced pancreatic beta-cell replication by partial pancreatectomy in $R e g R^{+/-}$mice. Both the BrdU incorporation and the PCNA positive cell numbers in insulinpositive cells of residual pancreas of $R e g R^{+/}$mice were significantly decreased compared to those of $R e g R^{+/+}$wild type mice after surgery.
Conclusion: These results indicate that the Reg protein-Reg receptor system is essential for normal mouse development as well as for the replication of pancreatic beta-cells for regeneration.

\section{8}

Antagonism between Hepatocyte Nuclear Factor 4 alpha (HNF4a) and Chicken Ovalbumin Upstream Promoter-Transcription Factor 2 (COUP-TFII) modulates Ped/Pea-15 gene expression

P. Ungaro, R. Teperino, P. Mirra, G. Botta, V. D’Esposito, S. Iovino, G. Lupoli, F. Oriente, C. Romano, R. Valentino, F. Beguinot; Dipartimento di Biologia e Patologia Cellulare e Molecolare \& IEOS-CNR, Università di Napoli Federico II, Napoli, Italy.

Background and Aims: Overexpression of the Ped/Pea-15 (Phosphoprotein enriched in diabetes/phosphoprotein enriched in astrocytes) gene has been found in tissues from type 2 diabetic patients and their healthy first degree relatives. In transgenic mouse models, ped/pea-15 overexpression determines insulin-resistance and impairs glucose-stimulated insulin secretion. In order to address the molecular mechanisms responsible for Ped/Pea-15 overexpression, we isolated the promoter of the gene. We identified a cisacting regulatory element (RE) between -307 and -322 bps upstream from the Ped/Pea-15 gene transcriptional start site $(+1)$. The RE is recognized by both the Hepatocyte Nuclear Factor 4 (HNF4a) and by the Chicken Ovalbumin Upstream Promoter Transcription Factor 2 (COUP-TFII), two orphan members of the steroid/thyroid receptor superfamily both of which are involved in lipid and glucose homeostasis.

Materials and Methods: We have investigated the role of HNF4 $\alpha$ and COUPTFII in the regulation of Ped/Pea-15 expression in cultured Hela and HepG2 cells.

Results: Based on luciferase assays HNF4 $\alpha$ overexpression in Hela cells reduced Ped/Pea-15 promoter activity in a dose-dependent and site-specific manner. Consistently, both Real Time PCR and Western Blot analysis revealed a decreased PED/PEA-15 expression in Hela cells transiently transfected with an HNF4 $\alpha$ expression vector. In addition, RNA interference (RNAi)mediated depletion of HNF4 $\alpha$ in HepG2 cells increased Ped/Pea-15 mRNA. Similar experiments demonstrated that COUP-TFII acts in the opposite way, as it increases the activity of the Ped/Pea-15 promoter, as well as that of the endogenous Ped/Pea-15 RNA by real time PCR in Hela cells. Cotransfection of Hela cells with the reporter construct containing the RE located between -307 and -322 bps and various combinations of HNF4 $\alpha$ and COUP-TFII expression vectors indicated that COUP-TFII antagonizes the repression of the Ped/Pea-15 gene by HNF4 $\alpha$. Finally, Chromatin Immuno-Precipitation (Chip) and Electrophoretic Mobility Shift Assay (EMSA) experiments indicate that both transcription factors directly bind to the responsive element between -307 and -322 bps on Ped/Pea-15 promoter with a similar affinity, indicating that these two proteins compete for the transcriptional control of the Ped/Pea-15 promoter.

Conclusion: Our data demonstrate that the antagonism between HNF4 $\alpha$ and COUP-TFII modulates Ped/Pea-15 gene expression. Alterations in the action of HNF $4 \alpha$ and COUP-TFII may lead to the dysregulation of Ped/Pea-15 gene expression, thereby contributing to susceptibility to type 2 diabetes.

\section{9}

Shedding light on the controversial role of Tmem27

J. Altirriba ${ }^{1}$, R. Gomis ${ }^{1}$, M. C. Ruiz de Villa ${ }^{2}$, M. Julià ${ }^{1}$, A. Barberà ${ }^{1}$; ${ }^{1}$ Endocrinology and Diabetes, Hospital Clinic / IDIBAPS, Barcelona, Spain, ${ }^{2}$ Department of Statistics, UB, Barcelona, Spain.

Background and Aims: Two independent groups have recently identified Tmem27 in the pancreatic $\beta$ cells as an HNF1a target. However, they have reached different conclusions about Tmem 27 role using similar transgenic studies. While one group concludes that an increase of its expression stimulates pancreatic $\beta$ cell proliferation without modifying insulin secretion, the other states that its rise enhances glucose-stimulated insulin exocytosis without changing the islet mass. To shed light on this controversy, without using a genetically modified model, we aim to assess the changes of Tmem 27 in animals treated with tungstate and reconfirm the results obtained in human islets. 
Materials and Methods: Wistar rats were treated with tungstate $(2 \mathrm{mg} / \mathrm{ml}$ drinking water) during 40 days. Insulin sensitivity (ITT) and intraperitoneal glucose tolerance (IGTT) tests were performed. Total pancreas was removed, fixed and islet proliferation was assessed. Islets were isolated from untreated and treated rats in order to study the glucose induced insulin secretion and Tmem 27 and insulin expression by qRT-PCR. In order to asses if the changes observed were due to a decrease in food intake induced by the treatment, a pair-fed experiment was conducted. At the end of this experiment, insulinemia and insulin and Tmem27 expression levels in the islets were determined. Finally, the levels of Tmem27, insulin, snapin, cyclin E1 and CDK2 were assessed in human islets by qRT-PCR, establishing its correlation.

Results: Treated (T) rats compared to the untreated (UT) were more glucose tolerant (glycaemia AUC UT 24576 vs T $21324 \mathrm{mg} / \mathrm{dL}$ x $120 \mathrm{~min}, \mathrm{p}<0.01$; insulinemia AUC UT 549 vs T $292 \mu \mathrm{g} / \mathrm{L}$ x $120 \mathrm{~min}, \mathrm{p}<0.05$ ), showed an increased insulin sensitivity (AUC UT 6246 vs T $5113 \mathrm{mg} / \mathrm{dL}$ x $120 \mathrm{~min}$, $\mathrm{p}<0.01$ ) and a decreased insulinemia levels (UT 2.28 vs T $1.46 \mu \mathrm{g} / \mathrm{L}, \mathrm{p}<0.01$ ). In isolated islets, glucose induced insulin secretion was decreased (UT $5.85 \mathrm{vs} \mathrm{T} 2.60 \mu \mathrm{g}$ /islet x $90 \mathrm{~min}, \mathrm{p}<0.01)$. Morphometric analysis revealed a significantly increased islet proliferation in T rats ( $\mathrm{T} 4.03$ vs UT 32.90 nuclei PCNA $+/ \mathrm{mm}^{2}$ islet, $\left.\mathrm{p}<0.001\right)$. Interestingly, in $\mathrm{T}$ animals, a decrease in mRNA levels of insulin (fold change $-2.26, \mathrm{p}<0.01$ ) and Tmem27 (fold change $-3.08, \mathrm{p}<0.01$ ) was detected. It is worth noting that expression levels of insulin and Tmem 27 showed a significant correlation $(\rho=0.724, p<0.001)$. Therefore, tungstate administration to rats led to a decrease in Tmem27 expression similarly to what happened with insulin expression, secretion and insulinemia; whereas the islet proliferation was increased. Furthermore, pair-fed animals showed no differences in insulinemia and insulin and Tmem 27 mRNA levels compared to UT animals, discarding the possibility that the changes observed were due to the decrease in food intake induced by tungstate. Finally, in order to check whether the correlation between Tmem27 and insulin expression levels also occurs in human islets, the expression of several key genes of $\beta$ cell function was assessed. The results showed that Tmem 27 strongly correlates with insulin $(\rho=0.866, p<0.01)$ and snapin (component of the SNARE complex) $(\rho=0.733, p<0.05)$ but no significant correlation was found with cyclinE1 and CDK2 (key genes in the progression of the cell cycle).

Conclusion: Our results show that Tmem 27 role in $\beta$ cell function is involved in insulin secretion but not in $\beta$ cell proliferation.

Supported by grants from MEC and REDIMET from ISC III, MSC

\section{0}

Affinity binding analysis shows that hnRNP K and hnRNP E, in cytosolic extracts from human islets, binds specifically to the stabilizing segment of insulin mRNA

R. G. Fred ${ }^{1}$, C. M. Adams ${ }^{2}$, N. Welsh ${ }^{1}$;

${ }^{1}$ Medical Cell Biology,

${ }^{2}$ Biological and Medical Mass Spectrometry, Uppsala University, Sweden.

Background and Aims: The increased production of insulin that occurs in response to a glucose challenge is in part due to an enhanced stability of insulin mRNA. The stability of insulin mRNA is in turn controlled by a pyrimidine-rich sequence located in the 3 '-untranslated region (UTR) of the messenger. We have previously shown that the polypyrimidine tract binding protein (PTB1) by binding to this sequence stabilizes the mRNA. Since the exact mechanisms of glucose-induced insulin mRNA stabiliziation are yet to be determined we presently investigated whether other proteins than PTB1, either directly or in complex with PTB1, specifically bind to the 3'-UTR of insulin mRNA.

Materials and Methods: Biotinylated 27-mer RNA oligonucleotides corresponding to the specific sequences of the rat and human insulin mRNA 3'-UTR were incubated with cytosolic extracts from INS-1 cells or naive human islets. The human islets were obtained from Prof. Olle Korsgren at the Akademiska Hospital, Uppsala, Sweden. Following a 60 min binding reaction, the oligonucleotide/protein complexes were recovered using streptavidin coated ferrite beads. The proteins were eluted from the beads by boiling in SDS-sample buffer and run on 1-D gels together with controls lacking or having mutated oligonucleotides. After commassie staining sequence-specific bands were excised and analyzed by Fourier Transform Mass Spectrometry.

Results: Mass Spectrometry revealed that in addition to the $55 \mathrm{kD}$ band, which contained PTB, specific $40 \mathrm{kD}$ and $70 \mathrm{kD}$ bands contained hnRNP E and hnRNP K, respectively. Further immunoblot analysis of the RNA-binding proteins, using antibodies recognizing hnRNP E and hnRNP K, showed that the two proteins, bind specifically to the polypyrimidine sequence of insulin mRNA in both human and rodent cell extracts.

Conclusion: Our studies provide ample verification that both hnRNP K and hnRNP E binds specifically to the stabilizing segment of insulin mRNA and that they therefore may be part of the glucose signaling pathway. Further experimentation will provide information on whether the hnRNP proteins bind directly or via protein/protein-interaction to the RNA-oligonucleotide, and whether glucose promotes post-translational modifications to the insulin mRNA-binding proteins.

\section{1}

Glucose and zinc-depletion stimulate beta cell expression of $\mathrm{ZnT} 3$, a zinc transporter, whereas $\mathrm{ZnT} 3 \mathrm{KO}$ mice have decreased beta cell capacity J. B. Jeppesen ${ }^{1}$, A. Larsen ${ }^{2}$, N. Jessen ${ }^{3}$, N. Magnusson ${ }^{1}$, L. Egefjord ${ }^{1}$,

L. Wogensen ${ }^{4}$, B. Brock ${ }^{5}$, O. Schmitz ${ }^{3}$, K. Smidt ${ }^{1}$, M. Stoltenberg ${ }^{2}$, J. Rungby ${ }^{1}$; ${ }^{1}$ Pharmacology and Endocrinology C, University of Aarhus,

${ }^{2}$ Dept. Neurobiology, Inst. of Anatomy, University of Aarhus, ${ }^{3}$ Pharmacology and Endocrinology M, University of Aarhus, ${ }^{4}$ Biochemical Pathology, University of Aarhus,

${ }^{5}$ Pharmacology and Pharmacy, University of Aarhus, Denmark.

Background: Zinc transporter ( $\mathrm{ZnT})$-proteins regulate zinc fluxes into beta-cell vesicles and recently ZnT8 was identified as a major risk factor for developing type 2 diabetes. Contrasting other $\mathrm{ZnTs}$, $\mathrm{ZnT} 3$ is upregulated by high glucose levels as well as by zinc depletion in $\beta$-cells. We here confirm the glucose dependence of ZnT3 and show by electron microscopy that ZnT3 is located in beta-cell cytoplasm. Further, we report the first results of experiments examining glucose metabolism in $\mathrm{ZnT} 3 \mathrm{KO}$ mice.

Methods: Ins1-E rat beta-cells were exposed to 2, 5 or $16 \mathrm{mM}$ glucose for 24 hrs. ZnT3 mRNA was measured by RT-PCR. Measurements were normalized to a mean of three house-keeping genes. Insulin secretion was measured by Elisa. For electron microscopy, INS1-E cells were fixed in paraformaldehyde and examined for the presence of ZnT3 by immunogold staining with a specific goat-rabbit antibody. To determine if knocking out the ZnT3 gene affects whole body glucose metabolism, twelve weeks old ZnT3 KO mice derived from the C57B16 mouse strain were compared to wild type mice after five days of treatment with intraperitoneal streptozotocin $50 \mathrm{mg} / \mathrm{kg}$ daily or saline. After two weeks of recovery, tail blood glucose levels were measured after a 14 hrs fast with a One Touch Ultra Meter.

Results: We confirmed that, relative to $5 \mathrm{mM}$ glucose, $2 \mathrm{mM}$ glucose caused a significant down-regulation of ZnT-3 and $16 \mathrm{mM}$ an up-regulation ( $\mathrm{p}$ $<0.01$ ). Insulin secretion was increased at $16 \mathrm{mM}$ glucose. By electron microscopy we could identify the presence of ZnT3 in the cytoplasm of INS1E cells. No signifant changes in blood glucose levels were found between ZnT3 KO- and WT mice. However, after treatment with streptozotozin, KO mice had significantly higher blood glucose levels compared with shaminjected KO mice $(\mathrm{p}<0.05)$ and streptozotocin-treated WT mice $(\mathrm{p}<0.01)$ (Figure).

Conclusion: This study identifies ZnT3 as a pivotal regulator of beta-cell zinc metabolism. Zinc ions have numerous roles in the islets as well as possible roles in the periphery. These include regulation of counter-regulatory hormones and beta-cell apoptosis as well as the formation of insulin crystals.

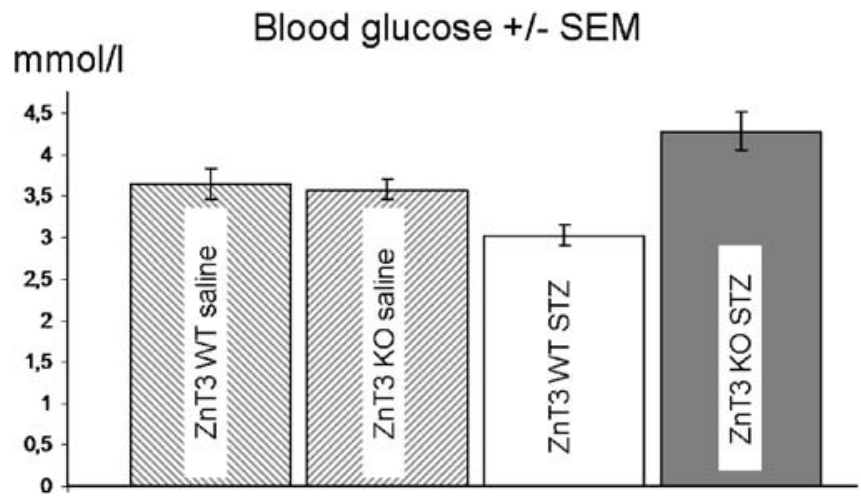


The fact that ZnT3 gene expression levels can be up-regulated by increasing glucose levels suggest a possible direct function in insulin storage or secretion of $\mathrm{ZnT} 3$ controlled zinc fluxes. Furthermore, the increase in glycaemia after moderate beta-cell stress by streptozotocin suggest that other ZnTs are unable to compensate for the lack of $\mathrm{ZnT} 3$ in otherwise healthy mice. Supported by Danish Diabetes Asssociation

\section{2}

High content isoflavone soy protein isolate increases insulin sensitivity and changes the gene expression of key insulin regulatory genes and $\beta$ cell transcription factors in islets of the diabetic KKAy-mouse I. Nordentoft, P. B. Jeppesen, J. Hong, R. Abudula, K. Hermansen; Dept. of Endocrinology and Metabolism C., Aarhus University Hospital, Aarhus Sygehus THG, Denmark.

Background and Aims: High content isoflavone soy protein (SBP) have a beneficial effect on the insulin resistance syndrome. The aim of this study was to investigate if SBP possesses beneficial effects on the metabolism in the diabetic KKAy-mouse. Moreover, we investigated the long term in vivo effect of SBP on the gene expression profile of key insulin regulatory genes in islets.

Material and Methods: 26 KKAy-mice, age 5 weeks, were divided into 2 groups and treated for 9 weeks with either: A: standard chow diet (control); B: chow $+50 \%$ SBP. Blood samples were collected and analysed before and after intervention. As a non diabetic control group (C) twenty normal C57BLmice were fed with standard chow diet. Gene expression was determined in islets by quantitative real-time RT-PCR and Affymetrix microarray.

Results: We demonstrated that long-term treatment with SBP improves glucose homeostasis, increase insulin sensitivity, lower plasma triglycerides and lower total plasma cholesterol and doubles the islet insulin content in the diabetic KKAy-mice strain. Furthermore, SBP markedly change the gene expression profile of key insulin regulatory genes including GLUT2, Ins1, Ins2, Beta/Neurod1, and 11- $\beta$-HSD-1, Nkx6-1, C/EBPalpha and Foxa2 in the islets isolated from the KKAy-mice.

Conclusion: The results indicate that SBP improves glucose homeostasis, increases insulin sensitivity, lowers plasma triglycerides and lowers weight in the diabetic KKAy-mice and up-regulates the gene expression of key beta cell insulin regulatory genes.

\section{3}

Gene expression regulated by pioglitazone and exenatide in normal and diabetic rat islets exposed to palmitate

H. Ghanaat-pour ${ }^{1}$, N. Welsh ${ }^{2}, \AA ̊$. Sjöholm ${ }^{1}$;

${ }^{1}$ Karolinska Institutet, Department of Internal Medicine,

Stockholm South Hospital, Stockholm, Sweden,

${ }^{2}$ Department of Medical Cell Biology, Uppsala University, Sweden.

Background and Aims: Hyperlipidemia has been suggested to contribute to the loss of $\beta$-cell mass (apoptosis), its secretory defects and thereby impaired $\beta$-cell function in type 2 diabetes. Treatment of genetically diabetic GK rats and also type 2 diabetic patients with pioglitazone, a PPAR- $\gamma$ agonist, lowers the fasting levels of plasma glucose and triglycerides. Another recently launched antidiabetic drug, exenatide, an incretin mimetic, has been suggested to stimulate insulin secretion, growth and proliferation of pancreatic $\beta$-cells.We aimed to investigate $\beta$-cell lipoapoptosis, and the possible protective effect of in vitro treatment with pioglitazone and exenatide against this toxicity.

Materials and Methods: Cell viability was examined in pancreatic islets of Wistar rats by utilizing fluorescence microscopy. Global gene expression profiling was applied to characterize genes differently regulated by palmitate, pioglitazone and exenatide in islets from non-diabetic Wistar rats and type 2 diabetic Goto-Kakizaki (GK) rats.

Results: The results from fluorescence microscopy indicated no significant protective effect of pioglitazone on $\beta$-cell lipoapoptotis. Gene expression profiling revealed significant changes in islet mRNAs involved in control of $\beta$ cell function, e.g. epigenetic regulation of gene expression, cell differentiation and morphogenesis, also metabolism, response to stimulus, transport and signal transduction. Pioglitazone and exenatide appear to significantly impact epigenetic processes, e.g. stable alterations in gene expression potential that arise during development and cell proliferation. Bcl2 family members may act as anti- or pro-apoptotic regulators and thereby play an important role in the fate of cells. Bcl2-like 1 (Bcl211), an anti-apoptotic member of this family, seems to exert a permissive role in cell proliferation and differentiation. Bcl2 modifying factor (Bmf), a pro-apoptotic member of this family, is required for binding to the $\mathrm{Bcl} 2$ family members to trigger apoptosis. Bcl2l1 and $\mathrm{Bmf}$ were both down-regulated by pioglitazone and exenatide in the presence of palmitate in diabetic GK islets. In contrast, Bmf was down-regulated by pioglitazone in the presence of palmitate in non-diabetic Wistar islets. The activation of PPAR $\beta / \delta$ seems to induce the expression of target genes involved in fatty acid metabolism. In this study, exposure of non-diabetic Wistar islets to palmitate led to a reduction in the expression of PPAR $\beta / \delta$. This suggests that palmitate may increase the accumulation of triglycerides by reducing PPAR signaling. Moreover, treatment with either pioglitazone or exenatide restored and increased the expression of PPAR $\beta / \delta$ in non-diabetic Wistar islets.

Conclusion: Taking into account that these drugs target different components of the epigenetic machinery, our findings suggest that they might participate in restoring normal gene activity in dysfunctional islets and that additive benefits may occur. Whether such events contribute to the $\beta$-cell sparing, proliferative and anti-apoptotic effects of these drugs in diabetes remains to be elucidated.

\section{4}

Pioglitazone modulates lipid metabolism, cell cycle and cell death gene expression profiles in pancreatic islets

R. N. Lamounier ${ }^{1,2}$, P. White ${ }^{2}$, C. N. Coimbra ${ }^{1}$, L. S. Oliveira ${ }^{1}$,

M. L. Correa-Giannella ${ }^{1}$, K. H. Kaestner';

${ }^{1}$ LIM-25, Endorinology Division, University of Sao Paulo, Brazil,

${ }^{2}$ Department of Genetics, University of Pennsylvania, Philadelphia, United States.

Background and Aims: Peroxisome proliferator-activator receptor- $\gamma$ (PPAR$\gamma$ ) is a target for thiazolidinedione (TZD) antidiabetic drugs and a regulator of adipose tissue differentiation and metabolism. TZD act as an insulin sensitizing agent on peripheral tissues. It has been speculated that TZD could play a role on beta-cell function, preventing loss and improving viability in the long-term. Nevertheless some studies also showed a lack or even a potential deleterious effect of TZD on islets. The role of PPAR- $\gamma$ target genes in pancreatic islets is still largely unclear. We studied the gene expression profile induced by the treatment with pioglitazone (PIO), an approved TZD for T2DM therapy, on rat pancreatic islets primary culture both at normal and high glucose medium concentrations.

Materials and Methods: Islets were obtained from 2 month-old wistar rats and isolated by the Ficoll gradient method and then cultured in $5.6 \mathrm{mM}$ or $23 \mathrm{mM}$ glucose for $24 \mathrm{~h}$, being treated with PIO $10 \mu \mathrm{M}$ or DMSO $0.1 \%$ (vehicle). Pioglitazone was provided by Takeda Pharmaceuticals, Osaka, Japan. RNA was extracted with Trizol (Sigma) and purified with RNeasy kit (Qiagen). The Ovation ${ }^{\mathrm{mm}}$ Aminoallyl RNA Amplification and labeling System was used for sample amplification. Samples were labeled and then hybridized on the Mouse PanChip 13k cDNA microarray, using 5 different biological replicates for each test condition. Statistical Analysis of the microarray data was performed using significance analysis of microarrays (SAM) with a false discovery rate of $10 \%$. Gene expression results were confirmed through Q-PCR. Pathways assessment was performed through Ingenuity Pathway Analysis (www.ingenuity.com).

Results: At $5.6 \mathrm{mM}$ glucose 101 genes were modulated by PIO, 49 upregulated and 52 downregulated. At $23 \mathrm{mM} 1235$ genes were affected, 612 upregulated and 623 downregulated. Comparison between both conditions revealed 74 genes that were similarly modulated at both glucose concentrations. Pathway analysis of perturbed genes revealed biologically relevant networks related to lipid metabolism at both glucose medium concentrations. At $23 \mathrm{mM}$, cell cycle and cell death pathways were significant modulated as well.

Conclusion: This data demonstrates that in addition to known effect in adipocytes, the insulin sensitizing agent Pioglitazone modulates gene expression in pancreatic islets, especially in the presence of supraphysiological glucose concentrations affecting especially lipid metabolism and mechanisms of cell death and cell cycle. 


\begin{tabular}{lll}
\multicolumn{3}{l}{ Genes confirmed through qPCR. Fold-change Pio vs. control ${ }^{*} \mathrm{p}<=0.05$} \\
\hline Gene & $5.6 \mathrm{mM}$ & $23 \mathrm{mM}$ \\
Ins1 & 1.55 & 1.02 \\
Hmox1 & 1.68 & $3.43^{*}$ \\
Insig1 & $1.97^{*}$ & 1.52 \\
Scd2 & $1.86^{*}$ & $1.53^{*}$ \\
Fabp4 & $5.83^{*}$ & $4.96^{*}$ \\
Srebf1 & $1.32^{*}$ & $1.33^{*}$ \\
Bax & 1.19 & $1.45^{*}$ \\
Tnf & 1.03 & $3.59^{*}$ \\
Sod2 & 1.58 & $4.46^{*}$ \\
Lipe & -1.04 & $2.03^{*}$ \\
Myc & 1.19 & $1.97^{*}$ \\
\hline
\end{tabular}

Supported by Fund. de Amparo à Pesquisa do Estado de São Paulo (FAPESP), Functional Genomics of the Beta Cell NIDDK grant and Diabetes and Endocrinology Research Center (DERC)

\section{PS 31 Beta cell neogenesis and replication}

\section{5}

Regulation of neonatal beta cell growth in rat. Role of Wnt/ beta-catenin signaling pathway

F. Figeyc, B. Portha, J. Movassat;

Physiology, University Paris Diderot, France.

Background and Aims: Wnt signalling pathway plays important roles in normal development of a variety of organs and in the pathogenesis of diseases such as cancer. Wnt signals are secreted glycoproteins which interact with a family of seven-pass transmembrane receptors called Frizzled. This interaction transduces distinct kinds of intracellular signalling pathways. The "canonical" Wnt pathway implicates changes of the intracellular levels of beta-catenin (Wnt/ beta-catenin pathways) and it is thought to be involved in cell fate specification and cell proliferation. The role of beta-catenin dependent Wnt signalling in the development of the normal pancreas has been investigated recently in few studies. However its specific role in the differentiation and growth of the endocrine pancreas remains controversial. In our study we addressed the importance of Wnt/ beta-catenin signalling in the endocrine pancreatic growth during the first post-natal week in Wistar rats. This is an important period during which the endocrine cell mass increases dramatically by both replication of existing cells and differentiation of precursor cells into beta cells.

Materials and Methods: First we assessed the expression of the active hypophosphorylated form of beta-catenin in the pancreas of 2 and 7 days old neonates by Western blot analysis of pancreatic lysates and by immunofluorescence staining of pancreatic sections. Next, in order to address the specific role of beta-catenin stabilization in beta cell proliferation, we established culture of islets of Langerhans isolated from the pancreas of 2 days old pups and assessed the rate of beta cell proliferation following inactivation of Glycogen Synthase Kinase 3 (GSK3 beta) by lithium chloride (LiCl). Cell proliferation was also assessed in culture of insulinoma cell line INS1 treated by LiCl.

Results: Immunostaining of pancreatic sections showed that nuclear betacatenin can be found in a subset of endocrine cells as well as ductal and acinar cells. Western blot analysis of pancreatic lysates revealed that the active form of beta-catenin is expressed in neonatal pancreases and its expression decreases significantly as a function of age. The greater levels of active beta-catenin in the pancreas at 2 days compared to 7 days of age are correlated with the higher rate of cell proliferation in the pancreas of younger pups. In primary cultures of neonatal islets, the GSK3 beta inhibitor $\mathrm{LiCl}(10 \mathrm{mM})$ significantly increased beta cell proliferation after 24, 48 or 72 hours exposure of islets. The proliferation of INS1 cells was also significantly stimulated by LiCl. The inhibition of GSK3 beta led to beta-catenin stabilization as evidenced by increased levels of hypophosphorylated beta-catenin in cells cultured with $\mathrm{LiCl}$. Moreover, we showed that upstream components of the Wnt signalling pathway such as Wnt 5A, Wnt 11 and Frizzled 10 are expressed in insulinoma INS1 cells and in neonatal pancreases.

Conclusion: Overall, our results show that in vivo, the active form of betacatenin is abundant in the neonatal rat pancreas during the intense phase of cell proliferation, and that stabilization of intracellular beta-catenin leads to the stimulation of beta cell proliferation in both primary culture of neonatal beta cells and in the beta cell line INS1. This suggests that Wnt/ beta-catenin pathway could be important in the regulation of neonatal beta cell growth.

\section{6}

Gastrin treatment stimulates beta cell replication, increases beta cell mass and improves metabolic control in $95 \%$ partially pancreatectomized rats

N. Téllez, G. Joanny, J. Escoriza, J. Soler, E. Montanya;

Lab. Diabetes and Experimental Endocrinology, IDIBELL, L'Hospitalet de Llobregat, Spain.

Background and Aims: The administration of the gastrointestinal hormone gastrin has been reported to induce beta cell neogenesis and to increase beta cell mass. These effects were found in particular when gastrin was coadministered with EGF. However, the effects of gastrin alone are less well 
defined, and the potential role of gastrin on beta cell replication remains to be established. The aim of the study was to determine the effects of the administration of gastrin to partially pancreatectomized rats, a well established model of beta cell regeneration.

Materials and Methods: SD rats underwent 95\% partial pancreatectomy $(95 \%-\mathrm{Px})$ and were treated with the gastrin analogue [15 leu] gastrin-17 $(150 \mu \mathrm{g} / \mathrm{kg} \cdot 12 \mathrm{~h}, \mathrm{sc})$ or with vehicle $(\mathrm{n}=5)$ from day 1 (when $95 \%$ Px was performed), to day 15 (when pancreatic remnants were harvested). Allocation of pairs of same day pancreatectomized animals to gastrin- and vehicle-treated groups was blinded. Blood glucose and body weight were monitored on days $1,3,7,10$ and 15. A group of animals of similar body weight underwent a $95 \%$ Px and had the pancreatic remnant harvested immediately after surgery. The pancreas of an additional control group of sham-Px rats $(n=6)$ was harvested after 15 days of follow up. Beta cell mass and cross-sectional individual beta cell size was determined by morphometry, and beta cell replication by BrdU incorporation, in insulin stained sections of harvested pancreatic remnants and pancreases.

Results: 95\%- Px rats treated with vehicle developed severe hyperglycemia (day 15: $516 \pm 41 \mathrm{mg} / \mathrm{dl}$ ) that was reduced by gastrin treatment (day 15: 318 $\pm 85 \mathrm{mg} / \mathrm{dl}, \mathrm{p}<0.01$ ). $50 \%$ of animals treated with gastrin, but none of those treated with vehicle, remained normoglycemic after surgery (day 15: $130 \pm 20$ $\mathrm{mg} / \mathrm{dl}$ ). Beta cell mass immediately after $95 \%$ Px was $0.34 \pm 0.09 \mathrm{mg}$, and was $34 \%$ higher $(0.45 \pm 0.13 \mathrm{mg}, \mathrm{p}: \mathrm{ns})$ in vehicle-treated rats. Gastrin treatment resulted in a dramatic $364 \%$ increment in beta cell mass $(1.24 \pm 0.24 \mathrm{mg})$ $(\mathrm{p}<0.05)$. Beta cell replication in sham-Px rats was $0.72 \pm 0.1 \%$, and was $40 \%$ higher in vehicle-treated Px rats $(1.02 \pm 0.2 \% ; \mathrm{p}=\mathrm{ns})$. Gastrin treatment further increased beta cell replication $(1.29 \pm 0.23 \% ; \mathrm{p}=0.07)$. The individual beta cell size was similar in gastrin- and vehicle-treated $95 \%$-Px rats $(244 \pm 13$ vs $\left.251 \pm 24 \mu \mathrm{m}^{2}\right)$ and higher than sham-Px rats $\left(197 \pm 16 \mu \mathrm{m}^{2}, \mathrm{p}<0.05\right)$, indicating that gastrin had no effects on beta cell hypertrophy. The number of scattered beta cells (groups of $<5$ beta cells), considered an indirect marker of islet neogenesis, was similar in gastrin-treated $(5.96 \pm 0.45)$ and vehicletreated $(6.16 \pm 0.74 \%) 95 \%$-Px rats.

Conclusion: Exogenous administration of gastrin enhanced beta cell regeneration in $95 \%$ partially pancreatectomized rats increasing beta cell replication and beta cell mass and, ameliorated blood glucose control. Gastrin might be potentially useful to stimulate regeneration of beta cells in the treatment of diabetes.

Supported by Fis PI-06089, ISCIII-RETIC RD06, Novo Nordisk

\section{7}

The ob/ob-mouse as a model for studying $\beta$ cell proliferation

J. Westerlund ${ }^{1}$, M. Hovsepyan ${ }^{2}$, P. Lindström ${ }^{3}$, P. Bergsten ${ }^{2}$;

${ }^{1}$ Department of Caring and Public Health Sciences, Mälardalen University, Västerås, Sweden,

${ }^{2}$ Department of Medical Cell Biology, Uppsala University, Sweden,

${ }^{3}$ Department of Integrative Medical Biology, Umeå University, Sweden.

Background and Aims: Loss of $\beta$-cells is part of the development of not only type 1 diabetes mellitus (T1DM), but also type 2 diabetes mellitus (T2DM). The ability of $\beta$-cells to regenerate is very limited, however. The $\beta$-cell population in $o b / o b$-mice provides an exception from this rule; as hyperglycaemia develops the $\beta$-cells increase. We have observed that whereas some animals are almost normoglycaemic (NG), others are hyperglycaemic (HG) despite a common ob/ob-background. We hypothesized that NG animals would show higher $\beta$-cell proliferation than HG animals. The first aim of the study was to determine under what conditions $o b / o b$-mice showed accentuated differences in $\beta$-cell proliferation. The second aim was to isolate islets from $o b / o b$-mice showing such differences, protein profile the islets and identify differentially expressed islet proteins.

Material and Methods: $o b / o b$-mice were kept on normal laboratory chow with free access to water. Three- and six-months-old animals were recruited to the NG or HG groups on the basis of blood sugar levels lower than 10 $\mathrm{mM}$ or higher than $12 \mathrm{mM}$, respectively. Body weight, food intake and blood sugar levels were monitored for three weeks, after which animals were killed and pancreata were removed and either fixated in formalin or used for islet isolation. Pancreatic sections were used to determine islet area in percentage of total tissue area and $\beta$-cell proliferation by Ki67 staining. Isolated islets were used for measuring insulin secretion in the presence of 3 or $20 \mathrm{mM}$ glucose. In addition, isolated islets were lysed and subjected to two-dimensional gel electrophoresis. Separation by iso-electric focusing was done on immobilized
$\mathrm{pH}$-gradient strips, followed by size separation with SDS-PAGE. Gels were stained by silver staining and analyzed for differential protein expression. Results: Food-intake and weight did not differ between NG and HG animals. In contrast, circulating insulin levels were higher in NG than HG animals, $87 \pm 8$ and $64 \pm 15 \mathrm{ng} / \mathrm{ml}$ at 3 months and $126 \pm 11$ and $90 \pm 15 \mathrm{ng} / \mathrm{ml}$ at 6 months. Elevated insulin levels were paralleled by increased islet-area in NG compared to HG animals. Islet area (expressed in percentage of total area) in NG and HG animals was 3.9 and 3.2 at 3 months and 11.9 and 6.3 at 6 months. Proliferation of $\beta$-cells was measured by counting average number of Ki67-positive cells per islet. Whereas proliferation in 3-month old NG and $\mathrm{HG}$ animals was $3.0 \pm 1.9$ and $0.37 \pm 0.21(\mathrm{p}<0.01)$, it was $2.13 \pm 1.39$ and $1.10 \pm 0.83$ (NS) in 6-month old NG and HG animals. Glucose stimulated insulin secretion from isolated islets increased essentially equally by 10 -fold in all groups when raising glucose concentration from 3 to $20 \mathrm{mM}$. Protein profiling of islets isolated from NG and HG revealed approximately 600 spots with approximately 80 differentially expressed proteins.

Conclusion: Findings suggest that animals with a high islet proliferative capacity have higher serum insulin levels and lower blood glucose values than animals with a smaller proliferative capacity. We conclude that $o b / o b$-mice offer an opportunity to explore mechanisms underlying $\beta$-cell proliferation. It is expected that identification of differentially expressed islet proteins will yield information that could make $\beta$-cells proliferate in individuals with T2DM and potentially T1DM.

Supported by Swedish Medical Research Council

\section{8}

Effects of islet transplantation on endogenous $\beta$ cell regeneration after partial pancreatectomy in rodents

H.-S. Jung ${ }^{1}$, J.-Y. Park ${ }^{1}$, Y.-R. Ahn², S.-H. Oh², M.-S. Lee ${ }^{1}$, M.-K. Lee ${ }^{1}$, K.-W. Kim ${ }^{1}$;

${ }^{1}$ Medicine, Samsung Medical Center,

${ }^{2}$ Lab251, Samsung Biomedical Reserach Institute, Seoul, Republic of Korea.

Background and Aims: Islet transplantation is one of regimens supplying insulin in diabetes patients, but the effects of islet grafts on the endogenous $\beta$ cells are not clear. In the present study, we examined the changes of endogenous $\beta$-cell mass after islet transplantation in partially pancreatectomized mice. Materials and Methods: Balb/c mice were $70 \%$ pancreatectomized, transplanted with syngeneic islets (group IV), and were compared with pancreatectomized mice treated with insulin (group III), no insulin (group II), and Sham operation group (I). Remnant pancreas was obtained 6 and 10 days after pancreatectomy, and immunohistochemical staining was done for the evaluation of $\beta$-cell mass and cell proliferation. Before sacrification, plasma blood and insulin levels were measured in each subject.

Results: Hyperglycemia and weight loss were induced after pancreatectomy. After islet transplantation or insulin treatment, blood glucose levels recovered to normal, and body weight started to increase. $\beta$-cell mass was larger in group IV than in group II or III until 10 days after pancreatectomy $(\mathrm{p}<0.05$, Fig.1). Cell proliferation evaluated using BrdU staining was increased in all the pancreatectomized groups 6 days after surgery. It was exhausted in group II soon, while it continued in treatment groups (III, IV) although there was a

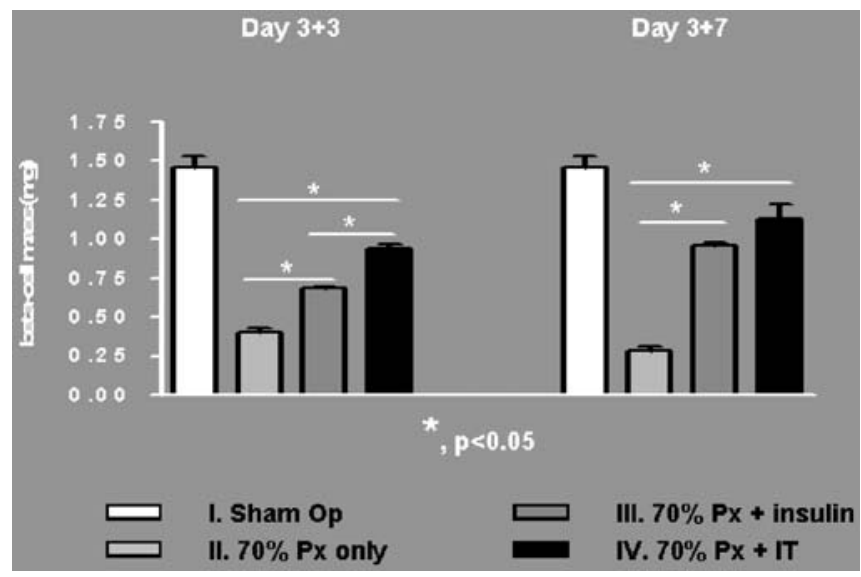

Fig. 1. Absolute $\beta$-cell Mass in the Pancreas 
trend to decrease in group IV (Fig. 2). After 10 days, group IV showed larger individual $\beta$-cell area than group II or III ( $\mathrm{p}<0.05$, Fig.3). Plasma insulin levels and intensity of insulin staining of islets were also most high in group IV.

Conclusion: These observations suggest that islet transplantation plays a role in enhancing remnant $\beta$-cell regeneration and insulin content of islets after partial pancreatectomy in mice. Induction of $\beta$-cell proliferation and supplement of adequate amount of insulin might contribute to these phenomena.

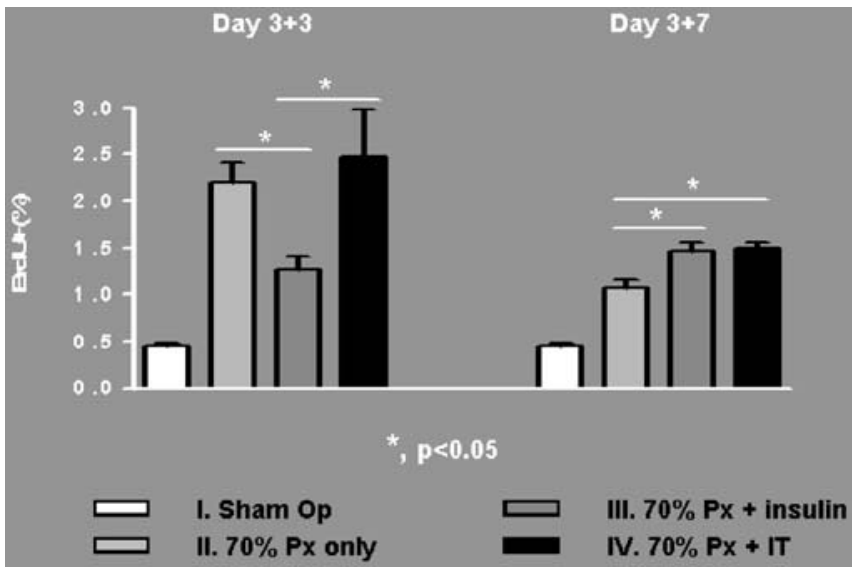

Fig. 2. BrdU-Positive Cells in the Pancreas

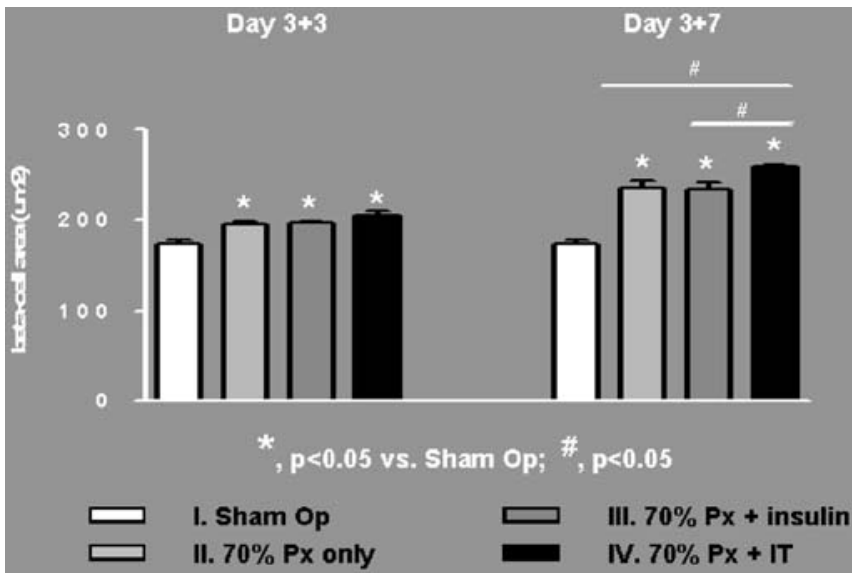

Fig. 3. Area of Individual $\beta$-cell in the Pancreas

Supported by grant from SBRI

\section{9}

The spontaneously decreased beta cell mass and pancreatic IGF2 production in the GK fetal rat are both improved in response to maternal undernutrition

E. Fernández ${ }^{1}$, M.-N. Gangnerau ${ }^{2}$, S. Calderari², L. de Miguel ${ }^{1}$, P. Serradas ${ }^{2}$, F. Escrivá ${ }^{1}$, B. Portha ${ }^{2}$, C. Álvarez;

${ }^{1}$ Bioquímica y Biología Molecular II, Facultad de Farmacia,

Universidad Complutense, Madrid, Spain, ${ }^{2}$ Lab. Physiopathol. Nutr.,

CNRS and University Paris Diderot, UMR 7059, Paris, France.

Background and Aims: The Goto-Kakizaki (GK) rat is a spontaneous model of type 2 diabetes with a defective beta-cell mass detectable in late fetal development (21.5 days postcoitum [dpc]) and the diminished IGF2 production seems to be involved in this effect. Since we have previously shown that early undernutrition increases beta-cell mass in foetuses at term, the aim of the present study was to investigate whether a condition of maternal malnutrition might similarly positively modulate the beta-cell mass in GK foetuses. In addition, the implication of the IGF system, which is highly responsive to nutritional status in these processes was evaluated. To this end, we have measured in $21.5 \mathrm{dpc}$ undernourished GK foetuses
(GK-U) 1) serum GH and IGF levels, 2) beta-cell mass, 3) replication and differentiation of beta-cells, and 4) IGF-1 and -2 protein content in liver and pancreas. All values were compared to those in control GK foetuses.

Materials and Methods: GK rats were obtained from our colony of GK/Par rats. Pregnant GK females were food restricted (65\% restriction) during the last week of gestation. Serum concentrations of GH, IGF-1 and -2 were measured by radioimmunoassay. Beta-cell mass, beta-cell differentiation and beta-cell replication were evaluated using quantitative immunohistochemistry methods. The protein content of IGF-1 and -2 was analyzed by western blot in pancreas and liver.

Results: Although in no case the GK-U values reached the levels of control Wistar rats, the results show that beta-cell mass increased by $90 \%$ in GK-U foetuses as compared to the GK group and that both beta-cell neogenesis and replication were stimulated as well. On the other hand, similar serum GH, IGF-1 and -2 levels were observed in GK and GK-U foetuses. Malnutrition affected IGF protein content in the various tissues studied. Particularly relevant was a 3 -fold increase of pancreatic IGF-2 protein levels in GK-U foetuses.

Conclusion: Our data suggest that 1) maternal malnutrition increased betacell mass and pancreatic IGF-2 protein levels in GK-U foetuses at $21.5 \mathrm{dpc}, 2$ ) the increased beta-cell mass could be related to the stimulation of both betacell neogenesis and beta-cell replication, 3) the locally increased pancreatic IGF-2 may be instrumental in these processes. They also illustrate for the first time that enhanced IGF-2 production in the pancreas can be triggered by a nutritional manipulation of the mother. So far it is not known whether or not undernutrition of the mother is sufficient to delay/decrease the risk of the GK/Par rat for developing overt hyperglycaemia at adult age.

Supported by grants from Ministerio de Ciencia y Tecnología and ISCIII-RETIC RD06, Spain.

\section{0}

Maternal low protein diet alters the regeneration capacity of the adult $B$ cells after streptozotocin destruction K. J. R. Goosse, T. Bouckenooghe, B. Reusens, C. Remacle; Institute of Life Sciences, Université Catholique de Louvain, Louvain-La-Neuve, Belgium.

Background and Aims: Maternal Low Protein diet alters the programming of the endocrine pancreas in the progeny. Fetal islets incubated with nitric oxide donor or with cytokines exhibit higher level of apoptosis. Maternal diet has long-term consequences since adult islets of early protein-restricted rats maintained a higher vulnerability toward cytokines. Streptozotocin (STZ) is well-known for its specific destructive action on the $\beta$ cells. In adult rodents, the endocrine pancreas regenerate after STZ injections. Regenerating protein 1 (Reg1) is expressed during experimental regenerative processes of pancreatic islets such as after $90 \%$ pancreatectomy or after STZ injections. Our aim was to determine whether early malnutrition increases the vulnerability of adult $\beta$ cells toward STZ and impairs their regeneration capacity.

Materials and Methods: Wistar rats were fed a 20\% (Control [C group]) or an isocaloric $8 \%$ protein diet (Recovery [R group]) during gestation and lactation. After weaning, offspring received the $20 \%$ protein diet. Three month-old rats were injected once a week during 5 weeks with $0.5 \mathrm{ml}$ Freund's Adjuvant (FA) injections followed 24h later by injection of $17.5 \mathrm{mg} /$ $\mathrm{kg}$ body weight STZ. Blood glucose level was monitored every week. Because we wanted to study the regeneration capacity of the endocrine pancreas, one half of the rats were sacrificed 4 days after the fifth STZ injection (d34), and the other half was maintained for two more weeks without treatment (d48). The pancreas was cut into two pieces, for quantification of insulin content and for morphometry. For that purpose, the piece was fixed with a $2 \%$ paraformaldehyde-glutaraldehyde solution and processed for immunohistochemistry. Beta cell surface area, the number of BrdU-positive cells and Reg1 surface area was estimated.

Results: The glycaemia in R group was increased after the second STZ injection, while in $\mathrm{C}$ group, rats became hyperglycaemic only after the last injection $(\mathrm{p}<0.05 v s \mathrm{C})$. When pancreases were analysed at $\mathrm{d} 34$, STZ reduced the pancreatic insulin content (PIC) by $90 \%$ and the $\beta$ cell surface area by $75 \%$ in both C and R group. Two weeks later (d48), when compared to the values obtained at $\mathrm{d} 34$, the PIC $(\mathrm{p}<0.01)$ and the $\beta$ cell surface area $(\mathrm{p}<0.05)$ were increased in $C$ animals. These two parameters remained unchanged in the R group during these two weeks. The number of $\mathrm{BrdU}$ positive cells/ $\beta$ cell surface area was 3-fold higher in STZ animals compared to untreated rats, but 
there was no difference between $\mathrm{C}$ and $\mathrm{R}$ groups as well as between $\mathrm{d} 34$ and d48. Reg1 immunostaining was absent in the endocrine pancreases of the FA animals. STZ induced however the expression of the protein in the islets of both groups. Reg1-positive cells were mainly located in the periphery of the islets and co-localized with glucagon. At d34, almost $50 \%$ of the islet surface area of the $\mathrm{C}$ group was immuno-reactive for Reg1, while in R pancreases only $25 \%$ of the islets surface area was Reg1-positive. At d $48,46.1 \pm 12.9 \%$ of the islets cells were reactive for Reg1 whereas in R group, the Reg1 positive surface area fell to $4.1 \pm 1.4 \%(\mathrm{p}<0.05 v s \mathrm{C})$.

Conclusion: Islets from the progeny that received a low protein diet during gestation and lactation are more prone to the destructive effect of STZ. Such islets have an altered capacity to regenerate new $\beta$ cells. An inappropriate diet during fetal and neonatal period programs a vulnerability of the adult $\beta$-cells toward cytotoxic molecules. This could predispose the progeny to diabetes.

\section{1}

Keratinocyte growth factor stimulates beta cell regeneration in diabetic newborn rats via activation of ductal cell proliferation. Implication of MAP kinase signaling pathway

B. Uzan, B. Portha, J. Movassat; Physiology, University Paris Diderot, Paris, France.

Background and Aims: Keratinocyte Growth factor (KGF) is a potent growth factor with high specificity for epithelial cells. KGF has been shown to promote epithelial growth and tissue homeostasis in various organs. In the pancreas, KGF and its receptor are overexpressed in pancreatic cancer. Organ culture of pancreatic rudiments with KGF promotes proliferation of epithelial cell precursors. We investigated the role of KGF in the compensatory growth of beta cells during the process of regeneration following their destruction by streptozotocin.

Materials and Methods: Wistar rats were injected with streptozotocin (STZ) at the day of birth. A group of STZ treated pups received a daily subcutaneous injection of KGF palifermin (provided by AMGEN, Thousand Oaks, CA, USA) from day 2 to day 6 after birth. Beta cell mass was measured at day 7 after birth. Beta cell replication, apoptosis and neogenesis from precursor cells were assessed in the pancreas of 2, 4 and 7 days old KGF treated and saline treated control neonates. The intracellular mechanism of action of KGF was also investigated using the human pancreatic duct cell line, Pancl.

Results: Daily subcutaneous treatment of STZ diabetic rat neonates with KGF palifermin induced a significant increase of beta cell mass compared to control STZ neonates. We showed that the mitotic index of existing beta cell was similar in both control and KGF treated groups. Moreover there was no difference in the rate of beta cells apoptosis between the two groups. KGF significantly increased ductal cell proliferation which subsequently differentiated into beta cells as evidenced by the increased number of single beta cells incorporated into the epithelium lining the ducts or in the close vicinity of the duct epithelium. KGF also stimulated the proliferation of serum-deprived Pancl cells. Stimulation of Pancl cells by KGF induced the activation of MAP kinases ERK 1/2. The MEK inhibitor PD98059 abolished the growth promoting effect of KGF on Pancl cells, indicating that the mitogenic effect of KGF are mediated mainly by ERK1/2 signalling in pancreatic ductal cells. Moreover, acute in vivo administration of KGF in STZ diabetic neonates stimulated rapid and transient phosphorylation of ERK 1/2 kinases.

Conclusion: We show that KGF stimulates beta cell regeneration in neonatal STZ diabetic rats through the activation of ductal cell proliferation and their subsequent differentiation into beta cell. The mitogenic effect of KGF on ductal cells is mediated mainly by ERK $1 / 2$ signalling.

\section{PS 32 Mediators of insulin secretion}

\section{2}

Ghrelin attenuates insulin release via $\mathrm{G} \alpha_{\mathrm{i} 2}$-mediated modulation of $\mathrm{Kv}$ channels and $\mathrm{Ca}^{2+}$ signaling in islet $\beta$ cells

K. Dezaki ${ }^{1}$, M. Kakei ${ }^{2}$, T. Yada ${ }^{1}{ }^{1}{ }^{1}$ Physiology, Jichi Medical University, Shimotsuke, ${ }^{2}$ Internal Medicine, Akita University, Akita, Japan.

Background and Aims: It has been shown that ghrelin is produced in stomach and pancreatic islets and serves as a physiological regulator of insulin release. However, the ghrelin's signaling mechanisms in islet $\beta$-cells downstream of its receptor, growth hormone (GH) secretagogue-receptor (GHS-R), are largely unknown. This study aimed to explore signaling mechanisms for insulinostatic ghrelin action in islet $\beta$-cells, with special attention to pertussis toxin (PTX)sensitive heterotrimeric GTP-binding proteins and $\mathrm{K}^{+}$channels.

Materials and Methods: Plasma insulin and GH concentrations in rats were measured by ELISA. Islets were isolated from rats, ghrelin-knockout (Ghr-KO) and wild-type mice by collagenase digestion, and insulin release was determined by ELISA. In rat single $\beta$-cells, cytosolic $\mathrm{Ca}^{2+}$ concentration $\left(\left[\mathrm{Ca}^{2+}\right]_{\mathrm{i}}\right)$ was measured by fura- 2 microfluorometry, while membrane potentials and whole cell currents were measured by patch-clamp technique.

Results: Systemic ghrelin administration decreased plasma insulin concentrations in rats and this effect was blocked by treatment with PTX, whereas stimulation of GH release was unaffected by PTX. Conversely, counteraction of endogenous ghrelin by specific GHS-R antagonist [D-Lys ${ }^{3}$ GHRP-6 markedly increased plasma insulin levels in a PTX-sensitive manner. In rat isolated islets, GHS-R antagonist significantly increased glucose (8.3 $\mathrm{mM}$ )-induced insulin release, while exogenous ghrelin suppressed it, both in a PTX-sensitive manner. Glucose (8.3 and $16.7 \mathrm{mM}$ )-induced insulin release in isolated islets from Ghr-KO mice was greater than that from wild-type mice. This augmented secretion in islets of Ghr-KO mice was blunted after treatment with PTX. Ghrelin increased voltage-dependent $\mathrm{K}^{+}(\mathrm{Kv})$ currents without affecting ATP-sensitive $\mathrm{K}^{+}$channel currents in rat $\beta$-cells, and this effect was abolished after PTX treatment. Ghrelin also attenuated glucoseinduced action potential firing and increase in $\left[\mathrm{Ca}^{2+}\right]_{\mathrm{i}}$ in $\beta$-cells. The effects of

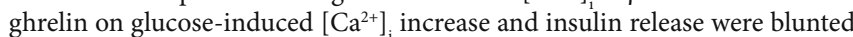
in the $\beta$-cells treated with PTX and with antisense oligonucleotide specific for $\mathrm{G} \alpha_{i 2}$ subunit of heterotrimeric G-proteins.

Conclusion: Ghrelin attenuates glucose-induced insulin release via PTXsensitive $\mathrm{G} \alpha_{\mathrm{i}_{2}}$-mediated activation of $\mathrm{Kv}$ channels and suppression of $\mathrm{Ca}^{2+}$ signaling in $\beta$-cells, which represents unique signaling of ghrelin distinct from that for $\mathrm{GH}$ release.

\section{3}

Kisspetin-13 inhibits insulin secretion without affecting glucagon or somatostatin release: study in the perfused rat pancreas R. A. Silvestre, E. M. Egido, R. Hernández, J. Marco; Experimental Endocrinology and Department of Physiology, Hospital Universitario Puerta de Hierro, Medical School, Universidad Autónoma de Madrid, Spain.

Background and Aims: Kisspeptins are a family of peptides encoded by the KISS1 gene, the most abundant of which is the amidated 54-amino acid form, known as kisspeptin-54 or metastin although shorter forms of 13 and 14 amino acids have also been identified. Kisspeptins have recently been shown to be ligands for an orphan G protein-coupled receptor, GPR54, a receptor structurally related to the galanin receptor. Kisspeptins and GPR54 mRNAs have been detected in both pancreatic B and A cells. GPR54 mRNA expression was also found in MIN6 and alphaTC1 pancreatic cell lines. Kisspeptin- 54 has been shown to stimulate the late phase of glucose-induced insulin secretion in mouse islets and to inhibit insulin secretion in MIN6 cells. In vitro, all kisspeptin fragments have a similar affinity and efficacy for GPR54, thus, indicating that the C-terminal part of the kisspeptin molecule is responsible for the activation of GPR54. To gain further insight into the effect of kisspeptin on islet cell secretion, we have investigated the effect of kisspeptin-13 on insulin, glucagon and somatostatin secretion.

Materials and Methods: The study was performed in the perfused rat pancreas. Kisspeptin-13 (Bachem, Switzerland) was infused at $1 \mu \mathrm{M}, 100 \mathrm{nM}$, 
$10 \mathrm{nM}, 1 \mathrm{nM}$ or $0.1 \mathrm{nM}$. Hormone secretion was stimulated by increasing perfusate glucose concentration (from 5.5 to $9 \mathrm{mM}$ ), and by infusing arginine $(10 \mathrm{mM})$ or exendin-4 $(1 \mathrm{nM})$. Insulin, glucagon and somatostatin were measured by RIA.

Results: Infusion of kisspeptin-13, at $1 \mu \mathrm{M}$, blocked both phases of the insulin response elicited by glucose $\left(1^{\text {st }}\right.$ phase: $-1.7 \pm 1.7$ [SEM] $\mathrm{ng} / 5 \mathrm{~min}$ vs. $21 \pm 8 \mathrm{ng} / 5 \mathrm{~min}$ in controls, $\mathrm{p}<0.025 ; 2^{\text {nd }}$ phase: $-1.8 \pm 5.6 \mathrm{ng} / 15 \mathrm{~min}$ vs. $41 \pm 14$ $\mathrm{ng} / 15 \mathrm{~min}$ in controls, $\mathrm{p}<0.025)$ and by arginine $\left(1^{\text {st }}\right.$ phase: $14 \pm 7 \mathrm{ng} / 5 \mathrm{~min}$ vs. $144 \pm 80 \mathrm{ng} / 5 \mathrm{~min}$ in controls, $\mathrm{p}<0.01,2^{\text {nd }}$ phase: $9.4 \pm 11 \mathrm{ng} / 15 \mathrm{~min}$ vs. $298 \pm 58$ $\mathrm{ng} / 15 \mathrm{~min}$ in controls, $\mathrm{p}<0.01)$. Exendin-4-induced insulin release $(88 \pm 13$ $\mathrm{ng} / 15 \mathrm{~min})$ was also reduced by infusion of $10 \mathrm{nM}$ kisspeptin-13 (15 \pm 8.7 $\mathrm{ng} / 15 \mathrm{~min}, \mathrm{p}<0.05)$. The inhibitory effect of kisspeptin- 13 on glucose-induced insulin secretion was dose-dependent, fitted a sigmoidal curve $\left(\mathrm{R}^{2}=0.9965\right)$ and the $\mathrm{IC}_{50}$ was $1.6 \mathrm{nM}$. Kisspeptin-13 $(1 \mu \mathrm{M})$ failed to significantly modify the glucagon or somatostatin responses to arginine $(14 \pm 4 \mathrm{ng} / 20 \mathrm{~min}$ vs. $15.7 \pm 1.3 \mathrm{ng} / 20 \mathrm{~min}$ in control perfusions, $\mathrm{p}=0.757$, and $397 \pm 119 \mathrm{pg} / 20 \mathrm{~min}$ vs. $479 \pm 106 \mathrm{pg} / 20 \mathrm{~min}$ in control perfusions, $\mathrm{p}=0.71$, respectively).

Conclusion: In view of the potent insulinostatic effect of kisspeptin-13, it is tempting to speculate that this family of peptides may be implicated in the regulation of $B$ cell secretion. The lack of effect of kisspeptin-13 on both glucagon and somatostatin secretion would indicate that it influences the $B$ cell directly, rather than through an A- or D-cell paracrine effect.

Supported by Ministerio de Educación y Ciencia, Fondo de Investigación Sanitaria, Instituto de Salud Carlos III, Fundación Médica MMA and Fundación Investigación Biomédica HUPT, Spain

\section{4}

The GLP-1 analogue liraglutide regulates glucose-stimulated insulin granule kinetics

L. $\mathbf{M a}^{1}$, A. Kuznetsov ${ }^{1}$, C. Labno ${ }^{2}$, V. P. Bindokas ${ }^{2}$, L. H. Philipson ${ }^{1}$;

${ }^{1}$ Medicine, The University of Chicago, ${ }^{2}$ BSD Light Microscopy Core Facility, The University of Chicago, United States.

Background and Aims: The glucagon-like peptide 1 (GLP-1) analog liraglutide is currently being investigated for use as a novel therapeutic agent in the treatment of type 2 diabetes. The glucose dependency of the action of GLP-1 has been well established. However, the precise mechanisms of these actions, including downstream changes in insulin granule dynamics, are less well studied.

Materials and Methods: We used total internal reflection fluorescence (TIRF) microscopy to image the motion and exocytosis of insulin granules near the plasma membrane in a MIN6 cell-line stably transfected with green fluorescent protein (GFP)-tagged insulin. We developed a method to photobleach previously docked granules (PDG) using TIRF (TIRFFRAP) and the appearance of newly docked granules (NDG) at the plasma membrane over the next 5-20 minutes was recorded.

Results: In the first five minutes (first phase of release) fusion events were seen mostly from previously docked granules. The results show a significant increase in disappearance of docked insulin granules from the plasma membrane with $t$ Liraglutide compared to control experiments $(8.6 \pm 0.93$ to $4.6 \pm 1.3 \%, P<0.01)$ in $8 \mathrm{mmol} / \mathrm{L}$ glucose. In the presence of liraglutide, there was an increase in the rate of appearance NDG, as expressed as a percentage of total PDG $(2.37 \pm 0.496$ per min. compared to $1.52 \pm 0.301$ per min. for controls, $\mathrm{P}<0.01)$. Our data illustrates that liraglutide evoked glucosedependent exocytosis consistent with a bi-phasic pattern.

Conclusion: These findings indicate that liraglutide both increases insulin granule exocytosis from the pre-docked pool and leads to the mobilization of more insulin secretory granules from the large reservoir pool to then dock at the plasma membrane. Both of these processes are glucose dependent. This new method of using TIRF and TIRF-FRAP can therefore evaluate both exocytosis of docked insulin granules as well as transport of insulin granules to the plasma membrane.

\section{5}

Potentiation of insulin secretion by obestatin, a peptide derived from preproghrelin

E. M. Egido, R. Hernández, J. Marco, R. A. Silvestre; Hospital Puerta de Hierro and Department of Physiology, Universidad Autónoma de Madrid, Spain.

Background and Aims: Obestatin is 23 -amino acid peptide derived from preproghrelin. In rats, this ghrelin-associated peptide has been identified and purified from stomach extracts an has also been detected in peripheral plasma. Contrary to ghrelin, obestatin reduces food intake and body-weight gain. Obestatin binds to the orphan G protein-coupled receptor GPR39, which belongs to the ghrelin receptor subfamily and is expressed in several tissues including pancreas. We have previously found that ghrelin inhibits insulin secretion. In the present study we have investigated the effect of obestatin on insulin secretion.

Materials and Methods: The study was performed in the isolated perfused rat pancreas. Obestatin (Peptides International Inc., Luisville, KY, USA) was infused at $3.14 \mathrm{nM}, 1 \mathrm{nM}$ or $0.314 \mathrm{nM}$. Insulin secretion was stimulated by increasing perfusate glucose concentration (from 5.5 to $9 \mathrm{mM}$ ), and by infusing arginine $(5 \mathrm{mM})$. Insulin was measured by RIA.

Results: Infusion of obestatin significantly potentiated the insulin response to glucose at $3.14 \mathrm{nM}$ (incremental area: $96 \pm 23, \mathrm{SEM}, \mathrm{ng} / 15 \mathrm{~min}$ vs. $41 \pm 8$ $\mathrm{ng} / 15 \mathrm{~min}$ in controls; $\mathrm{p}<0.05$ ) and at $1 \mathrm{nM}$ (incremental area: $109 \pm 23 \mathrm{ng} / 15$ $\mathrm{min}$ vs. $34 \pm 19 \mathrm{ng} / 15 \mathrm{~min}$ in controls; $\mathrm{p}<0.05$ ). No effect was observed at 0.314 $\mathrm{nM}$ (incremental area: $51 \pm 8 \mathrm{ng} / 15 \mathrm{~min}$ vs. $49 \pm 12 \mathrm{ng} / 15 \mathrm{~min}$ in controls). Obestatin also potentiated the first phase of arginine-induced insulin secretion (incremental area: $124 \pm 35 \mathrm{ng} / 5 \mathrm{~min}$ vs. $42 \pm 8 \mathrm{ng} / 5 \mathrm{~min}$ in controls; $\mathrm{p}<0.05)$.

Conclusion: The stimulating effect of obestatin on insulin secretion constitutes further evidence that its biological activity is opposite to that of ghrelin. Our results allow us to speculate about a role of obestatin in the control of B-cell secretion. Furthermore, as an insulinotropic agent its potential antidiabetic effect may be worthy of investigation.

Supported by Ministerio de Educación y Ciencia, Fundación Médica MMA and Fundación Investigación Biomédica HUPT, Spain

\section{6}

Role of the beta cell granulation state in drug-induced desensitization of insulin secretion

K. Hatlapatka ${ }^{1}$, A. Wienbergen ${ }^{1}$, A. Jörns ${ }^{2}$, I. Rustenbeck ${ }^{1}$;

${ }^{1}$ Institute of Pharmacology and Toxicology, University of Braunschweig, Braunschweig, ${ }^{2}$ Institute of Anatomy, Hannover Medical School, Germany.

Background and Aims: The decreased insulin secretion after a prolonged stimulation is often ascribed to an exhaustion of insulin stores. However, the relation between the B-cell granulation state and the secretory responsiveness is only poorly understood.

Methods: Insulin secretion was measured by perifusion and ELISA of the fractionated efflux. $\mathrm{K}_{\text {ATP }}$ channel activity and membrane potential were measured by patch clamping, the cytosolic calcium concentration $\left[\mathrm{Ca}^{2+}\right]_{\mathrm{i}}$ by microfluorometry. The granule content was assessed by electron microscopy of islet sections or by time lapse microfluorometry of MIN6 cells transfected with an insulin-EGFP fusion protein.

Results: An overnight culture of NMRI mouse islets in RPMI 1640 (5 mM glucose) containing either $500 \mu \mathrm{M}$ tolbutamide or $100 \mu \mathrm{M}$ efaroxan led to a strongly diminished secretory response upon re-exposure to either drug. After $4 \mathrm{~h}$ culture in RPMI alone ( $5 \mathrm{mM}$ glucose), an overshooting recovery of secretion resulted upon re-exposure to efaroxan, but only a partial recovery upon re-exposure to tolbutamide. After overnight exposure, tolbutamide and efaroxan were still effective to block B-cell $\mathrm{K}_{\mathrm{ATP}}$ channels and to depolarize the plasma membrane. The resulting pattern of $\left[\mathrm{Ca}^{2+}\right]_{\text {, response }}$ was not altered during desensitization or recovery. Immunohistochemically, the PDX1 expression was also unchanged. The B-cell granule content in contrast was clearly affected. Tolbutamide desensitization degranulated more strongly than efaroxan desensitization. After $4 \mathrm{~h}$ recovery, the regranulation of the tolbutamide-exposed islets was more extensive than that of the efaroxan-exposed islets. Use of MIN6 cells expressing EGFPinsulin showed that the tolbutamide-induced degranulation started after $1 \mathrm{~h}$ of perifusion and was more extensive than that by efaroxan or a strongly 
depolarizing $\mathrm{K}^{+}$concentration. Similar to B-cells within islets the degree of degranulation varied considerably between single MIN6 cells. Upon withdrawal of tolbutamide, regranulation started after $1 \mathrm{~h}$ of perifusion in basal glucose.

Conclusion: Drug-induced desensitization of insulin secretion is accompanied by a B-cell degranulation, but the total number of insulin granules does not determine secretory responsiveness. The regranulation is not regulated by transcription nor is the secretion level explained by changes in $\left[\mathrm{Ca}^{2+}\right]_{i \text {. This }}$ leaves translational control and/or metabolic signalling as possible regulators of regranulation and secretion.

\section{7}

Serotonin 2B receptor agonist $\mathrm{BW723C86}$ increases cytosolic $\mathrm{Ca} 2+$ in $\beta$ cells via PLC pathway and stimulates insulin and glucagon release at basal glucose in islets

A. Ando ${ }^{1,2}$, K. Dezaki $^{1}$, M. Nakata ${ }^{1}$, S. Nagasaka ${ }^{2}$, S. Ishibashi ${ }^{2}$, T. Yada ${ }^{1}$; ${ }^{1}$ Division of Integrative Physiology, Department of Physiology, Jichi Medical University, Shimotsuke, ${ }^{2}$ Division of Endocrinology and Metabolism, Department of Internal Medicine, Jichi Medical University, Shimotsuke, Japan.

Background and Aims: Localization of serotonin-containing nerve fibers in pancreas suggests that serotonin possibly has physiological function in pancreatic islets. The effect of serotonin $(5-\mathrm{HT})$ on glucose metabolism has been reported. However, whether it has direct effects on insulin secretion is not evident. We here aimed to study the effect of 5-HT and its receptor agonists, especially 5-HT-2B receptor selective agonist BW723C86 hydrochloride, on insulin secretion in rat islets and cytosolic $\mathrm{Ca} 2+$ concentration ([Ca2+]i) in $\beta$-cells and on glucagon secretion in islets.

Materials and Methods: Expression of 5-HT receptor subtypes in isolated islets from Wistar rats was determined by reverse transcription-PCR and Western-blotting. Localization of 5-HT receptor subtypes in rat pancreas was examined using immunofluorescent staining. Insulin and glucagon release from isolated rat islets under static incubation was determined by EIA. [Ca2+]i in single islet $\beta$-cells of rats was measured by fura- 2 microfluorometry.

Results: mRNAs for 5-HT-1A,-2B,-2C,-3,-5B,-6,-7 receptors were expressed in rat islets. Among the 5-HT-2 receptors, western blotting analysis revealed expression of 5-HT-2B and -2C receptor proteins in islets. Double immunofluorescence staining of rat pancreas revealed localization of 5HT-2B receptor in insulin-containing $\beta$-cells. In the presence of $8.3 \mathrm{mM}$ glucose, 5 -HT $(100 \mu \mathrm{M})$ increased [Ca2+] in $44.2 \%$ of glucose-responsive $\beta$-cells. A 5-HT-2B receptor selective agonist, BW723C86 hydrochloride, at $0.1-100 \mu \mathrm{M}$ dose-dependently increased [Ca2+]i in $\beta$-cells at $8.3 \mathrm{mM}$ glucose, and the $[\mathrm{Ca} 2+] \mathrm{i}$ increase was blocked by 5 -HT-2B receptor selective antagonist SB204741 $(1 \mu \mathrm{M})$. The 5-HT-2B agonist-induced [Ca2+]i increase was suppressed by phospholipase $\mathrm{C}$ inhibitor U73122(2 $\mu \mathrm{M})$, ER Ca2+ pump inhibitor thapsigargin $(500 \mathrm{~nm})$, and L-type $\mathrm{Ca} 2+$ channel blocker nitrendipine $(1 \mu \mathrm{M})$. Surprisingly, 5-HT-2B receptor agonist BW723C86 had no effect on insulin secretion at $8.3 \mathrm{mM}$ glucose, and 5-HT $(100 \mu \mathrm{M})$ suppressed glucose $(8.3 \mathrm{mM})$ induced-insulin release. Under a basal glucose concentration of $2.8 \mathrm{mM}$, BW723C 86 increased [Ca2+]i in islet $\beta$-cells and increased insulin release. On the other hand, immunoreactivities for both $5-\mathrm{HT}-2 \mathrm{~B}$ and $-2 \mathrm{C}$ receptors were observed in glucagon-containing $\alpha$-cell. BW723C86 stimulated glucagon secretion at $2.8 \mathrm{mM}$ glucose.

Conclusion: Rat islet $\beta$-cells express 5-HT-2B receptor and 5-HT-2B receptor agonist increases $[\mathrm{Ca} 2+] \mathrm{i}$ in $\beta$-cells and stimulates insulin release in islets at $2.8 \mathrm{mM}$ glucose. The $5-\mathrm{HT}-2 \mathrm{~B}$ receptor agonist also enhances $8.3 \mathrm{mM}$ glucose-induced $[\mathrm{Ca} 2+] \mathrm{i}$ increase in $\beta$-cells via PLC pathway but fails to enhance glucose-induced insulin release in islets. The apparent discrepancy between $[\mathrm{Ca} 2+] \mathrm{i}$ and insulin release suggests that an additional 5-HT-2B receptor signal downstream of [Ca2+]i could suppress distal processes of insulin secretion, and/or that BW723C86 could react with a yet-unidentified 5-HT receptor subtype other than $2 \mathrm{~B}$ that influences insulin secretion at $8.3 \mathrm{mM}$ glucose. The latter possibility is supported by the result that $5-\mathrm{HT}$ suppressed insulin secretion at $8.3 \mathrm{mM}$ glucose. Furthermore, 5-HT-2B agonist also stimulates glucagon release from rat islets.

\section{8}

Increased basal insulin secretion from isolated MIN6B1 cells is a consequence of elevated cytosolic calcium

F. Jaques ${ }^{1}$, H. Jousset ${ }^{2}$, A. Tomas ${ }^{1}$, C. Wollheim ${ }^{2}$, J.-C. Irminger ${ }^{1}$, N. Demaurex ${ }^{2}$, P. A. Halban ${ }^{1}$;

${ }^{1}$ Genetic, Medicine and Development, University medical center, Geneva, Switzerland, ${ }^{2}$ Cell Physiology and Metabolism, University medical center, Geneva, Switzerland.

Background and Aims: Cell-cell interaction is essential for proper function of islet beta-cells. We showed previously that dispersed MIN6B1 cells are poorly responsive to glucose compared with confluent cells with cell-cell contacts, due to the combination of a marked increase in basal and reduction in stimulated secretion. The aim of this study was to understand why basal insulin secretion is elevated in dispersed cells.

Materials and Methods: After $48 \mathrm{~h}$ in culture, cells were preincubated for $2 \mathrm{~h}$ in KRB-buffer at $2.8 \mathrm{mM}$ glucose and then incubated for $1 \mathrm{~h}$ in this same buffer (=basal secretion) followed by $1 \mathrm{~h}$ at $16.7 \mathrm{mM}$ glucose or different secretagogues (=stimulated secretion). In order to decrease intracellular calcium, a 15 min period in KRB-buffer without calcium supplemented with $0.1 \mathrm{mM}$ EGTA was performed at the beginning of the preincubation. To measure intracellular calcium, cells were loaded with $2 \mu \mathrm{M}$ FURA-2/AM and imaged using a frame transfer Micro-Max camera.

Results: The use of different secretagogues showed that stimulation with $\mathrm{Ca}^{2+}$ mobilizing agents (glucose, $\mathrm{KCl}$, arginine, leucine and glibenclamide) do not enhanced insulin secretion in dispersed cells (fold-stimulation nonsignificant). In contrast, dispersed cells were able to respond to secretagogues acting by a mechanism independent of changes in cytosolic $\mathrm{Ca}^{2+}$ (foldstimulation: IBMX $3.41+/-0.27$, mastoparan $4.9+/-1.44$ and PMA $16+/-3.42$, all $\mathrm{p}<0.005)$. These results suggested that dispersed cells had a disregulation in $\mathrm{Ca}^{2+}$ homeostasis. Intracellular $\mathrm{Ca}^{2+}$ measurements demonstrated that more than $20 \%$ of dispersed cells presented spontaneous $\mathrm{Ca}^{2+}$ oscillations whilst confluent cells did not. Furthermore, dispersed cells in $2.8 \mathrm{mM}$ glucose presented a depolymerized actin cytoskeleton and an activation of the MAPK ERK pathway, two events known to depend on intracellular $\mathrm{Ca}^{2+}$ elevation and to be essential for insulin exocytosis. By contrast, confluent cells presented these two characteristics only after glucose stimulation. Finally we showed that short-term deprivation of extracellular $\mathrm{Ca}^{2+}$ at the beginning of the preincubation in dispersed cells led to a reduction in the number of cells with $\mathrm{Ca}^{2+}$ oscillations at low glucose ( $10 \%$ instead of $22 \%$ in normal conditions) as well as a decrease in basal secretion to a level comparable to confluent cells $(0.87+/-0.22 \%$ vs. $0.73+/-0.23 \%$ respectively $)$. This reveals glucose responsiveness in dispersed cells (8-fold stimulation).

Conclusions: Dispersed MIN6B1 cells present spontaneous $\mathrm{Ca}^{2+}$ oscillations that trigger cytoskeleton depolymerization and ERK pathway activation, leading to insulin secretion in absence of glucose stimulation. This could be rectified by short-term (15 min) deprivation of extracellular $\mathrm{Ca}^{2+}$. These defects underlie the elevated basal insulin secretion from cells without normal intercellular contacts and emphasise the importance of such contacts in the normal regulation of secretion from beta cells.

\section{9}

Prolonged alanine exposure induces changes in metabolism, $\mathrm{Ca}^{2+}$ handling and desensitization of insulin secretion in clonal pancreatic beta cells

L. Brennan ${ }^{1}$, S. M. Scullion ${ }^{2}$, B. Mion ${ }^{1}$, C. Hewage ${ }^{1}$, J. P. G. Malthouse ${ }^{1}$, P. Flatt ${ }^{2}$, P. Newsholme ${ }^{1}$, N. H. McClenaghan ${ }^{2}$;

${ }^{1}$ UCD School of Biomolecular and Biomedical Science, Dublin, Ireland, ${ }^{2}$ School of Biomedical Sciences, University of Ulster, Coleraine, United Kingdom.

Background and Aims: Amino acids are an important class of insulinotropic nutrient, playing a key role in regulation of pancreatic beta-cell function. While the acute stimulatory actions of amino acids have been characterised in detail, little is known about the consequences of prolonged beta-cell exposure to elevated concentrations of this class of insulinotropic nutrient. This prompted the present study examining the effects of prolonged beta-cell exposure to the metabolizable amino acid, L-alanine.

Materials and Methods: Clonal pancreatic BRIN-BD11 beta-cells were incubated in culture for $18 \mathrm{~h}$ in the presence or absence of $10 \mathrm{mM} \mathrm{L}$-alanine 
followed by a $1 \mathrm{~h}$ incubation in the presence of $\left[3-{ }^{13} \mathrm{C}\right]$-alanine. Insulin secretion was measured, and cellular metabolites evaluated following perchloric acid extraction. Neutralised cell supernatants were then prepared for NMR analysis with ${ }^{13} \mathrm{C}$ NMR spectra acquired on a Bruker DRX 500 spectrometer. Determination of cellular membrane potential, intracellular $\mathrm{Ca}^{2+}\left(\left[\mathrm{Ca}^{2+}\right]_{\mathrm{i}}\right)$ and protein expression was also performed after prolonged alanine exposure.

Results: Prolonged exposure to $10 \mathrm{mM} \mathrm{L}$-alanine in culture did not significantly alter basal insulin release (at $1.1 \mathrm{mM}$ glucose) or cellular insulin content. However, alanine culture markedly suppressed (by $74 \%, \mathrm{p}<0.001$ ) the subsequent acute stimulatory insulinotropic action of alanine. An increase in glutamate labelled at position C4 (by $60 \%, \mathrm{p}<0.01$ ), arising as a result of an increase in the singlet peak was observed after prolonged alanine exposure. This indicates increased flux through pyruvate dehydrogenase $(\mathrm{PDH})$ of alanine derived carbon. Notably, protein expression of pyruvate dehydrogenase kinases (PDK2 and PDK4) was significantly reduced following prolonged alanine exposure, consistent with the observed increased flux through PDH. Interestingly however, prolonged alanine exposure resulted in decreased ATP levels from $5.8 \mathrm{pmol} / 10^{4}$ cells to $4.2 \mathrm{pmol} / 10^{4}$ cells $(\mathrm{p}<0.05)$. Alanine evoked marked membrane depolarization in cells after control culture (in the absence of alanine). Area-under-the-curve (AUC) analysis revealed that this alanineinduced depolarization response was significantly $(\mathrm{p}<0.01)$ suppressed in cells previously cultured with L-alanine. In accordance with this effect, cells cultured in the presence of $10 \mathrm{mM}$ L-alanine exhibited a marked decrease $(p<0.01)$ of $\left[\mathrm{Ca}^{2+}\right]_{i}$ in response to subsequent acute incubation with alanine compared with control culture. This is consistent with the insulin-releasing actions of this amino acid. Intact responses to acute alanine exposure were reestablished after $18 \mathrm{~h}$ culture of cells in the absence of alanine, demonstrating reversible amino acid-induced desensitization.

Conclusion: The present study extends understanding of nutrient induced beta-cell desensitization to include amino acids, importantly indicating that prolonged exposure to metabolizable L-alanine can induce reversible alterations in metabolic flux, membrane depolarisation, calcium handling and insulin secretion.

\section{PS 33 Intracellular signaling for insulin secretion}

\section{0}

AKAPs-mediated subcellular anchoring of PKA plays an essential role in the cAMP-dependent stimulation of pancreatic beta cells and potentiation of insulin secretion

M. O. Faruque ${ }^{1,2}$, D. Le-Nguyen ${ }^{3}$, A. D. Lajoix ${ }^{1}$, E. Vives ${ }^{4}$, L. Ali ${ }^{2}$, P. Petit ${ }^{1}$, D. Bataille ${ }^{1}$, E. H. Hani ${ }^{1}$;

${ }^{1}$ UMR-5230, CNRS, Montpellier, France, ${ }^{2}$ Dept Biochem \& Cell Biology, BIRDEM, Dhaka, Bangladesh, ${ }^{3}$ FRE-3009, CNRS, Montpellier, France, ${ }^{4}$ EMI0227, INSERM, Montpellier, France.

Background and Aims: Within the endocrine pancreas, hormones like glucagon and glucagon-like peptide-1 are well known to strongly amplify beta-cell mass, function and insulin secretion. Stimulation of their respective receptors leads to elevation of the intracellular concentrations of cAMP, which activates the cAMP-dependent protein kinase (PKA) cascade. The specificity of the PKA signaling module is determined by a sophisticated subcellular compartimentation machinery, which directs the spatio-temporal activation of the kinase. Subcellular localization of PKA pools occurs through highaffinity association with A-kinase anchoring proteins (AKAPs). Recently, a novel peptide, AKAPis, has been proposed to competitively disanchor PKAAKAPs interactions in vitro. The aim of the present study was to develop a cell-permeable construct of the AKAPis competitor, and use it as a toolmodel to characterize the impact of PKA compatimentation by AKAPs on beta-cell responses.

Materials and Methods: AKAPis peptide was chemically synthesized and linked to the HIV1 cell-penetrating TAT peptide to obtain a composite construction "TAT-AKAPis". This construct was used to disrupt endogenous protein-protein interactions between PKA and AKAPs expressed in INS-1. We then evaluated the effects of disrupting PKA-AKAPs complexes on: 1) glucagon-stimulated phosphorylation of key PKA downstream substrates (transcription factor, CREB and p44/p42 MAP kinases); 2) glucagonstimulated amplification of insulin secretion in intact INS-1 pancreatic betacells. PKA-AKAPs interactions were quantitatively determined using coimmunoprecipitation and western immunoblotting. Effects of TAT-AKAPis on PKA localization were also examined using immunofluorescence analysis. The effects of TAT-AKAPis on insulin secretion in response to glucose (with or without glucagon) were quantified using an insulin kit assay. TAT-AKAPis effects on glucagon-stimulated CREB and p44/p42 MAPKs phosphorylations were determined by western immunoblotting.

Results: TAT-AKAPis dose-dependently disrupted the endogenous PKAAKAPs interactions in INS-1 cells: at $20 \mathrm{uM}$ concentration of the TATAKAPis, more than $50 \%$ of PKA-AKAP95 complexes were disrupted. This was confirmed by immunofluorescence analysis showing that TAT-AKAPis induced a clear alteration of PKA subcellular localization within INS-1 cells. Moreover, exposure of INS-1 cells to TAT-AKAPis markedly reduced the glucagon-induced phosphorylation of CREB. Likewise, TAT-AKAPis drastically attenuated the glucagon-induced phosphorylation of p44/p42 MAPKs in INS1 cells, a double phosphorylation event that is dependent on PKA activation. Finally, we found that glucagon-induced potentiation of insulin release from INS- 1 cells was considerably reduced in the presence of TAT-AKAPis.

Conclusion: These data demonstrate that subcellular compartimentation of PKA, through interaction with AKAPs, is a key mechanism for PKAdependent phosphorylation events and potentiation of insulin secretion in pancreatic beta-cells. These PKA-AKAPs interactions represent an additional level of complexity in the regulation of pancreatic beta cells responses to physiological cAMP-elevating agents.

Supported by the French Government

\section{1}

PKCE's role in insulin biosynthesis and release in rat islets N. Warwar, E. Haber, A. Dov, E. Abramovitch, E. Cerasi, R. Nesher; Endocrinology and Metabolism, Hadassah - The Hebrew University Medical Center, Jerusalem, Israel.

Introduction: Glucose metabolism affects most major signal pathways in pancreatic $\beta$-cells, including insulin production, storage and exocytosis. 
Multiple protein kinases, mediate these effects; however, the role of most kinases is poorly defined. Here we examined the dynamics of glucose dependent activation and translocation of PKC $\varepsilon$ in $\beta$-cells of Wistar rats, and followed its role in the control of insulin secretion and biosynthesis.

Methods: (A) Isolated pancreata from Wistar and GK rats and were stimulated with glucose $(16.7 \mathrm{mmol} / \mathrm{l})$ and fixed at time points selected to resolve function in the biphasic dynamics of insulin release. Sections were immunostained with anti-PKC $\varepsilon$ and anti-insulin antibody and imaged using a three-gun laser-scanning confocal microscope. (B) Adenoviral vectors were used to inhibit PKC $\varepsilon$ translocation or to inhibit the enzyme's activity, and siRNA constructs were used to diminish PKCe levels. Isolated islets were infected or transfected and used in perifusion studies to study the dynamics of insulin response to glucose or in batch studies to follow total protein and insulin biosynthesis. Insulin precursors and intermediates were identified by HPLC.

Results: Glucose stimulus resulted in the concentration of PKCE near the nucleus, strongly associated with insulin staining and with dynamics resembling that of biphasic insulin response. However the signal persisted for $15 \mathrm{~min}$ after cessation of stimulation. Adenovirus or siRNA mediated depletion of PKC $\varepsilon$ function lead to partial inhibition of insulin secretion in isolated islets. No glucose-induce translocation of PKC $\varepsilon$ was observed in GK rats pancreas, suggesting a role in the defective insulin response in this model for T2DM. $\beta$ 'COP- 1 was identified as the anchoring protein for PKCE. The fact that $\beta^{\prime} \mathrm{COP}-1$, is a member of the COP complex that controls Golgi protein transport, lead us to examine the role of PKC $\varepsilon$ in production of $\beta$-cell proteins in general and that of insulin, specifically. In isolated islets, diminished PKC $\varepsilon$ activity by adenovirus or by siRNA, lead to specific inhibition of proinsulin biosynthesis and its conversion to insulin.

Conclusion: Our data suggest a role for PKC $\varepsilon$ in insulin biosynthesis and in granules exocytosis in pancreatic $\beta$-cell. Its diminished activity might be one of the defective signaling in the diabetic islet.

\section{2}

Glucose and dexamethasone regulation of serine/threonine protein phosphatase 5 in normoglycaemic and hyperglycaemic human and rat islets

N. Grankvist ${ }^{1}$, H. Ortsäter ${ }^{1}$, Q. Zhang ${ }^{1}$, R. Honkanen ${ }^{2}$, Å. Sjöholm ${ }^{1}$; ${ }^{1}$ Department of Clinical Science and Education, Karolinska Institutet, Stockholm, Sweden, ${ }^{2}$ Department of Biochemistry and Molecular Biology, University of South Alabama, Mobile, United States.

Background and Aims: Serine/threonine Protein Phosphatase 5 (PP5) is a single polypeptide chain containing a catalytic domain near its C-terminus and a regulatory domain at the $\mathrm{N}$-terminus end. The enzyme is present in the cytosol. PP5 is an okadaic acid/microcystin/calyculin A-sensitive phosphatase that belongs to the PPP family of enzymes. Like the other PPP-phosphatases (PP1, PP2A, PP2B/calcineurin, PP4, PP6, and PP7), PP5 is highly conserved among species, and PP5 is expressed in most, if not all, mammalian cells. Its biological function is only beginning to emerge, but PP5 is suggested to be involved in the regulation of glucocorticoid receptor signaling to promote cell growth and to terminate responses to oxidative stress. The influence of PP5 on $\beta$-cell function is currently unknown. The aim of the present study was to investigate if the cellular protein level of PP5 is regulated by glucose and dexamethasone.

Materials and Methods: Isolated islets of Langerhans from human donors with or without type 2 diabetes were cultured at 3 or $30 \mathrm{mM}$ glucose in the absence or presence of $500 \mathrm{nM}$ dexamethasone for 48 hours. Islets isolated from normoglycaemic Wistar and age- and sex-matched hyperglycaemic Goto-Kakizaki (GK) rats were cultured for 48 hours in the presence of either 3 or $30 \mathrm{mM}$ glucose. After exposure insulin secretion to the culture media was determined by ELISA. The islets content of PP5 and $\beta$-actin proteins were measured by western blot and their insulin content were measured by ELISA.

Results: Human pancreatic islets isolated from healthy donors released 6fold more insulin when cultured at $30 \mathrm{mM}$ glucose than islets cultured at 3 $\mathrm{mM}$ glucose. Dexamethasone did not affect basal insulin secretion at $3 \mathrm{mM}$ glucose but blunted the insulinotrophic effect of $30 \mathrm{mM}$ glucose. Human islets isolated from patients with type 2 diabetes showed elevated basal insulin secretion at $3 \mathrm{mM}$ glucose and thus an augmented secretory response to 30 $\mathrm{mM}$ glucose. In such islets dexamethasone reduced the secretory rate both at 3 and $30 \mathrm{mM}$ glucose. The islet protein levels of PP5 was positively regulated both by glucose and dexamethasone in healthy human islets whereas only dexamethasone were able to increase PP5 protein levels in islets isolated from patients with type 2 diabetes. We also investigated the capability of glucose to regulate PP5 protein levels in islets isolated from normoglycaemic Wistar and hyperglycaemic GK rats. As expected $30 \mathrm{mM}$ glucose caused a 5 -fold increase in insulin secretory rate in Wistar islets but only a 2 -fold increase in GK rat islets. Similar to the finding in human islets glucose caused an elevation (211 $\pm 43 \%$ ) in islets PP5 protein levels in normoglycaemic rats but failed to do so in islets obtained from hyperglycaemic rats. When comparing Wistar and GK rat islets with regard to their basal PP5 protein levels we observed that GK rats had higher basal PP5 protein levels.

Conclusion: We describe glucose and dexamethasone regulation of PP5 at the translational levels, and show quantitative and qualitative differences between normal and diabetic islets with regard to ability of glucose to regulated PP5 protein levels. PP5 may be a novel regulatory mechanism linking $\beta$-cell stimulation to biological responses such as proliferation, apoptosis and insulin exocytosis.

Supported by Filip Lundbergs stiftelse Eirs 50-Arsstiftelse

\section{3}

Type III phosphatidylinositol 4-kinases mediate plasma membrane PIP $_{2}$ synthesis in insulin-secreting cells

A. Wuttke, J. Sågetorp, N. Welsh, A. Tengholm;

Medical Cell Biology, Uppsala University, Sweden.

Background and Aims: The membrane phospholipid phosphatidylinositol 4,5-bisphosphate ( $\mathrm{PIP}_{2}$ ) is important in many types of cells as precursor for second messengers and as regulator of a plethora of cellular processes, including ion channel and transporter activity, cytoskeletal rearrangements and vesicle trafficking. Many of these functions are crucial for insulin secretion from pancreatic $\beta$-cells. The first step of PIP $_{2}$ synthesis involves formation of phosphatidylinositol 4-phosphate catalyzed by phosphatidylinositol 4kinases (PI4-kinases). These enzymes may act as metabolic sensors translating changes in the ATP/ADP ratio to phosphoinositide synthesis. This study aimed at identifying the PI4-kinases expressed in pancreatic $\beta$-cells and their importance for plasma membrane PIP ${ }_{2}$ production.

Materials and Methods: mRNA expression of PI4-kinases was analyzed in MIN6 $\beta$-cells with real-time PCR and isoform-specific primers. Plasma membrane $\mathrm{PIP}_{2}$ concentration was measured in individual cells expressing the PIP -binding PH-domain from PLC $\delta$ fused to GFP (PHPLC $\delta$-GFP). After plasma membrane permeabilization with $\alpha$-toxin, ATP-dependent PIP $_{2}$ synthesis was recorded as PHPLC $\delta$-GFP translocation with evanescent wave microscopy.

Results: Real-time PCR analysis of MIN6 $\beta$-cells demonstrated the presence of mRNA for all four known mammalian isoforms of PI4-kinase (type II $\alpha$, II $\beta$, III $\alpha$ and III $\beta$ ). Individual permeabilized MIN6 cells maintained in intracellular-like medium lacking ATP showed low and stable membrane PHPLC $\delta$-GFP fluorescence. Introduction of ATP resulted in a concentrationdependent rise of fluorescence. This effect reflected membrane translocation of the construct as PIP ${ }_{2}$ was synthesized and was prevented by $100 \mu \mathrm{M}$ of the PI4-kinase inhibitor phenylarsine oxide. PIP ${ }_{2}$ synthesis induced by 0.3-1 mM ATP was unaffected by wortmannin at $100 \mathrm{nM}$, a concentration sufficient to inhibit PI3-kinase. However, at $10 \mu \mathrm{M}$, a concentration known to inhibit type III PI4-kinases, wortmannin completely prevented ATP-induced PIP synthesis. Such complete inhibition was also obtained with $200 \mu \mathrm{M}$ LY294002, which like wortmannin affects only type III, but not type II PI4kinases. Addition of $0.5 \mathrm{mM}$ adenosine, which preferentially inhibits type II PI4-kinases, did not further affect membrane PIP 2 levels.

Conclusion: MIN6 $\beta$-cells express both type II and type III PI4-kinases. Intracellular ATP stimulates plasma membrane $\mathrm{PIP}_{2}$ synthesis mainly via type III isoforms. The various PI4-kinases may control phosphoinositide synthesis in distinct cellular comparments and thereby contribute to the variety of functions of PIP.

Supported by Novo Nordisk Foundation 


\section{4}

Selective insulin signaling in pancreatic beta cells involves different classes of PI3 kinases

B. Leibiger ${ }^{1}$, S. Uhles ${ }^{1}$, T. Moede ${ }^{1}$, J. Domin ${ }^{2}$, P.-O. Berggren ${ }^{1}$, I. B. Leibiger ${ }^{1}$; ${ }^{1}$ Department of Molecular Medicine \& Surgery, Karolinska Institutet, Stockholm, Sweden, ${ }^{2}$ Division of Medicine, Renal Section, Imperial College School of Medicine, London, United Kingdom.

Background and Aims: Pancreatic beta-cells are targets for positive feedback action by secreted insulin. We have shown that insulin activates the transcription of its own gene by signaling through A-type insulin receptor (IRA) while it activates transcription of the beta-cell glucokinase transcription unit (BGK) via the B-type, IR-B. Because both signaling pathways were differently sensitive towards Wortmannin and LY294002, we studied the PI3K involved.

Materials and Methods: As the readout for signaling via either IR-A or IR-B served insulin promoter-driven DsRed expression and BGK promoter-driven GFP expression, respectively, in co-transfected HIT-T15, MIN6 and primary mouse beta-cells. PI3K activity was measured in IR immunoprecipitates (IPs). By combining the use of pharmacological inhibitors with transient overexpression of insulin receptor variants, phosphatidylinositol phosphatases PTEN and SHIP, and siRNA based knockdown of PI3Ks, we now show that selective activation of insulin and glucokinase gene transcription can be simultaneously achieved by utilizing different classes of PI3K and thereby activating different signaling cascades.

Results: $25 \mu \mathrm{M}$ LY294002 or $50 \mathrm{nM}$ Wortmannin in the culture medium blocked insulin-stimulated insulin promoter activity. It needed $100 \mu \mathrm{M}$ LY294002 or $150 \mathrm{nM}$ Wortmannin to block insulin-stimulated BGK promoter activity. PI3K activity in IR-A IPs was inhibited by $5-10 \mathrm{nM}$ Wortmannin as described for PI3K classes I, II C2b and III. It needed 100 $\mathrm{nM}$ to block PI3K activity associated with IR-B, i.e. concentrations similar to those that inhibit PI3K class II C2a. Overexpression of the dominantnegative acting form of the class Ia adapter protein p85, i.e. Delta-p85, or siRNA against p85, abolished insulin-stimulated insulin promoter activity but not insulin-stimulated BGK promoter activity. Vice versa, siRNAmediated knockdown of PI3K-C2a abolished insulin-stimulated BGK activation without any effect on insulin promoter activity. The data show that PI3K class Ia is crucial in up-regulating insulin gene transcription, while the class II member PI3K-C2a is required to activate the BGK in the same cell. Moreover, we identified the juxtamembrane NPEY-motif in the insulin receptor B-type as being crucial for PI3K-C2a-mediated upregulation of the BGK promoter.

Conclusion: Our data suggest that one of the molecular mechanisms involved in the selective signaling via the two IR isoforms in the pancreatic beta-cell is the selective activation of different classes of PI3K.

Supported by Novo Nordisk Fonden, Swedish Research Council, Family Stefan Persson Foundation

\section{5}

Insulin release and islet nitric oxide synthase (NOS) activities in type 2 diabetes obese model, the ob/ob mouse. Interection with regulatory peptides and islet carbon monoxide $(\mathrm{CO})$ production

S. Meidute Abaraviciene ${ }^{1}$, J. Jimenez-Felstrom ${ }^{1}$, R. Henningsson ${ }^{2}$,

I. Lundquist ${ }^{2}$, A. Salehi ${ }^{1}$;

${ }^{1}$ Clinical Sciences, Malmoe, Lund University, Malmoe,

${ }^{2}$ Experimental Medical Science, Lund University, Lund, Sweden.

Background and Aims: We investigated the role of islet nitric oxide synthase (NOS) isoforms on insulin secretory mechanisms in the obese $o b / o b$ mouse, an animal model of type 2 obese diabetes.

Materials and Methods: Nitric oxide synthase (NOS) activity was determined by HPLC, carbon monoxide (CO) production was detected with a sensitive gas chromatographic method and insulin release with RIA.

Results: In isolated islets ncNOS activity was similar in $o b / o b$ mice and lean littermates and iNOS expression was negligible. Islets from $o b / o b$ or lean mice incubated at high glucose showed increased iNOS activity, while ncNOS activity was raised only in lean islets. Glucose injection induced strong expression and activity of islet iNOS in lean, but surprisingly not in obese mice. In $o b / o b$ islets the insulinotropic peptides glucagon, GLP-1, and GIP suppressed islet NOS activities and increased insulin release, while the insulinostatic peptide leptin induced the opposite effects. The production of heme oxygenase (HO)-derived carbon monoxide (CO), a NOS inhibitor, was impressively raised in $o b / o b$ islets and the $\mathrm{HO}$ inhibitor $\mathrm{ZnPP}$ suppressed while the $\mathrm{HO}$ activator hemin amplified glucose-stimulated insulin release.

Conclusion: The data suggest that islets from $o b / o b$ mice existing in an insulin resistant and hyperglycemic milieu maintain high rate of insulin secretion and protection from glucotoxic effects through being equipped with negligible iNOS activity, which apparently results from a general suppression of islet NOS activities induced by cyclic AMP-generating hormones, high $\mathrm{CO}$ production, and leptin deficiency. This beneficial effect on islet function and survival by suppressed iNOS coupled with leptin deficiency might have its clinical counterpart in human leptin-resistant type 2 obese diabetes with hyperinsulinemia.

\section{6}

NN414, a SUR1/Kir6.2-selective potassium channel opener prevents diabetes-induced structural changes of mitochondria: studies in a diabetic transplantation model

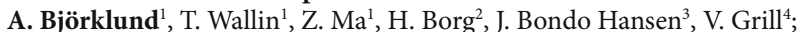

${ }^{1}$ Molecular Medicine and Surgery, Karolinska Institutet, Stockholm, Sweden, ${ }^{2}$ Medical Cell Biology, BMC, Uppsala, Sweden, ${ }^{3}$ Novo Nordisk Research and Development, Novo Nordisk Park, Målöv, Denmark,

${ }^{4}$ Institute of Cancer Research and Molecular Medicine,

Norwegian University of Science and Technology, Trondheim, Norway.

Background and Aims: We have previously shown that chronic overstimulation by moderate hyperglycaemia as commonly found in Type 2 diabetes can irreversibly desensitise beta cells and decrease glucoseinduced insulin secretion. In this study we tested for a role of mitochondrial dysfunction.

Materials and Methods: We transplanted islets from Wistar-Furth rats under the kidney capsule to neonatally streptozotocin-diabetic or non-diabetic recipients. Diabetic recipients received daily vehicle, or the selective activator of beta cell type $\mathrm{K}(+)$-ATP channels 6-chloro -3-(1-methylcyclopropyl) amino-4 H-thienol [3,2-e]-1,2,4-thiadiazine 1,1-dioxide (NN414) (3 $\mathrm{mg} / \mathrm{kg}$ ) intragastrically for 8 weeks. Non-diabetic rats received vehicle alone. Transplants were excised exactly 7 days after cessation of treatment (wash-out) and prepared for electron microscopy followed by quantitative morphometric analysis of mitochondria.

Results: Mean blood glucose was $4.6 \mathrm{mmol} / \mathrm{l}$ in the non-diabetic rats, 7.7 in vehicle-treated diabetic and 9.5 in NN414- treated animals. Non-diabetic recipients increased weight more markedly than vehicle-treated diabetic rats whereas there was almost no increase in the NN414-treated diabetic rats. The beta cells of islets from grafts from vehicle-treated diabetic rats displayed an extensive rough endoplasmic reticulum with numerous free ribosomes spread through the cytoplasm of the cells. Swollen, pale mitochondria with cristae only seen in the periphery of the organelle were apparent. Volume density of mitochondria $\left(V_{V M}\right)$, density of the mitochondrial outer surface area $\left(S_{V M}\right)$ and the number of mitochondria per unit volume of tissue $\left(N_{V M}\right)$ were measured. From these primary parameters secondary ones were calculated. In vehicle-treated diabetic rats the average mitochondrial volume $\left(V_{V M} / N_{V M}\right), \mathrm{p}<0.001$ as well as the average mitochondrial outer surface area $\left(S_{V M} / N_{V M}\right) \mathrm{p}<0.001$ was increased vs. non-diabetic rats. NN414-treatment of the diabetic rats partly but significantly normalized these diabetes-induced differences, $\mathrm{p}=0.003$ and $\mathrm{p}=0.001$ for effects on $\left(V_{V M} / N_{V M}\right)$ and $\left(S_{V M} / N_{V M}\right)$ respectively.

Conclusion: Long-term diabetes with over-stimulation by hyperglycemia leads to pathologically enlarged but fewer mitochondria and these effects are partly counteracted by treatment with NN414. Therapies that prevent overstimulation may have beneficial effects on beta cell functioning by way of preventing mitochondrial dysfunction.

Supported by Swedish Society of Medicine, Swedish Research Council 


\section{7}

$\mathrm{Ca}_{\mathrm{v}} 1.2$ channels, rather than $\mathrm{Ca}_{\mathrm{v}} 1.3$ and $\mathrm{Ca}_{\mathrm{v}} 2.3$ channels, permit insulin secretion from INS-1 832/13

M. Dekker Nitert ${ }^{1}$, A. Wendt ${ }^{2}$, L. Eliasson ${ }^{2}$, H. Mulder ${ }^{1}$.

${ }^{1}$ Diabetes, Metabolism \& Endocrinology, Experimental Medical Science,

Lund, ${ }^{2}$ Islet cell exocytosis, Clinical Science, Malmö, Sweden.

Background and Aims: An elevation of intracellular calcium mediated by voltage-gated calcium channels is required for insulin secretion from pancreatic $\beta$-cells. However, the voltage-gated calcium channels in the clonal $\beta$-cell line INS-1 832/13 have not been characterized. In other clonal $\beta$-cell lines and islets from different species, a variety of calcium channels has been found to influence insulin secretion. The aim of this study is to identify the voltage-gated calcium channels that control insulin secretion in INS-1 832/13 cells.

Materials and Methods: $\mathrm{Ca}_{\mathrm{v}} 1.2, \mathrm{Ca}_{\mathrm{v}} 1.3$, and $\mathrm{Ca}_{\mathrm{v}} 2.3$ mRNA levels were examined by real time-PCR. Using the calcium-indicator FURA-2AM, intracellular calcium influx into the cells was studied in response to glucose and potassium chloride. The calcium channel blockers isradipine, which blocks $\mathrm{Ca}_{\mathrm{v}} 1.2$ and $\mathrm{Ca}_{\mathrm{v}} 1.3$, and $\mathrm{SNX}-482$, which blocks $\mathrm{Ca}_{\mathrm{v}} 2.3$, were employed. $\mathrm{Ca}_{\mathrm{v}} 1.2$ expression was knocked down by RNA interference.

Results: Insulin secretion, which rose 10 -fold in response to $16.7 \mathrm{mM}$ glucose $(46 \pm 14 \mathrm{pg} / \mathrm{mg} / \mathrm{h})$, was completely abolished by isradipine $(5 \pm 2 \mathrm{pg} / \mathrm{mg} / \mathrm{h})$. Similarly, the increase in intracellular calcium in response to $15 \mathrm{mM}$ glucose was $61 \pm 15 \mathrm{nM}$ but decreased by $21 \pm 12 \mathrm{nM}$ in the presence of isradipine, and the frequency of calcium spikes was decreased to the level seen at 2.8 $\mathrm{mM}$ glucose. Isradipine also reduced insulin secretion in response to 16.7 $\mathrm{mM}$ glucose with $35 \mathrm{mM}$ potassium chloride from $143 \pm 10$ to $4 \pm 2 \mathrm{pg} / \mathrm{mg} / \mathrm{h}$. In contrast, inhibition of $\mathrm{Ca}_{\mathrm{v}} 2.3$ with SNX-482 did not significantly affect insulin secretion or intracellular calcium. While the cells expressed mRNA for all three calcium channels, the levels of $\mathrm{Ca}_{\mathrm{v}} 1.2$ exceeded those of $\mathrm{Ca}_{\mathrm{v}} 1.3$ and $\mathrm{Ca}_{v} 2.3$ by two orders of magnitude. $\mathrm{Ca}_{\mathrm{v}} 1.2 \mathrm{mRNA}$ was knocked down by RNA interference to $\approx 70 \%$ of that in control cells, reducing insulin secretion in response to $16.7 \mathrm{mM}$ glucose by $\approx 50 \%$. The remaining insulin secretion could be reduced to a level equivalent to that at $2.8 \mathrm{mM}$ glucose with isradipine. The calcium influx underlying this remaining insulin secretion could be accounted for by $\mathrm{Ca}_{\mathrm{v}} 1.2$ expressed on untransfected cells and/or $\mathrm{Ca}_{\mathrm{v}} 1.3$ expression.

Conclusions: INS-1 832/13 cells express $\mathrm{Ca}_{\mathrm{v}} 1.2, \mathrm{Ca}_{\mathrm{v}} 1.3$ and $\mathrm{Ca}_{\mathrm{v}} 2.3 . \mathrm{Ca}_{\mathrm{v}} 1.2$ is critical for insulin secretion in these cells although $\mathrm{Ca}_{\mathrm{v}} 1.3$ may also play a role. $\mathrm{Ca}_{\mathrm{v}} 2.3$ does not appear to play a significant role in insulin secretion in INS-1 832/13 cells, which confirms the previously reported findings in parental INS- $1 \beta$-cells. In primary mouse $\beta$-cells, however, $\mathrm{Ca}_{\mathrm{v}} 2.3$ accounts for $18 \%$ of insulin secretion. Taken together, this implies species differences in the role of $\mathrm{Ca}_{\mathrm{v}} 2.3$ in insulin secretion.

Supported by Swedish Research Council
PS 34 Metabolic regulation of insulin secretion

\section{8}

Loss of extracellular glucose detection by GLUT2 in pancreatic beta cells deeply alters mouse glucose tolerance

E. Stolarczyk, M. Le Gall, E. Brot-Laroche, P. Serradas, A. Leturque; Equipe 9, UMRS 872 INSERM/UPMC/UPD, Paris, France.

Background and Aims: We are investigating the mechanisms by which cells sense and adapt their functions to their nutritional environment, focusing on glucose detection. In hepatic cell line, the expression of a GLUT2 cytoplasmic loop-domain inhibits the stimulation of glucose-sensitive gene expression without modification of glucose metabolism. Our aim is to evaluate in vivo the importance of the detection of extracellular glucose triggered by GLUT2 independently of intracellular glucose metabolism on glucose tolerance and pancreatic functions.

Materials and Methods: We created transgenic mice that ubiquitously express the GLUT2 cytoplasmic loop-domain and measured the changes in glucose homeostasis that are resulting from the loss of sugar detection. Glucose tolerance tests (3.6g glucose $/ \mathrm{kg}$ ) were performed in $24 \mathrm{~h}$ fasted-mice. Insulin and glucagon labelling were performed in pancreas fixed in Bouin's solution and embedded in paraplast according to standard procedures. The number of small beta-cell clusters ( $<3$ insulin positive cells) and islets were quantified in serial pancreas sections.

Results: In the pancreas of GLUT2-loop transgenic mice, the stimulation of sugar-sensitive target genes such ChREBP was reduced in response to a sugar-rich meal. This was not due to decreased GLUT2 protein warranting a preserved glucose metabolism. An oral glucose tolerance test revealed glucose intolerance in transgenic mice. This was correlated with reduced plasma insulin levels. Further investigation of transgenic pancreas showed lower insulin stores (by 30\%) but normal proinsulin mRNA expression. Moreover, islet architecture was normal since glucagon cells surrounded insulin positive cells. Total number of islets was similar in transgenic and wild-type mice. However, pancreas weight and ratio of small beta-cell clusters $(<3$ insulin positive cells) over total number of islets were increased in transgenic compared to wild-type mice.

Conclusion: Taken together these data suggest that invalidation of GLUT2mediated glucose detection in GLUT2-loop transgenic mice perturbed significantly insulin response to oral glucose in the pancreas. Multiple steps in the process of insulin maturation, formation of insulin stores and islet size are dependent on GLUT2-mediated glucose detection. Thus extracellular glucose detection by GLUT2 controls many aspects of glucose homeostasis. Perturbation of this physiological process may participate in metabolic diseases.

Supported by doctoral fellowship from the Ministère de la Recherche (France)

\section{9}

Upregulation of key $\beta$ cell genes and improvement of function in rodent islets following chronic in vitro treatment with a glucokinase activator D. Gill, D. M. Smith;

AstraZeneca, Macclesfield, United Kingdom.

Background and Aims: Glucokinase (GK) is the glucose sensor in the $\beta$-cell and controls the threshold for glucose-stimulated insulin secretion (GSIS). Several small molecule direct activators of GK have been discovered which increase GSIS in $\beta$-cells and stimulate hepatic glucose metabolism. We have previously reported the acute effects of a glucokinase activator (GKA50) on the $\beta$-cell, demonstrating that GKAs stimulate insulin secretion by a "glucoselike" mechanism. Whether chronic GKA treatment would result in beneficial or "glucotoxic" effects on the islet has not previously been described. The aim here was to determine the chronic effects of one of these compounds, GKA71, on pancreatic $\beta$-cell function. Following chronic in vitro culture of mouse and rat islets in the presence of GKA71 gene expression was analysed. Perifusion was used to assess the effect of chronic GKA treatment on rat islet GSIS.

Materials and Methods: Islets were prepared from male Wistar rats and $\mathrm{C} 57 \mathrm{Bl} / 6$ mice by Liberase digestion. For acute assessment of insulin secretion islets were cultured overnight in $11 \mathrm{mM}$ glucose prior to $2 \mathrm{~h}$ static incubations. For chronic experiments, islets were allowed to recover overnight prior to $72 \mathrm{~h}$ 
incubation in the absence and presence of $10 \mu \mathrm{M}$ GKA71 (in $5 \mathrm{mM}$ and $25 \mathrm{mM}$ glucose), with daily media changes. Taqman low density arrays (TLDAs) (mouse islets, only changes $>3$ fold were considered biologically significant) and single gene Taqman RT-PCR (mouse and rat islets) were performed. All expression data was normalised to HPRT. Islets were perifused using a Brandel SF-12 Superfusion system, using 50 islets per channel. Insulin secretion was assessed at basal $(3 \mathrm{mM})$ and stimulated $(15 \mathrm{mM})$ glucose concentrations.

Results: In rat islets incubated with $3 \mathrm{mM}, 5 \mathrm{mM}$ and $8 \mathrm{mM}$ glucose $1 \mu \mathrm{M}$ GKA71 caused a 2.3, 4.6 and 2.6 fold increase in insulin release, respectively $(\mathrm{n}=4)$, but at $1 \mathrm{mM}, 10 \mathrm{mM}$ and $20 \mathrm{mM}$ glucose there was no effect of GKA71. In mouse islets cultured in $5 \mathrm{mM}$ glucose GKA71 $(10 \mu \mathrm{M})$ caused a 42 fold $(\mathrm{p}=0.002)$ increase in insulin mRNA $(\mathrm{n}=3)$. The TLDA results showed that GKA71 caused an increase in amylin (4.6 fold, $\mathrm{p}=0.0045)$, insulin (15.9 fold, $\mathrm{p}=0.0013$ ), PDX1 (4.5 fold, $\mathrm{p}=0.012$ ), PC1 (6.5 fold, $\mathrm{p}=0.0014$ ), and GLUT2 (29.3 fold, $\mathrm{p}<0.001$ ) expression and a decrease in CEBP $\beta$ ( 5.0 fold, $\mathrm{p}=0.04$ ) expression. In rat islets cultured in $5 \mathrm{mM}$ glucose $(\mathrm{n}=6)$ GKA71 caused an increase in insulin (2.7 fold, $\mathrm{p}<0.001)$, GLUT2 (2.3 fold, $\mathrm{p}<0.001)$, PC1 (2.5 fold, $\mathrm{p}<0.001)$ and amylin $(2.2$ fold, $\mathrm{p}<0.001)$ expression with no significant changes in PDX1 or GK expression. Rat islets treated chronically with GKA71 had an 8.5 fold increase $(n=3, p<0.001)$ in first phase insulin secretion and a 35 fold increase $(\mathrm{p}=0.001)$ in second phase insulin secretion compared with control-treated islets. The insulin secretion profile of islets treated with GKA in the presence of $5 \mathrm{mM}$ glucose was very similar to that of islets cultured in $25 \mathrm{mM}$ glucose for the same period.

Conclusion: GKA71 stimulates insulin release from rat islets in acute experiments. Here we show for the first time that chronic treatment of rodent islets with GKA71 in the presence of $5 \mathrm{mM}$ glucose significantly increased the expression of key $\beta$-cell genes, including insulin, and significantly increased first and second phase insulin secretion. These results demonstrate that in addition to the reported effects of GKAs on improving blood glucose homeostasis, they may also have an important role in $\beta$-cell preservation, and by increasing insulin mRNA be less likely to cause $\beta$-cell exhaustion.

\section{0}

Glucose-dependent interaction of glucokinase and PFK-2/FBPase-2 in insulin-producing cells

S. Langer, S. Lenzen, S. Baltrusch;

Institute of Clinical Biochemistry, Hannover Medical School, Germany.

Background and Aims: Glucokinase (GK) plays a pivotal role in the process of glucose-induced insulin secretion in pancreatic beta cells. In previous experiments we elucidated binding between GK and the bifunctional enzyme 6-phosphofructo-2-kinase/fructose-2,6-bisphosphatase (PFK2/FBPase-2). In pancreatic beta cells expressing the PFK-2/FBPase-2 brain isoform the enzyme acts as an endogenous activator of GK enzyme activity. By immunohistochemistry we demonstrated a glucose-dependent colocalization of GK and PFK-2/FBPase-2 accompanying the increase in GK enzyme activity. The aim of this study was to monitor GK and PFK-2/FBPase2 complex formation and separation in living cells.

Material and Methods: Interaction between GK and PFK-2/FBPase-2 was analyzed in insulin-producing MIN6 cells using a new fluorescence based mammalian two-hybrid system. Colocalization of GK and PFK-2/FBPase2 was determined via in vivo labeling with the fluorescent proteins ECFP (enhanced cyan fluorescent protein) and EYFP (enhanced yellow fluorescent protein). Association between these labeled proteins was measured via FRET (fluorescence resonance energy transfer).

Results: The mammalian two-hybrid system was established in insulinproducing cells using a new reporter vector containing two fluorescent protein genes. For quantitative analysis one fluorescent protein was necessary for normalization of unavoidable variability in the transfection efficiency while the second one served as the reporter. In contrast to the commonly used mammalian two-hybrid systems that require cell lysis prior to detection our system allows monitoring of the effects of environmental changes on the protein interaction. In medium supplemented with $25 \mathrm{mmol} / \mathrm{l}$ glucose a significant interaction between GK and PFK-2/FBPase-2 was measured. Lowering of the glucose concentration in the cell culture medium abolished the interaction between the enzymes. The fluorescent fusion proteins ECFPGK and EYFP-PFK-2/FBPase-2 were colocalized in a comparable manner as the endogenous proteins, demonstrated by immunohistochemistry. In agreement with the two-hybrid results colocalization between GK and PFK-2/ FBPase-2 was significantly higher in cells cultured in medium supplemented with $25 \mathrm{mmol} / \mathrm{l}$ glucose than in medium with $3 \mathrm{mmol} / \mathrm{l}$ glucose. Furthermore transfer of fluorescence energy (FRET) from ECFP-GK to EYFP-PFK-2/ FBPase-2 was detectable indicating a direct interaction between GK and PFK-2/FBPase-2.

Conclusion: The interaction between GK and PFK-2/FBPase-2 is promoted by glucose. Thus PFK-2/FBPase- 2 plays a major role in the glucose-dependent compartmentation and regulation of GK in insulin-producing cells. Our results may open the perspective for a therapeutic targeting of this interaction in type 2 diabetes therapy. Furthermore our established mammalian twohybrid system offers in insulin-producing cells the possibility to monitor changes in the association of the two key regulatory beta cell proteins in dependence on metabolic conditions as accruing in type 2 diabetes.

\section{1}

Long-chain fatty acyl coenzyme A inhibition of glucokinase in pancreatic islets from rats depleted in long-chain polyunsaturated $\omega 3$ fatty acids

Y. Zhang ${ }^{1}$, N. Bulur ${ }^{1}$, S. Peltier ${ }^{2}$, Y. A. Carpentier ${ }^{2}$, W. J. Malaisse ${ }^{1}$, A. Sener ${ }^{1}$; ${ }^{1}$ Lab of Experimental Hormonology, Université Libre de Bruxelles,

${ }^{2}$ Lab of Experimental Surgery, Université Libre de Bruxelles, Belgium.

Background and Aims: The metabolism of D-glucose, but not that of Lglutamine or palmitate, is impaired in pancreatic islets from $2^{\text {nd }}$ generation rats depleted in long-chain polyunsaturated $\omega 3$ fatty acids ( $\omega 3-\mathrm{D}$ rats). The possible participation of altered glucokinase kinetics in such an impairment was investigated in the present study.

Methods: The phosphorylation of $\mathrm{D}-\left[\mathrm{U}-{ }^{14} \mathrm{C}\right]$ glucose by islet homogenates from control animals and $\omega 3-\mathrm{D}$ rats was measured over $60 \mathrm{~min}$ incubation at $37^{\circ} \mathrm{C}$ in the presence of $5.0 \mathrm{mM}$ ATP.

Results: The apparent activity of glucokinase, as judged from the increase in D-glucose phosphorylation in response to a rise in the concentration of the hexose (e.g. from 1.0 to $20.0 \mathrm{mM}$ ), only represented, in $\omega 3$-D rats, 81.8 $\pm 4.8 \%(\mathrm{n}=11 ; \mathrm{p}<0.005)$ of the paired value recorded within the same experiment in control animals. Two series of findings suggest that these results reflect an altered kinetic behaviour of glucokinase, rather than a mere decrease of its islet content. First, the incorporation of exogenous D-glucose 6-phosphate (G6P, $3 \mathrm{mM}$ ) and D-fructose 1-phosphate (F1P, $1 \mathrm{mM}$ ) into the assay medium decreased the reaction velocity in $\omega 3$-D rats by only 93.2 $\pm 2.9 \%(\mathrm{n}=8 ; \mathrm{p}<0.05)$ of the inhibition found in control animals, as if glucokinase was already partially inhibited by some endogenous factor in the former rats. Second, glucokinase activity, as estimated from the increase in reaction velocity resulting from a rise in $\mathrm{D}$-glucose concentration from 6.0 to $20.0 \mathrm{mM}$ in the presence of exogenous G6P and FIP, was decreased to $62.0 \pm$ $6.0 \%(\mathrm{n}=4 ; \mathrm{p}<0.01)$ by palmitoyl $\operatorname{CoA}(50 \mu \mathrm{M})$ in control animals, whilst failing to be significantly affected $(85.9 \pm 12.5 \% ; \mathrm{n}=5 ; \mathrm{p}>0.3)$ by palmitoyl CoA in $\omega 3-\mathrm{D}$ rats.

Conclusion: Glucokinase activity in islet homogenates from $\omega 3$-D rats is apparently inhibited by endogenous long-chain fatty acyl CoA. This may account, in part at least, for the abnormally low increment in D-glucose utilization in response to a rise in hexose concentration and abnormally low ratio between oxidation and utilization of $\mathrm{D}$-glucose prevailing in intact islets from $\omega 3-\mathrm{D}$ rats.

\section{2}

Knock-down of pyruvate dehydrogenase kinase 1 in INS-1 832/13 cells increases insulin secretion

O. V. Kotova ${ }^{1}$, U. Fransson ${ }^{1}$, E. Hallgard ${ }^{1}$, M. C. Sugden ${ }^{2}$, H. Mulder ${ }^{1}$; ${ }^{1}$ Experimental Medical Science, Lund University, Lund, Sweden, ${ }^{2}$ Centre for Diabetes and Metabolic Medicine, Institute of Cell and Molecular Science, Barts, United Kingdom.

Background and Aims: Hyperglycemia and impaired glucose-stimulated insulin secretion are hallmarks of Type 2 Diabetes. High concentrations of glucose are toxic to pancreatic beta-cells, inducing changes in gene expression. Altered expression of genes encoding enzymes controlling the metabolic pathways involved in stimulus-secretion coupling may underlie impaired insulin secretion in Type 2 Diabetes. Here, we examined which genes coding for metabolic enzymes were affected by elevated glucose, and how they control insulin secretion. 
Materials and Methods: We cultured INS-1-derived rat 832/13 clonal betacells for $48 \mathrm{~h}$ at low $(2.8 \mathrm{mM})$ or high $(16.7 \mathrm{mM})$ glucose concentrations. Insulin secretion was measured, and cells were subjected to analysis by a custom-made array of 46 real time-PCR assays for genes involved in glycolysis, TCA cycle, oxidative phosphorylation, and lipid metabolism. A candidate gene was subsequently knocked down by RNA interference, and cellular function was evaluated by determination of insulin secretion, glucose oxidation, and the ATP/ADP ratio.

Results: After $48 \mathrm{~h}$ culture at $2.8 \mathrm{mM}$ glucose, insulin secretion increased 6.8fold upon acute $(1 \mathrm{~h})$ stimulation with $16.7 \mathrm{mM}$ glucose. In contrast, insulin secretion rose only 3.5-fold, when cells had been cultured for $48 \mathrm{~h}$ at 16.7 $\mathrm{mM}$ glucose. The pyruvate dehydrogenase kinase (PDK) 1 mRNA level was upregulated $(2.4$ fold; $\mathrm{P}<0.01)$ in cells cultured at $16.7 \mathrm{mM}$ glucose. When PDK1 was knocked down by RNA interference (PDK1 mRNA was reduced by $80 \% ; \mathrm{P}<0.001$; PDK1 protein expression was decreased, as assessed by Western Blot), insulin secretion in response to $16.7 \mathrm{mM}$ glucose was enhanced by $60 \%(\mathrm{P}<0.01)$. In contrast, glucose oxidation and the glucose-induced rise in ATP/ADP ratio were unaffected by PDK1 knock down.

Conclusion: We suggest that maintenance of low PDK1 expression, resulting in dephosphorylation of pyruvate dehydrogenase (PDH) and thus, an increase in its activity, is an essential factor for robust glucose-stimulated insulin secretion in pancreatic beta-cells. Based on the observation that PDK1 knock down affected neither glucose oxidation nor the ATP/ADP ratio, we further propose that augmented cycling of citrate to pyruvate may be responsible for the enhancement of insulin secretion.

Supported by Swedish Research Council

\section{3}

Ecto-nucleotidase activity in pancreatic islet cells

M.-H. Giroix ${ }^{1}$, K. Louchami ${ }^{2}$, E. Hupkens ${ }^{2}$, N. Bulur ${ }^{2}$, Y. Zhang ${ }^{2}$, B. Portha ${ }^{1}$, R. Beauwens ${ }^{3}$, W. J. Malaisse ${ }^{2}$, A. Sener';

${ }^{1}$ Laboratory of Nutrition Physiopathology, CNRS UMR 7059, Université

Paris 7 (Denis Diderot), Paris, France, ${ }^{2}$ Laboratory of Experimental

Hormonology, Université Libre de Bruxelles, Belgium, ${ }^{3}$ Laboratory of Cell

and Molecular Physiology, Université Libre de Bruxelles, Belgium.

Background and Aims: The purine nucleotide ATP is released intermittently in pancreatic islets from nerves, and from $\beta$-cells themselves together with insulin during exocytosis. This ATP is viewed as both a coordinator of the $\beta$ cell rhythmicity, and an autocrine regulator of insulin secretion acting at the intervention of purinoceptors. Such a view led us to investigate whether islet cells display ecto-nucleotidase activity.

Materials and Methods: Pancreatic islets isolated from rat, as well as BRIN-BD 11 and INS-1E cells, were incubated in a salt-balanced medium containing, as required, ATP or other nucleotides. The fall in nucleotide concentration of the incubation medium was monitored by bioluminescent or HPLC procedure. The nucleotide-hydrolyzing activity was also assessed in cell-free incubation media obtained after incubation of islets or islet cells in the absence of any exogenous nucleotide.

Results: After 60 min preincubation of pancreatic islets, BRIN-BD 11 or INS-1E cells in the absence of exogenous ATP, both the islet cells and cellfree medium collected after preincubation demonstrated ATP-hydrolyzing activity, the rate of ATP breakdown being about twice higher in the former than latter case. The release of the hydrolytic activity by the islets occurred at a fairly constant rate over two first preincubation periods of 15 min each and, thereafter, slowly decreased. It was independent of the glucose concentration, and was also detected after a prior 4-day culture of the islets. It apparently failed to differ significantly in islets from normal Wistar (W) rat and GotoKakizaki (GK) rat, which represents a model of type 2 diabetes. Thus, the hydrolase activity released over $60 \mathrm{~min}$ incubation at $37^{\circ} \mathrm{C}$ averaged, in $\mathrm{W}$ and GK rats respectively, $3.09 \pm 0.09$ and $3.14 \pm 0.15 \mathrm{pmol} /$ islet per $15 \mathrm{~min}$ at $1.0 \mu \mathrm{M}$ ATP, and $8.88 \pm 0.57$ and $9.05 \pm 0.62 \mathrm{pmol} /$ islet at $3.0 \mu \mathrm{M}$ ATP ( $\mathrm{n}=8$ in all cases). ADP, AMP and adenosine were generated during ATP breakdown. External ATP, ADP and AMP $(10 \mu \mathrm{M}$ each) were hydrolyzed at near equal rates. Hydrolysis of GTP, CTP, UTP and ITP (10 $\mu \mathrm{M}$ each) was also observed. The ATP hydrolytic activity was modulated by the ATP concentration and required the presence of divalent cations (residual activity $<20 \%$ in $\mathrm{Ca}^{2+} / \mathrm{Mg}^{2+}$-free medium containing $2.5 \mathrm{mM}$ EDTA). It was little affected by classic inhibitors of the P-, F-, and V-type ATPases such as ouabain $(1.0 \mathrm{mM})$, sodium orthovanadate $(0.5 \mathrm{mM})$, sodium azide $(1.0 \mathrm{mM})$ or sodium nitrate $(100 \mathrm{mM})$, but decreased by $50-80 \%$ by the $\mathrm{P}_{2}$ receptor antagonists pyridoxalphosphate-6-azophenyl-2','-disulfonic acid (PPADS) and suramin $(0.1 \mathrm{mM}$ each) reported as inhibitors of ecto-ATPases.

Conclusion: The present study reveals that pancreatic islet cells display ectonucleotidase activity, which is apparently expressed at the plasma membrane level and is also released from the cells in the extracellular medium. Such an activity, which could involved several ecto-enzymes, is obviously susceptible to affect the concentration of ATP released by nerve endings and insulinproducing cells in the islet interstitial fluid.

Supported by INSERM (France) / CFB and FNRS (Belgium)

\section{4}

The effect of over-expression of the mitochondrial Aspartate-Glutamate exchanger Aralarl on insulin secretion and metabolism in the BRIN-BD11 cell line

K. Bender ${ }^{1}$, P. Maechler' ${ }^{2}$, P. Newsholme ${ }^{1}$;

${ }^{1}$ Conway Institute, UCD School of Biomolecular and Biomedical Science, Dublin, Ireland, ${ }^{2}$ Department of Cell Physiology and Metabolism, University Medical Centre, Geneva, Switzerland.

Background and Aims: In the beta cell, mitochondrial Redox- NADHshuttles are important for coupling glycolysis to mitochondrial energy metabolism. Aralar1, an Aspartate- Glutamate exchanger in the MalateAspartate shuttle, has been found to play an important regulatory role in oxidative glucose metabolism and insulin secretion in rat derived INS- 1E cells as well as in the clonal BRIN- BD11 cell line. Certain amino acids, in particular L- alanine, L-leucine and L- glutamine, are known to stimulate both insulin secretion and glucose metabolism in the BRIN- BD11 cell line. These effects may have been due to stimulation of anaplerotic metabolism.

Materials and Methods: We have now over- expressed Aralar1 by the means of the recombinant Adenovirus AdCA- Aralar1 in clonal BRIN BD11 cells and have investigated the involvement of Aralar1 in stimulation of glucose and amino acid metabolism plus insulin secretion in the presence or absence of the aminotranferase inhibitor Aminooxyaxctetate or intracellular Calcium chelator BAPTA-AM. The following parameters were determined; glucose and glutamine consumption, lactate and glutamate generation, mitochondrial membrane potential, NADH and ATP levels, triglyceride and glycogen content and insulin secretion.

Results: Aralar1 over- expression in BRIN-BD11 cells resulted in enhanced glucose consumption $(\mathrm{p}<0.05)$, mitochondrial membrane hyperpolarization $(\mathrm{p}=0.03)$, NADH levels $(\mathrm{p}<0.05)$ and insulin secretion $(\mathrm{p}<0.01)$. Insulin secretion and glucose consumtion were further enhanced by the presence of $10 \mathrm{mM}$ alanine $(+30 \%, \mathrm{p}<0.01$ and $+25 \%, \mathrm{p}=0.02$ respectively) whereas both were significantly reduced by the presence of $10 \mathrm{mM} \mathrm{L}$ - aspartate $(\mathrm{p}<0.05)$. However, addition of L- glutamine did not significantly change any of these parameters. The presence of $5 \mathrm{mM}$ aminooxyactetate did not significantly inhibit insulin secretion or glucose consumption in Aralar1- over- expressing cells.

Conclusion: The Aspartate- Glutamate exchanger Aralar 1 may be an important site for metabolic control and stimulus-secretion coupling in the pancreatic beta cell.

Supported by Health Research Board Ireland

\section{5}

GAD65 and GABA-transaminase genes are more highly expressed in pancreatic islets than the $\alpha$-ketoglutarate dehydrogenase gene

I. Hernández-Fisac ${ }^{1}$, J. Pizarro-Delgado ${ }^{1}$, R. Martín-del-Río ${ }^{2}$,

J. Tamarit-Rodriguez;

${ }^{1}$ Biochemistry, Complutense University,

${ }^{2}$ Research Department, Hospital "Ramón y Cajal", Madrid, Spain.

Background and Aims: There is no complete information available in the literature on the expression of all the enzymes responsible of GABA metabolism in pancreatic islets. Direct evidence is missing that GABA can be metabolized either to succinic or to $\gamma$-hydroxybutyric acid.

Material and Methods: Semialdehyde succinic acid dehydrogenase and reductase (SSAdh and SSAred), and $\alpha$-ketoglutarate dehydrogenase ( $\alpha$ KGdh) activities were measured in rat islet homogenates as either NADH production or NADPH consumption in the presence of excess of substrates. Succinic 
acid dehydrogenase activity (SUCdh) was measured as the reduction of 2,6dichloroindophenol by respiratory complex II in the presence of rotenone and excess of succinic acid. Islet RNA isolation and retrotranscription were performed with RNeasy ${ }^{\oplus}$ Mini and Quantitect ${ }^{\circ}$ Reverse Transcription kits (Quiagen). Gene expression analysis was performed by real-time RT-PCR, using $18 \mathrm{~S}$ rRNA as an internal control and pre-designed TaqMan ${ }^{\circ}$ Gene Expressions Assays (Applied Biosystems).

Results: SSAdh and SSAred activities measured in islet homogenates were of a similar magnitude than $\alpha$ KGdh and SUCdh. SSAdh and $\alpha$ KGdh were strongly inhibited by $\alpha$-ketobutyric acid. SSAred and $\alpha$ KGdh were dosedependently inhibited by $\alpha$-ketoisocaproic acid. In proportion to $\alpha \mathrm{KGdh}$ $(100.0 \pm 24.3 \%, \mathrm{n}=6)$, GAD65 and GABA-transaminase (GABA-T) genes showed the highest expression in islets $(757.9 \pm 20.4 \%, \mathrm{n}=6$, and $556.3 \pm 22.2 \%$, $\mathrm{n}=6$, respectively), succinic acid dehydrogenase exhibited intermediate expression levels $(259.2 \pm 18.3 \%, \mathrm{n}=6)$ whereas SSAdh and SSAred were similarly expressed $(131.1 \pm 20.8 \%, \mathrm{n}=6$, and $52.9 \pm 27.6, \mathrm{n}=6$, respectively). This islet gene expression pattern was found different from those observed in cerebellum and liver.

Conclusions: Islets express the enzyme activity responsible for returning "GABA-shunt" metabolic flow into the Krebs cycle (SSA conversion into succinic acid by SSAdh) and another one diverting it out (SSA conversion into $\gamma$-hydroxybutyric acid by SSAred).The much higher gene expression of GAD65 and GABA-T than $\alpha$ KGdh suggests that GABA is synthesised at a high rate in $\beta$-cells and that its transamination might compete efficiently with $\alpha \mathrm{KG}$ decarboxylation in the Krebs cycle. We hypothesize that nutrient secretagogues decrease the intracellular concentration of GABA in $\beta$-cells supplying $\alpha$-KG to feed GABA-T hence facilitating its metabolism to succinic or $\gamma$-hydroxybutyric acid. Preliminary experiments seem to indicate that $\gamma$-hydroxybutyric acid is produced in pancreatic islets incubated with $\mathrm{L}$ glutamine and glucose.

Supported by Instituto de Salud Carlos III and Subprograma B, Universidad Complutense-Comunidad de Madrid

\section{PS 35 Stem cells and extracellular matrix in beta cell growth}

\section{6}

Human embryonic stem cell derived pancreatic endocrine islet cells G. S. Korbutt ${ }^{1}$, A. Eshpeter ${ }^{2}$, R. Rajotte ${ }^{1}$, J. Jiang ${ }^{3}$, M. Au ${ }^{1}$, A. S. Majumdar ${ }^{1}$; ${ }^{1}$ Surgery \& Surgical-Medical Research Institute, University of Alberta, Edmonton, Canada, ${ }^{2}$ Surgical-Medical Research Institute, University of Alberta, Edmonton, Canada,

${ }^{3}$ Cell Biology, Geron Corporation, Menlo Park, United States.

Background and Aims: Human embryonic stem cells (hES) have been proposed as a potential unlimited source of tissue for transplantation in the treatment of type 1 diabetes. A great deal of research has been conducted on the differentiation of mouse embryonic stem cells into insulin producing cells, much of which is controversial due to the addition of insulin to culture media. There has been new success in the field with a successful differentiation of hES into definitive endoderm.

Materials and Methods: Recently, we have developed a defined protocol to differentiate human embryonic stem cells (hES) into cellular aggregates that are partially composed of pancreatic islet endocrine cells. We have further characterized these islet-like clusters (ILCs), and found them to be immunoreactive for islet markers such as insulin, C-peptide, glucagon, somatostatin, prohormone convertase $1 / 3$ and 2, and PDX-1. We have observed that ILCs contain detectable insulin secretion by responding to a high glucose challenge in vitro. We have also transplanted hES derived ILCs under the kidney capsule of streptozotocin induced diabetic immunoincompetant mice, and although blood glucose levels were not normalized, human C-peptide was detected in the serum of these mice. One explanation for not achieving normoglycemia in these mice is that a cub-optimal number of insulin-positive beta cells were transplanted. Nonetheless, the expression of islet endocrine cell markers by immunohistochemistry and RT-PCR (insulin, glucagon, PDX-1) was also maintained even after 70 days in vivo.

Conclusion: These results show that ILCs differentiated from hES appear to be immature cells of pancreatic endocrine lineage, and it is possible that further purification, differentiation, and maturation of these cells could lead to the generation of a sufficient source of islets for transplant in patients with Type 1 diabetes.

Supported by Geron Corporation

\section{7}

Isolation, characterisation and prospects of endothelial progenitor cells to aid pancreatic beta cell regeneration

C. P. Khoo ${ }^{1,2}$, M. G. Valorani ${ }^{1,3}$, M. Brittan ${ }^{1}$, M. R. Alison ${ }^{1}$, C. Guglielmi ${ }^{2}$, G. Warnes ${ }^{4}$, U. Johansson ${ }^{5}$, M. I. Hawa ${ }^{1}$, P. Pozzilli ${ }^{1,2}$;

${ }^{1}$ Centre for Diabetes and Metabolic Medicine, Queen Mary University, London, United Kingdom, ${ }^{2}$ Area di Endocrinologia, Universita' Campus Bio-Medico, Rome, Italy, ${ }^{3}$ Stem Cell Research, Fondazione Livio Patrizi, Rome, Italy, ${ }^{4}$ Flow Cytometry \& Imaging, Queen Mary University, London, United Kingdom, ${ }^{5}$ Centre for Haematology, Queen Mary University, London, United Kingdom.

Background and Aims: Endothelial progenitor cells (EPCs) in the bone marrow and peripheral blood contribute to tissue repair in various pathological conditions, via the formation of new blood vessels, and thus have a degree of stem cell potential. It is now established that diabetic patients have reduced EPC number and dysregulated EPC function. Our aim was to isolate, characterise and compare EPCs from pre-diabetic and diabetic nonobese diabetic (NOD) mice, a well-established model of type 1 diabetes to delinate their role in tissue regeneration.

Material and Methods: Whole bone marrow was obtained by flushing femurs, tibias and illiac crests from pre-diabetic and diabetic NOD mice (5-30 weeks) in which the diabetic status was confirmed by measuring blood glucose levels $(\geq 11.5 \mathrm{mmol} / \mathrm{l})$; peripheral blood was collected in heparin coated tubes and lysed after incubation with antibodies directed agains EPCs. Pancreata from diabetic and pre-diabetic mice were acquired and paraffin embedded for immunohistochemical studies. Slides were stained with endothelial (Factor $\left.\mathrm{VIII}^{+}\right)$and vascular smooth muscle cell $\left(\mathrm{SMA}^{+}\right)$markers. 
Results: Morphological and immunohistochemical studies showed that in pancreata from diabetic mice compared to pre-diabetic mice, blood vessel density was significantly increased with vessels containing elevated numbers of both endothelial (Factor $\left.\mathrm{VIII}^{+}\right)$and vascular smooth muscle cells $\left(\mathrm{SMA}^{+}\right)$. FACS analyses revealed a significant decrease in EPC number $\left(\mathrm{CD} 31^{+}, \mathrm{c}-\mathrm{Kit}^{+}\right.$, $\left.\mathrm{Sca}^{+}{ }^{+}, \mathrm{Lin}^{-}\right)$in $\mathrm{BM}$ from diabetic compared to pre-diabetic mice (P-value: 0.021), which is consistent with human studies. Conversely, EPC number was significantly increased in peripheral blood from diabetic compared to prediabetic mice ( P-value: 0.015)

Conclusion: These preliminary data suggest that at the onset of diabetes, $\mathrm{BM}$-derived EPCs are stimulated to enter the systemic circulation in response to signals from the pancreas. Our findings of increased EPC-derived lineages and vessel density in the NOD mouse imply that circulating EPCs engraft within the pancreas. This latter observation is supported by many studies of BM-derived stem cell engraftment and contribution to neovasculogenesis in adult tissues, which is enhanced by increasing regenerative demand within a diseased or damaged tissue. The exact signalling pathways and molecular mechanism for this EPC recruitment are as yet unknown. However, our data demonstrates that EPCs could be a prospective source of autologous cells for transplantation with the aim to promote restoration of pancreatic function following the onset of type 1 diabetes by contributing to tissue regeneration via neovasculogenesis.

Supported by the Ministry of University (MIUR) Italy

\section{8}

Transdifferentiation of monocytes derived from healthy and type 1 diabetic donors into insulin-producing cells: development of an optimal and reproducible protocol

D. Chesnel ${ }^{1}$, V. Corcelle 2 , B. Boehm ${ }^{3}$, H. Zulewski ${ }^{4}$, L. Gruaz ${ }^{5}$, R. Schorer ${ }^{1}$, D. Burger ${ }^{5}$, C. B. Wollheim ${ }^{1}$, B. R. Gauthier ${ }^{1}$;

${ }^{1}$ Cell Physiology \& Metabolism, Geneva University, Switzerland, ${ }^{2}$ Faculty of Medecine, INSERM U568, Nice, France, ${ }^{3}$ Clinic of Internal Medicine, Ulm University, Germany, ${ }^{4}$ Division of Endocrinology, University Hospital Basel, Switzerland, ${ }^{5}$ Clin Immunology, GUH, Geneva, Switzerland.

Background and Aims: Although daily insulin injection is the treatment of choice for Type 1 diabetic (T1D) patients, control of glycaemia is problematic and results in long-term complications. The ultimate treatment of T1D would be the replacement of destroyed b-cells by insulin-producing cells. Proof of principle of this concept was shown by normalization of blood glucose of T1D patients subsequent to islets transplantation. However, worldwide use of this approach is hampered by the scarcity of pancreas donors. This has led to efforts into the development of alternative sources of human insulinproducing cells. We have shown that large amount of readily accessible human monocytes can be expanded in the presence of MCSF and partially differentiated into insulin-producing cells, using an islet differentiating medium (IDM) containing $17.5 \mathrm{mM}$ glucose. These conditions resulted in low insulin mRNA levels ( $0.5 \%$ as compared to human islets) and cytosolic rather than nuclear localisation of key transcription factors (TFs). The aim of the current study was to improve insulin yields using agents which are known to influence TFs nuclear/cytosolic shuttling: glucose, IBMX/forskolin and Leptomycin B. We also evaluated the capacity of monocytes derived from T1D donors to generate surrogate b-cells.

Material and Methods: Monocytes isolated from healthy and T1D donors were cultured for 9 days in MCSF and subsequently in either RPMI control media or IDM for an additional 11 days. Increasing concentrations of glucose $(5,8.5$ or $17.5 \mathrm{mM})$ were added to culture media. In parallel, $100 \mu \mathrm{M}$ IBMX/ $1 \mu \mathrm{M}$ forskolin or $37 \mathrm{nM}$ Leptomycin B was included in IDM for 4 and 9 days. RNA was extracted and insulin transcript levels were determined by QT RT/PCR. Immunocytochemistry was performed to detect insulin, NeuroD, Pdx1 and MafA.

Results: Altering glucose concentrations did not increase nuclear localization of TFs nor improved insulin transcript levels as compared to standard IDM. Exposure to either IBMX/forskolin or Leptomycin B in combination with $17.5 \mathrm{mM}$ glucose for 9 days targeted TFs to nuclei resulting in a 2 -fold increase in insulin mRNA levels. Lower glucose concentrations failed to improve insulin yields and TFs nuclear localization. Intriguingly, insulin could not be revealed by immunocytochemistry. Reducing exposure time to IBMX/ forskolin and Leptomycin B to 4 days did not ameliorate insulin detection. Monocytes maintained in MCSF/IDM for up to 56 days resulted in a 3-fold increase in the insulin transcript. More importantly, monocytes derived from
T1D donors and cultured in MCSF and standard IDM were re-programmed to express the insulin transcript as well as protein.

Conclusions: Nuclear localization of TFs by IBMX/forskolin and Leptomycin $\mathrm{B}$ improved insulin transcription yet decreased insulin protein. The latter suggests a potential long-term detrimental effect of the agents on insulin biosynthesis. Furthermore, we established proof of principle on the feasibility of using monocytes derived from T1D donors to generate surrogate b-cells for auto-transplantation.

Supported by EFSD/Lilly European Diabetes Research Programme

\section{9}

\section{Pancreatogenesis from embryonic stem cells in streptozocin-injured} mice

G. Quinn ${ }^{1,2}$, T. Ochiya ${ }^{1}$, S. Kanegasaki ${ }^{2}$, F. Takeshita ${ }^{1}$, M. Kodama ${ }^{1}$;

${ }^{1}$ Section for Studies on Metastasis, NCCRI,

${ }^{2}$ Dept of Regenerative Medicine, Effector Cell Institute, Inc., Tokyo, Japan.

Background and Aims: Pancreatic beta cell regeneration is unclear due to poor knowledge of both regenerative stimuli and endogenous responder cells. Previously we demonstrated that streptozocin (STZ)-induced beta cell injury promotes homing and pancreatic morphogenesis of i.p. transplanted ES cells in euglycemic mice. In this study, we demonstrate that similar injury induces pancreatogenesis in renal capsule transplanted embryonic stem cells (ES), in situ, and provide a more detailed analysis of pancreatic cell ontogeny at this site.

Methods: 7 week old female BALB/c nude mice were treated with $175 \mathrm{mg} /$ $\mathrm{kg} \mathrm{STZ}$, and transplanted with $1 \times 10^{\wedge} 5$ ES. Immunohistochemistry was performed on ES-derived pancreatic foci at 21 and 28 days following transplantation using antibodies against stage- and lineage-specific markers. Separately, the ability of transplanted ES to modulate blood glucose levels was measured by intraperitoneal glucose tolerance test in eugylemic mice treated with $200 \mathrm{mg} / \mathrm{kg}$ STZ.

Results: After 21 days, PDX-1+ pancreatic foci in the renal capsule expressed amylase and endocrine hormones. These foci increased in size by day 28 due to acinar and duct proliferation, while endocrine cells remained post-mitotic. PDX-1, Nkx6.1, Ngn3 and ISL-1 were expressed in the endocrine lineage. Unlike embryonic pancreatogenesis, Nkx6.1 was not detected before exocrine/endocrine lineage divergence, and multi-hormone expressing transitional cells were prevalent during endocrine cell ontogeny. Improved glucose tolerance was observed in ES transplanted mice after 4 weeks.

Conclusion: Regenerative stimuli are detectable using renal capsule transplanted ES, and induce both endocrine and exocrine pancreatic lineages resembling embryonic pancreas development.

Supported in part by: Grant-in-Aid for the Third-Term Comprehensive 10-Year Strategy for Cancer Control and Health Science Research Grants for Research on the Human Genome and Gene Therapy from the Ministry of Health, Labor and Welfare of Japan.

\section{0}

Generation of insulin-producing islet-like clusters from human embryonic stem cells

A. Sen Majumdar ${ }^{1}$, J. Jiang ${ }^{1}$, M. Au ${ }^{1}$, B. Cooper ${ }^{1}$, A. Eshpeter ${ }^{2}$, G. Korbutt ${ }^{2}$, G. Fisk ${ }^{1}, \mathrm{~K} . \mathrm{Lu}^{1}$, J. Lebkowski ${ }^{1}$;

${ }^{1}$ Cell Therapy Research, Geron Corporation, Menlo Park, United States, ${ }^{2}$ Department of Surgery, University of Alberta, Edmonton, Canada.

Recent success in pancreatic islet transplantation has energized the field to discover an alternative source of stem cells with differentiation potential to $\beta$ cells. Generation of glucose-responsive, insulin-producing $\beta$ cells from self-renewing, pluripotent human embryonic stem cells (hESCs) has immense potential for diabetes treatment. We report here the development of a novel serum-free protocol to generate insulin-producing islet-like clusters (ILCs) from hESCs grown under feeder-free conditions. In this 36day protocol, hESCs were treated with sodium butyrate (Na-butyrate) and activin A to generate definitive endoderm expressing Sox17 and Foxa2. The endoderm population was then converted into cellular aggregates and further differentiated to Pdx1-expressing pancreatic endoderm in the presence of 
EGF, bFGF and noggin. Soon thereafter, expression of Ptf1a and Ngn3 was detected indicative of further pancreatic differentiation. The aggregates were finally matured in the presence of IGFII and nicotinamide. The temporal pattern of pancreas-specific gene expression in the hESC-derived ILCs showed considerable similarity to in vivo pancreas development and the final population contained representatives of the ductal, exocrine and endocrine pancreas. The hESC-derived ILCs contained 2-8\% human C-peptide positive cells as well as glucagon- and somatostatin-positive cells. We observed as high as 70ng insulin / $\mu \mathrm{g}$ DNA of ILCs, which is higher than the insulin content of human fetal islets. In addition, the hESC-derived ILCs contained numerous secretory granules as determined by electron microscopy and secreted human C-peptide in a glucose-dependent manner.

Geron Corporation

\section{1}

Successful ex vivo differentiation of c-met and c-kit positive pancreatic precursor cells into insulin positive cells

S. T. Gangaram-Panday, M. M. Faas, P. de Vos;

Pathology and Laboratory Medicine, University Medical Center Groningen, The Netherlands.

Background and Aims: The endocrine pancreas has a high capacity for regeneration. Pancreatic precursor cells are suggested to contribute to this regenerative capacity. A few proteins are described to be markers for pancreatic stem cells of which the c-met and c-kit receptors have already been successfully applied. These c-met and c-kit positive cells can be found in both the endocrine and exocrine pancreas. In the present study we investigated whether c-met or c-kit positive cells have the potential to proliferate and differentiate into insulin producing cells after ex vivo manipulation.

Materials and Methods: Single cells of neonatal rat pancreata (collagenase digestion) were used and c-met and c-kit positive cells were sorted and characterised by studying the mRNA levels of c-met, c-kit, insulin, Pdx1, Pax4, Pax6, Beta2, Islet-1, ngn3, Glut-2, glucagon, amylase. C-met and c-kit sorted cells were also cultured in proliferation (containing EGF and bFGF) (10 and 20 days) or differentiation medium (containing betacellulin, ActivinA, GLP-1, and HGF) (10 days) or 10 days on proliferation medium followed by 10 days on differentiation medium, after which the cells were harvested for protein and mRNA expression of c-met and c-kit, insulin, and Pdx-1.

Results: C-met sorted cells expressed c-met, c-kit, Beta2, Pax6, ngn3, insulin, glucagon, and amylase on mRNA levels. After culture for 10 and 20 days in proliferation medium the cells did not express insulin on mRNA or protein level. The number of cells increased 3 fold and the cells remained positive for c-met. Interestingly, c-met positive cells cultured for 10 days in differentiation medium expressed insulin on mRNA level and protein level. The number of cells did not change during culture. When the 10 days proliferation protocol was followed by a 10 days differentiation protocol c-met positive cells did not express insulin (mRNA or protein), but did express Pdx-1 (mRNA), and c-met (mRNA and protein). C-kit sorted cells expressed c-kit, Beta2, Islet-1, Pax4, insulin, amylase on mRNA levels. C-kit positive cells cultured for 10 days in proliferation medium did not express insulin. The number of cells increased 3 fold and cells became c-kit negative on both protein and mRNA level. When cultured for 10 days on differentiation medium the cells were still weakly positive for c-kit, but did not express insulin or c-met on protein level.

Conclusion: Our data suggest that c-met and c-kit positive cells are pancreatic precursor cells, because they express transcription factors involved in differentiation into endocrine cell types, such as Pdx-1, Pax4, Pax6, Beta2, ngn3, and insulin. Furthermore, our data indicate that c-met positive cells and c-kit positive cells might be distinct populations of pancreatic precursor cells, because they differ in the expression of transcription factors involved in endocrine cell formation. C-met positive cells can be multiplied ex vivo and stimulated after proliferation to gain a beta-cell phenotype.

Supported by the QUIDE institute of Rijksuniversiteit Groningen and University Medical Center Groningen

\section{2}

Human pancreatic islet cells interact with their basement membrane laminin through Lutheran glycoprotein

M. Banerjee ${ }^{1}$, I. Virtanen ${ }^{2}$, A. Lukinius ${ }^{3}$, O. Korsgren ${ }^{4}$, M. Hukkanen ${ }^{2}$, Y. Kikkawa ${ }^{5}$, Y. T. Konttinen ${ }^{6}$, T. Otonkoski ${ }^{1}$;

${ }^{1}$ Biomedicum Stem Cell Center, University of Helsinki, Finland, ${ }^{2}$ Institute of Biomedicine/Anatomy, University of Helsinki, Finland, ${ }^{3}$ Department of Genetics and Pathology, University of Uppsala, Sweden, ${ }^{4}$ Department of Clinical Immunology, University of Uppsala, Sweden, ${ }^{5}$ Department of Internal Medicine, Washington University, St. Louis, United States, ${ }^{6}$ Department of Medicine, University of Helsinki, Finland.

Background and Aims: Basement membrane (BM) proteins play an important role in endocrine cell differentiation and function. It has been shown that mouse islets do not have a separate BM but instead interact with endothelial cell BM. The aim of this study was to investigate the BM structure of human islets and experimentally test the adhesion of islet cells to BM proteins.

Materials and Methods: We employed a panel of antibodies against human laminins and their receptors (integrins and Lutheran glycoprotein), as well as other BM components, collagen IV and nidogen, to study their expression by immunofluorescence in surgically obtained human pancreatic samples. Isolated and dissociated human islets were used for short-term in vitro adhesion studies.

Results: We found that human islets possess a unique organization pattern of $\mathrm{BM}$ components, which structurally resembles to the $\mathrm{BM}$ of central nervous system. The peri-islet $\mathrm{BM}$ invaginates into islets with vessels forming a duplex pattern of BM of intra-islet vascular channels, distinct from the regular vascular BM. The peri-islet BM mainly consist of laminin $\alpha 5, \beta 1$ and $\gamma 1$ chains (Lm-511), whereas intra-islet vascular BMs contain laminin chains suggestive of Lms-411/421 and -511/521. This duplex BM organization of the intra-islet vascular channels was further confirmed by collagen IV ( $\alpha$ $1 / 2$ ), nidogen immunostaining and electron microscopy. In vitro adhesion experiments demonstrated that insulin-positive beta cells adhered to Lm511 (5-fold compared to plastic alone). This binding could be inhibited both with an antibody against integrin $\beta 1$ (90\% block) or even more effectively by soluble recombinant Lutheran protein ( $94 \%$ block; mean of five separate experiments).

Conclusions: The present study discloses a hitherto unrecognized islet $\mathrm{BM}$ organization in the adult human pancreas, different from the mouse. Furthermore, we demonstrate an important role for a novel laminin receptor, Lutheran, in the adhesion of human beta cells to the laminin isoform of the islet BM.

\section{3}

Extracellular matrix, Rho-ROCK pathway and beta cell function E. B. Hammar, P. A. Halban; Genetic Medicine and Development, University Medical Center, Geneva, Switzerland.

Background and Aims: Extracellular matrix secreted by 804G cells (804GECM) has been shown previously to increase spreading, induce actin cytoskeleton remodeling and to improve glucose-stimulated insulin secretion (GSIS) of pancreatic beta cells. Actin cytoskeleton dynamics are known to play an important role in beta cell function and three main classes of the small GTPases of the Rho family - Rho, Cdc42 and Rac- are known to play a central role in such dynamics. The function of Rho and its effector ROCK (Rho kinase) in the beta cell has not been studied yet, but there are theoretical reasons for expecting that inhibition of the Rho-ROCK pathway might underlie the beneficial impact of 804G-ECM on beta cells. The aims of this work were to study 1) the impact of $804 \mathrm{G}$-ECM on RhoA activity and 2) the role of Rho and ROCK in the spreading and function of pancreatic beta cells.

Materials and Methods: All experiments were performed with primary rat pancreatic beta cells purified by FACS. Cells were cultured on 804G-ECMcoated dishes or on poly-L-lysine (pLL)-coated dishes (=control) with or without inhibitors: the Rho inhibitor C3 transferase (C3T, $2 \mu \mathrm{g} / \mathrm{ml})$ and the ROCK inhibitors Y-27632 $(50 \mu \mathrm{M})$ and H-1152 $(10 \mu \mathrm{M})$. RhoA activity was analyzed using the RhoA activation assay (G-LISA) from Cytoskeleton, results were normalized to the control (cells on pLL). Insulin secretion was measured 
by radioimmunoassay after static insulin secretion test and expressed as percentage of total insulin content. Actin cytoskeleton remodelling was analyzed by confocal immunofluorescence using phalloidin to stain F-actin. Data are means $\pm \operatorname{SD}(\mathrm{n}=3$ independent experiments).

Results: There was no significant difference in the RhoA activity in cells cultured on $804 \mathrm{G}-\mathrm{ECM}$ as compared to control cells (pLL) after $1 \mathrm{~h}$ of culture. However, after $4 \mathrm{~h}$ of culture, cells on $804 \mathrm{G}-\mathrm{ECM}$ displayed a $25 \pm 1 \%$ lowering of RhoA activity as compared to cells on $\mathrm{pLL}$ ( $\mathrm{p}<0.007$ ). Treatment of cells with Rho or ROCK inhibitors for $24 \mathrm{~h}$ or more led to a change in morphology, with cells appearing more spread and flatter on both pLL and 804G-ECM. Treatment of cells with the ROCK inhibitor Y-27632 (Y) led to a significant increase of glucose-stimulated insulin secretion (GSIS $=\%$ of content secreted at $16.7 \mathrm{mM}$ glucose): pLL control: $6.9 \pm 1.5$ vs. pLL Y: 14.3 $\pm 2.4, \mathrm{p}<0.003$; 804G-ECM control: $18.8 \pm 1.3$ vs. $804 \mathrm{G}$-ECM Y: $38.0 \pm 8.4$, $\mathrm{p}<0.005$. By contrast, basal insulin secretion was not affected by Y-27632: pLL control: $0.74 \pm 0.56$ vs. pLL Y: $1.3 \pm 0.95, p=0.4 ; 804 \mathrm{G}-\mathrm{ECM}$ control: $0.97 \pm$ 0.71 vs. $804 \mathrm{G}-\mathrm{ECM}$ Y: $1.91 \pm 1.25, \mathrm{p}=0.24$. These results were confirmed using H-1152, another ROCK inhibitor. To get insight into mechanisms involved in the positive impact of inhibition of ROCK on GSIS, the impact of ROCK inhibitors on the actin cytoskeleton of cells exposed for 10 minutes to $16.7 \mathrm{mM}$ glucose was visualized by confocal microscopy. The density of the cortical actin cytoskeleton was reduced by the ROCK inhibitors, suggesting that the negative impact of ROCK on GSIS is related to its impact on the state of polymerization of the actin cytoskeleton.

Conclusion: These results indicate that $804 \mathrm{G}-\mathrm{ECM}$ has a negative impact on the RhoA-ROCK pathway and that inhibition of this pathway leads to increased spreading and improved function of pancreatic beta cells. Taken together, these data suggest that the positive impact of the 804G-ECM on GSIS is related to its negative impact on RhoA activity, thereby providing important insight into the molecular basis of outside-in signaling in beta cells and its impact on their function.

Supported by the Swiss National Science Foundation and JDRF

\section{4}

Glucose-dependent stimulation of beta cell proliferation

by extracellular-matrix

G. Parnaud, P. A. Halban;

Department of Genetic Medicine and Development,

University Medical Center, Geneva, Switzerland.

Background and Aims: Extracellular matrix derived from 804G cells (804GECM) induces spreading, improves glucose-stimulated insulin secretion, and increases survival and proliferation of pancreatic beta cells. The aim of the study was to investigate the mechanisms by which the 804G-ECM increases beta cell proliferation.

Materials and Methods: Autofluorescence FACS-sorted rat pancreatic beta cells were plated on poly-L-lysine (PLL) or on matrix produced by $804 \mathrm{G}$ rat bladder carcinoma cell line (804G-ECM). One day later, cells were incubated with BrdU for $24 \mathrm{~h}$ under control conditions or in the presence of different inhibitors: PD98059 (a specific MAPK ERK pathway inhibitor), LY294002 (a phosphatidylinositol 3-kinase (PI3K) inhibitor), diazoxide (which opens K/ATP channels, thereby preventing depolarization and $\mathrm{Ca}^{2+}$ entry) and cyclosporin A (a calcineurin inhibitor). Cell replication was determined by BrdU incorporation and the identity of beta cells confirmed by insulin immunofluorescence. . Insulin secretion was measured by radioimmunoassay after static incubation ( $1 \mathrm{~h} 2.8 \mathrm{mM}$ glucose $+1 \mathrm{~h} 16.7 \mathrm{mM}$ glucose). Data for proliferation are expressed as percentage of BrdU positive beta cells. Data are mean \pm SEM for 3 or more independent experiments.

Results: As expected, rat beta cells spread on 804G-ECM but not on PLL. Rat beta cells proliferated on PLL and 804G-ECM with a significant beneficial effect of the matrix. As previously shown, after 2 days, $\%$ of BrdU positive beta cells was $1.8 \pm 0.4$ vs. $4.6 \pm 0.5$ ( $\mathrm{p}=0.006$ ) on PLL vs. 804G-ECM respectively. The stimulation of beta-cell proliferation was glucose-dependent (PLL at 2.8 $\mathrm{mM}$ glucose $=0.4 \pm 0.2 \mathrm{vs}$. PLL at $11.2 \mathrm{mM}$ glucose $=1.2 \pm 0.2, \mathrm{p}=0.02 ; 804 \mathrm{G}-$ $\mathrm{ECM}$ at $2.8 \mathrm{mM}$ glucose $=0.6 \pm 0.2$ vs. $804 \mathrm{G}-\mathrm{ECM}$ at $11.2 \mathrm{mM}$ glucose $=3.2$ $\pm 0.6, \mathrm{p}=0.005)$. Treatment of beta-cells with LY294002 $(50 \mu \mathrm{M})$ inhibited rat beta-cell proliferation by $90 \%$ on $804 \mathrm{G}-\mathrm{ECM}$ ( $\mathrm{p}=0.0021$ vs. control). By contrast, the MAPK inhibitor PD98059 $(50 \mu \mathrm{M})$ had no effect either on cells attached on PLL or on 804G-ECM. Diazoxide inhibited proliferation induced by the matrix (804G-ECM control $=2.8 \pm 0.5$ vs. $804 \mathrm{G}-\mathrm{ECM}+$ diazoxide $=$ $0.8 \pm 0.2(p=0.01)$. Finally, the $804 \mathrm{G}$-ECM-induced increase of proliferation was significantly inhibited in cells treated with cyclosporin A (804G-ECM control $=2.4 \pm 0.5$ vs. $804 \mathrm{G}-\mathrm{ECM}+$ cyclosporin $=1.0 \pm 0.2 \quad(\mathrm{p}=0.03) \quad$ whereas insulin secretion was not affected by this inhibitor.

Conclusion: Rat beta cells can proliferate in vitro and this is increased significantly by ECM in a glucose-dependent manner. Our results suggest that $\mathrm{PI} 3 \mathrm{~K}-\mathrm{Akt} / \mathrm{PKB}$ and elevated $\left[\mathrm{Ca}^{2+}\right] \mathrm{i}$ (possibly acting via activation of calcineurin) might be involved in this stimulation by $804 \mathrm{G}-\mathrm{ECM}$ whereas the MAP kinase ERK pathway is not.

Supported by the JDRF programme for regeneration of beta cell function 


\section{PS 36 Structural and functional aspects of beta cell regulation}

\section{5}

Characterisation of two $\beta$ cell subpopulations with different functional activity in normal and 48-h glucose-infused Wistar rats

M. Karaca, J. Castel, C. Magnan, C. Tourrel-Cuzin;

Université Paris 7 Denis Diderot, CNRS UMR France.

Background and Aims: We have previously shown that the sialylated form of the neural cell adhesion molecule (PSA-NCAM) was a good tool to sort $\beta$-cell subpopulations according to their functional activity: In high PSANCAM-labelled $\beta$-cells ( $\beta+$-cells), insulin release in response to glucose was significantly higher than that in low PSA-NCAM-labelled $\beta$-cells ( $\beta$--cells). The present study compares some aspects of the intracellular mechanisms that could explain this differential response to glucose in these two $\beta$ cell subpopulations in normal rat (control rat) but also in an endocrine pancreas plasticity model: the 48-h glucose-infused rat (HGHI rat) with increased $\beta$-cell mass and function. At this end, we examined the handling of intracytoplasmic $\mathrm{Ca} 2+$ concentrations $([\mathrm{Ca} 2+] \mathrm{i})$, the cAMP content and the response of these cells to various secretagogues.

Materials and Methods: Pancreatic islet $\beta$-cells from Wistar rats (control and HGHI) were sorted by flow cytometry either with or without PSA-NCAM labelling. Insulin release was measured in sorted $\beta$-cell subpopulations by a perifusion technique in a system allowing concomitant recording of [Ca2+]i movements by microfluorimetry. Insulin content was determined by RIA. Using batch-incubated $\beta$-cells, cAMP content and/or insulin release in response to glucose (5.5 and $16.7 \mathrm{mM}$ ) and/or 10nM GLP-1, 19mM arginine, $10 \mathrm{mM}$ leucine and $5 \mathrm{mM}$ dibutyryl cAMP were studied. The effect of $50 \mathrm{mM}$ $\mathrm{KCl}$ was also assessed in perifusion experiments.

Results: In control $\beta+$-cells, insulin release in response to $16.7 \mathrm{mM}$ glucose was highly increased compared to $\beta$--cells and was correlated with an increase in $[\mathrm{Ca} 2+] \mathrm{i}$ whereas there was almost no increase in $\beta$--cells. In HGHI $\beta$ cell subpopulations, the rise of [Ca2+]i in response to $16.7 \mathrm{mM}$ glucose is not bigger than that described in control $\beta$-cells despite a clear increase of insulin response to the same stimuli. The impaired functional activity in control or HGHI $\beta$--cells can't be explained by a decrease in their insulin content. The cAMP accumulation in control $\beta+-$ cells, in response to 5.5 and $16.7 \mathrm{mM}$ glucose with GLP-1 was increased compared to that with glucose alone. Insulin release in response to $16.7 \mathrm{mM}$ glucose was also potentiated in this population by adding GLP-1. In HGHI rats, glucose infusion increased glucose and/or GLP-1 effects in $\beta+-$ cells. Nevertheless, control or HGHI $\beta$ -cells were not responsive to any glucose or GLP-1 stimulation. In response to $16.7 \mathrm{mM}$ glucose with $5 \mathrm{mM}$ db-cAMP, insulin release was significantly increased in $\beta+$-cells and, in part, restored in $\beta$--cells in both control and $\mathrm{HGHI}$ rats. Addition of $\mathrm{KCl}$ at the end of perifusion experiments showed that $\beta$--cells were capable to secrete insulin in the same extent as $\beta+$-cells. Moreover, arginine elicited same insulin release in both $\beta+$ and $\beta$ - populations whereas leucine showed a profile similar to glucose.

Conclusion: Alteration of the functionality of $\beta$--cells could be explained by a blockage in the steps linking glucose metabolism to the insulin release process. The analysis of glucose-stimulated insulin release characteristics in $\beta$-cell subpopulations with a different secretory activity is a prerequisite to identify factors able to ameliorate the functional status of $\beta$-cells with low secretory capacities such as in advancing type 2 diabetes.

\section{6}

Effect of low and high glucose concentration on the insulin secretory cell line INS-1

R. D. Fernández, B. Torrejon-Escribano, J. Blasi;

Department of Pathology and Experimental Therapeutics,

University of Barcelona-IDIBELL, Spain.

Background and Aims: Chronic exposure of pancreatic islets to elevated glucose levels has been associated to $\beta$-cell dysfunction and the lost the glucose-stimulated insulin secretion. The final steps in insulin secretion are governed by a series of proteins that mediate the correct docking, priming and fusion of insulin containing secretory granules to plasma membrane.
In the present work, the expression of proteins related to insulin secretion was studied in the INS-1 insulin secretory cell line incubated for 1, 3 and 7 days in the presence of 3.3, 11.1 or $20 \mathrm{mM}$ glucose.

Materials and Methods: The protein expression and distribution were analyzed by immunofluorescence and confocal microscopy, ELISA, western blot and Real Time-PCR (RT-PCR).

Results: In addition to insulin, actin and connexin 36 (which has been shown to play a regulatory role in various $\beta$-cell functions), the rest of proteins analyzed comprised the SNARE proteins SNAP-25, syntaxin-1, VAMP-2 and cellubrevin and a series of proteins directly or indirectly involved in the regulation of insulin exocytosis: Munc 18, synaptotagmin I, complexin and munc 13. All together, the results show a consistent decrease in the expression of several proteins, in addition to insulin, with the increase of glucose concentration and time of incubation, these proteins include connexin 36, synaptotagmin I, syntaxin 1 and VAMP-2. Exceptionally, western blot analysis showed a slight but significant increase in the expression of SNAP-25 along the time and the increase of glucose concentration in the media. Low glucose concentration produced a consistent increase in the expression of connexin 36 and the formation of cell aggregates. Munc 18, munc 13, complexin and actin did not show any evident change in their expression in the conditions tested. Additionally, the distribution and colocalization of selected pairs of the above proteins were analyzed by confocal microscopy and the results are discussed. Conclusion: Prolonged exposure of INS-1 cells to high glucose concentration glucose produces evident changes in the expression of proteins directly involved in the secretion of insulin. Low glucose concentrations produce the increase in connexin 36 expression and the formation of cell aggregates, while high glucose concentrations had the opposite effect. These results suggest that elevated concentrations of glucose might disorganize islet cyto-architecture by impairing the formation of both functional cell contacts and cell aggregation. Supported by a grant from MEC of the Spanish Government

\section{7}

Impact of over-expression of iduronate-2-sulfatase (ids) on glucosestimulated insulin secretion

S. Piquer ${ }^{1}$, S. Casas ${ }^{1,2}$, A. Nadal ${ }^{3}$, I. Quesada ${ }^{3}$, A. Novials ${ }^{2}$, R. Gomis ${ }^{1}$; ${ }^{1}$ Laboratory of Experimental Diabetes, Institut d'Investigacions Biomediques August Pi i Sunyer (IDIBAPS), Barcelona,

${ }^{2}$ Institute of Diabetes, Sardà Farriol Foundation, Barcelona, Spain,

${ }^{3}$ Bioengineering Institute, Miguel Hernandez University, Elche, Spain.

Background and Aims: Iduronate-2-sulfatase (IDS) is a lysosomal enzyme expressed in pancreatic islets responsible for degradation of proteoglycans, such as perlecan and dermatan sulfate. Previous findings of our group demonstrated the involvement of IDS in the normal pathway of lysosomal degradation of secretory peptides suggesting a role of this enzyme in $\beta$-cell secretory functionality. The present work aims to characterise the effect of IDS over-expression on insulin release.

Materials and Methods: INS1E cells were transiently transfected with a pcDNA 3.1 Hygro(-) construct encoding human IDS fused with the 5' end of a c-myc epitope tag. Transfected cells with empty vector were used as controls. Western blot and immunofluorescence assays were performed to verify successful transfection. Total RNA was extracted using a mono-phasic solution of phenol and guanidine isothiocyanate and insulin 1 and 2 (INS1 and INS2) mRNA levels were estimated by quantitative real-time PCR. Release of insulin was measured during static incubation of transfected cells for $30 \mathrm{~min}$, and quantified by ELISA. Exocytosis was analyzed at the singlecell level by monitoring fluorescence changes from INS1-E cells exposed to the cell-impermeable dye FM1-43. The number of vesicles $/ \mu \mathrm{m}^{2}$ and the $\%$ of vesicles in membrane respect total ones were quantified from fifty cells selected at random from transmission electron microscope images.

Results: Successful transfections were confirmed by western blot and immunofluorescence using an antibody against the c-myc epitope tag. Overexpression of hIDS potentiated the secretory response at $22.7 \mathrm{mM}$ glucose compared to controls $(+69.15 \%, p<0.01)$ leading to a reduction on insulin content $(-54.54 \%, p<0.05)$. No significantly changes were observed at $2.5 \mathrm{mM}$ or $0 \mathrm{mM}$ glucose. Moreover, hIDS expressing cells showed no changes in insulin mRNA levels (arbitrary units) (INS1: 2.06 vs 1.81, INS2: 1.85 vs 1.70, n.s.) compared to controls. These results indicated that the increase in insulin secretion was not due to an effect of hIDS on insulin expression. Furthermore, ultramorfological analysis suggested a major number of vesicles at the membrane level in hIDS transfected cells compared to controls. Quantitation 
of vesicles demonstrated an increase in this value in hIDS transfected cells $(68.66 \%$ vs $59.97 \%, p<0.01)$ as well as a decrease in total vesicles $/ \mu \mathrm{m}^{2}(0.82$ vs $0.62, p<0.05$ ). Preliminary results indicated a higher exocytotic response at the single-cell level in hIDS transfected cells respect to control ones. Conclusion: Over-expression of hIDS on INS1E cells leads to the enhancement of glucose-stimulated insulin secretion by a mechanism that implies the activation of exocytosis processes.

\section{8}

Expression and functional activity of the putative endogenous imidazol(in)e receptor ligand, imidazole-4-acetic acid ribotide, in the endocrine pancreas

A. R. Oknianska ${ }^{1}$, V. L. Fredrich Jr², G. P. Martinelli², G. R. Holstein², G. D. Prell ${ }^{2}$, N. G. Morgan ${ }^{1}$;

${ }^{1}$ Institute of Biomedical and Clinical Science, Peninsula Medical School, Plymouth, United Kingdom, ${ }^{2}$ Department of Neurology, Mt Sinai Medical School, New York, United States.

Introduction: A variety of ligands with an imidazoline structure cause the glucose-dependent stimulation of insulin secretion from pancreatic $\beta$-cells and this action has been attributed to their binding to $\mathrm{I}_{3^{-}}$"imidazol(in)e" receptors. It has been proposed that they may reproduce the actions of a putative endogenous ligand, imidazole-4-acetic acid ribotide (IAA-RP) recently characterised in the central nervous system. In the present study, we have investigated the actions of IAA-RP on insulin secretion from islets of Langerhans and have studied the distribution of this ligand in pancreas to establish whether it might serve as a neuroregulator controlling insulin secretion.

Methods: Isolated rat and human islets were prepared by collagenase digestion of the pancreas and exposed to IAA-RP in vitro. Insulin secretion was measured by radioimmunoassay. The distribution of IAA-RP in normal rat and human pancreas was analysed by immunohistochemistry using a highly specific antiserum raised against synthetic IAA-RP.

Results: IAA-RP and the immediate product of its dephosphorylation, IAA$\mathrm{R}$, each exerted a dose-dependent stimulation of insulin secretion from both rat and human islets over the range $0.01-10 \mu \mathrm{M}$. The response to each agent was biphasic with concentrations of IAA-RP in the range 10-100nM eliciting a marked rise in insulin secretion from human islets incubated in $6 \mathrm{mM}$ glucose $(140 \pm 15 \%$ and $230 \pm 25 \%$ vs control, at $10 \mathrm{nM}$ and $100 \mathrm{nM}$ respectively; $\mathrm{p}<0.01)$ while stimulation was lost at higher concentrations (98 $\pm 20 \%$ vs control at $10 \mu \mathrm{M}$ IAA-RP). IAA-R increased secretion to maximum of $165 \pm 18 \%$ of control $(\mathrm{p}<0.01)$ at $100 \mathrm{nM}$. A highly specific anti-IAA-RP serum (which does not recognise IAA-R) labelled a sub-set of neurons in rat olfactory bulb, confirming the presence of IAA-RP within the CNS. Examination of sections of rat and human pancreas revealed the presence of specific IAA-RP immunoreactivity by both immunohistochemistry and immunofluoresence. The ligand was localised predominantly within islets of Langerhans but not all islets within a given section of pancreas stained positively for IAA-RP. Staining was only rarely seen outside the islets. Antibody binding was completely displaced by pre-incubation with excess ligand, confirming the specificity of labelling.

Conclusions: The putative endogenous imidazol(in)e receptor ligand, IAA$\mathrm{RP}$, is present in human and rat islets of Langerhans where it may serve as a local neuroregulator involved in the control of insulin secretion. Supported by Wellcome Trust

\section{9}

\author{
AMPK activation increases the expression and activity of PPAR $\alpha$ \\ in INS-1 cells \\ H. Guo ${ }^{1,2}$, Y. Sun ${ }^{1}$, W. Xin ${ }^{3}$, L. C. Wang ${ }^{3}$, J. J. Zhao ${ }^{4}$, L. Gao' ${ }^{1}$; \\ ${ }^{1}$ Centre Laboratory, Shandong Provincial Hospital,Shandong University, \\ ${ }^{2}$ Institute of Pharmacology, School of Medicine, Shandong University, \\ ${ }^{3}$ Centre Laboratory, Shandong Provincial Hospital, \\ ${ }^{4}$ Endocrinolgy and Metabolism,, Shandong Provincial Hospital, \\ Shandong University, Jinan, China.
}

Background and Aims: Peroxisome proliferator-activated receptor alhpa $(\mathrm{PPAR} \alpha)$ is a ligand-activated transcription factor involved in key metabolic pathways, such as lipid metabolism. In $\beta$ cells, PPAR $\alpha$ is reported to major contribute to fatty acid oxidation. Recently, several studies has addressed that AMP-activated protein kinase (AMPK), an cellular energy sensor that is responsible for cellular glucose and lipid metabolism, may affect PPAR $\alpha$ expression in peripheral tissues, such as skeletal muscle. Of these studies, most models were made with transgenic mice or with transfected-gene cells cultured in high-glucose or high-lipid conditions, because PPAR $\alpha$ occurs at relative very small quantities in tissues or cell lines under physiological status, which results in much difficulty to observe the PPAR $\alpha$ change in protein level. Thus, so far, the detection of PPAR $\alpha$ in physiological cells is limited to gene transcriptional levels, while data concerning protein levels and activity of PPAR $\alpha$ are very few. The present study was completed in physiological status, and aimed to investigate effects of AMPK on PPAR $\alpha$ in the levels of transcriptional, protein and binding activity with DNA via AMPK activator and inhibitor, mimicing AMPK activation and inactivation.

Materials and Methods: INS-1 cells were cultured in RPMI 1640 medium, and treated with and without $0.5 \mathrm{mM}$ AICAR (AMPK activator), in the presence and absence of $10 \mu \mathrm{M}$ Compound C (AMPK inhibitor). Treatment for 8,12 or 24 hours, total RNA was extracted for determining the levels of PPAR $\alpha$ mRNA by real-time PCR, protein was gained for analysis the expression of PPAR $\alpha$ protein by immunoprecipitation, and nuclear protein was extracted for detecting the PPAR $\alpha$ binding activity with DNA by PPAR $\alpha$ Complete Transcription Factor Assay Kit, respectivitly.

Results: Compared to control, PPAR $\alpha$ mRNA was increased by $52.25 \%$ (p $<0.05$ ) in the cells treated with AICAR alone for 8 hours, but this increase was oppressed and reduced by $33.88 \%$ in the cells treated with AICAR and Compound $\mathrm{C}$ together relative to AICAR alone. However, at 24 hours, we failed to detect any significant change of PPAR $\alpha$ mRNA levels in the observed cells, which could result from the bio-half life of AICAR in our experimental conditions. Consistent with the PPAR $\alpha$ mRNA changes at 8 hours, IP experiment showed a strong stained band $(p<0.05)$ representing PPAR $\alpha$ protein in the cells in the present of AICAR alone, while the weak band ( $\mathrm{p}$ $<0.05)$ was obtained in the present of AICAR and compound C together. Interestingly, the increase in PPAR $\alpha$ binding activity with PPRE sequence induced by AICAR did not change at 8 hours until 12 hours treatment, which was in agreement with the previous study showing that the change of DNA binding activity occurred later than its protein. AMPK activation enhanced the PPAR $\alpha$-DNA binding activity by $38.5 \%$ relative to control, while compound C -induced AMPK inactivation reduced the binding activity by $17.8 \%$ as compared with AICAR alone treatment.

Conclusion: AMPK activation can increase the PPAR $\alpha$ in gene transcriptional level, which leads to the increase in both levels of protein expression and binding activity with DNA in INS-1 cells under physiological conditions. Supported by National Natural Science Foundation of China and Foundation of Weisheng Ting Shandong Province

\section{0}

Influence of glucose and $\mathrm{Ca}^{2+} / \mathrm{Calmodulin}$-dependent protein Kinase II (CaMKII) on expression and activity of NAD(P)H-Oxidase in INS-1 rat insulinoma cells

M. A. Osterhoff ${ }^{1,2}$, K. Pütz ${ }^{1}$, E. Arslan ${ }^{1}$, F. Isken ${ }^{1,2}$, M. O. Weickert ${ }^{1,2}$, A. F. H. Pfeiffer ${ }^{1,2}$;

${ }^{1}$ Clinical Nutrition, German Institute of Human Nutrition, Nuthetal, ${ }^{2}$ Endocrinology, Diabetes and Nutrition, Charité - University Medicine Berlin Campus Benjamin Franklin, Berlin, Germany.

Background and Aims: The Incidence of type 2 diabetes mellitus (T2DM) increases world-wide with a trend to reduced age of manifestation. T2DM is characterized by insulin-resistance, an initially relative peripheral insulin deficiency, and a progressive loss of $\beta$-cell mass. This finally leads to an absolute lack of insulin. Chronically elevated glucose concentrations cause a damage of $\beta$-cells, partially by production of reactive oxygen species (ROS) in mitochondria but also by a $\mathrm{NAD}(\mathrm{P}) \mathrm{H}$-oxidase subtype expressed in $\beta$ cells. Goal of the present work was, to verify the expression of $\mathrm{NAD}(\mathrm{P}) \mathrm{H}-$ oxidase in INS-1 cells and to investigate the glucose- and CaMKII-dependent expression and activation of $\mathrm{NAD}(\mathrm{P}) \mathrm{H}$-oxidase.

Materials and Methods: RNA from INS-1 rat insulinoma and INS-W12 cells, where the expression of CaMKII is suppressed nearly completely, was extracted with Trizol(R) (Sigma). The expression of $\alpha$-tubulin (standard) and $\mathrm{NAD}(\mathrm{P}) \mathrm{H}$-oxidase subunits p65mox and gp91phox were measured by real-time PCR on an ABI 7900HT (“TaqMan"). The cells were stimulated for 
4, 24, 48 and 72 hours with $0,5,7.5,1116.5$ and $33 \mathrm{mmol} / \mathrm{l}$ glucose. The superoxide-production was measured by lucigenin- or DCF/DA-assays (Molecular Probes).

Results: The investigation of gene expression showed that p65mox but not gp91phox was expressed in INS-1 cells. In INS-W12 cells p65mox was expressed but only $4 \%$ of the amount expressed in INS-1 cells. After 48 hours with increased glucose concentrations $(16.5 \mathrm{mmol} / \mathrm{l})$ the expression of p65mox tended to higher values in INS-1 cells compared to the expression with glucose concentrations of 2 or $5 \mathrm{mmol} / \mathrm{l}$. After 72 hours the increase in expression was highly significant ( $\mathrm{p}=0.005$ and $\mathrm{p}=0.002$, respectively). Lucigenin- as well as DCF/DA-assays showed that the superoxide-production of $\mathrm{NAD}(\mathrm{P}) \mathrm{H}$-oxidase (suppression of the mitochondrial fraction by rotenone) increases with the glucose concentration. Up to a glucose concentration of $16.5 \mathrm{mmol} / \mathrm{l}$ the production of superoxide by $\mathrm{NAD}(\mathrm{P}) \mathrm{H}$-oxidase was predominant, while the mitochondrial part (suppression of the $\mathrm{NAD}(\mathrm{P}) \mathrm{H}$ oxidase fraction by DPI) was elevated using $33 \mathrm{mmol} / \mathrm{l}$ glucose. In INS-W12 cells the NAD(P)H-oxidase portion of superoxide production was barely detectable and resembled that of INS-1 cells when suppressed with DPI Conclusion: Experiments with CaMKII-suppressed INS-W12 cells demonstrated that the expression of the $\mathrm{NAD}(\mathrm{P}) \mathrm{H}$-oxidase subtype p65mox depended on the presence of CaMKII. The glucose stimulation experiments point to a predominant superoxide production by $\mathrm{NAD}(\mathrm{P}) \mathrm{H}$-oxidase in INS$1 \beta$-cells under physiological conditions. Only under super physiological glucose concentrations the mitochondrial superoxide production gained higher importance.

\section{1}

Live imaging of glucose uptake and metabolism in insulin-producing MIN6 cells by a fluorescent sensor

M. T. Kaminski, S. Lenzen, S. Baltrusch;

Institute of Clinical Biochemistry, Hannover Medical School, Germany.

Background and Aims: Glucose plays a key role in pancreatic beta cell signal transduction. After the uptake of the sugar via the low affinity glucose transporter GLUT2 inside the cell, glucose is phosphorylated in the first and rate limiting step of glycolysis by glucokinase. In the subsequent metabolism the stimulus for insulin secretion is generated through an increase in the ATP/ADP ratio. Furthermore, glucose mediates intracellular changes in protein expression and modification and chronically high glucose has been considered to facilitate development of type 2 diabetes. While different indirect techniques have been used to analyze changes in the intracellular glucose concentration, real time measurements in living cells are so far not available. The aim of this study was to monitor the intracellular glucose concentration in dependence upon metabolic changes.

Materials and Methods: Intracellular glucose concentration changes were analyzed using FRET (fluorescence resonance energy transfer) based sensors (FLIPglu) with specificity for glucose by fluorescence microscopy. Insulin-producing MIN6 cells or COS-7 as established control cells were transfected with FLIPglu. Thereafter, cells were incubated at various glucose concentrations either by static incubation or in a perifusion chamber. FRET was calculated as EYFP/ECFP emission intensity ratio.

Results: FLIPglu sensors for intracellular sensing of glucose dynamics were expressed in COS-7 and insulin-producing MIN6 cells. FLIPglu sensors exhibited a correlation between the glucose concentration and EYFP/ ECFP ratio. Static incubation at different glucose concentrations revealed a concentration-dependent shift in the EYFP/ECFP ratio. A 3-fold change in the ratio was observed at a physiologically relevant concentration range of 1 to $25 \mathrm{mmol} / \mathrm{l}$ glucose in the incubation medium. Thus, the intracellular glucose concentration can be calculated in living insulin-producing cells. In addition, these changes could be resolved temporarily on a single cell level. Upon perifusion of glucose deprived cells with 1, 5, 10 or $25 \mathrm{mmol} / \mathrm{l}$ glucose a rapid change in the EYFP/ECFP ratio could be detected, indicating a high capacity of MIN6 cells for glucose uptake via the GLUT2 glucose transporter from the medium. Upon removal of glucose the effects were reversible within $5 \mathrm{~min}$. This opens the possibility to elucidate the regulation of glucose homeostasis in insulin-producing MIN6 cells.

Conclusion: FLIPglu sensors could be successfully established to monitor in real time changes in the intracellular glucose concentration of insulinproducing MIN6 cells. To investigate effects of long-term glucose exposure with respect to glucose toxicity FLIPglu sensors are a promising tool to verify the dependency of the intra- to extracellular glucose concentration.

\section{2}

The effect of pseudoislet formation on insulin production in beta cells in vitro

T. Kopska, V. Fürstova, J. Kovar;

Division of Cell and Molecular Biology and Centre for Research of Diabetes, Metabolism and Nutrition, Third Faculty of Medicine, Charles University in Prague, Czech Republic.

Background and Aims: After the dispersion of islet cells the ability of beta cells to produce insulin is impaired. But after reorganisation into pseudoislets beta cells regain cell-to-cell contact important for their function and their ability to secrete insulin improves. Pseudoislets can be formed from isolated beta cells as well as from cells of beta cell lines. The aim of this study was to test the effect of pseudoislet formation and pseudoislet growth on insulin secretion by beta cells in vitro in the time course of cultivation.

Materials and Methods: Beta cells of the line MIN6 were cultured in DMEM medium containing $22 \mathrm{mM}$ glucose supplemented with $15 \%$ FBS. Cell culture plates for suspension cells (Sarstedt, Germany) were used for the induction of pseudoislet formation. The amount of insulin secreted into the culture medium during $1 \mathrm{~h}$ was determined by Insulin Mouse ELISA kit (DRG Diagnostics).

Results: The number of pseudoislets formed depended on the number of cells seeded. Pseudoislet number to seeded cell number ratio was highest for cell densities ranging $50-100 \times 10^{3}$ cells $/ \mathrm{ml}$ per well. The overall number of cells per well did not increase with time after induction of pseudoislet formation. However, number of cells per pseudoislet grew fast from about $200 \times 10^{3}$ cells/pseudoislet on the 2 nd day to about $600 \times 10^{3}$ cells/pseudoislet on the 6 th day after the induction. Then the growth ceased and pseudoislets had about $800 \times 10^{3}$ cells/pseudoislet on the 10th day. On the 2 nd day after the induction the insulin secretion by the cells in pseudoislets $\left(48.9 \pm 20.3 \mathrm{ng} / 10^{5}\right.$ cells $)$ was comparable to the secretion by the cells growing in monolayer $(57.5 \pm 5.8$ $\mathrm{ng} / 10^{5}$ cells). On the 4 th day the insulin secretion by the cells in pseudoislets increased approximately 2 -fold $\left(117.3 \pm 13.1 \mathrm{ng} / 10^{5}\right.$ cells) compared to 2 nd day. The highest insulin secretion $\left(167.1 \pm 20.6 \mathrm{ng} / 10^{5}\right.$ cells) was found on the 6th day after the induction. After the 6th day, i.e. on the 8th and 10th day the insulin secretion stayed on slightly lower levels (131.0 \pm 6.2 and $128.2 \pm 5.3$ $\mathrm{ng} / 10^{5}$ cells)

Conclusion: The ability of beta cells (MIN6) to secrete insulin in vitro gradually increases with time after the induction of pseudoislet formation and reaches its maximum within six days. The insulin secretion correlates with the growth of pseudoislet cell number. The secretion is near maximum when the pseudoislets have reached their full size. Our knowledge concerning insulin secretion by pseudoislets in vitro during the course of cultivation should be useful for the consideration of their involvement in transplantation.

Supported by research programme MSM0021620814 Third Faculty of Medicine, Charles University

\section{3}

Characterisation of islet morphology in pancreatic tissue from non-diabetic organ donors

C. Reers ${ }^{1}$, S. Erbel ${ }^{1}$, I. Esposito ${ }^{2}$, B. Schmied ${ }^{3}$, M. W. Buechler ${ }^{3}$, P. P. Nawroth ${ }^{1}$, R. A. Ritzel';

${ }^{1}$ Department of Internal Medicine I, University of Heidelberg, ${ }^{2}$ Institute of Pathology, University of Heidelberg,

${ }^{3}$ Department of Surgery, University of Heidelberg, Germany.

Background and Aims: Pancreatic beta-cell mass is an important parameter for overall islet function in the context of diabetes mellitus. Since human pancreatic tissue is difficult to obtain analysis of islet morphology is usually performed in autopsy tissue, which prior to fixation might be damaged by ischemia and autolytic processes. In contrast, pancreatic tissue from organ donors is processed directly after explantation with minimal ischemia time. Therefore we characterized islet morphology in human pancreatic tissue from organ donors and compared it to beta-cell volume in autopsy tissue.

Materials and Methods: Paraffin embedded pancreatic tissue (head, body and tail region) from $\mathrm{n}=14$ non-diabetic organ donors was sectioned and stained for insulin, glucagon and the replication marker Ki-67. Beta-cell and alpha-cell volume were determined by morphometric analysis and correlated to age and body-mass index (BMI). Beta-cell volume was compared to 
previously established beta-cell volume in autopsy tissue of non-diabetic humans ( $\mathrm{n}=48)$.

Results: Organ donors were $46.6 \pm 17.2$ years old (range $7-63$ years), BMI $25.5 \pm 4.3 \mathrm{~kg} / \mathrm{m}^{2}$ (range $17.1-33.1 \mathrm{~kg} / \mathrm{m}^{2}$ ). Islet architecture showed a typical location of beta-cells in the core and alpha-cells in the periphery of the islets. Mean beta-cell volume was $2.3 \pm 1.0 \%(0.4-4.1 \%)$, alpha-cell volume was $0.6 \pm 0.6 \%(0.1-2.0 \%)$. There were no regional differences in alpha- and beta-cell volume $(\mathrm{p}=\mathrm{n}$.s. for head vs body vs tail). Beta-cell volume was comparable in donor and autopsy pancreata $(2.3 \pm 1.0$ vs $2.3 \pm 0.3 \%$; $\mathrm{p}=\mathrm{n}$. s.). There was no relationship between alpha- or beta-cell volume with age or $\mathrm{BMI}$ of organ donors. Beta-cell replication was generally low $(0.2 \pm 0.2 \mathrm{Ki}-67$ positive cells/islet), regression analysis showed an age-dependent decrease of beta-cell replication $\left(\mathrm{r}^{2}=0.63, \mathrm{p}<0.001\right)$.

Conclusion: In the present group of non-obese and non-diabetic subjects alpha- and beta-cells are evenly distributed throughout the pancreas and overall alpha- and beta-cell volume is not affected by age or BMI. The present data provides evidence that in humans beta-cell replication becomes less important for islet turnover with aging. Finally, pancreatic tissue from donor organs and obtained at autopsy is equally suitable for analysis of beta-cell volume.

Supported by DFG

\section{PS 37 The signal transduction of cytokine-induced beta cell degeneration}

\section{4}

Gene modules and networks involved in cytokine-mediated death in human pancreatic islets

B. Kutlu ${ }^{1}$, D. Baxter ${ }^{1}$, D. Burdick ${ }^{1}$, A. K. Cardozo ${ }^{2}$, N. Goodman ${ }^{1}$ D. L. Eizirik ${ }^{2}$, N. Welsh ${ }^{3}$, L. Hood';

${ }^{1}$ JDRF Center for Bioinformatics, Institute for Systems Biology, Seattle, United States, ${ }^{2}$ Lab. of Experimental Medicine, Universite Libre de Bruxelles, Belgium, ${ }^{3}$ University of Uppsala, Dept. of Medical Cell Biology, Sweden.

Background and Aims: Type 1 Diabetes (T1D) is an autoimmune disease that leads to destruction of beta cells of the pancreatic islets of Langerhans. It is well established that cytokines are cytotoxic to pancreatic islets in vitro. With the aim of identifying networks and modules activated in response to cytokines, we performed a detailed transcriptome analysis of IL-1 $\beta$ and IFN$\gamma$ stimulated human islets.

Materials and Methods: High quality human islets $(\mathrm{n}=2)$ were exposed to IL- $1 \beta(50 \mathrm{U} / \mathrm{ml})+\mathrm{IFN}-\gamma(1000 \mathrm{U} / \mathrm{ml})$ for $48 \mathrm{~h}$ and gene expression was detected with Massively Parallel Signature Sequencing (MPSS: a signature sequencing technique that is able to detect low abundance transcripts). MPSS analysis of untreated islets was performed previously. A different set of human islet samples (from 3 separate donors, average purity $>90 \%$ and beta cell percentage $>58 \%$ ) were either left untreated or exposed to IL-1 $\beta$ $(50 \mathrm{U} / \mathrm{ml})+\mathrm{IFN}-\gamma(1000 \mathrm{U} / \mathrm{ml})$ for $1,2,4,8,12,24,36,48,60,72,84$ and $96 \mathrm{~h}$ and gene expression was detected at each time point with HGU133 plus 2.0 Affymetrix microarrays. Real Time PCR (RT-PCR) was performed on select transcripts and time points to complement and validate the MPSS and microarray results.

Results: Time series microarray analysis identified 2491 differentially expressed genes in islets in response to cytokine treatment (linear regression model analysis, $\mathrm{p}<0.05$ - corrected for multiple testing). In the MPSS dataset, a total of 6893 genes were expressed at a minimum level of 5 transcripts per million (tpm) in human islets after $48 \mathrm{~h}$ of cytokine treatment. Many of these genes (4624) were detected at less than $50 \mathrm{tpm}$ and 438 are not included on the Affymetrix microarrays. RT-PCR analysis of select transcripts confirm that MPSS-detected genes such as CD99, SAA1 and KREMEN are regulated by cytokines. Model based clustering of time series data resulted in 26 clusters that represent the main trends observed in the data. Two clusters contained most of the 22 significantly regulated chemokines in response to cytokines: all but 2 (CCL26, CXCL12) were up-regulated in an early and robust manner (2-9 fold change on log2 scale). Gene Ontology (GO) enrichment analysis of cluster members indicate that some strongly induced clusters are enriched in genes involved in apoptosis namely, CASP10, TRAF1, I-FLICE, PUMA etc. All these genes are putative targets of 169 significantly expressed transcription factors that form the backbone of the gene regulatory networks involved in beta cell apoptosis. Screening for a molecular interaction network to identify active subnetworks by integrating protein-protein interactions and gene expression data indicates the presence of a gene module that contains NF- $\mathrm{\kappa B}$ and STAT1 and their interacting partners.

Conclusion: We have identified the early changes in the gene networks active in human islets under cytokine assault. The detailed time course data allows us to refine the existing model of cytokine-induced beta cell death and to increase our understanding of the gene regulatory networks involved in this process.

Supported by Juvenile Diabetes Research Foundation

\section{5}

Characterisation of the pattern of gene expression induced by IL-1 $\beta$ and TNF- $\alpha$ in insulin-producing INS-1E cells

F. Ortis, N. Naamane, P. Pirot, A. Y. Kreins, D. L. Eizirik; Laboratory of Experimental Medicine, Université Libre de Bruxelles, Belgium.

Background and Aims: Cytokines such as IL- $1 \beta$ and TNF- $\alpha$ play an important role in the autoimmune destruction of pancreatic beta-cells in 
type 1 diabetes. IL-1 $\beta$ has a more pronounced pro-apoptotic effect in beta cells and insulin producing INS-1E cells than TNF- $\alpha$. Both cytokines induce the transcription factor NF- $\kappa \mathrm{B}$, but recent observations suggest that NF- $\kappa \mathrm{B}$ blocking is more effective in preventing IL- $1 \beta+$ IFN- $\gamma$ - than TNF- $\alpha+$ IFN$\gamma$-induced beta-cell apoptosis. We presently compared cell death and the pattern of NF- $\mathrm{\kappa B}$ activation and gene expression induced by IL- $1 \beta$ and TNF$\alpha$ in INS-1E cells in order to clarify the reasons behind the different effects of these cytokines on beta-cell apoptosis.

Material and Methods: INS-1E cells were exposed to either IL-1 $\beta$ (100U/ $\mathrm{ml})$ or TNF- $\alpha(1000 \mathrm{U} / \mathrm{ml})$ in time course experiments. Cell viability was evaluated by Hoechst 342 and propidium iodide, in the presence or absence of cycloheximide $(1 \mu \mathrm{g} / \mathrm{ml}-2 \mathrm{~h}$ pre-exposure). NF- $\kappa B$ translocation to the nucleus was measured by immunostaining using an anti-p65 antibody. RNA extracted from these cells was used in real-time RT-PCR and microarray analysis. Microarray analysis was performed using the ApoChip, a targeted oligonucleotide array designed by our group and containing 600 rat genes putatively implicated in beta cell dysfunction and death. Microarray experiments were replicated with dyes swapped in each hybridisation. Results of three independent array experiments were calculated as mean + standard error. mRNA expression was considered as modified by cytokines when they had a $\geq 1.2$-fold variation and $\mathrm{p}<0.025$ (t-test) in at least one time point as compared to control cells.

Results: Blocking of proteins synthesis by cycloheximide promoted TNF- $\alpha$ induced INS-1E cell apoptosis after $24 \mathrm{~h}$ (apoptotic index of $4.9 \pm 1.2 ; \mathrm{P}<0.05$ vs. control; $n=6$ ), while it did not affect IL-1 $\beta$-induced apoptosis (apoptotic index of $1.5 \pm 0.7$; N.S.; $\mathrm{n}=6$ ). IL-1 $\beta$ induced an earlier translocation of NF- $\kappa B$ to the nucleus as compared to TNF- $\alpha(77.4 \pm 4.2 \%$ vs $37 \pm 8.3 \%$ of nuclear positive cells for respectively IL- $1 \beta$ and TNF- $\alpha$ after $30 \mathrm{~min}$; $\mathrm{n}=7$ ). Microarray analysis of INS-1E cells exposed to IL- $1 \beta$ showed an up-regulation and down-regulation of respectively $46(7.7 \%)$ and $7(1.2 \%)$ genes, while in TNF- $\alpha$-treated cells there was up-regulation of 42 genes (7\%) and down regulation of 5 genes $(0.8 \%) .22 \%$ of the up-regulated genes, but only $8 \%$ of the down-regulated genes, were common for IL- $1 \beta$ and TNF- $\alpha$. IL- $1 \beta$ induced a higher expression of NF- $\kappa B$ target genes such as MCP-1, Mob- 1 and IкB $\alpha$, a finding confirmed by real time RT-PCR for some of the genes. Real time RT-PCR for some relevant genes not present and/or not detected in the ApoChip (A20, CD40, etc) also showed a higher induction by IL- $1 \beta$ as compared to TNF- $\alpha$.

Conclusions: Our results indicate that de novo protein synthesis has a protective role against TNF- $\alpha$ - but not against IL- $1 \beta$-induced INS-1E cell death. IL$1 \beta$ induces an earlier NF- $\mathrm{kB}$ translocation to the nucleus and an increased expression of some NF- $\kappa \mathrm{B}$ target genes as compared to TNF- $\alpha$. Interestingly, and in spite of the fact that both cytokines induce the same key transcription factor, there are more similarity regarding up-regulation than down-regulation of genes potentially contributing to beta cell dysfunction and death. Supported by EU-FP6-STREP SAVEBETA-036903 of the EC

\section{6}

Pro-inflammatory cytokines inhibit insulin secretion but increase glucose consumption, lactate production, triacylglycerol utilisation and AMPK activiation in a clonal pancreatic $\beta$ cell line

A. M. Kiely ${ }^{1}$, P. R. Flatt ${ }^{2}$, P. Newsholme ${ }^{1}$;

${ }^{1}$ Biochemistry Department, Conway Institute of Biomolecular and Biomedical Research, Dublin, ${ }^{2}$ Biochemistry Department, School of Biomedical Sciences, University of Ulster, Ireland.

Background and Aims: Islet associated pro-inflammatory cytokines (IL-1 $\beta$, TNF $\alpha$ and IFN $\gamma$ ) are involved in the pathogenesis of type I diabetes via the induction of apoptosis in pancreatic $\beta$-cells. The mechanism(s) by which cytokines stimulate apoptosis remain to be elucidated, but gene expression profiling data has determined significant alterations in gene expression related oxidative and endoplasmic reticulum stress and to intermediary metabolism in the $\beta$-cell. Our work aimed to elucidate the key metabolic processes that were altered after exposure of a clonal beta cell line to sublethal concentrations of pro-inflammatory cytokines.

Materials and Methods: Clonal rat BRIN BD11 beta cells were incubation in the presence of pro-inflammatory cytokines at sub-lethal concentrations for 24 hours (IL-1 $\beta \quad 0.3125 \mathrm{U} / \mathrm{ml}$, TNF $\alpha 31.25 \mathrm{U} / \mathrm{ml}$, IFN $\gamma 15.625 \mathrm{U} / \mathrm{ml}$ ). Glucose consumption, lactate production and intracellular triacylglycerol concentrations were determined by enzymatic assays. Insulin secretion was determined by ELISA. AMPK-P concentration was determined by Western blot.
Results: Glucose consumption after $24 \mathrm{hr}$ treatment with the cytokine cocktail increased from $20.47 \pm 5.31$ to $44.61 \pm 6.67 \mu$ moles glucose $/ \mathrm{mg}$ protein, an increase of at least 2 -fold, $\mathrm{p}<0.0001$. Lactate production increased from $15.87 \pm 6.09$ to $58.81 \pm 13.11 \mu$ moles lactate/mg protein, $\mathrm{p}=0.002$, an increase of almost 4-fold. Intracellular triglyceride levels were reduced from $0.12 \pm$ 0.02 to $0.06 \pm 0.04 \mathrm{mg}$ triglyceride/mg protein, a reduction of $49 \%, \mathrm{p}=0.008$. The concentration of AMPK-P increased almost 2-fold from $10 \pm 0.59$ to $1.75 \pm 0.45$ relative units $\mathrm{p}=0.03$. Insulin secretion was reduced from $16.09 \pm$ 2.78 to $6.62 \pm 1.04 \mu \mathrm{g}$ insulin/mg protein, $\mathrm{p}=0.0002$, a $59 \%$ reduction.

Conclusion: Our work clearly demonstrated that sub-lethal concentrations of pro-inflammatory cytokines appear to shift $\beta$-cell metabolism away from a primary role of stimulus-secretion coupling, and towards a catabolic state that may be related to cell defence. As each molecule of glucose generates 2 molecules of lactate, these results indicate that $89 \%$ of the increased glucose consumed was lost as lactate from the BRIN BD11 cells, indicating that anaerobic glycolytic metabolism was favoured at the expense of oxidative flux. One possible explanation for this is that increased generation of glycolytic intermediates e.g. ATP, triose-phosphates and possibly products of the pentose phosphate pathway, eg NADPH, are essential for cell defence. LDH would be required under these conditions to regenerate $\mathrm{NAD}^{+}$resulting in an increase in export of lactate. Increased intracellular generation of fatty acids from triglyceride would at the same time provide acetyl-CoA for oxidative metabolism in the TCA cycle.

Supported by HRB

\section{7}

Inhibitory effects of St. John's wort extract and its component hyperforin on cytokine-induced apoptosis and iNOS expression in pancreatic beta cells by down-regulation of STAT-1 and NF-kB

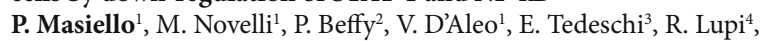

R. Zaffini ${ }^{3}$, P. Marchetti ${ }^{4}$, H. Suzuki ${ }^{3}$, M. Menegazzi';

${ }^{1}$ Dept. of Experimental Pathology, University of Pisa,

${ }^{2}$ Institute of Clinical Physiology, CNR, Pisa,

${ }^{3}$ Dept. of Morphological and Biomedical Sciences, University of Verona,

${ }^{4}$ Dept. of Endocrinology and Metabolism, University of Pisa, Italy.

Background and Aims: Type 1 diabetes is believed to result from betacell destruction induced by immune and inflammatory cytokines. No lowmolecular-weight compounds are known so far to block the cytokine-induced activation of the signal transducers and transcription factors STAT-1 and NF$\mathrm{kB}$ in pancreatic beta cells, leading to expression of inducible NO synthase (iNOS), beta-cell dysfunction and death. Based on previous results in other cell types, we tested the hypothesis that the extract of Hypericum perforatum (St. John's wort, SJW), commonly used as an antidepressant in phytotherapy, and its phloroglucinol component hyperforin (HPF) might interfere with the mechanisms of action of cytokines in beta cells, and consequently protect them from functional impairment and cytotoxicity.

Materials and Methods: Cultured INS-1E cells were exposed to cytokine mixtures (IFN- $\gamma+$ IL- $1 \beta+$ TNF- $\alpha$ ) for different time periods as appropriate, in the presence or absence of various concentrations of a hydro-alcoholic SJW extract or HPF. Apoptosis was evaluated by caspase-3 activity assay, iNOS expression by Northern blot, STAT-1 and NF-kB activation by electrophoretic mobility shift assay. Rat and human isolated pancreatic islets were also used to validate the effects of the vegetal compounds on cytokine-induced STAT-1 activation.

Results: Either SJW extract or HPF significantly and dose-dependently protected INS-1E cells against cytokine-induced beta-cell apoptosis (IC50 $36 \mu \mathrm{g} / \mathrm{ml}$ or $2.2 \mu \mathrm{mol} / \mathrm{l}$, respectively) and iNOS expression (IC50 $7 \mu \mathrm{g} / \mathrm{ml}$ or $0.5 \mu \mathrm{mol} / \mathrm{l}$ ), and inhibited both STAT-1 (IC50 $10 \mu \mathrm{g} / \mathrm{ml}$ and $0.4 \mu \mathrm{mol} /$ 1) and NF-kB (IC50 $30 \mu \mathrm{g} / \mathrm{ml}$ and $1.0 \mu \mathrm{mol} / \mathrm{l}$ ) activation. Constitutively expressed transcription factors such as CBF-1 or SP-1 were unchanged. The inhibitory effect of SJW extract on STAT-1 activation became greater with increasing time of incubation and was also apparent upon preincubation and subsequent removal from the medium during cytokine exposure. Impairment in glucose-stimulated insulin secretion was fully reversed when INS-1E cells were previously exposed to cytokines in the presence of $25 \mu \mathrm{g} / \mathrm{ml} \mathrm{SJW}$ or 1 $\mu \mathrm{mol} / \mathrm{l}$ HPF. The STAT-1 down-regulating effect of the vegetal compounds was confirmed in both rat and human islets. In the latter, SJW and HPF (in the range $50-200 \mu \mathrm{g} / \mathrm{ml}$ and $2.5-10 \mu \mathrm{mol} / \mathrm{l}$, respectively) dose-dependently inhibited cytokine-induced STAT-1 activation. 
Conclusions: SJW extract and hyperforin are novel non-peptidyl compounds targeting key mechanisms of cytokine-induced beta-cell injury, thereby improving beta-cell function and survival. Thus, they represent a promising approach to prevent or delay beta-cell destruction elicited by immune and inflammatory cytokines.

\section{8}

In utero exposure to the vacuolar-ATPase inhibitor bafilomycin increases susceptibility of pancreatic beta cells to cytokine-induced cell death S. Ling ${ }^{1}$, M. A. Myers ${ }^{1}$, H. E. Thomas ${ }^{2}$;

${ }^{1}$ Biochemistry and Molecular Biology, Monash University,

${ }^{2}$ Immunology and Diabetes, St. Vincent's Institute of Medical Research, Melbourne, Australia.

Background and Aims: Environmental agents are known to contribute to Type 1 Diabetes development. Bafilomycin, a potent inhibitor of the proton translocating vacuolar ATPase, is found in tuberous vegetables infected with common scab disease. When fed in drinking water to pregnant NOD mice, bafilomycin disrupts beta cell development and accelerates Type 1 diabetes onset in the offspring. Cytokines, in particular IFN gamma, TNF alpha and IL-1 are mediators of the autoimmune beta cell destruction that leads to Type 1 Diabetes. The aim of this study was to test the susceptibility of bafilomycinexposed beta cells to cytokine-induced cell death.

Materials and Methods: A severe combined immunodeficient mutant derived from NOD (NODscids) was used in these experiments to minimize potential complications due to insulitis. NODscids breeders were exposed to bafilomycin in drinking water $(60 \mathrm{ng} / \mathrm{ml})$ and pancreatic islets were isolated from the offspring at 4 to 6 weeks of age. Pancreatic islets were isolated by intraductal digestion of the pancreas with collagenase $\mathrm{P}$ and the separation on a density gradient. The islets were hand-picked to purity and cultured. 100 islets were exposed to IL-1, TNF alpha, IFN gamma, zvad and nmma at different combinations with known concentrations for 4 days at $37^{\circ} \mathrm{C}$ and apoptosis measured by propidium iodide staining and flow cytometry.

Results: Islet beta cells from control and exposed mice showed similar percentage cell death $(7 \pm 2$ and $11 \pm 6 \%$, mean \pm sem, $\mathrm{n}=3)$ when untreated. Similar degrees of cell death were observed for both groups when exposed to IL-1 and IFN gamma or TNF alpha alone. Exposure to TNF alpha and IFN gamma in combination showed significant increases in beta cell death in islets from 6 week-old NODscids exposed to bafilomycin in utero $(58 \pm 3 \%)$, as compared to non-bafilomycin exposed mice $(37 \pm 1 \%, p=0.002$, Students ttest). This increased susceptibility to TNF alpha and IFN gamma was inhibited by the caspase inhinitor zvad ( $26 \pm 7$ and $29 \pm 5 \%$ respectively) and the nitric oxide (NO) synthase inhibitor nmma ( $21 \pm 5$ and $23 \pm 4 \%$ respectively).

Conclusion: In utero exposure to bafilomycin increases susceptibility of pancreatic beta cells to cytokine-induced cell death in an insulitis-free environment. Increased cytokine susceptibility is mediated by both caspases and NO. This raises the possibility that accelerated diabetes development in NOD mice is caused by an increase in the rate of beta cell destruction leading to more rapid progression to insulin deficiency. Dietary exposure to bafilomycin during pregnancy in early life may be a risk factor for Type 1 Diabetes in those with genetic susceptibility to the disease.

Supported by International Diabetes Institute

\section{9}

Antiapoptotic action of exendin- 4 in ins- 1 beta cells: comparative protein-pattern analysis of isolated mitochondria

D. Tews, S. Lehr, S. Hartwig, A. Osmers, W. Paßlack, J. Eckel; Inst. of Clinical Biochemistry and Pathobiochemistry, German Diabetes Center, Duesseldorf, Germany.

Background and Aims: Besides its numerous physiological functions, Glucagon like peptide-1 (Glp-1) exhibits beneficial effects on the beta-cell mass by both enhancing proliferation and inhibiting apoptosis. However, the precise mechanisms of the antiapoptotic effect have remained elusive. Mitochondria play a key role in the regulation of apoptosis. We performed a comparative protein pattern analysis using two-dimensional difference gel electrophoresis (2D-DIGE) in order to get further insight into the antiapoptotic action of Glp-1 receptor agonists.
Materials and Methods: After a preincubation with the long acting glp-1 receptor agonist exendin- 4 for 5 hours, INS- 1 cells were cultured overnight with a cytokine cocktail (Interleukin- $1 \beta$, Interferon- $\gamma$ ). Apoptosis was determined by measuring caspase-3 activity or by Hoechst33342 / propidiumiodide staining. Mitochondria were enriched by sub-cellular fractionation using differential centrifugation monitored by marker enzyme assays. Subsequently, the proteins of the isolated organelles were labeled with Cy-dyes and separated on overlapping zoom 2D gels spanning a pH-range of 4-9. Differences between protein profiles were analyzed by Proteomewaever software, and protein spots with significant differences were identified by MALDI mass spectrometry.

Results: Cytokine incubation increased the number of apoptotic cells to $11 \%$ and caspase- 3 activity to about $300 \%$. Apoptotic events were reduced to $5 \%$ of the cells and caspase- 3 activity was reduced by $60 \%$ by incubation with exendin- 4 . $2 \%$ of the 3300 reproducibly detected protein spots showed significant differences after cytokine treatment, of which we were able to identify 17 proteins via mass spectrometry analysis. Interestingly, several proteins were counter-regulated by exendin- 4 .

Conclusion: This study allows further insights into the role of mitochondria in cytokine induced apoptosis. Induction of apoptosis by cytokines is regulated by interaction of several metabolic and signalling proteins. Analysis of the counter-regulation of some proteins by exendin- 4 may deliver new targets, which could play a role in the antiapoptotic action of Glp-1 receptor agonists.

\section{0}

Protective effects of GLP-1 receptor agonists on TNF $\alpha$-induced apoptosis in INS-1 beta cells

A. Natalicchio, F. De Stefano, E. Lauranzano, S. Perrini, M. R. Orlando, A. Leonardini, A. Cignarelli, M. Melchiorre, S. Martemucci, C. Caccioppoli, L. Laviola, F. Giorgino;

Endocrinology \& Met. Diseases, University of Bari, Italy.

Background and Aims: TNF $\alpha$ represents an important mediator of beta-cell dysfunction and death in diabetes. The objective of this study was to evaluate the mechanisms of TNF $\alpha$-induced beta-cell damage, and to investigate the potential protective effects of GLP-1 mimetics on beta-cell function and survival.

Materials and Methods: INS-1 beta-cells were stimulated with $20 \mathrm{ng} / \mathrm{ml}$ TNF $\alpha$ for several hours. The effects of GLP-1 receptor agonists was evaluated by pre-incubating INS-1 cells with GLP-1 $(10 \mathrm{nM})$ or Exenatide $(10 \mathrm{nM})$ for $18 \mathrm{~h}$. Protein content and phosphorylation of intracellular signaling intermediates were evaluated by immunoprecipitation and immunoblotting techniques. Beta-cell apoptosis was quantified by an ELISA assay evaluating oligosome release into the cytosol.

Results: Treatment with TNF $\alpha$ increased the phosphorylation levels of the serine-kinase JNK 2 -fold $(\mathrm{p}<0.05)$ and reduced $\operatorname{IkB} \alpha$ protein content by $50 \%(\mathrm{p}<0.05)$. Furthermore, TNF $\alpha$ stimulation resulted in increased serine phosphorylation levels of the IRS-1 protein $(\mathrm{p}<0.05)$, and a reduction of IRS-1 mRNA expression by $50 \%$, even though protein levels of both IRS-1 and IRS-2 appeared to be unaffected by the cytokine treatment. Moreover, TNF $\alpha$ reduced basal Akt phosphorylation by $30 \%$ and increased beta-cell apoptosis 7 -fold $(\mathrm{p}<0.05)$. Since the incretin hormone GLP-1 has been shown to promote beta-cell survival, we next investigated potential protective effects of GLP-1 receptor agonists on TNF $\alpha$-induced beta-cell apoptosis. Preincubation of INS-1 cells with GLP-1 $(10 \mathrm{nM})$ or Exenatide $(10 \mathrm{nM})$ for $18 \mathrm{~h}$ inhibited TNF $\alpha$-induced apoptosis by $50 \%(\mathrm{p}<0.05)$. Furthermore, both GLP1 and Exenatide prevented TNF $\alpha$-induced JNK phosphorylation and IRS-1 serine phosphorylation, and restored Akt phosphorylation, without affecting other MAP kinases such as p38. Finally, Exenatide treatment induced a 3-fold increase in IRS-2 protein expression.

Conclusion: TNF $\alpha$ induces apoptosis of INS-1 beta-cells, and this likely occurs by induction of JNK phosphorylation and impairment of IRS-1 and Akt signaling. GLP-1 and Exenatide prevent the signaling abnormalities and cell apoptosis induced by TNF $\alpha$. Thus, GLP-1 receptor agonists may promote beta-cell survival by directly antagonizing cytokine-dependent signaling reactions mediating beta-cell death.

Supported by Eli Lilly \& Co. 


\section{PS 38 Signal transduction I}

\section{1}

\author{
Factors in serum from type 2 diabetes patients cause cellular insulin \\ resistance \\ J. W. Eriksson ${ }^{1,2}$, F. Renstrom ${ }^{3}$, J. Burén ${ }^{3}$, M. Svensson ${ }^{3}$; \\ ${ }^{1}$ Lundberg Laboratory for Diabetes Research, Sahlgrenska University \\ Hospital, Gothenburg, ${ }^{2}$ AstraZeneca R\&D, Molndal, \\ ${ }^{3}$ Medicine, Umea University Hospital, Umea, Sweden.
}

Objective: This study was designed to investigate whether there are humoral factors, in addition to glucose, insulin and free fatty acids (FFA), in serum from type 2 diabetic patients that can induce or contribute to peripheral insulin resistance with respect to glucose transport.

Methods: Isolated subcutaneous adipocytes from 11 type 2 diabetic subjects and 10 non-diabetic control subjects were incubated for $24 \mathrm{~h}$ in medium supplemented with $25 \%$ serum obtained from non-diabetic (control) donors or with serum from type 2 diabetic donors. The glucose concentration was adjusted to predefined levels: a low, physiological $(5 \mathrm{mM})$ or a high, 'diabetic' $(15 \mathrm{mM})$ level, respectively. After the incubation period glucose uptake capacity was assessed in intact adipocytes in the presence of $0-1000 \mathrm{mU} / \mathrm{L}$ insulin, and Western blots were performed on cellular lysates.

Results: In adipocytes from control subjects, pretreatment with $25 \%$ serum from type 2 diabetic donors significantly reduced maximally insulinstimulated, but not basal, glucose uptake capacity (by $\sim 30 \%, \mathrm{p}<0.05$ ), as compared to control serum. This was seen at $5 \mathrm{mM}$ glucose, but not at 15 $\mathrm{mM}$. There were no differences in insulin and FFA levels, and neither in TNFalpha or IL-6, during the incubation that could account for the differences seen between serum from diabetic and control subjects, respectively. A similar impairment of glucose uptake capacity, as that seen with diabetic serum, was exerted by pretreatment with high glucose $(15 \mathrm{mM})$ together with control serum $(\mathrm{p}<0.05)$. There was no clear additive effect with high glucose in combination with diabetic serum. No significant alterations were found in cellular content of key proteins in the insulin signaling cascade (IRS-1, IRS2 and GLUT4) that could explain the impaired insulin-stimulated glucose transport in control adipocytes incubated with serum from type 2 diabetic donors.

In adipocytes isolated from type 2 diabetic subjects, insulin-stimulated glucose uptake capacity was impaired compared to control cells, and this was seen in both fresh cells (reduction by $~ 50 \%$ ) and, to a lesser extent, in cells cultured for $24 \mathrm{~h}(\mathrm{p}<0.01)$. No further changes were observed with different culture conditions, i.e. diabetic or control serum, 5 or $15 \mathrm{mM}$ glucose. The impairment in glucose uptake capacity seen in cultured 'diabetic' adipocytes was very similar to that found in control adipocytes cultured with diabetic serum.

Conclusion: These findings indicate the presence of biomolecules, besides glucose, insulin and FFA, in the circulation of type 2 diabetic patients, that can induce or maintain peripheral insulin resistance. This implies that even if glucose levels are normalised, other circulating factors can impair insulin sensitivity in type 2 diabetic patients.

Supported by Swedish Research Council, Swedish Diabetes Association, AstraZeneca R\&D, Västerbotten County Council

\section{2}

Insulin signaling in type 2 diabetes - role of the oxidative stress related protein p66shc: preliminary data

E. Pagnin, E. Murphy, E. Iori, S. Schiavo, L. Bertipaglia, L. A. Calo, A. Avogaro;

Dep. Clinical and Experimental Medicine, University of Padova, Italy.

Background and Aims: Oxidative stress plays an important role in endothelial cardiovascular dysfunction and atherogenesis. This is of particular relevance in diabetes, a condition characterized by oxidative stress, and increased prevalence of cardiovascular disease. The major role played by p66shc in oxidative stress-related response has been established by the resistance to and reduction of oxidative stress of p66shc knockout mice. We have demonstrated for the first time in type 2 diabetic patients an increased mononuclear cell p66shc gene expression compared to healthy controls. Abnormal regulation of the phosphatidylinositol 3-kinase (PI3-K)/Akt pathway, which plays an essential role in the insulin signalling, is another factor known to contribute to diabetic vascular dysfunction. No data are available on the role played by $\mathrm{p} 66 \mathrm{shc}$ on insulin signalling. This study evaluates in fibroblasts derived from skin biopsies of three type 2 diabetic patients the effect of p66shc gene silencing on activation of Akt upon insulin stimulation.

Materials and Methods: After incubation (15 min) with insulin (100nM), phospho-Akt (Thr308 and Ser473) were determined by western blot analysis. p66shc gene silencing was performed using siRNA methodology.

Results: Ser473 Akt phosphorylation was significantly lower after incubation with insulin in fibroblasts from type 2 diabetic patients compared to healthy subjects $(201,7 \mathrm{D} \%$ vs basal $\pm 36,2$ vs $260,9 \pm 47,9$, p $<0,03)$. p66shc silencing not only increased Ser473Akt phosphorylation in cells from type 2 diabetic patients compared to not silenced cells (457,0 D\% vs basal $\pm 31,5$ vs $201,7 \pm 36,2, p<0,03)$ but also compared to not silenced cells from healthy subjects $(457,0 \mathrm{D} \% \pm 31,5$ vs $260,9 \pm 47,9, \mathrm{p}<0,05)$. No significant differences in Thr308Akt phosphorylation were found in cells from type 2 diabetic patients and healthy subjects $(118,8 \mathrm{D} \% \pm 20,2$ vs $97,0 \pm 37,9$, ns). On the contrary, p66shc silencing caused an increase of Thr308Akt phosphorylation in cells from type 2 diabetic patients compared to not silenced cells from healthy subjects $(426,8 \mathrm{D} \% \pm 124,5$ vs $118,8 \pm 20,2, \mathrm{p}<0,03)$.

Conclusion: Our preliminary results demonstrate a role for p66shc in the altered insulin signalling of type 2 diabetic patients due to a p66shc mediated inhibition of PI3-K/Akt pathway which is removed by p66shc gene silencing. This is the first demonstration of a direct involvement of p66shc in insulin signalling in fibroblasts from type 2 diabetes.

\section{3}

Anti-inflammatory effect of insulin on IL-6 signaling in the human hepatoma cell line HepG2

E. Wallerstedt, C. X. Andersson, U. Smith;

Department of Molecular and Clinical Medicine/Diabetes, Medicine, Gothenburg, Sweden.

Background and Aims: It has previously been reported that infusion of insulin leads to anti-inflammatory effects including reduction of plasma concentrations of the acute-phase proteins $\mathrm{C}$-reactive protein (CRP), serum amyloid A (SAA) and plasminogen activator inhibitor-1 (PAI-1). We have recently shown that insulin can antagonize interleukin-6 (IL-6) signaling and exert anti-inflammatory effects in 3T3-L1 adipocytes by significantly reducing the tyrosine phosphorylation of its intracellular signaling molecule STAT3. This is also accompanied by reduced expression of the inflammatory marker genes SAA3 and haptoglobin. To study if the cross-talk between IL-6 and insulin also occurs in other cells, we have used the human hepatoma cell line hepG2.

Materials and Methods: The human hepatoma cell line, HepG2, was cultured and incubated with insulin (100 nM), IL-6 $(20 \mathrm{ng} / \mathrm{ml})$ and insulin+IL-6 for 30 minutes up to 24 hours. RNA was extracted and the gene expression was analyzed with real-time RT-PCR (TaqMan) while the proteins were analyzed by immunoblotting.

Results: Insulin exerts an inhibitory effect on IL-6 signaling in HepG2 cells by reducing the tyrosine phosphorylation of STAT3. However, the serine phosphorylation of STAT3 was not affected by insulin. A reduced gene expression of the inflammatory marker genes SAA1, SAA2, haptoglobin, orosomucoid and PA1-1 was observed in cells stimulated with both IL-6 and insulin compared to cells treated with IL- 6 alone. IL- 6 increased the gene expression of SOCS 3 but in contrast to the inflammatory markers, which were reduced by the addition of insulin, a synergistic effect of the combination was found. SOCS3 functions as a feed-back inhibitor of the IL-6 signaling pathway in order to clarify the mechanism for this; insulin could also activate STAT5 another inducer of SOCS3 expression. Importantly, we found that insulin increased the tyrosine phosphorylation of STAT5. Furthermore, coimmunoprecipitation experiments showed that STAT3 and STAT5 were able to heterodimerize when cells had been incubated with insulin. This finding can explain the positive effect of insulin and IL- 6 on SOCS3 expression.

Conclusion: Insulin antagonizes IL-6 signaling in the human hepatoma cell line, HepG2, by reducing the tyrosine phosphorylation of STAT3 and, thereby, inhibits the expression of inflammatory marker genes. Our data also suggest that the insulin-induced reduction of the tyrosine phosphorylation of STAT3 is due to an insulin-dependent induction of the feed-back inhibitor of IL-6 signaling, SOCS3.

Supported by grants from HEPADI), Inger Hultman Foundation, Eirs Foundation and Wilhelm and Martina Lundgren Foundation 


\section{4}

The inhibition of stress kinases, NF- $\mathrm{BB}$, and PKC $\theta$ by EGB 761 restored palmitate-induced insulin resistance in L6 Myotubes

J. Jung ${ }^{1}$, E.-S. Ha ${ }^{1}$, H.-J. Jang ${ }^{1}$, S.-E. Choi ${ }^{1}$, S. Jung ${ }^{2}$, H. Kim², D. Kim²,

Y. Kang ${ }^{1}$, K.-W. Lee ${ }^{2}$;

${ }^{1}$ Institutes for Medical Scienses, ${ }^{2}$ Endocrinology and Metabolism, Ajou University School of Medicine, Suwon-Si, Republic of Korea

Background and Aims: EGb 761, a standardized form of Ginkgo biloba leaf extract, was recently reported to increase pancreatic beta-cell function. However, the detailed effect of EGb 761 on insulin resistance has not been examined. We found that EGb 761 prevented palmitate-induced insulin resistance in L6 myotubes. In this study, we investigated the molecular and cellular mechanisms of the protective effect of EGb 761 on palmitate-induced insulin resistance.

Materials and Methods: We used differentiated L6 myotubes as an in vitro skeletal muscle model with glucose deprivation for 3 days. To determine the effect of EGb 761 on insulin resistance, before palmitate treatment, we pretreated differentiated L6 myotubes with EGb 761 and then measured the insulin-stimulate glucose uptake and insulin signaling.

Results: A 12 -h exposure of L6 myotubes to palmitate reduced the insulin stimulated glucose uptake by $67 \%$, insulin receptor substrate (IRS)- 1 tyrosine phosphorylation by $72 \%$, and protein kinase B phosphorylation by $70 \%$, and increased IRS-1 serine phosphorylation by $169 \%$. EGb 761 prevented the palmitate-induced reduction of insulin _stimulated glucose uptake, IRS-1 tyrosine phosphorylation, and protein kinase B phosphorylation in dosedependent manners. To elucidate the mechanism of the EGb 761-prevented palmitate-induced reduction of insulin resistance, we monitored the activation of stress kinases, NF- $\kappa \mathrm{B}$, and $\mathrm{PKC} \theta$. Palmitate stimulated the phosphorylation of JNK and p38 by 2 - and 1.9 -fold, respectively, the degradation of I $\mathrm{B} \alpha$ and $\mathrm{I} \kappa \mathrm{B} \beta$ by 1.9-and 1.6-fold, respectively, and the translocation of protein kinase $c \theta$ by 2.2 -fold. Treatment with EGb 761 reduced the activation of stress kinases, NF- $\kappa \mathrm{B}$, and protein kinase $\mathrm{c} \theta$ by palmitate, suggesting that EGb 761 prevents palmitate-induced insulin resistance in L6 myotubes via the inhibition of stress kinases, NF- $\kappa \mathrm{B}$, and PKC $\theta$.

Conclusion: Our data suggest that EGb 761 -prevented palmitate-induced insulin resistance is mediated by the inhibition of stress kinases, NF- $\kappa \mathrm{B}$, and protein kinase c $\theta$ and the subsequent restoration of insulin signaling. Supported by the "GRRC" project of the Gyeonggi Provincial Government and a grant from the Korean Health 21 R\&D Project, Ministry of Health and Welfare, Republic of Korea. (to K. W. Lee)

\section{5}

The AMPK-induced p70S6K inhibition correlates to the synergistic effect of biguanides and insulin on glucose uptake via the overphosphorylation of the PKB/AS160 axis

L. Bertrand ${ }^{1}$, A. Ginion ${ }^{1}$, C. Beauloye ${ }^{1}$, C. Mouton ${ }^{1}$, L. Hue ${ }^{2}$,

J.-L. Vanoverschelde ${ }^{1}$;

${ }^{1}$ Division of Cardiology, ${ }^{2}$ Hormone and Metabolic Research unit,

Université Catholique de Louvain, Brussels, Belgium.

Background and Aims: Biguanides, like metformin and phenformin, are known to lower blood glucose concentration by increasing muscle glucose disposal. Several findings suggest that the effects of biguanides could be mediated by the activation of AMP-activated protein kinase (AMPK). The aim of this work was to study the relationship between AMPK and the biguanide-stimulated glucose uptake in cardiomyocytes.

Materials and Methods: Cultured rat cardiomyocytes were incubated with insulin and phenformin, alone or in combination. Glucose uptake was measured by the detritriation rate of $[2-3 \mathrm{H}]$ glucose. The phosphorylation state of the different signalling elements was measured by immunoblot.

Results: The stimulation of glucose uptake by biguanides (phenformin $0.5 \mathrm{mM}$ : $0.77 \pm 0.17$ vs control: $0.09 \pm 0.03 \mu$ moles $/ \mathrm{mg} . \mathrm{h}, \mathrm{p} \leq 0.05)$ correlated to AMPK phosphorylation (control: $0.03 \pm 0.03$ vs phenformin: $1.00 \pm 0.00$, arbitrary unit, $\mathrm{p} \leq 0.05)$, whereas that by insulin $(1.07 \pm 0.14 \mu \mathrm{moles} / \mathrm{mg} . \mathrm{h}$, $\mathrm{p} \leq 0.05$ vs control) correlated with protein kinase $\mathrm{B}(\mathrm{PKB})$ phosphorylation (control: $0.0 \pm 0.0$ vs phenformin: $1.0 \pm 0.0$, arbitrary unit, $\mathrm{p} \leq 0.05$ ). When added together, insulin and phenformin induced a concomitant increase in AMPK phosphorylation and PKB overactivation $(2.27 \pm 0.27$, arbitrary unit, $\mathrm{p} \leq 0.05$ vs insulin alone), resulting to a synergistic overstimulation of glucose uptake $(2.24 \pm 0.32 \mu$ moles/mg.h, $\mathrm{p} \leq 0.05$ vs insulin alone $)$. This phenformin-induced $\mathrm{PKB}$ overactivation is accompanied by the inhibition of p70 ribosomal S6 kinase (p70S6K) (control: $0.0 \pm 0.0$, insulin: $1.0 \pm$ 0.0 and insulin+phenformin $0.6 \pm 0.1$, arbitrary unit, $\mathrm{p} \leq 0.05)$ and of the phosphorylation of insulin receptor substrate 1 (IRS-1) (control: $0.07 \pm 0.03$, insulin: $1.00 \pm 0.00$ and insulin + phenformin $0.36 \pm 0.09$, arbitrary unit, $\mathrm{p} \leq 0.05$ ) on serine, both presumed to be involved in the negative feedback inhibition of the insulin signalling. In all conditions, PKB and AMPK activation fully correlated to the phosphorylation of Akt-substrate 160 (AS160), known to participate in glucose uptake stimulation.

Conclusion: AMPK activators seem to induce overstimulation of glucose uptake in insulin-treated cardiomyocytes via the inhibition of the p70S6K negative feedback loop. This is accompanied by the overphosphorylation of the PKB/AS160 axis. Our results suggest that AMPK activation could be a potential therapeutic approach to treat insulin resistance.

\section{6}

Differential effects of 5'AMP-activated protein kinase (AMPK) activation by metformin and AICAR on glucose uptake and lipolysis in human adipocytes

T. Radimerski ${ }^{1}$, D. Seboek ${ }^{1}$, B. Kola ${ }^{2}$, P. Linscheid ${ }^{1}$, H. Zulewski ${ }^{3}$, A. Grossman², U. Keller ${ }^{3}$, B. Müller ${ }^{3}$, M. Korbonits ${ }^{2}$, M. Christ-Crain ${ }^{3,2}$. ${ }^{1}$ Department of Research, University Hospital Basel, Switzerland, ${ }^{2}$ Department of Endocrinology, Barts and the London Medical School, London, United Kingdom, ${ }^{3}$ Department of Endocrinology, Diabetes and Clinical Nutrition, University Hospital Basel, Switzerland.

Background and Aims: Controversy exists regarding the role of 5'AMP activated protein kinase (AMPK) on basal and insulin-mediated glucose uptake and on lipolysis. Data from human adipocytes are lacking.

Here we compared the effects of AMPK stimulator metformin and 5'phosphoribosyl-5-aminoimidazole-4-carboxamide (AICAR), respectively, on basal and insulin-mediated glucose uptake as well as on lipolysis in human mesenchymal stem cell (MSC)-derived adipocytes.

Materials and Methods: Cells were treated with different doses of metformin, which activates AMPK via reduction of cellular ATP levels or AICAR, an AMP-mimic known to stimulate AMPK, for 3 to 24 hours. mRNA expression was determined by quantitative RT-PCR. Lipolysis was assessed by measuring glycerol release into culture supernatant using a colorimetric method. Uptake of ${ }^{3} \mathrm{H}$-labelled 2-deoxy-glucose (GU) was assessed in the presence or absence of insulin. AMPK activity was measured using a kinase assay and by immunoblotting for phospho-Acetyl-CoA-Carboxylase (pACC).

Results: As compared to controls, AMPK activity was increased upon AICAR administration to $333 \pm 115 \%(n=5, p=0.005)$ after 24 hours. AMPK activation by AICAR significantly decreased both basal and insulin-stimulated glucose uptake in a time- and dose-dependent manner. In contrast, metformin significantly increased basal and insulin-stimulated glucose uptake, in a timeand dose-dependent manner. Lipolysis was significantly decreased by prestimulation with either metformin or AICAR for 24 hours. Insulin responsive glucose transporter 4 (GLUT4) mRNA expression was transiently increased upon 3 hours treatment with AICAR or metformin, in contrast to controls. Conclusion: The AMPK activating compounds metformin and AICAR exhibit similar effects regarding the inhibition of lipolysis and the stimulation of GLUT4 mRNA expression. Conversely, the different effects on basal and insulin-stimulated glucose uptake by metformin and AICAR suggest that other AMPK activation-independent pathways are involved in their regulatory effects on glucose uptake in adipose tissue.

\section{7}

Resveratrol improves insulin-responsiveness in insulin-resistant models of human primary muscle cells

S. Fröjdö, C. Durand, H. Vidal, L. Pirola;

Inserm u870, INRA U1235, INSA Lyon, HCL, University Claude Bernard, Lyon, France.

Background and Aims: Resveratrol, a polyphenol found in fruits, increases lifespan and protects mice on a high fat diet against the development of metabolic disease. These beneficial effects are mediated by the activation 
of the histone deacetylase SIRT1 and its target PCG-1 $\alpha$, resulting in improved mitochondrial function and insulin sensitivity. Paradoxically, we recently reported that short pre-treatment of cells with resveratrol inhibits insulin-induced PI3K, an observation that argues against a positive effect of resveratrol on insulin action. However, we hypothesize that mild pharmacological inhibition of the PI3K/PKB pathway, which is the major mediator of insulin action in insulin-responsive peripheral tissues, may have a beneficial effect on insulin sensitivity. This hypothesis is supported by ample genetic evidence - in metazoans from yeast to mammalians - indicating that mild repression of several components of the insulinIGF-1 receptor signalling improves insulin action and increases lifespan. Therefore, we evaluated the outcomes of resveratrol treatment of primary human myotubes and muscle-derived L6 cells subjected to treatments leading to insulin resistance.

Results: In starving human primary myotubes, L6 and CCL cells, insulininduced PI3K activity and $\mathrm{PKB} /$ FoxO4 activatory phosphorylation were fully inhibited by a 30 minute pre-treatment with resveratrol. On the contrary, resveratrol only mildly affected the sub-maximal activation of the PI3K/PKB cascade induced by a 24-hour insulin treatment, a treatment that also induced insulin resistance at subsequent insulin stimulation. In this experimental setup, we find that prolonged resveratrol treatments alleviate the insulinresist status of the cell and thus ameliorate their insulin responsiveness. This improvement was associated with decreased serine-phosphorylation events on IRS-1 and IRS-2, while tyrosine phosphorylation was unchanged after resveratrol treatment.

Conclusion: The improvement of insulin responsiveness induced by prolonged resveratrol treatment is dependent on the capability of resveratrol to inhibit $\mathrm{PI} 3 \mathrm{~K}$ and the consequential PI3K-induced serine/threonine phosphorylation events in IRS1/2 that impair their signalling potential. These data support the idea that a mild inhibition of the insulin signalling by resveratrol improves insulin action and could partially explain the beneficial effects seen after resveratrol treatment in vivo. Further experiments are ongoing to further clarify these effects.

Supported by an ARD/PNRD (Association pour la Recherche sur le Diabètel Programme national de recherche sur le diabète)

\section{8}

A natural occurring tetrapeptide GHTD-amide increases intracellular free zinc in $\mathrm{C} 2 \mathrm{C} 12$ myotubes

R. C. C. Lim, S. G. Paule, B. Nikolovski, R. E. Gray, P. Z. Zimmet,

M. A. Myers;

Biochemistry and Molecular Biology, Monash University, Victoria,

Australia.

Background and Aims: GHTD-amide is a naturally occurring tetrapeptide hormone first isolated from human urine that improves insulin action in animal models of Type 2 diabetes. GHTD-amide binds zinc ions and disperses hexameric insulin to monomers and this in part accounts for its enhancement of insulin activity in vivo. The known insulin-mimetic properties of zinc and deregulated zinc homeostasis in Type 2 diabetes implicate zinc in the processes underlying insulin resistance. The aim of this study was to investigate the effect of GHTD-amide on zinc homeostasis in muscle cells.

Materials and Methods: Detection of intracellular free zinc was by fluorozin$3 \mathrm{AM}$ ester fluorescence in $\mathrm{C} 2 \mathrm{C} 12$ myotubes by live cell imaging. Cells were loaded with $2 \mu \mathrm{M}$ of fluoZin-3 then washed and incubated for an additional 30 minutes in media supplemented with physiological concentration of $(20 \mu \mathrm{M})$ of $\mathrm{ZnCl}_{2}$. Time-lapse photography of the cells was performed after the addition of $10 \mu \mathrm{M}$ GHTD-amide or relevant positive and negative controls, including the zinc ionophore pyrithione. Zinc uptake and efflux were measured using ${ }^{65} \mathrm{Zn}$. Differentiated $\mathrm{C} 2 \mathrm{C} 12$ myotubes were incubated in uptake buffer containing $10 \mu \mathrm{M}$ GHTD-amide or $10 \mu \mathrm{M}$ of negative control peptide, $10 \mu \mathrm{M}$ of $\mathrm{ZnCl}_{2}$ and $50,000 \mathrm{cpm} /$ well of ${ }^{65} \mathrm{Zn}$. Media was collected every 5 minutes for 40 minutes and ${ }^{65} \mathrm{Zn}$ radioactivity was determined in a gamma-counter. The radioactivity data was normalized for protein content of cell lysates to obtain radioactivity per $\mu \mathrm{g}$ of protein.

Results: Addition to the culture media of GHTD-amide but not a negative control peptide increased fluozin- 3 fluorescence intensity in $\mathrm{C} 2 \mathrm{C} 12$ myotubes over time. Fluorescence intensity stimulated by GHTD-amide decreased after 11 minutes, unlike cells treated with pyrithione where fluorescence continued to increase with time. The increase of intracellular free zinc can be caused by influx of extracellular zinc or by efflux from intracellular compartments. ${ }^{65} \mathrm{Zn}$ uptake assay revealed similar uptake whether the cells were incubated with GHTD-amide or negative control peptides.

Conclusion: GHTD-amide causes a transient increase in intracellular free zinc concentration but does not stimulate the uptake of extracellular zinc. The increase of intracellular free zinc observed in fluozin-3 live cell imaging is likely to be due to release of zinc from intracellular storage compartments or zinc-binding proteins. Transient increases in intracellular free zinc may activate the insulin signalling pathway and this may be one mechanism by which GHTD-amide enhances insulin activity. Supported by Dia-B Tech and Monash Univerity

\section{9}

Glucagon causes HISS-dependent insulin resistance

R. S. Patarrao ${ }^{1,2}$, W. Lautt ${ }^{3}$, J. Schafer ${ }^{3}$, M. Macedo ${ }^{1,4}$;

${ }^{1}$ Dep. of Physiology, Faculty Medical Sciences - UNL, Lisbon, Portugal,

${ }^{2}$ Dep. of Biochemistry, Faculty of Medical Sciences - UNL, Lisbon, Portugal, ${ }^{3}$ Dep. of Pharmacology and Therapeutics, Faculty of Medicine, Winnipeg, Canada, ${ }^{4}$ Portuguese Diabetes Association, Lisbon, Portugal.

Background and Aims: Insulin action is associated with the release of the Hepatic Insulin Sensitizing Substance (HISS), which enhances peripheral glucose uptake in skeletal muscle. In the immediate postprandial state HISS release is maximal, decreasing with the duration of fasting. The prandial control of HISS action is mediated through hepatic parasympathetic - derived nitric oxide (NO) and hepatic glutathione (GSH). An important regulator of hepatic GSH levels is the cAMP/protein kinase A (PKA) pathway, since a rise in cAMP levels is associated with a decrease in GSH levels. In the liver, glucagon mediates its actions by interacting with specific receptors leading to an increase of the intracellular cAMP levels and PKA activity. We tested the hypothesis that, in fed rats, intraportal (ipv) administration of the cAMP analog, dibutyryladenosine 3', 5'-cyclic monophosphate (DBcAMP) and glucagon decrease HISS-dependent insulin action, in a dose-dependent manner.

Materials and Methods: Male Sprague-Dawley rats (210-230g) were fasted for $8 \mathrm{~h}$, refed for $2 \mathrm{~h}$ and anesthetized with sodium pentobarbital $(65 \mathrm{mg} / \mathrm{kg}$, ip). Insulin sensitivity was measured using the Rapid Insulin Sensitivity Test (RIST), with the RIST Index defined as the amount of glucose required to maintain euglycemia following intravenous insulin administration $(50 \mathrm{mU} /$ $\mathrm{kg}$ ). Insulin sensitivity was evaluated in the control fed state and after each drug administration. DBcAMP was administered ipv at doses from 0.01 to $1.0 \mathrm{mg} / \mathrm{kg}$. Glucagon was also administered ipv at doses from $0.5 \mathrm{ng} / \mathrm{kg}$ to $20 \mu \mathrm{g} / \mathrm{kg}$. In order to evaluate the role of glucagon on the HISS pathway, HISS release was blocked with ipv administration of $\mathrm{N}$-monomethyl-L-arginine (L-NMMA, $0.73 \mathrm{mg} / \mathrm{kg}$ ), a NO synthase competitive antagonist and then insulin sensitivity was evaluated after administration of glucagon, at a dose that produces a maximal inhibition on insulin sensitivity without affecting basal glycemia (200ng/kg, ipv).

Results: In the first group of animals, where DBcAMP was administered ipv $(0.01$ to $1.0 \mathrm{mg} / \mathrm{kg})$, a significant decrease in insulin sensitivity was observed, corresponding to an inhibition ranging from $27.2 \pm 2.1 \%$ to $48.8 \pm 7.6 \%$ ( $n=9$ ). In the second group of animals, ipv glucagon $(0.5 \mathrm{ng} / \mathrm{kg}-20 \mu \mathrm{g} / \mathrm{kg})$ also decreased insulin sensitivity in a dose-dependent manner, corresponding to an inhibition ranging from $22.1 \pm 8.7 \%$ to $66.6 \pm 4.2 \%(\mathrm{n}=14)$. In the third group of animals, the control RIST Index (177.1 $\pm 1.6 \mathrm{mg}$ glucose $/ \mathrm{kg} \mathrm{bw}, \mathrm{n}=5)$ was significantly reduced after abolishing HISS action with ipv infusion of L-NMMA (81.0 $\pm 8.3 \mathrm{mg}$ glucose $/ \mathrm{kg}$ bw, $\mathrm{n}=5, \mathrm{p}<0.001)$, corresponding to an inhibition of $54.2 \pm 4.9 \%$. Further ipv administration of glucagon $(200 \mathrm{ng} / \mathrm{kg})$ did not change insulin sensitivity (80.4 $\pm 5.9 \mathrm{mg}$ glucose/kg bw, $\mathrm{n}=5)$.

Conclusion: Our results are in agreement with the proposed hypothesis that increased levels of glucagon results in increased cAMP/PKA pathway activity leading to an impairment of insulin sensitivity, exclusively due to a decrease of HISS release.

Supported by Fundação para a Ciência e Tecnologia (FCT), Portuguese Diabetes Association and Canadian Institutes of Health Research. Rita S. Patarrão is supported by a FCT fellowship 


\section{PS 39 Signal transduction II}

\section{0}

Comparison of intracellular signaling properties induced by the longacting insulin analogues glargine and detemir in cultured cells

T. Sasaoka, H. Tsuneki, T. Wada;

Department of Clinical Pharmacology, University of Toyama, Japan.

Background and Aims: Insulin glargine and detemir are long-acting human insulin analogues. Although their binding affinity to the insulin receptor (IR) has been intensively studied, little is known about the signaling pathways activated after binding. We directly compared intracellular signaling properties of glargine, detemir, and human insulin leading to metabolic and mitogenic actions in cultured cells.

Materials and Methods: 3T3-L1 adipocytes, L6 myocytes, and rat primary hepatocytes were incubated with various concentrations of human insulin, glargine, and detemir with varying concentrations of bovine serum albumin (BSA). Immunoprecipitation was performed and phosphorylation of IR, IRS1, Akt, GSK3, and MAP kinase (MAPK) was detected by Western blot with anti-phosphospecific antibodies. 2-deoxyglucose uptake was measured in adipocytes and myocytes. Vascular smooth muscle cells (VSMCs) from rat aortas were incubated with insulin analogues, and 5-bromo-2'-deoxyuridine (BrdU) incorporation was measured by enzyme linked immunoassay.

Results: Phosphorylation kinetics were similar among insulins. In 3T3-L1 adipocytes, glargine induced phosphorylation of IR, IRS-1, Akt, GSK3 and MAPK in a dose-dependent manner similar to human insulin, whereas detemir-induced effects were reduced by 30 to $60 \%$. At maximal concentration of $170 \mathrm{nM}$, detemir-induced glucose uptake was decreased by $65 \%$ in 3T3L1 adipocytes compared with $170 \mathrm{nM}$ human insulin and glargine. In L6 myocytes, the effects of detemir were reduced by 30 to $50 \%$ compared to human insulin and glargine. In VSMCs, glargine-induced phosphorylation of IR, IRS-1, and Akt was slightly increased, whereas detemir-induced phosphorylation was 20 to $40 \%$ lower versus human insulin. Similarly, detemir-induced phosphorylation of GSK3 and MAPK was less than glargine or human insulin. Consistent results were observed in hepatocytes. BrdU incorporation in VSMCs was similar for all analogues, although for detemir the effect was mildly decreased. The phosphorylation kinetics of detemir was further decreased with increasing concentrations of BSA.

Conclusion: Glargine and human insulin have similar properties in activating intracellular signaling pathways related to metabolic and mitogenic action. In contrast, detemir appears less active in four cell types, and its activity is inhibited by increasing concentrations of albumin.

Supported by a sanofi-aventis research grant

\section{1}

Overexpression of protein tyrosine phosphatase sigma in mice induces hyperglycaemia

E.-C. Långberg, H. F. Gu, S. Nordman, S. Efendic, C.-G. Östenson;

Molecular Medicine and Surgery, Karolinska Institutet, Stockholm, Sweden.

Background and Aims: A number of protein tyrosine phosphatases (PTPs) that are expressed in insulin sensitive tissues are proposed to act as key regulators in insulin signaling. One of these proteins is receptor tyrosine phosphatase sigma (RPTP $\sigma$ ), which is highly expressed in all insulin sensitive tissues. Previously we have demonstrated that overexpression of RPTP $\sigma$ is linked to impaired glucose-induced insulin secretion in hereditary diabetic Goto-Kakizaki (GK) rats. We have now developed a transgenic (TG) mouse model overexpressing RPTP $\sigma$ to investigate its role in the development of type 2 diabetes.

Material and Methods: The TG construct consisting of ubiquitin promoter and rat RPTP $\sigma$ cDNA was used for random insertion on a $\mathrm{C} 57 \mathrm{Bl} / 6$ background. Mice were studied regarding body weight, plasma glucose, serum insulin and insulin secretion in vitro.

Results: TG mice had significantly increased body weight after 2 months of age compared to wildtype (WT) mice, $23.0 \pm 0.6(\mathrm{n}=32)$ vs. $21.3 \pm 0.4$ $(\mathrm{n}=50) \mathrm{g}(\mathrm{p}<0.05)$. TG mice showed increased fasting blood glucose levels compared to WT animals already after 2 months of age, $4.5 \pm 0.2(\mathrm{n}=32)$ vs. $3.3 \pm 0.1(\mathrm{n}=50) \mathrm{mmol} / \mathrm{l}(\mathrm{p}<0.001)$ and also at 5 months of age $5.6 \pm 0.2(\mathrm{n}=32)$ vs. $4.8 \pm 0.2(\mathrm{n}=49) \mathrm{mmol} / \mathrm{l}(\mathrm{p}<0.01)$. Serum insulin levels were decreased in TG mice compared to WT mice, $30.5 \pm 2.4(\mathrm{n}=18)$ vs. $39.9 \pm 3.2(\mathrm{n}=17)$ $\mu \mathrm{U} / \mathrm{ml}(\mathrm{p}<0.05)$. RPTP $\sigma$ TG mice also showed decreased glucose-induced insulin secretion in isolated pancreatic islets compared to wt animals. Thus, islet insulin responses to $16.7 \mathrm{mmol} / \mathrm{l}$ glucose were $153 \pm 16(\mathrm{n}=7)$ vs. $220 \pm 17$ $(\mathrm{n}=8) \mu \mathrm{U} / \mathrm{islet} / \mathrm{h}(\mathrm{p}<0.01)$.

Conclusion: Our results indicate that overexpression of RPTP $\sigma$ induces increased body weight and hyperglycemia due to impaired glucosestimulated insulin release. Hence, overexpression of RPTP $\sigma$ may play a role in the development of type 2 diabetes.

Supported by Swedish Diabetes Association and Swedish Research Council

\section{2}

Prevention of diabetes by attenuation of PKC $\varepsilon$ induced serine phosphorylation of IRS-1 in Psammomys obesus

E. Shafrir ${ }^{1}$, E. Mack ${ }^{1}$, H. Reuveni ${ }^{1}$, A. Joerns ${ }^{2}$, S. Lenzen ${ }^{2}$, E. Ziv';

${ }^{1}$ Diabetes Center, Hadassah University Hospital, Jerusalem, Israel,

${ }^{2}$ Institute of Clinical Biochemistry, Hannover Medical School, Germany.

Background and Aims: The gerbil Psammomys obesus exhibits hyperglycemia and hyperinsulinemia, beta cell lesions and overexpression of muscle PKCE on high energy (HE) diet. Insulin receptor function and tyrosine kinase activity were inhibited due to increased serine phosphorylation, eliciting marked insulin resistance. Our aim is to prevent the diabetes and PKCE induced phosphorylation of signalling proteins by treating with specific peptides.

Material and Methods: Peptides were copied from substrate-binding domain of the PKCE molecule, to prevent serine phosphorylation of IRS1. The peptides (PCT patent application IL2006/000755) were synthesized and purified to $98 \%$ by HPLC. The peptide $\mathrm{N}$ terminus was myristoylated to facilitate cell entry. Female Psammomys weighing 180-200 g, bred at the Animal Facility on low energy diet were fed high energy diet, then injected with peptide KCe-12 on days 1,8 and 14.Control animals received injections of peptide solvent.

Results: Food consumption and animal weight were unchanged by the treatment On day 16 non-fasting glucose levels were normalised to 4.8 vs $19.3 \mathrm{mmol} / \mathrm{l}$ in controls. Serum insulin was reduced from $584+/-55$ to $180+/-22 \mathrm{mu} / \mathrm{l}$. Western blot analyses show that the phosphoserine ${ }^{636,639}$ on IRS-1 in the gastrocnemius muscle of was reduced fourfold and muscle content of GLUT4 rose twofold. PKC $\varepsilon$ levels were not affected by KCe-12 administration. The damage and loss of insulin in beta-cells were prevented by KC- 12 treatment (shown in photomicrographs). The preserved secretion function enabled Psammomys to compensate for peripheral insulin resistance and restored glucose homeostasis.

Conclusions: KCE-12 derived from the catalytic region of the PKCe molecule prevents the serine phosphorylation of IRS- 1 and abrogates the impediment in insulin signalling, including the translocation of GLUT4, in type 2 diabetes model. Application of specific anti-diabetic peptides may lead to the development of a modality preventing the human over-nutrition induced insulin resistance and diabetes.

Supported by Nofar fund

\section{3}

Protein kinase C- $\boldsymbol{\delta}$-induced phosphorylation of ser-357 in insulin receptor substrate-1(IRS-1) attenuates insulin action

R. S. Waraich, C. Weigert, E. D. Schleicher, H. U. Häring, R. Lehmann; Department of Internal Medicine IV, Eberhard-Karls University, Tuebingen, Germany.

Background and Aims: The function of insulin receptor substrate-1 (IRS1), a key molecule of insulin signaling, is modulated by phosphorylation at multiple Ser/Thr residues. Recently, we identified Ser357 of rat IRS-1 as an in vitro PKC phosphorylation site. The present work was aimed to study the regulation and (patho) physiological function of this phosphorylation site in IRS-1.

Materials and Methods: To monitor phosphorylation of Ser357 in cellular extracts we generated a polyclonal affinity-purified phospho-site specific antibody. Furthermore, Ala357, Ala318, Ala358and Ala357/358 mutants were made by oligonucleotide mediated site-directed mutagenesis 
Results: Using the phospho-Ser357 specific antibody we observed in BHK, C2C12 and HepG2 cells that Ser357 is strongly phosphorylated by phorbolester (TPA) and insulin stimulation. Cross reactivity with the adjacent Ser358 phosphorylation site was eliminated by affinity-purification of the antiserum using a phosphoSer358 synthetic peptide. Phosphorylation on Ser357 is mediated, at least partially, by PKC-delta. Other stimuli tested as anisomycin, TNF-alpha and free fatty acids did not enhance the phosphorylation of Ser357. When we studied insulin signal transduction we found that the activation of Akt/PKB was more pronounced in IRS-1 Ala357 and Ala358 expressing skeletal muscle cells after 10 and 60 min of insulin stimulation, as compared to cells expressing wild type IRS-1. Mutation of both serine sites had no additive effect. Comparable results were obtained in liver cells.

Conclusion: The findings indicate that phosphorylation of Ser357 or Ser358 could lead to down-regulation of insulin action with an interchangeable function of both sites. Interestingly, Ser358 is not conserved between rodents and humans and this putative phosphorylation site is not found in human IRS-1.

Supported in part by Higher Education Commission, Pakistan \& Deutscher Akadamischer Austausch Dienst, Germany

\section{4}

JNK and the PI 3-K/mTOR pathway mediate the platelet-derived growth factor-induced IRS-1 phosphorylation at serine residues 307 and 318 in C2C12 myotubes

K. Müssig, H. Lönz, H. Staiger, H.-U. Häring;

Department of Endocrinology, Diabetology, Nephrology, Angiology, and Clinical Chemistry, University Hospital of Internal Medicine, Tübingen, Germany.

Background and Aims: Insulin signaling is negatively regulated by serine/ threonine phosphorylation of insulin receptor substrate-1 (IRS-1). Several hormones and cytokines, including platelet-derived growth factor (PDGF), have been shown to impair insulin signaling. The aim of the present study was to investigate whether PDFG induces phosphorylation of IRS-1 at serine 307 (Ser307) and serine 318 (Ser318), two phosphorylation sites that inhibit the interaction between the tyrosine-phosphorylated insulin receptor and IRS-1, and to identify the kinases that are involved in these phosphorylations.

Materials and Methods: C2C12 myotubes were incubated with PDGF for different time periods and with diverse concentrations. When indicated, cells were treated with $50 \mu \mathrm{M}$ c-jun N-terminal kinase (JNK) inhibitor SP600125, $25 \mathrm{nM}$ mammalian target of rapamycin (mTOR) inhibitor rapamycin, and $10 \mu \mathrm{M}$ phosphatidylinositol 3-kinase (PI 3-K) inhibitor LY294002 prior to PDGF stimulation. Protein expression and phosphorylation of IRS-1, c-jun, Akt, and p70S6 kinase (S6K) were analyzed by Western-Blotting using the appropriate antibodies.

Results: Treatment of C2C12 myotubes with PDGF increased IRS-1 phosphorylation at Ser307 as well as at Ser318. The maximal stimulating effect occurred after $30 \mathrm{~min}$ and with a concentration of $5 \mathrm{nM}$. Inhibition of the PDFG receptor by $5 \mu \mathrm{M}$ imatinib decreased time-dependently phosphorylation at Ser307 and Ser318 to the basal levels with the maximal inhibition after 90 min. Preincubation with JNK inhibitor SP600125, PI 3$\mathrm{K}$ inhibitor LY294002, and mTOR inhibitor rapamycin markedly decreased PDGF-induced Ser307 and Ser318 phosphorylation. PDGF treatment increased phosphorylation of c-jun, Akt, and S6K, substrates of JNK, PI 3-K, and mTOR, respectively.

Conclusion: PDGF induces IRS-1 phosphorylation at Ser307 and Ser318 in C2C12 myotubes. JNK, PI 3-K, and mTOR appear to be involved in these phosphorylation processes.

\section{5}

Subcellular localization of PKB/Akt substrate PRAS40

E. B. M. Nascimento ${ }^{1}$, M. M. L. Linssen ${ }^{1}$, A. B. Jonsdottir ${ }^{1}$, M. Fodor ${ }^{2}$, J. Kriek ${ }^{1}$, G. C. M. Van der Zon ${ }^{1}$, M. Diamant ${ }^{3}$, J. A. Maassen ${ }^{1}$,

D. M. Ouwens ${ }^{1}$;

${ }^{1}$ Molecular Cell Biology, Leiden University Medical Center, ${ }^{2}$ Anatomy and Embryology/Central Animal Facility, Leiden University Medical Center, ${ }^{3}$ Endocrinology/Diabetes Center, VU Medical Center, Amsterdam, The Netherlands.

Background and Aims: Insulin resistance is an early clinical hallmark of obesity and type 2 diabetes. This condition is associated with a decreased insulin-mediated activation of the PKB/Akt-pathway. Activation of PKB/ Akt has been linked to a variety of cellular responses, including glucose metabolism, cell survival and longevity. The physiological substrates for $\mathrm{PKB} / \mathrm{Akt}$ regulating these downstream responses are poorly characterized. Recently, we characterized proline-rich $\mathrm{PKB} / \mathrm{Akt}$ substrate 40 (PRAS40) as a predominant substrate for $\mathrm{PKB} / \mathrm{Akt}$ in insulin target tissues. Although PRAS40 has been linked to cell survival and nutrient sensing, its exact role in insulin action remains unclear. In the current study we studied the subcellular localization of PRAS40 to further detail the function of this protein.

Materials and Methods: Rat tissues and cultured cells were analyzed for localization and phosphorylation of PRAS40 by immnuofluorescence and immunohistochemistry.

Results: Immunohistochemical staining of rat cardiac muscle and liver displayed nuclear localization of phosphorylated PRAS40. Immunofluorescent staining of cultured cells showed nuclear and cytoplasmatic localization of total PRAS40 protein. After insulin stimulation, the immunoreactivity for the total PRAS40 antibody was increased for both the nucleus and the cytoplasm. When we used the phosphorylated PRAS40 antibody in immunofluorescent staining of cultured fibroblasts and cardiomyocytes, the antibody showed nuclear localization. Insulin stimulation of cultured cells increased the nuclear localization of phosphorylated PRAS40. Part of the phosphorylated PRAS40 signal was detected in the cytoplasm. The insulin stimulated effects were not detected when cells were pretreated with the PI3K inhibitors LY294002 or wortmannin. The signal for phosphorylated PRAS40 was in proximity of phosphorylated $\mathrm{PKB} / \mathrm{Akt}$. The immunoreactivity towards the phosphorylated $\mathrm{PKB} /$ Akt antibody was detected at the interface of cytoplasm and nuclear membrane. Interestingly, in the absence of insulin, we also identified a bright spot which was detected between dividing cells i.e. the midbody, using both the antibody against total PRAS40 and the phosphophorylated PRAS40. Double staining with antibodies recognizing aurora-B, which is known to localize to the midbody during cytokinesis, confirmed the midbody localization of PRAS40.

Conclusion: Phosphorylated PRAS40 was detected in the nucleus of the cell. After insulin stimulation an increased amount of phosphorylated PRAS40 was detected in the nucleus and to a small extent in the cytoplasm. Furthermore, the identification of PRAS40 as part of the midbody complex suggests a critical role for PRAS40 in cell cycle regulation. Supported by the Dutch Diabetes Research Foundation

\section{6}

cAMP-dependent signaling pathways suppress ERK activity but further augmented Akt/PKB activity stimulated by insulin in CHO-IR cells Z. Cui ${ }^{1}$, B. Kim ${ }^{1}$, J. Kim², D. Lee', G. Koh', Y. Lee' ${ }^{1}$, D. Park' ${ }^{1}$ Department of Medicine, Cheju National University, ${ }^{2}$ Jeju Biodiversity Reaech Institute, Jeju, Republic of Korea.

Background and Aims: Although the activation of receptor tyrosine kinase (RTK) is crucial to execute a number of physiological responses by insulin, the roles of signaling pathways derived by G protein-coupled receptors (GPCRs) are unravelled. We previously showed that insulin suppressed apoptotic cell death through signaling pathways including stimulation of PI3 kinase and ERK. The aim of the present study was to examine whether the activation of cAMP-dependent signaling pathways might affect insulin's anti-apoptotic function and RTK-dependent signaling components including ERK and Akt/ PKB upon insulin stimulation in CHO-IR cells.

Materials and Methods: cAMP-dependent signaling pathways were stimulated by treatments with cholera toxin (an activator of Gs protein), pertussis toxin (an inhibitor of Gi protein), and 8-bromo-cAMP (a cell 
membrane-permeable analogue of cAMP) together with- or without insulin in CHO-IR cells. Activities of ERK and Akt/PKB were analyzed by immunoblotting experiments using specific antibodies against activated ERK and Akt/PKB proteins. Apoptotic cell death was evaluated with fluorescence microscopy after H33342 staining and with cell cycle analysis with flow cytometer after propidium iodide staining.

Results: Addition of cholera toxin as well as pertussis toxin suppressed ERK activity stimulated by insulin treatment. However, insulin-induced Akt/PKB activity was more increased by the addition of pertussis toxin or cholera toxin. 8-bromo-cAMP treatment also led to a suppression of ERK activity and an additional increase in Akt/PKB activity stimulated by insulin. Regardless of an additional increase in Akt/PKB activity, insulin's anti-apoptotic function was blocked by activations of cAMP-dependent signaling pathways.

Conclusion: The present study showed that activation of cAMP-dependent signaling pathways regulated downstream steps of RTK after binding of insulin to its receptor in different manners, down-regulating the ERK activity and up-regulating Akt/PKB activity in CHO-IR cells. From the blockade of insulin's anti-apoptotic function even with the up-regulation of Akt/PKB activity by cAMP pathways, it is suggested that insulin's anti-apoptotic function is not totally dependent on Akt/PKB activity or, at least, is regulated at any downstream signaling steps of Akt/PKB activation.

Supported by second phase BK21 programme in Korea

\section{7}

Overexpression of Sam68 enhances metabolic and mitogenic effects of insulin by potentiating MAPK and PI3K pathways

V. Sanchez-Margalet, S. Najib, C. González-Yanes;

Medical Biochemistry and Molecular Biology, Virgen Macarena University

Hospital, Seville, Spain.

Background and Aims: Sam68, is an RNA binding protein with different domains for protein-protein interaction. Thus, a role for Sam68 as an adaptor protein in signal transduction has been proposed. We have previously found that Sam68 is tyrosine phosphorylated by the insulin receptor and recruited to signaling complexes in the PI3K and Ras pathways via interaction with $\mathrm{SH} 2$ and SH3 domain containing proteins. In the present study we have investigated the possible contribution of Sam68 in insulin action.

Materials and Methods: We have studied the role of Sam68 on metabolic and mitogenic insulin actions by overexpressing the protein in the cytosol of CHO-WT and CHO-IR cells, by using a construct lacking the C-terminal nuclear localization sequence cloned in pcDNA3.1 expression vector.

Results: Overexpression of a cytosolic Sam68 (lacking the C-terminal nuclear localization sequence) increased both basal and insulin-mediated metabolic (glucose uptake and glycogen synthesis) and mitotic (DNA and protein synthesis) effects in $\mathrm{CHO}-\mathrm{WT}$ and $\mathrm{CHO}-\mathrm{IR}$ cells. We also studied the effect of Sam68 overexpression in signaling pathways involved in metabolic and mitogenic effects of insulin: IR, IRS-1, PKB, GSK, MAPK, as well as p70S6K, 4E-BP1 and eIF4E. We found that Sam68 overexpression increased both basal and insulin-stimulated phosphorylation of all these signaling proteins. Moreover, the expression of the glucose transporter 1 (GLUT-1) was increased.

Conclusion: These results provide evidence for a role of Sam68 in insulinmediated metabolic and mitotic effects, and confirm the possible participation of Sam68 in the signal transduction pathways of the insulin receptor. Supported by Fondo de Investigación Sanitaria FIS 05/1751, Instituto de Salud Carlos III (ISCIII), Ministerio de Sanidad y Consumo, Spain

\section{8}

Insulin receptor mediates intracellular re-localization of Sam68, an RNA-binding protein that may participate in insulin signaling C. González-Yanes, S. Najib, V. Sánchez-Margalet;

Medical Biochemistry and Molecular Biology, Virgen Macarena University Hospital, Seville, Spain.

Background and Aims: Sam68, an RNA-binding protein involved in signal transduction, has been previously found to be tyrosine phosphorylated by insulin stimulation and recruited to insulin receptor signaling complexes. Sam68 has a nuclear localization signal, but can also localize in the cytoplasm.
Besides traffic of Sam68 from the nucleus to the cytoplasm and the other way round has been described depending on the Tyr phosphorylation. In the present work we sought to investigate the possible relocalization of Sam68 in response to insulin.

Materials and Methods: We employed Sam68-YFP construct to monitor the localization of Sam68 under basal and insulin-stimulated conditions. The transient transfections were carried out in CHO-WT and CHO-IR cells, and Sam68 localization was investigated by confocal microscopy.

Results: We found that Sam68 was present mainly in the nucleus in the CHOWT cells, but in $\mathrm{CHO}$ cells overexpressing insulin receptor (CHO-IR), Sam68 was found equally in the nucleus and the cytoplasm. In response to insulin, after 10 min stimulation, nuclear localization of Sam68 was lower, and some concentration in the cytoplasm/perinuclear area was observed. After $30 \mathrm{~min}$ the localization of Sam 68 was the same as basal conditions.

Conclusion: Sam68 is found in both the nucleus and the cytoplasm. Insulin receptor overexpression increased the cytosolic localization, and insulin stimulation mediates a transient relocalization of Sam68 in the cytoplasm/ perinuclear area.

Fondo de Investigación Sanitaria FIS CP05/00273, Instituto de Salud Carlos III (ISCIII), Ministerio de Sanidad y Consumo, Spain 


\section{PS 40 Transcription factors in metabolism}

\section{9}

Liver-specific knock down of liver X receptor [alpha] in KKAy diabetic mice improves lipid profile but induces organ toxicity

J. Prestle ${ }^{1}$, C. Schoelch ${ }^{1}$, A. van Marle ${ }^{2}$, H. Pavliska ${ }^{2}$, T. Nolte ${ }^{1}$,

J. F. Rippmann ${ }^{1}$;

${ }^{1}$ Boehringer-Ingelheim Pharma GmbH \& Co. KG, Biberach a.d.R.,

Germany, ${ }^{2} \mathrm{~A}$ Galapagos company, Biofocus DPI, CN Leiden,

The Netherlands.

Background and Aims: Nuclear hormone receptors liver X receptor (LXRalpha and LXRbeta) ligands are attractive approaches for the treatment of dyslipidemia and atherosclerosis. To further elucidate the function of LXRalpha in liver lipid metabolism in a disease relevant animal model, the KKAy mouse, we used different shRNAs in adenoviral vectors (Ad5) to selectively knock down LXRalpha gene expression.

Materials and Methods: Obese KKA $y$ mice were transduced with $1 \times 10^{11}$ virus genomes per animal via i.v. tail vein injection. Quantitative gene expression was analysed by real-time PCR. Metabolic parameters as well as liver histology were assessed at 8 days after virus transduction.

Results: The most effective shRNA reduced LXRalpha mRNA in liver by 66\%. LXRalpha-regulated lipogenic genes like SREBP1c, SCD1, and FASN were significantly downregulated, whereas ABCA1 and PCK1 expression was unchanged upon LXRalpha knock down. In addition, we show a LXRalpha gene dosage effect on lipid metabolism with reductions of both plasma and liver triglycerides by about $80 \%$ when compared to control virus transduction. Notably, although the animals did not show obvious signs of tolerability problems, LXRalpha knock down induced liver toxicity over time. Strong knock down of LXRalpha drastically increased serum activity of liver specific enzymes. Histological analysis of liver sections revealed hepatocellular degeneration and proliferation of Kupffer and oval cells. None of these changes were seen with control virus transduction.

Conclusion: Liver-specific knock down of LXRalpha in vivo by shRNA improved lipid profile of hypertriglyceridemic KKAy mice, but also causes serious liver damage.

\section{0}

Identification of a peroxisome proliferator responsive element (PPRE) in rat glucose 6-phosphatase gene promoter

J.-S. Bae ${ }^{1,2}$, S.-K. Kwon ${ }^{1,3}$, S.-S. Im $^{1}$, Y.-S. Park ${ }^{1,3}$, T.-H. Kim ${ }^{1,3}$, J.-M. Park ${ }^{1,3}$, Y.-H. Ahn ${ }^{1,2}$,

${ }^{1}$ Department of Biochemistry and Molecular Biology, ${ }^{2}$ Center for Chronic Metabolic Disease Research, ${ }^{3}$ Brain Korea 21 Project for Medical Sciences, Yonsei University College of Medicine, Seoul, Republic of Korea.

Background and Aims: Glucose-6-phosphatase (G6Pase) is known to play a key role of gluconeogenesis in the fasting liver. Peroxisome proliferators activated receptors (PPARs) are transcription factors involved in the regulation of numerous metabolic processes. The PPAR $\alpha$ isotype is abundant in liver and activated by fasting. However, it is not very clear that the expression of G6Pase gene was related to PPAR $\alpha$. In this study, we demonstrate that PPAR $\alpha /$ $\mathrm{RXR} \alpha$ complex also plays a role in carbohydrate metabolism via regulation of glucose 6-phosphatase gene expression. We have attempted to localize and characterize PPRE (PPAR responsive element) in the promoter regions of rat G6Pase, which plays the important role in the glucose homeostasis in fasting liver.

Materials and Methods: G6Pase mRNA expression was determined in liver tissue of Sprague-Dawley rats by northern blot analysis and RT-PCR. The effect of transiently overexpressed PPAR $\alpha / R X R \alpha$ was studied using G6Pase promoter reporter gene assay in HepG2 cell lines. PPRE was identified in the promoter regions of rat G6Pase by Electrophoretic mobility shift assay (EMSA).

Results: Treatment of HepG2 rat hepatoma cell lines as well as primary rat hepatocytes with PPAR $\alpha / R X R \alpha$ agonists led to stimulation of G6Pase mRNA expression. We examined the physiological significance of PPAR $\alpha /$ $\mathrm{RXR} \alpha$ agonist-induced enhancement of G6Pase expression in primary rat hepatocytes. The overexpression of PPAR $\alpha$ by transfection in HepG2 cell and the treatment of PPAR $\alpha$ agonist, Wy14,643 activated the promoter activity. Serial deletion of the construct revealed that the promoter activity was dramatically decreased when -231 region was deleted. More- over the promoter activity was decreased by the mutation of PPRE and EMSA study indicated that the putative PPRE is located in the region between bases -109 and -100 of this promoter.

Conclusion: These data suggest that the putative PPRE may be located in the rat G6Pase promoter between -268 and -256 . PPAR $\alpha / R X R \alpha$ agonist Wy14,643 is a direct transcriptional mediator of G6Pase gene.

Supported by Center for Chronic Metabolic Disease Research, Yonsei University College of Medicine

\section{1}

Effect of the Pro12Ala polymorphism of the PPAR $\gamma 2$ gene on hepatic glucose uptake

M.-J. Honka ${ }^{1,2}$, M. Vänttinen ${ }^{1,2}$, P. Iozzo ${ }^{1}$, K. A. Virtanen ${ }^{1}$, R. Lautamäki ${ }^{1}$, T. Takala ${ }^{1}$, A. P. M. Viljanen ${ }^{1}$, J. Kemppainen ${ }^{1}$, J. Knuuti ${ }^{1}$, P. Nuutila ${ }^{1}$,

M. Laakso2

${ }^{1}$ Turku PET Centre, University of Turku,

${ }^{2}$ Department of Medicine, University of Kuopio, Finland.

Background and Aims: The Pro12Ala polymorphism of the PPAR 2 gene (PPARG2) has been associated with reduced risk of type 2 diabetes. We have previously reported that the skeletal muscle insulin sensitivity is enhanced in lean but not in obese subjects carrying the Ala12 allele. The aim of this study was to investigate the effect of the Pro12Ala polymorphism on hepatic glucose uptake (GU), which is an important determinant of glucose tolerance, and liver fat content in non-diabetic and type 2 diabetic subjects.

Materials and Methods: Study population included 68 healthy and 95 diabetic individuals whose liver GU was measured using fluorine-18labelled deoxyglucose $\left(\left[{ }^{18} \mathrm{~F}\right] \mathrm{FDG}\right)$ and positron emission tomography during hyperinsulinemic euglycemic conditions. Liver fat percentage measured by proton spectroscopy was available for 58 diabetic and 16 healthy (obese) subjects. $5 \%$ was used as a cut-off point between groups with high $(n=50)$ and low liver fat content $(n=24)$. The Pro12Ala polymorphism of PPARG2 was genotyped with Taqman allelic discrimination assay. Body mass index of $27 \mathrm{~kg} / \mathrm{m}^{2}$ was used as a cut-off point between non-obese and obese subjects.

Results: In obese subjects with type 2 diabetes, hepatic GU was 39\% higher in carriers of the Ala12 allele compared to carriers of the Pro12Pro genotype (21.0 \pm 10.6 vs. $15.1 \pm 5.1 \mu \mathrm{mol} / \mathrm{tissue} \mathrm{kg} / \mathrm{min}, \mathrm{p}=0.002$ adjusted for gender and age), whereas in obese non-diabetic subjects the $28 \%$ difference in hepatic GU did not reach statistical significance $(25.3 \pm 11.5$ vs. $19.8 \pm 6.6 \mu \mathrm{mol} / \mathrm{kg} /$ min, $\mathrm{p}=0.093$, adjusted for gender and age, respectively). When subjects with high liver fat content were analysed separately, hepatic GU was $75 \%$ higher in subjects carrying the Ala12 allele than in subjects with the Pro12Pro genotype (26.2 \pm 11.4 vs. $15.0 \pm 8.4 \mu \mathrm{mol} / \mathrm{kg} / \mathrm{min}, \mathrm{p}<0.0005$, adjusted for gender and age). No effect of the Ala12 allele on hepatic GU was observed in non-obese subjects or in subjects with low liver fat content. No differences were found in liver fat percentage between carriers of the Ala12 allele and carriers the Pro12Pro genotype.

Conclusion: We conclude that the Ala12 allele of PPARG2 is associated with high hepatic GU in obese type 2 diabetic and non-diabetic subjects. Furthermore, this effect is more pronounced in subjects with high liver fat content.

\section{2}

Reciprocal regulation of peroxisome proliferator-activated receptor alpha and glucocorticoid receptor forms a new negative feedback loop X. Chen, W. Sun, Y. Bi, M. Li, M. Cai, H. liang, Q. Yu, X. He, J. Weng; Department of Endocrinology, The First Affiliated Hospital of Sun-Yat Sen University, Guangzhou, China.

Background and Aims: Peroxisome proliferator-activated receptor alpha (PPAR $\alpha$ ) and glucocorticoid receptor (GR) are all pleiotropic nuclear factors. Previous research suggested a negative feedback loop might exist between PPAR $\alpha$ and glucocorticoids. Glucocorticoids are rapid and potent stimulator of hepatic PPAR $\alpha$ expression. On the contrary, PPAR $\alpha$ showed negative 
regulation on glucocorticoids at pre-receptor level, inhibiting the expression and activity of the key enzyme type $111 \beta$-hydroxysteroid dehydrogenase (11/-HSD1) in liver. However, our previous study demonstrated a more direct and important action might exist in this feedback loop. We found PPAR $\alpha$ agonist fenofibrate decreased glucocorticoid receptor (GR) protein expression in liver of SD rats. Therefore, this study was design to further investigate the regulation of fenofibrate on GR.

Materials and Methods: Male SD rats were randomly divided into five groups: normal control rats (NC group), rats treated with fenofibrate (50mg/ kg.d for 1 month) (FE1 group), rats treated with fenofibrate (100mg/kg.d for 1 month) (FE2 group), rats treated with fenofibrate (100mg/kg.d for 2 months) (FE3 group), and rats treated with combination of fenofibrate $(100 \mathrm{mg} / \mathrm{kg} . \mathrm{d})$ and PPAR $\alpha$ antagonist MK886 (30mg/kg.d) for 1 month (MK886 group). The GR gene and protein expression levels were detected in liver, visceral fat (VF) and muscle tissues. Circular corticosterone (CORT) level was measured using RIA method.

Results: PPAR $\alpha$ agonist fenofibrate conferred significant tissue-specific downregulation of hepatic GR gene and protein expression of SD rats with increasing CORT level in circulation, and PPAR $\alpha$ antagonist MK886 could block these effects. The downregulation of fenofibrate on GR was dose- and time-dependent on fenofibrate a certain degree.

Conclusion: We further demonstrated a reciprocal regulation between PPAR $\alpha$ and GR, which forms a new negative feedback loop in liver of SD rats.

Supported by the National Basic Research Programme of China

\section{3}

A change of gluconeogenic gene expression following caloric restriction in liver of OLETF rats

H.-Y. Bae ${ }^{1}$, S.-Y. Kim ${ }^{1}$, J.-H. Kim²;

${ }^{1}$ Internal Medicine, College of Medicine, Chosun university,

${ }^{2}$ Internal Medicine, Dept. of Internal Medicine, Chosun University Hospital, Gwang-ju, Republic of Korea.

Background and Aims: Traditionally, gluconeogenesis is strongly stimulated during fasting and is aberrantly activated in diabetes mellitus. Carolic restriction(CR) has been shown to improve the glucose control in type 2 diabetes in human and animal models. However, the exact mechanism of CR on gluconeogenesis in the liver has not been well elucidated. In this study, we investigated the effects of CR on gluconeogenesis in the liver of OLETF rats.

Materials and Methods: OLETF rats(male 24 weeks) for diabetic model and LETO rats(male 24 weeks) for control group were used in this study. At 20th week, all OLETF rats underwent an oral glucose tolerance test after an overnight fast. From 24th week, all of these rats received an allotment of food equal to $70 \%$ of the food intake measures in the last weeks. The activity of the key gluconeogenic enzymes such as PEPCK, G-6-Pase were measured at 0,4 , 8,12 weeks in the liver of both groups. At the same time, we measured the mRNA expression levels of PGC- 1 and PPAR $\gamma$ in the liver of both groups by RT-PCR. Also we investigated the mRNA expression of AMPK in the liver of both groups.

Results: The fasting glucose levels and body weights were decreased progressively in OLETF rats. The mRNA expression of the gluconeogenic enzymes such as PEPCK, G-6-Pase were progressively decreased after caloric restriction in both groups and kept a high level in OLETF rats compared to LETO rats during all periods. A expression of PGC-1 were increased to $29 \%$ in the liver of non-diabetic LETO rats after 12 weeks, but there was no significant change in OLETF rats. On the other hand, the mRNA expression of PPAR $\gamma$ were progressively increased in OLETF rats compared to LETO rats. At the same time, we evaluated the mRNA levels of AMPK in both groups. The mRNA expression of AMPK were significantly increased after 12 weeks CR except $\beta 1$ subunit in OLETF rats.

Conclusion: The effects of caloric restriction on the expression of gluconeogenic genes in the liver are sufficiently predictable and resonable, but the PGC-1 expression after caloric restriction are altered in the liver of OLETF rats compared to LETO rats. These findings suggest that altered PGC1 expression may play some role on the aberrantly increased gluconeogenesis in the liver of OLETF rats.

Supported by the Chosun University

\section{4}

Insulin glargine alleviated fatty liver by down-regulation of Srebf 1 in the liver of high fat diet induced insulin resistant $\mathrm{C} 57 \mathrm{BL} / 6$ mice M. Li, Q. Yu, X. Chen, X. He, Y. Bi, W. Sun, J. Yan, M. Cai, H. Liang, W. Xu, J. Weng;

Department of Endocrinology, The 1st Affiliated Hospital of Sun Yat-sen University, Guangzhou, China.

Background and Aims: Insulin resistance in the liver is one of the main defects in the development of type 2 diabetes. Here we investigated the effect of insulin glargine on the liver of high fat diet induced insulin resistant mice. Materials and Methods: 30 5-week-old C57BL/6 mice were randomized into 3 groups: A, fed with normal diet (9.9\% calorie from fat); B and C, fed with high fat diet (60\% calorie from fat). After 12 weeks fed, mice in group $\mathrm{C}$ were injected with insulin glargine s.c. at $0.5 \mathrm{IU}$. Mice in group A and B were injected with saline s.c. at $0.5 \mathrm{ml}$. The intervention lasted for 4 weeks. After that, the mice were executed and the livers were frozen for mRNA extraction and fat content determination. The mRNA expression of genes involed in insulin signaling pathway and Srebf 1 were determined by gene chip. Fat content of the liver was determined by optical density after Sudan III staining.

Results: The fat content indicated by optical density was $7606 \pm 1230$, $11456 \pm 786,9802 \pm 524$ in Group A, B and C $(P<0.05$, between any two groups $)$ , respectively. If the mRNA expression in Group A was 100\%, the expression of genes involved in the insulin signaling pathway of Group B and C was as follow: Ptpn1 293\% and 143\%; Pik3r2 241\% and 97\%; Akt-2 26\% and 63\%; Akt-3 $17 \%$ and $71 \%$, respectively. The expression of Srebfl, which stimulated lipid synthesis and uptake in heptocytes, in Group B and C were 358\% and $161 \%$ of that in Group A, respectively.

Conclusion: High fat diet induced defects of gene expression in the insulin signaling pathway and increased Srebf 1 expression, caused insulin resistance and fat overload in the liver of C57BL/6 mice. The 4 weeks intervention of insulin glargine partially alleviated the defects and decreased the fat deposition by down-regulation of Srebf 1 in the liver. Supported by the National Basic Research Programme of China

\section{5}

Influence of metabolic stimuli on transcriptional regulation by Hepatocyte Nuclear Factor $4 \alpha$

A. S. Devonshire, N. J. Plant, A. E. Thumser;

School of Biomedical and Molecular Sciences, University of Surrey, United Kingdom.

Background and Aims: The nuclear receptor Hepatocyte Nuclear Factor $4 \alpha$ (HNF4 $\alpha$ ) (NR2A1) regulates the transcription of many genes involved in both glucose and lipid metabolism. Mutations in the HNF4a gene are responsible for MODY type 1, and genetic linkage analyses have implicated HNF4 $\alpha$ in the disease processes leading to late-onset type 2 diabetes and dyslipidaemia. HNF4 $\alpha$-dependent transcriptional activity is known to be modulated by a range of hormones associated with the fasting-fed cycle: glucagon and insulin, as well as their downstream effectors such as protein kinase A (PKA) and AMP-activated protein kinase (AMPK). In this study our aim was to investigate the role of HNF4 $\alpha$ in modulating the effects of physiological mediators on genes involved in carbohydrate and lipid metabolism.

Materials and Methods: Human liver-derived HepG2 cells were serumstarved for $24 \mathrm{~h}$ and then incubated for $6 \mathrm{~h}$ in the presence or absence of glucagon, dexamethasone, insulin, activators of PKA (8-bromoadenosine $3^{\prime}, 5^{\prime}$-cyclic monophosphate (8-Br-cAMP) or 3-isobutyl-1-methylxanthine (IBMX)), or 5-aminoimidazole-4-carboxamide 1- $\beta$-D-ribofuranoside (AICAR), an activator of AMPK. In further experiments, HepG2 cells were transfected with a dominant negative (DN) HNF4 $\alpha$ vector (pDGT23.1, a gift of Prof. T. Leff, Wayne State University School of Medicine, Detroit, MI) or an equal amount of control plasmid pcDNA3, $16 \mathrm{~h}$ prior to serum-starvation. The relative mRNA expression levels of HNF4 $\alpha$ and selected target genes were quantified using real-time PCR and normalized to the expression of a housekeeping gene, cytochrome c-1.

Results: In testing several hormones and physiological mediators, we found that insulin produced an increase in the expression of fatty acid synthase $(74 \%, p=0.05)$, and a decrease in apolipoprotein C3 $(42 \%, \mathrm{NS})$; however HNF4 $\alpha$ gene expression itself was not altered. Glucagon, dexamethasone and the AMPK activator, AICAR, did not produce significant changes in HNF4 $\alpha$ 
or target gene expression. In addition, peroxisome proliferator-activated receptor- $\alpha$ and sterol regulatory element-binding protein $1 \mathrm{c}$ expression was not significantly altered by any of the conditions after $6 \mathrm{~h}$ incubation.

The PKA activators, 8-Br-cAMP and IBMX, stimulated the expression of PEPCK $(210 \%, \mathrm{p}<0.001$, and $91 \%$ respectively, $\mathrm{p}<0.05)$ and inhibited the expression of liver-type pyruvate kinase (L-PK) $(56 \%$ by 8 -Br-cAMP, $\mathrm{p}<$ $0.01 ; 36 \%$ by IBMX, NS). Further investigation of the effect of 8 -Br-cAMP on HNF4 $\alpha$ activity in HepG2 cells over-expressing a DN-HNF4 $\alpha$ plasmid showed that 8-Br-cAMP caused a similar increase in PEPCK as in the control, i.e. had no effect, whilst the expression of DN-HNF4 $\alpha$ partially reversed the negative effect of 8-Br-cAMP on L-PK expression, producing only $27 \%$ inhibition vs. $56 \%$ in the control ( $\mathrm{p}<0.05)$.

Conclusion: Transfection of HepG2 cells with a DN-HNF4 $\alpha$ expression plasmid, which forms non-functional heterodimers with wild-type HNF4 $\alpha$ and prevents DNA-binding, ameliorated the inhibitory effect of cAMP on L-PK expression, whilst it did not prevent the stimulatory effect of cAMP on PEPCK expression. This real-time PCR data provides evidence for the role of HNF $4 \alpha$ in mediating the inhibitory effects of cAMP on the endogenous expression of the L-PK gene in HepG2 cells. Further work will address the role of co-activators and HNF4 $\alpha$ levels on expression of target genes. Supported by the School of Biomedical and Molecular Sciences PhD Studentship (University of Surrey, UK) and the Guildford Bench Methodology Fund

\section{PS 41 Incretin effects: clinical}

\section{6}

Contrasting actions of sitagliptin and exenatide on food intake, body weight, glucose stimulated insulin secretion and gastric emptying in rodents

B. Gedulin, K. Tatarkiewicz, P. Smith, J. Wilson, D. Bhole, D. Kendall, D. Hargrove, D. Parkes;

Amylin Pharmaceuticals, Inc., San Diego, United States.

Background and Aims: Exenatide (EXN) is an incretin mimetic that shares similar glucoregulatory properties to human GLP-1 including enhancement of glucose-dependent insulin secretion, regulation of gastric emptying (GE), decreased food intake (FI) and decreased postprandial glucagon secretion. Sitagliptin (SGP) is a recently approved DPP-IV inhibitor and acts by increasing the amount of active endogenous GLP-1 in the circulation. The present studies compared the pharmacological effects of acute treatment with EXN vs SGP.

Materials and Methods: To assess duration of action of SGP, C57BL/6J male mice received a single oral dose of 10 or $50 \mathrm{mg} / \mathrm{kg}$, and plasma DPPIV activity was measured up to $24 \mathrm{~h}$. OGTTs were performed in $d b / d b$ mice with EXN $(20 \mu \mathrm{g} / \mathrm{kg}$ IP) and SGP (50 mg/kg PO) administered $15 \mathrm{~min}$ before a glucose challenge $(2 \mathrm{~g} / \mathrm{kg})$. For measurement of FI and body weight (BW), single housed fed male SD rats received SGP $(60 \mathrm{mg} / \mathrm{kg}$ PO) or water 45 minutes prior to lights out ( $\mathrm{n}=7 /$ group); EXN ( $5 \mu \mathrm{g} / \mathrm{kg} \mathrm{IP}$ ) or $10 \% \mathrm{DMSO} 15$ minutes prior to lights out ( $\mathrm{n}=7 /$ group). The food hopper was weighed every 5 seconds for the following $24 \mathrm{~h}$. BW was measured $24 \mathrm{~h}$ post treatment. To assess GE, conscious fasted male SD rats were given an oral gavage of SGP $(60 \mathrm{mg} / \mathrm{kg})$ or water at $\mathrm{t}=-45$; a SC injection of saline or EXN $(10 \mathrm{nmol} / \mathrm{kg})$ at $\mathrm{t}=-5$; and an oral gavage of $33 \mathrm{mg}$ acetaminophen (ACET) at $\mathrm{t}=0(\mathrm{~N}=6-12$ per group). GE was measured by appearance of ACET in plasma 30 minutes after ACET gavage.

Results: Three hours after oral administration of SGP $(10$ or $50 \mathrm{mg} / \mathrm{kg})$, DPP-IV activity decreased by $75 \%$ and $90 \%$ from baseline, respectively, and returned to basal levels after 24h. In the EXN group, after an OGTT, plasma insulin (assessed as $\mathrm{AUC}_{0-120 \min }$ ) increased by $86 \%(\mathrm{P}<0.05)$ and plasma glucose $\left(\mathrm{AUC}_{0-120 \min }\right)$ decreased by $47 \%(\mathrm{P}<0.01)$ vs control. There was no difference in insulin or glucose AUC between SGP-treated mice and controls. SGP $(60 \mathrm{mg} / \mathrm{kg}$ PO) did not affect mean cumulative FI (CFI) measured hourly over a $24 \mathrm{~h}$ period, while EXN $(5 \mu \mathrm{g} / \mathrm{kg}$ IP) significantly decreased CFI at each hour over the $24 \mathrm{~h}$ period. Mean CFI at $12 \mathrm{~h}$ and $24 \mathrm{~h}$ was reduced in the EXN group by $45 \%$ and $33 \%$ respectively ( $\mathrm{P}<0.01$ vs control group). BW gain after $24 \mathrm{~h}$ was significantly reduced by $2.5 \%$ in the EXN group $(\mathrm{P}<0.01)$ but not in SGP-treated rats, compared to their respective control groups. Oral administration of SGP $(60 \mathrm{mg} / \mathrm{kg})$ decreased GE by $39 \pm 4 \%$ vs $90 \pm 2 \%$ inhibition with EXN $(10 \mathrm{nmol} / \mathrm{kg})$ administered SC $(\mathrm{P}<0.0001)$. In these GErelated studies, DPP-IV activity measured at $\mathrm{t}=30 \mathrm{~min}$ was reduced only in SGP-treated rats $(91 \%$ from control group, $\mathrm{P}<0.001)$.

Conclusion: These studies reveal significantly greater in vivo efficacy with exenatide vs sitagliptin for modulating several physiological parameters that contribute to improved glucoregulation.

\section{7}

The insulinotropic effect of GIP is impaired in patients with chronic pancreatitis and secondary diabetes as compared to normal glucose tolerant patients with chronic pancreatitis

F. K. Knop ${ }^{1}$, T. Vilsbøll' ${ }^{1}$, P. V. Højberg ${ }^{1}$, S. Larsen ${ }^{2}$, S. Madsbad ${ }^{3}$, J. J. Holst ${ }^{4}$, T. Krarup ${ }^{1}$;

${ }^{1}$ Department of Internal Medicine F, Gentofte University Hospital,

${ }^{2}$ Department of Internal Medicine M, Glostrup University Hospital,

${ }^{3}$ Department of Endocrinology, Hvidovre University Hospital,

${ }^{4}$ Department of Biomedical Sciences, University of Copenhagen, Denmark.

Background and Aims: The incretin effect is reduced and the insulinotropic effect of the incretin hormone glucose-dependent insulinotropic polypeptide (GIP) is lost in patients with type 2 diabetes mellitus (T2DM). We aimed to evaluate the causality of this deficiency.

Materials and Methods: We investigated 8 patients with chronic pancreatitis (CP) and normal glucose tolerance (NGT) (fasting plasma glucose (FPG): 
5.5 (4.5-6.0) $\mathrm{mM}$ (mean (range); $\mathrm{HbA}_{1 \mathrm{c}}: 5.8$ (5.4-6.3) \%) and 8 patients with $\mathrm{CP}$ and secondary non-insulin dependent diabetes (FPG: 7.1 (6.0-8.8) mM; $\mathrm{HbA}_{1 \mathrm{c}}: 7.0$ (5.8-10.0) \%) during three 15-mM hyperglycaemic clamps with continuous iv infusion of saline, glucagon-like peptide-1 (GLP-1) or GIP.

Results: The initial (0-20 minutes) insulin and C-peptide responses were enhanced significantly in both groups by GLP-1 and GIP, respectively, compared to saline $(P<0.05)$. In both groups GLP-1 infusion resulted in significantly greater insulin and C-peptide responses from 20-120 minutes compared with saline infusion. During GIP infusion the late-phase insulin response (20-120 minutes) was $3.1 \pm 1.0$ fold greater than during saline infusion in the group of patients with CP and NGT $(P<0.05)$, whereas there was no significant differences in patients with $\mathrm{CP}$ and DM.

Conclusion: The lack of GIP amplification of the late insulin response to iv glucose develops alongside the deterioration of glucose tolerance in patients with CP, suggesting that the same may be true for the loss of the GIP effect in patients with T2DM.

Supported by the Chief Physician Boserup's Foundation and the Danish Diabetes Association

\section{8}

The long-acting GLP-1 analogue, liraglutide, normalises olanzapine-induced weight gain and glucose tolerance K. Lykkegaard ${ }^{1}$, P. J. Larsen ${ }^{1}$, L. B. Knudsen ${ }^{2}$;

${ }^{1}$ Rheoscience, Roedovre, Denmark, ${ }^{2}$ Novo Nordisk, Maaloev, Denmark.

Background and Aims: Therapeutic use of atypical antipsychotic agents is often associated with weight gain and impaired glucose tolerance. The longacting GLP-1 analogue liraglutide has been found to reduce weight and improve glycemic control in both preclinical and clinical experiments. In the present experiment, we investigated whether liraglutide could decrease olanzapine induced weight gain and reduce glucose levels in female rats.

Materials and Methods: Female Sprague Dawley rats were implanted with subcutaneous osmotic mini pumps for delivery of olanzapine $(1.75 \mathrm{mg} / 24$ hours, $\mathrm{n}=20)$ or vehicle $(2.5 \mu \mathrm{l} / \mathrm{h}, \mathrm{n}=20)$ for 28 days. After 14 days, ten animals from each group received in addition doses of liraglutide $(0.2 \mathrm{mg} / \mathrm{kg}$ BID) or vehicle (BID) for the remainder of the study. Statistical significance is indicated: ${ }^{a} \mathrm{p}<0.05$ olan or lira vs. vehicle, ${ }^{b} \mathrm{p}<0.05$ lira vs. olan.

Results: Liraglutide treatment (lira) during the last two weeks of olanzapine treatment normalized olanzapine-induced (olan) elevation of cumulated food intake to the level of vehicle treated (veh) animals (lira, $601.8 \pm 20.4^{\text {b }}$ olan, $667.3 \pm 7.0^{\mathrm{a}}$; veh, 593.2 $\pm 13.2 \mathrm{~g}$ ). Likewise, liraglutide normalized olanzapineinduced body weight gain (lira, $280.2 \pm 5.6^{\mathrm{b}}$; olan $306.6 \pm 4.2^{\mathrm{a}}$; veh, $276.4 \pm 3.6$ g), decreased subcutaneous inguinal fat depot (lira, $2.4 \pm 0.2^{\text {ab }}$; olan $3.4 \pm 0.3^{\mathrm{a}}$; veh, $1.9 \pm 0.1 \mathrm{~g}$ ) and normalized mesenterial (lira, $1.8 \pm 0.1^{\mathrm{b}}$; olan $3.1 \pm 0.2^{\mathrm{a}}$; veh, $1.7 \pm 0.2 \mathrm{~g}$ ) and retroperitoneal fat depots (lira, $3.5 \pm 0.4^{\mathrm{b}}$; olan $6.2 \pm 0.6^{\mathrm{a}}$; veh, $2.8 \pm 0.3 \mathrm{~g}$ ). Glucose tolerance was normalized after treatment with liraglutide, measured as the area under the curve of the glucose concentration curve during an OGTT (lira, 1,764 $\pm 32^{\mathrm{b}}$; olan 1,906 $\pm 66^{\mathrm{a}}$; veh, $1,770 \pm 28 \mathrm{mM} \mathrm{x}$ min). Conclusion: In conclusion, liraglutide is a promising compound for alleviating the weight gain and glucose intolerance often seen during treatment with atypical antipsychotic agents.

\section{9}

The role of endogenous GLP-1 on insulin secretion during oral glucose tolerance test in healthy subjects and patients with type 2 diabetes mellitus

B. A. Aulinger, T. P. Vahl, D. A. D’Alessio, M. Salehi;

Internal medicine - Endocrinology, University of Cincinnati, United States.

Background and Aims: Glucagon-like peptide-1 (GLP-1) enhances insulin secretion after ingestion of carbohydrates $(\mathrm{CHO})$ glucose-dependently. The effect of endogenous GLP-1 on postprandial insulin secretion has been investigated using the GLP-1 receptor (GLP-1r) antagonist exendin-(9-39) (Ex-9). Although the independent effect of GLP-1 on insulin secretion could not be evaluated given that the blockade of GLP-1r resulted in hyperglycemia, we sought to determine the effect of endogenous GLP-1 on postprandial insulin secretion independent of circulatory glucose concentrations during intravenous-oral hyperglycemic clamp using Ex-9.
Materials and Methods: 8 healthy subjects ( $5 \mathrm{~F}$ and $3 \mathrm{M}$; age 29-45; BMI 19-31) were studied on two occasions, once with Ex-9 (750 pmol/ $/ \mathrm{kg} / \mathrm{min})$ and once with a saline control. This dose of Ex-9 was sufficient to abolish the effects of a supraphysiologic dose of GLP-1 on glucose-stimulated insulin secretion. After an overnight fast, a square wave of hyperglycemia was produced and maintained at a target level. At $90 \mathrm{~min}$, subjects ingested $75 \mathrm{~g}$ of oral glucose within $5 \mathrm{~min}$. The glucose clamp was maintained for 3 hours after oral glucose by adjustments in the rate of intravenous glucose infusion. The same principle was used in a group of 12 patients ( 6 female and 6 male; age 40-59 (52 \pm 1.8$)$; BMI 26-44 (33.9 \pm 1.6$) \mathrm{kg} / \mathrm{m} 2)$ with established T2DM (9 treated with metformin with or without sulfonylurea, 3 treated with diet) and good glycemic control (HbA1C $6.2 \pm 0.17 \%$ ) using a 300 -kcal mixed meal ingested within $10 \mathrm{~min}$ instead of oral glucose solution.

Results: In healthy subjects glucose levels were maintained at $161.9 \pm 2.2$ $\mathrm{mg} / \mathrm{dl}$ and $166.4 \pm 2.6$ (NS) during the 2 studies and diabetic patients were clamped at $215.9 \pm 0.9$ and $217.5 \pm 0.6$ (NS), respectively. Glucose levels were not different before and after oral $\mathrm{CHO}$ in all studies. Mean insulin levels increased significantly $(\mathrm{p}<0.001)$ after oral glucose by a factor of $4.4 \pm 0.8$ and $3.5 \pm 0.4$ in healthy subjects and about 3 -fold in diabetic subjects after ingestion of the mixed meal. Ex-9 decreased mean insulin levels after oral glucose in a healthy subject from $1083 \pm 63$ to $791 \pm 55(\mathrm{p}<0.05)$ in healthy subjects and from $1136 \pm 85$ to $872 \pm 54(\mathrm{p}<0.05)$ in diabetic patients.

Conclusion: Oral administration of $\mathrm{CHO}$ significantly enhances insulin secretion during a hyperglycemic clamp in both healthy subjects and patients with well-controlled T2DM. In our healthy and diabetic cohort, endogenous GLP-1 action accounted for $30 \%$ of postprandial insulin secretion. Supported by NIH

\section{0}

Separate impact of obesity and glucose tolerance on incretin effect in humans

E. Muscelli ${ }^{1}$, S. Camastra ${ }^{1}$, F. Tesi $^{1}$, A. Natali ${ }^{1}$, A. Casolaro ${ }^{1}$, B. D. Astiarraga $^{1}$, R. Femia ${ }^{1}$, F. Santini ${ }^{2}$, J. Holst ${ }^{3}$, A. Mari ${ }^{4}$, E. Ferrannini ${ }^{1}$; ${ }^{1}$ Department of Internal Medicine, University of Pisa, Italy, ${ }^{2}$ Department of Endocrinology, University of Pisa, Italy, ${ }^{3}$ Department of Medical Physiology, Panum Institute, Copenhagen, Denmark, ${ }^{4}$ Institute of Biomedical

Engineering, Consiglio Nazionale delle Ricerche, Padova, Italy.

Background and Aims: The separate impact of obesity and hyperlycemia on incretin effect, i.e. the gain in insulin secretion after oral glucose $v s$ intravenous glucose, has not been investigated.

Materials and Methods: Isoglycemic oral (75 g of glucose, 3 hours) and IV glucose administration was performed - on separate days - in 50 subjects ( 25 NGT, 16 IGT and 9 type $2 \mathrm{DM}$, mean age $=41 \pm 2$ years) with a wide range of BMI (19.6 to $61.2 \mathrm{~kg} \cdot \mathrm{m}^{-2}$, mean=35.4 \pm 3.6 ). C-peptide deconvolution was used to reconstruct insulin secretion rates, and $\beta$-cell function was evaluated by mathematical modelling. $\beta$-cell glucose sensitivity was calculated as the slope of the insulin secretion/glucose concentration dose-response curve. The incretin effect was defined as the ratio of oral to IV secretory responses. Results: The glucose area-under-curve $\left(\mathrm{AUC}_{\mathrm{G}}\right)$ during the oral load ranged 965-2624 mmol. $\mathrm{l}^{-1} .3 \mathrm{~h}$ (mean $\left.=1483 \pm 52\right)$ and was closely reproduced by intravenous glucose $\left(1504 \pm 51 \mathrm{mmol} \cdot \mathrm{l}^{-1} .3 \mathrm{~h}\right)$. Fasting insulin secretion was similar in both studies (95 [57] vs 110 [90] pmol. $\mathrm{min}^{-1} \cdot \mathrm{m}^{-2}$, median [interquartile range], oral $v s \mathrm{IV}, \mathrm{p}=\mathrm{ns})$, while total post-OGTT insulin secretion was significantly higher with oral than IV glucose (62 [32] vs 46 [36] nmol. $\mathrm{m}^{-2}$, oral/IV ratio $\left.=1.44 \pm 0.07, \mathrm{p}<0.0001\right)$. $\beta$-cell glucose sensitivity also was higher with oral than IV glucose $\left(100 \pm 9\right.$ vs $68 \pm 6 \mathrm{pmol} \cdot \mathrm{min}^{-1} \cdot \mathrm{m}^{-2} \cdot \mathrm{mM}^{-1}$, ratio $=1.68 \pm 0.12, \mathrm{p}<0.0001)$. In the whole group, both the incretin effect on total insulin output and that on glucose sensitivity were inversely related to $\mathrm{BMI}$ and $\mathrm{AUG}_{\mathrm{C}}$ (all $\mathrm{p} \leq 0.01$ ). In a multiple regression model controlling for sex and age, the incretin effect on total insulin output was reciprocally related to both BMI (standardised $\mathrm{r}=-0.58, \mathrm{p}<0.0001$ ) and $\mathrm{AUG}_{\mathrm{C}}$ (standardised $\mathrm{r}=-$ $0.35, p=0.01$ ) independently of one another. Similarly, the incretin effect on glucose sensitivity was independently related to BMI (standardised $\mathrm{r}=-0.39$, $\mathrm{p}<0.01$ ) and $\mathrm{AUG}_{\mathrm{C}}$ (standardised $\mathrm{r}=-0.36, \mathrm{p}=0.02$ ).

Conclusion:Incretin-mediated stimulation of insulin secretion and $\beta$-cell glucose sensitivity is independently downregulated by both obesity and acute hyperglycaemia.

Supported by the University of Pisa 


\section{1}

No increased risk of hypoglycaemic episodes during 48 hours of continous GLP-1 administration in fasting healthy subjects

S. Lerche ${ }^{1}$, L. Soendergaard ${ }^{1}$, B. Brock ${ }^{1}$, J. Rungby ${ }^{1}$, N. Moeller ${ }^{2}$, J. J. Holst ${ }^{3}$, O. Schmitz

${ }^{1}$ Department of Pharmacology, Aarhus University,

${ }^{2}$ Medical Research Laboratory, Aarhus University Hospital,

${ }^{3}$ Department of Medical Physiology, Copenhagen University, Denmark.

Background and Aims: During fasting the glycaemic level is orchestrated by numerous biochemical processes. The insulinotropic and glucagonostatic effects of glucagon-like-peptide-1 (GLP-1) are glucose dependent. However it is speculative whether the ability to sufficiently compensate hypoglycaemia during fasting is preserved, when pharmacological GLP-1 levels are present. Furthermore, it is uncertain whether an oral glucose stimulus after a 60 hour fasting period would lead to reactive hypoglycaemia during high GLP-1 levels. To date the effect of GLP-1 administration on glycaemia and the counter regulatory response for more than 12 hours of fasting has never been explored. Thus the aim of the study was to assess the safety of a pharmacological relevant dose of native GLP-1 during long term fasting with regard to glycaemia and counter regulation. Our hypothesis was that there was no increased risk of hypoglycaemia with GLP-1 infusion due to glucose dependency and preserved counter regulation and only a minor risk of reactive hypoglycaemia exists in the wake of a subsequent glucose load.

Materials and Methods: We conducted a randomized placebo controlled cross-over study in 8 healthy male subjects (age; $24 \pm 2$ years (mean $\pm \mathrm{SD}$ ), BMI $24.4 \pm 0.7 \mathrm{~kg} / \mathrm{m} 2)$. After an overnight fast a subcutaneous infusion of GLP-1 (7-36amide) or placebo was started followed by a fasting period of 48 hours and finally a 3-hour oral glucose tolerance test (OGTT) was performed. Using a portable insulin pump GLP-1/placebo was infused continuously in a step wise manner for 6 hours following which $4.8 \mathrm{pmol} / \mathrm{kg} / \mathrm{min}$ was given for the rest of the study. Each subject completed two study periods. At a plasma glucose $\leq 2.8 \mathrm{mM}$ and/or neuroglycopenic symptoms the subjects were withdrawn from the study period.

Results: 2 subjects in the GLP- 1 group and 1 subject in the placebo group were withdrawn due to the above criteria (after 25, 29 and 25 hours of GLP1 and placebo infusion, respectively). The average concentration of intact GLP-1 was $30.5 \pm 2.0$ vs. $5.6 \pm 0.9 \mathrm{pmol} / 1$ (GLP-1 vs. placebo, mean $\pm \mathrm{SEM}$ ), $(\mathrm{P}=0.004)$. During the fasting period plasma glucose $(\mathrm{PG})$ declined in a comparable manner from $4.9 \pm 0.09$ vs. $5.0 \pm 0.1$ to $3.7 \pm 0.2$ vs. $3.7 \pm 0.1 \mathrm{mmol} / \mathrm{l}$ $(\mathrm{P}=0.003)$. Levels of insulin $(\mathrm{P}=0.0006)$ and $\mathrm{C}$-peptide $(\mathrm{P}=0.0001)$ decreased with fasting time, the glucagon level increased $(\mathrm{P}=0.0001)$ as did the levels of growth hormone $(\mathrm{GH}),(\mathrm{P}=0.0001)$ and free fatty acids $(\mathrm{FFA})(\mathrm{P}=0.0001)$. No difference between GLP-1 and placebo was observed. Similarly, circulating insulin, C-peptide, FFA, glucagon, GH, cortisol and epinephrine did not differ. During the OGTT, PG was similar to GLP-1 and placebo $(\mathrm{P}=0.48)$ whereas circulating insulin $(\mathrm{P}=0.06)$ and $\mathrm{C}$-peptide $(\mathrm{P}=0.05)$ levels tended to be higher during GLP-1 infusion

Conclusion: The counter regulatory response during 48 hours of subcutaneous GLP-1 infusion was preserved during fasting. There was no increased risk of major hypoglycaemic episodes. No reactive hypoglycaemia was observed after a glucose load following the fasting period. These observations may be of relevance utilizing potential future long acting GLP1 analogues.

Supported by the Danish Diabetes Association and the NovoNordisk Foundation

\section{2}

The influence of gastric inhibitory polypeptide on ghrelin secretion in different metabolic conditions

A. F. H. Pfeiffer ${ }^{1,2}$, O. Goegebakan ${ }^{1,2}$, B. Otto ${ }^{3}$, M. Mohlig ${ }^{1,2}$, J. Spranger ${ }^{1,2}$, M. O. Weickert ${ }^{1,2}$, N. N. Rudovich ${ }^{1,2}$,

${ }^{1}$ Clinical Nutrition, DIFE Potsdam-Rehbrücke, Nuthetal,

${ }^{2}$ Endocrinology, Diabetes and Nutrition, Campus B. Franklin, Charite University Medicine, Berlin, ${ }^{3}$ Internal Medicine and Gastroenterology, Innenstadt University Hospital, Munich, Germany.

Background and Aims: Circulating ghrelin concentrations decrease after meal consumption. The effect incretins on ghrelin secretion in humans remains unclear. We quantified circulating ghrelin concentrations under different metabolic conditions combined with infusion of gastric inhibitory polypeptide (GIP).

Materials and Methods: 14 health obese men (age: $47.4 \pm 8.4$ yr.; BMI: $32.5 \pm 2.2 \mathrm{~kg} / \mathrm{m} 2$; mean \pm S.E.) participated in two or four of the following procedures: 1 . Placebo $(0.9 \% \mathrm{NaCl})$ and GIP-infusion for $240 \mathrm{~min}$ in crossover, randomized manner. 2. A hyperinsulinemic, euglycemic clamp (EC) ( $4.4 \mathrm{mmol} / \mathrm{L}$ ) combined with placebo or GIP-infusion for $240 \mathrm{~min}$ in crossover, randomized manner. 3. A hyperinsulinemic, hyperglycemic clamp (HC) $(7.8 \mathrm{mmol} / \mathrm{L})$ combined with placebo and GIP-infusion for $240 \mathrm{~min}$ in cross-over, randomized manner. In all tests GIP was infused at infusion rate of [2.0 $\cdot \mathrm{pmol} \mathrm{kg} 1 \cdot \min 1]$ for $240 \mathrm{~min}$.

Results: Ghrelin levels were unchanged during GIP- and placebo infusion test. In the EC with placebo, ghrelin levels decreased over time to $5.3 \pm 9.8 \%$ of baseline, from a mean of $81.96 \pm 25.6 \mathrm{pg} / \mathrm{ml}$ to $76.96 \pm 23.67 \mathrm{pg} / \mathrm{ml}$ during the clamp ( $\mathrm{p}=0.059$, NS). In the EC with GIP, ghrelin levels decreased over time to $12.6 \pm 8.7 \%$ of baseline, from a mean of $85.16 \pm 28.78 \mathrm{pg} / \mathrm{ml}$ to $77.09 \pm 20.33$ $\mathrm{pg} / \mathrm{ml}$ during the clamp $(\mathrm{p}=0.008)$. Basal and steady-state concentration of ghrelin, blood glucose and insulin were not different between EC with placebo and GIP. In the HC with placebo, ghrelin levels decreased over time to $18.8 \pm 12.7 \%$ of baseline, from a mean of $93.76 \pm 38.41 \mathrm{pg} / \mathrm{ml}$ to $73.01 \pm 25.15 \mathrm{pg} /$ $\mathrm{ml}$ during the clamp $(\mathrm{p}=0.028)$. In the HC with GIP, ghrelin levels decreased over time to $34.2 \pm 17.1 \%$ of baseline, from a mean of $100.45 \pm 50.31 \mathrm{pg} / \mathrm{ml}$ to $60.55 \pm 21.35 \mathrm{pg} / \mathrm{ml}$ during the clamp $(\mathrm{p}=0.018)$. Steady-state concentration of ghrelin were lower in the HC with GIP ( $\mathrm{p}=0.018)$. Basal and steady-state concentration of blood glucose and insulin were not different in the $\mathrm{HC}$ with GIP and placebo.

Conclusion: GIP has an additive suppressed effect on ghrelin secretion under hyperglycemic and hyperinsulinemic condition in uncomplicated obesity. 


\section{PS 42 Incretin effects: experimental I}

\section{3}

GLP-1 and Exendin-4 normalise D-glucose uptake in fat and muscle of insulin-resistant rats

P. Moreno, V. Sancho, J. Cancelas, B. Nuche-Berenguer, I. Valverde, M. L. Villanueva-Peñacarrillo;

Metabolims, nutrition, hormones, Fundación Jiménez Díaz, Madrid, Spain.

Background and Aims: GLP-1, incretin with insulin-independent antidiabetic actions, has shown positive effects upon the glucose metabolism of extrapancreatic tissues participating in the hexose homeostasis. Exendin4 (Ex-4), structurally GLP-1-homologous, shares with GLP-1 some of its in vitro stimulating effects such as that upon glucose transport in muscle and fat. Here we have explored the in vivo effect of GLP-1 and Ex-4 upon glucose transport (GT) in skeletal muscle and adipocytes from an insulin-resistant rat model.

Materials and Methods: Insulin resistance (IR) was induced in adult Wistar rats by chronic feeding during 8 weeks with standard chow combined with D-fructose (20\% in the drinking water). Groups of IR rats ( $n=5-10$ each) were treated (3 days) -through an osmotic pump- with saline solution (control), GLP-1 $(0.86 \mathrm{nmol} / \mathrm{kg} / \mathrm{h})$ or Ex-4 $(0.1 \mathrm{nmol} / \mathrm{kg} / \mathrm{h})$. Plasma samples were taken before (basal) and by the end of the treatment, for glucose concentration measurement; then, rats were sacrificed and the epididimal fat pads and soleous muscles were collected. GT was measured as 2-deoxi$\mathrm{D}-\left[1,2-{ }^{3} \mathrm{H}\right]$ glucose uptake during 18 -min incubation period in the case of isolated adipocytes -by enzymatic digestion-, or during 60-min in that of the whole soleous muscle samples. A group of 5 untreated normal rats were also included.

Results: In IR rats, basal plasma glucose $(89 \pm 3 \mathrm{mg} / \mathrm{dl}, \mathrm{n}=25)$ was not significantly affected after saline, GLP- 1 or Ex- 4 treatment. In the muscle samples of IR rats, control GT value $(0.192 \pm 0.04 \mathrm{nmol} / \mathrm{mg} / \mathrm{min}, \mathrm{n}=7)$ was lower $(p<0.03)$ than that in untreated normal rats $(0.418 \pm 0.09 \mathrm{nmol} / \mathrm{mg} /$ $\mathrm{min}, \mathrm{n}=5)$. After treatment with GLP-1, the GT $(0.516 \pm 0.025 \mathrm{nmol} / \mathrm{mg} / \mathrm{min}$, $\mathrm{n}=10)$ was much higher $(p<0.001)$ than that in control rats saline-treated; also, after treatment with Ex- 4 an increased value was observed $(0.753 \pm 0.017$ $\mathrm{nmol} / \mathrm{mg} / \mathrm{min}, \mathrm{n}=10, p<0.001$ vs control) which was even higher $(p<0.04)$ than that exerted by GLP-1 treatment. In adipocytes from IR rats treated with GLP-1, the levels of GT $\left(11.9 \pm 0.8 \mathrm{fmol} / 10^{5}\right.$ cells, $\left.\mathrm{n}=8\right)$ were higher $(p<0.001)$ than in those from the control rats $\left(8.1 \pm 0.8 \mathrm{fmol} / 10^{5}\right.$ cells, $\left.\mathrm{n}=5\right)$, and also an increase of GT was observed after Ex- 4 treatment $\left(12.2 \pm 0.8 \mathrm{fmol} / 10^{5}\right.$ cells, $\mathrm{n}=6, p<0.001 v s$ control); no difference was detected between the magnitude of the effect of GLP- 1 and that of Ex-4.

Conclusion: These findings suggest a beneficial effect of GLP-1 and exendin4 upon the deleterious glucose transport in muscle and fat in insulin-resistant states, and add support to the therapeutic role of this hormone/peptide in pathological situations running with deficient insulin action.

Supported by the Ministerio de Sanidad y Consumo

\section{4}

Effect of the glucagon-like peptide-1 receptor agonist AVE0010 on postprandial hepatic glucose metabolism in the conscious dog M. C. Moore ${ }^{1}$, U. Werner ${ }^{2}$, M. S. Smith ${ }^{1}$, T. D. Rodewald ${ }^{1}$,

A. D. Cherrington ${ }^{1}$;

${ }^{1}$ Molecular Physiology \& Biophysics, Vanderbilt University, Nashville, United States,

${ }^{2}$ Sanofi-Aventis Deutschland GmbH, Frankfurt am Main, Germany.

Background and Aims: Glucagon-like peptide-1 (GLP-1) receptor agonists are tools for control of hyperglycemia in type 2 diabetes, but little is known about their effects on postprandial hepatic metabolism. The aim was to determine the effect of acute administration of the synthetic GLP-1 receptor agonist AVE0010 on net hepatic glucose uptake (NHGU) and insulin secretion following a mixed meal.

Materials and Methods: Adult mongrel dogs underwent surgery $\approx 16$ days before study for insertion of femoral artery, hepatic portal vein, and hepatic vein sampling catheters and placement of ultrasonic blood flow probes on the hepatic artery and portal vein. On the study day, overnight-fasted dogs received a subcutaneous injection of AVE0010 (1.5 $\mu \mathrm{g} / \mathrm{kg}$; AVE0010 group; $n=5$ ) or vehicle (saline; Control group, $n=6$ ) at $-30 \mathrm{~min}$. A defined formula diet (93.4 kJ/kg; energy composition $19 \%$ protein, $71 \%$ CHO, $10 \%$ lipid) was given orally at $0 \mathrm{~min}$, and blood samples were collected until $510 \mathrm{~min}$.

Results: Basal arterial and portal blood glucose concentrations were $\approx 4.6$ $\mathrm{mmol}$ in both groups. The peak glucose concentrations were $7.7 \pm 0.8$ vs $5.6 \pm 0.6 \mathrm{mM}$ (artery; $p<0.05$ ) and $9.5 \pm 0.8$ vs $7.6 \pm 0.7 \mathrm{mM}$ (portal vein; $p<0.05$ ) in Control and AVE0010, respectively. Areas under the curve (AUCs) of blood glucose and plasma insulin excursions were greater in Control than AVE0010 (Table). Glucose absorption was evident by $15 \mathrm{~min}$ in Control but was delayed $\approx 1 \mathrm{~h}$ in AVE0010. Neither the AUC of net gut glucose output nor that of NHGU differed significantly between control vs AVE0010 (Table). Glucagon concentrations did not differ between groups, remaining nearbasal during the study. (Tab. 1)

Conclusion: AVE0010 reduced the postprandial glycemic excursion without increasing insulin concentrations. Slowing the rate of gastric emptying could be one mechanism involved in the effect. The glycemic improvement was achieved without stimulation of NHGU or insulin secretion, suggesting that the drug enhanced glucose clearance by nonhepatic tissues.

Supported by sanofi-aventis

\section{5}

Enzyme resistant $\mathrm{N}$-terminally modified cholecystokinin-8 exhibits enhanced satiety and insulinotrophism compared with exendin- 4 F. P. M. O'Harte, P. A. Frizelle, P. R. Flatt;

School of Biomedical Sciences, University of Ulster, Coleraine,

Northern Ireland, United Kingdom.

Background and Aims: Cholecystokinin octapeptide (CCK-8) is a neuropeptide hormone that regulates short-term feeding and satiety. Like glucagon-like peptide-1(7-36)amide (GLP-1), CCK-8 is also a strong meal-dependent stimulator of insulin secretion. Both peptides have short circulating biological half-lives due to the actions of peptidase enzymes. Here we examine the effects of two stable $\mathrm{N}$-terminally modified CCK- 8 analogues, and compare these actions to those of native CCK- 8 , GLP-1 and its stable mimetic exendin-4(1-39).

Material and Methods: Peptide degradation was examined following incubation $\left(0-240 \mathrm{~min}, 37^{\circ} \mathrm{C}\right)$ with mouse plasma $(20 \mu \mathrm{l})$ using reversedphase HPLC and quadripole time of flight (Q-TOF) mass spectrometry techniques. Food intake was examined using 12 week-old male Swiss TO mice, which were habituated over 3 -weeks to $3 \mathrm{~h}(10.00-13.00 \mathrm{~h})$ of voluntary food intake per day. Mice $(29.5 \pm 0.1 \mathrm{~g})$ were injected intraperitoneally $(10.00$ h) with either N-glucitol-CCK-8, pGlu-Gln-CCK-8, native CCK-8, GLP1 , exendin-4(1-39) (100 nmol/kg each) or saline control $(10 \mathrm{ml} / \mathrm{kg})$ and immediately allowed to feed. Cumulative food intake was measured at times $30,60,90,120,150$ and $180 \mathrm{~min}$. In another study, fasted $(18 \mathrm{~h})$ obese diabetic $o b / o b$ mice were acutely treated $(100 \mathrm{nmol} / \mathrm{kg})$ with $\mathrm{N}$-glucitol-CCK, native CCK-8 or saline (control) and voluntary food intake monitored at intervals from 30 to $300 \mathrm{~min}$. Acute insulin secretion studies were performed using clonal BRIN-BD11 cells comparing the insulinotropic effects of $10^{-12}$ to $10^{-6}$ M CCK-8, pGlu-Gln-CCK and exendin-4(1-39) at $5.6 \mathrm{mM}$ glucose.

Results: Native CCK- 8 was rapidly and sequentially degraded by plasma with C-terminal fragment peptides (CCK-7, CCK-6, CCK-5, CCK-4, CCK-3 and CCK-2) identified by de novo sequencing software using Q-TOF mass spectrometry. N-glucitol-CCK-8 and pGlu-Gln-CCK-8 were completely resistant to $\mathrm{N}$-terminal plasma degradation. In food intake studies, saline-

Table 1: AUCs (all values are calculated as change from basal over $510 \mathrm{~min}$ )

\begin{tabular}{lllllll}
\hline Group & Art Glc $(\mathrm{mmol} / \mathrm{l})$ & Por Glc $(\mathrm{mmol} / \mathrm{l})$ & Gut Glc Output $(\mathrm{mmol} / \mathrm{kg})$ & NHGU $(\mathrm{mmol} / \mathrm{kg})$ & Art Ins $(\mathrm{nmol} / \mathrm{l})$ & Hepatic Ins $(\mathrm{nmol} / \mathrm{l})$ \\
\hline Control & $70 \pm 17$ & $151 \pm 22$ & $17.5 \pm 3.1$ & $11.6 \pm 1.4$ & $91 \pm 15$ & $398 \pm 122$ \\
AVE0010 & $33 \pm 11^{*}$ & $103 \pm 16^{*}$ & $17.5 \pm 2.4$ & $9.0 \pm 2.4$ & $63 \pm 7^{*}$ & $172 \pm 36^{*}$ \\
\hline
\end{tabular}

Art, artery; Glc, glucose; Por, portal; Ins, insulin; Hepatic, hepatic sinusoidal; ${ }^{*} P<0.05$ vs Control 
treated control Swiss TO mice $(\mathrm{n}=8)$ consumed (mean \pm sem) between $1.5 \pm 0.1 \mathrm{~g}$ and $3.3 \pm 0.2 \mathrm{~g}$ per mouse at $30-180 \mathrm{~min}$. N-glucitol-CCK- 8 and pGlu-Gln-CCK- 8 were more effective than native CCK- 8 and reduced cumulative food intake by $65-85 \%$ and $72-97 \%$, respectively compared to controls $(\mathrm{P}<0.001)$. GLP-1 had no effect on food intake but the stable GLP-1 receptor mimetic exendin-4(1-39) reduced food intake by $36-48 \%$ $(\mathrm{P}<0.001)$, being significantly less effective at $30-90 \mathrm{~min}$ than native CCK$8(\mathrm{P}<0.05$ to $\mathrm{P}<0.001)$. In $o b / o b$ mice, $\mathrm{N}$-glucitol-CCK- 8 was more effective than native CCK-8 reducing food intake by $74-93 \%$ from $30-300$ min compared to saline controls. Concentration-dependent studies in BRINBD11 cells revealed that pGlu-Gln-CCK-8 and native CCK-8 $\left(10^{-12}-10^{-6} \mathrm{M}\right)$ were highly effective insulinotropic agents evoking 1.6-3.9-fold stimulation of insulin secretion $(\mathrm{P}<0.05-\mathrm{P}<0.001)$ compared to $5.6 \mathrm{mM}$ glucose alone. In comparison, exendin-4(1-39) stimulated insulin secretion by $1.2-2.3$-fold at $10^{-12}-10^{-8} \mathrm{M}(\mathrm{P}<0.05-\mathrm{P}<0.001)$.

Conclusion: These data indicate that $\mathrm{N}$-terminal modification of CCK-8 affords resistance to aminopeptidase degradation. Such analogues are potent stimulators of insulin secretion and exhibit significantly enhanced satiating activity. N-terminally modified analogues of CCK- 8 are more effective than exendin-4(1-39) in suppressing appetite and stimulating insulin secretion, indicating potential as future agents for treatment of type 2 diabetes. Supported by the Department of Education \& Learning, N. Ireland.

\section{6}

Exenatide reverses hypertension in a rat model of metabolic syndrome D. Parkes ${ }^{1}$, J. Landry ${ }^{1}$, S. Guss ${ }^{1}$, T. Coffey ${ }^{1}$, K. Laugero ${ }^{2}$;

${ }^{1}$ Amylin Pharmaceuticals, Inc., San Diego, ${ }^{2}$ Nutrition, Western Human

Nutrition Research Center, USDA-ARS, Davis, United States.

Background and Aims: Exenatide (exendin-4) is a 39-amino acid peptide incretin mimetic that has glucoregulatory actions with associated weight reduction. Previous reports demonstrated acute increases in blood pressure after systemic or intracerebroventricular administration of exenatide or GLP-1 in the rat. This acute pressor effect appears to be species-dependent and transient, but might also depend on the animal's physiological state, study conditions, or dosing regimen. To date, there are no reported studies testing the chronic effects of these peptides on blood pressure in the rat. To better understand the chronic hemodynamic effects of peripheral exenatide administration, we examined telemetry-assessed responses in conscious, unrestrained, male, Sprague-Dawley rats under normal conditions and in a model of glucocorticoid-induced hypertension and metabolic syndrome.

Materials and Methods: Rats were subcutaneously implanted with either corticosterone (CORT, $\mathrm{n}=16$ ) or wax (control) pellets $(\mathrm{n}=4)$ and then 14 days later were implanted with subcutaneous osmotic pumps to deliver vehicle $(\mathrm{n}=8)$ or exenatide $(1 \mathrm{micro} \mathrm{g} / \mathrm{kg} / \mathrm{d}, \mathrm{n}=8)$ for 7 days (wax-treated animals received exenatide only).

Results: In control rats implanted with wax pellets, exenatide reduced mean arterial blood pressure by $4.7 \pm 0.2 \mathrm{mmHg}(\mathrm{P}=0.044)$ below baseline levels by day 7. CORT treatment for 14 days produced hypertriglyceridemia, visceral fat deposition, hyperglycaemia and insulin resistance. CORT significantly $(\mathrm{P}<0.05)$ elevated average daily systolic, diastolic and mean arterial pressure by $18 \pm 2,13 \pm 2$ and $14 \pm 2 \mathrm{mmHg}$ above baseline, respectively. Compared to vehicle, exenatide ( $7 \mathrm{~d})$ significantly $(\mathrm{P}=0.015)$ reversed all measures of CORTinduced hypertension to levels within $3 \mathrm{mmHg}$ of baseline ( $95 \%$ confidence intervals: systolic $(-3,5)$, diastolic $(-2,5)$, MAP $(-2,5))$. Exenatide's ability to normalize blood pressure occurred independently of any change in body weight, as no effect was measured on either food intake or body weight at this dose $(1 \mathrm{micro} g / \mathrm{kg} / \mathrm{d}, \mathrm{P}>0.05)$ in the present study.

Conclusion: Our results are the first to show a durable anti-hypertensive effect of exenatide in a glucocorticoid-induced model of the metabolic syndrome, and reveal that the acute hypertensive actions of exenatide in rats do not extend to any long-term change in blood pressure.

\section{7}

Effect of Exendin-4 on bone remodelling in normal and type 2 diabetic rats

V. Sancho ${ }^{1}$, B. Nuche-Berenguer ${ }^{1}$, L. Arnés ${ }^{1}$, P. Esbrit ${ }^{2}$, M. L. Villanueva-Peñacarrillo ${ }^{1}$;

${ }^{1}$ Metabolism, Nutrition and Hormones, ${ }^{2}$ Laboratory of Bone and Mineral Metabolism, Fundación Jiménez Díaz, Madrid, Spain.

Background and Aims: It has been suggested that incretin hormones could participate in the changes in bone remodelling process occurring after nutrients absorption. Exendin-4 (Ex-4) shows GLP-1-like effects upon glucose metabolism in extrapancreatic tissues where it is agonist of the GLP-1 specific receptors; also, Ex-4 exerts an antidiabetic effect which is even more prolonged than that of GLP-1. Here we have explored the possible in vivo effect of Ex-4 on bone turnover markers in normal rats and in a streptozotocininduced type 2 diabetic model.

Materials and Methods: Type 2 diabetic rat model was developed by streptozotocin injection $(100 \mu \mathrm{g}$ streptozotocin/g body weight dissolved in $25 \mu$ citrate buffer) in neonatal Wistar rats (STZ-T2D). Adult rats, normal $(\mathrm{n}=5)$ and STZ-T2D $(\mathrm{n}=6)$, were treated (3 days) -through an osmotic pumpwith Ex-4 $(0.1 \mathrm{nmol} / \mathrm{kg} / \mathrm{h})$. In fed conditions, plasma samples were taken before (basal) and by the end of the treatment; then, rats were sacrificed and one tibia from each rat was collected. In plasma, osteocalcin and tartrateresistant acid phosphatase (TRAP) -by ELISA-, insulin -by RIA- and glucose concentrations, were measured. In the bone, after RNA extraction, the osteocalcin gene expression -by real time-PCR- was determined; untreated rats of both groups (3 rats each) were also included (control).

Results: In normal rats, Ex-4 exerted a reducing effect $(-6.4 \pm 1.8 \% \Delta, p<0.009)$ on basal plasma osteocalcin value $(438.9 \pm 7.4 \mathrm{ng} / \mathrm{ml})$, similar to that on the STZ-T2D group $(-12.3 \pm 8.8 \% \Delta$ of basal) which, initially, was showing an apparent lower than normal basal levels $(385.1 \pm 13.9 \mathrm{ng} / \mathrm{ml})$; basal plasma TRAP $(2.64 \pm 0.27 \mathrm{U} / \mathrm{l})$ was clearly increased by Ex-4 $(36.6 \pm 15.3 \% \Delta$ of basal, $p<0.01)$ in normal rats, while no effect was detected in STZ-T2D; neither plasma glucose nor insulin were significantly affected by Ex-4 in normal or in STZ-T2D rats. As judged from the paired difference between the primary values (cycles) for osteocalcin mRNA and 18S rRNA, and expressing results as a mean fold change from control data ( $2^{-\triangle \mathrm{CT}}$ values), the level of osteocalcin mRNA in the bone of STZ-T2D rats was about 3 times higher (range: 2.454.33) than in that of normal controls (range: 0.59-1.71); treatment with Ex4 in normal rats did not modify (range: $0.63-1.37$ ) the bone control value, and induced in that of STZ-T2D a somewhat lower levels of gene expression (range: 0.57-1.48) respect those in untreated control diabetic rats.

Conclusion: These data show that Ex-4 affects bone remodeling in an insulin-independent manner in normal and type 2 diabetic rats. Although the present data are preliminary, this novel finding suggests that Ex-4 appears to stimulate bone resorption in normal state, effect which does not seem to occur in this diabetic model.

Supported by the Ministerio de Sanidad y Consumo

\section{8}

Participation of GLP-1 in the bone remodelling process in normal and type 2 diabetic state

B. Nuche-Berenguer ${ }^{1}$, V. Sancho ${ }^{1}$, J. Cancelas ${ }^{1}$, B. Rodríguez-Frutos ${ }^{1}$, P. Esbrít ${ }^{2}$, M. L. Villanueva-Peñacarrillo ${ }^{1}$;

${ }^{1}$ Metabolism, Nutrition and Hormones, ${ }^{2}$ Laboratory of Bone and Mineral Metabolism, Fundación Jiménez Díaz, Madrid, Spain.

Background and Aims: The insulin-independent antidiabetic action of GLP1 , is now well documented also its positive effect upon glucose metabolism in liver, muscle and fat, and its neurotrophic and anoretic properties, among others. It has been suggested that hormones with incretin character could participate in the changes in bone turnover occurring after nutrient absorption. Here we have explored the possible in vivo modulating effect of GLP-1 on bone turnover markers in normal and streptozotocin-induced type 2 diabetic model

Materials and Methods: type 2 diabetic rat model was developed by streptozotocin injection ( $100 \mu \mathrm{g}$ streptozotocin/g body weight, dissolved in $25 \mu$ citrate buffer) in neonatal Wistar rats (STZ-T2D). Adult rats, normal $(n=5)$ and STZ-T2D $(n=6)$, were treated (3 days) -through an osmotic pumpwith GLP-1 $(0.86 \mathrm{nmol} / \mathrm{kg} / \mathrm{h})$. In fed conditions, plasma samples were taken 
before (basal) and by the end of the treatment; then, rats were sacrificed and one tibia from each rat was collected. In plasma, osteocalcin and tartrateresistant acid phosphatase (TRAP) -by ELISA-, insulin -by RIA- and glucose concentrations were measured. In the bone, after RNA extraction, the expression of osteocalcin gene -by real time-PCR- was determined; untreated rats of both groups ( 3 rats each) were included as controls.

Results: In normal rats, GLP-1 did not modify the basal plasma osteocalcin value $(440.5 \pm 6.0 \mathrm{ng} / \mathrm{ml})$, while in the STZ-T2D group, initially showing an apparently lower than normal level, GLP-1 induced a clear reduction $(-10.9 \pm 0.9 \% \Delta$ of basal, $p<0.001)$; basal plasma TRAP $(2.64 \pm 0.27 \mathrm{U} / \mathrm{l})$ was slightly increased by GLP-1 in normal $(2.87 \pm 0.24 \mathrm{U} / \mathrm{l})$ and also in STZ-T2D rats $(2.88 \pm 0.29 \mathrm{U} / 1$ vs basal: $2.45 \pm 0.27 \mathrm{U} / \mathrm{l})$; plasma glucose and insulin were not significantly affected in normal and in STZ-T2D rats. As judged from the paired difference between the primary values (cycles) for osteocalcin mRNA and $18 \mathrm{~S}$ rRNA, and expressing results as a mean fold change from control data ( $2^{-\triangle C T}$ values), the level of osteocalcin mRNA in the bone of STZT2D rats was over 3-times higher (range: $2.45-4.33$ ) than in that of normal controls (range: 0.59-1.71); treatment of normal rats with GLP-1 induced a moderate increase (range: 0.61-2.73) in normal controls, while in the STZT2D rats group the level achieved after GLP-1 was somewhat lower (range: $0.38-1.18$ ) than that in its corresponding untreated diabetic rats control.

Conclusion: These findings demonstrate that GLP-1 directly modulates bone turnover markers in both normal and type 2 diabetic states. Moreover, GLP-1 appears to promote an uncoupling of bone resorption and bone function in both conditions.

Supported by the Ministerio de Sanidad y Consumo

\section{PS 43 Incretin effects: experimental II}

\section{9}

The insulin response to oral glucose is almost entirely dependent on incretins in mice

B. Ahrén ${ }^{1}$, M. Sörhede Winzell ${ }^{1}$, G. Pacini²;

${ }^{1}$ Clinical Sciences, Medicine, Lund University, Lund, Sweden,

${ }^{2}$ Metabolic Unit, Institute of Biomedical Engineering, Padova, Italy.

Background and Aims: The entero-insular axis is the physiological regulation by the gut of islet function. A key component of this axis is the incretin effect, which is the augmented increase in insulin secretion following oral versus intravenous glucose. This effect is achieved by gastrointestinal hormones, which are released during meal ingestion and which augment glucosestimulated insulin secretion; the two most important being glucagon-like peptide-1 (GLP-1) and glucose-dependent insulinotropic polypeptide (GIP). In humans, a study with careful matching of glucose levels after oral versus intravenous administration has shown that the incretins contributes to $70-90 \%$ of the insulin response to oral glucose. The aim of this study was to estimate insulin secretion after oral and intravenous glucose administration when glucose levels were matched in these two conditions.

Materials and Methods: A variable rate intravenous glucose infusion was used to match glucose levels after oral glucose to estimate the contribution of incretins for insulin secretion after oral glucose through gavage in anesthetized C57BL/6J female mice. Insulin secretion was estimated from C-peptide levels. An initially established dose-response relationship between oral glucose load and plasma insulin revealed that $25 \mathrm{mg}$ glucose is a threshold dose whereas $75 \mathrm{mg}$ glucose is a maximal dose; these two doses were chosen for the matching studies.

Results: Comparison between oral versus intravenous glucose at low glucose (25mg) showed that insulin secretion (the $15 \mathrm{~min}$ C-peptide response) was $1272 \pm 138 \mathrm{nmol} / 1$ during the oral test versus $655 \pm 88 \mathrm{nmol} / 1$ during the intravenous test $(\mathrm{P}=0.016)$. Furthermore, at high glucose $(75 \mathrm{mg})$, the $15 \mathrm{~min}$ C-peptide response was $5105 \pm 587 \mathrm{nmol} / 1$ during the oral test versus $685 \pm 101$ $\mathrm{nmol} / \mathrm{l}$ during the intravenous test $(\mathrm{P}<0.001)$. This corresponds to an incretin contribution to insulin secretion by $50 \%$ after low glucose and more than $85 \%$ after high glucose loads. Furthermore, the total hepatic extraction of insulin, calculated from insulin and C-peptide data, was $71.2 \pm 1.2 \%$ after oral glucose and $74.4 \pm 1.1 \%$ after intravenous glucose at low glucose (NS) and $50.5 \pm 2.3 \%$ after oral glucose versus $69.9 \pm 2.8 \%$ after intravenous glucose at high glucose $(\mathrm{P}<0.001)$

Conclusion: We conclude that the insulin response to oral glucose at a maximal glucose load is almost entirely explained by incretins, and caused by marked augmentation of the hormone secretion in association with reduced total hepatic insulin extraction of insulin.

\section{0}

Exenatide reduces glycaemia via a mechanism mediated by portal glucagon-like peptide-1 receptors but independent of islet hormones and intestinal motility

V. Ionut, D. Zheng, D. Stefanovski, R. N. Bergman;

Physiology and Biophysics, USC Keck School of Medicine, Los Angeles, United States.

Background and Aims: Exenatide (synthetic exendin-4, EX) is a long acting Glucagon-like peptide-1 (GLP-1) mimetic that is currently used in the treatment of type 2 diabetes. EX lowers fasting and postprandial glucose, decreases $\mathrm{HbAl}$ and reduces body weight. The mechanism by which EX exerts these effects is not completely explained. The aim of our study was to investigate whether in addition to the effects on pancreatic hormones and slowing of gastro-intestinal motility EX lowers glycemia through an isletindependent effect, mirroring the native intestinal hormone GLP-1.

Materials and Methods: Experiments were performed in male mongrel conscious dogs $(n=8)$. In each animal several experiments were performed: 1) mixed (high-fat, high carbohydrate) meal tests, with or without preadministration of EX ( $20 \mathrm{mcg}$, s.c. injection, $15 \mathrm{~min}$ before the experiment); 2) intraportal glucose infusions, simulating the meal test glucose levels, in 
matched experiments, with or without EX; 3) intraportal glucose infusion with s.c. EX and intraportal infusion of antagonist exendin 9-39.

Results: 1) Meal tests: Administration of EX resulted in lower glycemia $(105 \pm 5 \mathrm{mg} / \mathrm{dl}$ vs. $90 \pm 3 \mathrm{mg} / \mathrm{dl}$ peak postprandial, $\mathrm{p}<0.01)$, without a corresponding increase in insulin $(80 \pm 21 \mathrm{pM}$ saline vs. $55 \pm 13$ pM EX, $\mathrm{p}=0.9)$, but with significant glucagon suppression $(73 \pm 10 \mathrm{ng} / \mathrm{l}$ vs. $54 \pm 9$ $\mathrm{ng} / \mathrm{l}, \mathrm{p}<0.001)$ and slowing of gastric emptying. 2) Intraportal glucose infusion with and without EX: To assess whether the hypoglycemic effect of exenatide is maintained in the absence of delayed intestinal absorption, in the same animals we infused glucose intraportally with or without EX. With EX we measured lower glycemia $(97 \pm 3 \mathrm{mg} / \mathrm{dl}$ vs. $92 \pm 3 \mathrm{mg} / \mathrm{dl}, \mathrm{p}<0.001)$, without compensatory hyperinsulinemia $(60 \pm 9 \mathrm{pM}$ saline vs. $53 \pm 8 \mathrm{pM} \mathrm{EX}$, $\mathrm{p}=0.9)$ or glucagon suppression $(57 \pm 12 \mathrm{ng} / \mathrm{l}$ vs. $61 \pm 16 \mathrm{ng} / \mathrm{l}, \mathrm{p}=0.1)$. Thus EX lowers glucose in the absence of delayed gastric emptying and without a corresponding insulinotropic effect or glucagon suppression. 3) Intraportal glucose with EX and antagonist: In a separate experiment we infused intraportally the GLP-1 receptor antagonist exendin 9-39, concomitant with s.c. EX and intraportal glucose infusion. For the period of antagonist infusion glucose levels increased to values that were significantly higher than those in the EX only experiment $(95 \pm 3 \mathrm{mg} / \mathrm{dl}$ vs. $92 \pm 3 \mathrm{mg} / \mathrm{dl}, \mathrm{p}<0.001)$; after the antagonist infusion was stopped at $60 \mathrm{~min}$, glycemic levels reverted to values that were no significantly different form the EX experiment $(92 \pm 3 \mathrm{mg} / \mathrm{dl} \mathrm{vs}$. $91 \pm 2 \mathrm{mg} / \mathrm{dl}, \mathrm{p}=0.4$ ) Blockade of GLP-1 receptors with exendin 9-39 indicates that portal GLP-1 receptors might be involved in EX's effect.

Conclusion: Similar to GLP-1, EX has a pancreatic hormone-independent effect on lowering glycemia. This effect appears to be mediated via GLP1 receptors situated in the porto-hepatic area. Activation of these portal receptors, via a neural mechanism, could increase glucose clearance independent of islet hormones.

Supported by Amylin Inc.

\section{1}

Effect of Exendin-4 upon glucotransporter GLUT-4 expression in the adipose tissue of normal and type 2 diabetic states

P. Tornero-Esteban, L. Arnés, V. Sancho, P. Moreno, M. Garcia -Arevalo,

I. Valverde, M. L. Villanueva-Peñacarrillo;

Metabolism, Nutrition and Hormones, Fundación Jiménez Díaz,

Madrid, Spain.

Background and Aims: Exendin-4 (Ex-4), a peptide of non-mammalian nature, shares with GLP-1 not only a 53\% of structural homology but also its antidiabetic action; also, Ex-4 shows GLP-1-like stimulatory effects upon parameters related with glucose and/or lipid metabolism in extrapancreatic tissues involved in glucose homeostasis. In this work, we explored whether Ex-4 modulates in vivo the GLUT-4 expression in the fat tissue of a streptozotocin-induced type 2 diabetic rat model as compared to normal.

Materials and Methods: Type 2 diabetic rat model was developed by streptozotocin injection (100 $\mu \mathrm{g}$ streptozotocin/g body weight, dissolved in $25 \mu \mathrm{l}$ citrate buffer) in neonatal Wistar rats (STZ-T2D). Adult rats, normal and STZ-T2D, were treated during 3 days -through an osmotic pump- with saline solution (control) -5 rats/group- or Ex-4 (0.1 nmol $/ \mathrm{kg} / \mathrm{h})-5$ rats/ per group-. In fed state, plasma samples were taken before (basal) and by the end of the treatment for glucose and insulin -by RIA- concentration measurements; then, rats were sacrificed and the epididimal fat pads were collected for plasma membranes and RNA extraction to determine GLUT-4 protein -by Western blot- and mRNA -by real time PCR-, respectively.

Results: In normal and in STZ-T2D rats, neither basal plasma glucose nor insulin were significantly affected by Ex- 4 treatment. As judged from the paired difference between the primary values (cycles) for GLUT-4 mRNA and $18 \mathrm{~S}$ rRNA, and expressing results as a mean fold change from control data $\left(2^{-\triangle C T}\right.$ values), the level of GLUT- 4 mRNA in the fat tissue of normal rats treated with Ex-4 was not different from that in the control group. Although in STZ-T2D rats treated with Ex-4, the GLUT-4 mRNA (range 0.66-1.01) showed a tendency toward lower values than those in STZ-T2D control group saline-treated (range $0.77-1.29$ ), the gene expression was not significantly different. In normal rats, GLUT-4-protein value after Ex-4 treatment was indistinct of that found in those saline-treated $(100 \pm 10 \%$ of control); yet, in STZ-T2D rats, Ex-4 induced a clear stimulation of GLUT-4-protein in STZT2D rats ( $151 \pm 13 \%$ control, $p=0.024)$, similar in magnitude to that previously detected after GLP-1 treatment.
Conclusion: These present data suggest that Ex- 4 could exert a control on the GLUT-4 expression, at least at the translational level, in pathologic situations running with insulin deficiency or action.

Supported by Ministerio de Sanidad y Consumo

\section{2}

Sustained GLP-1R-dependent control of glucose homeostasis by a GLP-1 mimetibody

K. M. Picha ${ }^{1}$, S. Adhikarakunnathu ${ }^{1}$, M. Cunningham ${ }^{1}$, D. Drucker ${ }^{2}$, D. Makropoulos ${ }^{1}$, A. Mathur ${ }^{1}$, C. McCauley ${ }^{1}$, T. Ort ${ }^{1}$, M. Scully $^{1}$, A. Soderman ${ }^{1}$, T. Spinka-Doms ${ }^{1}$, J. Sprenkle ${ }^{1}$, V. Stojanovic-Susulic ${ }^{1}$, B. Thomas ${ }^{1}$, K. O'Neil ${ }^{1}$;

${ }^{1}$ Discovery Research, Centocor R\&D, Radnor, United States,

${ }^{2}$ University of Toronto, Samuel Lunenfeld Research Institute, Canada.

Many biologic processes are regulated by small peptides acting in an endocrine or paracrine manner. Such peptides are generally rapidly metabolized so that although their utility as pharmacologic agents may be well validated, their therapeutic potential is limited. We have developed a novel platform for display and delivery of bioactive peptides that links the biological properties of the peptide to the pharmacokinetic properties of an antibody. Peptides engineered in the Mimetibody ${ }^{\text {mit }}(\mathrm{MMB})$ platform have significantly improved biochemical and biophysical properties that are distinct from those of Fcfusion proteins. CNTO736 is a GLP-1 MMB that retains many of the activities of native GLP-1 but exhibits a significantly enhanced pharmacokinetic profile. In mice, the terminal half-life of CNTO736 is 1000-fold longer relative to GLP-1 peptide. Acute studies in diabetic mice showed that CNTO736 dosedependently improved glucose tolerance, with a reduction in the AUC during a glucose tolerance test of more than $50 \%$ at the highest dose. CNTO736 treatment markedly reduced fasting glucose (FBG) in $\mathrm{db} / \mathrm{db}$ by $250 \mathrm{mg} / \mathrm{dL}$ after six weeks of dosing. Diet-induced obese mice dosed chronically with CNTO736 demonstrated a sustained dose-dependent reduction in FBG and up to a $20 \%$ reduction in body weight relative to control mice whereas daily treatment with an equimolar dose of exendin- 4 resulted in $2 \%$ reduction in body weight loss. The biological activities of CNTO736 were shown to be GLP-1 receptor-dependent as the glucose lowering and food intake effects were not observed in Glp1r-/- mice. Although CNTO736 is a 60kDa protein, peripheral administration activated c-fos expression in regions of the central nervous system protected by the blood brain barrier. Taken together, our data demonstrate that a GLP-1 MMB mimics a broad spectrum of GLP-1Rdependent actions while exhibiting significantly improved pharmacokinetics relative to the native GLP-1 peptide.

\section{3}

Structural studies of glucose-dependent insulinotropic polypeptide (GIP) in micellular media

C. M. Hewage ${ }^{1}$, I. Alana ${ }^{1}$, F. P. M. O’Harte ${ }^{2}$;

${ }^{1}$ School of Biomolecular and Biomedical Science, University College Dublin, Ireland, ${ }^{2}$ School of Biomedical Sciences, University of Ulster, Coleraine, Northern Ireland, United Kingdom.

Backgroundand Aims: GIP(YAEGTFISDYSIAMDKIHQQDFVNWLLAQK GKKNDWKHNI TQ) is an incretin hormone synthesised and secreted in the gut. GIP promotes pro-insulin gene expression, proliferation of $\beta$-cells and $\beta$-cell survival and mainly glucose lowering action. GIP is believed to play a major role in fat metabolism stimulating the synthesis of fatty acids. We are interested in assessing the importance of the structure of GIP and its analogues in determining their biological activity. Ongoing spectroscopic studies in our laboratory have identified the bioactive conformation of GIP. As a part of drug development program for type 2 diabetes currently running in our laboratory, we have studied the solution structure of GIP in membrane mimicking micellular media using NMR spectroscopy. The results presented here could help in the design of new drugs for diabetes and obesity related disorders.

Materials and Methods: Human GIP was sequentially synthesised on an Applied Biosystems 432A automated peptide synthesiser using standard solid-phase Fmoc procedure. The peptide was purified to homogeneity 
by RP-HPLC and structural identity confirmed by MALDI-TOF mass spectrometry. NMR experiments were performed using a $5 \mathrm{~mm}$ inverse probe with Bruker DRX 500 and 900 NMR spectrometers operating at a $1 \mathrm{H}$ resonance frequency of 500.13 and $900.27 \mathrm{MHz}$ respectively, at $298 \mathrm{~K}$. The peptide samples of GIP $(1.5 \mathrm{mM})$ were dissolved in micellular media $(600$ $\mu \mathrm{l})$. One and two dimensional (DQF-COSY, TOCSY and NOESY) proton NMR spectra were acquired with a standard parameter sets. All spectra were referenced internally to the TSP at $0 \mathrm{ppm}$ where necessary. Data were acquired and processed using the Bruker XWINNMR program.

Results: The DQF-COSY and TOCSY spectra provided a basis for the identification of individual residue spin-systems. Discrimination of nonunique residues and identification of all other unidentified spin systems was achieved by direct comparison of the TOCSY and NOESY spectra. The backbone nOe connectivities of were clearly identified using the $\alpha_{i} \mathrm{H} / \mathrm{N}_{\mathrm{i}+1} \mathrm{H}$ and $\beta_{i} \mathrm{H} / \mathrm{N}_{i+1} \mathrm{H}$ cross-peaks in the fingerprint region of the NOESY spectrum. Strong $\alpha_{i} \mathrm{H} / \beta_{\mathrm{i}+3} \mathrm{H}$ medium-range connectivities were observed suggesting the presence of an $\alpha$-helix for residues Ser ${ }^{8}$-Lys ${ }^{30}$. Clear medium-range $\alpha_{i} \mathrm{H} / \mathrm{N}_{\mathrm{it3}} \mathrm{H}$ connectivities were also identified for residues between $\mathrm{Ser}^{8}-\mathrm{Lys}^{30}$. Therefore the NMR data suggest that the structure of GIP in micellular medium consists of an $\alpha$-helical motif between residues $\operatorname{Ser}^{8}-\mathrm{Lys}^{30}$, unlike the other major incretin hormone, GLP-1, which mainly adopts a random coil conformation, under physiological conditions.

Conclusion: We have shown that GIP adopts an $\alpha$-helical conformation between residues Ser $^{8}$-Lys ${ }^{30}$ when dissolved in micellular media using NMR spectroscopic analysis. Molecular modelling studies of GIP in micellular media are currently being investigated. These results may help to gain further insight into the biologically relevant receptor-bound structure of GIP, and so facilitate the design of new analogues and peptidic drugs to treat type 2 diabetes and related diseases.

Supported by the Science Foundation Ireland

\section{4}

Liraglutide modulates expression of vascular adhesion molecules and

PAI-1 in an in vitro model of endothelial cell dysfunction

A. E. Dear ${ }^{1}$, H. Liu ${ }^{1}$, L. B. Knudsen ${ }^{2}$, R. W. Simpson 3 ;

${ }^{1}$ Medicine, Monash University, Melbourne, Australia,

${ }^{2}$ NovoNordiskA/S, Måløv, Denmark,

${ }^{3}$ Endocrinology, Box Hill Hospital, Melbourne, Australia.

Background and Aims: Endothelial cell dysfunction together with elevated levels of circulating proinflammatory cytokines, including TNF $\alpha$, and increased expression of endothelial cell adhesion molecules and PAI-1 are among the earliest detectable abnormalities identified in people at risk of atherosclerosis. Accelerated atherosclerosis is a major contributor to morbidity and mortality in type 2 diabetes mellitus. Liraglutide, a novel treatment for diabetes mellitus, is a long-acting human glucagon-like peptide1(GLP-1) analogue. Liraglutide improves glycaemic control and lowers body weight in subjects with type 2 diabetes. Previous studies have demonstrated that natural GLP-1 improves endothelial cell dysfunction in diabetic patients and attenuates TNF $\alpha$-mediated induction of PAI-1 expression in vascular endothelial cells. Our current study aims to explore the in vitro effect of liraglutide on expression of vascular endothelial cell adhesion molecules and PAI-1 under conditions of TNF $\alpha$ stimulation in the C11-STH vascular endothelial cell system.

Materials and Methods: PAI-1, VCAM-1, ICAM-1 and E-Selectin protein levels were determined in conditioned medium from C11-STH vascular endothelial cells using ELISA kits for PAI-1 (Biopool), VCAM-1, ICAM-1, and E-Selectin (R\&D systems). Northern Blot and Reverse Transcriptase (RT) PCR was used to determine mRNA expression levels for PAI-1, VCAM1, ICAM-1 and E-Selectin in C11-STH vascular endothelial cells.

Results: PAI-1 protein expression levels increased from 50 to $100 \mathrm{IU} / \mathrm{ml}$ with TNF $\alpha(10 \mathrm{ng} / \mathrm{ml})$ stimulation $(\mathrm{n}=3, \mathrm{P}<0.05)$. VCAM-1, ICAM-1 and E-Selectin protein expression levels were induced from undetectable levels to $8.0 \mathrm{ng} / \mathrm{ml}, 25.0 \mathrm{ng} / \mathrm{ml}$ and $0.60 \mathrm{ng} / \mathrm{ml}$ respectively post TNF $\alpha$ treatment $(\mathrm{n}=3, \mathrm{P}<0.05)$. Maximal response of PAI-1, VCAM-1, ICAM-1 and ESelectin protein expression to TNF $\alpha$ stimulation was observed at 16h. Basal and TNF $\alpha$-inducible PAI-1 mRNA expression were observed with maximal response identified at 16h. VCAM-1, ICAM-1 and E-Selectin mRNA expression was undetected in unstimulated cells whilst TNF $\alpha$ stimulation resulted in increased VCAM-1, ICAM-1 and E-Selectin mRNA expression with maximal response identified at $16 \mathrm{hrs}$. Addition of liraglutide (100nM) for $1 \mathrm{~h}$ did not significantly affect basal expression of PAI-1, VCAM-1, ICAM1 or E-Selectin mRNA or protein. Addition of liraglutide (100nM) for $1 \mathrm{~h}$ did significantly inhibit TNF $\alpha$-mediated induction of PAI-1 mRNA and protein expression with a reduction to control levels of PAI-1 protein and mRNA expression at the $6 \mathrm{hr}$ time point $(\mathrm{n}=3, \mathrm{P}<0.05)$. Liraglutide treatment (100nM) for $1 \mathrm{~h}$ also inhibited VCAM-1, ICAM-1 and E-Selectin protein and mRNA expression by between 30 and $40 \%$ over TNF $\alpha$-induced levels with the maximal effect observed at $16 \mathrm{hrs}$ post $\mathrm{TNF} \alpha$ stimulation $(\mathrm{n}=3, \mathrm{P}<0.05)$.

Conclusion: Taken together, these observations suggest that liraglutide inhibits TNF $\alpha$-mediated PAI-1, VCAM-1, ICAM- 1 and E-Selectin mRNA expression and protein secretion in an in vitro model of vascular endothelial cell dysfunction.

Supported by Novo Nordisk A/S 


\section{PS 44 Insulin secretion in vivo}

\section{5}

An assessment of the effect of high fat diet on pancreatic function in the female zucker diabetic fatty (ZDF) rat using the hyperglycaemic clamp R. Potter, J. L. Teague, S. M. Poucher;

AstraZeneca, Alderley Park, United Kingdom.

Background and Aims: Obese female ZDF rats fed with a specific $48 \%$ high fat diet develop marked hyperglycaemia, suggested to be due to progressive $\beta$-cell dysfunction. The primary aim of these studies was to use the hyperglycaemic clamp (HC) to assess and quantify the effect of $48 \%$ high fat diet on glucose stimulated insulin secretion (GSIS) and $\beta$-cell function in the female obese ZDF rat compared to chow-fed obese and lean controls. Furthermore we investigate the ability of an acute Exendin- 4 infusion to improve $\beta$-cell function.

Materials and Methods: Female obese ZDF rats ( 7 weeks old) were fed normal chow or $48 \%$ high fat diet for $\sim 15$ days. Aged matched chow-fed female lean ZDF rats were included as controls. Following an overnight fast, animals were anaesthetised with $\operatorname{Inactin}^{\star}(150-180 \mathrm{mg} / \mathrm{kg}$; i.p). Cannulae were placed in the carotid artery and jugular vein, and a tracheotomy performed. After stabilisation blood samples were taken before a 180-minute HC protocol. Plasma glucose (PG) concentrations were monitored at regular intervals, and maintained $\sim 5.4 \mathrm{mM}$ above basal concentrations for 90 minutes using a variable continuous infusion of $25 \%$ glucose. Exendin- 4 was then administered via i.v infusion (30pmol $/ \mathrm{kg} / \mathrm{min})$, for a further 90 -minutes and clamp re-established. Blood samples were obtained regularly for analysis of insulin and c-peptide concentrations.

Results: Basal fasting PG was higher in the OHF-ZDF $(7.95 \pm 0.86 \mathrm{mM})$ and OC-ZDF $(7.11 \pm 0.36 \mathrm{mM})$ groups compared to the LC-ZDF $(5.25 \pm 0.13 \mathrm{mM})$ controls $(\mathrm{p}<0.05)$. In all animals, a steady state HC $(5.44 \pm 0.02 \mathrm{mM}$ above basal PG) was achieved and maintained. GIR required to maintain hyperglycaemia was lower in the OHF-ZDF compared to the OC-ZDF and LC-ZDF controls $(\mathrm{p}<0.05)$. All animals demonstrated a bi-phasic insulin response. The OHFZDF and OC-ZDF groups had higher insulin and c-peptide concentrations compared to LC-ZDF controls $(\mathrm{p}<0.05)$. DI (a calculated measure of $\beta$-cell function related to insulin sensitivity) was reduced in the OHF and OC-ZDF compared to LC-ZDF controls $(\mathrm{p}<0.05)$. DI was $50 \%$ lower in the OHFZDF compared to the OC-ZDF $(\mathrm{p}<0.05)$. Exendin- 4 caused an increased insulin secretion in the LC-ZDF and OC-ZDF with a parallel increase in GIR $(p<0.05)$. Exendin-4 had no significant effect in the OHF-ZDF.

Conclusions: We have used the HC to demonstrated that the OC-ZDF and OHF-ZDF have a significantly reduced $\beta$-cell function following 15 -days on respective diets. Furthermore the high fat diet reduced $\beta$-cell function by $50 \%$ compared to that in the OC-ZDF. This may account for the marked progressive hyperglycaemia observed following high-fat diet administration. Exendin-4 infusion increased both GSIS and glucose disposal in LC-ZDF and OC-ZDF. No effect of Exendin-4 was observed in the OHF-ZDF. This may be due to an increased insulin resistance and reduced $\beta$-cell function. Prolonged treatment with Exendin-4 may improve $\beta$-cell function and GSIS in the OHF-ZDF.

\begin{tabular}{|c|c|c|c|c|c|c|}
\hline \multirow{5}{*}{$\begin{array}{l}\text { Plasma Glucose } \\
(\mathrm{mM}) \\
\text { GIR } \\
(\mu \mathrm{mol} / \mathrm{kg} / \mathrm{min})\end{array}$} & \multicolumn{2}{|c|}{$\begin{array}{l}\text { Lean Chow-Fed } \\
\text { (LC-ZDF) } n=3\end{array}$} & \multicolumn{2}{|c|}{$\begin{array}{l}\text { Obese Chow-Fed } \\
\text { (OC-ZDF) } n=5\end{array}$} & \multicolumn{2}{|c|}{$\begin{array}{l}\text { Obese High Fat-Fed } \\
(\text { OHF-ZDF) } n=4\end{array}$} \\
\hline & \multicolumn{6}{|c|}{ Hyperglycaemic Clamp } \\
\hline & $\cdot$ & + Exendin -4 & $\cdot$ & + Exendin -4 & $\cdot$ & - Exendin 4 \\
\hline & $10.2 \pm 0.0$ & $10.1 \pm 0.2$ & $13.1=0.3$ & $13.3 \pm 0.2$ & $13.3=1.0$ & $13.4=1.0$ \\
\hline & $87.5 \pm 5.2$ & $186.8+20.7^{5}$ & $57.7 \pm 3.5$ & $74.4 \pm 8.0^{6}$ & $39.4 \pm 3.1^{2 \mathrm{~b}}$ & $38.1=10.9$ \\
\hline $\begin{array}{l}\text { Plasma Insulin } \\
\text { (nM) }\end{array}$ & $0.2 \pm 0.0$ & $0.3 \pm 1.0$ & $1.0=0.1^{2}$ & $1.9 \pm 0.5$ & $1.5=0.2^{2 b}$ & $2.1=0.4$ \\
\hline $\begin{array}{l}\text { Plasma C-Peptide } \\
(\mathrm{nM})\end{array}$ & $1.2 \pm 0.2$ & $2.0 \pm 0.7$ & $4.9 \pm 0.5^{*}$ & $5.8 \pm 0.6$ & $5.5=0.3^{n}$ & $5.0 \pm 0.7$ \\
\hline $\begin{array}{l}\text { Deposition Index } \\
\text { (DI) }\end{array}$ & $0.5 \pm 0.1$ & $1.2 \pm 0.1^{\mathrm{F}}$ & $0.3=0.0^{*}$ & $0.3 \pm 0.0$ & $0.2=0.0^{\text {ab }}$ & $0.1=0.0$ \\
\hline
\end{tabular}

0596

Glucose intolerance associated to insulin resistance and increased insulin secretion in rats depleted in long-chain $\omega 3$ fatty acids J. Cancelas ${ }^{1}$, P. G. Prieto ${ }^{1}$, M. L. Villanueva-Peñacarrillo ${ }^{1}$, Y. Zhang ${ }^{2}$, L. Portois ${ }^{3}$, A. Sener ${ }^{2}$, Y. A. Carpentier ${ }^{3}$, I. Valverde ${ }^{1}$, W. J. Malaisse ${ }^{2}$; ${ }^{1}$ Metabolism, Nutrition and Hormones, Fundación Jiménez Díaz, Madrid, Spain, ${ }^{2}$ Laboratory of Experimental Hormonology, Brussels Free University, Belgium, ${ }^{3}$ Laboratory of Experimental Surgery, Brussels Free University, Belgium.

Background and Aims: Second-generation rats depleted in long-chain polyunsaturated $\omega 3$ fatty acids ( $\omega 3 \mathrm{D}$ rats) display several features of the metabolic syndrome including visceral obesity, liver steatosis, hypertension and cardiac hypertrophy. Pilot measurements made in fed $\omega 3 \mathrm{D}$ rats also suggest that these animals could display insulin resistance. This work meant to determine the insulinogenic index and HOMA values in $\omega 3 \mathrm{D}$ and normal rats during intragastric D-glucose tolerance test.

Materials and Methods: Female $\omega 3 \mathrm{D}$ rats (11-12 weeks of age) were compared to control rats of same age and gender. After overnight starvation, D-glucose ( $4 \mu \mathrm{l} / \mathrm{g}$ body wt. of a $30 \%(\mathrm{w} / \mathrm{v})$ solution, i.e. $1.2 \mathrm{mg}$ of D-glucose $/ \mathrm{g}$ body wt.) was administered as an intragastric bolus. Blood was collected from the severed end of the tail in chilled tubes before and up to $120 \mathrm{~min}$ after administration of the hexose, for later measurement of plasma D-glucose and insulin concentrations.

Results: The $\omega 3 \mathrm{D}$ rats displayed a higher body weight $(230 \pm 4 \mathrm{~g} ; \mathrm{n}=16$; $\mathrm{p}<$ $0.001)$ than the control animals $(189 \pm 8 \mathrm{~g} ; \mathrm{n}=8)$. After overnight starvation, the plasma D-glucose concentration was higher $(\mathrm{p}<0.05)$ in $\omega 3 \mathrm{D}$ rats $(5.28 \pm$ $0.10 \mathrm{mmol} / \mathrm{l} ; \mathrm{n}=16)$ than in control animals $(4.93 \pm 0.12 \mathrm{mmol} / \mathrm{l} ; \mathrm{n}=8)$, also, the plasma insulin concentration, insulinogenic index and HOMA were all significantly higher $(\mathrm{p}<0.02$ or less) in $\omega 3 \mathrm{D}$ rats than in control animals. Over the $120 \mathrm{~min}$ period following the intragastric administration of $\mathrm{D}$-glucose, the area under the curve of plasma D-glucose concentration was higher $(\mathrm{p}<0.05)$ in $\omega 3 \mathrm{D}$ rats $(931 \pm 24 \mathrm{mmol} / \mathrm{l} \cdot \mathrm{min} ; \mathrm{n}=16)$ than in control animals $(848 \pm 17$ $\mathrm{mmol} / \mathrm{l} \cdot \mathrm{min} ; \mathrm{n}=8)$, although the incremental area was not significantly higher in $\omega 3 \mathrm{D}$ rats $(296 \pm 29 \mathrm{mmol} / \mathrm{l} \cdot \mathrm{min} ; \mathrm{n}=16)$ than in control rats $(257 \pm 14$ $\mathrm{mmol} / \mathrm{l} . \mathrm{min} ; \mathrm{n}=8$ ). A comparable situation prevailed in the case of the plasma insulin concentration and insulinogenic index, which both yielded a higher area under the curve $(\mathrm{p}<0.001)$ over $120 \mathrm{~min}$, but no significant difference for the incremental area above basal value. In the case of HOMA, both the area under the curve $(\mathrm{p}<0.001)$ and incremental area above basal value $(\mathrm{p}<0.01)$ were significantly higher in $\omega 3 \mathrm{D}$ rats than in control animals.

Conclusion: The present study reinforces the view that second generation $\omega 3 \mathrm{D}$ rats represents a suitable animal model for investigating the molecular determinants of obesity and insulin resistance resulting from a depletion in long-chain polyunsaturated $\omega 3$ fatty acids. It indeed emphasizes the lesser peripheral insulin sensitivity and higher secretory responsiveness of pancreatic $\beta$ cells to D-glucose in these second generation $\omega 3 \mathrm{D}$ rats.

Supported by Ministerio de Sanidad y Consumo and a convention between the Région de Bruxelles-Capitale and Target Hit s.a. (Brussels, Belgium)

\section{7}

Copper administration prevents and reverses the diabetogenic state and abnormal fat metabolism observed in the Cohen diabetic rat S. W. Zangen, L. T. Chen, I. Raz;

Diabetes Unit, Hadassah University hospital, Jerusalem, Israel.

Background: Nutrition is an essential element in the manifestation of type 2 diabetes in susceptible individuals. Restricting dietary copper combined with high concentration of dietary sucrose induces lipid peroxidation, atrophy of pancreatic acinar tissue and also reduces mitochondrial function. The CDs rat is genetically susceptible to develop impaired glucose stimulated insulin secretion (GSIS) associated with exocrine lesions, lipid deposition and macrophage infiltration when fed a high-sucrose, copper-poor diet (HSD, copper $=1 \mathrm{ppm}$ ). Resistance of peripheral tissues to insulin and obesity were not observed in the hyperglycemic CDs rat :

Aim: To study if the reduced GSIS and exocrine lesions may be reverted and prevented by supplementing the HSD diet with an adequate level of copper (15ppm, HSD-Cu].

Materials and Methods: 3 groups of CDs rats were studied: rats fed 30 days HSD (CDs-HSD $n=5)$; rats fed 40 days HSD-Cu $(n=6)$ and CDs-HSD rats 
switched to HSD-Cu diet for an additional 40 days of feeding (HSD-reversal, $\mathrm{n}=6$ ). Blood glucose and insulin levels were assessed during intravenous glucose tolerance test (IVGTT). Fasting serum free fatty acids (FFA) levels, pancreatic triglyceride (TG) content and weight, were assessed in each treatment group. Sensitivity of peripheral tissues to exogenous insulin was assessed by intravenous insulin tolerance test (ITT $0.5 \mathrm{U} / \mathrm{kg} \mathrm{BW}$ ).

Results: The high glucose area under the IVGTT curve of CDs-HSD rats was significantly reduced in the HSD-Cu and HSD-reversal rats (1500 \pm 158 vs. $724 \pm 64$ and $659 \pm 66$, respectively $\mathrm{mM} / 120 \mathrm{~min}, \mathrm{P}<0.001$ ). In contrast, the insulin output was significantly lower in CDs-HSD compared to the HSD-Cu and HSD-reversal rats ( $87 \pm 34$ vs. $10603 \pm 6250$ and $1980 \pm 658 \mathrm{pM} / 120 \mathrm{~min}$, $\mathrm{P}<0.01)$. Pancreatic exocrine lesions were prevented by HSD-Cu and were milder in the HSD-reversal rats. Pancreatic weight of HSD-Cu and HSDreversal rats was significantly higher compared to CDs-HSD rats $(0.306 \pm 003$ vs. $0.217 \pm 0.01$ and $0.133 \pm 0.02 \mathrm{~g} P<0.001)$ while pancreatic TG content and serum FFA were markedly reduced in HSD-Cu compared to CDs-HSD $(0.25$ $\pm 0.03 \mathrm{vs} .1 .9 \pm 0.01 \mathrm{mg} / \mathrm{g}$ tissue for the TG and $0.225 \pm 0.00 \mathrm{vs} .1 .48 \pm 0.12 \mathrm{mM}$ for the FFA, $\mathrm{P}<0.001)$. No fat was found in the gastrocnemius muscle of either group. The high sensitivity to exogenous insulin exhibited by CDs-HSD rats was further augmented by HSD-reversal diet.

Conclusion: The CDs rat is a good model to study the sole effect of reduced copper intake on GSIS and exocrine lesions. The abnormalities induced by the HSD may be prevented and reverted by adequate dietary copper concentration. This may suggest that dietary copper concentration as a key element in the mechanism of insulin secretion and fat metabolism of the CDs-HSD rats.

Supported by Russell Berrie Foundation and D-Cure, Diabetes Care in Israel and by grants from AM Cohen Foundation

\section{8}

The diagnostic value of traditional insulin sensitivity and $B$ cell function indices

B. Buday ${ }^{1}$, L. Koranyi;

${ }^{1}$ Cardiology, Péterfy Sándor Hospital, Budapest, ${ }^{2}$ Metabolic Diseases,

DRC Ltd, Balatonfüred, Hungary.

Background and Aims: Improving insulin resistance is a powerful tool in prevention of type 2 diabetes, but only the complicated clamp method is sensitive enough for an early diagnosis. We investigated the utility of insulin sensitivity (IS) and beta cell function (BCF) indices derived from fasting blood and oral glucose tolerance test (OGTT).

Materials and Methods: Healthy (NGT, $n=47$ ), glucose intolerant (GIT, $n=$ 92, IFG/IGT: $n=67$;type 2 diabetic $(2 \mathrm{DM}) \mathrm{n}=25)$ patients were investigated by an OGTT, an iv.glucose tolerance test (IVGTT) and a hyperinsulinemic euglycemic clamp

Results: With the exception of older (age above median) NGT and GIT groups we found moderate $(\mathrm{r}=0.4-0.6, \mathrm{p}<0.001)$ correlations in most examined groups (formed by glucose tolerance, age, gender and BMI) between HOMA indices and whole body glucose utilization measured by clamp (M). The IS OGTT indices (MCRest, ISIcomp, OGIS) showed strong correlation with the $M(r=0.6-0.8, p<0.001$.). While their average sensitivity for insulin resistance was low $(50.42 \pm 4.65 \%$ for HOMA-S and $60.25 \pm 8.06 \%$ for OGTT IS indices), their specificity was fairly good $(83.37 \pm 1.62 \%$ for HOMA-S, and $87.29 \pm 1.87 \%$ for OGTT indices). Fasting BCF indices (HOMA-B) showed significant correlation only with the AIR of IVGTT in IFG/IGT ( $r=0.47$, $\mathrm{p}<0.001)$ and $2 \mathrm{DM}(\mathrm{r}=0.54, \mathrm{p}<0.02)$ groups, but not in the NGT groups, while amongst BCF indices derived from the OGTT insulogenic index and $\Delta \mathrm{I}_{30}$ showed strong correlation with AIR in the NGT $(\mathrm{r}=0.63,0.73, \mathrm{p}<0.001)$, moderate in IFG/IGT $(r=0.40, p<0.01$ and $r=0.57, \mathrm{p}<0.05)$ and moderate or non-significant $\left(\mathrm{r}=0.51, \mathrm{p}<0.05, \mathrm{r}=0.12 \mathrm{n} . \mathrm{s}\right.$.) in the $2 \mathrm{DM}$ group. $1^{\text {st }}$ and $2^{\text {nd }}$ phase insulin response of OGTT (Stumvoll indices) correlated weakly with the AIR only in the IFG/IGT $(r=0.33,0.36, p<0.01)$ but not in the NGT and $2 \mathrm{DM}$ groups. Their average sensitivity for reduced $\mathrm{BCF}$ was very low (36.99 $\pm 2,72$ for HOMA-B and $38.2 \pm 8.19 \%$ for OGTT BCF) while their specificity was better $(81.75 \pm 0.49$ for HOMA-B and $79.96 \pm 5.41 \%$ for OGTT BCF).

Conclusion: Even though some of the widely used simple IS or BCF indices show good correlation with the indices of more accurate clamp methods, their diagnostic value seems to be insufficient in terms of sensitivity. Therefore we still need to develop a simple but more accurate method, comparable to the clamp and IVGTT techniques.

\section{9}

Acute administration of a sulfonylurea compound restores the insulinotropic effect of glucose-dependent insulinotropic polypeptide in patients with type 2 diabetes

K. Aaboe ${ }^{1,2}$, F. K. Knop ${ }^{1,2}$, T. Vilsbøll' ${ }^{1}$, S. Madsbad ${ }^{3}$, C. F. Deacon ${ }^{2}$, J. J. Holst ${ }^{2}$, T. Krarup ${ }^{1}$;

${ }^{1}$ Department of Internal Medicine F, Gentofte University Hospital, Hellerup, ${ }^{2}$ Department of Medical Physiology, The Panum Institute, University of Copenhagen,

${ }^{3}$ Department of Endocrinology, Hvidovre University Hospital, Denmark.

Background and Aims: Mutations in the kir 6.2 gene, encoding the poreforming unit of the ATP-sensitive potassium channels (KATP-channels) of the beta-cell, are part of the genetic polymorphism seen in type 2 diabetes. Defects in the function the kir 6.2 subunit in mice are known to inhibit the insulinotropic effect of glucose-dependent insulinotropic polypeptide (GIP). Our aim with this study was to evaluate a possible role of the KATP-channels in the lost insulinotropic effect of GIP. We hypothesized that closing the KATP-channels in the beta-cell through acute administration of sulfonylurea (SU) would re-establish the insulinotropic effect of GIP.

Material and Methods: Twelve patients ( 8 men) with type 2 diabetes (Age: 54 [45-70] years; BMI: 30 [26-35] kg/m²; $\mathrm{HbA}_{1 \mathrm{c}}$ : 7.7 [7-8.6] \%; values mean

Fig. 1

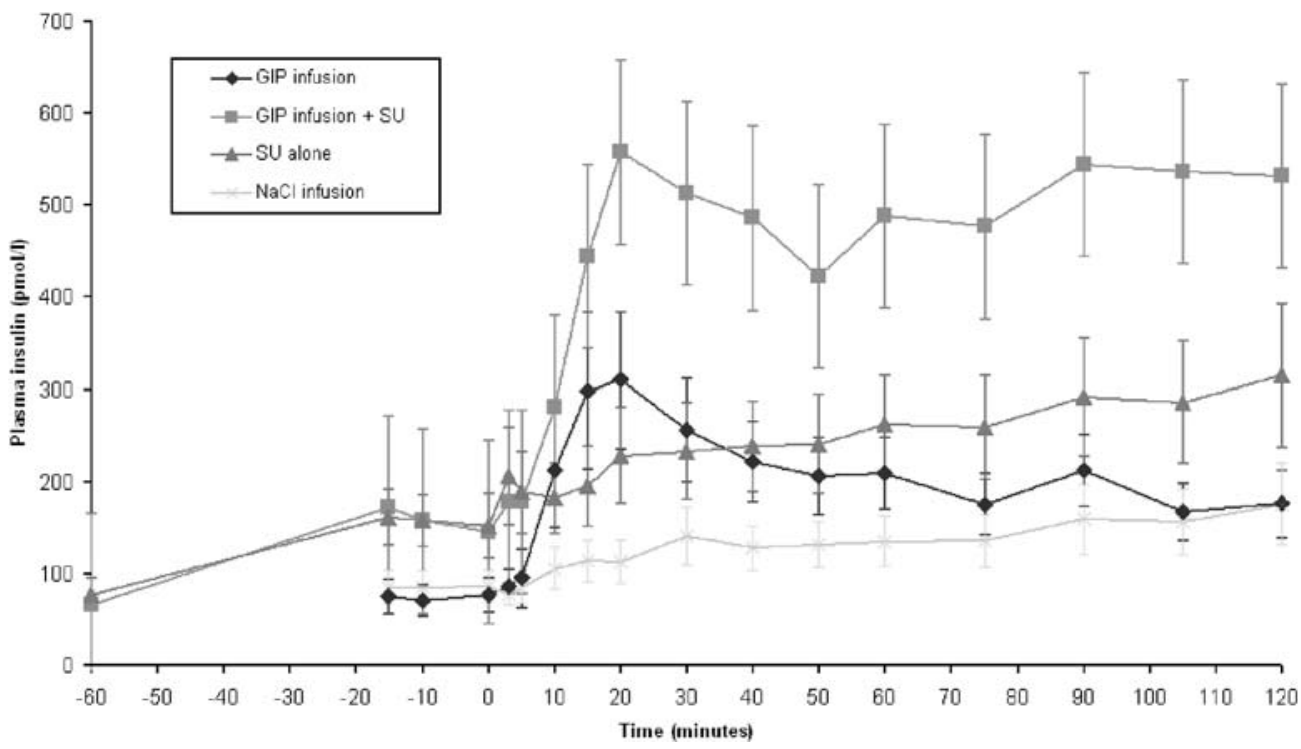


[range]) on mono-therapy with metformin (1 diet) were studied over the course of 4 days. Patients were examined using hyperglycaemic clamps (15 $\mathrm{mM}, 2$ hours) with concomitant infusion of GIP (4 pmol/kg body weight/ $\mathrm{min}$ ), GIP + administration of $10 \mathrm{mg} \mathrm{SU}$ (glipizide) as tablets 1 hour before the clamp, saline + administration of $10 \mathrm{mg} \mathrm{SU}$ as tablets 1 hour before the clamp or saline alone. During the examinations blood was sampled at intervals of 10 to 15 minutes to measure plasma concentrations of glucose, intact and total GIP, glucagon, insulin, C-peptide, free fatty acids and SU.

Results: Insulin secretion, calculated as the incremental area under the curve (iAUC) from time 0 to 120 minutes, was significantly higher during GIP+SU $(38 \pm 9 \mathrm{nmol} / \mathrm{l} \times 120 \mathrm{~min})$ compared to GIP alone $(15 \pm 3 \mathrm{nmol} / \mathrm{l} \times 120 \mathrm{~min}$; $p=0.0005)$, to SU alone $(12 \pm 5 \mathrm{nmol} / 1 \times 120 \mathrm{~min} ; p=0.001)$ and to infusion with saline alone $(6 \pm 2 \mathrm{nmol} / \mathrm{l} \times 120 \mathrm{~min} ; p=0.0005)$. The iAUC during examination with GIP+SU was also significantly higher than the sum of SU alone and GIP alone ( $38 \pm 9$ vs. $21 \pm 5 \mathrm{nmol} / \mathrm{l} \times 120 \mathrm{~min} ; p=0.02)$. There was no significant difference between examinations with GIP alone compared to SU alone ( $15 \pm 3$ vs. $12 \pm 5 \mathrm{nmol} / \mathrm{l}$ x $120 \mathrm{~min} ; p=\mathrm{NS}$ ).

Conclusion: Our results imply that in patients with type 2 diabetes, treated with metformin as mono-therapy, acute administration of a SU compound seems at least partially to restore the insulinotropic effect of GIP. This effect may be caused by SU closing the K-ATP channels in the beta cell, hereby uncoupling any influence from K-ATP channel malfunction on the effect of GIP. We suggest that K-ATP channel malfunction contributes to the lost insulinotropic effect of GIP observed in patients with type 2 diabetes. (Fig. 1) Supported by the Danish Diabetes Association

\section{0}

Suit index is a useful predictor of the benficial effect of short-period insulin therapy in type 2 diabetic patients

Y. Hamamoto ${ }^{1,2}$, S. Honjo ${ }^{2}$, H. Ikeda ${ }^{2}$, K. Nabe ${ }^{2}$, K. Nomura ${ }^{2}$, Y. Wada ${ }^{2}$, H. Koshiyama ${ }^{2}$;

${ }^{1}$ Department of Diabetes \& Clinical Nutrition, Graduate School of Medicine, Kyoto University, Kyoto,

${ }^{2}$ Center for Diabetes \& Endocrinology, The Tazuke Kofukai Foundation

Medical Research Institute Kitano Hospital, Osaka, Japan.

Background and Aims: Type 2 diabetic patients often exhibit decreased insulin secretion after a long-period treatment with Sulfonylurea drugs (SU). Insulin therapy has been reportedly shown to restore $\beta$ cell function in not only animal models but also humans. Although there have been several reports suggesting beneficial effects of insulin therapy on glycemic control in newly diagnosed type 2 diabetic patients, the effect of insulin on the patients who have taken SU drugs for a long period has not been well examined. Here we report the effects of short-period insulin (SPI) therapy on $\beta$ cell function assessed by the secretory units of islets in transplantation (SUIT) index in relation with the subsequent glycemic control after the therapy in Japanese diabetic subjects. SUIT index was developed as an index of $\beta$ cell function for islet transplanted patients but also was shown to be applicable to type 2 diabetic patients by Yamada et al., our colleagues.

Materials and Methods: Sixteen patients who had taken SU more than 5 (8.4 \pm 4.0$)$ years were examined. All the patients were hospitalized and were given insulin therapy 8 to 13 days, and the changes of blood glucose levels, plasma C-peptide (CPR) levels, glucagon-stimulated CPR responses before and after SPI therapy were evaluated in the hospital. HbAlc levels before, 6 months and 12month after hospitalization were also evaluated, and the effect of short-period insulin therapy on glycemic control was examined by comparing $\mathrm{HbAlc}$ levels with the patients who were treated without insulin during their hospitalization as a control group. SUIT index was calculated by the formula : $250 x F-C P R(n M) /(F-B G(m M)-3.43)$. It was developed by assuming that normal subjects aged $<40$ years have $100 \% \beta$ cell function.

Results: HbAlc levels six months after SPI therapy were decreased in all the patients. Although SU doses were reduced after SPI therapy in half of the patients, the average HbAlc level significantly decreased from $8.8 \pm 0.9$ $\%$ to $7.1 \pm 1.0 \%$, while that in the control group showed any statistically significant change ( $8.6 \pm 1.6 \%$ to $7.6 \pm 0.8 \%)$. Twelve months after SPI therapy, the average $\mathrm{HbAlc}$ level increased to $7.4 \pm 1.4 \%$. The rate of change in HbAlc levels from 6 to 12 months compared with that from before to 6 months after hospitalization could be divided into two groups by the border of $20 \%$ increase (preserved group: -333 to $18 \%$ vs. worsened group: 23 to $161 \%$ increase, $n=8$ for each). SUIT index before SPI therapy tended to be higher in the preserved group, but was not statistically different between the two groups (45.7 \pm 8.4 vs. $35.1 \pm 3.6$ ). However, SUIT index after SPI therapy was significantly higher in the preserved group compared to the worsened group $(70.8 \pm 14.2$ vs. $43.7 \pm 4.9)$. Increase in SUIT index after SPI therapy in the preserved group suggested a recovery of $\beta$ cell function, which might result in a better glycemic control after SPI therapy.

Conclusion: These results suggest that 1 ) SPI therapy may be beneficial to recover $\beta$ cell function in certain subsets of patients with failure of SU drugs, and that 2) SUIT index can be a useful indicator to predict the beneficial effect of SPI therapy on subsequent glycemic control.

\section{1}

Diabetes mellitus and $\beta$ cell function in patients with cystic fibrosis awaiting lung transplantation

C. Schmid, A. Klarer, M. Hofer, M. Faulenbach, P. Wiesli, A. Boehler; Internal Medicine, University Hospital of Zurich, Switzerland.

Background and Aims: To assess the frequency of diabetes mellitus (DM) and to characterize $\beta$-cell function and insulin sensitivity in patients with cystic fibrosis (CF) referred to our centre for lung transplantation (LTx). Materials and Methods: Over 5 years, consecutive patients were included; they had full evaluation with regard to LTx. Patients not known for previous fasting plasma glucose (FPG) $\geq 7 \mathrm{mM}$ and a control group of healthy subjects underwent an oral $(75 \mathrm{~g})$ glucose tolerance test (oGTT). Insulin sensitivity and insulin secretion were assessed by indices proposed by Matsuda and by Wareham, respectively, derived from the oGTT.

Results: 42 CF patients were evaluated. Only one patient was obese, and three did not have exocrine pancreatic insufficiency. 24 (14 female) patients had an established diagnosis of DM (57\%), and 18 (13 male) patients and 23 (15 male) healthy controls underwent oGTTs. Patients with known DM and patients undergoing oGTTs did not differ with regard to age (mean \pm SD, $26 \pm 6$ y) and had comparably reduced BMI $\left(18 \pm 2 \mathrm{~kg} / \mathrm{m}^{2}\right)$ and $\mathrm{FEV}_{1}(29 \pm 6 \%)$ but the former were more often female and had higher HbAlc values $(7.2 \pm 0.7 \mathrm{vs}$. $6.1 \pm 0.4 \%)$. Among those assessed by oGTT, FPG was similar in CF patients $(4.8 \pm 0.5 \mathrm{mM})$ and in healthy controls $(4.8 \pm 0.4 \mathrm{mM})$, and so was fasting insulin, fasting proinsulin, and insulin sensitivity. By contrast, $\mathrm{HbAlc}$ and peak (>11 mM in 13$)$ and $2 \mathrm{~h}$ PG $(10.4 \pm 3.6 \mathrm{mM}$ vs. $6.1 \pm 1.2 \mathrm{mM} ;>11 \mathrm{mM}$ in 7 patients, 6 male) during oGTTs were higher in the CF patients, and the insulinogenic index was significantly lower in the patients $(20.9 \pm 11.1)$ than in the controls (60.4 \pm 39.0$)$. Within the patients with CF, the insulinogenic index was highest $(>30)$ in those without exocrine pancreatic insufficiency whereas it was consistently low $(<30)$ in those with exocrine failure, irrespective of whether they met diagnostic criteria for DM or not.

Conclusion: $74 \%$ of the CF patients ( 15 of 19 female, 16 of 23 male) referred for LTx had DM. Increased energy expenditure and insulin-independent glucose clearance may account for completely normal FPG despite severely impaired $\beta$-cell function in an important subset (31\%) of these patients. FPG is an inappropriate tool to identify patients who might benefit (beyond control of glycaemia) from treatment with insulin, an anabolic hormone.

\section{2}

Is the disposition index law hyperbolic? Importance of the regression methodology

P. Denti ${ }^{1}$, M. Campioni ${ }^{1}$, G. Toffolo ${ }^{1}$, R. Rizza ${ }^{2}$, C. Cobelli ${ }^{1}$;

${ }^{1}$ Department of Information Engineering, University of Padova, Italy, ${ }^{2}$ Division of Endocrinology, Diabetes, Metabolism and Nutrition, Mayo Clinic, Rochester, United States.

Background and Aims: Glucose tolerance requires to express beta cell function in relation to insulin action. The most widely used measure is based on the Hyperbolic Law Disposition Index (DI), i.e. DI= beta-cell function $\mathrm{x}$ insulin sensitivity=constant. Recently the validity of this law has been questioned and a different description has been suggested: DI= beta-cell function $\mathrm{x}$ insulin sensitivity ${ }^{\text {alpha }}=$ constant. Aim of this work is to test the validity of the hyperbolic law.

Materials and Methods: To do so, IVGTT in 145 elderly healthy subjects (age $68.9 \pm 0.5$ years, BMI $27.5 \pm 0.3 \mathrm{~kg} / \mathrm{m}^{2}$ ) was interpreted with glucose and Cpeptide minimal models to estimate insulin sensitivity SI ${ }_{\mathrm{IVGTT}}$ and $1^{\text {st }}$ and $2^{\text {nd }}$ phase beta-cell responsivity, $\mathrm{Phi}_{1}$ and $\mathrm{Phi}_{2}$, with their estimation error. Two 
disposition indices, $\mathrm{DI}_{1}$ and $\mathrm{DI}_{2}$, were calculated by multiplying, respectively, $\mathrm{Phi}_{1}$ and $\mathrm{Phi}_{2}$ by $\mathrm{SI}_{\mathrm{IVGTT}}$ alpha. Four regression models have been compared, L$1 \mathrm{~V}$ : linear regression on log-data assuming error only on one variable (betacell responsivity); NL-1V: nonlinear regression assuming error only on one variable (beta-cell responsivity); L-2V: linear regression on log-data assuming error on both variables; NL-2V nonlinear regression assuming error on both variables.

Results: The table shows the results obtained by the four methods (subscripts 1 and 2 denote $1^{\text {st }}$ and $2^{\text {nd }}$ phase parameters, respectively and, within brackets, confidence intervals are displayed).

\begin{tabular}{lllll} 
& DI $_{1}$ & Alpha $_{1}$ & DI $_{2}$ & Alpha $_{2}$ \\
\hline L-1V & $94(66-122)$ & $0.18(0-0.35)$ & $9(7-11)$ & $0.20(0.09-0.3)$ \\
NL-1V & $135(107-164)$ & $0.19(0.06-0.32)$ & $14(12-16)$ & $0.30(0.21-0.39)$ \\
L-2V & $498(410-585)$ & $0.72(0.64-0.81)$ & $32(28-36)$ & $0.67(0.62-0.73)$ \\
NL-2V & $333(273-393)$ & $0.97(0.85-1.09)$ & $23(21-26)$ & $0.66(0.60-0.72)$ \\
\hline
\end{tabular}

Conclusion: We conclude that the choice of the regression method is crucial, since neglecting error-in-two-variables, but also performing linear regression on log-data, severely affect the results. When both error in-two-variables and the nonlinearity of the relationship are taken into account (NL-2V), results support the classical hyperbolic law for $\mathrm{DI}_{1}$ but not for $\mathrm{DI}_{2}$.

\section{PS 45 Adiponectin}

\section{3}

Peripheral monocyte Adipo-R1 and Adipo-R2 gene and protein expression in patients with CAD and type 2 diabetes

A. Kollias ${ }^{1,2}$, P. Tsiotra ${ }^{1}$, E. Maratou ${ }^{1}$, I. Halvatsiotis ${ }^{1,2}$, E. Kyriazi $^{1,2}$,

E. Boutati ${ }^{2}$, A. Raptis ${ }^{2}$, K. Fountoulaki ${ }^{3}$, I. Lekakis ${ }^{3}$, G. Dimitriadis ${ }^{2}$, D. Kremastinos ${ }^{3}$, S. A. Raptis ${ }^{1,2}$;

${ }^{1}$ Molecular Biology Lab., Division of Basic Sciences, Hellenic National Diabetes Center, ${ }^{2}$ 2nd Dept. of Internal Medicine, Research Institute \& Diabetes Center, "Attiko" University General Hospital, Medical School, ${ }^{3}$ 2nd Dept. of Cardiology, "Attiko" University General Hospital, Athens, Greece.

Background and Aims: Adiponectin, an adipose tissue produced molecule, has been known to have anti-diabetic and anti-atherogenic properties. Adiponectin levels are decreased in obesity, type 2 diabetes, and patients with coronary artery disease (CAD). Adiponectin receptors 1 (Adipo-R1) and 2 (Adipo-R2) are expressed ubiquitously in most organs and also in human peripheral monocytes and mediate adiponectin's signalling into its target cells. The aim of this study was to examine whether the relative mRNA and protein expression of adipo-R1 and -R2 in human monocytes is altered in patients with CAD with/without type 2 diabetes and whether this might relate to indices of obesity and insulin resistance.

Materials and Methods: We studied 20 subjects ( 9 with CAD and impaired glucose tolerance (CAD-IGT), 7 with CAD and type 2 diabetes (CAD-DM2), and four (4) without CAD but with impaired glucose tolerance (control-IGT). We developed a real-time quantitative RT-PCR using fluorecently labelled hybridization probes to detect relative adipo-R1 and adipo- $\mathrm{R} 2 \mathrm{mRNA}$ levels in isolated CD14+ blood monocytes (Light Cycler, Roche). mRNA levels were calculated as the relative ratio of the fluorescence acquisition of adipo- $\mathrm{R} 1$ and -R2 mRNA divided by that of $\beta$-actin. We also detected adipo-R1 and adipo$\mathrm{R} 2$ protein expression at the same cells using a FACSCalibur Flow Cytometer (BD Biosciences). We also measured fasting and 2 hour post-OGTT plasma insulin (RIA), and plasma glucose levels.

Results: Adipo-R1 mRNA levels tended to be higher in peripheral monocytes from CAD patients (IGT and DM2) compared to controls (Table), while adipo-R2 mRNA levels tended to be lower in CAD compared to controls. Both adipo- $\mathrm{R} 1$ and adipo- $\mathrm{R} 2$ protein levels tended to be lower in CAD patients compared to controls (Table). The adipo-R1/adipo- $\mathrm{R} 2$ protein ratio levels correlated positively with fasting and 2 hour post-OGTT glucose levels $(\mathrm{r}=0.587, \mathrm{p}=0.017$ and $\mathrm{r}=0.535, \mathrm{p}=0.033$, respectively) and HOMA-IR index $(\mathrm{r}=0.532, \mathrm{p}=0.034)$, while adipo- $\mathrm{R} 2$ protein levels correlated negatively with HOMA-IR ( $\mathrm{r}=-0.629, \mathrm{p}=0.009)$. (Tab. 1 )

Conclusion: The reduced adipo-R2 mRNA and protein levels in CAD(either IGT or DM2) patients compared to controls-IGT subjects, suggests that adiponectin receptor 2 regulation, possibly, is influenced by the elevated insulin and glucose levels observed in these conditions. This impaired adiponectin signaling which occurs in the peripheral monocytes of the above patients, could possibly contribute to their atherogenicity and their insulin resistance.

Supported by the 3rd Community Support Programme (Operational Programme Competiveness - PENED03E4597)

Tab. 1

\begin{tabular}{|c|c|c|c|c|c|c|c|}
\hline & $\begin{array}{l}\mathrm{BMI} \\
\mathrm{kgr} / \mathrm{m}^{2}\end{array}$ & WHR & $\begin{array}{l}\text { Glucose at } 0 \mathrm{~h} \\
\mathrm{mg} / \mathrm{dL}\end{array}$ & $\begin{array}{l}\text { Adipo-R1 } \\
\text { mRNA }\end{array}$ & $\begin{array}{l}\text { Adipo-R2 } \\
\text { mRNA }\end{array}$ & $\begin{array}{l}\text { Adipo-R1 } \\
\text { protein }\end{array}$ & $\begin{array}{l}\text { Adipo-R2 } \\
\text { protein }\end{array}$ \\
\hline Control-IGT & $31.5 \pm 0.5$ & $0.9 \pm 0.02$ & $99.0 \pm 6.0$ & $2.91 \pm 1.22$ & $0.91 \pm 0.18$ & $87.7 \pm 35.3$ & $94.6 \pm 35.4$ \\
\hline CAD-IGT & $27.9 \pm 0.9$ & $1.0 \pm 0.02$ & $112.2 \pm 2.6^{*}$ & $3.76 \pm 0.82$ & $0.79 \pm 0.15$ & $67.5 \pm 11.2$ & $86.0 \pm 15.1$ \\
\hline CAD-DM2 & $30.0 \pm 0.9$ & $1.0 \pm 0.04$ & $141.6 \pm 7.9^{*}$ & $3.62 \pm 1.11$ & $0.79 \pm 0.21$ & $67.0 \pm 12.2$ & $61.8 \pm 8.44$ \\
\hline
\end{tabular}

${ }^{*}, \mathrm{p}<0.05$ vs control-IGT 


\section{4}

Expression of adiponectin receptors in a model of insulin-resistance and type 2 diabetes: the diabetic Zucker rat

C. Metais ${ }^{1}$, F. Forcheron ${ }^{1}$, P. Del Carmine ${ }^{2}$, G. Bricca ${ }^{1}$, M. Beylot ${ }^{1}$;

${ }^{1}$ Agressions vasculaires et réponses tissulaires, INSERM ERI 22,

${ }^{2}$ Faculté Rockefeller, ANIPHY, Lyon, France.

Background and Aims: Adiponectin secreted by adipocytes has effects on glucose and lipid metabolism. Its expression is reduced in obesity and diabetes. Adiponectin reverses insulin-resistance in rodent models of type 2 diabetes. Therefore, it improves insulin sensitivity and has protective effects against the development of insulin-resistance and type 2 diabetes. Adiponectin exerts its metabolic effects via two receptors: AdipoR1 and AdipoR2 that have been cloned and characterized.They are expressed in liver, adipose tissue, skeletal muscle, macrophages and pancreatic beta cells. In addition to a decrease in the circulating levels of adiponectin, a decrease in the expression of adipoRs in target tissues could play a role in the development of type 2 diabetes. Actually, the expression of AdipoRs was found reduced in insulin-resistant and type 2 diabetic mice. A therapeutic strategy for the treatment of type 2 diabetes may include the up-regulation of AdipoRs. This could be achieved by metformin, known to improve insulin sensitivity, or fenofibrate, used in the treatment of hyperlipidemia. The aims of this study were 1) to investigate the expression of adipoRs in diabetic Zucker rats in three insulin-dependent tissues: white adipose tissue (WAT), skeletal muscle and liver 2) to test the effect of fenofibrate and metformin on the expression of adipoRs.

Materials and Methods: We studied 4 groups of animals, all receiving a high fat diet: lean Zucker Rats $(+/+) \mathrm{n}=15$, diabetic Zucker Rats (fa/fa) $n=15$, diabetic Zucker Rats ( $\mathrm{fa} / \mathrm{fa}$ ) treated by fenofibrate $\mathrm{n}=10$ and diabetic Zucker Rats $(\mathrm{fa} / \mathrm{fa}$ ) treated by metformin $\mathrm{n}=10$. The expression of adipoRs was studied at 7 weeks of age (insulin-resistance), 14 weeks of age (type 2 diabetes) and 21 weeks of age (type 2 diabetes with insulin deficiency). Liver, skeletal muscle and adipose tissue (peri-renal) samples were collected in anesthesized rats (post-absoptive state). The expression of adipoRs was appreciated by the measurement of their mRNA concentrations (real time PCR).

Results: 1) There was a trend for a decrease in AdipoR1/R2 expression with age in WAT and liver of Lean rats. 2) We found no decrease in the expression of adipoRs in the diabetic Zucker rat in any of the three tissues studied, either in 7 weeks, nor in 14 or 21 weeks old rats. There was on the contrary a trend for higher mRNA concentrations of AdipoR1 and R2 in WAT of diabetic Zucker rats. 3) fenofibrate increased significantly $(\mathrm{p}<0,001)$ the expression of adipoRs in the liver of the type 2 diabetic Zucker rats (14 weeks), no changes were found in the skeletal muscle and in WAT, 4) metformin increased significantly $(p<0,001)$ the expression of adipoRs in the skeletal muscle of the type 2 diabetic Zucker rats (14 weeks) and type 2 diabetic Zucker rats with insulin deficiency (21 weeks); metformin had no action on AdipoRs in the liver and in WAT.

Conclusion: We found no reduction in the expression of adiponectin receptors in this genetic model of type 2 diabetes. Our results do not support a role for decreased expression of adipoRs in the development of insulinresistance and type 2 diabetes, contrary to what was observed in type 2 diabetic mice. However, fenofibrate and metformin increased the expression of adipoRs in the liver and in the skeletal muscle, respectively, of the diabetic Zucker rats. This could contribute to the beneficial actions of fenofibrate and metformin in the treatment of type 2 diabetes.

\section{5}

Circulating high-molecular weight adiponectin is a strong determinant of ectopic fat in liver and skeletal muscle in humans

K. Kantartzis ${ }^{1}$, H. Staiger ${ }^{1}$, J. Machann ${ }^{2}$, F. Schick ${ }^{2}$, F. Machicao',

A. Fritsche ${ }^{1}$, H.-U. Häring ${ }^{1}$, N. Stefan ${ }^{1}$;

${ }^{1}$ Department of Internal Medicine,

${ }^{2}$ Section of Experimental Radiology, University of Tübingen, Germany.

Background and Aims: The adipokine adiponectin increases lipid oxidation in muscle and liver and has insulin-sensitizing and anti-inflammatory properties. Adiponectin circulates in several multimeric forms in human plasma, i.e. trimeric low molecular weight (LMW), hexameric middle molecular weight (MMW) as well as more complex high molecular weight (HMW) structures. Of the higher-ordered structures predominantly the HMW form is considered to have bioactivity. Accordingly, among all multimeric forms of adiponectin, low circulating HMW adiponectin was found to be the major determinant of insulin resistance, an atherogenic lipoprotein profile, and the metabolic syndrome. However, in a recent study these relationships could not be confirmed. Thus, it is still unsolved whether HMW adiponectin is a more sensitive marker of glucose and lipid metabolism than total adiponectin.

Materials and Methods: In the present study we addressed this issue in 54 subjects without type 2 diabetes applying state of the art measurements. Ectopic fat in liver and skeletal muscle were quantified by localized proton magnetic resonance spectroscopy and insulin sensitivity was determined during a euglycemic hyperinsulinemic clamp. Total-, HMW-, MMW- and LMW adiponectin were quantified using an enzyme immunoassay (ALPCO Diagnostics, Salem, NH, USA).

Results: High-density lipoprotein cholesterol levels, adjusted for age, gender and body fat correlated positively with circulating total- $(\mathrm{r}=0.37, \mathrm{p}=0.006)$ and HMW- $(\mathrm{r}=0.39, \mathrm{p}=0.005)$ adiponectin, but not with MMW- or LMW adiponectin (both $\mathrm{p}>0.08$ ). Adjusted insulin sensitivity also correlated positively with circulating total- $(\mathrm{r}=0.39, \mathrm{p}=0.004)$ and HMW $(\mathrm{r}=0.31$, $\mathrm{p}=0.02$ ) adiponectin, while no correlations of insulin sensitivity with MMWor LMW adiponectin were found (both $\mathrm{p}>0.13$ ). Only adjusted circulating HMW adiponectin ( $\mathrm{r}=-0.32, \mathrm{p}=0.03)$, but not adjusted total-MMW or LMW adiponectin (all $\mathrm{p}>0.12$ ) correlated with intramyocellular lipids in the tibialis anterior muscle in this group of subjects. In addition, adjusted liver fat significantly correlated with HMW adiponectin $(\mathrm{r}=-0.35, \mathrm{p}=0.01)$, while only a non significant relationship of liver fat with total- $(\mathrm{r}=-0.26, \mathrm{p}=0.06)$, and no associations with MMW- and LMW adiponectin (both $\mathrm{p}>0.70$ ) were observed. Moreover, when subjects were divided into a group with fatty liver (liver fat $>5.5 \%, \mathrm{n}=15)$ and controls $(\mathrm{n}=39)$, HMW adiponectin displayed a larger area under the receiver operating characteristic curve (0.77) than total adiponectin (0.73) to predict fatty liver disease.

Conclusion: We provide novel data showing that HMW adiponectin has a higher predictive power than total adiponectin to determine ectopic fat deposition in liver and skeletal muscle in humans.

Supported by Deutsche Forschungsgemeinschaft (KFO 114/2)

\section{6}

Upregulation of HMW adiponectin in visceral adipose tissue by pioglitazone associates with amelioration of insulin resistance syndrome in obese rats

J. Pita, A. Panadero, M. Gonzalo, J. Lara, O. Sanchez-Vilar, A. Rovira; Laboratory of endocrinology, Fundación Jiménez Díaz - Capio, Madrid, Spain.

Background and Aims: Adiponectin is an insulin-sensitizing hormone produced by adipocytes with anti-inflammatory properties which is reduced in insulin resistance states like obesity and type 2 diabetes. Adiponectin exists as truncated form (LMW adiponectin) and as a group of complexes or fulllength adiponectin (MMW and HMW adiponectin). Some studies suggest that among the circulating forms, HMW is the best indicator of insulin sensitivity during the treatment with PPAR- $\gamma$ agonists in human and in mice. Furthermore, they suggest that the HMW form could be the bioactive form of adiponectin responsible for the level of insulin sensitivity. Pioglitazone is a PPAR- $\gamma$ agonist that improves insulin resistance and upregulates adiponectin levels in plasma and adipose tissue. It has been proposed that adiponectin upregulation could be the mechanism used by PPAR- $\gamma$ agonists to improve insulin resistance linked to obesity. The aim of this study is to know if there is a different regulation of the adiponectin forms by pioglitazone in fatty Zucker rats, an animal model that associates extreme obesity, insulin resistance and disregulation of lipidic metabolism.

Materials and Methods: Fatty Zucker rats were treated with pioglitazone $(3 \mathrm{mg} / \mathrm{kg} / \mathrm{d}, \mathrm{n}=10)$ or placebo $(\mathrm{n}=10)$ for 12 weeks. Blood was collected weekly, and at the end of the treatment rats were sacrificed by intraperitoneal injection of sodium penthotal. Blood, visceral (VAT) and subcutaneous (SAT) adipose tissue were collected. Measurements of adiponectin complexes: western-blot analysis were performed in plasma and adipose tissue. Equal amounts of protein, $25 \mu \mathrm{g}$, were subjected to electrophoresis in $4-15 \%$ gradient polyacrylamide gels (SDS-PAGE) and immunoblotting-SDS-PAGE under non-reducing and non-denaturising conditions.

Results: Compared to placebo, rats treated with pioglitazone had a significant increase $(\mathrm{p}<0.01)$ of the HMW form of adiponectin in plasma $(1.03 \pm 0.06 \mathrm{vs}$ 
$1.43 \pm 0.08$ a.u, mean \pm SE) and in VAT ( $1.28 \pm 0.13$ vs $3.06 \pm 0.34$ a.u) but not in SAT ( $2.49 \pm 0.36$ vs $3.54 \pm 0.39$ a.u, $p=0.087)$.

No changes were found in the MMW adiponectin in plasma or in adipose tissue after the pioglitazone treatment. We studied the correlations between the molecular forms of adiponectin and other features that ameliorated with the pioglitazone treatment, such as, plasma insulin, HOMA, plasma cholesterol and plasma triglycerides. VAT HMW adiponectin significantly correlated with these measurements and plasma HMW adiponectin correlated with plasma insulin and plasma triglycerides. No correlations between the remaining adiponectin forms (HMW form of SAT and MMW form of plasma, VAT and SAT) and the different markers were observed.

Conclusion: HMW adiponectin seems to be upregulated by pioglitazone in visceral adipose tissue from fatty Zucker rats. The increase in the HMW form correlates with the amelioration of metabolic features and insulin resistance observed after the treatment, supporting a role of this form in the insulin sensitivity effects of pioglitazone.

Supported by FISS (Spain)

\section{7}

Globular adiponectin activates nuclear factor-kB and activating protein-1 and enhances angiotensin II-induced proliferation in cardiac fibroblasts

S. Hattori, Y. Hattori, K. Suzuki, K. Kasai;

Endocrinology and Metabolism, Dokkyo University School of Medicine, Mibu, Japan.

Adiponectin is present in the serum as a trimer, hexamer, or high molecular weight form. A proteolytic cleavage product of adiponectin, known as globular adiponectin (gAd), also circulates in human plasma. The biologic activities of these isoforms are not well characterized. Pressure overload in adiponectindeficient mice results in enhanced concentric cardiac hypertrophy and increased mortality, suggesting that adiponectin inhibits hypertrophic signaling in the myocardium. Therefore, we examined whether gAd exerts the same effects on myocardium signaling. Nuclear factor $(\mathrm{NF})-\kappa \mathrm{B}$ and activating protein (AP)-1 activation were examined using cardiac fibroblasts prepared from the ventricles of 1- to 2-day-old Wistar rats and grown in culture. Globular adiponectin activated NF- $\mathrm{KB}$ and enhanced tumor necrosis factor $\alpha$-induced NF- $\mathrm{KB}$ activity. Globular adiponectin also activated AP-1 and enhanced angiotensin II-induced AP-1 activity. Globular adiponectin induced mRNA expression of c-fos and c-jun and activated extracellular signal regulated kinase. Thus, gAd enhanced angiotensin II-induced DNA and collagen synthesis. Antibodies against adiponectin receptors (AdipoR1 and AdipoR2) elicit activation of NF- $\mathrm{KB}$ or AP-1, two redox-sensitive transcription factors. Thus, rather than having an anti-hypertrophic effect, gAd might contribute to the activation of myocardium signalling, leading to myocardial hypertrophy.

Supported in part by a grant from the Seki Minato Foundation

\section{PS 46 Insulin sensitivity}

\section{8}

Insulin action in adipose tissue and skeletal muscle in obesity P. I. Mitrou ${ }^{1}$, G. Dimitriadis ${ }^{1}$, V. Lambadiari ${ }^{1}$, E. Boutati ${ }^{1}$, E. Maratou ${ }^{2}$, A. Papakonstantinou ${ }^{3}$, V. Komesidou ${ }^{4}$, T. Economopoulos ${ }^{1}$, S. A. Raptis ${ }^{1,2}$; ${ }^{1}$ 2nd Department of Internal Medicine and Research Institute, Athens University Medical School, ${ }^{2}$ Hellenic National Diabetes Center, Attikon University Hospital, ${ }^{3} 1$ st Department of Surgery, Evangelismos Hospital, ${ }^{4}$ Department of Nutrition, Evangelismos Hospital, Athens, Greece.

Background and Aims: Although whole body insulin resistance in obesity is well documented, information on insulin action in vivo in peripheral tissues, especially adipose tissue, is limited. This study was undertaken in obese subjects to investigate insulin action on (a) glucose disposal and (b) lipolysis, in skeletal muscle and adipose tissue $(\mathrm{AD})$ after ingestion of a mixed meal. Materials and Methods: A mixed meal was given to 30 obese $(\mathrm{OB}$, age $34 \pm 1 \mathrm{yrs}, \mathrm{BMI} 47 \pm 1 \mathrm{~kg} / \mathrm{m}^{2}$ ) and 10 non-obese subjects (NOB, age $39 \pm 4 \mathrm{yrs}$, BMI $\left.23 \pm 1 \mathrm{~kg} / \mathrm{m}^{2}\right)$. Plasma samples for measurements of glucose $(G)$, insulin (I), triglycerides (TAG) and non-esterified fatty acids (NEFA) were taken for 360 min from veins $(\mathrm{V})$ draining the anterior abdominal subcutaneous $\mathrm{AD}$ depot and forearm muscle $(\mathrm{M})$ and from the radial artery (A). With each sample blood flow (BF) was measured in $\mathrm{AD}$ with ${ }^{133} \mathrm{Xe}$ and in $\mathrm{M}$ with straingauge plethysmography. Calculations: Glucose flux: $\left[\mathrm{G}_{(\mathrm{A}-\mathrm{V})}\right] \mathrm{x}[\mathrm{BF}]$; Glucose fractional extraction: $\left[\mathrm{G}_{(A-V)}\right] /\left[\mathrm{G}_{(A)}\right]$; LPL: $\left[\mathrm{TAG}_{(\mathrm{A}-\mathrm{V})}\right] \mathrm{x}[\mathrm{BF}]$; Hormone sensitive lipase (HSL):[GLY $\left.{ }_{(\mathrm{V}-\mathrm{A})} \mathrm{x}(\mathrm{BF})\right]-[\mathrm{LPL}]$.

Results: Postprandial changes (area under curve $0-360 \mathrm{~min}$ ) in $\mathrm{OB}$ vs NOB: (1) Arterial G was not different $(2173 \pm 73$ vs $2040 \pm 40 \mathrm{mMmin})$, but I was increased $(23578 \pm 2372$ vs $10331+608 \mathrm{mU} / \mathrm{Lmin}, \mathrm{p}<0.05)$. (2) $\mathrm{BF}$ was decreased $(1106 \pm 78$ vs $1589 \pm 129 \mathrm{ml} / 100 \mathrm{ml}$ tissue in $\mathrm{M}$ and $657 \pm 48$ vs $1345+102 \mathrm{ml} / 100 \mathrm{ml}$ tissue in $\mathrm{AD}, \mathrm{p}<0.05$ ). (3) Glucose flux was decreased in both tissues $(339 \pm 54$ vs $826 \pm 157 \mu \mathrm{mol} / 100 \mathrm{ml}$ tissue in $\mathrm{M}$ and $164 \pm 45$ vs $320 \pm 91 \mu \mathrm{mol} / 100 \mathrm{ml}$ tissue in $\mathrm{AD}, \mathrm{p}<0.05)$. (4) Fractional glucose uptake (which is independent of $\mathrm{BF})$ was decreased in $\mathrm{M}(20 \pm 3$ vs $33 \pm 5 \%$ per min, $\mathrm{p}<0.05)$, but was not different in $\mathrm{AD}(12 \pm 3$ vs $13 \pm 5 \%$ per min). (5) Arterial NEFA $(166 \pm 16$ vs $97 \pm 11 \mathrm{nmol} / \mathrm{Lmin}, \mathrm{p}<0.05)$ and TAG $(501 \pm 37$ vs $260 \pm 32 \mathrm{nmol} / \mathrm{Lmin}, \mathrm{p}<0.05)$ were increased. (6) In AD, HSL was increased $\left(16 \pm 2\right.$ vs $\left.7 \pm 4 \mathrm{pmol}_{\text {glycerol }} / \mathrm{Lmin}, \mathrm{p}<0.05\right)$, and LPL was decreased $(5 \pm 2$ vs $80 \pm 31 \mathrm{pmol}$ glycerol $/$ Lmin, $\mathrm{p}<0.05)$.

Conclusion: In obesity: (a) the sensitivity of glucose metabolism to insulin is impaired in both $M$ (because of defects in insulin-stimulated glucose utilization and decreased blood flow) and in $\mathrm{AD}$ (because of decreased blood flow); (b) in adipose tissue, impaired suppression of HSL and activation of LPL result in increased lipolysis and impaired TAG clearance, postprandially; this may increase the risk of cardiovascular disease.

\section{9}

Weight loss resulting from bariatric surgery in morbidly obese insulin resistant individuals leads to improvements in insulin signaling M. Z. Chen ${ }^{1}$, C. A. Hudson ${ }^{2}$, E. E. Vincent ${ }^{2}$, C. M. Dayan ${ }^{3}$, D. DeBerker', J. M. Tavaré2 ${ }^{2}$ R. C. Andrews';

${ }_{1}^{1}$ Joint Clinical Research Unit, Bristol Royal Infirmary, ${ }^{2}$ Department of Biochemistry, University of Bristol, ${ }^{3}$ Henry Wellcome Laboratories for Integrative Neuroscience \& Endocrinology, University of Bristol, ${ }^{4}$ Department of Dermatology, Bristol Royal Infirmary, United Kingdom.

Background and Aims: Insulin resistance (IR), worsened by weight gain, plays a major role in the pathogenesis of Type 2 Diabetes. Although the exact mechanism is not known, a likely explanation is that the decreased insulinstimulated glucose-uptake results from abnormalities in insulin signalling. In this study we have used the hyperinsulinaemic euglycaemic (HE) clamp method to compare in vivo glucose disposal rates (GDR) with insulin signalling in lean $(\mathbf{C})$ and obese $(\mathbf{O B})$ individuals, and within the OB before and after bariatric surgery.

Materials and Methods: Six C and four OB healthy volunteers participated in this study. The $\mathrm{C}$ were aged $28.7 \pm 3.9$ (mean $\pm \mathrm{SE})$, had a body mass index (BMI) of $19.8 \pm 0.4 \mathrm{~kg} / \mathrm{m}^{2}$ and were on no medication. The $\mathrm{OB}$ were aged $48.5 \pm 4.6$, had BMI of $49.4 \pm 2.2 \mathrm{~kg} / \mathrm{m}^{2}$ with 2 of them having diabetes. Written consent was obtained from all participants. 
All attended for a 10-hour HE "long" clamp, where insulin infusion was increased in a stepwise fashion every 2 hours $(0.2,0.5,1,2$ and $5 \mathrm{mU} / \mathrm{kg} / \mathrm{min})$ with blood glucose maintained at $5 \mathrm{mmol} / \mathrm{l}$ by simultaneous glucose infusion. An insulin response curve was constructed using GDR established in the last 30 minutes, during steady state, of each insulin rate, and used to calculate the insulin concentrations needed to induce half-maximum (ED50) and maximum (ED100) effects on glucose disposal. At the next visit, volunteers attended a 3-hour HE "short" clamp, when muscle biopsies were taken at baseline, and at the ED50 and ED100 insulin doses noted above. All muscle biopsies were examined for insulin-stimulated phosphorylation of PKB and PRAS-40, a potential biomarker of in vivo PKB activity. Both long and short clamp visits were repeated for the OB group after surgery.

Results: OB were substantially more IR than $\mathrm{C}$, with their glucose disposal curve shifted to the right; as demonstrated by an increased ED50 (126 \pm 43 vs $27+2 \mathrm{mIU} / \mathrm{L}, \mathrm{p}<0.05)$ and reduced $\mathrm{ED} 100(793+58$ vs $384+15 \mathrm{mIU} / \mathrm{L}$, $\mathrm{p}<0.05)$. Insulin-stimulated phosphorylation of PKB and PRAS40 were not significantly different between the two groups.

Post-surgery, mean weight loss was $34 \pm 7.4 \mathrm{~kg}(\mathrm{p}<0.01)$ in the $\mathrm{OB}$ and affected subjects were able to reduce their diabetes medication. IR improved with the glucose disposal curve shifted to the left and an improvement in ED50 (75 \pm 14 vs $126 \pm 43, p<0.05)$. Despite the major weight loss, no significant change in ED100 ( $709 \pm 102$ vs $793 \pm 58, p=0.17)$ was observed. Surprisingly, we found that there was a significant increase in the sensitivity of both PKB and PRAS40 phosphorylation to insulin, particularly at physiologically relevant serum insulin concentrations.

Conclusion: These findings suggest that the IR seen in OB as compared to $\mathrm{C}$ cannot be explained simply by reduced phosphorylation and activation of PKB. However, upon rapid weight loss the fact that improvements in insulin sensitivity occur in parallel with an improvement in PKB signalling, suggests that this pathway may be important in modulating acute responses to weight changes. Thus this signalling pathway may represent a therapeutic target for intervention in morbid obesity.

Supported by Diabetes Research \& Wellness Foundation, Medical Research Council

\section{0}

Intralipid dramatically reduces access of insulin to its target tissue J. D. Chiu ${ }^{1}$, C. Kolka' ${ }^{1}$, J. M. Richey ${ }^{1}$, L. N. Harrison ${ }^{1}$, E. Zuniga ${ }^{1}$,

E. Kirkman ${ }^{1}$, M. Ellmerer ${ }^{2}$, R. N. Bergman ${ }^{1}$;

${ }^{1}$ Physiology and Biophysics, Keck School of Medicine, Los Angeles,

United States, ${ }^{2}$ Internal Medicine, Medical University Graz, Austria.

Background and Aims: Insulin action on skeletal muscle cells includes 1)delivery of insulin to the target tissue via transendothelial transport, 2)diffusion through interstitium, 3)binding to cells, 4)insulin signaling, and 5)transporter mobilization. Several groups have focused on the effect of hyperlipidemia with intralipid to inhibit insulin signaling at the muscle cell, but less on access of insulin to sensitive tissues. The present study was undertaken to determine whether intralipid-induced resistance is a result of limited insulin transport to skeletal muscle.

Materials and Methods: We injected insulin into muscle interstitium directly and examined interstitial insulin levels (lymph) and glucose uptake (GU, a-v difference) $\mathrm{w} /$ or w/o intralipid. Basal insulin euglycemic clamps $(0.2 \mathrm{mU} /$ $\mathrm{min} / \mathrm{kg}$ ) were done on anesthetized dogs $(\mathrm{n}=14)$. The control group $(\mathrm{CON}$, $\mathrm{n}=10,31.3 \pm 3 \mathrm{~kg}$ ) received 5 sequential insulin doses (D1 to D5: 0.3, 0.5, 0.7, $1,3 \mathrm{U}$ ) with a $30 \mathrm{~g}$ needle directly into the interstitial space of the v. medialis of one hindleg. The intralipid group (INL, $\mathrm{n}=4,31.2 \pm 1 \mathrm{~kg}$ ) received the same injections, after $240 \mathrm{~min}$ infusion of intralipid/heparin (20\% fat emulsion at $1.5 \mathrm{~mL} / \mathrm{min})$.

Results: Intralipid caused insulin resistance (max. glucose infusion rate, $\mathrm{GINF}=2.2 \pm 1 \mathrm{v} . \mathrm{CON}$ GINF $=5.4 \pm 1 \mathrm{mg} / \mathrm{min} / \mathrm{kg} ; \mathrm{p}<0.05)$. Interstitial insulin in CON increased with each insulin injection (D1: $+28 \pm 6$, D2: $+139 \pm 7$, D3: $+237 \pm 12$, D4: $+345 \pm 13$, D5: $+389 \pm 13 \mathrm{pM}, \mathrm{p}<0.001)$. Surprisingly, systemic infusion of intralipid totally prevented the increase in interstitial insulin after intramuscular injection of insulin: (D1: $+0.7 \pm 5, \mathrm{D} 2:-0.5 \pm 7, \mathrm{D} 3:+0.7 \pm 7, \mathrm{D} 4$ : $+1.3 \pm 8, \mathrm{D} 5:+4 \pm 14 \mathrm{pM}, \mathrm{P}<0.001$ compared to $\mathrm{CON})$. That intramuscular insulin was diverted into the capillary circulation was evidenced by elevated hindlimb venous insulin when intralipid was present (CON v. INL; D1: $35 \pm 4$ v. $86 \pm 19$; D2: $71 \pm 4$ v. $255 \pm 5$; D3: $97 \pm 4$ v. $260 \pm 32$; D4: $99 \pm 6$ v. $290 \pm 57$; D5: $176 \pm 9$ v. $460 \pm 109 p M ; p<0.001)$. Average GU across the limb without intralipid was increased with each insulin dose, but we observed little increase with intralipid (CON: $13 \pm 4 \mathrm{v}$. INL: $6 \pm 2 \mathrm{mg} / \mathrm{min} / \mathrm{leg}$; INL $\mathrm{n}=2$ ). With systemic intralipid infusion, there was no apparent increase in interstitial (lymph) insulin and no measurable increase in local GU.

Conclusion: Intralipid's acute induction of resistance may relate to decreased availability of insulin in the interstitial space of skeletal muscle. With intralipid, it appears that insulin rapidly exits through the capillaries to the venous system to be redistributed systemically. Shunting of insulin away from insulin sensitive tissues may play a role in insulin resistance during FFA elevations during obesity or diabetes.

\section{1}

\section{WITHDRAWN}

\section{2}

Tissue specificity of insulin resistance in humans: fat in the liver rather than in the muscle associates with features of the metabolic syndrome A. Kotronen ${ }^{1,2}$, A. Seppala-Lindroos ${ }^{1}$, R. Bergholm ${ }^{1}$, H. Yki-Jarvinen ${ }^{1}$; ${ }^{1}$ Department of Medicine, University of Helsinki, ${ }^{2}$ Department of Medicine, Minerva Medical Research Institute, Helsinki, Finland.

Background and Aims: We determined how intrahepatic as compared to intramyocellular fat are related to features of the metabolic syndrome (MetS) and insulin resistance in the liver and muscle.

Materials and Methods: Hepatic (insulin infusion rate $0.3 \mathrm{mU} / \mathrm{kg} \cdot \mathrm{min}$ combined with $\left[3-{ }^{3} \mathrm{H}\right]$ glucose $)$ and muscle $(1.0 \mathrm{mU} / \mathrm{kg} \cdot \mathrm{min})$ insulin sensitivity were measured in 45 non-diabetic men (age $42 \pm 1$ yrs, BMI $26.2 \pm 0.6 \mathrm{~kg} / \mathrm{m}^{2}$ ) on two separate occasions by euglycemic hyperinsulinemic clamp. Liver fat (LFAT) and intramyocellular lipid (IMCL) were measured by ${ }^{1} \mathrm{H}-\mathrm{MR}$ spectroscopy. In addition, serum FFA concentrations during the $0.3 \mathrm{mU} /$ $\mathrm{kg} \cdot \mathrm{min}$ insulin clamp, fasting serum insulin and adiponectin, components of the metabolic syndrome, and $\mathrm{VO}_{2}$ max were determined.

Results: Serum triglycerides $(1.0 \pm 0.1$ vs. $1.6 \pm 0.2 \mathrm{mmol} / \mathrm{l}, \mathrm{p}=0.002)$ and $\mathrm{fS}-$ insulin $(5.3 \pm 0.4$ vs. $9.2 \pm 0.7 \mathrm{mU} / 1, \mathrm{p}<0.0001)$ were significantly higher, and $\mathrm{S}$ HDL cholesterol $(1.48 \pm 0.1$ vs. $1.26 \pm 0.1 \mathrm{mmol} / \mathrm{l}, \mathrm{p}=0.02)$ and fS-adiponectin $(12.2 \pm 1.2$ vs. $9.5 \pm 1.2 \mathrm{ug} / \mathrm{ml}, \mathrm{p}=0.05)$ significantly lower when the subjects were divided into low (median 2.0\% [interquartile range 1.0-2.0\%]) as compared to high $(12.0 \%$ [5.7-18.5\%]) LFAT groups. When divided into groups with low $(5.0 \%$ [2.3-7.5\%]) as compared to high (19.5\% [16.0-26.0\%]) IMCL, the groups were comparable with respect to all these parameters In addition, liver fat content did not differ between the IMCL groups. Liver fat was significantly higher in subjects with $(10.9 \pm 2.0 \%)$ than without $(5.3 \pm 1.1 \%, \mathrm{p}=0.017)$ the MetS. In contrast, IMCL was similar in subjects with and without the MetS. Insulin suppression of glucose $\mathrm{R}_{\mathrm{a}}$ (79\% [51-120\%] vs. 97\% [75-129\%] during 300-360 min, high $v$ s. low LFAT group, $\mathrm{p}=0.05$ ) and of serum FFA were impaired in the high LFAT group, as was muscle insulin sensitivity $(4.18 \pm 0.38$ vs. $6.26 \pm 0.39 \mathrm{mg} / \mathrm{kg} \mathrm{FFM} \cdot \mathrm{min} ; \mathrm{p}=0.0008)$. In the IMCL groups, only insulin suppression of serum FFA concentrations was impaired in the high as compared to the low IMCL group.

Conclusion: Fat accumulation in the liver rather than in the skeletal muscle is associated with features of the metabolic syndrome and insulin resistance both in the liver and muscle.

Supported by research grants from the Academy of Finland, Sigrid Juselius Foundation, Medical Society of Finland, and Novo Nordisk Foundation.

\section{3}

Metabolic MR imaging of human myocardial lipotoxicity

R. W. van der Meer ${ }^{1}$, S. Hammer ${ }^{2}$, J. W. A. Smit ${ }^{2}$, M. Frölich ${ }^{3}$, J. J. Bax ${ }^{4}$, M. Diamant ${ }^{5}$, L. J. Rijzewijk ${ }^{5}$, A. De Roos ${ }^{1}$, H. J. Lamb ${ }^{1}$, J. A. Romijn'; ${ }^{1}$ Radiology, Leiden University Medical Center, ${ }^{2}$ Endocrinology, Leiden University Medical Center, ${ }^{3}$ Clinical Chemistry, Leiden University Medical Center, ${ }^{4}$ Cardiology, Leiden University Medical Center, ${ }^{5}$ Endocrinology, VU University Medical Center, Amsterdam, The Netherlands.

Background and Aims: Type 2 diabetes mellitus (DM2) and obesity are associated with increased plasma non-esterified fatty acid (NEFA) levels and 
myocardial dysfunction. Using a very low calorie diet (VLCD) to increase plasma NEFA levels we studied the relation between plasma NEFA levels and myocardial lipid and high-energy-phosphate metabolism and cardiac function in healthy subjects.

Materials and Methods: Fourteen healthy non-obese men underwent ${ }^{1} \mathrm{H}$ magnetic resonance spectroscopy (MRS) to determine myocardial and hepatic triglyceride content (\%TG), ${ }^{31} \mathrm{P}-\mathrm{MRS}$ to assess myocardial highenergy-phosphate metabolism (PCr/ATP), and functional MR imaging of the heart at baseline and after a 3-day VLCD.

Results: After the diet-intervention, plasma NEFA levels increased significantly when compared to baseline (from $0.5 \pm 0.1$ to $1.1 \pm 0.1 \mathrm{mmol} / \mathrm{l}$, $\mathrm{p}<0.05)$. In addition, myocardial $\% \mathrm{TG}$ increased significantly when compared to baseline (from $0.38 \pm 0.05$ to $0.59 \pm 0.06 \%, \mathrm{p}<0.05$ ), whereas epatic \% TG decreased significantly during the VLCD as compared to baseline (from 2.2 \pm 0.5 to $1.5 \pm 0.4 \%, \mathrm{p}<0.05)$. In addition, the difference in myocardial \%TG between the VLCD and baseline in the myocardium was negatively correlated to the difference of the \%TG between the VLCD and baseline in the liver (Pearson $\mathrm{r}=-0.61, \mathrm{p}<0.05$ ). The VLCD did not change myocardial PCr/ATP $(2.33 \pm 0.15$ vs. $2.33 \pm 0.08, \mathrm{p}>0.05)$ or left ventricular systolic function. Interestingly, deceleration of the early diastolic flow across the mitral valve decreased significantly after the VLCD as compared to baseline (from $3.37 \pm$ 0.20 to $2.91 \pm 0.16 \mathrm{ml} / \mathrm{s}^{2} \times 10^{-3}, \mathrm{p}<0.05$ ). This change in diastolic function was significantly correlated with the increase in myocardial \%TG after the VLCD (Pearson $\mathrm{r}=-0.55, \mathrm{p}<0.05)$.

Conclusion: Short-term VLCD induces accumulation of myocardial triglycerides which is associated with altered left ventricular diastolic function, but has no impact on myocardial high-energy-phosphate metabolism. Furthermore, short term caloric restriction exerts differential tissue specific effects on triglyceride contents. These observations stress the physiological flexibility of ectopic triglyceride pools.

\section{4}

Increased epicardial fat and impaired left ventricular energy metabolism in young men with newly found fatty liver

G. Perseghin ${ }^{1}$, G. Lattuada ${ }^{2}$, F. De Cobelli ${ }^{3}$, A. Esposito ${ }^{3}$, E. Belloni ${ }^{3}$, F. Ragogna ${ }^{2}$, T. Canu ${ }^{3}$, P. Scifo ${ }^{4}$, A. Del Maschio ${ }^{5}$, L. Luzi ${ }^{1}$;

${ }^{1}$ Internal Medicine, Università degli Studi di Milano, Faculty of Exercise Sciences, Milano, ${ }^{2}$ Internal Medicine, Istituto Scientifico H San Raffaele, Milano, ${ }^{3}$ Diagnostic Radiology, Istituto Scientifico H San Raffaele, Milano, ${ }^{4}$ Nuclear Medicine, Istituto Scientifico H San Raffaele, Milano,

${ }^{5}$ Diagnostic Radiology, Università Vita e Salute San Raffaele, Milano, Italy.

Background and Aims: Fatty liver is characterized by metabolic abnormalities at the liver, but also at the skeletal muscle and adipose tissue sites. It is hypothesized that also the heart may be suffering metabolic alterations and this study was undertaken to ascertain whether individuals with fatty liver have left ventricular (LV) alterations of energy metabolism, structure and function and abnormal amount of epicardial fat as a specific marker of visceral fat accumulation.

Materials and Methods: To this end we studied young, non-diabetic men matched for anthropometric features with $(n=21)$ or without $(n=21)$ fatty liver by means of 1) cardiac magnetic resonance imaging (MRI) 2) cardiac ${ }^{31} \mathrm{P}-\mathrm{MR}$ spectroscopy (MRS) and 3) hepatic ${ }^{1} \mathrm{H}-\mathrm{MRS}$ to assess quantitatively the IHF content. Insulin sensitivity was determined by the updated HOMA-2 computer model.

Results: Individuals with fatty liver showed reduced insulin sensitivity, increased serum FFA and E-selectin, abnormal adipokines concentrations and higher blood pressure. LV morphology, systolic and diastolic functions were not different but in the scanned intra-thoracic region, the intrapericardial (7.8 \pm 3.1 vs. $\left.5.9 \pm 2.5 \mathrm{~cm}^{2} ; \mathrm{P}<0.05\right)$ and extrapericardial $(11.7 \pm 6.1$ vs. $7.8 \pm 3.2$ $\left.\mathrm{cm}^{2} ; \mathrm{P}<0.03\right)$ fat was increased in men with fatty liver compared to those without fatty liver. The phosphocreatine (PCr)/ATP ratio, a recognized in vivo marker of myocardial energy metabolism, was reduced in men with fatty liver in comparison with normals ( $1.85 \pm 0.35$ vs. $2.11 \pm 0.31 ; \mathrm{P}<0.016)$.

Conclusion: In newly found individuals with fatty liver, fat was accumulated in the epicardial area and despite of normal LV morphologic features and systolic and diastolic functions, they had abnormal LV energy metabolism. Supported by the Italian Ministry of Health
0615

Serum soluble E-selectin concentration in relation to the whole-body insulin resistance and carbohydrate and lipid oxidation in lean and obese women

M. Straczkowski, I. Kowalska, A. Adamska, A. Nikolajuk, M. Karczewska-Kupczewska, M. Karolczuk-Zarachowicz, M. Gorska; Department of Endocrinology, Diabetology and Internal Medicine, Medical University of Bialystok, Poland.

Background and Aims: Markers of endothelial dysfunction, including soluble E-selectin (sE-selectin), are related to insulin resistance and are independent predictors of type 2 diabetes. The explanation of these associations remains unclear. Insulin resistance might be related to the socalled metabolic inflexibility, i.e. to an impaired stimulation of carbohydrate oxidation and an impaired inhibition of lipid oxidation by insulin. The aim of the present study was to estimate the relationships of serum sE-selectin concentration with carbohydrate and lipid oxidation in the baseline state and during hyperinsulinemia in apparently healthy lean and obese women.

Materials and Methods: The study group consisted of 76 apparently healthy women with NGT: 30 lean and 46 with overweight or obesity. Insulin sensitivity was evaluated with the euglycemic hyperinsulinemic clamp. Carbohydrate and lipid oxidation was studied with indirect calorimetry within two 30-minute periods: in the baseline state and during the last 30 minutes of the clamp.

Results: Obese women had lower insulin sensitivity $(\mathrm{p}=0.0057)$ and higher serum $\mathrm{sE}$-selectin concentration $(\mathrm{p}=0.046)$. In the obese group, carbohydrate oxidation was increased in the baseline state $(\mathrm{p}=0.022)$ and decreased during hyperinsulinemia $(\mathrm{p}=0.032)$ in comparison to the lean controls. Lipid oxidation during hyperinsulinemia was higher in the obese in comparison to the lean group ( $\mathrm{p}=0.00028$ ). Obese women had also a lower increase in carbohydrate oxidation $(\mathrm{p}=0.0014)$ and a lower decrease in lipid oxidation $(p=0.021)$ in response to hyperinsulinemia. Serum $s E$-selectin concentration was negatively related to insulin sensitivity $(\mathrm{r}=-0.30, \mathrm{p}=0.009)$. Serum $\mathrm{sE}-$ selectin concentration was positively associated with carbohydrate oxidation in the baseline state $(\mathrm{r}=0.31, \mathrm{p}=0.007)$ and negatively related to carbohydrate oxidation $(\mathrm{r}=-0.27, \mathrm{p}=0.018)$ and positively - to lipid oxidation $(\mathrm{r}=0.29$, $\mathrm{p}=0.011$ ) during hyperinsulinemia. Additionally, higher serum $\mathrm{sE}$-selectin concentration was associated with a lower increase in carbohydrate oxidation $(\mathrm{r}=-0.41, \mathrm{p}<0.001)$ and a lower decrease in lipid oxidation $(\mathrm{r}=0.30, \mathrm{p}=0.008)$ in response to hyperinsulinemia.

Conclusion: Serum sE-selectin concentration is associated with both carbohydrate and lipid oxidation. Higher serum sE-selectin concentration might be linked to the metabolic inflexibility of obesity and insulin resistance.

Supported by the Polish Ministry of Science and Higher Education 


\section{PS 47 Carbohydrate metabolism: humans}

\section{6}

\begin{abstract}
Differential impact of fatness, fitness and physical activity energy expenditure on whole body, liver and fat insulin sensitivity C. D. T. Byrne ${ }^{1}$, H. B. Holt ${ }^{1}$, S. H. Wild ${ }^{2}$, N. Wareham ${ }^{3}$, U. Ekelund ${ }^{3}$, M. Umpleby ${ }^{4}$, F. Shojaee-Moradie ${ }^{4}$, R. I. G. Holt ${ }^{1}$, D. I. Phillips ${ }^{5}$; ${ }^{1}$ Endocrinology and Metabolism, University of Southampton, ${ }^{2}$ Public Health Sciences, University of Edinburgh, ${ }^{3}$ MRC Epidemiology Unit, MRC Epidemiology Unit, Cambridge, ${ }^{4}$ Diabetes and Endocrinology, Wolfson Centre for Translational Research, University of Surrey, ${ }^{5} \mathrm{MRC}$ Resource Centre, MRC Resource Centre, Southampton, United Kingdom.
\end{abstract}

Background and Aims: The relative contribution of fitness (maximal oxygen uptake, VO2 max), physical activity energy expenditure (PAEE) and fatness to influence whole body, liver and fat insulin sensitivity is uncertain. The aim of this study was to determine whether fatness, fitness and PAEE were independently associated with whole body, liver and fat insulin sensitivity in men.

Materials and Methods: 25 men (mean [SD] age 53 [6] years) were recruited. With a hyperinsulinaemic, euglycaemic clamp, whole body (M value) and liver (percentage suppression endogenous glucose output) insulin sensitivity were estimated. During an OGTT, insulin sensitivity in fat (insulin sensitivity index for non-esterified fatty acids, [ISI- NEFA]), was estimated. Total and truncal fat were measured by DEXA (dual X-ray absorptiometry), fitness by treadmill and PAEE by three day heart rate monitoring and by Baecke physical activity questionnaire.

Results: In univariate analyses, fitness was associated with whole body, liver and fat insulin sensitivity. PAEE was associated with insulin sensitivity in liver and fat but not whole body insulin sensitivity. Regression models that included PAEE, $\mathrm{VO}_{2}$ max and fatness as explanatory variables were established to describe associations with the various measures of insulin sensitivity (whole body, fat and liver). Only fatness was independently associated with whole body insulin sensitivity ( $B$ coefficient $-0.96, p<0.001$ ) and fat insulin sensitivity (B coefficient $-1.6 \times 10^{-5}, \mathrm{p}=0.004$ ). In contrast only PAEE was independently associated with liver insulin sensitivity ( $B$ coefficient 28.4, $\mathrm{p}=0.03$ ).

Conclusion: These data show for the first time that PAEE, including more vigorous physical activity explains most of the variance in liver insulin sensitivity. In contrast, fatness explains most of the variance in whole body and fat insulin sensitivity.

Supported by Wellcome Trust

\section{7}

Evidence that the transaldolase reaction and/or isotope effect at the triose-P-isomerase step occurs in humans: implications for measurement of gluconeogenesis and glucose turnover

G. Bock ${ }^{1,2}$, R. Basu' ${ }^{2}$, S. C. Burgess ${ }^{3}$, W. C. Schumann ${ }^{4}$, V. Chandramouli ${ }^{4}$, R. A. Rizza ${ }^{2}$, B. R. Landau

${ }^{1}$ Department of Internal Medicine, Division of Diabetes and Metabolism, Medical University Graz, Austria, ${ }^{2}$ Division of Endocrinology, Metabolism, Diabetes and Nutrition, Mayo Clinic, Rochester, United States, ${ }^{3}$ Department of Radiology, University of Texas Southwest, Dallas, United States, ${ }^{4}$ Department of Medicine, Case Western Reserve University, Cleveland, United States.

Background: Measurement of gluconeogenesis by the $2 \mathrm{H} 20$ method assumes that carbon 5 of glucose becomes labeled with $2 \mathrm{H}$ during gluconeogenesis whereas carbon 2 becomes labeled with $2 \mathrm{H}$ during both glycogenolysis and gluconeogenesis. Therefore the $\mathrm{C} 5 / \mathrm{C} 2$ deuterium enrichment ratio provides the fraction of circulating glucose derived from gluconeogenesis. To the extent that other factors besides gluconeogenesis contribute to C5 enrichment, the $2 \mathrm{H} 2 \mathrm{O}$ method would provide an over estimate of gluconeogenesis.

Methods: To determine if $\mathrm{C} 5$ enrichment can be influenced by processes other than gluconeogenesis, non-diabetic obese subjects were infused with [3,5$2 \mathrm{H} 2$ ] glucose and insulin $(0.5 \mathrm{mU} / \mathrm{kg} / \mathrm{min})$ while glucose was clamped at $\sim 5$ $\mathrm{mmol} / \mathrm{L}$. Protons on $\mathrm{C} 3$ and $\mathrm{C} 5$ should exchange with body water at identical rates if the triose-P-isomerase step in gluconeogenesis is the only pathway responsible for this exchange. Deuterium enrichment of C 3 and $\mathrm{C} 5$ of hepatic
UDP-glucose was measured by NMR analysis of urinary glucuronide under basal and clamp conditions.

Results: The $\mathrm{C} 5 / \mathrm{C} 3$ deuterium enrichment ratio in the infusate was confirmed to be 1.0 , but the C5/C3 ratio in the UDP glucose pool measured in 4 subjects prior to insulin infusion averaged $0.75 \pm 0.07$ and in six subjects during insulin infusion averaged $0.67 \pm 0.05$. This data indicates that the deuterium on C5 of UDP-glucose is lost more rapidly than the deuterium on C3 in all subjects. This divergence might occur by either selective loss of the C5 deuterium, which occurs when carbons of glucose are exchanged with unlabeled three carbon precursors during the transaldolase reaction of the pentose phosphate pathway, and/or slower removal of the C3 deuterium compared to the $\mathrm{C} 5$ deuterium (i.e. an isotope effect) at the level of the trioseP-isomerase reaction followed by resynthesis of glucose from the resultant selectively enriched three carbon precursors.

Conclusion: These data are consistent with loss of C5 enrichment by transaldolase activity, a $2 \mathrm{H}$ isotope effect in the gluconeogenic pathway or both in humans. The extent to which these processes introduce error in the $2 \mathrm{H} 2 \mathrm{O}$ method or whether the magnitude of the error differs in various disease states (e.g. diabetes and obesity) when the $2 \mathrm{H} 20$ method is used to measure gluconeogenesis or [3-3H] glucose is used to measure glucose turnover needs to be evaluated.

Supported by DK14507

\section{8}

Direct pathway metabolism of [U-d $]$ glucose in healthy humans during an oral glucose tolerance test reveals both G6P-isomerase and transaldolase exchanges

T. J. C. Delgado ${ }^{1}$, C. Silva ${ }^{2}$, M. Bastos ${ }^{3}$, C. Baptista ${ }^{3}$, A. Fagulha ${ }^{3}$,

L. Barros ${ }^{3}$, M. Carvalheiro ${ }^{3}$, A. Mota ${ }^{4}$, M. C. A. Castro ${ }^{1}$, C. F. G. Geraldes ${ }^{1}$, M. Caldeira ${ }^{5}$, C. Barosa ${ }^{2}$, J. G. Jones ${ }^{1}$;

${ }^{1}$ NMR Research Unit, Dept of Biochemistry, Faculty of Sciences and Technology, Center for Neurosciences and Cell Biology, University of Coimbra, ${ }^{2}$ Center for Neurosciences and Cell Biology, University of Coimbra, ${ }^{3}$ Department of Endocrinology and Diabetes, University Hospital of Coimbra, ${ }^{4}$ Department of Urology and Renal Transplantation, University Hospital of Coimbra, ${ }^{5}$ NMR Research Unit, Dept of Chemistry, Faculty of Sciences and Technology, University of Coimbra, Coimbra, Portugal.

Introduction: Hepatic glycogen is synthesized from ingested glucose by direct and indirect pathways. We developed a simple method for quantifying direct and indirect pathway contributions to glycogen synthesis based on the enrichment of positions 5 and 2 of glucuronide from deuterated water. This analysis assumes that: 1) there is complete exchange of G6P and F6P during metabolism of glucose to glycogen, and 2) that the position 5 hydrogen of hexose metabolized via the direct pathway undergoes no exchange with that of body water via transaldolase. The aim of this work was to examine the validity of these assumptions in healthy subjects during an oral glucose tolerance test.

Methods: Four healthy overnight-fasted subjects were given 75 grams of glucose enriched to $3.33 \%$ with [ $\mathrm{U}-\mathrm{d}_{7}$ ]glucose. Peppermint oil $(200 \mathrm{mg})$ was ingested 1 hour before and 1 hour after the glucose load. Urine was collected 2$4 \mathrm{hr}$ after the load. Menthol glucuronide was isolated by solid phase extraction and glucuronide positional ${ }^{2} \mathrm{H}$-enrichment was analyzed by ${ }^{2} \mathrm{H}$ NMR.

On metabolism of $\left[\mathrm{U}-\mathrm{d}_{7}\right]$ glucose to glycogen via the direct pathway, ${ }^{2} \mathrm{H}$ in position 2 will be lost due to G6P-F6P exchange while ${ }^{2} \mathrm{H}$ in position 3 is retained. Hence, the ratio of enrichment in position 2 relative to position 3 $(\mathrm{H} 2 / \mathrm{H} 3)$ reflects the extent of G6P-F6P exchange. Transaldolase will exchange carbons 4, 5 and 6 of F6P with a molecule of glyceraldehyde-3-phosphate. This results in a loss of enrichment in position 5 relative to position 3 hence the ratio of enrichment in position 5 relative to position $3(\mathrm{H} 5 / \mathrm{H} 3)$ of glucuronide reflects the extent of transaldolase exchange.

Results \& Conclusions: From the relative ${ }^{2} \mathrm{H}$-signal intensities of glucuronide hydrogens 2 and $3, \mathrm{H} 2 / \mathrm{H} 3$ was $0.17 \pm 0.04$ indicating that G6P-F6P exchange was largely complete $(83 \pm 4 \%)$. Glucuronide $\mathrm{H} 5 / \mathrm{H} 3$ was $0.81 \pm$ 0.06 indicating that $19 \pm 6 \%$ of glucose metabolized via the direct pathway underwent transaldolase exchange. This level of transaldolase activity would result in substantial underestimates of the direct pathway contribution to glycogen synthesis using the deuterated water measurement.

Supported by Juvenile Diabetes Research Foundation International, Portuguese Foundation of Science and Technology (to Teresa Delgado) and the University of Coimbra Faculty of Medicine Research Support Programme 


\section{9}

Inhibition of glycogen phosphorylase attenuates hepatic glucose disposal in normal and insulin resistant rats

G. Rakipovski ${ }^{1,2}$, M. W. Jensen ${ }^{2}$, K. E. Pedersen ${ }^{2}$, H. Andersen ${ }^{2}$, J. Breinholt ${ }^{2}$, K. Fosgerau ${ }^{1,2}$;

${ }^{1}$ Rheoscience A/S, Roedovre, ${ }^{2}$ Novo Nordisk A/S, Maaloev, Denmark.

Background and Aims: Type 2 diabetes is characterized by an increased hepatic glucose production as well as a decrease in whole body glucose uptake and disposal, which ultimately may lead to the postprandial hyperglycaemia. Accordingly, inhibition of hepatic glucose production by inhibition of glycogen phosphorylase (GP), the controlling enzyme in glycogen breakdown, has been suggested as a possible therapeutic area of development of new antihyperglycaemic drugs. Under normal conditions hepatic glycogenolysis and glycogenesis are tightly regulated to ensure no concurrent glycogen synthesis and breakdown. Thus, the active form of glycogen phosphorylase (GPa) is known as a powerful inhibitor of glycogen synthase (GS), the controlling enzyme of glycogen synthesis. Here we investigated to what extend pharmacological inhibition of hepatic glycogen phosphorylase might affect hepatic glucose utilization in normal and insulin resistant rats.

Materials and Methods: Rats previously fed a normal chow or a high fat diet (HFD) were acutely treated with or without the glycogen phosphorylase inhibitor, 1,4-dideoxy-1,4-imino-D-arabinitol (DAB, $100 \mathrm{mg} / \mathrm{kg}$ ), and subsequently subjected to an oral glucose tolerance test (OGTT, $2 \mathrm{~g} / \mathrm{kg}$ ). De novo glycogen synthesis was determined by nuclear magnetic resonance technique.

Results: As judged by the oral glucose tolerance test, acute inhibition of the liver GP by DAB significantly reduced the whole body glucose clearance both in chow and HFD fed animals. Interestingly the liver glycogen synthase activity ratio was lowered by $2-3$ fold by treatment with DAB both in chow and HFD fed rats, thus giving rise to a concomitant decrease in the rate of $d e$ novo synthesized liver glycogen in DAB treated animals. (Tab. 1)

The table summarizes the Area Under the Curve for glucose (AUC glucose) of the OGTT, the enzyme activity of glycogen synthase (GS a: activated form of GS, GS t: total activity of GS) and the rate of liver glycogen synthesis in HFD or chow rats treated with or without DAB and subsequently subjected to a glucose challenge. Data are given as mean $\pm \mathrm{SE}$ with $\mathrm{n}=7$ per group. Statistics: Two-way ANOVA; ${ }^{* *} \mathrm{p}<0.01$ or ${ }^{* * *} \mathrm{p}<0.001$ for treatment with $\mathrm{DAB},{ }^{\wedge} \mathrm{p}<0.05$ or ${ }^{\wedge \wedge \wedge} \mathrm{p}<0.001$ for HFD.

Conclusion: Inhibition of liver GP by DAB leads to a decrease in the liver glycogen synthesis and whole body glucose clearance in normal and insulin resistant rats.

\section{0}

Quantification of hepatic transaldolase exchange activity and its effects on tracer measurements of human hepatic glycogen synthesis by direct and indirect pathways

J. Jones ${ }^{1}$, C. Barosa ${ }^{1}$, P. Garcia ${ }^{2}$, T. Delgado ${ }^{3}$, M. Caldeira ${ }^{4}$, L. Diogo ${ }^{2}$;

${ }^{1}$ Dept of Biochemistry, Center for Neurosciences, ${ }^{2}$ Dept of Metabolic

Diseases, Coimbra Pediatric Hospital, ${ }^{3}$ Dept of Biochemistry, University of

Coimbra, ${ }^{4}$ Dept of Chemistry, University of Coimbra, Coimbra, Portugal.

Background and Aims: Exchange of hepatic glucose-6-phosphate (G6P) and glyceraldehyde-3-phosphate via G6P-isomerase and transaldolase (see Figure 1) modifies hepatic G6P enrichment from gluconeogenic tracers independently of gluconeogenic activity. Consequently, the indirect pathway or gluconeogenic contribution to hepatic glycogen synthesis may be overestimated with some current tracer methodologies. 13C-isotopomer enrichment of the triose units of G6P from gluconeogenic metabolism of [U-13C]glycerol is sensitive to transaldolase exchange and provides a basis for estimating the extent of this activity. Hepatic G6P can be noninvasively sampled in humans as urinary Paracetamol glucuronide following ingestion of Paracetamol.

Materials and Methods: To determine transaldolase exchange activity in human liver, five healthy fed subjects were given an oral bolus of [U13C] glycerol $(25-30 \mathrm{mg} / \mathrm{kg}$ ) and Paracetamol $(10-12 \mathrm{mg} / \mathrm{kg})$. 13C Isotopomers of G6P were quantified by 13C NMR spectroscopy of the monoacetone glucuronolactone derivative of urinary Paracetamol glucuronide.

Results: [1,2,3-13C3]-and [4,5,6-13C3] glucuronideisotopomers, representing the conversion of $[1,2,3-13 \mathrm{C} 3]$ glycerol to $\mathrm{G} 6 \mathrm{P}$ via dihydroxyacetone phosphate, were resolved from [1,2-13C2]- and [5,6-13C2]glucuronide 13C-isotopomers, derived from the conversion of [1,2,3-13C 3$]$ glycerol to G6P via pyruvate, phosphoenolpyruvate and glyceraldehyde-3-phosphate. Enrichment of $[1,2,3-13 \mathrm{C} 3]$ glucuronide was significantly less than that of [4,5,6-13C3] glucuronide $(1.30 \pm 0.57 \%$ versus $1.67 \pm 0.42 \%, \mathrm{p}<0.05)$. Also, [1,2-13C2]glucuronide enrichment was significantly less than that of [5,6-13C2] glucuronide $(0.28 \pm 0.08 \%$ versus $0.36 \pm 0.03 \%, p<0.05)$. The contributions of transaldolase and triose phosphate isomerase exchanges to the glucuronide 13C-isotopomer distributions were resolved by applying the 13C-isotopomer data to a model of hepatic G6P metabolism that features transaldolase and triose phosphate isomerase exchanges. Triose phosphate isomerase exchange was $\sim 99 \%$ complete and did not contribute significantly to the unequal 13C-isotopomer distributions of the glucuronide triose halves. Instead, these observations indicate that transaldolase exchange accounted for $25 \pm 23 \%$ of hepatic G6P flux. Consequently, the indirect pathway contribution to hepatic glycogen synthesis is overestimated by $25 \pm 23 \%$ with tracers such as $[5-3 \mathrm{H}]$ glucose and deuterated water.

Conclusion: Transaldolase-mediated exchange of hexose and triose phosphates is active in human liver. This results in substantial overestimates of indirect pathway contributions to hepatic glycogen synthesis by some tracer methods in current use.

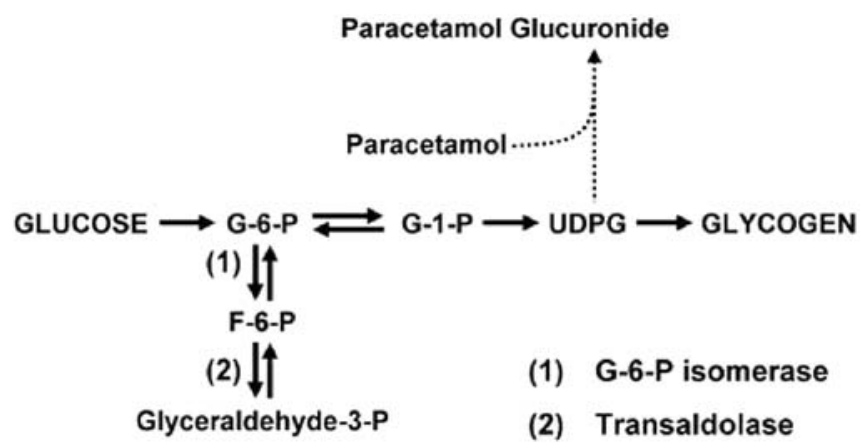

Fig. 1. Exchanges of hepatic glucose-6-phosphate (G6P) and sampling via Paracetamol glucuronide under fed conditions

Supported by POCTI QUI 55603

Tab. 1 Abstract 0619

\begin{tabular}{|c|c|c|c|c|c|c|c|c|c|c|c|}
\hline \multirow[b]{3}{*}{$\overline{\text { AUC glucose }\left(\mathrm{mM}^{\star} \mathrm{min}\right)}$} & \multicolumn{6}{|l|}{ HFD } & \multicolumn{5}{|c|}{ Chow } \\
\hline & \multicolumn{3}{|l|}{ Vehicle } & \multicolumn{3}{|l|}{ DAB } & \multicolumn{2}{|c|}{ Vehicle } & \multicolumn{3}{|l|}{$\mathrm{DAB}$} \\
\hline & 1078 & \pm & $26^{\wedge \wedge \wedge}$ & 1142 & \pm & $50^{* *}$ & 865 & $\pm 19^{\wedge \wedge \wedge}$ & 992 & \pm & $25^{\star *}$ \\
\hline GS a (U/g liver) & 0,44 & \pm & $0,08^{\wedge}$ & 0,17 & \pm & $0,02^{* * *}$ & 0,70 & $\pm 0,08^{\wedge}$ & 0,24 & \pm & $0,03^{* * *}$ \\
\hline GS t (U/g liver) & 0,50 & \pm & $0,08^{\wedge \wedge \wedge}$ & 0,57 & \pm & 0,03 & 0,91 & $\pm 0,06^{\wedge \wedge \wedge}$ & 0,72 & \pm & 0,07 \\
\hline GS a / GS t (Activity ratio) & 0,87 & \pm & 0,03 & 0,30 & \pm & $0,05^{* * *}$ & 0,77 & $\pm 0,08$ & 0,34 & \pm & $0,04^{* * *}$ \\
\hline Glycogen synthesis (mg/kg*min) & 0.23 & \pm & $0.14^{\wedge \wedge \wedge}$ & 0.14 & \pm & $0.04^{* *}$ & 0.93 & $\pm 0.12^{\wedge \wedge \wedge}$ & 0.37 & \pm & $0.02^{\star *}$ \\
\hline
\end{tabular}




\section{1}

Divergent relationship between circulating soluble CD36 and markers of liver injury according to glucose tolerance

E. Esteve ${ }^{1}$, A. Handberg ${ }^{2}$, J. Basols ${ }^{1}$, A. López-Bemejo ${ }^{1}$, J. Vendrell ${ }^{3}$, W. Ricart ${ }^{1}$, J. Fernandez-Real ${ }^{1}$;

${ }^{1}$ Endocrinology, Hospital of Girona, Spain, ${ }^{2}$ Department of Clinical Biochemistry, Aarhus University Hospital, Denmark,

${ }^{3}$ Endocrinology, Hospital of Tarragona, Spain.

Background and Aims: CD36-facilitated fatty acid uptake in different tissues has been demonstrated to constitute an important determinant of insulin action in animal models. Liver injury linked to insulin resistance is characterized by mild to moderate increases in aminotransferase activity. A soluble form of CD36 (sCD36) was recently identified in human plasma, and was proposed to reflect altered CD36 expression. The aim of this study was to evaluate the relationships among plasma sCD36, insulin sensitivity and indicators of liver health.

Materials and Methods: A cohort of consecutive middle-aged men from the general population ( $n=117), 73$ with normal and 44 with altered glucose tolerance. Insulin sensitivity ( $S_{I}$, minimal model), circulating sCD36 (ELISA) and serum markers of liver injury were measured.

Results: As expected, serum ALT, AST and $\gamma \mathrm{GT}$ were associated positively with body mass index (BMI), age and negatively with insulin sensitivity. Circulating sCD36 was positively associated with ALT, AST and $\gamma$ GT in subjects with altered glucose tolerance but not in those with normal glucose tolerance. Multiple regression analysis models were constructed to predict serum ALT activity, with age, BMI, alcohol intake, fasting triglycerides, insulin sensitivity and sCD36 as independent variables. Age, BMI and triglycerides contributed independently to $29 \%$ of ALT variance in subjects with normal oral glucose tolerance. In contrast, insulin sensitivity and sCD36 contributed independently to $39 \%$ of ALT variance in subjects with altered glucose tolerance.

Conclusion: Circulating sCD36 could represent a marker of liver injury in subjects with altered glucose tolerance independently of insulin resistance.

\section{2}

Evaluation of metabolic and nutritional parameters in cirrhotic patients before, 3 and 6 months after liver transplantation (OLT)

F. Donadio ${ }^{1}$, M. E. Lunati ${ }^{1}$, F. Agnelli ${ }^{2}$, E. Benetti ${ }^{1}$, B. Masserini ${ }^{1}$,

L. Sburlati ${ }^{1}$, F. Donato ${ }^{2}$, M. Colombo ${ }^{2}$, P. Beck-Peccoz ${ }^{1}$, E. Orsi ${ }^{1}$;

${ }^{1}$ Department of Medical Sciences, Endocrine and Diabetologic Unit, University of Milan, Fondazione IRCCS Ospedale Maggiore Policlinico,

Mangiagalli e Regina Elena, Italy, ${ }^{2}$ Department of Medical Sciences, Gastroenterologic Unit, University of Milan, Fondazione IRCCS Ospedale Maggiore Policlinico, Mangiagalli e Regina Elena, Italy.

Background and Aims: End-stage liver disease (ESLD) annually affects thousands of people. Cirrhosis is considered to be a catabolic disease that can lead to a profound alteration of metabolic and nutritional state. Furthermore, previous studies shown that after OLT patients may present diabetes, hypertension, obesity and dyslipidemia. The aim of the study was to evaluate changes in metabolic parameters and nutritional habits after OLT.

Materials and Methods: 28 cirrhotic patients (22M/6F, age 52.3 $\pm 9.4 \mathrm{yrs}$ ) were evaluated at baseline and 3 and 6 months after OLT by medical history, anthropometric parameters (BMI, waist and hip circumferences), biochemical parameters (glucose, $\mathrm{HbA} 1 \mathrm{C}$, lipid profile, urate, creatinine, azotemia) and bioelectrical impedance. Moreover nutritional habits were evaluated with 3-day-food records. Differences were analyzed using one-way analysis of variance and values of $P<0.05$ were considered statistically significant.

Results: We reported the onset of de novo diabetes mellitus in 3 patients (16.6\%). After 3 and 6 months a change in metabolic parameters (BMI: basal: 25 \pm 3.4 , 3rd mo.: $24 \pm 2.8$, 6th mo.: $25.3 \pm 3.1 \mathrm{Kg} / \mathrm{m}^{2}$; WC: basal: $93.7 \pm 10.6$, 3rd mo.: $90.1 \pm 8.9$, 6th mo.: $93.4 \pm 10 \mathrm{~cm}$ ) and in body composition (fat mass: basal:18.6 \pm 7.2 , 3rd mo.:16.8 \pm 6.3 , 6th mo.: $19.8 \pm 6.4 \mathrm{Kg}$ ) was documented; furthermore after OLT we observed a liver function improvement, and a worsening of renal index, the last one due to immunosuppressive therapy. We did not observe significant modification of other biochemical parameters. A change in caloric intake after OLT (basal: $1623 \pm 336.3$, 3rd mo.: $2410 \pm 468.3$, 6 th mo.: $1801 \pm 328.3 \mathrm{Kcal} / \mathrm{die}$ ) was seen, with an increase in lipid assumption (basal: $32.2 \pm 11.6,3 \mathrm{rd}$ mo.: $43.6 \pm 5.7,6$ th mo.: $37.5 \pm 7.1 \%$ ), despite carbohydrate intake (basal: $52.1 \pm 13$, 3rd mo.: $42.2 \pm 6.5$, 6th mo.: $45.6 \pm 7.4 \%$ ).

Conclusion: We reported a significant increase of de novo diabetes mellitus cases after OLT and a worsening of nutritional habits, which could lead to obesity, dyslipidemia and other metabolic diseases. Indeed, a multidisciplinary approach to these patients seems to be necessary in order to prevent the onset of obesity or other metabolic disease that often affects patients after OLT; moreover in non diabetic ESLD patients the improvement of nutritional habits, obtained by therapeutic education, could prevent also the onset of diabetes mellitus.

\section{3}

Arteriovenous concentration difference and carbon 13 NMR measurements reveal that intestinal gluconeogenesis is not demonstrable in diabetic rats

G. Baverel, R. Gadet, R. Nazaret, M. Martin, M. Boghossian, F. Saadé, G. Martin, B. Ferrier, A. Conjard; Metabolomics and Metabolic Diseases, INSERM U820, Lyon, France.

Background and Aims: Recent studies have reported that the small intestine of diabetic rats substantially contributes to systemic glucose production via gluconeogenesis. Since this would (i) represent a major change in the classical view that only the liver and kidney have a gluconeogenic capacity and (ii) have major consequences in the pathophysiology of diabetes, we have thoroughly re-examined this question.

Materials and Methods: For this, metabolically viable intestinal cells from adult fed and $48 \mathrm{~h}$-fasted diabetic ZDF rats, a classical animal model of type 2 diabetes, were incubated in the presence of $5 \mathrm{mM}\left[3-{ }^{13} \mathrm{C}\right]$ glutamine in the absence and the presence of $5 \mathrm{mM}$ unlabelled glucose. Substrate removal and product accumulation were measured at the end of incubation by enzymatic and ${ }^{13} \mathrm{C}$-NMR spectroscopy methods. Arterio-portal concentration difference measurements were also performed in $48 \mathrm{~h}$-fasted diabetic ZDF rats.

Results: Despite the removal of ${ }^{13} \mathrm{C}$-glutamine and the formation of glutamate, lactate, alanine, citrulline and ${ }^{13} \mathrm{CO} 2$ at high rates, glucose synthesis by isolated small intestinal cells from both fed and fasted diabetic animals was not demonstrable and this was not due to the re-utilization of glucose synthesized from glutamine. Similarly, no glucose synthesis occurred from $5 \mathrm{mM}$ glycerol or fructose. Arterio-portal concentration difference measurements for glucose, lactate, pyruvate, alanine, glycerol, glutamine, glutamate and ketone bodies in anesthetized $48 \mathrm{~h}$-fasted diabetic ZDF rats showed that the intestine neither added nor removed glucose in net amounts. Lactate, pyruvate, glutamate and alanine were released whereas glycerol and $\beta$-hydroxybutyrate but not acetoacetate were removed in net amounts by the intestine. Only $32 \%$ of circulating glutamine, which has been reported to be the main if not the exclusive intestinal gluconeogenic precursor in diabetic rats in vivo, was found to be taken up. Assuming that the glutamine plus glycerol taken up were completely converted into glucose, a very unlikely hypothesis, this would have diluted the circulating glucose by only $1.4 \%$, a percentage that is not measurable in a reliable manner by currently available analytical methods. Similar findings were made in fasted streptozotocindiabetic Sprague Dawley rats.

Conclusion: The present results, together with our recent demonstration that intestinal gluconeogenesis in 72h-fasted normal Wistar and Sprague Dawley rats remains to be demonstrated, strongly suggest that intestinal gluconeogenesis does not exist in adult diabetic rats. Supported by INSERM 


\section{PS 48 Adipose tissue biology and treatment}

\section{4}

Identification of adipose tissue secreted proteins as possible therapeutic targets for treatment of obesity

R. Oberauer ${ }^{1}$, W. Rust ${ }^{1}$, K. Quast ${ }^{1}$, W. Patsch ${ }^{2}$, D. Mennerich ${ }^{1}$, B. Hamilton ${ }^{1}$, H. Neubauer';

${ }^{1}$ Boehringer Ingelheim Pharma GmbH \& Co. KG, Biberach, Germany, ${ }^{2}$ Dept. of Laboratory Medicine, Salzburger Landeskliniken and Paracelsus Private Medical University, Salzburg, Austria.

Background and Aims: With increasing rates of obesity driving the incidence of diabetes to epidemic levels in both developed and developing countries, understanding the role of adipose tissue in the pathogenesis of morbidities is a focus of current research. The identification of adipocytokines such as leptin and adiponectin have confirmed the endocrine role of the fat cell. Identification of novel adipocytokines could provide further insights into the endocrine function of adipose tissue and additional means to therapeutically influence the de-regulated metabolic state of obese persons. Therefore, we compared expression profiles of adipose tissue of obese and lean humans with the aim to identify differentially expressed genes with signal peptide which are currently not associated with obesity. Expression of one candidate gene was further analysed in obese mice to estimate if the murine system could be used for further experiments.

Materials and Methods: Screening for differentially expressed genes in the adipose tissue of humans was performed from several perspectives. Affymetrix HG-U133 DNA Plus 2.0 chip data, accessed via the GeneLogic Gene Express 2.0 database, were used to compare mRNA expression profiles of human adipose tissue obtained from lean (BMI $\left.<25 \mathrm{~kg} \mathrm{~m}^{-2}, \mathrm{n}=20\right)$ and obese (BMI $\left.>30 \mathrm{~kg} \mathrm{~m}^{-2}, \mathrm{n}=45\right)$ individuals. Additionally Affymetrix chips were used for hybridisation of total RNA isolated from human adipose tissue biopsies of 9 obese patients (BMI $=42.3 \pm 4.25 \mathrm{kgm}^{-2}$ ) before and after massive weight loss $(29.7 \pm 5.4 \mathrm{~kg})$ achieved by gastric banding. Confirmation of the chip results was performed by quantitative RT-PCR on adipose tissues of lean (subcutaneous $n=4$, visceral $n=4$ ) and obese humans (subcutaneous $\mathrm{n}=5$, visceral $\mathrm{n}=6)$ as well as 16 weeks old lean $(\mathrm{C} 57 / \mathrm{Bl6} ; \mathrm{n}=4)$ and obese mice (ob/ob; $\mathrm{n}=4$ and $\mathrm{db} / \mathrm{db} ; \mathrm{n}=4$ ).

Results: Several proteins, not linked to obesity in current literature, showing an altered expression in the adipose tissue of obese humans comparing to lean controls were examined in detail ( 6 with fold change (FC) $>+1.8 ; 6$ with FC $<-1.8)$. Leptin, as positive control revealed a median FC of $+3.3(p=6.3 \mathrm{E}-$ 6). Expression of one candidate with enhanced median FC in obese $(+16.3$, $p=3.4 \mathrm{E}-5$ ) was further analysed in more detail. The increased expression in obesity was confirmed by Taqman analysis of another adipose tissue sample set (subcutaneous: mean $F C=+24.2$, visceral: mean $F C=+10$ ). By comparing the expression profile in adipose tissue of humans after weight loss we observed a decreased expression (subcutaneous: -4.2 fold, $p=1.2 \mathrm{E}-4$; visceral: -1.3 fold, $p=8.5 \mathrm{E}-3$ ). The murine homologue was weakly expressed in the adipose tissue of $\mathrm{C} 57 / \mathrm{Bl} 6$ lean mice and furthermore hardly detectable in the adipose tissue of the obesity mouse models ob/ob and $\mathrm{db} / \mathrm{db}$.

Conclusion: By expression profiling of human adipose tissue, several proteins with altered expression in obesity were identified, which are currently not known to be associated with obesity. For one secreted protein increased expression in obese humans was shown by three independent studies. The investigated mouse models of obesity did not show the observed human profile, therefore further model systems for functional validations will be studied.

\section{5}

Serum amyloid $A$ as a biomarker and a potential contributor to the development of insulin resistance

L. Scheja ${ }^{1}$, H. Zitzer ${ }^{1}$, B. Heese ${ }^{1}$, J. Heeren ${ }^{2}$, U. Beisiegel ${ }^{2}$, K. Seedorf ${ }^{1}$;

${ }^{1}$ Lilly Research Laboratories,

${ }^{2}$ IBM II, University Medical Center, Hamburg, Germany.

Background and Aims: Chronic low-grade inflammation is a hallmark of insulin resistance in the context of obesity. It has been reported that circulating levels of acute phase proteins, sensitive indicators of such inflammation, are elevated in insulin resistant subjects. The purpose of this study was to address the role of the acute phase protein serum amyloid A (SAA) in a mouse model of diet-induced obesity.

Materials and Methods: Blood and tissues were collected from 20 week old, fasted C57Bl/6 mice which were fed either a chow diet (controls) or a high fat diet (59\% fat by calories) for 1 week (1w HFD) and 16 weeks (16w HFD), respectively $(\mathrm{n}=8)$. An ELISA detecting SAA isoforms SAA1 and SAA2 (Biosource Inc.) was used for quantification in plasma. Gene expression in liver, muscle and adipose tissue was studied using TaqMan ${ }^{\circ}$ real-time PCR assays. Recombinant apoSAA1/2 (Peprotech Ltd.) was used for assessing SAA effects on 3T3.L1 adipocytes.

Results: 1w HFD induced significant hyperinsulinemia and hyperglycemia in the mice compared to controls $(\mathrm{p}<0.05)$, indicating mild insulin resistance already after a short period on the obesigenic diet. As expected, 16w HFD led to pronounced overweight and insulin resistance compared to controls (body weight: $44.9 \pm 0.7 \mathrm{~g}$ vs. $23.0 \pm 0.5 \mathrm{~g}$; plasma insulin: $357 \pm 58 \mathrm{pM}$ vs. $79 \pm 23 \mathrm{pM}$, plasma glucose: $202 \pm 6 \mathrm{mg} / \mathrm{dl}$ vs. $119 \pm 8 \mathrm{mg} / \mathrm{dl}$; Mean $\pm \mathrm{SEM}, \mathrm{p}<0.01)$. Plasma SAA was elevated in the $1 \mathrm{w}$ HFD group (controls $2.11 \pm 0.25 \mathrm{ug} / \mathrm{ml}$, 1w HFD $5.06 \pm 1.05 \mathrm{ug} / \mathrm{ml}, \mathrm{p}<0.05)$ and further increased in the 16w HFD group (31.4 $\pm 7.0 \mathrm{ug} / \mathrm{ml}$; Mean \pm SEM, $\mathrm{p}<0.01$ vs. controls). In order to understand the contribution of tissues to the observed SAA induction in plasma we performed real-time PCR experiments in liver, adipose and muscle. The mRNAs of both SAA1 and SAA2 were expressed almost exclusively in liver, as described previously, and were induced by the HFD ( $>3.5$-fold in 1w HFD, p < 1.50; $>8$-fold in 16w HFD vs. controls; $\mathrm{p}<0.001$ ). SAA3, although well-expressed in liver, was not significantly induced in this tissue. In contrast, SAA3 was strongly expressed in adipose tissue and markedly induced by the HFD ( $>8$ fold in $1 \mathrm{w}$ HFD and $16 \mathrm{w}$ HFD, $\mathrm{p}<0.05$ vs. controls). The strong correlation of SAA1 and SAA2 with insulin resistance in the mouse model prompted us to study a potential causal role of SAA in the development of insulin resistance. To this end, we incubated 3T3.L1 adipocytes with recombinant SAA. Interestingly, we observed a pronounced induction of the proinflammatory markers monocyte chemoattractant protein-1 (MCP-1), inhibitor of kappaB kinase epsilon (IKKe) and SAA3. In contrast, the genes of several proteins involved in insulin signaling, glucose tranport and mitochondriogenesis (adiponectin, IRS1, Glut4, PGC1 $\alpha$, PGC1 $\beta$ ) were significantly downregulated by SAA. The alterations in 3T3.L1 gene expression were very similar to the changes observed in adipose tissue in vivo after feeding HFD.

Conclusions: In C57Bl/6 mice serum amyloid A is elevated after induction of insulin resistance and obesity by a high fat diet and correlates well with the severity of the metabolic condition. The effects of recombinant SAA on gene expression in 3T3.L1 adipocytes suggest that SAA produced in liver has the potential to promote inflammation in adipose tissue thereby worsening insulin resistance.

Supported by the Department of Science and Health, Hamburg Government

\section{6}

Serum components mediate glucose-induced immune gene expression in leukocytes from patients with the metabolic syndrome

K. Kempf, R. Rütter, B. Rose, C. Herder, S. Martin;

Institute for Clinical Diabetes Research, German Diabetes Center, Düsseldorf, Germany.

Background and Aims: Type 2 diabetes mellitus (T2DM) and the Metabolic Syndrome (MetS) are associated with chronic, subclinical inflammation. Recently we could demonstrate that an oral glucose challenge significantly increased immune gene expression in leukocytes from MetS patients and that this increase in expression and secretion of immune mediators also occurred after glucose stimulation in vitro. Therefore, the aim of the study was to analyze if components in the serum of MetS patients mediate glucoseinduced immune gene expression.

Materials and Methods: Peripheral blood from subjects with $(\mathrm{n}=10)$ and without MetS $(\mathrm{n}=10)$ was cultured in low-glucose medium $(100 \mathrm{mg} / \mathrm{dl} \mathrm{D}$ glucose), high-glucose medium (500mg/dl D-glucose) and an osmotic control medium $(100 \mathrm{mg} / \mathrm{dl} \mathrm{D}$-glucose $+400 \mathrm{mg} / \mathrm{dl} \mathrm{L}$-glucose $)$ for 72 hours. The capacity of serum factors to prime leukocytes for glucose-induced immune gene expression was tested by incubation of leukocytes from MetS patients with serum from non-MetS subjects and vice versa ( $\mathrm{n}=5$ each). Expression of tumor necrosis factor alpha (TNF- $\alpha$ ), intercellular adhesion molecule 1 (ICAM-1) and interleukin 6 (IL-6) was determined by quantitative RT-PCR. For statistical analysis Wilcoxon rank sum test was used. 
Results: As shown before glucose significantly increased mean expression of ICAM-1 $(\mathrm{p}<0.0001)$, TNF- $\alpha(\mathrm{p}<0.0001)$ and IL-6 $(\mathrm{p}=0.014)$ during the OGTT as well as after in vitro glucose stimulation with autologous serum in the MetS group only. Serum factors from MetS patients were able to increase immune gene expression in leukocytes from non-MetS subjects $(+30 \%$ for TNF- $\alpha,+25 \%$ for ICAM- 1 and $+35 \%$ for IL- 6 ), whereas incubation of leukocytes from MetS patients with serum from non-MetS subjects reduced glucose-induced immune gene expression compared with incubation with MetS serum ( $-15 \%$ for TNF- $\alpha,-25 \%$ for ICAM- 1 and $-130 \%$ for IL- 6 ).

Conclusion: Presence of the MetS sensitizes leukocytes to up-regulate proinflammatory markers in response to glucose, and serum factors seem to be important mediators for this effect.

\section{7}

Macrophage migration inhibitory factor (MIF), adipocytokines and and insulin sensitivity in severe obese women with normal and impaired glucose tolerance

M. S. Sumarac-Dumanovic ${ }^{1}$, D. D. Micic ${ }^{1}$, V. Trajkovic ${ }^{2}$, D. Stevanovic ${ }^{3}$, J. Jorga ${ }^{4}$, M. Georgiev ${ }^{1}$, D. Stamenkovic-Pejkovic ${ }^{1}$, A. Kendereski ${ }^{1}$, G. Cvijovic ${ }^{1}$, S. Zoric ${ }^{1}$;

${ }^{1}$ Dept for Endocrinology, Institute of endocrinology, ${ }^{2}$ Department for immunology, School of Medicine, Institute of immunology, ${ }^{3}$ Dept for Physiology, School of Medicine, Institute of Physiology, ${ }^{4}$ Dept for Eating Disordes, School of Medicine, Institute of Eating Disordes, Belgrade, Serbia.

Background and Aims: There are data that hyperglycemia causes an increase in plasma visfatin levels in people with T2DM. This increase gets more prominent as the glucose intolerance worsens. Macrophage Migration Inhibitory Factor (MIF) is elevated in obesity and it was shown that metformin suppresses plasma MIF in the obese. The aim of the study was to determine level of plasma MIF and adipocytokine and insulin sensitivity in severely obese women with normal and impaired glucose tolerance.

Materials and Methods: Sixteen obese women (age: 34.56 $\pm 2.24 \mathrm{yrs}$; BMI $34.06 \pm 0.66 \mathrm{~kg} / \mathrm{m} 2$ ) with normal glucose tolerance (NGT) and 10 age and BMI matched obese women (age: $35.80 \pm 2.54$ yrs; BMI $36.98 \pm 1.66 \mathrm{~kg} / \mathrm{m} 2$ ) with normal fasting and impaired glucose tolerance during oral glucose tolerance test (OGTT) (IGT) were included in the study. Fasting plasma visfatin (EIA Phoenix, ng/ml), adiponectin (Linco RIA, ng/ml), leptin (Linco RIA, ng/ml) and insulin (RIA Inep, mU/l), MIF (ELISA, ng/l) were measured. OGTT (75 gr of glucose) were performed in all obese women. Insulin sensitivity (M index: $\mathrm{mg} / \mathrm{kgBW} / \mathrm{min}$ ) was determined using euglycemic $2 \mathrm{hr}$ clamp.

Results: There was no difference in fasting visfatin between NGT and IGT ( $74.85 \pm 4.40$ vs. $69.80 \pm 5.55, \mathrm{p}>0.05)$, fasting leptin ( $32.63 \pm 3.52$ vs.30.70 \pm 3.8 $8, \mathrm{p}>0.05)$ fasting adiponectin $(7.84 \pm 1.61 \mathrm{vs} .9 .65 \pm 4.59, \mathrm{p}>0.05)$ and plasma MIF (2252.75 $\pm 420.01 v s .2344 .80 \pm 481.80$, p $>0.05)$. Insulin senstitivity was reduced in obese women with IGT $(6.02 \pm 0.56 \mathrm{vs} .2 .74 \pm 0.38, \mathrm{p}<0.05)$. There were no significant correlation among investigated parameters neither with insulin sensitivity index.

Conclusion: In conclusion, decreased insulin sensitivity is confirmed in severely obese women with IGT. Our data suggest that impairment in insulin sensitivity precede change in adipocytokines and MIF during development of type 2 diabetes in obesity.

Ministry of Science, Republic of Serbia

\section{8}

Glycaemic variability (measured by $48 \mathrm{~h}$ continuous glucose monitoring) in subjects with metabolic syndrome, with or without diabetes, is independently associated with IL- 6 blood concentrations

S. Buscemi, S. Verga, S. Cottone, V. Azzolina, B. Buscemi, D. Gioia, M. R. Tranchina, G. Cerasola;

Internal medicine, cardiovascular and kidney diseases, University of Palermo, Italy.

Background and Aims: Subjects who develop diabetes have an increased cardiovascular risk even before the appearance of diabetes. It cannot be excluded that both fasting and postprandial plasma glucose and glycated haemoglobin do not describe adequately the glycaemic control and its impact on endothelial dysfunction and cardiovascular risk in diabetic subjects. Glycaemic variability is an interesting measurement concerning the frequency and duration of hyperglycaemic and hypoglycaemic excursions, another factor that is potentially able to induce endothelial dysfunction. Furthermore, adipose tissue is known to release adipokines that are able to influence both insulin sensitivity and endothelial dysfunction. The aim of this study was to investigate the glycaemic variability measured by continuous glucose monitoring (CGM CV\%) in subjects affected by metabolic syndrome (MS) and to explore if glycaemic variability was associated with circulating levels of IL-6, a proinflammatory cytokine, or with an anti-inflammatory factor like adiponectin. Materials and Methods: Three groups of obese subjects with (MS+: $6 \mathrm{~m}, 8$ f; BMI $33.1 \pm 1.4$ mean \pm sem) or without metabolic syndrome (MS-: $2 \mathrm{~m}, 4 \mathrm{f} ;$ BMI $29.2 \pm 2.2$ ) and with MS associated with type 2 diabetes (MS/T2D: $3 \mathrm{~m}, 5 \mathrm{f}$; BMI $32.9 \pm 1.4$ ) were investigated. The glycaemic variability was measured in all subjects in terms of CV\% of the glycaemic values obtained every 3 minutes during the course of a 48h CGM performed using a subcutaneous glucose sensor (Glucoday; Menarini Diagnostics, Italy). The circulating levels of both IL- 6 and adiponectin were measured in a subgroup of 18 subjects ( $n=5$ in SM-; $n=8$ in SM+; $n=5$ in SM+/T2D group).

Results: The average CGM CV\% increased from MS- group (21.1\%) to the MS+ group (23.9\%) and to the MS+/T2D group (27.4\%) but it was not correlated to the CGM mean glycaemia ( $r=0.20 ; \mathrm{p} \mathrm{ns})$. In some instances CGM CV\% was found higher in MS+ subjects than in some MS+T2D subjects. As expected, IL-6 blood concentrations increased across the three groups (MS-: $50.0 \pm 4.2$; MS+: $88.2 \pm 10.4$; MS+/T2D: $115.3 \pm 9.6 \mathrm{pg} / \mathrm{ml}$ ) while adiponectin concentrations decreased (MS-: $7.6 \pm 0.6$; MS+: $6.1 \pm 0.3$; MS+/T2D: $5.2 \pm 0.8 \mu \mathrm{g} / \mathrm{ml})$; IL-6 and adiponectin were inversely correlated $(\mathrm{r}=-0.63 ; \mathrm{p}<0.01)$. According to stepwise multiple correlation analysis, IL6 was a predictor of CGM CV\% $\left(\mathrm{R}^{2}=0.35, \beta=0.13 ; \mathrm{p}<0.05\right)$ independent from BMI, waist circumference, adiponectin and insulin concentrations; adiponectin concentrations predicted CGM mean glycaemia $\left(\mathrm{R}^{2}=0.63\right.$, $\beta=29.7 ; p<0.05)$ independently from BMI, waist circumference, IL-6 and insulin concentrations.

Conclusions: These results are in agreement with the hypothesis that microinflammation may have a central role in the pathogenesis of metabolic and cardiovascular complications of MS. The CGM CV\% may contribute to better describe the individual metabolic state and to understand the pathogenesis of endothelial dysfunction in subjects with MS.

Supported by Italian MURST 60\% funds

\section{9}

The effect of telmisartan on selected insulin-stimulated plasma and subcutaneous adipocytokines in patients with metabolic syndrome P. Wohl ${ }^{1}$, E. Krušinová ${ }^{1}$, S. Kratochvílová ${ }^{1}$, L. Kazdová ${ }^{1}$, M. Hill ${ }^{2}$, P. Mlejnek ${ }^{3}$, M. Pravenec ${ }^{3}$, T. Pelikánová1;

${ }^{1}$ Diabetes center, IKEM, ${ }^{2}$ Institute of Endokrinology, Institute of Endocrinology, ${ }^{3}$ Academy of Science, Academy of Science, Prague, Czech Republic.

Background and Aims: Telmisartan exerts a beneficial effect on lipid and glucose metabolism and improves insulin sensitivity, but mechanisms are still unclear. The aim of our study was to test the effect of telmisartan on selected plasma and subcutaneous adipocytokines and their insulin-stimulated changes.

Materials and Methods: 12 patients with impaired glucose tolerance or impaired fasting glucose were enrolled in randomized, placebo-controlled, cross-over study of 3 weeks treatment with telmisartan $(160 \mathrm{mg} / \mathrm{d})$ or placebo. The glucose disposal rate (M) was estimated by one-step hyperinsulinemic euglycemic clamp (120 minutes; $1 \mathrm{mU} \cdot \mathrm{kg}^{-1} \cdot \mathrm{min}^{-1,} 5 \mathrm{mmol} / \mathrm{l}$ ) conducted at the end of each treatment period. Before and during the clamp $(0,30,120$ minutes) plasma levels of adiponectin, resistin, tumor necrosis factor-alfa (TNF), leptin were measured and needle biopsy of abdominal subcutaneous adipose tissue $(0,30$ minutes) was performed to evaluate the expression of adipocytokines by the real-time PCR method.

Results: Despite fasting plasma glucose was lower after telmisartan compared to placebo $(5.1 \pm 0.6$ vs $5.6 \pm 0.4 \mathrm{mmol} / \mathrm{l}, \mathrm{p}<0.02)$, there were no significant differences in $\mathrm{M}$ and in basal plasma adiponectin, resistin, TNF, leptin levels after telmisartan as compared to placebo. However, telmisartan significantly increased plasma adiponectin $(\mathrm{p}<0.05)$, leptin $(\mathrm{p}<0.001)$ and resistin $(\mathrm{p}<0.001)$ during the clamp, while the decrease in insulin-stimulated TNF $(p<0.05)$ was found. Subcutaneous expressions of adiponectin, TNF and 
leptin were not affected by insulin or telmisartan. Expressions of resistin were higher after telmisartan compared to placebo $(\mathrm{p}<0.05)$.

Conclusion: We conclude that 3 weeks intervention with telmisartan has significant effect on insulin-stimulated increase in plasma adiponectin, leptin, resistin and decrease in plasma TNF during the clamp. These changes cannot be explained by their subcutaneous adipose tissue expressions. Interestingly, higher expression of resistin was observed after telmisartan treatment. The results support the hypothesis, that the changes in selected plasma adipocytokines might be involved in the beneficial metabolic effects of telmisartan in patients with metabolic syndrome. (Tab. 1) Supported by NR 8821-3, MZO 00023001

\section{0}

The anti-inflammatory effects of rosiglitazone in patients with type 2 diabetes mellitus

N. P. Kadoglou ${ }^{1}$, F. Iliadis ${ }^{1}$, C. D. Liapis ${ }^{2}$, D. Perrea ${ }^{3}$, T. P. Didangelos ${ }^{1}$, H. Tsanikidis ${ }^{4}$, M. Alevizos ${ }^{1}$;

${ }^{1} 1$ st Propedeutic Department of Internal Medicine, AHEPA University Hospital of Thessaloniki, Greece, ${ }^{2}$ Department of Vascular Surgery, University of Athens, Greece, ${ }^{3}$ Laboratory of Experimental Surgery and Surgical Research, University of Athens, Greece, ${ }^{4}$ Diabetic outpatients' department, General Hospital of Katerini, Greece.

Background and Aims: Growing evidence indicates that adipokines, interleukins, matrix metalloproteinases (MMPs) and their inhibitors (TIMPs) have all been implicated in atherosclerotic plaque development and vulnerability. The aim of our study was to evaluate the pharmacological effects of the addition of rosiglitazone to oral anti-diabetic regimen on the above variables in patients with type 2 diabetes mellitus.

Material and Methods: Seventy individuals with type 2 diabetes mellitus were randomized to either rosiglitazone group ( $8 \mathrm{mg} /$ day - RSG) or control group (CG) for 6 months. All participants were already on stable anti-diabetic regimen (gliclazide $160 \mathrm{mg}$ plus metformin $1700 \mathrm{mg}$ ), but with inadequate glycemic control ( $\mathrm{HbAl} 1 \mathrm{c}>7 \%)$. Patients receiving lipid lowering medications or with diabetic vascular complications were excluded. Anthropometric parameters, glycemic indexes, lipid profile, insulin resistance, adiponectin, IL-6, IL-10, IL-18, MMP-9, TIMP-1 and TIMP-2 were measured at baseline and after 6 months. We used Student's independent $t$-test and paired-samples $\mathrm{t}$-test for statistical analysis $(\mathrm{p}<0.05)$.

Results: Improvements in glycemic control (HbAlc:- $0.88 \pm 0.23 \%$, fasting plasma glucose:- $30.93 \pm 17.36 \mathrm{mg} / \mathrm{dl}), \mathrm{HDL}(3.28 \pm 1.08 \mathrm{mg} / \mathrm{dl})$, fasting insulin $(-2.46 \pm 0.94 \mathrm{mU} / \mathrm{L})$ and HOMA-IR $(-1.92 \pm 0.96)$ levels were noted only in RSG $(\mathrm{p}<0.05)$. The rest of lipid parameters remained unaltered in both groups. In comparison to baseline values and control group, rosiglitazone treatment increased adiponectin (from $7.19 \pm 2.77 \mu \mathrm{g} / \mathrm{ml}$ to $14.085 \pm 2.44 \mu \mathrm{g} / \mathrm{ml}$ ), IL10 (from $4.38 \mathrm{pg} / \mathrm{ml}$ to $5.45 \mathrm{pg} / \mathrm{ml}$ ) and TIMP-2 (from $132.83 \pm 9.39 \mathrm{ng} / \mathrm{ml}$ to $140 \pm 10.98 \mathrm{ng} / \mathrm{ml})$ concentrations significantly $(\mathrm{p}<0.05)$ after 6 months. Considerable decreases in IL-6, IL-18 and MMP-9 $(\mathrm{p}<0.01)$ were observed in the rosiglitazone-treated patients, while the latter variables slightly increased in CG. Plasma levels of TIMP-1 decreased in both groups, but its difference between groups was marginal $(\mathrm{p}=0.063)$. Finally rosiglitazone therapy elicited a pronounced increase of body weight in comparison to control group $(+2.6 \pm 1.08 \mathrm{~kg}$ vs $+0.3 \pm 1.06 \mathrm{~kg} ; \mathrm{p}<0.001)$.

Conclusion: Despite significant weight gain adjunctive treatment with rosiglitazone exerted beneficial effects on glycemic control, insulin sensitivity and plasma concentrations of cytokines, MMP-9 and TIMPs. Totally rosiglitazone suppressed inflammatory milieu, along with increments of anti-inflammatory markers indicating novel cardioprotective mechanisms in patients with type 2 diabetes mellitus.

Supported by Project "Pythagoras I" and Propondis Foundation

\section{1}

Synergistic effects of n-3 polyunsaturated fatty acids and rosiglitazone in the amelioration of insulin resistance and adipose tissue inflammation O. Kuda ${ }^{1}$, Z. Jilkova ${ }^{1}$, M. Rossmeisl ${ }^{1}$, P. Flachs ${ }^{1}$, N. C. Ogston ${ }^{2}$,

V. Mohamed-Ali ${ }^{2}$, S. Cinti ${ }^{3}$, J. Kopecky';

${ }^{1}$ Department of Adipose Tissue Biology, Institute of Physiology, Prague, Czech Republic, ${ }^{2}$ Adipokines and Metabolism Research Group, Centre for Clinical Pharmacology, University College London, United Kingdom, ${ }^{3}$ Institute of Normal Human Morphology, University of Ancona, Italy.

Background and Aims: Effective therapeutic strategies for the prevention and treatment of metabolic syndrome are being sought. Polyunsaturated fatty acids of $n-3$ series ( $n-3$ PUFA), especially eicosapentaenoic (EPA; 20:5 n-3) and docosahexaenoic (DHA; 22:6 n-3) acids, act as natural hypolipidemics and reduce risk of cardiovascular disease. In rodents, n-3 PUFA can prevent, but not reverse, obesity and insulin resistance induced by a high-fat diet. We investigated the combined effects of n-3 PUFA and rosiglitazone in the prevention of obesity and glucose intolerance in mice.

Materials and Methods: Experiments were conducted in C57BL/6 mice fed for 20 weeks either a corn oil-based high fat (HF) diet or HF diet containing: (i) $15 \%$ of its lipids replaced by n-3 PUFA concentrate - EPAX 1050TG (60\% EPA/DHA; Pronova Biocare a.s.); (ii) rosiglitazone (ROSI; $10 \mathrm{mg} / \mathrm{kg}$ diet); and (iii) EPA/DHA and rosiglitazone at doses as above (EPA/DHA-ROSI).

Results: Beneficial synergistic effects of EPA/DHA and ROSI on plasma triglycerides (HF: $2.09 \pm 0.13 \mathrm{mmol} / \mathrm{l}$; EPA/DHA: $1.75 \pm 0.20$; ROSI: $1.49 \pm$ 0.10 ; EPA/DHA-ROSI: $1.14 \pm 0.08 ; \mathrm{p}<0.01$ ), glucose tolerance and plasma levels of adiponectin were observed. The systemic effects of the treatment correlated with the significant reduction of low-grade inflammation of adipose tissue, namely accumulation of macrophages, which was nearly abolished by the EPA/DHA-ROSI combination. Additive impact of EPA/ DHA and ROSI in the prevention of hypertrophy of adipocytes was obvious and was in accord with the changes in the DNA tissue content.

Conclusions: The combination of n-3 PUFA and thiazolidinediones thus represents a promising strategy for the treatment of metabolic syndrome. Supported by research projects AV0Z50110509 and GA CR 303/03/H065

\section{2}

Chronic ethanol feeding induces visceral fat accumulation and GLUT4 impairment in rat adipose tissue via AMPKo, MEF2, and PPAR $\gamma$ L. Feng ${ }^{1}$, J. Zhang ${ }^{2}$, L. Gao ${ }^{2}$, X. L. Hou' ${ }^{1}$ J. J. Zhao ${ }^{3}$;

${ }^{1}$ Endo., Shandong Provincial Hospital, Shandong University,

${ }^{2}$ Central Laboratory, SPH, Jinan, China,

${ }^{3}$ Endo., Shandong Provincial Hospital, Jinan, China.

Background and Aims: Visceral fat accumulation and GLUT4 impairment are two major contributors to insulin resistance. The mechanism by which ethanol feeding influences them remains obscure. AMP-activated protein kinase $\alpha(\mathrm{AMPK} \alpha)$ regulates lipid oxidation and GLUT4 expression via peroxisome proliferator activated receptor $\gamma(\operatorname{PPAR} \gamma)$ and myocyte enhancer factor 2 (MEF2). To observe effect of chronic ethanol feeding on visceral fat accumulation and GLUT4 expression in rat adipose tissue and explore

Tab. 1

\begin{tabular}{|c|c|c|c|c|c|c|}
\hline \multirow[b]{2}{*}{ Time (min) } & \multicolumn{3}{|l|}{ placebo } & \multicolumn{3}{|l|}{ telmisartan } \\
\hline & 0 & 30 & 120 & 0 & 30 & 120 \\
\hline P-TNF (pg/ml) & $7.91 \pm 1.3$ & $8.42 \pm 1.4$ & $8.64 \pm 1.5$ & $7.43 \pm 1.2$ & $6.89 \pm 1.0$ & $7.22 \pm 1.1$ \\
\hline P-Resistin (ng/ml) & $2.70 \pm 0.1$ & $2.73 \pm 0.1$ & $3.01 \pm 0.1$ & $2.75 \pm 0.1$ & $2.98 \pm 0.1$ & $3.43 \pm 0.2$ \\
\hline P-adiponectin (ug/ml) & $6.41 \pm 0.8$ & $7.30 \pm 0.8$ & $6.01 \pm 0.9$ & $6.53 \pm 0.8$ & $6.52 \pm 0.8$ & $7.51 \pm 0.9$ \\
\hline P-leptin (ng/ml) & $11.71 \pm 1.2$ & $12.49 \pm 1.3$ & $12.42 \pm 1.3$ & $14,12 \pm 1,4$ & $13.77 \pm 1.4$ & $13.71 \pm 1.4$ \\
\hline E-TNF & $10.81 \pm 1.9$ & $4.94 \pm 0.7$ & & $12.23 \pm 2.2$ & $5.93 \pm 1.1$ & \\
\hline E-Resistin & $6.08 \pm 1.3$ & $4.73 \pm 0.8$ & & $7.34 \pm 2.0$ & $5.08 \pm 0.9$ & \\
\hline E-leptin & $38.04 \pm 4.6$ & $22.98 \pm 3.2$ & & $39.13 \pm 4.7$ & $26.1 \pm 3.4$ & \\
\hline E-adiponectin & $28 \pm 7.9$ & $25.9 \pm 7.7$ & & $30.3 \pm 10.9$ & $26.6 \pm 4.5$ & \\
\hline
\end{tabular}


possible underlying mechanism, we measured AMPK $\alpha$, MEF2A, and PPAR $\gamma$ in both mRNA and protein levels.

Materials and Methods: Thirty-six male Wistar rats were randomly divided into three groups, receiving either distilled water (group C) or ethanol with a single daily dose of 0.5 (group L), $5 \mathrm{~g} / \mathrm{kg}$ (group H). Body weights were monitored weekly and treatment lasted for 22 weeks. After anaesthetic, fasting blood samples were obtained for determining the concentration of blood glucose and serum insulin. Epididymal, perirenal and retroperitoneal fat pads were rapidly removed, weighed and saved. HOMA-IR index and the ratio of visceral fat weight to body weight were calculated. Gene transcription and protein expression were tested by RT-PCR (normalized against GAPDH) and western blotting, respectively.

Results: In the present study, body weights of ethanol-treated rats showed a slight decrease, but no significant differences were found. However, visceral adipose tissue was increased by $13.43-$ and $22.5 \%$ in group $\mathrm{L}$ and $\mathrm{H}$ when normalized for body weight. Accumulation of visceral adipose tissue is considered to be a positive contributor to insulin resistance, and we further calculated HOMA-IR index. As expected, group L and $\mathrm{H}$ had a higher HOMA-IR index compared with group C and increased by 5.11- and $9.56 \%$, respectively. Correlation analysis showed a positive relationship between the ratio of visceral adipose tissue to body weight and HOMA-IR index $(r$ $=0.7, P<0.05)$, indicating ethanol feeding induced visceral fat accumulation, and hence insulin resistance. We subsequently measured AMPK $\alpha$, MEF2A, PPAR $\gamma$, and GLUT4 expression in epididymal adipose tissue. Results showed no significant effect of ethanol on AMPK $\alpha 1, \alpha 2$ isoform mRNA, and totalAMPK $\alpha$ protein, but phosphorylated-AMPK $\alpha$ (P-AMPK $\alpha$ ) protein was reduced in ethanol feeding groups, indicating ethanol feeding impairs AMPK $\alpha$ activation. It is reported that activated-AMPK up-regulates GLUT4 expression via MEF2. In this study, we found the changes of MEF2A in rat adipose tissue after ethanol exposure was indeed consistent with that of P-AMPK, it was decreased in mRNA level by 7.29 14.77\%, and decline of MEF2A protein was also observed. Recently, several studies revealed activated-AMPK could also lessen the inhibition of GLUT4 via depressing PPAR $\gamma$ expression. Concurrent to these findings, we tested that ethanol exposure increased the expression of PPAR $\gamma$ in mRNA level by $7.67-, 46.54 \%$ and in protein level by $6.43-, 21.86 \%$ in group $\mathrm{L}, \mathrm{H}$, respectively. Co-regulated by MEF2A isoform and PPAR $\gamma$, GLUT4 was significantly declined in mRNA level by $6.1 \sim 27.23 \%$ and in protein level by $20.69 \sim 26.13 \%$ in the ethanoltreated group.

Conclusion: Chronic ethanol feeding induces visceral fat accumulation and GLUT4 impairment via P-AMPK $\alpha$, MEF2A, and PPAR $\gamma$, and hence contributes to insulin resistance.

Supported by the Natural Science Foundation of Shandong Province

\section{PS 49 Adipokines: effects of weight loss}

\section{3}

Bariatric surgery and regional adiposity; the relevance of insulin sensitivity

C. H. Thompson ${ }^{1,2}$, M. L. Heath ${ }^{2}$, J. P. Slavotinek ${ }^{3}$, R. Valentine ${ }^{3}$, L. Kow ${ }^{4}$, J. Toouli ${ }^{4,2}$;

${ }^{1}$ General Medicine, Flinders Medical Centre, Bedford Park,

${ }^{2}$ Medicine, Flinders University, Adelaide,

${ }^{3}$ Radiology, Flinders Medical Centre, Bedford Park, ${ }^{4}$ General and Digestive Surgery, Flinders Medical Centre, Bedford Park, Australia.

Background and Aims: Weight loss following bariatric surgery is usually less in the diabetic than in the non-diabetic subject. Studies including both diabetic and non-diabetic subjects indicate that relative visceral fat (VAT) loss exceeds abdominal subcutaneous fat (SAT) loss over 12 months. During significant weight loss, few studies have evaluated the relevance of insulin sensitivity to regional adiposity including hepatic steatosis. This study evaluates and relates regional adiposity and insulin sensitivity before and after laparoscopic adjustable gastric banding surgery (LAGB) in a non-diabetic population.

Materials and Methods: Eighteen obese women underwent anthropometry, proton magnetic resonance (MR) spectroscopy to assess liver fat (LFAT) and MR imaging to assess VAT and SAT volume. Fasting blood was taken for NEFA and for homeostasis model assessment (HOMA-R). Measurements were repeated 3 and 12 months after LAGB.

Results:

Table 1: Descriptive characteristics

\begin{tabular}{llll}
\hline & Baseline & $\begin{array}{l}\text { 3 months after } \\
\text { LAGB }\end{array}$ & $\begin{array}{l}\text { 12 months } \\
\text { after LAGB }\end{array}$ \\
\hline Body weight $(\mathrm{kg})$ & $104.3 \pm 12.5$ & $94.5 \pm 10.6^{\dagger}$ & $85.0 \pm 12.3^{\ddagger}$ \\
BMI $\left(\mathrm{kg} / \mathrm{m}^{2}\right)$ & $39 \pm 5$ & $3.5 \pm 4^{\dagger}$ & $32 \pm 5^{\ddagger}$ \\
Waist circumference $(\mathrm{cm})$ & $112.2 \pm 11.9$ & $102.8 \pm 8.3^{\dagger}$ & $96 \pm 10.3^{\ddagger}$ \\
LFAT (\%) & $12 \pm 13$ & $7.5 \pm 7.5$ & $9.3 \pm 14.9$ \\
VAT (ml) & $175 \pm 89$ & $142 \pm 71^{\dagger}$ & $111 \pm 54^{\ddagger}$ \\
SAT (ml) & $596 \pm 127$ & $506 \pm 108^{\dagger}$ & $421 \pm 124^{\ddagger}$ \\
NEFA (mM) & $0.77 \pm 0.021$ & $0.57 \pm 0.27^{\dagger}$ & $0.48 \pm 0.23$ \\
HOMA-R & $3.6 \pm 3.5$ & $2.7 \pm 1.5$ & $1.8 \pm 1.3^{\dagger}$ \\
\hline
\end{tabular}

Data presented are mean \pm SD; LFAT, liver fat; VAT, abdominal visceral adipose tissue; SAT, abdominal subcutaneous adipose tissue; HOMA-R, homeostasis model assessment insulin resistance index; ${ }^{\dagger} \mathrm{p}<0.05 \mathrm{cf}$ baseline; ${ }^{\ddagger} \mathrm{p}<0.01 \mathrm{cf} 3$ months after LAGB.

Descriptive characteristics of subjects before and after LAGB are shown in Table 1. Three months after LAGB, reductions had occurred in body weight, BMI, waist circumference, VAT, SAT (all $\mathrm{p}<0.01)$ and NEFA $(\mathrm{p}<0.05) .12$ months after LAGB, further reductions $(\mathrm{p}<0.01)$ occurred in these variables, except for NEFA. No significant reduction occurred in LFAT at either time point in the group as a whole. In those with pre-operative hepatic steatosis (LFAT $>5 \%, \mathrm{n}=8$ ), LFAT fell from $12 \%$ to $8 \%(\mathrm{p}=0.015) 3$ months after LAGB with no further reduction occurring by 12 months. There was an improvement in HOMA-R at $12(\mathrm{p}=0.04)$ but not 3 months.

Though there was progressive fat loss $(\mathrm{p}<0.01)$ from both VAT $(-11 \%$ at 3 months, $-29 \%$ at 12 months) and SAT (-13\% at 3 months, $-28 \%$ at 12 months) sites, the relative changes were similar at each timepoint $(p>0.05)$. At baseline, LFAT correlated both with VAT volume $(\mathrm{r}=0.68, \mathrm{p}<0.01)$ and HOMA-R $(\mathrm{r}=0.48, \mathrm{p}<0.05)$, as well as with the change in HOMA- $\mathrm{R}$ at $3(\mathrm{r}=$ $0.48, \mathrm{p}<0.05)$ and 12 months $(\mathrm{r}=0.47, \mathrm{p}<0.05)$ and with the change in VAT volume at 12 months $(\mathrm{r}=0.51, \mathrm{p}<0.05)$. Relative to VAT loss, SAT loss at 12 months was greatest in the most insulin sensitive patients $(r=0.70 ; p<0.01)$. Conclusions: After LAGB in the non-diabetic population, relative VAT and SAT loss were similar and progressive over 12 months. The ability to mobilise SAT relative to VAT related to insulin sensitivity postoperatively. Excess LFAT was lost in the first 3 months postoperatively. The pre-operative degree of hepatic steatosis may predict the post-operative loss of visceral adiposity and improvement in insulin resistance. 


\section{4}

Insulin secretion, $\beta$-cell glucose sensitivity and insulin resistance in severe obesity: effect of massive weight loss after gastric bypass D. M. Marin ${ }^{1}$, S. Monte Alegre ${ }^{1}$, J. N. M. Ortiz ${ }^{1}$ J. C. Pareja ${ }^{1}$, C. A. Silva ${ }^{1}$, A. Souza ${ }^{1}$, A. Gastaldelli ${ }^{2}$, E. Muscelli ${ }^{3,1}$;

${ }^{1}$ Department of Internal Medicine, Faculdade de Ciências Médicas, UNICAMP, Campinas, S. Paulo, Brazil,

${ }^{2}$ Istituto di Fisiologia Clinica, Consiglio Nazionale delle Ricerche, Pisa, Italy, ${ }^{3}$ Department of Internal Medicine, Università di Pisa, Italy.

Background and Aims: The aims of this study were to evaluate the contribution of insulin resistance and impaired $\beta$-cell function to glucose tolerance in severely obese subjects and to evaluate the effect of massive weight loss by Roux-en-Y Gastric bypass, RGB-Y, on the adaptation of insulin secretion to insulin resistance (IR).

Materials and Methods: 34 obese (17 OB-NGT, 11 OB-IGT and 6 type 2 diabetic - OB-T2D; age $37.3 \pm 1.4 y$; BMI $\left.50.0 \pm 1.5 \mathrm{~kg} / \mathrm{m}^{2}\right)$ and 10 healthy lean CT (BMI $22.3 \pm 0.6 \mathrm{~kg} / \mathrm{m}^{2} ; 35 \pm 4 \mathrm{y}$ ). Protocol: euglycemic hyperinsulinemic clamp (3h-insulin infusion at $240 \mathrm{pmol} / \mathrm{min} . \mathrm{m}^{-2}$ ) with indirect calorimetry, IVGTT and OGTT was used. 14 patients (11OB-NGT and 3OB-IGT) were re-evaluated post-surgery after weight stabilization $\sim 18$ months. Total (TISR) and fasting insulin secretion (f-ISR) were evaluated by C-peptide deconvolution and $\beta$-cell glucose sensitivity ( $\beta$-GS) as the slope of the insulin secretion/glucose concentration dose-response curve. The disposition index was calculated as ISRxMvalue of the clamp.

Results: Obese subjects were insulin resistant for oxidative, nonoxidative and whole body glucose disposal (OB-T2D, OB-IGT and OB-NGT were $~ 30 \%$, $60 \%$ and $43 \%$ of CT). During IVGTT the acute insulin response $\left(\mathrm{AIR}_{0-10 \min }\right)$ was significantly higher in OB-NGT than in CT and in the other obese groups (CT 5378 \pm 590 ; OB-NGT 9363 \pm 895 ; OB-IGT $6544 \pm 558$ and OBT2D $3893 \pm 622 \mathrm{pmol} \mathrm{min}^{-1} \mathrm{~m}^{-2}$ ), T-ISR was elevated in all obese groups (CT 20.2 \pm 1.9; OB-NGT 39.2 \pm 3.6 ; OB-IGT $39.2 \pm 2.5$ and OB-T2D $37.1 \pm 4.5 \mathrm{nmol}$ $\mathrm{min}^{-1} \mathrm{~m}^{-2} \mathrm{x} 60 \mathrm{~min}$ ) while $\beta$-GS was decreased in OB-IGT and -T2D but not in -NGT as compared to CT (OB-T2D $-4 \pm 3$; OB-IGT $31 \pm 4$; OB-NGT $56 \pm 6$ and CT $\left.43 \pm 6 \mathrm{pmol} \mathrm{min}^{-1} \mathrm{~m}^{-2} \mathrm{mM}^{-1}\right)$. The disposition index was reduced in OBIGT and -T2D but not in -NGT.

After weight loss BMI was $30.2 \pm 1.2 \mathrm{~kg} \cdot \mathrm{m}^{-2}$, f-ISR (from $148 \pm 12$ to $83 \pm 12$, $\mathrm{p}<0.05$ vs. pre; $\mathrm{p}=\mathrm{ns} v s$. CT $76 \pm 8 \mathrm{pmol} \mathrm{min}^{-1} \cdot \mathrm{m}^{-2}$ ) and insulin sensitivity (from $30 \pm 3$ to $55 \pm 4, \mathrm{p}<0.05$ vs. pre; $\mathrm{p}=\mathrm{ns} v s \mathrm{CT} 62 \pm 4 \mu \mathrm{molkg}^{-1} \mathrm{~min}^{-1}$ ) changed to normal values. The time-course evidenced a significant decrease in T-ISR, and an unchanged AIR. $\beta$-GS increased to levels higher than CT (from $49 \pm 7$ to $78 \pm 12$ pmol $\mathrm{min}^{-1} \mathrm{~m}^{-2} \mathrm{mM}^{-1}$ ). Before surgery the dispositionn index was similar to the CT group, but after weight loss increased to values higher than in CT.

Conclusion: T2D and IGT severe obese subjects show a progressive deterioration of glucose homeostasis, despite increased insulin secretion. This can be explained as an impairment of $\beta$-cell function for the prevailing IR. RGB-Y weight loss normalized IR and improved $\beta$-cell function in IGT and NGT obese to levels higher than in lean CT.

Supported by the Fundaçao de Amparo à Pesquisa do Estado de Sao Paulo, FAPESP

\section{5}

Expression of adipocytokines in adipose tissue of obese type 2 diabetic subjects before and after major weight reduction

M. Nannipieri ${ }^{1}$, E. Mancini ${ }^{1}$, M. Anselmino ${ }^{2}$, F. Cecchetti ${ }^{1}$, B. Solito ${ }^{2}$, F. Santini ${ }^{3}$, A. Bonotti ${ }^{1}$, D. Gandini ${ }^{4}$, A. Pinchera ${ }^{3}$, E. Ferrannini ${ }^{1}$; ${ }^{1}$ Internal Medicine, University of Pisa, ${ }^{2}$ Unit of Bariatric Surgery, S. Chiara Hospital of Pisa, ${ }^{3}$ Endocrinology, University of Pisa,

${ }^{4}$ Plastic Surgery, Cisanello Hospital of Pisa, Italy.

Background and Aims: Adiponectin (APM1) and visfatin have been linked with obesity and atherosclerosis in opposite manner, negatively the former, positively the latter. We examined the effect of weight loss (by gastric by-pass surgery) on the expression of APM1, its receptors (AdipoR1 and AdipoR2) and visfatin in abdominal subcutaneous adipose tissue (SAT) of morbidly obese subjects with or without diabetes.

Materials and Methods: In 10 obese non-diabetic subjects (NGT) $(39 \pm 9$ years, BMI $45.1 \pm 5.4 \mathrm{~kg} / \mathrm{m} 2$ ) and 10 obese diabetic patients (DM2) ( $45 \pm 5$ years, BMI $47.2 \pm 3.0 \mathrm{~kg} / \mathrm{m} 2$ ), SAT tissue was obtained during bariatric surgery and again one year after surgery (by biopsy). cDNA was synthesised and relative quantifications of APM1, AdipoR1, AdipoR2 and visfatin mRNA (target gene) were performed by RT-PCR. Results were expressed as the target to reference (hypoxantine-phosphorybosyl-transferase) ratio for each sample. Results: After surgery, BMI was similarly reduced in NGT and DM2 subjects $(-15.9 \pm 3.6$ and $-15.9 \pm 6.1 \mathrm{~kg} / \mathrm{m} 2$, respectively, $\mathrm{p}<0.0001$ for both). Likewise, fasting plasma insulin was reduced in both groups $(-15.3 \pm 11.3$ and $-13.1 \pm 9.4$ $\hat{a} \mu \mathrm{U} / \mathrm{ml}, \mathrm{p}<0.01)$. Fasting plasma glucose was reduced in DM2 $(-2.9 \pm 1.6$ $\mathrm{mmol} / \mathrm{l}, \mathrm{p}=0.001)$, but not in NGT $(-0.1 \pm 0.6 \mathrm{mmol} / \mathrm{l}, \mathrm{p}=\mathrm{ns})$. Following weight loss, APM1 did not change in NGT, $(3.83 \pm 1.25$ vs. $4.08 \pm 2.01$, $\mathrm{p}=\mathrm{ns})$, whereas it increased in DM2 (2.69 \pm 1.32 vs. $4.77 \pm 1.26, \mathrm{p}=0.0035)$. AdipoR1 was not significantly changed by weight loss in either group ( $3.49 \pm 1.18$ vs. $2.83 \pm 0.98$ in NGT and $2.73 \pm 1.29$ vs. $2.89 \pm 2.89$ in DM2, $\mathrm{p}=\mathrm{ns}$ ); this was also true of AdipoR2 (4.92 \pm 2.84 vs. $3.88 \pm 1.7$ in NGT and $4.18 \pm 3.02$ vs. $3.76 \pm 1.3$ in DM2, $\mathrm{p}=\mathrm{ns})$. In contrast, visfatin expression decreased both in NGT (2.12 \pm 1.15 vs. $1.02 \pm 0.27, \mathrm{p}=0.008)$ and DM2 (3.09 \pm 2.56 vs $1.02 \pm 0.24, \mathrm{p}=0.028)$ after weight loss.

Conclusion: We conclude that, whereas adiponectin receptor expression is not altered following weight loss, visfatin expression in subcutaneous fat falls markedly with weight reduction. In addition, adiponectin expression increases in weight-reduced diabetic subjects, possibly contributing to their improved insulin sensitivity.

\section{6}

Effect on plasma visfatin concentrations after bariatric surgery in morbidly obese patients

J. Garcia-Aleman ${ }^{1}$, J. M. Garcia-Almeida ${ }^{1}$, E. Garcia-Fuentes ${ }^{2}$, J. GarciaArnes $^{3}$, F. Vilches ${ }^{1}$, S. Garcia-Serrano ${ }^{2}$, J. Pinzon ${ }^{1}$, D. Fernandez ${ }^{1}$, M. Picon ${ }^{1}$, I. Mancha ${ }^{1}$, F. Tinahones ${ }^{1}$;

${ }^{1}$ Endocrinology, H. Virgen de la Victoria, Malaga, ${ }^{2}$ Endocrinology, Ciber Fisiopatologia Obesidad y Nutricion (CB06/03), Instituto de Salud Carlos III, Malaga, ${ }^{3}$ Endocrinology, H. Carlos Haya, Malaga, Spain.

Background and Aims: Adipose tissue has recently been shown to secrete a peptide, visfatin, which is high in obese persons and in persons with type 2 diabetes mellitus (DM2). The aim of this study was to determine the course of plasma visfatin in morbidly obese persons following weight loss after bariatric surgery in relation with the glycemia concentration.

Materials and Methods: Plasma levels of visfatin and leptin were studied in 53 morbidly obese persons (MO) before and seven months after bariatric surgery, and in 28 healthy persons. All the patients underwent bariatric surgery

Results: The basal levels of visfatin in the MO were greater than in the controls (55.9 \pm 39.9 vs. $42.9 \pm 16.6 \mathrm{ng} / \mathrm{mL}, \mathrm{P}=0.024)$. This increase was due to the MO with impaired fasting glucose (IFG) $(63.4 \pm 36.6 \mathrm{ng} / \mathrm{mL})$ and with diabetes $(60.0 \pm 46.0 \mathrm{ng} / \mathrm{mL})$. The MO with normal fasting glucose had similar levels of visfatin $(32.1 \pm 17.6 \mathrm{ng} / \mathrm{mL})$ to the controls. Seven months after surgery, visfatin levels were significantly increased $(84.8 \pm 32.8 \mathrm{ng} / \mathrm{mL}, \mathrm{P}<0.001)$. This increase was independent of the presurgical glucose levels. The type of bariatric surgery had no influence on visfatin levels. Post-surgical visfatin was significantly correlated with the percentage reduction in hip measurement, leptin and the change in plasma leptin concentrations (presurgery - postsurgery leptin).

Conclusion: Plasma visfatin in MO were increased, but only when accompanied by high glucose levels, even in the range of IFG. Bariatric surgery causes an increase in visfatin that is not wholly independent of the changes in the distribution of body fat.

Supported by CB06/03 


\section{7}

Circulating levels of preadipocyte factor-1 (PREF-1/FA1) decreased after massive weight lost and have a modulatory effect on pro-inflammatory cytokines

M. R. Chacón ${ }^{1}$, M. Miranda ${ }^{1}$, C. J. Jensen ${ }^{2}$, J. Fernández-Real ${ }^{3}$, N. Vilarrasa ${ }^{4}$, A. García-España ${ }^{1}$, W. Ricart ${ }^{3}$, S. Näf ${ }^{1}$, J. Gómez ${ }^{4}$, J. J. Vendrell';

${ }^{1}$ Research Unit, University Hospital Joan XXIII, Tarragona, Spain,

${ }^{2}$ Department of Immunology and MIcrobiology, University of Southern Denmark, Odense C, Denmark, ${ }^{3}$ Diabetes Unit, University Hospital "Dr. Josep Trueta", Girona, Spain, ${ }^{4}$ Endocrinology Unit, University Hospital of Bellvitge, Barcelona, Spain.

Background: PREF-1 gene is highly expressed in mouse preadipocyte cells and it has been proposed to be a negative regulator of adipogenesis. Transgenic mice showed substantial but not complete loss of adipose tissue, hypertriglyceridemia, impaired glucose tolerance and decreased insulin sensitivity. However, in human subcutaneous adipose tissue this gene is scarcely expressed. On the other hand, the soluble circulating form of this protein named "foetal antigen 1" (FA1) has been associated in obese subjects and in patients with type 2 diabetes with a worse cardiovascular profile.

Aim: We aimed to search for the cell type responsible for its scarce gene expression in adipose tissue. We also evaluated FA-1 circulating plasma levels in severely obese patients before and 6 months after bariatric surgery and related them to lipidic, glyceamic and inflammatory profile. Biological function was also assessed in vitro on THP-1 human monocytic/macrophage cells.

Materials and Methods: FA1 plasma levels were measured by ELISA technique on $n=61$ severely obese women. Gene expression levels were measured in several human samples: placenta, subcutaneous and visceral adipose tissue, human monocytes and lymphocytes, mature adipocytes, stromovascular fraction and preadipocytes. Real Time PCR was used to evaluate PREF-1 gene expression. THP-1 human monocytic cell line was stimulated in vitro with 1 , 3 and $5 \mu \mathrm{g} / \mathrm{ml}$ of FA1. Cytokine protein and gene expression for TNF $\alpha$, IL-6, MCP-1 and TNFR2 were evaluated.

Results: PREF-1 gene expression on human preadipocytes was quantifiable but the low levels detected were not considered to have any biological activity. Plasma levels of FA1 in the severely obese cohort decreased significantly $(\mathrm{p}<0.001)$ with the lost of weight after 6 months of surgery (before:27.13 $\pm 10.53 \mathrm{ng} / \mathrm{ml}$ and 6 months after surgery: 19.98 $\pm 9.34 \mathrm{ng} /$ $\mathrm{ml})$. A multivariate regression model was constructed with FA1 at basal state as dependent variable and hsCRP, IL-6, glucose, insulin, triglycerides as independent variables; FA1 levels at basal state were strongly related to triglyceride levels $(\beta=0.587, \mathrm{p}=0.008)$. A similar model was constructed for FA1 levels after 6 months and we found that age and triglycerides were the independent determinants of FA1 levels $(\beta=0.499, p=0.001$ and $\beta=0.457$ $\mathrm{p}=0.002$ respectively). The biological effect of FA1 protein on THP- 1 human monocytic cells observed in our study were a clear up-regulation of TNF- $\alpha$, MCP-1 and IL-6 pro-inflammatory cytokines.

Conclusion: PREF-1 gene is not a relevant gene in human preadipocytes. FA-1 circulating levels may be a marker of a worse metabolic profile in severely obese patients with a possible modulator effect on pro-inflammatory cytokines derived from monocyte/macrophage cells.

Supported by grants (FIS 04/0377, REDIMET) and by a fellowship from FIS CP06/00119 to Dr. M.R. Chacon

\section{PS 50 Animal models of insulin resistance}

\section{8}

New mouse models of impaired glucose tolerance generated using

$\mathrm{N}$-ethyl-N-nitrosourea mutagenesis

L. Bentley, W. Wang, C. Church, P.-S. S. Muller, R. D. Cox; Mammalian Genetics Unit, MRC HARWELL, Oxfordshire, United Kingdom.

Background and Aims: N-ethyl-N-nitrosourea (ENU) is a powerful chemical mutagen that induces point mutations at a per locus rate of approximately $1 / 1000$ and has been used effectively in a number of phenotype-driven screens in the mouse to produce new mouse mutants. Both dominant (F1) and recessive (G3) offspring of mutagenised males from the new Harwell ENU mutagenesis programme were assayed for impaired glucose tolerance and plasma and urine biochemistry with the aim of identifying new mouse models of metabolism and associated kidney disease.

Materials and Methods: C57BL/6J male mice were treated with ENU and upon regaining fertility were mated to $\mathrm{C} 3 \mathrm{H} / \mathrm{HeH}$ females to produce an $\mathrm{F} 1$ population of males that were assayed as part of the dominant phenotype screen. A proportion of these F1 males were backcrossed to $\mathrm{C} 3 \mathrm{H} / \mathrm{HeH}$ females and then intercrossed with their daughters to produce G3 recessive pedigrees. At 12 wks of age mice were tested using an intra-peritoneal glucose tolerance test (IPGTT) to ascertain their ability to respond to a glucose challenge. At 16wks of age, the same cohort of mice were put into metabolic cages for a period of $24 \mathrm{hrs}$ to facilitate collection of a $24 \mathrm{hr}$ urine sample and a fasted blood sample was also subsequently taken. Biochemical analysis of plasma and urine samples was performed using an Olympus AU400 clinical chemistry analyser. Outliers were identified as individuals with values for a parameter greater than $2 \mathrm{SD}$ from the population mean.

Results: A total of $821 \mathrm{~F} 1$ males from the dominant screen and 548 G3 males from 34 recessive pedigrees have been screened using IPGTT and plasma biochemistry at 12 and 16wks of age, respectively; as well as urine biochemistry from $598 \mathrm{~F} 1$ males at 16wks of age. Outliers were re-tested to confirm an abnormal test result and a selection of the confirmed outliers were put into inheritance testing. In the dominant screen we have identified 24 outliers with abnormal IPGTT. Nine of these outliers were put into inheritance testing and seven were found to be inherited. Two of these lines (SweetP and Dipdab) are currently being investigated to refine the phenotype data and produce progeny for mapping studies. SweetP mice tested at $12 \mathrm{wks}$ of age show fasting hyperglycaemia and elevated glucose levels $2 \mathrm{hrs}$ after a glucose challenge. At 16wks of age SweetP mice exhibit fasting hyperinsulinaemia, glycosuria and are significantly heavier than unaffected littermates. Dipdab mice tested at $12 \mathrm{wks}$ of age exhibit elevated glucose levels $2 \mathrm{hrs}$ after a glucose challenge. At 16wks of age Dipdab mice exhibit fasting hyperinsulinaemia and are significantly heavier than unaffected littermates. In both SweetP and Dipdab lines, females are affected but to a lesser extent than the males. In the recessive screen we have identified 10 pedigrees with abnormal IPGTT phenotypes. Three of these pedigrees (Sugarlump, Bonbon and Jazzie) are currently undergoing further investigation to confirm inheritance and refine phenotype data. Initial studies on Suagrlump mice have shown this to be a dominantly inherited phenotype with impaired glucose tolerance, hyperinsulinaemia and glycosuria.

Conclusion: We have identified a number of potentially interesting mouse models with impaired glucose tolerance and possible renal dysfunction using a phenotype driven approach. SweetP, Dipdab and Sugarlump are currently undergoing genome scans to facilitate the mapping and cloning of these mouse models.

Supported by EuReGene FP6 project 5085 


\section{9}

Effects of pioglitazone on fuel homeostasis, plasma adiponectin, and p70S6 kinase activity at different stages of the diabetes-like syndrome of the Zucker Diabetic Fatty (ZDF) rat

Z. Szöcs ${ }^{1}$, B. Brunmair ${ }^{1}$, K. Stadlbauer ${ }^{1}$, P. Nowotny ${ }^{1}$, L. Bauer ${ }^{2}$, A. Luger ${ }^{1}$, C. Fürnsinn ${ }^{1}$,

${ }^{1}$ Department of Medicine III, Division of Endocrinology \& Metabolism, Medical University of Vienna, Austria,

${ }^{2}$ 55pharma Drug Discovery \& Development AG, Vienna, Austria.

Background and Aims: Clinical studies suggest that the glucose lowering potential of glitazones fades in the course of the progressive development of type 2 diabetes. To analyse the interdependence of disease development and glitazone action, we examined the effects of pioglitazone treatment in ZDF rats being at different stages of their type 2 diabetes-like syndrome.

Materials and Methods: Male obese ZDF rats were subjected to daily oral treatment with $12 \mathrm{mg}^{\star} \mathrm{kg}^{-1 \star} \mathrm{d}^{-1}$ pioglitazone at an age of 7,11 , or 16 weeks (referred to as age groups I, II, and III). Blood glucose and body weight were documented weekly, other parameters were measured at the end of the respective treatment period and included blood glucose, fat pad weight, plasma adiponectin, plasma lipids, glycogen stores, and the activity of p70S6 kinase, which is known to impair insulin signalling.

Results: Early intervention with pioglitazone (age group I) completely prevented deterioration of glycaemia as seen in untreated ZDF rats (increase in blood glucose between 7 and 12.5 weeks of age, mmol/l: vehicle, from $6.2 \pm 0.4$ to $18.7 \pm 1.5, \mathrm{p}<0.001$; pioglitazone, from $6.0 \pm 0.3$ to $6.5 \pm 0.4$, ns). The same intervention at an age of 11 weeks (age group II) transiently ameliorated the prevailing hyperglycaemia (after 10 days of treatment: vehicle, $22.1 \pm 0.8$, vs pioglitazone, $17.8 \pm 1.1 \mathrm{mmol} / \mathrm{l}, \mathrm{p}<0.001)$, but this benefit faded during prolonged treatment (after 31 days: vehicle, $25.1 \pm 0.9$, vs pioglitazone, $23.5 \pm 0.7$ $\mathrm{mmol} / \mathrm{l}$, ns). In older animals (age group III), pioglitazone lacked any effect on blood glucose (after 10 days of treatment: $22.6 \pm 0.6$ vs $22.2 \pm 1.5 \mathrm{mmol} / \mathrm{l}$, ns). Pioglitazone-induced glucose lowering in age group I was accompanied by reduced plasma triglycerides $(715 \pm 94$ vs $126 \pm 5 \mathrm{mg} / \mathrm{dl}, \mathrm{p}<0.001)$ and reduced p70S6 kinase activity in extensor digitorum longus muscle (\% of kinase in phosphorylated state: $32 \pm 3$ vs $19 \pm 3$, p $<0.02$ ), but these effects were lost together with glucose lowering action in older rats (triglycerides: group II, $269 \pm 26$ vs $297 \pm 26$, ns; group III, $349 \pm 41$ vs $314 \pm 38$, ns; p70S6 kinase activity: group II, $25 \pm 5$ vs $25 \pm 2$, ns; group III, $22 \pm 3$ vs $20 \pm 1$, ns). In contrast, pioglitazone actions on weight gain, adiposity, and muscle glycogen persisted independently of metabolic improvement in all age groups. Furthermore, pioglitazone increased plasma adiponectin in all age groups, but this effect was clearly more distinct in group I (plasma adiponectin, $\mathrm{mg} / \mathrm{l}$ : group I, $4.6 \pm 0.4$ vs $17.8 \pm 0.4$; group II, $3.6 \pm 0.2$ vs $5.0 \pm 0.3$; group III, $4.1 \pm 0.2$ vs $7.1 \pm 0.6 \mathrm{mg} / \mathrm{l}$; $\mathrm{p}<0.01$ each), suggesting that the increase of adiponectin is in part a primary effect of the glitazone and in part secondary to metabolic improvement. Conclusion: In line with evidence from the clinic, pioglitazone can prevent hyperglycaemia in ZDF rats, but loses its antidiabetic potential during progressive disease development. Whereas effects on plasma triglyzerides and p70S6 kinase activity seem to be coupled to glucose lowering, effects on fat mass, muscle glycogen and, at least in part, plasma adiponectin, are primary effects that occur independently of metabolic improvement. Supported by the Austrian Research Promotion Agency (FFG)

\section{0}

Astaxanthin transiently improves insulin resistance and affect skeletal muscle Akt signaling in Otsuka Long-Evans Tokushima Fatty rats (OLETF)

H. Kobayashi ${ }^{1,2}$, H. Naito ${ }^{3}$, S. Kado ${ }^{1}$, D. Ohmori ${ }^{4}$, Y. Iida ${ }^{5}$;

${ }^{1}$ Dept of General Medicine, National Defense Medical College Hospital, Tokorozawa, ${ }^{2}$ Dept of Epidemiology and Environmental health, Juntendo University, Tokyo, ${ }^{3}$ Dept of Exercise Physiology, Juntendo University, Inba, ${ }^{4}$ Dept of Chemistry, Juntendo University, Inba, ${ }^{5}$ Research and Development Institute, Toyo Koso Kagaku Co.,Ltd, Yachimata, Japan.

Background and Aims: Insulin resistance is a key feature of type 2 diabetes. We hypothesize that supplementation of Astaxanthin, which is a potent Carotenoid antioxidant, in diabetic rats may prevent the onset of type 2 diabetes by antioxidant effects of Astaxanthin on skeletal muscle to restore insulin resistance.
Materials and Methods: Male Otsuka Long-Evans Tokushima Fatty (OLETF) and Long-Evans Tokushima Otsuka (LETO) rats at 5 weeks of age were obtained from the Otsuka Pharmaceutical Tokushima Research Institute and they were assigned to one of three groups: 1)LETO (non-diabetic control, $\mathrm{n}=32$ ), 2)OLETF control (diabetic control, $\mathrm{n}=30$ ), and 3)OLETF antioxidant (diabetic antioxidant, $\mathrm{n}=24$ ). OLETF antioxidant were given to standard rat chow supplemented with $0.2 \%$ Astaxanthin daily since 6 weeks of age. Body weight and food intake were measured. Glucose tolerance tests (GTT) were performed by infusing glucose $(1 \mathrm{mg} / \mathrm{kg} \mathrm{BW})$ intraperitoneally under anesthesia at $6,12,25$ and 52 weeks of age. Blood glucose and insulin level were measured at fasting and 30,60 and 120 minutes after glucose infusion. Hindlimb muscles (soleus, EDL, plantalis and gastrocnemius muscle) were removed and weighed at 25 and 52 weeks of age. Phosphorylated Akt signaling and carbonylation in hindlimb muscles were analyzed.

Results: There was no significant difference in body weight and food intake between OLETF control and OLETF antioxidant. GTT at 12 weeks shows that blood glucose at fasting in OLETF control were significantly increased, but not in OLETF antioxidant group. GTT at 25 weeks shows that blood glucose at fasting and 30 minutes in OLETF control were significantly increased and insulin level at 30 and 60 minutes were also significantly increased, but not in OLETF antioxidant group (Fig.1). Hindlimb muscles in OLETF control at 25 weeks shows significant decrease of muscle weight and reduction of Akt phosphorylation (Fig.2), but not in OLETF antioxidant group.

Conclusion: Antioxidant supplementation of Astaxanthin for OLETF rats before onset of type 2 diabetes transiently improves insulin resistance and attenuates atrophy and reduction of Akt phosphorylation in hindlimb muscles.
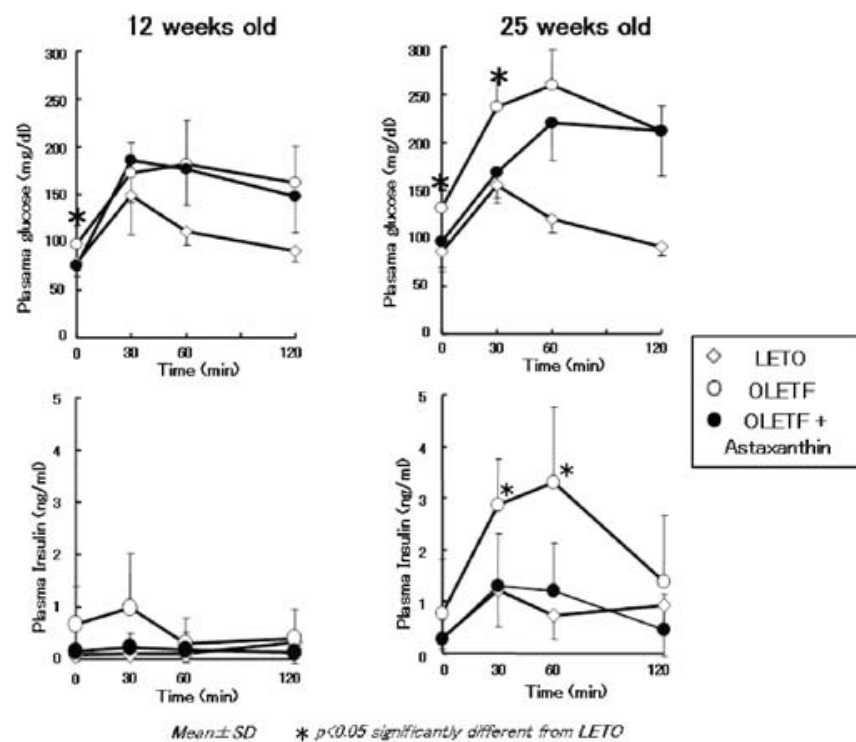

Fig. 1. Glucose and Insulin Level in GTT at 12 and 25 weeks

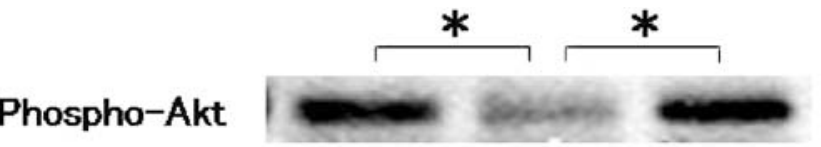

Total-Akt

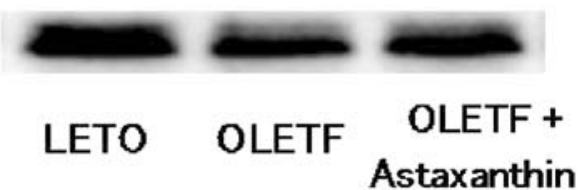

* p<0.05 significantly different from OLFTF

Fig. 2. Akt signaling in gastrocnemius muscle at 25 weeks 


\section{1}

High-fat diet decreases meal-induced insulin sensitivity by impairing hepatic parasympathetic pathway, which is responsible for insulin resistance

R. A. Afonso ${ }^{1,2}$, W. W. Lautt ${ }^{3}$, J. Schafer ${ }^{3}$, D. J. Legare ${ }^{3}$, M. P. Macedo ${ }^{2,4}$; ${ }^{1}$ Biochemistry, Faculty Medical Sciences, Lisbon, Portugal,

${ }^{2}$ Physiology, Faculty Medical Sciences, Lisbon, Portugal,

${ }^{3}$ Pharmacology \& Therapeutics, Faculty of Medicine, Winnipeg, Canada, ${ }^{4}$ Portuguese Diabetes Association, (apdp), Lisbon, Portugal.

Background and Aims: Although the association between obesity and insulin resistance is well documented, the mechanisms involved remain unclear. Meal-induced insulin sensitisation (MIS) depends not only on insulin action per se, but also on hepatic parasympathetic nerve activation (HPN pathway) and glutathione content, which are impaired in several pathological models. We tested the hypothesis that obesity induced by high-fat diet (HFD) leads to the impairment of MIS, accounting for the insulin resistance.

Materials and Methods: Male Sprague Dawley rats were fed either standard $\operatorname{diet}(\mathrm{STD}, \mathrm{n}=7$ ), HFD for 1 week (HFD-A, $\mathrm{n}=6$ ) or HFD for 4 weeks (HFD-B, n=7). All animals were tested in the fed state at the age of 9 weeks. MIS was evaluated by measuring the hepatic glutathione content and by quantification of the HPN pathway-dependent insulin sensitivity. Insulin sensitivity was assessed using the modified euglycemic clamp RIST (Rapid Insulin Sensitivity Test, mg glucose $/ \mathrm{kg}$ bw), in which glucose was given as a variable infusion (iv) in order to maintain euglycemia, after administration of a bolus of insulin $(50 \mathrm{mU} / \mathrm{kg}$, iv). Insulin sensitivity was measured before (control RIST) and after HPN pathway blockade (post-atropine RIST, $1 \mathrm{mg} /$ $\mathrm{kg}$, iv). HPN-dependent component of insulin action was determined by subtraction of the post-atropine from the control RIST. Whole-body fat mass was estimated by tetrapolar bioelectric impedance analysis and visceral fat (sum of perinephric, epididymal and perienteric fat pads) was collected and weighed, at the end of the experiment.

Results: Hepatic glutathione levels were similar in all groups (STD, $6.2 \pm 0.2$ $\mu \mathrm{mol} / \mathrm{g}$; HFD-A, $5.5 \pm 0.2 \mu \mathrm{mol} / \mathrm{g}$; HFD-B, $5.4 \pm 0.3 \mu \mathrm{mol} / \mathrm{g}$; non-significant). Insulin sensitivity decreased from the STD $(189.2 \pm 5.3 \mathrm{mg}$ glucose $/ \mathrm{kg} \mathrm{bw})$ to the HFD-A animals (111.0 $\pm 4.1 \mathrm{mg}$ glucose $/ \mathrm{kg} ; p<0.001)$; the decrease in insulin sensitivity was further aggravated in the HFD-B rats $(69.3 \pm 2.6 \mathrm{mg}$ glucose/kg bw; $p<0.001$ vs STD and HFD-A). The HPN-dependent insulin action was also impaired by HFD (STD, 113.3 $\pm 3.7 \mathrm{mg}$ glucose $/ \mathrm{kg}$ bw; HFDA, $43.3 \pm 5.2 \mathrm{mg}$ glucose $/ \mathrm{kg}$ bw; HFD-B, $5.4 \pm 1.7 \mathrm{mg}$ glucose $/ \mathrm{kg}$ bw; $p<0.001$ between all groups), whereas the HPN-independent component (postatropine RIST) was not affected by the diet. Both whole-body and visceral fat masses correlated negatively with total insulin action (control RIST, $\mathrm{r}^{2}=0.82$ and $\mathrm{r}^{2}=0.85$, respectively) and with HPN-dependent insulin action $\left(\mathrm{r}^{2}=0.81\right.$ and $\mathrm{r}^{2}=0.82$, respectively).

Conclusion: Our results suggest that HFD-induced obesity affects the MIS by impairing the HPN pathway specifically, since it affected neither hepatic glutathione content nor insulin action per se. Thus, the HPN-pathway impairment seems to be responsible for the insulin resistance observed in the obese animals. Furthermore, the increase in fat content induced by HFD correlates with the HPN-dependent insulin action impairment and consequently with the overall insulin resistance.

Supported by Portuguese Foundation for Science \& Technology, Portuguese Society of Endocrinology Diabetes \& Metabolism, Canadian Institutes of Health Research

\section{2}

Inverse relationship between triglycerides accumulation and glutathione production in the liver in nonobese and obese models of insulin resistance

O. Oliyarnyk, H. Malinska, M. Buresova, L. Kazdova;

Department of Metabolism and Diabetes, Institute for Clinical and Experimental Medicine, Prague, Czech Republic.

Background and Aims: Excessive hepatic accumulation of triglycerides (Tg) is one of metabolic disorders in insulin resistance syndrome, contributing to increased production and secretion of VLDL, glucose, C-reactive protein and fibrinogen. These factors aggravate the risk of diabetes and non-alcoholic fatty liver disease but knowledge about mechanisms are still missing. Some studies suggested that oxidative stress, obesity and advancing age might be involved. In this study we therefore investigated the relationship between $\mathrm{Tg}$ accumulation and glutathione (GSH) production in liver in an experimental model of insulin resistance - in old nonobese hereditary hypertriglyceridemic rats (HHTg) and in HHTg rats render obese by long term feeding with high sucrose diet (HHTg-OB). The liver is the main site of GSH synthesis from where GSH is transported to other tissues. GSH is not only the main intracellular antioxidant, but can affect prostaglandins synthesis, transmembrane transport of amino acids, gene expression and cytokine production.

Materials and Methods: The experiments were carried out in one year old male a) nonobese HHTg fed a standard laboratory chow and b) in HHTg rats with nutritional induced obesity by feeding with high carbohydrate diet (HS; $70 \%$ of sucrose) for 6 months (HHTg-OB). As a control group we used one year old Wistar rats (C). Liver Tg were measured by enzymatic assay (PlivaLachema, Brno, Czech Republic). GSH concentration and GSH-Px activity were determined spectrophotometrically by using Sigma assay kits.

Results: The body weight (HHTg: 380 $12 \mathrm{~g}$; HHTg-OB: $531 \pm 34 \mathrm{~g}$; C: $481 \pm$ 24g) and epididymal fat pad weight of HHTg rats fed the HS diet for six months were significantly higher that those of HHTg rats fed the standard diet (Table). Compared with controls, the HHTg rats exhibited elevated serum $\mathrm{Tg}$ concentration. The liver $\mathrm{Tg}$ content was higher in HHTg rats and was increased more than twofold in diet induced obesity.

Liver Tg accumulation was associated with declined GSH level. A negative linear correlation between liver Tg and GSH concentrations was observed ( $\mathrm{R}$ $=-0.77, p<0.001)$. We found reduced GSH-Px activity in the liver of obese and non-obese models of insulin resistance might be due to lower enzymes cofactor GSH concentration.

Conclucions: Our results suggest the association between liver Tag accumulation and decreased GSH production, which has an important function in antioxidant system, prostaglandin synthesis, and in the regulation of mitochondrial function and integrity. Elevated Tag accumulation in liver can contribute to decreased GSH transport to other tissues and so be involved in oxidative stress and its negative implications in insulin resistance and fatty acid liver disease.

\begin{tabular}{llll} 
& Wistar & Nonobese HHTg & Obese HHTg \\
\hline Serum Tg, mmol/l & $0.91 \pm 0.067$ & $1.89 \pm 0.27^{\mathrm{a}}$ & $4.62 \pm 0.27^{\mathrm{a}, \mathrm{b}}$ \\
Epididymal fat pad & $1.19 \pm 0.14$ & $1.26 \pm 0.09$ & $2.18 \pm 0.14^{\mathrm{a}, \mathrm{b}}$
\end{tabular}

weight, g/100g b.wt

Liver

$\begin{array}{llll}\mathrm{Tg}, \mu \mathrm{mol} / \mathrm{g} & 8.6 \pm 0.7 & 11.0 \pm 0.7^{\mathrm{a}} & 29.8 \pm 2.1^{\mathrm{a}, \mathrm{b}} \\ \text { GSH, mmol/g protein } & 20.2 \pm 1.7 & 16.2 \pm 0.7^{\mathrm{a}} & 10.1 \pm 1.4^{\mathrm{a}, \mathrm{b}} \\ \text { GSH-Px, } \mu \mathrm{mol} / \mathrm{GSH} / & 668 \pm 25 & 534 \pm 12^{\mathrm{a}} & 463 \pm 28^{\mathrm{a}, \mathrm{b}}\end{array}$

$\mathrm{min} / \mathrm{mg}$ protein

a) $\mathrm{p}<0.5$ Wistar vs HHTg, b) p $<0.5$ HHTg vs HHTg-OB

Supported by grant MZ 000023001: IGA MH CR NR-8495-3

\section{3}

Enhanced insulin action following subcutaneous co-administration of insulin and C-peptide

Y. Sato ${ }^{1}$, O. Khookhor ${ }^{1}$, M. Kubota ${ }^{2}$, M. Nagasaki ${ }^{1}$, T. Uno ${ }^{1}$, K. Ekberg ${ }^{3}$, J. Wahren ${ }^{3}$;

${ }^{1}$ Department of Health Science, Aichi Gakuin University, Nisshin, Japan, ${ }^{2}$ Department of Health Science, Kyoto University, Kyoto, Japan, ${ }^{3}$ Department of Molecular Medicine and Surgery, Karolinska Institutet, Stockholm, Sweden.

Background and Aims: Recent in vitro observations demonstrate that Cpeptide may interact with insulin, causing hexameric insulin to disaggregate into its biologically active monomeric form. Using the glucose clamp technique, we have evaluated the in vivo effect of C-peptide on whole body glucose utilization following s.c. co-administration of the two peptides in rats.

Material and Methods: Male Wistar rats (age 6 weeks, 145-165 g ) were randomly divided into eight different treatment groups ( $n=7$ per group ). Either regular insulin ( I ) ( $1.2 \mathrm{U} / \mathrm{kg}$ Humulin, Eli Lilly ) or an insulin analogue ( IA ) ( $1.2 \mathrm{U} / \mathrm{kg}$ Humalog, Eli Lilly ) were injected s.c. as a physical mixture or separately into two depots with either $29 \mathrm{nmol} / \mathrm{kg} \mathrm{C}$ - 
peptide ( C ) or its C-terminal pentapeptide ( P ) ( $29 \mathrm{nmol} / \mathrm{kg}$ ). The latter is known to exert effects similar to those of the full-length peptide. Rats were awake and allowed to move freely within a large cage throughout the study. During baseline ( $\sim 60 \mathrm{~min}$ ) the blood ambient glycemic level was established and subsequently clamped $\pm 0.3 \mathrm{mM}$ throughout the experiment by frequent glucose monitoring and administration of a variable i.v. glucose infusion ( GIR ).

Results: Injection of a mixture of C+I resulted in a 30\% greater amount of glucose being required $(p<0.01)$ and a glucose infusion of longer duration ( $+13 \%, p<0.05$ ) compared to when I and C were injected into separate depots. Likewise, after s.c. injection of a mixture of $\mathrm{P}+\mathrm{I}$, the amount of glucose infused was $54 \%$ greater $(p<0.01)$ and the duration of the glucose infusion was longer $(+27 \%, p<0.001)$ compared to the corresponding data after separate injections of $\mathrm{P}$ and I. Plasma concentrations of insulin were not significantly different after co-injection of $\mathrm{C}+\mathrm{I}$ or $\mathrm{P}+\mathrm{I}$ compared to subsequent separate injection of the peptides. Co-administration of either $\mathrm{C}+\mathrm{IA}$ or $\mathrm{P}+\mathrm{IA}$ into the same s.c. depot did not result in requirements of increased glucose infusion or prolonged infusion time compared to when the peptides were injected separately, possibly as a consequence of spontaneous disaggregation of the insulin analogue.

Conclusion: We conclude that co-administration of insulin plus C-peptide or its C-terminal pentapeptide causes enhanced insulin action, which may be attributed to augmented disaggregation of insulin hexamers into their active monomeric form.

\section{PS 51 Metabolism: children}

\section{4}

The decrease in insulin sensitivity is associated to the increase in blood pressure and post load glucose levels in prepubertal obese children C. Invitti, L. Gilardini, A. Girola, L. Pasqualinotto, M. Croci; Unit of Metabolic Diseases and Diabetes, Istituto Auxologico Italiano, Milan, Italy.

Background and Aims: It is unclear to what the decrease in insulin sensitivity (IS) is associated before puberty. We examined the associations between different indexes of IS and cardiovascular and metabolic risk factors in prepubertal obese children.

Materials and Methods: 173 obese children at Tanner I stage $(10 \pm 1.9 \mathrm{yr}$, BMI $30.4 \pm 4.8 \mathrm{~kg} / \mathrm{m}^{2}$, standard deviation score-BMI (SDS-BMI) $3.8 \pm 0.8$, waist circumference $91.8 \pm 10.8 \mathrm{~cm}$ ) underwent an OGTT and a bioelectrical impedance. Insulin sensitivity was estimated using HOMA-IR (homeostasis model assessment), QUICKI (quantitative insulin sensitivity check index), ISI Comp (composite whole body insulin sensitivity index) and OGIS (oral glucose insulin sensitivity index). Metabolic syndrome (MS) was defined using the National Cholesterol Education Program's Adult Treatment Panel III (ATPIII) criteria modified for children (using age and sex specific cut-off derived from a group of normal weight children to define abnormal waist, lipids and BP levels).

Results: $3 / 173$ had IGT, $1 / 173$ IFG and $7.3 \%$ the MS. In the logistic regression analysis, dyslipidemia, hyperglycaemia, hypertension, and central obesity explained $41.2 \%, 6 \%, 1.5 \%$ and $0.8 \%$ respectively of the MS. None of the indexes of IS was significantly correlated with waist and fat mass. Among the indexes of IS, HOMA-IR was that associated with the highest risk of having MS [OR for unit increase in HOMA-IR 1.36 (1.02-1.81), p <0.05; OR for unit increase in QUICKI $1.33(1.03-1.71), \mathrm{p}<0.05$; OR for unit increase in OGIS 1.01 (1.00-1.02), $\mathrm{p}<0.05$; OR for increase in ISI Comp NS]. The Receiver Operating Characteristic analysis evidenced that the best cut off in identifying MS was HOMA-IR $\geq 2.7$ (sensitivity $58.3 \%$ and specificity $68.6 \%$ ). The increase in HOMA-IR was associated to an increased risk of hypertension [OR 1.41 (CI 1.01-1.22), $\mathrm{p}<0.05$ ] and of increase in post load glycaemia [OR 1.35 (CI $1.01-1.62), \mathrm{p}<0.05]$ but not of dyslipidemia and high waist circumference. This differs from what was observed in a group of 724 pubertal children in whom the increase in HOMA-IR was significantly associated to the risk of having each of the components of MS [OR 2.2 (1.2-4.1), 1.2 (1.1-1.3), $1.1(1.0-1.2)$ and $1.1(1.0-1.2)$ for high waist, high triglycerides, low HDLcholesterol and high $B$ P respectively, $\mathrm{p}<0.0001$ for all].

Conclusion: In prepubertal obese children, the decrease in IS is associated to $\mathrm{BP}$ and post load glycaemia increase, but not to dyslipidemia and central distribution of fat, as observed after puberty.

\section{5}

Relationship between leptin level and insulin resistance in children and adolescents with type 1 diabetes

A. Szadkowska, K. Wyka, W. Młynarski, I. Pietrzak, B. Mianowska, J. Bodalski;

Department of Children Diseases, Institute of Pediatrics, Lodz, Poland.

Background and Aims: The aim of this study was to evaluate a relationship between leptin level and insulin resistance and components of metabolic syndrome in children and adolescents with type 1 diabetes.

Materials and Methods: 158 patients with type 1 diabetes mellitus (70 girls and 80 boys) aged from 8.2 to 18.4 years (mean \pm SD: $14.1 \pm 3.1$ years) were included into the study. The diabetes duration ranged from 1.6 to 14.7 years (3.8 \pm 2.5 years). The height, weight, waist circumference (WC), skin folds and blood pressure were measured. Body mass index (BMI-SDS), body fat (BF) according to Slaughter formula, and daily dose of insulin were calculated. HbA1c (HPLC) and serum lipids (enzymatic method) concentrations were examined. Serum leptin concentration was measured by ELISA method. Euglycemic-hyperinsulinemic clamp by de Fronzo was performed to estimate insulin resistance. Glucose disposal rate ( $M$ value) determined during the last 30 min of the test was calculated as a surrogate of insulin resistance.

Results: Serum leptin levels ranged from 1.47 to $57.39 \mathrm{ng} / \mathrm{ml}(8.25 \pm 8.27$ $\mathrm{ng} / \mathrm{ml}) . M$ values were between $2.10-15.19 \mathrm{mg} / \mathrm{kg} / \mathrm{min}(7.33 \pm 2.4 \mathrm{mg} / \mathrm{kg} /$ 
min). Leptin level positively correlated with BMI-SDS, WC, skin folds and $\mathrm{BF}$. Leptin level, normalized to BMI-SDS or BF, was significantly higher in females than males at Tanner stages IV $(p=0.04)$ and $V(p<0.001)$, but not at earlier stages of pubertal development. Moreover, a negative correlation between leptin level and $M$ value $(r=-0.26 ; p=0.001$ adjusted to sex and puberty) was found. However, after adjustment for BF or BMI-SDS this relationship became insignificant. No association between leptin level and HDL-cholesterol, SBP and DBP was also noted. Using the multivariate linear regression models, we found that plasma leptin level remained significantly associated with triglycerides.

Conclusion: It is likely that the observed relationship between leptin concentration and insulin resistance in young patients with type 1 diabetes is due to body fat composition rather than an independent association in this group. Supported by a grant of the Polish State Committee for Scientific Research

\section{6}

Analysis of adiponectin, leptin, TNF- $\alpha$ levels in children and adolescents with type 1 diabetes

O. Sysoyeva ${ }^{1}$, T. Mokhort ${ }^{2}$, N. Karlovich ${ }^{1}$;

${ }^{1}$ Endocrinology, State Centre of Medical Rehabilitation,

${ }^{2}$ Endocrinology, Belarusian State Medical University, Minsk, Belarus.

Background and Aims: Adiponectin and leptin are adipocytokines secreted by adipose tissue. Adiponectin has important insulin-sensitizing, antiinflammatory and cardioprotective properties. Adiponectin expression is regulated by TNF- $\alpha$, which is also involved in metabolism of adipose tissue and glucose homeostasis. Leptin plays a crucial role in the regulation of food intake and metabolism. Adipocytokines play an important role in obesity and type 2 dibetes, but little is known about their role in type 1 diabetes (T1D). The aim of our research was to assess adiponectin, leptin, TNF- $\alpha$ levels in T1D children and lean non-diabetic controls.

Material and Method: The study group included 56 patients. There were 34 patients with T1D and 22 lean non-diabetic persons (control group), age and sex matched. Serum levels of adiponectin, leptin and TNF- $\alpha$ in both groups were measured by ELISA.

Results: Leptin levels in T1D patients $(2.8 \mathrm{ng} / \mathrm{ml}[0.8 ; 8.1])$ were higher than in the control group $(1.8 \mathrm{ng} / \mathrm{ml}[0.3 ; 2.5]), \mathrm{p}=0.027$, while adiponectin levels were practically the same $(21.1 \mathrm{mcg} / \mathrm{ml}[14.9 ; 26.1]$ and $22.1[18.8 ; 26.0]$ respectively). Adiponectin to leptin ratio was lower in T1D patients (6.6 [3.1; $22.1])$ compared to lean non-diabetic controls (11.6 [10.0; 55.9]). Difference between T1D patients and controls was significant $(\mathrm{p}=0.032)$. In T1D patients significant correlation of adiponectin and weight $(\mathrm{r}=-0.35 ; \mathrm{p}=0.039)$ was found; adiponectin and adiponectin to leptin ratio negatively correlated with BMI $(\mathrm{r}=-0.37 ; \mathrm{p}=0.032, \mathrm{r}=-0.35 ; \mathrm{p}=0.049$ respectively). No significant difference in TNF- $\alpha$ levels between patients with T1D $(2.63 \mathrm{pg} / \mathrm{ml}[1.64 ; 4.22])$ and the control group $(2.98 \mathrm{pg} / \mathrm{ml}[1.84 ; 3.98])$ was observed. In the control group TNF- $\alpha$ correlated with the patients' age $(\mathrm{r}=-0.64 ; \mathrm{p}=0.001)$, as well as weight $(\mathrm{r}=-0.44 ; \mathrm{p}=0.039)$, while in T1D patients similar correlations were not found. Conclusion: There was no impairment of adiponectin and TNF- $\alpha$ secretion in T1D patients. The leptin level was significantly higher compared to controls, while the adiponectin to leptin ratio was lower. Further investigation is required to study the influence of leptin on sensitivity to exogenous insulin in patients with T1D. As age and weight increase, TNF- $\alpha$ level decreases in healthy patients, while in T1D patients no such correlation was revealed.

\section{7}

Opposing association of adiponectin and TNF-alpha versus IL-6 with clinical remission and glucose utilization in children with newly diagnosed type 1 diabetes mellitus

C. Pfleger ${ }^{1}$, H. B. Mortensen ${ }^{2}$, P. Hougaard ${ }^{3}$, L. Hansen ${ }^{4}$, H. Kolb ${ }^{1}$ N. C. Schloot ${ }^{1}$; the Hvidoere Study Group,

${ }^{1}$ Clinical Diabetes Research, German Diabetes Center, Düsseldorf, Germany, ${ }^{2}$ Department of Paediatrics, Glostrup University Hospital,, Glostrup, Denmark, ${ }^{3}$ Statistical Department, University of Southern Denmark, Odense, Denmark, ${ }^{4}$ Novo Nordisk A/S, Bagsværd, Denmark.

Background and Aims: Adiponectin, IL- 6 and TNF-alpha have been shown to modulate glucose utilisation in experimental models. The aim of the current study was to investigate whether circulating levels of adiponectin, IL-6 or TNF-alpha are associated with parameters of glucose metabolism in children with newly diagnosed type 1 diabetes (T1D) and during follow-up. Material and Methods: Serum was obtained from 256 newly diagnosed children with type 1 diabetes (122 males/ 134 females, aged 9.6 years, range $0.2-16.8$ years) one, six, and twelve months after diagnosis. Serum cytokines were measured by double sandwich ELISA and multiplex technology. Blood glucose was determined in the fasting state and 90 min after ingestion of a standard liquid mixed meal. We used a very strict definition of remission as $\mathrm{HbAlc}<6.5$ and insulin requirements $<0.5 \mathrm{U} / \mathrm{kg} /$ day. The difference of stimulated and fasting blood glucose levels reflecting glucose utilization was defined as delta blood glucose (dbg). For group comparison data were analysed by non parametric Wilcoxon test, for associations by multiple regression analyses adjusted for gender, age and BMI, adiponectin, IL-6, TNF-alpha.

Results: Adiponectin was positively associated with $\mathrm{dbg}$ at $\operatorname{six}(\mathrm{p}=0.03)$ months whereas IL-6 was negatively associated with $\mathrm{dbg}$ at $\operatorname{six}(\mathrm{p}=0.0389)$ and twelve $(\mathrm{p}=0.047)$ months. Adiponectin was the only cytokine showing associations (positive) with HbAlc at one $(p<0.0001)$, six $(p=0.007)$ and twelve ( $\mathrm{p}=0.002$ ) months. In support of these findings, children in clinical remission exhibited significantly different cytokine/adipokine levels than children not in remission. Adiponectin serum concentrations were lower at one $(\mathrm{p}=0.006)$, six $(\mathrm{p}=0.0005)$ and twelve $(\mathrm{p}=0.0004)$ months after diagnosis; IL- 6 concentrations were higher after twelve months $(\mathrm{p}=0.049)$, and TNFalpha concentrations were lower after one $(\mathrm{p}=0.012)$, six $(\mathrm{p}=0.016)$ and twelve ( $\mathrm{p}=0.013$ ) months.

Conclusion: IL- 6 and TNF-alpha concentrations show opposing associations with clinical remission, with IL-6 concentrations being positively associated with better metabolic control and negatively with dbg. Our findings of increased adiponectin in T1D non-remitters, which also might be an age effect, seem to be in contrast to reduced adiponectin concentrations observed in T2D. One explanation would be that due to overnutrition of cells in T2D cells secrete less adiponectin. This would be in contrast to T1D where cells in bad metabolic control have a deficiency in energy and secrete increased amounts of adiponectin to compensate for energy homeostasis. Therefore adiponectin and IL-6 could serve as marker for metabolic control, glucose utilization, and disease progression.

\section{8}

Inflammation, obesity and insulin resistance in adolescents

C. Herder ${ }^{1}$, S. Schneitler ${ }^{1}$, W. Rathmann ${ }^{2}$, B. Haastert ${ }^{2}$, H. Schneitler ${ }^{3}$, H. Winkler ${ }^{3}$, R. Bredahl ${ }^{3}$, E. Hahnloser ${ }^{3}$, S. Martin ${ }^{1}$;

${ }^{1}$ Institute for Clinical Diabetes Research,

${ }^{2}$ Institute of Biometrics and Epidemiology, German Diabetes Centre, ${ }^{3}$ Gesundheitsamt, Public Health Office, Duesseldorf, Germany.

Background and Aims: Subclinical inflammation is a characteristic feature of obesity, insulin resistance and type 2 diabetes in adults, but comparable data for youths are very limited. Therefore, it was the aim of this study to investigate the relationship between immune activation, indices of obesity and of insulin resistance in adolescents and to analyse whether the association between inflammation and insulin resistance is mainly explained by, or independent of, obesity.

Materials and Methods: In Düsseldorf (Germany), all school-leaving students from selected secondary schools are routinely invited to a medical check-up in their final year by the local Public Health Service. In 2005, we conducted a cross-sectional survey in 519 adolescents (293 boys, 226 girls) with a mean age of 15.5 years. We measured serum levels of cytokines, chemokines and adipokines that are related with obesity, insulin resistance and risk for type 2 diabetes in adults: interleukin (IL)-6, IL-18, tumour necrosis factor (TNF)- $\alpha$, monocyte chemoattractant protein (MCP)-1, IL-8, interferon- $\gamma$-inducible protein (IP)-10 and adiponectin. We investigated the association between these novel biomarkers and body mass index (BMI), waist circumference (WC), fasting glucose, fasting insulin and insulin resistance (HOMA-IR).

Results: In age and sex-adjusted linear regression analyses, IL-6, IL-18, IP10 and adiponectin (inversely) were significantly associated with both BMI and WC ( $\mathrm{p} \leq 0.001$ for all associations). None of the tested immune markers was related with fasting plasma glucose levels, but there were significant associations between IL-6, IL-18, IP-10 and adiponectin (inversely) with insulin and/or HOMA-IR. Most of these associations were considerably 
attenuated when BMI or WC were added to the model, whereas associations between TNF- $\alpha$ and MCP-1 with HOMA-IR appeared more pronounced. Adjustment for age, sex and BMI or age, sex and WC indicated that IL-6, TNF- $\alpha$ and MCP- 1 may be related with HOMA-IR ( $p$ between 0.03 and 0.08 ) independently of obesity.

Conclusion: We found a differential immune activation that is associated with parameters of obesity and insulin resistance in adolescents. Moreover, there is evidence that IL- 6 , TNF- $\alpha$ and MCP- 1 may be associated with insulin resistance independently of BMI or WC and may represent novel inflammatory risk factors for the development of type 2 diabetes in youths. It remains to be shown in prospective studies whether or not inflammation plays a similar role in the pathogenesis of type 2 diabetes in youths as in adults.

\section{PS 52 Inflammatory markers}

\section{9}

Changes in circulating levels of leptin and soluble leptin receptor during OGTT in overweight/obese postmenopausal women

E. Kassi, G. Hroussalas, I. Delimaris, M. Dalamaga, K. Kazanis, A. Zachari, A. Dionyssiou-Asteriou;

Dept of Biological Chemistry and Dept of Clinical Biochemistry,

University of Athens, Medical School, Greece.

Background and Aims: Adipose tissue plays a pivotal role in glucose homeostasis and insulin resistance by secreting adipokines. In overweight/ obese postmenopausal women with normal glucose metabolism (NGT) and with impaired glucose tolerance (IGT) we assessed serum leptin and the soluble leptin receptor (sOB-R) levels during OGTT in order to investigate their response to acute changes in glucose and insulin in the abnormal glucose metabolism, as it is detected early by IGT.

Materials and Methods: In total, thirthy overweight/obese postmenopausal women were included; fifteen with normal glucose metabolism (NGT), age 48-69 years, BMI 28-32, waist circumference $82-104 \mathrm{~cm}$ and fifteen with impaired glucose tolerance (IGT),age 53-69 years, BMI 29-40, waist circumference $87-115 \mathrm{~cm}$. OGTT with $75 \mathrm{gr}$ was performed in the morning. Leptin and sOB-R were evaluated by ELISA at time $0-60-120$ min of OGTT. Serum glucose and insulin were measured in an automated analyzer (Roche Diagnostics). Insulin resistance(HOMA)/sensitivity(QUICKI) indexes were also calculated.

Results: In fasting state: leptin and sOB-R did not differ between the two groups. In subjects with NGT leptin was positively correlated with BMI $(p=0.022)$, insulin $(p=0.021)$ and HOMA $(p=0.029)$ and negatively correlated with sOB-R $(p=0.04)$ and QUICKI ( $\mathrm{p}=0.05)$.sOB-R was negatively correlated with HOMA $(\mathrm{p}=0.032)$ and positively correlated with QUICKI $(\mathrm{P}=0.030)$. A negative correlation between insulin fasting levels and sOB-R in both NGT $(p=0.003)$ and IGT $(p=0.020)$ group was also observed. During OGTT: in both groups there was an overall significant increase in serum leptin between 0 and $120 \mathrm{~min}$, in subjects with normal OGTT from $44.4 \pm 41$ to $51.4 \pm 45 \mathrm{ng} /$ $\mathrm{ml},(p=0.007)$ and in subjects with impaired OGTT from $71.4 \pm 42$ to $87 \pm 49$ $\mathrm{ng} / \mathrm{ml},(p=0.001)$. There was no change in sOB-R levels. The difference in serum leptin levels between two groups at $120 \mathrm{~min}$ was significant $(p=0.042)$. Conclusion: The abnormal glucose metabolism as it is detected early by IGT is not accompanied by a disturbance in the basal secretion (baseline time in OGTT) of leptin and sOB-R. Leptin secretion is acutely regulated by insulin and glucose levels. Insulin seems to regulate leptin action via regulating the sOB-R levels. In the NGT group, there is an autoregulatory mechanism of leptin action through its receptor (sOB-R). The relationship between leptin and insulin or insulin resistance/sensitivity indexes in women with normal OGTT is not sustained in IGT women raising the hypothesis that a disruption of the normal communication between beta-cell and adipocyte has already occurred at this early stage of abnormal glucose metabolism.

Supported by the Special Research Accounts of Athens University

\section{0}

TNF-alpha induces a marked proinflammatory phenotype in undifferentiated human preadipocytes

P. Isakson, A. Hammarstedt, U. Smith;

Department of Molecular and Clinical Medicine, Center of Excellence for Cardiovascular and Metabolic Research, Göteborg, Sweden.

Background and Aims: The adipose tissue is important for storage and release of lipids. However, in recent years, it has been shown that the adipose tissue also is an endocrine and secretory organ. It releases a wide range of important adipokines like adiponectin, leptin and retinol-binding protein-4 (RBP-4) as well as cytokines such as interleukin-6 (IL6) and monocyte chemo-attractant protein-1 (MCP-1) that are related to the innate inflammatory response and inflammation. Furthermore, the adipokines crosstalk with other tissues of the body and modulate insulin sensitivity and action. The adipose tissue becomes inflamed during obesity with cells expressing markers of macrophages. It has also been described that the low-grade inflammation, with elevated levels of the acute phase-reactants CRP and serum Amyloid A, is associated with insulin resistance type 2 diabetes as well as risk of cardiovascular disease. We 
have examined if primary preadipocytes from the stromal vascular fraction of human adipose tissue are responsive to proinflammatory cytokines, in particular TNF-alpha, since this potent cytokine is released by infiltrating macrophages.

Materials and Methods: Abdominal subcutaneous adipose tissue was obtained from 11 different donors by needle biopsy. From the biopsy, the stromal vascular fraction was retrieved following collagenase digestion and removal of the primary adipocytes. The inflammatory cells were also removed by magnetic bead eluation of CD 14 and CD 45 positive cells. The remaining part of the fraction was seeded and grown in six-well plates. The cells were either left untreated or grown in the presence of TNF-alpha $(5 \mathrm{ng} / \mathrm{mL})$, IL-6 $(20 \mathrm{ng} / \mathrm{mL})$ or resistin $(50 \mathrm{ng} / \mathrm{mL})$ for 10 days. RNA was extracted and the gene expression was analyzed with real-time RT-PCR (Taqman). Protein secretion to the cell culture supernatant was analyzed with an enzyme-linked immunosorbent assay.

Results: The undifferentiated preadipocytes expressed various inflammatory cytokines as well as some macrophage related genes in the non-stimulated state. However, the secretion and gene expression of IL-6, IL-8 and MCP1 was markedly and significantly increased by the addition of TNF-alpha. Interestingly, CCR-2, the receptor for MCP-1, was expressed at extremely low levels in human preadipocytes indicating that these cells produce MCP-1, as well as other cytokines, but they are not major target cells for MCP-1. Moreover, TNF-alpha also induced the expression of several genes known to be highly expressed in macrophages such as CD68, TLR2, GMCSF, F4/80 and MIP-1a. Conclusion: Our data show that TNFa, which is released by macrophages, leads to a marked inflammatory phenotype in human undifferentiated preadipocytes. This is also associated with high secretion of MCP-1, IL-6 and IL-8. Furthermore, our data also show that TNFa induces macrophagespecific genes in these cells. Thus, cells expressing CD68, F4/80, MIP-1a in the adipose tissue may not be infiltrating macrophages but may emanate from activated preadipocytes.

Supported by Swedish Research Council, European Community's FP6 EUGENE), Swedish Diabetes Association, Novo Nordisk Foundation, Sonya Hedenbratt Memorial Fund, Martina and Wilhelm Lundgren Foundation

\section{1}

Evaluation of the D299G and T399I polymorphisms of toll-like receptor 4 on tumor necrosis factor (TNF)-alpha, TNF receptor 2 levels and insulin resistance

A. Jermendy ${ }^{1}$, A. Korner ${ }^{1}$, M. Kovacs ${ }^{2}$, E. Kaszas 33 , J. Balazsovics 3 , A. Szocs ${ }^{3}$, L. Madacsy' ${ }^{1}$, K. Cseh';

${ }^{1} 1$ st Department of Paediatrics, Semmelweis University,

${ }^{2} 3$ rd Department of Medicine, Semmelweis University,

${ }^{3} 1$ st Department of Medicine, Karolyi Sandor Hospital, Budapest, Hungary.

Background and Aims: The expression of Toll-Like Receptor 4 (TLR4) has recently been shown in adipose tissue. The hypomorphic mutations D299G and T399I of TLR4 may influence the cytokine secretion from adipocytes. The aim of our study was to compare Tumor Necrosis Factor (TNF)-alpha, soluble TNF Receptor 1 (sTNFR1), soluble TNF Receptor 2 (sTNFR2) levels in obese children, adults and control subjects. The assocation between allelic polymorphisms of TLR4 and cytokine levels as well as indirect parameters of insulin resistance was assessed.

Materials and Methods: The D299G and T399I allelic polymorphisms of TLR4 were determined with PCR-RFLP technique in 79 obese children (OCh group, age: $13.1 \pm 2.9$ years; $\mathrm{x} \pm \mathrm{SD}$; BMI: $31.1 \pm 5.4 \mathrm{~kg} / \mathrm{m} 2$ ), 171 obese adult patients (OAd group, age: $57.2 \pm 11.2$ years; BMI: $28.8 \pm 4.1 \mathrm{~kg} / \mathrm{m} 2$ ). As for controls, age and sex matched 169 non-obese adult subjects (NOAd group) and 42 non-obese children (NOCh group) were investigated. Serum TNF-alpha, sTNFR1, sTNFR2 levels, plasma insulin levels were measured by ELISA (Biosource).

Results: In OCh group, TNF-alpha $(5.5 \pm 0.9 \mathrm{pg} / \mathrm{ml})$ and sTNFR2 $(4.3 \pm 1.1$ $\mathrm{ng} / \mathrm{ml}$ ) levels were significantly $(\mathrm{p}<0.01)$ higher compared to those in NOCh group (TNF-alpha: $3.9 \pm 0.7 \mathrm{pg} / \mathrm{ml}$; sTNFR2: $0.5 \pm 0.3 \mathrm{ng} / \mathrm{ml}$, respectively). Cytokine levels in the OCh group differed significantly $(\mathrm{p}<0.01)$ from those in OAd group (TNF-alpha: $6.1 \pm 1.7 \mathrm{pg} / \mathrm{ml}$; sTNFR2: $6.7 \pm 1.9 \mathrm{ng} / \mathrm{ml}$ ). However no significant difference was found between the two groups regarding the ratio of sTNFR2/sTNFR1 (OCh group: 2.4 \pm 0.7 ; OAd group: 2.6 \pm 1.0 ). Significant $(\mathrm{p}<0.05)$ positive linear correlations were observed between TNF-alpha, sTNFR2 levels, BMI and insulin resistance measured by HOMAindex. Allelic frequencies of D299G and T399I of TLR4 tended to be lower in children as compared to control subjects (OCh group: 299G 3.79\%; 399I $3.79 \%$, versus NOAd group: 299G 5.0\%; 399I 4.5\%). In OAd group, the frequencies of the mutant alleles were $4.3 \%$ and $3.8 \%$, respectively. Analysing obese groups (OCh and OAd groups), patients carrying the mutant alleles of TLR4 (299G and 399I) had lower values of cytokines levels and HOMA indices when compared to those patients carrying wild type alleles (299D and 399T) (OCh group: TNF-alpha $4.4 \pm 0.7 \mathrm{pg} / \mathrm{ml}$ versus $5.5 \pm 0.9 \mathrm{pg} / \mathrm{ml}$; sTNFR2 $2.9 \pm 1.2 \mathrm{ng} / \mathrm{ml}$ versus $4.4 \pm 1.1 \mathrm{ng} / \mathrm{ml}$; HOMA-indices $1.1 \pm 0.9$ versus $2.6 \pm 2.3$; OAd group: TNF-alpha $5.0 \pm 1.2 \mathrm{pg} / \mathrm{ml}$ versus $6.2 \pm 1.7 \mathrm{pg} / \mathrm{ml}$; sTNFR2 $6.9 \pm 1.3$ $\mathrm{ng} / \mathrm{ml}$ versus $7.4 \pm 2.6 \mathrm{ng} / \mathrm{ml}$; HOMA-indices $3.5 \pm 1.3$ vs. $5.8 \pm 3.5$ ).

Conclusion: Elevated TNF-alpha and sTNFR2 levels may contribute to insulin resistance in obese children similarly to adults. In obesity, cytokine concentrations and insulin resistance might be influenced by TLR4 allelic polymorphisms both in children and adult subjects. Supported by OTKA TO46427KON and ETT 277/2003 grants

\section{2}

\section{Role of interleukin-6 in hepatic glucose production}

L. Immel, H. Runge, R. Lehmann, H.-U. Häring, E. Schleicher, C. Weigert; Department of Internal Medicine IV, University Hospital Tuebingen, Germany.

Background and Aims: Besides its function in inflammatory processes IL-6 is a potent player in metabolism. It is known to regulate insulin action and to stimulate lipolysis. The aim of our work is to elucidate the function of IL-6 in gluconeogenesis in hepatocytes.

Materials and Methods: All experiments were conducted in Fao rat hepatoma cells (Fao). Cells were starved overnight in a serum and glucose free medium containing $2 \mathrm{mM}$ lactate. On the following day cells were stimulated with various substances in a glucose- and serum-free medium containing 5 or 10 $\mathrm{mM}$ lactate. After indicated timepoints supernatant, protein and RNA was collected for subsequent analyses.

Results: Fao cells produced glucose when lactate as substrate was provided. Increasing insulin concentrations $(0.1-100 \mathrm{nM})$ reduced the glucose production gradually, while the rate of glucose production was increased by $67 \%$ and $40 \%$ under stimulation with dexamethasone and IL-6, respectively. Furthermore we found that IL- 6 counteracts the insulin action on gluconeogenesis. After the overnight starvation in glucose-free medium no glucose production derived from glycogenolysis was detectable. Studying the putative underlying mechanism, we observed that IL-6 did slightly reduce insulin-induced Foxo-1 phosphorylation with no inhibitory effect on phosphorylation of Ser-473 of Akt. However, IL-6 did not abolish the inhibitory action of insulin on the expression of the gluconeogenic enzymes PEPCK and Glucose-6-phosphatase.

Conclusion: IL-6 is able to induce glucose production in Fao cells and to counteract the inhibitory action of insulin. This effect is not mediated by altered expression of gluconeogenic enzymes.

Supported by DFG International Graduate School "The PI3K Pathway in Tumour Growth and Diabetes"

\section{3}

Dual role of interleukin-6 in regulating insulin sensitivity in murine skeletal muscle cells

M. Lorenzo, S. Fernandez-Veledo, C. De Alvaro, R. Vila, L. Garcia-Guerra, I. Nieto-Vazquez;

Bioquimica y Biologia Molecular II, Universidad Complutense, Madrid, Spain.

Background and Aims: Over the last decade it has been demonstrated that cytokines are elevated in various insulin-resistant states including type 2 diabetes and obesity. However, the role of the pro-inflammatory cytokine IL6 in the induction of these diseases is controversial.

Materials and Methods: In this study we analyzed the impact of IL- 6 on insulin action in murine skeletal muscle cell lines wild-type and PTP1Bdeficient, and primary myotubes, by studying glucose transport and insulin signaling after different exposure time to IL-6.

Results: A dual effect was observed when cells were exposed to this cytokine. IL-6 per se increases glucose uptake by activating the AMPK pathway, and 
whereas short-term IL-6 treatment had an additive effect on insulin-stimulated glucose uptake, chronic exposure produced insulin resistance. This effect was correlated with impaired GLUT4 translocation to the plasma membrane and defects in insulin signaling at the level of IRS- 1 and AKT. Because this cytokine activated stress kinases, we explored whether the sustained activation of this pathways could mediate insulin resistance. This study demonstrated that pretreatment with SP600125, inhibitor of JNK, restored insulin signaling and normalized insulin-induced glucose uptake in the presence of IL-6. Moreover, treatment of muscle cells with IL-6 significantly increased PTP1B activity. Accordingly, PTP1B-deficient myocytes were protected against insulin resistance by IL-6. Finally, we evaluated the potential role of nuclear receptor activation in alleviating insulin resistance. In this way, GW3965, a synthetic LXR agonist, restored insulin action by down-regulating PTP1B activation induced by IL-6.

Conclusion: These findings indicate the important role of IL- 6 in the pathogenesis of insulin resistance and further implicate PTP1B as a potential therapeutic target in the treatment of type 2 diabetes.

Supported by BFU2005-03054

\section{4}

Visceral obesity and plasma glucose-insulin homeostasis: contributions of IL- 6 and TNF-o

A. Cartier ${ }^{1}$, I. Lemieux ${ }^{1}$, N. Almeras ${ }^{1}$, A. Tremblay ${ }^{2}$, J. Bergeron ${ }^{3}$,

J.-P. Despres';

${ }^{1}$ Quebec Heart Institute, Hopital Laval Research Centre, ${ }^{2}$ Department of Social and Preventive Medicine, division of kinesiology, Universite Laval, ${ }^{3}$ Lipid Research Centre, CHUL Research Centre, Quebec, Canada.

Background and Aims: It is well known that abdominal obesity, especially excess visceral adiposity, is associated with metabolic alterations which include an inflammatory profile. It has been recently suggested that overexpression of proinflammatory molecules such as tumor necrosis factor (TNF)- $\alpha$ and interleukin (IL)-6 by adipose tissue in the context of visceral obesity could be due to macrophage infiltration. Many studies have also demonstrated that TNF- $\alpha$ and IL- 6 is strongly linked to the insulin resistance state of abdominal obesity. The aim of the present study was to quantify the associations of these inflammatory markers with specific adipose tissue depots assessed by computed tomography as well as with several total adiposity variables. We were therefore interested to test the potentially additive contribution of TNF$\alpha$ and IL- 6 to the variation in indices of plasma glucose-insulin homeostasis and their respective contributions to plasma glucose-insulin homeostasis beyond visceral adiposity.

Materials and Methods: Plasma levels of IL- 6 and TNF- $\alpha$ were measured in a sample of 189 untreated asymptomatic men covering a wide range of adiposity values (age: $43.7 \pm 7.8$ years; body mass index (BMI): $29.0 \pm 4.3 \mathrm{~kg} / \mathrm{m}^{2}$; waist girth: $98.6 \pm 10.3 \mathrm{~cm}$ ). Weight, height and waist girth were measured whereas cross-sectional areas of abdominal adipose tissue were assessed at L4-L5 by computed tomography. Fasting blood samples were collected to determine a complete lipoprotein-lipid profile and a $75 \mathrm{~g}$ oral glucose tolerance test was performed.

Results: Significant and positive associations were found between both cytokines, IL- 6 and TNF- $\alpha$, with total adiposity and adipose tissue distribution indices $(0.15 \leq \mathrm{r}<0.32 ; \mathrm{p}<0.05)$ as well as with plasma glucose-insulin homeostasis variables $(0.22 \leq \mathrm{r}<0.28 ; \mathrm{p}<0.05)$. Comparison of two subgroups of overweight men with similar BMI values $\left(\geq 25 \mathrm{~kg} / \mathrm{m}^{2}\right)$ but with markedly different levels of visceral adipose tissue $\left(<v s . \geq 130 \mathrm{~cm}^{2} ; \mathrm{n}=32\right)$ revealed significant differences only in IL-6 levels $(1.42 \pm 1.15 \mathrm{pg} / \mathrm{mL} v s .0 .86 \pm 0.52$; $\mathrm{p}<0.02$ for men with high $v s$. low visceral adipose tissue, respectively). Finally, when subjects were stratified on the basis of their respective concentrations of IL- 6 and TNF- $\alpha$ (using the $50^{\text {th }}$ percentile of the distribution of these cytokines), a $2 \mathrm{X} 2$ analysis of variance revealed an independent contribution of IL- 6 to the variation of fasting insulin $(\mathrm{p}<0.01)$ and of each of these two cytokines to the variation of post oral glucose load insulin levels $(\mathrm{p}<0.01$ for IL- 6 and $\mathrm{p}<0.05$ for TNF- $\alpha$ ).

Conclusion: These results support the notion that circulating IL-6 concentration is influenced to a greater extent by visceral than subcutaneous adiposity in contrast to circulating TNF- $\alpha$ concentration which appeared to be more influenced by the amount of subcutaneous fat. However, both cytokines contributed to the variation in plasma insulin levels beyond the known independent contribution of visceral adiposity.
0655

Changes of endocrine function of adipose tissue in anorexia nervosa: dissociation of circulating adipokine levels vs. subcutaneous mRNA expression

R. Dolezalova ${ }^{1}$, Z. Lacinova $^{1}$, M. Dolinkova ${ }^{1}$, P. Kleiblova ${ }^{2}$, D. Haluzikova ${ }^{1,3}$, M. Mraz ${ }^{1}$, D. Housa ${ }^{1}$, J. Housova ${ }^{1}$, H. Papezova ${ }^{4}$, M. Haluzik ${ }^{1}$;

${ }^{1} 3$ rd Department of Medicine,

${ }^{2}$ Institute of Biochemistry and Experimental Oncology,

${ }^{3}$ Department of Sports Medicine,

${ }^{4}$ Department of Psychiatry, 1st Faculty of Medicine, Prague, Czech Republic.

Background and Aims: Adipose tissue-derived hormones exert not only systemic endocrine effects but also local paracrine effects within the adipose tissue. A significant part of adipokines produced in adipose tissue may not be released to the systemic circulation and exert only local paracrine rather than endocrine effects. Therefore, serum concentrations of adipose tissuederived hormones can differ from its local concentrations in adipose tissue and their higher local levels can in turn affect adipose tissue metabolism without detectable changes of its circulating levels. The aim of this study was to evaluate the influence of chronic malnutrition in patients with anorexia nervosa on endocrine function of adipose tissue on both circulating and subcutaneous fat mRNA expression level.

Materials and Methods: 12 patients with anorexia nervosa (BMI $16.37 \pm$ $0.41 \mathrm{~kg} / \mathrm{m} 2$ ) and 18 normal weight age-matched women (BMI $22.96 \pm 0.67$ $\mathrm{kg} / \mathrm{m} 2$ ) underwent anthropometric examination, single blood drawing and subcutaneous adipose tissue biopsy. Serum concentrations of highsensitive CRP (hsCRP), leptin, soluble leptin receptor, adiponectin, resistin, interleukin- 6 and insulin were measured by Luminex, ELISA and RIA kits. Subcutaneous adipose tissue mRNA expression of the same adipokines, adiponectin receptors 1 and 2 and immunocompetent cells marker CD68 was measured by real time PCR.

Results: Decreased body fat content of patients with anorexia nervosa was accompanied by reduced hsCRP and leptin ( $1.01 \pm 0.60$ vs. $2.86 \pm 0.65 \mathrm{mg} / \mathrm{l}$, $\mathrm{p}<0.001 ; 1.94 \pm 0.57$ vs. $12.1 \pm 1.77 \mathrm{ng} / \mathrm{ml}, \mathrm{p}<0.05)$ and increased adiponectin, soluble leptin receptor, MCP- 1 and TNF- $\alpha$ levels. $(38.23 \pm 5.32$ vs. $24.94 \pm 2.92$ $\mu \mathrm{g} / \mathrm{ml}, \mathrm{p}<0.05 ; 39.32 \pm 2.63$ vs. $29.74 \pm 2.0 \mathrm{IU} / \mathrm{ml}, \mathrm{p}<0.05 ; 237.83 \pm 27.02$ vs. $158.26 \pm 23.26 \mathrm{pg} / \mathrm{ml}, \mathrm{p}<0.05 ; 4.22 \pm 0.4$ vs. $3.12 \pm 0.28 \mathrm{pg} / \mathrm{ml}, \mathrm{p}<0.05)$. Resistin, interleukin- 6 and insulin levels did not differ from the control group. Fat mRNA adiponectin, leptin, interleukin-6, MCP-1 and CD68 expression was reduced $(0.161 \pm 0.0344$ vs. $0.405 \pm 0.0484, \mathrm{p}<0.05 ; 8.21 \times 10-3 \pm 2.97 .10-3$ vs. $52.2 \times 10-3 \pm 8.21 \times 10-3, \mathrm{p}<0.05 ; 0.91 \times 10-5 \pm 0.419 \times 10-5$ vs. $5.38 \times 10-$ $5 \pm 2.3 \times 10-5, \mathrm{p}<0.05 ; 9.15 \times 10-4 \pm 2.93 \times 10-4$ vs. $34.4 \times 10-4 \pm 4.3 \times 10-4, \mathrm{p}<0.05$; $2.41 \times 10-2 \pm 0.266 \times 10-2$ vs. $8.56 \times 10-2 \pm 1.4 \times 10-2, \mathrm{p}<0.05)$, resistin mRNA expression was increased $(9.22 \times 10-4 \pm 2.4 \times 10-4$ vs. $1.83 \times 10-4 \pm 0.581 \times 10-4$, $\mathrm{p}<0.05)$ and adiponectin receptor 1 and 2 expression were unchanged as compared to the control group. Gene expression data are presented as relative values normalized to beta-2 microglobulin.

Conclusion: Local perturbations in resistin, adiponectin, MCP-1 and interleukin-6 mRNA expression in subcutaneous adipose tissue are not reflected by its circulating levels. These changes could be involved in some local metabolic disturbances in subcutaneous adipose tissue of anorexia nervosa patients.

Supported by grant IGA 8302-5 and MZO 000064165

\section{6}

LPIN1 expression in HIV-associated lipodystrophy syndrome is associated with insulin sensitivity

M. Miranda ${ }^{1}$, M. R. Chacón ${ }^{1}$, L. Gallart ${ }^{1}$, F. Vidal ${ }^{2}$, J. Vendrell';

${ }^{1}$ Research Unit,

${ }^{2}$ Internal Medicine, University Hospital Joan XXIII, Tarragona, Spain.

Background and Aims: HIV-associated lipodystrophic syndrome (HALS) is associated with morbidities normally found in obesity. It is characterized by metabolic abnormalities like hyperlipidemia and insulin resistance. LPIN1 gene was described as the mutated gene in the mouse model for fatty liver dystrophy (fld) with features of human lipodystrophy. The relation of LPIN1 with glucose homeostasis has been analysed in transgenic mice and in humans. Studies in humans suggest a positive association with insulin sensitivity and negative with body mass index (BMI).The aim of the study was to analyse LPIN1 adipose tissue gene expression levels in HIV-associated 
lipodystrophy and to study its relationship with insulin sensitivity, and adipogenic and inflammatory markers.

Materials and Methods: $37 \mathrm{HIV}$-positive patients (including 24 who developed lipodystrophy) and 21 HIV-negative subjects. Real Time PCR was used to evaluate LPIN1, and several inflammatory and adipogenic markers, gene expression. Frequently sampled intravenous glucose tolerance test (FSIGTT) was used to measure insulin sensitivity $\left(\mathrm{S}_{\mathrm{i}}\right)$.

Results: HIV-positive groups had received highly active antiretroviral therapy (HAART) for $51.8 \pm 28.7$ months and duration of antiretroviral combination therapy was comparable in HALS-negative and HALS-positive groups. Time of evolution to HALS was $24.41 \pm 15.09$ months. All patients in both HALSnegative and HALS-positive groups were currently receiving nucleoside reverse transcriptase inhibitors (NRTI). There were no significant differences between HALS groups in the frequency of receiving non-nucleoside reverse transcriptase inhibitors (NNRTI) and protease inhibitors (PI). There were no significant differences in the length of treatment of each specific agent between groups. LPIN1 mRNA levels were significantly higher (relative units in HIVnegative: $1.36 \pm 0.64$, HIV-positive without lipodystrophy: $4.24 \pm 2.68$ and with lipodystrophy: $3.82 \pm 2.40 ; \mathrm{p}<0.001)$ in HIV-positive patients compared to controls, but there were no differences between both HIV-positive groups. PPAR $\gamma$ mRNA tended to be lower in the HIV-positive patients; this tendency was more evident in HALS-positive patients, compared to HIV-negative subjects. HIV-positive subjects had significantly higher $I L-6$ mRNA and increased levels of TNF $\alpha$ compared to HIV-negative controls. HIV-positive patients who received PI showed a trend to have lower insulin sensitivity and expressed significantly lower levels of LPIN1 mRNA ( $\mathrm{p}=0.047)$. LPIN1 expression levels were positively correlated with $\mathrm{S}_{\mathrm{i}}(\mathrm{r}=0.496, \mathrm{p}=0.014)$ and $P P A R \gamma$ mRNA expression $(\mathrm{r}=0.636, \mathrm{p}<0.001)$; and negatively with $\mathrm{BMI}(\mathrm{r}=-$ $0.371, \mathrm{p}=0.037)$, and cytokine mRNA expression (IL-6: $\mathrm{r}=-0.458, \mathrm{p}=0.006$; $T N F \alpha: \mathrm{r}=-0.508, \mathrm{p}=0.002)$. In multiple regression analysis, both $P P A R \gamma$ $(\beta=0.527, \mathrm{p}=0.006)$ and TNF $\alpha$ mRNA expression $(\beta=-0.467, \mathrm{p}=0.011)$ were determinants of LPIN1 mRNA levels, independently of gender and presence of lipodystrophy.

Conclusion: LPIN1 mRNA expression levels were significantly higher in HIV-infected patients and positively correlated with insulin sensitivity. Moreover, pro-inflammatory cytokines such TNF $\alpha$ and IL- 6 were found strongly negatively determinants of LPIN1 expression. We suggest a possible role of LPIN1 as a modulator of insulin-resistance induced by PI treatments. Supported by grants FIS 04/0377, FIS 05/1994, FIPSE 36610/06, REDIMET RD06/0015/0011

\section{7}

The role of osteoprotegerin in type 2 diabetes mellitus

E. P. O'Sullivan ${ }^{1}$, D. Ashley ${ }^{2}$, N. Devlin' ${ }^{2}$, R. Crowley ${ }^{1}$, A. Agha ${ }^{1}$,

C. J. Thompson ${ }^{1}$, D. O'Gorman ${ }^{2}$, D. Smith ${ }^{1}$;

${ }^{1}$ Diabetes and Endocrinology, Beaumont Hospital,

${ }^{2}$ Metabolic Research Unit, School of Health and Human Performance,

Dublin City University, Ireland.

Background and Aims: Osteoprotegerin (OPG) is a glycoprotein which acts as an anti-resorptive agent in bone. It does so by inhibiting the binding of Receptor Activator of Nuclear factor Kappa beta ligand (RANKL) to its constitutive receptor Receptor Activator of Nuclear factor Kappa beta (RANK). This system also appears to exert a role in the vasculature, as illustrated by the fact that mice who are deficient in OPG exhibit excessive vascular calcification as well as severe osteoporosis. Additionally, increased levels of OPG have been found in patients with cardiovascular disease. The purpose of this study was to examine further the possible role of OPG in patients with diabetes - a condition characterised by exaggerated vascular calcification.

Materials and Methods: OPG levels were compared between a group of patients with type 2 diabetes $(\mathrm{n}=38)$ and an age, gender and BMI matched control group $(\mathrm{n}=37)$. Subjects $(23$ male) had a median age of 63 years (Interquartile range 54.3-66.3) and a mean ( \pm standard deviation) BMI of $30.2+3.7 \mathrm{~kg} / \mathrm{m}^{2}$. Median duration of diabetes was 5.4 (IQR 2-8) years, mean HbAlc was $7.1 \pm 0.8 \%$, and 7 patients had microvascular complications. Controls (22 male) had a median age of 55 years (IQR 50-65) and a mean BMI of $29.2 \pm 2.8 \mathrm{~kg} / \mathrm{m}^{2}$. OPG was measured using a commercial ELISA assay. Statistical analysis was performed with JMP IN 5.1.

Results: Serum OPG was significantly elevated in the patients with type 2 diabetes ( 6.03 vs. $5.15 \mathrm{pmol} / \mathrm{l} ; \mathrm{p}=0.03$ ). OPG was higher in women than men $(\mathrm{p}=0.02)$, and was highest in postmenopausal women $(\mathrm{p}=0.02)$. OPG correlated positively with age $(\mathrm{r}=0.5 ; \mathrm{p}<0.0001)$. There were no other correlations between OPG and anthropological measurements.

Conclusion: Our results demonstrate that diabetes is associated with higher levels of OPG. This may be an important mediator of arterial calcification and cardiovascular disease in these patients, especially in post-menopausal women. Further research is necessary to elucidate the exact role of OPG in type 2 diabetes. 


\section{PS 53 Obesity therapy}

\section{8}

Pharmacological blockade of the ACC system prevents obesity but only marginally improves insulin sensitivity in mice fed a high fat diet

M. Schreurs, M. H. Oosterveer, T. H. van Dijk, R. Havinga, D.-J. Reijngoud, F. Kuipers;

Research lab Pediatrics, UMCG, Groningen, The Netherlands.

Background and Aims: The acetyl-CoA carboxylases (ACC) are enzymes that are very important in both lipid and glucose metabolism. This enzyme system with two isoforms, ACC1 and ACC2, catalyses the conversion of acetyl-CoA into malonyl-CoA. Malonyl-CoA is used for synthesis of fatty acids (ACC1) but is also an allosteric inhibitor of mitochondrial $\beta$-oxidation (ACC2). Pharmaceutical agents that inhibit ACC have been developed to assess their potential benefit in the prevention and treatment of obesity and insulin resistance. The aim of this study was to assess whether CP-640186 (CP), a general ACC inhibitor, prevents obesity and/ or insulin resistance in mice fed a high fat (HF) diet.

Materials and Methods: Male C57Bl6/J mice were either fed a control (chow) diet, a high fat ( $60 \%$ energy) diet or a high fat diet containing CP $(50 \mathrm{mg} / \mathrm{kg} /$ d) for 6 weeks. The fourth group of mice received the HF diet for 4 weeks and the HF diet including the CP-compound for 2 weeks (intervention group). Mice were provided with a permanent jugular vein catheter ( 5 days before the end of the dietary period) for measuring basal glucose production ( $2 \mathrm{hrs})$ and glucose metabolism during a hyperinsulinemic euglycemic clamp ( $4 \mathrm{hrs)}$ at the end of the dietary period.

Results: Mice given the ACC inhibitor for 6 weeks showed a remarkable deficit in body weight gain from 32 days of feeding onwards compared to mice fed the diet. Such an effect was not seen for the intervention group: this group showed a similar weight gain as the HF-fed animals did. In liver, triglycerides and total cholesterol levels were not different between the HF and CP-fed animals, but significantly higher compared to the control mice. Plasma values of triglycerides in the four groups of mice did not differ. However, plasma cholesterol levels were significantly elevated in all HF-fed groups. Hepatic expression of genes involved in lipid metabolism was not affected in the intervention group compared to controls, while they were induced in the HF and CP-fed animals. Expression levels of genes involved in glycolysis were back at control levels in the intervention mice; in the other HF-fed animals no reduction was observed. During clamping, glucose infusion rate (GIR) was significantly lower in both HF, CP-fed and intervention animals than in controls, indicating impaired insulin sensitivity in the first three groups. However, in the CP-group the GIR was slightly but significantly (10\%) higher than in HF-fed mice. Glucose disposal was lower in the three HF-fed groups compared to control mice, indicating that these mice have developed insulin resistance. CP-fed mice have a tendency towards improvement of the disposal rate, but were not back at control levels.

Conclusion: Treatment with CP-640186 prevents development of obesity by as yet unidentified mechanisms and is associated with marginal improvements of insulin sensitivity in HF-fed mice.

Supported by the Dutch Diabetes Foundation

\section{9}

Effects of 11ß-hydroxysteroid dehydrogenase inhibition on body weight and glucose homeostasis in mice with diet-induced obesity

V. A. Gault, A. I. Taylor, N. Irwin, A. M. McKillop, P. R. Flatt;

School of Biomedical Sciences, University of Ulster, Coleraine, United Kingdom.

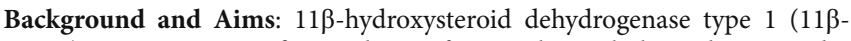
HSD1) is a tissue specific regulator of cortisol metabolism that is under consideration as a potential drug target for type 2 diabetes and obesity. The present study examines the metabolic effects of daily administration of carbenoxolone (11 $\beta$-HSD inhibitor) in mice with high-fat diet-induced insulin resistance and obesity.

Materials and Methods: Young (8 week-old) male Swiss TO mice were divided into two groups $(\mathrm{n}=8)$ and placed on a synthetic high-fat diet $(45 \%$ fat, $20 \%$ protein and $35 \%$ carbohydrate; $26.15 \mathrm{~kJ} / \mathrm{g}$ ) for 150 days until obesity and diabetes were clearly manifest as judged by body weight, plasma glucose and $\mathrm{HbA}_{1 \mathrm{C}}$ analyses. Age-matched control mice $(\mathrm{n}=8)$ with free access to standard rodent maintenance diet $(12.99 \mathrm{kj} / \mathrm{g}$ ) were used as untreated diet controls. High-fat fed mice received once daily intraperitoneal injections $(17: 00 \mathrm{~h})$ of either saline vehicle $(0.9 \%, \mathrm{w} / \mathrm{v}, \mathrm{NaCl})$ or carbenoxolone $(10 \mathrm{mg} /$ $\mathrm{kg}$ body weight) over a 16-day period. Food intake and body weight were recorded daily whilst plasma glucose concentrations were monitored at 2-3 day intervals. At the end of the treatment period, glucose tolerance $(18 \mathrm{mmol} /$ $\mathrm{kg}$ body weight glucose i.p.), insulin sensitivity (50 U insulin/kg body weight) and plasma corticosterone concentrations were assessed. Additionally, sections of omental adipose, gastrocnimus and liver were excised and snap frozen for measurement of gene expression.

Results: Daily administration of carbenoxolone had no effect $(p>0.05)$ on food intake. From Day 12 onwards, high-fat fed mice receiving carbenoxolone had significantly lowered (1.1-fold; $\mathrm{p}<0.05)$ body weights compared to saline-treated high-fat fed mice. However, body weights of high-fat fed mice receiving carbenoxolone were still significantly greater $(1.4$-fold; $\mathrm{p}<0.001)$ than mice on a standard rodent diet. Non-fasting plasma glucose levels were significantly decreased $(1.2$-fold; $\mathrm{p}<0.05)$ in mice receiving carbenoxolone treatment from Day 4 and remained significantly lower $(p<0.05$ to $p<0.01)$ throughout the treatment period. Plasma glucose concentrations were significantly reduced $(1.4$-fold; $\mathrm{p}<0.01)$ following an i.p. glucose load in highfat treated mice compared with saline-treated controls. Similarly, exogenous insulin injection following a 16-day treatment with carbenoxolone evoked a significant reduction in glucose concentrations ( 1.4 -fold to 1.8 -fold; $\mathrm{p}<0.05$ to $\mathrm{p}<0.001$ ) in terms of AUC measures and post-injection values. No significant effect $(p>0.05)$ on circulating plasma corticosterone concentrations was observed following treatment with carbenoxolone. Drug treatment also resulted in decreased expression of the glucocorticoid receptor in omental tissue, but not in gastrocnimus or liver

Conclusion: Once daily administration of the 11ß-HSD inhibitor carbenoxolone for 16 days resulted in improved body weight and metabolic control in mice with high-fat diet-induced obesity. These data indicate the potential value of selective $11 \beta-\mathrm{HSD} 1$ inhibition as a new target for the treatment of type 2 diabetes and obesity

\section{0}

Rosiglitazone as a therapeutic agent to reduce inflammatory risk in type 2 diabetic patients

A. L. Harte ${ }^{1}$, N. M. Al-Daghri ${ }^{2}$, K. Al-Rubeaan ${ }^{3}$, O. Al-Attas ${ }^{2}$, N. F. da Silva ${ }^{1}$, S. L. Sabico' ${ }^{2}$, S. Kumar' ${ }^{1}$, P. G. McTernan ${ }^{1}$;

${ }^{1}$ Unit for Diabetes and Metabolism, University of Warwick, United Kingdom, ${ }^{2}$ Biochemstry Department, King Saud University, Riyadh, Saudi Arabia, ${ }^{3}$ Diabetes Center, King Abdul-Aziz University Hospital, Riyadh, Saudi Arabia.

Background and Aims: Chronic low-grade inflammation is a significant factor in the development of obesity associated diabetes. This is supported by recent studies suggesting endotoxin, derived from gut flora, may be key to the development of inflammation by stimulating the secretion of an adverse cytokine profile from fat. The study investigated the relationship of endotoxin on various metabolic parameters of diabetic patients and aimed to determine if different anti-diabetic therapies exerted a significant effect on endotoxin levels and the adipocytokine profiles of subjects with Type 2 Diabetes Mellitus (T2DM).

Materials and Methods: Fasting blood samples were collected from consenting Saudi Arabian patients (BMI: $30.2 \pm 5.6 \mathrm{Kg} / \mathrm{m}^{2}, \mathrm{n}=413$ ), consisting of non-diabetics (controls, $n=67$ ) and T2DM subjects $(n=346)$. The diabetics were divided into 5 subgroups based on their 1 year treatment regimes, which included diet-control $(n=36)$, insulin $(n=47)$, metformin ( $\mathrm{n}=141$ ), rosiglitazone (RSG, $\mathrm{n}=22$ ) or a combined fixed dose of metformin/ rosiglitazone (Avandamet $\mathrm{n}=100$ ). Lipid profiles, fasting plasma glucose, insulin, adiponectin, resistin, TNF- $\alpha$, leptin, C-reactive protein (CRP) and endotoxin concentrations were determined.

Results: Regression analyses revealed significant correlations between endotoxin levels and triglycerides $\left(\mathrm{r}^{2}=0.40 ; \mathrm{p}<0.0001\right)$; total cholesterol $\left(\mathrm{r}^{2}=0.10 ; \mathrm{p}<0.0001\right)$, glucose $\left(\mathrm{r}^{2}=0.14 ; \mathrm{p}<0.0001\right)$ and insulin $\left(\mathrm{r}^{2} 0.12 ; \mathrm{p}=0.01\right)$. Endotoxin showed a strong inverse correlation with HDL-cholesterol $\left(r^{2}=0.10 ; p<0.0001\right)$. In addition, endotoxin levels were elevated in all of the treated diabetic subgroups compared to control, but only the RSG group did not differ significantly from control (Control:4.2 \pm 1.7 , RSG:5.6 $\pm 2.2 \mathrm{EU} /$ $\mathrm{mL}$ ). Both the Avandamet and RSG treated groups had significantly higher 
adiponectin levels than all the other groups, with the RSG group expressing the highest levels overall.

Conclusion: We conclude that sub-clinical inflammation in T2DM may be mediated by endotoxin in serum. Furthermore, that whilst the endotoxin and adipocytokine profiles of diabetic patients treated with different therapies were comparable, the RSG group demonstrated significant differences in both adiponectin and endotoxin levels. We confirm an association between endotoxin and serum insulin and triglycerides and an inverse relationship with HDL. Lower endotoxin and higher adiponectin levels in the RSG treated group may be related and indicate another potential mechanism through which RSG may be mediating its effects on insulin sensitivity.

\section{1}

Efficacy of a novel CB antagonist, GRC 10801, in improving glucose tolerance in combination with known antidiabetic drugs

R. Anupindi, B. Mahalingam, C. Misra, M. Natarajan, S. Narayanan;

Biological research, Glenmark Pharmaceuticals Ltd., Navi Mumbai, India.

Background and Aims: Endocannabinoids stimulate food intake in animals and humans through their action on CB-1 receptors located both centrally and peripherally. Thus, CB-1 receptor antagonists have been shown to be useful in the treatment of obesity. Hyperglycemia and insulin resistance are frequently associated with human obesity. Administration of selective CB1 antagonists, such as rimonabant, ameliorates obesity related glucose intolerance and insulin resistance and dyslipidemia. The present study describes the potential of a novel orally active CB-1 antagonist GRC 10801 in combination with known antidiabetic compounds metformin and rosiglitazone in DIO mice, a model widely used for the screening of antidiabetic and antiobesity compounds.

Materials and Methods: The ability of GRC $10801(1 \mathrm{mg} / \mathrm{kg})$ in reducing body weight, food intake and improving glycemic profile was evaluated in DIO mice alone and in combination with metformin $(300 \mathrm{mg} / \mathrm{kg})$ and rosiglitazone $(5 \mathrm{mg} / \mathrm{kg})$.

Results: GRC 10801 showed an excellent in vitro profile and a favorable pharmacokinetic profile in rats and in vivo efficacy models. In DIO model in C57BL/6J mice, GRC 10801 produced a significant reduction $(p<0.05)$ in body weight, alone (11.1\%) and in combination with metformin $(16.2 \%)$ and rosiglitazone (10.4\%), at the end of 14-day treatment. Greater improvement in glycemic control ( $>15 \%$ reduction in fed-state whole blood glucose) was seen in the animals that received a combination of therapies than either treatment alone. In a pair-fed study in DIO rats, GRC 10801 showed a significant reduction in body weight and food intake.

Conclusion: GRC 10801, a novel, highly selective, orally active CB-1 antagonist that has the potential in the treatment of obesity, may be of use in diabetes by inducing weight loss which may contribute to the improvement of glucose homeostasis.

\section{2}

Rimonabant prevents fat accumulation during a hypercaloric high-fat diet

O. O. Woolcott, J. M. Richey, L. N. Harrison, D. Stefanovski, D. Zheng, M. Lottati, S. P. Kim, K. J. Catalano, J. D. Chiu, I. R. Hsu, V. Ionut, M. Kabir, R. N. Bergman;

Physiology \& Biophysics, Keck School of Medicine, University of Southern California, Los Angeles, United States.

Background and Aims: Obesity is a known risk factor for diabetes. The accumulation of fat tissue, predominantly in the abdominal region, is correlated with insulin resistance. Rimonabant (RIM) is a new drug for the treatment of obesity. We evaluated the body distribution during RIM treatment in dogs fed a high-fat diet.

Materials and Methods: Nine dogs were fed a hypercaloric high-fat diet (HHFD) consisting of $\sim 5400 \mathrm{kcal} / \mathrm{day}$ ( $53 \% \mathrm{kcal}$ from fat), starting at week -6. After 6 weeks of HHFD (baseline: week 0), dogs were randomly divided in two groups: 5 dogs received RIM $1.25 \mathrm{mg} / \mathrm{kg} / \mathrm{day}$, P.O., and 4 dogs, placebo (PBO) during 16 weeks. HHFD was maintained until the end of the study. Magnetic resonance imaging was performed in the trunk and eleven slices 1$\mathrm{cm}$ thick were analyzed at weeks $-6,0,2,6,12$ and 16 . RIM and PBO groups had similar baseline body weights $(28.4 \pm 2.4$ and $30.1 \pm 2.9 \mathrm{~kg}(\mathrm{P}=0.67)$; respectively, mean \pm SEM) and abdominal fat distribution. Total fat tissue was $989.3 \pm 94.1$ and $991.0 \pm 256 \mathrm{~g}(\mathrm{P}=0.99)$, subcutaneous adipose tissue (SAT) $432.9 \pm 73.1$ and $474.0 \pm 124 \mathrm{~g}(\mathrm{P}=0.79)$, and VAT 556.4 \pm 35.8 and $518.0 \pm$ $139 \mathrm{~g}(\mathrm{P}=0.80)$, in RIM and $\mathrm{PBO}$ groups, respectively.

Results: After treatment, body weight was not significantly increased in the RIM group $(\mathrm{P}=0.22)$ compared with $11.2 \%$ in the $\mathrm{PBO}$ group $(\mathrm{P}=$ 0.07). Total tissue (total fat tissue plus non-fat tissue) estimated from the trunk was well correlated with body weight $\left(r^{2}=91.27 \%\right.$; $\left.<0.0001\right)$. In the RIM group, the increase in total tissue was accompanied by non-fat tissue accumulation $(2190.0 \pm 239 \mathrm{~g}$ at baseline vs. $2385.3 \pm 214 \mathrm{~g}$ at week $16 ; \mathrm{P}=$ $0.025)$. Conversely, in the PBO group there was no change in non-fat tissue $(2231.1 \pm 98.3 \mathrm{~g}$ at baseline vs. $2317.8 \pm 93.6 \mathrm{~g}$ at week $16 ; \mathrm{P}=0.11)$. There was a significant increase on VAT with PBO by $36.2 \%(\mathrm{P}=0.028)$ at week 16 . In contrast, VAT decreased by $5.8 \%$ with RIM $(\mathrm{P}=0.71)$. Surprisingly, RIM reduced the percentages of total fat from $31.8 \%$ to $29.5 \%(\mathrm{P}=0.017)$ and VAT from $18.2 \%$ to $16.7 \%(\mathrm{P}=0.034)$ during the first 2 weeks, and the percentage of SAT from $13.6 \%$ to $11.2 \%(\mathrm{P}=0.021)$ during the first 12 weeks. There was no relationship between food intake and weight gain.

Conclusion: RIM prevented fat accumulation during HHFD and consistently decreased the percentage of SAT and, to a lesser extent, VAT. These changes were independent of food intake changes. These results suggest that RIM might have an important regulatory effect on fat tissue by modifying energy expenditure.

Supported by sanofi-aventis

\section{3}

Cannabinoid receptor antagonist rimonabant increases insulin sensitivity independent of weight loss

J. M. Richey, O. Woolcott, D. Zheng, S. Kim, D. Stefanovski, V. Ionut, K. Catalano, J. Chiu, I. Hsu, L. Harrison, M. Lottati, M. Kabir, E. Zuniga, E. Kirkman, R. N. Bergman;

Physiology \& Biophysics, Keck School of Medicine of USC, Los Angeles, United States.

Background and Aim: Rimonabant (RIM), a canabinoid receptor CB1 antagonist, has been shown to have beneficial effects on glucose metabolism via promotion of weight loss and central adiposity reduction. However, recent data from clinical trials suggests that RIM's positive metabolic effects may not be due to weight loss alone. The aim of our study was to assess the effects of chronic RIM treatment on insulin sensitivity (SI) and adiposity in an animal model of obesity-induced insulin resistance.

Methods: Experiments were performed in male mongrel dogs $(n=9)$ fed a high fat hypercaloric diet ( $5400 \mathrm{kcal} / \mathrm{d}, 53 \% \mathrm{kcal}$ from fat) for 6 weeks and subsequently treated orally with $1.25 \mathrm{mg} / \mathrm{kg}$ RIM $(\mathrm{n}=5)$ or placebo $(\mathrm{n}=4)$ for an additional 16 weeks, while being maintained on the high fat diet. Euglycemic clamps and MRIs were performed to assess SI and body adiposity, respectively, during pre-fat (week -6), post-fat (week 0) and 16 weeks (week 16) of RIM.

Results: The initial 6 weeks of fat feeding increased body weight $(+2.5 \pm 0.3$ $\mathrm{kg}, \mathrm{p}=0.04)$ and reduced whole body SI by more than $40 \%(\mathrm{p}<0.001)$. Interestingly, by week 16 of RIM treatment, SI returned to normal prefat values in the treated group $(3.1 \pm 0.3 \mathrm{dl} / \mathrm{min} / \mathrm{kg} / \mathrm{uU} / \mathrm{ml}$ at week -6 vs. $2.6 \pm 0.5 \mathrm{dl} / \mathrm{min} / \mathrm{kg} / \mathrm{uU} / \mathrm{ml}$ at week $16, \mathrm{p}=\mathrm{NS}$ ). In contrast, the placebo group remained insulin resistant $(4.4 \pm 0.9 \mathrm{dl} / \mathrm{min} / \mathrm{kg} / \mathrm{uU} / \mathrm{ml}$ at week -6 vs. $1.9 \pm 0.4$ $\mathrm{dl} / \mathrm{min} / \mathrm{kg} / \mathrm{uU} / \mathrm{ml}$ at week $16, \mathrm{p}=0.01)$. The placebo group continued to gain additional weight throughout the 16 weeks $(+2.3 \mathrm{~kg}, \mathrm{p}=0.03)$, while the RIM group maintained body weight $(29.1 \pm 2.4$ vs $20.3 \pm 2.2 \mathrm{~kg}, \mathrm{p}=\mathrm{NS})$. Similarly, central adiposity, represented as omental fat content did not change in the RIM group ( $619 \pm 89$ to $583 \pm 156 \mathrm{~cm}^{3}, \mathrm{p}=\mathrm{NS}$ ) but was increased in the placebo group ( $572 \pm 308$ to $784 \pm 408 \mathrm{~cm}^{3}, \mathrm{p}=0.03$ ). Thus, RIM prevented further increases in fat accumulation and body weight even in the face of continued high fat feeding. RIM treatment on body fat was reflected in the plasma adiponectin levels. We found a rapid dramatic increase in plasma adiponectin in the RIM group, an increase that became significant by week $2(+32 \%, \mathrm{p}=0.04)$ and remained elevated throughout the 16 weeks of RIM treatment $(+57 \%, p=0.02)$. Conversely, in the placebo group there was no change in adiponectin levels.

Conclusion: That RIM improved insulin sensitivity without significant reductions in either body weight or omental fat content supports a bodyweight independent effect of rimonabant as an insulin sensitizer. Moreover, 
given that plasma adiponectin levels were elevated with RIM treatment suggests that it may play a role in mediating rimonabant's insulin sensitizing effect.

Supported by sanofi-aventis

\section{4}

Accumulation of large adipocytes in omental and subcutaneous fat depots during development of obesity in dogs is prevented by Rimonabant

I. R. Hsu, M. Kabir, D. Stefanovski, O. Woolcott, D. Zheng, K. J. Catalano, J. D. Chiu, S. P. Kim, L. N. Harrison, M. Lottati, V. Ionut, R. N. Bergman, J. M. Richey;

Physiology and Biophysics, Keck School of Medicine of the University of Southern California, Los Angeles, United States.

Background and Aims: The cannabinoid receptor antagonist rimonbant's (RIM) ability to reduce weight gain may be due to its direct effect on adipocytes. It has been shown that mild obesity leads to increased fat cell size, which is associated with insulin resistance. However, it is unknown whether RIM affects adipocyte morphology. In this study we determined the effects of RIM on adipocyte cell size and distribution in omental (OM) and subcutaneous (SQ) tissues in a well-characterized model of canine obesity.

Materials and Methods: Animals were fed a high fat diet fat-supplemented (52\% fat) diet for a period of 6 weeks to induce mild obesity in both OM and SQ adipose depots. Animals were then continued on the fat-supplemented diet (FF) and treated for an additional 16 wks with either 1) placebo (PL; $\mathrm{n}=4)$ or 2) rimonabant $(1.25 \mathrm{mg} / \mathrm{kg}$ per day; RIM; $\mathrm{n}=4)$. A separate group of animals fed only a standard diet ( $35 \%$ fat) were used as a control (CON; $n=6)$. Biopsies from OM and SQ adipose tissues were obtained in CON, RIM and PL groups. Cell diameter was quantified in $400-500$ cells per group. Cell size was defined as follows: small $(20-50 \mu \mathrm{m})$, medium $(50-120 \mu \mathrm{m})$, and large $(120-250 \mu \mathrm{m})$

Results: Fat cell size distribution was affected by both diet and drug treatment. After 6 weeks of FF, in OM adipocytes we observed the appearance of small cells in addition to medium sized cells seen in CON. In contrast, SQ adipocytes remained unchanged compared to CON. We observed the appearance of an additional population of large adipose cells in both OM and SQ tissue after 16 weeks of FF in the placebo group. Rimonabant prevented this increase in large cells in both OM and SQ depots. Analysis of cell numbers demonstrated that large cells contributed $9 \%$ to the total cell distribution for the OM depot after 16 wks of FF (PL) vs only $0.1 \%$ for the RIM group ( $<<0.001)$. Similarly, in the SQ depot large cells contributed 5\% to total distribution in PL vs $0 \%$ for the RIM group $(\mathrm{p}<0.001)$. Thus, rimonabant completely prevented the formation of large cells with fat feeding in both OM and SQ depots.

Conclusion: Rimonabant may exert beneficial effects on diet-induced obesity by preventing the formation of large adipocytes, which may lead to the improvement of insulin sensitivity in obesity.

Supported by sanofi-aventis

\section{5}

Functional analysis of newly identified obesity-related gene, secretogranin III (SCG3): possible role in regulation of appetite-related neuropeptides

K. Hotta, A. Tanabe;

Laboratory for Obesity, SNP Research Center, Riken, Kanagawa, Japan.

Background and Aims: Both genetic and environmental factors contribute to the development of obesity. Epidemiological studies showed that heritability of body weight is estimated to be between 60 and $70 \%$. We have performed a large-scale case-control association study using gene-based single nucleotide polymorphisms (SNP) and found that twelve SNPs in the secretogranin III (SCG3) gene were significantly associated with obesity. Among them, two SNPs (rs16964465, rs16964476) affected the transcriptional activity of SCG3: the DNA fragment containing minor alleles had higher transcriptional activity than those containing major alleles. Subjects with the minor allele seemed to be resistant to obesity (odds ratio $=9.23$, 95\% confidence interval $=2.77$ $\left.30.80, \chi^{2}=19.2, p=0.0000067\right)$. SCG3 immunoreactivity was detected in the paravetricular nucleus (PVN), lateral hypothalamic area (LHA) and arcuate nucleus (ARC), and the protein co-existed with orexin, melanin concentrating hormone (MCH), and neuropeptide Y (NPY). SCG3 formed a granulelike structure together with these neuropeptides. Our aim is to investigate functions of SCG3 in order to clarify how SCG3 causes obesity.

Materials and Methods: In situ hybridization and immuohistochemical analysis were performed in whole brain, pituitary gland, adrenal gland and pancreas. Mice were provided with regular chow or high-fat diet (33\% fat) for 4 weeks and hypothalamic regions were dissected from mice. The mRNA levels of SCG3 were measured using real time PCR. To isolate SCG3-binding proteins, we performed a yeast two-hybrid system.

Results: SCG3 was expressed mainly in the hypothalamus and pituitary gland and pancreatic islet. In pituitary gland, SCG3 were not detected in the cells expressing POMC. We also could not detected SCG3 in the adrenal gland. SCG3 may not play important roles in pituitary-adrenal axis. SCG3 was expressed both in $\beta$-cell and $\alpha$-cell of the islet. SCG3 may also affect insulin secretion as well as appetite-related neuropeptides secretion and contribute to obesity. The mRNA levels of SCG3 were reduced in hypothalamus of highfat diet induced obese mice. Reduced SCG3 expression would be related to food intake regulation at hypothalamus. Two genes that bound to SCG3 were isolated using yeast two-hybrid system. One of the genes was specifically expressed in the hypothalamus, especially in LHA. This gene was expressed in the orexin neuron, but not in the $\mathrm{MCH}$ neuron. The newly identified gene would interact with SCG3 and regulate orexin secretion.

Conclusion: SCG3 and newly identified SCG3-binding protein would play some important roles in regulation of appetite-related neuropeptides secretion.

Supported by the Japanease Millenium Projects and Takeda Science Foundation

\section{6}

Serotonin $2 \mathrm{C}$ receptor agonists improve type 2 diabetes via MC4 receptor signaling pathways

D. D. Lam ${ }^{1}$, L. Zhou ${ }^{1}$, G. Sutton ${ }^{2}$, M. J. Przydzial' ${ }^{1}$, P. G. Clifton ${ }^{3}$,

A. A. Butler ${ }^{2}$, L. K. Heisler ${ }^{1}$; J. K. Elmquist ${ }^{4}$,

${ }^{1}$ Clinical Biochemistry, University of Cambridge, United Kingdom,

${ }^{2}$ Pennington Biomedical Research Center, Louisiana State University,

Baton Rouge, United States, ${ }^{3}$ Psychology, Sussex University, Brighton,

United Kingdom, ${ }^{4}$ Center for Hypothalamic Research, University of Texas

Southwestern Medical Center, Dallas, United States.

Background and Aims: Substantial evidence exists for the regulation of energy balance and glucose homeostasis by central serotonin (5-HT) pathways. Mice lacking the 5-HT $2 \mathrm{C}$ receptor $\left(5-\mathrm{HT}_{2 \mathrm{C}} \mathrm{R}\right)$ develop obesity, hyperinsulinemia and glucose intolerance. Conversely, $5-\mathrm{HT}_{2 \mathrm{C}} \mathrm{R}$ agonists produce potent reductions in food intake and body weight. We hypothesize that $5-\mathrm{HT}_{2 \mathrm{C}} \mathrm{R}$ agonists also produce beneficial effects on glucose homeostasis independent of effects on food intake and body weight. Moreover, we hypothesize that the effects of $5-\mathrm{HT}_{2 \mathrm{C}} \mathrm{R}$ agonists on energy balance and glucose are mediated by central melanocortin pathways.

Materials and Methods: For food intake studies, diet-induced obese (DIO) mice received an i.p. bolus of the highly selective $5-\mathrm{HT}_{2} \mathrm{R}$ agonist BVT. $\mathrm{X}$ (various doses) 45 minutes before the onset of the dark cycle. Intake of palatable high fat diet was recorded for the first 6 hours of the dark cycle. For glucose homeostasis studies, DIO mice were treated with a subanorectic dose (causing no change in food intake) of the $5-\mathrm{HT}_{2 \mathrm{C}} \mathrm{R}$ agonist $\mathrm{mCPP}$ (i.p. bolus). Glucose tolerance and insulin tolerance were assessed by glucose tolerance test (GTT) and insulin tolerance test (ITT). To investigate the downstream pathways involved in effects on food intake and glucose homeostasis, in situ hybridization was used to demonstrate colocalization of $5-\mathrm{HT}_{2 \mathrm{C}} \mathrm{R}$ on melanocortin (POMC) neurons. Mice lacking either the melanocortin 3 (MC3R) or melanocortin 4 (MC4R) receptors were tested in the same paradigms as the DIO mice. To elucidate the peripheral mechanisms of 5-HT ${ }_{2 \mathrm{C}} \mathrm{R}$ agonist-induced improvements in glucose homeostasis, effects of $\mathrm{mCPP}$ on hepatic glucose production were assessed by RT-PCR for gluconeogenic genes.

Results: The selective 5-HT $\mathrm{R}$ agonist BVT.X produced a potent dosedependent reduction in food intake in DIO mice. Another $5-\mathrm{HT}_{2 \mathrm{C}} \mathrm{R}$ agonist, $\mathrm{mCPP}$, adminstered at subanorectic doses (producing no effect on food intake), improved glucose and insulin tolerance as assessed by GTT and ITT. In situ hybridization studies demonstrated that $5-\mathrm{HT}_{2 \mathrm{C}} \mathrm{R}$ are expressed on melanocortin (POMC) neurons of the hypothalamic arcuate nucleus, and thus are in a position to modulate the activity of melanocortin pathways. 
Assessments performed in mice lacking either of the centrally expressed melanocortin receptors, the MC3R and MC4R, showed that effects of 5$\mathrm{HT}_{2 \mathrm{C}} \mathrm{R}$ agonists on food intake and glucose homeostasis were replicated in MC3R-deficient mice, but were abolished in mice lacking the MC4R. Treatment with mCPP was associated with an inhibition of PEPCK and glucose-6-phosphatase, genes of hepatic gluconeogenesis.

Conclusion: These findings show that $5-\mathrm{HT}_{2 \mathrm{C}} \mathrm{R}$ agonists produce independent reductions in food intake and improvements in glucose homeostasis. Furthermore, these effects are mediated by the central melanocortin pathway, and depend specifically on downstream MC4R, but not MC3R. Moreover, $5-\mathrm{HT}_{2 \mathrm{C}} \mathrm{R}$ agonists inhibit genes of hepatic gluconeogenesis, which suggests a possible peripheral mechanism for the observed improvements in glucose homeostasis. Taken together, these data suggest that 5-HT 2C receptor agonists warrant consideration as therapeutic agents for obesity and type 2 diabetes.

Supported by NIDDK, NIMH, ADA, Gates Cambridge Trust

\section{PS 54 Insulin action in the critically ill}

\section{7}

Glycaemic and insulinaemic response during lower abdominal surgery under general anesthesia in type 2 diabetic subjects

L. Ali ${ }^{1}$, K. Sardar', U. H. S. Khatun 2 , M. H. Rahman ${ }^{3}$, M. A. Rashid ${ }^{1}$;

${ }^{1}$ Department of Biochemistry and Cell Biology, BIRDEM,

${ }^{2}$ Dept of Anesthesiology, Dhaka Medical College Hospital, ${ }^{3}$ Department of Physiology and Molecular Biology, BIRDEM, Dhaka, Bangladesh.

Background and Aims: Diabetic patients are at increased risk of preparative morbidity and mortality due to enhanced hemodynamic and metabolic alterations under a stress response. The metabolic alterations in diabetic patients undergoing surgery are still matters of investigation. We have investigated the glycemic and insulinemic responses during total abdominal hysterectomy, under general anesthesia, in a group of type 2 diabetic patients. Materials and Methods: A total number of 30 women (Age in yrs, $\mathrm{M} \pm \mathrm{SD}$, $44 \pm 6$; BMI, $\mathrm{kg} / \mathrm{m}^{2}, 24.4 \pm 3.0$ ) admitted in the BIRDEM hospital (the central hospital of the Diabetic Association of Bangladesh) in fit physical condition (ASA Class I \& II) were included in the present study. The anesthetic protocol was strictly maintained in all patients and consisted of an intravenous induction using thiopental sodium $5 \mathrm{mg} / \mathrm{kg}$ body weight, vecuronium 0.1 $\mathrm{mg} / \mathrm{kg}$ body weight and fentanyl $1.5 \mathrm{mg} / \mathrm{kg}$ body weight. Once intubated with an endotracheal tube, anesthesia was maintained with inhaled $\mathrm{O}_{2} / \mathrm{N}_{2} \mathrm{O}(1: 2)$ and appropriate dialing of halothane. Three samples were collected from each subject. The first sample (Control, PT ) was collected just before anesthesia, the second sample $\left(\mathrm{PT}_{1}\right)$ was collected 10 minutes after incision and the third sample $\left(\mathrm{PT}_{2}\right.$ ) was collected 10 minutes after extubation. Plasma glucose was measured by glucose-oxidase method, serum electrolytes by dry chemistry, and serum C-peptide and cortisol were measured by chemiluminesencebased ELISA technique.

Results: As judged by serial measurements of the pulse rate, systolic \& diastolic blood pressures and blood gas \& serum electrolytes the patients remained hemodynamically stable throughout the period of the surgery. Plasma glucose values showed a continuous rise with significantly higher level in $\mathrm{PT}_{2}$ (plasma glucose, $\mathrm{mmol} / \mathrm{l}, \mathrm{M} \pm \mathrm{SD}, 5.86 \pm 1.9$ in $\mathrm{PTo}, 6.0 \pm 1.85$ in $\mathrm{PT}_{1}$ and 9.55 \pm 2.7 in $\mathrm{PT}_{2} ; \mathrm{p}<0.001$ in $\mathrm{PT}_{0} \mathrm{vs}_{2} \mathrm{PT}_{2}$ ). The rise in plasma glucose was not accompanied by any change in serum $\mathrm{C}$-peptide (ng/ml, $\mathrm{M} \pm \mathrm{SD}, 2.16 \pm 0.90$ in $\mathrm{PT}_{0}, 2.0 \pm 0.95$ in $\mathrm{PT}_{1}$ and $2.0 \pm 1.2$ in $\mathrm{PT}_{2}$ ), but it was paralleled by a rise of serum cortisol which already showed significantly higher value in PT, (serum cortisol, mmol/l, 11.77 \pm 4.74 in $\mathrm{PT}_{0}, 14.27 \pm 6.0$ in $\mathrm{PT}_{1}$ and $33.13 \pm 7.70$ in $\mathrm{PT}_{2}, \mathrm{p}<0.003$ in $\mathrm{PT}_{0}$ vs $\mathrm{PT}_{1}$ ). The data was also analyzed after stratifying the patients into two groups based on their ongoing treatment by only insulin (the Insulin group) or by insulin combined with an oral hypoglycemic agents (the Insulin-OHA group). In contrast to $147 \%$ rise of blood glucose in the Insulin group at $\mathrm{PT}_{2}$, the rise of blood glucose in the Insulin-OHA group was $196 \%$ at the same time point. Such a difference between the two treatment groups was not observed in case of serum C-peptide or cortisol.

Conclusion: The data suggest that a) lower abdominal surgery under general anesthesia in well controlled type 2 diabetic subjects is accompanied by a hyperglycemic response which results from a rise of insulin antagonists like cortisol and not by a fall in insulin secretion; and b) insulin treatment may be more effective than insulin-OHA combination to control blood glucose in type 2 diabetic subjects undergoing surgery under general anesthesia. Supported by International Program in the Chemical Sciences (IPICS) and Diabetic Association of Bangladesh (DAB)

\section{8}

Insulin resistance during coronary surgery in diabetic and non-diabetic patients-parallel microdialysis and organ balance technique studying skeletal muscle (preliminary results)

Z. Szabó ${ }^{1}$, R. Andersson ${ }^{2}$, H. Arnqvist ${ }^{3}$;

${ }^{1}$ Cardiothoracic and Vascular Anaesthesia, Östergötlands Heart Centre, ${ }^{2}$ Clinical Pharmacology, Pharmacology, Medical University

${ }^{3}$ Diabetes, Endocrinology, University Hosp Linköping, Sweden.

Background and Aims: During cardiac interventions the elevated FFA (free fatty acids) and consecutive decreased carbohydrate utilisation characterize 
the intraoperative insulin resistance. Intraopeative insulin resistance is a dangerous milieu in case of any ischemic event because of higher tissue oxygen demand. Our aim was to combine the organ balance technique and microdialysis to study intra operative muscle metabolism and systematically relate the microdialysis data to the organ balance technique as golden standard.

Material and Methods: 9 diabetic and 9 non-diabetic patients after written informed consent were studied with microdialysis and organ balance technique using the brachio-radial muscle on their forearm and microdialysis in the pre-pectoral fat tissue. Concentration of glucose, lactate and glycerol was measured in the arterial / venous plasma and microdialysis rinse solution at time 3 points: before the heparin (and ECC), at the release of the aortic cross clamp and just before departure from the OR to the ICU.

Results: In the diabetic patients the changes in metabolism were more moderate having a pathological pattern compared with the non-diabetic group. The magnitude of the muscular blood flow was lower in the diabetics. The interstitial glucose level, glucose concentration in the muscle and plasma was higher from the beginning in diabetic patients.

No difference was noted in lipolysis between the groups as shown by the A-I concentration difference of glycerol concentration in the fat tissue, a reliable marker of lipolysis (Figure 1.).

Conclusion: Contrary to expectations the diabetic patients did not become more insulin resistant than the non diabetic group during cardiac operations. These preliminary data indicates that with regard to intra operative insulin resistance the non-diabetic patients behave as "diabetics" during coronary operations. Clinical implication: A strict blood glucose control already intraoperative seems advisable. We are not aware of similar studies in cardiac surgery.

\section{At conc. diff of the glycerol in the fett tissue}

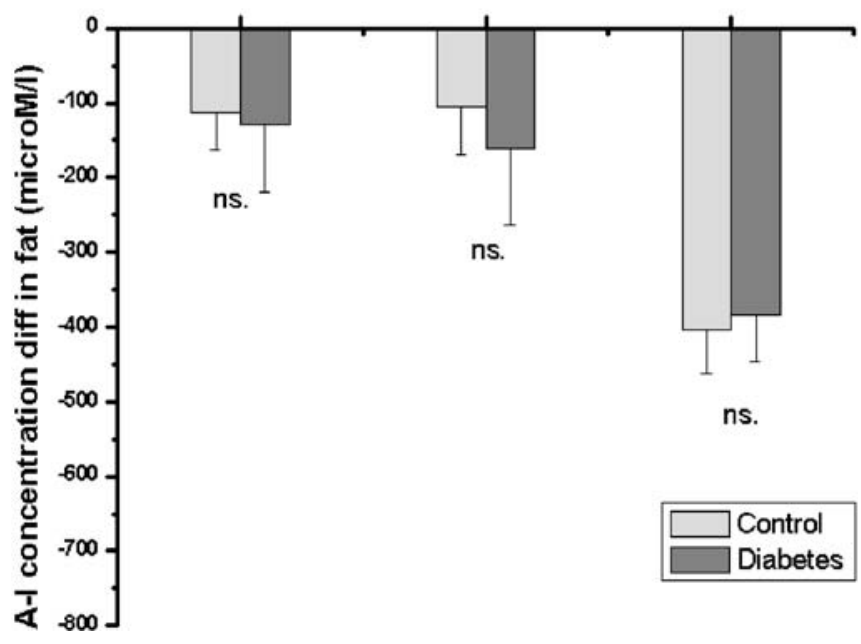

Figure1. A-I glycerol levels in the fat tissue

Supported by US forsk-stipendiestift Nr 03021022

\section{9}

\section{Insuline resistance in febrile states}

D. Z. Toljic Suluburic ${ }^{1}$, D. D. Micic 2 , M. Pavlovic ${ }^{3}$;

${ }^{1}$ Internal medicine section, Health Center Cacak, ${ }^{2}$ Internal medicine section, Institute for Endocrinology, Diabetes and Metabolic Disorders, Belgrade, ${ }^{3}$ Institute for Infectious and Tropical Diseases, Belgrade, Serbia.

Background and Aims: The occurrence of insulin resistance during fever is a well-known phenomenon, but underlying mechanisms are incompletely understood. We aimed to evaluate the efficacy of the Minimal Model technique to calculate insulin sensitivity in febrile patients and to investigate if there is any relationship between the degree of insulin resistance and duration or degree of fever.

Materials and Methods: We studied twenty one patients with fever (body temperature $38,7+/-0,8^{\circ} \mathrm{C}$, age $32,19+/-10,17 \mathrm{yr}$., $\mathrm{BMI}=22,4+/-2,17 \mathrm{~kg} / \mathrm{m}^{2}$, and normal values of fasting glycemia, serum lipids, AST, ALT and creatinine) and ten normal subjects matched for age and BMI as control group. Insulin sensitivity $\left(\mathrm{Si}, \mathrm{x} 10^{-4} \mathrm{~min} / \mathrm{microU} / \mathrm{ml}\right)$ and glucose effectiveness $\left(\mathrm{Sg}, \mathrm{min}^{-1}\right)$ were estimated using modified_Minimal Model technique. Patients were divided into groups according to degree (cut-off point of $38.5{ }^{\circ} \mathrm{C}$ ) and/or duration (cut- off point three months) of fever.

Results: In febrile group of patients insulin resistance was shown to exist in twenty patients out of twenty-one (Si $1,67+/-1,0$, Sg $0,32+/-0,14$ ). In control group it existed only in one patient. Patients with a body temperature higher than $38,5^{\circ} \mathrm{C}, \mathrm{n}=6$ had significantly $(\mathrm{p}<0,05)$ lower $\mathrm{Si}(0,99+/-0.85)$ than patients with a body temperature lower than $38,5{ }^{\circ} \mathrm{C}, \mathrm{n}=15(1,98+/-0,95)$. Differences in $\mathrm{Sg}$ were not statistically significant between these two patient groups (Sg $0,29+/-0,08 \mathrm{vs}$. $S g$ $0,33+/-0,15 \mathrm{mg} / \mathrm{min}, \mathrm{p}>0.05)$. The duration of fever itself did not significantly $(\mathrm{p}>0,05)$ affect insulin sensitivity nor $\mathrm{Sg}($ patients with fever longer than three months had Si 1,37+/-0,52,Sg $0,36+/-$ 0,07 vs. Si $1,85+/-1,19$ Sg $0,29+/-0.16$ in patients with fever less than three months). According to both the degree and duration of fever, we divided patients into four groups: The first group (patients with body temperature below $38,5{ }^{\circ} \mathrm{C}$, duration less than three months, $\mathrm{n}=6$ ) had Si $2,63+/-1,05$, Sg $0,32+/-0,21$. The second group (patients with body temperature below $38,5^{\circ} \mathrm{C}$, duration of fever over three months, $\mathrm{n}=8$ ) had $\mathrm{Si} 1,33+/-0,52, \mathrm{Sg}$ $0,38+/-0,06$. The third group (patients with body temperature above 38,5 ${ }^{\circ} \mathrm{C}$, duration of fever less than three months, $\mathrm{n}=6$ ) had Si $1,21+/-1,14, \mathrm{Sg}$ $0,25+/-0,086$. The fourth group (patients with body temperature over 38,5 ${ }^{\circ} \mathrm{C}$, duration over three months, $\mathrm{n}=1$ ) had Si $1,47+/-1,01$, Sg $0,245+/-0,06$. The first group had statistically significant higher values of $\mathrm{Si}$, but not $\mathrm{Sg}$, compared to other three groups. There were no significant differences between patients according cause of fever either in $\mathrm{Si}$ or $\mathrm{Sg}$ (bacterial: $\mathrm{Si}$ $1,4+/-0,83$, Sg $0,29+/-0,08$ vs. viral: Si $1,36+/-0,77$, Sg- $0,3+/-0,08$, and vs. others: Si 2,38+/-1,2.Sg-0,38+/-0,22).

Conclusion: It is concluded that the Minimal Model technique is valid for evaluation of insulin sensitivity in febrile patients. Also, it was found that degree, rather than duration of fever, influenced the level of insulin sensitivity. A possible explanation is that it is connected with higher increase of countraregulatory hormones, as well as higher increase of cytokines IL1 and TNF alpha. Finally, the degree of insulin resistance has not been found to be related to the causalities of infection.

\section{0}

Constancy of relative insulin sensitivity and importance of absolute insulin sensitivity shift to develop insulin infusion protocol in critically ill patients

M. Matsuda, M. Shigeto, Y. Akiyama, M. Masuzawa, T. Okabe; Diabetes and Endocrine Department, Kameda Medical Center, Kamogawa, Japan.

Background and Aims: To keep blood glucose conc. (BG) lower than $140 \mathrm{mg} /$ $\mathrm{dl}(\sim 8 \mathrm{mM})$ in critically ill patients, it is necessary to use an insulin infusion protocol like the Yale Protocol (Diabetes Care 27:461, 2004). However its application is difficult as demonstrated in randomized controlled trials. To reduce hypoglycemic risks, we have developed a unique algorithm.

Materials and Methods: The input $(\mathrm{X})$ and the output $(\mathrm{Y})$ of this system to adjust the insulin infusion rate to keep BG are the insulin infusion rate $(\mathrm{U} / \mathrm{hr})$, and the difference of BG in a fixed amount of time ( $\mathrm{mg} / \mathrm{dl}$ per $\mathrm{hr}$ ), respectively. These two values were plotted on a graph using $\mathrm{X}$ and $\mathrm{Y}$ axes. Glucose infusion rate $(\mathrm{g} / \mathrm{hr})$ is converted to the theoretical increase of BG (mg/dl per hr) assuming simple distribution in glucose space (20\% and $25 \%$ of body weight in obese and lean subjects), and is added on the graph. BG was measured from 1 to $6 \mathrm{hrs}$ intervals according to the expected $\mathrm{Y}$ value to have optimal $B G$ by reading the graph. To determine the next $\mathrm{X}$ value to achieve optimal expected $\mathrm{Y}$ value, we have observed 50 diabetic cases $(\mathrm{M} / \mathrm{F}=$ $36 / 14$, age $=64 \pm 12$ y.o., $\mathrm{BMI}=24 \pm 4 \mathrm{~kg} / \mathrm{m}^{2}$ ) before and/or after operation to keep BG from 90 to $140 \mathrm{mg} / \mathrm{dl}$, by using the graph, resulting in no hypoglycemia $(\mathrm{BG}<60 \mathrm{mg} / \mathrm{dl})$. Operations included those in coronary artery bypass graft, orthopedics, abdominal, gynecological, and obstetric surgery. BG was measured by PCx (Abbott, USA), and human regular insulin (1 U/ml saline) and glucose were infused by infusion pumps for clinical use calibrated routinely. In vivo, it is possible that the whole body insulin resistance (IR) is defined as absolute amount of insulin to keep fixed glucose turnover and keep BG constant (IRI-abs: absolute Insulin Resistance Index). On the other hand, insulin sensitivity is defined as a decremental conc. of BG by a fixed increment of insulin (or delta insulin) (ISI-delta: relative Insulin Sensitivity Index). Glucose infusion and change of BG were approximated to calculate 
insulin resistance by extrapolating the line of the graph, reading $\mathrm{X}(\mathrm{U} / \mathrm{hr})$ on $\mathrm{X}$-axis $(\mathrm{Y}=0)$ to calculate IRI-abs. ISI-delta $(\mathrm{mg} / \mathrm{dl}$ per $\mathrm{U})$ was the slope of the line on the graph, when IRI-abs became fixed.

Results: Total number of sampling of BG was 1626 . BG before insulin infusion was $163 \pm 58 \mathrm{mg} / \mathrm{dl}$, and the average BG after insulin infusion was $126 \pm$ $18 \mathrm{mg} / \mathrm{dl}$. IRI-abs shifted from $0.4 \pm 1.8$ to $3.4 \pm 10.2 \mathrm{U} / \mathrm{hr}$. When insulin sensitivity is defined as relative amount of insulin to suppress a fixed level of $\mathrm{BG}$, this ISI-delta was fairly constant during whole observation before and/or after operations. ISI-delta was roughly equal to the incremental BG $(\mathrm{mg} / \mathrm{dl})$ by basal endogenous glucose production $(2.2 \mathrm{mg} / \mathrm{kg}$ lean body weight per min) divided by 1 unit/hr of incremental insulin infusion rate in almost all subjects with glucose infusion less than $5 \mathrm{~g} / \mathrm{hr}$, and the average was $57 \pm 5$ $\mathrm{mg} / \mathrm{dl}$ per $\mathrm{U}$.

Conclusion: We concluded that rapidity of shifts of IRI-abs (instability) is necessary to be added to the previous protocols like the Yale Protocol that used only absolute $B G$, change of $B G$, and absolute insulin infusion rate to reduce hypoglycemic risks in critically ill patients. In addition, a decremental conc. of BG by a fixed increment of insulin (ISI-delta) was relatively constant despite the stress of operations. This phenomenon may be often masked by the shift of the IRI-abs, and neglect of emergence of ISI-delta resulting in an unexpected change of insulin sensitivity may cause sudden hypoglycemic accidents.

\section{1}

Evaluation of insulin action on glucose uptake and lipolysis in patients with ketosis-prone type 2 diabetes during remission

S. Choukem ${ }^{1}$, E. Sobngwi ${ }^{2}$, I. Hainault ${ }^{3}$, P. Vexiau ${ }^{1}$, F. Calvo ${ }^{4}$, J.-F. Gautier ${ }^{1,3}$, ${ }^{1}$ Endocrinology and Diabetes, Saint Louis Hospital, Paris, France,

${ }^{2}$ Obesity, Endocrinology and Diabetes, Yaounde Central Hospital, Yaounde, Cameroon, ${ }^{3}$ U671, INSERM, Paris, France, ${ }^{4}$ Clinical Investigations Centre,

Saint Louis Hospital, Paris, France.

Background and Aims: Ketosis-prone type 2 diabetes (KPD), also known as idiopathic type 1 or type $1 \mathrm{~B}$ diabetes, is a syndrome of unclear pathogenesis found mainly in people of sub Saharan African ancestry. It is characterised by an acute onset with sustained hyperglycaemia and ketosis or ketoacidosis that require urgent insulin treatment. This is followed by a prolonged long-term near-normoglycaemic remission without insulin treatment in more than $50 \%$ of patients. The aim of this study was to characterize insulin action during remission.

Materials and Methods: We studied insulin action on glucose uptake and lipolysis, and body composition after an overnight fast in 13 patients with $\mathrm{KPD}\left(12 \mathrm{M} / 1 \mathrm{~F}\right.$; age $=45.2 \pm 8.8(\mathrm{SD})$ years; $\mathrm{BMI}=29.8 \pm 1.2 \mathrm{~kg} / \mathrm{m}^{2} ; \mathrm{HbAlc}$ $=6.2 \%)$ in remission. They were compared to $13(11 \mathrm{M} / 2 \mathrm{~F}$; age $=43.2 \pm 9.5$ years; $\mathrm{BMI}=24.6 \pm 2.3 \mathrm{~kg} / \mathrm{m}^{2}$ ) normoglycaemic control subjects. All patients and controls were of sub Saharan African origin. The diagnosis of KPD was considered for patients with acute hyperglycaemia with ketosis at onset without precipitating factors, and remission was defined as maintenance of $\mathrm{HbAlc}$ level $\leq 6.5 \%$ on an oral antidiabetic drug or diet alone for at least 3 months. We performed a 2 -step euglycaemic clamp with a $10 \mathrm{mUI} / \mathrm{m}^{2} / \mathrm{min}$ insulin infusion step of $100 \mathrm{~min}$ to evaluate the antlipolytic action of insulin (decrease in non-esterified fatty acid (NEFA) concentrations) followed by a $80 \mathrm{mUI} / \mathrm{m}^{2} / \mathrm{min}$ insulin infusion step of $100 \mathrm{~min}$ to measure glucose uptake (M-value). Results are expressed as percentage or mean \pm standard error. Comparison between groups was performed using the non-parametric Mann-Whitney U test.

Results: Whole body glucose uptake (M-value) was $7.4 \pm 0.7$ and $10.2 \pm 1.1 \mathrm{mg} /$ $\mathrm{kg}$ of fat-free mass $/ \mathrm{min}$ in patients and controls respectively $(\mathrm{p}=0.04)$. Plasma insulin levels were higher in patients at baseline $(10.9 \pm 2.0 \mathrm{mU} / \mathrm{ml}$ vs $6.2 \pm 1.3$ in controls; $\mathrm{p}=0.03$ ), but not at the end of the first step (T100min: patients $24.1 \pm 5.6 \mathrm{mU} / \mathrm{ml}$, controls $19.4 \pm 1.9 \mathrm{mU} / \mathrm{ml} ; \mathrm{p}=0.46)$. Patients had a baseline NEFA concentration $84 \%$ higher than controls $(60.2 \pm 6.5$ vs. $32.8 \pm 5.4 \mathrm{mg} / \mathrm{dl}$ in patients and controls respectively; $\mathrm{p}=0.004$ ). It decreased in both groups from baseline to the first step, but remained higher in patients compared with controls ( $24.7 \pm 3.1$ vs. $10.3 \pm 2.9 \mathrm{mg} / \mathrm{dl}$ at $\mathrm{T} 100 \mathrm{~min}: \mathrm{p}=0.003)$. However, there was no difference in percent decrement of NEFA concentration from baseline between groups $(55.3 \%$ vs. $66.6 \%$ at $\mathrm{T} 100 \mathrm{~min}$ in patients and controls respectively, $\mathrm{p}=0.16$ ).

Conclusion: During remission, patients with KPD have lower whole body glucose uptake, higher NEFA concentrations, and higher fasting insulinaemia.

However the NEFA clearance rate in response to insulin infusion appears similar. This suggests that patients with KPD in remission may be similar to those with type 2 diabetes.

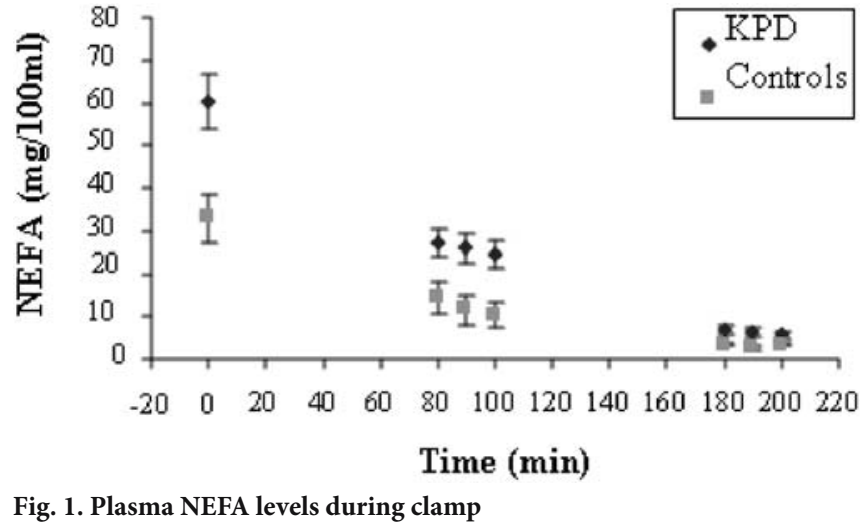

Supported by an institutional grant (PHRC) and Association Française des Diabétiques (AFD), a non-profit organisation 


\section{PS 55 ER stress and insulin resistance}

\section{2}

Ectopic expression of insulin receptor substrates 1 or 2 in 3T3-L1 adipocytes counteracts endoplasmic reticulum stress-induced insulin resistance

L. Xu' ${ }^{1}$, B. Joblin ${ }^{1}$, S. Boller ${ }^{1}$, A. Egli ${ }^{1}$, N. Boschetti ${ }^{1}$, T. Trüb ${ }^{2}$, G. A. Spinas ${ }^{1}$, M. Niessen ${ }^{1}$;

${ }^{1}$ Department of Internal Medicine, Endocrinology and Diabetes, ${ }^{2}$ Zentrale Dienste der Universität, Ausrüstung und Logistik, Zurich, Switzerland.

Background and Aims: Insulin receptor substrates (IRS) are important components of insulin signal transduction and indispensable regulators of glucose homeostasis. Endoplasmic reticulum (ER) stress has been shown to inhibit IRS via c-Jun N-terminal kinase (JNK)-dependent serine phosphorylation/downregulation thereby linking obesity with insulin resistance (1). ER stress has also been shown to impair insulin secretion (2). With this study we wanted to investigate the effects of ER stress on insulin signalling and function of 3T3-L1 adipocytes.

Materials and Methods: ER stress was induced with either tunicamycin or with thapsigargin. Expression levels of the chaperone Grp78/BiP were assessed to monitor ER stress. Intracellular insulin signal propagation was analysed by Western blotting with phospho-specific antibodies against the activated forms of the insulin receptor (IR), Akt/PKB and ERK1/2. The function of 3T3-L1 adipocytes was assessed by measuring the rate of insulin-dependent 2-deoxy$\mathrm{D}$-glucose uptake and the (constitutive) secretion of adiponectin, respectively. IRS1, IRS2 and GFP (as a control) were constitutively overexpressed from adenoviral constructs.

Results: ER stress strongly decreased the activation of the insulin receptor, Akt/PKB and ERK1/2 in 3T3-L1 adipocytes. However, total expression levels of the IR were also reduced under ER stress. Overexpression of fulllength IRS1 or IRS2 restored insulin-dependent activation of Akt/PKB and ERK1/2 under ER stress without restoring expression levels/activation of the IR. Secretion of adiponectin from 3T3-L1 adipocytes and insulin-induced 2deoxy-D-glucose uptake were both reduced by $50 \%$ under ER stress. Ectopic expression of IRS significantly improved insulin-induced 2-deoxy-D-glucose uptake by $30 \%$ under ER stress compared to controls (GFP), while secretion of adiponectin was improved by $25 \%$.

Conclusion: Our study confirms that ER stress induces insulin resistance in 3T3-L1 adipocytes. In addition to the previously described downregulation of IRS proteins we find that in 3T3-L1 adipocytes ER stress also impairs the expression of the IR. This observation might explain why in our experiments constitutive high-level expression of IRS1 or IRS2 can only partially rescue insulin sensitivity under ER stress. Furthermore, ER stress inhibited the secretion of adiponectin. Lower circulating levels of adiponectin due to obesity-induced ER stress could contribute to the development of insulin resistance and hyperglycemia in vivo. Ectopic expression of IRS proteins improved the production of adiponectin from 3T3-L1 adipocytes under ER stress indicating a link between insulin signalling and adiponectin production.

Supported by VELUX foundation, Jubiläumsstiftung der Schweizerischen Lebensversicherungs- und Rentenanstalt für Volksgesundheit und medizinische Forschung
0673

4-PBA(4-phenylbutyrate) reduced palmitate-induced endoplasmic reticulum stress and apoptosis in HUVEC

H.-J. Jang ${ }^{1}$, J. Jung ${ }^{1}$, E.-S. Ha ${ }^{1}$, S.-E. Choi ${ }^{1}$, S. Jung ${ }^{2}$, H. Kim² , D. Kim², Y. Kang ${ }^{1}$, K.-W. Lee';

${ }^{1}$ Institutes for Medical Sciences, ${ }^{2}$ Endocrinology and Metabolism, Ajou University School of Medicine, Suwon-Si, Republic of Korea.

Background and Aims: The death of endothelial cells may play a critical role in the development of various vascular diseases, including atherosclerosis. While free fatty acids (FFAs) may stimulate endothelial apoptosis, the detailed molecular and cellular mechanisms of this effect have not been studied. To elucidate the mechanisms involved in FFA-induced endothelial cell apoptosis, we investigated the effects of different pharmacological inhibitors on palmitate-induced apoptosis in endothelial cells. Interestingly, 4-PBA (4phenylbutyrate), a chemical chaperone, had a protective effect.

Materials and Methods: To examine endothelial cell apoptosis, endothelial cells were first treated with different concentrations of palmitate, and then endothelial cell apoptosis was measured using a Cell Death ELISA assay kit. Palmitate dramatically increased endothelial cell apoptosis in a mitochondria- and caspase -dependent manner. To examine the involvement of endoplasmic reticulum (ER) stress in palmitate-induced endothelial cell apoptosis, induction of the unfolded protein response (UPR) in response to palmitate treatment was monitored, using immunoblotting and RT-PCR.

Results: Endothelial cells exposed to palmitate for $12 \mathrm{~h}$ increased the protein levels of phosphorylated eukaryotic translation initiation factor 2 alpha (eIF2alpha), X box-binding protein 1 (XBP-1), and C/EBP homologous transcription factor (CHOP) compared to control cells. In addition, palmitate treatment markedly increased the levels of the ER chaperone proteins Grp78, suggesting that palmitate highly regulates the unfolded protein response (UPR). Interestingly, pretreatment with a chemical chaperone, such as 4-PBA, reduced palmitate-induced $\mathrm{CHOP}$ and BIP expression and had protective effects against palmitate-induced endothelial cell apoptosis.

Conclusion: ER-stress is involved in palmitate-induced endothelial cell apoptosis.

Supported by the "GRRC" project of the Gyeonggi Provincial Government and a grant from the Korean Health 21 R\&D Project, Ministry of Health and Welfare, Republic of Korea. (to K. W. Lee)

\section{4}

Effect of acute hyperinsulinaemia and AT-1 receptor blockade on androstane metabolites in men with impaired glucose tolerance and/or impaired fasting glucose

K. Zidkova ${ }^{1}$, E. Krusinova ${ }^{1}$, J. Vrbikova ${ }^{1,2}$, M. Hill ${ }^{2}$, T. Pelikanova ${ }^{1}$; ${ }^{1}$ Diabetes Center, Institute for Clinical and Experimental Medicine, ${ }^{2}$ Department for Steroid Hormones, Institute of Endocrinology, Prague, Czech Republic.

Background and Aims: The association between lower testosterone levels and type 2 diabetes in men was delineated; however the responsible mechanisms were not identified till now. It is not clear which steps of androgen metabolism could be influenced by hyperinsulinaemia/ insulin resistance in men. The aim was to study the effect of acute hyperinsulinaemia with and without AT-1 receptor blockade on the plasma

Tab. 1

Serum concentrations of androstane metabolites during HIC. Median (lower quartile;upper quartile).

\begin{tabular}{|c|c|c|c|c|}
\hline & \multicolumn{2}{|l|}{ HIC-Placebo } & \multicolumn{2}{|l|}{ HIC-Telmisartan } \\
\hline & $\mathbf{0} \mathrm{min}$ & $120 \mathrm{~min}$ & $0 \mathrm{~min}$ & $120 \mathrm{~min}$ \\
\hline Testosterone (nmol. $\mathrm{l}^{-1}$ ) & $21.7(11.9 ; 26.5)$ & $19.7(14.8 ; 30.4)$ & $18.3(14.9 ; 22.2)$ & $20.2(13.3 ; 29.6)$ \\
\hline Dihydrotestosterone (nmol. $\left.\mathbf{l}^{-1}\right)$ & $1.67(1.16 ; 2.8)$ & $1.81(1.18 ; 2.7)$ & $1.41(0.77 ; 1.69)$ & $1.22(0.87 ; 2.33)$ \\
\hline Etiocholanolone (nmol..$\left.^{-1}\right)$ & $0.42(0.31 ; 0.58)$ & $0.34(0.29 ; 0.43)$ & $0.27(0.22 ; 0.41)$ & $0.25(0.21 ; 0.48)$ \\
\hline $\begin{array}{l}\text { Etiocholanolone-conjugate } \\
\left(\text { nmol. }{ }^{-1}\right)\end{array}$ & $161(96.3 ; 213)$ & $103(77.9 ; 167)$ & $162(111 ; 215)$ & $118(81.6 ; 224)$ \\
\hline $\begin{array}{l}\text { Androsterone } \\
\left(\mathrm{nmol.1^{-1 } )}\right.\end{array}$ & $0.89(0.43 ; 1.18)$ & $0.83(0.28 ; 1.08)$ & $0.6(0.43 ; 1.06)$ & $0.57(0.4 ; 0.88)$ \\
\hline Androsterone-conjugate (nmol. $\left.\mathbf{l}^{-1}\right)$ & $1827(705 ; 2953)$ & $1757(610 ; 2212)$ & $2143(816 ; 3998)$ & $2106(689 ; 3339)$ \\
\hline
\end{tabular}


levels of androstanes in men with impaired glucose tolerance (IGT) and/or impaired fasting glucose (IFG).

Materials and Methods: 12 men with IGT and/or IFG underwent a doubleblind, cross-over study with 3 weeks of either telmisartan $160 \mathrm{mg}$ or placebo administration. At the end of each period, serum concentrations of selected androstane metabolites were measured using GC/MS method before and at 120 minutes of hyperinsulinaemic $\left(1 \mathrm{mU} \cdot \mathrm{kg}^{-1} \cdot \mathrm{min}^{-1}\right)$ euglycaemic $\left(5 \mathrm{mmol} . \mathrm{I}^{-}\right.$ $\left.{ }^{1}\right)$ clamp (HIC). ANOVA with repeated measures, followed by least significant difference multiple comparisons, were used for data evaluation, $\mathrm{p}<0.05$ was considered statistically significant.

Results: Testosterone levels did not differ either after telmisartan treatment or during HIC. Telmisartan treatment resulted in a decrease in dihydrotestosterone (DHT) levels $(\mathrm{p}<0.03)$, without any significant changes during HIC. Telmisartan decreased etiocholanolone $(\mathrm{p}<0.02)$ and increased etiocholanolone-conjugate levels $(\mathrm{p}<0.05)$, the latter one decreased significantly during HIC $(\mathrm{p}<0.002)$. AT-1 receptor blockade increased the androsterone-conjugate levels $(\mathrm{p}<0.0002)$, that also declined during HIC $(\mathrm{p}<0.02)$. There were no changes in androsterone levels.

Conclusion: Plasma levels of conjugated etiocholanolone and androsterone are decreased by acute hyperinsulinaemia. Telmisartan treatment, compared to placebo, resulted in a significant decrease of free androstane metabolites, whereas their conjugates were increased. Our results suggest a potential role of telmisartan in androgen metabolism. (Tab. 1)

Supported by IGA MH CZ No. NR/8991-3 and No.1A/8649-3

\section{5}

The role of endoplasmic reticulum stress on insulin-resistance induced by glucosamine in the L6 skeletal muscle cells

G. A. Raciti ${ }^{1}$, L. Ulianich ${ }^{1}$, F. Paturzo ${ }^{1}$, C. Iadicicco ${ }^{1}$, A. Lombardi ${ }^{2}$,

F. Andreozzi ${ }^{3}$, A. P. M. Barbagallo ${ }^{1}$, B. Di Jeso ${ }^{2}$, F. Beguinot ${ }^{1}$, P. Formisano ${ }^{1}$,

C. Miele ${ }^{1}$;

${ }^{1}$ Dip. Biologia e Patologia Cellulare e Molecolare \& IEOS-CNR, University

of Naples Federico II, Naples, I²Dip. Scienze e Tecnologie Biologiche e

Ambientali, University of Lecce, ${ }^{3}$ Dip. Medicina Sperimentale e Clinica,

University of Catanzaro Magna Graecia, Catanzaro, Italy.

Background and Aims: The endoplasmic reticulum (ER) is responsible for the quality control of proteins. When the productive folding process in the ER is perturbed by environmental stress conditions, eukaryotic cells activate a homeostatic response, termed the unfolded protein response (UPR). Dysfunction of the UPR plays an important role in several human diseases, including Type 2 diabetes (T2D). Indeed, recent discoveries reported that ER stress is involved both in pancreatic beta-cell dysfunction and in peripheral insulin resistance. Hyperglycaemia has been reported to cause insulin resistance and, in some tissues, this process appears to be linked to UPR activation. Even glucosamine, generated by the activation of the hexosamine pathway during hyperglycaemia, causes insulin resistance and disturbances similar to glucose toxicity in muscle cells. Thus, in the present work we sought to evaluate the possible role of ER stress on the insulin-resistance induced by glucosamine in L6 skeletal muscle cells.

Materials and Methods: L6 skeletal muscle cells were incubated either with $10 \mathrm{mM}$ glucosamine (GLC) or 0.5 microM thapsigargin (THA) in the presence or in absence of insulin (100nM). mRNA and protein expression were measured respectively by Real Time PCR and Western Blot. Glucose uptake was measured using the glucose analogue 2-deoxy-D-[3H]-glucose (2DG).

Results: We evaluated if GLC was able to activate the UPR in L6 cells. The mRNA levels of BiP and CHOP, two ER stress markers, were increased respectively up to 14 -fold and 12 -fold in a time-dependent manner upon GLC treatment. The same results were also obtained upon THA treatment, a commonly used ER stressor. Next we analyzed phosphorylation of protein kinases known to be activated by ER stress in several cellular systems. Among the MAPKs, p38, JNK and ERK were all activated upon both GLC and THA stimulation. Furthermore, phosphorylation of the translation initiation factor eIF2alpha by PERK was evident as early as after $15 \mathrm{~min}$ of GLC treatment. We then evaluated the effect of GLC- and THA-induced ER stress on the insulin signalling. Both GLC and THA blunted insulin induced activation of Akt measured as phosphorylation of Akt on both Thr308 and Ser473. Furthermore, insulin was unable to inhibit GSK3beta upon GLC as well as THA treatment. Finally, given that ER stress cause a transcriptional reprogramming of cells, we hypothesized that GLUT4 expression could be susceptible to variation in stressed L6 cells. Real Time PCR and Western blot experiments, indeed, showed that both GLC and THA induced respectively a $60 \%$ and $85 \%$ decrease of both GLUT4 mRNA and protein levels. Decrease of GLUT4 expression levels was paralleled by the reduced capability of L6 cells to uptake the 2DG, when measured in the presence of insulin stimulation.

Conclusion: We show for the first time that glucosamine is able to activate the UPR in L6 skeletal muscle cells. This pathway may play a role in insulin resistance through inhibition of Akt activity and Glut4 expression.

\section{6}

$\mathrm{N}^{\varepsilon}$-(Carboxymethyl)lysine (CML) is increased in human adipose tissue: Biological effects of AGE-adducts

K. H. J. Gaens ${ }^{1}$, M. P. H. van de Waarenburg ${ }^{1}$, J. Nijhuis ${ }^{2}$, J. Scheijen ${ }^{3}$,

C. D. A. Stehouwer ${ }^{1}$, C. G. Schalkwijk ${ }^{1}$;

${ }^{1}$ Internal Medicine / CARIM, ${ }^{2}$ General Surgery,

${ }^{3}$ Clinical Genetics, Maastricht University, The Netherlands.

Background: A dysregulation of adipocytokines in adipose tissue has been associated with an increased cardiovascular risk. However, factors by which obesity leads to such a dysregulation have not been elucidated. Because of local conditions in adipose tissue, i.e. increased fatty acids and oxidative stress, we speculate that an increased formation of the major advanced lipoxidation/ glycation endproduct (ALEs/AGEs) $\mathrm{N}^{\varepsilon}$-(carboxymethyl)lysine (CML) accumulates in adipose tissue and that this increased formation of CML causes dysregulation of adipocytokines production. We therefore examined the presence and accumulation of CML and the receptor for AGE (RAGE) in adipose tissue and we performed experiments in 3T3 cells to examine the biological effects of AGE-adducts on the expression of adipocytokines.

Methods: Accumulation and localisation of CML and RAGE in human adipose tissue was determined by immunohistochemistry. Subjects were recruited from a population of obese patients. To investigate colocalisation with macrophages and endothelial cells, serial sections of adipose tissue were analysed by antibodies against CD68 and CD31, resp. Plasma concentrations of CML and CEL were determined by HPLC-MSMS. For the gene expression experiments, we used 3T3-L1 murine cells that were differentiated to adipocytes. After incubation of 3T3 adipocytes with different AGE-adducts (CML-, glucose-, glucose-6-phosphate- and methylglyoxal-modified human serum albumin) $(0.5 \mu \mathrm{M}, 1.5 \mu \mathrm{M}$ and $4.5 \mu \mathrm{M})$ for $0 \mathrm{~h}, 8 \mathrm{~h}, 24 \mathrm{~h}$ and $48 \mathrm{~h}$, changes in gene expression of leptin, adiponectin, resistin, RAGE, PAI-1, PPAR- $\gamma$, IL6 and IK-B $\alpha$ were analysed by RT-PCR.

Results: Immunohistochemical staining demonstrated accumulation of CML and RAGE in adipocytes, macrophages and endothelial cells in adipose tissue of obese patients. Plasma CML concentrations did not, but CEL did, differ between obese patients and a control group $(1.46 \pm 0.4 \mu \mathrm{M}$ and $1.55 \pm 0.2 \mu \mathrm{M}$, resp and $1.69 \pm 0.6 \mu \mathrm{M}$ and $0.80 \pm 0.2 \mu \mathrm{M}$, resp, $\mathrm{p}<0.05)$. Moreover, weight loss had no effect on plasma AGE concentrations in obese patients. The CML concentration was significantly higher in 3T3 adipocytes as compared to 3T3 fibroblasts and oxidative stress further increased CML levels in adipocytes. Western blot analysis showed that exposure of 3T3 adipocytes to oxidative stress during $0 \mathrm{~h}, 2 \mathrm{~h}, 8 \mathrm{~h}$ and $24 \mathrm{~h}$ resulted in a progressive increase of CML in a major CML-modified protein of $36 \mathrm{kDa}$. Incubation of $3 \mathrm{~T} 3$ adipocytes with different AGE-adducts demonstrated positive and negative effects on the expression of adipocytokines.

Conclusions: This study provides evidence for the accumulation of CML in adipocytes, endothelial cells and macrophages in human adipose tissue. The accumulation of CML is a local process, since plasma CML concentrations showed no difference between obese patients and a control group. In contrast, plasma CEL was higher in obese patients. As shown in 3T3 adipocytes, incubation with AGE-adducts caused a dysregulated expression of adipocytokines. These experiments support the hypothesis that the accumulation of CML in adipose tissue of obese patients has important biological consequences regarding the dysregulation of adipocytokines. Supported by the Dutch Diabetes Research Foundation 


\section{PS 56 Other hormones}

\section{7}

Direct non-genomic actions of progesterone on isolated rat skeletal muscle: inhibition of cell respiration and induction of insulin resistance B. Brunmair, F. Gras, L. Quarré, Z. Szöcs, W. Waldhäusl, C. Fürnsinn; Department of Medicine III, Division of Endocrinology \& Metabolism, Medical University of Vienna, Austria.

Background and Aims: In search of mechanisms responsible for the adaptations of glucose metabolism to pregnancy, we have previously reported that progesterone, which circulates at increased concentrations during late pregnancy, affects fuel metabolim of rat skeletal muscle via a direct, rapid, and non-genomic mechanism. The present study was to better define this action by sorting out, how acute progesterone exposure modulates basal and insulin-stimulated substrate fluxes through the major pathways of cellular glucose metabolism.

Materials and Methods: Strips of freshly isolated soleus muscle from healthy Sprague-Dawley rats were exposed to 1,10 , or $50 \mu \mathrm{M}$ progesterone for $5 \mathrm{~h}$. During the last hour, rates glucose oxidation, lactate production, glucose transport, and pyruvate oxidation were measured under basal and insulinstimulated $(25 \mathrm{nM})$ conditions. Glycogen content was determined at the end of the experiments.

Results: Under basal conditions, progesterone distinctly and dose-dependently reduced the rate of glucose oxidation $\left(\mathrm{nmol}^{*} \mathrm{~g}^{-1 *} \mathrm{~h}^{-1}\right.$ : control, $819 \pm 76 ; 1 \mu \mathrm{M}$, $745 \pm 113 ; 10 \mu \mathrm{M}, 499 \pm 55, \mathrm{p}<0.002 ; 50 \mu \mathrm{M}, 230 \pm 18, \mathrm{p}<0.001$; all $\mathrm{p}$-values vs control), which was accompanied by an increase in lactate release $\left(\mu \mathrm{mol}^{*} \mathrm{~g}^{-}\right.$ ${ }^{1 *} \mathrm{~h}^{-1}$ : control, $8.0 \pm 0.5 ; 1 \mu \mathrm{M}, 9.8 \pm 0.5 ; 10 \mu \mathrm{M}, 13.2 \pm 0.7 ; 50 \mu \mathrm{M}, 20.6 \pm 0.5$; $\mathrm{p}<0.002$ each). Higher carbohydrate requirements for anaerobic than aerobic energy supply were reflected by increased glucose transport (cpm ${ }^{3} \mathrm{H}$-deoxyglucose transported ${ }^{*} \mathrm{mg}^{-1 *} \mathrm{~h}^{-1}$ : control, $279 \pm 10 ; 1 \mu \mathrm{M}, 378 \pm 29, \mathrm{p}=0.06 ; 10 \mu \mathrm{M}$, $364 \pm 29, \mathrm{p}<0.05 ; 50 \mu \mathrm{M}, 517 \pm 29, \mathrm{p}<0.001)$ and glycogen depletion (glycogen content after incubation, $\mu \mathrm{mol}$ glucosyl units/g: control, $9.7 \pm 0.9 ; 1 \mu \mathrm{M}$, $8.2 \pm 1.0, \mathrm{p}<0.05 ; 10 \mu \mathrm{M}, 3.9 \pm 0.8, \mathrm{p}<0.001 ; 50 \mu \mathrm{M}$, no detectable glycogen). This characteristic response clearly indicated progesterone-induced inhibition of cell respiration, which was likewise seen under insulin-stimulated conditions (nmol glucose oxidation ${ }^{\star} \mathrm{g}^{-1 \star} \mathrm{h}^{-1}$ : control, $1706 \pm 131 ; 1 \mu \mathrm{M}, 1566 \pm 93 ; 10 \mu \mathrm{M}$, $1030 \pm 72, \mathrm{p}<0.005 ; 50 \mu \mathrm{M}, 340 \pm 64, \mathrm{p}<0.001)$. Inhibition of respiration occurred obviously distal to glycolysis (i.e., at the mitochondrium), since progesterone also markedly inhibited the oxidation of pyruvate $\left(\mathrm{cpm}{ }^{14} \mathrm{C}\right.$-pyruvate oxidised ${ }^{\star} \mathrm{mg}^{-1 *} \mathrm{~h}^{-1}$ : control, $1411 \pm 31 ; 1 \mu \mathrm{M}, 1012 \pm 31 ; 10 \mu \mathrm{M}, 636 \pm 56 ; 50 \mu \mathrm{M}$, $184 \pm 37, \mathrm{p}<0.001 \mathrm{each})$. At variance to what was seen under basal conditions, progesterone did not increase and, at a concentration of $50 \mu \mathrm{M}$, even impaired insulin-stimulated glucose transport $\left(\mathrm{cpm}^{\star} \mathrm{mg}^{-1 \star} \mathrm{h}^{-1}\right.$ : control, $901 \pm 52 ; 1 \mu \mathrm{M}$, $892 \pm 42$, ns; $10 \mu \mathrm{M}, 1013 \pm 58$, ns; $50 \mu \mathrm{M}, 715 \pm 47, \mathrm{p}<0.05$ ), which obviously limited substrate availability for glycolysis (lactate release, $\mu \mathrm{mol}^{*} \mathrm{~g}^{-1 *} \mathrm{~h}^{-1 \text { : }}$ control, $18.1 \pm 0.5 ; 1 \mu \mathrm{M}, 23.1 \pm 0.7, \mathrm{p}<0.001 ; 10 \mu \mathrm{M}, 28.5 \pm 1.7, \mathrm{p}<0.001 ; 50 \mu \mathrm{M}$, $20.7 \pm 2.5$, ns). This effect was seen only under insulin stimulation and hence indicated that progesterone, in addition to its effect on the mitochondrium, impaired insulin-dependent glucose uptake.

Conclusion: Taken together, our results show that the acute non-genomic effects of progesterone on skeletal muscle have two overlapping effects on cellular fuel metabolism, which are (i) inhibition of cell respiration at the mitochondrium, and (ii) impairment of insulin-dependent glucose uptake (i.e., insulin resistance). These actions of progesterone could contribute to changes in glucose homeostasis seen during late pregnancy.

\section{8}

Dehydroepiandrosterone (DHEA) prevented obesity-induced acceleration of senescence in adipose tissue

K. Kajita, T. Ikeda, I. Mori, K. Fujioka, Y. Uno, H. Morita, T. Ishizuka; General Internal Medicine, Gifu University, Gifu, Japan.

Background and Aims: Numerous research indicates that DHEA administration decreases fat mass in human and rodent. We reported previously that DHEA decreased the expression of PPAR $\gamma$ and related genes in primary cultured adipocyte. These results can partially explain the mechanism of weight reducing effect of DHEA, however other mechanisms may be involved. On the other hand, some studies suggest the possibility that longevity might be associated with high blood level of DHEA, although conflict results have been published. Actually, few data have been provided whether DHEA administration affects life span or cell senescence. Moreover, biological mechanisms of anti-aging effects of DHEA have not been clarified. In this context, we evaluated the effects of DHEA treatment on biological markers in adipose tissue.

Materials and Methods: Male Otsuka Long Evans Fatty (OLETF) rats, hereditary obese animals derived from Long Evans Tokushima (LETO) rats, LETO rats and Wistar rats were fed with or without (control) 0.4\% DHEA containing food for $52 \mathrm{wk}$, and then killed by decapitation. Epididymal fats, livers, kidneys and skins were harvested and kept at $-80^{\circ} \mathrm{C}$. Epididymal fat pads were minced and digested with type 1 collagenase to obtain stromal vascular fraction (SVF). Frozen skin and SVF were cut, and thin $(5 \mathrm{~mm})$ sections were mounted on slide glass. These sections were stained for senescence-associated $\beta$-galactosidase (SA- $\beta$-Gal). Protein levels of CUG binding protein-1 (CUGBP-1), which bind to (CUG) triplet repeat RNA to modulate translation, in each organs were measured with Western blot. Cell proliferation was assessed with BrdU uptake in 3T3-L1 preadipocyte.

Results: Treatment with DHEA for 52 wk reduced body weight and fat weight, but not food consumption, in Wistar, LETO and OLETF rats. These results clearly indicated that treatment with DHEA for $52 \mathrm{wk}$ decreased fat mass without affecting food intake. Fasting plasma IRI, triglyceride and free fatty acid levels were significantly decreased in Wistar rats, but not in LETO and OLETF rats. SA- $\beta$-Gal was detected in SVF isolated from control OLETF rats, whereas it was not observed in other animals including Wistar and LETO rats, both fed with and without DHEA containing food and OLETF rats treated with DHEA. Considering the fact that SA- $\beta$-Gal is expressed in fibroblast sampled from aged animals or cultured cells in late passage, these results suggested that an obesity-induced elevated turnover of preadipocyte was prevented with DHEA treatment. CUGBP-1 protein levels were decreased in fat, liver and kidney in rats treated with DHEA. As it has been reported that aging results in CUGBP-1 accumulation in fat and liver, these results will support the idea that DHEA suppressed senescence. To investigate the possibility that DHEA might decrease cell proliferation, we evaluated the effects of DHEA on BrdU incorporation during DNA synthesis in 3T3-L1 cells. Treatment with insulin increased preadipocyte proliferation, whereas DHEA significantly decreased its proliferation in a dose response manner (100 nM DHEA, $\mathrm{p}<0.02)$.

Conclusion: These results suggested that DHEA -induced suppression of cell proliferation, including preadipocyte, might lead to anti-obesity and have an anti-senescence effect.

\section{9}

Estradiol treatment decreases body weight and improves insulin sensitivity in aged female mice fed on a high fat diet G. Bryzgalova ${ }^{1}$, L. Lundholm ${ }^{2}$, H. Gao ${ }^{2}$, A. Khan ${ }^{1}$, K. Dahlman-Wright ${ }^{2}$, J.-A. Gustafsson², S. Efendic ${ }^{1}$;

${ }^{1}$ Molecular Medicine and Surgery, Karolinska Institute, Stockholm, ${ }^{2}$ Biosciences and Nutrition, Karolinska Institute, Huddinge, Sweden.

Background and Aims: It has been shown that estradiol (E2) treatment in menopausal women reduced fasting levels of glucose and insulin, and in women with type 2 diabetes hormone replacement promoted insulin sensitivity and glycemic control. In many spontaneous rodent models of type 2 diabetes, E2 displays powerful antidiabetic properties. Therefore, in this study, we have explored the effect of E2 on phenotype and insulin sensitivity in female mice fed on a high fat diet (HFD).

Materials and Methods: C57BL female mice were fed on a HFD for 10 months, with daily $17 \beta$-estradiol $(50 \mu \mathrm{g} / \mathrm{kg}$, s.c.) or vehicle (sesame oil) given during the last month of feeding. Glucose and insulin tolerance tests were performed. Plasma hormone levels were determined by RIA. Real-time PCR was employed to measure gene expression profiles.

Results: Feeding mice with a HFD resulted in obesity (body weight was increased by $83 \%$ ) and severe insulin resistance (plasma glucose and insulin levels were elevated by 2.0 - and 4.8 -fold, respectively). Furthermore, mice on a HFD had significantly elevated plasma leptin and resistin levels. E2 administration to HFD mice decreased body weight by $22 \%$, improved glucose tolerance and increased insulin sensitivity. Plasma insulin levels, as well as levels of leptin and resistin, were significantly decreased after E2 treatment. The expression of the genes encoding resistin and leptin were reduced in WAT in E2-treated animals. Furthermore, TG levels were lowered in WAT, 
although they were not changed in the liver. Expression levels of lipogenic genes such as SCD1, FASN and SREBP1c were significantly decreased in WAT, whilst in liver, only expression of SCD1 was reduced to the same extent after E2 treatment.

Conclusion: HFD induces obesity and insulin resistance in aged female mice. Treatment with E2 markedly reduces these changes. These effects of E2 are probably mediated by down-regulation of lipogenic genes in WAT and liver. Supported by Swedish Research Council, KaroBio AB

\section{0}

Progesterone increases glucose tolerance in STZ-induced glucose intolerant rats

M. M. Faas, G. van der Schaaf, P. de Vos;

Division of Medical Biology, Dept of Pathology and Laboratory Medicine,

University Medical Centre Groningen, The Netherlands.

Background and Aims: The normal pancreas has a high regenerative capacity: during pregnancy the beta cell mass increases, which is due to an increased insulin demand, but also to stimulation by the pregnancy hormone, progesterone. Although studies into the regenerative capacity of the diabetic pancreas are scarce, it has been suggested that the diabetic beta cell mass can increase, but does not respond to an increased insulin demand. We hypothesize that the diabetic pancreas can grow, if appropriately stimulated. Therefore in the present study we treated STZ-induced glucose intolerant rats with progesterone.

Materials and Methods: Glucose tolerance was induced by STZ $(30 \mathrm{mg} / \mathrm{kg}$ b.w.) (GI-rats) and after 4 weeks rats were treated with progesterone implants or sham implants; control untreated rats were also used. One, 2 and 3 weeks after the start of the progesterone/sham treatment, IVGTT were done and 3 weeks after the start of the progesterone/sham treatment rats were sacrificed, pancreata snapfrozen and percentage proliferating beta cells (KI67 immunostaining), percentage beta cell area and stem cell number (nestin, c-kit and c-met positive cells) in the pancreas was evaluated.

Results: Progesterone treatment decreased the area under the curve (AUC) for glucose significantly after 3 weeks of treatment as compared with AUC before progesterone treatment (fig 1). The AUC for insulin after the IVGTT increased with time after treatment with progesterone as compared with before treatment (fig 1).

Despite the increase in insulin response during the IVGTT, 3 weeks after progesterone treatment the percentage beta cell area was only slightly increased (GI-rats: $0.55 \pm 0.11 \%$ vs GI-rats treated with progesterone $0.59 \pm 0.12 \%$ (not significant)). For comparison percentage beta cell area of control untreated rats was $0.72 \pm 0.074 \%)$. The slight increase in percentage beta cell area may be due to a significant increased number of proliferating beta cells in GI-rats treated with progesterone as compared with untreated GI-rats $(1.08 \pm 0.077 \%$ vs $0.58 \pm 0.1 \%$ respectively, $\mathrm{p}<0.05$; control rats: $0.13 \pm 0.05 \%$ ). Neogenesis was increased in progesterone treated GI rats, since the percentage of small beta cells clusters ( $1-5$ beta cells) was significantly increased in GI rats treated with progesterone as compared with untreated GI rats $(49.6 \pm 1.4 \%$ vs $40.6 \pm 3.9 \%$, respectively, $\mathrm{p}<0.05$; control rats: $43 \pm 2.1 \%$ ). Moreover, increased numbers of nestin or c-met positive cells in the islets and increased numbers of c-met positive cells around the ductules were found in progesterone treated GI-rats as compared to sham treated GI-rats.

Conclusion: The present results show that progesterone treatment of GI rats increased glucose tolerance and insulin responses. This may be due to proliferation of beta cells and neogenesis, resulting in a slightly increased beta cell mass at the time of sacrifice. It seems therefore that the beta cell mass of diabetic rats can grow with appropriate stimulation, such as progesterone. Supported by J.K. de Cockfoundation

\section{1}

Effects of estrogen replacement therapy on tissue antioxidant status: impact of OVX in diabetic state

A. Ceylan-Isik ${ }^{1}$, O. Erdogan-Tulmac ${ }^{2}$, F. Aktan ${ }^{3}$, N. Ari' ${ }^{1}$, G. Ozansoy ${ }^{1}$; ${ }^{1}$ Pharmacology, University of Ankara, Faculty of Pharmacy, ${ }^{2}$ Gynecology, Zekai Tahir Burak Women Helath, Education and Research Hospital, ${ }^{3}$ Biochemistry, University of Ankara, Faculty of Pharmacy, Ankara, Turkey.

Background and Aims: Diabetes is closely related with the generation of reactive oxygen species (ROS) and ovarian hormone deficiency is also related with this phenomenon. The excess production of ROS induces oxidative stress which results in cell damage or death. The aim of the present study is to investigate the effects of $17-\beta$ estradiol $\left(\mathrm{E}_{2}\right)$ on antioxidant status of aorta, uterus, liver and kidney from diabetic- and nondiabetic-ovariectomized (OVX) rats.

Materials and Methods: OVX $(n=8), \operatorname{OVX}^{2}+\mathrm{E}_{2}(\mathrm{n}=8)$, OVX+DIA $(\mathrm{n}=8)$, $\mathrm{OVX}+\mathrm{DIA}+\mathrm{E}_{2}(\mathrm{n}=8)$ were used as experimental groups. Bilateral ovariectomy was applied to the animals under anesthesia. Diabetes was induced by streptozotocin (STZ; $45 \mathrm{mg} / \mathrm{kg}$ i.p.) a week after the operation, and rats having blood glucose $\geq 150 \mathrm{mg} / \mathrm{dl}$ were accepted as diabetic. After 8 weeks rats were anesthesied and the tissues were removed, weighed and frozen at liquid nitrogen. Blood glucose (BG), total cholesterol $(\mathrm{CH})$, triglyceride (TG) and plasma $\mathrm{E}_{2}$ concentrations have been measured as final values. Malondialdehyde (MDA) levels, glutathione peroxidase (GPx) and catalase (CAT) activities were measured by fluorimetric and spectrophotometric methods.

Results: Although BG concentration was not affected ( $\mathrm{P}>.05$ vs. $\left.\mathrm{OVX}+\mathrm{DIA}+\mathrm{E}_{2}\right), \mathrm{CH}$ and $\mathrm{TG}$ concentrations were significantly reduced in diabetic group by the treatment $\left(\mathrm{P}<.05 v s\right.$. OVX+DIA $\left.+\mathrm{E}_{2}\right)$. The treatment also increased plasma $\mathrm{E}_{2}$ concentarions in the treated groups $(\mathrm{P}<.05$ vs. OVX and OVX+DIA). (Tab. 1-3)

Tab. 1

Tissue MDA concentrations ( $\mathrm{nmol} / \mathrm{mg}$ protein)

\begin{tabular}{|c|c|c|c|c|}
\hline & AORTA & UTERINE & LIVER & KIDNEY \\
\hline$\overline{\mathrm{OVX}}$ & $1.746 \pm 0.001^{\star}$ & $0.240 \pm 0.001^{\dagger \#}$ & $0.062 \pm 0.004^{\dagger \ddagger}$ & $0.129 \pm 0.002^{\dagger \ddagger}$ \\
\hline $\mathrm{OVX}+\mathrm{E}_{2}$ & $1.542 \pm 0.010^{\#}$ & $0.246 \pm 0.010^{\#}$ & $0.056 \pm 0.004$ & $0.110 \pm 0.004^{\#}$ \\
\hline OVX+DIA & $2.489 \pm 0.020^{\star \#}$ & $0.400 \pm 0.003^{\star \#}$ & $0.102 \pm 0.004^{\star \#}$ & $0.131 \pm 0.003^{\star \#}$ \\
\hline $\mathrm{OVX}+\mathrm{DIA}+\mathrm{E}_{2}$ & $1.751 \pm 0.025^{\star}$ & $0.272 \pm 0.018^{\star}$ & $0.055 \pm 0.004$ & $0.121 \pm 0.002^{*}$ \\
\hline
\end{tabular}

Tab. 2

Tissue GPx activation (nmol min-1/mg protein)

\begin{tabular}{lllll}
\hline & AORTA & UTERINE & LIVER & KIDNEY \\
\hline OVX & $0.088 \pm 0.002^{\dagger}$ & $0.238 \pm 0.002^{* *}$ & $0.258 \pm 0.004$ & $0.144 \pm 0.001^{\dagger \ddagger}$ \\
OVX+E & $0.074 \pm 0.001^{\ddagger}$ & $0.286 \pm 0.01$ & $0.261 \pm 0.004$ & $0.152 \pm 0.003$ \\
OVX+DIA & $0.097 \pm 0.003^{\star *}$ & $0.182 \pm 0.019^{* *}$ & $0.323 \pm 0.004^{\star *}$ & $0.228 \pm 0.003^{\star *}$ \\
OVX+DIA+E $_{2}$ & $0.082 \pm 0.002^{\dagger}$ & $0.296 \pm 0.005$ & $0.256 \pm 0.003$ & $0.159 \pm 0.003$ \\
\hline
\end{tabular}

${ }^{*} \mathrm{P}<.001$ and $\dagger \mathrm{P}<.05$ vs. $\mathrm{OVX}+\mathrm{E}_{2 ;} \# \mathrm{P}<.001$ and $\ddagger \mathrm{P}<.05$ vs. OVX $+\mathrm{DIA}+\mathrm{E}_{2}$
Fig. 1

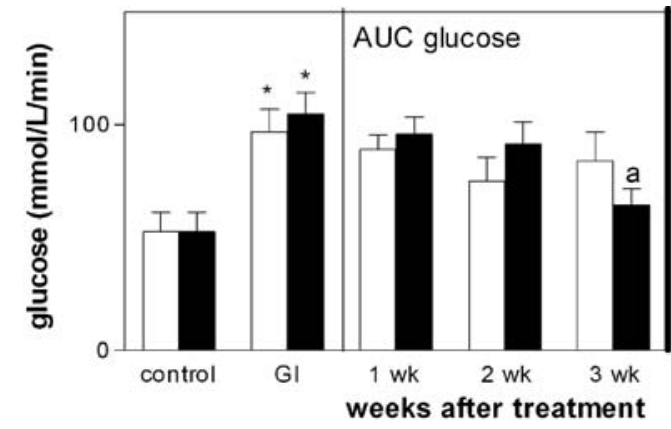


Tab. 3

Tissue CAT activity (ks-1/mg protein)

\begin{tabular}{|c|c|c|c|c|}
\hline & AORTA & UTERINE & LIVER & KIDNEY \\
\hline OVX & $0.017 \pm 0.001^{\dagger_{\#}}$ & $0.039 \pm 0.002$ & $0.231 \pm 0.003^{* \#}$ & $0.073 \pm 0.001^{*}$ \\
\hline $\mathrm{OVX}+\mathrm{E}_{2}$ & $0.015 \pm 0.001^{\#}$ & $0.042 \pm 0.001$ & $0.225 \pm 0.004^{\#}$ & $0.084 \pm 0.001 \#$ \\
\hline OVX+DIA & $0.032 \pm 0.001^{*^{\ddagger}}$ & $0.038 \pm 0.003$ & $0.319 \pm 0.003^{* \#}$ & $0.035 \pm 0.001^{\star} \#$ \\
\hline $\mathrm{OVX}+\mathrm{DIA}+\mathrm{E}_{2}$ & $0.029 \pm 0.001^{\star}$ & $0.0042 \pm 0.002$ & $0.253 \pm 0.002^{*}$ & $0.075 \pm 0.001^{*}$ \\
\hline
\end{tabular}

Conclusion: Ovarian hormone deficiency led to alteration in lipid peroxidation and free radical scavenging enzyme systems, and diabetes has a major contribution in this stiuation. This study demostrated that $\mathrm{E}_{2}$ replacement therapy has therapeutical importance to regulate in alteration of free radical-induced changes of OVX in both diabetic and non-diabetic condition.

\section{2}

A study of the serotoninergic tone in healthy subjects and diabetic patients treated by a clinical-paedagogic approach (Group Care) or traditional care

M. Trento ${ }^{1}$, P. Tibaldi ${ }^{1}$, M. Balbo ${ }^{2}$, C. Kucich ${ }^{1}$, M. Basile ${ }^{1}$, M. Tomelini ${ }^{1}$, P. Passera ${ }^{1}$, R. Giordano ${ }^{2}$, F. Cavallo ${ }^{3}$, E. Arvat ${ }^{2}$, M. Porta ${ }^{1}$;

${ }^{1}$ Internal Medicine, Laboratory of Clinical Paedagogy,

${ }^{2}$ Internal Medicine, Division of Endocrinology, Italy,

${ }^{3}$ Department of Public Health and Microbiology, Department

of Public Health and Microbiology, University of Turin, Italy.

Background and Aims: Alterations of mood and depression may be associated with type 2 diabetes (T2DM) and impaired glucose metabolism in non-diabetic people. Central serotoninergic activity may modulate glucose tolerance via neuroendocrine effectors. The serotoninergic tone was assessed by measuring the hormonal response to a selective serotonin reuptake inhibitor, citalopram, in non-diabetic subjects and patients with T2DM followed by either a traditional approach or a clinico-paedagogic intervention (Group Care) proven to improve quality of life and reduce anxiety.

Materials and Methods: Ten healthy subjects (age 53.20 \pm 3.92 ; males=5) and 13 patients with T2DM (age 60.92 \pm 3.52 , known duration $17.38 \pm 5.85$, males $=10$ ), 7 followed by Group Care and 6 by traditional care, underwent infusions of either citalopram $20 \mathrm{mg}$ or saline over $120 \mathrm{~min}$, in random order, at 8.30-9.00 am after overnight fasting. Samples were taken from an antecubital vein every $15 \mathrm{~min}$ for 4 hrs to measure circulating glucose, insulin, ACTH, free cortisol, DHEA, growth hormone $(\mathrm{GH})$ and prolactin. Hormonal responses were calculated as differences between the areas under the curves after saline and citalopram ( $\triangle$-AUC).

Results: Citalopram increased ACTH and cortisol more than saline in healthy subjects $(\mathrm{p}<0.026$ and $\mathrm{p}<0.011$, respectively) and diabetic patients treated by Group Care $(\mathrm{p}<0.056$ and $\mathrm{p}<0.038)$ but not in those followed by traditional care. The responses of DHEA, GH, prolactin, insulin and glucose to citalopram did not differ significantly from those to saline in any of the 3 groups. However, in the healthy subjects, basal glucose levels correlated directly with the GH $\Delta$-AUC $(\mathrm{r}=0.820 ; \mathrm{p}<0.004)$ and inversely with the insulin $\Delta$-AUC $(r=-0.822 ; \mathrm{p}<0.003)$. The correlation between basal glucose and $\mathrm{GH} \triangle$-AUC was preserved in diabetic patients $(\mathrm{r}=0.637 ; \mathrm{p}<0.026)$, in whom HbAlc correlated with basal glucose $(\mathrm{r}=0.657 ; \mathrm{p}<0.015)$ and the insulin $\Delta$-AUC $(\mathrm{r}=0.864 ; \mathrm{p}<0.012)$, and the glycaemic $\Delta$-AUC correlated with that of cortisol $(r=0.698 ; \mathrm{p}<0.012)$.

Conclusions: These results suggest that serotoninergic tone may modulate glucose metabolism through opposite effects on ACTH, cortisol, insulin and GH secretion. Diabetes may blunt the response of the pituitary-adrenal axis, though not in patients followed by a long-term intervention model that encompasses clinical as well as cognitive and emotional factors. Supported by a grant from the University of Turin (60\%)

\section{3}

Melatonin inhibits lipopolysaccharide-induced activities of cytokines and the hypothalamus-pituitary-adrenal axis in diabetic rats L.-Y. Zhong;

Department of Endocrinology, Beijing Tiantan Hospital,Capital Medical University, Beijing, China.

Background and Aims: The relationship between melatonin (MLT) and the neuroendocrine-immune network is well characterized; however, little is known regarding the effects of MLT on stress such as severe infection in diabetes. The aim of this study was to determine the effects of MLT on lipopolysaccharide (LPS)-induced activities of serum cytokines and the hypothalamus-pituitary-adrenal (HPA) axis in streptozotocin (STZ)-induced diabetic rats and to analyze the parameters related to diabetes and oxidative stress.

Materials and Methods: A total of 50 male Sprague-Dawley rats were assigned to 5 groups. Four groups simultaneously received a single dose of STZ (50 $\mathrm{mg} / \mathrm{kg}$ ) intraperitoneally (i.p.). The diabetic group received STZ alone; the LPS group received STZ plus LPS $(0.5 \mathrm{mg} / \mathrm{kg})$ i.p. after induction of diabetes with STZ; the LPS+MLT ${ }_{01 \mathrm{mg}}$ and LPS+MLT groups received 0.1 and $1 \mathrm{mg} /$ $\mathrm{kg}$ melatonin i.p., respectively, q6h for $12 \mathrm{~h}$ after LPS administration; and the remaining 10 rats served as control.

Results: LPS significantly increased the serum levels of tumor necrosis factoralpha (TNF- $\alpha$ ) and interleukin-6 (IL-6) in diabetic rats; it also dramatically increased the plasma concentrations of corticotropin-releasing hormone (CRH), adrenocorticotropic hormone (ACTH), and corticosterone. Both 0.1 and $1 \mathrm{mg} / \mathrm{kg}$ MLT doses significantly decreased the serum levels of TNF- $\alpha$ and IL-6. Significant inhibitory effects of melatonin $(1 \mathrm{mg} / \mathrm{kg})$ were observed on the plasma concentrations of $\mathrm{CRH}, \mathrm{ACTH}$, and corticosterone of the HPA axis. The beneficial effects of MLT, such as the antioxidative stress effect and maintaining glucose homoeostasis, were also observed in this study.

Conclusion: MLT significantly inhibited the increase in the serum levels of cytokines and appropriately ameliorated LPS-mediated HPA axis activation, which this resulted in a protective effect against the damages caused by LPS in STZ-induced diabetic rats. This finding provides a new approach for preventing the undesirable effects of the vicious cycle of hyperglycemia and stress factors such as severe infection in diabetic patients.

Supported by the Scientific Research Foundation for the Excellent Talent of Capital Medical University

\section{4}

Neuroendocrine markers in streptozotocin (STZ)-induced type 1 diabetes

Y. Revsin ${ }^{1,2}$, M. S. Oitzl ${ }^{1}$, F. E. Saravia ${ }^{2}$, A. F. De Nicola ${ }^{2}$, E. R. de Kloet ${ }^{1}$; ${ }^{1}$ Division of Medical Pharmacology, LACDR, Leiden University, The Netherlands, ${ }^{2}$ Laboratory of Neuroendocrine Biochemistry, IBYME, Buenos Aires, Argentina.

Background and Aims: Type 1 diabetes (T1D) is a common metabolic disorder characterized by profound dysregulations in the hypothalamicpituitary-adrenal (HPA) axis and severe disturbances in central nervous system (CNS) functions related to stress and adaptation. Reports from diabetic humans and animals showed HPA dysregulations, which become manifest as increased basal secretion of glucocorticoids. Among the alterations in the CNS functions, we previously described in uncontrolled streptozotocin (STZ)-induced diabetic mice changes in markers for astrogliosis and increased oxidative stress among others. Based on these results we hypothesize that glucocorticoids are the major trigger of these alterations.

Materials and Methods: Three months old C57BL/6J male mice were injected with STZ (170 mg/kg i.p.). Six days after diabetes onset, oral administration of high dose of RU486 (200mg/kg in $100 \mu \mathrm{l}$ p.o.) or vehicle was given during 4 days. We examined morphological-like numbers of hippocampal astrocytes and proliferative cells in the dentate gyrus, as well as molecular markers for key processes of HPA axis activation that control glucocorticoid hypersecretion such as MR, GR AVP and CRH mRNAs and circulating corticosterone and ACTH levels.

Results: In our model the HPA axis reached a new setpoint characterized by higher circulating corticosterone. Diabetic mice show increased plasma corticosterone, while plasma ACTH is decreased, and the expression mRNA of AVP in the paraventricular nucleus (PVN) of the hypothalamus is elevated 
and hippocampal MR is decreased. These changes in HPA axis regulation suggest adrenal hypersensitivity. Adrenal function analysis exhibited increased expression of ACTH receptors and corticosterone hypersecretion -from adrenal gland cell cultures challenged to ACTH- in diabetes. GR antagonist (RU486) treatment resulted in partial restoration of astrogliosis and neurogenesis alterations found in the brain of diabetic mice.

Conclusion: The profound disturbance in the HPA axis regulation, characterized by adrenal hypersensitivity to ACTH, provides evidence of the GR-mediated central role of glucocorticoids in diabetic neuropathology. A better understanding of these mechanisms may open up new avenues for therapeutically useful strategies to normalize neuronal disturbances and improve cognitive disabilities of diabetic patients.

Supported by NWO-WOTRO grant

\section{5}

Insulin resistance, beta cell function and glucose intolerance in acromegaly

R. Lichiardopol ${ }^{1}$, M. Coculescu ${ }^{2}$, D. Niculescu ${ }^{2}$, C. Pencea ${ }^{1}$, M. Vladica ${ }^{1}$;

${ }^{1}$ Clinic of Diabetes, Nutrition and Metabolic Diseases,

"N.C. Paulescu"Institute, ${ }^{2}$ Neuroendocrinology, "C.I. Parhon"

Institute of Endocrinology, Bucharest, Romania.

Background and Aims: Insulin resistance and glucose intolerance frequently occurrs in acromegaly. Although excess secretion of growth hormone (GH) can reduce insulin sensitivity, it is unclear if insulin resistance per se may induce glucose intolerance. We aimed to evaluate the insulin resistance and beta-cell ( $\beta$-cell) function, as the main mechanisms leading to glucose intolerance, in a group of patients with confirmed acromegaly.

Materials and Methods: This is a cross-sectional study of one hundred and twenty-six consecutive patients with acromegaly ( 80 women, 46 men) admitted in hospital between 2003-2006. Basal and 30 min plasma insulin (IRI), growth hormone (GH) levels (basal, 30, 60, and $120 \mathrm{~min}$ ) during a $75 \mathrm{~g}$ OGTT, $\mathrm{HbA}_{1 \mathrm{c}}$ and anthropometric measures were determined. Insulin resistance (HOMA-IR) was estimated using the HOMA2 (computersolved) model. The insulinogenic index (Ins index), normalized for insulin resistance $\left[\Delta\right.$ IRI $(30-0) \mu \mathrm{U} / \mathrm{ml}^{\star} 100 / \Delta$ BG $\left.(30-0) \mathrm{mg} / \mathrm{dl}\right] /$ HOMA-IR, was used to estimate the $\beta$-cell function. Data are presented as mean $( \pm \mathrm{SEM})$ values. Kruskal-Wallis or ANOVA (as appropriate) was used to compare data across groups. Simple and multiple regression between variables and logistic regression analysis were performed. The EpiInfo software was used for analysis. A $p<0.05$ was considered significant.

Results: According to WHO criteria, there were 41 subjects (23 women, 18 men) with glucose intolerance (GINT): 21 with IFG or IGT and 20 with diabetes. The remaining 85 subjects had normal glucose tolerance (NGT). As compared to NGT, the GINT group had greater mean values for age (56.1 \pm 1.7 vs. $49.2 \pm 1.4$ yrs; $p<0.003), \mathrm{HbA}_{1 c}(6.6 \pm 0.2$ vs. $5.4 \pm 0.07 \% ; p<0.001)$, waist to hip ratio $(0.917 \pm 0.01$ vs. $0.877 \pm 0.008 ; p<0.015)$ and reduced values for Ins index ( $40.8 \pm 7.1$ vs. $111.4 \pm 20.2 ; p<0.001)$. Compared to the third tertile, the patients within the first tertile of the Ins index distribution were seven times as likely to have prevalent glucose intolerance (Odds ratio $=7.4$; 95\% CI: $1.84-35.3 ; p<0.002$ ). Between the two groups (GINT vs. NGT), there were no significant differences regarding mean values for HOMAIR $(2.1 \pm 0.3$ vs. $1.7 \pm 0.1 ; p=0.31)$, basal GH $(15.7 \pm 2.7$ vs. $16.3 \pm 4.7 \mathrm{ng} / \mathrm{ml}$; $p=0.92)$ and minimal GH levels during OGTT $(13.1 \pm 2.4$ vs $13.6 \pm 2.2 \mathrm{ng} /$ $\mathrm{ml} ; p=0.88$ ), duration of the disease ( $5.9 \pm 1.4$ vs. $6.8 \pm 0.85 \mathrm{yrs} ; p=0.58$ ), BMI $\left(30.2 \pm 0.8\right.$ vs. $\left.29.1 \pm 0.6 \mathrm{Kg} / \mathrm{m}^{2} ; p=0.28\right)$, waist circumference $(988.4 \pm 22.4$ vs. $937.9 \pm 15.1 \mathrm{~mm} ; p=0.08$ ). In simple linear regression analysis, $\mathrm{HbA}_{1}$ was significantly correlated to area under the curve of plasma glucose levels during OGTT $(r=0.557 ; n=80 ; p<0.001)$, age $(r=0.224 ; n=95 ; p<0.035)$, Ins index $(r=-0.332 ; n=69 ; p<0.006)$ and HOMA-IR $(r=0.224 ; n=83 ; p<0.035)$. In multiple regression analysis with a change of $\mathrm{HbA}_{1 c}$ as the dependent variable, the following variables were significant: Ins index ( $p<0.03)$, HOMAIR $(p<0.001)$. In logistic regression analysis only Ins index was significantly correlated $(p<0.03)$ to glucose tolerance.

In conclusion, our data suggest that reduced insulin response, reflecting the failure of $\beta$-cells adaptation to increased insulin resistance, leads to glucose intolerance in acromegaly.

\section{6}

Experimental metabolic syndrome strengthens age-related hormonal changes in Syrian hamsters

A. L. Zagayko ${ }^{1}$, L. N. Voronina ${ }^{1}$, N. S. Krasova ${ }^{2}$, V. V. Poltorak ${ }^{2}$;

${ }^{1}$ Department of Biological Chemistry, National University of Pharmacy, ${ }^{2}$ Laboratory for Pathophysiology and Medical Genetics, Institute of Endocrine Pathology Problems, Kharkiv, Ukraine.

Background and Aims: It has been shown, that half unsaturated fat and fructose-enriched diet induces metabolic syndrome in Golden Syrian hamsters. The development of hyperinsulinaemia depends both of sex and age of animals: in the young, insulin resistance (IR) more significantly educes in males, but in the adult - in females. The aim of the study was to investigate the sex and age differences of steroid hormones content in Syrian hamsters with experimental metabolic syndrome (MS).

Materials and Methods: 72 Golden Syrian hamsters (males/females: 1/1) were investigated. The experimental groups (4-wk-old animals were formed the $1^{\text {st }}$ group, 20-wk-old animals - the $2^{\text {nd }}$ group and 1-y-old ones - the $3^{\text {rd }}, 12$ animals each) were submitted for 4 weeks to a diet containing $29 \%$ vegetableand animal oils and fructose ( $2 \mathrm{~g} / 100 \mathrm{~g} \mathrm{~b}$. w./ day) - MS groups. A control group ( $\mathrm{n}=36$, matched for age) was submitted to a standard diet. Plasma hydrocortisone, estradiol and testosterone levels were determined by ELISA. Fasting blood glucose and insulin (by RIA) were measured. Homeostasis Model Assessment (HOMA) was used to estimate IR. Statistical analyses were carried out using SPSS and Microsoft Excel.

Results: Hyperglycaemia, hyperinsulinaemia and IR were developed in hamsters fed a half unsaturated fat and fructose-enriched diet compared to controls. Both males and females of MS groups showed enhance of hydrocortisone levels (males: by $38 \%, 40 \%, 75 \%$; females: by $53 \%, 21 \%$ and $54 \%$ in the $1^{\text {st }}, 2^{\text {nd }}$ and $3^{\text {rd }}$ group, respectively, as compared to controls, $p<0.05$ ). Thus, the tendency to hypercortisolaemia strengthened during aging. Age changes of estradiol content in MS groups demonstrated a typical trend, but were more pronounced. Estradiol levels in 4- and 20-wk-old hamsters with MS were higher in comparison with controls $(\mathrm{p}<0.05)$, but in 1-y-old animals the estradiol levels were decreased (by 30 and $32 \%$ in males and females, respectively, $\mathrm{p}<0.01)$. In control males levels of testosterone were the highest at 20 weeks, whereas in MS males - at 4 weeks. Thus, 20-wk- and 1-y-old males with MS showed the reduction of testosterone content (by 18 and 16 $\%$, respectively, $\mathrm{p}<0.05$ ), that could be explained due to transformation of the hormone to estradiol in fat tissue at 20 weeks, and downstroke of secretion in 1 -y-old animals (that was accompanied by decrease in estradiol content). Conclusion: Thus, the experimental metabolic syndrome strengthens agerelated alterations of steroid hormones secretion in Golden Syrian hamsters of both sexes. The data suggest that not only an increase of predisposition to metabolic syndrome takes place with age, but also the development of the metabolic syndrome causes hormonal changes typical for aging. 


\section{PS 57 DPP-4}

\section{7}

OGTT modulates GLP-1 but not DPPIV levels in different glucose tolerance degree patients

I. Dicembrini ${ }^{1}$, L. Pala ${ }^{1}$, G. Bardini ${ }^{1}$, S. Ciani ${ }^{1}$, A. Pezzatini ${ }^{1}$, B. Cresci ${ }^{1}$,

L. Buci ${ }^{1}$, S. Giannini ${ }^{1}$, E. Mannucci ${ }^{2}$, C. M. Rotella ${ }^{1}$;

${ }^{1}$ Dep. of clinical Pathphysiology. Unit of Endocrinology,

${ }^{2}$ Geriatric Unit Dept of Critical Care, University of Florence, Italy.

Background and Aims: Glucagon-like peptide 1 (GLP-1) is a gut-derived incretin hormone that stimulates insulin and suppresses glucagons secretion, inhibits gastric emptying, and reduces appetite and food intake. A few minutes after its synthesis, GLP-1 is degraded by dipeptidylpeptidase-4 (DPP-IV). The reduced GLP-1 levels after an oral glucose load in type 2 diabetic patients could depend on a reduced synthesis or on an increased degradation; anyway GLP-1 and DPP-IV level during an OGTT are never studied. The aim of our study is to investigate GLP-1 and DPP-IV levels during a OGTT in different tolerance degree patients.

Materials and Methods: OGTT using $75 \mathrm{gr}$ of glucose in a 50\% water solution are performed in 46 subjects ( $30 \mathrm{~F} ; 16 \mathrm{M}$ ), aged $58,9 \pm 11,4 \mathrm{ys;} \mathrm{BMI} 31,08 \pm 6,15$ $\mathrm{KG} / \mathrm{m}^{2}$; Waist $104 \pm 13 \mathrm{~cm}$. After OGTT: Normal glucose tolerance (NGT) resulted in 22 subjects; Impaired glucose tolerance (IGT) in 10 subjects and Type 2 Diabetes (DMT2) in 14 subjects. GLP-1 assay was performed with an ELISA kit and DPPIV assay was performed with a colorimetric method.

Results: Our data show a significant difference in the Area Under Curve (AUC) for glycemia among the three groups: NGT vs IGT ( $p<0.0001)$, NGT vs DMT2 $(\mathrm{p}<0.0001)$ and IGT vs DMT2 $(\mathrm{p}=0,007)$. The AUC for GLP-1 results significantly different among the three groups, in particular there is a significant difference between NGT and IGT $\left(199,1 \mathrm{pmol} / \mathrm{L}^{*} \mathrm{~min}[117 ; 343]\right.$ vs $\left.105,2 \mathrm{pmol} / \mathrm{L}^{*} \min [28,8 ; 125,5] \mathrm{p}=0.004\right)$; between NGT and DMT2 $(199,1$ $\mathrm{pmol} / \mathrm{L}^{\star} \min [117 ; 343]$ vs $84,7 \mathrm{pmol} / \mathrm{L}^{\star} \min [52,03 ; 184,07]$, but not between IGT and DMT2. On the contrary, DPPIV AUC presents no significant difference among the groups.

Conclusion: These results could suggest a different role between impaired GLP-1 synthesis and degradation in the preclinical and clinical evolution of Type 2 Diabetes. In particular, a reduction of GLP-1 synthesis could be involved in the early stages of diabetes and perhaps also in the preclinical phases while an increase of GLP-1 degradation could have a role in the late stages of diabetes or in metabolic failure.

\section{8}

Metformin is a GLP-1 secretagogue, not a dipeptidyl peptidase-4 inhibitor

R. Sinha Roy ${ }^{1}$, R. Bergeron ${ }^{2}$, L. Zhu ${ }^{1}$, H. He ${ }^{1}$, G. Jiang ${ }^{1}$, F. Liu ${ }^{1}$, K. Lyons ${ }^{1}$, K. Pryor ${ }^{1}$, J. Yao ${ }^{1}$, B. B. Zhang ${ }^{1}$, N. Thornberry ${ }^{1}$;

${ }^{1}$ Merck \& Co., Inc., Rahway, United States,

${ }^{2}$ Department of Kinesiology, University of Montreal, Canada.

Background and Aims: Treatment of type 2 diabetic patients with a combination of metformin (MET) and a dipeptidyl peptidase-4 (DPP-4) inhibitor improves glucose control better than with either drug alone. The combined effects of MET and the DPP-4 inhibitor desfluorositagliptin (DFSITA) on plasma GLP-1 levels were studied in mice using immunoassays selective for active and inactive GLP-1 (truncated by DPP-4 cleavage).

Materials and Methods: Lean, diet-induced obese (DIO) C57BL/6N, or $d b /$ $d b$ mice were dosed orally with vehicle (0.5\% carboxy-methylcellulose), MET $(500 \mathrm{mg} / \mathrm{kg})$, DF-SITA $(100 \mathrm{mg} / \mathrm{kg})$, or MET+DF-SITA with or without oral administration of glucose ( $3 \mathrm{~g} / \mathrm{kg})$. Plasma DPP- 4 activity and active/inactive GLP-1 levels were measured for up to $6 \mathrm{hrs}$ post-dose.

Results: MET rapidly increased plasma inactive GLP-1 3-5 fold for as long as $6 \mathrm{hrs}$ in both fed and fasted animals. Active GLP-1 was not significantly increased, reflecting rapid cleavage by DPP-4. During this period, preproglucagon mRNA was increased in the gut but not in the pancreas of MET-treated animals. MET treatment appeared to inhibit plasma DPP4 activity in DIO mice. In vitro studies, however, demonstrated that MET does not directly inhibit DPP-4. MET+DF-SITA consistently increased active GLP-1 levels 2-3 fold in both fed and fasted animals, but some inactive GLP1 remained in circulation. Combination treatment produced lower levels of total GLP-1 than with MET alone. The improvement in glucose tolerance with MET treatment was not further improved by DPP-4 inhibition, and was not dependent on GLP-1 receptor signaling.

Conclusion: Metformin increases plasma GLP-1, which can be protected from rapid inactivation by co-administration of a DPP-4 inhibitor. Metformin reportedly decreases shedding of membrane-associated DPP-4, which can decrease plasma enzyme activity in vivo. MET increases plasma glucagon in mice, which counteracts the glucose lowering effects of elevated plasma GLP1. The absence of additive glucose lowering effects is likely a murine-specific phenomenon given the additive effects of metformin and DPP-4 inhibitors in the clinic.

\section{9}

Pharmacodynamic characteristics of SK-0403, a novel highly selective dipeptidyl peptidase IV inhibitor, in animal models

Y. Yasuda ${ }^{1}$, M. Goto ${ }^{1}$, K. Nakaya ${ }^{1}$, S. Kawamura ${ }^{1}$, S. Furuta ${ }^{1}$, M. Tamura ${ }^{1}$, N. Kato ${ }^{1}$, T. Ishida ${ }^{2}$, Y. Matsumoto';

${ }^{1}$ Pharmaceutical Research Laboratories, SANWA KAGAKU KENKYUSHO CO.,LTD., Mie, ${ }^{2}$ Project Development Dept., SANWA KAGAKU KENKYUSHO CO.,LTD., Nagoya, Japan.

Background and Aims: Dipeptidyl peptidase-IV (DPP-IV) inhibitor is well known as a new class of oral agent for type 2 diabetes (T2D). It is generally accepted that $80 \%$ inhibition of plasma DPP-IV is sufficient to elevate intact incretins and improve glycemic control. However, it is unknown whether the $\mathrm{IC}_{80}$ of DPP-IV inhibitor in systemic circulation is sufficiently high to protect the rapid degradation of glucagon-like peptide-1 (GLP-1), which is secreted from the intestine in response to nutrient ingestion. The aims of the present studies were to investigate the in vitro and in vivo profiles of SK-0403, a novel DPP-IV inhibitor currently under development for the treatment of T2D, and to clarify the relationship between higher DPP-IV inhibition (over $80 \%$ ) and degradation of intact GLP-1.

Materials and Methods: In an in vitro study, the inhibition for DPP-IV and selectivity for DPP-IV related peptidases including DPP8, DPP9, fibroblast activation protein $\alpha$ (FAP) and other enzymes were investigated. In an in vivo study, the effects of SK-0403 on plasma DPP-IV activity were assessed in rats and dogs. A glucose lowering action in rats and mice and an active GLP-1 enhancement in rats was investigated. To evaluate systemic metabolism of GLP- 1 by DPP-IV, GLP-1 was infused to rats from the femoral vein after oral administration of SK-0403. Plasma DPP-IV activity and active GLP-1 were measured before and after administration of SK-0403 and GLP-1.

Results: SK-0403 showed an $\mathrm{IC}_{50}$ of $3.3 \pm 0.3 \mathrm{nmol} / \mathrm{L}($ mean \pm SD) for recombinant human DPP-IV and more than 17,000-fold higher selectivity with recombinant human DPP8, DPP9, FAP and other enzymes. In dogs, a single oral dose of SK-0403 dose-dependently suppressed plasma DPP-IV activity, and DPP-IV inhibition was long-lasting compared with vildagliptin ( $66 \%$ for $10 \mathrm{mg} / \mathrm{kg} \mathrm{SK}-0403$ vs. $13 \%$ for $30 \mathrm{mg} / \mathrm{kg}$ vildagliptin at $24 \mathrm{~h}$ after administration). In C57BL6 mice, potency of SK-0403 in reduction of plasma glucose excursion during OGTT was almost equal to that of sitagliptin at doses of 0.3 to $3 \mathrm{mg} / \mathrm{kg}$. Furthermore, in diabetic rats, SK-0403 $(1,3$ and $10 \mathrm{mg} / \mathrm{kg}$ ) exhibited the dose-dependent improvements of plasma glucose and insulin during OGTT. When GLP-1 was intravenously infused to rats after oral administration of SK-0403, active GLP-1 in plasma significantly increased to $353 \pm 20 \mathrm{pmol} / \mathrm{L}$ at $\mathrm{IC}_{80}$ for DPP-IV of SK-0403 compared with control (129 $\pm 3 \mathrm{pmol} / \mathrm{L})$. In addition, active GLP-1 was increased with SK0403 (from 1- to 10 -fold $\mathrm{IC}_{80}$ ) in a concentration-dependent manner and reached a maximum of $445 \pm 33 \mathrm{pmol} / \mathrm{L}$ at 10 -fold $\mathrm{IC}_{80}\left(p<0.05 \mathrm{vs}\right.$. $\left.\mathrm{IC}_{80}\right)$. The concentration-dependent effect of SK-0403 on GLP-1 degradation was also confirmed based on the experiment in which endogenous active GLP-1 after an oral carbohydrate load increased 1.9 times at 10 -fold $\mathrm{IC}_{80}$ compared to the $\mathrm{IC}_{80}$.

Conclusion: SK-0403 is a potent and highly selective DPP-IV inhibitor. SK0403 at $\mathrm{IC}_{80}$ for DPP-IV activity in circulation may not be sufficient to protect degradation of GLP-1 because metabolism of GLP-1 is attributed to DPP-IV hydrolysis in tissues as well as plasma. These results show for the first time that over $80 \%$ inhibition of plasma DPP-IV is required to more effectively avoid systemic degradation of GLP-1, and they suggest that SK-0403 with appropriate dose-titration is promising for the treatment of T2D. 


\section{0}

Increased beta cell replication and mass in $95 \%$ pancreatectomized rats treated with a DPPIV stabilized GLP-1 analogue

G. Joanny, N. Téllez, E. Estil-les, M. Nacher, S. Rodríguez-Mulero,

E. Montanya;

Lab. Diabetes and Experimental Endocrinology, IDIBELL,

L'Hospitalet de Llobregat, Spain.

Background and Aims: The incretin hormone GLP-1 has a well known insulinotropic action and some more recently reported positive effects on beta cell viability and proliferation. The very short life of GLP-1 makes it unsuitable as a therapeutic agent, and has fostered the search for GLP-1 analogues with extended action. The aim of this study was to determine the effects of a new DPPIV stabilized GLP-1 analogue on beta cell growth and metabolic control in partially pancreatectomized rats, a model of beta cell regeneration.

Materials and Methods: The GLP-1 analogue used in this study is a DPPIV stablilised GLP-1 analogue which has been derivatised with a chemical moiety that binds to albumin in a reversible manner thereby extending the plasma half life to at least one day. SD rats underwent 95\% partial pancreatectomy (95\%-Px) and were treated with the GLP-1 analogue $(100 \mu \mathrm{g} / \mathrm{kg} \cdot \mathrm{day}$, IP once daily) or vehicle from day 1 (when 95\% Px was performed), to day 15 (when pancreatic remnants were harvested). Allocation of pairs of same day pancreatectomized animals to gastrin- and vehicle-treated groups was blinded. Blood glucose and body weight were monitored on days 1, 3, 7, 10 and 15. A group of animals of similar body weight underwent a 95\% Px and had the pancreatic remnant harvested immediately after surgery. An additional control group of sham-Px rats $(n=6)$, had the pancreas harvested after 15 days of follow up. Beta cell mass and cross-sectional individual beta cell size was determined by morphometry, and beta cell replication by BrdU incorporation, in insulin stained sections of harvested pancreatic remnants and pancreases.

Results: 95\%-Px rats treated with vehicle developed severe hyperglycemia (day 15: $516 \pm 41 \mathrm{mg} / \mathrm{dl}$ ) that was prevented in GLP-1 analogue-treated rats (day 15: $216 \pm 36 \mathrm{mg} / \mathrm{dl}, \mathrm{p}<0.05$ ). Beta cell mass after 95\% Px was $0.34 \pm 0.09$ $\mathrm{mg}$, and was $34 \%$ higher $(0.45 \pm 0.13 \mathrm{mg}$, p: $\mathrm{ns})$ in vehicle-treated rats. GLP-1 analogue treatment increased significantly the beta cell mass $(0.90 \pm 0.08 \mathrm{mg})$ $(\mathrm{p}<0.05)$. Beta cell replication in $95 \%$-Px rats treated with vehicle was $40 \%$ higher than in the normal pancreas of sham-Px rats (vehicle: $1.02 \pm 0.2 \%$; sham: $0.72 \pm 0.2 \%, \mathrm{p}=\mathrm{ns})$. GLP-1 analogue increased beta cell proliferation ( $1.64 \pm 0.2 \%, \mathrm{p}<0.05$ vs sham-Px and vehicle). The individual beta cell size was similar in GLP-1 analogue- and vehicle-treated 95\%-Px rats $(260 \pm 14$ $\mu \mathrm{m}^{2}$ vs $\left.251 \pm 24 \mu \mathrm{m}^{2}\right)$ and higher than sham-Px rats $\left(197 \pm 16 \mu \mathrm{m}^{2}\right)$, indicating that GLP-1 analogue had no effects on beta cell hypertrophy. The number of scattered beta cells (groups of $<5$ beta cells) considered an indirect marker of islet neogenesis, was similar in GLP-1 analogue-treated $(6.81 \pm 0.5 \%)$, vehicle-treated $(6.16 \pm 0.7 \%)$ Px rats.

Conclusion: The treatment with the long-acting GLP-1 analogue stimulated beta cell replication, increased beta cell mass in 95\%-Px rats, and prevented the development of severe hyperglycemia, supporting its potential in the treatment of diabetes.

Supported by FIS PI-060891, ISCIII-RETIC RD06, Novo Nordisk

\section{1}

The dipeptidyl peptidase 4 inhibitor vildagliptin has no acute effect on the endocrine secretion in the isolated perfused porcine pancreas K. R. Hjøllund ${ }^{1}$, T. E. Hughes ${ }^{2}$, J. J. Holst ${ }^{1}$;

${ }^{1}$ Department of Biomedical Sciences, The Panum Institute,

Copenhagen, Denmark,

${ }^{2}$ Novartis Institutes for biomedical research, Cambridge, United States.

Background and Aims: Inhibitors of dipeptidyl peptidase 4 (DPP-4) are being developed as a treatment for type 2 diabetes because they enhance the metabolic stability of glucagon-like peptide-1(GLP-1) and glucose-dependent insulinotropic polypeptide (GIP) in the body and improve glycemic control. It is not known, however, whether the inhibitors influence the pancreatic islets directly or influence the activity of pancreatic neuropeptides such as pituitary adenylate cyclase-activating peptide (PACAP) which is a known DPP-4 substrate. The effects of DPP-4 inhibition on direct metabolic stimulation of islet secretion by glucose and arginine are also unknown.
Material and Methods: The effect of DPP-4 inhibition was examined using isolated perfused porcine pancreas with $(n=10)$ or without $(n=15)$ intact vagal innervation. Insulin, glucagon, somatostatin and vasoactive intestinal peptide (VIP) secretion were measured in response to electrical vagus stimulation at $8 \mathrm{~Hz}$, and during PACAP $(1 \mathrm{nM})$, arginine $(5 \mathrm{mM})$ and glucose $(11 \mathrm{mM})$ infusion. All stimuli were applied 3 times namely before, during and after vildagliptin $(1 \mu \mathrm{M})$ infusion. In addition, control experiments $(\mathrm{n}=7)$ with identical protocols but no addition of vildagliptin during the middle stimulation period were carried out for comparison.

Results: Vagus stimulation and PACAP increased insulin, glucagon and VIP secretion $(\mathrm{p}<0.05)$, but the presence or absence of vildagliptin had no influence on these responses. Arginine strongly stimulated the release of all three islet hormones, which was not altered by vildagliptin. Glucose increased the secretion of insulin and somatostatin $(\mathrm{p}<0.05)$ and inhibited glucagon secretion, again unchanged by vildagliptin. A comparison of the control experiments with no vildagliptin infusion and that involving vildagliptin infusion during the middle stimulation period similarly indicated that addition of vildagliptin did not modify the responses to any applied stimulus.

Conclusion: Taken together, these findings indicate that DPP-4 expressed in the pancreas does not exert any influence of pancreatic endocrine secretion whether stimulated by cholinergic or noncholinergic neural mechanisms or by metabolic stimuli. Rather vildagliptin acts by prolonging the circulating half-life and thereby increasing the circulating plasma concentration of Land $\mathrm{K}$-cell derived incretin hormones. 


\section{PS 58 Incretin release}

\section{2}

Retrograde intracolonic infusion of free fatty acids as a novel approach to directly stimulate GLP-1 release by rat intestinal L-cells in vivo R. Iakoubov ${ }^{1,2}$, P. L. Brubaker ${ }^{1,3}$;

${ }^{1}$ Physiology, University of Toronto, Canada,

${ }^{2}$ Internal Medicine I, Merheim Medical Center, Cologne, Germany, ${ }^{3}$ Medicine, University of Toronto, Canada.

Background and Aims: Glucagon-like peptide-1 (GLP-1) is a potent stimulator of glucose-dependent insulin secretion that is secreted by the distal intestinal L-cell in response to nutrient ingestion. Our lab has identified longchain monounsaturated fatty acids (LC-MUFA) as particularly important regulators of GLP-1 secretion, not only initiating a neural pathway from the duodenum to enhance release, but also exerting direct stimulatory effects on the ileal and colonic L-cells. To avoid the complicating effects of the neural regulation of the L-cell in vivo, we have investigated the influence of LCMUFA on GLP-1 secretion in rats following intracolonic (IC) infusion of the LC-MUFA, oleic acid (OA).

Materials and Methods: Male Wistar rats (250-300 g) were fed a bowel cleansing solution (Klean-Prep ${ }^{\oplus}$ ) for 48 hours and fasted for the last 12 hours. The rats were then anesthetized with isoflurane and the bowel further cleansed with saline solution. The appropriate volume of the IC infusate and its distribution within the rat distal intestine was established by barium sulfate-assisted X-Ray imaging. The carotid artery was cannulated for blood sampling and an enema was then performed using a silicone catheter with $25 \mathrm{ml} \mathrm{OA}(125 \mathrm{mM})$ or vehicle (16\% TWEEN 80$)$; each group consisted of 4 animals. Blood samples were collected at $0,5,15,30$ and $60 \mathrm{~min}$, and plasma levels of bioactive GLP-1, insulin and glucose were determined by ELISA, RIA and glucose analyzer, respectively. Statistical analysis was performed with GraphPad Prism software using one-way ANOVA followed by Tukey's HSD post hoc test.

Results: IC infusion of barium sulfate followed by X-Ray imaging showed that administration of $25-30 \mathrm{ml}$ of solution allowed complete coverage of the colon, caecum and terminal ileum, the known locations of most intestinal L-cells, and did not cause any damage to the animals as determined by lack of leakage and normal recovery for several days thereafter. The basal plasma concentration of bioactive GLP- 1 in fasted rats was $7.5 \pm 3.3$ pM. IC infusion of OA induced a progressive increase in plasma GLP-1 levels, by up to $316 \pm$ $42 \%$ of basal at $60 \mathrm{~min}(\mathrm{p}<0.05)$. The OA treatment also increased the GLP1 AUC, as compared with controls $(\mathrm{p}<0.05)$. Vehicle alone did not increase GLP-1 levels. Plasma insulin and glucose concentrations did not differ between the groups, although insulin levels slightly decreased in both groups directly after initiation of the IC infusion.

Conclusion: Intracolonic OA infusion directly stimulates GLP-1 release in vivo, independent of changes in insulin and glucose levels. These experiments demonstrate that retrograde intracolonic infusion is a reliable and noninvasive approach to directly stimulate GLP-1 release by the intestinal L-cell. Supported by Banting and Best Diabetes Centre, the Canadian Diabetes Association and the Canada Research Chairs Programme

\section{3}

\section{Cyclic AMP triggers glucagon-like peptide-1 secretion from GLUTag} cells

F. Reimann, A. K. Simpson, P. S. Ward, A. M. Habib, K. Wong,

F. M. Gribble;

Dept. of Clinical Biochemistry, University of Cambridge, United Kingdom.

Background and Aims: Glucagon-like peptide-1 (GLP-1) is an incretin hormone secreted from enteroendocrine L-cells in response to food ingestion, which enhances insulin release from pancreatic $\beta$-cells. Understanding the pathways involved in GLP-1 release is essential for the development of oral GLP-1 secretagogues, which would add to the group of GLP-1 based therapies for the treatment of type 2 diabetes. Some of the most reliable stimuli for GLP-1 release in different model systems act by increasing intracellular cyclic AMP (cAMP) concentrations, but the downstream signalling mechanisms remain unclear. The aim of the current study was to investigate the pathways by which cAMP stimulates GLP-1 secretion, using the GLUTag cell line.
Materials and Methods: GLP-1 release from GLUTag cells was assayed by ELISA following 2 hour incubations in the presence of test agents. cAMP concentrations were measured by ELISA after 15 min test agent incubations in the presence of IBMX $(10 \mu \mathrm{M})$. Intracellular calcium $\left(\left[\mathrm{Ca}^{2+}\right]_{\mathrm{i}}\right)$ was monitored by single cell fluorescence imaging in cells loaded with fura-2 AM. Electrophysiology was performed by perforated patch experiments.

Results: Pituitary Adenylate Cyclase-Activating Polypeptide (PACAP) dose-dependently stimulated GLP-1 release to a maximum of $\sim 2$-fold from GLUTag cells, and at $100 \mathrm{nM}$ increased cAMP levels 1.5-fold. PACAP (100 $\mathrm{nM}$ ) or forskolin plus IBMX (fsk/IBMX, $10 \mu \mathrm{M}$ each) triggered a rise in $\left[\mathrm{Ca}^{2+}\right]_{i}$ and enhanced the response to glucose by increasing both the number of responsive cells and the magnitude of the calcium rise in individual cells. Fsk/IBMX also stimulated GLP-1 release and $\left[\mathrm{Ca}^{2+}\right]_{i}$ elevation in the absence of added nutrients. In patch clamp experiments no significant effect was observed on the magnitude of voltage gated calcium currents. Fsk/IBMX triggered membrane depolarisation of $+5.3 \pm 1.1 \mathrm{mV}(\mathrm{n}=6, \mathrm{p}<0.01)$ and an increase in the mean action potential frequency from $0.07 \pm 0.05$ to $0.4 \pm 0.1$ $\mathrm{Hz}(\mathrm{n}=6, \mathrm{p}<0.05)$. This was associated with changes in the properties of two ion conductances. Hyperpolarisation activated currents ( $\mathrm{I}_{\mathrm{L}}$ ) exhibited a right shift in their voltage-dependence: $\mathrm{V}_{0.5}$ shifting from $-85.7 \pm 1.9 \mathrm{mV}$ to $-72.1 \pm 1.7 \mathrm{mV}(\mathrm{n}=6 ; \mathrm{p}<0.001)$. Inhibition of $\mathrm{I}_{\mathrm{b}}$ currents by ZD7288 $(10 \mu \mathrm{M})$, did not however, prevent fsk/IBMX-triggered membrane depolarisation. In the presence of ZD7288 we found that fsk/IBMX also closed a background potassium conductance. This was distinct from $\mathrm{K}_{\mathrm{ATP}}$, as it was still observed in the presence of tolbutamide $(500 \mu \mathrm{M})$. In addition to its direct effect on the membrane potential, fsk/IBMX potentiated secretion downstream of membrane depolarisation, as it stimulated GLP-1 secretion $2.3 \pm 0.2$-fold $(\mathrm{n}=4)$ in the presence of $30 \mathrm{mM} \mathrm{KCl}$ plus diazoxide $(340 \mu \mathrm{M})$.

Conclusion: cAMP acts as both a trigger and a potentiator of GLP-1 release from GLUTag cells. The triggering pathway is associated with a shift in the voltage-dependence of hyperpolarisation activated currents and closure of a background potassium conductance, both of which tend to depolarise the membrane and make cells more responsive to other depolarising stimuli. The ability of cAMP to trigger GLP-1 release in the absence of nutrient could provide a pathway for the indirect stimulation of L-cells lower in the gut, where levels of luminal nutrients are low. Cyclic AMP elevating pathways and the cAMP modulated conductances in L-cells present important targets for the development of therapeutic GLP-1 secretagogues.

Supported by the Wellcome Trust, St John's College Cambridge and the Winston Churchill Foundation

\section{4}

Regulation of peptide hormone release from neuroendocrine cells in the gut and the pancreas

S. Leicht, P. Eickelmann, S. Mayet;

Boehringer-Ingelheim, Biberach, Germany.

Background and Aims: GLP-1 (Glucagon-like-peptide 1) is an important incretin hormone released by L-cells in the small intestine and upper colon in response to nutrients and hormones. GLP-1 has different metabolic effects; one important task is the stimulation of insulin release in the $\beta$-cells of the pancreas. L-cells and $\beta$-cells exhibit similarities in their receptor expression profiles and in their signaling pathways which are responsible for the GLP-1 and insulin release. Recently, a number of G-protein coupled receptors have been deorphanized as receptors for different nutrient factors like fatty acids, peptones or lipids and bile acids, which play a physiological role in GLP-1 and/or insulin release. Focus of the current study are GPR40, GPR120 and GPR119 and their signal transduction pathways in L-cells and $\beta$-cells for the GLP-1 and insulin release.

Materials and Methods: The receptor expression of the GPCRs was determined in STC-1, NCI-H716, INS-1E and Min6 cell lines as well as in rat ileum, colon and islets using Taqman analysis. Protein expression was investigated with Western blots as well as immunfluorescent staining in the various tissues. GLP-1 release from STC-1 cells and colon slices upon stimulation with different receptor ligands was measured using ELISA (Linco). Insulin release from INS-1E, Min6 cells and isolated rat islets was measured by ELISA as well. Direct measurements of receptor activation was measured using the Calcium 3 FLIPR kit (Molecular Devices) or the HTRF IP1 kit (GBO) for Gq-coupled receptors GPR40 and GPR120, elevation of cAMP by activation of GPR119 was examined by cAMP Alpha Screen (Perkin Elmer). 
Results: The Taqman analysis showed that GPR40, GPR119 and GPR120 are expressed in all investigated cell lines, in primary islets and in the intestine. The protein expression of the receptors could be confirmed by Western Blot analysis in colon, ileum and STC-1 cells. In the STC-1 cells as well as in colon slices stimulation of GPR119, GPR40 and GPR120 by their natural or small molecule ligands led to a GLP-1 release in a concentration-dependent manner. Activation of GPR 40 and GPR120 by their natural or small molecule ligands caused an increase in $\mathrm{Ca}^{2+}$ and IP1 levels in cell lines stably transfected with the human receptors. In STC-1 cells increase in IP1 concentration could also be measured. In INS-1E, Min6 cells and isolated islets, activation of the receptors led to an increased, glucose dependent insulin secretion. Whereas stimulation of the Gq signaling pathway with increase in intracellular $\mathrm{Ca}^{2+}$ seems to have direct effects on insulin and GLP-1 release, Gs-coupled increase of cAMP has only modulatory effects on either glucose-stimulated insulin secretion from $\beta$-cells or Calcium-mediated GLP- 1 secretion from Lcells.

Conclusion: These results show that GPR120 and/ or GPR40 contribute to the GLP-1 release from L-cells and insulin release from $\beta$-cells via generation of IP3 and release of intracellular Calcium. The Gs-coupled receptor GPR119 also plays an important role in modulation of primary secretagogue effects of nutrients and glucose, respectively.

\section{5}

Expression of glucose-sensing machinery in intestinal GLP-1 secreting cells

A. M. Habib, F. Reimann, H. E. Parker, F. M. Gribble;

Clinical Biochemistry, Cambridge University, United Kingdom.

Background and Aims: Glucagon-like peptide 1 (GLP-1) is secreted from intestinal L-cells in response to oral carbohydrate and promotes insulin secretion from pancreatic $\beta$-cells. The ability of L-cells to sense glucose directly is controversial because L-cells lie scattered throughout the intestinal epithelium, therefore restricting investigations to partially enriched primary cell cultures or cell lines. The aim of this study was to generate transgenic mice with fluorescently labeled L-cells, and to use this model to investigate the expression in L-cells of genes implicated in glucose sensing.

Materials and Methods: Transgenic mice expressing yellow fluorescent protein (Venus) driven by the proglucagon promoter were made using a bacterial artificial chromosome containing the proglucagon gene. Colocalization of Venus and GLP-1 was confirmed by immunofluorescence (IF) microscopy. Venus-positive and control negative gut epithelial cells were separated by FACS, and used to isolate RNA and cDNA. Expression of genes of interest in sorted cells was measured by real time PCR and expressed relative to that of $\beta$-actin.

Results: Several transgenic founders were obtained, from which six lines were established with different transgene copy number. All lines showed Venus-expression in the gut restricted to GLP-1 positive cells by IF. When colonic tissue was digested to single cells and sorted by FACS, $\sim 0.3 \%$ of the cell population exhibited Venus fluorescence. Real time PCR revealed that FACS-sorted positive cells contained 27000 -fold more glucagon mRNA than non-fluorescent (control) cells collected in parallel, confirming the identity of Venus positive cells as L-cells. Our previous studies on the GLP1 secreting cell line, GLUTag, showed that both ATP-sensitive potassium $\left(\mathrm{K}_{\mathrm{ATP}}\right)$ channels and sodium-glucose cotransporters (SGLTs) contribute to the glucose-triggered depolarisation that initiates GLP-1 release. In FACSpositive colonic cells, expression of the $\mathrm{K}_{\text {ATP }}$ channel subunit Kir6.2 was 250fold higher than that in negative cells, and 9-fold higher than in GLUTag cells. Expression of the sulphonylurea receptor (SUR1) was also enhanced 200-fold in L-cells relative to negative cells, and 14-fold relative to GLUTag cells. SGLT1 was expressed in negative cells from ileum and colon, consistent with its function in glucose absorption. Its expression was enhanced 5 -fold in ileal L-cells and 10-fold in colonic L-cells compared with respective negative cells, and 6-14-fold relative to GLUTag cells. SGLT3a expression in ileal L-cells was 2 -fold higher than in GLUTag cells, but was barely detectable in control ileal cells or in colonic cells. Of the facilitative transporters, GLUT1 was detectable in all cell types, GLUT2 and GLUT3 were expressed at low levels in all ileal and colonic cells, whilst GLUT5 expression was enhanced 58-fold in colonic L-cells relative to controls, and 1.5-fold relative to GLUTag cells. We were unable to detect the sweet taste receptor Tas1R2 in gut epithelial cells, while its partner, Tas1R3, was expressed at similar (low) levels in both positive and negative cells from ileum and colon.
Conclusion: We have developed transgenic mice exhibiting L-cell-specific expression of Venus. Real time PCR revealed expression in FACS-sorted L-cells of $\mathrm{K}_{\text {ATP }}$ channel subunits (Kir6.2 and SUR1), SGLTs (1 and 3a) and GLUT1 and 5. Our results indicate that glucose-sensing machinery similar to that found in GLUTag cells, is present in native murine L-cells. Supported by the Wellcome Trust and St John's College, Cambridge

\section{6}

Evidence for the role of the phosphodiesterase (PDE) isoform PDE4D in regulating glucagon-like peptide (GLP-1) secretion in the GLUTag cell line W. Ong ${ }^{1}$, M. D. Houslay², M. J. Lynch ${ }^{2}$, G. S. Baillie ${ }^{2}$, N. J. Pyne ${ }^{1}$,

B. L. Furman ${ }^{1}$;

${ }^{1}$ Strathclyde Institute of Pharmacy \& Biomedical Sciences, University of Strathclyde, United Kingdom, ${ }^{2}$ Institute of Biomedical and Life Sciences, University of Glasgow, United Kingdom.

Background and Aims: The reported increase in the synthesis and secretion of the major incretin GLP-1 in response to activation of adenylyl cyclase suggests a role for cyclic AMP (cAMP). Previous work indicated that PDE4 was probably the most important cAMP phosphodiesterases (PDE) relevant to GLP-1 secretion present in GLUTag cells, in which the PDE4 selective inhibitor rolipram potently inhibited PDE activity and augmented glucoseinduced GLP-1 secretion. The present study aimed to determine which PDE4 isoform was functionally important.

Materials and Methods: GLUTag cells were transfected with a dominant negative PDE4D5 (PDE4D5DN; Asp556Ala) construct, containing a neomycin resistance gene and selected in $0.9 \mathrm{mg} / \mathrm{ml}$ geneticin (G418) for stable PDE4D5DN-transfected clones.

Results: In one clone (C3), RT-PCR with PDE4 primers showed elevated levels of PDE4 amplicons. This clone showed marked elevation in basal GLP1 secretion relative to wild-type (WT) GLUTag cells (pmol/ $10^{6}$ cells $/ 2 \mathrm{~h} \mathrm{WT}$ $0.114 \pm 0.016$; C3 $0.427 \pm 0.028 \mathrm{P}<0.0001$ ). Evidence for the role of cAMP in mediating the effects of the PDE4D5DN was obtained using the protein kinase A (PKA) inhibitor H89. In WT GLUTag cells, H89 failed to modify basal or glucose elevated GLP-1 secretion during a $2 \mathrm{~h}$ incubation. GLP- 1 secretion was increased in response to glucose $(10 \mathrm{mM})$ in $\mathrm{C} 3$ and glucose-induced, but not basal, GLP-1 secretion was suppressed by incubation with the PKA inhibitor H89 $(10 \mu \mathrm{M})$ [GLP-1 secretion ( $\left.\mathrm{pmol} / 10^{6} \mathrm{cells} / 2 \mathrm{~h}\right)$ basal $0.34 \pm 0.017$; basal + H89 0.224 $\pm 0.034 ; 10 \mathrm{mM}$ glucose $0.493 \pm 0.045 ; 10 \mathrm{mM}$ glucose + H89 0.3 \pm 0.021 ; ANOVA $\mathrm{P}<0.0001 ; \mathrm{H} 89+$ glucose $10 \mathrm{mM}$ vs glucose $10 \mathrm{mM}$ $\mathrm{P}<0.01 ; \mathrm{n}=6-8$ wells from at least 2 separate experiments)]. In both WT cells and cells stably transfected with PDE4D5DN, 24h pre-incubation with H89 $(10 \mu \mathrm{M})$ markedly suppressed basal and glucose induced GLP-1 secretion (e.g. in WT pre-treated with $\mathrm{H} 89$ for $24 \mathrm{~h}$, GLP-1 secretion ( $\mathrm{pmol} / 10^{6}$ cells $/ 2 \mathrm{~h}$ ); basal 0.172 \pm 0.024 ; basal + H89 $0.058 \pm 0.03 \mathrm{P}<0.05$ - Dunnett's post hoc).

Conclusion: These data suggest that GLUTag cells express functional PDE4D5, since abrogation of the activity of this isoform using a stably transfected dominant negative construct markedly augments both basal and glucose-induced GLP-1 secretion. The inhibition of glucose-induced GLP1 secretion by $\mathrm{H} 89$ suggests that a role for PKA in the response to glucose in these cells. The marked inhibition of basal GLP-1 secretion by prolonged treatment with $\mathrm{H} 89$ in WT cells and in cells expressing the dominant negative PDE4D5 may suggest a role for cAMP in regulating GLP-1 synthesis.

\section{7}

Glucagon-like peptide 1 response to meals and post-prandial hyperglycaemia in type 2 diabetic patients

E. Mannucci ${ }^{1}$, M. Monami ${ }^{1}$, L. Pala ${ }^{2}$, S. Ciani ${ }^{2}$, L. Da Vico ${ }^{1}$, C. Lamanna ${ }^{1}$, G. Bardini ${ }^{2}$, N. Marchionni ${ }^{1}$, C. M. Rotella ${ }^{2}$;

${ }^{1}$ Critical Care Medicine and Surgery, Unit of Gerontology

${ }^{2}$ Clinical Pathophysiology, Section of Endocrinology -

University of Florence, Italy.

Background and Aims: The response of the intestinal insulinotropic hormone Glucagon-like peptide-1 (GLP-1) to meals is impaired in type 2 diabetic patients; this could contribute to the pathogenesis of impaired insulin secretion and post-prandial hyperglycemia. This study is aimed at 
the assessment of the relationship between meal-induced GLP-1 and postprandial hyperglycemia in type 2 diabetic patients.

Materials and Methods: 21 drug-naïve type 2 diabetic patients aged $(\mathrm{m} \pm \mathrm{SD})$ $58.2 \pm 11.1$ years and with a BMI of $32.1 \pm 7.2 \mathrm{~kg} / \mathrm{m}^{2}$ were studied. Blood glucose and active GLP-1 levels were measured 0, 30, 60, 90, and $120 \mathrm{~min}$ after a standard test meal. A continuous glucose monitoring (CGM) system with a subcutaneous sensor measuring glucose every 5 min was applied for the following 3 days, during which patients were invited to self-monitor accurately their food intake. Nutrient intake at each meal was calculated on the basis of patients' food records. For each patient, postprandial 120 min glucose incremental area under the curve (iAUC) was included in linear regression model exploring its relationship with total energy and carbohydrate intake, and the angular coefficient for total energy (EAC) and carbohydrate (CAC) was calculated.

Results: GLP-1 levels peaked 30 min after the test meal. Logarithmically transformed 60-min GLP-1 iAUC showed a significant inverse correlation with $\mathrm{HbAlc}(\mathrm{p}<0.01)$. A significant inverse correlation of 60 -min GLP1 iAUC was also observed with EAC and CAC (both $\mathrm{p}<0.01$ ), meaning that patients with a lower GLP-1 response to the test meal had a higher increment of postprandial glucose for each additional unit of total energy or carbohydrate intake.

Conclusion: In type 2 diabetic patients, a lower GLP-1 response to meals is associated with a higher $\mathrm{HbAlc}$, and with a greater degree of mealinduced hyperglycaemia, both in a meal test and during CGM in "real-life" conditions.

\section{PS 59 Adipocyte differentation}

\section{8}

Increased growth and elevated IGF-1 serum concentrations in mice with an adipose tissue specific deletion of the Igf- 1 receptor

N. Kloting ${ }^{1}$, L. Koch ${ }^{2}$, T. Wunderlich ${ }^{2}$, M. Kern ${ }^{1}$, K. Ruschke ${ }^{1}$, J. C. Bruning ${ }^{2}$, M. Bluher';

${ }^{1}$ Department of Internal Medicine III, University of Leipzig, Germany,

${ }^{2}$ Department of Mouse Genetics and Metabolism, University of Cologne and Center of Molecular Medicine Cologne, Germany.

Background and Aims: Insulin-like growth factor-1 (IGF-1) and the IGF-1 receptor have been implicated in the regulation of adipocyte differentiation and lipid accumulation in vitro. To investigate the role of IGF-1 receptor in vivo, we created mice with fat-specific disruption of the IGF-1 receptor gene (FIGF-1RKO mice) using the Cre-loxP system.

Materials and Methods: Ten mice of each genotype of both sexes were studied from an age of 4 weeks up to 32 weeks of life. Body weight was recorded weekly up to an age of 14 weeks, and thereafter in 2-week intervals, body length (naso-anal length) was measured once at week 32 . At an age of 16 weeks both sexes of a subgroup of twenty (10 FIGF-1RKO and 10 controls) underwent a food intake measurement over a time period of one week. The daily food intake was calculated as the average intake of chow within the time stated. Intraperitoneal (i. p.) glucose tolerance tests (GTT) and insulin tolerance tests (ITT) were performed at the age of 12 and 24 weeks.

Results: At an age of 14 weeks the FIGF-1RKO mice have remarkably increased somatic growth, most likely as a consequence of significant elevated IGF-1 serum concentrations compared with control mice. Higher circulating IGF-1 levels could be due to increased IGF-1 expression both in liver and epigonadal adipose tissue, suggesting that adipose tissue plays a role in the regulation of IGF-1 serum concentrations. FIGF-1RKO mice exhibit increased adipose tissue mass with a predominantly increased lipid accumulation in epigonadal fat pads. Insulin-stimulated glucose uptake into adipocytes was unaffected by the deletion of the IGF- 1 receptor.

Conclusion: Thus, IGF-1 receptor signalling in adipocytes is not crucial for the development and differentiation of adipose tissue, but seems to participate in the regulation of IGF-1 serum concentration.

\section{9}

Tumour necrosis factor (TNF) $\alpha$ induces canonical Wnt signaling and de-differentiation of mature 3T3-L1 adipocytes

B. Gustafson, U. Smith;

The Sahlgrenska Academy at Goteborg University, Lundberg Laboratory for Diabetes Research, Center of Exellence for Cardivascular Research, Department of Molecular and Clinical Medicine/Diabetes, Goteborg, Sweden.

Background and Aims: Obesity is a central and causal part of insulin resistance and type 2 diabetes. Enlargement of adipocytes induces inflammation and macrophage recruitment into the adipose tissue with induction of a variety of inflammatory markers which can exert local effects or systemic effects in other tissues, such as liver and muscle. The source of secreted TNFalfa in adipose tissue is probably infiltrated macrophages. We have recently shown that TNFalfa maintains the canonical Wnt-signalling during differentiation of preadipocytes and, thereby, keeps the preadipocytes in a proliferating and undifferentiated state. In mature adipocytes, TNFalfa is able to activate the Toll like receptor 4 and NFkappaB and, thereby, induce an inflammatory phenotype. In this study, we examined the effects of TNFalfa on the Wnt signalling pathway and inflammation in fully differentiated adipocytes.

Materials and Methods: Differentiated 3T3-L1 adipocytes (differentiation day 8) were incubated with or without TNFalfa $(20 \mathrm{ng} / \mathrm{mL})$ for 16 days. RNA was extracted and analysed with real-time PCR and immunoblots were performed on cell lysates. The effect of TNFalfa on adipocyte morphology was followed with microphotographs.

Results: TNFalfa induced de-differentiation of the adipocytes with a decrease in adipogenic genes such as peroxisome proliferator-activated receptor (PPAR)gamma2, CCAAT/enhancer binding protein (C/EBP)alfa, aP2, adiponectin and the glucose transporter (GLUT)4. During initiation of the 
normal preadipocyte differentiation, the canonical Wnt-signalling is downregulated combined with the degradation of the transcriptional activator beta-catenin. However, within $24 \mathrm{hrs}$, TNFalfa induced the expression of Wnt10b and stabilisation of beta-catenin in mature adipocytes. The Wnt signalling inhibitors Dickkopf (DKK)1 and soluble frizzled-related protein (sFRP)2 were down-regulated within 2 days of TNFalfa stimulation which is in agreement with the induction of beta-catenin. Surprisingly, however, the rate-limiting protein for beta-catenin degradation, Axin, was still present in spite of the fact that beta-catenin expression was stabilized. The presence of TNFalfa induced a number of proinflammatory molecules such as BMP4, Wisp2, MCP-1 as well as the macrophage-specific protein colony stimulating factor (CSF) 2 .

Conclusion: Unexpectedly, TNFalfa is able to induce canonical Wnt signalling with stabilisation of beta-catenin in mature 3T3-L1 adipocytes. At the same time, the adipocytes undergo de-differentiation with a decreased expression of adipocyte-specific markers and transcription factors such as PPARgamma2 and C/EBPalfa. A proinflammatory and insulin-resistant phenotype is achieved with decreased GLUT4 expression and induction of proinflammatory molecules such as MCP-1. Thus, induction of Wnt secretion by cells in the adipose tissue leads to a dysregulated state with impaired differentiation and function of the mature adipose cells.

\section{0}

Wnt-signaling is maintained and adipogenesis inhibited by TNFalpha but not MCP-1 and resistin

A. Hammarstedt, P. Isakson, B. Gustafson, U. Smith;

Dept of Molecular and Clinical Medicine/Diabetes, Medicine, Göteborg, Sweden.

Background and Aims: Type 2 diabetes and obesity with enlarged fat cells are associated with low-grade systemic inflammation and impaired adipogenesis, as well as the recruitment of inflammatory cells into the adipose tissue. Cytokines like TNFalpha and IL- 6 are secreted by the inflammatory cells and have been shown to impair normal adipocyte differentiation. An important mechanism whereby these cytokines inhibit adipogenesis is by maintaining an active Wnt-signaling pathway. Also other cytokines, like MCP-1 and resistin, are involved in the inflammatory process and are secreted by macrophages. If these cytokines also affect Wnt-signaling and adipocyte differentiation is currently unclear.

Materials and Methods: 3T3-L1 cells were differentiated to adipocytes under basal conditions or in the presence of TNFalpha, MCP-1 or resistin alone or in combination with a thiazolidinedione, pioglitazone. Cells were harvested at various time points during the adipocyte differentiation process and mRNA and protein expression levels quantified to study the effect of these cytokines on inflammation, Wnt-signaling and adipogenic markers. Each experiment was repeated 6 times and Wilcoxon's non-parameteric test for paired comparison was used to evaluate differences between the various conditions.

Results: In the presence of TNFalpha, there was a marked reduction in lipid accumulation after 4 days when the first lipid droplets are seen, and an almost complete absence of lipid droplets in fully differentiated adipocytes after 8 days. Furthermore, the gene expression of adipogenic markers, such as aP2, adiponectin, LPL, perilipin and GLUT4, was significantly reduced. In contrast, no significant effect was seen in the presence of MCP-1 or resistin. The presence of TNFalpha inhibited axin expression, which is the rate-limiting scaffold-protein for the degradation of the Wnt-signaling responsive transcriptional co-factor beta-catenin. As a result, Wnt-signaling and the expression of Wnt-signaling responsive genes were maintained. Again, no effect was seen in the presence of MCP-1 or resistin. The addition of pioglitazone antagonized the effect of TNFalpha on Wnt-signaling and adipogenesis. In addition, pioglitazone had beneficial effects on inflammation, Wnt-signaling and adipogenesis per se.

Conclusion: In the present study, we show that while TNFalpha is able to maintain an active Wnt-signaling, induce inflammation and completely block adipose cell differentiation, no effect was found by either MCP-1 or resistin on these processes. Addition of the thiazolidinedione, pioglitazone, was found to antagonize the effect of TNFalpha on the Wnt-signaling process and, consequently, promote adipogenesis.

Supported by Swedish Research Council, European Community's FP6 EUGENE2, Swedish Diabetes Association, Novo Nordisk Foundation, Sonya Hedenbratt Memorial Fund

\section{1}

The sumo-conjugating enzyme Ubc9 regulates adipocyte differentiation M. Melchiorre, A. Cignarelli, S. Miccoli, C. Caccioppoli, F. De Stefano, M. R. Orlando, A. Natalicchio, S. Perrini, L. Laviola, F. Giorgino; Endocrinology \& Met. Diseases, University of Bari, Italy.

Background and Aims: Ubc9, the E2 enzyme implicated in conjugation of the small ubiquitin-like modifier SUMO to intracellular substrates, has recently been shown to participate in the regulation of eukaryotic cell differentiation. The objective of this study was to examine the role of Ubc9 in the differentiation of adipose cells.

Materials and Methods: To evaluate potential changes in Ubc9 expression during adipogenesis, Ubc9 protein and mRNA levels, as well as the intracellular localization of Ubc9, were analyzed in 3T3-L1 cells by immunoblotting, real-time PCR, and immunofluorescence analyses, respectively, at different times after induction of differentiation. Selective knock-down of Ubc9 in 3T3-L1 preadipocytes was obtained by transfection of a Ubc9-specific siRNA at day -2 and +2 of the differentiation protocol. Achievement of adipocyte differentiation was assessed by visual inspection, Oil-O-Red staining, gene expression analysis by real-time PCR, and evaluation of adipocyte-specific metabolic responses, including FFA release into the culture medium and 2deoxy-D-glucose uptake.

Results: Ubc9 mRNA and protein levels were found to be increased at day $2(2.5$-fold vs. baseline, $\mathrm{p}<0.05)$ and then gradually declined to basal levels at day 8 of differentiation. In addition, the Ubc9 protein, visualized by immunofluorescence analysis, was expressed predominantly in the nucleus of preadipocytes, whereas, beginning from day 4 after induction of differentiation, the majority of the enzyme was evident in cytoplasmic compartments, in which it partially co-localized with the glucose transporters GLUT4. To evaluate the role of Ubc9 in adipogenesis, a Ubc9-specific siRNA to reduce Ubc9 expression was transfected in 3T3-L1 preadipocytes prior to induction of cell differentiation; this resulted in $80 \%$ reduction of both mRNA and protein levels of Ubc9 at day 2 after induction of differentiation compared to control. In the presence of Ubc9 gene silencing, mRNA levels of various adipocyte transcription factors and adipocyte-specific proteins, including C/EBP $\delta(\mathrm{p}<0.05$ at days 2 , and 4$), \operatorname{C} / \operatorname{EBP} \alpha(\mathrm{p}<0.001$ at day 4$)$, PPAR $\gamma(\mathrm{p}<0.001$ at day 4$)$, GLUT4 $(\mathrm{p}<0.05$ at day 8$)$ and FAS $(\mathrm{p}<0.05$ at day 8 ), were significantly decreased as compared to control. In addition, knock-down of Ubc9 resulted in inhibition of the clonal expansion that physiologically occurs during the early phases of differentiation, since the siRNA-treated 3T3-L1 cells were reduced by $30 \%$ compared to control cells $(p<0.05)$. At the end of differentiation, Oil-Red-O staining demonstrated accumulation of large triglyceride droplets in $>90 \%$ of control adipocytes, whereas cell triglycerides were evident as minute lipid droplets in only $60 \%$ of the 3T3-L1 adipocytes treated with the Ubc9-specific siRNA. Consistent with these results, following Ubc9 gene silencing, FFA release into the culture medium was reduced by $35 \%(\mathrm{p}<0.05)$ and both basal and insulinstimulated glucose uptake rates were reduced by $40 \%(\mathrm{p}<0.05)$ compared to control.

Conclusion: In conclusion, Ubc9 expression and localization are regulated during adipocyte differentiation. Ubc9 may play an essential role in the differentiation program by affecting mitotic clonal expansion of the preadipocytes and induction of adipogenic transcription factors.

\section{2}

Gene expression profiling identifies molecular pathways that cause decreased differentiation of preadipocytes in type 2 diabetes patients F. H. J. van Tienen ${ }^{1}$, C. J. H. van der Kallen ${ }^{2}$, P. J. Lindsey ${ }^{1}$, H. J. M. Smeets ${ }^{1}$; ${ }^{1}$ Department of Genetics \& Cell Biology,

${ }^{2}$ Department of Internal Medicine, Maastricht University, The Netherlands.

Background and Aims: The important role for white adipose tissue in the development of type 2 diabetes mellitus (T2DM) has only recently been recognized. Insulin resistance and T2DM are associated with increased adipocyte size, altered secretory patterns and decreased differentiation of preadipocytes. Molecular processes have already been identified by gene expression profiling in white adipose tissue and isolated adipocytes of T2DM patients. To characterize the role of preadipocytes in further detail we performed gene expression profiling in cultured subcutaneous preadipocytes of T2DM and BMI matched controls. 
Materials and Methods: Preadipocyte cell cultures were prepared from subcutaneous fat biopsies of T2DM patients $(\mathrm{n}=7$, age $52.7 \pm 12.0 \mathrm{yr}$, BMI $\left.34.2 \pm 5.3 \mathrm{~kg} / \mathrm{m}^{2}\right)$ to age and BMI matched control subjects $(\mathrm{n}=9$, age $50.5 \pm 12.1 \mathrm{yr}$, BMI $29.5 \pm 3.2 \mathrm{~kg} / \mathrm{m}^{2}$ ). Gene expression analysis was performed on two-colour 70-mer oligo arrays (21521 features, Operon) followed by linear regression modelling and gene ontology analysis using GenMAPP/ MAPPFinder and GOTree Machine.

Results: Normal linear regression modelling indicated 499 transcripts to be differentially expressed between T2DM patients and controls, 344 were down-regulated and 155 transcripts were up-regulated. Gene ontology analysis showed a decreased activity of genes involved in differentiation related processes, including adipogenesis, extracellular matrix adhesion, actin cytoskeleton and RNA polymerase II transcription factor activity. In addition, genes in pathways involved in lipid metabolism including biosynthesis and beta-oxidation were also down-regulated in preadipocytes of T2DM patients.

Conclusion: The down-regulation of genes involved in differentiation is possibly an explanation for the already earlier described decreased differentiation of preadipocytes of T2DM subjects, which leads to a reduction of newly formed adipocytes in subcutaneous fat depots, ultimately leading to ectopic fat storage, e.g. in muscle and liver. Since the cells are cultured for some weeks, our data indicate that these changes can be an intrinsic property of the cultured preadipocytes and, therefore, not related to changed in vivo signalling from macrophages and adipocytes in insulin resistant adipose tissue. Whether the down-regulation of lipid-metabolism is cause or consequence needs to be validated by functional studies, which will be performed for all the relevant processes.

Supported by the Dutch Diabetes Research Foundation

\section{3}

Orphan nuclear receptor DAX-1 interacts and represses PPARgamma transactivation

H.-S. Choi, G. Kim, B. Nedumaran;

Hormone Research Center, Chonnam National University, Gwangju,

Republic of Korea.

Background and Aims: DAX-1 (NROB1) is an atypical nuclear receptor (NR) which functions primarily as a transcriptional corepressor of other NRs through heterodimerization. Peroxisome proliferator-activated receptor (PPAR; NR1C3) gamma is a ligand-dependent NR that plays a key role in lipid metabolism, inflammation, and adipogenesis. In this study, we examined the novel cross-talk between DAX-1 and PPARgamma and elucidated the role of DAX-1 in adipocyte differentiation.

Materials and Methods: GST-pull down assay and immunopreicipation assay was used to confirm the direct interaction between DAX-1 and PPARgamma. Transient transfectin assay was used to examine the role of DAX-1 on PPARgamma transactivation. Adenoviral vector mediated overexpression of DAX-1 was used to the effect of DAX-1 on 3T3L1 adipocyte differentiation. Results: Transient transfection assay demonstrated that DAX-1 inhibited the transactivity of PPARgamma in a dose-dependent manner. Further, DAX1 directly interacted with DNA binding domain (DBD)/hinge region of PPARgamma in a ligand-independent manner, and showed the competitive interaction with PPARgamma coactivator (PGC)-1alpha in PPARgamma binding. Compared to preadipocytes or stromal vascular cells, the endogenous level of DAX-1 was significantly lower in differentiated 3T3-L1 adipocytes or primary adipocytes. Intriguingly, adenoviral overexpression of DAX-1 downregulates the expression of PPARgamma target genes, resulting in attenuation of adipogenesis in 3T3-L1 cells.

Conclusion: Overall, our results suggest that DAX-1 acts as a novel corepressor of PPARgamma via PGC-1alpha competition and has the potential role on the regulation of the PPARgamma-mediated cell differentiation.

\section{4}

Deficiency of the neuronal transcription factor NSCL-2 results in an increased number of preadipocytes in WAT

K. Ruschke ${ }^{1,2}$, M. Blüher ${ }^{1}$, N. Klöting ${ }^{1}$, T. Braun ${ }^{2}$;

${ }^{1}$ Medical Clinic III, University of Leipzig,

${ }^{2}$ Cardiac Development and Remodelling, Max-Planck-Institute for Heart and Lung Research, Bad Nauheim, Germany.

Background and Aims: The basic helix-loop-helix protein NSCL-2, a neuronal transcription factor, is widely expressed in different areas of the CNS and PNS during embryonic and fetal development. During postnatal life the expression decreases significantly but is specifically maintained in hypothalamic areas known for control of food intake and energy expenditure. In particular, the expression of NSCL-2 co-localizes with POMC and NPY in neurons of the arcuate and paraventricular nucleus. NSCL-2 mutant mice show adult onset of obesity and leptin resistance.

Materials and Methods: We measured serum insulin, adiponectin and resistin concentrations by ELISA Kit from Linco Research. Histological studies and analysis of isolated adipocytes were performed using four male mice at the age of 6 months of the four genotype groups: NSCL-2 -/-; ob/ob; NSCL-2 -/ $-\mathrm{x}$ ob/ob and C57/BL/6J WT controls.

Results: NSCL-2 -/- mice, unlike ob/ob mice, are characterized by low insulin $\left(\mathrm{F}=5.98 ; \mathrm{p}<10^{-4}\right)$, high adiponectin serum levels and normal glucose tolerance. We investigated glucose uptake, lipolysis and glucose metabolism in isolated adipocytes of NSCL- $2 \mathrm{x} \mathrm{ob} / \mathrm{ob}$ double mutants and in $\mathrm{ob} / \mathrm{ob}$ mice Interestingly, adipocytes derived from NSCL-2/ob/ob compound mice show a decreased insulin resistance compared to isulin resistant ob/ob or NSCL-2 mutant adipocytes. Epigonadal adipose tissue showed a bimodal distribution with two populations of adipocytes, a small preadipocyte $(<50$ $\mu \mathrm{m})$ and a large mature adipocyte $(>100 \mu \mathrm{m})$ form. Furthermore, the number of preadipocytes was 7 times higher compared to wild type and ob/ob mice $\left(\mathrm{F}=250.71 ; \mathrm{p}<10^{-4}\right)$. In addition, we found increased serum resistin level in NSCL-2 -/- and NSCL-2 -/ $\mathrm{x}$ ob/ob mice compared to WT and ob/ob mice when normalized to the bodyweight on the animals $(F=6.33 ; p<0.0009)$.

Conclusion: We anticipate that resistin might participate in the control of adipocyte differentiation.

Supported by DFG amd BMBF 


\section{PS 60 Insulin and vasculature}

\section{5}

Insulin vascular action in skeletal muscle is attenuated with insulin resistance and features of the metabolic syndrome

M. J. Turzyniecka ${ }^{1}$, J. C. C. Ung' ${ }^{2}$, A. J. Krentz', G. F. Clough ${ }^{2}$, C. D. Byrne'

${ }^{1}$ Endocrinology and Metabolism, University of Southampton,

${ }^{2}$ IRR Division, University of Southampton, United Kingdom.

Background and Aims: The relationship between total muscle blood flow, capillary recruitment and skeletal muscle insulin sensitivity remains unclear. The aim of this study was to investigate insulin-induced changes in muscle microvascular blood flow using a novel non-invasive laser Doppler surface probe in individuals with features of metabolic syndrome

Materials and Methods: Twenty one volunteers (age range 37 - 67years; mean $51.5 \pm 1.5 \mathrm{y} ; 8 \mathrm{men}$ ) were recruited. All gave written informed consent. Blood flux in muscle and skin was measured by laser Doppler fluximetry (DRT4 Moor Instruments Ltd, UK) using a $785 \mathrm{~nm}, 20 \mathrm{~mW}, 4 \mathrm{~mm}$ separation (muscle) and a $1 \mathrm{~mW}, 0.5 \mathrm{~mm}$ separation (skin) probe placed above the anterior tibialis muscle. Measurements were made at rest and during a reactive hyperaemia $(\mathrm{RH})$ to arterial occlusion, before and during stepped hyperinsulinaemic euglycaemic clamp at low $(0.2 \mathrm{mU} / \mathrm{kg} / \mathrm{min})$ and high dose insulin $(1.5 \mathrm{mU} / \mathrm{kg} / \mathrm{min})$. Insulin sensitivity was measured as the rate of glucose disposal $(\mathrm{M}=\mathrm{mg} / \mathrm{kg} / \mathrm{min})$ during the steady state of the clamp. Results: Resting blood flux in muscle and skin was $80 \pm 3$ and $9 \pm 0.3 \mathrm{AU}$ (arbitrary perfusion units), respectively. The insulin-induced increase in resting muscle blood flow was positively related to insulin sensitivity $(\mathrm{r}=0.45$ $\mathrm{p}<0.05)$. The relative increase in $\mathrm{RH}$ from baseline was negatively correlated with percentage body fat measured by DEXA $(r=-0.59, \mathrm{p}<0.02)$.

Conclusion: We conclude that insulin's vascular action within skeletal muscle, as measured by non- invasive laser Doppler fluximetry, is attenuated with insulin resistance and with increased body fat. We speculate that an impaired insulin response within skeletal muscle microvasculature may contribute to whole body insulin resistance and to decreased glucose disposal by muscle in these patients.

Supported by independent research grant from Pfizer

\section{6}

The influence of very low calorie diet on serum concentrations and subcutaneous mRNA expression of endothelial cell adhesion molecules in obese, insulin-resistant women

M. Haluzik, L. Bosanska, D. Haluzikova, R. Dolezalova, T. Roubicek, M. Mraz, M. Dolinkova, M. Matoulek, Z. Lacinova;

3 Department of Medicine, 1 Faculty of Medicine, Prague 2, Czech Republic.

Background and Aims: Endothelial cell adhesion molecules (CAM) are proteins expressed on the surface of endothelial cells that participate in the development of endothelial dysfunction and/or atherosclerosis. One of the possible sources of increased circulating levels of CAM in obese subjects is adipose tissue. The aim of our study was to test the hypothesis that adipose tissue significantly contributes to the increased levels of CAM in obesity and to explore how is this production influenced by diet intervention.

Material and Methods: Twelve obese women (mean BMI $45.7 \pm 6.7 \mathrm{~kg} / \mathrm{m}^{2}$ ) and twelve lean healthy subjects (C) (mean BMI $24 \pm 4.3 \mathrm{~kg} / \mathrm{m}^{2}$ ) were included into the study. None of the subjects suffered from diabetes. Blood drawing and subcutaneous adipose tissue (SAT) biopsy were performed after an overnight fasting. The same procedure was repeated in obese subjects after three weeks of very low calorie diet (VLCD) with energy intake of $550 \mathrm{kcal} /$ day. Serum concentrations of insulin, ICAM-1, VCAM-1 and E-selectin were measured using RIA kit - insulin (Cis Bio Internatinal, France) and Lincoplex kit - CAM (Linco Research, USA). CAM mRNA expression in SAT was measured using RT PCR (ABI PRISM 7500, USA) and normalized to $\beta$-2-microglobulin mRNA expression.

Results: At baseline, BMI, serum insulin levels, HOMA index, ICAM-1 and E-selectin concentrations of obese group were significantly higher relative to C (ICAM $138 \pm 72$ vs. $78 \pm 28 \mathrm{ng} / \mathrm{ml}$; E-selectin $23,7 \pm 10,8$ vs. $9,1 \pm 4,7 \mathrm{ng} /$ $\mathrm{ml}, \mathrm{p}<0.05)$. Serum concentrations of VCAM-1 levels did not differ. On the contrary, mRNA expression of E-selectin in SAT was significantly lower in obese relative to $\mathrm{C}$ group while ICAM-1 and VCAM-1 mRNA expression did not differ. Three weeks of VLCD significantly decreased BMI, serum insulin levels, HOMA index and E-selectin levels but it did not affect VCAM-1 and ICAM-1 levels. Three weeks of VLCD significantly increased SAT mRNA expression of ICAM-1 and VCAM-1. SAT mRNA expression of E-selectin was not significantly affected by VLCD.

Conclusion: We conclude that three weeks of VLCD had favorable effect on metabolic parameters and circulating levels of some of CAM in obese, insulinresistant women. SAT mRNA expression of CAM differed from its circulating levels and VLCD increased, rather than decreased its SAT mRNA expression. Our data suggest that SAT is not a principle source of increased CAM in obese patients and that the local regulation of CAM mRNA expression in SAT is different from its systemic concentrations.

Supported by MZO 00064165, IGA 8302-5

\section{7}

Identification of molecules underlying human aortic smooth muscle cells phenotypic change in hyperglycaemia and hyperlipidaemia

L.-D. Popov ${ }^{1}$, E. Constantinescu ${ }^{2}$;

${ }^{1}$ Vascular Dysfunction in Diabetes,

${ }^{2}$ Cerebrovascular dysfunction in ageing and disease, Institute of Cellular Biology and Pathology, Bucharest, Romania.

Background and Aims: Several lines of evidence suggest that in diabetes arterial smooth muscle cells (SMCs) play a key role in the atherosclerotic process. In this study we questioned on: (i) phenotypic changes of human aortic SMCs induced in vitro by high glucose concentration and by sera of obese type 2 diabetic patients, (ii) identification of signaling molecules underlying metabolic dysregulations of the cells subjected to either hyperglycemia or hyperlipidemia-hyperglycemia.

Materials and Methods: Human aortic SMCs were cultured to confluence in: (i) DMEM with $10 \%$ fetal calf serum and $30 \mathrm{mmol}$ glucose (in controls, $5 \mathrm{mmol}$ glucose), and (ii) DMEM with $10 \%$ serum of obese type 2 diabetic patients of BMIs $35-39 \mathrm{~kg} / \mathrm{m}^{2}$, mean plasma glucose: $298 \mathrm{mg} / \mathrm{dl}$, cholesterol: $294 \mathrm{mg} / \mathrm{dl}$, triglycerides (TGs): $234 \mathrm{mg} / \mathrm{dl}$ (in controls, $10 \%$ serum of healthy subjects of BMIs $19-21 \mathrm{~kg} / \mathrm{m}^{2}$, with normal plasma glucose, cholesterol and TGs levels). Electron microscopy was used to examine SMCs structure, fluorescence microscopy, to observe Oil red O stained lipid droplets and immunostained vinculin and type IV collagen, and immunoblotting, to evaluate expression and phosphorylation of STAT-3(Tyr 705), ERK1/2(Thr202/Tyr204), IRS1 (Tyr), Ob-R and HSL.

Results: The presence of $30 \mathrm{mM}$ glucose conducted to the following modifications of human aortic SMCs: (i) enrichment in biosynthetic organelles, indicating a secretory phenotype (in controls: a contractile phenotype), (ii) organization of vinculin plaques at the cells periphery, supporting spreading and focal adhesions formation (in controls: intracytoplasmic distribution), (iii) production of an extensive extracellular matrix, with intensely immunostained type IV collagen (in controls: a faint labeling), (iv) proliferation (as demonstrated by the presence of centriols) associated with significantly increased protein expression of pSTAT-3 $(\sim 2.54$ fold) and pERK1/2 ( 2.12 fold) above the levels in controls, (v) addition of 10-100 nM insulin reduced IRS-1 phosphorylation, in a time-dependent process, suggesting negative regulation of insulin receptor-mediated signaling, a process restricted to the SMCs with secretory and proliferative phenotype (controls: no insulin, and SMCs in $5 \mathrm{mmol}$ glucose). $30 \mathrm{mmol}$ glucose did not induced lipid loading of human aortic SMCs. The presence of serum of obese type 2 diabetic patients conducted to: (i) accumulation of Oil red O stained lipid droplets, indicating a phenotypic switch towards differentiation into foam cells, and storage of TGs and cholesteryl esters (CEs)(in comparison with controls), (ii) protein expression of HSL, an enzyme involved in hydrolysis of TGs and CEs within the lipid droplets, that was not detected in controls, (iii) protein expression and activation of Ob-R, STAT-3, and ERK1/2 underlying metabolic dysregulations in lipid laden-cells.

Conclusion: Although expression of transcription factors from insulin signaling is in common to phenotypic changes of human aortic SMCs grown in medium supplemented with either high glucose or serum of obese diabetic patients, each of these insults induced definite metabolic peculiarities: a biosynthetic metabolism in high glucose conditions, and an active lipid metabolism associated with cells differentiation into foam cells, in hyperglycemic-hyperlipidemic environment.

Supported by grants from the Romanian Academy and European SSA-SERA 


\section{8}

New insights into the molecular mechanisms involved in the resistance to nitric oxide occurring in vascular smooth muscle cells from an animal model of insulin-resistance

G. Doronzo, I. Russo, L. Mattiello, P. Del Mese, G. Anfossi, M. Trovati; Department of Clinical and Biological Sciences, University of Turin, Diabetes Unit, San Luigi Gonzaga Hospital, Orbassano-Turin, Italy.

Background and Aims: Nitric Oxide (NO) plays a critical role in the pathogenesis of atherosclerosis and arterial hypertension, by inhibiting steps of atherogenic process and by inducing vasodilation. In particular, NO stimulates production of guanosine 3', ''cyclic monophosphate (cGMP), which regulates several functions of Vascular Smooth Muscle Cells (VSMC). The major substrate for cGMP in VSMC is the cGMP-dependent Protein Kinase (PKG), that catalyzes phosphorylation of many signalling proteins. We previously observed that in aortic VSMC from lean, insulin-sensitive and obese, insulin-resistant Zucker rats (a classical animal model of insulin resistance), the NO donor DETA NONOate and the cGMP analog 8-Br-cGMP phosphorylate Akt (a key molecule the PI-3K/Akt pathway) and ERK1/2 (key molecules of MAPK family).We also demonstrated that activation of Akt and of ERK1/2 induced by the NO/cGMP pathway is impaired in VSMC from obese Zucker rats. Aim of this study is to evaluate whether NO and cGMP: i)activate molecules downstream PI-3K/Akt, such as p70S6K, involved in VSMC proliferation and post-angioplasty restenosis; ii)activate other molecules of the MAPK family, such as p38 MAPK and JNK1/2, correlated with cell stress, and whether PKG is involved in the NO/cGMP-induced activation of PI3K/Akt/p70S6K pathway and of MAPK family molecules. Materials and Methods: In cultured aortic VSMC from lean and obese Zucker rats, we measured the influence of the NO donor DETA NONOate and of the cGMP analog 8-Br-cGMP on the activating phosphorylation of p70S6K, p38 MAPK, JNK1/2 (western blots, $n=6$ ) and whether activation of all these signalling molecules are blunted by KT5823, a selective PKG inhibitor.

Results: In VSMC from lean Zucker rats, DETA NONOate (500 microM) and 8 -Br-cGMP (500 microM) increased phosphorylation of p70S6K (at 2 hours, $\mathrm{p}=0.007$ and 0.0001 ) and of $\mathrm{p} 38 \mathrm{MAPK}$ (at 2 hours, $\mathrm{p}=0.0001$ for both). Activation of p70S6K and p38 MAPK was induced by DETA NONOate and 8-Br cGMP also in VSMC from obese Zucker rats ( $\mathrm{p}=0.0001$ and 0.02$)$, but the extent of activation was significantly lower. In particular, i) at 2 hours of VSMC incubation with DETA NONOate: i)phosphorylation of p70S6K was $171.9 \pm 7.8 \%$ of baseline in lean vs $128.9 \pm 2.5 \%$ in obese Zucker rats ( $p=0.0001$ ); ii)phosphorylation of $\mathrm{p} 38$ MAPK was $273.3 \pm 4.6 \%$ of baseline in lean vs $181.6 \pm 5.4 \%$ in obese Zucker rats ( $p=0.0001)$; $b$ ) at 2 hours of VSMC incubation with 8-Br-cGMP: i)phosphorylation of p70S6K was $140.5 \pm 4.8 \%$ of baseline in lean vs $121.7 \pm .5 .5 \%$ in obese Zucker rats ( $\mathrm{p}=0.028)$; ii)phosphorylation of p38 MAPK was $371.5 \pm 20 \%$ of baseline in lean vs $195.8 \pm 3.5 \%$ in obese Zucker rats $(\mathrm{p}=0.0001)$. No influence was exerted by DETA NONOate and 8 -BrcGMP on JNK1/2 in VSMC from lean and obese Zucker rats. The influence of DETA NONOate and 8-Br-cGMP on Akt, ERK1/2, p70S6K and p38 are blunted by 1 hour pre-incubation with the PKG inhibitor KT5823 in VSMC from lean and obese Zucker rats ( $\mathrm{p}=0.0001)$.

Conclusions: The NO/cGMP pathway activates -via PKG- the PI3-K/Akt/ p70S6K pathway and the MAPK family molecules ERK1/2 and p38 -but not JNK1/2- in lean Zucker rats and the effects are reduced in VSMC from the obese Zucker rats. These results provide new insights in the molecular mechanisms characterizing the nitric oxide resistance occurring in VSMC in insulin resistant states.

Supported by the National Ministry of University

\section{9}

Palmitate and stearate are potent inducers of inflammatory genes in human coronary artery endothelial cells A. O. Krogmann;

Medizinische Klinik IV und Poliklinik, Universitätsklinikum Tübingen, Germany.

Background and Aims: Obesity-linked insulin resistance is associated with increased lipolysis and elevated plasma levels of free fatty acids (FFA). We asked whether common plasma FFA contribute to vascular inflammation via induction of inflammatory genes in human coronary artery endothelial cells (CAEC), as was previously shown for interleukin-6.
Materials and Methods: CAEC were treated with $0.5 \mathrm{mM}$ palmitate $(\mathrm{C} 16: 0)$, stearate $(\mathrm{C} 18: 0)$, oleate $(\mathrm{C} 18: 1 \omega 9)$, or linoleate $(\mathrm{C} 18: 2 \omega 6)$, respectively, for 20 hours. Gene expression was assessed by macroarrays, real-time RT-PCR, and/or Western blot.

Results: Using macroarrays, qualitative analysis of palmitate-treated CAEC revealed a broad inflammatory response. As quantified by RT-PCR, treatment of CAEC with palmitate or stearate, but not with oleate or linoleate, respectively, resulted in significant mRNA induction of IL8, CCL20, CXCL3, IL1 $\alpha$, and SPP1 $(\mathrm{p}<0.05$ for all, N=3). Similar results were obtained for the inflammatory transcription factor C/EBP $\beta$, as verified by Western blot. Using pharmacological inhibitors, we furthermore demonstrate that palmitateinduced expression of IL8, CCL20, and CXCL3 ( $\mathrm{p}<0.05$ for all, N=3) depends on the activation of the transcription factor NFkB. In addition, Palmitateinduced expression of IL8, CXCL3, IL1 $\alpha$, and SPP1 ( $p<0.001$ for all, N=3) is mediated by c-Jun kinase. P38 MAP kinase was not found to be involved in these gene regulations.

Conclusion:Here, we show that the saturated FFA palmitate and stearate, but not unsaturated FFA, such as oleate and linoleate, induce a broad inflammatory response in human coronary artery endothelial cells. These events are mediated by $\mathrm{NF \kappa B}$ and c-Jun kinase. Our findings point to a potential contribution of saturated FFA to vascular inflammation.

Supported in part by a grant from the Deutsche Forschungsgemeinschaft and the European Community's FP6 EUGENE 2

\section{0}

Action of insulin and insulin-like growth factors in human aortic smooth muscle cells

S. I. Chisalita, E. Liefvendahl, G. S. Johansson, H. J. Arnqvist;

Cell Biology, Biomedicine and Surgery, Linköping, Sweden.

Background and Aims: Vascular smooth muscle cells play an important role in the development of atherosclerosis. Circulating levels of insulin-like growth factor-I (IGF-I) and insulin-like growth factor-II (IGF-II) are lowered in diabetic subjects. Little is known about direct effects of IGF-II on human aortic smooth muscle cells (HASMC). Our aim was to characterize effects of IGF-II in relation to insulin and IGF-I in HASMC.

Materials and Methods: Cultured HASMC were used. Receptor mRNA expression was measured by quantitative real time- polymerase chain reaction (RT-PCR) analysis. Receptor proteins, hybrid IR/IGF-IR (hybrid insulin receptor/ insulin-like growth factor-I receptor) and phosphorylation of receptor $\beta$-subunits were analyzed by immunoprecipitation and Western blot. Metabolic and mitogenic effects were assessed by $\mathrm{D}-\left[\mathrm{U}_{-}{ }^{14} \mathrm{C}\right]$-glucose accumulation and ${ }^{3} \mathrm{H}$-thymidine incorporation into DNA, respectively.

Results: The mRNA expression of insulin-like growth factor-I receptor (IGF-IR) was approximately 5 fold higher than that of insulin receptor (IR) in HASMC $(\mathrm{P}<0.02)$. Insulin receptor isoform A (IR-A), which has approximately 10 fold higher affinity for IGF-I and IGF-II than IR-B, was 13 fold more expressed than insulin receptor isoform $B(I R-B)(P=0.01)$. By immunoprecipitation and Western Blot analysis both IGF-IR and IR and hybrid IR/IGF-IR could be demonstrated. Phosphorylation of the IGF-IR $\beta$ subunit was obtained by insulin $10 \mathrm{nM}$, IGF-I $0.1-10 \mathrm{nM}$ and IGF-II $10 \mathrm{nM}$. IR $\beta$-subunit was activated by IGF-II $10 \mathrm{nM}$ and IGF-I $1-10 \mathrm{nM}(\mathrm{P}<0.05)$. IGF-I and IGF-II at a concentration of 10-100 nM significantly stimulated ${ }^{3} \mathrm{H}$-thymidine incorporation $(\mathrm{P}<0.05)$, whereas insulin did not. Glucose accumulation was stimulated by IGF-I $10-100 \mathrm{nM}$, IGF-II $10-100 \mathrm{nM}$, and by insulin $100 \mathrm{nM}(\mathrm{P}<0.05)$.

Conclusion: Both IGF-I and IGF-II activated the IGF-IR and stimulated glucose metabolism and DNA synthesis in HASMC, whereas insulin had little effect. The lack of insulin effect could partly be due to the presence of hybrid IR/IGF-IR.

Supported by Landstinget Östergotland, Swedish Medical Research Council (04952), Swedish Diabetes Association and Barndiabetes Fonden 


\section{1}

Metacholine-induced vasodilation differentially modulates the vascular and the metabolic responses in skeletal muscle of insulin-sensitive and insulin-resistant obese individuals

G. Murdolo ${ }^{1}$, M. Sjostrand ${ }^{2}$, L. Strindberg ${ }^{3}$, S. Gudbjornsdottir ${ }^{4}$, L. Lind ${ }^{5}$, P. Lonnroth', P.-A. Jansson';

${ }^{1}$ Department of Internal Medicine, Perugia University, Italy,

${ }^{2}$ Astra Zeneca Research, Gothenburg University, Molndal, Sweden,

${ }^{3}$ Department of Molecular and Clinical Medicine, Gothenburg University,

Sweden, ${ }^{4}$ Department of Internal Medicine, Gothenburg University, Sweden, ${ }^{5}$ Department of Medicine, Uppsala University, Sweden.

Background and Aims: The impairment of insulin-mediated capillary recruitment in skeletal muscle has been indicated as an important contributor to the obesity-linked metabolic resistance to glucose uptake. The main aim of this study was to test whether a sustained vasodilation induced by intra-brachial metacholine infusion (Mch, $2 \mu \mathrm{g}^{*} \mathrm{~min}^{-1}$ for 60 min) improves muscle capillary recruitment and forearm glucose uptake during hyperinsulinemia.

Materials and Methods: Fifteen uncomplicated obese individuals $(\mathrm{M} / \mathrm{F}$ : 14/1; age: $45.5 \pm 2.4 \mathrm{yrs)}$ were divided into a group of insulin-sensitive (IS, $\mathrm{n}=8$; HOMA-IR <2.5; BMI: $32.6 \pm 1.2 \mathrm{~kg} / \mathrm{m}^{2}$ ) and insulin-resistant (IR, $\mathrm{n}=7$; HOMA-IR $\geq 2.5$; BMI: $31 \pm 0.9 \mathrm{~kg} / \mathrm{m}^{2}$ ) subjects. The double forearm technique (ie, infused $v s$ control forearm) was combined with the intramuscular microdialysis during an oral glucose tolerance test (OGTT).

Results: In the infused forearm, Mch significantly increased ( $\mathrm{p}<0.0001$ vs baseline) the forearm blood flow (FBF), the capillary permeability-surface area product (PS) for glucose (capillary recruitment) and the forearm glucose uptake (FGU). In the control forearm, FBF remained stable throughout the OGTT, whereas PS and FGU significantly rose over-time ( $\mathrm{p}<0.001)$. By design, FBF changes above the baseline were larger in the infused as compared with the control forearm $(p<0.0001)$, whereas comparable PS and FGU responses were seen between the two forearms. Moreover, in the control forearm, the IR subjects exhibited similar FBF, but lower PS and FGU changes $(\mathrm{p}<0.01)$, as compared with the IS controls. In contrast, in the infused forearm, FBF was slightly blunted in the IR individuals, but PS and FGU changes did not differ between the groups. Finally, we found a tight correlation between the FGU and the PS ( $\mathrm{R}^{2}$ adj. 0.74, $\left.\mathrm{p}<0.01\right)$, but neither FGU nor PS were significantly associated with the FBF.

Conclusion: We conclude that, during an OGTT, the forearm glucose uptake may be independent of increment in total blood flow. The differential effects on the vascular and the metabolic response between IS and IR individuals, as well as the lack of association between the PS for glucose or the FGU with the FBF implicate that, in the obese state, the metabolic resistance to glucose uptake in skeletal muscle might be overcome by circumventing the dysfunctional capillary recruitment rather than by increasing the bulk blood flow. Further efforts are required to better elucidate the role of NO-tone modulating agents in the regulation of both vascular and metabolic responses in insulin-resistant skeletal muscle.

Supported by Swedish Medical Research Council, Swedish Diabetes Association, Novo Nordisk Foundation

\section{2}

Impaired postprandial adipose tissue blood-flow may be an early marker of insulin resistance in type 2 diabetes

V. Lambadiari ${ }^{1}$, G. Dimitriadis ${ }^{1}$, P. Mitrou ${ }^{1}$, E. Boutati ${ }^{1}$, I. Maratou ${ }^{2}$,

D. Panagiotakos ${ }^{3}$, T. Economopoulos ${ }^{1}$, S. A. Raptis ${ }^{1,2}$;

${ }^{1}$ 2nd Department of Internal Medicine and Research Institute,

Athens University Medical School,

${ }^{2}$ Hellenic National Diabetes Center, Attikon University Hospital,

${ }^{3}$ Department of Statistics, Harokopion University, Athens, Greece.

Background and Aims: It is increasingly recognized that impairment of endothelial and vascular function is an integral part of the insulin resistance syndrome. In subcutaneous adipose tissue (AT), blood flow plays a decisive role in its metabolic and endocrine function, and normally increases after meal ingestion. In insulin resistant states, like obesity and overt type 2 diabetes (DM2), this response is blunted. The study was undertaken to investigate the existence of de novo impairment in AT endothelial function, in the various stages of type 2 diabetes.

Materials and Methods: A standard mixed meal was given to five groups of subjects, whose features are shown in the Table 1. Blood samples were drawn from the radial artery (A) at 30-60min intervals for $6 \mathrm{~h}$ for the measurement of glucose, insulin and adiponectin. Simultaneous measurements of blood flow in AT were done with ${ }^{133} \mathrm{Xe}$.

Results: (a) The pre-prandial values of glucose were lower in controls vs all other groups, (b) HOMA Index in controls was lower vs all other groups, (c) adiponectin was higher in controls and relatives vs other groups, (d) AT blood flow (AUC 0-360min) was higher in controls vs all other groups (Table1). Conclusions: After meal ingestion, no response of insulin-stimulated AT blood flow was detected in the subcutaneous AT of relatives and patients with IGT, DM2-A, DM2-B. This defect could represent another facet of insulin resistance that precedes the development of overt glucose intolerance.

RELATIVES: $1^{\text {st }}$ degree relatives of diabetic patients with a normal glucose tolerance test, IGT: subjects with impaired glucose tolerance, DM2-A: subjects with mild type 2 diabetes, with normal fasting plasma glucose and postprandial hyperglycemia, DM2-B: subjects with overt type 2 diabetes. FPG: Fasting Plasma Glucose

\section{3}

Leptin activates 5'-AMP-activated protein kinase and shifts insulinmediated vasoreactivity to vasodilatation in muscle resistance arteries E. C. Eringa ${ }^{1}$, I. Korstjens ${ }^{1}$, G. P. van Nieuw Amerongen ${ }^{1}$,

C. D. A. Stehouwer ${ }^{2}$, W. Bakker ${ }^{1}$, P. Sipkema ${ }^{1}$;

${ }^{1}$ Laboratory for Physiology, VU University Medical Centre, Amsterdam,

${ }^{2}$ Internal Medicine, Academic Hospital Maastricht, The Netherlands.

Resistance to the metabolic effects of leptin is associated with insulin resistance and hypertension. Effects of leptin on the muscle microcirculation may mechanistically explain these associations. 5'-AMP-activated protein kinase (AMPK) has been has been hypothesized to mediate the physiological effects of leptin.

Objective: To study the effects of leptin on vascular tone, insulin-mediated vasoreactivity and insulin-mediated cell signaling in muscle resistance arteries and the role of AMPK in these effects.

Methods/Results: Resistance arteries from the rat cremaster muscle were stimulated in vitro with leptin $(10 \mathrm{nM})$ alone or in combination with insulin

Tab. 1

\begin{tabular}{|c|c|c|c|c|c|}
\hline Table of Contents & $\begin{array}{l}\text { CONTROLS } \\
\mathrm{N}=10\end{array}$ & $\begin{array}{l}\text { RELATIVES } \\
\mathrm{N}=11\end{array}$ & $\begin{array}{l}\text { IGT } \\
\mathrm{N}=6\end{array}$ & $\begin{array}{l}\text { DM2-A } \\
\mathrm{N}=6\end{array}$ & $\begin{array}{l}\text { DM2-B } \\
\mathrm{N}=13\end{array}$ \\
\hline Age (yrs) & $36 \pm 3$ & $41 \pm 3$ & $43,5 \pm 3$ & $53 \pm 4$ & $56 \pm 2$ \\
\hline BMI $\left(\mathrm{kg} / \mathrm{m}^{2}\right)$ & $23,5 \pm 1$ & $25 \pm 1$ & $26 \pm 1,5$ & $24,5 \pm 1,5$ & $26 \pm 0,7$ \\
\hline FPG $(\mathrm{mM})$ & $4,7 \pm 0,1$ & $5 \pm 0,1^{\star}$ & $5,7 \pm 0,4^{*}$ & $5,8 \pm 0,2^{*}$ & $8 \pm 0,2^{\star}$ \\
\hline HOMA Index & $0,98 \pm 0,1$ & $1,43 \pm 0,1^{*}$ & $1,7 \pm 0,1^{*}$ & $1,8 \pm 0,2^{*}$ & $2,2 \pm 0,2^{*}$ \\
\hline HbA1c (normal 3,5-5,5\%) & $3,5 \pm 0,1$ & $4,2 \pm 0,1^{*}$ & $5 \pm 0,2^{*}$ & $5,3 \pm 0,1^{*}$ & $6,5 \pm 0,2^{*}$ \\
\hline Adiponectin (ng/ml) & $20,5 \pm 2,8$ & $22,9 \pm 3,1$ & $11,4 \pm 1,9^{*}$ & $14,8 \pm 3,9^{*}$ & $11,5 \pm 3,3^{*}$ \\
\hline Blood Flow (ml/100cctissue $\left.{ }^{\star} \min \right)$ & $1507 \pm 103$ & $845 \pm 123^{*}$ & $679 \pm 69^{*}$ & $765 \pm 60^{*}$ & $757 \pm 69^{*}$ \\
\hline
\end{tabular}

${ }^{\star} \mathbf{p}<\mathbf{0 , 0 5}$ vs controls. Data are means \pm SEM. 
(0.01-1 nM). Vascular diameter was not significantly altered by leptin alone $(10+7$ percent, $\mathrm{P}=0.3)$ and insulin alone $(0-2$ percent, $\mathrm{P}=0.9)$. In contrast, insulin induced a dose-dependent vasodilatation in the presence of leptin $(19+4$ percent, $\mathrm{P}<0.01$ vs. insulin alone). Western blotting of muscle resistance arteries revealed that the leptin-induced shift in vasoreactivity to insulin was associated with potentiation of insulin-mediated phosphorylation of Akt, which mediates insulin-stimulated NO production. Further examination of pAkt in the arteriolar wall by three-dimensional microscopy showed that phosphorylated Akt was predominantly located in the microvascular endothelium. Furthermore, leptin activated 5'AMP-activated protein kinase (AMPK) in endothelium of muscle resistance arteries, which was shown by increased phosphorylation of AMPK at its Threonine 172 residue. Finally, direct activation of AMPK with the AMPK agonist 5 amino-4imidazolecarboxamide riboside (AICAR; $2 \mathrm{mM}$ ) was sufficient to mimic the effect of leptin on insulin-mediated vasoreactivity. In the presence of AICAR, insulin induced NO-dependent vasodilatation in the presence of AICAR $(+20+5$ percent at $1 \mathrm{nM}, \mathrm{P}<0.05$ vs. insulin; $+3+1$ percent during NOS inhibition).

Conclusions: Leptin shifts insulin-mediated vasoreactivity of muscle resistance arteries towards vasodilatation by enhancing insulin-mediated activation of Akt and activation of AMPK. These findings provide a new mechanism for the association of leptin resistance with hypertension and insulin resistance.

\section{PS 61 Non-alcoholic steatohepatitis}

\section{4}

Fatty liver is strongly associated with a qualitative change in the serum HDL cholesterol sub-fractions

N. Stefan ${ }^{1}$, K. Kantartzis ${ }^{1}$, A. Cegan ${ }^{1}$, K. Rittig ${ }^{1}$, C. Thamer ${ }^{1}$, J. Machann ${ }^{2}$, B. Balletshofer ${ }^{1}$, A. Fritsche ${ }^{1}$, E. Schleicher ${ }^{1}$, H.-U. Häring ${ }^{1}$;

${ }^{1}$ Department of Internal Medicine,

${ }^{2}$ Section of Experimental Radiology, University of Tübingen, Germany.

Background and Aims: Fatty liver is strongly associated with atherosclerosis. Mechanisms explaining this relationship include a quantitative change in serum levels of triglycerides (TG), high-density lipoprotein cholesterol (HDLc) and an increase in small dense LDL particles. So far it is unknown whether a qualitative change in serum lipids links fatty liver and atherosclerosis. As particularly a high HDL2-c level was found to be protective of atherosclerosis, and the HDL2-c and HDL3-c serum levels are regulated by the liver, we investigated whether fatty liver is associated with different HDL2/3-c levels in humans.

Materials and Methods: Thirty-seven subjects were studied. Liver fat was measured by $1 \mathrm{H}$ magnetic resonance spectroscopy and insulin sensitivity was determined by the euglycemic hyperinsulinemic clamp. The HDL2/3-c levels were quantified after separation by ultracentrifugation.

Results: Subjects with fatty liver ( $\mathrm{n}=16$, liver fat $>5.5 \%$ ) had lower HDL2-c levels and a lower HDL2/3-c ratio compared to controls $(n=21, p=0.001$ and $\mathrm{p}=0.0001$ ). As expected, liver fat was negatively correlated with total HDL$c(r=-0.47, p=0.003)$. Of note, stronger negative correlations with HDL2$c(r=-0.56, p=0.0003)$ and the HDL2/3-c ratio $(r=-0.61, p<0.0001)$ were observed. After adjustment for gender, age and body fat, liver fat was still negatively correlated with total HDL-c $(r=-0.40, p=0.02)$, albeit weaker, and the correlation of liver fat with the HDL2/3-c ratio continued to be strong $(r=$ $-0.54, p=0.001)$. While additional adjustment for insulin sensitivity rendered the correlation of liver fat with total HDL-c non-significant $(\mathrm{p}=0.25)$, the correlation of liver fat with the HDL2/3-c ratio remained strong $(r=-0.43$, $\mathrm{p}=0.02)$.

Conclusion: We provide novel data showing that fatty liver is not only associated with a quantitative decline in HDL-c, but also with a qualitative change in the HDL-c composition. This may represent a new pathophysiological link between fatty liver and atherosclerosis.

Supported by Deutsche Forschungsgemeinschaft

\section{5}

Plasma adipocytokines in patients with metabolic syndrome and nonalcoholic fatty liver disease

L. Diaconu, V. Serban, R. Z. Timar, A. Vlad, B. Timar, C. Mazilu, V. Botea;

Diabetes Clinic, University of Medicine and Pharmacy, Timisoara, Romania.

Background and Aims: Nonalcoholic fatty liver disease (NAFLD) is a major cause of liver-related morbidity with possible progression to cirrhosis. NAFLD often represents a component of the metabolic syndrome (MS) characterized by obesity, hyperinsulinemia, peripheral insulin resistance, diabetes, hypertriglyceridemia and hypertension. Insulin resistance states are characterized by elevated production of several adipocytokines (leptin, resistin, TNF $\alpha$, IL-6, TGF $\beta$, PAI-1). Adiponectin is the only known adipocytokine which produces antiinflammatory and antiatherogenic effects. Low plasma adiponectin is considered a key factor in the development of the insulin resistance underlying MS. The aims of our study was to evaluate the prevalence of NAFLD in patients with MS, to assess plasma leves of adiponectin and proinflammatory state in patients with MS with and without NAFLD.

Materials and Methods: The study enrolled 224 subjects with MS. The diagnosis of MS was established according to IDF criteria. The diagnosis of NAFLD was based on detection of fatty liver by ultrasonography with or without chronically elevated aminotransferaze levels (ALT>1.5 times the upper normal values for 6 months or more). All subjects had negative hepatitis B and C viral markers, negative history of alcohol intake, absence of autoimmune hepatits, Celiac disease, no evidence of genetic, drug-induced or cholestatic liver disease. 
Results: The prevalence of NAFLD in patients with MS was $87 \%$. NAFLD with aminotransferaze levels was present in $44(19.64 \%)$ patients while NAFLD with normal liver enzymes was present in 151 (67.41\%) patients with MS. Plasma levels of adiponectin were significantly lower in patients with NAFLD with elevated aminotransferaze levels than in those with normal liver enzymes and than in subjects without NAFLD. Plasma levels of adiponectin were significantly lower in patients with NAFLD with elevated aminotransferaze levels than in those with normal liver enzymes and than in subjects without NAFLD. Plasma levels of TNF- $\alpha$, IL-6, leptin were significantly higher in patients with NAFLD and elevated aminotransferaze levels than in patients with NAFLD and normal liver enzymes and than in subjects without hepatic injury. HOMA-IR was higher in patients with low levels of adiponectin. (Tab. 1)

Conclusion: The prevalence of NAFLD is higher in patients with MS than in general population. Although insulin resistance is present in all patients with MS, those with NAFLD present higher insulin resistance than those without NAFLD. Plasma levels of adiponectin are lower while proinflammatory adipocytokines and leptin are higher in patients with MS and NAFLD than in those without liver injury, suggesting a possible contribution of these abnormalities in the pathogenesis of NAFLD.

\section{6}

Hepatic and systemic inflammation markers in patients with type 2 diabetes and NASH: decreased hepatic levels of TNF- $\alpha$ and IL-8 as increased severity of NASH

G. Kocabay' ${ }^{1}$, I. Satman ${ }^{1}$, A. Telci², Y. Tütüncü ${ }^{1}$, S. Ozel ${ }^{3}$, U. Cevikbaşş, B. Tiryaki ${ }^{5}$, A. Ökten ${ }^{6}$;

${ }^{1}$ Endocrinology\&Metabolism, ${ }^{2}$ Biochemistry, ${ }^{3}$ Statistics\&Public Health,

${ }^{4}$ Pathology, ${ }^{5}$ Radiology, ${ }^{6} \mathrm{Hepatology}$, Istanbul Faculty of Medicine, Turkey.

Objective: Nonalcoholic liver steatosis (NASH) is commonly accompanied with type 2 diabetes, and is considered as a proinflammatory state linked to insulin resistance (IR). It is unclear whether serum levels of inflammatory markers reflect intrahepatic pathology. We investigated the relationships between hepatic and systemic markers of inflammation and IR in NASH cases.

Methods: The study composed of 52 type 2 diabetes cases with NASH (male $58 \%$, mean age $53.2 \pm 7.3$ years, duration of diabetes $7.4 \pm 4.7$ years and BMI $31.9 \pm 4.2 \mathrm{~kg} / \mathrm{m}^{2}$. Diagnosis of NASH was based on biochemical markers and ultrasound scans. Cases were classified according to the Brunt Criteria. HsCRP, ferritin, leptin and adiponectin (ADPN), glucose, lipids, liver enzymes and HOMA-IR were investigated in the serum. TNF- $\alpha$ and IL- 8 levels were measured in biopsy specimens.

Results: Based on histopathological evaluation, $63.5 \%$ of cases had macrovesicular steatosis and the remaining had mixed type. Steatosis was mild $57.3 \%$, moderate in $30.8 \%$, and severe in $11.6 \%$ of cases. We did not find any infiltration in the majority, however $15.4 \%$ had mild and $5.8 \%$ had moderate infiltration. Fibrosis was mild in $50 \%$ and moderate in $7.7 \%$ cases. When the study group was stratified as mild-to-moderate (57.7\%) and severe steatosis (42.3\%) based on ultrasound evaluation and compared, in severe steatosis group serum levels of hs-CRP $(p=0.007)$, C-peptide $(p=0.006)$, ALP $(p=0.043)$ and GGT $(p=0.034)$ were significantly higher than in the mild-tomoderate group. Hepatic TNF- $\alpha$ levels in mild steatosis group was significantly higher than in the moderate and severe groups (mild vs. moderate and severe $10.78 \pm 8.76$ vs. $4.84 \pm 5.21$ and $6.83 \pm 8.63 \mathrm{pg} / \mathrm{mg}, \mathrm{p}=0.044$ ). Similarly hepatic IL-8 levels tended to be higher in mild steatosis than in the moderate and severe groups (mild vs. moderate and severe $5.08 \pm 3.39$ vs. $3.06 \pm 2.11$ and $3.98 \pm 2.17 \mathrm{pg} / \mathrm{mg}, \mathrm{p}=0.09)$. In the group with inflammation serum levels of ADPN was lower than the non-inflammation group $(p=0.006)$. Based on fibrosis, serum triglyceride $(\mathrm{p}=0.03)$ and ferritin $(\mathrm{p}=0.015)$ were lower, and ALP was higher $(\mathrm{p}=0.02)$ in the cases with fibrosis group compared with the non-fibrosis group. After controlling for age and duration of diabetes, we found a highly significant correlation between hepatic levels of TNF- $\alpha$ and IL-8 ( $\mathrm{r}=0.85, \mathrm{p}=0.0001)$. TNF- $\alpha$ negatively correlated with A1C, total iron binding capacity and AST whereas IL-8 negatively correlated with ferritin and hs-CRP.

Conclusion: To our knowledge for the first time in the literature, we quantitatively measured TNF- $\alpha$ and IL-8 levels in the liver tissue of NASH cases. Hepatic levels of these cytokines were related to IR and glucose metabolism. But limited relations were found between tissue cytokines and systemic inflammation markers. In contrast to our expectation, we found that as the liver pathology progressed, these cytokines tended to be decreased in the liver. In order to further clarify this topic, large prospective studies should be conducted in the future.

\section{7}

Impaired insulin-mediated non-esterified fatty acid suppression in patients with hepatic steatosis quantified using proton magnetic resonance spectroscopy

S. R. Mehta ${ }^{1}$, N. Barton ${ }^{1}$, I. F. Godsland ${ }^{1}$, S. Robinson ${ }^{1}$, J. D. Bell' E. L. Thomas ${ }^{2}$, N. Patel ${ }^{2}$, J. Fitzpatrick ${ }^{2}$, G. Durighel' ${ }^{2}$, D. Pavitt ${ }^{1}$, S. D. Taylor-Robinson ${ }^{3}$, D. G. Johnston ${ }^{1}$;

${ }^{1}$ Endocrinology \& Metabolic Medicine, ${ }^{2}$ Imaging Sciences,

${ }^{3}$ Hepatology, Imperial College, London, United Kingdom.

Background and Aims: Non-alcoholic fatty liver disease (NAFLD) is a condition characterised by triglyceride accumulation within the liver. It is frequently associated with features of the metabolic syndrome, with insulin resistance the most consistent underlying abnormality. The aim of the current study was to evaluate whether insulin-mediated suppression of adipose tissue lipolysis is impaired in subjects with NAFLD compared with healthy controls, in whom intrahepatocellular lipid (IHCL) content was measured by the safe and non-invasive method of proton magnetic resonance spectroscopy.

Material and Methods: We performed a frequently sampled intravenous glucose tolerance test (FSIVGTT) and proton magnetic resonance spectroscopy on 20 European males. 13 had known NAFLD with a median IHCL content of $17.87 \%$ and interquartile range $8.81-26.14 \%$ (normal IHCL

Table 1. Characteristics of patients with MS with and without NAFLD

\begin{tabular}{|c|c|c|c|c|c|c|}
\hline Parameter & $\begin{array}{l}\text { A. } \\
\text { MS without NAFLD }\end{array}$ & $\begin{array}{l}\text { B. } \\
\text { NAFLD with normal } \\
\text { liver enzymes }\end{array}$ & $\begin{array}{l}\text { C. } \\
\text { NAFLD with elevated } \\
\text { aminotransferaze levels }\end{array}$ & A vs. B & A vs.C & B vs. C \\
\hline Number & 29 & 151 & 44 & & & \\
\hline Waist circumference $(\mathrm{cm}) \mathrm{M} / \mathrm{F}$ & $\begin{array}{l}98.6 \pm 6.4 / \\
85.8 \pm 3.2\end{array}$ & $\begin{array}{l}102.6 \pm 7.2 / \\
92 \pm 6.4\end{array}$ & $\begin{array}{l}105.4 \pm 8.3 / \\
94 \pm 5.2\end{array}$ & $<0.001$ & $<0.001$ & $<0.001$ \\
\hline Triglycerides (mg/dL) & $210.5 \pm 36.2$ & $236.4 \pm 25.6$ & $235.8 \pm 29.2$ & $<0.001$ & $<0.001$ & NS \\
\hline HDL cholesterol (mg/dL) M/F & $\begin{array}{l}34.5 \pm 5.4 / \\
41 \pm 6.3\end{array}$ & $\begin{array}{l}31.2 \pm 4.8 / \\
39 \pm 5.6\end{array}$ & $\begin{array}{l}30.8 \pm 3.5 / \\
39 \pm 4.2\end{array}$ & $<0.001$ & $<0.001$ & NS \\
\hline Adiponectin $(\mu \mathrm{g} / \mathrm{mL})$ & $8.9 \pm 0.9$ & $7.8 \pm 0.5$ & $6.9 \pm 0.7$ & $<0.001$ & $<0.001$ & $<0.001$ \\
\hline TNF- $\alpha(p g / m L)$ & $7.5 \pm 1.2$ & $8.4 \pm 1.4$ & $8.9 \pm 1.5$ & 0.001 & $<0.001$ & 0.04 \\
\hline IL-6 (pg/mL) & $4.1 \pm 0.4$ & $4.9 \pm 0.6$ & $5.8 \pm 0.5$ & $<0.001$ & $<0.001$ & $<0.001$ \\
\hline Leptin (ng/mL) & $12.4 \pm 2.3$ & $14.2 \pm 2.6$ & $21.3 \pm 2.5$ & $<0.001$ & $<0.001$ & $<0.001$ \\
\hline hs-CRP(mg/L) & $7.2 \pm 0.7$ & $8.2 \pm 0.6$ & $8.4 \pm 0.9$ & $<0.001$ & $<0.001$ & NS \\
\hline HOMA-IR & $2.3 \pm 0.3$ & $2.9 \pm 0.2$ & $3.2 \pm 0.4$ & $<0.001$ & $<0.001$ & $<0.001$ \\
\hline
\end{tabular}

Data are mean \pm SD. $p$ was calculated with unpaired Student's $t$ test. 
content is $<5 \%$ ) and 7 were healthy controls with a median IHCL content of $0.12 \%$ (interquartile range $0.01-0.17 \%$ ). Sensitivity to the glucoregulatory effects of insulin was derived by mathematical modelling of glucose and insulin concentrations during the FSIVGTT using the minimal model of glucose disappearance. The rate of decline in NEFA concentrations during the IVGTT was quantified as the slope of the regression line passing through the log NEFA concentrations between 16 and 40 minutes, during which exponential decay was occurring in all participants. Sensitivity to the antilipolytic effects of insulin was expressed as a NEFA suppression index, calculated as the NEFA decay divided by the accompanying increment in the insulin concentration.

Results: Preliminary results of this ongoing study show no difference in basal NEFA concentrations between the 2 groups (median for cases with NAFLD $0.51 \mathrm{mEq} / \mathrm{L}$ [interquartile range $0.37-0.74$ ]; median for controls 0.63 $\mathrm{mEq} / \mathrm{L}$ [0.48-0.75]). The decrement in NEFA concentration at maximum suppression as a percentage of basal was 80.95 in the cases and 92.86 in the controls (Mann-Whitney $\mathrm{P}=0.001$ ) and the time to maximal suppression was 100 minutes in the cases and 80 minutes in the controls $(\mathrm{P}=0.15)$. The NEFA suppression index was substantially lower in the NAFLD group than in the control group (median $27.30 \mathrm{~min}^{-1} \cdot \mathrm{mU}^{-1} \cdot \mathrm{L} \mathrm{x}-10^{6}[15.86-31.66]$ versus $104.25 \mathrm{~min}^{-1} \cdot \mathrm{mU}^{-1} \cdot \mathrm{L} \mathrm{x}-10^{6}[60.92-138.72]$; Mann-Whitney $\left.\mathrm{P}<0.001\right)$. There was a significant positive correlation between glucoregulatory and antilipolytic effects of insulin (Spearman's rho $=0.80 ; \mathrm{P}<0.0001$ ).

Conclusion: Insulin-mediated NEFA suppression is impaired in subjects with NAFLD compared with healthy controls. The role of insulin-mediated NEFA suppression in contributing to hepatic fat accumulation requires further study.

Supported by the Novo Nordisk UK Research Foundation

\section{8}

Continuous glucose monitoring in non-diabetes, non-alcoholic fatty liver disease subjects

L. Czupryniak ${ }^{1}$, E. Szymanska-Garbacz ${ }^{1}$, M. Pawlowski ${ }^{1}$,

M. Saryusz-Wolska ${ }^{1}$, M. Jablkowski², J. Bialkowska², A. Borkowska ${ }^{1}$, A. Omulecka ${ }^{3}$, J. Loba ${ }^{1}$;

${ }^{1}$ Diabetology and Metabolic Diseases Dept, Medical University of Lodz, Lodz, Poland, ${ }^{2}$ Infectious Diseases Dept, ${ }^{3}$ Pathology Dept, Medical University of Lodz, Poland.

Background and Aims: Non-alcoholic fatty liver disease (NAFLD), a condition associated with insulin resistance, significantly increases the risk of development of type 2 diabetes. However, the natural history of glucose intolerance in NAFLD subjects is poorly understood. The aim of our study was to assess circadian glucose profile with the means of continuous glucose monitoring system in non-diabetes NAFLD subjects.

Materials and Methods: The study group consisted of 15 individuals with liver biopsy-confirmed NAFLD (mean age $37.5 \pm 8.7$ years, body mass index BMI $28.8 \pm 3.1 \mathrm{~kg} / \mathrm{m} 2$, plasma aspartate aminotransferase (AST) activity 60.1 $\pm 34.9 \mathrm{IU} / \mathrm{l}$, alanine aminotransferase (ALT) activity 104.6 $\pm 64.2 \mathrm{IU} / \mathrm{l}$, HbAlc 5.61 $\pm 0.52 \%$, HOMA index 4.13 \pm 2.49 ). The controls were 6 matched healthy subjects, with normal liver function tests and HOMA index $<2.0$. Diabetes was excluded in all studied persons upon the results of the oral 75 g glucose tolerance test (OGTT), which was performed at the beginning of the study. Continuous glucose monitoring (CGM) with the means of CGMS ${ }^{\bullet}$ System Gold (Medtronic MiniMed, Northridge, CA, USA) was conducted in all subjects. A glucose sensor was inserted into abdominal subcutaneous tissue for three days, and a CGMS monitor calibration was performed according to the CGMS use protocol. All subjects were asked to maintain their usual physical activity and food intake.

Results: In NAFLD subjects mean duration of continuous glucose monitoring was 75.6 hours (range 70-93 hours) with mean number of glucose readings 861 (range 564-1106). In the controls CGM was conducted for 76.2 hours (72-80.5 hours) with average 872 glucose readings (range 766-1062). Mean $( \pm$ SD) glucose for the whole monitored period was $104.5 \pm 11.3 \mathrm{mg} / \mathrm{dl}$ in NAFLD group and $85.5 \pm 5.9$ in healthy controls $(p<0.001)$. Mean lowest recorded glucose values in the study group and the controls were $68.9 \pm 13.5$ and $54.3 \pm 14.5 \mathrm{mg} / \mathrm{dl}(\mathrm{p}<0.05)$ and mean highest values were $153.1 \pm 16.5$ and $145.1 \pm 13.9 \mathrm{mg} / \mathrm{dl}(\mathrm{p}<0.05)$, respectively. Glucose variability calculated as the absolute value of measured glucose minus $99 \mathrm{mg} / \mathrm{dl}(5.5 \mathrm{mmol} / \mathrm{l})$ was similar in NAFLD subjects and in the controls (14.7 \pm 8.4 vs $14.8 \pm 4.9$ $\mathrm{mg} / \mathrm{dl})$.
Conclusion: Non-diabetes subjects with NAFLD present with significantly elevated diurnal glucose as assessed with CGMS, however its stability remains similar to healthy subjects.

Supported by the Medical University of Lodz

\section{9}

Glucose intolerance in non-alcoholic fatty liver disease - a possible role of impaired glucagon-like peptide-1 secretion M. Saryusz-Wolska ${ }^{1}$, L. Czupryniak ${ }^{1}$, E. Szymanska-Garbacz ${ }^{1}$, M. Pawlowski ${ }^{1}$, M. Jablkowski ${ }^{2}$, J. Bialkowska ${ }^{2}$, A. Omulecka ${ }^{3}$, A. Borkowska ${ }^{1}$, J. Loba ${ }^{1}$;

${ }^{1}$ Diabetology and Metabolic Diseases Department,

${ }^{2}$ Infectious Diseases Department, ${ }^{3}$ Pathology Department, Medical University of Lodz, Poland.

Background and Aims: Non-alcoholic fatty liver disease (NAFLD) is associated with insulin resistance and constitutes a significant risk factor for the development of diabetes mellitus. However, it is not clear why only a fraction of individuals with NAFLD become diabetic. The aim of the study was to identify factors predictive of glucose intolerance development in a group of NAFLD subjects.

Materials and Methods: The study group consisted of 56 otherwise healthy subjects with liver biopsy-confirmed NAFLD ( 42 males, 14 females, mean age $46.7 \pm 13.6$ years, BMI $28.8 \pm 3.7 \mathrm{~kg} / \mathrm{m} 2$ ), who were subject to oral $75 \mathrm{~g}$ glucose tolerance test (OGTT) with blood sampled at $0,60,120,180$ and $240 \mathrm{~min}$ following glucose ingestion for glucose, insulin and entero-insular axis (plasma glucagon-like peptide-1) assessment. Fasting plasma liver function enzymes activity as well as parameters of insulin resistance (plasma adiponectin, free fatty acids; FFA), endothelial dysfunction (adhesion molecules [VCAM, ICAM] and sE-selectin) and liver regeneration (hepatocyte growth factor; HGF) in a fasting state were also measured in all subjects.

Results: Impaired fasting glucose (IFG) or impaired glucose tolerance (IGT) were found in 21 (38\%) subjects, whilst $35(62 \%)$ subjects had normal glucose tolerance (NGT). Individuals with IFG/IGT were significantly older (57.0 \pm 11.2 vs $42.0 \pm 13.1$ years, $\mathrm{p}<0.01$ ), had lower GLP-1 levels at $0,60,120$ and $180 \mathrm{~min}$ of OGTT $(10.5 \pm 2.3 \mathrm{vs} 24.5 \pm 6.8 \mathrm{ng} / \mathrm{ml} ; 9.7 \pm 3.0 \mathrm{vs} 19.1 \pm 12.6 \mathrm{ng} /$ $\mathrm{ml} ; 8.1 \pm 2.9$ vs $13.6 \pm 3.5 ; 6.3 \pm 2.6$ vs $11.2 \pm 4.1 \mathrm{ng} / \mathrm{ml}$; all $\mathrm{p}<0.05)$, greater HOMA index $(5.3 \pm 3.7$ vs $2.9 \pm 1.5 ; \mathrm{p}<0.01)$, higher plasma FFA $(1.4 \pm 1.0$ vs $0.5 \pm 0.2$ $\mathrm{mmol} / \mathrm{l} ; \mathrm{p}<0.01)$, lower plasma adiponectin $(1526 \pm 1034$ vs $2452 \pm 1358 \mathrm{ng} /$ $\mathrm{ml} ; \mathrm{p}<0.05)$, higher plasma VCAM $(2144 \pm 956$ vs $1504 \pm 712 \mathrm{ng} / \mathrm{ml}, \mathrm{p}<0.05)$, ICAM $(511 \pm 336$ vs $363 \pm 143 \mathrm{ng} / \mathrm{ml} ; \mathrm{p}<0.05)$ and sE-selectin ( $85.8 \pm 24.1$ vs $54.1 \pm 21.3 \mathrm{ng} / \mathrm{ml} ; \mathrm{p}<0.05)$, and lower HGF $(835 \pm 234$ vs $1279 \pm 484 \mathrm{pg} / \mathrm{ml}$; $\mathrm{p}<0.05)$. Notably, no differences between IFG/IGT and NGT subjects were found in body weight, plasma insulin, lipids or liver function enzymes activity.

Conclusion: Increased risk of developing glucose intolerance in the course of NAFLD is associated not only with aging and higher insulin resistance, but also with reduced secretion of GLP-1, suggesting impaired incretin effect in NAFLD. Moreover, individuals with NAFLD and concomitant glucose intolerance present with apparent endothelial dysfunction and impaired stimulation of liver regeneration process.

Supported by the Medical University of Lodz

\section{0}

Long-term orlistat treatment reduces endotoxinaemia in fatty liver disease patients

N. F. da Silva ${ }^{1}$, A. L. Harte ${ }^{1}$, M. J. Hill' ${ }^{1}$, T. S. Billyard ${ }^{1}$, S. Kumar', C. P. Day², P. G. McTernan ${ }^{1}$,

${ }^{1}$ Clinical Sciences, University of Warwick,

${ }^{2}$ The Medical School, University of Newcastle, United Kingdom.

Background and Aims: Obesity-mediated diabetes is associated with insulin resistance/hyperinsulinaemia and chronic sub-clinical inflammation. Recent studies have highlighted fatty liver (steatosis) as a risk factor for diabetes. In addition, hepatic steatosis per se can lead to hepatic insulin resistance/ hyperinsulinaemia which may contribute significantly to the development of T2DM. Previous in vitro studies indicate liver may initiate an innate immune response which may be due to increased gut permeability through 
hyperinsulinaemia. This leads to increased bacterial absorption. Furthermore HDL has been shown to have a positive effect on endotoxin clearance. However, to date no study has confirmed whether there is a relationship between increased endotoxin levels and the progression of fatty liver disease (FLD), in vivo. Therefore for this study we (1) investigated the effect of disease status: FLD and T2DM compared to case controls (CC), (2) the impact of Orlistat, a lipase inhibitor, on endotoxin levels and (3) assessed inflammatory markers including soluble CD14 (an immunological receptor involved in the presentation of LPS to the TLRs), TNFRII, and the anti-inflammatory cytokine adiponectin.

Materials and Methods: Fasted human blood was collected from NAFLD and case control (CC) subjects (NAFLD: Age: $36.8 \pm 11.5 \mathrm{yrs}$ (mean \pm SD)yrs, BMI: $26.5 \pm 4.4($ mean $\pm \mathrm{SD}) \mathrm{kg} / \mathrm{m}^{2} \mathrm{n}=80$; CC: Age: $38.9 \pm 12.4 \mathrm{yrs}, \mathrm{BMI}: 26.5 \pm 4.4 \mathrm{~kg} /$ $\mathrm{m}^{2}, \mathrm{n}=35$ ) accordingly to LERC consent. Diabetic status was also ascertained by glucose and insulin levels. Endotoxin levels were measured by LAL Assay. Inflammatory markers were assessed by ELISA.

Results: The findings from this study showed that endotoxin levels were significantly higher in all patents with fatty liver disease compared with CC (FLD:11.06 $\pm 0.96 \mathrm{EU} / \mathrm{mL}$ (mean \pm SEM); CC:5.27 $\pm 0.45, \mathrm{p}<0.01)$. FLD alone produced comparable endotoxin levels to T2DM (FLD: T2DM: $10.82 \pm 1.15 \mathrm{EU} / \mathrm{mL}$; non-diabetic: $11.16 \pm 0.90 \mathrm{EU} / \mathrm{mL}$ ). In addition, Orlistat treated (1yr) FLD subjects had significantly reduced endotoxin levels compared to pre-treatment (pre: $15.8 \pm 1.6 \mathrm{EU} / \mathrm{mL}$; post: $11.1 \pm 1.4 \mathrm{EU} / \mathrm{mL}$, $\mathrm{p}<0.05)$. Furthermore, sCD14 and resistin were both raised in FLD subjects $(\mathrm{p}<0.05)$ whilst adiponectin was reduced in FLD compared with CC (FLD: $11.63 \pm 0.60 \mu \mathrm{g} / \mathrm{mL}$ vs CC: $17.06 \pm 1.74 \mu \mathrm{g} / \mathrm{mL}, \mathrm{p}=0.006)$.

Conclusion: In summary, our results suggest that FLD is associated with a 7 fold increase in endotoxin levels but ameliorated by Orlistat treatment. As such impaired liver metabolism may result in endotoxaemia, decreased metabolic clearance and increase susceptibility to T2DM. Therefore Orlistat, currently used in the treatment of T2DM could be implemented in FLD to reduce endotoxin and consequent inflammatory burden.

Supported by Wellcome Trust

\section{1}

Effects of metformin and fenofibrate on hepatic steatosis in zucker diabetic rats $(\mathrm{ZDF})$

F. Forcheron ${ }^{1}$, P. Abdallah ${ }^{2}$, A. Basset ${ }^{3}$, P. Del Carmine ${ }^{4}$, G. Haffar ${ }^{2}$, M. Beylot ${ }^{1,3}$;

${ }^{1}$ Faculté RTH Laennec, INSERM ERI 22,

${ }^{2}$ Faculté RTH Laennec, INSERM U499, ${ }^{3}$ Faculté Rockefeller, ANIPHY,

${ }^{4}$ Faculté Rockefeller, ANIPHY, Lyon, France.

Background and Aims: Non-alcoholic steatohepatitis (NASH) is frequent in type 2 diabetes and can result in liver fibrosis, cirrhosis and carcinoma. Besides dietary recommendations no specific treatment of NASH has been defined. Promising results have been obtained with metformin in ob/ob mice but studies in humans showed only a limited action. Therefore we tested the efficiency of metformin in an experimental model of NASH, the ZDF rats. Since ppar alpha agonists stimulate lipid oxidation and could reduce tissue TG stores, we investigated also the possible beneficial effect of a ppar alpha agonist, fenofibrate, on NASH.

Materials and Methods: ZDF $(n=35)$ and controls (Lean, $L, n=15)$ rats, all receiving a high fat diet, were studied at 7,14 and 21 weeks. After the initial study at 7 weeks, $10 \mathrm{ZDF}$ received no treatment, 10 received metformin and 10 fenofibrate (oral administrations) until final studies at 14 or 21 weeks. L rats received no treatment. Each study comprised measurements of plasma glucose, triacylglycerols (TAG), insulin levels, energy intake and expenditure and (5 rats of each group at 7,14 and 21 weeks) hepatic lipogenesis (deuterated water) and liver sampling (TAG content and relevant mRNA concentrations)

Results: Compared to L, ZDF were obese, hypertriglyceridemic and insulinresistant at 7 weeks, type 2 diabetic at 14 weeks and diabetic with insulin deficiency at 21 weeks. Steatosis was present at 7 weeks (liver TAG content $11.8+0.8$ vs $1.9+0.2 \mu$ moles/g $\mathrm{p}<0.001)$ with a large increase in hepatic lipogenesis and FAS and SCD mRNA levels $(\mathrm{p}<0.01)$. Steatosis was unchanged in $\mathrm{ZDF}$ at 14 and 21 weeks $(13.7 \pm 2.6$ and $12.9+0.9 \mu$ moles/g respectively, $\mathrm{p}<0.01 \mathrm{vs} \mathrm{L}$ ) despite less important rises in hepatic lipogenesis and FAS mRNA levels. Neither metformin nor fenofibrate modified significantly the evolution of body weight, energy intake or expenditure nor the evolution of diabetes. Plasma TAG were decreased by metformin at 14 ( $\mathrm{p}<0.01$ vs untreated ZDF) and $21(\mathrm{p}<0.05)$ weeks, but only at 21 weeks $(\mathrm{p}<0.05)$ by fenofibrate. Hepatic TAG content was reduced by fenofibrate at $14(5.6 \pm 1.4 \mu$ moles/g $\mathrm{p}<0.05$ vs untreated ZDF) and 21 weeks $(9.1 \pm 1.7 \mu$ moles/g $\mathrm{p}<0.05)$ but only at 21 weeks $(9.6 \pm 0.5 \mu$ moles/g $\mathrm{p}<0.05)$ by metformin. The contribution of lipogenesis to these hepatic TAG stores was not decreased at 14 or 21 weeks, neither by metformin nor by fenofibrate, despite a decrease in liver FAS mRNA levels at 14 weeks in ZDF receiving fenofibrate or metformin. Acyl-coA oxidase mRNA levels were increased by fenofibrate $(\mathrm{p}<0.05)$.

Conclusion: despite a clear lowering of plasma TAG, metformin had only a moderate beneficial effect on liver steatosis. The beneficial effect of fenofibrate was more marked but remained mild. These moderate actions on liver steatosis are not explained by decreases in hepatic lipogenesis and are probably more related to an increased in hepatic lipid oxidation rate. Supported by the Fondation de France

\section{2}

Effects of metformin on leptin, obesity and nonalcoholic fatty liver disease compared with lifestyle interventions in obese patients with type 2 diabetes mellitus

A. Nar Demirer ${ }^{1}$, O. Gedik²;

${ }^{1}$ Endocrinology and Metabolism, Baskent University Faculty of Medicine, ${ }^{2}$ Endocrinology and Metabolism, Hacettepe University Faculty of Medicine, Ankara, Turkey.

Background and Aims: Insulin resistance is a major feature of type 2 diabetes mellitus, obesity and nonalcoholic fatty liver disease (NAFLD). The pathogenesis of NAFLD is not clear and there is no universal treatment. Metformin is an oral anti-diabetic agent which improves hepatic insulin resistance and promotes weight-loss. It has been suggested that metformin also shows promising improvements in NAFLD. The mediators in these actions of metformin are not totally known. Leptin is an adipocytederived hormone that acts as a major regulator for food intake and energy homeostasis. Several studies pointed out the possible role of increased leptin in NAFLD in humans. The aim of this study is to determine the effects of metformin on plasma leptin levels, obesity and NAFLD in obese patients with type 2 diabetes mellitus.

Materials and Methods: Thirty four obese patients with newly diagnosed type 2 diabetes mellitus were prospectively followed for 6 months. All patients had ultrasonographic evidence of NAFLD at baseline. Other causes of liver disease were excluded. The patients were randomised into two groups: group $1(n=15)$ was followed by lifestyle changes only and group $2(n=19)$ received metformin (1700 mg/day) in addition to lifestyle changes. Body mass index (BMI), waist to hip ratio (WHR), fasting blood samples for the estimation of serum concentrations of glucose, HbAlc, insulin, leptin, aminotransferases and serum lipid profile were measured and liver ultrasonography was assessed at baseline and at the end of the study. There was no statistically significant difference between the groups at the beginning of therapy regarding age, sex, BMI, WHR, HbA1c, serum insulin, leptin, aminotransferases, lipid levels, and liver densities.

Results: At the end of treatment, BMI, WHR, $\mathrm{HbAlc}$, fasting glucose, leptin, alanine aminotransferase (ALT) values decreased in both groups $(\mathrm{p}<0.05 \mathrm{vs}$. baseline for all comparisons). No significant difference in the end-points was observed between two groups. Plasma leptin levels were found to correlate with BMI, but not with biochemical variables. In only group 2, who received metformin, serum LDL-cholesterol levels decreased and HDL-cholesterol levels increased significantly. Fasting insulin and triglyceride levels did not change in either group after the treatment period. Liver echogenity decreased and hepatomegaly reversed significantly at the end of study in both groups. The percentage of patients who no longer had evidence of NAFLD was not significantly different between the groups ( $20 \%$ of patients on lifestyle intervention vs. $16 \%$ of patients on metformin).

Conclusion: We conclude that, metformin and lifestyle interventions equally affected the plasma leptin levels, BMI and degree of NAFLD in obese patients with type 2 diabetes mellitus. In addition, the effects of metformin on the variables were not found to be mediated by leptin. 


\section{PS 62 Oxidative stress}

\section{3}

Redox stress parameters in type 2 diabetes mellitus

M. Vladica ${ }^{1}$, D. Margina ${ }^{2}$, D. Gradinaru ${ }^{2}$, S. Ioacara ${ }^{1}$, R. Danciulescu ${ }^{1}$; ${ }^{1}$ Diabetes 1, "N. Paulescu" Institute of Diabetes, ${ }^{2}$ Biochemistry, "Carol Davila" University, Faculty of Pharmacy, Bucharest 2, Romania.

Background and Aim: Hyperglycemia associated with type II diabetes mellitus determines oxidative stress that leads to important structural, functional and biochemical changes of the endothelial cells and also of the blood cells. The aim of the study was to assay the relationship between the oxidative stress and the biochemical profile of endothelial cells and also of the platelets, erythrocytes and granulocytes.

Materials and Methods: We have selected 40 patients: 20 patients with type II diabetes mellitus that do not respond properly to therapy (study group) and 20 normoglycemic patients (control group). à jeun venous blood samples were drawn in order to separate plasma, and (by differential centrifugation), the thrombocytes, granulocytes and erythrocytes. The following parameters were evaluated: fasting plasma glucose, superoxide dismutase activity SOD (using the Ransod method), the susceptibility to lipid peroxidation of granulocytes and platelets (induced by a non enzymatic system with $\mathrm{Fe}^{2+}$ and ascorbic acid and expressed as $\mathrm{mM}$ malondialdehyde-MDA/g proteins), the nitrites and nitrates level -NOx (with a global method using the Griess reagent).

Results: Our results pointed out a decrease of the antioxidantSOD activity at the study group ( $35.18 \pm 10.89 \mathrm{UI} / \mathrm{g}$ hemoglobin, vs. $53.45 \pm 6.66 \mathrm{UI} / \mathrm{g}$ hemoglobin, $\mathrm{p}=0.006$ ), associated with the increase of the susceptibility of granulocytes $(12.32 \pm 3.60 \mathrm{mM} \mathrm{MDA} / \mathrm{mg}$ proteins vs. $8.43 \pm 2.58 \mathrm{mM} \mathrm{MDA} / \mathrm{mg}$ proteins, $\mathrm{p}=0.01)$ and platelets $(20.16 \pm 4.19 \mathrm{mM} \mathrm{MDA} / \mathrm{mg}$ proteins vs. $15.21 \pm 3.30 \mathrm{mM}$ $\mathrm{MDA} / \mathrm{mg}$ proteins, $\mathrm{p}=0.01$ ) to peroxidation compared to control. The type II diabetes mellitus patients are characterized by a significantly higher NOx level compared to normoglycemic subjects $(43.13 \pm 3.89 \mu \mathrm{moles} / \mathrm{l}$ plasma vs. $37.04 \pm 5.52 \mu$ moles $/ 1$ plasma, $\mathrm{p}=0.04$ ).

Conclusions: Poor metabolic control of fasting plasma glucose in type II diabetic patients is associated with a decreased antioxidant protection at the erythrocyte' level, with oxidative changes of the blood cells and with an impairment of the endogenous NO synthesis.

\section{4}

Insulin reduces plasma enzymatic arginase activity in patients with type 2 diabetes

S. R. Kashyap ${ }^{1}$, A. Lara ${ }^{2}$, R. Zhang ${ }^{3}$, Y. M. Park ${ }^{4}$, R. A. Defronzo ${ }^{5}$;

${ }^{1}$ Endocrinology, Diabetes and Metabolism, Cleveland Clinic Foundation, Cleveland, ${ }^{2}$ Medicine, Cleveland Clinic Foundation, Cleveland,

${ }^{3}$ Cell Biology-Lerner Institute, Cleveland Clinic Foundation, Cleveland, ${ }^{4}$ Cell Biology, Cleveland Clinic Foundation, Cleveland, ${ }^{5}$ Diabetes, University of Texas Health Science Center, San Antonio, nited States.

Background and Aims: Nitric oxide (NO) bioactivity, critical for endothelial function, is dependent on substrate arginine bioavailability. Arginase enzyme converts L-arginine to urea and ornithine, and thereby decreases substrate availability for nitric oxide synthase to produce NO. We hypothesized that arginase activity is increased in patients with type 2 diabetes and that this is related to the severity of insulin resistance.

Materials and Methods: Therefore, we measured plasma arginase activity and arginine metabolites in 12 uncomplicated type $2 \mathrm{dm}(\mathrm{D})(49 \pm 2 \mathrm{y}$, BMI $=31 \pm 1 \mathrm{~kg} / \mathrm{m} 2$, FPG $136 \pm 13 \mathrm{mg} / \mathrm{dl}$, FPI $16 \pm 2 \mathrm{uU} / \mathrm{ml}, \mathrm{HbAlc}=6.7 \pm 1 \%$; diab dur 2y; diet/ oral sulfonylurea controlled) and 10 age/BMI matched non-diabetic subjects $(C)(49 \pm 3 \mathrm{y}, 29.4 \pm 1 \mathrm{~kg} / \mathrm{m} 2,89 \pm 1 \mathrm{mg} / \mathrm{dl}, 6 \pm$ $1 \mathrm{uU} / \mathrm{ml}$ )before and following 4 hour physiologic insulin infusion during a $80 \mathrm{mU} / \mathrm{m} 2 / \mathrm{min}$ euglycemic hyperinsulinemic clamp study to measure insulin sensitivity. Arginine metabolites were determined by tandem mass spectroscopy. Arginase activity was determined by conversion of $\left[{ }^{14} \mathrm{C}\right]$ guanidoinoarginine to $\left[{ }^{14} \mathrm{C}\right]$ urea. Liberated ${ }^{14} \mathrm{Co} 2$, trapped as $\mathrm{Na}_{2}{ }^{14} \mathrm{Co}_{3}$ was quantified by scintillation counting.

Results: Insulin-stimulated glucose disposal (Rd) in D was reduced by $50 \%$ vs. C, $\left(5.4 \pm 0.3\right.$ vs. $\left.10.4 \pm 0.5 \mathrm{mg} /\left(\mathrm{kg}^{\star} \mathrm{min}\right), \mathrm{P}<0.01\right)$. Basal arginine to ornithine ratio, (a surrogate marker of arginase activity) was markedly decreased in D vs. C subjects. Baseline plasma arginase activity was substantially elevated in $\mathrm{D}$ vs. $\mathrm{C}(0.48 \pm 0.11$ vs. $0.32 \pm 0.12 \mathrm{umol} / \mathrm{ml} / \mathrm{hr}, \mathrm{p}=0.05)$ and reduced significantly in $\mathrm{D}$ with $4 \mathrm{hr}$ insulin infusion (to $0.13 \pm .04 \mathrm{vs}$. basal, $\mathrm{p}<0.05$ ). In both groups collectively, plasma arginase activity correlated linearly with fasting plasma glucose $(\mathrm{r}=.32, \mathrm{p}=0.05)$ and $\operatorname{HbAlc}(\mathrm{r}=35, \mathrm{p}=0.05)$, but not with insulin sensitivity (Rd) or body mass index. Circulating soluble adhesion molecule, VCAM levels correlated linearly with arginase activity ( $\mathrm{R}=0.55, \mathrm{P}=0.05$ ).

Conclusion: In summary, plasma arginase activity is increased in subjects with T2DM, corresponds to the degree of hyperglycemia, and is markedly reduced by physiologic levels of insulin. Elevated arginase activity may contribute to endothelial dysfunction in type 2 diabetes and moreover, insulin may ameliorate endothelial dysfunction via reducing arginase activity. Arginase blockade may be a target for future therapy in treating diabetes. Supported by NIH K12

\section{5}

Thrombocyte function and oxidative stress markers in blood of type 1 and type 2 diabetic patients and healthy subjects: the in vitro effects of insulin

K. Maria ${ }^{1}$, E. Arato ${ }^{2}$, G. Jancso ${ }^{1}$, B. Cserepes ${ }^{1}$, J. Lantos ${ }^{1}$, S. Ferencz ${ }^{1}$, S. Bertok ${ }^{1}$, A. Ferencz ${ }^{1}$, E. Roth ${ }^{1}$;

${ }^{1}$ Surgical Research and Technique,

${ }^{2}$ Department of Surgery, Pecs University Medical School, Hungary.

Background and Aims: Insulin is one of the most intensively studied hormones with several physiological actions beyond the regulation of carbohydrate and lipid metabolism.

The aim of the present study was to investigate thrombocyte function and oxidative stress markers in the blood of type-1 (DM1, n=10) and type-2 $(\mathrm{DM} 2, \mathrm{n}=10)$ diabetic patients with peripheral arterial diseases and of healthy blood donors ( $\mathrm{HBD} \mathrm{n}=11$ ). In vitro effect of insulin on thrombocyte function and free radical production were also investigated.

Methods: Platelet aggregation was measured in platelet rich plasma (PRP) and in whole blood using ADP $(5$ and $10 \mu \mathrm{M})$ and collagen $(2 \mu \mathrm{g} / \mathrm{ml})$ as inductors. Prooxidant status was characterized by the oxygen free radical production (ROS), in response to phorbol-12-myristate 13-acetate (PMA), and plasma malondialdehide (MDA) levels. Reduced glutathion (GSH) level and superoxide dismutase (SOD) activity were measured to characterise the endogenous antioxidant status. Collagen induced aggregation, and ROS production were also measured in the presence of insulin $(0,40,80,160 \mu \mathrm{U} /$ $\mathrm{ml}$ ) in whole blood.

Results: ADP and collagen induced aggregation in PRP were suppressed in

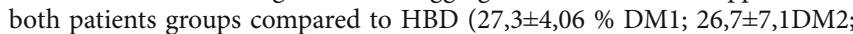
$66,87 \pm 9,6 \mathrm{HBD})(\mathrm{P}<0,01$ in both cases), signing the satisfactory antiplatelet therapy. In whole blood the aggregations were similar in the three groups. Preincubation of samples ( 3 minutes before administration of inductor), with the above doses of insulin, a U-shaped reduction in collagen induced aggregation occurred in healthy $(\mathrm{P}<0,05)$, and in DM1 $(\mathrm{p}<0,01)$ subjects, but insulin was ineffective in DM2 patient's blood. PMA induced ROS production were significantly higher in both patients groups, than in healthy subjects (DM1 $=194,2 \pm 61,11 ; \mathrm{DM} 2=61,19 \pm 9,53, \mathrm{HBD}=10,17 \pm 3,42$ ArbU,). Insulin reduced ROS production in DM1 patients $(\mathrm{p}<0,01)$, and in healthy subjects $(\mathrm{p}<0,05)$, but not in DM2 patients. SOD levels were reduced in DM1 $(583,1 \pm$ $63,6 \mu \mathrm{U} / \mathrm{ml} ; \mathrm{p}<0,01$ vs. HBD) and DM2 ( $704,4 \pm 81,6 \mu \mathrm{U} / \mathrm{ml} ; \mathrm{P}<0,05$ vs. HBD) patients compared to healthy people $(1008,75 \pm 91,97 \mu \mathrm{U} / \mathrm{ml})$. GSH level was decreased only in DM1 $(\mathrm{p}<0,05)$.

Conclusions: Platelets and white blood cells of healthy subjects were sensitive to insulin, which manifested in the insulin related reduction of collagen induced aggregation, and PMA induced ROS production in whole blood. A serious imbalance in endogenous antioxidant and prooxidants was observed in both patients groups and insulin resistance developed against antiplatelet and free radical decreasing effect of insulin in DM2 patients. The sensibility to insulin action was preserved in thrombocyte and white blood cells of DM1 patients.

Supported by OTKA T060227, OTKA T048854 Hungarian grants 


\section{6}

Effect of acute hyperinsulinaemia and acute AT-1 receptor blockade on the serum levels of oxidative stress markers in type 2 diabetes and healthy subjects

T. Neskudla ${ }^{1}$, E. Krusinova ${ }^{1}$, M. Kalousova ${ }^{2}$, J. Kopecky jr. ${ }^{1}$, T. Pelikanova ${ }^{1}$; ${ }^{1}$ Diabetes Center, Institute for Clinical and Experimental Medicine, ${ }^{2}$ Institute of Clinical Chemistry and Laboratory Diagnostics, First Faculty of Medicine and General University Hospital, Charles University, Prague, Czech Republic.

Background and Aims: It has been shown by recent studies that patients with cardiovascular risk or type 2 diabetes do benefit from AT-1 receptor blocker (ARB) therapy. One possible explanation might be the reduced production of reactive oxygen species (ROS). The relationship between hyperinsulinaemia and oxidative stress is still unclear. The aim of the study is to test the effect of acute hyperinsulinaemia with and without acute AT-1 receptor blockade (losartan) on the serum levels of advanced oxidation protein products (AOPP), pregnancy-associated plasma protein-A (PAPP-A), homocysteine (HCY) and total antioxidation capacity (TAC)

Material and methods: 11 type 2 diabetics (T2D) and 8 lean healthy male subjects $(\mathrm{H})$ underwent: 1) 4 hours lasting hyperinsulinaemic (1 mU.kg${ }^{1} . \mathrm{min}^{-1}$ ) euglycaemic $\left(5 \mathrm{mmol} . \mathrm{l}^{-1}\right)$ clamp (HIC); 2) the same protocol was performed after administration of losartan (two 100mg doses 12 hours before and in the morning of the test) (ARB-HIC). Serum concentrations of AOPP, PAPP-A, HCY and TAC were measured at $0 \mathrm{~min}, 30 \mathrm{~min}$ and $240 \mathrm{~min}$ of both clamp studies. AOPP and PAPP-A serum levels were assessed in ratio to serum albumin, to avoid the influence of haemodilution.

Results: Serum concentrations of AOPP were higher in T2D compared to $\mathrm{H}(\mathrm{p}<0.001)$. During HIC a decrease of AOPP was observed in both groups $(p<0.001)$, whereas during ARB-HIC a different trend was detected without any changes in T2D and with a decline in $H(p<0.01)$. Serum levels of TAC were in global higher in $\mathrm{H}$ compared to T2D $(\mathrm{p}<0.001)$ and they decreased during both clamps in both groups $(\mathrm{p}<0.001)$. During ARB-HIC lower TAC levels compared to HIC $(p<0.05)$ were detected in both groups. HCY levels were higher in T2D compared to $\mathrm{H}$ in both clamps $(\mathrm{p}<0.05)$. No effect of HIC on HCY was detected in either group, while during ARB-HIC serum HCY decreased significantly in $\mathrm{H}(\mathrm{p}<0.05)$. Serum levels of PAPP-A showed no changes in either group or clamp.

Conclusion: Acute hyperinsulinaemia is associated with reduction of total antioxidation capacity and unexpectedly of AOPP in both T2D and healthy subjects. T2D have unfavourable oxidative stress markers profile, which is not affected by acute losartan administration. In healthy subjects losartan reduced homocystein levels. (Tab. 1)

Supported by grant IGA MH CZ

\section{7}

Effect of antioxidants, Ricetorienol and $\alpha$-tocopherol, on the adipocytokine abnormalities and fatty liver in OLETF-diabetic rats K. Tatsumi ${ }^{1}$, H. Sasaki ${ }^{1}$, A. Fujita ${ }^{1}$, A. Doi ${ }^{1}$, Y. Kanaya ${ }^{2}$, K. Ogawa $^{1}$, H. Furuta $^{1}$, M. Nishi ${ }^{1}$, T. Tsuno ${ }^{2}$, H. Taniguti ${ }^{3}$, K. Nanjo ${ }^{1}$;

${ }^{1}$ The First Department of Medicine, Wakayama Medical University, Japan, ${ }^{2}$ Tsuno Food Industrial Co. Ltd, Wakayama, Japan, ${ }^{3}$ Industrial Technology Center, Wakayama Prefecture, Wakayama, Japan.

Background and Aims: Type 2 diabetes and metabolic syndrome are important risk factors of macrovascular disease. It has been reported that the adipocytokine abnormalities play a major role in the pathogenesis of atherosclerosis and oxidative stress may be one of causes of the abnormalities.
We therefore examined the effect of Ricetrienol, which is an antioxidants complex extracted from rice-bran and obtained 3\% tocotrienol, and $\alpha$ tocopherol on the adipocytokine abnormalites in OLETF-rats (Otsuka LongEvans Tokushima Fatty rat)

Materials and Methods: OLETF-rats $(n=18)$ were fed with $30 \%$ sugar water, as a model of type 2 diabetes mellitus with obesity. The other rats $(n=18)$ were fed with tap water, as a model of non-diabetes with obesity. After all rats fed with sugar water developed diabetes, the water and tap water fed rats were divided randomly into three groups. Then each group was fed with a standard chew (DM-S, OB-S group), $0.05 \%$ Ricetrienol mixed chew (DM-R, OB-R group) or $0.05 \% \alpha$-tocopherol mixed chew (DM- $\alpha, \mathrm{OB}-\alpha$ group), respectively. After twelve weeks' feeding, all rats were sacrificed and blood and liver tissue were collected. Body weight and blood glucose were monitored throughout the experiment. Glycohemoglobin was measured by HPLC. Plasma adiponectin, resistin and leptin concentrations were assayed by ELISA. Triglyceride contents of the liver tissue were also determined.

Results: Blood glucose and glycohemoglobin levels in sugar water fed rats were significantly higher than tap water fed rats. These parameters were not affected by Ricetrienol or $\alpha$-tocopherol supplimentation. Plasma adiponectin was significantly reduced in DM-S than OB-S $(2.39 \pm 0.69$ vs $3.43 \pm 0.42 \mathrm{ng} / \mathrm{dl}$, $\mathrm{p}<0.01)$. Plasma adiponectin was significantly higher in DM-R than DM-S ( $3.65 \pm 0.61$ vs $2.39 \pm 0.69, \mathrm{p}<0.01$ ). Plasma resistin was significantly increased in DM-S than OB-S $(29.9 \pm 10.2$ vs $16.2 \pm 5.2 \mathrm{ng} / \mathrm{dl}, \mathrm{p}<0.01)$. Plasma resistin was significantly lower in DM- $\alpha$ than DM-S $(19.6 \pm 11.0$ vs $29.9 \pm 10.2, \mathrm{p}<0.05)$. There was no significant difference in plasma leptin between all groups. Although triglyceride contents of the liver tissue in DM-S were significantly increased than OB-S, triglyceride contents were significantly lower in DM-R than DM-S.

Conclusion: Ricetrienol inhibited the reduction of plasma adiponectin and triglyceride accumulation in the liver. $\alpha$-tocopherol inhibited the increase of plasma resistin. Our study shows that antioxidants supplementation may prevent the adipcytokine abnormalities and fatty liver in OLETF-diabetic rats.

Tab. 1

Plasma levels of selected markers during HIC and ARB-HIC. Data are expressed as mean \pm SD; ANOVA

\begin{tabular}{llllllll}
\hline & & HIC & & \multicolumn{3}{c}{ ARB-HIC } \\
\hline Diabetics & AOPP $(\mu \mathrm{mol} / \mathrm{l}) /$ Alb. & $2.84 \pm 0.35$ & $2.61 \pm 0.34$ & $2.57 \pm 0.30$ & $2.74 \pm 0.33$ & $2.51 \pm 0.29$ & $2.72 \pm 0.33$ \\
& TAC $(\mathrm{mmol} / \mathrm{l})$ & $1.05 \pm 0.04$ & $1.14 \pm 0.05$ & $1.00 \pm 0.04$ & $1.04 \pm 0.04$ & $1.01 \pm 0.04$ & $0.94 \pm 0.03$ \\
& HYC $(\mu \mathrm{mol} / \mathrm{l})$ & $8.86 \pm 0.53$ & $8.84 \pm 0.53$ & $9.27 \pm 0.58$ & $9.25 \pm 0.58$ & $9.50 \pm 0.61$ & $9.79 \pm 0.65$ \\
Healthy Subjects & AOPP $(\mu \mathrm{mol} / \mathrm{l}) / \mathrm{Alb}$. & $1.63 \pm 0.17$ & $1.49 \pm 0.15$ & $1.38 \pm 0.13$ & $1.41 \pm 0.13$ & $1.36 \pm 0.13$ & $1.11 \pm 0.09$ \\
& TAC $(\mathrm{mmol} / \mathrm{l})$ & $1.35 \pm 0.1$ & $1.29 \pm 0.08$ & $1.25 \pm 0.08$ & $1.27 \pm 0.08$ & $1.25 \pm 0.08$ & $1.19 \pm 0.07$ \\
& HYC $(\mu \mathrm{mol} / \mathrm{l})$ & $7.34 \pm 0.43$ & $6.83 \pm 0.37$ & $7.3 \pm 0.42$ & $7.99 \pm 0.50$ & $7.66 \pm 0.46$ & $7.06 \pm 0.39$ \\
\hline
\end{tabular}




\section{PS 63 Fetal programming}

\section{8}

\section{Is there any evidence of foetal programming on insulin detectable at} birth?

J. Beltrand ${ }^{1,2}$, R. Verkauskern ${ }^{1,2}$, O. Sibony ${ }^{3}$, O. Claris ${ }^{4}$, P. Gaucherand ${ }^{5}$, D. Chevenne ${ }^{6}$, C. Lévy Marchal ${ }^{1,2}$;

${ }^{1}$ U690, Inserm, Paris, ${ }^{2}$ Paris 7, Université, Paris, ${ }^{3}$ Gynécologie et obstétrique, hopital Robert Debré, Paris, France, ${ }^{4}$ Neonatologie, Hopital Edouard Herriot, Lyon, France, ${ }^{5}$ Gynécologie et obstétrique, Hopital Edouard Herriot, Lyon, France, ${ }^{6}$ Biochimie, Hopital Robert Debré, Paris, France.

Background and Aims: Subjects born SGA (small for gestational age) are at increased risk of developing type 2 diabetes. Two major issues are not yet solved: Is the risk detectable at birth? Is small body size or foetal growth restriction more related to insulin metabolism? We studied the effect of body composition, foetal growth velocity (FGV) and birth weight (BW) on insulin secretion in newborns included in a prospective cohort of mothers at risk of delivering SGA babies (the CASYMIR cohort).

Materials and Methods: Foetal growth was monitored by monthly ultrasounds between 22-36 GW (gestational weeks) following a highly standardized procedure. FGV was calculated as the change in customized percentiles of the estimated fetal weight (EFW) over the same period. . Insulin was measured in cord blood. Fat mass was estimated with the sum of 4 skinfold thickness (STS) at postnatal day 3 .

Results: 201 newborns were included. BW was $2968 \mathrm{~g}+/-601 \mathrm{~g}$, gestational age $38,8+/-2,04 \mathrm{GW}$ and STS $16,57+/-3,5 \mathrm{~mm}$. Cord insulin was 3,65 $+/-2,8 \mathrm{mUI} / \mathrm{l}$. In a multivariate analysis, insulin levels (log transformed) were significantly correlated to $\mathrm{BW}$ (bêta $=0,0003, \mathrm{p}=0,048$ ) and FGV (bêta $=0,09, \mathrm{p}=0,005$ ) but not by gender ( $\mathrm{bêta}=-0,03, \mathrm{p}=0,58$ ) nor gestational age (bêta $=-0,01, p=0,10)$. When STS was included into the model, insulin level was no longer significantly associated to BW but to FGV (bêta=$0,09, \mathrm{p}=0,007$ ) and STS(bêta $=0,089, \mathrm{p}=0,05$ ).

Conclusion: Insulin secretion at birth is related to the quality of foetal growth and subsequently to birth weight. As in older subjects the degree of adiposity influences circulating levels of insulin. Insulin resistance described in children and adults born SGA cannot be detected at birth, when insulin reflects primarily foetal growth. The effect of programming on glucose metabolism is not detectable at the end of foetal life. This programming will be revealed later, under the influence of post natal nutrition and growth.

Supported by institut danone

\section{9}

Maternal diet induced obesity programmes the development of metabolic syndrome in the offspring

P. Nivoit ${ }^{1,2}$, C. Morens ${ }^{1,2}$, A. Piersma ${ }^{3}$, B. Reusens ${ }^{1}$, C. Remacle ${ }^{1}$,

F. A. Van Assche';

${ }^{1}$ Institute of life Sciences, Catholic University of Louvain, Louvain-la-Neuve, Belgium, ${ }^{2}$ Department of Obstetrics and Gynaecology, KULeuven, Belgium, ${ }^{3}$ Laboratory for Toxicology, Pathology and Genetics, National Institute for Public Health and the Environment, Bilthoven, The Netherlands.

Background and Aims: Pregravid and gravid obesity is a common situation with the worldwide explosion of obesity. Epidemiological studies suggest a close link between pregnancy and pre-pregnancy maternal body mass index and increased risk of obesity in children and young adults. Since obesity is a major risk factor for developing metabolic syndrome (MS), a situation usually considered as a prediabetic state, the aim of our study was to determine, in a rodent model, whether maternal diet induced obesity (DIO) could program the development of MS (obesity and impaired glucose homeostasis) in the offspring.

Materials and Methods: We developed a model in which female Wistar rats were rendered obese by consuming, from weaning on, a diet enriched in fat and/or sucrose (OB1: 45\% kcal fat, $15.5 \% \mathrm{kcal}$ sucrose and OB2: $45 \% \mathrm{kcal}$ fat + sweetened condensed milk). A group of females fed lab chow was also included as control (C). At 3 months, females were mated and allowed to deliver spontaneously. At 21 days of age, control, OB1 and OB2 offspring were weaned onto usual lab chow diet. Body weight (BW), food intake, plasma parameters, oral glucose tolerance and body composition were assessed in both male and female progeny.

Results: Offspring of DIO dams had a significant increased BW at weaning compared to control (C: $56.8 \pm 1.7 \mathrm{~g}$; OB1: $69.9 \pm 2.4 \mathrm{~g}$ and OB2 $68.1 \pm 5.0 \mathrm{~g}$, $\mathrm{p}<0.001)$. BW remained significantly higher at 3 and 6 months, except for OB1 females, and was associated with an increased food intake, especially in males. At 2 months, OB1 and OB2 offspring had higher leptin levels compared to C. Significance was observed for OB1 males ( $\mathrm{p}=0.023)$, OB2 males $(\mathrm{p}<0.001)$ and OB2 females $(\mathrm{p}=0.004)$ but not $\mathrm{OB} 1$ females $(\mathrm{p}=0.066)$. Free fatty acid circulating levels were also modified with a slight non significant increase in $\mathrm{OB} 1$ and $\mathrm{OB} 2$ females whereas a stronger increase was observed in $\mathrm{OB} 1$ and $\mathrm{OB} 2$ males reaching significance in OB2 males $(\mathrm{p}=0.004)$. Glycemia, insulinemia, triglyceridemia and total blood cholesterol remained unchanged in $\mathrm{OB} 1$ and OB2 offspring when compared to C. At 3 months, glucose response to the oral glucose load measured by the area under the glucose curve (AUG) was unchanged in OB1 males and females compared to $\mathrm{C}$ whereas OB2 males and females showed an increased AUG compared to $\mathrm{C}$, reaching significance only for $\mathrm{OB} 2$ males $(\mathrm{p}<0.001)$. Insulin response to the oral glucose load measured by the area under the insulin curve (AUI) was slightly but not significantly increased in $\mathrm{OB} 1$ and $\mathrm{OB} 2$ males compared to $\mathrm{C}$ whereas $\mathrm{OB} 1$ and $\mathrm{OB} 2$ females showed a stronger but not significant increase of the AUI compared to the $\mathrm{C}$ ones. At 6 months, animals were killed and body composition was determined, showing that $\mathrm{OB} 1$ and $\mathrm{OB} 2$ offspring had a significant increased fat mass when compared to $C$, especially mesenteric (males, $p=0.014$; females, $\mathrm{p}=0.044$ ) and retroperitoneal fat pads (males, $\mathrm{p}=0.009$; females, $\mathrm{p}=0.046$ ).

Conclusion: Our results show that maternal DIO induce development of obesity, altered plasma parameters and perturbations of glucose homeostasis in offspring. These results strongly support the idea that maternal DIO programs the development of MS in offspring. Experiments are running using hyperinsulinemic-euglycemic clamps in 6 months-old animals to confirm these results.

Supported by European project EARNEST, Food-CT-2005-007036

\section{0}

Offspring of diabetic pregnancy have reduced capacity to oxidize fat and become overweight

S. Lau $^{1,2}$, R. Stokes ${ }^{1}$, C. Scott ${ }^{1}$, M. McLean ${ }^{2}$, N. Cheung ${ }^{2}$, J. E. Gunton ${ }^{1,2}$; ${ }^{1}$ Immunology and Inflammation, Garvan Institute of Medical Research, ${ }^{2}$ Dept Diabetes and Endocrinology, Westmead Hospital, Sydney, Australia.

Background and Aims: Offspring of diabetic pregnancy (DP) are at higher risk of developing obesity and Type 2 diabetes (T2DM) than offspring of non-DP. We used a novel animal model of DP, the $\beta$-cell specific ARNT (aryl hydrocarbon receptor nuclear translocator) knockout ( $\beta$-ARNT) mouse to elucidate the effects on male offspring with and without a genetic predisposition to diabetes.

Materials and Methods: $\beta$-ARNT and floxed control (FC) females were mated with FC and $\beta$-ARNT males respectively, giving mixed litters of $\sim 50 \%$ $\beta$-ARNT and $\sim 50 \%$ FC mice. Male offspring were serially weighed and had glucose tolerance tests (GTT) and insulin tolerance tests (ITT) at 8, 16 and 31 weeks. Food consumption was measured at 24 weeks. At 28 weeks metabolic rate was measured using indirect calorimetry and DEXA was performed to measure fat mass.

Results: Non-pregnant $\beta$-ARNT females are mildly glucose intolerant owing to a defect in insulin secretion. In late pregnancy, $\beta$-ARNT but not FC dams show marked worsening of GTT with mean peak glucose of $21.8 \mathrm{vs} 14.4 \mathrm{mmol} /$ $\mathrm{L}(\mathrm{p}<0.01)$. Dams were systemically well. Litter size and birthweights did not differ from non-DP. Both groups of DP offspring gained more weight from weaning to 18 weeks of age (20.3 and $20.6 \mathrm{~g}$ versus 16.9 and $16.4 \mathrm{~g}$ respectively, $\mathrm{p}=0.05$ and $\mathrm{p}=0.027$ ). By 20 weeks of age $\beta$-ARNT offspring of DP were $\sim 10 \%$ heavier than $\beta$-ARNT non-DP offspring $(\mathrm{p}=0.008)$ despite consuming less food, and had a trend to lower energy expenditure. Floxed-control DP offspring were $1.2 \mathrm{~g}$ lighter at weaning $(\mathrm{p}=0.013)$ but weighed $\sim 1 \mathrm{~g}$ more by 18 weeks of age than non-DP floxed-control offspring despite greater energy expenditure. Area under the curve (AUC) of the maternal late pregnancy GTT was significantly correlated with offspring weight $(\mathrm{r}=0.53, \mathrm{p}=0.001$ at 15 wks). Overall, DP offspring were more insulin resistant than those from non$\mathrm{DP}(\mathrm{p}=0.023$ by ANOVA for repeated measures). For both FC and $\beta$-ARNT offspring, there was a trend to a higher respiratory exchange ratio (RER) during light hours in DP offspring. AUC of maternal GTT was significantly correlated with offspring RER $(\mathrm{r}=0.46, \mathrm{p}=0.01)$. 
Conclusion: The data suggest that DP is associated with a reduced capacity to oxidize fat compared to glucose both in offspring that do and do not have a genetic predisposition to diabetes. DP was only associated with a significantly higher weight in $\beta$-ARNT offspring, perhaps because it is also associated with lower energy expenditure. The correlation between maternal GTT and offspring RER may explain why human offspring of DP are at greater risk of developing obesity and T2DM than others who were exposed to a normal intrauterine environment.

Supported by Diabetes Australia Research Trust

\section{1}

Taurine prevented the changes of glucose metabolism and mitochondrial structure in the fetal protein-malnourished rat

Y. Y. Lee ${ }^{1}$, S. H. Park ${ }^{2}$, K. H. Yi ${ }^{1}$, S. S. Lee ${ }^{3}$, K. S. Park ${ }^{4}$, H. K. Lee ${ }^{4}$; ${ }^{1}$ Internal Medicine, Korea Cancer Center Hospital, ${ }^{2}$ Pathology, Seoul National University, ${ }^{3}$ Pathology, Korea Cancer Center Hospital, ${ }^{4}$ Internal Medicine, Seoul National University, Republic of Korea.

Intrauterine growth retardation has been linked to the development of type 2 diabetes in later life. The mechanisms underlying this phenomenon are unknown. Taurine, an amino acid, which is necessary for normal development, is implicated in this process. Also in the offspring of the protein-malnourished rats, decreased mitochondrial DNA levels in the pancreas accompanied by reduced insulin secretory responses to glucose load were reported. This study was aimed to investigate the pathogenic role of mitochondria as a link between poor nutrition in early life and type2 diabetes in later life, and the possible preventive role of taurine of these mitochondrial changes. Male offspring of Wistar rats fed a control diet (20\% casein, C group), or low-protein diet ( $8 \%$ casein, LP group), or a low protein diet supplemented with $2.5 \%$ taurine in the drinking water(LP-T group) during pregnancy and lactation were weaned onto the control diet. In each group, functional studies to measure insulin secretion and in vivo insulin action, and ultrastructural analysis of the pancreas were aimed at. Fetal protein-malnourished rats showed reduced insulin secretory responses to glucose load. Plasma glucose concentrations after intravenous glucose administration tended to be lower in LP and LP-T group than C group. The plasma insulin concentrations at 2 min, $6 \mathrm{~min}$, and $50 \mathrm{~min}$ were significantly lower in LP group than $\mathrm{C}$ group $(p<0.05)$. Taurine supplementation tended to restore insulin response to glucose challenge, and this difference was statistically significant at $6 \mathrm{~min}$ $(\mathrm{p}<0.05)(\mathrm{C} 3.73 \pm 0.68, \mathrm{LP} 1.67 \pm 0.43$, LP-T $2.27 \pm 0.43 \mathrm{ng} / \mathrm{ml}$ at $2 \mathrm{~min}, \mathrm{C}$ $2.05 \pm 0.44$, LP $0.89 \pm 0.22$, LP-T $1.48 \pm 0.29 \mathrm{ng} / \mathrm{ml}$ at $6 \mathrm{~min}, \mathrm{C} 2.02 \pm 0.04$, LP $0.85 \pm 0.11$, LP-T $0.96 \pm 0.26 \mathrm{ng} / \mathrm{ml}$ at $50 \mathrm{~min}$, respectively). There were no significant differences in glucose infusion rate during hyperinsulinemic clamp studies (C $14.52 \pm 0.69, \mathrm{LP} 19.15 \pm 0.55, \mathrm{LP}-\mathrm{T} 20.05 \pm 0.28 \mathrm{mg} / \mathrm{kg} / \mathrm{min}$ ) among three groups. In the electron microscopic examination of beta cells, the immature secretory granules were increased in $\mathrm{L}$ group than $\mathrm{C}$ group. In $\mathrm{L}$ group, mitochondria were slender elongated appearance with indistinct cristae. Taurine supplementation to the low protein diet restored all these changes. These findings indicate that long-lasting mitochondrial changes caused by fetal protein malnutrition may contribute to the development of type 2 diabetes in later life, and lack of taurine may be a key causative factor for these mitochondrial changes.

Supported by KIRAMS Grant

\section{2}

Gender differences of insulin resistance development in offspring of gestational diabetic mothers

Z. Leshchenko, V. Poltorak, N. Krasova, A. Gladkih;

Laboratory for Pathophysiology and Medical Genetics, Institute of

Endocrine Pathology Problems, Kharkiv, Ukraine.

Background and Aims: Previous studies have indicated that gestational streptozotocin diabetes (GD) induce insulin resistance and strengthened beta-cells dysfunction in Wistar rats adult offspring. But up to now it is unknown whether dysglycemia gender differences would exist in these offspring. The aim of the study was to compare the influence of maternal gestational streptozotocin diabetes on insulin resistance formation in male and female Wistar rats offspring of the first generation $\left(\mathrm{F}_{1}\right)$ at puberty.
Materials and Methods: The maternal cohort consisted of 25 pregnant rats exposed to GD and controls (C). GD was rendered by a single streptozotocin injection (45 mg/kg body weight, i.p.) on the second day of pregnancy. An i.p. glucose tolerance test (GTT, $3 \mathrm{~g}$ glucose/kg body weight; $0,30,60,120$ min) was performed after an overnight fast in male and female $\mathrm{F}_{1}$ groups (12 animals each) at 45 days of age. Fasting blood samples were used for glycemia, plasma insulin (IRI) and non-etherified fatty acids (NEFA) levels determination. Glycogen contents and glucose-6-phosphatase (G-6Pase) activity were measured in liver homogenates. Insulin resistance (IR) state was estimated using Homeostasis Model Assessment (HOMA).

Results: Decrease in glucose tolerance was observed in all GD offspring compared to corresponding $\mathrm{CF}_{1}$ (AUC/2h over GTT in male: $938.53 \pm 14.66 \mathrm{vs}$ $690.55 \pm 16.70 \mathrm{mmol} / \mathrm{l} / \mathrm{min}, \mathrm{p}<0.05$; in female: $1020.70 \pm 55.40$ vs $711.00 \pm 8.58$ $\mathrm{mmol} / \mathrm{l} / \mathrm{min}, \mathrm{p}<0.05)$. The reliable gender differences of above-mentioned data were absented. NEFA levels were enhanced in both GD-male $F_{1}$ and GD-female $F_{1}$ in comparison with corresponding $C_{1} F_{1}$ (male: $0.91 \pm 0.04$ vs $0.39 \pm 0.02 \mathrm{mmol} / \mathrm{l}, \mathrm{p}<0.001$; female: $0.77 \pm 0.05$ vs $0.36 \pm 0.03 \mathrm{mmol} / \mathrm{l}, \mathrm{p}<0.001$ ), but more pronounced in GD-male $\mathrm{F}_{1}(\mathrm{p}<0.05)$. Plasma IRI and HOMA-IR index were significantly increased in GD-male $\mathrm{F}_{1}$ only (IRI: $230.29 \pm 4.26$ vs $71.40 \pm 0.02 \mathrm{pmol} / \mathrm{l}$ in $\mathrm{C} \mathrm{F}_{1} \mathrm{p}<0.001$; HOMA-IR: $7.63 \pm 0.30$ vs $1.99 \pm 0.13$ in $\mathrm{C}$ $\left.\mathrm{F}_{1}, \mathrm{p}<0.001\right)$. Liver glycogen content was lower compared to control group in GD-male $\mathrm{F}_{1}(18.77 \pm 1.07$ vs $31.41 \pm 0.93 \mathrm{mg}$ glucose $/ \mathrm{kg}, \mathrm{p}<0.05)$. Maternal GD does not influence on G-6Pase activity in offspring at puberty as male as female.

Conclusion: Thus, the first generation rat offspring from gestational diabetic mothers demonstrate the gender differences of insulin resistance development at puberty, exactly more marked insulin resistance manifestation in male.

\section{3}

Lower nocturnal respiratory quotient in young healthy men who had low birth weight

S. K. Lilleøre ${ }^{1,2}$, C. Brøns ${ }^{1,2}$, C. B. Jensen ${ }^{1}$, S. Toubro ${ }^{2}$, A. Vaag',

A. V. Astrup ${ }^{2}$;

${ }^{1}$ Steno Diabetes Center, Gentofte, Denmark,

${ }^{2}$ Department of Human Nutrition, University of Copenhagen,

The Faculty of Life Sciences, Frederiksberg, Denmark.

Background and Aims: Low birth weight (LBW) is associated with obesity, insulin resistance and development of type 2 diabetes (T2D) later in life. We have previously shown increased abdominal fat accumulation and elevated fasting plasma glucose levels in healthy young lean men with LBW. Catchup growth, which often accompanies LBW, has been associated with reduced energy expenditure (EE) and increased fat mass. The aim of this study was to determine whether LBW was associated with changes in energy expenditure and/or whole-body substrate utilization.

Materials and Methods: Forty-six healthy men, mean age: 24.43 years, were included in the study, 20 subjects with LBW (BW $\leq 10^{\text {th }}$ percentile, BMI: $24.69 \pm 3.73 \mathrm{~kg} / \mathrm{m}^{2}$, lean mass (DEXA): $58.3 \pm 4.82 \mathrm{~kg}$, fat mass (DEXA): $16.74 \pm 9.24 \mathrm{~kg}(\mathrm{mean} \pm \mathrm{SD}))$, and 26 matched controls with normal birth weight $(\mathrm{NBW})\left(50^{\text {th }} \geq \mathrm{BW} \leq 90^{\text {th }}\right.$ percentile, BMI: $23.36 \pm 2.59 \mathrm{~kg} / \mathrm{m}^{2}$, lean mass (DEXA): $61.12 \pm 6.57 \mathrm{~kg}$, fat mass (DEXA): $14.30 \pm 9.24 \mathrm{~kg}$ (mean $\pm \mathrm{SD})$ ). Basal metabolic rate (BMR), 24-hr energy expenditure (EE), spontaneous physical activity (SPA), respiratory quotient (RQ), and substrate oxidation rates were assessed using respiratory chambers. Subjects were fed a weight maintenance diet ( $15 \mathrm{E} \%$ protein, $35 \mathrm{E} \%$ fat and $50 \mathrm{E} \%$ carbohydrate) during the 24-hr chamber measurements, following a 5-day standardized diet and physical activity schedule. Individual energy requirements were estimated according to FAO/WHO recommendations. Fasting plasma glucose levels were significantly elevated in the LBW subjects $(4.96 \pm 0.47 \mathrm{mmol} / \mathrm{l}$ vs. $4.59 \pm 0.57 \mathrm{mmol} / \mathrm{l}($ mean $\pm \mathrm{SD}), P=0.01)$.

Results: No difference in energy balance was observed between groups. Neither BMR, nor mean 24-hr EE, SPA, RQ or substrate oxidation were different between LBW and NBW groups. This remained true after adjustment for lean mass, fat mass, SPA and energy balance. Interestingly, when the 24-hr period was broken down in intervals, the LBW subjects exhibited significantly lower mean RQ during night time, defined as 1:00 am - 6:00 am, as compared to NBW controls (Fig. $1 \mathrm{~A}$ : $0.817 \pm 0.03$ vs. $0.844 \pm 0.05$ (mean \pm SD), $P=0.03$ ). $\mathrm{EE}$ was not different between groups during that period (Fig 1B). As a consequence, the relative contribution of lipid and glucose oxidation to EE was shifted in LBW, with significantly higher lipid oxidation (48.54 \pm 7.78 vs. $42.61 \pm 10.97 \%($ mean \pm SD), $P=0.05)$ as compared to NBW. 


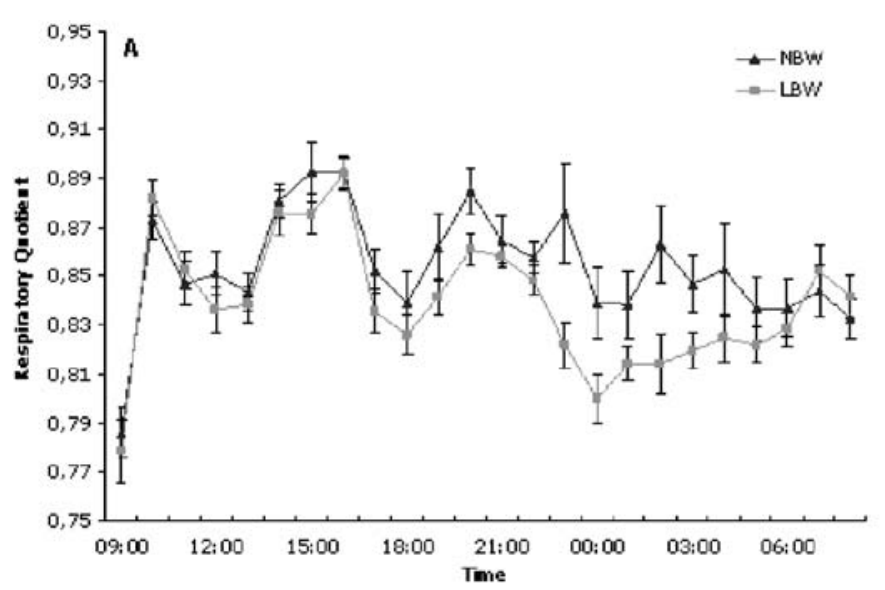

Conclusion: Healthy young lean LBW men had normal 24-hr EE when fed a weight maintenance diet. Interestingly, LBW subjects exhibited a significantly lower nocturnal RQ and, as a consequence, a higher contribution of lipid as compared to glucose oxidation to EE during the night. Reduced nocturnal glucose oxidation may via the glucose-fatty acid cycle, and possibly enhanced hepatic gluconeogenesis, contribute to the elevated fasting plasma glucose levels observed in LBW subjects.

The European Foundation for the Study of Diabetes, The Danish Diabetes Foundation, Aase and Ejnar Danielsens Foundation

\section{4}

An 11-year prospective study on the deterioration of glucose metabolism in adults born SGA

T. Meas ${ }^{1,2}$, D. Chevenne 3 , S. Deghmoun ${ }^{1}$, D. Collin ${ }^{4}$, C. Lévy-Marchal ${ }^{1,5}$; ${ }^{1}$ U690, INSERM, FRANCE, Paris, ${ }^{2}$ UMR 690, Université Paris 7, FRANCE, Paris, ${ }^{3}$ Hormonology, Hopital R Debré, Paris, ${ }^{4}$ Maternity, Haguenau City Hospital, Haguenau, ${ }^{5}$ UMR 690, Université, Paris 7, France.

Background and Aims: Insulin resistance observed in adults born SGA (small for gestational age) may result either from changes in body composition induced as early as during the catch-up period or from "programming" of peripheral tissues inducing IR, which would be more severe and more rapidly progressive in SGA-born subjects than in adults born AGA (adapted for gestational age).

Materials and Methods: These hypotheses were tested by prospectively observing two groups of young adults selected on birth data from a population-based Maternity registry: 88 subjects ( 43 women and 45 men) born full-term SGA (BW $<10^{\text {th }}$ percentile) have been compared to 133 (70 women and 63 men) AGA controls $\left(25^{\text {th }}<\right.$ AGA $<75^{\text {th }}$ percentile) at 21 and 32 years of age (T1 and T2). All subjects underwent OGTT and IR was assessed by QUICKI (derived from glucose and insulin fasting concentrations).

Results:

\begin{tabular}{llll} 
& SGA & AGA & $p$ \\
\hline $\mathrm{n}$ & 78 & 110 & \\
Age $(\mathrm{yr})$ at first observation & $21.9+/-2.1$ & $21.2+/-2.0$ & 0.05 \\
BMI $\left(\mathrm{kg} / \mathrm{m}^{2}\right)$ first observation & $22.4+/-3.9$ & $23.0+/-4.2$ & 0.3 \\
$\begin{array}{l}\text { Glucose } 120 \mathrm{~min}(\mathrm{mmol} / \mathrm{l}) \text { first } \\
\text { observation }\end{array}$ & $5.3+/-1.1$ & $5.2+/-1.0$ & 0.3 \\
QUICKI 1st observation & $0.17+/-0.01$ & $0.17+/-0.01$ & 0.2 \\
Age $(\mathrm{yr})$ at second observation & $31.9+/-1.2$ & $32.4+/-1.3$ & 0.4 \\
BMI $\left(\mathrm{kg} / \mathrm{m}^{2}\right)$ at second observation & $24.9+/-5.8$ & $24.7+/-5.0$ & 0.8 \\
Fat mass $(\%)$ at second observation & $24.06+/-8.3$ & $23.4+/-7.3$ & 0.5 \\
Glucose 120 min $(\mathrm{mmol} / \mathrm{l})$ 2nd & $5.9+/-1.6$ & $5.4+/-1.4$ & 0.05 \\
observation & & & \\
QUICKI at second observation & $0.16+/-0.01$ & $0.17+/-0.01$ & 0.07 \\
Diff BMI T2-T1 $\left(\mathrm{kg} / \mathrm{m}^{2}\right)$ & $2.6+/-3.2$ & $1.7+/-3.2$ & 0.06 \\
Diff QUICKI T2-T1 & $-0.007+/-0.002$ & $-0.005+/-0.001$ & $0.06^{*}$ \\
\hline
\end{tabular}

*: In a multivariate analysis, the progression of QUICKI over time was not significantly affected by gender and fat mass but was significantly associated with the change in BMI $(p=0.01)$ and marginally different between SGA and AGA $(p=0.1)$.

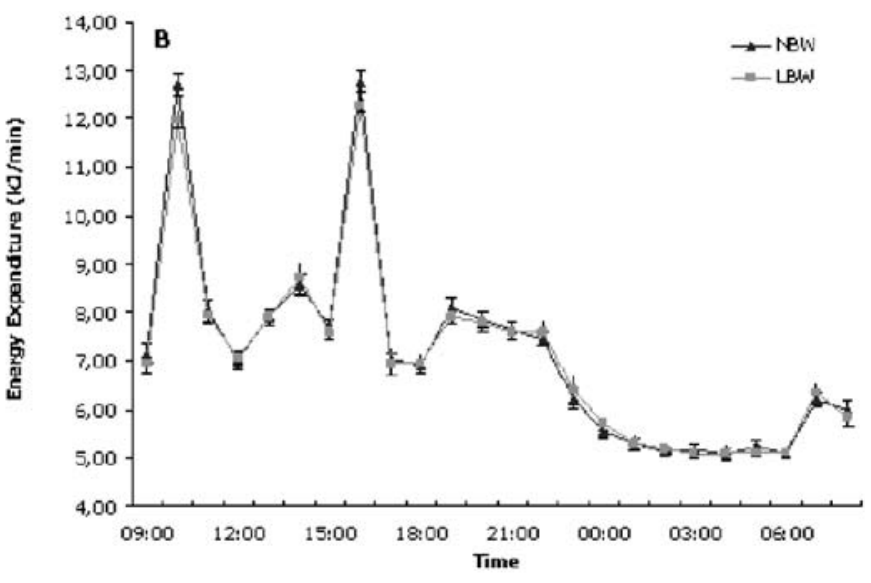

Conclusion: Over a 11-yr period, adults born SGA show a more rapid deterioration of glucose tolerance and insulin sensitivity due in part but not only to accretion of more adiposity. This observation suggests that foetal programming affects both body composition and glucose metabolism independently.

Supported by INSERM 


\section{PS 64 Nitric oxide}

\section{5}

\section{Hypothalamic nitric oxide synthase: a key component of lipid-induced} deregulation of glucose homeostasis

N. Marsollier, N. Kassis, C. Magnan, C. Cruciani-Guglielmacci;

Laboratoire de Physiopathologie de la Nutrition (LPPN), CNRS UMR 7059, Paris, France.

Background and Aims: Metabolic syndrome is a constellation of physiopathologic disorders often associated with a lipid rich diet. Recently the central nervous system, and particularly the hypothalamus, arises as a key player in etiology of type 2 diabetes (T2D). Indeed the hypothalamus integrates circulating signals of satiety and hunger, and is also a nutrient sensing device that monitors and exerts a control of glucose and lipid metabolism via the autonomic nervous system. We have previously shown that 24 hour infusion of triglyceride emulsion in carotid artery toward the brain, at a rate that does not modify plasma free fatty acid and triglyceride levels, results in a hepatic insulin resistance and enhances Glucose Induced Insulin Secretion (GIIS). These mechanisms could be an early step in the development of T2D.

Nitric Oxide (NO) acts as a neurotransmitter and is linked to mitochondrial metabolism, therefore we hypothesized that NO production by Nitric Oxide Synthase (NOS) could mediate central lipid sensing in the hypothalamus, and, at least in part, relay the perturbation of glucose homeostasis induced by central lipid infusion. To characterize the relevance of NO signalling in the effect of central lipid infusion on glucose homeostasis, we used different pharmacological approaches such as local injection of NOS inhibitors, or NO donors.

Materials and Methods: 2 groups of Wistar rats were infused $24 \mathrm{~h}$ at $2 \mu \mathrm{L} / \mathrm{min}$ into carotid, one with saline and heparin (C), and the other with triglycerides emulsion (Intralipide ${ }^{\circ}$ ) and heparin (IL). We have assayed NOS activity by measuring conversion of [3H]-L-Arginine into [3H]-L-Citrulline in both groups. We also performed Intravenous Glucose Tolerance Tests (IVGTT) and measurement of glucose turnover rate (euglycemic-hyperinsulinemic clamp), with or without pharmacologic modulators of NO pathway(L-NMMA as inhibitor of the 3 NOS isoforms, 7-Nitro-Indazole as specific inhibitor of neuronal NOS, 1400W as specific inhibitor of inducible NOS, L-Arginine as substrate of NOS, and Sodium Nitro Prusside, SNP, as pharmacologic NO donor). We also determined NOS activities with these different agents. At last inflammatory cytokines (IL-1 $\beta$, IL- 6 , TNF- $\alpha$, IFN- $\gamma$ ) mRNA expression were determined.

Results: Plasma triglycerides and free fatty acids are not augmented after central lipid infusion. Total NOS activities are significantly increased after $24 \mathrm{~h}$ of lipid infusion, with a greater augmentation of constitutive NOS (iNOS: $+56 \%$, cNOS: $+90 \%$ ). NOS activities were normalized after carotid injection of L-NMMA and 7-Ni. L-NMMA injected before glucose challenge normalizes insulin secretion in IL group, as well as hepatic glucose production, with no effect in $\mathrm{C}$ group. Treatments with specific 7-Nitro-Indazole (7-Ni) also restore normal insulin secretion and glucose production. Injection of L-Arginine before IVGTT increases insulin secretion to IL level in control rats. SNP treatments lead to increased GIIS and provoke glucose intolerance, indicating a state of insulin resistance in both groups. Inflammatory cytokine expressions were not modified after $24 \mathrm{~h}$ of lipid infusion.

Conclusion: We concluded that central NO production, and more particularly via neuronal NOS isoform, is implicated in hypothalamic lipid sensing and plays a key role in brain control of glucose homeostasis. This work could provide new molecular clues as potential target for designing anti-diabetic drugs.

\section{6}

Induction of abnormalities in neuronal NO synthase and its protein inhibitor PIN in rat pancreatic $\beta$ cells and skeletal muscle after a high-sucrose diet

K. Mezghenna, S. Péraldi-Roux, G. Dubois, M. Manteghetti, M. Tournier, F. Castex, B. Nguyen, J. Boyer, R. Gross, A.-D. Lajoix;

UMR 5232, CNRS, Montpellier, France.

Background and Aims: In our previous studies, we have shown that an isoform of neuronal NO synthase (nNOS) and its protein inhibitor PIN are expressed and control insulin secretion in rat pancreatic $\beta$-cells. Skeletal muscle is also known to express a variant of neuronal nitric oxide synthase, nNOS $\mu$, which modulates contractile force and glucose utilization. If a defect in the NOS pathway seems to be responsible for pancreatic $\beta$-cell dysfunction and insulin resistance in type 2 diabetes, the reasons for this remain to be determined. Our aim is thus to identify the nNOS and PIN abnormalities present in pancreatic $\beta$-cells and skeletal muscle of the high-sucrose diet fed rat, a nutritional model of insulin resistance. We therefore studied nNOS expression at the mRNA and proteic levels, its dimerization state as well as PIN expression in the pancreatic islets and the gastrocnemius skeletal muscle of rats fed a high-sucrose diet.

Materials and Methods: Male Wistar rats received sucrose (7\% in drinking water) during 8 weeks. nNOS and PIN expression has been analyzed by quantitative RT-PCR as well as by Western blotting using specific monoclonal antibodies. Dimerization of nNOS has been studied by low temperature SDSPAGE without boiling the samples, followed by a Western blotting. Subcellular localization of both proteins was determined by immunofluorescence.

Results: After 8 weeks of sucrose feeding, rats displayed a prediabetic state with a moderated hyperinsulinemia of $4.38 \mathrm{ng} / \mathrm{ml}$ versus $3.08 \mathrm{ng} / \mathrm{ml}$ in the control group $(\mathrm{P}<0.01)$, associated with a slight significant increase in glycemia ( 1.30 versus $1.20 \mathrm{~g} / \mathrm{l}$ in the control group, $\mathrm{P}<0.05)$. In pancreatic $\beta$-cells, we found an increased amount of nNOS dimers that correlates with the appearance of hyperinsulinemia in prediabetic rats. We also recorded a decrease in PIN expression which agrees with the enhanced nNOS dimerization state. In addition, we observed both increased nNOS mRNA and protein levels together with a stronger colocalization with insulin after immunofluorescence staining. Conversely, in skeletal muscle, nNOS expression was decreased in insulin resistant rats whereas PIN expression remained unchanged.

Conclusion: Abnormalities in the levels of both nNOS and PIN but also in dimer/monomer equilibrium of the enzyme in pancreatic $\beta$-cells and skeletal muscle are associated with the $\beta$-cell dysfunction and insulin resistance observed in the high-sucrose fed rat model.

\section{7}

Hepatic iNOS and ST6GalI inflammatory markers are increased in obese diabetic but not in nondiabetic obese rat models

R. T. Ribeiro ${ }^{1}$, A. B. Fernandes ${ }^{1}$, R. A. Afonso ${ }^{1,2}$, P. A. Videira ${ }^{3}$, M. Correia ${ }^{3}$, M. P. Macedo ${ }^{1,4}$;

${ }^{1}$ Physiology, Faculty Medical Sciences, ${ }^{2}$ Biochemistry, Faculty of Medical Sciences, ${ }^{3}$ Immunology, Faculty Medical Sciences,

${ }^{4} \mathrm{Apdp}$, Portuguese Diabetes Association, Lisbon, Portugal.

Background and Aims: Several inflammatory markers have been related to obesity, insulin resistance and type 2 diabetes mellitus. The liver has been shown to be paramount in the regulation of whole body insulin-stimulated glucose uptake, by a mechanism that involves hepatic nitric oxide. The liver is also the prime site of the production of acute-phase proteins, which include an increased $\alpha-2,6$ sialylation of glycoproteins and may play a role in the development of type 2 diabetes mellitus. Thus, we studied the expression of hepatic inflammatory markers related with these processes - inducible nitric oxide synthase (iNOS) and $\beta$-galactoside $\alpha-2,6$ sialyltransferase (ST6GalI) - in lean, obese and obese diabetic animals. Additionally, we measured whole body insulin sensitivity. In this study, we tested the hypothesis that hepatic iNOS and ST6GalI are increased in obesity and aggravated in type 2 diabetes mellitus.

Materials and Methods: We used male 9 weeks-old Zucker lean (LZR), obese fa/fa (OZR) and obese diabetic (ZDF) rats. Hepatic iNOS and ST6GalI expression were determined by Real Time-PCR, using the relative quantification method $-2^{\Delta \delta C t}$. Insulin sensitivity was determined, after an overnight fast and $1 \mathrm{~h}$ refeeding, by a transient euglycemic clamp performed after the administration of a bolus of insulin $(50 \mathrm{mU} / \mathrm{kg})$.

Results: In relation to the lean LZR controls $(n=8)$, iNOS expression was barely altered in the obese animals (OZR: $1.8 \pm 0.2$ times more expressed than LZR, $n=7$ ) but increased in the obese diabetic animals (ZDF: $532.1 \pm 123.4$ times more expressed than LZR, $\mathrm{n}=6, \mathrm{p}<0.001)$. Similarly, ST6GalI expression, whose basal level is particularly high in liver, was also only increased in the diabetic state (OZR: $1.3 \pm 0.1$ and ZDF: $2.0 \pm 0.3$ times more expressed than LZR, $\mathrm{p}<0.05)$. However, post-prandial insulin sensitivity decreased similarly in both obese and obese diabetic rats in relation to their lean littermates (OZR: $73.7 \pm 14.2$, and ZDF: $92.1 \pm 3.8$ vs LZR: $289.2 \pm 24.7 \mathrm{mg}$ glucose $/ \mathrm{kg}$ bw, 
$\mathrm{p}<0.001$ for both). Observed basal glycemias, as expected, were only raised in diabetic rats (LZR: $108.7 \pm 5.0$ and OZR: $117.3 \pm 5.7$ vs ZDF: $186.8 \pm 14.4 \mathrm{mg} / \mathrm{dl}$, $\mathrm{p}<0.001$ for both).

Conclusion: Although we observed an increase in both inflammatory markers only with declared diabetes, insulin sensitivity was decreased in both obese and obese diabetic Zucker rats. We conclude that the hepatic inflammatory markers iNOS and ST6GalI are associated not with insulin resistance, but with the hyperglycemia shown in type 2 diabetes mellitus.

Supported by Fundação da Ciência e Tecnologia (FCT) and APDP-Portuguese Diabetes Association

\section{8}

Hepatic nitric oxide synthase activity and expression related to insulin resistance in an ageing obesity animal model

A. B. Fernandes ${ }^{1}$, P. Videira ${ }^{2}$, M. P. Guarino ${ }^{1,3}$, R. Ribeiro ${ }^{1}$, R. A. Afonso ${ }^{1,4}$, M. P. Macedo ${ }^{1,5}$;

${ }^{1}$ Physiology, Faculty of Medical Sciences-UNL, ${ }^{2}$ Imunology,

Faculty of Medical Sciences-UNL, ${ }^{3}$ Pathophysiology, Faculty of Medical

Sciences-UNL, ${ }^{4}$ Biochemistry, Faculty of Medical Sciences-UNL,

${ }^{5}$ Associação Protectora dos Diabeticos de Portugal, APDP, Lisbon, Portugal.

Background and Aims: A number of studies indicate a central role of the liver in whole-body glucose disposal where a humoral factor, the Hepatic Insulin Sensitizing Substance - HISS - is released from the liver to potentiate glucose uptake in skeletal muscle. HISS synthesis is dependent of hepatic nitric oxide (NO) levels. Obesity and ageing are associated with insulin resistance. Our group described that a decrease of HISS release $(80.4 \pm 0.08 \%)$ is the major contributor to the insulin resistance associated with ageing. We also reported that insulin resistance presented in an animal model of obesity, the Zucker rat, is partly due to a decrease of HISS action $(40.1 \pm 6.5 \%)$. In this study we hypothesized that both obese and aged animals show a decrease in hepatic nitric oxide synthase (NOS) activity and expression, which correlates with decreased HISS action.

Material and Methods: The Zucker fa/fa rat (OZR) and its lean littermate, the Zucker fa/+ rat (LZR) were used. The animals were fasted for $18 \mathrm{~h}$ and refed for $1 \mathrm{~h}$. In the first group of experiments, male 9 weeks-old OZR and LZR rats were used. In a second group, male 52 weeks-old OZR and LZR animals were used. Hepatic NOS activity was determined by counting the radioactivity of the ${ }^{3} \mathrm{H}$-citruline formed by the biochemical conversion from ${ }^{3} \mathrm{H}$-arginine. Hepatic expression of NOS isoforms (eNOS, nNOS and iNOS) was determined by RT-PCR, using the relative quantification method $-2^{\Delta \delta \mathrm{Ct}}$.

Results:The 9 weeks-old obese rats showed an impaired hepatic NOS activity $\left(3.26 \pm 0.30 \mathrm{pmol}^{-1} \mathrm{mg}\right.$ protein $\left.\mathrm{min}^{-1}, \mathrm{n}=8\right)$ when compared with lean animals ( $7.53 \pm 0.52 \mathrm{pmol}^{-1} \mathrm{mg}$ protein $\left.\mathrm{min}^{-1}, \mathrm{n}=10 ; \mathrm{P}<0.001\right)$. At 52 weeks of age, the hepatic NOS activity of the OZR $\left(3.95 \pm 0.32 \mathrm{pmol}^{-1} \mathrm{mg}\right.$ protein $\mathrm{min}^{-1}$, $\mathrm{n}=5)$ was also impaired when compared with the LZR $\left(5.61 \pm 0.20 \mathrm{pmol}^{-1} \mathrm{mg}\right.$ protein $\min ^{-1}, \mathrm{n}=14, \mathrm{p}<0.05$ ). Moreover, ageing induced a significant decrease in NOS activity in the LZR but not in the OZR animals. The eNOS and nNOS expression were not altered by obesity or/and age. iNOS expression was significantly altered in the 52 weeks-old LZR animals (183.60土70.41 times more expressed, $\mathrm{n}=10, \mathrm{p}<0.05$ ) when compared to the 9 weeks-old LZR animals.

Conclusion: OZR showed an impairment of hepatic NOS activity when compared with LZR animals, independently of their age. There was no alteration in eNOS and nNOS expression, indicating that the impairment observed in NOS activity is a post-transcriptional modification. The iNOS expression was only altered in the 52 weeks-old LZR animals, suggesting an association between ageing and inflamatory process. In obese animals ageing does not aggravate the impairment in hepatic NOS activity, already observed at 9 weeks-old. In contrast, we observed that LZR animals showed a decrease of hepatic NOS activity with ageing which correlates with the age-dependent inhibition of HISS action.

Supported by Fundação para a Ciência e Tecnologia (FCT) and Portuguese Diabetes Association (APDP)

\section{9}

Ethanol acutely stimulates islet blood flow, amplifies insulin secretion, and induces hypoglycaemia through a NO-dependent mechanism Z. Huang, A. Sjöholm;

Internal Medicine, Karolinska Institutet, Stockholm, Sweden.

Background and Aims: Alcohol-induced hypoglycemia is a well known problem in diabetic patients, but the underlying mechanisms have largely remained elusive. Since insulin secretion in vivo can be rapidly tuned by changes in pancreatic microcirculation, we evaluated the influence of acute alcohol administration on pancreatic islet blood flow and dynamic changes in insulin secretion and glycemia in the rat.

Materials and Methods: Ethanol (10\%) was injected as a bolus into a catheter located in the left ventricle of the heart in Wistar rats, yielding serum ethanol levels of $\sim 8 \mathrm{mmol} / \mathrm{l}$. Measurements of pancreatic blood flow were performed by a microsphere technique in combination with a freeze-thawing technique after 10 minutes of injection.

Results: Ethanol preferentially and significantly increased pancreatic islet blood flow approx four-fold (Fig. 1A-B),

\section{$1 \mathrm{~A}$}

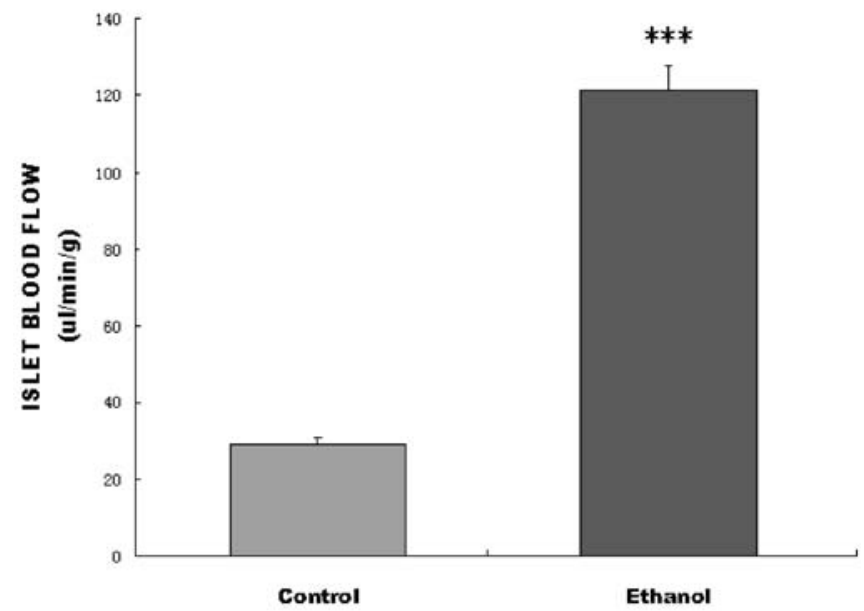

$1 \mathrm{~B}$

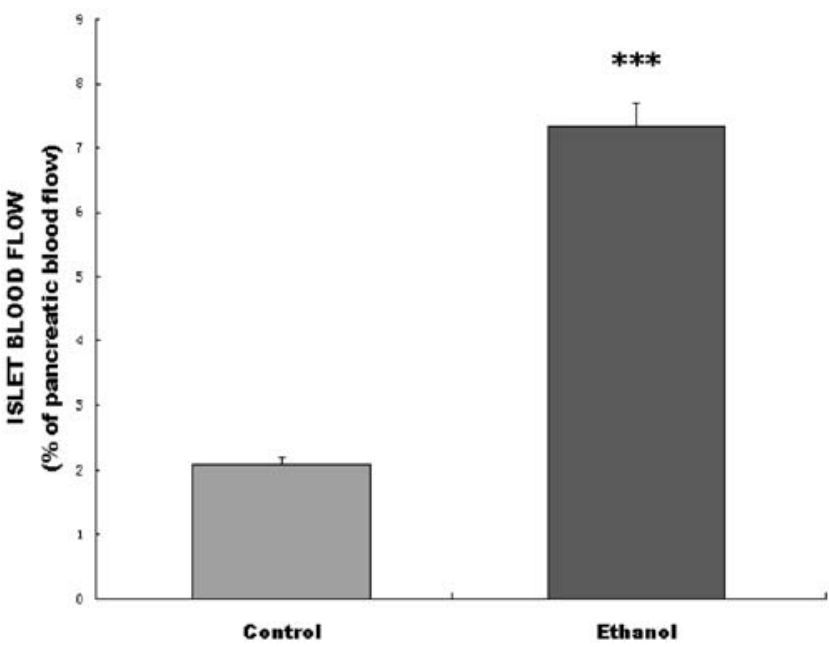

Fig. 1A-B

while not influencing whole pancreatic blood flow. The nitric oxide synthase inhibitor $N$ - $\omega$-nitro-L-arginine methyl ester completely prevented the increased pancreatic islet blood flow evoked by ethanol, while atropine caused a partial prevention. The alcohol also augmented late phase insulin secretion (Fig. 2A) and induced late hypoglycemia (Fig. 2B) upon intraperitoneal glucose tolerance tests. 


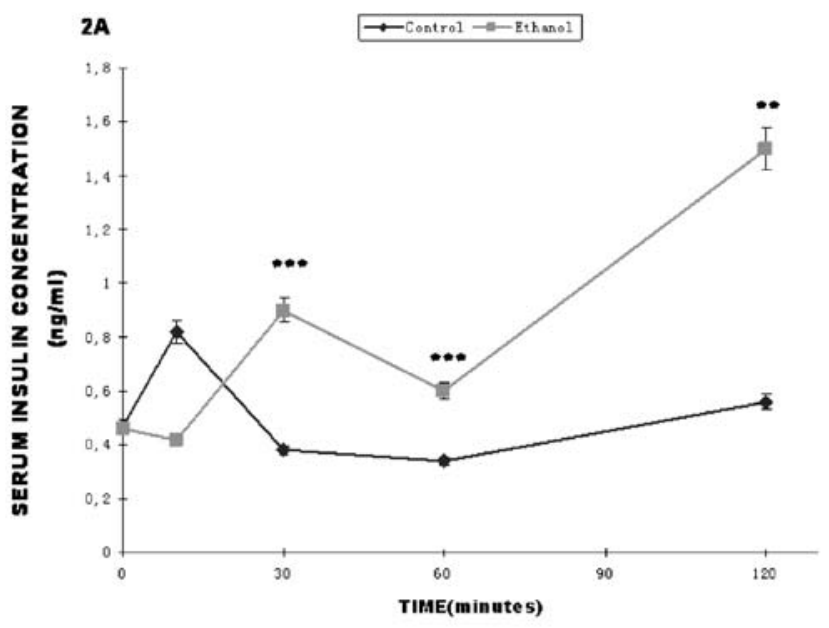

2B

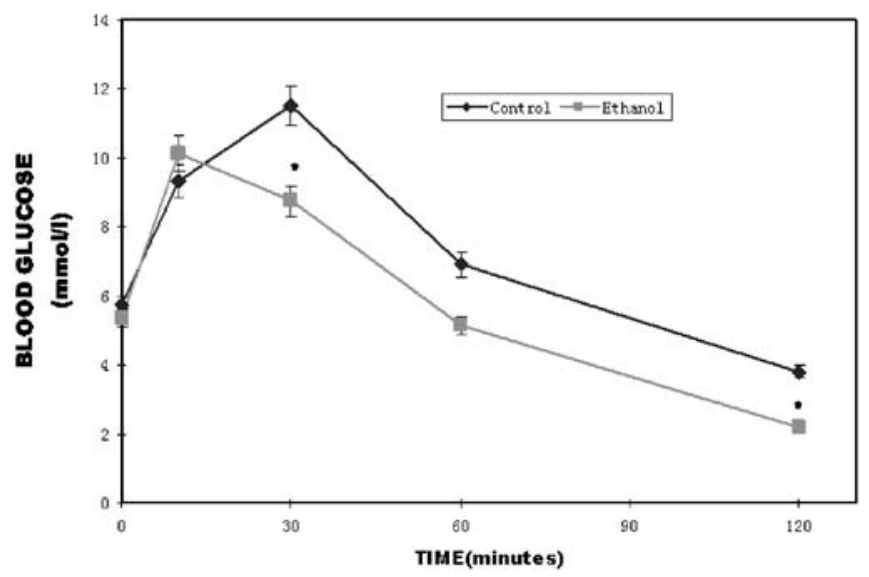

Fig. 2A-B

Conclusion: Ethanol acutely evokes a massive redistribution of pancreatic blood flow into islets via mechanisms mediated by nitric oxide and partly by vagal stimuli, augmenting late phase insulin secretion, and thereby evoking hypoglycemia. This effect may in part underlie the well known hypoglycemic properties of alcohol in diabetic patients or in alcoholics with hepatic failure. Financial support was provided by the Alcohol Research Council of the Swedish Alcohol Retailing Monopoly

\section{0}

The nitric oxide-donating derivative of acetyl-salicylic acid NCX 4016 increases glucose transport through translocation of glucose transporters to the plasma membrane in adipocytes

M. Cormont ${ }^{1}$, V. Kaddai ${ }^{1}$, T. Gonzalez ${ }^{1}$, M. Bolla ${ }^{2}$, Y. Le Marchand-Brustel ${ }^{1}$; ${ }^{1}$ Unit 568, INSERM, Nice, France,

${ }^{2}$ Research department, NicOx SA, Sophia-Antipolis, France.

Background and Aims: NCX 4016 is a nitric oxide (NO)-donating derivative of acetylsalicylic acid. NO and salicylate, which are in vivo metabolites of NCX 4016, are known to regulate glucose homeostasis. We have evaluated the activity of NCX 4016 on glucose transport in cultured adipocytes in basal conditions and following stimulation with insulin.

Materials and Methods: 3T3-L1 adipocytes were treated with NCX 4016, a reference NO-donor, or acetylsalicylic acid in presence of insulin or not. Effect of NCX 4016 on glucose uptake was measured using 2-deoxyglucose. To further characterize the NCX 4016 effect on adipocytes at molecular level we have assessed the amount of glucose transporters translocation at the plasma membrane by immunodetection, and studied the effect on different molecules of the insulin-signalling pathway.

Results: Following stimulation of 3T3-L1 adipocytes with 200 micromol/L NCX 4016 for 3 and 24 hours, glucose uptake was increased by 2 and 3 fold, respectively. In parallel, the translocation of the glucose transporters GLUT1 and GLUT4 to the plasma membrane was increased 2.5 fold without any change in the total expression levels of either protein. Importantly, NCX 4016 enhanced the effect of a physiological $(0.5 \mathrm{nmol} / \mathrm{L})$ concentration of insulin. In this specific model it seems that NO moiety is mostly major responsible for the effect on glucose uptake, considering that acetylsalicylic acid had no effect and that a NO scavenger (cPTIO) greatly reduced it. NCX 4016-stimulated glucose transport did not require the activation of the soluble cGMP cyclase/ protein kinase $\mathrm{G}$ pathway. It did not stimulate the insulin receptor, IRS, AS160 phosphorylation or PKB and ERK activity.

Conclusion: NCX 4016 induced a significant increase in glucose uptake in adipocytes. The effect of NCX 4016 and insulin was additive. Equimolar doses of acetylsalicylate did not have the same effect. Because NCX 4016 effectively promoted the translocation of both glucose transporters to the plasma membrane, it apparently acts on the endosomal compartment and not on the GLUT4-specific compartment. NCX 4016 may therefore represent a novel agent for treatment of hyperglycemia in patients suffering from type 2 diabetes.

\section{1}

nNOS is necessary for normal increases in glucose uptake during contraction of skeletal muscle

G. K. McConell, K. C. Linden, J. G. Ryall, C. Van der Poel, J. D. Schertzer,

G. S. Lynch;

Department of Physiology, The University of Melbourne, Parkville, Australia.

Background and Aims: People with type 2 diabetes have reduced skeletal muscle glucose uptake in response to insulin, but glucose uptake during

\section{EDL}

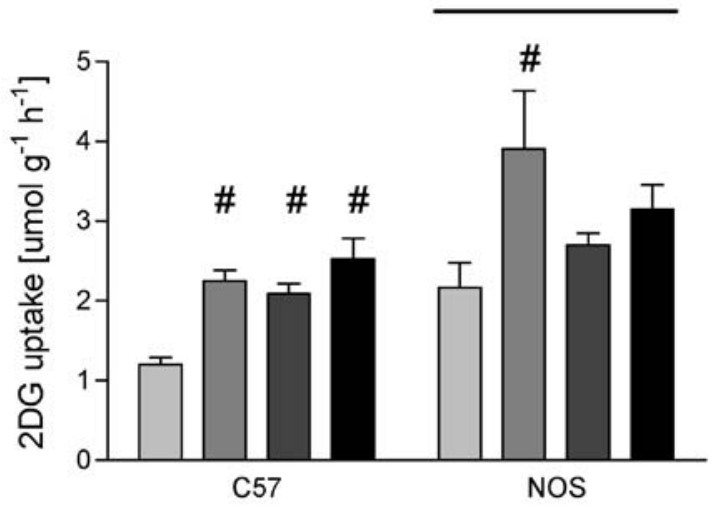

Soleus

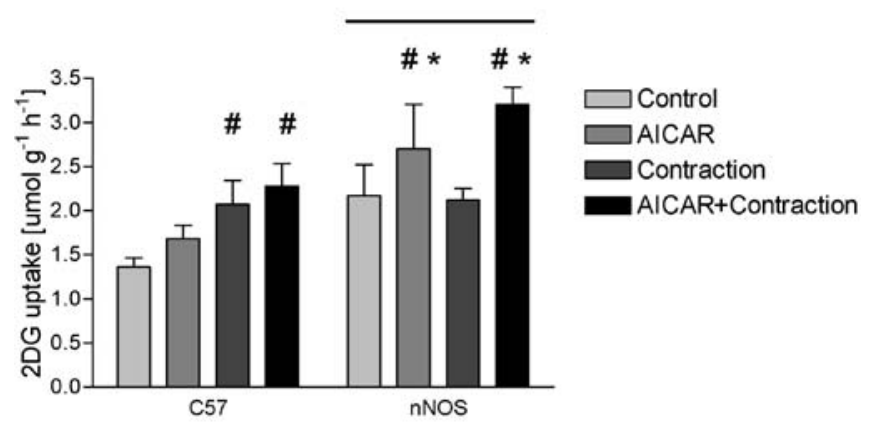

\#; Significantly different from basal value of the same genotype

*; Significantly different from contraction value of the same genotype

Horizontal line; main effect for genotype

Data presented as means \pm S.E. $N=7-9$ for all groups

Fig. 1 
exercise is normal. The factors regulating skeletal muscle glucose uptake during exercise are unclear. We have evidence that nitric oxide (NO) plays a role in humans, but evidence in rodents is conflicting. We examined glucose uptake in response to contraction and in response to the AMP-activated protein kinase (AMPK) activator AICAR in C57Bl/6 and nNOS null mice $\left(\mathrm{nNOS}^{--}\right)$. It has been shown the there is no increase in cGMP content in response to contraction in $\mathrm{nNOS}^{-/-}$mice.

Materials and Methods: 2-Deoxy-D-glucose (2DG) uptake was examined during contraction in extensor digitorum longus (EDL) and soleus muscles from adult $\mathrm{C} 57 \mathrm{Bl} / 6$ and $\mathrm{nNOS}^{-1-}$ mice $(\mathrm{n}=7-9 /$ group$)$. Following a 40 minute incubation, muscles were stimulated to contract for either 10 minutes (EDL, $60 \mathrm{~Hz}, 600 \mathrm{~ms}$, train rate 0.167 ) or 15 minutes (soleus, $60 \mathrm{~Hz}, 350 \mathrm{~ms}$, train rate 0.167 ).

Results: Basal 2DG was elevated in the $\mathrm{nNOS}^{-1-}(\mathrm{P}<0.05)$, however no increase in $2 \mathrm{DG}$ was observed during contraction in either the $\mathrm{EDL}(\mathrm{P}>0.05)$, or soleus $(\mathrm{P}>0.05)$, while contraction mediated 2DG was $\sim 1.5$ fold above basal in the $\mathrm{C} 57 \mathrm{Bl} / 6(\mathrm{P}<0.05)$. $\mathrm{nNOS}^{-/-}$mice have a normal increase $(\mathrm{P}<0.05)$ in glucose uptake with AICAR, and this increase is attenuated with contraction.

Conclusion: These results indicate that skeletal muscle nNOS is required for normal increases in glucose uptake in response to contraction, but not in response to AICAR. They suggest that NO is a critical regulator of skeletal muscle glucose uptake during exercise. (Fig. 1)

\section{2}

Insulin signaling induces p-AKT2 translocation and $\mathrm{nNOS}$ activation in muscle mitochondria: the NO-pathway to distribute glucose for oxidation or for glycogen synthesis

P. V. Finocchietto, F. Barreiro, S. Holod, J. G. Peralta, M. C. Franco,

D. P. Converso, M. C. Carreras, J. J. Poderoso;

University of Buenos Aires, Clinical Hospital, Argentina.

Post translational modifications as N- terminal acylation and Serine 1412 phosphorylation are associated to neuronal nitric oxide synthase traslocation to mitochondria in different tissues (mtNOS). mtNOS synthesizes nitric oxide $(\mathrm{NO})$ vectorially directed to the mitochondrial matrix and NO steadystate concentration modulates the electron transfer, $\mathrm{O}_{2}$ uptake and reactive oxygen species production.

Considering that Serine 1412 of NOS is in an AKT-dependent domain, we analyzed insulin/Akt effects on mitochondrial Nitric Oxide Synthase (mtNOS) from rat skeletal muscle.

Materials and Methods: Wistar rats (200-250g) were subcutaneously inoculated with $0.1 \mathrm{U} / \mathrm{Kg}$ insulin glargine or $\mathrm{ClNa} 0.9 \%$ and afterwards extensoris digitorum and gastrocnemius muscles were surgically excised at 3,12 and 24 hours. Purified muscle mitochondria from treated animals were obtained by differential centrifugation and tissue slices were incubated with insulin and LY294002 (PI3K specific inhibitor) as well. mtNOS activity was determined by conversion of $\left[{ }^{3} \mathrm{H}\right] \mathrm{L}$-arginine to $\left[{ }^{3} \mathrm{H}\right] \mathrm{L}$-citrulline. The expression of mtNOS, p-AKT, AKT 1 , AKT 2 and GSK $3 \alpha / \beta$ were analysed by Western Blot and p-AKT activity by formation of p-GSK-3 $3 \alpha /$ $\beta$. The mitochondrial p-AKT and NO were determined by flow cytometry. nNOS phosphorylation was evaluated in pure recombinant protein and mitochondria with ${ }^{32}$ PATP. Mitochondrial and muscular tissue oxygen uptake were determined polarographically. SiRNA (nNOS and Akt2) was injected into left gastrocnemius in rat under slight anaesthesia and electric pulses of $120 \mathrm{~V}, 1 \mathrm{~Hz}$ were applied. Metabolic activity was determined in electroporated muscles with deoxy-D glucose $2\left[1,2{ }^{3} \mathrm{H}(\mathrm{N})\right]$,glucose $\mathrm{D}$ $\left[{ }^{14} \mathrm{C}(\mathrm{U})\right]$ and glucose $\left.\mathrm{D}-\left[5-{ }^{3} \mathrm{H}(\mathrm{N})\right)\right]$. Data were expressed as means \pm s.em and analyzed by ANOVA and Dunnet's test. Statistical significance was accepted at $\mathrm{p}<0.05$.

Results: 1) Insulin increased the mtNOS activity at 12 and $24 \mathrm{~h}$ by $10-15$ fold (C: $30 \pm 1 ; 3 \mathrm{~h}: 35 \pm 3$; 12h: $291 \pm 9 ; 24 \mathrm{~h}: 145 \pm 3$ pmoles $/ \mathrm{min} / \mathrm{mg}$ prot) without changes in its expression. This activity was inhibited by LY294002 in muscle homogenates 2) AKT2 protein levels were increased in cytosol and mitochondria at 3 and $12 \mathrm{~h}$ but the AKT 1 expression was unaltered 3) Mitochondrial NO production rate was increased at 12 and $24 \mathrm{~h}$; and mitochondrial oxygen uptake was lower at 12 and $24 \mathrm{~h}$ respect to controls and $3 \mathrm{~h} \mathrm{4}$ ) p-AKT translocated to mitochondria increased at 3-12 $\mathrm{h}$ respect to controls but further decreased at $24 \mathrm{~h} \mathrm{5)} \mathrm{AKT} \mathrm{activity} \mathrm{was} \mathrm{higher} \mathrm{at} 3$ and $12 \mathrm{~h}$ in mitochondria and cytosol respect to controls and $24 \mathrm{~h} \mathrm{6)} \mathrm{mtNOS}$ was phosphorylated by p-AKT in vivo 7) Administration of siRNA nNOS to gastrocnemius muscle decreased by half $\mathrm{C}^{14}$-glucose $(\mathrm{U})$ incorporation to glycogen and increased oxidation to $\mathrm{C}^{14} \mathrm{O}_{2}$ and ${ }^{3} \mathrm{H}_{2} \mathrm{O}$. siRNA Akt2 reduced mtNOS activity and glycogen synthesis by $70 \%$.

Conclusions: p-AKT2 activated by Insulin-PI3K in mitochondria from skeletal muscle increases the mtNOS activity by phosphorylation in allosteric fashion. This effect reduces $\mathrm{O} 2$ uptake and promotes biosynthetic metabolic pathways, as glycogen synthesis. It is surmised that insulin deals out glucose utilization for energy production or glycogen store by modulating electron transfer rate through matrix NO.

We acknowledge to Dennis Stuehr for giving us cloned nNOS, to Daniel De Seta for insuline glargine and to Natalia Riobó and Juan C. Gagliardino for helpful comments. This work was supported with research grants of Agencia Nacional 


\section{PS 65 Adipocytes}

\section{3}

Protein array analysis reveals changes in the subcutaneous adipose tissue adipokines expression associated with the growth hormone deficiency J. Ukropec ${ }^{1}$, A. Penesová 2 , M. Škopková ${ }^{1}$, M. Pura ${ }^{3}$, M. Vlček ${ }^{2}$,

Ž. Rádiková 2 , R. Imrich², V. Belan ${ }^{4}$, P. Vaňuga ${ }^{3}$, J. Payer ${ }^{5}$, J. Eckel $^{6}$, I. Klimeš ${ }^{1}$, D. Gašperíkovái;

${ }^{1}$ Diabetes Laboratory, Institute of Experimental Endocrinology, Bratislava, Slovakia, ${ }^{2}$ Laboratory of Clinical Endocrinology, Institute of Experimental Endocrinology, Bratislava, Slovakia, ${ }^{3}$ Department of Endocrinology, National Institute for Endocrinology and Diabetes, Lubochňa, Slovakia, ${ }^{4}$ Radiodiagnistic clinic, Comenius University Hospital, Bratislava, Slovakia, 55th Department of Internal Medicine, Comenius University Hospital, Bratislava, Slovakia, ${ }^{6}$ Inst. of Clinical Biochemistry and Pathobiochemistry, German Diabetes Center, Duesseldorf, Germany.

Background and Aims: Adipokines and inflammatory cytokines from adipose tissue contribute to the regulation of metabolic processes. However, their specific role in pathogenesis of metabolic derangements related to the growth hormone deficiency (GHD) is not fully understood. This prompted us to investigate the protein expression of 120 adipokines in the subcutaneous adipose tissue of adult patients with untreated GHD.

Patients and Methods: Sixteen (10/6, M/F) young ( $30 \pm 2$ years), growth hormone deficient (ITT verified) individuals with BMI $\left(27.0 \pm 1.0 \mathrm{~kg} / \mathrm{m}^{2}\right)$ and 16 age, gender and BMI matched healthy control subjects were investigated. Subcutaneous adipose tissue biopsy was carried out between 8:00-10:00 AM. Protein expression was determined in the tissue lysates with aid of the "RayBio" Human Cytokine Antibody Array C Series 1000".

Results: GHD individuals had a higher waist circumference, elevated circulating hsCRP and $45 \%$ of them showed impaired glucose tolerance. Adipose tissue of the GHD patients showed elevated protein expression of IGFBP-1 $(+53 \%, p=0.05)$, and lowered expression of BDNF $(-29.5 \%$, $\mathrm{p}=0.015)$, NT-3 $(-37.3 \%, \mathrm{p}=0.027)$ a SDF-1 $(-25 \%, \mathrm{P}=0.045)$. Adipokine expression was significantly affected by minor changes in the obese phenotype. Multiple regression analysis using IGF-1 (GH deficiency) and BMI (obesity) as independent variables revealed some adipokines to be regulated by growth hormone deficiency, which was independent on obesity /BMI/ (IGFBP1, $\mathrm{p}=0.004 ;$ Interferon $\gamma, \mathrm{p}=0.014 ; \mathbf{M C P} 4, \mathrm{p}=0.04 ;$ IGF-1, $\mathrm{p}=0.05)$. Interestingly, some of the adipokines negatively associated with GH deficiency (GHD) were positively related to obesity (O) (TIMP2, GHD: $p=0.02 \mathbf{O}: \mathrm{p}=0.05$; HGF, GHD: $p=0.03$ O: $p=0.05$; Leptin, GHD: $p=0.05$ O: $p=0.001$ ) and, conversely, some adipokines positively associated with GHD were negatively related to obesity (b-NGF, GHD: $p=0.005$ O: $p=0.05$; IGFPB3, GHD: $p=0.04$ $\mathbf{O}: \mathrm{p}=0.05$ ).

Conculsions: According to our knowledge this is the first data on adipokine protein expression in adipose tissue of adult GH deficient individuals, which indicates specific changes in regulation of the adipose tissue growth and differentiation as well as in tissue inflammation. We hypothesize that this phenomenon could be related to early changes of the metabolic phenotype in young adults with yet untreated growth hormone deficiency. Supported by Grant APVV 51-040602 and VEGA 2/7110/27

\section{4}

\begin{abstract}
A role of lipin in depot specificity of peroxisome proliferator-activated receptor $\gamma$ action on adipose tissue metabolism in a diabetic animal model

S. Park ${ }^{1}$, J. Lee ${ }^{2}$, E. Park ${ }^{2}$, E. Kang ${ }^{1}$, C. Ahn ${ }^{1}$, H. Lee ${ }^{1}$, B. Cha ${ }^{1}$;

${ }^{1}$ Department of internal medicine, ${ }^{2}$ Brain Korea 21 Project for Medical Science, Yonsei University college of medicine, Seoul, Republic of Korea.
\end{abstract}

Background and Aims: Treatment with rosiglitazone, a potent peroxisome proliferator-activated receptor (PPAR)- $\gamma$ agonist, results in lipid storage coupled with reduced release of free fatty acids into the circulation. Many studies reported that PPAR- $\gamma$ agonism brings about redistribution of fat toward subcutaneous depots and away from visceral fat. Lipin, gene product of $L P I N 1$, is associated with increased adiposity in mature adipose tissue as well as differentiating adipocytes. Transgenic mice with enhanced lipin expression in adipose tissue become obese, but remain insulin sensitive, likely due to a redistribution of lipids from ectopic sites. Herein, we hypothesized that the depot-specific regulation of lipin by rosiglitazone may be associated with the fat-redistribution and insulin-sensitizing effects of rosiglitazone.

Materials and Methods: After 4 weeks of rosiglitazone treatment in Otusuka Long-Evans Tokushima Fatty rats, an animal model of type 2 diabetes mellitus, we measured changes in adiposity, adipocyte morphology, and lipin messenger RNA and protein expression in adipose tissue.

Results: Rosiglitazone increased subcutaneous adiposity, decreased plasma free fatty acids, decreased the cell diameters of adipocyte, and improved insulin resistance. Lipin messenger RNA expression increased in subcutaneous fat, but decreased in visceral fat by rosiglitazone treatment. In 3T3-L1 cell, rosiglitazone pretreatment decreased lipolysis and increased lipin mNRA and protein expression.

Conclusion: These findings demonstrate that depot-specific changes of lipin expression in adipose tissue after rosiglitazone treatment is likely to be a mediator of reduced lipolysis, resulting in fat redistribution and insulin sensitization.

\section{5}

Expression of KiSS-1 in rat adipose tissue and pituitary gland: regulation in diabetes and high fat diet

S. A. Imran ${ }^{1}$, R. Brown ${ }^{2}$, E. Ur 1 , M. Wilkinson ${ }^{2,3}$;

${ }^{1}$ Endocrinology \& Metabolism, Dalhousie University,

${ }^{2}$ Physiology, Dalhousie University,

${ }^{3}$ Obstetrics and Gynecology, IWK Hospital, Halifax, Canada.

KiSS-1, originally thought to be a metastasis suppressor gene, encodes a 145 amino acid precursor peptide that is proteolytically cleaved to a family of peptides known as kisspeptins. Kisspeptins appear to be the natural ligands of the previously orphan receptor GPR54. There is compelling evidence that hypothalamic kisspeptin is a critical component in sexual maturation. The kisspeptin receptor, GPR54,is widely distributed in the brain but is also found in peripheral sites such as pituitary, placenta, ovary and adipose tissue. Kisspeptin might therefore be a circulating hormone and/or be locally expressed in specific tissue sites. It is known that hypothalamic KiSS-1 mRNA levels are decreased by food restriction, stimulated by leptin and regulated by sex steroids. We have also provided evidence that the KiSS-1 gene is expressed in rat adipose tissue (FAT) and pituitary gland (PIT). We therefore hypothesised that KiSS-1 mRNA would be sensitive to changes in bodyweight and diabetic status in these tissues. Using realtime RT-PCR we detected a $56 \%$ reduction $(\mathrm{p}<0.01)$ in KiSS-1 mRNA in visceral fat from weanling male rats fed a high-fat diet for 20 days, although no changes in pituitary KiSS1 mRNA were observed. Adipose KiSS-1 mRNA levels in the adult, obese diabetic (fatty; fa/fa) Zucker rat were significantly reduced $(50 \% ; \mathrm{p}<0.05)$ in both visceral and subcutaneous fat, whereas it was increased in PIT tissue $(+60 \%, \mathrm{p}<0.05)$, relative to expression in lean littermates. In contrast an overnight fast increased KiSS-1 expression in FAT $(4.5$-fold; $\mathrm{p}<0.01)$ and significantly inhibited pituitary KiSS-1 $(-60 \%, \mathrm{p}<0.05)$. We conclude that KiSS-1 gene expression in fat tissue is sensitive to a high fat diet as well as diabetes. These novel findings suggest that adipose and pituitary kisspeptins may be implicated in the pathophysiology of diabetes.

Supported by NSHRF, IWK, UIMRF/Capital Health

\section{6}

Molecular cloning of a novel gene from human adipose tissue with its expression induced by fasting

Y. Kuroda, H. Kuriyama, K. Kishida, N. Maeda, H. Nishizawa, S. Kihara, T. Funahashi, I. Shimomura;

Department of Metabolic Medicine, Graduate School of Medicine, Osaka University, Japan.

Background and Aims: We made an expression profile in the human adipose tissue and we have isolated several novel adipose tissue-specific genes using the profile. "Adiponectin" and "Aquaporin adipose (AQPap)" correspond to those genes. We elucidated that Adiponectin is one of the important adipocytokines and a key molecule in the development of Metabolic syndrome and that AQPap is a glycerol channel in adipocytes and one of the important 
factors of determining fasting blood glucose levels. We tried to find another novel gene abundantly expressed in white adipose tissue.

Materials and Methods: The novel gene was isolated by 3'- directed cDNA library method. The mRNA expression was examined by Northern blotting and quantitative RT-PCR in the liver and white adipose tissue of mice and 3T3-L1 adipocytes. The mRNA change in feeding condition was investigated in mice.

Results: Recently we isolated a novel gene from human white adipose tissue and its expression in that tissue and liver was induced by fasting, therefore, we named the gene "fasting induced protein (FIP)". The human FIP gene encoded 214 amino acids which showed approximately $60 \%$ homology with mouse FIP at the amino acid level. The mRNA expression of FIP was increasing during differentiation in 3T3-L1 cells. The administration of insulin dramatically suppressed FIP mRNA expression in 3T3-L1 and HepG2 cells in dose- and time-course dependent manners. Compatible with this observation, the refeeding condition which means high insulin levels reduced the FIP mRNA expression in the liver and white adipose tissue of mice.

Conclusion: We isolated a novel gene named "FIP", from human adipose tissue of which expression was induced by fasting and reduced by refeeding.

\section{7}

\section{Adipocyte lipid-binding protein in subcutaneous adipose tissue in subjects with different categories of glucose intolerance \\ E. Krusinova ${ }^{1}$, J. Kopecky Jr. ${ }^{1}$, P. Mlejnek ${ }^{2}$, P. Wohl ${ }^{1}$, M. Pravenec ${ }^{2}$, \\ T. Pelikanova ${ }^{1}$; \\ ${ }^{1}$ Diabetes Center, Institute for Clinical and Experimental Medicine, Prague, \\ ${ }^{2}$ Institute of Physiology, Academy of Sciences of the Czech Republic, Prague, Czech Republic.}

Background and Aims: Adipocyte lipid-binding protein (ALBP) has been shown to regulate lipid metabolism and thereby to affect insulin sensitivity. However, its regulation in human obesity has not been clarified up to now. Our aims were to measure the expressions of ALBP in subcutaneous adipose tissue in subjects with different categories of glucose intolerance and to analyze possible relationships of ALBP with selected metabolic parameters. Materials and Methods: Eight healthy men (H), 12 subjects with impaired glucose tolerance (IGT) and 11 patients with type 2 diabetes (D) underwent 4-hour hyperinsulinaemic $\left(1 \mathrm{mU} \cdot \mathrm{kg}^{-1} \cdot \mathrm{min}^{-1}\right)$ euglycaemic $\left(5 \mathrm{mmol} \cdot \mathrm{l}^{-1}\right)$ clamp in order to verify their insulin sensitivity. Needle biopsy of abdominal subcutaneous fat was performed before the clamp to asses the ALBP expressions using the real-time PCR method. As a reference gene human cyclophilin was used.

Results: Insulin sensitivity, expressed as metabolic clearence rate of glucose (MCR), was significantly higher in H compared to D and IGT, whereas IGT did not differ from D: MCR (H vs. IGT vs. D: $8.68 \pm 0.55$ vs. $4.47 \pm 0.66$ vs. $4.88 \pm 0.49 \mathrm{ml} . \mathrm{kg}^{-1} \cdot \mathrm{min}^{-1} ; \mathrm{p}<0.001 ;$ ANOVA $)$. Other anthropometric and metabolic parameters (fasting serum lipids, waist circumference, BMI) were comparable between IGT and D, and lower in H compared to other groups. Relative expressions of ALBP were at the lowest level in IGT compared to D and $\mathrm{H}$, in $\mathrm{H}$ we detected the highest expression: ALBP mRNA/cyclophilin mRNA (H vs. IGT vs. D: $3498 \pm 761.7$ vs. $1133 \pm 126.1$ vs. $2024 \pm 194.8$; $\mathrm{p}<0.001$; ANOVA). Significant positive correlation was found between ALBP expression and MCR $(\mathrm{r}=+0.433 ; \mathrm{p}<0.02)$ and negative correlations between ALBP and fasting triglycerides $(r=-0.448 ; \mathrm{p}<0.02)$ or waist circumference $(\mathrm{r}=-0.473 ; \mathrm{p}<0.01)$.

Conclusion: Our results suggest, that ALBP expression in subcutaneous adipose tissue is positively associated with insulin sensitivity. Although having similar metabolic profile to type 2 diabetes, impaired glucose tolerance is associated with decreased expression of ALBP, which could not be explained by measured variables.

Supported by grant IGA $M H C Z$

\section{8}

Different expression of the adipocytokines in subcutaneous and visceral adipose tissue of extremely obese subjects

D. Gasperikova ${ }^{1}$, J. Stencl ${ }^{2}$, M. Skopkova ${ }^{1}$, M. Vlcek ${ }^{3}$, J. Pechan ${ }^{4}$, Z. Radikova ${ }^{3}$, R. Imrich ${ }^{3}$, B. Ukropcova ${ }^{1}$, J. Ukropec ${ }^{1}$, I. Klimes ${ }^{1}$, A. Penesova ${ }^{3}$;

${ }^{1}$ Diabetes Laboratory, Institute of Experimental Endocrinology, ${ }^{2}$ Department of Surgery, Faculty Hospital, Kramare, ${ }^{3}$ Laboratory of Human Endocrinology, Institute of Experimental Endocrinology,

${ }^{4}$ Department of Surgery, Comenius University, Bratislava, Slovakia.

Localized subclinical inflammation in the adipose tissue (AT) seems to play an important role in development of the negative metabolic consequences associated with obesity. Significance of different AT depots in this process has been intensively studied. Nevertheless, regulation of adipocytokine production at the molecular level particularly in extreme obesity has not yet been completely understood. Therefore, this study is aimed to investigate the gene expression of pro-inflammatory adipocytokines in subcutaneous $(\mathrm{Sc})$ AT and visceral (Vs) AT of extremely obese subjects.

Subjects and Methods: Twelve control (C) subjects (3M/9F) with $\mathrm{BMI}<25$ $\mathrm{kg} / \mathrm{m}^{2}$ and fifteen individuals $(4 \mathrm{M} / 11 \mathrm{~F})$ with extreme obesity $(\mathrm{O})(\mathrm{BMI}$ ranging from 35 to $60 \mathrm{~kg} / \mathrm{m}^{2}$ ) were recruited. ScAT and VsAT were obtained during laparoscopic gastric banding and/or during standard intra-abdominal surgical intervention in non-inflammatory diseases. The mRNA levels for selected adipokines and inflammatory cytokines were measured by the quantitative real-time RT-PCR.

Results: In ScAT of extremely obese patients, the mRNA levels for leptin (O: $2.7 \pm 0.3$ vs. $\mathrm{C}: 0.8 \pm 0.2 \mathrm{AU} ; \mathrm{p}<0.001$ ), PAI- 1 (O: $1.7 \pm 0.3$ vs. C: $0.5 \pm 0.1 \mathrm{AU}$; $\mathrm{p}<0.05)$ and $\mathrm{CD}-68(\mathrm{O}: 1.0 \pm 0.2$ vs. $\mathrm{C}: 0.5 \pm 0.2 \mathrm{AU} ; \mathrm{p}<0.05)$ genes were higher as in the control group. The VisAT of the obese subjects, contained higher mRNA levels for leptin (O: $1.4 \pm 0.2$ vs. C: $0.8 \pm 0.2 \mathrm{AU}$; $\mathrm{p}<0.01)$, NF-kB (O: $1.2 \pm 0.2$ vs. C: $0.5 \pm 0.1 \mathrm{AU} ; \mathrm{p}<0.01)$ and IL-6 (O: $6.4 \pm 1.4$ vs. C: $1.7 \pm 0.4 \mathrm{AU}$; $\mathrm{p}<0.01)$ and lower mRNA amount for adiponectin (O: $0.9 \pm 0.1$ vs. C: $1.2 \pm 0.1$ AU; $\mathrm{p}<0.05)$ as compared to the controls. Correlation analysis showed that expression of leptin in ScAT is positively associated with PAI-1 $\left(\mathrm{r}^{2}=0.39\right.$, $\mathrm{p}=0.0003)$, IL-6 $\left(\mathrm{r}^{2}=0.21, \mathrm{p}=0.016\right)$ and CD-68 $\left(\mathrm{r}^{2}=0.23, \mathrm{p}=0.07\right)$. A possible role of inflammation for the obese VisAT phenotype was further indicated by the positive correlation between NF-kB and PAI- $1\left(r^{2}=0,25, p=0,011\right)$ in the VsAT.

Conclusions: Results of this study indicates that the pro-inflammatory markers and regulators are expressed in extreme obesity mainly in the visceral adipose tissue. Moreover, different expression pattern for selected adipokines and inflammatory cytokines in the two AT depots investigated clearly advocates for distinct pathophysiological roles of the subcutaneous and visceral adipose tissue in extreme obesity.

Supported by APVT-51-040602 and VEGA 2/7110/27

\section{9}

The mechanisms of adrenaline-stimulated utilisation of glucose in rat adipose tissue

M. Cahova, H. Vavrinkova, H. Dankova, L. Kazdova;

PEM, Institute for Clinical and Experimental Medicine, Prague 4, Czech Republic.

Background and Aims: Besides its main effect, which is the stimulation of triglyceride breakdown we showed that in rat adipose tissue adrenalin also potentiates basal and insulin-stimulated utilisation of glucose in adipose tissue during in vitro incubation. The stimulation of $\beta$ adrenergic receptors by adrenalin results in the elevation of intracellular cAMP concentration and accentuated lipolysis. PKA (protein kinase A) has been considered as the main downstream target of cAMP but recently another PKA independent binding protein for cAMP called EPAC has been described. In muscle, adrenaline potentiates insulin-stimulated $\mathrm{PKB}$ activation via cAMP and EPAC but the role of EPAC in adipose tissue has not been elucidated yet. Our study was designed to assess the mechanism(s) of adrenalin stimulated glucose utilisation in adipose tissue. The adipose tissue was incubated in the presence of insulin, adrenalin and cAMP derivatives that stimulate selectively EPAC or PKA and the effects of these compounds on lipolysis and glucose utilisation was determined. The possible involvement of PKB in adrenalin stimulated glucose utilisation was also addressed. 
Materials and Methods: The epidydimal adipose tissue of fasting male Wistar rats $(300 \pm 20 \mathrm{~g})$ fed for two weeks by high sucrose diet (70 cal\% as sucrose) was incubated either in basal KRB medium or in medium supplemented with adrenaline $\left(5.10^{-6 \mathrm{M})}\right.$, insulin $(250 \mu \mathrm{U} / \mathrm{ml})$, EPAC activator (8-pCPT-2-O-MecAMP; $\left.5.10^{-4} \mathrm{M}\right)$ or PKA activator (6-Bnz-cAMP; $\left.1,7 \cdot 10^{-3} \mathrm{M}\right)$. The lipolysis was estimated according to the glycerol release, the utilisation of glucose was determined by incorporation ${ }^{14} \mathrm{C}$-glucose into $\mathrm{Tg}$. The $\mathrm{PKB}$ phosphorylation was determined by immunodetection using specific anti phosphoAkt (Ser 473) antibody.

Results: The lipolysis in adipose tissue was comparably increased by adrenaline, EPAC activator and PKA activator. Adrenaline increased the glucose incorporation into $\mathrm{Tg}$ and had no effect on lactate production. In contrast to this, EPAC had no effect on glucose metabolism and PKA activator the utilisation of glucose significantly decreased. The results are shown in the table; data are expressed \pm SEM, in $\mu \mathrm{mol} / \mathrm{g}$ w.v. The PKB phosphorylation on $\mathrm{Ser}^{473}$ after adrenalin stimulation in vitro increased by $60 \%$ over basal, insulin under the same condition stimulated the PKB phosphorylation by $130 \%$.

Conclusion: In adipose tissue, adrenalin concomitantly activates two opposite processes: lipolysis resulting in increased NEFA production and incorporation of glucose into glycerol of triglycerides (reesterification) that render part of the released NEFA in the tissue. Adrenaline, EPAC and PKA activators had similar positive effect on lipolysis but synthetic cAMP analogs in contrast to adrenaline did not stimulate glucose incorporation into Tg. Our experiments proved the stimulatory effect of adrenaline on PKB phosphorylation. This finding suggests the existence of possible cross talk between insulin receptor and $\beta$-adrenergic receptor signalling pathways in adipose tissue. (Tab. 1) Supported by IGA MH CR NR/8495-3

\section{0}

Whole-genome mRNA profiling in adipocytes from insulin-resistant subjects with familial combined hyperlipidaemia

C. J. H. van der Kallen ${ }^{1}$, S. Ghosh ${ }^{2}$, S. Meex ${ }^{1}$, M. van Greevenbroek ${ }^{1}$, C. Stehouwer ${ }^{1}$, D. Noel' ${ }^{2}$, T. de Bruin ${ }^{2}$;

${ }^{1}$ Department of Medicine, University of Maastricht, The Netherlands, ${ }^{2}$ GlaxoSmithKline, Research Triangle Park, United States.

Background and Aims: Insulin resistance is an underlying trigger for metabolic syndrome and is often associated with visceral adiposity, hypertension, hyperglycemia and dyslipidemia. The molecular mechanisms underlying insulin resistance are incompletely understood. We used wholegenome mRNA profiling to investigate transcriptional changes in adipocytes from insulin resistant subjects with Familial Combined Hyperlipidemia (FCHL) compared to controls. FCHL is the most common form of genetically determined dyslipidemia, present in $1-2 \%$ of the general population. FCHL patients share features of insulin resistance such as abdominal obesity, impaired glucose tolerance and hyperinsulinemia and is further characterized by familial aggregation of multiple dyslipidemic profiles in association with early-onset coronary artery disease. Our results shed light on the molecular mechanisms operative in the insulin resistant, FCHL adipocyte.

Materials and Methods: Adipocytes were isolated from periumbilical liposuction biopsies and purified by collagenase treatment and flotation after centrifugation. RNA was isolated using the Trizol reagent. RNA from 9 fasted controls and 21 fasted FCHL patients were hybridized to Affymetrix $\mathrm{HG}_{-}$ U133 Plus 2.0 microarrays. Gene signals were computed and normalized by GCRMA. Identification and interpretation of statistically significant differences in genes and pathways was accomplished by bioinformatic and statistical tools including Spotfire, Ermine J and JMP.

Results: Key genes related to fatty acid and cholesterol biosynthesis were downregulated in FCHL compared to controls (e.g. fatty acid synthase repressed $>2.5$-fold, $\mathrm{p}<0.02$; lanosterol synthase repressed $>3.5$-fold, $\mathrm{p}<0.0004)$. Phospholipase A2, a proinflammatory gene, was induced ( $>6.5$-fold, $\mathrm{p}<0.02)$ and leukotriene B4 12-hydroxydehydrogenase, an antiinflammatory gene, was repressed in FCHL $(>2.5$-fold, $\mathrm{p}<0.001)$. The T-cell specific CD28 antigen was upregulated $>11$-fold $(\mathrm{p}<0.001)$ and chemokine ligand 13 , involved in leukocyte accumulation in inflammation, was induced $>7.5$-fold $(\mathrm{p}<0.00001)$ in FCHL. Alpha 2 glycoprotein 1, a known lipolytic factor in cancer cachexia, was also downregulated in FCHL subjects ( $>4.5$ fold, $\mathrm{p}<0.001$ ). In addition to individual genes, differential regulation was also observed in gene sets encoding biological pathways. The most significant changes were observed in pathways related to humoral response, antigen presentation and fatty acid metabolism ( $\mathrm{p}<1 \mathrm{E}-12$ for each pathway). A majority of genes related to tricarboxylic acid cycle, glycolysis and pyruvate metabolism were also found to be downregulated in FCHL subjects based on visual analysis.

Conclusion: Initial analysis of whole genome transcript profiling point to a net inflammatory state in FCHL adipose tissue mediated by upregulation of pro-inflammatory and downregulation of anti-inflammatory genes. Genes related to fatty acid and cholesterol biosynthesis are repressed, possibly due to substrate feedback. The observed data offer a possible explanation for mechanisms underlying clinical observations in FCHL and insulin resistance.

Supported by GLAXOSMITHKLINE

Tab. 1 Abstract 0749

\begin{tabular}{llllll}
\hline & basal & insulin & adrenalin & EPAC activator & PKA activator \\
\hline lipolysis (glycerol production) & $15,6 \pm 1,9^{\mathrm{a}, \mathrm{b}, \mathrm{c}}$ & $16,4 \pm 0,9$ & $23,2 \pm 1,5^{\mathrm{a}}$ & $21 \pm 2,1^{\mathrm{b}}$ & $21,3 \pm 2,5^{\mathrm{c}}$ \\
incorporation of glucose into $\mathrm{Tg}$ & $2,0 \pm 0,2^{\mathrm{d}, \mathrm{f}, \mathrm{f}}$ & $2,9 \pm 0,1^{\mathrm{d}}$ & $3,8 \pm 0,5^{\mathrm{e}}$ & $2,5 \pm 0,3$ & $1,4 \pm 0,3^{\mathrm{f}}$ \\
production of lactate & $9,7 \pm 0,8^{\mathrm{g}}$ & $11,6 \pm 1,5$ & $9,3 \pm 1,1$ & $7,9 \pm 0,9$ & $5,6 \pm 0,3^{\mathrm{g}}$ \\
\hline
\end{tabular}




\section{PS 66 RBP4 and resistin}

\section{1}

RBP4 in glucose metabolism during fasting in lean healthy humans M. R. Soeters ${ }^{1}$, H. P. Sauerwein ${ }^{1}$, M. T. Ackermans ${ }^{2}$, E. Fliers ${ }^{1}$, M. J. Serlie ${ }^{1}$; ${ }^{1}$ Endocrinology and Metabolism, ${ }^{2}$ Clinical Chemistry, Laboratory of Endocrinology, Academic Medical Center, Amsterdam, The Netherlands.

Background and Aims: Plasma retinol binding protein 4 (RBP4) has been proposed as an adipokine involved in obesity-induced insulin resistance. Other reports suggested that the higher plasma RBP4 levels in insulin resistant subjects relate to the lower glucose influx in insulin resistant adipocytes. To test this hypothesis, we studied plasma RBP4 concentrations and glucose metabolism during fasting, since fasting is characterized by lower glucose uptake in adipocytes and insulin resistance.

Materials and Methods: We measured glucose metabolism and plasma RBP4 levels in the basal state and during a hyperinsulinemic euglycemic clamp after short term fasting. Data from 26 lean healthy volunteers were included. Six men were studied after 14 and $62 \mathrm{~h}$ of fasting (protocol 1), and 10 males and 10 females after 38 hours only (protocol 2).

Statistics: Data presentation median [min-max]. Differences: Wilcoxonsigned rank test (protocol 1) and Mann-Whitney U test (protocol 2). Correlations: Spearman's rank correlation coefficient (rho).

Results: RBP4 levels were $32 \%$ lower after $62 \mathrm{~h}$ compared to $14 \mathrm{~h}$ of fasting: $27.1 \mu \mathrm{g} / \mathrm{ml}[25.0-38.7]$ and $20.7 \mu \mathrm{g} / \mathrm{ml}[12.3-21.7]$ respectively, $(\mathrm{P}=0.046)$. Plasma RBP4 levels in women were $18 \%$ lower than in men: $18.4 \mu \mathrm{g} / \mathrm{ml}[13.0-$ $20.7]$ and $21.0 \mu \mathrm{g} / \mathrm{ml}[18.1-26.1]$ respectively, $(P=0.005)$. The endogenous glucose production tended to be lower in women $(\mathrm{P}=0.074)$. There was no statistically significant difference in peripheral insulin-mediated glucose uptake between women and men. Plasma RBP4 did not correlate with endogenous glucose production or insulin-mediated glucose uptake after fasting ( $\mathrm{rho}=0.2, \mathrm{p}=0.3$ and $\mathrm{rho}=0.2, \mathrm{p}=0.4$ respectively).

Conclusion: Plasma RBP4 levels decrease during fasting in lean healthy humans. Women have lower plasma RBP4 levels than men despite comparable glucose metabolism. Plasma RBP4 is not correlated to endogenous glucose production or peripheral glucose uptake. These findings contradict a role for RBP4 as an important regulator in glucose metabolism during fasting in lean healthy humans.

\section{2}

Serum retinol binding protein 4 levels are decreased in insulin treated type 1 diabetic patients

M. Jourdan ${ }^{1}$, A. Jaleel ${ }^{1}$, J. P. McConnell ${ }^{2}$, M. Bigelow ${ }^{1}$, H. Karakelides ${ }^{1}$, K. S. Nair';

${ }^{1}$ Endocrine Research Unit,

${ }^{2}$ Immunochemical Core Lab., Mayo Clinic, Rochester, United States.

Background and Aims: Retinol binding protein 4 (RBP-4) has been reported to be elevated in insulin resistant states such as in type 2 diabetes, as well as in obese and elderly people. In type 2 diabetic subjects, RBP-4 is negatively correlated to insulin sensitivity and insulin-sensitizing agents normalize RBP4 levels. RBP- 4 has therefore been proposed as an adipocyte-derived "signal" that may contribute to the pathogenesis of type 2 diabetes. No informations are available on RBP-4 levels in T1D patients or whether insulin per se has any effect on RBP- 4 levels. Thus, the purpose of this study was to determine whether serum RBP-4 levels are altered in type 1 diabetic subjects (T1D) and whether insulin treatment has an effect in serum RBP-4 levels in T1D.

Materials and Methods: We measured serum RBP-4, plasma glucose and insulin levels in 7 T1D subjects (age $=27.1 \pm 2.5 \mathrm{yr}, \mathrm{BMI}=24.8 \pm 0.79 \mathrm{~kg} / \mathrm{m}^{2}$ ) during insulin treatment (I+) (to maintain euglycemia) and following $8.6 \pm 0.6$ hours of insulin deprivation (I-). T1D were compared with 7 nondiabetic subjects (ND) matched for their BMI (age $=29.7 \pm 2.9 \mathrm{yr}, \mathrm{BMI}=24.9 \pm 1.1 \mathrm{~kg}$ / $\mathrm{m}^{2}$ ). RBP-4 was measured immunoturbidimetrically on the Hitachi 912 Chemistry analyzer using reagents from The Binding Site Inc.(Birmingham, UK).

Results: Insulin deprivation (I-) resulted in higher plasma glucose levels than the normal glycemia observed during insulin treatment $(\mathrm{I}+)(16.5 \pm 0.7 \mathrm{mmol} /$ 1 in I- vs. $5.3 \pm 0.1$ in $\mathrm{I}+, \mathrm{p}<0.01)$. Plasma insulin levels in T1D subjects during I+ were higher than in ND subjects $(29.8 \pm 5.2 \mathrm{pmol} / \mathrm{l}$ in ND vs $75.8 \pm 16.4$ in
$\mathrm{I}+, \mathrm{p}<0.05)$. During I+, RBP-4 levels in T1D subjects were lower than in ND $(35.9 \pm 2.6 \mathrm{mg} / \mathrm{l}$ in ND vs. in $26.2 \pm 1.8 \mathrm{I}+, \mathrm{p}<0.01)$. However, during I-, RBP-4 levels were not different in T1D than in ND subjects $(35.9 \pm 2.6 \mathrm{mg} / \mathrm{l}$ in ND vs in $30.1 \pm 3.2$ in I-).

Conclusions: We demonstrated that unlike in T2D patients, RBP-4 levels are not elevated in T1D patients during poor glycemic control. Moreover, insulin treatment caused a decline in RBP-4 levels clearly indicating that increased insulin action per se decreases RBP-4 levels.

Supported by NIH grant R01DK41973

\section{3}

Resistin reveals a separate evolution in placental mammals I. Nagaev;

Internal Medicine, Goteborg University, Sweden.

Background and Aims: To explain why homologous human and mouse resistins diverged radically, as specific immune and adipose cytokines, respectively.

Materials and Methods: Phylogenetic analysis in all major mammalian groups was corroborated by experimental data from NCBI and Ensembl. Gene and protein sequences were aligned using BLAST to reconstruct an evolutional tree based on a protein homology.

Results: Resistin and resistin like beta genes have orthologs in all general mammalian taxa: Prototheria or egg-laying mammals, Metatheria or marsupials and Eutheria or placentals. But homologous chromosomal loci in chicken outgroup void both genes, also missing in other eukaryotes. Thus, resistin gene family is a mammal-specific trait and its evolution can confer the speciation of mammals. Comparison of resistin and resistin like beta among all species revealed that these genes derived at once and deviated rapidly. However, they build identical topology, a branching pattern of phylogenetic tree, which mainly agrees with a current taxonomy. Human resistin has $72-98 \%$ identity with homologs in placentals except mouse and rat that show 55\%, like Prototheria and Metatheria outgroups. Also resistin like beta has $63-98 \%$ homology among placentals but least $59 \%$ to mouse with rat and Metatheria. The partition of mouse and rat from major mammalian groups was obvious here and consistent with other studies. Thus, mouse and rat constitute a monophyletic clade or separate branch in placentals. Descendants in all branches have ancestor's similarities but new parities developed separately in respond to similar challenges. Also common ancestor traits diverged adaptively; as murine and rat adipose-specific resistin holds energy balance, the resistin in man, pig, cow and dog has immuneintrinsic roles. Resistin like beta, that usually tagged to innate immunity of intestine in placentals, was copied in dog but vanished in cow and guinea pig. In mouse and rat, paralogous resistin like gamma and alpha genes derived in that order. They have immune qualities, albeit resistin like alpha is largely expressed in adipocytes. Resistin like gamma evolved as a full analog of human resistin and so proving the divergent-parallel-convergent evolution in Eutheria. Natural roles of resistin family suggest that adipose-immune or energy-defense development was critical to mammals and proper molecular 'fossils' must exist. Indeed, mast cell-expressed membrane protein 1 gene is not found in chickens, while mast cells exist in birds. Both mast and blood cells bolster inflammation by expressing resistin in man but resistin like gamma in mouse and rat. Also their interleukin 6 is really an analog of a similar human gene. Since they evolved from different ancestor genes, their proteins share only $41 \%$ identity. Both subunits of the interleukin 6 receptor are homologous in placentals, yet mouse and rat show the least similarity. Oncostatin $\mathrm{M}$ and its specific receptor once again confirm the root division of mouse and rat from all placental orders including Rodentia.

Conclusion: Mice and rats cannot be used as a congruent model of human biology. In contrast, the comparison of our independent evolutions, when different strategies have been employed in respond to similar challenges, can disclose both biological rewards and costs, i.e. evolutional basis of human diseases. In fact, mouse and rat developed the resistin adipose-immune traits rather evenly, because we do not have the adipose-derived resistin and resistin like alpha. Further bioinformatic inference is needed to clarify the evolutional intricacy in placental mammals. 


\section{4}

Murine resistin induces pro-angiogenic responses in endothelial cells and aortic arch explants

S. A. Robertson, C. Rae, A. Graham;

Vascular Biology Group, Glasgow Caledonian University, United Kingdom.

Background and Aims: Angiogenesis, the formation of new blood capillaries from existing vessels, is required for physiological processes such as embryonic development, wound healing, and female reproduction, but when dysregulated can contribute to tumour growth and atherosclerosis. Endothelial cell migration and proliferation, in response to angiogenic stimuli such as hypoxia, plays an essential role in this process. The novel cysteine-rich adipokine, resistin, which causes insulin resistance in murine hepatic and peripheral tissues, is found within human and murine atherosclerotic lesions, and induces inflammatory, proliferative and angiogenic responses in vascular cells. Here, we examine the mechanisms by which murine resistin induces angiogenic responses in murine aortic arch explants, primary endothelial cells and murine b.End.5 endothelioma cell line.

Materials and Methods: Murine endothelioma cells (b.End.5) or murine thoracic aortic arch explants were treated with murine resistin (50 $\mathrm{n} \mathrm{ml}^{-1}$; 0 $72 \mathrm{~h}$ ) and/or VEGF (10 $\mathrm{ng} \mathrm{ml}^{-1}$ ) or a combination. Migration and proliferation were then measured as indicated.

Results: Treatment with resistin $\left(50 \mathrm{ng} \mathrm{ml}^{-1}\right.$ ) increased cell growth and migration from aortic arch explants, when compared with control tissue $(758 \pm 40.36 \mu \mathrm{m}$ versus control $322 \pm 45.99 \mu \mathrm{m}, \mathrm{n}=4, p<0.01)$. The same concentration of resistin induced network tube formation when primary murine aortic endothelial cells, or b.End. 5 cells were cultured on Matrigel matrix. Resistin (50ng ml-1; 48h) significantly enhanced remodelling of an induced 'wound' or scratch in a confluent monolayer of b.End.5 endothelial cells (resistin $216 \mu \mathrm{m} \pm 19.8 \mu \mathrm{m}$ versus control $163 \pm 10.7 \mu \mathrm{m}, \mathrm{n}=6, p<0.001$ ) compared with the control. Under hypoxic conditions, resistin (50 $\mathrm{ng} \mathrm{ml}^{-1}$ ) stimulated a comparable level of growth, in the same assay $(158 \mu \mathrm{m} \pm 13.3 \mu \mathrm{m}$ versus control $131 \pm 10.1 \mu \mathrm{m}, \mathrm{n}=13, p<0.05)$, equivalent to that induced by

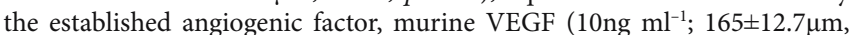
$\mathrm{n}=13, \mathrm{p}<0.05$ ); no additive response was noted when both resistin and VEGF were added together. Resistin induces a significant degree of endothelial cell migration, as judged by apical to basolateral migration (5h) across TransWell inserts $(8 \mu \mathrm{m}$ pore); murine resistin and VEGF increased endothelial cell migration by $48 \%(44.0 \pm 2.83$ versus control $30.0 \pm 5.45, \mathrm{n}=6, p<0.001)$ and $23 \%(37.0 \pm 4.67, n=6, p<0.001)$, respectively, compared with the control incubation, and by $66 \%(50.0 \pm 5.99 ; \mathrm{p}<0.01)$ in the presence of both resistin and VEGF. A mild increase in proliferation of b.End. 5 cells was also noted in the presence of resistin $\left(30-100 \mathrm{ng} \mathrm{ml}^{-1} ; 24 \mathrm{~h}\right)$, as judged by metabolic conversion of MTT to formazan $(17.5 \pm 0.7 \mu \mathrm{M}$ versus control $15.4 \pm 0.4 \mu \mathrm{M}$, $\mathrm{n}=8, p<0.05$ ). Using a real time PCR array (SuperArray, Tebu-Bio) to screen for angiogenic targets of resistin in bEnd5 cells, we have shown an increase in several factors involved in the angiogenic process: tumor necrosis factor (ligand) superfamily member 12 (TWEAK), tumor necrosis factor alphainduced protein 2 (Tnfaip2), interleukin-6, matrix metalloprotease 2 and VEGF-D increased after treatment with resistin $\left(50 \mathrm{ng} \mathrm{ml}^{-1} ; 48 \mathrm{~h}\right)$, while VEGF-A and endothelial PAS domain protein-1 were down-regulated under the same condition.

Conclusion: These data suggest that murine resistin can promote angiogenesis by increasing endothelial cell proliferation and migration, and may have an important role in pathological processes such as tumorigenesis and atherosclerosis.

\section{5}

Resistin-induced changes in fatty acid metabolism drive cholesteryl esterification and lipid accumulation in human macrophages

C. Rae, S. A. Robertson, A. Graham;

Vascular Biology Group, Glasgow Caledonian University, United Kingdom.

Background and Aims: Resistin is a novel cysteine-rich secretory adipokine, originally discovered in murine studies and proposed as a link between obesity and insulin-resistant diabetes. However, the (patho)physiological role of human resistin, secreted primarily from macrophages rather than adipocytes, is not confirmed, although it is known to exert inflammatory, angiogenic and proliferative effects in vascular cells. This study investigated the effect of resistin on lipid metabolism in macrophages, and in particular cholesterol efflux and cholesteryl ester accumulation - key elements of atherosclerosis.

Materials and Methods: Human THP-1 monocytes were differentiated into macrophages with phorbol 12-myristate 13-acetate (PMA; 250nM): these cells do not produce or secrete endogenous resistin. Macrophages were treated with human recombinant resistin (Phoenix) and parameters of lipid metabolism determined: these included triacylglycerol and cholesteryl ester mass; fatty acid uptake $\left(\left[{ }^{14} \mathrm{C}\right]\right.$ and $\left[{ }^{3} \mathrm{H}\right]$ oleate $)$ and oxidation $\left({ }^{14} \mathrm{CO}_{2}\right.$ production); $\left[{ }^{3} \mathrm{H}\right]$ cholesterol efflux and ester formation; expression of ACAT1 (Western blotting and PCR), ATP binding cassette transporter A1 (ABCA1) and acetyl CoA carboxylase phosphorylation status (Western blotting) and apolipoprotein (apo)E protein levels (ELISA).

Results: Chronic treatment with resistin $\left(5-100 \mathrm{ng} \mathrm{ml}^{-1} ; 24 \mathrm{~h}\right)$ increased triacyglycerol accumulation in THP-1 macrophages (143\% of untreated control cells, $\mathrm{p}<0.001$ ). Resistin increased cholesteryl ester mass (control, $3.51 \mu \mathrm{g} . \mathrm{mg}$ protein ${ }^{-1}$ versus resistin, $5.63 \mu \mathrm{g} . \mathrm{mg}$ protein $\left.^{-1}, \mathrm{p}<0.05\right)$ and incorporation of $\left[{ }^{3} \mathrm{H}\right]$ cholesterol and $\left[{ }^{3} \mathrm{H}\right]$ oleate into the cholesteryl ester pool (to $140 \%$ and $138 \%$ of control, respectively, $\mathrm{p}<0.05$ ). "Lipid-loading" of cells with oxidized LDL (50 $\left.\mu \mathrm{g} . \mathrm{ml}^{-1}\right)$ increased cholesteryl ester mass $(5.9$ $\mu \mathrm{g} . \mathrm{mg}$ protein ${ }^{-1}, \mathrm{p}<0.05$ from control), and this effect was further enhanced by co-incubation with resistin $\left(6.86 \mu\right.$ g.mg protein ${ }^{-1}, \mathrm{p}<0.001$ from control). Incorporation of $\left[{ }^{3} \mathrm{H}\right]$ oleate into the cholesteryl ester pool was reduced by an ACAT inhibitor $(10 \mu \mathrm{M}$ Sandoz $58-035+$ resistin, $87 \%$ of control, $\mathrm{p}<0.01$ from resistin). However, ACAT-1 expression was not affected by resistin. The 'anti-diabetic' agent C75, which inhibits fatty acid synthesis and promotes fatty acid oxidation, inhibited resistin-induced cholesteryl ester mass accumulation (control, $3.81 \mu \mathrm{g} . \mathrm{mg}$ protein $^{-1}$; resistin, $6.2 \mu \mathrm{g} . \mathrm{mg}$ protein $^{-1}$, $\mathrm{p}<0.05$ from control; $10 \mu \mathrm{M}$ C75 + resistin, $3.44 \mu \mathrm{g} . \mathrm{mg}$ protein $^{-1}, \mathrm{p}<0.05$ from resistin). Neither ApoA1-dependent or independent cholesterol efflux, ApoE secretion or ABCA1 expression were affected by resistin, indicating that decreases in cholesterol efflux were not responsible for cholesteryl ester accumulation. Fatty acid uptake was, however, increased by resistin $\left(\left[{ }^{3} \mathrm{H}\right]\right.$ oleate uptake increased by $29 \%$ after 5 mins, $\mathrm{p}<0.05 ;\left[{ }^{14} \mathrm{C}\right]$ oleate uptake increased by $52 \%$ after 2 hours, $\mathrm{p}<0.05)$. Oxidation of $\left[{ }^{14} \mathrm{C}\right]$ oleate was reduced by resistin (control, $5 \%$ intracellular $\left[{ }^{14} \mathrm{C}\right]$ oleate pool; resistin, $3.6 \%, \mathrm{p}<0.05$ ); acutely, these changes were accompanied by reduced phosphorylation of acetyl CoA carboxylase.

Conclusion: The combined increase in uptake, and decrease in oxidation of fatty acids induced by resistin, enhance triacylglycerol and cholesteryl ester accumulation. This could lead to macrophage 'foam cell' formation and atherosclerosis, the principal cause of mortality in diabetic individuals. Supported by Diabetes UK Project Grant 


\section{PS 67 Mitochondria}

\section{6}

Gene expression profiles in peripheral blood mononuclear cells reflect pathophysiology of type 2 diabetes

T. Takamura, H. Ando, A. Shimizu, H. Misu, S. Kurita, N. Matsuzawa, S. Kaneko;

Department of Disease Control and Homeostasis, Kanazawa University Graduate School of Medical Science, Kanazawa, Japan.

Background and Aims: Diagnosis and ideal treatment of diabetes require comprehensive understanding of hereditary aspects as well as habit and environmental effects as a cause of disease onset. Systemically circulating peripheral blood mononuclear cells (PBMCs) are considered to be unique tissue affected by the host condition and may reflect oxidative stress caused by high levels of glucose, insulin, free fatty acids and tissue-derived circulating bioactive mediators. To verify the hypothesis that PBMCs may change gene expression in response to diabetic circumstances, we comprehensively compared global gene expression profiles of PBMCs between people with and without type 2 diabetes, and between before and after glycaemic control by using a DNA chip technology.

Materials and Methods: PBMCs were obtained from 18 patients with type 2 diabetes and 16 young healthy volunteers. Antisense RNA from PBMC was amplified, and expression of genes was analyzed comprehensively by hybridization to an oligo-DNA chip (AceGene ${ }^{\circ}$ Human Oligo Chip 30K, HitachiSoft, Japan) followed by statistical analysis for individual genes and specific gene sets for biological categories. We screened a total of 535 human gene sets defined by BioCarta, KEGG and Broad/MIT pathways. The mean centroid is the mean of the normalized gene expression levels.

Results: (1) Supervised learning methods based on the compound covariate predictor revealed that, among various clinical parameters, only the two clinical parameters, glycaemic control and the presence of diabetes significantly classified these patients. (2) The specific unique group of 48 genes coordinately altered by the existence of diabetes and by glycaemic control was involved in the c-Jun N-terminal kinase (JNK) pathway. (3) JNK mean centroid was significantly higher in people with diabetes compared with healthy subjects, and was significantly decreased after glycaemic control (Figure). (4) JNK mean centroid in the PBMCs was significantly correlated with levels of fasting plasma glucose and $\mathrm{HbA}_{1}$. (5) In contrast, the only gene set coordinately altered by the existence of diabetes, but not by glycaemic control, consists of 92 genes involved in mitochondrial oxidative phosphorylation (OXPHOS) pathway. (6) OXPHOS mean centroid was significantly down-regulated in patients with diabetes compared with healthy young subjects, whereas it was not significantly altered after glycaemic control.

Conclusion: Genes involved in JNK and OXPHOS pathways in the PBMCs may be surrogate transcriptional markers for hyperglycemia-induced oxidative stress and morbidity of type 2 diabetes, respectively. This is the first evidence that gene expression profiles of PBMCs may reflect the systemic molecular signature of diabetes, suggesting the diagnostic potential of gene expression analyses of PBMCs for hereditay aspects and environmental effects in people with type 2 diabetes.

\section{7}

Mitochondrial function in skeletal muscle of arm and leg of type 2 diabetic patients

R. Rabol ${ }^{1}$, P. V. Højberg ${ }^{2}$, S. B. Haugaard ${ }^{3}$, T. Almdal', S. Madsbad ${ }^{3}$, F. Dela ${ }^{1}$; ${ }^{1}$ Dept of Biomedical Sciences, Faculty of Health Sciences, University of Copenhagen, ${ }^{2}$ Dept. of Internal Medicine, Gentofte Hospital, Hellerup, ${ }^{3}$ Dept. of Endocrinology, Hvidovre Hospital, Denmark.

Background and Aims: Skeletal muscle insulin resistance is central in the pathogenesis of type 2 diabetes. Insulin resistance has been linked to decreased mitochondrial function and accumulation of intramyocellular lipid which may interfere with insulin signaling. It has been shown that insulin sensitivity in skeletal muscle is higher in arm than in leg muscle, and we have therefore measured mitochondrial function in skeletal muscle of arm (m. deltoideus) and leg ( $\mathrm{m}$. vastus lateralis) in patients with type 2 diabetes (T2DM) and healthy control subjects.

Materials and Methods: Ten patients with T2DM (eight male) and 10 healthy control subjects (age 52.3 (8.6) yrs (mean (SD) and 54.3 (8.8) yrs; BMI $30.1(3.8) \mathrm{kg} / \mathrm{m}^{2}$ and $30.4(3.9) \mathrm{kg} / \mathrm{m}^{2}$, respectively) were included. Average duration of diabetes was 4.7 yrs (3.6), and patients were all treated with oral antidiabetic agents at inclusion. HbAlc was on average $9.0 \%$ (1.7) in the group of patients with T2DM and $5.4 \%(0.2)$ in the control group $(\mathrm{p}<0.001)$.

Results: Mitochondrial respiration was similar in arm and leg muscle of patients with T2DM, both in state 3 respiration with substrates for complex I (malate, pyruvate and glutamate + ADP) and substrates for complex I and II (malate, pyruvate, glutamate and succinate + ADP). Compared to controls, mitochondrial respiration was significantly reduced in leg muscle of patients with T2DM while mitochondrial respiration was comparable to controls in the arm muscle. Please see table, all data are expressed as mean (SE).

\begin{tabular}{|c|c|c|c|c|}
\hline \multirow{2}{*}{$\begin{array}{l}\text { O2 Flux } \\
\text { pmol }^{\star} \sec ^{-1} \\
{ }^{-m^{-1}}\end{array}$} & \multicolumn{2}{|l|}{ Arm } & \multicolumn{2}{|l|}{$\underline{\text { Leg }}$} \\
\hline & $\begin{array}{l}\text { State 3, } \\
\text { Complex I }\end{array}$ & $\begin{array}{l}\text { State 3, } \\
\text { Complex I + II }\end{array}$ & $\begin{array}{l}\text { State 3, } \\
\text { Complex I }\end{array}$ & $\begin{array}{l}\text { State } 3 \text {, } \\
\text { Complex I + II }\end{array}$ \\
\hline T2DM & $19(2)$ & $46(4)$ & $22^{\star}(3)$ & $50(5)$ \\
\hline Control & $22(2)$ & $48(4)$ & $30^{\star \star}(3)$ & $61(5)$ \\
\hline
\end{tabular}

${ }^{\star} \mathrm{P}<0.05$ compared to control leg; ${ }^{\star *} \mathrm{P}<0.05$ compared to arm

Conclusion: Even though insulin sensitivity is supposedly better preserved in arm muscle than leg muscle of type 2 diabetic patients, we were not able to detect differences in mitochondrial function between these two muscle groups. However, only leg muscle mitochondrial function per muscle mass is significantly reduced in T2DM compared to age and BMI matched controls. Supported by the Danish Diabetes Association
A

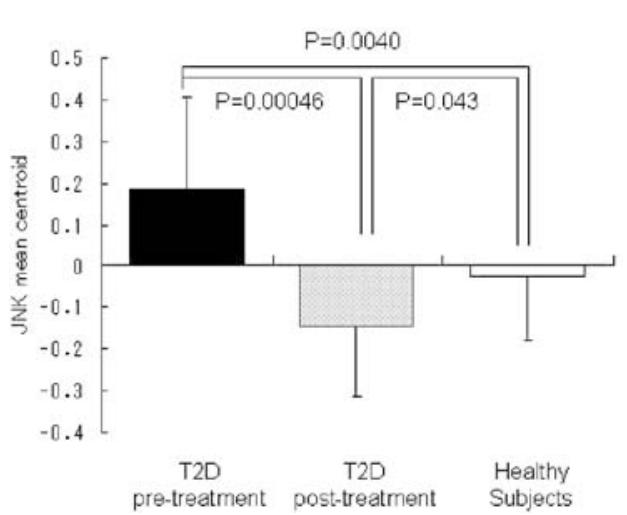

B

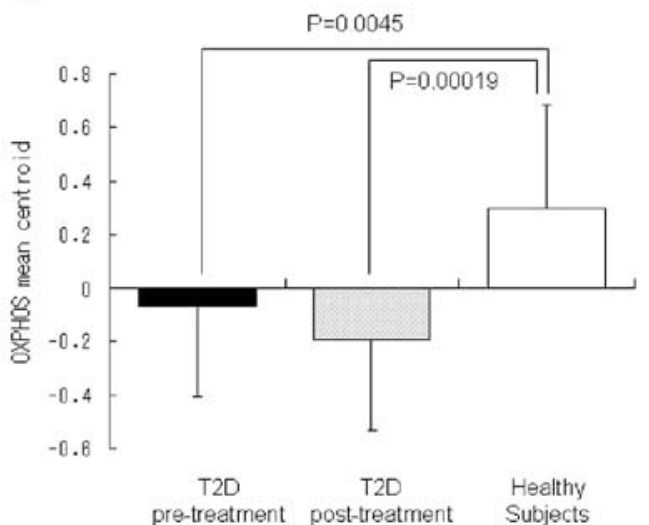

Fig. 1. Abstract $0756 \mathrm{JNK}$ mean centroid (A) and OXPHOS mean centroid (B) among people with type 2 diabetes (T2D) before and after glycemic control and healthy subjects. Values are mean \pm SD. 


\section{8}

Mitochondrial dysfunction and differentially expressed proteins in skeletal muscle of high-fat diet-fed mice

C. Bonnard ${ }^{1}$, S. Peyrol ${ }^{2}$, J. Garin ${ }^{3}$, H. Vidal ${ }^{1}$, J. Rieusset ${ }^{1}$;

${ }^{1}$ U870, Inserm, Lyon, France, ${ }^{2} \mathrm{CeCil}$, IFR62, Lyon,

${ }^{3}$ ERM0201, CEA, Grenoble, France.

Mitochondrial biogenesis and function are altered in type 2 diabetes, as well as in insulin-resistant relatives of type 2 diabetic subjects. In addition, excess dietary fat has been implicated in the development of obesity and diabetes. The purpose of this study was to identify mitochondrial dysfunction and differentially expressed mitochondrial proteins in skeletal muscle of highfat diet (HFD)-fed mice. Male mice were fed with a standard diet (SD) or a HFD (36\%) for 4 or 16 weeks, and mitochondrial structure and content of gastrocnemius muscle were analysed by electron microscopy and mitochondrial proteins levels by 2D electrophoresis. Metabolic analysis of mice revealed that 4 weeks HFD-fed mice were glucose intolerant whereas 16 weeks HFD-fed mice were insulin-resistant as assessed by intra-peritoneal insulin tolerance test. Muscle mitochondrial content was decreased in the 16 weeks HFD-fed mice, but this effect was not observed after 4 weeks of diet. Moreover, cellular mitochondrial DNA was also reduced in 16 weeks HFD-fed mice. In agreement, expression of genes implicated in mtDNA replication (POLG2 and SSBP1mRNA levels) or in mitochondrial biogenesis (PGC-1 $\alpha$ mRNA and protein levels) was decreased in muscle of 16 weeks HFD-fed mice compared to SD-fed mice. Also citrate synthase activity was reduced in muscle of 16 weeks HFD-fed mice. In addition, both subsarcolemmal and intermyofibrillar mitochondria were smaller and had altered internal structure membrane with disintegrated cristea and large low density vacuoles in muscle of 16 weeks HFD-fed mice. These changes were associated with an intramyocellular lipid accumulation, an increase of IRS1 Ser632 phosphorylation and a reduction in insulin-stimulated PKB activation, supporting the relation between mitochondrial defects and muscle insulin-resistant. To gain more information on the molecular mechanisms underlying the observed mitochondrial dysfunction in skeletal muscle, we have undertaken a differential proteomic analysis of mitochondria of SD-fed mice and 16 weeks HFD-fed mice. Identification by mass spectrometry of altered mitochondrial proteins in skeletal muscle in response of HFD should provide new insight in the pathogenesis of muscle insulin resistance in type 2 diabetes.

\section{9}

Insulin resistance in sepsis and mitochondrial dysfunction as markers for the outcome of a septic shock

P. G. Halvatsiotis ${ }^{1}$, S. Stefanopoulou ${ }^{1}$, A. Kotanidou ${ }^{2}$, S. Orfanos ${ }^{2}$, C. Martinos ${ }^{2}$, C. Roussos ${ }^{2}$, T. Economopoulos ${ }^{1}$, S. A. Raptis ${ }^{1,3}$;

${ }^{1}$ 2nd Dept of Int Med Propaedeutic, Athens University Medical School,

${ }^{2} 1$ st Dept of Critical Care, Athens University Medical School,

${ }^{3}$ Molecular Biology Lab, Hellenic National Diabetes Center, Athens, Greece.

Background and Aims: The cause of mortality in critically ill patients is considered the sepsis-induced multiple organ failure. Oxidative phosphorylation in mitochondria produces energy and accounts for more than $90 \%$ of total oxygen consumption. Furthermore the profound alterations in metabolic pathways occur in sepsis lead to the development of acute insulin resistance and to a decrease in the rates of muscle mitochondrial substrate oxidation. However remains unclear whether the mitochondrial dysfunction resulting in organ failure is attributed to hypermetabolism, which is known to inhibit mitochondrial respiration in vitro and is produced in excess during sepsis.

Materials and Methods: We determined in vastus lateralis needle biopsy samples, using a real-time PCR technique, gene transcript levels of representative mitochondrial enzyme such as citrate synthase (CS), Cytochrome C oxidase I \& III, NADH, and UCPs $2 \& 3$. We also measured the activity of the above enzymes by the means of a photometric assay. We studied 7 critically ill patients under irreversible septic shock (52 \pm 7 y) who finally died within 30 days (SS) and 8 age and gender matched septic patients $(46 \pm 7$ y) $(\mathrm{p}=0,51)$ who finally survived (SSV).

Results: All patients were non diabetic and SS group demonstrated lower insulin sensitivity than SSV by the means of HOMA test $(11,56 \pm 3,5$ vs $48,59 \pm 11,4)(\mathrm{p}=0,011)$. There was no difference in the enzyme activity of all mitochondrial enzymes measured between the 2 groups. The gene transcript of CS was significantly higher in SSV than SS $(2,62 \pm 0,3$ vs $1,29 \pm 0,6$ $\mathrm{AU})(\mathrm{p}=0,011)$ while there was no difference in the transcripts of the other mitochondrial enzymes.

Conclusion: In conclusion, in septic patients we found an association of the severity of sepsis with the development of insulin resistance and with mitochondrial dysfunction solely attributed to the citric cycle, whereas the enzymes of the electron transfer chain and the uncoupling proteins were unaffected. These data implicate the existence of a bioenergetic failure, which contributes to the pathophysiological mechanism underlying the multiorgan dysfunction.

\section{0}

The effect of HIV status and the impact of ARV therapy on mitochondrial metabolism in human adipose tissue M. J. Hill ${ }^{1}$, K. C. McGee ${ }^{1}$, M. Boothby ${ }^{2}$, P. Higgins ${ }^{1}$, C. M. Kusminski ${ }^{1}$, A. L. Harte', G. Tripathi ${ }^{1}$, L. L. Gathercole ${ }^{3}$, S. Kumar ${ }^{1}$, M. Shahmanesh², P. G. McTernan ${ }^{1}$;

${ }^{1}$ Clinical Sciences Research Institute, University of Warwick Medical School, Coventry, ${ }^{2} \mathrm{HIV}$ Medicine, University Hospital Birmingham,

${ }^{3}$ Division of Medical Sciences, University of Birmingham, United Kingdom.

Background and Aims: Emerging evidence suggests there is an association between mitochondrial dysfunction and the progression of the metabolic syndrome. However the mechanisms by which mitochondrial function becomes dysregulated, leading to defects in energy homeostasis and regulation of metabolism, have yet to be fully elucidated. We have previously demonstrated mitochondrial dysregulation in human adipose tissue (AT), in both centrally obese and diabetic patients. HIV infection is associated with lipodsytrophy and accumulation of central AT. Therefore, we investigated the effect of HIV status, in addition to antiretroviral therapy (ARV), in a number of mitochondrial and nuclear encoded genes involved in mitochondrial metabolism and energy homeostasis.

Materials and Methods: Subcutaneous fat biopsy samples were obtained from 10 controls and 18 HIV subjects (HIV: BMI: 24.3 (mean \pm SD) $\pm 3.2 \mathrm{~kg} /$ $\mathrm{m}^{2}$; Age: $37.5 \pm 10.2 \mathrm{yrs}$; Control: BMI: $26.7 \pm 4.5 \mathrm{~kg} / \mathrm{m}^{2}$; Age: $35.5 \pm 11.4 \mathrm{yrs}$ ) pre- and post- 6 month ARV therapy. Total RNA was extracted, reverse transcribed, and underwent Quantitative Real-Time PCR analysis assessing the following genes: PPAR $\gamma$ co-activator 1 (PGC-l $\alpha$ ), UCP2, UCP3, COX4, ND1 \& 4 (NADH dehydrogenase subunits 1 \& 4), hCS, NRF-1 (nuclear respiratory factor-1), and TFAM (mitochondrial transcription factor A). All reactions were multiplexed with the housekeeping gene $18 \mathrm{~S}$, and statistical analysis was carried out by student $\mathrm{t}$-test.

Results: Our findings determined that in human adipose tissue, following ARV treatment, there was a significant increase in expression of COX4 (Control: $\Delta \mathrm{Ct} 9.58 \pm(\mathrm{SEM}) 0.14$; Post 6 months ARV: $\Delta \mathrm{Ct} 8.32 \pm 0.22, \mathrm{p}=0.0001)$, NRF-1 (Control: $\Delta \mathrm{Ct} 18.56 \pm 0.11$; Post 6 months ARV: $\Delta \mathrm{Ct} 17.95 \pm 0.13, \mathrm{p}=0.0015$ ), PGC-1 $\alpha$ (Control: $\Delta$ Ct $20.43 \pm 0.24$; Post 6 months ARV: $\Delta$ Ct $19.75 \pm 0.19$, $\mathrm{p}=0.04$ ), TFAM (Control: $\Delta \mathrm{Ct} 16.30 \pm 0.10$; Post 6 months ARV: $\Delta \mathrm{Ct}$ $15.58 \pm 0.12, \mathrm{p}=0.00011)$, and human citrate synthase $(\Delta \mathrm{Ct} 14.98 \pm 0.16$; Post 6 months ARV: $\Delta$ Ct $13.96 \pm 0.18, p=0.0003)$. ND4 was increased in HIV naive patients compared with non HIV controls, (Control $\Delta \mathrm{Ct} 18.19 \pm 0.16$; Time 0 ARV: $\Delta$ Ct $17.24 \pm 0.26, p=0.008$ ), and increased further following treatment (Post 6 months ARV: $\Delta$ Ct $15.17 \pm 0.33, \mathrm{p}<0.0001$ ). Additionally, HIV naïve patients compared with post 6 month treatment showed a significant increase in TFAM ( $\mathrm{p}=0.009)$, UCP2 $(\mathrm{p}=0.03), \mathrm{hCS}(\mathrm{p}=0.01)$, however no effect was observed with either ND1 or UCP-3 between controls and HIV naïve or treated patients.

Conclusion: In summary, this study highlights that antiretroviral therapy significantly upregulates expression of genes involved in mitochondrial metabolism and regulation of energy homeostasis, and therefore improves overall mitochondrial function in HIV patients. This class of antiretroviral therapy may, therefore, have additional benefits to the HIV patient by improving oxidative phosphorylation, leading to increased sensitivity and lipid metabolism.

Supported by the Department of Health (UK) 


\section{1}

Supplementation with complex from mussels ameliorated oxidative processes in streptozotocin-induced diabetic rat's liver mitochondria V. V. Poltorak ${ }^{1}$, A. Shumeyko ${ }^{2}$, T. Ovsyannikova ${ }^{2}$, N. Krasova ${ }^{1}$, A. Gladkih ${ }^{1}$, O. Bityutska ${ }^{3}$, M. Gorshunska ${ }^{4}$;

${ }^{1}$ Experimental Endocrinology Department, Institute of Endocrine Pathology Problems, Kharkiv, ${ }^{2}$ Department of Biological and Medical Physics, Karazin Kharkiv National University, Kharkiv, ${ }^{3}$ Biology Department, South Institute of Marine Fishing and Oceanography, Kerch, ${ }^{4} \mathrm{Chair}$ of Endocrinology, Kharkiv Postgraduate Medical Academy, Kharkiv, Ukraine.

Background and Aims: The transport of glucose from the blood into the liver is insulin-independent process and there are not obvious causes for the decrease of oxidative phosphorylation in liver under streptozotocininduced diabetes. Mitochondria play not only a crucial role in cellular energy production, but also participate in reactive oxygen species (ROS) generation. Oxidative stress has been implicated in the etiology of diabetes mellitus and diabetic complications. We recently demonstrated antioxidant and hepatoprotective activities of extract from mussels, which include 20 amino acids, aminosaccharides, sialic acids, mucopolysaccharides, and micronutrients. The aim of the study was to investigate the effects of mussels extract supplementation on oxidative phosphorylation and glutathione peroxidase activity in liver mitochondria of the rat with streptozotocininduced diabetes.

Materials and Methods: 21 Wistar rats were randomized into 3 groups (7 animals each). Diabetes was induced by a single injection of streptozotocin (STZ, $45 \mathrm{mg} / \mathrm{kg}$ b.w. i.p.) - the $1^{\text {st }}$ and the $2^{\text {nd }}$ group. Rats of the $2^{\text {nd }}$ group received mussel extract $\left(0,4 \mathrm{mg} / \mathrm{kg}\right.$ b.w. per os) within $2^{\text {nd }}-25^{\text {th }}$ day of the experiment. Rats of the $1^{\text {st }}$ and the $3^{\text {rd }}$ group (controls) received placebo. Mitochondria were isolated from liver homogenates; there were measured state 3 and 4 of the respiration rates (substrates: malate+glutamate and succinate), respiratory control ratio, glutathione peroxidase (GSH-Px) activity.

Results: GSH-Px activity was significantly increased in mitochondria of the diabetics rats ( $1^{\text {st }}$ group: $91.94 \pm 5.42$ vs $50.40 \pm 6.07 \mathrm{nmol} \mathrm{NADPH} / \mathrm{mg}$ protein/ min, $\mathrm{p}<0.01)$ as compared to controls. The supplementation with mussel extract resulted in decrease of GSH-Px in diabetic rats $(63.80 \pm 4.55, \mathrm{p}<0.05)$. State 3 of the respiration rate (with malate as substrate) was increased in the $1^{\text {st }}$ group as compared to controls and the $2^{\text {nd }}$ group ( $42.25 \pm 4.13$ vs $61.07 \pm 5.95$ and $74.54 \pm 1.58$ nanoatom $\mathrm{O} / \mathrm{mg}$ protein $/ \mathrm{min}$, respectively, $\mathrm{p}<0.05)$. There were not significant changes in the state 3 and 4 of the respiration rates in all studied groups when succinate was used as substrate. The respiratory control ratio was enhanced in the $2^{\text {nd }}$ group when malate was used as substrate.

Conclusion:Our data suggest that ROS production was activated in STZdiabetic rats, and that resulting in GSH-Px activity elevation. NADHoxidize complex (I) was more vulnerable in STZ-diabetic rats, than the other complexes of the respiratory chain. The oral administration of mussel extract to diabetic rats resulted in normalizing of respiratory chain and antioxidative defense activity. It could associate with mussel extract composition - the large amount of amino acids that are precursors of the ubiquinone and glutathione.

\section{2}

Muscle mitochondrial dysfunction and reduced beta cell function in patients with previous acromegaly

J. M. Szendroedi ${ }^{1,2}$, E. Zwettler ${ }^{3,4}$, A. I. Schmid ${ }^{5,2}$, M. Chmelik ${ }^{5,2}$, G. Pacini ${ }^{6}$, G. Kacerovsky ${ }^{1}$, O. Wagner ${ }^{7}$, C. Schnack ${ }^{8}$, G. Schernthaner ${ }^{8}$, K. Klaushofer ${ }^{3,4}$, M. Roden ${ }^{1,2}$;

${ }^{1}$ Hanusch Hospital, Dept. Intern. Med. 1, Vienna, Austria, ${ }^{2}$ Hanusch Hospital, Karl-Landsteiner Institute for Endocrinology and Metabolism, Vienna, Austria, ${ }^{3}$ Hanusch Hospital, Dept. Intern. Med. 4, Vienna, Austria, ${ }^{4}$ Hanusch Hospital, Ludwig Boltzmann Institute of Osteology, Vienna, Austria, ${ }^{5}$ Med. Univ. Vienna, MR Centre of Excellence, Vienna, Austria, ${ }^{6}$ National Research Council, Institute of Biomedical Engineering ISIB-CNR, Padova, Italy, ${ }^{7}$ Medical University Vienna, Dept. of Medical and Chemical Laboratory Diagnostics, Vienna, Austria, ${ }^{8}$ Rudolfstiftung Hospital, Dept. Intern. Med. 1, Vienna, Austria.

Background and Aims: Mitochondrial gene mutations lead to mitochondrial diabetes with defects of insulin secretion and sensitivity. Mitochondrial dysfunction is also involved in common insulin resistance, but secondary metabolic events may be confounding the metabolic picture in overt type 2 diabetes. Acromegaly is associated with alterations of energy expenditure and insulin action and might serve as a model to investigate the role of mitochondrial function. Thus, we examined glucose metabolism, intracellular fat deposition and mitochondrial function in patients with a history of acromegaly (AM).

Materials and Methods: Six AM ( $4 \mathrm{f} / 2 \mathrm{~m}$, age: $49 \pm 10$ years, body mass index, BMI: $27 \pm 3 \mathrm{~kg} / \mathrm{m}^{2}$ ) with an at least 7-years history of successful treatment and age-/BMI-matched healthy volunteers (CON: $3 \mathrm{f} / 3 \mathrm{~m}, 43 \pm 12$ years, $26 \pm 4 \mathrm{~kg} /$ $\mathrm{m}^{2}$ ) were studied. Insulin sensitivity (OGIS) and first-phase insulin secretion were assessed from the frequently sampled OGTT (insulinogenic index, ISEC). Fasting mitochondrial function was assessed from ATP synthetic flux (fATP) using ${ }^{31} \mathrm{P}$ magnetic resonance spectroscopy (MRS) of calf muscle. Intracellular lipid contents of tibialis anterior (IMCLt) and soleus muscles (IMCLs) as well as liver (HCL) were measured with ${ }^{1} \mathrm{H}$ MRS.

Results: IGF-1 did not differ between groups (AM: $177 \pm 88 \mathrm{ng} / \mathrm{ml}$; CON: $145 \pm 51 \mathrm{ng} / \mathrm{l})$. Fasting plasma glucose was $\sim 16 \%$ higher in AM than in CON ( $99 \pm 8$ vs. $85 \pm 6 \mathrm{mg} / \mathrm{dl}, \mathrm{p}<0.05$ ), OGIS was comparable (395 \pm 74 , CON: $415 \pm 14$ $\left(\mathrm{ml} \cdot \mathrm{min}^{-1} \cdot \mathrm{m}^{-2}\right)$, whereas ISEC was $\sim 87 \%$ lower in AM $(0.9 \pm 0.9$, CON: $6.7 \pm 4.3$ $\mathrm{pmol} / \mathrm{mmol}, \mathrm{p}<0.05)$. fATP was $\sim 22 \%$ lower in AM ( $10.1 \pm 1.5$ vs. $12.9 \pm 2.4$ mmol. $\left.l^{-1} \cdot \mathrm{min}^{-1}, \mathrm{p}<0.05\right)$ and related positively to ISEC $(\mathrm{r}=0.687, \mathrm{p}<0.01)$. IMCLt, IMCLs and HCL were not different between groups. IMCLs related negatively to insulin sensitivity $(\mathrm{r}=-0.745, \mathrm{p}=0.005)$.

Conclusion: Patients with a history of acromegaly exhibit reduced $\beta$ cell and muscle mitochondrial function despite normal insulin sensitivity. Previous long-term exposure to high growth hormone and IGF concentrations and/ or chronic increases in plasma glucose concentrations could lead to similar abnormalities in both tissues.

Supported by the Austrian Science Foundation 


\section{PS 68 Carbohydrate metabolism - glucose transport}

\section{3}

Dapagliflozin (BMS-512148), a selective SGLT2 inhibitor, inhibits glucose resorption and reduces fasting glucose in patients with type 2 diabetes mellitus

B. Komoroski, E. Brenner, L. Li;

Bristol-Myers Squibb, Princeton, United States.

Background and Aims: The sodium-glucose reuptake cotransporter-2 (SGLT2), located in the proximal tubule, is a major pathway for renal glucose reabsorption. Dapagliflozin (BMS-512148), characterized by a stable C-aryl glucoside structure, is a rationally designed, potent, and selective inhibitor of SGLT2 that is being developed as an oral agent for the treatment of type 2 diabetes and represents a novel therapeutic approach for the treatment of hyperglycemia. This double-blind, placebo (PBO)-controlled, randomized, parallel-group, multiple-dose study assessed the safety, tolerability, pharmacodynamics, and pharmacokinetics of dapagliflozin in 47 patients with T2DM who were drug naive or on a stable dose of metformin for at least 4 weeks.

Materials and Methods: Patients were randomized to receive daily (QD): PBO $(\mathrm{n}=8)$, dapagliflozin $5 \mathrm{mg}(\mathrm{n}=11), 25 \mathrm{mg}(\mathrm{n}=12)$, or $100 \mathrm{mg}(\mathrm{n}=16)$ for 14 days. The amount of glucose excreted in urine was measured over $4 \mathrm{~h}$ intervals following dosing, as well as $24 \mathrm{~h}$ totals. The amount of renally-filtered glucose over the $0-4 \mathrm{~h}$ postdose interval and the amount of glucose excreted in the urine during the same interval were used to calculate the percentage inhibition of renal glucose reabsorption.

Results: Daily doses of 5, 25, or $100 \mathrm{mg}$ dapagliflozin administered alone or with metformin for 14 days appeared to be well tolerated. Exposure to dapagliflozin increased proportionally to the increment in dose. The mean amount of glucose eliminated in the urine (0-24h) after the first dose of 5, 25, and $100 \mathrm{mg}$ dapagliflozin was $45.2,75.3$, and $81.3 \mathrm{~g}$, respectively. Daily glucose excretion was consistent over the 14-day study. There were no apparent changes in body weight, urine volume, or urinary sodium excretion.

A total of 56 adverse events (AEs) were reported in 27 patients: 37 in 20/39 $(51.3 \%)$ who received dapagliflozin, and 19 in $7 / 8(87.5 \%)$ who received placebo. Most AEs were mild to moderate in intensity, although 1 severe case each of constipation and flank pain were reported, both in patients receiving metformin.

Dosing of dapagliflozin over a 14-day period inhibited glucose reabsorption to a somewhat greater extent than a single dose. Over a 14-day period, dapagliflozin at doses $\geq 25 \mathrm{mg}$ reduced renal glucose reabsorption by approximately $40 \%$ (Table 1). Similarly, dapagliflozin decreased fasting serum glucose (FSG) after 14 days of dosing.

Table 1.

\begin{tabular}{lllllll}
\hline & \multicolumn{3}{l}{$\begin{array}{l}\text { Mean \% Inhibition of Renal } \\
\text { Glucose Reabsorption }(\mathbf{0}-\mathbf{4 h})\end{array}$} & \multicolumn{2}{l}{ FSG (mg/dL) } \\
(\% change from Day -2)
\end{tabular}

No stats were done on these values

${ }^{\star} \mathrm{P}<0.05 ;{ }^{\dagger} \mathrm{P}<0.001$.

Conclusions: Dapagliflozin, in daily doses up to $100 \mathrm{mg}$, was shown to be safe when administered to T2DM patients for 14 days.

Patients who received 25 and $100 \mathrm{mg}$ dapagliflozin had increased urinary excretion corresponding to approximately $40 \%$ inhibition of renal glucose reabsorption as compared with baseline.

Based on glucose reabsorption inhibition and reductions in FSG observed in this study, dapagliflozin represents a promising novel treatment for the treatment of T2DM.

Supported by Bristol-Myers Squibb/AstraZeneca
0764

Safety, pharmacokinetics, and pharmacodynamics of Dapagliflozin (BMS-512148), a selective SGLT2 inhibitor, in an ascending, placebo-controlled, single-dose study in healthy adult subjects L. $\mathbf{L i}^{1}$, B. Komoroski ${ }^{1}$, D. Boulton ${ }^{1}$, E. Brenner ${ }^{1}$, N. Vachharajani ${ }^{2}$, D. Kornhauser ${ }^{3}$;

${ }^{1}$ Bristol-Myers Squibb, Princeton, United States, ${ }^{2}$ Dept. of Pharmaceuticals and Agrochemical Development, Advinus Therapeutics, Bangalore, India, ${ }^{3}$ ICON Clinical Research Drug Development Division, Globomax, Ellicott City, United States.

Background and Aims: In humans, the renal sodium-glucose cotransporter2 (SGLT2) is the major pathway by which plasma glucose is almost completely reabsorbed in the proximal tubule of the kidney. Dapagliflozin (BMS-512148), characterized by a stable $\mathrm{C}$-aryl glucoside structure, is a rationally designed, potent, and selective inhibitor of SGLT2 that is being developed as an oral agent for the treatment of type 2 diabetes and represents a novel therapeutic approach for the treatment of hyperglycemia. This study assessed the safety, tolerability, and pharmacokinetics/pharmacodynamics (PK/PD) of single oral doses of dapagliflozin $(2.5$ to $500 \mathrm{mg})$ in fasting healthy subjects and the effect of food on its $\mathrm{PK} / \mathrm{PD}$ parameters.

Materials and Methods: Eight healthy subjects were assigned to each of 8 sequential treatment panels $(2.5,5,10,20,50,100,250$, or $500 \mathrm{mg}$ dapagliflozin or placebo; $\mathrm{N}=64$ ). Each received either a single oral dose of dapagliflozin $(n=6)$ or placebo $(n=2)$ in a 3:1 ratio following an overnight fast. After each dose regimen was found to be safe and well tolerated, the next panel of 8 subjects received the next higher dose of dapagliflozin.

Following a 7 -day washout interval, all subjects in the 250 -mg group returned to the clinical facility and were given a high-fat breakfast approximately 5 minutes prior to an oral dose of $250 \mathrm{mg}$ dapagliflozin or matched placebo. Results were then compared to those obtained following fasting.

Results: Twenty-two adverse events (AEs) occurred in 14 subjects, including 15 AEs in 10/48 (21\%) who received dapagliflozin and 7 AEs in 4/16 (25\%) who received placebo. Most AEs were mild to moderate in intensity. Thirtyeight subjects had 49 laboratory abnormalities, including 11 hematologic and 7 blood chemistry variations and 31 cases of elevated urinary glucose, which were expected based on drug mechanism of action.

In the fasted state, $\mathrm{C}_{\max }$ increased slightly less, and $\mathrm{AUC}_{\text {inf }}$ increased slightly more than proportionally with the dapagliflozin dose.

Median $\mathrm{T}_{\max }$ was delayed by 2.5 hours when $250 \mathrm{mg}$ of dapagliflozin was taken with a high-fat meal compared to the fasted state. Geometric mean $C_{\max }$ was $39 \%$ lower, while $A U C_{\text {inf }}$ was $7 \%$ lower when $250 \mathrm{mg}$ of dapagliflozin was administered with food (Table 1).

Table 1. Summary of Dapagliflozin Pharmacokinetic Parameters for 250-mg Dose Administered to Fasted and Fed Subjects

\begin{tabular}{|c|c|c|c|}
\hline Pharmacokinetic Parameters & $\mathrm{n}$ & Fasted & Fed \\
\hline $\begin{array}{l}\mathrm{C}_{\max }(\mathrm{ng} / \mathrm{mL}) \\
\text { Geometric mean }(\mathrm{CV} \%)\end{array}$ & 5 & $2510(31)$ & $1532(24)$ \\
\hline $\begin{array}{l}\mathrm{AUC}_{\text {inf }}(\mathrm{ng} \cdot \mathrm{h} / \mathrm{mL}) \\
\text { Geometric mean }(\mathrm{CV} \%)\end{array}$ & 5 & $13,337(28)$ & $12,455(31)$ \\
\hline $\mathrm{T}_{\max }(\mathrm{h})$ median (min, max) & 5 & $1.50(1.00,2.00)$ & $4.00(4.00,4.00)$ \\
\hline $\mathrm{T} 1 \frac{1}{2}(\mathrm{~h})$ mean $(\mathrm{SD})$ & 5 & $17.33(19.75)$ & $18.25(15.94)$ \\
\hline \%UR (\%) mean (SD) & 6 & $1.54(0.40)$ & $1.90(0.60)$ \\
\hline CLR (mL/min) mean (SD) & 6 & $4.99(0.91)$ & $6.33(1.19)$ \\
\hline
\end{tabular}

$\mathrm{AUC}=$ area under concentration-time curve; $\mathrm{CLR}=$ renal clearance; $\mathrm{C}_{\max }=$ maximum observed concentration; $\mathrm{T}^{1 / 2}=$ half-life; $\mathrm{T}_{\max }=$ time to $\mathrm{C}_{\max } ; \% \mathrm{UR}=$ urinary recovery.

Conclusions: Single doses of dapagliflozin ranging from 2.5 to $500 \mathrm{mg}$ appeared to be safe and well tolerated. Giving a single 250-mg dose of dapagliflozin 5 minutes after a high-fat breakfast extended $\mathrm{T}_{\max }$, but did not substantially affect the AUC.

Supported by Bristol-Myers Squibb/AstraZeneca 


\section{5}

Safety, pharmacokinetics, and pharmacodynamics of Dapagliflozin (BMS-512148) in an ascending, placebo-controlled, multiple-dose study in healthy adult subjects

E. Brenner, B. Komoroski, D. Boulton, L. Li;

Bristol-Myers Squibb, Princeton, United States.

Background and Aims: Low-affinity sodium-glucose cotransporter-2 (SGLT2) plays a crucial role in glucose reabsorption in the proximal tubule of the kidney. Dapagliflozin (BMS-512148), characterized by a stable Caryl glucoside structure, is a rationally designed, potent, selective inhibitor of SGLT2 being developed as an oral agent for the treatment of type 2 diabetes and represents a novel therapeutic approach for the treatment of hyperglycemia. This study assessed the safety and tolerability of multiple oral doses of dapagliflozin administered to healthy subjects for 14 days. The study also assessed effects on urinary calcium excretion, safety markers in urine and serum, and impact of dosage on renal glucose resorption.

Materials and Methods: Healthy subjects received a 14-day regimen of once-daily oral administration of ascending doses of dapagliflozin $(2.5,10$, $20,50,100 \mathrm{mg}$ ). Each panel of 8 subjects consisted of 6 receiving drug and 2 receiving placebo $(\mathrm{N}=40)$. As each dose was shown safe and well tolerated, the next panel received the next higher dose of dapagliflozin. All subjects received diets that had fixed amounts of calcium and sodium chloride during the study. Serial ECGs were obtained on days 1, 7, and 14 with QT intervals corrected for heart rate (QTc).

Twenty-four-hour urine samples were analyzed for amino acids, calcium, chloride, creatinine, magnesium, oxalate, potassium, phosphate, sodium, uric acid, NAG, and $\beta 2-\mathrm{MG}$.

Results: A total of 24 adverse events (AEs) occurred in 16 subjects: 15 in 11/30 (36.7\%) who received dapagliflozin, and 9 in $5 / 10(50 \%)$ who received placebo. All events were of mild to moderate intensity. The most frequently reported $\mathrm{AE}$ was rash (5 subjects). There were no apparent differences in urinary excretion (absolute or corrected for creatinine) for magnesium, amino acids, calcium, chloride, oxalate, potassium, phosphate, sodium, uric acid, NAG, and $\beta 2$-MG. Dapagliflozin exposure (AUC) increased proportional to the increase in dose. An accumulation index of approximately 1.25 was observed for dapagliflozin upon repeated daily dosing. There was no apparent effect of dapagliflozin on renal safety markers or the QTc interval. The mean daily amount of glucose eliminated in the urine was similar after the first dose and after 14 days of dapagliflozin administration (Figure 1). Cumulative 24-h urine glucose excretion following 2.5 and $10 \mathrm{mg}$ dapagliflozin was approximately $50 \%$ and $70 \%$ of that excreted after doses $\geq 20 \mathrm{mg}$, respectively.

Conclusions: Multiple oral doses of dapagliflozin up to $100 \mathrm{mg} /$ day for 14 days were safe and generally well tolerated.

Doses of 20 to $100 \mathrm{mg}$ dapagliflozin appeared to provide the maximal inhibition of renal glucose resorption. Dapagliflozin is a potent inhibitor of SGLT2 with pharmacokinetic and pharmacodynamic behavior suitable for daily administration in humans.

Supported by Bristol-Myers Squibb/AstraZeneca

\section{6}

Gliclazide significantly increases cellular glucose transport (CGT) - studies in patients with type 2 diabetes mellitus using the lymphocyte model (PBL)

J. Taton, P. Piatkiewicz, A. Czech;

Chair and Department of Internal Medicine and Diabetology, Warsaw Medical University, Poland.

Background and Aims: The structural integration and functional efficiency of cellular glucose transport (CGT) is of great importance, as CGT constitutes one of the basic mechanisms involved in the regulation of blood glucose levels. Impairment of CGT is involved in the pathogenesis of diabetic hyperglycemia and may therefore be regarded as a target for the action of antidiabetic drugs. Studies devoted to this process may create new pharmacotherapeutic

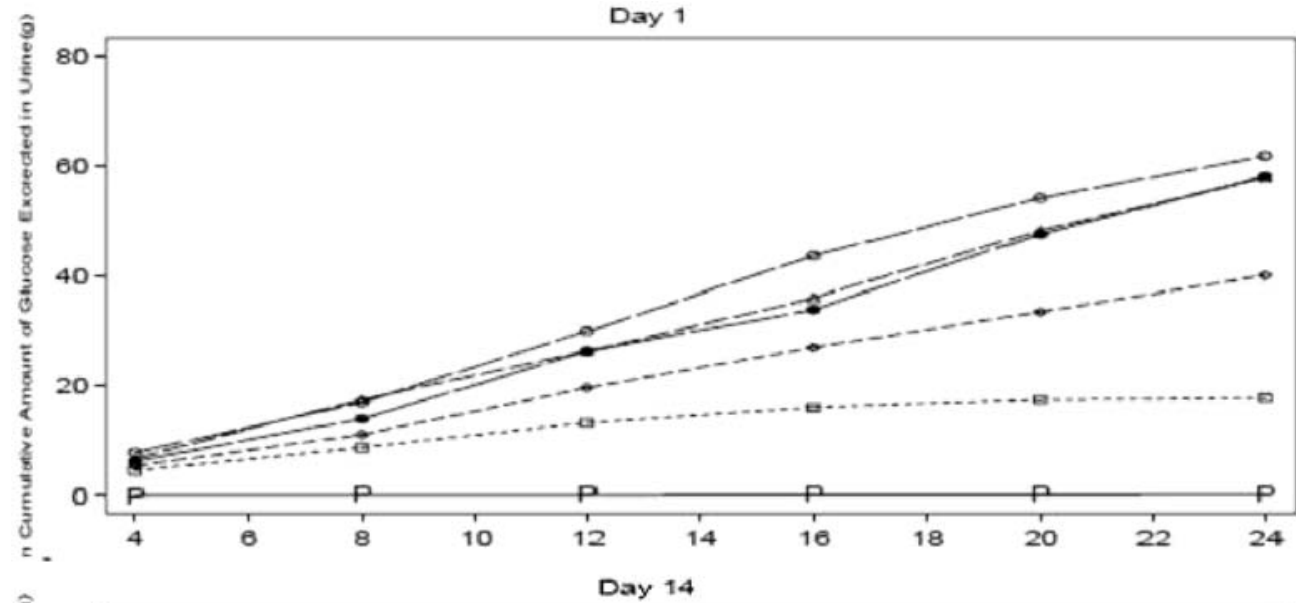

Figure 1. Mean cumulative amount of glucose excreted in urine on day 1 and day 14

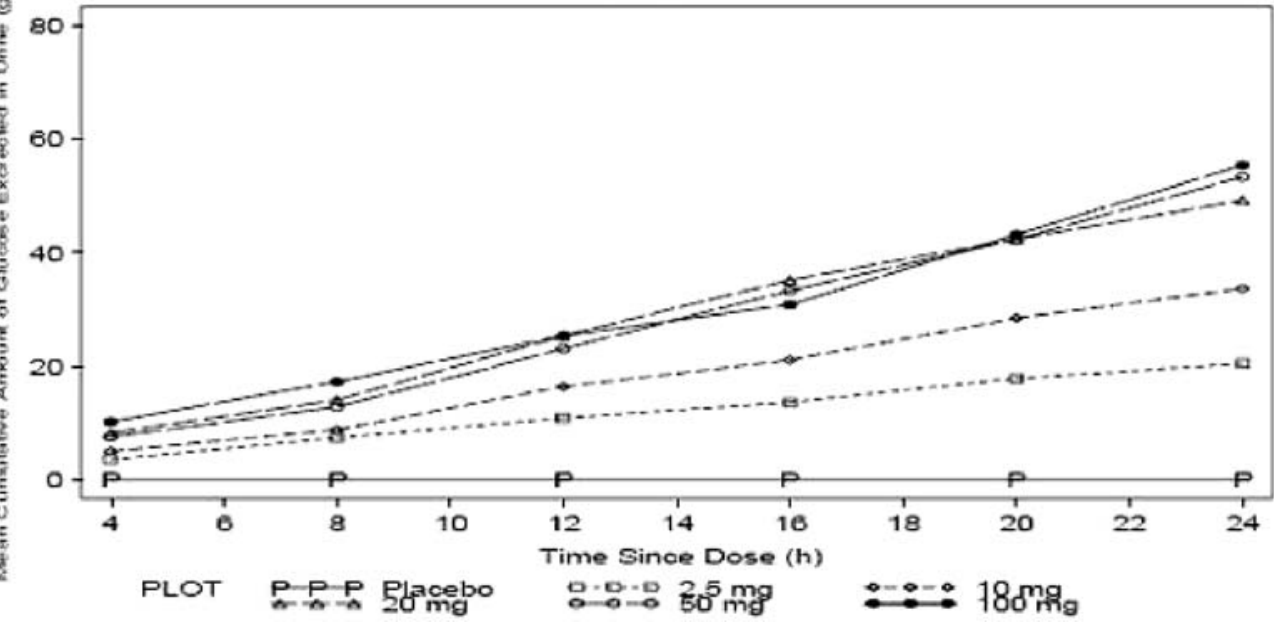


possibilities and interpretations. In order to explore this hypothesis, CGT was studied before and after gliclazide therapy in a group of type 2 diabetic patients who had previously not received any pharmacotherapy, and in a group of healthy subjects.

Materials and Methods: The CGT of peripheral blood lympocytes (PBLs) was assessed by timed incubation of cells with $2-\left[{ }^{3} \mathrm{H}(\mathrm{G})\right]$ glucose in basal conditions, and after the addition of gliclazide (in substantia) or gliclazide plus insulin. Incubation tests were performed at baseline in 28 newly-diagnosed type 2 diabetics and 20 control subjects; in the diabetic patients, the tests were repeated after 3 months of therapy with gliclazide. PBLs were separated from whole blood by Ficoll-Isopaque gradient centifugation. Incubation times were 15, 30 and 60 minutes. The scintillation of PBL lysate was measured with the Wallac 1450 MicroBeta counter.

Results: Treatment with gliclazide resulted in a statistically significant reduction in fasting plasma glucose of $1.77 \mathrm{mmol} / \mathrm{L}(P<0.001)$ and in $\mathrm{HbA}_{1 \mathrm{C}}$ of $0.69 \%(P<0.001)$. The magnitude of CGT in PBLs (pg/300 000 cells) in healthy subjects and in type 2 diabetics before and after 12-week therapy with gliclazide (mean dose at the end of the study, $39.6 \mathrm{mg}$ ) is presented in the table. In all samples of PBLs, a significant increase was found in CGT as a result of treatment with gliclazide.

\section{Table}

\begin{tabular}{lllll}
\hline Incubation model & $\begin{array}{l}\text { Time } \\
(\mathrm{min})\end{array}$ & $\begin{array}{l}\text { Healthy } \\
\text { subjects }\end{array}$ & $\begin{array}{l}\text { Type 2 diabetics } \\
\text { before therapy } \\
\text { with gliclazide }\end{array}$ & $\begin{array}{l}\text { Type 2 diabetics } \\
\text { after 12 weeks } \\
\text { of therapy with } \\
\text { gliclazide } \\
\text { Mean }\end{array}$ \\
\hline PBL without & 15 & 123.1 & 106.4 & 162.4 \\
additives & 30 & 228.7 & 179.4 & 308.1 \\
& 60 & 442.8 & 263.1 & 543.6 \\
PBL with addition & 15 & 203.6 & 113.5 & 210.3 \\
of gliclazide & 30 & 417.1 & 199.0 & 412.0 \\
& 60 & 664.1 & 316.2 & 727.2 \\
PBL with addition & 15 & 308.8 & 186.4 & 349.3 \\
of gliclazide and & 30 & 647.7 & 293.3 & 689.6 \\
insulin & 60 & 666.7 & 306.0 & 709.7 \\
\hline
\end{tabular}

ALL P VALUES $<0.001$

Conclusions: When studied in the PBL model, CGT was found to be significantly decreased in type 2 diabetes mellitus. It was partially and significantly increased by the addition of gliclazide to the incubation probe, and it was further increased by the addition of insulin to gliclazide. Gliclazide therapy increases the CGT process, as measured in diabetic PBLs. The influence of gliclazide on the CGT process constitutes a new pleiotropic action of this drug, and underlines the importance of CGT as a target of pharmacotherapy in type 2 diabetes mellitus.

Supported by Servier Poland

\section{7}

Evidence for sustained metabolic effects in Zucker diabetic fatty rats treated with GW869682, a sodium-dependent glucose transporter 2 antagonist

W. W. Harrington, N. O. Milliken, J. G. Binz, T. W. Balon, C. D. Smith; GlaxoSmithKline Research, Research Triangle Park, United States.

Background and Aims: Sustained hyperglycemia impairs insulin-stimulated glucose utilization and glycogen synthesis in human and rat skeletal muscle, a phenomenon referred to clinically as glucose toxicity. The kidneys play a major role in the regulation of plasma glucose (PG) levels via reabsorption along the convoluted segment of the proximal tubule through an active carriermediated transport process involving sodium-dependent glucose transporters (SGLTs). This has made SGLTs a target for the treatment of type 2 diabetes. Theoretically, inhibition of SGLTs would decrease glucose reabsorption by the kidney, decreasing PG and thus glucose toxicity. Ultimately, the reduction in glucose toxicity could lead to improvements in insulin resistance. The aim of these studies was to examine the in vivo efficacy of GW869682, an inhibitor of SGLT2, using Zucker Diabetic Fatty (ZDF) rats to model insulin resistance. Material and Methods: In Study 1, 10-week old, glucose-matched animals were dosed for 8 days with vehicle or GW869682 $(10,15$ or $30 \mathrm{mg} / \mathrm{kg})$
1-3 times/day. GW347845, a potent PPAR $\gamma$ agonist, was used as a positive control. Daily blood samples were obtained 2 hours post-dose and PG levels determined. On Day 8 an OGTT was performed; a final blood sample was obtained on Day 9. In Study 2, ZDF rats or their lean counterparts were dosed with vehicle or GW869682 (30 mg/kg twice daily), and the effects on basal and insulin-stimulated skeletal muscle glucose uptake rates were determined using the perfused hindquarter. Data were analyzed by ANOVA with repeated measures model, corrected with Dunnett's and Tukey's tests. Values were considered significant when $\mathrm{p}<0.05$.

Results: All ZDF rats were frankly diabetic when dosing began (mean PG, $470 \pm 10 \mathrm{mg} / \mathrm{dL}$ ). Two hours after the first dose, PG decreased in a dosedependent fashion $(\mathrm{p}<0.01)$. After 2 days of dosing, PG of rats administered GW869682 at $30 \mathrm{mg} / \mathrm{kg}$ b.i.d. were not different from rats treated with the PPAR $\gamma$ agonist. After 8 days of treatment, reductions in PG by dose were: $200 \mathrm{mg} / \mathrm{dL}$ at $10 \mathrm{mg} / \mathrm{kg}$ t.i.d.; $>225 \mathrm{mg} / \mathrm{dL}$ at $15 \mathrm{mg} / \mathrm{kg}$ b.i.d. or $30 \mathrm{mg} / \mathrm{kg}$ b.i.d. and t.i.d. ( $\mathrm{p}<0.01$ vs. vehicle). $\mathrm{HbA}_{1 \mathrm{c}}$ levels were also decreased $>1.5 \%$ at the higher doses. Rats treated with vehicle or GW869682 $10 \mathrm{mg} / \mathrm{kg}$ b.i.d. were unable to dispose of a glucose load during an OGTT after 120 minutes. ZDF rats administered GW869682 15 or $30 \mathrm{mg} / \mathrm{kg}$ b.i.d. disposed of the glucose load more rapidly than rats treated with vehicle $(\mathrm{p}<0.01)$ or GW347845 ( $<0.05)$. Eighteen hours after the final dose, PG had increased in a dose-related manner; however, all rats treated with GW869682 showed significantly smaller incremental increases in PG compared with vehicle ( $\mathrm{p}<0.05)$. Dosing GW869682 for 7 days had no effect on basal glucose uptake or insulin responsiveness in perfused ZDF hindquarter; however, insulinstimulated glucose uptake was enhanced $\sim 2.5$-fold vs. vehicle after 14 days of treatment $(\mathrm{p}<0.05)$.

Conclusion: Acute administration of an SGLT2 antagonist rapidly lowers PG in ZDF rats. Chronic administration may decrease glucotoxicity, resulting in long-term improvement in insulin-stimulated glucose disposal.

\section{8}

Lower reduction in free fatty acids in diabetic morbidly obese persons than the non-diabetic and controls during the frequently sampled intravenous glucose tolerance test

J. M. Garcia-Almeida ${ }^{1}$, E. Garcia-Fuentes ${ }^{2}$, S. Garcia-Serrano ${ }^{2}$, J. Garcia-Arnes ${ }^{3}$, J. Rivas-Marinn', J. Gallego-Perales ${ }^{5}$, B. Gonzalez ${ }^{6}$, F. Vilches ${ }^{1}$, B. Martinez-Alfaro ${ }^{1}$, J. Garcia-Aleman ${ }^{1}$, F. Tinahones ${ }^{1}$; ${ }^{1}$ Endocrinology, H. Virgen de la Victoria, ${ }^{2}$ Endocrinology, Ciber Fisiopatologia Obesidad y Nutricion (CB06/03), Instituto de Salud Carlos III, ${ }^{3}$ Endocrinology, H. Carlos Haya, ${ }^{4}$ Surgery, H. Virgen de la Victoria, ${ }^{5}$ Surgery, H. Carlos Haya, ${ }^{6}$ Psicology, H. Virgen de la Victoria, Malaga, Spain.

Background and Aims: Insulin sensitivity and free fatty acids (FFA) are increased in obese persons. The aims of this study are to assess the response of the FFA during a FSIGTT and its association with insulin sensitivity.

Materials and Methods: The study was undertaken in 38 morbidly obese persons before and seven months after undergoing bariatric surgery and in 12 healthy, non-obese persons. All persons underwent a FSIGTT and measurements of the concentration of FFA.

Results: The rate of decline of the concentration of FFA during the FSIGTT $\left(\mathrm{K}_{\mathrm{FFA} 1}\right)$ was significantly greater in the controls $(1.83 \pm 0.92)$ and in the morbidly obese persons with normal fasting glucose (NFG) (1.16 \pm 0.41$)$ than in the morbidly obese persons with impaired fasting glucose (IFG) $(0.97 \pm 0.29)$ or type 2 diabetes mellitus $(\mathrm{DM})(0.47 \pm 0.31)(\mathrm{P}<0.05)$. SI and glucose tolerance index $\left(\mathrm{K}_{\mathrm{G}}\right)$ were significantly reduced in the three groups of morbidly obese patients $(\mathrm{P}<0.05)$. The $\mathrm{K}_{\mathrm{FFA} 1}$ correlated significantly with glucose, insulin, SI and KG. Of all these, $\mathrm{K}_{\mathrm{G}}$ was the variable that was most significantly associated with $\mathrm{K}_{\mathrm{FFA} 1}$ before surgery $(\mathrm{P}=0.012)$. These variables improved after bariatric surgery in all the study groups. The $\mathrm{K}_{\mathrm{FFA} 1}$ in the morbidly obese persons was similar to that of the controls. The $\mathrm{K}_{\mathrm{FFA}}$ correlated significantly with the SI and KG, The variables that best explained the $\mathrm{K}_{\mathrm{FFA}}$ in a multiple linear regression model were $\mathrm{SI}(\mathrm{P}<0.001)$ and FFA $(\mathrm{P}=0.035)$.

Conclusion: During the FSIGTT, morbidly obese persons with IFG or DM experience a lower reduction in FFA than the NFG and controls, probably as a result of less inhibition of lipolysis and a reduced re-uptake of the FFA by the tissues. After bariatric surgery, the evolution of the FFA curve during the FSIGTT was similar to that of the controls. Supported by CB06/03/0018 


\section{9}

Possible role of alpha cell insulin resistance in exaggerated glucagon responses to arginine in type 2 diabetes

A. Shimizu, N. Tsuchiyama, H. Ando, K.-I. Kato, S. Kurita, M. Sakurai, S. Kaneko, T. Takamura;

Department of Disease Control and Homeostasis, Kanazawa University Graduate School of Medical Science, Kanazawa, Japan.

Background and Aims: Diabetes is associated with increased hepatic glucose production, which is linked to fasting and postprandial hyperglycemia. This is caused by the reduced suppression of glucagon along with the impairment of insulin secretion and insulin action. Inappropriate excessive secretion of glucagon is, therefore, a novel target for the treatment of diabetes. However, a valid way of identifying responders to such therapy has not yet been established. In this study, we sought to determine the factors associated with exaggerated glucagon secretion in response to an arginine challenge in patients with type 1 and type 2 diabetes.

Materials and Methods: Changes in circulating C-peptide immunoreactivity (CPR) and immunoreactive glucagon (IRG) after an arginine challenge were investigated in 35 patients with type 1 diabetes, 130 with type 2 diabetes and 35 with normal glucose tolerance. Arginine ( $30 \mathrm{~g}$ ) was administered by intravenous infusion of an L-arginine hydrochloride $10 \%$ solution over $30 \mathrm{~min}$.

Results: No significant differences were found in the basal level and the area under the concentration-time curve (AUC) of IRG (AUC ${ }_{\mathrm{IRG}}$ ) among between type 1 and type 2 diabetic patients and non-diabetic subjects. However, as shown in Figure 1, the correlation between $\mathrm{AUC}_{\mathrm{IRG}}$ and AUC of $\mathrm{CPR}\left(\mathrm{AUC}_{\mathrm{CPR}}\right)$ was inverse between type $1(\mathrm{r}=-0.388, P=0.023)$ and type 2 diabetic patients $(\mathrm{r}=0.396, P<0.0001)$, whereas $\mathrm{AUC}_{\mathrm{IRG}}$ was not correlated with $\mathrm{AUC}_{\mathrm{CPR}}$ in non-diabetic subjects $(\mathrm{r}=-0.079, P=0.655)$.
Type 1 diabetes

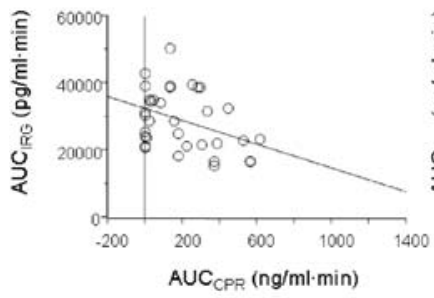

$\mathrm{AUC}_{\mathbb{R} \mathrm{RO}}=32,462-17.8 \mathrm{AUC}_{\mathrm{CPR}}$ $r=-0.388, P=0.023$
Type 2 diabetes

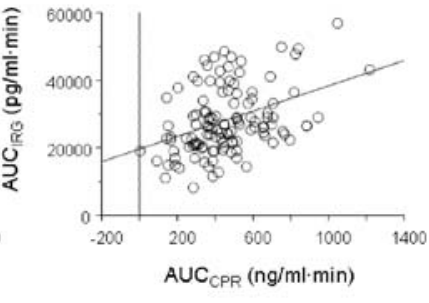

$A_{U U C}=19,418+18.8$ AUC $_{\mathrm{CPR}}$ $r=0.396, P<0.0001$
Fig. 1. The relationship between $A U C^{I R G}$ and $A U C^{C P R}$ in patients with type 1 and type 2 diabetes. AUC ${ }^{\mathrm{IRG}}$ was negatively correlated with $\mathrm{AUC}^{\mathrm{CPR}}$ in type 1 diabetes $(r=-0.388, P=0.023)$, whereas $A U C^{I R G}$ was positively correlated with $\mathrm{AUC}{ }^{\mathrm{CPR}}$ in patients with type 2 diabetes $(\mathrm{r}=0.396$, $P<0.0001$ )

In type 1 diabetic patients, the $\mathrm{AUC}_{\mathrm{CPR}}$ decreased and the $\mathrm{AUC}_{\mathrm{IRG}}$ increased with increasing disease duration. In type 2 diabetic patients, both $\mathrm{AUC}_{\mathrm{IRG}}$ and $\mathrm{AUC}_{\mathrm{CPR}}$ increased with increasing levels of BMI, basal CPR level (Figure 2), and HOMA-R.
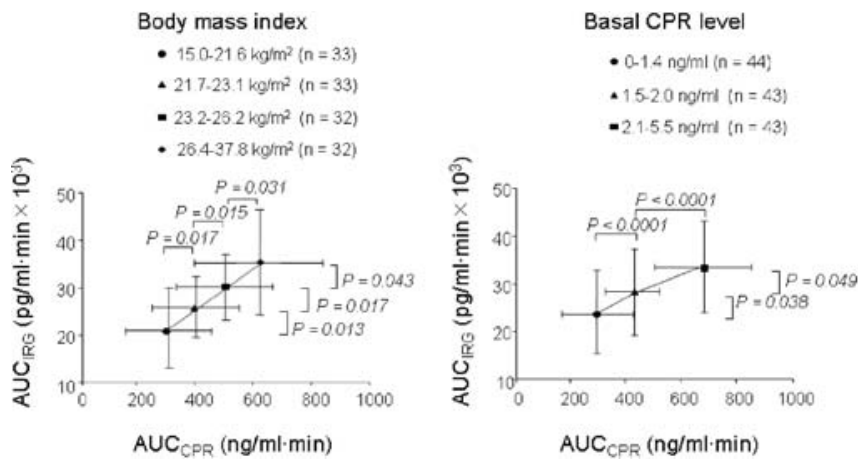

Fig. 2. The relationship between the means of AUC ${ }^{I R G}$ and AUC ${ }^{C P R}$ stratified by the class of body mass index and basal CPR level in patients with type 2 diabetes. The AUC ${ }^{\mathrm{IRG}}$ and $A \mathrm{AC}^{\mathrm{CPR}}$ significantly increased according to the increase of both body mass index and basal CPR level. Data are shown as mean values; error bars denote SD.
In non-diabetic subjects, $\mathrm{AUC}_{\mathrm{IRG}}$ was not correlated with $\mathrm{AUC}_{\mathrm{CPR}}$.

Conclusion: Our findings suggest that the pathophysiology of the exaggerated glucagon response differs between the types of diabetes. Intra-islet insulin deficiency and alpha cell insulin resistance may be the primary contributors to this condition in type 1 and type 2 diabetes, respectively. In addition, the arginine challenge test could be useful for assessing the heterogenous nature of diabetes and may be a valid method for identifying responders to therapy targeted at glucagon and its receptor, such as GLP-1 analogs.

\section{0}

Vascular endothelial growth factor increases during hypoglycaemia in patients with type 1 diabetes, independently of renin angiotensin system activity

L. G. Bie-Olsen ${ }^{1}$, P. L. Kristensen ${ }^{1}$, T. Høi-Hansen ${ }^{1}$, F. Boomsma ${ }^{2}$

U. Pedersen-Bjergaard ${ }^{3}$, B. Thorsteinsson ${ }^{1}$;

${ }^{1}$ Division of Internal Medicine I, Nordsjællands Hospital - Hillerød, Hilleroed, Denmark, ${ }^{2}$ Department of Internal Medicine, Erasmus MC, Rotterdam, The Netherlands, ${ }^{3}$ Department of Internal Medicine I, Bispebjerg Hospital, Copenhagen, Denmark.

Background and Aims: In healthy adults, levels of vascular endothelial growth factor (VEGF) rise significantly in response to hypoglycaemia. The rise is possibly positively correlated with preservation of cognitive function during hypoglycaemia. VEGF is implicated in glucose transport over the blood brain barrier, most likely due to translocation of the glucose transporter 1 (GLUT1) to the plasma membrane. High activity in the renin angiotensin system (RAS) is associated with increased risk of severe hypoglycaemia in patients with type 1 diabetes and a more pronounced deterioration of cognitive function during hypoglycaemia compared to patients with low RAS activity. RAS possibly exerts its mechanism in hypoglycaemia via VEGF. We studied the impact of mild hypoglycaemia on plasma levels of VEGF in patients with type 1 diabetes and high or low RAS activity and compared VEGF levels to cognitive functioning.

Materials and Methods: Nine patients with high and nine with low RAS activity, as classified by ACE activity, angiotensinogen concentration and genotype of the angiotensin 2 receptor subtype 2 underwent a single-blinded, placebo-controlled, cross-over study with either mild hypoglycaemia (intravenous insulin, 100\% of morning dose/hour) or stable glycaemia (intravenous glucose and insulin dependent on blood glucose; control study). Cognitive function was assessed by California Cognitive Assessment Package and Alzheimer Quick Test.

Results: Nadir plasma glucose was $2.3(0.3) \mathrm{mmol} / \mathrm{l}$ (mean (SD)) in the high RAS group and $2.5(0.2) \mathrm{mmol} / \mathrm{l}$ in the low RAS group $(\mathrm{p}=0.16)$. During the control study, p-VEGF did not change in either RAS-group. In high RAS group p-VEGF increased from 54 (34) pg/l to 93 (88) pg/l (42\% increment) ( $\mathrm{p}=0.008)$ and in low RAS group from 69 (26) pg/l to 104 (51) $\mathrm{pg} / \mathrm{l}(51 \%$ increment $)(\mathrm{p}=0.017)$. The increase did not differ between RAS

\section{p-VEGF during "hypo" day in the two RAS groups}

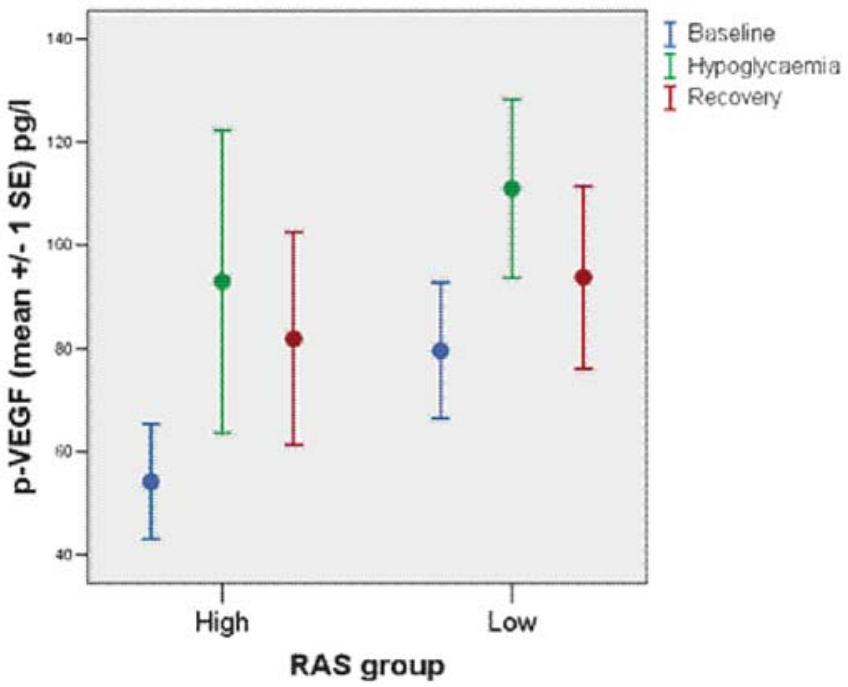


groups ( $\mathrm{p}=0.74$ ). The VEGF response was not correlated with the degree of deterioration of cognitive function during hypoglycaemia.

Conclusion: Plasma VEGF levels increase during mild, short-term hypoglycaemia in patients with type 1 diabetes. The mechanism behind this phenomenon is unknown. In this study, in contrast to a study with prolonged hypoglycaemia among healthy adults, the VEGF response was not correlated to deterioration of cognitive function during hypoglycaemia. The VEGF response was equal in both RAS groups. Hence, the association between low RAS activity and better cognitive performance during hypoglycaemia, does not seem to be mediated by VEGF.

Funded by grants from EFSD/JDRF/Novo Nordisk Programme for Research in Type 1 Diabetes, Foundation of Harald Jensen and wife, Foundation of Olga Bryde Nielsen, Foundation of Region 3, Research Foundation of Frederiks

\section{1}

Regulation of the insulin degrading enzyme in hepatocytes in normal and high glucose conditions

O. Pivovarova ${ }^{1,2}$, N. Rudovich ${ }^{1,2}$, I. Goering ${ }^{1}$, M. Osterhof ${ }^{1}$,

A. F. H. Pfeiffer ${ }^{1,2}$;

${ }^{1}$ Dept. of Clinical Nutrition, German Institute of Human Nutrition

Potsdam-Rehbruecke, Nuthetal, ${ }^{2}$ Dept. of Endocrinology and Nutrition,

Campus B. Franklin, Charite University Medicine of Berlin, Germany.

Background and Aims: The liver is the main site of insulin clearance removing approximately $75 \%$ during the first portal passage. Type 2 diabetes is characterized by decreased insulin clearance. Insulin-degrading enzyme (IDE) is thought to play a key role in insulin degradation. Little is known about IDE regulation in liver, however in brain IDE was shown to be a downstream target of the insulin signaling cascade. The aim of this study was to investigate the IDE regulation by insulin in hepatocyte cell culture.

Materials and Methods: Human hepatoma HepG2 cells were incubated in Dulbecco's modified Eagle's medium (DMEM) containing $10 \%$ fetal calf serum (FCS), $10 \mu \mathrm{g} / \mathrm{ml}$ tavanic and either normal $(5,5 \mathrm{mM})$ or high $(25 \mathrm{mM})$ concentrations of D-glucose (insulin resistance model). For the experiment cells were maintained in DMEM containing 2\% FCS for $24 \mathrm{~h}$ and stimulated with $0,1,1,10,100$ and $200 \mathrm{nM}$ insulin for $24 \mathrm{~h}$. Then total RNA or total cell lysate were prepared. The IDE mRNA level was determined by real-time PCR, the IDE protein level - by western blotting.

Results: We observed that insulin treatment of HepG2 cells maintained in normal glucose medium decreased IDE mRNA levels by 15-20\% with all insulin concentrations used in comparison to control. High glucose concentrations induced a 2-fold increase of IDE expression in the absence of insulin and altered the insulin effect: in high glucose conditions the IDE level was significantly increased after $24 \mathrm{~h}$ of insulin stimulation. Maximal increase ( 25\%) was observed with $10 \mathrm{nM}$ insulin. The results of real-time PCR were confirmed by western blotting.

Conclusion: In hepatocytes insulin downregulated the IDE level. High glucose concentrations significantly increased the IDE expression and inversed the regulation of IDE by insulin. The observed changes of IDE expression may contribute in the regulation of insulin degradation and pathogenesis of type 2 diabetes.

\section{PS 69 Iron / anaemia}

\section{2}

Insulin sensitivity and beta cell function in patients with hereditary haemochromatosis

M. Hatunic ${ }^{1}$, F. M. Finucane ${ }^{1}$, S. Norris ${ }^{2}$, G. Pacini ${ }^{3}$, J. J. Nolan ${ }^{1}$;

${ }^{1}$ Metabolic Research Unit, St. James's Hospital, Dublin, Ireland,

${ }^{2}$ Hepatology Department, St. James's Hospital, Dublin, Ireland,

${ }^{3}$ Metabolic Unit, ISIB-CRN, Padova, Italy.

Background and Aims: Diabetes affects $30-60 \%$ of patients with haemochromatosis. The underlying pathophysiology of diabetes in patients with haemochromatosis has not been fully elucidated. Initially thought to relate to iron induced beta cell failure, in reality many patients have features of insulin resistance. We prospectively studied the effects of iron overload on glucose metabolism in newly diagnosed patients with hereditary haemochromatosis $(\mathrm{HH})$.

Materials and Methods: Fifty-three recently diagnosed $\mathrm{HH}$ patients had a standard 75g-OGTT. Insulin sensitivity at fasting (QUICKI) and during oral glucose tolerance test (OGIS) was evaluated. Beta cell function was assessed as C-peptide concentration during OGTT in relation to ambient to insulin sensitivity.

Results: Fifteen patients (28\%) had abnormal glucose metabolism (AGM), seven (13\%) had type 2 diabetes mellitus (T2DM) and eight (15\%) had impaired glucose tolerance (IGT). Results are shown in the table below, with means \pm standard error of the mean. $\left({ }^{*}\right.$ denotes $\mathrm{p}<0.05$.)

\begin{tabular}{lll} 
& $\begin{array}{l}\text { Normal Glucose } \\
\text { Metabolism }\end{array}$ & $\begin{array}{l}\text { Abnormal } \\
\text { Glucose Metabolism }\end{array}$ \\
\hline $\mathrm{n}$ & $38(72 \%)$ & $15(28 \%)$ \\
Age $(\mathrm{yrs})$ & $46 \pm 1.7$ & $57 \pm 2.3^{*}$ \\
Male $(\%)$ & 71 & 68 \\
$\mathrm{BMI}\left(\mathrm{kg} / \mathrm{m}^{2}\right)$ & $26 \pm 0.8$ & $28 \pm 1.0$ \\
Waist/Hip ratio & $0.92 \pm 0.1$ & $0.99 \pm 0.2^{*}$ \\
Fasting Glucose $(\mathrm{mmol} / \mathrm{l})$ & $4.8 \pm 0.1$ & $6.0 \pm 0.3^{*}$ \\
HbAlc $(\%)$ & $5.4 \pm 0.1$ & $6.0 \pm 0.2^{*}$ \\
Fasting C-peptide $(\mu \mathrm{g} / \mathrm{l})$ & $2.3 \pm 0.1$ & $3.2 \pm 0.3^{*}$ \\
Fasting Insulin $(\mu \mathrm{U} / \mathrm{l})$ & $6.5 \pm 0.4$ & $10.6 \pm 0.9^{*}$ \\
OGIS(ml/min/m $)$ & $451 \pm 9$ & $365 \pm 13^{*}$ \\
QUICKI & $0.7 \pm 0.02$ & $0.5 \pm 0.02^{\star}$ \\
Beta Cell Function Index & $0.7 \pm 0.3$ & $0.6 \pm 0.1$ \\
$\left(\mathrm{nmol} / \mathrm{min} / \mathrm{m}^{2}\right)$ & & \\
Ferritin $(\mu \mathrm{l} / \mathrm{l})$ & $735 \pm 131$ & $1266 \pm 310^{*}$ \\
Transferrin saturation $(\%)$ & $78 \pm 3.8$ & $74 \pm 5.2$ \\
\hline
\end{tabular}

Conclusion: Haemochromatosis patients with AGM had higher fasting insulin and C-peptide levels than normal glucose tolerant controls, with higher waist/hip ratios. Patients with AGM were more insulin resistant at fasting and with OGTT. Our results challenge the traditional view that diabetes in $\mathrm{HH}$ is due primarily to iron induced beta cell failure. Our cohort of $\mathrm{HH}$ patients with AGM in comparison with those with normal glucose metabolism were more insulin resistant, hyperinsulinemic and with visceral obesity, similar to typical T2DM.

\section{3}

Excessive iron deposition in liver parenchyma imposes insulin resistance independent of oxidative stress pathway

C. Y. Yeung ${ }^{1}$, A. P. S. Kong ${ }^{1}$, C. S. $\mathrm{Ho}^{2}$, W. W. M. $\mathrm{Lam}^{3}$, M. $\mathrm{Ng}^{4}$, R. Ozaki ${ }^{1}$, W. Y. So ${ }^{1}$, C. C. Chow ${ }^{1}$, C. W. K. Lam², C. S. Cockram¹, J. C. N. Chan ${ }^{1}$, P. C. Y. Tong';

${ }^{1}$ Department of Medicine and Therapeutics, ${ }^{2}$ Department of Chemical Pathology, ${ }^{3}$ Department of Diagnostic Radiology and Organ Imaging, ${ }^{4}$ Department of Anatomical and Cellular Pathology, The Chinese University of Hong Kong, Hong Kong.

Epidemiological studies suggest that increasing iron body stores is associated with insulin resistance, metabolic syndrome and Type 2 diabetes mellitus. 
Possible mechanisms include direct effect of deposition in liver parenchyma and increase in oxidative stress associated with increase iron metabolism. We examined 50 pre-menopausal Chinese female (mean age $37.7 \pm 9.3$ years) with normal glucose tolerance confirmed by oral glucose tolerance test, normal serum iron saturation and a family history of diabetes in the first degree relatives. Subjects were divided into tertiles according to the serum ferritin level. Subjects in the top tertile (Group I: serum ferritin:363.5 \pm 144.9 $\mathrm{pmol} / \mathrm{L}$ ), when compared with subjects with the lowest tertile (Group III: $49.2 \pm 15.2 \mathrm{pmol} / \mathrm{L})$, had higher BMI $(24.9 \pm 4.7$ vs $20.6 \pm 1.9 \mathrm{~kg} / \mathrm{m} 2, \mathrm{p}<0.01)$, higher waist circumference $(82.4 \pm 12.7$ vs $74.5 \pm 5.3 \mathrm{~cm}, \mathrm{p}=0.03)$, higher percentage of body fat $(32.9 \pm 8.5$ vs $24.7 \pm 4.6 \%$, p $<0.01)$, higher systolic blood pressure (112.5 \pm 16.5 vs $110.0 \pm 9.1 \mathrm{mmHg}, \mathrm{p}=0.01)$, higher diastolic blood pressure $(75.5 \pm 12.7$ vs $66.3 \pm 5.3 \mathrm{mmHg}, \mathrm{p}=0.02)$, higher triglycerides $(1.25 \pm 0.50$ vs $0.74 \pm 0.4 \mathrm{mmol} / \mathrm{L}, \mathrm{p}<0.01)$, higher LDL-cholesterol $(2.94 \pm 0.98$ vs $2.3 \pm 0.55 \mathrm{mmol} / \mathrm{L}, \mathrm{p}=0.03)$, higher hsCRP $(2.79 \pm 3.69$ vs $0.38 \pm 0.4 \mathrm{mg} / \mathrm{L}$, $\mathrm{p}<0.01)$ and insulin resistance (HOMA-IR:12.7 \pm 8.3 vs $6.6 \pm 2.8, \mathrm{p}<0.01$ ). Subjects in Group I had more iron deposition in liver parenchyma as reflected by elevated ALP ( $55.8 \pm 25.9$ vs $44.4 \pm 9.0$ IU/L, p=0.045), ALT ( $26.4 \pm 17.0$ vs $14.8 \pm 4.8 \mathrm{IU} / \mathrm{L}, \mathrm{p}<0.01)$, GGT $(30.4 \pm 2.7 \mathrm{vs} 14.1 \pm 6.7, \mathrm{p}=0.01)$ and assessment of iron load in liver by MRI using reciprocal of T2 relaxation time $(0.039 \pm 0.007$ vs $0.031 \pm 0.003 \mathrm{~s}-1, \mathrm{p}<0.01)$. However, oxidative stress was not significantly different among two groups as measured by plasma isoprostanes $(320.0 \pm 101.1$ vs $328.6 \pm 101.1 \mathrm{pmol} / \mathrm{L}, \mathrm{p}=0.73)$ and membrane isoprostane $(25.5 \pm 6.8$ vs $22.8 \pm 4.3 \mathrm{pmol} / \mathrm{L}, \mathrm{p}=0.18)$. These results suggest that increase in iron load in the body may induce insulin resistance by effect of direct deposition in liver parenchyma without imposing significant oxidative stress. Supported by RGC Earmarked Grant

\section{4}

Iron metabolism parameters and their correlation with adipocytokines in type 2 diabetic patients against the background of metabolic syndrome

N. Krasova ${ }^{1}$, M. Gorshunska ${ }^{2}$, Y. Karachentsev ${ }^{2,1}$, E. Jansen ${ }^{3}$, A. Gladkih ${ }^{1}$, H. Cremers ${ }^{3}$, P. Beekhof ${ }^{3}$, R. v. Loenen ${ }^{3}$, V. Poltorak ${ }^{1}$;

${ }^{1}$ Laboratory for Pathophysiology and Medical Genetics, Institute of Endocrine Pathology Problems, Kharkiv, Ukraine, ${ }^{2}$ Chair of Endocrinology, Kharkiv Postgraduate Medical Academy, Kharkiv, Ukraine, ${ }^{3}$ Laboratory for Toxicology, Pathology and Genetics, National Institute for Public Health and the Environment, Bilthoven, The Netherlands.

Background and Aims: It is known that liver iron levels and expression of genes for iron transport are correlated with obesity in rodent models. Several studies have reported an association between serum ferritin concentration and insulin resistance or type 2 diabetes mellitus (T2DM), and it has been suggested that disturbances of iron metabolism are part of the metabolic syndrome (MS). The aim of the study was to evaluate the relationship of iron metabolism parameters with adipocytokines and inflammatory markers in obese T2DM patients.

Materials and Methods: Thirty one T2DM patients (females/males: 21/10, age $55.6 \pm 1.7$ years, diabetes duration $7.8 \pm 1.2$ years, BMI $31.8 \pm 0.9 \mathrm{~kg} / \mathrm{m}^{2}$, WHR $0.92 \pm 0.02$, fasting blood glucose (FBG) $11.5 \pm 0.4 \mathrm{mmol} / \mathrm{l}, \mathrm{HbA}_{1} \geq 7.0 \%$ ) were included. MS was diagnosed using the NCEP ATP III criteria. Seven healthy volunteers matched for age, sex and BMI served as controls. The studied subjects were assessed at the beginning and after 3 and 6 months periods. Plasma adiponectin, leptin, resistin, TNF- $\alpha$, high sensitive C-reactive protein (CRP), serum iron (Fe), ferritin, transferrin (Tf), percent saturation of Tf (FeTf), unsaturated iron binding capacity (UIBC), haptoglobin (Hp) and fasting insulin levels were determined. Insulin resistance (IR) was calculated using Homeostasis Model Assessment (HOMA). Statistical analysis was performed with ANOVA and the Spearman's rank order (SPSS, version 13.0).

Results: In T2DM patients, value of adiponectin was significantly reduced ( $\mathrm{p}=0.017$ ) whereas levels of Fe, Hp, leptin, TNF- $\alpha$, CRP, ferritin, insulin and IR-HOMA were significantly enhanced $(\mathrm{p}<0.05-0.001)$ compared to the healthy subjects. Serum ferritin was negatively correlated with Tf $(r=-0.266$, $\mathrm{p}=0.021)$. Positively correlated $(r=0.356, \mathrm{p}=0.002)$ both ferritin and Hp were positively associated with FBG $(r=0.309, \mathrm{p}=0.007$ and $r=0.503, \mathrm{p}<0.0005)$, IRHOMA $(r=0.381, \mathrm{p}=0.002$ and $r=0.343, \mathrm{p}=0.007)$ and CRP $(r=0.229, \mathrm{p}=0.049$ and $r=0.334, \mathrm{p}=0.003)$, respectively. It is known, that ferritin synthesis is TNF- $\alpha$-induced, thus ferritin concentration was strong positively correlated with TNF- $\alpha(r=0.400, \mathrm{p}<0.0005)$. We found a significant negative correlation of ferritin and adiponectin $(r=-0.262, \mathrm{p}=0.023)$. Strong negative correlations were revealed between leptin and such iron metabolism parameters as ferritin $(r=-0.312, \mathrm{p}=0.006), \mathrm{Fe}(r=-0.448, \mathrm{p}<0.0005)$, Fe-Tf $(r=-0.519, \mathrm{p}<0.0005)$, however, leptin concentrations were positively associated with Tf $(r=0.316$, $\mathrm{p}=0.006)$ and UIBC $(r=0.479, \mathrm{p}<0.0005)$. Finally, we report for the first time, that there was a strong negative correlation between $\mathrm{Hp}$ and circulating resistin $(r=-0.377, \mathrm{p}=0.003)$ in T2DM patients.

Conclusion: Our data support findings showing that iron metabolism parameters are strong associated not only with classical inflammatory markers, but also with adipocyte-derived hormones levels in type 2 diabetic patients with metabolic syndrome. Further work is required to establish a causative link between change of iron metabolism and the development of diabetic complications against the background of obesity.

\section{5}

Obesity is associated with iron deficiency in men with hyperferritinaemia genetically screened for hereditary haemochromatosis

J.-M. Gonzalez-Clemente ${ }^{1}$, N. Freixenet ${ }^{1,2}$, A. Caixàs ${ }^{1}$, O. Gimenez-Palop ${ }^{1}$, C. Vilardell ${ }^{1}$, F. Blanco-Vaca ${ }^{3}$, A. Remacha ${ }^{4}$, D. Mauricio ${ }^{5}$, C. Cabistañ ${ }^{2}$,

Y. Sánchez ${ }^{2}$, J. Tarrés ${ }^{2}$, V. Bach ${ }^{6}$, M. Baiget ${ }^{6}$, J. Felez ${ }^{2}$;

${ }^{1}$ Department of Diabetes, Endocrinology and Nutrition, Hospital de Sabadell, Sabadell, ${ }^{2} \mathrm{CAP}$ Canaletes, Institut Català de la Salut, Cerdanyola del Vallès, ${ }^{3}$ Servei de Bioquimica and Institut de Recerca, Hospital de Sant Pau, Barcelona, ${ }^{4}$ Hematology Department, Hospital de Sant Pau, Barcelona, ${ }^{5}$ Department of Diabetes, Endocrinology and Nutrition, Hospital Arnau de Vilanova, Lleida, ${ }^{6}$ Department of Genetics and Research Institute, Hospital de Sant Pau, Barcelona, Spain.

Background and Aims: Obesity is central in the pathogenesis of the metabolic syndrome (MS). In addition, an increase in serum ferritin (SF) concentrations has been associated with insulin resistance and the MS and, consequently, a pathogenic role of iron overload has been suggested in these conditions. Serum concentrations of the soluble transferrin receptor (sTfR) is considered a better index of body iron stores than SF, because it is not influenced by inflammatory conditions. Normally, the higher the concentrations of sTfR, the lower the body iron stores are. However, sTfR concentrations are influenced by the H63D mutation of the HFE gene of hereditary hemochromatosis $(\mathrm{HH})$, with lower concentrations in subjects bearing this mutation. Our aim was to evaluate whether iron overload (measured through sTfR) is associated with the components of the MS and insulin resistance or not, in men with hyperferritinemia, taking into account the gene mutations of the HFE gene. Materials and Methods: During 1 year-period, 150 men (age: 54.0 \pm 13.1 yrs), were genetically screened for HH (mutations of the HFE gene: C282Y, H63D, S65C) because of hyperferritinemia (SF > $200 \mu \mathrm{g} / \mathrm{L}$, twice, one month apart). They were evaluated for the components of the MS (ATP III criteria), insulin resistance (HOMA-R), highly-sensitive C-reactive protein (hs-CRP), transferrin saturation index and sTfR.

Results: In the whole group, $s$ TfR correlated positively with BMI $(r=0.173 ; \mathrm{p}=$ $0.046)$ and waist circumference (WC) $(r=0.246 ; p=0.005)$, but with no other other components of the MS, HOMA-R, hs-CRP and HFE gene mutations. Forty-seven subjects were obese (BMI $>30 \mathrm{~kg} / \mathrm{m} 2)$ and had significantly higher levels of triglycerides (median (interquartile range): 146 (85-118) vs $129(66-176) \mathrm{mg} / \mathrm{dl} ; \mathrm{p}=0.010)$, insulin resistance (HOMA-R: $1.81(0.46-2.69)$ vs $1.19(0.41-1.40) ; \mathrm{p}<0.001)$ and sTfR $(2.6(1.53-3.10)$ vs $2.3(1.57-2.73)$ $\mathrm{mg} / \mathrm{L} ; \mathrm{p}=0.014)$ than the non-obese. Also they had lower levels of HDL cholesterol (mean $\pm \mathrm{SD}: 49 \pm 13$ vs $53 \pm 13 \mathrm{mg} / \mathrm{dL} ; \mathrm{p}=0.047$ ), but no differences in HFE gene mutations were found between groups. On a multiple regression analysis sTfR and WC remained positively and independently associated after adjusting for potential confounders $(r=0.290 ; p=0.001)$.

Conclusion: In men with hyperferritinemia, obesity is associated with a moderate degree of iron deficiency, arguing against a role of iron overload in the pathogenesis of the MS.

Supported by Ministerio de Sanidad y Consumo, Instituto de Salud Carlos III, Associació Catalana de Diabetis 


\section{6}

C.E.R.A. twice monthly corrects anaemia and maintains stable $\mathrm{Hb}$ levels in patients with diabetes and CKD not on dialysis

M. F. Bexon ${ }^{1}$, F. C. Dougherty ${ }^{1}$, D. Halimi ${ }^{1}$, R. Beswick ${ }^{2}$;

${ }^{1}$ Hoffmann-La Roche Ltd., Basel, Switzerland,

${ }^{2}$ Hoffmann-La Roche Ltd., Nutley, United States.

Background and Aims: Anaemia is more prevalent and severe in chronic kidney disease (CKD) patients with diabetes than in those without diabetes, and is also associated with progression of diabetic complications. C.E.R.A. is a continuous erythropoietin receptor activator with a prolonged half-life, which makes it suitable for treatment of anaemia at extended administration intervals. Results from the Phase III study (ARCTOS), demonstrate that subcutaneous (SC) C.E.R.A. corrects anaemia in erythropoiesis-stimulating agent (ESA)-naive patients with CKD not on dialysis. Here we report the results from the sub-group of patients with diabetes in this study.

Materials and Methods: A total of 324 patients with CKD ( $\geq 18 \mathrm{yr}$ ) not on dialysis nor receiving treatment with ESAs were randomized $(1: 1)$ to SC C.E.R.A. twice monthly (Q2W) $(0.6 \mathrm{mg} / \mathrm{kg} / 2 \mathrm{wks})$ or SC DA once weekly (QW) $(0.45 \mathrm{mg} / \mathrm{kg} / \mathrm{wk})$. The dose was adjusted to achieve a $\mathrm{Hb}$ response $(\geq 1$ $\mathrm{g} / \mathrm{dL}$ increase and $\geq 11 \mathrm{~g} / \mathrm{dL}$ ) and to maintain Hb levels between 11-13 g/dL and $\pm 1 \mathrm{~g} / \mathrm{dL}$ of the response level throughout the 28 -week study.

Results: Of the 180 patients with diabetes, 86 received C.E.R.A. and 94 received DA. In those without diabetes, 76 received C.E.R.A. and 68 received DA. Mean $\mathrm{Hb}$ at baseline was $10.2 \mathrm{~g} / \mathrm{dL}$ and $10.0 \mathrm{~g} / \mathrm{dL}$ in patients with diabetes receiving C.E.R.A. and DA, respectively, and increased to 12.3 and $12.2 \mathrm{~g} / \mathrm{dL}$, respectively, during the evaluation period (wks 19-28). In patients without diabetes baseline $\mathrm{Hb}$ was $10.3 \mathrm{~g} / \mathrm{dL}$ in both treatment groups, and increased to 12.4 and $12.1 \mathrm{~g} / \mathrm{dL}$ in the C.E.R.A. and DA groups, respectively, during the evaluation period. Among patients with diabetes, $97.9 \%$ (95\% CI: 92.5-99.7) responded in the C.E.R.A group and 96.5\% (90.1-99.3) in the DA group, while $94.1 \%(85.6-98.4)$ and $98.7 \%(92.9-100)$ of patients without diabetes responded to C.E.R.A. and DA, respectively. The mean (95\% CI) differences in the change in $\mathrm{Hb}$ between C.E.R.A. and DA (from baseline to evaluation period) were $0.03 \mathrm{~g} / \mathrm{dL}(-0.25,0.31)$ in those with, and $0.38 \mathrm{~g} / \mathrm{dL}$ $(0.05,0.70)$ in those without, diabetes. The lower limit of the $95 \%$ CI was well above the pre-specified level of $-0.75 \mathrm{~g} / \mathrm{dL}$, demonstrating that C.E.R.A. Q2W is as effective as DA QW for anaemia correction in both patients with and without diabetes (both $P<0.0001$ ). C.E.R.A. was generally well tolerated. Some of the more frequent adverse events, including hypertension and peripheral oedema, were slightly more frequent in patients with diabetes in both treatment groups.

Conclusions: SC C.E.R.A administered twice monthly effectively corrects anaemia in ESA-naïve patients with CKD not on dialysis, regardless of diabetic status. This may help to simplify anaemia management in this important group of patients with diabetes.

Supported by F. Hoffmann-La Roche

\section{PS 70 Lipid metabolism}

\section{7}

Transgenic UCP1 in white fat protects against lipid accumulation in skeletal muscle of mice fed a high-fat diet

T. Jelenik, Z. Jilkova, K. Slamova, M. Rossmeisl, J. Kopecky; Department of Adipose Tissue Biology, Institute of Physiology, Prague 4, Czech Republic.

Background and Aims: The development of obesity-associated insulin resistance is closely related to the accumulation of lipids in non-adipose tissues. Transgenic mice (aP2-Ucp1 mice) with ectopic expression of mitochondrial uncoupling protein 1 (UCP1) in white adipose tissue are resistant to highfat diet-induced obesity and glucose intolerance. Respiratory uncoupling induced by transgenic UCP1 in white fat increases fatty acid (FA) oxidation and decreases in situ lipogenesis, which leads to a diminished release of FA from adipose tissue upon fasting. In this study, we investigated in detail the accumulation of lipids and FA metabolism in non-adipose tissues, especially in skeletal muscle of the aP2-Ucp1 mice fed a high-fat diet.

Materials and Methods: Male transgenic aP2-Ucp1 mice and non-transgenic littermate controls (C57BL/6J genetic background; $n=6-8$ ) were fed either a standard laboratory chow or high-fat diet (HF; 60\% calories as fat) from 3 mo of age. After 2 mo of HF feeding, glucose tolerance (expressed as incremental AUC) was assessed in mice following i.p. injection of glucose. Plasma levels of triglycerides were measured by coupled enzyme assay (Roche). Gene expression in skeletal muscle was quantitated by quantitative real-time PCR, while lipid content in tissues was estimated by measuring glycerol release from tissue triglycerides saponified by alcoholic $\mathrm{KOH}$. Oxygen consumption of skeletal muscle fragments incubated in Krebs-Ringer bicarbonate buffer $(\mathrm{pH}$ 7.4) at $37^{\circ} \mathrm{C}$ was measured using a high-resolution respirometry (Oroboros Oxygraph, Paar KG, Graz, Austria).

Results: Glucose tolerance was improved by transgenic UCP1 (AUC: transgenics, $1153 \pm 126$; controls, $1570 \pm 130 \mathrm{mmol} \times 180 \mathrm{~min} ; P<0.05)$ following 2 mo of HF feeding. Only in the fasting state, plasma triglycerides were decreased by $\sim 45 \%$ in the transgenics $(P<0.001)$. Accordingly, triglyceride content was significantly reduced by $\sim 40 \%$ in both skeletal muscle and liver. These changes were associated with a 2.6 -fold downregulation of gene expression for stearoyl-CoA desaturase-1, while GLUT4 gene expression was increased 1.6-fold in the skeletal muscle of transgenic mice. Preliminary results using a limited number of animals $(n=4)$ suggested that oxygen consumption in skeletal muscle of transgenic mice was increased by $20-30 \%$.

Conclusion: Respiratory uncoupling, brought about by transgenic UCP1 in white fat, limited the accumulation of triglycerides in skeletal muscle and liver, while improving glucose tolerance of mice fed a HF diet. In skeletal muscle, these changes were associated with a simultaneous inhibition of lipogenesis and induction of respiration, which could be associated with increased energy expenditure via futile cycling of fatty acids between their synthesis and oxidation. The enhancement of energy expenditure in muscle, in response to a lower release of fatty acids from white fat, may contribute to both, obesity-resistance and insulin sensitivity of the aP2-Ucp 1 transgenic mice.

Supported by research projects AV0Z50110509 and GA CR 303/03/H065

\section{8}

The PPAR $\alpha$ agonist fenofibrate does not improve insulin sensitivity in type 2 diabetes in man: a randomised controlled trial

N. Black ${ }^{1}$, C. N. Ennis ${ }^{1}$, I. S. Young ${ }^{2}$, S. J. Hunter ${ }^{1}$, B. Atkinson ${ }^{1}$, P. M. Bell' ${ }^{1}$ Regional Centre for Endocrinology and Diabetes, Royal Victoria Hospital, ${ }^{2}$ Nutrition and Metabolism Group, The Queen's University of Belfast, United Kingdom.

Background and Aims: Insulin resistance is a central feature of type 2 diabetes mellitus. Lipid and carbohydrate metabolism are inter-related and it is often assumed on the basis of studies in animal and human tissue models that PPAR alpha agonists can improve insulin sensitivity. However, this assumption is based mainly on surrogate measures of insulin sensitivity and the use of highaffinity PPAR alpha ligands which are not used in clinical practice. This study is designed to compare the effect on insulin sensitivity of the selective PPAR 
alpha agonist fenofibrate against that of the HMG CoA reductase inhibitor atorvastatin in human subjects with type 2 diabetes mellitus.

Materials and Methods: Thirteen subjects with type 2 diabetes mellitus were studied in a double-blind crossover design with 4-week placebo run-in and washout and 12-week treatment periods. Treatment was randomised to micronised fenofibrate $267 \mathrm{mg}$ or atorvastatin $10 \mathrm{mg}$ once daily followed by the alternate drug in the second period. Insulin resistance was measured using the isoglycaemic hyperinsulinaemic clamp method with an insulin infusion rate of $2 \mathrm{mU}^{-1} \mathrm{~kg}^{-1}$ minute ${ }^{-1}$ with isotope dilution $\left(\left[3{ }^{3} \mathrm{H}\right]\right.$-glucose) to determine glucose disposal rate and suppression of endogenous glucose production. The plasma glucose was maintained at a constant level during hyperinsulinaemia by an infusion of isotope-labelled $20 \%$ glucose at variable rate. The glucose infusion rate during the last 30 minutes of each clamp was used as an index of insulin sensitivity. Results are described as mean +/- standard error. Analysis was by the method of Hills and Armitage for 2-period crossover studies.

Results: Mean age was 61.3 (2.5), duration of diabetes 4.4 (0.7) years and body mass index was $29.5(0.9) \mathrm{kg} / \mathrm{m} 2$. Baseline fasting plasma glucose was $7.7(0.3) \mathrm{mmol} / \mathrm{L}, \mathrm{HbAlc} 7.1(0.2) \%$, fasting serum insulin $11.0(0.8) \mathrm{mU} /$ L. Ten subjects had been on lipid-lowering therapy prior to the study and this was withdrawn 4 weeks prior to the placebo run-in period. The baseline lipid profile was: total cholesterol 4.97 (0.25), LDL-cholesterol 2.95 (0.22), HDL-cholesterol $1.10(0.06)$ and triglycerides $2.03(0.27) \mathrm{mmol} / \mathrm{L}$. Weight, physical activity and other medications did not change during the study. Total cholesterol was $4.60+/-0.21$ versus $3.9+/-0.22 \mathrm{mmolL}^{-1}$ after fenofibrate and atorvastatin respectively, $\mathrm{p}<0.05$. LDL was $2.70+/-0.19$ versus 1.95 $+/-0.23 \mathrm{mmolL}^{-1}, \mathrm{p}<0.05$ and triglycerides were $1.64+/-0.23$ versus 1.84 $+/-0.26 \mathrm{mmolL}^{-1}, \mathrm{p}<0.05$. The glucose infusion rate required to maintain isoglycaemia was similar after each treatment $(30.3+/-1.9$ versus 26.3 $+/-2.4$ micromolkg ${ }^{-1} \mathrm{~min}^{-1}, \mathrm{p}=\mathrm{NS}$. Isotopically-determined rates of insulinstimulated whole-body glucose disposal $(35.4+/-3.1$ versus $33.2+/-3.0$ micromolkg $\left.{ }^{-1} \mathrm{~min}^{-1}\right)$ and nadir endogenous glucose production $(6.2+/-1.4$ versus $7.0+/-1.1$ micromolkg ${ }^{-1} \mathrm{~min}^{-1}$ ) revealed no significant differences in effects of the the treatments.

Conclusion: In human subjects with type 2 diabetes mellitus there were characteristic differences in lipid profile changes but no difference in insulin sensitivity after treatment with micronised fenofibrate compared to atorvastatin. Low-affinity PPAR alpha activators at doses used in clinical practice in type 2 diabetes in man do not improve insulin action.

Supported by a Fellowship Award from the R\&D Office of the Northern Ireland Health \& Social Sevices Central Services Agency

\section{9}

Fatty acid profile remodelling of epiploic and parametrial fat in longchain polyunsaturated $\omega 3$ fatty acid deficiency

W. J. Malaisse ${ }^{1}$, L. Portois ${ }^{2}$, C. Delporte ${ }^{3}$, A. Sener ${ }^{1}$, Y. A. Carpentier'; ${ }^{1}$ Laboratory of Experimental Hormonology, Brussels Free University, ${ }^{2}$ Laboratory of Experimental Surgery, Brussels Free University, ${ }^{3}$ Laboratory of Biological Chemistry and Nutrition, Brussels Free University, Belgium.

Background and Aims: A depletion of long-chain polyunsaturated $\omega 3$ fatty acids $(\omega 3)$, as often prevailing in Western populations, may favour the development of the metabolic syndrome. Likewise, in rats, an insufficient dietary supply of $\omega 3$ leads to hyperlipidemia, visceral obesity, liver steatosis, insulin resistance and hypertension. The present study deals with the agerelated alterations of the fatty acid profile in the epiploic and parametrial adipose tissue of $\omega 3$-depleted rats.

Materials and Methods: The fatty acid profile of epiploic and parametrial fat was assessed in 16 female control animals and 16 second generation $\omega 3$ depleted rats $(\omega 3-\mathrm{D})$ of same sex and comparable age. The time course of changes in $\omega 3$-D rats was monitored by comparing 16 young ( $8-9$ weeks), 16 middle-aged (15-16 weeks) and 9 older (22-23 weeks) rats.

Results: In both control and $\omega 3-\mathrm{D}$ rats, modest but significant differences were, as a rule, observed when comparing epiploic to parametrial fat. At variance with control animals, long-chain polyunsaturated $\omega 3$ fatty acid $(\mathrm{C} 18: 3 \omega 3, \mathrm{C} 20: 5 \omega 3, \mathrm{C} 22: 5 \omega 3, \mathrm{C} 22: 6 \omega 3)$ were below the limit of detection in $\omega 3$-D rats. The fat content of most long-chain polyunsaturated $\omega 6$ fatty acid (C18:2 $\omega 6, \mathrm{C} 20: 4 \omega 6, \mathrm{C} 22: 4 \omega 6)$ was also significantly lower in $\omega 3-\mathrm{D}$ rats than control animals, this coinciding with a lower $\mathrm{C} 20: 4 \omega 6 / \mathrm{C} 18: 2 \omega 6$ ratio in the former rats $(0.020 \pm 0.004)$ than in the latter animals $(0.036 \pm 0.001)$.

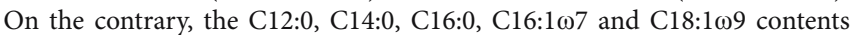

were increased in $\omega 3-\mathrm{D}$ rats. The $\mathrm{C} 16: 1 \omega 7 / \mathrm{C} 16: 0$ and $\mathrm{C} 18: 1 \omega 9 / \mathrm{C} 18: 0$ ratios were almost twice higher in $\omega 3-\mathrm{D}$ rats than in control animals, whilst the $\mathrm{C} 20: 1 \omega 9 / \mathrm{C} 18: 1 \omega 9$ ratio was lower in $\omega 3$-D rats $\left(9.09 \pm 0.2210^{-3}\right)$ than in control animals $\left(15.86 \pm 0.5210^{-3}\right)$. Several of these variables underwent agerelated changes in $\omega 3-\mathrm{D}$ rats often towards accentuation of the difference between these rats and control animals.

Conclusion: The lipid fatty acid pattern of epiploic and parametrial adipose tissue in $\omega 3-\mathrm{D}$ rats demonstrate, in addition to depletion in long-chain polyunsaturated $\omega 3$ and $\omega 6$ fatty acids and apparently impaired generation

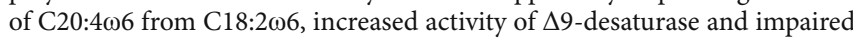
generation of C20:1 $\omega 9$, the immediate precursor of nervonic acid. Some of these features, e.g. the depletion in $\omega 6$ fatty acids, differ from those previously documented in the liver lipids of the same rats.

\section{0}

Effect of Acyl-CoA binding protein on sterol regulatory element-binding protein and AMP-activated protein kinase in transgenic rats S. Oikari ${ }^{1}$, T. Ahtialansaari ${ }^{1}$, K. Kiehne ${ }^{2}$, U. R. Fölsch ${ }^{2}$, L. Alhonen ${ }^{1}$,

J. Jänne ${ }^{1}$, K.-H. Herzigi,

${ }^{1}$ Department of Biotechnology and Molecular Medicine, A. I. Virtanen

Institute for Molecular Sciences, Kuopio, Finland,

${ }^{2}$ Dept. of Internal Medicine, UKSH Campus Kiel, Germany,

${ }^{3}$ Department of Physiology, Oulu University Medical School, Finland.

Background and Aims: Elevated free fatty acid levels are a central feature of metabolic syndrome which is a growing health problem in many societies. In cytosol, free fatty acids are converted to acyl-CoAs, important intermediates for many metabolic pathways. Acyl-CoA binding protein (ACBP) binds with high affinity and specificity to medium and long chain acyl-coAs and has been show to act as a pool former and transporter. Both acyl-CoAs and ACBP have emerged as important regulators of various intracellular functions including enzymes and transcription factors involved in carbohydrate and lipid metabolism. To further characterize the effects of ACBP on regulation of lipid and glucose metabolism we have used transgenic rats overexpressing the ACBP gene and studied effect on sterol regulatory element binding protein-1(SREBP-1) and AMP-activated protein kinase (AMPK). SREBP-1 has an important role in the regulation of lipogenic genes while AMPK is known to switching off lipogenic and switching on energy producing / catabolic pathways like B-oxidation. In addition, AMPK affects SREBP-1 expression and acyl-CoAs can regulate its function.

Materials and Methods: A rat line overexpressing ACBP was created by standard methods. Tissues were collected from adipose tissue, liver, muscle and brain and isolated hypothalamus of fed and fasted rats. Total RNA and protein was isolated and used to measure differences in either gene expression or protein levels. Gene expression analysis was performed by quantitative real-time RT-PCR using Sybr-green as fluorescent dye. Protein levels were investigated by Western blotting using specific antibodies to AMPK $\alpha$, phosphorylated AMPK $\alpha$, SREBP-1 and B-actin.

Results: Our results show that in fed state expression of SREBP-1 mRNA was significantly downregulated by $77 \%$ in liver and by $23 \%$ in adipose tissue of trangenic (tg) ACBP overexpressing rats when compared to wildtype (wt) litter mates. There was no significant change in mRNA levels between wt and $\operatorname{tg}$ rats in muscle tissue nor in the hypothalamus. These results were confirmed on the protein level in liver: tg rats had significantly reduced levels of SREBP-1 when compared to wt controls. In the fasting stage (16hrs) SREBP-1 expression was significantly downregulated in the livers of both wt and tg animals. In adipose tissue fasting reduced the SREBP-1 expression of wt rats but did not affect SREBP-1 levels in tg animals. To further characterize possible mechanisms how ACBP affects SREBP-1 we investigated protein levels of AMPK $\alpha$ in liver and brain. We found significantly elevated AMPK $\alpha$ levels in liver of the tg rats compared to wt controls. Furthermore, levels of the active, Thr172 phosphorylated form of AMPK $\alpha$ were also increased. On the contrary, there was no change between tg and wt animals in AMPK $\alpha$ protein levels in brain.

Conclusion: Our results showed that ACBP is reducing SREBP-1 levels and upregulating AMPK $\alpha$ levels in tissue specific manner. These results indicate that ACBP affects regulation of lipid metabolism in liver and adipose tissue either directly or through regulation of acyl-CoAs.

Supported by German Federal Ministry of Education and Reseach and Finnish Cultural Foundation of Northern Savo 


\section{1}

GLUT-4 mRNA and protein content of soleus muscle and parametrial fat in $\omega 3$ fatty-acid-depleted rats

L. Arnés ${ }^{1}$, P. Tornero-Esteban ${ }^{1}$, V. Sancho ${ }^{1}$, M. L. Villanueva-Peñacarrillo ${ }^{1}$, W. J. Malaisse ${ }^{2}$

${ }^{1}$ Metabolism, Nutrition and Hormones, Fundación Jiménez Díaz,

Madrid, Spain, ${ }^{2}$ Laboratory of Experimental Hormonology,

Brussels Free University, Belgium.

Background and Aims: Second generation long-chain polyunsaturated $\omega 3$ fatty acid-depleted rats are currently used as an animal model for the study of the metabolic and functional consequences of an insufficient exogenous supply of these $\omega 3$ fatty acids, as presently often prevailing in Western populations. In the light of recent findings on the perturbation of glucose metabolism in both skeletal muscle and visceral adipose tissue of $\omega 3$-depleted rats, the GLUT-4 expression in soleus muscle and parametrial fat was now compared in these rats and control animals.

Materials and Methods: The soleus muscle and parametrial adipose tissue were obtained from 16 female $\omega 3$-depleted $(\omega 3 \mathrm{D})$ rats and 16 control animals of same sex and age. Eight soleus muscle samples, each from 2 rats, and 5 adipose tissue samples, each from 3-4 rats, were examined for GLUT-4 protein -by Western blot- and mRNA -by RT-PCR- content.

Results: In soleus muscle and parametrial adipose tissue from $\omega 3 \mathrm{D}$ rats, the optimal density of GLUT-4 protein, normalized to that of ß-tubulin, averaged $103.2 \pm 5.2 \%(n=8)$ and $104.1 \pm 6.1 \%$ normal $(n=5)$, respectively. As judged from the paired difference between the primary values (cycles) for GLUT-4 mRNA and 18S rRNA, and expressing results as a mean fold change from

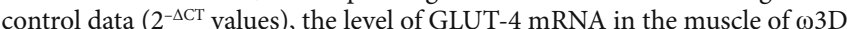
rats was somewhat higher (range 1.43-2.27) than in that of normal control rats (range 0.91-1.09); in the adipose tissue of $\omega 3 \mathrm{D}$ rats, the GLUT-4 gene expression was somewhat lower (range 0.49-0.73) than in that of normal group (range 0.75-1.34).

Conclusion: A defect in the expression of the GLUT-4 gene in skeletal muscle and visceral adipose tissue appears as an unlikely major determinant of the perturbation of glucose homeostasis in $\omega 3$ fatty acid deficiency. The data here collected in soleus muscle are consistent with previous observation of an unimpaired net basal uptake of $\mathrm{D}-\left[\mathrm{U}_{-}{ }^{14} \mathrm{C}\right]$ glucose by stretched soleus muscle examined in vitro; likewise, the present findings in adipose tissue are consistent with prior data indicating that the impairment of glucose uptake by adipocytes is not specific for the hexose, as identical results being obtained for the uptake of $\left[1,2-{ }^{14} \mathrm{C}\right]$ acetate.

Supported by Ministerio de Sanidad y Consumo and a convention between the Région de Bruxelles-Capitale and Target Hit s.a. (Brussels, Belgium)

\section{2}

Fish oil increases lipolysis and fat oxidation in healthy men with dexamethasone (D)-induced insulin-resistance

J. Delarue ${ }^{1}$, G. Allain ${ }^{1}$, C. Corporeau ${ }^{1}$, V. Le Guen ${ }^{1}$, S. Guillerm²;

${ }^{1}$ Laboratoire Régional de Nutrition Humaine \& EA-948, ${ }^{2}$ Laboratoire

Régional de Nutrition Humaine, CHU Cavale Blanche, Brest, France.

Background and Aims: Fish oil (FO) increases insulin sensitivity in healthy humans and decreases insulin response to OGTT after D treatment suggesting a partial prevention of $\mathrm{D}$-induced insulin resistance (IR). The aim of this study was to assess the effects of FO on insulin sensitivity, lipolysis and fatty acid oxidation in D-induced IR in healthy males.

Materials and Methods: 16 volunteers were studied 3 times. The first two tests, made at D0 and D15, were performed to study the effect of D (D: 2 $\mathrm{mg} / \mathrm{d}$ during 2 days vs placebo). The third test was performed at D36, after 3 weeks of either FO (1.5 g EPA+DHA) $(n=8)$ or placebo (controls, $n=8)$. The 2 groups received $2 \mathrm{mg} / \mathrm{d}$ of D at D35 and D36. Each test included of a 3-step (S) hyperinsulinaemic euglycaemic clamp (insulin rate: $\mathrm{S} 1: 0.2 \mathrm{mU} / \mathrm{kg} / \mathrm{min}$ from 0 to $90 \mathrm{~min}, \mathrm{~S} 2: 0.4 \mathrm{mU} / \mathrm{kg} / \mathrm{min}$ from 90 to $180 \mathrm{~min}$ and then S3: $1 \mathrm{mU}$ $/ \mathrm{kg} / \mathrm{min}$ from 180 to $270 \mathrm{~min}$ ). Insulin sensitivity was estimated from total plasma glucose disappearance (Rd) and hepatic glucose production (HGP) determined from infusion of 6,6-d2-glucose (hot clamp); carbohydrate (CHOox) and lipid (Lox) oxidations were determined by indirect calorimetry and lipolysis by d5-glycerol flux (Ra glycerol).

Results: (mean \pm SEM, ANOVA for repeated measures). D decreased Rd (S1: $1.92 \pm 0.12$ vs $2.33 \pm 0.16$; step 2 : $2.33 \pm 0.17$ vs. $4.41 \pm 0.52$; step $3: 5.66 \pm 0.43$ vs
$9.38 \pm 0.65 \mathrm{mg} / \mathrm{kg} / \mathrm{min}$; all $\mathrm{p}<0.01)$, reduced the inhibition of HGP by insulin ( $\mathrm{S} 1: 1.06 \pm 0.11$ vs $0.64 \pm 0.13 \mathrm{p}<0.05 ; \mathrm{S} 2: 0.39 \pm 0.07$ vs $0.36 \pm 0.07 \mathrm{NS} ; \mathrm{S} 3: 0.41 \pm 0.21$ vs $0.31 \pm 0.08 \mathrm{mg} / \mathrm{kg} / \mathrm{min}, \mathrm{NS})$, decreased CHOox (S1:2.26 \pm 0.12 vs $2.75 \pm 0.20 ; \mathrm{S} 2: 2.40 \pm 0.14$ vs $3.20 \pm 0.17 ; \mathrm{S} 3: 3.29 \pm 0.15$ vs $4.52 \pm 0.21 \mathrm{mg} / \mathrm{kg} /$ min; all $\mathrm{p}<0.01)$, increased Lox ( $1: 0.92 \pm 0.05$ vs $0.68 \pm 0.06$; $\mathrm{S} 2: 0.88 \pm 0.05$ vs $0.53 \pm 0.07 ; \mathrm{S} 3: 0.58 \pm 0.06$ vs $0.22 \pm 0.06 \mathrm{mg} / \mathrm{kg} / \mathrm{min}$; all $\mathrm{p}<0.01)$ and increased Ra glycerol (S1:1.92 \pm 0.36 vs1.22 $\pm 0.18 ; S 2: 1.5 \pm 0.28$ vs $0.87 \pm 0.11 ; S 3: 1.05 \pm 0.18$ vs $0.81 \pm 0.10 \mu \mathrm{mol} / \mathrm{kg} / \mathrm{min}$; all $\mathrm{p}<0.01)$. FO+D did not alter $\mathrm{Rd}$ vs $\mathrm{D}$, increased inhibition of HGP by insulin at S3: $0.03 \pm 0.02$ vs $0.67 \pm 0.37 \mathrm{mg} / \mathrm{kg} /$ min;p<0.01), increased Lox at S3:0.71 \pm 0.08 vs $0.47 \pm 0.07 \mathrm{mg} / \mathrm{kg} / \mathrm{min} ; \mathrm{p}<0.05$ ), decreased Gox at $\mathrm{S} 3: 2.66 \pm 0.38$ vs $3.55 \pm 0.28 \mathrm{mg} / \mathrm{kg} / \mathrm{min} ; \mathrm{p}<0.05)$ and increased Ra glycerol at S2:1.4 $\pm 0.34 \mathrm{vs} 1.22 \pm 0.30 \mu \mathrm{ol} / \mathrm{kg} / \mathrm{min} ; \mathrm{p}<0.05)$

Conclusion: D induced peripheral and liver IR and increased lipolysis and fat oxidation. FO+D enhanced the effects of $\mathrm{D}$ on lipolysis and Lox, did not alter peripheral IR but increased liver insulin sensitivity.

Supported by Région Bretagne

\section{3}

Type I Gaucher disease, a glycosphingolipid storage disorder, is associated with insulin resistance

M. J. Serlie ${ }^{1}$, M. Langeveld ${ }^{1}$, M. T. Ackermans ${ }^{2}$, K. J. Ghauharali ${ }^{3}$, J. E. Groener ${ }^{3}$, C. E. Hollak ${ }^{1}$, J. M. Aerts ${ }^{3}$, H. P. Sauerwein ${ }^{1}$;

${ }^{1}$ Endocrinology and Metabolism, Academic Medical Center, ${ }^{2} \mathrm{Clinical}$ Chemistry, Laboratory of Endocrinology, Academic Medical Center, ${ }^{3}$ Medical Biochemistry, Academic Medical Center, Amsterdam, The Netherlands.

Background and Aims: Glycosphingolipids (especially GM3) reside within lipid rafts in cell membranes. Therefore they may influence the function of membrane-bound receptors, like the insulin receptor. Gaucher disease is a lysosomal storage disorder in which glucosylceramide and more complex glycosphingolipids accumulate in macrophages and increase whole body macrophage burden. Glycosphingolipid concentrations in plasma are also elevated in this disorder. This may influence lipid raft composition in muscle cells, adipocytes and liver cells, thereby influencing insulin sensitivity. Furthermore, cellular storage causes activation of macrophages, which may lead to increased (non-insulin mediated) glucose uptake in these cells.

Materials and Methods: We studied the influence of glycosphingolipid storage on whole body glucose metabolism, by measuring basal glucose metabolism and insulin and non-insulin-mediated glucose uptake. We studied six Gaucher patients (either naive to treatment or with considerable remaining burden of disease despite treatment) and six matched healthy control subjects, during euglycemic and hyperglycemic clamps with somatostatin (non-insulin mediated glucose uptake (NIMGU)) and during a hyperinsulinemic euglycemic clamp (insulin-mediated glucose uptake (IMGU)), using stable isotopes. We also measured plasma FFA.

Results: IMGU was significantly lower in Gaucher patients, compared to controls, while NIMGU and hepatic insulin sensitivity did not differ between patients and controls. There was a trend for a decreased suppression by insulin of plasma FFA (lipolysis) in Gaucher patients.

Conclusion: Gaucher disease, a lysosomal glycosphingolipid storage disorder, is associated with peripheral insulin resistance. This may be explained by disturbed insulin signaling via an increase in membrane glycosphingolipids.

\section{4}

In a placebo-controlled clinical trial a novel Chinese herb formula increased insulin sensitivity and normalised postprandial dyslipidaemia in centrally obese men

C. $\mathrm{He}^{1}, \mathrm{~F} . \mathrm{Sun}^{2}$, B. $\mathrm{Li}^{1}$, Y. He ${ }^{1}$, W. Chen ${ }^{1}$, J. Ying ${ }^{1}$, W. Wang ${ }^{1}$;

${ }^{1}$ Diabetes \& Endocrinology, The Institute of Chinese Integrative Medicine, Shanghai, China, ${ }^{2}$ Diabetes and Endocrinology, Postgraduate Medical School, Guildford, United Kingdom.

Background and Aims: Central obesity is a well-recognized risk factor for the development of metabolic syndrome. The degree of insulin sensitivity has also been shown to be a major determinant of postprandial lipaemic excursions following a test meal. Although postprandial dyslipidaemia has been recognized as a risk factor for cardiovascular disease, there is little 
information regarding its origin in relation to metabolic syndrome and the early prevention of insulin resistance.

This study seeks to investigate the effects of a novel Chinese herb formula on a group of centrally obese subjects as described by IDF criteria in a Chinese population. Chinese herbs have historically been widely used to treat patients with various symptoms of metabolic syndrome and central obesity. Clinical effectiveness has been well demonstrated. However, the underlying mechanism of its pharmacological effect remains unknown.

Materials and Methods: A placebo-controlled clinical trial was performed in 43 centrally obese males (age $45.9 \pm 6.0 \mathrm{y} ;$ BMI $27.3 \pm 2.5 \mathrm{~kg} / \mathrm{m}^{2}$; waist circumference $97 \pm 6.4 \mathrm{~cm}$ ) and 31 lean males (age $43.7 \pm 7.6$ years; BMI $23.5 \pm 2.1 \mathrm{~kg} / \mathrm{m}^{2}$; waist circumference $81 \pm 6.9 \mathrm{~cm}$ ), who were free from any medication. The aim was to examine the effects of 10 weeks treatment of a novel Chinese herb formula (CHF) on insulin sensitivity and postprandial dyslipidaemia. Obese volunteers were randomized into a CHF treatment group or placebo group. An oral fat tolerance test (OFTT) was performed before and after treatment. Adiponectin, leptin, interleukin-6 (IL-6) and tumor necrosis factor- $\alpha$ (TNF- $\alpha$ ) were also measured.

Results: At baseline, the insulin sensitivity was assessed by the homeostasis model of insulin resistance (HOMA-IR) (obese $1.6 \pm 0.6$ vs $0.9 \pm 0.7$ lean, $\mathrm{P}=0.0001$ ). There were no differences in blood glucose, glucose tolerance test and fasting lipid levels between the lean and obese groups. The postprandial triglyceride (TG) response in the OFTT as determined by TG area under the curve (TG-AUC) was higher in the obese subjects $(10.52 \pm 6.17 \mathrm{mmol} / \mathrm{L} \cdot \mathrm{h})$ than the lean $(4.89 \pm 4.05 \mathrm{mmol} / \mathrm{L} \cdot \mathrm{h}, \mathrm{P}=0.0001)$.

After CHF treatment, there was a significant decrease in postprandial TGAUC following the fat load with no change in the placebo group (Table 1). In addition in the CHF group there was a 34\% decrease in HOMA-IR and $35.5 \%$ reduction in insulin concentration. Although there was a decrease in waist circumference in the treatment group, the change of body weight in the treatment group was small. (Table 1)

Conclusion: The Chinese herb formula significantly increased insulin sensitivity and normalized postprandial lipid excursions. Treatment also had beneficial effects on levels of adipocytokines. This treatment provides an early intervention for insulin resistance and metabolic syndrome.

Supported by National Natural Science Foundation of China and Special Science Research Foundation of Educational Ministry of China for Doctor Degree

\section{5}

Serum and glucocorticoid-inducible protein kinase (SGK)-1 protect from different apoptotic stimuli

D. Pastore, M. Lombardo, F. Ferrelli, B. Capuani, K. Basello, A. Coppola, M. Federici, P. Sbraccia, G. Donadel, D. Lauro;

Internal Medicine, University of Rome Tor Vergata, Italy.

Background: Different studies revealed that insulin resistance might be associated with the intracellular formation of ceramide. The Ceramide syntesis can be activated by different stimuli, such as TNF-alpha, IL-1beta, gamma irradiation, U.V. light, ischemia, serum deprivation and can induce cell necrosis and apoptosis.

Aims:It has been investigated the role of SGK-1 in modulating apoptotic stimuli induced by four different treatments: 1) serum deprivation; 2) UV treatment; 3) C2-Ceramide incubation and 4) TNF-alpha stimulation.

Matherials and Methods: HEK-293 and COS-7 cell lines has been used as experimental models, different cell lines has been stable transfected with SGK-1 and its dominant negative construct (SGK-1 dn), apoptosis was evaluated by FACS and Western Blot analysis.

Results: Serum deprivation, can increase apoptosis process in not transfected cells, differently in SGK-1 transfected cells, stimulated or not with insulin, has been detected lower levels of apoptosis compared to not transfected cell lines. Beside, It has been found that SGK-1 overexpression can protect from U.V. light-induced apoptosis and SGK-1 transfected cells are more resistant to C2-Ceramide-induced apoptosis. This process is mediated by reduced caspase- 3 and PARP cleavage and activation that is independent from insulin stimulation. C2-ceramide treatment induces a significant increase of SGK1 protein expression $(\mathrm{p}<0.05)$, phosphorylation and activity compared to dihydroceramide treated cells. Interestingly, it has been found that AKT-1 was not phosphorylated and activated, in the same conditions in which SGK1 activity and expression is induced. C2-ceramide inhibits AKT-1 kinase activation and induce apoptosis; in SGK-1 transfected cell it has been detected a reduction of apoptotic levels and an increased expression and activation of SGK-1 that is statistically signifcant $(\mathrm{p}<0.01)$ compared to mock and SGK-1dn transfected cell lines. In the present study it has been hypothesized that SGK1 expression induced by C2-ceramide stimulation is mediated by the cAMP/ PKA pathway. Western Blot analysis revealed that the induction of SGK-1 protein expression by $\mathrm{C} 2$-ceramide treatment is attenuated in the presence of the H-89 a cAMP/PKA inhibitor. TNF-alpha stimulation can rouse apoptosis process in not transfected cell lines, differently, SGK-1 transfected cells showed a higher resistance to apoptosis $(\mathrm{p}<0.001)$, inhibition of TNF-alpha signaling mediated by SGK-1 can potentially increase insulin sensibility.

Conclusions:

1) SGK-1 has an antiapoptotic effect after UV light, C2-ceramide and TNF alpha stimuli.

2) C2 Ceramide and TNF-alpha have a different effect on SGK-1 and AKT-1: a) C2 Ceramide reduce AKT-1 activity and, differently increase SGK-1 activity and expression, $b$ ) TNF-alpha can increase SGK-1 activity and, probably, expression.

3) SGK-1 expression can be activated by cAMP/PKA/CREB pathway.

Table 1: Data

\begin{tabular}{|c|c|c|c|c|c|c|c|c|c|}
\hline & \multicolumn{3}{|c|}{ Placebo(n=21) } & \multicolumn{3}{|l|}{$\mathrm{CHF}(\mathrm{n}=22)$} & \multirow{2}{*}{$\begin{array}{l}\text { P value } \\
\text { (CHF vs } \\
\text { placebo) }\end{array}$} & \multirow{2}{*}{$\begin{array}{l}\text { Controls } \\
(\mathrm{n}=31)\end{array}$} & \multirow{2}{*}{$\begin{array}{l}\text { P value } \\
\text { (CHF after treatment } \\
\text { vs controls) }\end{array}$} \\
\hline & $\begin{array}{l}\text { Before } \\
\text { treatment }\end{array}$ & $\begin{array}{l}\text { After } \\
\text { treatment }\end{array}$ & $P$ value & $\begin{array}{l}\text { Before } \\
\text { treatment }\end{array}$ & $\begin{array}{l}\text { After } \\
\text { treatment }\end{array}$ & $P$ value & & & \\
\hline Waist(cm) & $97.7 \pm 6.7$ & $96.9 \pm 6.8$ & 0.07 & $96.3 \pm 6.1$ & $92.8 \pm 5.4$ & 0.001 & 0.005 & $81.0 \pm 6.9$ & 0.001 \\
\hline Body weight (kg) & $80.4 \pm 11.8$ & $80.1 \pm 11.1$ & 0.653 & $76.4 \pm 7.6$ & $74.6 \pm 8.2$ & 0.001 & 0.009 & $68.3 \pm 6.8$ & 0.035 \\
\hline $\mathrm{HOMA}_{2}-\mathrm{IR}$ & $1.6 \pm 0.7$ & $1.6 \pm 0.7$ & 0.93 & $1.6 \pm 0.5$ & $1.0 \pm 0.4$ & 0.001 & 0.001 & $0.8 \pm 0.5$ & 0.396 \\
\hline Fast insulin $(\mathrm{mU} / \mathrm{L})$ & $12.2 \pm 4.4$ & $12.4 \pm 5.2$ & 0.848 & $12.2 \pm 4.4$ & $7.8 \pm 2.8$ & $<0.001$ & $<0.001$ & $6.3 \pm 3.8$ & 0.906 \\
\hline TGAUC (mmol/L·h) & $11.7 \pm 5.0$ & $13.3 \pm 9.1$ & 0.42 & $9.4 \pm 7.0$ & $5.0 \pm 2.9$ & 0.009 & 0.02 & $4.9 \pm 4.1$ & 0.997 \\
\hline $\operatorname{Adiponectin(mg/L)~}$ & $3.1 \pm 1.9$ & $2.8 \pm 1.4$ & 0.27 & $3.3 \pm 2.6$ & $4.1 \pm 2.3$ & 0.008 & 0.007 & $5.6 \pm 3.3$ & 0.142 \\
\hline Leptin $(\mu \mathrm{g} / \mathrm{L})$ & $15.3 \pm 7.1$ & $15.9 \pm 6.7$ & 0.62 & 13. $4 \pm 6.2$ & $10.5 \pm 4.6$ & 0.012 & 0.028 & $7.4 \pm 4.6$ & 0.108 \\
\hline $\mathrm{IL}-6(\mu \mathrm{g} / \mathrm{L})$ & $14.3 \pm 8.0$ & $15.4 \pm 7.0$ & 0.28 & $13.4 \pm 7.3$ & $9.8 \pm 5.1$ & 0.008 & 0.009 & $7.7 \pm 6.8$ & 0.497 \\
\hline TNF- $\alpha(\mu \mathrm{g} / \mathrm{L})$ & $102.6 \pm 84.7$ & $105.1 \pm 75.6$ & 0.64 & $126.4 \pm 89.1$ & $94.9 \pm 60.7$ & 0.047 & 0.041 & $69.5 \pm 59.9$ & 0.379 \\
\hline
\end{tabular}




\section{PS 71 Polycystic ovary syndrome}

\section{6}

\author{
Comparison of the NCEP and IDF definitions of metabolic syndrome \\ among Chinese subjects with polycystic ovary syndrome \\ R. C. W. Ma ${ }^{1}$, K.-H. Liu' ${ }^{2}$, W.-Y. So ${ }^{1}$, A. P. S. Kong ${ }^{1}$, P. C. Y. Tong ${ }^{1}$, \\ C.-C. Chow $^{1}$, J. C. N. Chan ${ }^{1}$; \\ ${ }^{1}$ Dept of Medicine \& Therapeutics, ${ }^{2}$ Dept of Diagnostic Radiology \\ and Organ Imaging, Prince of Wales Hospital, Hong Kong.
}

Background and Aims: Subjects with polycystic ovary syndrome (PCOS) have insulin resistance and are at increased cardiovascular risk. Carotid intimamedia thickness (IMT) has been shown to predict future cardiovascular events. There has been much recent debate as to the utility of the different definitions of the metabolic syndrome (MES). We recently demonstrated that among subjects with Type 2 DM, the IDF definition of MES failed to identify a subgroup of patients who were at highest risk for CHD. In this study, we aimed to compare the frequency of metabolic syndrome among a cohort of Chinese subjects with PCOS, and to compare their metabolic profile and cardiovascular risk as quantified by carotid IMT.

Materials and Methods: Ninety-eight Chinese subjects with PCOS were evaluated. All subjects fulfilled a diagnosis of PCOS according to the revised 2003 Rotterdam ESHRE/ASRM PCOS Consensus Workshop Group diagnostic criteria. Subjects with known cardiovascular or renal disease were excluded. Anthropometric measurements and metabolic risk profile, including a standard OGTT, were assessed in all subjects. All subjects were categorized by the criteria of IDF and NCEP. All subjects underwent an ultrasound examination for measurements of thickness of mesenteric, preperitoneal, and subcutaneous fat as well as carotid intima-media thickness.

Results: The subjects had a mean age of $27.5 \pm 6.2$. Mean BMI was $24.1 \pm 5.1$ $\mathrm{kg} / \mathrm{m}^{2}$. Fiveteen (15.3\%) of the subjects had metabolic syndrome according to the National Cholesterol Education Adult Treatment Panel III criteria whilst 12 subjects (12.2\%) fulfilled a diagnosis of metabolic syndrome according to the IDF definition, using the Asian definition of central obesity of waistcircumference $>80 \mathrm{~cm}\left(\mathrm{p}<0.01\right.$ by $\left.\chi^{2}\right)$. Subjects who had metabolic syndrome by either the NCEP or IDF defintion had a more adverse metabolic profile, as well as a higher ALT and creatinine. There was no significant difference in HOMA-IR among subjects with and without MES according to the IDF definition. Mesenteric fat was significantly associated with all components of the MES. IMT was increased among subjects who had metabolic syndrome by NCEP definition, but not among subjects who had MES by IDF definition alone.

Conclusion: Our preliminary data suggest that the NCEP definition identifies significantly more subjects with metabolic syndrome among subjects with PCOS. Subjects who had metabolic syndrome by NCEP definition had significantly increased carotid IMT compared to subjects without MES. Among Chinese subjects with PCOS, the NCEP definition of MES may be superior to the IDF definition in identifying subjects at increased risk of cardiovascular disease.

\section{7}

\author{
Rapid insulin pulsatile secretion in women with polycystic ovary \\ syndrome \\ T. Grimmichova ${ }^{1}$, J. Vrbikova ${ }^{1}$, P. Matucha ${ }^{1}$, K. Vondra $^{1}$, P. P. Veldhuis ${ }^{2}$, \\ M. L. Johnson'; \\ ${ }^{1}$ Laboratory of Functional Diagnostics, Institute of Endocrinology, Prague, \\ Czech Republic, ${ }^{2}$ Department of Pharmacology and Internal Medicine, \\ University of Virginia, Charlottesville, United States.
}

Background and Aims: Polycystic ovary syndrome (PCOS) is considered as a risk factor for impaired glucose tolerance and diabetes mellitus type 2. Hyperinsulinaemia secondary to insulin resistance, abnormal insulin secretion and decreased insulin clearance was reported in PCOS. The aim of our study was to evaluate rapid insulin pulses and insulin secretion regularity in fasting state in lean PCOS women in comparison to lean healthy controls. Materials and Methods: PCOS women ( $\mathrm{n}=8$; age 25.1 (21.8-29.5) years) fulfilling the diagnostic criteria as defined by ESHRE/ASRM. Healthy controls (C; $n=7$, age 30.2 (25.1-38.1) years). Blood samples for hormonal levels were performed in early follicular phase of menstrual cycle. After overnight fast PCOS and C underwent frequent blood sampling each minute for 60 minutes assessing rapid insulin pulses. Data analysis: Deconvolution software AutoDecon evaluating insulin pulsatility. Approximate entropy (ApEn) appraised insulin concentrations time series orderliness. Statistics: All data are given as retransformed means and 95\% confidence interval. Unpaired t-tests and Pearson correlation coefficients were used on transformed data ( $\mathrm{p}<0.05$ was considered statistically significant).

Results: PCOS had higher testosterone $(3.2(2.8-3.6)$ vs $2.3(0-3.3) \mathrm{nmol} /$ l; $\mathrm{p}<0.02)$, prolactin $(23.8(16.8-32.5)$ vs $13.7(6.3-25.4) \mathrm{ng} / \mathrm{ml} ; \mathrm{p}<0.05)$ and lower SHBG levels (46.3 (28.4-64.1) vs $98.1(83.1-113.1) \mathrm{nmol} / \mathrm{l} ; \mathrm{p}<0.0006)$ in comparison to C. PCOS had higher BMI than C (21.9 (20.2-23.5) vs 20.1(18.9$21.2) \mathrm{kg} / \mathrm{m} 2 ; \mathrm{p}<0.05)$. Fasting blood glucose (4.2 (4-4.35) vs $4.1(4-4.4) \mathrm{mmol} /$ 1), insulin (5.1 (3.9-6.5) vs $4.2(3.1-5.6) \mathrm{mIU} / \mathrm{l})$, C-peptide $(0.54(0.4-0.71)$ vs $0.51(0.23-0.96) \mathrm{nmol} / \mathrm{l})$ and cortisol concentrations (564 (442-729) vs 449 (306-680) nmol/l) did not differ between PCOS and C. Deconvolution analysis results: Insulin elimination kinetics were established with individual insulin half-life of $3.3(2.8-4.1)$ in PCOS and 3.2 (2.6-4.1) minutes in C. Both PCOS and C had comparable average frequency of 10 insulin pulses per 60 minutes. Insulin burst mass $(2.1(1.5-3)$ vs $1.4(1-2.4) \mathrm{mIU} / \mathrm{l})$, basal secretion (0.7 (0.4-1.0) vs $0.5(0.2-0.8) \mathrm{mIU} / \mathrm{l} / \mathrm{min})$, interpulse interval $(5.9(5-7.2)$ vs $5.6(4.6-7.1) \mathrm{min})$ and burst amplitude (1.1 (0.7-2.1) vs $1.5(1.0-2.6) \mathrm{mIU} / \mathrm{l} /$ $\mathrm{min}$ ] did not differ significantly between groups. PCOS women had a tendency to broader peaks determined by a common half-duration $(2.0(1.1-3.4)$ vs $0.9(0.5-1.5) \mathrm{min} ; \mathrm{p}<0.07)$ (duration of an algebraically Gaussian secretory pulse at half-maximal amplitude). The common-half duration was positively correlated with prolactin and cortisol concentrations $(\mathrm{r}=0.76 ; \mathrm{p}<0.008, \mathrm{r}=0.64$; $\mathrm{p}<0.03$, respectively). Higher testosterone levels were correlated with higher burst mass $(\mathrm{r}=0.61 ; \mathrm{p}<0.05)$. ApEn in PCOS was not different from $\mathrm{C}(1.14$ $(1.09-1.19)$ and $1.08(0.94-1.16)$, respectively; $p<0.17)$. Higher BMI and prolactin levels were correlated with more irregular insulin secretion (ApEn) $(\mathrm{r}=0.57 ; \mathrm{p}<0.04 ; \mathrm{r}=0.68 ; \mathrm{p}<0.03$, respectively).

Conclusion: We observed similar insulin pulsatile release and insulin secretion orderliness in lean PCOS women in comparison to healthy lean controls. Prolonged and intensive action of prolactin, androgens and cortisol could participate in alteration of insulin secretion patterns leading to glucose intolerance.

Supported by grant of MH CR NR 8759-3

\section{8}

Insulin resistance and beta cell function in polycystic ovary syndrome: the influence of family history of type 2 diabetes mellitus J. Vrbikova ${ }^{1}$, M. Hill ${ }^{2}$, B. Bendlová ${ }^{3}$, K. Vondra ${ }^{1}$, T. Grimmichova ${ }^{1}$, G. Pacini ${ }^{4}$;

${ }^{1}$ Functional diagnostic, Institute of Endocrinology, Prague, Czech Republic, ${ }^{2}$ Steroid, Institute of Endocrinology, Prague, Czech Republic, ${ }^{3}$ Molecular Endocrinology, Institute of Endocrinology, Prague, Czech Republic, ${ }^{4}$ Metabolic Modeling Unit, National Research Council, Padova, Italy.

Background and Aims: Polycystic ovary syndrome (PCOS) is often considered a risk factor for type 2 diabetes mellitus (DM2). It is not known if the family history of DM2 plays a role in the overall state of PCOS.The aim was to evaluate insulin sensitivity and beta cell secretory compensation in women with PCOS with respect to their family history of DM2.

Materials and Methods: 79 PCOS women (age 27.4 \pm 7.2 years, BMI $26.7 \pm 7.3 \mathrm{~kg} / \mathrm{m}^{2}$ ) according to NIH consensus and 94 healthy women (CT; age $26.6 \pm 5.7$ years, BMI $\left.23.1 \pm 5.2 \mathrm{~kg} / \mathrm{m}^{2}\right)$ free of family history of DM2 underwent oGTT (sampling at $0,30,60,90,120,180 \mathrm{~min}$ ) for blood glucose $(\mathrm{mg} / \mathrm{dl}), \mathrm{C}$ peptide (nmol/l) and insulin (mU/l). PCOS were divided into PCOS+ with first-degree relative affected by DM2 ( $\mathrm{n}=23$; age 29.9 \pm 6.5 , BMI 29.1 \pm 7.8$)$ and PCOS-, without family history of DM2 ( $\mathrm{n}=56$; age $26.8 \pm 6.6$, BMI $26.1 \pm 6.2)$. Insulin resistance was evaluated by oral glucose index (OGIS, $\mathrm{ml} / \mathrm{min} / \mathrm{m}^{2}$ ) and insulin secretion by the areas under the curve $\mathrm{AUC}_{\mathrm{I}}$ for insulin $(\mathrm{U} / \mathrm{l} 3 \mathrm{~h})$ and $\mathrm{AUC}_{\mathrm{CP}}$ for C peptide $(\mu \mathrm{mol} / \mathrm{l} 3 \mathrm{~h})$. $\mathrm{AUC}_{\mathrm{G}}$ for glucose $(\mathrm{g} / \mathrm{dl} 3 \mathrm{~h})$ was computed. Beta cell compensatory mechanism (adaptation index, $\mathrm{AI}$ ) was evaluated as OGIS $\times \mathrm{AUC}_{\mathrm{CP}}$. All data are as mean $\pm \mathrm{SD}$; BMI- and age-adjusted ANCOVA followed by least significant difference multiple comparisons $(\mathrm{p}<0.05)$ for evaluation of between-group differences.

Results: Impaired glucose tolerance was found in 9/23 PCOS+ and in 6/56 PCOS-. Basal blood glucose was higher in PCOS+ than in PCOS(90.5 \pm 12.8 vs. $82.5 \pm 11.4)$ or CT $(83.4 \pm 8.4)$. Basal insulin was significantly higher in PCOS+ $(14.9 \pm 12.7)$ than in PCOS- $(9.6 \pm 7.7)$ or in CT $(7.3 \pm 5.1)$. 
In comparison with CT, all PCOS have significantly higher basal C-peptide (0.89 \pm 0.45 vs. $0.60 \pm 0.27), \mathrm{AUC}_{\mathrm{G}}(20.1 \pm 5.9$ vs. $16.4 \pm 2.4), \mathrm{AUC}_{\mathrm{I}}(10.8 \pm 8.3$ vs. $5.7 \pm 4.6)$ and $\mathrm{AUC}_{\mathrm{CP}}(0.51 \pm 0.22$ vs. $0.36 \pm 0.12)$. Only PCOS+ have significantly lower OGIS (439.6 \pm 105.3$)$ than CT (524.9 \pm 69.8$)$. Hepatic insulin extraction was decreased in PCOS- $(64.3 \pm 11.4 \%)$ compared to CT $(71.2 \pm 12.4 \%)$ while did not differ in PCOS+ (61.6 $\pm 17.8 \%)$. Adaptation index was higher in PCOS- than in CT $(0.24 \pm 0.08$ vs. $0.19 \pm 0.06)$ and was not different between PCOS+ $(0.22 \pm 0.06)$ and CT.

Conclusion: The family history of DM2 plays an important role in the metabolic state of women with PCOS: in fact, elevated insulin resistance is present only in PCOS with first degree relative with DM2. On the other hand, insulin hypersecretion is present in PCOS irrespective of family history of DM2 and obesity. Despite this beta cell hypersecretion, PCOS women are not able to maintain similar blood glucose levels after oral glucose load as the healthy control subjects

Supported by IGA MH CR 8759/3

\section{9}

Abnormal glucose tolerance and metabolic syndrome in the first degree relatives of women with polycystic ovary syndrome

S. Yadav, P. Kalra, V. Bhatia, E. Bhatia;

Endocrinology, SGPGIMS, Lucknow, UP, India.

Background and Aims: Polycystic ovarian syndrome (PCOS) is a common problem affecting $6-8 \%$ of reproductive females. There is a high incidence of IGT, diabetes mellitus, insulin resistance, and hyperandrogenemia in first degree relatives of PCOS patients.

Our aim was to study prevalence of abnormal glucose tolerance (impaired glucose tolerance and or diabetes mellitus) and metabolic syndrome in first degree relatives of women with PCOS as compared to controls from north Indian population.

Materials and Methods: One hundred and seventy nine family members of 75 patients with PCOS [Mothers ( $\mathrm{n}=71$; mean age, $45.3 \mathrm{yr}$; mean body mass index $\left.(\mathrm{BMI}), 27.6 \mathrm{~kg} / \mathrm{m}^{2}\right)$, Fathers $(\mathrm{n}=70$; mean age, $50.7 \mathrm{yr}$; mean BMI, $\left.25.6 \mathrm{~kg} / \mathrm{m}^{2}\right)$, Sisters ( $\mathrm{n}=17$; mean age, $29.9 \mathrm{yr}$; mean BMI, $24.5 \mathrm{~kg} /$ $\left.\mathrm{m}^{2}\right)$, and Brothers $\left(\mathrm{n}=21\right.$; mean age, $22.9 \mathrm{yr}$; mean BMI, $\left.\left.22.5 \mathrm{~kg} / \mathrm{m}^{2}\right)\right]$ and 118 unrelated healthy control subjects without a family history of PCOS (4 age and BMI matched subgroups, i.e. Control Mothers, Control Fathers, Control Sisters, and Control Brothers) were evaluated by anthropometric measurements, OGTT, fasting and post glucose 2 hour insulin and fasting lipid profile.

Results: Prevalence of any degree of glucose intolerance (impaired glucose tolerance and or diabetes mellitus) was present in $52.9 \%$ of mothers of women with PCOS, and $27.5 \%$ of control mothers ( $<<0.05$; odd ratio 3.0 ); $48.5 \%$ fathers of women with PCOS and $24.5 \%$ control fathers $(\mathrm{p}<0.05$; odd ratio 2.9); $52.9 \%$ sisters of women with PCOS and $13.3 \%$ control sisters $(\mathrm{p}<0.05$; odd ratio 7.3 ); $19.0 \%$ brothers of women with PCOS and $14.3 \%$ control brothers ( $\mathrm{p}=\mathrm{NS}$ ). Metabolic syndrome as defined by NCEP-ATP III guidelines was present in $27.5 \%$ mothers of women with PCOS and $10.3 \%$ of control mothers $(p<0.05) ; 39.1 \%$ fathers of women with PCOS and $14.6 \%$ control fathers ( $\mathrm{p}<0.05), 23.5 \%$ sisters of women with PCOS and $6.6 \%$ control sisters $(\mathrm{p}=\mathrm{NS}), 23.8 \%$ brothers of women with PCOS and none of control brothers $(p<0.05)$. For analyzing insulin resistance, we matched the relatives of women with PCOS and healthy controls for age and BMI, till now we analyzed in 47 subjects in each group. HOMA-IR was significantly higher in relatives of $\mathrm{PCOS}$ subjects as compared to healthy controls $(\mathrm{P}=0.05)$. Univariate logistic regression in first degree relatives of PCOS subjects showed that metabolic syndrome, triglyceride and all parameters of obesity significantly predicted whether a subject would have abnormal glucose tolerance. In stepwise logistic regression analysis metabolic syndrome, waist hip ratio, and age were significant predictive factors for development of abnormal glucose tolerance in relatives of PCOS subjects.

Conclusions: Our results suggest that first degree relatives of PCOS subjects are at high risk for diabetes mellitus and glucose intolerance; there is an increased prevalence of metabolic syndrome in first degree relative of PCOS women. We propose that the high risks of these impairments warrant screening in first degree relatives of women with PCOS.

\section{0}

Elevated risk markers of atherosclerosis in polycystic ovary syndrome (PCOS) were significantly reduced during pioglitazone treatment in a randomised placebo controlled study

D. Glintborg ${ }^{1}$, K. Højlund ${ }^{1}$, M. Andersen ${ }^{1}$, J. Henriksen ${ }^{1}$, H. Beck-Nielsen ${ }^{1}$, A. Handberg';

${ }^{1}$ Endocrinology, Odense University Hospital,

${ }^{2}$ Clinical Biochemical department, Aarhus University Hospital, Denmark.

Background and Aims: Recent studies found increased levels of risk markers for atherosclerosis in polycystic ovary syndrome (PCOS), but the effect of insulin sensitizing treatment on these markers remains to be established. A soluble form of $\mathrm{CD} 36$ (sCD36) in plasma was recently identified, and shown to be strongly related to risk factors of accelerated atherosclerosis in type 2 diabetes including insulin resistance and glycemic control. In the present study, we investigated the relation between sCD36, oxidized LDL (oxLDL), high sensitive CRP (hsCRP), and interleukin 6 (IL-6 and measures of body composition and glucose metabolism in PCOS before and during pioglitazone treatment.

Materials and Methods: Thirty PCOS patients were randomized to pioglitazone, $30 \mathrm{mg} /$ day or placebo for 16 weeks. Fourteen weight-matched healthy females were included as controls. sCD36, oxLDL, hsCRP, IL-6, euglycemic hyperinsulinemic clamps, and measures of fat mass (whole body DXA-scans) were performed.

Results: PCOS patients had significantly decreased insulin-stimulated total (Rd), oxidative and non-oxidative glucose metabolism than controls, all $\mathrm{p}<$ 0.05 . $\mathrm{sCD} 36(2.87(0.88-9.36)$ vs. $1.67(0.72-3.89)$ relative units), oxLDL $(44.9(26.9-75.1)$ vs. $36.1(23.4-55.5) \mathrm{U} / 1)$, and hsCRP $(0.26(0.03-2.41)$ vs. $0.12(0.02-0.81) \mathrm{mg} / \mathrm{dl})$ were significantly increased in PCOS patients vs. control subjects (geometric mean (-2SD-2SD)). In PCOS patients, positive correlations were found between sCD36, hsCRP and IL- 6 and abdominal fat mass $(\mathrm{r}=0.40-0.43, \mathrm{p}<0.05)$ and between hsCRP, IL- 6 and total fat mass $(\mathrm{r}=0.59$ and $0.59, \mathrm{p}<0.05) . \mathrm{sCD} 36$ furthermore showed a positive correlation with lipid oxidation during insulin stimulation $(\mathrm{r}=0.53, \mathrm{p}=0.003)$ and an inverse correlation with insulin-stimulated oxidative glucose metabolism $(\mathrm{r}=-0.57, \mathrm{p}=0.001))$. Following pioglitazone treatment, insulin sensitivity and measures of insulin-stimulated glucose metabolism significantly increased (all $\mathrm{p}<0.05)$, whereas hsCRP $(0.36(0.06-2.20)$ vs. $0.24(0.04-1.58) \mathrm{mg} / \mathrm{dl})$ and sCD36 $(3.21(0.71-13.6)-2.33(0.84-6.46)$ relative units) significantly decreased. No significant changes were observed in oxLDL and IL6.

Conclusion: SCD36, oxLDL, hsCRP, and IL6 were correlated with abdominal and total fat mass, thus supporting the hypothesis that the high risk for the metabolic syndrome and possible future atherosclerosis is in part related to abdominal obesity in PCOS. sCD36 furthermore correlated positively with lipid oxidation and inversely with oxidative glucose metabolism. Pioglitazone treatment reduced $\mathrm{sCD} 36$ and hsCRP while improving insulin-stimulated glucose metabolism, thus suggesting that $\mathrm{sCD} 36$ may be used as a surrogate marker for insulin resistance and future risk for atherosclerosis in PCOS. Supported by Fonden for Leegevidenskabelig forskning ved Fyns Amt, Institute of Clinical Research, Odense University Hospital, A. J. Andersen's Foundation, Novo Nordisk Foundation, Overlagerådet Odense University Hospital, Danish Diabetes Association

\section{1}

Hyperphosphorylation and low protein content of muscle glycogen synthase contribute to insulin resistance in polycystic ovary syndrome: effects of pioglitazone treatment

K. Højlund ${ }^{1}$, D. Glintborg ${ }^{1}$, N. R. Andersen², J. B. Birk ${ }^{2}$, J. T. Treebak ${ }^{2}$, H. Beck-Nielsen ${ }^{1}$, J. F. P. Wojtaszewski²;

${ }^{1}$ Department of Endocrinology, University of Southern Denmark, Odense, ${ }^{2}$ Department of Human Physiology, University of Copenhagen, Denmark.

Background and Aims: Insulin resistance in skeletal muscle, characterized primarily by impaired insulin-stimulation of glycogen synthesis, is a major risk factor for type 2 diabetes in women with polycystic ovary syndrome (PCOS). The molecular mechanisms underlying reduced insulin action on glycogen synthesis in skeletal muscle of patients with PCOS have not been established.

Materials and Methods: We investigated protein content and multisite phosphorylation of glycogen synthase (GS) and its major upstream inhibitor, 
glycogen synthase kinase 3 (GSK3) in skeletal muscle of 24 PCOS patients and 14 matched control subjects, and examined the effects of 16 weeks treatment with pioglitazone in PCOS. Skeletal muscle biopsies from vastus lateralis were obtained before and after euglycemic-hyperinsulinemic clamp studies combined with indirect calorimetry.

Results: Reduced insulin-mediated total, oxidative and non-oxidative glucose disposal $($ all $\mathrm{p}<0.05)$ was associated with impaired insulin-stimulated GS activity in women with PCOS $(37 \pm 2 \%$ vs. $46 \pm 2 \%$ fractional velocity $(\mathrm{FV}) ; \mathrm{p}<0.05)$ compared with controls. This was caused by a $36 \%$ lower protein content of muscle GS (70 \pm 6 vs. $110 \pm 10 \mathrm{AU} ; \mathrm{p}<0.05)$, as well as a $\sim 2$ fold increase in the phosphorylation of GS at sites $2+2 a, 3 a+3 b$ and $1 b$ after correction for GS protein $($ all $\mathrm{p}<0.05)$. No significant abnormalities in protein content or phosphorylation of GSK3-alpha or -beta were found in PCOS. Pioglitazone treatment improved insulin-stimulated glucose metabolism in PCOS, and this was paralleled by a $30 \%$ increase in insulin-stimulated GS-activity ( $33 \pm 2 \%$ vs. $43 \pm 2 \% \mathrm{FV} ; \mathrm{p}<0.05)$. This was associated with a 40 $50 \%$ reduction in the phosphorylation of GS at sites $3 a$ and $3 a+3 b(p<0.05)$, whereas protein levels of GS remained reduced.

Conclusion: Impaired insulin activation of GS in part explains insulin resistance at the molecular level in skeletal muscle in PCOS. Both reduced protein levels and hyperphosphorylation at several sites of GS may contribute to this defect. The ability of pioglitazone to enhance insulin sensitivity may involve improved insulin-mediated dephosphorylation of GS. Supported by Novo Nordisk Foundation

\section{PS 72 Nutrition}

\section{2}

Effect of high-protein low-fat diet on cardiovascular disease risk factors of individuals with metabolic syndrome

E. Papakonstantinou ${ }^{1}$, D. Panagiotakos ${ }^{1}$, A. Koutsovasilis ${ }^{2}$, A. Manolis ${ }^{3}$, A. Melidonis ${ }^{2}$, A. Zampelas

${ }^{1}$ Dietetics and nutritional science, Harokopio University, ${ }^{2}$ Diabetes Clinic, Tzanio General Hospital of Piraeus, ${ }^{3}$ Hypertension Management Clinic, Tzanio General Hospital of Piraeus, ${ }^{4}$ Department of Food Science and Technology, Agricultural University of Athens, Athens, Greece.

Background and Aims: When substituted for carbohydrate in an diet, dietary protein improves blood pressure, glycemic control and markers of cardiovascular disease in people with metabolic syndrome. It is unknown whether the effect is due to increased protein or reduced carbohydrate. We compared the effects of 2 isocaloric, hypoenergetic diets that differed in protein and fat content on indices of glycemic control, body composition, blood pressure, and lipids.

Materials and Methods: 17 obese volunteers, age $46 \pm 3 y$, with mild type 2 diabetes in a random, blind, cross-over design study followed 2 diets: (a) a high protein, low fat diet (30\% protein, $50 \%$ carbohydrates, $20 \%$ fat) and (b) a low protein, high fat diet (15\% protein, $50 \%$ carbohydrates, $35 \%$ fat), for 4 weeks each with 3 weeks washout period without treatment. Diets included 2 meal replacements. Blood was collected in the beginning and end of treatments. At the beginning and end of each treatment, the volunteers consumed a meal (30\% protein, $50 \%$ carbohydrates, $20 \%$ fat) after 12 -hour fast. Glucose and insulin were measured at fasting, 30,60, 90, 120, 150, 180 minutes postprandially. Weight, waist circumference, blood lipids, and blood pressure were measured. Repeated measures analysis was used to test the effects of treatment, time of measurement, and their interactions on the dependent measures.

Results: High protein, low fat diet caused a significantly higher reduction in blood pressure $(\mathrm{p}=0.013)$ and triglyceride levels $(\mathrm{p}=0.042)$ compared to a low protein diet. High protein diet caused a significant reduction in glycated hemoglobin $(\mathrm{p}<0.001)$, whereas the low protein diet did not. Both diets reduced glucose and insulin levels, but no significant difference was found between them. Both diets led to a significant reduction of total, LDL, and HDL cholesterol but no significant difference was found between the diets. No statistically significant difference was found between the diets concerning BMI, waist, body fat, or fat-free mass.

Conclusion: A high protein low fat diet improves more blood pressure and plasma triglyceride levels compared to a low protein high fat diet. The improvements in glycated haemoglobin, insulin resistance, weight loss and blood lipids did not differ significantly between the two diets.

Supported by Novartis Hellas AEBE

\section{3}

The effect of a high-protein diet on parameters of glucose and lipid metabolism in patients with type 2 diabetes (T2DM) on insulin therapy B. Tripp, B. Ludvik, K. Schindler; Internal Medicine III, University of Vienna, Austria.

Background and Aims: The optimal macronutrient composition of a diet for T2DM remains a matter of controversy. This study investigated the metabolic effects of a high-protein diet predominantly from vegetables in type 2 diabetic patients on insulin therapy.

Materials and Methods: 44 patients with T2DM were randomly assigned either to a weight-maintaining high protein diet (PR: $n=22$; $\mathrm{P}: \mathrm{CHO}$ : $\mathrm{F}=30: 40: 30 \%$, age: $61.0 \pm 5.7$ years, body weight: $94.1 \pm 15.6 \mathrm{~kg}$, insulin dose: $57.3+26.6 \mathrm{IU} /$ day, $\mathrm{HbA}_{1}: 7.8+1.4 \%$ ), or a standard diet (ST: $\mathrm{n}=22, \mathrm{P}: \mathrm{CHO}$ : $\mathrm{F}=15: 55: 30 \%$, age: $63.7 \pm 5.2$ years, body weight: $91.5 \pm 20.2 \mathrm{~kg}$ insulin dose: $\left.52.7 \pm 28.8 \mathrm{IU}, \mathrm{HbA}_{1 \mathrm{c}}: 7.6 \pm 0.9 \%\right)$. Parameters of glucose- and lipid metabolism and anthropometric parameters were evaluated at baseline and after 3 months. The main outcome variables were daily insulin requirements and metabolic control.

Results: Body weight decreased in both groups after 3 months (PR: to $91.0 \pm 15.7$; ST: to $90.5 \pm 19.4 \mathrm{~kg}, \mathrm{p}<0.05)$, albeit to a higher extent in $\mathrm{PR}$ $(\mathrm{p}=0.0042)$. In $\mathrm{PR}$, insulin dose was reduced $(\mathrm{p}<0.05)$ from $57.3 \pm 26.6$ to 
$48.2 \pm 30.8 \mathrm{IU} /$ day, $\mathrm{HbA}$ from $7.8+1.4$ to $7.5+1.4 \%$, FPG from $203.2+56.9$ to $160.0 \pm 47.7 \mathrm{mg} / \mathrm{dl}$, triglycerides from $186.9 \pm 114.9$ to $140.7 \pm 55.6 \mathrm{mg} / \mathrm{dl}$. Diastolic blood pressure showed a significant decrease from $83.2 \pm 7.2$ to $76.9 \pm 8.7 \mathrm{mmHg}(\mathrm{p}<0.05)$. Fat mass was reduced from $29.1 \pm 8.2$ to $26.7 \pm 6.2 \mathrm{~kg}$ $(p<0.05)$. No specific changes were seen in ST except for an increase in insulin dose $(\mathrm{p}<0.05)$.

Conclusion: In this study we could demonstrate beneficial effects of a highprotein diet on glycemic parameters and insulin requirement in type 2 diabetic patients over 3 months compared to a standard diet. These changes seem to be related to the greater weight loss and require confirmation over longer observation periods.

\section{4}

High-CHO/high-fibre diet vs. low-CHO/high-MUFA diet: which is the better approach to improve postprandial lipid abnormalities in type 2 diabetic patients?

G. Annuzzi, C. De Natale, L. Patti, L. Bozzetto, O. Ciano, P. Massaro,

G. Costabile, G. Riccardi, A. A. Rivellese;

Department of Clinical and Experimental Medicine, University Federico II Naples, Italy.

Background and Aims: Postprandial lipid abnormalities may have an important role in increasing cardiovascular risk, especially in type 2 diabetes. Nonetheless, very few studies have evaluated the possibility to improve these abnormalities in type 2 diabetic patients by different dietary approaches. We compared the effects on postprandial lipaemia of the two diets generally recommended to individuals with diabetes (high-CHO/high-fibre diet and low-CHO/high-MUFA diet).

Materials and Methods: Six type 2 diabetic patients (age $58 \pm 5$ years; BMI $\left.27 \pm 7 \mathrm{~kg} / \mathrm{m}^{2}\right)(\mathrm{M} \pm \mathrm{SD})$ in satisfactory blood glucose control on diet or diet + oral hypoglycaemic drugs, have been studied so far, according to a randomised cross-over design. They followed for one month a diet relatively rich in $\mathrm{CHO}$ (52\% total energy) and fibre $(28 \mathrm{~g} / 1000 \mathrm{kcal})$ with low glycaemic index (GI) (58\%) or a diet low in CHO (45\% total energy) and fibre $(8 \mathrm{~g} / 1000 \mathrm{kcal})$ with high GI (88\%), and then crossed over to the alternate diet for another month. At the end of each period a test meal of the same composition as the corresponding diet was administered and plasma samples for determination of lipids and lipoprotein fractions (separated by discontinuous density gradient ultracentrifugation) were taken before and over $6 \mathrm{hrs}$ after meal.

Results: Fasting LDL cholesterol and postprandial blood glucose were lower at the end of the high-CHO/high-fibre/low-GI diet compared to the low-CHO/ high-MUFA diet, as was the postprandial response of plasma triglycerides (incremental AUC $136 \pm 99 v s .272 \pm 145 \mathrm{mg} / \mathrm{dl} \cdot 6 \mathrm{hrs}, \mathrm{p}=0.03$ ). At the end of the high-CHO diet, also cholesterol (Chol) and triglycerides $(\mathrm{Tg})$ concentrations were significantly lower in chylomicrons (incremental AUC Chol 1.8 \pm 0.6 vs. $4.0 \pm 1.8 \mathrm{mg} / \mathrm{dl} \cdot 6 \mathrm{~h}, \mathrm{p}<0.01$; Tg $55 \pm 25$ vs. $93 \pm 38 \mathrm{mg} / \mathrm{dl} \cdot 6 \mathrm{~h}, \mathrm{p}=0.009)$ and in large VLDL (incremental AUC Chol $10 \pm 16$ vs. $30 \pm 17 \mathrm{mg} / \mathrm{dl} \cdot 6 \mathrm{~h}, \mathrm{p}=0.03 ; \mathrm{Tg}$ $108 \pm 78$ vs. $214 \pm 120 \mathrm{mg} / \mathrm{dl} \cdot 6 \mathrm{~h}, \mathrm{p}=0.03)$.

Conclusion: Compared to a low-CHO/high-MUFA diet, a relatively high$\mathrm{CHO} /$ high-fibre/low-GI diet significantly improves the postprandial response of TG-rich lipoproteins. This kind of diet, also by virtue of its beneficial postprandial effects, could be the optimal approach for treating diabetic patients to reduce their cardiovascular risk.

\section{5}

Glycaemic index, glycaemic load and non-alcoholic fatty liver disease in Japanese adults

J. Oya ${ }^{1}$, T. Nakagami ${ }^{1}$, S. Jimba ${ }^{1}$, S. Sasaki ${ }^{2}$, T. Kasahara ${ }^{3}$, T. Wasada ${ }^{3}$,

Y. Iwamoto;

${ }^{1}$ Diabetes Center, Tokyo Women's Medical University, Tokyo, ${ }^{2}$ Nutritional Epidemiology Program, National Institute of Health and Nutrition, Tokyo, ${ }^{3}$ Internal Medicine, Saitama-ken Saiseikai Kurihashi Hospital, Saitama-ken, Japan.

Background and Aims: We have previously reported that non-alcoholic fatty liver disease (NAFLD) existed at $20 \%$ of non-obese Japanese adults and was closely associated with hyperglycemia and dyslipidemia, independent from age and abdominal obesity (Diabetologia 49 suppl1: 448, 2006). Recently, a study showed a favorable impact of supplementation with n-3 long-chain polyunsaturated fatty acids (n-3 PUFA) on NAFLD in Italian subjects. However, little is known about nutritional composition responsible for the development of NAFLD in Japanese living in Japan whose major resource of carbohydrate is white rice. Thus, we compared dietary habits and nutritional components between people with NAFLD and those with normal liver to establish a dietary recommendation to prevent NAFLD as one surrogate marker of the metabolic syndrome in Japan.

Subjects and Methods: The study subjects were 1282 men ( $51 \pm 9$ years) and 772 women ( $51 \pm 9$ years) in a cohort of middle-aged apparently healthy adults without medications for diabetes, hypertension or dyslipidemia. They took medical examinations including B-mode ultrasonography of the liver and measurement of waist circumference (Wc) at the height of umbilicus. We collected their dietary information of recent one month by brief-type self-administered diet history questionnaire developed by S.S and calculated nutritional intake. The diagnosis of NAFLD (147 subjects) was done on the basis of ultrasonographic findings and amount of alcohol intake less than 40 gram/week. The difference of foods and nutrition was compared between subjects with normal liver and NAFLD.

Results: The prevalence of NAFLD was $17.2 \%$ for men and $13.6 \%$ for women. The group with NAFLD had significantly higher intakes of grains, milk, fruits, soft drinks and lesser intake of fish for men ( $p<0.05)$, but no significant difference was found for women. In the group with NAFLD, intake of vegetable proteins, total fats, vegetable fats, saturated fatty acids, monounsaturated fatty acids, $n-6$ polyunsaturated fatty acids, dietary fiber, vitamin $C$, sugar was significantly higher than the group with normal liver only for men ( $p<0.05$ for all). However, no difference was found for intake of magnesium, animal proteins and fats, $n-3$ PUFA in both sexes. The group with NAFLD had significantly lesser intakes of alcohol for both sexes $(p<0.000)$. While the group with NAFLD had significantly higher intake of glycemic index (GI) (31.8 vs. $34.6 / 1000 \mathrm{kcal} ; \mathrm{p}<0.000)$ and glycemic load $(\mathrm{GL})(85.5$ vs.96.6 /1000 kcal; p <0.000) only for men. The logistic regression analysis showed that GI and GL were continuous and independent risk factors for NAFLD, after adjusting for age, body mass index, smoking, physical activity, dietary supplements, eating speed, total energy intake, and total dietary fiber intake in men.

Conclusion: Both GI and GL are continuous and independent risk factors for NAFLD in Japanese men whose major resource of carbohydrate is white rice.

Supported by a grant from the Japan Medical Women's Association for T.N.

\section{6}

The effect of daily consumption of b-glucan-enriched bread on serum cholesterol and plasma glucose levels of type 2 diabetic persons P. Tsapogas ${ }^{1}$, S. Liatis ${ }^{1}$, C. Dimosthenopoulos ${ }^{1}$, E. Chala ${ }^{2}$, E. Kapantais ${ }^{2}$, S. Grammatikou ${ }^{1}$, K. Makrilakis ${ }^{1}$, N. Katsilambros ${ }^{1}$;

${ }^{1} 1$ st Dep Propaedeutic Medicine, Laiko General Hospital, ${ }^{2}$ Department of Diabetes, Obesity, and Metabolism, Metropolitan Hospital, Athens, Greece.

Background and Aims: Certain studies have shown that the watersoluble dietary fiber beta-glucan, a natural component of oat, has hypocholesterolaemic actions, at least in normal subjects. The aim of this study was to evaluate whether the enrichment of bread with b-glucan can improve serum cholesterol levels in patients with type 2 diabetes mellitus (DM) and secondarily whether it affects glycaemic control and fasting insulin levels.

Materials and Methods: A double-blind, prospective, randomised study, was performed on 54 patients with type $2 \mathrm{DM}$ ( 31 men, 23 women, aged $62.76 \pm 9.6$ years, BMI $28.55 \pm 4.53 \mathrm{~kg} / \mathrm{m} 2$, total cholesterol $223.17 \pm 32.12 \mathrm{mg} / \mathrm{dl}$, LDL-C $150.16 \pm 28.58 \mathrm{mg} / \mathrm{dl}$ ), who completed the study. Each subject consumed 120 $\mathrm{g}$ of bread daily for 3 weeks, enriched with $3 \mathrm{~g}$ of b-glucan from oat (Group A, $\mathrm{n}=26$ ) or not-enriched [regular bread (Group B, $n=28$ )]. None of them were receiving hypolipidaemic treatment and their antidiabetic regimen remained stable throughout the study.

Results: At the end of the study, both groups showed a statistically significant reduction of BMI $(-0.365, \mathrm{p}=0.003$ and $-0.163 \mathrm{Kg} / \mathrm{m} 2, \mathrm{p}=0.030$ for Group A and Group B respectively) and waist circumference $(-1.354 \mathrm{p}=0.017$ and $1.036 \mathrm{~cm}$, and 0.027 for Group A and Group B respectively). Group A showed a statistically significant reduction of total-Cholesterol and LDL-Cholesterol compared to Group B (decrease of total-Cholesterol by $19.62 \mathrm{mg} / \mathrm{dl}$ vs. decrease by $1.18 \mathrm{mg} / \mathrm{dl},[\mathrm{p}=0.017]$, and decrease of LDL-C by $16.85 \mathrm{mg} / \mathrm{dl}$ 
vs. decrease by $0.01 \mathrm{mg} / \mathrm{dl},[\mathrm{p}=0.022]$ respectively). Group A also showed a greater decrease in fasting glucose and insulin levels compared to Group B (decrease of plasma glucose by $7.65 \mathrm{mg} / \mathrm{dl}$ vs. decrease by $2.82 \mathrm{mg} / \mathrm{dl}$, and of serum insulin decrease by $2.31 \mu \mathrm{U} / \mathrm{ml}$ vs. increase by $1.43 \mu \mathrm{U} / \mathrm{ml}$ ) but these differences did not reach statistical significance $(\mathrm{p}=0.61$ and $\mathrm{p}=0.23$, respectively). Both groups showed a significant reduction in $\mathrm{HbAlc}$ (group A: $7.07 \%$ baseline vs. $6.85 \%$ at the end of study, $p=0.002$, and group B: $7.01 \%$ baseline vs. $6.83 \%$. $\mathrm{p}=0.042)$. Mean differences in $\mathrm{HbAlc}$ in the two groups were -0.220 (group A) and -0.188 (group B), $\mathrm{p}=0.764$. The HOMA insulin resistance index was not statistically different in the 2 groups (Group A vs. Group B -1.776 vs. $+0.132, \mathrm{p}=0.29)$. Group A showed a significant reduction in systolic blood pressure $(141.7 \mathrm{mmHg}$ baseline vs. $134.4 \mathrm{mmHg}$ at the end of study, $\mathrm{p}=0.012$ ) while in group B there was a tendency for a lower systolic blood pressure $(131.2 \mathrm{mmHg}$ baseline vs. $126.9 \mathrm{mmHg}$ at the end of study, $\mathrm{p}=0.051$ ).

Conclusion: In persons with type $2 \mathrm{DM}$, the daily consumption of bread enriched with $3 \mathrm{~g}$ beta-glucan from oat leads to a significant reduction of total-Cholesterol and LDL-Cholesterol within 3 weeks.

\section{7}

Vinegar reduces postprandial glycaemia in patients with type 2 diabetes when added to a high glycaemic index (but not a low glycaemic index) carbohydrate-rich meal

S. Liatis ${ }^{1}$, S. Grammatikou ${ }^{1}$, K. A. Poulia' ${ }^{1}$, D. Perrea ${ }^{2}$, N. Apostolopoulos ${ }^{1}$, E. Diakoumopoulou ${ }^{1}$, N. Katsilambros';

${ }^{1}$ First Department of Internal Medicine, Athens University Medical School Laiko Hospital, ${ }^{2}$ Laboratory of Experimental Surgery and Surgical Research, Athens University Medical School Laiko Hospital, Greece.

Background and Aims: Previous studies have shown that the addition of vinegar in a carbohydrate-rich meal lowers glucose and insulin responses in both healthy individuals and patients with type 2 diabetes. The mechanism remains virtually unknown.

Aim of the present study was to examine the effect of vinegar in relation to the type of carbohydrate $(\mathrm{CHO})$ used in the meal

Materials and Methods: 16 patients with mild type 2 diabetes (treated either with diet alone or with metformin $\leq 850 \mathrm{mg}$ daily, mean age $59.5 \pm 9.15$ years, mean $\mathrm{HbA}_{1} \mathrm{c}: 6.2 \pm 1.05 \%$ ) participated in the study and were divided into two equal groups, matched for age, sex and $\mathrm{HbA}_{1} \mathrm{c}$. Patients in the first group (group A) were given a high-glycaemic index (GI) meal (mashed potatoes plus low-fat milk: $\mathrm{CHO} 52 \mathrm{~g}$, proteins $13 \mathrm{~g}$ and fat: $2 \mathrm{~g}$ ) on two different days, with and without the addition of vinegar $(20 \mathrm{ml}$, acidity $6 \%)$ respectively. In the second group (group B), patients were given an isocaloric meal with the same nutrient composition but a low GI (whole grain bread plus lettuce plus low-fat cheese), using the same protocol (with and without vinegar). Postprandial serum glucose and insulin values were measured every 30 minutes for a total period of 3 hours

Results: In group A (high GI meal), incremental area under the curve (iAUC) of glucose after meal ingestion was lower after the addition of vinegar (3574.75 $\pm 1628.32 \mathrm{mg} \mathrm{min} / \mathrm{dL}$ vs. $6300.25 \pm 3965.04 \mathrm{mg} \mathrm{min} / \mathrm{dL}, \mathrm{P}=0.04)$. iAUC of insulin was also reduced but the difference was of marginal statistical insignificance $(2455.16 \pm 1097.93 \mu \mathrm{U} \mathrm{min} / \mathrm{ml}$ vs. $3573.68 \pm 2023.74 \mathrm{~min} / \mathrm{ml}$, $\mathrm{P}=0.07$ ). In group $\mathrm{B}$, the addition of vinegar did not affect the iAUC of either glucose $(6594.37 \pm 1516.85 \mathrm{mg} \mathrm{min} / \mathrm{dL}$ with vinegar vs $7036.87 \pm 2103.81 \mathrm{mg}$ $\mathrm{min} / \mathrm{dL}$, without vinegar, $\mathrm{P}=0.69$ ) or insulin (with: $4680.66 \pm 1295.76 \mu \mathrm{U} \mathrm{min} /$ $\mathrm{mL}$ vs. without: $5267.99 \pm 2202.63 \mu \mathrm{U} \mathrm{min} / \mathrm{mL}, \mathrm{P}=0.38$ ).
Conclusion: We conclude that the addition of vinegar reduces postprandial glycaemia in patients with type 2 diabetes only when it is added to a high GI meal. Thus, vinegar may be useful for the reduction of postprandial hyperglycemia in patients with diabetes

\section{8}

Lard oil compared to olive oil impaires insulin-dependent glucose uptake in skeletal muscle

J. Lee ${ }^{1}$, S. Park ${ }^{2}$, E. Park ${ }^{1}$, J. Lee', E. Kang², C. Ahn², H. Lee², B. Cha²; ${ }^{1}$ College of Medicine, Brain Korea 21 Project for Medical Science, ${ }^{2}$ College of Medicine, Internal Medicine, Yonsei University, Seoul, Republic of Korea.

Background and Aims: Otsuka Long Evans Tokushima fatty (OLETF) rat is an animal model of human type 2 diabetes mellitus. Resistance of skeletal muscle to insulin-dependent glucose uptake and phosphorylation is an early step in the development of type 2 diabetes. The objective of this study is to confirm the difference in insulin signaling pathway between lard oil and olive oil.

Materials and Methods: Twenty nine-weeks-old OLETF rats were divided into 3 groups ( $n=10 /$ group) : normal diet $(N)$, high fat diet with lard oil (L) and high fat diet with olive oil (O). Rats were fed a diet for 3 weeks. We confirmed messenger RNA (mRNA) levels of insulin receptor substrate 1 (IRS-1) and insulin receptor substrate 2 (IRS-2) by reverse transcriptasepolymerase chain reaction (RT-PCR) in skeletal muscle (SKM). Protein levels of phosphatidylinositol 3-kinase (PI3K), extracellular-signal-regulated protein kinase (ERK1/2) and glucose transporter 4 (GLUT4) were analysis by western-blotting in SKM.

Results: The mRNA expression of IRS-1 was significantly increased in group $\mathrm{O}$ as compared with group $\mathrm{L}(\mathrm{p}<0.05)$. By contrast, the expression of IRS-2 was significantly increased in group $\mathrm{L}$ as compared with group $\mathrm{O}(\mathrm{p}<0.05)$. Insulin mediated PI3K and GLUT 4 translocation were significantly increased in group $\mathrm{O}$ but not in group $\mathrm{L}(\mathrm{p}<0.05)$. Group $\mathrm{L}$ showed significantly increased phospholylation of PI3K and ERK1/2 as compared with group O $(\mathrm{p}<0.05)$.

Conclusion: These observations show that diets with different fatty acid composition, lard oil versus olive oil, have important differences in insulin signaling pathway. Unlike group $\mathrm{O}$, which show stimulates glucose uptake of SKM via IRS-1, PI3K and GLUT4 translocation pathway, less translocation of GLUT4 was observed in group L. Interestingly, in group L, IRS-2, PI3K and ERK signaling axis were induced by lard oil. These suggest that lard oil induced insulin resistance impaired with IRS-1 dependent glucose uptake but not in olive oil in SKM.

\section{9}

Omega-3 fatty acid supplementation and vascular functions in type 2 diabetes

A. Gupta ${ }^{1}$, M. Matta ${ }^{2}$, N. Singh ${ }^{2}$, R. Gupta ${ }^{3}$;

${ }^{1}$ Diabetes, Jaipur Diabetes Research Centre,

${ }^{2}$ Foods and Nutrition, University of Rajasthan,

${ }^{3}$ Medicine, Monilek Hospital and Research Centre, Jaipur, India.

Background: Omega-3 fats are derived from marine (fish oils) and vegetable (flaxseed oil) sources and are useful to prevent diabetes complications.

Tab. 1

\begin{tabular}{|c|c|c|c|c|c|c|}
\hline \multirow[t]{2}{*}{ Variable } & \multicolumn{2}{|c|}{ Controls $(\mathrm{n}=10)$} & \multicolumn{2}{|c|}{ Flaxseed oil $(n=20)$} & \multicolumn{2}{|c|}{ Fish oils $(n=20)$} \\
\hline & Baseline & After 6 weeks & Baseline & After 6 weeks & Baseline & After 6 weeks \\
\hline Ankle-brachial index, right & $1.06+0.09$ & $1.08+0.09$ & $1.05+0.10$ & $1.07+0.09$ & $1.08+0.07$ & $1.08+0.07$ \\
\hline Ankle-brachial index, left & $1.03+0.12$ & $1.05+0.10$ & $1.04+0.12$ & $1.06+0.12$ & $1.08+0.09$ & $1.09+0.07$ \\
\hline Mean arterial pressure, brachial & $53.3+3.3$ & $53.0+2.7$ & $53.8+3.6$ & $53.7+1.7$ & $53.4+2.5$ & $52.7+1.8$ \\
\hline Mean arterial pressure, ankle & $43.0+2.3$ & $42.7+3.3$ & $40.4+4.6$ & $41.6+3.5$ & $41.6+3.0$ & $40.7+4.5$ \\
\hline Arterial stiffness, right & $42.0+15.4$ & $39.7+11.7$ & $47.3+20.9$ & $47.6+17.9$ & $44.8+23.2$ & $41.3+14.0$ \\
\hline Arterial stiffness, left & $43.5+16.7$ & $43.4+16.7$ & $59.3+23.7$ & $45.8+24.8^{*}$ & $47.9+25.3$ & $43.8+13.6^{*}$ \\
\hline Pulse wave velocity brachial, right & $1816.5+405$ & $1767.7+272$ & $1791.3+342$ & $1827.5+260$ & $1863.1+321$ & $1817.6+225$ \\
\hline Pulse wave velocity brachial, left & $1849.8+411$ & $1882.6+446$ & $1918.0+375$ & $1836.1+309$ & $1898.2+369$ & $1849.2+225$ \\
\hline
\end{tabular}

${ }^{*} \mathrm{P}<0.05$ 
Influence of short term supplementation on vascular function in type 2 diabetes is not well studied.

Methods: In fifty patients (age 53.3 \pm 7.9 ) with stable type 2 diabetes $\left(\mathrm{HbA}_{1} \mathrm{C}\right.$ $8.0 \pm 1.7$ ) we performed a randomised trial to evaluate vascular effects of supplementation of fish oil (300 mg, $n=20$ ) or flaxseed oil (1000mg, $n=20$ ) containing eicosapentanoic acid $180 \mathrm{mg}$ and docosahexanoic acid $120 \mathrm{mg}$. 10 age and sex matched controls were taken for comparisons. Vascular functions were measured using Vascular Profiler- $1000^{\circ}$ and included measurements of blood pressure, ankle-brachial index, arterial stiffness, and pulse wave velocities in upper and lower limbs at baseline and after 6 weeks of supplementation.

Results: The mean body mass index $(27.4 \pm 4.3 \mathrm{~kg} / \mathrm{m} 2)$, waist $(93.2 \pm 11.4$ $\mathrm{cm})$, waist-hip ratio $(0.92 \pm 0.09)$, serum cholesterol $(188.7 \pm 45.3 \mathrm{mg} / \mathrm{dl})$, triglycerides $(153.0 \pm 79.3 \mathrm{mg} / \mathrm{dl})$, HDL cholesterol $(45.1 \pm 7.5 \mathrm{mg} / \mathrm{dl})$ levels and dietary intake of calories $(1755 \pm 449 /$ day $)$, total fats $(79.5 \pm 24.1 \mathrm{~g} /$ day $)$, saturated fats $(24.5 \pm 8.0 \mathrm{~g} /$ day $)$, monounsaturated fats $(23.2 \pm 8.2 \mathrm{~g} /$ day $)$, polyunsaturated fats $(28.5 \pm 11.1 \mathrm{~g} /$ day $)$, and omega- 3 fats $(9.4 \pm 4.8 \mathrm{~g} /$ day $)$ were not significantly different in the three groups. Vascular functions are shown in the table. There is a significant decline in arterial stiffness in left arm in flaxseed and fish oil supplementation groups. (Tab. 1)

Conclusions: Omega-3 fatty acids supplementation using either marine or vegetable sources causes a significant decrease in arterial stiffness in type 2 diabetes subjects.

\section{0}

A fenugreek seed extract selectively reduces fat consumption in healthy volunteers and overweight subjects

H. Chevassus ${ }^{1,2}$, A. Farret ${ }^{1,2}$, N. Molinier ${ }^{1}$, E. Renard ${ }^{3}$, P. Petit ${ }^{4}$, F. Galtier ${ }^{1,2}$; ${ }^{1}$ Clinical Investigation Centre, CHU Montpellier, ${ }^{2} \mathrm{Cic} 0001$, Inserm,

Montpellier, ${ }^{3}$ Maladies Endocriniennes, CHU Montpellier,

${ }^{4}$ UMR 5232, Université Montpellier I / CNRS, Montpellier, France.

Background and Aims: Fenugreek (Trigonella foenum-graecum L.) is an herbaceous Mediterranean plant, whose seeds are traditionally assumed to have nutritive properties. A number of pharmacological and clinical studies have shown blood glucose and plasma lipid lowering properties of either the seed itself, seed extracts or purified components. Several animal studies have demonstrated modifications of the feeding behavior after repeated treatment with seed extracts. However, no clinical study has previously investigated an action on eating behavior and energy intake in human.

Materials and Methods: Healthy volunteers: 12 healthy men aged $19-26$, mean BMI $22.5 \mathrm{~kg} / \mathrm{m}^{2}$ (20.0-25.0), participated in a double-blind randomised placebo-controlled trial of two different doses (588 or $1176 \mathrm{mg} /$ day) of a fenugreek seed extract, according to a latin square design. The three 14-day treatment periods were separated by a 14-day washout. Food intake was assessed using a 3-day record (last 3 days of each period). On the last day of each period the following evaluations were performed: weight, lipid profile, blood glucose and insulin and visual analog scales for appetite and satiety before and 30, 60, 90, 120, 150, 180, 210 and $240 \mathrm{~min}$ after a standardised meal. Overweight subjects: 39 overweight men aged $18-59$, mean BMI $27.3 \pm 0.2$ $\mathrm{kg} / \mathrm{m}^{2}$ (24.9-29.4), participated in a 6-week double-blind, parallel, randomised placebo-controlled trial of $1176 \mathrm{mg} /$ day of a fenugreek seed extract. Dietary intake was assessed twice (prior to treatment start and during the last week of treatment) using a 7 -day record checked by a trained dietician. On the last day of treatment the following evaluations were performed: weight, bio-impedance analysis, visual analog scales for appetite and satiety before and 30, 60, 90, 120, 150, 180, 210 and $240 \mathrm{~min}$ after a standardised meal, and spontaneous food intake during an ad libitum test meal.

Results: Spontaneous fat intake was significantly decreased with fenugreek seed extract treatment, both for healthy volunteers (mean \pm SEM : $3.73 \pm$ 0.29 vs. $4.51 \pm 0.28 \mathrm{MJ} / \mathrm{d}$, i.e $-17.3 \%, \mathrm{p}=0,038$ ) and for overweight subjects $(3.41 \pm 0.20$ vs. $3.77 \pm 0.19 \mathrm{MJ} /$ d, i.e $-9.5 \%$, p = 0,032 for energy expenditureadjusted fat intake). There was a trend towards a decrease in total spontaneous energy intake for healthy volunteers $(9.97 \pm 0.76$ vs. $11.29 \pm 0.75 \mathrm{MJ} / \mathrm{d}$, i.e $11.7 \%, \mathrm{p}=0,1)$ but not for overweight subjects ( $8.73 \pm 0.52$ vs. $9.16 \pm 0.44$, NS). Basal plasma insulin/blood glucose ratio was decreased in overweight subjects treated with fenugreek seeds extract $(0.891 \pm 0.088$ vs. $1.60 \pm 0.095$ $\mathrm{mUI} / \mathrm{mmol}, \mathrm{p}=0.044)$. There was no difference concerning weight, body composition, appetite and satiety scores, plasma lipid profile, post prandial glucose and insulin values and spontaneous food intake during the ad libitum test meal.
Conclusion: These 2 clinical studies in healthy and overweight subjects yelded concordant results showing that a short- term administration of a fenugreek seeds extract modifies food consumption, with a specific reduction in fat intake. These results constitute a novel information which contrasts with the current use of fenugreek seeds and opens new perspectives for clinical applications.

Supported by the French Department of Health and Legras Laboratory

\section{1}

The effect of dietary choice on body size and insulin resistance in children: a longitudinal study

A. N. Jeffery, B. S. Metcalf, J. Hosking, D. S. Gardner, L. D. Voss,

T. J. Wilkin;

Department of Endocrinology and Metabolism, Peninsula Medical School, Plymouth, United Kingdom.

Background and Aims: Rates of childhood obesity are increasing but few longitudinal studies have examined the contribution of dietary choice. We examined the impact of dietary choice on the development of obesity and insulin resistance in healthy young children.

Materials and Methods: Participants were from The EarlyBird Diabetes Study (140 boys and 111 girls). This prospective, non-intervention, cohort study is following healthy children from age 5 to 16 , assessing factors leading to insulin resistance. Annual measures from 5 to 9 years: BMI(sds), sum of 5 skinfolds (SSF), insulin resistance (HOMA-IR). Dexa \% abdominal fat at 9 years. Food frequency questionnaires completed annually by a parent and three variables calculated (number of days consumed per week expressed as a percentage of maximum possible frequency): HS (composite of 6 high sugar foods and drinks including cola), HF (11 high fat foods), FV (3 foods: fruit, vegetables, salad). No food was assigned to more than one group.

Results:

1. Dietary reports were consistent year-on-year (in each food group year-onyear correlation $\mathrm{r}=0.3-0.5$ ).

2. From 5-9 years, BMI(sds) rose (0.34 to $0.48, \mathrm{p}=0.007)$; SSF rose $(3.98 \mathrm{~cm}$ to $5.62 \mathrm{~cm}, \mathrm{p}<0.001)$ yet frequency of HF fell $(25.5 \%$ to $22.1 \%, \mathrm{p}<0.001)$ and HS fell $54.4 \%$ to $49.3 \%, \mathrm{p}<0.001$ ).

3. Frequency of FV remained unchanged $(11.4 \%$ to $11.6 \%, \mathrm{p}=0.5)$ and was not associated with BMI or SSF in either gender, or with HOMA-IR in boys $(\mathrm{r}<0.1)$. In girls at $5 \mathrm{y}$ and $6 \mathrm{y}, \mathrm{FV}$ was positively associated with HOMA-IR $(\mathrm{r}=0.21,0.22, \mathrm{p}<0.05)$.

4. Frequency of HF correlated with HS foods at each age $(r=0.2-0.6$, $\mathrm{p}<0.01)$.

5. BMI at 8 and $9 y$ was inversely related to HF in both genders ( $r=-0.18$ to $-0.28, \mathrm{p}<0.05)$.

6. BMI and SSF were inversely related to HS at every age, significantly so in girls $(r=-0.18$ to $-0.29, \mathrm{p}<0.05)$.

7. HS at 5y predicted lower BMI, SSF, HOMA-IR and \% abdominal fat at 9y in girls $(\mathrm{r}=-0.26,-0.30,-0.24,-0.26, \mathrm{p}<0.05$ respectively).

Conclusion: Parental reports regarding children's dietary habits are consistent year-on-year. Body size appears unrelated to frequency of consumption of healthy foods. Unexpectedly, children whose diets are highest in fat and sugar seem to be the leanest and metabolically healthiest. This could be due to parents underestimating their child's intake of high fat and sugar foods, but it is also possible that meal size is more important than content in childhood obesity.

Supported by Novo Nordisk UK Research Foundation 


\section{PS 73 Exercise}

\section{2}

The effect of exercise training on lipopolysaccharide-induced cardiovascular dysfunction of type 1 diabetic rats

C. H. Hung ${ }^{1}$, C. N. Chang ${ }^{1}$, Y. W. Chen ${ }^{2}$, J. T. Cheng ${ }^{3}$;

${ }^{1}$ Department of Physical Therapy, National Cheng Kung University,

${ }^{2}$ Department of Medical Research, Chi-Mei Medical Center, ${ }^{3}$ Department of Pharmacology, National Cheng Kung University, Tainan, Taiwan.

Background and Aims: Diabetic patients usually develop serious infections such as sepsis which is often accompanied with severe hypotension. However, heat shock protein 72 (HSP72) overexpression could reduce the mortality rate and organ damage in septic shock and attenuate hemodynamic alterations due to lipopolysaccharide (LPS). Furthermore, through exercise training, HSP72 can be induced in various organs of normal rats. We validated the hypothesis that exercise preconditioning may induce the overexpression of HSP72 in the heart and nucleus tractus solitarii (NTS) of the brain and confer cardiovascular protection during endotoxemia in streptozotocin-induced diabetic rats.

Materials and Methods: Adult male Wistar rats were randomly assigned to one of the following groups: sedentary normal rats, sedentary diabetic rats, and diabetic rats with exercise training. The trained animals ran on a treadmill 5 days/week, 30-60 min/day with an intensity of $1.0 \mathrm{mile} /$ hour over a 3-week period. After $24 \mathrm{hrs}$ from the last training session, we compared the temporal profiles of mean arterial pressure, heart rate, cardiac output, stroke volume, total peripheral vascular resistance, and serum tumor necrosis factor-alpha level of urethane-anesthetized rats in all groups after injection of LPS. In addition, the HSP72 expressions in heart and NTS of the brain were determined in different groups by western blot with monoclonal anti-HSP72 antibody.

Results: The HSP72 expression in heart and NTS were significantly greater in diabetic rats with exercise training than in those without exercise training ( $7.5 \pm 1.2$ fold and $3.1 \pm 0.9$ fold, respectively). After administration of LPS, the survival times were significantly longer in diabetic rats with exercise training than those in sedentary diabetic rats $(489.17 \pm 23.47 \mathrm{~min}$ versus $316.40 \pm 39.55 \mathrm{~min}, \mathrm{P}=0.0004$ ). Tumor necrosis factor-alpha level in serum were significantly lower in diabetic rats with exercise preconditioning than those in diabetic rats without exercise training $(78.89 \pm 56.30 \mathrm{pg} / \mathrm{ml}$ versus $1080.30 \pm 489.49 \mathrm{pg} / \mathrm{ml}, \mathrm{P}=0.007)$. In addition, prior exercise training could significantly diminish cardiovascular dysfunction in diabetic rats during endotoxemia.

Conclusion: Exercise preconditioning could overexpress HSP72 in the heart and NTS, confer significant protection against a high mortality rate, and improve cardiopulmonary function of streptozotocin-induced diabetic rats during endotoxemia.

Supported by the National Science Council of Taiwan

\section{3}

Impact of diabetic status in the six-minute-walk-test

E. Marin, R. Fabregate, O. Sánchez, M. Fabregate, S. Redondo,

G. Nuñez de Arenas, N. de la Torre, J. Sabán-Ruiz;

Endothelial Pathology Unit, Ramon y Cajal Hospital, Madrid, Spain.

Introduction: Modern systems allow for the evaluation of cardiac and vascular adaptations to body demands of increased tissue blood flow during exercise, particularly at the muscle.

Aims: To evaluate the haemodynamic response and recovery to a standardized exercise test in diabetic and non-diabetic patients.

Methods: $\mathrm{N}=21$ patients, $40-80$ years $(56,10 \pm 2,71), 15 / 6$ male/female ratio, 10 hypertensives, and 5 smokers. They were distributed in two groups: Group A (non-diabetic group): $\mathrm{n}=14$, aged $41-71$ (59,89 $\pm 5,18), 8 / 4$ male/female ratio; Group B (diabetic group): $\mathrm{n}=9$, aged $40-80(59,89 \pm 5,18), 7 / 2$ male/female ratio, 2 type 1, 7 type 2 . Both groups were comparable by age, and hypertension and smoking status. Pulse Rate (PR)(beats/min), Systolic and Diastolic Blood Pressure (SBP, DBP) (mm Hg), Pulse pressure ( $\mathrm{PP}=\mathrm{SBP}-\mathrm{DBP})$. Large (C1) and small (C2) artery elasticity index ( $\mathrm{ml} / \mathrm{mmHgx} 100)$, Systemic Vascular Resistance (SVR)(dyne.sec.cm5), Total Vascular Impedance (TVI)(dyne.sec. $\mathrm{cm} 5)$. Stroke Volume (SV)(ml/beat); Stroke Volume Index (SVI)(ml/beat/ $\mathrm{m} 2)$. Cardiac Output $(\mathrm{CO})(\mathrm{L} / \mathrm{min})$. Cardiac Output Index (COI) $(\mathrm{L} / \mathrm{min} /$ $\mathrm{m} 2$ ). Cardiac Ejection Time (msec)(CET). HDI/PulseWave CR-2000. The Profilor test was performed at baseline and after two, five and ten minutes during recovery time. It was not possible to record data at all three different moments for all patients in the control group. Six-minute-walk (SMW) test: constant slope (7\%) and marching speed were equivalent in groups $\mathrm{A}$ and B (4,67 $\pm 0,50$ vs $4,78 \pm 0,53 \mathrm{mph}$, respectively; not statistically significant). Statistical analysis: Kolmogorov-Smirnov, Mann-Whitney and Wilcoxon Signed Rank non-parametric tests. Independent and Paired Student's T tests. Results: 1 - After two minutes post-SMW: both groups showed an increase in PR compared to baseline (Group A: $p=0,017)$ (Group B: $p=0,038$ ) and a decrease in SVI $(A: p=0,032)(B: p=0,012)$; in $A$, but not in $B$, both CO and COI showed significant increases $[(p=0,033)$ and $(p=0,017)$, respectively]. Besides, in B, blood pressure levels increased (SBP: $p=0,038)(D B P: p=0,012)$ as well as PP levels ( $p=0,05)$, whereas CET decreased ( $p=0,021) .2$ - After five minutes: significant haemodynamic changes were present only in Group A: increase in PR $(p=0,010)$, CO $(p=0,041)$, and COI $(p=0,021)$, and decrease in SVR ( $\mathrm{p}=0,021)$ and SVI ( $\mathrm{p}=0,028) .3$ - After ten minutes: in group A there was an increase in PR $(p=0,012)$, and a decrease in SVR $(p=0,028)$. In diabetic patients, no differences were found after ten minutes with respect to baseline.

Conclusions: 1- Two minutes after moderate exercise, only the non-diabetic group shows increases in $\mathrm{CO}$ and COI. On the contrary, only the diabetic group has significant increases in blood pressure and pulse pressure, and decreases in CET. 2- After five minutes, significant differences with respect to baseline were observed in the non-diabetic group compared to the diabetic group in terms of PR, SVR, CO, COI, SVI. 3- After 10 minutes of moderate exercise, the diabetic group shows different haemodynamic behaviour compared to that of the non-diabetic group, with an increase in PR and a decrease in SVR.

\section{4}

Relationship of oxygen consumption with beta cell function and insulin sensitivity in IGT and newly diagnosed type 2 diabetic subjects Q. Nahar ${ }^{1}$, M. O. Faruque ${ }^{2}$, M. A. Rashid ${ }^{2}$, M. H. Rahman ${ }^{3}$, K. Sultana ${ }^{1}$, S. Kasem ${ }^{1}$, A. K. Azad Khan ${ }^{4}$;

${ }^{1}$ Dept of Human Nutrition, ${ }^{2}$ Biochemistry \& Cell Biology, ${ }^{3}$ Dept of Endocrinology, ${ }^{4}$ Health Economics Unit, BIRDEM, Dhaka, Bangladesh.

Background and Aims: Physical fitness is a strong independent determinant of insulin sensitivity and it is determined by the measurement of oxygen consumption worldwide. The present study was planned to explore the relationship of oxygen consumption with Beta cell function and insulin sensitivity in IGT and newly diagnosed type 2 diabetic subjects.

Materials and Methods: A total number of 188 subjects were recruited of which 34 were Control, 92 were isolated IGT, 27 were combined IFG-IGT and 35 were type $2 \mathrm{DM}$. Plasma insulin was measured by chemiluminescene based immunoassay technique. Insulin secretary capacity HOMA B (\%) and insulin sensitivity HOMA $S$ (\%) were calculated from fasting glucose and fasting insulin values by homeostasis model assessment (HOMA) using HOMA-CIGMA software. For the estimation of oxygen consumption subjects were requested to run on a treadmill for $6 \mathrm{~min}$ at their maximum speed and the burning calorie including the weight of the subjects were used in a standard formula.

Results: BMI and waist-hip ratio did not differ among the Controls, IGT, IFG-IGT and DM subjects. DM subjects had significantly $(\mathrm{p}<0.05)$ higher neck circumference compared to controls. Significantly $(\mathrm{p}<0.05)$ higher TG and cholesterol levels were found in IGT and IFG-IGT subjects compared to controls. The data showed significantly $(\mathrm{p}<0.05)$ lower HOMA B in IGT [101 (29-288)], IFG-IGT [77 (25-121)] and DM [77 (27-161)] subjects compared to Controls [(112 (34-247)]. Insulin sensitivity (HOMA S) in diabetic subjects $[100(16-306)]$ was significantly $(p<0.05)$ lower compared to controls [122 (48-308)]. Oxygen consumption ( $\mathrm{ml} / \mathrm{kg} / \mathrm{min})$ in DM subjects [13.8 $(8.8-18.8)]$ was significantly $(\mathrm{p}<0.05)$ lower compared to Controls [17.8(8.8-33.5)]. The levels of oxygen consumption in IGT [16.2(8.2-33.5)] and IFG-IGT [16.2(9.5-28.2)] did not differ with Controls or DM subjects but a lowering tendency of oxygen consumption was observed in both IGT and IFG-IGT subjects compared to Controls. In regression analysis it was found that oxygen consumption was negatively associated $(\mathrm{r}=-0.71, \mathrm{p}=-$ 0.08 ,) with fasting serum glucose concentration in diabetic subjects. Oxygen 
consumption level in IGR (IFG and IFG-IGT) subjects was negatively associated $(\mathrm{r}=-0.29, \mathrm{p}=0.01)$ with HOMA B and it was positively associated $(\mathrm{r}=0.27, \mathrm{p}=0.02)$ with HOMA S.

Conclusion: Diabetes mellitus is associated with decreased oxygen consumption. In hyperglycemic condition oxygen consumption is positively correlated with insulin sensitivity.

Supported by BIRDEM, IPICS

\section{5}

The importance of light intensity physical activity in sedentary individuals with a family history of type 2 diabetes

U. Ekelund, S. J. Griffin, N. J. Wareham;

Institute of Metabolic Sciences, MRC Epidemiology Unit, Cambridge, United Kingdom.

Background and Aims: Increasing levels of energy expenditure devoted to physical activity (PAEE) is likely to be important in the prevention of type 2 diabetes, especially in those with a family history of the disease, as the risk is further magnified by physical inactivity. It is not clear whether the variation PAEE is due to the variation in light, moderate or vigorous intensity physical activity. Identifying the sub-dimensions of activity that contributes the most to the variation in PAEE is essential when promoting physical activity and when designing interventions.

Materials and Methods: Participants $(n=258)$ were selected from the ProActive trial, which is a randomised controlled trial of the efficacy of a family-based intervention to increase physical activity among individuals at high risk of diabetes. Physical activity energy expenditure was measured by individually calibrated heart rate monitoring and body movement was simultaneously measured by accelerometry. We analysed whether the time estimates (time spent sedentary, light, moderate and vigorous intensity activity) from accelerometry contributed to the explained variance in PAEE by linear regression analysis.

Results: PAEE was significantly correlated with time spent at light intensity physical activity $(\mathrm{r}=0.17, \mathrm{P}=0.007)$ and with time spent at moderate intensity activity $(r=0.20, P<0.001)$. In multiple regression analysis, sex, age and body weight explained $13.7 \%$ (adjusted $\mathrm{R}^{2}$ ) of the variance in PAEE. In our multivariate model, fraction of time spent at light $(\beta=0.015, \mathrm{P}<0.001)$ and at moderate intensity physical activity $(B=0.038, P=0.026)$, but not fraction of time spent sedentary $(B=0.0002, P=0.96)$ or at vigorous intensity activity $(B=0.05, P=0.50)$ contributed to the explained variance in PAEE, with a full model of $20.6 \%$ (adjusted $\mathrm{R}^{2}$ ). The magnitude of association between light intensity activity and PAEE was almost twice as strong as that between moderate intensity and PAEE.

Conclusion: Time spent at light and at moderate intensity physical activity, but not vigorous intensity activity, significantly contributes to an elevated PAEE. Therefore, incorporating activities of light and moderate intensity into daily life, such as walking, bicycling and household and garden work, which contributes to an elevated PAEE, is likely to be important in the prevention of type 2 diabetes and related metabolic disorders.

Supported by Medical Research Council, NHS R\&D, RCGP Scientific Foundation, and Diabetes UK

\section{6}

Prevalence of cardiovascular risk factors and physical capacity in cardiac rehabilitation: is there a difference between diabetic and non diabetic patients?

P. R. Blanc ${ }^{1}$, A. Boussuges ${ }^{2}$, S. Chopra ${ }^{1}$, X. Debussche ${ }^{3}$;

${ }^{1}$ Cardiac Rehabilitation, Sainte Clotilde, Réunion,

${ }^{2}$ IMNSSA, Mediterranean University, Toulon Marseille, France,

${ }^{3}$ Diabetology, Felix Guyon Hospital, Sainte Denis, Réunion.

Background and Aims: Patients with type 2 diabetes mellitus have typically elevated risk factors and low fitness. We compared the prevalence of cardiovascular risk factors and physical capacity between diabetic patients with coronary heart disease (CHD) and non diabetic patients (NDP) with CHD admitted in a 6-week multidisciplinary cardiac rehabilitation program. Materials and Methods: 351 type 2 diabetic patients (DP) were compared with 480 non diabetic patients (NDP). DP and NDP were not different in age
( 57 vs. 57 years), sex ratio (19\% vs. $16 \%$ women) and type of coronary event. Sixty two percent of DP was treated by insulin. Baseline $\mathrm{HbA}_{1 c}$ was greater than $6.6 \%$ in $63 \%$ of cases, retinopathy and microalbuminuria were detected in respectively $30 \%$ and $36 \%$ of subjects.

Results: Compared with NDP, DP had a higher prevalence of hypertension ( $70 \%$ vs. $45 \%$ - $<<0.001$ ), hypercholesterolemia ( $80 \%$ vs. $66 \%-\mathrm{p}<0.001$ ), hypertriglyceridemia ( $41 \%$ vs. $32 \%-p=0.01)$, obesity ( $33 \%$ vs. $15 \%-p<0.001)$ and excess abdominal fatness quantified by waist circumference measurement ( 98.5 vs. $93.7 \mathrm{~cm}$ in women, 97.5 vs. $95 \mathrm{~cm}$ in men - p<0.05). Furthermore, DP had a higher score (HAD scale) for depression $(6.52$ vs. $5.74-\mathrm{p}<0.027)$ with a higher prevalence of depression $(38.1 \%$ vs. $30.5 \%-\mathrm{p}<0.05)$. Anxiety (HAD scale) and stresses were similar in the two groups. Patients who continue to smoke were less frequent in DP compared with NDP $(9.8 \%$ vs. $16 \%$ $-\mathrm{p}=0.007$ ). At baseline, DP had a lower VO2 peak ( $14.4 \mathrm{vs.} 16.7 \mathrm{ml} / \mathrm{kg} / \mathrm{min}$ $-p<0.001)$ at the cardiorespiratory exercise testing, a lower time of anaerobic threshold ( 1.60 vs. $1.76 \mathrm{~min}-\mathrm{p}<0.001$ ), a lower distance at the 6 min walk test ( 325 vs. $400 \mathrm{~m}-\mathrm{p}<0.05$ ) and a lower, but non statistically significant, hand strength at the hydraulic dynamometer ( 34 vs. $37 \mathrm{KGs}$ for right hand 33 vs. $35 \mathrm{KGs}$ for left hand), when compared with NDP. At the end of the exercise program, the entire patients achieved significant improvements in physical capacity. Improvements were statistically similar in DP and NDP: peak VO2 $(+25 \%$ vs. $+27 \%)$, time of anaerobic threshold $(+70 \%$ vs. $+70 \%)$ and distance at the 6 min test $(+32 \%$ vs. $+34 \%)$. The comparison between DP and NDP after exercise training demonstrated lowers VO2 peak (18 vs. $21.3 \mathrm{ml} / \mathrm{kg} / \mathrm{min}$ $-\mathrm{p}<0.001)$, time of anaerobic threshold $(2.7 \mathrm{vs.} 3 \mathrm{~min}-\mathrm{p}<0.001)$, and distance at the 6 min walk test $(429$ vs. $530 \mathrm{~m}-\mathrm{p}<0.05)$ in DP.

Conclusion: In our study, DP with CHD had a higher cardiovascular risk including hypertension, dyslipidemia, abdominal fatness and depression as well as a lower physical capacity compared with NDP. The entire patient group demonstrated improvements in exercise capacity after rehabilitation. However, in DP, physical capacity was lower at the beginning of the program and remained significantly altered after the training. This study emphasizes the need to target DP for an aggressive and multidisciplinary program of risk factor management, including exercise training and educational programs. Greater efforts to improve their outcomes are needed.

\section{7}

Influence of combined exercise training on indices of obesity, diabetes and cardiovascular risk in diabetes type 2 patients P. Calders ${ }^{1}$, S. Lambers ${ }^{1}$, K. Van Acker ${ }^{2}$, C. Van Laethem ${ }^{1}$; ${ }^{1}$ Revalidatiewetenschappen en Kinesitherapie Gent, Arteveldehogeschool, Belgium, ${ }^{2}$ Endocrinologie, AZ Sint-Jozef, Bornem, Belgium.

Background and Aims: The influence of combined exercise training (strength and endurance training) on indices of obesity, diabetes and cardiovascular risk in type 2 diabetes patients was investigated compared to endurance training.

Materials and Methods: Forty six type 2 diabetes patients (female: 17, male: 29) were randomly assigned to three experimental groups (COM: combined exercise training $(n=17)$; END: endurance training $(n=18)$ and C: control $(n=11)$. Patients exercised for three months three times a week for 1 hour. Before and after the training period following parameters were assessed: 6 minute walk test (6MWT), muscular strength of upper limb (sum of 1 Repetitive maximum (1RM) of $\mathrm{m}$. Biceps brachii and $\mathrm{m}$. Triceps brachii) and lower limb (sum of 1RM of m. Quadriceps femoris and mm. Hamstrings), sit to stand test, length, weight, body mass index, body composition (fat mass), HbAlc, glycemia, triglycerides, HDL, total cholesterol and quality of life (SF36).

Results: Comparing combination training to no training, combination training had significant higher results on the sit to stand test $(\mathrm{P}<0,05), 6$ minute walk test $(\mathrm{p}<0,05)$, strength upper $\operatorname{limb}(\mathrm{p}<0,001)$ and strength lower $\operatorname{limb}(\mathrm{p}<0,05)$ compared to the control group. HbAlc $(\mathrm{p}<0,05)$ (figure 1$)$ and cholesterol $(\mathrm{p}<0,01)$ showed a significant decrease in the combination group and an increase in the control group. HDL increased in the combination group and decreased in the control group $(\mathrm{p}<0,05)$ (figure 2). Comparing the endurance group to the control group, the endurance group had significantly higher results on the 6 minute walk test $(\mathrm{P}<0,01)$ compared to the control group. Comparing combination training to endurance training, the combination group had significantly higher results on strength upper $(\mathrm{p}<0,005)$ and lower limb $(\mathrm{P}<0,05)$. The evolution of SF-36 items was not significantly different between the 3 groups. 
Conclusion: Combined exercise training had significant effects on indices of obesity, diabetes and cardiovascular risk in type 2 diabetic patients. Compared to endurance training, combined exercise gave a tendency towards better results.

We thank Dr. Luc Feyen, Mrs. Erna Feyen (general practice, Sas-Van-Gent, Holland), Mrs. Greet Vergauwen (AZ Sint-Jozef, Bornem, Belgium) for selecting and encouraging the participants and for their local support of this project

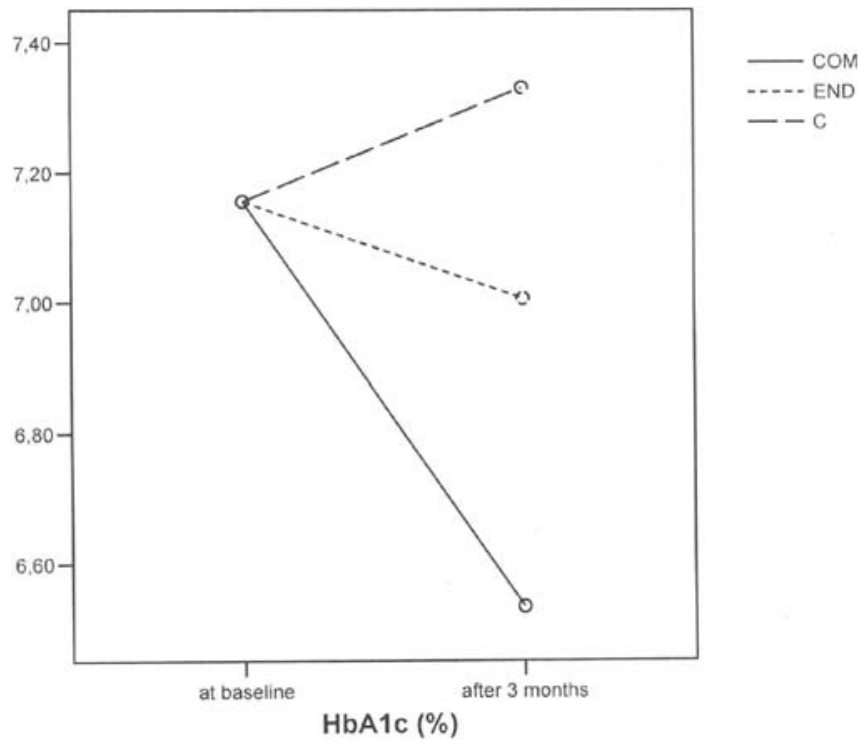

Fig. 1. HbAlc at baseline and after 3 months with baseline values as covariate

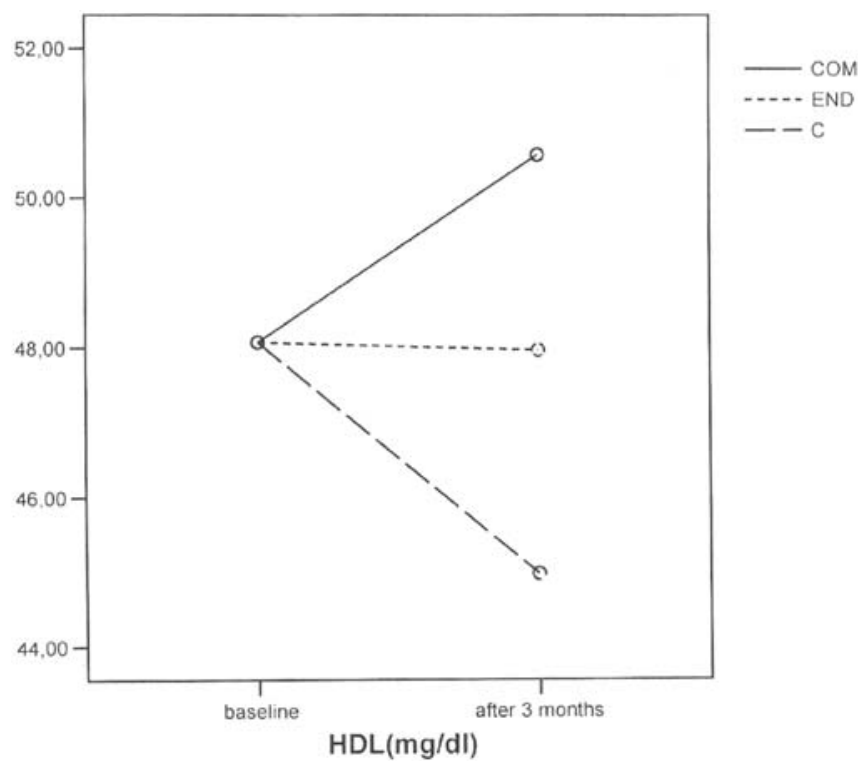

Fig. 2. HDL at baseline and after 3 months with baseline values as covariate

\section{8}

Educational intervention in a group of half-marathon runners with type 1 diabetes (The DIATLETIC team)

S. Murillo ${ }^{1}$, R. Arana ${ }^{2}$, M. Subirà ${ }^{1}$, A. Novials ${ }^{1}$;

${ }^{1}$ Institute of Diabetes, Fundació Sardà Farriol, Barcelona,

${ }^{2}$ Fundación para la Diabetes, Madrid, Spain.

Background and Aims: Little is known about the strategies that diabetic athletes use in relation to insulin and carbohydrate dosage and theirs glycemic fluctuations in a half-marathon competition. The aim of this study is to investigate the self-management with regard to a half-marathon race in patients with type-1 diabetes (T1DM) and the influence of an educational intervention

Materials and Methods: 14 well controlled T1DM men (age 35.6 7.4 years, IMC $23.9 \pm 2.3 \mathrm{~kg} / \mathrm{m}^{2}$ ) took part in the study. They were amateur athletes belonging to the DIATLETIC diabetes team. All the participants were taking insulin in multiple bouts a day and 3 of them were using an insulin pump.

The day before the race, they completed a questionnaire about their usual insulin dosage and carbohydrate intake (in reference to those days without exercise practice). These data were collected again a few days after, in reference to the half-marathon day. Moreover, values of glycemic control were gathered by means of capillary blood analyses at the following points: start, $\mathrm{km} 5, \mathrm{~km}$ $10, \mathrm{~km} \mathrm{15}$, arrival, 2 hours post-exercise (lunch), pre-dinner, 3 a.m. and prebreakfast of the following day. All these athletes took part in the same halfmarathon the following year. Again, data about insulin dosage, carbohydrate intake and glycemic control were collected, using the same protocol as in the previous year. Furthermore, the day before the competition, athletes were informed about the results obtained in the previous year in an educational session of 30 minutes duration. Results obtained in the first edition (FE) were compared with those of the second edition (SE).

Results: On average, participants realised a reduction in the total units of insulin of $14.13 \%$ in the $\mathrm{FE}$ and of $13.13 \%$ in the $\mathrm{SE}$. This reduction was greater in the delayed action or basal insulin in the FE than in the SE (5.1 14.6 units vs. $4.63 \pm 7.9$ units respectively, $\mathrm{p}<0.05)$. Also, the reduction of short action insulin in the breakfast before the competition was greater in the FE than in the SE ( $2.0 \pm 1.3$ units vs. $1.07 \pm 0.47$ units respectively, $\mathrm{p}<0.05)$. We did not find significant differences in the insulin doses used during the rest of the day. On the other hand, all runners needed to take supplements with carbohydrates to avoid hypoglycaemia during the competition. In comparison to their usual breakfast, they added $15.0 \pm 6.9 \mathrm{~g}(\mathrm{p}<0.05)$ in the FE and $18.0 \pm 4.9 \mathrm{~g}(\mathrm{p}<0.05)$ in the SE. As well, athletes needed additional carbohydrates during the course, this quantity being greater in the SE than in the FE $(59.1 \pm 11.2 \mathrm{~g}$ vs. $49.0 \pm 16.4 \mathrm{~g}$ respectively, $\mathrm{p}<0.05$ ). These carbohydrates were taken preferably as isotonic beverages or glucose gels. Before the competition, the mean value of blood glucose was of $254.6 \pm 121 \mathrm{mg} / \mathrm{dl}$ in the $\mathrm{FE}$ and of $187.3 \pm 57.9 \mathrm{mg} / \mathrm{dl}$ in the SE, going down to $167.7 \pm 67 \mathrm{mg} / \mathrm{dl}$ in the FE and until $147.74 \pm 75.5$ in the SE in the finish line. The percentage of values over $300 \mathrm{mg} / \mathrm{dl}$ was of $22.2 \%$ in the FE and of $5.0 \%$ in the SE $(\mathrm{p}<0.05)$ and below $100 \mathrm{mg} / \mathrm{dl}$ was of $33.3 \%$ in the FE and of $15 \%$ in the SE $(p<0.05)$.

Conclusion: Adaptations to a half-marathon competition in T1DM patients imply a reduction in the insulin doses and an increase in carbohydrate intake. However, it seems to be more successful if these carbohydrates extra are taken during the competition. Educational intervention in T1DM athletes group is an effective tool to improve glycemic control reducing glycemic fluctuations in a half-marathon competition.

Supported by ISCIII-RETIC RD06

\section{9}

Long-term brisk walking vs medical fitness in type 2 diabetes S. F. E. Praet ${ }^{1}$, L. S. J. van Rooij ${ }^{1}$, L. J. M. Boonman-de Winter ${ }^{2}$, A. P. Wijtvliet ${ }^{1}$, T. Enneking ${ }^{3}$, L. J. C. van Loon ${ }^{1}$;

${ }^{1}$ Movement Sciences, Nutrition and Research Institute Maastricht, ${ }^{2}$ SHL Contract Research, Stichting Huisartsen Laboratorium, Etten-Leur, ${ }^{3}$ General Practioner Group De Keen, Health Center De Keen, Etten-Leur, The Netherlands.

Background and Aims: Structured exercise is considered an important cornerstone to achieve good glycemic control and improve cardiovascular risk profile in type 2 diabetes (T2D). However, long-term adherence to medical fitness (MF) programs is generally poor, while costs are high. As such, brisk walking (BW) may be an attractive alternative, however, its longterm efficacy as an addendum to a primary health care based diabetes care program is unknown. Therefore, the effects of $1 \mathrm{yr} \mathrm{BW}$ versus $1 \mathrm{yr}$ MF in T2D patients in a primary health care setting was studied.

Materials and Methods: 92 T2D patients $(59.9+/-0.9 \mathrm{yrs})$ were randomised to structured exercise, consisting of either 3 times a week $45-60 \mathrm{~min}$ BW $(n=43)$ or MF $(n=49)$. Changes in metabolic profile, blood pressure regulation, physical fitness and BMI were monitored 12 months after start of the exercise intervention. 
Results: After $1 \mathrm{yr}, 39.1 \%$ of the participants (BW:n=17, MF:n=19, ns) were still actively participating, while only $25 \%$ of the participants adhered to $>70 \%$ of the exercise sessions. Besides motivational problems, co-morbidities, such as overuse injuries and subclinical osteoarthritis appeared an important reason for our relatively high drop out rate. Intention-to-treat analysis showed significant improvements in blood pressure regulation, HbAlc, TChol/ HDL-C ratio, LDL-C and BMI, while fasting glucose tended to improve as well $(p=0.09)$. Post-hoc analyses revealed that glycemic control at baseline (HbAlc_BL) correlates negatively with the change in HbAlc (Pearson's $\mathrm{R}=-0.39, \mathrm{p} 0.05$ ). Table 1 shows the results of this subgroup analysis. Overall, metabolic and cardiovascular improvements in BW were not statically different from MF (ANOVA, $\mathrm{p}>0.05$ ).

Conclusion: Long-term structured exercise interventions, as addendum to primary health care based diabetes care programs, improve cardiovascular risk profile, especially in patients with suboptimal glycemic control. Longterm program adherence may improve if the exercise intervention is tailored to the physical capabilities of the individual patient. (Fig. 1)

Supported by OZ/CZ Health Care Insurances, Breda, The Netherlands

Fig. 1

\begin{tabular}{|c|c|c|c|c|c|}
\hline \multirow{2}{*}{\multicolumn{2}{|c|}{$\begin{array}{c}\text { Intention to } \\
\text { Treat Analysis } \\
\mathrm{N}=57\end{array}$}} & \multicolumn{2}{|c|}{ Brisk Walking $(n=30)$} & \multicolumn{2}{|c|}{ Medical Fitness $(n=27)$} \\
\hline & & $\mathrm{Hb} A_{1 \mathrm{eBL}}<7.0 \%(\mathrm{n}=18)$ & $\mathrm{HbA}_{1 \mathrm{c} \_\mathrm{BL} .} \geq 7.0 \%(\mathrm{n}=12)$ & $\mathrm{HbA}_{10 \mathrm{BL}}<7.0 \%(\mathrm{n}=15)$ & $\mathrm{HbA}_{1 \mathrm{e}_{-} \mathrm{BL} .} \geq 7.0 \%(\mathrm{n}=12)$ \\
\hline \multirow{2}{*}{$\begin{array}{c}\mathrm{BMI} \\
\left(\mathrm{kg} / \mathrm{m}^{2}\right)\end{array}$} & T0 & $31.5 \pm 4.7$ & $32.5 \pm 3.9$ & $32.8 \pm 5.2$ & $31.3 \pm 4.0$ \\
\hline & $\mathrm{T} 12$ & $31.5 \pm 4.6$ & $31.8 \pm 2.9^{*}$ & $32.2 \pm 5.7$ & $30.3 \pm 4.1^{*}$ \\
\hline \multirow{2}{*}{$\begin{array}{l}\text { Fast.Glc } \\
(\mathrm{mmol} / \mathrm{L})\end{array}$} & T0 & $7.2 \pm 0.9$ & $9.4 \pm 2.7$ & $7.2 \pm 0.5$ & $10.0 \pm 3.7$ \\
\hline & $\mathrm{T} 12$ & $7.7 \pm 2.1$ & $8.3 \pm 3.3^{*}$ & $7.1 \pm 1.2$ & $8.7 \pm 2.1^{*}$ \\
\hline \multirow{2}{*}{$\begin{array}{c}\mathrm{Hb}_{\mathrm{le}} \\
(\%)\end{array}$} & T0 & $6.2 \pm 0.4$ & $7.7 \pm 0.7$ & $6.4 \pm 0.3$ & $8.4 \pm 1.3$ \\
\hline & $\mathrm{T} 12$ & $6.4 \pm 0.8$ & $7.5 \pm 1.3^{*}$ & $6.2 \pm 0.5$ & $7.6 \pm 1.2^{*}$ \\
\hline \multirow{2}{*}{$\begin{array}{c}\mathrm{SBP} \\
(\mathrm{mmHg})\end{array}$} & T0 & $151 \pm 21$ & $151 \pm 21$ & $151 \pm 14$ & $148 \pm 16$ \\
\hline & $\mathrm{T} 12$ & $132 \pm 12^{\#}$ & $144 \pm 20^{\#}$ & $137 \pm 14^{\#}$ & $138 \pm 16^{\#}$ \\
\hline \multirow{2}{*}{$\begin{array}{c}\text { DBP } \\
(\mathrm{mmHg})\end{array}$} & T0 & $83 \pm 13$ & $80 \pm 11$ & $83 \pm 9$ & $82 \pm 10$ \\
\hline & $\mathrm{T} 12$ & $74 \pm 9^{+\#}$ & $76 \pm 9$ & $75 \pm 6^{H}$ & $78 \pm 10$ \\
\hline \multirow{2}{*}{$\begin{array}{l}\text { TChol/ } \\
\text { HDL-C }\end{array}$} & T0 & $4.3 \pm 0.9$ & $4.0 \pm 0.9$ & $4.3 \pm 0.7$ & $4.3 \pm 0.9$ \\
\hline & $\mathrm{T} 12$ & $4.0 \pm 1.0$ & $3.9 \pm 0.9$ & $4.0 \pm 0.7$ & $3.8 \pm 0.9^{*}$ \\
\hline \multirow{2}{*}{$\begin{array}{l}\text { LDL-C } \\
(\mathrm{mmol} / \mathrm{L})\end{array}$} & T0 & $3.0 \pm 0.9$ & $2.3 \pm 0.5$ & $2.6 \pm 0.6$ & $2.7 \pm 0.6$ \\
\hline & $\mathrm{T} 12$ & $2.5 \pm 0.7^{*}$ & $2.1 \pm 0.6$ & $2.4 \pm 0.5^{\circ}$ & $2.6 \pm 0.7$ \\
\hline \multirow{2}{*}{$\begin{array}{l}\text { Triglyc. } \\
(\mathrm{mmol} / \mathrm{L})\end{array}$} & T0 & $1.9 \pm 1.1$ & $2.0 \pm 1.0$ & $1.8 \pm 0.8$ & $1.4 \pm 0.5$ \\
\hline & $\mathrm{T} 12$ & $1.8 \pm 0.8$ & $2.0 \pm 1.3$ & $1.6 \pm 0.5$ & $1.3 \pm 0.7$ \\
\hline
\end{tabular}

" $\mathrm{p}<0.01 \%$ p $<0.05$ ANOVA, after Bonferroni correction for multiple comparisons 


\section{0}

Regular use of pedometers does not enhance beneficial outcomes in a physical activity intervention study in type 2 diabetes M. R. Bjørgaas ${ }^{1,2}$, J. T. Vik ${ }^{3}$, T. Stølen ${ }^{4}$, S. Lydersen ${ }^{2}$, V. Grill ${ }^{2,1}$; ${ }^{1}$ Department of Internal Medicine, University Hospital of Trondheim, Trondheim, Norway, ${ }^{2}$ Department of Cancer Research and Molecular Medicine, Norwegian University of Science and Technology, ${ }^{3}$ Department of Physical Medicine and Rehabilitation, University Hospital of Trondheim, ${ }^{4}$ Department of Circulation and Medical Imaging, Norwegian University of Science and Technology, Trondheim, Norway.

Background and Aims: Use of pedometer is proposed to increase walking and improve physical fitness. We investigated whether use of pedometer can enhance beneficial outcomes in a physical intervention study in type 2 diabetes (T2DM).

Tab. 1

Clinical and laboratory variables at Visit 1 in Pedometer-group, Non-Pedometer-group and Droputs

\begin{tabular}{llll}
\hline & $\begin{array}{l}\text { Pedometer } \\
\text { group }(n=23)\end{array}$ & $\begin{array}{l}\text { Non-Pedometer } \\
\text { group }(\mathrm{n}=25)\end{array}$ & $\begin{array}{l}\text { Dropouts } \\
(n=22)\end{array}$ \\
\hline Age (years) & $56.4(11.0)$ & $61.2(9.7)$ & $57.7(14.0)$ \\
Weight $(\mathrm{kg})$ & $94.8(19.3)$ & $95.2(16.6)$ & $94.3(14.3)$ \\
BMI $\left(\mathrm{kg} / \mathrm{m}^{2}\right)$ & $31.2(5.3)$ & $31.5(5.6)$ & $31.0(4.4)$ \\
FPG $(\mathrm{mmol} / \mathrm{l})$ & $8.0(1.6)$ & $8.7(2.6)$ & $7.5(1.9)$ \\
HbA $_{1 \mathrm{c}}(\%)$ & $7.4(1.1)$ & $7.7(1.4)$ & $7.3(1.1)$ \\
Insulin C-peptide $(\mathrm{nmol} / \mathrm{l})$ & $1.1(0.5)$ & $1.1(0.6)$ & $1.3(0.5)$ \\
Cholesterol $(\mathrm{mmol} / \mathrm{l})$ & $5.0(1.2)$ & $5.0(0.8)$ & $4.8(1.2)$ \\
HDL-cholesterol $(\mathrm{mmol} / \mathrm{l})$ & $1.28(0.38)$ & $1.17(0.32)$ & $1.12(0.24)$ \\
Triglycerides $(\mathrm{mmol} / \mathrm{l})$ & $2.0(1.0)$ & $2.0(1.0)$ & $2.2(1.2)$ \\
VO2peak $(\mathrm{l} / \mathrm{min})$ & $2.70(0.70)$ & $2.65(0.88)$ & $2.19(0.60)$ \\
VO2peak $(\mathrm{ml} / \mathrm{kg} / \mathrm{min})$ & $29.6(8.3)$ & $28.1(7.5)$ & $22.4(5.3)$ \\
\hline
\end{tabular}

Materials and Methods: Seventy persons with T2DM were randomised to a pedometer- and a non-pedometer-group ( $\mathrm{P}$ - and Non-P-group). All participants were seen by a nurse at a baseline visit (V1), after 1 month (V2), 3 months (V3) and 6 months (V4) and were then encouraged to increase walking. Subjects in the P-group additionally registered pedometer steps 3 days twice per month for 6 months. After V1 and V4 aerobic capacity (VO2peak) was measured and subjects reported perceived physical fitness and activity. At V4 they were also asked if they could recognise any change in physical or mental state during the study.

Results: Twenty-two subjects did not complete the study (dropouts). VO2peak at V1 was lower in dropouts than in subjects who completed the study (completers) $(p=0.003)$. In the P-group, the number of steps/day was 7628 (3715) (mean (SD)) in month 1 and 8021(3368) in month 6 and did not increase during the study ( $p=0.65)$. In completers, taken together, there was a decrease in body weight $(p=0.005), \operatorname{HbAlc}(p=0.034)$, fasting blood glucose $(p=0.033)$, triglycerides $(p=0.002)$ and diastolic blood pressure $(p=0.048)$ and an increase in HDL-cholesterol (p0.38). Perceived improvement in physical and mental state correlated with improvement in VO2peak $(\mathrm{r}=0.45, p=0.008$ and $\mathrm{r}=0.38, p=0.03$, respectively, $n=34$ ).

Conclusion: Participation in the study entailed favourable changes in metabolic parameters. Improvement in VO2peak correlated with perceived improvement in health. Use of pedometer did not increase walking, nor enhance beneficial outcomes. The low VO2peak in dropouts indicates that persons most needy of physical exercise are the least compliant in exercise programmes. (Tab. 1)

Supported by Norwegian Foundation for Health and Rehabilitation, Central Norway Regional Health Authority and Norwegian University of Science and Technology, Trondheim, Norway

\section{PS 74 Hypoglycaemia}

\section{1}

Continuous glucose monitoring - what can we learn about nocturnal hypoglycaemia?

G. Roman ${ }^{1}$, M. Geamanu ${ }^{2}$, N. Hâncu ${ }^{1}$, A. Morosanu ${ }^{1}$, M. Gribovschi'; ${ }^{1}$ Clinical Center of Diabetes, Nutrition, Metabolic Diseases, "Iuliu Hatieganu" University of Medicine \& Pharmacy,

${ }^{2}$ Clinical Center of Diabetes, Nutrition, Metabolic diseases, County Hospital, ${ }^{3}$ Clinical Center of Diabetes, Nutrition, Metabolic diseases, "Motilor" Medical Center, Cluj-Napoca, Romania.

Background and Aims: Glycaemic control is a major therapeutic objective in the clinical management of diabetes: glycaemic values should be as near as possible to normal and A1c in target. First step should be to normalise fasting glycaemia without nocturnal hypoglycaemia. Optimising glucose control raises the risk of hypoglycaemia, a barrier to glycaemic control. Continuous glucose monitoring (CGM) is an ideal tool to assess glycaemic values during the night and to individualise therapeutic interventions. The aim of the study was to assess and interpret the nocturnal hypoglycaemic values in correlation with glyaemic control and therapy, in patients with type 1 and 2 diabetes who had CGM.

Materials and Methods: In persons with type 2 diabetes, 78 Medtronic CGM have been applied: age $55.6 \pm 10.3$ years, mean duration of diabetes $=8.6 \pm 7.3$ years, 22 women and in persons with type 1 diabetes, 46 CGM: median age 24 years, 39 women. Intial therapy: 2 on diet, 29 oral therapy, 47 on insulin. Among persons with type 1 diabetes, 13 were on insulin pump. The CGMS measures glucose every five minutes, 72 hours, around 850 glucose values. For this study only nocturnal values between $00-6$ am have been considered. Hypoglycaemia has been considered at a glucose value below $72 \mathrm{mg} / \mathrm{dl}$, as threshold for hormonal counter regulation. Basal glucose value has been considered at $6.00 \mathrm{am}$.

Results: The distribution according to Alc value was 36 patients $\leq 7 \%, 35$ : $7.1-8 \%, 22: 8.1-9 \%$ and $31:>9 \%$. In type 2 patients glucose values $\leq 72 \mathrm{mg} /$ $\mathrm{dl}$ represented $2.6 \%$ from all the values recorded, no severe hypos. While total period analysed summarised 822 hours, glucose values $\leq 72 \mathrm{mg} / \mathrm{dl}$ lasted 21.4 hours, $2.55 \%$. Mean fasting glucose was $141 \pm 46.1 \mathrm{mg} / \mathrm{dl}$. When A1c $\leq 7 \%$, the mean basal glucose value was $116.1 \pm 17.6 \mathrm{mg} / \mathrm{dl}$ compare to $142.2 \pm 54.7$ in patients with $\mathrm{A} 1 \mathrm{c} \geq 9 \%$. In patients with $\mathrm{Alc} \leq 7 \%$, glucose values $\leq 72$ $\mathrm{mg} / \mathrm{dl}$ occurred in $1.5 \%$, vs. $4.5 \%$ in patients with A1c $\geq 9 \%$. In patients on oral agents, mean A1c was $7.84 \pm 1.6 \%$ vs. $8.5 \pm 1.6 \%$ in those insulin treated. In patients on insulin glargine, mean basal glucose value was $128.42 \pm 45.4$ $\mathrm{mg} / \mathrm{dl}$, mean of all glucose values was $122.4 \pm 17 \mathrm{mg} / \mathrm{dl}$, with $3,4 \%$ glucose values $\leq 72 \mathrm{mg} / \mathrm{dl}$, compared to mean basal glucose of $157.6 \% \pm 52 \mathrm{mg} / \mathrm{dl}$, mean of all glucose values $142.7 \pm 20.7 \mathrm{mg} / \mathrm{dl}$ and $4.1 \%$ hypos in patients on other insulin. In persons with type 1 diabetes, total period summarised 852 hours with 75.6 hours glucose values $\leq 72 \mathrm{mg} / \mathrm{dl}, 8.9 \%$. Among the 13 patients with Alc $\leq 7 \%, 8$ experienced glucose values $\leq 72 \mathrm{mg} / \mathrm{dl}$, lasting 26 hours, $11 \%$ from a total of 234 hours of monitoring, while among the 7 patients with Alc $>9 \%$, one experienced glucose values $\leq 72 \mathrm{mg} / \mathrm{dl}$ with a duration of 8.2 hours/ 3 nights, $5.7 \%$ from a total of 144 hours.

Conclusion: Nocturnal hypoglycaemia can occur in patients with both type 1 and type 2 diabetes. Nocturnal hypoglycaemia was more frequent in patients with type 1 diabetes well controled and type 2 diabetes poorly controled. In type 2 diabetes, improvements in glycaemic control can be obtain with a low risk of hypos. Patients treated with insulin glargine had a better basal glucose value and lower prevalence of hypos. Identifying hypoglycemia with CGMS can minimise the frequency of hypoglycemia and help patients attain their glycemic goals.

\section{2}

Do high fasting glucose levels suggest nocturnal hypoglycaemia? The Somogyi effect - fact or fiction?

P. Choudhary ${ }^{1}$, C. J. Emery ${ }^{2}$, S. R. Heller;

${ }^{1}$ Department of Diabetes, King's College London,

${ }^{2}$ Department of Diabetes, University of Sheffield, United Kingdom.

Background and Aims: High fasting blood glucose levels can be a frustrating obstacle in achieving glycaemic targets. In this setting, it is common clinical 
practice to ask patients to check their blood glucose at 3 am to exclude the Somogyi effect (asymptomatic nocturnal hypoglycaemia causing counterregulatory hormone release leading to fasting hyperglycaemia). Patients are often advised to reduce their overnight insulin if fasting glucose levels are high. We used continuous glucose monitoring data to establish the prevalence of this phenomenon in clinical practice in patients with insulin treated diabetes.

Materials and Methods: We obtained Continuous Glucose Monitoring data from 226 patients with insulin treated diabetes (91 type 1); mean (sd) age 56.2(12.1)yrs; mean (sd) HbAlc 7.5(1.0) \%. We analysed the first complete valid night of data and identified capillary readings entered at bedtime and pre-breakfast. The lowest sensor glucose value between these two points was termed the nadir glucose. Nocturnal hypoglycaemia was defined as nadir sensor glucose $<3.0 \mathrm{mmol} / \mathrm{l}$.

Results: Nocturnal hypoglycaemia occurred on $28.1 \%$ of nights in type 1 diabetes and $23 \%$ of nights in type 2 diabetes. Nocturnal hypoglycaemia was more common in those with longer duration of diabetes. 3/56 episodes of nocturnal hypoglycaemia was followed by fasting glucose $>10 \mathrm{mmol} / \mathrm{l}$, although in 2 episodes the patient had been woken up by hypoglycaemia and had consumed carbohydrate. Fasting blood glucose was significantly lower following nights with hypoglycaemia compared to nights with no hypoglycaemia $5.9(2.0)$ vs. $8.7(3.1) \mathrm{mmol} / \mathrm{l}(\mathrm{p}<0.001)$. In $40 \%$ of episodes, fasting blood glucose was $<5 \mathrm{mmol} / 1$ following nocturnal hypoglycaemia. There was no difference in age, $\mathrm{HbAlc}$, duration of diabetes or self reported hypoglycaemia awareness between those who did or did not have nocturnal hypoglycaemia. There was a significant correlation between nadir glucose and fasting glucose $(r=0.7 ; \mathrm{p}<0.001)$ but there was no correlation between bedtime capillary glucose and nadir glucose $(r=0.25 ; \mathrm{p}=0.7)$.

Conclusion: Nocturnal hypoglycaemia is common in insulin treated diabetes and is usually associated with low fasting blood glucose levels. Our data refutes the existence of the Somogyi effect.

Supported by the Department of Transport, UK

\section{3}

How to classify hypoglycaemia awareness in type 1 diabetes?

T. Høi-Hansen, U. Pedersen-Bjergaard, B. Thorsteinsson;

Medical department 1, Nordsjællands Hospital-Hillerød, Denmark.

Background and Aims: Impaired hypoglycemia awareness (IHA) is a major risk factor for severe hypoglycemia (SH) in patients with type 1 diabetes. There is no consensus on classification of hypoglycemia awareness (HA). We compared three methods for assessment of self-estimated HA in a clinical setting.

Materials and Methods: A questionnaire including all three methods was mailed to all our outpatients with type 1 diabetes. Method A is a 7-point Likert scale (7: total IHA; 1: total HA) dividing patients into HA or IHA. Method $\mathrm{B}$ uses 8 questions and an additive symptom score dividing patients into HA or IHA. Method C uses the question: 'can you feel when you have an hypo ?', with 4 possible answers dividing patients into HA, IHA or unaware (HUA). All patients were divided into two groups with 0 or $\geq 1$ episode of severe hypoglycemia the preceding year.

Results: A total of $385(77 \%)$ patients returned the questionnaire. In the preceding year, $289(75 \%)$ patients had no episodes of severe hypoglycemia, and 91 have had $\geq 1$ episode ( 5 did not provide an answer). The mean number of episodes for all patients was 0.91 per patient-year while patients with $\geq 1$ episode had 3.8 episodes per patient-year.

Using method A, B and C, respectively, $100 \%, 79 \%$ and $53 \%$ of the patients could be classified as HA or IHA/HUA (C) whilst the rest of the patients were either classified as having impaired awareness (method C: $46 \%$ ) or could not be classified (method B: $21 \%$ ). The table shows number of patients with HA and IHA/HUA, as well as positive and negative predictive values in relation to retrospectively recalled episodes of severe hypoglycemia for the three methods.

Conclusion: In conclusion, method $\mathrm{C}$ had higher positive and negative predictive values, and was at the same time one of the simplest methods. However, both method B and C left many patients unclassified or classified as IHA. Consensus on the method for clinical classification of hypoglycaemia awareness is warranted.
Frequency and predictive values

\begin{tabular}{llll}
\hline & Method A & Method B & Method C \\
\hline HA (n)/ SH (mean) & $281 / 0.56$ & $195 / 0.34$ & $152 / 0.14$ \\
IHA(A+B) or HUA (C) (n)/ SH(mean) & $94 / 1.9$ & $106 / 1.91$ & $46 / 2.9$ \\
Positive predictive value & 0.37 & 0.42 & 0.54 \\
Negative predictive value & 0.81 & 0.85 & 0.91 \\
\hline
\end{tabular}

\section{4}

Glycaemic profile characteristics and frequency of impaired awareness of hypoglycaemia in subjects with T1D and repeated non-severe and severe hypoglycaemic episodes

M. Lara, M. Giménez, J. Nicolau, I. Conget;

Endocrinology Unit, Hospital Clinic i Universitari, Barcelona, Spain.

Background and Aims: Intensive insulin therapy significantly reduces the risk of complications in subjects with type 1 diabetes (T1D) and represents the standard treatment since the onset of the disease. However, this sort of therapy is unfailingly associated with a higher risk of non-severe (NSH) and severe hypoglycaemia (SH), precluding, in some cases, this type of therapy. Aim: To evaluate the prevalence of hypoglycaemia unawareness and continuous glucose profile characteristics in a group of T1D subjects with repeated NSH and SH.

Materials and Methods: Twenty subjects with T1D (aged $35.2+7.6$ years, 9 women, T1D duration $16.4 \pm 6.2$ years) receiving intensified insulin treatment fulfilling the following criteria: $>4 \mathrm{NSH}$ episodes/week (last 8 weeks) and $>2 \mathrm{SH}$ during the last 2-3 years, were included. General and metabolic characteristics, an evaluation of hypoglycaemia awareness (HA) and a 72-h continuous glucose monitoring (CGM) were performed. An acute induction of hypoglycaemia was performed with i.v. regular insulin and symptoms and signs of hypoglycaemia were evaluated after clamping of glycaemia at $2.5-2.8 \mathrm{mM}$.

Results: At entry, $\mathrm{HbA}_{1 \mathrm{c}}$ was $6.9 \pm 1.0 \%$. All the subjects were classified as having hypoglycaemia unawareness by the HA test and only 4 of them displayed normal symptoms and signs response during induced hypoglycaemia (coefficient of correlation $-0.61, \mathrm{p}<0.012$; sensitivity $100 \%$, specificity $25 \%$, PPV $80 \%$, kappa index 0.333 ). CGM of the whole group revealed $18 \%$ of measurements $<70$ $\mathrm{mg} / \mathrm{dl}$ and this percentage was correlated with HA test score $(\mathrm{r}=0.55 ; \mathrm{p}<0.035)$ and symptoms/signs during the induced hypoglycaemia $(\mathrm{r}=-0.57 ; \mathrm{p}<0.026)$. In those subjects with an abnormal symptoms/signs response during induced hypoglycaemia, CGM $\%$ of values $<70 \mathrm{mg} / \mathrm{dl}$ was higher $(22.6 \pm 8.4 \%)$ than in those with normal one $(10.2 \pm 9.0 \% ; \mathrm{p}<0.028)$.

Conclusion: In subjects with T1D and repeated NSH and SH, the prevalence of impaired awareness is substantially common. Its presence is related with a high proportion of ambulatory glycaemia profile below desirable range. The detection of this alteration has to be considered for a safe approach to optimising metabolic control.

\section{5}

Erythropoietin concentration and renin angiotensin system activity during hypoglycaemia in type 1 diabetes

P. L. Kristensen ${ }^{1}$, T. Høi-Hansen ${ }^{1}$, N. V. Olsen ${ }^{2}$, U. Pedersen-Bjergaard ${ }^{3}$, B. Thorsteinsson ${ }^{1}$;

${ }^{1}$ Division of Internal Medicin I, Nordsjællands Hospital - Hillerød, Denmark, ${ }^{2}$ The Department of Neuroanaesthesia, Copenhagen University Hospital, Denmark, ${ }^{3}$ Department of Internal Medicine I, Bispebjerg Hospital, Copenhagen, Denmark.

Background and Aims: High renin angiotensin system (RAS) activity is associated with increased risk of severe hypoglycaemia in patients with type 1 diabetes and more pronounced cognitive dysfunction during hypoglycaemia. An interaction between RAS and erythropoietin (EPO) has been established. EPO is neuroprotective in brain damage and may hypothetically improve cognitive function during hypoglycaemia. We therefore studied the impact of mild hypoglycaemia on EPO concentrations in patients with type 1 diabetes and high or low RAS activity.

Materials and Methods: Nine patients with high and nine with low RAS activity, as classified by ACE activity, angiotensinogen concentration and genotype of the angiotensin 2 receptor subtype 2, underwent a single-blinded, 
placebo-controlled, cross-over study with either hypoglycaemia induced by intravenous insulin (100\% of morning dose/hour) or stable glycaemia maintained by intravenous glucose and insulin administration dependent on blood glucose (control study).

Results: Nadir plasma glucose was $2.3(0.3) \mathrm{mmol} / \mathrm{l}$ (mean (SD)) in the high RAS group and $2.5(0.2) \mathrm{mmol} / \mathrm{l}$ in the low RAS group $(\mathrm{p}=0.16)$. During the control study, p-EPO did not change in either RAS group. During hypoglycaemia, pEPO increased from 9.7 (3.6) U/l to 12.5 (5.4) U/l (29\% increment) in the high RAS group, while p-EPO in the low RAS group increased from 9.0 (2.9) U/l to 9.6 (2.2) U/l (7\% increment). Intervention and RAS group both had impact on the EPO level, with hypoglycaemia and high RAS activity being associated with significantly higher levels of EPO: Hypoglycaemia: estimate: + $1.7 \mathrm{U} / 1$ (p $=0.02)$, high RAS group: estimate $1.6 \mathrm{U} / \mathrm{l}(\mathrm{p}=0.04)$.

Conclusion: Plasma EPO concentrations increase significantly during mild hypoglycaemia in patients with type 1 diabetes. Subjects with high RAS activity have higher EPO levels and a higher EPO response. The mechanism and the clinical significance are unknown. The association between low RAS activity and low frequency of hypoglycaemia/better cognitive performance during hypoglycaemia does not seem to be mediated by the hypoglycaemic EPO response.

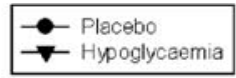

High RAS

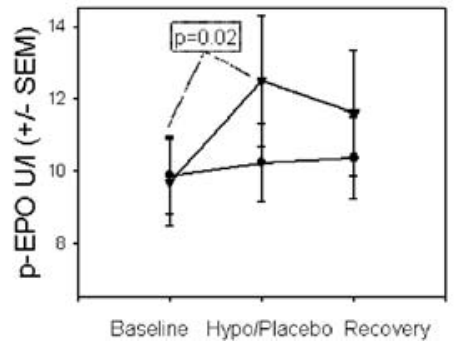

Baseline Hypo/Placebo Recovery
LOW RAS

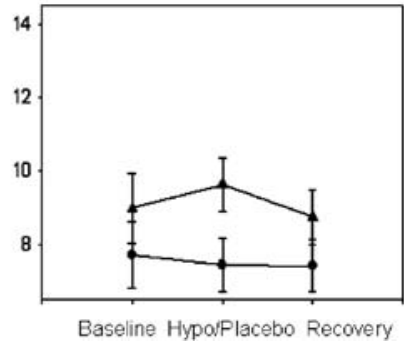

Baseline HyporPlacebo Recovery
Supported by EFSD/JDRF/Novo Nordisk Programme for Research in Type 1 Diabetes, Foundation of Harald Jensen and wife, Foundation of Olga Bryde Nielsen, Foundation of Region 3, Research Foundation of Frederiks

\section{6}

Experience of hypoglycaemic symptoms is associated with lower quality of life (EuroQoL) among patients with type 2 diabetes on oral antihyperglycaemic therapy in Europe

D. Yin ${ }^{1}$, C. Morillas ${ }^{2}$, R. Gomis ${ }^{3}$, G. Krishnarajah ${ }^{1}$, R. Lyu ${ }^{4}$, P. Mavros ${ }^{1}$; ${ }^{1}$ Merck \& Co, White house station, United States, ${ }^{2}$ Hospital Dr. Peset, Valencia, Spain, ${ }^{3}$ Hospital Clinic, Barcelona, Spain, ${ }^{4}$ Merck \& Co, Shanghai, China.

Background and Aims: Some OHA are often associated with hypoglycemia with varying degrees of severity. The impact of hypoglycemia on patients' health related quality of life (QoL) in patients with type 2 diabetes (T2DM) is not well established. This study was undertaken to evaluate the experience of hypoglycemia symptoms among patients with type 2 diabetes receiving OHA and its impact on QoL.

Materials and Methods: A retrospective clinical chart review and patient survey during physician visit (June 2006-Feb 2007) was conducted in 7 countries (Finland, France, Germany, Norway, Poland, Spain, UK). Patients recruited (aged $\geq 30$ years at T2DM diagnosis) added SU or PPAR $\gamma$ agonist to previous MF. Patients with gestational diabetes and those using insulin were excluded. Case report forms were used to collect clinical data from medical records. A1C refers to the most recent measurement within a year prior to enrollment. Questionnaires were used to ascertain patient reported experience of hypoglycemia, and QoL as measured by the EQ-5D VAS. Adequate glycemic control was defined according to the IDF (2005) recommendations as $\mathrm{A} 1 \mathrm{C}<6.5 \%$. Unadjusted differences in QoL by experience and severity of hypoglycemia was assessed using the t-test.

Results: 1709 patients were included in this analysis. Average age was 63 $(\mathrm{SD}=11)$ years, $45 \%$ were female, and $50 \%$ were diagnosed with diabetes for more than 7 years. The mean A1C level was $7.1 \%$ (SD=1.1), while $28 \%$ (477 patients) had adequate glycemic control. 652 (38\%) reported $\geq 1$ episode of hypoglycemia during the past year. The corresponding values by country are provided in the table below.

\begin{tabular}{lllll} 
Country & $\begin{array}{l}\text { Experience of } \\
\text { Hypoglycemia }-\%\end{array}$ & $\begin{array}{l}\text { Mild only } \\
\text { symptoms } \%\end{array}$ & $\begin{array}{l}\text { Moderate } \\
\text { symptoms }\end{array}$ & $\begin{array}{l}\text { Severe or Very } \\
\text { Severe symptoms }\end{array}$ \\
\hline Total & $38.0(652 / 1697)$ & $68.1(444 / 652)$ & $26.8(175 / 652)$ & $5.1(33 / 652)$ \\
Finland & $48.1(77 / 160)$ & $72.7(56 / 77)$ & $24.7(19 / 77)$ & $2.6(2 / 77)$ \\
France & $38.7(58 / 150)$ & $65.5(38 / 58)$ & $25.9(15 / 58)$ & $8.6(5 / 58)$ \\
Germany & $24.2(79 / 326)$ & $67.1(53 / 79)$ & $27.9(22 / 79)$ & $5.1(4 / 79)$ \\
Norway & $35.4(17 / 48)$ & $64.7(11 / 17)$ & $17.7(3 / 17)$ & $17.7(3 / 17)$ \\
Poland & $32.9(79 / 240)$ & $88.8(67 / 79)$ & $13.9(11 / 79)$ & $1.3(1 / 79)$ \\
Spain & $37.0(161 / 435)$ & $74.5(120 / 161)$ & $22.4(36 / 161)$ & $3.1(5 / 161)$ \\
UK & $53.6(181 / 338)$ & $54.7(99 / 181)$ & $38.1(69 / 181)$ & $7.2(13 / 181)$ \\
\hline
\end{tabular}

Patients experiencing hypoglycemic symptoms reported lower QoL values than those not (EQ-5D VAS: 68.7 vs. 73.5; $\mathrm{p}<0.0001)$. More severe hypoglycemia was significantly $(\mathrm{p}<0.0001)$ associated with lower QoL values (mean EQ-5D-VAS score: 70.9 for only mild symptoms, 65.8 for up to moderate symptoms, and 54.3 for severe or very severe symptoms).

Conclusions: Patient-reported experience of hypoglycemic symptoms was common among patients with T2DM on OHA in Europe. The experience of hypoglycemic symptoms in these patients was associated with lower health related quality of life.

\section{7}

A logistic regression model predicting those with diabetes treated with alternative short-acting insulin regimens who report hypoglycaemia to their GP

A. Simpson ${ }^{1}$, C. D. Poole 2 , A. J. Tynan ${ }^{1}$, C. J. Currie ${ }^{3}$;

${ }^{1}$ Eli Lilly and Company Limited, Windlesham, United Kingdom, ${ }^{2}$ Healthcare Research, $360^{\circ}$ Research, Penarth, United Kingdom, ${ }^{3}$ Department of Medicine, Cardiff University, United Kingdom.

Background and Aims: The pharmacokinetic profile of the rapid acting analogue insulins is known to reduce the likelihood of hypoglycaemia compared to short acting regular human insulin. The purpose of this study was to mathematically model those subjects who report hypoglycaemia to their GP.

Materials and Methods: Data was extracted from the General Practice Research Database (GPRD), a resource that describes the primary care histories of around 7\% of the UK population from 1990 to 2007. Subjects were selected having been treated with one short-acting insulin (SAI) regimen, although patients treated with animal insulin regimens were excluded here. GPRD records in detail a number of experiences, including: diagnoses, treatments, biochemistry and other laboratory test results, blood pressure, height, weight, and mortality. A logistic regression model (LRM) was derived that characterised people who either did or did not report hypoglycaemia to their GP for the duration of their treatment on a basal-bolus regime that included either short acting analogue insulin (AI) or soluble regular human insulin (RHI). A wide range of explanatory variables were explored including $\mathrm{HbAlc}$, age, sex and duration of diabetes.

Results: It was possible to identify 7,982 people treated with a single SAI regimen; 5,483 (68.7\%) AI, and 2,499 (31.3\%) RHI. The LRM is given in the table.

Conclusion: It was possible to model those people who were likely to report hypoglycaemia to their GP; and by inference, people who were more likely to experience hypoglycaemia. Those more likely to report hypoglycaemia had the following characteristics: type 1 diabetes, a longer duration of insulin therapy and used soluble regular human insulin.

TABLE. Logistic regression model predicting the odds of reporting hypoglycaemia to the GP.

\begin{tabular}{lllllll}
\hline Parameter & Beta & SEM & $\mathrm{p}$ & Odds ratio & $95 \%$ CI & \\
\hline T2DM (cf. T1DM) & -0.330 & 0.096 & $<0.001$ & 0.72 & 0.60 & 0.87 \\
SAI duration (months) & 0.021 & 0.002 & $<0.001$ & 1.02 & 1.02 & 1.02 \\
AI (cf. RHI) & -0.204 & 0.089 & 0.022 & 0.82 & 0.69 & 0.97 \\
Constant & -2.895 & 0.100 & $<0.001$ & 0.06 & & \\
\hline
\end{tabular}

Supported by Eli Lilly and Company Limited 


\section{8}

Detemir causes similar counter-regulatory hormone response but increased symptom awareness during hypoglycaemia compared to regular human insulin

S. A. Schäfer, O. Tschritter, A. Bury, J. Klett, A. Hennige, H.-U. Häring, A. Fritsche;

Medizinische Klinik IV, University Tübingen, Germany.

Background and Aims: The long acting insulin analogue Detemir has structural and physicochemical properties which differ from human insulin. The aim of the present study was, therefore, to test whether this leads to altered hormone and symptom response to hypoglycaemia.

Materials and Methods: $12(6 \mathrm{f} / 6 \mathrm{~m}$, age $32 \pm 6$ years (mean \pm SD), BMI $24.2 \pm 2.5$ $\mathrm{kg} / \mathrm{m}^{2}$ ) healthy subjects underwent a 200 min stepwise hypoglycaemic clamp ( $45 \mathrm{~min}$ steps of 4.4, 3.7, 3.0 and $2.3 \mathrm{mmol} / \mathrm{l}$ ) with either insulin Detemir or Actrapid in random order. Detemir (Actrapid) was infused at a rate of 5 (2) $\mathrm{mU} / \mathrm{kg} / \mathrm{min}$. At the start of the clamp, a bolus of $660(60) \mathrm{mU} / \mathrm{kg}$ was given over 12 (6) minutes. Assessment of this dose was done in pilot hyperinsulinaemic euglycaemic clamp experiments. Blood was drawn and a semi-quantitative symptom questionnaire (20 real and 9 dummy symptoms) were administered before and after each plateau of the hypoglycaemic clamp. A stroop- and reaction time test was performed during each step.

Results: Although Detemir infusion resulted in $\sim 35$ times higher peripheral total insulin levels, blood glucose levels and glucose infusion rates (GIR) were similar during both conditions except of the $2.3 \mathrm{mmol} / \mathrm{l}$ plateau with higher GIR under Detemir. No significant differences between Detemir and Actrapid in glucagon, epinephrine, norepinephrine, cortisol or growth hormone response during hypoglycaemia were observed (MANOVA for repeated measures design). There were also no significant differences in cognitive function tests. The total symptom score was higher with Detemir during the 3 and $2.3 \mathrm{mmol}$ step compared to Actrapid ( $\mathrm{p}=0.048$ ). Especially sweating was increased with Detemir versus Actrapid ( $\mathrm{p}=0.02)$, and showed an earlier and faster increase during the hypoglycaemic clamp with Detemir (interaction insulin $\mathrm{x}$ time: $\mathrm{p}=0.04$ ).

Conclusion: Hormonal counter-regulation and cognitive function during hypoglycaemia are not different with Detemir and human regular insulin. Symptom perception, especially sweating, is increased during hypoglycaemia induced by Detemir. Type 1 diabetic patients with hypoglycaemia unawareness may profit from increased sweating during hypoglycaemia.

Supported by Novo Nordisk

\section{9}

Relationship between hypoglycaemia, background oral antidiabetic agent, and glycaemic control in patients treated with exenatide or insulin glargine

M. Trautmann ${ }^{1}$, J. Burger ${ }^{1}$, D. Johns ${ }^{1}$, R. Brodows ${ }^{1}$, T. Okerson ${ }^{2}$,

A. Roberts ${ }^{3}$, A. Barnett ${ }^{4}$

${ }^{1}$ Eli Lilly \& Co., Indianapolis, United States, ${ }^{2}$ Amylin Pharmaceuticals, Inc., San Diego, United States, ${ }^{3}$ Endocrine Unit, Royal Adelaide Hospital, Australia, ${ }^{4}$ Dept of Diabetes/Endocrinology, University of Birmingham and Birmingham Heartlands and Solihull NHS Trust, United Kingdom.

Background and Aims: Hypoglycaemia is a limitation of many therapies for type 2 diabetes.

Materials and Methods: Incidence of hypoglycaemia and the relationship to changes in $\mathrm{HbA}_{1 c}$ were analysed from a two-period (16 wks each), open-label, crossover study comparing exenatide (EX, $5 \mu \mathrm{g}$ BID for 4 wks, then 10 $\mu \mathrm{g}$ BID for $12 \mathrm{wks}$ ) and insulin glargine (IG, QD targeting a fasting blood glucose $\leq 5.6 \mathrm{mmol} / \mathrm{L}$ ). Patients continued treatment with metformin (MET, 56\%) or a sulphonylurea (SU, $44 \%$ ).

Results: Patients treated with EX or IG ( $\mathrm{n}=114$ completers) had similar reductions in mean $\mathrm{HbA}_{1 c}(-1.43 \%$ vs. $-1.41 \%$, respectively, $\mathrm{P}=0.773)$. Reductions in $\mathrm{HbA}_{1 c}$ were similar in EX patients treated with MET or SU ($1.48 \pm 1.20 \%$ and $-1.38 \pm 0.14 \%$, respectively) or in IG patients treated with MET or SU $(-1.50 \pm 0.12 \%$ and $-1.29 \pm 0.14 \%$, respectively). EX-treated patients had a $2.6 \%$ decrease in body weight while IG-treated patients had a $1.8 \%$ increase in body weight (mean $\pm \mathrm{SEM},-1.95 \pm 0.36 \mathrm{~kg}$ vs. $0.53 \pm 0.36$, respectively; $\mathrm{p}<0.001)$. Mean weight was reduced in EX/SU-treated patients $(-0.5 \pm 0.5$ $\mathrm{kg}, \mathrm{p}=0.328)$ and in EX/MET-treated patients $(-3.1 \pm 0.5 \mathrm{~kg}, \mathrm{p}<0.001)$. Mean weight gain was observed in IG/SU-treated patients $(0.9 \pm 0.5 \mathrm{~kg}, \mathrm{p}=0.096)$ while weight was almost unchanged in IG/MET-treated patients $(-0.1 \pm 0.5$ $\mathrm{kg}, \mathrm{p}=0.860$ ). Incidence of hypoglycaemia was similar in EX/SU- and IG/SUtreated patients (EX, 30\% vs. IG, 34\%, p $>0.99$ ) but was significantly lower in patients treated with EX/MET compared with IG/MET (2.6\% vs.17.4\%, $\mathrm{p}=0.010$ ). Although the incidence of nocturnal hypoglycaemia tended to be lower in EX/SU- compared with IG/SU-treated patients (7\% vs. $16 \%$, $\mathrm{p}=0.152$ ), it was significantly less in patients treated with EX/MET compared with IG/MET $(1.3 \%$ vs. $13 \%, p=0.018)$. There were 7 episodes of severe hypoglycaemia in 3 IG-treated patients but none in EX-treated patients. In SU treated-patients who experienced hypoglycaemia, mean endpoint $\mathrm{HbA}_{1}$ was lower in patients treated with EX $(n=18,7.01 \pm 0.97 \%)$ compared with IG $(n=20,7.58 \pm 1.07 \%)$. Median IG doses were similar in MET- and SU-treated patients with or without hypoglycaemia (MET, 24 vs. 25 U/day; SU, 24 vs. $23 \mathrm{U} /$ day).

Conclusion: Higher incidences of overall and nocturnal hypoglycaemia in EX-treated patients tended to be associated with concomitant SU use; while in IG-treated patients, hypoglycaemia was observed independent of oral agent used. Severe hypoglycaemia was observed only in IG-treated patients. In addition, the risk of hypoglycaemia was greater in IG/SU-treated patients with poorer glycaemic control (higher $\mathrm{HbA}_{1 c}$ ). These data suggest that adding EX to a single oral agent can be an alternative to IG therapy resulting in similar $\mathrm{HbA}_{1 \mathrm{c}}$ improvements while reducing weight and hypoglycaemia risk, particularly in combination with MET.

Supported by Eli Lilly and Company and Amylin Pharmaceuticals, Inc. 


\section{PS 75 Lifestyle}

\section{0}

TTF-1, a homeodomain transcription factor-1, is associated with hyperphagia in the diabetic rat

I. Nam-Goong ${ }^{1}$, E. Kim², J. Kim², C. Yun², B. Lee², Y. Kim;

${ }^{1}$ Department of Internal Medicine, Ulsan University Hospital,

${ }^{2}$ Department of Biological Sciences, University of Ulsan, Republic of Korea

Background and Aims: Thyroid transcription factor-1 (TTF-1) is a member of the NKx family of homeodomain genes and plays an imporrant role in the control of neuroendocrine functions. Recently, our research group reported that a novel role for TTF-1 in the regulation of feeding behavior in the rat hypothalamus. Blockade of hypothalamic TTF-1 immensely reduced hyperphagia induced by food deprivation. Furthermore, expression of TTF-1 was significantly increased in the hypothalamus from fasted rats. Here, to determine whether TTF-1 is related with diabetic hyperphagia, we investigated the expression of TTF-1 in streptozotocin (STZ)-induced diabetic rats.

Materials and Methods: Two-month-old male Sprague-Dawley rats were used and diabetes was induced by a single intraperitoneal injection of $65 \mathrm{mg} /$ $\mathrm{kg} \mathrm{STZ}$. To determine if TTF-1 is involved in the control of food intake in the diabetic rats, a phosphorothioate antisense (AS) TTF-1 oligodeoxynucleotide (ODN) was delivered into the vicinity of the hypothalamus. Changes in food intake were analysed by repeated measures ANOVA.

Results: STZ-induced diabetic rats had significant hyperphagic and weight loss compared with control rats. Level of hypothalamic TTF-1 synthesis significantly increased in diabetic rats (enhanced mRNA (2.4-fold induction, $p<0.05)$ and protein $(3.3$-fold induction, $p<0.01)$ expression) as compared with control rats. In addition, blockade of hypothalamic TTF-1 synthesis by intracerebroventricular administration of an AS TTF-1 ODN greatly prevented diabetic hyperphagia ( $36.7 \mathrm{~g} /$ day vs. $25.8 \mathrm{~g} /$ day, $\mathrm{F}=13.029, p<0.05$ ) and changed expression of neuropeptides relate to feeding behavior.

Conclusion: These results suggest the connection between hypothalamic TTF-1 synthesis and diabetic hyperphagia.

Supported by Ulsan University Hospital (Biomedical Research Center Promotion Fund)

\section{1}

Influence of lifestyle change on Glucagon-Like Peptide-1 in new onset type 2 diabetes. Pilot study

T. Hart $^{1}$, D. Kerr' ${ }^{2}$, J. Begley

${ }^{1}$ Clinical Biochemistry, Royal Bournemouth Hospital,

${ }^{2}$ BDEC, Bournemouth Diabetes and Endocrine Centre,

${ }^{3}$ Clinical Biochemistry, Royal Bournemouth Hospital and Bournemouth

University, United Kingdom.

Background an Aims: Previously, Glucagon-like peptide-1 (GLP-1) levels and the GLP-1 responses to oral glucose have been shown to be attenuated in patients with established diabetes. Whether impaired secretion is a factor in the development of diabetes or is a consequence of chronic hyperglycaemia is unknown. The aim of this study was to evaluate GLP-1 levels in patients with newly diagnosed type 2 diabetes and to assess the effect of an initial period of lifestyle and dietary adjustment.

Methods: 9 newly-diagnosed type 2 diabetic (DM) patients (4 female, age 57.2 [8.1] years, BMI 27.6 [4.0)], time from diagnosis 24.7 [5.2] days) were compared with 9 controls, matched for age, sex and body weight. All participants had a standard $75 \mathrm{~g}$ oral glucose tolerance test with blood samples taken via an indwelling cannula at 30 min intervals for $2 \frac{1}{2}$ hours for glucose, GLP-1, insulin, glucagon, and intermediate metabolites. Blood was also taken at baseline for HbAlc and blood lipids. DM patients had repeat testing after approximately three months (93.7 [17] days) of lifestyle intervention.

Results: In the DM group, weight fell by 0.5 [4.2] kg with lifestyle changes. 5 patients achieved weight loss (range 0.2 to $5 \mathrm{~kg}$ ), the other four gained weight (range 2.7 to $6.5 \mathrm{Kg}$ ). $\mathrm{HbAlc}$ reduced from 7.31 [1.43] to 6.96 [1.19] $\%, \mathrm{p}=0.25$. When compared with controls, DM patients had higher glucagon levels (AUC 7164.5 vs. $73.94 \mathrm{ng} / \mathrm{l} . \mathrm{min}, \mathrm{p}=0.04$ ), lower active GLP-1 levels at baseline before the glucose load, although overall AUC levels were not different (1317.1 vs. $761.62 \mathrm{pmol} / \mathrm{l} . \mathrm{min}, \mathrm{p}=0.2)$. There were also elevated levels of Free Fatty Acids (AUC 19.3 vs. $43.1 \mathrm{mmol} / \mathrm{l} . \mathrm{min}, \mathrm{p}=0.001$ ) and $\beta$ OH Butyrate (AUC 2.19 vs. $7.02 \mathrm{mmol} / \mathrm{l} . \mathrm{min}, p=0.03$ ) in the diabetic group. After lifestyle changes active GLP-1 secretion was augmented in the diabetic group (AUC 855.9 vs. $971.7 \mathrm{pmol} / \mathrm{l} / \mathrm{min}, \mathrm{p}=0.018$ ) and this was independent of weight change. There was also a trend towards earlier insulin secretion at this time. Glucagon levels were unchanged.

Conclusion: Lifestyle treatment of new onset type 2 diabetes appears to improve GLP-1 responses to an oral glucose load without influencing glucagon levels.

\section{2}

Cholinergic regulation of meal induced ghrelin and PYY release is impaired in obesity

C. Maier ${ }^{1}$, M. Riedl ${ }^{1}$, G. Vila ${ }^{1}$, D. Haider ${ }^{1}$, M. Wolzt ${ }^{2}$, B. Ludvik ${ }^{1}$, A. Luger ${ }^{1}$; ${ }^{1}$ Clinical Dep. of Endocrinology \& Metabolism, Austria,

${ }^{2}$ Dep. of Clinical Pharmacology, Medical University of Vienna, Austria.

Background and Aims: Ghrelin and PYY are both hormones derived from the gastrointestinal tract involved in appetite regulation, ghrelin as orexigenic and PYY as anorexigenic agent. Ghrelin concentrations rise before expected meals and decrease upon food ingestion whereas PYY concentrations rise after meals. It has been shown repeatedly that the cholinergic part of the vagus nerve is involved in the regulation of glucose and insulin; the cholinergic antagonist atropine has been shown to decrease ghrelin plasma concentrations in lean, healthy subjects. The aim of the study was to examine the effects of atropine on ghrelin, PYY, glucose and insulin under basal conditions and after meal ingestion in lean and obese subjects.

Materials and Methods: The study design was randomised, double-blind, placebo controlled crossover with four study days. 8 obese (BMI $39.6 \pm 2$, 6 female $/ 2$ male, aged $28.7 \pm 2.4$, mean \pm s.e.m. $)$ and 8 lean $(B M I=22.9$ $\pm 1 \mathrm{~kg} / \mathrm{m}^{2}$ ), age and gender distribution matched, healthy subjects were scheduled for a prestudy visit including a 180min OGTT and four study days in randomised order: A, Atropine $1 \mathrm{mg}$ iv at time point $-30 \mathrm{~min}+$ breakfast ( 2 rolls with butter and cocoa drink, $550 \mathrm{kcal}$ ) at time point 0 ; B, Atropine $1 \mathrm{mg}$ iv at time point $-30 \mathrm{~min}+$ no breakfast; $\mathrm{C}$, placebo at time point -30 min + breakfast at time point $0 ; \mathrm{D}$, placebo at time point $-30 \mathrm{~min}+$ no breakfast. Blood samples were drawn at time points - 30, -15, 0, 15, 30, 60 and $90 \mathrm{~min}$ for measurements of plasma ghrelin, pyy, insulin and glucose. At time points - 30, -15, 0, 15, 30, 45, 60, 75 and 90 min subjects rated their hunger and satiety feelings on $10 \mathrm{~cm}$ visual analog scales. Blood pressure and heart rate were monitored throughout the study days. Differences between the four study days were analysed by ANOVA, followed by multiple t-tests with Bonferroni correction, if appropriate.

Results: Obese subjects were insulin resistant $(\mathrm{HOMA}=5.24 \pm 0.3$, OGIS $=$ $379.7 \pm 26.1)$ compared to lean controls $(\mathrm{HOMA}=2.58 \pm 0.4$, OGIS $=454.3 \pm$ 12.7). Atropine led in lean individuals to a decrease in ghrelin concentrations comparable to and non-additive with breakfast ingestion $(\Delta$ baseline/ $+90 \mathrm{~min}$ : A, $-261.9 \pm 59 ; \mathrm{B},-254.8 \pm 38 ; \mathrm{C},-196.8 \pm 31, \mathrm{D},+140.1 \pm 68 \mathrm{pg} / \mathrm{mL}, \mathrm{A}, \mathrm{B}$, $\mathrm{C} p<0.0001$ vs. $\mathrm{D}$, and $\mathrm{p}=$ n.s. from each other). In obese subjects atropine did not significantly change ghrelin concentrations and breakfast induced ghrelin decrease was impaired $(\mathrm{A},-130 \pm 44 ; \mathrm{B},-115 \pm 55$; $\mathrm{C},-162,9 \pm 52$; $\mathrm{D},+62,5 \pm 49$, C vs. $\mathrm{D} p=0.025$, all others $\mathrm{p}=\mathrm{n} . \mathrm{s})$. Atropine significantly decreased both basal and meal induced PYY concentrations in lean subjects $(A,-2 \pm 6 ; B,-35 \pm 5 ; C,+16.3 \pm 5 ; D,-5 \pm 10 \mathrm{pg} / \mathrm{mL}$ ) In obese subjects there were no significant changes of PYY concentrations at all. In contrast, atropine resulted in a comparable increase in heart rate in lean and obese subjects, and in a comparable decrease in meal induced glucose concentrations in lean and obese subjects. Only lean, but not obese subjects experienced sustained feelings of satiety (according to VAS ratings) after breakfast. Atropine did not significantly change hunger or satiety VAS ratings in either lean or obese subjects.

Conclusions: The impaired cholinergic regulation of the postprandial drop in (orexigenic) ghrelin concentrations and rise in (anorexigenic) PYY concentrations might be part of the deregulated food intake in obese subjects. 


\section{3}

Elucidating the minimum exercise requirements to overcome dietaryinduced insulin resistance

F. J. Spargo ${ }^{1}$, M. A. Febbraio ${ }^{2}$, J. A. Hawley';

${ }^{1}$ Exercise Metabolism Group, RMIT University, Bundoora,

${ }^{2}$ Cellular and Molecular Metabolism Laboratory,

Baker Institute of Heart Research, Melbourne, Australia.

Background and Aims: During the past 50 years, the prevalence of a cluster of interrelated metabolic disease states including obesity, insulin resistance and type 2 diabetes mellitus has reached epidemic proportions. While there is irrefutable evidence that physical training is an effective therapeutic intervention to increase insulin action in skeletal muscle from obese and insulin resistant individuals, the minimum amount of exercise required to restore insulin tolerance is not known.

Materials and Methods: Thirty male Wistar rats (age $\sim 9$ wk) were fed standard chow (CHOW, $n=6$ ) or a $60 \%$ fat diet (FAT, n=24) for $6 \mathrm{wk}$. Fat-fed animals were then assigned to a control group (FAT, $n=6$ ) or one of three exercise groups; F100 (60 min/d for $6 \mathrm{~d}$, n=6); F50 (50\% volume of F100; 60 $\mathrm{min} / \mathrm{d}$ for $3 \mathrm{~d}, \mathrm{n}=6$ ) or F25 (25\% volume of F100; 30 mind for $3 \mathrm{~d}, \mathrm{n}=6) .24$ $\mathrm{hr}$ after the last exercise session trained animals, as well as CHOW and FAT controls underwent an intraperitoneal insulin tolerance test (IPITT), before the red gastrocnemius was removed for analyses.

Results: CS activity was higher in all fat-fed groups compared to $\mathrm{CHOW}$ (all $\mathrm{P}<0.05$ ), while F50 CS activity was increased compared to FAT and F25. F100 CS activity was also increased compared to FAT, F25 and F50 (all P<0.05). Compared to FAT, IPITT was improved in all exercise groups $(\mathrm{P}<0.05)$. F100 also had greater insulin tolerance compared to both F25 and F50 $(\mathrm{P}=0.02$; Figure 1A). FAT glycogen was lower than CHOW $(\mathrm{P}=0.03)$, F50 $(\mathrm{P}=0.05)$ and F100 $(\mathrm{P}=0.05)$, while F25 glycogen was lower than F100 $(\mathrm{P}=0.04)$. There were no intramuscular triglyceride (TGm) differences between $\mathrm{CHOW}$, FAT and F25, however both F50 and F100 had higher TGm compared to all other groups (all $\mathrm{P}<0.05)$. There were no differences in total IRS1 or IRS1 tyrosine phosphorylation (relative to total IRS1). However, FAT, F25 and F50 had reduced IRS1-PI3 kinase association (relative to total IRS1) compared to CHOW and F100 (all P<0.05). FAT, F25 and F50 GLUT4 was lower than both CHOW and F100 (all P<0.05), while FAT GLUT4 was also lower than both F25 and F50 (both $\mathrm{P}<0.05$ ).

Conclusion: Insulin tolerance was improved in fat-fed animals after all exercise interventions. However, only the largest exercise dose $(60 \mathrm{~min} / \mathrm{d}$ for $6 \mathrm{~d}$ ) was sufficient stimulus to overcome high fat feeding-induced downregulation of the insulin signalling pathway. These data suggest that in order to overcome dietary-induced insulin resistance, daily moderate intensity activity of $\sim 1 \mathrm{hr}$ duration is recommended. (Fig. 1)
0824

Lifestyle-induced changes in transferrin are associated with changes in insulin sensitivity

E. E. Blaak ${ }^{1}$, C. Roumen ${ }^{1}$, E. J. M. Feskens ${ }^{2}$, W. H. M. Saris ${ }^{1}$;

${ }^{1}$ Human Biology, Maastricht University,

${ }^{2}$ Division of Human Nutrition, Wageningen University, The Netherlands.

Background and Aims: Indicators of iron status, like transferrin and ferritin levels may be related to diabetes risk. Ferritin and transferrin may be independent predictors of insulin resistance. Less is known on whether lifestyle intervention can improve serum levels of transferrin and ferritin. We evaluated the effect of a lifestyle intervention in a Dutch population with impaired glucose tolerance (IGT) and analysed the relationship between lifestyle-induced changes glucose tolerance, and insulin sensitivity and changes in transferrin and ferritin levels.

Materials and Methods: 147 IGT subjects were initially randomised into an intervention group; $n=74$ (INT) and control group; $n=73$ (CON). We studied 129 subjects after one year of study (INT $n=64$ and CON $n=65$ ). Subjects underwent measurements of body composition, body fat distribution, glucose tolerance (OGTT), and maximal aerobic capacity ( $\mathrm{VO}_{2}$ max). INT were individually guided with respect to diet and physical activity, based on general public health recommendations. We used simple linear regression to evaluate the relationship between lifestyle-induced changes in glucose tolerance, and insulin sensitivity and changes in transferrin and ferritin levels. We used stepwise and multiple linear regression to evaluate whether changes in transferrin were independently related to changes in insulin resistance (as assessed by HOMA-IR).

Results: INT decreased more in body weight and 2-hr glucose levels than CON, $-2.32 \pm 0.45 \mathrm{~kg}$ vs. $0.59 \pm 0.47 \mathrm{~kg}(\mathrm{p}=0.009)$ and $-0.63 \pm 0.22 \mathrm{mM}$ vs. $0.25 \pm 0.27 \mathrm{mM}(0.014)$. $\mathrm{VO}_{2}$ max increased more in INT compared to CON $(\mathrm{p}=0.033)$. HOMA-IR tended to decrease more in INT compared to CON $(p=0.075)$. At baseline, transferrin and ferritin were negatively associated $(\beta=$ $-0.524, \mathrm{p}<0.001)$ and lifestyle-induced changes in these parameters were also negatively associated $(\beta=-0.253, p=0.004)$. The lifestyle-induced decrease in fasting and 2-hr insulin and HOMA-IR were positively associated with decrease in transferrin $(p<0.05)$, but not with ferrtitin $(p>0.05)$. Multiple linear regression revealed that the change in transferrin was related to the change in HOMA-IR $(\beta=0.143, p=0.044)$, independent of changes in body mass index, age and sex with these factors accounting for $44.6 \%$ of the variation. Stepwise linear regression analysis adjusted for age and sex revealed that the most important predictors of change in HOMA-IR were change in BMI $(\beta=0.640, p<0.001)$ and change in transferrin $(\beta=0.144, p=0.035)$.

Conclusion: Transferrin and ferritin were negatively associated and did not change between INT and CON. Transferrin appeared to be an important independent determinant of the improvements in insulin sensitivity. These data suggest that the iron metabolism may be involved in the development of insulin resistance and type 2 diabetes.

Supported by grants from the Dutch Diabetes Research Foundation, the Netherlands Organisation for Health Research and Development and the Netherlands Organisation for Scientific Research

Fig. 1

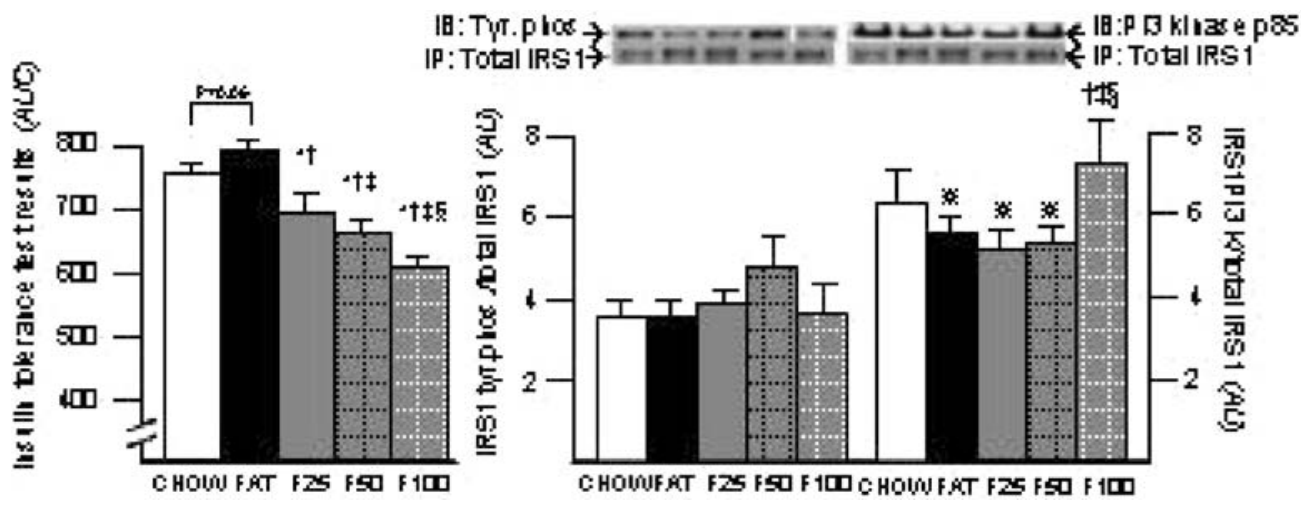




\section{5}

The effects of exercise- versus diet-induced weight reduction on the body fat composition and the vascular function in type 2 diabetes

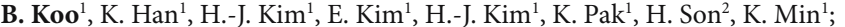
${ }^{1}$ Internal medicine, Eulji University School of Medicine,

${ }^{2}$ Internal medicine, The Catholic University of Korea College of Medicine, Seoul, Republic of Korea.

Background and Aims: Overweight individuals are at increased risk for developing cardiovascular disease. However, the effects of weight loss on the vascular structure and function and insulin resistance are inconclusive especially in regard to the difference in the modality of weight loss. The purpose of this study was to evaluate the effects of the increased daily usual physical activity versus diet restriction with comparable amount of weight loss on diverse metabolic parameters and markers of vascular functional or structural change in type 2 diabetic women.

Materials and Methods: We randomly assigned 70 women with type 2 diabetes to control (C), diet (D), exercise (E) or diet with exercise group (DE). The $\mathrm{C}$ were given the conventional education for diet and exercise, the $\mathrm{D}$ were restricted their calorie intake lower than $1400 \mathrm{kcal} / \mathrm{day}$, and the $\mathrm{E}$ were encouraged to increase their daily activity more than $2000 \mathrm{kcal} /$ day. For 12 weeks, we monitored their daily activities with Lifecorder ${ }^{\circledR}$ (Suzuken Co., Nagoya, Japan), their diet with diet records and body weight (BW) weakly, and excluded the subjects who could not reach their target level of diet or exercise.

Results: Finally, 55 subjects $(C, n=15 ; D, n=18 ; E, n=11 ; D E, n=11)$ were included for the analysis. The mean age of subjects was $56 \pm 8$ years, the duration of diabetes was $8 \pm 6$ years, BW was $67.8 \pm 6.6 \mathrm{~kg}$ and BMI was $27.7 \pm 2.4 \mathrm{~kg} / \mathrm{m}^{2}$ and there were no differences among 4 groups. After 12 week program, change and percent change of BW were significantly bigger in the D and the DE [D: $-4.8 \pm 1.4 \mathrm{~kg}(-7.2 \%), \mathrm{DE}:-5.1 \pm 2.7 \mathrm{~kg}(-7.5 \%)]$ than other two groups [C: $-1.5 \pm 1.6 \mathrm{~kg}(-2.2 \%), \mathrm{E}:-2.2 \pm 2.0 \mathrm{~kg}(-3.3 \%)]$.

There was significant reduction in subcutaneous and visceral fat in the all intervention groups $(P<0.05)$ except the $C$. However, the proportion of visceral fat to the total fat was decreased $(P<0.01$ in both) and insulin sensitivity calculated by $\mathrm{K}_{\mathrm{I}}$ was improved $(P=0.002$ and 0.008 , respectively) only in the $\mathrm{E}$ and $\mathrm{DE}$ but not in the $\mathrm{D}$. Flow mediated dilatation (FMD), endothelium-independent dilatation (EID) and augmentation index (AI) were significantly improved only in the DE $(P=0.001, P=0.010, P=0.008$, respectively) but there were no changes in carotid IMT, pulse wave velocity (PWV), and ankle-brachial index (ABI). In the case of $\mathrm{D}$, there were no differences in the FMD, EID and AI, but a deteriorated tendency in PWV and $\mathrm{ABI}(P=0.034$ and 0.021 , respectively). The changes of other parameters such as LDL cholesterol, free fatty acid, HbAlc, adiponectin and IL-6 were not significantly different among groups.

Conclusion: In conclusion, the increased daily physical activity with diet control for 12 weeks significantly reduced visceral fat $\%$ and improved insulin sensitivity, endothelial function and aortic stiffness, which were not observed in the diet control only in spite of the comparable amount of weight loss.

\section{6}

The relationship between lifestyles and prediabetes with metabolic syndrome in newly developed type 2 diabetes mellitus - based on the Diabetes Case Management Program 2001, Taiwan

M. M. Fuh ${ }^{1}$, H.-Y. Su' ${ }^{2}$ H.-Y. Chang ${ }^{3}$, C.-T. Chang ${ }^{4}$, R.-H. Chen ${ }^{4}$,

C.-C. Chen ${ }^{4}$, C.-C. Lee $^{5}$, C.-C. Lin $^{6}$;

${ }^{1}$ Diabetes Prevention and Control Center, China Medical University

Hospital, Taichung, ${ }^{2}$ Dietetics, Taipei Medical University Hospital,

${ }^{3}$ Division of Health Policy, National Health Research Institute, Hsinchu,

${ }^{4}$ Medicine, China Medical University Hospital, Taichung,

${ }^{5}$ Neurology, China Medical University Hospital, Taichung,

${ }^{6}$ Family Medicine, China Medical University Hospital, Taichung, Taiwan.

Background and Aims: In order to evaluate the relationship between lifestyles and metabolic syndrome in the prediabetes state in newly developed type 2 diabetes mellitus ( T2DM ) and ensuing development of primary preventive interventions in high risk people, a nationally standardised, multi-professionally integrated, evidence-based and patientcenter healthcare program - DCMP 2001 was implementing in a medical center, Mid-Taiwan.
Materials and Methods: From 2003 to Dec. 2006, 6928 diabetes were randomly recruited in DCMP 2001. Based on the number of metabolic syndrome criteria in the ATP III, all the newly developed T2DM $(n=1331)$ was classified into 3 Groups, GI ( 2 or less), GII (3criteria), and GIII (more than 3). Accordingly, the lifestyle measurements ( lifestyle I : no smoking, no alcoholic and regular exercise; lifestyle II : smoking and/or alcoholic and/ or no exercise ) were tri-monthly interviewed and recorded by the diabetes nurses following lifestyle intervention after seeing physician. From this cohort, all the newly developed T2DM were randomly sorted out and further divided up into 4 groups with different age ranges of onset of disease, $\leq 40$ years, $>40$ to $\leq 50$ years, $>50$ to $\leq 60$ years, and over 60 years for study. Comparisons between groups were performed by using Chi-Square test. The significant level was set at $\mathrm{p}<0.05$.

Results: In patients of younger onset of diabetes in GIII and GII, the lifestyle measures showed that almost $80 \%$ cases were living on lifestyle II. The older onset of diabetes in G III and GII, however, $50 \%$ cases were sticking on lifestyle I ( Table 1 and 2 respectively ). These differences in lifestyles between younger and older onset of T2DM with metabolic syndrome in prediabetes state at different age ranges were statistically significant not only in the GIII $(\mathrm{p}<0.0001)$ but also in the GII diabetes $(\mathrm{p}<0.0001)$. Comparison between exercisers and non-exercisers in GIII and GII also shown that non-exercisers developed diabetes were significantly earlier than the exercisers $(p=0.004$ and $\mathrm{p}<0.0001$ respectively).

Conclusion: The aforementioned results clearly indicated that lifestyles and regular physical activity would have significant impact on not only the ages of onset of diabetes but also the development of metabolic syndrome from the prediabetes state to newly developed T2DM.

Table 1. The case and percentage dirstributions of different liefestyles at different age ranges in newly developend type 2 diabetes mellitus with metabolic syndrome (GIII)

\begin{tabular}{lllllll}
\hline & \multicolumn{2}{l}{ Lifestyle I } & & \multicolumn{2}{l}{ Lifestyle II } & \multirow{2}{*}{ p-value } \\
\cline { 2 - 3 } & Case & $\%$ & & Case & $\%$ & \\
\hline$\leq 40$ & 12 & 22.2 & & 42 & 77.8 & $<0.001$ \\
$>40 \leq 50$ & 15 & 20.0 & & 60 & 80.0 & \\
$>50 \leq 60$ & 22 & 25.3 & & 65 & 74.7 & \\
$>60$ & 52 & 47.7 & & 57 & 52.3 & \\
\hline
\end{tabular}

GIII: MetS and prediabetes before T2DM diagnosed

Table 2. The case and percentage dirstributions of different liefestyles at different age ranges in newly developend type 2 diabetes mellitus with metabolic syndrome (GII)

\begin{tabular}{lllllll}
\hline & \multicolumn{2}{l}{ Lifestyle I } & & \multicolumn{2}{l}{ Lifestyle II } & \multirow{2}{*}{ p-value } \\
\cline { 2 - 3 } & Case & $\%$ & & Case & $\%$ & \\
\hline$\leq 40$ & 16 & 19.0 & & 68 & 81.0 & $<0.001$ \\
$>40 \leq 50$ & 30 & 22.4 & & 104 & 77.6 & \\
$>50 \leq 60$ & 67 & 44.4 & & 84 & 55.6 & \\
$>60$ & 96 & 55.5 & & 77 & 44.5 & \\
\hline
\end{tabular}

GIII: MetS in prediabetes

\section{7}

The long-term effects of lifestyle intervention in impaired glucose tolerant subjects: determinants of intervention outcome

C. Roumen ${ }^{1}$, E. J. M. Feskens ${ }^{2}$, E. Corpeleijn ${ }^{1}$, W. H. M. Saris ${ }^{1}$, E. E. Blaak ${ }^{1}$; ${ }^{1}$ Human Biology, Maastricht University,

${ }^{2}$ Division of Human Nutrition, Wageningen University, The Netherlands.

Background and Aims: Lifestyle interventions may be effective in improving glucose tolerance and preventing diabetes. Less is known on what factors determine intervention outcome. Here, we evaluated the efficacy of a lifestyle intervention in a Dutch population with impaired glucose tolerance (IGT) and analysed which factors were predictive of changes in glucose tolerance and insulin sensitivity (as assessed by HOMA-IR)

Materials and Methods: We studied 147 IGT subjects, initially randomised into an intervention group; $\mathrm{n}=74$ (INT) and control group; $\mathrm{n}=73(\mathrm{CON})$ during a mean of 4.2 years lifestyle intervention. Subjects underwent 
measurements of body composition, body fat distribution, glucose tolerance (OGTT), insulin sensitivity (estimated by HOMA-IR), and maximal aerobic capacity $\left(\mathrm{VO}_{2}\right.$ max). Subjects in the intervention group were individually guided with respect to diet and a physical activity. Guidance was based on general public health recommendations.

Results: INT decreased their total fat and saturated fat intake and increased their carbohydrate and fibre intake, whereas this did not change in CON ( $\mathrm{p}<0.05$ group $\mathrm{x}$ time interaction). $\mathrm{VO}_{2}$ max improved more in INT, compared to $\mathrm{CON}(\mathrm{p}=0.042)$. Body weight decreased in INT during the first 3 years, but increased again during the last part of the study, whereas body weight did not change in $\mathrm{CON}$ ( $\mathrm{p}=0.20$ group $\mathrm{x}$ time interaction). 2 -hr glucose levels increased less in INT compared to CON $(+0.24 \pm 0.39 \mathrm{mM}$ vs $0.95 \pm 0.38$ $\mathrm{mM}$, respectively, $\mathrm{p}=0.041$ ). Cumulative diabetes incidence increased less in INT $(n=18)$ than in CON ( $n=27, p=0.052$ group $x$ time interaction). At baseline, low body fat mass, plasma resistin levels, high 2-hr glucose and Apo lipoprotein A (ApoA) levels were most predictive of a decrease in 2hr glucose levels $(p<0.05)$. High fasting insulin and mono unsaturated fat intake at baseline were most predictive of a decrease in HOMA-IR ( $\beta=-0.540$, $p=0.001$ and $\beta=-0.329, p=0.009$, respectively). Stepwise regression analysis in INT revealed that lifestyle-induced change in fasting glucose was most predictive for $\Delta 2 \mathrm{~h}$ glucose $(\beta=0.436, \mathrm{p}=0.009)$ and $\Delta$ body fat mass was most predictive for $\triangle$ HOMA-IR $(\beta=0.577, \mathrm{p}=0.001)$.

Conclusion: In conclusion, our lifestyle intervention program, aiming at increasing physical activity and using a healthy diet, improved glucose tolerance and reduced diabetes incidence. INT subjects with high baseline 2hr glucose improved more in glucose tolerance Lifestyle-induced changes in body fat mass were most predictive for the improved insulin sensitivity.

Supported by grants from the Dutch Diabetes Research Foundation, the Netherlands Organisation for Health Research and Development and the Netherlands Organisation for Scientific Research

\section{8}

Saturated dietary fats intake and the leisure-time physical activity among persons with high risk of type 2 diabetes. Results from National type 2 diabetes prevention programme in Finland (FIN-D2D)

M. E. H. Poskiparta ${ }^{1}$, M. Vanhala ${ }^{2}$, J. Villberg ${ }^{1}$, S. Salmela ${ }^{1}$, K. Vähäsarja ${ }^{1}$;

${ }^{1}$ Department of Health Sciences, University of Jyväskylä,

${ }^{2}$ Central Finland Hospital Districts, University of Kuopio, Jyväskylä,

Finland.

Background: Lifestyle changes including increased physical activity, reduced fat and increased fibre have shown to be even more effective than medication in prevention of type 2 diabetes. The basis of behaviour change is the assessment of the need for change. FIN-D2D is a big prevention programme for type 2 diabetes in Finland and, in this, the use of the Transtheoretical model as a health counselling method is recommended.

Aims: To examine the baseline findings concerning the participants' selfreported use of saturated dietary fats and leisure-time physical activity and to determine which factors affect their need for change of use of saturated dietary fats and the leisure-time physical activity.

Materials and Methods: The data consists of 3397 adults (men $37 \%$, women $63 \%$, mean age $55 \mathrm{yrs}$, mean BMI $32.1 \mathrm{~kg} / \mathrm{m}^{2}$ (in men 31.5 and women 32.4), mean waist circumference $103.1 \mathrm{~cm}$ (in men $108.4 \mathrm{~cm}$ and women $100.4 \mathrm{~cm}$ ) with high risk of T2D, who voluntarily participated and were referred to lifestyle counselling by primary care providers. The assessment of person characteristics, self-reported leisure-time physical activity and consumption of saturated dietary fats was based on questionnaires completed by participants and nurses. The Pearson's $\chi^{2}$-test and multivariate logistic regression were used

Results: One third of all respondents were physically active. $27 \%$ percent reported having a diet with high saturated dietary fat content. According the logistic regression analysis, in women $(\mathrm{BMI}>30)$ overweight increased the probability to the self-reported need of change saturated dietary fats use. In men abundant saturated dietary fats use and an age less than 65 increased the probability of the need for change fats use. The need for changing physical activity was more prevalent among overweight women $<45$ years of age, and among physically in-active men with waist circumference $<94 \mathrm{~cm}$.

Conclusion: The amount of high-risk persons, who eat too much saturated dietary fats and are insufficiently physical active, is alarming. Most of the participants reported that they are consciousness of their needs of behaviour changes. An assessment of the participants' knowledge and stages of change should be carried out in more detail, making the content of counselling more effective, personal and relevant to the participants ' needs Supported by study group of National Type 2 Diabetes Prevention Programme in Finland (FIN-D2D)

\section{9}

Effectiveness of a national diabetes prevention campagn for high risk groups in The Netherlands

I. de Weerdt ${ }^{1}$, H. P. Kuipers ${ }^{1}$, G. Kok ${ }^{2}$;

${ }^{1}$ NDF, Netherlands Diabetes Federation, Amersfoort,

${ }^{2}$ Psychology, University of Maastricht, The Netherlands.

Background and Aims: Currently 850.000 people in the Netherlands have diabetes and about 3.2 million is at risk for developing the disease in the coming years. Research has shown that a healthy lifestyle can delay the onset of diabetes or even prevent it. Determining which individuals are at risk for diabetes type 2 and stimulating these individuals to live healthily is thus imperative. Consequently, these goals are the main focus of the national prevention campaign entitled 'Look at Diabetes'. This campaign targets highrisk groups (people older than 45 years of age with overweight or obesity) and endeavours to make them aware that they are at risk for developing diabetes type 2. Special attention has been paid to people with a risk higher than average: people with a Turkish, Maroc or Hindu background and with a lower educational level. Those at risk are stimulated to perform the Dutch Diabetes Risk Test. When as a result of this test, people have a high risk of developing diabetes, they are advised to visit their GP for further diagnosis and advice. The campaign itself (April-December 2006) is based on behavioural theories and consists of national and local activities, both for target groups ans intermediaries (GP's, practice nurses, dieticians, municipal health organisations).

Material and Methods: In order to asses the efficacy of the campaign on knowledge, risk perception (perceived seriousness and perceived vulnerability), perceived avoidability of risk and number of high risk persons detected, a study was performed using the best available design for evaluating mass media campaigns. This study consisted of an effect and process evaluation among target groups and professionals. In total 911 people older than 45 years of age and overweight were recruited for this study: 645 person with an autochthonous and 266 persons with an allochtonous background. To correct for the Hawthorne effects, $50 \%$ was asked to participate in the study three times (panel group) and $50 \%$ was asked to participate only once (new sample). Data collection took place prior and 3 months after the start, and at the end of the campaign using a combination of face to face interviews (allochtonous respondents) and interviews by phone (autochtonous respondents). Professionals completed a questionnaire concerning attitudes, self-efficacy and perceived barriers to diabetes prevention prior and after the campaign.

Results: The campaign was successful in reaching all target groups, especially those with an allochtonous background. Generally the campaign was effective in increasing knowledge concerning diabetes, perceived 'avoidability of risk' and risk perception. Due to the campaign a significant number of people were diagnosed as having diabetes or prediabetes. The study among professionals showed a very positive attitude to diabetes prevention interventions in general and to the campaign in specific. After the campaign the perceived barriers for taking an active role in diabetes prevention were significantly decreased.

Conclusion: The campaign was effective in reaching high risk groups with respect to their risk of diabetes and concerning this risk, taking adequate follow up, especially for allochtonous groups. The campaign was also effective in stimulating professionals to take an active role in accomplishing the main targets of the campaign. Based on the results, the national campaign will be further improved and continued for the forthcoming years.

Supported by ZonMw 


\section{PS 76 Antiobesity drugs}

\section{0}

Effects of rimonabant on metabolic parameters and pancreatic function in male ZDF rats: comparison with rosiglitazone

P. Chamiot-Clerc, C. Duquenne, J. Ludop-Maignel, V. Delion, F. Petoux,

V. Duvivier, M.-P. Pruniaux, A.-M. Galzin;

Sanofi-aventis R\&D, Rueil-Malmaison, France.

Background and Aims: Recent clinical studies have shown evidence of a beneficial effect of Rimonabant (RIMO) in type 2 diabetic patients. The aim of the study was to compare the effects of RIMO and Rosiglitazone (ROSI) following chronic administration in male Zucker Diabetic Fatty (ZDF) rats, an animal model known to spontaneously develop type 2 diabetes from the age of 8 weeks, characterised by a rapid increase in glycaemia, concomitant with a decrease in insulin secretion and a loss in $\beta$ cell function.

Materials and Methods: Male ZDF rats (8 week-old) were orally treated with RIMO (10 mg/kg/d), ROSI ( $3 \mathrm{mg} / \mathrm{kg} / \mathrm{d})$ or vehicle (VEH) during either 2 or 6 weeks. Glycaemia, insulinemia and body weight were measured weekly. At the end of each period (i.e. 2 or 6 weeks), pancreata were recovered from all groups for islet isolation. Insulin secretion was assessed by static incubation, in the presence of $2.8 \mathrm{mM}$ and $16.7 \mathrm{mM}$ glucose, and by perifusion with media containing the same glucose concentrations.

Results: In contrast to VEH-treated rats which developed a marked hyperglycaemia from the beginning until the end of the experiment $(470 \pm$ $13 \mathrm{mg} / \mathrm{dl}$ after 6 weeks), RIMO- like ROSI-treated animals maintained a close to normal glycaemia with values after 6 weeks of $126 \pm 11 \mathrm{mg} / \mathrm{dl}$ ( $\mathrm{p}<0.01 \mathrm{vs}$. $\mathrm{VEH})$ and $157 \pm 34 \mathrm{mg} / \mathrm{dl}$ ( $<<0.01 \mathrm{vs.} \mathrm{VEH})$ respectively. Similarly, while insulinemia dramatically decreased from $13.2 \pm 0.8$ at the beginning of the study to $1.0 \pm 0.1 \mathrm{ng} / \mathrm{ml}$ after 6 weeks in VEH, insulin levels in RIMO and ROSI animals, despite a decrease during the first 2 weeks, started to increase again to reach values at 6 weeks of $8.3 \pm 1.2(\mathrm{p}<0.01 \mathrm{vs}$. VEH) and $4.5 \pm 0.5$ $\mathrm{ng} / \mathrm{ml}(\mathrm{p}<0.01$ vs. VEH) respectively. As usually observed, ROSI induced a marked increase in body weight ( $+100 \%$ vs. baseline) while RIMO animals increased their body weight by $50 \%$ only over the 6 weeks study similar to body weight gain of healthy animals. Noteworthy, VEH rats displayed a very flat body weight curve ( $+20 \%$ vs. baseline after 6 weeks) due to their severe diabetic status. In static incubation experiments, RIMO and ROSI treatment increased isolated islets stimulation index $(+507 \%$ and $+553 \% \mathrm{p}<0.001$ respectively vs. VEH at 6 weeks). This effect was mainly due to an increase in glucose-induced insulin secretion $(21.3 \pm 3.2 \mu \mathrm{g} / \mathrm{l}, \mathrm{p}<0.0005,22.0 \pm 3.7 \mu \mathrm{g} / \mathrm{l}$, $\mathrm{p}<0.0005$, and $4.7 \pm 0.4 \mu \mathrm{g} / \mathrm{l}$ in RIMO, ROSI and VEH group respectively). Perifusion results showed that in islets from VEH-treated animals the insulin secretion profile were altered with a low first phase together with the absence of a second phase insulin response to $16.7 \mathrm{mM}$ glucose. Conversely RIMO and ROSI treatments restored glucose-induced insulin secretion with the characteristic biphasic insulin secretion in isolated islets.

Conclusion: In conclusion, these results show that treatment with Rimonabant in $\mathrm{ZDF}$ rats prevents the development of hyperglycaemia, improves $\beta$ cell function and maintains a normal profile of insulin secretion with a much lower impact on body weight compared to Rosiglitazone treatment.

\section{1}

Brain CB-1 receptor occupancy of Taranabant in lean subjects using CB-1R PET

I. De Lepeleire ${ }^{1}$, C. Addy ${ }^{2}$, P. Rothenberg ${ }^{3,4}$, D. Burns ${ }^{5}$, S. Sanabria ${ }^{5}$, W. Eng ${ }^{5}$, G. Bormans ${ }^{6}$, M. Depré 7 , L. Mortelmans ${ }^{8}$, P. Dupont ${ }^{8}$, A. Stoch ${ }^{3}$, K. Gottesdiener ${ }^{3}$, R. Hargreaves ${ }^{5}$, J. Wagner ${ }^{3}$, K. Van Laere ${ }^{8}$;

${ }^{1}$ Clinical Pharmacology, MSD (Europe) Inc., Brussels, Belgium,

${ }^{2}$ Clinical Pharmacology, Merck \& Co..Inc., Boston, United States,

${ }^{3}$ Clinical Pharmacology, Merck \& Co.,Inc., Rahway, United States,

${ }^{4}$ Experimental Medicine/Clinical Pharmacology, Johnson\&Johnson, Raritan, United States, ${ }^{5}$ Imaging Research, Merck \& Co.,Inc., West Point, United States, ${ }^{6}$ Laboratory for Radiopharmacy, K.U.Leuven, Belgium, ${ }^{7}$ Center for Clinical Pharmacology, K.U.Leuven, Belgium, ${ }^{8}$ Division of Nuclear Medicine, MIRACLE, University Hospital K.U.Leuven, Belgium.

Background and Aims: The endocannabinoid system has been implicated in the central regulation of body weight. Cannabinoid-1 receptors (CB-1R) are expressed throughout the human brain, and inverse agonists of these receptors have demonstrated weight loss efficacy in clinical trials. This study was designed to evaluate human brain CB-1R occupancy after SD and MD administration of taranabant, a structurally distinct CB-1R inverse agonist, as assessed by PET using the CB-1R ligand, $\left[{ }^{18} \mathrm{~F}\right] \mathrm{MK}-9470$.

Materials and Methods: This was a randomised, double-blind, placebocontrolled study in which 15 healthy, lean male subjects were randomised to receive 1 of 5 14-day MD treatments (placebo, taranabant 1, 4 and 7.5 $\mathrm{mg}$, or SD of taranabant $12 \mathrm{mg}$ followed by $6 \mathrm{mg} \mathrm{MD}$ ). PET scans using $\left[{ }^{18} \mathrm{~F}\right]$ MK-9470 were performed at predose and at $\sim 2$ hours and/or $\sim 24$ hours following taranabant SD or MD administration. Blood samples were obtained for taranabant plasma concentration pre- and post-PET. The brain receptor occupancy data were fit with a curve described by a Hill equation: Occupancy $(\%)=\mathrm{A} \mathrm{y}^{\mathrm{nH}} /\left[\left(\mathrm{Occ}_{50}\right)^{\mathrm{nH}}+\mathrm{y}^{\mathrm{nH}}\right]$, where $y$ corresponds to taranabant plasma concentration, $A$ was set to $100 \%$ (maximum occupancy) and the Hill coefficient $n H$ set to 1 .

Results and Conclusion: Taranabant was generally well tolerated and demonstrated dose-dependent occupancy of brain CB-1Rs after SD and MD administration as assessed by PET of the CB-1R ligand, $\left[{ }^{18} \mathrm{~F}\right] \mathrm{MK}-9470$. PET may facilitate dose selection and help to define the therapeutic index for taranabant and other CB-1R inverse agonists in the treatment of overweight and obese patients.

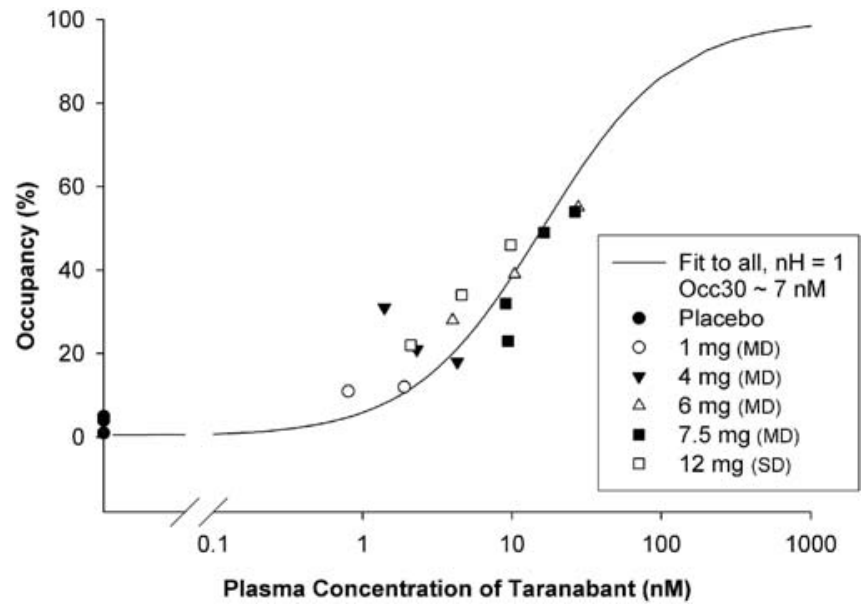

Supported by Merck \& Co.,Inc.

\section{2}

Taranabant increases energy expenditure in overweight and moderately obese subjects

C. Addy $^{1}$, S. Heymsfield ${ }^{2}$, C. Cilissen ${ }^{3}$, I. N. Gendrano III', M. De Smet ${ }^{3}$, M. De Decker ${ }^{5}$, J. Yuan ${ }^{6}$, P. Larson ${ }^{6}$, S. Dunbar ${ }^{6}$, A. Stoch ${ }^{4}$, J. Wagner ${ }^{4}$; ${ }^{1}$ Clinical Pharmacology, Merck \& Co., Inc., Boston, United States, ${ }^{2}$ Clinical Research - Metabolism, Merck \& Co., Inc., Rahway, United States, ${ }^{3}$ Clinical Pharmacology, MSD (Europe), Brussels, Belgium, ${ }^{4}$ Clinical Pharmacology, Merck \& Co., Inc., Rahway, United States, ${ }^{5}$ Life Science Services, SGS Biopharma, Antwerp, Belgium, ${ }^{6}$ Clinical Biostatistics, Merck \& Co., Inc., Rahway, United States.

Background and Aims: Cannabinoid-1 receptor (CB-1R) inverse agonists are associated with weight loss efficacy in clinical trials. Whether this is due to reduction in food intake, an increase in energy expenditure, or a combination of the two remains to be determined. This study was designed to evaluate the effect of single doses of taranabant, a structurally distinct CB-1R inverse agonist, compared to placebo on resting energy expenditure (REE) in overweight and moderately obese male volunteers.

Materials and Methods: This was a double-blind, double-dummy, active comparator- and placebo-controlled, 4-period crossover study in which 17 overweight and moderately obese male subjects were randomised to receive 1 of 4 single-dose treatments (placebo, taranabant $4 \mathrm{mg}$ and $12 \mathrm{mg}$, and sibutramine $30 \mathrm{mg}$ as a positive control). REE was assessed using indirect calorimetry over a 5 hour postdose period and at 24 hours postdose. Peak REE was obtained as the maximum of the average of 2 consecutive 30 -minute measurements within 2-5 hours postdose. 
Results: The ANOVA model contained the factors: subject, period, treatment, and 30 -minute predose baseline (the average of the two 30 minute readings) REE. Baseline REE was detected as a significant covariate. A test for first order carryover effect was also performed, and no significant effect was observed. The peak REE geometric mean ratio (GMR) and 90\% confidence interval (CI) for sibutramine $30 \mathrm{mg}$, taranabant $4 \mathrm{mg}$, and taranabant $12 \mathrm{mg}$ versus placebo were $1.03(0.99,1.07), 1.02(0.98,1.05)$, and $1.06(1.02,1.09)$, respectively. Single doses of taranabant and sibutramine were generally well tolerated.

Conclusion: This appears to be one of the first clinical studies to demonstrate that a CB-1R inverse agonist is associated with increases in REE, which may, in part, explain the basis for weight loss efficacy with this mechanism. Taranabant was generally well tolerated and may show promise as a medication to induce weight loss in overweight and obese patients.

\section{3}

The CB-1 $\mathrm{R}$ inverse agonist taranabant reduces acute food intake in overweight/obese male volunteers

D. Wright ${ }^{1}$, C. Stevens ${ }^{1}$, C. Cilissen ${ }^{2}$, M. De Smet ${ }^{2}$, D. Krupa ${ }^{3}$, P. Larson ${ }^{3}$, M. De Decker ${ }^{4}$, G. Herman ${ }^{1}$, J. Wagner ${ }^{1}$;

${ }^{1}$ Clinical Pharmacology, Merck \& Co., Inc., Rahway, United States,

${ }^{2}$ Clinical Pharmacology, Merck \& Co., Inc., Brussels, Belgium,

${ }^{3}$ Biostatistics, Merck \& Co., Inc., Rahway, United States,

${ }^{4}$ Research Unit Stuivenberg, SGS Biopharma, Antwerp, Belgium.

Background and Aims: Endogenous cannabinoids and exogenous agonists of the brain cannabinoid 1 receptor (CB-1R) are implicated in promoting appetite and food intake. We have previously described an experimental medicine paradigm to test anti-obesity compounds for acute anorexic effects. Herein, we illustrate the use of this paradigm as a platform to assess the mechanism of action, in part, of a CB-1R inverse agonist.

Materials and Methods: This study evaluated the potential in the clinic for dose-dependent anorexic effects as mediated by taranabant, an investigational CB-1R inverse agonist. A randomised, placebo-controlled, double-blind, 4period, crossover study in 36 overweight/obese male subjects assessed the effects of taranabant and sibutramine (positive control) on ad libitum ('free choice') energy intake. Subjects received single doses of taranabant $12 \mathrm{mg}$ or $4 \mathrm{mg}$, sibutramine $30 \mathrm{mg}$ or placebo.

Results: At 3, 10, 13 and 24 hrs postdose, respectively, lunch, dinner, snack and breakfast meals were provided in $\sim 5$-fold excess over standard portions; subjects consumed these ad libitum. The amount of food consumed was transformed into caloric and macronutrient data. Sibutramine $30 \mathrm{mg}$ significantly reduced energy intake $(p<0.001)$ by $\sim 12 \%$ over $24 \mathrm{hrs}$ and by

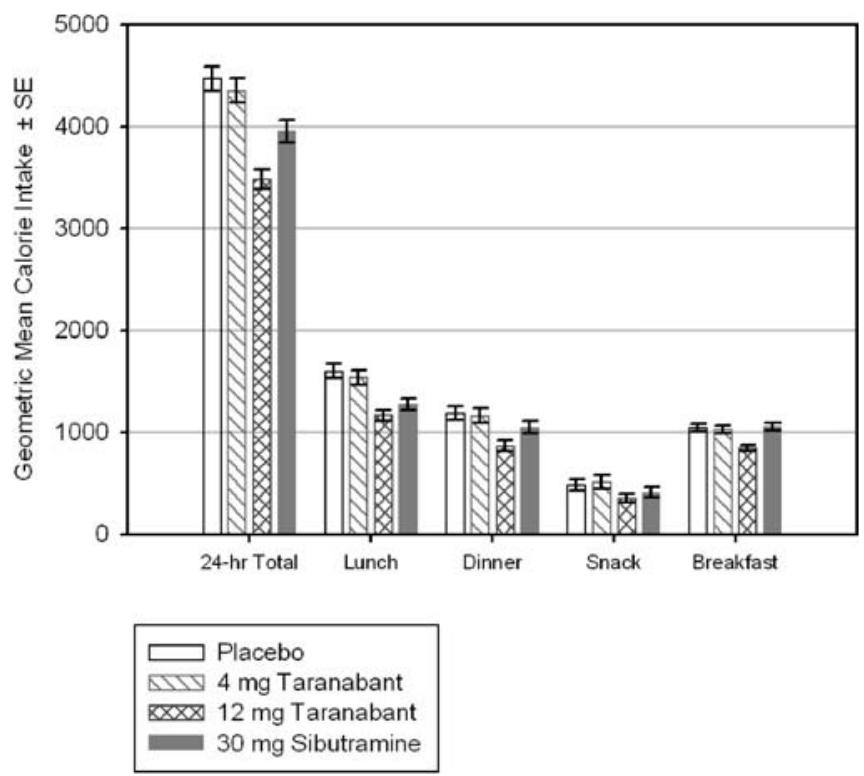

Figure 1. 24-hour \& Meal Specific Energy Intake (kcal) After SD Administration of Placebo, Taranabant, or Sibutramine $\sim 20 \%$ at the lunch meal vs. placebo. Taranabant $12 \mathrm{mg}$ significantly reduced energy intake $(p<0.0001)$ by $\sim 22 \%$ over 24 hrs and by $\sim 27 \%$ at the lunch meal vs. placebo (Figure 1). The mean change (95\% confidence interval) in energy intake over 24 hours, relative to placebo, was $-3 \%(+5 \%,-10 \%),-22 \%$ $(-16 \%,-28 \%)$, and $-12 \%(-5 \%,-18 \%)$ for taranabant $4 \mathrm{mg}$ and $12 \mathrm{mg}$, and sibutramine $30 \mathrm{mg}$, respectively. Effects were equally distributed across all macronutrients. Taranabant $4 \mathrm{mg}$ had no significant effect on energy intake. Both compounds were well-tolerated.

Conclusion: Clinical administration of a CB-1R inverse agonist mediates acute, dose-dependent anorexic effects, as measured by short-term food intake reduction.

\section{4}

\section{Rimonabant reduces visceral fat in obese Japanese patients with cardiometabolic risk factors \\ K. Shirai;}

Department of Internal Medicine, Toho University Sakura Medical Center, Chiba, Japan.

Background and Aims: We performed a dose-response, multicentre, doubleblind, parallel-group study to evaluate the effect of rimonabant on body weight and multiple cardiometabolic risk factors, and to study for the first time its effect on visceral fat area (VFA) (assessed by computed tomography scan), in obese Japanese patients.

Materials and Methods: Patients were randomised to placebo $(n=131)$ or rimonabant $5 \mathrm{mg}(\mathrm{n}=133), 10 \mathrm{mg}(\mathrm{n}=129)$ or $20 \mathrm{mg}(\mathrm{n}=131)$ for 24 weeks. Inclusion criteria were body mass index $\geq 25 \mathrm{~kg} / \mathrm{m}^{2}, V F A \geq 100 \mathrm{~cm}^{2}$ and two of the following: impaired glucose tolerance or type 2 diabetes; untreated dyslipidaemia; hypertension. Efficacy endpoints included change from baseline in body weight, VFA, waist circumference, HDL-C and triglycerides.

Results: Rimonabant 20 mg significantly reduced body weight, VFA, waist circumference and triglycerides and increased HDL-C compared with placebo (table). A similar incidence of adverse events (AEs) was reported between groups, with no dose effect. Withdrawals due to AEs were lower with rimonabant $20 \mathrm{mg}(4.5 \%)$ than placebo (7.6\%). Completion rate was $86 \%$. Rimonabant was generally well tolerated.

\begin{tabular}{llllll} 
& & Placebo & \multicolumn{3}{l}{ Rimonabant } \\
\cline { 4 - 6 } & & & $5 \mathrm{mg}$ & $10 \mathrm{mg}$ & $20 \mathrm{mg}$ \\
\hline Body weight & Baseline & 87.1 & 85.9 & 88.1 & 85.5 \\
$(\mathrm{~kg})$ & Change & -1.7 & -2.4 & -2.7 & -4.5 \\
& & & $\mathrm{NS}$ & $(\mathrm{p}=0.03)$ & $(\mathrm{p}<0.0001)$ \\
$\mathrm{VFA}$ & Baseline & 171.6 & 181.6 & 168.3 & 175.3 \\
$\left(\mathrm{~cm}^{2}\right)$ & Change & -20.3 & -24.4 & -30.4 & -40.6 \\
& & & $\mathrm{NS}$ & $(\mathrm{p}=0.04)$ & $(\mathrm{p}<0.0001)$ \\
& \% change & -11.3 & -13.7 & -16.7 & -23.2 \\
& & & $\mathrm{NS}$ & $(\mathrm{p}=0.04)$ & $(\mathrm{p}<0.0001)$ \\
Waist & Baseline & 102.1 & 101.2 & 102.9 & 100.7 \\
circumference & Change & -2.5 & -3.1 & -3.6 & -4.7 \\
$(\mathrm{~cm})$ & & & $\mathrm{NS}$ & $(\mathrm{p}=0.05)$ & $(\mathrm{p}=0.0001)$ \\
HDL-C & Baseline & 1.15 & 1.18 & 1.17 & 1.20 \\
$(\mathrm{mmol} / \mathrm{L})$ & \% change & 5.8 & 7.9 & 11.2 & 16.4 \\
& & & $\mathrm{NS}$ & $(\mathrm{p}=0.005)$ & $(\mathrm{p}<0.0001)$ \\
Triglycerides & Baseline & 2.95 & 2.77 & 2.71 & 2.67 \\
$(\mathrm{mmol} / \mathrm{L})$ & \% change & -2.8 & -3.5 & -1.5 & -15.6 \\
& & & $\mathrm{NS}$ & $\mathrm{NS}$ & $(\mathrm{p}=0.04)$ \\
\hline
\end{tabular}

Least-square mean change from mean baseline at Week 24

(p-value vs. placebo); NS, non-significant.

Conclusion: This is the first study to demonstrate that rimonabant $20 \mathrm{mg}$ achieves reduction in VFA. Improvements in cardiometabolic risk factors, such as waist circumference, HDL-C and triglycerides, reported in the obese Japanese population were similar to those previously observed in the Caucasian population. This study confirmed the efficacy and tolerability of rimonabant $20 \mathrm{mg}$.

Supported by sanofi-aventis 


\section{5}

$\mathrm{HbA}_{1 \mathrm{c}}$ reductions plus weight loss and improved lipid profile with rimonabant in overweight/obese drug-naive type 2 diabetes: the SERENADE trial in patients with BMI $>27 \mathrm{~kg} / \mathrm{m}^{2}$

J. Rosenstock ${ }^{1}$, P. A. Hollander ${ }^{2}$, A. Iranmanesh ${ }^{3}$;

${ }^{1}$ Dallas Diabetes and Endocrine Center, Dallas, ${ }^{2}$ Endocrinology Center, Baylor University Medical Center, Dallas, ${ }^{3}$ Endocrine Section, Veterans Administration Medical Center, Salem, United States.

Background and Aims: The first selective $\mathrm{CB}_{1}$ receptor blocker, rimonabant $20 \mathrm{mg}$, significantly improved glycaemic control, body weight, waist circumference and lipid parameters in drug-naive type 2 diabetes patients, as shown in the SERENADE trial $(\mathrm{n}=278)$. The primary endpoint was $\mathrm{HbAlc}$ and increased body weight was not an inclusion criterion. The aim of this additional analysis was to extend the initial report of SERENADE, to specifically assess the efficacy of rimonabant in overweight or obese patients (baseline BMI $>27 \mathrm{~kg} / \mathrm{m}^{2}, \mathrm{n}=249$ ) who participated in this study.

Materials and Methods: SERENADE was a 6-month, randomised, doubleblind, placebo-controlled trial of rimonabant $20 \mathrm{mg}$ /day in drug-naive type 2 diabetes patients. For this analysis, the efficacy of rimonabant in overweight/obese patients was assessed by examining changes from baseline to 6 months in glycaemic control, body weight, waist circumference and lipid parameters.

Results: At 6 months, rimonabant significantly improved glycaemic control, body weight, waist circumference and lipid parameters (table). A larger effect on $\mathrm{HbAlc}$ occurred in overweight/obese patients with $\mathrm{HbAlc} \geq 8.5$ at baseline $(-1.9 \%$ from baseline $[\mathrm{p}=0.0019$ vs. placebo]). Rimonabant was generally well tolerated.

Conclusion: Overweight or obese drug-naive type 2 diabetes patients on rimonabant $20 \mathrm{mg} /$ day had clinically meaningful $\mathrm{HbAlc}$ lowering plus weight loss, with improvements in HDL-C and triglyceride levels similar to those observed in the overall population in the SERENADE trial.

\begin{tabular}{llll} 
Mean change & $\begin{array}{l}\text { Placebo } \\
(\mathbf{n}=\mathbf{1 2 5})\end{array}$ & $\begin{array}{l}\text { Rimonabant } \\
\mathbf{2 0 ~} \mathbf{~ m g}(\mathbf{n}=\mathbf{1 2 4})\end{array}$ & p vs. placebo \\
\hline HbAlc (\% units) & & & \\
Baseline & $7.9(0.7)$ & $7.9(0.8)$ & \\
6 months & $7.5(1.3)$ & $7.1(1.1)$ & \\
Change from baseline & $-0.4(1.2)$ & $-0.9(1.2)$ & 0.0009 \\
Body weight (kg) & & & \\
Baseline & $98.9(20.2)$ & $99.2(20.5)$ & \\
6 months & $96.0(19.6)$ & $92.2(19.3)$ & \\
Change from baseline & $-2.9(5.0)$ & $-7.0(5.6)$ & $<0.0001$ \\
Waist (cm) & & & \\
Baseline & $110.3(14.8)$ & $111.0(13.5)$ & \\
6 months & $107.9(15.1)$ & $104.6(12.6)$ & \\
Change from baseline & $-2.4(4.7)$ & $-6.4(6.4)$ & $<0.0001$ \\
HDL-C (mmol/L) & & & \\
Baseline & $1.3(0.3)$ & $1.3(0.3)$ & \\
6 months & $1.3(0.3)$ & $1.4(0.4)$ & \\
Change from baseline (\%) & $3.1(12.0)$ & $10.4(17.0)$ & 0.0001 \\
Triglycerides (mmol/L) & & & \\
Baseline & $2.0(1.0)$ & $2.4(1.6)$ & \\
6 months & $2.0(1.2)$ & $1.8(1.0)$ & \\
Change from baseline (\%) & $4.5(60.0)$ & $-18.3(28.2)$ & 0.0024 \\
\hline
\end{tabular}

Intention to treat population; results are expressed as mean (standard deviation).

Supported by sanofi-aventis

\section{6}

Treatment with rimonabant for overweight or obese patients with diabetes: health economic estimates for the UK

D. Getsios ${ }^{1}$, J. Möller ${ }^{2}$, P. McEwan ${ }^{3}$, K. Ishak ${ }^{4}$, C. Nicholls 5 , J. J. Caro ${ }^{6}$; ${ }^{1}$ Research, Caro Research Institute, Halifax, Canada, ${ }^{2}$ Research, Caro Research Institute, Eslov, Sweden, ${ }^{3}$ Research, University Hospital of Wales, Cardiff, United Kingdom, ${ }^{4}$ Biostatistics, Caro Research Institute, Montreal, Canada, ${ }^{5}$ Sanofi-Aventis, Surrey, United Kingdom,

${ }^{6}$ Research, Caro Research Institute, Concord, United States.

Background and Aims: The RIO Diabetes trial demonstrated rimonabant's benefits in the treatment of overweight or obese patients with diabetes. Not only did patients lose weight, other cardiometabolic risk factors, including glycaemic and lipid levels, also improved. A discrete event simulation was developed to evaluate the cost-effectiveness of rimonabant in patients with diabetes and a BMI $>27$ in the UK.

Materials and Methods: Adding rimonabant 20mg to patients' existing antidiabetic treatments and a diet and exercise regimen was evaluated. Individual patients are assigned characteristics based on data from the Health Survey of England (HSE). Two populations were studied in the simulations: high risk patients in the HSE population and the overall HSE population. Predicted changes in risk factors are estimated using equations developed from RIO Diabetes. Treatment persistence and adverse events are also simulated based on analysis of clinical trial data. Individuals are followed over their lifetimes and their risk factor profiles are used to predict cardiovascular disease, microvascular disease and death, based on UKPDS, UK life tables and other published risk equations. In addition, consequent direct medical costs in $2005 £$ and effects on health-related quality of life were based on data from Cardiff and the Vale National Health Service Trust, NHS reference costs, and other published sources.

Results: After 1 year of rimonabant, patients lose more weight and have better risk factor profiles compared with diet and exercise alone, with a mean weight loss over $3 \mathrm{~kg}$ higher with rimonabant. Patients on diet and exercise alone experience little change in lipid levels, while for those on rimonabant, mean HDL increases by an average of $0.11 \mathrm{mmol} / \mathrm{L}$ and triglycerides fall by $0.33 \mathrm{mmol} / \mathrm{l}$. $38 \%$ of patients have $\mathrm{HbA}_{1 \mathrm{c}}$ levels $>7 \%$ at baseline. While this falls to $30 \%$ with diet and exercise, it drops to $20 \%$ when rimonabant is added. Over the course of their lifetimes, 1,000 patients treated with rimonabant for 5 years avoid 45 cardiovascular events and 38 microvascular complications, with survival increasing by 234 years and by 320 when quality-adjusted. At a cost of $£ 1.97$ per day, the cost per discounted quality-adjusted life year is $£ 8,700$. Analyses restricted to HSE patients at the higher levels of CVD risk result in increased reductions in CVD risk. The proportion of patients with $\mathrm{HbA}$ levels $>7 \%$ falls from $34 \%$ at baseline to $19 \%$ with rimonabant. While CVD events avoided increases to 56, gains in quality adjusted survival are somewhat lower because of the older age of the population. Quality adjusted survival increases by 257 years, resulting in a cost per discounted quality-adjusted life year of $£ 8,800$, equivalent to that when all patients are treated.

Conclusions: These simulations indicate that weight loss and improvements in lipid and glycaemic levels for patients on rimonabant are likely to translate into meaningful reductions in disease in overweight and obese patients with diabetes. In the UK, these gains can be attained at reasonable costs, with costeffectiveness estimates below $£ 10,000 /$ QALY gained.

Supported in part by an unrestricted grant from sanofi-aventis 


\section{7}

Initial blood pressure response of high-risk patients with diabetes plus cardioavascular disease after 6-weeks Sibutramine treatment - an analysis of the Sibutramine Cardiovascular Outcomes (SCOUT) Trial I. Caterson ${ }^{1}$, W. Coutinho ${ }^{2}$, N. Finer ${ }^{3}$, L. Van Gaal ${ }^{4}$, A. P. Maggioni ${ }^{5}$, A. M. Sharma ${ }^{6}$, C. Torp-Pedersen ${ }^{7}$, W. P. T. James ${ }^{8}$;

${ }^{1}$ Human Nutrition Unit, Univ of Sydney, Australia, ${ }^{2}$ Obes \& Eating Dis Res Gp, Catholic Univ. of Rio de Janeiro, Brazil, ${ }^{3}$ Wellcome Trust Clin. Res. Facility, Univ of Cambridge, United Kingdom, ${ }^{4}$ Dept. of Diab, Metabol \& Clin Nutr, Univ Hosp of Antwerp, Belgium, ${ }^{5}$ Res Center, ANMCO Res Center, Florence, Italy, ${ }^{6}$ Obes Res, McMaster Univ Hosp, Hamilton, Canada, ${ }^{7}$ Dept of Cardiol, Bispebjerg Univ Hosp, Copenhagen, Denmark,

${ }^{8}$ Dept of Nutrition, London Sch of Hyg \& Trop Med, United Kingdom.

Background: Type 2 diabetes mellitus (DM) is a common cause of morbidity/ mortality in obese subjects. DM subjects may be 4 times more likely to have cardiovascular $(\mathrm{CV})$ disease than those without DM; in this disease state, obesity plays a major role in contributing to this outcome. Lifestyle changes are important, but weight reduction is often unsuccessful without pharmacotherapy. Sibutramine (SIB) is an effective weight-management medication, but due to its potential to increase blood pressure (BP), patients with DM and associated CV disease may be contraindicated. Data from the 6-week single-blind period of the ongoing SCOUT trial are utilised to assess SIB plus weight management on BP in this hypertensive, high-risk DM population.

Method: SCOUT is a double-blind, randomised, placebo-controlled outcome trial. Eligible patients included those with CV disease and/or DM with another risk factor. Patients with BP $>160 / 100 \mathrm{mmHg}$ were excluded. All patients entered a single-blind, 6-week lead-in period and received SIB. At entry, BP was stratified according to BP levels: Normotensive [NT] /Controlled HT <130/80mmHg; Grade 1 HT 130-<160/80-<100mmHg, and Grade 2 HT $\geq 160 / 100 \mathrm{mmHg}$. Anti-hypertensive BP-medication class use (none, 1, $\geq 2$ ) at entry was to be held constant and determined for patients according to their BP category. Changes in BP in DM patients were assessed over this period.

Results: Of the 10,742 patients who received SIB $10 \mathrm{mg}, 9015$ (84\%) had preexisting DM. At week $-6,20 \%, 78 \%$, and $2 \%$ of DM patients were classified as BP category: NT/Controlled HT, Grade 1 HT or Grade 2 HT, respectively. The majority (63\%) of NT/Controlled HT patients required $\geq 2$ anti-hypertensive medication classes to achieve BP goals. The majority (72\%) with Grade 1 HT was also receiving $\geq 2$ anti-hypertensive medication classes. Median change in body weight and waist circumference to endpoint was $-2.1 \mathrm{~kg}(-2.3 \%)$ and $-2.0 \mathrm{~cm}$
Median SBP/DBP Changes from Week -6 to Lead-in Period Endpoint

\begin{tabular}{|c|c|c|c|}
\hline \multirow[t]{2}{*}{ Week -6 BP category } & \multirow{2}{*}{$\begin{array}{l}\text { Anti-HT Medication: } \\
\text { No. of Classes }\end{array}$} & \multicolumn{2}{|c|}{ Median SBP/DBP $(\mathrm{mmHg})$} \\
\hline & & Initial & Change \\
\hline \multirow[t]{3}{*}{ NT/Controlled HT } & None & $121.0 / 69.0$ & $2.0 / 2.0$ \\
\hline & 1 & $122.5 / 70.0$ & $1.0 / 1.5$ \\
\hline & $\geq 2$ & $122.5 / 69.5$ & $2.5 / 2.0$ \\
\hline \multirow[t]{3}{*}{ Grade 1 HT } & None & $139.5 / 80.0$ & $-4.5 /-1.0$ \\
\hline & 1 & $142.0 / 80.0$ & $-3.5 /-1.0$ \\
\hline & $\geq 2$ & $142.5 / 80.5$ & $-5.0 /-2.0$ \\
\hline \multirow[t]{3}{*}{ Grade $2 \mathrm{HT}$} & None & $163.5 / 89.5$ & $-16.0 /-8.5$ \\
\hline & 1 & $162.0 / 84.0$ & $-9.5 /-2.5$ \\
\hline & $\geq 2$ & $161.5 / 84.5$ & $-13.0 /-3.0$ \\
\hline
\end{tabular}

Further analysis showed BP reduction in HT patients even in the absence of weight loss.

Conclusion: High-risk DM patients with increased BP ( $\geq$ Grade $1 \mathrm{HT})$ on entry to the 6-week period had an appreciable reduction in SBP and DBP, regardless of weight loss, even in those patients receiving $\geq 2$ classes of antihypertensive medication. Normotensive/Controlled HT patients had small median increases in BP similar to those previously observed.

Supported by Abbott Laboratories

\section{8}

Sustained weight loss following 1-year pramlintide treatment as an adjunct to lifestyle intervention in obesity

A. Halseth ${ }^{1}$, S. Smith ${ }^{2}$, E. Klein ${ }^{3}$, C. Burns ${ }^{1}$, N. Kesty ${ }^{1}$, C. Weyer ${ }^{1}$; ${ }^{1}$ Amylin Pharmaceuticals, Inc., San Diego, ${ }^{2}$ Pennington Biomedical Research Center, Louisian State University System, Baton Rough, ${ }^{3}$ Capital Clinical Research Center, Olympia, United States.

Background and Aims: In a previously reported 4 mo, randomised, placebocontrolled, dose-ranging study, treatment with the amylin analog pramlintide $(120,240,360 \mu \mathrm{g}, \mathrm{BID} / \mathrm{TID})$ used in conjunction with lifestyle intervention (LSI) resulted in additional weight loss than observed with LSI alone. To assess the durability of this effect, we instituted a single-blind, placebocontrolled extension protocol that assessed body weight and safety for an additional $8 \mathrm{mo}$.
Fig. 1

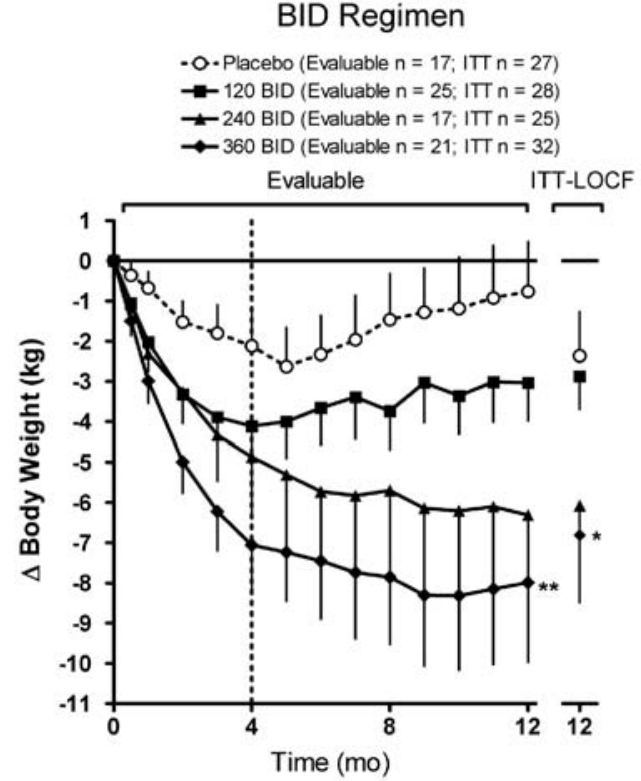

TID Regimen

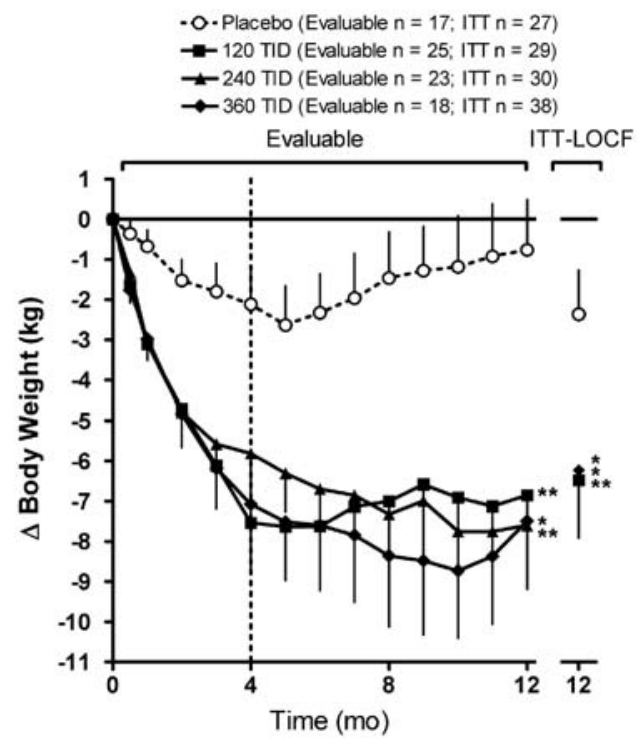

${ }^{*} P<0.05 ; * P<0.01 ; * * P<0.001$ from baseline to Mo-12 for each pramlintide treatment group vs. placebo Only Mo-12 significance depicted in figures for clarity 
Materials and Methods: Of subjects completing 4 mo, $75 \%$ of subjects (ITT $\mathrm{n}=209$; $76 \%$ female; weight $106.0 \pm 18.4 \mathrm{~kg}$; mean $\pm \mathrm{SD}$ ) opted to continue treatment with either pramlintide $(120,240,360 \mu \mathrm{g}$, BID/TID) or placebo administered 15 min before meals. Throughout the extension, LSI was geared towards weight maintenance (not loss).

Results: By Mo 12, the withdrawal rate was $26 \%$ for pramlintide- vs. $37 \%$ for placebo-treated subjects (Evaluable $\mathrm{N}=146$ ). Initial weight loss was largely regained in the placebo group, but was maintained in all but one pramlintide group (see figure, below)

At 12 mo, 40 and $43 \%$ of evaluable subjects treated with $120 \mu \mathrm{g}$ TID and 360 $\mu \mathrm{g}$ BID, respectively, achieved $\geq 10 \%$ weight loss (vs. $12 \%$ for placebo). The incidence of nausea was low and similar between pramlintide- $(0-9 \%)$ and placebo-treated subjects (7\%).

Conclusion: In this study, pramlintide treatment, as an adjunct to LSI, elicited sustained weight loss and helped obese subjects maintain lost weight over time compared to LSI alone. These findings support further development of pramlintide as a potential treatment for obesity. (Fig. 1)

\section{PS 77 Obesity-related disorders}

\section{9}

Cardiovascular risk factors characteristic for the metabolic syndrome in patients with type 1 diabetes

J. Nadas ${ }^{1}$, Z. Putz ${ }^{1}$, J. Fövényi ${ }^{2}$, Z. Gaál ${ }^{3}$, A. Gyimesi ${ }^{4}$, T. Hídvégi ${ }^{5}$,

N. Hosszúfalusi ${ }^{6}$, G. Neurwith ${ }^{7}$, T. Oroszlán ${ }^{8}$, P. Pánczél' ${ }^{6}$, G. Vándorfi, G. Winkler ${ }^{10}$, I. Wittmann ${ }^{11}$, G. Jermendy ${ }^{1}$;

${ }^{1}$ Med Dept, Bajcsy Hospital, Budapest, ${ }^{2}$ Med Dept, Péterfy Hospital, Budapest, ${ }^{3}$ Med Dept, Jósa Hospital, Nyíregyháza,

${ }^{4}$ Med Dept, Réthy Hospital, Békéscsaba, ${ }^{5}$ Med Dept, Petz Hospital, Győr, ${ }^{6}$ Med Clinic, Semmelweis University, Budapest, ${ }^{7}$ Med Dept, Kenézy Hospital, Debrecen, ${ }^{8} \mathrm{Med}$ Dept, County Hospital, Zalaegerszeg, ${ }^{9} \mathrm{Med}$ Dept, Cholnoky Hospital, Veszprém, ${ }^{10} \mathrm{Med}$ Dept, János Hospital, Budapest,

${ }^{11}$ Med Clinic, Medical University, Pécs, Hungary.

Background and Aims: The prevalence rate and the clinical significance of the metabolic syndrome in type 1 diabetic patients remain to be established. The aim of this study was to estimate the prevalence rate of the metabolic syndrome in Hungarian type 1 diabetic patients.

Materials and Methods: Adult patients with type 1 diabetes ( $\mathrm{n}=533 ; 256$ men, 277 women; age: $35.6 \pm 11.6$ years; duration of diabetes: $18.0 \pm 11.1$ years; BMI: $25.3 \pm 4.3 \mathrm{~kg} / \mathrm{m} 2$, HbAlc: $8.29 \pm 1.59 \%$; $\mathrm{x} \pm \mathrm{SD}$ ) were consecutively enrolled from 11 diabetes outpatient departments. Data on medical history, actual treatment, anthropometric and laboratory parameters as well as actual blood pressure were registered while eating habits, education level and physical activity were evaluated by standardised questionnaires. The metabolic syndrome was defined according to the IDF and ATPIII criteria. All subjects fulfilled the criteria for hyperglycaemia by definition.

Results: The prevalence rate of the metabolic syndrome according to the ATPIII criteria was $31.1 \%$ (29.7\% men, $32.7 \%$ women; $p>0.05)$ while the prevalence rate was higher according to the IDF criteria (36.2\% men: $32.8 \%$, women $39.4 \% ; p>0.05$ ). According to ATP III criteria elevated blood pressure was detected in $55.5 \%$, high triglycerides level in $22.3 \%$, abdominal obesity in $19.3 \%$ and eleveted HDL-cholesterol in $18.0 \%$ of patients. Patients with the metabolic syndrome were significantly $(\mathrm{p}<0.01)$ older $(40.2 \pm 11.8$ vs. $33.6 \pm 10.9$ years), had longer duration of diabetes ( $19.9 \pm 10.8$ vs. $17.2 \pm 11.2$ years), used higher daily insulin dose $(59.9 \pm 22.6$ vs. $46.9 \pm 15.8 \mathrm{IU})$ and had higher BMI $(28.7 \pm 4.8$ vs. $23.7 \pm 3.0 \mathrm{~kg} / \mathrm{m} 2)$ than those without the metabolic syndrome. The HbAlc and calculated GFR values did not differ significantly in patients with versus without the metabolic syndrome. The prevalence rate of the metabolic syndrome increased with albuminuria $(p<0.001)$. There was an inverse relationship between the prevalence rate of the metabolic syndrome and patients' education level $(\mathrm{p}<0.001)$. According to the stepwise logistic regression analysis, the prevalence rate of the metabolic syndrome was associated in a decreasing tendency with waist circumference, serum triglycerides, antihypertensive medication, HDL-cholesterol $(\mathrm{p}<0.0001)$, diastolic blood pressure and serum creatinine $(\mathrm{p}<0.001)$, and systolic blood pressure, duration of diabetes $(\mathrm{p}=0.05)$.

Conclusion: In Hungary the metabolic syndrome can be frequently detected among adult patients with type 1 diabetes. The prevalence rate of the metabolic syndrome is predominantly associated with the higher waist circumference. In order to reduce cardiovascular risk in adult patients with type 1 diabetes, prevention of abdominal obesity is of great importance.

\section{0}

Endogenous cortisol homeostasis affects glucose metabolism and fibrinogen levels in patients with metabolic syndrome

S. Ayturk, N. B. Tutuncu, A. N. Demirer, A. Gursoy, N. G. Demirag; Endocrinology and Metabolism, Baskent University Faculty of Medicine, Ankara, Turkey.

Background and Aims: Cushing's syndrome and metabolic syndrome share many common findings which make one to think that metabolic syndrome represents a varient form of hypercortisolism. In this regard we aimed at screening the presence of endogenous hypercortisolism in a group of patients diagnosed as having metabolic syndrome.

Materials and Methods: A total of 375 (335 female, 40 male) patients diagnosed with metabolic sydrome using the criteria of NCEP-2001 (ATP- 
III) guidelines were enrolled for this study. Patients were analysed in detail to find out their degree of metabolic derangements. Blood was drawn for the determination of glucose tolerance (by $75 \mathrm{gm}$ oral glucose tolerance test), fasting insulin, fibrinogen and lipoprotein levels including lipoprotein a. HOMA-IR calculation, blood pressure monitoring, and anthropometric measurements were done for each patient. Screening for the presence of endogenous hypercortisolism was done by $1 \mathrm{mg}$ Dexamethasone supression test and $1.8 \mu \mathrm{g} / \mathrm{dl}$ of fasting morning plasma cortisol level was the cut-off limit. Patients were analysed with respect to their degree of fasting cortisol supression and metabolic and anthropomtric parameters.

Results: Mean age of the patients was $48.4 \pm 13.3$ years. Glucose intolerance was detected in 201 patients ( 49 overt diabetic, 90 with impared fasting glucose, 20 with impaired glucose tolerance and 41 with both impaired fasting glucose and glucose intolerance). There were 17 patients (4.5\%) with nonsupressed cortisol levels. Those having insulin resistance revealed similer degrees of cortisol supression. Fasting glucose levels were significantly correlated with the degree fasting cortisol levels after dexamethasone (pearson=0.227; $\mathrm{p}=$ 0.000 ). As the degree of glucose intolerance increased, the level of fasting cortisol after dexamethasone also increased. This difference was especially prominent for those with overt diabetics (pearson $=0.148, \mathrm{p}=0.007$ ). Similar positive correlation was also seen for the plasma fibrinogen levels and the supressed cortisol levels after dexamethasone (pearson $=0.174 ; \mathrm{p}=0.000$ ). Conclusion: Endogenous hypercortisolism in patients with diagnosed metabolic syndrome seems to be important for the metabolic homeostasis especially with regard to glucose metabolism and acute phase reactants like fibrinogen.

\section{1}

The effect of CLA on endothelial function and traits of the metabolic syndrome

K. Fielitz ${ }^{1,2}$, U. Helwig' ${ }^{1}$ M. Pfeuffer ${ }^{1}$, P. Winkler ${ }^{2}$, C. Laue ${ }^{2}$, E. Schwedhelm², R. H. Böger ${ }^{3}$, D. Bell' ${ }^{4}$ J. Schrezenmeir ${ }^{1}$;

${ }^{1}$ Institute of Physiology and Biochemistry of Nutrition, Federal Research Centre for Nutrition and Food (BfEL), Kiel, Germany, ${ }^{2}$ Tecura GmbH Medizin \& Biotechnik, Kiel, Germany, ${ }^{3}$ Institute for Experimental and Clinical Pharmacology and Toxicology, University Medical Center Hamburg-Eppendorf, Germany, ${ }^{4}$ Nutrition \& Health - Global R \& D, Cognis Germany GmbH \& Co. KG, Monheim, Germany.

Background and Aims: Conjugated linoleic acid (CLA) has several effects e.g. on insulin sensitivity, lipid metabolism, body composition, particularly shown in animal studies. However, in human studies these findings were inconsistent and the dietary fat used as control differed. It was mostly olive oil, which may have effects of its own. Moreover, the content of antioxidative substances in the preparations used was not declared. The aim of the study was to assess the effect of CLA supplementation on endothelial function and risk factors of the metabolic syndrome compared to 3 different control fats: native safflower oil, oxidised safflower oil, and native olive oil. All fats contained the identical amount of tocopherol $(1051 \mu \mathrm{g} / \mathrm{g})$.

Materials and Methods: 81 healthy overweight men (mean \pm SD: age $61.5 \pm 5.3 \mathrm{y}$, BMI $28.3 \pm 2.1 \mathrm{~kg} / \mathrm{m}^{2}$ ) were randomly allocated to either a total of $4.5 \mathrm{~g} / \mathrm{d}$ CLA isomeric mixture $(\mathrm{c} 9, \mathrm{t} 11: \mathrm{t} 10, \mathrm{c} 12)$, native safflower oil, oxidised safflower oil, or olive oil in form of $2 \times 4$ capsules daily in a 4 week doubleblind study. The endothelial function (primary parameter) was assessed using finger volume pulse waves (PAT index) fasting and 4 hours after intake of a liquid standardised mixed meal (Oral Metabolic Tolerance Test $=$ OMTT). Blood samples for safety, inflammatory and postprandial (pp) parameters were taken before, during and 9 hours after the OMTT before and after intervention. Data were analysed using SPSS software. All data are expressed by mean \pm SEM.

Results: The fasting PAT index remained unaltered by CLA $(\Delta-0.03 \pm 0.1)$ while all other groups tended to impair endothelial function (safflower oil native $\Delta-0.08 \pm 0.13$, safflower oil ox. $\Delta-0.15 \pm 0.15$, olive oil $\Delta-0.18 \pm 0.12$ ). The pp PAT index improved in the CLA group compared to olive oil $(\Delta 0.2 \pm 0.1 \mathrm{vs.}$ $\Delta-0.14 \pm 0.07 ; \mathrm{p}<0.05)$. Body weight decreased by $\Delta-1.13 \pm 0.36 \mathrm{~kg}$ within the CLA group $(\mathrm{p}<0.01)$ and compared to all other groups $(\mathrm{p}<0.05)$. Consistent with these results the alteration of BMI differed within the CLA group $(\Delta-0.35 \pm 0.11, \mathrm{p}<0.01)$ and compared to all other groups $(\mathrm{p}<0.05)$. Systolic blood pressure (RR) decreased within the CLA group $(\Delta-10.81 \pm 3.81 \mathrm{mmHg}$, $\mathrm{p}<0.05)$ and the native safflower oil group $(\Delta-9.14 \pm 2.44, \mathrm{p}=0.001)$. Diastolic RR also decreased within the CLA group $(\Delta-6.86 \pm 2.37 \mathrm{mmHg}, \mathrm{p}<0.01)$ and compared to the oxidised safflower oil group $(\mathrm{p}<0.05)$. The alterations of fasting glucose $(\Delta-1.73 \pm 1.49 \mathrm{mg} / \mathrm{dl})$ were more pronounced in the CLA group compared to the group receiving oxidised safflower oil $(\mathrm{p}<0.05)$. LDL-C $(\Delta-10.6 \pm 4.18 \mathrm{mg} / \mathrm{dl}, \mathrm{p}<0.05)$ and cholesterol $(\Delta-10.97 \pm 4.99 \mathrm{mg} / \mathrm{dl}$, $\mathrm{p}<0.05)$ decreased within the CLA group. Triglycerides and HDL-C were unchanged. $\mathrm{F}_{2}$-isoprostanes were increased by CLA $(\Delta 146.96 \pm 19.49 \mathrm{pg} / \mathrm{ml}$ Crea, $\mathrm{p}<0.001)$. This alteration differed from all other groups $(\mathrm{p}<0.001)$.

Conclusion: These results suggest that CLA induces beneficial metabolic effects. The fasting endothelial function remained constant and was postprandially even improved by CLA. The impact of increased $\mathrm{F}_{2}$-isoprostane levels needs to be clarified.

Supported by the Federal Ministry of Education and Research (BMBF) and Cognis GmbH \& Co. KG

\section{2}

Subjects affected by central obesity are resistant to the platelet antiaggregating effects exerted by the AMP-activated protein kinase (AMPK) via nitric oxide

I. Russo, P. Del Mese, G. Doronzo, P. Poy, L. Mattiello, M. Trovati, G. Anfossi;

Department of Clinical and Biological Sciences, University of Turin, Orbassano Italy.

Background and Ams:We previously observed that platelets from patients affected by central obesity -a condition characterised by an increased atherothrombotic risk- are resistant to the anti-aggregating effects of insulin, nitric oxide and prostacyclin. This study aims to present new molecular aspects of the reduced platelet sensitivity to anti-aggregating agents observed in central obesity. AMP-activated Protein Kinase (AMPK) is an intracellular enzyme system which acts as a sensor of cell energy balance and it is involved in the cell signalling of the adipocytokines leptin and adiponectin and in the mechanism of action of the insulin sensitisers metformin and thiazolidinediones. Aim of this study is to investigate whether AMPK influences platelet function by evaluating the effect of a specific AMPK activator on the ADP-induced aggregation and on the nitric oxide (NO)/ cGMP/PKG/VASP pathway in platelets from lean subjects and patients affected by central obesity. It is known that NO increases cGMP production, and cGMP, via the cGMP-dependent Protein Kinase (PKG), phoshorylates VASP, a protein deeply involved in platelet anti-aggregation.

Materials and Mthods: Platelet-rich plasma and washed platelets were obtained from 18 lean (7 M, $11 \mathrm{~F}$; age:33.2 \pm 1.4 yrs; BMI: $22.2 \pm 0.4 \mathrm{~kg} / \mathrm{m}^{2}$; waist circumference $81.2 \pm 1.4 \mathrm{~cm}$; HOMA IR: $1.3 \pm 0.3)$ and 10 obese subjects (4 M, 6 F; age: $36.3 \pm 2.8$ yrs; BMI: $33.5 \pm 1.9$; waist circumference $108.9 \pm 2.1 \mathrm{~cm}$; HOMA IR: $5.1 \pm 0.9$ ). In platelet samples incubated for $10 \mathrm{~min}$ with the AMPK activator 5-amino-4-imidazolecarboxamide riboside phosphate (AICAR;0.01-1mmol/l) we evaluated: i) the response to $4 \mu \mathrm{mol} / \mathrm{l}$ ADP both in the absence and in the presence of the specific AMPK inhibitor Compound C $(5 \mu \mathrm{mol} / \mathrm{l})$ and of NO synthase (ecNOS) inhibitor L-NAME (100 $\mu \mathrm{mol} / \mathrm{l})$; ii) the phosphorylation of ecNOS) at Ser ${ }^{177}$ and of Vasodilator-Stimulated Phosphoprotein (VASP) at Ser $^{239}$ (cGMP-dependent) (Western blots with monoclonal antibodies).

Results: AICAR decreased platelet response to ADP in a concentrationdependent way in lean subjects (IC-50:0.81 $\pm 0.16 \mathrm{mmol} / \mathrm{l}$ ) but not in obese subjects (IC-50 not detectable). In lean subjects, the effect of AICAR ( $1 \mathrm{mmol} /$ 1) on ADP-induced aggregation was significantly reduced by Compound $C$ (Maximal aggregation:78.0 \pm 16.0 vs. $35.6 \pm 9.6$ with AICAR alone; $\mathrm{p}<0.05$ ) and by L-NAME (Maximal aggregation:74.8 \pm 13.9 vs. $35.6 \pm 9.6$ with AICAR alone; $\mathrm{p}<0.05$ ). Platelet exposure to AICAR induced phosphorylation of eNOS at Ser ${ }^{177}$ and of VASP at $\operatorname{Ser}^{239}$ both in lean and in obese subjects; at each concentration AICAR, however, the effect was much lower in platelets from obese subjects as far as phosporylation of both eNOS and VASP are concerned.

Conclusions: In platelets from lean, insulin sensitive subjects AICAR exerts antiaggregating effects through activation of the NO/cGMP pathway via eNOS phosphorylation. These effects were deeply reduced in obese subjects, likely owing to a reduced AMPK-induced phosphorylation of NOS and, consequently, a reduced NO-dependent activation of the cGMP/PKG/VASP pathway. These results suggest that AMPK exerts antiaggregating mechanisms via endogenous NO. The lack of sensitivity to AMPK effects in obese subjects can be involved in their increased atherothrombotic risk.

Supported by the Italian Ministry of University (MIUR) 


\section{3}

Thrombocyte surface P-selectin, soluble P-selectin levels and the formation of microparticles of thrombocyte origin in relation to the intima media thickness of the carotid artery in obesity

É. Csongrádi ${ }^{1}$, B. Nagy ${ }^{2}$, A. Juhász ${ }^{1}$, Z. Karányi ${ }^{1}$, J. Kappelmayer ${ }^{2}$, G. Paragh ${ }^{1}$, M. Káplár ${ }^{1}$.

${ }^{1} 1$ st. Department of Internal Medicine, ${ }^{2}$ Department of Clinical

Biochemistry and Molecular Pathology, University of Debrecen, Hungary.

Background: Increased thrombocyte activation analogous to type 2 diabetes mellitus and the shift in prothrombotic coagulation is observed in relation to obesity. While the role of thrombocyte hyperfunction in the formation of atherosclerosis is known, in obesity it is an important risk factor of type 2 diabetes mellitus that is rarely examined. A sensitive marker of thrombocyte activation is P-selectin. Soluble P-selectin levels vary in healthy individuals in accordance with T715P polymorphism; according to literature data the protective effect of lower levels associated with the homo- and heterozygote forms is presumed in atherosclerotic processes. The hemostatic effect of microparticles dislodging from thrombocytes can be considerable in the formation of thrombus and inflammatory processes.

Aim: To determine the activation of thrombocytes and the number and activity of microparticles of thrombocyte origin. To determine the corelation between thrombocyte activation parameters, vascular risk factors and intima media thickness of the carotid artery, as well as the examination of the influence of T715P polymorphism of P-selectin on these factors.

Patients and methods: In our examination 142 obese patients (age: $41.6 \pm$ 11.1 years; BMI: $37.8 \pm 7.83 \mathrm{~kg} / \mathrm{m}^{2}$ ) and 64 healthy control individuals (age: $38.5 \pm 10.0$ year; BMI: $22.1 \pm 1.97 \mathrm{~kg} / \mathrm{m}^{2}$ ) were involved. The examination of the thrombocyte surface P-selectin, the number of microparticles of thrombocyte origin and the microparticles expressing P-selectin too were investigated by cytometric flow analysis using monoclonal antibodies. The soluble P-selectin was determined by the ELISA procedure. The genotype examination was carried out by PCR-RFLP analysis.

Results: The thrombocyte surface and soluble P-selectin levels in obesity were significantly increased $(\mathrm{p}<0.05)$, in contrast to the control group (median and quartile values of surface P-selectin: obese group: $1.29(0.70-2.24) \%$, control: 0.75 (0.40-1.21) \%; soluble P-selectin: obese group: 44.9 (38.7-55.0) $\mathrm{ng} / \mathrm{ml}$, control: $36.0(25.1-48.6) \mathrm{ng} / \mathrm{ml})$. The number of microparticles of thrombocyte origin (obese group: $82.0(52.0-136.0) / \mu 1$, control: $48.5(21.0-$ $73.0) / \mu \mathrm{l}$ ) as well as the number of activated microparticles (obese group: $34.0(18.0-59.0) / \mu \mathrm{l}$, control: $16.5(8.0-26.0) / \mu \mathrm{l})$ were significantly higher in the obese group compared to the control group also. Both the thrombocyte activation parameters and the microparticles of thrombocyte origin showed a significant positive relation to waist circumference, BMI, CRP and HbAlc values. We found a significant positive correlation between the thrombocyte surface P-selectin and the intima media thickness of the carotid artery. In the homo- and heterozygote forms of T715P polymorphism the soluble Pselectin levels was significantly lower in comparison to the wild type; this difference could not be observed in obesity.

Conclusions: Our results show the close relationship between increased thrombocyte activity and intima media thickness, which highlights the higher risks of developing vascular complications. In obesity the presumed protective effect of soluble P-selectin levels in relation to P-allele could not be observed as compared to healthy individuals.

\section{4}

Gender-specific hypertriglyceridaemic waist phenotype significantly associates with glycosylated haemoglobin $\left(\mathrm{HbA}_{1 \mathrm{c}}\right)$ in subjects with normal glucose tolerance and perfectly normal $\mathrm{HbA}_{1 \mathrm{c}}$ values

E. Voidonikola, C. Papamichael, K. Stamatelopoulos, A. Protogerou, G. Kollias, E. Chryssochoou, N. Zakopoulos, M. Alevizaki, M. Theodorakis; Dept. Clinical Therapeutics, University of Athens Medical School, Athens, Greece.

Background and Aims: Much attention has been drawn to the association between phenotype characteristics of subjects exhibiting features of the metabolic syndrome and its functional correlates that could translate into earlier detection of populations at risk by using readily available markers. Waist circumference (WC), especially of the "hypertriglyceridemic" phenotype has recently emerged as a key determinant of augmented propensity towards cardiovascular morbidity later in life as it strongly associates with early manifestations of coronary artery disease in patients with impaired glucose tolerance and type 2 diabetes. We sought to investigate this relationship further in euglycemic subjects with perfectly normal values of glycosylated hemoglobin $\left(\mathrm{HbA}_{1} \mathrm{c}\right)$

Materials and Methods: We screened 352 healthy volunteers, without concurrent disease or history for diabetes, hypertension, dyslipidemia and on no medications by carrying out an OGTT for 2 hours with two blood samples per subject (baseline and 2 hours post-glucose (75 grams) ingestion). From those we recruited 252 subjects ( 100 men and 152 women, age $46 \pm 1$ yrs, BMI $25 \pm 1$, WC $85 \pm 1 \mathrm{~cm}$ ), who all had normal glucose tolerance (both fasting and $2 \mathrm{~h}$-plasma glucose levels were $<100 \mathrm{mg} / \mathrm{dl}(88 \pm 1)$ and $<140 \mathrm{mg} / \mathrm{dl}(99 \pm 2)$, respectively, for assessment of features of the metabolic syndrome according to the latest IDF definition.

Results: In the population as a whole, waist circumference, plasma triglyceride (TG) concentration and the homeostatic model assessment (HOMA-IR) insulin resistance index, significantly associated with glycosylated hemoglobin across its normal range of values $\left(r^{2}=0.045 ; p=0.0009, r^{2}=0.051 ; p=0.0003\right.$, and $r^{2}=0.035 ; p=0.003$, respectively). This association was also retained when we only looked at men or women (men: $\left(r^{2}=0.061 ; p=0.0016, r^{2}=0.12\right.$; $p=0.0006$, and $r^{2}=0.088 ; p=0.0041$ - women: $r^{2}=0.032 ; p=0.0030, r^{2}=0.041$; $p=0.0012$, and $r^{2}=0.028 ; p=0.003$, respectively). Also, when the IDF-defined WC cut-off points were applied, apart from the TG and HOMA-IR, only $\mathrm{HbA}_{1} \mathrm{c}$ was significantly different between groups, both in women $(4.70 \pm 0.04$ vs. $4.83 \pm 0.05 ; \mathrm{p}<0.05$ for women with a WC $<82$ or $>82 \mathrm{~cm}$, respectively) and in men ( $4.74 \pm 0.05$ vs. $4.93 \pm 0.09$; $\mathrm{p}<0.0001$ for men with a WC $<90$ or $>90$ $\mathrm{cm}$, respectively). Fasting and $2 \mathrm{~h}-$ plasma glucose, insulin, total-, HDL- and LDL cholesterol, and arterial blood pressure did not differ between groups within genders.

Conclusion: In otherwise healthy non-obese individuals with perfectly normal glucose tolerance and $\mathrm{HbA}_{1}$, emergence of elements of the hypertriglyceridemic waist phenotype (increased waist circumference, elevated plasma triglyceride levels) and the insulin resistance index strongly associate with increasing values even within its normal range. An $\mathrm{HbA}_{1} \mathrm{c}$ value of 4.7 appears to be on the critical threshold. Detecting altered $\mathrm{HbA}_{1} \mathrm{C}$ might therefore be of particular significance early in the pathophysiology of insulin resistant states such as the metabolic syndrome.

\section{5}

Evaluation of TNFa, IL-6 and adiponectin in obese patients with and without obstructive sleep apnea syndrome (OSAS)

E. Benetti ${ }^{1}$, C. Baldessari ${ }^{2}$, B. Masserini ${ }^{1}$, L. Sburlati ${ }^{1}$, F. Donadio ${ }^{1}$, M. E. Lunati ${ }^{1}$, R. Bossi ${ }^{2}$, P. Beck-Peccoz ${ }^{1}$, E. Orsi ${ }^{1}$;

${ }^{1}$ Department of Medical Sciences, Endocrine and Diabetologic Unit, University of Milan, Fondazione IRCCS Ospedale Maggiore Policlinico, Mangiagalli e Regina Elena, ${ }^{2}$ Department of Cardiovascular and Respiratory Disease, Center of Sleep Respiratory Disorders, University of Milan, Fondazione IRCCS Ospedale Maggiore Policlinico, Mangiagalli e Regina Elena, Milan, Italy.

Background and Aims: The prevalence of OSAS among obese patients is $40-50 \%$ and these patients have an increased risk of myocardial infarction, stroke and mortality as compared to those without OSAS. Aim of the study was to investigate if an increased cardiovascular risk could be explained by alterated inflammatory response and reduced adiponectin levels.

Materials and Methods: 46 obese patients (24 F, 22 M, age $54 \pm 10$ yrs, BMI $38.5 \pm 6.1 \mathrm{~kg} / \mathrm{m}^{2}$ ) were evaluated for the presence of OSAS (polysomnography), anthropometric parameters, biochemical parameters (lipid profile, $\mathrm{HbAlC}$, urate, HOMA-IR, oral glucose tolerance test, adiponectin, TNF $\alpha$, IL-6).

Results: according to polysomnography results, patients were subdivided in two groups: I. OSAS ( $\mathrm{n}=25$, age $55.2 \pm 12.9 \mathrm{yrs}$, BMI $\left.39.6 \pm 7.1 \mathrm{~kg} / \mathrm{m}^{2}\right)$, II. non OSAS ( $\mathrm{n}=21$, age $55.4 \pm 9.6 \mathrm{yrs}$, BMI $\left.37.5 \pm 4.7 \mathrm{~kg} / \mathrm{m}^{2}\right)$. Group I shown higher level of apnea/hypopnea index (AHI) $(40.62 \pm 27.51$ vs. $1.54 \pm 1.37$, $P<0.0001)$, waist circumference $(121.5 \pm 14.5$ vs. $113.3 \pm 10.3 \mathrm{~cm}, P<0.05)$, HOMA-IR (6.1 \pm 5.07 vs. $5.02 \pm 2.96, P<0.05)$, IL-6 $(9.33 \pm 2.4 v s .5 .77 \pm 2.14 \mathrm{ng} /$ $\mathrm{ml}, P<0.001), \mathrm{TNF} \alpha(68.18 \pm 13.41$ vs. $50.75 \pm 15.42 \mathrm{pg} / \mathrm{ml}, P<0.001)$ and lower levels of total cholesterol $(200.62 \pm 57.92$ vs. $231.25 \pm 36.86 \mathrm{mg} / \mathrm{dl}, P<0.05)$ and adiponectin $(7.3 \pm 2.93 v s .9 .25 \pm 3.87 \mathrm{mg} / \mathrm{L}, P<0.05)$ than Group II. No difference in the other parameters between the two groups was observed. Moreover, we observed a negative correlation between adiponectin levels and AHI, a positive correlation between IL- 6 and AHI and between TNF $\alpha$ and 
AHI. No correlation between HOMA-IR and TNF $\alpha$ and IL-6 was found. In a subgroup of 13 OSAS patients adiponectin , IL- 6 and TNF $\alpha$ was evaluated also after one whole night Continous Positive Air Pressure therapy. No differences in adiponectin levels were observed, but a significant reduction in TNF $\alpha$ and IL-6.

Conclusion: the present study shows altered inflammatory response in OSAS patients characterised by elevated cytokine levels and insulinresistance and reduced adiponectin level. Therefore, hypoxia seems to be an additional factor to cause alterations of cytokine levels, but its role is less clear about adiponectin. These metabolic features contribute to the elevated cardiovascular risk and mortality in patients with OSAS.

\section{6}

Insulin resistance and aminoterminal pro-brain natriuretic peptide (NT-proBNP) is associated with sleep-disordered breathing in morbid obesity

J. Ybarra' ${ }^{1}$, F. Planas ${ }^{2}$, E. Escudero 3 , C. Ballesta-Lopez 2 , M. Isart ${ }^{2}$,

S. Torrejon ${ }^{4}$, J. Jurado ${ }^{5}$, J. Merce ${ }^{3}$, F. Navarro-Lopez ${ }^{2}$, J. M. Pou ${ }^{4}$;

${ }^{1}$ Centro Medico Teknon, Instituto de Cardiología Avanzada y

Medicina(ICAMED), Barcelona, ${ }^{2}$ Instituto Cardiologia Avanzada,

ICAMED, Centro Medico Teknon, ${ }^{3}$ Dept. Biochemistry,

Autonomous University of Barcelona, Hospital de la Sta. Creu i S. Pau,

${ }^{4}$ Dept. Endocrinology and Nutrition, Autonomous University of Barcelona,

Hospital de la Sta. Creu i S. Pau, ${ }^{5}$ Primary Care Center, Catalan Health

Institute, Olot, Girona, Spain.

Background and Aims: Sleep-disordered breathing and obstructive sleep apnea are encountered in morbid obesity (MO) patients in conjunction with insulin resistance and several cardiovascular risk factors. Aminoterminal pro-brain natriuretic peptide (NT-proBNP) is secreted by myocytes in the ventricular wall in response to increased wall stress stands and could become a promising marker for left ventricular disfunction. We wanted to explore the relationships between NT-proBNP in morbid obesity patients with sleepdisorders breathing and obstructive sleep apnea (SDB) and other parameters as hypertension and insulin resistance.

Materials and Methods: Cross-sectional pilot-study involving $110 \mathrm{MO}$ young female patients which underwent a detailed clinical, biochemical and echo-cardiography study and completed the Berlin Questionnaire (BQ) to assess risk of SDB pre-operatively (bariatric surgery). Insulin Resistance (IR) was assessed using fasting insulin concentrations (IRI). Echo-cardiograms were performed to quantify ejection fraction values (EF), cardiac output (CO), left ventricular mass (LVM), inter-ventricular (IVWTh) and posterior wall thickness (PWTh) and LV diastolic indexes such as the Em/Am and E/ Em ratios, left atria size (LA), and inter-ventricular relaxation time (IVRT). Serum NT-proBNP was measured using an electrochemiluminescence immunoassay (Elecsys ${ }^{\circledR}$, Roche Diagnostics, Indianapolis, Inc.).

Results: Age $=37.4 \pm 1.0, \mathrm{BMI}=45.5 \mathrm{Kg} \cdot \mathrm{m}-2$. Hypertension and type 2 diabetes mellitus prevalence averaged 25 and $4 \%$ (respectively). Log-transformed NT-proBNP levels correlated with $\log$ IRI $(\mathrm{r}=-0.208 ; \mathrm{P}=0.023)$ and $\mathrm{BQ}$ categories $(r=0.492 ; \mathrm{P}<0.0005)$ and the relationship was even more robust if only hypertensive participants were considered $(\mathrm{r}=0.618 ; \mathrm{P}=0.008)$. All patients disclosed normal EF. NT-proBNP levels, diastolic indexes and LVMI increase significantly along with $\mathrm{BQ}$ categories $(\mathrm{P}<0.0001)$. Stepwise multiple regression analysis identified log IRI and $\mathrm{BQ}$ as the independent variables predicting as much as $43.6 \%$ of log-transformed NT-proBNP's variability (dependent variable).

Conclusion: Sleep-disordered breathing worsens along with left ventricular hypertrophy and diastolic dysfunction. NTpro-BNP levels increase proportionally with SDB and are independently predicted by insulin resistance and the $\mathrm{BQ}$ score in female $\mathrm{MO}$ patients. Larger prospective trials are warranted.

Supported by Spanish Network CO3/08 Instituto Carlos III
0847

Variability of nocturnal glycaemia related to severity of sleep apnea in type 2 diabetics: results of continuous glucose monitoring M. Pallayova, V. Donic, Z. Tomori;

Department of Physiology and Sleep Laboratory, Faculty of Medicine, PJ Safarik University, Kosice, Slovakia.

Background and Aims: It is suggested that severe sleep-disordered breathing (SDB) with recurrent episodes of airflow obstruction and hypoxemia may adversely affect glucose control in patients who also suffer from type 2 diabetes. Our aim was to assess and evaluate nocturnal glycemic variability in type 2 diabetics with various degrees of SDB using the continuous glucose monitoring system (CGMS; Medtronic MiniMed).

Materials and Methods: Twenty-nine type 2 diabetic patients on diet/oral hypoglycemic therapy (19 men, 10 women; mean age 55.41 \pm 7.5 years, BMI $35.64 \pm 8.46$, duration of diabetes $3.14 \pm 1.48$ years, HbAlc/HPLC method $6.85 \pm 0.98 \%$ ) were tested for SDB using sleep questionnaire, ApneaLink sleep screener with oximetry (ResMed), and/or overnight polysomnography (Alice 3; Respironics). Subjects were divided according to their apnea-hypopnea index (AHI) into three groups: $[\mathrm{A}]$ normal with an $\mathrm{AHI}<10 / \mathrm{h}$, $[\mathrm{B}]$ with mild to moderate sleep apnea and $\mathrm{AHI}$ greater than or equal to $10 / \mathrm{h}$ but less than or equal to $25 / \mathrm{h}$, and [C] with severe sleep apnea and AHI $>25 / \mathrm{h}$. All patients were monitored using the CGMS. Overnight glucose standard deviation (SD) and mean of nocturnal glucose differences (MOND) were used to assess the degree of glycemic variability during the night. The secondary parameters assessed included AHI and mean nocturnal glucose levels. The differences between groups were tested using ANOVA.

Results: 86 nocturnal CGMS glucose profiles (mean length of sensor wear $82.72 \pm 31.96 \mathrm{~h}$; correlation coefficient $0.89 \pm 0.15$; mean absolute difference $11.96 \pm 6.0 \%)$ showed the most stable overnight glucose control in $[\mathrm{A}](\mathrm{n}=15$; 46 nocturnal CGMS profiles; AHI $<10 / \mathrm{h}$; BMI $31.73 \pm 8.26$, HbA1c $6.41 \pm 0.68 \%$ ) with overnight glucose SD $0.41 \pm 0.13$ and MOND $1.7 \pm 0.57 \mathrm{mmol} / 1$. Less stable diabetes control was found in [B] $(n=6 ; 19$ nocturnal CGMS profiles; AHI $12.73 \pm 3.56 / \mathrm{h}$; BMI $39.53 \pm 7.5$, HbAlc $7.4 \pm 1.09 \%$ ) with overnight glucose SD $0.63 \pm 0.22$ and MOND $2.52 \pm 0.77 \mathrm{mmol} / \mathrm{l}$. The most labile nocturnal glucose control was observed in $[\mathrm{C}](\mathrm{n}=8 ; 21$ nocturnal CGMS profiles; AHI $42.08 \pm 21.65 / \mathrm{h}$; BMI $40.04 \pm 6.48$, HbA1c $7.26 \pm 1.1 \%$ ) with overnight glucose SD $1.05 \pm 0.53$ and MOND $4.07 \pm 2.12 \mathrm{mmol} / \mathrm{l}$. ANOVA showed significant differences in overnight glucose SD between $[A]$ and $[B](p=0.0015),[A]$ and $[C](p<0.0001)$, and significant differences in MOND between $[A]$ and $[B](p=0.0036)$, and $[A]$ and $[C](p<0.0001)$. Mean nocturnal glucose values were significantly higher in $[\mathrm{C}]$ as compared to $[\mathrm{A}](9.01 \pm 1.74 \mathrm{mmol} / \mathrm{l} \mathrm{vs}$. $5.84 \pm 1.03 \mathrm{mmol} / \mathrm{l} ; \mathrm{p}<0.0001)$ and significantly higher in $[\mathrm{B}]$ as compared to [A] $(8.51 \pm 2.42 \mathrm{mmol} / \mathrm{l}$ vs. $5.84 \pm 1.03 \mathrm{mmol} / 1 ; \mathrm{p}<0.0001)$. Despite no significant difference neither in mean nocturnal glycemia nor in $\mathrm{HbAlc}$ between [B] and [C], ANOVA showed significant differences in MOND and in overnight glucose $\mathrm{SD}$ between these groups (both $\mathrm{p}<0.0001$ ) indicating that $\mathrm{HbAlc}$ and average glucose levels does not provide information about fluctuations or peaks in glycemia.

Conclusion: Although glucose variability is usually less pronounced in type 2 diabetic patients in whom endogenous insulin secretion exists, our CGMS results indicate that sleep apnea in type 2 diabetics may lead to enhanced nocturnal glycemic variability, which increases with increasing severity of SDB, as compared to type 2 diabetic state without sleep apnea. Glucose variability seems to be an important feature of a nocturnal glucose pattern distinguishing between type 2 diabetics with and without sleep apnea. 


\section{PS 78 GLP-1 receptor agents}

\section{8}

Efficacy and safety of continous, intravenous glucagon-like-peptide 1 (GLP-1) in comparison to insulin infusion for normalisation of hyperglycaemia in patients with type 2 diabetes

I. Schmoelzer, E. Kettler-Schmutt, H. Pressl, H. Sourij, T. C. Wascher; Diabetic Angiopathy Research Group, Medical University, Graz, Austria.

Background and Aims: In critical situations such as acute vascular events or during surgery normalisation of glucose concentrations should be obtained in patients with type 2 diabetes. Usually it is tried to achieve normoglycemia by continuous intravenous administration of normal human insulin (NHI) at a glucose concentration-driven, variable dose. This treatment demands manpower-intensive tests of glycemia with subsequent adjustment of insulin dose and hypoglycaemia may occur as a side effect of therapy. Glucagonlike-Peptide-1 (GLP-1) lowers plasma glucose by a glycemia-dependent stimulation of pancreatic insulin secretion and continuous administration of fixed doses of GLP-1 normalise blood glucose in type 2 diabetic without any reported hypoglycaemia. Efficacy of both therapies in comparison has not been investigated before. The aim of the present study was to compare an established treatment regimen of intravenous normal insulin in variable doses with continuous intravenous GLP-1 infusion $(1.2 \mathrm{pmol} / \mathrm{kg} / \mathrm{min})$.

Materials and Methods: 8 patients with type 2 diabetes were included in this prospective, open, randomised study. Each subject participated in two examinations separated by 3 days. 30 minutes after a standardised breakfast they were treated either with a dose-adapted infusion of normal insulin or continuous infusion of GLP-1 $(1.2 \mathrm{pmol} / \mathrm{kg} / \mathrm{min})$ over a period of 8 hours. Serum samples for determination of glucose and insulin were drawn every 30 minutes. Following parameters were targeted: maximum glycemia, glycemia after 2 and 4 hours and time until glycemia $<115 \mathrm{mg} / \mathrm{dl}$. Statistical analyses were done with ANOVA for repeated measurements and paired student's t-test. All data are $\mathrm{MW} \pm \mathrm{SD}$, level of significance was defined as $\mathrm{p}<0.05$.

Results: Blood glucose levels before start of therapy were comparable on both examination days (NHI: $252 \pm 42$, GLP-1 $244 \pm 24 \mathrm{mg} / \mathrm{dl}$; ns). Maximum glycemia $(312 \pm 51$ vs. $254 \pm 48 \mathrm{mg} / \mathrm{dl} ; \mathrm{p}=0.007)$ and glycemia after $2(208 \pm 51$ vs. $137 \pm 48 \mathrm{mg} / \mathrm{dl} ; \mathrm{p}=0.012)$ and 4 hours $(155 \pm 51 \mathrm{vs} .116 \pm 27 \mathrm{mg} / \mathrm{dl} ; \mathrm{p}=0.021)$ were significantly lower under therapy with GLP-1. Time until achievement of glycemia $<115 \mathrm{mg} / \mathrm{dl}$ was $252 \pm 51$ minutes under GLP- 1 treatment and $321 \pm 43$ minutes under therapy with normal insulin $(\mathrm{p}=0.008)$. After the observation period of 8 hours glycemia was comparable in both groups (NHI: 110 \pm 24 , GLP-1: $103 \pm 22 \mathrm{mg} / \mathrm{dl}$; ns). Under treatment with NHI we observed one symptomatic hypoglycaemia ( $48 \mathrm{mg} / \mathrm{dl}$ ), under GLP-1 no hypoglycaemia occurred.

Conclusion: Both, intravenous infusion of normal insulin as with GLP1 infusion effectively normalise glycemia in type 2 diabetic patients. Achievement of normoglycemia, however, is faster and no dose adjustments are needed by use of GLP-1. Therefore we conclude that intravenous application of GLP-1 is a safe and effective alternative to intravenous NHI. Supported by an unrestricted research grant of Novo Nordisk

\section{9}

Pharmacokinetics and pharmacodynamics of exenatide long-acting release after single- and multiple-dosing

L. MacConell ${ }^{1}$, K. Taylor ${ }^{1}$, D. Zhuang' ${ }^{1}$, P. Kothare ${ }^{2}$, K. Mace ${ }^{2}$, W.-I. Li ${ }^{3}$, S. Flanagan ${ }^{1}$, M. Fineman ${ }^{1}$;

${ }^{1}$ Amylin Pharmaceuticals, Inc., San Diego, United States,

${ }^{2}$ Eli Lilly and Company, Indianapolis, United States,

${ }^{3}$ Alkermes, Inc., Cambridge, MA, United States.

Background and Aims: Glucagon-like peptide-1 (GLP-1) is an incretin hormone secreted by the gut in response to ingestion of food. It has several effects, including stimulating glucose-dependent insulin secretion and reducing appetite. The incretin mimetic, exenatide, shares several effects with GLP-1, leading to improved glycaemic control. Current studies assessed the pharmacokinetics (PK) and pharmacodynamics (PD) of single and repeated weekly subcutaneous injections of exenatide long-acting release (LAR) formulation in patients with type 2 diabetes (T2DM).
Materials and Methods: 62 and 45 patients with T2DM were treated in single and repeat dose exenatide LAR trials, respectively. Single dose patients received LAR (2.5 mg, $5.0 \mathrm{mg}, 7.0 \mathrm{mg}$ or $10.0 \mathrm{mg}$ ) or placebo (PBO). Doses were escalated based on safety and tolerability of the preceding lower dose. Weekly dose PK was predicted from non-parametric superposition of single dose data. Weekly dose patients received LAR $(0.8 \mathrm{mg}$ or $2.0 \mathrm{mg}$, targeting the low and high concentrations achieved by therapeutic doses of exenatide BID) or PBO for 15 weeks. Plasma exenatide, fasting (FPG) and postprandial plasma glucose (PPG), and body weight were assessed. Data were analysed non-compartmentally.

Results: Plasma exenatide concentrations were measurable for approximately 9 weeks after a single injection; exposure increased dose dependently. Reductions in FPG and PPG correlated with increasing exenatide concentrations. Repeat doses of LAR predicted plasma exenatide concentration profiles consistent with observed data. Weekly dosing yielded mean steady-state exenatide plasma concentrations spanning the targeted therapeutic range, leading to significant reductions in mean PPG and body weight with 2.0mg LAR. Mean FPG was significantly reduced independent of dose. Generally, adverse events were transient and mild to moderate in intensity.

Conclusion: Single dose PK supported and predicted weekly dose PK. Weekly LAR doses targeting the therapeutic exenatide plasma concentration range were well tolerated. Dose-dependent effects on PPG and body weight, but not FPG, suggested differential exposure-response relationships across the pharmacology of exenatide. These data indicated LAR prolonged the rate of exenatide delivery to the circulation, while retaining the PD action of exenatide.

\section{0}

Pharmacokinetic modelling of the once-daily human glucagon-like peptide-1 analogue, liraglutide, in healthy volunteers and comparison to exenatide

D. M. Jonker, E. Watson, A. D. Toft, P. Kristensen, L. B. Knudsen,

S. H. Ingwersen;

Novo Nordisk A/S, Bagsvaerd, Denmark.

Background and Aims: Glucagon-like peptide-1 (GLP-1) is an incretin hormone that has been shown to stimulate insulin secretion in a glucosedependent manner, to inhibit glucagon secretion and to delay gastric emptying. These are promising properties for treatment of type 2 diabetes. Liraglutide is a long-acting human GLP-1 analogue that was designed to be eliminated considerably slower than the natural hormone. The aim of this modelling was to develop a pharmacokinetic model on available data on liraglutide and exenatide in man, and thereby compare their pharmacokinetic properties.

Material and Methods: The pharmacokinetics of liraglutide were studied in 72 healthy male subjects using a dose escalation design. Each subject received a single sc injection of 1.25 up to $20 \mu \mathrm{g} / \mathrm{kg}$ liraglutide or vehicle and 22 venous blood samples were collected up to 48 hours after dosing. A population-based modelling approach was taken to describe the time course of liraglutide concentrations and its between-subject variability (BSV) using NONMEM V. During model building, goodness-of-fit was assessed by the objective function value and a range of graphical procedures. The estimates from the final model were used to generate a predicted PK profile for once daily sc dosing. Similarly, a PK profile for twice daily sc dosing of exenatide was generated from literature data.

Results: The absorption of liraglutide following subcutaneous (sc) administration was slow, with peak concentrations occurring at 9-12 hours post-dosing. Liraglutide absorption was adequately described by a dose proportional zero order process and a subsequent first order process. A onecompartment model with a central volume amounting to $0.086 \mathrm{~L} / \mathrm{kg}$ (rSE $11 \%$, BSV 33\%) was estimated, showing that after absorption, liraglutide is mainly confined to the central circulation. Clearance was independent of dose and was estimated to be $0.0060 \mathrm{~L} / \mathrm{hr} / \mathrm{kg}$ (rSE 6.8\%, BSV 13\%). The mean elimination half-life after sc dosing was $13 \mathrm{hr}$ (by non-compartmental analysis). The absolute bioavailability was found to be $51 \%$ (rSE $8 \%$, BSV $30 \%$ ). The Figure shows the PK profiles for $20 \mu \mathrm{g} / \mathrm{kg}$ liraglutide once daily sc (solid line) and for $10 \mu \mathrm{g} / \mathrm{kg}$ exenatide twice daily sc (dashed line).

Conclusion: The model and data demonstrate suitable pharmacokinetic properties for $24 \mathrm{~h}$-coverage with once daily sc administration of liraglutide. In addition, the PK profile of once daily liraglutide was found to have a lower peak to trough variation than that of twice-daily exenatide. 
Fig. 1 Abstract 0850

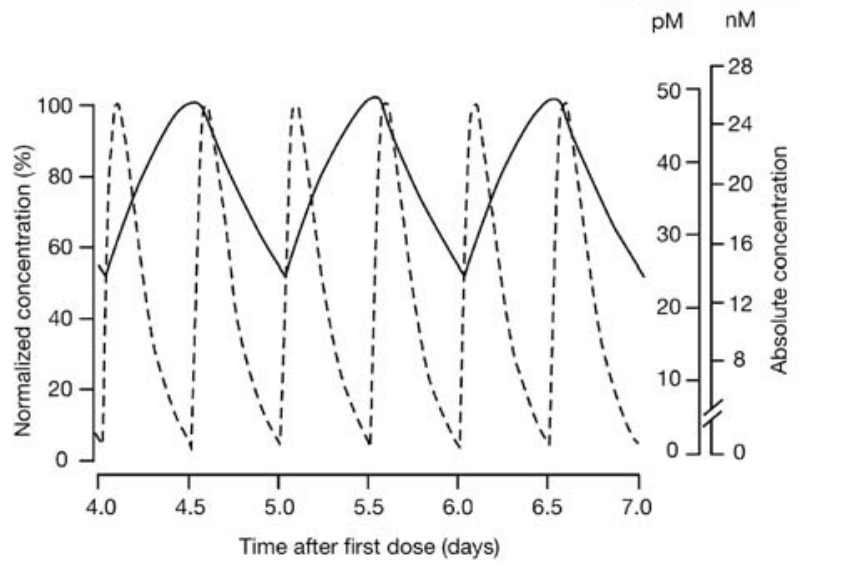

Supported by Novo Nordisk A/S

\section{1}

The long-acting human GLP-1 analogue liraglutide given once daily significantly improves glycaemic control by reducing fasting and postprandial glucose levels in subjects of Japanese origin with type 2 diabetes Y. Seino ${ }^{1}$, K. Kaku' ${ }^{2}$, K. Nishijima ${ }^{3}$, M. F. Rasmussen ${ }^{4}$, M. Zdravkovic ${ }^{4}$, P. Kristensen ${ }^{4}$;

${ }^{1}$ Division of Diabetes and Clinical Nutrition, Kansai-Denryoku Hospital, Osaka, Japan, ${ }^{2}$ Endocrinology, Kwasaki Medical School, Okayama, Japan, ${ }^{3}$ Novo Nordisk Pharma Ltd, Tokyo, Japan,

${ }^{4}$ Novo Nordisk A/S, Bagsvaerd, Denmark.

Background and Aims: In this 14-week study we assessed the efficacy and safety of the long-acting daily GLP-1 analogue liraglutide in 226 Japanese subjects with type 2 diabetes treated with diet $(54 \%)$ or a single oral antidiabetic drug.

Material and Methods: Subjects were randomised after an 8-week wash out period to four doses of liraglutide $(0.1,0.3,0.6$ and $0.9 \mathrm{mg})$ or placebo. The two highest doses were reached by weekly increments of $0.3 \mathrm{mg}$ until final dose was reached.

Results: $\mathrm{HbA}_{1 \mathrm{c}}$ decreased dose-dependently in all treatment groups compared to placebo from a baseline value of $8.3 \%$ by $-0.8,-1.2,-1.6$ and $-1.9 \%$, respectively $(\mathrm{p}<0.0001)$. $\mathrm{HbA}_{1<}<7.0 \%$ was achieved by $22,43,62$ and $75 \%$ of the subjects in the liraglutide groups compared to $9 \%$ in the placebo group. A dose-dependent decrease of fasting plasma glucose by -14 , $-23,-41$, and $-45 \mathrm{mg} / \mathrm{dL}$ vs. placebo, respectively $(\mathrm{p}<0.0001)$ was seen and postprandial glucose ( $2 \mathrm{~h}$ post-meal) decreased by $-26,-51,-76$ and -94 $\mathrm{mg} / \mathrm{dL}$, respectively compared to placebo. Liraglutide was well tolerated and there were no reports of major or minor hypoglycaemic events. Main gastrointestinal system adverse events in the high dose group vs. placebo were: constipation $7 \%$ vs. $9 \%$; diarrhoea $9 \%$ vs. $4 \%$; gastritis $7 \%$ vs. $0 \%$; nausea $7 \%$ vs. $2 \%$. No increase in liraglutide antibodies was seen.

Conclusion: Liraglutide given once-daily as monotherapy improves glycaemic control by reducing fasting and post-prandial glucose levels without risk of major or minor hypoglycaemia in Japanese subjects with type 2 diabetes and is well tolerated.

Supported by Novo Nordisk A/S

\section{2}

Pharmacokinetics of the long-acting human GLP-1 analogue liraglutide in subjects with renal impairment

L. V. Jacobsen ${ }^{1}$, C. Hindsberger ${ }^{1}$, R. Robson ${ }^{2}$, M. Zdravkovic ${ }^{1}$;

${ }^{1}$ Novo Nordisk A/S, Bagsværd, Denmark,

${ }^{2}$ Christchurch Clinical Studies Trust, Christchurch, New Zealand.

Background and Aims: Liraglutide is a long-acting GLP-1 analogue to be used as a once-daily treatment in patients with type 2 diabetes.
Materials and Methods: The effect of renal impairment on the pharmacokinetics (PK) of liraglutide was investigated in a single centre, open label trial. Six subjects with normal renal function (creatinine clearance $>80$ $\mathrm{mL} / \mathrm{min}), 6$ with mild renal impairment $(>50-\leq 80 \mathrm{~mL} / \mathrm{min}), 7$ with moderate ( $>30-\leq 50 \mathrm{~mL} / \mathrm{min}), 5$ with severe renal impairment $(\leq 30 \mathrm{~mL} / \mathrm{min})$ and 6 subjects with end-stage renal disease requiring dialysis entered the trial. All subjects received a single $0.75 \mathrm{mg} \mathrm{sc}$ dose of liraglutide and completed a 72 $\mathrm{h}$ blood sampling period for plasma liraglutide concentrations. An ANOVA of the log transformed PK endpoints adjusted for renal group, age and log (weight) was performed. Equivalence was declared if the $90 \%$ CI for an endpoint ratio was contained in the interval 0.70 to 1.43.

Results: While the comparison between subjects with normal renal function and severe renal function did not show equivalence (AUC ${ }^{\text {severe }} / A^{\text {AUn }}{ }^{\text {normal }}$ ratio: 0.73 [ $90 \%$ CI: $0.57-0.94]$ ) a regression analysis on the other hand failed to show a significant effect of decreasing creatinine clearance on AUC of liraglutide. Furthermore, the expected AUC ratio (based on the regression model) between two subjects with the lowest (severe) and highest (normal) creatinine clearance in the study (132 and $14 \mathrm{~mL} / \mathrm{min}$ ) with the same age and weight was estimated to be 0.88 [95\%CI: 0.58-1.34] (NS). The comparison between subjects with normal renal function and subjects in dialysis showed an estimated AUC dialysis/AUC normal $_{\text {b }}$ ratio of 0.74 [90\% CI: 0.56-0.97]. The liraglutide concentration in urine and in dialysis fluid was negligible. The degree of renal impairment did not appear to be associated with increased risk of adverse events.

Conclusion: Patients with diabetes and renal impairment should use standard treatment regimens of liraglutide and be dosed according to their glycaemic control.

\section{3}

Predictors of achieving glycaemic targets in patients with type 2 diabetes treated with exenatide or insulin

R. Brodows ${ }^{1}$, D. Milton ${ }^{1}$, J. Mao', M. Wintle ${ }^{2}$, M. Trautmann ${ }^{1}$;

${ }^{1}$ Eli Lilly and Company, Indianapolis, United States,

${ }^{2}$ Amylin Pharmaceuticals, San Diego, United States.

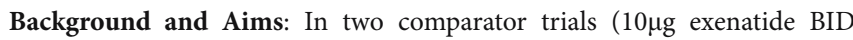
vs. either insulin glargine or biphasic insulin aspart), both exenatide and insulin treatment achieved similar reductions in $\mathrm{HbA}_{1 \mathrm{c}}$ for approximately 6 months, while exenatide was associated with weight reduction and insulin was associated with weight gain. The aim of this analysis was to identify characteristics associated with achievement of glycaemic targets in the pooled sample of patients.

Materials and Methods: Data were pooled from these two studies $(\mathrm{N}=1050)$ to examine whether patients' baseline characteristics $\left(\mathrm{HbA}_{1 \mathrm{c}}\right.$, fasting serum glucose [FSG], weight, age, duration of diabetes, origin, and gender) were associated with an endpoint $\mathrm{HbA}_{1 \mathrm{c}}$ of $\leq 6.5 \%$ and $\leq 7 \%$, for exenatide or insulin treatment on background of metformin (MET) and a sulphonylurea (SU).

Results: Mean $( \pm \mathrm{SD})$ baseline $\mathrm{HbA}_{1 c}(8.4 \pm 1.0 \%)$ was similar for both treatment groups, and treatment was not a significant predictor for achieving an $\mathrm{HbA}_{1 \mathrm{c}}$ $\leq 7 \%$. However, exenatide-treated patients were 1.69 (odds ratio, $95 \% \mathrm{CI}$ $1.21-2.35, \mathrm{p}=0.002$ ) times more likely to have achieved an $\mathrm{HbA}_{1 \mathrm{c}} \leq 6.5 \%$ than insulin-treated patients. In the exenatide group, lower baseline $\mathrm{HbA}_{1 \mathrm{c}}$ (odds ratio 0.40 [95\% CI 0.29-0.54]) and lower baseline FSG (odds ratio 0.87 [95\% CI 0.78-0.96]) were significant predictors for achieving $\mathrm{HbA}_{1 \mathrm{c}} \leq 6.5 \%$; lower baseline $\mathrm{HbA}_{1 \mathrm{c}}$ (odds ratio 0.47 [95\% CI 0.36-0.62]), lower baseline FSG (odds ratio 0.87 [95\% CI 0.79-0.96]), and older age (odds ratio 1.04 [95\% CI 1.01-1.06]) were significant predictors for achieving $\mathrm{HbA}_{1 \mathrm{c}} \leq 7 \%$. For insulin patients, lower baseline $\mathrm{HbA}_{1 \mathrm{c}}$ was the only significant factor associated with reaching an $\mathrm{HbA}_{1 \mathrm{c}} \leq 6.5 \%$ (odds ratio 0.36 [95\% $\left.\mathrm{CI} 0.27-0.50\right]$ ) or $\mathrm{HbA}_{1 \mathrm{c}} \leq 7 \%$ (odds ratio 0.30 [ $95 \% \mathrm{CI} 0.23-0.40]$ ).

Conclusion: In patients with type 2 diabetes failing on SU and MET for whom the next step may be insulin, exenatide treatment was associated with a greater percentage of patients achieving an $\mathrm{HbA}_{1 \mathrm{c}} \leq 6.5 \%$ than insulin, with no increase in overall hypoglycaemia. In these trials, exenatide was associated with a lower rate of nocturnal hypoglycaemia and mean weight reduction, while insulin patients gained weight. Irrespective of treatment, a lower baseline $\mathrm{HbA}_{1 c}$ value was associated with a higher probability of achieving glycaemic targets. Supported by Eli Lilly and Company and Amylin Pharmaceuticals, Inc. 


\section{PS 79 Type 2 diabetes: mechanisms}

\section{4}

\section{Potent antidiabetic effects of rivoglitazone, a novel PPAR $\gamma$ agonist, in} obese diabetic rodent models

J. Ohsumi, S. Kanda, J. Tanaka, K. Takahashi, R. Nakashima, N. Koieyama, Y. Hagisawa, K. Araki;

Biological Research Laboratories II, Daiichi Sankyo Co., Ltd., Tokyo, Japan.

Background and Aims: Rivoglitazone (Rivo) is a novel peroxisome proliferator-activated receptor $\gamma$ (PPAR $\gamma)$ agonist. The antidiabetic effects of Rivo were characterised and compared with the effects of rosiglitazone (Rosi) and pioglitazone (Pio) in Zucker Diabetic Fatty (ZDF) rats and $d b / d b$ mice. Also, the mechanism of action of Rivo was assessed in ZDF rats and nondiabetic Zucker Fatty (ZF) rats.

Materials and Methods: Male ZDF rats and $d b / d b$ mice were orally administered Rivo $(0.03-1 \mathrm{mg} / \mathrm{kg} / \mathrm{day})$, Pio $(3-100 \mathrm{mg} / \mathrm{kg} / \mathrm{day})$ or Rosi $(0.3-$ $100 \mathrm{mg} / \mathrm{kg} /$ day) for 10 or 14 days. Subsequently, plasma glucose (PG), free fatty acid (FFA), triglyceride (TG) and adiponectin levels and pancreatic insulin content were determined. A hyperinsulinemic-euglycemic clamp study was performed in ZF rats given Rivo $(0.1 \mathrm{mg} / \mathrm{kg} /$ day $)$ for 8 days. Also, the effects of Rivo on plasma TG production and clearance, and expression of PPAR $\alpha$ target genes and genes implicated in cardiac function, were assessed in ZF rats and ZDF rats, respectively, with Wy-14,643 (Wy), a PPAR $\alpha$ agonist, as a comparator.

Results: In ZDF rats, Rivo was approximately 100 -fold more potent at reducing PG than either Rosi $\left(\mathrm{ED}_{50}=0.20\right.$ [Rivo] vs. $28 \mathrm{mg} / \mathrm{kg}$ [Rosi] $)$ or Pio $\left(\mathrm{ED}_{50}=0.19\right.$ [Rivo] vs. $34 \mathrm{mg} / \mathrm{kg}$ [Pio]). Rivo also lowered plasma TG and FFA levels, and increased pancreatic insulin content, more potently than either Rosi or Pio. Similarly, in $d b / d b$ mice, Rivo reduced PG and TG over 100 -fold more potently than Pio (PG reduction: $\mathrm{ED}_{50}=0.47$ [Rivo] vs. $70 \mathrm{mg} / \mathrm{kg}$ [Pio]; TG reduction: $\mathrm{ED}_{50}=0.093$ [Rivo] vs. $4.6 \mathrm{mg} / \mathrm{kg}$ [Pio]) after 14 days of treatment. After 10 days, Rivo was approximately 9 -fold more potent at lowering PG than Rosi. Interestingly, in $d b / d b$ mice, Rivo increased plasma adiponectin levels more effectively than Pio. In the hyperinsulinemic-euglycemic clamp study, Rivo increased the glucose infusion rate to $36.6 \pm 1.1 \mathrm{mg} / \mathrm{kg} / \mathrm{min}$ compared with $26.0 \pm 0.4 \mathrm{mg} / \mathrm{kg} / \mathrm{min}$ in vehicle-treated controls. In ZF rats, Wy and Rivo reduced plasma TG, primarily by decreasing hepatic TG production and by accelerating TG clearance, respectively. Consistent with this, Wy decreased hepatic expression of Apolipoprotein C-III (ApoCIII; 26 $\pm 2 \%$ of control), and increased expression of PPAR $\alpha$ target genes implicated in fatty acid $\beta$-oxidation such as acyl-coenzyme A oxidase $1(A C O ; 1054 \pm 30 \%)$ and carnitine palmitoyltransferase $1 b$ (CPT1b; $3574 \pm 196 \%)$ in ZDF rats. Rivo had no detectable effects on the expression of ApoCIII and, in contrast to Wy, decreased hepatic expression of $A C O(72 \pm 4 \%)$ and $C P T 1 b(65 \pm 5 \%)$. Rivo decreased cardiac expression of ACO (66 $2 \%$ of control), pyruvate dehydrogenase kinase $4(10 \pm 1 \%)$ and medium chain acyl-CoA dehydrogenase $(46 \pm 6 \%)$ and increased expression of glucose transporter $4(147 \pm 8 \%)$. Furthermore, Rivo did not alter the expression of genes involved in cardiac function, such as myosin heavy chain $\alpha$ and $\beta$, or sarco/endoplasmic reticulum $\mathrm{Ca}^{2+}$ ATPase 2 .

Conclusions: In obese diabetic rodents, Rivo modifies glucose and lipid metabolism by enhancing insulin sensitivity without affecting expression of PPAR $\alpha$ target genes. The gene expression profile suggests that Rivo may enhance glucose utilisation and reduces oxidative stress in the diabetic heart. In the models examined, Rivo demonstrated greater potency than both Rosi and Pio, suggesting that it may be more efficacious in clinical practice.

\section{5}

Effect of rosiglitazone and exercise on $\mathrm{Na}^{+} \mathrm{K}^{+} \mathrm{ATPase}$ function in insulin resistant rat skeletal muscle

S. F. Fraser, S. L. La Vita, S. Lessard, J. A. Hawley;

School of Medical Science, RMIT University, Bundoora, Australia.

Background: Rosiglitazone is an oral antidiabetic drug, that improves hyperglycaemia and hyperinsulinemia in insulin resistant and type 2 diabetic patients via insulin sensitising mechanisms but may also have a detrimental effect on membrane lipids which is integral to the functioning of $\mathrm{Na}^{+}-\mathrm{K}^{+}$ATPase. Chronic exercise training has been shown to decrease skeletal muscle insulin resistance in insulin resistant animal models and diabetics as well as upregulate $\mathrm{Na}^{+}-\mathrm{K}^{+}$-ATPase content in healthy skeletal muscle. The main purpose of the present study was to evaluate the effects of rosiglitazone and exercise on $\mathrm{Na}^{+}-\mathrm{K}^{+}$-ATPase function and membrane phospholipids in the skeletal muscle of high fat fed Sprague Dawley rats.

Methodology: Rats were assigned into a high fat (D12330) diet group High Fat (HF), rosiglitazone treatment groups HF Rosi (HF R), while the exercise groups (HFX) and Exercise + Rosi (HFXR) undertook 6 weeks of endurance training on a rat treadmill. Glucose uptake was measured using $\left[{ }^{14} \mathrm{C}\right]$-glucose. Red gastrocnemius (RG) was extracted from the rat hind limb and analysed for 3-O-MFPase activity and [3H]-ouabain binding site content. RG was further analysed for determination of the fatty acid (FA) profile and molecular species composition of phospholipid in the membrane using Electrospray ionisation mass spectrometry.

Results: With insulin stimulation, there was still no significant difference in glucose uptake in RG between HF and HFIns (HF $2.32 \pm 0.85$ and HFIns $4.11 \pm 0.56 \mathrm{mmol} . \mathrm{g}^{-1} \cdot \mathrm{hr}^{-1}$ ), indicating established insulin resistance. There was a significant increase in 3-O-MFPase activity when insulin resistant rats were treated with exercise. Compared to the HF, exercise training caused a significant 58\% $(P<0.001)$ and $46 \%(P<0.01)$ rise in maximal 3-O-MFPase activity in HFX and HFRX groups respectively (HF $412 \pm 27$, HFX 652 \pm 38 and HFRX $600 \pm 24$ nmol.min ${ }^{-1} \cdot \mathrm{g}^{-1}$ wet wt). Exercise training had a significant effect on rats with HFX and HFRX showing increases in $\mathrm{Na}^{+} \mathrm{K}^{+}$ATPase content when compared to HF control (HF $307 \pm 18$, HFX $471 \pm$ 28and HFRX $549 \pm 23$ pmol. $\mathrm{mg}^{-1}$ wet wt). The phospholipid fatty acid (FA) composition in RG membranes was determined by measuring the content of individual FA types and representing them as a percentage of the total FA content. Mixed results were shown which potentially impacted in membrane fluidity and membrane bound enzyme function.

Conclusions: The results investigated the effect of a therapeutic dose of Rosiglitazone, and exercise training in high fat fed rats. The most important finding was the positive effect exercise training had on the reduced $\mathrm{Na}^{+} \mathrm{K}^{+}$ATPase capacity found with insulin resistance. Exercise training significantly increased skeletal muscle $\mathrm{Na}^{+} \mathrm{K}^{+}$-ATPase content and its corresponding maximal activity but had a mixed effect on the membrane phospholipid profile. Rosiglitazone did not have the same desired effect, furthermore at therapeutic doses, did not have any effect.

Supported by RMIT Faculty grant

\section{6}

Pioglitazone inhibit high-glucose-induced human umbilical vein endothelial cells apoptosis: involvement of intracellular ceramide signaling event

D. Huang ${ }^{1}$, M. Lei ${ }^{1}$, X. He ${ }^{2}$, E. H. Schuchman ${ }^{2}$, B. Chen ${ }^{3}$;

${ }^{1}$ Department of Endocrinolog, Xiangya Hospital, Changsha, China, ${ }^{2}$ Department of Human Genetics, Mount Sinai School of Medicine, New York, United States, ${ }^{3}$ Modern Analytical Testing Center,

Central South University, Changsha, China.

Background and Aims: Ceramide (CER) is an important second messenger to play a role in the induction of cell apoptosis. Thiazolidinediones has been shown to exert direct effects on vascular cells to potentially retard theatherosclerotic process. The aim of the present study was to examine the effect of pioglitazone on high-glucose-induced apoptosis in human umbilical vein endothelial cells (HUVECs) and the role of the ceremide signal pathway involved.

Materials and Methods: HUVECs were incubated with $5 \mathrm{mM}, 30 \mathrm{mM}$ glucose, and with $30 \mathrm{mM}$ glucose+ pioglitazone $(0.1,1,10 \mu \mathrm{M}), 30 \mathrm{mM}$ glucose $+2 \mu \mathrm{M}$ Desipramine, $30 \mathrm{mM}$ glucose $+10 \mu \mathrm{M}$ pioglitazone $+50 \mu \mathrm{M}$ exogenous C2-CER, 30mM glucose $+10 \mu \mathrm{M}$ pioglitazone $+1 \mathrm{U} / \mathrm{ml}$ exogenous acidic sphingomyelinase (A-SMase), $30 \mathrm{mM}$ glucose $+2 \mu \mathrm{M}$ Desipramine $+50 \mu \mathrm{M}$ C2-CER, and with C2-CER $(25,50 \mu \mathrm{M})$, A-SMase in a series of concentrations $(0.01,0.1,1 \mathrm{U} / \mathrm{ml})$ respectively for $24,48 \mathrm{~h}$, apoptosis of HUVEC were observed by terminal deoxynucleotidyl transferase-mediated dUTP nick end-labeling (TUNEL) assay, and quantitated by flow cytometry using Annexin-FITC/ propidiumiodid(PI)staining. HUVECs were incubated with $5 \mathrm{mM}, 30 \mathrm{mM}$ glucose respectively for $2,4,8,12,16,24 \mathrm{~h}$, and with glucose in a series of concentrations $(5,10,20,30 \mathrm{mM}), 30 \mathrm{mM}$ glucose + pioglitazone $(0.1,1,10 \mu \mathrm{M})$, $30 \mathrm{mM}$ glucose $+2 \mu \mathrm{M}$ Desipramine respectively for $16 \mathrm{~h}$, cellular ceramide (cell membrane fraction and cytosol plus organelle fraction) were detected by liquid chromatography/ion spray ionization tandem mass spectrometry (LC/ 
MS/MS). HUVECs were incubated with $5 \mathrm{mM}, 30 \mathrm{mM}$ glucose respectively for $0.5,1,2,4,8,12 \mathrm{~h}$, and with $30 \mathrm{mM}$ glucose $+2 \mu \mathrm{M}$ Desipramine, $30 \mathrm{mM}$ glucose+ pioglitazone $(0.1,1,10 \mu \mathrm{M})$ respectively for $2 \mathrm{~h}$, intracellular A-SMase activity were determined using the fluorescent substrate BODIPY C12sphingomyelin and reverse-phase high-performance liquid chromatography (HPLC).

Results: High glucose increase the apoptosis in HUVECs in 48h. Pioglitazone can inhibit the high glucose-induced apoptosis in manner of concentrationdependent in HUVECs, exogenous CER and A-SMase can reverse the inhibition of pioglitazone. Desiparmine also shows the same effect on HUVECs as Pioglitazone, exogenous ceramide can reverse the inhibition of Desiparmine. Exogenous 50 $\mu \mathrm{M}$ C2-CER and $1 \mathrm{U} / \mathrm{ml}$ A-Smase can increase the apoptosis in HUVECs in $48 \mathrm{~h}$ significantly. High glucose caused a concentration-dependent increase of intracellular ceramide, and the increase in $16 \mathrm{~h}$ is the most significant, but the contents of membranous ceramide remained unaltered. High glucose caused the increase in the intracellular ASMase activity, and the increase in $2 \mathrm{~h}$ is the most significant. Pioglitazone and Desiparmine can inhibit both the intracellular A-SMase activity and ceramide increase.

Conclusion: High glucose prominently utilizes the lysosomal acidic SMase-ceramide signal pathway in the induction of apoptosis in HUVECs. Pioglitazone attenuates high glucose-induced HUVEC apoptosis through its inhibition on intracellular A-SMase and ceramide.

\section{7}

Role of adiponectin in thiazolidinedione mediated plasminogen activator inhibitor-1 suppression in type 2 diabetic subjects W. S. Chow ${ }^{1}$, R. L. C. Hoo ${ }^{1}$, M. H. Yau ${ }^{1}$, A. Xu ${ }^{1,2}$, H. F. Tse ${ }^{1,2}$, A. W. K. Tso ${ }^{1}$, C. H. Y. Fong ${ }^{1}$, S. Tam ${ }^{2,3}$, L. Chan ${ }^{4}$, K. S. L. Lam ${ }^{1,2}$;

${ }^{1}$ Department of Medicine, The University of Hong Kong, China,

${ }^{2}$ Research Centre of Heart, Brain, Hormone, and Healthy Aging,

The University of Hong Kong, China, ${ }^{3}$ Clinical Biochemistry Unit,

Queen Mary Hospital, Hong Kong, China,

${ }^{4}$ Baylor College of Medicine, University of Texas, United States.

Background and Aims: Circulating levels of plasminogen activator inhibitor-1 (PAI-1), which correlate with cardiovascular events in clinical studies, are increased in type 2 diabetes and, by reducing fibrinolysis, may contribute to the increased cardiovascular mortality in patients with type 2 diabetes. Recent studies suggest that plasma PAI-1 levels can be lowered by the thiazolidinedione insulin-sensitizers. In this study, we examined the effect of rosiglitazone, relative to sulfonylurea, on plasma PAI-1 levels, in type 2 diabetic subjects with suboptimal glycemic control. We also investigated whether this beneficial effect was mediated through adiponectin, a fatderived hormone with various metabolic activities, in clinical and animal studies.

Materials and Methods: The effects of 24-week add-on therapy with rosiglitazone $(n=32)$ or sulfonylurea $(n=32)$ on glycemic control (HbA1c), insulin resistance (homeostasis model assessment, HOMA-IR), circulating PAI- 1 level, serum adiponectin, markers of inflammation and oxidative stress were studied in 64 orally treated type 2 diabetic subjects in a randomised controlled trial. We also evaluated the inter-relationship between PAI- 1 and adiponectin by using an adiponectin-deficient mouse model and adenovirusmediated over-expression of adiponectin.

Results: Our clinical data showed that after 24-week add-on therapy, both the rosiglitazone- and sulfonylurea-treated groups had significant reductions in PAI-1 levels ( $<<0.001$ vs. baseline), with the reduction being significantly greater in the rosiglitazone group ( $\mathrm{p}=0.002$ vs. sulfonylurea treatment). Stepwise multiple linear regression analysis, including all independent variables with significant changes only in the rosiglitazone group $(p<0.05$ after Bonferonni correction), showed that only the reduction in plasma fasting glucose and increase in plasma adiponectin levels were independently associated with the reduction in PAI-I concentration. Consistent with the clinical findings, adipose tissue PAI-1 mRNA expression was significantly decreased in high-fat (HF) induced $\mathrm{C} 57 \mathrm{BL} / 6 \mathrm{~N}$ mice after rosiglitazone treatment. This suppressive effect on PAI-1 expression was attenuated in high-fat induced adiponectin (ADPN) knockout (KO) mice. Similar trend was observed in its plasma concentration. Plasma glucose concentration, however, was significantly decreased in both HF-C57BL/6N mice and HFADPN-KO mice to a similar extent after rosiglitazone treatment. We also found that adenovirus-mediated over-expression of adiponectin alone could lead to a suppression of adipose tissue PAI-1 expression in both lean and $\mathrm{db} / \mathrm{db}$ diabetic mice.

Conclusion: Both the human and animal studies showed that, in addition to its glucose-lowering action, rosiglitazone, a thiazolidinedione, can also reduce the prothrombotic tendency in type 2 diabetes by suppressing PAI- 1 production, in part via adiponectin.

Supported by CRCG grant, RGC grants and the Strategic Research Fund on Healthy Aging, the University of Hong Kong

\section{8}

A one-year follow-up study: comparing the cardiovascular safety of rosiglitazone and metformin with plasma BNP levels and myocardial performance index in type 2 diabetes mellitus

Y. T. Kemal' ${ }^{1}$, N. G. Demirağ ${ }^{1}$, A. Ylldırır ${ }^{2}$, A. Atar ${ }^{2}$, S. T. Eroğlu², A. D. Unal ${ }^{1}$, N. B. Tütünc $\ddot{u}^{1}$, Z. Biyıklığlu'

${ }^{1}$ Department of Endocrinology and Metabolism, Baskent University, ${ }^{2}$ Department of Cardiology, Baskent University, ${ }^{3}$ Department of Biostatistics, Ankara University, Faculty of Medicine, Ankara, Turkey.

Background and Aims: Thiazolidinediones (TZDs) are powerful tools that specifically augment insulin sensitivity and counter insulin resistance in patients with type 2 diabetes mellitus. There has been increasing discussion about whether TZDs affect cardiovascular function, and evidence suggests that those agents exert both negative and positive effects on myocardial performance. We aimed to evaluate the long term effects of rosiglitazone on cardiac functions and fluid dynamics.

Materials and Methods: Thirty-nine type 2 diabetic patients -without any symptoms and findings of heart failure were randomised to three treatment groups after receiving approval from the local Ethical Committee. Group $1(\mathrm{n}=12)$ received $8 \mathrm{mg}$ rosiglitazone per day and performed diabetic diet, group $2(\mathrm{n}=14)$ received $1700 \mathrm{mg}$ metformin per day and performed diabetic diet and group $3(n=13)$ only performed diabetic diet. Before the treatment all subjects were evaluated for fasting and postprandial plasma glucose levels, HbAlc, plasma brain natriuretic peptide (BNP) levels, body mass index (BMI) and myocardial performance index (MPI: a parameter of left ventricular global function, measured by tissue Doppler imaging echocardiography). After twelve months all these parameters were repeated. During that 12-month study period, the patients were asked to report any adverse effects and underwent a physical examination and biochemical analyses each month.

Results: Before the treatment there were no significant differences between the groups in the duration of diabetes, HbAlc and BNP levels, body mass index and MPIs. After one year follow-up period plasma BNP levels, interventricular septum and lateral wall MPIs didn't change significantly in all treatment groups (all $\mathrm{p}>0.05$ ). During the study period no patient exhibited congestive heart failure, elevated levels of liver enzymes or creatinine.

Conclusion: In patients with type 2 diabetes, long term TZD treatment might be as safe as metformin treatment. However, these findings must be supported and strengthened with large scale clinical trials.

\section{9}

Rosiglitazone does not affect the diuretic response to furosemide or amiloride in insulin-resistant subjects

A. J. M. Rennings ${ }^{1}$, Y. Li $^{2}$, P. M. T. Deen ${ }^{2}$, R. Masereeuw ${ }^{1}$, B. Rossier ${ }^{3}$, F. Russel ${ }^{1}$, C. J. Tack ${ }^{4}$, P. Smits';

${ }^{1}$ Pharmacology-Toxicology, Radboud University Nijmegen Medical Center, The Netherlands, ${ }^{2}$ Physiology, Radboud University Nijmegen Medical Center, The Netherlands, ${ }^{3}$ Institut de Pharmacologie et de Toxicologie, Universite de Lausanne, Switzerland, ${ }^{4}$ Internal Medicine, Radboud University Nijmegen Medical Center, The Netherlands.

Background and Aims: Treatment with thiazolidinedione derivatives (TZD) is associated with fluid retention. Animal studies suggest that TZD-related fluid retention is caused by upregulation of the epithelial sodium channel $(\mathrm{ENaC})$ in the distal part of the nephron. So far, this has not been studied in humans. Besides, several case reports and one clinical trial suggest that TZDrelated edema is resistant to loop diuretic therapy, but again, this hypothesis has not been addressed appropriately. Therefore, we investigated both at 
the functional and molecular level whether rosiglitazone (RSG) induces furosemide resistance and/or upregulation of ENaC.

Materials and Methods: We conducted a randomised, double blind, placebo-controlled, cross-over study in 12 subjects with insulin resistance, characterised by two features of the metabolic syndrome but with normal glucose intolerance. Subjects were treated for 9 weeks with either placebo or rosiglitazone $4 \mathrm{mg}$ bid. The wash out period was 4 weeks. At the end of each treatment period, the response to an intravenous dose of furosemide and amiloride was quantified. Total sodium excretion was measured after a bolus injection of furosemide $40 \mathrm{mg}$. In order to construct concentration response curves, the urinary excretion rates of furosemide and sodium were calculated in all urine samples over an 8 hour-period. Amiloride, a direct inhibitor of $\mathrm{ENaC}$, was administered as a bolus injection followed by a continuous infusion, to obtain a target plasma amiloride concentration for 5 hours. Again, total sodium excretion over 8- and 24-hour-periods were measured. Separately, the morning urine before furosemide injection was freshly collected in buffer and centrifuged for isolation of urinary exosomes, in which $\mathrm{ENaC}$ expression was analysed by Western Blotting.

Results: RSG did not alter the furosemide concentration response curve ( $\mathrm{E}_{\text {: }}$ : RSG $3166 \pm 1201 \mu \mathrm{mol} \mathrm{Na} / \mathrm{min}$ versus PLAC $3263 \pm 1065 \mu \mathrm{mol} \mathrm{Na} / \mathrm{min} P=0.49$

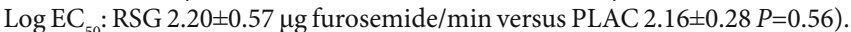
There was no difference in 8 hour total sodium excretion [RSG $257 \pm 46 \mathrm{mmol}$ vs. PLAC $251 \pm 64 \mathrm{mmol}(P=0.66)]$. In addition, we did not observe differences in the 8 and 24-hour sodium excretion after start of the amiloride infusion [RSG $313 \pm 73 \mathrm{mmol}$ vs. PLAC $315 \pm 68 \mathrm{mmol}(P=0.90)]$. Finally, rosiglitazone did not change $\mathrm{ENaC}$ expression in the urinary exosomes.

Conclusion: Rosiglitazone did not induce furosemide resistance or upregulate EnaC, neither at a functional nor at a molecular level. The results of this wellcontrolled accurate clinical study challenges previous findings in animals and suggest that fluid retention by rosiglitazone should respond normally to diuretic treatment.

\section{0}

Oral antidiabetics thiazolidinediones directly inhibit hepatic gluconeogenesis in perifused liver cells C. Sanchez ${ }^{1}$, J. M. Recio ${ }^{2}$, N. Sanz ${ }^{1}$, G. R-Villanueva ${ }^{1}$, J. M. Lopez-Novoa ${ }^{1}$ D. Detaille ${ }^{1}$, X. Leverve ${ }^{3}$, M. El-Mir ${ }^{1}$,

${ }^{1}$ Fisiología y Farmacología, Universidad de Salamanca, Spain,

${ }^{2}$ Servicio de Endocrinología, Hospital Universitario de Salamanca, Salamanca, Spain, ${ }^{3}$ Univ. Grenoble 1, INSERM E0221, Grenoble, France.

Background and Aims: The Thiazolidinediones (TZDs) are new antidiabetic oral agents which exert indirectly their hipoglycemic effects by selective activation of the peroxisome proliferator-activated receptor-gamma (PPARgamma). But it's also known that TZDs could exert some of their antidiabetic effects in the liver and the skeletal muscle where usually the PPARgamma receptors are not very abundant. So, it's possible that the TZDs could exert DIRECT EFFECTS on the hepatic metabolism in independent way not mediated by activation of the PPAR gamma receptors. We decided to investigate the direct effects (hepatic gluconeogenesis and glycolysis) of the thiazolidinediones in perifused liver cells.

Materials and Methods: Hepatocytes were isolated from 24h starved rats according to the method of Berry and Friend. They were perifused ( $5 \mathrm{ml} /$ $\mathrm{min}$ ) at $37^{\circ} \mathrm{C}$ with Krebs-bicarbonate-calcium satured with $\mathrm{O}_{2} / \mathrm{CO}_{2}$ at a flow rate of $5 \mathrm{ml} / \mathrm{min}$ and titrated with increasing substrate concentrations of dihydroxiacetone $(0.15 ; 0.3 ; 0.6 ; 1.2 ; 2.4 ; 4.8 \mathrm{mM})$ in the presence or absence of $25 \mu \mathrm{M}$ Rosiglitazone (RGZ) or Pioglitazone (PGZ) to get seven consecutive steady states. For each steady state we measured glucose, pyruvate and lactate concentrations in cellular perifusate. Dihydroxyacetone phosphate (DHAP), glucose-6-phosphate (G6P) and fructose-6-phosphate (F6P) concentrations were measured fluorimetrically in the cellular fraction.

Results: In perifused hepatocytes, Pioglitazone and Rosiglitazone inhibited hepatic glucose production $\left(J_{\text {glusse }}\right)$ by $40 \%$ (DHA $2.4 \mathrm{mM}$; p $<.001$ ) and $57 \%$ (DHA $2.4 \mathrm{mM}$; p <.001) compared with control. Nevertheless, both PGZ and RGZ stimulated hepatic glycolysis by similar way (15\%; $<<.05)$. Both molecules inhibited total DHA metabolism by $8 \%$ in case of PGZ $(\mathrm{p}<.05)$ and by $16 \%$ in case of RGZ $(\mathrm{p}<.001)$. TZDs increased cytosolic NADH/NAD ${ }^{+}$ redox state, estimated as lactate/pyruvate ratio, by $24 \%$ in case of RGZ (DHA $2.4 \mathrm{mM} ; \mathrm{p}<.001$ ) and by $10 \%$ in case of PGZ (DHA $2.4 \mathrm{mM} ; \mathrm{p}<.05)$. Also, Rosiglitazone and Pioglitazone changed the relationship between $\mathrm{J}_{\mathrm{CInce}}$ and DHAP, F6P or G6P compared with control. The TZDs decreased DHAP, F6P and G6P concentrations at each steady state, obtaining a double relationship between $\mathrm{J}_{\text {Glucose }}$ and DHAP, F6P or G6P.

Conclusions: The TZDs Pioglitazone and Rosiglitazone, besides their known antidiabetic effects by activation of PPAR gamma receptors, exert DIRECT and ACUTE hypoglycaemic effects on the liver by inhibition of hepatic gluconeogenesis. This decrease in presence of TZDs could occur by a possible inhibition of the enzyme glucose-6-phosphatase.

\section{1}

Comparison of the effects of pioglitazone and metformin on hepatic and extra-hepatic insulin action in people with type 2 diabetes R. Basu' ${ }^{1}$, B. Norby ${ }^{1}$, A. Basu ${ }^{1}$, V. Chandramouli ${ }^{2}$, B. Dicke ${ }^{1}$, O. Cohen ${ }^{3}$, P. Shah ${ }^{4}$, B. Landau ${ }^{2}$, R. Rizza ${ }^{1}$;

${ }^{1}$ Endocrinology, Mayo Clinic College Of Medicine, Rochester, United States, ${ }^{2}$ Clinical and Molecular Endocrinology, Case Western Reserve, Cleveland, United States, ${ }^{3}$ Endocrinology, Chaim Sheba Medical Center, Tel Aviv, Israel, ${ }^{4}$ Endocrinology, MD Anderson Cancer Center, Houston, United States.

Background and Aims: Pioglitazone (Pio) and metformin (Met) are both considered "insulin sensitisers". We wished to determine if these drugs have different effects on hepatic and extra-hepatic insulin action in the presence of insulin concentrations that are commonly present under the conditions of daily living.

Materials and Methods: In order to do so 31 subjects with type 2 diabetes treated with either metformin alone $(n=24)$, diet alone $(n=3)$ or a sulfonylurea $(\mathrm{n}=4)$ were randomly assigned to Pio (45 mg qd) or Met (2000 mg qd) for four months.

Results: Glucose was clamped before and after therapy at $\sim 5 \mathrm{mmol} / \mathrm{l}$, insulin raised to $\sim 180 \mathrm{pmol} / \mathrm{l}$, C-peptide suppressed with somatostatin, glucagon replaced at $\sim 75 \mathrm{pg} / \mathrm{ml}$ and glycerol maintained at $\sim 270 \mu \mathrm{mol} / \mathrm{l}$ to insure comparable and equal portal concentrations on all occasions. $\mathrm{HbAlc}$ remained unchanged ( $6.5 \pm 0.2$ vs. $6.2 \pm 0.2 \%)$ during Pio and but increased slightly ( $6.3 \pm 0.2$ vs. $7.0 \pm 0.3 \%$; $\mathrm{p}<0.05)$ during Met. The glucose infusion rate (GIR) required to maintain euglycemia increased during Pio ( $17 \pm 3$ vs. $24 \pm 3$ $\mu \mathrm{mol} / \mathrm{kg} / \mathrm{min} ; \mathrm{p}<0.01)$ but did not change $(19 \pm 4$ vs. $22 \pm 4 \mu \mathrm{mol} / \mathrm{kg} / \mathrm{min})$ during Met. The increase in GIR with Pio was entirely explained by enhanced insulin induced suppression ( $6.0 \pm 1$ vs. $0.2 \pm 2 \mu \mathrm{mol} / \mathrm{kg} / \mathrm{min} ; \mathrm{p}<0.001)$ of endogenous glucose production (EGP) since glucose uptake (GU) did not differ ( $23 \pm 3$ vs. $24 \pm 2 \mu \mathrm{mol} / \mathrm{kg} / \mathrm{min})$. Neither EGP $(5.6 \pm 1.0$ vs. $5.0 \pm 0.8 \mu \mathrm{mol} /$ $\mathrm{kg} / \mathrm{min})$ nor GU ( $25 \pm 4$ vs. $24 \pm 3 \mu \mathrm{mol} / \mathrm{kg} / \mathrm{min})$ differed before and during Met. Pio enhanced $(\mathrm{p}<0.001)$ insulin induced suppression of gluconeogenesis measured with the ${ }^{2} \mathrm{H}_{2} 0$ method ( $\left.\mathrm{n}=7: 4.3 \pm 0.8 \mathrm{vs} .1 .6 \pm 0.9 \mu \mathrm{mol} / \mathrm{kg} / \mathrm{min}\right)$. On the other hand, there was no change in suppression of gluconeogenesis during Met ( $\mathrm{n}=6: 4.4 \pm 1.1$ vs. $3.3 \pm 1.2 \mu \mathrm{mol} / \mathrm{kg} / \mathrm{min})$. Pio also enhanced $(\mathrm{p}<0.001)$ insulin induced suppression of free fatty acids $(0.14 \pm 0.03 \mathrm{vs.} 0.06 \pm 0.01 \mu \mathrm{mol} /$ 1) whereas Met had no effect $(0.12 \pm 0.04$ vs. $0.15 \pm 0.07 \mu \mathrm{mol} / \mathrm{l})$.

Conclusion: Thus relative to metformin, pioglitazone improves hepatic insulin action in people with type 2 diabetes by enhancing insulin induced suppression of glucose production, gluconeogenesis and free fatty acids. On the other hand, extra-hepatic insulin action is comparable following treatment with pioglitazone or metformin.

Supported by DK29953 


\section{PS 80 Type 2 diabetes: clinical}

\section{2}

\section{Determinants of monotherapy failure in ADOPT}

(A Diabetes Outcome Progression Trial)

R. R. Holman ${ }^{1}$, S. E. Kahn', S. M. Haffner ${ }^{3}$, B. Zinman', G. F. Viberti', W. H. Herman ${ }^{6}$, J. M. Lachin ${ }^{7}$, B. G. Kravitz ${ }^{8}$, M. A. Heise ${ }^{8}$;

${ }^{1}$ Diabetes Trials Unit, OCDEM, Churchill Hospital, Oxford, United Kingdom, ${ }^{2}$ Department of Medicine, VA Puget Sound Health Care System, Seattle, United States, ${ }^{3}$ Medicine/Clinical Epidemiology, University of Texas Health Science Center at San Antonio, United States, ${ }^{4}$ Medicine, Mount Sinai Hospital, Toronto, Canada, ${ }^{5}$ Department of Diabetes \& Endocrinology, King's College London School of Medicine, United Kingdom, ' Internal Medicine - Metabolism, Endocrinology and Diabetes (MEND), University of Michigan, Ann Arbor, MI, United States, ${ }^{7}$ The Biostatistics Center, George Washington University, Rockville, United States, ${ }^{8} \mathrm{GlaxoSmithKline,}$ King of Prussia, United States.

Background and Aims: ADOPT (A Diabetes Outcome Progression Trial), a double-blind, randomised clinical trial in 4360 recently diagnosed type 2 diabetic patients, assessed the efficacy of rosiglitazone (RSG), glyburide (GLY) and metformin (MET) as initial therapy. This 4-year study showed that, compared with GLY or MET, RSG slowed progression to monotherapy failure (MTF), defined as the time to the first of two successive fasting plasma glucose (FPG) values $>180 \mathrm{mg} / \mathrm{dl}$. To address whether tailoring glucoselowering medication based on individual phenotypes would promote the achievement and maintenance of glycemic targets, data from ADOPT were analysed to assess which phenotypic features at baseline most influenced time to MTF.

Materials and Methods: Patient demographic and baseline characteristics by allocated therapy were analysed using a multivariate proportional hazards model.

Results: Significant factors $(\mathrm{p}<0.05)$ with hazard ratios $(\mathrm{HR}), 95 \%$ confidence intervals and Madalla $\mathrm{R}^{2}$ values were:

\begin{tabular}{|c|c|c|c|c|c|c|}
\hline & Rosiglitazone & & Metformin & & Glyburide & \\
\hline & HR & $\mathrm{R}^{2} \%$ & HR & $\mathrm{R}^{2} \%$ & HR & $\mathrm{R}^{2} \%$ \\
\hline $\begin{array}{l}\text { Age } \\
\text { (per year) }\end{array}$ & $\begin{array}{l}0.92 \\
(0.90-0.94)\end{array}$ & 4.7 & $\begin{array}{l}0.96 \\
(0.94-0.97)\end{array}$ & 2.1 & $\begin{array}{l}0.96 \\
(0.95-0.97)\end{array}$ & 3.6 \\
\hline $\begin{array}{l}\mathrm{HbA}_{1 \mathrm{c}} \\
\text { (per 1\%) }\end{array}$ & $\begin{array}{l}1.50 \\
(1.21-1.86)\end{array}$ & 1.1 & $\begin{array}{l}1.46 \\
(1.25-1.71)\end{array}$ & 1.7 & $\begin{array}{l}1.25 \\
(1.09-1.44)\end{array}$ & 0.8 \\
\hline $\begin{array}{l}\text { FPG } \\
\text { (per mg/dl) }\end{array}$ & & & $\begin{array}{l}1.01 \\
(1.00-1.02)\end{array}$ & 0.6 & $\begin{array}{l}1.01 \\
(1.01-1.02)\end{array}$ & 1.7 \\
\hline $\begin{array}{l}\text { HOMA\%B } \\
\text { (per unit) }\end{array}$ & $\begin{array}{l}0.98 \\
(0.97-0.99)\end{array}$ & 1.1 & & & & \\
\hline $\begin{array}{l}\text { HOMA\%S } \\
\text { (per unit) }\end{array}$ & $\begin{array}{l}1.01 \\
(1.006-1.014)\end{array}$ & 1.5 & & & $\begin{array}{l}0.99 \\
(0.98-1.00)\end{array}$ & 0.3 \\
\hline $\begin{array}{l}\text { Proinsulin: } \\
\text { insulin } \\
\text { (per unit) }\end{array}$ & & & & & $\begin{array}{l}2.06 \\
(1.37-3.09)\end{array}$ & 1.0 \\
\hline
\end{tabular}

For all therapies, increasing age was associated with decreasing risk of MTF, with time to MTF increasing; the greatest effect of age $\left(R^{2}\right)$ was seen in the RSG group $(\mathrm{p}<0.05)$. High levels of $\mathrm{HbA}_{1 \mathrm{c}}$ and $\mathrm{FPG}$ were the next greatest influences in the MET and GLY treatment groups, with both reducing the time to MTF. In the RSG group, HOMA\%S had the next greatest impact, with higher levels increasing time to MTF. Lower HOMA\%B levels and higher $\mathrm{HbA}_{1 \mathrm{c}}$ levels reduced time to MTF in the RSG group. For GLY, a higher proinsulin: insulin ratio (another measure of beta-cell function) increased time to MTF, and higher HOMA\%S levels reduced time to MTF.

Conclusion: Hyperglycaemic progression is slower in older patients. The next most important determinants for the rate of progression to MTF were initial hyperglycaemia in the MET and GLY groups, and lower insulin resistance (assessed by HOMA\%S) in the RSG group.

Supported by GlaxoSmithKline

\section{3}

The effect of combining rosiglitazone and metformin on insulin resistance in women with polycystic ovary syndrome

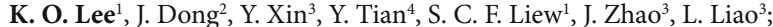
${ }^{1}$ Medicine, National University of Singapore, Singapore, ${ }^{2}$ Obstetrics and Gynecology, Shandong Provincial Hospital, Jinan, China, ${ }^{3}$ Endocrinology, Shandong Provincial Hospital, Jinan, China, ${ }^{4}$ Endocrinology, Qilu Hospital, Medical School of Shandong University, Shandong, China.

Background and Aims: Women with the polycystic ovary syndrome (PCOs) are often insulin resistant and predisposed to developing type 2 diabetes. Thiazolidinediones (TZDs) and metformin both improve insulin sensitivity but studies on the efficacy and safety of TZDs in PCOs have been limited. We performed this randomised open-label parallel two group pilot study to compare the impact of metformin alone (Group $\mathrm{M}$ ) versus combining metformin with rosiglitazone (Group $\mathrm{M}+\mathrm{R}$ ) in reducing insulin resistance. Materials and Methods: We enrolled 58 patients with PCOs (Inclusion criteria: hyperandrogenaemia, chronic menstrual abnormality, ultrasound proven polycystic ovaries) and insulin resistance. Patients were assigned to receive metformin only $(1500 \mathrm{mg}, \mathrm{n}=29)$, or metformin $(1500 \mathrm{mg})$ and rosiglitazone $(4 \mathrm{mg})(\mathrm{n}=29)$, daily for 6 months. Oral glucose tolerance tests (OGTT), with venous glucose and insulin measurements, and other endocrine parameters, were assessed before and after the treatment period. Treatment was discontinued immediately if a patient became pregnant. Results: The study had 3 drop-outs (1 in Group M, 2 in Group M+R). There was no significant difference in the OGTT glucose concentrations between the two groups pre or post-treatment, but fasting and post-OGTT insulin concentrations decreased significantly after treatment (table with Mean + Standard Deviation) and more significantly in Group $\mathrm{M}+\mathrm{R}$ compared to Group M (see table; $\mathrm{p}<0.01)$.

\begin{tabular}{|c|c|c|c|c|}
\hline & \multicolumn{2}{|l|}{ Metformin } & \multicolumn{2}{|c|}{$\begin{array}{l}\text { Metformin PLUS } \\
\text { Rosiglitazone }\end{array}$} \\
\hline & Pre & Post & Pre & Post \\
\hline $\begin{array}{l}\text { GLUCOSE } \\
0 \text { min }\end{array}$ & $4.2+1.03$ & $4.1+0.96$ & $4.5+0.85$ & $3.9+0.73$ \\
\hline 60 & $7.9+2.15$ & $7.4+2.10$ & $7.8+1.91$ & $7.0+1.03$ \\
\hline 120 & $6.7+1.81$ & $6.3+1.76$ & $6.2+1.84$ & $5.5+0.82$ \\
\hline $\begin{array}{l}\text { INSULIN } \\
0 \mathrm{~min}\end{array}$ & $26.4+7.4$ & $15.8+5.4^{*}$ & $27.1+8.2$ & $12.7+6.7^{\star}$ \\
\hline 60 & $141.8+47.4$ & $90.7+46.5$ & $140.0+43.6$ & $76.7+34.0^{*}$ \\
\hline 120 & $195.6+57.6$ & $101.1+52.3^{*}$ & $198.7+47.0$ & $65.2+19.1^{*}$ \\
\hline HOMA-IR & $4.97+4.15$ & $2.88+3.54$ & $5.42+4.21$ & $2.20+3.41^{*}$ \\
\hline BMI & $27.7+2.9$ & $22.5+2.2$ & $27.8+2.7$ & $26.7+3.3$ \\
\hline
\end{tabular}

${ }^{*} \mathrm{p}<0.05$ between pre and post values within groups.

There were 2 pregnancies in the $M$ group and 6 in the $M+R$ group. All the babies have been followed for more than 12 months and do not show any abnormality.

Conclusions: Rosiglitazone and metformin combination is more effective in decreasing hyperinsulinaemia in PCOs than metformin alone. Rosiglitazone during the first trimester of pregnancy did not show any abnormality in the 6 babies followed up to 12 months in our study patients. 


\section{4}

The effects of pioglitazone vs glimepiride on adipose tissue distribution in patients with type 2 diabetes: results from the CHICAGO Trial M. Davidson ${ }^{1}$, R. D'Agostino, Sr², S. Feinstein ${ }^{1}$, S. Haffner ${ }^{3}$, G. Kondos ${ }^{4}$, P. Meyer ${ }^{5}$, A. Perez ${ }^{6}$, T. Mazzone ${ }^{7}$;

${ }^{1}$ Section of Cardiology, Rush Presbyterian-St. Luke's Medical Center, Chicago, ${ }^{2}$ Statistics and Consulting Unit, Boston University, Boston, ${ }^{3}$ Department of Medicine, University of Texas Health Science Center, San Antonio, ${ }^{4}$ Section of Cardiology, University of Illinois, Chicago, ${ }^{5}$ Department of Preventive Medicine, University of Illinois College of Medicine, Chicago, ${ }^{6}$ Takeda Global Research and Development Center, Inc., Deerfield, ${ }^{7}$ Section of Diabetes and Metabolism, University of Illinois at Chicago, Chicago, United States.

Background and Aims: Weight gain is a known side effect of most type 2 diabetes (T2D) therapies, including insulin, sulfonylureas, and thiazolidinediones (TZDs). Weight gain that occurs with TZDs is likely due to fluid retention, increases in adipose tissue, or both. During the recently completed CHICAGO study, there was a mean weight gain of $3.2 \mathrm{~kg}$ with pioglitazone (PIO) and $1.0 \mathrm{~kg}$ with the comparator glimepiride (GLM). Here we report changes in BMI, waist-hip ratio, and abdominal adipose tissue distribution observed during the trial.

Materials and Methods: CHICAGO was a randomised, double-blind, multicenter, 72-week study designed to compare carotid intima-media thickness during treatment with PIO $(\mathrm{N}=230) v s$. GLM $(\mathrm{N}=228)$ in patients with T2D. Abdominal adipose tissue content and distribution (as measured by electron beam tomography) and BMI were determined at baseline and the final visit (week 72 or the time of withdrawal).

Results: There were significantly greater increases in BMI with PIO vs.GLM, whereas waist-hip ratio decreased with PIO and increased with GLM. Total abdominal adipose tissue volume increased for both PIO and GLM. For PIO, the increase was largely accounted for by accretion of subcutaneous tissue, which increased to a significantly greater extent with PIO $v s$. GLM. Therefore, the ratio of visceral to total adipose tissue volume tended to increase with GLM and decrease with PIO.

Changes in BMI, Waist Measurements, and Adipose Tissue Distribution

\begin{tabular}{llll}
\hline & & GLM Mean (SD) & PIO Mean (SD) \\
\hline BMI $\left(\mathbf{k g} / \mathbf{m}^{2}\right)$ & BL & $31.9(4.9)$ & $32.1(5.2)$ \\
& Change & $0.4(1.4)$ & $1.1(1.8) \dagger$ \\
Waist circumference (in) & BL & $41.7(5.1)$ & $42.0(5.1)$ \\
& Change & $0.3(2.4)$ & $0.6(2.7)$ \\
Waist/hip ratio & BL & $0.953(0.078)$ & $0.956(0.073)$ \\
& Change & $0.008(0.054)$ & $-0.006(0.059)^{*}$ \\
Abdominal Adipose & & LS mean (SE) & LS mean (SE) \\
Tissue Volume & & & \\
\hline Total $\left(\mathrm{cm}^{3}\right)$ & BL & $309.48(7.84)$ & $321.85(7.74)$ \\
& Change & $14.11(6.05)$ & $25.52(6.03)$ \\
Visceral $\left(\mathbf{c m}^{3}\right)$ & BL & $126.27(3.73)$ & $129.38(3.66)$ \\
& Change & $7.94(3.12)$ & $7.35(3.13)$ \\
Subcutaneous $\left(\mathbf{c m}^{3}\right)$ & BL & $186.95(6.34)$ & $196.71(6.26)$ \\
& Change & $4.12(4.88)$ & $18.93(4.84)^{\star}$ \\
Visceral/Total ratio & BL & $0.41(0.01)$ & $0.39(0.01)$ \\
& Change & $0.01(0.01)$ & $-0.01(0.01)$ \\
\hline
\end{tabular}

$\mathrm{BL}=$ Baseline; $\mathrm{P}$-value is for the treatment-group difference; change is from baseline to Final Visit (Week 72 or time of withdrawal). ${ }^{\star} \mathrm{P}<0.05, \uparrow \mathrm{P}<0.0001$

Conclusion: These results indicate that subcutaneous rather than visceral abdominal fat largely accounts for the increase in adipose tissue noted with PIO therapy. This altered distribution of visceral to subcutaneous fat may contribute to improved insulin sensitivity.

Supported by Takeda Pharmaceuticals North America, Inc.

\section{5}

Effects of $30 \mathrm{mg}$ of pioglitazone $v \mathrm{~s} \mathbf{\mathrm { mg }}$ of rosiglitazone on hyperglycaemia and dyslipidaemia: results from a head-to-head trial T. McCall ${ }^{1}$, S. Kupfer ${ }^{2}$, S. Gbenado 2 , S. Jacober 3 , M. H. Tan ${ }^{3}$.

${ }^{1}$ Takeda Pharmaceuticals North America, Inc., Deerfield,

${ }^{2}$ Takeda Global Research \& Development Center, Inc., Deerfield,

${ }^{3}$ Eli Lilly and Company, Indianapolis, United States.

Background and Aims: The GLAI study supported the hypothesis that the thiazolidinediones pioglitazone (PIO) and rosiglitazone (ROSI) have similar glucose-lowering effects but differing effects on lipids. This head-to-head study showed that PIO and ROSI lowered HbAlc to a similar extent, but that PIO was associated with more favorable changes in triglycerides (TG), HDL$\mathrm{C}$, and non-HDL-C. The purpose of the present analysis was to evaluate the effectiveness of the starting doses of PIO $(30 \mathrm{mg})$ and ROSI $(4 \mathrm{mg})$ used in GLAI.

Materials and Methods: GLAI was a 6-month, randomised, double-blind trial in patients with type 2 diabetes (treated with diet or oral monotherapy only) and dyslipidemia (TG $\geq 150,<600 \mathrm{mg} / \mathrm{dL}$ and LDL-C $\leq 130 \mathrm{mg} / \mathrm{dL}$ ) who were not receiving lipid-altering drugs. Patients were assigned to treatment with PIO $(\mathrm{N}=369)$ or ROSI $(\mathrm{N}=366)$, both of which were titrated to their respective maximum effective dose: patients received 3 months of PIO $30 \mathrm{mg}$ QD or ROSI 4mg QD, followed by an additional 3 months of PIO 45mg QD or ROSI $8 \mathrm{mg}$ (4mg BID). The primary variable was change in TG at the final visit (ie, month 6 or the time of withdrawal). For the present analysis, change from baseline in lipid and $\mathrm{HbAlc}$ values were evaluated at month 3 (ie, before dose titration).

Results: As observed for maximum doses, the starting doses of PIO and ROSI differed significantly in their effects on TG $(\mathrm{P}<0.001)$, HDL-C $(\mathrm{P}<0.001)$, and non-HDL-C $(\mathrm{P}=0.003)$ in favor of PIO. However, unlike for the maximum doses, the $30 \mathrm{mg}$ dose of PIO led to a significantly greater reduction in $\mathrm{HbAlc}$ than the $4 \mathrm{mg}$ dose of ROSI ( $\mathrm{P}=0.017)$. (See table).

Conclusion: Initial treatment with the $30 \mathrm{mg}$ dose of PIO was more effective than use of the $4 \mathrm{mg}$ dose of ROSI in terms of improving measures of hyperglycemia and dyslipidemia.

Change from Baseline to Month 3 in Lipid and HbAlc Levels

\begin{tabular}{lllll}
\hline & & PIO & ROSI & P-Value \\
& & N=330 & N=320 & \\
\hline TG (mg/dL) & Baseline (SE) & $257.8(8.2)$ & $235.3(6.6)$ & $<0.001$ \\
& LS mean \% change (SE) & $-11.95(2.63)$ & $19.32(2.64)$ & \\
HDL-C (mg/dL) & Baseline (SE) & $38.8(0.5)$ & $39.8(0.6)$ & $<0.001$ \\
& LS mean \% change (SE) & $13.30(1.09)$ & $4.26(1.10)$ & \\
non-HDL-C & Baseline (SE) & $154.8(1.6)$ & $153.6(1.6)$ & 0.003 \\
$(\mathbf{m g / d L )}$ & LS mean \% change (SE) & -6.53 & 12.67 & \\
HbA1c (\%) & Baseline (SE) & $7.6(0.1)$ & $7.5(0.1)$ & 0.017 \\
& LS mean change (SE) & $-0.46(0.05)$ & $-0.31(0.05)$ & \\
\hline
\end{tabular}

Supported by Takeda Pharmaceuticals North America and Eli Lily and Company

\section{6}

Insulin sensitivity and metabolic control in response to pioglitazone treatment differ between patients with type 2 diabetes with and without the Pro12Ala variant in the PPAR $\gamma 2$ gene

H. Vestergaard ${ }^{1}$, J. Clausen ${ }^{2}$, R. Benediktsson ${ }^{3}$, V. A. Lyssenko ${ }^{4}$, D. Larsson ${ }^{5}$, K. N. Valnes ${ }^{6}$, M. Lilja ${ }^{7}$, P. J. Dahl'

${ }^{1}$ Department of Endocrinology, Herlev Hospital, Univeristy of Copenhagen, Herlev, Denmark, ${ }^{2}$ Eli Lilly A/S, Lyngby, Denmark, ${ }^{3}$ Department of Endocrinology and Metabolism, Landspítali - University Hospital, Reykjavík, Iceland, ${ }^{4} 4$ Department of Clinical Sciences, Diabetes and Endocrinology, University Hospital Malmö, Wallenberg Laboratory, Malmö, Sweden, ${ }^{5}$ General practitioner, Degeberga, Sweden, ${ }^{6}$ Senior Consultant, Oslo, Norway, ${ }^{7}$ The Research and Development Unit, Jämtland, Sweden.

Background and Aims: To evaluate the possible modifying effect of the peroxisome-proliferator-activated receptor $\gamma$ gene (PPARG) polymorphism on the efficiency of pioglitazone (Actos ${ }^{\oplus}$ ) treatment with regard to glycaemic control in both men and women with type 2 diabetes (T2D). 
Materials and Methods: A total of 326 patients with T2D, in a normal clinical practice setting, were treated with pioglitazone at the dose of 15-30 $\mathrm{mg} /$ day for 26 weeks, added to existing oral anti-diabetic medication. All were genotyped for the Pro12Ala variant of the PPARG. Insulin resistance and $\beta$-cell function was calculated using HOMA model.

Results: There were 248 patients with the Pro12Pro genotype and 78 with the Pro12Ala genotype, no difference between females and males ( $p=0.78)$. $\mathrm{HbA}_{1 c}$ decreased significantly between visits, in both females and males $(p<0.001)$. The difference between visits across gender depended on genotype, with a significantly larger decrease in the Pro12Pro genotype $(p=0.026)$. Insulin resistance fell significantly across gender and genotype between visits $(p<$ 0.001 ), but the change across gender was significantly greater for the Pro12Pro genotype ( $p=0.034)$, compared to baseline.

Conclusion: In patients with T2D, the effects of pioglitazone treatment on metabolic control and insulin sensitivity may vary dependent on the wild type Pro12Pro variant of the PPAR $\gamma$ gene. Therefore, assessment of the Pro12Pro variant may in future prove to have clinical significance in the identification of patients with T2D who will benefit from pioglitazone treatment. Supported by Eli Lilly and Company

\section{7}

Comparison of resources utilization (RU) and cost in drug-naive type 2 diabetes (T2D) patients treated with rosiglitazone (RSG) vs. sulfonylurea (SU) monotherapy

M. S. Duh ${ }^{1}$, A. Gosselin ${ }^{1}$, N. Beaulieu ${ }^{1}$, M. Arana ${ }^{1}$, B. Arondekar²;

${ }^{1}$ Health Care, Analysis Group inc., Boston,

${ }^{2}$ GlaxoSmithKline, Philadelphia, United States.

Background and Aims: The objective of this study was to compare RU and costs for drug-naïve patients (pts) treated with RSG versus SU first-line monotherapy using real-world claims data.

Material and Methods: Based on medical, pharmacy (Rx), and disability insurance claims data between 10/2001 and 12/2004, patients with a diagnosis $\mathrm{T} 2 \mathrm{D}$ who were newly initiated on an OAD (no OAD in previous 6 months), $\geq 18$ years old, and had $\geq 60$ days of uninterrupted treatment were analysed. Frequency of inpatient (inpt) and outpatient (outpt) visits and average direct (inpt, outpt, and $\mathrm{Rx}$ ) and indirect (workloss) costs were compared between the RSG and SU groups.

Results: 3,377 RSG and 11,778 SU pts met the inclusion criteria with RSG pts being younger ( 63.8 vs. 66.9 years, $p<.001$ ) with less comorbidities (Charlson comorbidity index 0.95 vs. $1.23, \mathrm{p}<.01)$ at baseline. During treatment, the RSG group incurred statistically significantly less frequent inpt visits $(0.47 \mathrm{vs.}$ 0.77 visits per pt per year (PPPY), $\mathrm{p}<.001$ ), outpt visits (17.0 vs. 17.9 visits PPPY, $\mathrm{p}<.001$ ), and number of hospital days (1.6 vs. 2.9 days PPPY, $\mathrm{p}<.001)$ than the $\mathrm{SU}$ group. The total direct medical cost was significantly lower in the RSG group ( $\$ 1,065$ vs. $\$ 1,315$ per pt per month (PPPM), $\mathrm{p}<.001)$ than the SU group, including significantly lower inpt and outpt cost ( $\$ 717$ vs. $\$ 1,046$ PPPM, $\mathrm{p}<.001$ ) but higher Rx cost ( $\$ 348$ vs. $\$ 270$ PPPM, $\mathrm{p}<.001$ ). After taking into account the indirect workloss cost, the total direct and indirect cost was significantly lower in the RSG group ( $\$ 1,103$ vs. $\$ 1,355$ PPPM, $\mathrm{p}<.001)$. Multivariate analysis controlling for age, gender, comorbidities, and other covariates confirmed that the RSG group was associated with a significantly lower total cost than the SU group (cost difference: $\$ 92.75$ PPPM, $\mathrm{p}=0.012$ ).

Conclusion: This observational study of over 15,000 pts initiated on firstline monotherapy shows that RSG pts incur significantly lower resource utilisation and costs than SU pts, outweighing higher pharmacy cost. Supported by GlaxoSmithKline PLC (to Mei Sheng Duh, Antoine Gosselin, Nicolas Beaulieu and Mateo Arana)

\section{8}

Economic analysis of thiazolidinedione add-on therapy relative to insulin add-on therapy in individuals treated with sulfonylureas: a third party payer's perspective

S. Balu ${ }^{1}$, W. Lee ${ }^{2}$, B. Arondekar ${ }^{3}$, R. Horblyuk ${ }^{3}$, C. Pashos ${ }^{1}$;

${ }^{1}$ HERQuLES, Abt Associates Inc., Lexington, ${ }^{2}$ HERQuLES, Abt Associates

Inc., Bethesda, ${ }^{3}$ GlaxoSmithKline, Philadelphia, United States.

Background and Aims: This study evaluated the economic impact to a managed care organisation's budget of adding a thiazolidinedione versus insulin to sulfonylurea monotherapy for the management of type 2 diabetes. Materials and Methods: Using an integrated medical and pharmacy claims database from $07 / 01$ to $06 / 02$, we identified type 2 diabetes patients treated with sulfonylurea monotherapy, who subsequently received a thiazolidinedione or insulin in addition to sulfonylurea monotherapy. Permember per-month (PMPM) total healthcare costs and pharmacy costs during two-years of follow-up were assessed. Multivariate generalised linear models were used to assess the cost differential of thiazolidinedione add-on versus insulin add-on accounting for differences in baseline demographics, comorbidities, and pre-index health care costs.

Results: A total of 994 individuals (Mean [SD]: 53.4 [10.4] years; 51.0\% males) were identified, with 384 initiating insulin add-on and 610 initiating thiazolidinedione add-on to sulfonylurea monotherapy. There were no significant differences in age or gender across the two groups, however, insulin add-on patients had greater cardiovascular comorbidities and other complications. Although overall PMPM pharmacy costs were lower for the thiazolidinedione add-on group (\$262 [\$3,739] vs. \$360 [\$4,026], p<0.01), diabetes-related pharmacy costs were greater for thiazolidinedione-add on group (\$109.4 [\$3,336] vs. \$96.1 [\$2,067]; $\mathrm{P}=0.04$ ). Despite higher PMPM diabetes-related pharmacy costs, patients with thiazolidinedione-add on therapy realised cost savings of $\$ 96$ PMPM over two years as compared to patients with insulin $(\$ 229[\$ 6,448$.] vs. $\$ 325[\$ 9,159] ; p=0.01)$. After adjusting for differences in baseline characteristics, patients with insulin addon therapy incurred $22 \%(\mathrm{p}<0.05)$ higher total health care costs compared to patients on thiazolidinedione add-on therapy.

Conclusion: The findings from this study demonstrate that the addition of thiazolidinedione to sulfonylurea monotherapy was associated with a significant 22\% reduction in PMPM total health care costs versus add-on therapy with insulin.

Supported by GlaxoSmithKline

\section{9}

Economic impact of add-on therapy with thiazolidinedione versus insulin to metformin monotherapy

B. Arondekar ${ }^{1}$, S. Balu², W. Lee ${ }^{3}$, R. Horblyuk ${ }^{1}$, C. Pashos ${ }^{2}$; ${ }^{1}$ GlaxoSmithKline, Philadelphia, United States,

${ }^{2}$ HERQuLES, Abt Associates Inc., Lexington, United States,

${ }^{3}$ HERQuLES, Abt Associates Inc., Bethesda, United States.

Background and Aims: Changes to pharmacotherapy for type 2 diabetes are generally based on stability of glycemic control and therapy tolerability. Differences in glycemic control often influence resource utilisation which in-turn impacts health care costs. This study assessed the economic impact of addition of a thiazolidinedione versus insulin to type 2 diabetes patients receiving monotherapy with metformin.

Materials and Methods: Administrative claims data from a large integrated medical and pharmacy claims database was used. Type 2 diabetes adults on metformin monotherapy who received add-on therapy with thiazolidinedione or insulin were identified based on pharmacy claims from 07/2001 through 06/2002. Primary endpoints included differences in baseline comorbidities and unadjusted two-year follow-up total cost. Multivariate generalised linear models were used to assess the cost differential of thiazolidinedione add-on versus insulin add-on accounting for differences in baseline demographics, comorbidities, and pre-index health care costs.

Results: A total of 1,608 individuals were identified with 1,153 receiving add-on therapy with thiazolidinedione versus 455 receiving insulin addon therapy. Although there were no significant differences in age or gender between thiazolidinedione (mean $=51.4$ years, $46.4 \%$ males) and insulin (mean $=51.5$ years, $47.9 \%$ males) add-on cohorts, patients with thiazolidinedione-add on had decreased prevalence of cardiovascular disease 
( $15.4 \%$ vs. $19.6 \% ; \mathrm{p}<0.01)$ and increased prevalence of hypertension $(45 \%$ vs. $39.3 \% ; \mathrm{p}=0.04)$ at baseline. The unadjusted two-year follow-up total costs were significantly lower among thiazolidinedione users as compared to insulin users $(\$ 17,339.80$ vs. $\$ 21,150.30 ; P=0.03)$. This difference persisted after adjusting for differences in baseline characteristics, with patients on insulin plus metformin incurring $20 \%(\mathrm{p}<0.05)$ higher total health care costs compared to patients on thiazolidinedione plus metformin.

Conclusion: Controlling for differences in comorbidities, this analysis showed significant reduction in costs with thiazolidinedione versus insulin as add-on therapy to metformin monotherapy. These differences indicate that selection of a thiazolidinedione as the second agent can have an important economic impact on healthcare costs for type 2 diabetes.

Supported by GlaxoSmithKline

\section{PS 81 Metformin and sulphonylurea}

\section{0}

Patient characteristics of new users of oral antidiabetic drugs in daily clinical practice

S. van der Bij ${ }^{1}$, J. Koerselman ${ }^{1}$, J. A. Erkens ${ }^{1}$, S. Kessabi², M. T. Groot ${ }^{2}$, F. J. A. Penning-van Beest ${ }^{1}$, R. M. C. Herings ${ }^{1}$;

${ }^{1}$ PHARMO Institute, Utrecht, The Netherlands,

${ }^{2}$ Novartis Pharma AG, Basel, Switzerland.

Background and aim: Pharmacological treatment of type 2 diabetes mellitus consists of oral antidiabetic drugs (OADs) and, in case of insufficient effect, insulin therapy. Currently, metformin, sulphonylureas (SU), and thiazolidinediones (TZD), are the most frequently used OAD classes. Channeling of drugs into patients may occur. The aim of this study was to characterise new users of OADs in daily clinical practice.

Methods: From the PHARMO record linkage system, comprising among others linked drug-dispensing, clinical laboratory, and hospital data of $>2.3$ million inhabitants in The Netherlands, new users of OADs were identified in the period between January 1, 1999 and December 31, 2005. Patient characteristics, i.e. gender, age, type of prescriber of first OAD, year of start OAD-therapy, and $\mathrm{HbAlc}$ level at start, were determined per type of initial OAD. Also, patients were classified into sub-cohorts of high-risk patients: A) elderly, age $\geq 65$ years; B) elderly with a history of cardiac, hepatic, or renal failure; and C) any age with a history of cardiac, hepatic, or renal failure.

Results: The study included 35,514 new users of OADs. Of these, $40 \%$ started on monotherapy with metformin $(\mathrm{n}=14,277), 53 \%$ with SU-monotherapy $(\mathrm{n}=18,876), 0.9 \%$ with TZD-monotherapy $(\mathrm{n}=310)$, and 1,499 patients (4\%) started on combination therapy with metformin $+\mathrm{SU}(\mathrm{n}=1,426)$, or metformin+TZD $(n=73)$. For each initial OAD, male and female gender was about equally divided, and a general practitioner was the most frequent prescriber, ranging from $67 \%$ of first TZD-dispensings to $88 \%$ in case of SU. The percentage of patients $\geq 65$ years of age, ranged from $33 \%$ for metformin+TZD to $54 \%$ in case of SU. Also, for each initial OAD, many patients were classified as high-risk patient; e.g. the percentage of patients with a history of cardiac, hepatic, or renal failure ranged from $58 \%$ for metformin+TZD to $66 \%$ in case of TZD, alone. Of a subpopulation $(4,051$ patients), baseline $\mathrm{HbAlc}$-measurements were available. Mean baselineHbAlc was lowest for patients on initial TZD $(7.4 \pm 1.5 \%)$, and highest for patients who started on metformin $+\mathrm{SU}(8.7 \pm 2.2 \%)$.

Conclusion: The majority of patients starting with OAD-therapy in daily clinical practice are high-risk patients, marked by age $\geq 65$ years and symptomatic cardiovascular, renal or hepatic disease, and started their therapy by a GP. Differences between initial OADs were observed with respect to age and baseline-HbAlc indicating that combination therapy is started more often in younger patients and in patients with higher HbAlc.

Supported by an unrestricted grant from Novartis Pharma AG, Basel, Switzerland

\section{1}

One-third of new users of oral antidiabetic drugs fail to attain $\mathrm{HbA}_{1 \mathrm{c}}$-goal in the first year after start of therapy

J. A. Erkens ${ }^{1}$, J. Koerselman ${ }^{1}$, S. van der Bij ${ }^{1}$, S. Kessabi ${ }^{2}$, M. T. Groot ${ }^{2}$,

F. J. A. Penning-van Beest ${ }^{1}$, R. M. C. Herings ${ }^{1}$;

${ }^{1}$ PHARMO Institute, Utrecht, The Netherlands,

${ }^{2}$ Novartis Pharma AG, Basel, Switzerland.

Background and Aims: The major objective of the treatment of type 2 diabetes mellitus (T2DM) is to control glycemic parameters in order to minimise the risk of long-term complications and to alleviate any symptoms present. Within the Dutch guidelines for treatment of T2DM, the target value of $\mathrm{HbAlc}$ has been set at $<7 \%$. The aim of this study was to determine mean levels of $\mathrm{HbAlc}$ over time among new users of oral antidiabetic drugs (OADs) in daily clinical practice, and the proportion of patients attaining goal within the first year of follow-up.

Methods: From the PHARMO record linkage system, comprising among others linked drug-dispensing, clinical laboratory, and hospital data of $>2.3$ million inhabitants in The Netherlands, new users of OADs were identified in the period between January 1, 1999 and December 31, 2005. Patients 
who started on monotherapy with metformin, a sulphonylurea (SU), or a thiazolidinedione (TZD), or on combination therapy with metformin+SU, or metformin+TZD, with a baseline HbAlc-measurement, and at least one HbAlc-measurement in the one-year period after treatment onset, were included in the study-cohort. Patients were not required to be still on initial OAD-therapy, at the time of each HbAlc-measurement. Mean HbAlc-levels over time were assessed per quarter of the first year of follow-up. Kaplan Meier survival analyses were used for the assessment of time to goal (HbA1c $<7 \%$ ). For the latter analysis, only patients with a baseline HbAlc-value $\geq$ $7 \%$ were included.

Results: The study included 4,051 new T2DM-patients with a HbAlcmeasurement at baseline. Of these, 3,398 (84\%) had at least one measurement during the first year of follow-up. Across all OAD classes mean HbAlc ( \pm sd) at baseline was $8.2 \pm 1.8 \%$, and ranged from $7.4 \pm 1.5 \%$ among patients who started on TZD-monotherapy, to $8.7 \pm 2.2 \%$ for patients who started on metformin+SU combination therapy (patients on initial metformin+TZD were not included due to too low numbers). Overall, about $75 \%$ of the patients had a baseline $\mathrm{HbAlc} \geq 7 \%$, except for the TZD-monotherapy group, in which $53 \%$ of the patients had a HbAlc $\geq 7 \%$ at baseline. During followup, overall, the mean level of $\mathrm{HbAlc}( \pm \mathrm{sd})$ was $7.6 \pm 1.4 \%$ in the first quarter, and $6.9 \pm 0.9 \%$ in subsequent quarters of the first year of follow-up. Mean HbA1c-levels during follow-up were lower in TZD-patients (e.g. 7.0 $\pm 1.3 \%$ in the first quarter, and $6.5 \pm 0.7 \%$ in the fourth quarter of follow-up). Six months after start of therapy, the proportion of patients who had attained goal was $15 \%$ for metformin alone and metformin+SU, 19\% for TZD alone, and $20 \%$ for SU alone. After one-year of follow-up, these proportions on goal were between $60 \%$ and $75 \%$.

Conclusion: The results of this study indicate that $75 \%$ of new users of OADs in daily clinical practice have a HbAlc-level $\geq 7 \%$ at start of therapy, and that about two-thirds of these patients attain goal, i.e. HbAlc $<7 \%$, in the first year of follow-up. This percentage includes both patients still on initial therapy, and with a change in OAD-treatment. However, it is not known whether these patients remained at goal after goal-attainment.

Supported by an unrestricted grant from Novartis Pharma AG, Basel, Switzerland

\section{2}

In too many patients a change in initial therapy with oral antidiabetic drugs is already required in the first year of treatment

F. J. A. Penning-van Beest ${ }^{1}$, J. Koerselman ${ }^{1}$, S. van der Bij ${ }^{1}$, S. Kessabi ${ }^{2}$, M. T. Groot ${ }^{2}$, J. A. Erkens ${ }^{1}$, R. M. C. Herings ${ }^{1}$;

${ }^{1}$ PHARMO Institute, Utrecht, The Netherlands,

${ }^{2}$ Novartis Pharma AG, Basel, Switzerland.

Background and aim: Oral antidiabetic drugs (OADs) are first choice in the pharmacological treatment of type 2 diabetes mellitus (T2DM). OADtherapy is tailored to the patient's needs dependent on its observed effect in glycemic control. In case of insufficient effect, an add-on with, or a switch to another OAD or even insulin, may occur. Accordingly, the aim of this study was to determine the persistence and treatment pattern with initial OADtherapy in the first year of treatment in daily clinical practice.

Methods: From the PHARMO record linkage system, comprising among others linked drug-dispensing, and hospital data of $>2.3$ million inhabitants in The Netherlands, new users of OADs were identified in the period between January 1, 1999 and December 31, 2005. Patients starting with monotherapy of metformin, a sulphonylurea (SU), or a thiazolidinedione (TZD), or on combination therapy with metformin+SU, or metformin+TZD, and with at least one year of follow-up, were included in the study cohort. One-year persistence with initial treatment was determined by using episodes of each OAD-treatment, based on the method of Catalan allowing for drug holidays. For non-persistent patients, the first change in OAD-treatment (add-on, switch, or discontinuation) in the one-year period after starting treatment was determined.

Results: The study included 34,962 new users of OADs. Of these, $40 \%$ started on monotherapy with metformin $(\mathrm{n}=14,277), 53 \%$ with SU-monotherapy $(\mathrm{n}=18,876)$, and $0.9 \%$ with TZD-monotherapy $(\mathrm{n}=310)$, and 1,499 patients (4\%) started on combination therapy with metformin + SU $(n=1,426)$, or metformin+TZD ( $n=73)$. Overall, after one year, $59 \%$ of the OAD-users was persistent with the initial OAD-treatment. One-year persistence-rates ranged from $46 \%$ among patients who started on TZD-monotherapy, to $60 \%$ for patients who started on SU-monotherapy. Among the non-persistent patients starting on OAD-monotherapy, add-on and discontinuation were the most frequent first treatment changes in the one-year period after starting treatment (38-43\% and $38-50 \%$, respectively). In patients starting on combination therapy, a switch was the most frequent first treatment change (67-69\% of the non-persistent patients). Overall, mean duration ( \pm sd) to first add-on or switch among non-persistent patients was $4.0( \pm 3.4)$ months. The most frequent add-on to metformin monotherapy was SU (81\%), and the most frequent add-on to SU-monotherapy was metformin (91\%). Patients starting on metformin+SU combination therapy most often switched to mono-use of either SU (46\%) or metformin (35\%).

Conclusion: The results of this study indicate that in many T2DM-patients (about $40 \%$ ) a change in initial OAD-therapy is already required in the first year of treatment, particularly in case of initial TZD-monotherapy. Among non-persistent patients, initial OAD-monotherapy most often changed to combination therapy with OADs, and initial OAD combination therapy most often changed to monotherapy with an OAD.

Supported by an unrestricted grant from Novartis Pharma AG, Basel, Switzerland

\section{3}

How should patients with diabetes and heart failure have their blood sugars controlled? A systematic review and meta-analysis of the available literature

D. T. Eurich ${ }^{1,2}$, F. A. McAlister ${ }^{3,1}$, D. F. Blackburn', S. R. Majumdar ${ }^{3,1}$,

R. T. Tsuyuki ${ }^{5,1}$, J. Varney ${ }^{2}$, J. A. Johnson ${ }^{1,2}$;

${ }^{1}$ School of Public Health, University of Alberta, Edmonton,

${ }^{2}$ Institute of Health Economics, Edmonton, ${ }^{3}$ Division of Internal Medicine, Department of Medicine, University of Alberta, Edmonton,

${ }^{4}$ College of Pharmacy and Nutrition, University of Saskatchewan, Saskatoon, ${ }^{5}$ Division of Cardiology, Department of Medicine, University of Alberta,

Edmonton, Canada.

Background and Aims: To systematically review the literature on the relationship between different antidiabetic therapies and morbidity and mortality in people with heart failure (HF) and diabetes.

Materials and Methods: Literature search from 1966 to 2007 (e.g., Medline, Embase, trial registries) identified controlled studies (either randomised trials or cohort studies) evaluating antidiabetic agents and outcomes (i.e., death, hospitalizations) in patients with HF and diabetes. References were hand-searched and investigators of included studies contacted. Two reviewers independently extracted data. Risk estimates for specific therapies were abstracted, and pooled estimates derived by meta-analysis only when appropriate.

Results: Of 9,68 references initially identified, only six studies (four cohort studies, two subgroup analyses of randomised trials) were included. In four studies of 19,205 patients, use of insulin was associated with a marked increased risk for all-cause mortality in 3 studies and no effect in 1 study, risk estimates ranging from $0.96-3.42$. In three studies of 21,125 patients, use of metformin was associated with a significant reduction in all-cause mortality in two studies (risk estimate from 0.61-0.86), and a similar trend in the third. Metformin was not associated with increased all-cause or HF-specific hospitalisations in any studies. There were two studies of thiazolidinediones in 19,022 patients; one study demonstrated a significant reduction in allcause mortality (risk estimate 0.87 ), and the other found no association. Thiazolidinediones were associated with an increased risk of HF-specific hospitalisations in both studies (pooled odd ratio 1.14, 95\%CI 1.04-1.25, $\mathrm{I} 2=0 \%, \mathrm{p}=0.004)$. There were no studies of nonsulfonylurea secretagogues or acarbose in this setting, and no randomised trials that addressed the question.

Conclusion: Our systematic review suggests that metformin is the only agent studied that is not associated with measurable harm in patients with $\mathrm{HF}$ and diabetes; in fact, it was associated with reduced all-cause mortality. Unfortunately, it is also contraindicated in this population. It is imperative; therefore, that further valid evidence is generated. In the meantime, consideration to labeling changes for metformin should be strongly considered. 


\section{4}

Impact of type of sulfonylureas on survival in diabetic patients with acute myocardial infarction

N. Danchin ${ }^{1}$, M. Zeller ${ }^{2}$, T. Simon ${ }^{3}$, A. Vahanian ${ }^{4}$, Y. Cottin ${ }^{2}$, J. Berland ${ }^{5}$, P. Gueret ${ }^{6}$, P. Wyart ${ }^{7}$, R. Deturck ${ }^{8}$, J.-P. Cambou ${ }^{9}$;

${ }^{1}$ Cardiology, HEGP, Paris, France, ${ }^{2}$ Cardiology, CHU Dijon,

${ }^{3}$ Pharmacology, Hopital St Antoine, Paris, ${ }^{4}$ Cardiology, CHU Bichat, Paris, ${ }^{5}$ Cardiology, Clinique St Hilaire, Rouen, ${ }^{6}$ Cardiology, CHU Mondor, Créteil, ${ }^{7}$ Cardiology, Villeneuve St Georges, Paris, ${ }^{8}$ Cardiology, CHG, Lens,

${ }^{9}$ Société Française de Cardiologie, Paris, France.

Background and Aims: Questions have been raised as to the cardiovascular safety of sulfonylureas (SU). Glibenclamide is a non-selective sulfonylurea known to inhibit ischemic preconditioning of the myocardium, unlike the newer SU (gliclazide, glimepiride). Aim: To assess early and 6-month mortality according to the previous use and type of SU in diabetic patients admitted for AMI in a nationwide French registry.

Materials and Methods: The FAST-MI registry included consecutive patients admitted for ST-elevation or non-ST elevation myocardial infarction $\leq 48$ hours of symptom onset, in 223 French intensive care units over 2 months from October 2005. In the 1316 diabetic patients, 3 groups were defined according to their antidiabetic treatment before admission: no SU (SU0, $\mathrm{n}=851$ ), Glibenclamide (SU1, $\mathrm{n}=120$ ), new SU with pancreatic beta-cell selectivity (glimepiride/gliclazide, $n=339$ ) (SU2).

Results: Age was not significantly different between the 3 groups ( $70 \pm 12$, $71 \pm 10,69 \pm 11$, respectively for SU0, SU1 and SU2); $39 \%, 37 \%$ and $35 \%$, respectively had a diabetes duration $>5$ years (NS). Concomitant risk factors were similar in the 3 groups and the GRACE risk score was not significantly different $(170 \pm 38,162 \pm 35,166 \pm 38$, respectively). Patients with SU2 were less often co-treated with insulin (SU2: 9\%, SU1: 16\%, SU0: 40\%, p<0.001) and those without SU received less metformin (SU0: 30\%, SU1: 53\%, SU2: $40 \%, \mathrm{p}<0.001)$. Entry glycaemia was not significantly different for SU1 patients $(2.32 \pm 1.06 \mathrm{~g} / \mathrm{L})$ and SU2 patients $(2.19 \pm 1.07)$ but was lower in SU0 patients $(2.02 \pm 1.05, \mathrm{p}<0.005)$. HbAlc was available in half of the patients; it was higher in SU1 $(8.0 \pm 1.5 \%)$ than in SU0 or SU2 $(7.5 \%)(\mathrm{p}<0.05)$. Time from onset of symptoms to admission was similar in the 3 groups and reperfusion therapy was used in a similar proportion of the patients. Five-day mortality was $5.3 \%$ in SU0, $2.5 \%$ in SU1, and $0.9 \%$ in SU2 patients $(\mathrm{p}<0.001)$. 6-month mortality was significantly higher in SU0 (18.3\%) and SU1 (13.4\%) compared with SU2 $(8.7 \%)(\mathrm{p}<0.001)$. Mortality was the lowest in patients on SU2, both in those on metformin or insulin and in those not receiving these medications. Using Cox multivariate analysis, the odds ratio for 6-month mortality versus patients without SU was 0.82 ; (95\% CI: $0.47-1.43, \mathrm{p}=\mathrm{NS})$ in patients on SU1, and 0.42 (95\% CI: $0.27-0.65, \mathrm{p}<0.001)$ in patients on SU2. Conclusion: Patients on new sulfonylureas have an improved early and 6month survival, compared with patients with non-selective sulfonylureas or no SU.

Supported by Pfizer, Servier, CNAM

\section{5}

Functional and molecular effects of gliclazide and glibenclamide on isolated human islets cultured at high glucose concentration

S. Del Guerra ${ }^{1}$, M. Grupillo ${ }^{1}$, R. Lupi ${ }^{1}$, M. Bugliani ${ }^{1}$, S. Torri ${ }^{1}$, R.

Mancarella ${ }^{1}$, M. Galli ${ }^{1}$, V. D’Aleo ${ }^{1}$, M. Del Chiaro ${ }^{2}$, U. Boggi ${ }^{3}$, F. Filipponi ${ }^{3}$,

F. Mosca ${ }^{3}$, S. Del Prato ${ }^{1}$, P. Marchetti ${ }^{1}$;

${ }^{1}$ Endocrinology and Metabolism, ${ }^{2}$ Oncology, Transplantation and

Advanced Technologies in Med, ${ }^{3}$ Oncology, Transplantation and Advanced

Technologies, University of Pisa, Italy.

Background and Aims: Decreased beta-cell functional mass is crucial for the development and progression of type 2 diabetes. Chronic exposure to high glucose levels (glucotoxicity) is a probable underlying mechanism, whereas the role of oral anti-diabetic agents is still unsettled. We have recently found that gliclazide protected human beta-cells from apoptosis induced by intermittent high glucose, an effect that was due, at least in part, to the anti-oxidant properties of the molecule. The aim of the present study was to further investigate on this issue at functional and molecular levels.

Materials and Methods: For this study 9 pancreata from non-diabetic multiorgan donors (age $58.5 \pm 14.5$, gender $5 \mathrm{M} / 4 \mathrm{~F}$, body mass index $24.8 \pm 2.9$ $\mathrm{kg} / \mathrm{m}^{2}$ ) were used. Islet isolation was performed by collagenase digestion and gradient purification. The islets were then cultured for 5 days in continuous normal glucose concentration (NG, $5.5 \mathrm{mmol} / \mathrm{L}$ ) or normal and high (HG, $16.7 \mathrm{mmol} / \mathrm{L}$ ) glucose levels (alternating every $24 \mathrm{~h}$ ), with or without the addition of therapeutical concentration of gliclazide $(10 \mu \mathrm{mol} / \mathrm{l})$ or glibenclamide $(1.0 \mu \mathrm{mol} / \mathrm{l})$. At the end of incubation, islet function as well as gene and protein expression of molecules involved in islet function, survival and regeneration were assessed by glucose-stimulated insulin secretion, quantitative Real-Time RT-PCR and Western Blot experiments.

Results: Insulin secretion, expressed as stimulation index, SI, differed significantly between the groups (NG: $3.4 \pm 1.5$, HG: $2.1 \pm 0.8$, HG+gliclazide: $2.3 \pm 1.0, \mathrm{HG}+$ glibenclamide: $1.4 \pm 0.2, \mathrm{p}<0.01$ by ANOVA), and the value with glibenclamide was lower compared to NG ( $<<0.05$ by the Bonferroni correction). Compared to NG, insulin expression decreased significantly at HG $(-70.5 \pm 3.1 \%)$, and increased similarly with gliclazide or glibenclamide $(+144 \pm 50$ and $+118 \pm 52 \%$, respectively). In addition, gliclazide, but not glibenclamide exposure significantly induced the expression of PDX-1, a fundamental beta-cell differentiation transcription factor $(+44.0 \pm 14.5 \%)$ and Ki67, a marker of proliferation $(+86.9 \pm 36.2 \%)$. These results were confirmed by Western Blot experiments $(+65.4 \pm 20.1$ and $+19.0 \pm 8.3 \%$ for PDX- 1 and Ki67 protein expression, respectively). Gliclazide and glibenclamide did not differ as for the effects on gene expression of the antiapoptotic molecule $\mathrm{Bcl} 2$ (increased significantly with both) and the proapoptotic molecule Bax (decreased significantly with both).

Conclusion: Gliclazide could counteract part of the deleterious effects of glucotoxicity on human pancreatic islets at both the functional and molecular level.

Supported by sanofi-aventis

\section{6}

Impact of gliclazide on DNA damage and repair in type 2 diabetic patients - a preliminary report

J. Drzewoski ${ }^{1}$, A. Sliwinska ${ }^{1}$, J. Blasiak ${ }^{2}$, J. Kasznicki ${ }^{1}$;

${ }^{1}$ Department of Internal Diseases, Diabetology and Clinical Pharmacology, Medical University of Lodz, Zgierz,

${ }^{2}$ Department of Molecular Genetics, University of Lodz, Poland.

Background and Aims: Type 2 diabetes mellitus (T2DM) is associated with increased oxidative stress and a reduced antioxidant defense system. Reactive oxygen species can cause damage to biological molecules, including DNA. It is also documented that in diabetic patients, the efficacy of DNA repair mechanisms is reduced. DNA damage and repair play a primary role in neoplastic transformation. Mutations in DNA repair genes may be associated with cancer, and the efficacy of DNA repair may determine the susceptibility to carcinogens. Gliclazide is an oral hypoglycemic drug with antioxidant properties that may diminish oxidative stress and its consequences in patients with T2DM. The aim of our study was to assess the impact of gliclazide on: (i) endogenous DNA damage (ii) DNA damage induced by hydrogen peroxide and (iii) the efficacy of the removal of DNA damage induced by hydrogen peroxide in patients with T2DM and in healthy individuals.

Materials and Methods: The DNA damage and the kinetics of DNA repair in peripheral blood lymphocytes of 15 (6 male) T2DM patients (age 59.5 \pm 11.1 years; body mass index, $31.5 \pm 6.8$; glucose level, $227 \pm 72 \mathrm{mg} / \mathrm{dL}$; duration of diabetes, $9.6 \pm 5$ years) and 15 ( 8 male) healthy individuals (age 64 \pm 14.9 years; body mass index, $25.5 \pm 2.9$; glucose level, $92 \pm 18 \mathrm{mg} / \mathrm{dL}$ ) were evaluated by alkaline single cell electrophoresis (comet) assay. Oxidative DNA damage was assayed with the use of the DNA repair enzyme endonuclease III (Endo III) and formamidopyrimidine-DNA glycosylase (Fpg), recognizing oxidized DNA bases.

Results: Basal endogenous and oxidative DNA damage was higher in T2DM patients, although not significantly. Gliclazide significantly decreased the level of DNA damage induced by hydrogen peroxide in both groups (Table 1). We did not observe any impact of gliclazide on the repair of hydrogen peroxideinduced DNA damage during the 120 minutes of the repair incubation (data not shown). Table1: Impact of gliclazide on endogenous basal DNA damage and oxidative DNA damage induced by hydrogen peroxide $\left(\mathrm{H}_{2} \mathrm{O}_{2}\right)$ in human peripheral blood lymphocytes of healthy individuals and type 2 diabetic patients. Cells were treated or not with $10 \mu \mathrm{M} \mathrm{H}_{2} \mathrm{O}_{2}$ for 10 minutes at $4^{\circ}$ $\mathrm{C}$ in the presence or absence of $20 \mu \mathrm{M}$ gliclazide, and received subsequent treatment with endonuclease III (Endo III) or formamidopyrimidine-DNA glycosylase (Fpg) at $1 \mu \mathrm{g} / \mathrm{mL}$. The damage was measured as the percentage of tail DNA in the alkaline comet assay. 


\begin{tabular}{|c|c|c|c|c|c|c|}
\hline \multirow[t]{2}{*}{ Abstract 0876} & \multirow{2}{*}{$\begin{array}{l}\text { Healthy individuals } \\
(\mathrm{n}=15) \\
\text { No enzyme }\end{array}$} & \multicolumn{5}{|l|}{$\begin{array}{l}\text { Type } 2 \text { diabetes mellitus } \\
\text { patients }(n=15)\end{array}$} \\
\hline & & Endo III & Fpg & No enzyme & Endo III & Fpg \\
\hline Endogenous basal DNA damage & $0.635 \pm 0.152$ & $0.580 \pm 0.375$ & $0.907 \pm 0.398$ & $\begin{array}{l}2.670 \pm 0.387 \\
* * *\end{array}$ & $3.254 \pm 0.785$ & $3.871 \pm 0.876$ \\
\hline $\begin{array}{l}\text { Impact of } 20 \mu \mathrm{M} \text { gliclazide on } \\
\text { endogenous basal DNA damage }\end{array}$ & $0.692 \pm 0.165$ & $0.804 \pm 0.321$ & $0.760 \pm 0.275$ & $1.945 \pm 0.326$ & $\begin{array}{l}2.749 \pm 0.673 \\
*\end{array}$ & $\begin{array}{l}2.397 \pm 0.594 \\
*\end{array}$ \\
\hline $\begin{array}{l}\text { Oxidative DNA damage induced } \\
\text { by } 10 \mu \mathrm{M} \mathrm{H}_{2} \mathrm{O}_{2}\end{array}$ & $18.889 \pm 2.068$ & $23.072 \pm 2.727$ & $21.689 \pm 2.836$ & $20.955 \pm 1.612$ & $29.952 \pm 2.448$ & $\begin{array}{l}30.291 \pm 2.405 \\
*\end{array}$ \\
\hline $\begin{array}{l}\text { Impact of } 20 \mu \mathrm{M} \text { gliclazide on oxidative } \\
\text { DNA damage induced by } 10 \mu \mathrm{M} \mathrm{H}_{2} \mathrm{O}_{2}\end{array}$ & $\begin{array}{l}2.993 \pm 0.548 \\
\#\end{array}$ & $\begin{array}{l}2.922 \pm 0.740 \\
\#\end{array}$ & $\begin{array}{l}3.521 \pm 0.858 \\
\#\end{array}$ & $\begin{array}{l}4.751 \pm 0.662 \\
\#\end{array}$ & $\begin{array}{l}5.884 \pm 1.172 \\
* \\
\#\end{array}$ & $\begin{array}{l}5.040 \pm 1.155 \\
\#\end{array}$ \\
\hline
\end{tabular}

${ }^{\star} P<0.05,{ }^{\star \star} P<0.01,{ }^{\star *} P<0.001$ as compared with a sample of healthy individuals; $\# P<0.001$ as compared with a gliclazide-untreated sample

Conclusion: We confirm that the level of basal endogenous DNA damage is higher in T2DM patients. Our results indicate that gliclazide may reduce the toxic effect of free radicals on DNA, and thereby decrease the risk of genomic instability in T2DM patients.

Supported by a grant from the Medical University of Lodz and in part by an unrestricted grant from Servier, Poland

\section{7} ADOPT: The effectiveness of metformin versus glyburide in type 2
diabetes

W. H. Herman ${ }^{1}$, S. M. Haffner', S. E. Kahn', B. Zinman', R. R. Holman ${ }^{5}$, G. F. Viberti' , J. M. Lachin 7 , B. G. Kravitz ${ }^{8}$, M. A. Heise ${ }^{8}$;

${ }^{1}$ Internal Medicine - Metabolism, Endocrinology and Diabetes (MEND), University of Michigan, Ann Arbor, United States, ${ }^{2}$ Medicine/Clinical Epidemiology, University of Texas Health Science Center at San Antonio, United States, ${ }^{3}$ Department of Medicine, VA Puget Sound Health Care System, Seattle, United States, ${ }^{4}$ Medicine, Mount Sinai Hospital, Toronto, Canada, ${ }^{5}$ Diabetes Trials Unit, OCDEM, Churchill Hospital, Oxford, United Kingdom, ${ }^{6}$ Department of Diabetes \& Endocrinology, King's College London School of Medicine, United Kingdom, ${ }^{7}$ The Biostatistics Center, George Washington University, Rockville, United States, ${ }^{8}$ GlaxoSmithKline, King of Prussia, United States.

Background and Aims: UKPDS reported similar $\mathrm{HbA}_{1 \mathrm{c}}$ responses in overweight ( $\geq 120 \%$ ideal body weight, mean BMI $31.4 \mathrm{~kg} / \mathrm{m}^{2}$ ) type 2 diabetic patients treated with metformin (MET) or sulphonylurea. ADOPT (A Diabetes Outcome Progression Trial) compared initial oral monotherapies in recently diagnosed type 2 diabetic patients and found rosiglitazone (RSG) to be superior to MET and glyburide (GLY), as assessed by time to monotherapy failure (time to the first of two successive fasting plasma glucose [FPG] values $>180 \mathrm{mg} / \mathrm{dl}$ ). Here, we present a prespecified secondary analysis from ADOPT comparing the efficacy of MET and GLY.

Materials and Methods: Eligible patients (diagnosed with type 2 diabetes within 3 years) were $30-75$ years of age and had FPG levels $126-180 \mathrm{mg} / \mathrm{dl}$ with lifestyle management alone. Mean BMI was $32.2 \mathrm{~kg} / \mathrm{m}^{2}$. Treatment was randomised and study medication was titrated to a total daily dose of $2 \mathrm{~g}$ $\operatorname{MET}(\mathrm{n}=1454)$ or $15 \mathrm{mg}$ GLY ( $\mathrm{n}=1441)$.

Results: Compared with GLY, the risk of monotherapy failure was reduced by $46.5 \%$ with MET treatment $(\mathrm{p}<0.01)$. At 4 years, $36 \%$ of patients in the MET group had $\mathrm{HbA}_{1 \mathrm{c}}<7 \%$ and remained on monotherapy compared with $26 \%$ in the GLY group $(\mathrm{p}<0.01)$. A longitudinal linear model showed that MET maintained a group mean $\mathrm{HbA}_{1 \mathrm{c}}$ of $<7 \%$ until month 45 , while GLY did so until month 33. Higher incidences of gastrointestinal adverse events were reported with MET treatment ( $38 \%$ vs. $22 \%, \mathrm{p}<0.01)$; however, the occurrence of hypoglycemia was lower $(12 \%$ vs. $39 \%, p<0.01)$. In contrast to patients treated with MET, GLY-treated patients gained weight at 4 years $(-2.53 \mathrm{~kg}$ vs. $1.81 \mathrm{~kg}$, respectively, $\mathrm{p}<0.01)$.

Conclusion: ADOPT demonstrated that in patients with recently diagnosed type 2 diabetes, MET is more effective than GLY, providing more durable glycemic control with less hypoglycemia and without weight gain. Supported by GlaxoSmithKline

\section{PS 82 DPP-4 inhibitors: session I}

\section{8}

\author{
Alogliptin (SYR-322) is a novel, potent, highly selective dipeptidyl \\ peptidase-IV inhibitor that improves glucose tolerance and $\beta$ cell \\ function in $o b / o b$ mice \\ K. Takeuchi ${ }^{1}$, Y. Moritoh ${ }^{1}$, T. Asakawa ${ }^{1}$, O. Kataoka ${ }^{1}$, L. Shi ${ }^{2}$, H. Odaka ${ }^{1}$; \\ ${ }^{1}$ Takeda Pharmaceutical Company, Ltd, Osaka, Japan, \\ ${ }^{2}$ Takeda San Diego, Inc, San Diego, United States.
}

Background and Aims: Alogliptin (ALO; SYR-322) is a novel, oral dipeptidyl peptidase-IV (DPP-4) inhibitor under development for the treatment for type 2 diabetes (T2D). DPP-4 inhibitors are a new class of antihyperglycemic agents that increase active glucagon-like peptide 1 (GLP-1) and glucosedependent insulinotropic peptide levels in plasma by inhibiting their rapid degradation by DPP-4. These incretin hormones induce glucose-dependent insulin secretion and play an important role in glucose homeostasis. Nonselective DPP-4 inhibitors that also target DPP-8 and DPP-9 have been associated with vascular/skin lesions in monkeys and other toxic effects in rats and dogs, suggesting that high selectivity for DPP-4 over other, related serine proteases is an important characteristic for this class of agents. The aim of this work was 1) to assess the potency and selectivity of ALO for DPP4 in vitro; and 2) to assess the pharmacodynamics and efficacy of chronic administration of ALO in $o b / o b$ mice, an obese model of T2D.

Materials and Methods: The selectivity of ALO for recombinant human DPP-4 over DPP-2, DPP-8, DPP-9, FAP/seprase, PREP, and tryptase was determined in vitro. In the first in vivo study, ALO was administered to ob/ob mice as a dietary admixture at 2.8 and $14.1 \mathrm{mg} / \mathrm{kg} /$ day for 4 weeks. In the second study, ALO was administered to $o b / o b$ mice as a dietary admixture at $42.2 \mathrm{mg} / \mathrm{kg} /$ day for 4 weeks; the mice were then fed a compound-free diet for 24 hours, fasted for a further 18 hours, and subjected to an oral meal tolerance test (OMTT).

Results: ALO potently inhibited human DPP-4 enzymatic activity with an IC $_{50}$ of $\sim 6.9 \mathrm{nM}$ and exhibited $>10,000$ selectivity for DPP-4 over other, related serine proteases in vitro $\left(\mathrm{IC}_{50}>100,000 \mathrm{nM}\right.$ vs. DPP-2, DPP-8, DPP9, FAP/seprase, PREP, and tryptase). In the first $o b / o b$ mouse study, ALO dose dependently inhibited plasma DPP-4 (24\% and 62\% for 2.8 and 14.1 $\mathrm{mg} / \mathrm{kg} / \mathrm{day}$, respectively) and significantly increased active GLP-1 (2.3- and 5.3-fold, respectively) after 4 weeks. In addition, decreased glycosylated hemoglobin ( $0.4 \%$ and $0.7 \%$, respectively), increased plasma insulin (1.5and 1.8-fold, respectively), increased pancreatic insulin content (1.5- and 1.9 -fold, respectively), decreased glucagon ( $26 \%$ and $23 \%$, respectively), and significantly decreased plasma triglycerides ( $51 \%$ and $42 \%$, respectively) were observed. In the second $o b / o b$ mouse study, ALO significantly inhibited plasma DPP-4 (80\%), significantly decreased glycosylated hemoglobin $(0.9 \%)$, and significantly increased plasma insulin (2-fold) and pancreatic insulin content (2.5-fold) after 4 weeks. Despite the lack of measurable DPP- 4 inhibition before the OMTT, significantly increased early phase insulin secretion (2.4-fold) and improved glucose tolerance ( $25 \%$ decrease in glucose AUC) were observed in the ALO-treated mice. Immunohistochemical analysis revealed increased insulin staining in the $\beta$-cells of ALO-treated mice compared with non-treated mice, suggestive of improved $\beta$-cell function with ALO. No significant changes in body weight or food consumption were observed during the 2 studies. Conclusion: Taken together, these results indicate that ALO is a potent and highly selective inhibitor of DPP-4 that has the potential to improve glycemic control and preserve $\beta$-cell function in patients with T2D. 


\section{9}

BI 1356, a novel and selective xanthine based DPP-IV inhibitor, exhibits a superior profile when compared to sitagliptin and vildagliptin L. Thomas, F. Himmelsbach, M. Eckhardt, E. Langkopf, M. Mark; ${ }^{1}$ Boehringer Ingelheim Pharma GmbH \& Co. KG, Biberach an der Riss, Germany.

Background and Aims: BI 1356 is a novel xanthine based DPP-IV inhibitor with a high potency and a low $\mathrm{k}_{\text {off }}$ rate in vitro that has been demonstrated to have a long duration of action also in vivo. Here, we compare the in vivo efficacy and duration of action of BI 1356 after single oral dosing with that of sitagliptin and vildagliptin.

Results: All three compounds dose-dependently inhibited plasma DPP-IV activity in HanWistar rats. ED50 values for inhibition of DPP-IV activity 7 hours post dose were $0.3 \mathrm{mg} / \mathrm{kg}$ for BI $1356,0.7 \mathrm{mg} / \mathrm{kg}$ for vildagliptin and $3.5 \mathrm{mg} / \mathrm{kg}$ for sitagliptin. However, while the inhibitory potency remained high even 24 hours post dose with BI 1356 (ED50 $0.7 \mathrm{mg} / \mathrm{kg}$ ), the ED50 increased to $>10 \mathrm{mg} / \mathrm{kg}$ for vildagliptin and sitagliptin. The effects of BI 1356 , vildagliptin and sitagliptin on glucose tolerance in $\mathrm{C} 57 \mathrm{Bl} / 6 \mathrm{~J}$ mice were compared after giving the compounds either $45 \mathrm{~min}$ or 16 hours before an oral glucose challenge. All three compounds reduced glucose AUC to a similar extent when given shortly before the glucose load (BI 1356 by 45\%, sitagliptin by $53 \%$, vildagliptin by $47 \%$; all at $1 \mathrm{mg} / \mathrm{kg}$ ). In contrast, only BI 1356 still improved glucose tolerance after administration of the same dose 16 hours before the test (AUC by 25\%). While sitagliptin improved glucose tolerance when a 10 -fold higher dose $(10 \mathrm{mg} / \mathrm{kg})$ was administered, vildagliptin even with that dose was without effect. Thus, the duration of action for enhancing glucose tolerance in vivo follows the order BI $1356>$ sitagliptin > vildagliptin.

Conclusion: We conclude that BI 1356 can be preclinically differentiated from vildagliptin and sitagliptin by a longer lasting inhibition of plasma DPPIV activity and a longer lasting improvement of glucose tolerance at the same doses. These data predict BI 1356 to have a truly once daily dose regimen in humans. This profile of BI 1356 has been confirmed in clinical trials in healthy volunteers and type 2 diabetic patients.

\section{0}

Improvements in $\beta$ cell function parameters during 52-week treatment with vildagliptin in patients with mild hyperglycaemia

J. E. Foley ${ }^{1}$, A. Schweizer ${ }^{2}$, W. A. Scherbaum ${ }^{3}$, P. M. Nilsson ${ }^{4}$, G. Lalanne ${ }^{5}$, B. E. Dunning ${ }^{6}$, A. Mari ${ }^{7}$;

${ }^{1}$ Novartis, E Hanover, United States, ${ }^{2}$ Novartis, Basel, Switzerland,

${ }^{3}$ Endo, Diabetes, Rheum, Univ Düsseldorf, Germany, ${ }^{4} \mathrm{Med}$, Lund Univ, Malmö, Sweden, ${ }^{5}$ Medical Group, Mont De Marsan, France, ${ }^{6}$ PharmaWrite, Princeton, United States, ${ }^{7}$ ISIB, National Research Council, Padova, Italy.

Background and Aims: Vildagliptin is a potent and selective DPP-4 inhibitor that improves glycemic control in patients with type 2 diabetes (T2DM) and moderate-to-marked hyperglycemia by increasing $\alpha$ - and $\beta$-cell responsiveness to glucose. This study was undertaken to assess the effects of vildagliptin on model-assessed $\beta$-cell function during 52 -week treatment of patients with less advanced T2DM.

Materials and Methods: This multicenter, randomised, parallel-group placebo-controlled study enrolled 306 mildly hyperglycemic (A1C 6.2-7.5\%) patients with T2DM. Standard meal tests were performed at Week 0 and Week 52 of treatment with vildagliptin $50 \mathrm{mg}$ qd $(\mathrm{n}=156)$ or placebo $(\mathrm{n}$ $=150$ ). Insulin secretory rate (ISR) was calculated by deconvolution of Cpeptide levels, and $\beta$-cell function was evaluated with a model that describes ISR as a function of absolute glucose levels (insulin secretory tone and glucose sensitivity), the rate of change of glucose (rate sensitivity), and a potentiation factor.

Results: At baseline, mean age $=63.1 \mathrm{y}, \mathrm{BMI}=30.2 \mathrm{~kg} / \mathrm{m}^{2}, \mathrm{FPG}=7.1 \mathrm{mM}$ and $\mathrm{A} 1 \mathrm{C}=6.7 \%)$. As shown below, vildagliptin significantly increased insulin secretory tone (+17\%), glucose sensitivity $(+40 \%)$ and rate sensitivity $(+32 \%)$. (Fig. 1)

The excursion of the potentiation factor and total insulin secretion (ISR AUC $240 \mathrm{~min}$ ) during meals was unchanged. The enhancements of $\beta$-cell function were accompanied by a decrease in the glucose $\mathrm{AUC}_{0-2 \mathrm{~h}}$ (between-group difference in the adjusted mean change $[\mathrm{AM} \Delta]=-1.7 \pm 0.5 \mathrm{mM} \bullet \mathrm{h}, \mathrm{P}=0.002$ ) and in $\mathrm{AlC}$ (between-group difference in $\mathrm{AM} \Delta=-0.3 \pm 0.1 \%, \mathrm{P}<0.001$ ).

Conclusions: In patients with mild hyperglycemia, improved $\beta$-cell function is maintained throughout 52-wk treatment and underlies sustained improvement in glycemic control.

Supported by Novartis

\section{1}

Vildagliptin is safe and well tolerated in patients with mild or moderate renal impairment

T. Thuren ${ }^{1}$, S. Byiers ${ }^{2}$, P. Mohideen ${ }^{2}$;

${ }^{1}$ Novartis, E Hanover, United States, ${ }^{2}$ Novartis, Basel, Switzerland.

Background and Aims: Vildagliptin is a potent and selective DPP-4 inhibitor that improves glycemic control in patients with type 2 diabetes (T2DM) by increasing both $\alpha$ - and $\beta$-cell responsiveness to glucose. Clinical data have shown that vildagliptin, at doses of 50 or $100 \mathrm{mg}$ daily, effectively reduces $\mathrm{HbA}_{1 \mathrm{c}}$, with a tolerability profile similar to placebo. Among patients with T2DM, the prevalence of kidney disease ranges from $20-40 \%$. The purpose of this abstract is 1) to report on the prevalence of mild or moderate renal impairment in the safety database for the vildagliptin global development program and 2) to examine the safety profile of vildagliptin monotherapy in patients with mild or moderate renal impairment in comparison to those with normal kidney function. Metabolism is the major elimination pathway for vildagliptin, with a minority of unchanged drug (23\%) excreted in the urine.

Material and Methods: Glomerular filtration rate (GFR) was calculated by the MDRD method: cutoffs of 60 to $<90 \mathrm{~mL} / \mathrm{min} / 1.73 \mathrm{~m}^{2}$ and 30 to $<60$ $\mathrm{ml} / \mathrm{min} / 1.73 \mathrm{~m}^{2}$ were used to define mild and moderate renal insufficiency, respectively. Phase 3 safety data from 8 pooled monotherapy and 7 pooled add-on therapy studies with vildagliptin were used.

Results: A total of 1864 patients receiving vildagliptin (either as monotherapy or in combination with other agents) had MDRD-estimated mild $(\mathrm{n}=1650)$

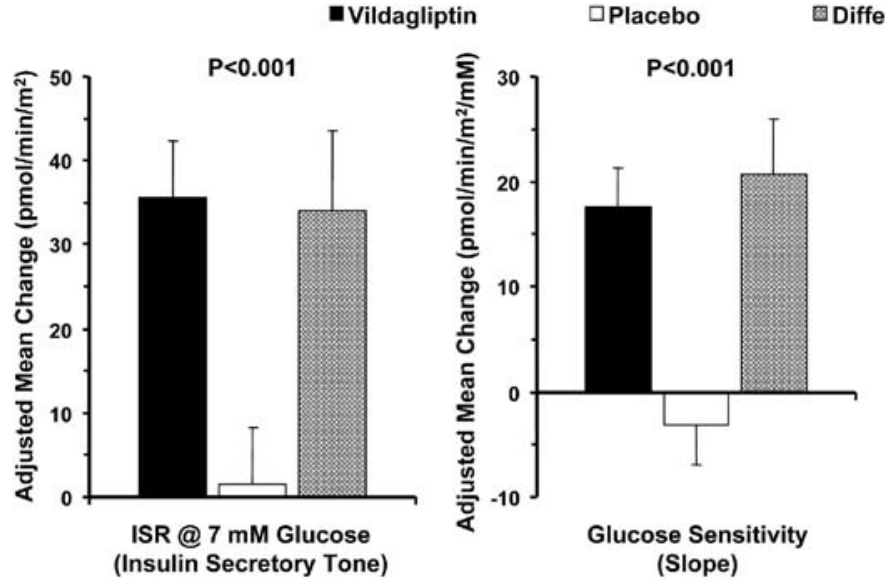

(Slope)
Difference

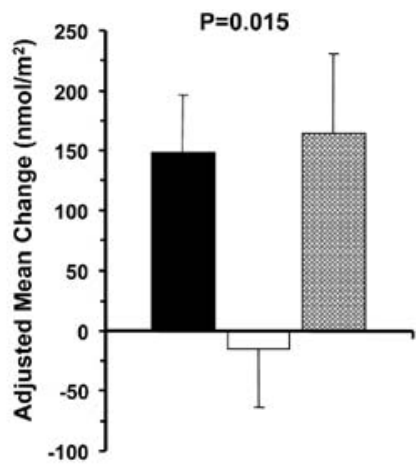

Rate Sensitivity (Derivative factor)
Fig. 1 Abstract 0880 
or moderate $(\mathrm{n}=214)$ renal impairment, vs. 1913 vildagliptin-treated patients with normal renal function. As shown below, in monotherapy studies the incidence of any adverse event (AE) was similar among patients receiving vildagliptin 50 or $100 \mathrm{mg}$ daily, rosiglitazone, pioglitazone or placebo, but lower than that in patients receiving metformin. In patients with moderate renal insufficiency, the incidence of any $\mathrm{AE}$ was similar among all active treatment groups, but higher than in the placebo group.

\begin{tabular}{|c|c|c|c|c|c|c|}
\hline & $\begin{array}{l}\text { Vilda } \\
50 \mathrm{mg} / \mathrm{d}\end{array}$ & $\begin{array}{l}\text { Vilda } \\
100 \mathrm{mg} / \mathrm{d}\end{array}$ & $\begin{array}{l}\text { Met up to } \\
2 \mathrm{~g} / \mathrm{d}\end{array}$ & $\begin{array}{l}\text { Rosi } \\
8 \mathrm{mg} / \mathrm{d} \\
\end{array}$ & $\begin{array}{l}\text { Pio } \\
30 \mathrm{mg} / \mathrm{d}\end{array}$ & $\mathrm{PBO}$ \\
\hline \multicolumn{7}{|c|}{ Normal renal function $\left(\mathrm{GFR} \geq 90 \mathrm{~mL} / \mathrm{min} / 1.73 \mathrm{~m}^{2}\right)$} \\
\hline $\mathrm{n}$ & 223 & 873 & 67 & 151 & 137 & 160 \\
\hline Any AE & 54.3 & 59.9 & 74.6 & 64.9 & 50.4 & 63.8 \\
\hline \multicolumn{7}{|c|}{ Mild renal insufficiency (GFR $60-<90 \mathrm{~mL} / \mathrm{min} / 1.73 \mathrm{~m}^{2}$ ) } \\
\hline $\mathrm{n}$ & 163 & 873 & 165 & 104 & 75 & 162 \\
\hline Any AE & 55.2 & 63.7 & 76.4 & 61.5 & 48.0 & 57.4 \\
\hline \multicolumn{7}{|c|}{ Moderate renal insufficiency (GFR $30-<60 \mathrm{~mL} / \mathrm{min} / 1.73 \mathrm{~m}^{2}$ ) } \\
\hline & 23 & 107 & 20 & 12 & 4 & 25 \\
\hline Any AE & 82.6 & 67.3 & 70.0 & 75.0 & 75.0 & 44.0 \\
\hline
\end{tabular}

In monotherapy studies, the overall incidence of serious AEs and discontinuations due to AEs showed generally similar levels between mild and moderate renal impairment groups. Subjects with normal renal function tended to have somewhat lower incidences of these events, as would be expected, since renal impairment is generally associated with more comorbid illness.

Conclusions: Nearly half of all patients exposed to vildagliptin (50mg and $100 \mathrm{mg}$ daily doses) during its global development program had mild or moderate renal impairment. Assessment of safety in these renally-impaired subjects reveals a safety profile versus (placebo or active) control, similar to that observed in patients with no renal impairment.

Supported by Novartis

\section{2}

Antidiabetic action of nateglinide includes augmentation of glucagon-like peptide-1 activity mediated partly through inhibition of DPP IV mediated degradation

A. M. McKillop, N. A. Duffy, B. D. Green, N. Irwin, V. A. Gault,

F. P. M. O'Harte, P. R. Flatt;

School of Biomedical Sciences, University of Ulster, Coleraine,

United Kingdom.

Background and Aims: Dipeptidyl peptidase (DPP IV) inactivates glucagonlike peptide-1(7-36)amide (GLP-1) by removing the dipeptide $\mathrm{His}^{7}-\mathrm{Ala}^{8}$ from the N-terminus. Due to the rapid enzymatic degradation of GLP-1, recent diabetic therapeutic approaches have focussed on the inhibition of DPP IV to ameliorate insulin release and metabolic control. This study examined the effect of a range of antidiabetic drugs on DPP IV activity and incretin hormone degradation in type 2 diabetes.

Materials and Methods: The effects of rosiglitazone, troglitazone, glibenclamide, tolbutamide, nateglinide, repaglinide and BTS 67582 on DPP IV activity were investigated in pooled normal human plasma over a range of drug concentrations. Reverse-phase HPLC and electrospray ionisation mass spectrometry (ESI-MS) were used to determine the effects of nateglinide on DPP IV-mediated degradation of GLP-1. Further in vitro experiments using clonal beta cells assessed the effects of nateglinide on GLP-1 stimulated insulin release and in vivo studies determined the metabolic effects of nateglinide and GLP-1 in obese diabetic $(o b / o b)$ mice.

Results: DPP IV inhibitory effects of the thiazolidinediones, troglitazone $(p<0.001)$ and rosiglitazone $(p<0.001)$ were concentration-dependent from 125 to $1000 \mu \mathrm{M}$. The strongest inhibitor nateglinide (67\% inhibition observed at $200 \mu \mathrm{M})$ was effective at low therapeutically relevant concentrations (25 $\mu \mathrm{M} ; \mathrm{p}<0.001)$. HPLC and ESI-MS analysis revealed that after $8 \mathrm{~h}$ incubation only $14 \%$ of GLP-1 $(7-36)$ amide remained intact $\left(\mathrm{t}^{1} / 21.4 \mathrm{~h}\right)$ in plasma whereas in the presence of $1 \mathrm{mM}$ nateglinide, no GLP-1 degradation was detected $\left(\mathrm{t}^{1} / 2\right.$ $>8 \mathrm{~h}$ ). Inhibition of DPP IV mediated degradation of GLP-1 by nateglinide was both concentration and time dependent. At a concentration of $62.5 \mu \mathrm{M}$, nateglinide inhibited GLP-1 degradation by $45 \%(\mathrm{p}<0.001)$. Concentration dependent stimulation of insulin secretion was evident with nateglinide (2.02.4-fold; $\mathrm{p}<0.001$ ) in BRIN-BD11 cells and further enhanced up to 3.6-fold with $10^{-7} \mathrm{M}$ GLP-1 $(\mathrm{p}<0.001)$. In obese diabetic $o b / o b$ mice, nateglinide (630 $\mu \mathrm{M} / \mathrm{kg}$, ip) improved the glucose-lowering $(\mathrm{p}<0.001)$ and insulin-releasing $(\mathrm{p}<0.001)$ activity of $25 \mathrm{nM} / \mathrm{kg} \mathrm{GLP}-1$ given together with $18 \mathrm{mM} / \mathrm{kg}$ glucose. The glucose lowering activity of GLP-1 $(53 \%$; $\mathrm{p}<0.001)$ was significantly enhanced when administered with nateglinide $(67 \%$; $<<0.001)$ in contrast to nateglinide alone $(11 \% ; \mathrm{p}<0.05)$. Combined administration of GLP-1 and nateglinide increased insulin levels after 15, 30 and $60 \mathrm{~min}$ and AUC values were significantly greater than either glucose alone $(529 \%$; $<<0.001)$ or glucose plus GLP-1 (103\%; $\mathrm{p}<0.001)$. This was accompanied by significantly enhanced circulating concentrations of active GLP-1(7-36)amide (61\% increase at $30 \mathrm{~min}$ ). Similarly nateglinide augmented the glucose and insulin responses to feeding and such actions were abolished by co-administration of $25 \mathrm{nM} / \mathrm{kg}$ exendin(9-39) and (Pro $\left.{ }^{3}\right)$ GIP to block incretin hormone action. Conclusion: The action of nateglinide as a prandial insulin-releasing agent may partly rely on inhibition of GLP-1 degradation as well as beta cell $\mathrm{K}_{\text {ATP }}$ channel inhibition.

\section{3}

The correlation among the serum dipeptidyl peptidase IV activity the serum HDL-C and $\mathrm{HbA}$ levels in patients with type 2 diabetes

T. Varga ${ }^{1}$, A. Somogyi ${ }^{1}$, G. Lengyel ${ }^{1}$, B. Sármán ${ }^{1}$, Z. Tulassay ${ }^{1}$, L. Selmeci ${ }^{2}$, G. Firneisz';

${ }^{1}$ 2nd Department of Medicine,

${ }^{2}$ Laboratory of Heart and Vessel Surgery Clinic, Semmelweis University, Budapest, Hungary.

Background and Aims: Serum dipeptidyl peptidase IV (DPP IV, CD26) is a cell surface ectoenzyme widely distributed in the human body, that is also detectable in the human serum. The significant efforts from the DPP IV inhibitors enhance the incretin system put the molecule on the map in the treatment of patients (patients) with type 2 diabetes mellitus (T2DM). Although previous studies found correlations between the serum DPP IV activity and the $\mathrm{HbAlC}$ furthermore with the fasting glucose levels, no data were available on the potential correlation with other clinically important factors such as the serum lipid profile. We aimed to study the serum dipeptidyl peptidase IV activities in patiens with T2DM and compare them with healthy controls. Correlations among the serum DPP IV activity and laboratory data were also evaluated.

Materials and Methods: Serum DPP IV activity was measured by microplatebased (Multiskan-Plus-MKII, Labsystem) kinetic assay in 49 patients with T2DM, and 18 healthy controls. Gly-Pro-pNA (Bachem, Bubendorf, Switzerland) was used as substrate. Results are expressed in $\mathrm{nmol} / \mathrm{ml} / \mathrm{min}$ (U/ L) pNA hydrolysed. T-test and Pearson correlation were used for statistical analysis. Glucose, HbAlc, CRP, HOMA-R, TG, LDL-C, HDL-C, Creatinin, Cystatin-C, ALAT, ASAT, gGT, ALP, BI, UA were determined according to the standard methods. Patients with T2DM received a standard test meal $(50 \mathrm{~g} \mathrm{CH})$ to observe postprandial DPP IV activities.

Results: The fasting serum DPP IV activity was not different in patients with T2DM (mean $=25,78 \mathrm{U} / \mathrm{L}[\mathrm{SD} 6,44]$ ) compared to healthy controls $(26,05[3,93])$. Changes in serum DPP IV activity were neither detectable $(0$, $\left.60,180^{\prime}\right)$ in patients with T2DM nor in controls after test meal. No correlation was found between DPP IV activity and glucose $\left(0,60,120,180^{\prime}\right)$, HOMA$\mathrm{R}$ index, CRP, LDL-C, TG levels. The serum DPP IV activities correlated positively with $\mathrm{HbA1C}$ values at borderline significance (Pearson Corr.: $0,253 \mathrm{p}<(2$-tailed signif.): 0,0797) in patients with T2DM. Furthermore a significant negative correlation was found between the DPP IV activity and the serum HDL-C levels in type 2 diabetic patients (Pearson Correlation:0,366, $\mathrm{p}<$ (2-tailed signif.): 0,0105).

Conclusion: The serum DPP IV activity remains relatively unwavering. The determination of the serum DPP IV activity probably does not provide clinical benefit in decision making about the initiation of the DPP IV inhibitor therapy in patients with 2TDM. The effect of the DPP IV enzyme is likely to be exerted via a paracrin route, however in the clinical parctice this can not be assessed on a routine basis. In this set of type 2 diabetic patients the serum DPP IV activities showed the strongest (negative) correlation with the serum HDL-C levels. Further studies are needed to clarify the exact molecular pathomechanism of the correlation between the serum DPP IV activities and the serum HDL-C levels, that we report here for the first time. Supported by the Székelyhidi Miklós awald of the Hungarian liver research society, and 448/2006ETT 


\section{PS 83 DPP-4 inhibitors: session II}

\section{4}

Initial combination therapy with sitagliptin, a selective DPP-4 inhibitor, and metformin leads to marked improvement in $\beta$ cell function in patients with type 2 diabetes

L. Xu, D. E. Williams-Herman;

Clinical \& Quantitative Sciences, Merck Research Laboratories, Rahway, United States.

Background and Aims: GLP-1 improves $\beta$-cell function in patients with type 2 diabetes. Sitagliptin (SITA) inhibits the degradation of intact (active) incretins, resulting in 2- to 3-fold increases in active GLP-1 levels, and has also been demonstrated to improve $\beta$-cell function. Metformin (MF) augments active and total GLP-1 levels. With potentially additive effects on incretins, co-administration of SITA and MF on $\beta$-cell function was examined in patients with type 2 diabetes.

Material and Methods: Data were from a 24-week, placebo (PBO)controlled randomised study in which 1091 patients were randomised to 1 of 6 treatments: SITA $100 \mathrm{mg} / \mathrm{MF} 1000 \mathrm{mg}$, SITA $100 \mathrm{mg} / \mathrm{MF}$ 2000mg, MF $1000 \mathrm{mg}$, MF $2000 \mathrm{mg}$ (all as divided doses administered b.i.d.); SITA $100 \mathrm{mg}$ q.d.; or PBO. A subset $(\mathrm{n}=500)$ of patients underwent frequently sampled (10-points collected over $3.5 \mathrm{hrs)} \mathrm{meal} \mathrm{tolerance} \mathrm{tests.} \beta$-cell function was assessed using the C-peptide minimal model. Parameters of $\beta$-cell function from this model allow for the estimation of the insulin secretion rate (ISR) and the characterisation of the ISR into static $\left(\Phi_{\mathrm{s}} ; \beta\right.$-cell responsiveness to abovebasal glucose following a meal), and dynamic $\left(\Phi_{d} ; \beta\right.$-cell responsiveness to the rate of increase in above-basal glucose following a meal) components. Results: After 24 weeks, the changes in static and dynamic $\beta$-cell responsiveness $\left(\Phi_{\mathrm{s}}\right.$ and $\left.\Phi_{\mathrm{d}}\right)$ and disposition indices (ISR in the context of changes in insulin sensitivity) were increased with all active treatments relative to $\mathrm{PBO}$ and appeared to be approximately additive with co-administered SITA and high dose MF relative to the monotherapies (Table).

Table. Change from Baseline at Week 24 in Indices of Beta-Cell Function

\begin{tabular}{|c|c|c|c|}
\hline$\Phi_{\mathrm{s},}\left(10^{-9} / \mathrm{min}\right)$ & No MF & MF $1000 \mathrm{mg}$ & MF $2000 \mathrm{mg}$ \\
\hline No SITA & -1.3 & 5.9 & 13.0 \\
\hline SITA $100 \mathrm{mg}$ & 10.6 & 18.4 & 18.9 \\
\hline$\Phi_{\mathrm{d}},\left(10^{-9}\right)$ & No MF & MF $1000 \mathrm{mg}$ & MF $2000 \mathrm{mg}$ \\
\hline No SITA & 5.2 & 31.4 & 54.1 \\
\hline SITA 100 & 24.2 & 21.8 & 132.1 \\
\hline $\mathrm{DI}_{\mathrm{s}}$ & No MF & MF $1000 \mathrm{mg}$ & MF $2000 \mathrm{mg}$ \\
\hline No SITA & -6.9 & 19.0 & 48.5 \\
\hline SITA $100 \mathrm{mg}$ & 21.7 & 63.9 & 90.7 \\
\hline $\mathrm{DI}_{\mathrm{d}}$ & No MF & MF 1000 mg & MF $2000 \mathrm{mg}$ \\
\hline No SITA & -48.5 & 204.2 & 483.5 \\
\hline SITA 100 & 62.2 & 271.1 & 1087.9 \\
\hline
\end{tabular}

Data are median change from baseline at Week 24; DI = disposition index

Conclusions: There were marked and approximately additive improvements in $\beta$-cell function with co-administered sitagliptin and metformin, consistent with their effects on post-meal active GLP-1 levels.

Supported by Merck

\section{5}

Achievement of glycaemic targets with vildagliptin

S. Dejager ${ }^{1}$, A. Schweizer ${ }^{2}$, A. Couturier ${ }^{1}$, R. E. Pratley ${ }^{3}$;

${ }^{1}$ Novartis, E Hanover, United States, ${ }^{2}$ Novartis, Basel, Switzerland,

${ }^{3}$ Diabetes Metab Trans Med, Univ Vermont, Burlington, United States.

Background and Aims: The ADA recommends a target $\mathrm{A} 1 \mathrm{C}<7.0 \%$ to minimise risk of microvascular complications in patients with type 2 diabetes (T2DM), but a minority achieve this goal. Vildagliptin is a potent and selective DPP-4 inhibitor that improves glycemic control by increasing both $\alpha$ - and $\beta$ cell responsiveness to glucose. The aim of this work is to summarise findings from the global Phase 3 program regarding the efficacy of vildagliptin in bringing patients to an A1C goal of $<7 \%$.

Materials and Methods: Data are reported from two 24-week, placebocontrolled monotherapy trials in drug-naive patients (pooled), one study of vildagliptin (100mg daily) added to metformin (MET, 2000mg daily) and one study of initial combination therapy with pioglitazone (PIO, 30mg qd). The proportion of patients reaching goal is dependent on the baseline $\mathrm{AlC}$, therefore data are presented for all subjects and for those with a baseline A1C $<8 \%$.

Results: As reported below, target A1C was achieved by $40 \%$ of patients receiving vildagliptin $100 \mathrm{mg}$ daily vs. $20 \%$ of patients receiving placebo; in patients with baseline (BL) A1C $\leq 8.0 \%$, more than half of those receiving vildagliptin reached goal. These data were confirmed in a larger pooled analysis of all (5) Phase 3 monotherapy trials; in patients with BL A1C $\leq 8.0 \%$ (mean $\approx 7.6 \%$ ) $60 \%$ of patients receiving vildagliptin $100 \mathrm{mg}$ daily achieved A1C $<7.0 \%$. When vildagliptin $100 \mathrm{mg}$ qd was given as initial combination with PIO $30 \mathrm{mg} \mathrm{qd}, 65 \%$ of patients receiving the combination achieved endpoint $\mathrm{A} 1 \mathrm{C}<7.0 \%$ vs. $43 \%$ of patients receiving either PIO or vildagliptin alone. As add-on to MET, 4-fold more patients got to goal with vildagliptin $100 \mathrm{mg} / \mathrm{d}$ than with placebo; in patients with BL AlC $\leq 8.0 \%, 54 \%$ of patients receiving vildagliptin attained the goal vs. $13 \%$ of those receiving placebo added to MET.

\begin{tabular}{|c|c|c|}
\hline & Placebo & $\begin{array}{l}\text { Vildagliptin } \\
100 \mathrm{mg} / \mathrm{d}\end{array}$ \\
\hline $\begin{array}{l}\text { Monotherapy } \\
\text { All pts, BL A1C } \approx 8.4 \% \\
\text { BL } \leq 8.0 \% \text {, mean } \approx 7.6 \% \%\end{array}$ & $\begin{array}{l}19.8 \%(36 / 182) \\
25.7 \%(19 / 74)\end{array}$ & $\begin{array}{l}39.7 \%^{\mathrm{a}}(71 / 179) \\
54.2 \%^{\mathrm{a}}(39 / 72)\end{array}$ \\
\hline $\begin{array}{l}\text { Add-on to MET } \\
\text { All pts, BL A1C } \approx 8.4 \% \\
\text { BL } \leq 8.0 \% \text {, mean } \approx 7.5 \%\end{array}$ & $\begin{array}{l}9.4 \%(12 / 128) \\
13.3 \%(8 / 60)\end{array}$ & $\begin{array}{l}35.5 \% \%^{\mathrm{a}}(50 / 141) \\
54.1 \%^{\mathrm{a}}(33 / 61)\end{array}$ \\
\hline $\begin{array}{l}\text { Initial combination } \\
\text { All pts, mean } \mathrm{BL} \approx 8.7 \% \\
\mathrm{BL} \leq 8.0 \% \text {, mean } \mathrm{BL} \approx 7.6 \%\end{array}$ & $\begin{array}{l}\text { PIO or Vildagliptin alone } \\
43 \%(62 / 146 \text { or } 67 / 156) \\
67 \% \text { or } 70 \%(32 / 48 \text { or } 30 / 43)\end{array}$ & $\begin{array}{l}\text { Combination } \\
65 \%^{\mathrm{a}}(93 / 143) \\
77 \%(34 / 44)\end{array}$ \\
\hline
\end{tabular}

${ }^{a} \mathrm{P}<0.001$ vs $\mathrm{PBO}$ or monotherapy

Conclusions: Twice as many patients achieved the ADA goal A1C of $<7.0 \%$ with vildagliptin monotherapy compared to placebo, whereas 4-times more patients reached goal when vildagliptin was added to MET. Two-thirds of patients achieved an endpoint $\mathrm{AlC}<7.0 \%$ when initially treated with the combination of vildagliptin/PIO (100mg/30mg qd).

Supported by Novartis

\section{6}

Sitagliptin, a DPP-4 inhibitor, administered once- or twice-daily, provides improvement throughout 24-hour glucose control in Japanese patients with type 2 diabetes mellitus

K. Nonaka ${ }^{1}$, H. Tsubouchi ${ }^{1}$, K. Okuyama ${ }^{1}$, Y. Fukao ${ }^{1}$, P. P. Stein ${ }^{2}$; ${ }^{1}$ Clinical Development Institute, Banyu Pharmceutical Co., Ltd., Tokyo, Japan, ${ }^{2}$ Merck \& Co., Inc., Whitehouse Station, N.J., Rahway, United States.

Background and Aims: Sitagliptin is an orally-active, potent and selective DPP-4 inhibitor for the treatment of patients with type 2 diabetes. This 4 week, randomised, double-blind, placebo-controlled, parallel group study evaluated the 24-hour glucose profile with sitagliptin $100 \mathrm{mg} /$ day dosed once-daily (100 mg q.d.) or twice-daily (50 mg b.i.d.) in Japanese patients with type 2 diabetes.

Materials and Methods: Following an 8-week diet/exercise including a 2 -week placebo run-in, and wash-off period required for those on oral antihyperglycemic agents, patients with an $\mathrm{A} 1 \mathrm{C}$ of $\geq 6.5 \%$ and $<10.0 \%$ were randomised to sitagliptin $100 \mathrm{mg}$ q.d., $50 \mathrm{mg}$ b.i.d. or placebo in a 1:1:1 ratio for 4 weeks. Frequent blood sampling over 24 hours was performed at baseline and Week 4 and a weighted average was calculated (the 24 hour weighted mean glucose [24-hr WMG]); change in the $24 \mathrm{hr}-\mathrm{WMG}$ was the primary endpoint. Efficacy analysis was based on patients who completed 24-hr sampling at baseline and Week 4.

Results: Seventy-six (of 80 randomised) patients, ages 31-69 years, were analysed for efficacy, including 25, 24 and 27 patients in the sitagliptin $100 \mathrm{mg}$ q.d. (mean baseline A1C 7.8\%), 50mg b.i.d. (7.5\%) and placebo (7.9\%) groups, respectively. At Week 4, LS mean change in 24-hr WMG from baseline $(95 \%$ 
CI) was significantly $(\mathrm{p}<0.001)$ greater with $100 \mathrm{mg}$ q.d. $(-34.9 \mathrm{mg} / \mathrm{dL}[-40.9$, $-28.9])$ and $50 \mathrm{mg}$ b.i.d. $(-28.6 \mathrm{mg} / \mathrm{dL}[-34.7,-22.4])$ compared to placebo $(-9.0 \mathrm{mg} / \mathrm{dL}[-14.8,-3.2])$. No significant difference was observed between sitagliptin groups (i.e., $100 \mathrm{mg}$ q.d. vs. $50 \mathrm{mg}$ b.i.d.) in the changes of $24-\mathrm{hr}$ WMG ( $\mathrm{p}=0.146)$. Two-hour postprandial plasma glucose (2-hr PPG) was measured after breakfast, lunch and dinner in this study. The LS mean change in 2-hr PPG from baseline after breakfast was significantly greater with $100 \mathrm{mg}$ q.d. $(-54.5 \mathrm{mg} / \mathrm{dL})$ and $50 \mathrm{mg}$ b.i.d. $(-42.6 \mathrm{mg} / \mathrm{dL})$ compared to placebo $(-8.2$ $\mathrm{mg} / \mathrm{dL}$ ). Similar results were observed in the LS mean change in 2-hr PPG from baseline after lunch and dinner: $-43.9 \mathrm{mg} / \mathrm{dL},-31.0 \mathrm{mg} / \mathrm{dL}$ and -14.8 $\mathrm{mg} / \mathrm{dL}$ after lunch, and $-33.2 \mathrm{mg} / \mathrm{dL},-34.1 \mathrm{mg} / \mathrm{dL}$ and $-12.7 \mathrm{mg} / \mathrm{dL}$ after dinner, in $100 \mathrm{mg}$ q.d., $50 \mathrm{mg}$ b.i.d. and placebo groups, respectively. Fasting plasma glucose was significantly reduced with sitagliptin $100 \mathrm{mg}$ q.d. and $50 \mathrm{mg}$ b.i.d. compared to placebo with LS mean change from baseline of -22.3 $\mathrm{mg} / \mathrm{dL},-16.0 \mathrm{mg} / \mathrm{dL}$, and $-3.1 \mathrm{mg} / \mathrm{dL}$, respectively ( $\mathrm{p}<0.001 \mathrm{vs}$. placebo). Glycosylated albumin was also decreased with sitagliptin $100 \mathrm{mg}$ q.d. and $50 \mathrm{mg}$ b.i.d. compared to placebo: $-2.7 \%,-2.3 \%,-0.4 \%$, respectively $(\mathrm{p}<0.001$ vs. placebo). For all parameters measured, there was no significant difference between sitagliptin dosing regimens. Moreover, both sitagliptin dosing regimens were well tolerated with no significant between-group differences in the incidence of adverse events and drug-related adverse events. There were neither serious drug-related $\mathrm{AE}$ nor events of hypoglycemia reported. Conclusion: In Japanese patients with type 2 diabetes who had inadequate glycemic control on diet and exercise, sitagliptin $100 \mathrm{mg} /$ day provides substantial glucose lowering efficacy over a 24-hour period following 4 weeks of treatment. No additional efficacy was observed with the twice-daily dose regimen compared to the once-daily dose regimen.

\section{7}

Efficacy and safety of vildagliptin in the elderly: pooled analysis of 5 monotherapy studies

R. E. Pratley ${ }^{1}$, J. Rosenstock ${ }^{2}$, F. X. Pi-Sunyer ${ }^{3}$, A. Couturier ${ }^{4}$, A. Schweizer ${ }^{5}$, S. Dejager

${ }^{1}$ Diabetes Metab Trans Med, Univ Vermont, Burlington, United States,

${ }^{2}$ Diabetes, Dallas Diabetes Endo Ctr, Dallas, United States,

${ }^{3}$ Medicine, Columbia Univ, New York, United States,

${ }^{4}$ Novartis, E Hanover, United States, ${ }^{5}$ Novartis, Basel, Switzerland.

Background and Aims: Vildagliptin is a potent and selective DPP-4 inhibitor that improves glycemic control in patients with type 2 diabetes (T2DM) by increasing $\alpha$ and $\beta$ cell responsiveness to glucose.

Materials and Methods: Phase 3 trials with vildagliptin enrolled a substantial number of persons age $\geq 65$ years, allowing us to examine whether there were any age-associated differences in efficacy or tolerability with this new agent. Data were pooled from the vildagliptin monotherapy arms (100mg daily; $50 \mathrm{mg}$ bid or $100 \mathrm{mg} \mathrm{qd}$ ) of 2 placebo-controlled trials and 3 active-controlled trials

Results: Summarised below are baseline (BL) values and adjusted mean $\Delta$ in A1C, FPG and body weight (BW), and the general safety profile of vildagliptin monotherapy.

\begin{tabular}{|c|c|c|c|c|c|c|}
\hline \multirow{3}{*}{ Efficacy Population } & \multicolumn{2}{|c|}{$\begin{array}{l}\text { Age } \geq 65 y \\
(\text { mean }=70 y)\end{array}$} & \multicolumn{2}{|c|}{$\begin{array}{l}\text { Age }<65 \text { y } \\
(\text { mean }=50 y)\end{array}$} & \multicolumn{2}{|c|}{$\begin{array}{l}\text { All } \\
(\text { mean }=53 y)\end{array}$} \\
\hline & \multicolumn{2}{|c|}{$\mathrm{N}=238$} & \multicolumn{2}{|c|}{$\mathrm{N}=1231$} & \multicolumn{2}{|c|}{$\mathrm{N}=1469$} \\
\hline & $\mathrm{BL}$ & $\Delta \pm \mathrm{SE}$ & $\mathrm{BL}$ & $\Delta \pm \mathrm{SE}$ & $\mathrm{BL}$ & $\Delta \pm \mathrm{SE}$ \\
\hline $\mathrm{AlC}(\%)$ & 8.3 & $-1.2 \pm 0.1^{\star}$ & $8.7^{\ddagger}$ & $-1.0 \pm 0.0^{*}$ & 8.6 & $-1.0 \pm 0.0^{*}$ \\
\hline FPG (mg/dL) & 173 & $-26.1 \pm 3.0^{*}$ & $189^{\ddagger}$ & $-19.3 \pm 1.4^{* \neq}$ & 186 & $\begin{array}{l}-20.7 \pm \\
1.3^{*}\end{array}$ \\
\hline BW (kg) & 83.4 & $-0.9 \pm 0.2^{*}$ & $92.0 \ddagger$ & $-0.2 \pm 0.1^{\ddagger}$ & 90.6 & $-0.3 \pm 0.1^{\star}$ \\
\hline Safety Population & \multicolumn{2}{|c|}{$\mathrm{N}=270$} & \multicolumn{2}{|c|}{$\mathrm{N}=1395$} & \multicolumn{2}{|c|}{$\mathrm{N}=1665$} \\
\hline Any AE: n (\%) & \multicolumn{2}{|c|}{$181(67.0)$} & \multicolumn{2}{|c|}{$816(58.5)$} & \multicolumn{2}{|c|}{997 (59.9) } \\
\hline SAE: n (\%) & \multicolumn{2}{|c|}{$13(4.8)$} & \multicolumn{2}{|c|}{$39(2.8)$} & \multicolumn{2}{|c|}{$52(3.1)$} \\
\hline DC due to AE: n (\%) & \multicolumn{2}{|c|}{$8(3.0)$} & \multicolumn{2}{|c|}{$34(2.4)$} & \multicolumn{2}{|c|}{$42(2.5)$} \\
\hline Hypoglycemia: n (\%) & \multicolumn{2}{|c|}{$2(0.7)$} & \multicolumn{2}{|c|}{$4(0.3)$} & \multicolumn{2}{|c|}{$6(0.4)$} \\
\hline Death: n (\%) & \multicolumn{2}{|c|}{$2(0.7)$} & \multicolumn{2}{|c|}{$1(0.1)$} & \multicolumn{2}{|c|}{$3(0.2)$} \\
\hline
\end{tabular}

${ }^{*} \mathrm{P}<0.05$ vs $\mathrm{BL}$ (within subgroup), ${ }^{\ddagger} \mathrm{P}<0.05$ vs Age $\geq 65$ y (between subgroups)

Relative to the younger subgroup, in older patients vildagliptin decreased $\mathrm{A} 1 \mathrm{C}$ and FPG to a somewhat greater degree, from significantly lower baseline values, and vildagliptin significantly decreased body weight only in the older subgroup. Vildagliptin was well tolerated in older patients, with a low risk of hypoglycemia (and no severe events). Specific AEs occurring in $\geq 5 \%$ of older or younger subgroups, respectively, were constipation $(5.6 \%, 2.2 \%)$, nasopharyngitis $(5.9 \%, 6.8 \%)$, dizziness $(6.3 \%, 5.2 \%)$ and headache $(3.7 \%$, $6.2 \%)$.

Conclusion: Vildagliptin represents a good therapeutic option for treating elderly patients with T2DM.

Supported by Novartis

\section{8}

Dose-response relationship for glycaemic efficacy, safety and tolerability of sitagliptin after 12 weeks of treatment in Japanese patients with type 2 diabetes mellitus

Y. Iwamoto ${ }^{1}$, K. Nonaka ${ }^{2}$, A. Okada ${ }^{2}$, K. Okuyama ${ }^{2}$, P. P. Stein ${ }^{3}$; ${ }^{1}$ Diabetes Center, Tokyo Women's Medical University, Tokyo, Japan, ${ }^{2}$ Banyu Pharmaceutical CO., LTD., Tokyo, Japan,

${ }^{3}$ Merck\&Co., Inc., Rahway, United States.

Background and Aims: Sitagliptin (SITA) is an orally active, potent, selective dipeptidyl peptidase-4 (DPP-4) inhibitor. This randomised, doubleblind, placebo-controlled, parallel-group study evaluated the dose response relationship for efficacy and tolerability of SITA in Japanese patients with type 2 diabetes mellitus (T2DM).

Materials and Methods: Japanese patients with T2DM either not on an antihyperglycemic agent or on an antihyperglycemic agent in monotherapy (discontinued at entry into the observation period), entered an 8 week diet and exercise observation period including 2 weeks placebo (PBO) run-in period. Patients with an $\mathrm{A} 1 \mathrm{C}$ of $6.5 \%$ to $10 \%$ after the observation period were eligible to be randomised to 1 of 5 treatment groups (PBO, SITA $25 \mathrm{mg}, 50 \mathrm{mg}$, $100 \mathrm{mg}$ or $200 \mathrm{mg}$ q.d.) in a 1:1:1:1:1 ratio for a 12 weeks treatment period. The primary endpoint was the change from baseline of A1C at week 12 and the efficacy analysis was based on a full analysis set with last observation carried forward.

Results: 363 patients, ages 28 to 75 years, were randomised. Mean baseline A1C ranged from $7.49 \%$ to $7.74 \%$. LS mean A1C change from baseline was $+0.28 \%,-0.41 \%,-0.71 \%,-0.69 \%$, and $-0.76 \%$ in PBO, SITA $25 \mathrm{mg}, 50 \mathrm{mg}, 100$ $\mathrm{mg}$, and $200 \mathrm{mg}$ groups, respectively. A significant dose-response relationship across PBO to SITA $200 \mathrm{mg}$ was demonstrated $(\mathrm{p}<0.001)$. Between group differences compared to PBO in LS mean change from baseline $(95 \% \mathrm{CI})$ were, $-0.69 \%(-0.85,-0.52),-0.99 \%(-1.16,-0.82),-0.96 \%(-1.14,-0.79)$, and $-1.04 \%(-1.21,-0.86)$, in SITA $25 \mathrm{mg}, 50 \mathrm{mg}, 100 \mathrm{mg}$, and $200 \mathrm{mg}$ groups, respectively. The mean baseline fasting plasma glucose (FPG), ranged from $142.6 \mathrm{mg} / \mathrm{dL}$ to $156.5 \mathrm{mg} / \mathrm{dL}$, the LS mean change from baseline was +6.3 $\mathrm{mg} / \mathrm{dL},-9.6 \mathrm{mg} / \mathrm{dL},-11.4 \mathrm{mg} / \mathrm{dL},-14.6 \mathrm{mg} / \mathrm{dL}$, and $-16.9 \mathrm{mg} / \mathrm{dL}$ in $\mathrm{PBO}$ to SITA $200 \mathrm{mg}$, respectively. In this study, a meal tolerance test (MTT) was conducted with all randomized patients to assess an effect on postprandial glucose lowering. The LS mean 2-hour postprandial glucose (2hr-PPG) change from baseline was $+1.7 \mathrm{mg} / \mathrm{dL},-38.6 \mathrm{mg} / \mathrm{dL},-50.5 \mathrm{mg} / \mathrm{dL},-56.6 \mathrm{mg} /$ $\mathrm{dL}$, and $-63.4 \mathrm{mg} / \mathrm{dL}$ in PBO to SITA $200 \mathrm{mg}$ groups, respectively. While the effect of SITA on A1C after 12 weeks plateaued at doses $>=50 \mathrm{mg}$, other key endpoints such as FPG and 2hr-PPG showed numerically better efficacy at doses $>=50 \mathrm{mg}$. During the MTT, glucagon levels were suppresses at all doses of SITA. DPP-4 inhibition rate was also assessed at week 12. On safety, SITA was generally well-tolerated. There were no significant differences between any SITA treatment groups compared to PBO with respect to the incidences of drug-related clinical and laboratory AEs. No drug-related serious AEs were reported. The incidence of hypoglycemia observed in the SITA treatment groups was low and comparable to that observed in $\mathrm{PBO}$ group. The effect of SITA on body weight was neutral.

Conclusion: In this study, in Japanese patients with T2DM who were inadequately controlled on a diet/exercise therapy alone, dose-response relationship was established across groups (PBO to SITA $200 \mathrm{mg}$ ) in glycemic control efficacy for both fasting and postprandial glucose. A1C reduction was similar for doses from 50 to $200 \mathrm{mg}$ and ranged from $0.96 \%$ to $1.04 \%$. SITA up to $200 \mathrm{mg}$ was generally well-tolerated without any serious drug-related AEs. The effect on body weight was neutral. 


\section{9}

Saxagliptin improves glycaemic control as monotherapy in type 2 diabetes

J. List ${ }^{1}$, J. Rosenstock ${ }^{2}$, S. Sankoh ${ }^{3}$, R. Chen ${ }^{1}$;

${ }^{1}$ Global Clinical Research, Bristol-Myers Squibb, Princeton,

${ }^{2}$ Diabetes \& Endo, Dallas Diabetes \& Endocrine Ctr., Dallas,

${ }^{3}$ Global Biometric Sciences, Bristol-Myers Squibb, Princeton, United States.

Background and Aims: The incretin hormones have a number of beneficial effects on blood glucose regulation, including increasing glucose-stimulated insulin secretion and suppressing glucagon secretion. A new approach to the treatment of type 2 diabetes is to enhance the physiologic actions of incretins by inhibiting the enzyme responsible for their inactivation, dipeptidyl peptidase-4 (DPP-4). Saxagliptin is a potent, oral DPP-4 inhibitor. This phase 2 study evaluated the efficacy and safety of a range of once-daily doses of saxagliptin for the treatment of type 2 diabetes in drug-naive subjects.

Materials and Methods: Drug-naive subjects with inadequately controlled type 2 diabetes $\left(\mathrm{HbA}_{1 \mathrm{~b}} \geq 6.8 \%\right.$ and $\leq 9.7 \%$ ) were studied in a phase 2 , multicenter, randomised, parallel-group, double-blind, placebo-controlled trial. The trial included 2 distinct cohorts: a low-dose cohort (LDC) $(2.5,5$, 10, 20, and 40mg once daily vs. placebo), with 338 subjects treated for 12 weeks, and a high-dose cohort (HDC) (100mg once daily vs. placebo), with 85 subjects treated for 6 weeks.

Results: In the LDC (mean baseline $\mathrm{HbA}$ 7.9\%), treatment with saxagliptin led to significant reductions in $\mathrm{HbA}_{1 \mathrm{c}}$ at all doses compared with placebo $(\mathrm{P}<$ $0.007)$. The adjusted change from baseline in $\mathrm{HbA}_{1 \mathrm{c}}$ at Week 12 ranged from $0.72 \%$ to $-0.90 \%$ for doses of 2.5 to $40 \mathrm{mg}$ daily vs. $-0.27 \%$ for placebo. Fortyone to $53 \%$ of saxagliptin-treated subjects achieved $\mathrm{HbA}_{1 \mathrm{c}}$ of $<7.0 \%$ vs. $20 \%$ of placebo-treated subjects. Fasting serum glucose (FSG) decreased by 11 to 22 $\mathrm{mg} / \mathrm{dL}$ in the saxagliptin treatment arms compared with an increase of $3 \mathrm{mg} /$ $\mathrm{dL}$ for placebo. Postprandial glucose 60 minutes after a liquid meal, a major determinant of overall glycemic control, decreased by 24 to $41 \mathrm{mg} / \mathrm{dL}$ in the saxagliptin treatment arms compared with a decrease of $1 \mathrm{mg} / \mathrm{dL}$ for placebo. Similar overall results were seen in the high-dose cohort (mean baseline $\mathrm{HbA}_{1 \mathrm{c}} 7.7 \%$ ), with an adjusted change from baseline in $\mathrm{HbA}_{1 \mathrm{c}}$ at Week 6 of $-1.09 \%$ for saxagliptin $100 \mathrm{mg}$ daily vs. $-0.36 \%$ for placebo. In both cohorts, beta cell function, as measured by HOMA- $\beta$, increased in all saxagliptin treatment arms. Saxagliptin had a neutral effect on weight. The incidence of adverse events was similar across all treatment groups, including placebo. The most common adverse events in the LDC were headache, upper respiratory tract infection, and urinary tract infection; the most common adverse events in the HDC were headache, urinary tract infection, and constipation. No cases of confirmed hypoglycemia were observed in the LDC.

Conclusions: Saxagliptin, across a range of doses, improved glycemic control in drug-naive subjects with type 2 diabetes. Treatment with saxagliptin led to improvements in $\mathrm{HbA}_{1 \mathrm{c}}$, fasting serum glucose, postprandial glucose, and beta cell function. Saxagliptin was generally safe and well-tolerated. Supported by Bristol-Myers Squibb/AstraZeneca

\section{0}

BI 1356, a novel xanthine-based DPP-IV inhibitor, exhibits high potency with a wide therapeutic window and significantly reduces postprandial glucose excursions after an oGTT

K. A. Dugi ${ }^{1}$, T. Heise ${ }^{2}$, A. Ring ${ }^{1}$, U. Graefe-Mody ${ }^{1}$, A. Ritzhaupt ${ }^{1}$, S. Huettner ${ }^{1}$;

${ }^{1}$ Boehringer Ingelheim Pharma GmbH \& Co KG, Biberach, Germany, ${ }^{2}$ Profil Institut, Neuss, Germany.

Background and Aims: BI 1356 is a novel, orally available, selective inhibitor of DPP-IV in development for the treatment of type 2 diabetes. Safety, tolerability, pharmacokinetics, and pharmacodynamics of BI 1356 were investigated in a single-rising dose trial (SRD, 2.5 to $600 \mathrm{mg}$ ) in healthy men ( 47 on BI 1356, 16 on placebo, mean age 38.3 years, BMI $24.8 \mathrm{~kg} / \mathrm{m}^{2}$ ), and in a multiple rising dose trial (MRD, 1 to $10 \mathrm{mg}$ ) in men with type 2 diabetes $(35$ on BI 1356, 12 on placebo, age 56.0 years, BMI $28.6 \mathrm{~kg} / \mathrm{m}^{2}$, baseline $\mathrm{HbA1c}$ $6.7 \%)$ treated once daily for 12 days.

Materials and Methods: Both trials were double-blind, randomised, and placebo controlled. In the MRD trial, patients entered a 14-day washout period of previous antidiabetic medication before randomisation. DPPIV activity and BI 1356 plasma concentrations were measured at regular intervals. An oGTT was performed prior to first drug administration and 24 hours after the last dose to assess postprandial glucose excursions $\left(\mathrm{AUC}_{0-2 \mathrm{~h}}\right)$ and GLP-1 levels.

Results: In both studies, $\mathrm{C}_{\max }$ was reached 1-3h post-dose. The exposure of BI 1356 increased less than proportionally in the relevant therapeutic dose range from 1 to $10 \mathrm{mg}$. Time to steady state decreased from 6 to 2 days with increasing doses. Renal excretion was low and does not constitute the main pathway of BI 1356 elimination. In the SRD study, DPP-IV activity was inhibited by $>80 \%$ with $5 \mathrm{mg}$ BI 1356 . After administration of $25-600 \mathrm{mg}$ BI 1356 , mean plasma DPP-IV activity was still below $10 \%$ at $24 \mathrm{~h}$ post-dosing. In the MRD study, 24h post-dose, the activity of DPP-IV was inhibited by $>50 \%$ and $>80 \%$ with BI 1356 plasma concentrations as low as $3.2 \mathrm{nmol} / \mathrm{L}$ and $5.3 \mathrm{nmol} / \mathrm{L}$, respectively. Compared to baseline, the mean GLP-1 plasma concentrations measured at $24 \mathrm{~h}$ after the last dose had increased by 12.4 to $14.0 \mathrm{pmol} / \mathrm{L}$ for $2.5,5$, and $10 \mathrm{mg}$ BI 1356 , but only by $2.8 \mathrm{pmol} / \mathrm{L}$ with placebo. After 12 days of treatment, postprandial glucose excursions $\left(\mathrm{AUC}_{0}\right.$ after oGTT $24 \mathrm{~h}$ post-dose) were reduced dose-dependently by $-53.0 \mathrm{mg} /$ $\mathrm{dL}(1 \mathrm{mg}),-106 \mathrm{mg} \cdot \mathrm{h} / \mathrm{dL}(2.5 \mathrm{mg}),-82 \mathrm{mg} \cdot \mathrm{h} / \mathrm{dL}(5 \mathrm{mg})$, and $-111 \mathrm{mg} \cdot \mathrm{h} / \mathrm{dL}$ (10mg) for BI 1356 and $-25.1 \mathrm{mg} / \mathrm{dL}$ for placebo compared with baseline. Despite the small group sizes, the reduction was statistically significant compared to baseline and compared to placebo $(\mathrm{p}<0.05)$ for the $2.5,5$, and $10 \mathrm{mg}$ dose groups. In both studies, treatment with BI 1356 up to a single dose of $600 \mathrm{mg}$ and multiple doses of up to $10 \mathrm{mg}$ for 12 days was well tolerated with no signs of hypoglycemia. The overall incidence of adverse events (AEs) in subjects or patients treated with BI 1356 was not higher than with placebo. There were no signs of QTc prolongation in either study. No serious adverse events and no discontinuations due to AEs were observed.

Conclusion: Administration of BI 1356 in single doses of up to $600 \mathrm{mg}$ in healthy men and in multiple doses of up 10mg for 12 days in patients with type 2 diabetes was well tolerated and safe. BI 1356 plasma concentrations correlated well with DPP-IV activity, achieving DPP-IV inhibition of $>80 \%$ with a plasma concentration of $5.3 \mathrm{nmol} / \mathrm{L}$. The results confirm the high potency, $24 \mathrm{~h}$ duration of action, and very wide $(>100$-fold) therapeutic window of BI 1356.

Supported by Boehringer Ingelheim Pharma GmbH \& Co KG

\section{1}

Single-rising-dose pharmacokinetics, pharmacodynamics, and tolerability of alogliptin benzoate (SYR-322), a novel, dipeptidyl peptidase-IV inhibitor, in healthy male subjects

R. Christopher ${ }^{1}$, P. Covington ${ }^{2}$, M. Davenport ${ }^{3}$, P. Fleck ${ }^{4}$, Q. Mekki ${ }^{4}$, E. Wann ${ }^{5}$, A. Karim ${ }^{4}$;

${ }^{1}$ Takeda San Diego, ${ }^{2}$ Development, Pharmaceutical Product Development, Inc., Wilmington, ${ }^{3}$ Clinical Pharmacology, Pharmaceutical Product Development, Inc., Richmond, ${ }^{4}$ Takeda Global Research \& Development Center, Inc., Deerfield, ${ }^{5}$ Takeda Pharmaceuticals North America, Inc., Deerfield, United States.

Background and Aims: Alogliptin benzoate (ALO; SYR-322) is a novel, oral, potent, and highly selective dipeptidyl peptidase-IV (DPP-4) inhibitor under development to improve glycemic control in patients with type 2 diabetes. DPP-4 rapidly degrades glucagon-like peptide-1 (GLP-1) and glucose-dependent insulinotropic peptide, incretin hormones that induce glucose-dependent insulin secretion and play an important role in glucose homeostasis. This study was designed to assess the pharmacokinetics (PK), pharmacodynamics (PD), and tolerability of single rising doses of ALO in healthy male subjects.

Materials and Methods: In this randomised, double-blind, placebocontrolled study, 36 subjects (6 cohorts of 6 subjects each) received either a single rising dose of $\operatorname{ALO}(25,50,100,200,400$, or $800 \mathrm{mg} ; \mathrm{n}=5$ per cohort) or placebo ( $\mathrm{n}=1$ per cohort); all subjects completed the study. Serial PK blood and urine samples were collected up to 72 hours postdose. The PD effects of ALO were determined over 72 hours postdose based on ex vivo plasma DPP4 inhibition and plasma intact GLP-1 levels.

Results: ALO was absorbed rapidly with a median Tmax of 1 to 2 hours across all doses. Mean AUC and Cmax of ALO increased with increasing dose. Mean T1/2 of ALO ranged from 12.4 to 21.4 hours across all doses. The fraction of dose excreted unchanged in urine over 72 hours postdose ranged from $60 \%$ to $71 \%$ across all ALO doses. Alogliptin resulted in rapid, almost complete, and sustained inhibition of plasma DPP-4 activity (Figure). Mean inhibition ranged from $74.3 \%$ to $97.0 \%$ at 24 hours postdose and from $47.5 \%$ to $83.0 \%$ 
at 72 hours postdose. Peak and total exposure of plasma intact GLP-1 were 2 - to 3-fold greater for the ALO dose groups than placebo. Alogliptin was well tolerated across all dose levels. No serious adverse events were reported and no subject discontinued due to an adverse event. The proportion of subjects who experienced at least 1 adverse event did not increase with increasing ALO dose and was similar for all ALO groups combined and the placebo group.

Conclusion: Administration of a single dose of ALO resulted in rapid, potent, and sustained inhibition of plasma DPP-4 activity and increased intact GLP-1 levels in healthy male subjects. The PK and PD profiles of ALO are supportive of a once-daily dosing regimen.

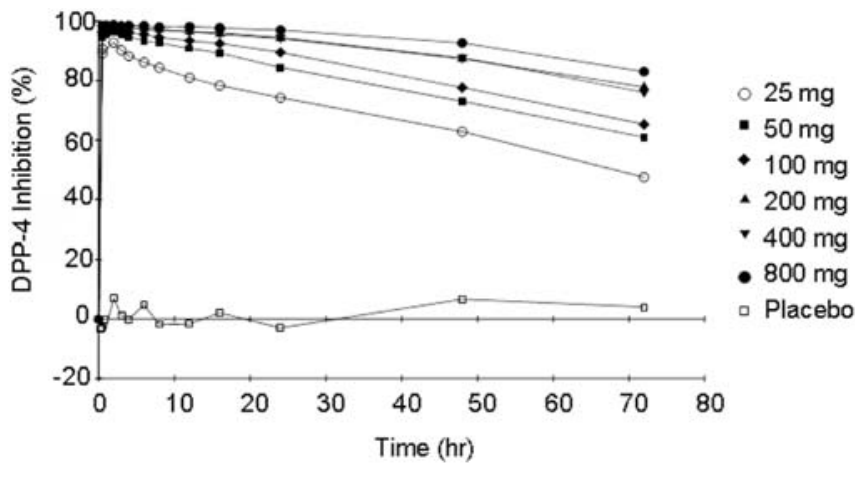

Supported by Takeda Global Research \& Development Center Inc.

\section{PS 84 Body weight regulation}

\section{2}

Polymorphisms of the TUB gene are associated with anthropometry and eating behaviour in middle-age women

J. V. van Vliet-Ostaptchouk ${ }^{1,2}$, N. C. Onland-Moret ${ }^{3,4}$, R. Shiri-Sverdlov ${ }^{2}$, P. J. J. van Gorp 2 , P. H. M. Peeters ${ }^{4}$, C. Wijmenga ${ }^{3}$, M. H. Hofker ${ }^{1,2}$, Y. T. van der Schouw ${ }^{4}$;

${ }^{1}$ Department of Pathology and Laboratory Medicine, University Medical Center Groningen, ${ }^{2}$ Molecular Genetics, Maastricht University, ${ }^{3}$ DBG-Department of Medical Genetics, University Medical Center Utrecht, ${ }^{4} J u l i u s$ Center for Health Sciences and Primary Care, University Medical Center Utrecht, The Netherlands.

Background and Aims: The TUB gene, encoding an evolutionary conserved protein, is highly expressed in the hypothalamus and might act as a transcription factor. Mutations in TUB cause late-onset obesity, insulinresistance and neurosensory deficits in mice. An association of common variants in the TUB gene with body weight in humans has been reported. The aim was to investigate the relationship of single nucleotide polymorphisms (SNPs) of the TUB gene (rs2272382, rs2272383 and rs1528133) with both anthropometry and macronutrient intake.

Materials and Methods: The association of SNPs in the TUB gene with anthropometrical measurements and self-reported macronutrient intake from a validated food frequency questionnaire was studied in a populationbased, cross-sectional study of 1680 middle-aged Dutch women, using linear regression analysis.

Results: The minor allele $\mathrm{C}$ of the rs $1528133 \mathrm{SNP}$ was significantly associated with increased weight $(+1.88 \mathrm{~kg}, \mathrm{P}=0.022)$ and $\mathrm{BMI}(+0.56$ units, $\mathrm{P}=0.05)$. Compared with non-carriers, both AG heterozygotes and AA homozygotes of the rs2272382 SNP derived less energy from fat (AG: $-0.55 \pm 0.28 \%, \mathrm{P}=$ 0.05 , AA: $-0.95 \pm 0.48 \%, \mathrm{P}=0.047$ ). However, both genotypes were associated with an increased energy intake from carbohydrates $(0.69 \pm 0.33 \%, \mathrm{P}=0.04$ and $1.68 \pm 0.56 \%, \mathrm{P}=0.003$, respectively), mainly because of a higher intake of mono- and disaccharides $(0.86 \pm 0.31 \%, \mathrm{P}=0.005$, and $1.08 \pm 0.52 \%, \mathrm{P}=$ 0.038 , respectively). Both these SNPs, rs2272382 and rs1528133, were also associated with a higher glycemic load (GL) in the diet. The GL was higher among those with AG and AA genotypes for the variant rs2272382 than among the wild types $(+1.49$ (95\% CI: $-0.27-3.24)$ and +3.89 (95\% CI: $0.94-6.85$ ) units, respectively). Carriers of the minor allele $\mathrm{C}$ of rs 1528133 were also associated with an increased GL of 1.85 units compared with noncarriers.

Conclusion: We found that genetic variation of the TUB gene was associated with both body composition and macronutrient intake, suggesting that TUB might influence eating behavior.

Supported by Dutch Diabetes Foundation, European Vascular Genomics Network, SenterNovem (IOP Genomics IGE05012), European Commission Europe Against Cancer (WHO AEP/90/05), Dutch Ministry of Health, Dutch Prevention Fund

\section{3}

Differential gene expression in adipose tissue during weight loss and weight maintenance

L. E. Johansson, H. Parikh, L. Groop, M. Klintenberg, F. Norström, M. Ridderstråle;

Department of Clinical Sciences Malmö, Lund University, Malmö, Sweden.

Background and Aims: Weight loss has previously been associated with a down regulation of inflammatory genes in the adipose tissue. In order to better understand the physiology of weight loss, and also weight maintenance, it is important to identify the pathways involved in these processes. The aim of this study was to investigate the effect on gene expression in adipose tissue after weigh loss and weight maintenance after weight loss, respectively.

Materials and Methods: Eleven obese patients referred to the Department of Endocrinology at UMAS were included in the study. Three subcutaneous adipose tissue needle biopsies were collected (at baseline, after $\geq 10 \%$ body weight reduction by low calorie diet and after two months of group therapy for weight maintenance) and RNA extracted. The transcriptomes were analysed using HG-U133 Plus 2 GeneChip from Affymetrix. The robust multi-array 
average (RMA) method was used for background adjustment, quantile based normalisation and calculation of expression values. Probe sets for which percentage present calls were less than $50 \%$ of all scans were excluded, which left 27,400 probe sets for further analysis. We applied the Patterns from Gene Expression (PaGE) method for log-transformed data with the use of modified $t$ statistics to identify differentially expressed probe sets between different groups. We considered only those probe sets as differentially expressed between two groups with a confidence level of at least 0.99 . WebGestalt was used for further analysing and visualising the data.

Results: During weight loss 50 genes were upregulated and included mostly ribosomal genes. 185 genes were down regulated during weight loss. These genes were mostly involved in lipid metabolism, oxidoreductase activity genes and cytoplasmic genes. Genes coding for proteins involved in Wnt signalling were also among these genes. During weight maintenance 73 genes were upregulated. They were mostly genes participating in lipid metabolism, carbohydrate metabolism and angiogenesis. Interestingly, 330 genes were downregulated during weight maintenance and most of them were inflammatory genes. Between baseline and weight maintenance only two genes were upregulated but 69 were downregulated.

Conclusion: This study indicates that weight loss and weight maintenance after weight loss should be viewed as two separate processes in the adipose tissue. In both cases, more genes were down regulated than up regulated. This may indicate that many genes are up regulated due to resistance to negative regulation in the obese state. Finally, previous findings of a down regulation of inflammatory genes seem to be associated with weight maintenance rather than weight loss.

Supported by Novo Nordisk, Påhlsson, Craaford, Borgström, Bergvall, Zoegas, Hierta, and Swedish Diabetes Foundation. We would like to acknowledge SCIBLU Microarray Resource Center in Lund for performing the microarrays.

\section{4}

Factors associated with change in status of non-alcoholic fatty liver disease (NAFLD) in Korea

H.-K. Kim ${ }^{1}$, C.-H. Kim ${ }^{2}$, G.-E. Lee ${ }^{1}$, S.-H. Chun ${ }^{1}$, H.-S. Lee ${ }^{1}$, H.-C. Cho', K.-R. Kim ${ }^{1}$, I.-Y. Pae ${ }^{1}$, J.-H. Kim ${ }^{1}$;

${ }^{1}$ Health Promotion Center, Asan Medical Center, Seoul,

${ }^{2}$ Endocrinology \& Metabolism, Internal Medicine, Soonchunhyang

University Bucheon Hospital, Bucheon, Republic of Korea.

Background and Aims: Nonalcoholic fatty liver disease (NAFLD) is a common hepatic disorder characterised by fat accumulation in the liver without personal history of heavy drinking. However, there has been little data about long-term changes in status of NAFLD for large number of subjects. To know the factors associated with changing status of NAFLD, we analysed life style factors, biochemical data, and abdominal ultrasonography from subjects who could be followed for 5 -years (between 2000 and 2005) at the Health Promotion Center of Asan Medical Center.

Materials and Methods: Among 6096 subjects whose follow-up data were available, 2895 subjects (mean age $47 \pm 8.7$, range 20-79 years; 1,502 men and 1,393 women) were analyzed after strict exclusion. Subjects who were heavy alcohol consumers, positive for hepatitis B virus surface antigen or hepatitis $C$ virus antibody, had hepatic enzyme concentrations higher than three times the upper normal limit, overt hypo- or hyperthyroidism, any cancer, or sonographic evidence of other chronic liver disease at baseline were excluded.

Results: Fatty liver on ultrasonography was graded into four categories (normal, mild, moderate, and severe) by the degree of echogenisity. At baseline, 1965 subjects were classified as normal, 622 as mild, 288 as moderate, and 20 as severe fatty liver. At follow-up examination after 5 years, 1719 were normal, 690 had mild, 383 had moderate, and 66 had severe fatty liver. After 5 years, $2012(70 \%)$ subjects remained at the same ultrasonographic grade of fatty liver. However, 263 (9\%) subjects showed decreased grade of echogenisity and $155(5 \%)$ subjects among them were normalised. In contrast, $583(20 \%)$ subjects were changed into higher grade of fatty liver. Improved group showed $1.6 \pm 3.0 \mathrm{~kg}$ reduction of body weight from baseline while aggravated group gained $1.7 \pm 2.9 \mathrm{~kg}$ of weight. Improvement of fatty liver was associated with decrease of serum AST, ALT, triglyceride, cholesterol, and fasting glucose. Conclusion: In conclusion, mild degree of weight reduction is associated with improvement of NAFLD and related metabolic abnormalities such as dyslipidemia and hyperglycemia in the long-term.

\section{5}

Insulin resistance and insulin secretion in morbidly obese patients after bariatric surgery

M. Promintzer ${ }^{1}$, G. Prager ${ }^{2}$, G. Pacini ${ }^{3}$, C. Anderwald ${ }^{1}$, M. Mandl ${ }^{1}$, S. Shakeri Manesch ${ }^{2}$, M. Stadler ${ }^{4}$, P. Nowotny ${ }^{1}$, M. G. Bischof ${ }^{1}$, B. Ludvik ${ }^{1}$, A. Luger ${ }^{1}$, M. Krebs ${ }^{1}$;

${ }^{1}$ Department of Internal Medicine III, Division of Endocrinology and Metabolism, Medical University of Vienna, Austria, ${ }^{2}$ Department of Surgery, Medical University of Vienna, Austria, ${ }^{3}$ Metabolic Unit, ISIB-CNR, Padua, Italy, ${ }^{4}$ Third Medical Department of Metabolic Diseases and Nephrology, Hospital of Hietzing, Vienna, Austria.

Background and Aims: The increase in the prevalence of type 2 diabetes is closely linked to the rise in obesity. However, severe obesity is a refractory condition and, at present, surgical intervention is the only treatment yielding long term results. Bariatric surgery not only induces lasting weight loss but also leads to improvements or resolution of comorbid diseases, particularly type 2 diabetes. Improvements in insulin sensitivity and insulin resistance might contribute to observed improvements in glucose metabolism. The aim of the study was to assess the effects of bariatric surgery on these parameters.

Materials and Methods: We performed a frequently sampled oral glucose tolerance test $\left(75 \mathrm{~g}, 3 \mathrm{~h}\right.$ OGTT) and a hyperinsulinemic $\left(40 \mathrm{mU} / \mathrm{min} / \mathrm{m}^{2}\right)$ isoglycemic clamp test in 12 morbidly obese non diabetic patients before bariatric surgery (OBpre: 10f/2m, age: $38.9 \pm 2.8 \mathrm{a}, \mathrm{BMI}: 48.8 \pm 1.5 \mathrm{~kg} / \mathrm{m}^{2}$ ) and in 10 matched healthy controls (CON: $8 \mathrm{f} / 2 \mathrm{~m}$, age: $41.6 \pm 1.3 \mathrm{a}$, BMI: $22.1 \pm 0.4$ $\left.\mathrm{kg} / \mathrm{m}^{2}\right)$. Glucose turnover was determined using DD-[6,6- $\left.{ }^{2} \mathrm{H}_{2}\right]$ glucose. Adaptation index characterising the relationship between insulin sensitivity and direct $\beta$-cell function was calculated. Examinations were repeated $\sim 6$ months after surgery in 5 ( 3 Roux-en-Y gastric bypass, 2 gastric bypass) of these obese patients (OBpost: $4 \mathrm{f} / 1 \mathrm{~m}$, age:47.8 $\pm 2.4 \mathrm{a}$, BMI: $37.7 \pm 3.4$ $\mathrm{kg} / \mathrm{m}^{2}$ ) and compared to $6 \mathrm{BMI}-$, age- and sex- matched obese patients without history of gastrointestinal surgery (CONob: $5 \mathrm{f} / 1 \mathrm{~m}$, age:50.9 $\pm 2.7 \mathrm{a}$, BMI: $35.3 \pm 0.7 \mathrm{~kg} / \mathrm{m}^{2}$ ).

Results: Surgery: Bariatric surgery resulted in a weight loss of $25.9 \mathrm{~kg}$ $\left(\triangle \mathrm{BMI}-10.6 \mathrm{~kg} / \mathrm{m}^{2}\right)$. OGTT: While fasting plasma glucose concentrations were comparable between OBpre and CON fasting insulin and c-peptide were higher in OBpre. During the oGTT peak plasma glucose and insulin concentrations were significantly higher in OBpre compared to CON.

In OBpost fasting and early postprandial glucose concentrations were unchanged compared to OBpre. However, lower glucose concentrations were observed in the late postprandial period. Fasting insulin and c-peptide concentrations decreased in response to bariatric surgery. However, the postprandial increase in insulin and c-peptide concentrations was markedly augmented in OBpost compared to all other groups (insulin and c-peptide at 60 min.: OBpost vs. OBpre/ CON/ CONob: $\mathrm{p}<0.001$ respectively).

Insulin and c-peptide secretion following glucose load were adapted to insulin resistance prior surgery but were inadequately elevated after bariatric surgery (adaptation index: OBpost:5.0 $\pm 0.7 /$ OBpre: $2.6 \pm 0.8 /$ CONob:2.8 $\pm 0.5 /$ CON:3.3 $\pm 0.6 ; \mathrm{p}<0.05$ for OBpost vs. OBpre and CONob).

Clamp: As expected the insulin stimulated glucose infusion rate (GIR) was markedly lower in OBpre (M: $2.1 \pm 0.5 \mathrm{mg} / \mathrm{kg} / \mathrm{min}$ ) compared to CON (M: $9.5 \pm 1.6 \mathrm{mg} / \mathrm{kg} / \mathrm{min} ; \mathrm{p}<0.001)$. Despite dramatic weight loss GIR was comparable between OBpre and OBpost (M: $2.8 \pm 0.4 \mathrm{mg} / \mathrm{kg} / \mathrm{min}$ ).

Conclusion: Our data indicate that rather augmented insulin secretion than changes in insulin resistance are responsible for the reported improvements in glycaemic control after bariatric surgery.

M.P. is employed on a grant of the Austrian Society of Internal Medicine (JosefSkoda-Award) which was competitively awarded to M.K.

\section{6}

Bariatric surgery results in a significant decline in sensory nerve function

R. A. Gandhi ${ }^{1}$, D. Selvarajah ${ }^{1}$, C. N. MacDermott ${ }^{1}$, C. J. Emery ${ }^{1}$, R. Ackroyd ${ }^{2}$, S. Tesfaye ${ }^{1}$;

${ }^{1}$ Diabetes Research Unit, ${ }^{2}$ Department Of Surgery, Royal Hallamshire Hospital, Sheffield, United Kingdom.

Background: Lack of efficacy of conventional treatments of obesity has led to increasing popularity of bariatric surgery as a therapeutic option. Little is known about possible long-term effects. Although an increased incidence of 
peripheral neuropathy after surgery has been reported, there is an absence of any prospective studies.

Aims: The aim of this study was therefore to objectively assess peripheral and autonomic nerve function in subjects before and 1 year after bariatric surgery

Methods: 12 subjects (10 females) underwent detailed quantitative sensory testing, nerve conduction studies (radial, sural, common peroneal and tibial nerves), and autonomic function tests.

Results: Sensory nerve conduction velocity (SNCV) fell from $66.7 \pm 7.3$ to $56.3 \pm 4.2 \mathrm{~m} / \mathrm{s}($ mean $\pm \mathrm{SD})$ in the radial nerve $(\mathrm{p}<0.001)$ and from $51.3 \pm 7.0$ to $41.9 \pm 5.2 \mathrm{~m} / \mathrm{s}$ in the sural nerve $(\mathrm{p}<0.001)$. This was accompanied by a rise in amplitude in both nerves (radial nerve: $42.4 \pm 11.0$ to $55.1 \pm 16.2 \mu \mathrm{V}, \mathrm{p}=0.06$; sural nerve: $16.0 \pm 4.7$ to $22.6 \pm 10.4 \mu \mathrm{V}, \mathrm{p}<0.05)$. No significant changes were seen in parameters of motor nerve function. On quantitative sensory testing, significantly greater numbers of subjects had hyperalgesia during heat-pain testing at 1 year. Autonomic function testing showed a fall in the resting heart rate from $79.7 \pm 9.1$ to $65.8 \pm 9.8$ beats $/ \mathrm{min}(\mathrm{p}<0.01)$ and a rise in beat-to-beat variation $(\mathrm{R} / \mathrm{R}$ ratio) on standing from $1.28 \pm 0.19$ to $1.56 \pm 0.25(\mathrm{p}<0.001)$. No other significant changes were noted in autonomic function.

Conclusion: This study shows a large decline in SNCV after bariatric surgery. Substantial reductions in body fat may be having an impact on the composition of myelin, a major determinant of SNCV, which consists of $70 \%$ lipid. The role of nutritional deficiencies after surgery, as well as inflammatory and immune mechanisms also deserves consideration. The improvements in sensory nerve amplitude are probably due to improved signal detection as a result of reductions in subcutaneous fat. The increase in hyperalgesia is consistent with the development of an early peripheral neuropathy.

Bariatric surgery is increasingly being employed to treat obesity. It is clear, however, that sensory nerve dysfunction is common sequelae and may herald the development of more overt peripheral neuropathy.

\section{PS 85 Prediction, detection and biomarkers}

\section{7}

Could $\mathrm{HbA}_{1 \mathrm{c}}$ have a role in the diagnosis of type 2 diabetes? S. E. Manley ${ }^{1}$, I. M. Stratton ${ }^{2}$, P. G. Nightingale ${ }^{3}$, R. A. Round ${ }^{1}$, J. M. Smith ${ }^{1}$, S. C. L. Gough ${ }^{4}$;

${ }^{1}$ Clinical Biochemistry, University Hospital Birmingham NHS Foundation Trust, Birmingham, ${ }^{2}$ Oxford Centre for Diabetes, Endocrinology and Metabolism, University of Oxford, ${ }^{3}$ Wellcome Trust Clinical Research Facility, University Hospital Birmingham NHS Foundation Trust, ${ }^{4}$ Selly Oak Hospital Diabetes Centre, University Hospital Birmingham NHS Foundation Trust, United Kingdom.

Background and Aims: Diabetes is increasing worldwide, currently 250 million patients, with International Diabetes Federation (IDF) predicting 380 million in 2025, the majority living in the developing world. Diabetes is diagnosed by measurement of fasting plasma glucose (FPG) with the oral glucose tolerance test (OGTT) restricted to those with impaired fasting glucose (IFG). The OGTT is poorly reproducible, time consuming and expensive. The recent IDF clinical guideline suggests $\mathrm{HbA}_{1 c}$ testing at diagnosis.

Materials and Methods: 700 patients with IFG (WHO criteria) were referred by community physicians in South Birmingham, UK, to a hospital diabetes centre. FPG and $\mathrm{HbA}_{\text {c }}$ were measured in capillary blood with 'DCCT aligned' $\mathrm{HbA}_{1 \mathrm{c}}$ reported from an ion exchange, high performance liquid chromatography (IE HPLC) analyser that detects variant haemoglobin. Diabetes (DM) was identified if FPG $\geq 7.0 \mathrm{mmol} / \mathrm{L}$ or capillary 2 hour plasma glucose $(2 \mathrm{hPG}) \geq 12.2 \mathrm{mmol} / \mathrm{L}$ on $75 \mathrm{~g}$ OGTT. A cut-point for $\mathrm{HbA}$, for use with FPG to best identify those with DM, was calculated using the first 500 patients' (cohort A) results and subsequently validated in the next 200 (cohort B).

Results: Patients were aged 61(12) years, mean (1SD), 51\% male and $11 \%$ Asian Indian. FPG from venous blood collected prior to referral was lower than capillary FPG at OGTT, 6.5(0.4) $\mathrm{mmol} / \mathrm{L} \mathrm{v} 6.7(0.8) \mathrm{mmol} / \mathrm{L}, \mathrm{p}<0.001$. $\mathrm{HbA}_{1 \mathrm{c}}$ was 6.3(0.7)\% with 4 patients in $\mathrm{A}$ and $1 \mathrm{in} \mathrm{B}$ omitted because of variant haemoglobin. One patient was excluded from B with no $2 \mathrm{hPG}$ value. $2 \mathrm{hPG}$ on OGTT was $9.8(2.8) \mathrm{mmol} / \mathrm{L}$ with OGTT not performed in 35\% (245/700) since FPG was $\geq 7.0 \mathrm{mmol} / \mathrm{L}$ ( $66 / 198$ for B). There were no significant differences between data from each cohort or for Asian Indians versus the rest. ROC curves derived from cohort $\mathrm{A}$ indicated that $\mathrm{HbA}_{1 c} \geq 6.0 \%$ can be used as a cut-point where FPG $<7.0 \mathrm{mmol} / \mathrm{L}$ to identify those patients requiring OGTT for diagnosis with resulting sensitivity $96 \%$ and specificity $100 \%$ for DM. When the algorithm was applied to cohort B, it identified $86 / 88$ cases diagnosed conventionally i.e. sensitivity $98 \%$ and specificity $100 \%$. Use of this algorithm would reduce the number of OGTT required in B by $39 \%$ from 132 to 81 .

Conclusion: Use of this algorithm, $\mathrm{HbA}_{1 \mathrm{c}} \geq 6.0 \%$ (by IE HPLC) in combination with FPG $<7.0 \mathrm{mmol} / \mathrm{L}$, to identify IFG patients requiring OGTT would reduce the number of OGTT required by $40 \%$. Such a modification to current diagnostic criteria for DM would not impair sensitivity or specificity but reduce costs and the use of resources. It would simplify procedures for diagnosis of DM in those with IFG, as $\mathrm{HbA}_{1 c}$ could be measured with FPG prior to referral for OGTT.

\begin{tabular}{|c|c|c|c|c|}
\hline$n=198$ & $\begin{array}{l}\mathrm{HbA}_{1 \mathrm{c}}<6 \% \\
\mathrm{FPG} \\
<7 \mathrm{mmol} / \mathrm{L}\end{array}$ & $\begin{array}{l}\mathrm{HbA}_{1 \mathrm{c}}>\mathbf{6 \%} \\
\mathrm{FPG} \\
<7 \mathrm{mmol} / \mathrm{L}\end{array}$ & $\begin{array}{l}\mathrm{HbA}_{1 \mathrm{c}}<6 \% \\
\mathrm{FPG} \\
\geq 7 \mathrm{mmol} / \mathrm{L}\end{array}$ & $\begin{array}{l}\mathrm{HbA}_{1 \mathrm{c}} \geq 6 \% \\
\mathrm{FPG} \\
\geq 7 \mathrm{mmol} / \mathrm{L}\end{array}$ \\
\hline Not DM & 49 & 61 & - & - \\
\hline $\begin{array}{l}\text { DM on } \\
\text { FPG alone }\end{array}$ & - & - & 11 & 55 \\
\hline $\begin{array}{l}\text { DM on } \\
\text { 2hPG alone }\end{array}$ & 2 & 20 & - & - \\
\hline
\end{tabular}




\section{8}

Population screening for metabolic syndrome - the IJsselstein Screening for Central Obesity study

I. W. G. Bobbink ${ }^{1}$, P. L. Salome ${ }^{2}$, K. J. Gorter ${ }^{1}$, M. van den Donk ${ }^{1}$, G. E. H. Rutten ${ }^{1}$;

${ }^{1}$ Julius Center, University Medical Center Utrecht,

${ }^{2}$ General Practice, Medische Maatschap IJsselstein, The Netherlands.

Background and Aims: Instead of screening for diabetes type 2 in the general population, focussing on groups at high risk for cardiovascular diseases may be preferred. In The Netherlands it is unknown how often cardiovascular risk factors cluster and in how many cases metabolic syndrome (MetS) is present. This study aims to investigate whether GP-mediated population-based screening for central obesity can be used to identify subjects with MetS and to assess the prevalence of MetS.

Materials and Methods: Cross-sectional study among 11,872 adults (20-70 year) on the list of 12 GPs in a city in the center of The Netherlands. Subjects, who were not known with MetS, diabetes, hypertension, or dyslipidaemia, received an invitation from their GP to measure their waist circumference (WC) with an enclosed tape measure. Subjects with a WC $>88 \mathrm{~cm}$ (women) or $>102 \mathrm{~cm}$ (men) were invited for further examinations to determine MetS according to the NCEP/ATPIII criteria (WC, blood pressure, fasting glucose, HDL and triglycerides). In addition, a $3 \%$ random sample of subjects with a WC $\leq 88 / 102 \mathrm{~cm}$ was invited for further examinations to study the sensitivity of the procedure.

Results: A total of 7253 subjects (61\%) responded, 6773 subjects (57\%) had measured their WC. 2206 of them (33\%) had a WC > 88/102 cm and 1655 of these subjects participated in further examinations. In 450 (27\%) of these participants MetS was detected. Additionally, in $4(3.3 \%)$ of the 122 randomly selected subjects with a WC $\leq 88 / 102 \mathrm{~cm}$ who underwent examinations MetS was detected. The prevalence of MetS assessed by screening subjects with central obesity is $7 \%$. The total prevalence of MetS in the source population will be assessed by adding these numbers to the prevalence of MetS in 3500 patients known with MetS, diabetes, hypertension or dyslipidaemia (data available at the EASD meeting). Both the response rate and the participation rate were age-dependent. The sensitivity of the used screening method (MetS in WC $>88 / 102$ group / (MetS in WC $>88 / 102$ group $+33^{*}$ MetS in WC $\leq$ $88 / 102$ group)) was $77 \%$. Self-measured WC was overestimated by $76 \%$ of the subjects. The mean difference between self-measured and investigator values was $4 \mathrm{~cm}$.

Conclusions: A GP-mediated population screening for central obesity with self-measurement of WC can be used to identify adults with MetS. The prevalence of MetS assessed by such a screening is $7 \%$. This creates possibilities for targeted prevention and treatment of these persons who are at an increased cardiovascular risk.

Supported by an unrestricted grant from MSD

\section{9}

\section{Abnormal glucose regulation in a community-based population with coronary artery disease; are routine oral glucose tolerance tests necessary? \\ O. A. Mongan ${ }^{1}$, A. El-Helali ${ }^{2}$, M. Glacken ${ }^{3}$, A. W. Murphy ${ }^{4}$, F. Dunne ${ }^{1}$; ${ }^{1}$ Department of Diabetes and Endocrinology, University College Hospital Galway, ${ }^{2}$ Department of Medicine, National University of Ireland, Galway, ${ }^{3}$ Department of Public Health, Merlin Park Regional Hospital, ${ }^{4}$ Department of General Practice, National University of Ireland Galway, Ireland.}

Background: Ischaemic heart disease accounts for $25 \%$ of all deaths in Ireland. Studies have shown that without an oral glucose tolerance test (OGTT), abnormal glucose regulation (AGR), including impaired fasting glucose (IFG), impaired glucose tolerance (IGT) and diabetes, remains undiagnosed in many people with coronary artery disease. The Heartwatch programme is the national programme in general practice for the secondary prevention of cardiovascular disease in Ireland. A fasting glucose is performed annually as part of this programme, however an OGTT is not routinely recommended.

Aim: This community based study aims to provide reliable evidence of glucose dysregulation, by universal screening, using a 75 gram oral glucose tolerance test, in a defined high risk Irish population with established coronary artery disease.
Materials and Methods: Using the Heartwatch database described, we invited general practitioners (GPs) in the West of Ireland (counties Galway, Mayo and Roscommon) to take part in the study. Participating GPs were asked to invite all of their patients on the database for an OGTT to be carried out in the practice, using a protocol provided by the study group. Training on this protocol was provided to the individual phlebotomists. Informed consent was obtained. To aid recruitment, practices were reimbursed for every OGTT carried out. The OGTT results were classified as normal, IFG, IGT, both IFG and IGT or diabetes according to American Diabetes Association Guidelines.

Results: At the time of the study there were 969 patients enrolled on the Heartwatch database in the selected area. These patients were spread across 33 practices. 23 out of 33 practices agreed to participate. 19 practices comprising of a total of 633 patients on Heartwatch took part in OGTT testing. The baseline prevalence of diagnosed diabetes was $13 \%(n=83)$ leaving 550 people eligible for OGTT. Baseline prevalence of diagnosed IFG was $1 \%$ and 2 cases out of 633 had IGT. Of the 550 eligible patients, $56 \%$ had an OGTT performed. Of the 305 tests $7.2 \%$ had IFG $(n=22), 8.2 \%$ had IGT $(n=25)$, $4.9 \%$ had both IFG and IGT $(n=15)$ and $4.6 \%$ were diagnosed with diabetes mellitus ( $\mathrm{n}=14)$.

In total we found that $25 \%$ of those tested had AGR $(\mathrm{N}=76)$, of these 25 had isolated IGT and 4 of those diagnosed with diabetes had a normal fasting glucose level therefore $37 \%$ of those with AGR would have remained undiagnosed without OGTT. This study resulted in the prevalence of abnormal glucose regulation (AGR) increasing from $14.5 \%$ to $26.5 \%$ in the population of 633 patients.

Conclusion: The baseline prevalence of diabetes and the prevalence of AGR after OGTT are much lower in this study of a high risk Irish population with coronary artery disease, than in similar international studies. Almost 10\% of those tested would have remained undiagnosed if an OGTT was done only when indicated by an elevated fasting glucose. We therefore conclude that routine oral glucose tolerance testing, in patients with coronary artery disease, is feasible and necessary.

Supported by the Department of Public Health, Galway University Hospitals, Merlin Park Hospital, Galway

\section{0}

Intramuscular injection of a vector encoding sRAGE decreases the incidence of diabetes mellitus induced by low dose streptozotocin in rats fed high fat diet

B. Su${ }^{1}, \mathrm{X} . \mathrm{Su}^{1}, \mathrm{X} . \mathrm{Meng}^{2}$;

${ }^{1}$ Endocrinology and Metabolism, Dalian Medical University Second Affiliated Hospital, ${ }^{2}$ Hematology, Dalian Medical University School of Laboratory Medicine, Dalian, China.

Background and Aims: Studies implicated that dietary AGE could induce body redox and inflammatory cytokines production. We aimed to study if the intramuscular injection of a vector encoding sRAGE could decrease the incidence of diabetes mellitus induced by low dose streptozotocin (STZ) in rats fed on high fat diet.

Materials and Methods: sRAGE was RT-PCR amplified from rat lung total mRNA and cloned to a pLNCX expression vector. Germ-free vectors were injected in hind leg muscles, and tranduction was facilitated with electroproation. Mice were grouped into two groups. Mice fed high fat and received sRAGE were designed as treatment group and mice fed high fat that received pLNCX without SRAGE were designed as control group. After 8 weeks of high fat diet, mice were injected with sRAGE or empty vectors. One serial of mice ( $n=6$ in each group) were to observe changes in serum MDA and SOD levels. A second serial ( $\mathrm{n}=10$ in each) were to study of STZ-induced incidence of diabetes. One week after injection of vectors, a dose of $30 \mathrm{mg} / \mathrm{kg}$ STZ was injected intraperitoneally and tail venous glucose was detected one week later.

Results: MDA levels were found decreased one week after injection of sRAGE and lasted for at least three weeks. SOD activities were found increased slowly in the second week and significantly in the third week. As determined with fasting glycemia and random glycemia two mice were in diabetic level $(>7.0)$ in treatment group while 8 mice in diabetic level in control group.

Conclusion: Intramuscular injection of sRAGE decreases the oxygen reactive level and increases anti-reactive activities, and decreases the STZ-induced incidence of diabetes in rats in high fat-diet.

Supported by a grant from Liaoning Previncial Grant for Natural Sciences 


\section{1}

Type 2 diabetes phenotype and progression is significantly different if diagnosed before versus after 65 years of age

H. Kolb ${ }^{1}$, B. Schneider ${ }^{2}$, T. Heise ${ }^{3}$, L. Heinemann ${ }^{3}$, V. Lodwig ${ }^{4}$, W. Scherbaum ${ }^{1}$, S. Martin ${ }^{1}$;

${ }^{1}$ Institute for Clinical Diabetology, German Diabetes Center, Düsseldorf, Germany, ${ }^{2}$ Institute of Biometrics, Medical University of Hannover, Germany, ${ }^{3}$ Institute for Metabolic Research, Profil, Neuss, Germany, ${ }^{4} \mathrm{IMIB}$, Institute for Medical Informatics and Biostatistics, Basel, Switzerland.

Background and Aims: The incidence of type 2 diabetes is increasing preferentially in younger persons ( $<65$ years of age). It is not known whether disease characteristics in these younger patients are similar to the "classic" late-onset type 2 diabetes.

Materials and Methods: The data base of the German multicentre, retrospective cohort study ROSSO was used to address this question. In ROSSO, 3268 people with type 2 diabetes, from randomly contacted primary care practices, were followed from diagnosis between 1995 and 1999 for a mean of 6.5 years. All newly diagnosed patients of these practices were included, with a lower age limit of 45 years

Results: At diagnosis, $64.2 \%$ of the patients had an age between 45 and 65 years. In this younger age group, $57.2 \%$ were males, whereas in the age group $>65$ years only $35.0 \%$ were males $(\mathrm{p}<0.001)$. There was more severe metabolic deterioration at diagnosis and in the following years in the younger cohort, as determined by fasting blood glucose (FBG) and HbAlc levels ( $<<0.001$ for both), and by a higher BMI ( 30.5 vs $28.5, \mathrm{p}<0.001$ ). Conversely, the prevalence of cardiovascular risk factors (coronary heart disease, heart insufficiency of hypertension, either diagnosed or concluded from the prescription of antihypertensives or from increased systolic blood pressure) was significantly higher in the older patient cohort $(\mathrm{p}<0.001$, each). Self monitoring of blood glucose (SMBG) was more prominent in the younger cohort (50 vs $36 \%, \mathrm{p}<0.001$ ). Both age cohorts experienced a lower risk of the combined endpoint myocardial infarction, stroke and all-cause mortality during the observation period when performing SMBG ( $\mathrm{p}=0.003$ in the younger cohort, $\mathrm{p}=0.039$ in the older cohort).

Conclusion: There are considerable differences in disease characteristics between people diagnosed with type 2 diabetes during $45-65$ years of age and patients with diagnosis at later age. Late-onset type 2 diabetes preferentially affects women and is characterised by consistently lower mean BMI, FBG and $\mathrm{HbAlc}$, but exhibits more advanced cardiovascular risk factors. Conversely, younger patients exhibit consistently poorer metabolic but fewer cardiovascular risk factors. The use of SMBG is associated with better clinical outcome in both age cohorts.

Supported by a non-restricted research grant from Roche Diagnostics

\section{2}

Latent autoimmune diabetes in adults as a part of polyendocrine syndrome

M. Bernas, S. M. Zawada-Targoni, A. Czech, J. Taton;

Chair and Department of Internal Medicine and Diabetology, Medical University of Warsaw, Poland.

Background and Aims: Many cases of recently recognized diabetes can have autoimmunological background. Autoantibodies against pancreatic antigens (as it has been proven in the number of studies in diabetes type 1) are not the only one that are present in those patients. It is known that autoimmune thyroiditis is the most common autoimmune disease coexisting with diabetes type 1. Little is known about this problem in subjects with LADA. Latent autoimmune diabetes in adults (LADA) is characterized by clinical features of type 2 diabetes, initial euglycemia (during at least 6 months of treatment) achieved with diet or oral hypoglycemic agents, and presence of autoantibodies.

The aim of the study was to compare the frequency of autoimmune thyroiditis (AT) in adult subjects with newly recognized diabetes type 1, type 2 and LADA.

Materials and Methods: The study included 147 patients with recently recognized diabetes type 1 ( 29 patients, mean age 46 yrs), LADA (39 patients, mean age $48 \mathrm{yrs}$ ) and diabetes type 2 (79 patients, mean age $46.1 \mathrm{yrs}$ ). Patients were qualified to the LADA group due to WHO criteria. To asses the type of diabetes the anamnesis was taken, the patients were examined to BMI, ketoacidosis, concentration of C-peptide (basic and - in the $6^{\text {th }}$ minute after glucagon stimulation) and to autoantibodies: glutamic acid decarboxylase antibodies - GADA, Islet Cell Antibodies -ICA and protein tyrosine phosphatase-like protein IA2-antibodies (ELISA test) at the time of diagnosis. Autoimmune Thyroiditis (AT) was diagnosed using thyroid peroxidase antibodies (TPO), thyreoglobulin-antibodies (TG), TSH and fT4 levels.

Results: High titers of autoantibodies were observed in the LADA (ICA: $31 \%$, IA-2: 62,5\%/ mean level: $6.69 \mathrm{ng} / \mathrm{ml}$, GADA: 56\%/mean: $1082 \mathrm{ng} / \mathrm{ml}$ ) and type 1 diabetes (ICA: $71.42 \%$, IA-2: $42.85 \% /$ mean level: $1.67 \mathrm{ng} / \mathrm{ml}$, GADA: $100 \% /$ mean: $1408 \mathrm{ng} / \mathrm{ml}$ ). Low levels of GADA were present in $20 \%$ of type 2 diabetic patients. Clinically overt hypothyroidisms was estimated in 7 cases of LADA patients (18\%), but high levels of anti-thyroid antibodies were observed more common: ATG:15\%, mean level $3000 \mathrm{IU} / \mathrm{ml}(\mathrm{N}<180 \mathrm{IU} / \mathrm{ml})$, TPO: in $46 \%$, mean level $1428,5 \mathrm{U} / \mathrm{ml}(\mathrm{N}<100 \mathrm{U} / \mathrm{ml})$ comparing to type 1 where ATG were present in $20 \%$, mean concentration $621.3 \mathrm{IU} / \mathrm{ml}$, and TPO in $33 \%$, mean concentration $1558,5 \mathrm{u} / \mathrm{ml}$ and in contrast to type 2 patients, were there was no case of overt hypothyroidisms. Anti-thyroid antibodies were present mostly in the presence of more than one diabetic antibodies (58\%).

Conclusion: The study has evaluated that the highest coexistence of antithyroid and antipancreatic antibodies is present in patients with LADA, whereas in type 2 and type 1 is not as common. Thus it seems that there is a need for autoantibody screening for autoimmune thyroiditis especially in patients with LADA.

Supported by the Medical Academy, Warsaw

\section{3}

Transcriptome analyses reveals potential novel biomarkers for the development of type 2 diabetes

P. Ikonomi ${ }^{1}$, S. Zangen ${ }^{2}$, Q. Li ${ }^{1}$, S. Hacohen ${ }^{2}$, I. Raz ${ }^{2}$, C. Gelber';

${ }^{1}$ SCIENCE, ATCC, Manassas, United States,

${ }^{2}$ Diabetes Unit, Hadassah Hebrew UMC, Jerusalem, Israel.

Background and Aims: The Cohen diabetic (CD) rat is a versatile animal model comprised of 2 rodent strains manifesting many of the common features of type 2 diabetes (T2D) in humans. The sensitive strain (CDs) develops diabetes within 30 days when maintained on a high sucrose/copper poor diet (HSD), whereas the resistant strain (CDr) retains normal blood glucose levels. Neither strain shows any signs of diabetes when provided regular rodent diet (RD). To further characterize the genomic and proteomic changes responsible for either resistance or predisposition to T2D, pancreatic transcriptome changes of these two strains were studied by microarray analyses using the rat expression arrays.

Materials and Methods: Pancreatic RNA extracted for each of the strains was analyzed using the Rat Expression Arrays (Affymetrix) containing oligonucleotide probes for over 30,000 transcripts. The data was analyzed using GeneSpring GX (Agilent, CA) and expression of selected transcripts was confirmed by Real Time PCR.

Results: We have isolated a total of 82 unique transcripts expressed differently in the rat models. Comparisons between the CDs strain and CDr strain when exposed to high sucrose diet, shows that expression of 19 transcripts is reduced by 3 fold or more in CDr-HSD compared to CDs-HSD. These transcripts belong to genes involved in inflammation and immuno responses, such as CD38, CD132, CD53 and CCL21. Alternatively, in CDr strain, the expression of 62 transcripts is increased over 3 fold. Regenerating islet-derived 3 gamma (REG3G) and regenerating islet-derived 3 alpha were increased over 20 fold in CDr strain compared to CDs strain, both exposed under a high sucrose diet. These results indicate that both REG3G and REG3A could play protective roles in pancreatic islets. Increased protein and amino acid metabolism was also indicated as shown by the upregulation of methionine adenosyltransferase (MAT1A), glycine amidinotransferase (GATM), phenylalanine hydroxylase (PAH) and asparagines synthetase. Transporter transcripts such as solute carrier family 7 member 3 (SLC7A3), solute carrier family 30 (zinc transporter) member 2 (SLC30A2) and solute carrier family 7 (cationic amino acid transporter member 5 (SLC7A5) as well as the eukaryotic translation initiation factor $4 \mathrm{~B}$ (EIF4B) were also increased in $\mathrm{CDr}$ strain. Sucrose metabolism-related genes including fucosyltransferase 1 (FUT1) were shown to be upregulated by at least 3 fold. Moreover, serine proteinases such as proteinase serine 3 , and serine protease inhibitor- kazal type 1 (SPINK1), serine proteinase inhibitor clade I member 2 (SERPINI2), 
serine peptidase inhibitor clade A member 10 (SERPINA10) were significantly upregulated in CDr rats. Several unidentified transcripts, whose involvement in T2D remains unknown also show differentiated expression in the resistance and sensitive strains of Cohen Diabetic rat, these transcripts are in the process of further characterization.

Conclusion: Using the Cohen rat models, our microarray analysis indicated the potential involvement of novel pathways in T2D. Quantitative analyses of individual transcripts as well as modifications of their respective proteins will determine the role of such transcripts in the development of the disease and will contribute to the selection of a cluster of predictive markers for the progression of or resistance to T2D.

\section{4}

Liver enzymes and risk of diabetes and cardiovascular disease. Results of the FIBAR Study

C. Lamanna ${ }^{1}$, M. Monami ${ }^{1}$, G. Bardini ${ }^{2}$, L. Pala ${ }^{2}$, B. Cresci ${ }^{2}$, C. M. Rotella ${ }^{2}$, E. Mannucci ${ }^{1}$;

${ }^{1}$ Critical Care Medicine and Surgery, Unit of Gerontology, ${ }^{2}$ Clinical

Pathophysiology, Section of Endocrinology, University of Florence, Italy.

Background and Aims: To assess $\gamma$-glutamil-transpeptidase ( $\gamma$-GT), alanine amino-transferase (ALT), and aspartate amino-transferase (AST) in the prediction of diabetes and cardiovascular disease (CVD) in subjects free from hepatic diseases other than non-alcoholic fatty liver disease (NAFLD)

Materials and Methods: The present analysis was performed on the cohort of subjects enrolled in the FIBAR study, a screening program for diabetes performed between $1^{\text {st }}$ March 2001 and $31^{\text {st }}$ December 2003 in the city of Florence on 3124 subjects who underwent a oral glucose tolerance test. Incident cases of diabetes in nondiabetic subjects $(\mathrm{N}=2662)$ were obtained through databases of drug prescriptions, hospital admissions, and lists of subjects eligible for reimbursement. Incident CVD in subjects free of diabetes and CVD at enrolment $(\mathrm{N}=2617)$ was identified through hospital admissions and through the register of causes of death. Mean follow-up was 39.6 \pm 12.0 months and 39.8 \pm 11.4 months for diabetes and CVD, respectively

Results: Yearly incidence of diabetes and CVD was $0.4 \%$ and $0.2 \%$, respectively. After adjustment for age and sex, $\gamma-\mathrm{GT}>40 \mathrm{U} / \mathrm{l}$ was associated with increased incidence of diabetes and CVD (HR [95\% CI] 2.54 [1.26-5.11], $\mathrm{p}<0.05$ and 2.21 [0.98-5.43], $\mathrm{p}<0.10$, respectively). Risk of diabetes, but not of CVD, was increased in patients with $\gamma$-GT in the 25-40 U/l range. After adjustment for confounders, AST $>40 \mathrm{U} / 1$ predicted CVD (HR 6.5 [1.5-28.1]), but not diabetes.

Conclusion: Elevated $\gamma$-GT or AST are independent predictors of CVD. An increase of $\gamma$-GT levels above the normal range, or also in the upper normal range, is an independent predictor of incident diabetes

Supported by Menarini Diagnostic International, Florence, Italy

\section{5}

Body iron stores and the risk of type 2 diabetes

J. Montonen, P. Knekt, A. Reunanen;

Department of Health and Functional Capacity, National Public Health Institute, Helsinki, Finland.

Background and Aims: Epidemiological studies suggest that high body iron stores may be associated with insulin resistance and type 2 diabetes. The aim of this study was to examine serum ferritin concentration and the ratio of the concentrations of transferrin receptors to ferritin in relation to risk of type 2 diabetes.

Materials and Methods: The study design was a nested case-control study within a longitudinal population study. Serum levels of ferritin and transferring receptors were determined in 234 incident cases with type 2 diabetes identified from the nation wide register of drug reimbursements during the follow-up of 21 years and 464 controls matched for sex, age and study region.

Results: Serum ferritin concentration was associated with an increased risk of type 2 diabetes. The relative risks across the quartiles of serum ferritin concentration were $1.00,1.76(95 \%$ confidence interval $(\mathrm{CI})=0.92-3.35)$, $2.93(\mathrm{CI}=1.49-5.79)$ and $2.08(\mathrm{CI}=1.03-4.17$; $\mathrm{P}$ for trend $(\mathrm{P})=0.08)$ when adjusted for body mass index, smoking, leisure time physical activity, and alcohol consumption. The corresponding multivariate relative risks across increasing quartiles of transferrin receptors to ferritin ratio were $1.84(\mathrm{CI}=$ $0.95-3.55), 1.77(\mathrm{CI}=0.94-3.33), 1.17(\mathrm{CI}=0.63-2.18)$ and $1.00(\mathrm{P}=$ 0.26 ). Stronger associations were observed among persons with higher serum concentrations of vitamin $\mathrm{E}$ or selenium.

Conclusion: The result of the present study suggest that higher iron stores measured as higher serum ferritin concentration and a lower ratio of transferrin receptors to ferritin are associated with an increased risk of type 2 diabetes.

\section{6}

Fatty liver is associated with risk for development of type 2 diabetes mellitus in Korea

C.-H. Kim ${ }^{1}$, H.-K. Kim², K.-E. Lee ${ }^{2}$, S.-H. Chun², I.-Y. Bae ${ }^{2}$, J.-Y. Park K.-U. Lee ${ }^{3}$, J.-H. Kim²;

${ }^{1}$ Endocrinology \& Metabolism, Internal Medicine, Soonchunhyang

University Bucheon Hospital, Bucheon, Kyeonggi-do,

${ }^{2}$ Health Promotion Center, Asan Medical Center, Seoul, ${ }^{3}$ Endocrinology \&

Metabolism, Asan Medical Center, Seoul, Republic of Korea.

Background and Aims: Fatty liver is the most commonly found abnormality on liver ultrasonography in general health check-up. Non-alcoholic fatty liver disease is known to be associated with obesity, diabetes, and metabolic syndrome. A limited number of studies have reported associations of markers liver injury, such as alanine aminotransferase (ALT), aspartate aminotransferase (AST), and $\gamma$-glutamyltransferase (GGT), with prospective risk of type 2 diabetes. However, it is not established whether hepatic steatosis itself is an independent predictor of incident type 2 diabetes. To investigate the associations of ultrasonographic fatty liver with incident type 2 diabetes, we performed a retrospective longitudinal study.

Materials and Methods: We examined the clinical and laboratory data of 6096 subjects (age, $46.8 \pm 8.4$, range $20-79$ years; 4234 men and 1862 women) who visited the Health Promotion Center at Asan Medical Center for a medical check-up in 2000 and underwent a follow-up examination in 2005. Subjects who had diabetes at baseline, were positive for hepatitis B virus surface antigen or hepatitis $\mathrm{C}$ virus antibody, had hepatic enzyme concentrations higher than three times the upper normal limit, or had sonographic evidence of other chronic liver disease (total 724 subjects) were excluded.

Results: Among 5372 included subjects ( 3670 men and 1702 women), 233 individuals $(4.3 \%)$ had developed type 2 diabetes after 5 years. In univariate analysis, risk of development of diabetes was associated with male sex, family history of diabetes, smoking, older age, higher BMI, systolic and diastolic blood pressure, fasting glucose, triglyceride, lower HDL cholesterol, and presence of fatty liver on ultrasonography at baseline. In multiple logistic regression models adjusting for age, sex, and alcohol consumption, participants with fatty liver were at significantly increased risk of incident type 2 diabetes compared with those without (risk ratio [RR] 3.92, 95\% CI 2.89 - 5.31). After further adjustment for smoking, BMI, triglyceride, HDL, fasting glucose, and GGT, fatty liver remained significantly associated with incident type 2 diabetes (RR 1.84, 95\% CI $1.28-2.64$ ).

Conclusion: Fatty liver predicts development of type 2 diabetes mellitus independently of classical metabolic syndrome components and markers of hepatic dysfunction in Korean. 


\section{PS 86 Novel therapies, drugs}

\section{7}

In-vitro evaluation of new pharmacodynamic hybrids with insulin secretagogue and NO-donor activity

S. Torri ${ }^{1}$, M. Galli ${ }^{1}$, V. Calderone ${ }^{2}$, S. Rapposelli ${ }^{3}$, M. Digiacomo ${ }^{3}$, A. Martelli ${ }^{2}$, V. D’Aleo ${ }^{1}$, U. Boggi ${ }^{4}$, A. Balsamo ${ }^{3}$, P. Marchetti ${ }^{1}$;

${ }^{1}$ Endocrinology and Metabolism, Metabolic Unit,

${ }^{2}$ Psychiatry, Neurobiology, Pharmacology and Biotechnologies, ${ }^{3}$ Pharmaceutics Sciences, Faculty of Pharmacy, ${ }^{4}$ Oncology, Transplantation and Advanced Technologies, University of Pisa, Italy.

Background and Aims: Diabetes is associated with beta-cell damage. Vascular alterations are a frequent consequence of the diabetic syndrome, mainly due to endothelium dysfunction. Exogenous nitric oxide (NO) can compensate for the reduced levels of endogenous NO, possibly improving cardiovascular function. Hence we assessed the potential of a molecule combining the properties of insulin secretagogue and NO-donor.

Materials and Methods: Human islets were isolated by collagenase digestion and density gradient purification from the pancreas of 11 multiorgan donors (age $61 \pm 15$ years; gender 3/8 M/F; BMI $25.0 \pm 4.1 \mathrm{Kg} / \mathrm{m}^{2}$ ). The glibenclamide active metabolite 4-trans-hydroxyglibenclamide (M-1) and its nitrooxy derivative DK 315 were obtained through usual synthetic routes. Glibenclamide (GB) was choosen as reference drug. Insulin secretion was studied in response to glucose $(\mathrm{G}, 16.7 \mathrm{mM}), \mathrm{GB}(10$ and $100 \mu \mathrm{M}), \mathrm{M}$ $1(10$ and $100 \mu \mathrm{M})$ and DK $315(10$ and $100 \mu \mathrm{M})$. In order to evaluate the vasorelaxing effects, the drugs were also tested on isolated rat aortic rings.

Results: Insulin release at basal glucose $(3.3 \mathrm{mM})$ was $41.0 \pm 22.3 \mu \mathrm{U} / \mathrm{ml}$. Stimulation index (S.I., insulin release in response to a tested compound over basal release) was $4.1 \pm 2.5$ at $16.7 \mathrm{mM} \mathrm{G} ; 3.8 \pm 2.4$ and $3.9 \pm 2.6$ at 10 and 100 $\mu \mathrm{M} \mathrm{GB} ; 4.8 \pm 3.0$ and $5.7 \pm 4.4$ at 10 and $100 \mu \mathrm{M} \mathrm{M}-1$, and $4.9 \pm 4.0$ and $8.7 \pm 5.8$ at 10 and $100 \mu \mathrm{M}$ DK 315, respectively (all N.S. vs $16.7 \mathrm{mM} \mathrm{G}$ ). GB and M1 were almost devoid of any significant vasorelaxing effect. DK 315 caused concentration dependent vasorelaxing responses $\left(\mathrm{pIC}_{50}=7.240 \pm 0.012\right)$ which were antagonised by ODQ ( $1 \mu \mathrm{M}$, inibitor of guanilate-cyclase), indicating that the activity was due to the release of NO.

Conclusion: This study shows that M-1 and DK 315 possess, on human islets, insulinotropic effects comparable to that exhibited by GB. Furthermore, DK 315 showed NO relaxing properties, which can be considered as a complementary pharmacodynamic feature, of potential usefulness for cardiovascular complication associated with the diabetic status.

\section{8}

A novel antioxidant L-2501 ameliorates metabolic disturbances and attenuates weight gain in rats with fructose-induced insulin resistance N. Gorbenko' ${ }^{1}$, V. Poltorak ${ }^{2}$, A. Gladkih ${ }^{2}$, O. Ivanova ${ }^{1}$, E. Khudyakova ${ }^{1}$, V. Lipson ${ }^{1}$, N. Krasova ${ }^{2}$, J. Leschenko ${ }^{2}$, K. Gorbenko';

${ }^{1}$ Pharmacology, ${ }^{2}$ Pathophysiology, Institute of Endocrine Pathology

Problems, Kharkiv, Ukraine.

Background and Aims: Accumulating evidence suggests a link between increased oxidative stress and insulin resistance. We have previously shown that the low-toxic pyrimido[1,2-a] benzimidazole derivative L-2501 possesses potent antioxidant, anti-inflammatory activity and improves lipid profile in diabetic rabbits. The aim of the study was to explore the impact of L-2501 on metabolic derangements in rats with fructose-induced insulin resistance.

Materials and Methods: Male Wistar rats were divided into three groups: the control group $(\mathrm{C}, \mathrm{n}=8)$, the high fructose-fed group $(\mathrm{F}, \mathrm{n}=8)$, which had free access to $250 \mathrm{~g} / \mathrm{L}$ solutions of fructose for 8 weeks and fructose-fed group treated with L-2501(F+L) for 8 weeks ( $50 \mathrm{mg} / \mathrm{kg} /$ day per os). At the end of the study fasted rats were subjected to the glucose tolerance test (GTT). Serum levels of non-etherified fatty acids (NEFA), triglycerides (TG) and alanine aminotransferase (ALT) were measured as parameters of insulin resistance. Activities of hepatic glucose-6-phosphatase (G6P), glucose-6-phosphatedehydrogenase (G6PDH) and liver glycogen content were also measured in all experimental groups. Oxidative status was estimated by plasma thiobarbituric acid reactive substances (TBARS) and total antioxidant activity (TAA).

Results: Fructose feeding induced insulin resistance, as indicated by higher HOMA-IR index (F: $4.52 \pm 0.70$ vs C: $1.49 \pm 0.01, \mathrm{p}<0.001$ ) and lower QUICKI $(\mathrm{p}<0.01)$ indexes. The fructose group also developed glucose intolerance, hypertriglyceridemia, significantly elevated fructosamine and NEFA levels, ALT and G6P activities $(\mathrm{p}<0.01)$. Administration of L-2501 protected against the forming of fructose-induced glucose intolerance. Area under curve over the GTT was (F+L: $919 \pm 34$ vs F: $1420 \pm 90$, p $<0.01$; C: $851 \pm 25 \mathrm{mM}$ x hour). The treatment with L-2501 decreases insulin resistance (HOMA-IR index F+L: $2.29 \pm 0.19, \mathrm{p}<0.01)$, triglyceride concentration $(\mathrm{F}+\mathrm{L}: 0.424 \pm 0.094$ vs $\mathrm{F}$ : $0.941 \pm 0.97 \mathrm{mmol} / \mathrm{l}, \mathrm{p}<0.01$ ), fructosamine concentrations ( $\mathrm{F}+\mathrm{L}: 1.23 \pm 0.07$ vs F: $1.94 \pm 0.17 \mathrm{mmol} / \mathrm{l}, \mathrm{p}<0.01)$, NEFA levels $(2.93 \pm 0.13$ vs F: $4.20 \pm 0.35 \mathrm{mmol} /$ $1, \mathrm{p}<0.01)$ and serum ALT activity $(\mathrm{p}<0.02)$ in compared with fructose-fed rats treated with placebo. Administration of L-2501 was accompanied by suppression of G6P activity by $44 \%(\mathrm{p}<0.01)$, elevation of G6PDH activity $(\mathrm{p}<0.02)$ and glycogen content $(\mathrm{p}<0.01)$ in liver of fructose-fed animals compared to placebo. In addition, the use of L-2501 was associated with decrease in TBARS concentration 2 - fold $(\mathrm{p}<0.02)$ and increase in TAA by $75 \%(\mathrm{p}<0.01)$ compared to control fructose-fed rats. L-2501 administration resulted in a significant decrease in body weight gain in fructose-fed rats (F+L: $18.9 \pm 1.6$ vs F: $33.1 \pm 2.0 \%$; $\mathrm{p}<0.01$ ).

Conclusion: These data demonstrate that L-2501 administration can ameliorate the deleterious effects of high-fructose diet such as insulin resistance, hypertriglycerdemia, increased gluconeogenesis, oxidative stress and weight gain. These results substantiate the potential of L-2501 as a new therapeutic agent for the correction of multiple risk factors of metabolic syndrome.

\section{9}

Effect of dual (alpha/gamma) peroxisome proliferator-activated receptor activator muraglitazar (MURA) therapy in patients with type 2 diabetes mellitus

M. Fernandez ${ }^{1}$, E. Cersosimo ${ }^{1}$, A. Gastaldelli², R. A. DeFronzo ${ }^{3}$; ${ }^{1}$ Texas Diabetes Institute, University of Texas Health Science Center, San Antonio, United States, ${ }^{2}$ Stable Isotope Laboratory, Institute of Clinical Physiology, Pisa, Italy, ${ }^{3}$ Diabetes Division, University of Texas Health Science Center, San Antonio, United States.

Background and Aims: We evaluated the effect of muraglitazar, a dual (alpha/gamma) peroxisome proliferator-activated receptor (PPAR) activator within the new glitazar class, on glucose and lipid metabolism.

Materials and Methods: A double-blind, randomized controlled trial was performed in 27 patients with type 2 diabetes. Patients received once-daily doses of either $5 \mathrm{mg}$ muraglitazar or placebo plus diet for 16 weeks. Before and after treatment patients underwent: (i) euglycemic hyperinsulinemic clamp combined with tracer infusion to assess endogenous glucose production (EGP) and disposal, and to estimate adipose tissue (Adip-IR=fasting plasma FFA X insulin conc (FPI)) and liver (Hep-IR=EGP $\mathrm{x}$ FPI) indices of insulin resistance (IR); (ii) lean and fat body mass quantification by DXA; (iii) liver and visceral fat measurement by MRI/MRS.

Results: MURA treatment improved fasting glycemia $(-43 \mathrm{mg} / \mathrm{dl}, \mathrm{p}<0.001)$, HbAlc $(-1.9 \%, \mathrm{p}=0.003)$, Hep-IR $(-37 \%, \mathrm{p}<0.01)$ and Adip-IR $(-50 \%$, $\mathrm{p}<0.001$ ). Compared to placebo MURA improved total body (primarily reflects muscle)disposal $(+2.2 \mathrm{mg} / \mathrm{kg} \cdot \mathrm{min}, \mathrm{p}=0.01)$, increased plasma adiponectin $\sim 2$ fold ( $<<0.0001$ vs placebo) and improved insulin-mediated suppression of FFA during the clamp $(\mathrm{p}<0.002)$. MURA treatment was associated with a significant increased body weight $(+2.5 \mathrm{~kg})$, primarily due to increased body fat $(+1.7 \mathrm{~kg})$ but it was associated with significant decreases in both liver fat $(-9 \%, \mathrm{p}<0.001)$ and visceral fat $(-1.5 \%$, form 158 to 134 $\mathrm{cm}^{2}, \mathrm{p}<0.001$ ); subcutaneous fat increased by $+9 \%$ ( from 393 to $428 \mathrm{~cm}^{2}$, $\mathrm{p}<0.001)$.

Conclusion: Muraglitazar significantly (i) improved insulin sensitivity in muscle, liver and adipose tissue, despite a modest increase in body fat, and (ii) decreased hepatic and visceral fat content. 
0910

CS-917, a fructose 1,6-bisphosphatase inhibitor, combined with a thiazolidinedione or insulin decreases plasma glucose and improves lactate metabolism more effectively than CS-917 monotherapy T. Yoshida, J. Ogawa, Y. Hagisawa, S. Kanda, K. Takahashi, A. Okuno; Biological Research Laboratories II, Daiichi Sankyo Company, Limited, Tokyo, Japan.

Background and Aims: CS-917 is a novel, oral inhibitor of fructose 1,6bisphosphatase, a rate-limiting enzyme in gluconeogenesis. We have previously shown that CS-917 reduces plasma glucose (PG) in cynomolgus monkeys and rodent models of type II diabetes such as Zucker diabetic fatty (ZDF) rats and Goto-Kakizaki rats. Elevated plasma lactate (LA) levels have been previously reported following CS-917 administration in old ZDF rats with advanced disease, but not in young ZDF rats. Here, we describe the effect of CS-917 on LA metabolism in rats pretreated with antidiabetic agents.

Materials and Methods: Male, 16-week-old ZDF rats were pretreated for 1 week with rivoglitazone (Rivo; a thiazolidinedione [TZD; $1 \mathrm{mg} / \mathrm{kg}$ once daily]), insulin (INS [implant]) or acarbose (AC; a glucosidase inhibitor $[0.03 \%$ in the diet]). Male, 9 -week-old streptozotocin (STZ)-treated SpragueDawley rats, a model of INS-deficient diabetes, were pretreated with INS, Rivo or dichloroacetic acid (DC; an inhibitor of pyruvate dehydrogenase kinase [50mg/kg, twice daily]). After the pretreatments, CS-917 (100mg/kg) was administered to both ZDF and STZ rats. After administration of CS-917, PG and LA were measured during 8 hours in ZDF rats and LA was measured during 6 hours in STZ rats. The change in the area under the plasma concentration-time curve ( $\triangle \mathrm{AUC}$ ) for LA was calculated.

Results: PG and the $\triangle \mathrm{AUC}$ for LA after CS-917 administration to ZDF and STZ rats with various pretreatments are shown in the table below. CS-917 reduced $P G$ in $Z D F$ rats at 6 hours post administration compared with control. This decrease was more pronounced in ZDF rats that had been pretreated with Rivo, INS and AC compared with non-pretreated ZDF rats. The $\triangle \mathrm{AUC}$ for LA was increased after CS-917 administration in ZDF and STZ rats. In ZDF rats, pretreatment with INS and Rivo, but not AC, suppressed the increase of $\triangle \mathrm{AUC}$ for LA after CS-917 administration. In STZ rats, pretreatment with INS and DC, but not Rivo, suppressed the increase in $\triangle \mathrm{AUC}$ for LA observed after CS-917 administration.

PG and $\triangle \mathrm{AUC}$ for LA after CS-917 administration in pretreated ZDF and STZ rats

\begin{tabular}{|c|c|c|c|c|c|}
\hline & \multicolumn{3}{|c|}{ ZDF rats } & \multicolumn{2}{|c|}{ STZ rats } \\
\hline & & $\Delta \mathrm{AUC}_{0-8} \mathrm{LA}$ & $\begin{array}{l}\text { PG at } 6 \text { hours } \\
\text { post CS-917 } \\
\text { administration }\end{array}$ & & $\Delta \mathrm{AUC}_{0-6} \mathrm{LA}$ \\
\hline & $\mathrm{n}$ & $(\mathrm{h} \bullet \mathrm{mg} / \mathrm{dL})$ & $(\mathrm{mg} / \mathrm{dL})$ & $\mathrm{n}$ & $(\mathrm{h} \bullet \mathrm{mg} / \mathrm{dL})$ \\
\hline $\begin{array}{l}\text { Control } \\
\text { (non-CS-917) }\end{array}$ & 8 & $-9.0 \pm 2.6$ & $448 \pm 13$ & 8 & $-9.8 \pm 2.9$ \\
\hline \multicolumn{6}{|l|}{ Pretreatment } \\
\hline None & 8 & $54.6 \pm 5.1^{\mathrm{a}}$ & $311 \pm 40^{\mathrm{a}}$ & 8 & $415.8 \pm 44.0^{\mathrm{a}}$ \\
\hline INS & 8 & $24.4 \pm 5.8^{\mathrm{a}, \mathrm{b}}$ & $128 \pm 16^{\mathrm{a}, \mathrm{b}}$ & 8 & $0.4 \pm 7.7^{\mathrm{b}}$ \\
\hline RIVO & 7 & $28.2 \pm 5.5^{\mathrm{a}, \mathrm{b}}$ & $149 \pm 16^{\mathrm{a}, \mathrm{b}}$ & 8 & $395.1 \pm 53.4^{\mathrm{a}}$ \\
\hline $\mathrm{AC}$ & 8 & $61.4 \pm 5.9^{\mathrm{a}}$ & $246 \pm 14^{\mathrm{a}}$ & - & ND \\
\hline $\mathrm{DC}$ & - & $\mathrm{ND}$ & ND & 7 & $150.0 \pm 25.1^{\mathrm{a}, \mathrm{b}}$ \\
\hline
\end{tabular}

$\mathrm{a}=\mathrm{P}<0.05$ versus control; $\mathrm{b}=\mathrm{P}<0.05$ versus non-pretreated control ( $\mathrm{t}$-test).

Mean \pm standard error, ND $=$ not determined.

Conclusion: These results suggest that enhancement of INS signalling, but not insulin sensitisation, improves LA metabolism. The combination of CS917 with a TZD or INS may decrease PG and improve LA metabolism more effectively than CS-917 monotherapy in patients with type II diabetes.

\section{1}

A novel glucagon antagonist inhibits glucagon-stimulated glucose production in human hepatocytes and lowers blood glucose in animal models of type 2 diabetes

P. D. van Poelje ${ }^{1}$, S. C. Potter ${ }^{1}$, J. Hou ${ }^{1}$, R. Wu ${ }^{1}$, J. M. Fujitaki ${ }^{1}$, D. W. Reeder ${ }^{1}$, J. Gomez ${ }^{1}$, D. L. Linemeyer ${ }^{1}$, M. D. Erion ${ }^{1}$

${ }^{1}$ Metabasis Therapeutics Inc., San Diego, United States.

Background and Aims: Elevated levels of circulating glucagon contribute to fasting and postprandial hyperglycemia in patients with T2DM by inappropriately increasing hepatic glucose production. The aim of these studies was to assess the antidiabetic potential of a novel, small molecule glucagon antagonist (Cpd G) by determining its potency and efficacy in relevant cellular and in vivo models.

Materials and Methods: Receptor antagonism studies were performed with mouse or rat liver cell membrane or recombinant human glucagon receptor-containing cell membrane preparations by ${ }^{125}$ I-glucagon $(125 \mathrm{pM})$ displacement. Extracellular glucose and/or intracellular cAMP levels were determined in cultured human or rat hepatocytes exposed to glucagon (1 $\mathrm{nM}$ ) and varying concentrations of Cpd G. Expression of the PGC-1 $\alpha$ and PEPCK genes was assessed by the real time-polymerase chain reaction. Blood glucose was measured in freely feeding $\mathrm{db} / \mathrm{db}$ mice ( $\mathrm{n}=8$ /group) or overnight fasted Zucker fa/fa (ZF) rats (n=6/group) following IP administration of Cpd $\mathrm{G}$ for 1or 7 days. Pharmacokinetic parameters were determined in Sprague Dawley rats ( $\mathrm{n}=8$ /group) following $\mathrm{PO}$ and IV administration of 30 and 5 $\mathrm{mg} / \mathrm{kg}$ doses of Cpd G, respectively.

Results: The $\mathrm{IC}_{50}$ values of Cpd G in the mouse, rat and human glucagon receptor assays were $75 \mathrm{nM}, 260 \mathrm{nM}$ and $45 \mathrm{nM}$, respectively. In human hepatocytes, Cpd G fully inhibited glucagon-stimulated glucose production with an $\mathrm{EC}_{50}$ value of $230 \mathrm{nM}$. Inhibition of glucose production was reversed by increasing concentrations of glucagon. Cpd G also inhibited $\left(\mathrm{EC}_{50} 300\right.$ $\mathrm{nM})$ the $\sim 7$-fold increase in PGC- $1 \alpha$ and PEPCK mRNA levels elicited by glucagon in human hepatocytes. In rat and human hepatocytes, Cpd G inhibited glucagon-stimulated cAMP production with $\mathrm{EC}_{50}$ values of 550 $\mathrm{nM}$ and 210nM, respectively. Single administrations of Cpd G $(3,10$, or 30 $\mathrm{mg} / \mathrm{kg}$ ) to $\mathrm{db} / \mathrm{db}$ mice lowered blood glucose in a dose-dependent manner with a reduction from $\sim 450 \mathrm{mg} / \mathrm{dL}$ to $\sim 300 \mathrm{mg} / \mathrm{dL}$ observed at the highest dose, while an increase in blood glucose levels in vehicle-treated mice. In ZF rats, administration of Cpd G lowered blood glucose from $\sim 100 \mathrm{mg} / \mathrm{dL}$ to levels observed in lean controls, $\sim 70 \mathrm{mg} / \mathrm{dL}$, within 3 hours following a single dose $(30 \mathrm{mg} / \mathrm{kg})$, and to a similar extent following 7 days of treatment ( $30 \mathrm{mg} / \mathrm{kg}$, b.i.d.). Blood glucose levels in vehicle-treated animals were unchanged. The 7-day treatment with Cpd G did not alter body weight, food consumption, or plasma glucagon levels. Plasma Cpd G concentrations were similar on Day 1 and Day 7. Key pharmacokinetic parameters of Cpd $\mathrm{G}$ in rats were as follows: oral bioavailability, $21 \%$; terminal half-life, $1.6 \mathrm{~h}$; clearance, $6.4 \mathrm{~L} / \mathrm{h} / \mathrm{kg}$.

Conclusion: Cpd G demonstrated all the expected effects of a potent glucagon antagonist in rodent and human hepatocytes: inhibition of glucagonstimulated cAMP production, glucose production, and gluconeogenic gene expression. Moreover, Cpd G markedly lowered fed and fasting blood glucose levels in animal models of overt or mild T2DM. Sub-chronic administration to ZF rats did not result in tachyphylaxis or elevation of plasma glucagon levels. Pharmacokinetic optimization of the Cpd G series may lead to a promising new antidiabetic agent.

\section{2}

Pramlintide improved glycaemic control and reduced weight without increased hypoglycaemia in patients with type 2 diabetes inadequately controlled with insulin glargine \pm oral agents

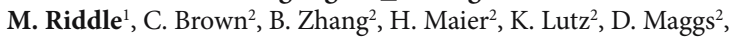
O. Kolterman ${ }^{2}$

${ }^{1}$ School of Medicine, Oregon Health \& Science University, Portland,

${ }^{2}$ Amylin Pharmaceuticals, Inc., San Diego, United States.

Background and Aims: When type 2 diabetes (T2DM) is inadequately controlled with basal insulin \pm oral agents, the usual options are increasing basal insulin and/or adding mealtime insulin. Limitations of these options include weight gain and increased risk of hypoglycemia. Pramlintide, an analog of the beta-cell hormone amylin, has been shown to reduce $\mathrm{HbA}_{1 c}$, 
postprandial glucose (PPG), and body weight in patients with T2DM using basal + mealtime insulin. In the current study, we examined the efficacy and safety of pramlintide as an alternative, rather than an addition, to mealtime insulin.

Materials and Methods: In this 16-week, randomised, double-blind study, 211 patients with T2DM using insulin glargine \pm oral agents were treated with pramlintide or placebo with major meals. Patient demographics at baseline (age $55 \pm 9$ y, diabetes duration $11 \pm 6$ y, $\mathrm{HbA}_{1 \mathrm{c}} 8.5 \pm 0.9 \%$, BMI $35 \pm 5 \mathrm{~kg} / \mathrm{m}^{2}$; mean \pm SD) were well balanced between treatment groups. Baseline insulin doses (mean $\pm \mathrm{SD}$ ) were $48 \pm 25 \mathrm{U}$ (pramlintide) and $54 \pm 42 \mathrm{U}$ (placebo). After optimisation of pramlintide dosage (60 or $120 \mu \mathrm{g})$, insulin was titrated weekly, targeting a fasting glucose concentration of $70-100 \mathrm{mg} / \mathrm{dl}$.

Results: Week 16 insulin doses (mean $\pm \mathrm{SD}$ ) were $61 \pm 32 \mathrm{U}$ (pramlintide) and $70 \pm 50 \mathrm{U}$ (placebo). Reductions in $\mathrm{HbA}_{1 \mathrm{c}}$ and PPG increments from baseline (mean $\pm \mathrm{SE}$ ) were greater in pramlintide- vs. placebo-treated patients $\left(\mathrm{HbA}_{1 \mathrm{c}}\right.$ : $-0.7 \pm 0.1 \%$ vs. $-0.4 \pm 0.1 \%, \mathrm{P}<0.05$; PPG increments: $24.4 \pm 3.6 \mathrm{mg} / \mathrm{dl}$ vs. $-0.4 \pm 3.0 \mathrm{mg} / \mathrm{dl}, \mathrm{P}<0.0001)$. Improvements in glycemia were accompanied by progressive weight loss with pramlintide and weight gain with placebo $(-1.6 \pm 0.3 \mathrm{~kg}$ vs. $+0.7 \pm 0.3 \mathrm{~kg}, \mathrm{P}<0.0001)$. More patients reached a composite outcome measure at Week $16\left(\mathrm{HbA}_{1 \mathrm{c}} \leq 7.0 \%\right.$ or $\mathrm{HbA}_{1 \mathrm{c}}$ reduction $\geq 0.5 \%$ AND no weight gain AND mean PPG increments $\leq 40$ $\mathrm{mg} / \mathrm{dl}$ AND no severe hypoglycemia) with pramlintide than with placebo ( $25 \%$ vs. $7 \%, \mathrm{P}<0.001)$. To further explore treatment responses, the study population was divided into two subgroups according to the mean baseline $\mathrm{HbA}_{1 \mathrm{c}}(\leq 8.5 \%$ or $>8.5 \%)$.

Post-hoc patient stratification according to baseline $\mathrm{HbA}$

\begin{tabular}{|c|c|c|c|c|}
\hline \multirow{2}{*}{$\begin{array}{l}\Delta \text { from baseline to } \\
\text { Week } 16\end{array}$} & \multicolumn{2}{|c|}{ Baseline $\mathrm{HbA}_{1 c} \leq 8.5 \%$} & \multicolumn{2}{|c|}{ Baseline $\mathrm{HbA}_{1 c}>8.5 \%$} \\
\hline & $\begin{array}{l}\text { Placebo } \\
(\mathrm{N}=58)\end{array}$ & $\begin{array}{l}\text { Pramlintide } \\
(\mathrm{N}=63)\end{array}$ & $\begin{array}{l}\text { Placebo } \\
(\mathrm{N}=48)\end{array}$ & $\begin{array}{l}\text { Pramlintide } \\
(\mathrm{N}=42)\end{array}$ \\
\hline$\Delta \mathrm{HbA}_{1 \mathrm{c}}(\%)$ & $-0.1 \pm 0.1$ & $-0.4 \pm 0.1$ & $-0.7 \pm 0.1$ & $-1.2 \pm 0.1$ \\
\hline $\begin{array}{l}\triangle \text { PPG increments } \\
(\mathrm{mg} / \mathrm{dl})\end{array}$ & $-3.6 \pm 3.7$ & $-24.9 \pm 4.4$ & $3.2 \pm 4.6$ & $-23.7 \pm 5.9$ \\
\hline$\Delta$ weight $(\mathrm{kg})$ & $0.4 \pm 0.4$ & $-2.0 \pm 0.4$ & $1.1 \pm 0.4$ & $-1.0 \pm 0.3$ \\
\hline
\end{tabular}

Data are reported as mean $\pm \mathrm{SE}$

Regardless of baseline $\mathrm{HbA}_{1 \mathrm{c}}$, pramlintide + basal insulin titration produced benefits not provided by

basal insulin titration alone. Pramlintide was generally well tolerated, with the most frequent adverse events being mild-to-moderate hypoglycemia (pramlintide 44\%; placebo $47 \%$ ) and nausea (pramlintide 31\%; placebo $10 \%$ ). No treatment-related severe hypoglycemia occurred.

Conclusion: In summary, pramlintide + basal insulin titration improved $\mathrm{HbA}_{1 \mathrm{c}}$ and PPG without weight gain or increased hypoglycemia compared to placebo + basal insulin titration in patients with T2DM. These findings support pramlintide as a potential option for the next therapeutic step when patients with T2DM are not achieving glycemic targets with basal insulin therapy.

\section{3}

Metabolic responses to a sodium-glucose transporter-2 inhibitor, GW869682, assessed by whole-genome transcript profiling

S. Ghosh ${ }^{1}$, Y. Shen ${ }^{1}$, W. W. Harrington ${ }^{1}$, J. Schroeck ${ }^{1}$, R. Boyle ${ }^{1}$, T. Fox ${ }^{1}$, T. DeBruin ${ }^{1}$, C. D. Smith ${ }^{1}$;

${ }^{1}$ GlaxoSmithKline Research, Research Triangle Park, United States.

Background and Aims: Enhanced urinary glucose excretion by selective inhibition of the sodium-glucose transporter 2 (SGLT2) in the proximal tubules of the kidney represents a novel therapeutic approach in the treatment of type 2 diabetes. However, it is unclear whether direct removal of glucose by this mechanism improves sensitivity to insulin. We investigated the effects of the SGLT2 inhibitor GW869682 on the transcription of genes involved in key metabolic tissues in the Zucker Diabetic Fatty (ZDF) rat.

Materials and Methods: We profiled whole genome transcripts from adipose tissue, muscle (soleus), liver and kidney of ZDF rats. Eight groups, comprising five animals each, were treated with either a single dose of GW869682 at 30 $\mathrm{mg} / \mathrm{kg}$ or vehicle for $1,3,7$ and 14 days. Amplified RNA was hybridised to Affymetrix rat RAE230A chips according to standard procedures. Gene signals were computed using the MAS5.0 software and normalised by the global scaling method in MAS5.0. Genes with an ANOVA $p<0.05$ were retained for additional analysis. Statistical comparisons were by 2 -sample, heteroscedastic $\mathrm{t}$-tests followed by estimation of false discovery rates.

Results: In the adipose tissue, gene expression by principal components analysis from Day 0 to Day 14 suggested a delay in the progression from glucose intolerance to frank diabetes in drug-treated animals compared with vehicle-treated controls. Analysis of relevant biological pathways in muscle, liver and white adipose tissue indicated statistically significant $(\mathrm{p}<0.05)$ upregulation of several genes involved in glucose transport and glucose utilization in drug-treated animals, with concomitant reductions in the expression of genes regulating fatty acid utilization in liver and adipose tissue. The expression of genes encoding key bypass enzymes of gluconeogenesis - glucose-6-phosphatase and fructose-1,6-bisphosphatase - was reduced in the kidney $(\mathrm{p}<0.05)$. No changes were observed in the expression of genes normally used as markers for renal damage.

Conclusion: Treatment with GW689682 regulated the expression of key genes involved in glucose and fatty acid metabolism in several metabolic tissues. The patterns of gene expression point to an overall enhancement of insulin sensitivity and better glucose utilization in animals treated with GW689682. Confirmatory in vivo physiologic data are needed to support the findings from this transcriptomics study.

\section{4}

Clinical studies to assess safety, pharmacokinetics and pharmacodynamics of sergliflozin, a novel inhibitor of glucose reabsorption

L. Kler ${ }^{1}$, E. K. Hussey ${ }^{2}$, R. L. Dobbins ${ }^{2}$, R. L. O’Connor-Semmes ${ }^{2}$, A. Kapur ${ }^{3}$, S. C. Murray ${ }^{4}$, R. V. Clark², R. R. Stoltz ${ }^{5}$, N. L. Stockman ${ }^{5}$, D. M. Amin ${ }^{6}$, M. S. Kipnes ${ }^{7}$, E. C. O’Driscoll ${ }^{8}$, J. Leong ${ }^{3}$, D. J. R. Nunez ${ }^{9}$;

${ }^{1}$ Clinical Pharmacology and Discovery Medicine, GSK, Greenford, United Kingdom, ${ }^{2}$ Clinical Pharmacology and Discovery Medicine, GSK, RTP, United States, ${ }^{3}$ Clinical Study Sciences and Operations, GSK, RTP, United States, ${ }^{4}$ Clinical Pharmacology Statistics and Programming, GSK, RTP, United States, ${ }^{5}$ Clinical Operations, Covance-Evansville, Evansville, United States, ${ }^{6}$ Global Medical Services, Quintile Global CRO, Bracknell, United Kingdom, ${ }^{7}$ Endocrinology, DGD Research Inc, San Antonio, United States, ${ }^{8}$ Clinical Pharmacology and Discovery Medicine, GSK, King of Prussia, PA, United States, ${ }^{9}$ Metabolic Experimental Medicine, GSK, RTP, NC, United States.

Background and Aims: Sergliflozin (S; GW869682) is a novel selective inhibitor of the renal low-affinity sodium-glucose cotransporter (SGLT2). S causes dose-dependent urinary glucose excretion (UGE) in animals and may provide a new treatment for type 2 diabetes (T2D) independent of insulin secretion and sensitivity. Clinical studies have been undertaken to evaluate the effects SGLT2 inhibition in humans.

Materials and Methods: Two double-blind, randomised, placebocontrolled, single-dose escalation crossover studies were conducted. In Study 1, S doses ranged from 5-500mg in 14 healthy males. In Study 2, doses ranged from $50-500 \mathrm{mg}$ in 8 subjects with T2D. A 14-day repeat-dose study in 18 healthy overweight and obese subjects (BMI $25-35 \mathrm{~kg} / \mathrm{m}^{2}$ ) was also conducted. Subjects received S 500mg, 1000mg or placebo ( $n=6$ for each) three-times daily. Efficacy endpoints included UGE and glucose response during OGTT.

Results: Following single doses, dose proportionality was observed for pharmacokinetic (PK) parameters with no differences observed between healthy and T2D subjects. There was a dose-dependent increase in UGE which plateaued, suggesting that glucose reuptake was maximally inhibited at higher S doses. Duration of UGE paralleled plasma concentrations of the active moiety. S 500mg decreased plasma glucose concentrations over $4 \mathrm{~h}$ from 18.3 to $11.2 \mathrm{mmol}^{\star} \mathrm{h} / \mathrm{L}$ following OGTT in T2D subjects. There were small transient increases in urinary excretion of electrolytes, but no overall increase over $24 \mathrm{~h}$. The most common adverse effects (AEs) were headache and sore throat in healthy subjects, and headache and dyspepsia in T2D subjects. Multiple dosing was well tolerated; most frequently reported AEs were headache, dizziness, flatulence and nausea. PK parameters were stable with no accumulation of $\mathrm{S}$ or its major metabolites in plasma. $\mathrm{S}$ caused a considerable dose-dependent UGE increase. High doses of S did not provoke hypoglycemia in nondiabetic subjects. There was a $\sim 1.5 \mathrm{~kg}$ body weight reduction in S-treated subjects from baseline to Day 15 vs. placebo. S caused increases in urine electrolyte levels on Day 1 which resolved by Day 14 
without an effect on serum electrolytes or calculated creatinine clearance. S caused a dose-dependent negative fluid balance on Day 1 which had resolved by Day 14 in the $500 \mathrm{mg}$ group and was less marked in the 1000mg group. Conclusion: Single and multiple doses of $S$ were well tolerated, resulted in pharmacodynamic changes consistent with renal SGLT2 inhibition and lowered plasma glucose concentrations in T2D subjects. These are the first clinical data to support SGLT2 inhibition as a unique mechanism for glycemic control

\section{PS 87 Novel therapies, plant-derived}

\section{5}

Acacia catechu aqueous bark extract stimulates insulin secretion and insulin action and inhibits starch digestion

Y. H. A. Abdel-Wahab, G. C. Clarke, P. R. Flatt;

School of Biomedical Sciences, University of Ulster, Coleraine, Northern Ireland, United Kingdom.

Background and Aims: Higher plants such as Acacia catechu, also known as Cutch tree or Indian liquorice root, provide therapeutic opportunities and represent a possibly rich source of potential antidiabetic agents. The aim of the present work was to investigate at the cellular level the antidiabetic activity of Acacia catechu on insulin secretion using clonal pancreatic BRINBD11 cells, glucose uptake using adipocyte differentiated 3T3-L1 fibroblasts and starch digestion using in vitro model.

Materials and Methods: Dried bark/wood was homogenised to a fine powder and a decoction was prepared by bringing $25 \mathrm{~g} / \mathrm{L}$ of material to the boil in water. The suspension was filtered and $1 \mathrm{~mL}$ aliquots of the filtered plant solution were brought to dryness under vacuum. Aliquots were reconstituted in incubation buffer for subsequent experiments as required.

Results: An aqueous extract of Acacia catechu stimulated insulin secretion 24 fold in a dose dependent manner from BRIN-BD11 cells at $5.6 \mathrm{mM}$ glucose $(1-25 \mathrm{mg} / \mathrm{ml}, \mathrm{P}<0.001, \mathrm{n}=8)$. Removal of extracellular calcium reduced insulin secretion at both 1 and $5 \mathrm{mg} / \mathrm{ml}$ Acacia catechu by $57 \%(\mathrm{P}<0.001)$ and $32 \%(\mathrm{p}<0.05)$, respectively. Incubation with verapamil $(50 \mu \mathrm{M})$ and diazoxide $(300 \mu \mathrm{M})$ abolished Acacia catechu $(1 \mathrm{mg} / \mathrm{ml})$ induced insulin secretion. Furthermore, the extract significantly increased insulin secretion in depolarised cells $(30 \mathrm{mM} \mathrm{KCl})$. Addition of extract $(5 \mathrm{mg} / \mathrm{ml})$ to BRINBD11 cells produced a sharp rise, which was followed by a gradual sustained increase in intracellular calcium compared to cells incubated with $5.6 \mathrm{mM}$ glucose alone. Furthermore, the extract induced a transient rise in BRINBD11 membrane polarization which was reduced by addition of diazoxide $(300 \mu \mathrm{M})$. Exposure of adipocyte differentiated 3T3-L1 fibroblasts to Acacia catech $u(1 \mathrm{mg} / \mathrm{ml}$ for $20 \mathrm{~min})$ produced a 1.8 -fold increase $(\mathrm{P}<0.001)$ in glucose uptake compared to unsupplemented controls $(\mathrm{P}<0.001, \mathrm{n}=4)$. Incubation of insulin $\left(10^{-9} \mathrm{M}\right)$ with plant extract $(1 \mathrm{mg} / \mathrm{ml})$ induced a $141 \%$ increase in glucose uptake compared to insulin alone ( $29800 \pm 1663$ vs. $14420 \pm 421.5$ DPM, $\mathrm{P}<0.001, \mathrm{n}=4)$. This effect was significantly greater than the actions of either alone $(\mathrm{P}<0.001)$. Incubation of aqueous extract of Acacia catechu $(1-50$ $\mathrm{mg} / \mathrm{ml})$ with starch produced a $2-16 \%$ decrease $(\mathrm{P}<0.001)$ in carbohydrate digestion in vitro.

Conclusion: The study has revealed that aqueous extract of Acacia catechu contains natural molecules that stimulate insulin secretion, insulin action and inhibit starch digestion in vitro. Future work directed at the purification and characterization of active components may reveal new agents for type 2 diabetes therapy.

Supported by University Research Strategy Funds

\section{6}

Antidiabetic effect of a plant material Murraya koenigii on nondiabetic and diabetic model rats

S. Sarma ${ }^{1}$, S. Khan ${ }^{2}$, B. P. Sarma ${ }^{1}$, N. Nahar ${ }^{3}$, M. Mosihuzzaman ${ }^{3}$, B. Rokeya ${ }^{2}$;

${ }^{1}$ Dept of Pharmacology, Govt Ayurvedic College, Assam, India,

${ }^{2}$ Dept of Pharmacology, BIRDEM, Dhaka,

${ }^{3}$ Dept of Chemistry, Dhaka University, Bangladesh.

Background and Aims: Murraya koenigii (popularly known as Curry leave plants) is available in South India, Bangladesh, Sri-Lanka and Tropical Asia. It is being used extensively in Indian cuisine as special sauces. It has been claimed by the Ayurvedic physicians that curry leaves may control hyperglycemia in type 2 diabetic subjects with special reference to obese type 2 diabetics. We screened the hypoglycemic activity of Murraya koenigii in nondiabetic and diabetic model rats following a standardised approach. Murraya koenigii leaves were collected from around Dhaka city and identified in the Bangladesh National Herbarium.

Materials and Methods: Freeze-dried $80 \%$ ethanol extract of Murraya koenigii leaves was used for the study at a dose of $1.25 \mathrm{mg} / \mathrm{kg}$ body weight. 
Male Long-Evans rat bred at BIRDEM Animal House, weighing between (180-200) grams were used to carry out the experiment. Type 1 and type 2 model rats were produced with intraperitoneal injection of streptozotocin using conventional methods and following the procedures standardized in BIRDEM. Acute experiments were done in nondiabetic and in both diabetic model rats with a single feeding in different prandial states. Blood was collected by cutting the tail tips under mild ether anesthesia. Glucose was measured by Glucose-Oxidase (GOD-PAP) method. Statistical Analysis was done by using one-way ANOVA.

Results: Screening result for hypoglycemic activity of $80 \%$ ethanol extract of PE075 leaves in nondiabetic rats showed no effect in the fasting state. On the other hand, the extract showed significant hypoglycemic effect $(\mathrm{p}=0.03)$ in the fasting state in type 2 model rats. Moreover the extract significantly lowered serum glucose level of type 2 diabetic rats in both prandial states (simultaneously with oral glucose load $\mathrm{p}=0.002$ and 30 minutes prior to oral glucose load $\mathrm{p}=0.002$ ). Reduction in glucose level was comparable with Glibenclamide treated group. In type 1 diabetic model rats ethanol extract $(80 \%)$ significantly $(\mathrm{p}=0.03)$ opposed the rise of serum glucose when the extract was fed simultaneously with glucose load.

Conclusion: The data suggest that $80 \%$ Murraya koenigii leaves extract has significant hypoglycemic effect in normal rats and significant antihyperglycemic effect in both types of diabetic model rats. Therefore, the plant merits further exploration both chemically and biologically to identify the active principle(s) and mechanism of action.

Supported by International Programme in the Chemical Sciences (IPICS)

\section{7}

DC250188, a natural compound isolated from Chinese herb with potent efficacy on the treatment of type 2 diabetes and metabolic syndrome in animal models

Y. Leng ${ }^{1}$, J. H. Chen ${ }^{2}$, Y. Feng ${ }^{1}$, Z. Zhou ${ }^{1}$, H. Zhang ${ }^{1}$, Y. Shen ${ }^{1}$, J. H. Shen ${ }^{2}$; ${ }^{1}$ Pharmacology Department I, ${ }^{2}$ DDDC, Shanghai Institute of Materia Medica, Chinese Academy of Sciences, Shanghai, China.

Background and Aims: Type 2 diabetes mellitus (T2DM) is a major cause of morbidity and mortality worldwide. Current pharmacological therapies are insufficient and new strategies to treat and prevent T2DM are urgently needed. The aim of present study is to investigate the anti-diabetic effect of DC250188, a natural compound isolated from Chinese herb on obese diabetic $\mathrm{ob} / \mathrm{ob}$ and diet induced obese (DIO) mice. The possible mechanisms of antidiabetic effect of DC250188 were also explored.

Materials and Methods: DC250188 was administered orally to ob/ob or DIO mice at different doses for 26 or 45 days. Metformin and rosiglitazone were used as positive control. Blood glucose level, body weight and food intake were measured regularly, and insulin, lipid profiles, fructosamine, uric acid and other blood chemistry were analysed after treatment. OGTT, ITT and 2-deoxyglucose uptake assay were also performed on ob/ob mice. The phosphorylation of AMPK in liver and skeletal muscle of ob/ob mice after DC250188 treatment was evaluated by Western Blot. The effects of DC250188 on phosphorylation of AMPK and ACC were also studied on 3T3-L1, L6 and HepG2 cells.

Results: DC250188 dose dependently reduced the hyperglycemia of ob/ $\mathrm{ob}$ and DIO mice. The hypoglycemic efficacy of DC250188 is similar with metformin and rosiglitazone. The serum fructosamine level in DC250188 treated group is much lower than that of metformin or rosiglitazone treated group, which suggested it had more beneficial effect on the long term glycemic control. In ob/ob mice, DC250188 showed significant reduction in serum triglyceride, free fatty acid, total cholesterol and LDL-C level, whereas metformin and rosiglitazone were less potential in lowering those lipid profiles. The triglyceride deposit in liver and serum uric acid level were reduced significantly after DC250188 treatment, suggested its beneficial effect in the T2DM associated with fatty liver and hyperuricemia. DC250188 could improve OGTT and ITT, and increase insulin stimulated glucose uptake in isolated EDL muscle, which suggested its insulin sensitising effect. DC250188 could also decrease the body weight of both ob/ob and DIO mice. Treatment with DC250188 on ob/ob mice for 26 days could increase the phosphorylation of AMPK in liver and skeletal muscle. DC250188 could also stimulate the phosphorylation of AMPK and ACC in 3T3-L1 adipocyte, L6 myotube and HepG2 cells.

Conclusion: DC250188 exerts potent and efficacious antidiabetic and hypolipidemic effect, and improves metabolic abnormalities such as fatty liver and hyperuricemia, which suggested it would be an excellent therapeutic candidate for treatment of T2DM and metabolic syndrome. Activation of AMPK might be one of the possible mechanisms of this effect.

\section{8}

Effects of a plant material PE053 on glycaemic and lipidaemic status of type 2 diabetic Long-Evans rats

T. Bandara ${ }^{1}$, E. R. Jansz ${ }^{1}$, S. Ekanayeka ${ }^{1}$, K. Balasubramanium ${ }^{2}$, B. Rokeya ${ }^{3}$; ${ }^{1}$ Dept.of Biochemistry, University of Sri Jayawardanapura, Colombo, Sri Lanka, ${ }^{2}$ Dept.of Biochemistry, University of Jaffna, Sri Lanka, ${ }^{3}$ Dept of Pharmacology, BIRDEM, Dhaka, Bangladesh.

Background and Aims: The use of the leaves of the plant PE 053 has a long folkloric history in the treatment of diabetes all over the South Asian countries. Due to its proven hypoglycemic activity on various diabetic animals and humans PE053 has become increasingly popular as a supportive treatment for diabetes. Recent reports have indicated that in addition to hypoglycemic effect the plant PE053 has got beneficial effects on dyslipidemia. The present study evaluates the effect of PE053 on glycaemic and lipidemic status of type 2 diabetic rats.

Materials and Methods: Male Long -Evans rats, bred at BIRDEM animal house and weighing between 150-200g, were used in the study. Type 2 diabetes was induced by a single intraperitoneal injection of Streptozotocin to $48 \mathrm{~h}$ old pups and experiments were carried out three months later. Leave powder of PE053 was prepared from mature leaves obtained from Jaffna, Sri Lanka. Acute experiment was done in type 2 model rats with a single feeding. For chronic experiment type 2 rats were divided into 3 groups: 1 ) Vehicle ( receiving only water, $n=6$ ); 2) Treated with PE 053 leave powder (once daily for 28 consecutive days at a dose of $1.25 \mathrm{~g} / \mathrm{kg}$ body weight, $\mathrm{n}=6$ ) and 3) Treated with standard drug glibenclamide $(5 \mathrm{mg} / \mathrm{kg}$ body weight, $\mathrm{n}=6)$. Body weight was checked every week. Blood was collected by cutting the tail tip at the beginning and on the days $7,14,21$, and by decapitation on the $28^{\text {th }}$ day. The parameters measured were serum glucose (by glucose oxidase), serum insulin (by ELISA), lipid profile (enzymatic-colorimetric), serum creatinine (by picric acid-colorimetric) and serum ALT (by enzymatic-colorimetric methods).

Results: Results of the acute experiment showed no significant blood glucose lowering effect of PE053 in type 2 diabetic model rats. On the other hand, chronic and ministration of PE053 leave powder caused a gradual fall of serum glucose level on day $14(15.45 \%)$. A significant reduction in serum glucose level was noticed on the 28th day (serum glucose in $\mathrm{mmol} / \mathrm{l} ; \mathrm{M} \pm \mathrm{SD}$, $8.95 \pm 1.36$ vs. $5.56 \pm 0.72, \mathrm{p}<0.001)$. Reduction in glucose level was comparable with the glibenclamide treated group. Fasting serum insulin content did not change in the PE 053 treated group whereas $26.3 \%$ increase was observed in glibenclamide treated group. Serum total cholesterol level of the treated group was reduced significantly $(\mathrm{p}<0.05)$. Serum TG, and LDL-cholesterol were reduced by $23.5 \%$ and $12.3 \%$ respectively and HDL-cholesterol level increased by $39.42 \%$. Serum ALT and creatinine level remained unchanged throughout the study period among the Control and as well as PE053 treated groups. ALT level decreased significantly $(\mathrm{p}=0.01)$ in the glibenclamide treated group. Body weight did not change significantly in any of the groups during the treatment period.

Conclusion: PE053 leave powder improves the glycemic and lipidemic status of type 2 diabetic model rats.

Supported by International Program in the Chemical Sciences (IPICS)

\section{9}

Impact of cinnamon vs. placebo on surrogate markers of metabolic control in patients with type 2 diabetes mellitus

R. Weitgasser ${ }^{1}$, A. Pfützner ${ }^{2}$, B. Kerschgens ${ }^{2}$, H. Drexel ${ }^{3}$, R. Prager ${ }^{4}$, B. Ludvik ${ }^{4}$, T. Wascher ${ }^{5}$, F. Hoppichler 6 , S. Berger ${ }^{7}$, T. Forst ${ }^{2}$, P. Grafinger ${ }^{8}$; ${ }^{1}$ Diabetes, University Hospital, Salzburg, Austria, ${ }^{2}$ R\&D, IKFE Institute for Clinical Research and Development, Mainz, Germany, ${ }^{3} \mathrm{R} \& \mathrm{D}$, Institute for vascular research, Feldkirch, Austria, ${ }^{4}$ Diabetes, University Hospital, Vienna, Austria, ${ }^{5}$ Diabetes, University Hospital, Graz, Austria,

${ }^{6}$ Diabetes, General Hospital, Salzburg, Austria, ${ }^{7}$ Diabetes, Rehabilitation Hospital, Aflenz, Austria, ${ }^{8}$ Diabetes, Public Hospital, Linz, Austria.

Background and Aims: Preliminary work with cinnamon as nutritional supplement has pointed to a beneficial effect of this spice in reducing 
postprandial glucose excursions. However, further evidence is lacking and some potentially carcinogenic cumarine derivatives in the cinnamon formulations have lead to some controversy among scientists worldwide about the use of cinnamon in supporting dietary treatment in type 2 diabetes.

Materials and Methods: In order to evaluate these effects further, we performed a randomized double-blind prospective study with a cinnamon extract deprived from all potentially dangerous components (Alsidiabet, Alsitan) vs. placebo, in compliance with the regulations of Good Clinical Practice. In total, 109 patients (treated with diet and exercise) were included (62 men, 47 women, age (mean $\pm \mathrm{SD}$ ): $59 \pm 9$ years, , HbAlc: $7.8 \pm 0.8 \%$, BMI: $\pm \mathrm{xx} \mathrm{kg} / \mathrm{m}^{2}$ ). They were randomised to either receive $360 \mathrm{mg}$ of a cinnamon extract or a similarily shaped and smelling placebo once daily in the morning over a period of 3 months. No change in anti-diabetic medication was allowed during the study. Fasting blood samples were drawn for measurement of $\mathrm{HbAlc}$, glucose, insulin (for calculation of the HOMA $\mathrm{IR}_{\mathrm{R}}$ score), intact proinsulin, hsCRP, adiponectin, Lipids, and oxLDL at baseline and after 6 and 12 weeks.

Results: The HbA1c improved in both groups, but the change was more pronounced in the verum group (verum vs. placebo: $-0.6 \pm 1.0 \%$ vs. $-0.3 \pm 1.0$ $\%, \mathrm{p}=0.070)$. Insulin resistance was improved with verum and further deteriorated with placebo $\left(\mathrm{HOMA}_{\mathrm{IR}}:-0.8 \pm 3.1\right.$ vs. $\left.+0.6 \pm 2.5, \mathrm{p}<0.05\right)$. Allmost all other observation parameters showed slight improvement with verum while a further impairment was seen with placebo (adiponectin: $+1.1 \pm 2.3$ vs. $+0.1 \pm 2,7 \mathrm{mg} / \mathrm{l}, \mathrm{p}=0.052$; hsCRP: $-0.4 \pm 1.6$ vs. $-0.2 \pm 1.2 \mathrm{mg} / \mathrm{l}$, n.s.; intact proinsulin: $-2.0 \pm 9.4$ vs. $+1.5 \pm 7.4 \mathrm{pmol} / \mathrm{l}, \mathrm{p}<0.05$; Cholesterol: $-1 \pm 26$ vs. $+9 \pm 27 \mathrm{mg} / \mathrm{dl}, \mathrm{p}=0,051$; LDL: $-1 \pm 20$ vs. $+5 \pm 18 \mathrm{mg} / \mathrm{dl}$, n.s.; HDL: $+2 \pm 4$ vs. $0 \pm 5$ $\mathrm{mg} / \mathrm{dl}, \mathrm{p}=0.071$; triglycerides: $-16 \pm 64$ vs. $+27 \pm 87 \mathrm{mg} / \mathrm{dl}, \mathrm{p}<0.005$; oxLDL: $-7 \pm 103$ vs. $+7 \pm 54 \mathrm{~nm} / \mathrm{ml}$, n.s.). There were no changes in BMI from baseline to endpoint in both groups. Both treatments were well tolerated and there were no differences between the groups regarding adverse events.

Conclusion: We found a consistent improvement in markers of glycemic control, insulin resistance, $\beta$-cell dysfunction and cardiovascular risk when testing cinnamon extract vs. placebo in a double-blind randomized study protocol over three months. Whether this efficacy can be attributed to a direct sensitizing effect of cinnamon on the insulin receptor as postulated in the literature or through other mechanisms (e.g. appetite regulation) needs to be further elucidated. The effects were less pronounced to the results that can be obtained with anti-diabetic drugs, but our study results clearly support the value of cinnamon when used as nutrition supplement in the course of a special diabetes diet.

Supported by Alsitan $\mathrm{GmbH}$

\section{0}

Ergothioneine protection against lipotoxic effects

I. Laurenza ${ }^{1}$, R. Colognato ${ }^{2}$, A. Bertacca ${ }^{1}$, S. Del Prato ${ }^{1}$, L. Benzi ${ }^{1}$;

${ }^{1}$ Dept. Endocrinology and Metabolism,

${ }^{2}$ Dept. of Human and Env. Sciences, University of Pisa, Italy.

Background and Aims: Inflammation and reactive oxygen species (ROS) have been claimed to contribute to the development of micro- and macrovascular diabetic complications. Though this may suggest that use of anti-oxidant agent could provide some protection, so far treatment with classic free-radical scavengers and anti-oxidants has been disappointing. Therefore, more potent agents are under search. We have, then assessed in vitro the anti-oxidant capacity of a natural product, Ergothioneine (EGT).

Materials and Methods: To this purpose $\mathrm{C} 2 \mathrm{C} 12$ myoblasts have been incubated for $24 \mathrm{hrs}$ in presence of $250,500,750$, and $1000 \mu \mathrm{M}$ palmitic acid (PA), conjugated with $10 \%(\mathrm{w} / \mathrm{v})$ FFA-free BSA with or without $24-\mathrm{hr}$ pre-incubation with 500 and $1000 \mu \mathrm{M}$ EGT for assessment of cell viability, p38 and JNK activity by Western blot, and IL- 6 both mRNA expression and protein synthesis.

Results: PA incubation led to dose dependent cell viability reduction, while EGT pre-incubation resulted in greater cell viability at each PA concentration

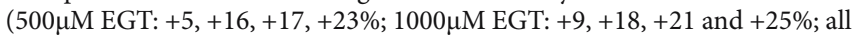
$P<0,05$ or less). Whether this protective effect could be mediated by specific effect on intracellular redox signalling was evaluated by assessment of p38 and JNK activity. As compared to control cells, exposure to PA $(250,500$, $750 \mu \mathrm{M})$ significantly increased p38 $(+50,+131,+261 \%$ respectively $)$ and JNK $(+261 \%,+396 \%$ and $+441 \%$ respectively) activities (both $P<0.001)$. In contrast, pre-treatment with EGT was associated with reduction of their activity (p38: with $500 \mu \mathrm{M}$ EGT: $-12,-13,-31 \%$ and with $1000 \mu \mathrm{M}$ EGT: -1 ,
$-8,-33 \%$; for JNK: with $500 \mu \mathrm{M}$ EGT: $-15,-6,-34 \%$ and with $1000 \mu \mathrm{M}$ EGT: $-15,-18,-46 \%$ all $P<0,01)$. Moreover, EGT prevented PA-induced IL- $6 \mathrm{mRNA}$ expression evaluated at the IC50 $(500 \mu \mathrm{M})$ production $(P<0.001)$ both at the transcriptional ( -8 and $-44 \%$ for 500 and $1000 \mu \mathrm{M}$ of EGT) and translational $(-0.6$ and $-1.2 \mathrm{ng} / \mathrm{mg}$ protein for 500 and $1000 \mu \mathrm{M}$ of EGT) level.

Conclusion: Our results indicate that EGT can effectively protect viability of myoblasts to deleterious metabolic environment such as increase FFA concentration possibly due to inhibition of the activation of the MAPK cascade and reduced inflammatory response.

Supported by the Ministero dell'Universita' e della Ricerca 


\section{PS 88 Diabetes in childhood}

\section{1}

Treatment with insulin aspart versus human insulin in children and adolescents with newly diagnosed type 1 diabetes

J. Ludvigsson ${ }^{1}$, H. Mersebach ${ }^{2}$, K. Kanc Hanzel ${ }^{2}$, H. Holmberg';

${ }^{1}$ Dept of Clinical and Molecular Medicine, Linköping University, Linköping, Sweden, ${ }^{2}$ Novo Nordisk A/S, Bagsvaerd, Denmark.

Background and Aims: Rapid-acting modern insulin may offer advantages for the patients but potentially induce antibodies of immunological and clinical interest. Studies of the immunogenicity of modern insulin have mainly been performed in adults. The aim of this study was therefore to compare therapy with IAsp with human insulin (HI) both in combination with NPH insulin in insulin-naive children and adolescents. Insulin antibodies, $\mathrm{HbAlc}$, insulin dose and serious adverse events (SAEs) were investigated.

Materials and Methods: Seventy-two newly diagnosed children and adolescents, aged $2-17$ years treated with HI $(n=30)$ or IAsp $(n=42)$, both in combination with NPH insulin, were included. IAsp specific antibodies (IAsp-Ab) and antibodies cross-reacting with $\mathrm{HI}$ and IAsp (HI-cross- $\mathrm{Ab}$ ) were analyzed by radioimmunoassay at diagnosis of diabetes and every 3-6 months for 30 months. Data on HbAlc, insulin dose and SAEs were collected retrospectively.

Results: At diagnosis, the mean total daily insulin dose was $1.02 \mathrm{U} / \mathrm{kg}$ in the $\mathrm{HI}$ group and $0.98 \mathrm{U} / \mathrm{kg}$ in the IAsp group. After 18 months treatment the insulin dose was $0.93 \mathrm{U} / \mathrm{kg}$ and $0.89 \mathrm{U} / \mathrm{kg}$ in the $\mathrm{HI}$ and IAsp group, respectively. The total daily bolus doses were $0.58 \mathrm{U} / \mathrm{kg}$ of $\mathrm{HI}$ and $0.56 \mathrm{U} /$ $\mathrm{kg}$ of IAsp at diagnosis and $0.51 \mathrm{U} / \mathrm{kg}$ of $\mathrm{HI}$ and $0.52 \mathrm{U} / \mathrm{kg}$ of IAsp after 18 months treatment. The mean (SD) HbAlc in the HI and IAsp groups were similar at diagnosis, 9.52\% (1.97) and 9.57\% (1.62), respectively. The HbAlc then decreased to $5.45 \%(1.40)$ and $4.81 \%(0.93)$ at 6 months and thereafter stabilised at about $6 \%$ in both groups for the remaining period. The level of IAsp-Ab remained low throughout the study. The level of HI-cross- $\mathrm{Ab}$ increased from a low baseline level at diagnosis to a mean (SD) of $48.75 \%$ (21.53) and $40.20 \%$ (17.92) after nine months of treatment in the HI and IAsp group, respectively and remained elevated. In a repeated measurement analysis, no statistical significant difference in $\mathrm{HI}$-cross $\mathrm{Ab}$ levels was found between treatment groups $(\mathrm{p}=0.16)$. HI-cross- $\mathrm{Ab}$ were significantly associated with total insulin dose $(\mathrm{U} / \mathrm{kg})$ at 18 months $(\mathrm{p}=0.001)$, but not with $\mathrm{HbAlc}$ at 18 months ( $\mathrm{p}=0.24$ ). Only few SAEs were reported (HI group: $\mathrm{n}=5,14$ events and IAsp group: $n=3,3$ events), the majority being hypoglycemic episodes Conclusion: The insulin doses and HbAlc were similar in patients using HI or IAsp from diagnosis. Both treatments were associated with a similar increase in $\mathrm{HI}$-cross- $\mathrm{Ab}$ in insulin naive children, but with no influence on treatment efficacy or safety. These results support the safe use of IAsp in children and adolescents with type 1 diabetes.

Supported by Novo Nordisk A/S

\section{2}

Low risk of poor metabolic control in pre-pubertal diabetic children treated with insulin pump. The results of the Polish Prospective Pump Study

E. Pankowska ${ }^{1}$, A. Szypowska ${ }^{1}$, E. Czerniawska ${ }^{2}$, B. Surdej ${ }^{3}$, L. Lisowicz ${ }^{3}$, E. Kolender ${ }^{4}$, M. Myśiwiec

${ }^{1}$ Pediatric Diabetology and Birth Defects, Medical University, Warsaw, ${ }^{2}$ Pediatric, Medical University, Lodz, ${ }^{3}$ Pediatrics, The Specialist Hospital, Rzeszów, ${ }^{4}$ Endocrinology, The Specialist Pediatric Hospital, Olsztyn,

${ }^{5}$ Pediatrics Hematology, Oncology and Endocrinology, Medical University, Gdańsk, Poland.

Background and Aims: Epidemiological study has shown that the incidence rate of T1DM increased four times among the youngest children during last decade. Continues subcutaneous insulin infusion (CSII) as an alternative method is becoming one of the common method uses in pediatric patients; however the best option of replacement insulin therapy in young T1MD is still disputed. To asses the efficacy of pump therapy in pre-pubertal children comparing with multiply daily injection.

Materials and Methods: The cohort, multi center study was conducted in 17 centers in Poland according to the protocol of the Polish Prospective Pump
Study. The data was collected electronically from November 2005 to February 2007 during follow-up visits. HbA1c was assessed in central laboratory using BioRad method (HPLC).

Results: A study group consisted of 538 pre-pubertal children treated with CSII; mean age was $8.03 \pm 2.4$; ; mean diabetes duration $3.6 \pm 2.1$ years; mean pump treatment duration was $2.0 \pm 1.3 y$ s. 245 subjects were enrolled into the control group; there are no significant differences in age and diabetes duration between two groups.HbAlc for study group was $7.38 \pm 0.9 \%$ vs. $7.33 \pm 1.1 \% \mathrm{~ns}$. The analysis was given in three age related groups: $\mathrm{I}<5$ years, II 5-9 years; III 9-12 years it shows the best control in preschoolers $7.24 \pm 0.7$ vs. $7.39 \pm 0.9$ vs. $7.42 \pm 1.0 \%$ ns. The risk of poor glycemic control was $23 \%$ in individuals treated with CSII. The significant differences were noticed in growth development parameters. In study group the mean BMI in group

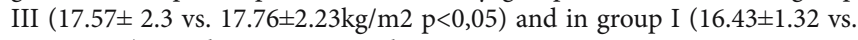
$18.16 \pm 4.83)$ were lower, respectively.

Conclusion: Continuous subcutaneous insulin infusion is an effective method of insulin treatment for pre-pubertal children with type 1 diabetes and the risk of high HbAlc is lower if the CSII is used. The growth development consists with physiology was only in patients treated with pump. Supported by Foundation Great Orchestra of Christmas Charity

\section{3}

Trends in the management of childhood type 1 diabetes in Romania between 1998 and 2006

V. Serban ${ }^{1}$, A. Enache ${ }^{2}$, M. Rosu' ${ }^{1}$ A. Lacatusu ${ }^{3}$, L. Barna ${ }^{3}$, A. Sima ${ }^{1}$; ${ }^{1}$ Diabetes Clinic, University of Medicine and Pharmacy, Timisoara, ${ }^{2}$ Diabetes Clinic, Clinical Medical Center "Cristian Serban", Buzias, ${ }^{3}$ Diabetes Clinic, Clinical Medical Center "Cristian Serban" Buzias, Romania.

Background and Aims: In Romania, a country with a low incidence of type 1 diabetes mellitus (DM), the original feature of the management of childhood type $1 \mathrm{DM}$ consists in the existence of a unique modern hospital, specialized in the evaluation and therapy optimization of type $1 \mathrm{DM}$ patients aged between 0 and 30 years, from all over Romania: the Clinical Medical Center,"Cristian Serban" from Buzias.

The present paper has two aims: 1) to assess the changes over time (between 1998 and 2006) in insulin strategies 2) to evaluate the variations in glycemic control at the country level before and after admission in the Clinical Center ,"Cristian Serban", depending on the insulin regimens and age-group, between 1998 and 2006.

Materials and Methods: We enrolled in the study 1191 patients aged between 1 and 18 years, admitted once, twice or three times in the Clinical Center ,Cristian Serban" from Buzias, between 1998 and 2006. We investigated the following: a) time trends of insulin regimens using 2, 3 and 4 daily insulin injections b) the quality of glycemic control expressed by glycated hemoglobin $\left(\mathrm{HbA}_{1 \mathrm{c}}\right.$ ) (measured using the turbidimetric method, with a normal range of 4.5-5.7\%): mean $\mathrm{HbA}_{1 \mathrm{c}}$ was computed for the first hospital admission (reflecting the glycemic control at home), as well as for the second and the third admission; c) glycemic control depending on age-group ( $<4$ years, 5-9 years, 10-14 years and 15-18 years). Statistical analysis was performed using GraphPad software, a p value of less than 0.05 was considered significant. Results: Between 1998 and 2006, the percentage of patients using 2 and 3 daily insulin injections decreased from $13.7 \%$ to $3.6 \%$ ( $\mathrm{p}=0.0041$ ) and from $60.0 \%$ to $21.4 \%$ ( $<<0.0001)$, respectively, while the proportion of patients using 4 insulin injections increased from $26.3 \%$ to $75.0 \%$ ( $\mathrm{p}<0.0001$ ). Changes in glycemic control between 1998 and 2006, depending on insulin regimen and on age-group are presented in table 1.

Table 1

\begin{tabular}{llllllll}
\hline & \multicolumn{3}{l}{$\begin{array}{l}\text { HbA } \\
\text { on } \\
\text { on no. of injections }\end{array}$} \\
\cline { 2 - 8 } & 2 inj & 3 inj & 4 inj & $<4$ yrs & $5-9$ yrs & $10-14$ yrs & $15-18$ yrs \\
\hline 1998 & $11.4 \pm 2.9$ & $10.8 \pm 2.6$ & $10.0 \pm 2.4$ & $8.8 \pm 1.7$ & $10.8 \pm 2.7$ & $10.7 \pm 2.6$ & $11.2 \pm 2.9$ \\
$2006^{* *}$ & $9.8 \pm 2.4$ & $9.4 \pm 2.6$ & $9.2 \pm 2.3$ & $8.4 \pm 1.5$ & $8.1 \pm 1.8$ & $9.5 \pm 2.1$ & $10.2 \pm 2.4$ \\
p & 0.04 & 0.018 & 0.06 & 0.5 & $<0.0001$ & 0.0005 & 0.019 \\
\hline
\end{tabular}

* patients at first admission in the center ${ }^{* *}$ for 2 injections group: data from 2001

The analysis of glycemic control depending on age-group has showed a reduction of mean $\mathrm{HbA}_{1 c}$ as follows (the first figure represents the 1998 
value and the second the 2006 value): in children younger than 4 years from $8.8 \pm 1.7 \%$ to $8.4 \pm 1.5 \%(\mathrm{p}=0.5)$; in age-group $5-9$ years from $10.8 \pm 2.7 \%$ to $8.1 \pm 1.8 \%$ ( $<<0.0001)$; in age-group $10-14$ years from $10.7 \pm 2.6 \%$ to $9.5 \pm 2.1 \%$ $(\mathrm{p}=0.0005)$, and in age-group $15-18$ years from $11.2 \pm 2.9 \%$ to $10.2 \pm 2.4 \%$ $(\mathrm{p}=0.019)$.

Conclusion: 1. In Romania, in 2006, most children with type 1 DM use multiple insulin injections regimens. 2. Glycemic control $\left(\mathrm{HbA}_{1 \mathrm{c}}\right)$ has improved starting 1998, but remained unsatisfactory, regardless of insulin regimens and insulin types used.

\section{4}

Insulin antibodies correlate negatively with C-peptide levels and positively with insulin sensitivity in young type 1 diabetic patients during the first two years of diabetes

B. Mianowska, A. Szadkowska, O. Wegner, I. Pietrzak, E. Czerniawska, A. Heinrich, W. Młynarski, J. Bodalski;

Department of Pediatrics, Medical University, Lodz, Poland.

Background and Aims: This study was aimed at evaluating the impact of insulin antibodies (IA) on clinical and metabolic characteristics of children and adolescents with type 1 diabetes (T1DM).

Materials and Methods: 33 patients with T1DM (aged 7.6-17.6 years, 24 males) treated with human insulin or human insulin analogs participated in the study. IA (radioimmunoassay, IA-AIA Cis bio international, normal value $0-7 \%$ ), fasting C-peptide (C-pep, radioimmunoassay, C-PEP-CT2 Cis bio international), HbAlc (HPLC, Variant Bio-Rad) and glucose disposal rate (M-index determined by euglycemic hyperinsulinaemic clamp technique by de Fronzo) were measured at diabetes diagnosis (0-3rd day), 6 months after diagnosis and 24 months after diagnosis. Daily insulin doses were calculated.

Results: IA levels significantly raised within the first 6 months of treatment $(6,6+/-1.2 \%$ vs. $32,1+/-17.0 \%$ at diagnosis and at $6 \mathrm{mth}$ respectively, $\mathrm{P}<0.0001)$, and remained stable thereafter $(33.8+/-18.2 \%$ at $24 \mathrm{mths}, \mathrm{P}=0.23$ vs. $6 \mathrm{mths}$ ). At $6 \mathrm{mths}$ and at $24 \mathrm{mths}$ IA levels correlated negatively with C-pep at diabetes diagnosis $(\mathrm{r}=-0.33, \mathrm{P}=0.05$ at $6 \mathrm{mths}, \mathrm{r}=-0.39, \mathrm{P}=0.04$ at $24 \mathrm{mths}$ ). A negative correlation was found between IA levels and C-pep at 6 $\mathrm{m}$ ths $(\mathrm{r}=-0.35, \mathrm{P}=0.04)$ and at $24 \mathrm{mths}(\mathrm{r}=-0.39, \mathrm{P}<0.05)$. IA levels positively correlated with insulin sensitivity measured as $\mathrm{M}$-index $(\mathrm{r}=0.34, \mathrm{P}=0.05$ at $6 \mathrm{mths}$ and $\mathrm{r}=0.37, \mathrm{P}<0.05$ at $24 \mathrm{mths}$ ). At $6 \mathrm{mths}$, but not at $24 \mathrm{mths}$, IA correlated with daily insulin dose per kilogram $(\mathrm{r}=0.43, \mathrm{P}=0.01)$. A slight negative correlation between C-pep at diagnosis and daily insulin dose per kilogram at $6 \mathrm{mths}(\mathrm{r}=-0.31, \mathrm{P}=0.06)$ and a significant negative correlation between C-pep at diagnosis and daily insulin dose per kilogram at $24 \mathrm{mths}$ were observed ( $r=-0.39, P=0.03)$. No correlation between IA and HbAlc was found.

Conclusion: Currently used human insulin preparations and human insulin analogs induce immune response to exogenous insulin. Patients with lower residual beta cell function at diagnosis require higher insulin doses, and this can promote more intense IA production. The positive correlation between IA and insulin sensitivity can be related either to IA ability to act as a reservoir of free insulin, and can disclose an uncontrolled insulin release from IA-insulin immune complexes, or IA-insulin complexes may not be "inactive" but may participate in total hypoglycemic effects of insulin.

Supported by the State Committe for Scientific Research

\section{5}

Determinants of insulin resistance development in teenage boys

O. O. Khyzhnyak, T. N. Sulima, E. I. Plekhova;

Pediatric Endocrinology, Scientific Research Institute of Children Health Care, Kharkov, Ukraine.

Background and Aims: It is well known that early onset of adult diseases may be triggered by metabolic disturbances during pubertal development. Nowadays, metabolic disorders such as type 2 diabetes mellitus (DM) and metabolic syndrome keep a leading position in increased prevalence among the world's population. In our studies we were interested in factors that determine predisposition to early onset of metabolic disorders in which resistance to insulin play crucial role.
Materials and Methods: 123 teenage boys with clinical manifestation of the metabolic syndrome were under investigation in the Department of Pediatric Endocrinology. Expanded anamnesis including prenatal and early development period, family history of type $2 \mathrm{DM}$, hypertension, thyroid dysfunctions, traumas of the head and neck, pediatric infections was collected. Physical examination included height, weight, body temperature and blood pressure measurements, skin, eyes and thyroid gland examination, pubertal (sexual) development (Tanner stage) estimation. Blood samples for insulin, cortisol, thyrotropin (TTH) and leptin measurements were taken in fasting state. Selective participants underwent an OGTT. Blood samples were obtained $0,30,60,90$, and 120 minutes after oral glucose administration. Surrogate measure of the insulin resistance - HOMA was calculated according to Matthews et al (1985).

Results: Multiple regression analysis was performed to identify the predictors of the gradually increase in HOMA value from 0.29 to 36.3 in overall sample of subjects. Basic multiple regression model consists of cortisol, TTH and leptin as predictors which explain up to $58.6 \%$ of the HOMA dispersion. It was assumed that cutoff point for diagnosis of the insulin resistance divides investigated patients into groups with physiologically derivated decrease in insulin sensitivity and genetically predisposed to insulin resistance individuals. In our study HOMA cutoff point was found at the level of 3.9. In sample with $\mathrm{HOMA}<3.9$, combination of cortisol $(360.9 \pm 260.6 \mathrm{nmol} / \mathrm{L}$; $\mathrm{M} \pm \mathrm{SD})$ and TTH $(2.83 \pm 1.17 \mathrm{mIU} / \mathrm{L})$ significantly describe HOMA variability $\left(\mathrm{R}^{2}=87 \%\right)$ in teenage boys with subtle endocrine dysfunctions. As mentioned by several investigators, the fall in insulin sensitivity during puberty is associated with a compensatory increase in insulin secretion. The regulatory purpose of these changes in insulin action and secretion is not clear but is thought to provide a mechanism for increasing the anabolic effects of insulin and growth hormone during a period of rapid somatic growth.

In sample with $\mathrm{HOMA} \geq 3.9$ gradual increase in HOMA significantly was associated $\left(\mathrm{R}^{2}=64.4 \%\right)$ with rise in cortisol $(434 \pm 197.6 \mathrm{nmol} / \mathrm{L})$ and leptin $(40 \pm 30.59 \mathrm{mkg} / \mathrm{L})$ concentration in fasting blood and serious metabolic disorders. In addition, relative deposit of cortisol to HOMA value is higher in overweight individual ( $30 \geq \mathrm{BMI} \geq 25)$, but in obese boys (BMI $>30)$ - leptin mainly predetermines HOMA value. These resultes may be explained by recent finding that leptin modulates glucose homeostasis by acting as an insulin-sensitizing factor in most insulin target tissues.

Conclusion: Positive family history of the type $2 \mathrm{DM}$ and obesity create background for metabolic disorder development, while early puberty and a longer period of maturation may be additional risk factors for early onset of type $2 \mathrm{DM}$.

Supported by the Academy of Medical Science of Ukraine

\section{6}

A case of type 1 diabetes mellitus with insulin resistance secondary to anti-insulin antibodies treated with plasmapheresis, rituximab and cyclophosphamide

D. R. Franco ${ }^{1}$, I. L. Noronha ${ }^{2}$, M. H. B. Kiss ${ }^{3}$, J. G. H. Vieira ${ }^{4}$,

F. G. Eliaschewitz;

${ }^{1}$ Clinical research, CPCLIN, ${ }^{2}$ Laboratory of Molecular and Cellular

Nephrology, USP, ${ }^{3}$ Reumatology, USP,

${ }^{4}$ Endocrinology, Fleury Laboratory, São Paulo, Brazil.

Aims: To describe a case of type 1 diabetes (T1DM) in whom the presence of anti-insulin antibodies presented a treatment challenge

Case History: Diagnosed at the age of nine, patient R.A.B. was initially well controlled on four shots daily of NPH insulin and Lispro. Two years after the diagnosis the patient was also diagnosed with hypothyroidism and subcutaneous requirements increased reaching $150 \mathrm{U} / \mathrm{day}(3.2 \mathrm{U} / \mathrm{kg} /$ d). Anti-insulin antibodies [ $>20 \%$ (normal range $<3 \%$ )] were identified, and the patient was initially controlled with sc insulin as an outpatient, but presented several episodes of ketoacidosis in the first year of uncontrolled glycemia. His insulin regimen was changed to Glargine, and for 11 months glycemia control improved. However, as insulin requirement again increased and control worsened the patient was switched to i.v. insulin. The patient continued to need more than $1000 \mathrm{IU}$ insulin i.v./day without exhibiting any nocturnal hypoglycemia. We postulated the patient could be producing antiinsulin antibodies, which were rendering him resistant to insulin. Various modalities of immunosuppression were tried out, beginning with $60 \mathrm{mg}$ prednisone daily, which had no effect on the patients' insulin requirements (1200 IU/day). He subsequently received a cycle of gammaglobulin and 
methylprednisolone, in an imunossupressive dose with no effect on insulin requirement. Hospitalisation to perform plasmapheresis for one week was the following step; indeed, glycemia control improved and insulin requirement was reduced to $500 \mathrm{IU} /$ day. However, one week after plasmapheresis insulin requirement increased again and plasmapheresis was repeated concomitant with a new cycle of methylprednisolone and Rituximab. This time, insulin requirement diminished within several weeks and a sustained response to treatment was obtained. The patient was transferred to a s.c. pump receiving $100 \mathrm{IU} /$ day, and remained stable for one year on prednisone and cyclophosphamide. The patient remains now with insulin pump therapy, at $1 \mathrm{U} / \mathrm{kg} /$ day of insulin requirement and without any immunossupression.

Conclusion: This unusual case of an adolescent boy with anti-insulin antibodies and T1DM illustrates the use of immunosuppressant therapy and plasmapheresis in improving glycemia control. Rituximab and cyclophosphamide may be considered as an effective means of immunosuppression in patients with markedly raised anti-insulin antibodies with diabetes uncontrollable with insulin alone

\section{7}

Exploratory study on the long-term outcome in children aged up to age 9 years born to mothers with type 2 diabetes mellitus

H. W. de Valk ${ }^{1}$, N. H. G. van Nieuwall ${ }^{1}$, W. Lasfar ${ }^{1}$, G. H. A. Visser²

${ }^{1}$ Internal Medicine, Univ. Medical Centre,

${ }^{2}$ Obstetrics, University Medical Centre, Utrecht, The Netherlands.

Background and aims: The number of women with pregestational type 2 diabetes mellitus (PGDM2) is increasing as part of the global diabetes epidemic. Studies have shown that these pregnancies are associated with an increased incidence of adverse outcomes and meticulous obstetric and metabolic care is mandatory. Little is known about long-term outcome of children born to mothers with PGDM2. This study was aimed at exploring evolution of body weight, neuropsychological development and school achievements in a group of these children.

Materials and Methods: Fifty-three children were identified as born to 44 women with PGDM2. Follow-up was available for 41 children (77.4\%) ranging from 4 to 156 months; this analysis is limited to data up to 9 years. Development was assessed by attainment of milestones and is expressed as normal or abnormal "van Wiechenscore". This scheme is evaluated at 15 (W15) and 48 months (W48). Weight was assessed monthly during the first year, at 15, 24 and 45 months and at toddler's age (4 years) and at the age of nine years. Differences between groups in continuous data were tested with (non-)parametric tests, in categorical data with $\mathrm{X}^{2}$-test or single proportion test; in longitudinal changes with repeated measures analysis.

Results: Maternal characteristics: mean age at conception is $33 \pm 4$ years; body mass index: $32.3 \pm 7.5 \mathrm{~kg} / \mathrm{m}^{2}$; mean HbAlc $6,1 \%$; median duration of disease: 3 years. $51 \%$ Caucasian, $34 \%$ of Moroccan descent, $15 \%$ of other origin. Neonatal characteristics: macrosomia $\left(\geq 90^{\text {th }}\right.$ percentile) $45.3 \%$, severe hypoglycaemia $58.5 \%$, preterm delivery $18.9 \%$. Maternal and neonatal were similar in Caucasians and Moroccans. W15 was abnormal in $67.5 \%$ and W48 in $72.4 \%$ with most cases having one abnormal item. Presence of an abnormal score and number of abnormal items were comparable for both sexes and not related to neonatal glucose levels or gestational age at delivery. However, mean $\mathrm{HbAlc}$ levels during the first or second trimester were lower with an abnormal than with a normal W15 ( $6.5 \pm 0.8 \%$ vs. $5.8 \pm 0.7 \%(\mathrm{p}<0.05)$ and $6.3 \pm 0.6 \%$ vs. $5.7 \pm 0.6 \%(\mathrm{p}<0.05)$ respectively). The frequency of abnormal W48 was higher in Moroccan compared to Caucasian children (91.7 vs. $50.5 \%, \mathrm{p}<0.05)$ with no difference in W15. This difference at W48 was not attributable to speech or language problems. From 4 to 9 years, 3 of the 22 children (13.6\%) required special education compared to $5.5 \%$ in the normal population $(\mathrm{p}>0.05)$. Speech therapy was required in $29 \%$ compared to $3.7 \%$ in the normal population $(\mathrm{p}<0.05)$ and specific follow-up examination because of delayed motor-development was required in $26.3 \%$ compared to $2.0 \%$ in the normal population $(\mathrm{p}<0.05)$. Macrosomic neonates retained a significantly higher mean weight percentile during the first six years of age compared to non-macrosomic children $(\mathrm{p}<0.001$, repeated measures analysis).

Conclusion: Maternal pregestational type 2 diabetes is possibly associated with abnormal offspring development to the van Wiechenscore. Surprisingly, abnormal van Wiechenscore at 15 months was associated with lower HbAlclevels during early pregnancy. Children have a higher mean weight percentile during follow-up. Although this is a relatively small patient group, these results indicate that the consequences of maternal type 2 diabetes stretch for the developing child further than the immediate neonatal period and that more developmental research in large groups is needed.

\section{8}

Characteristics of adolescents with type 1 diabetes who exhibit adverse outcomes

M. S. Faulkner' ${ }^{1}$, C. Johns², L. Quinn';

${ }^{1}$ College of Nursing, University of Arizona, Tucson,

${ }^{2}$ Medical-Surgical Nursing, University of Illinois at Chicago, United States.

Background and Aims: Adolescents with type 1 diabetes are challenged to balance their therapeutic regime of diet, exercise, and insulin management with developmental changes to achieve optimal glycemic control. The goal of this study was to identify characteristics among adolescents with type 1 diabetes who have poor outcomes from their disease. Poor outcomes were defined as inadequate glycemic control, hypoglycemic events, and hospitalizations due to diabetes. A secondary aim of this study examined differences in glycemic control or insulin dose among subjects receiving insulin via pump versus multiple daily injections.

Materials and Methods: Adolescents with type 1 diabetes ages 13-18 years ( $\mathrm{n}=108 ; 55 \%$ males; 67\% Caucasian; 27\% Black; 6\% Hispanic) were recruited from a diabetes care center in a large urban setting in the United States. Subjects completed a survey quantifying frequency of insulin injections, blood glucose monitoring, hypoglycemic reactions requiring assistance from another person, and hospitalisations within the past year because of diabetes. Parents completed a demographic questionnaire pertaining to income level, marital status, and highest educational level completed. Adolescents kept a 3-day food record that was later reviewed with them by a registered dietician for accuracy and entered into Nutrionist Pro ${ }^{\text {max }}$ software for macronutrient analysis. A lab draw was performed to obtain current $\mathrm{HbA}_{1 c}$. Subjects were grouped according to level of glycemic control ( $<8 \%$ vs. $\geq 8 \% \mathrm{HbA}_{1 c}$ ), hypoglycemia requiring assistance in the past year (none vs. $\geq 1$ episodes), and hospitalisations over the past year (none vs. $\geq 1$ ) to determine differences in the following variables: $\mathrm{BMI}$, insulin dose, self blood glucose checks, $\mathrm{HbA}_{1 \mathrm{c}}$, total caloric intake, \% calories from carbohydrates, protein, and fats, parents' educational level, parents' marital status, annual family income, race, and gender.

Results: Subjects receiving insulin via pump on average had better glucose control $\left(\mathrm{HbA}_{1 \mathrm{c}} 7.9 \%\right.$ vs. $\left.9.0 \%, \mathrm{p}<0.001\right)$ and were on lower insulin doses $(0.7$ $\mathrm{u} / \mathrm{kg} /$ day vs.1.1 u/kg/day, $\mathrm{p}<0.001)$. Those with adequate glucose control $\left(\mathrm{HbA}_{1 \mathrm{c}}<8 \%\right)$ were on a lower insulin dose $(0.9 \mathrm{u} / \mathrm{kg} /$ day vs. $1.1 \mathrm{u} / \mathrm{kg} /$ day, $\mathrm{p}=$ 0.005 ), checked their blood sugar more frequently ( 4.9 vs. 3.9 times per day, $\mathrm{p}<0.001)$, and had fathers with a higher education level (14.8 years vs. 13.2 years, $p=0.007$ ) when compared to counterparts with poor glucose control $\left(\mathrm{HbA} \geq_{1 \mathrm{c}} 8 \%\right)$. In addition, those with poor control were more likely to come from a single parent home $(p=0.02)$, lower income family $(p=0.05)$, and from an ethnic minority $(\mathrm{p}=0.01)$. No significant differences were found between those hospitalized and those not hospitalized as well as those who experienced hypoglycemic reactions versus those who did not.

Conclusion: Findings from this analysis supports using pump therapy for adolescents when appropriate. It also identifies groups of adolescents who are in need of more guidance and supervision to manage their disease appropriately. Further research is needed to explore what interventions will most likely bring about more favorable outcomes for such groups. Supported by NINR/NIH R01 NR07719

\section{9}

Social disparity in clinical presentation of childhood onset type 1 diabetes in Germany

J. Rosenbauer, A. Icks, G. Giani;

Institute of Biometrics and Epidemiology, German Diabetes Centre, Düsseldorf, Germany.

Background and Aims: Aim of the study was to describe the clinical presentation of Type 1 diabetes at diagnosis in a population-based cohort of children in particular with respect to social status.

Materials and Methods: During 2002-2006, 2735 new cases of Type 1 diabetes under 15 years of age were prospectively registered by the diabetes 
register of the German federal state of North-Rhine-Westphalia (completeness of registration $\geq 95 \%$ ). Data on demographics, clinical symptoms preceding diagnosis, and clinical presentation and hospital stay at diagnosis were obtained from hospital records and from parents of diabetic children by means of mailed standardised questionnaires. Social status of families was assessed by the parents' school education (Low: $\leq 9$ years, middle: 10-11 years, high: $\geq 12$ years). Besides descriptive statistics data were analysed by logistic regression.

Results: Data of 1642 patients (60\% of registered cases, 901 boys) could be included in the analysis The age distribution at onset was $22.7 \%, 36.1 \%$ and $41.2 \%$ for the age groups $0-4,5-9$ and $10-14$ years, respectively. The most frequently reported symptoms were polydipsia (93.6\%), polyuria $(92.4 \%)$, weight loss (76.6\%), fatigue (69.7), and abdominal pain (37.1\%). Mean duration of symptoms before diagnosis was 31.6 days. While the reported frequency of clinical symptoms did not depend on families' social status, lower social status families reported a shorter duration of symptoms. At clinical examination $46.7 \%$ of the children presented dehydration, $11.8 \%$ impaired consciousness, and $0.7 \%$ coma. Mean levels of glucose and glycosylated haemoglobin (relative to upper normal value) at diagnosis was $464.5 \pm 193.0$ $\mathrm{mg} / \mathrm{dl}$ and $1.87 \pm 0.43$ respectively. Ketonuria, bicarbonate values $\leq 10 \mathrm{mmol} / \mathrm{l}$, and severe ketoacidosis $(\mathrm{pH} \leq 7.2)$ were present in $79.0 \%, 14.1 \%$, and $15.6 \%$ of the children, respectively. Children of lower social status families presented significantly increased metabolic decompensation than children of upper class families. Prevalence of severe ketoacidosis was $18.2 \%, 14.6 \%$ and $12.8 \%$ for low, middle and high social status $(\mathrm{p}<0.05)$, respectively. Accordingly, children of low social status were hospitalised for a longer period (in average 15.4 days) than children of middle (13.8 days) or high social status (13.5 days) ( $\mathrm{p}<0.05) .13 .6 \%$ of deprived children were hospitalised for at least 3 weeks compared to $6.5 \%$ and $5.3 \%$ of children of middle and high social status $(\mathrm{p}<0.05)$.Adjusted for sex, age, familial diabetes and family situation (single parent vs. complete family), relative risk of low social status children compared to high social status children for severe ketoacidosis was 1.78 (95\%CI: $1.21-2.62 ; \mathrm{p}=0.003$ ), and for a long time hospitalisation ( $>=3$ weeks) relative risk was 2.89 (95\%-CI: $1.72-4.86$; $\mathrm{p}<0.001$, additionally adjusted for ketoacidosis)

Conclusion: This population-based survey showed that deprived children present with increased metabolic derangement at onset of type 1 diabetes and stay at hospital for a longer time. Activities aiming at increasing public awareness of presenting characteristics of childhood diabetes in particular in the target group of low social status families might help to reduce the social disparity in clinical presentation of childhood onset Type 1 diabetes.

Supported by the German Research Foundation

\section{PS 89 Care and treatment of diabetes in pregnancy}

\section{0}

Pre-Pregnancy Care (PPC) in women with diabetes mellitus resident in the West Midlands UK

F. P. Dunne ${ }^{1,2}$, D. Jenkins ${ }^{2}$, N. Shah², W. MacKenzie' ${ }^{2}$, W. Oakley ${ }^{2}$, J. Benn², P. Brydon ${ }^{2}$, C. Shuter ${ }^{2}$, P. McGeown' ${ }^{2}$ J. Gardosi²;

${ }^{1}$ National University of Ireland, University College Hospital, Newcastle Road Galway, Ireland,

${ }^{2}$ West Midlands Perinatal Institute, Birmingham, United Kingdom.

Aim: A prospective cohort study as part of the National CEMACH collection to examine pre-pregnancy care in a multi ethnic population of women with established Diabetes Mellitus.

Methods: The study period extended for 12 months between March 2002 and Feb 2003. Information was collected on uptake and location of pre-pregnancy care, uptake of folic acid, achievement of target HbA1C.

Results: Information on 418 women, 269 (type 1) 149 (type 2), mean age 31 was collected. $25.1 \%$ overall were of non-European origin rising to $57 \%$ in those with type 2 diabetes. $55 \%$ of non European women with type 1 and $78 \%$ with type 2 disease were associated with the highest quintile of deprivation compared to $45 \%$ nationally. $28 \%$ of the total group received prepregnancy care (PPC), lower in type 2 than type 1 women ( $24 \mathrm{v} 30 \%$ ). $41 \%$ were receiving folic acid, less in type 2 than type 1 ( $33 \mathrm{v} 45 \%) .41 \%$ had a recorded PPC $\mathrm{HbAlC}$, less in type 2 than type 1 ( $32 \mathrm{v} 46 \%$ ). Only $12.5 \%$ had an $\mathrm{HbA} 1 \mathrm{C}<7 \% .60 \%$ of PPC was delivered in the adult hospital diabetes clinic with the other $40 \%$ receiving PPC in GP and community services. PPC was significantly associated with achieving a target $\mathrm{HbAlC}<7 \%$ in the prepregnancy and first trimester periods $(\mathrm{P}<0.01)$. Achievement of a $\mathrm{HbAlC}<$ $7 \%$ in trimester 1 was significantly associated with infant survival at 28 days $(\mathrm{P}=0.01)$

Conclusion: In the West Midlands we fall well short of achieving the prepregnancy goals for women with diabetes. Women with type 2 diabetes appear to have a lower standard of care. Increasing the number of women who achieve these targets is likely to improve their pregnancy outcomes. A combined primary and secondary delivery of a PPC service is likely to have a more successful outcome than either alone.

\section{1}

Continuous subcutaneose insulin infusion (CSII) reduces the rate of hypoglycaemic episodes throughout pregnancy

N. Ignatova ${ }^{1}, \mathrm{~N}$. Arbatskaya ${ }^{1}$, E. Melnikova ${ }^{2}$;

${ }^{1}$ Clinical - diagnostic centre, City Clinical Hospital №1,

${ }^{2}$ Department of Endocrinology and Diabetology,

Russian State Medical University, Moscow, Russian Federation.

Background and Aims: Pregnant women with type 1 diabetes mellitus (DM) have an increased risk of experiencing adverse maternal and fetal outcomes. Improving glycaemic control can minimise these risks, but must be balanced against increasing the rate of severe hypoglycaemia, which can prove fatal. The aim of study was to evaluate the effectiveness of CSII therapy in good metabolic control.

Materials and Methods: 41 women with type $1 \mathrm{DM}$ and unplanned pregnancy were divided by two groups. CSII group included 21 women transferred on CSII therapy at $14 \pm 9,9$ weeks of pregnancy. Group of insulin analog (Ian) included 21 women treated with insulin lispro or aspart and NPH before and during pregnancy. Insulin dose was adjusted based on 8 capillary meter readings daily. Safety and efficacy of CSII vs. Ian was evaluated by $\mathrm{HbA}_{1 \mathrm{C}}$, maternal hypoglycaemia (major, minor and nocturnal), pregnancy outcomes.

Results: Mean $\mathrm{HbA}_{1 \mathrm{C}}$ values in the start of study were $6,8+/-0,7$ (CSII) vs. $7,4+/-1,8$ (Ian). At end of study, women in both groups had mean $\mathrm{HbA}$ values of $6,3 \%$ ( $p>0.5$, not significant), however $\mathrm{HbA}_{1 \mathrm{C}}$ was lower in the $3 \mathrm{rd}$ trimester of pregnancy in the CSII group. Only one major hypoglycaemic event was recorded throughout the study in Ian group. Rates of minor hypoglycaemia were lower in CSII treatment group in comparing Ian group: $6,1 \pm 1,7$ vs. $11,6 \pm 2,3$ episodes in the $1^{\text {st }}$ trimester of pregnancy; $4,8 \pm 1,4$ vs. $12,2 \pm 2,7$ and $3,4 \pm 1,1$ vs. $10,0 \pm 3,0$ episodes in the $2^{\text {d }}$ and $3^{\text {rd }}$ trimesters. Night 
time hypoglycemia occurs more rarely in CSII group then in Ian one in each trimesters of pregnancy: $1,0 \pm 0,3$ vs. $2,8 \pm 0,6$ / 1,4 40,4 vs. $1,7 \pm 0,3$ / 1,8 $8 \pm 0,6$ vs. $2,5 \pm 0,7$. Pregnancy outcomes were similar in both groups.

Conclusion: Changing therapy on CSII treatment during pregnancy assists to improve metabolic control and reduce the rate of hypoglycaemic episodes.

\section{2}

Insulin pump therapy in pregnant women with diabetes mellitus type 1: a systematic review

T. W. Gratzer ${ }^{1,2}$, K. J. Jeitler ${ }^{1,2}$, A. Berghold ${ }^{3}$, K. Horvath ${ }^{1}$, T. R. Pieber ${ }^{2}$,

A. Siebenhofer ${ }^{1}$;

${ }^{1}$ Dept. of Internal Medicine, EBM Review Center, Medical University,

${ }^{2}$ Institute of Medical Technologies and Health Management, Joanneum

Research, ${ }^{3}$ Institute for Medical Informatics, Statistics and Documentation,

Medical University, Graz, Austria.

Background and Aims: Due to the need of a more stringent metabolic control during pregnancy continuous subcutaneous insulin infusion (CSII) therapy is thought to have advantages in the treatment of pregnant women with diabetes mellitus type 1 (DMT1). Our aim was to investigate possible clinical benefits of CSII therapy compared to subcutaneous injection therapy (IT) in pregnant women with DMT1 with regard to glycaemic control, insulin requirements and adverse events.

Material and Methods: We systematically searched databases (MEDLINE, EMBASE, CENTRAL) up to March 2007 for randomised controlled trials (RCT). Two reviewers independently evaluated the relevance of studies and their quality after inclusion. Discrepancies were solved by consensus and a systematic review was performed.

Results: The search yielded 608 references of which 6 RCTs were relevant to our question and included in the review. The number of patients ranged from 10 to 89 and publication dates from 1986 to 1993 . Methodological quality was judged low for all studies. We did not perform meta-analysis because most of the studies did not provide sufficient data.

$\mathrm{HbA1} / \mathrm{HbAlc}$ was reported in 4 RCTs. No statistically significant differences between treatment groups were found at any time (one study, Laatikainen 1987, did not report a formal statistical test). The findings on percentage of blood glucose values outside of target range did not provide conclusive data, showing advantages for CSII therapy in some studies and for IT therapy in others.

One investigation found statistically significant lower insulin requirements in the CSII vs. IT group ( 1.3 vs. $1.6 \mathrm{IU} / \mathrm{kg} / \mathrm{day} ; \mathrm{p}=0.041)$ at the end of the $3^{\text {rd }}$ trimester.

No statistically significant differences in the incidence of severe maternal hypoglycaemia and the number of maternal ketoacidodic events were reported in any of the studies.

None of the studies found statistically significant differences between CSII and IT groups on intrauterine/perinatal death or birth weight under the $10^{\text {th }}$ and above the $90^{\text {th }}$ percentile.
Conclusions: No clinically important advantages for CSII therapy in pregnant women with DMT1 can be deduced from the available RCTs. The fact that the majority of the studies were conducted some 20 years ago raises doubts as to their current relevance. Well conducted RCTs employing new CSII systems are needed. (Tab. 1)

Supported by Roche Diagnostics

\section{3}

Hypoglycaemia in pregnant women with type 1 diabetes: predictors and role of metabolic control

L. Ringholm Nielsen ${ }^{1}$, U. Pedersen-Bjergaard ${ }^{2}$, B. Thorsteinsson ${ }^{2}$,

M. Johansen ${ }^{3}$, P. Damm³ ${ }^{3}$ E. R. Mathiesen ${ }^{1}$;

${ }^{1}$ Department of Endocrinology, Rigshospitalet, Copenhagen,

${ }^{2}$ Endocrinology Section, Nordsjællands Hospital - Hillerød,

${ }^{3}$ Department of Obstetrics, Rigshospitalet, Copenhagen, Denmark.

Background and aims: Women with type 1 diabetes (T1DM) are at particular risk of severe hypoglycaemia during pregnancy. We evaluated frequencies of mild and severe hypoglycaemia and analysed influence of strict metabolic control, nausea, vomiting and other potential predictors on occurrence of severe hypoglycaemia in these subjects.

Materials and Methods: Prospective, observational study of 108 consecutive pregnant women with T1DM. At 8, 14, 21, 27 and 33 weeks gestation the women performed self-monitoring plasma glucose (SMPG) (8/day) for 3 days and completed a questionnaire on nausea causing change in diet, vomiting in the previous week, history of mild (managed by the patient herself) and severe hypoglycaemia (requiring assistance from others), and self-estimated awareness of hypoglycaemia.

Results: Forty-nine (45\%) women experienced 178 events of severe hypoglycaemia throughout pregnancy, corresponding to an incidence of 5.3, 2.4 and 0.5 events/patient-year in first, second and third trimester, respectively, compared to 1.1 event/patient-year the year preceding pregnancy. Sixty-five (37\%) severe hypoglycaemic events occurred at night (0-8 a.m.). Eighty percent of all severe hypoglycaemic events occurred before 20 weeks with a peak (15 events) at 9 weeks. The incidence of mild hypoglycaemia was 5.5, 5.1, 4.2, 3.8, and 3.8 events/patient-week at 8,14 , 21,27 and 33 gestational weeks, respectively, with a significant decrease from 8 to 33 weeks $(\mathrm{p}<0.0001)$ and no difference between women with and without severe hypoglycaemia. Biochemical hypoglycaemia $(<3.9 \mathrm{mM})$ was present in $15 \%$ of the SMPG throughout pregnancy both in women with and without severe hypoglycaemia. Nausea or vomiting was only reported as a possible provoking cause in $4(3 \%)$ severe hypoglycaemic events. Nor prevalence of nausea and vomiting, median SMPG, fraction of SMPG values $\leq 3.9 \mathrm{mM}, \mathrm{HbAlc}$ or insulin dosage differed significantly between women with and without severe hypoglycaemia. SMPG fluctuated significantly more at 8 vs. 33 weeks in women with and without severe hypoglycaemia $(\mathrm{p}<0.0001)$. Throughout pregnancy, HbAlc and median SMPG decreased and insulin dosage increased with no differences between women with and without severe hypoglycaemia. Women with severe hypoglycaemia

Summary of Results (Tab. 1)

\begin{tabular}{|c|c|c|c|c|c|c|c|}
\hline \multicolumn{8}{|l|}{ CSII versus IT } \\
\hline Endpoint & & Botta 1986 & Burkart 1988 & Carta 1986 & Coustan 1986 & Laatikainen 1987 & Nosari 1993 \\
\hline $\mathrm{HbA} 1 / \mathrm{HbAlc} 1^{\mathrm{st}}$ trimester & (\%) & - & - & 7.8 vs. 8.5 & - & 8.5 vs. 8.2 & 6.0 vs. 6.2 \\
\hline $\mathrm{HbA} 1 / \mathrm{HbA} 1 \mathrm{c} 3^{\text {rd }}$ trimester & (\%) & - & - & 7.1 vs. 7.0 & 6.3 vs. 6.4 & 6.7 vs. 7.1 & 6.3 vs. 6.2 \\
\hline \multirow[t]{2}{*}{ Insulin dose $1^{\text {st }}$ trimester } & $(\mathrm{IU} / \mathrm{d})$ & - & - & 34 vs. 30 & - & - & 40 vs. 36 \\
\hline & $(\mathrm{IU} / \mathrm{kg} / \mathrm{d})$ & - & - & - & 0.7 vs. 1.0 & - & - \\
\hline \multirow[t]{2}{*}{ Insulin dose $3^{\text {rd }}$ trimester } & $(\mathrm{IU} / \mathrm{d})$ & - & - & 50 vs. 58 & - & 49 vs. 54 & 57 vs. 55 \\
\hline & $(\mathrm{IU} / \mathrm{kg} / \mathrm{d})$ & - & - & - & 1.3 vs. $1.6^{\dagger}$ & - & \\
\hline Maternal severe hypoglycaemia & (events) & - & 5 vs. 3 & 0 vs. 1 & - & - & 3 vs. 1 \\
\hline Maternal ketoacidosis & (events) & 1 vs. 0 & 1 vs. 0 & - & 0 vs. 0 & - & 3 vs. 0 \\
\hline Intrauterine / perinatal death & & 1 vs. 0 & 1 vs. 2 & 0 vs. 0 & - & - & 2 vs. 1 \\
\hline Birth weight $<10^{\text {th }}$ percentile & & - & 3 vs. 1 & 0 vs. 0 & 2 vs. 0 & - & 0 vs. 2 \\
\hline Birth weight $>90^{\text {th }}$ percentile & & - & 7 vs. 12 & 2 vs. 0 & 1 vs. 0 & - & 1 vs. 0 \\
\hline
\end{tabular}

$\dagger=$ statistically significant difference; $-=$ not available 
had longer duration of T1DM (median 19 vs. 13 years), more often severe hypoglycaemia in the year before pregnancy ( $47 \mathrm{vs} .17 \%$ of the patients), more often self-estimated impaired awareness (75 vs. 43\%), and more frequently SMPG outside the range of $4.0-9.9 \mathrm{mM}$ at 8 weeks ( $48 \mathrm{vs.} 34 \%$ ), all $\mathrm{p}<0.01$. Logistic regression analysis identified self-estimated impaired awareness at 8 weeks (OR [95\% CI]: $3.8[1.3-8.8]$ ) and history of severe hypoglycaemia during the previous year (OR: $3.1[1.1-8.5]$ ) as independent predictors for severe hypoglycaemia.

Conclusion: In pregnant women with T1DM, the incidence of mild and severe hypoglycaemia was highest in early pregnancy although the metabolic control was stricter and insulin dosage was higher in last part of pregnancy. Predictors for severe hypoglycaemia were self-estimated impaired awareness and a history of severe hypoglycaemia during the previous year.

Supported by grants from the Danish Diabetes Association, Rigshospitalet and Novo Nordisk

\section{4}

Have pregnancy outcomes in type 1 diabetes improved over the past 13 years?

S. Pye ${ }^{1}$, S. Gennatas ${ }^{1}$, V. Dawson ${ }^{1}$, M. Halliday ${ }^{2}$, S. Hodgett ${ }^{2}$, I. Scudamore ${ }^{2}$, M.-F. Kong ${ }^{1}$, R. Gregory ${ }^{1}$;

${ }^{1}$ Diabetes \& Endocrinology,

${ }^{2}$ Obstetrics \& Gynaecology, Leicester General Hospital, United Kingdom.

Background and Aims: In 1989 one of the goals of the St Vincent declaration was to achieve pregnancy outcomes in women with diabetes that approximates that of women without diabetes over the next 5 years but this target has not been achieved. The aim of this study was to compare pregnancy outcomes in women with type 1 diabetes mellitus (T1DM) over the past 13 years.

Materials and Methods: We undertook a retrospective survey of all pregnancies complicated with T1DM in our centre between 1994 and 2006. There were 147 pregnancies, 124 were in caucasians. As we were expecting an improvement in outcomes over the years we divided the women into two groups.

Results: There were 61 pregnancies between 1994-2000 (group A) and 86 between 2001-2006 (group B). Mean age was $28.7 \pm 4.6$ in both groups. Gestational age at booking was 8.6 weeks in group A and 7.9 weeks in group B. $64 \%$ received preconception counselling in group A vs. $50 \%$ in group B. $43 \%$ of women were taking folic acid at booking in group A and $71 \%$ in group B. The duration of diabetes was $10.9 \pm 7.7$ years in group A and 13.9+ 10.1 years in group $\mathrm{B}$. Mean $\mathrm{HbA}_{1} \mathrm{c}$ at booking was $7.9 \pm 1.5 \%$ in group $\mathrm{A}$ vs. $7.6 \pm 1.5 \%$ in group $\mathrm{B}$. Mean $\mathrm{HbA}_{1} \mathrm{c}$ during pregnancy was $6.7 \pm 0.7 \%$ in group A vs. $6.5 \pm 0.9 \%$ in group B. Mean gestational age was $37.1 \pm 2$ weeks in group A, $36.5 \pm 3.7$ weeks in group B. Mean birth weight was $3399.7 \pm 714$ $\mathrm{g}$ in group $\mathrm{A}$ and $3441.4 \pm 850 \mathrm{~g}$ in group B. $49.2 \%$ in group $\mathrm{A}$ had induction of labour vs. $31.4 \%$ in group B. $24.6 \%$ in group A and $27.9 \%$ in group B had a normal vaginal delivery. $16.4 \%$ had instrumental delivery in group A vs. $9.3 \%$ in group B. $23 \%$ had emergency caesarean section in group A vs. $36 \%$ in group B. $26.2 \%$ had elective caesarean section in group A vs. $23.3 \%$ in group B. There were 5 miscarriages in group A and 3 in group B. There was one stillbirth and one IUGR in group A. $14.8 \%$ had large for gestational age babies in group A vs. $22.1 \%$ in group B. No pregnancy was complicated by shoulder dystocia in group A but there were 2 in group B. Eleven women had polyhydramnios in group A and 4 in group B. Eleven babies (18\%) in group A had hypoglycaemia and 7 (8\%) in group B. 24 women (39.3\%) in group A had babies who needed admission to the neonatal intensive care unit (NICU) vs. $14(16.3 \%)$ in group B. Two babies in group B had congenital anomalies but none in group A. There was 1 neonatal death in group $\mathrm{B}$ and none in group A.

Conclusion: There were some improvements in a number of key outcomes (more women taking folic acid, reduced admission rate to NICU, reduced neonatal hypoglycaemia and less cases of polyhydramnios) over recent years. However, preconception counselling has not improved; $\mathrm{HbA} \mathrm{c}$ at booking was $7.9 \pm 1.5 \%$ in group $\mathrm{A}$ vs. $7.6 \pm 1.5 \%$ in group $\mathrm{B}$, and more women were delivered by emergency caesarean section in group B. The percentage of women delivered by elective caesarean section has not changed. There seems to be an increase in the number of large for gestational age babies over recent years. Women with T1DM of child-bearing age should receive intensified medical treatment during preconception and pregnancy and should be targeted to receive information about the potential impact of poorly controlled diabetes on the outcome of pregnancy.

\section{5}

The effectiveness of glibenclamide in women with gestational diabetes: preliminary experience

R. I. G. Holt ${ }^{1}$, P. Clarke 2 , E. Parry ${ }^{2}$, M. A. G. Coleman²;

${ }^{1}$ Endocrinology \& Metabolism Sub-division, University of Southampton, ${ }^{2}$ Fetal Maternal Medicine, Princess Anne Hospital, Southampton, United Kingdom.

Background and Aims: Several studies have suggested that glibenclamide may be used safely and effectively in women with gestational diabetes (GDM). The aim of our study was to assess effectiveness and safety of glibenclamide for GDM in UK clinical practice.

Materials and Methods: Women with GDM requiring pharmacological therapy were offered a choice of insulin or glibenclamide. Maternal and fetal outcomes were assessed in women treated with insulin (45) or glibenclamide (44) and compared with those treated with diet alone (55).

Results: Women choosing glibenclamide were more likely to be Asian ( $\mathrm{p}=0.002)$ and had higher fasting $(6.3 \pm 0.2 \times 5.8 \pm 0.1 \mathrm{mmol} / \mathrm{l}, \mathrm{p}=0.04)$ and $2 \mathrm{hr}$ glucose $(10.9 \pm 0.4 \times 9.5 \pm 0.4 \mathrm{mmol} / \mathrm{l}, \mathrm{p}=0.02)$ at diagnosis than those choosing insulin. There was no difference in maternal age or parity. The gestation at the start of treatment was $31^{+1} \pm 0^{+3}$ weeks (range $21^{+5} \pm 37^{+5}$ ) with no difference between those treated with insulin or glibenclamide. The mean dose of glibenclamide was $7.8 \pm 0.8 \mathrm{mg}$ (median $5 \mathrm{mg}$, range 2.5 $15 \mathrm{mg}$ ). Ten glibenclamide women switched to insulin (inadequate control (7), unpredictable hypoglycaemia (1), other (2)). These women had higher baseline body mass index than those who continued with glibenclamide $\left(33.2 \pm 1.7\right.$ vs. $\left.28.8 \pm 1.0 \mathrm{~kg} / \mathrm{m}^{2} \mathrm{p}=0.02\right)$.

There was no difference in mode of birth, birth weight or birth weight centile between groups. One unrelated stillbirth occurred with glibenclamide. Despite higher baseline glycaemia in the glibenclamide group, maternal and neonatal outcomes were not significantly different between glibenclamide and insulin treated women except for lower APGAR scores at 1 minute in the glibenclamide group. Neonatal jaundice and hypoglycaemia occurred more frequently in women treated pharmacologically compared with women treated with diet $(\mathrm{p}<0.03)$.

Conclusion: The use of glibenclamide in pregnancy is associated with adequate glycaemic control in $77 \%$ and equivalent fetal outcomes to women treated with insulin.

\section{6}

How can we improve postnatal glucose tolerance testing in the gestational diabetic population? M. Wu ${ }^{1}$, N. De Mendonca ${ }^{2}$ J. Girling 2 , C. Cotzias ${ }^{2}$; ${ }^{1}$ Clinical Effectiveness/Obstetrics and Gynaecology, ${ }^{2}$ Obstetrics and Gynaecology, West Middlesex University Hospital NHS Trust, Isleworth, United Kingdom.

Background and Aims: Women with gestational diabetes (GDM) in our unit are managed in a multidisciplinary obstetric endocrine clinic and counselled about the importance of a postnatal glucose tolerance test (GTT) to identify ongoing glucose intolerance. All women with GDM are given a GTT appointment at 6-8 weeks postnatal. Ongoing glucose intolerance in a multi-ethnic population in the United States has been reported to be as high as $36 \%$. The obstetric population in our unit is multi-ethnic with a significant proportion of women of South Asian or Black African/Caribbean background (only $54 \%$ White European). The aims of this study are to describe the demographic and clinical characteristics of our GDM population and to determine the association between these and postnatal glucose testing.

Materials and Methods: Women attending the obstetric endocrine clinic for GDM from 2000-2005 inclusive were identified from clinic records and a retrospective case note review performed.

Results: $419 / 474$ (88\%) women were included in the analysis. Mean body mass index (BMI) was $29 \mathrm{~kg} / \mathrm{m}^{2}$ and 160/398 (40\%) women were classified as obese $\left(\mathrm{BMI}>30 \mathrm{~kg} / \mathrm{m}^{2}\right) .300 / 419(72 \%)$ women were aged 30 years and above and only 105/415 (25\%) were of White European background, while 218/415 (53\%) were of Asian ethnicity, 64/415 (15\%) of Black African/ Caribbean ethnicity, and 28/415 (7\%) of Oriental or other ethnicity. 221/419 (53\%) required insulin to achieve target glucose levels during pregnancy and 240/419 (57\%) were induced. 172/419 (41\%) women delivered by caesarean section. 
254/419 (61\%) women attended for postnatal GTT: Of those, 55/254 (22\%) women had an abnormal result; (36 women had impaired glucose tolerance or impaired fasting glucose and19 had diabetes mellitus). There was no difference in ethnicity, age, or BMI between women who attended for postnatal glucose testing and those who did not. More women who required insulin in addition to dietary modification to control glucose levels during pregnancy attended for a postnatal GTT 147/221 (66.5\%) vs. 107/198 (54\%), $\mathrm{p}=0.006$. Not only did women on insulin therapy have a greater likelihood of attending for their postnatal GTT (OR 1.69, 95\% CI, 1.14-2.50), they were also more likely than those managed by diet alone to have an abnormal result $46 / 147$ (31\%) vs. $9 / 107$ (8.5\%), $\mathrm{p}<0.000$.

Conclusion: The association between insulin therapy during pregnancy and rate of postnatal GTT attendance is consistent with health behaviour that is motivated by the belief that one is genuinely ill and that the consequences of the illness will impact one's well being. Given that nearly one-quarter of our GDM population who return postnatally for a GTT have an abnormal result, with reported incidences of more than one-third in the literature, it is crucial to encourage women to attend for postnatal glucose testing. Women with GDM in our unit need to be informed that almost 1 in 3 needing insulin treatment during pregnancy and 1 in 10 managed with diet alone will have ongoing abnormal carbohydrate metabolism. This counselling strategy should be implemented to improve our postnatal glucose testing rates.

\section{PS 90 Diabetes in pregnancy: education and diet}

\section{7}

Gestational diabetes mellitus: advantages of an intensive educational programme using E-consulting

J.-J. Altman ${ }^{1}$, M. M. Agarwal' ${ }^{2}$, S. Parat ${ }^{3}$, R. Ducloux ${ }^{1}$, M. Duval ${ }^{1}$, A. Yamgnane ${ }^{4}$, Y. Dumez ${ }^{4}$, M. F. Safraou';

${ }^{1}$ Diabetology - Nutrition - Endocrinology, Hopital Européen Georges Pompidou, Paris, France, ${ }^{2}$ Pathology, Faculty of Medecine, UAE University, Al-Ain, United Arab Emirates, ${ }^{3}$ Pediatrics, Hopital Necker, Paris, France, ${ }^{4}$ Obstetrics and Gynecology, Hopital Necker, Paris, France.

Background: Despite four decades of resarch, there is still no consensus on the optimal screening, diagnosis and follow-up strategy for gestational diabetes mellitus (GDM). However, there is agreement that strict control of maternal hyperglycaemia results in better outcome for both, the mother and her neonate in the index pregnancy.

Aim: To optimise the control of maternal hyperglycemia using an educational program via E-consulting for GDM.

Materials and Methods: One hundred consecutive pregnant women were followed prospectively. Fasting plasma glucose (FPG) and post prandial glucose (PPG) were performed at booking and at 24- 28 weeks, if the initial testing was normal. If the FPG was $\geq 90 \mathrm{mg} / \mathrm{dl}$ and/or PPG $\geq 120 \mathrm{mg} / \mathrm{dl}$, women were educated for self monitoring capillary glucose (SMPG). Six SMPG per day, an appropriate diet and physical therapy were recommended. The SMPG results were reported weekly to the physician who advised her within 24 hours, both being done via E-mail.

Results: Of the 100, 30 women were followed by this intensive E-consulting program while the remaining 70 women were managed by the traditional hospital approach. Overall, 27 (27\%) women from the entire cohort needed insulin therapy. The women undergoing e-consulting were treated earlier by $10+/-2$ days. Optimal glycemic targets were obtained in $95 \%$ women.

Conclusion: E-consulting is a reliable, quick and good approach to achieve glycemic control for GDM. Moreover, these women being at high-risk for type 2 diabetes mellitus, can be better motivated for long-term follow-up after delivery.

\section{8}

Gestatational diabetes mellitus (GDM): a comparison between women who achieve target capillary blood glucose levels (CBG's) with education, diet and exercise alone and those who also require insulin

R. Kaushal ${ }^{1}$, N. I. M. De Mendonca ${ }^{2}$, T. J. J. Bracewell-milnes ${ }^{2}$, M. $\mathrm{Wu}^{3}$, J. C. Girling ${ }^{4}$, C. S. Cotzias ${ }^{2}$;

${ }^{1}$ Diabetes and Endocrinology, ${ }^{2}$ Obstetrics and Gynaecology,

${ }^{3}$ Clinical Governance and Risk, ${ }^{4}$ Obstetrics and Gynaecology,

West Middlesex University Hospital, Isleworth, Middlesex, United Kingdom.

Aim: To identify demographic and pregnancy outcome differences between women who achieve target CBGs with diet and exercise alone and those who need insulin to enable accurate risk counselling for women with GDM.

Introduction: Gestational diabetes is a common, poorly characterised condition. The method of screening, criteria for diagnosis, and the target CBG's to be achieved are not universally agreed.

Methods: All women with GDM managed in our combined obstetric diabetes clinic between 2000-2005 inclusive were identified and a retrospective case note review performed.

Women with the following risk factors;

- $1^{\text {st }}$ degree family history of diabetes

- Non-Caucasian

- Age or Body Mass Index (BMI) $>30$ years or $\mathrm{kg} / \mathrm{m} 2$

- Poor obstetric history - previous; unexplained SB, NND, congenital abnormalities

- Previous large baby $(>4.5 \mathrm{~kg})$

In Current pregnancy:

- Large fetus

Polyhydramnios

- Fasting glycosuria $\mathrm{x} 2$

- Multiple pregnancy 
- Hyper / hypothyroidism

were identified and offered a $50 \mathrm{gm}$ oral glucose challenge test (OGCT) at 28 weeks gestation. If the blood glucose at 1 hour $>/=7.8 \mathrm{mmol} .1$ a formal $75 \mathrm{~g}$ glucose tolerance test (OGTT) was undertaken. GDM was diagnosed with fasting blood glucose $>6 \mathrm{mmol} / \mathrm{l}$ or $>9 \mathrm{mmol} / \mathrm{l}$ at $120 \mathrm{~min}$. Women were counselled about feto-maternal implications of GDM, importance of postnatal (PN) OGTT and implications of GDM on long- term health and subsequent pregnancies. All received formal diabetes lifestyle and CBG monitoring education, with target levels of $<6 \mathrm{mmol} / \mathrm{l}$ fasting and $<8 \mathrm{mmol} /$ $12 \mathrm{hr}$ post-prandially. Women unable to achieve these targets with diet and exercise were commenced on insulin.

Results: From 2000-2005, of 474 women with GDM, 419 (88\%) notes were reviewed: (47.3\%) achieved target BMs with diet and exercise alone and (52.7\%) required insulin. No differences were observed in age, ethnicity or parity, but women requiring insulin had a higher BMI, Demographic and pregnancy outcome data are shown in Table 1. Discussion: We have demonstrated that women requiring insulin to control GDM are more obese and carbohydrate intolerant at diagnosis. These women have high caesarean section rates (almost 50\%) and almost $10 \%$ of those achieving a vaginal delivery will have an associated shoulder dystocia, a potential cause of serious perinatal complications. Such data have not been reported extensively before. This robust information will allow more focussed counselling of our women with GDM according to their treatment needs.

\begin{tabular}{|c|c|c|c|}
\hline & $\begin{array}{l}\text { Diet Group, } \\
\mathrm{N}=198\end{array}$ & $\begin{array}{l}\text { Insulin group, } \\
\mathrm{N}=\mathbf{2 2 1}\end{array}$ & $P$ value \\
\hline $\mathrm{BMI} \mathrm{m} / \mathrm{kg} 2^{*}$ & $27(24-31)$ & $29(26-33)$ & 0.001 \\
\hline $\mathrm{BMI}>30 \mathrm{~m} / \mathrm{kg} 2^{* *}$ & $62(34 \%)$ & $98(46 \%)$ & 0.004 \\
\hline OGCT result $\mathrm{mmol} / \mathrm{l}^{*}$ & $9.3(8.5-9.3)$ & $10.7(9.2-2.2)$ & $<0.000$ \\
\hline Fasting OGTT result $\mathrm{mmol} / \mathrm{l}^{*}$ & $5.7(4.8-6.3)$ & $6.2(5.7-6.9)$ & $<0.000$ \\
\hline 2 hr OGTT result ${ }^{*}$ & $9.2(7.4--10.3)$ & $10.1(8.8-11.7)$ & $<0.000$ \\
\hline $\mathrm{HBA} 1 \mathrm{C} \geq 7^{\star *}$ & $28(14.1 \%)$ & $50(22.8 \%)$ & 0.015 \\
\hline Caesarean section (CS) total ${ }^{\star *}$ & $67(34 \%)$ & $105(48 \%)$ & 0.005 \\
\hline $\begin{array}{l}\text { Shoulder dystocia n/(vaginal } \\
\text { deliveries)** }\end{array}$ & $3 / 131=2.3 \%$ & $11 / 116=9.5 \%$ & 0.015 \\
\hline Birthweight $>90$ th centile ${ }^{* *}$ & $35(19 \%)$ & $70(33 \%)$ & 0.001 \\
\hline OGTT PN done ${ }^{* *}$ & $106(54 \%)$ & $147(66.5 \%)$ & 0.009 \\
\hline Abnormal OGTT PN ${ }^{* *}$ & $9(8.5 \%)$ & $46(31.3 \%)$ & 0.000 \\
\hline
\end{tabular}

${ }^{\star}$ median(interquartile range); ${ }^{* *} \mathrm{~N}(\%)$

Supported by NovoNordisk

\section{9}

Gestational diabetes in a multiethnic London population; the demographics, treatment requirements and pregnancy outcomes and the implications of the ACHOIS trial for them

N. I. M. De Mendonca ${ }^{1}$, T. J. J. Brace-well-Milnes ${ }^{1}$, R. Kaushal ${ }^{2}$, J. C. Girling ${ }^{1}$, M. Wu ${ }^{3}$, C. S. Cotzias ${ }^{1}$;

${ }^{1}$ Obstetrics and Gynaecology, ${ }^{2}$ Endocrinology and Diabetes,

${ }^{3}$ Clinical Governance, West Middlesex University Hospital, Isleworth, Middlesex, United Kingdom.

Aim: To compare the demographic and pregnancy outcomes of our GDM population with our general antenatal population and consider the relevance of the ACHOIS trial for these women.

Introduction: ACHOIS recently showed that treating GDM significantly reduces serious perinatal morbidity. Our unit differs from ACHOIS in terms of diagnostic criteria for GDM and target Capillary Blood Glucose (CBG) levels. The screening and Diagnostic Criteria of ACHOIS suggest Risk identification - refer for $75 \mathrm{gm}$ Oral Glucose Tolerance Test (OGTT) Or selective 50gm Oral Glucose Challenge Test (OGCT) - refer for 75gm OGTT if $>/=7.8 \mathrm{mmol} / \mathrm{l}$ at $1 \mathrm{hr}$. Diagnostic values are fasting $<7.8 \mathrm{mmol} / \mathrm{l}$, 2hour $7.8-11.0 \mathrm{mmol} / \mathrm{l}$. CBG monitoring targets are $<5.5 \mathrm{mmol} / \mathrm{l}$ fasting/ pre-prandial and $<7 \mathrm{mmol} / \mathrm{l} 2 \mathrm{hrs}$ post prandial. In our unit, if risk factor positive, a 50gm OGCT is performed (refer for $75 \mathrm{gm}$ OGTT if $>7.8 \mathrm{mmol} / 1$ at 1 hour). Diagnostic values at OGCT are: Fasting $>6 \mathrm{mmol} / \mathrm{l}, 2 \mathrm{hr}>9 \mathrm{mmol} / \mathrm{l}$. CBG monitoring targets are $<6$ $\mathrm{mmol} / \mathrm{l} \mathrm{fFasting}$,/ preprandial and $<8 \mathrm{mmol} / \mathrm{l} 2$ hour post prandial.

Methods: A retrospective review of all women with GDM in our unit from 2000-2005 inclusive was performed. Demographic, treatment and outcome data were collected.

Results: During the study period, there were 474 women with GDM $(2.6 \%$ incidence). Our GDM population was older, more obese, more insulin resistant and had an Asian and Afro Caribbean representation almost 75\% and $50 \%$ respectively, greater than in the ACHOIS group. Furthermore, 53\% of our GDM population required insulin compared with $20 \%$ in the ACHOIS intervention group despite lower target BMs. Our GDM group were more likely to need an induction of labour, have a caesarean section, suffer from shoulder dystocia and have a baby $>4 \mathrm{~kg}$ at birth. (Tab. 1 )

Conclusion: The ACHOIS trial demonstrated that treating GDM reduces serious perinatal complications. The data presented highlight the higher risk in our women with GDM compared with the general population and the disparity between them and the women in the ACHOIS study. In view of this the outcomes achieved by ACHOIS should only be extrapolated with caution to such multi-ethnic populations. The screening and diagnostic criteria of ACHOIS are not without significant economic and clinical implication and it must be considered that the outcome improvements demonstrated may not be replicated in other populations.

Supported by NovoNordisk

TABLE1. Major Demographic and clinical characteristics and outcomes

GDM women N=419 General Population N=17971

Maternal Age yrs
BMI:*kg/m2
$\%$ BMI $>30$
Ethnicity
White (British \& other)
Asian
Afro/Caribbean
Other
OGCT (50gm) result mmol// ${ }^{\star}$
Ohrs OGGT (75gm) result $\mathrm{mmol} / 1$
2hr OGTT (75gm) result ${ }^{\star} \mathrm{mmol} / 1$
Number requiring induction (\%)
Caesarean delivery Total
Number affected by shoulder dystocia
If delivered vaginally
Still birth

Still birth

33yrs (29-36)

30yrs (25-34)

$\begin{array}{ll}28(25-32) & 24(22-28) \\ 40 \% & 17 \% \\ \mathbf{N}=415 & 9771(54 \%) \\ 105(25 \%) & 4915(27 \%) \\ 218(53 \%) & 1969(11 \%) 1318(7 \%) \\ 64(15 \%) & \\ 28(7 \%) & \\ 9.9(8.7-11.6) & \\ 6.0(5.2-6.6) & \\ 9.5(8.1-11.0 & 2772(15 \%) \\ 240(57 \%) & 3784(21 \%) \\ 172(41 \%) & 86(0.6 \%) \\ 14(5.7 \%) & 101(0.6 \%) \\ 3(0.7 \%) & 1740(10 \%) \\ 64(15 \%) & \end{array}$

${ }^{*}$ median and (inter-quartile range)
OR $(95 \% \mathrm{CI})$ ACHOIS intervention group $\mathrm{N}=490$ Mean + SD yrs

$30.9+-5.4$

$26(23-31)$

$3.2(2.6-3.9)$

Asian ethnicity $356(73 \%)$

2.9(2.4-3.5) $72(14 \%)$

$42(9 \%)$

$8.8(8.2-9.7)$

$4.8+-0.7$

$8.6(8.1-9.3)$

7.6 (6.2-9.3) $189(39 \%)$

$2.6(2.1-3.2) \quad 152(31 \%)$

6.9(3.9-12.2) $7(1 \%)$

Not signif

$1.7(1.3-2.2)$
0 
0940

Fetal outcomes and improved $\mathrm{HbA}_{1 \mathrm{c}}$ values in pregnant women with type 1 diabetes using real-time continuous glucose sensors

M. Voelmle, S. L. Ellis, L. Gerard, P. Gottlieb, S. Garg;

Young Adult Clinic, Barbara Davis Center for Childhood Diabetes, Aurora, United States.

Background and Aims: Optimising glucose control in pregnant patients with diabetes continues to be a challenge. Intensive insulin management that includes increased glucose monitoring improves glycemic control.

Materials and Methods: The purpose of this retrospective pilot study was to evaluate the glycemic control of pregnant patients with Type $1 \mathrm{DM}$ who used Real-time Continuous Glucose Monitors (RTCGM ) in addition to self monitored blood glucose (SMBG) as compared to women who only preformed SMBG. Secondary outcomes included fetal size.

Results: A total of 6 patients used either a DexCom STS or Medtronic Paradigm RT for approximately $50 \%$ of the time during the remainder of the pregnancy after mean initiation at 16.8 weeks (12-24 weeks gestation) gestation. A computer matched control group for age, gender, duration of diabetes, ethnicity, and normal AERs ( $<20 \mathrm{ug} / \mathrm{min})$ was used for data analysis. Mean $( \pm$ SD) age and duration of diabetes was $32.2 \pm 5.4$ yrs and $17.8 \pm 3.2$ yrs for the study group The baseline characteristics were similar in the two groups. Baseline A1C values were $6.6 \pm 1.1 \%$ and $6.8 \pm 0.5 \%$ in the sensor and control groups respectively ( $>0.05)$. Significant reductions in mean A1C values were seen at all time points in the sensor group (Fig 1.). There was a tendency towards worsening of $\mathrm{A} 1 \mathrm{C}$ at 7 months of gestation ( $\mathrm{p}=0.08$, figure 1 ) in the control group. All babies born to the women in the sensor wearing group were average for gestational age. The mean birth weight was 3309 grams and matched in the $50-90^{\text {th }}$ percentile for gestational age. The women in the sensor group wore the sensors at least $50 \%$ of the time since initiation and throughout gestation. The mean weight of the babies in the control group was 3688 grams and included 2 large for gestational age (LGA) babies.

Conclusion: We conclude that use of real-time continuous glucose monitors in pregnant moms with diabetes can improve Alc values with improved fetal outcomes. This pilot study supports the need for larger randomized control trials to evaluate the significance and clinical implications of RTCGM in the management of pregnant women with Type 1 DM. (Fig. 1)

\section{1}

Nutritional beliefs and practices among diabetic pregnant mothers in a tertiary care hospital in Bangladesh

U. S. Munni ${ }^{1}$, S. Hossain ${ }^{1}$, T. Khan ${ }^{1}$, K. R. Ahmed ${ }^{2}$;

${ }^{1}$ Dept of Human Nutrition, BIRDEM, ${ }^{2}$ Health Economics Unit,

Diabetic Association of Bangladesh, Dhaka, Bangladesh.

Background and Aims: Food beliefs and practices in a particular society are crucial for the dietary management of diabetic patients. Pregnant mothers with diabetes, specially in developing countries, are particularly prone to suffer from dietary imbalances due to superstitions and irrational beliefs and practices. This may, consequently, have deleterious effect on the health of both mother and child. The present study was designed to explore the existing beliefs and practices related to food intake during pregnancy among a group of Bangladeshi diabetic pregnant mothers.

Materials and Methods: One hundred diabetic pregnant mothers (age in yrs, $\mathrm{M} \pm \mathrm{SD}, 30 \pm 4$ ) were purposively selected from the Out-Patient Department of BIRDEM (the tertiary care hospital of the Diabetic Association of Bangladesh). Majority of the diabetic pregnant mothers were literate (96\%) and by profession, housewives (86\%). A pre-designed, pretested, intervieweradministered questionnaire was used to explore the beliefs and practices about the time of intake and the nature of food. $\chi^{2}$ - test was done for performing significant test.

Results: Forty three percent of the pregnant mother believed that food should not be taken during the 'call for prayer' and $91 \%$ of these mothers practiced it. For 'eclipse' the percentage of such kind of belief was much higher $(80 \%)$ and $90 \%$ of these mothers practiced it. Mentally and physically abnormal child and different kinds of child illness were among the reasons for these beliefs. With varying proportion for particular fishes $44-58 \%$ respondents believed that some fishes like Macrognathus aculeatus (local name Baim), Cirrhinus mrigala (Mrigal) and Wallago attu (Boal) should be avoided due to the increased movement of child, fetal malformation and disease. Many of them (93\%) actually practiced it. Twenty eight percent had adverse beliefs about Anas platyrhyncha_(Duck), $78 \%$ about Ananas spp (Pineapple), 4\% about Rubus fruiticosus_(Black berry), $8 \%$ about Cocos mucifera (Coconut), $10 \%$ about 'Tea \& coffee. The corresponding percentages for practice were $82 \%, 99 \%, 75 \%, 50 \%$ and $90 \%$. The reasons included maternal and fetal abnormality, appearance of the child (like eye spot with pineapple, black skin with black berry and tea/coffee, and white eyes with coconut) and threat of abortion.

Conclusion: There are a number of nonscientific beliefs and prejudices among diabetic pregnant mother of Bangladesh regarding the intake of food. Supported by International Programme in the Chemical Sciences (IPICS) and Diabetic Association of Bangladesh (DAB)

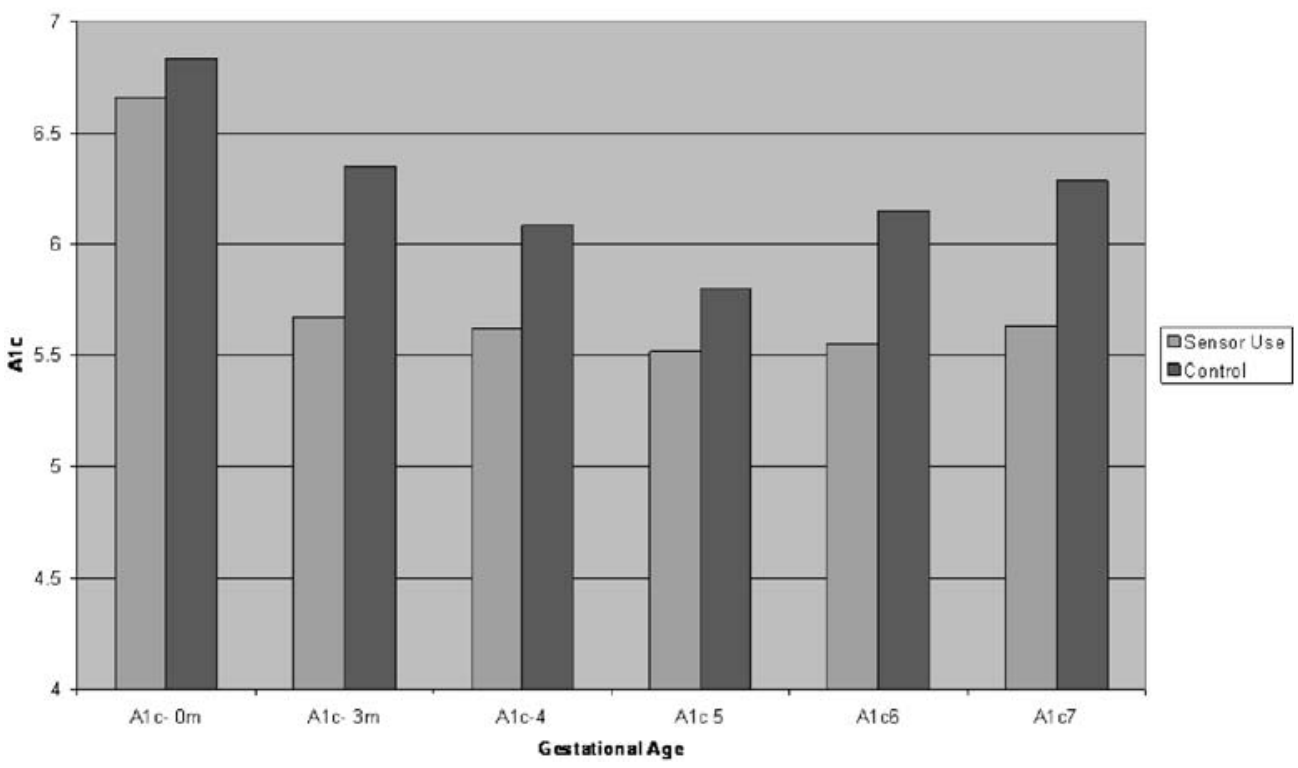




\section{2}

Dietary habits during pregnancy and their influence on glucose challenge test

A. Bertolotto $^{1}$, A. Calianno ${ }^{2}$, M. C. Pugliese ${ }^{2}$, G. Pucci ${ }^{2}$, S. Vignali ${ }^{2}$, C. Lencioni ${ }^{1}$, L. Volpe ${ }^{1}$, L. Benzi ${ }^{2}$, G. Di Cianni ${ }^{1}$;

${ }^{1}$ Department of Endocrinology and Metabolism, University of Pisa, ${ }^{2}$ Department of Endocrinology and Metabolism, Section of Clinical Nutrition, University of Pisa, Italy.

Background and Aims: Dietary therapy during pregnancy, is the cornerstone to maintain normal weight gain in order to decrease risk of maternal morbidity and adverse perinatal outcomes. The nutritional counselling needs informations on dietary and lifestyle habits. The Glucose Challenge Test (50 g-1 h GCT) is recommended as a screening test for gestational diabetes (GDM). The aims of the present study were to: 1 . evaluate energy and nutrient content of diet of pregnant women; 2 . verify whether dietary patterns could affect the result of the GCT test.

Materials and Methods: A cohort of pregnant women were interviewed before the execution of GCT to obtain their dietary habit informations. A food frequency questionnaire that included a list of 85 foods was performed by three trained dieticians. Photographs were used to compare different servings for each item. Women were asked to report how often a day and how many days each food was used during the week before GCT. The energy intake and the nutrient content of their diets were calculated using a nutritional analysis program based on data of the European Institute of Oncology.

Results: We studied 166 pregnant women that underwent a GCT at $26 \pm 1.5$ weeks of gestation; their clinical characteristics were: age $32.4 \pm 3.7$ years; prepregnancy BMI $22.5 \pm 4.3 \mathrm{Kg} / \mathrm{m}^{2}$; weight gain $7.4 \pm 3 \mathrm{Kg}$; positive family history of diabetes mellitus $13.2 \%$; primiparous $56 \%$; smokers $8 \%$. GCT was found positive (GCT + if blood glucose concentration $\geq 140 \mathrm{mg} / \mathrm{dl}$ after 1 hour of glucose load) in 42 (25.3\%) and negative (GCT -) in 124 cases (74.7\%). No difference in demographic and clinical data resulted between GCT+ and GCT- cases. The mean daily energy intake of the cohort was $2136 \pm 555 \mathrm{Kcal}$ : carbohydrates (CHO) 47\% (complex CHO 32\%; refined CHO 15\%), total fats $38 \%$ (satured fatty acids (FA) $7 \%$; monounsatured FA (MUFA) $28 \%$; polyunsatured FA (PUFA) 3\%), proteins 15\%. The cholesterol intake resulted $267 \pm 78 \mathrm{mg} / \mathrm{d}$ and that of fibers was $21.6 \pm 9.5 \mathrm{~g} / \mathrm{d}$. All parameters referring energy and macronutrient intake were not different between GCT + and GCT-. Only fiber intake resulted significantly higher in GCT- $(22 \pm 8.4 \mathrm{~g} / \mathrm{d}$ vs. $18.9 \pm 6.5 \mathrm{~g} / \mathrm{d}, \mathrm{p}=0.03$ ) than in GCT+ women.

Conclusion: This study gives informations about dietary habits in Mediterranean pregnant women. A diet with low fiber intake could be associated with an increased risk of minor abnormalities of glucose metabolism during pregnancy.

\section{PS 91 Causes and consequences of gestational diabetes}

\section{3}

Suitability of the new diagnosis criteria of the American Diabetes Association for gestational diabetes in Maresme area (Barcelona). Is it adequate to apply these criteria to our population?

E. Ballestar ${ }^{1}$, E. Pizarro ${ }^{1}$, J. Solé ${ }^{2}$, J. Sargatal ${ }^{2}$, E. Palomera ${ }^{3}$, C. Monfort ${ }^{4}$; ${ }^{1}$ Endocrinology and Nutrition, ${ }^{2}$ Obstetrics and Gynecology, ${ }^{3}$ Research Unit, ${ }^{4}$ Diabetes Education Unit, Hospital of Mataró, Barcelona, Spain.

We present the results of a Gestational Diabetes Mellitus (GDM) Program in the Maresme area (215,000 inhabitants) of Barcelona (1994 - 2004).

Aims: Evaluation of: (a) GDM prevalence according to National Diabetes Data Group (NDDG) criteria, (b) clinical, obstetrical and neonatal results in GDM global group and GDM with pregravid body mass index (BMI) $\geq 30$ and (c) insulin therapy in GDM 100-g glucose tolerance test (GTT) with basal plasmatic glycemia (BPG) $\geq 95 \mathrm{mg} / \mathrm{dL}$ (new American Diabetes Association BPG criterion).

Method: 1 or 2 screenings with 1hour-50 g of glucose loading test at the beginning of gestation, selective screening initial and/or between 24-30 weeks of gestation. If positive (glycemia $\geq 140 \mathrm{mg} / \mathrm{dL}$ ), GTT fasting and 1-, 2, 3-hour postprandial was indicated. GDM was diagnosed by NDDG criteria. Diet and self-monitoring of capillary blood glucose (CBG) at home (1 fasting and 3 postprandial / 1 hour) were required. Optimum control: fasting $\mathrm{CBG}<$ $95 \mathrm{mg} / \mathrm{dL}$ and postprandial $<130 \mathrm{mg} / \mathrm{dL}$. If $\geq 2$ CBG in a week were elevated, insulin therapy was indicated.

Results: 12,705 pregnancies were screened for GDM over 11 years. 962 had GDM (prevalence $7.6 \%$ ). Age was higher in GDM group (31.6 \pm 4.9 y.) compared with non-GDM (27.8 \pm 4.8 y.).Insulin therapy was required in $23.4 \%$ of GDM, being more frequent when BPG was $\geq 95 \mathrm{mg} / \mathrm{dL}$ ( $51.1 \%$ vs. $15.5 \%$ ), and when pregravid BMI was $\geq 30(40.2 \% v s .20 .5 \%, \mathrm{p}<0.001)$. Weight increase was similar in GDM treated with only diet $(9.45 \pm 4.5 \mathrm{~kg}) v \mathrm{~s}$. insulin therapy $(9.50 \pm 4.55 \mathrm{~kg})$. Gestational age at delivery $(39.2 \pm 1.6$ vs. $39.5 \pm 1.5$ weeks $)$ and neonatal weight $(3210 \pm 490 \mathrm{~g} v s .3206 \pm 436 \mathrm{~g})$ were similar in GDM and nonGDM.Cesarean and macrosomia were more frequent in GDM $(23.5 \% v s$. $19 \%$ and $5 \%$ vs. $3 \%$, respectively), particularly when pregravid BMI was $\geq$ 30 (cesarean: $39 \%$ vs. $20.6 \%, \mathrm{p}<0.001$; macrosomia: $9.1 \%$ vs. $4.3 \%$ ). In GDM global group, 5 minutes Apgar $\leq 6$ and neonatal hypoglycemia were observed in $0.3 \%$ and $5.4 \%$,respectively.

Conclusions: Our Gestational Diabetes Mellitus Program was successfully applied during 11 years. According to our results, we can conclude that : (a) because of $50 \%$ of GDM with BPG $\geq 95 \mathrm{mg} / \mathrm{dL}$ of GTT required insulin therapy, a reduction of BPG level ( 95 instead of $105 \mathrm{mg} / \mathrm{dL}$ ), maintaining the rest of NDDG criteria would be appropriate in our area, and (b) the elevated rate of insulin therapy, cesarean and macrosomia in GDM with pregravid $\mathrm{BMI} \geq 30$ suggest that obesity prevention programmes before pregnancy should be implemented.

\section{4}

The effect of paternal and maternal history of diabetes mellitus on the development of gestational diabetes mellitus (GDM) A. G. Tabák ${ }^{1}$, G. Tamás ${ }^{1}$, A. Péterfalvi² ${ }^{2}$ Z. Bosnyák ${ }^{3}$, E. Madarász ${ }^{1}$, J. Szánthó ${ }^{4}$, I. Rákóczi ${ }^{4}$, Z. Kerényi;

${ }^{1} 1$ st Department of Medicine, Semmelweis University, Faculty of Medicine, ${ }^{2}$ Diabetology, Health Service of Csepel,

${ }^{3}$ Outpatient Clinic, Health Service of Ferencváros, ${ }^{4}$ Department of Obstetrics and Gynaecology, Szent Imre Hospital, Budapest, Hungary.

Background and Aims: Previously several studies reported an excess of maternal diabetes among type 2 diabetes patients. Much less is known about the differential role of paternal or maternal diabetes on the development of gestational diabetes mellitus (GDM). Our aim was to explore the risk of GDM associated with paternal and maternal diabetes, and to describe the unique characteristics of GDM women with maternal diabetes.

Materials and Methods: A nested case-control study was performed using data of a population-based screening program of GDM (75g oGTT, WHO criteria) in an urban area of Hungary between May 2002 and October 2003. All Caucasian women with GDM ( $\mathrm{n}=134)$ and an age-matched control group 
$(\mathrm{n}=134)$ was selected. Family history data was ascertained during a direct interview with the patient.

Results: GDM women were more obese (BMI $25.4 \pm 6.4$ vs. $23.0 \pm 4.7 \mathrm{~kg} / \mathrm{m}^{2}, \mathrm{P}$ $=0.001)$, and had a higher fasting $(5.3 \pm 3.4$ vs. $4.5 \pm 0.4 \mathrm{mmol} / \mathrm{l}, \mathrm{P}=0.016)$ and postload blood glucose ( $8.6 \pm 0.8$ vs. $5.5 \pm 1.0 \mathrm{mmol} / \mathrm{l}, \mathrm{P}=0.0001)$ compared to controls. Neither paternal history nor the father's ancestry with diabetes mellitus was a risk factor of GDM. Mothers (and the mothers' ancestry) of the GDM women had diabetes mellitus more frequently than those of the control group (odds ratio [OR] $=3.25295 \%$ confidence interval [CI] 1.147-9.222, OR $=2.9295 \%$ CI 1.455-5.859). Using a logisitic regression the only significant and independent predictors of GDM were higher BMI $(\mathrm{P}=0.003)$ and a positive maternal history of diabetes mellitus $(\mathrm{P}=0.077)$. Positive maternal history of diabetes mellitus of GDM women was independently associated with higher post-load blood glucose $(120 \mathrm{~min})(9.0 \pm 1.0$ vs. $8.5 \pm 0.9 \mathrm{mmol} / \mathrm{l}, \mathrm{P}=0.004)$, higher weight $(76.0 \pm 18,1$ vs. $67.9 \pm 14.7 \mathrm{~kg}, \mathrm{P}=0.087)$, and higher systolic blood pressure ( $117 \pm 21$ vs. $110 \pm 13 \mathrm{mmHg}, \mathrm{P}=0.086)$ compared to $\mathrm{GDM}$ women without a maternal history of diabetes mellitus.

Conclusion: Maternal history of diabetes mellitus seems to be a stronger predictor of gestational diabetes than paternal history. This association was independent of the effect of age and BMI. GDM women with a maternal history of diabetes mellitus might have a more severe disease than those GDM cases without.

\section{5}

Circulating visfatin in women with gestational diabetes

B. Telejko ${ }^{1}$, A. Zonenberg ${ }^{1}$, K. Niedziolko-Bagniuk ${ }^{1}$, M. Kuzmicki ${ }^{2}$,

A. Nikolajuk ${ }^{1}$, J. Szamatowicz ${ }^{3}$, M. Gorska';

${ }^{1}$ Department of Endocrinology, Diabetology and Internal Medicine,

${ }^{2}$ Department of Pathophysiology of Pregnancy, ${ }^{3}$ Department of Gynecology, Medical University of Bialystok, Bialystok, Poland.

Background and Aims: Visfatin - a new adipokine exhibiting insulin - like activity - has been shown to be increased in patients with type 2 diabetes and long - standing type 1 diabetes, whereas in women with gestational diabetes (GDM) the opposite results, namely increased and decreased levels have been demonstrated. In this study we compared visfatin concentrations between pregnant women with normal glucose tolerance (NGT), GDM and non-pregnant healthy women.

Materials and Methods: The group studied consisted of 61 patients with GDM at $28.8 \pm 3.8$ week of gestation, 63 pregnant women with NGT at $29.7 \pm 3.2$ week of gestation and 36 non-pregnant healthy women. Serum visfatin concentration was measured using an enzyme - linked immunosorbent assay.

Results: Median visfatin concentrations did not differ significantly between women with GDM (median 14.8 [interquartile range 10.8-17.3] ng/ml) and NGT (15.3 [11.8-19.4] ng/ml), but were markedly higher than those found in non-pregnant women $(11.4$ [8.6-15.2] ng/ml, $\mathrm{p}=0.0008$ vs NGT and $\mathrm{p}=0.0083$ vs. GDM group). Visfatin levels correlated slightly with insulin $(R=2001$, $\mathrm{p}=0.0111)$ and HOMA-IR values $(\mathrm{R}=0.1783, \mathrm{p}=0.0241)$. No association with glucose, gestational age or body mass index was observed. Stepwise multiple regression analysis revealed that visfatin concentrations were significantly predicted only by $\mathrm{HbAlc}$ values ( $\mathrm{Beta}=0.2630, \mathrm{p}=0.0046$ )

Conclusion: Elevated visfatin levels in pregnant women may be connected with increased insulin or insulin resistance but not with GDM.

\section{6}

Visfatin response to glucose is reduced in women with gestational diabetes mellitus

A. Handisurya ${ }^{1}$, D. G. Haider ${ }^{1,2}$, A. Storka ${ }^{2}$, E. Vojtassakova ${ }^{2}$, A. Luger ${ }^{1}$, M. Wolzt ${ }^{1,2}$, A. Kautzky-Willer';

${ }^{1}$ Department of Internal Medicine III, Division of Endocrinology and Metabolism, ${ }^{2}$ Department of Clinical Pharmacology,

Medical University of Vienna, Austria.

Context: The insulin-mimetic adipocytokine visfatin has been associated with insulin resistance and is regulated by glucose. Recently, retinol binding protein-4 (RBP-4) was related to the development and progression of insulin resistance.
Objective: We hypothesized that glucose-induced plasma visfatin and RBP-4 regulation are different in women with gestational diabetes mellitus (GDM). Design and Patients: Plasma visfatin and RBP-4 concentrations were investigated in 10 women with GDM and 10 age-matched healthy women in pregnancy week 24-28 during a standard oral glucose tolerance test (OGTT).

Results: There was no difference between fasting plasma RBP-4 or the appetite-regulating peptide obestatin, which remained unchanged during OGTT. In contrast, women with GDM had lower systemic visfatin concentrations than controls $(1.9 \pm 0.8 \mathrm{ng} / \mathrm{ml}$ vs. $5.2 \pm 4.4 \mathrm{ng} / \mathrm{ml}$, respectively, $\mathrm{p}<0.05)$, which were associated with fasting glucose $(\mathrm{p}<0.05)$. The glucoseinduced increase in plasma visfatin over baseline was smaller in GDM, with an area under the curve of $409 \pm 106 \mathrm{ng}^{\star} \mathrm{min} / \mathrm{ml}$ versus $780 \pm 345 \mathrm{ng}^{\star} \mathrm{min} / \mathrm{ml}$ in controls $(\mathrm{p}<0.05)$

Conclusion: Glucose-induced increases in circulating visfatin are reduced in women with GDM. This mitigated response may be associated with impaired glucose tolerance. RBP-4 or obestatin plasma levels are not acutely affected by changes in plasma glucose concentrations.

\section{7}

Early development of cardiovascular risk in women with prior gestational diabetes

L. Volpe ${ }^{1}$, I. Cuccuru' ${ }^{1}$, C. Lencioni ${ }^{1}$, V. Napoli ${ }^{2}$, A. Ghio ${ }^{1}$, C. Fotino ${ }^{1}$, F. Romero ${ }^{1}$, G. Penno ${ }^{1}$, S. Del Prato ${ }^{1}$, G. Di Cianni ${ }^{1}$;

${ }^{1}$ Department of Endocrinology and Metabolism, University of Pisa,

${ }^{2}$ Department of Radiology, University of Pisa, Italy.

Background and Aims: We have previously shown that both the Metabolic Syndrome and low-grade inflammation are common findings in women with previous gestational diabetes ( $\mathrm{pGDM}$ ) suggesting these women may have not only greater risk for diabetes but also for cardiovascular disease (CVD). As such they are expected to have earlier signs of sub-clinical atherosclerosis as compared to women without pGDM. The aim of present work was, then, to verify such a hypothesis.

Materials and Methods: 28 clinically healthy women with pGDM and 24 controls women with a history of normal pregnancy were evaluated at 36 months after delivery. A $75 \mathrm{~g}-2 \mathrm{~h}$ oral glucose tolerance test (OGTT) was performed to determine area under glucose curve $\left(\mathrm{AUC}_{\mathrm{gluc}}\right)$, insulin sensitivity index (ISI) and resistance (HOMA-R). Moreover, blood pressure (BP), BMI, waist circumference, lipid profile, oxidised LDL (Ox-LDL), C-Reactive Protein (CRP), and fibrinogen were determined. In all women Intimal Media Thickness (IMT) was measured at 4 segments of left and right carotid, and mean carotid IMT value calculated.

Results: Family history of type 2 Diabetes ( 61.3 vs $8.1 \%, \mathrm{p}<0.0001$ ), and cardiovascular diseases ( 54.8 vs. $20.8 \%, \mathrm{p}<0.001)$ was more common amongst pGDM than control women. The former also were older $(37.75 \pm 4.4$ vs. $34.12 \pm 3.2$ years, $\mathrm{p}<0.001)$, had larger waist circumference $(85.9 \pm 9.3$ vs. $79.9 \pm 9.7 \mathrm{~cm} \mathrm{p}<0.03)$, and higher diastolic BP $(70.6 \pm 7.3$ vs. $66.6 \pm 5.7 \mathrm{mmHg}$ $\mathrm{p}<0.03)$, while there was no difference in BMI $(24.22 \pm 3.2$ vs. $23.1 \pm 3.5 \mathrm{Kg} /$ $\left.\mathrm{m}^{2}\right)$. Fasting plasma glucose levels $(84.3 \pm 9.6$ vs. $79.5 \pm 4.3 \mathrm{mg} / \mathrm{dl}), \mathrm{AUC}_{\mathrm{gluc}}$ $(873.5 \pm 147,4$ vs. $690.6 \pm 114.4 \mathrm{mg} / \mathrm{dlxmin})$, triglycerides (91.28 \pm 67.7 vg. $62.7 \pm 21,4 \mathrm{mg} / \mathrm{dl})$, fibrinogen ( $356.7 \pm 69.2$ vs $298.45 \pm 60 \mathrm{mg} / \mathrm{dl})$, CRP $(3.4 \pm 1.8$ vs $0.8 \pm 0.9 \mathrm{mg} / \mathrm{L})$, and HOMA-R $(1.9 \pm 1.08$ vs. $1.39 \pm 0.7)$ were all greater ( $\mathrm{p}<0.05$ or less $)$ in $\mathrm{pGDM}$, while ISI $(6.4 \pm 4$ vs. $11.7 \pm 10, \mathrm{p}<0.01)$ was lower than controls. Mean common carotid IMT value was higher in pGDM women than in control women $(0.57 \pm 0.058$ vs. $0.51 \pm 0.051 \mathrm{~mm}, \mathrm{p}<0.01)$ with no difference in the values at the artery bulb level. On simple regression IMT was related with age $(\mathrm{r}=0.43, \mathrm{p}<0.0009)$, BMI $(\mathrm{r}=0.37, \mathrm{p}<0.0043)$, waist circumference $(r=0.32, p<0.019)$, systolic $(r=0.36, p<0.007)$ and diastolic $B P$ $(\mathrm{r}=0.3, \mathrm{p}<0.0013)$, HOMA R $(\mathrm{r}=0.29, \mathrm{p}<0.037), \mathrm{AUC}_{\text {gluc }}(\mathrm{r}=0.28, \mathrm{p}<0.004)$, Ox-LDL $(r=0.34, p<0.004)$ and CRP $(r=0.33, p<0.001)$. However when all latter variables were introduced in a multiple regression analisys only $\mathrm{Ox}-$ LDL (f-test $5.3, p<0.02$ ) remained significantly associated with IMT.

Conclusion: Our results show that sub-clinical carotid artery atherosclerosis is present three years after delivery in PGDM women. IMT is associated with multiple CVD risk factors, but independently associated only with Ox-LDL. Therefore our data suggest that pGDM women are at greater CVD risk and indicate Ox-LDL as a target for early preventative intervention. 


\section{8}

Women with pregnancy-induced hypertension have an increased incidence of subsequent diabetes

A. Dhawan ${ }^{1}$, P. Baker ${ }^{2}$, S. Dissanayake ${ }^{3}$, S. Khan ${ }^{1}$, P. Lewis ${ }^{1}$;

${ }^{1}$ Cardiology, Stepping Hill Hospital, Stockport,

${ }^{2}$ Maternal foetal health, CMMC, United Kingdom,

${ }^{3}$ Diabetes, Stepping Hill Hospital, Stockport, United Kingdom.

Introduction: Studies have shown that hypertension in pregnancy is associated with a higher incidence of hypertension in the later life. We investigated whether there is a relationship between hypertension in pregnancy and the subsequent incidence of diabetes.

Methods: The current diabetic status of 200 women with a history of pregnancy-induced hypertension was compared with that of 100 women delivered during the same period in the past. Data from this index pregnancy including age, family history of diabetes, gestational diabetes, BMI and blood pressure was collected. Current diabetic status was established by direct contact, questionnaire and general practitioner and hospital records.

Results: These women were followed up over a mean period of 15 years (range 11-20 years). There was no significant difference between the mean age of the women (43 years, range 37-50 Years) in the two groups. Incidence of diabetes was significantly higher in women with pregnancy-induced hypertension compared with normotensive pregnancy (39/200 vs. 7/100, $\mathrm{p}<0.001$ ). Using a multivariate binary logistic model (age, hypertension in pregnancy, gestational diabetes, family history of diabetes and BMI), pregnancy induced hypertension (OR 3.904, 95\% CI: 1.41-10.79, p=0.009) and gestational diabetes (OR 6.06, 95\% CI: 2.39-15.36, $\mathrm{p}=0.0001$ ) were sole significant independent predictors of future diabetes.

Conclusion: Pregnancy induced hypertension is associated with increased future risk of developing diabetes.

Supported by Stockport NHS Foundation Trust

\section{PS 92 Pumps and analogues}

\section{9}

Efficacy of continuous subcutaneous insulin infusion in type 1 diabetes: a 2-year perspective under the established guidelines and indications for funding from a national health service

M. Giménez, I. Levy, M. Jansà, M. Vidal, G. Chiganer, I. Conget; Endocrinology and Diabetes Unit, Hospital Clínic i Universitari/IDIBAPS, Barcelona, Spain.

Background and Aims: To determine the 2-year efficacy of CSII following the current established indications and guidelines of the Catalan National Health Service compared to previous conventional MDI therapy.

Materials and Methods: Longitudinal, prospective, observational unicenter study. 153 T1D subjects previously treated with MDI in whom CSII was indicated following the guidelines of the Catalan National Health Service Authorities were included in the study. At baseline, we registered data on age, gender, duration of the disease, BMI, insulin dose and indications for CSII. $\mathrm{HbA}_{1 c}$ and the frequency of hypoglycaemic events, classified into mild and severe, assessed the quality of glycaemia control. For evaluation of the quality of life, three different self-report questionnaires were assessed. After 24 months of follow-up all subjects were evaluated including the same items measured before the initiation of CSII. Serious adverse events and injection site complications were also recorded.

Results: In 96\% of subjects, CSII indication included inability to obtain optimal glycaemic profile using MDI. $\mathrm{HbA}_{1 \mathrm{c}}$ fell from $7.9 \pm 1.3$ at baseline to $7.3 \pm 1.1$ $(\mathrm{p}<0.001)$ after 24 months of CSII, and this diminution depended on the initial value of $\mathrm{HbA}_{1 \mathrm{c}}$ and the number of severe hypoglycaemia episodes reported during the year before CSII initiation. Insulin requirements were significantly lower at the end of follow-up $(0.55 \pm 0.21 \mathrm{U} / \mathrm{kg}$.body weight $)$ in comparison to before using CSII $(0.70 \pm 0.20, \mathrm{p}<0.001)$. BMI increased from $24.1 \pm 3.1 \mathrm{~kg} / \mathrm{m}^{2}$ at baseline to $24.4 \pm 3.2$ after 24 months of CSII treatment $(\mathrm{p}=0.024)$. The rate of diabetic ketoacidosis episodes per year remained unchanged during follow-up. Mild and severe hypoglycaemic episodes were significantly reduced. The overall DQoL scores significantly improved in all aspects after 24 months of CSII.

Conclusion: The use of CSII improves metabolic control and quality of life outcomes with fewer hypoglycaemia episodes in T1D subjects previously conventionally treated with MDI under indications for funding from National Health Service guidelines.

\section{0}

Insulin pump therapy in adults allows metabolic control at lower rates of hypoglycaemia along with reduced insulin doses - results from the nationwide DPV-survey among German diabetes centers

W. Quester ${ }^{1}$, W. Kerner ${ }^{2}$, E. M. Fach ${ }^{3}$, K. Edel ${ }^{4}$, S. Wüchner ${ }^{5}$, K. Badenhoop ${ }^{6}$, T. Klaes ${ }^{7}$, H. R. Engels ${ }^{8}$, A. Gordalla ${ }^{9}$, H. J. Ziegelasch ${ }^{10}$, H. R. Henrichs ${ }^{11}$, R. W. Holl'

${ }^{1}$ Heart- and Diabetes Center NRW, Bad Oeynhausen, ${ }^{2}$ Klinikum Karlsburg, ${ }^{3}$ Schwerpunktpraxis, Stephanskirchen, ${ }^{4}$ Klink Hermannsborn, Bad Driburg, ${ }^{5}$ Diabetes Center, Klinikum Darmstadt, ${ }^{6}$ University Clinic, Frankfurt, ${ }^{7}$ Gemeinschaftsklinikum Koblenz-Mayen, ${ }^{8}$ Krankenhaus Hellersdorf, Berlin, ${ }^{9}$ Abt. Endokrinologie Stoffwechselerkrank., Klinik für Innere Medizin Universität Rostock, ${ }^{10}$ Innere Medizin, Klinikum Schwerin,

${ }^{11}$ Diabets Zentrum, Christliches Krankenhaus Quakenbrück, ${ }^{12}$ Institut für Epidemiologie, Universität Ulm, Germany.

Background and Aims: In adult patients with type 1 diabetes, intensified insulin therapy and insulin pump therapy are options for insulin substitution. Data on pump therapy under real-life-conditions in large multicenter databases are rare.

Materials and Methods: The DPV diabetes documentation system allows prospective documentation of parameters relevant for the process of diabetes care, as well as outcome indicators. For each individual patient, records during the most recent year were averaged. HbAlc values were mathematically standardised to the DCCT normal range. The SAS 9.2 analysis software was used for nonparametric comparisons, using Holm adjustment for multiple testing, and generalised mixed models.

Results: By March 2007, data on 8483 adult subjects with type 1 diabetes (mean age $36.1 \pm 0.2$ years, range $18-88$ years) were available for analysis. 
Gender ratio was $51 \%$ males and $49 \%$ females, the mean age at onset of diabetes was $20.2 \pm 0.2$ years (mean diabetes duration $15.9 \pm 0.1$ years). 6245 patients $(73.6 \%)$ used multiple daily injections, while 2238 (26.4\%) used CSII. Diabetes duration was longer in pump patients (20.1 versus 14.3 years, $\mathrm{p}<0.0001$ ), and more women opted for CSII ( $57.5 \%$ versus $45.8 \%$, $\mathrm{p}<0.0001)$. Daily insulin requirement was lower in pump patients $(43.9 \pm 0.4$ Units compared to $53.6 \pm 0.3$ Units, $\mathrm{p}<0.0001$ ). $50.4 \%$ of subjects on multiple injections used insulin analogs, compared to $72.7 \%$ of CSII patients $(\mathrm{p}<0.0001)$. Mean $\mathrm{HbAlc}$ was $7.8 \%$ in both groups, and no difference was present after adjustment for age, duration of diabetes, gender, insulin dose, insulin preparation, degree of overweight and center heterogeneity. In contrast, the rate of severe hypoglycaemic episodes was lower in CSII patients (4.1 events per 100 patients-years) compared to MIT patients (6.6 events, $\mathrm{p}<0.0001)$. This difference remained significant after adjustment for potentially confounding variables $(p<0.02)$. CSII patients were slightly more overweight with a BMI of 25.7 compared to $24.9 \mathrm{~kg} / \mathrm{m}^{2}$ in MIT patients. Micro- and macroalbuminuria were more prevalent in MIT compared to CSII patients ( $p<0.0001$ and $\mathrm{p}<0.01$, respectively, after adjustment for confounders).

Conclusion: Insulin requirement is significantly lower in CSII patients compared to MIT and the rate of severe hypoglycemia is significantly lower, while BMI is slightly higher. No significant difference in $\mathrm{HbAlc}$ values was detected, but the rate of micro- and macroalbuminuria were lower in CSIIpatients.

\section{1}

Long-term results of continuous subcutaneous insulin infusion (CSII) treatment - national register in the Czech Republic

Z. Jankovec, D. Cechurova, M. Krcma, S. Lacigova, M. Zourek, Z. Rusavy; Medical Department, University Hospital, Plzen, Czech Republic.

Background and Aims: National Register of patients treated by insulin pump are available during treatment period 1998-2007 obtained from nearly 2.550 patients from 52 departments of diabetology in the Czech Republic. The aim of our study is to present the current results of data evaluation.

Materials and Methods: The data was collected continuously utilising special database software and evaluated in regular intervals. For statistical evaluation the common statistical methods, Wilcoxon rank-sum test, Spearman rank correlatin coefficient and Kruskal-Wallis analysis were used.

Results: The average age of patients was $40,6 \pm 15,66$ years, the average history of diabetes at the start of CSII treatment was 13,0 $0 \pm 9,42$ years, and the average duration of insulin pump treatment was $4,9 \pm 3,55$ years, BMI $35,2 \pm 15,76$ $\mathrm{kg} / \mathrm{m}^{2}$. Main reasons for the treatment by CSII: $63 \%$ for bad control, $18 \%$ for hypoglycemia, 22\% for neuropathy, 19\% for dawn Phenomenon, and 14 $\%$ for other chronic complications (each of the patients could state multiple reasons). The results have shown a significant improvement of values of $\mathrm{HbA}_{1 \mathrm{C}}$ in the insulin pump treatment compared to the values before the start: $9,60 \pm 2,12 \%$ vs. $8,47 \pm 1,71$ after 1 year $(p<0,001)$. Simultaneously, the average daily insulin dose was reduced: $49,6 \pm 18,2$ vs. $40,2 \pm 14,4$ IU/day $(p<0,05)$. During the CSII treatment a permanent and statistically significant increase of basal doses while bolus doses do not change. Likewise, there has been a significant weight increase (BMI) after the $2^{\text {nd }}$ year of treatment $(p<0,05)$. The values of $\mathrm{HbA}_{1 \mathrm{C}}$ during CSII treatment have been directly proportional to the $\mathrm{HbA}_{1 \mathrm{C}}$ values at the start of the treatment. The predictive factors of success of the CSII therapy seem to be bad control of diabetes and hypoglycemia as reasons for the start of CSII treatment.
Conclusion: Our results show an enduring character of improvement of monitored parameters from a long-term point of view. CSII treatment has been effective especially in very badly controlled diabetic patients.

Supported by a grant from the Ministry of Education, Charles University Prague, Faculty of Medicine in Pilsen, Czech Republic

\section{2}

Insulin requirement profiles of type 2 diabetic patients after achieving stabilised glycaemic control with short-term continous subcutaneous insulin infusion

Y. H. Noh ${ }^{1}$, S. Y. Park ${ }^{1}$, J. H. Lee ${ }^{1}$, D. Y. Kim¹ ${ }^{1}$, S. B. Choi ${ }^{2}$;

${ }^{1}$ Biochemistry,

${ }^{2}$ Internal Medicine, Konkuk University, Chungju, Republic of Korea.

Background and Aims: Large prospective studies have demonstrated that optimum glycemic control is not routinely achieved in clinical practice. Recently CSII therapy for T2DM patients have emerged as a promising therapeutic strategy, for several studies have reported a better glycemic control, fewer complications and more remission cases with CSII in T2DM patients. However, barriers to optimal insulin therapy including fear of hypoglycemia, suboptimal initiation and dose titration have kept physicians from initiating CSII therapy for T2DM patients who have endogenous insulin secretion with wide spectrum. Moreover, few studies have reported the actual insulin does requirement profiles of T2DM patients in CSII therapy. In the present study, to find predicting factors for determining the insulin dose requirement profiles of T2DM patients to be used on the initiation of CSII therapy, we analysed clinical parameters and laboratory data at baseline and total daily dose (TDD) infused in T2DM patients after achieving stabilised glycemic control with CSII treatment.

Materials and Methods: We analysed insulin requirement profiles of 300 T2DM patients [male $50 \%$, age $57.9 \pm 11.4$, duration of T2DM $11.0 \pm 7.9$ years, fasting plasma glucose (FPG) $155 \pm 54.4$ and postprandial plasma glucose (PP2) $236 \pm 81.7 \mathrm{mg} / \mathrm{dl}, \mathrm{HbA}_{1 \mathrm{c}} 9.1 \pm 2.2 \%$ ] after stabilisation of blood glucose level achieved (FPG $110 \pm 21.2 \mathrm{mg} / \mathrm{dl}$ and PP2 $121 \pm 17.4 \mathrm{mg} / \mathrm{dl}$ ) by two weeks of CSII therapy and performed statistical analyses including $t$-tests, Pearson correlation, and multiple linear regression tests. Carbohydrate to insulin ratio [CIR, carbohydrate intake amount (g/day) divided by insulin bolus dose(IU/ day)] was calculated.

Results: Insulin requirement profiles and CIR data from 300 T2DM patients are summarised in Table 1. Means of TDD, basal insulin dose, CIR, and BMI were significantly different between male and female patients $(\mathrm{p}<0.01)$. TDD was associated with sex, height, $\mathrm{BMI}, \mathrm{HbA}_{1 c}, \mathrm{FPG}, \mathrm{PP} 2$, fasting C-peptide, and HOMA-IR ( $\mathrm{p}$ 0.05). CIR was associated with age, sex, duration of DM, height, BMI, HbA, FPG, PP2, fasting C-peptide, and HOMA-IR $(\mathrm{p}<0.05)$. After adjustment for age, sex, and BMI, TDD was associated with $\mathrm{HbA}_{1 \mathrm{c}}$, FPG, PP2, fasting C-peptide, and HOMA-IR ( $<0.05)$. After adjustment for age, sex and BMI, CIR was associated with weight, height, $\mathrm{HbA}_{1 \mathrm{c}}$ PP2, HOMA-IR $(\mathrm{p}<0.05)$.

Conclusion: From these analyses we could make equations to predict TDD, CIR, bolus and basal profiles using clinical and laboratory data before the initiation of CSII therapy. Theses equations may give more practical guidelines for the initiation and dose titration of CSII therapy on T2DM patients than the present guideline based on weight in determination of insulin dosage. (Tab. 1)

Table1. Insulin Requirement Profiles of 300 Type 2 Diabetic Patients 2 weeks after CSII therapy

\begin{tabular}{|c|c|c|c|c|c|c|c|c|}
\hline & $\begin{array}{l}\text { TDD } \\
\text { (IU/day) }\end{array}$ & $\begin{array}{l}\text { Bolus } \\
\text { (IU/day) }\end{array}$ & $\begin{array}{l}\text { Basal } \\
\text { (IU/day) }\end{array}$ & $\begin{array}{l}\text { Carbohydrate intake } \\
\text { (g/day) }\end{array}$ & $\begin{array}{l}\text { CIR } \\
(\mathrm{g} / \mathrm{IU})\end{array}$ & $\begin{array}{l}\mathrm{BMI} \\
\left(\mathrm{kg} / \mathrm{m}^{2}\right)\end{array}$ & $\begin{array}{l}\text { HbAlc } \\
(\%)\end{array}$ & $\begin{array}{l}\text { Fasting C-peptide } \\
(\mathrm{ng} / \mathrm{ml})\end{array}$ \\
\hline Total $(n=300)$ & $44.9 \pm 24.7$ & $29.7 \pm 19.6$ & $15.1 \pm 7.5$ & $246.8 \pm 38.8$ & $15.1 \pm 7.5$ & $24.4 \pm 3.3$ & $9.1 \pm 2.2$ & $1.6 \pm 1.0$ \\
\hline $\begin{array}{l}\text { (proportion to TDD } \\
\text { in total patients) }\end{array}$ & $(100 \%)$ & $(64 \%)$ & $(36 \%)$ & & & & & \\
\hline Male $(n=150)$ & $42 \pm 23.4^{\star}$ & $28.5 \pm 18.9$ & $13.7 \pm 6.4^{*}$ & $271.6 \pm 31.8^{*}$ & $14.5 \pm 12.2^{*}$ & $23.8 \pm 3.3^{*}$ & $9.0 \pm 2.3$ & $1.6 \pm 1.1$ \\
\hline $\begin{array}{l}\text { (proportion to TDD } \\
\text { in male patients) }\end{array}$ & $(100 \%)$ & $(65 \%)$ & $(35 \%)$ & & & & & \\
\hline Female $(n=150)$ & $47.8 \pm 25.6^{*}$ & $31.3 \pm 20$ & $16.4 \pm 7.7^{*}$ & $223.2 \pm 23.6^{*}$ & $11.1 \pm 8.9^{*}$ & $25.0 \pm 3.2^{*}$ & $9.1 \pm 2.1$ & $1.6 \pm 0.8$ \\
\hline $\begin{array}{l}\text { (proportion to TDD } \\
\text { in female patients) }\end{array}$ & $(100 \%)$ & $(63 \%)$ & $(37 \%)$ & & & & & $\begin{array}{l}{ }^{*} \mathrm{p}<0.01 \\
\text { male vs famale }\end{array}$ \\
\hline
\end{tabular}




\section{3}

Insulin pump treatment in the elderly: a closer look at its feasibility and effectiveness

R. Hoogma ${ }^{1}$, S. Matthaei ${ }^{2}$, M. A. Schweitzer ${ }^{3}$, S. Grunder ${ }^{3}$, A. Buhr ${ }^{3}$; ${ }^{1}$ Interne Geneeskunde/Diabetes, Groene Hart Ziekenhuis, Gouda, The Netherlands, ${ }^{2}$ Diabetes Zentrum Quakenbrueck, Germany, ${ }^{3}$ Roche Diagnostics GmbH, Mannheim, Germany.

Background and Aims: Insulin pump therapy is considered the gold standard for optimised glucose control and increasingly the preferred way to intensified treatment for the younger patients for whom the long-term benefits are of utmost importance. There might be a number of patients getting older with the pump and others looking for the short-term benefits CSII offers to them. However, not much data is available for this age group on pumps.

Materials and Methods: A structured questionnaire was distributed to 36,450 CSII patients with T1 and T2 diabetes from Europe and North America to determine patient characteristics, medical data, complication rates and treatment satisfaction. In total, there were 25 structured questions with additional information on socio-demographic background, insulin pump indication, initiation and follow-up of treatment. We report the analysis of the sub-set of data of patients older than 65 years and compare them with the younger ones.

Results: 902 (6.4\%) of the 14,015 respondents were $\geq 65$ years old. Overall, elderly patients on CSII had a longer duration of diabetes (29.6 \pm 14.4 vs. $20.2 \pm 11.4$ years $)$ and their time on insulin treatment was longer $(26.3 \pm 15.6$ vs. $19.5 \pm 11.5$ years), the difference to the duration of diabetes most likely explained by the higher percentage of T2 patients $(21.6 \%$ vs. $4.2 \%)$. Remarkably, the mean age at start of CSII was $63.6 \pm 6.5$ vs. $35.2 \pm 12.8$ years; on average they were $5.5 \pm 5.1$ vs. $4.7 \pm 4.3$ years on CSII. Reasons for starting CSII therapy differed between the two groups mainly in: neuropathy ( 30.8 vs. $13.3 \%$ ), diabetic complications (22.2 vs. $13.0 \%$ ) and bG fluctuations (59.5 vs. $39.8 \%$ ), whereas more flexible lifestyle ( 44.5 vs. $67.5 \%)$, variable nature of work (16.2 vs. $31.3 \%$ ) and Dawn phenomenon (32.6 vs. $49.3 \%$ ) were less important reasons in the elderly. Both groups reached good glycaemic control with a mean $\mathrm{HbAlc}$ of $6.9 \pm 1.1 \%$ vs $7.0 \pm 1.1 \%$. All patients reported equally high treatment satisfaction levels for CSII therapy.

Conclusion: Whereas elderly patients on CSII differ in their indication for this form of intensified insulin therapy, they reach equally good glycaemic control and high treatment satisfaction based on self-reported figures in this observational study. Improvement in symptoms of diabetic complications is important indications for CSII in patients $\geq 65$ years and can be addressed successfully. Higher age therefore should not be a contra-indication to start diabetic patients on pumps.

Supported by Disetronic Medical Systems AG, Switzerland

\section{4}

Pharmacokinetic and pharmacodynamic effects of an s.c. infusion of insulin lispro by a disposable basal insulin pump in healthy volunteers K. Rave ${ }^{1}$, L. Heinemann ${ }^{1}$, P. Gravesen ${ }^{2}$;

${ }^{1}$ Profil, Institute for Metabolic Research, Neuss, Germany,

${ }^{2}$ Danfoss A/S, Microtechnology, Nordborg, Denmark.

Background and Aims: Basal infusion of insulin with a constant rate over a period of at least 3 days is an attractive option for patients with type 2 diabetes. In comparison to a subcutaneous (sc) injection of a long-acting insulin analogue once daily a continuous infusion of a fast-acting insulin analogue should led to a more constant metabolic effect. A novel disposable Microfluidic Elastomer Patch Pump (MEPP) was developed to allow a high accurate insulin infusion at a constant flow rate.

Materials and Methods: By means of the glucose clamp technique we measured the pharmacokinetic and pharmacodynamic responses to a continuous sc infusion of insulin lispro (IL) either applied via a MEPP or by a commercially available insulin pump (Accu-Chek Spirit, A-CS, Roche Diagnostics). This study was conducted in an open-labelled cross-over design. Eight healthy male volunteers (mean age 36 years (range 30 - 44); BMI $26.7 \mathrm{~kg} \cdot \mathrm{m}^{-2}(23.0-29.9)$ ) were enrolled. Insulin lispro was infused at a rate of $2 \mathrm{U}$ per hour for 24 hours, using the MEPP for 3 out of 4 experiments performed in each volunteer; the ACS was used during the other experiment. Blood glucose of volunteers was kept constant at a target value of $4.5 \mathrm{mmol} \cdot \mathrm{l}^{-1}$ by a glucose clamp procedure. Blood samples were taken in regular intervals for specific measurement of serum IL levels. Twentyseven individual MEPPs were employed during the 24 glucoseclamps, among which 21 constantly delivered IL and 6 failed.

Results: When taking data from the successful experiments only, total serum insulin lispro area under curve (AUC-INS ${ }_{0-24 \mathrm{~h}} 44.7 \pm 11.7$ vs. $38.8 \pm 7.5$ $\mathrm{mU} \cdot \mathrm{min} \cdot \mathrm{ml}^{-1}$ (mean $\pm \mathrm{SD}, \mathrm{n}=19$ for MEPP and $\mathrm{n}=8$ for A-CS)), fractional serum IL AUC (AUC-INS ${ }_{0-6 \mathrm{~h}} 7.6 \pm 2.5$ vs. $7.3 \pm 1.4 \mathrm{mU} \cdot \mathrm{min} \cdot \mathrm{ml}^{-1}$ ), peak serum IL levels (INS ${ }_{\max } 43.1 \pm 11.7$ vs. $36.4 \pm 6.0 \mu \mathrm{U} \cdot \mathrm{ml}^{-1}$ ) and time to peak levels (TINS $853 \pm 253$ vs. $559 \pm 308 \mathrm{~min}$ ) were comparable after infusion with MEPP or A-CS. Likewise, total glucose infusion rate AUC (AUC-GIR ${ }_{0-24 h} 5533 \pm 2233$ vs. $5499 \pm 1865 \mathrm{mg} \cdot \mathrm{kg}^{-1}$ ), fractional GIR AUC (AUC-GIR ${ }_{0-6 \mathrm{~h}} 755 \pm 505$ vs. $\left.845 \pm 520 \mathrm{mg} \cdot \mathrm{kg}^{-1}\right)$, maximum GIR $\left(\mathrm{GIR}_{\max } 5.7 \pm 2.0\right.$ vs. $\left.5.7 \pm 2.1 \mathrm{mg} \cdot \mathrm{kg} \cdot \mathrm{min}^{-1}\right)$ and time to maximum GIR (T-GIR ${ }_{\max } 933 \pm 462$ vs. $978 \pm 472 \mathrm{~min}$ ) were similar during infusion of IL via the MEPP or the A-CS. Mean weight difference of insulin pumps after $24 \mathrm{~h}$ of insulin pumping was $0.63 \pm 0.07 \mathrm{~g}$ for the MEPP and $0.49 \pm 0.01 \mathrm{~g}$ for the A-CS, which is well the theoretical amount of pumped insulin. Analysis of failing MEPPs revealed that remains from the production process obstructing the flow capillary were responsible for pump failures. Neither adverse events or adverse device effects were observed during the study, and there were no adverse findings of safety laboratory tests and vital sign measurements which could be attributed to the study treatment. Examination of the application site revealed no noticeable skin reactions.

Conclusion: In summary, continuous sc infusion of the fast-acting insulin analog insulin lispro by means of a disposable pump result in a pharmacokinetic and pharmacodynamic response comparable to that observed with a commercially available pump. Given that a more reliable performance of this functional model is possible, this Microfluidic Elastomer Patch Pump is an attractive alternative to conventional insulin pumps. Supported by Danfoss A/S

\section{5}

Reduction of $\mathrm{HbA}_{1 \mathrm{c}}$ using the "bolus wizard" in patients with type 1 diabetes on CSII therapy

J. Ferri, F. J. Ampudia-Blasco, L. Sorribes, C. Argente, R. Carmena; Endocrinology, Clinic University Hospital Valencia, Valencia, Spain.

Aims: To assess the benefit of using the "bolus wizard" (BW) in the metabolic control of patients with type 1 diabetes on continuous subcutaneous insulin infusion therapy (CSII).

Methodology: Observational, prospective study conducted on a group of patients with type 1 diabetes who were using the BW, after a variable period of CSII therapy and/or multiple dose insulin (MDI). A comparison was made by means of mean $\mathrm{HbA}_{1 \mathrm{c}}$ values during the 6 months before and after using this pump utility. All patients used a pump model with a BW (Paradigm ${ }^{\circ}$ 512/712 or 515/715, Medtronic Inc., USA). To draw comparisons, the Wilcoxon test was performed with SPSS 12.0. Data were expressed using mean \pm SD.

Results: 38 patients, of a total of 49 users (77.5\%), used the BW routinely (27 women, aged $35.3 \pm 6.9$ years $\left(\mathrm{X} \pm \mathrm{DE}\right.$ ), BMI $24.3 \pm 3.7 \mathrm{~kg} / \mathrm{m}^{2}$, diabetes since $18.7 \pm 8.9$ years, on CSII therapy for $2.83 \pm 1.89$ years, with "bolus wizard" for $8.9 \pm 5.2$ months). Of these, $60.5 \%$ were patients who had been on CSII therapy previously but used earlier models without this function (Minimed 507C and 508, Medtronic Inc., USA). The difference between the mean $\mathrm{HbA}_{1 c}$ before and after using the BW, was $-0.31 \%$ (CI $95 \%:-0.03 \%,-0.59 \%, \mathrm{p}=0.031$ ). However, when an analysis was made only of patients who had been on CSII previously, the incorporation of the BW did not lead to a significant reduction in $\mathrm{HbA}_{1 c}$ (difference in $\mathrm{HbA}_{1 c}$ of $-0.12 \%$ (CI 95\%: $-0.50 \%, 0.26 \%, \mathrm{p}=0.432$ ). Conclusions: Use of the BW in patients with type 1 diabetes on CSII therapy leads to improved metabolic control, and this is more significant in patients who had been on MDI prior to starting the new therapy.

\section{6}

Insulin detemir provides superior glycaemic control compared with $\mathrm{NPH}$ insulin

J. J. Larsen ${ }^{1}$, S. Heller ${ }^{2}$, C. B. Pedersen ${ }^{1}$;

${ }^{1}$ Novo Nordisk A/S, Bagsværd, Denmark, ${ }^{2}$ School of Medicine and Biomedical Sciences, University of Sheffield, United Kingdom.

Background and Aims: To investigate the glycaemic control achieved with the long-acting analogue insulin, detemir (detemir) and intermediate-acting 
$\mathrm{NPH}$ insulin (NPH), using a meta-analysis based on three randomised, multinational, parallel, open-label trials that included 1555 men (60.5\%) and women (39.5\%) with type 1 diabetes.

Materials and Methods: Subjects received detemir or NPH once-daily plus human soluble insulin (HSI) at meals in one 26-week trial, and received detemir or NPH twice-daily plus insulin aspart at meals in two 16-week trials. At baseline, subjects had a mean (SD) duration of diabetes of 16.3 (10.6) yrs, with a mean age of $40.3(12.2)$ yrs. Average $\mathrm{HbA}_{1 \mathrm{c}}$ was $8.4 \%$ and $8.3 \%$, FPG was 11.4 and $11.5 \mathrm{mmol} / \mathrm{L}$, body weight was 75.9 and $76.0 \mathrm{~kg}$ and BMI 25.1 and $25.3 \mathrm{~kg} / \mathrm{m}^{2}$, for subjects randomised to detemir and $\mathrm{NPH}$, respectively. Results: Glycaemic control, as measured by $\mathrm{HbA}_{1 \mathrm{c}}$ and FPG, and change in body weight were analysed across the three trials. There was a significant improvement in $\mathrm{HbA}_{1 \mathrm{c}}$ after treatment with detemir superior to that seen with $\mathrm{NPH}$, with an estimated mean difference of $-0.13 \%$, 95\% CI [-0.22,0.04], $p=0.006$ and mean reductions from baseline to end of trial of 0.4 and 0.2 percent points, respectively. FPG also decreased in both groups, with an estimated mean difference of $-1.53 \mathrm{mmol} / \mathrm{L}, 95 \% \mathrm{CI}[-1.99,-1.07], \mathrm{p}<0.0001$ in favour of detemir. When using detemir, no increase in body weight was observed, whereas use of NPH resulted in weight gain, with an estimated mean difference between treatments of $-0.64 \mathrm{~kg}$, 95\% CI [-0.94,-0.34], $\mathrm{p}<0.0001$. In each trial, these results were obtained with similar basal and bolus insulin doses for the detemir and NPH groups. Over the 24-hour period, the relative risk of hypoglycaemia was similar between the detemir and NPH groups in each of the three trials. Reduced risk of minor nocturnal hypoglycaemia was observed with detemir in the 26-week trial and in one of the 16-week trials. The safety profiles, described by the incidence of adverse events in each trial, were similar with detemir and NPH.

Conclusion: Use of detemir resulted in a modest improvement in glycaemic control and a beneficial effect on weight compared with NPH in subjects with type 1 diabetes treated on a basal-bolus insulin regimen.

Supported by Novo Nordisk A/S

\section{7}

Italian experience trial for the implementation of insulin glargine in basal-bolus regimen in patients with type 1 diabetes

P. Brunetti ${ }^{1}$, M. Muggeo ${ }^{2}$, L. Cattin ${ }^{3}$, A. Arcangeli ${ }^{4}$, P. Pozzilli ${ }^{5}$,

V. Provenzano ${ }^{6}$, A. Francesconi ${ }^{7}$, P. Calatola ${ }^{8}$, F. Santeusanio ${ }^{1}$;

${ }^{1}$ Department of Internal Medicine, University of Perugia, ${ }^{2}$ Division of Endocrinology and Metabolic Disease, University of Verona, ${ }^{3}$ Department of Clinical, Morphological and Technological Sciences, University of Trieste, ${ }^{4}$ USL 4, Diabetes and Metabolic Disease Unit, Prato, ${ }^{5}$ Division of Diabetes and Metabolic Disease, University Campus Bio-Medico, Rome, ${ }^{6}$ Division of Diabetes and Metabolic Disease, Hospital of Partinico, Palermo, ${ }^{7}$ Diabetologia and Metabolic Disease Unit, Hospital of Santa Maria Annunziata, Florence, ${ }^{8}$ ASL SA/2, Diabetology Centre, Salerno, Italy.

Background and Aims: Once-daily (OD) basal insulin glargine can be used as part of a multiple daily injection regimen in patients with type 1 diabetes mellitus (T1DM). This randomised, multicentre study compared OD glargine (GLA)+prandial regular human insulin (RHI) with OD GLA+prandial lispro (LIS) in reducing the incidence of severe nocturnal hypoglycaemia at endpoint.

Materials and Methods: Patients with T1DM $(n=489)$ previously on a basalbolus regimen (NPH insulin+RHI or LIS) were switched to OD GLA as basal insulin. The daily dose of OD GLA was titrated (Fasting blood glucose $[\mathrm{FBG}]=5.0-6.7 \mathrm{mmol} / \mathrm{L}[90-120 \mathrm{mg} / \mathrm{dL}]$ ) for 8 weeks (run-in) prior to randomisation; patients continued with their previous bolus insulin. Patients $(n=395)$ were randomised to LIS $(n=193)$ or RHI $(n=202)$ and treated for 16 weeks. Data are mean \pm standard deviation.

Results: Both OD GLA+RHI and OD GLA+LIS regimens reduced $\mathrm{HbA}_{1 c}$ from baseline (with NPH insulin) to the end of run-in (both $\mathrm{p}<0.01$ ) and were similar at endpoint, although the LIS arm tended towards a lower value (Table).

\begin{tabular}{lll}
$\mathbf{H b A}_{1 \mathrm{c}}(\%)$ & RHI $\mathbf{a r m}$ & LIS arm \\
\hline Baseline (with NPH insulin) & $7.39 \pm 0.88$ & $7.39 \pm 0.97$ \\
8 weeks with GLA (run-in) & $7.10 \pm 0.82^{\star}$ & $7.00 \pm 0.88^{\star}$ \\
16 weeks with GLA (endpoint) & $7.10 \pm 0.83 \dagger$ & $6.95 \pm 0.78 \dagger$ \\
\hline
\end{tabular}

${ }^{*} \mathrm{p}<0.01 ;{ }^{\dagger} \mathrm{p}<0.001$, both vs baseline
For the patient subgroup who switched from RHI to LIS at randomisation, there was an additional reduction of $\mathrm{HbA}_{1 \mathrm{c}}$ at endpoint $(-0.14 \%, \mathrm{p}<0.05 \mathrm{vs}$. run-in). Overall, OD GLA+LIS showed a greater improvement in $\mathrm{HbA}_{1 \mathrm{c}}$ $(-0.41 \%$ vs. NPH insulin at baseline; $\mathrm{p}<0.001)$. FBG were similar at endpoint in both arms $(\mathrm{RHI}=9.1 \pm 2.4 \mathrm{mmol} / \mathrm{L}[164.6 \pm 42.4 \mathrm{mg} / \mathrm{dL}]$ vs. $\mathrm{LIS}=9.4 \pm 2.3$ $\mathrm{mmol} / \mathrm{L}[169.2 \pm 41.8 \mathrm{mg} / \mathrm{dL}] ; \mathrm{p}=\mathrm{NS}$ ). The incidence of nocturnal severe hypoglycaemia was similar in both groups at endpoint ( $\mathrm{RHI}=0.015 \pm 0.009$ vs. LIS $=0.016 \pm 0.008$ episodes/patient/month; $\mathrm{p}=\mathrm{NS}$ ).

Conclusion: These results suggest that the OD GLA+LIS regimen is more effective in achieving good glycaemic control when switched from NPH insulin+RHI using a stepwise approach of changing basal insulin first followed by bolus insulin.

Supported by sanofi-aventis 


\section{PS 93 Pharmacokinetics and physiology of insulin therapy}

\section{8}

Long-term follow-up of patients on U-500 Human Actrapid

V. Nayyar, I. Lawrence, M.-F. Kong, A. Gallagher, R. Gregory, S. Hiles,

S. Jackson, P. McNally, M. Davies;

Diabetes \& Endocrinology, University Hospitals Of Leicester NHS Trust, Leicester, United Kingdom.

Background and Aims: Patients with severe insulin resistance need to inject large volumes of insulin, the discomfort of which may lead to poor concordance. In addition the absorption of insulin may be erratic. U500 Human Actrapid is available on 'named patient' basis directly from the manufacturer, and has been used in insulin-treated diabetic patients who are severely insulin resistant.

Materials and Methods: We undertook retrospective case record review of 81 patients who had received U500 Human Actrapid and looked at ethnic background, changes in weight, amount of insulin injected, HbA1c, lipid profile and blood pressure. These were analysed in pre group (parameters before initiation of U500 insulin) and post group (latest parameters till February 2007).

Results: Eighty-one patients received U500 Human Actrapid (73 type 2 DM and 8 type $1 \mathrm{DM})$. U500 insulin used for a mean duration $30 \pm 22.6$ months (range 1-98months). Sixty one patients were White European, (36 male, 25 female), 17 Asian ( 11 male, 6 female) and 3 patients Afro-Caribbean ( 2 female, 1 male).Twenty-five patients were also receiving metformin, 5 were receiving a thiazolidinedione, 2 on combination of metformin and thiazolidinedione, and 2 were on a combination of metformin and acarbose. The mean insulin dose was $311.3 \pm 111.5$ vs. $368.4 \pm 179.9$ units/day. The mean HbAlc improved from $10.0 \pm 1.8 \%$ to $8.9 \pm 2.0 \%(\mathrm{p}=<0.0001)$. However, the number of patients achieving an $\mathrm{HbAlc}<7.5 \%$ increased from $5.1 \%$ (4 patients) to $27.5 \%$ (23 patients), $p=<0.0001$. Similarly more patients achieved a target HbAlc $<7 \%$ : $0 \%$ vs. $22.5 \%$ (18 patients), $\mathrm{p}=<0.0001$. Patients who were on U500 insulin for a longer period ( $>36$ months) showed a greater reduction in HbA1c compared to those who had taken U500 insulin for a shorter period ( $<36$ months): $1.8 \%+1.5 \%$ vs. $0.99 \%+1.8 \%, p=<0.05 \%$. There was also a reduction in Total Cholesterol $(4.9 \pm 1.4$ vs $4.3 \pm 1.0 \mathrm{mmol} / \mathrm{l}, \mathrm{p}=<0.0001)$ and Triglycerides $(4.3+4.5$ vs. $3.0+3.0 \mathrm{mmol} / 1, \mathrm{p}=<0.001)$. However, there was no

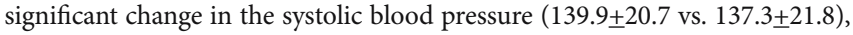
diastolic blood pressure $(76 \pm 14.5$ vs. $74 \pm 12.2)$, LDL -cholesterol $(2.7 \pm 1.3$ vs. $2.3 \pm 1.0 \mathrm{mmol} / \mathrm{l})$ and HDL-cholesterol $(0.9 \pm 0.3 \mathrm{vs} .1 .0 \pm 0.3 \mathrm{mmol} / \mathrm{l})$. There was significant weight gain: $116.2 \mathrm{~kg} \pm 27.1 \mathrm{~kg}$ vs. $121.3 \mathrm{~kg} \pm 29.3 \mathrm{~kg}(\mathrm{p}=<0.001)$. Two patients received U500 Human Actrapid via an insulin pump. 11 patients have died (4 from sepsis, 3 from myocardial infarction, 1 due to opiate overdose, 1 from an acute exacerbation of COPD, 1 from post operative pneumonia \& 1 cause of death was unknown). U500 insulin was discontinued in 14 patients (6 due to improved glycaemic control, 1 following bariatric surgery, 2 at patient's requests, 2 due to poor compliance and 3 due to unclear reasons).

Conclusion: Long-term glycaemic control improved with the use of U500 Human Actrapid in all ethnic groups $(\mathrm{p}=<0.05)$. There was also an improvement in lipid parameters, but most patients were on a statin. However, there was associated increased weight gain and patients were taking larger insulin doses. U500 Human Actrapid is a valuable treatment option in insulin-treated diabetic patients who are severely insulin resistant.

\section{9}

Self-association of insulin glulisine in solution - results of a comparative circular dichroism study

R. H. A. Becker ${ }^{1}$, A. Wollmer ${ }^{2}$, G. Seipke ${ }^{1}$;

${ }^{1}$ sanofi-aventis, Frankfurt, Germany,

${ }^{2}$ Biochemistry, RWTH Aachen University, Germany.

Background and Aims: Pure monomeric insulin analogues are vulnerable to denaturation and require zinc for shelf-life stability. This promotes selfassociation that compromises the gain in rapid absorption, compared with regular human insulin. This study compared the association state of insulin glulisine in pharmaceutical solutions with insulin aspart and insulin lispro using Circular Dichroism (CD) spectroscopy.
Materials and Methods: Pure insulin glulisine, insulin aspart, insulin lispro and wild-type human insulin were dissolved in $25 \mathrm{mmol} / \mathrm{L}$ Tris/HCl buffer, pH 7.8 with or without phenol $(10 \mathrm{~mol} / \mathrm{monomer})$, with or without $\mathrm{ZnCl}_{2}$ $(0.4 \mathrm{~mol} / \mathrm{monomer})$ and thus included insulin solutions that simulated the 'marketed' formulations (SMF) of insulin lispro, insulin aspart and wild-type insulin (all with added zinc) and insulin glulisine (zinc-free). The association state of each insulin at concentrations of $0.02-1.00 \mathrm{~g} / \mathrm{L}$ was assessed by $\mathrm{CD}$ spectroscopy as mean residue weight ellipticity at $275 \mathrm{~nm}$, which reflects the quaternary structure of insulin complexes in solution.

Results: In the absence of zinc with or without phenol (Figure), insulin lispro and insulin aspart remained mainly monomeric whereas insulin glulisine (SMF) and, to a greater extent, wild-type insulin assemble as dimers and/or higher-order aggregates at increasing concentrations. In the presence of $\mathrm{ZnCl}_{2}$, both insulin lispro and insulin aspart, to a greater extent insulin glulisine and, above all, wild-type insulin formed higher-order aggregates. The addition of phenol further promotes formation of higher-order aggregates of all insulins (SMF), except insulin glulisine.

Conclusions: The results show that pure insulin glulisine is less dissociated than pure insulin lispro and insulin aspart in solution, while showing less higher-order aggregates when compared with insulin lispro and insulin aspart formulated with zinc and phenol (Figure). This may help to explain why insulin glulisine without zinc meets the stability criteria for a viable product, while concomitantly presenting with a more rapid early deployment compared with other monomeric insulin products.

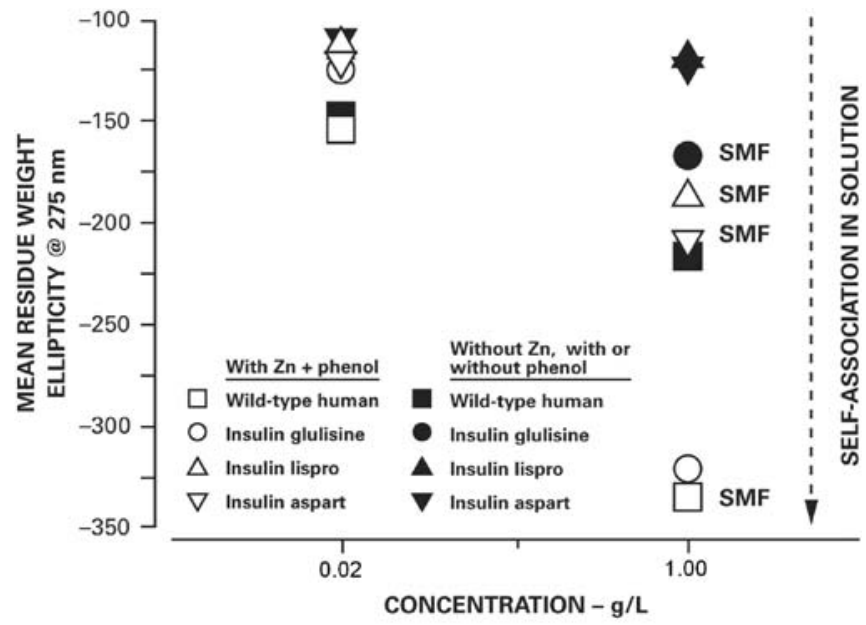

SMF: simulated 'marketed' formulation

(Insulin glulisine=without $\mathrm{Zn}$ with phenol; Insulin lispro, insulin aspart, wild-type human=all with $\mathrm{Zn}+$ phenol)

Supported by sanofi-aventis

\section{0}

Insulin glulisine improves postprandial metabolism and microvascular blood flow compared with regular human insulin in type 2 diabetic patients

C. Hohberg' ${ }^{1}$, M. Larbig ${ }^{2}$, A. Pfützner ${ }^{1}$, B. Jenke ${ }^{1}$, C. Hartmann ${ }^{1}$, M. Weber ${ }^{3}$, T. Forst ${ }^{1,3}$;

${ }^{1}$ Institute for Clinical Research and Development, Mainz,

${ }^{2}$ sanofi-aventis Deutschland GmbH, Berlin,

${ }_{3}^{3}$ Johannes Gutenberg University, Mainz, Germany.

Background and Aims: Increasing attention is being directed on the causal association between the postprandial state and the development of micro- and macrovascular complications. Several studies have demonstrated associations between postprandial metabolism, endothelial function and microvascular blood flow. The aim of our study was to compare the effect of insulin glulisine with regular human insulin on postprandial metabolism and microvascular function in patients with type 2 diabetes (T2DM).

Materials and Methods: Fifteen T2DM patients treated with oral antidiabetic drugs ( 9 male, 6 female; mean $\pm \mathrm{SD}$ age $57.9 \pm 8.2$ years; $\mathrm{HbA}_{1 \mathrm{c}} 7.1 \pm 0.5 \%$; duration of diabetes $11.2 \pm 10.9$ years) received insulin glulisine immediately 
prior or regular human insulin 15 minutes before consuming a standardised liquid meal (Ensure Plus ${ }^{\infty}, 56 \%$ carbohydrates, $29 \%$ fat, $15 \%$ proteins) in a randomised sequence on two different study days. At baseline and after 30, $60,120,180$, and 240 minutes, blood was taken for the measurement of glucose, insulin, intact proinsulin, asymmetric dimethylarginine (ADMA) and the peroxidative status using enzyme-linked or chemiluminescent immunoassays. In addition, microvascular skin blood flow was measured using laser doppler fluxmetry. Exploratory statistical analyses were conducted to compare insulin glulisine with regular human insulin, with calculation of two-sided 95\% confidence intervals for between-group differences.

Results: Insulin glulisine resulted in significantly higher insulin levels for the first 120 minutes following the meal (Table), and glucose levels and intact proinsulin were significantly lower over the entire 240-minute postprandial observation time compared with regular human insulin. A suppression of ADMA levels by $-15 \pm 6 \%$ (mean \pm SEM; $p=0.02$ ) was observed during insulin glulisine treatment, while regular human insulin was associated with a nonsignificant (n.s.) increase of $14 \pm 8 \%$. In addition, insulin glulisine increased postprandial microvascular blood flow by $38.2 \pm 21.9 \%(\mathrm{p}=0.008)$, whereas no change was observed with regular human insulin. The peroxidative status remained unchanged with both insulins.

Conclusion: Prandial treatment with insulin glulisine resulted in improved postprandial metabolic control including suppression of postprandial intact proinsulin secretion. In addition, treatment with insulin glulisine reduced postprandial endothelial stress and improves microvascular blood flow compared with regular human insulin.

\begin{tabular}{llll} 
& $\begin{array}{l}\text { Insulin } \\
\text { glulisine }\end{array}$ & $\begin{array}{l}\text { Regular human } \\
\text { insulin }\end{array}$ & p value \\
\hline AUC-Insulin $_{0-120} \min (\mathrm{mU} / \mathrm{L})$ & $51.0 \pm 6.8$ & $38.2 \pm 5.4$ & 0.004 \\
AUC-Glucose $_{0-240} \min (\mathrm{mg} / \mathrm{dL})$ & $158 \pm 8.8$ & $180 \pm 8.9$ & 0.03 \\
AUC-Proinsulin $_{0-240} \min (\mathrm{pmol} / \mathrm{L})$ & $26.2 \pm 3.5$ & $31.1 \pm 4.3$ & 0.002 \\
\hline
\end{tabular}

Results are means \pm SEM

Supported by sanofi-aventis

\section{1}

The use of rapid-acting insulin analogue as the baseline infusion during glucose clamping improves pharmacokinetic evaluation

J. Cassidy ${ }^{1}$, R. Baughman ${ }^{1}$, G. Tonelli ${ }^{1}$, A. Boss ${ }^{1}$, B. Hernandez ${ }^{2}$,

S. Schwartz';

${ }^{1}$ MannKind Corporation, Danbury, ${ }^{2}$ Clinical Research,

Diabetes \& Glandular Disease Clinic, San Antonio, United States.

Background and Aims: In the glucose clamp technique, a glucose infusion maintains concentrations within a target range and an i.v. insulin infusion is used to suppress endogenous insulin secretion and/or provide basal insulin. However, separating endogenous from exogenously administered regular human insulin remains problematic. A potential solution uses lispro insulin (lispro) as the insulin infusate. An insulin analog-specific assay enables the independent quantification of the infused lispro (Lispro Insulin RIA Kit, Linco) and regular human insulin (E170 RHI Assay, Roche Diagnostics).

Materials and Methods: This procedure was used in a euglycemic clamp study evaluating a pulmonary regular human insulin formulation (PI) in type 1 diabetes mellitus. Subjects received an i.v. insulin infusion to establish glucose concentrations of $90-110 \mathrm{mg} / \mathrm{dL}$.

Results: The insulin infusion rate was fixed 1 hour before dosing with PI at $\mathrm{t}=0$, and remained constant thereafter. Regular human insulin concentrations from PI dosing were established by 3 methods (see figure): 1) lispro infusate (circle, no correction); 2) regular human insulin infusion, corrected by subtracting the $\mathrm{t}=0$ value from all values (triangle); or 3 ) regular human insulin infusion, corrected by subtracting the mean of the 240-360 minute values from the preceding data (square). A fixed insulin infusion can still generate $\mathrm{t}=0$ insulin concentrations that are higher (see figure) or lower than the end-of-study values (240-360 $\mathrm{min}$ ), and using either correction method will generate erroneous kinetics.

Conclusion: The data demonstrate that the use of lispro as the insulin infusate allows for direct regular human insulin determination and reduces data variability. This modified procedure can also be used to suppress endogenous insulin in type 2 diabetes mellitus, avoiding the commonly used discontinuation of the baseline insulin infusion at dosing, a situation that often generates regular human insulin concentrations $<0$ when normalized.

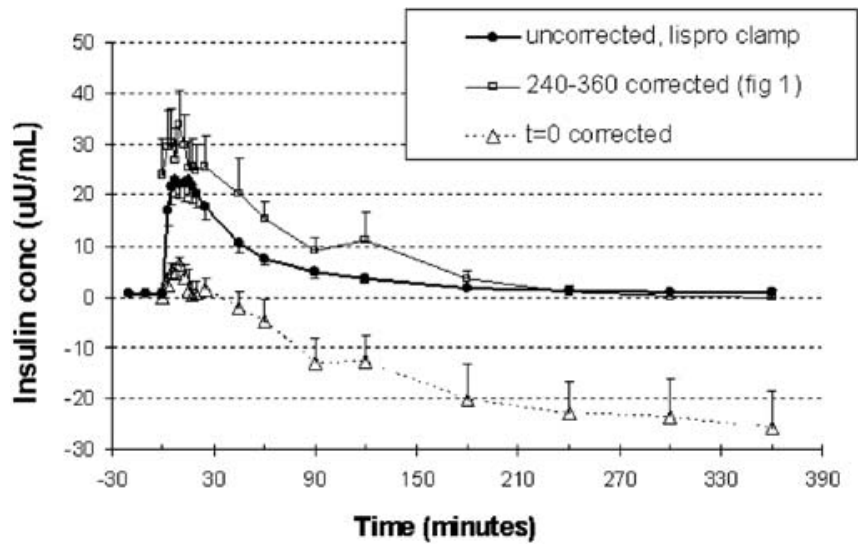

Supported by MannKind Corporation

\section{2}

Comparing within-patient blood glucose variability in analog vs human insulin self-prepared mixture regimens (insulin lispro + insulin lispro protamine suspension vs regular human insulin $+\mathrm{NPH}$ )

S. D. Beattie, L. N. Kerr, S. J. Jacober, C. A. Jones, M. H. Tan; Eli Lilly and Company, Indianapolis, United States.

Background and Aims: Day-to-day variability in glucose response to the same subcutaneously delivered insulin dose can challenge insulin-requiring diabetes patients (pts). This analysis examined intra-subject variability (ISV) of mean BG values in a clinical trial, rather than PK/PD, setting.

Materials and Methods: These post-hoc analyses were conducted on data from a published, randomized 12-month, open-label, 2-arm parallel study of 166 pts with type 1 or 2 diabetes mellitus (102 T1DM, 64 T2DM). In that study, after a 2-4 wk lead-in with self-mixed regular human insulin $+\mathrm{NPH}$ $[\mathrm{RI}+\mathrm{NPH}]$ before breakfast and dinner, pts were randomised to insulin lispro+insulin lispro protamine suspension [LP+ILPS] or RI+NPH bid for 12 mos; insulins were titrated to achieve premeal $\mathrm{BG} \leq 7.8 \mathrm{mM}$ and postprandial (pp) $B G \leq 10 \mathrm{mM}$. At $3 \mathrm{mos}$, investigators could intensify treatment if needed. In the post-hoc analyses, ISV was defined as day-to-day BG variability within pts at different time points during the day or across different time points during the day among 3 reading days. To compare variances between treatments in the ISV analyses at different time points during the day, a likelihood ratio (LR) test was based on an analysis of variance (ANOVA) model for BG values incorporating fixed effects for treatment and random effects for pts within treatment and measurements within pts. To compare variances between treatments in the ISV analyses across different time points during the day, a LR test was based on an ANOVA model for BG values incorporating fixed

\begin{tabular}{lll} 
LP+ILPS (mM) & RI+NPH (mM) & $\begin{array}{l}\text { P-value } \\
\text { Endpoint SD }\end{array}$ \\
Endpoint SD & (LR test) \\
$\mathrm{N}=85$ & $\mathrm{~N}=80$ & \\
\hline
\end{tabular}

Intra-subject variability (among 3 BG reading days)

8-pt BG Profile Time Points: *

2-h post-breakfast BG

\subsection{7}

Pre-lunch BG

2-h post-lunch BG

Pre-dinner BG

2-h post-dinner BG

Bedtime BG

3 AM BG

All Pre-meal $\mathrm{BG}^{\dagger}$

All Post-meal BG ${ }^{\dagger}$

Overall $\mathrm{BG}^{\ddagger}$

3.19

2.40

$$
3.31
$$

3.28

2.91

3.07

3.08

2.80

3.16

3.00

\subsection{7}

3.78

3.11

3.55

3.51

3.51

3.28

2.91

3.29

3.57

3.25

0.03

0.03

0.001

0.38

0.39

0.007

0.41

0.50

$<0.001$

*3 measurements per subject contributed to the analysis ${ }^{\dagger} 9$ measurements per subject contributed to the analysis

${ }^{\ddagger} 24$ measurements per subject contributed to the analysis 
effects for treatment, time points, and their interaction and random effects for pts within treatments, time-by-patient interaction, measurements across days within pts, and measurements across times within days.

Results: From the published results, at endpoint the analog insulins group showed lower $\mathrm{HbAlc}(7.5 \pm 0.1$ vs. $7.9 \pm 0.1 \%, \mathrm{p}=0.02)$, lower BG levels 2 -h post-breakfast and post-dinner, pre-dinner, and at bedtime, and no difference in FBG ( 8.4 vs. $8.5 \mathrm{mM})$ or hypoglycemia rate $(1.1$ vs. 1.1 episodes/pt/30 days) vs the human insulins group. Additionally, $16 \%$ of the LP+ILPS group and $6 \%$ of the RI+NPH group intensified treatment at 3 mos. The present post-hoc analyses demonstrated the analog insulins resulted in lower BG ISV pre-breakfast, 2-h post-breakfast, pre-lunch, and 2-h post-dinner and lower all pre-meal, all post-meal, and overall BG ISV (table 1).

Conclusions: Analog insulin regimens (lispro+IPLS) provided better $\mathrm{HbAlc}$ control than human insulins (RI+NPH) with lower pp BG and no difference in FBG or hypoglycemia rate. BG ISV was also lower with the analog insulins (including all pre-meal and all post-meal BG), however the relative contributions of lispro and IPLS to lower ISV could not be identified. Funded by Eli Lilly and Company

\section{3}

Clear differentiations in onset and duration of action are shown with different premixed ratios of biphasic insulin aspart (BIAsp)

U. Eckers ${ }^{1}$, T. Heise ${ }^{1}$, K. Kanc ${ }^{2}$, J. N. Nielsen ${ }^{2}$, L. Nosek ${ }^{1}$;

${ }^{1}$ Profil Institut für Stoffwechselforschung, Neuss, Germany,

${ }^{2}$ Novo Nordisk A/S, Bagsvaerd, Denmark.

Background and Aims: Most diabetes mellitus patients can achieve good glycaemic control using once-, twice- or three times daily biphasic insulin aspart (BIAsp 30), but some may benefit from a higher proportion of fastacting insulin aspart to reduce prandial glucose levels. We investigated the time-action profiles of different premixed ratios of $0.4 \mathrm{U} / \mathrm{kg}$ BIAsp (BIAsp 30, 50 and 70 ; containing $30 \%, 50 \%$ and $70 \%$ insulin aspart [IAsp], respectively) and IAsp in people with type 1 diabetes.

Materials and Methods: This was a randomised, single-centre, four-period crossover trial comprising 32 type 1 diabetes patients ( 11 female, 21 male); mean $( \pm \mathrm{SD})$ age $37 \pm 8$ years; $\mathrm{HbA}_{1 c} 7.6 \pm 0.7$; BMI $25 \pm 3 \mathrm{~kg} / \mathrm{m}^{2}$. Each patient took part in four glucose clamp experiments, one for each insulin (clamp level $5 \mathrm{mmol} / \mathrm{l}$, with a clamp duration of $28 \mathrm{~h}$ [ $12 \mathrm{~h}$ for IAsp]). Each experiment was 7-14 days apart.

Results: Higher 'early' metabolic activity (area under the glucose infusion rate curve from $0-6 \mathrm{~h}\left[\mathrm{AUC}_{\mathrm{GIR}}, 0-6 \mathrm{~h}\right]$ ) correlated with a higher percentage of soluble IAsp (BIAsp 30: 1155, BIAsp 50: 1448, BIAsp 70: 1718, IAsp: $1968 \mathrm{mg} / \mathrm{kg} ; \mathrm{p}<0.01$ for each neighbouring ratio comparison). A higher proportion of soluble IAsp also correlated with a faster onset of action. Protaminated IAsp was inversely correlated to the lower 'late' metabolic activity (AUC ${ }_{\mathrm{GIR}}$ 12-28h: BIAsp 30: 159, BIAsp 50: 84, BIAsp 70: $18 \mathrm{mg} / \mathrm{kg}$; $\mathrm{p}<0.05$ for each neighbouring ratio comparison). Peak metabolic activity, inferred from maximum glucose infusion rate $\mathrm{GIR}_{\max }$, increased linearly with higher ratio of soluble IAsp (BIAsp 30: 4.7, BIAsp 50: 6.0, BIAsp 70: 7.1, IAsp: $8.9 \mathrm{mg} / \mathrm{kg} / \mathrm{min} ; \mathrm{p}<0.01$ between insulin ratios) (Figure), although

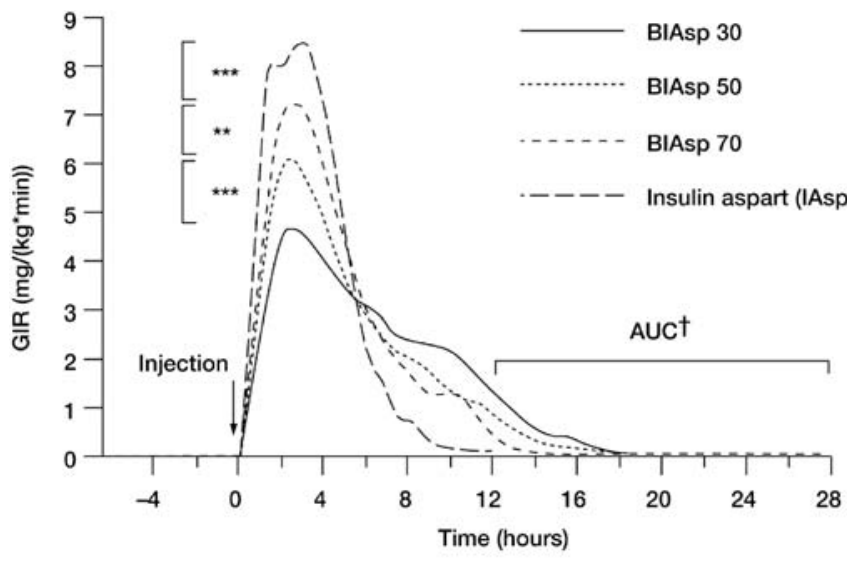

$* p<0.01 ; \cdots p<0.001 ;{ }^{\dagger} p<0.05$ (excluding IAsp) time to GIR was comparable between formulations. The duration of action (time until blood glucose exceeded $8.9 \mathrm{mmol} / \mathrm{l}$ ) decreased as the proportion of soluble IAsp increased (70.2 vs. 57.4 vs. 55.4 vs. $35.2 \mathrm{~min}$, respectively; between-treatment differences for each neighbouring ratio comparison: $\mathrm{p}<0.05)$. Pharmacokinetic endpoints supported the pharmacodynamic data; higher early IAsp levels $\left(\mathrm{AUC}_{\text {IAsp }} 0-6 \mathrm{~h}\right)$ correlated with increasing proportion of IAsp, and significant differences were observed between the preparations $(\mathrm{p}<0.001)$. Late levels decreased with increasing soluble IAsp. Maximum serum IAsp concentrations $\left(\mathrm{C}_{\mathrm{max}}\right)$ were higher in formulations with a higher proportion of IAsp (BIAsp 30: 67, BIAsp 50: 98, BIAsp 70: 152, IAsp: 212 $\mathrm{m} / \mathrm{U} / \mathrm{l})$, though time to $\mathrm{C}_{\max }$ was not notably different.

Conclusion: Supporting previous research, greater early-phase metabolic effects and a shorter duration of action were shown with increasing ratios of rapid-acting IAsp in BIAsp preparations. A longer metabolic effect was observed with an increased proportion of protaminated IAsp. The differences in action profile between BIAsp preparations should help physicians to optimise insulin therapy according to individual patients' needs. (Fig. 1) Supported by Novo Nordisk A/S

\section{4}

The modest weight gain observed in the Belgian and French ADAPT ${ }^{\mathrm{TM}}$ Study correlates with the mealtime bolus insulin dose but not with the basal insulin detemir

J.-P. Le Floch ${ }^{1}$, M. Lévy ${ }^{2}$, H. Mosnier-Pudar ${ }^{3}$, R. Mira ${ }^{4}$, F. Nobels ${ }^{5}$, S. Laroche ${ }^{6}$, S. Gonbert ${ }^{6}$, E. Eschwege ${ }^{7}$, P. Fontaine ${ }^{8}$;

${ }^{1}$ Diabetology Dept, Clinique de Villecresnes, Villecresnes, France, ${ }^{2}$ Diabetology Dept, Hôpital Max Fourestier, Nanterre, France, ${ }^{3}$ Diabetology Dept, Hôpital Cochin, Paris, France, ${ }^{4}$ Diabetology Dept, Diabetology, Antibes Juan les Pins, France, ${ }^{5}$ Endocrinology Dept, O.L. Vrouwziekenhuis, Aaslt, Belgium, ${ }^{6}$ Novo Nordisk, La Defense Puteaux, France,

${ }^{7}$ U780, INSERM, Villejuif, France, ${ }^{8}$ Diabetology Dept, clinique Marc Linquette, Lille, France.

Background and Aims: Treatment guidelines emphasise the importance of achieving $\mathrm{HbA}_{1 \mathrm{c}}$ targets using intensified insulin therapy in type 1 diabetes. However, this type of treatment can result in weight gain. This study is based on data from a French and Belgian multicentre randomised, parallel-group study (ADAPT ${ }^{\mathrm{sx}}$ ) comparing once- with twice-daily insulin detemir, and using insulin aspart before meals, in type 1 diabetic patients over a 7 month period (including 1-month run-in). These analyses aimed to investigate possible reasons of weight gain for these patients

Materials and Methods: The study was completed by 457 out of 520 randomised patients. Completers comprised men 52\% aged (mean \pm SD) 42 \pm 13 years, with a diabetes duration of $17 \pm 11$ years, $42 \%$ with degenerative complications. Weight change was analysed as a quantitative and qualitative value. Factors associated with weight change were screened using linear regression, ANOVA and chi-square tests. Multivariate analyses were performed using multiple linear regression and logistic regression models.

Results: End-of-study $\mathrm{HbA}_{1 \mathrm{c}}$ was $7.9 \pm 1.0 \%$ (an improvement of $0.6 \pm 0.8 \%$ ) and the BMI was $25.6 \pm 4.1 \mathrm{~kg} / \mathrm{m}^{2}$. Over 6 months, weight increased by $1.1 \pm 3.1$ $\mathrm{kg}$ and $\mathrm{BMI}$ increased by $0.4 \pm 1.1 \mathrm{~kg} / \mathrm{m}^{2}$, with $55 \%$ of patients gaining weight. Similar results were shown in the patient subgroups receiving once- or twicedaily detemir injections $(1.0 \pm 3.0$ versus $1.1 \pm 3.2 \mathrm{~kg}, 0.4 \pm 1.1$ versus $0.4 \pm 1.1$ $\mathrm{kg} / \mathrm{m}^{2}$, respectively, NS). The weight gain was significantly associated with the absence of diabetes complications $(1.9 \pm 0.3$ versus $1.0 \pm 0.3 ; \mathrm{p}<0.05)$, the improvement in $\mathrm{HbA}_{1 \mathrm{c}}(\mathrm{r}=0.22 ; \mathrm{p}<0.001)$, the aspart dose $(\mathrm{r}=0.13 ; \mathrm{p}<0.01)$ and the total insulin dose $(\mathrm{r}=0.10 ; \mathrm{p}<0.05)$, but not with the frequency of hypoglycaemia or with the detemir dose. The multivariate analyses showed that the improvement in $\mathrm{HbA}_{1 c}$ and the aspart dose were explanatory factors for weight gain (multiple regression: $R^{2}=0.08$; logistic: $R^{2}=0.02 ; p<0.001$ ). Similar results were found with BMI.

Conclusion: These exploratory analyses suggest that the modest weight gain observed in patients treated with a basal-bolus detemir plus aspart insulin regimen can be attributed to the meal time short-acting insulin dose and not to the detemir dose. These analyses did not show evidence of a relationship between weight change and hypoglycaemia.

Fig. 1 


\section{5}

Contribution of fasting and postprandial hyperglycaemia to haemoglobin $A_{1 c}$ levels in insulin-treated patients

H. Shimizu, S. Oh-I, Y. Ariyama, T. Tsuchiya, Y. Uehara, S. Okada, M. Mori; Department of Medicine and Molecular Science, Gunma University Graduate School of Medicine, Maebashi, Japan.

Background and Aims: The relative contribution of postprandial glucose decreased progressively from the lowest to the highest quintile of haemoglobin $\mathrm{HbAlc}$, whereas the relative contribution of fasting glucose increased gradually with increasing levels of $\mathrm{HbAlc}$ in non-insulin- and nonacarbose-treated diabetic subjects. However, the exact contribution of fating and postprandial glucose concentrations to $\mathrm{HbAlc}$ levels remains unclear in insulin-treated patients.

Materials and Methods: Fifty seven insulin-treated, outcoming diabetic patients were included in the present study. We analysed the diurnal glycaemic profiles of diabetic patients conventionally (twice a day; b.i.d., $\mathrm{n}=24$ ) or intensively treated (basal-bolus; $\mathrm{n}=33$ ) with insulin. Over $10 \%$ of insulin dose was not changed for at least 8 weeks before the start of the study. Their fasting and postprandial glucose concentrations were determined at home by themselves with glucose self-monitoring apparatus. HbAlc levels were measured by HPLC method on the visit to our hospital. Correlation of $\mathrm{HbAlc}$ levels to self monitored blood glucose levels at home were calculated in each measuring point.

Results: The average of HbAlc levels in all subjects included was $7.83 \%$. In conventionally treated group (b.i.d.), there was a significant correlation between $\mathrm{HbAlc}$ levels and fasting glucose levels only before lunch (L), but at $2 \mathrm{hr}$ after all meals (B-PP, L-PP, D-PP). In intensively treated group (basalbolus), a significant correlation between $\mathrm{HbAlc}$ levels and fasting glucose levels was found before breakfast (B), lunch (L) and at $2 \mathrm{hr}$ after breakfast (B$\mathrm{PP}$ ) and dinner (D-PP). When we analysed all subjects together, there was no significant correlation between $\mathrm{HbAlc}$ levels and fasting glucose levels before breakfast (B) or dinner (D), while only fasting glucose level before lunch (L) was significantly correlated with $\mathrm{HbAlc}$ levels. In contrast, postprandial glucose levels were significantly correlated with HbAlc levels in all measuring points (B-PP, L-PP, D-PP). The correlation was highest at $2 \mathrm{hr}$ after breakfast and dinner (B-PP, D-PP). The sum of fasting and postprandial glucose levels was also significantly correlated with HbAlc levels $(\gamma=0.626, p<0.001)$.

Conclusion: These data indicate that postprandial blood glucose levels are more important determinants of HbAlc levels than fasting glucose levels in insulin-treated patients. It is concluded that postprandial hyperglyceamia after breakfast and dinner should be important to more improve HbAlc levels in insulin treated diabetic patients.

Correlation of blood glucose and haemoglobin Alc levels

\begin{tabular}{lllllll}
\hline & b.i.d. & \multicolumn{3}{l}{ basal-bolus } & total \\
\cline { 2 - 7 } & $\gamma$ & p-value & $\gamma$ & p-value & $\gamma$ & p-value \\
\hline number & 24 & & 33 & & 57 & \\
\hline B & 0.044 & n.s. & 0.359 & 0.05 & 0.238 & n.s. \\
B-PP & 0.669 & 0.001 & 0.525 & 0.01 & 0.576 & 0.001 \\
L & 0.559 & 0.01 & 0.504 & 0.01 & 0.513 & 0.001 \\
L-PP & 0.480 & 0.05 & 0.319 & n.s. & 0.389 & 0.05 \\
D & 0.413 & n.s. & 0.183 & n.s. & 0.273 & n.s. \\
D-PP & 0.707 & 0.001 & 0.602 & 0.01 & 0.646 & 0.001 \\
\hline
\end{tabular}

\section{6}

Influence of treatment with insulin analogs on pancreatic reserve one year after type 1 diabetes diagnosis

M. Ferrer-Camps, M. Perez-Maraver, J. Caballero-Corchuelo,

S. Martin-Grillo, A. Boltaña-Lorenzo, R. Insa-Soria, E. Montanya-Mias,

J. Soler Ramon;

Endocrinology, HU Bellvitge, Hospitalet Llobregat. Barcelona, Spain.

Background and Aims: At the diagnosis of type 1 diabetes there is a remanent pancreatic reserve with beneficial metabolic effects that tends to decline progressively. Insulin analogs have been used for ten years and they have pharmacokinetic advantages with respect to human insulins. However it is not known whether they have a beneficial effect on the evolution of pancreatic reserve at the begining of type 1 diabetes. The aim was to compare the evolution of pancreatic reserve during the first year from type 1 diabetes diagnosis between patients treated with insulin analogs and those with a human insulin regimen.

Materials and Methods: Our study was a retrospective analysis. Every patient discharged from our Endocrinology Unit with a diagnosis of new-onset type 1a diabetes mellitus in the period 2002-2005 were included $(n=78)$. Those diagnosed in 2002-2003 ( $\mathrm{n}=41)$ were treated with an intensive human insulin protocol (regular and NPH, group 1). Patients diagnosed in 2004-2005 $(\mathrm{n}=37)$ were treated with an intensive insulin analogs therapy (aspart and glargine, group 2). At the begining the following variables were analysed: age, gender, BMI, biochemical and clinical situation, $\mathrm{HbAlc}$, insulin dose at discharge as well as basal and stimulated C-peptide (glucagon test). During the follow-up (at six and twelve months) evolution of weigth, insuline dose, $\mathrm{HbAlc}$ and C-peptide were also evaluated. Both groups were compared with parametric tests. Statistical significance was defined as $p<0,05$.

Results: At the initial point both groups were comparable in terms of clinical and biochemical parameters. Initial basal and stimulated C-peptide in both groups were also comparable (basal: $0,23 \pm 0,18$ vs. $0,18 \pm 0,17 \mathrm{nmol} / \mathrm{l}$; stimulated $0,38 \pm 0,26$ vs. $0,32 \pm 0,28 \mathrm{nmol} / 1$, groups 1 and 2 respectively). Nine and six patients were lost of follow-up (group 1 and 2 respectively). At the end of the study groups were comparable in terms of BMI, $\mathrm{HbAlc}(5,6 \% \pm 1,2 \mathrm{vs}$. $6,1 \% \pm 2,4$, groups 1 and 2 respectively) and insuline dose $(0,40 \pm 0,18 \mathrm{UI} / \mathrm{kg}$ versus $0,42 \pm 0,15 \mathrm{UI} / \mathrm{kg}$, groups 1 and 2 respectively). Basal and stimulated C-peptide was statistically different between groups, with a better evolution in group 2 (basal: $0,18 \pm 0,15$ vs. $0,31 \pm 0,27 \mathrm{nmol} / 1$; stimulated $0,32 \pm 0,28$ vs. $0,51 \pm 0,44 \mathrm{nmol} / 1$; groups 1 and 2 respectively, $\mathrm{p}<0,05$ ).

Conclusion: our results suggest that, in type 1 diabetes, treatment with insulin analogs from the diagnosis could be beneficial for the evolution of pancreatic reserve compared with traditional human insulin therapy. 


\section{PS 94 Novel insulins}

\section{7}

\section{Effectiveness of oral insulin delivery by means of solid lipid nanoparticles}

B. Sarmento, S. Martins, A. Silva, D. Santos, E. Souto, D. Ferreira; Department of Pharmaceutical Technology, Faculty of Pharmacy, University of Porto, Portugal.

Background and Aims: The aim of this work was to produce and characterise cetyl palmitate-based solid lipid nanoparticles (SLN) containing insulin, and to evaluate the potential of these colloidal carriers for oral insulin administration. The new carrier is composed of a lipid core aimed to protect and to control the release of insulin.

Materials and Methods: SLN were prepared by a modified solvent emulsification-evaporation method based on a w/o/w double emulsion. The particle size, zeta potential and association efficiency of unloaded and insulin-loaded SLN were determined by photon correlation spectroscopy, laser doppler anemometry and HPLC, respectively. Insulin pharmacological activity was determined in diabetic rats in accordance with the FELASA and the European Union (Council Directive 86/609/EEC). Plasma glucose levels were plotted against time to evaluate the cumulative hypoglycemic effect over time after insulin administration.

Results: The attempts to develop an insulin-loaded SLN formulation for oral administration produced nanoparticles with spherical shape showed and homogenous size distribution of $361 \pm 30 \mathrm{~nm}$ and slight negative zeta potential values. The microscopic appearance and the structural characterization of cetyl palmitate based SLN showed that these particles exhibited a spherical shape and a dense lipid matrix. The association efficiency of insulin in was around $45 \%$, and is noteworthy that being a hydrophilic molecule a much lower association efficiency of insulin within the lipid matrix of SLN was expected. Nonetheless, the modified w/o/w double emulsion method was shown to be a suitable production procedure to achieve relatively high loading for insulin. Insulin-loaded SLN decreased glycemia by comparison with rats treated with oral insulin solution and empty nanoparticles This hypoglycaemic effect was observed to occur in a biphasic way, with an initial peak between 4 and $8 \mathrm{~h}$ with a decrease of $25 \%$ of the initial glucose level and later after $12 \mathrm{~h}$ of assay prolonged for up to $24 \mathrm{~h}$. This may be related with the typical biphasic drug release pattern with an initial burst and prolonged release from SLN, characterised by a microcrystal matrix structure. Such biphasic pattern can originate an immediate release of insulin but also retain a significant fraction of the drug entrapped into the lipid matrix during prolonged time. The solid matrix of SLN was able to partially protect insulin against chemical degradation in the gastrointestinal tract and to promote the intestinal absorption of insulin.

Conclusion: In conclusion, SLN were found to be suitable carrier systems for the administration of insulin through the oral route. This study may contribute for the development of an optimized oral insulin formulation. Supported by Lilly Portugal, FCT Portugal, Abbot Portugal

\section{8}

Sustained weight reduction and improved glycemic control with inhaled Technosphere $\%$ insulin

N. Tu, D. Kramer, R. Baughman;

MannKind Corporation, Danbury, United States.

Background and Aims: Improved glycemic control with insulin treatment is generally associated with weight gain. Two open-label randomised trials were conducted to compare the effect of inhaled Technosphere /Insulin (T/I) with that of subcutaneous insulin aspart on glycemic control and weight.

Materials and Methods: Diabetic patients receiving glargine as basal insulin were randomised to receive T/I or aspart, with glargine as basal insulin in two separate trials: a 12 -week study of 110 patients with type 1 diabetes and a 24 -week study with a 22 -week follow-up period of 309 patients with type 2 diabetes.

Results: Type 1 diabetic patients receiving T/I experienced a mean weight loss of $0.45 \mathrm{~kg}$ over 12 weeks versus a mean gain of $0.80 \mathrm{~kg}$ in the aspart group $(P=.0017)$. In the 24 -week treatment period, type 2 diabetic patients receiving T/I experienced a weight loss of $0.78 \mathrm{~kg}(P=.0016)$ compared with a mean gain of $0.23 \mathrm{~kg}$ in the aspart group. When type 1 and type 2 patients were combined for analysis, the T/I patients experienced a mean loss of 0.69 $\mathrm{kg}(P=.0001)$ at Week 12 . Type 2 diabetic patients who participated in the 22week follow-up resumed conventional therapy. Patients initially randomised to receive aspart had a mean weight gain of $0.81 \mathrm{~kg}$ during follow-up. Patients originally in the T/I group similarly gained a mean $0.74 \mathrm{~kg}$ following resumption of conventional therapy $(P=.8125)$. In addition to treatment, baseline BMI, categorised into three groups $(<27,27-29,>29)$, was found to be a significant factor in weight change at 24 weeks (weight gain, no weight gain) based on multivariate logistics regression model for subjects with type 2 diabetes. Subjects with BMI 27-29 are 3.09 (95\% CI: 1.285-7.420) times more likely to have weight loss or no weight gain than those with BMI $<27$.

Conclusion: T/I and aspart produced similar improvement in glycemic control as prandial insulin together with a basal insulin. There was a weight increase in the group receiving aspart, while there was no increase in weight during T/I treatment; when returning to conventional therapy, these patients gained weight. We conclude that in contrast to aspart, T/I offers the advantage of similar glycemic control without associated weight gain. Supported by MannKind Corporation

\section{9}

Pharmacokinetic/glucodynamic effects and safety of $\mathrm{AIR}^{\circledast}$ inhaled insulin in asthmatic subjects

M. Wolzt ${ }^{1}$, A. de la Peña ${ }^{2}$, F. Tibaldi ${ }^{3}$, P. Berclaz 2 , D. Muchmore²;

${ }^{1}$ Dept of Clinical Pharmacology, Medical University of Vienna, Austria, ${ }^{2}$ Eli Lilly and Co., Indianapolis, United States,

${ }^{3}$ Eli Lilly and Co., Mont-Saint-Guibert, Belgium,

Background and Aims: Pharmacokinetic (PK) and glucodynamic (GD) response to $\mathrm{AIR}^{\circ}$ inhaled insulin (Lilly/Alkermes inhaled insulin system), relative to subcutaneous insulin lispro, has not yet been evaluated in asthmatic patients.

Materials and Methods: Non-smoking, non-diabetic healthy $(n=13)$, mild persistent asthmatic $(n=13)$, and moderate asthmatic $(n=13)$ subjects (age 19-58 yrs) participated in this phase I, open-label, cross-over study. Subjects received either 12U-equivalent of AIR Insulin (replicate treatments), or $12 \mathrm{U}$ of SC insulin lispro, or salbutamol pretreatment $+12 \mathrm{U}$-equivalent of AIR Insulin (moderate asthma group only), prior to undergoing up to 4 euglycemic glucose clamps.

Results:

\begin{tabular}{|c|c|c|c|c|c|c|c|}
\hline & \multicolumn{2}{|c|}{ Healthy } & \multicolumn{2}{|c|}{ Mild Asthma } & \multicolumn{3}{|c|}{ Moderate Asthma } \\
\hline & AIR $^{\circ}$ & $\begin{array}{c}\mathrm{SC} \\
\text { lispro }\end{array}$ & AIR $^{\circ}$ & SC lispro & AIR $^{6}$ & SC lispro & $\begin{array}{c}\text { AlR }^{\circ} \\
+ \text { Salbutamol }\end{array}$ \\
\hline \multicolumn{8}{|l|}{ Pharmacokinetic } \\
\hline $\mathrm{N}_{p K}$ & 25 & 13 & 26 & 11 & 22 & 12 & 11 \\
\hline$A \cup C_{D r f}(\mathrm{nmol} \cdot \mathrm{min} / \mathrm{L})$ & $\begin{array}{c}35.2 \\
(46.1)\end{array}$ & $\begin{array}{l}61.1 \\
(25.4)\end{array}$ & $\begin{array}{l}24.0 \\
(59.5)\end{array}$ & $\begin{array}{l}65.2 \\
(26.6)\end{array}$ & $\begin{array}{l}21.1 \\
(74.8)\end{array}$ & $\begin{array}{c}59.5 \\
(17.1)\end{array}$ & $\begin{array}{c}31.3 \\
(36.8)\end{array}$ \\
\hline $\mathrm{C}_{\operatorname{mx}}(\mathrm{pmol} / \mathrm{L})$ & $\begin{array}{c}248 \\
(46.0)\end{array}$ & $\begin{array}{l}421 \\
(38.7)\end{array}$ & $\begin{array}{l}185 \\
(41.3)\end{array}$ & $\begin{array}{c}432 \\
(36.8)\end{array}$ & $\begin{array}{c}183 \\
(29.0)\end{array}$ & $\begin{array}{c}374 \\
(35.8)\end{array}$ & $\begin{array}{c}221 \\
(27.0)\end{array}$ \\
\hline$t_{\max }(\min )^{*}$ & $\begin{array}{c}50 \\
11-131\end{array}$ & $\begin{array}{c}48 \\
34-64\end{array}$ & $\begin{array}{c}51 \\
12-124\end{array}$ & $\begin{array}{c}62 \\
32-125\end{array}$ & \begin{tabular}{|c|}
48 \\
$12-124$
\end{tabular} & $\begin{array}{c}62 \\
32-191\end{array}$ & $\begin{array}{c}60 \\
22 \cdot 121\end{array}$ \\
\hline \multicolumn{8}{|l|}{ Glucodynamic } \\
\hline $\mathrm{N}_{\infty}$ & 25 & 12 & 25 & 10 & 18 & 11 & 10 \\
\hline $\mathrm{G}_{m t}(\mathrm{~g})$ & $\begin{array}{c}38.7 \\
(108.0)\end{array}$ & $\begin{array}{c}59.7 \\
(43.6)\end{array}$ & $\begin{array}{c}23.4 \\
(156.0)\end{array}$ & $\begin{array}{r}73.5 \\
(33.2)\end{array}$ & $\begin{array}{c}10.7 \\
(159.0)\end{array}$ & $\begin{array}{c}47.8 \\
(88.5)\end{array}$ & $\begin{array}{c}15.5 \\
(180.0)\end{array}$ \\
\hline$R_{\max }(\mathrm{mg} / \mathrm{min})$ & $\begin{array}{c}355 \\
(59.5)\end{array}$ & $\begin{array}{l}471 \\
(30.8)\end{array}$ & $\begin{array}{c}290 \\
(70.1)\end{array}$ & $\begin{array}{c}526 \\
(41.8)\end{array}$ & $\begin{array}{c}183 \\
(68.3)\end{array}$ & $\begin{array}{c}401 \\
(56.1)\end{array}$ & $\begin{array}{c}261 \\
(69.5)\end{array}$ \\
\hline$t_{\text {max }}(\min )^{\prime}$ & $\begin{array}{c}47 \\
33-174\end{array}$ & $\begin{array}{c}79 \\
44.130\end{array}$ & $\begin{array}{c}47 \\
26-104\end{array}$ & $\begin{array}{c}67 \\
25-153\end{array}$ & \begin{tabular}{|c|}
52 \\
$33-132$
\end{tabular} & $\begin{array}{c}81 \\
46-184\end{array}$ & $\begin{array}{c}62 \\
34-110\end{array}$ \\
\hline
\end{tabular}

AIR Insulin was rapidly absorbed in all subject groups, comparable with SC insulin lispro. Overall mean AIR Insulin exposure in mild and moderate asthmatic subjects was reduced by approximately $30 \%$ and $40 \%$ respectively $(p<0.01)$ compared to healthy subjects. Overall mean GD response $\left(G_{t o}\right)$ in mild asthmatic subjects approximated that of healthy subjects $(\mathrm{p}=0.16)$, despite lower insulin exposure. In moderate asthmatic subjects, overall GD response was approximately $72 \%$ lower $(\mathrm{p}<0.01)$. In the moderate asthma group, pretreatment with salbutamol resulted in an approximate $50 \%$ increase in overall insulin exposure $(\mathrm{p}<0.02)$ and metabolic response $(\mathrm{p}=0.03)$, compared to AIR Insulin treatment alone. During the course of the study, there were no deaths, serious adverse events, or clinically significant alterations in laboratory values. No significant treatment-group effects were 
noted from either bedside spirometry tests, or from laboratory pulmonary function tests. Adverse reactions related to AIR Insulin were mild to moderate (cough, headache, and dizziness) and have been reported in previous studies or are typical of insulin studies involving glucose clamp procedures. No subject discontinued the study because of an adverse event.

Conclusions: Data from this study suggests that pulmonary disease severity influences the metabolic effect of AIR Insulin in non-diabetic individuals with asthma, but does not affect the profile of AIR Insulin safety and tolerability. Furthermore, these data suggest that patients with diabetes and comorbid moderate asthma may require an increase AIR Insulin dosage, while patients with mild asthma would not likely require dosage adjustment. Pre-treatment with salbutamol increased both the overall exposure to and metabolic response of AIR Insulin.

$\mathrm{AIR}^{\otimes}$ is a registered trademark of Alkermes Inc., Cambridge, Massachusetts

\section{0}

Sustained long-term efficacy and safety of inhaled human insulin (Exubera ${ }^{\star}$ ) following at least 8 years of continuous therapy

M. R. Burge ${ }^{1}$, J. M. Reis ${ }^{2}$, A. S. Krasner ${ }^{2}$, P. F. Schwartz' ${ }^{2}$ J. G. Teeter²; ${ }^{1}$ Department of Internal Medicine, University of New Mexico School of Medicine, Albuquerque, ${ }^{2}$ Pfizer Inc, Groton, United States.

Background and Aims: The efficacy and safety of inhaled human insulin (EXU; Exubera (insulin human [rDNA origin]) Inhalation Powder) has been demonstrated previously in controlled clinical trials of up to 2 years duration. The aim of this study was to examine the trends in diabetes control and lung function in a trial of 8 years duration.

Materials and Methods: Continued open-label EXU therapy was offered to patients with diabetes who had completed any of three 3-month randomised controlled Phase 2 clinical trials. A total of 173 adult patients with type $1(\mathrm{~N}$ $=61)$ or type $2(\mathrm{~N}=112)$ diabetes were treated with EXU as their exclusive preprandial insulin. Concomitant diabetic therapies were not otherwise specified in this study. Fifty-two patients have received at least 8 years of EXU treatment $(\mathrm{n}=23$, type $1 ; \mathrm{n}=29$, type 2$)$. $\mathrm{HbA}_{1 c}$ and forced forced expiratory volume in 1 second $\left(\mathrm{FEV}_{1}\right.$ ) were measured every 3 months.

Results: Mean $( \pm \mathrm{SD}) \mathrm{HbA}_{1 \mathrm{c}}$ was $7.9 \pm 1.2 \%$ after 8 years compared with $8.5 \pm$ $1.4 \%$ at the start of EXU treatment. Overall rates of hypoglycemia decreased from 2.9 episodes/subject-month after 4 weeks of EXU treatment to 1.7 episodes/subject month after 8 years as measured over the final 6 months. Annualised change in forced expiratory volume in 1 second $\left(\mathrm{FEV}_{\mathrm{1}}\right)$ after 4 years was $-0.054 \pm 0.004 \mathrm{~L} / \mathrm{y}(\mathrm{N}=88)$ and $-0.049 \pm 0.004 \mathrm{~L} / \mathrm{y},(\mathrm{N}=54)$ after 8 years of continuous EXU treatment. This annualised change in lung function is similar to that observed in subjects with diabetes who have received comparator therapies as part of controlled clinical trials elsewhere in the EXU development program.

Conclusion: Long-term EXU use is effective in maintaining glycemic control for at least 8 years. Moreover, declines in $\mathrm{FEV}_{1}$ are stable and predictable after 4 and 8 years of EXU therapy and are similar to those expected in an adult diabetic population.

Supported by Pfizer Inc

\section{1}

Effects of inhaled human insulin (Exubera ${ }^{\circledR}$ ) on postprandial glucose profiles compare favorably to those of glargine in patients with type 2 diabetes

M. Hompesch ${ }^{1}$, C. Bowden ${ }^{1}$, T. Strack ${ }^{2}$, M. Mitnick ${ }^{2}$, W. Canovatchel ${ }^{2}$, S. Davies², P. Maheux';

${ }^{1}$ Profil Institute for Clinical Research, San Diego, United States, ${ }^{2}$ Pfizer Inc, New York, United States.

Background and Aims: Interventions aimed at reducing glucose excursions in patients with diabetes are potentially important as failure to do so may increase risk of vascular complications. This exploratory study is the first trial comparing inhaled human insulin (EXU; Exubera ${ }^{\circ}$ [rDNA origin] Inhalation Powder) with glargine as the first insulin added to oral agents (OAs) in patients with inadequately controlled type 2 diabetes.

Materials and Methods: The single-site, 2 -arm cross-over, open-label study of 40 patients on 2 OAs (baseline Alc, 7.9\%) compared the effects of prandial
EXU to morning basal glargine on 24-hr CGMS. Patients were randomised to receive either treatment for 8 consecutive in-patient days separated by a wash-out phase of 7 to 10 days. The 24-hr CGMS means were calculated for the final $72 \mathrm{hrs}$ of each treatment period.

Results: The 72-hr mean glucose, area under the curve (AUC) for 72-hrglucose, and glucose AUCs (0-4 hrs) around individual meals were significantly lower for EXU than for glargine $(P<0.0001$ to 0.0318$)$. Standard deviation, mean amplitude of glycemic excursions, and mean of daily differences in glucose concentration over $72 \mathrm{hrs}$ were lower for EXU but did not reach statistical significance. Glucose AUC from midnight to $6 \mathrm{AM}$ was similar between EXU and glargine (Figure). Hypoglycemia was the most frequent adverse event (EXU, 28; glargine,13), but no events were rated severe.

Conclusions: In addition to lowering postprandial glucose, EXU achieved midnight-to-6-AM glucose concentrations similar to glargine. In managing both postprandial and overnight glucose, prandial EXU may be an attractive alternative to basal insulin for patients with type 2 diabetes inadequately controlled on OAs.

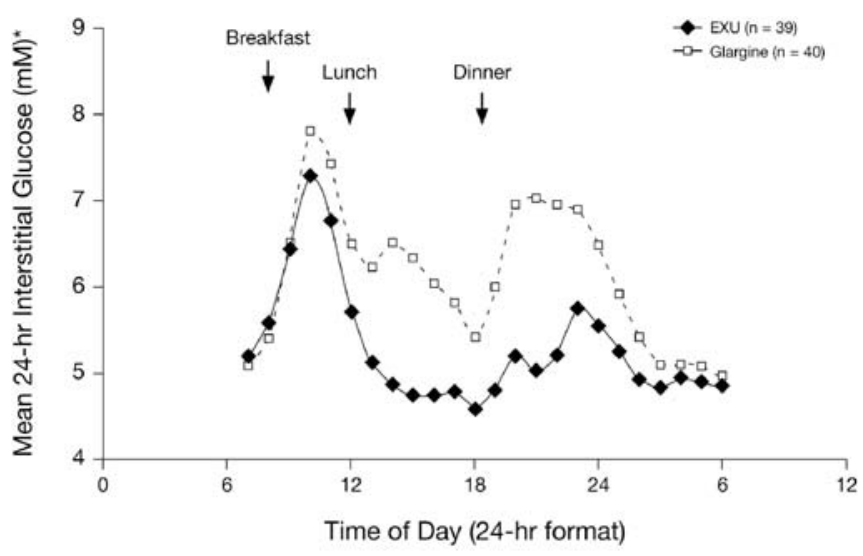

"Calculated from the final $72 \mathrm{hrs}$ of treatment.

EXU, inhaled human insulin; CGMS, continuous glucose monitoring system.

Supported by Pfizer Inc

\section{2}

Discontinuation and readministration of inhaled human insulin $\left(\right.$ Exubera $\left.^{\circ}\right)$ in adults with type 1 diabetes: 3 -year pulmonary safety data J. Skyler ${ }^{1}$, P. Hollander ${ }^{2}$, L. Jovanovic ${ }^{3}$, S. Klioze ${ }^{4}$, A. Krasner ${ }^{4}$, R. Riese ${ }^{4}$, J. Reis ${ }^{4}$, P. Schwartz

${ }^{1}$ Division of Endocrinology, Diabetes \& Metabolism, University of Miami Miller School of Medicine, Miami,

${ }^{2}$ Baylor Endocrine Center, Baylor University Medical Center, Dallas, ${ }^{3}$ Division of Diabetes, Sansum Diabetes Research Institute, Santa Barbara, ${ }^{4}$ Pfizer Inc, New London, CT, United States.

Background and Aims: Clinical trials in patients with type 1 diabetes have demonstrated that Exubera (insulin human [rDNA origin]) Inhalation Powder (EXU)) is as effective as subcutaneous (SC) regular insulin and well tolerated when administered for up to 2 years. This interim analysis assessed pulmonary safety following discontinuation and readministration of EXU therapy in adults with type 1 diabetes.

Materials and Methods: Adults with type 1 diabetes (18-65 years) received SC insulin for 4 weeks and were then randomised and received either premeal EXU $(n=290)$ or a short-acting SC insulin $(n=290)$, plus NPH insulin or Ultralente $^{\circ}$ once or twice daily, or insulin glargine once daily (comparative phase) for 2 years, followed by 6 months when all patients received SC insulin (follow-up phase) and a 6-month extension phase during which all patients received their original randomised therapy. Comprehensive lung function tests were performed.

Results: Small, nonprogressive treatment group differences favouring SC insulin in change from baseline forced expiratory volume in 1 second $\left(\mathrm{FEV}_{1}\right)\left(1 \%\right.$ of baseline) and carbon monoxide diffusing capacity $\left(\mathrm{DL}_{\mathrm{CO}}\right)$ (1.5\% of baseline) (Figure 1) occurred early during the comparative phase, and were not driven by outliers. These differences completely resolved upon discontinuation of EXU, and recurred by the same magnitude during the 
extension phase. Both treatment groups maintained glycaemic control, and hypoglycaemic event rates were similar. Increased median insulin antibody levels were detected in the EXU group within a few weeks of commencing therapy, peaking at 12 months before decreasing somewhat during the remainder of the initial comparative phase. During the follow-up phase when EXU was discontinued, median insulin antibody levels declined to near baseline levels. When EXU therapy was resumed during the extension phase, median insulin antibody levels again increased to levels similar to those observed in the original comparative phase. In patients receiving SC insulin, insulin antibody levels remained stable.

Conclusions: $\mathrm{FEV}_{1}$ and $\mathrm{DL}_{\mathrm{CO}}$ changes observed during discontinuation and readministration of EXU therapy are consistent with a reversible, nonprogressive and non-pathologic effect on lung function in adults with type 1 diabetes. EXU readministration is not associated with an augmented insulin antibody response.
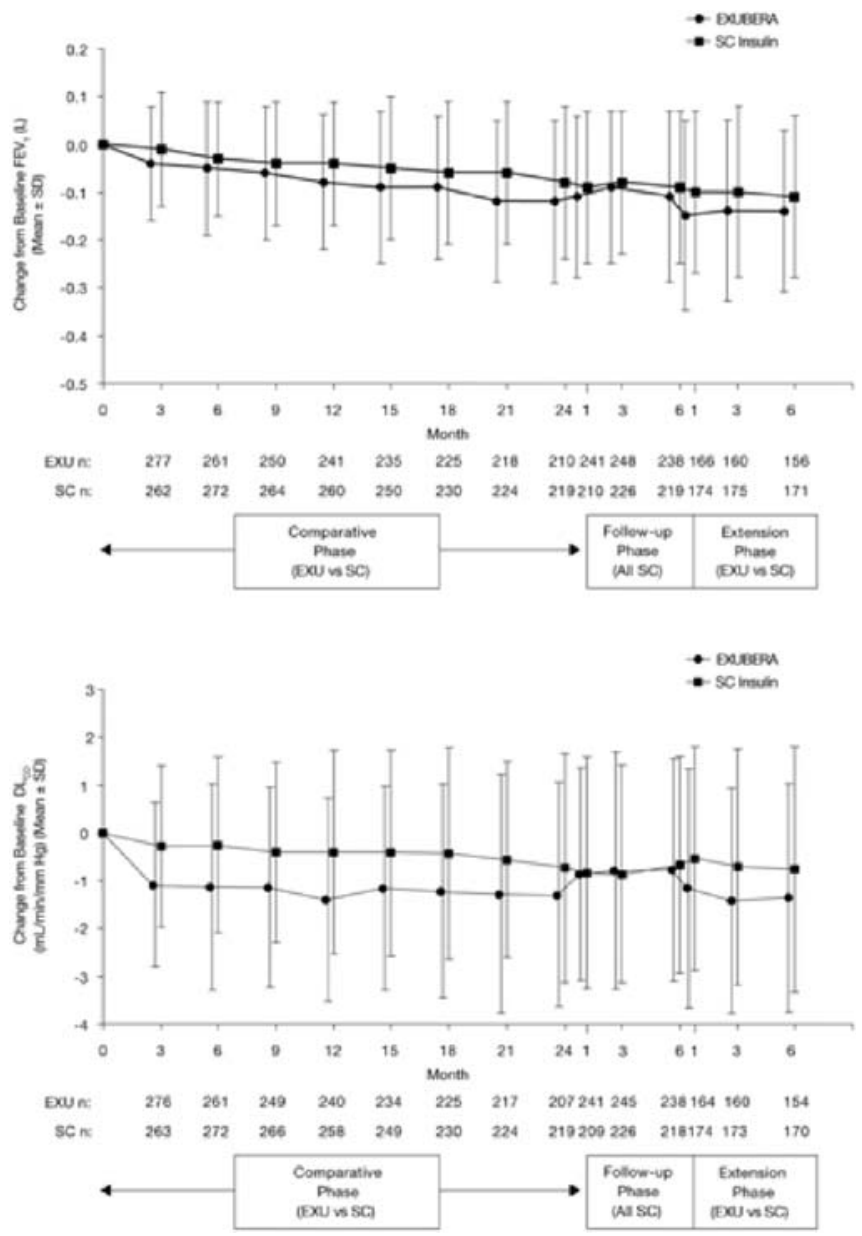

0973

Insulin antibody responses to 2 years of inhaled human insulin $\left(\right.$ Exubera $\left.^{\circ}\right)$ followed by discontinuation and readministration after 6 months

S. E. Fineberg ${ }^{1,2}$, D. Finco-Kent ${ }^{3}$, T. T. Kawabata ${ }^{3}$, P. F. Schwartz ${ }^{3}$, J. G. Teeter ${ }^{3}$, A. S. Krasner

${ }^{1}$ Medicine, Indiana University School of Medicine, Indianapolis,

${ }^{2}$ School of Medicine, University of Alabama Birmingham,

${ }^{3}$ Pfizer, Groton, United States.

Background and Aims: Inhaled human insulin therapy (EXU; Exubera ${ }^{\circ}$ (insulin human [rDNA origin]) Inhalation Powder) has been shown to be more immunogenic than subcutaneous (SC) comparator insulins in patients with type 1 diabetes and in insulin-using patients with type 2 diabetes. Previous studies have raised concerns that discontinuation and reinstitution of SC insulin therapy after EXU treatment might lead to enhanced immunologic responses with undesirable clinical effects.

Materials and Methods: In open-label trials lasting up to 24 months, patients with type 1 diabetes (aged $18-65$ years) received EXU $(n=290)$ or SC human insulin $(n=290)$, and patients with type 2 diabetes received EXU $(n=316)$ or SC $(\mathrm{n}=311)$. Following 24 months of therapy, EXU was discontinued (and replaced with SC) for 6 months, then the original therapy was resumed for an additional 6 months. Throughout, insulin antibody (IAb) responses were assessed using a quantitative assay (reported in $\mu \mathrm{U} / \mathrm{mL}$ ); pulmonary function was assessed by standardised measures of forced expiratory volume in 1 second $\left(\mathrm{FEV}_{1}[\mathrm{~L}]\right)$ and carbon monoxide diffusing capacity $\left(\mathrm{DL}_{\mathrm{CO}}[\mathrm{mL} /\right.$ $\mathrm{min} / \mathrm{mm} \mathrm{Hg}]$ ); and clinical outcomes, including hypoglycemic events, insulin dose, glycemic control, and evidence for immunological sequelae, were monitored periodically.

Results: No significant correlations with IAb response could be shown with changes in pulmonary function, insulin dose, hypoglycemic events, or glycemic control. Antibody levels in the EXU group increased initially, decreased from 12 months, then decreased further by 6 months from discontinuation (Table). An exaggerated anamnestic response was not seen after EXU resumption. Antibody levels upon readministration did not correlate with changes in glycemic control. Samples with the highest IAb levels were found to consist predominantly of IgG for both EXU- and SC-insulin-treated patients. A small mean treatment group difference in change from baseline $\mathrm{FEV}_{1}$ was apparent after the first 3 months of EXU treatment, remained stable throughout the remainder of the initial 24-month comparative phase, resolved upon discontinuation, and recurred to the same magnitude upon readministration of EXU.

Conclusions: Immunologic safety was demonstrated with long-term EXU therapy, discontinuation and readministration. There did not appear to be any impact on safety parameters as a result of IAbs. (Tab. 1) Supported by Pfizer Inc

Supported by Pfizer Inc

Tab. 1

IAb Response and PFT Measurements

\begin{tabular}{|c|c|c|c|c|c|c|}
\hline & Baseline & 3 Mos & 12 Mos & 24 Mos & DC/6 Mos & RA/6 Mos \\
\hline \multicolumn{7}{|c|}{ Median IAb binding $(\mu \mathrm{U} / \mathrm{mL})$} \\
\hline TIDM $(\mathrm{EXU})^{*}$ & $4.5(\mathrm{n}=285)$ & $31.0(\mathrm{n}=272)$ & $142.0(\mathrm{n}=235)$ & $63.0(\mathrm{n}=211)$ & $22.0(\mathrm{n}=238)$ & $42.0(\mathrm{n}=159)$ \\
\hline T2DM $(E X U)^{\dagger}$ & $1.1(\mathrm{n}=310)$ & $12.0(\mathrm{n}=286)$ & $19.0(\mathrm{n}=263)$ & $20.0(n=223)$ & $6.6(n=246)$ & $12.0(\mathrm{n}=86)$ \\
\hline \multicolumn{7}{|c|}{ Treatment group difference (EXU - SC) in change from baseline in mean FEV1 $(\mathrm{L})(90 \% \mathrm{CI})$} \\
\hline All subjects & - & $\begin{array}{l}-0.036 \\
(-0.049,-0.023\end{array}$ & $\begin{array}{l}-0.029 \\
(-0.045,-0.013)\end{array}$ & $\begin{array}{l}-0.030 \\
(-0.049,-0.011)\end{array}$ & $\begin{array}{l}0.011 \\
(-0.008,0.031)\end{array}$ & $\begin{array}{l}-0.034 \\
(-0.058,-0.010)\end{array}$ \\
\hline
\end{tabular}

${ }^{*}$ All subjects with type 1 diabetes who had an FEV1 measurement at baseline and at least once post baseline.

† All subjects with type 2 diabetes who had an FEV1 measurement at baseline and at least once post baseline.

IAb, insulin antibody; PFT, pulmonary function test; DC, discontinue; RA, readministration; T1DM, type 1 diabetes mellitus;

EXU, Exubera (inhaled human insulin); T2DM, type 2 diabetes mellitus; FEV1, forced expiratory volume in 1 second; CI, confidence interval. 


\section{4}

Significant predictors of preference for inhaled versus injectable insulin in patients with type 2 diabetes

R. P. Hayes ${ }^{1}$, T. E. Stump ${ }^{1}$, D. B. Muchmore ${ }^{1}$, J. Schmitke ${ }^{2}$;

${ }^{1}$ Eli Lilly and Company, Indianapolis,

${ }^{2}$ Product Dev., Alkermes, Inc., Cambridge, United States.

Background and Aims: As new insulin therapy options become available for the patient with type 1 diabetes (T1D), information from the patient perspective may assist clinicians and their patients in treatment decisionmaking. The aim of this study was to determine patient factors that contribute to preference for inhaled insulin in patients with T1D.

Materials and Methods: This randomised, open-label, crossover trial (12 weeks each sequence) compared glycemic control (A1C) of mealtime human insulin inhalation powder (HIIP) with mealtime injectable (SC) in patients with T1D. At a screening visit prior to lead-in (week -6), crossover (week 12), and endpoint (week 24), patient reported outcomes (PROs) were assessed using the SF-36 Vitality Scale; Cognitive Distress, Fatigue, Hyperglycemia, and Hypoglycemia Subscales of the Diabetes Symptom Checklist-Revised; and the Insulin Delivery System Questionnaire. A1C was assessed at randomisation (week 0), crossover, and endpoint. Preference for mealtime insulin delivery system (inhaled or injectable) was assessed at endpoint. A logistic regression model was developed with preference as the dependent variable, and PROs and clinical variables after treatment with inhaled insulin that were significantly $(\mathrm{p}<0.05)$ associated with preference as independent variables. Baseline variables that differed significantly $(\mathrm{p}<0.05)$ between the two preference groups (inhaled or injectable) were entered first. Then PRO scores and A1C after treatment with HIIP were entered into the model.

Results: Of 137 patients, 119 (87\%) completed the study; 46\% were male; mean age was $41 \mathrm{yrs}$; mean baseline $\mathrm{A} 1 \mathrm{C}$ was $8.1 \%$. At the end of the study, $80 \%$ of patients preferred inhaled insulin for their mealtime insulin. Univariate testing indicated that the group that preferred injectable insulin reported significantly $(\mathrm{p}<0.01)$ more troublesome cognitive distress and hyperglycemia symptoms at baseline than the group that preferred inhaled. After controlling for these two baseline variables in the logistic regression model, only lifestyle impact of inhaled insulin and cognitive distress symptoms were significant predictors of insulin delivery system preference. After treatment with HIIP, patients who rated HIIP more positively on aspects of lifestyle impact were approximately 2.5 times more likely to prefer HIIP $(p=0.015)$ than those who rated it less positively; patients with greater cognitive distress were approximately $89 \%$ less likely to prefer HIIP $(\mathrm{p}=0.003)$ than those with less symptom burden.

Conclusion: Many patients with T1D who are not at treatment goal with their injectable insulin therapy may prefer an inhaled insulin to assist them in meeting glycemic goals. However, similar patients with more severe symptom burden may feel more comfortable using their familiar insulin delivery system, but possibly could benefit from additional education about managing symptoms and, perhaps, a change in insulin regimen to reach treatment goals.

Supported by Eli Lilly

\section{5}

Albulin-G, a sustained-exposure basal insulin intended for once-daily administration

A. C. Bell ${ }^{1}$, J. B. Bock ${ }^{1}$, P. Palanisamy Kanakaraj ${ }^{1}$, B. L. Osborn ${ }^{1}$, L. Pukac ${ }^{1}$, M. A. Reitman ${ }^{1}$, V. Roschke ${ }^{1}$, P. Strange ${ }^{2}$, D. Woods ${ }^{1}$;

${ }^{1}$ CoGenesys, Inc., Rockville, ${ }^{2}$ Clinical Development Strategy, Poul Strange

Consulting, LLC, Princeton Junction, United States.

Background and Aims: Current systemic insulin therapy results in low portal concentrations of insulin compared to the normal state resulting in an inadequate suppression of hepatic glucose production and a skewed GH/ IGF-1 axis. In addition to this problem, the advances of long acting insulins including the reduced variability of insulin detemir and longer duration of action with insulin glargine, while good, are still not adequate to meet the characteristics of an ideal basal insulin, primarily because the action beyond 16-20 hours wanes and the peripheral to hepatic exposure ratio is reversed relative to natural insulin. Albulin-G was developed to address these issues by providing low variability, a longer duration of action than insulin glargine and detemir, and a potentially greater hepatic exposure than is typical of subcutaneous insulin.

Materials and Methods: Albulin-G is a fully recombinant fusion protein generated by creating a single open reading frame that encodes a single-chain mini human insulin fused at its $\mathrm{C}$-terminus to the $\mathrm{N}$-terminus of human serum albumin. This protein product is engineered so that it is secreted from yeast in fully-functional form at near gram/liter titers, following a simple purification using conventional and scalable means. It can be formulated for convenient, low volume, subcutaneous injection with a 31-gauge needle. In vitro potency was assessed by measuring glucose uptake in 3T3-L1 adipocytes and mitogenicity was assessed using multiple IGF-1-responsive cell lines. The pharmacokinetic and pharmacodynamic properties of Albulin-G have been assessed in mice, rats, monkeys and Type 1 diabetic mini-pigs.

Results: The pharmacologic properties of Albulin-G are consistent with its composition as an insulin analog. Albulin-G increases glucose uptake in vitro and in vivo but is less mitogenic than other insulin preparations. In animals, Albulin-G had a longer and flatter time/action profile than insulin glargine and insulin detemir. The terminal half-life of Albulin-G in monkeys was 24 hrs and following repeated daily administration a steady-state with a daily peak-to-trough ratio of only $\sim 1.2$-fold was achieved. The pharmacokinetics and pharmacodynamic properties of Albulin-G predict once-daily dosing in man will provide continuous basal insulin coverage in man with low variation in daily insulin exposure and thus potential for consistent control of fasting blood glucose.

Conclusion: With the combined attributes of sustained exposure and low variability following daily administration and potential for greater liver exposure following subcutaneous delivery, Albulin-G has features potentially ideal for basal insulin treatment in Type 1 and Type 2 diabetics. 


\section{PS 95 Insulin analogues in type 2 diabetes}

\section{6}

PREDICTIVE 303 Study: insulin detemir improves glycaemic control, weight and hypoglycaemia in patients previously treated with insulin glargine or NPH insulin

C. Koenen ${ }^{1}$, L. Meneghini ${ }^{2}$, W. Weng ${ }^{3}$, J.-L. Selam ${ }^{4}$;

${ }^{1}$ Novo Nordisk A/S, Bagsværd, Denmark, ${ }^{2}$ Diabetes Research Institute, University of Miami Miller School of Medicine, Miami, United States, ${ }^{3}$ Novo Nordisk Inc., Princeton, United States,

${ }^{4}$ Endocrinology, Diabetes Research Center, Tustin, United States.

Background and Aims: The PREDICTIVE 303 study (N=5604) evaluated the effectiveness of insulin detemir in patients with type 2 diabetes, using a simplified self-adjusted dosing algorithm (G1) compared to standard-of-care physician-driven adjustments (G2).

Materials and Methods: The study was conducted in predominantly primary care settings, over a period of 6 months. Study sites were randomised to either G1 or G2. Patients at G1 sites adjusted their detemir dose every 3 days based on mean fasting blood glucose (FBG) values using a simplified algorithm: mean $\mathrm{FBG}<4.4 \mathrm{mmol} / \mathrm{L}$, reduce dose by $3 \mathrm{U}$; between $4.4-6.1 \mathrm{mmol} / \mathrm{L}$, no change; $>6.1 \mathrm{mmol} / \mathrm{L}$, increase by $3 \mathrm{U}$. The detemir dose for patients from G2 was adjusted by physicians according to the standard of care. This subgroup analysis included patients who were on pre-study basal insulin only (glargine or NPH) or on basal + oral anti-diabetic medications (OADs).

Results: A total of 1663 patients on glargine \pm OADs and 310 patients on $\mathrm{NPH} \pm$ OADs were switched to detemir \pm OADs following randomisation. In patients switched from glargine to detemir, $\mathrm{HbA}_{1 c}$ decreased from $8.4 \%$ at baseline to $8.0 \%$ at 26 weeks for G1, and from 8.4 to $8.1 \%$ for G2 (p<0.0001, vs. baseline for both groups). In patients switched from NPH to detemir, $\mathrm{HbA}_{1 \mathrm{c}}$ decreased from 8.2 to $8.0 \%$ for G1 and from 8.3 to $8.1 \%$ for G2 (p=0.046 vs. baseline for G1, $p=0.112$ for G2). The rates of hypoglycaemia (event/patient/ year) were calculated based on episodes reported for the four weeks prior to each visit. In the glargine-switch group, the overall rate of hypoglycaemia decreased from 10.1 at baseline to 7.1 at 26 weeks for G1, and from 10.3 to 5.8 for G2. The group switched from NPH insulin showed a reduction in the rates of hypoglycaemia that did not reach statistical significance. Patients in both groups showed significant weight lost after switching to detemir insulin (see table).

Table. HbAlc, Weight, and Rate of Hypoglycaemia at 26 Weeks (G1 and G2 combined)

\section{Baseline 26 weeks Change from p value} baseline

\begin{tabular}{lllll}
\hline $\begin{array}{l}\text { Mean HbA } \\
\text { Glargine to detemir }(\mathrm{n}=1663)\end{array}$ & 8.4 & 8.1 & -0.3 & $<0.0001$ \\
NPH to detemir $(\mathrm{n}=310)$ & 8.3 & 8.1 & -0.2 & 0.0126 \\
Mean Body Weight $(\mathbf{k g})$ & & & & \\
Glargine to detemir $(\mathrm{n}=1663)$ & 98.9 & 98.2 & -0.6 & $<0.0001$ \\
NPH to detemir $(\mathrm{n}=310)$ & 97.2 & 95.7 & -1.6 & $<0.0001$ \\
Hypoglycaemia & & & & \\
$\begin{array}{l}\text { (event/patient/year) } \\
\text { Glargine to detemir }(\mathrm{n}=1663)\end{array}$ & 10.1 & 6.6 & -- & 0.0001 \\
NPH to detemir $(\mathrm{n}=310)$ & 12.1 & 7.0 & -- & 0.2754 \\
\hline
\end{tabular}

Conclusion: Patients switched from glargine or $\mathrm{NPH} \pm \mathrm{OADs}$ to detemir \pm OADs in this primary care setting trial showed improvements in glycaemic control, associated with both modest weight loss and a reduced risk of hypoglycaemia.
0977

Insulin detemir improves glycaemic control and reduces hypoglycaemia without weight gain, in type 2 diabetes: comparison of 26-week data from two PREDICTIVE ${ }^{\mathrm{m}}$ studies

A. Robinson ${ }^{1}$, J. B. Hansen ${ }^{2}$, C. Koenen², F. Hernandez ${ }^{3}$, L. Meneghini ${ }^{4}$; ${ }^{1}$ Department of Diabetes and Endocrinology, The Royal Bolton Hospital, Bolton, United Kingdom, ${ }^{2}$ Novo Nordisk A/S, Bagsværd, Denmark, ${ }^{3}$ Diabetes and Endocrinology, Endocare, Hialeah, United States, ${ }^{4}$ Kosow Diabetes Treatment Center, University of Miami School of Medicine, Miami, United States.

Background and Aims: The efficacy and safety of insulin detemir has been examined in several randomised controlled trials and observational studies. These include PREDICTIVE - a multinational, prospective, observational study - and PREDICTIVE 303, a randomised, controlled trial conducted in the US. While the primary endpoint of PREDICTIVE was to assess safety, PREDICTIVE 303 investigated the efficacy two titration approaches. The PREDICTIVE 303 sites were randomised to either standard of care or a patient self-titration arm. Here we examine 26-week data from the two studies to assess similarities and variation between them.

Materials and Methods: The inclusion criteria for the PREDICTIVE 303 trial were: type 2 diabetes, $\mathrm{HbA}_{1} \leq 12 \%, \mathrm{BMI} \leq 45 \mathrm{~kg} / \mathrm{m}^{2}$ and age $\geq 18$ years. In order to accurately compare the two studies, these inclusion criteria were applied to the data collected from 6 European countries in the PREDICTIVE observational study ('EU'). One thousand nine hundred and ninety $(52 \%$ male; mean age 59.9 years; BMI $31.0 \pm 5.2 \mathrm{~kg} / \mathrm{m}^{2}$; diabetes duration 11.9 years) EU patients were compared with 5,604 (51.6\% male; mean age 58.6 years; BMI $33.8 \pm 6.3 \mathrm{~kg} / \mathrm{m}^{2}$; diabetes duration 11.4 years) patients in the PREDICTIVE 303 trial ('US'). All patients were treated with insulin detemir as an add-on to other glucose-lowering regimens and/or as a replacement for previous basal insulin.

Results: Total hypoglycaemia was significantly reduced from 13.0 to 6.6 episodes per patient-year for EU and 8.9 to 6.0 episodes per patient-year for US $(P<0.0005$ for both groups). Major hypoglycaemia was also significantly reduced from 0.9 to 0.1 and 0.9 to 0.2 episodes per patient-year for EU and US respectively $(P<0.0005$ for EU and $P<0.0005$ for US). Nocturnal hypoglycaemia was reduced from 3.5 to 0.9 and 2.4 to 2.0 episodes per patient-year in the EU and US respectively $(P<0.0005$ in the EU and $P=$ 0.222 in the US). In the EU study, $59 \%$ of patients maintained or reduced their body weight while in the US study this value was $53 \%$. Glycaemic control was significantly improved in both studies (Table).

\begin{tabular}{|c|c|c|c|c|}
\hline Parameter & Baseline & 26-weeks & $\begin{array}{l}\text { Change from } \\
\text { baseline at } \\
\text { week } 26\end{array}$ & $P$-value \\
\hline \multicolumn{5}{|c|}{ Total hypoglycaemia, episodes/patient-year } \\
\hline EU & 13.0 & 6.6 & -6.4 & $<0.0005$ \\
\hline us & 8.9 & 6.0 & -2.9 & $<0.0005$ \\
\hline \multicolumn{5}{|c|}{ Major hypoglycaemia, episodes/patient-year } \\
\hline EU & 0.9 & 0.1 & -0.8 & $<0.0005$ \\
\hline us & 0.9 & 0.2 & -0.7 & $<0.0005$ \\
\hline \multicolumn{5}{|c|}{ Nocturnal hypoglycaemia, episodes/patient-year } \\
\hline EU & 3.5 & 0.9 & -2.6 & $<0.0005$ \\
\hline us & 2.4 & 2.0 & -0.4 & 0.222 \\
\hline \multicolumn{5}{|c|}{ Mean $\mathrm{HbA}_{1,} \% \mathrm{o} \pm \mathrm{SD}$} \\
\hline EU & $8.4 \pm 1.5$ & $7.7 \pm 1.6$ & $-0.7 \pm 1.5$ & $<0.0005$ \\
\hline us & $8.5 \pm 1.6$ & $7.9 \pm 1.5$ & $-0.5 \pm 1.4$ & $<0.0005$ \\
\hline \multicolumn{5}{|c|}{ Mean fasting glucose, $\mathrm{mmol} / \mathrm{L} \pm \mathrm{SD}$} \\
\hline EU & $10.1 \pm 2.8$ & $8.2 \pm 2.4$ & $-1.9 \pm 2.9$ & $<0.0005$ \\
\hline us & $9.7 \pm 4.0$ & $8.1 \pm 3.5$ & $-1.5 \pm 4.5$ & $<0.0005$ \\
\hline \multicolumn{5}{|c|}{ Mean body weight, $\mathrm{kg} \pm \mathrm{SD}$} \\
\hline EU & $88.8 \pm 16.7$ & $88.4 \pm 16.9$ & $-0.4 \pm 6.1$ & 0.024 \\
\hline us & $97.6 \pm 20.7$ & $97.4 \pm 20.7$ & $0.0 \pm 5.7$ & 0.370 \\
\hline
\end{tabular}

Conclusion: Despite the different settings (observational study vs randomised, controlled trial), the 26-week data for EU and US are similar and support the previously reported 12 -week data for PREDICTIVE. The data show that insulin detemir improves glycaemic control and reduces hypoglycaemia, while maintaining a stable body weight in patients with type 2 diabetes. Supported by Novo Nordisk $A / S$ 


\section{8}

Less weight gain and hypoglycaemia with once-daily insulin detemir than with NPH insulin in basal-bolus therapy of overweight type 2 diabetes patients: The PREDICTIVE-BMI trial

C. Fajardo Montanana ${ }^{1}$, C. Hernandez Herrero ${ }^{2}$, M. Rivas Fernandez ${ }^{3}$; ${ }^{1}$ Diabetes Unit, Hospital de la Ribera, Alzira,

${ }^{2}$ Endocrinology, Hospital Virgen de Macarena, Sevilla,

${ }^{3}$ Endocrinology, Hospital Infanta Luisa, Sevilla, Spain.

Background and Aims: The majority of people with type 2 diabetes are overweight, yet, to maintain glycaemic control, many require intensified insulin therapy, which is well known to cause weight gain. The prospect of gaining further weight can act as a barrier to patients' acceptance of insulin intensification, potentially affecting prognosis. Insulin detemir (IDet), a long acting basal insulin analogue, has been shown to cause less weight gain than NPH insulin (NPH) and insulin glargine when added to OADs in type 2 diabetes. This weight-sparing effect increases with baseline BMI. The PREDICTIVE-BMI trial was conducted at 41 centres in Spain and aimed to assess weight change caused by IDet or NPH used as part of a basal-bolus regimen in already overweight type 2 diabetes patients.

Materials and Methods: This 26-week, parallel-group, randomised, controlled trial included men or women aged $\geq 18$ years with type 2 diabetes who had been receiving 2 daily doses of insulin (at least one of them a premix) for $\geq 3$ months. $7.5 \% \leq \mathrm{HbA}_{1 \mathrm{c}} \leq 11.0 \%, 25 \mathrm{~kg} / \mathrm{m}^{2} \leq \mathrm{BMI} \leq 40 \mathrm{~kg} /$ $\mathrm{m}^{2}$. These subjects were randomised to receive either once-daily (evening) IDet ( $\mathrm{n}=125$ exposed) or NPH ( $\mathrm{n}=146$ exposed). All subjects also received insulin aspart at main meals. Concomitant treatment with metformin was also allowed. The basal insulin dose was continually and individually titrated, aiming for a pre-breakfast plasma glucose level of $\leq 6.1 \mathrm{mmol} / \mathrm{L}$ without levels of hypoglycaemia considered unacceptable to the patient. Insulin aspart was also individually titrated aiming for postprandial glucose levels of $\leq 10.0$ $\mathrm{mmol} / \mathrm{L}$ without unacceptable levels of hypoglycaemia. Endpoints included weight and BMI change, glycaemic control and frequency of hypoglycaemia. Results: The two groups were well matched for demographic characteristics. Metformin was used by $\sim 50 \%$ and $\sim 58 \%$ of patients on IDet and NPH, respectively, but despite this non-significant slight excess in the NPH group, weight gain at 26 weeks was significantly lower with IDet $(0.4 \mathrm{~kg})$ than with NPH $(1.9 \mathrm{~kg}$ ), between-treatment difference: $1.5 \mathrm{~kg}, \mathrm{p}<0.0001$ (mean baseline values were 79.5 and $82.2 \mathrm{~kg}$, respectively). Similarly, BMI increased significantly less with IDet $\left(0.17 \mathrm{~kg} / \mathrm{m}^{2}\right)$ than with $\mathrm{NPH}\left(0.77 \mathrm{~kg} / \mathrm{m}^{2}\right)$, between-treatment difference: $0.60 \mathrm{~kg} / \mathrm{m}^{2}, \mathrm{p}<0.0001$ (mean baseline values were 31.6 and $32.2 \mathrm{~kg} / \mathrm{m}^{2}$, respectively). $\mathrm{HbA}_{1 \mathrm{c}}$ changed from $8.9 \%$ to $7.8 \%$ in the IDet group and from $8.8 \%$ to $7.8 \%$ in the NPH group (ns for betweentreatment difference in change or final value). Incidences of all hypoglycaemic events and nocturnal events were lower in the IDet group (relative risks 0.62 and 0.43 , respectively, $\mathrm{p}<0.0001$ for both). Major hypoglycaemic episodes (requiring third party assistance) occurred too infrequently for statistical analysis (no episodes with IDet, three with NPH).

Conclusion: When used as the basal component of basal-bolus therapy for overweight or obese patients with type 2 diabetes, IDet reduces weight gain in comparison with NPH despite equivalent improvement in glycaemic control. At equivalent $\mathrm{HbA}_{1 \mathrm{c}}$, patients using IDet have a lower incidence of hypoglycaemia. We therefore conclude that IDet is a better choice than NPH for overweight type 2 diabetes patients requiring intensive insulin therapy. Supported by Novo Nordisk

\section{9}

Use of insulin detemir once-daily results in less weight gain compared with the use of NPH insulin, particularly in obese subjects with type 2 diabetes

A. Philis-Tsimikas ${ }^{1}$, C. Koenen ${ }^{2}$, C. B. Pedersen², B. Thorsteinsson ${ }^{3}$; ${ }^{1}$ Diabetes Department, The Whittier Institute for Diabetes-Scripps Health, La Jolla, United States, ${ }^{2}$ Novo Nordisk A/S, Bagsvaerd, Denmark, ${ }^{3}$ Diabetes Department, Hillerod Hospital, Denmark.

Background and Aims: Obese patients with type 2 diabetes mellitus (T2DM) have a higher risk of cardiovascular complications than normalweight patients. Therefore, potential weight gain is of particular concern once insulin therapy is initiated. Many trials suggest that insulin detemir (detemir) is associated with less weight gain than NPH insulin (NPH). The aim was to investigate if there are any differences in weight development when using detemir or NPH, according to subjects' BMI.

Materials and Methods: This exploratory analysis was based on data from insulin-naive subjects with $\mathrm{T} 2 \mathrm{DM} \geq 1$ year poorly controlled on $1 \geq \mathrm{OAD}(\mathrm{s})$ who were randomised to receive detemir $(n=168)$ or NPH ( $n=163)$ once-daily in the evening as part of a 20 -week multinational, open-label, randomised parallel group trial. Baseline characteristics for detemir- and NPH-treated subjects, respectively, were: mean age 58.7 vs. 58.4 yrs, $\mathrm{HbA}_{1 c} 8.9$ vs. $9.2 \%$, weight 83.5 vs. $85.3 \mathrm{~kg}$ and BMI 29.7 vs. $30.4 \mathrm{~kg} / \mathrm{m}^{2}$ at baseline. Insulin treatment, initiated at 10 units/day as an add-on to OADs, was titrated individually based on fasting plasma glucose (FPG) with pre-breakfast and pre-dinner PG targets of $6.0 \mathrm{mmol} / \mathrm{L}$. The trial demonstrated similar glycaemic control as measured by $\mathrm{HbA}_{1 \mathrm{c}}$ and FPG and less weight gain $(0.7$ vs. $1.6 \mathrm{~kg}, \mathrm{p}=0.005)$ with detemir than with $\mathrm{NPH}$ when administered oncedaily in the evening at equal doses $(0.4$ units $/ \mathrm{kg})$. To further explore any difference in weight development, weight changes were compared between detemir and NPH by baseline BMI, using linear regression (lr) with BMI at baseline as a covariate.

Results: The linear regression confirmed the overall weight benefit of detemir. Furthermore, it demonstrated less weight gain with increasing baseline BMI when detemir was used, whereas more weight gain with increasing baseline BMI was seen when using NPH, as illustrated in the figure. This difference between detemir and NPH in weight change by BMI was statistically significant $(\mathrm{p}=0.004)$. For detemir and NPH respectively, there was a mean weight increase of $2.2 \mathrm{~kg}$ and $1.2 \mathrm{~kg}$ for lean patients $\left(B M I<=25 \mathrm{~kg} / \mathrm{m}^{2}\right), 1.2$ $\mathrm{kg}$ and $1.8 \mathrm{~kg}$ for overweight patients (BMI $\left.25-30 \mathrm{~kg} / \mathrm{m}^{2}\right), 0.6 \mathrm{~kg}$ and $2.2 \mathrm{~kg}$ for obese patients (BMI $30-35 \mathrm{~kg} / \mathrm{m}^{2}$ ) and $0.2 \mathrm{~kg}$ and $1.8 \mathrm{~kg}$ for very obese patients (BMI $\left.>35 \mathrm{~kg} / \mathrm{m}^{2}\right)$.

Conclusion: Once-daily detemir when added to OADs in T2DM improves glycaemic control with less weight gain compared with NPH, particularly in overweight or obese subjects initiating insulin therapy.

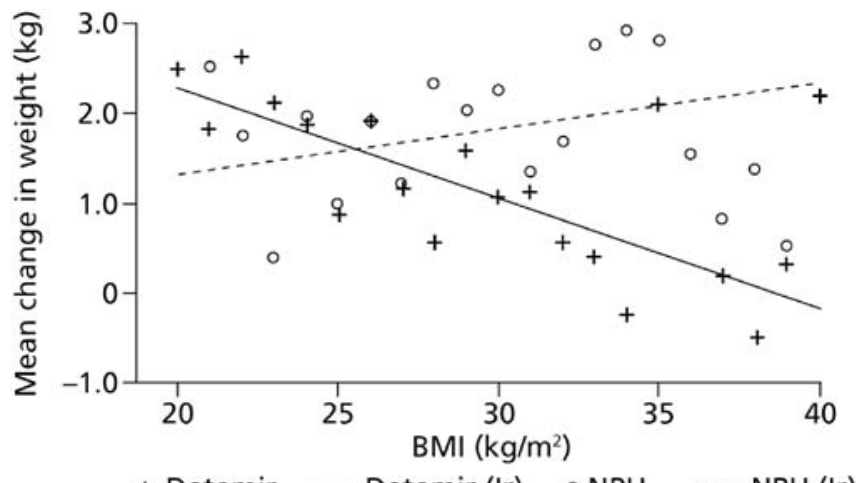

Supported by Novo Nordisk A/S

\section{0}

Basal insulin and oral antidiabetic therapy (BOT) plus a single dose of insulin glulisine (BOT+) reduces $\mathrm{HbA}_{1 \mathrm{c}}$ and blood glucose values in patients with type 2 diabetes

M. Lankisch ${ }^{1}$, K. Ferlinz ${ }^{2}$, W. A. Scherbaum ${ }^{3}$; for the OPAL study group, ${ }^{1}$ Medizinische Klinik III, Kardiologie, Herzzentrum Wuppertal, ${ }^{2}$ sanofi-aventis Deutschland GmbH, Berlin, ${ }^{3}$ German Diabetes Clinic, Heinrich-Heine-University Düsseldorf, Germany.

Background and Aims: Oral antidiabetic drugs (OADs) may initially be used to control hyperglycaemia in patients with type 2 diabetes (T2DM). However, most patients with T2DM will ultimately require insulin therapy to maintain tight glycaemic control. Basal insulin and oral antidiabetic drug therapy (BOT), a single daily injection of basal insulin and OAD therapy, has been shown to be highly effective when using long-acting insulin glargine as the basal insulin. However, a more intensive insulin regimen may be required if $\mathrm{HbA}$ targets are not reached. We show the results of a single daily prandial dose of insulin glulisine plus BOT (BOT+).

Materials and Methods: A 26-week, randomised, multicentre study assessed the efficacy of BOT + in patients with T2DM ( $\mathrm{n}=316$ per protocol set), poorly controlled $\left(\mathrm{HbA}_{1 c}>6.5 \%-\leq 9.0 \%\right)$ on previous BOT. Patients (fasting blood 
glucose $[\mathrm{FBG}] \leq 6.7 \mathrm{mmol} / \mathrm{L}[\leq 120 \mathrm{mg} / \mathrm{dL}]$ ) were stratified by the main meal (breakfast, lunch or dinner), determined by the highest postprandial blood glucose (BG) level, and randomised to BOT+ (insulin glargine as basal insulin) with insulin glulisine given at breakfast $(\mathrm{n}=162)$ or to BOT+ with insulin glulisine taken at the main meal $(n=154)$. We aimed to show equivalence in baseline to endpoint $\mathrm{HbA}_{1 \mathrm{c}}$ change between both arms.

Results: Baseline demographics were similar in both groups. The regimens were equivalent (equivalence margin $=0.4 \%$; Table), with overall $\mathrm{HbA}_{1 c}$ reduced at endpoint ( $6.99 \pm 0.83$ vs. $7.32 \pm 0.70 \%$ at baseline, $\mathrm{p}<0.0001)$. Most BG values improved significantly within each group (Table). Overall, $84.2 \%$ of patients reached titration target ( $2 \mathrm{~h}$ postprandial $\mathrm{BG} \leq 7.5 \mathrm{mmol} / \mathrm{L}[\leq 135$ $\mathrm{mg} / \mathrm{dL}]$ ) on $\geq 1$ visit and $30.7 \%$ of patients achieved $\mathrm{HbA}_{1 \mathrm{c}} \leq 6.5 \%$ at endpoint ( $27.8 \%$ breakfast group and $33.8 \%$ main meal group, $\mathrm{p}=0.21)$. Mean insulin glargine dose was unchanged whereas insulin glulisine dose increased (starting dose of $5 \mathrm{U} /$ day). The rate of on-treatment hypoglycaemia ( $\mathrm{BG} \leq 3.3$ $\mathrm{mmol} / \mathrm{L}[\leq 60 \mathrm{mg} / \mathrm{dL}]$ ) was 3.21 per patient year ( $\mathrm{n}=393$ safety set).

Conclusions: This is the first prospective study to assess the benefits of adding a single dose of rapid-acting insulin to BOT. We show that insulin glulisine given at breakfast is equivalent to insulin glulisine given at the main meal in lowering $\mathrm{HbA}_{1 \mathrm{c}}$. However, taking insulin glulisine at the main meal shows a slightly better responder rate.

\begin{tabular}{lll} 
Parameter & Breakfast & Main meal \\
\hline $\mathrm{HbA}_{1 \mathrm{c}}(\%)$ & $7.35 / 7.03^{*}$ & $7.29 / 6.94^{*}$ \\
$\begin{array}{l}\text { Adjusted mean difference in } \mathrm{HbA}_{1 \mathrm{c}} \\
\text { (\%) change between groups }(95 \% \mathrm{CI})\end{array}$ & $0.048(-0.115 ; 0.211)$ \\
Blood glucose $(\mathrm{mmol} / \mathrm{L})[\mathrm{mg} / \mathrm{dL}]$ & & \\
FBG & $6.1 / 6.6[110 / 118]^{*}$ & $6.0 / 6.3[108 / 113]^{\dagger}$ \\
Post-breakfast & $9.6 / 7.6[173 / 137]^{*}$ & $9.6 / 8.6[173 / 154]^{*}$ \\
Pre-lunch & $7.2 / 6.3[129 / 114]^{*}$ & $7.0 / 6.9[126 / 124]$ \\
Post-lunch & $9.2 / 8.2[166 / 147]^{*}$ & $9.5 / 8.4[171 / 152]^{*}$ \\
Pre-dinner & $8.2 / 7.8[148 / 141]$ & $7.9 / 7.7[143 / 139]$ \\
Post-dinner & $10.1 / 9.5[181 / 171]^{\dagger}$ & $10.4 / 8.5[188 / 153]^{*}$ \\
Bedtime & $8.9 / 8.6[161 / 154]^{\dagger}$ & $9.3 / 7.9[167 / 143]^{*}$ \\
0300 hr & $6.7 / 6.8[121 / 122]$ & $6.7 / 6.3[120 / 113]^{\dagger}$ \\
Insulin dose (U/day) & & \\
Insulin glulisine & $0 / 11$ & $0 / 12$ \\
Insulin glargine & $31 / 32$ & $27 / 27$ \\
\hline
\end{tabular}

Within-group change: ${ }^{*} \mathrm{p}<0.0001 ;{ }^{\dagger} \mathrm{p}<0.05$

Data are mean (baseline/endpoint)

Supported by sanofi-aventis

\section{1}

A comparison of insulin dosing algorithms in a study of prandial premixed therapy vs. basal bolus therapy in type 2 diabetes patients inadequately controlled by glargine plus OAMs

S. A. Martin ${ }^{1}$, H. Jiang ${ }^{1}$, M. Palaisa ${ }^{1}$, S. Jacober ${ }^{1}$,

${ }^{1}$ Eli Lilly and Company, IndianapolisUnited States.

Background and Aims: Little information has been published comparing the effectiveness of dosing algorithms in insulin regimens. In a study comparing prandial premixed therapy (PPT) to basal bolus therapy (BBT) in type 2 diabetes (T2D) patients, 2 algorithms differing in the aggressiveness of dose escalation were also evaluated. This analysis aims to compare the efficiency of 2 dosing algorithms (See Algorithms Table: ALGO1, ALGO2) as measured by the weeks to reach the maximum dose (with no subsequent dose modification of more than $5 \%$ ).

Materials and Methods: This 24-week, randomised, open-label, activecontrolled trial was conducted at 58 centers in the US and Puerto Rico. Patients with T2D and inadequate glycemic control (defined by A1C level $\geq$ $7.5 \%$ and $\leq 12 \%$ ) were randomised to PPT (lispro mix 50; 50\% insulin lispro, $50 \%$ insulin lispro protamine suspension; $\mathrm{n}=187$ ) tid with meals or BBT (glargine at bedtime + mealtime lispro; $\mathrm{n}=187$ ). Within each therapy patients were then randomized to one of 2 recommended dosing algorithms. Strict adherence to dosing requirements of the algorithms was not mandatory or specifically documented by sites.

Results: Baseline characteristics were similar (mean $\pm \mathrm{SD}$ ): age $(53.8 \pm 9.1$ vs. $55.6 \pm 9.9$ years, $\mathrm{P}=0.076)$, weight $(99.0 \pm 20.0$ vs. $99.9 \pm 21.1 \mathrm{~kg}, \mathrm{P}=0.666)$,
A1C ( $8.9 \pm 1.0$ vs. $8.8 \pm 1.1 \%, P=0.336)$, and duration of diabetes $(10.9 \pm 6.1$ vs. $11.3 \pm 6.3$ years, $\mathrm{P}=0.525)$ for $\mathrm{ALGO} 1$ and $\mathrm{ALGO}$, respectively. There was no difference in time to reach maximum dose (ALGO1 18.8 \pm 6.0 weeks vs. ALGO2 18.0 \pm 7.0 weeks, $\mathrm{P}=0.278$ ). ALGO1 was associated with a higher endpoint insulin dose $(1.3 \pm 0.8 \mathrm{u} / \mathrm{kg}$ vs. $1.2 \pm 0.6 \mathrm{u} / \mathrm{kg}, \mathrm{P}=0.015)$ and more weight gain $(4.8 \pm .9 \mathrm{~kg}$ vs. $3.7 \pm 3.6 \mathrm{~kg}, \mathrm{P}=0.015)$. ALGO1 resulted in greater reduction from baseline of $\mathrm{A} 1 \mathrm{C}(-2.1 \pm 1.0 \%$ vs. $-1.85 \pm 1.2 \%, \mathrm{P}=0.028)$, lower fasting plasma glucose (PG), $(7.9 \pm 2.2 \mathrm{mmol} / \mathrm{L}$ vs. $8.2 \pm 2.1 \mathrm{mmol} / \mathrm{L}$, $\mathrm{P}=0.037)$, and lower mean 2-hour postprandial PG $(8.6 \pm 2.3 \mathrm{mmol} / \mathrm{L}$ vs. $9.4 \pm 2.6 \mathrm{mmol} / \mathrm{L}, \mathrm{P}=0.005)$. Percent of patients reaching glycemic targets were similar: $\mathrm{A} 1 \mathrm{C} \leq 6.5 \%(47.0 \%$ vs. $38.1 \%, \mathrm{P}=0.129)$ and $\mathrm{A} 1 \mathrm{C}<7.0 \%(64.2 \%$ vs. $57.8 \%, \mathrm{P}=0.286$ ) for ALGO1 and ALGO2, respectively. Both algorithms were generally well tolerated with a low incidence of severe hypoglycemia $3.2 \%$ vs. $2.2 \%, \mathrm{P}=0.52)$ and similar rates of overall hypoglycemia (54.8 \pm 50.7 vs. $45.0 \pm 47.3$ events/pt/yr, $\mathrm{P}=0.055$ ) for ALGO1 and ALGO2, respectively.

Conclusion: Both algorithms were equally efficacious in time to reach maximum dose. Randomization to the more aggressive dosing algorithm (ALGO1) yielded a slightly greater reduction of A1C levels, a larger endpoint insulin dose, more weight gain, and a slight but not significant increase in rates of overall hypoglycemia.

\section{Dosing Algorithms}

\begin{tabular}{lll}
\hline $\begin{array}{l}\text { Preprandial Blood Glucose } \\
(\mathrm{mg} / \mathrm{dl}[\mathrm{mM}])\end{array}$ & $\begin{array}{l}\text { ALGO1 (TDI < 100) } \\
\text { (Units Dose Increased) }\end{array}$ & $\begin{array}{l}\text { ALGO1 (TDI } \geq 100) \\
\text { (Units Dose Increased) }\end{array}$ \\
\hline $110-150(6.1-8.3)$ & 2 & 4 \\
$151-200(8.4-11.1)$ & 4 & 8 \\
$201-250(11.2-13.9)$ & 6 & 12 \\
$251-300(13.9-16.7)$ & 8 & 16 \\
$>300(>16.7)$ & 10 & 20 \\
Preprandial Blood Glucose & ALGO2 & \\
$(\mathrm{mg} / \mathrm{dl}[\mathrm{mM}])$ & (Units Dose Increased) & \\
$110-200(6.1-11.1)$ & 2 & \\
$201-300(11.2-16.7)$ & 3 & \\
$>300(>16.7)$ & 4 &
\end{tabular}

TDI, total daily insulin

Supported by Eli Lilly and Company

\section{2}

Efficacy and safety of insulin glargine added to ongoing therapy with multiple oral antidiabetic drugs (OADs) in patients with type 2 diabetes mellitus (T2DM)

P. Raskin ${ }^{1}$, R. J. Tanenberg 2 , A. Vlajnic ${ }^{3}$, P. Hollander';

${ }^{1}$ Department of Internal Medicine, University of Texas Southwestern Medical Center, Dallas, ${ }^{2}$ Division of Endocrinology, Brody School of Medicine, Greenville, ${ }^{3}$ sanofi-aventis U.S., Bridgewater, ${ }^{4}$ Endocrinology Center, Baylor University Medical Center, Dallas, United States.

Background and Aims: Intensive glycemic control can reduce the microvascular and macrovascular complications associated with diabetes. Owing to the progressive nature of the disease, patients often progress from treatment with OADs to basal insulin therapy and ultimately, prandial insulin therapy. The objective of the current analysis is to assess the efficacy, as measured by $\mathrm{AlC}$ and fasting plasma glucose (FPG), and safety of basal insulin added to OADs.

Material and Methods: We conducted an open-label, parallel, 3-arm study in adult patients with T2DM who had basal insulin glargine added to existing OADs, and were 1:1:1 randomised to 1,2 , or 3 injections of prandial insulin glulisine. Efficacy and safety were analyzed during the 14 -week run-in phase of this study ( $\mathrm{N}=643)$. Inclusion criteria were an $\mathrm{AlC} \geq 8.0 \%$ while on a combination of $\geq 2$ OADs for $\geq 3$ months. Oral therapy subgroups consisted of patients treated with combinations of metformin (MET), sulfonylureas (SUs), and/or thiazolidinediones (TZDs). Data pertaining to medication dose were obtained from self-report diaries.

Results: Values for demographic characteristics (50.5\% male, $65.3 \%$ white, mean $\pm \mathrm{SD}$ age $52.3 \pm 10.2 \mathrm{y}$; body mass index (BMI) $35.8 \pm 14.2 \mathrm{~kg} / \mathrm{m}^{2} ; \mathrm{T} 2 \mathrm{DM}$ duration $8.5 \pm 6.2 \mathrm{y}$; mean A1C 9.8\% \pm 1.4 ; mean FPG $231.8 \mathrm{mg} / \mathrm{dL} \pm 71.4$ ) were relatively similar among 3 of the OAD subgroups. Subjects initially received 
$10 \mathrm{U}$ of glargine while continuing their oral regimens; dose adjustments were made based on mean morning FPG to a target of $<96 \mathrm{mg} / \mathrm{dL}$. After 14 weeks of insulin glargine treatment, but prior to randomisation to prandial insulin, adjusted mean A1C decreased among all subgroups by $2.47 \%$; the adjusted mean FBG decreased by $104.8 \mathrm{mg} / \mathrm{dL}$; and $40.6 \%$ of patients achieved an A1C $<7.0 \%$. (Tab. 1)

Conclusion: The subjects in the MET+TZD+GLAR subgroup experienced the greatest adjusted mean reduction in A1C ( $P=0.003$ vs MET+SU+GLAR) and the highest adjusted percentage of subjects reaching $\mathrm{A} 1 \mathrm{C}<7.0 \%(P=0.0001$ vs MET+SU+GLAR). This group also reported the lowest event rate of hypoglycemia. These results demonstrate that addition of insulin glargine to ongoing OAD combinations yields excellent glycemic results.

Editorial support for this abstract is provided by sanofi-aventis U.S.

\section{3}

Advancing insulin therapy in T2DM: emerging dynamics among parameters affecting tight glycaemic control

M. Johnson ${ }^{1}$, M. A. Powers ${ }^{1}$, P. Hollander ${ }^{2}$, A. Vlajnic ${ }^{3}$, R. Bergenstal ${ }^{1}$;

${ }^{1}$ International Diabetes Center, Park Nicollet Institute, Minneapolis,

${ }^{2}$ Endocrinology Center, Baylor University Medical Center, Dallas, ${ }^{3}$ sanofi-

aventis U.S., Bridgewater, United States.

Background and Aims: An insulin-to-carbohydrate ratio allows flexibility in food choices and accuracy in dosing of mealtime insulin. However, insulin-to-carbohydrate ratio is complex and often difficult for patients to implement. Thus, there is a need for a simple, effective method that can be more easily executed. The purpose of this study was to compare glycemic control, as measured by $\mathrm{A} 1 \mathrm{C}$, in subjects receiving a basal-bolus insulin regimen, wherein prandial insulin was dosed based on either carbohydrate (CARB) counting or using a fixed algorithm. This subanalysis focused on the relationship among Body mass index (BMI), and $\mathrm{AlC}$, the method of insulin titration, and evaluated the implications for weight gain and hypoglycemia.

Material and Methods: This was an open-label, controlled, parallel-group, randomised study. Subjects with uncontrolled type 2 diabetes mellitus (T2DM) on $\geq 2$ insulin injections were switched to basal-bolus therapy with insulin glargine and insulin glulisine \pm metformin. Glargine was adjusted for 24 weeks based on fasting plasma glucose and glulisine by using a simple algorithm (ALG) based on preprandial glucose patterns (ALG; $n=136$ ) or by carbohydrate (CARB) counting (CARB; $n=137$ ).

Results: Total insulin doses increased from baseline (102U) to 12 weeks in both groups; the largest increase was in the ALG (174 U) versus CARB (149 U) group. By 12 weeks, the mean AlC decreased to $<7.0 \%$ (ALG 6.9\%, CARB 6.7\%). From 12 to 24 weeks, A1C improved incrementally (ALG $6.6 \%$, CARB $6.5 \% ; P=0.15$ ) but required $21 \%$ more total insulin in the ALG group (210 U) versus $9 \%$ in the CARB group (163 U) (Figure). There was a similar pattern of weight gain in the 0- to12-week period (ALG gained 2.1 $\mathrm{kg}$, CARB gained $1.2 \mathrm{~kg}$ ) compared with the 12 - to 24 -week period. Rates of moderate hypoglycemia were not significantly different between the 0 - to
12- and 12- to 24-week periods. We also noted that longer T2DM duration increased the risk of hypoglycemia $(P=0.025)$ and higher BMI showed a trend toward lower risk of hypoglycemia $(P=0.055)$. Following a weekly titration regimen with basal-bolus (glargine-glulisine) insulin therapy, the greatest $\mathrm{A} 1 \mathrm{C}$ reduction was in the first 12 weeks of titration; subsequent A1C lowering was associated with continued weight gain, with no increase in hypoglycemia.

Conclusion: This is one of the few clinical trials where obese (baseline BMI $36.7 \mathrm{~kg} / \mathrm{m}^{2}$ ) patients with T2DM reached near-optimal glycemic control. In obese patients with T2DM, clinicians need to be aware of interactions among insulin dose, weight, and hypoglycemia over time to achieve optimal glycemic control (A1C 6.5\%-6.6\%).

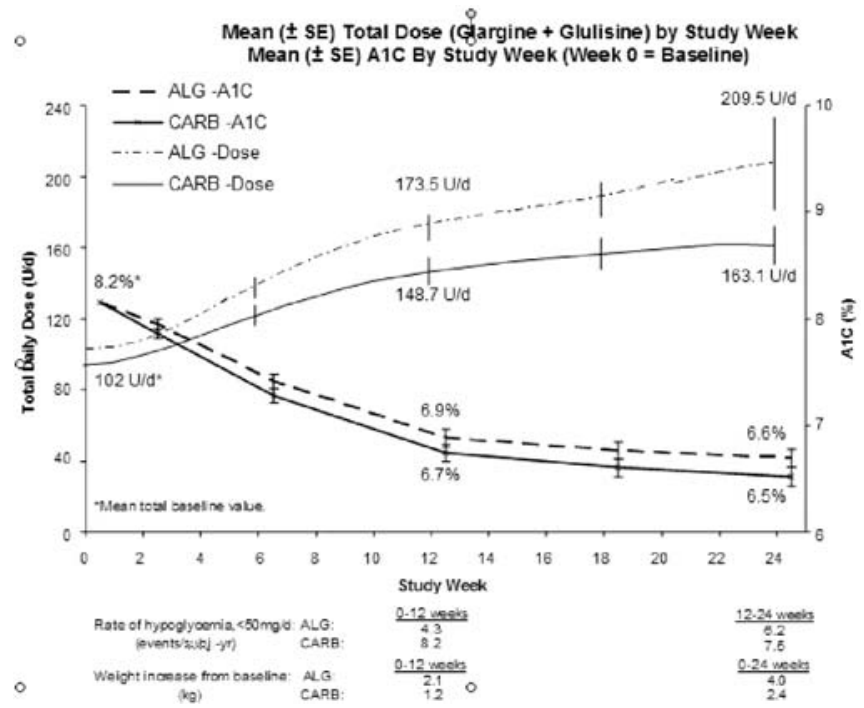

Supported by sanofi-aventis U.S.

Tab. 1

\begin{tabular}{|c|c|c|c|c|c|}
\hline & $\begin{array}{l}\text { Total } \\
(\mathrm{N}=643)\end{array}$ & $\begin{array}{l}\text { MET+SU + GLAR } \\
(n=388)\end{array}$ & $\begin{array}{l}\text { MET+TZD+ GLAR } \\
(\mathrm{n}=147)\end{array}$ & $\begin{array}{l}\text { SU+TZD +GLAR } \\
(\mathrm{n}=52)\end{array}$ & $\begin{array}{l}\text { MET+SU+TZD +GLAR } \\
(\mathrm{n}=56)\end{array}$ \\
\hline \multicolumn{6}{|l|}{ Baseline Characteristics } \\
\hline $\operatorname{AlC}^{\dagger}(\%)$ & $9.8 \pm 1.4$ & $9.7 \pm 1.4$ & $9.9 \pm 1.5$ & $9.9 \pm 1.4$ & $9.9 \pm 1.2$ \\
\hline $\mathrm{BMI}^{\dagger}\left(\mathrm{kg} / \mathrm{m}^{2}\right)$ & $35.8 \pm 14.2$ & $35.7 \pm 16.9$ & $36.2 \pm 9.3$ & $34.6 \pm 8.4$ & $36.4 \pm 6.9$ \\
\hline $\mathrm{FPG}^{\dagger}(\mathrm{mg} / \mathrm{dL})$ & $231.8 \pm 71.4$ & $228.8 \pm 70.6$ & $236.9 \pm 72.5$ & $232.6 \pm 75.2$ & $238.1 \pm 71.1$ \\
\hline \multicolumn{6}{|l|}{$\begin{array}{l}\text { Week } 14 \text { (end of run-in period) } \\
\text { Characteristics }\end{array}$} \\
\hline Change in $\mathrm{AlC}^{\ddagger}(\%)$ & $-2.47 \pm 0.05$ & $-2.42 \pm 0.06$ & $-2.71 \pm 0.09$ & $-2.45 \pm 0.15$ & $-2.34 \pm 0.14$ \\
\hline $\mathrm{A} 1 \mathrm{C}<7.0 \%(\%)$ & 40.6 & 34.5 & 57.3 & 47.0 & 38.6 \\
\hline Weight change $^{\ddagger}(\mathrm{kg})$ & $3.5 \pm 0.2$ & $3.1 \pm 0.2$ & $3.9 \pm 0.4$ & $5.3 \pm 0.6$ & $4.5 \pm 0.6$ \\
\hline FPG change $^{\ddagger}(\mathrm{mg} / \mathrm{dL})$ & $-104.8 \pm 1.7$ & $-101.5 \pm 2.3$ & $-112.0 \pm 3.4$ & $-107.9 \pm 6.0$ & $-108.8 \pm 6.0$ \\
\hline $\begin{array}{l}\text { Mean insulin glargine dose:* } \\
\mathrm{U} / \mathbf{k g}^{\dagger} \\
\mathrm{U} / \mathbf{d}^{\ddagger}\end{array}$ & $\begin{array}{l}0.58 \pm 0.26 \\
64.8 \pm 1.8\end{array}$ & $\begin{array}{l}0.60 \pm 0.26 \\
68.8 \pm 2.2\end{array}$ & $\begin{array}{l}0.61 \pm 0.26 \\
68.7 \pm 3.6\end{array}$ & $\begin{array}{l}0.49 \pm 0.23 \\
61.3 \pm 6.5\end{array}$ & $\begin{array}{l}0.46 \pm 0.25 \\
56.9 \pm 6.2\end{array}$ \\
\hline $\begin{array}{l}\text { Hypoglycemia with } \mathrm{SMBG}<50 \mathrm{mg} / \mathrm{dL}^{\ddagger} \\
\text { (events/patient-y) }\end{array}$ & $1.06 \pm 0.14$ & $1.24 \pm 0.20$ & $0.45 \pm 0.17$ & $1.73 \pm 0.62$ & $0.70 \pm 0.34$ \\
\hline
\end{tabular}

${ }^{\dagger}$ Values are \pm SD. ${ }^{\ddagger}$ Values are mean \pm SE. ${ }^{*}$ medication dose data were obtained from self-report diaries. SMBG $=$ self-monitored blood glucose. 


\section{4}

Relationship between $\mathrm{HbA}_{1 c}$ and hypoglycaemia in type 2 diabetes patients treated with prandial+basal insulin mixture or basal insulin regimens

J. Chan ${ }^{1}$, B. M. Frier², M. Leyk ${ }^{1}$, C. A. Jones ${ }^{1}$, M. H. Tan';

${ }^{1}$ Eli Lilly and Company, Indianapolis, United States,

${ }^{2}$ Dept of Diabetes, Royal Infirmary of Edinburgh, United Kingdom.

Background and Aims: Achieving glycaemic targets with minimal hypoglycaemia is a challenge for insulin-requiring patients with type 2 diabetes (T2D). Understanding the different effects insulin formulations have on glycaemic targets and hypoglycaemia risk helps to optimise treatment.

Materials and Methods: Data comparing a prandial+basal insulin lispro mixture +metformin (25\% lispro/75\% insulin lispro protamine suspension (IPLS) bid [2 studies] or 50\% lispro/50\% IPLS tid [1 study]; LM+Met) with basal insulin glargine bedtime (G+Met) were pooled. Data were analyzed using ANCOVA model for responses with data from the last visit having an $\mathrm{HbAlc}$; treatment and study were model covariates. Baseline values were included in $\mathrm{HbAlc}, \mathrm{BG}$, and insulin dose models. Hypoglycaemic episodes were defined as BG $<3.5 \mathrm{mM}(63 \mathrm{mg} / \mathrm{dL})$, calculated as episodes/pt/yr, and analyzed (a) at endpoint and (b) over the study period.

Results: At baseline, both groups (LM+Met, $n=253$; G+Met, $n=241$ ) had similar mean $\mathrm{HbAlc}(8.3 \%)$, age, sex, BMI, T2D duration, and fasting and postprandial BG (FBG, PBG). At endpoint, there was an inverse relationship between hypoglycaemia and HbAlc in the combined treatment cohort. For a $1 \%$ reduction in $\mathrm{HbAlc}$, the increase (slope) was 1.4 episodes $(\mathrm{p}=0.04)$ for all hypoglycaemia, 1.1 episodes ( $\mathrm{p}=0.04$ ) for daytime hypoglycaemia, and not significant for nocturnal hypoglycaemia. At endpoint, the LM+Met group had lower HbA1c than the G+Met group $(7.2 \pm 0.1$ vs. $7.7 \pm 0.1 \%, \mathrm{p}<0.0001)$ and all-meals combined PBG (8.9 \pm 0.1 vs. $10.2 \pm 0.1 \mathrm{mM}, \mathrm{p}<0.0001)$, but higher FBG $(8.1 \pm 0.1$ vs. $6.8 \pm 0.1 \mathrm{mM}, \mathrm{p}<0.0001)$ and insulin dose $(0.7 \pm 0.01$ vs. $0.6 \pm 0.01 \mathrm{U} / \mathrm{kg}, \mathrm{p}<0.0001)$. For hypoglycaemia at endpoint, the $\mathrm{LM}+\mathrm{Met}$ group had a higher rate for all ( 8.1 vs. 4.4 episodes, $\mathrm{p}=0.01$ ) and daytime (6.2 vs. 1.4 episodes, $\mathrm{p}<0.0001$ ) hypoglycaemia. Nocturnal hypoglycaemia was similar between groups (1.9 vs. 3.0 episodes). Over the study period, no difference was observed between groups for all hypoglycaemia (12.4 vs. 9.5 episodes), the LM+Met group had a higher daytime rate (10.3 vs. 3.5 episodes, $\mathrm{p}<0.0001)$, and the $\mathrm{G}+$ Met group had a higher nocturnal rate $(3.4$ vs. 6.6 episodes, $\mathrm{p}=0.003)$. In both groups, severe hypoglycaemia was rare. About $78 \%$ of all patients had no hypoglycaemia at endpoint and $55 \%$ had none over the study period.

Conclusions: As HbAlc decreased, all and daytime hypoglycaemia episodes increased. The lispro mixture+Met group had lower HbAlc and PBG and higher FBG and insulin dose. Daytime hypoglycaemia was higher in the lispro mixture+Met group, both at endpoint and over the study period. Nocturnal hypoglycaemia was similar between groups at endpoint and higher in glargine+Met over the study period. Providing prandial+basal insulins in 2-3 injections daily vs. basal insulin (both + Met) resulted in different glycaemic control and hypoglycaemia profiles.

Funded by Eli Lilly and Company

\section{PS 96 Pre-mixed insulins}

\section{5}

Patients with poorly controlled early type 2 diabetes show improved treatment results with biphasic insulin aspart 30: PRESENT study results D. Khutsoane ${ }^{1}$, S. Lee ${ }^{2}$, S. Güler ${ }^{3}$, S. K. Sharma ${ }^{4}$, M. A. S. Al-Mustafa ${ }^{5}$, N. Christiansen ${ }^{6}$, S. T. Azar ${ }^{7}$, R. Danciulescu ${ }^{8}$, M. Shestakova 9 ,

N. M. A. Ayad ${ }^{10}$, O. M. Bech ${ }^{11}$;

${ }^{1}$ Med, Medi-Clinic, Bloemfontein, South Africa, ${ }^{2}$ Novo Nordisk, Seoul, Republic of Korea, ${ }^{3}$ Endocrinology and Metabolism, Ankara Numune Hospital, Turkey, ${ }^{4}$ Med, M G Medical College, Jaipur, India, ${ }^{5}$ National Diabetes Center, Al Mstansiriya University, Baghdad, Iraq, ${ }^{6}$ Novo Nordisk, Vouliagmeni, Greece, ${ }^{7}$ Endocrinology, American University of Beirut, Lebanon, ${ }^{8} \mathrm{~N}$. Paulescu Institute, Bucharest, Romania, ${ }^{9}$ Institution of Diabetology, Russian Academy of Medical science, Moscow, Russian Federation, ${ }^{10}$ Internal Medicine Department, Taiba Hospital, Kuwait, ${ }^{11}$ Med, Novo Nordisk, Beijing, China.

Background and Aims: The efficacy and safety of biphasic insulin aspart 30 (BIAsp 30) in clinical practice in T2DM of different duration were investigated in a multi-national, observational study (PRESENT study).

Materials and Methods: Patients (mean BMI $27 \mathrm{~kg} / \mathrm{m}^{2}$ ) were categorised by diabetes duration (years/yrs): $<5(\mathrm{n}=4779) ; 5-10(\mathrm{n}=6332) ; 10-20$ $(\mathrm{n}=6776) ; \geq 20(\mathrm{n}=1999)$. Mean age increased with duration from 50.1 yrs ( $<5$ yrs group) to 63.4 yrs ( $\geq 20$ yrs group). Prior treatment with only OAD was more common in patients with shorter duration $(<5 \mathrm{yrs})(41.6 \%)$, compared with those with longer duration ( $\geq 20 \mathrm{yrs})(27.5 \%)$, conversely for insulin-only prior treatment: $<5$ yrs group (21.9\%), $\geq 20$ yrs group $(39.1 \%)$; and OAD + insulin prior treatment: $<5$ yrs group (15.3\%), $\geq 20$ yrs group (28.1\%). Treatment followed physician discretion. Most initiated treatment at breakfast and dinner (75.6-81.6\%). Hypoglycaemia incidence at baseline was based on patient recollection over 3 months prior to the first visit, and from the last visit for 3 and 6 month data points.

Results: Mean $\mathrm{HbA}_{1 \mathrm{c}}$, FPG and PPPG decreased significantly in all groups (Table). The greatest reductions were observed in $<5$ yrs group, which also reported the lowest insulin dose (Figure). At 6 months, the proportion of

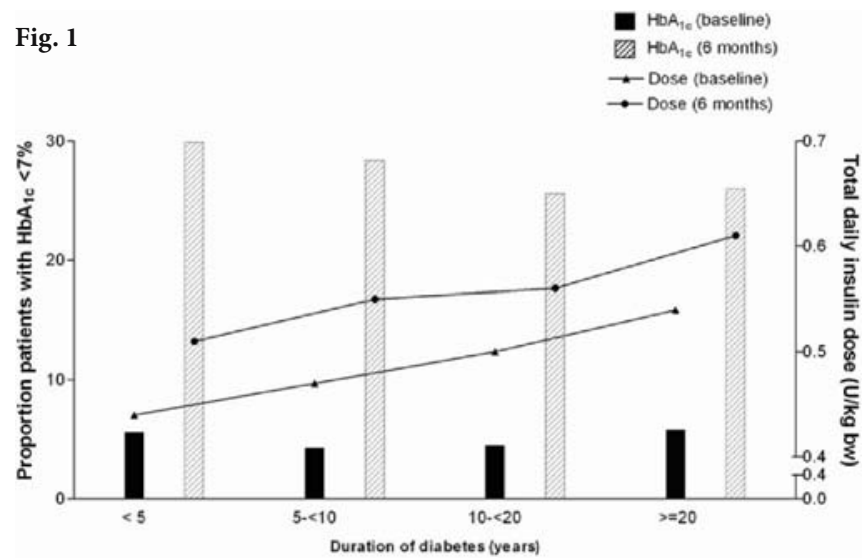

Tab. 1 Abstract 0985

\begin{tabular}{|c|c|c|c|c|}
\hline & $<5$ years & $5-<10$ years & $10-<20$ years & $\geq 20$ years \\
\hline $\begin{array}{l}\text { Mean } \mathrm{HbA}_{1 \mathrm{c}}, \% \pm \mathrm{SD} \\
\text { Baseline } \\
\text { Change (baseline- } 3 \text { months) } \\
\text { Change (baseline- } 6 \text { months) }\end{array}$ & $\begin{array}{l}9.65 \pm 2.12 \\
-1.47 \pm 1.84^{*} \\
-1.95 \pm 2.01^{*}\end{array}$ & $\begin{array}{l}9.49 \pm 1.82 \\
-1.33 \pm 1.61^{*} \\
-1.81 \pm 1.76^{*}\end{array}$ & $\begin{array}{l}9.48 \pm 1.80 \\
-1.27 \pm 1.54^{*} \\
-1.75 \pm 1.78^{*}\end{array}$ & $\begin{array}{l}9.35 \pm 1.86 \\
-1.11 \pm 1.64^{*} \\
-1.55 \pm 1.82^{*}\end{array}$ \\
\hline $\begin{array}{l}\text { Mean FPG, } \mathrm{mM} \pm \mathrm{SD} \\
\text { Baseline } \\
\text { Change (baseline- } 3 \text { months) } \\
\text { Change (baseline- } 6 \text { months) }\end{array}$ & $\begin{array}{l}12.18 \pm 4.63 \\
-3.44 \pm 4.37^{*} \\
-4.10 \pm 4.41^{*}\end{array}$ & $\begin{array}{l}11.65 \pm 3.80 \\
-2.95 \pm 3.47^{*} \\
-3.71 \pm 3.68^{*}\end{array}$ & $\begin{array}{l}11.74 \pm 3.90 \\
-2.83 \pm 3.65^{\star} \\
-3.54 \pm 3.72^{\star}\end{array}$ & $\begin{array}{l}11.44 \pm 4.13 \\
-2.42 \pm 3.86^{*} \\
-3.22 \pm 3.91^{*}\end{array}$ \\
\hline $\begin{array}{l}\text { Mean PPPG, } \mathrm{mM} \pm \mathrm{SD} \\
\text { Baseline } \\
\text { Change (baseline- } 3 \text { months) } \\
\text { Change (baseline- } 6 \text { months) }\end{array}$ & $\begin{array}{l}16.78 \pm 5.60 \\
-5.16 \pm 5.35^{\star} \\
-6.20 \pm 5.48^{\star}\end{array}$ & $\begin{array}{l}16.22 \pm 4.94 \\
-4.71 \pm 4.72^{*} \\
-5.82 \pm 4.97^{\star}\end{array}$ & $\begin{array}{l}16.29 \pm 5.01 \\
-4.60 \pm 4.79^{\star} \\
-5.64 \pm 4.93^{\star}\end{array}$ & $\begin{array}{l}16.10 \pm 5.40 \\
-4.19 \pm 5.04^{*} \\
-5.36 \pm 5.23^{*}\end{array}$ \\
\hline
\end{tabular}

${ }^{*} p<0.001$ (paired t-test) 
patients in all groups having $\mathrm{HbA}_{1 c}<7 \%$ increased significantly from baseline $(p<0.001)$, with the highest in $<5$ yrs group. $21 \%$ in this group used the oncedaily regimen, compared with $15.7 \%$ (5-10 yrs); $13.2 \%$ (10-20 yrs); $12.1 \%$ ( $\geq$ 20 yrs). Overall hypoglycaemia (events/patient-yr) was low at 6 months: 2.1 (< 5 yrs); 2.0 (5-10 yrs); 2.3 (10-20 yrs); 2.8 ( $\geq 20$ yrs).

Conclusions: BIAsp 30 treatment initiation in patients with poorly controlled early T2DM improved glucose control to a greater extent, with fewer injections and lower doses, compared with patients having longer diabetes duration. (Fig. 1, Tab. 1)

Supported by Novo Nordisk

\section{6}

Biphasic insulin aspart is associated with a reduced risk of hypoglycaemia compared with biphasic human insulin

J. Davidson ${ }^{1}$, A. Liebl' ${ }^{2}$, J. S. Christiansen ${ }^{3}$, G. Fulcher ${ }^{4}$, R. J. Ligthelm ${ }^{5}$,

A. Toft ${ }^{6}$, T. Gylvin ${ }^{6}$, H. Frimer-Larsen ${ }^{7}$, R. Kawamori ${ }^{8}$;

${ }^{1}$ Diabetes Dpt, Endocrine \& Diabetes Associates of Texas, Dallas, United

States, ${ }^{2}$ Diabetes Dpt, Center for Diabetes and Metabolism, Bad Heilbrunn,

Germany, ${ }^{3}$ Endocrinology, Aarhus University Hospital, Denmark,

${ }^{4}$ Endocrinology, Royal North Shore Hospital, Sydney, Australia,

${ }^{5}$ Diabetes Dpt, EHM, Hoofddorp, The Netherlands, ${ }^{6}$ Novo Nordisk,

Bagsvaerd, Denmark, ${ }^{7}$ Statistics, Larix Aps, Værløse, Denmark,

${ }^{8} \mathrm{~S} c h o o l$ of Medicine, Juntendo University, Tokyo, Japan.

Background and Aims: To compare the risk of hypoglycaemia (all, major, minor, nocturnal, daytime) associated with once- or twice-daily use of the premixed insulin aspart (BIAsp 30) and premixed human insulin (BHI 30) using a meta-analysis including all Novo Nordisk-sponsored studies comparing these insulins. A total of nine randomised, parallel or crossover, trials (duration 12 weeks to 4 years) were included involving 151 type 1 (BIAsp 30, $\mathrm{n}=79$; BHI 30, $\mathrm{n}=72$ ) and 1674 type 2 (BIAsp 30, $\mathrm{n}=1142$; BHI 30, $\mathrm{n}=849$ ) diabetes patients.

Materials and Methods: At baseline, subjects (58\% male) had a mean (SD) age of $59.4(12.0)$ years, mean BMI of $26.5(4.6) \mathrm{kg} / \mathrm{m}^{2}$, and average $\mathrm{HbA}_{1 c}$ was $8.2(1.4) \%$. Hypoglycaemia was classified as major (requiring third party assistance) or minor (symptoms of hypoglycaemia confirmed by $\mathrm{BG}<2.8$ $\mathrm{mmol} / \mathrm{L}[\mathrm{PG}<3.1 \mathrm{mmol} / \mathrm{L}]$ ). In one Japanese trial, minor hypoglycaemia was not defined by BG values. Analysis of minor hypoglycaemia was therefore performed with and without data from this trial. All hypoglycaemic endpoints were analysed using a generalised linear regression model assuming a negative binomial distribution and accounting for exposure time.

Results: Over the 24-hour period, the relative risk (RR) for all major hypoglycaemia was lower with BIAsp 30 than with BHI 30 (RR, 0.41 [0.22; 0.75], $\mathrm{p}<0.01)$ across trials. The RRs for major daytime and nocturnal hypoglycaemia were also lower for BIAsp 30 . The estimated RR for minor hypoglycaemia, excluding data from Japanese trial was 1.11 (95\% CI, 0.85; 1.45 ; NS). Results of the result of the analysis were unchanged when including this trial. The RR for nocturnal hypoglycaemia was statistically significantly lower with BIAsp 30 than BHI 30 across trials (RR, 0.65 [0.47; 0.88], p=0.005) and was statistically significantly lower in subjects on BIAsp 30 with type 2 (RR, $0.64[0.45 ; 0.90], \mathrm{p}<0.01)$ but not type 1 (RR, 0.73 [0.34;1.56], NS) diabetes. The estimated risk of daytime hypoglycaemia was significantly higher in BIAsp 30- than in BHI 30-treated subjects (RR, 1.23 [1.03; 1.46] $\mathrm{p}<0.05$ ). In each trial, results for nocturnal hypoglycaemia were consistent with these findings but heterogeneity was observed between trials for daytime hypoglycaemia.

Conclusion: Use of BIAsp 30 was associated with a reduced risk of major and nocturnal hypoglycaemia but increased risk of daytime minor hypoglycaemia compared with BHI 30 . The higher estimated risk for daytime hypoglycaemia with BIAsp 30 may be a consequence of the dose regimens employed in these trials that gave $70 \%$ of the total daily dose in the morning as opposed to currently recommended, more physiological, dose regimens for BIAsp 30 that provide $50 \%$ of the daily insulin dose in the morning due to its improved pharmacokinetics relative to $\mathrm{BHI} 30$.

Supported by Novo Nordisk A/S

\section{7}

Efficacy and safety of dietary counseling coupled with initiation of biphasic insulin aspart 30 in type 2 diabetes patients - The INITIATE plus Study

D. S. Oyer ${ }^{1}$, M. D. Shepherd ${ }^{2}$, F. C. Coulter ${ }^{3}$, A. Bhargava ${ }^{4}$, J. Brett ${ }^{5}$, P.-L. Chu ${ }^{5}$, B. S. Trippe ${ }^{6}$;

${ }^{1}$ Endocrinology, Associates in Internal Medicine, LTD., Chicago,

${ }^{2}$ Diabetes Treatment Center, North Mississippi Health Services, Tupelo, , ${ }^{3}$ Endocrinology, Coulter Clinic, Orangeburg, ${ }^{4}$ Endocrinology, Iowa Diabetes and Endocrinology Center, Des Moines, ${ }^{5}$ Novo Nordisk Inc., Princeton, ${ }^{6}$ Endocrinology, Endocrinology Associates of Montgomery, Montgomery, United States.

Background and Aims: The impact of nutritional counseling on glycaemic control and the risk of hypoglycaemia were studied in a 24-week, openlabel, Phase IV trial, conducted predominantly in the primary care setting (934 sites in the United States) in 4820 insulin-naive patients with poorly controlled T2DM.

Material and Methods: Patients initiated BID biphasic insulin aspart 30 (30\% soluble and $70 \%$ protaminated insulin aspart; BIAsp 30) with $6 \mathrm{U}$ prebreakfast and $6 \mathrm{U}$ pre-supper. Doses were titrated according to self-measured blood glucose (SMBG) values by the following algorithm: $-3 \mathrm{U}$ if $\mathrm{BG}<4.4$ $\mathrm{mmol} / \mathrm{L}$; no change if $\mathrm{BG}=4.4$ to $6.1 ;+3 \mathrm{U}$ if $\mathrm{BG}=6.2$ to $7.7 ;+6 \mathrm{U}$ if $\mathrm{BG}$ $=7.8$ to 10.0 ; and $+9 \mathrm{U}$ if $\mathrm{BG}>10.0 \mathrm{mmol} / \mathrm{L}$. The breakfast dose was titrated to pre-supper SMBG; evening dose was titrated to pre-breakfast SMBG. Patients were randomised (1:1:1) to 1 of 3 dietary counseling groups provided by a registered dietician: no dietary counseling (NC), 1 counseling session (1C), or 3 counseling sessions (3C).

Results: The mean baseline $\mathrm{HbA}_{1 \mathrm{c}}$ was $9.9 \%$, BMI was $34 \mathrm{~kg} / \mathrm{m}^{2}$, and age was 53.6 years. At the end of the study, mean $\mathrm{HbA}$ values decreased approximately $2.5 \%$ in each group to $7.49 \% \pm 1.48,7.48 \% \pm 1.50$, and $7.44 \% \pm 1.46$ in the NC, $1 \mathrm{C}$, and $3 \mathrm{C}$ groups, respectively. Within these respective groups, an end-ofstudy $\mathrm{HbA}_{1 \mathrm{c}}$ value $<7 \%$ was achieved by $40.2,41.6$ and $41.8 \%$ of patients; and a value $\leq 6.5 \%$ was achieved by $28.6,27.8$, and $28.3 \%$ of patients (between group differences not significant). Hypoglycaemia (major and minor) was experienced by a similar percentage of patients in each group $(10.2 \%$ to $11.4 \%)$. In general, the rates of minor and major hypoglycaemia were low but decreased as dietary counseling increased (minor hypoglycaemia: 0.56 vs. 0.50 vs. 0.45 episodes per pt-yr; major hypoglycaemia, 0.09 vs. 0.06 vs. 0.04 episodes per pt-yr, for the NC vs. $1 \mathrm{C}$ vs. 3C groups, respectively). The rates of major and minor hypoglycaemia in the $3 \mathrm{C}$ group were significantly less than those in the NC group. At the end of the study, all counseling groups had a weight increase $(3.13,3.40$, and $2.88 \mathrm{~kg}$ for the NC, $1 \mathrm{C}$, and $3 \mathrm{C}$ groups, respectively).

Conclusion: In the primary care setting, biphasic insulin aspart 30 was safe and effective in achieving recommended $\mathrm{HbA}_{1 \mathrm{c}}$ targets even with minimal dietary counseling.

Supported by Novo Nordisk

\section{8}

Comparison of the efficacy and safety of three different premixed combinations of biphasic insulin aspart - a randomised controlled clinical trial in subjects with type 2 diabetes

D. Cucinotta ${ }^{1}$, A. Liebl ${ }^{2}$, J. Sandahl Christiansen ${ }^{3}$, K. Kanc Hanzel ${ }^{4}$, C. Le Dèvèhat ${ }^{5}$;

${ }^{1}$ Policlinico Universitario, Dipartimento Malattie Metaboliche, Messina, Italy, ${ }^{2}$ Fachklinik Heilbrunn, Diabeteszentrum, Bad Heilbrunn, Germany, ${ }^{3}$ Department of Endocrinology, Aarhus University Hospital, Denmark, ${ }^{4}$ NovoNordisk A/S, Bagsvaerd, Denmark,

${ }^{5}$ URCHB, Hopital Pierre Beregovoy, France.

Background and Aims: Biphasic insulin aspart (BIAsp) is a premixed insulin analogue, containing soluble, rapid-acting insulin aspart (IAsp) and intermediate-acting protamine-bound IAsp. We compared the efficacy and safety of three different BIAsp formulations: BIAsp 30, BIAsp 50 and BIAsp 70 , the numbers denoting the percentage of the rapid-acting fraction, in a 36week three-arm, open-label treat-to-target study, with algorithm controlled insulin titration.

Materials and Methods: A total of 599 subjects currently treated with insulin and metformin with or without other oral antidiabetic drugs (OADs) were 
randomised 1:1:1 to treatment with BIAsp 30 twice daily (BID), BIAsp 50 three times daily (TID) or BIAsp 70 TID, all in combination with metformin. Insulin was injected immediately before the meals. All OADs except metformin were discontinued at randomisation. After 12 weeks of treatment subjects in the TID regimens with fasting plasma glucose (FPG) $>7.0 \mathrm{mmol} /$ $\mathrm{L}$ were switched to BIAsp 30 at dinner. This was needed for $~ 50 \%$ of the TID treated subjects. The changed regimen was maintained for the rest of the treatment (24 weeks). Primary endpoint was $\mathrm{HbA}_{1 \mathrm{c}}$ after 36 weeks.

Results: $\mathrm{HbA}_{1 \mathrm{c}}$ decreased between 1.5 and $1.8 \%$-points in the three arms. The study was designed to test for non-inferiority of the TID regimens versus BIAsp $30 \mathrm{BID}$ and to test for superiority if non-inferiority was shown. Both TID regimens were non-inferior and the BIAsp 50 regimen was superior to BIAsp 30 BID (difference BIAsp 50 BIAsp30: -0.3\%, 95\% CI [-0.47;-0.14] $\mathrm{p}=0.004$; difference BIAsp 70 BIAsp 30: $-0.07 \%$, CI $[-0.25 ; 0.10], \mathrm{p}=0.404)$. Mean PG decreased with all regimens and significantly more with the two TID regimens than with BIAsp 30 BID (difference BIAsp 50 BiAsp 30: -0.55 $\mathrm{mmol} / \mathrm{L}, \mathrm{p}=0.0001$; difference BIAsp 70 BIAsp 30: $-0.32 \mathrm{mmol} / \mathrm{L}, \mathrm{p}=0.03$ ). Also prandial PG increase was lower with both TID regimens (difference BIAsp 50 BIAsp 30: $-0.60 \mathrm{mmol} / \mathrm{L}, \mathrm{p}=0.0002$; difference BIAsp 70 BIAsp 30: $-0.69, \mathrm{p}<0.0001)$. Insulin dose increased significantly more with the TID regimens than with BIAsp 30. In general, the profile of adverse events was similar with all three regimens; most events were of mild or moderate severity and most were considered not related to treatment. The frequency of hypoglycaemic episodes was 15.8, 21.6, and 13.7 episodes/patient-year for BIAsp 50, BIAsp 70, and BIAsp 30 respectively (BIAsp 50 vs. 30 relative risk was $1.2, \mathrm{p}=0.16$; BIAsp 70 vs. 30 relative risk was $1.58, \mathrm{p}=0.0002$ ).

Conclusion: A TID regimen with BIAsp 50 provided a superior glycaemic control than BIAsp 30 BID without increasing the risk of hypoglycaemia; BIAsp 70 was non-inferior with respect to $\mathrm{HbA}_{1 \mathrm{c}}$ but the rate of hypoglycaemic episodes was higher.

Supported by NovoNordisk A/S

\section{9}

Efficacy and safety of BIAsp 30 (Biphasic insulin aspart 30) TID in combination with oral hypoglycaemic agents in non-obese patients with type 2 diabetes

S. S. Lund ${ }^{1}$, L. Tarnow ${ }^{1}$, B. B. Nielsen ${ }^{1}$, H.-H. Parving ${ }^{2}$, O. Pedersen ${ }^{1}$,

A. A. Vaag $^{1}$;

${ }^{1}$ Steno Diabetes Center, Gentofte,

${ }^{2}$ Dept of Medical Endocrinology, Rigshospitalet, Copenhagen, Denmark.

Background and Aims: To investigate the efficacy and safety of BIAsp 30 injected three times daily (TID), we performed a post-hoc analysis on the subset of patients that received BIAsp 30 TID at end of study of the 12-month REFORM study.

Materials and Methods: Patients inadequately controlled on OAD combination therapy were enrolled in REFORM $(n=101)$. At baseline patients were randomised to repaglinide $6 \mathrm{mg}$ /day or metformin $2 \mathrm{~g} /$ day (double-masked) both in combination with BIAsp 30. BIAsp 30 was initiated with $6 \mathrm{U}$ once-daily pre-dinner. Patients self-adjusted insulin dose according to an algorithm aiming for fasting plasma-glucose (FPG) of 4.0-6.0 mmol/1 and $\mathrm{HbA}_{1 \mathrm{c}}<6.5 \%$. Patients intensified to 2 or 3 daily injections after 3,6 or 9 months if target glycaemic goals were not reached, or earlier if hypoglycaemia prevented an increase in insulin dose. At end of trial, 42 patients (42\%) were receiving BIAsp 30 TID in combination with OAD.

Results: Baseline data for patients on BIAsp 30 TID at end of study were mean (SD) BMI $24.5(2.5) \mathrm{kg} / \mathrm{m}^{2}$, mean age 64.6 (7.4) years, and mean duration of disease 12.0 (6.4) years. The patients were $54.8 \%$ male, and $45.2 \%$ were insulin-naive. Mean $\mathrm{HbA}_{1 c}$ was $8.7(1.5) \%$ at baseline and $7.0(0.7) \%$ at the 12-month end-of-trial visit (difference from baseline, $-1.7 \%$ : $\mathrm{p}<0.0001$ ). In comparison, mean $\mathrm{HbA}$ for all patients who finished the REFORM study receiving BIAsp 30 dropped from 8.1 (1.4) \% at baseline to $6.8(0.7) \%$ at endof-trial visit. Among patients on BIAsp 30 TID at end of study, 11 (26.2\%) had met the glycaemic target of $\mathrm{HbA}_{1 c}<6.5 \%$ at end-of-trial visit. FPG and postprandial plasma glucose (PPG) levels after each meal were reduced. In the majority of patients, intensification from BID to TID occurred at the 6 or 9 month visit (7\% and 45\% of subjects used TID at 6 and 9 months, respectively). From 6 months to 12 months, mean $\mathrm{HbA}_{1 \mathrm{c}}$ improved by $0.4 \%$, from $7.4 \%$ to $7.0 \%$, and patients achieving $\mathrm{HbA}_{1 c}<6.5 \%$ increased from $7 \%$ to $26 \%$. The mean total daily insulin dose was $0.40(0.24) \mathrm{U} / \mathrm{kg}$ after 3 months and $0.59(0.30) \mathrm{U} / \mathrm{kg}$ at study end. The dose distribution was 0.20
(0.12) U/kg, $0.10(0.07) \mathrm{U} / \mathrm{kg}, 0.29(0.17) \mathrm{U} / \mathrm{kg}$, at breakfast, lunch and dinner, respectively. Six patients reported a total of 9 major hypoglycaemic episodes over 12 months, a rate of 0.2 major episodes/subject/year. Minor episodes were reported by 38 patients (561 episodes, 12.8 episodes/subject/year). Mean weight increased by $4.8 \mathrm{~kg}$ during the first 9 months of the study, but stabilised during the final 3 months.

Conclusions: For non-obese patients with T2DM and poor glycaemic control, initiation of BIAsp 30 in combination with OADs, and adherence to a strict dose-escalation algorithm over 12 months significantly reduces $\mathrm{HbA}_{1}$. Results suggest that adding a third injection of BIAsp 30 to a BID regimen was important in improving glycaemic control. At study end, more than a quarter of the cohort met the aggressive target for $\mathrm{HbA}_{1 c}$ of $<6.5 \%$, and the overall frequency of major hypoglycaemia was low, indicating that BIAsp 30 TID is both efficacious and well tolerated in this non-obese population. Supported by Novo Nordisk A/S

\section{0}

Thrice-daily biphasic insulin aspart $\mathbf{3 0}$ is more effective and as safe as a twice-daily regimen in subjects with type 2 diabetes inadequately controlled with OADs

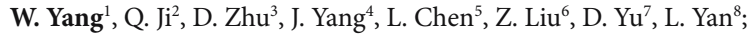
${ }^{1}$ Dept.Endocrinology, China-Japan Friendship Hospital, Beijing,

${ }^{2}$ Dept.Endocrinology, Xijing Hospital, Xían, ${ }^{3}$ Dept.Endocrinology, Nanjing Drum Tower Hospital, ${ }^{4}$ Dept.Endocrinology, Beijing Tongren Hospital, Beijing, ${ }^{5}$ Dept.Endocrinology, Wuhan Union Hospital, ${ }^{6}$ Dept.Endocrinology, Shanghai Changzheng Hospital, ${ }^{7}$ Dept.Endocrinology, Metabolic Disease Hospital, Tianjin, ${ }^{8}$ Dept.Endocrinology, Sun Yixian Commemorative Hospital, Guangzhou, China.

Background and Aims: Twice-daily (BID) treatment with biphasic insulin aspart (BIAsp 30) immediately before breakfast and dinner is a commonly used regimen to initiate insulin therapy in insulin naïve patients with type 2 diabetes inadequately controlled with oral antidiabetic drugs (OADs). Knowing the short duration of action of IAsp we hypothesised that adding a third injection (a TID regimen) could be more effective and as safe as a BID regimen. This trial was designed to evaluate the efficacy and safety of BIAsp 30 TID or BID in patients with type 2 diabetes.

Materials and Methods: In this 24-week randomised, open-labelled, parallelgroup, treat-to-target trial (pre-meal BG target: $4.4-6.1 \mathrm{mmol} / \mathrm{L}$ ), a total of 321 Chinese subjects with type 2 diabetes (FBG $\geq 7.8 \mathrm{mmol} / \mathrm{L} ; \mathrm{HbA}_{1 \mathrm{c}} \geq 7.5 \%$ ) were randomised 1:1 to receive BIAsp 30 BID or TID and with algorithmcontrolled insulin titration throughout the trial. Previous OAD treatments were discontinued at randomisation. Initially, daily dose distribution was 50$50 \%$ in the BID group and $25-25-50 \%$ in the TID group. Change in $\mathrm{HbA}_{1 \mathrm{c}}$, pre-meal blood glucose, hypoglycaemic episodes, insulin dose and weight were assessed.

Results: Demographic characteristics were comparable between the two groups and approximately half of the subjects in both groups were treated with more than two OADs at trial entry. Baseline $\mathrm{HbA}$ was $9.5 \%$. BIAsp 30 TID reduced $\mathrm{HbA}_{1 \mathrm{c}}$ significantly more after 24 weeks (primary endpoint) compared to BIAsp $30 \mathrm{BID}(2.8 \%$ versus $2.5 \%$, difference: $-0.33 \%, 95 \% \mathrm{CI}$ $[-0.527 ;-0.127], \mathrm{p}<0.01)$. A significantly better effect of the TID regimen was registered already after the first 12 weeks $(\mathrm{p}<0.01)$ and this was maintained until the end of treatment. Decrease in pre-breakfast self-measured BG was significantly lower with the TID regimen $(-3.59 \mathrm{mmol} / \mathrm{L})$ than the $\mathrm{BID}$ regimen $(-3.24 \mathrm{mmol} / \mathrm{L}) \quad$ (difference:-0.35mmol/L,95\%CI[-0.604;-0.098], $\mathrm{p}<0.01$ ). Pre-dinner self-measured BG decreased more in the TID group $(-4.75 \mathrm{mmol} /$ $\mathrm{L})$ than in BID group $(-3.84 \mathrm{mmol} / \mathrm{L}$, difference: $-0.91 \mathrm{mmol} / \mathrm{L}, 95 \% \mathrm{CI}[-$ $1.262 ;-0.560], \mathrm{p}<0.01)$. No difference was observed for pre-lunch BG between the two groups. Most hypoglycaemic episodes were reported in the first 12week treatment period. No significant difference in rates of major \& minor (relative risk=0.75, $\mathrm{p}=0.32$ ) and nocturnal (relative risk $=0.81, \mathrm{p}=0.51$ ) hypoglycaemic episodes per patient year between the treatment groups was reported. No significant difference in adverse events, weight gain and mean daily insulin doses between two groups were observed.

Conclusion: In subjects with type 2 diabetics, both twice-daily and thricedaily BIAsp 30 were efficacious and safe after 24 weeks of treatment. BIAsp 30 TID offered a better long-term glycaemic control than BIAsp 30 BID, without an increased rate of hypoglycaemia. 


\section{1}

Advancing insulin therapy in type 2 diabetes previously treated with glargine plus oral agents: prandial premixed (Lispro/ILPS) vs basal/bolus (Glargine/Lispro) therapy

A. J. Ahmann ${ }^{1}$, J. Rosenstock ${ }^{2}$, G. Colon ${ }^{3}$, J. Scism-Bacon ${ }^{4}$, H. H. Jiang ${ }^{4}$, S. Martin ${ }^{4}$;

${ }^{1}$ Diabetes and Clinical Nutrition, OHSU, Portland, ${ }^{2}$ Dallas Diabetes and Endocrine Center, Dallas, ${ }^{3}$ The San Juan Health Centre, San Juan, Puerto Rico, ${ }^{4}$ Eli Lilly and Company, Indianapolis, United States.

Background and Aims: Initiation of insulin therapy with a single bedtime injection of intermediate-acting insulin, or a bedtime or morning injection of long-acting insulin is a common approach to insulin initiation. Further treatment advancement is recommended via the addition of mealtime insulin injections, such that basal/bolus therapy is eventually achieved. Premixed insulin is a convenient alternative if the proportion of rapid and basal insulin used is similar to fixed proportions available. However, there is a lack of clinical trial data providing head-to-head comparisons of prandial premixed therapy (PPT) to analog basal bolus therapy (BBT), thus limiting the ability to clearly recommend one insulin regimen over the other. Therefore, we conducted a head-to-head comparison of two insulin therapies (PPT vs. BBT) in T2D patients previously treated with insulin glargine $(\geq 30 \mathrm{U} / \mathrm{d})+$ oral agents, with the aim of demonstrating non-inferiority of PPT to BBT.

Materials and Methods: Patients with $\mathrm{A} 1 \mathrm{C} \geq 7.5$ and $\leq 12 \%$ were randomised to PPT (lispro mix 50; 50\% lispro, 50\% ILPS: insulin lispro protamine suspension; $\mathrm{n}=187$ ) tid with meals or BBT (glargine at bedtime + mealtime lispro; $\mathrm{n}=187$ ) in a 24 -wk, multicenter, open-label, non-inferiority trial using structured insulin titration to achieve preprandial plasma glucose (PG) values $<110 \mathrm{mg} / \mathrm{dL}(<6.1 \mathrm{mM})$. Patients on PPT not reaching fasting PG target could switch to lispro mix 25 (25\% lispro, 75\% ILPS) at the evening meal.

Results: Baseline characteristics were similar (mean): age 55yrs, diabetes duration $11 \mathrm{yrs}$, BMI $34 \mathrm{~kg} / \mathrm{m}^{2}$ and A1C (Table). At wk 24, A1C was lower with BBT than PPT, but A1C was reduced significantly from baseline for both therapies $(\mathrm{P}<0.0001)$, resulting in final $\mathrm{A} 1 \mathrm{C}<7.0 \%$ for both insulin regimens. The difference in AlC change from baseline to endpoint (BBT minus PPT) was $-0.22 \%$ with a $90 \%$ CI from $-0.38 \%$ to $-0.07 \%$. Non-inferiority was not demonstrated based on the pre-specified margin of $0.3 \%$. The $\%$ of patients achieving target $\mathrm{A} 1 \mathrm{C}$ values $<7.0 \%$ and $\leq 6.5 \%$ was greater for BBT than PPT (Table), but did not differ for target $\leq 6.0 \%$ ( $14.3 \%$ vs. $14.6 \%, \mathrm{P}=1.0$ ) or for target $<7.5 \%$ ( $83 \%$ vs. $81 \%, \mathrm{P}=0.76)$. Treatment with $\mathrm{BBT}$ was associated with higher insulin dose, however both groups had similar weight gain (PPT: $3.98 \mathrm{~kg}$ vs. BBT: $4.55 \mathrm{~kg}, \mathrm{P}=0.22$ ). Rates of overall, nocturnal, severe, or hypoglycemia confirmed by PG values were also similar.
Conclusion: In conclusion, as non-inferiority of PPT to BBT was not demonstrated, findings on A1C reduction, \% achieving specific A1C targets, hypoglycemia, insulin dosing complexity, and number of injections need to be considered in the individual decision-making process of selecting PPT vs BBT in T2D necessitating advancement of insulin therapy.

\begin{tabular}{llll} 
& PPT & BBT & P-value \\
\hline A1C (\%), baseline & $8.8 \pm 1.0$ & $8.9 \pm 1.1$ & 0.6041 \\
A1C (\%), endpoint & $6.9 \pm 0.1$ & $6.8 \pm 0.1$ & 0.0208 \\
A1C (\%), change from baseline & $-1.87 \pm 0.07$ & $-2.09 \pm 0.07$ & 0.0208 \\
A1C $<7.0 \%(\mathrm{n}[\%])$ & $81[54]$ & $101[69]$ & 0.0075 \\
A1C $\leq 6.5 \%(\mathrm{n}[\%])$ & $53[35]$ & $74[50]$ & 0.0072 \\
Daily insulin dose (U/kg) & $1.17 \pm 0.54$ & $1.38 \pm 0.79$ & 0.0019 \\
Overall hypoglycemia & $51.2 \pm 50.1$ & $48.7 \pm 48.4$ & 0.6189 \\
(episodes/patient/year) & & & \\
Nocturnal hypoglycemia & $4.8 \pm 7.2$ & $6.2 \pm 10.7$ & 0.1390 \\
Severe hypoglycemia (n[\%]) & $6[3.21]$ & $4[2.14]$ & 0.7506 \\
\hline
\end{tabular}

Supported by Eli Lilly and Company

\section{2}

Efficacy of combining insulin glulisine with basal insulin glargine in patients with type 1 and type 2 diabetes previously on premix insulin therapy

S. Schreiber ${ }^{1}$, K. Ferlinz ${ }^{2}$, B. Donaubauer ${ }^{3}$;

${ }^{1}$ Diabetes Zentrum Schreiber, Quickborn, ${ }^{2}$ sanofi-aventis Deutschland

$\mathrm{GmbH}$, Berlin, ${ }^{3}$ Schwerpunktpraxis Diabetes, Oschatz, Germany.

Background and Aims: A 12-week, observational, cohort study - LADI (Lantus and ApiDra in an Intensified insulin [INS] regimen) - assessed the efficacy of introducing INS glargine (GLAR) as basal INS and INS glulisine (GLU) as the prandial INS therapy in patients (pts) with type 1 diabetes (T1DM; $\mathrm{n}=2325$ ) and type 2 diabetes (T2DM, $\mathrm{n}=9001$ ), poorly controlled on their previous INS therapy. A sub-analysis of pts previously on premix INS was conducted.

Materials and Methods: Both T1DM $(n=123)$ and T2DM $(n=1674)$ pts previously on premixed INS were included in this sub-analysis. The majority of pts received regular INS premixes (90.5\%) and a minority were on premixed analogues $(9.5 \%)$. A small percentage of pts on premix INS were

Tab. 1

\begin{tabular}{|c|c|c|c|c|c|}
\hline & $\begin{array}{l}\text { FBG (mmol/L) } \\
{[\mathrm{mg} / \mathrm{dL}]}\end{array}$ & $\begin{array}{l}\text { lh-ppBG }(\mathrm{mmol} / \mathrm{L}) \\
{[\mathrm{mg} / \mathrm{dL}]}\end{array}$ & $\begin{array}{l}\text { 2h-ppBG }(\mathrm{mmol} / \mathrm{L}) \\
{[\mathrm{mg} / \mathrm{dL}]}\end{array}$ & $\begin{array}{l}\text { Total daily glargine } \\
\text { dose }(\mathrm{U})\end{array}$ & $\begin{array}{l}\text { Total daily glulisine } \\
\text { dose (U) }\end{array}$ \\
\hline \multicolumn{6}{|l|}{ T1DM } \\
\hline Study start & $\begin{array}{l}9.7 \pm 2.9 \\
{[174.0 \pm 52.8]} \\
\mathrm{n}=99\end{array}$ & $\begin{array}{l}11.3 \pm 2.9 \\
{[203.3 \pm 52.0]} \\
\mathrm{n}=29\end{array}$ & $\begin{array}{l}10.7 \pm 2.9 \\
{[192.9 \pm 51.4]} \\
\mathrm{n}=55\end{array}$ & - & - \\
\hline 12 weeks & $\begin{array}{l}6.5 \pm 1.5 \\
{[116.6 \pm 27.8]} \\
n=100\end{array}$ & $\begin{array}{l}7.3 \pm 1.6 \\
{[131.3 \pm 28.7]} \\
n=27\end{array}$ & $\begin{array}{l}7.7 \pm 1.7 \\
{[138.2 \pm 29.7]} \\
n=49\end{array}$ & $\begin{array}{l}21.8 \pm 9.1 \\
\mathrm{n}=115\end{array}$ & $\begin{array}{l}28.5 \pm 13.4 \\
\mathrm{n}=97\end{array}$ \\
\hline Pre-post difference & $\begin{array}{l}-3.1 \pm 2.9 \\
{[-56.2 \pm 51.5]} \\
n=97\end{array}$ & $\begin{array}{l}-3.2 \pm 3.2 \\
{[-57.8 \pm 57.9]} \\
n=26\end{array}$ & $\begin{array}{l}-3.4 \pm 2.7 \\
{[-61.0 \pm 49.3]} \\
n=50\end{array}$ & $\begin{array}{l}+2.1 \pm 4.6 \\
\mathrm{n}=116\end{array}$ & $\begin{array}{l}+3.2 \pm 9.0 \\
\mathrm{n}=95\end{array}$ \\
\hline \multicolumn{6}{|l|}{ T2DM } \\
\hline Study start & $\begin{array}{l}9.9 \pm 2.2 \\
{[178.7 \pm 40.3]} \\
n=1457\end{array}$ & $\begin{array}{l}12.2 \pm 2.2 \\
{[220.4 \pm 40.1]} \\
\mathrm{n}=510\end{array}$ & $\begin{array}{l}11.7 \pm 2.3 \\
{[210.5 \pm 42.1]} \\
\mathrm{n}=635\end{array}$ & - & - \\
\hline 12 weeks & $\begin{array}{l}6.8 \pm 1.4 \\
{[122.2 \pm 24.6]} \\
n=1443\end{array}$ & $\begin{array}{l}8.4 \pm 1.6 \\
{[150.7 \pm 29.4]} \\
n=476\end{array}$ & $\begin{array}{l}8.0 \pm 1.6 \\
{[144.1 \pm 29.0]} \\
n=615\end{array}$ & $\begin{array}{l}22.8 \pm 11.6 \\
\mathrm{n}=1506\end{array}$ & $\begin{array}{l}38.6 \pm 18.7 \\
n=1502\end{array}$ \\
\hline Pre-post difference & $\begin{array}{l}-3.2 \pm 2.2 \\
{[-57.7 \pm 40.2]} \\
\mathrm{n}=1426\end{array}$ & $\begin{array}{l}-3.9 \pm 2.3 \\
{[-70.2 \pm 41.6]} \\
\mathrm{n}=441\end{array}$ & $\begin{array}{l}-3.9 \pm 2.5 \\
{[-70.5 \pm 45.0]} \\
\mathrm{n}=541\end{array}$ & $\begin{array}{l}+3.3 \pm 6.8 \\
\mathrm{n}=1587\end{array}$ & $\begin{array}{l}+6.2 \pm 10.5 \\
\mathrm{n}=1570\end{array}$ \\
\hline
\end{tabular}

1h-ppBG=1-hour postprandial (breakfast) blood glucose; $2 \mathrm{~h}-\mathrm{ppBG}=2$-hour postprandial (breakfast) blood glucose; FBG=fasting blood glucose (breakfast)

Data are mean \pm SD 
also taking basal (3.5\%) or bolus (6.8\%) INS. At study start, all pts changed from their previous premix therapy and placed onto a basal-bolus regimen with 1-3 daily injections of GLU as the prandial INS and once-daily INS glargine (GLAR) as the basal INS. All treatment decisions were made by the physician. At study start $>97 \%$ of pts were on GLAR.

Results: Data are T1DM and T2DM, respectively. Demographics at study start $($ mean $\pm \mathrm{SD})$ were: age $=47.5 \pm 14.5$ and $63.8 \pm 10.0$ years; body mass index $=26.1 \pm 3.9$ and $30.7 \pm 5.3 \mathrm{~kg} / \mathrm{m}^{2}$; diabetes duration $=15.0 \pm 12.8$ and $10.8 \pm 7.1$ years. $\mathrm{HbA}_{1 \mathrm{c}}$ improved significantly (study start vs. 12 weeks) in the T1DM ( $8.7 \pm 1.4$ vs. $7.2 \pm 0.8, \mathrm{n}=116 ; \mathrm{p}<0.0001$ Wilcoxon signed rank test) and T2DM ( $8.6 \pm 1.2$ vs. $7.3 \pm 0.9, \mathrm{n}=1470 ; \mathrm{p}<0.0001)$. Other blood glucose parameters improved after 12 weeks for both groups (Table). At previous therapy, the total daily dose of INS (including premix, basal and bolus INS) was similar for T1DM (46.8 U) and T2DM (48.5 U) pts. There were small increases in GLAR and GLU dose after 12 weeks in both groups (Table). At study end, the majority of pts took GLU three times daily in the T1DM ( $82 \%$, $\mathrm{n}=101)$ and T2DM $(88 \%, \mathrm{n}=1466)$ groups. Body weight was stable during the study for T1DM $(-0.5 \pm 3.1 \mathrm{~kg})$ and T2DM $(-0.8 \pm 4.8 \mathrm{~kg})$ pts. Treatment satisfaction (TS) was assessed by the Diabetes Treatment Satisfaction Questionnaire (maximum 36=most satisfied). The TS scores were improved (study start vs. last available questionnaire) in T1DM (17.2 \pm 9.2 vs. $30.9 \pm 4.2$, $\mathrm{p}<0.0001$; Wilcoxon signed rank test) and T2DM (17.6 \pm 7.2 vs. 29.3 \pm 5.3 , $\mathrm{p}<0.0001)$ pts. There was no specific question for recording hypoglycaemia on the documentation forms (only adverse events [AEs] were noted) and thus hypoglycaemia was under-reported (a total of 27 pts reported $43 \mathrm{AEs}$; of which 10 were hypoglycaemia).

Conclusions: Switching from a premix INS therapy to an intensive basalbolus INS regimen, with GLU as the prandial INS and GLAR as the main basal INS, is accompanied by improvements in $\mathrm{HbA}_{1 c}$ and blood glucose control in T1DM and T2DM pts in everyday clinical practice. (Tab. 1) Supported by sanofi-aventis

\section{PS 97 Insulin therapy in routine practice and special situations}

\section{3}

A comparison of analogue and human short acting insulin for the treatment of type 1 and type 2 diabetes in UK general practice A. J. Tynan ${ }^{1}$, C. J. Currie ${ }^{2}$, A. Simpson ${ }^{1}$, C. D. Poole;

${ }^{1}$ Eli Lilly and Company Limited, Windlesham, United Kingdom, ${ }^{2}$ Department of Medicine, Cardiff University, United Kingdom, ${ }^{3}$ Healthcare Research, $360^{\circ}$ Research, Penarth, United Kingdom.

Background: There exists two general classes of short acting insulins (SAI) for the treatment of people with types 1 and 2 diabetes (T1DM and T2DM, respectively), these are the analogue insulins (AI) and the soluble regular human insulins (RHI). The purpose of this study was to compare outcome using these diabetes regimens using routinely available data from general practice.

Methods: Data were extracted from the GPRD, that describes the primary care of around 7\% of the UK population from 1990 to 2007. Subjects were selected if treated with an SAI. GPRD records detail a number of experiences including: diagnoses, treatments, biochemistry/other tests, blood pressure, height and weight, and mortality. Comparisons were made using a variety of appropriate statistical tests to compare these classes of treatment for typical diabetes-related outcomes over the first year (by quarterly period) following switching to SAI. Patients who had switched between alternative SAI regimens were excluded for homogeneity.

Results: In total 4400 cases with T1DM and 2582 cases with T2DM met the selection criteria. The patient characteristics in each group with T1DM were as follows for AI and RHI, respectively: age, 35.0 years vs. $39.1(\mathrm{p}<0.001)$; percent female, $45.1 \%$ vs. $44.8 \%$ ( $\mathrm{p}=0.844$ ); mean HbAlc in the Quarter prior to switching, $9.0 \%$ vs. $8.7 \%$ ( $\mathrm{p}=0.015)$; mean time from first observation to SAI switch, 7.1 years vs. $6.8(\mathrm{p}=0.088)$; and percent with a history of macrovascular disease, $5.1 \%$ vs. 8.9 (RR 1.73, p<0.001). For T2DM and for $\mathrm{AI}$ and RHI, respectively: age, 55.3 years vs. $58.4(\mathrm{p}<0.001)$; percent female, $43.7 \%$ vs. $49.4 \%$ ( $\mathrm{p}=0.008)$; mean HbAlc in the Quarter prior to switching, $9.3 \%$ vs. $9.2 \%$ ( $p=0.40$ ); mean time from first observation to SAI switch, 8.8 years vs. $8.0(\mathrm{p}<0.001)$; and percent with a history of macrovascular disease, $23.3 \%$ vs.34.1\% (RR 1.48, $\mathrm{p}<0.001$ ). Both alternative regimens showed improvement in HbAlc in the year post switching (table) and in T1DM subjects, after standardising for age, prior $\mathrm{HbAlc}$, and macrovascular disease history, subjects treated with AI showed consistently greater reduction in $\mathrm{HbA1c}$ from baseline than those treated with RHI (Q1, $-0.18 \%[\mathrm{p}=0.005]$; Q2, $-0.15 \%$ [ $\mathrm{p}=0.059$ ]; $\mathrm{Q} 3,-0.17 \%$ [ $\mathrm{p}=0.072$ ]; $\mathrm{Q} 4,-0.12 \%$ [p=0.274]). Increases in weight were similar between people treated with either AI or RHI (table). The risk ratio (RR) for glycaemic coma was notably higher in cases using RHIs compared to AI (T1DM, RR=1.64, $\mathrm{p}=0.007$; T2DM, $\mathrm{RR}=2.12 ; \mathrm{p}=0.06$ ). Conclusion: SAI regimens were effective in improving control. In T1DM, AI resulted in consistently better improvement in glycaemic control than did RHI over the year following switching, with similar weight gain. These data suggest that the incidence of glycaemic coma was reduced using AI regimens.

TABLE. Glucose control, weight gain, glycaemia-related coma following switching to a SAI regimen.

\begin{tabular}{lllllll}
\hline & & \multicolumn{3}{c}{ T1DM } & & T2DM \\
\cline { 3 - 4 } \cline { 6 - 7 } & & AI & RHI & & AI & RHI \\
\hline Number (n) & & 3591 & 1809 & & 1892 & 690 \\
Mean diff HbA1c (\%) & Q1 & -0.27 & -0.12 & & -0.39 & -0.42 \\
& Q2 & -0.35 & -0.22 & & -0.59 & -0.66 \\
& Q3 & -0.37 & -0.27 & & -0.61 & -0.68 \\
& Q4 & -0.34 & -0.26 & & -0.64 & -0.76 \\
Mean diff weight (Kg) & Q1 & 0.53 & 0.53 & & 0.80 & 1.00 \\
& Q2 & 0.86 & 0.71 & & 1.62 & 1.62 \\
& Q3 & 1.24 & 1.05 & 2.10 & 2.17 \\
& Q4 & 1.30 & 1.56 & 2.30 & 2.33 \\
Glycaemic coma & & 1.33 & 3.70 & 0.53 & 2.04 \\
(reports/1000 pt-years) & & & & & \\
\hline
\end{tabular}

Supported by Eli Lilly and Company Limited 


\section{4}

Near normoglycaemic control under supplementary insulin therapy in type 2 diabetes with insulin aspart and insulin detemir - is a fixed dose regimen as effective as a flexible therapy regimen?

K. Milek ${ }^{1}$, E. Wizemann'2 S. Martin ${ }^{3}$, K. Böhme ${ }^{4}$, T. Rendschmidt'; ${ }^{1}$ Diabetologist practice, Hohenmölsen, ${ }^{2}$ Diabetologist practice, Herrenberg, ${ }^{3}$ Krankenhaus Gerresheim, Duesseldorf, ${ }^{4}$ Novo Nordisk Pharma GmbH, Mainz, Germany.

Background and Aims: Prandial insulin therapy with short acting insulin is known to have a high potential of effective $\mathrm{HbA}_{1 c}$ reduction in type 2 diabetes. This study compares two clinical concepts of insulin supplementation both common in Germany: A type 1 derived flexible therapy regimen with extended patient education, daily blood glucose profiles, carbohydrate count and patient driven insulin dose adjustment (FLEX) vs. a simple fixed dose regimen with rare blood glucose monitoring up to two profiles per week (FIX). Main endpoints are efficacy in terms of blood glucose control, safety and quality of life.

Materials and Methods: In this 52 weeks open labelled, randomised multicentre (61 diabetologists across Germany) parallel group trial 373 patients with insufficiently controlled type 2 diabetes previously treated with OAD, long acting or premixed insulin or a combination of those were randomised to either FIX or FLEX with insulin aspart at mealtimes. Patient characteristics (FIX vs. FLEX): $54 \%$ vs. $57 \%$ males, age $63.4 \pm 9.2$ vs. $61.8 \pm 8.7$ years, BMI $31.6 \pm 4.5$ vs. $31.4 \pm 4.6 \mathrm{~kg} / \mathrm{m}^{2}$, diabetes duration both $10.1 \pm 7.0$ years; $\mathrm{HbA}_{1 \mathrm{c}}$ $8.20 \pm 0.81$ vs. $8.24 \pm 0.95 \%$ (all ns). In both regimen insulin detemir and metformin could be added. Efficacy parameters were assessed at baseline and after 12, 26, 39 and 52 weeks, quality of life by DTSQ and WHO- 5 at baseline and after 26 and 52 weeks. Safety assessment was performed continuously. Results: Primary objective $\mathrm{HbA}_{1 c}$ showed a significant difference of $0.25 \%$ in ITT group (FIX 6.97 \pm 0.066 ; FLEX 6.72 $\pm 0.066 ; \mathrm{p}=0.0074$ ) and $0.21 \%$ in PP group (FIX 6.81 \pm 0.063 ; FLEX $6.60 \pm 0.061 ; p=0.0191$ ). However, only ANCOVA of the PP group showed a non-inferiority of FIX vs. FLEX (mean difference $0.207 \%, 95 \% \mathrm{CI}[0.034,0.379]$ ) based on the assumption of $0.4 \%$ $\mathrm{HbA}_{1 \mathrm{c}}$ difference as clinically relevant. $61.1 \%$ of the FIX and $69.2 \%$ (ns) of the FLEX group reached $\mathrm{HbA} \leq 7.0 \%$. Postprandial blood glucose control did not differ on a near normoglycaemic level. Education was longer in FLEX $(11.40 \pm 6.29$ vs. FIX $6.70 \pm 4.14$ hours; $p<0.0001)$. Mean total insulin aspart dose was FIX $48.6 \pm 27.9$ vs. FLEX $45.2 \pm 34.4$ units (ns). Insulin detemir once daily at dinner or bedtime was added in FIX $78 \%$ vs. FLEX $75 \%$ (ns) of the patients. Mean total insulin detemir dose was FIX $27.1 \pm 16.4$ vs. FLEX $25.4 \pm 17.2$ units (ns). No difference in the incidence of total hypoglycaemic events (FIX 0.08 vs. FLEX 0.106 events per subject week; $p=0.3073$ ) could be observed. Only one case of severe hypoglycaemia occurred in each treatment group. No significant difference or change in quality of life could be detected.

Conclusion: Supplementary insulin therapy reaches safely $\mathrm{HbA}_{1 c}$ targets $\leq 7.0 \%$ in previously uncontrolled type 2 diabetics under conditions close to daily practice. A flexible regimen is associated with more resources in terms of education, therapy monitoring and patient compliance. However, it offers a significant advantage in $\mathrm{HbA}_{1 \mathrm{c}}$ reduction over a fixed dose regimen. Preprandial administration of insulin aspart ensures an effective control of postprandial glucose regulation in both study arms.

Supported by Novo Nordisk

\section{5}

More subjects achieve an $\mathrm{HbA}_{1 \mathrm{c}} \leq 7 \%$ without hypoglycaemia or weight gain when treated with insulin detemir in comparison with NPH insulin K. Hermansen ${ }^{1}$, J. Larsen², S. C. Tamer², C. Mathieu;

${ }^{1}$ Endocrinology and Metabolism, Aarhus University Hospital, Denmark, ${ }^{2}$ Novo Nordisk A/S, Bagsvaerd, Denmark,

${ }^{3}$ Medical Department, Katholieke Universiteit, Leuven, Belgium.

Background and Aims: To avoid macro- and micro-vascular complications, type 2 diabetes patients are continuously encouraged to aim for as normal a $\mathrm{HbA}_{1 \mathrm{c}}$ level as possible. As the disease progresses, most patients need insulin treatment to achieve good glycaemic control. However, the most important barriers to the initiation of insulin therapy are hypoglycaemia and weight gain, which may also discourage compliance once insulin treatment has been started. It is therefore most important to identify insulin regimens that can limit such harmful effects, whilst ensuring good glycaemic control. The aim of this study was to investigate whether the $\mathrm{HbA}_{1 \mathrm{c}}$ improvement with detemir could be obtained without the compromise of having a high risk of hypoglycaemia and weight gain.

Materials and Methods: This explorative analysis was based on a multicentre, open-labelled, parallel-group, randomised trial involving 475 insulin-naive subjects with type 2 diabetes treated with detemir (237) or NPH (238) for 24 weeks. Detemir or NPH was added to oral antidiabetic drugs and administered twice daily using a treat-to-target regimen. Baseline $\mathrm{HbA}_{1}$ was $8.6 \%$ and $8.5 \%$ with detemir and NPH, respectively. Baseline weight was 82.7 and $82.5 \mathrm{~kg}$. Responders were defined as subjects treated $\geq 12$ weeks who achieved an $\mathrm{HbA}_{1 c} \leq 7 \%$, without any confirmed hypoglycaemia within the last 3 months of treatment ( $\mathrm{PG}<3.1 \mathrm{mmol} / \mathrm{L} \pm$ symptoms or symptomatic episode with $\mathrm{PG}<4.0 \mathrm{mmol} / \mathrm{L}$ ) and a weight gain of no more than $2 \%$ of baseline value. A comparison of the treatments using Fishers exact test was made with respect to responders.

Results: Excellent glycaemic control, with an $\mathrm{HbA}_{1 \mathrm{c}}$ of around $6.6 \%$, was achieved in both groups at end of the trial. The risk of hypoglycaemia was $47 \%$ lower with detemir than with NPH and body weight gain was less with detemir in comparison with NPH (1.2 versus $2.8 \mathrm{~kg}, \mathrm{p}<0.001)$. However, a significantly larger proportion of subjects treated with detemir $(23 \%)$ than with $\mathrm{NPH}(15 \%)$ achieved an $\mathrm{HbA}_{1 c} \leq 7 \%$ without confirmed hypoglycaemia, and with less than $2 \%$ gain in weight, $(\mathrm{p}=0.024)$.

Conclusion: This explorative analysis showed that more subjects achieved good glycaemic control without unwanted side-effects by adding insulin detemir rather than NPH insulin to oral agents. It remains to be investigated what characterises these subjects are and what it takes to increase the proportion of responders.

Supported by Novo Nordisk A/S

\section{6}

Non-glycaemic effects of insulin-therapy: a comparison between insulin aspart and normal insulin during two consecutive meals in patients with type 2 diabetes

A. DeCampo ${ }^{1}$, I. Schmoelzer ${ }^{1}$, E. Kettler-Schmut ${ }^{1}$, H. Sourij ${ }^{1}$, T. Forst ${ }^{2}$, T. C. Wascher';

${ }^{1}$ Metabolism and Vascular Biology Research Group, Medical University, Graz, Austria,

${ }^{2}$ Institute for Clinical Research and Development, Mainz, Germany.

Background and Aims: To control postprandial hyperglycemia in insulintreated type 2 diabetic patients prandial therapy with normal insulin or short acting insulin-analogues is used. Postprandial hyperglycemia is reduced more effectively with insulin analogues, but there are no data concerning the effect on lipolysis or pancreatic insulin- and proinsulin-secretion of normal insulin in comparison to insulin analogues. The aim of our study was, thus, to compare prandial insulin therapy with normal insulin or insulin aspart regarding the effectiveness on postprandial lipolysis and secretion of insulin and proinsulin in a "near-physiological" situation of two consecutive meals (breakfast and lunch) in type 2 diabetic patients.

Materials and Methods: 14 patients with type 2 diabetes mellitus (7 men; age $62.2 \pm 2.7$ years) with pre-existing insulin therapy were included in this prospective, open, randomised study. Examinations were performed after a fasting period of at least 12 hours on two different occasions. Each participant was treated either with normal insulin or insulin aspart in the setting of a standardised breakfast ( $634 \mathrm{kcal} ; 100 \mathrm{~g} \mathrm{KH}, 35 \mathrm{~g}$ fat, $13.6 \mathrm{~g}$ protein) with 1.8 $\mathrm{IE} / 12 \mathrm{~g} \mathrm{KH}$ and 4 hours later a standardised lunch (608 kcal; $84 \mathrm{~g} \mathrm{KH}, 21 \mathrm{~g}$ fat, $21.5 \mathrm{~g}$ protein) with $1.5 \mathrm{IE} / 12 \mathrm{~g} \mathrm{KH}$. The second test meal was performed 3 days later. Blood samples for determination of free fatty acids (FFA), cpeptide $(\mathrm{cP})$ and specific proinsulin (PI) were drawn fasting and every 30 minutes until 4 hours after the second test meal. Statistical analysis was done with ANOVA for repeated measurements and paired student's t-test.

Results: Both therapies reduced the total amount of FFA after the consecutive meals but mean reduction of FFA was significantly higher with insulin aspart in comparison to normal insulin (0.47/0.04 vs. $0.35 / 0.04 \mu \mathrm{mol} / \mathrm{l}, \mathrm{p}<0.001)$. This effect was seen after breakfast $(p=0.004)$ and after lunch $(p=0.001)$. Increase of $\mathrm{cP}$, as a marker of insulin-secretion, was significantly lower after insulin aspart $(1.34 / 0.17$ vs. $2.3 / 0.25 \mathrm{ng} / \mathrm{ml}, \mathrm{p}<0.001)$ than after normal insulin. The mean increase of PI was significantly lower after insulin aspart than normal insulin (12.3/1.4 vs. $16.7 / 2.3 \mathrm{pmol} / \mathrm{l}, \mathrm{p}=0.002)$. This effect was more pronounced after lunch $((\mathrm{p}<0.001)$ than after breakfast $(\mathrm{ns}, \mathrm{p}=0.152)$. 
Conclusion: In the setting of two consecutive meals insulin aspart on the one hand reduces lipolysis more effective than normal insulin, on the other hand pancreatic secretion of insulin is reduced and therefore also the secretion of potentially proatherogen proinsulin. These effects of insulin aspart should be further investigated concerning their importance for insulin resistance and atherogenesis in type 2 diabetics.

Supported by Novo Nordisk

\section{7}

Factors predicting long partial remission of new onset type 1 diabetes S. Pilacinski, D. Zozulinska, P. Uruski, A. Sporna, A. Uruska,

P. Niedzwiecki, M. Kasprzak, B. Wierusz-Wysocka;

Department of Internal Medicine and Diabetology,

Poznan University of Medical Sciences, Poland.

Background and Aims: Factors predicting prolonged clinical remission of type 1 diabetes are not definitely identified. Studies have demonstrated that low initial insulin requirement is a predictor of entering remission, but its usefulness in predicting long remission is not established. Therefore, the aim of this study was to assess the value of insulin dose in the beginning of diabetes in predicting length of partial remission of the disease.

Materials and Methods: In this cohort study we followed prospectively 104 patients (47 women and 57 men, aged 16-34, mean age $23 \pm 4$ ), admitted to the Department with newly diagnosed type 1 diabetes and then attending the outpatient clinic. Mean hospitalisation time was $7 \pm 2$ days. 16 patients presented with diabetic ketoacidosis (DKA), mean glycaemia at diagnosis was $24.1 \pm 8.9 \mathrm{mmol} / \mathrm{l}, \mathrm{HbAlc} 11.5 \pm 2.5 \%$ and BMI $21.3 \pm 3.2 \mathrm{~kg} / \mathrm{m}^{2}$. In all patients intensive functional insulin therapy in multiple injections was introduced. Partial remission was defined as an insulin dose $\leq 0.3 \mathrm{U} / \mathrm{kg} / 24 \mathrm{~h}$ and an $\mathrm{HbA1c}$ value $<7 \%$ with baseline serum C-peptide concentration over $0.5 \mu \mathrm{g} / \mathrm{l}$. Length of remission was counted from the onset of disease. Statistical analysis was performend using Statistica 5.1 software. Mann-Whitney U test, Fisher's exact test and multivariate logistic regression were used.

Results: Fifty-four patients remained in partial remission after one year of observation, in 28 patients partial remission ended before 12 months of disease and 22 patients did not enter remission. Group with long remission did not differ from the remaining patients (with no or short remission) in age, gender, presence of DKA at diagnosis, initial: glycaemia, $\mathrm{HbAlc}$, serum TC, LDL-C and HDL-C and serum C-reactive protein (hsCRP) concentration. Daily insulin dose $(\mathrm{U} / \mathrm{kg} / 24 \mathrm{~h})$ at the end of initial hospitalization was significantly lower in patients with remission exceeding 1 year $(0.22 \pm 0.14$ vs. $0.32 \pm 0.13 \mathrm{U} / \mathrm{kg} / 24 \mathrm{~h}, \mathrm{p}<0.0001)$. Significantly lower mean fasting and postprandial glycaemia in the last 3 days of initial hospitalisation $(7.7 \pm$ 1.9 vs. $8.5 \pm 1.8 \mathrm{mmol} / \mathrm{l}, \mathrm{p}<0.05$ and $11.2 \pm 3.9$ vs. $12.5 \pm 2.9 \mathrm{mmol} / \mathrm{l}, \mathrm{p}<0.05$, respectively) and lower serum triglyceride concentration at diagnosis ( $1.11 \pm 0.57$ vs. $2.03 \pm 2.05 \mathrm{mmol} / \mathrm{l}, \mathrm{p}<0.05)$ were also noted in this group. In the multivariate logistic regression model, insulin requirement at the end of initial hospitalisation was the predictor of long partial remission of diabetes, independent from age, BMI, and presence of DKA at diagnosis (RR: 18.5; 95\%CI: $2.1-162.7, \mathrm{p}<0.01$ )

Conclusion: Low insulin requirement in the early days after diagnosis may be considered as predictor of long partial remission of type 1 diabetes.

\section{8}

Insulin glargine provides good glycaemic control in those diabetic patients on gastrostomy feeding

N. B. Tutuncu, S. Ayturk, A. D. Unal, A. N. Demirer, A. Gursoy, N. G. Demirag;

Endocrinology and Metabolism, Baskent University Faculty of Medicine, Ankara, Turkey.

Background and Aims: Percutaneous endoscopic gastrostomy (PEG) tube feeding is a critically important mode of nutritional support for diabetic patients with cerebrovascular events which end up with impairment of swallowing reflex. Achievement of the optimal glycemic conntrol in the long term is a big problem for these cases due to the frequent bedside capillary glucose monitoring and continous insulin infusion or frequent subcutaneous insulin regimens for each gastrostomy feeding session. Our aim was to evalute the effectiveness of once daily injection of subcutaneous insulin glargine for the control of glycemia in these patients.

Materials and Methods: We enrolled 13 adult patients receiving PEG tube feeding due to cerebrovascular sequela. All these patients staying in the intensive care unit were receiving five times daily $200-420 \mathrm{cc}$ of commercially prepared enteral nutritional products specific for the diabetic patients. Their glycemic control was done by continous insulin infusion or frequent subcutaneous short acting insulin regimen before each feeding session. After achievement of the optimal glycemic regulation for each patient, $75 \%$ of the total daily insulin requirements were given as once daily insulin glargine. Glycemic monitoring was done each week and the HbAlc was determined after 3 months of insulin glargine regimen.

Results: Mean age of the patients was $70.2 \pm 6.9$ years ( 4 female, 9 male). Mean insulin requirement and mean daily blood glucose levels during insulin infusion were $3.0 \pm 1.3$ Units/hour, $170.7 \pm 30.4 \mathrm{mg} / \mathrm{dl}$, respectively. Total daily insulin requirement during the frequent subcutaneous insulin regimen was $57.1 \pm 27.4$ Units/day, and the mean daily blood glucose was $177.8 \pm 39.7 \mathrm{mg} / \mathrm{dl}$. Mean HbA1c level at the beginning of the gastrostomy feeding was $7.2 \pm 1.5 \%$. At the end of the 3 months of insulin glargine regimen mean daily insulin requirements and capillary glucose levels, as well as the HbAlc decreased significantly (43.9 \pm 21.8 Units/day, $138.4 \pm 25.3 \mathrm{mg} / \mathrm{dl}$ and $6.7 \pm 0.8 \%$, respectively).

Conclusion: Insulin glargine is a good alternative to frequent subcutaneous insulin regimens in those with gastrostomy feeding. While achieving the optimal glycemic goal it has the advantage of ease of administration for once a day.

\section{9}

Tranference from intravenous to subcutaneous insulin in critically ill patients with artificial nutrition

S. Torrejon ${ }^{1}$, L. Zapata ${ }^{2}$ J. M. Cubero ${ }^{1}$, B. Biagetti ${ }^{1}$, P. Vera ${ }^{2}$, A. J. Betbesé2 A. Pérez ${ }^{1}$;

${ }^{1}$ Endocrinology, ${ }^{2}$ Intensive Care Unit, Hospital Sant Pau, Barcelona, Spain.

Background and Aims: To evaluate a strategy for the transference from intravenous insulin infusion to subcutaneous long acting insulin analogue (glargine) in critical care patients receiving artificial nutrition.

Materials and Methods: Between June to December 2006, 120 consecutive patients hospitalised in Intensive Care Unit (ICU) were included in an intensive glycemic control with intravenous insulin infusion protocol (IIT). We studied a subgroup of 74 patients (mean age $65.3 \pm 14.6,74.3 \%$ men, Apache II score $19.7 \pm 11.5$, 69\% without diabetes) receiving parenteral and/or enteral nutrition. Transference to subcutaneous insulin was started after patient's clinical status improve (off pressor agents or corticoids) and the blood glucose was stable and in target range. The 24-hour long lasting insulin glargine dose was estimated as a $50 \%$ of average amount of insulin infused during previous 12 hours. Blood glucose was checked $\geq 4$ times daily and insulin dose was adjusted each evening. Correction doses of rapid acting insulin analogue for hyperglycemia were administered every 4-6 hours.

Results: Basal subcutaneous insulin was started at $6 \pm 5$ days after admission in ICU and mean duration of treatment in ICU was $10 \pm 9$ days. Initial insulin dose was $27 \pm 15$ UI and increased to $33 \pm 21$ at $10^{\text {th }}$ day. The mean blood glucose during the period of treatment was $138 \pm 34 \mathrm{mg} / \mathrm{dL}$, the percentage of glycemias in the target range $(80-120 \mathrm{mg} / \mathrm{dl})$ was $54.7 \%$ and only $6.7 \%$ were $>$ $200 \mathrm{mg} / \mathrm{dL}$. Only 3 hypoglycemic episodes were observed (4.1 episodes/1000 days of treatment). As compared to the subgroup of patients with total enteral nutrition (TEN) $(\mathrm{n}=27)$, patients with total parenteral nutrition (TPN) $(\mathrm{n}=14)$ had higher mean plasma glucose ( $147 \pm 37 \mathrm{vs.} 136 \pm 35 \mathrm{mg} / \mathrm{dL}$ ) and a $40 \%$ dose increase was necessary to achieve the target blood glucose levels.

Conclusion: Long acting insulin glargine is an effective and safety alternative in the transference from intravenous to subcutaneous insulin on patients with artificial nutrition. Estimation of total subcutaneous dose as $50 \%$ of average insulin infused during the last 12 hours is suitable for patients with TEN but insufficient for patients with TPN. 


\section{0}

Intensive intravenous insulin treatment in patients admitted to an intensive care unit

J. M. Cubero ${ }^{1}$, L. Zapata ${ }^{2}$, B. Biagetti ${ }^{1}$, S. Torrejón ${ }^{1}$, I. Vinagre ${ }^{1}$, P. Vera $^{2}$, A. Betbesé ${ }^{2}$, A. Pérez ${ }^{1}$;

${ }^{1}$ Endocrinology and Nutrition,

${ }^{2}$ Intensive Care Unit, Hospital Santa Creu i Sant Pau, Barcelona, Spain.

Aims: To evaluate the efficacy and safety of an intensive insulin treatment protocol (IIT) in patients with hyperglycaemia admitted to an Intensive Care Unit (ICU)

Materials and Methods: We compared a prospective cohort in whom an IIT was used to maintain the blood glucose at a level between 80 and $120 \mathrm{mg} / \mathrm{dl}$ with a retrospective cohort (control group). We studied 272 patients (158 as IIT group, and 114 as control group). The IIT consisted in 6 algorithms with a decision table for the adjustment of continuous intravenous insulin infusion according to glycaemia measurements. Once patients were stabilised without needing inotropic or vasopressor drugs or corticoids, and receiving any type of nutrition, we started subcutaneous insulin treatment (bolus-basal with lispro and glargine insulin). Total initial dose was estimated at $50 \%$ of the intravenous insulin requeriments in the previous 12 hours. Glycaemias $<40$ $\mathrm{mg} / \mathrm{dl}$ were considered as severe hypoglycaemias. In the control group no predefined protocol was used in the management of hyperglycaemia.

Results: No differences were found in the mean age (62.9+/-16 vs. $62.2+/-16$ years) and APACHE II score $(20.1+/-8$ vs. $18.5+/-8)$ between control and ITT groups. We collected 2600 capillary glucose measurements in the control group and 12036 in the IIT group. The mean glucose level during the stay in ICU was $143.1+/-32 \mathrm{mg} / \mathrm{dl}$ in the control group vs. $118+/-16 \mathrm{mg} / \mathrm{dl}$ in the IIT group $(\mathrm{p}<0.001)$. In the IIT group, the percentage of glycaemias at a level between $80-120 \mathrm{mg} / \mathrm{dl}$ was higher $(59.3+/-15 \%$ vs. $36.3+/-25 \%$; p $<0.001)$ while the percentage of glycaemias $>200 \mathrm{mg} / \mathrm{dl}$ was lower $(4.7+/-8 \%$ vs. $12.6+/-19 \% ; \mathrm{p}<0.001)$. Severe hypoglycaemias were similar in both groups (6 vs. 9 episodes). Mortality in ICU was $30.1 \%$ in the control group vs. $21.1 \%$ in the IIT group $(\mathrm{p}=0.08)$ and in-hospital death $37.2 \%$ in the control group vs. $26.8 \%$ in the IIT group ( $\mathrm{p}=0.06)$. No significant differences were observed in morbidity except for hyperbilirrubinemia (39\% in the control group vs. $24.4 \%$ in the IIT group ; $\mathrm{p}<0.01$ )

Conclusion: The IIT protocol established allows us to maintain a target blood glucose range of $80-120 \mathrm{mg} / \mathrm{dl}$ without increasing severe hypoglycaemic episodes.

\section{1}

Glycaemic control in type 2 diabetic patients during long-term tube feeding with a disease specific enteral formula: final results of a randomised, controlled multicentre trial

P. Mayr ${ }^{1}$, M. Mertl-Roetzer ${ }^{2}$, F. Lauster ${ }^{2}$, M. Pohl ${ }^{3}$, B. Hipper ${ }^{4}$, D. Steube ${ }^{5}$, J. Eriksen ${ }^{6}$, V. W. Rahlfs ${ }^{7}$;

${ }^{1}$ Health Care Centre, Stockach, Germany, ${ }^{2}$ Schoen-Clinic, Bad Aibling, Germany, ${ }^{3}$ Clinic Bavaria, Kreischa, Germany, ${ }^{4}$ Median Clinic, Magdeburg, Germany, ${ }^{5}$ Clinic, Bad Neustadt/Saale, Germany, ${ }^{6}$ County Hospital, Herning, Denmark, ${ }^{7}$ Data Analysis and Planning, idv, Gauting, Germany.

Background and Aims: Achieving optimal glycaemic control in tube-fed diabetic patients is complicated by metabolic stress, immobility and feeding difficulties. Results of stage I of a pre-planned two-stage procedure in type 2 diabetics after brain injury have provided good evidence for improved glycaemic control with a disease specific enteral formula low in carbohydrates, high in monounsaturated fatty acids, fish oil, chromium and antioxidants (Diben, Fresenius Kabi) as compared to a standard formula. Since the confirmatory $\mathrm{P}$ value of stage I (summarising Wei-Lachin procedure) was lying between the pre-planned benchmarks, the study was continued to confirm beneficial effects with a reduced set of primary variables and adjusted sample size.

Material and Methods: Randomised, double-blind, controlled multicentre trial. Insulin treated type 2 diabetic patients $\left[\mathrm{HBA}_{1 \mathrm{C}} \geq 7.0 \%\right.$ and/or fasting blood glucose $(\mathrm{FG})>120 \mathrm{mg} / \mathrm{dl}]$ with neurological disorders and indication for long-term enteral tube feeding were randomised to receive either $27 \mathrm{kcal} /$ $\mathrm{kg} \mathrm{BW} / \mathrm{d}(\max .2025 \mathrm{kcal} / \mathrm{d}$ ) of the disease specific formula (test, $\mathrm{n}=53$ ) or an isoenergetic, isonitrogenous enteral standard formula (control, $n=52$ ) for up to 84 days. Total insulin requirements (TI), FG and afternoon blood glucose
(AG) were assessed daily and expressed as changes from baseline, $\mathrm{HBA}_{1 \mathrm{C}}$ (changes from baseline) and safety criteria on days $1,28,56$, and 84 . The Wilcoxon-Mann-Whitney test was used for statistical analysis. Results are presented as effect sizes of the univariate Mann-Whitney test (MW).

Results: 105 patients were included in stage II (53 test/52 control). A total of 55 patients (33 test/22 control) participated until day 84 , most frequent reasons for premature discontinuation were "regained swallowing" and "referral to other institution". After 84 days, median changes of TI with data as available were -8.0 vs. $+2.0 \mathrm{U}$ (test vs. control, $\mathrm{MW}=0.8326, \mathrm{P}<0.0001$ ) and of FG -39.0 vs. $-12.1 \mathrm{mg} / \mathrm{dl}$ (test vs. control, $\mathrm{MW}=0.8030, \mathrm{P}<0.0001$ ); the effect sizes denote a large superiority of the test group for both variables as well as for the combined efficacy criteria (days 56/84, MW=0.7899, $\mathrm{P}<0.0001$, $\mathrm{P}$ value to be reached in stage II: $\mathrm{P}<0.0309)$. For $\mathrm{HBA}_{1 \mathrm{C}}$, relevant and for $\mathrm{AG}$ large superiority of the test group could be demonstrated on day 84/days 56 and 84 , respectively. Feeding tolerance was comparable in both groups. The number of relevant hypoglycaemic episodes $(\mathrm{FG}<60 \mathrm{mg} / \mathrm{dl})$ was 1 (test) vs. 5 (control), suggesting a slight superiority of the test group.

Conclusion: Statistically significant beneficial effects on total insulin dosages and fasting blood glucose with disease specific compared to standard formula could be shown for the formal combination of the two stages. Long-term treatment with a disease specific enteral formula improves and stabilises glycaemic control. The formula is safe and well tolerated in tube-fed type 2 diabetes patients with neurological disorders.

Supported by Fresenius-Kabi, Bad Homburg, Germany 


\section{PS 98 Devices and information technology}

\section{2}

Pilot study of the effects of gastric electrical stimulation (Tantalus ${ }^{\circ}$ ) on glycaemic control in obese patients with type 2 diabetes (T2DM) C. Rosak ${ }^{1}$, B. Ludvik ${ }^{2}$, R. Jung ${ }^{1}$, M. Schramm ${ }^{3}$, A. Bohdjalian ${ }^{4}$, G. Prager ${ }^{4}$, R. Weiner ${ }^{3}$;

${ }^{1}$ Dept of Diabetology, Sachsenhausen Hospital, Frankfurt a. Main, Germany, ${ }^{2}$ Dept of Internal Medicine III, Medical University of Vienna, Austria, ${ }^{3}$ Dept of Surgery, Sachsenhausen Hospital, Frankfurt a. Main, Germany, ${ }^{4}$ Dept of Surgery, Medical University of Vienna, Austria.

Background: The TANTALUS System (MetaCure Ltd.) is a minimally invasive, implantable device that automatically detects food intake and delivers non-excitatory electrical stimulation to the gastric wall during meals. Previous studies showed that this modality can induce weight loss in morbidly obese subjects.

Aim: To investigate the potential effect of the TANTALUS System on glycemic control and weight in obese subjects with T2DM.

Patients and Methods: In this European multi-center, open label study, 24 T2DM obese $\left(9 \mathrm{~m}, 15 \mathrm{f}, \mathrm{BMI}: 41.7 \pm 0.9 \mathrm{~kg} / \mathrm{m}^{2}\right)$ subjects treated either with insulin (7) or oral anti-diabetic medications (17) were implanted laparoscopically with the TANTALUS System. The system includes a pulse generator, three bipolar leads and delivers a non-excitatory signal initiated upon automatic detection of food intake.

Results: Sixteen subjects initially on oral anti-diabetic medications that have completed one year follow up show a significant $(\mathrm{p}<0.05)$ decrease in weight of $5.5 \pm 2 \mathrm{~kg}$. A subset of eight patients initially not well controlled (A1c $>7 \%)$ on stable oral medications decreased $\mathrm{HbAlc}$ from $8.34 \pm 0.3 \%$ to $7.44 \pm 0.4 \%$, FBG dropped from $206 \pm 12 \mathrm{mg} / \mathrm{dl}$ to $158 \pm 15 \mathrm{mg} / \mathrm{dl}(\mathrm{p}<0.05)$. Self glucose monitoring data in this subset available at 9 months showed a significant $(\mathrm{p}<0.05)$ decrease in 2-hour post-prandial glucose $(176 \pm 12 \mathrm{mg} / \mathrm{dl}$ to. $141 \pm 13 \mathrm{mg} / \mathrm{dl})$. The other eight patients on oral meds either changed drug regime during treatment or were initially well controlled $(\mathrm{A} 1 \mathrm{c}<7 \%)$. The four insulin subjects that have completed one year showed no significant changes in $\mathrm{HbAlc}$ and weight.

Conclusion: Interim results with the TANTALUS System suggest that nonexcitatory stimulation applied during food intake can potentially improve glucose levels and induce weight loss in obese T2DM subjects on oral antidiabetic therapy. Further evaluation is required to determine underlying mechanisms of glucose lowering and weight loss.

\section{3}

The relationship between self-monitoring of blood glucose results and glycosylated haemoglobin in type 2 diabetes: the Fremantle Diabetes Study

T. M. E. Davis, K. Van-Minnen, D. G. Bruce, W. A. Davis;

School of Medicine and Pharmacology, University of Western Australia, Fremantle, Australia.

Background and Aims: Self-monitoring of blood glucose (SMBG) and its frequency did not predict glycosylated haemoglobin $\left(\mathrm{HbA}_{1}\right)$ in patients with type 2 diabetes participating in the observational community-based Fremantle Diabetes Study (FDS), regardless of treatment modality. This could reflect problems with one or more steps in the complex process of obtaining and applying SMBG data. The aim of the present FDS sub-study was to assess whether the SMBG results documented by the patients correlated with $\mathrm{HbA}_{1 \mathrm{c}}$ and thus could, if properly applied, be used to guide therapeutic change.

Materials and Methods: 900 out of the 1294 type 2 FDS patients performed SMBG, and 554 of these (61.6\%) provided documented SMBG results from which were abstracted the highest and lowest results before and after meals during the week before assessment. We assessed the associations between the averages of these levels and $\mathrm{HbA}_{1 c^{\circ}}$

Results: There was a highly significant correlation between both pre- and post-prandial average SMBG results and $\mathrm{HbA}_{1 c}(P<0.0001$ in each case $)$. The $95 \%$ confidence intervals for $\mathrm{HbA}_{1 \mathrm{c}}$ (normal reference range $<6.0 \%$ ) at three SMBG levels are shown in the Table.

Conclusion: These data suggest that, despite wide confidence intervals, pre-prandial SMBG data could be used to justify intensification of therapy, especially at levels above $10 \mathrm{mmol} / \mathrm{L}$. Post-prandial levels had a flat relationship with $\mathrm{HbA}_{1 c}$ and are thus of limited value in optimising management.

Glycosylated haemoglobin $95 \%$ c.i. for three pre- and post-prandial average SMBG results

\begin{tabular}{llll}
\hline & SMBG & SMBG & SMBG \\
& $6 \mathrm{mmol} / \mathrm{L}$ & $10 \mathrm{mmol} / \mathrm{L}$ & $14 \mathrm{mmol} / \mathrm{L}$ \\
\hline Pre-prandial average & 4.2 to 9.9 & 5.6 to 11.3 & 7.0 to 12.7 \\
Post-prandial average & 2.4 to 7.9 & 2.5 to 8.1 & 2.6 to 8.2 \\
\hline
\end{tabular}

Supported by Raine Foundation/University of Western Australia

\section{4}

Reliability of self-recorded blood glucose data in patient logbooks compared with SMBG data saved in device memory and printed out with Accu-Chek Smart Printer

J. K. Tshiang Tshiananga ${ }^{1}$, D. Franke ${ }^{2}$, M. Luebker ${ }^{3}$, C. Weber ${ }^{1}$, K. Neeser ${ }^{1}$;

${ }^{1}$ IMIB: Institute for Medical Informatics and Biostatistics, Basel, Switzerland,

${ }^{2}$ Medical clinic, VS-Villingen, Germany,

${ }^{3}$ Roche Diagnostics, Mannheim, Germany.

Objectives: This study aimed to examine the reliability of self-recorded blood glucose (BG) values in a handwritten diary compared with memory data of SMBG devices printed out with Accu-Chek Smart Printer, to investigate possible sources of errors and to deduce predictive factors in order to avoid recording errors in the future.

Methods: Patients in this study were followed during 28 days by a diabetologist for their disease management in south of Germany. The discrepancies in the BG-values were determined as difference between diary correct documented values and the averages resulting from corresponding device memory values (one or four) of measurements performed in the morning, at midday, in the evening and at bedtime. ANOVA models were built to determine the influences of variables on discrepancies at the different measuring points.

Results: 94 of 176 patients (44\% females) aged between $16-90$ years were performing SMBG daily. The mean age was $66.38 \pm 13.92$ and the mean duration of diabetes $11.37 \pm 8.99$ (range:1-44) years. Device BG-values were likely to be higher than self-recorded diary-values for the corresponding measurements $(\mathrm{p}<0.001)$. Only $8 \%$ of patients could properly transfer BGvalues in their handwritten logbooks $(\mathrm{p}<0.001)$. This proportion was most elevated in the morning and was fading at the following measuring points $(p<0.001)$. Diary-values were higher than values in device memory during the bedtime measurement. Five recording errors were detected: 1)fully different values in both diary and device memory for the same measurement period, 2)inversed numbers, 3)same value but different recording times, 4)missing values in diary but available in memory or vie versa and 5)missing values as well as missing dates. ANOVA models have shown influences of various sociodemographic and metabolic characteristics, number of SMBG measurements and frequency of errors on BG-discrepancies at different measurement periods.

Conclusion: This study has shown a higher reliability of patients to record correctly the measured SMBG-values in their logbooks during the morning time than at the other measuring points. The discrepancies are related to Sociodemographic features as well as to reasons varying from cognitive capacity, visual impairment and compliance to their physician. Our findings have shown a trend maybe also existing beyond our particular study population and would have serious implications in disease management (patient guidance and adjustment of therapy). Therefore we would recommend not to rely on only handwritten logbooks but to favorize recorded BG-values and analysed with electronic systems like the Smart Printer or similar devices.

Data: Medical clinic Dr. Franke, VS-Villingen and Roche Diagnostics Diabetes care (Mannheim); Analysis: IMIB (Basel) 


\section{5}

Evaluating the effect of miscoding on a large number of glucose meter results collected over 4-years

J. M. Ellison ${ }^{1}$, A. Cariski ${ }^{1}$, C. Davis ${ }^{1}$, K. Delaney ${ }^{2}$, F. Urquhart ${ }^{2}$;

${ }^{1}$ LifeScan, Inc., Milpitas, United States, ${ }^{2}$ LifeScan Scotland, Inverness, United Kingdom.

Background and Aims: Results from 4 published studies suggest that the incidence of patient meter miscoding ranges from 3-16\%. As with other sources of procedural error such as blood collection and application, miscoding can result in glucose measurement errors. The magnitude of the error depends on the degree of miscoding (how many codes the meter setting differs from the correct value) and the glucose meter system. The objective of this study was to evaluate the impact of miscoding the OneTouch ${ }^{\circledast}$ Ultra $^{\oplus}$ (Ultra) Meter on measured blood glucose readings taken from a very large data set.

Materials and Methods: The data set comprises blood glucose results from ongoing clinical evaluations conducted at three clinical sites in the UK. During each evaluation, capillary blood samples were collected from donors with diabetes and tested on an Ultra meter. Additional capillary blood was then collected and a reference value determined from duplicate tests performed on the YSI 2300 Glucose Analyzer. A random sample of 45,343 glucose results collected over a 4-year period (October 2000 to December 2004) from 552 lots of Ultra Test Strips was obtained and the Ultra glucose values were recalculated by applying each of the 49 calibration codes to each of these results.

Results: Of the original 45,343 samples measured with the correct calibration code, $98.6 \%$ of values were in zone A, $1.4 \%$ were in zone B, and 3 results were in zone $\mathrm{C}$ of the Consensus Error Grid (Table). The calculations produced over 2.2 million glucose values, of which only 65 fell outside zones A and B: only these 65 zone $\mathrm{C}$ results were likely to affect clinical outcome. No values were sufficiently inaccurate to cause a treatment decision with significant medical risk (zone D) or dangerous consequences (zone E).

Conclusion: Our study confirms that correct meter coding optimises system accuracy and reinforces the need to code in accordance with label instructions. However, if the code on the meter does not match the code on the test strip vial, there is a low risk of adverse health outcomes in the Ultra meter system.

\begin{tabular}{lll} 
Summary of Consensus Error Grid Analysis \\
\hline Error Grid Zone & $\begin{array}{l}\text { Meter results coded } \\
\text { correctly }\end{array}$ & $\begin{array}{l}\text { Meter results all codes } \\
\text { applied }\end{array}$ \\
\hline A & $98.6 \%$ & $78.8 \%$ \\
B & $1.4 \%$ & $21.2 \%$ \\
C & $0.0 \%$ (3 results) & $0.0 \%(65$ results $)$ \\
D & $0.0 \%(0$ results $)$ & $0.0 \%(0$ results $)$ \\
E & $0.0 \%(0$ results $)$ & $0.0 \%(0$ results $)$ \\
\hline
\end{tabular}

\section{6}

Differences in dose accuracy of insulin pens

A. Pfützner, A. Weise, W. Sun, H. Hänel, T. Forst;

IKFE Institute for Clinical Research and Development, Mainz, Germany.

Background and Aims: Modern insulin injection pens provide a convenient and accurate way for diabetes patients to inject insulin. Their use is widespread among children and adults with type 1 diabetes as well as those with type 2 diabetes in Europe. A previous study in Japan brought the accuracy of an insulin pen system in question. This investigation was performed to establish whether this could be reproduced on insulin pens available in Europe. This study compared the dosing accuracy of two commonly available insulin pens, the OptiClik pen from sanofi-aventis which is available with insulin glulisine and insulin glargine, and the FlexPen pen from Novo Nordisk which is available with insulin aspart, biphasic insulin aspart and insulin detemir. Materials and Methods: Insulin and pens were obtained from the stock of a local pharmacy in Düsseldorf, Germany and the dosing accuracy was tested for both pens with $24 \times 10 \mathrm{U}$ and $9 \times 30 \mathrm{U}$ injection volumes to investigate, whether both pens comply within the acceptable limits of $10 \%(+/-1 \mathrm{U})$ for $10 \mathrm{U}$ and $5 \%(+/-1.5 \mathrm{U})$ for $30 \mathrm{U}$. These limits are based on the limits set forth by ISO. The needles used were chosen following the recommendations of both manufacturers of the insulin pens (NovoFine 31 gauge $(\mathrm{G}) 6 \mathrm{~mm}$ needles for the FlexPen and BD Micro-Fine $31 \mathrm{G} 5 \mathrm{~mm}$ needles for the OptiClik). A new needle was used for every dose, which was delivered according strictly according to the instructions for use for both devices. A sensitive pharmaceutical balance was used for the assessment of the applied volumes, and the results were corrected for the specific density of the insulin formulations. Four insulin pens from 2 different lots were used for the two volumes, respectively, resulting in a total of 192 dosings per pen with $10 \mathrm{U}$ and 72 doses per pen with $30 \mathrm{U}$.

Results: FlexPen was more accurate than the OptiClik insulin pen at both doses as shown in the table. Each OptiClik pen underdosed at least on one occasion, while all doses from the FlexPen were within the specified accuracy range.

Conclusion: A direct head-to-head comparison of both insulin pens with a standardised protocol resulted in a more stable accuracy of the FlexPen in comparison to the OptiClik pen. Even though all insulin delivery systems undergo rigorous testing before being approved for sale, it seems there may be reason to be attentive to the performance of the devices in practical use. As an increasing amount of delivery systems are introduced by all the major insulin producers (i.e. NovoPen 4, Novo Nordisk; SoloSTAR, sanofi-aventis; HumaPen Luxura, Lilly) in response to an ever increasing amount of patients treated with insulin, emphasis must be put on device manufacturers to ensure an important but evident task; delivering the correct amount of insulin to the patients in an easy and convenient way.

Table: \% of doses outside accuracy range

\begin{tabular}{lll}
\hline Selected dose & OptiClik & FlexPen \\
\hline $\mathbf{1 0 U}(+/-\mathbf{1 U}$ max $)$ & $8.33 \%$ & $0 \%$ \\
$\mathbf{3 0 U}(+/-\mathbf{1 . 5 U}$ max $)$ & $16.66 \%$ & $0 \%$ \\
\hline
\end{tabular}

Supported by Novo Nordisk

\section{7}

A silicon beta cell: towards an intelligent closed loop insulin delivery system

N. S. Oliver, P. Georgiou, C. Toumazou;

Institute of Biomedical Engineering, Imperial College, London, United Kingdom.

We have developed a bioinspired electronic version of the beta cell which replicates the normal bursting behaviour of the beta cell. Beta cells show electrical excitability which is coupled to insulin secretion by exocytosis. The membrane potential oscillates in slow wave bursts with action potentials superimposed on the active phase. These bursts do not appear unless blood glucose concentrations rise above $7 \mathrm{mM}$ and, as glucose concentration rises, the pulse width of the bursts increases up to a concentration of $20 \mathrm{mM}$ at which the mechanism is saturated and a continuous train of action potentials remains. Implementation of the beta cell in silicon requires accurate modelling of the dynamics of the ionic currents. The silicon beta cell uses an ion-sensitive field effect transistor (ISFET) coupled with glucose oxidase as the glucose sensor. Glucose is metabolised by glucose oxidase to produce hydrogen ions which are detected by the ISFET sensor. Glucose may be sensed up to $25 \mathrm{mmol} / \mathrm{L}$. Mathematical modelling of the ionic behaviour of the beta cell has been replicated using low power MOS transistors and the circuit has been fabricated in silicon. Simulation results confirm that the membrane dynamics of the silicon beta cell are comparable to those seen in actual beta cell data. The resting potential is $-68 \mathrm{mV}$ and appropriate bursting behaviour is observed at varying glucose concentrations.

Results mimic the pancreatic beta cell and, as bursting behaviour is associated with insulin release by exocytosis, physiological insulin delivery may be driven by the output from the silicon cell. This circuit is an important step towards creating an artificial bionic pancreas utilising an insulin pump for blood glucose regulation in type 1 diabetes. The silicon beta cell shows bursting behaviour similar to that observed in beta cells in vitro as well as demonstrating low power consumption making it ideal as an implantable or wearable device. 


\section{8}

Outpatient assessment of KADIS ${ }^{\oplus}$-based decision support P. Augstein, L. Vogt, K.-D. Kohnert, E.-J. Freyse, P. Heinke, E. Salzsieder; Institute of Diabetes, Karlsburg, Germany.

Objective: To assess the benefit of the Karlsburg Diabetes Management System $\left(\mathrm{KADIS}^{\circledR}\right)$ in conjunction with continuous glucose monitoring $\left(\mathrm{CGMS}^{\mathrm{m}}\right)$ in an outpatient setting.

Research Design and Methods: A multicentric trial was performed in insulin-treated outpatients $(n=49)$, aged $21-70$ years with a mean diabetes duration of 14.2 years. Subjects were recruited from 5 outpatient centers and randomised for $\mathrm{CGMS}^{\mathrm{m}}$ - or $\mathrm{CGMS}^{\mathrm{m}} / \mathrm{KADIS}^{\oplus}$-based decision support and followed up for 3 months. After two CGMS ${ }^{\text {mat }}$ monitorings, the outcome parameters $\mathrm{HbA}_{1 c}(\%)$, mean sensor glucose of the CGMS ${ }^{\mathrm{mat}}$ profile (MSG, $\mathrm{mmol} / \mathrm{L}$ ) and duration of hyperglycemia ( $\mathrm{h} /$ day) were evaluated.

Results: In contrast to the CGMS ${ }^{m}$ group $(0.27 \pm 0.67 \%)$, mean change in $\mathrm{HbA}_{1 \mathrm{c}}\left(\triangle \mathrm{HbA}_{1 \mathrm{c}}\right)$, decreased in the CGMS ${ }^{\mathrm{m}} / \mathrm{KADIS}^{\circ}$ group during the follow up $(-0.34 \pm 0.49 \% ; \mathrm{p}<0.01)$. MSG levels were not affected in the CGMS ${ }^{\text {mo }}$ group ( $7.75 \pm 1.33$ vs. $8.45 \pm 2.46 \mathrm{mmol} / \mathrm{L})$, but declined in the CGMS ${ }^{\text {mit }} / \mathrm{KADIS}^{\varpi}$ group $(8.43 \pm 1.33$ vs. $7.59 \pm 1.47 \mathrm{mmol} / \mathrm{L} ; \mathrm{p}<0.05)$. Net KADIS ${ }^{\circledast}$ effect $[-0.60$ ( $95 \%$ CI $-0.96--0.25 \%) ; \mathrm{p}<0.01]$ was associated with reduced duration of hyperglycemia ( 4.6 vs. $1.0 \mathrm{~h}$ /day; $\mathrm{p}<0.01$ ) without increasing hypoglycemia. Multiple regression revealed that the $\mathrm{HbA}_{1 \mathrm{c}}$ outcome was dependent on KADIS $^{\circledR}$-based decision support. Age, sex, physician's specialty, diabetes type and BMI had no measurable effect.

Conclusions: If physicians were supported by CGMS ${ }^{m / 1} / \mathrm{KADIS}^{\circledR}$ in therapeutic decisions, they achieved a better glycemic control for their patients compared to $\mathrm{CGMS}^{\mathrm{rm}}$ alone. KADIS ${ }^{\oplus}$ is a suitable decision support tool for physicians in outpatient diabetes care and has the potential to improve evidence-based management of diabetes.

Supported by grants from the German Federal Ministry of Education and Research and the Ministerium für Bildung, Wissenschaft und Kultur Mecklenburg-Vorpommern

\section{PS 99 Continuous glucose monitoring systems}

\section{9}

Detection of hypoglycaemia in patients with T1DM using the FreeStyle Navigator ${ }^{\circ}$ continuous monitoring system and traditional blood glucose monitoring

G. V. McGarraugh;

Abbott Diabetes Care, Alameda, United States.

Background and Aims: The FreeStyle Navigator Continuous Glucose Monitoring System (CGM), a 5-day, subcutaneously inserted electrochemical sensor, was compared to blood glucose monitoring (BG) at an average testing frequency of 8/day for the detection of hypoglycemia. A single measurement indicating hypoglycemia could occur immediately after the onset of an episode or many hours later. In order to understand hypoglycemia detection, the onset and duration of the hypoglycemic event must be defined - very frequent or continuous reference values are necessary. CGM was evaluated in a 5-day clinic study where YSI reference glucose tests were performed at 15-minute intervals. BG testing was studied in the home environment where testing regimens were integrated into normal daily activities. The reference in the home environment was CGM with results masked from the patients. CGM does not have the accuracy of a laboratory reference, but the performance of CGM was well characterised in the clinic study, and the home use study of BG can be interpreted with an understanding of the CGM reference method limitations.

Materials and Methods: The clinic study included 58 subjects with T1DM where reference readings were taken for 50 hours, and subject schedules were staggered to include the sensor's 120 hour lifetime. Hypoglycemia was defined as at least two successive YSI readings $<70 \mathrm{mg} / \mathrm{dL}-$ a duration $>15$ minutes. The 20-day home use study included 91 adult subjects with T1DM. A hypoglycemic event was defined as successive CGM readings $<70 \mathrm{mg} / \mathrm{dL}$ for $>15$ minutes, similar to the clinic study. A BG reading of $\leq 85 \mathrm{mg} / \mathrm{dL}$ was considered detection of hypoglycemia since this indicated necessary treatment to reverse or avoid hypoglycemia.

Results: Using the CGM threshold alarm set at $85 \mathrm{mg} / \mathrm{dL}, 90.6 \%(155 / 171)$ of hypoglycemic events were detected within \pm 30 minutes of onset. When a CGM alarm was activated, the YSI reference glucose was $\leq 85 \mathrm{mg} / \mathrm{dL}$ for 77.2 $\%(217 / 281)$ of alarms, indicating treatment was necessary to reverse or avoid hypoglycemia. In actual use, a BG test would be performed to determine if treatment was necessary. Projected alarms give advanced indication of impending hypoglycemia, and more alarms occur when these are used. Detection was $93.5 \%(160 / 171)$ when high sensitivity projected alarms were employed and $72.2 \%(281 / 389)$ of the alarms occurred when YSI was $>70$ $\mathrm{mg} / \mathrm{dL}$. Projected alarms would be used in order to avoid rather than reverse hypoglycemia. Using CGM $<70 \mathrm{mg} / \mathrm{dL}$ for $>15$ minutes to identify events in the clinic study, $52.0 \%$ of the YSI-defined events were also identified by CGM. When CGM indicated an event, YSI was $\leq 70 \mathrm{mg} / \mathrm{dL} 85 \%$ of the time and $\leq 85$ $\mathrm{mg} / \mathrm{dL} 97.5 \%$ of the time. CGM will not identify all events in the home use study - mild hypoglycema of short duration is less likely to be identified. When an event is indicated, however, it is very likely to be true or impending hypoglycemia. With an average testing frequency of 7.9/day in the home use study, $27.5 \%(303 / 1103)$ of events were detected within \pm 30 minutes, and $12.7 \%(49 / 385)$ were detected during nocturnal hours. If an hour was allowed for detection the rate was $35.3 \%$ (389/1103) or 19.0\% (73/385) during nocturnal hours. Since the actual number of events was underestimated by CGM, the true detection rates by BG are likely to be lower.

Conclusion: CGM provides superior detection of hypoglycemia and patients can customize projected and threshold alarm settings to allow a suitable balance of alarm frequency and treatment strategy.

Supported by Abbott Diabetes Care 


\section{0}

Improved glycaemic control with real-time continuous glucose sensors in patients with type 1 diabetes

S. L. Ellis, M. Voelmle, P. Gottlieb, R. Gutin, S. Garg;

Department of Clinical Pharmacy, School of Pharmacy, Barbara Davis

Center for Diabetes, Aurora, United States.

Background and Aims: Continuous glucose sensors have been shown to decrease glycemic excursions in short-term studies but data are lacking on their effects on long term glycemic control.

Materials and Methods: We performed a retrospective study of patients initiated on either the DexCom STS or Medtronic Paradigm RT glucose sensors for 3 months. The primary outcome of this study was change in A1C values. Data were also collected on hypoglycemic episodes, insulin use, and weight and mean home blood glucose during monitoring (SMBG) before, during and after sensor use.

Results: A total of 34 patients had both baseline and 3 month data available. Baseline characteristics included 25 (74\%) females, duration of diabetes was $23.7 \pm 16.2$ years and mean A1c was $7.36 \% \pm 1.01 \%$.

Average sensor use was $17 \pm 9$ days/month (range 1-30 days). A1C values decreased significantly at 3 months (Figure 1a; $\mathrm{p}<0.0001$ ), despite no change in overall insulin dose $(43.0 \mathrm{U}$ vs. $42.7 \mathrm{U}, \mathrm{p}=0.58)$. Self-reported total hypoglycemic events decreased 23\% (Figure 1b; $<<0.0001$ ) whereas nocturnal hypoglycemia was reduced by $41 \%$ ( 1.16 vs. $0.69 \mathrm{p}=0.01$ ). Severe hypoglycemic events were 0.173 and 0.088 events/patient before and after sensor use, respectively ( $\mathrm{p}=0.15)$. Mean SMBG was reduced from 164.3 \pm 74.5 to $153.2 \pm 69.9(\mathrm{p}=0.053)$ at 3 months. Frequent sensor usage ( $>15$ days each month) was associated with a $30 \%$ increase in the number of patients achieving an ADA target A1c of $<7 \%$ compared to patients using the sensors less often.

Conclusion: The use of a real-time continuous glucose sensor significantly improved $\mathrm{A} 1 \mathrm{C}$ values while reducing overall and nocturnal hypoglycemic events after 3 months.

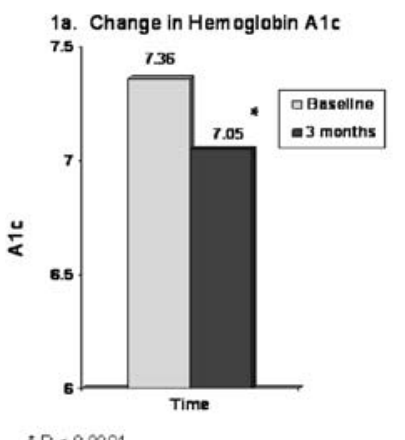

1b. Change in Self-Rep orted Hypoglycemia

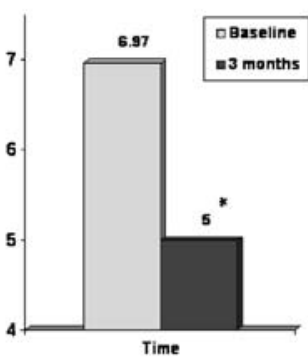

$* P<00001$

\section{1}

Evaluation of continuous use of real-time glucose monitoring, alone and after combined insulin pump therapy, as a mean to normalize glycaemic control in IDDM

C. S. Zoupas ${ }^{1}$, N. Kefaloyannis ${ }^{2}$, A. Pappas ${ }^{2}$, O. Kepaptzoglou ${ }^{1}$, E. Kyrlaki², I. Taraoune ${ }^{1}$, F. Giannakopoulos ${ }^{1}$, P. Garoutsou ${ }^{1}$, H. Vasilopoulos ${ }^{3}$;

${ }^{1}$ Diabetes Center, "Hygeia" General Hospital, Athens,

${ }^{2}$ Diabetes Center, "Venizelio-Pananio", General Hospital, Heraklion,

${ }^{3}$ Diabetes Center, "Evagelismos" General Hospital, Athens, Greece.

Background and Aims: It is well known that insulin pumps improve glycemic control in adults and children. The Paradigm REAL- Time System (PRT, Medtronic Minimed) is an Insulin Infusion Pump combined with a Continuous Glucose Monitoring System (CGMS) and a subcutaneously self-inserted electroenzymatic glucose sensor with continuous telemetered display of real time glucose values. The aim of this pilot study was to evaluate the impact on glycemic control of PRT Pump in IDDM for a long period of time.

Materials and Methods: A total of 9 patients, mean age $32.9+11.2$ years and diabetes duration $16.7 \pm 9.1$ years $(\mathrm{X} \pm \mathrm{SD})$, wore the Sensor-Augmented Pump system continuously for 6 months. For educational purposes all subjects were on real time CGM for the 1st month without the Pump function (remained on MDI). The Pump therapy was added in a second stage. HbAlc, average daily and monthly glucose and hypo/hyperglycemic excursions were evaluated at baseline, 4 and 6 months respectively.

Results: $\mathrm{HbA1c}(\%)$ decreased from $8.79 \pm 1.49$ at baseline,to $6.91 \pm 0.67$ after 4 and $6.98 \pm 0.73$ after 6 months $(\mathrm{X} \pm \mathrm{SD}),(\mathrm{p}=0.009)$. Glycemic control improved from the beginning and the average Daily and Monthly Glucose Values did not significantly differ after 1,4 and 6 months (Table 1). The average Area Under the Curve (AUC) above $180 \mathrm{mg} / \mathrm{dl}^{\star} 24 \mathrm{~h}$ was $11.07 \pm 6.77 \mathrm{mg} / \mathrm{dl}^{\star} 24 \mathrm{~h}$ after the 1st month use of real time CGM without Pump. Tend to be reduced after the introduction of pump and remained lower, $9.23 \pm 4.79$ and $9.22+3.15$ $\mathrm{mg} / \mathrm{dl}^{*} 24 \mathrm{~h}$ at 4 and 6 months respectively. Average monthly AUC below $70 \mathrm{mg} / \mathrm{dl}$ remained constant throughout the study period (Table 1), and severe hypoglycemia occurred only once! No significant weight gain were noticed. There was a statistically significant decrease of Total Daily Insulin Dose from $56.1 \pm 16.1$ to $48.7 \pm 19.0$ and $45.4 \pm 16.8$ units $(\mathrm{X} \pm \mathrm{SD})$ at 4 and 6 months respectively with increased number of bolus (Table 1). All patients remained on the combined therapy after the end of the study.

Conclusion: Our results indicate that continuous use of real time CGM provide a useful tool to optimize glycemic control in patients with IDDM, on both MDI (after 1 month) and pump therapy thereafter. The additional use of CSII leads to a decrease of Total Insulin Dose less hyperglycemic excursions due to increased number of bolus and coupled with the information from real time CGM, it significantly improves glycemic profiles without increasing the risk of hypoglycemia.

TABLE 1

\begin{tabular}{llllllll}
\hline MONTHS & $\begin{array}{l}\text { BASE- } \\
\text { LINE }\end{array}$ & 1 & 2 & 3 & 4 & 5 & 6 \\
\hline $\begin{array}{l}\text { MONTHLY AVERAGE } \\
\text { GLUCOSE (mg/dl) }\end{array}$ & & 146.4 & 144.4 & 146.1 & 144.0 & 142.4 & 141.3 \\
$\begin{array}{l}\text { DURATIONS WITHIN } \\
\text { LIMITS }\end{array}$ & & $71 \%$ & $72 \%$ & $71 \%$ & $71 \%$ & $72 \%$ & $72 \%$ \\
$\begin{array}{l}\text { DURATIONS ABOVE } \\
\text { HIGH LIMITS }\end{array}$ & & $24 \%$ & $22 \%$ & $24 \%$ & $23 \%$ & $22 \%$ & $22 \%$ \\
$\begin{array}{l}\text { DURATIONS BELOW } \\
\text { LOW LIMITS }\end{array}$ & & $5 \%$ & $6 \%$ & $5 \%$ & $6 \%$ & $6 \%$ & $6 \%$ \\
AREA ABOVE HIGH & & 11.1 & 9.7 & 10.6 & 9.2 & 9.9 & 9.2 \\
LIMITS (mg/dl $\left.{ }^{*} 24 \mathrm{~h}\right)$ & & & & & & & \\
AREA BELOW LOW & & 0.6 & 0.6 & 0.5 & 0.5 & 0.6 & 0.6 \\
LIMITS (mg/dl $\left.{ }^{*} 24 \mathrm{~h}\right)$ & & & & & & & \\
HbAlc (\%) & 8.79 & & & & 6.91 & & 6.98 \\
DIF-WEIGHT (Kg) & 0 & & & & 1.6 & & 0.0 \\
TDD (U) & 56.1 & & & & 48.7 & & 45.4 \\
BASAL/BOLUS (\%) & $59 / 41$ & & & & $49 / 51$ & & $46 / 54$ \\
No BOLUS & 2.9 & & & & 6.4 & & 7.4 \\
\hline
\end{tabular}

1012

Use and acceptability of two minimally invasive continuous blood glucose monitors

D. Cooke ${ }^{1}$, L. Steed ${ }^{1}$, S. J. Hurel ${ }^{2}$, A. Nunn ${ }^{3}$, J. Ahlquist ${ }^{4}$, J. Weaver ${ }^{5}$,

D. Kerr' ${ }^{6}$, M. Barnard ${ }^{7}$, S. Newman ${ }^{1}$;

${ }^{1}$ Centre for Behavioural \& Social Sciences in Medicine, University College London, ${ }^{2}$ Dept of Diabetes \& Endocrinology, UCLH, LONDON, ${ }^{3} \mathrm{Clinical}$ Trials Unit, MRC, LONDON, ${ }^{4}$ Dept of Diabetes \& Endocrinology, Southend Hospital, Southend, ${ }^{5}$ Dept of Diabetes \& Endocrinology, Queen Elizabeth Hospital, Newcastle, ${ }^{6}$ Royal Bournemouth Hospital, BDEC, Bournemouth, ${ }^{7}$ Dept of Diabetes \& Endocrinology, Whittington Hospital, LONDON, United Kingdom.

Background and Aims: Continuous glucose monitoring has been developed to overcome the limitations of traditional blood glucose monitoring methods that only provide a snapshot view of blood glucose levels over time. Widespread use of new technology in healthcare, such as these devices is predicated on their acceptability to patients. Although a number of devices have been introduced in diabetes care, relatively little work has been performed on their acceptability. The aim of this study was to examine the relative use and acceptability of two such devices. 
Materials and Methods: As part of a randomised controlled trial, 102 people were randomised to the CGMS (Minimed) and 100 to the Biographer (Animas). All were insulin-requiring people with diabetes who had an $\mathrm{HbAlc}$ of at least $7.5 \%$. The acceptability of the devices was assessed over 3 -months, using a measure developed specifically for the trial. This examined the relative level of interference of the devices in participants' lives as well as the degree to which they were willing to accept this interference. Both the levels of interference and acceptability were compared between the groups using Mann-Whitney U Tests. Frequency of use was treated as a further indicator of acceptability and analysed using t-tests and ANOVAs.

Results: Four CGMS and 6 Biographer participants chose never to wear the devices. More Biographer than CGMS participants had stopped wearing the device by follow-up ( $48 \%$ v. $10 \%, \mathrm{p}<.001)$. In the CGMS arm, reasons for stopping use included fear of using the monitor $(n=1)$, psoriasis requiring treatment $(\mathrm{n}=1)$, and being too uncomfortable to sleep $(\mathrm{n}=1)$. Skin reactions $(\mathrm{n}=21)$ and difficulty using the device $(\mathrm{n}=8)$ were the main reasons for stopping use in the Biographer group. Mean usage over 3 months was 2.5 in the CGMS group ( $83 \%$ of that requested) and 10 times in the Biographer group (all less than requested). Skin problems were reported in 7\% of the CGMS and $98 \%$ of the Biographer group. The Biographer group reported greater interference with work $(\mathrm{p}=0.046)$ and skin care routine $(\mathrm{p}=0.001)$ while the CGMS group rated more interference with mobility $(\mathrm{p}=0.001)$. No association between ratings of interference and acceptability was found. Participants in the highuse Biographer group had higher fear of hypoglycemia scores but this was not significant.

Conclusion: Overall, the CGMS was more acceptable to participants than the Biographer in terms of both discontinuation rates and interference with lifestyle. It is notable that many participants continued to use both of these devices despite reporting significant interference with daily living. The use of these devices in the face of significant interference suggests that this is balanced by patients' perception of the potential value or importance of the devices in their care. This study demonstrates that it is possible to assess the relative acceptability of devices in diabetes. This is a crucial aspect in determining whether a device can be routinely incorporated into diabetes management.

Supported by NHS Health Technology Assessment Programme

\section{3}

Postprandial instead of preprandial calibration allows better accuracy of real-time continuous glucose monitoring (CGM)

I. Capel ${ }^{1}$, B. Pons ${ }^{1}$, M. E. Hernando ${ }^{2,3}$, G. García ${ }^{2}$, E. Brugués ${ }^{1}$, A. de Leiva ${ }^{1,3}$, E. Gómez ${ }^{2}$, A. Prados ${ }^{1}$, L. Erdozain ${ }^{1}$, M. Rigla ${ }^{1,3}$;

${ }^{1}$ Endocrinology, Hospital de Sant Pau, Barcelona, ${ }^{2}$ GBT, Universidad

Politécnica de Madrid, ${ }^{3}$ Isciii, CIBER BBN, Madrid, Spain.

Background and Aims: The last generation of CGM systems have the advantage of showing glucose data in real-time but their accuracy seem to be worse than the previous CGM systems with retrospective data download. The aim of our study was to find out calibration strategies for improving the agreement of both monitoring systems.

Materials and Methods: Eight patients with type 1 diabetes treated with insulin pumps were requested to use simultaneously the blind CGMSGold and the real time Guardian RT from Minimed-Medtronic ( 72 hours) in 3 different periods. Both needles were placed in the same abdominal side (distance $<3 \mathrm{~cm}$ ) and monitor's time was synchronised with the pump and the glycometer. The CGMS-Gold was calibrated preprandially along the study while, for the Guardian RT, 3 strategies were tested: 3 pre-meal calibrations, calibration at 1 hour postprandial and, finally, pre- and 1 hour postprandial. The simultaneously measured glucose levels were analysed with Pearson correlation and their mean absolute differences with the Wilcoxon test.

Results: Correlation between capillary glucose (used and not used for calibration) and glucose values of the monitor was better for the CGMS-Gold than for the Guardian RT (0.937 vs. 0.784). Calibrations including glucose at 1 hour after meals achieved a stronger correlation between Guardian RT and capillary glycemia and also a significant decrease in the mean absolute difference (see table).

Conclusion: One-hour postprandial calibration of the Guardian RT achieves a better accuracy than preprandial time. No benefit was shown when adding pre- to postprandial values.

\begin{tabular}{llll} 
& $\begin{array}{l}\text { Before meals } \\
\text { calibration }\end{array}$ & $\begin{array}{l}1 \text { hour } \\
\text { after meals } \\
\text { calibration }\end{array}$ & $\begin{array}{l}\text { Before and 1 } \\
\text { hour after meals } \\
\text { calibration }\end{array}$ \\
\hline $\begin{array}{l}\text { Correlation GuardianRT- } \\
\text { Capillary glucose }\end{array}$ & 0.784 & 0.950 & 0.964 \\
$\begin{array}{l}\text { Mean absolute difference } \\
\text { GuardianRT-CGMS }\end{array}$ & 38.27 & $23.4(\mathrm{p}<0.05)$ & 27.6 \\
$\begin{array}{l}\text { Gold (mg/dl) } \\
\begin{array}{l}\text { Correlation GuardianRT- } \\
\text { CGMS Gold }\end{array}\end{array}$ & 0.775 & 0.928 & 0.920 \\
\hline
\end{tabular}

\section{4}

Performance of FreeStyle Navigator against standards for Continuous Glucose Monitoring Systems and ISO15197 system accuracy blood glucose meter guidelines

J. R. Bugler, H. Pryor, C. Harris;

Abbott Diabetes Care, Witney, United Kingdom.

Materials and Methods: The FreeStyle ${ }^{\oplus}$ Navigator Continuous Glucose Monitoring System is an investigational continuous glucose monitor (CGM) based on Wired Enzyme ${ }^{\mathrm{mix}}$ glucose transduction technology. The FreeStyle Navigator system includes a disposable subcutaneous sensor that can be worn up to 5 days, measuring interstitial glucose concentrations. The accuracy and performance of the sensor were evaluated in a multi-center, prospective, single-arm study. Fifty-eight subjects aged 18 to 64 were enrolled, and each wore two sensors, one on the arm and one on the abdomen. Venous blood glucose measurements were taken using a standard laboratory reference (YSI ${ }^{\mathrm{m}}$ 2300) every 15 minutes for 50 hours distributed across the 5-day sensor wear, a total of 20,362 comparative measurements were made with the reference method. In the absence of specific ISO performance requirements for CGM's, performance, assessments are likely to be made against the ISO 15197 performance requirements for blood glucose meters. These requirements are designed for evaluation of the same sample on the test device and reference method and generally at times of stable glucose (as subjects attend a clinic appointment). It has not been possible to compare the same interstitial glucose sample for the FreeStyle ${ }^{\oplus}$ Navigator measurements to a reference method, hence a venous sample is drawn and glucose measurement made for comparison.

Results: The results demonstrate that at times of relative glucose stability (glucose changing at rates of $< \pm 30 \mathrm{mg} / \mathrm{dL} / \mathrm{hr}$ ) $87.0 \%$ (8984/10331) of FreeStyle ${ }^{\circ}$ Navigator CGM readings were within $\pm 0.83 \mathrm{mmol} / \mathrm{L}( \pm 15 \mathrm{mg} / \mathrm{dL})$ and within $\pm 20 \%$ of YSI venous reference values. By glucose range, system accuracy was $64.8 \%$ (263/406) within $\pm 15 \mathrm{mg} / \mathrm{dL}$ for glucose concentrations of less than $4.2 \mathrm{mmol} / \mathrm{L}(75 \mathrm{mg} / \mathrm{dL})$ and $87.2 \%(8721 / 9925)$ within $\pm 20 \%$ for glucose concentrations of at least $4.2 \mathrm{mmol} / \mathrm{L}(75 \mathrm{mg} / \mathrm{dL})$. The data set is considerably larger (50-fold) than recommended by the ISO15197 guidelines.

To date, the most widely quoted proposals on CGM performance assessment criteria is the Continuous Error Grid Analysis (Kovatchev, Clarke et al, Diabetes Care Aug 2004). Considering readings at all glucose levels and rates of change $(n=19,627)$, FreeStyle ${ }^{\oplus}$ Navigator provided accurate readings of $53.2 \%(\leq 70 \mathrm{mg} / \mathrm{dL}), 95.7 \%(70-180 \mathrm{mg} / \mathrm{dL})$ and $95.4 \%(>180 \mathrm{mg} / \mathrm{dL})$. If just stable glucose results (glucose changing at rates of $< \pm 30 \mathrm{mg} / \mathrm{dL} / \mathrm{hr}$ ) are assessed accurate readings of $68.0 \%(\leq 70 \mathrm{mg} / \mathrm{dL}), 99.5 \%(70-180 \mathrm{mg} / \mathrm{dL})$ and $99.6 \%(>180 \mathrm{mg} / \mathrm{dL})$ are calculated $(\mathrm{n}=9950)$.

Conclusion: The study results support the accuracy and clinical performance of the FreeStyle Navigator Continuous Glucose Monitoring System and demonstrate it as approaching ISO15197 expectations. However a full judgment against the ISO15197 criteria needs to account for the comparison of different samples (blood vs. ISF) and physiologic rates of change when evaluating CGM's. Any future 'standards' developed on performance requirements for CGM's need to account for this issue Supported by Abbott Laboratories 


\section{5}

Continuous glucose monitoring system glycaemic variability in pediatric type 1 diabetes patients on basal insulin plus prandial insulin: utility of Fourier analysis

M. Miller ${ }^{1}$, N. H. White ${ }^{2}$, H. Chase ${ }^{3}$;

${ }^{1}$ sanofi-aventis U.S., Bridgewater, United States, ${ }^{2}$ Pediatrics and Medicine, Washington University School of Medicine, St. Louis, United States,

${ }^{3}$ Barbara Davis Center, University of Colorado, Aurora, United States.

Background/Aims: Glycemic variability may be an important contributor to the risk of diabetes-related complications and glycemic control, independent of A1C level. In a subset $(\mathrm{n}=90)$ of pediatric patients with type 1 diabetes (T1D) participating in a trial comparing insulin glargine (GLAR) $(n=45)$ vs intermediate acting insulin (NPH/Lente) $(n=45)$ as part of a basal-prandial regimen, Continuous Glucose Monitoring System (CGMS) was used to assess glycemic variability. Each patient had complete 24-hour CGMS records at baseline and the results have been reported previously (EASD 2006). Notably, the CGMS standard deviation was significantly reduced from its baseline value for the GLAR group $(-13.4 \pm 3.1 \mathrm{mg} / \mathrm{dL}, P<0.001)$ but not for NPH/Lente $(-2.4 \pm 3.0 \mathrm{mg} / \mathrm{dL}, P=\mathrm{NS})$ at week 24 ( $P=\mathrm{NS}$, between groups). The current analysis applies Fourier methodology as a novel approach to assess whether components of glycemic variability at baseline are predictive for A1C.

Material/Methods: Each subject's 24-hour CGMS profile was approximated using the baseline 24-hour glucose mean plus a sum of trigonometric functions having integral frequencies of oscillation (starting from 1 cycle per 24 hours, then 2 cycles, then 3 cycles, etc). Amplitudes of each cycle can be considered components of the overall standard deviation. Patients' baseline data yielded sufficient precision for discerning cycles 1 through 5 . The 24hour glucose mean, $\mathrm{A} 1 \mathrm{C}$, and amplitudes of the first 5 harmonic cycles assessed at baseline for each subject were used in a multiple regression model predicting week $24 \mathrm{AlC}$.

Results: Baseline factors that were significant predictors of week 24 A1C were $\mathrm{A} 1 \mathrm{C}$ and the amplitudes of cycles 2 and 3. Combination of cycle 2 and cycle 3 baseline sinusoidal functions yielded a harmonic amplitude that was the most predictive factor for week-24 A1C $(P<0.0025)$. This amplitude was associated with baseline $\mathrm{A} 1 \mathrm{C}$ and baseline mean 24 -hour glucose (both at $P<0.01$ ). The higher this amplitude at baseline, the higher the week-24 AlC (even after adjusting for baseline $\mathrm{A} 1 \mathrm{C}$ ).

Conclusions: These data support that glucose variability may contribute to A1C outcome. The baseline amplitude of the second and third harmonic functions is not a component of the 24-hour mean glucose, but a component of variability about the mean, and thus likely most affected by patient meals (size and timing). Fourier analysis is a novel method for examining the relationships between glucose variability and clinical outcome. Further study will determine the ultimate utility of this approach.

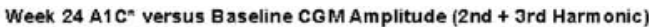

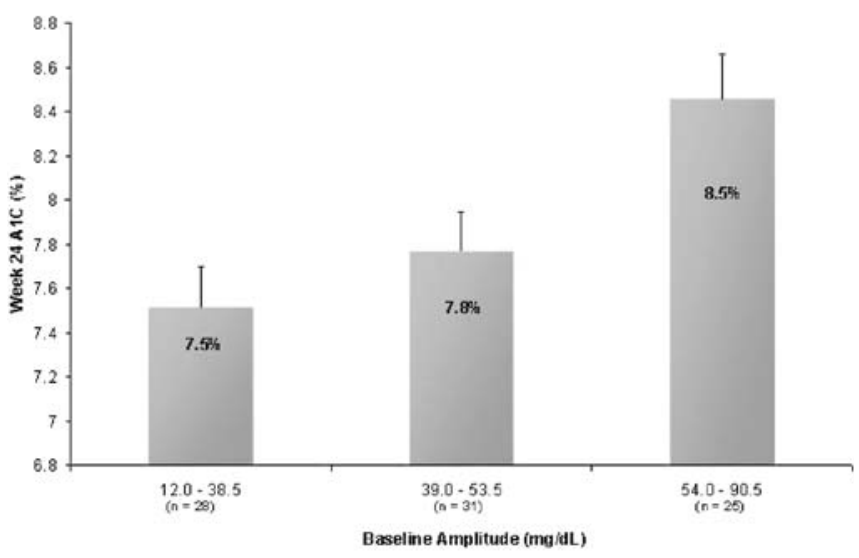

Week $24 \mathrm{~A} 1 \mathrm{C}$ estimates are least squares means djusted for baseline $\mathrm{A} 1 \mathrm{C}(\rho=0,03)$, age $(\rho=0.06)$ study arm ( $P=$ NS). The $P$ value for the second and third harmonic amplitude was $P=0.003$.

Editorial support provided by sanofi-aventis U.S.

\section{PS 100 Health care delivery: national variations in practice}

\section{6}

Comparison of an American and a Swedish diabetes population: Better glucose but worse blood pressure control in the Swedes

U. Lindblad ${ }^{1}$, A. Melander ${ }^{2}$, R. D. Langer ${ }^{3}$;

${ }^{1}$ Clinical Sciences, CRC, University Hospital, Malmö, Sweden, ${ }^{2}$ Nepi Foundation, CRC, University Hospital, Malmö, Sweden, ${ }^{3}$ Geisinger Center for Health Research, Geisinger Health System, Danville, United States.

Background and Aims: The hyperglycæmia of diabetes promotes both vascular and neuronal damage. Hypertension is common in diabetes patients and is a major contributor to the vascular complications. Hence, optimum control of both glucose and blood pressure levels is important, and it is of interest to assess and compare the extent of glucose and blood pressure control in populations in different countries. The populations served by the Geisinger Health System (GHS) in Pennsylvania, USA, and the Skaraborg Primary Health Care (SPH) in southwest Sweden differ geographically but have important demographic similarities. Both are primarily rural, have similar ethnic mix, health care infrastructure and physical environment, and minor immigration and emigration. Both have experienced a shift from physically demanding jobs to more sedentary occupations, and their prevalence of obesity is higher than their country average. Comprehensive longitudinal electronic health care records are available in both areas. Our aim was to compare glucose and blood pressure control in these two diabetes populations.

Materials and Methods: Diabetes patients seen within two preceding years were identified from the electronic records. Equivalent data on demographics, height, weight, HbAlc, systolic/diastolic blood pressure and medication were extracted. Uncontrolled glucose was defined as $\mathrm{HbAlc} \geq$ $7.0 \%$ and uncontrolled blood pressure as $\geq 140 / 90 \mathrm{~mm} \mathrm{Hg}$. Logistic regression analyses were carried out with age, gender, body mass index (BMI), number of antihyperglycæmic drugs, insulin dependence, treatment with an ACE inhibitor (ACEI) or angiotensin II receptor blocker (ARB), and type of health insurance as covariates when appropriate, and differences calculated as Odds Ratios (OR).

Results: Diabetes was present in 26,443 GHS and 10,391 SPH patients. The US diabetes population had a higher proportion of women (51.5 vs. $47.3 \%$; $\mathrm{p}<0.001$ ), was younger (mean 62.7 vs. 67.9 years; $\mathrm{p}<0.001$ ), more overweight (mean BMI 33.2 vs. $29.5 \mathrm{~kg} / \mathrm{m}^{2}$; p $<0.001$ ), treated with more antihyperglycæmic drugs ( $\mathrm{n}=1.6$ vs. 0.9 ; $\mathrm{p}<0.001)$, and had worse glucose control (proportion with HbA1c $\geq 7.0 \% 59.9$ vs. $25.4 \%$; $p<0.001$, and mean HbAlc 7.54 vs. $6.29 \%$; $<<0.001$ ). The difference in glucose control remained after adjustments for differences in gender distribution, age, BMI, insulin dependence, antihyperglycæmic drug treatment and access to care. In contrast to their worse glucose control, the US diabetes population had better blood pressure control. Mean blood pressure was lower (126/70 vs. 141/78 mm Hg; $\mathrm{p}<0.001$ ); more had antihypertensive medication (78 vs. $60 \%$; $\mathrm{p}<0.001$ ), including an ACEI or ARB (48.8 vs. 37.4\%; $\mathrm{p}<0.001$ ) and a diuretic (36.6 vs. $31.1 \% ; \mathrm{p}<0.001)$, and more of those on antihypertensive drug treatment had blood pressure < 140/90 mm Hg (67 vs. 32\%; p < 0.001). The difference in blood pressure control remained after adjustments for differences in gender distribution, age, BMI, and type of antihypertensive treatment.

Conclusion: In comparable American and Swedish diabetes populations, glucose control was better among the Swedes but blood pressure control better among the Americans. While differences in diet, alcohol, physical activity and gene-environment interactions may contribute, differences in screening, physician attitudes and treatment compliance are more likely.

\section{7}

Does Quality and Outcomes Framework (QoF) reflect a good standard of care for individual patients with diabetes?

J. Pachaiappan ${ }^{1}$, S. Koganti ${ }^{1}$, R. Gadsby ${ }^{2}$, J. Morrissey ${ }^{1}$, V. Patel ${ }^{1}$;

${ }^{1}$ Diabetes Centre, George Eliot Hospital, Nuneaton,

${ }^{2}$ Red Roofs Surgery, Nuneaton, United Kingdom.

Background and Aims: The UK GMS (General Medical Services) contract is devised to improve services to patients in primary care.The QoF (Quality 
and Outcomes framework) is the mechanism to assess GP (General Practice) performance. It enables payment for the quality of care given to patients not just the size of the list. Most practices achieved maximum points, reflecting good standards of care. The aim of this study is to find out whether QoF reflects a good standard of care for individual patients with diabetes.

Materials and Methods: 3 diabetes centres (one each from general practice, community and hospital) wre selected. From GP, all patients with diabetes (who were included for QoF, $n=537$ ) were selected.From community and hospital centres 99 and 100 patients were randomly selected respectively. QoF points for diabetes (except Flu indicator) for each of these centres and individualised $Q O F(\underline{i-Q O F})$ points for every patients in the study were calculated. Results were analysed.

Results: GP centre achieved 89 QoF points (maximum of 90 points excluding "Flu" indicator) compared to community 69.7 points and hospital 63.5 points reflecting different cohorts of patients looked after in each setting. $i-Q O F$ varied widely between the three settings, the average $i$-QoF being 79.9 for GP, 65.5 for community and 64.8 for hospital. Only $42 \%(n=270)$ of patients in GP, $8 \%$ in community and $3 \%$ in hospital achieved maximum $i$-QoF. The two poorly performed clinical indicators in the three (GP;community;hospital) settings were $\mathrm{HbA}_{1} \mathrm{c}$ of 7.5 or less $(75 \% ; 39 \% ; 35 \%)$ and $\mathrm{BP}$ of $145 / 85 \mathrm{mmHg}$ or less ( $78 \% ; 63 \% ; 71 \%)$.

Conclusion: Although QoF reflects improved standard of care for patients with diabetes, $i-Q o F$ showed the need for better standard of care for individual patients.

\begin{tabular}{llll} 
QoF vs i-QoF & & & \\
\hline Diabetes Centre & QoF points & average i-QoF & $\begin{array}{l}\text { i-QoF points } \\
\text { (inter-quartile range) }\end{array}$ \\
\hline GP & 89 & 79.9 & $73-90$ \\
Community & 69.7 & 65.4 & $52-73$ \\
Hospital & 63.5 & 64.8 & $54-75$ \\
\hline
\end{tabular}

\section{8}

Quality and Outcomes Framework (QOF) and GMS Contract: are they really representative of quality of service delivered in primary care in the UK

L. Varadhan ${ }^{1}$, S. Sankaranarayanan ${ }^{1}$, P. O Hare ${ }^{1}$, V. Patel ${ }^{2}$;

${ }^{1}$ Diabetes Centre, University Hospital Coventry and Warwickshire, Coventry, ${ }^{2}$ Diabetes Centre, George Eliot Hospital NHS Trust, Nuneaton, United Kingdom.

Background: The General Medical Services contract in the UK is a novel method of investing in primary care to facilitate flexibility of care delivery and reward practices based on their clinical and organisational quality. Apart from providing essential services, the practices could chose what services to provide, and get scored and paid based on depth of quality and breadth of framework delivered, as measured by the QOF indicators. A maximum of 99 point could be scored in diabetes care as shown in the table below, calculated as 3 points if $90 \%$ of patients had HbAlc checked and 16 points if $50 \%$ of them were $<7.4 \%$. However this system does not consider the proportion of patients beyond the threshold percentage needed to reach to score.

The aim of our study was to assess if the overall QOF score was actually representative of the individual diabetes care every patient was receiving, by calculating and correlating with an individualised QOF score(i-QOF).

Methods: Records of 404 patients with type 2 diabetes were collected from 6 different general practices from socially deprived area in Coventry, UK. The overall QOF score was calculated. However we calculated a maximum of 79 points only as data on smoking and feet examination were not available. Individual QOF scores were then calculated based on the same criteria and its average achieved.

Results: The overall QOF score achieved on this cohort of patients was 78.6/79.

\begin{tabular}{llll} 
Parameter & Target & GP/Max & $\%$ to target \\
\hline DM1 - Record keeping for patients & $100 \%$ & $6 / 6$ & $100 \%$ \\
DM11 - BP measured & $25-90 \%$ & $3 / 3$ & $97.5 \%$ \\
DM12 - BP $\leq 145 / 85$ & $25-55 \%$ & $17 / 17$ & $67.3 \%$ \\
DM16 - Total cholesterol (TC) & $25-90 \%$ & $3 / 3$ & $98.5 \%$ \\
measured & & & \\
DM17 - TC $\leq 5 \mathrm{mmol} / \mathrm{L}$ & $25-60 \%$ & $6 / 6$ & $81.7 \%$ \\
DM13 - Microalbuminuria & $25-90 \%$ & $2.6 / 3$ & $80.9 \%$ \\
DM14 - Record of creatinine & $25-90 \%$ & $3 / 3$ & $98.5 \%$ \\
DM5 - HBA1c record & $25-90 \%$ & $3 / 3$ & $98.3 \%$ \\
DM6 - HbA1 $\leq 7.4 \%$ & $25-50 \%$ & $16 / 16$ & $59.2 \%$ \\
DM7 - HbA1c $\leq 10 \%$ & $25-85 \%$ & $11 / 11$ & $91.1 \%$ \\
DM8 - Eye screening record & $25-90 \%$ & $5 / 5$ & $100 \%$ \\
DM15 - MA on ACE/ARB & $25-70 \%$ & $3 / 3$ & $76.2 \%$ \\
Total QOF & & $78.6 / 79$ & 62.95 \\
\hline
\end{tabular}

Apart from record of retinopathy, 100\% was not achieved in any of the above targets recommended. $2.5 \%$ of the patients did not have a proper record of blood pressure and 33\% failed to reach the target range recommended. 1.5\% had not had their cholesterol or HbAlc checked in the previous 15 months. $40.8 \%$ had an $\mathrm{HbAlc}$ above $7.4 \%$ and $8.9 \%$ had above $10 \%$. The average of $\mathrm{i}$ QOF calculated was 62.95/79, ranging from 11 to 79 , with $9 \%$ of the patients scoring less than 40 and only $39.1 \%$ reaching more than 70 . Considering $\mathrm{HbAlc} \leq 7.4 \%, \mathrm{BP} \leq 145 / 85$ and $\mathrm{TC} \leq 5 \mathrm{mmol} / \mathrm{L}$ as essential indicators of good diabetes management, $3.2 \%$ of the patients had all 3 parameters out of range, $17.3 \%$ had at least 2 out of range and $41.8 \%$ were not treated to target in atleast one of them.

Conclusion: The QOF scoring method may be overestimating the quality of care provided to patients with diabetes in the community. A significant number of patients may still not be treated to targets and require better service, which could be overlooked by the QOF scoring method. Individualised strategies are required to optimise diabetes service delivery in the community to achieve comprehensive results in terms of prevention of complications.

\section{9}

Influence of the national programme "Diabetes in the Community" on the diabetes patients' cost in Israel between the years 2003 - 2004 M. Goldfracht ${ }^{1,2}$, R. Adler ${ }^{3}$, N. Lieberman ${ }^{4}$, A. Porath ${ }^{4}$;

${ }^{1}$ Quality Improvement Department, Medicine Section, Community Division, Clalit Health Services, Tel-Aviv, ${ }^{2}$ Department of Family Health Care,, Bruce Rappaport Faculty of Medicine, The Technion, Haifa, ${ }^{3}$ Economics Revision Department, Community Division, Clalit Health Services, Tel-Aviv, ${ }^{4}$ Medicine Section, Community Division, Clalit Health Services, Tel-Aviv, Israel.

Background and Aims: The 'Diabetes in the Community' program has been in operation for over 10 years, the aims of which are to improve control and management of diabetes patients in Clalit, Israel. This study reviewed the relationship between quality of care and cost of diabetes in patients under the management of the Clalit Health Maintenance Organization (HMO), Israel during the years 2003 and 2004. Clalit is Israel's largest HMO and insures 70\% of diabetes patients in Israel, according to the National Indicators Program. Materials and Methods: The study analysed the cost impact of improved control of diabetes in approximately 200,000 diabetes patients in Clalit from 2003-2004, taking factors such as age, gender, ethnicity, socio-economic status, type of treatment, additional diagnoses, serious illnesses and oncology, into account.

Results: During the study period, the prevalence of diabetes in Israel was $3.6 \%$ of the population, and $5.0 \%$ for Clalit. This population has multiple illnesses (20\% having diabetes alone) and were older, $80 \%$ over 55 years of age. The low socio-economic status of this population was mirrored by the $39 \%$ of the diabetes population who relied on social security benefits, the costs for whom were, on average, $34 \%$ more than for those not receiving social security. Interestingly, the incidence of diabetes was $10 \%$ more in the non-Jewish sectors compared with the Jewish population. The financial burden of serious illness or oncology was such that these patients (11\%) 
were not included in the calculations. It is accepted that the costs for diabetes patients are likely to increase over time due to complications of the disease; however, despite the projected increase in weighted per-capita costs for diabetes patients between 2003 and 2004, the report found a 3.3\% decline in costs, with a saving of approximately corrected for purchasing power parity, PPP, US $\$ 3.46$ million per year. The decline in costs, observed in all age groups and districts, was primarily attributed to a reduction of $6.4 \%$ in the number of hospitalization days. Fewer hospitalization days and more ambulatory services were required for patients with well-controlled diabetes $(\mathrm{HbAlc}<=7)$, who used $21.2 \%$ fewer resources than those whose diabetes was poorly controlled $(\mathrm{HbAlc}=>9)$. This study showed that the average diabetes patient incurred costs 1.54 times more than the average HMO patient. Between 2003 and 2004 , the weighted per capita cost of diabetes patients declined by $3.3 \%$, in contrast with stable costs for all policyholders. The annual savings totaled PPP US\$ 3.45 million, primarily due to the decline in hospitalization costs, as a result of better control of the disease. An inverse correlation was established between diabetes patient costs and level of diabetes control, in that the wellcontrolled group used $21.2 \%$ fewer resources in comparison with the poorly controlled group.

Conclusion: The 'Diabetes in the Community' program has resulted in better control of diabetes in Clalit and, as well as improving the quality of life, morbidity and mortality of diabetes patients, has reduced associated costs by PPP US\$2.56-3.45 million per year.

The work has been translated into English and edited by support of Novo Nordisk

\section{0}

Improvements in the quality of clinical diabetes care in Norwegian general practice 1995 - 2005

J. G. Cooper ${ }^{1}$, T. Claudi' 2 A. K. Jenum³ ${ }^{3}$, M. F. Hausken4, W. Ingskog ${ }^{3}$; ${ }^{1}$ Department of Medicine, Stavanger University Hospital,

${ }^{2}$ General practice, Rønvik Medical Centre, Bodø, Norway, ${ }^{3}$ Diabetes

Research Centre, Aker University Hospital, University of Oslo, Norway,

${ }^{4}$ Stavanger Health Research, Stavanger University Hospital, Norway.

Background and Aims: Norway has had national guidelines for the care of diabetes in general practice since 1988. The guidelines have been revised in 1995,2000 and 2005. The aim of this study is to assess changes in quality of care for patients with type 2 diabetes between 1995 and 2005 .

Materials and Methods: 33 general practices in two geographical areas of Norway (Rogaland and Salten) participated in the study. Medical record data were collected from 1470 patients with type 2 diabetes in 1995 and 2792 patients in 2005. All known patients with diabetes in the practices were included in the study. Most of the practices were rural serving defined geographical populations. The same 33 practices participated in 1995 and 2005. Quality of care was assessed against predefined review criteria based on key recommendations in the national guidelines. Data collection from computerised records was manually validated by a research nurse/doctor in 1995 and by a research nurse in 2005 .

Results: Between 1995 and 2005 mean age decreased from 69.1 to 67.4 years, mean weight increased from 81.1 to $86.5 \mathrm{~kg}$, mean diabetes duration increased from 6.6 to 7.0 years. The quality of care improved markedly from 1995 to 2005. Important changes occured in several variables potentially related to improved health outcomes. These included significantly improved glycaemic control $\left(\mathrm{HbA}_{1 c} \leq 7.5 \%\right)$ from $52.1 \%$ to $69.0 \%$, control of systolic blood pressure $(\leq 140 \mathrm{mmHg})$ from $40.6 \%$ to $59.4 \%$ and control of serum cholesterol $(\leq 5.0$ $\mathrm{mmol} / \mathrm{l}$ ) from $18.1 \%$ to $54.0 \%$. Mean values improved significantly $\mathrm{HbA}_{1 \mathrm{c}}$ $7.74 \%$ to $7.15 \%$, systolic blood pressure 150.0 to $140.4 \mathrm{mmHg}$ and serum cholesterol 6.28 to $5.0 \mathrm{mmol} / \mathrm{l}$. There was an improvement in the percentage of patients with a recorded $\mathrm{HbA}_{1 c} 88.5 \%$ to $92.5 \%$, recorded blood pressure $86.5 \%$ to $89.2 \%$ and recorded serum cholesterol $50.0 \%$ to $87.8 \%$. Significant improvements also occured in referral to ophthalmological examination $52.6 \%$ to $76.1 \%$, documentation of smoking habits $21.4 \%$ to $60.0 \%$, recording of height $14.6 \%$ to $42.0 \%$ and weight $41.6 \%$ to $57.4 \%$. The percentage of patients treated with diet alone $30.4 \%$ vs. $28.3 \%$, oral agents alone $43.0 \%$ vs. $46.1 \%$ and insulin (with or without oral agents) $26.6 \%$ vs. $25 \%$ was similar in 1995 and 2005. Drug treatment of associated conditions was intensified in the same period, hypertension $33.1 \%$ vs. $59.8 \%$, dyslipidemia $4.8 \%$ vs. $45.7 \%$ and $19.7 \%$ vs. $33.9 \%$ received ASA. The number of patients with diabetes in the practices increased by $90 \%$ from 1995 to 2005 . Even after taking into account new diagnostic criteria in 1998 (with a lower cutoff for fasting plasma glucose) and increased efforts to diagnose diabetes early, our study suggest that there has been a striking increase in the prevalence of diabetes between 1995 and 2005.

Conclusion: Substantial improvements were seen in the quality of diabetes care in Norwegian general practice between 1995 and 2005.

Supported by Norwegian Diabetes Association, University of Tromsø, Norwegian Heath Region North and West, Novo Nordisk, Sanofi-Aventis, Eli Lilly

\section{1}

Current diabetes care status in China: an observational survey study (DIABCARE 2006)

C. Pan ${ }^{1}$, W. Yang ${ }^{2}$, W. Jia ${ }^{3}$, J. Weng ${ }^{4}$, H. Tian ${ }^{1}$;

${ }^{1}$ Dept.Endocrinology, Chinese PLA General Hospital, Beijing,

${ }^{2}$ Dept.Endocrinology, China-Japan Friendship Hospital, Beijing,

${ }^{3}$ Dept.Endocrinology, Shang Hai No. 6 People’s Hospital, Shanghai,

${ }^{4}$ Dept.Endocrinology, The First Affiliated Hospital,Sun Yet San University, Guangzhou, China.

Background and Aims: China is one of the countries with largest diabetes population. Surveillance of diabetes is an urgent and necessary step for the diabetes prevention and control. DIABCARE studies were designed to describe the current status of diabetes care in China and explore the relationships among diabetes control, diabetes management and late diabetic complications status, which may affect the quality of diabetes management. Four studies were initiated in the year of 1998, 2001, 2003 and 2006, providing an opportunity to demonstrate the change in diabetes management in China.

Materials and Methods: In 2006, a total of 2779 subjects with diabetes (mean age:61.5 years;BMI:24.6kg/ $\mathrm{m}^{2}$ ), who registered for management of diabetes for more than 12 months at 60 hospitals in 18 cities, were participated in this surveillance. The subjects were enrolled in an order of visit. No more than 10 consecutive subjects would be screened every day. Data were collected on a retrospective manner by reviewing patient medical records, interview and laboratory assessments. $\mathrm{HbA}_{1 \mathrm{c}}$ was measured at central laboratory. All data were tabulated and the descriptive statistical analysis was used.

Results: Mean age at the time when diabetes being diagnosed, diabetes duration, sex distribution and type of diabetes were comparable with previous DIABCARE studies. Of all, $97.8 \%$ of subjects were diagnosed as type 2 diabetic patients in DIABCARE 2006 project. The mean age at the time when diabetes being diagnosed was 52.8 years, and the mean diabetic duration was 8.6 years. Furthermore, BMI was increased from $23.7 \mathrm{~kg} / \mathrm{m}^{2}$ in 1998 to $24.6 \mathrm{~kg} / \mathrm{m}^{2}$ in 2006 . The mean $\mathrm{HbA}_{1 \mathrm{c}}$ was decreased from $8.8 \%$ in 1998 to $7.6 \%$ in 2006. Geographical difference in $\mathrm{HbA}_{1 c}$ levels was observed in cities. The the biggest gap of $\mathrm{HbA}_{1 c}$ levels among cities was more than $1 \%$. In 2006 , only one fourth of subjects achieved the target of $\mathrm{HbA}_{1 c} \leq 6.5 \% .40 \%$ of subjects met the $\mathrm{HbA}_{1 c}$ target $<7 \%$. An increase in the frequency of self blood glucose monitoring was observed: 9 times per month in 2006 compared to 4 times per month in $1998.96 .9 \%$ and $87.5 \%$ of subjects had at least one time of FPG and PPG monitoring in the past 12 months respectivley. In subjects treated with OADs, most of subjects had combination therapy (55.9\%). More subjects were treated with insulin either in combination with OAD or insulin alone in 2006. This increase was associated with diabetic duration. In 2006, $22.8 \%$ of subjects were treated with insulin. Eighty-eight percent of insulin treated patients achieved the treatment with human insulin, which was the most popular insulin therapy. More than half of insulin treated patients used with premixed human insulin (58.2\%).

Conclusion: The glycaemic control was improved in the majority of patients with diabetes during 1998-2006. However, glycaemic control was still unsatisfactory mainly due to lacking of more comprehensive and effective therapy. The gap between therapeutic guideline and daily clinical practice strongly indicates that there is an urgent requirement for dramatic improvements in diabetes management in China. 


\section{2}

A nationwide quality improvement project in Belgium: evolution over 5 years

N. Debacker ${ }^{1}$, F. Nobels ${ }^{2}$, P. Van Crombrugge ${ }^{2}$, A. Scheen ${ }^{3}$, C. Mathieu ${ }^{4}$, V. Van Casteren ${ }^{1}$;

${ }^{1}$ Epidemiology, Scientific Institute Public Health, Brussel,

${ }^{2}$ Endocrinology, OLV Ziekenhuis, Aalst, ${ }^{3}$ Endocrinology, CHU Sart Tilman, Liege, ${ }^{4}$ Endocrinology, UZ Gasthuisberg, Leuven, Belgium.

Background and Aims: Describe the organisation of the Initiative for Quality improvement and Epidemiology for Diabetes (IQED), a quality assurance system that was set up in 2001 in Belgian hospital-based multidisciplinary diabetes centres, and its effects on the quality of care in the 5 following years. Material and Methods: Up to now four cross-sectional data collections (2001, 2002, 2004 and 2006) were organised. Approximately 120 diabetes centres provided data on a random sample of $10 \%$ of their adult diabetes patients on $\geq 2$ daily insulin injections. By using the DiabCare basic information sheet at start and the new IQED-software from 2006 on, data on patient characteristics, glycemic control, cardiovascular risk profile, diabetes complications, blood glucose self-monitoring, follow-up procedures and treatment were obtained. Local quality evaluation and promotion were encouraged by returning an elaborate feedback (benchmarks) regarding process and outcomes, by a generation of a global report and by organising information meetings.

Results: The pooled sample consists of 9,194 (32\%) type 1 and 19,828 (68\%) type 2 diabetes patients, with a mean age of respectively 46 and 66 years and a mean diabetes duration of respectively 17 and 14 years. Especially the type 2 diabetes population presents a high prevalence of complications as expected in hospital-based centres. At start quality of care was much better in terms of risk factor testing rates, than in terms of patients meeting goals for risk factor management (table 1). Indeed except for the determination of LDL cholesterol, the other processes of care were carried out in at least $80 \%$ of the patients. After 5 years significant improvements were seen in almost all risk factor testing rates. Improvements in intermediate outcomes were less obvious, except for a clear reduction in LDL-cholesterol (from a mean of 121 $\mathrm{mg} / \mathrm{dl}$ in 2001 to $99 \mathrm{mg} / \mathrm{dl}$ in 2006). An enquiry revealed that at least $80 \%$ of the centres value the tools offered by this study. Moreover at least $50 \%$ of the centres initiated local quality promoting initiatives using the feedback data. Conclusions: The obligatory participation to the quality assurance system has led to a large sample of representative data on intensively treated diabetes patients in Belgium. The results show that it is feasible to implement a continuous quality improvement project on a nation-wide scale, with clear improvements in process indicators and to a lesser extent in intermediate outcome indicators.

Table 1: Risk factor control at start and in 2006

\begin{tabular}{|c|c|c|c|c|c|}
\hline & & \multicolumn{2}{|c|}{ Type 1 diabetes } & \multicolumn{2}{|c|}{ Type 2 diabetes } \\
\hline & & 2001 & 2006 & 2001 & 2006 \\
\hline \multirow[t]{2}{*}{ HbAlc (\%) } & $\%$ tested & 96.4 & $99.6^{*}$ & 95.4 & $99.3^{*}$ \\
\hline & $\%<7 \%$ & 28.5 & $21.3^{*}$ & 32.5 & $29.7^{*}$ \\
\hline \multirow{2}{*}{$\begin{array}{l}\text { LDL-cholesterol } \\
(\mathrm{mg} / \mathrm{dl})\end{array}$} & $\%$ tested & 42.2 & $48.2^{*}$ & 48.6 & $53.2^{*}$ \\
\hline & $\%<130 \mathrm{mg} / \mathrm{dl}$ & 68.1 & $84.7^{*}$ & 64.0 & $82.7^{*}$ \\
\hline \multirow{2}{*}{$\begin{array}{l}\text { Blood pressure } \\
(\mathrm{mmHg})\end{array}$} & $\%$ tested & 95.5 & $98.7^{\star}$ & 97.3 & $99.3^{*}$ \\
\hline & $\begin{array}{l}\%<140 / 90 \\
\mathrm{mmHg}\end{array}$ & 63.0 & $69.8^{*}$ & 37.0 & $47.2^{*}$ \\
\hline \multirow[t]{2}{*}{ BMI $\left(\mathrm{kg} / \mathrm{m}^{2}\right)$} & $\%$ tested & 82.4 & $94.7^{\star}$ & 79.4 & $91.9^{*}$ \\
\hline & $\begin{array}{l}\text { result } \\
(\text { mean } \pm \mathrm{SD})\end{array}$ & $25.4 \pm 4.4$ & $25.7 \pm 5.8^{*}$ & $30.4 \pm 5.9$ & $30.9 \pm 5.9^{*}$ \\
\hline \multirow[t]{2}{*}{ Smoking status } & $\%$ tested & 92.6 & $96.7^{*},{ }^{(1)}$ & 91.0 & $95.5^{*},{ }^{(1)}$ \\
\hline & $\begin{array}{l}\text { result } \\
\text { (\% smoking) }\end{array}$ & 22.6 & 22.1 & 15.0 & $12.9^{*}$ \\
\hline $\begin{array}{l}\text { Retinal } \\
\text { examination }\end{array}$ & $\%$ tested & 81.1 & $83.8^{*},{ }^{(1)}$ & 74.8 & $75.6^{(1)}$ \\
\hline $\begin{array}{l}\text { Foot sensory } \\
\text { examination }\end{array}$ & $\%$ tested & 82.7 & $90.3^{*},{ }^{(1)}$ & 86.0 & $87.5^{(1)}$ \\
\hline Renal screening & $\%$ tested & 81.7 & $84.2^{(1)}$ & 80.2 & $82.5^{*},{ }^{(1)}$ \\
\hline
\end{tabular}

${ }^{*} \mathrm{p}<0.05$; (1) data from 2004 since data from 2006 cannot be used for comparison because of a slightly changed questionnaire

\section{3}

Quality indicators of diabetes care: the Italian experience

M. C. Rossi ${ }^{1}$, F. Pellegrini ${ }^{1}$, A. Cimino ${ }^{2}$, C. Giorda ${ }^{3}$, I. Meloncelli', G. Vespasiani ${ }^{4}$, G. De Bigontina ${ }^{5}$, U. Valentini ${ }^{2}$, A. Nicolucci ${ }^{1}$;

${ }^{1}$ Clinical Pharmacology and Epidemiology, Consorzio Mario Negri Sud, S. Maria Imbaro (CH), ${ }^{2}$ Diabetes Unit, A.O. Spedali Civili, Brescia,

${ }^{3}$ Diabetes and Metabolism Unit, Local Health Unit and Maggiore Hospital, Chieri (TO), ${ }^{4}$ Diabetes Unit, Madonna del Soccorso Hospital,

S. Benedetto del Tronto (AP), ${ }^{5} \mathrm{UOS}$ di Diabetologia, Ospedale del Cadore, Dolomiti - Belluno, Italy.

Background and Aims: In the context of a national quality improvement program developed by the Associazione Medici Diabetologi (AMD), a comprehensive yet parsimonious set of process and outcomes indicators (www. aemmedi.it) identified to describe the performance of Diabetes Outpatient Clinics (DOCs) - AMD members - in Italy.

Materials and Methods: Information on quality indicators was derived from computerised medical records using a software specifically developed, allowing the creation of a standardised dataset to be analysed on an annual basis (AMD Data File). The first analysis was performed on all the patients who had had at least one encounter with the DOC during 2004. Process measures included frequency of testing of $\mathrm{HbAlc}$, lipids, blood pressure, microalbuminuria, and serum creatinine, and foot examination. Outcome measures included $\mathrm{HbAlc}$, blood pressure, and lipid levels. The percentage of patients achieving specific therapeutic goals were also evaluated. Centres performance was compared to a gold standard, that was based on the $75^{\circ}$ percentile of the values distribution of each process or favourable outcome indicator (or $25^{\circ}$ percentile for unfavourable outcomes) obtained in those centres with adequate completeness of their AMD Data File.

Results: Overall, 77 centres were included in the analysis. The gold standard was calculated on 30 centres. The final database contained 123,863 patients (median=1,286 per centre; range $=149-6,076)$; of these, $6,999(5,7 \%)$ had T1DM, 114,249 (92,2\%) had T2DM, and the remaining had other conditions. Among patients with diabetes, $53 \%$ were males and $56 \%$ were aged $>65$ years. Over 16,000 patients had been referred to a DOC for the first time during the examination year. Results show an acceptable metabolic control in both T1DM and T2DM (over $90 \%$ of patients had HbAlc measured every year and mean $\mathrm{HbAlc}$ values were $8.0 \pm 1.5$ and $7.4 \pm 1.5$, respectively), but process indicators document an unsatisfactory low rate of renal function monitoring (59\% in T1DM and 48\% in T2DM) and foot examination (23\% in T1DM and $22 \%$ in T2DM). Outcomes indicators highlight a significant rate of patients with uncontrolled hypertension (>130/85 mmHg: 56\% in T1DM and 70\% in T2DM) and dyslipidemia (LDL cholesterol $>100 \mathrm{mg} / \mathrm{dl}: 68 \%$ in T1DM and $70 \%$ in T2DM). A wide geographic variation was also documented, and the performance of individual centres showed marked differences in comparison with the gold standard for most of the quality indicators utilised.

Conclusion: Patterns of diabetes care will be re-analysed on annual basis and published in AMD Annals. Each centre will be able to compare its own performance against the gold standard and longitudinally across years, as a benchmarking activity. The use of "real" reference targets as a gold standard as opposed to theoretical guidelines could greatly help in improving the quality of diabetes care, by setting realistic objectives.

Supported by Lifescan 


\section{PS 101 Health care delivery: keeping up with the guidelines}

\section{4}

Primary care physicians identify and act on glycaemic abnormalities found in episodic, intensive blood glucose monitoring data from noninsulin treated type 2 diabetics

W. H. Polonsky' ${ }^{1}$, R. S. Wagner';

${ }^{1}$ Psychiatry, University of California, San Diego, Del Mar, United States, ${ }^{2}$ Roche Diagnostics, Indianapolis, IN, United States.

Background and Aims: Primary care physicians (PCPs) often make therapy adjustments for non-insulin treated (NIT) type 2 diabetes (T2D) on the basis of Alc alone without considering self-monitoring blood glucose (SMBG) data. The purpose of this study was to determine if PCPs could accurately identify glycemic abnormalities in episodic (short term), intensive SMBG data from NIT T2D and if their therapeutic decisions would be changed by evaluation of SMBG data.

Materials and Methods: Twenty-five case studies demonstrating a range of glycemic states were prepared from data from subjects with T2D who participated in a prior study on episodic, intensive SMBG. Case studies included patients' history, current medications, current A1c values, and 3-7 days of SMBG data. Five diabetes care experts evaluated the case studies, identified glycemic abnormalities, and determined if and how the patients' therapy should be changed. Subsequently, board certified Family Practitioners $(\mathrm{n}=36)$ and Internists $(\mathrm{n}=25)$ evaluated the case studies first without SMBG data and then with SMBG data. Their interpretations of the SMBG data were compared to the interpretations of the experts. The PCPs' therapeutic recommendations for case studies without SMBG data were compared to those the PCPs made for case studies with SMBG data to determine if the availability of SMBG data in addition to Alc values changed the PCPs' therapeutic recommendations. At the end of the study, a survey questionnaire evaluating the value of episodic SMBG data in addition to Alc results was administered.

Results: Two case studies representing normoglycemia were discarded after review due to poor design. For the 23 remaining case studies with SMBG data, $78 \%$ of the PCPs correctly identified normoglycemia, hypoglycemia, hyperglycemia, elevated fasting values and elevated postprandial values. When the PCPs assessed case studies with SMBG data, 77\% of them changed the therapeutic decisions they had made previously when evaluating the case studies without SMBG data. When hypoglycemia was identified, PCPs decided to change the time the current medication was given, reduce the dose of the current medication, discontinue the medication altogether, or change to a new medication. When frequently elevated fasting levels or elevated postprandial SMBG levels were apparent, PCPs elected to change the time the current medication was given, increase the dose of the current medication, switch to a new medication, or add a new medication to the current treatment regime. PCPs were asked to assess the added value of SMBG data when presented with Alc data. The PCPs (86\%) found the BG information to be of equal or greater value than the Alc while less than $14 \%$ found it to be of lesser or no value.

Conclusion: These data show that PCPs can correctly identify glycemic abnormalities in episodic, intensive SMBG data. Indeed, PCP's assessment of the SMBG data prompted most of them to change the therapeutic choices made initially when the case studies did not include the SMBG data. In addition, the vast majority of participating PCPs found the SMBG data to be valuable when evaluating the case studies. These findings suggest that PCPs can effectively utilize SMBG data in addition to Alc when managing NIT T2D. Additional studies may be worthwhile to determine the impact of episodic, intensive SMBG monitoring on glycemic control in actual clinical practice.

Supported by Roche Diagnostics

\section{5}

Effect of nurse-led intensified management of hypertension in patients with and without advanced diabetic nephropathy

S. Anandarajah, R. Squires, K. Kular, T. Khong, D. Harry, K. Zitouni, K. A. Earle;

Cellular and Molecular Medicine, Thomas Addison Diabetes Centre, London, United Kingdom.

Background and Aims: Lowering blood pressure is the key strategy for preserving renal function in patients with diabetes. Recent reports suggest that a nurse-led approach is more effective than conventional care in achieving this aim. It is unknown to what extent this strategy is applicable or advantageous in patients with advanced nephropathy in secondary care Materials and Methods: Consecutive patients with diabetes and a systolic (SBP) and/or diastolic blood (DBP) pressure $>130$ and/or $>80 \mathrm{mmHg}$ with either advanced nephropathy (ADN) from a nephrology clinic, or with preserved renal function (PRF) from a diabetes clinic were eligible to enter a 6 month programme of nurse-led, intensified care for hypertension.

Patients received a baseline assessment and standardised nonpharmacological advice. Sitting BP was measured after 5 minutes rest using a digital oscillometric device. Venous blood was sampled to measure total cholesterol (TC) by enzymatic method, glycated haemoglobin (HbAlc) by HPLC and creatinine by a Jaffe rate-reaction. Patients were reviewed monthly by a nurse not specialised in diabetes care who adjusted treatment according to an algorithm and addressed issues of concordance.

Results: One hundred patients (57\% male) were studied. There were no differences in mean [SD] age and baseline SBP, DBP and TC between the PRF $(\mathrm{n}=39)$ and ADN $(\mathrm{n}=61)$ groups $(61.6[13.1]$ vs. $64.8[12.6] \mathrm{yrs}$; $\mathrm{p}=0.23,143.2[18.0]$ vs. $146.3[20.7] \mathrm{mmHg} ; \mathrm{p}=0.42,78.8[12.7]$ vs. $76.1[12.4]$ $\mathrm{mmHg} ; \mathrm{p}=0.30$ and $4.5[1.3]$ vs $4.7[1.0] \mathrm{mmol} / \mathrm{l} ; \mathrm{p}=0.58$ ) respectively. Plasma creatinine (89.4[38.8] vs. 123.1[59.5] umol/l; $\mathrm{p}=0.002)$ and HbAlc (7.1[1.3] vs. $8.2[1.7] \% ; \mathrm{p}=0.001$ ) were significantly lower in PRF compared with the ADN group. At the end of the programme there were significant decrements in the PRF and ADN groups for mean [95\% confidence interval] SBP (- 9.9 $[-2.1$ to -17.7$] ; \mathrm{p}=0.02$ and $-9.6[-3.3$ to -16.0$] ; \mathrm{p}=0.003)$ and $\mathrm{DBP}(-5.5[-0.2$ to -10.9$] ; \mathrm{p}=0.04$ and $5.2[-1.4$ to- 9.1$] ; \mathrm{p}=0.009)$ and a tendency for HbAlc to be lower $(-0.5[0.0$ to -1.0$] ; \mathrm{p}=0.05$ and -0.42 [0.03 to -0.87$] ; \mathrm{p}=0.07$ respectively). There was a significant fall in total cholesterol in the ADN group $(-0.60[-0.3$ to -0.9$] ; \mathrm{p}=0.001)$

Conclusion: Patients with advanced diabetic renal disease benefit from a nurse-led approach in the management of uncontrolled hypertension similarly to less complex patients. This intervention also positively impacts on cardiovascular disease risk and diabetes control. A regimen of monthly review by nurses is a useful adjunct to secondary care.

\section{6}

Variation in efficiency of diabetes care across 28 medical groups: potential for improved care and reduced costs P. J. O’Connor, T. J. Flottemesch, R. R. Paskach, G. J. Kitching; Research, HealthPartners Research Foundation, Minneapolis, United States.

Background and Aims: The objectives of this analysis are: 1) to explore methods of measuring the efficiency of diabetes mellitus (DM) care across medical groups (MG)s, 2) to quantitate variation in the provision of diabetes care provided by MGs, and 3) to estimate potential resource savings and quality improvements.

Materials and Methods: Study subjects were 2,511 adults with DM, insured by a single payer during 2003-2004, and receiving care at one of 33 Minnesota medical groups. Chart abstractions assessed Alc $<8 \%, \mathrm{BP}<130 / 85$, and $\mathrm{LDL}<130 \mathrm{mg} / \mathrm{dl}$. Standard methods were used to calculate resource utilisation by converting all billing into resource value units (RVUs) scaled by a single cost factor. Quality of care was quantified as the proportion of patients achieving two or more clinical goals (Alc $<8 \%$, BP $<130 / 85$, or LDL $<130 \mathrm{mg} / \mathrm{dl}$ ). Non-parametric Data Envelopment Analysis(DEA) was used to model the relationship between resource utilisation and quality of care, to rank MGs in terms of efficiency of diabetes care, and to identify performance benchmarks for MGs.

Results: MGs with fewer than $30 \mathrm{DM}$ patients $(\mathrm{n}=5)$ were excluded. The remaining 28 MGs averaged 109 sampled DM patients (range: 174 to 38 ). Median per patient costs were $\$ 19,045$ with MG-specific medians ranging 
from $\$ 8,822$ to $\$ 38,842.73 \%$ of all patients $(n=1,824)$ achieved two or more goals with MG-specific range from $57 \%$ to $82 \%$. After controlling for patient age and comorbidities, three MGs were identified as relatively efficient "benchmarks", four were classified as outliers, and twenty-one were found inefficient. If inefficient MGs performed at benchmark levels an estimated $35 \%$ decrease in resource use OR an $8 \%$ increase in patients achieving two or more goals could be realized.

Conclusions: There is considerable variation in efficiency of diabetes care across the medical groups in this study. Health plans or national health care systems can use existing data sources to assess efficiency of diabetes care across medical groups. Improved efficiency of diabetes care could lead to either cost reduction or quality improvement and could have substantial benefits for both patients and payers.

Supportd by HealthPartners Research Foundation

\section{7}

EuroAction: the achievement of lifestyle and risk factor targets in high risk individuals and their partners

K. Kotseva ${ }^{1}$, S. B. Connolly ${ }^{1}$, C. Jennings ${ }^{1}$, A. Mead ${ }^{1}$, J. Jones ${ }^{1}$, A. Holden ${ }^{1}$, T. Collier', D. De Bacquer ${ }^{3}$, G. De Backer ${ }^{3}$, D. A. Wood';

${ }^{1}$ Cardiovascular Medicine, Imperial College, London, United Kingdom, ${ }^{2}$ Epidemiology and Public Health, London School of Hygiene and Tropical medicine, London, United Kingdom,

${ }^{3}$ Public Health, University of Ghent, Belgium.

Background and Aims: EuroAction is a cluster randomised controlled trial of a nurse-led multi-disciplinary preventive cardiology programme which aims to manage high risk patients and their partners to the European lifestyle, risk factor and therapeutic targets for cardiovascular disease prevention.

Materials and Methods: In each of 6 European countries, pairs of comparable general practices were randomised to provide the EuroAction cardiovascular prevention programme (INT) or to be monitored for usual care (UC). High risk individuals $\geq 50$ years with no history of cardiovascular disease were identified in 3 groups: (I) at high multifactorial risk (HeartScore $\geq 5 \%$ over 10 years); (II) treated hypertension/dyslipidaemia; and (III) diabetes mellitus < 3 years. Patients and their partners were invited to attend the EUROACTION programme with a follow up assessment at one year. Using random effects meta-analysis (REML estimation) differences in proportions achieving goal were compared.

Results: 1019 patients in INT and 1005 patients in UC attended the one year assessment. 225 partners (63\% of those contactable) in INT and 335 partners in UC (67\% of those contactable) attended the one year assessment. Despite patients in Groups I and II being proactively screened for diabetes, there was no significant difference in the overall prevalence of diabetes at one year $(32.5 \%$ INT, $26.8 \% \mathrm{UC}, \mathrm{p}=0.46)$. There was a trend towards a higher prevalence of impaired glucose tolerance in INT compared with UC $(10.0 \%$ INT, $2.0 \%$ in UC, $\mathrm{p}=0.08$ ). Tables 1 shows the proportions of patients and their partners achieving the European lifestyle, risk factor and therapeutic targets.

Conclusion: Although EUROACTION programme did not result in significant reduction in central obesity, it did help high risk individuals and their partners to significantly increase their physical activity levels in comparison with usual care. In addition, there was a significant improvement in their blood pressure control with a trend towards improved glycaemic control. There was also an improvement in the use of cardioprotective medication particularly ACE inhibitors/ARBs and statinsin high risk patients. Therefore EUROACTION achieved its overall aim in raising standards of preventive cardiology care for high risk patients and their partners in everyday clinical practice.
Proportions of patients and partners achieving European targets at one year

\begin{tabular}{lllll}
\hline Proportions achieving target & Patients INT & $\begin{array}{l}\text { Patients } \\
\text { UC }\end{array}$ & $\begin{array}{l}\text { Partners } \\
\text { INT }\end{array}$ & $\begin{array}{l}\text { Partners } \\
\text { UC }\end{array}$ \\
\hline $\begin{array}{l}\text { Waist circumference: } \\
\text { M }<94 \mathrm{~cm}, \mathrm{~W}<80 \mathrm{~cm}\end{array}$ & 27 & 21 & 34 & 30 \\
$\begin{array}{l}\text { European physical activity } \\
\text { guidelines }\end{array}$ & $50^{*}$ & 22 & $44^{*}$ & 25 \\
BP $<140 / 90 \mathrm{~mm} \mathrm{Hg}$ & & & & \\
$(<130 / 85$ in diabetes $)$ & $58^{*}$ & 41 & $71^{*}$ & 53 \\
TC $<5$ mmol/L & & & & \\
LDL-C $<3$ mmol/L & 36 & 32 & 33 & 30 \\
HbA1c $<7.0 \%$ & 45 & 35 & 39 & 38 \\
Antiplatelet & 80 & 65 & $* *$ & $* *$ \\
Beta-blocker & 13 & 10 & 14 & 9 \\
ACE inhibitor/ARBs & 17 & 16 & 19 & 12 \\
Statins & $38^{*}$ & 24 & 12 & 11 \\
\hline * & $38^{*}$ & 22 & 22 & 15 \\
\hline
\end{tabular}

${ }^{*} \mathrm{p}<0.05 ;{ }^{* *}$ to few for statistical analysis

Supported by Astra Zeneca

\section{8}

$\mathrm{HbA}_{1 \mathrm{c}}$ : the gap between guidelines and clinical reality across eight countries

E. S. Kilpatrick ${ }^{1}$, C. Ørskov ${ }^{2}$, K. Berntorp 3 , T. Koblik ${ }^{4}$;

${ }^{1}$ Clinical Biochemistry, Hull Royal Infirmary, United Kingdom,

${ }^{2}$ Novo Nordisk A/S, Copenhagen, Denmark,

${ }^{3}$ Endocrinology, Malmö University Hospital, Sweden,

${ }^{4}$ Clinic of Metabolic Diseases, Jagiellonian University, Krakow, Poland.

Background and Aims: Expert guidelines highlight that good glycaemic control is vital to improve quality of life and reduce the risk of complications in patients with diabetes. Despite this, glycaemic control is sub-optimal, with $\mathrm{HbA}$ levels $>6.5 \%$ in the majority of patients. For example, $83 \%$ of patients in India have reported $\mathrm{HbA}_{1 \mathrm{c}}$ levels $>7 \%$ and approximately half of patients in the UK have levels $\geq 7.5 \%$. We present data from a recent, global survey that evaluated $\mathrm{HbA}_{1 \mathrm{c}}$ awareness, attitudes and behaviours among healthcare professionals (HCPs) and patients across 8 countries, with the aim of identifying factors responsible for the gap between guidelines and clinical reality.

Materials and Methods: $185 \mathrm{HCPs}$ and 95 patients with type 2 diabetes in Canada, Poland, Russia, Sweden, Turkey and the UK (Aequus Research), China (Synovate) and India (AC Nielsen) took part in 1-hr face-to-face interviews to provide qualitative data on the underlying causes of the difference between the guidelines and clinical reality. A larger sample of HCPs $(n=1357)$ and patients $(n=1006)$ participated in 20-min telephone interviews to obtain quantitative data, including medication, information sources, glycaemic control and frequency of consultation. Patients and HCPs had tailored questions.

Results: The patient interviews showed differences between countries in terms of awareness of $\mathrm{HbA}_{1 c}$ (Table) and communication with their HCP. Patient awareness of $\mathrm{HbA}_{1 \mathrm{c}}$ ranged from $15 \%$ in China and $19 \%$ in India, to $92 \%$ in Sweden, with $62 \%$ of Swedish patients regarding $\mathrm{HbA}_{\text {c }}$ as very important on a scale of 1 to 5 ( 5 being 'very important'). These findings suggest that the least $\mathrm{HbA}_{1 \mathrm{c}}$ awareness seemed to occur in countries likely to experience largest growth in diabetes numbers. The interviews also showed that patients in China have only 5-10 mins to consult with their HCPs, compared with 19 mins in India (defined as length of time explaining $\mathrm{HbA}_{1 \mathrm{c}}$ test) and $30 \mathrm{mins}$ on average in Sweden. In China approximately $80 \%$ of HCPs stated that they routinely tested $\mathrm{HbA}_{1}$, while approximately $25 \%$ of patients reported having their $\mathrm{HbA}_{1 \mathrm{c}}$ levels tested at every 3-month visit. Other findings from the HCP interviews were more positive. In Sweden, $70-87 \%$ of HCPs regarded $\mathrm{HbA}_{1 c}$ as highly important, and took frequent measurements (2-3 times a year). Similarly, in Canada, India and the UK, the HCPs reported taking 2-4 measurements a year on average.

Conclusion: These findings indicate a need for greater awareness of the importance of $\mathrm{HbA}_{1 c}$ among patients, and for changes in the way many HCPs and patients think and act with respect to this measurement. The next step will be to identify what practical solutions are needed to facilitate these changes 
and address the gap between guidelines and clinical reality. Data from some countries, for example Sweden and the UK, indicate that improvements are achievable.

\begin{tabular}{ll} 
Table & \\
\hline Country & Percentage of patients aware of $\mathrm{HbA}_{1 \mathrm{c}}(\%)$ \\
\hline China & 15 \\
Canada & 45 \\
India & 19 \\
Poland & 33 \\
Russia & 57 \\
Sweden & 92 \\
Turkey & 55 \\
UK & 61 \\
\hline
\end{tabular}

Supported by Novo Nordisk A/S

\section{9}

Patterns of glycaemic control across Europe: has clinical practice kept pace with recommended targets for blood glucose management? P. D. Ambery ${ }^{1}$, B. Pandya ${ }^{1}$, M. Benford ${ }^{2}$, M. Evans ${ }^{3}$;

${ }^{1}$ Metabolic Centre of Excellence, Glaxosmithkline, Uxbridge,

United Kingdom, ${ }^{2}$ Adelphi Europe, Adelphi, Bollington, United Kingdom,

${ }^{3}$ Department of Medicine, University of Cardiff, United Kingdom.

Introduction: Liebl described in 2002 that more than 2/3rds of patients were missing the $\mathrm{HbA}_{1 c}$ target of $6.5 \%$. Since then this target has been reiterated by IDF guidelines, and regular $\mathrm{HbA}_{1 \mathrm{c}}$ testing 3-4 times/year has been endorsed. This Independent patient record study, (Adelphi Diabetes Tracker 2006), examines parameters relating to glycaemic control across 5 major European Countries.

Methods: 622 randomly selected Primary Care Physicians (PCPS) in UK, France(Fr), Germany(Ger), Italy(It) and Spain(Sp) submitted questionnaires on management of their 8 most recent consulting T2DM patients receiving $\mathrm{OAD}$ therapy $+/$ - insulin. Most recent actual $\mathrm{HbA}_{1 c}$ was recorded along with an individualised target $\mathrm{HbA}_{1 \mathrm{c}}$, frequency of $\mathrm{HbA}_{1 \mathrm{c}}$ testing during previous year $(12 / 12)$ and previous and current therapies.

Results: See results table, P for UK differences shown in the table $<0.001$. In addition we found that $29 \%$ of UK patients had $\mathrm{HbA}_{1 \mathrm{c}}>8 \%,(14 \%$ across other 4 countries) and that $68 \%$ of oral monotherapy patients across all 5 countries have $\mathrm{HbA}_{1 \mathrm{c}}>6.5 \%$.

Conclusion: Glycaemic control across Europe remains sub-optimal, with over $2 / 3$ rds of patients receiving oral hypoglycaemic monotherapy failing to achieve target $\mathrm{Hba}_{1 \mathrm{c}}$. Whilst the frequency of $\mathrm{HbA}_{1 \mathrm{c}}$ testing across all 5 countries gives grounds for optimism, aspirations for $\mathrm{HbA}_{1 \mathrm{c}}$ achievement are above $6.5 \%$ in every country apart from Italy. This is associated with achieved mean $\mathrm{HbA}_{1 c}$ levels above $7 \%$ in every case. If we are to impact on the future burden of complications in type 2 diabetes then more aggressive intensive management in line with guidelines is essential.

\begin{tabular}{lllll}
\multicolumn{2}{l}{ Results Table } \\
\hline Country & $\begin{array}{l}\text { Number } \\
\text { Evaluable } \\
\text { HbA }_{1 c} \text { tests }\end{array}$ & $\begin{array}{l}\text { Average } \\
\text { Target } \\
\text { HbA }_{1 c}\end{array}$ & $\begin{array}{l}\text { Mean } \\
\text { achieved } \\
\text { HbA }_{1 c}\end{array}$ & $\begin{array}{l}\text { Average } \\
\text { number HbA } \\
\text { tests prev year }\end{array}$ \\
\hline UK & 515 & 7.1 & 7.7 & 2.0 \\
France & 588 & 6.8 & 7.3 & 3.1 \\
Spain & 353 & 6.7 & 7.2 & 2.8 \\
Germany & 594 & 6.6 & 7.2 & 2.9 \\
Italy & 481 & 6.2 & 7.2 & 2.5 \\
\hline
\end{tabular}

Funded by GSK

\section{0}

Do we correctly follow type 2 diabetes mellitus therapeutic guidelines in daily clinical practice?

A. Calderón ${ }^{1}$, V. Barrios ${ }^{2}$, G. Romero ${ }^{3}$, V. Pérez ${ }^{1}$;

on behalf of the Post-Adelante study group,

${ }^{1}$ Family Medicine, CS Rosa de Luxemburgo, Madrid, ${ }^{2}$ Cardiology Institute, Hospital Ramon y Cajal, Madrid, ${ }^{3}$ sanofi-aventis S.A., Barcelona, Spain.

Background and Aims: The guidelines for the diagnosis and treatment of patients with type 2 diabetes (T2D) are based on algorithms derived from randomized controlled clinical trials. Currently, it is not known if these guidelines are followed and applied in daily clinical practice. Accordingly, we sought to determine profiles of patients with T2D poorly controlled with two or more oral antidiabetic agents (OAD) who would benefit from initiating insulin treatment according to the $2005 \mathrm{ADA} / \mathrm{EASD}$ guidelines included in the Post-Adelante study, as well as evaluating the degree of compliance with the guidelines.

Materials and Methods: The Post-Adelante study is a 4-month observational multicentre study, designed to examine the metabolic and cardiovascular profiles of patients with T2D inadequately controlled with OADs who initiated insulin therapy. It aims to evaluate the efficacy of adding insulin to OADs after 4 months in a primary care setting. The study was approved by a local Ethics Committee. All patients completed a written consent form. Results: Of 1714 patients included, 1500 were valid for the analysis. 55.8\% were women, age $64.7 \pm 10.8$ years, body mass index $29.2 \mathrm{~kg} / \mathrm{m}^{2}, \mathrm{HbA}_{1 \mathrm{c}} 8.6$ $\pm 1.1 \%$, morning fasting plasma glucose (FPG) $10.5 \pm 2.8 \mathrm{mmol} / \mathrm{L}(189.9$ $\pm 50 \mathrm{mg} / \mathrm{dL}$ ), and duration of T2D was $10.5 \pm 6.6$ years. Metformin (Met) was the most used drug ( $80.6 \%$ of patients) followed by sulfonylureas (SU) (75.3\%), $\alpha$-glucosidase inhibitors (GI) (22.1\%), rapid-acting secretagogues (RAS) (9.2\%) and glitazones (GTZ) (5.6\%). The most frequent daily dose of Met was $2550 \mathrm{mg}$ ( $47 \%$ of patients taking Met), SU: $15 \mathrm{mg}$ of glibenclamide (40.8\% of patients taking SU), GI: $300 \mathrm{mg}$ acarbose $(59.05 \%$ of patients taking GI), RAS: $6 \mathrm{mg}$ of repaglinide (49.6\%), and GTZ: $4 \mathrm{mg}$ rosiglitazone (29.4\%). $17.3 \%$ of patients used one ( $46.2 \%$ of them Met, $43.1 \%$ SU), $69.3 \%$ two and $13.2 \%$ three OADs. The most frequent combinations were Met $+\mathrm{SU}$ for double therapy $(70.5 \%$ of patients using 2 OADs) and Met + SU + GI for triple therapy (72.1\% of patients using 3 OADs). Although $71.2 \%$ of patients had $\mathrm{HbA}_{1 c} \geq 8 \%$ and $87 \%$ had FPG $>7.8 \mathrm{mmol} / \mathrm{L}(>140 \mathrm{mg} / \mathrm{dL})$, only $13.2 \%$ were on intensive therapy with three OADs.

Reasons why physicians altered treatment were: bad glycaemic control $(97.7 \%$ of cases); poor compliance (18.4\%); hypoglycaemia (6.6\%); and treatment change initiated at the patient's request (4.0\%). Upon insulin initiation, $28.4 \%$ of patients discontinued OAD therapy, $44.4 \%$ of patients used basal insulin (BI) added to one OAD, 25.4\% used two OADs + BI, 1.6\% used three OADs $+\mathrm{BI}$ and $0.08 \%$ used four OADs + BI. In addition to insulin, Met was used by $57.9 \%$ of patients, SU by $30.7 \%$, GI by $9.9 \%$, RAS by $5.4 \%$ and GTZ by $3.0 \%$. The most frequent daily dose of Met was also reduced to $1.7 \mathrm{mg}(42.8 \%$ of patients taking Met), while no changes were observed in relation with the most frequent dose of the other OADs. The most frequent therapies were Met + BI (31.3\% of patients), followed by BI (28.4\%), Met + SU + BI (17.3\%) and $\mathrm{SU}+\mathrm{BI}(6.9 \%)$.

Conclusion: According to ADA/EASD 2005 guidelines, the choice of OADs and dosages were correct, however, in order to achieve adequate glycaemic control, the add-on treatment effect was clearly insufficient. According to the new ADA/EASD 2006 guidelines, it appears necessary to add or change the choice of drugs and the combination strategy when starting basal insulin. Supported by sanofi-aventis

\section{1}

Evaluation of a complex intervention: a cluster randomised controlled trial of enhanced diabetes care - the United Kingdom Diabetes Study (UKADS)

N. T. Raymond ${ }^{1}$, S. Bellary ${ }^{2,3}$, P. J. O'Hare ${ }^{1}$, S. Mughal ${ }^{2}$, A. Szczepura ${ }^{1}$, S. Kumar' ${ }^{1}$ A. H. Barnett ${ }^{2,3}$; on behalf of the UKADS Study Group, ${ }^{1}$ Health Sciences Research Institute, Warwick Medical School, Coventry, ${ }^{2}$ Diabetes, Heart of England NHS Foundation Trust, Birmingham, ${ }^{3}$ Diabetes, University of Birmingham, United Kingdom.

Background and Aims: Intensive management in type 2 diabetes can improve clinical outcomes, but delivering healthcare to achieve targets remains a 
challenge for health care professionals. Within a complex interventions framework and following a pilot study, we investigated the effectiveness of a culturally sensitive enhanced care package designed to improve cardiovascular risk factors in patients of South Asian ethnicity with type 2 diabetes.

Materials and Methods: In Coventry and Birmingham, UK, 21 practices were randomised to either intervention (protected practice nurse time, link worker sessions and diabetes specialist nurse support) or standard, care groups. Treatment protocols, including detailed prescribing algorithms for control of diabetes, blood pressure and lipids were provided for both groups. Outcome measures were blood pressure, total cholesterol, HbAlc and proportions of patients achieving internationally recommended and Quality and Outcomes Framework (QOF) targets after 1 year. Analyses used multiple linear and mixed regression methods to estimate intervention effects allowing for confounding factors and cluster randomisation.

Results: 1494 consenting patients of South Asian ethnicity with type 2 diabetes were included in analyses. Baseline differences between intervention and control groups were significant for systolic and diastolic BP, gender and diabetes duration. After one year, significant decreases in systolic blood pressure $(4 \mathrm{mmHg})$, diastolic blood pressure $(2 \mathrm{mmHg})$, total cholesterol $(0.3 \mathrm{mmol} / \mathrm{l})$ and proportions of patients achieving blood pressure and total cholesterol targets were achieved for the whole study group. With adjustment for confounding factors, the intervention group achieved statistically significantly lower systolic $(-2.1 \mathrm{mmHg}(-3.7$ to -0.4$), \mathrm{P}=0.013)$ and diastolic $(-1.4 \mathrm{mmHg}(-2.4$ to -0.4$), \mathrm{P}=0.004)$ blood pressures. After adjustment for clustering, only diastolic blood pressure remained significant. There were no significant differences between groups for total cholesterol or HbAlc. In the sub-group of patients with blood pressure $>145 / 85 \mathrm{mmHg}$ at baseline, the intervention group achieved significantly greater reductions in systolic $(-4.7,-8.2$ to $-1.1, \mathrm{P}=0.012)$ and diastolic $(-2.3,-4.6$ to $-0.1, \mathrm{P}=0.041)$ blood pressures after adjustment for confounding factors and clustering.

Conclusion: Whilst improvements in blood pressure and cholesterol were achieved, limited effects of the intervention were observed. There were no significant improvements in glycaemic control, despite the QOF initiative plus our intervention. In high risk groups, there was some evidence of added benefits from our culturally sensitive initiative. Innovative measures to motivate patients and evidence based clinical targets may be needed to maximise healthcare outcomes in patients of South Asian ethnicity.

Supported by Pfizer, Aventis UK, Servier Laboratories UK, Merck Sharp \& Dohme/Schering-Plough, Takeda UK, Sanofi-Aventis, Roche, Merck Pharma, Daiichi-Sankyo UK, Boehringer Ingleheim, Eli Lilly, NovoNordisk, Bristol Myers Squibb, Solvay Health Care and Assurance

\section{PS 102 Costs of diabetes}

\section{2}

INSTIGATE Study: Differences in initial insulin regimes initiated in patients with type 2 diabetes in 5 European countries

S. Cleall ${ }^{1}$, L. Timlin ${ }^{1}$, M. Benroubi ${ }^{2}$, A. Charles ${ }^{3}$, A. Tynan ${ }^{4}$;

${ }^{1}$ Eli Lilly, Windlesham, United Kingdom, ${ }^{2}$ Diabetes Centre, Athens General Hospital, Greece, ${ }^{3}$ Dept de Recherche, INSERM, Paris, France,

${ }^{4}$ Eli Lilly, Surrey, United Kingdom.

Background and Aims: The objectives of the INSTIGATE study are to assess the direct costs, resource utilisation and clinical outcomes associated with the first 6 months of insulin therapy in people with type 2 diabetes and to observe patient outcomes over the first 24 months of insulin therapy. This abstract presents data on initial insulin regimes prescribed to enrolled patients in five European countries.

Materials and Methods: INSTIGATE is an ongoing prospective observational study in five European countries (UK, Germany, Greece, Spain and France) studying patients with type 2 diabetes who have initiated insulin as part of usual care. Patients were enrolled into the study following the independent clinical decision to initiate insulin. Data on medications for diabetes, clinical outcomes, health related quality of life and resource use are collected when patients initiate insulin and at regular intervals over the following 24 months.

Results: 1172 patients were enrolled across 5 countries. At insulin initiation mean (SD) HbAlc was 10.2 (1.7)\% in the UK, $9.2(2.0) \%$ in Germany, 9.6 $(1.6) \%$ in Greece, $9.2(1.6) \%$ in Spain and $9.6(1.9) \%$ in France. Insulin regimes were summarised into five categories; long or intermediate acting alone, mixture alone, short acting alone, basal/bolus and other. The initial insulin regimes prescribed varied across countries as can be seen from table 1. Long or intermediate insulin alone and mixture alone were the two most commonly prescribed regimes in UK, Greece, Spain and France. Between $77.6 \%$ and $99.1 \%$ of the study patients in these countries received one of these regimes. Short acting insulin alone and a basal bolus regime were the two most commonly prescribed regimes (excluding "other") in the German sample, accounting for $55.6 \%$ of starting regimes prescribed. Overall the highest starting insulin dose, mean (SD) $0.420(0.20) \mathrm{IU} / \mathrm{kg}$, was prescribed to patients initiated on a basal/bolus regime. The lowest starting insulin dose, mean (SD) $0.219(0.13) \mathrm{IU} / \mathrm{kg}$, overall was for a long or intermediate only regime.

Conclusion: The observed initial insulin regimes prescribed across the 5 participating European countries varied substantially with simpler regimes of long/intermediate insulin alone or mixtures alone preferred as starter insulin regimes in most countries. More complex multi-injection regimes were prescribed less frequently as starting regimes in most countries. The opposite is observed in Germany with short acting alone and basal/bolus regimes initiated in the majority of patients. Further investigation is required as to how much the choice of starter insulin regime is influenced by patient characteristics and how much by country specific guidelines and site preferences. (Tab. 1)

Supported by Eli Lilly and Amylin

Table 1

\begin{tabular}{|c|c|c|c|c|c|c|}
\hline Insulin Regime & & Long/intermediate & Mixture & Short acting & Basal/bolus & Other \\
\hline \multirow[t]{2}{*}{ Germany $(n=254)$} & $\%$ of patients & 10.2 & 8.3 & 34.7 & 20.9 & 21.3 \\
\hline & Mean starting dose (IU/kg) & 0.097 & 0.200 & 0.295 & 0.372 & 0.261 \\
\hline $\mathrm{UK}(\mathrm{n}=253)$ & $\%$ of patients & 37.2 & 48.2 & 0.8 & 5.9 & 0.4 \\
\hline Long/intermediate & Mean starting dose (IU/kg) & 0.130 & 0.264 & 0.177 & 0.303 & 0.222 \\
\hline \multirow[t]{2}{*}{ Greece $(n=263)$} & $\%$ of patients & 47.2 & 30.4 & 6.5 & 12.9 & 2.7 \\
\hline & Mean starting dose (IU/kg) & 0.306 & 0.604 & 0.537 & 0.500 & 0.601 \\
\hline Spain $(n=224)$ & $\%$ of patients & 60.3 & 18.8 & 9.4 & 1.8 & 1.3 \\
\hline Short acting & Mean starting dose (IU/kg) & 0.225 & 0.373 & 0.343 & 0.358 & 0.206 \\
\hline \multirow[t]{2}{*}{ France $(n=178)$} & $\%$ of patients & 78.7 & 12.4 & 1.1 & 5.6 & 0.6 \\
\hline & Mean starting dose (IU/kg) & 0.211 & 0.419 & 0.262 & 0.603 & 0.597 \\
\hline
\end{tabular}




\section{3}

Cost-effectiveness of detemir based basal-bolus therapy versus NPH for type 1 diabetes in a Spanish setting

J. A. Ray ${ }^{1}$, W. J. Valentine ${ }^{1}$, G. Goodall ${ }^{1}$, M. Aagren ${ }^{2}$, R. W. Kotchie ${ }^{3}$;

${ }^{1}$ Health Economics Department, IMS Health, Basel, Switzerland,

${ }^{2}$ Novo Nordisk A/S, Bagsværd, Denmark,

${ }^{3}$ Health Economics Department, IMS Health, London, United Kingdom.

Background and Aims: A meta-analysis of results from three clinical trials in type 1 diabetes patients showed that insulin detemir (IDet) based basalbolus treatment compared to protamine Hagedorn human (NPH) based basal-bolus therapy in type 1 patients led to improved $\mathrm{HbA}_{1 \mathrm{c}}(0.13 \%$ points lower), a decrease in hypoglycaemic events (by $4 \%$ ) and lower body mass index (BMI) $\left(0.21 \mathrm{~kg} . \mathrm{m}^{-2}\right)$.

Materials and Methods: A published, validated, peer-reviewed computer simulation model of diabetes (the CORE Diabetes Model) was used to project short-term results obtained from the fixed-effects (weighted average) metaanalysis to long-term clinical and cost outcomes (including life expectancy, quality adjusted life expectancy, incidence of complications, and direct medical costs) for IDet versus NPH in type 1 diabetes patients, when used in combination with either insulin aspart (IAsp) or human soluble insulin (HIS) as the bolus component of therapy. Probabilities of complications were derived from landmark clinical and epidemiological studies and the costs of treating complications in Spain were retrieved from published sources. Total direct costs (complications + treatment costs) for each arm were projected over patient lifetimes from a Spanish Heath Service perspective. Both costs and clinical outcomes were discounted at $3.5 \%$ annually.

Results: Improved glycaemic control, decreased hypoglycaemic events and lower BMI with IDet-based basal/bolus therapy led to fewer diabetes-related complications, an increase in quality-adjusted life expectancy of 0.173 years, increased total lifetime costs/patient of $€ 4,254$, and an incremental costeffectiveness ratio of $€ 24,616$ per quality-adjusted life years (QALYs) gained. Results were stable under variation in a range of reasonable assumptions.

Conclusion: Short-term improvements seen with IDet versus NPH as part of a basal-bolus regimen were projected to lead to decreased complications, improvements in QALYs, and reductions in complication costs leading to a cost-effectiveness ratio which fell within a range considered to represent excellent value for money ( $<€ 50,000 / \mathrm{QALY}$ gained).

Supported by Novo Nordisk A/S

\section{4}

Cost-effectiveness of pioglitazone in type 2 diabetes in Switzerland based on the PROactive study

M. Brandle ${ }^{1}$, W. Valentine ${ }^{2}$, G. Goodall ${ }^{2}$, N. Dumont ${ }^{3}$, F. Pang ${ }^{4}$,

M. Massi Benedetti ${ }^{5}$;

${ }^{1}$ Division of Endocrinology and Diabetes, Kantonsspital St Gallen, Switzerland, ${ }^{2}$ Center for Outcomes Research, IMS Health, Allschwil, Switzerland, ${ }^{3}$ Takeda Pharma AG, Lachen, Switzerland,

${ }^{4}$ Takeda Global Research \& Development, London, United Kingdom,

${ }^{5}$ Dipartimento di Medicina Interna, Universita di Perugia, Italy.

Background and Aims: Type 2 diabetes is recognised as a chronic progressive disease that exerts a considerable burden on healthcare resources due to the increased risk of macrovascular and microvascular complications. The PROspective pioglitAzone Clinical Trial In macroVascular Events (PROactive) Study is the first large-scale outcomes study to investigate prospectively the effect of a PPAR $\gamma$-agonist on macrovascular outcomes in patients with type 2 diabetes with a history of cardiovascular disease and demonstrated that pioglitazone (PIO) was associated with a $10 \%$ reduction in the primary endpoint $(\mathrm{p}=0.09)$ and a $16 \%$ reduction in the key secondary endpoint $(\mathrm{p}=0.03)$ vs placebo (PLB) when administered in addition to existing diabetes and CV therapies, including statins. The objective of this health economic analysis was to determine the cost-effectiveness of PIO versus PLB over a lifetime horizon from the Swiss healthcare perspective using a validated modelling framework - the CORE Diabetes Model (CDM).

Materials and Methods: The CDM was modified to accommodate patientlevel data from PROactive and included 18 relevant disease and procedure specific interdependent Markov sub-models to extrapolate costs and effects over time beyond the duration of the trial. Direct medical costs (treatment, management and complication) were derived from published Swiss sources and expressed in 2005 Swiss Francs (CHF). Health utilities were taken from the Cost of Diabetes in Europe - Type 2 study of diabetes (CODE-2). Clinical and economic outcomes were discounted at $2.5 \%$ per annum in line with the Swiss Federation Office of Social Security guidelines, and univariate and probabilistic sensitivity analyses (PSA) were performed to estimate the impact of uncertainty surrounding input parameters on the results.

Results: Over a lifetime horizon, PIO treatment was projected to have an additional life expectancy (discounted) of 0.258 years over PLB at an additional direct cost of CHF10,914 giving an incremental cost-effectiveness ratio (ICER) of CHF42,274 per life year gained. PIO was also associated with an additional 0.180 QALYs generating an ICER of CHF60,596 per QALY gained. Probabilistic sensitivity analysis demonstrated that, at a willingness to pay of CHF 80,000 per QALY gained, there was a $62.5 \%$ probability of PIO being cost-effective. Univariate sensitivity analyses demonstrated that the results were most sensitive to the time horizon and duration of PIO benefit. Conclusion: The addition of pioglitazone to existing therapy in patients with type 2 diabetes at high risk of further cardiovascular events is a cost-effective treatment option and represents good value for money in the Swiss setting. Supported by Takeda Global Research and Development

\section{5}

Cost of treatment and metabolic profile of patients with type 2 diabetes during the last decade

S. Papaoikonomou, S. Liatis, S. Dimopoulos, P. Thomakos, I. Tombrou,

N. Katsilambros;

First Department of Internal Medicine, Athens University Medical School Laiko Hospital, Athens, Greece.

Background and Aims: During the last decade, several new agents have been introduced for the treatment of type 2 diabetes, and at the same period of time, a multifactorial approach including more strict control of glycemia, blood pressure (BP) and lipid profile as well as the frequent use of antiplatelet agents has been established. Aim of the present study was to compare the metabolic and BP profile of type 2 diabetic patients between the years 1998 and 2006 and to estimate the change in the cost of medications used for their treatment.

Materials and Methods: We compared the files of a random sample of patients $(n=244)$ who visited the Diabetes outpatient Clinic of our Department during 1998 with a sample of 336 patients matched for sex, age and duration of diabetes who visited our Clinic during 2006. The cost of medications was calculated in Euros, using the 01-01-2006 official Greek market prices (as indicated in each medication's label).

Results: The total cost of drug treatment increased by 245\% (from 23.08 Euros per patient/month in 1998 to 79.84 Euros per patient/month in 2006, $\mathrm{P}<0.001)$. The expenses for antidiabetic medications increased by $124 \%$, for hypolipidemic medications by $1,105 \%$, for antihypertensive agents by $162 \%$ and for antiplatelet agents by $3,930 \%$ ( $\mathrm{P}<0.00001$ for all comparisons). The median number of medications taken per patient, increased from 2 (min 0, max. 7) to 4 ( $\min 0, \max .9)$, mainly due to an increase in the use of statins (from $7.8 \%$ of patients in 1998 to $49.8 \%$ in 2006) and antiplatelet agents (from $14.8 \%$ of patients in 1998 to $41.7 \%$ in 2006). The mean value of $\mathrm{HbA}_{1} \mathrm{c}$ decreased from $7.39 \% \pm 1.36$ in 1998 to $6.95 \% \pm 1.05$ in $2006(\mathrm{P}<0.001)$. This difference was significant for insulin treated patients $(7.91 \% \pm 1.36$ vs. $7.27 \% \pm 1.08, \mathrm{P}=0.001)$, whereas for patients receiving only OADs, the difference was not significant $(7.11 \% \pm 1.25$ vs. $6.90 \% \pm 0.97, \mathrm{P}=0.09)$. LDLcholesterol decreased from $144.63 \pm 49.73 \mathrm{mg} / \mathrm{dL}$ to $110.54 \pm 36.33 \mathrm{mg} / \mathrm{dL}$ but no significant difference was observed in triglycerides and HDL-C. There was no significant difference in systolic BP, but diastolic BP decreased from $81.12 \pm 8.91 \mathrm{mmHg}$ to $75.15 \pm 10.82 \mathrm{mmHg}$.

Conclusion: In our Diabetes Outpatient Clinic, the cost spent for antidiabetic, hypolipidemic, antihypertensive and antiplatelet medications of type 2 diabetic patients increased by $245 \%$ during the last 8 years. At the same time we observed a significant reduction in $\mathrm{HbA}_{1} \mathrm{c}$ of insulin treated patients and a significant reduction in LDL-C and diastolic blood pressure. 


\section{6}

Economic evaluation of improved diabetes care to patients of South Asian ethnicity: the United Kingdom Asian Diabetes Study (UKADS) A. Gumber ${ }^{1}$, A. Szczepura ${ }^{1}$, N. Raymond ${ }^{2}$, J. O’Hare ${ }^{3}$, S. Bellary ${ }^{4}$, S. Mughal ${ }^{4}$, S. Kumar' ${ }^{1}$ A. Barnett ${ }^{4}$;

${ }^{1}$ Clinical Sciences, Warwick Medical School, Coventry,

${ }^{2}$ Health Sciences, Warwick Medical School, Coventry, ${ }^{3}$ Clinical Education, Warwick Medical School, Coventry, ${ }^{4}$ Heartlands Hospital, Heart of England NHS Trust, Birmingham, United Kingdom.

Background and Aims: Diabetes affects over 2 million people in England and accounts for nearly $9 \%$ of the annual NHS budget, or $£ 5.2$ billion per year. The South Asian population raises major challenges for type II diabetes care due to higher disease incidence, early onset, higher risk of developing complications, and cultural \& language barriers to accessing care. The United Kingdom Asian Diabetes Study (UKADS) is assessing the costs and benefits of a community-based, culturally sensitive enhanced care package specifically designed to improve general practice (GP) care for South Asian patients with type II diabetes. A national Quality and Outcomes Framework (QOF) has also been introduced into general practices in the UK. The QOF sets practice targets linked to financial incentives, with $39 \%$ of payments for diabetes care associated with achievement of designated blood pressure (BP), HbAlc and cholesterol levels. In 2004/5 an estimated £98.6 million was spent in England on QOF payments to GP practices for diabetes.

Method: 21 general practices in two English cities (Coventry and Birmingham) were randomised to either UKADS intervention (protected practice nurse time, South Asian link-worker sessions and diabetes specialist nurse support) or standard care. Treatment protocols provided for both groups included prescribing algorithms for control of diabetes, BP and lipids. Clinical outcomes were measured after 1 year to include BP, total cholesterol and HbA1c. Health related quality of life (HRQL) assessed using EQ5D. Intervention costs were calculated to include staff time, capital (accommodation \& equipment), overheads and consumables (including prescribing costs), and QOF payments. Cost-effectiveness was assessed by comparing incremental costs and marginal benefits.

Results: Data from 1,494 South Asian patients with type II diabetes showed significant decreases in systolic BP $(4.6 \mathrm{mmHg})$, diastolic BP $(2.3 \mathrm{mmHg})$ and total cholesterol $(0.3 \mathrm{mmol} / \mathrm{l})$ after 1 year for the whole study group. After adjusting for confounding factors and clustering, the control group achieved $11 \mathrm{mmHg}$ fall in systolic BP in high risk patients $(>145 / 85 \mathrm{mmHg})$, while the intervention group achieved a further decrease of $5 \mathrm{mmHg}$. There was no significant net difference in HRQL between intervention and control group. UKADS intervention costs were estimated at $£ 105$ per patient per year (including prescribing). Average QOF payment for diabetes were approximately $£ 57$ per patient per year. Initial analysis estimates a costeffectiveness ratio of approximately $£ 5.2$ per $\mathrm{mmHg}$ fall in BP for QOF incentives plus treatment protocol; and an incremental cost-effectiveness ratio for the tailored intervention is $£ 21.0$ per additional $1 \mathrm{mmHg}$ fall in BP. Further results will be presented.

Conclusions: Our results confirm decreased cardiovascular risk factors (BP, cholesterol) for South Asian patients with type II diabetes following the introduction of QOF financial incentives. Also, that further improvements can be achieved at a reasonable cost through use of a tailored community intervention for this disadvantaged population. However, changes in glycaemic control remain a major challenge, as do improvements in HRQL. Supported by various pharmaceutical industries

\section{7}

Cost-effectiveness analysis of medical intervention in patients with early detection of diabetic foot in a tertiary care hospital in Bangladesh S. H. Habib ${ }^{1}$, K. B. Biswas ${ }^{2}$, S. Akter ${ }^{3}$, A. K. Azad Khan';

${ }^{1}$ Health Economics Unit, DAB, ${ }^{2}$ Dept of Physiology \& Molecular Biology, BIRDEM, ${ }^{3}$ Dept of Biochemistry \& Cell Biology, BIRDEM, Dhaka, Bangladesh.

Background and Aims: The economic burden resulting from diabetic foot complications consume a major portion of resources allocated for healthcare services. Cost-effectiveness of various interventions on diabetic foot \& its complications have relatively been well explored in developed countries, but these are almost absent in developing countries. The present study was undertaken to assess the cost-effectiveness of medical intervention in patients with diabetic foot.

Materials and Methods: Two hundred patients with diabetic foot, with at least $1 \mathrm{yr}$ of follow-up, were purposively selected from BIRDEM (tertiary diabetes care hospital) of Bangladesh. Of them 100 were late in detection \& poorly managed (Late diabetic foot or LDF) and 100 were early detected \& properly managed (Early diabetic foot or EDF). The degree \& extent of complications like peripheral neuropathy, retinopathy, cardiopathy and vasculopathy, treatment outcome, clinical effectiveness of interventions and direct, indirect $\&$ incremental cost of complications were calculated. Comparison was made between the groups. Cost included drugs, hospitalizations, diagnostics \& visits.

Results: A total of 200 patients were considered for an average of 365 days, amounted to 651 person-yrs of observation in total. The mean \pm SD total cholesterol of the groups was $205.5 \pm 41.6 \mathrm{mg} / \mathrm{dl}$ in LDF \& $103.2 \pm 34.5 \mathrm{mg} / \mathrm{dl}$ in EDF, HDL cholesterol was $56.2 \pm 20.3 \& 39.0 \pm 14.1 \mathrm{mg} / \mathrm{dl}$ and $\mathrm{HbA}_{1 \mathrm{c}}$ was $8.2 \pm 1.5 \%$ \& $4.9 \pm 1.3 \%$ respectively. About $19 \%$ patients in LDF \& $36 \%$ in EDF were free of diabetic complications other than diabetic foot. In LDF, $32 \%$ had one complication, $29 \%$ had two \& $20 \%$ had more than two complications. On the other hand, in EDF the corresponding values were $48 \%, 10 \%$ \& $6 \%$ respectively. The most frequent complication was peripheral neuropathy, which affected $33 \%$ patients in LDF \& $27 \%$ in EDF, followed by cardiopathy $21 \%$ \& $18 \%$, retinopathy $17 \% \& 13 \%$, and vasculopathy $10 \% \& 6 \%$ respectively. The average annual cost of care was US\$ 26846 (direct US\$ 17893 \& indirect US\$ 8953), with an average US\$ 134 per patient. Among the average annual cost LDF consumed US\$ 18918 (US\$ 189 per patient) and EDF US\$ 7929 (US\$ 79 per patient). $50 \%$ (US\$13473) of costs were attributable to drugs for both groups of which US\$ 10419 (77\%) was for LDF \& US\$ 3054 (23\%) for EDF, $29 \%$ (US\$ 7653) to hospitalisations of which US\$ 4914 (64\%) for LDF \& 2739 (36\%) for EDF. In case of diagnostics and visits the corresponding values were US\$ 1953 (55\%) \& $1580(44 \%)$ and US\$ 1631 (75\%) \& 556 (25\%) for LDF \& EDF respectively. The annual medical costs increased with the number of complications from US\$ 1,320 to 2,296 \& to 3,989 in LDF with one, two \& more than two complications (other than diabetic foot) which is increasing at a rapid speed and US\$ 917 to 1556 \& to 2372 in EDF respectively, increasing at a diminishing marginal rate. The regression equation showed that medical cost is significantly related to complications tested in both univariate $(\mathrm{P}<$ $0.0001) \&$ multiple linear regression analyses $\left(R^{2}=0.51 ; F-81.5, P<0.0001\right)$. Conclusion: Proper management with regular follow up substantially reduces the expenditure related to care of patients with diabetic foot \& related complications even in a developing country. Strategies aimed at preventing \& early detection of the onset of foot complications will reduce medical costs in a substantial way.

\section{8}

Impact of increasing body mass index on medical resources for people with type 2 diabetes - a retrospective analysis in United States M. Aagren ${ }^{1}, \mathrm{H}$. Colclough ${ }^{2}, \mathrm{M}$. Hammer ${ }^{3}$;

${ }^{1}$ Novo Nordisk A/S, Virum, Denmark, ${ }^{2}$ Adelphi Group, Bollington, United Kingdom, ${ }^{3}$ Novo Nordisk A/S, Bagsværd, Denmark.

Background and Aims: The risk of developing cardiovascular and other life threatening diseases is strongly linked to increased body weight. This is particularly relevant to people with type 2 diabetes (T2D) of whom over $80 \%$ are overweight or obese. However, limited data exist on the impact on obesity on the medical resources and lost productivity. The aim of this study was to investigate the annual use of medical resources (drug use and outpatient visits) and lost productivity (days off work) for people with T2D with increasing BMI.

Materials and Methods: A retrospective analysis was conducted in United States based on a random sample of T2Ds from the Adelphi Metabolic Syndrome Disease Specific Programme (a large cross sectional study) in 2006 with 643 people diagnosed with T2D, aged between 35 to 64 years and a $\mathrm{BMI} \geq 20 \mathrm{~kg} / \mathrm{m}^{2}$ (based on physician reported height and weight). People were stratified according to their BMI; normal/overweight $(20-29.99 \mathrm{~kg} /$ $\mathrm{m} 2 ; \mathrm{n}=110)$, obese $(30-34.99 \mathrm{~kg} / \mathrm{m} 2 ; \mathrm{n}=178))$; very obese $(35-39.99 \mathrm{~kg} / \mathrm{m} 2$; $\mathrm{n}=170)$ and morbidly obese $(>39.99 \mathrm{~kg} / \mathrm{m} 2 ; \mathrm{n}=185)$. The cohort had an average age of 53 years, $58 \%$ were male, and $69 \%$ were Caucasians.

Results: For people with T2D with a BMI of 20 to $29.99 \mathrm{~kg} / \mathrm{m} 2$, mean number of total drugs used for any condition was 4.7 and 5\% used more than 2 diabetes drugs. The number of annual visits to health professionals (primary 
care physician, cardiologist, diabetes specialist, diabetes nurse, other doctor/ nurse) was 5.9. The rate ratio i.e. the mean annualized resource utilization relative to the utilization in the normal/overweight group for people with T2D with a BMI of $30-34.99 \mathrm{~kg} / \mathrm{m} 2,35-39.99 \mathrm{~kg} / \mathrm{m} 2$ and $\geq 39.99 \mathrm{~kg} / \mathrm{m} 2$, respectively, were $1.08,1.15$ and 1.32 for total drug use, $1.75,2.37$ and 3.37 for percentage of people using more than 2 diabetes drugs, and 1.18, 1.23 and 1.37 for annual number of visits to health professionals. People not retired had an average annual number of days off work due to CV or diabetes complications of 1.5 for the normal/overweight group, with 3.2 for the obese group (BMI $\geq 30 \mathrm{~kg} / \mathrm{m} 2)$.

The proportion of people achieving HbAlc $<7 \%$ (ADA target for good glyceamic control) was $54 \%$ among the normal/overweight, and $45 \%$ in the morbidly obese group $(\geq 39.99 \mathrm{~kg} / \mathrm{m} 2)$. Furthermore statistical tests showed that people in the normal/overweight group had statistically significant better glyceamic control $(\mathrm{p}<0.05)$ than people in the morbidly obese group $(\geq 39.99$ $\mathrm{kg} / \mathrm{m} 2)$.

Conclusion: These findings suggest a positive correlation between BMI and medical resources. The impact of obesity on resource use is particularly evident in people with T2D in the high obesity groups where a significantly lower glyceamic control also is seen.

Supported by Novo Nordisk A/S

\section{9}

\author{
Hyperglycaemia-related complications and medical care costs of \\ intermediate hyperglycaemia \\ G. A. Nichols ${ }^{1}$, B. Arondekar ${ }^{2}$, W. H. Herman ${ }^{3}$; \\ ${ }^{1}$ Center for Health Research, Kaiser Permanente, Portland, \\ ${ }^{2}$ GlaxoSmithKline, Inc, Philadelphia, ${ }^{3}$ Endocrinolgy and Metabolism, \\ University of Michigan, Ann Arbor, United States.
}

Background and Aims: There is a lack of published studies that estimate the overall disease and economic burden of intermediate hyperglycemia (impaired fasting glucose [IFG] or impaired glucose tolerance [IGT]). The objectives of this study were to identify the prevalence of hyperglycemiarelated complications and compare the medical care costs of IFG and/or IGT and its associated complications.

Materials and Methods: We used laboratory test results from Kaiser Permanente Northwest, a large HMO in Northwestern USA, to identify patients who had both a random and fasting plasma glucose (FPG) test result no more than 365 days apart between 1 January 1998 and 31 December 2004. Those with evidence of diabetes (chart diagnosis, FPG $>125 \mathrm{mg} / \mathrm{dl}$, or use of an anti-hyperglycemic), cancer, chronic renal failure or pregnancy were excluded. We then applied a validated algorithm that uses random glucose to screen for dysglycemia and results from the FPG test to assign patients to one of four categories: 1) isolated IFG (FPG $100-125 \mathrm{mg} / \mathrm{dl}$ but did not screen as dysglycemic, $\mathrm{n}=5,713$ ); 2) isolated IGT (FPG $<100 \mathrm{mg} / \mathrm{dl}$ but screened as dysglycemic, $\mathrm{n}=2,552$ ); 3) IFG/IGT (FPG $100-125 \mathrm{mg} / \mathrm{dl}$ and screened as dysglycemic, $\mathrm{n}=2,217) ; 4$ ) normoglycemia (FPG $<100 \mathrm{mg} / \mathrm{dl}$ and did not screen as dysglycemic, $n=15,629)$. Using electronic medical records, we identified and compared the prevalence of hyperglycemia-related complications. We then constructed multivariable regression models to compare age and sex adjusted annual medical costs across the four groups and to estimate the costs associated with the complications.

Results: Subjects averaged 62.5 years of age and $41 \%$ were male. Mean annual age/sex adjusted costs were $\$ 6208, \$ 6171, \$ 7250$, and $\$ 7003$ for normoglycemic, isolated IFG, isolated IGT, and IFG/IGT subjects, respectively. Compared to normoglycemics, age/sex adjusted complications were more prevalent among each of the dysglycemic groups, and were greatest among the isolated IGT and the IFG/IGT groups (see table). Presence of complications added significantly and independently to costs, ranging from $\$ 993$ for estimated GFR $<60 \mathrm{ml} / \mathrm{min}$ to $\$ 5324$ for peripheral vascular disease. After controlling for complications, costs among the isolated IGT group were $\$ 489$ higher than those with normoglycemia. No other comparisons were significantly different.

\begin{tabular}{|c|c|c|c|c|c|}
\hline & $\begin{array}{l}\text { Normo- } \\
\text { glycemia }\end{array}$ & $\begin{array}{l}\text { Isolated } \\
\text { IFG }\end{array}$ & $\begin{array}{l}\text { Isolated } \\
\text { IGT }\end{array}$ & $\begin{array}{l}\text { IFG/ } \\
\text { IGT }\end{array}$ & $\begin{array}{l}\text { Estimated } \\
\text { Annual Cost }\end{array}$ \\
\hline $\begin{array}{l}\text { Cardiovascular } \\
\text { Disease }\end{array}$ & $17.5 \%$ & $21.5 \%$ & $23.2 \%$ & $22.8 \%$ & $\$ 2669$ \\
\hline Stroke & $7.4 \%$ & $7.9 \%$ & $9.8 \%$ & $8.6 \%$ & $\$ 2262$ \\
\hline $\begin{array}{l}\text { Peripheral Vascular } \\
\text { Disease }\end{array}$ & $2.5 \%$ & $3.1 \%$ & $3.2 \%$ & $2.8 \%$ & $\$ 5324$ \\
\hline $\begin{array}{l}\text { Congestive Heart } \\
\text { Failure }\end{array}$ & $5.2 \%$ & $6.7 \%$ & $8.6 \%$ & $10.5 \%$ & $\$ 4479$ \\
\hline $\begin{array}{l}\text { Any Macrovascular } \\
\text { Complication }\end{array}$ & $25.0 \%$ & $30.0 \%$ & $33.6 \%$ & $33.3 \%$ & $\$ 4020$ \\
\hline Retinopathy & $0.2 \%$ & $0.2 \%$ & $0.4 \%$ & $0.3 \%$ & $\$ 4005$ \\
\hline Macular Edema & $0.3 \%$ & $0.3 \%$ & $0.3 \%$ & $0.5 \%$ & $\$ 2334$ \\
\hline $\begin{array}{l}\text { Peripheral } \\
\text { Neuropathy }\end{array}$ & $4.5 \%$ & $4.8 \%$ & $5.0 \%$ & $5.1 \%$ & $\$ 3014$ \\
\hline $\begin{array}{l}\text { GFR }<60 \mathrm{ml} / \mathrm{min} \\
\text { per } 1.73 \mathrm{~m} 2\end{array}$ & $12.3 \%$ & $13.7 \%$ & $17.5 \%$ & $16.8 \%$ & $\$ 993$ \\
\hline $\begin{array}{l}\text { Any Microvascular } \\
\text { Complication }\end{array}$ & $16.2 \%$ & $18.0 \%$ & $21.5 \%$ & $21.2 \%$ & $\$ 1945$ \\
\hline Any Complication & $33.9 \%$ & $39.1 \%$ & $44.1 \%$ & $44.4 \%$ & $\$ 3700$ \\
\hline
\end{tabular}

Conclusion: Intermediate hyperglycemia is associated with increased medical costs. Hyperglycemia-related complications are highly prevalent among patients with intermediate hyperglycemia. Medical costs are correspondingly greater, especially when macrovascular complications are present. The highest costs were found among those with isolated IGT, a form of intermediate hyperglycemia that would seldom be identified. Complications did not fully account for the cost differences between this group and those with normoglycemia.

Supported by GlaxoSmithKline, Inc.

\section{0}

Estimating the cost of complications of diabetes in Sweden using administrative hospitalization data and the National Diabetes Register P. M. Nilsson ${ }^{1}$, U. Gerdtham ${ }^{2}$, P. Clarke ${ }^{3}$, A. Hayes ${ }^{3}$, B. Eliasson ${ }^{4}$, S. Gudbjörnsdottir ${ }^{4}$;

${ }^{1}$ Medicine, Clinical Sciences, Malmö, Sweden, ${ }^{2}$ Health Economy, Clinical Sciences, Malmö, Sweden, ${ }^{3}$ Health Economy, School of Public Health, Sidney, Australia, ${ }^{4}$ Medicine, Diabetes Centre, Göteborg, Sweden.

Background and Aims: Diabetes is a major public health problem with large, but varying financial costs at different stages of the disease. We aimed to estimate acute and long-term costs of hospitalization in Sweden for major diabetes-related complications, using administrative health care data.

Materials and Methods: The costs were estimated using administrative information on hospital data from the Swedish National Board of Health Welfare which have been linked to the Swedish National Diabetes Register (NDR). Data were available for a total of 179,749 patients with diabetes in Sweden from 1998-2003 (mean duration of 5.9 years of follow-up). Costs of inpatient admissions was based on Nordic Diagnostic Related Groups (DRG). Multiple regression analysis was used to estimate inpatient care costs controlling for age, sex and co-morbidities. The data on hospitalisations were converted to costs ( $€ 2004)$ using exchange rates.

Results: For a male aged 70 years the average annual costs associated with inpatient admissions in the year when the event first occurred were for coma: $€ 6,275$ (95\%CI: 3,500, 9,050); heart failure: €6,432 (5,359, 7,505); non-fatal myocardial infarction (MI) $€ 6,863(5,642,8,084)$; non-fatal stroke $€ 7,706$ $(6,580,8,832)$; renal failure $€ 9,371(7,097,11,644)$; ischaemic heart disease $€ 9,524(8,452,10,596)$; chronic leg ulcer $€ 10,261(7,301,13,221)$; dialysis $€ 13,686(10,722,16,651)$; and amputation $€ 16,174(14,316,18,033)$. The costs were on average higher when also taking co-morbidities into account. For example, a diagnosis of MI with a co-morbidity was twice as costly as for MI alone.

Conclusion: Estimates of the hospitalization costs associated with diabetesrelated complications can be used in modelling the long-term costs of diabetes and in evaluating the cost-effectiveness of improving care.

Supported by Svenska Diabetesförbundet and Novo Nordisk Ltd. 


\section{PS 103 Socio-economic aspects of diabetes}

\section{1}

Using the chronic care model as a framework to improve diabetes care in a large U.S. health system

L. M. Siminerio ${ }^{1}$, F. X. Solano ${ }^{2}$, J. Zgibor ${ }^{3}$, G. Piatt ${ }^{4}$;

${ }^{1}$ Endocrinology, University of Pittsburgh, ${ }^{2}$ Center for Quality Improvement and Innovation, University of Pittsburgh, ${ }^{3}$ Graduate School of Public Health, University of Pittsburgh, ${ }^{4}$ University of Pittsburgh Medical Center, United States.

Background and Aims: A Chronic Care Model (CCM) organised around elements (health system, community, decision support, clinical information systems, self-management, and delivery system design) has been shown to improve outcomes. It was our objective to use the CCM as a framework to improve quality and outcomes for the University of Pittsburgh Medical Center (UPMC), a large US health system, by gaining organisational and community support; implementing American Diabetes Association (ADA) medical and education standards for decision support; using clinical information systems to characterise target populations and track outcomes for continuous quality improvement (CQI), and integrating diabetes educators to provide point of service education in primary care practices for system re-design.

Materials and Methods: UPMC health system leadership provided organizational support for tracking of outcomes, negotiating with insurers and physician education. Programs were promoted through public awareness campaigns throughout all communities served by the health system. Target populations were characterised and CQI outcomes improved throughout the health system from $2 / 02-9 / 06$.

Results: The proportion of patients in the health system with A1c levels $<7 \%$ $(60.4 \%$ to $72 \%, \mathrm{p}=<0.0001)$ and LDLc levels $<100 \mathrm{mg} / \mathrm{dL}(42.6 \%$ to $52.3 \%$, $\mathrm{p}<0.0001)$ significantly increased over time, as did the proportion of patients on statin (36\% to $48 \%, \mathrm{p}<0.0001)$ and ace inhibitor $(48.1 \%$ to $50.7 \%, \mathrm{p}<0.0001)$ therapy. Additionally, improvements in mean A1c ( $7.6 \%$ to $7.3 \%, \mathrm{p}<0.0001)$ and LDLc $(116.7 \mathrm{mg} / \mathrm{dL}$ to $100.6 \mathrm{mg} / \mathrm{dL}, \mathrm{p}<0.0001)$ levels occurred over time (2/03-6/03) in patients receiving education in the primary care setting. Additionally, a significantly greater proportion of people accessed diabetes education when provided at point of service in the primary care office than in the traditional hospital-based setting $(14.5 \%$ vs. $10.4 \%, p<0.0001)$.

Conclusion: Using the CCM as a framework to improve diabetes care in a large health system resulted in organizational and community attention, ability to establish an effective system for monitoring ADA standards and CQI, and provided a novel approach for access. By using and coordinating the many resources of the health system, there have been significant improvements in health delivery processes and outcomes.

Partially sponsored by funding from the US Air Force administered by the US Army Medical Research Acquisition Activity, Fort Detrick, Maryland

\section{2}

Cost-effectiveness of self-monitoring of blood glucose for the management of patients with non-insulin treated type 2 diabetes: economic evaluation of the DiGEM trial

J. Simon ${ }^{1}$, A. M. Gray 1 , A. N. Wade 2 , H. A. W. Neil ${ }^{3}$, A. J. Farmer'; ${ }^{1}$ Department of Public Health, University of Oxford, United Kingdom, ${ }^{2}$ Johns Hopkins Bayview Medical Centre, Johns Hopkins University School of Medicine, Baltimore, United States, ${ }^{3}$ Division of Public Health and Primary Care, University of Oxford, United Kingdom, ${ }^{4}$ Department of Primary Health Care, University of Oxford, United Kingdom.

Background and Aims: Current guidelines promote the use of selfmonitoring of blood glucose (SMBG) for patients with non-insulin treated diabetes without robust clinical and economic evidence. Although the additional resources required for the technology have long been recognised, no previous study has attempted to estimate the real health care cost impact of the intervention. The aim of this economic evaluation is to assess the incremental cost-effectiveness of using SMBG either alone (less intensive monitoring) or with training in interpretation of the results of testing (more intensive monitoring) compared to standardised usual care for patients with non-insulin treated type 2 diabetes.
Materials and Methods: Patient level resource use information was collected retrospectively at baseline and prospectively for the 12-month trial period for 453 patients in the randomised, controlled DiGEM study. Data were available on frequency of blood glucose testing, nurse involvement, diabetes and nondiabetes related medications, and primary and secondary health care use. Costs (UK $£, £ 1 \approx € 1.47$ ) were calculated from a health care perspective using national-level unit cost data for the year 2004/05. Costing was carried out on an intention-to-treat basis. Within-trial health care costs were compared between the three trial arms and to baseline costs where applicable. The within-trial change in the level of HbAlc is used to predict the long-term health outcomes and health care costs using the UKPDS outcome model. Results: The DiGEM trial showed a mean difference of $-0.14 \%$ in $\mathrm{HbA1c}$ level between the less intensive monitoring and the control arms, and a mean difference of $-0.17 \%$ between the more intensive monitoring and the control arms. The overall intervention costs, including the costs of SMBG and nurse time, were $£ 160$ (SD 45.95), £267 (SD 60.70) and £264 (SD 81.09) in the control, less intensive and more intensive monitoring arms respectively. Only the difference between the control and the less intensive monitoring arms ( $£ 107,95 \%$ C.I. $£ 95$ to $£ 119$ ) and between the control and the more intensive monitoring arms ( $£ 104,95 \%$ C.I. $£ 88$ to $£ 119)$ were significant $(\mathrm{p}<0.001)$. There was also significant increase in the costs of both diabetes-related and other medications compared to baseline in all three trial arms. The size of increase, however, was independent of the type of intervention received. Conclusion: The increase in the overall medication costs compared to baseline is likely to be a consequence of the closer follow-up of patients in the trial compared with their previous management. No differences are shown in the cost of diabetes medications between patients using SMBG and those receiving standardised usual care. Analysis of the effect of SMBG on other health care resource use, long-term life expectancy, quality-adjusted life years and health care costs, and estimation of cost-effectiveness, is in progress. Supported by the NHS Health Technology Assessment Programme

\section{3}

Long-term clinical outcomes of insulin detemir versus NPH based basal-bolus therapy for type 1 diabetes patients in Germany: A modeling analysis using results from a meta-analysis of three clinical trials R. W. Kotchie ${ }^{1}$, M. Aagren ${ }^{2}$, W. J. Valentine ${ }^{3}$, G. Goodall'; ${ }^{1}$ IMS Health, London, United Kingdom, ${ }^{2}$ Novo Nordisk A/S, Bagsværd, Denmark, ${ }^{3}$ IMS Health, Basel, Switzerland.

Background and Aims: The aim of this study was to estimate the longterm clinical outcomes of type 1 diabetic patients treated with detemir (IDet) based basal-bolus therapy versus NPH based basal-bolus therapy in a German setting using the short-term results from a meta-analyses of three clinical trials.

Materials and Methods: A published and validated diabetes model was used to project long-term cumulative incidence (CI) of complications, life expectancy, quality-adjusted life expectancy, and time to onset of complications in a German setting. The model comprises a series of semiMarkov sub-models that simulate the long-term incidence and progression of diabetes-related complications and utilized second order Monte Carlo simulation. The probabilities of complications were derived from landmark clinical and epidemiological studies. Baseline cohort characteristics (mean age 40.3 years, duration of diabetes 16.3 years, HbA1c $8.3 \%$, BMI $25.2 \mathrm{~kg} . \mathrm{m}^{-2}$ ) and intervention effects were derived from a fixed-effects (weighted average) meta-analysis of three clinical trials $(n=1555)$ where patients received either human insulin (neutral protamine Hagedorn, NPH) or insulin determir as the basal component of their therapy. Clinical benefits were discounted at a rate of $5 \%$ annually.

Results: Treatment with IDet was projected to improve discounted life expectancy by approximately 0.05 years $(12.27 \pm 0.13$ versus $12.22 \pm 0.12$ years) and quality-adjusted life expectancy by 0.14 quality-adjusted life years (QALYs) (6.23 \pm 0.07 versus 6.09 \pm 0.06 QALYs) compared to NPH. Improved glycaemic control with IDet based basal-bolus therapy led to a delayed onset of the first diabetes-related complication, approximately 0.04 years $(0.90$ versus 0.86 years). IDet was associated with a reduction in the $\mathrm{CI}$ of diabetesrelated complications; a $2.0 \%$ reduction of the $\mathrm{CI}$ of end stage renal disease (from $20.73 \%$ to $20.32 \%$ ), and a $4.6 \%$ reduction of proliferative diabetic retinopathy (from $19.87 \%$ to $18.96 \%$ ). In addition IDet was also associated with a $3.5 \%$ reduction in the CI of peripheral vascular disease (from $14.63 \%$ to $14.12 \%$ ), but a projected increase of CI of stroke events of approximately 
$1.0 \%$ (from $12.20 \%$ to $12.27 \%$ ). This increase in stroke events was due to the survival paradox, whereby patients living longer on IDet were exposed to the risk of stroke for a longer period than those on NPH.

Conclusion: Projections based on short-term (3-month) improvements in glycemic control and reduced weight gain with IDet versus NPH basal insulin in a recent meta-analysis indicate that IDet can be reasonably expected to yield long-term survival benefits, reduce the incidence of complications and improve quality-adjusted life expectancy. The data from this modeling study suggest that use of IDet as the basal component of a basal-bolus regimen in German type 1 diabetes patients will lead to long-term clinical benefits, making it an attractive treatment option for this patient group. Supported by Novo Nordisk A/S

\section{4}

Quality of metabolic control in geriatric patients with diabetes mellitus A. Braun, A. Abel, C. Wittmann-Jennewein, T. Zieschang, P. Oster; Department of Geriatrics at the University of Heidelberg, Bethanien Hospital, Germany.

Background and Aims: For elderly people with diabetes mellitus maintenance of autonomy is an important therapeutic goal influencing quality of life. Objective: Systematic assessment of the diabetes treatment quality and perceptions of elderly patients admitted to an acute geriatric hospital from different home environments (nursing home residents, home care, family carer, auto-sufficient).

Research Design and Methods: Quality of diabetes treatment, metabolic control (HbAlc), nutrition, patients perceptions, treatment satisfaction and level of dependency (Barthel activities of daily living) were assessed in 130 patients with diabetes (age 81.2 $\pm 6.5 \mathrm{y}$., HbAlc $7.6 \pm 1.7 \%$, diabetes duration 9.8 (0.01-51.7)y., body-mass-index $25.7 \pm 4.8 \mathrm{~kg} / \mathrm{m}^{2}$, waist-hip-ratio $0.99 \pm 0.08$ ). 42 patients were on diet therapy, 38 received oral antidiabetics (OAD), 38 insulin therapy and 12 insulin+ OAD therapy.

Results: Eigthy-six out of 130 patients $(66.2 \%)$ showed an HbA1c $\leq 8 \%$ according to the guidelines for ageing people with diabetes of the German Diabetes Association (DDG). Compared to patients living independently at home $(n=28)$, the metabolic control in nursing home residents $(n=36)$ (nursing home residents vs. autosufficient: HbAlc $7.6 \pm 1.6 \%$ vs. $8.0 \pm 2.0 \%$, n.s.) and their treatment satisfaction ( $26.9 \pm 6.8$ vs. $30.8 \pm 5.2$ points, n.s.) were as good. They had a higher degree of dependency though (Barthel $5(0-80)$ vs. $65(5-100)$ points, $\mathrm{p}<0.001)$, more strongly impaired mobility (Tinetti $1(0-$ 17) vs. 11(0-27) points, $\mathrm{p}<0.01)$, less knowledge about diabetes knowledge $(2.1 \pm 2.9$ vs. $5.1 \pm 3.3$ points, $p<0.001)$, inferior cognitive performance (MMSE, SPMSQ, $\mathrm{p}<0.01)$ and a higher prevalence of depression (GDS) $(\mathrm{p}<0.01)$. Better cognitive function correlated with better diabetes knowledge $(r=0.49$; $\mathrm{p}<0.001$ ), but not with better metabolic control.

Conclusion: Metabolic control of nursing home residents seems to be as good as that of patients caring for themselves at home. $66 \%$ of the nursing home residents showed $\mathrm{HbAlc} \leq 8 \%$ according to the national guidelines.

Supported by Robert Bosch Foundation

\section{5}

Does intensive treatment of people with screen-detected diabetes improve outcomes? Impact on cardiovascular risk factors at one year in the ADDITION (Cambridge) trial

S. J. Griffin ${ }^{1}$, T. Prevost ${ }^{2}$, A. L. Kinmonth ${ }^{2}$, K. M. Williams ${ }^{2}$, N. J. Wareham ${ }^{1}$; ${ }^{1}$ Epidemiology Unit, Medical Research Council, ${ }^{2}$ Public Health and Primary Care, University of Cambridge, United Kingdom.

Background and Aims: A key determinant of the cost-effectiveness of screening for type 2 diabetes is the magnitude of cardiovascular (CVD) risk reduction associated with early detection and treatment of diabetes and related risk factors. However, there are few published data concerning the effects of simultaneous treatment of multiple risk factors, and none among patients with diabetes detected by screening. We assessed the effects on cardiovascular risk factors of a program of screening and intensive, multifactorial treatment compared with a program of screening followed by routine general practice care.

Materials and Methods: In a pragmatic, parallel group, cluster randomised trial, 55 general practices in East Anglia were allocated to screening and intensive treatment (IT:28), or screening followed by routine care according to local and national guidelines (RC:27). Six practices were unable to commence the study (IT:2, RC:4). Stepwise screening of 135,825 people aged $40-69$ years included a risk score, random capillary blood glucose and HbAlc tests $(24,654)$, fasting capillary test, and finally oral glucose tolerance tests $(1,481)$. 867 people were diagnosed with diabetes (1999 WHO criteria). 13.9 months (SD:2.9) post-diagnosis, $85 \%$ of patients returned for re-measurement by staff unaware of study group. In the IT group, management of blood glucose, blood pressure, cholesterol and microalbuminuria was optimised through practice-based educational meetings, guidelines, target setting, academic detailing, audit and feedback, alongside provision of a pack of theory-based educational materials for newly diagnosed patients.

Results: Patients with screen-detected diabetes had high levels of cardiovascular risk factors at diagnosis which improved over the first year. Reduction in levels of risk factors was significantly greater in the intensive treatment group.

Conclusion: Screening for type 2 diabetes is feasible and identifies people with high levels of modifiable cardiovascular risk factors which can be improved through routine general practice diabetes care. Optimisation of intensive multi-factorial treatment was associated with clinically important improvements in intermediate outcomes 14 months after diagnosis. Whether such a program will be a cost-effective means of reducing CVD events over five years is being addressed in the ADDITION (Europe) study in Denmark, the Netherlands and the UK. (Tab. 1)

Supported by Wellcome Trust, MRC, NHS R\&D

Tab. 1

Cardiovascular risk factors at diagnosis and follow-up (13.9 months)

\begin{tabular}{|c|c|c|c|c|c|c|}
\hline & \multicolumn{2}{|c|}{ Routine care group } & \multicolumn{4}{|c|}{ Intensive treatment group } \\
\hline & $\begin{array}{l}\text { Mean (SD) at } \\
\text { diagnosis }\end{array}$ & $\begin{array}{l}\text { Mean (SD) at } \\
\text { follow-up }\end{array}$ & $\begin{array}{l}\text { Mean (SD) at } \\
\text { diagnosis }\end{array}$ & $\begin{array}{l}\text { Mean (SD) at } \\
\text { follow-up }\end{array}$ & $\begin{array}{l}\text { Difference in change } \\
(95 \% \mathrm{CI})\end{array}$ & P value \\
\hline HbA1c (\%) & $7.33(1.65)$ & $6.62(0.95)$ & 7.35 (1.77) & $6.42(0.77)$ & $-0.20(-0.33$ to -0.06$)$ & 0.009 \\
\hline BMI $\left(\mathrm{kg} / \mathrm{m}^{2}\right)$ & $33.6(5.9)$ & $32.6(6.0)$ & $33.1(5.3)$ & $31.9(5.2)$ & $-0.26(-0.55$ to +0.03$)$ & 0.084 \\
\hline Systolic BP (mmHg) & $142.1(20.0)$ & $138.0(18.6)$ & $141.5(19.8)$ & $134.6(18.3)$ & $-3.18(-6.26$ to -0.11$)$ & 0.048 \\
\hline Diastolic BP (mmHg) & $81.4(10.3)$ & $79.6(9.9)$ & $82.1(10.4)$ & $77.9(9.2)$ & $-1.81(-3.30$ to -0.32$)$ & 0.022 \\
\hline Total cholesterol $(\mathrm{mmol} / \mathrm{l})$ & $5.42(1.18)$ & $4.74(0.96)$ & $5.36(1.06)$ & $4.36(0.95)$ & $-0.32(-0.51$ to -0.12$)$ & 0.002 \\
\hline HDL cholesterol (mmol/l) & $1.21(0.32)$ & $1.25(0.36)$ & $1.17(0.34)$ & $1.19(0.33)$ & $-0.03(-0.07$ to +0.003$)$ & 0.082 \\
\hline LDL cholesterol (mmol/l) & $3.30(1.02)$ & $2.64(0.82)$ & $3.33(0.91)$ & $2.38(0.82)$ & $-0.25(-0.42$ to -0.09$)$ & 0.004 \\
\hline
\end{tabular}




\section{6}

Clinical utility of indentifying metabolic syndrome (MetS) in patients with type 2 diabetes for primary cardiovascular disease (CVD) prevention: a population-based study

U. Bulugahapitiya ${ }^{1,2}$, S. Siyambalapitiya ${ }^{1,2}$, J. Sithole ${ }^{3}$, D. J. S. Fernando ${ }^{1,2}$, I. Idris ${ }^{1,2}$;

${ }^{1}$ Diabetes and Endocrinology, Sherwood Forest Hospitals Foundation Trust, Nottinghamshire, ${ }^{2}$ Academic Unit of Diabetes, Endocrinology and Metabolism, University of Sheffield,

${ }^{3}$ Trent RDSU, University of Nottingham, United Kingdom.

Background and Aims: In patients with type 2 diabetes (T2D) without overt cardiovascular disease, we aimed to determine whether identifying patients with MetS (IDF criteria) would affect the decision to prescribe statin for primary CVD prevention, based on currently available public health guidelines

Materials and Methods: We analysed last recorded CVD risk profiles obtained from The Health Improvement Network (THIN) primary care database which contains inputed patients dataset using the shared VISION software from $229 \mathrm{GP}$ practices in England and Wales. Out of 60258 patients with diabetes, 11005 (10\%) men and women aged 30-74 years fulfils criteria for primary CVD prevention and were not on lipid lowering drugs. Outcome data was extrapolated to an estimated national diabetes prevalence of $3.6 \%$. Results: The prevalence of MetS is $67.3 \%$. Females, but not males with MetS have a significantly higher LDL-cholesterol compared with non-MetS patients. Mean framingham 10-year CVD risk was significantly higher in MetS as was the proportion of patients with 10 -year risk $>20 \%$ (56\% vs. $34.5 \%$, pearson chi square $0.2, \mathrm{p}<0.001)$. Identifying MetS in this group of patients would produce an additional $29,536(8.4 \%)$ and $104,288(29.6 \%)$ patients nationally, who would require statin therapy based on the Joint British Society (JBS -treat all patient above the age of 40 years) and the National Institute of Clinical Excellence (NICE - treat all patient whose 10-year CVD risk exceeds 20\%) guidelines respectively.

Conclusion: Framingham risk-score appears to reflect the heightened CVD risk among patients with T2D and MetS. However, identifying patients with MetS may further increase the use of statins in this high risk group of patients.

\section{7}

Acute complications continue as a major cause of death for children and young adults with type 1 diabetes

R. G. Feltbower ${ }^{1}$, H. J. Bodansky ${ }^{2}$, C. R. Stephenson ${ }^{1}$, R. C. Parslow ${ }^{1}$, C. C. Patterson ${ }^{3}$, P. A. McKinney ${ }^{1}$;

${ }^{1}$ Paediatric Epidemiology Group, University of Leeds, ${ }^{2}$ Paediatric

Epidemiology Group, The General Infirmary at Leeds, ${ }^{3}$ Department of Epidemiology \& Public Health, Queen's University Belfast, United Kingdom.

Background and Aims: There is a paucity of information on cause of death in subjects diagnosed with type 1 diabetes (T1D) between 15-29 years of age compared with that available for individuals diagnosed aged $0-14$ years. The aim of the study was to examine mortality rates and cause of death amongst subjects diagnosed with T1D between the ages of 0-29 years.

Materials and Methods: Subjects were identified from the populationbased Yorkshire Register of Diabetes, a high quality database with a $98 \%$ ascertainment rate. Patients were selected with T1D aged $0-14$ years at diagnosis from all Yorkshire (1978-2004) and 15-29 years from West Yorkshire only (1991-2004). Subjects were traced on the UK National Health Service Central Register to provide details of death notifications. Deaths were coded according to ICD-9 (1979-2000) and ICD-10 (2001-2005) classification schemes. A person-years at risk analysis calculated expected numbers of deaths using UK mortality rates. Standardised mortality ratios (SMR) with 95\% confidence intervals were derived using the Poisson distribution.

Results: 3,349 and 897 patients diagnosed aged $0-14$ years and 15-29 years represented 50,471 person years of follow-up. Mean length of follow-up was 12.6 years for $0-14$ and 9.1 years for 15-29 year olds. Only ten patients $(0.2 \%)$ were untraceable. Overall $107(2.5 \%)$ patients died, of whom $76(71 \%)$ were male. $73(2.2 \%)$ deaths occurred in the younger diagnosed group and $34(3.8 \%)$ occurred in the older group. The SMR was 4.6 (95\%CI 3.8-5.6) overall and identical for males and females. The SMR rose with increasing duration of diabetes: 3.7 for a duration of $0-4$ years compared with 6.8 for 15-19 years. Only 11 deaths occurred in children before age 15 , with the majority occurring between 20-39 years of age, most likely due to increasing disease duration. 47 deaths (44\% of the total) occurred from diabetes complications: 32 acute and 15 chronic. Acute complications comprised 14 from DKA, 2 from hyperglycaemia (excluding DKA), 8 from hypoglycaemia and 8 unspecified cases. Males constituted 25/32 (78\%) deaths from acute complications. Acute complication fatalities occurred evenly throughout the cohort by age at death. Deaths associated with chronic complications included: 6 from ischaemic heart disease, 1 stroke and 8 renal ( 6 deaths being female). 24 out of the 107 (22\%) deaths were attributed to accidents or violence (SMR $2.1 ; 95 \%$ CI 1.4-3.2), 5 of which were suicides. Within this group, the SMR was non-significantly higher for females $(3.4 ; 1.4-6.9)$ compared to males $(1.9$; $1.1-3.0) .10 \%$ of all deaths (11/107) were related to drug misuse compared to $6 \%$ in the general population $(\mathrm{p}=0.09)$. There were 19 deaths of other or unknown causes: SMR=3.3 (2.1-5.2).

Conclusion: Follow-up of subjects diagnosed under 30 with T1D since the 1970s has shown a four-and-a-half-fold excess mortality risk compared to the background population, an effect which was similar for males and females although increased with disease duration. Almost half the deaths (44\%) were due to acute or chronic complications of T1D and with continued vigilance and education most of such future deaths should be preventable. There may be an emerging trend for young people with T1D to misuse drugs which warrants further investigation.

Supported by Department of Health, England, UK

\section{8}

Direct costs of diabetic ketoacidosis in children and adolescents with diabetes

A. Rewers ${ }^{1}$, M. Rewers ${ }^{2}$;

${ }^{1}$ Department of Pediatrics, University of Colorado, Denver,

${ }^{2}$ The Barbara Davis Center for Childhood Diabetes, University of Colorado, Denver, United States.

Background and Aims: DKA remains the costliest complication of diabetes in young people. The goal of this work was to estimate the direct cost of managing diabetic DKA among children and adolescents and to provide national estimates.

Materials and Methods: Administrative hospital data were analysed for all cases of DKA treated at The Children's Hospital in Denver, between1995-2006. DKA was defined by the ICD-9 diagnosis code $250.10-250.13$, validated by chart review $(\mathrm{pH}<7.35$ arterial or $<7.30$ venous or bicarbonates $<15$ in presence of hyperglycemia and ketonuria). Direct medical cost of DKA included hospital charges and professional fees. The cost was adjusted for inflation using the U.S. consumer price index and expressed in 2006 dollars. The cost estimates for specific patient groups were compared using Kruskal-Wallis test.

Results: There were 1093 DKA events in 777 patients during the study period. Median age at DKA was 12 yrs (range $0-19) ; 55 \%$ of the patients were females. DKA at onset of diabetes accounted for $49 \%$ of the episodes. Overall $23 \%$ of the patients were treated only in emergency department, $12 \%$ in an observation unit $(<24 \mathrm{~h})$ and $65 \%$ were hospitalised for a median of 1 day (range 1-61 days). Insurance status included private/HMO insurance in 66\%, government/indigent coverage in $27 \%$ and $7 \%$ the patients had no insurance. The median cost of the DKA treatment was higher $(\mathrm{p}<0.0001)$ at the onset of diabetes - $\$ 10890$ (interquartile range $\$ 6830-16023$ ) than in patients with long standing diabetes - \$8010 (\$3656-13237). In multivariable regression analysis, the cost of DKA at onset of diabetes was 67\% (95\% CI 45\%-93\%) higher than that of DKA in established patients adjusting for age and gender. Median cost of DKA increased 20\% between 1995-96 (\$8836) and 2005-06 ( $\$ 10551)(\mathrm{p}=0.008)$, adjusting for inflation. We previously reported prevalence of DKA in newly diagnosed youth $(25.5 \%)$ and DKA incidence in patients with established diabetes ( $8 / 100$ person-year). Using these estimates, the SEARCH estimates of the incidence and the prevalence of diabetes in the U.S. youth and the U.S. census population data we calculated the cost of DKA in the U.S. population younger than 20. In 1995, direct medical cost of DKA in the U.S. youth (expressed in 2006) dollars was $\$ 184$ million ( $\$ 48$ million at onset and \$136 million in established cases), increasing to \$258 million (\$73 million and \$185 million, respectively) in 2006.

Conclusion: In conclusion, children with newly diagnosed diabetes incur higher cost of DKA treatment and longer LOS, probably due to more severe presentation and lower family resources for transition to outpatient management. The economical burden of DKA among children and adolescents with is very high and increasing. 


\section{PS 104 Psychological aspects} 1049

Depressive symptoms increase the risk of all-cause mortality in men with high postload glucose levels: results of a 30 year prospective study J. Kruse ${ }^{1}$, N. Schmitz ${ }^{2}$, K.-H. Ladwig ${ }^{3}$, J. Heinrich;

${ }^{1}$ Psychosomatic Medicine and Psychotherapy, University Duesseldorf, Germany, ${ }^{2}$ Douglas Hospital Research Centre, McGill University, Montreal, Canada, ${ }^{3}$ Department of Epidemiology, GSF, München, Germany.

Background and Aims: About 25\% of people with diabetes have coexisting depressive symptoms. The aim of the present study was to evaluate the effect of depressive symptoms on all cause mortality among men with and without postload hyperglycemia $(\mathrm{PH})$ over a period of 30 years.

Materials and Methods: We examined mortality in the population based Erfurt cohort study in Germany. Between September 1973 and August 1975, 1160 men aged 40-59 years were surveyed. At baseline a 1-hour postload venous blood sample was obtained after an oral glucose tolerance test. Depressive symptoms were assessed by an exhaustion scale. The mortality follow up continued until death or the end of 2003.

Results: Over 30 years, 595 study participants (51.3\%) died. Mortality rates were highest in men with depressive symptoms and coexisting postload hyperglycemia. Hazard ratio for mortality was 0.81 (95\%CI $0.64-1.02)$ for men with depressive symptoms alone, 1.29 (95\% CI 0.96-1.73) for men with postload hyperglycemia alone, and 2.02 (95\% CI 1.24-3.30) for men with both, postload hyperglycemia and depressive symptoms. These associations remained significant even after controlling for age, smoking, body mass index, education, hypertension, and cholesterol level.

Conclusion: The results suggest that the coexistence of depressive symptoms and postload hyperglycemia is associated with an increased risk of mortality in men.

\section{0}

Influence of diabetes on cognitive function and physical impairments in the elderly

M. Lechleitner, U. Benvenuti-Falger, M. Kufner;

Internal Medicine, Hospital Hochzirl, Zirl, Austria.

Background and Aims: The prevalence of type 2 diabetes increases with increasing age. Diabetes seems to be related to an earlier manifestation of cognitive dysfunction and physical impairments, resulting in a worse metabolic control, loss of autonomy and thus quality of life. Published study results of systematic and comprehensive test programs for elderly are hardly available for diabetic patients. We have applied the routinely performed geriatric assessment test program to study the impact of diabetes on cognitive and motoric abilities in elderly diabetics and an age-matched non-diabetic population.

Patients and Methods: A total of 506 patients participated in the evaluation, 112 with diabetes (mean age $80.5 \pm 6.7$ years) and 394 without diabetes (mean age $78.7 \pm 0.0$ years). In order to study an age-dependency of the impairments 3 subgroups were created, group I: $\leq 74.9$ years, group II: 75.0-84.9 years, group III: $\geq 85$ years. In addition to clinical and laboratory routine controls all patients underwent a structured test program summarised as the geriatric assessment (Mini Mental State Examination (MMSE), geriatric depression scale, Activity of Daily Living (ADL), Mobility Tests).

Results: The mean HbAlc value of all diabetic patients was $7.2 \pm 1.4 \%$. Compared with non-diabetic patients diabetics revealed a less favourable outcome in the various tests of the geriatric assessment. Especially in group II (age 75.0-84.9 years) ADL score (66.7 \pm 22.1 points), MMSE (25.8 \pm 3.8 points) and mobility tests (Tinetti score with $13.8 \pm 6.2$ points and Up and Go test with $67.7 \pm 48.8$ seconds) were significantly worse than in non-diabetic patients (ADL-score 77.0 $\pm 18.0, \mathrm{p}<0.001$; MMSE $26.8 \pm 3.0$ points, $\mathrm{p}<0.041$; Tinetti score 16.8 $\pm 5.2, \mathrm{p}<0.001$; Up and Go test $43.9 \pm 32.8$ seconds, $\mathrm{p}<0.0001$ ). Diabetic patients in group II revelaed similar results as non-diabetic patients, who were 10 years older (group III $\geq 85$ years). The depression score in all diabetic patients was $30 \%$ higher than in non-diabetic patients $(\mathrm{p}<0.005)$.

Conclusion: Elderly diabetic patients are at an increased risk for a more pronounced manifestation of age-associated impairments, especially with respect to mobility, cognitive function and depressive disorders. Test programs, like the geriatric assessment, should thus belong to clinical routine care in order to modify the therapy according to the individual need of the elderly diabetic patient.

\section{1}

Cardiovascular risk factors and cognitive impairment in type $\mathbf{2}$ diabetes L. A. Chugunova ${ }^{1}$, I. Semenova ${ }^{1}$, E. Tarasov ${ }^{1}$, A. Vorontsov ${ }^{2}$, L. Nikankina ${ }^{3}$, P. Kamchatnov ${ }^{4}$, A. Chugunovi ${ }^{4}$, M. Shestakova ${ }^{1}$;

${ }^{1}$ Nephrology, National Research Center for Endocrinology,

${ }^{2}$ Radiology, National Research Center for Endocrinology,

${ }^{3}$ Biochemistry, National Research Center for Endocrinology,

${ }^{4}$ Neurology, Russian State Medical University, Moscow, Russian Federation.

Background and Aims: Over the past years the cerebrovascular disease was identified as the major cause of mortality and disability, particularly in type 2 diabetes. Patients with type 2 diabetes have a 2-6 fold increased risk of developing cerebrovascular disease not only strokes but also cognitive impairment. This study evaluated the relationship of cardiovascular risk factors, neuropsychological tests for cognitive impairment and cerebral MRI scan in type 2 diabetes.

Materials and Methods: The examination included glycated haemoglobin (HbAlc), body mass index (BMI), systolic (SBP) and diastolic blood pressure (DBP) by 24-h ambulatory blood pressure monitoring (24-h ABPM), homocysteine plasma levels, cerebral MRI scan (T1 and T2 weighted), neuropsychological tests: Frontal Assessment Battery (FAB), Mini Mental State Examination (MMSE), Global Deterioration Scale (GDS), Clock Drawing Test (CDT). The study involved 91 (40 men and 50 women) patients with type 2 diabetes.

Results: Mean age of patients was $61 \pm 8,4$ years. Mean duration of diabetes was $9,2 \pm 4,2$ years. Mean $\mathrm{HbAlc}$ was $8,3 \pm 1,8 \%$. The most of MRI scan abnormalities were subcortical and periventricular white matter lesions - 49\%. GDS scores showed significant correlation with total area of white matter lesions $(\mathrm{R}=0,514 ; \mathrm{p}=0,003)$. MMSE showed a significant negative correlation with diabetes duration $(\mathrm{R}=0,408 ; \mathrm{p}=0,019)$. $\mathrm{FAB}$ scores were negatively associated with diabetes duration $(\mathrm{R}=0,355 ; \mathrm{p}=0,049)$, BMI $(\mathrm{R}=0,271 ; \mathrm{p}=0,041), \mathrm{SBP}(\mathrm{R}=0,402 ; \mathrm{p}=0,028)$ and homocysteine plasma levels $(\mathrm{R}=0,353 ; \mathrm{p}=0,048)$. CDT scores exhibited a significant negative correlation with homocysteine plasma levels $(R=0,459 ; \mathrm{p}=0,021)$. Association between HbAlc and neuropsychological test scores was not estimated.

Conclusion: Our study confirms strong association of cognitive impairment in type 2 diabetes with vascular MRI abnormalities, cardiovascular risk factors, especially SBP, BMI, diabetes duration and homocysteine plasma levels as marker of endothelial dysfunction.

Supported by National Programme for Diabetes

\section{2}

Increased glucose levels are associated with episodic memory in nondiabetic women

O. Rolandsson ${ }^{1}$, A. Backeström ${ }^{1}$, S. Eriksson ${ }^{2}$, L.-G. Nilsson ${ }^{3}$;

${ }^{1}$ Department of Public Health and Clinical Medicine, Family Medicine, Umeå, ${ }^{2}$ Department of Community Medicine and Rehabilitation, Geriatric Medicine, Umeå, ${ }^{3}$ Department of Psychology, Stockholm, Sweden.

Background and Aims: Patients with type 2 diabetes have an increased risk of a reduction in cognitive function. We investigated the hypothesis that plasma glucose is associated with a reduction in episodic and/or semantic memory already in nondiabetic subjects.

Materials and Methods: We linked and matched two large population based data sets in Sweden. First, the Västerbotten Intervention Program, a health survey with subjects aged 40, 50 and 60 years. It includes measuring of fasting and 2-hour plasma glucose, and other risk factors for diabetes and cardiovascular disease. The second database was the Betula study where a random sample from the population aged $35-85$ years was investigated for cognitive function by an elaborate memory testing including episodic and semantic memory. We identified 411 (M/F 179/232, mean age $50.6 \pm 8.0$ years) nondiabetic subjects, free from dementia who had participated in the two surveys within six months (mean time difference between surveys 14 \pm 68 days). 
Results: Women had better episodic and semantic memory compared to men ( $\mathrm{p}<0.001$ for both). Both quartiles of fasting (figure 1) and $2 \mathrm{~h}$ plasma glucose (figure 2) among women were correlated to episodic memory. In a multivariate linear regression fPG $(B-0.195$, SE 0.067 , Beta $-0.206, p=0.004)$ and $2 \mathrm{hPG}$ ( $\mathrm{B}-0.071$, SE 0.031 , Beta $-0.170, \mathrm{p}=0.025$ ) were significantly negatively associated to episodic memory among women, but not in men. This was found after adjustment for age, systolic and diastolic blood pressure, hypertension, total choletsterol, body mass index, educational level, depression, smoking and history of cardiovascular disease. The association was not found in relation to semantic memory.

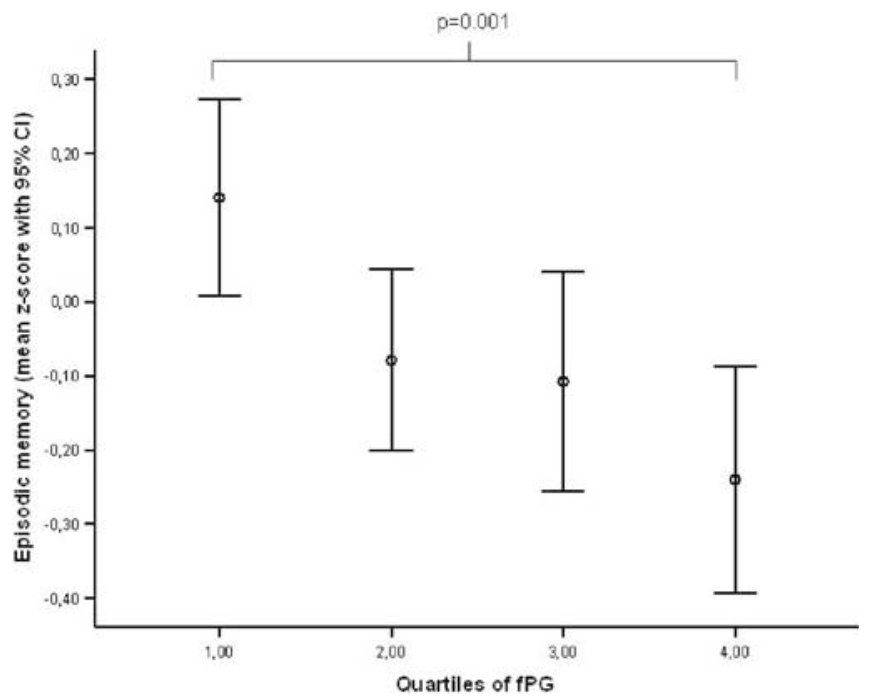

Fig. 1. Mean (95\% CI) episodic memory (z-store) among women by quartiles of fasting plasma glucose.

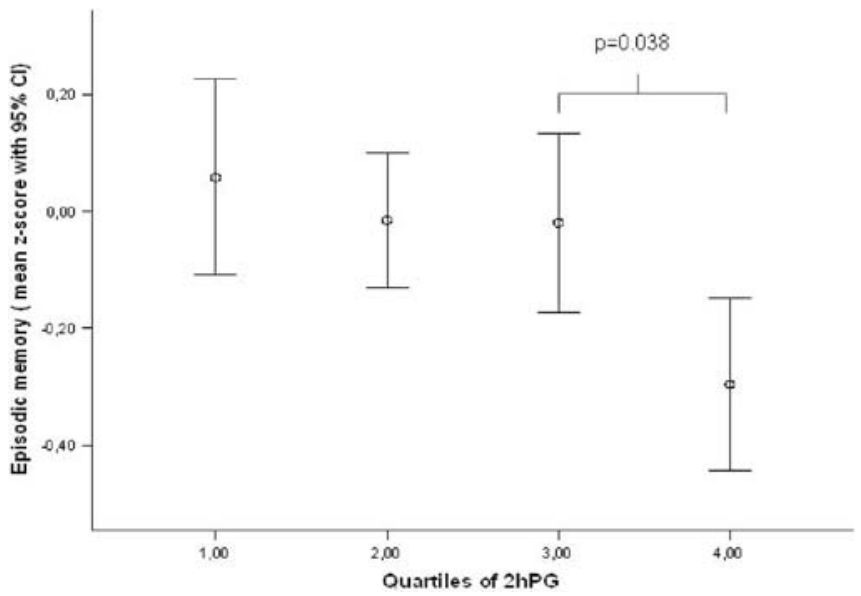

Fig. 2. Mean ( $95 \%$ CI) episodic memory (z-store) among women by quartiles of $2 \mathrm{~h}$ plasma glucose.

Conclusion: We conclude that an increase in plasma glucose is associated with impairment in episodic memory in women. This could be explained by a negative effect on hippocampus by raised plasma glucose levels.

Supported by the County Council of Västerbotten, Sweden

\section{3}

Diabetes, chronic diseases and well-being: the WHO 5 into practice G. Hochberg;

DELF, Paris, France.

Aims: In 2001, the Dawn Study helped for the first time to understand more fully diabetic patient attitudes worldwide in order to improve their outcomes. In the same perspective, French Dawn experts decided in 2005 to use the
WHO 5 questionnaire as a barometer to have a clearer picture of the wellbeing state of diabetic patients in comparison with patients suffering from other chronic diseases (CD) and through the results, to make practitioners more aware of psychosocial aspects of diabetes.

Methods: The WHO-5 Questionnaire was proposed to 4965 general practitioners and 1702 diabetologists in hospitals and cities for distribution to their chronic patients. The practitioners' geographic area was specified. Patients were also invited to give socio-demographic and medical information as sex, age, type of $\mathrm{CD}$, type of diabetic treatment (insulin or not), then to answer a question on the possible link between their well being and their CD. Patients fulfilled questions alone and returned questionnaires back directly. Results: 2253 were returned, 2213 could be analysed. $49 \%$ males, $51 \%$ females. $41 \%$ suffered from diabetes, $34 \%$ from diabetes and other CD, $25 \%$ from other $\mathrm{CD}$ than diabetes. $57 \%$ of diabetics were treated by insulin. WHO 5 : mean score $=14.1($ males $=14.97$; females $=13.5 ; \mathrm{p}<0.001) .1 / 3$ of patients could be considered as depressed $(<13)$. Significant difference was found between patients with diabetes alone and patients with diabetes and other $\mathrm{CD}(\mathrm{p}<0.005)$. Diabetics have better well-being than patients with other CD $(p=0.002)$. Among diabetics only, insulin treatment is associated with worse well-being $(\mathrm{p}=0.03)$.

Conclusions: Globally, diabetics seem to have better well-being than patients with other CD. Negative factors influencing well-being are female sex, insulin treatment within diabetics. These results tend to highlight that HCP have to consider more systematically well-being of patients with CD. Supported by Novo Nordisk

\section{4}

Psychosocial adjustment and outcomes of glycaemic control with basal insulin therapy in patients with type 2 diabetes mellitus (T2DM) R. J. Tanenberg ${ }^{1}$, Q. Zhang ${ }^{2}$, A. Zisman ${ }^{3}$;

${ }^{1}$ Division of Endocrinology, Brody School of Medicine, Greenville, ${ }^{2}$ sanofi-aventis U.S., Bridgewater,

${ }^{3}$ Endocrine Center of Aventura, Miami, United States.

Background and Aims: Psychosocial adjustment may influence glycemic control in patients with T2DM using insulin therapy. A 24-week, randomised, open-label study that compared the rate of severe hypoglycemic events (primary endpoint) and the proportion of patients reaching $\mathrm{AlC}<7.0 \%$ was used to examine the relationship between poor psychosocial adjustment and increased risk of hypoglycemia. Patients $(\mathrm{N}=4163)$ with T2DM failing oral therapy (mean $\mathrm{A} 1 \mathrm{C}=9.2$ ) were randomised to 5 insulin glargine treatment algorithms with target fasting blood glucose of $80,90,100,110$, and $120 \mathrm{mg} /$ $\mathrm{dL}$, respectively.

Material and Methods: A 46-item Psychosocial Adjustment to Illness Scale (PAIS) was administered at baseline and end of the study period to assess psychosocial adjustment to diabetes in each of the 7 domains that include health care orientation; vocational, domestic, social environment; sexual and extended family relationships; and psychological distress.

Results: Patients were 55 years, $48 \%$ male, $70 \%$ white, with mean T2DM duration of 8.3 years and BMI 34.7. Poor psychosocial adjustment was consistently associated with lower risk of hypoglycemia and higher studyend $\mathrm{AlC}$ independent of baseline A1C and BMI. In addition, effect of psychosocial adjustment was constant among titration algorithms (ie, different target fasting blood glucose). Study-end A1C was lower $(P<0.0001)$ in patients with best psychosocial adjustment (7.3 and 7.0 in patients with and without hypoglycemia, respectively) compared with those with poorer adjustment ( 8.0 and 7.5 , respectively). Of the 7 domains, poor adjustment was associated with higher A1C over 6 domain scores $(P<0.05)$. At the item level, poorly-adjusted patients recorded higher A1C in 30 of 46 items and poor adjustment was associated significantly with lower incidence of hypoglycemia in 18 of 46 items $(P<0.05)$. Patients' age, sex, and T2DM duration interacted with hypoglycemia and psychosocial adjustment to yield an impact on A1C. Specifically, the effect of adjustment (poor vs. excellent) to hypoglycemia and A1C control is different in younger individuals with poor adjustment being associated with lower risk of hypoglycemia and lower A1C compared to older individuals, where poor adjustment predicts higher risk for hypoglycemia and higher A1C. Gender also interacted with A1C with a decrease in studyend A1C observed among better vs. worse adjusted female patients ( $7.9 \%$ vs. $7.5 \%$, respectively) and only a minimal impact of adjustment among males ( $7.3 \%$ vs. $7.4 \%$, respectively). 
Conclusion: This study demonstrated that better psychosocial adjustment contributed to lower A1C during the treatment of patients with T2DM with basal insulin therapy. Patient treatment plans that incorporate the consideration of psychosocial factors may achieve more desirable glycemic outcomes.

Editorial support provided by sanofi-aventis U.S.

\section{5}

Monitoring health-related quality of life in adolescents with type 1 diabetes improves psychosocial well-being and satisfaction with care M. de Wit ${ }^{1}$, R. Gemke ${ }^{2}$, J. Bokma ${ }^{3}$, B. Boersma ${ }^{4}$, A. Derksen ${ }^{5}$, A. Felius ${ }^{2}$, K. Haasnoot ${ }^{4}$, M. Houdijk ${ }^{5}$, E. van Lie-Peters ${ }^{4}$, E. van Mil $^{2}$, M. Reeser ${ }^{5}$, J. Rotteveel ${ }^{2}$, T. Sas ${ }^{5}$, H. Delemarre-van de Waal ${ }^{2}$, F. Snoek ${ }^{1}$;

${ }^{1}$ Medical Psychology, VU University medical center, Amsterdam,

${ }^{2}$ Pediatrics, VU University medical center, Amsterdam, ${ }^{3}$ Pediatrics, Spaarne Ziekenhuis, Hoofddorp, ${ }^{4}$ Pediatrics, Medical Center Alkmaar, Alkmaar,

${ }^{5}$ Pediatrics, Juliana Kinderziekenhuis, Den Haag, The Netherlands.

Background and Aims: Systematic monitoring of psychosocial functioning in adolescents with diabetes may help to improve physical and psychosocial well-being and glycaemic control. In an ongoing RCT, we investigate the effects of monitoring health related quality of life (HRQoL) on physical and psychosocial well-being, $\mathrm{HbAlc}$ and satisfaction with care in adolescents with type 1 diabetes.

Materials and Methods: Ninety-one adolescents with type 1 diabetes (age $14.9 \pm 1.1, \mathrm{HbAlc} 8.8 \pm 1.7$, BMI $21.1 \pm 3.2$ and duration $6.4 \pm 4.2$ years) were recruited from four outpatient paediatric diabetes clinics in The Netherlands. Clinics were randomised over either control or monitoring condition. The clinics in the control condition provided care as usual, while in the monitoring condition the patients completed a 3-monthly computerised HRQoL assessment (using the PedsQL) and discussed the outcomes with their paediatrician or nurse. Both groups were followed for 12 months, assessing physical and psychosocial well-being using validated questionnaires (Child Health Questionnaire (CHQ), Center for Epidemiological Studies scale for Depression (CES-D), diabetes specific family conflict (DFCS), satisfaction with care (PEQ-D) and medical data (HbAlc, BMI, treatment regimen, diabetes duration) at baseline and follow-up. MANCOVA analyses, correcting for baseline differences, were used to explore the effect of the intervention in 80 patients. Multiple linear regression was used to identify factors that explained (variation in) HbAlc levels.

Results: A significant improvement was found in the psychosocial and physical summary scales of the CHQ for the adolescents in the monitoring group, compared to those in the control group $\left(\mathrm{p}<0.001, \mathrm{R}^{2}=0.37\right.$ and $\mathrm{R}^{2}=$ 0.41 ). This effect was most profound in reduction of behavioural problems and improvement in self-esteem. There were no changes in HbAlc levels, depression and diabetes-specific family conflict for either of the groups. However, the patients' satisfaction with care significantly improved in the monitoring group in contrast to the control group, which remained the same. For the group as a whole, HbAlc levels at follow up were predicted by glycaemic control at baseline and by the amount of diabetes specific family conflicts $\left(\mathrm{p}<0.001, \mathrm{R}^{2}=0.42\right)$.

Conclusion: This is the first study to demonstrate monitoring health related quality of life improves psychosocial and physical well-being in adolescents with diabetes as well as their satisfaction with care but not glycaemic control. Additional targeted interventions are required to decrease HbAlc levels. Supported by Dutch Diabetes Research Foundation

\section{6}

The association of weight worry with quality of life and diabetes self-management

M. Peyrot ${ }^{1}$, S. E. Skovlund ${ }^{2}$;

${ }^{1}$ Sociology, Loyola College, Baltimore, United States,

${ }^{2}$ Novo Nordisk, Bagsvaerd, Denmark.

Background and Aims: This study examined the epidemiology of worry about weight among adults with diabetes and whether this weight worry (WW) was associated with lower quality of life and poorer diabetes selfmanagement.
Material and Methods: Participants were 5104 adults with type 1 or type 2 diabetes from the multi-national DAWN study. Random samples of approximately 500 were obtained from each of 11 countries/regions in Asia, Australia, Europe, and North America. All measures were self-reported data obtained during structured interviews. $32.8 \%$ of participants had type 1 diabetes, $26.9 \%$ had insulin-treated type 2 diabetes, $40.4 \%$ had non-insulin treated type 2 diabetes. Median age was 55 years, median duration of diabetes was 10 years, median age at termination of education was 17 years. $21.8 \%$ lived in a rural area. $54.0 \%$ were female.

Results: $50.2 \%$ of participants reported some WW; $25.1 \%$ reported high WW. The study did not contain a measure of obesity, but there were measures of several obesity risk factors. High WW was significantly $(\mathrm{p}<.05)$ higher among women (31.0\%), those with type 2 diabetes $(27.0 \%$ of those not on insulin, $30.3 \%$ of those on insulin), those who had diabetes less than 10 years (26.9\%) and those who were older than $54(27.8 \%)$, less physically active (no regular exercise, 26.1\%) and terminated education before age 18 (28.7\%). In the full study population high WW was associated with significantly $(\mathrm{p}<$ $.05)$ more diabetes-related distress, more diabetes related symptoms, lower self-rated health, lower vitality, poorer psychological well-being, and more psychological treatment. High WW was associated with significantly $(\mathrm{p}<.05)$ lower diet and exercise adherence and poorer self-rated diabetes control. The only significantly $(\mathrm{p}<.05)$ positive correlate of high WW in the full study population was appointment-keeping. Among insulin-naive respondents high WW was associated with significantly $(\mathrm{p}<.05)$ more worry about starting insulin and more self-blame about having to start insulin. Among those taking anti-hyperglycaemic medication, high WW was associated with significantly $(\mathrm{p}<.05)$ more worry about hypoglycaemia. Among those whose only antidiabetic medication was insulin, high WW had a complex relationship with medication adherence; among type 1 patients WW was associated with significantly $(\mathrm{p}<.05)$ lower insulin adherence, among type 2 patients $\mathrm{WW}$ was associated with significantly $(\mathrm{p}<.05)$ higher insulin adherence.

Conclusion: Weight worry was common among adults with diabetes. Although it was not possible to examine the relationship between weight and weight worry, those at greater risk for being overweight experienced higher levels of weight worry. Weight worry was associated with a plethora of negative outcomes, including multiple indicators of diabetes-related distress, poor physical and psychological well-being, and regimen non-adherence. Weight worry is associated with worry about starting insulin and having hypoglycaemia, which may lead patients to resist initiation of insulin therapy. These findings suggest that health care providers should assess weight worry among their patients with diabetes, and take steps to prevent negative impacts on patient outcomes.

Supported by Novo Nordisk

\section{7}

Can a theory-based behaviour change programme improve outcomes for people with recently diagnosed type 2 diabetes? - the ADDITION Plus trial

A. L. Kinmonth ${ }^{1}$, N. J. Wareham ${ }^{2}$, S. Sutton ${ }^{1}$, W. Hardeman ${ }^{1}$, T. Prevost ${ }^{1}$, K. M. Williams ${ }^{1}$, S. J. Griffin ${ }^{2}$;

${ }^{1}$ Public Health and Primary Care, University of Cambridge, ${ }^{2}$ Epidemiology Unit, Medical Research Council, Cambridge, United Kingdom.

Background and Aims: Recent trials emphasise the value of intensive treatment of multiple risk factors among people with diabetes. This consists of poly-pharmacy, combined with efforts to achieve healthy behaviours, and presents a considerable burden to patients and a challenge to practitioners. In an explanatory, parallel group, individual randomised trial we assessed the efficacy of a theory-based intervention to promote health-related behaviours (physical activity, dietary change, medication adherence and smoking cessation) and to achieve clinically important changes in cardiovascular disease (CVD) risk factors, among patients with diabetes detected by screening, or recently clinically diagnosed, and offered intensive drug treatment.

Materials and Methods: 239/422 patients newly diagnosed through a stepwise screening program in primary care, and 239/684 patients clinically diagnosed within the previous three years were recruited from 34 general practices in the east of England. Following baseline measurement participants were independently randomised to intervention (I:239) or control (C:239) groups using a partial minimisation procedure to balance covariates. Intensive drug treatment of all study patients and general support in selfmanagement was facilitated through practice-based educational meetings, 
guidelines, target setting, academic detailing and educational materials for patients. Intervention patients also received a theory-based behavioural intervention delivered by trained and quality-assured facilitators at six practice-based appointments over 10 months. After 13.2 months (SD:1.6) $93 \%$ of participants attended for objective measurement of the principal outcomes: physical activity (individually calibrated heart rate monitoring), diet (plasma Vitamin C), medication adherence (serum drug levels) and smoking status (serum cotinine); self-reported health behaviours, clinical, biochemical, functional status and psychological outcomes.

Results: CVD risk factors (secondary outcomes) improved among intensively managed patients in both study groups. There was no additional benefit associated with the behavioural intervention. Newly diagnosed patients demonstrated a small advantage for the intervention group for bmi (difference $(95 \% \mathrm{CI}):-0.49 \mathrm{~kg} / \mathrm{m}^{2}(-0.91$ to -0.07$\left.), \mathrm{p}=0.021\right)$, and HDL cholesterol $(+0.05 \mathrm{mmol} / \mathrm{l}(+0.004$ to +0.10$), \mathrm{p}=0.034)$.

Conclusion: A theory-based intervention to promote changes in health behaviours was not associated with improvements in CVD risk factors among recently diagnosed patients over and above that achieved though educational materials and intensive general practice management. Behavioural interventions may be more successful if delivered soon after diagnosis. Results for principal behavioural outcomes will be presented. (Tab. 1) Supported by MRC, NHS, R\&D

\section{8}

Disordered eating behaviour and metabolic control in young subjects with type 1 diabetes transferred from a paediatric to an adult diabetes unit I. Conget ${ }^{1}$, M. Lara ${ }^{1}$, M. Vidal ${ }^{1}$, M. Jansa ${ }^{1}$, J. Castro ${ }^{2}$, M. Giménez ${ }^{1}$; ${ }^{1}$ Endocrinology and Diabetes Unit, ${ }^{2}$ Paediatric Psychiatry-Psycology Unit, Hospital Clínic i Universitari/IDIBAPS, Barcelona, Spain.

Background and Aims: Eating disorders are a significant health problem in young people and they could be especially troublesome and a determinant factor to achieve an optimal metabolic control in young subjects with type 1 diabetes (T1D) transferred to adult diabetes units. Our study aimed to evaluate the prevalence of disordered eating behaviour and its relationship with metabolic control among young subjects with T1D transferred from a paediatric to an adult diabetes unit.

Subjects and Methods: Seventy-four consecutive young subjects with T1D (aged 17.6 \pm 1.4 years, 40 women, T1D duration $9.0 \pm 1.3$ years) transferred from a paediatric to an adult diabetes unit were included. In addition to clinical and physical exam, we evaluated: the metabolic control, the Eating attitude Test (EAT), the eating behaviour, the self-management abilities and knowledge (DKQ2 test), the Diabetes Quality-of-Life questionnaire (DQoL) and the SF-12 health survey questionnaire.

Results: At entry $\mathrm{HbA}_{1 c}$ was $8.7 \pm 1.6 \%$. EAT score was significantly correlated with $\mathrm{HbA}_{1 c}(r=0.32, p<0.008)$ and it was higher in girls than in boys $(12.2 \pm 6.0$ vs. $5.7 \pm 3.1 ; p<0.000$ ). In 10 subjects (9 girls) we found a highly/moderately disordered eating behaviour and in those subjects $\mathrm{HbA}_{1 c}$ tended to be higher ( $9.3 \pm 2.3$ vs. $8.5 \pm 1.4 \%, p=0.178)$. DQoL Diabetes-related issues worrying subscale score was significantly higher in girls $(9.1 \pm 2.9$ vs. $7.5 \pm 2.1 ; p<0.015)$. The perception of health assessed by SF-12 test and DKQ2 score were better in boys than in girls $(p<0.015$ and $p<0.05$, respectively).

Conclusion: At the time of transfer of young people with T1D from a paediatric to an adult diabetes unit the presence of a significant disordered eating behaviour is far from uncommon, particularly in girls, and it is related with a worse metabolic control.

Tab. 1

Cardiovascular risk factors at baseline and follow-up

\begin{tabular}{|c|c|c|c|c|c|c|}
\hline & \multicolumn{2}{|l|}{ Control group } & \multicolumn{2}{|c|}{ Intervention group } & \multirow[b]{2}{*}{$\begin{array}{l}\text { Difference in change } \\
(95 \% \mathrm{CI})\end{array}$} & \multirow[b]{2}{*}{$P$ value } \\
\hline & $\begin{array}{l}\text { Mean (SD) at } \\
\text { baseline }\end{array}$ & $\begin{array}{l}\text { Mean }(\mathrm{SD}) \text { at } \\
\text { follow-up }\end{array}$ & $\begin{array}{l}\text { Mean (SD) at } \\
\text { baseline }\end{array}$ & $\begin{array}{l}\text { Mean (SD) } \\
\text { at follow-up }\end{array}$ & & \\
\hline HbA1c (\%) & $7.01(1.23)$ & $6.67(0.95)$ & $7.23(1.62)$ & $6.66(0.94)$ & $-0.10(-0.24$ to +0.04$)$ & 0.17 \\
\hline BMI $\left(\mathrm{kg} / \mathrm{m}^{2}\right)$ & $32.8(5.7)$ & $32.3(5.7)$ & $32.7(5.3)$ & $32.1(5.2)$ & $-0.16(-0.46$ to +0.14$)$ & 0.29 \\
\hline Systolic BP (mmHg) & $134.4(19.3)$ & $128.2(17.2)$ & $138.2(19.1)$ & $132.1(17.4)$ & $+1.96(-0.67$ to +4.59$)$ & 0.14 \\
\hline Diastolic BP (mmHg) & $79.1(10.9)$ & $75.1(9.8)$ & $81.6(10.0)$ & $77.7(8.8)$ & $+1.40(-0.09$ to +2.90$)$ & 0.066 \\
\hline Total cholesterol (mmol/l) & $4.90(1.16)$ & $4.31(0.86)$ & $4.96(0.98)$ & $4.33(0.91)$ & $-0.01(-0.15$ to +0.14$)$ & 0.94 \\
\hline HDL cholesterol (mmol/l) & $1.20(0.35)$ & $1.19(0.32)$ & $1.17(0.35)$ & $1.19(0.31)$ & $+0.02(-0.02$ to +0.05$)$ & 0.33 \\
\hline LDL cholesterol (mmol/l) & $2.87(0.99)$ & $2.34(0.76)$ & $2.89(0.91)$ & $2.29(0.76)$ & $-0.06(-0.19$ to +0.07$)$ & 0.35 \\
\hline
\end{tabular}

\section{PS 105 Education and psychological aspects}

\section{9}

\section{Characteristics and eating habits of diabetic patients during the fasting} month of Ramadan

Y. Altuntas, S. Ucak, H. Tokel, T. Erdem, O. Basat, T. Saler, A. Kol; Endocrinology\&Metabolism, Sisli Etfal Research and Training Hospital, Istanbul, Turkey.

Background and Aims: Ramadan is an absolute fast (no food or water) month between dawn and sunset. Duration is 1 lunar month in a year. It is a Holy Month for Muslims. The aim of this study was to evaluate the characteristics and eating habits of patients with type 2 diabetes during Ramadan.

Materials and Methods: This was a single-centre, retrospective study. A total number of 300 patients attending to our diabetes outpatient clinic were screened. They were under follow and applied for their routine visit during the month of Ramadan. All patients were type 2 diabetics and at least 35 years of age. A standard questionnaire-based interview was conducted by the physician. Interviews were made one to one and face to face. Patients were asked if they were fasting during Ramadan. And also sweet food intake frequency in a month, hypoglycemic episodes, duration of diabetes mellitus, treatment, change in treatment during Ramadan were questioned. For qualitative data group comparisons were performed using chi-square test, otherwise Fisher's exact test was performed. For quantitative data analysis of variance or nonparametric tests were performed. A p value less than 0.05 was considered significant.

Results: A total number of 300 diabetic patients were recruited fort he study. During Ramadan 51.7 \% of patients fasted. 168 patients treated with OAD's and 132 patients treated with insulin. In OAD group there were 123 female and 45 male patients while there were 91 female and 41 male patients in insulin group. In OAD group $69.6 \%$ of subjects and in insulin group $21.2 \%$ of subjects fasted $(\mathrm{p}<0.01)$. In OAD group 107 patients $(63 \%)$ and in insulin group 84 patients $(63 \%)$ ate sweet foods during Ramadan. In OAD group $23.8 \%$ of patients had baklava (a special Turkish desert), $35.1 \%$ of patients had cookies, $47.6 \%$ of patients had dry persimmon (high glycaemic index), $19,6 \%$ had honey and $31 \%$ had jam. In insulin group $48 \%$ of patients had baklava, $34.8 \%$ had cookies, $34.8 \%$ had dry persimmon and $18.2 \%$ had jam. Hypoglycemia rate was greater in insulin group than OAD group ( $40.2 \%$ vs. $9.5 \% \mathrm{p}<0.01)$. Duration of disease was longer in insulin group $(10.45 \pm 7.05$ vs. $5.22 \pm 5.10, \mathrm{p}<0.01)$. Patients in insulin group were fasting longer than in OAD group ( $5.40 \pm 4.51$ vs. $3.87 \pm 3.15, \mathrm{p}<0.05)$. Only 10 patients in insulin group and 5 patients in $\mathrm{OAD}$ group made changes in their treatment during Ramadan. Although in insulin group $\mathrm{HbAlc}$ levels did not change significantly after 3 months ( $6.47 \pm 1.15$ vs. $6.10 \pm 1.16, \mathrm{p}=0.001)$.

Conclusion: A high number of patients with diabetes mellitus still fast during the month of Ramadan. Especially patients using OAD's seem to fast more. Although fasting may cause dangerous conditions in insulin treated patients, they persist on fasting. Patients with diabetes mellitus also consume large amounts of sweet foods in contrary to physicians advices. Although fasting does not seem to effect metabolic control in patients using OADs, more time and effort should be put in on patients education impressing the importance of lifestyle changes in the management of diabetes. significantly 3 months after Ramadan, in OAD group HbAlc levels decreased 


\section{0}

Multifactor approach associated with orlistat for 2 years weight loss maintenance

V. Makoundou ${ }^{1}$, F. Habicht ${ }^{1}$, E. Harsch-Bobbioni ${ }^{1}$, J.-P. Gachoud ${ }^{2}$, A. Golay ${ }^{1}$;

${ }^{1}$ S.E.T.M.C., Geneva University Hospital, Geneva 14, Switzerland, ${ }^{2}$ Roche Pharma, Reinach, Switzerland.

Background and Aims: Weight loss maintenance management over time after weight loss is difficult and important. We have considered a multifactor approach in order to achieve weight loss maintenance: diet and physical activity counseling associated with a cognitive-behavioral therapy and an orlistat "on-off" treatment program when the weight relapse is higher than $2.5 \%$

Materials and Methods: 50 obese patients (mean BMI $36.4 \mathrm{~kg} / \mathrm{m}^{2}$ ) were enrolled in the weight loss maintenance study after a weight loss of more than $10 \%$ (mean $-17 \%$ ). Patients came to the clinic every 2 months for the first year and then every 3 months until year 2 . In order to maintain their weight loss, patients received a diet and physical activity counseling during the study. If a weight relapse of $\geq 2.5 \%$ was measured during a study visit, the following procedures were added: food questionnaire for the patient, cognitivebehavioral therapy, orlistat $120 \mathrm{mg}$ tid. This procedure was maintained as long as the weight relapse was of $\geq 2.5 \%$ ("on-off" treatment program). In order to manage so called risky situations on special occasions such as birthdays, restaurants, social events, orlistat was also prescribed at patient request. Body weight was measured and weight relapse (if any) calculated at each visit, body composition and self assessment eating questionnaire (EDI2) were performed once a year; anxiety and depression, physical activity selfassessment questionnaires were performed every 6 months.

Results: At baseline the mean age was $43 \pm 11$ years, and BMI $30.1 \pm 5.7 \mathrm{~kg} / \mathrm{m}^{2}$. Five patients dropped during the study. After 2 years, the mean body weight was very well maintained: $80.7 \mathrm{~kg} \pm 17.1$ at 2 years compared to $82.1 \mathrm{~kg} \pm 15.6$ at baseline. $58 \%$ of the patients had at least an additional weight loss of $>$ $2.5 \%$ or stable weight and $38 \%$ had at least one episode of weight gain of $>$ $2.5 \%$ during the 2 years of follow up. The orlistat was prescribed in $48 \%$ for special occasions only, in $34 \%$ for special occasions and continuous intake, in $8 \%$ for continuous intake only and in $10 \%$ no intake. $18 \%$ of the patients had at least once a cognitive behavioral therapy, 22\% physical and diet motivational workshops, $16 \%$ new diet counseling. For body composition, EDI2, HAD, parameters, no change were observed after the 2 years weight loss/maintenance program.

Conclusion: This new multifactor approach with an "on-off” orlistat program appears to be promising. These results should be confirmed with the weight loss maintenance program at 4 years which is actually ongoing.

Supported by Roche Pharma (Switzerland)

\section{1}

Short-term effects of an educational program on health seeking behavior for infections in patients with type 2 diabetes: a randomized controlled trial in primary care

K. J. Gorter, L. M. A. Venmans, E. Hak, G. E. H. Rutten;

Julius Center for Health Sciences and Primary Care, University Medical Center Utrecht, The Netherlands.

Background and Aim: Patients with type 2 diabetes mellitus (DM2) have an increased risk of urinary tract infections (UTIs) and lower respiratory tract infections (LRTIs). Also, common infections may be more difficult to treat, often recur and diabetes increases the risk of hospitalization and mortality from infections. However, results of focus group interviews and a questionnaire study showed that patients with DM2 are lacking basic background knowledge about risk factors and symptoms of infections and risks are underestimated. Together with barriers like being afraid that insulin will be prescribed, this may cause substantial delay in health seeking behavior and consequently lead to increased morbidity and mortality in patients, economic costs for health care and loss of productivity to society. Based on these findings, we developed an educational program on common infections in diabetes. This study aims to asses the short-term effects of an educational program on health seeking behavior for UTIs and LRTIs in patients with DM2.

Material and Methods: In a randomised controlled trial 1,124 patients with DM2 aged between 44 and 85 years participated. The intervention consisted of an educational program with an interactive meeting, a leaflet, a website and a consult with the diabetes care provider. The program focussed on the patients' needs, apparent from a prior focus group and questionnaire study. The primary outcome measure was an indicator of health seeking behavior for UTI and LRTI, defined as the proportion of participants with a positive score on at least seven out of nine determinants of health seeking behavior according to the Health Belief Model and the additional domains knowledge, need for information and intention. Variables were measured with questionnaires at baseline and after five months. Before-after comparisons were made according to the intention-to-treat and on-treatment principle by use of General Equation Estimation modeling to account for baseline levels and clustering.

Results: The mean age of the patients was 64 years (SD 9), 53\% of the participants were male, co-morbidity was present in $44 \%$ of the patients and insulin was used by $13 \%$ of the patients. Mean duration of diabetes was 6 years (SD 7). Complete outcome data were available for 468 intervention group patients and 472 control patients. In all, $68 \%$ of the intervention group patients attended the meeting. At baseline, $28 \%$ of the participants of the intervention group had a positive score on seven of the nine determinants, compared to $27 \%$ of the controls. After the educational program, these percentages were $53 \%$ and $32 \%$, respectively $(\mathrm{p}<0.001)$. In the intervention group, especially knowledge about symptoms of LRTI ( $31 \%$, compared with $4 \%$ in controls) and UTI ( $11 \%$ compared with $0 \%$ ) improved. Besides, there was an improvement in the perception of an increased risk of infections (18\% compared with $7 \%$ ). Conclusion: Our educational program positively influenced determinants of health seeking behavior for common infections in patients with DM2. Especially knowledge and risk perception were improved. Besides, the proportion of participants with a positive score on seven of nine determinants of health seeking behavior increased significantly. By improving this behavior, complications of common infections in patients with DM2 may be prevented. Supported by Public Health Fund, The Hague; Diabetic Fund, Amersfoort, The Netherlands

\section{2}

Predicting return for a long-term follow-up diabetes self management education visit following a chronic care model based diabetes care intervention

G. A. Piatt ${ }^{1}$, J. C. Zgibor ${ }^{2}$;

${ }^{1}$ University of Pittsburgh Diabetes Institute, University of Pittsburgh Medical Center, Pittsburgh, ${ }^{2}$ Epidemiology, University of Pittsburgh Graduate School of Public Health, Pittsburgh, United States.

Background and Aims: Evidence supports the effectiveness of diabetes selfmanagement education (DSME) in the short term; however mixed results are reported with follow-up greater than one year, possibly due to attrition. We therefore aimed to determine the patient factors that best predict return for a long term follow-up DSME visit (FUV) following a Chronic Care Model based intervention (CCMI) that took place in an underserved community between 2002 and 2003 .

Materials and Methods: Eleven primary care practices, along with their patients, (mean age: 66.6 yrs, $47.6 \%$ male, $10.7 \%$ non-white) were randomly assigned to 3 groups: patient and provider education $(\mathrm{CCM})(\mathrm{n}=30)$, provider education only (PROV) $(n=38)$, and usual care $(\mathrm{UC})(\mathrm{n}=51)$. CCM received all elements of the CCMI. Subjects were followed for 3 years post CCMI.

Results: $54 \%(57$ of 105$)$ subjects (mean age: $66.4 \pm 8.7,49.1 \%$ male, $8.8 \%$ non-white, $19.3 \%$ insulin user), who were evaluated at one year post CCMI, chose to be reevaluated at three year post CCMI. Follow-up rate was similar when examined by intervention group (CCM: 56\%, PROV: 56\%, UC: $52 \%$ ). Overall, a significantly greater proportion of subjects who returned for a FUV were not using insulin $(56.1 \%$ vs. $29.7 \%, \mathrm{p}=0.008)$ and had not seen a healthcare provider in the previous 12 months $(61.8 \%$ vs. $38.5 \%, \mathrm{p}=0.02)$. Additionally, those who returned for a FUV had higher diabetes knowledge test scores ( $72 \pm 14.4$ vs. $60.9 \pm 19.2, \mathrm{p}=0.002)$ and lower systolic blood pressure levels $(138.9 \pm 18.5 \mathrm{mmHg}$ vs. $145.9 \pm 19.3 \mathrm{mmHg}, \mathrm{p}=0.06$. Multivariate analyses controlled for age and insulin use at study entry and intervention group. Significant associations remained as subjects not taking insulin were more likely to return for a FUV (OR=0.18, 95\% CI: $0.06,0.54)$. Additionally, with every $5 \%$ increase in diabetes knowledge test scores $(\mathrm{OR}=1.3,95 \% \mathrm{CI}$ : $1.1,1.5$ ) and every $5 \mathrm{mmHg}$ decrease systolic blood pressure (OR=0.87, 95\% CI: 0.76, 0.99), the likelihood of returning for a FUV increased significantly. Conclusion: These results demonstrate that a variety of factors predict return for a FUV. This type of information may aid researchers in targeting specific 
populations, and may be translated into the clinical practice setting to guide changes in scheduling patterns or to recognise patients appropriate for additional diabetes or non-diabetes related interventions to change behavior. Partially sponsored by funding from the US Air Force administered by the US Army Medical Research Acquisition Activity, Fort Detrick, Maryland

\section{3}

Cardiovascular risk awareness and metabolic control are correlated with disease specific knowledge in patients with type 2 diabetes

A. H. Pfützner ${ }^{1}$, C. Hohberg ${ }^{1}$, G. Lübben ${ }^{2}$, E. Karagiannis ${ }^{2}$, S. Gauert ${ }^{3}$, W. Roth ${ }^{1}$, T. Forst ${ }^{1}$, A. Pfützner ${ }^{1}$;

${ }^{1}$ IKFE Institute for Clinical Research and Development, Mainz, ${ }^{2}$ Takeda Pharma, Aachen, Germany, ${ }^{3}$ Health Care, BKK, Hannover, Germany.

Background and Aims: A high incidence of cardiovascular disease (CAD) in type 2 diabetes mellitus is seen even in patients with good glycemic control. Increasing the CAD risk awareness may be a helpful tool to implement an acceptance for life style changes and other therapeutic measures in routine health care.

Materials and Methods: In order to evaluate the risk awareness of diabetic patients and their physicians in correlation with patients knowledge about their disease, we performed a brief 9-item diabetes knowledge and CAD risk estimation test in 157 patients with type 2 diabetes ( 49 women, 108 men, age(mean $\pm \mathrm{SD}): 65 \pm 8$ years, disease duration: $9 \pm 8$ years) and applied other clinical and laboratory means for CAD risk assessment (e.g. Intima-mediathickness (IMT), PROCAM-Score, UKPDS risk score).

Results: Based on the tertiles of the knowledge test, patients were stratified into low (LK, 64 pts., 40\%), medium (MK, 45 pts. 29\%) and high knowledge (HK, 49 pts. 31\%) groups. The knowledge test outcome was independent from the intensity of previous diabetes trainings as reported by the patients. The $\mathrm{HK}$ patients had a better $\mathrm{HbAlc}$ and lower IMT than the LK group (HbAlc: $6.7 \pm 0.8 \%$ vs. $7.1 \pm 1.5 \%, p<0.05$; IMT: $0.774 \pm 0.128 \mathrm{~mm}$ vs. $0.864 \pm 0.181 \mathrm{~mm}$, $\mathrm{p}<0.05)$. They also had a lower $\mathrm{HOMA}_{\mathrm{IR}}$ score $(4.7 \pm 1.5$ vs. $7.1 \pm 3.2, \mathrm{p}<0.05)$ and better metabolic profile with regard to lipids and other laboratory risk markers. When the individual risk for a $\mathrm{CAD} /$ stroke event was estimated by the patients and their physicians, the agreement was better in the HK group than in the LK or MK group. The UKPDS-risk engine revealed a low CAD risk in $8.8 \%, 13.8 \%$, and $13.8 \%$ (LK,. MK, HK; Procam: $8.3 \%, 15 \%$, and $17.8 \%)$. LK patients underestimated their CAD risk to a much higher extend (no risk: $40.3 \%$ ), than MK $(25.6 \%)$ or HK patients $(8.3 \%)$. The treating physicians estimated that $12.2 \%, 9.1 \%$ and $6.1 \%$ of the patients in the three groups had no current CAD risk.

Conclusion: Patients who had more knowledge about their disease were better controlled and had a better understanding of their own vascular risk. Their risk assessment was in better agreement with the risk estimate by their physicians and with the UKPDS risk engine or the PROCAM score. The findings of our evaluation that were independent from the previous diabetes training status, nevertheless, underline the importance of a constant flow of patient education to increase the knowledge level of the diabetic patient and to increase the risk awareness, which is in our opinion of major importance to achieve adherence to therapeutic measures of any kind.

Supported by Takeda Pharma

\section{4}

Structured treatment and teaching programmes (DTTP) are very efficient tools to improve glucose metabolism in insulin treated younger and older patients with diabetes type 2 - an analysis of data from AKD (Arbeitsgemeinschaft Klinische Diabetologie) hospitals from 1999 to 2006 one year after participation in a DTTP

C. S. Kloos ${ }^{1}$, A. Sämann ${ }^{1}$, N. Müller ${ }^{1}$, D. Tessmann², H. Fischer ${ }^{3}$,

U. A. Müller ${ }^{1}$;

${ }^{1}$ Internal Medicine, Klinikum für Innere Medizin III, Jena,

${ }^{2}$ Internal Medicine, Medizinische Klinik Passau,

${ }^{3}$ Internal Medicine, Krankenhaus Düren, Germany.

Background and Aims: Structured teaching in small groups is the standard intervention and basis to improve blood glucose in diabetes. In Germany, DTTP are an essential part of disease management programmes. We examined, if DTTP are equally effective in younger and older patients with diabetes type 2 .

Materials and Methods: The AKD is a nationwide circle to assure treatment quality in Germany. Participation is on a voluntary basis. An unselected sample of at least 50 (till 2003) or 60 (from 2004 on) patients is examined personally 12 to 15 months after participation in a DTTP. Markers of treatment quality (HbAlc, blood pressure, body weight) and further information to characterise treatment modalities (e.g. number of injections, age) are collected. Data of 67 AKD-hospitals with a total of 5985 patients from 1999 to 2006 are analysed. All patients were treated with insulin. The data were divided in equally distributed thirds (all data before participation in the DTTP) Third $1(\mathrm{n}=2021)$ : mean age 50years, body weight $93.8 \mathrm{~kg}$, BMI $32.3 \mathrm{~kg} / \mathrm{m}^{2}$, RR syst. 138.5 , RR diast. $82.8 \mathrm{mmHg}$, Diabetes duration 8.1y.; Third 2(n=1927): mean age $61.9 \mathrm{y}$, body weight $87.8 \mathrm{~kg}$, BMI $30.6 \mathrm{~kg} /$ $\mathrm{m}^{2}$, RR syst. 142.6, RR diast. $81.7 \mathrm{mmHg}$, diabetes duration 11.3 years; Third $3(\mathrm{n}=2037)$ : mean age $71.9 \mathrm{y}$, body weight $81.6 \mathrm{~kg}, \mathrm{BMI} 29.1 \mathrm{~kg} / \mathrm{m}^{2}$, RR syst. 144.9 , RR diast. $80.4 \mathrm{mmHg}$, diabetes duration 13.5 years. The thirds of the youngest and oldest patients were compared. In those thirds body weight, BMI, Diabetes duration and RR were significantly different $(\mathrm{p}<0.01)$. Not all variables were complete.

Results: HbAlc improved in both groups significantly without any difference between younger and older patients (Third 1 : from $8.5 \%^{*}$ to $7.2 \%^{*} /$ Third 3 : from $8.5 \%^{*}$ to $\left.7.3 \%^{*} ; \mathrm{p}<0.001\right)$. Younger as well as older patients experienced a weight gain (Third $1:+1.8 \mathrm{~kg} /+0.5 \mathrm{~kg} / \mathrm{m}^{2}$; Third $3:+0.7 \mathrm{~kg} /+0.4 \mathrm{~kg} / \mathrm{m}^{2}$; $\mathrm{p}<0.001)$. Blood pressure in younger patients was normal and remained normal. Blood pressure in older patients before DTTP was significantly higher compared with the younger patients and improved in older patients performing blood pressure self-control (Third 3: from 142.9/80.1 mmHg to $138.9 / 78.4 \mathrm{mmHg} ; \mathrm{p}<0.001$ ) but not if the measurements were carried out by the medical staff

Conclusion: Structured teaching and treatment programmes (DTTP) are very efficient tools to improve glucose control. The effectiveness is independent from age.

${ }^{*} \mathrm{HbAlc}$ DCCT normalised

\section{5}

Factors driving medication satisfaction in the patient with diabetes: an analysis using the Perceptions About Medications for Diabetes Questionnaire

B. Curtis ${ }^{1}$, R. P. Hayes ${ }^{1}$, P. O. Monahan 2 , K. A. Lane ${ }^{2}$, D. G. Marrero ${ }^{3}$; ${ }^{1}$ Eli Lilly and Company, Indianapolis, ${ }^{2}$ Department of Medicine, Indiana University School of Medicine, Indianapolis, ${ }^{3}$ Division of Endocrinology \& Metabolism, Indiana University School of Medicine, Indianapolis, United States.

Background and Aims: Treatment satisfaction has been shown to be associated with adherence, and in turn with better glycemic control. The purpose of this study was to investigate factors that are associated with patient satisfaction with diabetes medication.

Materials and Methods: To validate The Perceptions About Medications for Diabetes (PAM-D) Questionnaire, 67 items developed from 18 focus groups of patients with diabetes were administered via a mailed survey to 1000 randomly selected inner-city patients in Indianapolis, Indiana. Psychometric analysis indicated 10 clinically meaningful PAM-D subscales: flexibility of timing, convenience of transport, negative impact of diabetes medications (i.e., hassle, interferes with daily life, takes too much time), medication effectiveness, trouble remembering medications, perceived (gastrointestinal, hypoglycemic, and weight/edema) physical side effects, and perceived (mood impact and cognitive distress) emotional side effects. The survey included questions about demographics, types of diabetes medications, and 5 items on a 7-point scale measuring satisfaction with aspects of diabetes medications (e.g., ease of taking) summed to create a treatment satisfaction scale (Cronbach's alpha $=0.91$ ). A step-wise linear regression model with treatment satisfaction as the dependent variable included any demographic variables and PAM-D subscales as independent variables that were univariately significant at alpha $=.25$.

Results: Response rate was 36\% ( $\mathrm{n}=362) ; 5 \%$ of respondents had type 1 diabetes (mean age at diagnosis $25.0 \pm 14.3 \mathrm{yrs}$ ), $95 \%$ had type 2 diabetes (mean age at diagnosis $47.7 \pm 12.8 \mathrm{yrs}$ ). $49 \%$ of patients reported use of oral medication alone, $22 \%$ insulin alone, and $26 \%$ a combination of oral medication and insulin. $71 \%$ of subjects were female and $40 \%$ were Caucasian. 
The best predictors of treatment satisfaction were four PAM-D subscales; medication effectiveness $\left(R^{2}=0.13\right)$, followed by negative impact $\left(R^{2}\right.$ change $=0.05)$, perceived mood side effects $\left(R^{2}\right.$ change $\left.=0.01\right)$, and flexibility of timing $\left(R^{2}\right.$ change $\left.=0.01\right)$, for a total $R^{2}=0.20($ all $p<0.05)$. No other variables including demographics, duration of disease, bodily pain, vitality, mental health, or health distress reached significance for inclusion in the final model. After adjusting for these four significant predictors, no significant differences were found between medication groups $(\mathrm{p}=0.42)$ in mean satisfaction scores ( $95 \% \mathrm{CI}$ ): oral medications alone 26.3 (25.0 to 27.6 ); insulin alone 25.6 (23.7 to 27.5), oral medications and insulin 24.8 (23.0 to 26.6).

Conclusions: These results indicate that patients with diabetes are more likely to be satisfied with medications that have clinical effectiveness, less negative impact such as interfering with daily life, lower perceived mood side effects, and greater flexibility of application timing. Treatment satisfaction may, in turn, drive adherence to treatment regimens and thereby improve glycemic control.

Supported by Eli Lilly

\section{6}

Monitoring individual needs in persons with diabetes: results of an ongoing, nurse-led well-being assessment as part of the annual review N. Zandbelt ${ }^{1}$, M. de Wit ${ }^{1}$, C. H. C. Lubach ${ }^{2}$, N. Breas ${ }^{2}$, F. J. Snoek ${ }^{1}$; ${ }^{1}$ Medical Psychology, ${ }^{2}$ Diabetes Centre, VU University Medical Centre, Amsterdam, The Netherlands.

Background and Aims: International guidelines advocate systematic monitoring of well-being as integral part of diabetes care. In previous research we demonstrated feasibility and effectiveness of a nurse-led computer-assisted assessment procedure. The aim is to implement and evaluate pc-assisted monitoring of psychological well-being by the diabetes nurse specialist (DNS) as integral part of ongoing outpatient care for adults with diabetes. Data on well-being and glycaemic control of the first 301 patients included in this ongoing project, are presented here.

Materials and Methods: A computerised assessment programme was developed, including the WHO-5 Well-being Index and the Problem Areas in Diabetes (PAID) scale. The DNSs received training to interpret and discuss psychological outcomes. The computerised assessment takes 5-7 minutes and is scheduled as part of the annual review, prior to the consultation with the DNS. A report on psychological status is automatically generated and discussed with the patient. All adult outpatients with diabetes type 1 or 2 are eligible.

Results: So far, 308 diabetes patients participated in the period between September 2005 and October 2006. Five patients dropped out (1.6\%; reasons: language problems or new patients), and 2 patients $(0.6 \%)$ came for their second annual review, resulting in a total of 301 patients $(\mathrm{N}=301$; mean age was 52.3 years $\pm 15.7,50.2 \%$ male, $47.2 \%$ type 1 ). Overall mean $\mathrm{HbAlc}$ was $7.9 \% \pm 1.1$ (range: $5.1-11.5 \%$ ). No significant differences in HbAlc were found between males and females nor between type 1 and type 2 diabetes. Seventy patients (23.3\%) reported poor well-being (WHO-5 score $\leq 50)$, and 23 patients $(7,6 \%)$ had scores indicative for depression (WHO- 5 score $\leq 28$ ). Fifty-nine patients $(19,6 \%)$ reported seriously elevated diabetes distress (PAID $\geq 40$ ). WHO- 5 and PAID showed a significant but moderate correlation ( $\mathrm{r}=$ $-.457, \mathrm{p}<.01)$. In total, 32 patients $(10,6 \%)$ had high diabetes-specific distress (PAID score $\geq 40$ ) and low emotional well-being (WHO-5 score $\leq 50$ ), while 14 patients $(4,7 \%)$ had high diabetes-specific distress (PAID score $\geq 40)$ and likely depression (WHO-5 score $\leq 28)$. Psychological functioning was not significantly associated with $\mathrm{HbAlc}$. Patients who indicated low well-being and/or high diabetes-distress did not have significantly higher (or lower) HbAlc's. From PAID/WHO-5 scores, 14 'cases' $(4,7 \%)$ were identified, of whom 2 patients were already known and had received psychological support prior to the assessment. Nine patients were referred for professional psychological treatment as a result of the monitoring. Four of them had not (yet) agreed to psychological counselling although they had high depression and distress scores.

Conclusion: Monitoring well-being as part of ongoing diabetes care is feasible and well appreciated by both health care professionals as well as patients. It provides valuable information on patient's psychological status. Approximately 1 in 4 patients has (serious) psychological issues, warranting attention. Glycaemic control is not a good marker for psychological functioning, underscoring the need to assess well-being next to clinical parameters. An international collaborative study (DAWN MIND) has just started to promote further dissemination of this procedure in secondary care.

\section{7}

No relation between treatment modality and quality of life in type 1 diabetes after 11-year follow-up

S. J. J. Logtenberg ${ }^{1}$, N. Kleefstra ${ }^{1,2}$, E. G. Gerrits ${ }^{1}$, E. van Ballegooie ${ }^{2,3}$, H. J. G. Bilo ${ }^{1,4}$;

${ }^{1}$ Diabetes Research Centre, Isala Clinics, Zwolle, ${ }^{2}$ Langerhans, Medical Research Group, Zwolle, ${ }^{3}$ Internal Medicine, Bethesda General Hospital, Hoogeveen, ${ }^{4}$ Internal Medicine, UMCG, Groningen, The Netherlands.

Background and Aims: Over the last decades intensified insulin therapy with multiple daily injections (MDI) or continuous insulin infusion (CII) has become the treatment of choice in patients with type 1 diabetes mellitus (T1DM). CII improved health related Quality of Life (QoL) in randomized trials with limited follow-up. The purpose of this study is to evaluate metabolic control and QoL in a T1DM population in relation to intensive insulin regimens during an 11-year follow-up.

Materials and Methods: As part of a prospective cohort study that was initiated in 1995, a cohort of patients with T1DM was examined annually according to protocol. To determine long term changes in QoL (as assessed with the SF-36 questionnaire) and metabolic control ( $\mathrm{HbA}_{t c}$ ) data from 184 subjects who completed baseline and follow-up measurements were analysed. Paired t-tests and analysis of variance were used for comparisons with correction applied for multiple testing.

Results: Final follow-up measurements were performed in 2005 and 2006 (follow-up 11.1 \pm 0.3 years). Baseline characteristics (\% or mean \pm SD): $57.6 \%$ male, age $37.5 \pm 10.9$ years, diabetes duration $16.0 \pm 9.7$ years.

Significant changes (mean $\Delta(95 \% \mathrm{CI}))$ in $\mathrm{HbA}_{1 \mathrm{c}}(-0.25(-0.50,-0.01)$ and QoL (Physical Component Summary (CS); $-3.5(-4.7,-2.2)$, Mental CS; -2.0 $(-3.6,-0.4))$ over time were observed (table). Regarding mode of therapy, 50 patients progressed from MDI to CII at some point during follow-up (median time on CII in 20066.8 years). 87 patients remained on MDI and 45 remained on CII throughout follow-up. Two patients discontinued CII in favour of MDI. There were no significant differences in change or final follow-up measurements of metabolic control and QoL parameters between patients that remained on either therapy or patients that switched from MDI to CII (ANOVA; $\mathrm{p}>0.05$ ). At baseline, no differences in $\mathrm{HbA}_{1 c}$ were observed that could possibly identify patients that would progress in the years to come from MDI to CII. However, at baseline some SF-36 subscores and the Mental CS were lower in patients that would switch in the following years (time on CII $>$ median) compared to patients that would switch later in follow-up $(\mathrm{p}<0.05)$.

Values at baseline and 11-year follow-up in cohort of patients with type 1 diabetes $(\mathrm{n}=184)$

\begin{tabular}{lllll}
\hline Parameter & Baseline & Follow-up & Mean $\Delta(\mathbf{9 5} \% \mathrm{CI})$ & p-value \\
\hline $\mathrm{HbA}_{1 \mathrm{c}}(\%)$ & $8.0 \pm 1.7$ & $7.7 \pm 1.0$ & $-0.25(-0.50,-0.01)$ & 0.04 \\
Total cholesterol $(\mathrm{mmol} / \mathrm{L})$ & $4.9 \pm 0.9$ & $4.9 \pm 0.9$ & $0.06(-0.08,0.20)$ & 0.40 \\
eGFR $(\mathrm{mL} / \mathrm{min})$ & $109.2 \pm 25.5$ & $97.7 \pm 27.1$ & $-11.5(-13.8,-9.1)$ & $<0.001$ \\
$\begin{array}{l}\text { Systolic blood pressure } \\
(\mathrm{mm} H \mathrm{H})\end{array}$ & $138.4 \pm 17.4$ & $134.5 \pm 17.2$ & $-3.8(-6.8,-0.9)$ & 0.01 \\
Body mass index $\left(\mathrm{kg} / \mathrm{m}^{2}\right)$ & $24.8 \pm 3.1$ & $26.9 \pm 4.4$ & $2.1(1.7,2.6)$ & $<0.001$ \\
& & & & \\
$\begin{array}{l}\text { SF-36: Physical } \\
\text { Component Summary }\end{array}$ & $53.1 \pm 6.5$ & $49.6 \pm 8.5$ & $-3.5(-4.7,-2.2)$ & $<0.001$ \\
$\begin{array}{l}\text { SF-36: Mental } \\
\text { Component Summary }\end{array}$ & $52.3 \pm 8.8$ & $50.3 \pm 9.8$ & $-2.0(-3.6,-0.4)$ & 0.01 \\
\hline
\end{tabular}

Conclusion: These long term results show improved metabolic control over time in patients with T1DM, regardless of treatment mode and regardless of treatment intensification during the years. Interestingly, differences in QoL between treatment modes could not be identified in this cohort at long term follow-up. Prospective trials will need sufficient follow-up time to verify their results of improving QoL with CII. If not verified, improvement of QoL with CII could be a short term effect, which could also be due to bias because of the impossibility of blinding. 


\section{PS 106 Neuropathy: diagnosis and prevalence}

\section{8}

\author{
A comparison of the new indicator test for sudomotor function \\ $\left(\right.$ Neuropad $\left.^{\circ}\right)$, the vibration perception threshold and the clinical \\ examination in the diagnosis of peripheral neuropathy in type 2 diabetic \\ patients \\ N. Papanas ${ }^{1}$, K. Papatheodorou ${ }^{1}$, D. Papazoglou ${ }^{1}$, C. Monastiriotis ${ }^{1}$, \\ D. Christakidis ${ }^{2}$, E. Maltezos ${ }^{1}$; \\ ${ }^{1}$ Second Department of Internal Medicine, Democritus University of Thrace, \\ Alexandroupolis, \\ ${ }^{2}$ Diabetic Department, University Hospital of Alexandroupolis, Greece.
}

Background and Aims: Peripheral neuropathy remains a major cause of morbidity and a cardinal factor in the pathogenesis of diabetic foot ulceration. Clinical examination is the cornerstone of diagnosis. The Vibration Perception Threshold (VPT) is a reliable tool not only for the diagnosis of neuropathy, but also for the estimation of the risk for foot ulceration. Thus, the aim of the present study was to evaluate the new indicator test for sudomotor function (Neuropad ${ }^{\star}$ ) in the diagnosis of peripheral neuropathy in patients with type 2 diabetes mellitus, as compared with clinical examination and with the VPT. Materials and Methods: This study included 154 type 2 diabetic patients ( 76 men, 78 women) with a mean age of $64.3 \pm 7.3$ years and a mean diabetes duration of $12.8 \pm 4.3$ years. Recruitment was consecutive and performed in a tertiary care setting. Neuropathy was diagnosed clinically using the Neuropathy Disability Score (NDS). The VPT was measured with a neurothesiometer (Horwell Scientific Laboratory Supplies, London, UK) whose tractor was applied vertically on the pulp of the hallux. The test was repeated three times and the mean voltage was recorded. The VPT was considered abnormal in patients with a VPT $>25$ Volts. Patients were also examined with the indicator test, applied on the plantar aspect of the feet. Time until complete colour change of the test was recorded and sudomotor impairment was diagnosed in patients with time until complete colour change exceeding 10 minutes in at least one foot.

Results: Neuropathy was diagnosed in 90 patients (58.4\%). Sudomotor impairment was diagnosed in 88 patients $(97.8 \%)$ with neuropathy and in 21 patients $(32.8 \%)$ without neuropathy $(p<0.001)$. Sensitivity of the indicator test for diagnosing neuropathy was $97.8 \%$ and specificity was $67.2 \%$, while positive and negative prognostic values were $80.7 \%$ and $95.6 \%$ respectively. Abnormal VPT was detected in 71 patients with neuropathy and 9 patients without neuropathy $(p<0.001)$. Sensitivity, specificity and positive and negative prognostic values of VPT were $78.9 \%, 84.4 \%, 88.8 \%$ and $74.3 \%$ respectively. Time until complete colour change of the indicator test was $21.8 \pm 6$ minutes in patients with neuropathy and $9.1 \pm 3.6$ minutes in patients without neuropathy $(p<0.001)$. A significant correlation was shown between time until complete colour change and VPT (Spearman's rank coefficient $r_{s}=$ $0.889, p<0.001$ ).

Conclusions: Both the indicator test and the VPT have a high sensitivity for neuropathy. Sensitivity is slightly higher with the indicator test, but specificity is higher with VPT. Time until complete colour change of the indicator test shows a positive correlation with VPT. These results suggest that, while VPT is a validated test to estimate the risk of foot ulceration, Neuropad may prove valuable as an additional diagnostic tool of neuropathy in type 2 diabetes. Neuropad appears to be suitable for screening, self-examination and education. Its potential contribution to the estimation of risk for foot ulceration could only be investigated in a prospective study.

\section{9}

Evaluation of the self-administered indicator plaster neuropad for the diagnosis of neuropathy in diabetes

N. Tentolouris, V. Achtsidis, K. Marinou, S. Liatis, E. Diakoumopoulou, N. Katsilambros;

1st Department of Propaedeutic Medicine, Athens University Medical School, Laiko General Hospital, Greece.

Background and Aims: Chronic peripheral sensorimotor neuropathy (PN) is a common complication of diabetes and it is the main cause for foot problems. Sudomotor dysfunction develops early in the course of diabetes. Previous data demonstrated that sudomotor dysfunction-assessed with the use of the indicator plaster neuropad (IPN)-has a high sensitivity and specificity for the diagnosis of the PN. The advantage of the IPN is that it is an easy to perform test by the patient. In the present study we evaluated the inter-rater reliability of the IPN between patient and provider sensory evaluations.

Materials and Methods: A total of 156 consecutive subjects (age 59.6 \pm 15.4 years, duration of diabetes $13.8 \pm 9.2$ years) with both type 1 and type 2 diabetes were recruited from the outpatient diabetes clinic of our hospital. Inclusion criteria required that they were able to read and understand the written instructions on the use and evaluation the IPN. Patients with dyschromatopsia or severe visual loss as well as severe peripheral vascular disease (ankle-brachial pressure index $<0.5$ ) were excluded. Participants were assessed for neuropathy at the first visit. Diagnosis of neuropathy was based on neuropathy symptom score, neuropathy disability score, and vibration perception threshold. The IPN was applied for $10 \mathrm{~min}$ and evaluated as normal or abnormal by the doctor at the same visit. Afterwards, the IPN together with written instructions for its use and evaluation at home were given to the patient. In addition, a special questionnaire was provided asking for the easiness to understand the instructions, the easiness to use and the easiness to evaluate the IPN (visual analogue scales $0-10$, with 0 the most difficult and 10 the easiest for each question). All participants returned the results of the IPN and the completed questionnaires in a second visit to another participating doctor, who was blind to results of the tests of the first visit.

Results: Neuropathy was diagnosed in 93 subjects (59.6\%). The sensitivity of the IPN for the diagnosis of PN was $87 \%$ and the specificity $68.9 \%$. The agreement between patient and doctor in the evaluation of neuropad as normal or abnormal was $90.3 \%$ (in 141 out of 156 subjects). The $k$ statistic to measure overall agreement between patients and health-care provider was very good: 0.88 (95\% confidence intervals $0.85-0.91$ ). Also, the evaluation of the instructions and the test by the patients was as follows: easiness to understand the instructions $9.1 \pm 1.4$, easiness to use the IPN 9.0 1.5 , and easiness to evaluate the test as normal or abnormal 8.9 \pm 1.4 .

Conclusion: Self-administered IPN provides patients an opportunity to share in the responsibility for the identification of the PN. The test can be used easily by the patients when clear, written instructions for its use and evaluation are provided.

\section{0}

In vivo corneal confocal microscopy and nerve growth factors in the assessment of microvascular complications in diabetic patients

J. Nam ${ }^{1}$, J. Park ${ }^{1}$, M. Cho ${ }^{1}$, H. Kim ${ }^{1}$, H. Chung ${ }^{1}$, E. Kang ${ }^{1}$, Y. Lee ${ }^{1}$, C. Ahn ${ }^{1}$, Y. Song ${ }^{2}$, B. Cha ${ }^{1}$, E. Lee ${ }^{1}$, S. Lee ${ }^{1}$, K. Kim ${ }^{1}$, H. Lee';

${ }^{1}$ Internal Medicine, Yonsei University Medical College, Seoul,

${ }^{2}$ Internal Medicine, NHIC Hospital, Koyang, Republic of Korea.

Background and Aims: In vivo corneal confocal microscopy (IVCCM) is being recognised as a non-invasive, early diagnostic tool for diabetic neuropathy, for it provides a clear image of corneal subbasal nerve plexus in detail. Nerve growth factors (NGF) are believed to regulate peripheral and central nervous system, neuronal differentiation, and regeneration of damaged nerves, and their role in diabetic neuropathy is being emphasised these days. Moreover, NGFs and receptors are also expressed in retina and renal mesangial cells, suggesting their possible role in the common pathogenesis of diabetic microvascular complications. We plan to examine corneal structures of diabetic patients and compare those with conventional tools and analyze their serum and tear NGF levels.

Materials and Methods: IVCCM, nerve conduction velocity (NCV), and serum, urine, and tear samplings were done in 42 diabetic patients. From IVCCM, we measured corneal nerve density, branch, and tortuosity, total corneal/epithelial thickness, and the number of endothelial/keratocyte cells, and we checked patients' biochemical profiles and serum and tear NGF levels.

Results: Patients with more severe neuropathy had less corneal endothelial cells ( $3105 \pm 218$ vs. $2537 \pm 142$ vs. $2350 \pm 73$ vs. $1914 \pm 465, P=0.02$ ), higher serum NGF ( $35.51 \pm 15$ vs. $59.86 \pm 57.66$ vs. $79.67 \pm 57.63$ vs. $109 \pm 60.81, P=$ $0.39)$ and tear NGF levels ( $135 \pm 11.94$ vs. $304.29 \pm 242.22$ vs. $538.5 \pm 251.92$ vs. $719.5 \pm 92.63, P=0.01)$. There was a positive correlation between neuropathy and corneal nerve tortuosity $\left(\mathrm{r}^{2}=0.479, P=0.044\right)$ and negative correlation between neuropathy and endothelial cell count $\left(r^{2}=-0.709, P=0.002\right)$. Interestingly, similar changes were seen in other microvascular complications as well. 
Conclusion: Our results provide a possibility of using novel tools, IVCCM and NGF, as common diagnostic tools for diabetic microvascular complications, but it should be followed by a large population study. Supported by Seoul R\&BD Programme, Republic of Korea

\section{1}

Assessment of the risk of falling in postmenopausal women with diabetes using a foot mat system

S. L. Hyer ${ }^{1}$, M. Plank ${ }^{1}$, A. Rodin ${ }^{1}$, S. Patel ${ }^{2}$;

${ }^{1}$ Diabetes, ${ }^{2}$ Rheumatology, St Helier University Hospital, Surrey, United Kingdom.

Background: Older women with diabetes have an increased risk of falls which is only partly explained by known risk factors.

Objective: To assess postural stability in women with diabetes compared with healthy controls

Methods: Post-menopausal women were randomly selected from a hospitalbased diabetes database and invited to participate. We studied 147 patients (aged 55-91) with diabetes and 18 age-matched controls. All subjects were assessed for neuropathy and falls risk from detailed history, clinical examination and vibration perception threshold (VPT). Postural stability (sway) was assessed by the motion of the centre of force (COF) during quiet standing on a foot mat system $\left(\right.$ Tekscan $\left.{ }^{\mathrm{R}}\right)$. Force transducers in the foot mat detect pressure changes under the foot from which COF trajectories can be displayed.

Results: Mean VPT (volts) in those who had fallen 3 or more times (22.7 \pm 2.3 ) was greater than in those who had not fallen at all in the last 12 months (18.4 \pm 0.9$) ; p=0.05)$. Mean (SD) COF centroid area (area of sway) $\left(\mathrm{mm}^{2}\right)$ was significantly increased in the diabetic group versus controls; 505 (317) vs. 360 (128); $p<0.05$ and was highest in the 65 neuropathic patients; 513 (273). Asymmetric loading with lateralisation of the COF to one side was observed in 19 patients with diabetes and only 1 control. Mean time (hours) on feet was inversely correlated with severity of neuropathy and sway area $(r=-0.28$; $p=001$ ).

Conclusion: Increased postural sway and hence postural instability occurs frequently in older women with diabetes even when VPT is below the conventional threshold for significant neuropathy (i.e. $<25 \mathrm{~V}$ ). Patients with instability adopt a strategy of spending less time on their feet in order to reduce their risk of falling. Assessment of postural stability in addition to clinical examination and VPT measurement provides useful additional information on falls risk in this group of patients.

Supported by grant from R\&D department at Epsom and St Helier University Hospitals

\section{2}

Diagnostic aspects of diabetic somatic and sudomotor neuropathy in general practice

Z. A. Kamenov, V. G. Christov, T. Yankova, V. N. Karamfilova; Medical university, Clinic of Endocrinology, Sofia, Bulgaria.

Background and Aims: Epidemiological data for the most common late diabetic microvascular complication - diabetic neuropathy (DN) vary considerably among different studies depending on the method. Large questionnaires and sophisticated electrophysiological testing are inappropriate for everyday practice. Simple devices are usually used for somatic DN, but autonomic DN is often under diagnosed. A new diagnostic test - Neuropad ${ }^{\circ}$ has been developed as a simple tool for early detection of sudomotor neuropathy and for early recognition of the diabetic foot syndrome.

The aim of this study was to evaluate the utility of Neuropad and to compare the results with other tests for DN.

Materials and Methods: In this cross-sectional study were included 264 consecutive diabetic men/women (126/138) with mean age \pm SD $55.4 \pm 12.0$ years, diabetes type 1 or $2(61 / 203)$ and diabetes duration of $9,3 \pm 7,1$ years. The patients were divided according to the presence or absence of neuropathic symptoms. For somatic DN a modified Neuropathy Disability Score (NDS) was used including vibration perception (VP) with $128-\mathrm{Hz}$ graduated in 8 stages Rydel-Seiffer tuning fork; temperature perception (TP) (Thio-Term, Germany); 10g monofilament (MF); Achilles reflex (AR). The maximum
NDS was 10 points ( 5 points for each foot). For sudomotor DN Neuropad was used - an adhesive indicator test that changes color when applied to the skin of the sole. The color change was rated as normal - grop 0 - complete change from blue to pink $(n=81)$, borderline - group $1-$ mottled blue/pink color $(\mathrm{n}=164)$, abnormal - group 2 - no color change $(\mathrm{n}=19)$.

Results: 187 (70.8\%) from all patients had neuropathic symptoms. Of them positive for DN were only: for VP $(\leq 6 / 8)-158$ patients $(84.5 \%)$; TP (no feeling)-109(58.3\%); MF (no feeling)-80(42.8\%); AR (abnormal)165(88.2\%); NDS ( $\geq 6$ points)-123(65.8\%); NDS ( $\geq 1$ point)-182(97.3\%); Neuropad ( $\geq 1)-152(81.3 \%)$. On the contrary, in the group of $77(29.2 \%)$ asymptomatic patients positive neuropathic tests had VP-42 (54.5\%); TP-26 (33.8\%); MF-13 (16.9\%); AR-47 (61.0\%); NDS( $\geq 6$ )- 22 (28.6\%); NDS( $\geq 1)$ 58 (75.3\%); Neuropad-44(57.1\%) The three Neuropad-groups differed significantly in their NDS and in each test in particular.

Conclusion: The objective differences in test results, caused by separate neuronal modality engagement, require a complex diagnosis of $\mathrm{DN}$ in the general practice, including medical history, use of simple devices for somatic $\mathrm{DN}$, and examination for autonomic neuropathy with tests like Neuropad. We would like to thank our colleagues Stefan Dimitrov, Victoria Andreeva and Petya Staikova for technical assistance. The diagnostic test Neuropad was a kind gift from miro Verbandstoffe GmbH.

\section{3}

The prevalence and current therapeutic management of painful polyneuropathy in diabetes clinics in Belgium

K. Van Acker ${ }^{1}$, I. Colin ${ }^{2}$, D. Bouhassira ${ }^{3}$, S. Weiss ${ }^{4}$, K. Matthys ${ }^{4}$,

H. Raemen ${ }^{4}$, C. Mathieu,

${ }^{1}$ Endocrinology and Diabetes, St. Jozefkliniek, Bornem, Belgium,

${ }^{2}$ Endocrinology, CHR. St.-Joseph-Hôpital de Warquignies, Mons, Belgium, ${ }^{3}$ Centre d'Evaluation et de Traitement de la douleur, CHU Ambroise Paré, Paris, France, ${ }^{4}$ Pfizer Inc., Brussels, Belgium,

${ }^{5}$ Endocrinology and diabetology, UZ gasthuisberg, Leuven, Belgium.

Background and Aims: Painful diabetic polyneuropathy (DPN) is a common and debilitating, but still under-recognized and undertreated complication of diabetes. The aim of this study was to evaluate the prevalence of DPN and painful DPN (PDPN) in diabetes clinics throughout Belgium. In addition, PDPN-associated conditions, impact on quality of life (QoL) and current therapeutic management of neuropathic pain were investigated.

Materials and Methods: A cross-sectional multi-centre study including 1111 patients (767 type 2, 344 type 1 ) in 40 diabetes clinics was carried out. Validated tools were used for the diagnosis of DPN (Neuropen ${ }^{*}$-diagnosed sensory loss) and PDPN (score $\geq 4$ on DN4 questionnaire applied in patients with DPN and pain in the legs). Pain intensity was measured by a Visual Analog Scale (VAS). Participants completed the Short Form-12 (SF-12) to evaluate the physical and mental QoL. Multivariate models were used to analyse patient data.

Results: The prevalence of both DPN and PDPN increased with age and diabetes duration $(\mathrm{p}<0.0001)$, not with gender or $\mathrm{HbAlC}$. Prevalences were $43 \%$ for DPN and $14 \%$ for PDPN, with a significantly higher prevalence in type 2 than in type 1 (DPN $43,5 \%$ vs. $38,4 \%$ and PDPN $15,4 \%$ vs. $10,8 \%$ resp.). Multivariate analysis showed that age, diabetes duration, nephropathy, obesity, low HDL-C and high triglycerides were all independently associated with PDPN. In the subgroup of patients with pain in their legs $(n=531)$, the prevalence of PDPN proved to be $30 \%$. The median VAS score was twice as high in patients with neuropathic pain vs. non-neuropathic pain $(50 \mathrm{vs} .25 \mathrm{~mm}$, $\mathrm{p}<0.0001)$. Multivariate analysis of the impact on QoL showed that PDPN independently affected the physical QoL, next to age, BMI, nephropathy and sensory loss neuropathy. PDPN also affected the mental QoL, next to female gender, smoking, BMI and sensory loss neuropathy. Further adjustment for pain intensity did not alter these findings.

Nearly half of the PDPN patients $(46,3 \%)$ were treated for their pain. Only $25,7 \%$ of those treated for pain received anticonvulsants or antidepressants.

Conclusion: PDPN is a frequent long-term complication of diabetes, especially in type 2 patients with metabolic syndrome characteristics. It profoundly diminishes the quality of life in affected persons. Diagnosis can be easily made with cheap tools, not requiring special expertise and should therefore be used more extensively. More adequate treatment of neuropathic pain should be considered in PDNP patients. 


\section{4}

Does motor nerves impairment predominate in children with short duration type 1 diabetes and poor metabolic control?

E. Piontek ${ }^{1}$, M. Wysocka-Mincewicz ${ }^{1}$, I. Gromek ${ }^{2}$, J. Skwarzec ${ }^{1}$;

${ }^{1}$ Department of Metabolic Diseases, Endocrinology and Diabetology,

${ }^{2}$ Department of Neurology and Epileptology, The Children's Memorial Health Institute, Warsaw, Poland.

Background and Aims: Diabetic neuropathy is serious and burdensome and with no effective treatment till now. The aim of the study was to detect early abnormalities to distinguish the group of risk developing peripheral neuropathy.

Materials and Methods: 45 children with type 1 diabetes were included in the study (25 girl and 20 boys, mean age 14,68 years $+/-3,07 \mathrm{SD}$, mean $\mathrm{HbA1c} 8,38 \%+/-1,63 \mathrm{SD}$, mean duration of diabetes $4,96+/-3,28)$. In all cases neurologic examination and nerve conduction studies of motor (median, ulnar, tibial and peroneal) and sensory nerves (median, ulnar and sural) with standard techniques were done. Chi-square frequency analysis were performed.

Results: In the studied group polyneuropathy was recognised in 8 children $(17,8 \%$, in 5 person motor, and in 3 sensory-motor), with only 2 person showing no clinical signs and symptoms. Abnormalities in conduction tests of $31,1 \%$ of peroneal nerves, $24,4 \%$ tibial, $6,7 \%$ in sural nerves and $4,4 \%$ in sensory ulnar and $2,2 \%$ in motor median, were recorded. In motor nerves primary axonal changes with decreasing of potential amplitude were detected (abnormalities in 37/180 tested nerves, 20,5\%) and elongation of wave $\mathrm{F}$ latency, which indicates injury of nerves trunk (50/180, 27,8\%). In sensory nerves decrease of conduction velocity $(22 / 135,16,3 \%)$ was dominant. Significant differences between frequency of motor and sensory nerves impairment were recorded $(26 / 180$ vs. 5/135 respectively, $\mathrm{p}<0,005)$ and nerves of legs and arms (28/135 vs. $3 / 180$ respectively, $\mathrm{p}<0,00001)$.

Conclusion: In poor metabolic controlled children with type 1 diabetes with short duration of diabetes axonal changes and trunk damage of motor nerves were predominating. In sensory nerves demyelinating lesions with the decrease in conduction velocity were observed.

\section{5}

Diabetic neuropathy in children and adolescents: a multicentre study L. Caracotche $e^{1,2}$, S. Segal ${ }^{1}$, L. Trifone ${ }^{1}$, M. Ferraro ${ }^{3}$, I. Moreno ${ }^{4}$, A. Figueroa Sobrero $^{5}$, S. Lopez 6 , M. Rópolo ${ }^{7}$, G. Freijoo ${ }^{8}$, D. González ${ }^{9}$ L. Tartaglino ${ }^{10}$, D. Fernandez ${ }^{11}$;

${ }^{1}$ R. Gutiérrez, Children Hospital, Buenos Aires, ${ }^{2}$ N. López Hospital, Buenos Aires, ${ }^{3}$ Elizalde Children Hospital, Buenos Aires, ${ }^{4}$ Sor Ludovica Children Hospital, La Plata, Buenos Aires, ${ }^{5}$ Hospital San Roque, Parana, Santa $\mathrm{Fe},{ }^{6}$ Municipal Children Hospital of Córdoba, Buenos Aires, ${ }^{7}$ Children Hospital Sta. Ma. Trinidad, Córdoba, ${ }^{8}$ Children Hospital Jesús of Praga, Salta, ${ }^{9}$ Regional Hospital de Neuquén, ${ }^{10}$ Minister Health, Posadas, Misiones, ${ }^{11}$ Hospital Enrique V. Barrios, La Rioja, Argentina.

Background: Neuropathy is well recognized as a major complication of type 1 diabetes mellitus in adults, resulting in significant morbidity and mortality. Symptomatic peripheral neuropathy is uncommon in children and adolescents with type 1 diabetes mellitus, although a high prevalence of subclinical peripheral nerve conduction abnormalities has been reported in these patients. Michigan neurophatic inspection is a clinical examination easy to use in the detection of neuropathy, and has been validated in epidemiological studies in adults.

Aims: To asses the availability of a clinical method screening (Michigan test) to detect peripheral neuropathy in children and adolescents with type1 Diabetes Mellitus (DM), and to estimate the prevalence and associated risk factors.
Methods: Two hundred and fifty five asymptomatic patients, aged 10-20 years diagnosed with type $1 \mathrm{DM}$ and a control group of fifty two healthy agematched subjects participated in the multicentre study, during 2005 / 2006. The patients included in the study had been diagnosed as type $1 \mathrm{DM}$ for more than one year, without any evidence of neurological illness, and drugs that would disturb the nerve conduction velocity, without cetoacidosis or severe hypoglycaemia in the last 6 month before and at the moment of the study. Clinical examination: weight, height, BMI (body mass index) pubertal stage (Tanner), alcohol and tobacco consumption in both. In patients with DM we considered: duration of disease (years), HbAlc (mean value of the previous 12 months), insulin dose (U/ $\mathrm{kg})$. Microangiophatic complications: microalbuminuria and retinopathy.

Michigan tests: 1) examination of the foot appearance (skin lesion, hallux), 2) foot ulceration, 3) vibration perception (128hz tuning fork), 4) sensibility (10 grams Semmes Weinstein monofilament pressure), 5) Achilles tendon reflex. Classification score: <3: normal; 4-5: risk score; $>6$ pathologic.

In addition, thermal sensibility (warm, cool), pedals pulses, muscle strength testing (assessing finger spread) and associated symptoms were evaluated.

Results: Michigan normal: DM: $97.2 \%$ vs. control: $100 \%$; risk score: $1.96 \%$ pathologic score: $0.78 \%$.with more than 9 years duration of disease (or: 11.28 $\mathrm{p}=0.003$ ) Associated symptoms were cramps with normal score $23.8 \%$ risk score $40 \%$ and in patologic score $100 \%$. The decrease of muscle strength on the $13.3 \%$ and pedal pulse abnormal: $2.8 \%$; abnormal thermal sensibility for cold, $5.5 \%$ warm: $5.9 \%$ vs control:0\%. Characteristics of the diabetic and control populations in table 1

Conclusions: While it is an uncommon problem during childhood, it needs to be considered in adolescents with long duration of diabetes. Michigan score is a simple method, easy to apply and has high effectiveness in order to diagnose DM neurophaty.

\begin{tabular}{llllllllll} 
& $\begin{array}{l}\text { Age/ } \\
\text { years }\end{array}$ & Sex F/M & Puberty & Alcohol & Tobacco & $\begin{array}{l}\text { Insulin } \\
\text { UI/kg }\end{array}$ & $\begin{array}{l}\text { DM } \\
\text { duration }\end{array}$ & $\begin{array}{c}\text { Hb Alc } \\
\text { Microalbuminuria }\end{array}$ & $\begin{array}{l}\text { Retino- } \\
\text { pathy }\end{array}$ \\
\hline $\begin{array}{l}\text { DM255 } \\
\text { (median } \pm \text { SD range }\end{array}$ & $13.9 \pm 2.53$ & $134 / 121$ & 223 & $11 \%$ & $4.7 \%$ & $1.07 \pm 0.34$ & $5.6 \pm 3.4$ & $9 \pm 1.89$ & $7.9 \%$ \\
$\begin{array}{l}\text { Control:52 } \\
\text { (median } \pm \text { SD range }\end{array}$ & $13.19 \pm 2.4$ & $30 / 24$ & 43 & $19.2 \%$ & $7.7 \%$ & - & - & - & - \\
\hline
\end{tabular}




\section{PS 107 Nephropathy: assessing renal function}

\section{6}

\section{Evaluation of intrarenal resistance index for the identification and prediction of diabetic nephropathy}

M. Masulli ${ }^{1}$, M. Mancini², R. Liuzzi ${ }^{2}$, S. Daniele ${ }^{3}$, P. Mainenti' ${ }^{2}$, E. Vergara ${ }^{3}$, S. Genovese ${ }^{1}$, A. A. Rivellese ${ }^{1}$, M. Salvatore ${ }^{3}$, G. Riccardi ${ }^{1}$, O. Vaccaro ${ }^{1}$; ${ }^{1}$ Department of Clinical and Experimental Medicine, "Federico II" University, ${ }^{2}$ Institute of Biostructure and Bioimage, National Council of Research (CNR), ${ }^{3}$ Department of Biomorphological and Functional Science, "Federico II" University, Naples, Italy.

Background and aims: Ultrasound (US) measured intrarenal resistance index (RI) predicts renal function in several conditions. No standard cutoffs have been identified to date; however values above $0.75-0.85$ have been associated with chronic kidney disease and deterioration of renal function over time. The use of RI in routine clinical practice for the identification and prediction of diabetic nephropathy (DN) is virtually unexplored, particularly in patients with normoalbuminuria. Aims of the study are: 1) to compare the distribution of RI in control subjects and in type 2 diabetic patients; 2) to validate the use of RI in the identification and prediction of DN

Methods: Design: observational, prospective. Participants: 88 type 2 diabetic patients aged 40 to 70 and 37 non diabetic controls age and gender matched; both groups included people with moderate hypertension and normal renal function. Measurements: BP, fasting glucose, lipids, creatinine, HbAlc, urinary albumin and creatinine on spot collection; creatinine clearance and urinary albumin excretion rate (AER) were calculated. RI was measured by US with a standard protocol. Diabetic patients were re-examined after $48 \pm 5$ months; the progression of albuminuric state was defined as - i.e. transition from baseline normoalbuminuria to micro/macroalbuminuria at follow-up or from baseline normo/microalbuminuria to macroalbuminuria at followup.

Results: Diabetic patients had significantly higher RI than controls $(0.69 \pm 0.04$ vs. $0.59 \pm 0.05, p<0.001)$. RI values were normally distributed in both groups with little overlapping: ranging from 0.61 to 0.80 and from 0.48 to 0.67 respectively in diabetic and non diabetic participants. We used the $80^{\text {th }}$ percentile of the distribution in diabetic patients (i.e. $\geq 0.73$ ) to define high RI; this value is lower than arbitrary cut-offs used in previous studies. Patients with high RI had significantly higher AER ( $48.9 \pm 74.8$ vs. $31.7 \pm 45.3 \mu \mathrm{g} / \mathrm{mg}$, $\mathrm{p}<0.001)$. Age, HbAlc, lipids, smoking habits and use of renin/angiotensin system inhibitors were not significantly different in the two groups. At followup AER increased significantly more in patients with high RI (follow-up minus baseline was $290.8 \pm 469.4$ vs. $34.9 \pm 140.3 \mu \mathrm{g} / \mathrm{mg}, \mathrm{p}<0.001$ ). Progression of the albuminuric state was observed in $52.9 \%$ and $9.5 \%, \mathrm{p}<0.001$, of those with high or low RI respectively. Unadjusted Odds Ratio for progression was 10.7 (95\%CI 3.0-38.0) for patients with $\mathrm{RI} \geq 0.73$ vs. $<0.73$. After correction for established predictors of DN (i.e. age, BP, renin/angiotensin system inhibitors, HBA1c, microalbuminuria and creatinine clearance) multivariate OR calculated by exponentiation of regression coefficients was 6.0 (95\% CI 1.7-21.2). By confining the analysis to patients with normoalbuminuria, OR for progression was 7.2 ( $95 \%$ CI 1.6-32.3) for patients with $\mathrm{RI} \geq 0.73$ vs $<0.73$.

Conclusions: Ultrasound measured RI is associated with clinical signs of DN and predicts its progression overtime. Values above 0.73 predict progression of albuminuric stage in the total group as well as in normoalbuminuric patients. Thus RI is an early and reliable marker of risk of DN

\section{7}

Intrarenal arterial resistance index is a good predictor of chronic kidney disease in type 2 diabetes

A. P. S. Kong ${ }^{1,2}$, E. K. H. Liu ${ }^{3}$, W. C. W. $\mathrm{Chu}^{3}$, W. Y. So ${ }^{1}$, P. C. Y. Tong ${ }^{1}$, J. C. N. Chan ${ }^{1}$;

${ }^{1}$ Medicine and Therapeutics, ${ }^{2} \mathrm{Li}$ Ka Shing Institute of Health Science, ${ }^{3}$ Diagnostic Radiology and Organ Imaging, The Chinese University of Hong Kong, Shatin, Hong Kong.

Background and Aims: Intrarenal arterial resistance index (RI) is associated with various renal diseases, notably obstructive uropathy and hypertensive renal disease. Despite the growing incidence of diabetes-associated chronic kidney disease (CKD), there is a paucity of data of RI in diabetes. We examined the relationship between RI and CKD and defined its optimal cutoff value to predict CKD in a consecutive cohort of Chinese male patients with type 2 diabetes.

Materials and Methods: 113 Chinese men with type 2 diabetes (mean age $=54.7 \pm 9.9$ years, mean body mass index $\left.(B M I)=25.9 \pm 5.2 \mathrm{~kg} / \mathrm{m}^{2}\right)$ had RI measured by real-time Doppler ultrasound in addition to detailed phenotyping. Estimated glomerular filtration rate (eGFR) was calculated using Modified Modification of Diet in Renal Disease (MDRD) formula calibrated for Chinese.

Results: RI was correlated with eGFR $(\mathrm{r}=-0.61, \mathrm{p}<0.001)$, urinary albumincreatinine ratio $(\mathrm{ACR})(\mathrm{r}=0.38, \mathrm{p}<0.001)$ and serum creatinine levels $(\mathrm{r}=$ $0.49, \mathrm{p}<0.001)$ using Pearson's correlation analysis. There was progressive increase of RI with advancing stages of CKD ( $p$ for trend $<0.001$ ). After adjustment for age, BMI, disease duration, hypertension, dyslipidaemia and urinary ACR, RI remained an independent predictor for CKD $(<60 \mathrm{ml} / \mathrm{min} /$ $1.73 \mathrm{~m}^{2}$ ). Using Receiver Operating Characteristics (ROC) curve analysis, the cutoff value of RI $\geq 0.75$ had a sensitivity of $82 \%$ and a specificity of $89 \%$ to predict CKD.

Conclusion: RI is an independent predictor of CKD in Chinese men with type 2 diabetes and is potentially useful to identify high risk subjects for close monitoring.

Supported by Li Ka Shing Institute of Health Science, The Chinese University of Hong Kong

\section{8}

Performance of the new ' 175 ' MDRD equation using 'gold standard' creatinine values in persons with newly diagnosed type 2 diabetes R. A. Chudleigh, J. N. Harvey, G. Dunseath, R. Peter, S. Luzio, D. R. Owens; Diabetic Medicine, Diabetes Research Unit, Cardiff, United Kingdom.

Introduction: The Modification of Diet in Renal Disease (MDRD) equation has been revised for use with Isotope Dilution Mass Spectrometry (IDMS) traceable 'gold standard' creatinine values. This revision is proposed to improve the performance of four variable-MDRD equation. We compared the performance of the traditional ' 186 ' MDRD and new ' 175 ' MDRD equations to predict Glomerular Filtration Rate (GFR) measured by ${ }^{51} \mathrm{CrEDTA}$ in a cohort of newly diagnosed persons with type 2 diabetes.

Methods: 293 subjects newly diagnosed with type 2 diabetes were recruited and studied fasting at 0830 hours. Serum creatinine concentration was measured on a Vitros analyser using OCD dry slide method. Creatinine was aligned to ID-MS traceable values using United Kingdom National External Quality Assessment Service (UK NEQAS) recommendations. Estimated Glomerular Filtration Rate (eGFR) was calculated by the '186' MDRD equation using non aligned creatinine values, and the ' 175 ' MDRD equation using ID-MS traceable creatinine values. GFR was measured by single plasma injection clearance of $1 \mathrm{MBq}$ of ${ }^{51} \mathrm{CrEDTA}$ using the four sample method. Results: In the study population $75 \%$ of subjects were male with a mean (SD) age of $55.2(9.4)$ years, BMI of $31.52(5.58) \mathrm{kg} / \mathrm{m}^{2}$, fasting plasma glucose of $9.67(3.04) \mathrm{mmol} / \mathrm{l}$ and $\mathrm{HbA}_{1 \mathrm{c}}$ of $7.79(2.00) \% .89 \%$ of subjects had normoalbuminuria.

Mean (SD) creatinine and ID-MS traceable creatinine values for the study population were significantly different at $80.0(14.8)$ and $73.0(14.9) \mu \mathrm{mol} / \mathrm{l}$ respectively $(\mathrm{p}<0.05)$. Mean (SD) Isotopic GFR of the population was $115(22)$ $\mathrm{ml} / \mathrm{min} / 1.73 \mathrm{~m}^{2}$, this was significantly underestimated by the ' 186 'MDRD and ' 175 ' MDRD equations at 89 (19) and $95(22) \mathrm{ml} / \mathrm{min} / 1.73 \mathrm{~m}^{2}$ respectively $(\mathrm{p}<0.05)$. The ' 186 ' MDRD equation had a significantly greater negative bias than the ' 175 ' MDRD equation, mean [SE] bias were -25.0 [1.0] and -20.1 [1.0] $\mathrm{ml} / \mathrm{min} / 1.73 \mathrm{~m}^{2}$ respectively $(\mathrm{p}<0.05)$. The ' 186 ' MDRD equation was less accurate than the ' 175 ' MDRD equation with $79 \%$ [2\%] of results within $30 \%$ of measured GFR compared with $86 \%$ [2\%] using the ' 175 ' MDRD equation.

Conclusion: In persons with newly diagnosed type 2 diabetes using IDMS traceable creatinine values in the ' 175 ' MDRD equation improves performance of the MDRD equation to estimate GFR. 


\section{9}

Both Cockcroft-Gault and MDRD4 estimated GFR are markedly lower than $24 \mathrm{hr}$ creatinine clearance in subjects with diabetes mellitus type 2 A. G. Lieverse ${ }^{1}$, S. J. J. Logtenberg ${ }^{2}$, N. Kleefstra ${ }^{2,3}$, R. T. Gansevoort ${ }^{4}$, H. J. G. Bilo ${ }^{2,5}$;

${ }^{1}$ Internal Medicine, Maxima Medical Centre, Eindhoven,

${ }^{2}$ Diabetes Centre, Isala Clinics, Zwolle, ${ }^{3}$ Langerhans, Medical Reseach Group, Zwolle, ${ }^{4}$ Nephrology, UMCG, Groningen,

${ }^{5}$ Internal Medicine, UMCG, Groningen, The Netherlands.

Background and Aims: Decreased renal function has been identified as a risk indicator for both cardiovascular mortality and all cause mortality in subjects with diabetes mellitus (DM) type 2. In national and international guidelines, it is advised for reasons of practicality to abandon measurement of $24 \mathrm{hr}$ creatinine clearance $(\mathrm{CrCl})$, and to estimate renal function by using either the CockcroftGault (CG) formula or the four-component Modification of Diet in Renal Disease (MDRD4) formula. Furthermore, it is advised to consider referral of subjects with an estimated glomerular filtration rate (eGFR) $<60$ to an internist or nephrologist. We compared estimates of GFR obtained with both formulas to GFR as calculated with $\mathrm{CrCl}$ in a population of patients with DM type 2 .

Materials and Methods: As part of the local protocol for medical care of the diabetes outpatient clinic of the Maxima Medical Centre in Eindhoven, 24hr urine samples and venous blood samples are collected. For the purpose of this study analysis was performed on data from 1087 consecutive patients who visited the clinic between June 2005 and December 2006. Serum and urinary creatinine was measured using the enzymatic method. To estimate GFR, $\mathrm{CrCl}$ was calculated from $24 \mathrm{hr}$ urine samples, and by using the CG formula and the MDRD4 formula. Body surface area (BSA) was calculated using the method of Mosteller.

Results: Patient characteristics (mean \pm SD): Age $61.1 \pm 13.3$ years, body mass index $28.7 \pm 5.7 \mathrm{~kg} / \mathrm{m}^{2}$, BSA $2.0 \pm 0.2 \mathrm{~m}^{2}$ and $\mathrm{HbA}_{1 \mathrm{c}} 6.9 \pm 1.2 \%$. $56 \%$ was male. Mean eGFR \pm SD was with $\mathrm{CrCl} 115 \pm 50 \mathrm{~mL} / \mathrm{min}$, with CG $99 \pm 42 \mathrm{~mL} / \mathrm{min}$, and with MDRD4 $82 \pm 27 \mathrm{~mL} / \mathrm{min} / 1.73 \mathrm{~m}^{2}$. Figure 1 shows boxplots of the 3 methods. Mean difference (95\% confidence interval) was for CrCL and CG 16.4 (14.4, 18.5), for $\mathrm{CrCl}$ and MDRD4 $32.6(30.4,34.9)$ and for CG and MDRD4 16.2 (14.6, 17.8); all $\mathrm{p}<0.01$. When using $\mathrm{CrCl}$ as proxy for GFR $12 \%$ should be considered for referral, whereas these numbers are considerably higher when GFR is estimated according to the CG $(17 \%, \mathrm{p}<0.01 \mathrm{vs.} \mathrm{CrCl})$ or MDRD4 formula (20\%, $\mathrm{p}<0.001$ vs. $\mathrm{CrCl})$.

Conclusion: Practical tools to estimate renal function are needed in the care for patients with DM. However, our results show marked differences in estimated GFR when formulas are used compared to $\mathrm{CrCl}$ calculated from $24 \mathrm{hr}$ urine samples. This could maybe be expected for the MDRD4 due to its BSA correction, but is also true for the standard CG. This results in large differences in numbers of diabetic patients to be considered for referral. In our opinion, the changes in methodology for assessing renal function advocated

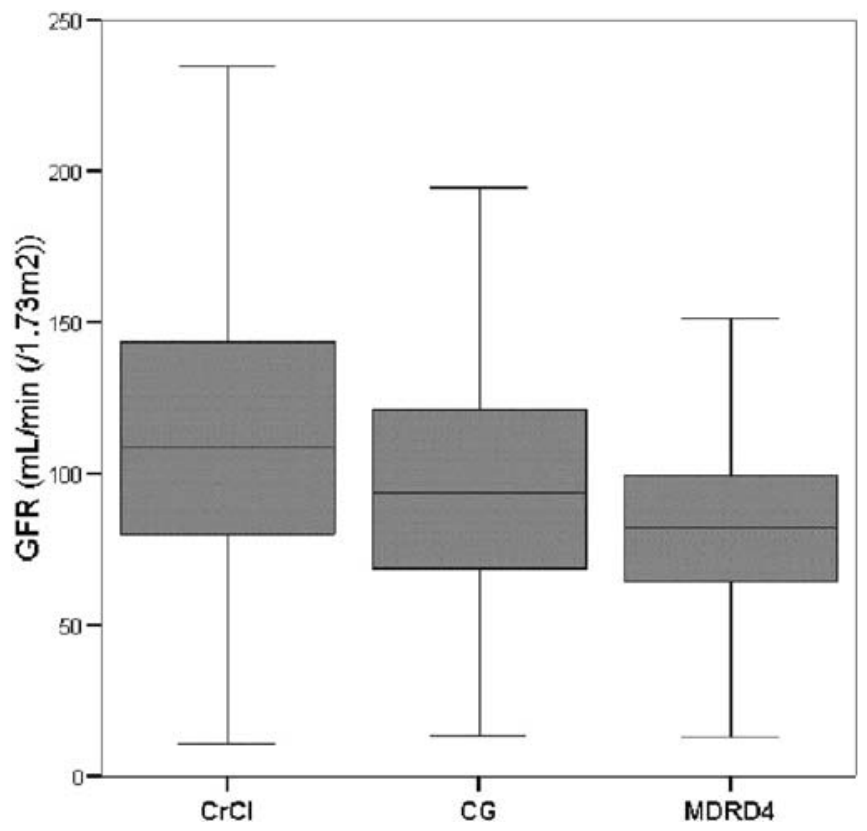

by present clinical guidelines, and the possible consequences attached to the resulting (e)GFR, need careful reconsideration.

\section{0}

Is creatinine clearance valid during renin angiotensin system blockade in diabetic kidney disease?

P. Rossing ${ }^{1}$, L. Tarnow ${ }^{1}$, S. Andersen ${ }^{1}$, J. Bröchner-Mortensen ${ }^{2}$,

H.-H. Parving 3,4 .

${ }^{1}$ Steno Diabetes Center, Gentofte, Denmark,

${ }^{2}$ Aalborg Syd: Nuclear Medicine, University of Aarhus, Aalborg, Denmark, ${ }^{3}$ Medical Endocrinology, Rigshospitalet, Copenhagen, Denmark,

${ }^{4}$ Faculty of Health Science, University of Aarhus, Denmark.

Background and Aims: It has been suggested that chronic ACE inhibition in patients with diabetic kidney disease increases organic ion clearance e.g. creatinine. This implies that creatinine based indices of kidney function would give different results from true GFR based indices in patients treated with an ACE inhibitor. Consequently, it is possible that at least some of the benefit attributed to therapy blocking the renin angiotensin system (RAS)may be related to improved tubular secretion of creatinine rather than a beneficial effect on renal function. Our aim was to perform a post hoc analysis of studies of type 1 diabetic patients with diabetic nephropathy randomised to ACE inhibition (lisinopril 10-20 mg/day) or calcium channel blocker (CCB) (nisoldipine $20-40 \mathrm{mg} /$ day) and followed for 4 years (4 investigations) and type 2 diabetic patients with microalbuminuria randomised to placebo on top of conventional antihypertensive treatment or angiotensin II receptor blocker (ARB) (irbesartan 150-300 mg/day) and followed for 2 years with GFR determination at baseline and at the end.

Materials and Methods: 48 type 1 diabetic patients with diabetic nephropathy randomised to ACE inhibition or CCB and 46 type 2 diabetic patients with microalbuminuria randomised to placebo or ARB on top of conventional antihypertensive treatment. The main outcome measure was change in GFR (51Cr-EDTA plasma clearance) and Cockroft Gault estimated creatinine clearance $(\mathrm{CrCl})$ (s-creatinine measured with enzymatic method, results adjusted for body surface area)

Results: In the type 1 diabetic patients the mean baseline GFR was 85 (26) (SD) $\mathrm{ml} / \mathrm{min} / 1.73 \mathrm{~m}^{2}$ (ns between groups) and CG $\mathrm{CrCl} 96$ (30) (ns). For patients treated with nisoldipine the rate of decline in GFR was $6.0(1.2)$ (SE) $\mathrm{ml} / \mathrm{min} /$ year and decline in $\mathrm{CG} \mathrm{CrCl} 6.1$ (1.2), for lisinopril group it was 6.8 (1.1) and 6.5 (1.3) respectively (ns between groups and methods). In type 2 diabetic patients GFR was 110 (25) (SD) in 15 patients randomised to placebo and 117 (21) in 31 patients randomised to $\mathrm{ARB}$. $\mathrm{CrCl}$ was 104 (31) and 115(35) respectively. For patients treated with ARB the change in GFR was 16 (2) (SE) $\mathrm{ml} / \mathrm{min} / 1.73$ after 2 years and in $\mathrm{CrCl} 10$ (3), and in patients treated with placebo it was 14 (2) vs 7 (2) respectively (ns between groups). If all patients were combined (decline in GFR-decline in $\mathrm{CrCl}$ ) was $1.3(0.7)$ $\mathrm{ml} / \mathrm{min} /$ year in non-RAS vs. $1.7(0.8) \mathrm{ml} / \mathrm{min} /$ year in patients treated with ACE inhibitor or ARB $(\mathrm{p}=0.70)$.

Conclusion: estimated creatinine clearance is valid during chronic RAS blockade in diabetic kidney disease.

\section{1}

Both eGFR and albuminuria are predictors of mortality in type 2 diabetes mellitus: 6-year follow-up of the ZODIAC study L. J. Ubink-Veltmaat ${ }^{1}$, S. J. J. Logtenberg ${ }^{2}$, G. W. D. Landman ${ }^{3}$, S. T. Houweling ${ }^{4}$, N. Kleefstra ${ }^{2,4}$, H. J. G. Bilo ${ }^{2,5}$;

${ }^{1}$ General practice, Huisartsenpraktijk ,t Veen, Hattem,

${ }^{2}$ Diabetes Centre, Isala Clinics, Zwolle, ${ }^{3}$ Internal Medicine, Isala Clinics,

Zwolle, ${ }^{4}$ Langerhans, Medical Research Group, Zwolle,

${ }^{5}$ Internal Medicine, UMCG, Groningen, The Netherlands.

Background and Aims: Micro- or macroalbuminuria are considered as an indicator for the risk on cardiovascular complications in patients with type 2 diabetes mellitus (T2DM). Recently, decreased renal function has been added as a potentially important risk indicator for all cause mortality. We investigated the impact of the presence of micro- or macroalbuminuria and renal function separately and combined on all cause mortality in a cohort of subjects with T2DM treated in primary care. 
Materials and Methods: The ZODIAC study (Zwolle Outpatient Diabetes project Integrating Available Care) started in 1998. A group of 1145 patients with T2DM was seen annually, and data on mortality were assessed 5.8 years later on average. Data of 1142 subjects were available at follow-up. Presence of micro- or macroalbuminuria at baseline was assessed by albumin creatinine ratio (ACR); ACR was considered normal when $<3.5 \mathrm{mg} / \mathrm{mmol}$ in females and $<2.5 \mathrm{mg} / \mathrm{mmol}$ in males. Baseline renal function was assessed by estimating glomerular filtration rate (eGFR) using the Cockcroft-Gault formula (CG); CG was considered normal when $\geq 60 \mathrm{~mL} / \mathrm{min}$. Comparisons of mortality were made for the total group and between age categories $(<70$ and $\geq 70$ years). Data were analysed using Chi-square test.

Results: Baseline characteristics: age $68.7 \pm 11.5$ years, $42 \%$ male, median $(\mathrm{P} 25, \mathrm{P} 75)$ diabetes duration $5.3(2.5,10.4)$ years, serumcreatinine $96 \pm 22$ $\mu \mathrm{mol} / \mathrm{l}$. Baseline eGFR could be calculated for all subjects; baseline ACR was available for 1096 subjects (96\%), with 34\% microalbuminuria and 8\% macroalbuminuria.

Both low eGFR (CG< $60 \mathrm{~mL} / \mathrm{min})$ and the presence of micro- or macroalbuminuria were associated with increased mortality (table). When combining both risk indicators, the all cause mortality rate further increased to $56 \%$ compared $14 \%$ in patients without these two risk factors.

\begin{tabular}{|c|c|c|c|c|c|c|}
\hline \multirow{2}{*}{$\begin{array}{l}6 \text { year } \\
\text { mortality }\end{array}$} & \multicolumn{2}{|l|}{ CG } & \multicolumn{2}{|l|}{ ACR } & \multicolumn{2}{|c|}{$\mathrm{ACR}+\mathrm{CG}$} \\
\hline & normal & abnormal & normal & abnormal & normal & abnormal \\
\hline total & $\begin{array}{l}138 / 744 \\
18 \%\end{array}$ & $\begin{array}{l}195 / 398 \\
49 \%^{* *}\end{array}$ & $\begin{array}{l}143 / 503 \\
21 \%\end{array}$ & & $\begin{array}{l}66 / 456 \\
14 \%\end{array}$ & $\begin{array}{l}108 / 194 \\
56 \%^{* *}\end{array}$ \\
\hline$<70 \mathrm{yrs}$ & $\begin{array}{l}48 / 497 \\
10 \%\end{array}$ & $\begin{array}{l}9 / 51 \\
18 \%\end{array}$ & $\begin{array}{l}24 / 355 \\
7 \%\end{array}$ & & $\begin{array}{l}22 / 324 \\
7 \%\end{array}$ & $\begin{array}{l}7 / 22 \\
32 \% * *\end{array}$ \\
\hline$\geq 70 \mathrm{yrs}$ & $\begin{array}{l}90 / 247 \\
36 \%\end{array}$ & $\begin{array}{l}186 / 347 \\
54 \%^{* *}\end{array}$ & $\begin{array}{l}110 / 282 \\
39 \%\end{array}$ & $\begin{array}{l}140 / 279 \\
50 \%^{*}\end{array}$ & $\begin{array}{l}44 / 132 \\
33 \%\end{array}$ & $\begin{array}{l}101 / 172 \\
59 \%^{* *}\end{array}$ \\
\hline
\end{tabular}

Data presented as ratio and percentage of deceased patients per total patients per group. $C G$ is defined normal $\geq 60 \mathrm{~mL} / \mathrm{min}$; ACR was considered normal when $<3.5 \mathrm{mg} / \mathrm{mmol}$ in females and $<2.5 \mathrm{mg} / \mathrm{mmol}$ in males. Chi-square tests; ${ }^{*}=p<0.01,{ }^{* *}=p<0.001$ normal vs. abnormal

Conclusion: Decreased eGFR and the presence of (micro) albuminuria predict all cause mortality risk in a cohort of subjects with T2DM in primary care. Combined presence of these two "renal" risk factors predicts a poor prognosis in T2DM in primary care.

\section{2}

Development of a prediction model for early onset of microalbuminuria in type 1 diabetes mellitus

S. S. Soedamah-Muthu ${ }^{1,2}$, Y. Vergouwe ${ }^{1}$, J. Zgibor ${ }^{3}$, N. Chaturvedi ${ }^{4}$, T. J. Orchard 3 , J. H. Fuller', K. G. M. Moons';

${ }^{1}$ Julius Center for Health Sciences and Primary Care, UMC Utrecht, The Netherlands, ${ }^{2}$ Department of Epidemiology and Public-Health, Royal Free and University College London Medical School, United Kingdom, ${ }^{3}$ Department of Epidemiology, Graduate School of Public Health, University of Pittsburgh, ${ }^{4}$ National Heart and Lung Institute, Imperial College London, United Kingdom, ${ }^{5}$ Department of Epidemiology and Public Health, Royal Free and University College London Medical School, United Kingdom.

Background and Aims: Microalbuminuria is an important early marker of risk for diabetic renal disease and for cardiovascular disease in patients with type 1 diabetes. Early prediction of microalbuminuria in patients with type 1 diabetes will help clinicians to target these patients to prevent longterm chronic complications. Prediction models for this purpose have not been developed yet. We therefore developed prediction model to predict the individual risk of developing microalbuminuria in patients with type 1 diabetes.

Materials and Methods: The EURODIAB Prospective Complications Study (PCS, European multicentre study) data were used to develop the prediction model. Microalbuminuria was defined as an albumin excretion rate (AER) between 20 and $200 \mu \mathrm{g} / \mathrm{min}$, based on the average of two 24-hour urine collections at follow-up. The model was developed with multivariable logistic regression analysis. Performance of the model was corrected for overoptimism with a bootstrap procedure.

Results: In the EURODIAB PCS, 1134 type 1 diabetic patients were normoalbuminuric (AER $<20 \mu \mathrm{g} / \mathrm{min}$ ) at baseline, out of which 972 patients were still normoalbuminuric and 143 patients became microalbuminuric (13\%) during the follow-up. The prediction equation was as follows: logodds (microalbuminuria $)=-8.59+1.66^{*} \ln ($ glycated haemoglobin $)+0.075^{*} A E R$ $+1.62^{*}$ waist-hip ratio $+0.058^{\star}$ body mass index $+0.30^{*}$ ever-smoking.

This model had a reasonable discriminative value (c-statistic 0.71 after correction for optmism) and good calibration (Hosmer-Lemeshow test pvalue $=0.42$ ).

Conclusions: With relatively easily obtainable predictors such as glycated haemoglobin, albumin excretion rate, WHR, BMI and smoking the individuals' risk of microalbuminuria can be estimated in patients with type 1 diabetes.

Supported by grants from the Wellcome Trust, the European Community and Diabetes UK

\section{3}

The relation between plasma creatinine level and lactic acid in type 2 diabetic patients with normal renal function

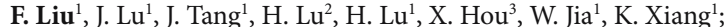

${ }^{1}$ Endocrinology \& Metabolism, ${ }^{2}$ Nuclerology, ${ }^{3}$ Shanghai Research Institute for Diabetes, Shanghai 6th People's hospital, Shanghai, China.

Background and Aims: To study whether the fasting lactic acid(LA) levels change with the creatinine and GFR levels in type 2 diabetic (T2DM)patients with normal renal function, the effect of metformin administration on it and to find a predictive clinical index for hyperlactic acidemia.

Materials and Methods: The whole venous blood was collected from 723 T2DM inpatients (include 275 cases with metformin administration as treatment group and 448 cases without merformin as control group) in the morning without meal and physical activity. The LA was measured with enzyme-electrode assay (EKF diagnostic Company, Germany). The fasting glucose (FPG), fasting insulin (FINS), Creatinine (Cr), alanine aminotransferase (ALT), and HbA levels were determined. Total glomerular filtration rate (GFR) was detected by ${ }^{99}$ Technetium isotope scan. BMI was calculated, and HOMA-IR was evaluated based on FPG and FINS levels. The data were analysed with SPSS13.0 software.

Results: 1 . The fasting LA levels of 448 cases of control group was $1.17 \pm$ $0.47 \mathrm{mmol} / 1$, and it was in the normal range. 2 . The LA levels of treatment group $(1.33 \pm 0.57 \mathrm{mmol} / \mathrm{l})$ was higher significantly than control group ( $p=0.000)$, and the FINS levels $(p=0.003)$ and $\operatorname{BMI}(p=0.000)$ decreased significantly. 3 . The incidence of hyperlactatemia $(9.45 \%)$ in metformin group was higher than control group (4.91\%), and no lactic acidosis was found in all patients. 4. The LA levels increase with the Cr levels and decrease with total GFR, especially when $\mathrm{Cr}$ was over $90 \mathrm{umol} / \mathrm{l}$ and the latter was below $80 \mathrm{ml} / \mathrm{min}$, and the AUC of Cr to predict the LA was $67.2 \%$ (95\% confidence $0.534 \sim 0.810, p=0.008)$ and the cutpoint of $\mathrm{Cr}$ level causing hyperlactic acidemia was $95.35 \mathrm{umol} / 1.5$. Based on the lactic acid as dependent variable, and the others as independent variable, the correlation analysis showed that the $\operatorname{Cr}(r=0.36, p=0.000), \operatorname{ALT}(r=0.201, p=0.000)$ and BMI $(r=0.137, p=0.004)$ was positively correlated with LA in control group, and GFR was negatively correlated with $\mathrm{Cr}(r=-0.30, p=0.000)$ in these patients.

Conclusion: The LA level of type 2 diabetic patients was similar to healthy adults, it is influenced mainly by the function of renal and liver, BMI, and administration of metformin. The risk of hyperlactic acidemia increase when plasma $\mathrm{Cr}$ level is above $95 \mu \mathrm{mol} / \mathrm{l}$, and $\mathrm{Cr}$ is a good index to predict the hyperlactatemia.

\section{4}

Risk of contrast-induced nephropathy in patients with diabetes mellitus type 2

N. V. N. Zaytseva ${ }^{1}$, M. S. Shamkhalova ${ }^{1}$, E. N. Drozdova ${ }^{1}$, M. V. Shestakova ${ }^{1}$, S. T. Matskeplishvili², E. F. Tugeeva ${ }^{2}$, U. O. Buziashvili², A. D. Deev ${ }^{3}$;

${ }^{1}$ Diabetic Nephropathy, Endocrinology Research Center,

${ }^{2}$ Clinical Diagnostic, Bakoulev Scientific Center of Cardiovascular Surgery, ${ }^{3}$ Laboratory of Biostatistics, State Research Center for Preventive Medicine, Moscow, Russian Federation.

Background and Aims: To determine the incidence of contrast-induced nephropathy (CIN) after percutaneous coronary intervention (PCI), 
characterise independent predictors and determine the prognostic implications of CIN among patients with and without diabetes mellitus (DM) type 2.

Materials and Methods: We have studied 121 patients (98 males, 23 females) with DM and 42 patients ( 33 males, 9 females) without DM who was under coronarography to determine further intervention on the heart vessels. Mean age was $57.7 \pm 0.7$ and was not different between groups. The CIN defined as an increase in serum creatinine (SCr) of at least $25 \%$ or $44 \mu \mathrm{mol} / \mathrm{L}$ over baseline within 48 hours of PCI.

Results: Baseline SCr and glomerular filtration rate (GFR) were similar in diabetics and non-diabetics $(90.6 \pm 2.2$ vs. $91.6 \pm 3.3 \mu \mathrm{mol} / \mathrm{L}$ and $80.4 \pm 1.9$ vs. $80.5 \pm 2.8 \mathrm{ml} / \mathrm{min}$, respectively). The incidence of CIN was significantly higher among the diabetics, than non-diabetics ( $36.4 \pm 4.4$ vs. $14.3 \pm 5.5$ percent respectively, $\mathrm{p}<.01)$. CIN was associated with baseline creatinine, contrast volume administrated $-\mathrm{OR}=2.57$ per $100 \mathrm{ml}$ of medium $95 \% \mathrm{CI}(1.59,4.16)$, low $(<40)$ ejection fraction $-\mathrm{OR}=3.44$ with $95 \% \mathrm{CI}(1.25,9.49)$, other heart failure indicators, systolic dysfunction, diuretics use $-\mathrm{OR}=3.09$ with $95 \% \mathrm{CI}$ $(1.26,7.59)$

Conclusion: Although similar baseline renal function, the overall incidence of CIN after PCI was significantly higher among diabetics in comparison with non-diabetics. Efforts to control risk factors should be intensified to limit the impact of injury and protect them from future adverse events.

\section{PS 108 Eye and brain}

\section{5}

Retinal vascular lesions in patients with type 2 diabetes mellitus of Caucasian and Asian origin: baseline results from the AdRem study R. P. Stolk ${ }^{1,2}$, M. J. van Schooneveld ${ }^{3}$, K. Cruickshank ${ }^{4}$, A. D. Hughes ${ }^{5}$, A. Stanton ${ }^{6}$, L. Juming ${ }^{7}$, A. Patel ${ }^{8}$, S. A. M. Thom ${ }^{5}$, J. R. Vingerling', D. E. Grobbee ${ }^{2}$;

${ }^{1}$ Epidemiology, University Medical Center Groningen, The Netherlands, ${ }_{2}^{2} J u l i u s$ Center, University Medical Center Utrecht, The Netherlands, ${ }^{3}$ Ophthalmology, University Medical Center Utrecht, The Netherlands, ${ }^{4}$ Clinical Epidemiology, University of Manchester Medical School, United Kingdom, ${ }^{5}$ International Centre for Circulatory Health, Imperial College, London, United Kingdom, ${ }^{6}$ ASCOT Clinic, Beaumont Hospital, Dublin, Ireland, ${ }^{7}$ Opthalmology, Chinese PLA General Hospital, Beijing, China, ${ }^{8}$ Cardiovascular Medicine, George Institute for International Health, Sydney, Australia, ${ }^{9}$ Ophthalmology, Erasmus Medical Center, Rotterdam, The Netherlands.

Background and Aims: The ADVANCE Retinal measurement study (AdRem), a sub-study of the international Action in Diabetes and Vascular disease (ADVANCE) clinical trial, provides data on diabetic retinal lesions in a large study population of patients with type 2 diabetes in 14 countries.

Materials and Methods: Seven-field stereoscopic photographs of both eyes were taken at baseline. Lesions were graded in one reading center. Gradable retinal images were received from 1605 patients.

Results: The number of patients with any retinopathy (ETDRS score $>20$ ) was $610(38.0 \%, 95 \% \mathrm{CI} 35.6-40.4)$, of these $35(2.2 \%, 1.6-3.0)$ had severe diabetic retinopathy (ETDRS $>50$ ). Focal arterial narrowing was present in $3.8 \%$, venous beading in $5.1 \%$, and arteriovenous (AV) nicking was found in $9.8 \%$. Prevalence of retinal abnormalities did not differ significantly between men and women. Among participants included in this study, Chinese and South-Asian patients had more retinopathy than Caucasians, as defined both by ETDRS score $(49.4 \%, 46.0 \%, 31.3 \%$; $\mathrm{p}<0.001$ adjusted for age, gender, $\mathrm{HbAlc}$ and duration of diabetes) and after comparing specific vascular lesions (e.g. AV nicking 12.3\%, 8.5\%, 7.5\%; adjusted p-value <0.005).

In patients with and without retinopathy (ETDRS score $>20$ ) mean $\mathrm{HbAlc}$ levels were 7.6\% (SD 1.6) and 7.2\% (1.4), duration of diabetes 8.6 (6.4) and 6.5 (5.7) years, and systolic blood pressure 138 (21) and 135 (20) $\mathrm{mmHg}$ (all comparisons: $\mathrm{p}<0.01)$. Focal arterial narrowing was associated with a mean HbAlc difference of $0.3(p<0.05)$ whereas macular oedema, venous beading and AV nicking were not associated with differences in current Hbalc levels. Systolic and diastolic blood pressure were higher in patients with venous beading and macular edema ( $<0.05$, adjusted for age, gender and duration of diabetes). Smoking was not associated with retinal lesions. All these associations were similar in Chinese, South-Asian, and Caucasians patients. Conclusion: Using a sensitive diagnostic procedure, more than one third of patients with type 2 diabetes enrolled in a clinical trial had retinal lesions at study entry. HbAlc, diabetes duration, and blood pressure were increased in patients with retinal vascular lesions irrespective of ethnic group.

Supported by Institut de Recherches Internationales Servier and Sydney University

\section{6}

A diabetic retinopathy screening program detecting non-diabetic eye problems

K. P. Shotliff ${ }^{1}$, G. Duncan²;

${ }^{1}$ Beta Cell Diabetes Centre, Chelsea and Westminster Hospital,

${ }^{2}$ Medical Illustration, First Retinal Screening, London, United Kingdom.

Background and Aims: As part of the United Kingdom National Screening Committee's grading criteria for digital retinal photographs in people with diabetes there are several grades for the level of diabetic retinopathy and a single grade of 'O' / 'Other' for all non-diabetic lesion. We wished to see which 'other' diagnoses were most commonly seen and whether any action was needed on the basis of diagnosing them.

Method: A prospective observational study of 4,083 consecutive people offered screening in a community based diabetic retinopathy screening program examined over a 12 month period.(3,659 attended) All images were 
dual graded by trained Ophthalmologists to give a diagnosis for these nondiabetic pathology which would all be classified by the UK National Screening Committee as 'O / Other'.

Results: Non-diabetic conditions were found in 1.313 (35.9\%) of people or $956(26.1 \%)$ if cataracts were excluded. Only $3 \%$ of patients required referral for non-diabetic retinopathy findings alone. The most common conditions seen were media opacities $(20.8 \%)$, drusen $(16.2 \%)$, age related macula degeneration (6.1\%), pigmented lesions (4.9\%) and abnormalities of the optic $\operatorname{disc}(2.2 \%)$.

Conclusion: Non-diabetic conditions were a common finding in this population based diabetic retinopathy screening program. If $35.9 \%$ of patients with a non-diabetic lesion were referred rather than the $3 \%$ our Ophthalmologist graders felt required referral we would need 140 extra eye clinics per year instead of just 12 clinics. We believe that all individuals grading these images should be aware of the more common non-diabetic conditions seen as this may reduce inappropriate referrals to Ophthalmology Clinics.

\section{7}

Natural history of beta cell destruction in type 1 diabetes influences development and progression of diabetic retinopathy: Protective effect of sustained residual beta cell function

K. Nakanishi ${ }^{1,2}$, C. Watanabe ${ }^{1,2}$;

${ }^{1}$ Dept. of General Internal Medicine and Metabolism, Toranomon Hospital, Kawasaki, ${ }^{2}$ Dept. of General Internal Medicine and Metabolism, Okinaka Memorial Institute for Medical Research, Tokyo, Japan.

Background and Aims: Presence of residual beta-cell function in type 1 diabetes delayed the development of diabetic retinopathy. However, how long residual beta-cell function needs to exist for protective effect on the development and progression of diabetic retinopathy is not yet known. We investigated time course of retinopathy findings in relation to time course of beta-cell destruction.

Materials and Methods: Subjects were 254 type 1 diabetic patients (men/ women: $145 / 109$, age at onset: $34 \pm 14$ years, mean \pm SD). Serum C-peptide was assessed longitudinally $4.7 \pm 3.7$ times during $12 \pm 11$ years. Serum Cpeptide level less than $0.017 \mathrm{nmol} / 1$ at fasting or $0.033 \mathrm{nmol} / 1$ at $2-3$ hours after meal was regarded as complete beta-cell destruction. Optic fundi were evaluated longitudinally $21 \pm 17$ times during $17 \pm 9$ years. Mean $\mathrm{HbAlc}$ value during entire illness was calculated as index of long-term glycemic control. Results: The patients were stratified into those who lost beta-cell function completely within $20,15,10$ and 5 years from the onset of diabetes and those who retained residual $\beta$-cell function even after the corresponding years from the onset of diabetes (patient number: 104 vs. 42,84 vs. 76,60 vs. 105 , and 37 vs. 152, respectively). Cumulative incidence of background retinopathy was higher in the patients who lost beta-cell function completely within 20,15 , and 10 years after the onset of diabetes than those who retained residual $\beta$-cell function even after the corresponding years (5.41 vs. $4.25 / 100$ patient-years, $\mathrm{P}=0.013$; 5.22 vs. $4.18 / 100$ patient-years, $\mathrm{p}=0.006$; and 4.77 vs. $4.15 / 100$ patient-years, $\mathrm{p}=0.048$, respectively). Cumulative incidence of preproliferative retinopathy was higher in the patients without beta-cell function within 20 years after the onset of diabetes than those with residual $\beta$-cell function even after 20 years ( 2.61 vs. $2.60 / 100$ patient-years, $\mathrm{P}=0.031$ ). Cumulative incidence of any type of retinopathy did not differ between the patients without $\beta$-cell function within 5 years and those with residual $\beta$ cell function even after the 5 years. Mean HbAlc value was higher in the patients without $\beta$-cell function within 20 or 15 years from the onset of diabetes than those with residual $\beta$-cell function even after corresponding years $(8.46 \pm 1.01$ vs. $7.87 \pm 0.73 \%, p=0.030$, and $8.54 \pm 1.03$ vs. $8.10 \pm 1.02$ $\%, \mathrm{p}=0.042$, respectively), whereas mean $\mathrm{HbAlc}$ value no longer differed between the patients without $\beta$-cell function within 10 or 5 years and those with residual $\beta$-cell function even after the corresponding years. After adjusting possible confounders (sex, mean $\mathrm{HbAlc}$ value, age at onset and period until insulin therapy) by Cox's proportional hazards model, complete loss of beta-cell function until 20,15, and 10 years from the onset of diabetes was an independent risk factor for the development of diabetic retinopathy [relative hazard (95\% confidence interval): $1.58(1.05-2.52), \mathrm{p}=0.029 ; 1.47$ (1.09-2.00), $\mathrm{p}=0.011$; and 1.39 (1.04-1.84), $\mathrm{p}=0.024$, respectively].

Conclusion: Sustained presence of residual beta-cell function in type 1 diabetes (more than 10 years) protects the patients from early development and progression of diabetic retinopathy through the attainment of better glycemic control during a long period.

Supported by Japanese Ministry of Education, Science and Culture

\section{8}

Retinal thickness changes after insulin therapy initiation in patients with type 2 diabetes. An Optical Coherence Tomography (OCT) based study F. Gomez-Peralta ${ }^{1}$, P. Pujante ${ }^{1}$, C. Abreu ${ }^{2}$, E. Santos ${ }^{3}$, A. Garcia-Layana ${ }^{4}$, A. Salinas ${ }^{4}$, N. Varo;

${ }^{1}$ Endocrinology and Nutrition Department, University Clinic of Navarra, Pamplona, ${ }^{2}$ Endocrinology and Nutrition Department, N.S La Candelaria Hospital, Santa Cruz de Tenerife, ${ }^{3}$ Endocrinology and Nutrition Department, Complejo Hospitalario, Burgos, ${ }^{4}$ Ophthalmology Department, University Clinic of Navarra, Pamplona, ${ }^{5}$ Center for Applied Medical Research, University Clinic of Navarra, Pamplona, Spain.

Background and Aims: Diabetic macular oedema is a diabetes-related complication with a high risk of vision loss. It is produced by fluid leakage due to blood-retinal barrier breakdown. Macular oedema is more frequent in patients with type 2 diabetes (DM2) after initiation of insulin therapy (IT). Local and systemic factors have been involved in this specific complication of DM. Optical Coherence Tomography (OCT) is the most sensitive, clinically available non-invasive method to measure retinal thicness (RT), able to detect even very small changes. OCT was previously shown to be useful in detecting and quantify diabetic macular oedema.

Aims:

1.- Measure changes in retinal thickness (RT) by means of OCT after IT initiation in DM2 patients.

2.- Evaluate the relationship of changes in RT with metabolic changes induced by IT.

3.- Study the implication of several inflammatory, proangiogenic or growth factors.

Materials and Methods: Prospective study in 12 DM2 patients (8 males, age $62,4 \pm 8.2$ years). OCT-measured RT and glycosylated haemoglobin; (A1c), lipid profile, blood pressure (BP), IGF-1, IGF-BP3; VEGF y sCD40L were recorded before and 6 months after IT initiation.

Results: Expressed as mean \pm SD, 1: pre, 2:post. A1c1 $=9.9 \pm 1.8$; $\mathrm{A} 1 \mathrm{c} 2=$ 8.2 $\pm 1.1(\%)(p=0.039)$. Peso1: 75.6 \pm 11.7 ; Peso2: $77.8 \pm 11.4(\mathrm{~kg})(p=0.01)$. HDL-Coll $=47.3 \pm 9.1 ; \mathrm{HDL}-\mathrm{Col} 2=49.3 \pm 8.7(\mathrm{mg} / \mathrm{dl})(p=0.3) . \mathrm{Tgl}: 195 \pm 155$; Tg2: $169 \pm 106(\mathrm{mg} / \mathrm{dl})(p=0.5)$. IGF-1 $(1)=0,18 \pm 0.17 ;$ IGF-1 $(2)=0,26 \pm 0.21$ $(p=0.043)$ IGF-BP3 $(1)=40.7 \pm 3.4$; IGF-BP3 $(2)=41.7 \pm 8.9(\mathrm{ng} / \mathrm{ml})(p=0.23)$. $\operatorname{VEGF}(1)=393 \pm 42 ; \operatorname{VEGF}(2)=528 \pm 48(\mathrm{pg} / \mathrm{ml})(p=0.23) ., \mathrm{CD} 40 \mathrm{~L}=4.9 \pm 2.9$; $\mathrm{sCD} 40 \mathrm{~L}=0.52 \pm 1.7(\mathrm{ng} / \mathrm{ml})(p=0.35)$. Retinal thickness difference: +7.025 microns; Retinal volume difference: $+0.025 \mathrm{~mm}^{3}$. No statistically significant correlation between A1c, BP and RT was observed.

Conclusion: Insulin therapy increases retinal thickness of patients with DM2 in a short time. This change seems to be independent of metabolic and BP improvement. Retinal thickness increase is associated with increases of IGF-1 and VEGF, suggesting a possible role of trophic and proangiogenic effect of insulin therapy in the development of diabetic macular oedema.

\section{9}

A functional magnetic resonance imaging study demonstrating alterations in brain responses to acute pain stimulation in diabetic neuropathy

D. Selvarajah ${ }^{1}$, R. A. Gandhi ${ }^{1}$, M. D. Hunter ${ }^{2}$, C. J. Emery ${ }^{1}$, P. D. Griffiths ${ }^{3}$, S. Tesfaye ${ }^{1}$, I. D. Wilkinson ${ }^{3}$;

${ }^{1}$ Diabetes Research Unit, ${ }^{2}$ Academic Psychiatry,

${ }^{3}$ Academic Radiology, University of Sheffield, United Kingdom.

Background and Aims: Chronic distal symmetrical painful diabetic neuropathy $(\mathrm{DN})$ is a common complication of diabetes resulting in significant patient morbidity. There is increasing evidence that the central nervous system is involved although its role remains unclear.

This study examines the functional neuroanatomical correlates to acute pain in subjects with type 1 diabetes at different stages of neuropathy.

Materials and Methods: Blood oxygen-level dependent (BOLD) functional magnetic resonance imaging (fMRI) datasets have been acquired in a high 
field system (3T) on 12 subjects with diabetes (4 No DN, 4 Painful DN and 4 Painless DN) and 4 healthy volunteers. Heat pain stimulus to the dorsum of the foot was provided by an MR compatible peltier type device (MEDOC TSA-II) in a box-car functional paradigm design. Whole brain fMRI datasets (EPI; TE $=35 \mathrm{~ms}, \mathrm{TR}=3000 \mathrm{~ms} ; 190$ time points) were acquired during baseline and hot stimulus presentation over $570 \mathrm{sec}$ for each subject. Functional analysis was performed using Statistical Parametric Mapping (SPM2, IoN, UCL).

Results: There was a significantly lower $(\mathrm{p}<0.05)$ BOLD response to acute heat pain stimulation in the diabetic cohort compared to healthy volunteers. The brain's BOLD haemodynamic response to heat pain also varied between diabetic subject sub-groups $(\mathrm{p}<0.05)$. Those with No DN showed greater response than those painful and painless DN. And those with painful DN showed significantly greater response than those with painless DN. The neuroanatomical areas that showed a greater response in the painful DN group include the primary somatosensory cortex, lateral frontal and cerebellar regions.

Conclusion: BOLD fMRI can be used to study the spatial distribution of the brain's response to acute heat stimulation in diabetes. This allows further investigations into the physiological CNS responses (plasticity) to alterations of acute pain sensation and perception in DN. Better understanding of these processes will help targeted drug development and also provides an objective tool for monitoring response to interventions.

Supported by Diabetes UK

\section{0}

Patients with clinical manifest vascular disease and diabetes have more atrophy and vascular lesions on brain MRI than non-diabetic patients with vascular disease

A. M. Tiehuis ${ }^{1}$, F. L. J. Visseren ${ }^{2}$, A. P. A. Appelman ${ }^{1}$, K. L. Vincken ${ }^{3}$,

G. Biessels ${ }^{4}$, L. Kappelle ${ }^{4}$, W. P. T. Mali ${ }^{1}$, Y. van der Graaf';

${ }^{1}$ Radiology, ${ }^{2}$ Internal Medicine, ${ }^{3}$ Image Sciences Institute,

${ }^{4}$ Neurology, ${ }^{5}$ Clinical Epidemiology, University Medical Center Utrecht, The Netherlands.

Background and Aims: Diabetes (DM) can lead to accelerated cognitive decline, dementia and structural brain abnormalities (atrophy and vascular lesions). It is still debated whether these cerebral changes are solely due to co-morbid vascular disease or if other DM-specific factors, such as chronic hyperglycemia, play an additional role.

Materials and Methods: Brain MRI was performed in 1032 participants (217 DM) of the SMART-study, an ongoing cohort study in patients with clinical manifest vascular disease (mean age $58.6+/-10.2$ ). Quantitative measurements of brain parenchyma, ventricle and white matter hyperintensities (WMH) volumes were obtained with an automated segmentation technique. The presence of cortical and lacunar infarcts was scored visually. Linear and logistic regression analyses with adjustment for age and gender were performed to estimate the effect of DM on brain MRI abnormalities and to identify determinants for brain abnormalities within the DM group.

Results: Vascular patients with DM had significantly more global ( $-1.1 \%$ brain volume; $95 \% \mathrm{CI}-1.4--0.7)$ and subcortical brain atrophy $(+0.2 \%$ ventricular volume; $95 \%$ CI $0.03-0.3)$ compared with non-DM vascular patients. Moreover, DM patients had larger WMH volumes ( $0.16 \mathrm{ml}(\mathrm{LN}) ; 0.03-0.30)$. Adjustment for hypertension or excluding patients with cortical infarcts did not affect these associations. Within DM patients, determinants of decreased brain volume were high fasting serum glucose levels $(\beta-0.13 \%$ per $1 \mathrm{mmol} / \mathrm{l}$ glucose; -0.23- -0.03$)$, diabetes duration ( $\beta-0.05 \%$ per 1 diabetes-year; -0.11 $-0.002)$, use of glucose-lowering agents $(\beta-1.43 \% ;-2.73--0.14)$ and presence of hypertension $(\beta-0.63 \% ;-1.22--0.04)$. Determinants of increased WMH volume were presence of hypertension $(\beta 0.29 \mathrm{ml}(\mathrm{LN}) ; 0.03-0.55)$ and a history of ischemic stroke ( $\beta 0.47 \mathrm{ml}(\mathrm{LN}) ; 0.17-0.76)$. Smoking (OR 2.82; $1.21-6.57$ ), history of ischemic stroke (OR $3.59 ; 1.69-7.66$ ) and carotid artery disease (OR $3.39 ; 1.36-8.45)$, or the presence of more than 2 vascular diseases (OR 4.84; 2.12-10.61) increased the risk for lacunar infarcts in DM patients. Conclusion: In patients with clinical manifest vascular disease, DM has an added detrimental effect on the brain. Within DM patients, diabetes-related factors contribute to brain atrophy, while vascular risk factors are related to both atrophy and vascular lesions.

\section{1}

Diabetes-specific determinants of regional brain volumes and white matter abnormalities in young adults with type 1 diabetes S. C. Ferguson ${ }^{1}$, J. Geddes ${ }^{1}$, J. M. Wardlaw ${ }^{2}$, A. Blane ${ }^{2}$, I. J. Deary ${ }^{3}$, B. M. Frier ${ }^{1}$;

${ }^{1}$ Department of Diabetes, Royal Infirmary of Edinburgh, ${ }^{2}$ Department of Clinical Neuroscience, Western General Hospital, Edinburgh,

${ }^{3}$ Department of Psychology, University of Edinburgh, United Kingdom.

Background and Aims: Type 1 diabetes (T1DM) is characterised by intermittent severe hypoglycaemia, chronic hyperglycaemia and the development of microangiopathy, and frequently develops before neurodevelopment is complete. These diabetes-specific factors may impact upon brain structure. We examined the associations of these factors with aspects of brain structure in young adults with T1DM.

Materials and Methods: 133 normotensive people with T1DM [mean (range): age 32.3yrs (20-45), 51.9\% male, diabetes duration 16.1yrs (9-31), onset age 16.1 yrs $(1-36 ; 19.8 \%$ diagnosed < 8yrs), $36.1 \%$ background retinopathy] participated in a cross-sectional study. Participants had no neuropsychological pathology and showed a range of severe hypoglycaemia exposure and microangiopathy status. Correlation was used to identify associations between diabetes-related factors (severe hypoglycaemia, retinopathy, diabetes duration, diabetes onset age) and brain structure (volumetric MRI analysis). Linear Modelling (brain volumes) and Multiple Logistic Regression (atrophy, white-matter abnormalities) techniques identified independent predictors of outcomes.

Results: Mild ventricular atrophy was common (30.8\%) but Sulcal atrophy $(10.5 \%)$ was infrequent. An early diabetes onset age $(<8 \mathrm{yrs})$ independently predicted ventricular atrophy $(\mathrm{p}=0.01)$ and greater Lateral Ventricular volumes $(p=0.01)$. No significant associations were observed between other diabetes-related factors and volumetric measures. Peri-ventricular white-matter lesions were common $(60 \%)$, mild and not significantly associated with diabetes-related factors. Small Punctate White-Matter Lesions (SPWML) were common $(45.1 \%)$, located in distinct brain areas [Hippocampus (20.3\%), Basal Ganglia (39.3\%), Centrum Semiovale $(31.1 \%)]$ and significantly associated with some diabetes-specific factors (retinopathy, onset age, duration). Logistic regression identified diabetes onset age as an independent predictor of Hippocampal $(\mathrm{p}=0.004)$ and Centrum Semiovale $(\mathrm{p}=0.01)$ SPWML and the overall presence/absence of SPWML $(p=0.02)$ : SPWML were more frequently observed in those with later onset diabetes. Retinopathy status independently predicted Hippocampal SPWML $(\mathrm{p}=0.03)$. No significant independent associations between diabetes duration, severe hypoglycaemia exposure and SPWML were identified.

Conclusion: Subtle brain structural abnormalities are common in young adults with T1DM. These are characterised by mild brain atrophy, mild peri-ventricular white-matter abnormalities and a specific type of whitematter lesion, the Small Punctate White-Matter Lesion. Early diabetes onset appears to be the most important diabetes-specific determinant of the differences observed, predisposing to central brain atrophy yet conferring relative resistance to SPWML. Intracranial microangiopathy, as background retinopathy, may also predispose to SPWML in specific brain regions, whereas diabetes duration and severe hypoglycaemia exposure do not appear to confer obvious disadvantage to brain structure in the subjects examined by the present study.

Supported by the Robertson Trust

\section{2}

Correlations between severity of diabetic neuropathy and impaired visual-evoked potentials at prospective evaluation in patients with longstanding type 1 diabetes

T. T. Várkonyi ${ }^{1}$, R. Takács ${ }^{1}$, É. Börcsök ${ }^{1}$, C. Lengyel ${ }^{1}$, Z. Fülöp ${ }^{2}$, M. Janáky ${ }^{2}$, P. Kempler ${ }^{3}$, J. Lonovics';

${ }^{1} 1$ st Department of Internal Medicine, University of Szeged,

${ }^{2}$ Department of Ophthalmology, University of Szeged, ${ }^{3} 1$ st Department of Internal Medicine, Semmelweis University, Budapest, Hungary.

Background and Aims: Our earlier studies revealed correlations between the severity of diabetic neuropathy and central afferent dysfunctions. The aim of our present study was to characterise the progression of impaired visual- 
evoked potentials, cardiovascular autonomic and peripheral sensory nerve dysfunction in patients with long-standing type 1 diabetes (DM).

Patients and Methods: 12 patients were studied (age: $46.3 \pm 3$ years, duration of DM: $24.1 \pm 1.9$ years; mean \pm SE). Every patient was tested twice, the second tests being performed 36 months after the initial ones. The five standard cardiovascular reflex tests were performed for the assessment of autonomic neuropathy (AN). Sensory nerve integrity was studied with a Neurometer (Neurotron Inc., Baltimore). The visual pathways were measured by analysis of the latencies (P100) of visual-evoked potentials.

Results: The AN score increased during the follow-up period (3.9 \pm 0.4 v.s $5.7 \pm 0.5, \mathrm{p}<0.05$ ). The results of three of the five reflex tests worsened (heart rate response to breathing: $12.1 \pm 1.7 v s .8 .4 \pm 1.5$ beats $/ \mathrm{min}, \mathrm{p}<0.05$; Valsalva ratio: $1.33 \pm 0.05$ vs. $1.18 \pm 0.03, \mathrm{p}<0.05$; orthostatic hypotension: $15 \pm 2.5$ vs. $33.4 \pm 3.8 \mathrm{~mm} \mathrm{Hg}, \mathrm{p}<0.01)$. The severity of sensory neuropathy and the latencies of the P100 waves did not change significantly. Evaluation of the follow-up data revealed several associations between the severity of the neuropathy and the impairment of the P100 waves 3 years after the first examinations (AN score-P100 latency: $\mathrm{r}=0.74, \mathrm{p}<0000.1$; heart rate response to breathing-P100 latency: $\mathrm{r}=-0.61, \mathrm{p}<0.01$; $\mathrm{n}$. peroneus: $\mathrm{CPT}$ at $2000 \mathrm{~Hz}-$ P100 latency: $r=0.48, p<0.05$; CPT at $250 \mathrm{~Hz}-\mathrm{P} 100$ latency: $\mathrm{r}=0.56, \mathrm{p}<0.01$; $\mathrm{CPT}$ at $5 \mathrm{~Hz}-\mathrm{P} 100$ latency: $\mathrm{r}=0.65, \mathrm{p}<0.001)$.

Conclusion: Progression of parasympathetic and sympathetic autonomic neuropathy was observed during the 3 -years follow-up period among patients with long-standing type 1 diabetes, while there were no significant changes in the peripheral sensory nerve function and visual-evoked potentials. The correlations between the cardiovascular autonomic and peripheral sensory neuropathy just as the impairment of the visual-evoked potentials confirmed by the follow-up data provide further evidence of an association between peripheral and central manifestations of diabetic neuropathy.

\section{PS 109 Neuropathy: cardiovascular links}

\section{3}

Correlation between cardiac autonomic neuropathy (CAN) and left ventricular mass (LVM) in type 2 diabetes

A. Ferrière, M. Ueffing, M. Pfohl, S. Zimny;

Medical Department I, EVK Bethesda, Duisburg, Germany.

Background and Aims: Studies in diabetic patients undergoing haemodialysis suggest that mechanisms of apoptosis triggered by the vegetative nervous system might lead to a myocardial remodelling. Concerning the CAN in diabetic patients undergoing haemodialysis an association between CAN and the left ventricular mass index (LVMI) as a marker of a left ventricular hypertrophy (LVH) might exist. We studied the relationship between CAN and LVM respectively LVMI in view of the prevalence of microvascular longterm consequences in type 2 diabetes.

Materials and Methods: The indices of LVH and heart rate variability (HRV) have been analysed as well in patients with type 2 diabetes as in non-diabetic patients. Standardised wall thickness measurement has been made and diameters of the left ventricle have been determined on the basis of the ASEconvention by echocardiography. The LVM has been calculated according to the formula described by Devereux et al. The LVMI has been calculated from $\mathrm{LVMI}=\mathrm{LVM} / \mathrm{KOF}$ (body surface). Additionally the relative left ventricular wall thickness (rLVWT) has been established from $r L V W T=2 x P W T d$ (left ventricular end-diastolic posterior wall thickness)[mm]/LVIDd (left ventricular internal end-diastolic dimension) $[\mathrm{mm}]$. The parameters of the HRV (SD, SDANN5, ASDNN5, RMSSD and pNN50) have been measured using a 24-hour ECG with computer-aided analysis.

Results: 103 patients with type 2 diabetes and 107 non-diabetic patients (control group) with the same gender ratio in both groups were included. The mean age was $65 \pm 12$ (mean \pm standard deviation) and $66 \pm 16$ years respectively. In the diabetes group the mean value of $\mathrm{HbAlc}$ was $8.5 \pm 2.3 \%$. In comparison to the control group the diabetic patients revealed significantly lower values of the time domain measurement of the HRV ( $p=0.01$ for SD,

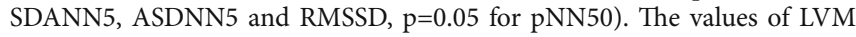
and LVMI were significantly greater in diabetic patients compared to the control group $\left(304.3 \pm 117.0 \mathrm{~g}\right.$ and $158.6 \pm 54.4 \mathrm{~g} / \mathrm{m}^{2}$ versus $261.9 \pm 94.9 \mathrm{~g}$ and $\left.140.4 \pm 46.2 \mathrm{~g} / \mathrm{m}^{2}(\mathrm{p}=0.01)\right)$. However there was no significant correlation between the parameters of HRV, LVM and LVMI respectively.

Conclusion: The variables of the time domain measurement of HRV as indices of CAN revealed significantly lower values in type 2 diabetic patients compared to non-diabetic patients. In contrast to a previous minor study in diabetic patients undergoing haemodialysis a correlation between CAN, LVM and LVMI respectively could not be shown. We assume that CAN and LVH describe independent variables accumulating in type 2 diabetes.

\section{4}

Cardiac autonomic neuropathy in painless post myocardial infarction patients with type 2 diabetes

T. Akhobadze ${ }^{1}$, R. Kurashvilii' ${ }^{1}$ L. Dzneladze ${ }^{2}$, M. Dundua ${ }^{1}$, E. Shelestova ${ }^{1}$, K. Paghava ${ }^{1}$, G. Kurashvili ${ }^{1}$, L. Tsutskiridze ${ }^{1}$; ${ }^{1}$ Georgian Diabetes Center,

${ }^{2}$ Clinical Pharmacology, National Center of Therapy, Tbilisi, Georgia.

Background and Aims: Asymptomatic coronary artery disease (CAD) and painless myocardial infarction (PMI) are common in patients (pts) with diabetes mellitus and are assumed as cardiac autonomic neuropathy (CAN) manifestation. Abnormalities of left ventricular repolarisation and heart rate variabilities (HRV) - markers of autonomic dysfunction, are associated with increased mortality in type 2 diabetic (T2DM) pts. The aim of the study was to assess CAN and QTc interval dispersion (QTcd) in T2DM pts with PMI in history.

Materials and Methods: We examined $67 \mathrm{~T} 2 \mathrm{DM}$ pts, who were divided into 3 groups (Gr): Cr.1 (22pts, mean age $56.7 \pm 7.3 \mathrm{yrs}, 17 \mathrm{~m} / 5 \mathrm{f}$.) who had PMI more than a month ago; Gr.2. (23pts, mean age $54.9 \pm 8.3 \mathrm{yrs}, 16 \mathrm{~m} / 7 \mathrm{f}$ ) with typical MI in hystory; Gr.3 (22pts, mean age $57.4 \pm 8.1 \mathrm{yrs}, 15 \mathrm{~m} / 7 \mathrm{f}$ ) without clinical signs of CAD (no changes on Holter and/or exercise ECG). Time domain HRV parameters - SDNN and triangular index (TI) were assessed on Holter 
ECG. QTcd was measured on surface ECG. CAN severity was evaluated using Ewing's five standard tests (severe CAN-scores 7-10, moderate-scores 4-6, mild-scores 2-3, no CAN -scores 0-1).

Results: HRV parameters in study groups were: SDNN $89 \pm 14.7 \mathrm{msec}$ (Gr. 1), $93 \pm 16.3 \mathrm{msec}$ (Gr. 2), and $114 \pm 15.6 \mathrm{msec}$ (Gr. 3); TI $21 \pm 7.3$ (Gr. 1), $22 \pm 5.1$ (Gr. 2), and $35 \pm 9.3$ (Gr. 3). Compared to Gr. 3, HRV parameters - SDNN and TI, were statistically lower in Gr. 1 and Gr. 2 ( $\mathrm{p}=0.000 ; \mathrm{p}=0.000$, respectively). No statistically evident difference in these parameters was observed between

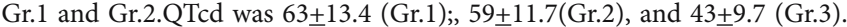
QTcd was higher in Gr.1 when compared to Gr.2, through this difference was not statistically evident; while difference in QTcd between Gr. 1/Gr. 2 and Gr. 3 was statistically significant $(\mathrm{p}=0.000)$. We revealed following CAN prevalence in the study groups: severe $-31.8 \%$ (Gr. 1), 8.7\% (Gr. 2), 0\% (Gr. 3); moderate $54.5 \%$ (Gr. 1), 52.2\% (Gr. 2), 45.5\% (Gr. 3); mild - 13.6\% (Gr. 1), $30.4 \%$ (Gr. 2), 36.3\% (Gr. 3), no CAN - 0\% (Gr. 1), 8.7\% (Gr. 2), 18.2\% (Gr. 3). Severe CAN was significantly higher in Gr. 1 when compared to Gr. 2 and 3; moderate CAN was also higher in Gr. 1; only $13.6 \%$ in this group had mild CAN, while CAN absence was not found in any of the pts with PMI.

Conclusion: There was no significant difference in the HRV parameters and QTcd between typical and PMI pts. While compared to Gr. 3 there was statistically evident prolongation of QTcd in Gr. 1 and 2, and decrease in HRV. It can be explained by the dysfunction of sympathetic nervous system. In addition, disorders of parasympathetic functional tests in pts with PMI may explain the high prevalence of severe and moderate CAN in this group. All this indicates to more profound damage of vegeto-neural system in T2DM pts with PMI.

\section{5}

Cardiac autonomic neuropathy (CAN) in T2 diabetics with or without chronic kidney disease: correlations with insulin resistance (IR), NT-proBNP and hsCRP

M. Mylonopoulou ${ }^{1}$, N. Tentolouris ${ }^{2}$, S. Antonopoulos ${ }^{3}$, S. Mikros ${ }^{3}$,

P. Anastasiades ${ }^{3}$, E. Konstantellou ${ }^{4}$, G. Papadakis ${ }^{1}$, A. Melidonis ${ }^{3}$,

N. Katsilambros

${ }^{1}$ Nephrology Dept, Tzanio General Hospital, Piraeus,

${ }^{2} 1$ st Dept of Propaedeutic Medicine, University Medical School, Athens,

${ }^{3}$ Diabetes Center, Tzanio General Hospital, Piraeus,

${ }^{4}$ Biochemistry Dept, General Hospital, Nikaia, Greece.

Background and Aims: CAN and IR are independent risk factors for cardiac mortality in type 2 Diabetes Mellitus (T2DM) and in Chronic Kidney Disease (CKD). Heart Rate Variability (HRV) is an established non-invasive evaluation method of CAN. N-terminal pro-B-type Natriuretic Peptide (NTproBNP) is a marker of congestive heart failure. Another biomarker for CVD is high-sensitivity $\mathrm{C}$ - reactive protein (hsCRP). Aim of this study was to assess CAN in T2DM patients with and without CKD, in comparison with non-diabetics with CKD and healthy controls. We measured NT-proBNP and hsCRP and we estimated IR by HOMA. We investigated their correlation and their relation with HRV parameters.

Materials and Methods: 100 patients divided into four age-matched groups (G): G1: 25 patients with T2DM and established stage 4 CKD, G2: 25 T2DM patients without CKD, G3: 25 non-diabetics with stage 4 CKD and G4: 25 healthy controls. CAN was assessed in all patients using 24-hours ECGHolter monitoring with HRV analysis in both time and frequency domain. Results: Marked differences in HRV were noticed between the four groups. G1 displayed significantly lower values in both time-domain maxRR, SD, SDANN, SDNN $(p<0,001)$, and frequency domain parameters Total Power $(\mathrm{p}<0,001), \operatorname{VLF}(\mathrm{p}<0,001), \operatorname{LF}(\mathrm{p}<0,001)$ and $\mathrm{LF} / \mathrm{HF}$ ratio $(\mathrm{p}=0,005)$ in comparison to the other 3 groups collectively. Independent comparison of G2 and G3 showed no differences. NT-proBNP and HOMA were significantly higher in $\mathrm{G} 1(\mathrm{p}<0,001$ and $\mathrm{p}=0,003)$ compared with the other 3 groups. There were no significant differences in NT-proBNP and HOMA between G2 and G3. hsCRP was significantly elevated in G1 compared with G2 $(p=0,02)$, but not with G3. Partial correlation analysis controlling for the four groups suggests significant correlations between NT-proBNP and hsCRP ( $\mathrm{p}=0,031)$ as well as between HOMA and hsCRP $(\mathrm{p}=0,018)$. Bivariate analysis within each group separately displayed significant correlations of HRV with HOMA or NT-proBNP, only in G3. In those patients, NT-proBNP was significantly correlated with time domain (PNN30 $p=0,023$, ASDNN $\mathrm{p}=0,013$ ) and frequency domain parameters (Total Power $\mathrm{p}=0,002$, LF $\mathrm{p}=0,001$, HF $\mathrm{p}=0,012)$, while HOMA was correlated with PNN50 $(\mathrm{p}=0,015)$ and VARINDEX ( $\mathrm{p}=0,013)$. HRV displayed no correlation with hsCRP in either group.

Conclusion: Uremic $\mathrm{T} 2$ diabetics present significantly important $\mathrm{CAN}$ as well as elevated CVD markers (NT-proBNP, hsCRP and HOMA) in comparison with all other groups. No such differences were observed between uremic non-diabetics and diabetics without $\mathrm{CKD}$, suggesting the synergistic role of T2DM and CKD to the development of CAN and CVD. Analysis of the four groups displays an overall correlation of hsCRP with HOMA and NT-proBNP. A significant correlation - predictive of CAN - of NT-proBNP and HOMA with time and frequency domain parameters of HRV existed only in nondiabetics with CKD. All correlations of these markers with HRV are vanished in the presence of T2DM in G1 and G2. We hypothesize that diabetes plays such a major disruptive role in CAN, irrespective of all other co-morbidities, that deprives any biochemical marker of its possible predictive value in this context.

\section{6}

Is the aminoterminal pro-brain natriuretic peptide (NT-proBNP) a marker for diabetic polyneuropathy?

J. M. Pou ${ }^{1}$, J. Jurado 2 , E. Escudero ${ }^{3}$, J. Merce ${ }^{3}$, M. Ferrandiz ${ }^{4}$, L. Comerma ${ }^{2}$, J. Ybarra

${ }^{1}$ Dept. Endocrinology and Nutrition, Autonomous University of Barcelona, Hospital de la Sta. Creu i S. Pau, ${ }^{2}$ Primary Care Center, Catalan Health Institute, Olot, Girona, ${ }^{3} \mathrm{Dpt}$. Biochemistry, Autonomous University of Barcelona, Hospital de la Sta. Creu i San Pau, ${ }^{4}$ Dept. Neurophysiology, Catalan Health Institute, Hospital Josep Trueta, Spain,

${ }^{5}$ Dept. Endocrinology, ICAMED, Centro Medico Teknon, Spain.

Background and Aims: The plasma levels of the N-terminal fragment of the brain natriuretic peptide (NT-proBNP), have recently gained extreme importance as marker of myocardial dysfunction in type 2 diabetes mellitus (T2DM) patients. Interestingly, T2DM patients with cardio-vascular disease (CVD) and/or nephropathy exhibit an elevation of plasma NT-proBNP levels related to those without CVD. NT-proBNP levels are independently associated with coronary artery disease and overt nephropathy. Scarce data is available regarding the association between diabetic polyneuropathy (DPN) and NT-proBNP levels.

Materials and Methods: Hence, we set forth a cross-sectional pilot study, to assess the hypothetical relationships between NT-proBNP levels, CVD, and DPN in a group of T2DM patients $(\mathrm{N}=140 ; \mathrm{M} 89 / \mathrm{F} 51)$ randomly selected from the North Catalonia Diabetes Study (NCDS). CVD and DPN (clinical and neurophysiology evaluation) prevalence were evaluated. Serum NTproBNP was measured using an electrochemiluminescence immunoassay (Elecsys ${ }^{\circ}$, Roche Diagnostics, Indianapolis, Inc.).

Results: Diabetic patients age: $63.5 \pm 7.6$, T2DM duration: $10.2 \pm 1.2$ years, HbA1c: 7.1 \pm 1.6 ; DPN: 47\%. Log-transformed NT-proBNP levels (logNTproBNP) were significanlly higher in T2DM patients with CVD $(P<0.0005)$ and DPN $(P<0.0005)$. LogNT-proBNP correlated with age $(\mathrm{r}=0.369$; $P<0.0005)$, CVD $(\mathrm{r}=0.377 ; P<0.0005)$ and DPN (r: 0.442; $P<0.0005)$-even after correction for age and CVD $(\mathrm{r}=0.334 ; P=0.001)$-. Log NT-proBNP was also correlated with neurothesiometer values $(\mathrm{r}=0.406: P<0.0005)$. No significant associations were found for gender, hypertension prevalence and micro-albuminuria

Conclusion: NT-proBNP levels display a significant association with the presence of DPN, even after correction for age and CVD.

NT-proBNP levels appear as promising molecules for T2DM micro and macro-angiopathic complications screening. Further prospective trials are warranted.

Supported by Fondo de Investigación Sanitaria: FIS 04/0181 and Spanish Network CO3/08: Instituto Carlos III 


\section{7}

Relationship between erectile dysfunction and cardiovascular risk factors in patients with type 2 diabetes in primary care is confounded by age and medication

M. Van den Donk, F. G. W. Cleveringa, M. G. G. Meulenberg,

K. J. Gorter, G. E. H. Rutten;

Julius Center for Health Sciences and General Practice,

University Medical Center Utrecht, The Netherlands.

Background and Aims: Erectile dysfunction (ED) is about 3 times more common in male patients with diabetes than in those without diabetes; its prevalence ranges between $34-89 \%$. It is associated with both diabetes and according to most authors also with cardiovascular disease. ED prevalence is mostly based on questionnaires, which are not feasible in daily diabetes care. We measured ED prevalence when patients were asked a single question by a practice nurse during a routine diabetes control visit. Besides we assessed if routinely asking patients with diabetes mellitus type 2 (DM2) about ED identifies patients at high risk for cardiovascular disease. The aims of the study are to asses the prevalence of ED using a single question asked by a practice nurse and to asses the association between ED and cardiovascular risk factors in men with DM2 in primary care.

Materials and Methods: In a cross-sectional study, 55 general practices throughout The Netherlands participated. All patients with DM2 treated by their general practitioner were invited. During the annual check-up, the practice nurse asked male patients after an introducing sentence whether they suffered from erection problems or not. Age, duration of diabetes, ethnicity, history of cardiovascular disease (HCVD), current smoking, alcohol consumption, medication use, $\mathrm{HbAlc}$, blood pressure, total cholesterol, HDL-cholesterol and BMI were also registered. The ED prevalence rate was calculated. Through univariate analysis (odds ratios (OR) and Student's Ttests), the association between the above-mentioned variables, ED and a HCVD was explored, to assess which variables were potential confounders. The association between ED and a HCVD was then corrected for confounders, in multiple logistic regression analysis. For patients without a HCVD we followed the same procedure for ED and 10 year UKPDS coronary heart disease risk estimate (ORs, Mann-Whitney tests, and multiple linear regression)

Results: The prevalence of ED in DM2 patients was $41.3 \%$, increasing with age. After correction for confounders, there was no association between ED and a history of cardiovascular disease, OR: 0.9 (95\% CI: $0.7-1.1)$, or a higher 10 year UKPDS CHD risk estimate, risk difference: 0.8\% (95\% CI: -1.3 - 2.9). For both associations age and medication were the most important confounders.

Conclusion: Measuring the prevalence of ED with a single question for $\mathrm{ED}$ asked by a practice nurse during diabetes consultation, gives an ED prevalence comparable to standardised questionnaire prevalence rates. After adjusting for confounders, the relationship between ED and cardiovascular disease could not be established. The high prevalence of ED in DM2 may be explained by a cluster of variables, among which age and medication use. Supported by Pfizer b.v.

\section{8}

Autonomic neuropathy and hypertension in type 1 and type 2 diabetes mellitus: is there a relationship?

K. Keresztes, I. Istenes, É. Sipos, Z. Putz, P. Vargha, P. Kempler;

1st Department of Medicine, Semmelweis University, Budapest, Hungary.

Background and Aims: Cardiovascular autonomic neuropathy carries a fivefold risk for mortality in diabetic patients, but the factors leading to increased mortality have not been clarified entirely up to now. The aim of our study was to evaluate a possible connection between autonomic neuropathy (AN) and 24-hour blood pressure profile in patients with type 1 and type 2 diabetes mellitus.

Materials and Methods: The five standard cardiovascular reflex tests were used to assess the autonomic function in 102 diabetic patients who had no history of hypertension. We examined 32 patients with type 1 diabetes (mean age: $37.8 \pm 12.7$ years), 70 normoalbuminuric patients with type 2 diabetes (mean age: $52.3 \pm 7.6$ years) and 29 healthy control subjects (mean age: $51.8 \pm 9.5$ years). The severity of autonomic neuropathy was characterised by autonomic score. Ambulatory 24-hour blood pressure monitoring (ABPM) was performed by MEDITECH ABPM 04 device. Among the ABPM parameters we evaluated the blood pressure load (\%-time when day-time blood pressure was above $140 / 90 \mathrm{mmHg}$ and night-time blood pressure was above $120 / 80 \mathrm{mmHg}$ ) and the hyperbaric impact values (the area under the blood pressure curve exceeding 140/90 $\mathrm{mmHg}$ during day-time and 120/80 mmHg during night-time).

Results: There was a significant correlation between the severity of autonomic neuropathy and the blood pressure load (systolic: $p<0.001$; diastolic: $\mathrm{p}<0.01$ ), the hyperbaric impact values (systolic: $p<0.01$; diastolic: $p<0.05$ ) and microalbuminuria $(\mathrm{p}<0.001)$ in type 1 diabetic patients. Autonomic neuropathy as well as microalbuminuria were independently associated with hypertension among type 1 diabetic patients. Among patients with type 2 diabetes, the severity of AN correlated positively with the 24-hour mean blood pressure, the blood pressure load (systolic: both $\mathrm{p}<0.0001$; diastolic: both $\mathrm{p}<0.01)$, just as with systolic $(\mathrm{p}<0.001)$ and diastolic $(\mathrm{p}<0.05)$ hyperbaric impact values. Analyzing the relationship between ABPM parameters and the five cardiovascular reflex tests separately, in type 1 diabetic patients a significant negative correlation was found with two tests only - 30/15 ratio and Valsalva ratio - both tests reflect mainly parasympathetic function. Among patients with type 2 diabetes, also two predominantly parasympathetic tests - the heart rate variation during deep breathing and the Valsalva ratio - were inversely related to the ABPM parameters. The Valsalva ratio correlated negatively with the 24-hour systolic blood pressure standard deviation $(\mathrm{p}<0.01)$. These relationships remained significant after adjustment for age, sex, body mass index and smoking.

Conclusion: Autonomic neuropathy is independently associated with elevated 24-hour ABPM parameters in patients with both type 1 and type 2 diabetes. Our data suggest that a relative sympathetic overactivity due to predominantly parasympathetic neuropathy might be responsible for the higher ABPM indices and increased blood pressure variability also in type 1 and type 2 diabetic patients. ABPM is suggested to be performed for the early assessment of hypertension in diabetic patients with autonomic neuropathy and vice versa: autonomic function should be screened as part of the cardiovascular risk assessment in diabetic patients with hypertension. Higher 24-hour-long blood pressure values just as increased blood pressure variability may contribute to the poor prognosis of cardiovascular autonomic neuropathy in diabetic patients.

\section{9}

Early signs of autonomic dysfunction in patients with uncomplicated type 1 diabetes: blunted baroreflex sensitivity M. Rosengård-Bärlund ${ }^{1,2}$, J. Fagerudd $^{1,2}$, M. Mäntysaari ${ }^{3}$, C.-G. af Björkesten ${ }^{1,2}$, H. Lindholm ${ }^{4}$, C. Forsblom ${ }^{1,2}$, L. Thorn ${ }^{1,2}$, J. Wadén ${ }^{1,2}$, M. Saraheimo ${ }^{1,2}$, P.-H. Groop ${ }^{1,2}$;

${ }^{1}$ Folkhälsan Institute of Genetics, Folkhälsan Research Center, Helsinki University, ${ }^{2}$ Div. of Nephrology, Dept. of Medicine, HUCH, Helsinki, ${ }^{3}$ Aeromedical Centre (AMC), The Finnish Defence Forces, Helsinki, ${ }^{4}$ Department of Health and Work Ability, Finnish Institute of Occupational Health, Helsinki, Finland.

Background and Aims: Cardiac autonomic neuropathy (CAN) is a serious long-term complication of type 1 diabetes (T1D), associated with increased morbidity. Numerous studies have confirmed an association between CAN and other diabetic complications, but their temporal relationship is still unclear. Recent advances have enabled identification of early stages of CAN using spectral analysis of heart rate variability (HRV) and blood pressure variability (BPV), and assessment of spontaneous baroreflex sensitivity (BRS). Although autonomic dysfunction seems to be an early phenomenon on the path leading to diabetic micro- and macrovascular complications, the data supporting this view is limited. Thus, our aim was to study if signs of autonomic dysfunction are detectable at an early stage in a homogeneous population-based sample of uncomplicated T1D patients with short duration.

Materials and Methods: The IDEAL Study (IDentification of EArly mechanisms in the pathogenesis of diabetic Late complications), launched in 2003, is a population-based, observational follow-up study with the aim to identify early markers of late diabetic complications. At baseline we studied 116 young (age $26.3 \pm 5.7 \mathrm{yrs}) \mathrm{T} 1 \mathrm{D}$ patients $(62 \mathrm{males})$ with short duration ( $8.9 \pm 1.6 \mathrm{yrs})$, and 36 age- and sex-matched healthy controls. $24 \mathrm{~h}$ ambulatory blood pressure measurement and conventional autonomic function tests were performed. Spectral analysis of HRV and BPV were obtained from a 10-minute supine recording of ECG and non-invasive, continuous blood 
pressure. Spontaneous BRS was estimated from the same recording by sequence method (BRS+/+ and BRS-/-), alfa coefficient (alfa LF and alfa HF) and transfer function (BRSpc). Data are cross-sectional.

Results: Patients with T1D had higher office SBP $(132 \pm 13$ vs. $122 \pm 13$, $\mathrm{p}<0.001)$ and S-LDL cholesterol $(2.4 \pm 0.8$ vs. $2.0 \pm 0.7, \mathrm{p}=0.040)$, than the control subjects. There was no difference in urinary albumin excretion rate, but patients with T1D had higher estimated GFR ( $\mathrm{p}=0.01)$. $24 \mathrm{~h}$ blood pressure was higher in the T1D group (24h SBP $123 \pm 7$ vs. $119 \pm 8, \mathrm{p}<0.010$; $24 \mathrm{~h}$ DBP $73 \pm 6$ vs. $70 \pm 6, \mathrm{p}<0.007)$. Heart rate was consistently higher in the patients ( $24 \mathrm{~h}$ HR $73 \pm 10$ vs. $69 \pm 8, \mathrm{p}=0.012$ ). The proportion of non-dippers was equal in both groups. All BRS estimates except BRS-/- were lower in T1D patients as shown in the figure.

Conclusion: In uncomplicated T1D with short duration there are already early signs of autonomic dysfunction. Collectively, these observations indicate sympathetic predominance that may predispose to future cardiovascular complications.

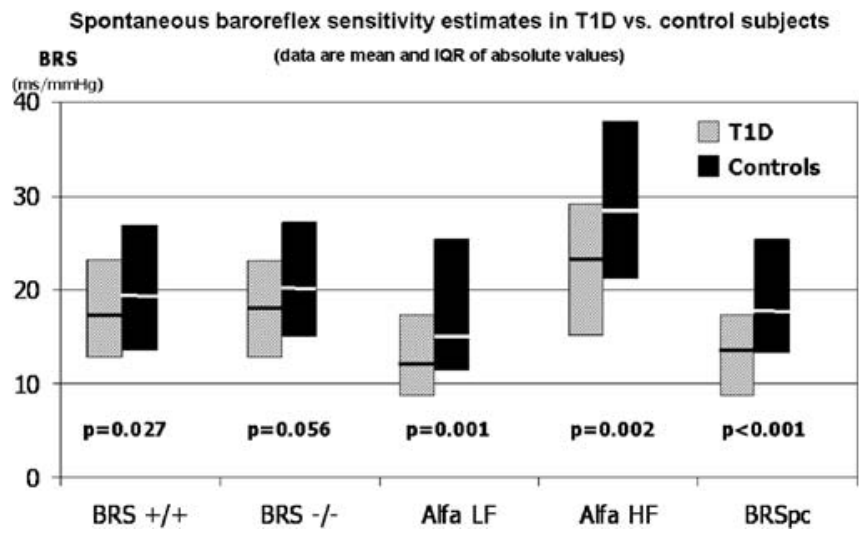

1100

Circadian blood pressure variability: cardiovascular autonomic dysfunction and cardiovascular risk as influencial factors in diabetes mellitus

J. Cabezas-Cerrato ${ }^{1,2}$, R. C. Hermida ${ }^{3,4}$, D. E. Ayala ${ }^{5}$,

J. M. Cabezas-Agricola ${ }^{2}$, C. Calvo

${ }^{1}$ Medicine, University Hospital of Santiago, Santiago de Compostela,

${ }^{2}$ Medicine (Servicio de Endocrinologia y Nutricion), University Hospital of Santiago, Santiago de Compostela, ${ }^{3}$ Bioengineering, University of Vigo, Santiago de Compostela, ${ }^{4}$ Unidad de Hipertension, University Hospital of Santiago, Santiago de Compostela, ${ }^{5}$ Bioengineering, University Hospital of Santiago, Santiago de Compostela, ${ }^{6}$ Medicine (Unidad de Hipertension), University Hospital of Santiago, Santiago de Compostela,

Background and Aims: The relationship between diabetes mellitus (DM) and circadian blood pressure variability (CBPV) is yet controversial. This research was designed to study the influential factors in CBPV in diabetes. We report the first 61 consecutive diabetic patients (6!\% type $2 \mathrm{DM})$ studied for two of this possible factors: cardiovascular autonomic function (CAF) and cardiovascular risk (CVR) estimated for 10-year.

Materials and Methods: BP was measured at 20-min intervals from 0.7:00 to $23: 00 \mathrm{~h}$ and at 30 -min intervals at night for $48 \mathrm{~h}$. Physical activity was simultaneously monitored every minute by wrist actigraphy to accurately calculate the diurnal and nocturnal means of $\mathrm{BP}$ on a pear subject basis. CAF was evaluated for the three main standard tests: HRDB, VM and Standing-up by a computer aided system. Subjects were classified according to the number of abnormal parameters into two groups: with no-abnormal autonomic parameters (GAP, 0$)$ and with 1 or more abnormal autonomic parameter (GAP, 1). CVR for coronary heart disease (CHD) and Stroke for 10-year was stimated by the UKPDS Risk Enginee v.2.

Results: 33 (54\%) diabetic patients were non-dippers (rate of $46 \%$ of 24 type 1 , and $59 \%$ of 37 type 2). Pulse pressure in $\mathrm{mmHg}(\mathrm{PP})$ was higher in GAP, 1 than in GAP, 0 both during the day $(55.6+/-1,5$ vs. $42.9+/-2.2, \mathrm{p}<0.001)$ and during the night $(53+/-1.5$ vs. $45+/-1.4, \mathrm{p}<0.001)$. When patients were divided according to their $\mathrm{CVB}$ for $\mathrm{CHD}$ whether $<$ or $>10 \%$, the mean value of the $24 \mathrm{~h}$ data-ajusted SBP curve (mesor) was $6 \mathrm{mmHg}$ higher $(\mathrm{p}<0.001)$ in the group of CVR $>10 \%$. When patients were divided according the CVR for
Stroke, whether $<$ or $>4 \%$, the messor was $8.4 \mathrm{mmHg}(\mathrm{p}<0.001)$ higher in the latter group toguether with an unexplained DBP mesor of $7 \mathrm{mmHg}$ lower. Conclusion: 1) More than half of diabetic patients were non-dippers (a 13\% higher in type 2). 2) The PP was statistically and clinically significantly higher in diabetic patients with cardiac autonomic dysfunction. 3) This higher value seems related to a higher $\mathrm{CHD}$ risk at 10 -year.

Supported by Vicerrectorado de Investigación USC $y$ UV, y Hospital Universitario de Santiago 


\section{PS 110 Complications: role of glycation}

\section{1}

\section{Possible involvement of AGE and MMP-2 in podocyte detachment and loss}

K. Fukami ${ }^{1}$, Y. Takamiya ${ }^{1}$, S. Ueda ${ }^{1}$, S.-I. Yamagishi ${ }^{2}$, T. Fujimura ${ }^{1}$, A. Hayashida ${ }^{1}$, K. Koike ${ }^{1}$, Y. Matsumoto ${ }^{1}$, M. Takeuchi ${ }^{3}$, S. Okuda ${ }^{1}$; ${ }^{1}$ Department of Medicine, Division of Nephrology, Kurume University School of Medicine, Fukuoka, ${ }^{2}$ Department of Internal Medicine, Division of Cardiovascular Medicine, Kurume University School of Medicine, Fukuoka, ${ }^{3}$ Department of Biochemistry, Hokuriku University, Kanazawa, Japan.

Background and Aims: Podocyte effacement is considered to be an important phenomenon for the progression of proteinuria in diabetic nephropathy (DN). Recently, loss of slit membrane protein, nephrin is involved in the detachment of podocytes from glomerular basement membrane (GBM) in DN. Metalloproteinases-2 (MMP-2) is decreased in glomeruli in advanced diabetic kidneys, and thereby, plays a central role in the pathogenesis of diabetic glomerulosclerosis. However, the role of MMP-2 in podocyte detachments in early diabetic nephropathy remains to be elucidated. Further, there is a growing body of evidence that AGE could participate in the progressive alteration in renal architecture and loss of renal function in diabetes as well. Therefore, we first examined here whether MMP-2 expression and activity were increased in early phase of diabetic nephropathy in rats. We next studied the pathological involvement of AGE and MMP-2 on podocyte detachment and loss in vitro.

Materials and Methods: Diabetes was induced by streptozotosin $(50 \mathrm{mg} /$ $\mathrm{kg}$ ) in Sprague Dawley (SD) rats. After 8 or 16 weeks, MMP-2 expression and activity were measured by immunohistochemistry and zymography, respectively. Nephrin gene expression was evaluated by real-time RT-PCR. AGE were prepared by incubating BSA with glyceraldehyde for 7 days. DNA flagmentation and caspase-3 levels in murine immortalized podocytes were examined by commet assay and western blots, respectively.

Results: Plasma level of glucose, $\mathrm{HbAlc}$ and kidney to total body weight ratio were increased by about 2-3-folds in 8- and 16-week diabetic rats, compared with non-diabetic control rats (plasma glucose; $589+/-38 \mathrm{mg} / \mathrm{dl}$, HbAlc; $8.8+/-0.3$ in 16 -week diabetic rats). MMP-2 expression and activity were increased to about 4-5-folds in the cortex of whole kidney, isolated glomeruli and the urine in 8 - and 16 -week diabetic animals $(\mathrm{p}<0.05)$. Compared with control rats, albumin excretion rate was increased by 4 -folds in 16-week diabetic rats, while nephrin gene expression was decreased to about $50 \%$ $(\mathrm{p}<0.01)$. In vitro, $100 \mu \mathrm{g} / \mathrm{ml}$ AGE significantly increased MMP- 2 and caspase3 expression to about 1.5-2-folds and subsequently induced apoptotic cell death in cultured immortalized podocytes $(\mathrm{p}<0.05)$. Human recombinant MMP-2 at $1 \mathrm{nM}$ and $100 \mu \mathrm{g} / \mathrm{ml}$ AGE were also found to cause podocyte detachment from basement membrane produced by endothelial cells.

Conclusion: The present study demonstrated for the first time that MMP-2 expression and activity are increased rather than decreased in early phase of experimental diabetic nephropathy. AGE may play a role in podocyte detachment and loss via MMP-2 activation in vitro.

\section{2}

Regulation of VEGF by pentosidine and glycated serum

G. L. Viviani, A. Puddu, A. Durante, D. Storace, R. Sanguineti, P. Odetti; DIMI, Universita’ di Genova, Italy.

Background and Aims: Proliferative retinopathy, one of the major long term complications of diabetes, is characterized by increased neovascularization in the retina. Relative hypoxia in retina, resulting in the induction of vascular endothelial growth factors (VEGFs) and other vascular mediators that stimulate proliferation of retinal endothelial cells, has been suggested as one of multiple mechanisms. VEGF-A is costitutively produced by retinal pigmented epithelium (RPE) cells. Exposure to high glucose and advanced glycation end-products (AGEs), a common situation found in diabetes, increases VEGF A secretion. Up-regulation of VEGF A gene expression is mediated by the transcription factor HIF-1. Moreover VEGF A has been reported to increase secretion of VEGF C, another angiogenic factor, and to act sinergistically with it to promote neovascolarization. The aim of this study was to investigate whether pentosidine (PENT), a well characterized AGE, and glycated serum (GS) are able to modulate the secretion of VEGF-A and VEGF-C in the human RPE cells ARPE-19.

Materials and Methods: PENT was synthesized from N-alpha-t-Boc-lysine, $\mathrm{N}$-alpha-t-Boc-arginine and D-ribose, and purified by HPLC. GS was obtained by incubation of FBS with $50 \mathrm{~mol} / \mathrm{l}$ ribose at $37^{\circ} \mathrm{C}$ for 7 days. ARPE19 cells were incubated in DMEM/F12 medium supplemented with $10 \% \mathrm{FBS}$, containing $0.25 \mathrm{pM}$ of PENT, a comparable concentration to plasma levels in diabetes, or in DMEM/F12 1:1 medium supplemented with 10\% GS. A set of cells was lysed after $4 \mathrm{~h}$ for evaluation of HIF- $1 \alpha$ expression by immunoblot. Another set was cultured for $24 \mathrm{~h}$ and then surnatants were collected for VEGF analysis. Levels of secreted VEGF-A and VEGF-C were evaluated by ELISA and normalized to protein content of the corresponding lysate.

Results: GS, but not pentosidine, was able to increase HIF- $1 \alpha$ expression and VEGF-A secretion (respectively $212.6 \pm 15.83 \mathrm{p}<0.05 ; 157.2 \pm 10.01 \mathrm{p}<0.05$ ). VEGF-C secretion was increased in both conditions, but the increment was more marked in presence of GS (PENT $155.7 \pm 5.364 \mathrm{p}<0.001$; GS: $220.4 \pm$ $3.037 \mathrm{p}<0.0001$ ).

Conclusion: Our results demonstrate that: a) PENT, independently from VEGF-A and HIF-1 $\alpha$, is able to increase VEGF-C in ARPE-19 cells; b) increment of VEGF-A by GS is associated to HIF- $1 \alpha$ increase; c) VEGF-A, elevated in GS condition, further increases VEGF-C secretion. Therefore, both GS and PENT, by increasing VEGF secretion, may play a detrimental role in stimulating angiogenesis.

\section{3}

Interaction between the receptor for advanced glycation end products (RAGE) and estrone (E1) in the development of type 2 diabetic nephropathy

B. E. Harcourt ${ }^{1}$, A. Mibus ${ }^{1}$, A. Gasser ${ }^{1}$, S. Andikopoulos ${ }^{2}$, M. Thomas ${ }^{1}$, M. T. Coughlan ${ }^{1}$, K. Sourris ${ }^{1}$, G. Rice ${ }^{3}$, A. Bierhaus ${ }^{4}$, M. E. Cooper ${ }^{1}$, J. M. Forbes ${ }^{1}$;

${ }^{1}$ Diabetes Complications, Baker Heart Research Institute, Melbourne, Australia, ${ }^{2}$ Department of Medicine, University of Melbourne, Australia, ${ }^{3}$ Proteomics, Baker Heart Research Institute, Melbourne, Australia, ${ }^{4}$ Department of Medicine, University of Heidelberg, Germany.

Background and Aims: Some 25-40\% of persons with Type 2 diabetes, of which obesity is a major risk factor, will develop nephropathy. We hypothesize that estrone (E1) secreted from white adipose tissue (WAT), worsens the progression of nephropathy via up-regulation of the receptor for advanced glycation end products (RAGE) through the estrogen receptor $\beta$ (ER $\beta)$.

Materials and Methods: Healthy RAGE deficient (RAGE-/-) and C57Bl/6 (WT, wild type) mice $(\mathrm{N}=10)$, fed diets either low in AGEs (LAGE) or High in AGEs and saturated fat (HFF) were studied for 16 weeks. A separate WT group was given the HFF diet in conjunction with the AGE cross-link breaker alagebrium (ALA, $1 \mathrm{mg} / \mathrm{kg} /$ day).

Results:Healthy RAGE deficient (RAGE-/-) and C57Bl/6 (WT, wild type) mice $(\mathrm{N}=10)$, fed diets either low in AGEs (LAGE) or High in AGEs and saturated fat (HFF) were studied for 16 weeks. A separate WT group was given the HFF diet in conjunction with the AGE cross-link breaker alagebrium (ALA, $1 \mathrm{mg} / \mathrm{kg} /$ day). WT mice fed a HFF diet had significantly increased WAT at the peri-renal and visceral fat depots when compared to LAGE fed WT mice (Peri-renal; $73 \pm 27$ vs $179 \pm 28 \mathrm{mg}, \mathrm{p}<0.0001$ ). Although visceral WAT accumulation was increased in both HFF fed RAGE-/- mice (743 $\pm 114 \mathrm{mg})$ and WT mice $(698 \pm 175 \mathrm{mg})$, there was no increase in peri-renal fat seen in HFF fed RAGE-/- mice $(91 \pm 40 \mathrm{mg})$ as compared to HFF WT mice (179 \pm 28 , $\mathrm{p}<0.01$ vs LAGE WT; $\mathrm{p}<0.01$ vs HFF RAGE-/-). Visceral fat accumulation was however decreased in ALA treated HFF fed mice $(462 \pm 107 \mathrm{mg}, \mathrm{p}<0.05$ vs HFF WT). The visceral WAT gene expression of ER $\beta$ was lower in all RAGE/- mice when compared to WT LAGE mice (RAGE -/- $\mathrm{p}<0.01$; RAGE-/HFF $p<0.05)$. HFF WT had increased visceral WAT gene expression of ER $\beta$ $(\mathrm{p}<0.05$ vs WT LAGE), which as ameliorated with ALA therapy ( $\mathrm{p}<0.01$ vs HFF WT mice).

Conclusion: This data suggests a link between estrone and RAGE in renal disease in HFF mice.

Supported by Juvenile Diabetes Research Foundation and National Health and Medical Research Council of Australia 


\section{4}

Fibrin gel porosity relates to glycosylated haemoglobin in patients with type 1 diabetes mellitus

A. M. Antovic, N. H. Wallen, A.-C. Salomonsson, P.-E. Lins, U. Adamson, G. Jörneskog;

Dept of Clinical Sciences, Danderyd Hospital, Karolinska Institute, Stockholm, Sweden.

Background and Aims: We have previously demonstrated tight and less permeable fibrin clot structure in patients with type 1 diabetes, which may contribute to increased atherothrombotic risk in these patients. However, the mechanisms behind these alterations are unclear and therefore the present study was aimed to investigate the relation between the fibrin clot structure and glycaemic control in patients with type 1 diabetes.

Materials and Methods: Fifty-five patients (age: $38 \pm 11$; diabetes duration $17 \pm 12$ years) and 15 controls (age: $40 \pm 8$ ) were investigated. Fibrin clot properties were analysed in recalcified plasma samples after addition of thrombin in vitro, using liquid permeation technique, and permeability coefficient $(\mathrm{Ks})$ and fiber mass-length ratio $(\gamma)$ were calculated.

Results: Glycaemic control, measured by glycosylated haemoglobin level $\left(\mathrm{HbA}_{1 \mathrm{c}} \%\right)$ was significantly elevated in patients compared to healthy control subjects $(\mathrm{p}<0.001)$. Ks was decreased in diabetic patients $\left(7.4 \pm 2.4 \mathrm{~cm}^{2} ; \mathrm{p}<0.01\right)$ as compared to healthy subjects $\left(10.0 \pm 3.4 \mathrm{~cm}^{2}\right)$ while $\gamma$ was not significantly altered. Plasma fibrinogen levels in patients were similar to those in healthy controls. An inverse correlation was found between permeability coefficient (Ks) and $\mathrm{HbA}_{1 \mathrm{c}}(\mathrm{r}=0.32 ; \mathrm{p}=0.006)$, whereas no correlation was found between fiber mass-length ratio $(\gamma)$ and $\mathrm{HbA}_{1 c}$.

Conclusion: Fibrin clots created in plasma samples from patients with diabetes type 1 are tighter, less porous than those from healthy individuals and these unfavourable changes in fibrin clot structure are significantly related to the glycaemic control of the investigated subjects. Despite normal plasma fibrinogen levels, the patients with poor glycaemic control (higher $\mathrm{HbA}_{1 \mathrm{c}}$ levels) have less porous fibrin network structure (lover Ks values), indicating that the changes in fibrin network permeability may be the result of the modified fibrinogen clotting property involving glycosylation of the fibrinogen molecule.

Supported by David \& Astrid Hagelens Stiftelse

\section{5}

Pigment epithelium-derived factor protects against AGE- or diabetesinduced retinal derangement through its anti-oxidative properties expression

Y. Yoshida ${ }^{1,2}$, S.-I. Yamagishi ${ }^{1}$, T. Matsui ${ }^{1}$, R. Yamakawa ${ }^{2}$, T. Imaizumi ${ }^{1}$; ${ }^{1}$ Department of Internal Medicine, ${ }^{2}$ Department of Ophthalmology, Kurume University School of Medicine, Japan.

Background and Aims: Pigment epithelium-derived factor (PEDF) is the most potent inhibitor of angiogenesis, suggesting that loss of PEDF contributes to proliferative diabetic retinopathy. However, the role of PEDF against retinal leukostasis and vascular hyperpermeability, the characteristic features of early diabetic retinopathy, remains to be elucidated. Since AGE, the senescent macroprotein derivatives formed at an accelerated rate under diabetes, are involved in the pathogenesis of diabetic retinopathy, we investigated here whether and how PEDF could inhibit the AGE- or diabetesinduced retinal leukostasis and vascular hyperpermeability in rats.

Materials and Methods: AGE were prepared by incubating BSA with Dglyceraldehyde for 7 days. Adherent leukocytes to retinal vasculatures were imaged with FITC-coupled concanavalin A lectin. Gene expression was analyzed by RT-PCR. Retinal vascular permeability was evaluated by leakage of FITC-conjugated dextran from retinal vasculature.

Results: Retinal levels of an oxidative stress marker, 8-hydroxydeoxyguanosine (8-OHdG), ICAM-1 gene expression and leukostasis were increased to about 1.5 -folds in streptozotocin-induced diabetic rats $(\mathrm{p}<0.01)$. Intravenous administration of PEDF or pyridoxal phosphate, an AGE inhibitor, completely inhibited the increase in 8-OHdG levels and subsequently suppressed ICAM-1 gene expression and retinal leukostasis in diabetic rats $(\mathrm{p}<0.01)$. The amplitudes of $a$ - and $b$-waves in the electroretinogram, one of the most sensitive markers for early diabetic retinopathy, were significantly reduced to about $70 \%$ of control rats, which were completely prevented by the treatment of PEDF. Further, intravenous injection of $1 \mathrm{mg}$ AGE to normal rats increased retinal levels of 8-OHdG, gene expression levels of p22phox and gp91phox, two membrane components of NADPH oxidase $(\mathrm{p}<0.05)$ and ICAM-1, retinal leukostasis and vascular permeability to about 1.5 -folds $(\mathrm{p}<0.01)$, all of which were also completely blocked by simultaneous treatment of PEDF $(\mathrm{p}<0.05)$. In addition, AGE injection to normal rats decreased retinal PEDF levels to about $70 \%$ of those in control rats.

Conclusion: These results suggest that PEDF may protect against early diabetic retinopathy by suppressing the deleterious signaling of AGE via its anti-oxidative properties. Substitution of PEDF may offer a promising strategy for halting the development of early diabetic retinopathy. Supported by grants of Venture Companies Project from the Education, Culture, Sports, Science and Technology, Japan

\section{6}

Hypertension increases pro-oxidant generation and decreases antioxidant defense in the kidney in the early stage of experimental diabetes

S. K. Biswas, E. B. M. Peixoto, D. S. Souza, J. B. Lopes de Faria; Division of Nephrology, Faculty of Medical Sciences, State University of Campinas, Brazil.

Background and Aims: Oxidative stress contributes to the pathogenesis of nephropathy resulting from both diabetes mellitus and hypertension. Diabetes and hypertension frequently coexist in humans, and individually both of them can induce oxidative stress in the kidney. Previously, we demonstrated that the combination of hypertension and diabetes exaggerates renal oxidative stress. The aim of the present study was to assess pro-oxidant and antioxidant mechanisms for the induction of oxidative stress in the kidney in presence of hypertension and diabetes mellitus.

Materials and Methods: Diabetes was induced in spontaneously hypertensive rats (SHR) and their genetically normotensive control Wistar Kyoto (WKY) rats by streptozotocin at 12 weeks of age. After 10 days, rats were sacrificed and kidneys were collected. Renal cortical nicotinamide adenine dinucletide phosphate (NADPH) oxidase activity was assessed by lucigenin-enhanced chemiluminescence, and the expressions of gp91phox, Nox4, nitrotyrosine, extracellular-superoxide dismutase (EC-SOD), heme-oxygenase-2 (HO-2) and receptor for advanced glycation end products (RAGE) were evaluated by Western blot analysis. Renal cortical 8-hydroxy-2'-deoxyguanosine (8$\mathrm{OHdG}$ ) was assessed by immunohistochemistry, and the reduced form of glutathione (GSH) was measured by colorimetry.

Results: Systolic blood pressure was higher $(\mathrm{p}<0.001, \mathrm{n}=15)$ in control $(\mathrm{C})$ and diabetic (D) SHR than the WKY groups. Plasma glucose levels were similar in WKY-D and SHR-D groups. NADPH-dependent superoxide generation in the renal cortex was significantly elevated in WKY-D and SHR-D groups compared to the respective control groups $(\mathrm{p}<0.01, \mathrm{n}=5)$. Superoxide generation was further elevated in SHR-D group compared to WKY-D group ( $\mathrm{p}=0.024)$. The expression of gp91phox subunit of NADPH oxidase was significantly elevated in SHR-D group $(p<0.05)$, but not in WKY$\mathrm{D}$ group, compared to the respective control groups. However, RAGE and Nox4 levels were found similar between control and diabetic groups of both WKY and SHR strains. Renal cortical EC-SOD level was found markedly decreased in SHR compared to WKY groups. However, EC-SOD level was non-significantly increased in WKY-D group and decreased in SHR-D group compared to respective control groups. Non-enzymatic antioxidant GSH level was slightly increased in WKY-D than WKY-C, but it was significantly decreased $(\mathrm{p}=0.033, \mathrm{n}=10)$ in SHR-D than SHR-C. However, antioxidant HO2 levels did not differ among the groups. Finally, nitrotyrosine and 8-OHdG, markers of oxidative stress, were found similar in the kidney in WKY-C and WKY-D, but they were found elevated in SHR-D compared to SHR-C.

Conclusion: Pro-oxidant generation increases in the kidney in the early stage of diabetes, which further increases in presence of hypertension. On the other hand, antioxidant system remains unaltered in early diabetes, but the presence of hypertension depletes antioxidant protection in the kidney in early diabetes. Consequently, oxidative stress appears in the kidney in early diabetes in hypertensive animals but not in normotensive animals. We therefore conclude that hypertension increases pro-oxidant generation and decreases antioxidant defense in the kidney in the early stage of experimental diabetes.

Supported by FAPESP, CAPES 


\section{7}

Increased skin autofluorescence: a more pronounced marker of mortality in haemodialysis patients than diabetes mellitus?

E. G. Gerrits ${ }^{1,2}$, H. L. Lutgers ${ }^{3}$, G. H. W. Smeets ${ }^{1}$, A. J. Smit ${ }^{3}$, H. J. G. Bilo ${ }^{2,3}$; ${ }^{1}$ Department of Medicine, Isala Clinics, Zwolle,

${ }^{2}$ Diabetes Center, Isala Clinics, Zwolle, ${ }^{3}$ Department of Medicine,

University Medical Center Groningen, The Netherlands.

Background and Aims: Advanced glycation endproducts (AGEs), measured in tissue, represent cumulative glycemic and oxidative stress. In addition to diabetes mellitus (DM), accelerated formation and tissue accumulation of AGEs occurs in patients with renal failure and dialysis which progresses during time. Skin autofluorescence (AF) non-invasively measures tissue accumulation of AGEs and is suitable for clinical application. Recent studies have already demonstrated its predictive value in overall and cardiovascular mortality in type 2 diabetic patients and hemodialysis patients. We investigated whether skin AF has an additional predictive value concerning mortality in diabetic hemodialysis versus non-diabetic hemodialysis patients.

Materials and Methods: Patients were recruited from our hemodialysis center and baseline skin AF was assessed at the lower (non-shunt) arm of the included patients. Skin AF measurement is based on the fluorescence characteristics of certain AGEs and is expressed in arbitrary units (AU). After a follow up period of 3.2 years we assessed mortality in relation to the presence or absence of DM, and to the level of baseline skin AF.

Results: Baseline skin AF was obtained in 106 hemodialysis patients, mean age of 65 years, $68 \%$ male and a mean renal replacement therapy duration of 3.6 years. There were 83 non-diabetic patients and 23 type 2 diabetic patients (mean diabetes duration 16.1 years; mean HbA1c 6.8\%). At the end of follow up 38 patients had died: 12 diabetic patients (52\%) and 26 non-diabetic patients $(31 \%)$, which was borderline non-significant, $p=0.065\left(\chi^{2}\right)$. Baseline skin AF was higher in the deceased independant of the presence or absence of DM. In the diabetes group baseline skin AF was increased, but $t$ test showed no significance in the non-survivors compared to the survivors (table).

Table. Skin autofluorescence - mean \pm SD (AU)

\begin{tabular}{lllll}
\hline $\begin{array}{l}\text { Hemodialysis } \\
\text { group }\end{array}$ & Survivors & Non-survivors & $\begin{array}{l}\mathbf{p} \text { (non-survivors } \\
\text { versus survivors) }\end{array}$ & Total \\
\hline Non-diabetic & $\begin{array}{l}2.94 \pm 0.91 \\
(\mathrm{n}=57)\end{array}$ & $3.56 \pm 0.67(\mathrm{n}=26)$ & 0.002 & $\begin{array}{l}3.14 \pm 0.88 \\
(\mathrm{n}=83)\end{array}$ \\
Diabetic & $\begin{array}{l}3.00 \pm 0.81 \\
(\mathrm{n}=11)\end{array}$ & $\begin{array}{l}3.43 \pm 0.89 \\
(\mathrm{n}=12)\end{array}$ & 0.24 & $\begin{array}{l}3.22 \pm 0.86 \\
(\mathrm{n}=23)\end{array}$ \\
Total & $\begin{array}{l}2.95 \pm 0.89 \\
(\mathrm{n}=68)\end{array}$ & $3.52 \pm 0.74(\mathrm{n}=38)$ & 0.001 & $\begin{array}{l}3.15 \pm 0.88 \\
(\mathrm{n}=106)\end{array}$ \\
\hline
\end{tabular}

Conclusion: Our study confirms a higher mortality rate in diabetic hemodialysis patients compared to non-diabetic hemodialysis patients. Mortality was increased in hemodialysis patients with a higher baseline skin $\mathrm{AF}$, independant of the presence or absence of DM. This might implicate that skin AF is a better predictor for mortality in hemodialysis patients than the presence or absence of DM.

\section{8}

Experimental diabetes treated with stobadine: effects on kidney oxidative/nitrosative stress parameters

A. Cumaoglu ${ }^{1}$, C. Cevik ${ }^{1}$, M. Kavutcu ${ }^{1}$, N. Ari ${ }^{2}$, U. Bagriacik $^{3}$, L. Rackova ${ }^{4}$, C. Karasu';

${ }^{1}$ Biochemistry, Gazi University, Ankara, Turkey, ${ }^{2}$ Pharmacology, Ankara University, Ankara, Turkey, ${ }^{3}$ Immunology, Gazi University, Ankara, Turkey, ${ }^{4}$ Experimental Pharmacology, Slovak Academy of Sciences, Bratislava, Slovakia, ${ }^{5}$ Medical Pharmacology, Gazi University, Ankara, Turkey.

Background and Aims: Glycation, generation of advanced oxidation/ nitration protein products and formation of protein carbonyl groups play important roles in diabetes, its secondary complications, and renal failure. Stobadine, a synthetic pyridoindole anti-oxidant, has the ability to reduce tissue injury induced by mechanisms involving reactive oxygen species in different oxidative stress models. Stobadine has been shown to reduce renal proteinuria, albuminuria and enzymuria, and decreases conjugated diene level and histochemical changes in kidney of diabetic animals. In addition, stobadine is able to antagonize the negative effects of diabetes on renal $\mathrm{Na}, \mathrm{K}$ ATPase, catalase and glutathione peroxidase. Stobadine has also been shown to have hypoglycemic, antihyperlypidemic and lipid peroxidation lowering effects on experimental diabetic animals. The aim of the present study was to investigate the effect of dietary supplementation with stobadine on renal markers of oxidative/nitrosative stress and antioxidant capacity of diabetic rats.

Materials and Methods: Wistar rats were made diabetic by streptozotosin ( 55 $\mathrm{mg} / \mathrm{kg}$, i.p.). Some of them (D) and control (C) rats were treated orally for 4 months with stobadine (STB; $24.7 \mathrm{mg} / \mathrm{kg} /$ day) starting at 1st week of diabetes induction (CSTB and DSTB) prevention study). The levels of advanced oxidation protein products (AOPP) (by a spectrophotometric method), protein carbonyl content (PCC), 3-nitrotyrosine (3-NT) -a marker specific for protein modification by nitric oxide derived oxidants-, and antioxidant potential (AOP) in kidney homogenates (by ELISA) were studied.

Results: STB treatment associated with decreased blood glucose levels seen in D rats. AOPP, 3-NT, PCC and AOP were found to be significantly higher in D group. Administration of STB was accompanied by a decrease of the all parameters studied in kidney of diabetic animals as shown in the table (Data \pm SEM; ANOVA, ${ }^{*} \mathrm{p}<0.05$ vs. C).

Conclusion: The ability of STB as an antioxidant and a factor reducing protein nitrozylation and oxidative modification, and a protecting antioxidant tissue defense may account, at least partly, for it's previously observed histological protection of diabetic kidney.

\begin{tabular}{lllll} 
& C & CSTB & D & DSTB \\
\hline $\begin{array}{l}\text { AOPP } \\
\text { (nmol/mg protein) }\end{array}$ & $6.23 \pm 0.43$ & $5.37 \pm 0.47$ & $9.31 \pm 0.17^{*}$ & $7.63 \pm 0.32^{*}$ \\
PCC & $5.77 \pm 0.33$ & $4.75 \pm 0.40$ & $8.01 \pm 0.66^{*}$ & $n=9$ \\
(nmol/mg protein) & $\mathrm{n}=7$ & $\mathrm{n}=5$ & $\mathrm{n}=7$ & $\mathrm{n}=7$ \\
3-NT & $248.48 \pm 9.91$ & $256.21 \pm 7.02$ & $299.10 \pm 6.91^{*}$ & $263.32 \pm 4.81$ \\
(nmol/mg protein) & $\mathrm{n}=5$ & $\mathrm{n}=5$ & $\mathrm{n}=6$ & $\mathrm{n}=8$ \\
AOP (mM uric & $0.133 \pm 0.0006$ & $0.133 \pm 0.0013$ & $0.138 \pm 0.0014^{*}$ & $0.134 \pm 0.0008$ \\
acid equivalents) & $\mathrm{n}=7$ & $\mathrm{n}=7$ & $\mathrm{n}=8$ & $\mathrm{n}=7$ \\
\hline
\end{tabular}

Supported by TUBITAK-SBAG-SLOVAK 2

\section{9}

Atorvastatin not only decreases serum levels of AGE in diabetic patients, but also inhibits the AGE-induced C-reactive protein expression in hepatoma cells

Y. Jinnouchi ${ }^{1}$, S.-I. Yamagishi ${ }^{1}$, M. Takeuchi ${ }^{2}$, T. Imaizumi ${ }^{1}$;

${ }^{1}$ Internal Medicine, Kurume University School of Medicine, Japan,

${ }^{2}$ Department of Pathophysiological Science, Hokuriku University,

Kanazawa, Japan.

Background and Aims: Lipid-lowering agents, statins, have been recently shown to reduce the risk for cardiovascular events in diabetic patients. Indeed, a multicentre randomized placebo-controlled trial revealed that atorvastatin prevented first cardiovascular events including stroke, in patients with type 2 diabetes without high LDL-cholesterol levels. In addition, statin treatment is associated with less risk of cardiovascular events than placebo treatment even in overlap groups where patients exhibited equivalent LDL-cholesterol levels. These observations support the concept that beyond lipid-lowering effects of statin could contribute at least in part to cardiovascular event reduction in diabetes. In this study, we examined whether atorvastatin treatment decreased serum AGE levels in diabetic patients. We also investigated whether atorvastatin could inhibit the AGE-induced CRP expression in Hep3B cells, a human hepatoma cell.

Materials and Methods: Sixteen hypercholesterolemic type 2 diabetic patients (mean age of $63.6+/-2.1$ years, 8 males and 8 females) without any cardiovascular disease who were previously untreated with statins, were enrolled for the present study. The patients were treated with $10 \mathrm{mg}$ atorvastatin once daily for 4 weeks. Age- and sex-matched 9 hypercholesterolemic diabetic patients who were treated with diet therapy only for 4 weeks served as control subjects. Serum levels of AGE were measured with ELISA before and after the treatment of atorvastatin. In vitro, AGE were prepared by incubating BSA with $0.1 \mathrm{~mol} / \mathrm{l} \mathrm{D}$-glyceraldehyde for 7 days. Reactive oxygen species (ROS) generation was measured by using the fluorescent probe CM-H2DCFDA. 
Gene expression was analyzed by quantitative real-time RT-PCR. CRP levels in the medium were measured with an ELISA.

Results: There were no significant differences of fasting glucose and HBA1c levels between before and after atorvastatin treatment. However, serum levels of AGE were significantly decreased after 4-week treatment with atorvastatin, whereas those of control subjects remained unchanged; AGE levels before and after atorvastatin treatment were $15.0+/-0.5$ and $13.6+/-0.1$ $\mathrm{U} / \mathrm{ml}$, respectively $(p<0.05)$. Although atorvastatin treatment significantly decreased total cholesterol, LDL-cholesterol and triglycerides levels in our patients, there were no significant correlations between changes of AGE and those of lipid parameters. In vitro, AGE significantly increased the ROS generation in Hep3B cells by about 1.3 -fold $(p<0.05)$. Atorvastatin dosedependently inhibited the AGE-induced increase in ROS generation $(p<0.05)$. In addition, AGE increased CRP mRNA levels in Hep3B cells to about 6-folds $(p<0.01)$, which was significantly blocked by atorvastatin or an anti-oxidant, $\mathrm{N}$-acetylcysteine $(p<0.05)$. Atorvastatin inhibited the AGE-induced CRP secretion by Hep3B cells in a dose-dependent manner as well.

Conclusion: Atorvastatin not only decreased serum AGE levels in patients with type 2 diabetes in a cholesterol-lowering independent manner, but also blocked the AGE-signaling to CRP expression through its anti-oxidative property in vitro. Our present study suggests that atorvastatin may have pleiotropic effects against atherosclerosis by blocking both AGE formation and its downstream signaling to inflammation.

Supported by grants of Venture Companies Project from the Education, Culture, Sports, Science and Technology, Japan

\section{PS 111 Diabetic foot: natural history and intervention}

\section{0}

Longitudinal association of glucose metabolism with neuropathy and peripheral arterial disease: the AusDiab study

R. Tapp ${ }^{1,2}$, P. Zimmet ${ }^{2}$, A. Tonkin ${ }^{1}$, J. Shaw ${ }^{2}$;

${ }^{1}$ Department of Epidemiology and Preventive Medicine, Monash University, ${ }^{2}$ Epidemiology Department, International Diabetes Institute, Melbourne, Australia.

Aims: We determined the incidence of peripheral neuropathy and peripheral arterial disease (PAD) across categories of glucose metabolism in an Australian population and assessed risk factors for these outcomes.

Materials and Methods: The Australian Diabetes Obesity and Lifestyle study (AusDiab), is a national, longitudinal study of adults aged $\geq 25$ years from 42 randomly selected areas of Australia. Neuropathy and PAD were assessed in 1999-2000 and 2004-2005 in participants identified as having diabetes (based on self report and oral glucose tolerance test), impaired glucose metabolism (IGM) and in a random sample with normal glucose tolerance (NGT). Complications data from both 1999-2000 and 2004-2005 were available for 1294 participants.

Results: The five year incidence of peripheral neuropathy was $16.5 \%$ in those with known diabetes at baseline (KDM), 7.7\% in those with newly diagnosed diabetes (NDM), $7.3 \%$ in those with IGM and 5\% in those with NGT. The five year incidence of PAD was 7.3\% in those with KDM, 3.2\% in those with NDM, 3.1\% in those with IGM and $1.6 \%$ in those with NGT. At baseline $19.6 \%$ of those with diabetes were at risk of foot ulceration and at followup $27.6 \%$ were at risk of foot ulceration. Those with KDM at baseline were four times more likely to develop PAD compared to those with NGT after adjustment for age, sex, systolic BP, cholesterol and smoking (3.90 [1.1513.26]). Independent risk factors for peripheral neuropathy were height (1.74 [1.05-2.88] per $10 \mathrm{~cm})$, age (2.16 [1.53-3.05] per 10 years) and HbAlc $(1.24$ [1.01-1.53] per $1 \%)$.

Conclusion: This is one of the first national, longitudinal population-based studies of neuropathy and one of the very few in which diabetes was defined by an oral glucose tolerance test. The present study identified a substantial proportion of the diabetic population at high risk of foot ulceration.

RTAPP is supported by a Sidney Sax Fellowship from the National Health and Medical Research Council of Australia

\section{1}

Randomised controlled trial of education in the prevention of foot ulcer recurrence in diabetes

W. Jeffcoate ${ }^{1}$, K. Radford ${ }^{1}$, P. Ince ${ }^{1}$, M. Smith ${ }^{1}$, F. Game ${ }^{1}$, N. Lincoln ${ }^{2}$; ${ }^{1}$ Diabetes and Endocrinology, Foot Ulcer Trials Unit, Nottingham, ${ }^{2}$ Institute of Work, Health and Organisations, University of Nottingham, United Kingdom.

Background and Aims: Although the importance of education in the prevention of foot ulcers is accepted, there are few data to substantiate its effectiveness. Nevertheless, a single previous randomised trial has suggested that a one-hour educational intervention may be associated with a significant reduction in incidence of both new ulcers and of amputation in the group at highest risk: those with previous ulcers (Malone et al, 1989). We have sought to confirm these findings by undertaking an observer-blind, randomised controlled trial of targeted education in such a population.

Materials and Methods: Patients with ulcers which were newly epithelialised, and which remained healed at 28 days, were randomised to receive either usual care or a single hour-long, semi-structured but personalised, educational interview in their own home with a specialist health care professional. The interview was based on standard educational material, refined following discussion with a focus group of ulcer-sufferers and supplemented by illustrations of different types of foot problems. An educational leaflet was given to reinforce the principles of good foot care behaviour. The interview was followed after one month by a telephone interview in which issues raised earlier were revisited. The control group received the educational leaflet only. Ulcer recurrence and Hospital Anxiety and Depression (HAD) scale scores were documented at 6 and 12 months, and foot care behaviour (Nottingham 
Assessment of Functional Footcare) at 12 months. New ulceration at 12 months was the primary endpoint and was determined by questionnaire sent to both GP and patient, and examination of hospital records by an observer blind to randomisation. In order to detect a reduction in the 12 month incidence of new ulceration from $35 \%$ to $15 \%$ (two-sided, $80 \%$ power, $\mathrm{p}<0.05), 69$ patients were required in each group.

Results: Of 259 patients attending one of three specialist multidisciplinary foot clinics between April 2003 and September 2005, who fulfilled the inclusion criteria, 87 were excluded $(52$ - withheld consent; 11 - serious medical problems; 5 - non-self caring; 19 - other reasons). 172 patients were randomised: 87 to the intervention group with 85 controls. Reported foot care behaviour was significantly better in the intervention group at 12 months $(t=2.09, p=0.04)$. There were slight discrepancies in the number of new ulcers reported by patients and those documented by hospital records, and every effort was made to establish which was correct. There were, however, no significant differences between the intervention and control groups in either case (12 months: patient report $-33 \%$ versus $41 \%$, respectively (OR 1.38 , CI 0.69-2.796); medical records $-41 \%$ versus $41 \%$ (OR 1.01, CI $0.55-1.85$ ). There were similarly no differences between the groups in the number of amputations (minor - intervention 8, control 8; major - intervention 1, control 1). There were no significant differences in HAD (Anxiety, Depression) scores.

Conclusion: We conclude that although the educational programme was associated with an apparent improvement in protective footcare behaviour, there was no evidence of any effect on either ulcer incidence or amputation. Further work is needed to establish the role of formal education programmes in this group.

Supported by Diabetes UK

\section{2}

Ethnic differences in risk of foot diseases in persons with diabetes on dialysis

A. Ndip ${ }^{1}$, J. Williams ${ }^{2}$, H. LaFontaine ${ }^{3}$, L. A. Lavery ${ }^{2}$, A. J. M. Boulton ${ }^{1}$; ${ }^{1}$ Diabetes and Medicine, Manchester Diabetes Centre, Manchester, United Kingdom, ${ }^{2}$ Surgery, Texas A\&M University Health Sciences Centre, Georgetown, United States, ${ }^{3}$ Health Sciences Centre, University of Texas, San Antonio, United States.

Background and Aims: Hispanics with diabetes in the USA are known to be at increased risk of foot ulceration and amputation compared to nonHispanics, whilst in the UK, Indo-Asians and Afro-Caribbeans have lower rates of ulceration. Whether these differences persist when these patients reach end-stage renal disease (ESRD) is unknown. We therefore sought to examine ethnic differences in diabetic foot ulceration in persons on dialysis. Materials and Methods: We examined foot problems according to ethnic origin in diabetic patients on dialysis in centers in the UK and USA. A consecutive sample of all persons with diabetes on dialysis in Texas (US) and Manchester (UK) were assessed for foot problems including neuropathy, peripheral arterial disease, past and present foot ulcers, Charcot foot, and amputation. Risk for foot diseases was assessed based on the international diabetic foot risk classification. Chi-squared test was used for comparing categorical data.

Results: A total of 208 diabetic patients were included (UK 92, USA 116), $53 \%$ males, predominantly with type 2 diabetes ( $82 \%$ ) and mean age of 59.8 (range 26-86) years. Peripheral neuropathy was present in 67\% of patients, peripheral vascular disease in $48 \%$, while $32.4 \%$ had a history of previous foot ulcers and $15.9 \%$ were already amputees. Among the UK patients, 4 were bilateral amputees whilst $20.5 \%$ of those with at least one limb had active ulcers including 22.6\% Caucasians and 22.2\% Indo-Asians ( $\mathrm{P}>0.05)$. Seven percent had had a diagnosis of Charcot foot. Further, no significant differences were observed in any of the 4 risk categories between Indo-Asians and Caucasians. In the US cohort, Hispanics were at significantly higher risk in all categories compared to non-Hispanics $(19.6 \%, 7.8 \%, 43.2 \%, 29.4 \%$ vs. $17.5 \%, 27.5 \%, 17.5 \%$ and $37.5 \%$ respectively) $(\mathrm{p}=0.016)$.

Conclusion: Thus, the high risk of diabetic foot diseases within ethnic minorities in the US appears to persist when they enter ESRD whereas in the UK, ethnic minorities known to be at low risk of diabetic foot diseases appear to lose this protection when in ESRD. The very high levels of foot pathology in all diabetic patients in ESRD emphasize the need for a comprehensive foot care program for these patients.

Supported by American Diabetes Association

\section{3}

Reduced incidence of lower-limb amputations in the diabetic population of a German city, 1990-2005

C. Trautner ${ }^{1,2}$, B. Haastert ${ }^{3}$, P. Mauckner ${ }^{4}$, L.-M. Gaetcke ${ }^{1}$, G. Giani ${ }^{3}$; ${ }^{1}$ Medicine Science Consulting, Berlin, ${ }^{2}$ Department of Public Health, University of Applied Sciences, Wolfsburg, ${ }^{3}$ Institute of Biometrics and Epidemiology, German Diabetes Center, Duesseldorf, ${ }^{4}$ Department of Internal Medicine, Remigius Hospital, Leverkusen, Germany.

Background and Aims: A reduction of diabetes-related amputations by at least one-half within 5 years was declared a primary objective for Europe (St. Vincent Declaration, 1989). We collected data in one German city (Leverkusen, approximately 160000 inhabitants) to ascertain a potential change in incidence rates of amputations. This goal had not been achieved until the end of 1998. In this study, we evaluated whether the incidence of amputations in Leverkusen had decreased until the end of 2005.

Materials and Methods: From all three hospitals in Leverkusen, we obtained complete lists of lower-limb amputations of the years 1990/1991 and 19942005. Only the first observed amputation was counted for the analysis. Only non-traumatic amputations in residents of Leverkusen were considered. We estimated incidence rates of amputations in the entire population, the diabetic population, and the nondiabetic population. Data about the population stucture, separately for each year of the observation period, were received from the city administration and the Federal Office of Statistics. To test for time trend, we fitted Poisson regression models, adjusting for age and sex.

Results: 692 patients met the inclusion criteria. Of all subjects, $58 \%$ were male, and $72 \%$ were known to have diabetes. Mean age was 71.7 years. Incidence rates in the diabetic population (standardised to the estimated German diabetic population, per 100000 person-years) varied considerably between years and were as follows: 1990: 549; 1991: 356; 1994: 544; 1995: 386; 1996: 426; 1997: 433;1998: 463;1999: 474; 2000: 415; 2001: 304; 2002: 335; 2003: 360; 2004: 281; 2005: 428.

The Poisson models showed that in the diabetic population, the estimated relative risk (RR) per year was $0.976(p=0.0164)$. The same trend was observed when only amputations above the ankle $(n=352 ; \mathrm{RR}=0.970 ; p=0.0318)$ were considered. Over 15 years, an estimated reduction of major amputations by $36.7 \%$ results. The Poisson models showed no significant change of incident amputations over time in the nondiabetic population. $(\mathrm{RR}=1.022$; $p=0.1981$ ).

Conclusion: Although possible sources of bias cannot be excluded with certainty, the data indicate a reduction of the incidence of amputations. This finding is likely to be due to improved management of the diabetic foot syndrome (e. g. wound treatment and metabolic control) after a network of specialised physicians (a foot clinic and two physicians' offices specialised in diabetes) and defined clinical pathways for patients with diabetes were introduced.

Supported by KCI, Novo Nordisk, Smith \& Nephew

\section{4}

Predictors of amputation in diabetic foot disease L. Prompers; on behalf of the Eurodiale Group,

Internal Medicine, University Hospital Maastricht, The Netherlands.

Aims: Amputation is a serious outcome of diabetic foot ulcers. Data on the determinants of major(=lower leg) and minor amputation are scarce. In the multi-center Eurodiale study performed by 14 European centers, prospective data were collected on clinical outcome and its determinants, including data on determinants of major and minor amputation.

Methods: Data were obtained on 1232 consecutive patients with diabetes and a new foot ulcer. Patients were categorized in 4 stages: A;(PAD- / infection-), $\mathrm{B} ;(\mathrm{PAD}-$ / infection+), $\mathrm{C} ;(\mathrm{PAD}+/$ infection-), $\mathrm{D} ;(\mathrm{PAD}+/$ infection+). Patients were followed until final outcome (healing, major amputation or death) or up to one year. Predictors of major and minor amputation were determined by means of multiple logistic regression analyses (manual stepwise backward procedure).

Results: Major amputation was performed in $5 \%$ of the total population; ranging from 2 to $10 \%$ in stage A to stage $\mathrm{D}(\mathrm{p}<0.001)$. Minor amputation was performed in $18 \%$; ranging from 8 to $31 \%(\mathrm{p}<0.001)$ respectively. In group D $34 \%$ underwent a major or minor amputation. Independent predictors of a major amputation were ulcer size $(\mathrm{OR}=7.71), \mathrm{PAD}(\mathrm{OR}=4.12)$, 
visual impairment $(\mathrm{OR}=2.32), \mathrm{PAD}(\mathrm{OR}=4.12)$, gender ( $\mathrm{F}$ vs. $\mathrm{M}-\mathrm{OR}=0.39)$, and a neurological disorder $(\mathrm{OR}=0.18)$. Independent predictors of a minor amputation were depth $(\mathrm{OR}=6.22), \mathrm{PAD}(\mathrm{OR}=1.98)$ and infection $(\mathrm{OR}=1.78)$. In both models an interaction is present between PAD and infection $(\mathrm{p}<0.001)$. Analyzing PAD+ and PAD- patients separately, showed that gender, ulcer size and infection were predictors of major amputation in $\mathrm{PAD}+$ and age and gender in PAD-. Predictors of minor amputation in PAD+ were depth and infection, and in PAD- heart failure, depth and PNP.

Discussion: Although the major amputation rate is relatively favorable, minor amputation is a frequent outcome of a diabetic foot ulcer. Patients with both PAD and infection are significantly more likely to undergo a major or minor amputation. When analyzing the determinants of amputation a clear interaction is present between the presence of PAD and infection. More insight into this relation and data on the optimal treatment for patients with both PAD and infection are urgently needed.

\section{5}

Evaluation of the impact of chiropodist care in the secondary prevention of foot ulcerations in diabetic subjects over a period of 6 years G. Köhler ${ }^{1}$, W. Haas ${ }^{1}$, I. Rakovac ${ }^{2}$, M. Schintler ${ }^{3}$, G. Bock ${ }^{1}$, S. Korsatko' J. Plank $k^{1}$, T. R. Pieber ${ }^{1}$;

${ }^{1}$ Department of Internal Medicine, Diabetes and Metabolism, Medical University Graz, ${ }^{2}$ Institute of Medical Technologies and Health Management, Joanneum Research, ${ }^{3}$ Department of Plastic Surgery, Medical University Graz, Austria.

Background and Aims: Diabetic foot ulcers after healing are associated with a high risk for recurrence of ulcers and amputations. Prevention of foot related complications is a major challenge in diabetes care. We have recently demonstrated in a randomised controlled trial with duration of 1 year that chiropodist care can reduce recurrence rate of foot ulcers within one year. The aim of this follow up study was to investigate the impact of chiropodist care in the original study population after 6 years.

Materials and Methods: After healing of a diabetic foot ulcer, 91 patients [female $(n=40)$, diabetes type $1(n=6)$ and type $2(n=85)$, age [mean $(\hat{A} \pm S D)$ ] $65 \hat{\mathrm{A}} \pm 11$ years, BMI $28.5 \hat{\mathrm{A}} \pm 4.4$, diabetes duration $16 \hat{\mathrm{A}} \pm 11$ years, $\mathrm{HbA1c}$ $8.4 \hat{\mathrm{A}} \pm 1.6 \%$ ] were initially randomized to regular chiropodist care, which was free of charge for patients, or a control group for the period of one year. The whole study cohort was invited to participate in a follow up evaluation 5 years after end of the randomised control trial. Outcome was analysed to asses the impact of self-paid chiropodist care (at least once every 8 weeks) and the recurrence rate of ulcers, amputation, and death.

Results: 89 out of 91 patients ( 2 lost to follow up) could be analysed after 6 years. 40 underwent regular chiropodist care. 26 in the chiropodist care group vs. 38 patients in the control group developed a new ulcer within the six years [HR 0.60 (95\%CI 0.36-0.97), p 0.04] (Fig.1), 4 vs.10 amputations [HR 0.39 (95\%CI $0.14-1.16)$, p 0.09] were recorded, 11 vs. 21 patients died [HR 0.59 (95\%CI 0.30-1.21), p 0.15] in the chiropodist vs. the control group, respectively. Analyses for the aggregated endpoint (ulceration, amputation, death) showed a reduction in favour of the chiropodist care group vs. the control group ( 29 vs. 46 events [HR 0.54 (95\%CI 0.33-0.83), p<0.01]).

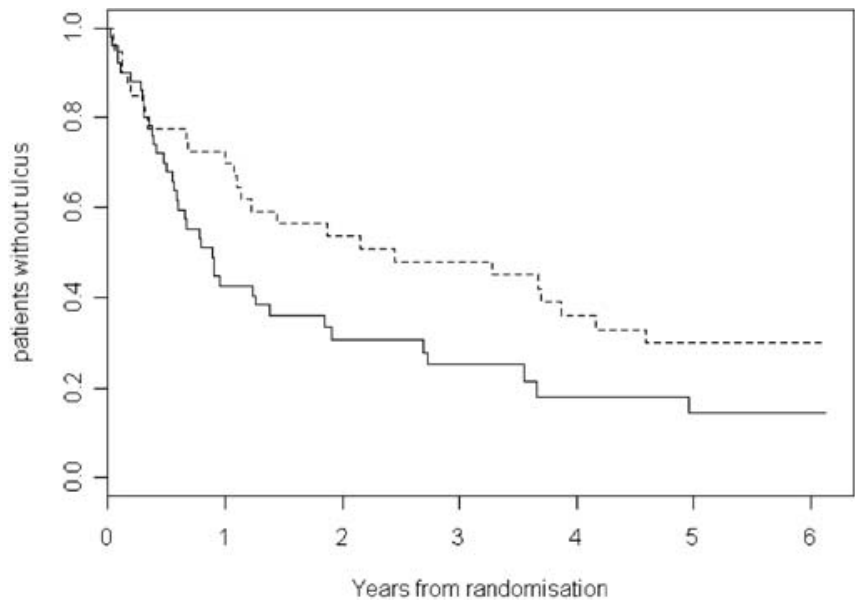

Conclusion: Our follow up investigation confirms the high rate of adverse outcome in patients with a diabetic foot syndrome. More than every second patient developed a new ulcer or amputation within the six years of follow up. Furthermore, our data suggests that regular chiropodist care may reduce the recurrence of ulcers and amputations.

\section{6}

CDUK: a UK-wide, web-based survey of the management of the acute Charcot foot of diabetes

F. Game ${ }^{1}$, R. Catlow ${ }^{2}$, W. Jeffcoate ${ }^{1}$, E. Jude ${ }^{3}$, M. Edmonds ${ }^{4}$, N. Baker ${ }^{5}$, G. Jones';

${ }^{1}$ Diabetes and Endocrinology, Foot Ulcer Trials Unit, Nottingham, ${ }^{2}$ Diabetes, CDUK Management Committee, Blackburn, ${ }^{3}$ CDUK Management Committee, Tameside, ${ }^{4}$ CDUK Management Committee, London, ${ }^{5} \mathrm{CDUK}$ Management Committee, Ipswich, ${ }^{6} \mathrm{CDUK}$ Management Committee, Blackburn, United Kingdom.

Background and Aims: The acute Charcot foot is a rare, but serious, complication of diabetic neuropathy - which may lead to progressive deformity of the affected foot, and often to limb loss. Pathogenesis is uncertain and current therapy is mainly reliant on reduced weight bearing until the active phase of the disease remits. It is recommended that this is achieved by the use of non-removable below knee casts or walkers. There are few data available on the median time to disease remission and whether outcome is affected by other treatments, such as bisphosphonates. For this reason we set up a web-based survey of active cases of Charcot disease in the UK (CDUK), in order to define baseline characteristics, precipitating factors, management details and outcome, in as large a cohort as possible.

Materials and Methods: Participating clinicians were invited to register anonymised details of patients with active disease being managed between $1^{\text {st }}$ June 2005 and $31^{\text {st }}$ December 2006, and further details were requested at 3-monthly intervals for 12 months.

Results: A total of 279 (73\% male) cases were registered to January 2007; mean age 57 years (range 21-90); $72 \%$ Type 2 diabetes. Outcome data are available for 200 patients at 3 months post registration, 142 patients at 6 months and 61 patients at 12 months. $61.6 \%$ were registered as having had a likely precipitating event within the 6 months prior to onset (accident 36\%, previous ulcer $35 \%$, surgery to affected foot $12 \%$, osteomyelitis $7 \%$ ). Initial management included offloading specified as non-removable below knee $34.1 \%$, removable below knee $45.5 \%$, non-removable foot $1.4 \%$, removable foot $2.9 \%$ and other (eg. bed rest, crutches) $8.9 \% .40 .1 \%$ had a non-removable below knee offloading device at some stage during the follow up period. $25.4 \%$ of patients received treatment with intravenous bisphosphonates, $19.4 \%$ with oral bisphosphonates, some in combination. 5.3\% had more than one treatment with intravenous bisphosphonates. 131 patients went into remission from their acute Charcot during follow up in a median of 10 (range 2-40) months. Those who had a non-removable offloading device at some stage in their management had a median time to resolution of 8.5 months compared to 12 months in those who had a removable offloading device (Mann Whitney U 1543, p=0.006). Those who were treated with any bisphosphonates had a median time to resolution of 12 months compared to 9 months in those who had none (Mann Whitney U 1598, p=0.013).

Conclusion: Whilst the possibility of confounders cannot be excluded these data support the use of non-removable offloading devices in the management of the acute Charcot foot of diabetes. The role of bisphosphonates requires further investigation in placebo controlled trials.

Supported by Diabetes UK 


\section{PS 112 Nephropathy: clinical treatment}

\section{7}

Impact of the basal activity in the renin angiotensin system (RAS) on NT-proBNP during hypoglycaemia and hypoxaemia in normal subjects R. Due-Andersen ${ }^{1}$, U. Pedersen-Bjergaard ${ }^{1}$, C. E. Larroude ${ }^{2}$, J. K. Kanters ${ }^{2}$, C. Kistorp ${ }^{3}$, J. Faber ${ }^{3}$, N. V. Olsen ${ }^{4}$, B. Thorsteinsson ${ }^{1}$;

${ }^{1}$ Endocrinology Section, Department of Internal Medicine, Hillerød Sygehus, ${ }^{2}$ Biomedical sciences, University of Copenhagen, ${ }^{3}$ Department of Cardiology and Endocrinology, Frederiksberg University Hospital, Copenhagen, ${ }^{4}$ Department of Neuroanaesthesia, Copenhagen University Hospital, Denmark.

Background and Aims: There is a slightly increased risk of sudden death among young patients with insulin treated diabetes compared to healthy young subjects. Malignant cardiac arrhythmias originating due to hypoglycaemia induced QT prolongation could be responsible for some of these deaths. Brain type natriuretic peptide (BNP) is thought to be a cardioprotective peptide. The peptide is released (together with the inactive N-terminal part of the prohormone, NT-proBNP) form the cardiac myocytes in response to different kinds of myocardial stress. BNP antagonise many of the classic effects of angiotensin II and a significant interplay exists between BNP and the RAS.

The aims of this study were to explore if myocardial stress induced by hypoglycaemia and hypoxaemia affected NT-proBNP levels and if basal RAS activity had impact on changes in NT-proBNP levels during the stimuli. Additionally we wanted to investigate if the cardiac repolarisation (measured as the QTc) was correlated to the NT-proBNP levels.

Materials and Methods: From a cohort of 303 healthy young men, 8 subjects with high RAS activity and 9 subjects with low RAS activity (age $26 \pm 4$ years) were studied on three different occasions separated by at least three weeks: 1) hypoglycaemia (mean plasma-glucose $2.7 \pm 0.5 \mathrm{mmol} / \mathrm{l}), 2$ ) hypoxaemia (mean $\mathrm{pO}_{2} 5.8 \pm 0.5 \mathrm{kPa}$ ), and 3) normoglycaemic normoxia (control day). NT-proBNP was measured at baseline, during the stimuli and in the recovery phase.

Results: Hypoglycaemia did not affect the NT-proBNP level. Hypoxaemia was significantly associated with an increase in NT-proBNP: baseline 2.2 $(+/-1.5) \mathrm{pmol} / \mathrm{l}$ - hypoxaemia $2.4(+/-1.5) \mathrm{pmol} / \mathrm{l}, \mathrm{p}=0.001$ during the stimulus but not in the recovery phase. RAS activity had no impact on the NTproBNP level neither at baseline, nor during hypoglycaemia nor hypoxaemia. Hypoglycaemia and hypoxia induced QTc prolongation compared with Qtc on the control day ( $\mathrm{p}<0.001$, both stimuli). The QTc prolongation did not correlate with the NT-proBNP level during either of the stimuli.

Conclusion: Hypoxaemia but not hypoglycaemia caused small increases in NT-proBNP. The basal activity in RAS had no impact on NT-proBNP levels at any point. The QTc prolongation during both hypoglycaemia and hypoxaemia was not correlated to the NT-proBNP level.

Supported by Foundation of Harald Jensen and wife, Foundation of Olga Bryde Nielsen, Foundation of Region 3, Research Foundation of Frederiksborg Amt

\section{8}

Time course of the antiproteinuric and the antihypertensive effect of aliskiren in patients with type 2 diabetes and albuminuria

F. Persson ${ }^{1}$, P. Rossing ${ }^{1}$, K. J. Schjødt ${ }^{1}$, T. R. Juhl ${ }^{1}$, L. Tarnow ${ }^{1}$, C. D. A. Stehouwer ${ }^{2}$, C. Schalkwijk ${ }^{2}$, F. Boomsma ${ }^{3}$, E. Frandsen ${ }^{4}$, H.-H. Parving ${ }^{1}$ Steno Diabetes Center, Gentofte, Denmark, ${ }^{2}$ Department of Medicine, University Hospital, Maastricht, The Netherlands, ${ }^{3}$ Department of Internal Medicine, Section Vascular Medicine, Erasmus MC, Rotterdam, The Netherlands, ${ }^{4}$ Department of Clinical Physiology and Nuclear Medicine, Glostrup University Hospital, Denmark, ${ }^{5}$ Department of Medical Endocrinology, University Hospital of Copenhagen, Denmark.

Background and Aims: The renin inhibitor aliskiren lowers blood pressure in both non-diabetic and diabetic hypertensive subjects, but it is not known whether this treatment can decrease albuminuria and if so, if this is done independently of its blood pressure lowering capability. We evaluated the time course of the antihypertensive and antialbuminuric effect of renin inhibition. We also evaluated the effect on both variables after 4 weeks of cessation of treatment.
Materials and Methods: Fifteen patients with type 2 diabetes and albuminuria were included. After a 4-week washout period, patients received aliskiren 300 mg once daily for 28 days. Early morning urine samples were collected for daily determination of UACR during 56 days. Mean of three consecutive days were analysed. Twenty-four hour blood pressure was measured at baseline and after 3,7,14 and 28 days of treatment and 3,7,14 and 28 days after cessation of treatment. Estimated GFR (revised MDRD) was calculated at baseline, during treatment and during washout.

Results: Baseline UACR was 173 (39-1725) mg/g (median and range) and mean ambulatory blood pressure (BP) was $142 / 75 \mathrm{mmHg}(\mathrm{SD} 13 / 10)$, eGFR was $76 \mathrm{ml} / \mathrm{min} / 1.73 \mathrm{~m}^{2}$. UACR was significantly reduced after $5-7$ days of treatment by $29 \%$ (14-41) (95\% CI) with further reduction to $44 \%$ (32-54) during the 28 day treatment period ( $\mathrm{p}=0.001$ vs. baseline). 24 - hour mesn systolic BP was significantly lowered by $6 \mathrm{mmHg}$ after 7 days ( $\mathrm{p}=0.037$ vs. baseline) with no further reduction after 28 days. BP returned towards baseline levels 7 days after cessation of treatment, whereas UACR was still $22(2-37) \%$ reduced after 4 weeks of withdrawal. There was no association between individual decline in UACR and $\mathrm{BP}$ at the end of the treatment period. No significant change in eGFR.

Conclusion: Renin inhibition with aliskiren $300 \mathrm{mg}$ once daily reduces UACR and arterial blood pressure in patients with type 2 diabetes and microand macroalbuminuria. The study demonstrates a significant effect on both variables already after one week. In addition, a further reduction in UACR was seen after 4 weeks, which was in part sustained after 4 weeks of wash-out and was independent of the reduction in blood pressure. This suggests that the reduction in UACR by aliskiren is explained both by haemodynamic and non-haemodynamic factors.

Supported by Novartis

\section{9}

Optimal dose of lisinopril for renoprotection in type 1 diabetic patients with diabetic nephropathy

K. J. Schjoedt ${ }^{1}$, A. Astrup ${ }^{1}$, F. Persson ${ }^{1}$, L. Tarnow ${ }^{1}$, P. Rossing ${ }^{1}$,

H.-H. Parving ${ }^{2,3}$;

${ }^{1}$ Steno Diabetes Center, Gentofte,

${ }^{2}$ Department of Medical Endocrinology, Rigshospitalet, Copenhagen,

${ }^{3}$ Faculty of health science, University of Aarhus, Denmark.

Background and Aims: ACE inhibitors are first-line therapy for renoprotection in type 1 diabetic patients with diabetic nephropathy. The optimal dose for renoprotection of the ACE-inhibitor lisinopril has not been established. The purpose of this study was to evaluate the optimal renoprotective effect, as reflected by short-term changes in urinary albumin excretion rate (UAER), of ultrahigh doses of lisinopril in type 1 diabetic patients with diabetic nephropathy.

Materials and Methods: A total of 56 type 1 diabetic patients with diabetic nephropathy were included in this double-masked randomized crossover trial consisting of three treatment periods. At inclusion, ongoing antihypertensive treatment was discontinued and replaced with slow-release furosemide in individual but fixed doses [median (range)] $60(0-360) \mathrm{mg}$ daily, for the entire studyperiod. After this 2 months wash-out period (baseline), patients were treated in random order with lisinopril 20,40 , and $60 \mathrm{mg}$ once daily, each dose for 2 months. UAER (three 24-hour urine collections), 24-hour ambulatory blood pressure (ABP) and estimated GFR (MDRD) were determined after the wash-out period and after each treatment period. GFR was measured as ${ }^{51} \mathrm{Cr}$-EDTA clearance at baseline.

Results: Forty-nine of 56 randomized patients completed the study. Two patients (who were on lisinopril $60 \mathrm{mg}$ and $20 \mathrm{mg}$ resp.) were excluded due to dizziness combined with an (reversible) increase in plasma creatinine (39\% and $31 \%$ resp.). One patient on lisinopril $60 \mathrm{mg}$ was excluded due to high blood pressure $(180 / 90 \mathrm{~mm} \mathrm{Hg})$, unchanged plasma creatinine and potassium. Four patients with unchanged plasma creatinine and potassium withdrew consent due to mild dizziness (lisinopril 60 and $40 \mathrm{mg}$ resp.), mild diarrhoea $(40 \mathrm{mg})$ and restless legs $(20 \mathrm{mg})$. Baseline values were: 24 -hour UAER [geometric mean ( $95 \% \mathrm{CI})$ ] 362 (240 to 545$) \mathrm{mg} / 24$ hours, ambulatory blood pressure [mean (SD)] 142(14)/74 (8) mm Hg, and GFR 81 (30) mL/ $\mathrm{min} / 1.73 \mathrm{~m}^{2}$. All doses of lisinopril significantly reduced UAER, ambulatory blood pressure, and eGFR from baseline. Reductions in UAER from baseline were $63 \%$ (55\% to $69 \%), 71 \%$ (66\% to $76 \%$ ), and $70 \%$ (64\% to $75 \%)$ with increasing doses of lisinopril $(P<0.001)$. Compared to lisinopril $20 \mathrm{mg}$ there was a significant further reduction in UAER with lisinopril $40 \mathrm{mg}$ of $23 \%$ (8 
to 35 ) and $60 \mathrm{mg} \mathrm{19 \%} \mathrm{(3} \mathrm{to} \mathrm{31)} \mathrm{p}<0.05$. After adjustment for changes in 24hour ABP, the difference in UAER between 20 and $40 \mathrm{mg}$ was not significant $(P=0.14)$. There was no difference in UAER between lisinopril 40 and $60 \mathrm{mg}$. With increasing doses of lisinopril, systolic ABP was reduced from baseline by 10 (6 to 15 ), 13 (9 to 19 ), and 12 ( 8 to 17 ) $\mathrm{mm} \mathrm{Hg}$, and diastolic ABP by 6 (4 to 8 ), 8 (6 to 10), and 7 (5 to 10$) \mathrm{mm} \mathrm{Hg}(\mathrm{P}<0.05$ for diastolic ABP 20 vs. $40 \mathrm{mg}$, otherwise NS between doses). There was a positive correlation between changes in blood pressure and changes in UAER on lisinopril 20 $\mathrm{mg}$ compared to lisinopril $40 \mathrm{mg}$ ( $\Delta 24$-h diastolic BP vs. $\triangle$ UAER $20-40 \mathrm{mg}$, $\mathrm{R}=0.54, P<0.001$ )

Conclusion: High dosing of lisinopril (40 mg once daily) is generally safe and offers additional renoprotection as reflected by short-term changes in UAER in comparison to the currently recommended dose of $20 \mathrm{mg}$. In contrast ultrahigh doses of lisinopril ( $60 \mathrm{mg}$ once daily) does not provide further renoprotection as compared to lisinopril $40 \mathrm{mg}$ once daily.

\section{0}

Irbesartan reduces urinary albumin excretion in hypertensiveand non-hypertensive Chinese patients with type 2 diabetes S. Shang ${ }^{1}$, C. Pan ${ }^{2}$;

${ }^{1}$ Sanofi-Aventis China, Shanghai,

${ }^{2}$ Endocrinology, Military General Hospital, Beijing, China.

Background and Aims: Albuminuria is an early sign of damage to both the kidney and the heart. As data from China (MAPS study) shows a high prevalence of MAU and albuminuria (around $40 \%$ and $17 \%$ respectively), preventing or delaying the progression of diabetic nephropathy is therefore an essential management goal. Two large studies (IRbesartan MicroAlbuminuria type 2 diabetes mellitus in hypertensive patients [IRMA 2] and the Irbesartan Diabetic Nephropathy Trial [IDNT]) showed irbesartan exerting a renoprotective effect (slowing the progression of renal disease) in hypertensive patients with type 2 diabetes at both, early and later stages of diabetic nephropathy. The current study was aiming to evaluate the efficacy and safety of irbesartan in Chinese hypertensive- and non-hypertensive patients with type 2 diabetes.

Materials and Methods: Three hundred thirty patients, aged 30-70 years with type 2 diabetes and albuminuria (UAER between 20 and $500 \mathrm{ug} / \mathrm{min}$ ), with $(n=241)$ or without $(n=89)$ hypertension, were randomly assigned to $300 \mathrm{mg} / \mathrm{d}$ irbesartan or placebo for 24 weeks. Adjunctive antihypertensive therapies (excluding ACE inhibitors and angiotensin II receptor antagonists) could be added in order to achieve appropriate blood pressure reduction. The primary end point was the change in urinary albumin excretion rate (UAER) from baseline to 24 weeks.

Results: In hypertensive type 2 diabetes patients, irbesartan significantly reduced albuminuria (mean change of UAER (log transformed) 0.14 versus 0.000 in placebo groups $(\mathrm{p}<0.05)$. The reductions in SBP and DBP observed in the irbesartan group were greater than in the placebo group $(7.0 / 4.1 \mathrm{mmHg}$ for irbesartan, $1.5 / 1.9 \mathrm{mmHg}$ for placebo, $\mathrm{p}<0.05$ ). In normotensive Type 2 diabetes patients, irbesartan also significantly reduce albuminuria (mean change of UAER (log transformed) 0.18 versus 0.000 in placebo groups, $\mathrm{p}<0.05)$. Covariance analysis by ANCOVA showed significant UAER reduction independent of BP reduction. Overall adverse event-rate was similar between irbesartan (44.26\%) and placebo group (51.69\%). Drug related adverse events were also similar in both groups (placebo 9.64\%; irbesartan 13.66\%).

Conclusions: Irbesartan $300 \mathrm{mg}$ is effective in reducing urinary albumin excretion rate in Chinese hypertensive- or normotensive patients with type 2 diabetes. The observed effects on albuminuria occured partially BPindependent. Irbesartan showed a comparable safety profile as placebo. Supported by sanofi aventis China

\section{1}

Prevalence of ACE inhibition escape phenomenon in patients with type 2 diabetes end diabetic nephropathy

N. Trubutsyna, L. Nikankina, G. Katsya, A. Ilin, M. Shamkalova, A. Deev, S. Kuharenko, A. Aleksandrov, N. Goncharov, M. Shestakova;

Nephropathy, Endocrinology research center, Moscow, Russian Federation.

Background and Aims: We evaluated the prevalence of the ACE inhibition escape phenomenon during long-term ACE inhibitors (ACEI) treatment in patients with type 2 diabetes and diabetic nephropathy (DN).

Materials and Methods: A total of 62 patients ( 37 men, 25 women) with type 2 diabetes and DN who received ACEI were studied. Escape of ACE inhibition was defined as an increase in plasma angiotensin II (AII) more than $50,6 \mathrm{pg} / \mathrm{ml}$. Upper limit of normal range was calculated (mean $\pm 1 \mathrm{SD}$ ) from values of control subjects, who involved age-matched normotensive subjects without diabetes. Dietary salt intake was controlled.

Results: Duration of ACEI treatment was 6.6 3.4 years. Plasma AII level in the control group was $34.5 \pm 16.1 \mathrm{pg} / \mathrm{ml}$. Patients were divided into two groups according to their levels of AII: more than $50,6 \mathrm{pg} / \mathrm{ml}$ - escape group (EG), less than 50,6 pg/ml - non-escape group (NEG). ACE inhibition escape occurred in 24 patients (39\%). There were no significant differences between the groups in age, duration of diabetes, albuminuria, lipid levels, 24$\mathrm{h}$ blood pressure, duration of ACEI treatment, diabetes control, hypertension management, 24-h urinary sodium. Patients in EG had a significantly higher cardiac interventricular septum thickness $(\mathrm{p}=0.02)$, more often had hypoand akinetic segments of myocardium $(\mathrm{p}=0.03)$. Also they showed lower 24$\mathrm{h}$ urinary potassium excretion ( $\mathrm{p}=0.03)$, higher level of plasma endothelin, serum creatinin and glomerular filtration rate (not statistically significant). Plasma renin concentration was higher in the EG ( $\mathrm{p}=0.004)$. AII did not correlate with aldosterone levels in both groups.

Conclusion: A relatively large percentage of type 2 diabetics with DN is affected by ACE inhibition escape. It may explain the lack of reno-and cardioprotective effect of renin-angiotensin-aldosterone system blockade. Supported by Federal programme of diabetes

\section{2}

Glucose fluctuations and activation of oxidative stress in type 1 diabetes patients

I. M. E. Wentholt ${ }^{1}$, W. Kulik², R. P. J. Michels ${ }^{1}$, J. B. L. Hoekstra ${ }^{1}$,

J. H. DeVries ${ }^{1}$;

${ }^{1}$ Internal Medicine, Academic Medical Center, Amsterdam,

${ }^{2}$ Laboratory for Genetic Metabolic Diseases, Academic Medical Center, Amsterdam, The Netherlands.

Background and Aims: Hyperglycemia is a major risk factor for microvascular complications and accelerated atherosclerosis in diabetes. Hyperglycemiainduced oxidative stress is seen as a key pathofysiological factor in these processes. In contrast to $\mathrm{HbAlc}$, short-term glucose fluctuations showed a strong correlation $(\mathrm{r}=0.86)$ with oxidative stress in 21 type 2 diabetes patients (Monnier, JAMA 2006), and may have predictive value for development of diabetic complications. We evaluated the relation between glucose fluctuations and oxidative stress in type 1 diabetes patients.

Materials and Methods: Continuous glucose monitors (CGMS gold) were inserted subcutaneously in 25 patients with a mean (SD) HbAlc of 8.1 (0.9) \% for a period of $48 \mathrm{hrs}$. During the measurement, patients collected two subsequent 24 -h urine samples, while 24 age and sex-matched healthy controls collected one 24-h urine for determination of 15(S)-8-iso-PGF $\alpha$ using HPLC MS/MS. Mean Of the Daily Differences (MODD), Mean Amplitude of Glycemic Excursions (MAGE) and Continuous Overlapping Net Glycemic Action (CONGA) over $1 \mathrm{~h}$ were calculated as markers for interday, intra-day and rapid glucose variability, and correlation with 15(S)-8-iso$\mathrm{PGF}_{2} \alpha$ excretion was sought.

Results: Median (IQR) urinary excretion of 15(S)-8-iso-PGF2 $\alpha$ was significantly higher in the patients than in the healthy controls: 159 (137 - 216) pg/mg of creatinine versus $118(101-146) \mathrm{pg} / \mathrm{mg}$ of creatinine $(P$ $=0.001)$. Data on glucose variability were: median (IQR) MODD $3.7(3.2$ - 5.0) $\mathrm{mmol} / \mathrm{L}$, MAGE 7.6 (6.4 - 9.0) mmol/L, CONGA-1 2.3 (2.1 - 2.8) $\mathrm{mmol} / \mathrm{L}$. Univariate regression did not reveal an association for any marker of glucose variability with 15(S)-8-iso-PGF2 $\alpha$ excretion, (maximum $r^{2}=0.01$, 0.08 and 0.07 for inter, intra-day and rapid glucose variability, respectively), 
neither did analysis after correction for HbAlc, age, gender and smoking in a multivariate model. Spearman's rho correlation coefficients between 15(S)-8iso-PGF2 $\alpha$ excretion and MODD, MAGE and CONGA-1 were -0.148, -0.399 and -0.207 , respectively, all non-significant.

Conclusion: We report no relationship between inter-, intra-day and rapid glucose variability and urinary 15 (S)-8-iso-PGF2 $\alpha$, determined with a highly specific method of HPLC MS/MS. Our data confirm that patients with type 1 diabetes have higher levels of urinary 15(S)-8-iso-PGF2 $\alpha$ than age and sex matched healthy controls, suggesting the existence of an oxidative stress favouring factor, other than glucose variability. We did not see a relation between high glucose variability and elevated levels of oxidative stress in patients with type 1 diabetes.

\section{3}

The therapeutic efficacy of prostglandin E1 in diabetic patients with nephropathy

L. Liao' ${ }^{1}$, J. Dong ${ }^{2}$, S. C. Liew ${ }^{3}$, K. O. Lee ${ }^{4}$, J. Zhao';

${ }^{1}$ Endocrinology, Shandong Provincial Hospital, Jinan, China,

${ }^{2}$ Endocrinology, Qilu Hospital, Jinan, China, ${ }^{3}$ Endocrinology, The National University Hospital, Singapore, ${ }^{4}$ Endocrinology, The National University

Hospital, Singapore.

Background and Aims: To investigate the efficacy of prostglandin E1 (PGE1) in diabetic patients with diabetic nephropathy (DN).

Materials and Methods: 120 patients, 60 with microalbuminuria (30 to $300 \mathrm{mg} / 24 \mathrm{hrs}$ ) and 60 with clinical nephropathy $(>300 \mathrm{mg} / 24 \mathrm{hrs})$ were studied. The patients had a mean (SD) age of $58 \pm 10.6$ yrs (Range:28-78 yrs), mean duration of diabetes of $10.5 \pm 5.0$ yrs (Range:2 -24 yrs) and all had acceptable $\mathrm{BP}<130 / 80 \mathrm{~mm} \mathrm{Hg}$. The 60 patients with microalbuminuria were randomly assigned to two treatment groups, microalbuminuria - ACEI group, treated with ACEI (benazpril, Lotensin) 10mg qd for 12 months, and microalbuminuria - PGE1 group who were treated with benazpril 10mg qd for 12 months AND had intravenous lipo-prostaglandin E1 (alprostadil injection) daily for 15 days. Similarly, the 60 patients with clinical nephropathy were also randomly assigned to nephropathy- ACEI group and nephropathy - PGE1 group.

Results: Urine albuminuria was significantly decreased in all the patients at the end of the one year study period $(\mathrm{P}<0.01)$. The decrease in the PGE1 - treated groups was more significant compared with the benazpril alone groups $(\mathrm{P}<0.01)$. Urine albuminuria in some patients decreased to normal range ( $<30 \mathrm{mg} /$ day) immediately after 15 days' PGE1 treatment. At the end of $12 \mathrm{mths}$, all the patients who had received PGE1 treatment had significantly less proteinuria compared to benazpril alone patients (Tab.1).

Conclusion: PGE1 is effective and rapid in decreasing albuminuria, and is significantly more effective than therapy of benazpril alone.

Supported by Doctor's fund of Shandong Province
PS 113 Diabetic foot: skin and bone

\section{4}

Patients with type 1 diabetes and recurrent Charcot osteoarthropathy have reduced bone mass in the calcaneum and osteopaenia of the femoral neck and need supplementation with vitamin $D$ therapy

M. E. Edmonds ${ }^{1}$, M. Buxton-Thomas ${ }^{2}$, C. Moniz ${ }^{3}$, N. L. Petrova ${ }^{1}$;

${ }^{1}$ Diabetic Foot Clinic, ${ }^{2}$ Nuclear Medicine Department, ${ }^{3}$ Department of Clinical Biochemistry, King's College Hospital, London, United Kingdom.

Background and Aims: We have noted a group of young diabetic patients with type 1 diabetes who presented with Charcot osteoarthropathy and continued to have recurrent Charcot osteoarthropathy at different sites associated with fracture. The aim of this study was to perform a bone evaluation in this group of patients by measuring calcaneal bone mass, bone mineral density (BMD) of the femoral neck and lumbar spine and biochemical parameters of bone health.

Materials and Methods: There were 8 patients with type 1 diabetes $(7 \mathrm{females}$ and 1 male). Mean age was $40 \pm 7.7$ years (mean \pm SD) and mean duration of diabetes was $25 \pm 5.5$ years. After their initial presentation, 7 patients developed fractures in the contralateral foot leading to Charcot osteoarthropathy and 2 of these also developed fractures of the tibial condyle leading to Charcot knee. One further patient sustained a pelvic fracture. Bone mineral density was measured in the lumbar spine and femoral neck by using DEXA scan. Bone mineral mass of the calcaneum was assessed by quantitative ultrasound. Bone mineral mass and density were expressed as a T-score. Serum vitamin $\mathrm{D}$, parathyroid hormone (PTH), calcium and creatinine were also measured. Estimated glomerular filtration rate (eGFR) was calculated.

Results: Although BMD was within normal range in the spine (T-score: $0.8 \pm 0.4 \mathrm{SD}$ ), there was osteopaenia in the femoral neck (T-score:- $1.7 \pm 0.8 \mathrm{SD}$ ) and T-score in the calcaneum was $-2.7 \pm 0.4 \mathrm{SD}$, which was very low. There was evidence of vitamin D insufficiency with a mean level of $14 \pm 6.8 \mathrm{ug} / \mathrm{L}$ (reference range: $10-42 \mathrm{ug} / \mathrm{L}$ ). Corrected calcium level was $2.26 \pm 0.1 \mathrm{mMol} / \mathrm{L}$ at the lower limit of the reference range $(2.20-2.60 \mathrm{mMol} / \mathrm{L})$. Serum PTH was $56 \pm 38.6 \mathrm{ng} / \mathrm{L}$ and was at the upper limit of the reference range (13-75 ng/L). Serum creatinine was raised at $131 \pm 26.8 \mathrm{uMol} / \mathrm{L}$ (reference range: $45-120$ $\mathrm{uMol} / \mathrm{L}$ ). Estimated GFR was $41 \pm 15 \mathrm{ml} / \mathrm{min}$ (moderate impairment of renal function $<60 \mathrm{ml} / \mathrm{min}$ ).

Conclusion: These patients with type 1 diabetes and recurrent Charcot osteoarthropathy had reduced calcaneal bone mass, osteopaenia of the femoral neck and normal BMD in the lumbar spine. They also had vitamin $\mathrm{D}$ insufficiency associated with a compensatory rise in PTH and moderate impairment of renal function. This indicates that bone health in patients with type 1 diabetes and recurrent Charcot osteoarthropathy should be investigated and optimised with vitamin D supplementation. Possible osteoporosis prevention therapies should also be considered.

Supported by Diabetes UK

Table 1: The characteristics before therapy and at the end of 12th month in patients with DN

\begin{tabular}{|c|c|c|c|c|c|}
\hline Group & & $\begin{array}{l}\mathrm{BUN} \\
(\mathrm{mmol} / \mathrm{L})\end{array}$ & $\mathrm{C}_{\mathrm{Cr}}{ }^{(\mathrm{mg} / \mathrm{min})}$ & $\begin{array}{l}\text { Microalbumin } \\
\text { (mg/24h) }\end{array}$ & $\begin{array}{l}\text { ARE } \\
\text { (ug/min) }\end{array}$ \\
\hline \multicolumn{6}{|c|}{ microalbuminuria } \\
\hline PGE1 group & $\begin{array}{l}\text { Before therapy } \\
\text { At the end of } 12 \text { th month }\end{array}$ & $\begin{array}{l}6.8 \pm 2.3 \\
5.9 \pm 1.8\end{array}$ & $\begin{array}{l}74.7 \pm 21.9 \\
84.9 \pm 24.0\end{array}$ & $\begin{array}{l}124.6 \pm 10.2 \\
24.9 \pm 5.7\end{array}$ & $\begin{array}{l}84.5 \pm 7.8 \\
16.5 \pm 3.6\end{array}$ \\
\hline ACEI group & $\begin{array}{l}\text { Before therapy } \\
\text { At the end of } 12 \text { th month }\end{array}$ & $\begin{array}{l}6.5 \pm 2.0 \\
6.2 \pm 2.1\end{array}$ & $\begin{array}{l}75.6 \pm 18.8 \\
80.2 \pm 20.5\end{array}$ & $\begin{array}{l}118.4 \pm 9.2 \\
55.4 \pm 6.8\end{array}$ & $\begin{array}{l}82.2 \pm 6.6 \\
38.5 \pm 4.8\end{array}$ \\
\hline \multicolumn{6}{|c|}{ Clinical diabetic nepropathy } \\
\hline PGE1 group & $\begin{array}{l}\text { Before therapy } \\
\text { At the end of 12th month }\end{array}$ & $\begin{array}{l}9.8 \pm 2.8 \\
9.1 \pm 2.5\end{array}$ & $\begin{array}{l}51.6 \pm 24.9 \\
60.2 \pm 30.4\end{array}$ & $\begin{array}{l}1698 \pm 387.1 \\
593.3 \pm 212.3\end{array}$ & $\begin{array}{l}1125 \pm 350.6 \\
391.8 \pm 201\end{array}$ \\
\hline ACEI group & $\begin{array}{l}\text { Before therapy } \\
\text { At the end of 12th month }\end{array}$ & $\begin{array}{l}9.5 \pm 2.2 \\
9.2 \pm 2.0\end{array}$ & $\begin{array}{l}50.2 \pm 22.5 \\
56.4 \pm 25.6\end{array}$ & $\begin{array}{l}1720 \pm 352.0 \\
1020.6 \pm 250.2\end{array}$ & $\begin{array}{l}1146.7 \pm 320.2 \\
780.5 \pm 225.2\end{array}$ \\
\hline
\end{tabular}




\section{5}

Biochemical markers of bone turnover in patients with type 2 diabetes and their correlations with $\mathrm{HbA}_{1 \mathrm{c}}$ levels

B. Ozkan ${ }^{1}$, A. Ozkan ${ }^{2}$, I. Capoglu ${ }^{3}$, Z. Umudum², Y. Sahin ${ }^{2}$, H. Alp²; ${ }^{1}$ Pediatric Endocrinology and Metabolism, ${ }^{2}$ Clinical Biochemistry, ${ }^{3}$ Endocrinology and Metabolism, Ataturk University Faculty of Medicine, Erzurum, Turkey.

Background and Aims: In this study, the probable effects of metabolic control on bone turnover markers in patients with type 2 diabetes mellitus (Type $2 \mathrm{DM}$ ) were prospectively carried out.

Materials and Methods: Thirty-five cases with type 2 DM aged between 37 and 66 years ( 18 female, 17 male) were included in this study. All cases were evaluated with particular respect to diabetic ages, current ages, follow-up periods, body mass indexes, treatment protocols, complications and bone turnover markers (bone specific alkaline phosphatase (B-ALP), osteocalcine (OC), urine deoxypyridinoline (DPyr), urine n-telopetit (NTx) both at the beginning and end of the study (mean $4.9 \pm 1.2$ months).

Results: Mean $\mathrm{HbA}_{1 \mathrm{c}}(\%)$ concentration of the cases was $10.6 \pm 1.6 \%$, and this rate decreased to the level of $7.7 \pm 1.0 \%$ nearly 5 months follow-up period $(p=0.000)$. In parallel with decrease of $\mathrm{HbA}_{1 \mathrm{c}}$ level, the bone turnover markers also were reduced significantly at the same period. While mean concentration of urinary DPyr (nmolDPyr/mmolCr), which is one of the markers of bone resorption, was $28.6 \pm 11.6$ in the beginning of the study, it decreased to the level of $17.9 \pm 7.1$ at the end of the follow-up period $(p=0.000)$. Similarly, mean urinary NTx (nmolBCE/mmolCr) concentration, that is also marker of bone resorption, showed signifcantly decrease from the level of $93 \pm 13.7$ to $67.1 \pm 12.8$ at the same period $(p=0.000)$. On the other hand, the reduce of bone formation parameters was also found in parallel with $\mathrm{HbA}_{1 \mathrm{c}}$ level in posted time-interval. While mean serum B-ALP levels were $129.5 \pm 37.3 \mathrm{U} / \mathrm{l}$ in the beginning of the study, it was reduced to the level of $94.8 \pm 35.1 \mathrm{U} / \mathrm{l}$ at the end of the study. Similarly, serum OC $(\mathrm{ng} / \mathrm{ml})$ concentration decreased from $94.6 \pm 16.8 \mathrm{ng} / \mathrm{ml}$ to $66.3 \pm 17.3 \mathrm{ng} / \mathrm{ml}$ and significantly was correlated with the $\mathrm{HbA}$ levels during study period.

Conclusion: We concluded that effective management of the metabolic disorder in cases with type 2 diabetes is the one of the most important contributors for improvement of the bone turnover.

\section{6}

RANKL plays an important role as activator of osteoclastic bone resorption in acute Charcot osteoarthropathy

N. L. Petrova ${ }^{1}$, G. Mabilleau ${ }^{2}$, M. E. Edmonds ${ }^{1}$, A. Sabokbar ${ }^{2}$;

${ }^{1}$ Diabetic Foot Clinic, King's College Hospital, London,

${ }^{2}$ Botnar Research Centre, Nuffield Department of Orthopaedic Surgery, University of Oxford, United Kingdom.

Background and Aims: Charcot osteoarthropathy is characterised by increased bone resorption, but the cellular mechanisms are not fully understood. It is possible that receptor activator of nuclear factor $\kappa \beta$ ligand (RANKL), a recently described essential cytokine for the formation and activation of osteoclasts, may play an important role as an activator of osteoclastic bone resorption in acute Charcot osteoarthropathy.

The aim of this study was to investigate the involvement of RANKL in the pathogenesis of Charcot osteoarthropathy by determining and comparing the extent of osteoclast formation and their bone-resorbing activity in patients with Charcot osteoarthropathy and age/sex-matched controls.

Materials and Methods: We studied 8 male subjects, mean age of $59 \pm 5$ years (mean \pm SD). These included 3 patients presenting with acute Charcot osteoarthropathy, and 5 control patients (3 with diabetes and 2 without diabetes). We used a technique to generate functional human osteoclasts in vitro from peripheral blood monocytes. It is known that in the presence of macrophage-colony stimulating factor (M-CSF), RANKL induces osteoclast formation from a subpopulation of monocyte cells, which are capable of differentiation into osteoclasts. Peripheral blood monocytes isolated from the EDTA-treated whole blood taken on the same day from all patient and control subjects, were cultured on dentine slices and glass coverslips in the presence of M-CSF and soluble RANKL. Osteoclasts were evident after 10-14 days on coverslips as multinucleated cells which stained positive for tartrate resistant acid phosphatase (TRAcP); one of the osteoclast-specific markers. The number of osteoclasts formed on glass coverslips was compared between the patients with Charcot osteoarthropathy and controls. Functional evidence of osteoclastic resorption was determined on dentine slices after 21 days in culture and the extent of lacunar resorption was expressed as the percentage of eroded surface by the number of formed TRAcP-positive osteoclasts.

Results: Although the mean number of newly-formed osteoclasts in vitro in the presence of M-CSF and sRANKL in the patients with Charcot osteoarthropathy was similar with that of controls $(31 \pm 25.9$ vs. $44 \pm 41.2$, $\mathrm{p}=0.654$ ), the osteoclasts in the Charcot patients were more aggressive and demonstrated significant increased resorbing activity. The extent of lacunar resorption in the patients with Charcot osteoarthropathy and the percentage of eroded surface by osteoclasts was significantly greater compared with controls ( $11.52 \pm 6.2 \%$ vs. $4.05 \pm 1.3 \%$; $\mathrm{p}=0.036)$.

Conclusion: Using this technique we were able to generate osteoclasts from circulating osteoclast precursors in vitro, by the addition of sRANKL in the presence of M-CSF in both Charcot patients and controls. Although the number of osteoclasts in the patients with Charcot osteoarthropathy was similar to that in the control subjects, the osteoclasts which were formed from the patients with Charcot osteoarthropathy were functionally more aggressive, exhibiting a significant increase in the extent of resorbing activity.

This is the first scientific evidence that RANKL plays an important role as an activator of osteoclastic bone resorption in acute Charcot osteoarthropathy. Supported by EFSD/AstraZeneca Clinical Travel Fellowship and Diabetes UK

\section{7}

Impaired reaction to hypoxia as a mechanism for development of diabetic foot ulcers

S.-B. Catrina ${ }^{1,2}$, I. Botusan ${ }^{1,2}$, V. Sunkari ${ }^{1}$, O. Savu ${ }^{1,3}$, J. Grünler ${ }^{1}$, K. Brismar ${ }^{1}$; ${ }^{1}$ Molecular Medicine and Surgery, Karolinska Institute, Stockholm, Sweden, ${ }^{2}$ Endocrinology, University of Medicine and Pharmacy "Carol Davila", Bucharest, Romania, ${ }^{3}$ Diabetes, N.C. Paulescu Institute, Bucharest, Romania.

Background: Diabetic foot ulcers represent major therapeutic concern, where chronic hyperglycemia and hypoxia are suggested to play essential roles. Hypoxia-inducible factor 1 (HIF-1) is a transcription factor expressed in response to physiologically relevant levels of hypoxia by stabilization of its alpha subunit. It induces more then 70 genes, which adapt the cell to low oxygen tension. We have proposed that hyperglycemia induce a deficient reaction of the cells to hypoxia and this deficit is a central mechanism in the development of diabetic foot ulcers .

Material and Methods: The effects of hyperglycemia on HIF-1alpha stability and function were performed in primary dermal fibroblasts and primary dermal endothelial cells from humans and mice. Protein expression was evaluated by Western blot while function and translocation by transient transfection with GFP tagged constructs or by luciferase assay. Evaluation of the expression of target genes was done by quantitative RT-PCR. The animal experiments were based on a full thickness wound model in $\mathrm{db} / \mathrm{db}$ mice. HIF 1 alpha was induced locally by chemical inhibition of its degradation or by adenoviral transfer and the healing process was followed by taking pictures, histology, immunohistochemistry, qRT-PCR.

Results: We have demonstrated that hyperglycemia is able to interfere with the hypoxia-dependent stabilization of HIF- $1 \alpha$ protein at the degradation level. High glucose levels decrease as well the transcription activation potency of HIF-1 $\alpha$, as assessed by transient hypoxia-inducible reporter gene assays and RT-PCR of its target genes. The interference of hyperglycemia with hypoxiadependent stabilization of HIF $\alpha$ protein levels was confirmed in vivo where only very low levels of HIF $1 \alpha$ protein could be detected diabetic wounds, as compared to chronic venous ulcers. As a proof of concept we were able to show that induction of HIF- $1 \alpha$ by a chemical approach or by viral transfer in experimental wounds in $\mathrm{db} / \mathrm{db}$ mice is able to normalize the healing rate.

Conclusion: In conclusion, we demonstrate that hyperglycemia, impairs hypoxia-dependent protection of HIF-1 $\alpha$ against proteosomal degradation and we were able to show that by correcting this defect the wound healing can be normalized in a well established diabetic wound model.

Supported by Family Stefan Persson Foundation, Juvenile Diabetes Research Found, Fredrik och Ingrid Thurings Stiftelse, Berth von Kantzows Stiftelse 


\section{8}

Autologous tissue repair cells in the treatment of critical limb ischaemia (CLI) induced chronic foot ulcer on diabetic foot patients without option of revascularisation: a new therapeutic approach?

S. Kirana ${ }^{1}$, B. Stratmann ${ }^{1}$, D. Lammers ${ }^{1}$, T. Quast ${ }^{1}$, P. Minartz ${ }^{1}$, M. Negrean ${ }^{1}$, A. Stirban' 1 , S. Petrule ${ }^{1}$, M. H. Gastens ${ }^{2}$, H. Körperich ${ }^{3}$, C. Götting ${ }^{2}$, W. Prohaska ${ }^{2}$, C. Prante ${ }^{2}$, K. Kleesiek ${ }^{2}$, D. Tschöpe ${ }^{1}$;

${ }^{1}$ Heart and Diabetes Center NRW, ${ }^{2}$ Institute for Laboratory and Transfusion Medicine, Heart and Diabetes Center NRW, ${ }^{3}$ Institute for Radiology,

Nuclear Medicine and Molecular Imaging, Heart and Diabetes Center NRW, Bad Oeynhausen, Germany.

Background and Aims: Diabetic Foot Syndrome (DFS) appears as a concomitant illness of diabetes mellitus. Diabetes is one of the main causes of non-traumatic amputation in Germany due to severe peripheral arterial occlusive disease with chronic critical limb ischemia being the most abundant problems. Ulceration results as a failure of the microcirculation and occlusive disease of larger blood vessels exacerbating pre-existing microvascular abnormalities.

The aim of this study is to assess the improvement of blood perfusion induced by expanded bone marrow stem cells for wound healing.

Materials and Methods: Eleven DFS patients with CLI and without option of surgical or interventional revascularisation were recruited and randomized to either the transplant group or the control group. About $40 \mathrm{ml}$ of bone marrow was harvested from the iliac crest, followed by preparation and culture of mononuclear cells. Application sites of the enriched mesenchymal bone marrow cells (BM) or expanded autologous stem cells - tissue repair cells (TRC) are intramuscular (i.m.) into the M. gastrocnemius or intraarterial (i.a.) into the A. femoralis communis. Three patients were excluded (due to hypoplastic bone marrow, withdrawal of patient's consent and amputation of affected lower limb).

Results:

\begin{tabular}{|c|c|c|c|c|c|c|c|c|}
\hline \multirow[t]{2}{*}{ Pt No. } & \multirow[t]{2}{*}{ Group } & \multicolumn{2}{|c|}{ Before } & \multicolumn{2}{|c|}{20 wks } & \multicolumn{2}{|c|}{52 wks } & \multirow{2}{*}{$\begin{array}{l}\text { Complete wound } \\
\text { healing (wks) }\end{array}$} \\
\hline & & LD & BOLD & LD & BOLD & LD & BOLD & \\
\hline 1 & TRC i.a & 2.23 & 1.5 & 2.2 & -1.3 & 6.7 & 3.6 & 44 \\
\hline 2 & TRC i.m & 1.27 & 5.3 & 1.48 & 7.1 & 1.08 & 6.6 & 40 \\
\hline 3 & Control & 1.17 & 12.6 & 3.0 & 4.6 & 1.28 & 6.3 & $\begin{array}{l}\text { No wound } \\
\text { healing }\end{array}$ \\
\hline 4 & BM i.m & 1.33 & 1.7 & 5.39 & 2.8 & 1.18 & * & 20 \\
\hline 5 & BM i.m & 4.04 & 1.5 & 3.06 & 1.3 & 2.62 & 0.4 & 27 \\
\hline 6 & TRC i.a & 1.08 & 13.3 & 1.26 & 13.0 & $* *$ & $* *$ & 20 \\
\hline 7 & BM i.a & 0.71 & -1.6 & 1.76 & 2.3 & 1.51 & 3.6 & $\begin{array}{l}\text { No wound } \\
\text { healing }{ }^{1)}\end{array}$ \\
\hline 8 & BM i.m & 2.82 & 4.3 & 1.30 & 1.6 & $* * *$ & $* * *$ & 48 \\
\hline
\end{tabular}

LD: Laser Doppler; BOLD: Blood Oxygen Level Dependency, measurement using magnetic resonance tomography technique; ${ }^{*}$ Pat refused the diagnostic; ${ }^{* *}$ Analysis will be performed in June 2007; ${ }^{* *}$ Analysis will be performed in April 2007; 1) No wound healing because of osteomyelitis

Independent from application methods, all patients treated with BM or TRC show tendential improvements of microcirculation (reactive hyperemia) of the affected foot as well as complete primary wound healing. No serious adverse event related to the treatment was noted. No calcification of soft tissue has been observed.

Conclusions: The transplantation of expanded autologous bone marrow stem cells as well as mesenchymal bone marrow cells in diabetic patients with critical limb ischemia induced chronic tissue ulcers proved to be safe and shows tendential improvement of microcirculation and complete wound healing.

Aastrom Biosciences, Inc., Ann Arbor
1129

Non-viral EGF (Epidermal Growth Factor) gene therapy accelerated cutaneous wound healing in streptozotocin diabetic $\mathrm{C57} \mathrm{BL} / 6 \mathrm{~J}$ mice J. Park ${ }^{1}$, H. Chung ${ }^{1}$, C. Yoon ${ }^{1}$, M. Kwon ${ }^{1}$, S. Lee ${ }^{1}$, M. Kim²;

${ }^{1}$ Division of Endocrinology and Metabolism, Department of Internal Medicine, Paik Memorial Institue for Clinical Research, Pusan Paik Hospital, Inje University, Busan, ${ }^{2}$ Division of Endocrinology and Metabolism, Department of Internal Medicine, Maryknoll General Hospital, Busan, Republic of Korea.

Background and Aims: EGF (Epidermal Growth Factor) is one the important growth factors involved in the healing of wounds. Topical peptide EGF has been used for the treatment of diabetic foot ulcer in several countries, but its high cost and the inconvenience of daily application due to its short half life have limited its wide spread clinical usage. EGF gene therapy might have potential to overcome these limitations. Continuous production of EGF at the transfected tissue at the margin just beneath the cutaneous wound might bypass the hostile degradation of EGF by over expressed proteinases at the wound bed, and the long term gene expression could enable so-called "one dose for cure". In addition the direct and indirect cross talking among various growth factors involved in wound healing might facilitate the sound healing.

Materials and Methods: We cloned novel mini-circle plasmid DNA containing one of the isoform of human EGF EGF ${ }^{828}$ driven by the chicken $\beta$-actin promoter with furin recognition sequence to enhance the release from the Golgi apparatus. The biologic competence of this novel plasmid was verified using cultured HEK293 and A549 cells via the increased phosphorylation of PI3K and GSK3 3 . Ultrasound micro-bubble destruction method with SonoVue $\left(\mathrm{SF}_{6}\right)$ as a bubbling agent was used for non-viral gene delivery. Streptozotocin was injected intraperitoneally to induce diabetes mellitus into total $20 \mathrm{C} 57 \mathrm{BL} / 6 \mathrm{~J}$ mice, and the another 20 mice were served as control group. Expressed EGF tissue concentrations were measured via ELISA and semi-quantitative RT-PCR. VEGF, KGF and TGF- $\beta$ expressions were also traced via Western blot, ELISA and RT-PCR. The degrees of neoangiogenesis were assessed by qualitative laser Doppler flowmetry (Periscan, PIM).

Results: Wound healing in diabetic animals was markedly enhanced after EGF gene therapy. The level of EGF and the degree of EGF mRNA expression were markedly increased in the gene therapy group. The level of EGF gradually decreased 10 days after gene injection. VEGF, KGF and TGF$\beta$ concentrations were also changed after EGF gene therapy. The histologic analysis revealed that the healing process was characterized by increased neovascularization and epithelization at the wound. No side effects were noticed during this experiment.

Conclusion: In conclusion, non-viral EGF gene therapy could accelerate cutaneous wound healing in stretpzotocin diabetic mice without any significant side effects. This strategy could be used in actual human trial not only due to its superior efficacy but also appreciable safety. Supported by KDA(Korean Diabetes Association)

\section{0}

Different expression of Connexin 43 during wound healing in an ex-vivo skin organ culture model transplanted with diabetic and non-diabetic keratinocytes

R. Lobmann ${ }^{1}$, S. Zacheja ${ }^{1}$, P. Houdeck ${ }^{2}$, J. Brandner ${ }^{2}$;

${ }^{1}$ Endocrinology and Metabolism, University of Magdeburg,

${ }^{2}$ Dept. of Dermatology, University Hamburg-Eppendorf, Germany.

Background and Aims: Wound healing is known to require a well organized balance of numerous cytokines, growthfactors, as well as proteases (MMP's) and their inhibitors (TIMP's). Moreover, direct cell-cell communication via gap junctions plays an important role in the dynamics of keratinocytes during reepithelialization. Disruption of these biological processes may lead to the formation of chronic wounds, such as diabetic foot ulcers.

Materials and Methods: To assess the differences in mRNA expression of connexin (Cx) 43, an important gap junctional protein of the epidermis, during wound healing of diabetic and non-diabetic patients, an $8 \mathrm{~mm}$ punch biopsy was taken from 14 diabetic and 11 non-diabetic intact lateral thighs. Keratinocytes were cultured and transplanted into a skin organ culture model. mRNA-expression of Cx 43 was analyzed after $0 \mathrm{hrs}, 7 \mathrm{hrs}$ and $18 \mathrm{hrs} / 24 \mathrm{hrs}$ of wound healing by using quantitative real-time PCR (TaqMan). 
Results: We demonstrate significant different dynamics of $\mathrm{Cx}$ expression during the wound healing process whereas in our previous studies no statistical relevant differences of mRNA-expression of MMP's, TIMP's, and cytokines (TNF $\alpha$, IL-1 $\beta$ ) were shown between diabetic and non-diabetic patients at the various points of time. RT-PCR indicated a delayed decrease of $\mathrm{Cx} 43(\mathrm{p}=0.001)$ during wound healing of diabetic keratinocytes in contrast to non-diabetics who showed an early loss of $\mathrm{Cx} 43(\mathrm{p}=0.009)$ after 7 hours.

Conclusion: Our results suggest the importance of a well-regulated balance of $\mathrm{Cx}$ such as an early downregulation of $\mathrm{Cx} 43$ to initiate the migration of keratinocytes. A delayed decrease of $\mathrm{Cx} 43$ may lead to chronification of diabetic wounds.

Supported by German Diabetes Society

\section{1}

Assessment of the activity of Pédimed ${ }^{\circ}$ cream on bacterial flora and skin hydratation in diabetic feet

C. Roques ${ }^{1}$, S. Trentin ${ }^{1}$, C. Panizzutti ${ }^{1}$, C. Saint Martory ${ }^{2}$, C. Dupont ${ }^{3}$,

E. Garrigue ${ }^{3}$, A. Degouy²;

${ }^{1}$ Microbiology and Virology, Fonderephar, Toulouse, ${ }^{2}$ European Research

Center on the Skin, Pierre Fabre Research Institute, Toulouse,

${ }^{3}$ Pierre Fabre Santé, Pierre Fabre Laboratories, Castres, France.

Background and Aims: Diabetes is a common disease which generates various complications. Among these, feet lesions which may become serious if left untreated and therefore call for amputation. The objective of this study was to assess the activity of Pédimed ${ }^{\circledR}$ cream on bacterial flora and skin hydration in diabetic feet.

Materials and Methods: Randomized open label comparative monocentric study among 30 diabetic patients ( 20 women and 10 men, mean age : $60.4 \pm 10.7$ years).

Treatment: Randomized application of Pédimed ${ }^{\circledR}$ cream (ruscus aculeatus, tocopheryl nicotinate, olamine piroctone, urea, lactamid, petrolatum jelly and glycerin) to one foot twice daily over a 3 weeks period.

Protocol: Comparative evaluation between treated side (TS) and non-treated side (NTS) at D0 and D21 of the activity on bacterial flora by microbiological testing as well as the hydrating effect by cutaneous clinical score, hydration index (corneometry) and squame samples analysis (D-Squame test).

Results: At D0, there was no significant difference between the 2 application sites as regards the bacterial flora and skin hydration.

At D21, a significant reduction in total aerobic $(-1.75 \log ; p<0.001)$ and aeroanaerobic flora $(-1.60 \log ; p<0.001)$ was observed for TS, with no significant evolution for NTS. Inter-group analysis revealed significant differences between TS and NTS, in favor of TS.

The clinical hydration score was significantly improved for TS $(-52.8 \%$; $p<0.001)$ whereas it decreased for NTS $(+10.5 \% ; p=0.03)$, confirming the significant inter-group difference $(p<0.001)$. The hydration index was significantly increased for TS $(+66.0 \% ; p<0.001)$ and NTS $(+21.6 \%$; $p=0.003)$, with a more significantly marked improvement for TS $(p<0.001)$. Finally, desquamation was significantly reduced for TS $(p<0.001)$, with no significant disease progression for NTS, and a significant inter-group difference $(p<0.002)$.

Conclusion: During the study period, Pédimed ${ }^{\circledR}$ cream demonstrated an antimicrobial activity, expressed by a reduction in the bacterial flora, as well as a clinical improvement in skin dryness, an emollient effect and a regulation or reduction of desquamation.

\section{PS 114 Complications: new markers and inflammation}

\section{2}

Effect of natriuretic peptide clearance receptor exon $8 \mathrm{~A} / \mathrm{G}$ polymorphism on urinary albumin excretion and blood pressure responses to dietary salt reduction

S. Hadjadj;

Endocrinology-Diabetology, Poitiers University Hospital, France.

Introduction: There is a familial clustering for the renal complications associated with type 2 diabetes. The natriuretic peptide clearance receptor (NPCR) was shown to be a risk factor for diabetic nephropathy in longitudinal epidemiological studies. The functional rule and clinical relevance of this polymorphism are currently unknown.

Setting: Secondary tertiary diabetic clinic.

Objective of the Study: to analyse the response on urinary albumin expression (UAE) and blood pressure (BP) to the reduction in sodium intake, in type 2 diabetic micro macro albuminuric patients.

Patients and Methods: Type 2 diabetic men with micro macro albuminuria and diuretic treatment were matched for age, RAS blocking medication (ACE inhibitor or ARB) and NPCR polymorphism. They were studied twice in usual dietary sodium intake and after $50 \%$ reduction of sodium intake. The dietary salt reduction lasted 10 days. In the 3 consecutive days preceding the clinical visit, patients collected urines for 24-hour determination of sodium, creatinine and albumin.

Results: 8 AA versus 7 non AA patients were studied. Their clinical characteristics did not differ at baseline (table 1). The dietary sodium reduction was similar in the two groups and resulted in a significant decrease in UAE ( $\mathrm{p}=0.009)$ and systolic $\mathrm{BP}(\mathrm{p}=0.0063)$. However, only those patients with the AA genotype yielded a significant response to dietary salt reduction on UAE ( $\mathrm{p}=0.0117)$ and SBP $(\mathrm{p}=0.0057)$.

AA patients
$(\mathrm{n}=8)$$\quad \begin{aligned} & \text { non-AA patients } \\ & (\mathrm{n}=7)\end{aligned}$

\begin{tabular}{llll}
\hline Baseline data & & & \\
Âge (years) & $61+/-10$ & $66+/-6$ & 0.2363 \\
SBP (mmHg) & $143+/-14$ & $137+/-7$ & 0.3982 \\
DBP (mmHg) & $79+/-6$ & $73+/-6$ & 0.0807 \\
estimated GFR (ml/min) & $88+/-36$ & $72+/-24$ & 0.3596 \\
Weight $(\mathrm{kg})$ & $89.2+/-11.5$ & $89.7+/-15.7$ & 0.9456 \\
Natriuresis (mmol/24h) & $231+/-69$ & $231+/-104$ & 0.9994 \\
UAE (mg/24h) & $231(134-526)$ & $282(60-465)$ & 0.7285 \\
Change between the 2 visits & & & \\
Natriuresis Reduction & $107+/-62$ & $111+/-69$ & 0.9105 \\
(mmol/24 h ) & & & \\
SBP Reduction (mmHg) & $18+/-13$ & $3+/-9$ & 0.0262 \\
UAE Reduction $(\mathrm{mg} / 24 \mathrm{~h})$ & $93(48 ; 250)$ & $20(45 ;-8)$ & 0.0372 \\
\hline
\end{tabular}

Conclusion: The short-term response to dietary sodium reduction is related to the NPCR exon $8 \mathrm{~A} / \mathrm{G}$ polymorphism. This could lead to potential modification of therapeutic strategies. Supported by AFD RECHERCHE 2003

\section{3}

The endothelial thrombomodulin - protein C system modulates diabetic nephropathy: protective effect of the prothrombotic factor $\mathrm{V}$ Leiden mutation

B. Isermann, I. Vinnikov, B. Hummel, T. Madhusudhan, M. Kashif, S. Herzog, A. Bierhaus, P. P. Nawroth;

Department of Internal Medicine I and Clinical Chemistry, University of Heidelberg, Germany.

Background and Aims: Activation of the coagulation system in diabetes mellitus has been established. Recently we have been able to show that the 
endothelial thrombomodulin (TM) protein C (PC) system, which mediates antiinflammatory and antiapoptotic effects in addition to anticoagulation, protects against diabetic nephropathy in mice. These data established a crucial role of endothelial dysfunction and associated loss of the TM-PC system for diabetic nephropathy (manuscript in revision). The factor V Leiden (FVL) mutation, the most frequent prothrombotic risk factor in Caucasians, disrupts the anticoagulant function of the TM-PC system at the substrate level, while other, receptor dependent effects of the TM-PC system (such as inhibition of inflammation and apoptosis) remain functional. We have previously shown that the FVL mutation is associated with increased levels of activated PC and improved survival in severe inflammation in mice and men (Kerlin, Blood, 2003). Since diabetes is associated with inflammation we addressed experimentally the question whether the FVL mutation (1) results in higher levels of PC activation in diabetic mice in vivo and (2) protects against diabetic nephropathy.

Materials and Methods: Persistent hyperglycemia was induced using streptozotocin in wild-type (WT), heterozygous (FVL ${ }^{\text {wt/q }}$ ) and homozygous $\left(\mathrm{FVL}^{\mathrm{q} / \mathrm{q}}\right) \mathrm{FVL}$ mice. Blood coagulation (TAT, D-Dimer), inflammation (IL-1b, IL-6), apoptosis (TUNEL, p53, Bax, Bcl-2) and nephropathy (albuminuria, histology) were analyzed after 6 months of persistent hyperglycemia.

Results: In diabetic WT mice markers of diabetic nephropathy, including albuminuria, kidney weight, glomerular size, and extracellular matrix deposition (PAS-staining), were significantly increased in comparison to non-diabetic WT mice. Conversely, diabetic FVL ${ }^{q / 9}$ mice were partial and $\mathrm{FVL}^{\text {wt/q }}$ mice were significantly protected against diabetic nephropathy. The protection against diabetic nephropathy in FVL-positive (FVL ${ }^{\text {wt/q }}$ or $\mathrm{FVL}^{\mathrm{q} /}$ q) mice was associated with a normalized $\mathrm{Bax} / \mathrm{Bcl}-2$ ratio. Hyperglycemia induced a significant pro-coagulant response (TAT, D-Dimer) in diabetic FVL-positive mice. The increased thrombin generation was associated with an enhanced PC-activation in diabetic FVL positive mice. These data imply that increased thrombin generation in diabetic FVL-mice results in increased activation of PC. Together with our previous data showing that activated PC protects against diabetic nephropathy through inhibition of glomerular apoptosis these data show that the FVL mutation inhibits apoptosis and diabetic nephropathy through modulation of activated PC dependent signalling via PARs (protease activated receptor).

Conclusion: These results establish that the coagulation system modulates diabetic complications. The cytoprotective effects of the endothelial TM-PC system protect against diabetic nephropathy in vivo. These results show that endothelial dysfunction crucially modulates diabetic nephropathy. Further experiments extending these in vivo findings to humans have been initiated. Supported by DFG IS 67/2-2

\section{4}

Proton NMR metabonomics of type 1 diabetes reveals multivariate biochemical characteristics of diabetic complications

V.-P. Mäkinen ${ }^{1,2}$, P. Soininen ${ }^{3}$, C. Forsblom ${ }^{1,4}$, M. Parkkonen ${ }^{1,4}$, P. Ingman ${ }^{5}$, K. Kaski ${ }^{2}$, M. Ala-Korpela ${ }^{2}$, P.-H. Groop ${ }^{1,4}$;

${ }^{1}$ Folkhälsan Research Center, Folkhälsan Insititute of Genetics, Helsinki, Finland, ${ }^{2}$ Laboratory of Computational Engineering, Helsinki University of Technology, Espoo, Finland, ${ }^{3}$ Department of Chemistry, University of Kuopio, Kuopio, Finland, ${ }^{4}$ Division of Nephrology, Helsinki University Central Hospital, Helsinki, Finland, ${ }^{5}$ Department of Chemistry, University of Turku, Helsinki, Finland.

Background and Aims: Macrovascular disease in connection to diabetic nephropathy accounts for most of the premature deaths in type 1 diabetic patients. Metabolic syndrome is common in the subset that develop these serious complications, which suggests that the risk factors are similar to those in the general population. The definitions of risk criteria, however, may not be well suited for type 1 diabetes nor be accurate enough to detect subtle metabolic changes. For this reason, we have measured proton nuclear magnetic resonance (NMR) spectra of serum for a subset of type 1 diabetic patients from the Finnish Diabetic Nephropathy (FinnDiane) study. Our goal is to determine the multi-variate metabolic characteristics of type 1 diabetes that will serve as the baseline for the prospective phase of FinnDiane, and to evaluate the role of proton NMR as an analytical tool in this effort.

Materials and Methods: Proton NMR spectra of serum for 613 type 1 diabetic patients was measured on a Bruker AVANCE 500Mhz spectrometer. Ageand sex-matched patients were selected from the FinnDiane cohort based on nephropathy status. Proton NMR data was obtained from two molecular windows: a standard spectrum with wide lipoprotein lipid and albumin signals and a T2-filtered spectrum that better reveals the smaller metabolites with lower molecular weights. A double-tube system for reference substance was used to enable absolute quantification of metabolites. The complex spectral data was analysed by constructing a self-organising map, an unsupervised neural network analysis method.

Results: The self-organising map of the spectra identified multi-variate spectral profiles that are linked to clinical criteria such as nephropathy, retinopathy, metabolic syndrome and macrovascular complications. It also revealed specific connections between the diagnoses (e.g. nephropathy) and the corresponding serum biochemistry. The observations were validated by non-spectroscopic data from the FinnDiane database and interpreted with the help of statistical map colourings (figure).

Conclusion: A single proton NMR analysis of serum for type 1 diabetic patients gave enough information for a multi-metabolite characterisation of diabetic complications that is likely to be of great value in the sub-clinical screening of metabolically vulnerable patients. (Fig. 1)

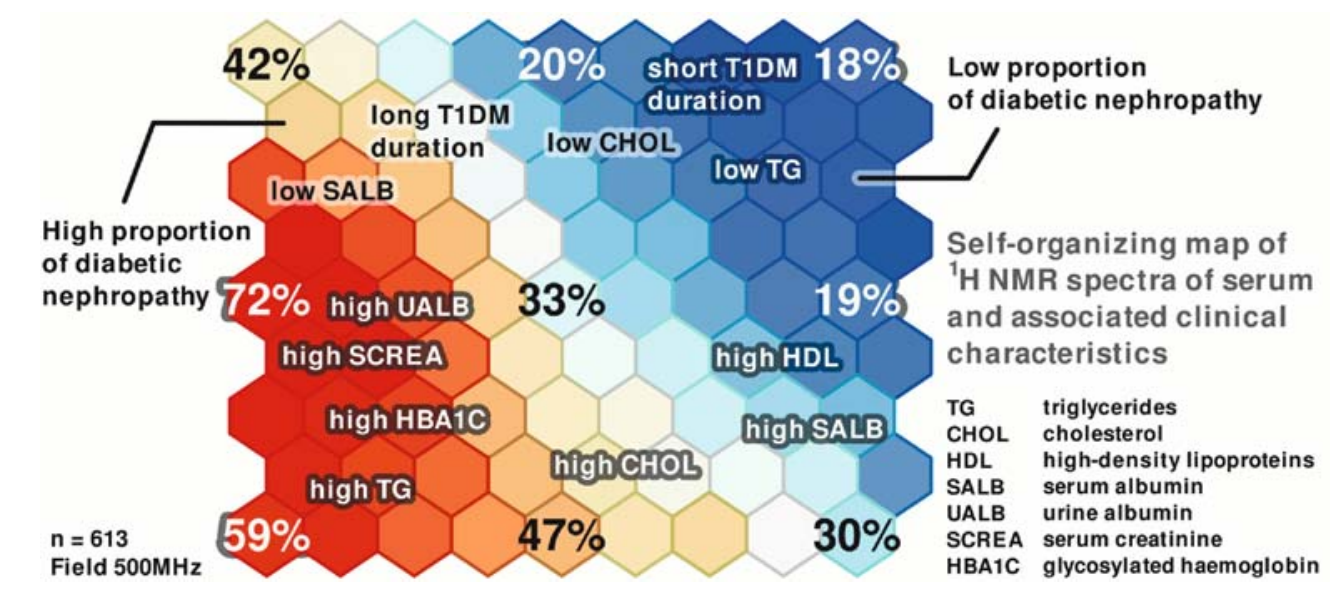

Fig. 1 


\section{5}

New protein biomarkers for diabetic nephropathy

J. McGuire, M. Lajer, L. Tarnow, P. Rossing, F. Pociot;

Steno Diabetes Center, Gentofte, Denmark.

Background and Aims: If early signs of diabetic nephropathy are identified and treatment is initiated early and aggressively it is possible to prevent or at least postpone its development. We applied proteomics to discover new and better protein biomarkers from serum for several aspects of diabetic nephropathy using a significant cohort of type 1 diabetes (T1D) patient samples.

Materials and Methods: The sample cohort contained 168 patient samples split into four groups (normal, micro- and macroalbuminuria plus nondiabetic controls; $\mathrm{n}=42 \mathrm{each}$ ) and was used for discovery of serum-based protein biomarkers for the early detection of diabetic nephropathy. Some of the patients were on anti-hypertensive medication at the time of sampling and are expected to be a representative diabetic sample set. Aliquots $(50 \mu \mathrm{L})$ of serum were processed using parallel, filter plate-based anion exchange fractionation. Four of the six fractions were analyzed in a top-down proteomics approach on CM10, H50 and IMAC30 arrays in a SELDI-TOFMS system. Reference human serum samples were run alongside the patient samples to ascertain intra-assay reproducibility. All steps were performed on a liquid handling robot. Univariate statistical methods were chosen for feature reduction: student's t-test for comparison of normo- and macroalbuminuria groups and ANOVA of a linear regression model of normo-, micro- and macroalbuminuria groups. Peaks showing highly significant $\mathrm{P}$ values $(\leq$ 0.001) in either test were carried forward for further analysis. Peak quality was checked manually to ensure robustness.

Results: We have analysed a large set of diabetic nephropathy serum samples using a top-down proteomics approach based on SELDI-TOF-MS that produced about 7000 mass spectra. The study has to date identified approximately 200 protein peaks in the mass range $2-100 \mathrm{kDa}$ with significant $\mathrm{P}$ values using an ANOVA test among three albuminuria groups (normo-, micro- and macroalbuminuria; $\mathrm{n}=42$ for each group) and among the proteins that are already identified, beta-2-microglobulin is significantly increased $(P=0.002)$ in the macroalbuminuria group $(>300 \mathrm{mg}$ albumin $/ 24$ $\mathrm{h})$ versus the normoalbuminuria group $(<30 \mathrm{mg}$ albumin $/ 24 \mathrm{~h})$ and this is in agreement with what has previously been reported in the literature. Transferrin, an $80 \mathrm{kDa}$ protein, is an important circulating source of iron in the body and was found to be significantly decreased $(\mathrm{P}=0.0002)$ in the macroalbuminuria versus the micro- and normoalbuminuria groups. The identities of the other candidate biomarkers are still under investigation.

Conclusion: With the help of proteomics we have identified over 200 protein peaks that show highly significant differences between normo- and macroalbuminuria groups or ANOVA $\mathrm{P}$ values among the three diabetic groups (normo-, micro- and macroalbuminuria). Two of the proteins have already been identified as beta-2-microglobulin and transferrin. Further markers and a model for prediction of diabetic nephropathy using a panel of serum proteins will be presented.

Supported by SDC

\section{6}

Increased urinary excretion of proinflammatory cytokines is associated with renal structural changes in type 1 diabetic patients

V. V. Klimontov ${ }^{1}$, I. A. Bondar ${ }^{1}$, A. P. Nadeev;

${ }^{1}$ Department of Endocrinology, ${ }^{2}$ Department of Pathology,

Novosibirsk State Medical University, Novosibirsk, Russian Federation.

Background and Aims: Inflammatory mechanisms are believed to be involved in the pathogenesis of diabetic nephropathy. Recent experimental studies have shown that cytokines synthesized by resident renal cells and migrating inflammatory cells, such as monocytes/macrophages, contribute to progression of glomerular and interstitial sclerosis in diabetes. The aim of our study was to assess the relation between urinary excretion of proinflammatory cytokines and diabetic nephropathy in type 1 diabetic patients.

Materials and Methods: 57 patients with type 1 diabetes were examined, including 22 normoalbuminuric (group DNO), 23 microalbuminuric (group DN1) and 12 macroalbuminuric ones (group DN2). Urinary excretion of interleukin-1beta (IL-1beta), monocyte chemoattractant protein-1 (MCP1) and chemokine regulated upon activation, normal T-cell expressed and secreted (RANTES) was determined by ELISA and compared to control (10 healthy subjects, group C). A kidney biopsy was performed in 8 normoalbuminuric and 10 microalbuminuric patients. Mesangial fractional volume $[\mathrm{Vv}(\mathrm{mes} / \mathrm{glom})]$, the width of glomerular and tubular basement membrane (GBM and TBM) was estimated by morphometric analysis. The presence of CD-68-positive cells (macrophages) in glomeruli and interstitium was studied immunohistochemically.

Results: (median, percentile 25-75). IL-1beta excretion was increased significantly in patients with micro- and macroalbuminuria as compared to control (DN0: $0.38,0.24-0.57, p=0.23$; DN1: $0.64,0.31-0.98, p=0.02$; DN2: $1.37,0.97-1.58, p=0.00008$; C: $0.25,0.19-0.48 \mathrm{pg} / \mu \mathrm{mol}$ creatinine). MCP1 excretion exceeded control in all diabetic groups (DN0: 14.7, 5.9-21.6, $p=0.04$; DN1: $14,5.2-37.9, p=0.03$; DN2: 64.3, 42.4-109.3, $p=0.0006$; C: 5.1 , $1.4-16.8 \mathrm{pg} / \mu \mathrm{mol}$ creatinine). RANTES excretion was increased significantly in patients with macroalbuminuria, but not in normo- and microalbuminuric patients (DN0: $0,0-0.1, \mathrm{p}=0.84$; DN1: $0,02,0-0.13, p=0.21$; DN2: 0.32 , $0.22-0.44, p=0.0005$; C: $0,0-0.06 \mathrm{pg} / \mu \mathrm{mol}$ creatinine). IL-1beta and MCP-1 correlated negatively with glomerular filtration rate $(r=-0.42, p=0.002$ and $r=-0.38, p=0.006$ respectively). In normoalbuminuric and microalbuminuric patients excretion of cytokines correlated with the width of GBM (IL-1beta: $r=0.69, p=0.002$; RANTES: $r=0.43, p=0.07$ ) and TBM (IL-1beta: $r=0.73$, $p=0.002$; MCP- $1: r=0.47, p=0.05$; RANTES: $r=0.53, p=0.04)$. Correlations between cytokines and $\mathrm{Vv}(\mathrm{mes} / \mathrm{glom})$ were weak and non-significant (all $p \geq 0.05)$. CD-68-positive macrophages were revealed in the renal interstitium (but not in glomeruli) in one normoalbuminuric patient and in six patients with microalbuminuria.

Conclusion: In type 1 diabetic patients increased urinary excretion of proinflammatory cytokines (IL-1beta, MCP-1 and RANTES) is associated with albuminuria, basement membrane width and macrophage appearance in the renal interstitium. This supports the hypothesis that inflammation is involved in pathogenesis of diabetic nephropathy.

\section{7}

Inflammatory markers predict all cause mortality in type 1 diabetic patients with diabetic nephropathy in a 10-year follow-up study A. S. Astrup ${ }^{1}$, L. Tarnow ${ }^{1}$, L. Pietraszek ${ }^{1}$, C. G. Schalkwik ${ }^{2}$, C. D. A. Stehouwer ${ }^{2}$, P. Rossing ${ }^{1}$, H.-H. Parving ${ }^{3,4}$; ${ }^{1}$ Steno Diabetes Center, Gentofte, Denmark, ${ }^{2}$ Dept of Internal Medicine, Academic Hospital Maastricht, The Netherlands, ${ }^{3}$ Dept of Medical Endocrinology, Rigshospitalet, Copenhagen, Denmark,

${ }^{4}$ Faculty of Health, Aarhus University, Denmark.

Background and Aims: To evaluate the significance of cardiovascular biomarkers on mortality in type 1 diabetic patients with and without diabetic nephropathy.

Material and Methods: We prospectively followed 199 type 1 diabetic patients with diabetic nephropathy and 192 patients with persistent normoalbuminuria for 10 years. Biomarkers were measured at baseline in 1993.The endpoint was all-cause mortality. In order to combine the biomarkers we constructed two combined z-scores, one for inflammation and one for endothelial dysfunction. The inflammatory Z-score combined Creactive protein (hsCRP), interleukin-6 (IL-6), soluble intercellular adhesion molecule-1s-(sICAM-1), and secreted phospholipase A(2) (sPLA2). The Zscore for endothelial dysfunction combined vascular cell adhesion molecule 1 (sVCAM-1), plasminogen activator inhibitor-1 (PAI-1), and sICAM-1, since sICAM-1 is both an inflammatory marker and a marker of endothelial dysfunction. For each individual, the values of each marker were expressed as a Z-score: (value in the individual minus the mean value in the study population) / standard deviation; a value that thus ranged from approximately -2.5 to +2.5 . Thus the inflammatory $Z$-score was calculated as $(Z$-score of hsCRP + Z-score of IL- $6+Z$-score of sICAM+Z-score of sPLA2)/4 and the Z-score for endothelial dysfunction (Z-score of PAI-1 + Z-score of sVCAM-1 + Z-score of sICAM-1)/3.

Results: During follow-up 60 patients (30\%) with nephropathy died versus 16 $(8 \%)$ of normoalbuminuric patients, $\log$ rank test $\mathrm{p}<0.0001$. In unadjusted Cox regression analysis the Z-score for inflammation was significantly predictive of death in patients with diabetic nephropathy (Hazard Ratio (HR) $1.9(95 \%$ CI $1.3-2.8 ; \mathrm{p}=0.001$ ) but not in patients with persistent normoalbuminuria HR $0.5(0.2-1.2 ; \mathrm{p}=0.11)$. After adjusting for traditional cardiovascular risk factors (age, sex, systolic blood pressure, smoking, cholesterol, hbalc, urinary albumin excretion, heart rate variation) the Z-score was still significantly 
predictive of death in patients with diabetic nephropathy: HR 1.7(1.1-2.6; $\mathrm{p}=0.025)$. In unadjusted analysis endothelial dysfunction was associated with death in patients with diabetic nephropathy, HR $1.6(1.0-2.3 ; \mathrm{p}=0.031)$ but not in patients with persistent normoalbuminuria; HR $0.9(0.4-2.3 ; \mathrm{p}=0.9)$. After adjusting for traditional risk factors the association was no longer significant in patients with diabetic nephropathy; HR $1.2(0.8-1.8 ; \mathrm{p}=0.4)$. Conclusions: Our 10-year follow-up study in type 1 diabetic patients with and without diabetic nephropathy indicates inflammatory biomarkers are significantly predictive of all-cause mortality in patients with diabetic nephropathy.

\section{8}

Plasma concentration of asymmetrical dimethylarginine (ADMA) predicts cardiovascular morbidity and mortality in type 1 diabetic patients with diabetic nephropathy

M. Lajer ${ }^{1}$, L. Tarnow ${ }^{1}$, A. Jorsal ${ }^{1}$, T. Teerlink ${ }^{2}$, H.-H. Parving ${ }^{3,4}$, P. Rossing ${ }^{1}$; ${ }^{1}$ Steno Diabetes Center, Gentofte, Denmark, ${ }^{2} \mathrm{VU}$, University Medical Center, Amsterdam, The Netherlands, ${ }^{3}$ Department of Medical

Endocrinology, Rigshospitalet, University Hospital of Copenhagen,

Denmark, ${ }^{4}$ Faculty of Health Science, University of Aarhus, Denmark.

Background and Aims: Asymmetrical dimethylarginine (ADMA) is an endogenous nitric oxide synthase inhibitor elevated in conditions associated with endothelial dysfunction and atherosclerosis as well as in patients with diabetic nephropathy. ADMA levels have been shown to be predictive of cardiovascular events (CVD) and total mortality in non-diabetic kidney disease, and of coronary artery disease.

Therefore, the aim of this study was to investigate whether circulating ADMA levels are predictive of cardiovascular events, decline in glomerular filtration rate (dGFR), end-stage renal disease (ESRD), and all-cause mortality in type 1 diabetic patients.

Materials and Methods: This study was a prospective observational follow-up study; including 397 type 1 diabetic patients with overt diabetic nephropathy (247 men; age $42.1 \pm 10.5$ years (mean $\pm S D$ ); duration of diabetes $28.3 \pm 8.8$ years; GFR $76 \pm 33.5 \mathrm{ml} / \mathrm{min} / 1.73 \mathrm{~m}^{2}$ ) and a control group of 175 patients with longstanding type 1 diabetes and persistent normoalbuminuria (106 men; age $42.6 \pm 9.7$ years; duration of diabetes $27.6 \pm 8.2$ years. Patients were followed for $11.0(0.0-12.9)$ years (median ([range)) with yearly measurements of GFR (51Cr-EDTA plasma clearance)

Results: Among patients with diabetic nephropathy, plasma ADMA levels above the median predicted fatal and non-fatal CVD $(p<0.001)$ during followup, persisting after adjusting for conventional CVD covariates including baseline GFR (adjusted hazard ratio (HR) of $2.05(1.31-3.20), p=0.002$ ). Furthermore, elevated ADMA levels predicted increased rate of decline in GFR, development of ESRD, and overall mortality $(p<0.001,=0.004$, and $<$ 0.001 , respectively). However, only dGFR and ESRD tended to be significant after adjustment for well known progression promoters including baseline GFR (adjusted HR of $-0.1(-1.70 ; 0.10) ; p=0.082$ and adjusted HR of 1.77 (0.95-3.33); $p=0.075$ for dGFR and ESRD, respectively).

Among the 175 patients with persisting normoalbuminuria 6 patients with ADMA levels below the median for this group of patients compared to 14 (16\%) patients with the highest ADMA levels died during the follow-up period ( $p=0.050)$ and an unadjusted hazard ratio of 2.52 (0.97-6.56), ( $p=$ 0.058 ). ADMA levels were not associated with fatal or non-fatal CVD in this low risk population (NS).

Conclusion: Plasma ADMA levels predicts fatal and non-fatal cardiovascular events in patients with type 1 diabetic nephropathy. Furthermore, increased ADMA levels tended to be predictive of increased risk of progressive diabetic kidney disease. In patients with persisting normoalbuminuria elevated ADMA levels predicted an increased overall mortality rate.

\section{9}

Asymmetric dimethylarginine (ADMA) and microvascular complications in type 2 diabetes patients: the Hoorn Study A. M. van Sijl ${ }^{1,2}$, A. M. W. Spijkerman ${ }^{1}$, J. M. Dekker ${ }^{1}$, C. D. A. Stehouwer ${ }^{3}$, R. J. Heine $\mathrm{e}^{1,4}, \mathrm{~T}$. Teerlink

${ }^{1}$ EMGO-Institute, VU University Medical Center, Amsterdam, ${ }^{2}$ Department of General Practice, VU University Medical Center, Amsterdam, ${ }^{3}$ Department of Internal Medicine, Academic Hospital Maastricht, Maastricht, ${ }^{4}$ Department of Endocrinology/Diabetes Center, VU University Medical Center, Amsterdam, ${ }^{5}$ Department of Clinical Chemistry, VU University Medical Center, Amsterdam, The Netherlands.

Background and Aims: High levels of asymmetric dimethylarginine (ADMA), an endogenous inhibitor of nitric oxide synthase, have been recognized as an important marker of cardiovascular disease and diabetes. Inhibition of nitric oxide synthase results in less nitric oxide production, precipitating vasoconstriction and oxidative stress, which may induce microvascular complications in diabetes. The aim of the present study was to investigate the associations of ADMA and ADMA-to-arginine ratio with microvascular complications.

Materials and Methods: The population consisted of 544 elderly participants with and without type 2 diabetes. ADMA and arginine were determined with high performance liquid chromatography. Peripheral neuropathy was assessed with the vibration perception threshold and with the temperature discrimination threshold. Microalbuminuria was measured by the albuminto-creatinine ratio and retinopathy with ophtalmoscopy and fundus photography. Linear and logistic regression analyses were performed.

Results: ADMA and ADMA-to-arginine ratio were not statistically significantly associated with microalbuminuria and retinopathy. High levels of ADMA (3rd tertile) were statistically significantly associated with high temperature discrimination threshold in patients with diabetes, regression coefficient (95\% confidence interval): RC (CI), 0.52 (0.003 to 1.03). A moderate ADMA-to-arginine ratio (2nd tertile) was statistically significantly associated with low vibratory perception threshold in participants with diabetes: RC (CI), -10.51 ( -21.01 to -0.01$)$.

Conclusion: Overall, there was no consistent evidence that ADMA or ADMA-to-arginine ratio were associated with microvascular complications. In patients with diabetes, high levels of ADMA were significantly associated with high temperature discrimination threshold. These equivocal results warrant prospective studies to investigate the associations of ADMA with microvascular complications.

\section{0}

Plasma asymetric dimethylarginine (ADMA) is associated with retinopathy in type 2 diabetes mellitus

M. T. Malecki ${ }^{1}$, A. Undas ${ }^{2}$, K. Cyganek ${ }^{1}$, B. Mirkiewicz-Sieradzka ${ }^{1}$, P. Wolkow ${ }^{3}$, G. Osmenda ${ }^{3}$, M. Walus-Miarka ${ }^{1}$, T. J. Guzik ${ }^{3}$, J. Sieradzki ${ }^{1}$; ${ }^{1}$ Department of Metabolic Diseases, ${ }^{2}$ Institute of Cardiology, ${ }^{3}$ Department of Pharmacology, Jagiellonian University, Medical College, Krakow, Poland.

Background and Aims: Asymmetric dimethylarginine (ADMA), an endogenous inhibitor of nitric oxide synthase, has been reported to be associated with coronary artery disease, hypertension in non-diabetic populations and also nephropathy in type 1 diabetes mellitus. It is not known whether there is any association of ADMA with diabetic retinopathy (DR). The objective of the study was to evaluate the relationship between plasma ADMA and the occurrence of DR in type 2 diabetes mellitus (T2DM).

Materials and Methods: We studied 111 T2DM patients without DR (NDR) and 71 T2DM subjects with DR (mean age $55.7 \pm 6.2$ and $57.1 \pm 7.0$ years, respectively). The control group consisted of 52 apparently healthy individuals matched for age and gender. Plasma ADMA, SDMA, and arginine levels were measured by high-performance liquid chromatography. The diagnosis of DR was based on ophthalmic examination. Potentially important clinical covariables were also measured. Determinants of DR were identified via stepwise multivariable logistic regression analysis.

Results: The ADMA level was highest in T2DM patients with DR $(0.60 \pm 0.06$ $\mu \mathrm{mol} / \mathrm{L})$, intermediate in the subjects with T2DM and NDR $(0.51 \pm 0.06 \mu \mathrm{mol} /$ $\mathrm{L})$, and lowest among controls $(0.45 \pm 0.05 \mu \mathrm{mol} / \mathrm{L})(\mathrm{p}<0.001$ for all intergroup comparisons). In stepwise multivariable logistic regression, plasma ADMA levels remained an independent predictor of the presence of $D R(p<0.0001$, 
$\mathrm{OR}=1.65,95 \% \mathrm{CI}: 1.39-1.97)$ in T2DM patients. DR was also independently associated with age at examination ( $\mathrm{p}<0.0001, \mathrm{OR}=0.79,95 \% \mathrm{CI}$ : $0.70-0.88$ ) and duration of T2DM ( $\mathrm{p}=0.032, \mathrm{OR}=1.1,95 \% \mathrm{CI}$ : $1.008-1.2)$. DR was not associated with smoking status, creatinine levels, glycated hemoglobin or lipids in our study.

Conclusion: Our findings suggest that elevated ADMA level is an independent predictor of DR in T2DM.

\section{PS 115 Neuropathy: symptoms and treatment}

\section{1}

Painful neuropathy is associated with greater autonomic dysfunction compared to painless neuropathy in diabetes mellitus

J. L. B. Marques, R. A. Gandhi, G. L. Ferrari, C. J. Emery, S. Tesfaye; Diabetes Research Unit, Royal Hallamshire Hospital, Sheffield,

United Kingdom.

Background:Although a clear relationship between peripheral diabetic neuropathy (DPN) and cardiac autonomic neuropathy (CAN) is recognised, the relationship of CAN with sub-types of DPN is less clear.

Aims: The aim of this study was to investigate the relationship of CAN to painless and painful neuropathy.

Methods: Seventy-six subjects (20 healthy volunteers (HV), 20 no DPN, 18 painful DPN, 18 painless DPN) underwent detailed neurophysiological investigations and spectral analysis of short-term heart rate variability (HRV). HRV is a highly sensitive measure of autonomic function and was calculated according to the frequency-domain method (total power (TP), low frequency (LF) and high frequency (HF) for assessment of sympathovagal modulation of heart rate) and results logarithmically transformed to correct for skewness.

Results: There were no significant differences in subjects with no DPN compared to $\mathrm{HV}$ in $\mathrm{HF}(4.6 \pm 1.6($ mean $\pm \mathrm{SD})$ vs. $5.4 \pm 0.9), \operatorname{LF}(5.1 \pm 1.5$ vs. $5.5 \pm 1.0)$ or $\mathrm{TP}(6.6 \pm 1.2$ vs. $7.1 \pm 0.8), \mathrm{p}>0.1$. Subjects with painless $\mathrm{DPN}$ had a reduction in all 3 measures (compared to $\mathrm{HV}) ; 3.8 \pm 1.0(\mathrm{HF}), 4.2 \pm 1.5(\mathrm{LF})$, and $5.9 \pm 1.1(\mathrm{TP}), \mathrm{p}<0.05$. There was a further reduction in subjects with painful DPN; $2.4 \pm 1.2(\mathrm{HF}), 3.0 \pm 1.3(\mathrm{LF})$, and $4.8 \pm 1.1(\mathrm{TP}), \mathrm{p}<0.05$. After correcting for age and drug therapy, there were no longer any significant differences between HV, and subjects with no DPN and painless DPN. The painful DPN group, however, continued to have significantly lower levels in all 3 parameters.

Conclusion: This study shows that subjects with painful DPN have significantly greater autonomic dysfunction than subjects with painless DPN. It suggests there are differing profiles of nerve fibre involvement in different types of diabetic neuropathy. The greater autonomic dysfunction seen in painful DPN may be reflecting more predominant small fibre involvement. The role that autonomic dysfunction may play in the pathogenesis of painful DPN also needs consideration.

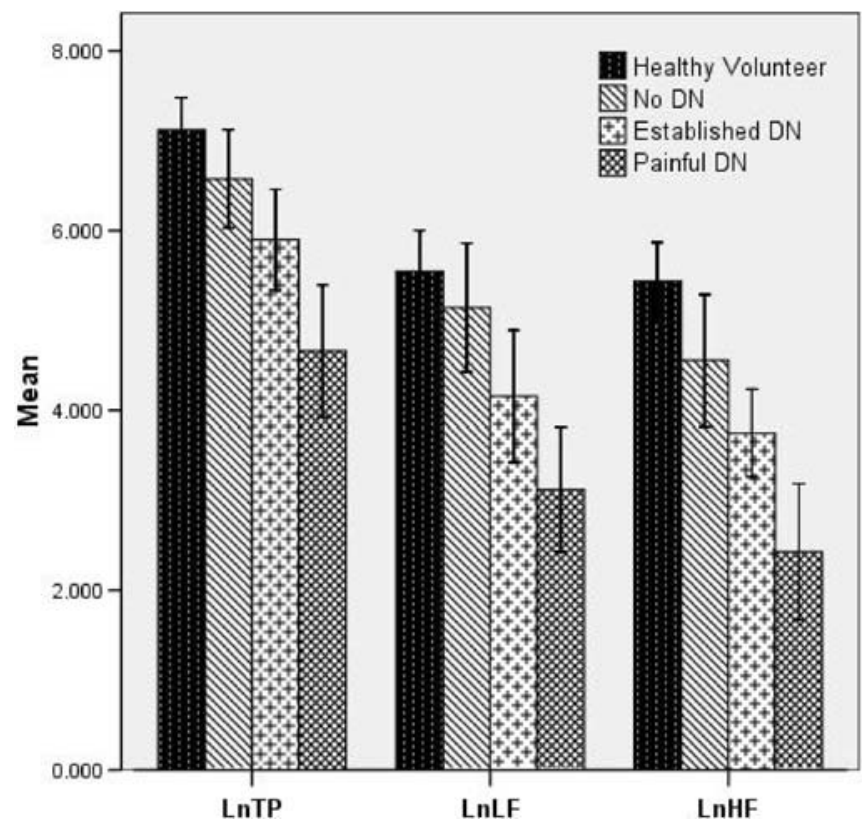




\section{2}

Upper abdominal symptoms in patients with type 1 diabetes are not related to impaired gastric emptying caused by autonomic neuropathy J. Punkkinen ${ }^{1}$, M. Färkkilä ${ }^{1}$, S. Mätzke ${ }^{2}$, T. Korppi-Tommola ${ }^{2}$, T. Sane ${ }^{3}$, P. Piirila ${ }^{4}$, J. Koskenpato ${ }^{1}$

${ }^{1}$ Department of Gastroenterology,

${ }^{2}$ Laboratory of Nuclear Medicine, ${ }^{3}$ Department of Endocrinology,

${ }^{4}$ Laboratory of Clinical Physiology, Helsinki University Hospital, Finland.

Background and Aims: Diabetic gastroparesis is a common condition occurring in about $30-50 \%$ of diabetic patients with a long disease history. Studies examining the relation of autonomic neuropathy and diabetic gastroparesis have been contradictory whereas acute changes in plasma glucose concentrations affect gastric emptying more clearly. The objective was to examine the causal relation of upper abdominal symptoms to gastric emptying and autonomic nerve function in patients with type 1 diabetes.

Materials and Methods: Gastric emptying of solids and liquids were measured with standardized scintigraphy in type 1 diabetic patients (age $42 \pm 8$ and diabetes duration $29 \pm 11$ years, $\mathrm{HbA}_{1 \mathrm{c}} 8.4 \pm 1.4 \%$ ) with upper abdominal symptoms for at least 3 months (upper abdominal pain, fullness, early satiation, nausea or vomiting) but with no findings in gastroscopy or abdominal ultrasound. Helicobacter pylori infection was excluded by negative findings in the gastroscopy biopsies. Autonomic nerve function was examined by standardized cardiovascular tests (heart rate variation during controlled and deep breathing and Valsalva manoeuvre, diastolic blood pressure rise during isometric handgrip excercise, and blood pressure and pulse reactions during active orthostatic test with measurement of maximum/minimum ratio of heart rate variation). Glucose concentrations were measured during scintigraphy. Severity of abdominal symptoms and quality of life were examined by validated questionnaires (SF-36).

Results: $7 / 27$ (26\%) of patients with upper abdominal symptoms had delayed gastric emptying of solids and 3/27 (11\%) of liquids. Gastric emptying rate of solids correlated positively with the autonomic neuropathy score $(r=0.56$, $\mathrm{P}<0.01$ ), but not to abdominal symptom score or actual plasma glucose concentrations during scintigraphy. Using the Cox regression model for an increment of one in the autonomic neuropathy score, the gastric emptying rate of solids emptying was $31 \%$ smaller $(10-4795 \%$ CI; P = 0.006). Considering emptying rate of liquids, for an increment of one in the autonomic neuropathy score, the gastric emptying rate of liquids rate was $20 \%$ smaller $(-1-3795 \%$ $\mathrm{CI}$; $\mathrm{P}=0.06)$. Neither gastric emptying rate of solids nor liquids correlated with age, duration of diabetes, glucose concentrations during scintigraphy, $\mathrm{HbA}_{1 \mathrm{C}}$ or abdominal symptoms. All of eight aspects of quality of life in diabetic patients with abdominal symptoms were significantly lower than in the normal population.

Conclusion: Impaired gastric emptying of solids in patients with type 1 diabetes is related to autonomic neuropathy but not to actual glycemic control during scintigraphy. However, upper abdominal symptoms observed in these patients can not be explained by impaired gastric emptying.

Supported by Finnish Diabetes Association

\section{3}

High prevalence of urinary incontinence in diabetic women is independent of age; preliminary results of a case-control study P. Topsever ${ }^{1}$, M. T. Filiz' 1 , C. Uludag 2 , N. Dede Cinar';

${ }^{1}$ Department of Family Medicine, Kocaeli University, Istanbul,

${ }^{2}$ Department of Nursing, SakaryaUniversity, Sakarya, Turkey.

Background and Aims: Urinary incontinence (UI) is an important health problem with psychological, social and hygienic effects on women. UI can also have considerable impact on quality of life of the affected individual and has been reported to be associated with the presence of some chronic diseases. The aim of the present study was to explore the association between Diabetes Mellitus (DM) and urinary incontinence in a sample of adult women.

Materials and Methods: In total, 954 women (mean age $38.4 \pm 13.0$ years, BMI $26.0 \pm 5.1 \mathrm{~kg} / \mathrm{m}^{2}$, prevalence of gravidity $85.5 \%$, prevalence of parity $82.2 \%$ ) composed of 344 diabetic cases (fasting plasma glucose $145.3 \pm 36.2 \mathrm{mg} / \mathrm{dL}$, systolic blood pressure $129.3 \pm 13.9 \mathrm{mmHg}$ diastolic blood pressure $76.4 \pm 8.5$ $\mathrm{mmHg}$, DM complications $12.4 \%$, insulin therapy $17.0 \%$, oral antidiabetic therapy $83.0 \%$ ) and 610 non-diabetic controls (chronic diseases 15.2\%) attending six Primary Health Care Centers and one State Hospital diabetes outpatient clinic were enrolled in this study. After giving their informed consent, all participants filled in a questionnaire inquiring "any kind of urinary leakage", related symptomatology and personal medical history. Reproductive and medical history was verified from patient records. The subjects were dichotomized into cases and controls according to presence of DM. Except for descriptives (median/range and mean \pm SD, \%), Independent Samples t-test and Chi-Square test were used; binary logistic regression analysis with the presence of UI as dependent and variables of interest as covariates was performed producing odds ratios (OR); areas under the curve (AUC) were calculated with a sensitivity of $\geq 90 \%$, a statistical significance of $<0.05$ and a confidence interval (CI) of $95 \%$.

Results: When diabetic cases were compared with non-diabetic controls, they were older ( $49.3 \pm 10.3$ years vs. $32.3 \pm 10.1$ years), more overweight (BMI $27.9 \pm 4.9 \mathrm{~kg} / \mathrm{m}^{2}$ vs. $\left.24.9 \pm 4.8 \mathrm{~kg} / \mathrm{m}^{2}\right)$, had experienced more pregnancies (mean number of pregnancies $3.1 \pm 1.0$ vs. $2.0 \pm 1.3$ ) and more deliveries (mean number of deliveries $2.8 \pm 1.1$ vs. $1.8 \pm 1.3$ ) and displayed a higher prevalence of UI $(41.9 \%$ vs. $13.5 \%)$ with $p<0.001$ for all. After adjustment for plausible confounders (age, reproductive history like number of deliveries and medical history like presence of chronic comorbidities, diabetic complications), logistic regression revealed the presence of DM (OR 2.9, 95\%CI 2.1 to 4.0 , $p<0.001$ ), BMI over $22.5 \mathrm{~kg} / \mathrm{m}^{2}$ (sensitivity $\geq 90 \%$, AUC $0.67,95 \% \mathrm{CI} 0.63$ to $0.71, p<0.001$, OR $1.1,95 \%$ CI 1.0 to $1.1, p=0.002$ ), and history of more than one pregnancy (sensitivity $\geq 90 \%$ AUC $0.72,95 \% \mathrm{CI} 0.68$ to $0.75, p<0.001$, OR $1.6,95 \%$ CI 1.4 to $1.9, p<0.001$ ) as independent determinants of urinary incontinence in adult females.

Conclusion: Even after adjustment for plausible confounders like age, UI was more prevalent in adult diabetic women. The fact that determinants of UI identified in the present sample were modifiable, is important in terms of its prevention. Relationship of UI with diabetes related parameters like metabolic and hemodynamic parameters and disease related quality of life need to be elucidated further.

\section{4}

Anxiety, depression and neuropathic pain in diabetes

E. V. Salgami ${ }^{1}$, L. Vileikyte ${ }^{1}$, J. S. Gonzalez ${ }^{2}$, C. Waterman ${ }^{1}$, K. Breislin ${ }^{1}$,

A. J. M. Boulton ${ }^{1}$, M. Peyrot ${ }^{3}$;

${ }^{1}$ Diabetes, University of Manchester, United Kingdom,

${ }^{2}$ Psychology, Harvard Medical School, Boston, United States,

${ }^{3}$ Sociology, Loyola College, Baltimore, United States.

Background and Aims: As new medications for diabetic neuropathic pain are potent mood modulators, exhibiting either anxiolytic or anti-depressant properties, it is important when prescribing therapies, to determine whether the patient with neuropathic pain is predominantly anxious or depressed. Theoretical and empirical research suggests that whether a person is anxious or depressed is a function of duration of the medical disorder.

Materials and Methods: We therefore studied 46 patients (mean age 61 years; $73 \%$ male) with diabetes (71\% type 2 ) and chronic neuropathic pain ( $>6$ months duration, mean $=47$ months), and examined the association between two indices of pain severity (pain intensity and duration) and negative affect. Neuropathy was diagnosed using Neuropathy Disability Score (mean 6.7) and Vibration Perception Threshold (mean 26.7 volts). Pain severity was assessed with the Visual Analog Graphic Rating Scale, VAS ( $>3 \mathrm{~mm}$; mean $=8.7$ ).

Results: Consistent with the tripartite model of mood, negative affect was measured with four scales from the Mood and Anxiety Symptom Questionnaire, MASQ, two of which assessed symptoms of generalized distress that are non-specific, i.e., common to both anxiety (11 items; alpha $=0.81)$ and depression (12 items; alpha $=0.93)$; while the other two scales measured symptoms unique to anxiety (anxious arousal: 17 items, alpha $=0.88$ ) and depression (anhedonic depression: 22 items; alpha $=0.95$ ). Of demographic and disease variables, only age significantly correlated with MASQ scales at the bivariate level and was therefore retained in subsequent regression analyses. Pain duration was not significantly associated with MASQ scales, whereas pain severity was a significant independent predictor of specific measures of anxiety and depression symptoms (anxious arousal: beta $=0.41 ; \mathrm{p}=0.018$; anhedonic depression: beta $=0.45 \mathrm{p}=0.007$ ).

Conclusion: These findings suggest that anxiety and depression are both associated with chronic painful neuropathy, and that pain intensity and not duration is associated with negative affect. This highlights the importance 
of measuring symptoms that are unique to anxiety and depression when examining the association between neuropathic pain and negative affect.

\section{5}

Depression is a major confounder of outcomes in clinical intervention trials of therapeutic compounds in painful diabetic neuropathy S. Tesfaye ${ }^{1}$, R. A. Gandhi ${ }^{1}$, C. J. Emery ${ }^{1}$, H. Bowler ${ }^{2}$, D. Selvarajah ${ }^{1}$; ${ }^{1}$ Diabetes Research Unit, University of Sheffield,

${ }^{2}$ Department of Pharmacy, Clinical Trial Unit, Sheffield, United Kingdom.

Background and Aims: Painful diabetic neuropathy causes great distress and disability to sufferers and affects quality of life immensely. Drug treatments are often ineffective and complicated by side effects. Some recent clinical drug trials comparing the efficacy of new therapeutic interventions with placebo have been complicated by a large placebo response. We conducted a post hoc analysis to assess the effects of depression on outcomes in a randomised placebo control trial evaluating the efficacy of sativex (a cannabis based medicinal product) in painful diabetic neuropathy.

Materials and Methods: Following a 1 week screening phase, 28 diabetic subjects with painful diabetic neuropathy were randomised to receive either sativex or matching placebo. Doses of study medication were titrated up over a two week titration phase until either a maximum tolerated dose was achieved or adequate pain relief obtained. During the screening phase, baseline level of depression was assessed by using the Hospital Anxiety and Depression Score (HADS) and daily pain assessed by completion of a pain diary. Following the two week titration phase, subjects proceeded to a ten week maintenance phase which terminated on a final visit. Primary outcome measure was change in total pain scores from baseline between the two treatment arms. For the post hoc analysis we divided the subjects into two groups based on the HADS [no depression $(\mathrm{HADS}<10)$ and depression $(\mathrm{HADS}>10)$ ]. Using univariate analysis of covariance we compared outcomes between depressed and non depressed subjects after adjusting for study medication received.

Results: There was no significant difference in primary outcome measures between the active and placebo arms of the study $(\mathrm{p}=0.74)$. Mean (SD) HADS for the depression $(\mathrm{n}=10)$ and no depression $(\mathrm{n}=18)$ groups were $13.4(3.5)$ and 5.94 (2.2) respectively. Subjects with depression had higher baseline total pain scores compared to those without depression [186.8(66.3) vs 130.3(72.8); $\mathrm{p}=0.050]$. Subjects with depression had a significant improvement in the primary outcome measure compared to those without depression, after adjusting for allocation to the active or placebo arm of the study [mean change in total pain scores (standard error of the mean) $-94.7(23.4)$ vs $-30.9(17.5)$; $\mathrm{p}=0.039]$.

Conclusion: Depression may be a major confounder of outcomes in clinical trials assessing the efficacy of therapeutic interventions in painful diabetic neuropathy. Subjects with even moderate levels of depression are more likely to have higher baseline pain scores and respond favourably to any intervention, whether in the placebo or active arm of a study.

Supported by Diabetes UK

\section{6}

Effects of pregabalin on pain and nerve conduction in patients with painful diabetic peripheral neuropathy (DPN): a 13-week randomized controlled trial and 6-month open-label safety extension

J. Arezzo ${ }^{1}$, J. Rosenstock ${ }^{2}$, L. LaMoreaux ${ }^{3}$, L. Pauer ${ }^{3}$, U. Sharma ${ }^{4}$;

${ }^{1}$ Neuroscience and Neurology Dept., Albert Einstein College of Medicine, Bronx, ${ }^{2}$ Diabetes and Endocrinology Dept., Dallas Diabetes and Endocrine Center, Dallas, ${ }^{3}$ Pfizer Inc, Ann Arbor, ${ }^{4}$ Consultant, Pfizer Inc, Canton, United States.

Background and Aims: This 13-week randomized clinical trial evaluated pregabalin $600 \mathrm{mg} / \mathrm{d}$ (BID) as treatment of painful DPN.

Materials and Methods: Primary efficacy measure was endpoint (EP) mean pain score (MPS) on an 11-point numeric rating scale (NRS) derived from patients' daily pain diaries. Secondary efficacy measures included weekly MPS and EP and weekly sleep-interference scores (on an 11-point NRS). Primary safety endpoints were amplitude and conduction velocity for 4 nerves (median-motor, median-sensory, ulnar-sensory, and peroneal-motor) assessed at baseline (BL), EP, and 2-week follow-up.
Results: 82 patients were randomized to pregabalin, 85 to placebo (PBO). After 1-week dose escalation, pregabalin patients received $600 \mathrm{mg} / \mathrm{d}$ for 12 weeks. EP MPS was significantly lower in pregabalin-treated patients than in PBO-treated patients (mean difference, $-1.28 ; P=.0003$ ). Significant pain improvement among pregabalin patients was evident at Week 1 and every weekly timepoint thereafter. Pregabalin was statistically significantly superior to $\mathrm{PBO}$ for reducing pain-related sleep-interference score at every weekly timepoint (except Weeks 9, 10) and at EP (mean difference, $-1.08 ; P=.0019$ ). No statistically significant differences in amplitude or velocity were observed for any of the 4 nerves tested. In an open-label (OL) extension of this trial, patients received $150-600 \mathrm{mg} / \mathrm{d}$ pregabalin (BID), adjusted to optimize efficacy and tolerability, for up to 6 months. $97 \%$ of patients received dosages of $\geq 300 \mathrm{mg} / \mathrm{d}$. Pregabalin was generally well tolerated, with $70 / 104$ patients completing 24 weeks of treatment. 15 patients $(14 \%)$ withdrew from OL treatment because of AEs. Peripheral edema, dizziness, and somnolence were the most common AEs associated with treatment.

Conclusion: Pregabalin $600 \mathrm{mg} / \mathrm{d}$ (BID) showed robust and sustained efficacy in pain improvement with no meaningful effect on nerve conduction during 13 weeks of double-blind treatment. Pregabalin was generally well tolerated during both 13-week double-blind and 6-month OL treatment. Supported by Pfizer Inc

\section{7}

Effect of gabapentin on impaired brain GABA transmission associated with type 1 diabetes

I. Shymanskyy, T. Kuchmerovska, V. Kopelevich, A. Klimenko; Coenzymes, O.V. Palladin Institute of Biochemistry, Kyiv, Ukraine.

Background and Aims: The anticonvulsant drug gabapentin (an amino acid chemically related to gamma-aminobutyric acid, GABA) has been shown recently to exhibit antinociceptive, anxiolytic and neuroprotective activity. Several animal and clinical studies have investigated the use of the drug as the management of neuropathic pain associated with diabetes. Although the binding site for gabapentin in the nervous system has been identified as the alpha-2-delta subunit of voltage-gated calcium channels, it is not yet clear how this finding relates to the modulatory action of gabapentin on various neurotransmitter systems in the brain. In view of the data showing GABAmimetic properties of gabapentin, we have now evaluated the role of GABAergic system in the mechanism of its action in diabetic brain.

Materials and Methods: All studies were carried out after 5 weeks of STZinduced diabetes $(60 \mathrm{mg} / \mathrm{kg}$, i.p.) in rats treated for 14 days with or without gabapentin $(50 \mathrm{mg} / \mathrm{kg}$, i.p.). GABA content was assayed in brain by cation exchange chromatography. The uptake and the release of $\left[\mathrm{U}-{ }^{14} \mathrm{C}\right] \mathrm{GABA}$ were determined in purified brain synaptosomes. Reactive oxygen species (ROS) production was measured quantitatively in synaptosomes using cell permeable dye 2',7'-dichlorofluorescein diacetate (DCF-DA).

Results: It was shown that diabetes lowered the brain level of GABA to $1.79 \pm 0.09$ from $2.36 \pm 1.24 \mu \mathrm{mol} / \mathrm{g}$ tissue in control $(\mathrm{p}<0.05)$. This may account for $53.4 \pm 4.9 \%$, probably compensatory, elevation of GABA uptake by diabetic synaptosomes vs control. To evaluate the possibility of diabetes-induced influence on quantal mode of the neurotransmitter secretion we examined the effect of the depletion of cytosolic GABA pool by $1 \mathrm{mM}$ nipecotic acid (competitive inhibitor of the GABA transporter) on neurosecretion evoked by $\mathrm{KCl}(30 \mathrm{mM})$ depolarization. Diabetic synaptosomes pretreated with nipecotic acid exhibited significant, more then two-fold, increase in unstimulated GABA release, whereas $\mathrm{K}^{+}$-evoked $\mathrm{Ca}^{2+}$-dependent neutransmitter release was much lower as compared to control suggesting diabetes-induced inhibition of GABA exocytosis from synaptic vesicles, $\mathrm{p}<0.05$. Following gabapentin administration, the content of brain GABA was almost completely restored with simultaneous $33.5 \pm 3.7 \%$ decrease in GABA uptake by synaptosomes, $\mathrm{p}<0.01$. It was found that drug treatment diminished synaptosomal response to nipecotic acid but augmented GABA secretion evoked by high $\mathrm{KCl}, \mathrm{p}<0.05$. As oxidative stress could be implicated in the diabetes-associated impairment of GABA transmission, gabapentin efficacy to counteract ROS generation in synaptosomes was assessed. Indeed, administration of the drug attenuated increased DCF-DA-sensitive cellular ROS production in diabetic rats. At the same time, gabapentin had no lowering effect on blood glucose and brain sorbitol levels.

Conclusion: The data suggest that diabetes may cause physiologically drastic failure in realization of GABA-mediated inhibitory effects in CNS. The decrease in GABA uptake by gabapentin as well as its facilitatory action 
on GABA exocytosis may result in elevation of GABA concentration in the synaptic cleft leading to better synaptic inhibition and thereby contributing to its neuroprotective effects. This, together with the antioxidant action of gabapentin might deserve further investigation for evaluating the link between the observed findings at the level of GABAergic transmission and the clinical effects of the drug.

\section{8}

$\mathbf{N}^{1}$-methylnicotinamide in prevention of diabetes-associated brain disorders

T. Kuchmerovska, I. Shymanskyy, S. Stepanenko, A. Klimenko; Coenzymes, O.V. Palladin Institute of Biochemistry, Kyiv, Ukraine.

Background and Aims: It was previously revealed that vitamin PP exerts strong neuroprotective action and can be efficient in brain abnormalities induced by type 1 diabetes. More evidences have been emerged indicating that besides being essential for energy transduction, the intermediates of vitamin PP metabolism have a variety of significant regulatory roles, some of which are involved in fundamental cellular processes. The potential function of $\mathrm{N}^{1}$-methylnicotinamide (MNM), the end product of niacin metabolism, in regulatory events has not yet attracted considerable attention. The aim of the study was to evaluate the potential benefits from $\mathrm{N}^{1}$-methylnicotinamide treatment on brain disorders associated with diabetes.

Materials and Methods: The studies were carried out after 6 weeks of STZinduced diabetes $(60 \mathrm{mg} / \mathrm{kg}$, i.p.) in rats treated for 5 weeks with or without MNM dissolved in drinking water $(100 \mathrm{mg} / \mathrm{kg}$ of body weight) after 1 week of diabetes induction. The uptake and the release of $\left[2-{ }^{14} \mathrm{C}\right]$ serotonin $(5 \mathrm{HT})$ were determined in purified synaptosomes. The synaptosomal plasma membrane potential was measured by accumulation of ${ }^{3} \mathrm{H}$-labelled tetraphenylphosphonium bromide ion.

Results: In diabetes, the hepatic vitamin PP content was shown to be significantly lower $(540 \pm 37$ vs $795 \pm 52 \mathrm{nmol} / \mathrm{g}$ tissue in control, $\mathrm{p}<0.05)$. Diabetes caused $34.8 \pm 2.4 \%$ decrease in the activity of $\mathrm{Na}^{+}, \mathrm{K}^{+}$-ATPase that indicates lowered efficacy of $\mathrm{Na}^{+}, \mathrm{K}^{+}$-pump, $\mathrm{p}<0.01$. To further support the role of $\mathrm{Na}^{+}, \mathrm{K}^{+}$-ATPase in neuropathophysiology of diabetic complications, we examined the possibility of alterations in transmembrane plasmatic potential of rat brain synaptosomes related to impaired ion transportation via synaptic membranes. It was shown more than two-fold decrease in the membrane potential of synaptic endings in diabetes vs control. Compared to control, diabetes led to $45.3 \pm 3.2 \%$ elevation of the spontaneous release and $37.0 \pm 2.8 \%$ decrease in the uptake of 5HT in isolated synaptic endings, $\mathrm{p}<0.05$. In addition, these changes were associated with 1.62 -fold elevation of brain sorbitol concentration compared with control rats. NAD ${ }^{+}$contents and free cytosolic $\mathrm{NAD}^{+} / \mathrm{NADH}$ ratios were approximately to the same extent reduced in brain and liver in diabetes vs. control. MNM supplementation to diabetic rats elicited slight lowering effect on blood glucose level. The content of vitamin PP in liver was completely restored in MNM-treated rats. Following the MNM treatment the activity of $\mathrm{Na}^{+}, \mathrm{K}^{+}$-ATPase was $13.5 \pm 1.2 \%$ increased vs diabetes, $\mathrm{p}<0.05$. The membrane potential of rat brain synaptosomes was reversed to initial level in MNM-supplemented diabetic rats. It is most likely that attenuation of synaptosomal membrane potential after MNM administration contributed to slight currently established improvement of 5HT uptake and spontaneous release. These changes correlated with 33.1 \pm 2.9 $\%$ reduction in brain sorbitol content, $\mathrm{p}<0.05$. MNM treatment partially normalized brain and liver $\mathrm{NAD}^{+}$levels and exhibited strong normalizing effect on redox state of free cytosolic $\mathrm{NAD}^{+} / \mathrm{NADH}$-couples only in rat liver. Conclusion: MNM treatment can be used as a strategy to improve brain cell function in diabetes. The mechanisms of MNM-related neuroprotection involve glucose lowering action, extra-brain metabolic effects and do not exclude direct MNM modulatory action on synaptic membrane properties. MNM ability to attenuate brain abnormalities related to diabetes deserves prospective study.

\section{9}

Benfotiamine and alpha-lipoic acid in the treatment of type 1 diabetic patients with autonomic gastroenteropathy

A. A. Serhiyenko, V. A. Serhiyenko, L. M. Serhiyenko; Endocrinology, Medical University of Lviv, Lviv, Ukraine.

Background and Aims: The perspective direction in the treatment of diabetic autonomic gastroenteropathy (DAGP) is the use of antioxidant and 'neurotrophic' B-group vitamins, in particular, benfotiamine (BET) - lisosoluble vitamin $\mathrm{B}_{1}$ form. The aim of this study was to assess the effects of BET, alpha-lipoic acid (ALA) and their combinations on clinical peculiarities, dynamics of instrumental and biochemical parameters in Type 1 diabetic patients with DAGP.

Materials and Methods: 64 patients with Type $1 \mathrm{DM}$ and moderate/severe DAGP (23,6 $\pm 2,9$ years), BMI $17,2 \pm 1,79 \mathrm{~kg} / \mathrm{m}^{2}$, DM duration $5,2 \pm 1,3$ years, HbA1c $8,5 \pm 1,6 \%$ were allocated to three groups. Patients of group A received BET $150 \mathrm{mg}$ in dragee three times; B - ALA $600 \mathrm{mg}$ in tab. tid; C - BET 150 $\mathrm{mg}$ in dragee three times plus ALA $600 \mathrm{mg}$ in tab. tid. The duration of the study was 2 months. Gastric electrical activity was evaluated by cutaneous electrogastrography (EGG) spectral analysis by distribution of the 3 spectra of gastric slow-wave frequency: bradygastria, normo-, and tachygastria; function of autonomous nervous system - by short-term spectral analysis of heart rate variability (HRV); contraction activity of the gallbladder by real-time ultrasonography. We investigated also the activities of superoxide dismutase (SOD), gluthation peroxidase (GPO), gluthatione (GSH), malonedyaldegide (MDA), conjugated dienes (CD). contents in the RBC's. Parameters were observed at baseline state and at the end of 2 month period. Statistics: ANOVA.

Results: Progressing of DAGP is accompanied by increase in percentage of tachygastria $(25,9 \pm 12,8 \%$ vs $17,3 \pm 7,6 \%, p<0,01)$, reduction in percentage of normogastria $(60,2 \pm 9,7 \%$ vs $72,3 \pm 10,1 \%, \mathrm{p}<0,01)$, bradygastria $(3,5 \pm 0,9 \%$ vs $5,7 \pm 0,7 \% \mathrm{p}<0,05)$. This disturbances, can testify about vagal damage. DAGP is accompanied by decrease in gallbladder ejection fraction at 30,40 and 60 min after egg yolk; depression of SOD $(p<0,001)$, GPO $(\mathrm{p}<0,01)$ activities, GSH content $(\mathrm{p}<0,01)$ with simultaneous increase MDA $(p<0,001)$, CD concentration $(p<0,05)$. Results between treatment group at 2 th month were the following: the HbAlc did not changed during the trial in investigated groups $(p>0,05)$. More expressed positive dynamics of investigated parameters, including, increase in gallbladder ejection fraction, improve of HRV parameters were observed in group C (table).

\begin{tabular}{llll}
$\begin{array}{l}\text { Mean \% change from baseline } \\
\text { at 3 months }\end{array}$ & BET $(\mathrm{n}=23)$ & ALA $(\mathrm{n}=21)$ & $\begin{array}{l}\text { BET/ALA } \\
(\mathrm{n}=20)\end{array}$ \\
\hline Bradygastria & $+49,7^{*}$ & $+12,3$ & $+74,2^{* *}$ \\
Normogastria & $+32,9$ & $+14,7$ & $+69,4^{* *}$ \\
Tachygastria & $-31,2$ & $-18,4$ & $-64,8^{* *}$ \\
SOD & $+30,1$ & $+49,2^{*}$ & $+67,3^{* *}$ \\
GPO & $+11,3$ & $+54,3^{*}$ & $+72,3^{* *}$ \\
GSH & $+21,4$ & $+42,3^{*}$ & $+62,7^{* *}$ \\
MDA & $-29,2$ & $-52,7^{*}$ & $-79,4^{* *}$ \\
CD & $-32,4$ & $-49,8^{*}$ & $-71,8^{* *}$ \\
Postprandial fullness & $-64,3^{*}$ & $-23,9$ & $-87,3^{* *}$ \\
Weight & $+18,4$ & $+16,9$ & $+49,3^{*}$ \\
Dyspepsia & $-39,8^{*}$ & $-18,4$ & $-74,3^{* *}$ \\
Dysphagia & $-42,4^{*}$ & $-26,2$ & $-84,3^{* *}$ \\
Expressive of abdominal pain & $-57,9^{*}$ & $-42,6^{*}$ & $-93,8^{*}$ \\
Night diarrhoea & $-60,3^{*}$ & $-39,5^{*}$ & $-92,1^{* *}$ \\
Constipation and obstipation & $-58,3^{* *}$ & $-12,6$ & $-82,7^{* *}$
\end{tabular}

Treatment difference presented with $95 \%$ CI. ${ }^{*} \mathrm{p}<0,01,{ }^{*} \mathrm{p}<0,001$

Conclusion: Thus, the combined treatment sick of BET and ALA during two months is accompanied by more expressed improvement of clinical current of DAGP, instrumental and biochemical parameters. The received results allow to assume, that efficiency of treatment by BET and ALA has no connections with improvement of compensation of DM, and cause direct influence of the last on the function of autonomous nervous system. So, combination of BET and ALA might be clinically useful for treatment, prevention from the progression of DAGP. 


\section{PS 116 New mechanisms in diabetic retinopathy}

\section{0}

\author{
Small populations of progenitor cells present in adult rodent retina \\ are modified by diabetes and hypertension \\ J. M. Lopes de Faria, T. C. Cavalcanti, K. C. Silva, P. A. Boer, \\ J. B. Lopes de Faria; \\ Faculty of Medical Sciences, University of Campinas, Brazil.
}

Background and Aims: The mature mammalian retina displays regenerative capacity. Stem/progenitor cells involved in this process may have important clinical applications in degenerative retinal disease as diabetic retinopathy (DR), retinitis pigmentosa and glaucoma. These observations prompt us to search for retinal cell replication in vivo in a model that combines diabetes and hypertension, a well-recognized factor associated with exacerbation of DR. Additionally, we characterized the nature of these replicating cells, assessed the retinal expression of a cell-cycle inhibitor (p27kip1) and search for earlier markers of DR.

Materials and Methods: Spontaneously hypertensive rats (SHR) and their normotensive control Wystar Kyoto (WKY) rats were rendered diabetic by streptozotocin injection and after 15 days the rats were sacrificed and the retinas collected.

Results: Although a rare phenomenon, positively stained BrdU cells attesting their proliferative nature were present in the retina. The number of BrdU positive cells in the retina was higher in SHR than in WKY $(p=0.01)$ and after 15 days of $\mathrm{DM}$, there was a marked reduction in cell replication only in diabetic SHR rats $(\mathrm{p}=0.007)$. The BrdU positive cells were also colabeled for neuron or glial or vascular progenitor cell markers as demonstrated by the presence of nestin, GFAP and Flk-1 antigens. The abundance of p27Kip1 was higher in ganglion cell layer in both diabetic groups $(\mathrm{p}=0.05)$ and also in inner nuclear layer only in diabetic SHR $(\mathrm{p}=0.02)$. There was a marked increment of VEGF expression in diabetic SHR $(\mathrm{p}=0.02)$ accompanied by blood-retinal barrier breakdown ( $\mathrm{p}=0.01$ ). Conclusion: We observed a small number of progenitor cells in the retina that are affected by both diabetes and hypertension. The concomitance of these two conditions markedly reduce the number of these progenitor cells. These findings may launch a new understanding of diabetic retinal neurodegeneration raising the possibility of regenerative therapy.

Supported by FAPESP (Fundação de Amparo à Pesquisa do Estado de São Paulo, Brazil)

\section{1}

Changes of different types of endothelial progenitor cells in relation to stages of diabetic retinopathy in type 1 diabetic patients

G. H. Schernthaner ${ }^{1}$, S. Brunner ${ }^{2}$, F. Zeiler', S. Binder ${ }^{2}$, G. Schernthaner'; ${ }^{1}$ Internal Medicine II - Angiology, Medical University of Vienna,

${ }^{2}$ Opthalmology, Rudolfstiftung Hospital,

${ }^{3}$ Medicine I, Rudolfstiftung Hospital, Vienna, Austria.

Background and Aims: Bone marrow-derived endothelial progenitor cells (EPC) have been suggested to be involved in vascular repair following ischemia and tumour neo-angiogenesis. It is tempting to speculate that EPC might also be involved in advanced diabetic retinopathy (DR) by directly going to the site of ischemia to differentiate and induce new vessel formation. In type 1 diabetic (T1D) patients only an in vitro augmented clonogenical potential of progenitor cells has been demonstrated so far. Thus, an in vivo study of circulating progenitor cells (CPC), EPC, and activated EPC (actEPC) was performed in T1D patients.

Materials and Methods: Ninety patients were studied and staged for DR according to the Early Treatment Diabetic Retinopathy Study (ETDRS) Group classification: no retinopathy (NoDR; $\mathrm{n}=30$ ), mild non-proliferative DR (mNPDR; $\mathrm{n}=30)$, moderate-severe NPDR (msNPDR; $\mathrm{n}=10)$, mild-moderate proliferative diabetic retinopathy $(\mathrm{mPDR} ; \mathrm{n}=10)$ and severe PDR (sPDR; $\mathrm{n}=10$ ). CPC, EPC and actEPC were defined by a direct 4-colour immunefluorescence staining (CPC being CD34+/CD133+, EPC being CD34+/ CD133+/CD309+ and actEPC being CD34+/CD133+/CD309+/CD31+) and enumerated by flow cytometry.

Results: Baseline characteristics (mean \pm STD) of the study population were an age of $41 \pm 12$ years, diabetes duration of $21 \pm 2$ years and an $\mathrm{HbAlc}$ of
$7.8 \pm 1.5$ rel\%. EPC were significantly lower in mNPDR $(114 \pm 66 ; \mathrm{p}<0.001)$ and msNPDR $(77 \pm 40 ; \mathrm{p}=0.042)$ vs No-DR $(244 \pm 115)$. In contrast, EPC were unchanged in mPDR $(248 \pm 155)$ vs No-DR. In contrast, EPC were significantly augmented in sPDR $(389 \pm 124)$ vs all other stages. Numbers of undifferentiated progenitor cells (CPC) did not differ between No-DR, $\mathrm{mPDR}$ and sPDR. The most impressive finding was the 3 -fold augmentation of actEPC in sPDR $(325 \pm 118 ; \mathrm{p}<0.001)$ vs No-DR $(100 \pm 49)$, but also in comparison with mNPDR, msNPDR and mPDR (158 $\pm 119 ; \mathrm{p}=0.006)$.

Conclusion: EPC show a stage-related regulation in type 1 diabetic patients with or without DR. Advancing stages of non-proliferative retinopathy were associated with a significant reduction of EPC vs. patients without retinopathy. In contrast, proliferative retinopathy showed a strong increase in EPC and actEPC numbers. In summary, EPC and actEPC, but not CPC are associated with proliferative diabetic retinopathy. Prospective studies are needed to define whether these findings have diagnostic or even therapeutic impact in diabetic retinopathy.

Supported by Österreichische Diabetesgesellschaft

\section{2}

High glucose-conditioned extracellular matrix enhances apoptosis of human retinal pericytes

E. Berrone ${ }^{1}$, E. Beltramo ${ }^{1}$, K. Nizheradze ${ }^{2}$, S. Tarallo ${ }^{1}$, M. Porta $^{1}$;

${ }^{1}$ Dept. Internal Medicine, University of Turin, Italy, ${ }^{2}$ Laboratory of Biomarkers, Institute of Colloid and Water Chemistry, Kiev, Ukraine.

Background and Aims: Thickening of the basement membrane and selective loss of pericytes are among the first events in the onset of diabetic retinopathy. We reported reduced adhesion of bovine retinal pericytes (BRP) cultured on extracellular matrix (ECM) produced by endothelial cells (HUVEC) in high hexose concentrations, probably due to glycation of matrix proteins, but no changes in their apoptosis. Some observations suggest, however, that human and bovine pericytes may behave differently in experimental conditions mimicking the diabetic milieu. The aim of this study was to verify human retinal pericytes (HRP) behaviour, when cultured on altered ECMs.

Materials and Methods: Conditioned ECMs were obtained by growing HUVEC for 7 days in culture media containing normal $(5.6 \mathrm{mmol} / \mathrm{l})$ or high $(28 \mathrm{mmol} / \mathrm{l}) \mathrm{D}$-glucose. Cells were then lysed and ECM fixed by $\mathrm{NH}_{4} \mathrm{OH}$. HRP were cultured in normal or high glucose on these conditioned ECMs or plastic alone. Pericyte adhesion was evaluated after 12-18 hours. After 7 days of culture, cell proliferation was assessed by cell counts and BrdU incorporation. DNA fragmentation was evaluated by ELISA, as a marker of early apoptosis. The expression of three genes involved in apoptosis, p53, Bax and $\mathrm{Bcl}-2$, was determined by RT-PCR and their concentration in cell lysate by ELISA.

Results: HRP adhered less on high glucose-conditioned ECM and plastic than on normal glucose-conditioned ECM, while there were no significant differences, after 7 days, in their number, nor in DNA synthesis, when grown in physiological glucose. In contrast, DNA synthesis was impaired in HRP cultured in high glucose, in comparison with HRP cultured in normal glucose on the three different matrixes. Apoptosis was greatly enhanced by high glucose-conditioned matrix, both in HRP grown in normal and high glucose and this was confirmed by $p 53, B c l-2$ and Bax mRNA expression and protein concentration.

Conclusion: Human pericyte apoptosis seems to be strongly affected by matrix produced in high glucose and this could play a major role in the development of diabetic retinopathy. Moreover, this behaviour is different from that observed with bovine pericytes, underlining the importance of establishing a cell model for diabetic retinopathy as similar as possible to the human diabetic eye.

\section{3}

Crosstalk between tnf-alpha, nadph oxidase, pkcbeta1/2, and core 2 transferase in diabetic leukocytes

R. Chibber, B. M. Ben-Mahmud, E. M. Kohner;

Cardiovascular Division, King's College London, United Kingdom.

Background and Aims: Increasing evidence suggests that chronic, subclinical inflammation plays an important role in diabetic retinopathy. 
We have recently reported that a glycosylating enzyme, core $2 \beta-1,6-\mathrm{N}$ acetylglucosaminyltransferase (core 2 GlcNAc-T), is implicated in increased leukocyte-endothelial cell adhesion in retinopathy via an up-regulation mechanism controlled by tumour necrosis factor-alpha (TNF- $\alpha$ ) (BenMahmud et al., 2006). The aim of the present study was to elucidate the potential role of NADPH oxidase signalling pathway in the raised activity of core 2 GlcNAc-T in diabetic leukocytes. NADPH oxidase is the most important source of cellular reactive oxygen species (ROS) in leukocytes and blood vessels.

Materials and Methods: Human leukocytes (U937 cells) and a lymphoblastoid cell-line deficient in p47phox were cultured in RPMI medium containing norml $(5.6 \mathrm{mM}$ glucose). The cells were exposed to TNF- $\alpha(8 \mathrm{pg} / \mathrm{ml})$ for $24 \mathrm{~h}$ in the presence and absence of (i) NADPH oxidase inhibitors, $30 \mu \mathrm{M}$ apocynin and $1 \mu \mathrm{M}$ of scrambled and unscrambled gp91ds-tat (ii) $50 \mathrm{nM}$ LY379196, a specific PKC $\beta 1 / 2$ inhibitor and (iii) antioxidants, $15 \mathrm{mM} \mathrm{N}$ acetyl cysteine (NAC, $15 \mathrm{mM}$ ) and $5 \mathrm{mM}$ Tiron. NADPH oxidase activity was measured using cytochrome $\mathrm{c}$ reduction assay. PKC $\beta 1 / 2$ activity was measured using TruLight ${ }^{\text {txx }}$ PKC- $\beta 1 / 2$ assay kit (Calbiochem, UK).

Results: Compared to control medium, exposure to TNF- $\alpha$ (8pg/ml; $24 \mathrm{~h})$ raised core 2 GlcNAc-T activity in human leukocytes $[1722 \pm 255.3(n=10)$ vs. $374 \pm 80.3(\mathrm{n}=10), \mathrm{P}<0.0001]$ that was significantly reversed with apocynin and LY379196. These findings were further supported with (i) significant reversal of TNF- $\alpha$-induced core 2 GlcNAc-T activity with unscrambled and not scrambled gp91ds-tat, a specific NADPH oxidase inhibitor, (ii) use of a lymphoblastoid cell-line deficient in p47phox, a major subunit of NADPH oxidase, and (iii) reversal with NAC and Tiron.

Conclusion: Our results demonstrate a novel signaling crosstalk between TNF- $\alpha$, core 2 GlcNAc-T, NADPH oxidase and PKC $\beta 1 / 2$ in diabetic leukocytes.

Supported by EFSD/Servier

\section{4}

Pro-Angiogenic Growth Factors, including VEGF, inhibit expression of the Anti-Angiogenic Protein PEDF: implications for new vessel formation in diabetes

R. Donnelly ${ }^{1}$, P. Atherton ${ }^{2}$, G. Manning ${ }^{1}$, B. Wang ${ }^{1}$;

${ }^{1}$ Vascular Division,

${ }^{2}$ Clinical Physiology, Nottingham University, Derby, United Kingdom.

Background and Aims: New vessel formation is a key component of diabetesrelated complications, and is influenced by the local inter-play between proand anti-angiogenic factors. Vascular Endothelial Growth Factor (VEGF), basic Fibroblast Growth Factor (bFGF) and Tumour Necrosis Factor- $\alpha$ (TNF$\alpha)$ are important pro-angiogenic growth factors, while Pigment Epithelium Derived Factor (PEDF) is a powerful inhibitor of angiogenesis associated inversely with retinopathy severity; PEDF replacement has been explored as a therapeutic option in ocular neovascularisation. This study explored the regulatory interactions between these cytokines, in particular the effects on PEDF expression.

Materials and Methods: Human umbilical smooth muscle cells were cultured to confluence, starved and quiesced in a low-serum medium for 24 hours. After incubation either untreated or treated with PEDF $10 \mathrm{nM}$, TNF- $\alpha 10 \mathrm{ng} /$ $\mathrm{ml}$, VEGF $10 \mathrm{ng} / \mathrm{ml}$ and bFGF $10 \mathrm{ng} / \mathrm{ml}$ respectively for 24 hours, cells were lysed. Following RNA extraction, a cDNA pool was synthesised for real time RT-PCR of VEGF and PEDF mRNA in a Bio-Rad iQ iCycler.

Results: In comparison with the untreated cells, treatment with PEDF inhibited PEDF expression by $50 \%$, indicating negative feedback. ( $\mathrm{p}=0.003$, $\mathrm{n}=4$ ); TNF- $\alpha$, bFGF and VEGF inhibited the expression of PEDF by $62 \%$ $(\mathrm{p}=0.006, \mathrm{n}=4) 57 \%(\mathrm{p}=0.004, \mathrm{n}=4)$ and $33 \%(\mathrm{p}=0.018, \mathrm{n}=4)$, respectively. No significant effects on the expression of VEGF mRNA were identified with any above treatment.

Conclusion: There is negative feedback in the control of PEDF expression, an important inhibitor of angiogenesis. In addition, pro-angiogenic cytokines that are often elevated in diabetes complications (VEGF, TNF and bFGF) have powerful inhibitory effects on expression of PEDF but not VEGF.

\section{5}

Lower somatostatin expression is an early event in diabetic retinopathy and is associated with retinal neurodegeneration

E. Carrasco ${ }^{1}$, C. Hernández ${ }^{1}$, A. Miralles ${ }^{2}$, P. Huguet ${ }^{3}$, J. Farrés ${ }^{4}$, R. Simo ${ }^{1}$; ${ }^{1}$ Diabetes Research Unit, Institut de Recerca Hospital Vall d'Hebron,

${ }^{2}$ Tissue Bank and Cell Therapy Center, Hospital Vall d'Hebron, ${ }^{3}$ Pathology Department, Hospital Vall d'Hebron, ${ }^{4}$ Department of Biochemistry and Molecular Biology, Universitat Autonoma de Barcelona, Barcelona, Spain.

Background and Aims: To test the hypothesis that a reduction of somatostatin (SST) exists in patients without clinically detectable diabetic retinopathy and that it is associated with retinal neurodegeneration.

Materials and Methods: Human diabetic postmortem eyes $(n=10)$ without clinically detectable retinopathy were compared with eyes $(n=10)$ from non diabetic donors matched by age. SST mRNA (RT-PCR) and SST-28 immunoreactivity (confocal laser) were measured separately in neuroretina and retinal pigment epithelium (RPE). In addition, SST-28 (RIA) was measured in the vitreous fluid. Glial fibrillar acidic protein (GFAP) was assessed by immunofluorescence and Western-blot. Apoptotic cells were quantified using terminal dUTP nick-end labeling (TUNEL).

Results: A higher expression of SST was detected in RPE than in neuroretina in both groups. SST mRNA levels and SST-28 immunoreactivity were significantly lower in both RPE and the neuroretina from diabetic donors in comparison with non-diabetic donors. These results were in agreement with those obtained by measuring SST-28 in the vitreous fluid of the same donors. Increased GFAP and a higher degree of apoptosis were observed in diabetic retinas in comparison with non-diabetic retinas. These changes were most evident in those patients with the higher deficit of SST.

Conclusion: Underproduction of SST is an early event in the eyes of diabetic patients and is associated with glial activation and neural death. In addition, our results suggest that RPE is an important source of SST in the human eye. The possible role of the lower production of SST in the pathogenesis of diabetic retinopathy deserves to be investigated.

Supported by grants from Fundación para la Diabetes, Novo Nordisk, the Ministerio de Sanidad y Consumo. Instituto de Salud Carlos III (REDIMET), and the Ministerio de Ciencia y Tecnología 


\section{PS 117 Nephropathy and end-stage renal failure}

\section{6}

\section{Association between diabetic nephropathy and carotid intima-media thickness and arterial stiffness \\ T. Otani, M. Higa, A. Yoshihara, M. Sue, Y. Tsuchida, N. Hiroi; Internal medicine, Saiseikai kanagawaken hospital, Yokohama, Japan.}

Background and Aims: The link between nephropathy, particularly microalbuminuria, and cardiovascular disease, is becoming increasingly apparent. Atherosclerosis involves a combination of fatty degeneration (atherosis) and of vessel stiffening (sclerosis) of the arterial wall. The aim of this study was to assess associations between diabetic nephropathy and structural changes in arteries, such as carotid intima-media thickness (IMT) and functional changes, such as arterial stiffness and the ankle-brachial index (ABI).

Materials and Methods: The subjects of this study were 56 type 2 diabetic patients (age $56.7 \pm 11.7$ years, 27 males and 29 females). They were divided into 3 groups according to level of urinary albumin excretion: a group of 28 patients with normoalbuminuria (N-group), a group of 17 patients with microalbuminuria (M-group), and a group of 11 patients with macroalbuminuria (MA-group). The IMT of the common carotid artery was measured by ultrasonography. Arterial stiffness and ABI were measured using the cardio ankle vascular index (CAVI) (VaSera VS-1000, Fukuda Denshi). Blood pressure, plasma glucose, and lipid levels, and serum adiponectin levels were compared in the 3 groups.

Results: No significant differences in age, duration of diabetes, BMI, smoking, or kind of antihypertensive agent used were found between the 3 groups. Systolic blood pressure in the M-group was $138.2 \pm 13.3 \mathrm{~mm} \mathrm{Hg}$ and significantly higher $(p<0.01)$ than in the N-group $(128.7 \pm 9.6 \mathrm{~mm} \mathrm{Hg})$ and MA-group (128.7 $\pm 7.9 \mathrm{~mm} \mathrm{Hg}$ ). IMT level in the M-group was $1.19 \pm 0.44 \mathrm{~mm}$ and significantly higher than in the $\mathrm{N}$-group and MA-group $(0.87 \pm 0.28 \mathrm{~mm}$, $0.99 \pm 0.23 \mathrm{~mm}$, respectively). There were no significant differences in CAVI levels between the 3 groups. The ABI levels in the M-group was $1.06 \pm 0.26$ and significantly lower than those in the N-group and MA-group $(1.17 \pm 0.06$, $1.16 \pm 0.04$, respectively). There were no significant differences in $\mathrm{HbA}_{1 c}$ lipid, serum creatinine or adiponectin levels between the 3 groups.

Conclusion: The carotid IMT was significantly higher in the patients with microalbuminuria, while no significant difference was found in CAVI levels. Microalbuminuria is associated with structural changes in arteries, but no association between microalbuminuria and functional changes in arteries was found in this study. The risk factors for structural changes and functional changes in arteries might be partly different.

\section{7}

Prevalence of anaemia in patients with diabetic nephropathy S. A. Martynov, M. V. Shestakova;

Department of Diabetic Nephropathy, Endocrinology Scientific Center, Moscow, Russian Federation.

Background and Aims: Diabetes mellitus is the one of most common cause of chronic renal failure (CRF) and therefore the most common cause of renal anemia. The aim of the study is to compare the prevalence of anemia in patients with diabetic nephropathy (DN) and chronic glomerulonephrities (GN). Materials and Methods: 107 patients with DN and 249 patients with biopsy proven GN were studied. Anemia was defined as hemoglobin $(\mathrm{Hb})<12 \mathrm{~g} / \mathrm{dl}$ in men and for women aged $>50$ years and $\mathrm{Hb}<11 \mathrm{~g} / \mathrm{dl}$ for women $\leq 50$ years. Glomerular filtration rate (GFR) was calculated using the CockcroftGault formula. Comparison of the prevalence of anemia based on stages of chronic kidney disease (CKD) of Kidney Disease Outcome Quality Initiative guidelines. Patients with GFR $<15 \mathrm{ml} / \mathrm{min} / 1.73 \mathrm{~m}^{2}$ and treated by erythropoietin-stimulating agents were not included. Differences between $\mathrm{DN}$ and GN patients were in age $(49.1 \pm 17.2$ vs $31.2 \pm 13.2$ years $(\mathrm{p}<0.001)$, duration of renal damage (13.6 \pm 6.9 vs $3.4 \pm 4.0$ years, $(\mathrm{p}<0.001), \mathrm{Hb}(12.7 \pm 2.2$ and $13.2 \pm 1.7 \mathrm{~g} / \mathrm{dl},(\mathrm{p}<0.01)$, proteinuria $2.2 \pm 2.7$ vs $6.8 \pm 5.0 \mathrm{~g} / 24 \mathrm{~h}(\mathrm{p}<0.001)$ and GFR $\left(80.5 \pm 44.5\right.$ vs $\left.88.7 \pm 35.7 \mathrm{ml} / \mathrm{min} / 1.73 \mathrm{~m}^{2}, \mathrm{p}<0.01\right)$

Results: The prevalence of anemia in total $\mathrm{DN}$ patients was more frequent than in $\mathrm{GN}$ patients $(29.9 \%$ vs $12.8 \%, \mathrm{p}<0.001)$. No significant differences in anemia prevalence in DN and GN patients were observed at GFR $\geq 90 \mathrm{ml} /$ $\mathrm{min} / 1.73 \mathrm{~m}^{2}-7.1 \%$ vs $5.2 \%,(\mathrm{p}=0.64)$ and at GFR level of $60-89 \mathrm{ml} / \mathrm{min} / 1.73$ $\mathrm{m}^{2}-10.5 \%$ vs $14.1 \%$, $(\mathrm{p}=0.68)$. Anemia prevalence was significantly greater in patients with DN compared to patients with GN in the CKD categories $30-59 \mathrm{ml} / \mathrm{min} / 1.73 \mathrm{~m}^{2}-50 \%$ vs $25.5 \%(\mathrm{p}=0.02)$ and $15-29 \mathrm{ml} / \mathrm{min} / 1.73 \mathrm{~m}^{2}$ - $83.3 \%$ and $37.5 \%(\mathrm{p}=0.03)$. Mean $\mathrm{Hb}$ values were significantly lower in DN patients than GN patients only at GFR level below $30 \mathrm{ml} / \mathrm{min} / 1.73 \mathrm{~m}^{2}$ $-9.8 \pm 1.7$ vs. $11.9 \pm 1.9 \mathrm{~g} / \mathrm{dl}(\mathrm{p}=0.02)$.

Conclusion: We conclude that anemia develops early in patients with DN compared to patients with chronic GN. The higher prevalence of anemia in diabetes related with advanced kidney damage in $\mathrm{DN}$ and contributes to progression CRF and cardiovascular complications.

\section{8}

Serum prohepcidin is decreased in progressive diabetic nephropathy and related to iron storage in patients with type 2 diabetes

J. Brix ${ }^{1}$, K. Krzyzanowska ${ }^{1}$, S. Siller ${ }^{1}$, W. Krugluger ${ }^{2}$, F. Mittermayer ${ }^{3}$, G. Schernthaner';

${ }^{1}$ Department of Internal Medicine I, Rudolfstiftung Hospital, ${ }^{2}$ Department of Clinical Chemistry, Rudolfstiftung Hospital,

${ }^{3}$ Department of Clinical Pharmacology, Medical University Vienna, Austria.

Background and Aims: Hepcidin, which is expressed by the liver, regulates the human iron metabolism and is increased in chronic inflammation. Furthermore, it has been suggested that it is responsible for the development of anemia in chronic disease. Its precursor prohepcidin was positively related to hematocrit in patients with end-stage renal failure. Microalbuminuria, a well known risk factor for the development and progression of cardiovascular disease in type 2 diabetes signifies incipient diabetic nephropathy while macroalbuminuria reflects progressive diabetic renal disease. To investigate if prohepcidin is related to diabetic nephropathy, red blood count, iron status or chronic inflammation we determined this parameter in patients with type 2 diabetes and different stages of albuminuria.

Materials and Methods: We included 92 type 2 diabetes patients (57 males) in a cross-sectional study. Serum prohepcidin was determined by ELISA. Micro- and macroalbuminuria were defined as 24 hour urinary protein excretion of more than $30 \mathrm{mg}$ or $300 \mathrm{mg}$, respectively.

Results: Micro- and macroalbuminuria were present in 42 and 22 type 2 diabetes patients. Median circulating prohepcidin concentrations were 147.5 (IQR: 111.3 - 177.8) ng/ml, 172.3 (IQR: 106.1 - 242.7) ng/ml and 109.7 $(67.0-164.1) \mathrm{ng} / \mathrm{ml}$ in patients with normo-, micro- and macroalbuminuria ( $\mathrm{P}=0.049$ for normo- vs. macroalbuminuria; $\mathrm{P}=0.003$ for micro- vs. macroalbuminuria). Prohepcidin correlated with ferritin $(\mathrm{R}=0.22 ; \mathrm{P}=0.04)$, HOMA-IR $(\mathrm{R}=-0.27 ; \mathrm{P}=0.03)$, diastolic blood pressure $(\mathrm{R}=0.21 ; \mathrm{P}=0.049)$ and HDL-cholesterol $(\mathrm{R}=0.22 ; \mathrm{P}=0.03)$ but not with red blood count (hemoglobin: $\mathrm{R}=0.12, \mathrm{P}=0.31$; hematocrit: $\mathrm{R}=0.08, \mathrm{P}=0.47)$ or hsCRP $(\mathrm{R}=-$ $0.09 ; \mathrm{P}=0.40)$. Multiple regression analysis revealed that ferritin was the only independent predictor of prohepcidin (Beta $=0.25 ; \mathrm{P}=0.049$ ).

Conclusions: Patients with type 2 diabetes and macroalbuminuria had lower prohepcidin concentrations compared to those with normo- or microalbuminuria. Prohepcidin was related to ferritin but not to red blood count or chronic inflammation. Low levels of prohepcidin in progressive diabetic nephropathy could indicate a potential mechanism counteracting the developement of anemia by increasing intestinal iron uptake.

\section{9}

Diabetic patients on chronic dialysis: complications and quality of life V. R. Sørensen ${ }^{1}$, E. R. Mathiesen ${ }^{2}$, T. Watt', J. B. Bjørner ${ }^{3}$, M. V. N. Andersen ${ }^{4}$, B. Feldt-Rasmussen ${ }^{1}$;

${ }^{1}$ Department of Nephrology, Rigshospitalet, Copenhagen,

${ }^{2}$ Department of Endocrinology, Rigshospitalet, Copenhagen,

${ }^{3}$ Department of Health Promotion, National Research Center for the working environment, Copenhagen,

${ }^{4}$ Department of Ophalmology, Rigshospitalet, Copenhagen, Denmark.

Background and Aims: Despite the improved treatment of diabetic patients during the last 20 years, diabetic patients, who are treated with chronic maintenance dialysis, often suffer from one or more diabetic complications 
besides kidney failure and have an increased morbidity and mortality. Health related quality of life has become an important parameter, when evaluating the wellbeing of patients with end-stage renal disease. The aims of the study were to describe the prevalence of diabetic complications and to evaluate health related quality of life in diabetic dialysis patients. In addition we investigated predictors of physical and mental health and the importance of beliefs about control over health.

Materials and Methods: All diabetic patients treated with dialysis by January 2004 in our clinic $(n=65)$ were characterised regarding cardiovascular diseases and diabetic complications and were asked to complete a kidneyspecific (KDQOL) and a generic (SF-36) questionnaire. Non-diabetic dialysis patients $(n=65)$, who were matched by age, sex and duration of end-stage renal disease and normo-albuminuric diabetic patients with normal kidney function $(n=76)$, who were matched by age, type and duration of diabetes, served as controls. Generic quality of life was also compared with matched data from a survey in the Danish general population $(n=2248)$. Diabetic patients were also asked to fill in a questionnaire regarding belief about control over health.

Results: Micro- and macrovascular complications were significantly more frequent in diabetic dialysis patients than in diabetic patients without renal disease with a frequency of current foot ulcers of $33 \%$ vs. $13 \%$, amputations $14 \%$ vs. $4 \%$ and impaired vision $22 \%$ vs. $2 \%$. Non-diabetic dialysis patients had a low frequency of these complications $(4 \%, 3 \%, 0 \%)$. Diabetic dialysis patients reported significantly shorter school education compared with diabetic patients with normal kidney function. There were no significant differences in the kidney disease quality of life among diabetic versus nondiabetic dialysis patients. Generic physical health was however significantly worse in diabetic dialysis patients (mean (SD)):(35(9) $n=38$ ) compared with non-diabetic dialysis patients (41(10) $n=40)$, diabetic patients with normal kidney function (45(12) n=38) and the matched general population (47(19) $(\mathrm{p}<0.01)$. Mental health was comparable in the three patients groups: 50(8) vs. $49(10)$ vs. $49(11)$, whereas the score in the general population was 55(9). Reduced physical health was predicted by presence of end-stage renal disease, diabetes and lower education. Diabetic dialysis patients believed significantly more in chance in relation to their diabetes than diabetic patients with normal kidney function. Diabetic patients, who believed more on their own ability to control their diabetes and less on chance, reported better mental health.

Conclusion: Diabetic dialysis patients were characterised by a high prevalence of diabetic complications. Generic physical health was significantly reduced and the presence of end-stage renal disease was the most important predictor of physical health.

Supported by the Danish Society of Nephrology

\section{0}

Remarkable differences in the prevalence of diabetics in dialysis in developed countries

H. J. G. Bilo ${ }^{1,2}$, S. H. A. Diepeveen ${ }^{3}$, S. J. J. Logtenberg ${ }^{1}$, R. T. Gansevoort ${ }^{4}$, K. J. Jager ${ }^{5}$, N. Kleefstra ${ }^{1}$;

${ }^{1}$ Diabetes Centre, Isala Clinics, Zwolle, ${ }^{2}$ Internal Medicine, UMCG,

Groningen, ${ }^{3}$ Internal Medicine, Isala Clinics, Zwolle,

${ }^{4}$ Nephrology, UMCG, Groningen, ${ }^{5}$ Stichting RENINE, Academic Medical

Centre, Amsterdam, The Netherlands.

Background and Aims: Diabetes mellitus (DM) is considered one of the main causes of end stage renal disease (ESRD) necessitating renal replacement therapy (RRT; dialysis and kidney transplantation). It has passed virtually all other causes in many countries. The accelerating growth in ESRD and DM not only contribute to a worse quality of life and early death, but also to a vast increase in health-care expenditure worldwide. Still, there are large differences in prevalence, varying from less than $20 \%$ to over $40 \%$. The aim of this search was to compare different developed countries regarding the prevalence of DM as cause of dialysis for ESRD.

Materials and Methods: Data concerning the percentage of DM as cause of dialysis for ESRD in the United States, Belgium, Germany, Denmark, Finland, Norway, Japan, and The Netherlands were derived from several relevant national and international databases (RENINE-, USRDS- and WHOdatabases). In addition, data were derived from websites of various national diabetes and nephrological organizations. For 2004, number of patients on dialysis per million inhabitants per country are reported. Furthermore, prevalence of DM in that group and the number of patients on dialysis due to DM per million inhabitants are reported.
Results: The number of patients on dialysis in 2004 ranged from 270 per million in Norway to 1855 per million in Japan (Table). Number of patients per million with DM as primary cause for dialysis is 16 times higher in Japan than in Norway. Prevalence of renal transplantation (\% of total RRT in ESRD) as treatment modality in ESRD was less than 2\% in Japan, $26 \%$ in Germany, $31 \%$ in the US, $33 \%$ in Denmark, $40 \%$ in Belgium, $54 \%$ in The Netherlands, $60 \%$ in Finland, and $62 \%$ in Norway.

\begin{tabular}{llll} 
& $\begin{array}{l}\text { number of } \\
\text { patients on dialysis } \\
\text { (n/million) }\end{array}$ & $\begin{array}{l}\text { DM as primary cause } \\
\text { of ESRD requiring } \\
\text { dialysis }(\%)\end{array}$ & $\begin{array}{l}\text { number of patients } \\
\text { on dialysis with } \\
\text { DM as primary } \\
\text { cause (n/million) }\end{array}$ \\
\hline Japan & 1855 & 41.0 & 761 \\
US & 1099 & 45.6 & 501 \\
Finland & 275 & 33.1 & 253 \\
Germany & 740 & 34.2 & 237 \\
Belgium & 585 & 22.5 & 146 \\
Denmark & 325 & 20.8 & 68 \\
Netherlands & 325 & 17.0 & 55 \\
Norway & 270 & 17.3 & 47 \\
\hline
\end{tabular}

Conclusion: These results show dramatic differences in the prevalence of patients on dialysis and in the prevalence of DM as the primary cause between developed countries. The low prevalence in The Netherlands and some Scandinavian countries can only partly be explained by the higher transplantation rate. Another reason for the differences might be the variation in DM prevalence in the general population, reported in 2004 to vary between $3.7 \%$ in The Netherlands and $10.2 \%$ in Germany. Finding explanations and workable solutions for these differences might help to alleviate the human and financial burden of ESRD in DM in the developed world. 


\section{PS 118 Hypertension: clinical management}

\section{1}

Evaluation of artificial intelligence and clinical decision making in the management of patients with type 2 diabetes and uncontrolled hypertension

K. A. Earle $^{1}$, R. Squires ${ }^{1}$, S. Anandarajah ${ }^{1}$, P. Taylor ${ }^{2}$, D. Sutton ${ }^{3}$; ${ }^{1}$ Cellular and Molecular Medicine, St George's Hospital NHS Trust, London, ${ }^{2}$ Centre for Health Informatics and Medical Education, Royal Free \& University College London Medical School,

${ }^{3}$ Computer Science, Oxford Brokkes University, United Kingdom.

Background and Aims: Hypertension is a major, modifiable risk factor for cardiovascular disease. However, target blood pressure in the United Kingdom is achieved in $<30 \%$ of patients. Recent data suggests a nurseled approach is more effective than conventional care in the attainment of target blood pressure. It is unclear how these approaches vary in relation to concordance with published guidance for hypertension. Therefore, we analysed the therapeutic decisions for patients with diabetes and uncontrolled hypertension in these settings against those in a virtual clinic using artificial intelligence.

Materials and Methods: We studied 219 patients with type 2 diabetes and uncontrolled hypertension - systolic (SBP) >130 and/or diastolic (DBP) blood pressure $>80 \mathrm{mmHg}$ respectively receiving antihypertensive therapy. Evaluations of 143 patients attending a nurse-led (NLC) and 76 patients in conventional, physician-led (PLC) clinics were made. Seated BP was measured using a digital oscillometric device. Treatment, BP, glycated haemoglobin (HbAlc) by HPLC and clinical decisions were reported on a standardised proforma.

The artificial intelligence system was written in the PROforma language. It contained expressions representing clinical criteria to influence and plan treatment based on the National Institute for Clinical Excellence guidelines. Data of all 219 patients were uploaded to the VLC database. The variables for decisions were cardiovascular risk score (CVD) determined by the Joint British Societies calculator, SBP, DBP and use of first line drugs (FLD). The FLDs were classed as angiotensin converting enzyme inhibitors (ACEI) or receptor blockers (ARB), beta blocker, calcium channel blocker and thiazide diuretic and entered according to whether they were prescribed at the maximal dose. Therapeutic decisions were coded as: $1=$ monitor only, $2=$ increase FLD, 3 = start a new FLD or $4=$ refer for second-line agent. Target SBP and DBP was $<130$ and $<80 \mathrm{mmHg}$ respectively.

Results: The patients seen the NLC and PLC clinics had similar mean [SD], age, SBP, DBP and HbA1C (63.2[12.8] vs. 61.6[13.5] yrs; $\mathrm{p}=0.38,144.9$ [19.4] vs. $142.2[12.6] \mathrm{mmHg} ; \mathrm{p}=0.29,77.6[11.8]$ vs. $78.0[10.2] \mathrm{mmHg} \mathrm{p}=0.79$ and $8.0[1.8]$ vs. 8.1[1.5] \%; $\mathrm{p}=0.09$ ). All patients had a CVD score of $>20 \%$. There were no differences between the groups in the prescription of any class of FLD, second line agent or the proportion on a maximal dose within any class. Decisions to monitor only in the PLC group was significantly higher and treatment altering decisions 2, 3 and 4 significantly lower than in the NLC group (67.6 vs. 32.8 and 10.8 vs. $35.1,13.5$ vs. $21.6,8.110 .4 ; \mathrm{p}=0.0001$ ). Decisions in VLC did not recommend to monitor only, but suggested decisions 2 and 3 respectively for $81 \%$ and $19 \%$ of the PLC group. VLC decisions 1, 2, 3 and 4 were made for $15 \%, 63 \%, 19 \%$ and $3 \%$ respectively of the NLC group. The K estimate for agreement with the artificial intelligence decisions of VLC was 0.1 (poor) in the PLC group and 0.3 (fair) for the NLC group.

Conclusion: This artificial intelligence system consistently recognises the impact of CVD risk and exposes FLD under-treatment by nurses and physicians. Use of such decision support could possibly improve practice and outcomes.

Supported by MSD
1162

Blood pressure lowering-independent improvement of central arterial stiffness by valsartan, but not amlodipine, in type 2 diabetic patients with systolic hypertension

J. Karalliedde ${ }^{1}$, A. Smith ${ }^{1}$, L. De Angelis ${ }^{1}$, V. Mirenda ${ }^{1}$, A. Kandra ${ }^{2}$,

P. Ferber ${ }^{2}$, G. Viberti ${ }^{1}$;

${ }^{1}$ Diabetes, King's College London, United Kingdom,

${ }^{2}$ Novartis Pharma AG, Basle, Switzerland.

Background and Aims: Raised systolic and pulse pressures (PP) and increased central arterial stiffness, as estimated from aortic pulse wave velocity(Ao-PWV), are powerful risk factors for cardiovascular disease (CVD) morbidity and mortality. Systolic hypertension is often associated with microalbuminuria (MA) and both are strong predictors of CVD and progressive renal failure in type 2 diabetic patients (T2D). In these patients angiotensin receptor blockers (ARBs) have cardio-renal benefits which seem independent of blood pressure (BP) lowering. Whether ARBs obtain these beneficial effects by improving central arterial stiffness to a greater extent than other equipotent anti-hypertensive medications remains unclear.We compared the effect of valsartan (Val), an ARB, with that of amlodipine, a calcium channel blocker, on Ao-PWV in 131 T2D patients with systolic hypertension, raised PP and MA in a 24 week randomized, double-blind parallel group active-controlled study.

Materials and Methods: In a 4-week run in wash out phase all patients received moxonidine ( $400 \mathrm{mcg} /$ day), which has minimal impact on the vessel wall properties, to avoid uncontrolled hypertension ( $\mathrm{SBP} \geq 180 \mathrm{mmHg}$ ). One hundred and thirty one $(41 \% \mathrm{M}, 59 \% \mathrm{~F})$ patients with $\mathrm{PP} \geq 60 \mathrm{mmHg}$ and systolic blood pressure $(\mathrm{SBP}) \geq 140 \mathrm{mmHg}$ were then randomized to receive Valsartan ( $\mathrm{n}=66) 160 \mathrm{mg}$ or amlodipine $5 \mathrm{mg}(\mathrm{n}=65)$. After 4 weeks $25 \mathrm{mg} /$ day hydrochlorothiazide (HCTZ), which has minimal effect on Ao-PWV, was added to valsartan and amlodipine up-titrated to $10 \mathrm{mg}$ to ensure equivalent BP control.

Results: Mean (SD) age was 59.5 (9.4) years, SBP 158.0 (10.7) mmHg, PP $95.6(11.0) \mathrm{mmHg}$, and duration of diabetes 10.0 (7.1) years. Albumin excretion rate (AER) median (interquartile range) was $27.5(7-97) \mu \mathrm{g} / \mathrm{min}$. All patients had serum creatinine below $\leq 115 \mu \mathrm{mol} / \mathrm{l}$. At baseline there were no significant differences in age, duration of diabetes, HbA1c, SBP, PP, AER and Ao-PWV between the two groups. Brachial PP and SBP fell similarly in both groups after 24 weeks with attained mean (SD) PP of 61.6 (13.6) $\mathrm{mmHg}$ and 61.5(12.2) $\mathrm{mmHg}$ and SBP of 135.3(18.8) $\mathrm{mmHg}$ and $137.2(13.8) \mathrm{mmHg}$ in the Val/HCTZ and amlodipine groups respectively. AER fell by $35 \%$ in the Val/HCTZ group as compared to a rise of $24 \%$ in the amlodipine group $(\mathrm{p}=0.0004)$. Glycaemic control was stable and similar in the two groups throughout the study. Following 24 weeks Ao-PWV fell from mean (SD) 12.5 (2.5) to $10.7(2.5) \mathrm{m} / \mathrm{s}$ in the Val/HCTZ group and from 12(2.5) to $11.3(2.6)$ $\mathrm{m} / \mathrm{s}$ in the amlodipine group. Between treatment comparison for change from baseline in mean Ao-PWV [by ANCOVA which included treatment, gender, age and operator as fixed factors and baseline mean Ao-PWV, baseline mean $\mathrm{PP}$ as covariates] at week 24 showed a significantly greater reduction of, mean (95\% CI), $-1.1 \mathrm{~m} / \mathrm{s}(-1.8$ to -0.5$)$ for Val/HCTZ compared to amlodipine ( $\mathrm{p}=$ 0.001). This magnitude of Ao-PWV difference translates to approximately 10 years of vascular ageing.

Conclusion: Our findings suggest that in this high CVD risk group of T2D, treatment for 24 weeks with valsartan improves central arterial stiffness significantly more compared to amlodipine despite similar brachial BP control. This and the significantly greater reduction of albuminuria by valsartan may explain the specific cardio-renal protective effects seen with ARBs.

Supported by Novartis 


\section{3}

Clinical utility of single-pill amlodipine besylate/atorvastatin calcium for the attainment of blood pressure and lipid goals in patients of diverse ethnicity with diabetes: Results from the GEMINI-AALA Study C. A. Aguilar-Salinas ${ }^{1}$, Y. Ro ${ }^{2}$, H.-F. Tse ${ }^{3}$, L. G. Howes ${ }^{4}$, H. Chaves ${ }^{5}$, S. Erdine ${ }^{6}$, R. Guindy ${ }^{7}$, P. Chopra ${ }^{8}$, R. A. Moller ${ }^{8}$, I. M. Schou'; ${ }^{1}$ Department of Endocrinology and Metabolism, Instituto Nacional de Ciencias Medicas y Nutricion, Mexico City, Mexico, ${ }^{2}$ Sejong Medical Research Institute, Korea University, Bucheon, Republic of Korea, ${ }^{3}$ Department of Medicine, University of Hong Kong, ${ }^{4}$ Griffith and Bond University Medical Schools, Gold Coast, Australia, ${ }^{5} \mathrm{Health}$ Science Centre, Federal University of Pernambuco, Boa Vista, Brazil, ${ }^{6}$ Cerrahpasa School of Medicine, Istanbul University, Turkey, ${ }^{7}$ Faculty of Medicine, Ain Shams University Cairo, Egypt, ${ }^{8}$ Pfizer Inc, New York, United States,

${ }^{9}$ Pfizer Australia, West Ryde, Australia.

Background and Aims: Patients with diabetes frequently have additional risk factors such as hypertension (HTN) and dyslipidemia (DYS), greatly increasing their risk of cardiovascular disease. This planned analysis of the GEMINI-AALA study evaluated the utility of single-pill amlodipine besylate/ atorvastatin calcium for the attainment of blood pressure (BP) and lipid goals in diabetic patients of diverse ethnicity with HTN and DYS.

Materials and Methods: GEMINI-AALA was a 14-week, multicenter, openlabel study in patients with HTN/DYS from 27 countries across 5 continents. Eight dosages of single-pill amlodipine besylate/atorvastatin calcium (5/10$10 / 80 \mathrm{mg}$ ) were administered and electively titrated at Weeks 6, 10 (titration optional), and 12. The percentage of patients who attained JNC 7 and NCEP ATP III goals for BP $(<130 / 80 \mathrm{~mm} \mathrm{Hg})$ and low-density lipoprotein cholesterol (LDL-C) $(<100 \mathrm{mg} / \mathrm{dL})$ at Week 14 was assessed. Changes from baseline in BP and LDL-C were also measured.

Results: Of the 1638 patients included in the intent-to-treat (ITT) population, 605 patients had type 2 diabetes. For diabetic patients, the mean dose of amlodipine besylate/atorvastatin calcium during the study was $6.2 \mathrm{mg} / 18.3$ mg. Mean BP and LDL-C at baseline were $145.0 / 86.2 \mathrm{mmHg}$ and 120.6 $\mathrm{mg} / \mathrm{dL}$, respectively. After 14 weeks treatment, $41.3 \%$ of diabetic patients reached both $\mathrm{BP}$ and LDL-C goals $(0.0 \%$ at baseline), $46.3 \%$ reached $\mathrm{BP}$ goal ( $0.2 \%$ at baseline), and $84.6 \%$ reached LDL-C goal (30.6\% at baseline). Mean BP reduction was $18.1 / 10.2 \mathrm{mmHg}$, and mean decrease in LDL-C was $40.5 \mathrm{mg} / \mathrm{dL}(27.5 \%)$. Attainment of both goals for the Middle East/Africa, Asia-Pacific, and Latin America was 33.7\%, 45.1\%, and 40.6\%, respectively. Among diabetic patients, $4.3 \%$ discontinued due to adverse events.

Conclusion: Amlodipine besylate/atorvastatin calcium single-pill therapy was an effective and safe intervention that helped diabetic patients of diverse ethnicity with combined HTN/DYS to achieve BP and LDL-C goals. Supported by Pfizer Inc

\section{4}

Activity of renin-angiotensin-aldosterone axis in salt-sensitive and salt-resistant hypertensive type 2 diabetic patients S. D. Jelic ${ }^{1}$, M. N. Zamaklar², Z. Caparevic ${ }^{1}$, V. Ilic ${ }^{1}$, S. Ilic ${ }^{1}$;

${ }^{1}$ University Clinic for Internal Medicine, CHC"Dr Dragisa Misovic"Dedinje, ${ }^{2}$ Institute for endocrinology, diabetes and metabolic diseases, School of Medicine, University of Belgrade, Belgrade, Serbia.

Background: Most of the investigators agree that plasma renin activity and plasma aldosterone levels are lowered or suppressed in hypertensive type 2 diabetic patients, compared with healthy controls. However, possible interrelations between the activity of renin-angiotensin-aldosterone axis and salt-sensitivity in these patients are not still fully elucidated.

Aim: To assess the activity of renin-angiotensin-aldosterone axis in hypertensive type 2 diabetic patients and its relationship with the saltsensitivity.

Methods: Subjects: 1) 45 (24 female/21 male) hypertensive type 2 diabetic patients mean (SD) age 48.91 (6.49) years; mean (SD) duration of diabetes 10.87 (5.33) years and mean (SD) duration of hypertension 11.53 (6.30) years. 2) 10 healthy controls ( 5 female/5 male), mean (SD) age 36 (4) years. The increment of mean arterial pressure on high salt ( $300 \mathrm{mmol} \mathrm{Na} /$ day) diet vs. low salt ( $40 \mathrm{mmol} \mathrm{Na}$ /day) diet exceeding $10 \mathrm{~mm} \mathrm{Hg}$ was used as the criterion for salt sensitivity (SS). According to the given definition 23 (51.11\%) of studied hypertensive type 2 diabetic patients were salt-sensitive.
Assays: Plasma renin activity (PRA) and urine and plasma aldosterone (U-A and P-A) levels were measured by CIS radioimmunoassay. Statistical analysis was performed using the STATISTICA 4.5 program (StatSoft, Tulsa, OK, USA).

Results: PRA [0.23 (0.12) vs. 0.30 (0.17) vs. $0.28(0.08) \mathrm{pmol} / \mathrm{L} / \mathrm{s} ; \mathrm{F}=1.45$, $\mathrm{p}=0.24]$ and P-A [0.16 (0.07) vs. $0.22(0.17)$ vs. $0.24(0.08) \mathrm{nmol} / \mathrm{l} ; \mathrm{F}=2.04$, $\mathrm{p}=0.14]$ levels did not differ significantly between salt-sensitive and saltresistant (SR) hypertensive type 2 diabetic patients, as compared with healthy controls. Urinary aldosterone excretion was significantly lower in salt-resistant patients than in healthy controls [SS: 18.15 (7.73) vs. SR: 17.97 (7.00) vs. controls: $24.75(4.73) \mathrm{nmol} / 24 \mathrm{~h} ; \mathrm{F}=3.77, \mathrm{p}=0.03$ ]. At the same time, positive correlation of borderline significance that existed between PRA and P-A levels among healthy controls $(\mathrm{r}=0.63014, \mathrm{P}=0.050839)$ was lost among salt-resistant hypertensive type 2 diabetic patients $(\mathrm{r}=-0.035806, \mathrm{P}=$ 0.874305). Among salt-sensitive patients correlation between PRA and P-A levels was significant but negative $(\mathrm{r}=-0.442672, \mathrm{P}=0.034405)$.

Conclusions: This study demonstrated that activity of renin-angiotensinaldosterone axis in hypertensive patients with type 2 diabetes did not differ significantly between salt-sensitive and salt-resistant subjects. However, urinary aldosterone excretion was lower especially in salt-resistant patients and there were signs of distorted feed-back between renin and aldosterone in hypertensive type 2 diabetic patients, when compared with healthy controls. Whether this disturbance represents one of the possible causes of diabetic renal lesion or its consequence remains to be elucidated.

\section{5}

Salt sensitivity is associated with insulin resistance, sympathetic overactivity in human essential hypertension

M. S. Yatabe ${ }^{1}$, H. Sanada ${ }^{1}$, J. Yatabe ${ }^{1}$, Y. Kusano ${ }^{1}$, S. Hashimoto ${ }^{1}$, M. Otsuki ${ }^{2}$, T. Watanabe ${ }^{1}$;

${ }^{1}$ Internal Medicine III, Fukushima Medical University, Fukushima, ${ }^{2}$ Internal Medicine, Asaka Medical clinic, Koriyama, Japan.

Background and Aims: Derangement of glucose metabolism and high blood pressure are two of the hallmarks of metabolic syndrome, however the association between these metabolic parameters still remains unclear. As both high blood pressure and impaired glucose metabolism are heterogeneous conditions, focusing on the salt sensitivity and insulin resistance may yield more information to delineate and unravel this problem. In this study, we aimed to clarify the relationship between salt sensitivity and insulin resistance, along with the effects of sympathetic nervous system and renin-angiotensin system on non-obese, non-diabetic essential hypertensive individuals.

Materials and Methods: Twenty-four subjects with untreated essential hypertension (12 males and 12 females, aged between 29-67 years) were enrolled into the study. The state of glucose metabolism was assessed by the means of hyperinsulinemic, euglycemic glucose clamp and $75 \mathrm{~g}$ OGTT. Glucose infusion rate (GIR) from the glucose clamp was used as an index for insulin resistance. The subjects were classified as salt-sensitive (SS) or salt-resistant (SR) according to the 24-hour ambulatory blood pressure monitoring performed during the periods of low-salt $(34 \mathrm{mmol} / \mathrm{day})$ diet and high-salt diet $(252 \mathrm{mmol} / \mathrm{day})$. Urinary norepinephrine was measured by HPLC method.

Results: Of the 24 hypertensive subjects, 10 were found to be SS, whereas 14 were classified as SR. Compared to SS group, SR group showed higher serum insulin levels after glucose ingestion ( $\mathrm{p}<0.01$ at $60,90,120$ minutes). Euglycemic glucose clamp revealed lower GIR in SS group compared with SR group $(5.1 \pm 0.7$ vs. $6.7 \pm 0.4 \mathrm{mg} / \mathrm{kg} / \mathrm{min}, \mathrm{p}<0.01)$. Salt sensitivity index, which is the MBP increase from low- to high-salt diet divided by the MBP upon a low-salt diet, showed an inverse linear relationship with GIR $(r=0.64)$. GIR also had a positive correlation with the change in urinary sodium excretion $(\mathrm{r}=0.66)$, and SS subjects have significant lower sodium excretion compared to SR with a similar GIR. Decline in hematocrit $(r=0.65)$ upon low-to highsalt diet showed an inverse correlation between SS and SR. GIR showed a significant inverse correlation with the change in urinary norepinephrine excretion $(r=0.55)$. Sodium loading was considered to suppress the sympathetic activity, however, significantly higher activity was still remained in SS subjects than in SR upon a high-salt diet.

Conclusion: We have demonstrated that salt sensitivity of blood pressure is strongly associated with insulin resistance. Hyperinsulinemia, sympathetic overactivation may play a role in the relationship between salt sensitivity of blood pressure and insulin resistance. 


\section{6}

Predictive value of insulinaemic status, insulin resistance, urinary total protein and urinary calcium in the subsequent development of pregnancy induced hypertension

R. Helal, F. Jebunnesa, A. Akhter, S. Akhter, L. Ali;

Dept of Biochemistry \& Cell Biology, Bangladesh Institute of Research \& Rehabilitation in Diabetes, Endocrine and Metabolic Disorders, Dhaka Bangladesh.

Background and Aims: Pregnancy induced hypertension (PIH) is known to be an insulin resistant condition. There are still controversies regarding the suitable predictive markers for this disorder. Prospective studies are needed but these are relatively scarce and still inconclusive due to technical and ethical issues in measuring appropriate parameters during pregnancy. In the present study we have investigated whether insulinemic status, insulin resistance, urinary calcium (UCa) and urinary total protein (UTP) can be used as predictive markers for the future development of PIH.

Materials and Methods: A total of 127 Bangladeshi pregnant women participated in the study up to their end of pregnancy. Among them 15 women (age in yrs, $\mathrm{M} \pm \mathrm{SD}, 26.2 \pm 5.1$ ) developed PIH. By a nested casecontrol design, 48 age- and parity-matched normotensive pregnant women as controls were randomly selected from among the Non-PIH subjects. Serum was collected in early pregnancy and stored at $-70^{\circ} \mathrm{c}$ for future analysis. PIH was diagnosed by standard criteria; UCa and UTP were measured by photometric methods. Insulinemic status was assessed by fasting serum Cpeptide (by chemiluminescent immunoassay). Insulin sensitivity (HOMA\% S) was assessed by Homeostasis Model Assessment. The cut-off value for higher serum C-peptide was determined from the $75^{\text {th }}$ percentile and the cut-off value for lower HOMA $S$ was calculated from the $25^{\text {th }}$ percentile. The Sensitivity, Specificity, Positive Predictive Value (PPV) and Negative predictive value (NPV) were assessed by using the McNemar test.

Results: The PIH group showed significantly higher $\mathrm{C}$-peptide $(\mathrm{mmol} / \mathrm{l}$; $\mathrm{M} \pm \mathrm{SD}, 2.04 \pm 0.54)$, UTP (mg/dl, 1.2 \pm 0.29$)$ and $\mathrm{UCa}(\mathrm{mg} / \mathrm{l} \mathrm{2.9 \pm 1.05)}$ as compared to Control [C-peptide, $1.6 \pm 0.42$, p $<0.02$; UTP, $0.91 \pm 0.36, \mathrm{p}<0.01$; $\mathrm{UCa}, 1.56 \pm 0.73, \mathrm{p}<0.01]$ in the early pregnancy. HOMA-S was significantly lower in $\mathrm{PIH}(\%, \mathrm{M} \pm \mathrm{SD}, 24.4 \pm 8.7)$ as compared to Control (29.7 \pm 7.2 , $\mathrm{p}<0.04)$. For predicting the future development of $\mathrm{PIH}$ in later stages the early pregnancy serum C-peptide showed a Sensitivity of $53 \%$, Specificity $81 \%$, PPV $47 \%$ and NPV $84 \%$. The corresponding values for UTP were $46 \% ; 79 \%$, $41 \%$ and $82 \%$; and those for UCa were $(60 \%, 83 \%, 52 \%$ and $86 \%$.

Conclusion: The positive and negative values of various parameters suggest that hyperinsulinemia, insulin resistance, urinary calcium, and urinary total protein in early pregnancy may be useful tools for predicting PIH in late pregnancy. Among the four predictive markers UCa seems to be the most reliable one.

Supported by IPICS and DAB

\section{PS 119 Vascular biology: in vitro studies}

\section{7}

Chronic hyperglycaemia increases TNF-alfa-stimulated adhesion molecule expression in human umbilical vein endothelial cells (HUVEC) from gestational diabetic women

A. Pandolfi ${ }^{1}$, P. Di Tomo ${ }^{1}$, A. Giardinelli ${ }^{1}$, P. Di Fulvio ${ }^{2}$, S. Di Silvestre ${ }^{1}$, N. Di Pietro ${ }^{1}$, G. Formoso ${ }^{2}$, P. Lanuti ${ }^{1}$, M. Marchisio ${ }^{1}$, A. Consoli ${ }^{2}$; ${ }^{1}$ Department of Biomorpholgy, ${ }^{2}$ Department of Medicine and Aging Sciences, University G. d'Annunzio, Aging Research Center, Ce.S.I., Chieti, Italy.

Background and Aims: Diabetes mellitus is associated with inflammatory endothelium activation and monocyte interactions with vascular adhesion molecules exert a relevant role in the development of vascular complications. Inflammatory cytokines, such as $\mathrm{TNF} \alpha$, can upregulate adhesion molecules expression and, in diabetic patients, their effects might be enhanced by hyperglycemia. To test this hypothesis, we evaluated $\mathrm{TNF} \alpha$-stimulated adhesion molecules expression in cultured Human Umbilical Vein Endothelial Cells (HUVEC) from umbilical cords obtained at delivery from healthy women and women with gestational diabetes used as a model of chronic exposure to hyperglycemia in vivo.

Materials and Methods: $\mathrm{TNF} \alpha(1 \mathrm{ng} / \mathrm{mL})$-stimulated adhesion molecules expression were determined in cultured HUVEC from umbilical cords obtained at delivery from 10 healthy women (C) and 10 women with gestational diabetes (GD). Vascular cell adhesion molecules (VCAM-1) and intercellular cell adhesion molecules (ICAM-1) protein levels were evaluated by Western Blot; adhesion molecules surface expression was measured by biparametric flow cytometry analysis.

Results: After 12 hours of stimulation, TNF $\alpha$-induced VCAM- 1 protein levels were increased 8.8 fold in GD- and 3.5 in C-HUVEC (GD- expressing $37 \%$ more VCAM-1 then C-HUVEC); in the same conditions, GD- and CHUVEC did not show any differences in ICAM-1 protein levels (4.6 and 4.7 folds increased in GD- and C-HUVEC respectively). Flow cytometry analysis demonstrated that 16 hours exposure to TNF $\alpha$ induced higher percentage of positive cells for VCAM-1 in GD-HUVEC (39\%) as compared to C-HUVEC; in the same conditions no differences in percentage of positive cells for ICAM-1 were detected.

Moreover, VCAM-1 MFI Ratio (Mean Fluorescence Intensity) was higher in GD-HUVEC as compared to C-HUVEC after both 12 (26\%) and $16(48 \%)$ hours of TNF $\alpha$ stimulation; a similar result was obtained for ICAM-1 (21\% and $56.8 \%$ respectively).

Conclusion: These data demonstrated that chronic exposure to hyperglycemia in vivo results in enhanced TNF $\alpha$ ability to elicit expression of potentially proatherogenic molecules such as VCAM-1 and ICAM-1 in cultured endothelial cells.

Supported by PRIN 2006, Ministero Italiano Università e Ricerca to AC

\section{8}

Linoleic acid increases TNF- $\alpha$-induced monocyte adhesion to human aortic endothelial cells via Cox-2 and PKC

N. Matesanz, V. Jewhurst, L. A. Powell, E. R. Trimble;

Clinical Biochemistry and Metabolic Medicine Department, Queen's University Belfast, United Kingdom.

Background and Aims: Atherosclerosis is the main cause of increased mortality and morbidity in diabetes. It is initiated by endothelial dysfunction which involves the recruitment of circulating monocytes to the endothelium. This process is regulated by inflammatory mediators including adhesion molecules (E-selectin, VCAM-1, ICAM-1), cytokines (e.g. TNF- $\alpha$ ), and signalling molecules (e.g. Cox-2, PKC). In diabetes, in addition to disturbances in glucose homeostasis, there is impairment of fatty acid metabolism which results in increased free fatty acids in the bloodstream. The aim of this work was to analyze the effects of linoleic acid, the main dietary fatty acid in a Western diet, on glucose-treated endothelial cells.

Materials and Methods: Human endothelial cells were cultured in $5 \mathrm{mM}$ (NG) or $25 \mathrm{mM}$ (HG) glucose (5\% FCS) for six days. On the third day, linoleic acid (LA; $0.2 \mathrm{mM}$ ) was added using fatty acid free-bovine serum albumin 
as a carrier. To mimic an inflammatory state, cells were stimulated with TNF- $\alpha(10 \mathrm{ng} / \mathrm{ml} ; 4 \mathrm{hrs})$ before extraction. Cells were also treated with Cox2 (indomethacin $10 \mu \mathrm{M} ; 4 \mathrm{hrs}$ ) or PKC inhibitors (Ly397196 $30 \mathrm{nM}, 5 \mathrm{hrs}$; rottlerin $1 \mu \mathrm{M}, 4 \mathrm{hrs})$. Monocyte adhesion was assessed using fluorescently labelled THP-1 and fluorescence microscopy. Protein expression was quantified by ELISA and western blotting.

Results: TNF- $\alpha$ induced an increase in monocyte adhesion in NG and HG conditions $(p<0.05)$. This was accompanied by increased cell surface expression of E-selectin, ICAM-1 and VCAM-1 $(\mathrm{p}<0.05)$. Treatment with LA further increased endothelial cell surface adhesion molecule expression $(\mathrm{p}<0.05)$. The Cox 2-inhibitor indomethacin $(10 \mu \mathrm{M})$ prevented LA-induced monocyte adhesion $(\mathrm{p}<0.05)$. LA enhanced PKC- $\delta$ isoform protein levels in HG $(p=0.05)$. Finally, PKC- $\delta$ inhibitor rottlerin $(1 \mu \mathrm{M})$ and PKC- $\beta \mathrm{I}$ inhibitor Ly397196 (30 nM) inhibited the LA-induced increase in E-selectin in HG and ICAM-1 surface expression in HG both glucose conditions ( $\mathrm{p}<0.05)$, and on VCAM-1 and E-selectin expression in NG $(\mathrm{p}<0.05)$, respectively, confirming the role of $\mathrm{PKC}$ in this process.

Conclusion: The pro-inflammatory effects of linoleic acid may be mediated via Cox-2 and PKC isoforms.

\section{9}

\author{
Aspirin inhibit adhesion molecules expression and MMP-2 activity in \\ resistin-induced endothelial dysfunction \\ W. H. H. Sheu ${ }^{1}$, C.-M. Wu ${ }^{1}$, W.-J. Lee ${ }^{2}$, C.-S. Wu ${ }^{1}$, I.-T. Lee ${ }^{1}$; \\ ${ }^{1}$ Endocrinology and Metabolism, ${ }^{2}$ Research and Medical Education, \\ Taichung Veterans Gen Hospital, Taichung, Taiwan.
}

Background and Aims: Obese subjects are characterised by dysregulated adipocytokines which cause endothelial dysfunction and vascular inflammation. Resistin, one of adipocytokines, has been shown to induce endothelial proliferation and upregulate several proinflammatory cytokines and adhesion molecules. However, the beneficial effects of aspirin, a wellknown anti-inflammatory agent, on resistin associated with increased inflammation cytokines and adhesion molecules expressions, NO synthase and matrix metalloproteinase 2 (MMP-2) activity from endothelial cells are largely unstudied.

Materials and Methods: NF- $\kappa \mathrm{B}$ activity, measured by EMSA, expression of mRNA and protein levels of MCP-1, IL-6, IL-8, ICAM, VCAM, E-selectin and eNOS, detected by real time PCR and/or ELISA, MMP-2 activity, measured by zymography, were studied with resistin $(100 \mathrm{ng} / \mathrm{ml})$ in human umbilical vein endothelial cells (HUVEC) pretreated with aspirin $500 \mu \mathrm{g} / \mathrm{ml}$.

Results: Resistin induced increased activity of NF- $\kappa$ B by $34.5 \%(\mathrm{p}<0.05)$ in HUVEC, which was inhibited when pretreated with aspirin for 2 hours. Expression of mRNA of MCP-1, IL-6, IL-8, ICAM, VCAM and E-selectin were elevated by stimulation of resistin (all $\mathrm{p}<0.05)$. All these increased expressions reduced significantly after pretreated with aspirin except in MCP-1. Protein concentrations of MCP-1, IL-6, IL-8, ICAM, VCAM and E-selection in the supernatant of HUVEC increased by $58.8 \%, 47.5 \%, 27.0 \%, 51.4 \%, 58.3 \%$ and $263.0 \%$, respectively (all $\mathrm{p}<0.05)$ after incubated with resistin, whereas these proteins reduced significantly after aspirin administration. Resistin reduced eNOS protein expression by $26.9 \%$ and elevation of MMP- 2 activity by $21.3 \%$ in HUVEC, which could be reversed after administration of aspirin.

Conclusion: We demonstrated that resistin induced enhancing expression of mRNA and proteins levels of MCP-1, IL-6, IL-8, VCAM, ICAM, and Eselectin presumably through activation of NF- $\mathrm{kB}$. Except in mRNA of MCP1 , all these detrimental effects could be reversed by treatment of aspirin. Aspirin also ameliorated resistin reduced eNOS protein and higher MMP-2 activity in human endothelial cells. Our findings further establish the crosslink between adipocytokines and endothelial dysfunction and shed light on protective effects of aspirin in obese individuals.

Supported by National Science Council, Taiwan, ROC
1170

The eliminating effects of a prescribed traditional Chinese medicine preparation on endothelium derived superoxide anion and its molecular mechanism

X. Gao, B. Zhang;

Endocrinology \& Metabolism, Zhongshan Hospital, Shanghai, China.

Background and Aims: Our previous study has revealed that a prescribed traditional Chinese medicine preparation (TCMP) can potently eliminate oxygen radicals in vitro and can improve microvascular endothelial cells growth in high glucose medium with similar effect to NAC. This study was to investigate its effect on endothelium-derived superoxide anion and possible molecular mechnism.

Materials and Methods: Microvascular endothelial cells (bEnd.3 strain) were cultured in $10 \%$ fetal bovine serum media with $5.6 \mathrm{mM}$ glucose (NG group), $35 \mathrm{mM}$ glucose(HG group), $35 \mathrm{mM}$ glucose and TCMP with working concentration at 1:100 in volume(TCMP group), $35 \mathrm{mM}$ glucose and $10 \mathrm{mM}$ NAC(NAC group), and 35mM glucose and SOD (working concentration: 400 $\mathrm{IU} / \mathrm{ml}$ ) (SOD group) respectively. Electronic paramagnetic resonance (EPR) together with spin trap technique was applied to determine reactive oxygen species (ROS) in each group. ROS in $35 \mathrm{mM}$ glucose culture medium without endothelial cells (blank group) and methylated free radicals in endothelial cells cultured in a medium with $35 \mathrm{mM}$ glucose and $10 \mathrm{mM}$ DMSO (DMSO group) were also determined to differentiate the origin of ROS. Spin trapper DMPO with a working concentration of $100 \mathrm{mM}$ were used in this study. The gene expression of MAPK, TNF- $\alpha$, UCP-1,UCP- 2 , and UCP-3 in NG, HG, TCMP, and NAC group was quantitively analyzed by real-time RT-PCR and the content of UCP- 2 protein molecule in each group was analyzed by Western blot analysis.

Results: Apparenthydroxyl free radical signals with an peak-to-peak amplitude of 2.0 $\times 104$ were detected in HG group cells in EPR spectrometer examination while no obvious ROS signal in NG group cells or blank group was observed. This indicated that the detected signals of hydroxyl free radicals were from endothelial cells and due to high glucose level in culture media. The peakto-peak amplitude of hydroxyl free radical signals in SOD group decreased to $9.63 \times 103$ and no identifiable signal of methylated free radicals appeared in DMSO group. This suggested the detected hydroxyl free radical by EPR spectrometer came from superoxide anions produced by endothelial cells in high glucose media. The peak-to-peak amplitude of hydroxyl free radical signals in TCMP and NAC group were $6.97 \times 103$ and $1.11 \times 104$ respectively, indicating TCMP had an effect similar to SOD. Levels $(m e a n \pm S D)$ of UCP2 gene expression in NG, HG, TCMP, and NAC group were $2.920 \pm 0.028$, $3.500 \pm 0.212,4.070 \pm 0.099$, and $3.875 \pm 0.050$ respectively (ANVOA, $\mathrm{p}=0.002$; HG vs NG, $p=0.009$; TCMP vs NG, $p=0.001$; TCMP vs HG, $p=0.009$; TCMP vs NAC, $\mathrm{p}=0.181$ ) whileas no significant difference was observed in gene expression of MAPK, TNF- $\alpha$, UCP-1 and UCP-3 among the four groups (ANVOA, $\mathrm{p}=0.57,0.223,0.129$, and 0.447 respectively). Western blot analyses also showed that UCP-2 in HG group, compared with NG group, was upregulated, and was further up-regulated in TCMP group.

Conclusion: TCMP can extinguish superoxide anion produced by endothelial cells cultured in high glucose milieu and this effect may partially relate to upregulation of UCP- 2 molecule, which plays a role in reducing mitochondrial superoxide anion production through lowering transmembrane electrochemical proton gradient.

Supported by Shanghai Science and technology Foundation

\section{1}

Mitochondrial DNA oxidation and MnSOD activity in peripheral blood mononuclear cells from type 2 diabetic patients

M. Garcia-Ramirez ${ }^{1}$, G. Francisco ${ }^{1}$, E. Garcia-Arumi ${ }^{2}$, C. Hernandez ${ }^{1}$, A. L. Andreu ${ }^{2}$, R. Simo

${ }^{1}$ Diabetes Research Unit, ${ }^{2}$ Centre d'Investigacions en Bioquimica i Biologia Molecular, Institut de Recerca Hospital Universitari Vall d'Hebron, Barcelona, Spain.

Background and Aims: Mitochondrial DNA (mtDNA) is markedly vulnerable to oxidative stress, resulting in both qualitative and quantitative changes. These changes can impair cell function and participate in the apoptosis mediated by hyperglycemia that occurs in the target tissues suffering from late diabetic complications. The aim of the study was to investigate the 
balance between parameters of oxidative stress and antioxidant defenses in the mitochondria of peripheral blood mononuclear cells (PBMCs) of type 2 diabetic patients with late complications

Materials and Methods: Ten type 2 diabetic patients with late diabetic complications and ten age-matched healthy volunteers (control group) were prospectively recruited. Mitochondrial DNA (mtDNA) oxidative damage and mtDNA content were measured as indices of oxidative stress. Manganese superoxide dismutase (MnSOD) activity has been used as an index of mitochondrial antioxidant defense. Finally, mitochondrial respiratory chain function (cytochrome $\mathrm{C}$ oxidase activity) was also assessed.

Results: mtDNA oxidation was significantly higher in the PBMCs of diabetic patients than in control subjects $(\mathrm{p}<0.0001)$. mtDNA content was lower in the diabetic group but without statistical significance. MnSOD activity was significantly increased in PBMCs of type 2 diabetic patients as compared with healthy controls $(1366 \pm 187 v s .686 \pm 167 \mathrm{U} / \mathrm{g}$ protein, $\mathrm{p}=0.01)$, and it was related to mtDNA oxidative damage. No differences in mitochondrial respiratory chain function were found between diabetic patients and the control group.

Conclusion: PMBCs from type 2 diabetic patients with late diabetic complications exhibit high mtDNA oxidative damage. The degree of mtDNA oxidation was associated with a reduction of mtDNA content as well as an increase of MnSOD as an adaptive response against oxidative stress. The consequences of mtDNA oxidative damage on PBMC function and in the progression of diabetic complications remain to be elucidated.

Supported by Lilly S.A. and the Ministerio de Sanidad y Consumo. Instituto de Salud Carlos III (REDIMET)

\section{2}

Glucose-potentiated vascular smooth muscle cell (VSMC) migration to angiotensin $\mathrm{II}$ is modulated through $\mathrm{PKC}_{\beta \mathrm{II}}$-dependent protein phosphatase (PP) 2A activation

M. Campbell ${ }^{1}$, J. Mussen ${ }^{1}$, L. A. Powell ${ }^{1}$, E. R. Trimble ${ }^{1,2}$;

${ }^{1}$ Clinical Biochemistry \& Metabolic Medicine, Queen's University,

${ }^{2}$ Clinical Biochemistry, Royal Group of Hospitals, Belfast, United Kingdom.

Background and Aims: Atherosclerosis occurs prematurely in diabetic subjects. A key event in atheroma formation is VSMC migration into, and subsequent retention within, the cytokine-rich plaque. In vitro, a rise in glucose concentration lowers the threshold at which angiotensin II causes VSMC migration; thus, with glucose $\geq 10 \mathrm{mM}$, VSMC migrate to circulating levels of angiotensin II $(10 \mathrm{nM})$. However as angiotensin II levels increase migration is lost. The aim of this work is to identify those glucose-potentiated pathways which prevent VSMC migration to elevated levels of angiotensin II, which may therefore promote VSMC retention within hyperglycaemic plaques.

Materials and Methods: Explant-derived VSMC were exposed to normal glucose (5 mM: NG) or high glucose (10-25 mM for 6-18 h: HG) conditions and circulating or raised $(<100 \mathrm{nM})$ angiotensin II levels \pm inhibitors. Migration was assessed using the Dunn chamber (cyclical statistics). Western blots and commercial activity assays were carried out on whole cell lysates (ANOVA and Student's t-test).

Results: Migration in NG without/with $20 \mathrm{mM}$ L-glucose was only detected for cells exposed to high angiotensin II levels (20-30 nM: $\mathrm{p}<0.01)$. Migration in $\mathrm{HG}(10-25 \mathrm{mM})$ conditions occurred to circulating angiotensin II levels $(10 \mathrm{nM}: \mathrm{p}<0.01)$ but was lost at higher levels $(\geq 15 \mathrm{nM}$ : $\mathrm{p}=\mathrm{ns})$.

Migration to angiotensin II (1-100 nM) was prevented by inhibition of Angiotensin II Type 1 receptor (Losartan: $10 \mathrm{nM}$ ) or ERK/MAPK pathway (PD98059: $10 \mu \mathrm{M}$; Pravastatin: $10 \mathrm{nM}$ ) in NG or HG. Glucose, through aldose reductase-dependent de novo synthesis of diacylglycerol, activates $\mathrm{PKC}_{\beta \mathrm{II}}$ in VSMC. As expected aldose reductase pathway inhibition (Alrestatin: $12 \mu \mathrm{M}$ )

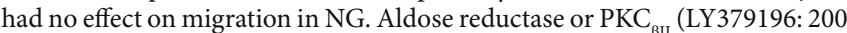
$\mathrm{nM}$ ) inhibition prevented migration to $10 \mathrm{nM}$ angiotensin II in HG; crucially both inhibitors restored migration at $15 \mathrm{nM}$ angiotensin II in $\mathrm{HG}(\mathrm{p}<0.01)$. Phosphatases are key negative regulators of signalling pathways and may be responsible for the loss of migration at high angiotensin II levels. PP2A is a target of Angiotensin II type 2 receptor signalling, can be activated by PKC, and inhibits ERK MAPK signalling. Angiotensin II type 2 receptor (PD123319: $10 \mathrm{nM}$ ) or PP2A (Endothall: $90 \mathrm{nM}$ ) inhibition permitted migration at both 10 and $15 \mathrm{nM}$ angiotensin II in $\mathrm{HG}$ and at 30 and $35 \mathrm{nM}$ in NG. Other protein phosphatase or protein tyrosine phosphatase inhibitors had no effect compared to solvent controls.
It was predicted that in $\mathrm{HG} \mathrm{PKC}_{\beta \mathrm{II}}$ may augment the Angiotensin II type 2 receptor-dependent activation of $\mathrm{PP} 2 \mathrm{~A}$ and so inhibit ERK signalling and migration. PP2A activity (activity assay) was significantly decreased in $15 \mathrm{nM}$ angiotensin II conditions in $\mathrm{HG}$ by $\mathrm{PKC}_{\beta \mathrm{II}}$ or Angiotensin II type 2 receptor inhibition ( $\mathrm{p}<0.01$ for both) while PP2A inhibition significantly increased ERK activity under these conditions (ERK activity assay: $\mathrm{p}<0.01$ ).

Conclusion: Loss of glucose-potentiated migration at moderately raised levels of angiotensin II is due to $\mathrm{PKC}_{\beta \mathrm{II}}$-dependent PP2A activation which inactivates ERK1/2 and prevents migration.

\section{3}

Multifactorial treatment increases circulating endothelial progenitor cell number in type 2 diabetic patients

H. Reinhard ${ }^{1}$, P. K. Jacobsen ${ }^{1}$, M. Lajer ${ }^{1}$, N. Billestrup ${ }^{1}$,

T. Mandrup-Poulsen ${ }^{1,2}$, J. Kastrup ${ }^{3}$, H.-H. Parving ${ }^{4}$, P. Rossing ${ }^{1}$;

${ }^{1}$ Steno Diabetes Centre, Gentofte, Denmark, ${ }^{2}$ Department of Molecular

Medicine, Karolinska Institutet, Stockholm, Sweden, ${ }^{3}$ The Heart Centre, Rigshospitalet, University Hospital of Copenhagen, Denmark,

${ }^{4}$ Department of Medical Endocrinology, Rigshospitalet, University Hospital of Copenhagen, Denmark.

Background and Aims: Circulating bone marrow-derived endothelial progenitor cells (EPC) augment vascular repair and neovascularisation. A reduced number of circulating EPC has been suggested to play a role in the progression of cardiovascular disease. Type 2 diabetic patients suffer from an increased risk of cardiovascular disease; this risk is reduced with multifactorial intervention. The aim of our study was to evaluate how EPC numbers in type 2 diabetic patients are influenced by multifactorial treatment.

Materials and Methods: We enrolled 8 type 2 diabetic patients (mean age: 55, known diabetes duration: 4.6 years) referred to Steno Diabetes Centre for initiation of multifactorial treatment. This included improving glycaemic control, lipid lowering, antihypertensive and antitrombotic therapy and lifestyle modification. The number of EPC was assessed by in vitro cultures and by flow cytometry analysis at baseline and after 30 days of treatment. Mononuclear cells were isolated from peripheral blood, using a Ficoll density gradient centrifugation.

1) Cells were seeded on plates coated with human fibronectin in endothelial basal medium. After 7 days in culture, we performed fluorescent chemical detection of the attached cells using 1,1_-dioctadecyl- 3,3,3_3_tetramethylindocarbocyanine-labelled acetylated low density lipoprotein (acLDL-DiI) and fluorescein isothiocyanate-labelled Ulex europaeus agglutinin-1 (UEA-1) for cell staining. We counted double-stained cells for both UEA-1 and acLDL-DiI as EPC in 10 randomly selected fields in each patient at each visit.

2) Numbers of EPC were also assessed by flow cytometry. Flourochrome labelled monoclonal antibodies against human CD34Fitc, CD133APC, VEGFR-2 and CXCR4PE-CY5 were used to identify EPC.

Results: All patients started $75 \mathrm{mg}$ aspirin and $40 \mathrm{mg}$ simvastatin therapy between first and second visit. Two patients started angiotensin II receptor blockers. Haemoglobin $\mathrm{A}_{1 \mathrm{c}}$ declined from 7.8\% (SD 1) to 7.5\% (SD 0.9) $\mathrm{p}<$ 0.012 total cholesterol decreased from $4.9 \mathrm{mmol} / 1$ (SD 0.77 ) to $3.7 \mathrm{mmol} / \mathrm{l}$ (SD $0.7) \mathrm{p}<0.01$, systolic blood pressure from $137 \mathrm{mmHg}$ (SD 9) to $133 \mathrm{mmHg}$ (SD 12) (ns) and diastolic blood pressure from $90 \mathrm{mmHg}$ (SD 5) to $85 \mathrm{mmHg}$ (SD 6) $\mathrm{p}<0.066$. There were no changes in body weight or smoking habits. Multifactorial intervention significantly increased the EPC number in vitro cultures by $41 \%$ from 83 (SD 43) cells per field to 117 (SD 39) $(\mathrm{p}<0.037)$. EPC defined by CD34/CD133 increased non-significantly by $58 \%$ from 1.54 (SD $0.98)$ to 2.43 (SD 1.9) $\times 10^{3} / \mathrm{ml}(\mathrm{p}=0.29)$ and EPC defined by CD34/CXCR-4 increased non-significantly by $21 \%$ from $1.11(0.43)$ to $1.34(0.88) \mathrm{x} 10^{3} / \mathrm{ml}$ $(\mathrm{p}=0.46)$. EPC defined by CD34/VEGF-2 was unchanged from 0.76 (SD 0.53) to 0.77 (SD 0.57) $\times 10^{3} / \mathrm{ml}$ (ns).

Conclusion: Our preliminary data demonstrates that EPC assessed by in vitro cultures increased significantly by multifactorial intervention in type 2 diabetic patients. These changes may contribute to the beneficial effects of multifactorial intervention on cardiovascular risk profile. The changes observed by flowcytometri were not significant, possibly due to a low number of experiments. 


\section{4}

Diabetes impairs the number of Bradykinin-sensitive circulating stem cells

N. Kraenkel ${ }^{1,2}$, V. Adams ${ }^{3}$, M. Schönauer ${ }^{4}$, P. Madeddu ${ }^{1,2}$;

${ }^{1}$ Bristol Heart Center, University of Bristol, United Kingdom,

${ }^{2}$ Instituto Nazionale di Biosistemi e Biostrutture, Roma, Italy,

${ }^{3}$ Dept. of Cardiology, University of Leipzig, Germany,

${ }^{4}$ Diabetes-Schwerpunktpraxis Leipzig, University of Leipzig, Germany.

Background and Aims: Reduced availability of vascular and endothelial progenitor cells (VPC) has been associated with an increased incidence of ischaemic complications in diabetic patients. In the past we demonstrated that adenoviral overexpression of human tissue kallikrein (hTK) is able to rescue diabetes-induced impairment of revascularisation in a mouse model of hindlimb ischaemia. However, the underlying mechanisms of hTK-facilitated neovessel growth are not well understood. We therefore investigated the hypothesis that bradykinin (BK), the physiologic product of hTK serves as a chemoattractant for VPC and that BK-signalling in VPC is impaired in diabetic patients.

Materials and Methods: Mononuclear cells (MNC) were isolated from peripheral blood of healthy $(H ; n=10)$ and diabetic donors $(D ; n=7)$. Expression of components of the kallikrein-kinin system were analysed by RT-PCR and flow cytometry. Furthermore, migration of VPC towards BK was studied in the presence or absence of BK-receptor antagonists using a modified Boyden chamber assay and scratched wound assay. The abundance of VPC expressing the BK-B2-receptor (B2R) was analysed by flow cytometry. $\mathrm{BK}$-responsive cells were isolated from total blood MNC and the ability to give rise to endothelial cells was studied in an outgrowth assay in the BKresponsive and non-responsive cell fractions.

Results: Expression of kininogens, B2R and TK was present in VPC. In healthy subjects, the $\mathrm{B} 2 \mathrm{R}$ was coexpressed on $71.5 \pm 3.6 \%$ of circulating $\mathrm{CD} 133^{+}$cells and in $53.3 \pm 4.4 \%$ of circulating $\mathrm{CD} 34^{+}$cells. The majority of B2R expressing VPC obtained from healthy individuals was also positive for the SDF-1 receptor CXCR4 $\left(72.5 \pm 4.9 \%\right.$ of $\mathrm{CD} 133^{+} \mathrm{B} 2 \mathrm{R}^{+}$cells and $78.1 \pm 3.0$ $\%$ of $\mathrm{CD}_{34}{ }^{+} \mathrm{B} 2 \mathrm{R}^{+}$cells). KDR was coexpressed in a smaller subfraction of B2R expressing VPC $\left(26.5 \pm 7.8 \%\right.$ of $\mathrm{CD}^{2} 33^{+} \mathrm{B}^{2} \mathrm{R}^{+}$cells and $3.6 \pm 0.7 \%$ of $\mathrm{CD} 34^{+} \mathrm{B} 2 \mathrm{R}^{+}$cells). In diabetic patients, coexpression of $\mathrm{B} 2 \mathrm{R}$ on VPC was decreased $\left(65.3 \pm 4.6 \%\right.$ of $C D 133^{+}$cells, $\mathrm{p}=$ n.s. vs. (H group) and $35.1 \pm 2.8 \%$ $\mathrm{CD}_{3} 4^{+}$cells; $\mathrm{p}=0.002$ vs. H). A reduced number of circulating $\mathrm{CXCR} 4^{+}$ coexpressing $\mathrm{B}_{2} \mathrm{R}^{+}$cells was furthermore detected in $\mathrm{D}$ subjects $(\mathrm{p}=0.01$ vs. $\mathrm{H})$. VPC migrated dose-dependently towards BK. BK-induced migration was significantly reduced in the presence of B2R antagonist icatibant and PI3kinase inhibitor LY294002. In MNC migrating towards BK, a larger number of cells was able to give rise to endothelial cells (17.7 $\pm 3.0 \%)$ as compared to the non-BK-responsive subpopulation ( $7.1 \pm 1.7 \%$; $\mathrm{p}=0.01$ vs. BK-responsive).

Conclusion: $\mathrm{BK}$ induced migration of VPC might represent a novel alternative signalling pathway mediating circulating stem cell recruitment to facilitate vasculogenesis. Reduction of BK sensitive circulating VPC in diabetic patients might contribute to the development of ischaemic complications via reduction of neovascularisation.

Supported by EFSD/JDRF/Novo Nordisk (European Programme in Type 1 Diabetes Research)

\section{PS 120 Endothelium, arterial stiffness and platelets: human studies}

\section{5}

Effects of C-peptide on several parameters of endothelial cell function in non diabetic healthy controls and in patients with type 1 diabetes during euglycaemic normo- and hyperinsulinaemic clamps

B. Wilhelm ${ }^{1}$, T. Forst ${ }^{1}$, K. Ekberg ${ }^{2}$, A. Pfuetzner ${ }^{1}$, C. Ries ${ }^{1}$, M. Kugler ${ }^{1}$, K. P. Kreisselmeier, J. Wahren ${ }^{2}$, M. M. Weber ${ }^{3}$;

${ }^{1}$ IKFE, Mainz, Germany, ${ }^{2}$ Creative Peptides, Stockholm, Sweden,

${ }^{3}$ Endocrinology, University Hospital, Mainz, Germany.

Background and Aims: During the recent years, C-peptide, which is released from the pancreatic beta cells, has been assigned a couple of biological activities. C-peptide was shown to exert endothelial, vascular, renal and metabolic effects in patients with type 1 diabetes. The aim of this study was to determine the effect of $\mathrm{C}$-peptide on several markers of endothelial cell function at different insulin concentrations.

Materials and Methods: 13 patients with diabetes mellitus type 1 (mean age $36.6 \pm 11.8$ years; diabetes duration $23.5 \pm 11.0$ years; $\left.\mathrm{HbA}_{1 \mathrm{c}} 7.3 \pm 0.7 \%\right)$ and 13 non-diabetic healthy controls (C: age $27.3 \pm 5.5$ years) were randomized to an euglycemic clamp procedure with either low $(0.25 \mathrm{mU} / \mathrm{kg} /$ body weight $)$ or high dose $(1 \mathrm{mU} / \mathrm{kg} /$ body weight $)$ insulin infusion on two different days. After stabilizing blood glucose to a target level of $100 \mathrm{mg} / \mathrm{dl}$ for 120 minutes, C-peptide was infused in a concentration of $8 \mathrm{pmol} / \mathrm{kg} / \mathrm{min}$ for another $120 \mathrm{~min}$. At baseline (tc0), after 60 (tc60) and 120 (tc120) minutes blood was drawn for the determination of asymmetric dimethylarginin (ADMA) and cyclic guanosinmonophosphat (cGMP) as parameters of endothelial function.

Results: C-peptide infusion resulted in comparable plasma levels of C-peptide in either group (DM 14.9 $\pm 4.9 ; \mathrm{C} 16.1 \pm 2.9 \mathrm{ng} / \mathrm{ml} ; \mathrm{n} . \mathrm{s}$, mean $+\mathrm{SD})$. In nondiabetic healthy controls cGMP and ADMA decreased especially during low dose insulin infusion (low dose insulin cGMP: $-0.67 \pm 0.7 \mathrm{pmol} / \mathrm{ml}, p<0.01$; ADMA: $-29.7 \pm 107.3 \mathrm{nmol} / 1, p<0.001$; high dose insulin cGMP: $-0.54 \pm$ $1.06 \mathrm{pmol} / \mathrm{ml}, p=0.08$; ADMA: $-42.8 \pm 81.8 \mathrm{nmol} / \mathrm{l}, p=0.07)$. In patients with diabetes mellitus type 1 similar, but less pronounced effects were detectable (low dose insulin cGMP: $-0.36 \pm 0.96 \mathrm{~mol} / \mathrm{ml}, \mathrm{n} . \mathrm{s}$; ADMA: $-56.1 \pm 190.2$ $\mathrm{nmol} / \mathrm{l}, \mathrm{n} . \mathrm{s} ;$ high dose insulin cGMP: $-0.47 \pm 0.74 \mathrm{pmol} / \mathrm{ml}, p<0.05$; ADMA: $-9.6 \pm 158.8 \mathrm{nmol} / \mathrm{l}, \mathrm{n} . \mathrm{s})$.

Conclusion: In the setting of an euglycemic clamp procedure the substitution of C-peptide resulted in a decrease of cGMP and ADMA plasma concentrations. These effects were more pronounced in the non diabetic healthy control subjects and partly antagonized by insulin.

\section{6}

Postprandial endothelial dysfunction, hyperglycaemia and hyperinsulinaemia in insulin resistance: is there a link? A. Major-Pedersen ${ }^{1}$, N. Ihlemann ${ }^{1}$, T. S. Hermann ${ }^{1}$, B. Christiansen ${ }^{1}$, H. Dominguez ${ }^{1}$, B. Kveiborg ${ }^{1}$, D. Nielsen ${ }^{1}$, C. R. Madsen², L. Køber C. Torp-Pedersen ${ }^{1}$;

${ }^{1}$ Cardiology, Bispebjerg Hospital, Copenhagen, Denmark,

${ }^{2}$ Joslin Diabetes Center, Boston, United States,

${ }^{3}$ Cardiology, Rigshospitalet, Copenhagen, Denmark.

Background and Aims: Postprandial hyperglycemia is considered an independent risk factor for cardiovascular disease, even before the development of overt diabetes. We aimed to determine if attenuation of postprandial hyperglycemia (with nateglinde administration - an oral antidiabetic of ultra-rapid action that stimulates postprandial first-phase insulin secretion) during three months could diminish endothelial dysfunction, one of the earliest markers of atherosclerosis.

Materials and Methods: We assessed post-oral glucose load endothelial function in 56 insulin-resistant subjects with impaired glucose tolerance (IGT) [Homeostasis Model Assessment (HOMA) $6.41 \pm 0.7$; body mass index (BMI) $34.4 \pm 5.68 \mathrm{~kg} / \mathrm{m}$; Oral Glucose Tolerance Test (OGTT): 0 min $6.2 \pm 1.0 \mathrm{mmol} / \mathrm{l}$ and $120 \mathrm{~min} .11 .9 \pm 2.7 \mathrm{mmol} / \mathrm{l} ; \mathrm{HbAlC} 6.4 \pm 0.82$ $\%$; waist circumference $115.8 \pm 20.5 \mathrm{~cm}$; age $57.5 \pm 7.3$ years], randomized 
to either intervention or time control group, at baseline (day $\mathbf{1}$ ) and after 3 months (day 4). Subjects in the intervention group received an initial dose of nateglinide (NAT) on a second day (day 2), $10 \mathrm{~min}$. before oral glucose load and during a 3 month study period. Thereafter, these subjects met for a last NAT dose (day 3) on which acute and chronic effect of NAT was measured. Chronic NAT effect was measured on day 4. Flow-mediated dilation (FMD) technique was used to measure endothelial function.

Results: The following postprandial Delta FMD values (fasting FMD value subtracted from postprandial FMD value) \pm SEM were seen in intervention and control groups respectively: $[(-3.42 \pm 1.67 \%) \mathrm{p}=0.04$ and $-2.20 \pm 1.19 \%)$ $\mathrm{p}=0.07]$. On day 2 , when NAT was administered $10 \mathrm{~min}$. prior to glucoseload in the intervention group, Delta FMD values increased to $2.32 \pm 1.45 \%$, $\mathrm{p}=0.02$. After 3 months NAT treatment postprandial delta FMD values were significantly impaired both on day 3 and day $4[(-2.09 \pm 0.80) \mathrm{p}=0.01$ and $(-4.25 \pm 1.15) \mathrm{p}=0.001]$. The following delta glucose values (fasting glucose value subtracted from postprandial glucose value) were seen on day 1 , day 2 , day 3 and day 4 respectively in the intervention group: [ $6.41 \pm 0.5),(4.22 \pm$ $0.45),(4.12 \pm 0.5)$ and $(5.83 \pm 0.5)$. Blood glucose levels in the control group resembled values on dayl and day 4 in the intervention group. We found no influence of the different blood glucose concentrations on FMD. There was a steep rise in postprandial insulin concentrations on day 2 and day 3 in the intervention group compared to day 1 and day 4 in both groups. On day 2 the fall in insulin concentration was sharper after 2 hours post glucose load, while insulin fall on day 3 was blunted.

Conclusion: We found no link between postprandial hyperglycemia and postprandial endothelial function. The enhancement of endothelial function observed after the initial NAT dose could be due to restoration of first phase insulin secretion, while deterioration of endothelial function observed after 3 months NAT treatment, could be due to sustained hyperinsulinemia. These surprising findings suggest that hyperinsulinemia might be tighter linked to endothelial dysfunction and atherogenesis, than is hyperglycemia.

Supported by Novartis, Bayer, Danish Diabetes Association

\section{7}

Treatment of endothelial dysfunction with glimepiride + metformin in patients with type 2 diabetes mellitus: evaluation with positron emission tomography

E. Alexanderson ${ }^{1}$, G. Alexanderson ${ }^{2}$, C. Sierra ${ }^{1}$, P. Cruz ${ }^{1}$, J. Talayero ${ }^{1}$, L. Garcia-Rojas ${ }^{1}$, M. Rodriguez ${ }^{1}$, A. Meave ${ }^{3}$;

${ }^{1}$ PET Ciclotron Unit, Mexico National Autonomous University School of Medicine, ${ }^{2}$ Internal Medicine Department, Mexico General Hospital, ${ }^{3}$ Magnetic Resonance and Computed Tomography Department, Mexico National Cardiology Institute, Mexico.

Background: Diabetes Mellitus (DM) has been considered an important risk factor for coronary artery disease. Elevated levels of blood glucose are responsible for endothelial dysfunction (ED) in the coronary arteries. ED is considered the first step in the development of atherosclerosis. Therefore, its opportune identification and treatment can importantly change the natural course of the disease. Glimepiride + Metformin has been used for the treatment of DM2 with excellent results in the achievement of glicemic goals and prevention of chronic complications. 13N-ammonia PET has become a useful non invasive method for the identification of ED by quantifying the coronary blood flow (CBF) during rest, cold pressor test (CPT), and pharmacologic stress with adenosine. With these data it is possible to calculate the endothelial dependent vasodilation index (EDVI) which is the most important marker for $\mathrm{ED}(\mathrm{EDVI}=\mathrm{CPT} \mathrm{CBF} /$ rest $\mathrm{CBF})$, the coronary flow reserve $(\mathrm{CFR}=$ stress $\mathrm{CBF} /$ rest $\mathrm{CBF})$ and the percentage of the change between rest and $\mathrm{CPT}(\% \triangle \mathrm{CBF})$. The aim of this study was to evaluate the effect of Glimepiride + Metformin in the endothelial function of patients with DM2.

Method: We enrolled 16 patients with recent diagnosis of DM2. The patients were randomized into two different groups, one group was treated with Glimepiride + Metformin (4mg/1000mg) daily for eight weeks and the other group was treated with Metformin $1000 \mathrm{mg}$ daily for 8 weeks. $13 \mathrm{~N}$-ammonia PET coronary blood flows were assessed after and before treatment. We compared the results of EDVI and MFR between both groups after treatment. Statistical analysis were performed using SPSS software v 13.0

Results: $62.5 \%$ (10) of all patients enrolled in this study showed ED prior to any treatment (EVDI Pre-Treatment was $1.21 \pm 0.37$ in the glimepiride/ metformin group and $1.30 \pm 0.29$ in the metformin group with no significant differences between both groups). Patients treated with glimepiride/ metformin combination or metformin alone showed improvement in their endothelial function. The group treated with the combination had a greater improvement than those treated with metformin alone (EVDI Post Treatment $1.87 \pm 1.02$ and $1.51 \pm 0.61$ respectively, $\mathrm{p}<0.001$ ). We observed only a slight improvement in CFR after treatment with combination or metformin alone $(2.74 \pm 1.33$ to $3 \pm 1.21$ and $2.42 \pm 0.84$ to $2.99 \pm 0.96 \mathrm{p}=$ n.s. $)$

Conclusions: ED is present in the majority of DM2 patients, even in the initial phases of the disease. Efective glicemic control is crucial to improve endothelial function. Combination therapy with glimepiride/metformin can improve the endothelial function in DM2 patients, offering better results than those achieved with metformin alone therapy. 13N-Amonnio PET is an excellent method for non invasive assessment of ED in patients with cardiovascular risk factors.

\section{8}

Rosiglitazone improves postprandially endothelial dysfunction in patients with type 2 diabetes

G. Ioannidis' ${ }^{1}$, S. Pastromas ${ }^{2}$, S. Koulouris 2 , D. Sakellariou², D. Tousoulis ${ }^{3}$, N. Zakopoulos ${ }^{4}$, A. Nikou' 1 , C. Stefanadis ${ }^{3}$, A. Manolis²;

${ }^{1}$ Endocrinology, Diabetes and Metabolism, Hosp. "Evangelismos",

${ }^{2} 1$ st Cardiology, Hosp. "Evangelismos", ${ }^{3} 1$ st Cardiology of the University of Athens, Hosp. "Hippokration", ${ }^{4}$ Therapeutic Clinics of the University of Athens, Hosp. "Alexandra", Athens, Greece.

Background and Aims: An oral fat load impairs brachial artery flow mediated dilatation (FMD) of both in healthy individuals and in patients with diabetes. Rosiglitazone ameliorates endothelial dysfunction (ED) in patients with type 2 diabetes. The aim of this study was to assess the effect of rosiglitazone on postprandially induced ED in these patients.

Materials and Methods: We enrolled 15 patients ( 7 men, 8 women) $62 \pm 4$ years old, with adequately controlled type 2 diabetes $\left(\mathrm{HbA}_{1 \mathrm{c}}<7 \%\right)$ with sulfonylureas or metformin and without clinical symptoms of cardiovascular disease. In all patients we assessed the lipidemic profile and the endothelial function before and after ingestion of an oral fat load (35\% weight/volume fat emulsion at a dose of $50 \mathrm{gr} \mathrm{fat} / \mathrm{m}^{2}$ body surface). The endothelial function was estimated by ultrasound measurement of brachial artery FMD. Then, in all patients rosiglitazone was added to their regimen at a dose of $4 \mathrm{mg}$ / day and the same measurements of lipids and brachial artery FMD were repeated after 12 weeks of therapy.

Results: After oral fat meal ingestion, there was a postprandial reduction in HDL cholesterol levels which was significant during the baseline measurement (from $53.0 \pm 12.2 \mathrm{mg} / \mathrm{dl}$ to $48.6 \pm 11.8 \mathrm{mg} / \mathrm{dl} \mathrm{p}<0.001$ ) but non - significant after the 12 - week rosiglitazone therapy $(51.8 \pm 12.9 \mathrm{mg} / \mathrm{dl}$ to $49.3 \pm 10.7 \mathrm{mg} / \mathrm{dl}, \mathrm{p}=\mathrm{ns}$ ). Postprandial FMD was significantly impaired during baseline measurements (from $11.5 \pm 5.2 \%$ to $7.7 \pm 3.4 \%, \mathrm{p}=0.03$ ) but remained essentially unchanged after rosiglitazone treatment (12.8 $4.4 \%$ to $11.0 \pm 5.3 \%$, p=ns).

Conclusion: Rosiglitazone seems to have beneficial effect in postprandial endothelial dysfunction as it appears to attenuate postprandial HDL reduction and prevents FMD impairment in patients with type 2 diabetes. This may have clinical implications in cardiovascular risk reduction conferred by the addition of rosiglitazone in these patients.

\section{9}

Peripheral endothelial dysfunction and cardiac autonomic neuropathy in patients with type 2 diabetes

A. Belhadj-Mostefa ${ }^{1}$, D. Roula ${ }^{1}$, P. Valensi ${ }^{2}$;

${ }^{1}$ Internal Medicine, CHU Constantine, Algeria,

${ }^{2}$ Department of Endocrinology-Diabetology-Nutrition, Jean Verdier Hospital, Bondy, France.

Background and Aims: Autonomic nervous system plays an important role in vasomotricity. Some data also suggest that it may change endothelium function. The aim of the present study was to examine the relationship between peripheral endothelium-dependent vasodilation and cardiac autonomic neuropathy in patients with type 2 diabetes.

Materials and Methods: 82 patients were included, mean age : $54.7 \pm 8.4$ years with a high cardiovascular risk : hypertension $61 \%$, lipid disorders 
$52.4 \%$, obesity $48 \%$. Mean (SD) HbAlc was $8.1 \pm 1.9 \%$. Cardiac autonomic neuropathy was assessed on three standardised tests for heart rate variations (deep-breathing, lying-to-standing, Valsalva). These tests were interpreted with age being taken into account. Peripheral endothelial function was assessed by post-occlusive hyperhemia of the bracchial artery and evaluated by the changes in blood flow velocity : V2/V1 = one minute after occlusion removal/basal.

Results: Cardiac autonomic neuropathy was found in 64 patients. V2/V1 was $4.63 \pm 1.75$. V2/V1 was significantly lower in the patients with cardiac autonomic neuropathy than in those without : $4.36 \pm 1.68$ vs $5.58 \pm 1.70$ (p $=0.006$ ). There was no significant correlation between V2/V1 and HbAlc, age or BMI.

Conclusion: The present results suggest that cardiac autonomic neuropathy is associated with peripheral endothelial dysfunction in patients with type 2 diabetes. Since the alterations in heart rate variations during the tests we used are mainly indicative of a parasympathetic defect, a relative sympathetic override might be involved in the impairment of endothelial function.

\section{0}

Daily glycaemic variability and arterial stiffness in type 1 diabetes

D. Gordin ${ }^{1,2}$, C. Forsblom ${ }^{1,2}$, M. Rönnback ${ }^{1,2}$, V. Mäkinen ${ }^{1,2}$, M. Saraheimo ${ }^{1,2}$, P.-H. Groop ${ }^{1,2}$;

${ }^{1}$ Folkhälsan research center, Folkhälsan Institute of Genetics,

${ }^{2}$ Helsinki University Central Hospital, Department of Medicine,

Division of Nephrology, Helsinki, Finland.

Background and Aims: The DCCT demonstrated that glycaemic control is important in order to prevent diabetic microvascular complications. In contrast, hyperglycaemia has not unequivocally been proven a risk factor for macrovascular complications. Furthermore, it is not yet known whether it is the total glucose exposure or the glucose variability that is linked to diabetic complications. Therefore the objectives of this study were to investigate whether daily glycaemic variations influence blood pressure and arterial stiffness, an early sign of macrovascular disease, at baseline and during a hyperglycaemic clamp in patients with T1D.

Materials and Methods: Twenty-two male patients with T1D without any diabetic complications, and who were non-smokers participated in the study. The patients were monitored for $72 \mathrm{~h}$ using the continuous glucose monitoring system (CGMS). The CGMS records glucose concentrations in each patient every fifth minute. As an index of hyperglycaemic episodes we calculated the area under the curve for values exceeding $10 \mathrm{mmol} / \mathrm{l}$ (AUC10) of each 24-h glucose profile. For low glucose excursions we used the area under the curve below $5 \mathrm{mmol} / \mathrm{l}$ (AUC-5). The mean amplitude of glycemic excursions (MAGE) was calculated to describe glucose fluctuations during the day. During a $2 \mathrm{~h}$ hyperglycaemic clamp ( $15 \mathrm{mmol} / \mathrm{l})$, pulse wave analysis and pulse wave velocity (PWV) were performed. Correlation coefficients were calculated using the Pearson's, Kendall's tau-b or Spearman's tests. Values were adjusted for BMI and duration of diabetes in a multiple regression analysis.

Results: The subjects were young males $(25.9 \pm 5.6$ years) with an intermediate glycaemic control $\left(\mathrm{HbA}_{1} 7.4 \pm 0.9 \%\right)$. All of them had normal kidney function [eGFR $117 \pm 6 \mathrm{ml} / \mathrm{min}$ per $1.73 \mathrm{~m}^{2}$ and AER $2.0(0.1-13.2) \mathrm{mg} / 24 \mathrm{~h}$ ] and their duration of diabetes was $9.5 \pm 4.4$ years. There was no correlation between MAGE and arterial stiffness at baseline. However, there was a univariate correlation between MAGE and aortic DBP $(\mathrm{R}=-0.48 ; P=0.039)$ at baseline but it disappeared after adjustment for BMI and duration of diabetes. Notably, there was no correlation between any of the other indices of glycaemic control and BP at baseline. There was also a correlation between mean daily glucose and aortic PWV after adjusting for BMI and duration of diabetes $(\mathrm{r}=0.46 ; P=0.033)$. Similarly, there was an independent correlation between AUC-5 and aortic PWV ( $\mathrm{r}=-0.62 ; P=0.001)$, but not between AUC10 and aortic PWV. MAGE correlated independently with differences in aortic SBP $(\mathrm{r}=0.52 ; P=0.014)$ and aortic DBP $(\mathrm{r}=0.49 ; P=0.037)$, and AUC-10 with aortic DBP $(\mathrm{r}=0.61 ; P=0.010)$ during the clamp. None of the measures of glucose control correlated with any of the variables of arterial stiffness during the clamp.

Conclusion: This study suggests that high mean daily blood glucose but not glycaemic variability per se increases arterial stiffness in patients with T1D. Daily glycaemic variability and episodes of hyperglycaemia are positively associated with the central blood pressure during a hyperglycaemic clamp. Supported by Finska Läkaresällskapet, Nylands Nation

\section{1}

Vascular reactivity of brachial artery is altered in type 2 diabetes but unaltered by hyperchylomicronaemia

L. A. Groisne ${ }^{1}$, A. Sérusclat ${ }^{2}$, M. Ronze ${ }^{1}$, A. Sassolas ${ }^{3}$, P. Moulin ${ }^{1}$;

${ }^{1}$ Endocrinologie-Diabétologie, ${ }^{2}$ Département d'Imagerie,

${ }^{3}$ Laboratoire de Biochimie, Groupement Hospitalier Est, Lyon-Bron, France.

Background and Aims: Vascular reactivity of brachial artery is considered as an early step in atherosclerosis and as an independent marker of cardiovascular risk. We studied hyperchylomicronemic patients with and without type 2 diabetes (D2) in order to establish the relative impact of both metabolic disease on early vascular alteration.

Materials and Methods: Prospective, unblinded study with longitudinal follow-up. Four groups were compared. Group CM $(n=10)$ : hyperchylomicronemic patients (history of TG $>15 \mathrm{mM}$, TG $>11 \mathrm{~mm}$ the day of the test) with metabolic syndrome (according NCEP-ATPIII), and insulin treated D2 (5/10), group CN $(n=7)$ : hyperchylomicronemic patients (history of TG $>15 \mathrm{~mm}, \mathrm{TG}>11 \mathrm{~mm}$ the day of the test) without metabolic syndrome: genetic LPL deficiency or alcohol ingestion, group ND $(n=12)$ : normo triglyceridemic ( $\mathrm{TG}<1.7 \mathrm{~mm}$ ) patients with type 2 diabetes, group NN $(n=14)$ : normo triglyceridemic subjects free of cardiovascular risk factors. Flow mediated dilation response to hyperaemia of brachial artery (FMD) and carotid intima-media thickness (cIMT) were measured using a 8.0 MHz linear transducer.

\section{Results:}

\begin{tabular}{lllll} 
& CM & CN & ND & NN \\
\hline Age years & $47 \pm 9$ & $47 \pm 13$ & $58 \pm 7$ & $47 \pm 12$ \\
FMD \% & $7.08 \pm 3.9$ & $6.1 \pm 3.2$ & $3.9 \pm 1.6$ & $5.8 \pm 2.4$ \\
cIMT mm & $0.71 \pm 0.11$ & $0.65 \pm 0.06$ & $0.85 \pm 0.14$ & $0.66 \pm 0.10$ \\
\hline
\end{tabular}

FMD was only decreased in group ND versus group CM $(p=0.016)$. Additionally cIMT was significantly increased only in group ND (ANOVA $p<0.001)$. In univariate and multivariate analysis no correlation was found between triglyceridemia and FMD after adjustment for metabolic syndrome, age, tobacco, systolic arterial blood pressure and LDL cholesterol. In the longitudinal follow up ( $n=\operatorname{six} \mathrm{CM}$ or $\mathrm{CN}$ ), following the decrease of triglyceridemia $<5.6 \mathrm{mmol} / \mathrm{l}$, FMD remained unchanged $(6.25+/-3.7 \%$ vs $5.27+/-3.7 \% \mathrm{p}=0.23)$.

Conclusion: Whereas FMD and cIMT are altered in type 2 diabetes, there is no evidence for an FMD alteration in hyperchylomicronemic subjects with or without metabolic syndrome and D2. Our data suggest that A) hyperchylomicronemia does not induce a major alteration in vascular reactivity and B) that acute insulin treatment upon hyperchylomicronemia might alleviate the alteration in vascular reactivity observed in type 2 diabetes.

Supported by Hospices civils de Lyon

\section{2}

Mechanisms of platelet hyperreactivity in type 1 and type 2 diabetes

S. Giubilato ${ }^{1}$, D. Pitocco ${ }^{2}$, A. Leo ${ }^{1}$, S. Riondino ${ }^{3}$, T. Musella ${ }^{2}$, L. M. Biasucci ${ }^{1}$, F. Crea ${ }^{1}$, F. Pulcinelli ${ }^{3}$, G. Ghirlanda ${ }^{2}$;

${ }^{1}$ Cardiology, Catholic University, ${ }^{2}$ Internal Medicine, Catholic University, ${ }^{3}$ Biochemistry, La Sapienza University, Rome, Italy.

Background and Aims: Evidences from clinical trials suggest that aspirin is less effective in reducing cardiovascular events in both type I and II diabetic patients, compared to nondiabetics. Despite of different pathogenetic and atherothrombotic implications, chronic hyperglycemia, in both diabetes types, could affect response to aspirin (ASA) in terms of impaired platelet membrane acetylation and protein glycation.

Materials and Methods: We sought to investigate TXA2 production following platelet stimulation with collagen and platelet COX-1 and COX-2 expression in 50 type II diabetics, in 15 type I diabetics and in 50 nondiabetics, all under chronic aspirin treatment

Results: TXA2 production in response to collagen was significantly higher $(\mathrm{p}<0.005)$ both in type I $\left(807.4 \pm 280.5 \mathrm{pg} / 10^{8}\right.$ cells $)$ and in type II $(896.4 \pm$ $232.3 \mathrm{pg} / 10^{8}$ cells $)$ diabetic patients compared to nondiabetics $(130.5 \pm 31.5$ $\mathrm{pg} / 10^{8}$ cells). The in vitro addition of aspirin prior to stimulation reduced 
collagen-induced TXA2 formation $\left(32.3+10.2 \mathrm{pg} / 10^{8}\right.$ cells in type I and 46.3 $+9.6 \mathrm{pg} / 10^{8}$ cells in type II). Expression of both isoforms of cyclooxygenase, demonstrated that while COX-1 was highly expressed in all groups, COX-2 was expressed in all diabetic patients, both of type I and type II, analyzed but only in $40 \%$ nondiabetics, suggesting that the residual TXA2 produced, might be consequent to COX-2 activation.

Conclusion: Our study demonstrates that both type I and type II diabetes are less sensitive to aspirin, due to a persistent TXA2 production, compared to nondiabetics, suggesting a reduced inhibitory action on platelet COX-1 and the expression of platelet COX-2. Our data strongly suggest that an extensive protein glycation secondary to hyperglicemia may reduce aspirin ability to acetylate target platelet proteins in diabetic patients of both type I and II. Supported by Hospices civils de Lyon

\section{PS 121 Glycation and vascular disease}

\section{3}

In vivo catabolism of glycated and glycoxidized LDL: a comparative study with LDL modified in vitro or LDL isolated from prediabetic and diabetic patients

J. Pietzsch ${ }^{1}$, C. Haase ${ }^{1}$, S. Hoppmann ${ }^{1}$, S. Richter ${ }^{2}$, J. Graessler ${ }^{3}$,

S. Kopprasch ${ }^{3}$, U. Julius';

${ }^{1}$ Radiopharmaceutical Biology, Institute of Radiopharmacy, Research Center Dresden-Rossendorf, ${ }^{2}$ Radiopharmaceutical Chemistry, Institute of Radiopharmacy, Research Center Dresden-Rossendorf, ${ }^{3}$ Internal Medicine III, Carl Gustav Carus Medical School,

University of Technology, Dresden, Germany.

Background and Aims: Modified LDL (modLDL) have been implicated in the pathogenesis of accelerated atherosclerosis in diabetes. Since atherogenesis already may occur at an early stage of diabetes, we investigated whether circulating LDL isolated from subjects with IGT (LDL-IGT) and newly diagnosed diabetes mellitus (DM; LDL-DM) showed an altered metabolic fate in vivo when compared with LDL isolated from subjects with NGT (nLDL) or LDL obtained from in vitro glycation (glycLDL-low/high) and glycoxidation (glycoxLDL-low/high) experiments.

Materials and Methods: We used a well-characterized methodology for radiolabeling of apolipoprotein B-100 of $\mathrm{nLDL}$ and modLDL with the positron emitter fluorine-18 (18F) by conjugation with $\mathrm{N}$-succinimidyl4 -[18F]fluorobenzoate $([18 \mathrm{~F}] \mathrm{SFB})$ and the use of $18 \mathrm{~F}$-radiolabeled LDL particles in dynamic positron emission tomography (PET) studies in male rats using a dedicated small animal PET camera. The extent of LDL apoB100 modification was evaluated by sensitive gas chromatography-mass spectrometry analysis of highly specific products of oxidation and glycation of amino acid side chain residues.

Results: 18F-radiolabeling of nLDL and modLDL caused neither additional oxidative structural modifications of LDL constituents nor alteration of their biological activity and functionality in vitro, respectively. Dynamic PET data in rats demonstrated a significantly delayed catabolism of glycLDL when compared with nLDL (mean plasma retention time, RT; glycLDLlow/glycLDL-high: $5.2 \pm 0.6 / 6.1 \pm 0.7$ h vs. nLDL: $3.4 \pm 0.5 \mathrm{~h} ; \mathrm{p}<0.05 ; \mathrm{n}=10$ ). In contrast, glycoxLDL showed a significantly enhanced catabolism when compared with nLDL (RT; glycoxLDL-low/glycoxLDL-high: $0.5 \pm 0.2 / 0.3 \pm 0.1$ $\mathrm{h} ; \mathrm{p}<0.05 ; \mathrm{n}=10)$. Imaging and functional characterization of in vivo distribution and kinetics of both nLDL and modLDL correlated well with the anatomical localization of LDL receptors, scavenger receptors, and receptors for advanced glycation end products in rats. Different interaction of modLDL with these receptors may discriminate modLDL particles in vivo into particles that show fast or low plasma clearance. In this line, LDL-IGT (RT; $4.2 \pm 0.4 \mathrm{~h} ; \mathrm{p}<0.05$ vs. $\mathrm{nLDL} ; \mathrm{n}=10$ ) and LDL-DM (RT; $4.7 \pm 0.6 \mathrm{~h} ; \mathrm{p}<0.05$ vs. $\mathrm{nLDL} ; \mathrm{n}=10$ ) mimicked the metabolic properties obtained with in vitro glycated LDL species, which underwent a similar extent of glycation and oxidation, respectively, as those isolated from subjects with IGT and newly diagnosed DM.

Conclusion: Radiolabeling of LDL with $18 \mathrm{~F}$ and the use of small animal PET provides a valuable tool to discriminate the kinetics and the metabolic fate of different modLDL particles in animal models in vivo. We suggest that, in particular, glycation of LDL even in an early stage of diabetes contributes to a diminished catabolism of LDL and, thus, to accelerated atherogenesis in IGT and newly diagnosed DM.

Supported by Deutsche Forschungsgemeinschaft

\section{4}

Glycated elastin and anti-elastin antibodies in patients with type 2 diabetes mellitus

M. K. Petkova ${ }^{1}$, S. Ganeva ${ }^{1}$, G. Nikoloff ${ }^{2}$;

${ }^{1}$ Clinic of Endocrinology, University Hospital,

${ }^{2}$ Department of Biology, Medical University, Pleven, Bulgaria.

Background: Accumulation of advanced glycation end-products (AGE) in the arterial wall plays a key role in the pathogenesis of micro-and macrovascular complications in patients with diabetes mellitus (DM). Identification 
of glycated proteins with different half-life may be used for evaluation of glycemic control during certain time.

Aim of the study was to investigate the levels of: circulated elastin peptides (CEP), glycated elastin as a marker of AGE deposition, and anti-elasin antibodies (AEAB) in sera of patients with different duration of type $2 \mathrm{DM}$ and in non-diabetic subjects.

Subjects and Methods: The levels of: circulated elastin peptides, glycated elastin, and $\mathrm{AEAB}$ in the sera of 32 patients with newly-diagnosed Type 2 $\mathrm{DM}$ and in 23 patients with 3 years-duration of Type $2 \mathrm{DM}$ were measured. The results of diabetic patients were compared with these of 22 non-diabetic control subjects.

Results: Mean age of the patients with Type $2 \mathrm{DM}$ and control subjects was not statistically different. The level of HbAlc was statistically significant higher in patients with newly-diagnosed type $2 \mathrm{DM}$ compared to the patients with 3 -years duration of the disease ( $11.5 \pm 0.6$ vs. $8.08 \pm 1.62 \%$; $\mathrm{p}<0.005)$. The levels of glycated elastin were significantly higher in both groups of diabetic patients compared to the controls $(\mathrm{p}<0.05)$. There were not any statistically significant differences between the levels of glycated elastin in diabetic patients from both groups. The levels of AEAB were statistically significant higher in patients with 3-years duration of DM compared to the patients with newly-diagnosed DM and controls $(\mathrm{p}<0.05)$. We found statistically significant positive correlation between the levels of HbAlc and glycated elastin in patients with type $2 \mathrm{DM}$ from both groups.

Conclusion: The higher levels of glycated elastin in our patients with newlydiagnosed type $2 \mathrm{DM}$ compared to the control subjects suggested that the processes of the protein glycation preceded the diagnosis of the disease. The positive correlation between levels of $\mathrm{HbAlc}$ and glycated elastin show that improvement of glycemic control may prevent synthesis and accumulation of AGE `s. Investigated glycated protein was long-lived and may be used for long-term glycemic control evaluation.

Supported by grant from Medical University, Pleven, Bulgaria

\section{5}

Increased Ne-(carboxymethyl)-lysine (CML) levels in cerebral blood vessels of diabetic patients and in a (streptozotocin-treated) rat model of diabetes mellitus

A. W. van Deutekom ${ }^{1}$, H. W. Niessen ${ }^{2,3}$, C. G. Schalkwijk ${ }^{4}$, R. J. Heine ${ }^{1}$, S. Simsek ${ }^{1}$;

${ }^{1}$ Department of Endocrinology/Diabetes Center, VU University Medical Center, Amsterdam, ${ }^{2}$ Department of Pathology, VU University Medical Center, Amsterdam, ${ }^{3}$ Department of Cardiac Surgery, VU University Medical Center, Amsterdam, ${ }^{4}$ Internal Medicine, Maastricht University Hospital, Maastricht, The Netherlands.

Objective: To evaluate the association between diabetes mellitus and the accumulation of advanced glycation end-products (AGE) in cerebral vessels. Materials and Methods: Brain tissue samples were obtained by autopsy from $23 \mathrm{DM}$ patients and 13 age-matched controls. In addition, we investigated brain tissue samples of 7 streptozotocin-induced diabetic rats and 6 nondiabetic control rats. We used an immunohistochemical staining method to examine the $\mathrm{N} \varepsilon$-(carboxymethyl)-lysine (CML) immunoreactivity in the cerebral vessels.

Results: Staining intensity of CML was significantly higher in cerebral vessels of diabetic patients than in nondiabetic subjects. ( $1.16 \pm 0.68$ vs. $0.55 \pm 0.18$ score $/ \mathrm{cm} 2 ; \mathrm{p}<0.005)$ Furthermore, there was a similar significant difference in CML staining pattern between diabetic rats and nondiabetic control rats. ( $1.19 \pm 0.42$ vs. $0.27 \pm 0.17$ score $/ \mathrm{cm} 2 ; \mathrm{p}<0.05$ )

Conclusion: Accumulation of CML-modified proteins is significantly greater in the cerebral vessels of the diabetic patients than their age-matched controls. This association has been confirmed in the rat model. It may be possible that the excessive accumulation of AGE-modified proteins in the cerebral vasculature alters the local environment and microcirculation and thereby contributes to the development of cognitive impairments in diabetes. Therefore, additional study on the causal link between AGE accumulation and cognitive dysfunction and the potential benefits of AGE-blocking reagents is indicated.

\section{6}

Human EPCs and HUVECs exposed to high glucose: expression of antioxidative enzymes

L. Pucci ${ }^{1}$, D. Lucchesi ${ }^{1}$, M. Barsotti ${ }^{2}$, R. Di Stefano ${ }^{2}$, A. Balbarini ${ }^{2}$, R. Miccoli' ${ }^{1}$ S. Del Prato ${ }^{1}$, G. Penno ${ }^{1}$;

${ }^{1}$ Dept Endocrinology and Metabolism,

${ }^{2}$ Dept Cardiac, Thoracic and Vascular, University of Pisa, Italy.

Background and Aims: Adult bone marrow is a rich reservoir of tissue-specific stem and progenitor cells. Among these, a population of cells known as EPCs, endothelial progenitor cells, the precursors of mature endothelial cells, can be mobilized to the circulation and contribute to the neoangiogenic processes. Vascular diseases, including hypertension, diabetes and atherosclerosis, are characterized by elevated levels of reactive oxygen species (ROS). When the rate of ROS formation excedes the capacity of the antioxidative defense system, a condition of oxidative stress occurs which can lead to cell death and genetic instability. Since recent studies demonstrated that EPCs are more resistant to oxidative stress than HUVECs, we analysed the expression of intracellular antioxidative enzymes catalase (CAT), glutathione peroxidase (GPX1) superoxide dismutase (SOD2) and expression of the transforming growth factor-beta 1 (TGF-beta1) in human EPCs and HUVECs exposed to constant high glucose.

Materials and Methods:We studied the expression profile of the antioxidative enzymes by real-time PCR. Human EPCs, isolated from peripheral blood mononuclear cells of healthy donors, and HUVECs, isolated from fresh umbilical cords, were exposed over a 96 hours period to following experimental conditions: $\mathrm{C}$, control, constant exposure to $5 \mathrm{mM}$ D-glucose; LG20, osmotic control, constant exposure to $20 \mathrm{mM} \mathrm{L}$-glucose + 5mM Dglucose; HG25, high constant exposure to $25 \mathrm{mM} \mathrm{D}$-glucose.

Results: The results are expressed as means $+/-S D$ as fold increase in control mRNA. In EPCs the transition from 5 (C) to $25 \mathrm{mM}$ D-glucose (HG25) induced an increase of $1.87+/-0.39$ fold in the expression of GPX1; $1.40+/-$ 0.55 in SOD2 mRNA levels; the levels of catalase mRNA was not significantly affected $(1.32+/-0.25)$. No increase in TGF-betal mRNA expression was observed $(1.06+/-0.23)$. The exposure of HUVECs to $25 \mathrm{mM}$ D-glucose or $20 \mathrm{mM}$ L-glucose induce a weakly increase in TGF-betal mRNA expression $(1.32+/-0.88 ; 1.56+/-1.05$ respectively). The exposure to $25 \mathrm{mM}$ induces a decrease of GPX1 mRNA $(0.67+/-0.07)$ and of SOD2 mRNA $(0.53+/-0.26)$ and of catalase mRNA $(0.61+/-0.08)$ in HUVECs. Exposure to LG20 did not induce differences in enzymes expression from control (C).

Conclusion: In conclusion, comparison of EPCs and HUVECs antioxidative enzymes expression highlights that CAT, SOD2, GPX1 are more strongly expressed in EPCs. So, these cells seem to be better equipped to be protected against oxidative stress.

\section{7}

Delayed intervention with AGE cross-link breaker alagebrium and aceinhibitor quinapril attenuates diabetes-accelerated atherosclerosis in apoE-/- mice

A. Soro-Paavonen, A. M. D. Watson, K. Sheehy, L. Jiaze, A. C. Calkin, M. C. Thomas, M. E. Cooper, K. A. Jandeleit-Dahm;

Diabetic Complications, Baker Heart Research Institute, Melbourne, Australia.

Background and Aims: Advanced glycation end-products (AGEs) participate in the development of microvascular and macrovascular damage in diabetes by forming "cross-links" between and within proteins, changing structure and function. Cross-link breakers such as alagebrium chloride (ALT-711) can prevent the development of atherosclerosis in diabetic mice when administered directly after the induction of diabetes. Clinically, however, patients are likely to already have some degree of atherosclerosis before initial treatment, thus studies using delayed intervention of treatment are urgently needed. The aim of this study was to investigate whether delayed treatment with ALT-711 (ALT) or the ACE-inhibitor quinapril (Q) decreases established diabetes-accelerated atherosclerosis in apolipoprotein E-deficient (apoE-/-) mice.

Materials and Methods: Mice were rendered diabetic with streptozotozin and followed for 20 weeks. The mice received either no treatment, ALT-711 (1mg/ $\mathrm{kg} /$ day), or quinapril $(30 \mathrm{mg} / \mathrm{kg} /$ day) from week $10-20$ of diabetes $(\mathrm{n}=15 \mathrm{per}$ group). Atherosclerotic lesion area was quantified by en face analysis. Gene 
expression of the 2 angiotensin II receptor subtypes, AT1a and AT2, which reflect and are likely to participate in the atherogenic process were examined by RT-PCR.

Results: The delayed intervention with ALT-711 significantly decreased aortic atherosclerosis when compared to the untreated diabetic mice (total plaque area; ALT, $9.12 \pm 1.6 \%$ vs untreated, $15.1 \pm 1.5 \%, \mathrm{P}<0.05$ ). The significant reduction in the plaque area was observed in the aortic arch as well as the abdominal aorta. Mice treated with quinapril from 10-20 weeks also showed a significant reduction of atherosclerosis $(\mathrm{Q}, 9.5 \pm 1.6 \%$ vs untreated, $15.1 \pm 1.4 \%$, $\mathrm{P}<0.05)$. The treated animals also had reduced aortic gene expression of the AT1a (control, $1 \pm 0.3$; diabetic, 7.0 \pm 2.1 , diabetic + Q, $1.5 \pm 0.6^{*}$, diabetic + ALT, $1.6 \pm 0.5^{* *},{ }^{*} \mathrm{P}<0.05,{ }^{* *} \mathrm{P}<0.01$ vs diabetic) and the AT2 receptor (control, $1 \pm 0.2$; diabetic, $4.8 \pm 1.0$, diabetic $+\mathrm{Q}, 2.4 \pm 0.4^{\star}, \mathrm{D}+\mathrm{ALT}, 2.8 \pm 0.4^{* *},{ }^{\star} \mathrm{P}<0.05$, ${ }^{\star *} \mathrm{P}<0.01$ vs diabetic). These changes were independent of glycaemic control or serum lipids.

Conclusions: Delayed intervention with both ALT-711 as well as ACEinhibitor quinapril significantly decreased established atherosclerosis in the diabetic aorta. Indeed, ALT-711 may partly confer its anti-atherosclerotic effects via effects on the intra-aortic renin-angiotensin system. These findings re-affirm the importance of ACE-inhibitor treatment in diabetes, and provide evidence that targeting AGEs could be effective in combating established diabetic macrovascular disease.

Supported by Juvenile Diabetes Research Foundation International, National Health \& Medical Research Council, Academy of Finland, the Helsingin Sanomat Centennial Foundation, the Paavo Nurmi Foundation and Alteon

\section{8}

In vitro selection of DNA aptamers that block toxic effects of AGE on cultured retinal pericytes

Y. Higashimoto ${ }^{1}$, S.-I. Yamagishi ${ }^{2}$, M. Takeuchi ${ }^{3}$, M. Noguchi ${ }^{1}$, H. Inoue ${ }^{4}$; ${ }^{1}$ Department of Medical Biochemisty, Kurume University School of Medicine, ${ }^{2}$ Department of Medicine, Kurume University School of Medicine, ${ }^{3}$ Department of Pathophysiological, Hokuriku University, Kanazawa, ${ }^{4}$ Radioisotope Institute for Basic and Clinical Medicine, Kurume University School of Medicine, Japan.

Background and Aims: Microvessels are composed of two types of cells, endothelial cells (ECs) and pericytes. We have previously shown that AGE not only inhibit DNA synthesis, but also induce apoptotic cell death in cultured retinal pericytes, thereby being involved in pericyte loss, the earliest histopathological hallmark of diabetic retinopathy. Since pericytes play a central role in the maintenance of microvascular homeostasis in the retina, blockade of the harmful effects of AGE on retinal pericytes may become a novel therapeutic strategy for the treatment of diabetic retinopathy. In this study, we selected DNA aptamers directed against AGE in vitro and then examined their cytoprotective effects on AGE-exposed retinal pericytes.

Materials and Methods: AGE were prepared by incubating human serum albumin (HSA) with D-glyceraldehyde for 7 days. Synthetic DNA templates (106-mer) with 56 random nucleotides, 5'-AGCTCAGAATGGATCCAAACGCTCA-(N) ${ }_{56}$-TTCGACATGAGAATTCGGCCGGATC-3', were amplified over 12 cycles of PCR. From the purified PCR products, single-stranded DNAs were obtained by an additional PCR using 5'-primer only. The singlestranded DNA pool was then loaded onto an AGE- HSA-immobilized column. Bound DNA was eluted, recovered, and amplified by PCR. After repeating this procedure five times, the sequences of AGE specific DNA aptamers were determined.

Results: DNA aptamers specific for AGE-HSA were isolated by an in vitroselection process from a pool of $\sim 10^{15}$ different nucleic acid sequences. We identified 15 DNA aptamers directed against AGE-HSA, but not non-glycates HSA, using combinatorial chemistry techniques in vitro. Structural analysis revealed that they had bulge-loop structures with cytosine-rich sequences. One hundred $\mu \mathrm{g} / \mathrm{ml}$ AGE-HSA for $24 \mathrm{~h}$ decreased DNA synthesis in cultured retinal pericytes to about $20 \%$ of control cells ( $<<0.01$ ). Further, $100 \mu \mathrm{g} / \mathrm{ml}$ AGE-HSA for 10 days increased apoptotic cell death in pericytes to about 3 -folds, compared with the control $(\mathrm{p}<0.01)$. All of the cloned aptamers, but not non-binding control ones, were found to significantly inhibit the AGE-induced decrease in DNA synthesis as well as increase in apoptosis in retinal pericytes $(\mathrm{p}<0.01)$. Among the selected aptamers, the clone 9 aptamer completely blocked the toxic effects of AGE, and its dissociation constant was $1 \mu \mathrm{mol} / \mathrm{l}$. The sequence of clone 9 aptamer was TAACTCACTCCATACTCACTTGCTGATTCGCCAACAACACACCCTTAAACAGTCCC.
Conclusion: These results indicate that DNA aptamers are a useful tool for inhibiting the cytotoxic effects of AGE on cultured retinal pericytes. Our study suggests that blockade of the AGE effects by DNA aptamers may lead to a novel therapeutic strategy for the treatment of diabetic retinopathy.

\section{9}

Effect of AGEs and rosiglitazone on the expression of galectin 3 in human renal mesangial cells

Z. Sun, C. Ma, H. Jin, N. Liu;

Department of Endocrinology, Zhongda hospital, Southeast University, Nanjing, China.

Background and Aims: To investigate the effects of advanced glycation end products (AGEs) and rosiglitazone on the expression and secretion of galectin-3 in cultured human renal mesangial cells (HRMCs).

Materials and Methods: 1. HRMCs were incubated with different concentrations of AGE-bovine serum albumin (AGE-BSA) for different time, and exposed to AGE-BSA $(200 \mathrm{mg} / \mathrm{L})$ in presence of different concentrations of rosiglitazone. 2. The mRNA and protein expression of galectin-3 in HRMCs were analyzed by use of RT-PCR and Westernblot respectively. 3 . The culture medium of HRMCs was collected and concentrated. Then the content of galectin-3 in the culture medium was detected by Westernblot.

Results: 1. Both RT-PCR and Westernblot revealed that AGE-BSA upregulated the expression of galectin-3 in HRMCs in a concentration- and timedependent manner compared with DMEM and BSA $(p<0.05)$. 2. Compared with DMEM and BSA, AGE-BSA elevated the content of galectin- 3 in the culture medium of HRMCs $(p<0.05)$ time- and concentration- dependently. 3. Exposure of HRMCs to different concentrations of rosiglitazone in presence of AGE-BSA but not to rosiglitazone alone and, to a lesser extent, AGE-BSA alone, increased both protein and mRNA expression of galectin-3, and its secretion in the medium $(p<0.05)$. Rosiglitazone in presence of AGEBSA increased the expression and secretion of galectin- 3 dose-dependently $(p<0.05)$.

Conclusion: 1. AGEs upregulates the expression and secretion of galectin-3 in HRMCs. These results indicate that galectin-3 might serve as a protective mechanism toward AGE-induced injury by favoring the removal of AGEs. 2. Rosiglitazone further upregulates the expression and secretion of galectin-3 in HRMCs. The data suggests rosiglitazone plays their role of reno-protection via upregulation of galectin-3.

\section{0}

Expression of glycolysis-derived advanced glycation endproducts (AGEs) in human cancer; regulation of tumor progression by AGE-AGE receptor interactions

T. Atsumi ${ }^{1}$, T. Umino ${ }^{1}$, T. Kondo ${ }^{1}$, H. Miyoshi ${ }^{1}$, N. Yoshioka ${ }^{1}$, M. Takeuchi ${ }^{2}$, T. Koike';

${ }^{1}$ Department of Medicine II, Hokkaido University Graduate School of Medicine, Sapporo, ${ }^{2}$ Department of Pathophysiological Science, Hokuriku University, Kanazawa, Japan.

Background and Aims: The association between diabetes mellitus and cancer risk has been suggested in recent reports. However, molecular mechanisms have not been well understood. Advanced glycation endproducts (AGEs) and receptor for AGE (RAGE) have been demonstrated to play a role in the development of diabetic vascular complications. RAGE is a cell surface receptor belonging to the immunoglobulin superfamily and is a recognized factor regulating cancer invasion and metastasis. Amphoterin is one of the ligands for RAGE and amphoterin-RAGE interactions link to the migration of cancer cells. We hypothesize that cancer cells produce AGEs and AGERAGE interactions are associated with the progression of cancer. The aim of this study is to determine the association between AGEs and cancer.

Materials and Methods: Glyceraldehyde-3-phosphate is an intermediate of glycolytic pathway and glyceraldehyde-derved AGEs, termed AGE2, have been demonstrated to play an important role in the pathogenesis of diabetic complications. Malignant tumors are characterized by increased glucose uptake accompanied with accelerated glycolysis. We have studied the expression of AGE2 in human tumor tissue by immunohitstochemistry using anti AGE2 antibody. 
6-phosphofructo-2-kinase/fructose-2,6-bisphosphatase (PFK-2/F2,6BPase) is the enzyme responsible for the synthesis and degradation of fructose-2,6bisphosphate (F2,6BP). F2,6BP in turn is a powerful allosteric activator of 6-phosphofructo-1-kinase (PFK-1), which is the rate-limiting enzyme for glycolysis. An inducible isoform of PFK-2 (iPFK-2) have been discovered to mediate $\mathrm{F} 2,6 \mathrm{BP}$ production in cancer cells. We analyzed the correlation of the expression iPFK-2 and AGE2 in human tumor by immunohistochemistry. To investigate the association between the progression of cancer and diabetes, we measured serum concentration of AGE2 in tumor bearing $\mathrm{db} / \mathrm{db}$ mice. B16 mouse melanoma cells were subcutaneously injected to $\mathrm{db} / \mathrm{db}$ mice. After 14 days, the serum concentration of AGE2 was determined by ELISA.

Results: High expression of AGE2 was observed in human tumor tissue, such as colon and breast carcinoma. The expression of AGE2 in tumor tissue is significantly higher than in normal epithelium. The positive correlation of the expression of AGE2 and the expression of iPFK-2 in human tumor tissue was observed. High concentration of serum AGE-2 level was observed in tumor bearing $\mathrm{db} / \mathrm{db}$ mice compared to normal control mice.

Conclusion: These findings suggest that increased glucose uptake by tumor cells leads to excess formation of AGE2 via F2,6BP mediated activation of glycolysis. Moreover, elevated serum glucose further increases the formation of AGE2 in tumor cells and AGE-RAGE interactions maybe associated with tumor progression.

\section{PS 122 Cardiovascular complications: animal studies}

\section{1}

Systemic osteoprotegerin delivery shows pro-atherosclerotic activity in diabetic apolipoprotein-E null mice

R. Candido ${ }^{1}$, B. Toffoli ${ }^{2}$, P. Secchiero ${ }^{3}$, S. Bernardi ${ }^{2}$, F. Corallini ${ }^{3}$, E. Manca ${ }^{1}$, E. Caroli ${ }^{1}$, D. Belgrado ${ }^{2}$, P. Da Col' ${ }^{1}$, R. Carretta ${ }^{2}$, G. Zauli ${ }^{4}$, B. Fabris ${ }^{2}$;

${ }^{1}$ Diabetic Centre, A.S.S. 1 Triestina, Trieste,

${ }^{2}$ Department of Clinical Medicine and Neurology, University of Trieste,

${ }^{3}$ Department of Morphology and Embryology, University of Ferrara,

${ }^{4}$ Department of Normal Human Morphology, University of Trieste, Italy.

Background and Aims: Diabetes mellitus is associated with a marked increase in atherosclerosis. Although clinical studies have suggested that systemic levels of osteoprotegerin (OPG) are elevated in diabetic vascular disease, the specific role of OPG in the pathogenesis of diabetic atherosclerosis has not been investigated.

Materials and Methods: Three groups of apolipoprotein E (apoE)-null mice ( $\mathrm{n}=12$ for each group) were obtained at 6 weeks of age and studied for 16 weeks: 1 . Non-diabetic apoE control (C), 2. Untreated streptozotocininduced ( $55 \mathrm{mg} / \mathrm{kg}$ daily for 5 days) diabetic apoE mice (D), and 3. Diabetic apoE mice treated with recombinant human OPG injected every 15 days intraperitoneally (D-OPG). Tail cuff systolic blood pressure, glycated haemoglobin, plasma lipid levels, atherosclerotic lesion area of the aorta and atheroscletic lesion complexity were evaluated.

Results: Diabetes in apoE-null mice was associated with a significant increase in total atherosclerotic plaque area (D: $12.2 \pm 4.12 \%$ vs C: $1.8 \pm 0.85 \%$; $p<0.01$ ) and complexity in the aorta, as assessed by a marked increase in interstitial collagen, cellular proliferation, macrophage and smooth muscle cell infiltration. Repeated intraperitoneal injections of recombinant human OPG significantly increased the development of atherosclerotic plaques in diabetic apoE-null animals (D-OPG: $20.7 \pm 2.65 \%$ vs. D: $12.2 \pm 4.12 \%$; $\mathrm{p}=0.034$ ). OPG also markedly affected the cellular composition of plaque lesions by increasing plaque collagen content, cellular proliferation, macrophage and vascular smooth muscle cell infiltration without influencing blood pressure, lipids or glycaemic control.

Conclusion: The present study shows that OPG delivery leads to significant acceleration of atherosclerotic lesion formation in diabetic apoE-null mice, thus suggesting an important role for OPG in the accelerated progression of atherosclerosis in diabetes.

\section{2}

The effects of angiotensin II receptor antagonist on left ventricular diastolic function in OLETF rats J.-H. Noh, J.-H. Doh, N.-Y. Jang, D.-J. Kim; Internal medicine, Inje medical school, Ilsanpaik Hospital, Koyang, Republic of Korea.

Background and Aims: Angiotensin II has been shown to involve the development of diabetic cardiomyopathy via promoting myocardial fibrosis, myocyte apoptosis, endothelial dysfunction, inflammation, increased insulin resistance and oxidative stress. The objective of the present study is to examine the effects of angiotensin II receptor antagonist on left ventricular (LV) diastolic function in obese diabetic OLETF rats.

Materials and Methods: The rats were divided into 4 groups: untreated OLETF rats $(\mathrm{n}=10)$, temisartan $(10 \mathrm{mg} / \mathrm{kg} /$ day; oral $)$ treated OLETF rats $(n=9)$, insulin treated OLETF rats $(n=8$; s.c.), or both telmisartan and insulin treated OLETF rats $(n=10)$ at 30 weeks of age. After 12 weeks of treatment, LV diastolic function examined by doppler echocardiography.

Results: The frequency of DM or IGT was $95 \%$ without differences among the four groups at 42 weeks of age. Echocardiographic study of left ventricle showed that a ratio of the peak early diastolic velocity wave to the late diastolic wave (E/A ratio) was increased $(2.25+/-0.30$ vs. $1.53+/-0.14, \mathrm{P}=0.03)$, and isovolumic relaxation time $(18.2+/-1.7$ vs. $25.2+/-0.4 \mathrm{~ms}, \mathrm{P}=0.004)$ and deceleration time (DT, $54.7+/-3.2$ vs. $42.5+/-3.1 \mathrm{~ms}, \mathrm{P}=0.012$ ) was decreased in both telmisartan and insulin treated rats compared with untreated OLETF rats at 42 weeks of age. E/A ratio and DT in telmisartan only or insulin only treated rats were not different to those of control. There were no significant 
differences in percent fractional shortening of LV diameter or ejection fraction among the four groups.

Conclusion: These data suggested that early simultaneous treatment of angiotensin II receptor antagonist and insulin could be protective for diabetic cardiomyopathy.

\section{3}

Rimonabant inhibits platelet activation, positively modulates circulating leukocytes, and reduces RANTES in type 2 diabetic Zucker-Rats

A. Schäfer, J. Pfrang, G. Ertl, J. Bauersachs;

Department of Internal Medicine, Cardiology, University of Würzburg, Germany.

Background and Aims: Diabetes is characterised by vascular inflammation and enhanced platelet reactivity. Both phenomena contribute to the exaggerated cardiovascular morbidity and mortality of diabetic patients. The first $\mathrm{CB}_{1}$-antagonist rimonabant has been shown to improve cardiometabolic risk factors such as waist circumference, HbAlc, HDL-cholesterol and triglycerides in overweight/obese patients. We investigated the effect of rimonabant on platelet activity in type-II Zucker rats.

Materials and Methods: We fed rimonabant for 2 weeks to 3-month-old male obese Zucker rats as a model for impaired glucose tolerance and for 10 weeks to 6-month-old male obese Zucker rats as a model for the metabolic syndrome. Treatment effects were compared to lean Zucker rats of both ages as the respective control strains. RANTES serum levels were determined by ELISA. Platelet activation was assessed by flow cytometry for platelet-bound fibrinogen, platelet aggregation and adhesion of isolated platelets to immobilized fibrinogen in a flow-chamber under arterial flow conditions. Leukocyte populations were quantitatively assessed using a veterinary differential blood cell counter. The activity of endogenous platelet inhibition was assessed by the basal phosphorylation state of platelet vasodilator-stimulated protein (VASP). Results: RANTES serum levels were increased in obese vs. lean Zucker rats and significantly reduced by chronic treatment with rimonabant, which slowed body weight gain, in rats with the metabolic syndrome. Circulating numbers of neutrophils and monocytes were significantly increased in young and old obese vs. lean Zucker rats. Rimonabant significantly reduced both leukocyte subpopulations. Platelet-bound fibrinogen was significantly increased in obese vs. lean Zucker rats of both age, which was reduced by rimonabant. Platelets from obese rats were more sensitive to thrombininduced aggregation as well as adhesion to fibrinogen, which was decreased by rimonabant therapy. Basal platelet VSAP phosphorylation was significantly reduced in obese vs. lean Zucker rats of both ages and partially improved by chronic treatment with rimonabant.

Conclusion: Rimonabant demonstrates positive modulation of circulating neutrophil and monocyte numbers, reduces platelet activation and lowers RANTES levels in an animal model of impaired glucose tolerance as well as of the metabolic syndrome. Clinical studies are underway to evaluate the effect of rimonabant on reduction of cardiovascular risk.

Supported by sanofi-aventis

\section{4}

Evaluation of the relationship between depression, diabetes and ischaemia/reperfusion injury following global ischaemia in rat hearts L. Solskov ${ }^{1}$, B. Lofgren ${ }^{2}$, H. E. Botker ${ }^{2}$, T. T. Nielsen ${ }^{2}$, D. H. Overstreet ${ }^{3}$, O. Schmitz ${ }^{1,4}$, G. Wegener ${ }^{5}$, S. Lund ${ }^{4}$;

${ }^{1}$ Department of Pharmacology, Aarhus University, Denmark, ${ }^{2}$ Department of Cardiology, Aarhus University, Denmark, ${ }^{3}$ Department of Psychiatry and Centre for Alcohol Studies, University of North Carolina, Chapel Hill, United States, ${ }^{4}$ Medical Research Laboratory and Medical Department M (Endocrinology and Diabetes), Aarhus University Hospital, Denmark, ${ }^{5}$ Centre for Basic Psychiatric Research, Aarhus University, Risskov, Denmark.

Background and Aims: Depression is among the leading global causes of years lived with disability and is often associated with medical comorbidities such as heart disease and diabetes. Depression may accelerate the onset of diabetes complications and thereby has a significant impact on patients with diabetes. Therefore it is of considerable importance to get insight into the medical comorbidities associated with depression. The Flinders Sensitive Line (FSL) rat is a genetic animal model of depression, bred from the SpragueDawley rat, which exhibits behavioral, neurochemical, and pharmacological features similar to depressed individuals. The aim of the study was to elucidate if the FSL rats were more susceptible to ischemia and reperfusion injury compared to the healthy Sprague-Dawley (SD) control rats and furthermore to determine if the FSL rats were pre-diabetic.

Materials and Methods: Hearts were perfused in a Langendorff model and subjected to 40 minutes of global ischemia followed by 120 minutes of reperfusion. Myocardial infarct size was measured and used as a marker of susceptibility to ischemia. As the FSL and SD rats had comparable fasting glucose and insulin levels an oral glucose tolerance test (OGTT) was performed to determine if the FSL rats also exhibited normal glucose tolerance. Rats were fasted for 12 hours prior to the test and blood was drawn from the tail vein.

Results: Infarct size was significantly larger in the FSL rats (I/R: $62.4 \pm 4.2 \%$ vs. $46.9 \pm 2.9 \%, \mathrm{p}<0.01, \mathrm{n}=9$ ) compared to the SD rats. No significant difference was found in blood glucose levels between the two groups during the OGTT although there was a tendency towards higher plasma insulin levels in the FSL compared to the SD rats though not statistically significant.

Conclusion: The data indicates that the depressed FSL rats are more susceptible to ischemia and reperfusion injury compared to the healthy SD rats. This animal model may be useful in future studies when looking more detailed at mechanisms that contribute to the medical complications associated with depression.

\section{5}

MB07811, a liver-targeted prodrug of a novel thyroid hormone receptor agonist, does not cause hyperglycaemia in Sprague Dawley rats or dietinduced obese mice

D. L. Linemeyer ${ }^{1}$, E. E. Cable ${ }^{1}$, X. Yang ${ }^{1}$, B. R. Ito ${ }^{1}$, P. D. van Poelje ${ }^{1}$, M. D. Erion ${ }^{2}$;

${ }^{1}$ Biological Sciences, ${ }^{2}$ Research \& Development, Metabasis Therapeutics, La Jolla, CA, United States.

Background and Aims: MB07811, a HepDirect prodrug of a phosphonatecontaining thyroid hormone receptor agonist, MB07344, is under clinical investigation for the treatment of hyperlipidemia. First-pass extraction of MB07811 arising from CYP 3A-dependent activation to MB07344 and limited distribution of MB07344 to extrahepatic tissues confers a high degree of liver selectivity to the pharmacological effects of this novel agent. Liver selectivity was incorporated into the drug design because triiodothyronine (T3) exposure, in addition to beneficial liver-mediated lipid lowering effects, can adversely affect cardiac function, the thyroid hormone axis, lean muscle mass, and bone integrity. Excess T3 can also cause hyperglycemia by accelerating endogenous glucose production via increased gluconeogenic gene expression and extrahepatic effects including increased free fatty acid (FFA), amino acid and glycerol flux to the liver. The purpose of these studies was to compare the effects of MB07811 and T3 on glycemic control and to assess the cholesterol and triglyceride lowering activity of MB07811.

Materials and Methods: In protocol 1, male Sprague Dawley (SD) rats ( $\mathrm{n}=6 /$ group) were dosed daily for 8 days with vehicle, T3 (650 $\mu \mathrm{g} / \mathrm{kg} /$ day, s.c.), or MB07811 (1, 10 or $50 \mathrm{mg} / \mathrm{kg} /$ day, p.o.). Blood glucose was measured after a 9-h fast on days 1, 4, 5, 6 and 8. Plasma insulin and FFA levels were analyzed prior to the first dose and on day 5 . On day 6 , plasma insulin and blood glucose levels were measured prior to and at selected times after administration of an oral glucose load (2 g/ $\mathrm{kg}$ ). In protocol 2, diet-induced obese (DIO) mice were produced by feeding 4 -week-old C57BL/6 mice a high-fat diet $(60 \%$ fat by kcal) for $\sim 90$ days. Groups of mice ( $\mathrm{n}=8 /$ group) were then gavaged daily for two weeks with MB07811 (0.3, 1, 3, 10 and $30 \mathrm{mg} / \mathrm{kg} /$ day) or vehicle and maintained on the same high-fat diet. Plasma cholesterol, triglycerides and blood glucose were measured at baseline and on days 7 and 14 of the treatment period. Liver triglyceride content was assessed at sacrifice.

Results: SD rats treated with T3 for 8 days showed increased fasting blood glucose (up to $\sim 17 \mathrm{mg} / \mathrm{dL}$ ) and plasma FFA (by $540 \mu$ mole/L), with no change in plasma insulin. In contrast, MB07811 treatment did not affect fasting blood glucose, plasma FFA, or plasma insulin. Neither T3 nor MB07811 treatment changed the blood glucose excursion or the plasma insulin response to an oral glucose challenge performed on day 6 . Treatment with T3, but not MB07811, resulted in weight loss (16\%). In DIO mice treated for 2 weeks with MB07811, plasma cholesterol was reduced dose-dependently with the highest 
dose resulting in $61 \%$ lowering relative to vehicle controls. Furthermore, MB07811 treatment reduced plasma and liver triglycerides (up to $40 \%$ and $42 \%$, respectively) and did not increase blood glucose levels at any dose.

Conclusions: MB07811, unlike T3, did not cause fasting hyperglycemia in SD rats. In addition, MB07811 lowered plasma cholesterol and plasma and liver triglycerides without increasing blood glucose levels in DIO mice. T3induced hyperglycemia may be due primarily to its extrahepatic actions. Targeting of thyroid hormone receptor agonists to the liver appears to be a promising strategy for harnessing the antihyperlipidemic activity of T3 while avoiding extrahepatic side effects.

\section{6}

A direct effect of ghrelin to augment cardiac AMPK activity is compromised by hyperglycaemia following ischaemia/reperfusion S. Samsuddin ${ }^{1}$, M. Glyn ${ }^{2}$, B. Kola ${ }^{3}$, M. Korbonits ${ }^{3}$, M. J. Holness ${ }^{1}$, M. C. Sugden ${ }^{1}$;

${ }^{1}$ Institute of cell and molecular science, London,

${ }^{2}$ Leukocyte Biology, Imperial College, London, ${ }^{3}$ Department of

Endocrinology, Barts and the London Medical School, United Kingdom.

Background: 5'AMP activated protein kinase (AMPK) is a serine/threonine kinase that is activated through AMP and upstream kinases. Both ghrelin gene expression and ghrelin receptors are found in the heart. Increasing evidence indicates that AMPK may exert an important cardioprotective function in the heart during ischaemia and reperfusion (I/R). Use of mice expressing a dominant negative AMPK mutant in the heart suggested that AMPK activation protects cardiac ATP levels and reduces infarct size and damage to myocytes during ischaemia.

Aims: Hyperglycaemia is a major cause of cardiac and vascular complications associated with diabetes. Therefore, hyperglycaemia may represent an important predictive risk factor for myocardial infarction and hypertension in patients with diabetes. The aim was to investigate whether direct cardioprotective effects of ghrelin could be mediated through AMPK activation and whether this action is adversely affected by hyperglycaemia.

Materials and Methods: Langendorff heart perfusions with low $(5 \mathrm{mM})$ and high $(18 \mathrm{mM})$ glucose were performed in the absence or presence of $10^{-4} \mathrm{pM}$ des-acyl ghrelin (60 mins or 30 mins for I/R hearts), with or without a 20 min period of ischaemia, with subsequent $15 \mathrm{~min}$ reperfusion (I/R). Cardiac AMPK was analysed in extracts of hearts freeze-clamped at the end of the perfusions using immunoprecipitate kinase assays. AMPK activity in the immune complex was determined by phosphorylation of SAMS, a synthetic peptide substrate of AMPK.

Results: Ghrelin addition to the perfusate augmented cardiac AMPK activity, especially at low glucose (377\% of control) and low glucose with I/R (439\% of control). AMPK activity following perfusion with high glucose was also greatly enhanced by ghrelin compared to the control $(339 \% \mathrm{P}<0.05)$. However, the ability of ghrelin to activate AMPK was greatly impaired with perfusion at high glucose and subjected to I/R, with $<50 \%$ of the activation of AMPK observed with I/R hearts in the presence of low glucose.

Conclusions: Ghrelin exerts a direct acute effect to activate AMPK in the heart, suggesting that ghrelin could be an endogenous cardioprotective factor. Targeting ghrelin receptors, ghrelin signalling and/or AMPK may provide novel means for treating myocardial ischemia. Importantly, the putative cardioprotective effect of ghrelin exerted via AMPK activation is opposed by high glucose following ischaemia and reperfusion. These data highlight the importance of good glycemic control, particularly in diabetic patients with macrovascular complications, to optimise cardioprotection via AMPK. Supported by British Heart Foundation

\section{7}

Peculiarities of heart function and coronary circulation changes provoked by sympathovagal balance modification in diabetic dogs

\section{A. Nescheret;}

Laboratory of Epidemiology of Diabetes Mellitus, Institute of Endocrinology and Metabolism of AMSUkr, Kyiv, Ukraine.

Background and Aims: Recent studies suggest a close relation between abnormal nervous autonomic function and diabetic cardiohaemodynamic complications. Impaired cardiac autonomic activity, coronary heart disease and myocardial infarction are associated with worse outcome in diabetic patients. So there exists a very important question concerning the tactics of sympathetic-vagal balance modulation in diabetological practice. The aim of our study was to determine peculiarities of heart function and coronary artery (CA) regulation changes after cholinergic and adrenergic receptors blockade during progressive development of hyperglycemia in diabetic dogs (DD).

Materials and Methods: Our investigations were carried out on dogs under alpha-chloralose anaesthesia $(50-100 \mathrm{mg} / \mathrm{kg}$, i.v.). Catheterization and programmed autoperfusion of left CA, heart and main vessels catheterization were performed in experiments without opening chest. Cardiohaemodynamics, CA perfusion pressure (CAPP), with another clinical and biochemical parameters were registered. In metabolically healthy dogs (CD, level of glycemia: $3.8 \pm 0.5 \mathrm{mmol} / \mathrm{l} ; \mathrm{n}=27)$ and alloxane-diabetic dogs $(75 \mathrm{mg} / \mathrm{kg}$ of alloxane, i.v.) beta-adrenoreceptors (beta-AR) and Mcholinergic receptors (M-ChR) blockades were induced respectively by propranolol $(2.0 \mathrm{mg} / \mathrm{kg}$, i.v. $)$ and atropine $(0.5 \mathrm{mg} / \mathrm{kg}$, i.v. $)$. Due to different levels of hyperglycemia development in DD all of them were divided into three groups: DD1: hyperglycemia $7.2 \pm 1.7 \mathrm{mmol} / 1$ (mild form; $\mathrm{n}=9$ ); DD2: 9.8+1.7 mmol/l (moderate form; $\mathrm{n}=7$ ) and DD3: $15.6 \pm 3.4 \mathrm{mmol} / \mathrm{l}$ (severe form; $\mathrm{n}=10$ ).

Results: The typical cardiohaemodynamics reaction 5-10 min after M-ChR blockade (at the nadir of the action) in $\mathrm{CD}$ due to a rise of sympathetic heart nerves activation was followed by an increase of heart rate (HR, $+59.5 \pm 2.5$ beats/min, $\mathrm{p}<0.001)$ and CAPP decrease $(-1.5 \pm 0.1 \mathrm{kPa}, \mathrm{p}<0.001)$. In all DD groups $\mathrm{M}-\mathrm{ChR}$ blockade was associated with significantly diminished degree of HR increase (DD1: to +32.2 \pm 2.7 ; DD2: to $+19.9 \pm 6.4$; DD3: to $+1.3 \pm 0.1$ beats/min; $\mathrm{p}<0.001$ for all), CAPP decrease (DD1: to $-3.2 \pm 0.1$; DD2: to $3.4 \pm 0.2$; DD3: to $-3.5 \pm 0.2 \mathrm{kPa}$; $<<0.001$ for all) compared with $\mathrm{CD}$. On the contrary, beta-AR blockade in $\mathrm{CD}$ due to depression of sympathetic heart nerves activity induced HR decrease $(-25.3 \pm 1.3$ beats $/ \mathrm{min} ; \mathrm{p}<0.001)$ and CAPP increase $(+1.2 \pm 0.1 \mathrm{kPa} ; \mathrm{p}<0.01)$. But in DD2 and DD3 blockade of beta-AR induced more pronounced $\mathrm{HR}$ depression (to $-39.4 \pm 2.2$ and to $-39.2 \pm 3.9$ beats/min resp.; $\mathrm{p}<0.01$ for both). In all $\mathrm{DD}$ groups beta-AR blockade caused CAPP augmentation (DD1: to $+3.6 \pm 0.5$; DD2: to $+4.1 \pm 0.6$; DD3: to $+2.6 \pm 0.5 \mathrm{kPa} ; \mathrm{p}<0.001$ for all). Both blockade of M-ChR and beta-AR after bolus intracoronary injection of epinephrine $(5.0 \mathrm{mcg})$ in $\mathrm{CD}$ provoked alpha-adrenergic CA constriction and elevation of CAPP $(+2.1 \pm 0.2$ and $+3.3 \pm 0.3 \mathrm{kPa}$, resp.; $\mathrm{p}<0.001$ for both). Nevertheless, in DD2 and DD3 the degrees of alpha-adrenergic CA constriction induced by epinephrine after $\mathrm{M}-\mathrm{ChR}$ and beta-AR blockades were diminished (to $+2.0 \pm 0.1$ and $+2.9 \pm 0.1$ $\mathrm{kPa}$, resp., $\mathrm{p}<0.001$ for both).

Conclusion: Our data suggest that continuing progression of hyperglycemia in DD seems to represent the most important factor in influencing the heart's autonomic nervous system activity. The obtained results demonstrate dynamics of the relationship between sympathetic and vagal nervous systems in regulation of heart function and coronary circulation in diabetes.

\section{8}

Dysregulated inflammation predicts reduced connective tissue growth factor expression in a baboon wound healing model of type 1 diabetes S. E. Thomson ${ }^{1}$, S. V. McLennan ${ }^{2}$, A. Hennessy ${ }^{3}$, J. Bonner ${ }^{2}$, H. Zoellner ${ }^{4}$, D. K. Yue ${ }^{5}$, S. M. Twigg

${ }^{1}$ Renal Medicine, Royal Prince Alfred Hospital, ${ }^{2}$ Endocrinology, Royal Prince Alfred Hospital, ${ }^{3}$ Medicine, University of Western Sydney, ${ }^{4}$ Oral Pathology and Medicine, Westmead Hospital,

${ }^{5}$ Endocrinology, University of Sydney, Australia.

Background and Aims: Transient macrophage infiltration is important early in normal wound healing, as is resolution of neutrophil infiltration and progressive induction of growth factors. Macrophage infiltration is impaired in diabetic wounds and while connective tissue growth factor (CTGF) is induced during normal wound healing its regulation in diabetic wounds is unknown. The aim of this study was to determine whether early abnormalities in macrophage and neutrophil infiltration in diabetic wound healing predict subsequent CTGF protein levels in a baboon wound healing model.

Materials and Methods: Wound tissue was obtained from drums placed subcutaneously in the thighs of 8 diabetic (D:average age $13.2 \mathrm{yr}$, wt $18.8 \mathrm{~kg}$, HbAlc 9.2\%) and 7 control (C:average age $12.8 \mathrm{yr}$, wt $25.1 \mathrm{~kg}$, HbAlc $3.8 \%$ ) baboons. Drums were removed after 2 and 4 wks for histological examination 
of wound tissue volume using Massons Trichrome stain. Macrophages, neutrophils and CTGF were detected with specific antibodies. Aspirated drum fluid was used to measure wound protease activity (total and active MMP-9 levels) by zymography and MCP-1 levels by ELISA. The CTGF staining intensity was graded from $0-3$, and the number of macrophages and neutrophils were counted and averaged/20 fields, by 2 observers blinded to tissue source. Data was expressed as Mean \pm SEM and analysed by either, TTest or where appropriate analysis of variance (ANOVA).

Results: At 2 wks the diabetic wound tissue volume and MCP-1 was not different to control, the macrophage infiltration was reduced and active MMP-9 was increased. At 4 weeks, the diabetic wound tissue volume tended to be less than control $(\mathrm{p}=0.06)$ and it contained less CTGF, active MMP-9 persisted and neutrophil clearance was delayed. For the entire group MCP-1 concentration at 4 wks correlated with macrophage number $(\mathrm{r}=0.56, \mathrm{p}=0.05)$ at 4 wks. In addition macrophage number at 2 wks correlated inversely with active MMP-9 $(\mathrm{r}=-0.77, \mathrm{p}<0.02)$, and positively with CTGF $(\mathrm{r}=0.64, \mathrm{p}=0.01)$ each at 4 wks.

Conclusion: As there is a decrease in wound tissue formation and alterations in cellular infiltration this drum model of wound tissue formation appears to be a valid model for the study of wound healing in diabetes. In addition this data supports the hypothesis that delayed and sustained macrophage infiltration predicts reduced CTGF levels in diabetic wound tissue. Whether macrophage chemoattractant therapy will normalise wound healing in experimental diabetes requires further study.

\begin{tabular}{lllllll} 
Animal & Week & $\begin{array}{l}\text { Macrophage } \\
\text { number }\end{array}$ & $\begin{array}{l}\text { Active } \\
\text { MMP-9 } \\
\text { \% of total) }\end{array}$ & $\begin{array}{l}\text { Total } \\
\text { MMP-9 } \\
\left(\times 10^{3}\right)\end{array}$ & $\begin{array}{l}\text { Neutrophil } \\
\text { number }\end{array}$ & $\begin{array}{l}\text { CTGF } \\
\text { Score }\end{array}$ \\
\hline Control & 2 & 5.99 & 13.57 & 34.95 & 2.56 & 1.63 \\
& & \pm 0.57 & \pm 2.71 & \pm 8.83 & \pm 0.48 & \pm 0.24 \\
& \multirow{2}{*}{4} & 1.95 & 24.69 & 74.69 & 0.67 & 2.36 \\
& & \pm 0.57 & \pm 2.71 & \pm 8.83 & \pm 0.48 & \pm 0.22 \\
Diabetic & 2 & 2.38 & 40.34 & 31.87 & 2.3 & 0.96 \\
& & \pm 0.54 & \pm 3.03 & \pm 9.87 & \pm 0.45 & \pm 0.21 \\
& \multirow{2}{*}{4} & 2.17 & 37.19 & 36.57 & 1.02 & 1.40 \\
& & \pm 0.54 & \pm 3.03 & \pm 9.87 & \pm 0.45 & \pm 0.21 \\
\hline
\end{tabular}

Supported by Juvenile Diabetes Research Foundation International and Rebecca Cooper Foundation

\section{PS 123 Cardiac complications I}

\section{9}

Insulin stimulation of myocardial perfusion and glucose uptake in patients with diabetes and idiopathic dilated cardiomyopathy: a PET study

S. Masi ${ }^{1}$, P. Iozzo ${ }^{1}$, C. Simi ${ }^{1}$, F. Frassi ${ }^{1}$, A. Sironi ${ }^{1}$, P. Pisani ${ }^{1}$, S. Pardini ${ }^{1}$, C. Porciello ${ }^{1}$, A. Giorgetti ${ }^{1}$, D. Petroni ${ }^{1}$, D. Giannessi ${ }^{1}$, P. Di Cecco ${ }^{1}$, P. A. Salvadori ${ }^{1}$, D. Neglia ${ }^{1}$, E. Ferrannini ${ }^{1,2}$;

${ }^{1}$ Institute of Clinical Physiology, CNR - National Research Council, ${ }^{2}$ Department of Internal Medicine, University of Pisa School of Medicine, Pisa, Italy.

Background and Aims: Idiopathic dilated cardiomyopathy (IDC) is associated with a high prevalence of diabetes, insulin-resistance, chronic neurohormonal activation and myocardial microcirculatory dysfunction. Insulin has mild vasodilatory properties in healthy individuals. We sought to evaluate the effect of an acute euglycaemic insulin infusion on myocardial blood flow and glucose uptake in patients with IDC and diabetes.

Materials and Methods: Seven male patients (age [mean \pm standard deviation]: $65 \pm 6$ years, body mass index $27.8 \pm 2.4 \mathrm{~kg} / \mathrm{m}^{2}$ ) with a diagnosis of IDC and diabetes underwent the current baseline characterization on myocardial responses to acute insulin stimulation, before entering a pilot study (insulin glargine/4053) on insulin treatment and cardiovascular risk. Positron emission tomography (PET) was used to quantify myocardial blood flow at rest (r-MBF), during euglycaemic insulin stimulation (i-MBF) and during dipyridamole stress (d-MBF), and to assess insulin-stimulated myocardial glucose uptake (MGU). MBF and MGU were estimated in 13 myocardial wall segments per patient $(\mathrm{n}=91)$ according to standardized segmentation procedures. Patients underwent an echocardiographic examination and repeated biohumoral characterization. Coronary flow reserve (CFR) was calculated as the ratio of $\mathrm{d}-\mathrm{MBF} / \mathrm{r}-\mathrm{MBF}$. Myocardial glucose extraction (MGE\%) was calculated as MGU divided by the product of steady-state plasma glucose and i-MBF (x 100).

Results: Left ventricular ejection fraction and end-diastolic diameter were 39 $\pm 11 \%$ and $63 \pm 9 \mathrm{~mm}$, respectively. Whole-body insulin-stimulated glucose uptake was $3.4 \pm 0.3 \mathrm{mg} / \mathrm{kg} / \mathrm{min}$. Insulin and dipyridamole increased MBF in a similar fashion (i-MBF $0.732 \pm 0.384 \mathrm{~mL} / \mathrm{min} / \mathrm{g}$ vs. d-MBF $0.750 \pm 0.223$ $\mathrm{mL} / \mathrm{min} / \mathrm{g}, \mathrm{p}=\mathrm{ns})$ relative to $\mathrm{r}-\mathrm{MBF}(0.520 \pm 0.143 \mathrm{~mL} / \mathrm{min} / \mathrm{g}, \mathrm{p}<0.05)$. Thus, values of CFR and $\mathrm{i}-\mathrm{MBF} / \mathrm{r}-\mathrm{MBF}$ were correlated $(\mathrm{r}=0.671, \mathrm{p}<0.0001)$. The increment in $\mathrm{MBF}$ during insulin infusion and CFR were inversely related to MGE\% ( $r=0.465, p<0.0001 ; r=0.492, p<0.0001$, respectively). Consistently, myocardial segments with lower CFR had an augmented MGU ( $\mathrm{p}=0.0014)$. Whole-body glucose uptake was negatively associated with glycosylated haemoglobin levels $(\mathrm{r}=0.91, \mathrm{p}=0.01)$. Neither MGU nor MBF showed independent associations with left-ventricular function or diameter.

Conclusion: Insulin stimulated MBF by $40 \%$ in patients with IDC and diabetes, equalling the maximal microcirculatory response to dipyridamole. Segments with a lesser blood flow response showed a compensatory increase in glucose extraction, which may help preserve the global left-ventricular systolic function in patients with diabetes and IDC.

Supported by sanofi-aventis

\section{0}

LPL und PPAR $\gamma$ in the pathogenesis of "diabetic cardiomyopathy" M. Lüttig-Rakowske ${ }^{1}$, B. Stratmann ${ }^{1}$, H. Milting ${ }^{2}$, G. Tenderich ${ }^{3}$, R. Körfer ${ }^{3}$, D. Tschöpe ${ }^{1}$;

${ }^{1}$ Heart and Diabetes Center NRW, ${ }^{2}$ Erich and Hanna Klessmann Institute, ${ }^{3}$ Clinic for Thoracic and Cardiovascular Surgery, Heart and Diabetes Center NRW, Bad Oeynhausen, Germany.

Cardiomyopathy may be triggered by metabolic influences associated with diabetes. However, the molecular mechanisms remain to be elucidated. Amongst others the lipoprotein lipase (LPL) and the peroxisome proliferator activated receptor $\gamma(\operatorname{PPAR} \gamma)$ are important proteins in the myocardial lipid metabolism. LPL is a key enzyme of lipid metabolism that hydrolyses triglycerides, providing free fatty acids for cells and affecting the maturation of circulating lipoproteins. Triglyceride-rich lipoproteins are known to play an important role in the progression of atherosclerosis. 
LPL requires Apolipoprotein-CII as a cofactor and LPL deficiency leads to hypertriglyceridemia. Insulin is known to enhance LPL synthesis in adipocytes and its placement in the capillary endothelium. High fat diets have been shown to cause tissue specific overexpression of LPL which has been implicated in tissue specific insulin resistance and consequent development of type 2 diabetes mellitus. To explore the role of LPL and PPAR $\gamma$ in the diabetic myocardial metabolism for the pathogenesis of dilated cardiomyopathy, the mRNA-expression of LPL and the ligand activated transcription factor PPAR $\gamma$ was analysed by real-time RT-PCR. Left ventricular samples of 14 patients with cardiomyopathy $(\mathrm{CM})$ and 13 with cardiomyopathy and diabetes $(\mathrm{CM}+\mathrm{DM})$ were investigated and compared to 7 non-failing donor hearts (NF), which could not be transplanted for technical reasons.

The left ventricular LPL mRNA level was increased in CM $(211 \pm 13 \%$ vs. NF, $\mathrm{p}=0.003)$ and $\mathrm{CM}+\mathrm{DM}(173 \pm 14 \%$ vs. NF, $\mathrm{p}=0.023)$ compared to NF. The PPAR $\gamma$ mRNA level of CM shows neither a significant difference in comparison to NF nor to group $\mathrm{CM}+\mathrm{DM}$. The PPAR $\gamma$ mRNA level of CM tended to be decreased in comparison NF whereas the PPAR $\gamma$ mRNA level of $\mathrm{CM}+\mathrm{DM}$ was non-significantly decreased compared to NF. The increase of the myocardial LPL mRNA level in terminal failing hearts might be associated with heart failure, since ongoing myocardial damage could be through an increased fatty acid uptake and oxidative stress which could result in cardiomyopathy. The alterations of the LPL mRNA expression are not in line with the PPAR $\gamma$ mRNA expression indicating that the expression of LPL is not only regulated through PPAR $\gamma$. In addition to the proven alterations in mRNA expression, further studies concerning the protein expression are necessary. A decreased protein expression of PPAR $\gamma$ could hind to a participation of PPAR $\gamma$ in the pathogenesis of cardiomyopathy.

\section{1}

Metabolic effects of co-administered simvastatin and $\omega-3$ polyunsaturated fatty acids in type 2 diabetic patients with cardiomyopathy

V. A. Serhiyenko, A. M. Urbanovich, A. A. Serhiyenko;

Endocrinology, Medical University of Lviv, Ukraine.

Background and Aims: Long-term administration of statins can be accompanied by essential infringements of function of a liver. $\omega-3$ polyunsaturated fatty acids ( $\omega$-3-PUFA), such as eicosapentaenoic acid (EPA) and docosahexaenoic acid (DHA) possess essential positive properties, including hepatoprotective effects. The aim of this study was to assess the effects of simvastatin (SIM), $\omega-3$ PUFA and their combinations on dynamics of such biochemical parameters in Type 2 diabetic patients with diabetic cardiomyopathy (DCMP).

Materials and Methods: 86 patients with Type 2 DM and DCMP $(51,2 \pm 4,3$ years), BMI $26,3 \pm 2,79 \mathrm{~kg} / \mathrm{m}^{2}$, DM duration 7,6 $\pm 2,4$ years, HbA1c $8,8 \pm 2,7 \%$ were allocated to three groups. Patients of group A $(n=25)$ received SIM 20 mg tid; B ( $n=37)$ - capsules of fish oil (1,0 g EPA, 1,0 g DHA and 0,1\% $\alpha$ tocopherol acetate tid), C ( $\mathrm{n}=24)$ - SIM $10 \mathrm{mg}$ tid plus capsules of fish oil. All patients were on the same diet. Research joined patients with the diagnosed lipid abnormalities (NCEP ATP III). The duration of the study was 3 months. We investigated total cholesterol (TC), low (LDL-C), high density lipoproteincholesterol (HDL-C), triglycerides (TG), the ${ }^{125} \mathrm{I}$-6-ketoprostaglandin $\mathrm{F}_{1 \alpha}$ (6-ketoPGF $\left.{ }_{1 \alpha}\right),{ }^{125}$ I-thromboxane $\mathrm{B}_{2}\left(\mathrm{TXB}_{2}\right)$ concentrations, activities of alaninaminotransferase, $\gamma$-glutamiltranspeptidase in the blood plasma; $\mathrm{Na}^{+}$, $\mathrm{K}^{+}$-ATPase, fatty acids in the membranes of RBC's; activities of superoxide dismutase (SOD), gluthation peroxidase (GPO) in the RBC's, level of conjugated dienes (CD). Statistics: ANOVA.

Results: Lipid disorders [high level of total TC, LDL-C, TG $(7,3 \pm 10,1 ; 4,5 \pm 0,3$; $2,87 \pm 0,2 \mathrm{mmol} / \mathrm{l}, \mathrm{p}<0,05)$ and decrease level of HDL-C] in the patients with DCMP is accompanied by a decrease of the activities of $\mathrm{Na}^{+}, \mathrm{K}^{+}$- ATPase in the RBC's membranes, SOD, GPO in the RBC's. There is a considerable increase in the $\mathrm{TXB}_{2}$ level, level of $\mathrm{CD}$ and a decrease in serum 6-keto-PGF${ }_{10}, \omega-3-$ PUFA in the RBC's membranes. It has been discovered that the monotherapy by SIM is accompanied by negative dynamics of activity of enzymes of a liver that proved by of ultrasonic scanning. After 3 months of treatment there was a more significant decrease in LDL-C, TC, TG concentration, TXB ${ }_{2}$ level $(152,5$ $\pm 16,9 \mathrm{pg} / \mathrm{ml}, \mathrm{p}<0,001)$, level of $\mathrm{CD}(\mathrm{p}<0,01)$ with simultaneous increases in EPA level, EPA/arachidonic acid ratio, $\mathrm{Na}^{+}, \mathrm{K}^{+}$-ATPase $(0,04 \pm 0,003$ vs $0,09 \pm 0,004 \mathrm{mMol} \mathrm{PI} / \mathrm{mg}$ protein per 1 hour, $\mathrm{p}<0,001)$ and the concentration of 6 -ketoPGF ${ }_{1 \alpha}$, increases activity of SOD, GPO $(10,94 \pm 0,22 ; 321,7 \pm 19,2$ $\mathrm{mcmol} \mathrm{GSH} / \mathrm{min} 1 \mathrm{~g} \mathrm{Hb}, \mathrm{p}<0,001$ ) in the 3-rd group (table).

\begin{tabular}{llll}
$\begin{array}{l}\text { Mean \% change from } \\
\text { baseline at 3 months }\end{array}$ & $\begin{array}{l}\text { SIM } \\
(\mathrm{n}=25)\end{array}$ & $\begin{array}{l}\omega-3 \text { PUFA } \\
(\mathrm{n}=37)\end{array}$ & $\begin{array}{l}\text { SIM } / \omega-3 \text { PUFA } \\
(\mathrm{n}=24)\end{array}$ \\
\hline LDL-C & $-34,2^{*}$ & $-12,8$ & $-52,9^{* *}$ \\
HDL-C & $+15,3$ & $+7,1$ & $+26,8^{*}$ \\
TC & $-8,2$ & $-12,5$ & $-19,5$ \\
TG & $-12,6$ & $-35,4^{*}$ & $-57,4^{* *}$ \\
6-ketoPGF & $+13,6$ & $+35,6^{*}$ & $+46,1^{* *}$ \\
$\mathrm{TXB}_{2}$ & $-18,6$ & $-51,5^{* *}$ & $-59,5^{* *}$ \\
$\mathrm{Na}^{+}, \mathrm{K}^{+}-$ATPase & $+5,9$ & $+89,4^{* *}$ & $+92,7^{* *}$ \\
SOD & $+12,7$ & $+36,8^{*}$ & $+48,4^{* *}$ \\
GPO & $+7,9$ & $+31,9$ & $+45,7^{* *}$ \\
\hline
\end{tabular}

Treatment difference presented with $95 \%$ CI. ${ }^{*} \mathrm{p}<0,01,{ }^{* *} \mathrm{p}<0,001$

Conclusion: SIM and $\omega$-3-PUFA treatment following 3 months significantly improve the lipid profile, state of prostacyclin $\mathrm{I}_{2}$-thromboxane $\mathrm{A}_{2}$ system, activeness of membrane-bound enzymes, some indexes of the oxidative stress. The combined purpose SIM and $\omega$-3 PUFA allows to lower a doze SIM, that allows to recommend their combination in the rational-proved treatment of lipid disorders in patients with DCMP.

\section{2}

Microalbuminuria is correlated with early right ventricular diastolic function abnormalities in patients with type 1 diabetes mellitus D. K. Levisianou ${ }^{1}$, A. Melidonis ${ }^{1}$, A. Koutsovasilis' ${ }^{1}$, D. Beldekos ${ }^{2}$, G. Tsiaousis ${ }^{2}$, I. Protopsaltis ${ }^{1}$, S. Handanis ${ }^{2}$, S. Foussas ${ }^{2}$;

${ }^{1}$ Diabetes Center, ${ }^{2}$ Cardiology Dpt., Tzanio Gen Hospital, Piraeus, Greece.

Background and Aims: Left ventricular dysfunction (systolic and diastolic) is well documented in patients with Type 1 Diabetes Mellitus (T1DM). There are not enough data for the function of the right ventricular in these patients. The aim of the study was the early detection of right diastolic dysfunction in asymptomatic patients with Type 1 Diabetes Mellitus (T1DM) by the use of tissue Doppler imaging (TDI) indices and its correlation with diabetological parameters

Materials and Methods: We examined 45 patients (aged 39,2 $\pm 9,9$ years) with T1DM (Group A) and 31 normal controls (Group B) of comparable age and gender. All participants underwent a standard 2-D and color Doppler echocardiographic study. Pulsed TDI was performed in apical 4-chamber view. We measured the $\mathrm{LV}$ ejection fraction $(\mathrm{EF})$, transmitral flow velocity (E, A, E/A) and Tran tricuspid flow velocity $\left(\mathrm{E}^{\prime}, \mathrm{A}^{\prime}, \mathrm{E}^{\prime} / \mathrm{A}^{\prime}\right)$. In addition, TDI measurements of mitral (mitral Em, Am, Sm) and tricuspid annulus velocities (tricuspid $\mathrm{Em}, \mathrm{Am}, \mathrm{Sm}$ ) were evaluated. A possible correlation was examined for age, diabetes duration, $\mathrm{HbAlc}$, microalbuminuria, heart rate, Body Mass Index (BMI), peripheral neuropathy, retinopathy and echocardiographic measurements of left and right ventricle with univariate analysis. The volume of right atrium, (RA volume) was calculated from the apical 4-chamber view using Simpson's method and subsequently indexed to body surface area (RAVI). Statistical analyses were performed using the SPS software version 13.0. All continuous variables are expressed as mean \pm standard deviation. Comparisons of echocardiographic variables of T1DM subjects and NC were performed using Student $t$ test for unpaired data. Chi-square tests were used for comparison of categoric variables. Pearson's correlation coefficients were used to assess the strength of the relationship between continuous variables.

Results: All participants had normal ejection fraction, dimensions and wall thickness of the ventricle. Group A patients had impaired right diastolic function compared to the controls of group $B\left(E^{\prime} / A^{\prime}=0.94 \pm 0.34\right.$ vs $1,77 \pm 0.25$, $\mathrm{p}<0.001, \mathrm{Em} / \mathrm{Am}=0.89 \pm 0.39$ vs $1.38 \pm 0.22, \mathrm{p}<0.001, \mathrm{E} / \mathrm{A}=0.95 \pm 0.18$ vs $1.8 \pm 0.16, \mathrm{p}<0.001)$. The presence of T1DM significantly correlated with all of the measures of LA and RA function:E/A, E'A', Mitral Em/Am, Tricuspid $\operatorname{Em} / \operatorname{Am}(\mathrm{p}<0.0001, \mathrm{p}<0,001, \mathrm{p}<0.0001, \mathrm{p}<0.001$, respectively). In the multivariate model most significant was the presence of microalbuminuria $(p<0.001)$. T1DM duration and heart rate were the only other variables in the univariate analyses that correlated significantly with all measures of LA and RA function $(\mathrm{p}<0.01)$. 
Conclusion: Asymptomatic microalbuminuric patients with T1DM have early right diastolic dysfunction that can be reliably detected using indices of tissue Doppler echocardiography. Our data suggest that microalbuminuria is associated with significant changes of right ventricular diastolic function. Microalbuminuria may be an early biochemical marker of these abnormalities. Therefore TDI should be used in all microalbuminuric patients with T1DM.

\section{3}

Nt-BNP as a short-term predictor of cardiovascular hospitalization or death in diabetic patients without history of cardiac disease compared to classical risk-factors

S. Neuhold ${ }^{1}$, M. Hülsmann ${ }^{1}$, M. Clodi ${ }^{2}$, M. Riedl ${ }^{2}$, D. Mörtl ${ }^{1}$, R. Berger ${ }^{1}$, R. Pacher ${ }^{1}$, A. Luger ${ }^{2}$;

${ }^{1}$ Department of Cardiology,

${ }^{2}$ Department of Endocrinology, Medical University Vienna, Austria.

Background and Aims: Nt-BNP is known to be an excellent marker for screening patients with a suspected cardiac disease. Cardiovascular morbidity contributes to the main causes of hospitalisation and death in diabetic patients. Also there is some information, that Nt-BNP predict worse outcome in diabetic patients on long-term, no data are available on short-term, especially in the subset of patients with no cardiac history. We compared NtBNP, beside known risk-markers for developing a cardiac disease in diabetic patients..

Material and Methods: We investigated 441 patients out of a tertiary care hospital diabetes out-patient unit. No patient had a history of cardiac disease. A number of clinical variables and Nt-BNP (Roche Diagnostics) was obtained in all patients. If Nt-BNP levels were increased over the normal limit $(125 \mathrm{pg} / \mathrm{ml})$ the treating physician was informed, that further investigations and probably optimization of treatment might be necessary. Patients were followed up 7.7 months on average (range 0.5-13 months). Unplanned cardiovascular hospitalisation or death was used as the endpoint.

Results: All over 13 patients experienced unplanned cardiovascular hospitalisation and 7 patients died. 19 patients reached the combined endpoint. Of the subset of patients with increased levels of Nt-BNP we received 69 records of echocardiography. Systolic dysfunction was present in $6 \%$, diastolic dysfunction in $55 \%$ and $39 \%$ had no evidence of heart disease. Patients reaching the endpoint were significantly older $(57 \pm 14$ vs $66 \pm 15$ years $(\mathrm{p}<0.01)$, had statistical significant, but not clinical relevant higher levels of creatinine ( $1 \pm 0.4 \mathrm{vs} 1.3 \pm 0.6 \mathrm{mg} / \mathrm{dl}$ py0.05) and higher Nt-BNP $(169 \pm 262$ vs $536 \pm 701 \mathrm{pg} / \mathrm{ml} \mathrm{p}<0.0001)$. There was no difference in therapy, blood pressure, LDL-Cholesterine or HBAlc. Outcome was similar, whether patients were above or below the cut-point as follows : $135 \mathrm{mmHg}$ as a threshold for blood pressure (4\%vs $4 \%), 100 \mathrm{mg} / \mathrm{dl}(4 \% \mathrm{vs} 4 \%)$ or $130 \mathrm{mg} / \mathrm{dl}$ (3\%vs5\%) for LDLCholesterine, and $7 \%$ for HBAlc ( 3\%vs5\%) for below and above the cutpoint, respectively. There was only a significant difference for Nt-BNP (for $125 \mathrm{pg} / \mathrm{ml} 1$ vs $9 \%$ event-rate $\mathrm{p}<0.0001$ ) and for creatinine (for $1.5 \mathrm{mg} / \mathrm{dl}$ 3 vs $13 \%$ p $<0.01$ ) This held true in Kaplan-Meyer analysis only for Nt-BNP $(\mathrm{p}<0.001)$.

Conclusion: Beside a number of variables only age, creatinine and Nt-BNP are significant higher in patients with an event. Using a cut-point of $125 \mathrm{pg} /$ $\mathrm{ml}$, Nt-BNP appears to unmask diabetic patients with a cardiac risk. This was not true for markers like HBAlc, blood pressure, kidney dysfunction or LDLCholesterine.

\section{4}

Rosiglitazone, but not glimepiride, improves myocardial diastolic function in association with a reduction of oxidative stress and inflammation in patients with type 2 diabetes mellitus H. von Bibra ${ }^{1}$, M. Diamant ${ }^{2}$, P. Scheffer ${ }^{2}$, T. Siegmund ${ }^{1}$,

P.-M. Schumm-Draeger ${ }^{1}$;

${ }^{1}$ Endocrinology, Academic Hospital Bogenhausen, München, Germany, ${ }^{2}$ Endocrinology, VU University Medical Centre, Amsterdam,

The Netherlands.

Background and Aims: Recent evidence indicates that thiazolidinediones (TZD) reduce cardiovascular disease (CVD) in high-risk patients with type 2 diabetes mellitus (DM2), however, their effect on cardiac function is under debate. On one hand, TZD improve lipotoxic diabetic cardiomyopathy in animal models, whereas their use in patients has been associated with an increased rate of congestive heart failure. In animals, direct and indirect actions of TZD may favourably affect cardiac function by improving insulin sensitivity and reducing inflammation and oxidative stress. Given the high occurrence of diastolic myocardial dysfunction in DM2 and its prognostic relevance, we tested the hypothesis, that rosiglitazone $(\mathrm{R})$, versus glimepiride (G), may improve diastolic myocardial function in patients with DM2 by simultaneously reducing oxidative stress and inflammation.

Materials and Methods: This randomised cross-over study investigated 12 patients with DM2 (aged 59 13 years) on monotherapy with metformin with normal systolic ventricular function (2-d echocardiography) and without history and signs of coronary artery disease or congestive heart failure before and after 4 months additional therapy with R (8mg daily) and with G (3mg daily) in the fasting state and 2 hours after a standardized breakfast (48 g carbohydrates). Left ventricular function was assessed by tissue Doppler as systolic (Vs) and diastolic myocardial velocity (Ve) averaged from six basal myocardial segments and also by standard 2-d echocardiography. Oxidative stress was evaluated by measuring levels of plasma malondialdehyde (MDA). Additionally, the inflammatory markers myeloperoxidase (MPO) and highsensitive C-reactive protein (CRP) were determined.

Results: With $\mathrm{R}$, Ve improved significantly from $7.9 \pm 1.7$ to $8.8 \pm 1.2$ $\mathrm{cm} / \mathrm{s}(\mathrm{p}=0.04)$ both in the fasting and the postprandial state $(\mathrm{p}=0.04)$. Concomitantly, MDA decreased significantly $(8.1 \pm 2.1$ to $7.1 \pm 1.5 \mu \mathrm{M}, \mathrm{p}<0.01)$. The change of Ve correlated significantly with the change of MDA $(r=-0.67$, $\mathrm{p}=0.03$ ). The improvement of $\mathrm{HbAlc}$ was not significant (from $7.0 \pm 1.0$ to $6.8 \pm 0.8 \%, p=0.15)$ but postprandial blood glucose decreased $(\mathrm{p}=0.007)$ and so did CRP $(\mathrm{p}=0.01)$, whereas MPO reduction missed significance $(\mathrm{p}=0.09)$. With G, patients had similar effects on HbAlc (from $6.7 \pm 0.6$ to $6.5 \pm 0.5 \%$, $\mathrm{p}=0.19$ ) but no significant reduction of fasting or postprandial blood glucose, of Ve $(8.5 \pm 1.3$ to $8.3 \pm 1.6 \mathrm{~cm} / \mathrm{s}), \mathrm{MDA}, \mathrm{CRP}$ and MPO. Other metabolic, haemodynamic and cardiac parameters remained unchanged in both therapy regimens.

Conclusion: In patients with type 2 diabetes mellitus, additional therapy with rosiglitazone improved diastolic myocardial function, in association with reduced oxidative stress and inflammation. These observed effects are prognostically important and could be relevant in the therapy choice for patients with type 2 diabetes mellitus without a history of heart disease. Supported by GlaxoSmithKline

\section{5}

Prevalence and determinants of $\mathrm{T}$-wave alternans in type 2 diabetes D. T. Martin ${ }^{1}$, A. Shoraki ${ }^{1}$, R. W. Nesto ${ }^{1}$, M. K. Rutter ${ }^{2}$;

${ }^{1}$ Dept of Cardiology, Lahey Clinic Medical Center, Burlington, United States, ${ }^{2}$ Dept of Medicine, University of Manchester, United Kingdom.

Background and Aims: Microvolt T-wave alternans (MTWA) positivity is defined as abnormal beat-to-beat variability in the T-wave amplitude of the ECG. Subjects with MTWA in association with structural heart disease have an increased risk for ventricular arrhythmias and sudden cardiac death. The presence of diabetes (DM) is associated with an increased risk of sudden death but there is limited data on the impact of DM and previous myocardial infarction (MI) on MTWA prevalence. In this study we describe the prevalence and clinical correlates of MTWA in a large cohort of outpatients with and without a history of DM and/or MI.

Materials and Methods: We studied 143 patients (mean (SD) age 58 (12), $20 \%$ female) referred to a large general hospital for treadmill exercise testing. Classification of DM and MI status was based on patient history: group 1 ( $n=54$ : DM -, MI -); group 2 ( $n=47: \mathrm{DM}+, \mathrm{MI}-)$; group 3 ( $n=26: \mathrm{MI}+, \mathrm{DM}$ -); group $4(n=13: \mathrm{DM}+, \mathrm{MI}+)$. Exclusion criteria included: ectopic beats, atrial fibrillation, unstable angina, severe hypertension, inability to exercise, severe aortic stenosis or digoxin therapy. A symptom-limited exercise test was performed using a standardized protocol. MTWA was assessed by the spectral method using commercially available equipment (Cambridge Heart Inc). Sustained alternans ( $>2$ minutes) in the absence of electrical interference or ectopy at heart rate $<110 \mathrm{bpm}$ in contiguous orthogonal or precordial ECG leads was required for definition of a positive test; unequivocally negative tests were those in which adequate heart rate was achieved and both electrical interference and ectopy were absent. Test interpretation was performed blind to patient status; using conventional criteria for MTWA assessment all indeterminate tests $(18 \%)$ were considered positive in this analysis. 
Results: Age and gender were similar in all groups. The prevalence of positive MTWA in groups $1-4$ was $24 \%, 20 \%, 48 \%$, and $62 \%$ respectively (between group $p=0.002$ ). Logistic regression analysis in all patients showed that positive MTWA was related to prior MI (OR (95\% CI): 4.0 (1.8-8.9), $p<0.001)$ but not to prevalent DM $(0.9(0.4-1.8), p<0.001)$. The power of the study was $80 \%$ for a $16 \%$ between-group ( 1 and 2 ) difference in MTWA prevalence. Echocardiographic assessment of left ventricular ejection fraction was available for $92(64 \%)$ patients. Mean (SD) ejection fraction was 61 (6)\%, $63(7) \%, 53(16) \%$, and $44(11) \%$ in groups $1-4$ respectively. In patients with DM the prevalence of MTWA was related to ejection fraction $(p=0.034)$ but not to BMI, DM duration, glycemic control, insulin use, lipid profile or the presence of microvascular complications.

Conclusion: MTWA positivity is related to a history of MI and impaired LV function, but is not strongly related to a history of DM, diabetes complications, or glycemic control in this cohort of patients referred for treadmill testing. Prospective studies are required to establish the prognostic value of MTWA in the diabetic population.

Supported by Robert E Wise Research and Education Institute

\section{6}

Strong association between cardiac autonomic dysfunction and reduced pulmonary diffusing capacity (DLCO) in type 1 diabetes

P. Santangeli ${ }^{1}$, D. Pitocco ${ }^{2}$, L. Fuso ${ }^{2}$, F. Zaccardi ${ }^{2}$, G. Sgueglia ${ }^{1}$, F. Infusino ${ }^{1}$, L. Maugeri ${ }^{2}$, E. Navarese ${ }^{1}$, G. Lanza ${ }^{1}$, F. Crea ${ }^{1}$, G. Ghirlanda ${ }^{2}$;

${ }^{1}$ Cardiology, ${ }^{2}$ Internal Medicine, Catholic University, Rome, Italy.

Background and Aims: Type 1 diabetes produces damage in small blood vessels characterized by morphologic and biochemical alterations which may result in pulmonary microvascular dysfunction leading to a loss of alveolar microvascular reserve. Abnormal peripheral autonomic function is detectable in type 1 diabetes mellitus. In this study we aimed at assessing the relation between cardiac autonomic function and subclinical loss of alveolar microvascular reserve in patients with type 1 diabetes.

Materials and Methods: We studied 20 patients with type 1 diabetes (age $50.1 \pm 9$ years, 13 men) without any clinical evidence of pulmonary abnormalities and systemic autonomic dysfunction. Carbon monoxide diffusing capacity (DLCO) was measured non-invasively by single-breath method. Cardiac autonomic function was assessed by time- and frequencydomain heart rate variability (HRV) analysis on 24-hour electrocardiographic Holter recordings

Results: A significant direct correlation was found between DLCO and several HRV variables, with the strongest association being found with SDNN-index (i.e. standard deviation of RR intervals for all 5-minute segments in the 24 hour) $(\mathrm{r}=0.61, \mathrm{p}=0.006)$ in the time domain and with low frequency (LF) power $(\mathrm{r}=0.65, \mathrm{p}=0.002)$ in the frequency domain (Table 1$)$.

\begin{tabular}{lll} 
& R coefficient & $\mathrm{P}$ \\
\hline RR & 0.22 & 0.36 \\
SDNN & 0.54 & 0.016 \\
SDANN & 0.52 & 0.023 \\
SDNN-i & 0.61 & 0.006 \\
rMSSD & 0.33 & 0.16 \\
pNN50 & 0.49 & 0.035 \\
TP & 0.54 & 0.017 \\
VLF & 0.60 & 0.006 \\
LF & 0.65 & 0.002 \\
HF & 0.36 & 0.12 \\
\hline
\end{tabular}

Conclusion: Our data show a strong association between cardiac autonomic dysfunction and reduced pulmonary diffusing capacity in patients with type 1 diabetes without any clinical evidence of pulmonary abnormalities or peripheral autonomic dysfunction. Our findings further support the hypothesis that autonomic dysfunction is linked to subclinical reduction of alveolar microvascular reserve, as assessed by DLCO. DLCO may be an early sensitive marker of autonomic neuropathy in type 1 diabetes.

\section{PS 124 Cardiac complications II}

\section{7}

UKPDS risk engine and French guidelines to select the diabetic patients to screen for silent myocardial ischaemia: a follow-up study E. Cosson, C. Balta, M. Nguyen, J. Pariès, S. Balta, P. Valensi; Department of Endocrinology-Diabetology-Nutrition, Jean Verdier Hospital, Bondy, France.

Background and Aims: French Societies of Diabetology and Cardiology recommended in 2004 to screen for silent myocardial ischemia (SMI) the diabetic patients selected on the following criteria: those with nephropathy or carotid or peripheral artery disease, those with $\geq 2$ other cardiovascular risk factors if type 1 diabetic patients older than 45 yrs or with diabetes duration $>15$ yrs, and if type 2 diabetic patients older than 60 yrs or with diabetes duration $>10$ yrs. The aim of the study was to evaluate these criteria and also the UKPDS risk engine for the 10-yrs risk of coronary events (UKPDSCHD).

Materials and Methods: Between 1992 and 2006, a total of 781 diabetic patients were screened for SMI, defined as an abnormal stress myocardial scintigraphy, and for significant $(>70 \%)$ coronary stenoses (CS) on angiography in those with SMI.. We followed up 643 patients for cardiac events (cardiac death, myocardial infarction, cardiac failure, coronary revascularisation, unstable angina). In this population, 406 patients fulfilled the French screening criteria $(\mathrm{C}+), 345$ did not (C-) and 30 were unclassified.

Results: SMI was present in 227 (29\%) patients. The prevalence of SMI was higher in the $\mathrm{C}+(133 / 406,32.8 \%)$ than in the C- group $(85 / 345$, $24.6 \%)$. Odds ratio 1.49 confidence interval 95\% [1.08-2.05] $\mathrm{p}<0.05)$, and was associated with a higher UKPDS-CHD score $(32 \pm 19 \%$ versus $24 \pm 16 \%$, $\mathrm{p}<0.0001)$. Coronary angiography was performed in 209 patients with SMI, and CS were identified in 83 patients $(40 \%)$. The positive predictive value of SMI for identifying the patients with CS was unchanged considering French recommendations or UKPDS-CHD score. A total of 55 cardiac events occurred follow-up 4.4 \pm 3.9 yrs. SMI was predictive of events (Kaplan Meier: $\log$ rank 29, p < 0.0001). In the patients with SMI, the presence of CS was associated with a poorer prognosis as compared with the patients without CS (Kaplan Meier: log rank 18.5, $\mathrm{p}<0.0001)$. UKPDS-CHD score correlated with the incidence of cardiac events $(\mathrm{p}=0.03)$.

Conclusion: SMI and silent CS are the main predictive factors of cardiac events in the diabetic population. The current French guidelines and UKPDSCHD risk are helpful in identifying the diabetic patients with a high risk of SMI.

\section{8}

Leptin predicts glucose perturbations and future risk after acute myocardial infarction in patients without previously known diabetes M. Wallander ${ }^{1}$, S. Söderberg ${ }^{2}$, K. Malmberg ${ }^{1}$, A. Norhammarr ${ }^{1}$, L. Rydén ${ }^{1}$; ${ }^{1}$ Dep. of Medicine, Cardiology Unit, Karolinska Institutet, Stockholm, ${ }^{2}$ Department of Internal Medicine, Umeå University, Sweden.

Background and Aims: High levels of leptin and low adiponectin are associated with type 2 diabetes (T2DM) and cardiovascular (CV) disease. We studied the prognostic importance of leptin and adiponectin for glucose tolerance (GT) and future CV events in patients with acute myocardial infarction (AMI) without known T2DM.

Materials and Methods: 168 AMI-patients (67\% men) were classified after an OGTT at hospital discharge (DIS; day 4-5) as having normal or abnormal GT (AGT), defined as impaired GT or T2DM. 185 sex- and age matched healthy persons served as controls. Fasting serum-leptin and adiponectin at hospital admission $(\mathrm{ADM})$ and discharge were related to AGT and CV events (CV mortality (8), non-fatal stroke (6), reinfarction (15) or severe heart failure (10)) during a median follow up of 34 months. Values are presented as means. All analyses are adjusted for age, sex and BMI.

Results: Leptin values were higher in patients at admission than in controls (women: 21.1 vs. $17.0 \mathrm{ng} / \mathrm{ml}$; $\mathrm{p}=0.029$; men: 9.1 vs. $6.4 \mathrm{ng} / \mathrm{ml} ; \mathrm{p}<0.001$ ) but decreased before hospital discharge to 18.1 (women) and 7.4 (men) $\mathrm{ng} / \mathrm{ml}$ respectively. High levels of leptin at admission and discharge related to AGT $\left(\mathrm{OR}_{\mathrm{ADM}}=2.2 ; \mathrm{p}=0.016 ; \mathrm{OR}_{\mathrm{DIS}}=2.1 ; \mathrm{p}=0.040\right)$ and to increased risk for $\mathrm{CV}$ events during follow up $\left(\mathrm{HR}_{\mathrm{ADM}}=1.8 ; \mathrm{p}=0.014 ; \mathrm{HR}_{\mathrm{DIS}}=1.7 ; \mathrm{p}=0.040\right)$. The 
relationship to $\mathrm{CV}$ events at admission but not at discharge was significant after multiple adjustment. The figure presents Kaplan-Meier curves of CV events in patients above or below the median of leptin (women: 22.6, men: 8.9 $\mathrm{ng} / \mathrm{ml}$ ) at admission. Adiponectin levels did not differ between patients and controls and did not relate to AGT or CV events.

Conclusion: Elevated levels of leptin at admission and discharge in patients with AMI where related to AGT and independently of the glucometabolic state, leptin at admission related to a more serious prognosis.

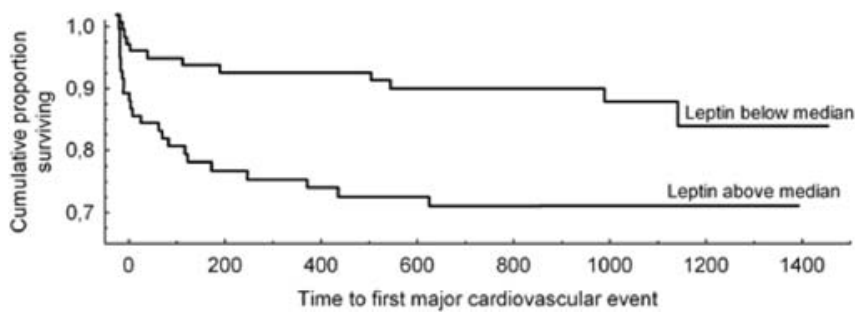

Supported by AFA Insurance, the Swedish Heart-Lung Foundation, King Gustaf the V, Queen Victoria Foundation, the Family Erling-Persson Foundation and Signe and Olof Wallenius Foundation. These grants were totally unrestricted and unconditional

\section{9}

Prevalence of abnormal glycaemia in patients with acute cardiacsounding chest pain

A. A. Tahrani ${ }^{1}$, J. Geen ${ }^{2}$, P. W. Jones ${ }^{3}$, F. W. F. Hanna ${ }^{1}$;

${ }^{1}$ Diabetes and Endocrinology, University Hospital of North Staffordshire,

Stoke-On-Trent, ${ }^{2}$ Diabetes and Endocrinology, North Glamorgan NHS

Trust, Merthyr Tydfil, ${ }^{3}$ Department of Mathematics, Keele University,

Stoke-On-Trent, United Kingdom.

Background and Aims: To assess the prevalence of diabetes, impaired glucose tolerance (IGT) and impaired fasting glucose (IFG) based on random plasma glucose (RPG), fasting plasma glucose (FPG) and oral glucose tolerance test (OGTT) on admission in patients with acute cardiac-sounding chest pain.

Materials and Methods: 200 consecutive patients admitted to a district general hospital in the UK with cardiac-sounding chest pain were included. Data collected on admission included: RPG, FPG, fasting lipid profile, troponin I, waist measurement, BMI, smoking status. OGTT was done during the admission.

Results: Diabetes prevalence was $4.5 \%$ and $3 \%$ based on admission RPG and FPG respectively. $89.5 \%$ had normal FPG on admission and 7.5\% had IFG. OGTT on admission showed that $20.5 \%$ had diabetes, $31.5 \%$ had IGT, $2 \%$ had IFG and $45 \%$ were normal. The OGTT samples from 2 patients (1\%) were not usable.

In the raised troponin sub-group: $45.5 \%$ of our patients had raised troponin I levels. Diabetes prevalence was $6.5 \%$ and $4.4 \%$ based on admission RPG and FPG respectively. $89 \%$ had normal FPG and $6.6 \%$ had IFG. OGTT on admission showed that $26.4 \%$ had diabetes, $38.5 \%$ had IGT, $1.1 \%$ had IFG and $33 \%$ were normal. OGTT sample from 1 patient (1.1\%) was not usable. $66.7 \%$ of patients with raised vs $44.4 \%$ with normal troponin had abnormal OGTT on admission ( $\mathrm{p}=0.002$ ). $11 \%$ of patients with elevated vs $10.1 \%$ with normal troponin had abnormal FPG on admission ( $\mathrm{p}=0.83$ ). Admission RPG also showed no statistically significant difference between high and normal troponin groups $(\mathrm{p}=0.55)$.

Conclusion: In patients admitted with cardiac sounding chest pain:

1. More than half of the patients had abnormal OGTT.

2. Fasting and random plasma glucose underestimate the prevalence of diabetes in our cohort considerably compared to OGTT.

3. Fasting/random glucose should not be used to rule out diabetes in this high-risk group of patients.

4. It is important not to miss the diagnosis of diabetes in this group as early treatment could delay the development of microvascular complications.

In patients with elevated troponin:

1. Only one third of the patients had normal OGTT.

2. Fasting and random plasma glucose underestimate the prevalence of diabetes considerably compared to OGTT.
Comparing the raised to normal troponin groups:

1. The prevalence of abnormal OGTT was significantly higher in the raised troponin group.

2. The prevalence of abnormal fasting and random plasma glucose levels was similar between both groups.

\section{0}

Prolonged corrected QT interval is associated with microalbuminuria and left ventricular hypertrophy in male subjects with type 2 diabetes mellitus

A. Jawa, M. Hasan, U. Chaudhary, S. Abaidullah, J. Zaheer, S. Iqtadar; Medicine, King Edward Medical University, Lahore, Pakistan.

Background and Aims: Patients with type 2 diabetes mellitus are at high risk for future cardiovascular events Microalbuminuria is a strong predictive risk factor for all-causes and cardiovascular mortality in type 2 diabetes. Prolonged QTc interval is an independent predictor of cardiovascular mortality in type 2 diabetic patients. Both microalbuminuria and prolonged QTc interval are risk factors for cardiovascular disease in type 2 diabetic subjects. The link between microalbuminuria and premature death in type 2 diabetes is not fully explained by conventional cardiovascular risk factors. We investigated whether QT prolongation is associated with microalbuminuria and Left Ventricular Hypertrophyin patients with type 2 diabetes.

Materials and Methods: We conducted a case-control study from 1st September 2006 to 31st January 2007 at Diabetes Clinic of King Edward Medical University and allied hospitals. Microalbuminuria was defined to be present as two of three urinary albumin excretion rate of $20-200 \mu \mathrm{g} /$ min. Prolonged QT interval was defined as $>440$ milliseconds(ms) and was corrected with Bazzet's formula. All male subjects with type 2 diabetes aged between 35-75 years with no clinical evidence of coronary disease were offered participation in the study. None of the subjects were on QTc interval prolonging drugs. After obtaining informed consent, fifty subjects were enrolled and tested for fasting blood sugar, $\mathrm{HbAlC}$, creatinine, triglycerides and HDL-cholesterol and blood pressure were measured. Urine was tested for the presence of microalbuminuria using standardized semi quantitative dipstick technique (MICRAL,Roche,USA). Electrocardiograms were performed and two independent trained physicians blinded to the subject's medical history analysed these electrocardiograms for rate, rhythm, axis, ST segment changes and corrected QT interval. Data was analysed using statistical software SPSS 14.0.

Results: Subjects enrolled were divided in two groups. Group A consisted of 25 male subjects who did not have microalbuminuria and Group B consisted of 25 male subjects who had microalbuminuria. The mean age for Group A was $51.24 \pm 12.02$ years and $56.36 \pm 11.06$ years for Group B $(p$-value $=0.1)$. $\mathrm{BMI}$, pulse, systolic and diastolic blood pressures $\mathrm{HbAlc}$, creatinine, serum triglycerides and HDL-cholesterol levels of both the groups were compared and did not differ in statistically significant manner. Eight percent $(2 / 25)$ of the subjects in Group A had prolonged QTc interval whereas in group B 60 $\%(15 / 25)$ of subjects had prolonged QTc interval. In Group A mean QTc interval was $420 \pm 20 \mathrm{~ms}$ where as it was $460 \pm 40 \mathrm{~ms}$ in Group B ( $p$-value = 0.0001). The correlation coefficient using Pearson's Correlation coefficient for microalbuminuria and prolonged QTc was statistically significant $(r=0.38$, 1 - tailed significance $=0.003$, Table 2)

Conclusion: Our study clearly shows that rate-corrected QT interval (QTc) is associated with presence of microalbuminuria and Left Ventricular Hypertrophy in type 2 diabetic subjects. Our findings support the hypothesis that QT prolongation and microalbuminuria have common determinants such as Left Ventricular Hypertrophy in Type 2 diabetes Mellitus. While treating patient with type 2 diabetes mellitus, physicians should pay special attention to non-conventional cardiovascular risk factors such as prolonged QT interval, an indicator of increased risk of sudden cardiac death. Supported by Higher Education Commission, Government of Pakistan 


\section{1}

Relationship of prolonged QTc interval with other risk factors than glycaemic control in individuals with type 2 diabetes

C. Bala, C. Nita, A. Rusu, G. Nicolescu, D. Giurgiuman, G. Roman, N. Hancu;

Department of Diabetes, Nutrition, Metabolic Diseases, Iuliu Hatieganu University of Medicine and Pharmacy, Cluj-Napoca, Romania.

Background and Aims: Ventricular myocardial depolarization and repolarization are reflected in QT interval. QT prolongation in patients with diabetes may contribute to ventricular fibrillation and sudden death. In individuals with diabetes, cardiac autonomic neuropathy is well known to be associated with QT prolongation. Hyperglycemia per se can also affect QT interval. The effect of other factors frequently associated with diabetes on the duration of QT interval is less investigated. The aim of the study is to examine the relationship between prolonged QTc interval and other risk factors such as waist circumference, microalbuminuria, lipid parameters, diabetes duration, and presence of hypertension.

Materials and Methods: 100 consecutive patients with diabetes attending the outpatient clinic from Clinical Center of Diabetes, Nutrition and Metabolic Diseases Cluj-Napoca have been included in a larger epidemiological study aiming to assess the prevalence of heart failure among persons with diabetes. A complete medical history, physical exam (weight, waist, blood pressure) and questionnaire for the presence of suggestive heart failure symptoms and signs have been performed. Blood sampling for HbAlc, lipid profile, a urine exam for microalbuminuria and ECG were performed for all patients who agreed to participate. Statistical methods consisted in a factor analysis followed by a linear regression model.

Results: The study group had a median age of 59 years (min 34, max 77 years), $60 \%$ females, median duration of diabetes 3 years (min 0 , max 13 years), stratification by antidiabetic treatment: $14 \%$ diet alone, $14 \%$ metformin, $10 \%$ sulphonylurea, $10 \%$ combined oral therapy, $45 \%$ insulin alone, $7 \%$ insulin+ oral therapy. $76 \%$ had metabolic syndrome according to IDF criteria. By factor analysis we extracted three factors that explained $86 \%$ of the variance in QTc interval [Factor 1 with positive loadings of diabetes duration, waist circumference, systolic blood pressure, and microalbuminuria, Factor 2 with positive loadings of age, HDL-cholesterol, presence of hypertension and Factor 3 with positive loadings of $\mathrm{HbAlc}$, total cholesterol and serum triglycerides]. In the linear regression model including the three factors identified, the model was significantly associated with QTc interval $(\mathrm{p}=0.017)$. Within the model, Factor 1 displayed a $\mathrm{p}$ value of 0.263 and was excluded from the analysis. The second regression included Factor 2 and 3, the model remaining statistical significant $(\mathrm{p}=0.01, \mathrm{~F}$ change 5,979 , significance of $\mathrm{F}$ change $=0.01$ ).

Conclusion: In a linear regression model, QTc interval was significantly associated with $\mathrm{HbAlc}$, HDL-cholesterol, serum triglycerides, presence of hypertension and total cholesterol. Our results suggest that controlling other factors than hyperglycaemia might result in less cases of sudden death in people with type 2 diabetes.

\section{2}

Fasting glucose predicts development of restenosis after PCI in patients with type 2 diabetes

C. A. Hage ${ }^{1}$, A. Norhammar ${ }^{1}$, N. Sarkar ${ }^{1}$, B. Svane ${ }^{1}$, L. Grip ${ }^{2}$, J. Öhrvik ${ }^{3}$, K. Malmberg ${ }^{3}$, L. Rydén

${ }^{1}$ Dep. of Cardiology, Karolinska University Hospital, Stockholm,

${ }^{2}$ Dept. of Cardiology, Sahlgrenska University Hospital, Göteborg, ${ }^{3}$ Dept. of

Medicine, Cardiology Unit, Karolinska Institutet, Stockholm, Sweden.

Background and Aims: Diabetic patients are at increased risk for restenosis after percutaneous coronary intervention (PCI). We investigated if insulin (I) based glucose control (GC) may reduce target lesion restenosis (TLRest) after PCI in patients with type 2 diabetes (T2DM).

Materials and Methods: 93 consecutive T2DM patients accepted for PCI were randomised to I-based GC (I-group; $\mathrm{n}=44$ ) or to continue ongoing treatment (C-group; $n=49$ ). Glucose target was fasting blood glucose (FBG) 5-7 mmol/ $\mathrm{L}$ and $\mathrm{HbAlc}<6.5 \%$. Information on TLRest after 6 months was available in 80 patients via angiography $(n=71)$ or angio and/or revascularisation before that time $(n=9)$ while a second angiography was not performed in 13 patients ( medical reasons $=5$; refusal $=6$; data missing $=2$ ).
Results: At baseline there was no difference in $\mathrm{HbAlc}$ (6.5 vs. 6.5\%) or FBG ( 7.0 vs. $7.4 \mathrm{mmol} / \mathrm{L}$; $\mathrm{p}=0.2$ ) in the I- and C-groups respectively. Physical findings, previous medical history or medication at baseline did not differ. At end of follow up HbAlc ( -0.2 vs. $-0.1 \%$; $=0.2)$ and FBG (+0.2 vs. $-0.3 \mathrm{mmol} /$ $\mathrm{L} ; \mathrm{p}=0.2$ ) did not change significantly in the I- and C- groups. TLRest did not differ after 6 months (I- vs. C-group $=39 \%$ vs. $43 \%$; $p=0.8$ ). Significant unadjusted predictors for TLRest were previous MI (OR 4.1; $\mathrm{p}=0.003)$ and previously known hyperlipidemia (OR 3.2; $\mathrm{p}=0.03$ ). FBG at baseline (OR 1.2; $\mathrm{p}=0.06$ ) was almost significant. In a multiple analysis including univariate predictors with $\mathrm{p}$-value $<0.2$, remaining predictors of restenosis were previous MI (OR 7.7; $\mathrm{p}=0.001)$ and FBG at baseline (OR1.5; $\mathrm{p}=0.013$ ).

Conclusion: Insulin based GC did not reduce TLRest in T2DM patients which, however, was predicted by baseline FBG. This suggests that future trials should target glucose normalisation and that the tool to achieve this seems to be of secondary importance.

\section{3}

The association between left ventricular mass and adipocytokine in type 2 diabetic patients

M. Harada, A. Sato, T. Onai, N. Aki, Y. Iwamoto;

Diabetes Center, Tokyo Women's Medical University, Tokyo, Japan.

Background and Aims: Left ventricular hypertrophy (LVH) is an independent risk factor for cardiovascular morbidity and mortality. It is well-known that obesity affects the development of LVH. Metabolic syndrome (Met S) results from the accumulation of abdominal fat which secretes adipocytokine, and composes atheroscrelosis which in turn leads to cardiovascular diseases. However, it is not clear whether adipocytokine has a direct effect on LVH. The purpose of this study is to identify the relationship between LV mass and adipocytokine in type 2 diabetic patients.

Materials and Methods: Type 2 diabetic patients without history of coronary disease, stroke, chronic kidney disease, or taking antihypertensive drug were recruited. Echocardiography was performed in 46 type 2 diabetes [ 30 men, age $51 \pm 12$ y.o., $($ mean $\pm S D)]$ and $L V$ internal diameter was measured by using M-mode, and fractional shortening and LV mass were calculated. LV mass was indexed by body surface area (LVMI) and body height to the power of 2.7. Met S was defined, according to the Japanese criteria of 2005, as a waist circumference level $\geq 85 \mathrm{~cm}$ in men and $\geq 90 \mathrm{~cm}$ in women, and two or three of the following three risk factors: hypertension (blood pressure level $\geq 130 / 85 \mathrm{mmHg}$ ), dyslipidemia (HDL cholesterol level $<40 \mathrm{mg} / \mathrm{dl}$ or TG level $\geq 150 \mathrm{mg} / \mathrm{dl}$ ), and glucose intolerance (fasting glucose level $\geq 110$ $\mathrm{mg} / \mathrm{dl})$. Subjects taking medications for any of the three components were also considered to have the corresponding risk factor. In addition, we also measured visceral $(\mathrm{V})$ subcutaneous(S) fat area and total fat area using computed tomography. Fasting plasma glucose, $\mathrm{HbA}_{1 \mathrm{c}}$, fasting insulin, serum total cholesterol, triglycerides, HDL cholesterol and LDL cholesterol were measured . Adipocytokine such as fasting leptin, adiponectin, total PAI-1, IL- 6 and TNF- $\alpha$ were measured. Atherosclelosis was evaluated through pulse wave velocity (PWV) and ankle-brachial index (ABI).

Results: The average metabolic parameters of 46 type 2 diabetes were BMI: $26 \pm 5 \mathrm{~kg} / \mathrm{m}^{2}, \mathrm{HbA}_{1} 9.0 \pm 2.0 \%$,waist circumference: $95.3 \pm 9.2 \mathrm{~cm}$,blood pressure: $124 \pm 11 / 77 \pm 10 \mathrm{mmHg}, \mathrm{TG}: 157 \pm 77 \mathrm{mg} / \mathrm{dl}$,and HDL-C: $43 \pm 11$ $\mathrm{mg} / \mathrm{dl}$. Met S was present in 29 patients (63\%). Patients with Met S had a

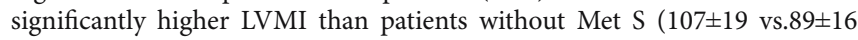
$\mathrm{g} / \mathrm{m}^{2}$, respectively). $(\mathrm{p}<0.05)$. LVMI was increased in the patients with the higher BMI and increased subcutaneous fat area $(\mathrm{p}<0.05)$. In contrast, LVMI had no correlation with visceral fat, V/S, blood pressure, lipid and waist circumference. Among adipocytokines, PAI- 1 and IL-6 level were significantly related to LVMI $(p<0.005)$. The increasing of LVMI tended to correlate with the increasing of leptin and the decreasing of adiponectin, although there was no significant correlation. The elevated IRI was the most significant factor for the LV mass increase. In multiple regression analysis, IL-6 and IRI were independent risk factors for increased LVMI.

Conclusion: Met $S$ was associated with the increased LV mass in type 2 diabetes. The results suggest that adipocytokine directly affects progression of LVH in type 2 diabetic patients with Met S. 


\section{4}

Macrovascular disease in type $\mathbf{1}$ diabetes in children and adolescents: subclinic atherosclerosis?

L. Trifone ${ }^{1}$, V. Osta ${ }^{2}$, A. Sardella ${ }^{3}$, H. Vazquez ${ }^{3}$, H. Cozzani ${ }^{4}$, V. Vaccarezza ${ }^{1}$; ${ }^{1}$ Nutrition and Diabetes Section, ${ }^{2}$ Central laboratory, ${ }^{3}$ Service of Cardiology, ${ }^{4}$ Radiology, Children Hospital R. Gutiérrez, Buenos Aires, Argentina.

Background and Aims: Diabetes mellitus is an established risk factor for atherosclerosis. Vascular complications of diabetes are not clinically evident in diabetic children. Aims: to detect subclinical atherosclerosis and risk factors in type 1 diabetic children and adolescents and its association with microangiopathyc complications, metabolic control and markers of subclinical inflammation

Materials and Methods: Ninety children and adolescents with type 1 diabetes were evaluated: medical history, familial history of obesity, high blood pressure (HBP), cardiovascular disease and dislypidaemia, diabetes duration, physical examination (weight and height, Tanner pubertal stage and blood pressure). Metabolic control: HbAlc; microangiopathyc complications: microalbuminuria and direct ophthalmoscopy. Laboratoy analysis: lipid profile, white blood cells count (WBC), fibrinogen and hs-CRP.Cardiovascular evaluation: electrocardiogram, color Doppler electrocardiography, tread mill and spect. Measurements of intimal-media thickness of both carotids with ultrasound scanning

Results: $51 \%$ (54) of the evaluated patients were male. Median age $15.4 \pm 3.0$ years. Overweight $12 / 90$ (13\%), diabetes duration $6.8 \pm 3$ years; HbAlc median $9.9 \pm 1.9 \%$, with $62,3 \%$ poor control ( $\mathrm{HbAlc}>8 \%$ ); high total cholesterol 10/82 (12\%), high tryglicerides 13/82 (16\%) combined dyslipidaemia 4/82(5\%), high blood pressure 5/89 (6\%), microalbuminuria $>20 \mu \mathrm{g} / \mathrm{min}$ $15 / 90$ (17\%); direct ophthalmoscopy normal in all.Hs-CRP > 3mg/l in 5\% (n); $1-3 \mathrm{mg} / \mathrm{l} 28.3 \%$ (n); fibrinogen $>400 \mathrm{mg} \% 28.8 \%$ and $\mathrm{WBC}>10 \times 10$ $3 / \mathrm{ml} 12.3 \%$. Abnormal ECG in 2/86. Diastolic dysfunction was present in $28 \%$ (19/67), 6 out of 19 were severe; tread mill was abnormal in 23\% (8/34)with hypertension during maximal effort ; Spect only in 27 patients all of them normal.Those patients with WBC $>10 \times 10^{3}$ had increase IM thickness $(\mathrm{p}<$ $0.05)$ as well as those with microalbuminuria $(\mathrm{p}<0.05)$. Tread mill induced hypertension was significantly associated with the increase in hs-CRP ( $<<$ $0.05)$. Basal hypertension was associated to fibrinogen $>400 \mathrm{mg} \%(\mathrm{p}<0.05)$. Conclusion: Early structural and functional cardiac alterations and elevated plasma markers of endothelial activation and injury are presents in children with diabetes. This data provides the best currently available non invasive marker of preclinical atherosclerosis in children with type 1 diabetes.

\section{PS 125 Epidemiology of cardio- vascular risk factors in diabetes}

\section{5}

Asymmetric dimethylarginine is associated with incident cardiovascular disease in the general population. The Hoorn Study

T. Teerlink ${ }^{1}$, R. J. Heine ${ }^{2}$, C. D. A. Stehouwer ${ }^{3}$, J. M. Dekker';

${ }^{1}$ Department of Clinical Chemistry, VU University Medical Center, Amsterdam, ${ }^{2}$ Department of Endocrinology / Diabetes Center, VU

University Medical Center, Amsterdam, ${ }^{3}$ Department of Internal Medicine, Academic Hospital Maastricht, ${ }^{4}$ Institute for Research in Extramural Medicine, VU University Medical Center, Amsterdam, The Netherlands.

Background and Aims: Asymmetric dimethylarginine (ADMA), an endogenous inhibitor of nitric oxide synthase (NOS), is a novel risk marker for cardiovascular disease (CVD). ADMA independently predicts the incidence of cardiovascular events in patients with renal failure and patients with coronary or peripheral artery disease. Only limited data exist regarding the association of ADMA and incident CVD in the general population. To address this question, we investigated the predictive value of ADMA in the Hoorn Study, a prospective community-based cohort study.

Materials and Methods: ADMA was measured by HPLC in baseline plasma samples from participants of the Hoorn Study (age 50 to 75 years). The main outcome measure was the combined incidence of fatal and non-fatal CVD in 1715 subjects ( 159 with type 2 diabetes) during a 10-year follow-up period. Results: In a multivariate linear regression model, higher age and BMI, and female sex and smoking were associated with higher levels of ADMA, whereas diabetes and higher glomerular filtration rate, serum albumin, and HDL-cholesterol were associated with lower levels. Together these variables explained only $10 \%$ of the variation in ADMA concentration. During followup, 379 subjects (63 with diabetes) experienced a cardiovascular event. Separate analyses for non-diabetic and diabetic individuals were performed, because of a significant interaction term in the Cox proportional-hazards models between ADMA and diabetes $(\mathrm{p}=0.002)$. After adjustment for age, sex and established CVD risk factors, the hazard ratio for CVD in the highest quintile of ADMA versus the combined lowest four quintiles was 1.38 (95\% CI, 1.08 to 1.77 ) in individuals without diabetes and 0.37 (95\% CI 0.17 to 0.81 ) in individuals with diabetes. In non-diabetic individuals the increased CVD risk associated with high ADMA did not significantly differ between subjects with normal and impaired glucose metabolism. Likewise, there were no significant differences in hazard ratios between males and females, smokers and non-smokers, and between individuals with and without prevalent CVD (all $\mathrm{p}>0.4$ ).

Conclusion: This study shows that in the general population a high plasma concentration of ADMA independently predicts CVD in individuals without type 2 diabetes. The inverse association between high ADMA and incidence of CVD in individuals with diabetes was unexpected and needs confirmation. Possibly, uncoupling of NOS, leading to a shift from NO production to superoxide production, plays a role in this paradoxical observation. As opposed to the anti-atherogenic effects of normally functioning NOS, uncoupled NOS accelerates atherosclerosis, and its inhibition by high ADMA may be beneficial.

Supported by Merck \& Co. Inc

\section{6}

Human complement $\mathrm{C} 3$ as a risk factor for coronary artery disease: relation with insulin, but not inflammation (the CODAM study) M. M. J. van Greevenbroek ${ }^{1}$, C. J. H. van der Kallen ${ }^{1}$, E. J. M. Feskens ${ }^{2}$, E. H. J. Jansen ${ }^{3}$, C. G. Schalkwijk ${ }^{1}$, C. D. A. Stehouwer ${ }^{1}$; also on behalf of the CODAM study group,

${ }^{1}$ Department of Internal Medicine / Cardiovascular Research Institute Maastricht, Maastricht University, ${ }^{2}$ Division of Human Nutrition, Wageningen University, Wageningen, ${ }^{3}$ Laboratory for Toxicology, Pathology en Genetics, National Institute for Public Health and the Environment, Bilthoven, The Netherlands.

Background: Complement $\mathrm{C} 3$ is an emerging risk factor for coronary artery disease (CAD), and several studies have indicated that their relation is independent of, at least some, other known CAD risk associates. 
Hypothesis: Since the larger part of plasma C3 is derived from liver and the presence fatty liver is, by itself, related to CAD, we hypothesize that the relation between $\mathrm{C} 3$ and $\mathrm{CAD}$ may be related to presence of fatty liver.

Materials: The relation between $\mathrm{C} 3, \mathrm{CAD}$ and an indicator of fatty liver (plasma ALT concentration) was studied in the CODAM study (Cohort on Diabetes and Atherosclerosis Maastricht). This cohort consists of 301 normal glucose tolerant, 127 impaired glucose tolerant and 146 type 2 diabetes mellitus (T2DM) subjects, invited from a large population-based cohort on basis of an elevated risk for T2DM. The relation between C3 and a selfreported history of CAD (defined as myocardial infarction, bypass surgery of the coronary arteries, balloon dilatation and / or stent placement) was evaluated by logistic regression. The relation between plasma C 3 and fatty liver was evaluated in linear regression analysis.

Results: Plasma C3 levels were significantly associated with increased $\mathrm{CAD}$ prevalence, also after adjusting for age, gender, smoking and glucose tolerance status (model 1, OR per unit C3 is 3.2 [95\% CI: 1.6-6.5], $\mathrm{p}<0.001$ ). Additional adjustment for known CAD risk factors showed that this relation was independent of: the metabolic syndrome, markers of inflammation (CRP, VCAM, ICAM, IL-6), markers of lipid metabolism (total and HDLcholesterol and triglycerides), and markers of an acute phase / complement response (C4, ceruloplasmin, haptoglobin, transferrin, serum amyloid A). It was, however, affected by fasting insulin level (OR C3 is 2.1 ([CI 95\% 1.0-4.6], $\mathrm{p}=0.06)$, with a significant contribution of insulin in this model $(\mathrm{p}=0.011)$. The partial correlation between C 3 and ALT was 0.364 ( $\mathrm{p}<0.001$; adjusted for age, gender, alcohol intake and glucose tolerance status). This association was affected by adjustment for fasting insulin but not, or considerably less, by adjustment for markers of inflammation, lipid metabolism, acute phase response / complement or kidney function. These data imply that the relation between insulin and $\mathrm{C} 3$ is affected by the presence of fatty liver, or vice versa. However, when the association between C3 and CAD (as shown in model 1) was additionally adjusted for ALT, the association between C3 and CAD was not substantially affected (OR is 3.2 [95\% CI: 1.5-6.8], $\mathrm{p}<0.002$ ).

Conclusion: Both the association between plasma C3 levels and CAD, and the relation between plasma C3 levels and fatty liver (as represented by plasma ALT) were, at least partly, explained by insulin and/or insulin resistance, but adjustment for ALT did not affect the relation between C3 and CAD. The data suggest that hyperinsulinemia and/or insulin resistance, rather than fatty liver by itself, explain part of the CAD risk associated with high levels of plasma C3.

Supported by grants of the Dutch Organisation for Scientific Research and the Dutch Diabetes Research Foundation

\section{7}

Low-grade inflammation and cardiovascular risk in the metabolic syndrome: the CODAM study

M. Jacobs ${ }^{1}$, M. M. J. van Greevenbroek ${ }^{1}$, C. J. H. van der Kallen ${ }^{1}$,

E. H. J. Jansen², E. E. Blaak ${ }^{3}$, E. J. H. Feskens ${ }^{4}$, C. G. Schalkwijk',

C. D. A. Stehouwer ${ }^{1}$; also on behalf of the CODAM study group,

${ }^{1}$ Department of Internal Medicine / CARIM, Maastricht University,

${ }^{2}$ Laboratory for Toxicology, Pathology and Genetics, National Institute for

Public Health and the Environment, Bilthoven, ${ }^{3}$ Department of Human

Biology / NUTRIM, Maastricht University, ${ }^{4}$ Human Nutrition, Wageningen

University, The Netherlands.

Background: The Metabolic Syndrome (MetS) is associated with an increased risk for cardiovascular disease and type 2 diabetes (T2DM). Abdominal obesity, dyslipidemia, hypertension and insulin resistance are the main determinants that define the MetS, but low-grade inflammation is also increasingly recognized as an underlying trait in the cardiovascular risk associated with the MetS.

Aim: To identify inflammation markers that are determinants of coronary heart disease (CHD) and to establish their association with the individual criteria of the metabolic syndrome.

Methods: The CODAM (Cohort study Diabetes and Atherosclerosis Maastricht) cohort was used. The cohort includes 574 subjects who were invited from a large population based cohort on basis of an elevated risk for T2DM. As determined by oral glucose tolerance test, 301 subjects were normal glucose tolerant, 127 were impaired glucose tolerant or had impaired fasting glucose levels and 146 had T2DM. Relevant anthropometric and blood parameters were determined. First, inflammation markers were identified that were elevated in subjects with a self-reported history of CHD. Subsequently, logistic regression analyses was used to determine if the relation between the inflammation markers and CHD was independent of the MetS. Finally, the relations between the inflammation makers and the individual determinants of the MetS were determined in linear regression analyses.

Results: C-reactive protein (CRP), interleukin 6 (IL6) and soluble vascular cell adhesion molecule (VCAM) were elevated in CHD. Logistic regression showed that the MetS was a significant determinant of CHD (odds ratio $\left[\mathrm{OR}_{\text {Mets }}\right]$ is $1.82, \mathrm{P}_{\text {Mets }}: 0.019$ adjusted for age, gender and glucose tolerance status). Addition of IL6 to the above described logistic regression model, reduced the association of MetS with $\mathrm{CHD}\left(\mathrm{OR}_{\mathrm{Mets}}: 1.62, \mathrm{P}_{\mathrm{Mets}}: 0.064 ; \mathrm{OR}_{\mathrm{IL} 6}\right.$ : $\left.3.05, \mathrm{P}_{\mathrm{IL}}: 0.005\right)$. Analysis with CRP resulted in similar results $\left(\mathrm{OR}_{\mathrm{Mets}}: 1.64\right.$, $\left.\mathrm{P}_{\text {Mets }}: 0.056 ; \mathrm{OR}_{\mathrm{CRP}}: 1.78, \mathrm{P}_{\mathrm{CRP}}: 0.061\right)$. On the other hand, addition of VCAM to the model did not affect the association of MetS and CHD ( $\mathrm{OR}_{\mathrm{Mets}}: 1.78$, $\left.\mathrm{P}_{\text {Mets }}: 0.026 ; \mathrm{OR}_{\mathrm{VCAM}}: 43.8, \mathrm{P}_{\mathrm{VCAM}}: 0.001\right)$. Multiple linear regression adjusted for age, gender and glucose tolerance status showed that CRP, IL6 and VCAM were all independently associated with lipids and HOMA-IR $\left(\mathrm{P}_{\mathrm{CRP}}: 0.039, \mathrm{P}_{\mathrm{IL}}\right.$ : $\left.0.035, \mathrm{P}_{\mathrm{yCAM}}<0.001\right)$. CRP $(\mathrm{P}<0.001)$ and IL6 $(\mathrm{P}<0.001)$ were also associated with waist circumference. Only CRP was related to blood pressure.

Conclusion: MetS was a significant determinant of CHD. This relation was reduced if CRP or IL6 were included in the model, and remained unaltered if VCAM was added. CRP and IL6 were mainly associated with waist and lipids, whereas VCAM was associated with insulin resistance and lipids. Our data suggest that low-grade inflammation may be an important pathway linking the MetS to CHD. Furthermore it suggests that the association of CRP and IL6 with CHD may occur via a different mechanism than between VCAM and CHD.

Supported by The Netherlands Organisation for Scientific Research and the Dutch Diabetes Research Foundation

\section{8}

Metabolic syndrome and goals of therapy for cardiovascular disease (CVD) risk

G. Bardini ${ }^{1}$, L. Pala ${ }^{1}$, B. Cresci ${ }^{1}$, M. Monami ${ }^{2}$, I. Targioni ${ }^{1}$, I. Sposato ${ }^{1}$, E. Mannucci ${ }^{2}$, C. M. Rotella ${ }^{1}$;

${ }^{1}$ Section of Endocrinology, Department of Clinical Pathophysiology,

${ }^{2}$ Geriatric unit, Department of Critical Care, University of Florence, Italy.

Background and Aims: Metabolic Syndrome (MS) is a cluster of risk factors for CVD. Recently, goals of therapy of blood pressure, glucose, and lipids were defined, categorizing patients with MS in 3 classes of Framinghambased estimates of $10 \mathrm{yr}-\mathrm{CVD}$ risk: low-to-moderate risk $(<10 \%)$, moderately high risk (10-20\%), and high CVD risk (>20\%). Aim of this study is to verify the achievement of treatment goals in patients with MS.

Materials and Methods: A cohort of 2945 individuals, with no history of diabetes, living in Florence and in the nearby Bagno a Ripoli municipality, enrolled in a screening program for diabetes, were studied. Of these, 672 (aged $60.5 \pm 9.7 \mathrm{yrs}$, BMI $29.5 \pm 4.0 \mathrm{~kg} / \mathrm{m}^{2}$ ) were affected by MS according to the revised (2004) National Cholesterol Education Program (NCEP) criteria. Anthropometric measures, smoking habits, glucose tolerance (120 minutes after a $75 \mathrm{~g}$ oral glucose load), lipid profile, and blood pressure were assessed. 10 yrs CVD risk was computed by Framingham score model based on sex, age, smoking, systolic and diastolic blood pressure, total HDL cholesterol, and diabetes.

Results: 281 (41.8\%) subjects had a low to moderate CVD risk, 187 (27.8\%) a moderately high risk, and 204 (30.3\%) a high CVD risk; normal weight was observed in $9.4 \%$ of subjects of the first class, $11.2 \%$ in the second class, and only $7.3 \%$ in the high risk class. Coronary heart disease (CHD) at enrolment was observed in $5.6 \%$ subjects. Considering LDL cholesterol, levels $<130$ $\mathrm{mg} / \mathrm{dl}$ were found in $47.0 \%$ and $44.9 \%$ subjects with low to moderate and moderately high risk, respectively, while LDL levels $<100 \mathrm{mg} / \mathrm{dl}$ were found in only $13.7 \%$ of high risk patients. CHD patients with $\mathrm{LDL}<70 \mathrm{mg} / \mathrm{dl}$ were $5.4 \%$, and those with non-HDL cholesterol $<100 \mathrm{mg} / \mathrm{dl}$ were $2.6 \%$. The prevalence of non-HDL cholesterol $<160 \mathrm{mg} / \mathrm{dl}$ was $46.3 \%$ in low risk individuals and $40.6 \%$ in subjects with moderately high risk class. Few patients were treated with lipid drugs as statins, fibrates or fish oil: $3.5 \%$ in the low risk, $4.3 \%$ in the medium risk class, and only $6.4 \%$ in the high risk class. Patients with blood pressure $<130 / 80 \mathrm{~mm} \mathrm{Hg}$ were $44.8 \%$ and $24.0 \%$ in low and moderately high risk, respectively, while high risk subjects with optimal blood pressure control were $14.7 \%$ only. $423(62.9 \%)$ subjects had normal glucose tolerance, 119 (17.7\%) impaired glucose tolerance, and 140 (20.8\%) type 2 diabetes; fasting plasma glucose $>100 \mathrm{mg} / \mathrm{dl}$ was found in $24.9 \%$ of low risk subjects, $18.2 \%$ 
of those with moderately high risk, and in $12.2 \%$ of high risk individuals. The higher prevalence of smokers was found in moderately high risk patients (24.6\%) and in high risk subjects (23.5\%), versus $13.2 \%$ of individuals with low CVD risk. Antiplatelet drugs were assumed in $4.5 \%$ of subjects with MS. Conclusion: Despite recommendations from guidelines, lipid lowering therapy is underutilized in subjects with MS, particularly among those at higher CVD risk and with known CHD. Similarly, blood pressure targets are seldom achieved in individuals with MS. Taken together, these data suggest that MS is not considered by many physicians as a condition of elevated CVD risk, requiring a special attention in the management of each individual risk factor.

\section{9}

\section{Diabetic dyslipidaemia: blood lipid profiles in $\mathbf{5 4 . 8 8 5}$ type 2 diabetic} patients

K. Eeg-Olofsson ${ }^{1}$, S. Gudbjornsdottir ${ }^{1}$, J. Cederholm², P. M. Nilsson ${ }^{3}$, B. Eliasson ${ }^{1}$, M. Eriksson ${ }^{4}$.

${ }^{1}$ Department of Medicine, Goteborg University, ${ }^{2}$ Department of Public Health and Caring Sciences, Family Medicine and Clinical Epidemiology Section, Uppsala, ${ }^{3}$ Department of Clinical Sciences Medicine, Division of Medicine, Lund, ${ }^{4}$ Department of Medicine and Molecular Nutrition Unit, Karolinska Institutet at Karolinska University Hospital in Huddinge, Stockholm, Sweden.

Background and Aims: Dyslipidemia is considered an integral part of the insulin resistance syndrome associated with type 2 diabetes and a strong risk factor for cardiovascular morbidity and mortality. The aim of this study was to describe blood lipid profiles in a large cohort of type 2 diabetic patients and to examine the role of gender, age, and association with cardiovascular risk factors such as obesity.

Material and Methods: Blood lipid values from 26413 patients without and from 28472 with lipid lowering agents (LLA) were included in the study based on yearly registrations of clinical data to the Swedish National Diabetes Register (NDR). Around one third of all diabetic patients in Sweden were reported to NDR in 2005.

Results: Patients treated with LLA (48\%) had lower total and LDL-cholesterol (4.6 vs. $5.1 ; 2.5$ vs. $3.0 \mathrm{mmol} / \mathrm{L}$, respectively), but slightly higher triglycerides (TG) than the untreated patients ( $1.7 \mathrm{vs} 1.6 \mathrm{mmol} / \mathrm{L})$. HDL levels were similar in the two groups ( $1.34 \mathrm{vs} 1.37 \mathrm{mmol} / \mathrm{L}$ ) (all differences $\mathrm{p}<0.001$ ). $56 \%$ of the patients treated with LLA reached LDL $<2.5 \mathrm{mmol} / \mathrm{L}$ and $28 \%$ reached LDL $<2.0 \mathrm{mmol} / \mathrm{L}$.

In patients not treated with LLA, women exhibited higher total, LDL, and HDL cholesterol levels as well as TG than men. Total and LDL cholesterol increased with age in women, and HDL levels were highest in the oldest patients. In men, LDL levels were highest between 40-60 years of age. In men and women, total and LDL cholesterol were highest in patients with BMI 2539 , while TG increased and HDL decreased with higher BMI. Patients with low HDL are obese, smoke, exhibit microalbuminuria and are more often treated with blood pressure lowering agents.

Conclusion: In this large type 2 diabetic population, TG and HDL levels are surprisingly normal, HDL being higher in women than in men at all ages. However, many patients are not treated with LLA and many treated patients do not reach the LDL goal. The results from this study raise questions: how should type 2 diabetic patients be treated adequately; is it justifiable to adopt the current goals that we have for all high risk individuals to patients with type 2 diabetes? Indirectly these results support the importance of glucose control in order to reduce glucosylation of the different lipoprotein fractions.

\section{0}

Characteristics of individuals with dyslipidaemia and hyperglycaemia in Shanghai communities

Y. Bao, W. Jia, J. Lu, J. Zhou, M. Yang, J. Pan, K. Xiang;

Shanghai Diabetes Institute, Department of Endocrinology and Metabolism, Shanghai Clinical Center for Diabetes, The Sixth People's Hospital Affiliated to Shanghai Jiao Tong University, Shanghai, China.

Background and Aims: To investigate the distribution of different kinds of dyslipidemia and characteristics among individuals with dyslipidemia and hyperglycemia in Shanghai communities.
Materials and Methods: Totally 5628 individuals aged over 20 years old were enrolled. According to the 1997's Recommendations for Prevention and Treatment of Dyslipidemia in China, the prevalences of different kinds of dyslipidemia were analyzed. The clinical characteristics of subjects with dyslipidemia and hyperglycemia were studied. All the individuals were divided into four groups, normal ones, isolated dyslipidemia (iDYL), iDYL with impaired glucose tolerance (IGR) and iDYL with diabetes mellitus (DM). Subjects with hypertension or overweight/obesity were excluded.

Results: 1 .The prevalence of dyslipidemia was $71.78 \%$. The most common kind of dyslipidemia washypertriglyceridemia with hypercholesterolemia/highlowdensity lipoprotein cholesterol (LDL-C) and isolated hypercholesterolemia/ high LDL-C. 2.The frequency of dyslipidemia increased with age in both men and women $(P$ for trend $<0.01)$. 3.The frequencies of IGR and DM in individuals with dyslipidemia were significantly higher compared with those with normolipidemia $(P<0.01)$. 4. Isolated postprandial hyperglycemia was the most common kind of hyperglycemia in the individuals with dyslipidemia and hyperglycemia. The frequency of dyslipidemia was $74 \%$ in IGR and 50\% in DM. 5.Body mass index (BMI), waist circumference (W), blood pressure, fasting plasma glucose (FPG), postprandial glucose (2hPG), fasting insulin (FIN), postprandial insulin (2hINS), C-reactive protein (CRP) and HOMAIR in iDYL group were all higher than normal group $(P<0.01)$. Age, BMI, W, TG, FIN, 2hINS, CRP and HOMA-IR in IDYL with IGR and iDYL with DM were higher than iDYL $(P<0.01)$. HOMA-IR in iDYL with DM further increased compared with iDYL with IGR.

Conclusion: 1.The prevalence of dyslipidemia in community was relatively high. 2 . The frequency of hyperglycemia in the individuals with dyslipidemia was higher. The most common kind of hyperglycemia in the individuals with dyslipidemia and hyperglycemia was isolated postprandial hyperglycemia. 3 . Total body fat, regional body fat, and CRP in the subjects with dyslipidemia increased independent of hyperglycemia. Total body fat and regional body fat further increased among the subjects with both dyslipidemia and hyperglycemia. 4. Individuals with dyslipidemia had insulin resistance. The extent of insulin resistance further increased in individuals with both dyslipidemia and hyperglycemia.

Supported by Shanghai Medical Developing Grant, Research Grant of Shanghai Educational Committee

\section{1}

Increase of gamma-glutamyltransferase as a predictor of established cardiovascular risk factors and diabetes in Japanese adults

S. Jimba ${ }^{1}$, T. Nakagami ${ }^{1}$, J. Oya ${ }^{1}$, T. Wasada ${ }^{2}$, Y. Iwamoto'; ${ }^{1}$ Diabetes Center, Tokyo Women's Medical University, Tokyo, ${ }^{2}$ Department of Internal Medicine, Saitama-ken Saiseikai Kurihashi Hospital, Saitama-ken, Japan.

Objective: Recently, associations between fatty liver disease and risk of cardiovascular diseases (CVD) have been accumulated. While Nakanishi et al. have reported that gamma-glutamyltransferase (GGT) is an important predictor for CVD and diabetes (DM) in Japanese. However, subjects with fatty liver disease existed in those with normal liver enzymes in our study. Thus, we assess the relation between the changes in GGT over 3-years and the development of DM, impaired fasting glucose (IFG), dyslipidemia and hypertension (HT), in Japanese.

Subjects and Methods: A total of 1,950 subjects were enrolled in a general health examination programme at SSK Hospital from Sep '02 to Feb '03. Among them 784 subjects who had pharmacological treatment for DM, HT or dyslipidemia were excluded. Of the remaining 1,166 subjects at baseline, 1,004 subjects (mean age: $48 \pm 7$ years old) were followed up until Oct 2006 (follow-up rate: $85.1 \%$ ). The relation between 3 -year change in GGT against baseline and 3-year changes in anthropometric and metabolic variables were examined by Spearman's correlation coefficients. Subjects were classified into three groups according to GGT changes, such as an increased $(>+5 \mathrm{U} /$ $\mathrm{L})$, unchanged $(-5$ to $+5 \mathrm{U} / \mathrm{L})$ or decreased $(<-5 \mathrm{U} / \mathrm{L})$ group. The logistic regression model was used to analyse the relation between changes in GGT levels and incident of DM (fasting plasma glucose [FPG] $\geq 7.0 \mathrm{mmol} / \mathrm{l}$ ), IFG (FPG: 6.1-6.9 mmol/l), HT (systolic $[\mathrm{sBP}] \geq 130 \mathrm{mmHg}$ and/or diastolic blood pressure $[\mathrm{dBP}] \geq 85 \mathrm{mmHg}$ ), hypertriglycemia (triglyceride $[\mathrm{TG}] \geq 1.69$ $\mathrm{mmol} / \mathrm{L}$ ), low HDL-cholesterol (HDL-C $<1.03 \mathrm{mmol} / \mathrm{L}$ ), and obesity (BMI $\geq 25 \mathrm{~kg} / \mathrm{m}^{2}$ ).

Results: The median follow-up period was 3 years and the average of 3year GGT change was $-4.56 \pm 33.86 \mathrm{IU} / \mathrm{L}$. The 3 -year GGT change was 
significantly correlated with baseline values for GGT (correlation coefficient: $-0.319)$, AST (-0.250), ALT (-0.272), BMI (-0.148), FPG (-0.078), uric acid $(-0.174)$, triglyceride $(-0.121)$, sBP $(-0.097)$ and $\mathrm{dBP}(-0.103)$. Moreover, the 3-year GGT change was positively correlated with the 3-year change in ALT (0.586), BMI (0.388), FPG (0.178), UA (0.241), TG (0.196), sBP (0.110) and $\mathrm{dBP}(0.132)$. The proportion of subjects with hypertriglycemia, lowHDL-cholesterol, obesity, IFG, and HT at baseline was $24.1 \%, 5.3 \%, 29.8 \%$, $7.2 \%$, and $24.8 \%$. Whereas the proportions of people who newly developed DM, hypertriglycemia, low-HDL cholesterol, obesity, IFG and HT were: $1.7 \%$ (16/967), 10.0\% (74/743), 7.4\% (69/929), 6.2\% (43/689), 6.3\% (57/912) and $29.7 \%(219 / 737)$, respectively. The group of subjects with an increased GGT over 3 years had an increased risk of developing hypertriglycemia, HT, obesity, IFG and DM, in comparison to those with a decreased GGT, with odds ratio of 5.78 (95\% confidence interval: 2.96-11.29), 2.02 (1.23-3.31), 8.81 (2.66-29.22), 5.10 (2.45-10.62) and 5.76 (1.82-18.18), after adjusting for age and sex, respectively. Further additional adjustment for alcohol habits, the 3-year change in BMI, and smoking did not change these associations except HT and obesity.

Conclusions: Increase of GGT over 3 years seems to be an independent predictor of newly developed DM, IFG, and hypertriglyceride in Japanese. It may be important to pay attention to increase of GGT as well as GGT level to prevent future CVD at primary health care settings.

This analysis has been carried out with the support of a grant from the Japan Medical Women's Association for T.N.

\section{2}

Population-based assessment of diabetes and major cardiovascular risk factors among adults in Syria (AlepDiab study)

N. Albache;

Diabetes Research, Aleppo University, Syrian Arab Republic.

Background: The prevalence of Diabetes is increasing in many countries in the world, particularly in some countries like the Arab gulf countries where the prevalence of DM reaches a high level. Syria, a country of Middle East with 18 million inhabitants, was also witness to a lifestyle modification \& diet changes, with probably an increase in the prevalence of DM as well other metabolic diseases during the last decades. We still lack population-based standardised prevalence to estimate the prevalence of diabetes and major cardiovascular disease CVD risk factors. We need also to have an accurate and updated idea about the metabolic diseases in general in Syria and to input that data on a regional map.

Objectives: The aim of this study is to estimate the population-based prevalence of diabetes and associated disorders and cardiovascular risk factors including obesity, hypertension and lipid profile abnormalities.

Methods: The first cross-sectional household survey on diabetes mellitus prevalence and associated risk factors among people aged $\geq 25$ years residing is Aleppo-Syria. It was carried out in 2006 involving 1168 household representatives ( $51.1 \%$ men, mean age is $44.6 \pm 10.7$ years). The study included a household interview of administration of computerized questionnaires and physical examinations (blood pressure, height, weight and waist and hip circumferences) and blood examination included fasting blood glucose, $\mathrm{HbAlc}$, lipid profile and CRP. Response rate for completed blood examination was $69.1 \%$. The data was standardised for age and gender.

Results: The prevalence of previously diagnosed diabetes was $8.9 \%$ and undiagnosed diabetes was $5.5 \%$, so $14.5 \%$ of participants had diabetes. The prevalence of impaired fasting glucose was $25.6 \%$. The prevalence of obesity, central obesity and hypertension was $43.9 \%, 72.4 \%, 46.9 \%$ respectively. Serum total cholesterol and triglyceride were high in $22.4 \%, 42.9 \%$ respectively. Mean values (SD) in CRP concentrations were $2.13(0.87) \mathrm{mg} / \mathrm{L}$ for men and $2.20(1.14) \mathrm{mg} / \mathrm{L}$ for women. There was a significant difference in CRP levels between men and women $(\mathrm{t}=1.095, \mathrm{p}<0.05)$.

Conclusions: AlepDiab, which is the first national study on diabetes prevalence in Syria, demonstrated that Syria has a high prevalence of diabetes (higher than published on Diabetes Atlas 2006) and associated risk factors. This increase could be due to the rapid urbanization and occidentalization of lifestyle. More studies in other urban and rural Syrian regions may be needed to confirm these results.

\section{PS 126 Cardiovascular risk in diabetes: prospective studies}

\section{3}

After a high-fat mixed meal, postprandial hyperglycaemia, but not postprandial insulin, impacts postprandial increases in inflammatory markers and nitrosative stress in patients with type 2 diabetes

A. Ceriello ${ }^{1}$, W. V. Brown ${ }^{2}$, N. A. Le ${ }^{2}$, R. B. Goldberg ${ }^{3}$, P. J. Beisswenger ${ }^{4}$, J. Cooke , D. C. Robbins ${ }^{6}$, R. G. Moses , H. B. Yuan ${ }^{8}$, S. Sarwat ${ }^{8}$, C. A. Jones ${ }^{8}$, Z. Milicevic ${ }^{8}$, M. H. Tan

${ }^{1}$ Dept of Clinical Sciences, Warwick Medical School, United Kingdom, ${ }^{2}$ Dept of Medicine, Emory University, Atlanta, United States, ${ }^{3}$ School of Medicine, University of Miami, United States, ${ }^{4}$ Section of Endocrinology, Diabetes, and Metabolism, Dartmouth Medical School and DartmouthHitchcock Medical Center, Hanover, United States, ${ }^{5}$ School of Medicine, Stanford University, United States, ${ }^{6}$ Section of Endocrinology, Kansas University Medical School, Kansas City, United States, ${ }^{7}$ Illawarra Diabetes Service, Wollongong Hospital, SESIAHS, Sydney, Australia,

${ }^{8}$ Eli Lilly and Company, Indianapolis, United States.

Background and Aims: In patients with type 2 diabetes (T2D), blood glucose (BG), serum insulin (INS), hs-C reactive protein (hs-CRP), tumour necrosis factor $\alpha$ (TNF- $\alpha$ ), interleukin 6 (IL-6) and nitrotyrosine (NT) increase in the postprandial (pp) period after a high-fat mixed meal. We studied the impact of pp BG or pp INS on pp increase in inflammatory markers (pp hs-CRP, pp TNF- $\alpha$, pp IL-6) and nitrosative stress (pp NT) in T2D patients.

Materials and Methods: A subgroup of T2D patients participating in a prospective, open-label, parallel group study comparing the effects of prandial+basal lispro mix 50 (LM50; 50\% lispro/50\%insulin lispro protamine suspension) ac tid with basal insulin glargine $(G)$ hs, both plus metformin (Met) participated in the test-meal substudy after 24 weeks of treatment. The night before the test meal, patients (LM50+Met, $n=25 ; \mathrm{G}+$ Met, $\mathrm{n}=21$ ) ate their supper, took their assigned insulin, and fasted overnight. Met was taken 30 min and LM50 injected 5 min before a standardized McDonald's $\mathrm{s}^{\circledR}$ breakfast (fat $39 \mathrm{~g}, \mathrm{CHO} 78 \mathrm{~g}$, protein $24 \mathrm{~g}$ ) followed by sampling at $0,1,2,3,4,6,8 \mathrm{hrs}$. The pp increase was measured as area under the curve (AUC) and AUC excursion above baseline 0-8 hours. Multiple linear regression approach was applied to assess the possible independent effect of pp BG or pp INS (independent variables) on pp hs-CRP, pp TNF- $\alpha$, pp IL- 6 and pp NT (dependent variables) respectively after adjusting for all other covariates (HbAlc, fasting BG/INS, age, gender and BMI). Hs-CRP, TNF- $\alpha$, IL- 6 and NT were measured by ELISA.

Results: The pp BG AUC and AUC excursion were significantly associated with pp hs-CRP, pp TNF- $\alpha$, pp IL- 6 and pp NT AUC and AUC excursion. The strength of the associations was weak for pp INS (see table).

\begin{tabular}{|c|c|c|c|c|c|c|}
\hline Variable & $P p B G$ & R square & p-value & $p p B G E x c$ & $\mathrm{R}$ square & p-value \\
\hline$p p h s-C R P$ & & 0.54 & $<0.001$ & & 0.63 & $<0.001$ \\
\hline$p p$ TNF- $\alpha$ & & 0.30 & 0.018 & & 0.41 & 0.009 \\
\hline$p p I L-6$ & & 0.57 & $<0.001$ & & 0.31 & 0.003 \\
\hline \multirow[t]{2}{*}{$p p N T$} & & 0.48 & $<0.001$ & & 0.43 & $<0.001$ \\
\hline & $p p$ INS & & & pp INS Exc & & \\
\hline$p p h s-C R P$ & & 0.23 & 0.06 & & 0.27 & 0.013 \\
\hline$p p$ TNF- $\alpha$ & & 0.10 & 0.29 & & 0.23 & 0.016 \\
\hline$p p I L-6$ & & 0.18 & 0.14 & & 0.15 & 0.14 \\
\hline$p p N T$ & & 0.13 & 0.28 & & 0.21 & 0.016 \\
\hline
\end{tabular}

Conclusion: In T2D patients after a high-fat mixed meal, the pp increases in inflammatory markers (pp hs-CRP, pp TNF- $\alpha$, pp IL-6) and nitrosative stress (pp NT) were strongly impacted by the increase in pp BG but not by the increase in pp INS. Control of pp BG may be important in decreasing the pp inflammation and nitrosative stress seen in T2D patients.

Supported by Eli Lilly and Company 


\section{4}

The Munich Myocardial Infarction Registry: impact of CRP and creatinine on hospital mortality

K. F. Braun ${ }^{1}$, E. Standl ${ }^{1}$, W. Otter ${ }^{2}$, O. Schnell';

${ }^{1}$ Department for Cardiovascular Disease, Diabetes Research Institute, Munich, Oberschleißheim, ${ }^{2}$ Department of Cardiology, Center of Internal Medicine, Unterschleißheim, Germany.

Background and Aims: To study the impact of CRP and creatinine in diabetic (D) and non-diabetic patients (ND) with acute myocardial infarction (AMI) on hospital mortality.

Materials and Methods: All patients of the Munich Myocardial Infarction Registry $(1999-2004, \mathrm{n}=2015)$ were assessed. In both D $(38.2 \%)$ and ND (61.2\%), CRP and creatinine levels on admission were analyzed with regard to hospital outcome.

Results: In the entire group of patients with AMI, hospital mortality was $15.2 \%(n=307)$. The median CRP level was $7 \mathrm{mg} / \mathrm{dl}$. In patients with CRP levels above the median, hospital mortality was higher compared to those with CRP levels equal or below the median $(21 \%$ vs. $9 \%$; $p<0.001)$. The median CRP level was higher in D than in ND: 9 vs. $6 \mathrm{mg} / \mathrm{dl}(\mathrm{p}<0.001)$. In both $\mathrm{D}$ and ND, CRP levels remained a significant predictor for hospital mortality after correction for age, impaired kidney function $(\mathrm{IKF}=$ creatinine $>1.2 \mathrm{mg} / \mathrm{dl})$ and hypertension: OR (95\% CI) highest vs. lowest CRP quintile: D $1.9(1.1-3.2)(\mathrm{p}<0.05)$, ND $2.4(1.4-4.3)(\mathrm{p}<0.01)$. In the entire group, 864 patients $(66.7 \%)$ had normal creatinine levels $(\leq 1.2 \mathrm{mg} / \mathrm{dl})$. IKF occurred in 432 patients (33.3\%). Hospital mortality was higher in patients with IKF than in those with normal creatinine levels $(31.5 \%$ vs. $6.9 \%$; $<<0.001)$. Creatinine levels were higher in D than in ND: $1.4 \pm 0.9 \mathrm{mg} / \mathrm{dl}$ vs. $1.1 \pm 0.7$ $\mathrm{mg} / \mathrm{dl}(\mathrm{p}<0.001)$. In both, $\mathrm{D}$ and ND, creatinine levels remained a significant predictor for hospital mortality after correction for age, CRP levels and hypertension: highest vs. lowest quintile: D $3.6(2.1-6.4)(\mathrm{p}<0.001)$, ND 4.9 $(2.8-8.7)(\mathrm{p}<0.001)$. In patients with CRP levels equal or below the median, absence of diabetes and IKF, hospital mortality was $4.4 \%$ whereas in patients with CRP levels above the median, presence of diabetes and IKF, hospital mortality increased significantly to $37.9 \%(\mathrm{p}<0.001)$.

Conclusion: The Munich Myocardial Infarction Registry emphasises the impact of CRP and creatinine levels on the hospital mortality of AMI. It demonstrates that the combined presence of diabetes, elevated CRP and creatinine on admission is associated with a highly increased hospital mortality in AMI. An intensification of early diagnostic and therapeutic approaches is required to potentially overcome the unfavourable prognosis.

\section{5}

Familial predisposition to cardiovascular disease contributes to the prevalence of cardiovascular risk and cardiovascular disease - from baseline features of a diabetic large-scale multicenter-based cohort H. Yokoyama, K. Kawai, H. Sugimoto, K. Hirao, H. Takeda, M. Oishi, M. Minami, M. Kudo, K. Doi, K. Iwasaki, Y. Fukumoto, H. Sone, M. Matsushima, H. Takagi, M. Kobayashi;

Japan Diabetes Data Management Study Group, Jiyugaoka Clinic, Obihiro, Japan.

Background and Aims: Diabetic population is affected by 2-6 folds higher cardiovascular morbidity and mortality than non-diabetic population. Effects of cardiovascular risk factors cannot fully account for the excess morbidity and effects of genetic predisposition remain elucidated.

Materials and Methods: A diabetic large-scale $(n=3611)$ multicenter-based (17 medical clinics (i.e. general practitioners) or general/university-affiliated hospitals from different areas in Japan) cohort was recruited. Cardiovascular risk factors, a first-degree family history $(\mathrm{FH})$ of diabetes, hypertension, coronary heart disease (CHD) and stroke, and a previous history of CHD, stroke (ischemic cerebrovascular disease), and peripheral vascular disease (PVD) were investigated. Cross-sectional associations of risk factors with prevalence of cardiovascular disease were explored by multiple logistic regression analysis.

Results: Among 3611 diabetic patients, 181 (5.0\%) had CHD, 118 (3.3\%) had stroke, and 37 (1.0\%) had PVD; accounting for 315 patients (8.7\%) who had at least one of them, i.e. cardiovascular disease. Age, male, hypertension, hyperlipidemia, and smoking were significant determinants of the prevalence of cardiovascular disease. After adjustment for these conventional cardiovascular risk factors, $\mathrm{FH}$ of $\mathrm{CHD}$ was independently associated with $\mathrm{CHD}$ and $\mathrm{FH}$ of stroke was independently associated with stroke. FH of hypertension was significantly associated with presence of hypertension. FH of diabetes, hypertension, $\mathrm{CHD}$ and stroke was significantly associated with $\mathrm{FH}$ of each disease, indicating clustering of $\mathrm{FH}$.

Conclusion: $\mathrm{FH}$ of $\mathrm{CHD}$ and stroke is an independent determinant of the prevalence of $\mathrm{CHD}$ and stroke, respectively. Clustering of $\mathrm{FH}$ of diabetes, hypertension, CHD, and stroke may indicate interrelation of genetic predisposition.

\section{6}

Use of oral contraceptives and coronary artery disease in type 1 diabetes: The Pittsburgh Epidemiology of Diabetes Complications (EDC) Study T. Costacou, T. J. Orchard;

Epidemiology, University of Pittsburgh, United States.

Background and Aims: Results from the Women's Health Initiative, a large clinical trial, raised concerns that hormone replacement therapy may pose increased health risks, including increased heart disease. Oral contraceptives (OC) may also result in the activation of the renin angiotensin system, increasing renal disease risk. As women with type 1 diabetes are often prescribed OC, there is concern that such use may further increase the already elevated risk of coronary artery disease (CAD) in these women. Thus, we assessed the association between use of OC and CAD incidence in a cohort of women with type 1 diabetes.

Materials and Methods: Female participants of the Pittsburgh Epidemiology of Diabetes Complications study, a prospective investigation of childhood onset type 1 diabetes, were selected for study (baseline mean age 27 and diabetes duration 18 years). Two hundred ninety-nine women free of CAD (defined as EDC physician diagnosed angina, confirmed myocardial infraction, stenosis $\geq 50 \%$, ischemic ECG, or revascularization) provided information on OC use throughout 16 years of follow-up. Repeated measures mixed model logistic regression analyses with a binomial error distribution and a logit link function were conducted.

Results: At baseline, adjusting for age, OC users were more likely to report having ever smoked ( $\mathrm{p}=0.005)$; no other differences were observed. During follow-up, higher blood pressure $(\mathrm{p}<0.0001)$, non-HDL cholesterol $(\mathrm{p}=0.002)$, pulse rate $(\mathrm{p}=0.05)$, albumin excretion rate $(\mathrm{p}=0.006)$, and white blood cell count $(\mathrm{p}=0.03)$ but lower HDL $(\mathrm{p}=0.07)$ and insulin sensitivity $(p<0.0001)$ were observed in those women who subsequently developed CAD $(n=62)$ compared to non-cases $(n=237)$. Moreover, compared to noncases, a greater proportion of subsequent $\mathrm{CAD}$ cases had overt nephropathy $(\mathrm{p}=0.02)$ and proliferative retinopathy $(\mathrm{p}=0.02)$. However, the proportion of OC users did not differ by subsequent CAD status $(\mathrm{p}=0.97)$ and OC use was not associated with $\mathrm{CAD}$ incidence $(\mathrm{OR}=1.32,95 \% \mathrm{CI}=0.77-2.25)$ in multivariable analyses.

Conclusion: Among women with type 1 diabetes, there was no association between OC use and CAD. However, as length of use was relatively short (mean length of use among users $=4$ years, median $=3$ years), definitive conclusions on the effects of long term OC use cannot be made. Spported by NIH

\section{7}

A study of cardiovascular risk management in adult patients with type 1 diabetes mellitus in Birmingham, United Kingdom

A. A. Syed ${ }^{1}$, S. Hussain ${ }^{1}$, A. H. Barnett ${ }^{2}$, M. Charlton ${ }^{2}$, P. De ${ }^{3}$, P. Narendran ${ }^{1}$; ${ }^{1}$ Division of Medical Sciences, University of Birmingham, ${ }^{2}$ Department of Diabetes, Heart of England NHS Trust, Birmingham, ${ }^{3}$ Department of Diabetes, City Hospital NHS Trust, Birmingham, United Kingdom.

Background and Aims: Cardiovascular disease (CVD) is the commonest cause of death in adult patients with type 1 diabetes (T1D). Aggressive management of CVD risk factors is therefore an important aspect of the care we provide for them. We studied the management of cardiovascular risk factors adult T1D patients attending hospital outpatient diabetes clinics in the City of Birmingham, UK.

Materials and Methods: Data was collected between June 2006 to February 2007 for age, T1D duration, smoking history, BP, BMI, lipids and HbAlc. 
CVD risk factor targets were those recommended by Joint British Societies 2005

Results: 1282 patients were analysed with mean age 46yrs (range 18-95). A minimum dataset, defined as BP, total cholesterol, smoking history and $\mathrm{HbA} 1 \mathrm{c}$, was available in $72 \%$ of patients, of whom only $1.3 \%$ achieved all 4 targets (BP130/80, total cholesterol 4mmol/1, not smoking, HbA1c 6.5\%). Using the previously applied criteria of HbA1c 7.5\%, BP 140/90, total cholesterol $5 \mathrm{mmol} / \mathrm{l}, 14 \%$ of patients achieved all 4 targets. BP, HbA1C, cholesterol, smoking status and BMI was documented in 93\%, 95\%, 94\%, 84\% and $74 \%$ respectively. Overall 56\%, 93\%, 63\%, $24 \%$ and $60 \%$ failed to achieve JBS 2005 targets for BP, HbA1C, total cholesterol, smoking and BMI $(<25 \mathrm{Kg} /$ $\mathrm{m}^{2}$ ) respectively. Patients over the age of $40 \mathrm{yrs}$ were less likely to achieve target blood pressure than younger patients (64\% Vs $41 \%)$. Otherwise there was no difference in target attainment. Of those patients over the age of $40,43 \%$ were not on lipid lowering therapy, and 58\% were not on aspirin, even though these are now recommended therapies. $19 \%$ of patients had microalbuminuria. Of these, $62 \%, 93 \%, 66 \%, 22 \%$, and $57 \%$ failed to achieve targets for $\mathrm{BP}, \mathrm{HbA} 1 \mathrm{C}$, total cholesterol, smoking and BMI respectively. Of those patients who failed to achieve lipid targets, 59\% were not on lipid treatment.

Conclusion: Cardiovascular risk factors are poorly recorded and managed in T1D patients. The low target attainment may be explained by complications of therapy, for example hypoglycaemia preventing attainment of HbAlc of $6.5 \%$; however acquisition and recording of data can and should be improved. We conclude that better data acquisition and treatment of CVD risk factors in T1D patients is required, and suggest in the long term this will reduce their macrovascular complications and mortality rates. Patients over the age of 40 , and those with microalbuminurea in particular need to be targeted.

\section{8}

\section{WITHDRAWN}

\section{9}

Five-year mortality in congestive heart failure patients with undiagnosed diabetes

J. Flores ${ }^{1}$, J. Comin ${ }^{2}$, J. F. Cano ${ }^{1}$, D. Benaiges ${ }^{1}$, J. J. Chillaron ${ }^{1}$, A. Goday ${ }^{1}$, J. Bruguera ${ }^{2}$, M. Fernandez ${ }^{1}$, J. Puig ${ }^{1}$;

${ }^{1}$ Endocrinology, Hospital del Mar,

${ }^{2}$ Cardiology, Hospital del Mar, Barcelona, Spain.

Background and Aims: Little is known about the prevalence and prognostic importance of undiagnosed diabetes mellitus (UDM) among individuals with heart failure (HF). To determine five-year mortality rate in heart failure hospitalized patients suffering UDM. To assess the prevalence of UDM and the relevant clinical parameters of this population. To evaluate the number of hospital re-admissions during a one year period due to cardiovascular and non-cardiovascular events.

Materials and Methods: A prospective study of patients admitted in the Heart Failure Unit of our hospital between January-2000 and December-2002. Patients suffering diagnosed DM at the time of admission were identified as well as those fulfilling the diagnosis criteria for diabetes according to ADA (outside the acute complication episode). A five-year survey was carried out. Results: During the study period, 410 patients were admitted at the Heart Failure Unit. The prevalence of diagnosed diabetes (DDM) was 36,8\% against $16 \%$ for UDM.

\begin{tabular}{lllll} 
& Non Diabetic & $\begin{array}{l}\text { Undiagnosed } \\
\text { diabetes }\end{array}$ & $\begin{array}{l}\text { Diagnosed } \\
\text { diabetes }\end{array}$ & $\mathrm{p}$ \\
\hline $\mathrm{N}$ & 193 & 66 & 151 & \\
Age (years,mean+SD) & $71,7(+-10,9)$ & $72,9(+-10)$ & $71,5(+-6,5)$ & n.s. \\
Sex (female \%) & 49,2 & 54,4 & 52,5 & n.s. \\
Ever smoker (\%) & 41 & 42.7 & 43,2 & n.s. \\
Hypertension (\%) & 68,9 & 72,1 & $\mathbf{8 3 , 3}$ & $\mathrm{P}<0,05$ \\
Dyslipidemia (\%) & 29,0 & 19,1 & $\mathbf{4 2 , 2}$ & $\mathbf{P}<\mathbf{0 , 0 1}$ \\
Chronical renal failure (\%) & 16,4 & 29,4 & 21,6 & n.s. \\
Peripheral arterial disease (\%) & 8,7 & 5,9 & $\mathbf{2 1}$ & $\mathrm{P}<0,01$ \\
Stroke (\%) & 10,9 & 14,7 & 14,8 & n.s. \\
CAD (\%) & 34,8 & 38,2 & $\mathbf{5 4 , 3}$ & $\mathrm{P}<0,01$ \\
Ejection fraction (\%, mean+SD) & $46,53(+17,6)$ & $47,29(+18,3)$ & $45,23(+17,8)$ & n.s. \\
\hline
\end{tabular}

The proportion of patients treated with ACEi, ARA-II, beta-blockers, spironolactone, diuretics and anti-thrombotic agents was similar across the three groups. Use of statins, however, was more common among patients with DDM. Nevertheless, total cholesterol, LDL and HDL levels were comparable. All patients with DM (diagnosed and undiagnosed) were more likely to be readmitted due to cardiovascular-events during the one year period ( 43 \& $41,8 \%$ vs $33,2 \% \mathrm{p}<0,01)$. No differences were observed for readmissions caused by non-cardiovascular episodes.

One-year mortality rate in DM patients (DDM \& UDM) was higher than among non-diabetics $(19,3-21,8 \%$ vs $13,1 \%)$ differences were not, however, statistically significant. During the five-year survey, mortality rates were considerably higher among the UDM group (66,7\%) than among non-diabetics $(40,7 \%, p<0,01)$. The former group also showed a higher rate when compared with DDM patients, although the difference is not statistically significant. Conclusion: UDM is common (16\%) in patients hospitalized as a result of heart failure and entails a five-year mortality rate almost twice as high than in the case of non-diabetics.

Patients with UDM, in spite of having lower rate of comorbidities and cardiovascular risk factors, showed a trend of higher mortality rate than DDM. Further studies are warranted to define this issue.

\section{0}

Are measures of adiposity associated independently with incident cardiovascular disease in individuals with newly diagnosed type 2 diabetes?

R. L. Coleman, R. R. Holman;

Diabetes Trials Unit, OCDEM, University of Oxford, United Kingdom.

Background and Aims: Obesity is associated with an increased risk of cardiovascular disease (CVD) and of type 2 diabetes (T2DM). The prevalence 
of obesity has tripled in most European countries in the past twenty years. We investigated whether measures of adiposity are associated with incident CVD in people with newly-diagnosed T2DM.

Materials and Methods: Of the 5102 patients with newly-diagnosed T2DM recruited into the UK Prospective Diabetes Study, 4478 did not have CVD and had the requisite data available for these analyses. Discrete time survival models were used to examine time to the first occurence of CVD, defined as any of fatal or non-fatal myocardial infarction, ischaemic heart disease, fatal or non-fatal stroke, coronary revascularization or fatal peripheral vascular disease. At diagnosis $59 \%$ of patients were male, $82 \%$ White caucasian, $30 \%$ current smokers and $1.1 \%$ had atrial fibrillation (AF). Their mean (SD) age was 52 (9) years, systolic blood pressure $136(20) \mathrm{mmHg}$, total cholesterol $5.4(1.0) \mathrm{mmol} / \mathrm{l}$, HDL-cholesterol $1.11(0.25) \mathrm{mmol} / \mathrm{l}$, total to HDL ratio (Tot:HDL) 5.1 (1.4) and median (IQR) HbAlc 6.5 (5.8-7.5) \%. Measures of adiposity were weight $80.9(16.4) \mathrm{kg}$, body mass index (BMI) $28.9(5.6) \mathrm{kg} /$ $\mathrm{m}^{2}$, waist circumference (WC) $94.9(13.2) \mathrm{cm}$ and hip circumference (HC) $87.0(17.6) \mathrm{cm}$

Results: 787 first CVD events occurred over median (IQR) 10 (8-13) years. All measures of adiposity were associated univariately $(p<0.001)$ with an increased risk of CVD but none remained significant after adjusting for traditional CVD risk factors (weight $\mathrm{p}=0.258$, BMI $\mathrm{p}=0.255$, waist circumference $\mathrm{p}=0.266$, waist:hip $\mathrm{p}=0.850$ ). Correlations between these measures of adiposity and traditional CVD risk factors were:

\begin{tabular}{lllllllll} 
& Age & Sex & Ethnicity & Smoker & HbAlc & SBP & Tot:HDL & AF \\
\hline BMI & -0.065 & 0.223 & -0.054 & -0.053 & 0.023 & 0.148 & 0.145 & 0.022 \\
Weight & -0.117 & -0.181 & -0.060 & -0.003 & -0.018 & 0.144 & 0.092 & 0.041 \\
WC & -0.025 & -0.117 & -0.080 & -0.007 & 0.035 & 0.123 & 0.199 & 0.017 \\
WC:HC & -0.007 & 0.500 & 0.039 & -0.065 & 0.028 & -0.024 & -0.200 & 0.013 \\
\hline
\end{tabular}

None of these measures of adiposity correlated significantly with any of the traditional CVD risk factors.

Conclusion: The increased risk of CVD seen with increased adiposity in the general population is not evident in individuals with newly-diagnosed T2DM. Although the measures of adiposity examined here are associated individually with future risk of CVD, the survival analysis suggests their effects are absorbed by the combined effects of the traditional CVD risk factors. The correlation patterns between these adiposity measures and the traditional CVD risk factors are, however, not significant. Although increased adiposity at diagnosis does not affect future CVD risk in this cohort, weight reduction after diagnosis may well influence CVD risk and should remain an important focus in the management of diabetes.

\section{1}

Cardiovascular risk and metabolic syndrome in patients with obstructive sleep apnea

R. Tkacova ${ }^{1}$, Z. Dorkova $^{1}$, A. Molcanyiova ${ }^{2}$, Z. Radikova ${ }^{3}$, I. Klimes ${ }^{4}$,

I. Tkac';

${ }^{1}$ Dept. for Respiratory Disorders, Medical Faculty, PJ Safarik University, Kosice, ${ }^{2}$ Dept. of Biochemistry, LABMED, Kosice, ${ }^{3}$ Laboratory of Human Endocrinology, Institute of Experimental Endocrinology, Bratislava, ${ }^{4}$ Diabetes and Nutrition Research Laboratory, Institute of Experimental Endocrinology, Bratislava, Slovakia.

Background and Aims: Obstructive sleep apnea (OSA) has been linked to the metabolic syndrome, insulin resistance, cardiovascular and cerebrovascular diseases in previous studies. Our aim was to determine whether OSA relates to National Cholesterol Education Program (Adult Treatment Panel III) (NCEP ATP III) cardiovascular risk independently of insulin resistance and other counfounders.

Materials and Methods: At the sleep unit in the tertiary referral teaching hospital, a cross-sectional analysis was undertaken on 98 subjects studied by attended polysomnography: 28 without OSA (apnea-hypopnea index, <5), 39 with mild-moderate (apnea-hypopnea index, $\geq 5$ to $<30$ ), and 31 with severe OSA (apnea-hypopnea index, $\geq 30$ ). Insulin resistance index (HOMA-IR) was based on the homeostasis model assessment method. Metabolic syndrome was defined according to the International Diabetes Federation criteria.

Results: From patients without to those with mild-moderate, and to those with severe OSA, significant increases were observed in the NCEP ATP III risk [from $2.0(1.0,8.0)$ to $3.0(1.0,12.0)$ to $16.0(5.3,20.0) \%, p$ for trend
$<0.001]$, in association with increases in HOMA-IR $[1.24(0.69,2.47)$ to 1.46 $(0.99,3.06)$ to $5.02(2.25,7.35), \mathrm{p}<0.001]$, mean blood pressure $(97.7 \pm 12.4$ to $103.2 \pm 12.0$ to $107.2 \pm 13.1 \mathrm{~mm} \mathrm{Hg}, \mathrm{p}=0.039)$, apolipoprotein $\mathrm{B}[0.95(0.86$, $1.07)$ to $0.91(0.78,1.08)$ to $1.10(0.91,1.16) \mathrm{g} / \mathrm{l}, \mathrm{p}=0.013]$, serum fibrinogen [2.73 $(2.28,3.08)$ to $2.74(2.21,3.12)$, to $3.28(2.62,3.89) \mathrm{g} / \mathrm{l}, \mathrm{p}=0.013)$, and fasting glucose levels $[4.8(4.4,5.3)$ to $5.0(4.6,5.4)$ to $5.8(5.0,6.6) \mathrm{mmol} /$ $1, \mathrm{p}=0.002]$. The proportion of patients treated for arterial hypertension increased from subjects without OSA to those with mild-moderate and to those with severe OSA [from $10(36 \%)$ to $18(46 \%)$ to $22(71 \%), \mathrm{p}=0.019$ ]. Metabolic syndrome was present in 13 (46\%) subjects without OSA, in 20 (51\%) patients with mild-moderate, and in 24 (77\%) patients with severe OSA $(\mathrm{p}=0.029)$. Patients with severe OSA had significantly higher odds for the presence of metabolic syndrome compared to those without OSA, after adjustments for age and gender (odds ratio, 3.60; 95\% CI, 1.18-11.00; $\mathrm{p}=0.025$ ). In addition, compared to subjects without sleep apnea, patients with severe OSA had significantly higher odds for the NCEP ATP III risk higher than 10\% (moderately high and high cardiovascular risk) both in the unadjusted model (odds ratio, 8.36; 95\% confidence interval, 2.48-28.19; $\mathrm{p}=0.001$ ), and after adjustments for body mass index and insulin resistance (odds ratio, 4.06; 95\% confidence interval, 1.02-16.25; $\mathrm{p}=0.048$ ).

Conclusion: The present findings suggest that severe OSA is related to higher level of several components of the metabolic syndrome. In addition, severe OSA is related to higher global cardiovascular risk, independently of obesity and insulin resistance.

Supported by the Ministry of Health, Slovakia 


\section{PS 127 Lipids, lipoproteins and macrovascular disease}

\section{2}

\author{
More than $20 \%$ of European dyslipidaemic patients have undiagnosed \\ dysglycaemia \\ M. Baccara-Dinet ${ }^{1}$, E. Eschwege ${ }^{2}$, E. Bruckert ${ }^{3}$; \\ ${ }^{1}$ Merck Santé, ${ }^{2}$ U780, INSERM, ${ }^{3}$ Service d'Endocrinologie Métabolique, \\ Groupe Hospitalier Pitié-Salpétrière, Paris, France.
}

Background: The Pan-European Survey of HDL-cholesterol (HDL-C) collected data on HDL-C and other cardiometabolic parameters from 8545 patients treated for dyslipidaemia in 11 European countries. Patients had a high prevalence of low HDL-C $(<1.03 \mathrm{mmol} / \mathrm{L}$ in $33 \%$ of men and $<1.29$ $\mathrm{mmol} / \mathrm{L}$ in $40 \%$ of women), type 2 diabetes $(45 \%)$ and coronary heart disease (45\%). Recent guidelines from the European Association for the Study of Diabetes/European Society of Cardiology and the World Health Organisation/International Diabetes Federation (WHO/IDF) recommend screening for undiagnosed dysglycaemia in patients at high cardiovascular risk. We used fasting plasma glucose (FPG) measurements to determine the prevalence of undiagnosed impaired fasting glucose (IFG) or diabetes in the survey population.

Patients and methods: The population included 2327 patients from the Pan-European Survey of HDL-C who had FPG measurement and no prior diagnosis of diabetes. IFG was defined as FPG $\geq 6.1 \mathrm{mmol} / \mathrm{L}$ and $<7.0 \mathrm{mmol} / \mathrm{L}$ (WHO/IDF definition).

Results: Undiagnosed diabetes was found in 159 patients $(6.8 \%)$ and IFG was found in 361 patients (15.5\%). The total prevalence of undiagnosed dysglycaemia (diabetes + IFG) was $22.3 \%$. Patients with previously diagnosed diabetes, undiagnosed diabetes, IFG or normoglycaemia had similar mean age (60-64 years). Overall, patients with previously diagnosed diabetes were obese $(\mathrm{BMI} \geq 30)$; patients with undiagnosed diabetes or IFG had a higher BMI $\left(29 \mathrm{~kg} / \mathrm{m}^{2}\right)$ compared to normoglycaemic patients $\left(27 \mathrm{~kg} / \mathrm{m}^{2}\right)$. The proportion of men with IFG $(71 \%)$ was higher than the proportion of men with diagnosed type 2 diabetes (58\%), undiagnosed diabetes (62\%), or normoglycaemia $(64 \%)$. Mean waist circumference was higher in patients with a prior type 2 diabetes diagnosis $(104 \mathrm{~cm})$, undiagnosed diabetes $(102 \mathrm{~cm})$ or IFG $(100 \mathrm{~cm})$ than in normoglycaemic patients $(96 \mathrm{~cm})$. The prevalence of hypertension was similar in pre-diabetic subjects and patients with diabetes and higher than that in normoglycaemic patients (see table); prevalence rates of myocardial infarction (MI) or coronary heart disease (CHD) were similar between groups. The prevalence rates of low HDL$\mathrm{C}$ and/or high triglycerides (TG), were highest in patients with diagnosed type 2 diabetes, and elevated in patients with IFG or undiagnosed diabetes relative to normoglycaemic patients. Mean values of lipid parameters, and the prevalence of the metabolic syndrome (IDF or US National Cholesterol Education Program [NCEP] criteria), followed a similar pattern.

Conclusions: Undiagnosed dysglycaemia (diabetes or IFG) is common $(>20 \%)$ among patients under treatment for dyslipidaemia in Europe and these patients display adverse cardiometabolic risk profiles relative to normoglycaemic patients. These data support recent recommendations for screening for undiagnosed dysglycaemia of patients at high cardiovascular risk. (Tab. 1)

Supported by an unrestricted educational grant from Merck Serono

\section{3}

Effects of $20 \mathrm{mg}$ rosuvastatin on VLDL1-, VLDL2-, IDL- and LDL-apoB kinetics in type 2 diabetes

B. Vergès ${ }^{1}$, L. Duvillard ${ }^{2}$, R. Chadarevian ${ }^{3}$, S. Baillot-Rudoni ${ }^{1}$, J.-M. Petit ${ }^{1}$, E. Florentin ${ }^{2}$, L. Princep ${ }^{2}$, P. Gambert ${ }^{2}$;

${ }^{1}$ Endocrinology-Diabetology, Hôpital du Bocage, Dijon, ${ }^{2}$ CRI 866, INSERM, Dijon, ${ }^{3}$ Astrazeneca, Laboratoires, Rueil-Malmaison, France.

Background and Aims: Rosuvastatin is a statin which, in addition to its efficacy to reduce LDL-cholesterol, has been shown to decrease significantly plasma triglyceride levels. However, the effects of rosuvastatin on the metabolism of triglyceride-rich lipoproteins remains unknown. This prompted us to perform an in vivo kinetic study of apoB containing lipoproteins with $\mathrm{C} 13$ leucine in patients with type 2 diabetes.

Materials and Methods: We performed a randomized double-blind crossover trial of six-week treatment period with placebo or rosuvastatin 20 mg in 8 patients with type 2 diabetes treated orally by metformin alone or in combination with sulfonylureas. In each patient, a kinetic study was performed at the end of each treatment period.

Results: Mean $\mathrm{HbAlc}$ was $6.88 \pm 1.6 \%$ and remained stable throughout the study. Rosuvastatine $20 \mathrm{mg}$ significantly reduced LDL cholesterol ( $60 \pm 18 \mathrm{vs.}$ $122 \pm 32 \mathrm{mg} / \mathrm{dl}, \mathrm{p}=0.012)$, plasma triglycerides $(170 \pm 70 \mathrm{vs} .274 \pm 122 \mathrm{mg} / \mathrm{dl}$, $\mathrm{p}=0.012)$ and total apoB $(67 \pm 12$ vs. $112 \pm 16 \mathrm{mg} / \mathrm{dl}, \mathrm{p}=0.012)$. Rosuvastatin significantly induced a reduction of VLDL1- $(p=0.049)$, VLDL2- $(p=0.012)$, IDL- $(\mathrm{p}=0.012)$ and $\mathrm{LDL}-(\mathrm{p}=0.012)$ apoB pool sizes. This reduction was associated with a significant increase in the FCR of VLDL1-apoB $(6,71 \pm$ 3.24 vs. $4.43 \pm 2.34 \mathrm{pool} /$ day, $\mathrm{p}=0.049)$, VLDL2-apoB ( $8.71 \pm 3.36$ vs. $5.39 \pm$ $2.62, \mathrm{p}=0.012)$, IDL-apoB $(6.97 \pm 1.56$ vs. $4.21 \pm 1.51, \mathrm{p}=0.012)$ and LDLapoB $(1.02 \pm 0.26$ vs. $0.58 \pm 0.13, \mathrm{p}=0.012)$. Rosuvastatin did not change the production rates of VLDL2, IDL nor LDL but tended to decrease VLDL1apoB production rate $(12.7 \pm 5.3$ vs. $19.1 \pm 8.5 \mathrm{mg} / \mathrm{kg} /$ day, $\mathrm{p}=0.08)$.

Conclusion: Treatment with rosuvastatin $20 \mathrm{mg}$, in type 2 diabetes, induces not only a significant increase of LDL-apoB catabolism $(+76 \%)$ but also favorable effects on the catabolism of triglyceride-rich lipoproteins such as a significant increase in the catabolism of VLDL1-apoB (+51\%), VLDL2-apoB $(+61 \%)$ and IDL-apoB $(+65 \%)$ as a borderline significant reduction in the production rate of VLDL1-apoB $(-34 \%)$. The effects of rosuvastatin on the metabolism of trigyceride-rich lipoproteins may have positive effects on the prevention of atherosclerosis, in type 2 diabetic patients.

Supported by AstraZeneca

\section{4}

Effects of achieving very low LDL-cholesterol concentrations with simvastatin or atorvastatin on steroidogenesis in high-risk diabetic patients

N. Demirag ${ }^{1}$, Z. Berberoglu ${ }^{1}$, M. Asik ${ }^{1}$, N. Bayraktar ${ }^{2}$, A. C. Yazici ${ }^{3}$, N. Tutuncu';

${ }^{1}$ Endocrinology, ${ }^{2}$ Biochemistry, ${ }^{3}$ Biostatistics, Baskent University, Ankara, Besevler, Turkey.

Background and Aims: The present study had 2 main objectives: (1) to compare the effect of achieving a low-density lipoprotein cholesterol (LDL-

\begin{tabular}{lllll} 
Tab. 1 & Prior T2D diagnosis $(\mathbf{n}=\mathbf{3 8 6 6})$ & Undiagnosed diabetes $(\mathbf{n}=\mathbf{1 5 9})$ & IFG $(\mathbf{n}=\mathbf{3 6 1})$ & Normal glycaemia $(\mathbf{n}=\mathbf{1 8 0 7})$ \\
\hline Hypertension (\%) & 82 & 65 & 76 & 64 \\
MI (\%) & 23 & 21 & 27 & 25 \\
CHD (\%) & 42 & 40 & 51 & 44 \\
Metabolic syndrome (IDF) (\%) & 79 & 58 & 63 & 30 \\
Metabolic syndrome (NCEP) (\%) & 80 & 63 & 64 & 25 \\
Low HDL-C (\%) & 43 & 31 & 36 & 30 \\
High TG (\%) & 55 & 44 & 51 & 39 \\
Low HDL-C + high TG (\%) & 30 & 22 & 25 & 17 \\
Mean total-C (mmol/L) & 5.12 & 5.32 & 5.39 & 5.44 \\
Mean LDL-C (mmol/L) & 3.02 & 3.25 & 3.31 & 3.32 \\
Mean HDL-C (mmol/L) & 1.22 & 1.34 & 1.26 & 1.38 \\
Mean TG (mmol/L) & 2.32 & 1.86 & 2.09 & 1.88 \\
\hline
\end{tabular}

T2D: type 2 diabetes; see text for other abbreviations. 
C) $<70 \mathrm{mg} / \mathrm{dL}$ vs achieving an LDL-C $<100 \mathrm{mg} / \mathrm{dL}$ on adrenal and testicular steroidogenesis in high-risk diabetic patients; and (2) to compare simvastatin with atorvastatin with respect to the abovementioned goal.

Materials and Methods: This was an open-label, randomized, controlled trial. High-risk diabetic males and postmenopausal females were randomly assigned to simvastatin $10 \mathrm{mg}$ or atorvastatin $10 \mathrm{mg}$. LDL-C was monitored at 30 days intervals. If LDL-C was $\geq 100 \mathrm{mg} / \mathrm{dl}$, the doses of the 2 agents were up-titrated. The study protocol allowed the doses of simvastatin and atorvastatin to be increased up to the maximum dose of $40 \mathrm{mg}$ and $80 \mathrm{mg}$, respectively. Patients within each treatment group were stratified by achieved LDL-C goals. Thereafter, they were followed up for 12 weeks. Before and after the intervention, lipids and hormones were assesed.

Results: Of the 120 subjects initially enrolled in the study, 114 patients completed all treatment and testing. After simvastatin therapy, mean LDL-C levels were $60.8 \mathrm{mg} / \mathrm{dl}$ in the subgroup with achieved LDL-C $<70 \mathrm{mg} / \mathrm{dL}$ (n = 29) compared to $87.8 \mathrm{mg} / \mathrm{dl}$ in the subgroup with achieved LDL-C $<100$ $\mathrm{mg} / \mathrm{dL}(\mathrm{n}=28)(P=0.000)$. After atorvastatin therapy, mean LDL-C levels were $55.6 \mathrm{mg} / \mathrm{dl}$ in the subgroup with achieved LDL-C $<70 \mathrm{mg} / \mathrm{dL}(\mathrm{n}=30)$ compared to $88.8 \mathrm{mg} / \mathrm{dl}$ in the subgroup with achieved LDL-C $<100 \mathrm{mg} / \mathrm{dL}$ (n $=27)(P=0.000)$. At 12 weeks, there were no statistically significant differences among the four groups nor any statistically significant within-group changes from baseline in cortisol, androstenedione and total testosterone. Significant reduction in DHEA-S was observed in subjects with an LDL-C $<70 \mathrm{mg} / \mathrm{dL}$ in both simvastatin and atorvastatin groups at 12 weeks compared with baseline $(P<0.05$ and $P<0.01$, respectively). DHEA-S fell by $17 \%$ and by $21 \%$ after simvastatin and atorvastatin administration, respectively. On the other hand, no significant effect was observed in the other two groups. The differences in the changes in this parameter between the LDL-C $<70 \mathrm{mg} / \mathrm{dL}$ groups and LDL-C $<100 \mathrm{mg} / \mathrm{dL}$ groups were significant $(P<0.01$ for both simvastatin and atorvastatin groups, respectively).

Conclusion: These results indicate that achieving an LDL-C $<70 \mathrm{mg} / \mathrm{dL}$ with simvastatin or atorvastatin have no considerable negative impact on adrenocortical and testicular steroidogenesis in diabetic patients. Whereas, the decrease in secretion of DHEA-S might reflect a decrease in substrate concentration causing compensatory alteration in the activity of DHEA sulphotransferase and steroid sulphatase in the adrenal cortex and an increased ratio of DHEA activation to inactivation. Additional data are required to determine whether there is a level of LDL-C beyond which downstream conversion of DHEA to sex steroids is affected.

\section{5}

Triglycerides and glycaemic control have influence on the small artery elasticity in diabetic patients

S. Redondo, O. Sánchez, R. Fabregate, M. Fabregate, E. Marin,

G. Nuñez de Arenas, J. Sabán-Ruiz;

Endothelial Pathology Unit, Ramon y Cajal Hospital, Madrid, Spain.

Introduction: The HDI/PulseWave CR-2000 obtains upper-arm blood pressure values and waveform data by non-invasive methods.

Aims: 1 - To determine the prevalence of abnormal elasticity index in a type 2 DM. 2- To determine its correlation with hemodynamic evaluation and glycemic control.

Methods: $\mathrm{N}=106,22-81 \mathrm{yrs} ; \mathrm{M} / \mathrm{F}$ ratio: 57/49, Group A: $\mathrm{N}=50$ (non-diabetic); group B: $\mathrm{N}=56$ (diabetic); Glyc, creat, HDL, LDL, total chol, trig, HbAlc. Hemodynamic evaluation: HDI/PulseWave. Statistical: t-Student, ChiSquare, Pearson correlation and Multivariate Linear Regression.

Results: 1- The prevalence of abnormal large artery elasticity (C1) was $37,7 \%$ in groups $A+B ; 28,0 \%$ in $A$ and $46,4 \%$ in $B(p<0,05)$. Abnormal subjects were older $(p=0,001)$ and had higher values of SBP $(p<0,001)$, DBP $(p=0,004)$, MBP $(p<0,001)$ and PP $(p<0,001)$ than those with normal elasticity. 2 - In relation to C1, significant differences in SVR ( $<<0,001)$, TVI ( $<<0,001)$, CO $(p<0,001)$, CO Index $(p=0,003), S V(p<0,001)$ and SVI $(p=0,001) .3$ - The prevalence of abnormal small artery elasticity (C2) was $43,4 \%$ in groups $A+B$; $40,0 \%$ in $A$ and $46,4 \%$ in B. 4-Abnormal subjects were older ( $p<0,001)$, and had higher SBP $(p=0,02)$, DBP $(p=0,014), \operatorname{MBP}(p<0,001)$, and PP $(p=0,010)$. 5 - In relation to $\mathrm{C} 2$, significant differences in the determination of $\mathrm{SV}$ Resistence $(p<0,001)$, TV Impedance $(p=0,007)$, C Output $(p=0,001)$, CO Index $(\mathrm{p}<0,001)$, Stroke V $(\mathrm{p}=0,004)$ and SVIndex $(\mathrm{p}=0,002) .6$ - There is a correlation between small artery elasticity and triglyceride levels $(p=0,007)$. 7- We found an age-independent linear correlation between $\mathrm{C} 2$ and metabolic control in the diabetic population $[\mathrm{p}=0,013]$, in the multivariate analysis.
Conclusion: 1- The prevalence of stiff large and small arteries was of 38,3\% and $43,4 \%$. 2- Age is more important than the diabetic status in vascular elasticity. 3- Both phenomena present with changes in other cardiovascular parameters. 4- The stiffness of small arteries is related to levels of triglycerides in all subjects and to glycemic control in diabetic patients.

\section{6}

Free fatty acid changes with insulin glargine vs thiazolidinediones according to baseline $\mathrm{HbA}_{1 \mathrm{c}}$ in type 2 diabetes mellitus

N. Rosenberg ${ }^{1}$, S. Schwartz ${ }^{2}$, A. Cohen ${ }^{3}$, L. Traylor ${ }^{1}$; J. Rosenstock ${ }^{4}$

${ }^{1}$ sanofi-aventis US, Bridgewater, ${ }^{2}$ dgd Research Inc, San Antonio,

${ }^{3}$ Endocrinology, Endocrine Clinic, Memphis,

${ }^{4}$ Dallas Diabetes and Endocrine Center, United States.

Background and Aims: Dyslipidemia, including elevated free fatty acids (FFA's), triglycerides (TGs), and low-density lipoprotein and reduced highdensity lipoprotein, greatly increases the cardiovascular risk associated with diabetes. Elevated FFA has a confirmed role in insulin resistance while thiazolidinediones (TZDs) have demonstrated effects on reducing lipotoxicity and FFA levels, resulting in improved insulin sensitivity. Additionally, insulin has well known effects on reducing hyperglycemia-associated lipolysis. This analysis examined the relationship between glycemic control and lipid variables.

Material and Methods: Two randomized, open label, parallel group studies comparing the effects of insulin glargine (GLAR) vs a TZD on glycemic control as add-on therapy in patients inadequately controlled on a sulfonylurea and/or metformin were conducted. We assessed FFA and TG changes according to baseline $\mathrm{HbA}_{1 c}$ in this post-hoc analysis. Study 1: GLAR ( $n=165)$ vs pioglitazone (PIO; $n=182$ ). Baseline and Wk 24 lipid values were available for 121 and 128 patients, respectively. Patients were separated into 2 groups based upon baseline $\mathrm{HbA}_{1 \mathrm{c}}$ values: $\leq 8.5 \%$ and $>8.5 \%$. Study 2:GLAR $(\mathrm{n}=105)$ versus rosiglitazone (RSG; $\mathrm{n}=112)$. Baseline and $\mathrm{Wk}$ 24 lipid values were available for 87 and 83 patients, respectively, who were likewise separated into two groups based upon baseline $\mathrm{HbA}_{1 c}$ values. Safety assessments, including hypoglycemia and adverse events, were monitored throughout the study.

Results: In study 1 , decreases in mean \pm SE FFA were significantly greater with GLAR vs PIO in the patient group with baseline $\mathrm{HbA}$ values $>8.5 \%$ $(-0.21 \pm 0.02 \mathrm{vs}-0.12 \pm 0.02 \mathrm{mmol} / \mathrm{L}, P=0.006)$. In study 2 , FFA decreases were similar with GLAR and RSG. Decreases in TGs were similar for GLAR and PIO in study 1 and significantly greater with GLAR versus RSG in the patient group baseline $\mathrm{HbA}_{1 \mathrm{c}}$ values $\leq 8.5 \%(-0.34 \pm 0.11$ vs $0.20 \pm 0.13, P=0.002)$ in study 2. All 3 therapeutic agents were safe and tolerable. There were no statistically significant differences in treatment-emergent adverse events between the GLAR and PIO groups in study 1 or the GLAR and RSG groups in study 2 .

Conclusion: Overall, the baseline $\mathrm{HbA}_{1 c}$-dependent effect on FFA and TG appears to be more pronounced with GLAR, possibly reflecting greater $\mathrm{HbA}_{1}$ reductions in patients with higher baseline $\mathrm{HbA}_{1 c}$. The data discovered in this post-hoc analysis suggest that insulin glargine exhibits greater FFA-lowering effects than TZDs when baseline $\mathrm{HbA}_{1 c}$ is $>8.5 \%$ and exhibits reductions similar to those observed with TZDs at lower baseline $\mathrm{HbA}_{1 c}$ levels. Since elevated FFA levels contribute to glucose metabolism dysregulation and have detrimental effects on cytokines and adiponectin, the FFA-lowering effect of

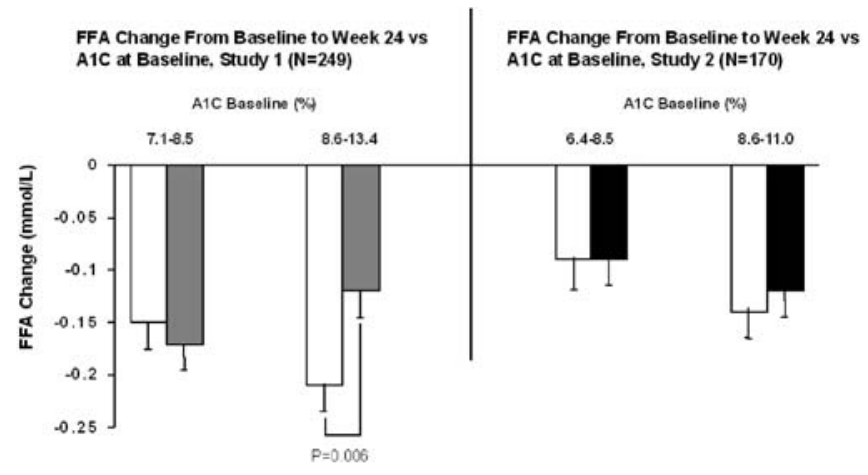


GLAR at elevated $\mathrm{HbA}_{1 c}$ values may be beneficial in type 2 diabetes mellitus patients.

Supported by sanofi-aventis U.S.

\section{7}

Change in lipid variables with insulin glargine versus pioglitazone in T2DM patients inadequately controlled on a sulfonylurea or metformin J. B. McGill ${ }^{1}$, L. Traylor ${ }^{2}$, A. Dwarakanathan ${ }^{3}$;

${ }^{1}$ Division of Endocrinology, Metabolism and Lipid Research, Washington University School of Medicine, St. Louis, ${ }^{2}$ sanofi-aventis U.S., Bridgewater, ${ }^{3}$ Diabetes Center, St James Diabetes Center, Chicago Heights, United States.

Background and Aims: The progressive nature of type 2 diabetes mellitus (T2DM) often requires increasingly aggressive therapy over time in order to maintain adequate glycemic control. Pharmacologic treatment is typically introduced with a sulfonylurea or metformin, with thiazolidinediones or insulin added for persistent hyperglycemia. The aim of this study was to examine the impact of pioglitazone versus insulin glargine when added to sulfonylurea or metformin monotherapy. This analysis focused on the change in lipid profiles.

Material and Methods: This randomized, multicenter, 48-week, parallelgroup, open-label study compared glargine (GLAR) $(n=129)$ versus pioglitazone (PIO) $(n=130)$ as add-on therapy in T2DM patients inadequately controlled with $\geq 50 \%$ of the maximum labeled dose of a sulfonylurea (SU) or $\geq 1000 \mathrm{mg}$ metformin (MET). We compared lipid variables by strata (SU vs MET) over 48 weeks focusing on free fatty acid (FFA), triglyceride (TG), and high-density lipoprotein (HDL) changes. Safety assessments including hypoglycemia and adverse events were monitored throughout the study.

Results: A1C decrease from baseline was significantly greater in the SU strata of the GLAR versus the PIO at 48 weeks $(P=0.035)$. FFA decrease from baseline with GLAR was significantly greater than PIO at 24 weeks in the MET strata $(-0.19 \pm 0.03 \mathrm{mmol} / \mathrm{L}, P=0.002)$ and 48 weeks in the SU strata $(-0.29 \pm 0.04 \mathrm{mmol} / \mathrm{L}, P=0.026)$. While TG reduction was substantial (ranging from -0.49 to $-0.59 \mathrm{mmol} / \mathrm{L}$ at 24 weeks and -0.42 to $-0.67 \mathrm{mmol} / \mathrm{L}$ at 48 weeks) with GLAR and PIO, differences in the MET and SU strata were not significant. GLAR and PIO increased HDL in both strata but the increase was significantly higher with PIO relative to GLAR in both strata at 24 and 48 weeks $(P<0.01$ in both strata with GLAR and PIO. Changes from baseline to week 24 and week 48 were statistically significant $(P<0.05)$ for all variables except for week $48 \mathrm{HDL}$ in the GLAR + SU strata $(\mathrm{p}=0.09)$. Both treatments were safe and tolerable, with no statistically significant differences in adverse events between GLAR and PIO groups. Hypoglycemia $(\mathrm{BG}<70 \mathrm{mg} / \mathrm{dL}$ ) occurred more frequently in patients treated with GLAR (45.7\%) relative to patients treated with PIO $(19.9 \%)(P<0.001)$. Conclusions: This study shows that both GLAR and PIO lower TGs when used as add-on therapy to SU or MET, however PIO raised HDL to a greater extent in both groups. At week 48, GLAR had a significantly greater A1C-lowering effect than PIO when used in combination with SU. Additionally, the FFA- lowering benefit of GLAR is greater than PIO when used in combination with MET (24 weeks) or SU (48 weeks). The FFA-lowering effect of GLAR may be an important contributor to its metabolic benefit in patients failing single, oral agent therapy. (Fig. 1) Supported by sanofi-aventis U.S.

\section{8}

Circulating interleukin-8 levels are selectively determined by plasma non-esterified myristate, oleate, and $\omega 3$-polyunsaturated fatty acids H. Staiger, F. Machicao, A. Fritsche, N. Stefan, H.-U. Häring; Internal Medicine IV, Medical Clinic, Eberhard-Karls-University Tübingen, Germany.

Background and Aims: Elevated plasma levels of non-esterified fatty acids (NEFA), due to high fat intake and/or dysregulated adipose tissue lipolysis, are a hallmark of the metabolic syndrome and play a role in the pathogenesis of atherosclerosis. In this study, we assessed whether certain plasma NEFA species contribute to the chronic low-grade inflammatory state seen in the metabolic syndrome.

Materials and Methods: We determined the individual fasting plasma NEFA patterns of 75 overweight non-diabetic subjects and analysed the relationship of single NEFA species with plasma inflammatory parameters.

Results: After adjustment for gender, age, body fat, and waist-hip ratio, no strong correlations of single NEFA species with plasma leukocyte number, C-reactive protein, interleukin (IL)- 6 , tumour necrosis factor (TNF)- $\alpha$, or

\begin{tabular}{|c|c|c|c|c|c|c|}
\hline \multicolumn{7}{|c|}{ AlC,FFA, $\mathrm{T} \mathrm{G}$, and HDL at baseline, week 24 , md week 48 for MET/SU Patients } \\
\hline & \multicolumn{3}{|c|}{ MET } & \multicolumn{3}{|c|}{ su } \\
\hline & $\begin{array}{l}\text { Thalin Glargine } \\
\text { Me ent" }\end{array}$ & $\begin{array}{l}\text { Pioglitazone } \\
\text { Meenn } * \pm S E\end{array}$ & $P$-wable & $\begin{array}{l}\text { Iroulin } \\
\text { Glargine } \\
\text { Mean" }{ }^{2} \pm \text { SE }\end{array}$ & $\begin{array}{l}\text { Pioglitrame } \\
\text { Meam }{ }^{*} \pm S E\end{array}$ & P.vahue \\
\hline \multicolumn{7}{|l|}{ Alc $(\%)+$} \\
\hline Baseline & $9.28 \pm 0.12$ & $9.40 \pm 0.14$ & 0.04 & $9.45 \pm 0.14$ & $9.37 \pm 0.14$ & 0.62 \\
\hline Week 24 & $6.96 \pm 0.12$ & $7.21 \pm 0.12$ & 0.15 & $6.86 \pm 0.12$ & $7.05 \pm 0.12$ & 0.22 \\
\hline Week 48 & $6.73 \pm 0.11$ & $6.97 \pm 0.13$ & 0.19 & $6.54 \pm 0.16$ & $6.97 \pm 0.16$ & 0.035 \\
\hline \multicolumn{7}{|c|}{ FFA (monolli): } \\
\hline Baseline & $0.56 \pm 0.02$ & $0.55 \pm 0.02$ & 0.96 & $0.62 \pm 0.03$ & $0.59 \pm 0.02$ & 0.24 \\
\hline Week 24 & $0.37 \pm 0.03$ & $0.45 \pm 0.04$ & 0.0021 & $0.43 \pm 0.04$ & $0.41 \pm 0.04$ & 0.997 \\
\hline Week 48 & $0.38 \pm 0.04$ & $0.42 \pm 0.06$ & 0.140 & $0.33 \pm 0.04$ & $0.41 \pm 0.05$ & 0.026 \\
\hline \multicolumn{7}{|c|}{ TG (mmoll) } \\
\hline Baseline & $2.29 \pm 0.16$ & $2.14 \pm 0.12$ & 0.10 & $2.26 \pm 0.21$ & $2.24 \pm 0.16$ & 0.22 \\
\hline Week 24 & $1.80 \pm 0.08$ & $1.63 \pm 0.07$ & 0.62 & $1.75 \pm 0.08$ & $1.65 \pm 0.07$ & 0.52 \\
\hline Week 48 & $1.79 \pm 0.09$ & $1.72 \pm 0.11$ & 0.75 & $1.59 \pm 0.09$ & $1.59 \pm 0.11$ & 0.90 \\
\hline \multicolumn{7}{|c|}{$\operatorname{HDL}(\mathrm{mmol} / \mathrm{L})^{*}$} \\
\hline Baseline & $1.13 \pm 0.03$ & $1.11 \pm 0.03$ & 0.89 & $1.12 \pm 0.03$ & $1.09 \pm 0.03$ & 0.33 \\
\hline Week 24 & $1.17 \pm 0.02$ & $1.26 \pm 0.02$ & 0.0003 & $1.18 \pm 0.03$ & $1.28 \pm 0.03$ & 0.005 \\
\hline Week 48 & $1.19 \pm 0.02$ & $1.28 \pm 0.03$ & 0.004 & $1.18 \pm 0.05$ & $1.34 \pm 0.05$ & 0.007 \\
\hline
\end{tabular}

Fig. 1

"Mean value adjusted by baseline for each variable

t A1C:

Week 24: for GLAR+MET, $n=84$; for PIO+MET, $n=68$; $G L A R+S U n=57$; for $P I O+S U, n=59$

Wieek 48: for GLAR+MET, $n=54$; for PIO+MET, $n=42$; GLAR+SU $n=30$; for $P I O+8 U, n=40$

FFF, TG and HDL:

Wieek 24: for GLAR+MET, $n=70$; for PIO+MET, $n=76$; GLAR+SU $n=59$; for $P 10+S U, n=64$

Wieek 48: tor GLAR+MET, $n=54$ : tor PIO+MET, $n=43$ : GLAR+SU $n=39$; to PIO+SU, $n=40$ 
monocyte chemoattractant protein-1 were detected. However, oleate was negatively $(r=-0.36, p=0.0015)$ and myristate $(r=0.41, p=0.0003)$ as well as the $\omega 3$-polyunsaturated NEFA (PUFA) $\alpha$-linolenate $(r=0.37, p=0.0011)$, eicosapentaenoate $(r=0.40, p=0.0003)$, and docosahexaenoate $(r=0.40$, $p=0.0004$ ) were positively associated with plasma IL-8 levels. The other NEFA species as well as the total plasma NEFA concentration did not correlate with plasma IL-8. The correlations of myristate, oleate, and the sum of all $\omega 3$-PUFA with plasma IL-8 levels were independent of plasma TNF- $\alpha$ concentration and overall adiposity.

Conclusion: Our data do not provide evidence for an association of single plasma fasting NEFA species with overall inflammatory state, but demonstrate close and selective associations of oleate, myristate, and $\omega 3$-PUFA with plasma levels of the pro-inflammatory chemokine IL-8. Thus, these NEFA species may represent specific determinants of plasma IL-8.

Supported by the German Research Foundation and the European Community's Frame Programme 6 EUGENE 2.

\section{9}

New capillary electrophoresis method for the analysis of glycated triacylglycerol-rich lipoproteins in diabetics

S. Sharma

Dept of Diabetes, UHI Millennium Institute, Inverness, United Kingdom.

In addition to hyperglycaemia and hyperinsulinaemia, diabetic subjects often present with hypertriacylglycerolaemia, which is associated with an increased risk of atherosclerosis and coronary heart disease (CHD). The combination of high plasma glucose and triacylglycerol (TAG)-rich lipoproteins increases the risk of lipoprotein modification (including glycation). This modification (glycation) has been hypothesised to increase the risk of CHD. To investigate the association and extent of TAG-rich lipoprotein modification, suitable routine assays are required. Conventional electrophoresis and immunochemical methods, which have been used for the analysis of glycated lipoproteins, are often inaccurate, time consuming and semi-quantitative. Aim: The aim of this study was to develop new, robust and rapid capillary electrophoresis (CE) methods for the separation of TAG-rich lipoproteins and their modified forms. A procedure has been developed that uses high performance $\mathrm{CE}$ to monitor the electrophoretic mobility of native chylomicrons (CMs), very low density lipoproteins (VLDL1 and VLDL2) isolated by ultracentrifugation and their modified forms in-vitro and in-vivo. For in-vitro modifications, native lipoproteins were incubated with sugar concentrations corresponding to the levels in normal and diabetic state. Separation of native lipoproteins and modified lipoproteins was successfully achieved within $10 \mathrm{~min}$ by this method called Micellar Electrokinetic Chromatography (MEKC). Changes in peak profile (shape, width, height) and emergence of new peaks during the incubation of these lipoproteins reflects the progressive change in mass, associated with the slight increase in electronegativity of these compounds due to modifications. For in-vivo study lipoproteins from 6 diabetic and 6 non diabetic volunteers were isolated and analysed by newly developed MEKC method. As expected from the in-vitro study and because diabetic subjects possess higher postprandial levels of TRLs (triglyceride rich lipoproteins), in MEKC the total peak area readily discriminated between lipoprotein samples from the diabetic and control groups. Gel electrophoresis data support the differences observed by MEKC. This new procedure has the ability to discriminate between TRLs from diabetic and control subjects, as well as TRLs modified by sugars in-vitro. This novel MEKC method will further facilitate the quantification of modified lipoproteins and aid in the research of factors influencing modification and glycation of lipoproteins in-vitro and in-vivo.

Supported by British Heart Foundation, The University of Reading

\section{PS 128 Biomarkers for cardiovascular disease}

\section{0}

Novel risk factors for atherosclerosis and silent myocardial ischaemia in diabetic patients: osteoprotegerin a useful marker to select patients who should benefit from screening?

A. Avignon ${ }^{1}$, A. Sultan ${ }^{1}$, C. Piot ${ }^{2}$, D. Marino-Goulart ${ }^{3}$,

J. Thuan dit Dieudonné 1 , A. Dupuy ${ }^{4}$, J. Cristol';

${ }^{1}$ Metabolic Disease, ${ }^{2}$ Cardiology, ${ }^{3}$ Nuclear Medicine, ${ }^{4}$ Biochemistry, University Hospital, Montpellier, France.

Background and Aims: Silent myocardial ischemia (SMI) is frequent in diabetic patients and is associated with a worse outcome, supporting efforts to identify and intervene in the preclinical phase of coronary artery disease. A process whereby the patients at highest risk could be selected for screening would optimize the cost effectiveness of the screening strategy. The aim of the present study was to evaluate osteoprotegerin, an inhibitor of osteoclastogenesis involved in atherosclerosis and additional novel risk factors for the prediction of SMI.

Materials and Methods: 465 consecutive diabetic patients with $>1$ additional risk factor were evaluated for SMI using stress myocardial perfusion imaging. Association of SMI (positive stress-ECG and/or abnormal scintigraphic image) with 1) osteoprotegerin, 2) other novel risk factors (liporotein(a), homocystein, adiponectin, C-reactive protein and fibrinogen) and 3) conventional risk factors (total cholesterol, LDL-cholesterol, HDL-cholesterol, triglycerides) was evaluated.

Results: 92/465 patients were diagnosed with SMI. Of the 6 novel markers measured, OPG was the only one to be associated with SMI; the relative risk of SMI for patients above the $75^{\text {th }}$ percentile for that marker was 3.19 (95\% CI, 1.99 to 5.18) in comparison to those who were below that percentile. In univariate analyses, the other plasma markers significantly associated with SMI were higher triglyceride levels $(\mathrm{p}=0.04)$ and lower levels of HDL$\mathrm{C}(\mathrm{p}=0.02)$. The association of OPG with SMI remained significant after correcting for other variables associated with SMI at $\mathrm{p}<0.15$ in univariate analysis (RR 3.59; 95\% CI 1.95-6.60; $\mathrm{p}<0.0001$ ). The association of OPG with SMI was observed in males $(\mathrm{p}<0.0001)$, in females $(\mathrm{p}=0.03)$, in type $1(0.002)$ and type $2(0.0004)$ diabetic patients, in patients with (0.0004) or without $(0.03)$ nephropathy and in patients without $(\mathrm{p}<0.0001)$ but not in those with $(\mathrm{p}=0.2)$ peripheral arterial disease.

Conclusion: Addition of the measurement of OPG to other factors may provide an improved method of identifying diabetic patients susceptible to benefit from SMI screening.

\section{1}

Suboptimal glycaemic control, plasma osteoprotegerin and interleukin 6 predict progression of coronary artery calcification in asymptomatic type 2 diabetic subjects

D. F. C. Hopkins ${ }^{1}$, D. V. Anand ${ }^{2}$, E. Lim², D. Lipkin², D. Darko', R. Corder ${ }^{4}$, A. Lahiri ${ }^{2}$;

${ }^{1}$ Diabetic Medicine, King's College Hospital, London,

${ }^{2}$ Cardiac Imaging and Research Centre, Wellington Hospital, London,

${ }^{3}$ Jeffrey Kelson Centre, Central Middlesex Hospital, London,

${ }^{4}$ William Harvey Research Institute, Barts and The London,

Queen Mary's School of Medicine \& Dentistry, London, United Kingdom.

Background and Aims: Coronary artery calcification (CAC) imaging enables non-invasive detection of coronary atherosclerosis and quantification of the extent of calcified atherosclerotic plaque. We have previously demonstrated that there is a high prevalence of CAC in type 2 diabetic subjects with no symptoms or history of coronary artery disease and that CAC predicts both abnormal myocardial perfusion and incident cardiovascular disease. In the present study we have followed a prospective cohort of subjects with type 2 diabetes to determine the progression of CAC and the factors that influence progression.

Materials and Methods: 398 asymptomatic patients (mean age $52 \pm 8$ years, HbA c $8.0 \pm 1.5 \%$ ) were evaluated by serial CAC imaging (mean follow-up $2.5 \pm 0.4$ years). Progression/regression of CAC was defined using previously published criteria. Demographic data, anthropometric measurements, 
medical and treatment history were recorded at baseline and follow up together with determination of biochemical parameters including HbAlc, microalbuminuria status, lipids, serum hs-CRP, IL-6 and plasma osteoprotegerin (OPG) levels.

Results: At baseline, CAC was present in 211 patients (53\%). Progression occurred in $118(29.6 \%)$ and regression in $3(0.8 \%)$. Age, male gender, hypertension, baseline $\mathrm{HbA}_{1} \mathrm{c}$, baseline CAC score, IL- 6 and OPG were univariate predictors of progression. In a multivariate logistic regression model adjusted for baseline $\mathrm{CAC}$, serum $\mathrm{HbA}_{1} \mathrm{c}$ (Odds ratio $[\mathrm{OR}]=10.5$ [95\% CI: 2.04, 53.9], $\mathrm{p}=0.02)$, plasma OPG $(\mathrm{OR}=2.50[1.19,5.22], \mathrm{p}=0.02)$ and IL-6 $(\mathrm{OR}=2.06[1.13,3.75], \mathrm{p}=0.05)$ were independent predictors of progression.

Conclusions: Suboptimal glycaemic control is a risk factor for progression of atherosclerosis in type 2 diabetes as are elevated concentrations of the cytokines IL-6 and OPG. Uniquely, OPG predicted both the extent of CAC at baseline and its subsequent progression and may prove useful as a biomarker for atherosclerosis in diabetes.

Supported by Harrow Cardiovascular Research Trust, Michael Tabor Foundation

\section{2}

Lipoprotein-associated phospholipase (Lp-PLA $)$, C-reactive protein (CRP) and coronary artery disease (CAD) among persons with type 1 diabetes

T. J. Orchard, R. G. Miller, T. Costacou, R. W. Evans;

Epidemiology, University of Pittsburgh, United States.

Background and Aims: The enzyme Lp-PLA may be implicated in processes leading to vascular inflammation. CRP, an acute phase protein, is elevated during inflammatory states. Recent evidence suggests that both Lp-PLA and CRP independently predict future cardiovascular events and together may be particularly effective. However, little is known about their effect on CAD risk in type 1 diabetes. We thus assessed the association between LpPLA (activity and mass) and CRP with CAD among individuals with type 1 diabetes who are at particularly high risk for cardiovascular events by also having overt nephropathy.

Materials and Methods: Participants of the Pittsburgh Epidemiology of Diabetes Complications (EDC) study, a prospective investigation of childhood onset type 1 diabetes (baseline mean age was 28 and diabetes duration 19 years), with overt nephropathy (confirmed albumin excretion rate $>200 \mu \mathrm{g} / \mathrm{min})$ were selected for study $(\mathrm{n}=96)$. CAD was defined as EDC physician diagnosed angina, confirmed myocardial infarction, stenosis $>50 \%$, ischemic ECG, or revascularization. CRP was measured using a high sensitivity turbidimetric method utilizing reagents developed by Carolina Liquid Chemicals (Brea, CA). Samples were assayed for Lp-PLA mass using a microplate-based ELISA (PLAC ${ }^{\mathrm{m}}$ assay, diaDexus, Inc., South San Francisco, CA) whereas for levels of Lp-PLA activity, samples were assayed using a colorimetric assay (diaDexus, Inc., South San Francisco, CA). Cox proportional hazards models were constructed.

Results: No correlations between CRP and Lp-PLA activity and mass were observed (Pearson's $r=0.03, p=0.76$ and $r=0.09, p=0.34$, respectivly). During 16 years of follow-up, 38 incident $\mathrm{CAD}$ cases developed. In univariate analyses, Lp-PLA 2 activity $(\mathrm{p}=0.007)$, but neither mass $(\mathrm{p}=0.38)$ nor CRP $(\mathrm{p}=0.60)$, was increased among subsequent $\mathrm{CAD}$ cases compared to noncases. Multivariable models allowed for duration, gender, blood pressure and blood pressure medication, smoking status, waist-hip ratio, and lipid levels. While neither Lp-PLA activity nor mass was independently associated with CAD incidence, a significant interaction between Lp-PLA 2 mass and CRP levels was noted $(\mathrm{p}=0.002)$, with Lp-PLA risk increasing with elevated CRP levels. No interactions were observed with gender or LDL cholesterol. Conclusion: In this cohort of type 1 diabetes persons at high risk for CAD, the predictive role of Lp-PLA 2 appears conditional on elevated CRP despite their concentrations not being correlated.

Supported by NIH

\section{3}

Platelet-activating factor acetylhydrolase activity in patients with type 1 diabetes mellitus

M. B. Gomes ${ }^{1}$, E. Nunes ${ }^{2}$, H. C. C. Faria Neto ${ }^{2}$, M. F. Bevilacqua ${ }^{1}$, M. N. Silva ${ }^{1}$;

${ }^{1}$ Diabetes, State University of Rio de Janeiro, ${ }^{2}$ Immunopharmacology, Laboratory of Immunopharmacology, FIOCRUZ, Rio de Janeiro, Brazil.

Background and Aims: Platelet-activating factor acetyhydrolase (PAF-AH) is a $\mathrm{Ca}^{2+}$ independent phospholipaseA that catalyses the conversion of platelet-activating factor (PAF) to lyso-PAF inactivating it. Plasma PAF-AH binds to lipoproteins mainly with LDL-cholesterol and HDL-cholesterol.The relationship between PAF-AH and atherosclerosis is currently controversial. Considering that PAF is a lipid mediator involved in inflammatory disease as well in atherogenesis, its inactivation by PAF- AH would resut in an antinflammatory effect. On the other hand, PAF-AH may also generate bioactive oxidized free fatty acids and lysophosphatidylcoline that have deleterious effect on the arterial wall, thus having a proinflamatory and proatherogenic action. The aims of the present study were to evaluate if type 1 diabetic patients with and without clinical microvascular complications matched to subjects without diabetes have altered levels of PAF-AH activity and whether its level is related to clinical and metabolic variables .

Materials and Methods: We studied cross-sectionally 118 type 1 diabetic outpatients (54 males) aged $27.1 \pm 11.3$ years with a duration of diabetes of $12.3 \pm 8.4$ years, witht $(n=38)$ and without $(n=80)$ clinical chronic complications and 94 non-diabetic subjects (48 males) aged $25.8+7.2$ years. PAF-AH activity was measured with PAF-AH assay kit (Cayman Chemical, Ann Arbor, MI, USA). Human PAF-AH standard or human serum and 2thio PAF, substrate for PAF-AH (Aarsman et al., 1991), were added to the wells, followed by DTNB ((5,5'-dithiobis (2-nitrobenzoic acid)). One unit of enzyme hydrolyzes one $\mu \mathrm{mol}$ of 2-thio-PAF per minute at $25^{\circ} \mathrm{C}$. Absorbance was read over a period of time $(0,1,2,3,4,5,10,15,20,30$ minutes) using a plate reader. A mimimum of two time points was used to determine enzyme activity. The reaction was determined using the DTNB extinction coefficient and results were represented as $\mu \mathrm{mol} / \mathrm{min} / \mathrm{ml}$.

Results: Diabetic patients had higher PAF-AH [ $(0.016 \pm 0.004)$ vs $(0.014 \pm$ $0.005-\mu \mathrm{mol} / \mathrm{min} / \mathrm{ml} p<0.05$ ] levels than nondiabetic subjects. No difference was noted between patients with and without microvascular complications. In diabetic patients PAF-AH was correlated with age, $\mathrm{HbAlc}$, uric acid and HDL-cholesterol No correlation was found ith LDL-Cholesterol. Stepwise multiple regression with PAF-AH as the dependent variable showed that HbAlc was the only significant independent variable.

Conclusion: According to our results, PAF-AH AFP, a lipide mediator involved in inflammatory diseases and atherogenesis, are increased in patients with type 1 diabetes probably independently of the presence of clinical microvascular disease. PAF-AH could be another inflammatory marker in patients with diabetes. Considering the importance of cardiovascular disease in patients with type 1 diabetes the influence of PAF-AH on the development of this complication in these patients must be addressed in prospective studies.

Supported by $\mathrm{CNPq}$

\section{4}

Implications of lipocalin-2 and visfatin levels in patients with coronary artery disease

K. Lee', K. Choi ${ }^{1}$, T. Kim ${ }^{1}$, H. Yoo 1 , S. Park², H. Kim², J.-A. Seo ${ }^{3}$, N. Kim³, Y. Kim ${ }^{4}$, D. Choi ${ }^{2}$, C. Park ${ }^{5}$, S. Baik ${ }^{1}$;

${ }^{1}$ Endocrinology, Korea University Guro Hospital, Seoul, ${ }^{2}$ Endocrinology, Korea University Anam Hospital, Seoul, ${ }^{3}$ Endocrinology, Korea University Ansan Hospital, Seoul, ${ }^{4}$ Endocrinology, Jae Saeng Hospital, Seung Nam, ${ }^{5}$ Cardiology, Korea University Guro Hospital, Seoul, Republic of Korea.

Background and Aims: Visfatin and lipocalin-2 have been implicated in insulin resistance and obesity-associated metabolic disorders.

The aim of this study was to examine lipocalin-2 and visfatin concentrations in patients with coronary artery disease (CAD) compared with those of control subjects, and evaluated their association with cardiovascular risk factors for atherosclerosis.

Materials and Methods: We examined visfatin, lipocalin-2 levels and cardiovascular risk factors in 91 subjects (49 patients with angiographically 
confirmed CAD vs. 42 age- and gender-matched control participants).Crosssectional study.

Results: Circulating lipocalin-2 levels were significantly higher in patients with CAD compared to the control subjects $(82.6 \pm 38.7 \mathrm{ng} / \mathrm{ml}$ vs. $43.8 \pm 27.8$ $\mathrm{ng} / \mathrm{ml} ; \mathrm{P}<0.001)$. However, visfatin levels were not significantly different between patients with CAD and control subjects. Serum lipocalin-2 levels were positively associated with weight $(\mathrm{r}=0.22 ; \mathrm{P}=0.036)$, fasting insulin $(\mathrm{r}=0.42 ; \mathrm{P}<0.001)$ and insulin resistance $(\mathrm{r}=0.44 ; \mathrm{P}<0.001)$, whereas these levels showed a negative correlation with HDL cholesterol $(\mathrm{r}=-0.30$; $\mathrm{P}=0.005$ ). The multiple regression analysis showed that lipocalin-2 levels were independently associated with HDL cholesterol and insulin resistance $\left(\mathrm{R}^{2}=0.199\right)$. In addition, the multiple logistic regression analysis showed that systolic blood pressure, insulin resistance and lipocalin-2 levels were independently associated with CAD.

Conclusion: Serum lipocalin-2 levels were significantly elevated in patients with CAD and were independently associated with CAD. The present findings suggest that measurement of serum lipocalin-2 levels may be useful for assessing CAD risk.

\section{5}

Visceral fat thickness measured by ultrasonography is associated with metabolic risk factors of cardiovascular disease in Korean type 2 diabetic patients

D.-H. Cho, G.-H. Jeong, J.-O. Chung, D.-J. Chung, M.-Y. Chung;

Department of Internal Medicine, Chonnam National University Medical School, Gwang-Ju, Republic of Korea.

Background and Aims: It was already demonstrated that visceral fat accumulation is a major contributor for multiple risk factors clustering in patients with type 2 diabetes mellitus. Several studies demonstrated stronger positive relationships between the amount of visceral abdominal fat and insulin resistance and several cardiovascular risk factors than that of total body fat in patients with type 2 diabetes. Evaluation of visceral fat has been conducted using waist circumference and several imagings using computed tomography, magnetic resonance imaging, or ultrasonography (US). Ultrasound measurements have been recently shown to correlate better with cardiovascular risk factors than those derived from anthropometric measures. The aim of our study was to compare visceral fat thickness measured by ultrasonography with other anthropometric indicies in order to assess the relationship between these indicies and cardiovascular risk factors in type 2 diabetic subjects.

Materials and Methods: 217 type 2 diabetic patients participated in the study: 108 men (mean age $62.4 \pm 9.6$ years) and 109 women (mean age 62.0 \pm 10.1 years). Anthropometric, clinical, and laboratory data including body mass index (BMI; $\mathrm{kg} / \mathrm{m}^{2}$ ), waist circumference (WC), systolic and diastolic blood pressure (SBP and DBP; $\mathrm{mmHg}$ ), lipid profiles [total cholesterol (TC), fractions, and triglycerides (TG)] were measured. Insulin resistance was calculated by using HOMA-IR [fasting plasma insulin $(\mathrm{mU} / \mathrm{ml}) \mathrm{X}$ fasting plasma glucose $(\mathrm{mmol} / \mathrm{L}) / 22.5$ ]. All ultrasonographic procedures were performed by the same examiner using a $3.5-\mathrm{MHz}$ probe located $1 \mathrm{~cm}$ from umbilicus. Two US measurements of visceral (VFT) and subcutaneous fat (SFT) were taken. US-determined VFT was defined as the distance between the internal face of the rectus abdominis muscle and the anterior wall of the aorta.

Results: VFT was positively correlated with body mass index $(\mathrm{r}=0.457$, $\mathrm{p}<0.001)$, waist circumference $(\mathrm{r}=0.516, \mathrm{p}<0.001)$, HOMA-IR $(\mathrm{r}=0.391$, $\mathrm{p}<0.05)$, and triglycerides $(\mathrm{r}=0.328, \mathrm{p}<0.05)$, whereas it was negatively correlated with HDL-cholesterol $(\mathrm{r}=-0.342, \mathrm{p}<0.05)$. The intraobservational reproducibility of the ultrasonographic estimations was $1.8-2.4 \%$ for the VFT. The patients were classified by the tertile on the basis of their VFT; the low tertile ( $<42.6 \mathrm{~mm}$ in men and $<40.7 \mathrm{~mm}$ in women), the middle tertile ( $42.6-$ $60.3 \mathrm{~mm}$ in men and $36.0-57.9 \mathrm{~mm}$ in women), and the high tertile (> 60.3 $\mathrm{mm}$ in men and $>57.9 \mathrm{~mm}$ in women). Men in the middle and high tertiles had a greater prevalence of coronary heart disease, low-HDL cholesterol, and metabolic syndrome than those in the low tertile. In women, the prevalence of coronary heart disease and metabolic syndrome was higher in the middle and high tertiles than in the low tertile. The high tertile of VFT had higher odd ratio of hypertension ( $\mathrm{OR}=4.34,95 \%$ CI 1.23 12.87), dyslipidemia (OR=9.51, 95\% CI 1.38 62.12), and metabolic syndrome (OR=15.29, 95\% CI 2.52 69.16) than did those in the low tertile, after adjustment for age, sex, and body mass index.
Conclusion: This study suggested that visceral fat thickness measured by ultrasonography may be the reliable and simple index for quantifying metabolic risk factors of cardiovascular disease in Korean type 2 diabetic patients.

\section{6}

Association of subclinical carotid atherosclerosis with ultrasonographic non-alcoholic liver steatosis in diabetic and non-diabetic subjects

D. Torres, G. Parrinello, S. Paterna, G. Licata;

Internal Medicine, University of Palermo, Italy.

Background and Aims: Non-alcoholic fatty liver disease (NAFLD) is the most common cause of elevated liver enzymes in adults. Ultrasonography of the liver is able to identify it with high sensitivity and specificity. NAFLD is frequently associated with type 2 diabetes (DM) and metabolic syndrome. In this study we investigate the relations of ultrasonographyc NAFLD with carotid artery intima-media thickness (IMT, a powerful predictor of cardiovascular (CV) and cerebrovascular complications defined by a thickness $\geq 0,9 \mathrm{~mm}$ and $\leq 1,5 \mathrm{~mm}$ ) and plaques (defined by a tickness $>1,6 \mathrm{~mm}$ ) and C-reactive protein (CRP) levels.

Materials and Methods: We studied a cohort of 54 patients (pts) $(32 \mathrm{M}$, mean age $54 \pm 11$ years) referred to ultrasound laboratory with diagnosis of NAFLD (defined by presence of bright liver echo pattern according to Saverymuttu criteria and by absence of alcohol abuse and other liver diseases) and subgrouped them in two sex- age-matched groups on the basis of presence $(n=27)$ or absence $(n=27)$ of DM and compared with 25 sex- age-matched diabetic pts (C) without ultrasonographic NAFLD. There were not significant differences in hypertension, central obesity and dyslipidemia prevalence between groups. Pts with inflammatory, infective, neoplastic, kidney and CV diseases or events were excluded.

Results: A positive correlation between IMT and CRP levels $(\mathrm{p}<0.01)$ only in all the patients with NAFLD and DM was found. When compared with C, all the enrolled patients with NAFLD had higher levels of CRP $(0,64 \pm 0,36$ $\mathrm{mg} / \mathrm{dl}$ vs. $0,28 \pm 0,27 \mathrm{mg} / \mathrm{dl}, \mathrm{p}<0,01)$, a greater IMT $(1.1 \pm 0.3 \mathrm{~mm}$ vs. $0.98 \pm 0.24$ $\mathrm{mm}, \mathrm{p}<0,01)$ and also greater prevalence of ACP ( $54 \%$ vs. $33 \%, \mathrm{p}<0.01)$. This association was more relevant in the soubgroup with DM $(\mathrm{p}<0,01)$. IMT was associated with degree of hepatic steatosis defined ultrasonographically $(p<0,05)$. If we considered all the diabetic and non-diabetic pts after multiple regression analysis NAFLD and its severity, adjusted for all confounders factors, are predictors of higher carotid IMT $(\mathrm{p}<0.01)$. Carotid IMT was significantly higher in NAFLD pts when associated with hypertension $(p<0.05)$. Diabetes, central obesity and hypetrigliceridaemia were the most predictive factors of NAFLD in all the pts.

Conclusion: Our results suggest that NAFLD and its severity are significantly associated with early asymptomatic carotid atherosclerosis and CRP serum levels, independently by the presence of DM. These data indicate that NAFLD, per se, may contribute to higher risk of $\mathrm{CV}$ disease suggesting a potential inflammatory pathogenic loop. This clinical-ultrasonographic condition must be considered, in our opinion, an additional criterium to classical CV risk factors for an early metabolic and CV stratification.

\section{7}

Insulin inhibits tissue factor expression in monocytes

J. Gerrits, J. W. Akkerman;

Department of Clinical Chemistry and Haematology, University Medical Center Utrecht, The Netherlands.

Background and Aims: Patients with diabetes mellitus (DM) have a 2-4 fold increased risk of coronary artery disease. They suffer from both microvascular and macrovascular complications. DM2 patients have hyperactive platelets in the circulation and elevated plasma levels of many clotting factors leading to augmented activity of the coagulation system. We demonstrated previously in healthy individuals that insulin inhibits platelets through inactivation of the inhibitory G-protein of adenylyl cyclase Gi, thereby interfering with P2Y12mediated suppression of cAMP formation. Platelets from DM2 patients have lost insulin signaling to cAMP, which explains the hyperaggregability in this disease. In the present study we investigated whether insulin also interferes with tissue factor (TF) expression in monocytes, since TF is the key initiator of the blood coagulation cascade. 
Materials and Methods: Human monocytes were stimulated with $1 \mu \mathrm{g} / \mathrm{ml}$ lipopolysaccharide (LPS) for $2-5$ hours at $37^{\circ} \mathrm{C}$ and expression of TF-mRNA (Taqman PCR), -protein (western blotting) and -activity (coagulation factor $\mathrm{X}$ activation) were analyzed. TF expression was measured in the soluble and membrane compartments. Insulin signaling though the receptor, IRS-1 / IRS- 2 and Gi was measured by western blotting. Monocytic THP- 1 cells were incubated with the fluorescent dye Fura-2/AM and after stimulation with 10 $\mu \mathrm{M}$ ATP and $10 \mathrm{ng} / \mathrm{mL}$ monocyte chemoattractant protein-1 (MCP-1) $\mathrm{Ca}^{2+}$ mobilization and influx were measured.

Results: LPS induced a 3-4 fold increase of TF activity in soluble and membrane compartements, which was set at $100 \%$. Preincubation for 15 minutes with insulin $(0.1-100 \mathrm{nM})$ dose-dependently inhibited TF upregulation (mRNA, protein) leading to a $34.4 \pm 3.1 \%$ (means \pm SEM, $n=4$ ) reduction of TF activity at $100 \mathrm{nM}$ insulin. Inhibition was accompanied by Tyr phosphorylation of the insulin receptor, IRS-1, Gi and formation of an IRS-1 - Gi complex, suggesting that insulin signaling leads to an increase in cAMP formation by interfering with the action of Gi. Indeed, increased cAMP levels induced by incubation with iloprost (a stable $\mathrm{PGI}_{2}$ analog), forskolin (an adenylyl cyclase activator), IBMX (an inhibitor of phosphodiesterases) and membrane permeable dibutyryl cAMP inhibited TF upregulation. For incubation with iloprost this inhibition was $75.7 \pm 5.0 \%$ (means \pm SEM , $\mathrm{n}=4$ ). Although LPS-induced TF upregulation occurred without an increase in cytosolic $\mathrm{Ca}^{2+}\left\{\left[\mathrm{Ca}^{2+}\right]_{i n}\right\}$, it was inhibited by $80.5 \pm 4.5 \%$ (means \pm SEM, $\mathrm{n}=4$ ) by $\mathrm{Ca}^{2+}$ sequestration (BAPTA-AM), indicating that TF upregulation is $\mathrm{Ca}^{2+}$ dependent. Activation of the P2X7 channel with ATP and addition of ionophore A23187 $\left(1 \mathrm{mM}\left[\mathrm{Ca}^{2+}\right]_{\text {out }}\right)$ increased $\left[\mathrm{Ca}^{2+}\right]_{\text {in }}$ to $500 \mathrm{nM}$ and 1 $\mathrm{mM}$ respectively and enhanced TF expression respectively by $143.9 \pm 58.7 \%$ $\mathrm{n}=4$ and $231.8 \pm 15.7 \% \mathrm{n}=3$ (means \pm SEM). Insulin neither changed these $\mathrm{Ca}^{2+}$ increases nor TF expression. Stimulation with MCP-1 increased $\left[\mathrm{Ca}^{2+}\right]_{\text {in }}$, which was inhibited by preincubation with iloprost and insulin. Monocytes from a limited number of DM2 patients failed to show suppression of TF upregulation by insulin.

Conclusion: These results show that in monocytes from normal subjects insulin suppresses the upregulation of TF expression by LPS, possibly by interfering with the regulation of cAMP and cytosolic $\mathrm{Ca}^{2+}$. Preliminary data suggest that this suppression is absent in patients with DM2.

Supported by the Dutch Diabetes Research Foundation

\section{PS 129 Genetic determinants of dyslipidaemia and cardiovascular disease}

\section{8}

AHSG tagSNPs associate with type 2 diabetes and dyslipidaemia in a large-scale population-based sample of 7,683 Danish whites G. Andersen ${ }^{1}$, K. S. Burgdorf ${ }^{1}$, T. Spars $\emptyset^{1}$, K. Borch-Johnsen ${ }^{1,2}$, T. Jørgensen ${ }^{2}$, T. Hansen ${ }^{1}$, O. Pedersen ${ }^{1}$;

${ }^{1}$ Steno Diabetes Center, Gentofte, Denmark, ${ }^{2}$ Glostrup University Hospital, Research Centre for Prevention and Health, Glostrup, Denmark.

Background and Aims: The gene encoding the $\alpha 2$ Heremans-Schmid glycoprotein $(A H S G)$ is a credible biological and positional candidate for type 2 diabetes and the metabolic syndrome, and previous attempts to relate AHSG variation with type 2 diabetes, obesity and correlated phenotypes in Swedish and French Caucasians have been largely successful. We sought to investigate seven frequent $A H S G$ tagSNPs in relation to a range of metabolic traits including type 2 diabetes, obesity, dyslipidaemia, and hypertension. Materials and Methods: The polymorphisms were genotyped in 7,683 Danish whites, providing a statistical power of more than $99 \%$ to replicate previous findings. Genotyping was performed using Taqman allelic discrimination or matrix-assisted laser desorption/ionisation time-of-flight mass spectrometry of PCR-generated primer extensions. All subjects underwent a standard $75 \mathrm{~g}$ oral glucose tolerance test.

Results: The rs2077119 and rs2518136 polymorphisms were associated with type 2 diabetes, and in a combined analysis of the present and a previous study the rs2077119 remained significant (odds ratio 0.90 [0.84-0.97], $p=0.007$ ). Furthermore, two AHSG haplotypes were associated with dyslipidaemia $(p=0.003)$. rs4917 was associated with lower fasting and postprandial serum insulin secretion and improved insulin sensitivity estimated by the homeostasis model assessment of insulin resistance. Indications of epistatic effects of AHSG variants with the IRS1 Gly971Arg polymorphism were observed for fasting serum triglyceride concentrations.

Conclusion: Common variation in AHSG may contribute to the interindividual variation in metabolic phenotypes, specifically type 2 diabetes and dyslipidaemia.

\section{9}

A common variant (T94A) of L-FABP is associated with fasting triglycerides and LDL-cholesterol in women

E. Fisher ${ }^{1}$, C. Weikert ${ }^{1}$, I. Lindner ${ }^{2}$, M. Klapper ${ }^{2}$, M. Möhlig ${ }^{3}$, J. Spranger ${ }^{3}$, A. Pfeiffer ${ }^{3}$, H. Boeing ${ }^{1}$, F. Döring ${ }^{2}$;

${ }^{1}$ Epidemiology, German Institute of Human Nutrition Potsdam-Rehbruecke, Nuthetal, ${ }^{2}$ Molecular Nutrition, Institute of Human Nutrition and Food Science, Kiel, ${ }^{3}$ Clinical Nutrition, German Institute of Human Nutrition Postdam-Rehbruecke, Nuthetal, Germany.

Background and Aims: A common variant (c.340A $>G$ ) of the human liver fatty acid binding protein $(F A B P 1)$ gene, that alters a threonine $(\mathrm{T})$ to alanine (A) at position 94 (T94A), had previously been shown to be associated with altered triglyceride levels after treatment with fenofibrate. Carriers of the A94 allele also had higher baseline plasma-free fatty acid levels, lower body mass index (BMI), and lower waist circumference than T94 homozygotes. To determine the possible role of the FABP1 T94A polymorphism in modulating susceptibility to traits of the metabolic syndrome and accomplished diseases, we genotyped the polymorphism in the EPIC-Potsdam type 2 diabetes (T2D) case-control study and the EPIC-Potsdam cardiovascular disease (CVD) case-cohort study.

Methods: Genotyping of the c.340A $>\mathrm{G}$ polymorphism (rs2241883) was performed with the TaqMan system (ABI, Foster City, CA, USA), and fluorescence was measured with ABI Prism $7900 \mathrm{HT}$ sequence detection system. Analysis of covariance (ANCOVA) was used to assess the association between genotypes and quantitative traits of the metabolic syndrome in men and women from the EPIC-Potsdam-CVD subcohort $(\mathrm{N}=826)$. Cox proportional hazard regression analysis was used for the examination of the relationship between FABP1 T94A and risk of CVD in 220 incident cases of myocardial infarction and ischemic stroke and 820 non-cases. To estimate the risk of T2D associated 
with FABP1 T94A, logistic regression analysis was applied in a study sample of 192 incident cases and 384 sex- and age-matched controls. All analysis were conducted with multivariate adjustments. Power calculation revealed a possible risk determination for T2D of OR 1.7/1.9 (males/females) and for CVD of RR 1.5/1.7 (males/females) in additive inheritance models.

Results: The overall frequency of the minor allele was 0.373 in EPIC-Potsdam study subjects. In women, a significant trend of higher plasma triglyceride $(P=0.01)$ and LDL-cholesterol $(P=0.02)$ concentrations were seen for A-allele carriers after adjustment for age, menopausal status, hormone intake and Apo E genotype. BMI, waist circumference, HDL-cholesterol, fasting glucose levels, and blood pressure measurements showed no association with FABP1 T94A genotypes. Also, no significant association with disease risk could be attained in both EPIC-Potsdam studies.

Conclusion: The study provides first evidence for an association of the FABP1 T94A polymorphism and fasting plasma triglycerides and LDL-cholesterol concentration in females. These results support previous findings about the functional role of the L-FABP protein in fatty acid metabolism in the liver. We thank Y. Dignal, D. Stengel, S. Kaschner and M. Steinke and Wolfgang Bernigau for excellent technical assistance

\section{0}

\author{
Association of variants in upstream transcription factor 1 (USF1) \\ with type 2 diabetes in the Dutch population \\ S. J. R. Meex ${ }^{1}$, J. V. van Vliet-Ostaptchouk ${ }^{2,3}$, C. J. H. van der Kallen ${ }^{1}$, \\ M. M. J. van Greevenbroek ${ }^{1}$, C. G. Schalkwijk ${ }^{1}$, E. J. M. Feskens ${ }^{4}$, \\ E. E. Blaak ${ }^{5}$, T. W. van Haeften ${ }^{6}$, T. W. A. de Bruin ${ }^{1,7}$, C. Wijmenga ${ }^{8,9}$, \\ M. H. Hofker ${ }^{2,3}$, C. D. A. Stehouwer ${ }^{1}$; \\ ${ }^{1}$ Department of Internal Medicine, Maastricht University, The \\ Netherlands, ${ }^{2}$ Department of Molecular Genetics, Maastricht University, \\ The Netherlands, ${ }^{3}$ Department of Pathology and Laboratory Medicine, \\ University Medical Center Groningen and University of Groningen, The \\ Netherlands, ${ }^{4}$ Human Nutrition, Wageningen University, The Netherlands, \\ ${ }^{5}$ Human Biology, Maastricht University, The Netherlands, ${ }^{6}$ Department of \\ Internal Medicine, University Medical Center Utrecht, The Netherlands, \\ ${ }^{7}$ GlaxoSmithKline, Research Triangle Park, United States, ${ }^{8}$ DBG-Dept. of \\ Medical Genetics, University Medical Center Utrecht, The Netherlands, \\ ${ }^{9}$ Department of Genetics, University Medical Center Groningen and \\ University of Groningen, The Netherlands.
}

Background and Aims: Type 2 diabetes shares substantial genetic and phenotypic overlap with Familial Combined Hyperlipidemia. Upstream stimulatory factor 1 (USF1), a basic helix-loop-helix leucine zipper transcription factor which regulates expression of genes involved in lipid- and glucose metabolism is postulated to be such a shared genetic determinant. Although two common polymorphisms in USF1 are consistently associated with Familial Combined Hyperlipidemia and lipid traits, their impact on type 2 diabetes susceptibility is less well established. Replication studies in large cohorts are required to provide conclusive evidence concerning the true contribution of USF1 to type 2 diabetes susceptibility, and allow robust assessment of effect size. We evaluated these variants for association with type 2 diabetes in 2003 Dutch Caucasians comprising 700 patients with type 2 diabetes and 1300 healthy controls from two independent cohorts.

Materials and Methods: The first case-control sample comprised 502 subjects with type 2 diabetes from the Breda cohort and 920 healthy blood bank donors of Dutch Caucasian origin. The second case-control sample included 211 subjects with type 2 diabetes $(\mathrm{N}=211)$, and 379 normoglycaemic control subjects. These subjects were selected from a group of 2700 Caucasian subjects with one or more cardiovascular risk factors, which were screened for type 2 diabetes.

Results: SNP rs2073658 and SNP rs3737787 were in complete LD $\left(D^{\prime}=1\right.$, $\mathrm{r}=1$ ). In the first case-control sample, prevalence of the major allele was higher in patients than in controls ( $75 \%$ versus $71 \%$, OR $1.25, \mathrm{p}=0.015)$. In the second case control sample, a similar frequency difference and concomitant odds ratio was observed: $76 \%$ in patients versus $72 \%$ in control subjects, $(\mathrm{OR}=1.22)$. Insufficient power of the second case control sample prevented this frequency difference to meet the threshold for statistical significance $(p=0.15)$. The effect-size and -direction were identical in both case-control samples and a combined analysis strengthened the evidence for association $(\mathrm{OR}=1.24, \mathrm{p}=0.005)$. Importantly, the increased risk for type 2 diabetes susceptibility could be ascribed to the major allele, and its high frequency translated in a substantial population attributive risk of $14.5 \%$.
Conclusion: These data suggest that the major allele of rs2073658 is associated with a modestly increased risk for developing type 2 diabetes in Dutch Caucasians, with considerable impact at the population level.

\section{1}

Lipoprotein lipase HindIII polymorphism influences HDL-cholesterol in statin-treated patients with coronary artery disease

M. Javorsky ${ }^{1}$, D. Gašperíková2 , J. Ukropec ${ }^{2}$, B. Sedláková ${ }^{3}$, I. Riečanský, O. Križanová ${ }^{3}$, E. Šeboková ${ }^{2}$, M. Dobríková ${ }^{1}$, I. Klimeš ${ }^{2}$, I. Tkáč́c;

${ }^{1} 4$ th Department of Medicine, Medical School PJ Safarik University, Kosice, ${ }^{2}$ Diabetes Research Laboratory and DIABGENE, Institute of Experimental Endocrinology, Bratislava, ${ }^{3}$ Institute of Molecular Physiology and Genetics, Slovak Academy of Sciences, Bratislava, ${ }^{4}$ Department of Cardiology, National Institute of Cardiovascular Diseases, Bratislava, Slovakia.

Background and Aims: HDL-cholesterol (HDL-C) is a recognized atheroprotective factor and low HDL-C levels occur frequently in patients with coronary artery disease (CAD). Regulation of HDL-C level most likely results from the interaction of genes involved in the lipoprotein metabolism and nongenetic factors. We studied associations and interactions among HindIII polymorphism of the lipoprotein lipase gene $(L P L)$ and selected non-genetic factors with respect to HDL-C levels in patients with CAD.

Materials and Methods: 288 Slovak (Caucasian) patients (35\% women, mean \pm SEM age $60 \pm 1$ years, BMI $29 \pm 0.3 \mathrm{~kg} / \mathrm{m}^{2}, 25 \%$ with type 2 diabetes) with documented coronary artery disease were examined and genotyped for LPL HindIII (rs320) by PCR/RFLP method. HDL-C was determined by a direct enzymatic method.

Results: In the total sample, there were no significant differences across the LPL genotypes in the adjusted HDL-C levels or other lipids, although a trend toward higher HDL-C and lower triglycerides in $\mathrm{H}-\mathrm{H}$ - homozygotes was observed. Multiple linear regression identified a significant interaction between LPL HindIII and statin treatment, which together with gender and diabetes explained $12.1 \%$ of HDL-C variance. Accordingly, in statin treated patients we observed a significant stepwise increments of the HDL-C level related to the increasing number of $\mathrm{H}$-alleles ( $\mathrm{p}=0.04$ for linear trend), whereas no such association was observed in patients without hypolipidemic treatment. Within the group of statin-treated patients, $\mathrm{H}-\mathrm{H}$ - homozygotes had the level of HDL-C by $16 \%(0.19 \mathrm{mmol} / \mathrm{l})$ higher when compared with the $\mathrm{H}+\mathrm{H}+$ homozygotes $(\mathrm{p}=0.06)$.

Conclusion: HDL-C may be influenced by an interaction between statin treatment and LPL HindIII genotype. However, effect of this interaction appears to be small when compared with the effect of non-genetic factors. Our findings require replication in a pharmacogenetic study.

Supported by State Programme SP51/0280800/0280802, Safarik University

\section{2}

Galectin 2 but not Lymphotoxin- $\alpha$ polymorphisms predicts cardiovascular mortality in families with type 2 diabetes

A. Jonsson ${ }^{1}$, M. Sjögren ${ }^{1}$, M. Svensson ${ }^{1}$, P. Almgren ${ }^{1}$, B. Isomaa ${ }^{2}$, L. Groop ${ }^{1,3}$, M. Orho-Melander';

${ }^{1}$ Department of Clinical Sciences, Diabetes \& Endocrinology, Lund University, Malmö, Sweden, ${ }^{2}$ Folkhälsan Research Center, Biomedicum, Folkhälsan Genetic Institute, Helsinki, Finland, ${ }^{3}$ Department of Medicine, Helsinki University Central Hospital, Finland.

Background and Aims: A single nucleotide polymorphism (SNP) in the gene coding for the inflammatory cytokine lymphotoxin- $\alpha$ (LTA, rs1041981, AA-genotype) has been shown to be associated with myocardial infarction (MI) in some but not all previous studies. One reason for this controversy could be that LTA is located in the HLA gene cluster on chromosome $6 \mathrm{p} 21$ making it difficult to determine if the association is due to LTA itself or other members of the cluster. Galectin 2, the principal ligand of LTA up-regulates the secretion of LTA and a polymorphism in intron 1 of the galectin 2 gene on chromosome 22q13 (LGALS2; rs7291467, C3279T) has been associated with $50 \%$ reduced transcriptional activity of LGALS2 and protection from MI. Our aim was to investigate if the two polymorphisms in LTA and LGALS2 are associated with cardiovascular or total mortality in type 2 diabetes (T2D) families. 
Materials and Methods: The SNPs rs1041981 and rs7291467 were genotyped using Sequenom iPLEX ${ }^{\text {st }}$ in 4658 subjects above the age of 35 years (2144 males, 2514 females, aged $58.2 \pm 13.8$ years) from the Botnia T2D family study, followed for a mean time of 11 years. Cause of mortality was divided into cardiovascular (predominantly coronary) or other causes. Survival analyses were performed using Cox regression analyses, stratified by sex and T2D and adjusted for family correlations.

Results: During the follow-up time 1091 subjects died (23.4\%; 528 males, 563 females) of which 337 due to cardiovascular events (7.2\%; 183 males, 154 females). The frequency of the LGALS2 rs7291476 CC-genotype was higher among individuals who died due to cardiovascular disease compared to those who remained alive or died due to other causes $(20.0$ vs. $15.4 \%$, $\mathrm{p}=0.028$ ) while the frequency of the LTA rs1041981 AA-genotype did not differ between the groups ( 15.9 vs. $16.2 \%, \mathrm{p}=0.87$ ). In line with this, the LGALS2 rs7291467 CC-genotype but not the LTA rs1041981 AA-genotype predicted cardiovascular mortality (HR 1.38 [1.02-1.85] $\mathrm{p}=0.035$ and 0.98 [0.71-1.35] $\mathrm{p}=0.89$, respectively). None of the two SNPs predicted other than cardiovascular mortality nor were they associated with total mortality ( $\mathrm{p}=0.94$ and $\mathrm{p}=0.69$ ).

Conclusion: Our data provide strong support for the earlier described association between LGALS2 rs7291467 by for the first time demonstrating that it also influences cardiovascular mortality. This could be explained by up-regulation of the inflammatory pathway mediated by LTA in carriers of the CC-genotype of LGALS2.

\section{3}

Reduced risk of coronary artery disease in type 2 diabetic men associated with the A-allele of the $-866 \mathrm{G}>\mathrm{A}$ variant in the promoter of UCP2 gene: the DIABHYCAR prospective study

G. Velho ${ }^{1}$, N. Cheurfa ${ }^{1}$, D. A. F. Ferrarezi ${ }^{1}$, G. M. Brenner ${ }^{1}$, F. Fumeron ${ }^{1,2}$, M. Marre ${ }^{1,2}$;

${ }^{1}$ Unité 695, INSERM, ${ }^{2}$ Faculté de Médecine Xavier Bichat, Université Paris 7 - Denis Diderot, Paris, France.

Background and Aims: Uncoupling protein 2 (UCP2) inhibits insulin secretion, decreases lipogenesis and increases adiponectin gene expression in adipocytes, decreases the production of reactive oxygen species, notably in macrophages, and is associated with protection against atherosclerosis. A common G/A variant at position -866 in the UCP2 promoter region (SNP rs659366) was shown to modulate mRNA expression, with increased expression associated with the A-allele in adipocytes and pancreatic beta-cells. Allelic associations with several phenotypes related to obesity, glucose homeostasis and dyslipidemia were observed. In the present study, we investigated the association of the $-866 \mathrm{G}>\mathrm{A}$ SNP with coronary artery disease (CAD) in a cohort of subjects with type 2 diabetes (T2DM) followed prospectively for cardiovascular and renal events.

Materials and Methods: We studied 3122 unrelated French type 2 diabetic subjects with high cardiovascular risk from the 6 year prospective DIABHYCAR study. The total prevalence of myocardial infarction at the latest follow-up of DIABHYCAR was $8.6 \%$, comprising 267 cases ( 172 baseline and 95 incident cases) in 257 subjects. When considering the 295 subjects who underwent coronary artery bypass graft (CABG) and 137 cases of sudden death, the prevalence of CAD was $18.5 \%$, (577 subjects). The variant was genotyped using an Assay by Design kit from Applied Biosystems. Genotype associations with CAD were assessed by logistic regression analyses and odds ratios (OR) were computed.

Results: We observed an inverse association of the A-allele with incident cases of CAD in a dominant model $(\mathrm{OR}=0.74,95 \%$ C.I. $0.61-0.91, \mathrm{p}=0.0036)$ as well as in a codominant model ( $\mathrm{OR}=0.63,95 \%$ C.I. $0.47-0.85, \mathrm{p}=0.0027)$. The association persisted after adjustment for age, BMI, smoking, total cholesterol, HDL-cholesterol, triglycerides, urinary albumin excretion, creatinine clearance, $\mathrm{HbAlc}$, duration of diabetes and arterial hypertension. Similar results were observed when we considered the total prevalence of CAD. A significant interaction between genotype and sex was observed in these associations. Stratification by sex showed the allelic associations with CAD to be stronger in men, while only a trend towards an association in a recessive model was observed in women. To assess which CAD phenotypes contributed to these associations we compared genotype frequencies of cases of myocardial infarction, CABG or sudden death to that of subjects with no CAD. Odds ratio for the A-allele in a dominant model was $0.72(95 \%$ C.I. $0.55-0.93, \mathrm{p}=0.01)$ for myocardial infarction, 0.76 (95\% C.I. 0.60-0.97, $\mathrm{p}=0.03)$ for $\mathrm{CABG}$, and 0.81 (95\% C.I. 0.57-1.14, $\mathrm{p}=0.22)$ for sudden death These associations were stronger in men: 0.64 (95\% C.I. 0.48-0.85, $\mathrm{p}=0.002)$ for myocardial infarction, 0.73 ( $95 \%$ C.I. $0.56-0.96, \mathrm{p}=0.024$ ) for $\mathrm{CABG}$, and 0.64 (95\% C.I. $0.43-0.95, p=0.03)$ for sudden death. No associations were observed in women and similar results were observed with a codominant model.

Conclusion: The A-allele of the-866G $>\mathrm{A}$ variant of UCP2 was associated with reduced risk of CAD in men with T2DM. Decreased risk of myocardial infarction, $\mathrm{CABG}$ and sudden death contributed individually and significantly to the reduction of CAD risk. This association was independent from other common CAD risk factors.

\section{4}

A polymorphism at the protein tyrosine phosphatase receptor type $F$ (PTPRF) locus is associated with increased risk of coronary artery disease in type 2 diabetes

C. Menzaghi ${ }^{1}$, G. Paroni ${ }^{1}$, C. De Bonis ${ }^{1}$, A. Coco ${ }^{1}$, S. Mastroianno ${ }^{1}$, G. Miscio ${ }^{1}$, V. Tassi ${ }^{1}$, S. Bacci ${ }^{1}$, V. Trischitta ${ }^{1,2}$;

${ }^{1}$ Research Unit of Diabetology and Endocrinology, IRCCS, San Giovanni Rotondo, ${ }^{2}$ Clinical Sciences, Sapienza University, Rome, Italy.

Background and Aims: PTPRF is known to down regulate insulin receptor signalling and downstream insulin action. PTPRF is overexpressed in insulinresponsive tissues of obese, insulin-resistant individuals, and may contribute to the pathogenesis of insulin resistance. A polymorphism in the PTPRF gene has been previously associated with insulin resistance, implicating variability at this locus as a possible etiologic factor in the development of insulin resistance-related abnormalities, including coronary artery disease (CAD). The aim of our study was to comprehensively evaluate the role of PTPRF polymorphisms in determining CAD risk.

Materials and Methods: In silico analysis using genotyping data downloaded from the HapMap database (Data Release 21/Jun 06) of the chromosomal region evenly spanning the PTPRF gene $(93 \mathrm{~kb})$, revealed three linkage disequilibrium blocks that could be tagged by four SNPs (htSNPs) which account for $87 \%$ of all possible haplotypes. The four htSNPs (rs11590627 for the first block, rs2782641 and rs 10890257 for the second block, and rs516790 for the third block) were typed in a sample of 592 type 2 diabetic patients comprising $248 \mathrm{CAD}$ cases (by stenosis $>50 \%$ at coronary angiography and/ or previous myocardial infraction) and $344 \mathrm{CAD}$ controls (i.e. asymptomatic patients with no signs of myocardial ischemia at resting and at stress ECG or with stenosis $<50 \%$ at coronary angiography.

Results: A significant association was observed between CAD and rs 2782641 (minor allele frequency $=0.202$ in CAD-positive vs. 0.137 in CAD-negative, $\mathrm{p}=0.035$ ). Minor allele homozygous had significantly increased CAD risk $(\mathrm{OR}=1.70,95 \%$ C.I. $=1.03-2.74, \mathrm{p}=0.037$ after adjusting for age, gender and smoking status). The association was still significant also after adjusting for other known risk factors such as adiposity measures, hypertension and dyslipidemia (data not shown).

Conclusion: In conclusion, these findings point to PTPRF gene as a modulator of CAD risk in type 2 diabetes.

Supported by the Italian Ministry of Health 


\section{PS 130 Cardiovascular complications: therapeutic interventions}

\section{5}

EuroAction: the achievement of lifestyle, risk factor and therapeutic targets at one year in coronary patients and their partners

S. B. Connolly ${ }^{1}$, K. Kotseva ${ }^{2}$, C. Jennings ${ }^{2}$, A. Mead ${ }^{2}$, J. Jones ${ }^{2}$, A. Holden ${ }^{2}$, T. Collier ${ }^{3}$, D. De Bacquer, , G. De Backer, D. Wood ${ }^{2}$;

${ }^{1}$ Cardiovascular Medicine, Imperial College, London, United Kingdom, ${ }^{2}$ Cardiovascular medicine, Imperial College, London, United Kingdom, ${ }^{3}$ Epidemiology and Public Health, London School of Hygiene and Tropical Medicine, London, United Kingdom, ${ }^{4}$ Public Health, University of Ghent, Belgium.

Background and Aims: EUROACTION is a cluster randomised controlled trial of a nurse-led multi-disciplinary preventive cardiology programme which aims to manage coronary patients and their families to the European lifestyle, risk factor and therapeutic targets for cardiovascular disease prevention. Materials and Methods: In each of 6 European countries, a pair of matched general hospitals was randomised to receive the Euroaction intervention programme (INT) or to be monitored for usual care (UC). Consecutive coronary patients were proactively identified by the study nurse and invited with their partners to attend the 16 week EUROACTION programme with a repeat assessment at one year.Using random effects meta-analysis (REML estimation) differences in proportions achieving goal were compared.

Results: 946 patients in INT (60\% of all eligible) and 994 patients in UC (66\% of all eligible) attended the one year assessment. 401 partners $(57 \%$ of those identified) in INT and 335 partners in UC (45\% of all identified) attended the one year assessment. Table 1 shows the proportions of patients and their partners achieving the European risk factor and therapeutic targets. Despite proactive screening for diabetes in INT, there was no significant difference in the prevalence of diabetes between INT and UC (18.4\% INT, $17.7 \%$ UC) at one year. More patients in INT were diagnosed with impaired glucose tolerance ( $8.9 \%$ INT, $2.3 \% \mathrm{UC}, \mathrm{p}=0.25)$ although not significantly so. However, there was a trend for improved glycaemic control in INT with more patients achieving HbA1c $<7.0 \%$ compared with UC, (61\% INT, 50\% $\mathrm{UC}, \mathrm{p}=0.29$ ). Moreover, patients with diabetes in INT were significantly more likely to have a lower fasting glucose at one year compared with UC (OR 0.43 , $\mathrm{p}<0.05)$.

Conclusion: The EUROACTION programme helped coronary patients and their families to reduce abdominal obesity and become more physically active. In addition, there was an improvement in blood pressure and lipid control although significant for blood pressure only. In those with diabetes, there was evidence for improved glycaemic control. There was also more use of statins in the intervention arm. These changes should help coronary pateints and their families reduce their cardiovascular risk in the longer term.

Proportions of patients and partners achieving European targets at one year

Proportions achieving target (\%) Patients Patients Partners Partners

\begin{tabular}{lllll} 
& INT & UC & INT & UC \\
\hline Waist circumference: $\mathrm{M}<94 \mathrm{~cm}$, & $35^{\star}$ & 22 & 32 & 26
\end{tabular}

$\mathrm{W}<80 \mathrm{~cm}$

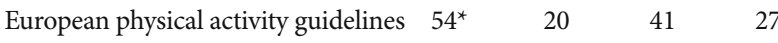

$\mathrm{BP}<140 / 90 \mathrm{~mm} \mathrm{Hg}$

$(<130 / 85$ in diabetes $)$

$\mathrm{TC}<5 \mathrm{mmol} / \mathrm{L}$

LDL-C $<3 \mathrm{mmol} / \mathrm{L}$

$\mathrm{HbAlc}<7.0 \%$

Antiplatelet

$65^{*}$

55

67

63

Beta-blocker

ACE inhibitor

$78 \quad 71$

$81 \quad 74$

$61 \quad 50$

$93 \quad 92$

$76 \quad 80$

75
74

$35 \quad 33$

Statin

$52 \quad 56$

${ }^{*} \mathrm{p}<0.05,{ }^{* *}$ to few for statistical analysis

Supported by AstraZeneca
1256

Addition of pioglitazone to atorvastatin exerts pleiotrophic and anti-inflammatory effects in non-diabetic patients at cardiovascular risk - the PIOVASC study

T. Forst ${ }^{1}$, M. Hanefeld ${ }^{2}$, W. Fuchs ${ }^{3}$, B. Wilhelm ${ }^{1}$, C. Köhler ${ }^{4}$, U. Lehmann ${ }^{3}$, J. Müller ${ }^{5}$, A. Pfützner ${ }^{1}$;

${ }^{1}$ Medical Dept., Institute for Clinical Research and Development, Mainz,

${ }^{2}$ Medical Dept., GWT, Dresden, ${ }^{3}$ Takeda-Pharma GmbH, Aachen,

${ }^{4}$ Laboratory Dept., GWT, Dresden, ${ }^{5}$ Acromion GmbH, Frechen, Germany.

Background and Aims: Insulin resistance and low-grade inflammation are important risk factors for atherosclerosis and the development of cardiovascular disease. We investigated the effect of pioglitazone in addition to atorvastatin compared to single treatment with atorvastatin on several established laboratory risk markers for cardiovascular disease, in a nondiabetic cardiovascular risk population.

Materials and Methods: 148 non-diabetic patients with increased cardiovascular risk, defined as hypertension and/or a previous cardiovascular event (76 male, 72 female; age $61.4 \pm 6.5$ years; BMI $29.2 \pm 4.1 \mathrm{~kg} / \mathrm{m}^{2}$; mean \pm SD) were included in our double blinded, placebo controlled parallel study. Patients were randomised to $40 \mathrm{mg}$ atorvastatin and placebo or 40 $\mathrm{mg}$ atorvastatin and $45 \mathrm{mg}$ pioglitazone. At baseline and after 6 months, fasting blood samples were taken for the measurement of Intact Proinsulin, Adiponectin, IL-6, MCP-1, MMP-9, sCD40L, P-Selectin and t-PA. In addition, the HOMAs Score was calculated from the measurement of fasting glucose and insulin plasma levels.

Results: In association with an improvement in HOMAs Score, combined treatment with atorvastatin and pioglitazone resulted in a reduction in Intact Proinsulin, MCP-1, P-Selectin, and t-PA levels, and an increase in Adiponectin levels (table 1). No significant effect of the combined treatment could be observed on IL-6, MMP-9, or sCD40L plasma levels. Single atorvastatin treatment did not effect any of the above mentioned laboratory parameters in our study.

Conclusion: The addition of pioglitazone to atorvastatin treatment exerts multiple pleiotrophic effects in non-diabetic patients with increased cardiovascular risk. Further investigations have to clarify if this kind of combination therapy might be able to improve the clinical outcome in this special risk population.

Laboratory parameters

( $m e a n \pm S D ;{ }^{\star} \mathrm{p}<0.05$ vs baseline; $\# \mathrm{p}<0.05$ in between treatments)

\begin{tabular}{|c|c|c|c|c|}
\hline \multirow[t]{2}{*}{ Parameter: } & \multicolumn{2}{|c|}{ Atorvastatin+Placebo } & \multicolumn{2}{|c|}{$\begin{array}{l}\text { Atorvastatin+ } \\
\text { Pioglitazone }\end{array}$} \\
\hline & Baseline & 6 months & Baseline & 6 months \\
\hline HOMAs & $2.9 \pm 2.2$ & $2.9 \pm 1.6$ & $3.0 \pm 2.3$ & $2.5 \pm 2.1 *$ \\
\hline $\begin{array}{l}\text { Intact Proinsulin } \\
(\mathrm{pmol} / \mathrm{l})\end{array}$ & $6.4 \pm 7.3$ & $5.9 \pm 4.5$ & $6.3 \pm 6.3$ & $5.3 \pm 4.3$ * \\
\hline Adiponectin $(\mu \mathrm{g} / \mathrm{ml})$ & $14.8 \pm 11.0$ & $14.3 \pm 11.5$ & $15.7 \pm 9.6$ & $32.0 \pm 19.0^{\star} \#$ \\
\hline IL-6 (pg/ml) & $3.23 \pm 0.2$ & $3.20 \pm 0.0$ & $3.37 \pm 1.0$ & $3.63 \pm 2.9$ \\
\hline MCP-1 (pg/ml) & $435 \pm 120$ & $432 \pm 119$ & $431 \pm 119$ & $409 \pm 107^{*}$ \\
\hline MMP-9 (ng/ml) & $277 \pm 126$ & $291 \pm 160$ & $291 \pm 117$ & $279 \pm 154$ \\
\hline sCD40 (pg/ml) & $2925 \pm 2135$ & $2532 \pm 1928$ & $3035 \pm 2161$ & $2792 \pm 2108$ \\
\hline P-Selectin (ng/ml) & $98.1 \pm 26.7$ & $96.6 \pm 28.5$ & $100.9 \pm 28.3$ & $95.3 \pm 25.9^{*}$ \\
\hline t-PA (ng/ml) & $13.6 \pm 6.4$ & $13.6 \pm 6.0$ & $12.9 \pm 5.3$ & $10.1 \pm 4.4^{\star} \#$ \\
\hline
\end{tabular}

Supported by Takeda Pharma GmbH Aachen, Germany

\section{7}

Risk of stroke and myocardial infarction is reduced in patients with type 2 diabetes treated with pioglitazone: results of a retrospective, claimsbased study

Y. Xu ${ }^{1}$, C. Vallarino ${ }^{1}$, R. W. Baran ${ }^{1}$, R. Spanheimer ${ }^{1}$;

${ }^{1}$ Takeda Pharmaceuticals North America, Inc., Deerfield, United States,.

Background and Aims: Patients with type 2 diabetes mellitus (T2DM) have a two- to four-fold increased risk of having a macrovascular event compared to age-matched individuals without T2DM. A recent controlled, randomized 
study suggested that the antidiabetes treatment pioglitazone $\mathrm{HCl}$ (PIO) may decrease the risk for such events, including stroke and myocardial infarction (MI). The aim of this retrospective, claims-based study was to determine whether the event-reducing treatment effects of PIO observed in the clinical trial setting are observed in clinical practice.

Materials and Methods: This analysis included data from patients enrolled in US healthcare plans affiliated with i3 Innovus. The i3 Innovus database includes eligibility, pharmacy, and medical claims data from more than 24 million patients. Using data from a $31 / 2$ year period (1 January 2003 to 30 June 2006), we extracted information for patients with a diagnosis of T2DM (ICD-9 codes 250.x0 and 250.x2). Two treatment groups were defined: (1) a group consisting of patients receiving the thiazolidinedione (TZD) PIO, with or without any other antidiabetes treatment (excluding the other clinically available TZD, rosiglitazone) and (2) a non-TZD-treated group consisting of patients receiving any antidiabetes treatments (excluding any TZD use). The index date was defined as the start date for the initial antidiabetes medication. Patients were included for analysis if they were 45 years of age or older at the index date, had continuous enrollment data available for at least 6 months before and 1 month after the index date, and did not have a record of stroke or MI in the 6 months before the index date. The definition of stroke included the following events (ICD-9 code): occlusion and stenosis of precerebral arteries (433.xx), occlusion of cerebral arteries (434.xx), transient cerebral ischemia (435.x), and acute cerebrovascular disease (436). MI was defined as an event of acute myocardial infarction (410.xx). The study endpoints were the first recorded occurrences of stroke or MI or last date of enrollment, whichever came first.

Results: The adjusted relative risk of stroke was 0.800 (95\% CI: $0.716-0.893$ ) for PIO $(\mathrm{N}=11,433)$ versus the non-TZD group $(\mathrm{N}=55,273)$ after controlling for age; gender; the status of hypertension, hyperlipidemia, heart failure, and edema; and use of other concurrent antidiabetic agents, clopidogrel, warfarin, aspirin, and statin. The adjusted relative risk of MI was 0.621 (95\% CI: 0.503 - 0.766) for PIO $(\mathrm{N}=11,917)$ versus the non-TZD group $(\mathrm{N}=57,956)$ using identical controls.

Conclusion: We conclude that, in a clinical practice setting in patients with T2DM, therapies that include PIO are associated with significant reductions in the risk for stroke or MI compared to non-TZD therapies.

Supported by Takeda Pharmaceuticals North America, Inc.

\section{8}

Effect of pramlintide treatment on markers of cardiovascular risk in patients with type 2 diabetes using basal insulin

C. Lush, B. Zhang, D. Maggs, T. Darsow, O. Kolterman; Amylin Pharmaceuticals, Inc., San Diego, United States.

Background and Aims: Cardiovascular disease (CVD) is a major cause of morbidity and mortality in patients with type 2 diabetes (T2DM). Pramlintide, an analog of the beta-cell hormone amylin, improves glycemic control and reduces body weight in patients with diabetes using mealtime insulin. A recent study examined the effects of pramlintide in patients with T2DM using basal insulin \pm oral agents (no mealtime insulin). This post-hoc analysis assessed the effects of pramlintide treatment on various cardiovascular (CV) risk factors in these patients.

Materials and Methods: In this 16-week, double-blind study, 211 patients with T2DM using insulin glargine \pm oral agents were randomized to treatment with pramlintide or placebo with major meals. Baseline demographics $(\mathrm{n}=$ 211, age $55 \pm 9 \mathrm{y}$, diabetes duration $11 \pm 6 \mathrm{y}, \mathrm{HbA}_{1 \mathrm{c}} 8.5 \pm 0.9 \%$, weight $103 \pm$ $18 \mathrm{~kg}$, BMI $35 \pm 5 \mathrm{~kg} / \mathrm{m}^{2}$; mean $\pm \mathrm{SD}$ ) were well-balanced between the two treatment groups. After optimization of pramlintide dosage (60 or $120 \mu \mathrm{g})$, insulin was titrated weekly, targeting a fasting plasma glucose concentration of $<100 \mathrm{mg} / \mathrm{dl}$.

Results: At Week 16, $\mathrm{HbA}_{1 \mathrm{c}}$, mean postprandial glucose (PPG) increments and body weight were significantly reduced in pramlintide- vs. placebotreated patients $\left(\mathrm{HbA}_{1 \mathrm{c}}:-0.7 \pm 0.1 \%\right.$ vs. $-0.4 \pm 0.1 \%, \mathrm{P}<0.05$; mean $\mathrm{PPG}$ excursions: $-24.4 \pm 3.6 \mathrm{mg} / \mathrm{dL}$ vs. $-0.4 \pm 3.0 \mathrm{mg} / \mathrm{dL}, \mathrm{P}<0.0001$; weight: $-1.6 \pm$ $0.3 \mathrm{~kg}$ vs. $+0.7 \pm 0.3 \mathrm{~kg}, \mathrm{P}<0.0001$; mean $\pm \mathrm{SE}$ ).

After 16 weeks of pramlintide treatment, hsCRP concentrations were also significantly reduced when compared to placebo (pramlintide $-0.85 \pm 0.21$ $\mathrm{mg} / \mathrm{L}$ vs. placebo $0.12 \pm 0.20 \mathrm{mg} / \mathrm{L} ; \mathrm{P}<0.01$; mean $\pm \mathrm{SE}$ ). The reduction in hsCRP was most pronounced in patients who were at high CV risk $(\geq 3$ $\mathrm{mg} / \mathrm{dl}$ ) at baseline (pramlintide $-1.57 \pm 0.35 \mathrm{mg} / \mathrm{L}(-24 \%)$ vs. placebo $0.55 \pm 0.37 \mathrm{mg} / \mathrm{L}(-9 \%) ; \mathrm{P}<0.05)$. Multiple regression analysis revealed that baseline hsCRP, BMI, and change from baseline in body weight were each significantly associated with changes in hsCRP. Positive trends were also noted in pramlintide-treated patients who had high risk lipid profiles or blood pressure at baseline. Pramlintide was generally well-tolerated, with the most frequent adverse events being mild-to-moderate hypoglycemia (pramlintide $44 \%$; placebo $47 \%$ ) and nausea (pramlintide $31 \%$; placebo $10 \%$ ). There was no treatment-related severe hypoglycemia.

Conclusion: Pramlintide, as an adjunct to basal insulin, improved glycemic control and reduced body weight in patients with type 2 diabetes. Pramlintideinduced weight loss was associated with a significant reduction in hsCRP levels, with the greatest decrease occurring in patients at high risk for $\mathrm{CV}$ disease at baseline. While long-term studies are needed, these results suggest that pramlintide treatment may have beneficial effects on CV risk factors, particularly in patients at high risk.

\section{9}

South Danish Diabetes Study: Effect of antidiabetic treatment on cardiovascular risk factors

J. Gram ${ }^{1}$, J. Henriksen ${ }^{1}$, H. Beck-Nielsen², T. SDDS Study Group²;

${ }^{1}$ Department of Endocrinology, University of Southern Denmark, Esbjerg, ${ }^{2}$ Department of Endocrinology, University of Southern Denmark, Odense, Denmark.

Type 2 diabetes is associated with an increased risk of cardiovascular disease. Intensive anti hyperglycaemic treatment may reduce macrovascular disease but is only proven for metformin. Postprandial hyperglycaemia as well as hyper-cholesterolemia, hypertension and microalbuminuria are well known cardiovascular risk factors. The effects of different antidiabetic treatments on these risk factors were studied in an investigator driven, 2 year randomised, intention to treat (ITT), and treat to target study, comparing the effect of NHP insulin given at bedtime with insulin aspart given at the 3 main meals. Beside insulin all patients received blinded treatment with metformin or placebo and rosiglitazone or placebo giving a total of 8 subgroups (factorial design). All participants received antihypertensive and cholesterol lowering treatment according to international guidelines. In total 371 patients with type 2 diabetes for at least 2 years and not sufficiently controlled on at least one antidiabetic treatment, were included in the study. Age $56.3 \pm 8.4 \mathrm{yr}$ (mean \pm SD), BMI 33.9 $\pm 5.7 \mathrm{~kg} / \mathrm{m} 2, \mathrm{HbAlc} 8.6 \pm 1.2 \%$, blood pressure $143 \pm 18 / 84 \pm 9.311$ patients completed the study but data analysis is based on 369 ITT subjects. Overall, following 2 years treatment, Hbalc was significantly lower in patients treated with aspart as compared to NPH insulin $(-0.41 \%, \mathrm{p}<0.001)$. Moreover, the add on treatment with metformin was associated with a further decrease in $\mathrm{Hbalc}$ as compared to placebo $(-0.60 \%, \mathrm{p}<0.001)$ as was rosiglitazone $(-0.55 \%$, $\mathrm{p}<0.001)$. Blood pressure was similar in insulin aspart, NPH insulin and metformin treated groups. However, rosiglitazone treatment reduced systolic blood pressure by $-3.7 \mathrm{mmHg}(\mathrm{p}<0.001)$ and diastolic blood pressure by -2.9 $\mathrm{mmHg}(\mathrm{p}<0.001)$ as compared to the non-rosiglitazone treatment group. Total cholesterol (T-CHOL) decreased in the metformin treated group $(-0.21$ $\mathrm{mmol} / \mathrm{l}, \mathrm{p}<0,012)$, and increased in the rosiglitazone group $(0.33 \mathrm{mmol} / \mathrm{l}$, $\mathrm{p}<0.001)$. HDL cholesterol increased in the insulin aspart treated group $(0.05$ $\mathrm{mmol} / \mathrm{l}, \mathrm{p}<0.012)$ as well as in the rosiglitazone group $(0.11 \mathrm{mmol} / \mathrm{l}, \mathrm{p}<0.001)$. LDL cholesterol decreased in the aspart group $(-0.18 \mathrm{mmol} / \mathrm{l}, \mathrm{p}<0.02)$ and the metformin group $(-0.17 \mathrm{mmol} / \mathrm{l}, \mathrm{p}<0.035)$ but increased in the rosiglitazone group $(0.30 \mathrm{mmol} / \mathrm{l}, \mathrm{p}<0.001)$. There were no differences in the effect on microalbuminuria between treatments.

In conclusion, intensive anti hyperglycaemic treatment regimes were followed by a significant reduction in blood pressure if rosiglitazone treatment was included. There were significant effects on lipid variables in treatment regimes including insulin aspart, metformin and rosiglitazone.

Supported by Novo Nordisk Scandinavia, GlaxoSmithKline, Denmark

\section{0}

Use of alpha-lipoic acid in patients with type 1 diabetes mellitus D. Najmutdinova, N. Sadikova; Endocrinology, the Tashkent Medical Academy, Tashkent, Uzbekistan.

Background and Aims: The aim of the research was to study the effect of alpha-lipoic acid on structural-functional remodeling of the left ventricle in patients with type I diabetes mellitus. 
Materials and Methods: The study enrolled 30 patients with type 1 DM complicated by the cardiovascular form of diabetic autonomic neuropathy. Twenty patients took alpha lipoic acid at a dose of $600 \mathrm{mg}$ a day against the background of conventional therapy (group I). The second group (10 patients) received only conventional treatment including individually dosed insulin and inhibitors of angiotensin converting enzyme, enapril. Before and in 3 months after treatment all the patients underwent cardiovascular tests, subjective and objective assessment of peripheral innervation, echocardiography and ECG with determination of heart rate variability and dispersion of QT-interval.

Results: Significant positive changes of parasympathetic cardiovascular tests were revealed in both therapeutic groups: inhalation/exhalation by $120.46 \%$ and $109.20 \%$, Valsalva test $-7.26 \%$ and $1.76 \%$ ), $30 / 15$ test $-4.85 \%$ and $2.13 \%$, respectively in groups I and II $(p<0.05)$. Differences of dynamics between groups were significant for Valsalva and $30 / 15$ tests $(p<0.05)$. Cardiovascular tests with isometric load did not change in both groups. Scores characterizing the condition of peripheral innervation did not change in group II and significantly decreased in group I - neuropathic symptomatic count (NSC) by $22.35 \%$ and neuropathic dysfunctional count (NDC) by $17.52 \%(p<0.05)$. Dynamics of parameters of the structure, systolic and diastolic functions of the left ventricle did not significantly differ in both groups. The diameter of the left atrium reduced by $2.82 \%$ and $1.80 \%$, respectively, end-diastolic volume of the left ventricle by $2.56 \%$ and $2.22 \%$, the thickness of the interventricular septum by $1.41 \%$ and $2.82 \%$, the thickness of the posterior wall of the left ventricle by $3.16 \%$ and $4.36 \%$, relative thickness of the walls by $1.28 \%$ and $2.66 \%$ in groups I and II respectively. Decrease of the wall thickness and internal volume of the left ventricle resulted in significant decrease of the left ventricle myocardium weight index by $5.28 \%$ and $6.75 \%$, respectively $(p<0.05)$.

Study of the diastolic function of the left ventricle demonstrated significant increase of PE by $12.06 \%$ and $8.32 \%$ and decrease of PA by $7.83 \%$ and $4.26 \%$, respectively. The phase of isometric relaxation was decreased by $6.59 \%$ and $4.75 \%$, DT by $4.77 \%$ and $4.29 \%$, respectively. The positive dynamics of parameters of active and passive diastolic relaxation of the left ventricle myocardium resulted in increasing integral parameters of diastolic function - filling spectrum by $21.89 \%$ and $13.98 \%$ and phase of early filling by $9.39 \%$ and $4.80 \%$ respectively.

Variability of RR interval on ECG significantly increased in both groups but more in the group on alpha lipoic acid $(90.81 \%$ against $32.79 \%)(p<0.05)$. QT interval dispersion decreased by $45.12 \%$ and $15.39 \%(p<0.05)$.

Conclusion: Use of alpha lipoic acid in addition to conventional therapy in patients with type I diabetes mellitus positively influenced results of parasympathetic cardiovascular tests, functional condition of peripheral innervation and electrophysiologic properties of the myocardium. The effect of the preparation on the diastolic function of the left ventricle was comparable in both groups.

\section{1}

In vivo and in vitro study of the effect of high glucose levels on immune system cells: treatment with lutein

M. Muriach ${ }^{1}$, E. Arnal ${ }^{1}$, M. Miranda ${ }^{1}$, R. Blomhoff ${ }^{2}$, D. Silvestre ${ }^{3}$,

F. Bosch-Morell ${ }^{1}$, J. Romero ${ }^{1}$;

${ }^{1}$ Physiology, Universidad Cardenal Herrera-CEU, Valencia, Spain,

${ }^{2}$ Institute for nutrition research, University of Oslo, Norway,

${ }^{3}$ Food Technology, Universidad Cardenal Herrera-CEU, Valencia, Spain.

Background and Aims: Diabetic patients present an increased susceptibility to frequent and protracted infections. Several in vitro functional defects of the immune system have been correlated with the metabolic control of diabetic patients, and the recognition of an impaired immune system has implications for the diagnosis, treatment and outcome of infections. Hyperglycaemia induces an oxidative stress situation in diabetic condition which is involved in the pathogenesis of late diabetes complications. NFkB is a redox sensitive transcription factor involve in immune response, cell proliferation and apoptosis that has been associated to development of diabetic complications. The aim of this study was to study the effect of high glucose levels on oxidative stress parameters and NFkB activity, in vitro in U937 cells, and in vivo in T cells of diabetic rats in an experimental model of diabetes. We also assay a treatment with lutein, a natural antioxidant without hypoglicemiant properties.

Materials and Methods: In vitro study: Human promonocytic cells (U937) transfected with luciferase reporter gene with NF-kB sites from immunoglobulin $\mathrm{k}$ chain promoter were use for this experiment Cells were incubated 24 hours with $50 \mathrm{mM}$ glucose. Also was assayed LPS, which is known to induce NFkB activity. Lutein was added to the medium solved in DMSO 0,1\%). In vivo study: Rats were induced diabetic by streptozotocin injection and divided into subgroups: control, diabetic, d+lutein and $\mathrm{d}+$ insulin. Treatments (lutein $0.5 \mathrm{mg} / \mathrm{kg}$ body weight and insulin $50 \mathrm{mUI} / \mathrm{g}$ body weight s.c.) were administered daily since day 4 after streptozotocin injection, until the end of the experiment, 1 month after diabetes induction. Lymphocytes were separated from normal whole blood by Ficoll density gradient separation. Malondialdehyde (MDA), a lipid peroxidation product, and glutathione (GSH), were measured by HPLC. NFkB activity was determined by adding luciferin to the medium and measuring the light generated at $530 \mathrm{~nm}$ with an optical system.

Results: In vitro study: MDA increased significantly after incubation with glucose, LPS, and both together, while GSH concentration was decreased. LPS induced NFkB activity in U937 cells. This activity was even higher when incubated together with glucose, being this increase statistically significant when compared to control or LPS treated cells. Lutein treatment was able to prevent all of these alterations. In vivo study: MDA concentration increased significantly in lymphocytes of diabetic rats when compared to controls, while GSH decreased. Again, lutein prevented these alterations.

Conclusion: This study confirms that oxidative stress is involved in the impairment observed in diabetic immune system and that a treatment with lutein, which has been suggested to regulate NFkB activity, and it is known to stimulate both cell-mediated and humoral immune responses, could be an appropriate treatment for the impairments observed in the study. Supported by from Fondo de Investigación Sanitaria and Universidad CEUCardenal Herrera. 


\section{Author Index}

Aaboe, K. 0599

Aagren, M. 1033, 1038, 1043

Aalto, J. 0083

Aanstoot, H. 0255

Aarnisalo, J. 0358

Abaidullah, S. 1210

Abdallah, P. 0207, 0721

Abdel-Wahab, Y. H. A. 0915

Abderrahmani, A. 0199, 0203, 0419

Abdul-Ghani, M. A. 0179

Abel, A. 1044

Abicht, A. 0148

Abrahamsson, N. 0393

Abramovitch, E. 0511

Abreu, C. 1088

Abudula, R. 0217, 0492

Accili, D. 0085

Achenbach, P. 0462

Achtsidis, V. 1069

Ackermans, M. 0145

Ackermans, M. T. 0751,0783

Ackermann, S. 0422

Ackroyd, R. 0896

Aczel, S. 0343

Adachi, H. 0387

Adachi, T. 0023

Adams, C. M. 0490

Adams, V. 1174

Adamska, A. 0615

Adamson, U. 1104

Addy, C. 0831, 0832

Adhikarakunnathu, S. 0592

Adlem, E. C. 0294

Adler, A. 0067

Adler, A. I. 0388

Adler, R. 1019

Aebert, H. 0252

Aerts, J. M. 0783

af Björkesten, C. 1099

Afonso, R. A. $\quad 0641,0737,0738$

Aftring, R. P. 0077

Agardh, C. 0317

Agarwal, M. M. 0937

Agha, A. 0657

Agnelli, F. 0622

Aguilar-Bryan, L. 0024

Aguilar-Salinas, C. A. 1163

Ahlquist, J. 0261, 1012

Ahlzén, M. 0318

Ahmann, A. J. 0991

Ahmed, A. T. 0342

Ahmed, K. R. 0941

Ahmed, M. 0195

Ahn, C. $\quad 0213,0315,0744,0798$, 1070

Ahn, Y. R. 0498

Ahn, Y. H. 0570

Ahrén, B. 0053, 0589

Ahrén, J. 0053

Ahtialansaari, T. 0780

Aimone, B. 0333

Akatsuka, H. 0235

Akhobadze, T. 1094

Akhter, A. 1166

Akhter, S. 1166

Aki, N. 1213

Akira, S. 0415

Akiyama, Y. 0670
Akkerman, J. W. 1247

Aktan, F. 0681

Akter, S. 1037

Akusjärvi, G. 0406

Al-Attas, O. 0660

Al-Daghri, N. M. 0660

Al-Hasani, H. $\quad 0003,0314$

Al-Khalili, L. 0130

Al-Mustafa, M. A. S. 0985

Al-Rubeaan, K. 0660

Ala-Korpela, M. 1134

Alana, I. 0593

Alaveras, A. E. G. 0334

Albache, N. 1222

Alberobello, A. T. 0013

Alberti, G. 0269

Aldington, S. J. 0225

Aleksandrov, A. 1121

Alemanno, I. 0254, 0303

Alevizaki, M. 0117, 0844

Alevizos, M. 0630

Alexanderson, E. 1177

Alexanderson, G. 1177

Alexopoulou, L. 0421

Alfieri, O. 0004

Alhenc-Gelas, F. 0365

Alhonen, L. 0780

Ali, L. $\quad 0394,0510,0667$

Ali, L. 1166

Alibegovic, A. C. 0242

Alison, M. R. 0527

Allagnat, F. $\quad 0095,0199,0419$

Allain, G. 0782

Allemann, S. 0149

Allen, J. E. 0294

Allen, T. J. 0209

Almdal, T. 0757

Almeras, N. 0654

Almgren, P. $\quad 0173,0287,1252$

Alonso, N. 0458

Alp, H. 1125

Alssema, M. 0001

Altirriba, J. $\quad 0125,0489$

Altman, J. 0937

Altshuler, D. $\quad 0081,0082$

Altuntas, Y. 0331, 1059

Álvarez, C. 0014, 0499

Alvarez-Guisasola, F. 0329

Amat, G. 0243

Amayo, A. 0374

Ambery, P. D. 1029

Amiel, S. A. 0041

Amin, D. M. 0914

Amoretti, R. 0158

Amoruso, R. 0240

Ampudia-Blasco, F. J. 0955

Anand, D. V. 1241

Anandarajah, S. 1025

Anandarajah, S. 1161

Anastasiades, P. 1095

Anastasiou, E. 0117

Andelova, K. 0465

Andersen, G. 1248

Andersen, H. 0619

Andersen, M. 0790

Andersen, M. V. N. 1159

Andersen, N. H. 0227

Andersen, N. R. 0791

Andersen, S. 1080
Andersson, C. X. 0553

Andersson, R. 0668

Anderwald, C. $\quad 0270,0895$

Andikopoulos, S. 1103

Ando, A. 0507

Ando, H. 0131, 0132, 0756, 0769

Andreozzi, F. 0026, 0675

Andreu, A. L. 1171

Andrews, R. C. 0609

Andrikopoulos, S. 0088

Anfossi, G. $\quad 0239,0708,0842$

Angelis, A. 0334

Anil, K. 0219

Annuzzi, G. 0794

Anselmino, M. 0635

Antonopoulos, S. 0011, 1095

Antovic, A. M. 1104

Anupindi, R. 0661

Apostolopoulos, N. 0797

Appelman, A. P. A. 1090

Araki, K. 0854

Araki, R. 0235

Arana, M. 0867

Arana, R. 0808

Arato, E. 0725

Arbatskaya, N. 0931

Arbit, E. 0112

Arcangeli, A. 0957

Archimandritis, A. 0380

Ardigò, D. 0296

Ardilouze, J. 0048

Arezzo, J. 1146

Argente, C. 0955

Ari, N. 0681,1108

Ariyama, Y. 0965

Armanet, M. 0436

Arnal, E. 1261

Arndt, T. 0448

Arnés, L. 0587, 0591, 0781

Arnqvist, H. 0668

Arnqvist, H. J. 0710

Arnstein, M. 0212

Arondekar, B. 0867, 0868, 0869, 1039

Arsenault, B. J. 0008

Arslan, E. 0540

Artwohl, M. 0030

Arutchelvam, V. 0043

Arvat, E. 0682

Asakawa, T. 0878

Asano, M. 0487

Asatiani, N. G. 0120

Aschner, P. 0325

Ashley, D. 0657

Asik, M. 1234

Astiarraga, B. D. 0580

Astrup, A. 1119

Astrup, A. S. $\quad 0242,1137$

Astrup, A. V. 0733

Atanassova, I. 0378

Atar, A. 0858

Atherton, P. 1154

Atignal, A. 0219

Atkinson, B. 0778

Atsumi, T. 1190

Au, M. 0526, 0530

Aubert, R. 0036, 0260

Augstein, P. 1008

Aulinger, B. A. 0579
Aumann, N. 0440

Avignon, A. 1240

Avogaro, A. 0552

Avram, D. 0423,0429

Axelsson, S. 0060, 0453

Ayad, N. M. A. 0985

Ayala, D. E. 1100

Aydin, K. 0331

Ayturk, S. $\quad 0840,0998$

Azad, K. 0394

Azad Khan, A. K. $\quad$ 0394, 0804, 1037

Azar, S. T. 0985

Azzolina, V. 0628

B

Biyıklığlu, Z. 0858

Baan, B. 0089

Babazono, T. 0065

Baccara-Dinet, M. 1232

Bacci, S. 1254

Bach, V. 0775

Bache, M. 0249

Backeström, A. 1052

Bacos, K. 0201

Badenhoop, K. 0304, 0950

Badet, L. 0478

Bae, H. 0573

Bae, I. 0906

Bae, J. 0570

Baek, H. 0134

Baertschiger, R. 0478

Bagriacik, U. 1108

Baiget, M. 0775

Baik, S. 1244

Baik, S. H. 0326

Bailbé, D. 0414

Baillargeon, J. 0048

Bailleux, V. 0015

Baillie, G. S. 0696

Baillot-Rudoni, S. 1233

Baj, A. 0360

Baker, A. R. 0109, 0153

Baker, N. 1116

Baker, P. 0948

Bakhtadze, E. 0253

Bakker, S. J. 0032

Bakker, W. 0237, 0713

Bal de Kier Joffé, E. 0232

Bala, C. 1211

Balas, B. 0073

Balasubramanium, K. 0918

Balazsovics, J. 0651

Balbarini, A. 1186

Balbo, M. 0682

Baldessari, C. 0845

Baldrich, M. 0338

Balkau, B. 0036, 0270, 0284, 0307, 0313

Ball, K. 0283

Ballesta-Lopez, C. 0846

Ballestar, E. 0943

Balletshofer, B. 0714

Balon, T. W. 0767

Balsamo, A. 0907

Balsells, M. 0119

Balta, C. 1207

Balta, S. 1207 
Baltrusch, S. 0086, 0166, 0427, 0520, 0541

Balu, S. $\quad 0868,0869$

Balzi, D. 0244

Banas, S. M. 0015

Bandara, T. 0918

Bandyopadhyay, G. K. 0152

Banerjee, M. 0532

Bao, Y. 1220

Baptista, C. 0618

Barak, L. 0392

Baran, R. W. 1257

Barbagallo, A. P. M. $\quad 0013,0675$

Barbera, A. 0125, 0489

Barceló, S. 0229

Bardini, G. 0687, 0697, 0904, 1218

Barg, S. 0054

Barna, L. 0923

Barnard, M. 0261, 1012

Barnett, A. 0302, 0316, 0819

Barnett, A. 1036

Barnett, A. H. 1031, 1227

Barosa, C. 0618, 0620

Barr, E. L. M. 0290, 0354

Barreiro, F. 0742

Barret, J. C. 0079

Barrett, A. 0274

Barrett, J. C. 0080

Barrios, V. 1030

Barros, L. 0618

Barrow, B. 0172

Barsotti, M. 1186

Barsukov, I. A. 0265

Bartaskova, D. 0465

Bartlett, J. 0162

Bartley, C. 0397

Bartley, P. C. 0222

Bartolini, B. 0090

Barton, N. 0717

Bartsch, W. 0304

Barutta, F. 0212

Basat, O. 0331, 1059

Basello, K. 0785

Basile, M. 0682

Basols, J. 0621

Basora, J. 0338

Basset, A. 0207, 0721

Bastarrachea MD, R. 0435

Bastos, M. 0618

Bastyr, III, E. J. 0135

Basu, A. 0861

Basu, R. 0617, 0861

Bataille, D. 0510

Bauduceau, B. 0365

Bauer, L. 0639

Bauersachs, J. 1193

Baughman, R. 0961, 0968

Baumgartner-Parzer, S. M. 0030

Baumstark, A. 0148

Baverel, G. 0623

Bax, J. J. 0613

Baxter, D. 0544

Bayle, F. 0478

Bayraktar, N. 0078, 1234

Bazarra-Castro, A. 0091

Bazzigaluppi MA, E. 0435

Beatson, C. 0263

Beattie, S. D. 0962

Beaulieu, N. 0867

Beauloye, C. 0555

Beauwens, R. 0523

Bech, O. M. 0985

Beck, C. A. 0352
Beck-Nielsen, H. 0790, 0791, 1259

Beck-Peccoz, P. 0622, 0845

Becker, D. J. 0188

Becker, R. H. A. 0959

Beekhof, P. 0774

Beffy, P. 0547

Begic, D. 0160

Begley, J. 0821

Beglinger, C. 0112

Begu, A. 0051

Beguinot, F. 0013, 0488, 0675

Beisiegel, U. 0625

Beisswenger, P. J. 1223

Bek, T. 0227

Belan, V. 0743

Beldekos, D. 1202

Belfort, R. 0073

Belgrado, D. 1191

Belhadj-mostefa, A. 1179

Beliaeva, N. 0098

Bell, A. C. 0975

Bell, D. 0841

Bell, J. D. 0717

Bell, P. M. 0778

Bellary, S. 0316, 1031

Bellary, S. 1036

Bellili, N. 0260, 0284, 0365

Bellmann, K. 0457

Belloni, C. 0435

Belloni, E. 0614

Beltramo, E. 1152

Beltrand, J. 0728

Ben-Mahmud, B. M. 1153

Benaiges, D. 1229

Bender, K. 0524

Bendlova, B. 0273, 0390, 0312, 0788

Benediktsson, R. $\quad$ 0292, 0866

Benetos, A. 0066

Benetti, E. 0622, 0845

Benford, M. 1029

Benhamou, P. 0478

Benke, I. 0107

Benmezroua, Y. 0307

Benn, J. 0930

Bennett, A. J. 0171

Benroubi, M. 1032

Bentley, L. 0638

Benvenuti-Falger, U. 1050

Benzi, L. 0920, 0942

Benzinou, M. 0287

Berberoglu, Z. $\quad 0078,1234$

Berclaz, P. 0969

Bergenstal, R. 0983

Berger, R. 1203

Berger, S. 0919

Bergeron, J. 0654

Bergeron, R. 0480, 0688

Bergert, H. 0020

Berggren, P. 0481, 0514

Berghold, A. $\quad 0221,0932$

Bergholdt, R. 0084

Bergholm, R. 0612

Berglund, G. $\quad 0173,0287$

Berglund, L. 0393

Bergman, R. N. 0050, 0590, 0610, 0662, 0663, 0664

Bergsten, P. 0396, 0404, 0406, 0407, 0416, 0497

Berland, J. 0874

Bernardi, G. 0230

Bernardi, S. 1191

Bernas, M. 0902
Berndt, J. 0309

Berne, C. 0393

Berney, T. 0021, 0052, 0436, 0478

Berntorp, K. 1028

Berrone, E. 1152

Bertacca, A. 0920

Berthou, F. 0015

Bertipaglia, L. 0552

Bertok, S. 0725

Bertolotto, A. 0942

Bertrand, L. 0555

Beswick, R. 0776

Betbesé, A. 1000

Betbesé, A. J. 0999

Bevilacqua, M. F. 1243

Bexon, M. F. 0776

Beyan, H. 0450

Beylot, M. 0207, 0604, 0721

Bezzi, P. 0202

Bhargava, A. 0987

Bhatia, E. 0789

Bhatia, V. 0789

Bhole, D. 0576

Bi, Y. 0572, 0574

Biagetti, B. 0999, 1000

Bialkowska, J. 0718, 0719

Bianchi, G. 0360

Biasucci, L. M. 1182

Biden, T. 0408

Bie-Olsen, L. G. 0770

Bierhaus, A. 1103, 1133

Biessels, G. 0161, 1090

Bigelow, M. 0752

Billestrup, N. 1173

Billyard, T. S. $\quad 0153,0720$

Bilo, H. J. 0100

Bilo, H. J. G. $\quad 0105,0157,0223$, 0345, 1067, 1079, 1081, 1107, 1160

Bilous, R. 0064

Binder, S. 1151

Bindokas, V. P. 0504

Bingley, P. J. 0462

Binz, J. G. 0767

Birk, J. B. 0791

Biroccio, A. 0090

Bischof, M. G. 0895

Biswas, K. B. 0394, 1037

Biswas, S. K. 1106

Bityutska, O. 0761

Bjerre Knudsen, L. 0144

Bjerregaard, P. 0362

Bjørgaas, M. R. 0810

Björklund, A. 0403, 0443, 0516

Björkqvist, M. 0201

Bjørner, J. B. 1159

Blaak, E. E. $\quad 0194,0310,0824$, 0827, 1217, 1250

Black, N. 0778

Blackburn, D. F. 0873

Blair, A. 0088

Blanc, P. R. 0806

Blanco-Vaca, F. 0775

Blane, A. 1091

Blasi, J. 0536

Blasiak, J. 0876

Blazhev, A. 0468

Bleich, D. 0446

Bloigu, A. 0348, 0381

Blomhoff, R. 1261

Blondeau, B. 0485

Blüher, M. 0309, 0704

Bluher, M. 0698

Blum, M. 0226

Bobbink, I. W. G. 0898

Bock, G. 0617, 1115

Bock, J. B. 0975

Bodalski, J. 0645, 0924

Bodansky, H. J. 1047

Boehler, A. 0601

Boehm, B. 0528

Boeing, H. 0003, 0170, 1249

Boekholdt, S. M. $\quad$ 0008, 0257

Boeni-Schnetzler, M. 0417

Boer, P. A. 1150

Boersma, B. 1055

Boesch, C. 0149

Boetker, H. E. 0016

Böger, R. H. 0841

Boggi, U. 0410, 0875, 0907

Boghossian, M. 0623

Bogoev, M. 0222

Bohdjalian, A. 1002

Böhme, K. 0994

Bohmova, K. 0465

Bokma, J. 1055

Bolla, M. 0740

Boller, S. 0672

Bolli, G. B. 0044, 0046

Boltaña-Lorenzo, A. 0966

Bolton, K. 0122

Bonadonna, R. C. 0370

Bondar, I. A. 1136

Bondo Hansen, J. 0516

Bonifacio, E. $0020,0059,0462$, 0477

Bonnard, C. 0758

Bonner, J. 1198

Bonner-Weir, S. 0418

Bonnet, F. 0313

Bonomo, M. 0215, 0246

Bonora, E. 0370

Bonotti, A. 0635

Bookout, T. 0263

Boomsma, D. I. 0389

Boomsma, F. 0770, 1118

Boonman-de Winter, L. J. M. 0809

Boothby, M. 0760

Borch-Johnsen, K. 0034, 0070, 0362, 1248

Börcsök, é. 1092

Borg, H. 516

Borkowska, A. 0718, 0719

Bormans, G. 0831

Bornstein, S. 0020

Boronat, M. 0319

Bosanska, L. 0706

Bosch-Morell, F. 1261

Boschetti, N. 0672

Bosco, D. 0021, 0052, 0436

Bosco, D. 0478

Bosi, E. 0004

Bosi MD, E. 0435

Bosnyák, Z. 0944

Boss, A. 0961

Bossi, R. 0845

Bot, S. 0104

Botea, V. 0384, 0715

Botker, H. E. 1194

Botta, G. 0488

Böttcher, Y. 0309

Botusan, I. 1127 
Boussuges, A. 0806

Boutati, E. 0603, 0608, 0712

Bouter, L. M. 0001, 0031

Bouwman, R. 0088

Bouzakri, K. 0400

Bova, L. 0090

Bowden, C. 0971

Bowler, H. 1145

Bowlus, C. 0110

Boyer, J. 0736

Boyko, E. J. 0290

Boyle, R. 0913

Bozaoglu, K. 0122

Bozzetto, L. 0794

Bracewell-Milnes, T. J. J. $\quad 0118$, 0938, 0939

Bradescu, O. 0335

Brammer, M. 0041

Brand, C. L. 0038

Brandle, M. 1034

Brandner, J. 1130

Brassard, P. 0048

Braun, A. 1044

Braun, K. F. 1224

Braun, T. 0704

Brauner, H. 0447

Breant, B. 0485

Breas, N. 1066

Bredahl, R. 0648

Breinholt, J. 0619

Breislin, K. 1144

Brennan, L. 0509

Brenner, E. 0763, 0764, 0765

Brenner, G. M. 1253

Brett, J. 0987

Bretzel, R. G. 0185

Bricca, G. 0207, 0604

Brichard, S. M. 0154

Brismar, K. 0366, 1127

Brittan, M. 0527

Britten, A. 0302, 0316

Brix, J. 1158

Bröchner-Mortensen, J. 1080

Brock, B. $\quad$ 0016, 0144, 0491, 0581

Brodows, R. 0819, 0853

Brøns, C. 0733

Brorsson, C. A. 0084

Brot-Laroche, E. 0518

Brouwers, M. 0007

Brown, C. 0912

Brown, K. 0073

Brown, L. C. 0352

Brown, R. 0745

Brown, W. V. 1223

Brubaker, P. L. 0692

Bruce, D. G. 1003

Brück, P. 0304

Bruckert, E. 1232

Brugaletta, S. 0459

Bruguera, J. 1229

Brugués, E. 1013

Bruining, G. J. 0068

Brundin, P. 0201

Brunelle, P. 0291

Brunetti, P. 0957

Bruning, J. C. 0698

Brunmair, B. 0639,0677

Brunner, S. 1151

Bruno, G. 0072, 0333, 0353

Bruno, N. E. 0235

Bruttomesso, D. 0158, 0215

Bryan, J. 0024

Brydon, P. 0930

Bryzgalova, G. 0679
Bucci, M. 0017

Buci, L. 0687

Buday, B. 0598

Buechler, M. W. 0543

Buesching, D. 0099

Bugler, J. R. 1014

Bugliani, M. 0093, 0174, 0410, 0416, 0875

Bühler, L. 0052

Buhr, A. 0953

Bulugahapitiya, U. 1046

Bulur, N. 0521, 0523

Bunck, M. C. 0251

Burcelin, R. G. 0151

Burdick, D. 0544

Burdick, D. B. 0294

Burén, J. 0551

Buresova, M. 0642

Burgdorf, K. S. 1248

Burge, M. R. 0970

Burger, D. 0528

Burger, J. 0819

Burgess, S. C. 0617

Burkart, V. 0056, 0457

Burns, C. 0838

Burns, D. 0831

Burren, O. S. 0294

Burt, D. 0072

Burtt, N. 0081, 0082

Bury, A. 0818

Buscemi, B. 0628

Buscemi, S. 0628

Busch, A. 0408

Busciantella Ricci, N. 0044, 0046

Busetto, E. 0158

Bussey, C. T. 0238

Butler, A. A. 0666

Buxton-Thomas, M. 1124

Buziashvili, U. O. 1084

Buzzetti, R. 0254, 0303

Byiers, S. 0881

Byrne, C. D. 0705

Byrne, C. D. T. 0616

C

Caballero, A. 0319

Caballero-Corchuelo, J. 0966

Cabezas-Agricola, J. M. 1100

Cabezas-Cerrato, J. 1100

Cabistañ, C. 0775

Cable, E. E. 1195

Cabre, J. J. 0338

Cabrera-Rode, E. 0359

Caccioppoli, C. 0193, 0550, 0701

Cahova, M. 0749

Cai, M. 0572, 0574

Caillard, S. 0369

Caille, D. 0095

Caixàs, A. 0775

Calatola, P. 0957

Caldeira, M. 0618, 0620

Calderari, S. 0499

Calderón, A. 1030

Calderone, V. 0907

Calders, P. 0807

Calegari, F. 0054

Calianno, A. 0942

Calkin, A. C. 1187

Calo, L. A. 0552

Calvo, C. 1100

Calvo, F. 0671

Camastra, S. 0580

Cambien, F. 0061
Cambou, J. $\quad$ 0243, 0874

Cameron, F. 0187

Campbell, M. 1172

Campioni, M. 0602

Camussi, G. 0212

Cancelas, J. 0583, 0588, 0596

Candeloro, P. $\quad 0044,0046$

Candido, R. 1191

Cani, P. D. 0151

Cano, J. F. 1229

Canovatchel, W. 0971

Canu, T. 0614

Capani, F. 0158

Caparevic, Z. 1164

Capel, I. 1013

Capizzi, M. 0254, 0303

Capoglu, I. 1125

Capuani, B. $\quad$ 0090, 0785

Capuano, G. 0246

Caracotche, L. 1075

Cardellini, M. 0234

Cardon, L. R. 0169

Cardozo, A. K. 0544

Cariski, A. 1005

Carlsson, S. 0297

Carmena, R. 0955

Caro, J. Jaime. 0836

Caroli, E. 1191

Carpentier, Y. A. 0521, 0596, 0779

Carrasco, E. 1155

Carreras, M. C. 0742

Carretta, R. 1191

Carrie, D. 0243

Carstensen, B. 0070

Cartier, A. 0257, 0654

Carvalheiro, M. 0618

Carvalho, D. 0268

Casas, R. 0060, 0453

Casas, S. 0433, 0434, 0537

Casbard, A. 0261

Casolaro, A. 0580

Cassader, M. 0476

Cassen, V. M. 0294

Cassese, A. 0013

Cassidy, J. 0961

Castel, J. 0535

Castex, F. 0736

Castiglioni, A. 0004

Castro, J. 1058

Castro, M. C. A. 0618

Catalano, K. 0050, 0663

Catalano, K. J. $\quad$ 0662, 0664

Caterson, I. 0837

Catlow, R. 1116

Catrina, S. 1127

Cattin, L. 0957

Caubet, E. 0198

Cavalcanti, T. C. 1150

Cavallari, G. 0473

Cavallo, F. 0682

Cavallo Perin, P. $\quad 0072,0212$ 0333, 0476

Cecchetti, F. 0635

Cechurova, D. 0951

Cederholm, J. 0033, 1219

Cegan, A. 0714

Ceperuelo-Mallafré, V. 0198

Cerasi, E. 0511

Cerasola, G. 0628

Ceriello, A. 1223

Cerna, M. 0465

Cersosimo, E. 0250, 0909

Cerutti, F. 0333

Cervin, C. $\quad 0253,0318$

Cevik, C. 1108

Cevikbaș, U. 0716

Ceylan-Isik, A. 0681

Cha, B. $\quad 0315,0744,0798,1070$

Cha, B. Y. 0301

Chacón, M. R. $\quad$ 0198, 0637, 0656

Chadarevian, R. 1233

Chai, R. 0460

Chakarova, N. 0378

Chala, E. 0796

Chalder, T. 0162

Chamiot-Clerc, P. 0830

Chan, J. 0984

Chan, J. C. N. 0322, 0375, 0773, 0786, 1077

Chan, L. 0857

Chandramouli, V. $\quad 0617,0861$

Chang, C. 0188

Chang, C. N. 0802

Chang, C. T. 0826

Chang, H. 0826

Chang, W. 0356

Charles, A. 1032

Charles, M. A. 0336

Charlton, M. 1227

Charollais, A. 0095

Charpentier, G. 0307, 0365

Charron, M. 0480

Chase, H. 1015

Chaturvedi, N. 0072, 1082

Chatzimichelakis, E. 0117

Chaudhary, U. 1210

Chaussade, C. 0085

Chaves, H. 1163

Chavez MD, A. O. 0435

Chavez-Velazquez, A. 0179

Cheatham, W. W. 0005

Chen, B. 0856

Chen, C. 0826

Chen, J. Hua. 0917

Chen, L. 0990

Chen, L. T. 0597

Chen, M. Z. 0609

Chen, R. 0826

Chen, R. 0889

Chen, W. 0784

Chen, X. 0572, 0574

Chen, Y. Wen. 0802

Cheng, C. 0135

Cheng, H. 0398

Cheng, J. Tang. 0802

Chernyshova, A. 0305

Cherrington, A. D. 0584

Chesnel, D. 0528

Chessler, S. D. $\quad 0204,0466$

Cheung, N. 0730

Cheurfa, N. 1253

Chevassus, H. 0800

Chevenne, D. 0728, 0734

Chiarle, R. 0212

Chibber, R. 1153

Chiganer, G. 0949

Chillaron, J. J. 1229

Chimienti, F. 0142

Chisalita, S. I. 0710

Chitson, P. 0269

Chittari, M. V. 0126

Chiu, J. 0050, 0663

Chiu, J. D. 0610, 0662, 0664 
Cho, Y. 0236

Choi, D. 1244

Choi, H. 0703

Choi, K. 1244

Choi, S. $\quad$ 0554, 0673

Choi, S. B. 0952

Chopra, P. 1163

Chopra, S. 0806

Choudhary, P. 0041, 0812

Choukem, S. 0671

Chow, C. 0375,0786

Chow, C. C. 0773

Chow, W. S. 0857

Christ, E. R. 0149

Christ-Crain, M. 0556

Christakidis, D. 1068

Christiansen, B. 1176

Christiansen, J. S. 0986

Christiansen, N. 0985

Christopher, R. 0891

Christov, V. G. 1072

Chryssochoou, E. 0844

Chu, P. 0987

Chu, W. C. W. 1077

Chudleigh, R. 0484

Chudleigh, R. A. 1078

Chudoba, D. 0465

Chugunov, A. 1051

Chugunova, L. A. 1051

Chun, S. 0315

Chun, S. 0894, 0906

Chung, D. 1245

Chung, H. 0424

Chung, H. 1070

Chung, H. 1129

Chung, J. 1245

Chung, K. W. 0096

Chung, M. 1245

Church, C. 0638

Ciani, S. 0687, 0697

Ciano, O. 0794

Ciardi, C. 0483

Cifcibasi, A. 0331

Cignarelli, A. 0193, 0550, 0701

Cilissen, C. $\quad 0832,0833$

Cimino, A. 1023

Cinek, O. $\quad 0068,0463$

Cinti, S. 0631

Cioli, P. 0044

Claris, O. 0728

Clark, A. 0434

Clark, M. G. 0238

Clark, R. V. 0914

Clarke, G. C. 0915

Clarke, P. 0935

Clarke, P. 1040

Claudi, T. 1020

Clausen, J. 0866

Cleall, S. 0336, 1032

Cleveringa, F. G. W. $\quad$ 0264, 1097

Clifton, P. G. 0666

Clodi, M. 1203

Clough, G. F. 0705

Cnop, M. 0051, 0091, 0093

Cobelli, C. 0602

Cockram, C. S. $\quad 0375,0773$

Coco, A. 1254

Coculescu, M. 0685

Coffey, T. 0586

Cohen, A. 1236

Cohen, O. 0861

Coimbra, C. N. 0494

Colagiuri, S. 0034

Colclough, H. 1038
Coleman, M. A. G. 0935

Coleman, R. L. 1230

Colin, C. 0478

Colin, I. 1073

Collier, G. 0122

Collier, T. 1027,1255

Collin, D. 0734

Colognato, R. 0920

Colombo, M. 0622

Colomé, E. 0458

Colon, G. 0991

Comerma, L. 1096

Comin, J. 1229

Comoletti, D. 0204

Comuzzie PhD, A. G. 0435

Conarello, S. 0480

Conget, I. 0814, 0949, 1058

Conjard, A. 0623

Connolly, S. B. 1027,1255

Consoli, A. 1167

Constantine, G. R. 0382

Constantinescu, E. 0707

Contreras, R. 0116

Converso, D. P. 0742

Cooke, D. 0261, 1012

Cooke, J. 1223

Cooper, B. 0530

Cooper, J. G. 1020

Cooper, M. E. $\quad 0209,0212,1103$, 1187

Coppola, A. 0785

Corallini, F. 1191

Corcelle, V. 0528

Corcoy, R. 0119

Corder, R. 1241

Corkey, B. 0402

Corkey, B. E. 0168

Corkey, R. 0168

Corlett, P. R. 0042

Cormont, M. 0740

Cornér, A. 0251

Cornu, M. 0203

Corpeleijn, E. 0310, 0827

Corporeau, C. 0782

Correa-Giannella, M. L. 0494

Correia, M. 0737

Corsi, A. 0215

Cosson, E. 1207

Costa, B. 0338

Costa, G. 0353

Costa, S. 0004,0215

Costabile, G. 0794

Costacou, T. 1226, 1242

Costigan, C. 0190

Cote, M. 0257

Cottin, Y. 0874

Cottone, S. 0628

Cotzias, C. 0936

Cotzias, C. S. $\quad 0118,0938,0939$

Coughlan, M. T. 1103

Coulaud, J. 0414,0415

Coulter, F. C. 0987

Coustan, D. R. 0115

Coutinho, W. 0837

Couturier, A. $\quad 0885,0887$

Covington, P. 0891

Cowley, A. W. 0291

Cox, R. 0061

Cox, R. D. 0638

Cozzani, H. 1214

Crea, F. $0459,1182,1206$

Creed, F. $\quad 0162,0162$

Creely, S. J. $\quad 0126,0153$

Cremers, H. 0774
Cresci, B. $\quad 0687,0904,1218$

Cristol, J. 1240

Croci, M. 0644

Cronstein, B. N. 0446

Crowley, R. 0657

Cruciani-Guglielmacci, C. 0735

Cruickshank, K. 1085

Cruz, P. 1177

Cseh, K. 0651

Cserepes, B. 0725

Csoka, B. 0446

Csongrádi, é. 0843

Cuba, I. 0359

Cubero, J. M. 0999

Cubero, J. M. 1000

Cuccuru, I. 0947

Cucinotta, D. 0988

Cui, Z. 0566

Cumaoglu, A. 1108

Cunha, D. A. 0091

Cunningham, M. 0592

Currie, C. J. $\quad$ 0330, 0817, 0993

Currie, P. J. 0039

Curtis, B. 1065

Cusi, K. 0073

Cvijovic, G. 0627

Cyganek, K. 1140

Czech, A. 0456, 0766, 0902

Czerniawska, E. 0922,0924

Czerwiński, E. 0191

Czupryniak, L. 0718, 0719

\section{D}

D'Agostino, G. 0058

D’Agostino Jr., R. B. 0350

D'Agostino, Sr., R. 0864

D'Aleo, V. 0174, 0547, 0875, 0907

D’Alessio, D. A. 0579

D'Esposito, V. 0488

Da Col, P. 1191

da Silva, N. F. $0124,0126,0153$, 0660, 0720

Da Vico, L. 0697

Dabelea, D. 0350, 0385

Dahiya, S. 0418

Dahl, P. J. 0866

Dahlén, E. M. 0010

Dahlman-Wright, K. 0679

Dahm, S. 0314

Daimon, M. 0288, 0379

Dal, S. 0331

Dalamaga, M. 0649

Dallinga-Thie, G. M. 0012

Damm, P. 0247, 0248, 0933

Danchin, N. 0243, 0874

Danciulescu, R. 0723

Danciulescu, R. 0985

Daniele, S. 1076

Dankova, H. 0749

Danne, T. 0189

Darko, D. 1241

Darsow, T. 1258

Darvasi, A. 0083

Datz, N. 0189

Davalli MD, A. 0435

Davenport, M. 0891

Davey-Smith, G. 0040

Davidson, H. W. 0139, 0140, 0141, 0143

Davidson, J. 0986

Davidson, M. 0864

Davies, M. 0958

Davies, S. 0971

Davis, B. 0064

Davis, C. 1005

Davis, T. M. E. 1003

Davis, W. A. 1003

Dawczynski, J. 0226

Dawson, V. 0934

Day, C. P. 0720

Dayan, C. M. 0609

De, P. 1227

De Alvaro, C. 0653

De Angelis, L. 1162

De Backer, G. 1027, 1255

De Bacquer, D. 1027, 1255

de Bakker, P. 0081, 0082

de Beaufort, C. 0187, 0192

De Bigontina, G. 1023

De Bonis, C. 1254

de Bruin, T. 0007, 0750

de Bruin, T. W. A. 1250

De Cobelli, F. $\quad$ 0477, 0614

De Decker, M. 0832

De Decker, M. 0833

De Fazio, M. 0193

De Feyter, H. M. M. 0178

De Flines, J. 0075

de Geus, E. 0389

De Grijse, J. 0469

de Haan, A. 0475

de Haan, B. J. $\quad 0472,0475$

de Jong, P. E. 0032

de Kloet, E. R. 0684

de la Peña, A. 0969

de la Torre, N. 0803

de Leiva, A. 1013

De Lepeleire, I. 0831

De Mendonca, N. 0936

De Mendonca, N. I. M. 0938, 0939

de Miguel, L. 0014, 0499

De Munter, J. S. L. 0363

De Natale, C. 0794

De Nicola, A. F. 0684

De Paoli, A. 0360

De Pauw, P. 0469

De Roos, A. 0613

De Smet, D. 0469

De Smet, M. 0832, 0833

De Stefano, F. $\quad 0193,0550,0701$

De Tata, V. 0361

de Valk, H. W. 0927

de Vos, P. 0472, 0475, 0531, 0680

de Vries, A. P. 0032

de Vries, R. 0012

de Weerdt, I. 0829

de Wit, M. 1055, 1066

De Witt, H. J. 0450

De-Mendonca, N. I. M. 0118

Deacon, C. F. 0111

Deacon, C. F. 0599

Deal, T. 0135

Dear, A. E. 0594

Deary, I. J. 1091

Debacker, N. 1022

DeBerker, D. 0609

DeBruin, T. 0913

Debussche, X. 0806

DeCampo, A. 0996

Decochez, K. 0469

Dede Cinar, N. 1143 
DeFronzo, R. 0073

DeFronzo, R. A. $\quad$ 0179, 0724, 0909

DeFronzo MD, R. A. 0435

Deghmoun, S. 0734

Degouy, A. 1131

Dei Cas, A. $\quad 0064,0296$

Deitch, E. A. 0446

Dejager, S. $\quad 0885,0887$

Dekker, J. M. 0001, 0007, 0031, 0104, 0161, 0272, 0389, 1139, 1215

Dekker, R. $\quad 0145,0210$

Dekker Nitert, M. 0517

Del Carmine, P. 0207, 0604, 0721

Del Chiaro, M. 0875

del Guerra, S. 0019, 0174, 0410, 0875

Del Maschio, A. $\quad 0477,0614$

Del Mese, P. $\quad$ 0239, 0708, 0842

del Prato, S. 0019, 0093, 0174, 0410, 0418, 0875, 0920, 0947, 1186

Dela, F. 0757

Delaney, K. 1005

Delarue, J. 0782

Delemarre-van de Waal, H. 1055

Delgado, T. 0620

Delgado, T. J. C. 0618

Delimaris, I. 0649

Delion, V. 0830

Dellweg, S. 0043

Deloukas, P. 0169

Delplanque, J. 0083

Delporte, C. 0779

Delzenne, N. M. 0151

Demaurex, N. 0094, 0167, 0508

Demirag, N. $\quad 0078,1234$

Demirağ, N. G. $\quad 0840,0858,0998$

Demirer, A. N. $\quad 0840,0998$

Demponeras, C. 0380

Deng, W. 0251

Denti, P. 0602

Depondt, C. 0051

Depré, M. 0831

Derksen, A. 1055

Derlindati, E. 0296

Desai, S. 0219

Deshmukh, A. 0130

Després, J. 0008, 0257, 0654

Detaille, D. 0860

Deturck, R. 0874

Devlin, N. 0657

Devonshire, A. S. 0575

DeVries, J. H. 1122

Dezaki, K. 0502, 0507

Dhawan, A. 0948

Dhayal, S. 0395

Di Bartolo, P. 0158

Di Benedetto, A. 0246

Di Cecco, P. 1199

Di Cianni, G. 0246, 0942, 0947

Di Fulvio, P. 1167

Di Jeso, B. 0675

Di Pietro, N. 1167

Di Silvestre, S. 1167

Di Stefano, R. 1186

Di Tomo, P. 1167

Diaconu, L. 0384, 0715

Diakogiannaki, E. $\quad 0395,0405$

Diakoumopoulou, E. $\quad 0797,1069$

Diamant, M. 0009, 0206, 0251, 0282, 0389, 0565, 0613, 1204

Diaz, A. 0359

Diaz-Horta, O. 0359
Dicembrini, I. $\quad 0244,0687$

Dick Jr., E. J. 0435

Dicke, B. 0861

Dickens, C. 0162

Didangelos, T. P. 0630

Diem, P. 0149

Diepeveen, S. H. A. 1160

Digiacomo, M. 0907

Dimitriadis, G. $\quad 0603,0608,0712$

Dimopoulos, S. 1035

Dimosthenopoulos, C. 0796

Diogo, L. 0620

Dionyssiou-Asteriou, A. 0649

Dissanayake, S. 0948

Dittert, D. 0020

Dixon, A. 0316

Djuricic, B. 0449

Dobbins, R. L. 0914

Dobríková, M. 1251

Dodesini, A. R. 0215

Dogusan, Z. 0421

Doh, J. 1192

Doi, A. 0727

Doi, K. 1225

Dolci, M. 0246

Dolezalova, R. $\quad 0655,0706$

Dolinkova, M. 0655, 0706

Dolman, G. E. 0294

Domin, J. 0514

Dominguez, H. 1176

Donadel, G. $\quad$ 0090, 0785

Donadio, F. 0622, 0845

Donath, M. Y. $\quad 0412,0415,0417$, 0437

Donato, F. 0622

Donaubauer, B. 0992

Dong, J. $\quad 0863,1123$

Donic, V. 0847

Donnelly, R. 1154

Döring, F. 1249

Dorkova, Z. 1231

Doronzo, G. $\quad$ 0239, 0708, 0842

Dotta, F. 0230

Dougherty, F. C. 0776

Dov, A. 0511

Dova, L. 0455

Dreval, A. V. 0265

Drewe, J. 0112

Drews, G. 0024

Drexel, H. 0343, 0919

Drexhage, H. A. 0450

Drozdova, E. N. 1084

Drucker, D. 0592

Drzewoski, J. 0876

Dubois, G. 0736

Ducloux, R. 0937

Due-Andersen, R. 1117

Düfer, M. 0024

Duffy, N. A. 0882

Dugal, T. 0355

Dugi, K. A. 0890

Duh, M. S. 0867

Dullaart, R. P. F. 0012

Dumez, Y. 0937

Dumont, N. 1034

Dunbar, S. 0832

Duncan, G. 1086

Duncker, D. J. 0210

Dundua, M. 1094

Dundua, M. G. 0120

Dunn, T. C. 0262

Dunne, F. $\quad 0248,0899$

Dunne, F. P. 0930

Dunning, B. E. 0880
Dunseath, G. $\quad 0484,1078$

Dunstan, D. W. $\quad 0150,0283$

Dupont, C. 1131

Dupont, O. 0167

Dupont, P. 0831

Dupuy, A. 1240

Duquenne, C. 0830

Durand, C. 0557

Durand, E. 0243

Durante, A. 1102

Durighel, G. 0717

Durilova, M. 0465

Durso-Decruz, E. 0137

Duval, M. 0937

Duvillard, L. 1233

Duvivier, V. 0830

Dwarakanathan, A. 1237

Dyachok, O. 0163, 0164

Dyck, P. J. 0138

Dyer, A. R. 0115

Dzivite, I. 0068

Dzneladze, L. 1094

\section{E}

Earle, K. A. 1025

Earle, K. A. 1161

Eckel, J. $\quad 0549,0743$

Eckers, U. 0963

Eckhardt, M. 0879

Economopoulos, T. 0608, 0712, 0759

Edel, K. 0950

Edghill, E. 0392

Edmonds, M. 1116

Edmonds, M. E. 1124,1126

Eeg-Olofsson, K. $\quad 0033,1219$

Eekhoff, E. M. W. 0389

Efendic, S. 0346, 0351, 0366, 0561, 0679

Egeberg, K. W. 0443

Egefjord, L. 0144, 0491

Egido, E. M. $\quad 0503,0505$

Egli, A. 0672

Ehala-Aleksejev, K. 0154

Ehehalt, F. 0020

Ehses, J. 0412

Ehses, J. A. 0415, 0417, 0437

Eickelmann, P. 0694

Eidem, I. 0347

Eisenbarth, G. S. $\quad 0139,0140$, 0143

Eizirik, D. L. $\quad 0091,0093,0421$, 0544, 0545

Ekanayeka, S. 0918

Ekberg, K. 0643, 1175

Ekbom, A. 0351

Ekelund, U. 0616, 0805

Ekholm, B. 0302

El-Helali, A. 0899

El-Mir, M. 0860

Elbein, S. C. 0169

Elbers, C. C. 0286,0293

Elbroend, B. 0043

Eliaschewitz, F. G. 0926

Eliasson, B. 0033,1219

Eliasson, B. 0251

Eliasson, B. 1040

Eliasson, L. 0517

Ellard, S. $\quad 0172,0281,0391,0392$

Ellard, S. E. 0068

Elliot, K. 0392

Elliot, K. S. 0295

Elliott, K. 0172

Elliott, K. S. $\quad 0040,0079,0080$

Ellis, S. L. $\quad 0263,0940,1010$

Ellison, J. M. 1005

Ellmerer, M. 0610

Elsner, M. 0409, 0439

Emery, C. J. $\quad 0812,0896,1089$, 1141,1145

Emir, B. 0137

Emre, Y. 0431

Enache, A. 0923

Endahl, L. 0189

Eng, W. 0831

Engel-Nitz, N. 0099

Engels, H. R. 0950

Engvall, J. 0010

Enigk, B. 0309

Enneking, T. 0809

Ennis, C. N. 0778

Enomoto, M. 0387

Eprinchard, L. 0369

Epstein, D. 0205

Erbel, S. 0543

Erdem, T. 1059

Erdine, S. 1163

Erdmann, E. 0340

Erdmann, K. 0020

Erdogan-Tulmac, O. 0681

Erdozain, L. 1013

Eriksen, J. 1001

Eriksson, A. 0346, 0351

Eriksson, J. G. 0320

Eriksson, J. W. $\quad 0196,0551$

Eriksson, K. 0173

Eriksson, M. 1219

Eriksson, S. 1052

Eringa, E. C. 0237,0713

Erion, M. D. $\quad 0911,1195$

Erkens, J. A. $\quad 0870,0871,0872$

Eroğlu, S. T. 0858

Erqou, S. 0388

Ertl, G. 1193

Esbrit, P. 0587

Esbrít, P. 0588

Eschwege, E. $\quad 0964,1232$

Escoriza, J. 0496

Escrivá, F. 0014, 0499

Escudero, E. 0846, 1096

Eshpeter, A. 0526, 0530

Esposito, A. 0477,0614

Esposito, I. 0013

Esposito, I. 0543

Esteve, E. 0621

Estil.les, E. 0690

Eto, M. 0062

Ettinger, R. A. 0454

Eurich, D. T. 0873

Evans, M. 0126

Evans, M. 0484, 1029

Evans, M. L. $\quad 0042,0182,0183$

Evans, R. W. 1242

F

Faas, M. M. 0472

Faas, M. M. $0475,0531,0680$

Faber, J. 0233, 1117

Fabiano, C. 0459

Fabregate, M. $\quad 0803,1235$

Fabregate, R. 0803, 1235

Fabris, B. 1191

Fach, E. M. 0950 
Fahlman, R. 0130

Fajardo Montanana, C. 0978

Fam, B. C. 0088

Fanelli, C. G. $\quad 0044,0046$

Farcasiu, E. 0335

Faresjö, M. 0060, 0453

Faresjo, M. 0465

Faria Neto, H. C. C. 1243

Färkkilä, M. 1142

Farmer, A. J. $\quad 0103,0280,1042$

Farrall, M. 0061

Farrés, J. 1155

Farret, A. 0800

Faruque, M. O. 0394, 0510, 0804

Faulenbach, M. 0601

Faulkner, M. S. 0928

Fava, F. 0151

Favaloro, J. 0088

Favaro, E. 0476

Favre, D. 0419

Febbraio, M. A. 0823

Federici, M. 0090, 0234, 0240, 0785

Feinstein, S. 0864

Feldt-Rasmussen, B. 1159

Felez, J. 0775

Felius, A. 1055

Feltbower, R. G. 1047

Femia, R. 0580

Feng, L. 0632

Feng, Y. 0917

Ferber, P. 1162

Ferdaoussi, M. 0203

Ferencz, A. 0725

Ferencz, S. 0725

Ferguson, S. C. 1091

Ferlinz, K. 0980, 0992

Fernandes, A. B. $\quad 0737,0738$

Fernandez, D. 0636

Fernandez, D. 1075

Fernández, E. 0014, 0499

Fernandez, M. 0909

Fernandez, M. 1229

Fernández, R. D. 0536

Fernández-Real, J. M. 0198, 0621, 0637

Fernandez-Veledo, S. 0653

Fernando, D. J. S. 1046

Ferrandiz, M. 1096

Ferrannini, E. $0049,0580,0635$, 1199

Ferrarezi, D. A. F. 1253

Ferrari, G. L. 114

Ferraro, M. 1075

Ferreira, D. 0967

Ferreira, G. B. 0471

Ferrelli, F. 0785

Ferrer-Camps, M. 0966

Ferretti, E. 0230

Ferri, J. 0955

Ferrier, B. 0623

Ferrière, A. 1093

Fery, F. 0051

Feskens, E. J. H. 1217

Feskens, E. J. M. 0824, 0827, 1216, 1250

Field, L. 0084

Fielitz, K. 0841

Figal, C. 0263

Figeyc, F. 0495

Figueroa Sobrero, A. 1075

Filipponi, F. $\quad$ 0410, 0875

Filiz, M. T. 1143

Finco-Kent, D. 0973
Fineberg, S. E. 0973

Fineman, M. 0849

Finer, N. 0837

Finocchietto, P. V. 0742

Finucane, F. M. 0772

Fiorentino, L. 0240

Firneisz, G. 0883

Fischer, H. 1064

Fisher, E. $\quad 0170,1249$

Fisk, G. 0530

Fitzpatrick, J. 0717

Flachs, P. 0631

Flacke, F. 0047

Flajsmanova, K. 0465

Flamez, D. 0421

Flanagan, S. 0849

Flanagan, S. E. 0068

Flatt, P. 0509

Flatt, P. R. 0546, 0585, 0659, 0882, 0915

Fleck, P. 0891

Fletcher, P. 0042

Fliers, E. 0751

Flodström-Tullberg, M. 0447

Florentin, E. 1233

Flores, J. 1229

Flottemesch, T. J. 1026

Flyvbjerg, A. 0259

Fodor, M. 0565

Foffi, C. 0254

Foley, J. E. 0880

Folli, F. 0179

Folli, F. 0435

Fölsch, U. R. 0780

Fong, C. H. Y. $\quad 0002,0857$

Fonseca, M. 0359

Fonseca, V. 0099

Fontaine, P. 0964

Foody, J. M. 0097

Forbes, J. 0212

Forbes, J. M. 1103

Forcheron, F. 0207, 0604, 0721

Foretz, M. 0241

Formisano, P. $\quad 0013,0675$

Formoso, G. 1167

Forsblom, C. 0061, 0216, 0228, 0259, 0364, 1099, 1134, 1180

Forst, T. 0340, 0919, 0960, 0996, 1006, 1063, 1175, 1256

Fosgerau, K. 0619

Foster, J. 0042

Fotino, C. 0947

Fountoulaki, K. 0603

Foussas, S. 1202

Fövényi, J. 0839

Fowler, A. E. 0153

Fox, T. 0913

Francesconi, A. 0957

Francini, F. 0231

Franciosi, M. 0158

Francisco, G. 1171

Franco, D. R. 0926

Franco, M. C. 0742

Frandsen, E. 1118

Frandsen, M. 0242

Franke, D. 1004

Franks, P. W. 0306

Franks, S. 0381

Fransson, U. 0522

Franz, M. 0363

Franzetti, I. 0246

Franzini, L. 0296

Fraser, S. F. 0855

Frassi, F. 1199
Frayling, T. M. 0040, 0080, 0171, 0295

Frayling, T. M. 0079

Frayn, K. N. 0194

Freathy, R. 0295

Freathy, R. M. 0040, 0079, 0080, 0171

Freckmann, G. 0148

Fred, R. G. 0490

Frederiksen, C. A. 0227

Fredrich Jr., V. L. 0538

Freeman, R. 0137, 0138

Freijoo, G. 1075

Freixenet, N. 0775

Freudenthaler, A. 0030

Freyse, E. 1008

Frier, B. M. 0984, 1091

Friery, K. F. 0294

Frigerio, F. 0397

Frimer-Larsen, H. 0986

Fritsche, A. 0003, 0252, 0605, 0714, 0818, 1238

Frizelle, P. A. 0585

Froguel, P. 0083, 0169, 0307, 0310

Frohlich, V. 0179

Fröjdö, S. 0557

Frölich, M. 0613

Frougel, P. 0287

Frystyk, J. 0259

Fuchs, W. 1256

Fuh, M. M. 0826

Fujimoto, K. 0328

Fujimura, A. $\quad 0131,0132$

Fujimura, T. 1101

Fujioka, K. 0678

Fujita, A. 0727

Fujitaki, J. M. 0911

Fukami, A. 0387

Fukami, K. $\quad 0028,1101$

Fukao, Y. 0886

Fukumoto, Y. 1225

Fulcher, G. 0986

Fuller, J. H. $\quad 0072,1082$

Fuller, M. 0408

Fülöp, Z. 1092

Fumeron, F. 0036, 0260, 0284

Fumeron, F. 0365

Fumeron, F. 1253

Funahashi, T. 0746

Furman, B. L. 0696

Fürnsinn, C. $\quad 0639,0677$

Fürstova, V. 0542

Furukawa, Y. 0289, 0344

Furuki, K. 0387

Furuta, H. $\quad 0289,0344,0727$

Furuta, S. 0689

Fuso, L. 1206

Fysekidis, M. 0365

G

Gašperíková, D. $\quad 0743,1251$

Gaál, Z. 0839

Gabazza, E. C. 0235

Gabriel, R. 0270

Gach, A. 0068, 0281

Gachoud, J. 1060

Gadet, R. 0623

Gadsby, R. 1017

Gæde, P. 0101

Gaens, K. H. J. 0676

Gaetcke, L. 1113

Gagliardino, J. J. 0324

Gagnon-Auger, M. 0048

Gale, E. A. M. 0462

Gallagher, A. 0958

Gallart, L. 0656

Gallego-Perales, J. 0768

Galli, M. 0174, 0875, 0907

Gallo, R. 0230

Galluccio, E. 0004

Gallwitz, B. 0252

Galstyan, G. R. 0367

Galtier, F. 0800

Galzin, A. 0830

Gambert, P. 1233

Game, F. 1111,1116

Gammeltoft, S. 0255

Gandhi, R. A. 0896, 1089, 1141, 1145

Gandini, D. 0635

Ganeva, S. 1184

Gangaram-Panday, S. T. 0531

Gangnerau, M. 0499

Gans, R. O. 0032

Gansevoort, R. T. 0032, 1079, 1160

Ganzoni, C. 0112

Gao, H. 0679

Gao, L. $\quad 0539,0632$

Gao, X. 1170

Gao, Z. G. 0087

Garcia-Arnes, J. $\quad 0636,0768$

Garcia-Fuentes, E. $\quad 0636,0768$

García, G. 1013

García, M. 0421

Garcia, P. 0620

Garcia -Arevalo, M. 0591

García Domínguez, M. 0245

Garcia-Aleman, J. 0636, 0768

Garcia-Almeida, J. M. 0636, 0768

Garcia-Arumi, E. 1171

Garcia-Cuenca, R. 0142

García-España, A. 0637

Garcia-Guerra, L. 0653

Garcia-Layana, A. 1088

García-Patterson, A. 0119

Garcia-Ramirez, M. 1171

Garcia-Rojas, L. 1177

Garcia-Serrano, S. $\quad 0636,0768$

Gardner, D. S. 0801

Gardosi, J. 0930

Garg, S. $0263,0940,1010$

Garin, J. 0758

Garoutsou, P. 1011

Garrigue, E. 1131

Garruti, G. 0193

Gasperikova, D. $\quad$ 0392, 0748

Gass, S. 0112

Gasser, A. 1103

Gastaldelli, A. 0073, 0634, 0909

Gastens, M. H. 1128

Gathercole, L. L. 0760

Gaucherand, P. 0728

Gaudet, D. 0291

Gauert, S. 1063

Gauguier, D. 0061

Gault, V. A. $\quad 0659,0882$

Gauthier, B. R. 0528

Gautier, J. 0365, 0671

Gautier, N. 0085

Gbenado, S. 0865

Geamanu, M. 0811

Geddes, J. 1091 
Geen, J. 1209

Gehrmann, W. 0409

Gelber, C. $\quad 0005,0903$

Gemke, R. 1055

Gendrano III, I. N. 0832

Gennatas, S. 0934

Genovese, S. 1076

George, S. 0190

Georgiev, M. 0627

Georgieva, A. 0007

Georgiou, P. 1007

Geraldes, C. F. G. 0618

Gerard, L. 0940

Gerber, P. A. 0474

Gerdtham, U. 1040

Germaschewski, L. 0457

Gerozissis, K. 0015

Gerrard, J. 0176

Gerrits, E. G. $\quad 0100,1067,1107$

Gerrits, J. 1247

Gerwien, R. 0006

Getsios, D. 0836

Geyer, M. 0309

Ghanaat-Pour, H. $\quad 0420,0493$

Ghauharali, K. J. 0783

Ghio, A. 0947

Ghirlanda, G. 0459, 1182, 1206

Ghosh, S. 0292

Ghosh, S. 0750, 0913

Giacco, F. 0013

Giani, G. $\quad 0929,1113$

Giannakopoulos, F. 1011

Giannessi, D. 1199

Giannini, S. 0687

Giardinelli, A. 1167

Giaretta, F. 0476

Gibson, G. R. 0151

Gibson, M. 0083

Gich, I. 0119

Gier, B. 0024

Gilardini, L. 0644

Gill, D. 0519

Giménez, M. 0814, 0949, 1058

Gimenez-Palop, O. 0775

Ginion, A. 0555

Gioia, D. 0628

Giorda, C. 1023

Giordano, R. 0682

Giorgetti, A. 1199

Giorgino, F. 0193, 0550, 0701

Giorgino, R. 0193

Giotaki, E. 0455

Girelli, A. 0158

Giri, M. 0469

Girling, J. 0936

Girling, J. C. $\quad 0118,0938,0939$

Giroix, M. 0415, 0523

Girola, A. 0644

Giubilato, S. 1182

Giunti, S. 0212

Giurgiuman, D. 1211

Gjedde, A. 0016

Glacken, M. 0899

Gladkih, A. 0732, 0761, 0774, 0908

Glintborg, D. $\quad 0790,0791$

Gloyn, A. L. $\quad 0172,0274,0391$, 0392

Glümer, C. 0034

Glyn, M. 1196

Gnavi, R. 0353

Gnudi, L. 0064

Goday, A. 1229

Godsland, I. F. 0717
Goegebakan, O. 0582

Goering, I. 0771

Golay, A. 1060

Goldberg, R. B. 1223

Goldfracht, M. 1019

Goldman, M. 0421

Gomes, M. B. 1243

Gómez, E. 1013

Gómez, J. 0637

Gomez, J. 0911

Gómez, J. M. 0198

Gomez-Orellana, I. 0112

Gomez-Peralta, F. 1088

Gomis, R. 0125, 0229, 0433, 0434, 0489, 0537

Gomis, R. 0816

Gonbert, S. 0964

Goncharov, N. 1121

Gonelle-Gispert, C. 0052

Gonzalez, B. 0768

González, D. 1075

Gonzalez, J. S. 1144

Gonzalez, P. 0359

Gonzalez, T. 0740

Gonzalez-Clemente, J. 0775

González-Sánchez, J. L. 0285

González-Yanes, C. 0567, 0568

Gonzalo, M. 0606

Gonzaludo, N. P. 0314

Goodall, G. 1033, 1034, 1043

Goodman, N. 0294, 0544

Goodyear, S. J. 0124

Goosse, K. J. R. 0500

Goossens, G. H. 0194

Gorbenko, K. 0908

Gorbenko, N. 0908

Gordalla, A. 0950

Gordin, D. 1180

Gorshunska, M. 0761, 0774

Gorska, M. 0615, 0945

Gorski, A. 0456

Gorter, K. J. 0106, 0264, 0898, 1061

Gorter, K. J. 1097

Gorus, F. 0469

Gosmain, Y. 0482

Gossard, F. 0291

Gosselin, A. 0867

Goto, M. 0689

Gottesdiener, K. 0831

Götting, C. 1128

Gottlieb, P. $\quad 0263,0940,1010$

Gough, S. C. L. 0897

Goyder, E. 0103

Gradinaru, D. 0723

Graefe-Mody, U. 0890

Graessler, J. 1183

Grafinger, P. 0919

Graham, A. 0754, 0755

Graham, T. E. 0309

Gram, J. 1259

Grammatikou, S. 0796, 0797

Granada, M. 0458

Granath, F. 0351

Granhall, C. 0019

Granier, C. 0059

Grankvist, N. 0512

Gras, F. 0677

Gratzer, T. W. $\quad 0221,0932$

Gravesen, P. 0954

Gray, A. M. 1042

Gray, R. E. 0558

Greeley, S. A. W. 0279

Green, B. D. 0882
Gregory, R. 0934, 0958

Gribble, F. M. 0433, 0693, 0695

Gribovschi, M. 0811

Griffin, S. J. 0383, 0805, 1045, 1057

Griffiths, P. D. 1089

Grill, V. 0297, 0368, 0443, 0516, 0810

Grimmichova, T. $\quad 0787,0788$

Grip, L. 1212

Grivennikov, S. 0152

Grobbee, D. E. 1085

Grodzinsky, E. 0010

Groen, A. K. 0012

Groener, J. E. 0783

Groenier, K. H. 0105, 0157, 0223, 0345

Groisne, L. A. 1181

Gromek, I. 1074

Groop, L. 0006, 0019, 0081, 0082, $0173,0174,0253,0287,0893$, 1252

Groop, P. $\quad 0061,0216,0228,0259$, $0364,1099,1134,1180$

Groot, M. T. $\quad 0870,0871,0872$

Gross, R. 0736

Grossman, A. 0556

Groves, C. 0274

Groves, C. J. 0040, 0079, 0080, 0169, 0295

Gruaz, L. 0528

Gruden, G. 0072, 0212

Grunder, S. 0953

Grünler, J. 1127

Grupillo, M. 0875

Gu, H. F. 0366, 0561

Guan, X. 0110

Guardado-Mendoza, R. 0435

Guarino, M. P. 0738

Gudbjornsdottir, S. 0033, 0711, 1219

Gudbjörnsdottir, S. 1040

Gueret, P. 0874

Guglielmi, C. 0527

Guigas, B. 0241

Guillerm, S. 0782

Guindy, R. 1163

Guja, C. 0335

Gulcher, J. R. 0292

Gülden, E. 0056

Guler, H. 0114

Güler, S. 0985

Gulino, A. 0230

Gumber, A. 1036

Gunton, J. E. 0730

Guo, H. 0539

Gupta, A. 0799

Gupta, R. 0799

Gurau, A. 0291

Gurgul-Convey, E. 0439

Gurisik, E. 0408

Gurney, M. 0292

Gurr, W. 0057

Gursoy, A. 0078, 0840, 0998

Guss, S. 0586

Gustafson, B. 0699, 0700

Gustafsson, J. 0679

Gut, I. 0061

Gutiérrez, C. 0198

Gutierrez, M. J. 0111

Gutierrez-Aguilar, R. 0307

Gutin, R. $\quad 0263,1010$

Guzik, T. J. 1140

Gyimesi, A. 0839

Gylfe, E. $\quad 0163,0164$

Gylling, H. 0037

Gylvin, T. 0986

Gysemans, C. 0421

H

Ha, E. 0554,0673

Haahr, H. 0189

Haaparanta, M. 0018

Haas, W. 1115

Haase, C. 1183

Haasnoot, K. 1055

Haastert, B. 0648,1113

Haber, E. 0511

Habib, A. M. 0693, 0695

Habib, S. H. 1037

Habicht, F. 1060

Hacibekiroglu, I. 0331

Hacibekiroglu, T. 0331

Hacohen, S. 0005, 0903

Hadden, D. R. 0115

Hadjadj, S. 0036, 0061, 0260, 0365,1132

Haefliger, J. 0095, 0199, 0419

Haffar, G. 0721

Haffner, S. 0864

Haffner, S. M. 0074, 0077, 0862, 0877

Hage, C. A. 1212

Hager-Vionnet, N. 0061

Hagisawa, Y. 0218, 0854, 0910

Hagopain, W. 0298, 0299

Hahnloser, E. 0648

Hai Ha, N. 0486

Haider, D. 0822

Haider, D. G. 0946

Hainault, I. 0671

Hak, E. 1061

Håkansson, R. 0317

Halban, P. A. 0400, 0417, 0437, 0508, 0533, 0534

Halff, G. 0435

Halimi, D. 0776

Hallgard, E. 0522

Halliday, M. 0934

Hallmans, G. 0373

Halseth, A. 0838

Haluzik, M. 0655, 0706

Haluzikova, D. $\quad 0655,0706$

Halvatsiotis, I. 0603

Halvatsiotis, P. G. 0759

Hämäläinen, H. 0320

Hamamoto, Y. 0600

Hambrock, A. 0422

Hamet, P. 0291

Hamilton, B. 0624

Hamman, R. F. 0350

Hammar, E. B. $\quad 0437,0533$

Hammarstedt, A. 0197, 0650, 0700

Hammer, M. 1038

Hammer, S. 0613

Hammersley, D. 0172

Han, K. 0825

Han, S. 0315

Hancu, N. 0339, 0811, 1211

Handanis, S. 1202

Handberg, A. 0621, 0790

Handisurya, A. 0946 
Hannedouche, T. 0369

Hansen, J. B. 0977

Hansen, L. $\quad$ 0255, 0647

Hansen, T. 1248

Hanson, R. L. 0169

Hanssen, K. F. 0347

Harada, M. 1213

Harcourt, B. E. 1103

Hardeman, W. 1057

Hardies, J. 0073

Hargreaves, R. 0831

Hargrove, D. 0576

Häring, H. $\quad 0003,0129,0252$, 0423, 0429, 0563, 0564, 0605, $0652,0714,0818,1238$

Harman-Bohem, I. 0147

Harrington, W. W. 0767, 0913

Harris, A. G. 0039

Harris, C. 1014

Harrison, L. 0663

Harrison, L. N. 0050, 0610, 0662, 0664

Harrison, S. A. 0073

Harry, D. 1025

Harsch-Bobbioni, E. 1060

Hart, T. 0821

Harte, A. L. 0109, 0124, 0126, 0153, 0660, 0720

Harte, A. L. 0760

Hartikainen, A. 0171, 0348

Hartmann, C. 0960

Hartwig, S. 0549

Harvey, J. N. 1078

Hasan, M. 1210

Hasegawa, N. 0430

Hashimoto, S. 0271, 1165

Hashiramoto, M. 0413

Hasko, G. 0446

Hassan, Z. 0394

Hatlapatka, K. 0506

Hattersley, A. 0281

Hattersley, A. T. 0040, 0068, 0079, 0080, 0171, 0295

Hattori, S. 0607

Hattori, Y. 0607

Hatunic, M. 0772

Hatziagelaki, E. 0300

Haug, C. 0148

Haugaard, S. B. 0757

Hausken, M. F. 1020

Havinga, R. 0658

Hawa, M. I. 0527

Hawley, J. A. 0823, 0855

Hayashi, T. 0065

Hayashida, A. $\quad 0028,1101$

Hayes, A. 1040

Hayes, R. P. $\quad 0974,1065$

Hayward, A. 0064

Hazelwood, S. 0425

He, C. 0784

He, H. 0688

He, Q. 0087

He, X. $\quad 0572,0574$

He, X. 0856

He, Y. 0784

Healy, B. C. 0294

Healy, G. 0283

Healy, G. N. 0150

Heath, M. L. 0633

Hedman, M. 0060, 0453

Hedman, M. H. 0373

Hedner, T. 0287

Hedrich, H. J. 0448

Heeren, J. 0625
Heese, B. 0625

Heine, R. J. 0001, 0007, 0009, 0031, 0251, 0272, 0389, 1139 , 1185,1215

Heinemann, L. $\quad$ 0047, 0901, 0954

Heininger, A. 0252

Heinke, P. 1008

Heinrich, A. 0924

Heinrich, J. 1049

Heise, M. A. 0074, 0077, 0862, 0877

Heise, T. 0043, 0890, 0901, 0963

Heisler, L. 0182

Heisler, L. K. 0666

Hekerman, P. 0091

Hekkala, A. 0461

Helal, R. 1166

Helbecque, N. 0307

Heller, S. 0247, 0956

Heller, S. R. 0812

Helwig, U. 0311, 0841

Heng-Hung, L. 0359

Hennessy, A. 1198

Hennige, A. 0818

Hennige, A. M. 0423

Hennings, S. 0383

Henningsson, R. 0515

Henrichs, H. R. 0950

Henriksen, E. J. 0128

Henriksen, J. 0790, 1259

Henriksen, T. 0347

Herder, C. $0386,0626,0648$

Herings, R. M. C. $\quad 0870,0871$, 0872

Herman, G. 0833

Herman, G. A. 0111

Herman, W. H. $0074,0077,0862$, 0877, 1039

Hermann, T. S. 1176

Hermansen, K. 0217, 0492, 0995

Hermida, R. C. 1100

Hernandez, B. 0961

Hernández, C. 1155

Hernandez, C. 1171

Hernandez, F. 0977

Hernández, R. 0503, 0505

Hernandez Herrero, C. 0978

Hernández-Fisac, I. 0525

Hernando, M. E. 1013

Herranz de la Morena, L. 0245

Herwig, J. 0304

Herzig, K. $\quad 0146,0780$

Herzog, S. 1133

Hesselink, M. 0177

Hesselink, M. K. 0206

Hesselink, M. K. C. 0180

Hewage, C. 0509

Hewage, C. M. 0593

Heymsfield, S. 0832

Hickey, L. 0220

Hídvégi, T. 0839

Hietala, K. 0228

Higa, M. 1156

Higashimoto, Y. 1188

Higgins, P. 0760

Hilding, A. 0346, 0351

Hiles, S. 0958

Hill, M. 0629, 0674, 0788

Hill, M. J. $\quad 0126,0720$

Hill, M. J. 0760

Hiller, S. 0422

Hilliard, D. 0111

Hillier, T. A. 0267

Hillman, M. 0302
Hilton, C. 0108

Himmelsbach, F. 0879

Hindsberger, C. 0852

Hipper, B. 1001

Hirao, K. 1225

Hiroi, N. 1156

Hirschhorn, J. 0081

Hirschorn, J. N. 0082

Hirvonen, J. 0017, 0018

Hitman, G. A. 0394

Hiukka, A. 0216

Hjøllund, K. R. 0691

Hladikova, Z. 0465

Ho, C. S. 0773

Hoashi, S. $\quad 0042,0183$

Hochberg, G. 1053

Hod, M. 0115, 0120, 0247, 0248

Hodgett, S. 0934

Hoekstra, J. B. L. 0181

Hoekstra, J. B. L. 1122

Hoey, H. M. V. 0190

Hofer, M. 0601

Hoffmann, K. 0003

Hofker, M. H. 0286, 0293, 0892, 1250

Höglund, P. 0447

Hohberg, C. 0960

Hohberg, C. 1063

Høi-Hansen, T. $\quad 0770,0813,0815$

Højberg, P. V. $\quad 0249,0577$

Højberg, P. V. 0757

Højlund, K. 0790, 0791

Holcombe, J. 0110

Holden, A. 1027, 1255

Holl, R. W. 0950

Hollak, C. E. 0783

Hollander, P. 0972, 0982, 0983

Hollander, P. A. 0835

Holleman, F. 0181

Hollingsworth, K. 0176

Holman, R. R. 0074, 0077, 0225, $0391,0862,0877,1230$

Holmberg, H. 0921

Holness, M. J. 1196

Holod, S. 0742

Holst, J. J. 0016, 0111, 0249, 0252, 0577, 0580, 0581, 0599, 0691

Holstein, G. R. 0538

Holt, H. B. 0616

Holt, R. I. G. $\quad 0616,0935$

Home, P. D. 0043

Homo-Delarche, F. $\quad 0414,0415$

Hompesch, M. 0047, 0971

Hong, J. 0217, 0492

Honjo, S. 0600

Honka, M. 0017

Honka, M. 0571

Honkanen, R. 0512

Hoo, R. L. C. 0857

Hood, L. 0544

Hoogma, R. 0953

Hopkins, D. F. C. 1241

Hopp, S. 0423

Hoppichler, F. 0919

Hoppmann, S. 1183

Horblyuk, R. $\quad 0868,0869$

Horvath, K. 0221, 0932

Horváth, R. 0275

Hosking, J. 0801

Hossain, S. 0941

Hosszúfalusi, N. 0275, 0839

Hotta, K. 0665

Hou, J. 0911

Hou, X. 1083

Hou, X. L. 0632

Houdeck, P. 1130

Houdijk, M. 1055

Hougaard, P. $\quad 0255,0647$

Housa, D. 0655

Houslay, M. D. 0696

Housova, J. 0655

Houweling, S. T. $\quad 0105,0157$, 0223, 1081

Hoverfält, A. 0364

Hovind, P. 0242

Hovsepyan, M. 0396, 0497

Howard, C. 0188

Howes, L. G. 1163

Hribal, M. L. 0026

Hroussalas, G. 0649

Hsu, I. 0050, 0663

Hsu, I. R. $\quad 0662,0664$

Hsu, T. H. 0460

Hu, F. B. 0363

Huang, D. 0856

Huang, G. 0298, 0299, 0445

Huang, J. 0211

Huang, P. 0211

Huang, Z. 0739

Hubbard DVM, MS, G. 0435

Hudson, C. A. 0609

Hue, L. 0241, 0555

Huettner, S. 0890

Hughes, A. D. 1085

Hughes, T. 0081

Hughes, T. E. 0082,0691

Huguet, P. 1155

Huho, G. 0374

Hukkanen, M. 0532

Hulbert, E. M. 0294

Hülsmann, M. 1203

Hummel, B. 1133

Humphreys, R. 0058

Hung, C. Hsia. 0802

Hunt, S. E. 0169

Hunter, M. D. 1089

Hunter, S. J. 0778

Hupkens, E. 0523

Hur, K. 0315

Hurel, S. 0261

Hurel, S. J. 1012

Hurtaud, C. 0431

Hussain, S. 1227

Hussey, E. K. 0914

Husson, B. 0485

Huttner, W. 0054

Hutton, J. 0143

Hutton, J. C. $\quad 0139,0140,0141$

Hyer, S. L. 1071

I

Iacobini, C. 0063

Iadicicco, C. 0675

Iakoubov, R. 0692

Iania, P. 0026

Iardino, P. 0013

Ibrahim, I. M. 0313

Ibrahim, S. 0055

Icks, A. 0929

Idevall Hagren, O. 0165

Idris, I. 1046

Iezzi, M. 0403

Ignatova, N. 0931 
Ikeda, H. 0600

Ikeda, T. 0487

Ikeda, T. 0678

Ikonomi, P. 0903

Ilanne-Parikka, P. 0320

Ilardi, A. 0013

Iliadis, F. 0630

Ilic, S. 1164

Ilic, V. 1164

Ilin, A. 1121

Ilkova, H. 0323

Ilonen, J. $\quad 0358,0461$

Im, S. 0570

Imaizumi, T. $\quad 0387,1105,1109$

Imamura, S. 0056

Imbergamo, M. 0246

IMDIAB Group, 0058, 0254 0303

Immel, L. 0652

Imran, S. A. 0745

Imrich, R. 0743, 0748

Ince, P. 1111

Infusino, F. 1206

Ingerslev, J. 0227

Ingman, P. 1134

Ingskog, W. 1020

Ingwersen, S. H. 0850

Inoue, H. 1188

Inoue, $\mathrm{Y} . \quad 0371$

Insa-Soria, R. 0966

Invitti, C. 0644

Ioacara, S. 0335, 0723

Ioannidis, G. 1178

Ionescu-Tirgoviste, C. 0335

Ionut, V. 0050, 0590, 0662, 0663, 0664

Iori, E. 0552

Iovino, S. 0488

Iozzo, P. 0017, 0018, 0571, 1199

Iqtadar, S. 1210

Iranmanesh, A. 0835

Irminger, J. 0437, 0508

Irwin, N. 0659, 0882

Isakson, P. 0650, 0700

Isart, M. 0846

Isermann, B. 1133

Ishak, K. 0836

Ishibashi, S. 0507

Ishida, T. 0689

Ishizuka, T. 0678

Isken, F. 0540

Islam, R. 0394

Ismail, K. 0162

Isomaa, B. 0006, 0081, 0082 0287, 1252

Istenes, I. 1098

Ith, M. 0149

Ito, B. R. 1195

Ivanova, O. 0908

Iwamoto, Y. 0065, 0795, 0888, 1213,1221

Iwasaki, K. 1225

Iyer, H. 0219

\section{J}

Jaana, L. 0320

Jablkowski, M. 0718, 0719

Jackson, N. 0045

Jackson, S. 0958

Jacober, S. 0865, 0981

Jacober, S. J. 0962

Jacobs, M. 1217

Jacobsen, L. V. 0852
Jacobsen, P. K. $\quad 0242$

Jacobsen, P. K. 1173

Jaeger, C. 0185

Jager, K. J. 1160

Jäger, M. 0020

Jaleel, A. 0752

James, N. M. 0479

James, W. P. T. 0837

Janáky, M. 1092

Jancso, G. 0725

Jandeleit-Dahm, K. 0209

Jandeleit-Dahm, K. A. 1187

Jang, H. $\quad$ 0554, 0673

Jang, N. 1192

Jani, R. 0179

Jankovec, Z. 0951

Jankovic, J. 0332

Jänne, J. 0780

Jansà, M. 0949

Jansa, M. 1058

Jansen, E. 0774

Jansen, E. H. J. M. 1216

Jansen, E. H. J. 1217

Janssen, G. C. M. 0308

Janssen, P. G. H. 0106

Jansson, P. 0197, 0711

Jansz, E. R. 0918

Jaques, F. 0508

Järvelin, M. 0171, 0348, 0381

Javorsky, M. 1251

Jawa, A. 1210

Jaziri, R. $\quad 0260,0284$

Jebunnesa, F. 1166

Jeffcoate, W. 1111, 1116

Jeffery, A. N. 0801

Jeitler, K. J. $\quad 0221,0932$

Jelenik, T. 0777

Jelic, S. D. 1164

Jelsing, J. 0038

Jendrike, N. 0148

Jenke, B. $\quad 0340,0960$

Jenkins, D. 0930

Jenkinson, C. P. 0179

Jenni, S. 0149

Jennings, C. 1027,1255

Jensen, A. C. 0034

Jensen, C. B. $\quad 0127,0733$

Jensen, C. J. 0637

Jensen, J. 0139

Jensen, L. 0248

Jensen, M. W. 0619

Jenum, A. K. 1020

Jeong, G. 1245

Jeppesen, J. B. $\quad 0144,0491$

Jeppesen, P. 0227

Jeppesen, P. B. $\quad 0217,0492$

Jermendy, A. 0651

Jermendy, G. 0839

Jesic, M. D. 0277

Jesic, M. M. 0277

Jessen, L. 0038

Jessen, N. 0491

Jewhurst, V. 1168

Ji, Q. 0990

Jia, W. $1021,1083,1220$

Jiang, G. $\quad 0480,0688$

Jiang, H. 0981

Jiang, H. H. 0991

Jiang, J. 0526, 0530

Jiaze, L. 1187

Jiazhong, S. 0451

Jilkova, Z. 0631, 0777

Jimba, S. 0795, 1221

Jimbu, Y. 0288
Jimenez-Felstrom, J. 0515

Jin, H. 0134

Jin, H. 1189

Jinnouchi, Y. 1109

Joanny, G. 0496, 0690

Joblin, B. 0672

Jocken, J. W. E. 0194

Joerns, A. 0448

Joerns, A. 0562

Johansen, M. 0933

Johansson, G. S. 0710

Johansson, L. 0318

Johansson, L. E. 0893

Johansson, S. 0278

Johansson, U. 0527

Johns, C. 0928

Johns, D. 0819

Johnsen, S. P. 0035

Johnson, J. A. 0352,0873

Johnson, J. R. 0113

Johnson, M. 0983

Johnson, M. L. 0787

Johnston, D. G. 0717

Jolivet, P. 0142

Jonas, J. 0428

Joner, G. 0347

Jones, C. A. $\quad 0962,0984,1223$

Jones, G. 1116

Jones, J. 0620

Jones, J. 1027, 1255

Jones, J. G. 0618

Jones, K. 0302

Jones, P. W. 1209

Jones, R. H. 0045

Jonker, D. M. 0850

Jonsdottir, A. B. 0565

Jonsson, A. 1252

Joost, H. $\quad 0003,0314$

Jorga, J. 0627

Jörgensen, I. 0403

Jørgensen, I. 0443

Jørgensen, M. E. 0362

Jørgensen, T. 1248

Jörneskog, G. 1104

Jörns, A. 0055, 0231, 0444, 0506

Jorsal, A. $\quad 0258,1138$

Jotic, A. 0449

Jourdan, M. 0752

Jousset, H. 0508

Jovanovic, L. 0972

Jowett, J. 0122

Jude, E. 1116

Juhász, A. 0843

Juhl, K. 0139

Juhl, T. R. 1118

Julià, M. 0489

Julius, U. 1183

Juming, L. 1085

Juncà, J. 0458

Jung, H. 0213

Jung, H. 0498

Jung, H. S. 0096

Jung, J. 0554, 0673

Jung, R. 1002

Jung, S. 0554, 0673

Jurado, J. 0846, 1096

Juvonen, K. R. 0146

\section{K}

Kaaja, R. 0248

Kabir, M. 0662, 0663, 0664

Kacerovsky, G. 0762

Kaddai, V. 0740

Kado, S. 0640

Kadoglou, N. P. 0630

Kadowaki, T. 0022

Kaestner, K. H. 0494

Kahles, H. 0304

Kahn, B. B. 0309

Kahn, S. E. $\quad$ 0074, 0077, 0862, 0877

Kaiser, D. 0481

Kajita, K. 0678

Kakei, M. 0502

Kakimoto, T. 0289

Kaku, K. 0413, 0851

Kallinteris, N. 0058

Kalousova, M. 0726

Kalra, P. 0789

Kamchatnov, P. 1051

Kameda, W. 0288

Kamenov, Z. A. 1072

Kaminski, M. T. 0541

Kamps, J. A. M. 0472

Kanaya, Y. 0727

Kanc, K. 0963

Kanc Hanzel, K. $\quad$ 0247, 0921, 0988

Kanda, S. 0218, 0854, 0910

Kanda, Y. 0413

Kandra, A. 1162

Kanegasaki, S. 0529

Kaneko, S. 0131, 0132, 0756, 0769

Kang, E. $\quad 0315,0744,0798,1070$

Kang, J. 0213

Kang, M. 0301

Kang, Y. 0554,0673

Kantartzis, K. $\quad$ 0605, 0714

Kanters, J. K. 1117

Kanzaki, M. 0121, 0123

Kapantais, E. 0796

Káplár, M. 0843

Kappelle, L. 1090

Kappelmayer, J. 0843

Kapur, A. 0914

Karaca, M. 0431, 0535

Karachentsev, Y. 0774

Karadeniz, S. 0372

Karadi, I. 0275

Karaghiosoff, L. 0353

Karagiannis, E. $\quad 0340,1063$

Karakelides, H. 0752

Karalliedde, J. 1162

Karamanos, B. 0380

Karamfilova, V. N. 1072

Karanauskaine, J. 0054

Karányi, Z. 0843

Karashima, S. 0133

Karasu, C. 1108

Karcagi, V. 0275

Karczewska-Kupczewska, M. 0615

Karhunen, L. J. 0146

Karim, A. 0891

Karin, M. 0152

Karin, N. 0464

Karlovich, N. 0646

Karmi, A. 0018

Karolczuk-Zarachowicz, M. 0615

Karpe, F. 0195

Kärre, K. 0447

Karter, A. J. 0342

Kasahara, T. 0795

Kasai, K. 0607 
Kaski, K. 1134

Kasprzak, M. 0997

Kassi, E. 0649

Kassis, N. 0414, 0735

Kastelein, J. J. P. $\quad 0008,0257$

Kastrup, J. 1173

Kaszas, E. 0651

Kasznicki, J. 0876

Kataoka, O. 0878

Kathiresan, S. 0082

Kato, K. 0769

Kato, N. 0689

Kato, T. 0092

Kato, T. 0288

Katsarou, A. 0317

Katsilambros, N. 0011, 0796, 0797, 1035, 1069, 1095

Katsuki, A. 0235

Katsya, G. 1121

Katulanda, P. 0274, 0382

Katz, A. 0220

Katz, L. S. 0482

Kaupper, T. 0141

Kaushal, R. 0118, 0938, 0939

Kautzky-Willer, A. 0946

Kavutcu, M. 1108

Kawabata, T. T. 0973

Kawai, K. 1225

Kawamori, R. 0986

Kawamura, S. 0689

Kawasaki, F. 0413

Kazanis, K. 0649

Kazdová, L. 0629

Kazdova, L. $\quad 0642,0749$

Kazeem, G. 0061

Kazim, S. 0210

Keane, D. 0399

Kefaloyannis, N. 1011

Keinänen-Kiukaanniemi, S. 0320

Keller, U. 0556

Kelly, A. 0302, 0316

Kelly, D. P. 0175

Kemal, Y. T. 0858

Kempf, K. 0626

Kempler, P. $\quad 1092,1098$

Kemppainen, J. 0018, 0571

Kendall, D. 0576

Kendall, D. M. 0251

Kendereski, A. 0627

Kendir, S. 0432

Kepaptzoglou, O. 1011

Kerényi, Z. 0944

Keresztes, K. 1098

Kern, M. 0698

Kerner, W. 0950

Kerr, D. 0261

Kerr, D. $\quad 0821,1012$

Kerr, L. N. 0962

Kerschgens, B. 0919

Kersting, S. 0020

Kerver, W. 0210

Kessabi, S. $\quad 0870,0871,0872$

Kessler, L. $\quad 0369,0478$

Kesty, N. 0838

Kettler-Schmut, E. 0996

Kettler-Schmutt, E. 0848

Keymeulen, B. 0469

Khalangot, M. D. 0327

Khan, A. 0679

Khan, M. 0205, 0428

Khan, S. 0916

Khan, S. 0948

Khan, T. 0941

Khanolkar, M. 0126
Khatun, U. H. S. 0667

Khaw, K. $\quad 0008,0257$

Khedkar, A. 0219

Khokha, R. 0240

Khong, T. 1025

Khoo, C. P. 0527

Khookhor, O. 0643

Khudyakova, E. 0908

Khutsoane, D. 0985

Khyzhnyak, O. O. 0925

Kiechl, S. 0370

Kiehne, K. 0780

Kiely, A. M. 0546

Kihara, S. 0746

Kikkawa, R. 0065

Kikkawa, Y. 0532

Kilic, B. 0159

Kilicdagi, T. 0331

Kilpatrick, E. S. 1028

Kilpeläinen, T. O. 0320

Kilpikari, R. 0364

Kim, B. 0566

Kim, C. 0134

Kim, C. 0213

Kim, C. 0894,0906

Kim, D. 0134

Kim, D. 0554,0673

Kim, D. 1192

Kim, D. Y. 0952

Kim, E. 0820

Kim, E. 0825

Kim, G. 0703

Kim, H. 0096

Kim, H. $\quad 0554,0673$

Kim, H. $\quad$ 0894, 0906

Kim, H. 1070

Kim, H. 1244

Kim, H.-J. 0825

Kim, H.-J. 0825

Kim, J. 0301

Kim, J. 0566

Kim, J. 0573

Kim, J. 0820

Kim, J. 0894, 0906

Kim, K. 0096

Kim, K. 0498

Kim, K. 0894

Kim, K. 1070

Kim, M. 0315

Kim, M. 1129

Kim, M. K. 0424

Kim, N. 1244

Kim, S. 0096

Kim, S. 0134

Kim, S. 0236

Kim, S. 0315

Kim, S. 0573

Kim, S. 0663

Kim, S. P. $\quad 0050,0662,0664$

Kim, T. 0570

Kim, T. 1244

Kim, Y. 0315

Kim, Y. 0820

Kim, Y. 1244

King, A. 0054

Kinmonth, A. 0103

Kinmonth, A. L. 1045,1057

Kinsey, E. 0425

Kipnes, M. S. 0914

Kirana, S. 1128

Kirkman, E. 0610,0663

Kishida, K. 0746

Kiss, M. H. B. 0926

Kistorp, C. 1117
Kistorp, C. N. 0233

Kitching, G. J. 1026

Klaes, T. 0950

Klapper, M. 1249

Klarer, A. 0601

Klaushofer, K. 0762

Klee, P. 0095

Kleefstra, N. 0105, 0157, 0223 , 0345, 1067, 1079, 1081, 1160

Kleesiek, K. 1128

Kleiblova, P. 0655

Klein, E. 0838

Klein, P. 0201

Kleinjung, F. 0314

Kler, L. 0914

Kles, K. A. 0135

Klett, J. 0818

Klimeš, I. $\quad 0743,1251$

Klimenko, A. 1147, 1148

Klimes, I. 0392, 0748, 1231

Klimontov, V. V. 1136

Klintenberg, M. 0893

Klioze, S. 0972

Klioze, S. S. 0136

Kloos, C. S. $\quad 0226,1064$

Klöting, I. 0467

Klöting, N. $\quad$ 0309, 0698, 0704

Klupa, T. 0281

Knauf, C. 0151

Knekt, P. 0905

Kniotek, M. 0456

Knip, M. 0358, 0461

Knoch, K. 0020

Knoops, B. 0441

Knop, F. K. $\quad$ 0249, 0577, 0599

Knudsen, L. B. $\quad 0578,0594$

Knudsen, L. B. 0850

Knudsen, S. T. 0227

Knuuti, J. 0571

Ko, G. T. C. 0375

Kobayashi, H. 0640

Kobayashi, M. 1225

Køber, L. 1176

Koblik, T. 1028

Kocabay, G. 0716

Koch, I. S. 0148

Koch, L. 0343

Koch, L. 0698

Kodama, M. 0529

Koehler, C. 0107

Koenen, C. 0224, 0976, 0977, 0979

Koerselman, J. $\quad$ 0870, 0871, 0872

Koganti, S. 1017

Koh, G. 0566

Koh, P. 0209

Köhler, C. 1256

Köhler, G. 1115

Kohner, E. M. 1153

Kohnert, K. 1008

Koieyama, N. 0854

Koike, K. 1101

Koike, T. 1190

Koitka, A. 0209

Kok, G. 0829

Kol, A. 1059

Kola, B. 0556, 1196

Kolaitis, N. 0455

Kolb, H. 0056, 0457, 0647, 0901

Kolberg, J. 0006

Kolehmainen, M. 0037

Kolender, E. 0922

Kolka, C. 0050, 0610

Kollias, A. 0603

Kollias, G. 0844

Kolterman, O. 0912, 1258

Komesidou, V. 0608

Komoroski, B. 0763, 0764, 0765

Kondo, T. 1190

Kondos, G. 0864

Kong, A. 0292

Kong, A. P. S. 0375, 0773, 0786, 1077

Kong, M. 0934, 0958

Kongkam, P. 0276

König, W. 0386

Konstantellou, E. 0011, 1095

Konttinen, Y. T. 0532

Koo, B. 0825

Kooi, M. E. 0180

Koopmans, S. J. $\quad 0145,0210$

Kopecky, J. 0631, 0777

Kopecky jr., J. 0726, 0747

Kopelevich, V. 1147

Kopp, M. 0377

Kopprasch, S. 1183

Kopska, T. 0542

Koranyi, L. 0598

Korbonits, M. 0556, 1196

Korbutt, G. 0530

Korbutt, G. S. 0526

Kordonouri, O. 0189

Körfer, R. 1200

Korner, A. 0651

Kornhauser, D. 0764

Körperich, H. 1128

Korppi-Tommola, T. 1142

Korsatko, S. 1115

Korsgren, O. 0532

Korsheninnikova, E. 0089

Korstjens, I. 0713

Kos, K. $\quad$ 0109, 0124

Koshiyama, H. 0600

Koskenpato, J. 1142

Koskinas, J. 0380

Kotanidou, A. 0759

Kotchen, T. A. 0291

Kotchie, R. W. 1033, 1043

Kothare, P. 0849

Kotova, O. V. 0522

Kotronen, A. 0612

Kotseva, K. $\quad 1027,1255$

Koulouris, S. 1178

Koureta, P. S. 0334

Koutsovasilis, A. 0792, 1202

Kovacs, M. 0651

Kovacs, P. 0309

Kovar, J. 0542

Kow, L. 0633

Kowalska, I. 0615

Kozlowska-Boszko, B. 0337

Kraenkel, N. 1174

Kramer, D. 0968

Kramvis, A. 0334

Kranebitter, M. 0483

Krarup, T. 0249, 0577, 0599

Krasner, A. 0972

Krasner, A. S. $\quad 0970,0973$

Krasova, N. 0732, 0761, 0774, 0908

Krasova, N. S. 0686

Kratochvílová, S. 0629 
Kremastinos, D. 0603

Krentz, A. J. 0705

Križanová, O. 1251

Kriek, J. 0089, 0206, 0565

Krippeit-Drews, P. 0024

Krishnarajah, G. $\quad 0329,0816$

Kristensen, J. K. 0070

Kristensen, P. $\quad$ 0850, 0851

Kristensen, P. L. $\quad$ 0770, 0815

Krogh, K. 0232

Krogmann, A. O. 0709

Kron, M. 0129

Kroon, K. 0389

Krušinová, E. 0629

Krugluger, W. 1158

Krupa, D. 0833

Kruse, J. 1049

Krusinova, E. $\quad 0674,0726,0747$

Krzyzanowska, K. 1158

Kubota, M. 0643

Kubota, N. 0022

Kuchmerovska, T. 1147,1148

Kucich, C. 0682

Kuda, O. 0631

Kudo, M. 1225

Kudo, T. 0430

Kuehn, N. 0420

Kufner, M. 1050

Kugelmeier, P. 0474

Kugler, M. 1175

Kuharenko, S. 1121

Kuhlisch, E. 0020

Kuipers, F. 0658

Kuipers, H. P. 0829

Kular, K. 1025

Kulchinska, Y. B. 0327

Kulik, W. 1122

Kumagae, S. 0387

Kumar, S. 0109, 0124, 0126, 0153 , 0302, 0316, 0660, 0720, 0760, 1031,1036

Kupfer, S. 0865

Kuraeva, T. L. 0305

Kurashvili, G. 1094

Kurashvili, R. 1094

Kurashvili, R. B. 0120

Kurita, S. 0756,0769

Kuriyama, H. 0746

Kuroda, Y. 0746

Kusano, Y. 0271, 1165

Kusminski, C. M. 0153

Kusminski, C. M. 0760

Kusuyama, A. $\quad 0289,0344$

Kutlu, B. 0544

Kuzmicki, M. 0945

Kuznetsov, A. 0504

Kvasnickova, H. 0273,0390

Kveiborg, B. 1176

Kwon, H. 0301

Kwon, M. 0424, 1129

Kwon, S. 0570

Kyriazi, E. 0603

Kyrlaki, E. 1011

$\mathbf{L}$

La Canna, G. 0004

La Vita, S. L. 0855

Laakso, M. 0049, 0320, 0571

Laaksonen, D. E. $\quad 0037,0146$, 0320

Labno, C. 0504

Labriola, L. 0232

Lacatusu, A. 0923
Lachin, J. M. $\quad 0074,0077,0862$, 0877

Lacigova, S. 0951

Lacinova, Z. 0655,0706

Lacraz, G. 0414

Ladriere, L. 0091

Ladwig, K. 1049

Laffel, L. M. B. 0188

LaFontaine, $\mathrm{H} . \quad 1112$

Laga, M. 0029

Lahiri, A. 1241

Lähteenmäki, L. 0146

Lai, L. 0175

Laino, I. 0026

Lajer, M. 0066, 0258, 1135, 1138, 1173

Lajoix, A. 0736

Lajoix, A. D. 0510

Lakka, T. A. 0320

Lalanne, G. 0880

Lalic, K. 0449

Lalic, N. M. $\quad 0270,0449$

Lam, C. W. K. 0773

Lam, D. D. 0666

Lam, K. S. L. $\quad 0002,0155,0857$

Lam, W. W. M. 0773

Lamanna, C. 0244, 0697, 0904

Lamb, H. J. 0613

Lamb, M. M. 0385

Lambadiari, V. 0608, 0712

Lambers, S. 0807

Lammers, D. 1128

Lamont, B. J. 0088

LaMoreaux, L. 1146

Lamounier, R. N. 0494

Lampasona, V. 0059

Landau, B. 0861

Landau, B. R. 0617

Landman, G. W. D. $\quad 0157,0345$, 1081

Landry, J. 0586

Lane, K. A. 1065

Lang, F. 0423, 0429

Långberg, E. 0561

Langer, P. 0343

Langer, R. D. 1016

Langer, S. 0520

Langeveld, M. 0783

Langkopf, E. 0879

Lango, H. 0040, 0079, 0080, 0295

Lankisch, M. 0980

Länne, T. 0010

Lantos, J. 0725

Lanuti, P. 1167

Lanza, G. 1206

Lapolla, A. 0246

Lara, A. 0724

Lara, J. 0606

Lara, M. 0814,1058

Laratta, E. 0026

Larbig, M. 0960

Laroche, S. 0964

Larroude, C. E. 1117

Larsen, A. 0491

Larsen, J. 0222, 0995

Larsen, J. J. 0956

Larsen, P. J. $\quad 0038,0578$

Larsen, S. 0577

Larson, P. $\quad 0832,0833$

Larson, P. J. 0111

Larsson, D. 0866

Lasfar, W. 0927

Lathrop, M. 0061

Lattuada, G. 0614
Lau, I. T. Y. 0155

Lau, S. 0730

Laue, C. 0841

Laugero, K. 0586

Laune, D. 0059

Lauranzano, E. 0550

Laurenza, I. 0920

Lauro, D. $\quad 0090,0785$

Lauro, R. 0234, 0240

Lauster, F. 1001

Lautamäki, R. 0571

Lautt, W. 0559

Lautt, W. W. 0641

Lavery, L. A. 1112

Laville, M. 0313

Laviola, L. 0193, 0550, 0701

Lawrence, I. 0958

Lawrence, J. M. 0116

Laybutt, R. 0408

Lazaroski, S. 0438

Lazarova, M. 0378

Le, N. A. 1223

Le Dèvèhat, C. 0988

Le Floch, J. 0964

Le Gall, M. 0518

Le Guen, V. 0782

Le Marchand-Brustel, Y. 0740

Le-Nguyen, D. 0510

Lebkowski, J. 0530

Leblanc, H. 0365

Lechleitner, M. 1050

Lee, B. 0820

Lee, C. 0826

Lee, C. C. 0388

Lee, D. 0566

Lee, E. 1070

Lee, G. 0894

Lee, H. $\quad 0315,0744,0798$

Lee, H. 0894

Lee, H. 1070

Lee, H. K. 0731

Lee, I. 1169

Lee, J. 0744,0798

Lee, J. 0798

Lee, J. H. 0952

Lee, K. $\quad 0554,0673$

Lee, K. 0906

Lee, K. 0906

Lee, K. 1244

Lee, K. O. 0863

Lee, K. O. 1123

Lee, M. 0096

Lee, M. 0096, 0498

Lee, M. 0498

Lee, S. 0041

Lee, S. 0213

Lee, S. 0301

Lee, S. 0424

Lee, S. 0985

Lee, S. 1070

Lee, S. 1129

Lee, S. S. 0731

Lee, W. 0868,0869

Lee, W. 1169

Lee, Y. 0566

Lee, Y. 1070

Lee, Y. Y. 0731

Lefebvre, P. 0365

Legare, D. J. 0641

Lehmann, R. 0129

Lehmann, R. 0473, 0474

Lehmann, R. 0563, 0652

Lehmann, U. 1256

Lehr, S. 0549

Lei, M. 0856

Leibiger, B. $\quad 0481,0514$

Leibiger, I. B. $\quad$ 0481, 0514

Leicht, S. 0694

Leinonen, E. 0216

Leinonen, M. 0381

Lekakis, I. 0603

Lekuleni, L. 0359

Lemieux, I. 0008, 0257, 0654

Lencioni, C. $\quad 0942,0947$

Leng, Y. 0917

Lengyel, C. 1092

Lengyel, G. 0883

Lenzen, S. 0166, 0231, 0409, 0427, 0439, 0442, 0444, 0448, 0520, 0541, 0562

Leo, A. 1182

Leonardini, A. 0550

Leone, T. C. 0175

Leonetti, F. 0158

Leong, J. 0914

Lepore, G. 0215

Lerche, S. 0016, 0581

Lernmark, Å. 0317

Lernmark, B. 0317

Leschenko, J. 0908

Leshchenko, Z. 0732

Leslie, R. D. G. 0298, 0299

Leslie, R. D. G. 0450

Lessard, S. 0855

Lethagen, A. 0173

Leto, G. 0303

Leturque, A. 0518

Leverve, X. 0860

Levisianou, D. K. 1202

Levy, I. 0949

Lévy, M. 0964

Lévy Marchal, C. 0728, 0734

Lewis, P. 0948

Leyk, M. 0984

Li, B. 0784

Li, C. 0480

Li, G. 0200

Li, J. 0201

Li, L. 0156

Li, L. $\quad 0763,0764,0765$

Li, M. 0572, 0574

Li, N. 0426

Li, N. 0451

Li, Q. 0903

Li, W. 0849

Li, Y. 0057

Li, Y. 0398

Li, Y. 0859

Li, Z. 0480

Liang, H. $\quad 0572,0574$

Liao, L. 0863,1123

Liapis, C. D. 0630

Liatis, S. $\quad 0796,0797,1035,1069$

Libert, C. 0421

Libert, S. 0142

Licata, G. 1246

Lichiardopol, R. 0685

Lieberman, N. 1019

Liebl, A. 0986, 0988

Liefvendahl, E. 0710

Liese, A. D. 0350

Lietz, T. 0337

Lieverse, A. G. 1079

Liew, D. 0354 
Lilleøre, S. K. 0733

Lim, E. 0176

Lim, E. 1241

Lim, R. C. C. 0558

Lin, C. 0826

Lin, Y. 0298

Lincoln, N. 1111

Lind, L. 0711

Lindahl, B. 0373

Lindauer, P. 0252

Lindblad, U. 1016

Linden, K. C. 0741

Lindgren, C. M. $\quad$ 0040, 0080, 0171, 0172, 0295

Lindholm, E. 0253

Lindholm, H. 1099

Lindi, V. 0320

Lindmark, S. 0196

Lindner, I. 1249

Lindqvist, A. 0054, 0201

Lindsey, P. J. 0702

Lindström, J. 0386

Lindström, P. 0497

Lindström, T. 0010

Linemeyer, D. L. $\quad 0911,1195$

Ling, C. $\quad$ 0019, 0174

Ling, S. 0548

Lingdren, C. M. 0079

Lins, P. 1104

Linscheid, P. 0556

Linssen, M. M. L. 0565

Lipinski, H. L. 0225

Lipkin, D. 1241

Lipson, V. 0908

Lisowicz, L. 0922

List, J. 0889

Liu, C. 0470

Liu, C. P. 0470

Liu, E. K. H. 1077

Liu, F. 0688

Liu, F. 1083

Liu, H. 0594

Liu, J. Y. 0342

Liu, K. 0786

Liu, L. 0005

Liu, L. 0168

Liu, N. 1189

Liu, Z. $\quad 0298,0299$

Liu, Z. 0990

Liuzzi, R. 1076

Livesey, D. 0479

Ljubicic, S. 0202

Lledo, A. 0135

Loba, J. $\quad 0718,0719$

Löbig, M. 0340

Lobmann, R. 1130

Lodwig, V. 0901

Loenen, R. van. 0774

Lofgren, B. 1194

Logtenberg, S. J. J. 0223, 0345, $1067,1079,1081,1160$

Lombardi, A. 0675

Lombardo, M. 0785

Lonie, L. 0391

Lonnroth, P. 0711

Lonovics, J. 1092

Lönz, H. 0564

Loos, R. J. F. 0306

Lopes de Faria, J. B. $\quad 1106,1150$

Lopes de Faria, J. M. 1150

Lopez, S. 1075

López-Bemejo, A. 0621

Lopez-Novoa, J. M. 0860

Lorenzo, M. 0653
Lortz, S. 0442

Losio, C. 0477

Lottati, M. 0050, 0662, 0663, 0664

Louchami, K. 0523

Low, P. A. 0138

Lowe, L. P. 0115

Loweth, A. 0425

Lu, H. 1083

Lu, H. 1083

Lu, J. $\quad 1083,1220$

Lu, K. 0530

Lu, W. 0398

Lubach, C. H. C. 1066

Lübben, G. $\quad 0340,1063$

Luben, R. 0008

Lucas, M. 0229

Luccarini, J. 0485

Lucchesi, D. 1186

Lucidi, P. 0046

Lucotti, P. C. G. 0004

Ludop-Maignel, J. 0830

Ludvigsson, J. 0060, 0453, 0921

Ludvik, B. 0030, 0793, 0822, 0895, 0919, 1002

Luebker, M. 1004

Luedemann, J. 0083

Luger, A. 0030, 0639, 0822, 0895, 0946, 1203

Lukasova, P. $\quad 0273,0312,0390$

Lukic, L. 0449

Lukic, T. 0039

Lukinius, A. 0532

Lunati, M. Elena. 0622

Lunati, M. E. 0845

Lunceford, J. K. 0113

Lund, S. 1194

Lund, S. S. $\quad 0242,0989$

Lund Nilsen, T. I. 0368

Lundgren, M. 0196

Lundholm, L. 0679

Lundquist, I. 0515

Lundqvist, I. 0054

Luo, J. 0152

Luo, R. 0200

Luo, X. 0256

Lupi, R. 0019, 0093, 0174, 0410, 0547, 0875

Lupoli, G. 0488

Lush, C. 1258

Lutgers, H. L. $\quad 0100,1107$

Luthman, H. 0019

Lüttig-Rakowske, M. 1200

Lutz, K. 0912

Luzi, L. 0614

Luzio, S. $\quad 0484,1078$

Lydersen, S. 0810

Lykkegaard, K. 0578

Lynch, G. S. 0741

Lynch, K. 0317

Lynch, M. J. 0696

Lyons, K. 0688

Lyssenko, V. 0006, 0081, 0082, 0173, 0174

Lyssenko, V. A. 0866

Lyu, R. 0816

\section{M}

Młynarski, W. 0645, 0924

Ma, C. 1189

Ma, J. 0366

Ma, L. 0504

Ma, R. C. W. $\quad 0375,0786$
Ma, Z. $\quad 0403,0443,0516$

Maassen, J. A. 0089,0272, 0308, 0565

Mabilleau, G. 1126

Mabley, J. G. 0446

MacConell, L. 0849

MacDermott, C. N. 0896

Mace, K. 0849

Macedo, M. 0559, 0641

Macedo, M. P. $\quad 0737,0738$

Machado, M. C. C. 0232

Machann, J. 0605, 0714

Machicao, F. $\quad 0605,1238$

Mack, E. 0562

MacKenzie, W. 0930

Madacsy, L. 0651

Madani, S. 0365

Madarász, E. 0944

Madeddu, P. 1174

Madhusudhan, T. 1133

Madsbad, S. 0127, 0249, 0577, 0599, 0757

Madsen, C. Rask. 1176

Maechler, P. 0199, 0397, 0426, 0524

Maeda, N. 0746

Maeda, S. 0065

Maffi, P. 0477

Mager, U. 0037

Maggioni, A. P. 0837

Maggs, D. 0110, 0912, 1258

Magliano, D. J. 0354

Magnan, C. $\quad 0535,0735$

Magnus, P. 0463

Magnusson, N. $\quad$ 0144, 0491

Mahalingam, B. 0661

Maheux, P. 0971

Mahoney, W. 0083

Maier, C. 0030, 0822

Maier, H. 0912

Maimaitiming, S. 0036, 0260, 0284

Mainenti, P. 1076

Maione, A. 0158

Maissi, E. 0162

Major-Pedersen, A. 1176

Majumdar, A. S. 0526

Majumdar, S. R. 0873

Mäkelä, M. 0358

Mäkinen, V.-P. 1134

Mäkinen, V. 1180

Makino, H. 0121, 0123, 0214

Makoundou, V. 1060

Makrilakis, K. 0796

Makropoulos, D. 0592

Malaisse, W. J. 0521, 0523, 0596, 0779, 0781

Malecki, M. 0068

Malecki, M. T. $\quad 0281,1140$

Mali, W. P. T. 1090

Malinska, H. 0642

Malloy, J. L. 0251

Malmberg, K. 0186, 1208, 1212

Malogajski, J. 0332

Malosio, M. L. 0477

Maltezos, E. 1068

Malthouse, J. P. G. 0509

Manca, E. 1191

Mancarella, R. 0174, 0410, 0875

Mancha, I. 0636

Mancini, E. 0635

Mancini, M. 1076

Mandecka, A. 0226

Mandl, M. 0895

Mandrup, S. 0397

Mandrup-Poulsen, T. 1173

Manesis, E. 0380

Manicardi, V. 0246

Mankovsky, B. 0376

Manley, S. E. 0897

Manning, G. 1154

Mannino, D. 0246

Mannucci, E. 0244, 0687, 0697, 0904, 1218

Manolis, A. 0792

Manolis, A. 1178

Mansour, A. A. 0266

Manteghetti, M. 0736

Manteiga, R. 0005

Manto, A. 0459

Mäntysaari, M. 1099

Mao, J. 0853

Mao, X. D. 0470

Maratou, E. 0603,0608

Maratou, I. 0712

Marchetti, P. $0019,0093,0174$, 0230, 0410, 0416, 0418, 0547, 0875, 0907

Marchionni, N. 0244, 0697

Marchisio, M. 1167

Marco, J. 0503, 0505

Margina, D. 0723

Mari, A. $\quad 0049,0580,0880$

Maria, K. 0725

Marin, D. M. 0634

Marin, E. 0803, 1235

Maringa, M. 0277

Marino-Goulart, D. 1240

Marinou, K. 1069

Mark, M. 0879

Markiewicz, P. 0337

Markovic, I. 0449

Marques, J. L. B. 1141

Marre, M. 0036, 0061, 0260 ,

0284, 0307, 0365, 1253

Marrero, D. G. 1065

Marselli, L. 0093, 0417, 0418

Marshall, S. 0064

Marsollier, N. 0735

Martano, M. 0410

Marte, T. 0343

Martelli, A. 0907

Martemucci, S. 0550

Marthinet, E. 0482

Martikainen, H. 0171

Martin, D. 0095, 0199

Martin, D. T. 1205

Martin, E. 0048

Martin, F. 0338

Martin, G. 0623

Martin, M. 0623

Martin, S. 0099, 0626, 0648, 0901, 0991, 0994

Martin, S. A. 0981

Martín-del-Río, R. 0525

Martin-Grillo, S. 0966

Martin-Gronert, M. S. 0127

Martinelli, G. P. 0538

Martinez-Alfaro, B. 0768

Martínez-Cáceres, E. 0458

Martínez-Calatrava, M. J. 0285

Martínez-Honduvilla, C. J. 0014

Martinez-Larrad, M. T. 0285 
Martynov, S. A. 1157

Masereeuw, R. 0859

Masi, S. 1199

Masiello, P. 0019, 0361, 0547

Masini, M. 0093, 0230, 0361

Massano-Cardoso, S. 0268

Massaro, P. 0794

Masserini, B. $\quad 0622,0845$

Massi Benedetti, M. 1034

Mastroianno, S. 1254

Masulli, M. 1076

Masuzawa, M. 0670

Matesanz, N. 1168

Mathiesen, E. $\quad 0247,0248$

Mathiesen, E. R. $\quad 0933,1159$

Mathieu, C. 0421, 0469, 0471, 0995, 1022, 1073

Mathur, A. 0592

Mato, E. 0229

Matoulek, M. 0706

Matskeplishvili, S. T. 1084

Matsuda, M. 0670

Matsudaira, T. 0069

Matsui, J. 0430

Matsui, T. 1105

Matsuki, M. 0413

Matsumoto, H. 0371

Matsumoto, K. 0235

Matsumoto, Y. 0028

Matsumoto, Y. 0689

Matsumoto, Y. 1101

Matsuno, S. 0289, 0344

Matsushima, M. 1225

Matsuzaka, T. 0092

Matsuzaki, M. 0102

Matsuzawa, N. $\quad 0131,0132,0756$

Matsuzawa, Y. 0102

Matta, M. 0799

Mattenberger, Y. 0094

Matthaei, S. 0953

Matthews, D. R. 0274, 0382

Matthey-Doret, D. 0021

Matthys, K. 1073

Mattiello, L. $\quad 0239,0708,0842$

Matucha, P. 0787

Mätzke, S. 1142

Mauckner, P. 1113

Maugeri, L. 1206

Mauricio, D. 0775

Maury, E. 0154

Maus, J. 0138

Mavrogiannaki, A. 0380

Mavros, P. 0816

Mayer-Davis, E. J. 0350

Mayet, S. 0694

Mayr, P. 1001

Mazilu, C. 0384,0715

Mazzone, T. 0864

McAlister, F. A. 0873

McCall, T. 0865

McCance, D. 0248

McCarthy, M. I. 0040, 0079, 0080, 0169, 0171, 0274, 0280, 0295, 0382

McCauley, C. 0592

McClenaghan, N. H. 0509

McConell, G. K. 0741

McConnell, J. P. 0752

McEwan, P. 0836

McGarraugh, G. V. 1009

McGee, K. C. $\quad 0126,0760$

McGeown, P. 0930

McGill, J. B. 1237

McGuire, J. 1135
McKenna, M. P. 0006

McKillop, A. M. $\quad 0659,0882$

McKinney, P. A. 1047

McLean, M. 0730

McLennan, S. V. 1198

McMillan, J. 0122

McNally, P. 0958

McTernan, P. G. $\quad$ 0109, 0124, 0126, 0153, 0660, 0720, 0760

Mead, A. 1027,1255

Meas, T. 0734

Meave, A. 1177

Meda, P. 0095, 0199

Meex, S. 0750

Meex, S. J. R. 1250

Megía, A. 0198

Mehmeti, I. 0442

Mehta, S. R. 0717

Meidute Abaraviciene, S. 0515

Meikle, P. 0408

Mekki, Q. 0891

Melander, A. 1016

Melander, O. $\quad 0082,0287$

Melchiorre, M. 0193, 0550, 0701

Melidonis, A. 0011

Melidonis, A. $\quad 0792,1095,1202$

Mellbin, L. G. 0186

Mello, V. D. F. 0037

Melnikova, E. 0931

Meloncelli, I. 1023

Melzi, R. 0477

Memeo, V. 0193

Ménard, J. 0048

Mendes, E. 0268

Menegazzi, M. 0547

Meneghini, E. 0215

Meneghini, L. 0224, 0976, 0977

Meng, X. 0220

Meng, X. 0900

Menghini, R. 0234, 0240

Menini, S. 0063, 0240

Mennerich, D. 0624

Mensink, M. 0177, 0180

Menzaghi, C. 1254

Merce, J. 0846, 1096

Meredith, S. 0261

Merletti, F. 0353

Merlo, E. 0291

Mersebach, H. 0248, 0921

Mertl-Roetzer, M. 1001

Metais, C. 0207,0604

Metcalf, B. S. 0801

Metelko, Z. 0160

Metzger, B. E. 0115

Meulenberg, M. G. G. 1097

Meyboom-de Jong, B. $\quad$ 0105, 0157

Meyer, P. 0864

Meyer zu Vilsendorf, A. 0444

Meyre, D. 0083

Mezghenna, K. 0736

Mianowska, B. $\quad 0645,0924$

Mibus, A. 1103

Miccoli, R. 1186

Miccoli, S. 0701

Miceli, I. 0476

Michalska, M. 0440

Michels, R. P. J. 1122

Micic, D. D. 0277, 0627, 0669

Mickovski, N. 0332

Midthjell, K. $\quad$ 0297, 0368

Miele, C. 0675

Miettola, S. 0348

Migoya, E. M. 0111

Mikiya Usukura, M. 0133
Mikros, S. 0011, 1095

Milek, K. 0994

Milicevic, Z. 1223

Milicic, T. 0449

Miljus, D. 0332

Miller, J. L. 0111

Miller, M. $\quad 0181,0184,1015$

Miller, R. G. 1242

Milliat-Guittard, L. 0478

Milliken, N. O. 0767

Milting, H. 1200

Milton, D. 0853

Min, K. 0825

Minami, M. 1225

Minartz, P. 1128

Mindell, J. 0071

Minns, I. 0043

Mion, B. 0509

Mira, R. 0964

Miralles, A. 1155

Miranda, M. 0198, 0637, 0656

Miranda, M. 1261

Mirenda, V. 1162

Mirkiewicz-Sieradzka, B. 1140

Mirra, P. 0488

Miscio, G. 1254

Misnikova, I. V. 0265

Misovicova, N. 0392

Misra, C. 0661

Misu, H. 0756

Mitchell, B. D. 0169

Mitnick, M. 0971

Mitrakou, A. 0049

Mitrou, P. 0712

Mitrou, P. I. 0608

Mittermayer, F. 1158

Miyashita, Y. $\quad 0069,0328$

Miyazaki, H. 0028

Miyoshi, H. 1190

Mlejnek, P. $\quad 0629,0747$

Mlynarski, W. $\quad 0068,0281$

Moede, T. 0481, 0514

Moehlig, M. 0170

Moeller, N. 0016, 0581

Moffet, H. H. 0342

Mogensen, C. E. 0227

Mohamed, H. 0402

Mohamed-Ali, V. 0631

Mohammedi, K. 0036

Mohideen, P. 0881

Mohlig, M. 0582

Möhlig, M. 1249

Mokhort, T. 0646

Molcanyiova, A. 1231

Moler, E. 0006

Molina, A. J. A. 0402

Molina, G. 0359

Molinier, N. 0800

Möller, J. 0836

Moller, R. A. 1163

Molven, A. 0278

Monahan, P. O. 1065

Monami, M. 0244, 0697, 0904, 1218

Monastiriotis, C. 1068

Monfort, C. 0943

Mongan, O. A. 0899

Moniz, C. 1124

Monroy, A. 0435

Montanya, E. 0496,0690

Montanya-Mias, E. 0966

Monte Alegre, S. 0634

Monti, L. D. 0004

Montonen, J. 0905

Moons, K. G. M. 1082

Moore, M. C. 0584

Moore, S. 0182, 0183

Moreau, F. 0369

Morel, P. $\quad$ 0021, 0052, 0436

Morelon, E. 0478

Moreno, I. 1075

Moreno, P. 0583, 0591

Morens, C. 0729

Morgan, N. G. $\quad$ 0395, 0405, 0538

Mori, I. 0678

Mori, M. 0965

Morillas, C. 0816

Morimoto, A. 0069

Morin-Papunen, L. C. 0381

Morita, H. 0678

Moritoh, Y. 0878

Moritz, W. 0473

Morosanu, A. 0811

Morris, A. D. $\quad 0079,0080$

Morrissey, J. 1017

Mortelmans, L. 0831

Mortensen, H. 0255

Mortensen, H. B. 0647

Mörtl, D. 1203

Morviducci, L. 0158

Mosca, F. 0875

Moses, R. G. 1223

Mosihuzzaman, M. 0916

Mosnier-Pudar, H. 0964

Mota, A. 0618

Moua, O. $\quad$ 0140, 0143

Moulin, B. 0369

Moulin, P. 1181

Moustakas, A. K. 0454

Mouton, C. 0555

Movassat, J. 0495, 0501

Moya Chimenti, E. 0245

Mraz, M. 0655, 0706

$\mathrm{Mu}, \mathrm{J} . \quad 0480$

Muchmore, D. 0969

Muchmore, D. B. 0974

Mucsi, I. 0377

Muendlein, A. 0343

Muggeo, M. 0370, 0957

Mughal, S. 1031, 1036

Muise, E. 0480

Mulak, G. 0243

Mulder, H. $\quad$ 0201, 0517, 0522

Müller, B. 0556

Müller, J. 1256

Müller, N. 0226, 1064

Muller, P. S. 0638

Müller, U. 0226

Müller, U. A. 1064

Mullins, P. 0181

Munni, U. S. 0941

Munzel, U. 0138

Murakami, K. 0121,0123

Murdolo, G. 0711

Muriach, M. 1261

Murillo, S. 0808

Murphy, A. W. 0899

Murphy, E. 0552

Murray, S. C. 0914

Muscelli, E. $\quad 0580,0634$

Musella, T. 0459,1182

Mussen, J. 1172 
$\mathbf{N}$

Naamane, N. 0545

Nabe, K. 0600

Nacher, M. 0690

Nadal, A. 0537

Nadas, J. 0839

Nadeev, A. P. 1136

Näf, S. 0637

Nagaev, I. 0753

Nagasaka, S. 0507

Nagasaki, M. 0643

Någren, K. 0018

Nagy, B. 0843

Nahar, N. 0916

Nahar, Q. 0804

Naïmi, M. 0085

Nair, K. S. 0130, 0752

Naito, H. 0640

Najib, S. $\quad 0567,0568$

Najmutdinova, D. 1260

Nakagami, T. $\quad 0379,0795,1221$

Nakakuki, M. 0092

Nakamura, A. 0022

Nakanishi, K. 1087

Nakashima, K. 0413

Nakashima, R. 0854

Nakata, M. 0507

Nakatsuka, A. 0121,0123

Nakaya, K. 0689

Nam, J. 0213, 1070

Nam-Goong, I. 0820

Nanjo, K. 0289, 0344, 0727

Nannipieri, M. 0635

Nano, R. 0477

Nansalmaa, E. 0030

Napoli, A. 0246

Napoli, V. 0947

Nar Demirer, A. 0722

Naraoka, M. 0430

Narayan, K. 0350

Narayanan, S. 0661

Narendran, P. 1227

Nascimento, E. B. M. 0565

Nata, K. 0487

Natali, A. 0580

Natalicchio, A. $0193,0550,0701$

Natarajan, M. 0661

Nathanson, D. 0025

Naugler, W. 0152

Naujok, O. 0231

Navarese, E. 1206

Navarro-Lopez, F. 0846

Navon, R. 0296

Nawroth, P. P. $\quad 0543,1133$

Nayyar, V. 0958

Nazaret, R. 0623

Nazim, J. 0191

Ndip, A. 1112

Nedumaran, B. 0703

Neels, J. G. 0152

Neeser, K. 1004

Neglia, D. 1199

Negrean, M. 1128

Neil, H. A. W. 0103, 1042

Nemeth, Z. H. 0446

Nescheret, A. 1197

Nesher, R. 0511

Neskudla, T. 0726

Nespoli, L. 0360

Nesterov, S. 0017

Nesto, R. W. 1205

Nestoris, C. 0189

Neubauer, H. 0624
Neuhold, S. 1203

Neurwith, G. 0839

Neve, B. 0307

Newman, S. 0261, 1012

Newsholme, P. 0399, 0509, 0524 0546

Neyrinck, A. M. 0151

Ng, M. 0773

Nguyen, B. 0736

Nguyen, M. 1207

Nicholls, C. 0836

Nichols, G. A. 0267,1039

Nicolau, J. 0814

Nicolay, K. 0178

Nicolescu, G. 1211

Nicoletti, F. 0438

Nicoloff, G. 0468

Nicolucci, A. $\quad 0158,1023$

Niculescu, D. 0685

Niederhauser, G. $\quad$ 0203, 0419

Niederwanger, A. 0483

Niedziolko-Bagniuk, K. 0945

Niedzwiecki, P. 0997

Nielsen, A. R. 0306

Nielsen, B. B. 0989

Nielsen, D. 1176

Nielsen, J. N. 0963

Nielsen, L. 0110

Nielsen, L. B. 0255

Nielsen, T. T. 1194

Niessen, H. W. 1185

Niessen, M. 0672

Nieto-Vazquez, I. 0653

Nightingale, P. G. 0897

Nijhuis, J. 0676

Nijpels, G. 0001, 0031, 0104, 0161, 0272

Nikankina, L. 1051,1121

Nikolajuk, A. $\quad 0615,0945$

Nikoloff, G. 1184

Nikolov, A. 0468

Nikolovski, B. 0558

Nikou, A. 1178

Nillson, J. 0270

Nillson, P. M. 0270

Nilsson, A. 0317

Nilsson, L. 1052

Nilsson, P. 0173

Nilsson, P. M. 0033, 0880, 1040, 1219

Nishi, M. $\quad 0289,0344,0727$

Nishijima, K. 0851

Nishimura, R. 0069, 0328, 0379

Nishizawa, H. 0746

Niskanen, L. 0037

Nita, C. 1211

Nivoit, P. 0729

Nizheradze, K. 1152

Njolstad, P. R. 0278

Nobels, F. $\quad 0964,1022$

Nocea Pulfer, G. 0329

Noel, D. 0750

Noël, L. 0154

Noguchi, M. 1188

Noguchi, N. 0487

Noh, J. 1192

Noh, Y. H. 0952

Nolan, J. 0049

Nolan, J. J. 0772

Nolop, K. 0220

Nolte, T. 0569

Nomura, K. 0600

Nonaka, K. $\quad$ 0886, 0888

Noordam, K. 0068
Norby, B. 0861

Nordentoft, I. 0217, 0492

Nordman, S. 0561

Norhammar, A. 1208, 1212

Noronha, I. L. 0926

Norris, J. 0385

Norris, S. 0772

Norström, F. 0893

Nosari, I. 0215

Nosek, L. 0963

Nosikov, V. V. 0305

Novak, M. 0377

Novelli, G. 0333

Novelli, M. 0361, 0547

Novials, A. $0229,0433,0434$, 0537, 0808

Nóvoa, F. J. 0319

Nowotny, P. $\quad 0639,0895$

Nubel, T. 0431

Nuche-Berenguer, B. $\quad$ 0583, 0587, 0588

Nunes, E. 1243

Nunez, D. J. R. 0914

Nuñez de Arenas, G. 0803, 1235

Nunn, A. 0261, 1012

Nusselder, W. J. 0354

Nuutila, P. $\quad 0017,0018,0571$

Nwokolo, C. 0124

Nyamdorj, R. 0269

Nyblom, H. K. 0416

Nyström, F. 0010

Nyström, T. 0025

Nzietcheung, R. 0066

\section{O}

O Hare, P. 1018

O'Connor, P. J. 1026

O'Connor-Semmes, R. L. 0914

O'Driscoll, E. C. 0914

O'Gorman, D. 0657

O'Hare, J. 1036

O'Hare, J. P. 0109

O'Hare, J. P. 0124

O'Hare, J. P. 0153

O'Hare, P. 0316

O'Hare, P. J. 1031

O'Harte, F. P. M. 0585, 0593, 0882

O’Neil, K. 0592

O'Riordan, S. M. P. 0190

O‘Sullivan, E. P. 0657

Oakley, W. 0930

Oates, P. J. 0136

Oats, J. N. 0115

Oberauer, R. 0624

Obermüller, S. 0054, 0201

Ochiya, T. 0529

Odaka, H. 0878

Odetti, P. 1102

Oetliker, C. 0149

Ogata, H. 0403, 0443

Ogawa, J. 0218, 0910

Ogawa, K. 0727

Ogawa, Y. 0430

Ogston, N. C. 0631

Oguzhan, B. 0372

Oh, S. 0498

Oh-I, S. 0965

Ohmori, D. 0640

Ohmori, K. 0214

Ohnuma, H. 0288

Öhrvik, J. 1212

Ohsumi, J. 0854

Oikari, S. 0780

Oikawa, S. 0102

Oikonen, V. 0018

Oishi, M. 1225

Oitzl, M. S. 0684

Oizumi, T. $\quad 0288,0379$

Oka, Y. 0452

Okabe, T. 0670

Okada, A. 0888

Okada, S. 0965

Okamoto, H. 0487

Okar, D. A. 0086

Okerson, T. $\quad 0110,0819$

Oknianska, A. R. 0538

Ökten, A. 0716

Okuda, S. 0028,1101

Okuno, A. 0218, 0910

Okuyama, K. $\quad 0886,0888$

Oldani, M. 0004

Olefsky, J. M. 0152

Oliveira, L. S. 0494

Oliver, N. S. 1007

Oliyarnyk, O. 0642

Olmez, B. 0372

Olsen, N. V. $\quad 0815,1117$

Omer, A. 0418

Omulecka, A. $\quad 0718,0719$

Onai, T. 1213

Öncü, A. 0252

Ong, W. 0696

Onland-Moret, N. C. $\quad 0286,0293$, 0892

Oono, K. 0371

Oosterveer, M. H. 0658

Orchard, T. J. $\quad 1082,1226,1242$

Orfanos, S. 0759

Orho-Melander, M. 0081, 0082, 0173, 0287, 1252

Oriente, F. 0488

Origasa, H. 0102

Orlando, M. R. 0193, 0550, 0701

Oroszlán, T. 0839

Orsi, E. $\quad 0622,0845$

Ørskov, C. 1028

Ort, T. 0592

Ortis, F. 0091, 0545

Ortiz, J. N. M. 0634

Ortsäter, H. 0512

Osawa, N. 0065

Osborn, B. L. 0975

Osmenda, G. 1140

Osmers, A. 0549

Osswald, H. 0422

Osta, V. 1214

Östenson, C. 0346, 0351, 0561

Oster, P. 1044

Osterhof, M. 0170, 0771

Osterhoff, M. A. 0540

Ostgren, C. J. 0010

Osundiji, M. 0182

Osundiji, M. A. 0183

Otani, T. 1156

Oterdoom, L. H. 0032

Otieno, F. C. F. 0374

Otonkoski, T. 0532

Otsuka, M. 0387

Otsuki, M. 1165

Ott, P. 0107

Otter, W. 1224

Otto, B. 0582 
Overbergh, L. 0471

Overstreet, D. H. 1194

Ovsyannikova, T. 0761

Owen, K. R. 0280

Owen, N. 0150, 0283

Owens, D. R. $\quad 0484,1078$

Oya, J. $\quad 0795,1221$

Oyer, D. S. 0987

Oz, G. 0432

Ozaki, R. 0773

Ozanne, S. E. 0127

Ozansoy, G. 0681

Ozel, S. 0716

Ozkan, A. 1125

Ozkan, B. 1125

\section{$\mathbf{P}$}

Pachaiappan, J. 1017

Pacher, P. 0446

Pacher, R. 1203

Pacini, G. 0049, 0589, 0762, 0772, 0788, 0895

Pade Hansen, A. 0247

Padma, S. 0179

Pae, I. 0894

Pagano, G. 0333

Pagava, K. 0120

Paghava, K. 1094

Pagnin, E. 0552

Pak, K. 0825

Pakbiers, M. 0194

Pala, L. $\quad 0687,0697,0904,1218$

Pala, M. G. 0004

Palaisa, M. 0981

Palanisamy Kanakaraj, P. 0975

Palau, N. 0125

Paldanius, M. 0381

Palik, E. 0275

Pallardo Sánchez, L. 0245

Pallayova, M. 0335, 0847

Palomera, E. 0943

Pan, C. 1021,1120

Pan, J. 1220

Panadero, A. 0606

Panagiotakos, D. 0712

Panagiotakos, D. 0792

Panczel, P. 0275

Pánczél, P. 0839

Pande, A. 0350

Pandolfi, A. 1167

Pandolfo, M. 0051

Pandya, B. 1029

Pang, F. 1034

Panizzutti, C. 1131

Pankowska, E. 0922

Paolini, M. 0410

Papadakis, G. $\quad 0011,1095$

Papadopoulos, G. K. 0454,0455

Papakonstantinou, A. 0608

Papakonstantinou, E. 0792

Papamichael, C. 0844

Papanas, N. 1068

Papaoikonomou, S. 1035

Papas, K. K. 0432

Papatheodoridis, G. 0380

Papatheodorou, K. 1068

Papazoglou, D. 1068

Papezova, H. 0655

Pappas, A. 1011

Paragh, G. 0843

Parat, S. 0937

Pardini, S. 1199

Pareja, J. C. 0634
Pariès, J. 1207

Parikh, H. 0893

Park, C. 1244

Park, D. 0566

Park, E. 0744,0798

Park, J. 0134

Park, J. 0424

Park, J. 0498

Park, J.-M. 0570

Park, J.-Y. 0906

Park, J. 1070

Park, J. 1129

Park, K. $\quad$ 0094, 0167

Park, K. S. 0731

Park, S. 0236

Park, S. 0744,0798

Park, S. 1244

Park, S. H. 0731

Park, S. Y. 0952

Park, T. 0134

Park, Y. 0570

Park, Y. M. 0724

Parker, H. E. 0695

Parker, M. M. 0342

Parkes, D. 0576, 0586

Parkkonen, M. 1134

Parnaud, G. 0417, 0534

Paroni, G. 1254

Parrinello, G. 1246

Parry, E. 0935

Parslow, R. C. 1047

Parving, H. 0061, 0066, 0101, 0242, 0258, 0989, 1080, 1118, $1119,1137,1138,1173$

Parving, I. 0242

Pascal, S. M. A. 0428

Paschou, S. A. 0455

Pashos, C. $\quad 0868,0869$

Paskach, R. R. 1026

Pasqualinotto, L. 0644

Passera, P. 0682

Paßlack, W. 0549

Pastore, D. 0785

Pastromas, S. 1178

Patarrao, R. S. 0559

Patch, A.-M. $\quad 0068,0281$

Patel, A. 0162

Patel, A. 1085

Patel, N. 0717

Patel, S. 0313

Patel, S. 1071

Patel, V. $\quad 1017,1018$

Paterna, S. 1246

Patsch, J. R. 0483

Patsch, W. 0624

Patterson, C. C. 1047

Patti, L. 0794

Paturzo, F. 0675

Pauer, L. 1146

Paule, S. G. 0558

Pauvaday, V. 0269,0484

Pavitt, D. 0717

Pavlicek, V. 0474

Pavliska, H. 0569

Pavlovic, M. 0669

Pawlowski, M. 0718, 0719

Payer, J. 0743

Péan, F. 0260

Pearce, M. 0349

Pechan, J. 0748

Pechova, M. 0465

Pedersen, B. K. 0306

Pedersen, C. B. $\quad 0956,0979$

Pedersen, K. E. 0619
Pedersen, O. $\quad$ 0101, 0310, 0989, 1248

Pedersen-Bjergaard, U. $\quad 0770$, 0813, 0815, 0933, 1117

Pedrini, M. T. 0483

Pedula, K. L. 0267

Peeters, A. 0354

Peeters, P. H. M. 0892

Peixoto, E. B. M. 1106

Pelengaris, S. 0205,0428

Pelikánová, T. 0629

Pelikanova, T. $0674,0726,0747$

Pellegrini, F. 1023

Pellme, F. 0197

Peltier, S. 0521

Pencea, C. 0685

Pendleton, N. 0083

Penesová, A. 0743

Penesova, A. 0748

Penfornis, F. 0478

Peng, H. 0298, 0299

Peng, J. 0445

Penna-Martinez, M. 0304

Penning-van Beest, F. J. A. 0870, 0871, 0872

Penno, G. 0947, 1186

Pentel, J. 0336

Peraldi-Roux, S. 0059

Péraldi-Roux, S. 0736

Peralta, J. G. 0742

Perez, A. 0864

Pérez, A. 0999, 1000

Pérez, V. 1030

Perez-Maraver, M. 0966

Perfetti, A. 0013

Pernes, J. 0243

Pernet, A. 0041

Perrea, D. 0630,0797

Perrini, S. 0193, 0550, 0701

Perruolo, G. 0013

Perry, J. R. B. 0040, 0079, 0080, 0295

Perseghin, G. 0614

Persson, B. 0115

Persson, F. 1118,1119

Pesce, C. 0063

Peschard, C. 0485

Peter, R. $\quad 0484,1078$

Péterfalvi, A. 0944

Peterkova, V. A. 0305

Peters, M. G. 0232

Petersen, E. 0443

Petersen, L. 0310

Petit, J. 1233

Petit, P. 0510

Petit, P. 0800

Petkova, M. K. 1184

Petoux, F. 0830

Petracca, M. 0244

Petrie, J. 0049

Petrone, A. 0254, 0303

Petroni, D. 1199

Petrova, C. 0468

Petrova, N. L. 1124,1126

Petrule, S. 1128

Pettersson-Fernholm, K. 0364

Peyrol, S. 0758

Peyrot, M. 1056, 1144

Peyrou, M. 0095

Peyrou, V. 0485

Peyser, T. A. 0262

Pezzatini, A. 0687

Pfeiffer, A. F. H. 0170, 0329, 0540, 0582, 0771, 1249

Pfeuffer, M. 0841

Pfleger, C. $\quad 0255,0647$

Pfohl, M. 1093

Pfrang, J. 1193

Pfützner, A. $\quad 0047,0340,0919$, 0960, 1006, 1063, 1175, 1256

Pfützner, A. H. 1063

Phielix, E. 0177

Philippe, J. 0482

Philipson, L. H. 0279, 0504

Philis-Tsimikas, A. 0979

Phillips, D. I. 0616

Philotheou, A. 0222

Pi-Sunyer, F. X. 0887

Piano, S. 0333

Piatkiewicz, P. 0766

Piatkiewicz, P. J. 0456

Piatt, G. 1041

Piatt, G. A. 1062

Piatti, P. 0004

Pibernik-Okanovic, M. 0160

Picha, K. M. 0592

Picon, M. 0636

Pieber, T. R. $0221,0932,1115$

Piemonti, L. 0477

Pierce, M. B. 0071

Piersma, A. 0729

Pietraszek, L. $\quad 0242,1137$

Pietrzak, I. 0281, 0645, 0924

Pietzsch, J. 1183

Piirila, P. 1142

Pilacinski, S. 0997

Pinach, S. 0072, 0212, 0476

Pinchera, A. 0635

Pinget, M. 0369

Piñol, J. L. 0338

Pinzon, J. 0636

Piontek, E. 1074

Piot, C. 1240

Pipeleers, D. 0469

Piquer, S. 0125, 0537

Pirola, L. 0557

Pirot, P. 0545

Pirskanen, M. 0083

Pisani, P. 1199

Pita, J. 0606

Pithova, P. 0465

Pitkäniemi, J. 0269

Pitocco, D. 0459, 1182, 1206

Pivovarova, O. 0170, 0771

Pizarro, E. 0943

Pizarro-Delgado, J. 0525

Planas, F. 0846

Plank, J. 1115

Plank, M. 1071

Plant, N. J. 0575

Plavsic, S. 0332

Plekhova, E. I 0925

Plengpanich, W. 0276

Pociot, F. 0084, 1135

Poderoso, J. J. 0742

Poerksen, S. 0255

Pohl, A. K. 0129

Pohl, M. 1001

Pohl, R. 0047

Pointecouteau, T. 0142

Polak, S. 0464

Poletti, A. 0477

Poller, B. 0112 
Ponzi, P. 0158

Poole, C. D. $0330,0817,0993$

Popov, L. 0707

Porath, A. 1019

Porcellati, F. $\quad 0044,0046$

Porciello, C. 1199

Porta, M. 0682, 1152

Portha, B. 0414, 0495, 0499, 0501, 0523

Portois, L. 0596, 0779

Poskiparta, M. E. H. 0828

Potter, R. 0595

Potter, S. C. 0911

Pou, J. M. 0846, 1096

Poucher, S. M. 0595

Poulia, K. A. 0797

Poulsen, P. L. 0227

Pouta, A. 0171, 0348, 0381

Poutanen, K. S. 0146

Pouwer, F. 0159

Powell, L. A. 1168, 1172

Powers, M. A. 0983

Poy, P. 0842

Pozzan, T. 0167

Pozzilli, P. 0058, 0254, 0303,

Pozzilli, P. 0527

Pozzilli, P. 0957

Prados, A. 1013

Praet, S. F. E. $\quad 0178,0809$

Prager, G. 0895, 1002

Prager, R. 0919

Prante, C. 1128

Pratley, R. E. $\quad 0885,0887$

Pravenec, M. 0629, 0747

Prazak, R. 0412

Prell, G. D. 0538

Pressl, H. 0848

Presta, I. 0026

Prestle, J. 0569

Preston, A. 0408

Prevost, T. 1045,1057

Pricci, F. 0063

Price, K. L. 0135

Prieto, P. G. 0596

Princep, L. 1233

Pritchard, L. E. 0479

Procopio, C. 0026

Prohaska, W. 1128

Prohaszka, Z. 0275

Proietto, J. 0088

Prokopenko, I. 0169

Promintzer, M. 0895

Prompers, J. J. 0178

Prompers, L. 1114

Protogerou, A. 0844

Protopsaltis, I. 1202

Provenzano, V. 0957

Pruniaux, M. 0830

Pryor, H. 1014

Pryor, K. 0688

Prysyazhna, O. D. 0208

Przydzial, M. J. 0666

Pucci, G. 0942

Pucci, L. 1186

Puddu, A. 1102

Pugliese, F. 0063

Pugliese, G. $\quad 0063,0240$

Pugliese, M. C. 0942

Puig, J. 1229

Puig, R. 0458

Pujante, P. 1088

Pukac, L. 0975

Pulcinelli, F. 1182

Pulkkinen, L. 0037, 0386
Punkkinen, J. 1142

Pura, M. 0743

Purcell, S. 0084

Puricelli, L. 0232

Pütz, K. 0540

Putz, Z. 0839

Putz, Z. 1098

Pye, S. 0934

Pyne, N. J. 0696

\section{Q}

Qiao, Q. 0269

Quadri, R. 0476

Quandalle, P. 0243

Quarré, L. 0677

Quast, K. 0624

Quast, T. 1128

Quesada, I. 0537

Quester, W. 0950

Quinn, G. 0529

Quinn, L. 0928

\section{R}

R-Villanueva, G. 0860

Rabøl, R. 0249

Rabol, R. 0757

Raciti, G. A. 0675

Rackova, L. 1108

Rader, H. 0278

Radford, K. 1111

Rádiková, Ž. 0743

Radikova, Z. $\quad 0748,1231$

Radimerski, T. 0556

Radtke, M. 0368

Rae, C. 0754,0755

Raemen, H. 1073

Ragogna, F. 0614

Rahlfs, V. W. 1001

Rahman, M. 0383

Rahman, M. H. 0667, 0804

Rajczy, K. 0275

Rajkovic, N. 0449

Rajotte, R. 0526

Rakipovski, G. 0619

Rakocevic, I. 0332

Rákóczi, I. 0944

Rakovac, I. 1115

Ramos-Lopez, E. 0304

Ranta, F. 0423, 0429

Rantzau, C. 0088

Rapacki, K. 0084

Rapposelli, S. 0907

Raptis, A. 0603

Raptis, S. A. 0603, 0608, 0712, 0759

Rashid, M. A. $\quad 0667,0804$

Raskin, P. 0982

Rasmussen, M. F. 0851

Rasmussen, T. 0463

Rasschaert, J. 0421

Rathcke, C. N. 0233

Rathmann, W. 0648

Rattigan, S. 0238

Rauramaa, R. 0037

Rave, K. 0954

Ray, J. A. 1033

Raymond, I. 0233

Raymond, N. 1036

Raymond, N. T. 1031

Rayner, N. W. 0040, 0079, 0080, 0169, 0172, 0295

Raz, I. 0005, 0147, 0597, 0903
Rebrin, K. 0262

Recio, J. M. 0860

Reddy, S. 0460

Redondo, S. 0803,1235

Reed, L. J. 0041

Reeder, D. W. 0911

Reers, C. 0543

Rees, S. 0316

Reeser, M. 1055

Regazzi, R. 0199, 0202, 0419

Reijngoud, D. 0658

Reiling, E. $\quad 0272,0308$

Reimann, F. 0433, 0693, 0695

Rein, P. 0343

Reinhard, H. 1173

Reis, J. 0972

Reis, J. M. 0970

Reitman, M. A. 0975

Remacha, A. 0775

Remacle, C. 0411, 0441, 0500, 0729

Renar, I. 0160

Renard, E. 0478,0800

Rendschmidt, T. 0994

Renier, G. 0156

Rennings, A. J. M. 0076, 0859

Renström, E. 0053

Renstrom, F. 0196, 0551

Rerknimitr, R. 0276

Reunanen, A. 0905

Reusens, B. 0411, 0441, 0500, 0729

Reuveni, H. 0562

Revsin, Y. 0684

Rewers, A. 1048

Rewers, M. 0140, 0357, 0385, 1048

Reynisdottir, I. 0292

Riahi, Y. 0027

Ribaux, P. 0437

Ribeiro, R. 0738

Ribeiro, R. T. 0737

Ribel, U. 0038

Ricart, W. 0621, 0637

Riccardi, G. $\quad 0794,1076$

Ricci, C. 0063

Rice, G. 1103

Richards, S. M. 0238

Richey, J. M. 0050, 0610, 0662, 0663,0664

Richter, S. 1183

Rickenbach, K. 0400

Ricquier, D. 0431

Ridderstråle, M. 0318, 0893

Riddle, M. 0912

Riečanský, I. 1251

Riedl, M. 0822, 1203

Ries, C. 1175

Riese, R. 0972

Rieusset, J. 0758

Rigla, M. 1013

Rijkelijkhuizen, J. M. 0031

Rijzewijk, L. J. 0613

Ring, A. 0890

Ringholm Nielsen, L. 0933

Rinne, J. O. 0017

Riondino, S. 1182

Rippmann, J. F. 0569

RISC investigators. 0049

Rittig, K. 0714

Ritzel, R. A. 0543

Ritzhaupt, A. 0890

Rivas Fernandez, M. 0978

Rivas-Marin, J. 0768

Rivellese, A. A. $\quad 0794,1076$

Rizza, R. 0602, 0861

Rizza, R. A. 0617

Rizza, S. 0234

Ro, Y. 1163

Robbins, D. C. 1223

Roberts, A. 0819

Robertson, K. 0255

Robertson, S. A. $\quad 0754,0755$

Robinson, A. 0977

Robinson, E. 0460

Robinson, S. 0717

Robson, R. 0852

Roche, E. F. 0190

Rodell, A. 0016

Roden, M. 0762

Rodewald, T. D. 0584

Rodin, A. 1071

Rodrigues, J. A. 0460

Rodríguez, J. C. 0319

Rodriguez, M. 1177

Rodríguez-Frutos, B. 0588

Rodríguez-Mulero, S. 0690

Rohrer, L. 0412

Rokeya, B. 0916, 0918

Rolandsson, O. 0373, 1052

Romagnoli, R. 0476

Roman, G. 0811, 1211

Romano, C. 0488

Romanus, P. 0441

Romero, F. 0947

Romero, G. 1030

Romero, J. 1261

Romics, L. 0275

Romijn, J. A. $\quad 0089,0613$

Rönn, T. S. 0019

Rönnback, M. 1180

Rønningen, K. S. 0463

Ronze, M. 1181

Roos, C. 0287

Rópolo, M. 1075

Roques, C. 1131

Rorsman, P. 0054

Rosa, P. 0054

Rosak, C. 1002

Rosberg, L. 0287

Roschke, V. 0975

Rose, B. 0626

Rosenbauer, J. 0929

Rosenberg, N. 0097, 1236

Rosengård-Bärlund, M. 1099

Rosenstock, J. 0137, 0835, 0887, 0889, 0991, 1146

Rossetti, M. 0476

Rossetti, P. 0044, 0046

Rossi, M. C. 1023

Rossier, B. 0859

Rossing, P. $\quad 0242,0258,1080$, $1118,1119,1135,1137,1138$, 1173

Rossmeisl, M. 0631, 0777

Rossodivita, A. 0004

Rosu, M. 0923

Rotella, C. M. 0244, 0687, 0697, 0904, 1218

Roth, E. 0725

Roth, W. 1063

Rothenberg, P. 0831

Rotteveel, J. 1055

Roubicek, T. 0706 
Roussel, R. $\quad 0036,0260,0284$, 0365

Roussos, C. 0759

Rovira, A. 0606

Rubin, D. 0311

Rudich, A. 0147

Rudovich, N. 0771

Rudovich, N. N. 0170, 0582

Ruiz de Villa, M. C. 0489

Rungby, J. 0016, 0035, 0144, 0491, 0581

Runge, H. 0129, 0652

Ruokonen, A. 0171, 0348, 0381

Rusavy, Z. 0951

Ruschke, K. 0698, 0704

Ruscio, G. 0026

Russel, F. 0859

Russell-Jones, D. $\quad 0045,0108$

Russo, I. 0239, 0708, 0842

Rust, W. 0624

Rustemeijer, C. 0009

Rustenbeck, I. 0506

Rusu, A. 1211

Rutten, G. E. H. 0106, 0264, 0898, 1061, 1097

Rutter, M. K. 1205

Rütter, R. 0626

Rütti, S. 0412

Ryall, J. G. 0741

Rydén, L. $\quad 0186,1208,1212$

Saadé, F. 0623

Sabán-Ruiz, J. 0803, 1235

Sabico, S. L. 0660

Sabokbar, A. 1126

Sadikot, S. M. 0341

Sadikova, N. 1260

Saeger, H. 0020

Saely, C. H. 0343

Sáez de Ibarra, L. 0245

Safraou, M. F. 0937

Sagach, V. F. 0208

Sågetorp, J. $\quad$ 0163, 0164, 0513

Sahin, Y. 1125

Saikku, P. 0381

Saint Martory, C. 1131

Saito, M. 0062

Saito, Y. 0102

Sajic, S. 0277

Sakamoto, Y. 0371

Sakellariou, D. 1178

Sakurai, M. 0769

Salaun-Martin, C. 0336

Salehi, A. 0054, 0515

Salehi, M. 0579

Salehzadeh, F. 0130

Saler, T. 1059

Salgami, E. V. 1144

Salinas, A. 1088

Salinas, I. 0458

Sallinen, R. 0364

Salmela, S. 0828

Salmenkallio-Marttila, M. 0146

Salmon, J. 0283

Salome, P. L. 0898

Salomonsson, A. 1104

Salonen, J. T. 0083

Salonsaari, T. 0461

Salvadori, P. A. 1199

Salvatoni, A. 0360

Salvatore, M. 1076

Salzsieder, E. 1008

Sämann, A. 1064
Samarasinghe, Y. P. 0041

Samigullin, R. 0138

Samsuddin, S. 1196

Sanabria, S. 0831

Sanada, H. $\quad 0271,1165$

Sanchez, C. 0860

Sánchez, O. 0803, 1235

Sánchez, Y. 0775

Sanchez-Margalet, V. $\quad 0567,0568$

Sanchez-Vilar, O. 0606

Sancho, V. 0583, 0587, 0588, 0591, 0781

Sandahl Christiansen, J. 0988

Sandrikova, V. 0392

Sane, T. 1142

Sanguineti, R. 1102

Sanjeevi, C. B. 0256

Sankaranarayanan, S. 1018

Sanke, T. 0289,0344

Sankoh, S. 0889

Sanmartí, A. 0458

Sansoni, V. 0063

Santangeli, P. 1206

Santeusanio, F. 0957

Santini, F. $\quad 0580,0635$

Santos, D. 0967

Santos, E. 1088

Sanz, N. 0860

Saraheimo, M. 1099, 1180

Saraheimo, M. T. 0259

Sarai, K. 0214

Saravia, F. E. 0684

Sardar, K. 0667

Sardella, A. 1214

Sargatal, J. 0943

Sargin, M. 0372

Sargsyan, E. 0404, 0406

Saris, W. H. M. $\quad 0310,0194,0824$, 0827

Sarkar, N. 1212

Sarkar, S. 0139

Sarma, B. P. 0916

Sarma, S. 0916

Sármán, B. 0883

Sarmento, B. 0967

Sarmiento, L. 0359

Sarwat, S. 1223

Saryusz-Wolska, M. 0718, 0719

Sas, T. 1055

Sasaki, H. 0289, 0344, 0727

Sasaki, S. 0795

Sasaki, Y. 0235

Sasaoka, T. 0560

Sassolas, A. 1181

Sasson, S. 0027

Satir, E. 0331

Satman, I. 0716

Sato, A. 1213

Sato, Y. 0643

Satoh, A. 0387

Satoh, J. 0452

Sauerwein, H. 0145

Sauerwein, H. P. $\quad 0751,0783$

Saumell, J. 0338

Savale, S. 0219

Savkovic, S. 0332

Savu, O. 1127

Sawyer, O. 0484

Saxena, R. 0081, 0082

Sbraccia, P. $\quad 0090,0785$

Sburlati, L. 0622, 0845

Scanlon, P. H. 0225

Scarpelli, D. 0026

Scarpello, J. 0425
Schaart, G. 0206

Schaeffer, P. J. 0175

Schäfer, A. 1193

Schafer, J. 0559, 0641

Schäfer, S. A. 0818

Schalkwijk, C. G. 0676, 1118,

$1137,1185,1216,1217,1250$

Scheele, C. 0306

Scheen, A. J. $\quad 0075,1022$

Scheffer, P. 1204

Scheffer, P. G. 0009

Scheijen, J. 0676

Scheja, L. 0625

Schenker, S. 0073

Scherbaum, W. A. 0880, 0901 0980

Schernthaner, G. $\quad 0762,1151$, 1158

Schernthaner, G. H. 1151

Schertzer, J. D. 0741

Schiavo, S. 0552

Schick, F. 0605

Schindler, K. 0793

Schintler, M. 1115

Schjødt, K. J. 1118

Schjoedt, K. J. 1119

Schleicher, E. $\quad 0652,0714$

Schleicher, E. D. $\quad 0129,0563$

Schloot, N. C. $\quad 0255,0457,0647$

Schlosser, M. 0467

Schmid, A. I. 0762

Schmid, C. 0601

Schmid, F. 0343

Schmidt, C. 0314

Schmidt, U. 0162

Schmied, B. 0543

Schmitke, J. 0974

Schmitt, H. 0427

Schmitz, N. 1049

Schmitz, O. 0016, 0144, 0491, 0581, 1194

Schmoelzer, I. $\quad$ 0848, 0996

Schnack, C. 0762

Schneider, B. 0901

Schneitler, H. 0648

Schneitler, S. 0648

Schnell, O. 1224

Schoelch, C. 0569

Schön, M. R. 0309

Schönau, E. 0185

Schönauer, M. 1174

Schöndorf, T. 0340

Schorer, R. 0528

Schou, I. M. 1163

Schramm, M. 1002

Schrauwen, P. 0177, 0180, 0206

Schrauwen-Hinderling, V. B. 0177, 0180

Schreiber, S. 0992

Schreurs, M. 0658

Schrezenmeir, J. 0211, 0311, 0841

Schroeck, J. 0913

Schuchhardt, J. 0314

Schuchman, E. H. 0856

Schuette, K. 0138

Schulze, M. 0170

Schulze, M. B. 0003

Schumann, W. C. 0617

Schumm-Draeger, P. 1204

Schuurman, T. 0145

Schwab, U. 0037

Schwartz, P. 0972

Schwartz, P. F. $\quad$ 0970, 0973

Schwartz, S. 0961, 1236

Schwedhelm, E. 0841

Schweitzer, M. A. 0953

Schweizer, A. $\quad 0880,0885,0887$

Sciacca, L. 0246

Scifo, P. 0614

Scipioni, A. 0063

Scism-Bacon, J. 0991

Scott, C. 0730

Scudamore, I. 0934

Scullion, S. M. 0509

Scully, M. 0592

SDDS Study Group, T. 1259

Seboek, D. 0556

Šeboková, E. 1251

Secchi, A. 0477

Secchiero, P. 1191

Seda, O. 0291

Sedimbi, S. K. 0256

Sedláková, B. 1251

Seedorf, K. 0625

Seewi, O. 0185

Segal, D. 0122

Segal, S. 1075

Segre, O. 0353

Seidinger, D. 0030

Seino, Y. 0851

Seipke, G. 0959

Selam, J. 0224, 0976

Selmeci, L. 0883

Selvarajah, D. $\quad 0896,1089,1145$

Selwood, M. P. 0280

Semenova, I. 1051

Semperboni, L. 0476

Sen Majumdar, A. 0530

Sener, A. 0521, 0523, 0596, 0779

Seneviratne, R. D. 0382

Seo, J. 1244

Seppala-Lindroos, A. 0612

Serban, V. $\quad 0384,0715,0923$

Serhiyenko, A. A. 1149,1201

Serhiyenko, L. M. 1149

Serhiyenko, V. A. 1149,1201

Serino, M. 0240

Serlie, M. J. $\quad 0751,0783$

Serradas, P. 0499, 0518

Serrano-Ríos, M. 0285

Serre-Beinier, V. 0052

Sérusclat, A. 1181

Sesti, G. 0026

Setola, E. 0004

Sgroi, D. C. 0418

Sgueglia, G. 1206

Shaat, N. 0317

Shafrir, E. 0562

Shaginian, R. M. 0251

Shah, N. 0930

Shah, P. 0861

Shahmanesh, M. 0760

Shai, I. 0147

Shakeri Manesch, S. 0895

Shamkalova, M. 1121

Shamkhalova, M. S. 1084

Shang, S. 1120

Sharma, A. 0418

Sharma, A. M. 0837

Sharma, S. 1239

Sharma, S. K. 0985

Sharma, U. 1146 
Sheehy, K. 1187

Shehadeh, N. 0464

Shelestova, E. 1094

Shelestova, E. L. 0120

Shen, J. Hua. 0917

Shen, Y. 0913

Shen, Y. 0917

Shepherd, M. 0172

Shepherd, M. D. 0987

Sheriff, M. H. R. 0382

Shervani, N. J. 0487

Sherwin, R. 0057

Shestakova, M. 0985, 1051, 1121

Shestakova, M. V. $\quad 1084,1157$

Sheu, W. H. H. 1169

Shi, L. 0878

Shields, B. 0068

Shields, B. 0295

Shigeto, M. 0670

Shigetoh, Y. 0387

Shikata, K. 0121, 0123, 0214

Shikata, Y. 0214

Shima, K. $\quad 0131,0132$

Shimada, T. 0289,0344

Shimano, H. 0092

Shimizu, A. 0756,0769

Shimizu, H. 0965

Shimizu, T. 0023

Shimomura, I. 0746

Shine, B. 0067

Shirai, K. 0834

Shirasawa, T. 0023

Shirasawa, T. 0069

Shiri-Sverdlov, R. 0892

Shirihai, O. 0402

Shojaee-Moradie, F. $\quad$ 0045, 0616

Shoraki, A. 1205

Shotliff, K. P. 1086

Shuldiner, A. R. 0169

Shumeyko, A. 0761

Shuter, C. 0930

Shymanskyy, I. 1147, 1148

Sibony, O. 0728

Siebenhofer, A. 0221, 0932

Siegmund, T. 1204

Sieradzki, J. $\quad 0281,1140$

Sierra, C. 1177

Sigurdsson, G. 0292

Siljander, H. T. A. 0461

Siller, S. 1158

Silva, A. 0967

Silva, C. 0618

Silva, C. A. 0634

Silva, K. C. 1150

Silva, M. N. 1243

Silvestre, D. 1261

Silvestre, R. A. $\quad 0503,0505$

Sima, A. 0923

Simell, O. $\quad 0358,0461$

Simell, S. 0461

Simell, T. 0461

Simi, C. 1199

Siminerio, L. M. 1041

Simms, P. 0047

Simo, R. $\quad 1155,1171$

Simon, J. 1042

Simon, T. 0243,0874

Simonis-Bik, A. M. C. 0389

Simpson, A. $\quad$ 0330, 0817, 0993

Simpson, A. K. 0693

Simpson, R. W. 0594

Simsek, S. 1185

Singh, N. 0799

Sinha Roy, R. $\quad 0480,0688$
Sipkema, P. $\quad 0237,0713$

Sipos, é. 1098

Sironi, A. 1199

Sithole, J. 1046

Siyambalapitiya, S. 1046

Sjögren, M. $\quad 0173,0287,1252$

Sjöholm, Å. 0025, 0420, 0493, 0512, 0739

Sjostrand, M. 0711

Skinner, T. 0187, 0192

Skljarevski, V. 0135

Skoglund, G. 0220

Škopková, M. $\quad$ 0743, 0748

Skorpen, F. 0443

Skovlund, S. E. $\quad$ 0192, 1056

Skupien, J. 0281

Skwarzec, J. 1074

Skyler, J. 0972

Sladek, R. 0307

Slamova, K. 0777

Slavotinek, J. P. 0633

Slingerland, A. S. 0068

Sliwinska, A. 0876

Slominski, P. 0170

Sluiter, W. J. 0012

Smaldone, C. 0459

Smeeton, F. J. 0045

Smeets, G. H. W. 1107

Smeets, H. J. M. 0702

Smelt, M. J. 0475

Smidt, K. 0491

Smink, L. J. 0294

Smirnov, S. 0120

Smit, A. J. $\quad 0100,1107$

Smit, J. W. A. 0613

Smith, A. 1162

Smith, C. D. 0767,0913

Smith, D. 0657

Smith, D. M. 0479, 0519

Smith, J. M. 0897

Smith, M. 1111

Smith, M. S. 0584

Smith, P. 0576

Smith, R. 0201

Smith, S. 0838

Smith, U. 0197, 0251, 0553, 0650, 0699, 0700

Smits, P. 0076, 0859

Snabboon, T. 0276

Snoek, F. 1055

Snoek, F. J. 0159

Snoek, F. J. 1066

Snyder, B. 0263

So, W. $\quad 0375,0786$

So, W. Y. 0773

So, W. Y. 1077

Sobngwi, E. 0671

Soddu, A. 0333

Söderberg, S. $\quad 0269,0373,1208$

Söderlund, J. Maria. 0364

Soderman, A. 0592

Soedamah-Muthu, S. S. 1082

Soendergaard, L. 0581

Soeters, M. R. 0751

Sogayar, M. C. 0232

Soininen, P. 1134

Sokal, E. 0241

Sol, E. M. $\quad 0404,0406$

Solano, F. X. 1041

Soldevila, B. 0458

Solé, J. 0943

Soler, J. 0496

Soler Ramon, J. 0966

Soleti, A. 0410
Solimena, M. 0020

Solin, O. 0018

Solinas, G. 0152

Solito, B. 0635

Solskov, L. 1194

Somogyi, A. 0883

Son, H.-Y. 0301

Son, H. 0825

Søndergaard, F. 0035

Sone, H. 1225

Song, Y. 1070

Sørensen, T. 0310

Sørensen, V. R. 1159

Sörhede Winzell, M. 0589

Soro-Paavonen, A. 1187

Sorop, O. 0210

Sorribes, L. 0955

Soulet, D. 0201

Sourij, H. 0848,0996

Sourris, K. 1103

Souto, E. 0967

Souza, A. 0634

Souza, D. S. 1106

Souza, K. L. A. 0439

Sovio, U. 0171

Spalova, I. 0465

Spanheimer, R. 1257

Spargo, F. J. 0823

Sparsø, T. 1248

Spiegelman, D. 0363

Spigoni, V. 0296

Spijkerman, A. M. W. 1139

Spinas, G. A. 0474

Spinas, G. A. 0672

Spinelli, W. 0220

Spinka-Doms, T. 0592

Spoletini, M. 0254, 0303

Sporna, A. 0997

Sposato, I. 1218

Spranger, J. $\quad$ 0170, 0582

Spranger, J. 1249

Sprenkle, J. 0592

Squires, R. 1025

Squires, R. 1161

Stadlbauer, K. 0639

Stadler, M. 0895

Staiger, H. $\quad 0564,0605,1238$

Stamatelopoulos, K. 0844

Stamenkovic-Pejkovic, D. 0627

Stampfer, M. J. 0147

Standl, E. 1224

Stanik, J. 0392

Stanton, A. 1085

Starzyk, J. 0191

Stechova, K. 0465

Steed, L. 0261

Steed, L. 1012

Steel, N. 0071

Stefan, N. 0605, 0714, 1238

Stefanadis, C. 1178

Stefanopoulou, S. 0759

Stefanovski, D. 0050, 0590, 0662, 0663, 0664

Stefansson, K. 0292

Steg, P. 0243

Stehouwer, C. D. A. $\quad 0001,0007$ 0031, 0072, 0237, 0676, 0713, $0750,1118,1137,1139,1215$, $1216,1217,1250$

Stein, P. P. $\quad 0111,0886,0888$

Steiner, S. S. 0047

Steiner-Böker, S. 0030

Steinfeld, I. 0296

Steinthorsdottir, V. 0292

Stencl, J. 0748

Stene, L. C. 0347,0463

Stepanenko, S. 1148

Stephenne, X. 0241

Stephenson, C. R. 1047

Stettler, C. 0149

Steube, D. 1001

Stevanovic, D. 0627

Stevens, C. 0833

Stewart, M. M. W. 0076

Stigliano, I. 0232

Stirban, A. 1128

Stirling, R. J. 0225

Stoch, A. $\quad 0831,0832$

Stoch, S. A. 0111

Stockman, N. L. 0914

Stojanovic, I. 0438

Stojanovic-Susulic, V. 0592

Stokes, R. 0730

Stolarczyk, E. 0518

Stølen, T. 0810

Stolk, R. P. $\quad 0106,1085$

Stoltenberg, M. 0491

Stoltz, R. R. 0914

Storace, D. 1102

Storgaard, H. 0127

Storka, A. 0946

Stosic-Grujicic, S. 0438

Stoy, J. 0279

Strack, T. 0971

Straczkowski, M. 0615

Strakhova, G. Y. 0367

Strange, P. 0975

Stratmann, B. 1128,1200

Stratton, I. 0391

Stratton, I. M. 0225, 0897

Straub, S. G. 0401

Strindberg, L. 0711

Strongin, L. G. 0098

Stump, T. E. 0974

Stumvoll, M. 0309

Sturis, J. 0038

Su, B. 0900

Su, H. 0826

Su, X. 0900

Subirà, M. 0808

Suckow, A. T. 0204, 0466

Suda, T. 0430

Sue, M. 1156

Suematsu-Urawa, M. 0235

Sugawara, A. 0487

Sugden, M. C. $\quad 0522,1196$

Sugihara, K. 0487

Sugimoto, H. 1225

Sulima, T. N. 0925

Suliman, S. G. I. 0392

Sultan, A. 1240

Sultana, K. 0804

Sumarac-Dumanovic, M. S. 0627

Sumida, Y. 0235

Summanen, P. 0228

Sumnik, Z. 0068

Sun, F. 0784

Sun, G. 0211

Sun, P. 0099

Sun, S. 0445

Sun, W. 0572, 0574

Sun, W. 1006 
Sunkari, V. 1127

Sunram-Lea, S. 0042

Sunthornyothin, S. 0276

Surdej, B. 0922

Suryanarayan, S. 0219

Susa, S. 0288

Sutton, D. 1161

Sutton, G. 0666

Sutton, S. 1057

Suwanwalaikorn, S. 0276

Suzuki, H. 0547

Suzuki, K. 0607

Suzuki, T. 0235

Svane, B. 1212

Svenson, L. W. 0352

Svensson, M. 0287,1252

Svensson, M. 0551

Swamy, A. 0042

Swan, D. 0280

Swiergala, E. 0084

Swift, P. 0192

Swift, P. G. F. 0187

Swinnen, S. G. H. 0181

Syed, A. A. 1227

Sysoyeva, O. 0646

Syvänen, A. 0393

Szabo, C. 0446

Szabo, S. 0160

Szabó, Z. 0668

Szadkowska, A. 0645, 0924

Szamatowicz, J. 0945

Szánthó, J. 0944

Szczepura, A. $\quad 1031,1036$

Szendroedi, J. M. 0762

Szocs, A. 0651

Szöcs, Z. 0639, 0677

Szymanska-Garbacz, E. 0718, 0719

Szypowska, A. 0922

T

Tabák, A. G. 0944

Tack, C. J. 0859

Tack, C. J. J. 0076

Tahrani, A. A. 1209

Tai, T. 0356

Taivankhuu, T. 0444

Tajima, N. 0069, 0328, 0379

Takács, R. 1092

Takagi, H. 1225

Takahashi, A. 0092

Takahashi, E. 0069

Takahashi, I. 0487

Takahashi, K. 0218

Takahashi, K. 0452

Takahashi, K. $\quad 0854,0910$

Takala, T. 0571

Takamiya, Y. 1101

Takamoto, I. 0022

Takamura, T. 0131, 0132, 0756, 0769

Takasawa, S. 0487

Takata, H. 0133

Takeda, H. 1225

Takeda, Y. 0133

Takeshita, F. 0529

Takeuchi, K. 0878

Takeuchi, M. 1101, 1109, 1188, 1190

Taki, K. 0328

Talayero, J. 1177

Tam, S. 0857

Tamarit-Rodriguez, J. 0525
Tamás, G. 0944

Tamasawa, N. 0430

Tamer, S. C. $\quad 0222,0995$

Tammara, B. 0220

Tamura, M. 0689

Tan, M. H. $\quad 0865,0962,0984$, 1223

Tanabe, A. 0665

Tanaka, J. 0218, 0854

Tando, Y. 0430

Tanen, M. R. 0111

Tanenberg, R. 0184

Tanenberg, R. J. $\quad$ 0982, 1054

Tang, J. 1083

Taniguti, H. 0727

Tankova, T. 0378

Taouis, M. 0015

Tapp, R. 1110

Tappy, L. 0149

Tarallo, S. 1152

Taraoune, I. 1011

Tarasov, E. 1051

Targioni, I. 1218

Tarnow, L. 0061, 0066, 0242, 0258, 0989, 1080, 1118, 1119, $1135,1137,1138$

Tarrés, J. 0775

Tartaglino, L. 1075

Taskinen, M. 0082, 0216, 0251

Tassi, V. 1254

Tatarczyk, T. 0483

Tatarkiewicz, K. 0576

Taton, J. 0456, 0766, 0902

Tatsumi, K. 0727

Tavaré, J. M. 0609

Taylor, A. I. 0659

Taylor, K. 0849

Taylor, P. 0204

Taylor, P. 1161

Taylor, R. 0176

Taylor-Robinson, S. D. 0717

Tcherny-Lessenot, S. 0336

Teachey, M. K. 0128

Teague, J. L. 0595

Tedeschi, E. 0547

Teerlink, T. $1138,1139,1215$

Teeter, J. G. 0970,0973

Teh, M. 0041

Telci, A. 0716

Telejko, B. 0945

Teller, S. 0486

Téllez, N. 0496, 0690

Tellone, V. 0230

Tenderich, G. 1200

Tengholm, A. $\quad 0163,0164,0165$, 0513

Tentolouris, N. 0011, 1069, 1095

Teperino, R. 0488

Terauchi, Y. 0022

Ternand, C. 0188

Terzidis, K. 0117

Tesfaye, S. $\quad 0896,1089,1141,1145$

Teshigawara, S. $\quad 0121,0123$

Tesi, F. 0580

Tessmann, D. 1064

Tews, D. 0549

Thamer, C. $\quad 0003,0714$

't Hart, L. M. 0272, 0308

Theodorakis, M. 0844

Theys, N. H. 0411

Thisted, H. 0035

Thivolet, C. 0478

Thom, S. A. M. 1085

Thomakos, P. 1035
Thomas, B. 0592

Thomas, E. L. 0717

Thomas, H. E. 0548

Thomas, J. E. 0097

Thomas, L. 0879

Thomas, M. 1103

Thomas, M. C. 1187

Thomas, S. M. 0162

Thompson, C. H. 0633

Thompson, C. J. 0657

Thompson, J. 0480

Thomson, S. E. 1198

Thorens, B. 0203

Thorleifsson, G. 0292

Thörn, K. 0407

Thorn, L. 0259, 1099

Thornberry, N. 0688

Thorne, J. 0418

Thorsteinsdottir, U. 0292

Thorsteinsson, B. 0770, 0813, $0815,0933,0979,1117$

Thuan dit Dieudonné, J. 1240

Thumser, A. E. 0575

Thuren, T. 0881

Tian, H. 1021

Tian, Y. 0863

Tibaldi, F. 0969

Tibaldi, P. 0682

Tichet, J. 0284

Tiedge, M. 0055, 0448

Tiehuis, A. M. 1090

Timar, B. $\quad 0384,0715$

Timar, R. Z. $\quad 0384,0715$

Timlin, L. 0336, 1032

Timmons, J. A. 0306

Timpson, N. J. 0040, 0079, 0080, 0171, 0295

Tinahones, F. 0636,0768

Tiryaki, B. 0716

Tkáč, I. 1251

Tkac, I. 1231

Tkacova, R. 1231

Tobler, K. 0030

Todd, C. 0108

Todd, J. A. 0294

Todorova, B. 0083

Tofe Povedano, S. 0329

Toffoli, B. 1191

Toffolo, G. 0602

Toft, A. 0986

Toft, A. D. 0850

Tokel, H. 1059

Tokmakova, A. Y. 0367

Tokumasa, Y. 0371

Toljic Suluburic, D. Z. 0669

Tolonen, N. 0259

Tolppanen, A. 0386

Tomas, A. 0508

Tombrou, I. 1035

Tomelini, M. 0682

Tominaga, M. $\quad 0288,0379$

Tomori, Z. 0847

Tomotsune, K. 0430

Tonelli, G. 0961

Tong, P. C. Y. $\quad 0375,0773,0786$, 1077

Toniolo, A. 0360

Tönjes, A. 0309

Tonkin, A. 1110

Tonutti, L. 0246

Toouli, J. 0633

Topsever, P. 1143

Torjesen, P. A. 0463

Torlone, E. 0246

Törmäkangas, L. 0381

Törn, C. 0302

Tornero-Esteban, P. $\quad 0591,0781$

Torp-Pedersen, C. $\quad 0837,1176$

Torrejon, S. $\quad$ 0846, 0999

Torrejón, S. 1000

Torrejon-Escribano, B. 0536

Torres, D. 1246

Torri, S. $\quad 0174,0418,0875,0907$

Torvinen, A. 0381

Toso, C. 0052

Toubro, S. 0733

Toumazou, C. 1007

Tourli, P. 0117

Tournier, M. 0736

Tourrel-Cuzin, C. 0535

Tousoulis, D. 1178

Towia, L. 0418

Trajkovic, V. 0627

Tranchina, M. R. 0628

Trautmann, M. 0819, 0853

Trautner, C. 1113

Traylor, L. 1236, 1237

Treasure, J. 0162

Treebak, J. T. 0791

Trégouët, D. A. 0061

Tremblay, A. 0654

Tremblay, J. 0291

Trenell, M. I. 0176

Trentin, S. 1131

Trento, M. 0682

Trevisan, R. 0215

Trifone, L. 1075,1214

Trimble, E. R. $\quad 0115,1168,1172$

Tringali, C. 0333

Tripathi, G. 0760

Tripathy, D. 0179

Triphaty, D. 0435

Tripp, B. 0793

Trippe, B. S. 0987

Trischitta, V. 1254

Tritschler, H. 0138

Tronko, M. D. 0327

Trovati, M. $\quad$ 0239, 0708, 0842

Trüb, T. 0672

Trubutsyna, N. 1121

Truyen, I. 0469

Tsanikidis, H. 0630

Tsapogas, P. 0796

Tsatsoulis, A. 0455

Tschöpe, D. 1128, 1200

Tschritter, O. 0818

Tse, H. 1163

Tse, H. F. 0857

Tshiang Tshiananga, J. K. 1004

Tsiaousis, G. 1202

Tsiotra, P. 0603

Tsitlidze, N. M. 0305

Tso, A. W. K. $\quad 0002,0857$

Tsubouchi, H. 0886

Tsuchida, Y. 1156

Tsuchiya, T. 0965

Tsuchiyama, N. 0769

Tsuda, K. 0023

Tsuda, M. 0023

Tsuneki, H. 0560

Tsuno, T. 0727

Tsutskiridze, L. 1094

Tsuyuki, R. T. 0873

Tu, N. 0968 
Tuomainen, T. 0083

Tuomi, T. 0006, 0081, 0082, 0173

Tuomilehto, J. $\quad$ 0269, 0320, 0386

Tura, A. 0049

Tural, D. 0331

Turzyniecka, M. J. 0705

Tushuizen, M. E. 0009

Tutuncu, N. 0078,1234

Tutuncu, N. B. $\quad 0840,0998$

Tütüncü, N. B. 0858

Tütüncü, ö. 0025

Tütüncü, Y. 0716

Twig, G. 0402

Twigg, S. M. 1198

Tynan, A. 1032

Tynan, A. J. $\quad$ 0330, 0817, 0993

\section{$\mathbf{U}$}

Ubink-Veltmaat, L. J. $\quad 0157,1081$

Ucak, S. 0331, 1059

Ueda, S. $\quad 0028,1101$

Ueffing, M. 1093

Uehara, Y. 0965

Uhles, S. 0514

Uimari, P. 0083

Ukropcova, B. 0748

Ukropec, J. $\quad$ 0743, 0748, 1251

Ulianich, L. 0675

Ullrich, S. 0423, 0429

Uludag, C. 1143

Umino, T. 1190

Umpleby, A. M. 0045

Umpleby, M. 0616

Umudum, Z. 1125

Unal, A. D. $\quad 0858,0998$

Undas, A. 1140

Unertl, K. 0252

Ung, J. C. C. 0705

Ungaro, P. 0488

Unno, M. 0487

Uno, T. 0643

Uno, Y. 0678

Unwin, N. 0349

Ur, E. 0745

Urbani, A. 0090

Urbanovich, A. M. 1201

Urdea, M. 0006

Urquhart, F. 1005

Uruno, A. 0487

Uruska, A. 0997

Uruski, P. 0997

Uusitupa, M. 0037, 0320, 0386

Uusitupa, M. I. J. 0146

Uzan, B. 0501

Uzu, T. 0065

\section{V}

Vaňuga, P. 0743

Vaag, A. 0733

Vaag, A. A. $\quad 0127,0242,0989$

Vaarala, O. 0358

Vaccarezza, V. 1214

Vaccaro, O. 1076

Vachharajani, N. 0764

Vadacca, M. 0058

Vahanian, A. 0874

Vähäsarja, K. 0828

Vahl, T. P. 0579

Vainstein, J. 0147

Vaiserman, A. M. 0327

Valensi, P. 1179,1207

Valente, L. 0058
Valentine, R. 0633

Valentine, W. 1034

Valentine, W. J. 1033, 1043

Valentini, U. 1023

Valentino, R. 0488

Valera, L. 0059

Valgimigli, L. 0410

Vallarino, C. 1257

Valle, T. T. 0320

Valnes, K. N. 0866

Valorani, G. 0058

Valorani, M. G. 0527

Valtuena, S. 0296

Valverde, A. M. 0085

Valverde, I. 0583, 0591, 0596

Vamos, E. P. 0377

Van Acker, K. $\quad 0807,1073$

Van Assche, F. A. 0729

van Baak, M. 0194

van Ballegooie, E. 0223, 1067

van Beusekom, H. M. M. 0210

Van Casteren, V. 1022

Van Crombrugge, P. 1022

Van Dam, R. M. 0363

van de Bunt, M. 0391

van de Waarenburg, M. P. H. 0676

van den Berg, E. 0161

van den Broek, N. M. A. 0178

van den Broek, P. J. A. 0308

van den Brom, C. E. 0206

van den Donk, M. 0264, 0898, 1097

van den Heuvel, M. 0210

van der Bij, S. 0870, 0871, 0872

van der Giessen, W. J. 0210

van der Graaf, Y. 1090

van der Kallen, C. J. H. 0007 ,

0702, 0750, 1216, 1217, 1250

van der Meer, R. W. 0613

Van der Poel, C. 0741

van der Schaaf, G. 0680

van der Schouw, Y. T. 0286, 0892

van der Zon, G. C. M. 0089, 0565

van Deutekom, A. W. 1185

van Dijk, T. H. 0658

Van Etten, E. 0471

Van Gaal, L. 0469, 0837

van Gorp, P. J. J. 0892

van Greevenbroek, M. M. J. 0007,

0750, 1216, 1217, 1250

van Haeften, T. W. $\quad 0293,1250$

van Hees, A. 0194

Van Herreweghe, F. 0029

van Hinsbergh, V. W. M. 0237

van Hove, E. C. $\quad$ 0272, 0308

Van Laere, K. 0831

Van Laethem, C. 0807

van Lie-Peters, E. 1055

van Loon, L. J. C. $\quad 0178,0809$

van Marle, A. 0569

van Mil, E. 1055

van Nieuw Amerongen, G. P. 0713

van Nieuwaal, N. H. G. 0927

Van Obberghen, E. 0085

van Poelje, P. D. 0911, 1195

van Rooij, L. S. J. 0809

van Schooneveld, M. J. 1085

Van Schravendijk, C. 0469

van Sijl, A. M. 1139

van Tienen, F. H. J. 0702

van Tol, A. 0012

van ,t Riet, E. 0031

van Veen, M. 0104 van Vliet, M. 0282

van Vliet-Ostaptchouk, J. V. 0286, 0293, 0892, 1250

Van-Minnen, K. 1003

Vancompernolle, K. 0029

Vandemeulebroucke, E. 0469

Vandewalle, B. 0441

Vándorfi, G. 0839

Vangen, S. 0347

Vanhala, M. 0828

Vankova, M. 0273, 0312, 0390

Vanoverschelde, J. 0555

Vänttinen, M. 0571

Varadhan, L. 1018

Vardarli, I. 0321

Varga, T. 0883

Vargha, P. 1098

Varillas, F. 0319

Varkonyi, J. 0275

Várkonyi, T. T. 1092

Varney, J. 0873

Varo, N. 1088

Vartholomatos, G. 0455

Vasilopoulos, H. 1011

Vasselli, J. R. 0039

Vavrinkova, H. 0749

Vaxillaire, M. 0169

Vazquez, H. 1214

Vcelak, J. 0273, 0291, 0312, 0390

Vedala, A. 0219

Veijola, R. 0358

Vejrazkova, D. $\quad 0273,0312,0390$

Veldhuis, P. P. 0787

Velho, G. 1253

Venditti, C. 0254

Vendrell, J. J. $\quad$ 0198, 0621, 0637, 0656

Venmans, L. M. A. 1061

Vera, P. 0999, 1000

Verga, S. 0628

Vergara, E. 1076

Vergès, B. 1233

Vergouwe, Y. 1082

Verkauskern, R. 0728

Vermeulen, S. U. 0352

Vermue, R. P. 0009

Vesa, J. 0364

Vespasiani, G. 1023

Vestergaard, H. $\quad 0233,0866$

Vesterhus, M. 0278

Vexiau, P. 0671

Viberti, G. F. 0064, 0074, 0077, 0862, 0877, 1162

Vidal, F. 0656

Vidal, H. $\quad$ 0557, 0758

Vidal, M. 0949

Vidal, M. 1058

Videira, P. A. $\quad 0737,0738$

Vieira, J. G. H. 0926

Vignali, S. 0942

Vik, J. T. 0810

Vila, G. 0822

Vila, R. 0653

Vilardell, C. 0775

Vilarrasa, N. 0198, 0637

Vilches, F. $\quad 0636,0768$

Vilcu, C. 0152

Vileikyte, L. 1144

Viljanen, A. 0018

Viljanen, A. P. M. 0571

Viljanen, T. 0018

Villanueva-Peñacarrillo, M. L. 0583, 0587, 0588, 0591, 0596, 0781

Villberg, J. $\quad 0828$

Vilsbøll, T. $\quad 0249,0577,0599$

Vilser, W. 0226

Vinagre, I. 1000

Vincent, E. E. 0609

Vincken, K. L. 1090

Vingerling, J. R. 1085

Vinik, A. I. 0138

Vinnikov, I. 1133

Viollet, B. 0241

Virally, M. 0365

Viretto, M. 0239

Virtanen, I. 0532

Virtanen, K. 0018

Virtanen, K. A. $\quad$ 0017, 0571

Visinoni, S. 0088

Visser, G. H. A. 0927

Visseren, F. L. J. 1090

Vistisen, D. 0034

Vitacolonna, E. $\quad 0158,0246$

Vives, E. 0510

Viviani, G. L. 1102

Vizi, S. 0446

Vlček, M. 0743

Vlad, A. $\quad 0384,0715$

Vladica, M. 0685,0723

Vlajnic, A. 0982, 0983

Vlasblom, R. 0206

Vlasov, V. 0120

Vlcek, M. 0748

Voelmle, M. 0940, 1010

Vogt, L. 1008

Voidonikola, E. 0844

Voight, B. 0081, 0082

Vojtassakova, E. 0946

Volpe, L. 0942, 0947

Volpi, E. 0392

von Bibra, H. 1204

von Eckardstein, A. 0412

von Rosenstiel, I. A. 0282

Vondra, K. 0273, 0312, 0390, 0787, 0788

Vorasuk Shotelersuk, V. 0276

Voronina, L. N. 0686

Vorontsov, A. 1051

Voshol, P. J. 0089

Voss, L. D. 0801

Vrbikova, J. 0312, 0674, 0787, 0788

\section{W}

Wada, J. $\quad$ 0121, 0123, 0214

Wada, K. 0288

Wada, T. 0560

Wada, Y. 0600

Wade, A. N. $\quad 0103,1042$

Waden, J. 0259

Wadén, J. 1099

Waeber, G. 0199, 0203, 0419

Wägner, A. M. 0319

Wagner, J. 0831, 0832, 0833

Wagner, J. A. 0111

Wagner, O. 0762

Wagner, R. S. 1024

Wahren, J. 0643, 1175

Waldenström, A. 0186

Walder, K. 0122

Waldhäusl, W. 0677

Waldrop, M. 0204

Waldrop, M. A. 0466 
Wallerstedt, E. 0553

Wallin, T. 0403,0516

Walschus, U. 0467

Walter, M. 0141

Walther, R. 0440, 0467, 0486

Walus-Miarka, M. 1140

Wang, B. 1154

Wang, H. 0403

Wang, L. C. 0539

Wang, S. 0211

Wang, W. 0638

Wang, W. 0784

Wang, Y. 0097

Wann, E. 0891

Waraich, R. S. 0563

Ward, P. S. 0693

Wardlaw, J. M. 1091

Wareham, N. J. $\quad 0008,0257,0306$ 0383, 0616, 0805, 1045, 1057

Wareing, M. 0447

Warnes, G. 0527

Warwar, N. 0511

Wasada, T. 0795,1221

Wascher, T. C. $\quad 0848,0919,0996$

Wat, N. M. S. 0002

Watanabe, C. 1087

Watanabe, N. 0214

Watanabe, T. 0271, 1165

Watelet-Jardin, B. 0059

Waterman, C. 1144

Watson, A. M. D. 1187

Watson, D. 0425

Watson, E. 0850

Watt, T. 1159

Weaver, J. 0261, 1012

Weber, C. 1004

Weber, M. 0473, 0474

Weber, M. M. $\quad 0960,1175$

Wedekind, D. 0448

Wedel, H. 0186

Weedon, M. N. $\quad 0040,0079,0080$, 0171, 0295

Weekers, L. 0365

Weets, I. 0469

Wegener, G. 1194

Wegner, O. 0924

Weickert, M. O. 0540, 0582

Weigert, C. $\quad 0129,0563,0652$

Weikert, C. 1249

Weiner, R. 1002

Weinzimer, S. A. 0188

Weir, G. 0093

Weir, G. C. $\quad 0417,0418$

Weise, A. 1006

Weisel, K. 0220

Weiss, H. 0055, 0448

Weiss, S. 1073

Weitgasser, R. 0919

Welschen, L. 0104

Welsh, N. 0490, 0493, 0513, 0544

Welters, H. J. 0395, 0405

Wendt, A. 0517

Weng, J. 0572, 0574, 1021

Weng, J. P. 0087

Weng, W. 0224, 0976

Wentholt, I. M. E. 1122

Wenzlau, J. M. 0139, 0140, 0141, 0143

Werner, U. 0584

Wessman, M. 0364

Wester Rosenlöf, L. 0055

Westergaard, L. 0189

Westerlund, J. 0497

Wetlesen, T. 0463
Weyer, C. 0838

White, A. 0479

White, K. 0064

White, N. H. 1015

White, P. 0494

Wicker, L. S. 0294

Widmann, C. 0203

Wiederkehr, A. $\quad 0094,0167$

Wienbergen, A. 0506

Wierusz-Wysocka, B. 0997

Wiesli, P. 0601

Wijesuriya, M. 0382

Wijmenga, C. 0286, 0293, 0892, 1250

Wijtvliet, A. P. 0809

Wikstrom, J. 0402

Wild, S. H. 0616

Wildbaum, G. 0464

Wildey, G. M. 0432

Wilhelm, B. 1175, 1256

Wilkin, T. J. 0801

Wilkinson, I. D. 1089

Wilkinson, M. 0745

Willeit, J. 0370

Willemsen, G. 0389

Williams, J. 0108

Williams, J. 1112

Williams, K. M. 1045, 1057

Williams, S. C. R. 0041

Williams-Herman, D. E. 0113, 0884

Wilson, B. 0041

Wilson, J. 0576

Wilson, N. 0392

Winkler, G. 0839

Winkler, H. 0648

Winkler, P. 0841

Wintle, M. 0110, 0853

Winzell, M. S. 0053

Witsø, E. 0463

Witte, D. 0072

Wittmann, I. 0839

Wittmann-Jennewein, C. 1044

Wizemann, E. 0994

Wogensen, L. 0144, 0491

Wohl, P. 0629, 0747

Wojtaszewski, J. F. P. 0791

Wojtusciszyn, A. 0436, 0478

Wolf, G. 0226

Wolf, G. 0440

Wolfram, S. 0420

Wolkow, P. 1140

Wollheim, C. B. 0094, 0167, 0403, 0508, 0528

Wollmer, A. 0959

Wolzt, M. 0030, 0822, 0946

Wolzt, M. 0969

Wong, K. 0480

Wong, K. 0693

Wong, L. S. 0124

Wood, D. 1255

Wood, D. A. 1027

Woods, D. 0975

Woods, J. 0480

Woolcott, O. O. $\quad 0050,0662,0663$, 0664

Wright, D. 0833

Wronka, M. 0337

Wu, C. M. 1169

Wu, C. S. 1169

Wu, M. 0118, 0936, 0938, 0939

$\mathrm{Wu}, \mathrm{R} .0911$

Wüchner, S. 0950

Wuerth, S. 0149
Wunderlich, T. 0698

Wuschke, S. 0314

Wuttke, A. 0513

Wyart, P. 0874

Wyka, K. 0645

Wysocka-Mincewicz, M. 1074

\section{$\mathrm{X}$}

Xiang, K. $\quad 1083,1220$

Xiang, Y. $\quad 0298,0299$

Xie, F. 0200

Xie, Z. 0005

Xin, W. 0539

Xin, Y. 0863

Xu, A. $\quad 0002,0155,0857$

$\mathrm{Xu}, \mathrm{L}, 0672$

$\mathrm{Xu}, \mathrm{L} . \quad 0884$

Xu, M. 0398

$\mathrm{Xu}, \mathrm{S} . \mathrm{H} . \quad 0470$

Xu, W. 0574

Xu, Y. 0470

Xu, Y. 1257

\section{Y}

Ylldırır, A. 0858

Yada, T. 0502, 0507

Yadav, S. 0789

Yakhini, Z. 0296

Yakir, B. 0083

Yamada, N. 0092

Yamagishi, M. 0133

Yamagishi, S. 0028, 0387, 1101, $1105,1109,1188$

Yamaguchi, H. 0288

Yamakawa, R. 1105

Yamamoto, Y. 0133

Yamauchi, A. 0487

Yamgnane, A. 0937

Yan, J. 0574

Yan, J. H. 0087

Yan, L. $\quad 0398,0990$

Yan, X. 0298, 0299

Yancheng, X. 0451

Yang, C. 0398

Yang, J. 0203

Yang, J. 0990

Yang, L. 0299

Yang, M. 1220

Yang, W. $\quad$ 0990, 1021

Yang, X. 0375

Yang, X. 1195

Yankova, T. 1072

Yano, Y. 0235

Yao, J. 0688

Yasuda, Y. 0689

Yasuhara, A. 0121, 0123

Yatabe, J. 0271, 1165

Yatabe, M. Sasaki. 0271

Yatabe, M. S. 1165

Yau, M. H. 0857

Yazici, A. C. 0078,1234

Ybarra, J. 0846, 1096

Ye, H. 0155

Ye, J. P. 0087

Yeung, C. Y. 0773

Yi, K. H. 0731

Yilmaz, T. 0372

Yin, D. $\quad 0329,0816$

Yin, X. 0385

Ying, J. 0784

Yki-Jarvinen, H. $\quad 0251,0612$

Yokoyama, H. 1225

Yokoyama, M. 0102

Yoneda, T. 0133

Yoo, H. 0213

Yoo, H. 1244

Yoo, S. 0301

Yoon, C. $\quad 0424,1129$

Yoon, K. 0301

Yoshida, T. $\quad 0218,0910$

Yoshida, Y. 1105

Yoshihara, A. 1156

Yoshiike, N. 0379

Yoshikawa, R. $\quad 0121,0123$

Yoshikawa, T. 0487

Yoshioka, N. 1190

Young, I. S. 0778

Yu, D. 0990

Yu, G. 0087

Yu, L. $\quad 0139,0140$

Yu, Q. 0572, 0574

Yu, X. 0055

Yuan, H. B. 1223

Yuan, J. 0832

Yudkin, P. 0103

Yue, D. K. 1198

Yueh, C. $\quad 0182,0183$

Yun, C. 0820

Z

Zabena, C. 0285

Zaccardi, F. $\quad 0459,1206$

Zachari, A. 0649

Zacheja, S. 1130

Zaffini, R. 0547

Zagayko, A. L. 0686

Zaheer, J. 1210

Zajickova, K. 0312

Zakopoulos, N. $\quad 0844,1178$

Zamaklar, M. 0449

Zamaklar, M. N. 1164

Zampelas, A. 0792

Zampetti, S. 0254, 0303

Zandbelt, N. 1066

Zangen, S. 0005, 0903, 0597

Zangger, K. 0086

Zaninotto, P. 0071

Zanone, M. 0476

Zapanti, E. 0117

Zapata, L. 0999, 1000

Zauli, G. 1191

Zavala, F. 0431

Zavarella, S. $\quad 0254,0303$

Zavaroni, I. 0296

Zawada-Targoni, S. M. 0902

Zaytseva, N. V. N. 1084

Zdravkovic, M. 0851, 0852

Zechner, C. 0175

Zeggini, E. $0040,0079,0080$, 0169, 0171, 0295

Zeiler, F. 1151

Zelaya, F. 0041

Zeller, M. 0243, 0874

Zemanova, A. 0312

Zervou, S. 0205

Zethelius, B. 0393

Zgibor, J. $\quad 1041,1082$

Zgibor, J. C. 1062

Zhang, B. $\quad 0912,1258$

Zhang, B. 1170

Zhang, B. B. $\quad 0480,0688$

Zhang, D. 0366

Zhang, H. 0917 
Zhang, L. 0211

Zhang, M. 0470

Zhang, Q. $\quad 0025,0512$

Zhang, Q. 0329

Zhang, Q. 1054

Zhang, R. 0724

Zhang, S. 0398

Zhang, S. 0445

Zhang, Y. 0211

Zhang, Y. 0398

Zhang, Y. 0445

Zhang, Y. 0521, 0523, 0596

Zhao, J. 0863,1123

Zhao, J. J. 0539

Zhao, J. J. 0632

Zheng, D. 0050, 0590, 0662,

0663, 0664

Zhong, L. 0683

Zhou, J. 1220

Zhou, L. 0666

Zhou, P. 0445

Zhou, Z. $0298,0299,0445$

Zhou, Z. 0917

Zhu, A. 0133

Zhu, D. 0990

Zhu, L. 0480,0688

Zhuang, D. 0849

Zidkova, K. 0674

Ziegelasch, H. J. 0950

Ziegler, A. 0141

Ziegler, D. 0138

Zieschang, T. 1044

Zilberman, L. I. 0305

Zimmet, P. 0122, 0150, 0269, 0283, 0290, 0354, 0558, 1110

Zimny, S. 1093

Zinman, B. 0074, 0077, 0862, 0877

Zisman, A. $\quad 0184,1054$

Zitouni, K. 1025

Zitzer, H. 0625

Ziv, E. 0562

Zivkovic, S. 0332

Zoellner, H. 1198

Zonenberg, A. 0945

Zoric, S. 0627

Zoupas, C. S. 1011

Zourek, M. 0951

Zozulinska, D. 0997

Zucco, C. 0353

Zuellig, R. 0473, 0474

Zulewski, H. $\quad 0528,0556$

Zuniga, E. 0610, 0663

Zwettler, E. 0762

Zycband, E. 0480 


\title{
European Association for the Study of Diabetes
}

\author{
EASD Executive Committee
}

President:

Vice-President:

Vice-President:

Honorary Secretary:

Honorary Treasurer:

Chairman of the Postgraduate Education Sub-Committee:

Editor-in-Chief, DIABETOLOGIA:

\author{
E. Ferrannini, Pisa (retires 2008) \\ U. Smith, Göteborg (retires 2008) \\ C. Boitard, Paris (retires 2009) \\ D.L. Eizirik, Brussels (retires 2007) \\ G. Spinas, Zurich (retires 2009) \\ J. Nolan, Dublin (retires 2009) \\ E. Gale, Bristol (retires 2008)
}

EASD Council comprises of the Officers above and the following members:

Term Expiring September 2007

L. Czupryniak, Lodz

L. Gardete Correia, Lisbon

D. Mauricio, Barcelona

T. Otonkoski, Helsinki
Term Expiring September 2008

K. Dahl Jørgensen, Oslo

R.J. Heine, Amsterdam

B. Mankovsky, Kiev

S. Sasson, Jerusalem
Term Expiring September 2009

V. Pirags, Riga

F. Pociot, Gentofte

M. Porta, Turin

M. Stumvoll, Leipzig

The Past President, P.A. Halban, and the Secretary of the Postgraduate Education Sub-Committee, L. Czupryniak, are members of the Council ex officio.

HONORARY AUDITORS

N. Katsilambros, Athens and M. Tiedge, Rostock

\section{HONORARY MEMBERS}

G. Alberti, Newcastle; D. Andreani, Rome; E. Cerasi, Jerusalem; A. Czyzyk, Warsaw; J.K. Davidson, Atlanta; T. Deckert, Hellerup; P. Freychet, Nice; L.G. Heding, Copenhagen; H. Keen, London; E. Kohner, London; P. Lefèbvre, Liège; R. Luft, Stockholm; C. Lurie, New York; J. Pirart, Brussels; S. Rahbar, Duarte; J. Roth, Whitestone; E. Shafrir, Jerusalem; D. Steiner, Chicago; E. von Wasielewski, Munich

\section{GOLD MEMBERS}

Astra Zeneca, Macclesfield, UK; Bayer HealthCare, Leverkusen, Germany; Eli Lilly \& Co., Indiana, USA; GlaxoSmithKline, Middlesex, UK; LifeScan, Inc., California, USA; Merck \& Co. Inc., New Jersey, USA; Novartis Pharma AG, Basel, Switzerland; Novo Nordisk A/S, Bagsvaerd, Denmark; Pfizer Inc., New York, USA; sanofi-aventis, Paris, France; Servier, Neuilly-sur-Seine, France; Takeda Chemical Industries Ltd., Osaka, Japan

\section{SILVER MEMBERS}

Abbott Laboratories GmbH, Wiesbaden, Germany; Daiichi Sankyo Europe GmbH, Munich, Germany; Janssen-Cilag, Issy-les-Moulineaux, France; Merck KGaA, Darmstadt, Germany; Roche Diagnostics GmbH, Mannheim, Germany; Sooil Development Co., Ltd., Seoul, Korea

\section{ASSOCIATE MEMBERS}

Amylin Europe Ltd., Oxford, UK; Animas Corporation, Pennsylvania, USA; A. Menarini Diagnostics, Grassina, Italy; Becton Dickinson Consumer Healthcare Europe, Pont-de-Claix, France; Berlin Chemie AG, Berlin, Germany; Boehringer Ingelheim GmbH, Ingelheim,

Germany; HemoCue AB, Angelholm, Sweden; Medtronic Europe, Brussels, Belgium; Nipro Diabetes Systems, Florida, USA; Owen Mumford Ltd., Oxford, UK; Smiths Medical MD, Inc., Minnesota, USA; Ypsomed GmbH, Sulzbach, Germany

The EASD Executive Committee is also the Executive Committee of the European Foundation for the Study of Diabetes (EFSD).

\section{EASD/EFSD office: Rheindorfer Weg 3, 40591 Düsseldorf, Germany - www.easd.org}

